\title{
ESICM LIVES 2020
}

\author{
Virtual. 06-09 December 2020
}

\section{Best Oral Presentations}

\section{5}

Cross-sectional validity of the Chelsea Critical Care Physical Assessment tool (CPAx) in critically ill adults

S. Eggmann ${ }^{1}$; ML. Verra ${ }^{1}$; V. Stefanicki ; A. Kindler ${ }^{1}$; D. Seyler ${ }^{1}$; R. Hilfiker ${ }^{3}$; JC. Schefold ${ }^{4} ; \mathrm{CH}$. Bastiaenen ${ }^{5} ;$ B. Zante ${ }^{4}$

${ }^{1}$ Department of Physiotherapy, Inselspital, Bern University Hospital, Insel Group, Bern, Switzerland; ${ }^{2}$ Physiotherapie, Schweizer Paraplegiker-Zentrum Nottwil, Nottwil, Switzerland; ${ }^{3}$ School of Health Sciences, HES-SO Valais-Wallis, Leukerbad, Switzerland; ${ }^{4}$ Department of Intensive Care Medicine, Inselspital, Bern University Hospital, University of Bern, Bern, Switzerland; ${ }^{5}$ Department of Epidemiology. Research Line Functioning and Rehabilitation Caphri, Maastricht University, Maastricht, Netherlands

Correspondence: S. Eggmann

Intensive Care Medicine Experimental 2020, 8(2): 000085

Introduction: The Chelsea Critical Care Physical Assessment tool (CPAx) is a performance-based measurement instrument to evaluate respiratory function, functional mobility and grip strength in critically ill adults [1]. The CPAx has established construct validity and high interrater reliability in a general ICU population [2], but little is known about its validity to measure change over time (cross-sectional-validity).

Objectives: The current study investigated cross-sectional validity at three relevant, pre-specified timepoints across the ICU and hospital stay in critically ill adults on prolonged mechanical ventilation.

Methods: This prospective, longitudinal, clinimetric study was conducted in a mixed ICU of a Swiss academic hospital. Participants were assessed by certified physiotherapists at three predefined timepoints: ICU baseline (between 72-144 h after the onset of mechanical ventilation), ICU discharge and hospital discharge. We hypothesized that the relationship of the CPAx with other measurement tools would differ between these timepoints due to temporal changes in the measured characteristics. Based on this theoretical model we determined 22 a-priori defined hypotheses about the relationship of the CPAx-GE with other instruments such as the Medical Research Council sum score (MRC-SS), ICU Mobility Scale (IMS) or Sequential Organ Failure Assessment (SOFA)

Results: CPAx data of 58 participants ( $70.7 \%$ male) with a median age of 68 years (IQR 56-73), APACHE II score of 32 (28-36) and ICU stay of 7.97 days $(6.69-12.85)$ were analysed. Cross-sectional validity was excellent with $86 \%$ ( 6 out of 7), $89 \%$ (8 out of 9 ) and $83 \%$ (5 out of 6 ) of the a-priori hypotheses accepted at ICU baseline, ICU discharge and hospital discharge, respectively. Floor (10\%) and ceiling effects $(6 \%)$ were likewise highly acceptable.

\begin{tabular}{|c|c|c|c|}
\hline Hypothesis & $\mathbf{n}$ & $\begin{array}{c}\text { Spearman's rank } \\
\text { correlation coefficient (r) }\end{array}$ & confirmed \\
\hline \multicolumn{4}{|l|}{ ICU Baseline } \\
\hline CPAx with MRC-SS (r $=0.5$ to 0.8 ) & 58 & 0.639 & yes \\
\hline CPAx with IMS ( $r=0.6$ to 0.9 ) & 58 & 0.711 & yes \\
\hline CPAx with SOFA ( $r=-0.4$ to -0.7$)$ & 58 & -0.341 & no \\
\hline \multicolumn{4}{|l|}{ ICU Discharge } \\
\hline CPAx with MRC-SS ( $r=0.6$ to 0.9 ) & 50 & 0.809 & yes \\
\hline CPAx with IMS $(r>0.7)$ & 50 & 0.883 & yes \\
\hline CPAx with SOFA ( $r=-0.4$ to -0.7$)$ & 50 & -0.482 & yes \\
\hline \multicolumn{4}{|l|}{ Hospital Discharge } \\
\hline CPAx with MRC-SS ( $r=0.5$ to 0.8 ) & 31 & 0.674 & yes \\
\hline CPAx with IMS (r>0.7) & 32 & 0.856 & yes \\
\hline
\end{tabular}

Conclusion: The CPAx demonstrated cross-sectional validity as an indicator of change over time. Floor and ceiling effects were low enabling therapists to detect change across the ICU and hospital stay. The CPAx can therefore be recommended to assess physical function and activity in critically ill adults from ICU baseline to ICU and hospital discharge.

\section{Reference(s) and grant ackowledgment(s)}

1. 1. Corner EJ, Wood H, Englebretsen C, Thomas A, Grant RL, Nikoletou D et al. The Chelsea critical care physical assessment tool (CPAx): validation of an innovative new tool to measure physical morbidity in the general adult critical care population; an observational proof-of-concept pilot study. Physiotherapy. 2013;99(1):33-41.

2. 2. Parry SM, Granger CL, Berney S, Jones J, Beach L, El-Ansary D et al. Assessment of impairment and activity limitations in the critically ill: a systematic review of measurement instruments and their clinimetric properties. Intensive Care Med. 2015;41(5):744-62.

3. Research grant from physioswiss

4. PhD Grant from the Swiss Foundation for Physiotherapy Science

\section{4}

Efficacy and Safety of Imipenem/Cilastatin/Relebactam Versus Piperacillin/Tazobactam in Patients With Hospital-Acquired Bacterial Pneumonia/Ventilator-Associated Bacterial Pneumonia by Geographic Region

M. Losada' ; J. Du' ; M. Brown ${ }^{1}$; K. Young ${ }^{1}$; P. Moise ${ }^{1}$; R. Tipping ${ }^{1}$; A. Paschke'; J. Butterton'; LF. Chen'

${ }^{1}$ Department of Infectious Diseases, Merck \& Co., Inc., Kenilworth, United States of America

Correspondence: $\mathrm{G}$. Cohen

Intensive Care Medicine Experimental 2020, 8(2): 000074

\section{Springer Open}

(c) The Author(s) 2020. Open Access This article is licensed under a Creative Commons Attribution 4.0 International License, which permits use, sharing, adaptation, distribution and reproduction in any medium or format, as long as you give appropriate credit to the original author(s) and the source, provide a link to the Creative Commons licence, and indicate if changes were made. The images or other third party material in this article are included in the article's Creative Commons licence, unless indicated otherwise in a credit line to the material. If material is not included in the article's Creative Commons licence and your intended use is not permitted by statutory regulation or exceeds the permitted use, you will need to obtain permission directly from the copyright holder. To view a copy of this licence, visit http://creativecommons.org/licenses/by/4.0/. 
Introduction: Imipenem/cilastatin/relebactam (IMI/REL) is a fixeddose combination of imipenem, a carbapenem antibacterial agent, cilastatin, a renal dehydropeptidase inhibitor, and relebactam, a novel $\beta$-lactamase inhibitor.

Objectives: To analyze the efficacy and safety of IMI/REL and piperacillin/tazobactam (PIP/TAZ) in patients with hospital-acquired bacterial pneumonia (HABP) and ventilator-associated bacterial pneumonia (VABP) from the phase 3 RESTORE-IMI 2 trial (NCT02493764) grouped by geographic region.

Methods: Randomized patients received IMI/REL (500 mg/500 $\mathrm{mg} / 250 \mathrm{mg}$ ) or PIP/TAZ (4000 mg/500 mg) intravenously every 6 hours for 7-14 days; dosing was adjusted for renal impairment. Efficacy endpoints included all-cause mortality (ACM) through day 28 , clinical response at early follow-up (EFU) in the modified intentionto-treat population (MITT), and microbiological response at end of treatment (EOT) in the microbiological MITT population (mMITT). The MITT included patients who received $\geq 1$ dose of study treatment and did not have only gram-positive cocci on the baseline respiratory specimen Gram stain; the mMITT comprised patients from the MITT with $\geq 1$ baseline pathogen, excluding monomicrobial infection with organisms known to be intrinsically resistant to IMI/REL (methicillinresistant Staphylococcus aureus, Enterococcus faecium, Legionella spp., Stenotrophomonas spp., or Burkholderia spp.). Differences in efficacy endpoints and $95 \% \mathrm{Cls}$ were adjusted by the Miettinen \& Nurminen method. The safety population included patients who received $\geq 1$ dose of study treatment. Adverse events (AEs) were collected for 14 days posttreatment. Efficacy and safety were analyzed by geographic region; patients were not randomized based on geographic blocks.

Results: Of $531 \mathrm{MITT}$ patients, 264 received IMI/REL and 267 received PIP/TAZ. Most patients were from Eastern Europe (52\%), followed by Asia (14\%), South America (13\%), North/Central America (11\%), and Western Europe (9\%). Mean age ranged from 47-71 years, and $57-80 \%$ were male. At baseline, $39-72 \%$ had APACHE II scores $\geq 15$, $44-80 \%$ had Clinical Pulmonary Infection Scores $\geq 6$, and $13-47 \%$ had moderate to severe renal impairment (creatinine clearance $<60$ to $\geq 15 \mathrm{~mL} / \mathrm{min}$ ). Across all regions, day $28 \mathrm{ACM}$ was lower withIMI/REL vs PIP/TAZ and clinical response at EFU and microbiological response at EOT was higher with IMI/REL vs PIP/TAZ (Table). IMI/REL and PIP/ TAZ had a comparable safety profile; $\geq 85 \%$ of patients reported $\geq 1$ $A E$, and serious $A E s$ ranged from $20-59 \%$. No regional differences in $\mathrm{AE}$ incidence were noted.

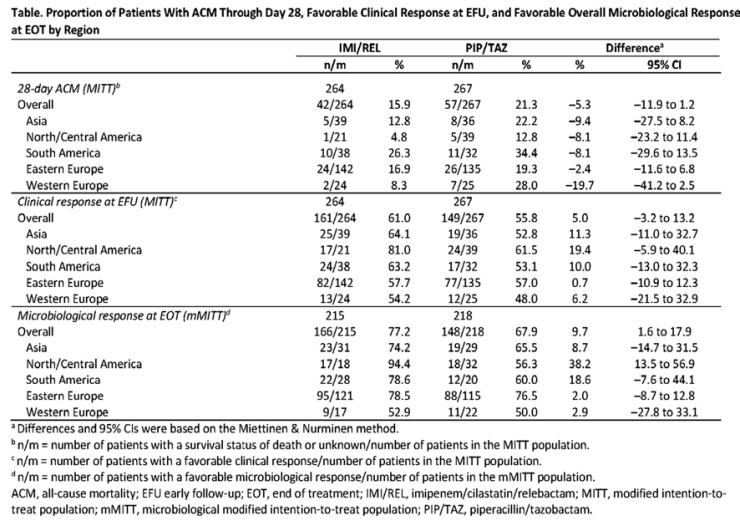

Conclusion: In clinically complex patients, the efficacy and safety of IMI/REL were comparable across treatment groups and regions, supporting the use of IMI/REL as an effective treatment for HABP/VABP in all regions. The incidence of AEs was typical of patients with HABP/ VABP who receive antibacterials.
000114

Involvement of $\beta$-adrenergic modulation in lymphocyte balance during experimental septic shock

M. Durand'; B. Levy²; A. Kimmoun ${ }^{2}$

${ }^{1}$ Faculté de médecine, UMR_S1116 Defaillance Cardiovasculaire Aigue et Chronique, Nancy, France; ${ }^{2}$ Réanimation médicale, institut lorrain du cœur et des vaisseaux, CHRU Nancy, Nancy, France

Correspondence: $M$. Durand

Intensive Care Medicine Experimental 2020, 8(2): 000114

Introduction: Septic shock (SS) is usually responsible of a dysautonomic syndrome including an impairment of cardiovascular variability, the onset of inappropriate tachycardia, an immunosuppression and an increased mortality. In experimental SS, $\beta 1$-adrenergic blockade is associated with cardiovascular benefits and possibly immune modulations.

Objectives: The main goal of this study was to investigate the effect of $\beta 1$-adrenergic modulation on lymphocyte subpopulations in a rodent experimental model of SS.

Methods: SS was induced by cecal ligation and puncture (CLP) in wild type (WT) mice with or without esmolol (ultra-selective $\beta 1$-blocker) and in $\beta 1$-AR knock out mice $(\beta 1 \mathrm{KO})$. Assessment 18 hours after surgery included in vivocardiac function assessed by echocardiography. Blood was collected for cytokine measurements. Splenocytes were isolated to assess lymphocyte subpopulations by flow cytometry and cultured $72 \mathrm{~h}$ with a cell trace to measure cell proliferation capacities.

Results: SS was characterized in WT CLP compared to WT sham animals by a decrease in heart rate (HR) $(318 \pm 31$ in CLP vs. $360 \pm 48$ $\mathrm{bpm}$ in sham animals, $\mathrm{p}=0.01$ ) and in mean arterial pressure (MAP) (mean \pm SD) $(62.2 \pm 11.8$ vs. $84.8 \pm 6.9 \mathrm{mmHg}, \mathrm{p}<0.0001)$. Compared to WT sham, WT CLP animals showed an increase in both proand anti-inflammatory cytokines (IL6: $10.58 \pm 15.01$ vs. $0.05 \pm 0.03$ $\mathrm{ng} / \mathrm{ml}, \mathrm{p}=0.004, \mathrm{IL} 10: 2.28 \pm 1.22$ vs. $0.04 \pm 0.01 \mathrm{ng} / \mathrm{ml}, \mathrm{p}=0.004)$ a decrease in Th4 lymphocytes (10.7 \pm 3.1 vs. $16.1 \pm 1.8 \%$ of T lymphocytes, $\mathrm{p}=0.0001$ ) and an increase in regulatory $T$ cell percentage $(12.49 \pm 2.74$ vs. $7.55 \pm 1.75 \%$ of T helper, $\mathrm{p}<0.0001)$. Compared to WT sham, WT CLP animals showed a decrease of lymphocytes proliferation ( $66.7 \pm 9.5$ vs. $78.6 \pm 7.1 \%$ of proliferative lymphocytes, $p=$ 0.025).

Compared to WT CLP, HR in WT CLP + esmolol, was decreased $(273 \pm 45$ in WT CLP + esmolol vs. $318 \pm 31$ bpm in WT CLP animals, $p$ $=0.005)$. There was no difference in MAP between WT CLP + esmolol and WT CLP(67.4 \pm 17.3 vs. $62.2 \pm 11.8 \mathrm{mmHg}, \mathrm{p}=0.20)$. In WT CLP + esmolol, pro- and anti-inflammatory cytokinesdecreased compared to WT CLP (IL6: $0.64 \pm 0.52$ vs. $10.58 \pm 15.01 \mathrm{ng} / \mathrm{ml}, \mathrm{p}=0.004$, IL10: $0.41 \pm 0.28$ vs. $2.28 \pm 1.22 \mathrm{ng} / \mathrm{ml}, \mathrm{p}=0.002$ ). The $\mathrm{T}$ helper lymphocytes percentage in the spleen increase in WT CLP + esmolol compared to WT CLP $(14.7 \pm 2.9$ vs. $10.7 \pm 3.1 \%$ of $T$ helper, $p=0.007)$. The regulatory $\mathrm{T}$ lymphocytes percentage was decreased in WT CLP + esmolol compared to WT CLP (10.09 \pm 3.03 vs. $12.49 \pm 2.74 \%$ of T helper, $p=0.03)$. There is a trend increase in splenocyte proliferation percentage for WT CLP + esmolol animals ( $72.0 \pm 4.2$ vs. $66.7 \pm 9.5 \%$ of proliferative lymphocytes, $p=0,72$ ). Hemodynamic and immune pattern results were similar in $\beta 1 \mathrm{KO}$ septic animals compared to $\beta 1 \mathrm{KO}$ sham animals.

Conclusion: $\beta 1$-adrenergic modulation by esmolol reduce septic shock-related immunosuppression by decreasing regulatory $\mathrm{T}$ lymphocyte percentage, increasing lymphocyte counts and trend to increase proliferation.

Reference(s) and grant ackowledgment(s)

1. This work was supported by the Fondation pour la Recherche Médicale (FRM grant number ECO20170637495 to MD.) and the Institut National de la Santé et de la Recherche Médicale 
000170

Randomized Clinical Trial Comparing Gram Stain-guided Initial Antibiotic Treatment with Guidelines-based Treatment in Patients with Ventilator-associated Pneumonia: GRam stain-guided Antibiotics ChoicE for Ventilator-Associated Pneumonia (GRACE-VAP) Trial

Y. Jumpei ${ }^{1}$;. Kazuma ${ }^{1}$; O. Yoshinori ${ }^{2}$; N. Kensuke 3 ; H. Hideki ${ }^{3}$; K. Masahiro ${ }^{4}$; T. Hiroki ${ }^{5}$; Y. Takeshi ${ }^{6}$; K. Akira $;$ K. Miyamoto ${ }^{8}$; F. Satoshi ${ }^{1}$; M. Takeshi ${ }^{9}$ ${ }^{1}$ Division of trauma and surgical critical care, Osaka General Medical Center, Osaka, Japan; ${ }^{2}$ Division of general medicine, Department of internal medicine, Hyogo College of Medicine, Hyogo, Japan; ${ }^{3}$ Department of emergency and critical care medicine, Hitachi Genaral Hospital, Hitachi, Japan; ${ }^{4}$ Department of emergency and critical care medicine, Kansai Medical University Medical Center, Osaka, Japan; ${ }^{5}$ Department of emergency and critical care medicine, Kansai Medical University Hospital, Osaka, Japan; ${ }^{6}$ Department of emergency medicine, Ebina General Hospital, Kanagawa, Japan; ${ }^{7}$ Department of emergency and critical care medicine, Sapporo City General Hospital, Hokkaido, Japan; ${ }^{8}$ Department of emergency and critical care medicine, Wakayama Medical University Hospital, Wakayama, Japan; ${ }^{9}$ Department of clinical epidemiology, Hyogo College of Medicine, Hyogo, Japan

Correspondence: $Y$. Jumpei

Intensive Care Medicine Experimental 2020, 8(2): 000170

Introduction: Restrictive use of antibiotics is required to prevent the rapid emergence and spread of multidrug-resistant pathogens but practice is challenging for critically ill patients. Gram staining should provide immediate information for predicting pathogenic bacteria, however, the effectiveness of Gram staining for initial antibiotic choice on clinical outcomes is not investigated in intensive care units (ICUs).

Objectives: To compare the clinical response between initial antibiotics selection based on Gram staining information and guidelines recommendation in patients with ventilator-associated pneumonia (VAP), which is one of the most common hospital-acquired infection in ICUs.

Methods: The GRam stain-guided Antibiotics ChoicE for VentilatorAssociated Pneumonia (GRACE-VAP) trial was a multicenter, randomized, open-label non-inferiority clinical trial conducted in 12 ICUs in Japan from April 2018 to April 2020. Eligibility criteria was aged > = 15 years, with mechanical ventilation for at least 48 hours and diagnosed as VAP with a modified clinical pulmonary infection score $>=$ 5. Patients were randomly assigned to receive Gram stain-guided initial antibiotic treatment (Gram-stain group) or guidelines-based treatment (standard group). The primary endpoint was clinical response rate which was defined as that fulfilling all four of the following components: completion of antibiotic therapy within 14 days, improvement or lack of progression of baseline radiographic findings at the end of therapy, resolution of signs and symptoms of pneumonia 7 days after the end of therapy, lack of antibiotic agent re-administration due to pneumonia within 7 days. We set non-inferiority margin at $20 \%$ for primary endpoint with one-sided alpha level of 0.025 . Secondary endpoints included the coverage rates of initial antibiotic therapies, the selected rates of anti-pseudomonal agents and anti-MRSA agents as initial antibiotic therapies, 28-day all-cause mortality, ICU-free days, ventilator-free days, and adverse events.

Results: A total of 206 patients underwent randomization (103 patients to the Gram-stain group and 103 patients to the standard group). The median age was 69 (range: 16-93)years, and men accounted for $68.4 \%$. The median hospital stay from ICU admission to enrollment was 4 (range: 3-34) days. The backgrounds were similar between groups. In a per-protocol analysis, the clinical response was observed in 79/103 patients (76.2\%) with the Gram-stain group and $74 / 103(71.7 \%)$ with the standard group (risk difference, $0.04995 \%$ confidence interval, -0.07 to $0.17 ; p<0.001$ for non-inferiority). Both anti-pseudomonal agents and anti-MRSA agents were significantly less administered in Gram-stain group than standard group 72 cases vs. 103 cases, $p<0.001 ; 63$ cases vs. 103 cases, $p<0.001$, respectively). The coverage rates of initial antibiotic therapies and 28-day all-cause mortality were similar $(86.4 \%$ vs. $92.2 \% ; p=0.18,13.6 \%$ vs. $17.5 \%, p=0.39$, respectively), and all other secondary outcomes were also similar between groups.
Conclusion: The GRACE-VAP trial showed Gram stain-guided treatment significantly restricted the use of broad-spectrum antibiotics than guidelines-based treatment with similar clinical responses in patients with VAP in ICU. This reinforced the utility of Gram staining in the setting of critical care to ameliorate the spread of multidrug-resistant pathogens. (Trial Registration; Clinicaltrials.gov NCT03506113)

\section{Reference(s) and grant ackowledgment(s)}

1. Funding was provided by Public Trust Foundation of Marumo ER Medicine \& Research Institute. However, the funding source had no role in 1) study design; 2) the collection, analysis, and interpretation of data; 3) the writing of the report; and 4) the decision to submit the manuscript for publication.

\section{6}

Role of endothelial micro RNA 155 on capillary leakage in systemic inflammation

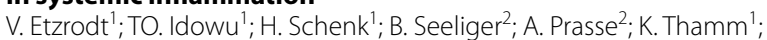

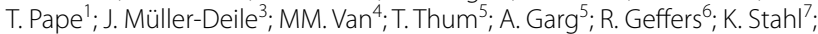
H. Haller'; S. David

${ }^{1}$ Department of Nephrology and Hypertension, Hannover Medical School, Hannover, Germany; '2Department of pneumology, Hannover Medical School, Hannover, Germany; ${ }^{3}$ Department of medicine, nephrology, University of Erlangen-Nuremberg, Erlangen, Germany; ${ }^{4}$ Department of critical care, University of Groningen, Groningen, Netherlands; Institute of molecular and translational therapeutic strategies, Hannover Medical School, Hannover, Germany; ${ }^{6}$ Genome analytics, Helmholtz Centre for Infection Research, Braunschweig, Germany; ${ }^{7}$ Department of gastroenterology, hepatology and endocrinology, Hannover Medical School, Hannover, Germany

Correspondence: $\mathrm{V}$. Etzrodt

Intensive Care Medicine Experimental 2020, 8(2): 000186

Introduction: Sepsis is a life-threatening pathological host response to an infection often leading to significant vascular leakage. The elementary molecular mechanisms remain incompletely understood and specially the role of the micro RNAs (MIR) has not been investigated further in detail. We hypothesized that specific MIRs might be upregulated in the septic endothelium thereby contributing to vascular barrier breakdown.

Objectives: To identify MIRs, regulated in the septic endothelium and to elucidate their role in endothelial permeability.

Methods: Possible regulated MIRs were screened by an unbiased MIR array approach from murine lung endothelial cells (CD146 + magnetic isolation). In vivo, the effect of specific MIRs on permeability was assessed in a transgenic zebrafish larvae (flk:mCherry) and a murine knockout (KO B6.Cg-Mir155tm1.1Rsky/J) model. Both Lipopolysaccharides (LPS) and cecal ligation and puncture (CLP) were used for the induction of systemic inflammation. Readouts were pulmonary permeability (Evans Blue, Wet-to-Dry), activity scoring and survival. Mechanistic studies were done in human umbilical vein endothelial cells (HUVECs) using standard molecular biology tools (RT-PCR, Immunoblots) and functional real-time permeability measurements (ECISTER). Furthermore, immediate post mortem kidney biopsies $(n=16)$ were performed to evaluate the expression of MIRs in a septic human organism.

Results: MIR155 was significantly upregulated in the array $(25 \times)$. Transgenic overexpression of MIR155 in zebrafish - as a screening tool - led to a extensive induction of vascular permeability (eye assay, $p<0.01$ ). The same was observed when MIR155 was experimentally overexpressed in human ECs in vitro and permeability was measured $(p<0,04)$. Moreover, MIR155 inhibition protects against classical permeability mediators (i.e. thrombin, $p<0,03$ ). We identified Claudin-1 (a tight junction protein) both on protein and mRNA level as putative MIR155 target both by overexpression and inhibition. Interestingly, in vivo the heterozygote MIR155 knockout mouse showed a protected phenotype with regard to capillary leakage and survival ( $40 \%$ benefit, $p<0.01$ ). MIR155 was also increased in septic human renal tissue compared to kidney biopsies from non-septic patients $(p=0.01)$.

Conclusion: Using an unbiased MIR-array approach, we found that MIR155 is upregulated in septic endothelium in mouse and men. It 
might contribute to the pathophysiology of vascular leakage in a Claudin-1 dependent manner. Future studies have to clarify if MIR155 could be a potential therapeutic target.

\section{Reference(s) and grant ackowledgment(s)}

1. Deutsches Zentrum für Lungenforschung (DZL)

2. German Research Foundation (DA 1209/4-3)

\section{7}

Long-term developmental impact of withholding parenteral nutrition in paediatric-ICU: a 4-year follow-up of the PEPaNIC randomized controlled trial

A. Jacobs'; K. Dulfer'2; R. Eveleens²; J. Hordijk²; H. Van Cleemput';

I. Verlinden'; P. Wouters'; L. Mebis'; G. Garcia Guerra ${ }^{3}$; K. Joosten ${ }^{2}$

S. Verbruggen ${ }^{2}$; F. Güiza'; I. Vanhorebeek'; G. Van den Berghe

${ }^{1}$ Department and laboratory of intensive care medicine, KU Leuven, Leuven, Belgium; ${ }^{2}$ Department of paediatrics and paediatric surgery, Erasmus MC-Sophia, Rotterdam, Netherlands; ${ }^{3}$ Department of paediatrics, intensive care unit, University of Alberta Hospital, Edmonton, Canada

Correspondence: A. Jacobs

Intensive Care Medicine Experimental 2020, 8(2): 000237

Introduction: Between 2012-2015, the PEPaNIC randomized controlled trial, which included 1440 critically ill infants and children, showed that withholding parenteral nutrition during the first week in the paediatric intensive care unit (PICU) (late-PN), as compared with initiating supplemental PN early (early-PN), improved PICU outcomes (1) and improved neurocognitive development assessed 2 years later (2). The latter was explained by avoiding early-PN induced adversely altered DNA-methylation of $37 \mathrm{CpG}$ sites (3). As a large number of patients were younger than 1 year of age at randomization and given that assessment of most neurocognitive domains is only possible from 4 years of age onwards, we performed a 4-year follow-up to determine the impact of late-PN versus early-PN on physical, neurocognitive, and emotional/behavioural development. This pre-planned, 4-year follow-up study of the 1440 PEPaNIC patients and of 369 matched healthy children was blinded for treatment allocation (ClinicalTrials. gov-NCT01536275).

Methods: Studied clinical outcomes included anthropometrics, health status, parent/caregiver-reported executive functions, and emotional/ behavioural problems, and clinical tests for intelligence, visual-motor integration, alertness,motor coordination and memory. Univariable and multivariable linear and logistic regression analyses adjusted for risk factors assessed the impact of late-PN versus early-PN on the outcomes and investigated a potential mediation role of the adversely altered DNA-methylation of $37 \mathrm{CpG}$ sites previously shown to be evoked by late-PN as compared with early-PN (3).

Results: Overall, at 4 years follow-up, patients (356 late-PN patients, 328 early-PN patients) could be tested neurocognitively. They revealed worse anthropometric, health status, neurocognitive and emotional/behavioural developmental outcomes than the healthy control children. Outcomes of late-PN patients were never worse than those of early-PN patients. In contrast, late-PN patients had fewer internalizing $(P=0.042)$ and externalizing problems $(P=0.046)$, and fewer total emotional/behavioural problems $(P=0.007)$ than earlyPN patients, which were normalized by late-PN. Avoiding the early-PN induced adversely altered DNA-methylation status of the $37 \mathrm{CpG}$ sites statistically explained its impact on the behavioural outcomes.

Conclusion: Four years after randomization to late-PN or early-PN in the PICU, late-PN did not show harm, and was found to protect against emotional/behavioural problems, with altered DNA-methylation as a potential biological mediator hereof. These data further support deimplementation of PN-use early during critical illness in infants and children.

\footnotetext{
Reference(s) and grant ackowledgment(s)

1. 1. Fivez et al. N Eng J Med 2016

2. 2. Verstraete et al. Lancet Respir Med 2019

3. 3. Guiza et al. Lancet Respir Med 2020

4. European Society for Clinical Nutrition and Metabolism (ESPEN) research grant (to SV)
}

5. Erasmus Trustfonds (to SV)

6. Nutricia Research BV (to SV)

7. Stichting Agis Zorginnovatie (to SV)

8. Sophia Foundation (to SV)

9. Institute for Science and Technology, Flanders, Belgium (IWT/070695/ TBM to GVdB)

10. Methusalem program of the Flemish Government (METH/08/07 to GVdB and METH14/06 to GVdB and IV)

11. ERC Advanced Grant (AdvG-2012-321670 to GVdB)

\section{7}

PEEP titration with electrical impedance tomography and pressure-volume curve: a randomized trial in ARDS patients HJ. Hsu ${ }^{1}$; HT. Chang ${ }^{2}$ : DHT. Yen ${ }^{3}$

${ }^{1}$ Department of Internal Medicine, Far Eastern Memorial Hospital, New Taipei, Taiwan; ${ }^{2}$ Critical care medicine, Far Eastern Memorial Hospital, New Taipei City, Taiwan; ${ }^{3}$ Emergency medicine, Taipei Veterans General Hospital, Taipei, Taiwan

Correspondence: $\mathrm{H} . J$. Hsu

Intensive Care Medicine Experimental 2020, 8(2): 000277

Introduction: The mortality rate of ARDS is high (35-46\% from mild to severe) [1]. The acute phase is usually accompanied with moderate to severe hypoxemia. Positive end-expiratory pressure (PEEP) may improve oxygenation but at the same time may induce barotraumas. The best PEEP selection method is still under debate.

Objectives: The aim of the study was to compare PEEP titration with electrical impedance tomography (EIT) and with ventilator-embedded pressure-volume (PV) loop in moderate to severe ARDS.

Methods: This randomized control trials study was conducted from April 2017 to February 2019 in the ICU of FEMH. Patients with moderate to severe ARDS according to Berlin criteria were included. Total eighty-seven ARDS patients were randomized to either EIT group ( $n=$ $42)$ or PV group $(n=45)$. The exclusion criteria were clinical contraindications applying EIT or high PEEP level.

All patients were total sedated to prevent any spontaneous breathing and ventilated under volume-control mode $(\mathrm{IBW} / 6 \mathrm{ml} / \mathrm{kg})$ and $\mathrm{FiO}_{2}$ $100 \%$. The PV group used Hamilton G5 to measure the PV loop. The lungs were inflated with an increase pressure of $3 \mathrm{cmH}_{2} \mathrm{O} / \mathrm{sec}$ from $5 \mathrm{cmH}_{2} \mathrm{O}$ to $40 \mathrm{cmH}_{2} \mathrm{O}$. The pressure was kept constant at $40 \mathrm{cmH}_{2} \mathrm{O}$ for 1 second. Subsequantly, the lungs were passively deflated down to $5 \mathrm{cmH}_{2} \mathrm{O}$. PEEP with maximal hysteresis (volume difference in the PV loop) was defined as optimal. In the EIT group, optimal PEEP was defined according to the regional compliance method published elsewhere [2]. The methods are illustrated in Fig. 1.

Parameters including optimal PEEP, airway pressures, compliance (Crs), and APACHE II, $\mathrm{PaO}_{2} / \mathrm{FiO}_{2}, \mathrm{ICU}$ day, resistance of the respiratory system (R) were measured and recorded.
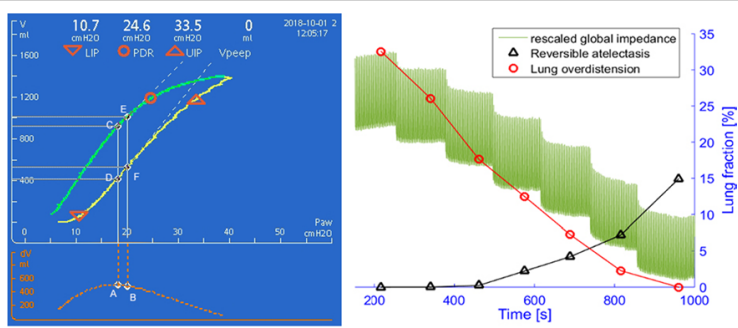

Figure 1: PEEP titration with G5 ventilator using maximal hysteresis in PV loop (Left) and EITbased regional compliance (Right).

Results: After optimization, PEEP was significantly higher in the PV group (17.4 \pm 1.7 vs. $16.2 \pm 2.6 \mathrm{cmH}_{2} \mathrm{O}$, PV vs. EIT groups, $\left.\mathrm{p}=0.02\right)$. After 48 hours, driving pressure was significantly higher in the PV group ( $12.4 \pm 3.6$ vs. $\left.10.9 \pm 2.5 \mathrm{cmH}_{2} \mathrm{O}, \mathrm{p}=0.04\right)$. Lung mechanics and oxygenation did not differ between the groups. The survival rate was 
lower in the PV group(44.4\% vs. $69.0 \%, \mathrm{p}=0.02$; hazard ratio 2.1 , confidence interval 1.1 to 3.9 ).

Conclusion: In moderate to severe ARDS, PEEP titration guided with EIT, compared with PV curve, was associated with improved driving pressure and survival rate. These findings support the use of EIT-based PEEP titration in ARDS.

\section{Reference(s) and grant ackowledgment(s)}

1. [4]. Muders T, et al. Curr Opin Crit Care, 16: 269-75, 2010

2. [3]. Zhao Z, et al. Engineering,5: 103-107,2013

3. [2]. E Costa, et al. Intensive Care Med,35: 1132-37, 2009

4. [1]. E Rezoagli, et al. Ann Transl Med,5:282-93,2017

5. This study was supported by grants of Far Eastern Memorial Hospital (FEMH-2018-C-077 and FEMH-2019-C-071)

\section{2}

\section{Moderately prolonged permissive hypotension results} in reversible cerebral metabolic perturbations evaluated by intracerebral microdialysis

RP. Jakobsen ${ }^{1}$;TH. Nielsen ${ }^{2}$; S. Mölström ${ }^{3}$; CH. Nordström²; P. Toft ${ }^{4}$

${ }^{1}$ Department of Anaestesiology and Intensive Care, Odense University Hospital, Odense, Denmark; ${ }^{2}$ Neurosurgery, Odense University Hospital, J. B. Winsløws Vej, Odense Municipality, Denmark, Odense, Denmark; ${ }^{3}$ Department of anesthesiology and intensive care, Odense University Hospital, J. B. Winsløws Vej, Odense Municipality, Denmark, Odense, Denmark; ${ }^{4}$ Department of anesthesiology and intensive care, Odense University Hospital, Odense, Denmark

Correspondence: R.P. Jakobsen

Intensive Care Medicine Experimental 2020, 8(2): 000462

Introduction: Trauma remains a leading cause of mortality. The leading cause of preventable deaths in trauma patients is uncontrollable hemorrhage. During recent years' Damage Control Resuscitation (DCR) and Damage Control Surgery (DCS) has been the main strategy in the management of major hemorrhage in trauma patients. One aspect of DCR is permissive hypotension. However, the duration of hypotension that can be tolerated without affecting the brain is unknown. The authors have in a previously publishedexperimental study shown that prolonged (90 minutes) and severe hypotension (mean arterial pressure (MAP) of $40 \mathrm{mmHg}$ ) results in irreversible metabolic perturbation evaluated by a marked and lasting increase in the lactate to pyruvate ratio (LP ratio) measured by intracerebral microdialysis (MD). Furthermore, the same study showed that the cerebral biochemical changes in response to severe hypotension was reflected in the cerebral venous outflow.

Objectives: In the present study we investigate the effect of $60 \mathrm{~min}$ severe hypotension (MAP equal $40 \mathrm{mmHg}$ ) on the brain's energy metabolism and seek to verify earlier findings that venous cerebral blood can be used as a marker of global cerebral energy state. The hypothesis is that hemorrhagic shock for 60 min results in less severe metabolic changes evaluated as a temporarily increase in the hemisphere LP ratio.

Methods: The study was approved by the Danish Animal Experiments Inspectorate. 10 pigs were anaesthetized, and vital parameters recorded. Probes for monitoring brain tissue oxygenation $\left(\mathrm{PbtO}_{2}\right)$ and intracranial pressure was placed in the right and left hemisphere respectively. Microdialysis catheters were placed in the left parietal lobe, femoral artery and superior sagittal sinus for analysis of lactate, pyruvate, glucose, glycerol and glutamate. The superior sagittal sinus was chosen for analysis of cerebral venous blood due to the anatomic characteristics of the pig. In the pig, most of the cerebral blood is drained via paraspinal venous plexa and only a minor part passes into the internal jugular vein. Hemorrhagic shock was induced by bleeding the animal until MAP of $40 \mathrm{mmHg}$ was achieved. The MAP was kept around $40 \mathrm{mmHg}$ by further withdrawing or infusing shed blood. After $60 \mathrm{~min}$ the pigs was resuscitated with autologous blood and observed for 3 hours.
Results: The volume of shed blood was (median (interquartile range)) $1469 \mathrm{~mL}(1378.4-1583.44 \mathrm{~mL}$ ) and median blood loss per kilogram was $35 \mathrm{~mL} / \mathrm{Kg}(31.9-36.8 \mathrm{~mL} / \mathrm{Kg})$.

During the period of hemorrhagic shock, MAP decreased to a median value of $39 \mathrm{mmHg}$ (35-42). Accordingly, CPP decreased to low levels of $33 \mathrm{mmHg}(28-39)$. Similarly, $\mathrm{PbtO}_{2}$ decreased to near critical levels of $19 \mathrm{mmHg}(8-23)$. After resuscitation with shed blood MAP increased to near baseline levels $(p<0.05)$. The CPP showed a similar response, normalizing, but not reaching baseline levels $(\mathrm{p}<0.05)$. In contrast, $\mathrm{PbtO}_{2}$ increased to levels exceeding baseline values and stayed at that level during the rest of the observation period $(p<0.05)$

At baseline the lactate to pyruvate ratio ( $\mathrm{LP}$ ratio) in the hemisphere, artery and sagittal sinus were $13(8-16), 21(18-24)$ and $9(6-22)$, respectively. After induction of hemorrhagic shock, the LP ratio from the left hemisphere in 9 pigs increased to levels indicating a reversible perturbation of cerebral energy metabolism 19 (12-30). The same pattern was seen in LP measurements from the femoral artery 28 (20-35) and sagittal sinus 22 (19-26). At the end of the experiment hemisphere, artery and sinus LP ratios were 16 (10-23), 17 (15-25), and 17 (10-27), respectively. Although hemisphere and sinus LP ratios decreased, they did not reach baseline levels $(p<0.05)$. In one pig hemisphere LP ratio increased to a level indicating irreversible metabolic perturbation (LP ratio $>200$ )

Conclusion: During 60 min of severe hemorrhagic shock intracerebral microdialysis shows signs of perturbations of cerebral energy metabolism, and these changes trends towards baseline values after resuscitation. Since time apparently is an important factor in the prevention of irreversible brain damage future studies should focus on methods (e.g. vasoactive drugs) to prolong the period in which permissive hypotension is safe for the brain. Sagittal sinus microdialysis values followed hemisphere values but were not distinguishable from systemic arterial values. Venous (jugular bulb) microdialysis might have a place in monitoring conditions were global cerebral ischemia is a risk, but further studies are required.

\section{Reference(s) and grant ackowledgment(s)}

1. Granfeldt A et al. (2012) Adenocaine and Mg(2+ ) reduce fluid requirement to maintain hypotensive resuscitation and improve cardiac and renal function in a porcine model of severe hemorrhagic shock* Critical care medicine 40:3013-3025 https://doi.org/10.1097/CCM.0b013e3182 $5 \mathrm{fd} 4 \mathrm{ed}$

2. Lavoie P et al. (2008) Functional cerebral venous outflow in swine and baboon: feasibility of an intracranial venous hypertension model Journal of investigative surgery : the official journal of the Academy of Surgical Research 21:323-329 https://doi.org/10.1080/0894193080 2438880

3. Jakobsen R, Halfeld Nielsen T, Granfeldt A, Toft P, Nordstrom CH (2016) A technique for continuous bedside monitoring of global cerebral energy state Intensive care medicine experimental 4:3 https://doi.org/10.1186/ s40635-016-0077-2

4. Gutierrez G, Reines HD, Wulf-Gutierrez ME (2004) Clinical review: hemorrhagic shock Critical care (London, England) 8:373-381 https://doi. org/10.1186/cc2851

5. Cecconi M et al. (2014) Consensus on circulatory shock and hemodynamic monitoring. Task force of the European Society of Intensive Care Medicine Intensive care medicine 40:1795-1815 https://doi. org/10.1007/s00134-014-3525-z

6. Cothren CC, Moore EE, Hedegaard HB, Meng K (2007) Epidemiology of urban trauma deaths: a comprehensive reassessment 10 years later World J Surg 31:1507-1511 https://doi.org/10.1007/s00268-007-9087-2

7. Intensiv Symposium Hindsgavl, Denmark

8. Aase and Ejnar Danielsens Foundation, Denmark

9. A. P. Møller Foundation for the Advancement of Medical Science, Denmark

10. Danish Society of Anaesthesiology and Intensive Care Medicine 


\section{3}

Impact of oral chlorhexidine de-adoption and implementation of an oral care bundle on mortality for mechanically ventilated adults: A stepped wedge cluster randomized controlled trial

C. Dale'; L. Rose 2 ;. Carbone ${ }^{1}$; R. Pinto ${ }^{3}$; O. Smith ${ }^{4}$; L. Burry ${ }^{5}$; E. Fan ${ }^{6}$; AC. Amaral ${ }^{3}$; V. Mccredie”; D. Scales ${ }^{3} ;$ B. Cuthbertson ${ }^{3}$

${ }^{1}$ Lawrence S. Bloomberg Faculty of Nursing, University of Toronto - St. George Campus, Toronto, Canada; ${ }^{2}$ Nursing \& midwifery, King's College London, London, United Kingdom; ${ }^{3}$ Critical care medicine, Sunnybrook Health Sciences Centre, Toronto, Canada; ${ }^{4}$ Critical care, St. Michael's Hospital, Toronto, Canada; ${ }^{5}$ Critical care, Mount Sinai Hospital, Toronto, Canada; ${ }^{6}$ Critical care medicine, Toronto General Hospital, Toronto, Canada; ${ }^{7} \mathrm{Critical}$ care medicine, Toronto Western Hospital, Toronto, Canada

Correspondence: $C$. Dale

Intensive Care Medicine Experimental 2020, 8(2): 000463

Introduction: Oral chlorhexidine is used widely for mechanically ventilated patients to prevent ventilator-associated pneumonia but recent studies show an association with excess mortality $[1,2]$.

Objectives: The primary objective of this study was to determine whether de-adoption of oral chlorhexidine and parallel implementation of a standardized oral care bundle reduces mortality in mechanically ventilated patients. Secondary outcomes were infection-related ventilator-associated complications (IVACs), oral health dysfunction and oral procedural pain.

Methods: A stepped wedge cluster randomized controlled trial withintegrated process evaluation conducted December 2017 to January 2019 in 6 academic intensive care units (ICUs) in Toronto, Canada [3] Clusters were randomized to de-adopt oral chlorhexidine and implement a standardized 5-part oral care bundle comprising oral assessment, tooth brushing, mouth moisturization, lip moisturization and secretion removal every two months. We used an integrated knowledge translation strategy including point-of-care education, audit and feedback, and reminders to facilitate change in oral care [4]. Analyses were adjusted for patient characteristics, clustering within sites and secular trends. Trained observers conducted random evaluation of fidelity to the oral care bundle.

Results: A total of 3260 patients were enrolled; 1560 baseline (control), 1700 intervention phase. We found no difference in crude ICU mortality, 332 (21.2\%) (control) and 400 (23.5\%) (intervention) (adjusted odds ratio [aOR], 1.13; 95\% confidence interval $[\mathrm{Cl}], 0.82$ $1.54 ; P=0.46$ ) or the time to IVACs (adjusted hazard ratio, $1.06 ; 95 \% \mathrm{Cl}$ $0.44-2.57 ; P=0.90)$. Oral procedural pain was not significantly different (aOR, 0.62; 95\% Cl, 0.34-1.10; $P=0.10)$, however, oral health dysfunction scores significantly improved in the intervention phase $(-0.96$ $95 \% \mathrm{Cl}_{x}-1.75$ to $-0.17 ; P=0.02$ ). Among 348 randomly observed oral care encounters, we identified $100 \%$ compliance with oral chlorhexidine de-adoption between control and intervention periods $(P<0.0001)$ and delivery of 4 out 5 elements of the oral care bundle increased $(P<0.0001)$ in the intervention phase.

Conclusion: Among patients receiving mechanical ventilation, deadoption of oral chlorhexidine and implementation of a standardized oral care bundle was not associated with lower mortality, IVACs or oral procedural pain, but did improve oral care. Our results indicate active de-adoption of oral chlorhexidine for IVAC prevention from clinical practice is needed due to lack of benefit and a standardized oral care bundle results in clinically important improvements in oral health and should be adopted into practice.

\section{Reference(s) and grant ackowledgment(s)}

1. 4. Sinuff T, Muscedere J, Adhikari NK, et al. Knowledge translation interventions for critically ill patients: a systematic review. Crit Care Med. 2013:41(11):2627-2640.

2. This study was funded by the Network for Canadian Oral Health and Research and the Canadian Lung Association.

3. 1. Klompas M, Speck K, Howell MD, Greene LR, Berenholtz SM. Reappraisal of routine oral care with chlorhexidine gluconate for patients receiving mechanical ventilation: systematic review and meta-analysis. JAMA Intern Med. 2014;174(5):751-761.

4. 2. Price R, MacLennan G, Glen J. Selective digestive oropharyngeal decontamination and topical oropharyngeal chlorhexidine for prevention of death in general intensive care: systematic review and network metaanalysis. BMJ. 2014;348:g2197

5. 3. Dale CM, Rose L, Carbone S, et al. Protocol for a multi-centered, stepped wedge, cluster randomized controlled trial of the de-adoption of oral chlorhexidine prophylaxis and implementation of an oral care bundle for mechanically ventilated critically ill patients: the CHORAL study. Trials. 2019:20(1):603

000512

Effects of high flow nasal cannula (HFNC) in patients with sepsis and septic shock of extrapulmonary origin MC. Basile ; I. Ottaviani2; T. Mauri'; S. Spadaro ${ }^{2}$; DL. Grieco ${ }^{3}$; E. Spinelli ${ }^{1}$; A. Galazzi'; G. Grasselli'i CA. Volta ${ }^{1}$; A. Pesenti

${ }^{1}$ Department of anesthesia, critical care and emergency, Fondazione IRCCS Ca'Granda Ospedale Maggiore Policlinico, Milano, Italy; ${ }^{2}$ Department of morphology, surgery and experimental medicine, University of Ferrara, Ferrara, Italy: ${ }^{3}$ Department of anesthesiology and intensive care medicine, Catholic University of The Sacred Heart, IRCCS Fondazione Policlinico A. Gemelli, Roma, Italy

Correspondence: M.C. Basile

Intensive Care Medicine Experimental 2020, 8(2): 000512

Introduction: High Flow Nasal Cannula (HFNC) provides heated and humidified high gas flow ( 30 to $60 \mathrm{~L} / \mathrm{min}$ ) that reduces the inspiratory effort in hypoxemic patients [1]. No study showed the effects of HFNC in patients with increased respiratory drive from systemic inflammation and acidosis.

Objectives: HFNC might decrease effort measured by esophageal pressure swing ( $\triangle \mathrm{P}$ es) in non-intubated patients with diagnosis of extra-pulmonary sepsis and septic shock.

Methods: We performed a prospective, multicentric physiological study in non-intubated sepsis and septic shock patients admitted to the Intensive Care Units of Maggiore Policlinico Hospital in Milan and of Sant'Anna Hospital in Ferrara. Exclusion criteria were: diagnosis of pneumonia, GCS $<12$ and contraindication to the positioning of the esophageal catheter. Study protocol consisted of 3 consecutive phases of 20 minutes: first low flow oxygen (LFO), then HFNC and lastly $\mathrm{LFO}$. $\mathrm{FiO}_{2}$ was titrated to maintain $\mathrm{SpO}_{2}$ of $92-96 \%$. During all phases, esophageal pressure and electrical impedance tomography waveforms were continuously recorded.

Results: We present preliminary results on 22 patients ( $68 \pm 14$ yo) $11(50 \%)$ with diagnosis of septic shock, with median serum lactate level of 2.30 [1.20-3.37] mmol/L and SOFA score 5 [4-9]. During HFNC, spontaneous effort measured as $\triangle$ Pes (see Figure 1) and as esophageal Pressure-Time Product (PTP) decreased, together with respiratory rate (RR), in comparison to LFO. Positive airway pressure generated by HFNC is showed by increased End Expiratory Lung Impedance ( $\Delta$ EELI) (see Table). Interestingly these effects were maintained, in part, also when patients were switched back to LFO.

\begin{tabular}{|c|c|c|c|c|}
\hline & LFO & HFNC & LFO & $\begin{array}{l}\text { ANOVA P } \\
\text { VALUE }\end{array}$ \\
\hline FiO $\square(\%)$ & $32[28-36]$ & $24[21-29]^{*}$ & $24[22-37]$ & 0.01 \\
\hline $\mathrm{SpO} \square(\%)$ & $96[94-96]$ & $96[95-96]$ & $96[95-96]$ & 0.526 \\
\hline $\mathrm{PaO} \square / \mathrm{FiO} \square$ & $\begin{array}{l}256[204- \\
355]\end{array}$ & $\begin{array}{l}312[250- \\
360]\end{array}$ & $\begin{array}{l}278[229- \\
327]\end{array}$ & 0.232 \\
\hline Rox Index & $13[9-21]$ & $19[15-28]^{*}$ & $17[11-25]$ & $<0.001$ \\
\hline RR (bpm) & $22[17-24]$ & $19[14-23]^{* *}$ & $20[15-24]$ & $<0.001$ \\
\hline$\triangle \operatorname{EELI}($ au) & baseline & $\begin{array}{l}728[22- \\
1496]^{*}\end{array}$ & $\begin{array}{l}348[23- \\
1338]^{*}\end{array}$ & $<0.01$ \\
\hline$\triangle$ Pes (cmH $\square$ O) & $8[6-9]$ & $5[4-7]^{*}$ & $7[5-12]$ & $<0.01$ \\
\hline $\begin{array}{l}\text { PTP } \\
\text { (cmH } \square O^{*} \text { s/min) }\end{array}$ & $\begin{array}{l}185[143- \\
256]\end{array}$ & $\begin{array}{l}130[82- \\
180]^{*}\end{array}$ & $165[90-237]$ & 0.01 \\
\hline
\end{tabular}

*Post-Hoc Tuckey $\mathrm{p}<0.05$ vs phase $1 \quad * *$ Post-Hoc Tuckey $\mathrm{p}<0.05$ vs phase 1 and phase 3 


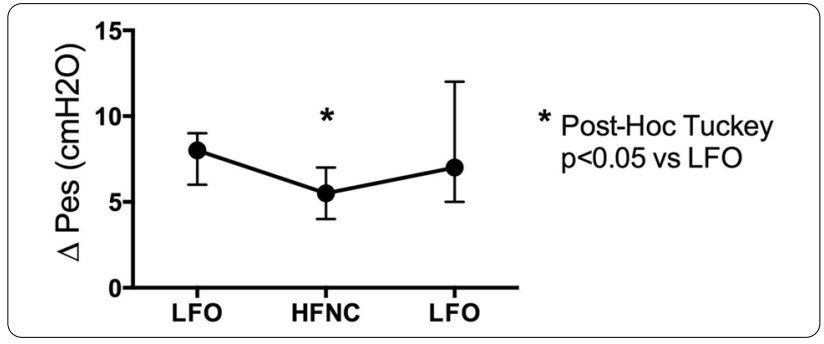

Conclusion: HFNC reduces the inspiratory effort and provides positive airway pressure in patients with sepsis and septic shock.

Reference(s) and grant ackowledgment(s)

1. [1] Mauri T, Alban L, Turrini $C$ et al. Optimum support by high-flow nasal cannula in acute hypoxemic respiratory failure: effects of increasing flow rates. Intensive Care Med. 2017 Oct;43(10):1453-1463. https://doi. org/10.1007/s00134-017-4890-1. Epub 2017 Jul 31

\section{4}

Evidence of heart decoupling between work, metabolism and microcirculation in endotoxic shock: [11C] acetate PET approach

N. Visinoni'; A. Mansart ${ }^{2}$; G. Pottier ${ }^{3}$; P. Merlet ${ }^{4}$; D. Annane'; D. Silva'; J. Aboab'

${ }^{1}$ Intensive care unit, Delafontaine Hospital, Saint-Denis, France; ${ }^{2}$ Neuroendocrine response laboratory - e4342, Versailles Saint-Quentin-en-Yvelines University, Versailles, France; ${ }^{3}$ Experimental molecular imaging laboratory - inserm u1203, cea, dsv, i2bm, French Atomique Energie Comission (CEA), Orsay, France; ${ }^{4}$ Nuclear medicin, Saint Louis Hospital - Assistance Publique Hopitaux de Paris, Paris, France; ${ }^{5}$ Intensive care unit, Raymond Poincaré Hospital -Assistance Publique Hôpitaux de Paris, Garches, France Correspondence: $\mathrm{N}$. Visinoni

Intensive Care Medicine Experimental 2020, 8(2): 000544

Introduction: Septic shock associated with cardiovascular failure is one ofthe most important reasons of death in Intensive Care Units. The mechanisms of the heart failure associated to sepsis remain unclear. Some ex-vivo data suggested a hypothetical mitochondrial failure inducted by the pro-inflammatory mediators released during septic shock. However, those anomalies were not confirmed in vivo.

Objectives: This experiment aims at exploring the cardiac oxidative metabolism and its relation with perfusion and work through positron emission tomodensitometry (PET) imaging with [11C] acetate tracer, during an endotoxic shock in a pig model.

Methods: Forty anesthetized and mechanically ventilated pigs (mean weight $=25 \mathrm{~kg}$ ) were randomized into two groups: controls and those challenged with intravenous lipopolysaccharid (LPS-150 $\mu \mathrm{g} / \mathrm{kg} / \mathrm{min}$ during $30 \mathrm{~min}$ ) to achieve a status of profound hypodynamic shock. No vasopressors were used. Animals were exclusively cristalloid fluid resuscitated as needed, following standard hemodynamic parameters. Heart rate (HR) and arterial blood pressure were continuously monitored throughout the study period. As a direct myocardial work reflection, the rate pressure product (RPP, in $\mathrm{mmHg}^{*} \mathrm{bpm}$ ) was calculated as the product between HR and systolic blood pressure (SBP).

Subjects were submitted to PET-scan at three different time frames: before LPS infusion, during the early stage of the endotoxic shock $(T=60$ minutes after infusion) and at a late time ( $T=450 \mathrm{~min}$ from the beginning of the experiment). After the second PET-scan, animals in both groups were randomized to receive an infusion of ESMOLOL (dosed enough to lower the HR of $20 \%$ ) in order to evaluate the impact of beta-blockers in the endotoxic shock. For each PET-scan, [11C] acetate was used as tracer for the combined assessment of myocardial mean blood flow $(F$, in $\mathrm{mL} / \mathrm{min} / \mathrm{g})$ and oxidative metabolism ( $\mathrm{k} 2$, acetate clearance rate, a measure of $\mathrm{MVO} 2$ rate, in $\mathrm{mL} / \mathrm{min}$ ). Definition of myocardial regions of interests (ROIs) and kinetic modeling were performed using PMOD software (Pmod 3.4).
Results: In the LPS group ( $\mathrm{n}=20$ ), the RPP increased respectively from 11708 to $14473 \mathrm{mmHg}^{*} \mathrm{bpm}(p=0,03)$ between the baseline $(T$ $=0 \mathrm{~min}$ ) and the LPS early stage ( $T=60 \mathrm{~min}$ after infusion). In the same time, quantification of PET images performed before and after the endotoxic shock does not reveal significant changes in values of microcirculation or oxidative metabolism. These results show a dissociation during LPS challenge between the myocardial work that increased and the cardiac oxydative metabolism and the cardiac microcirculation which remained stable.

As expected, the adjunction of beta-blockers reduced the RPP (18097 vs $\left.13613 \mathrm{mmHg}^{*} \mathrm{bpm} ; p=0,02\right)$ and increased the microcirculation $(1,408$ vs $2,101 \mathrm{~mL} / \mathrm{min} / \mathrm{g} ; p=0,02)$ between the second and the last PET. No impact on the oxidative metabolism has been identified.

Conclusion: (1) These results suggest that despite the increased myocardial work caused by the LPS challenge, no significant changes in myocardial flow nor oxidative metabolism were found. These suggest a decoupling between work and PET results. The disregulation of these adaptive phenomena may participate in the onset of myocardial involvement in sepsis. (2) The beta-blockers increased the microcirculation but had no effects on the oxidative metabolism.

\section{Reference(s) and grant ackowledgment(s)}

1. Lindner O, Sörensen J, Vogt J, Fricke E, Baller D, Horstkotte D, Burchert W. Cardiac efficiency and oxygen consumption measured with 11Cacetate PET after long-term cardiac resynchronization therapy. J Nucl Med (2006); 47: 378-383.

2. Klein LJ, Visser FC, Knaapen P, Peters JH, Teule GJ, Visser CA, Lammertsma AA. Carbon-11 acetate as a tracer of myocardial oxygen consumption. Eur J Nucl Med (2001); 28: 651-668

3. The authors have no conflict of interest with regards to the topic of this article. The funding source is public (Assistance Publique - Hôpitaux de Paris and Commissariat à l'énergie atomique et aux énergies alternatives) and had no impact on study design, data collection, data interpretation, or writing

000555

Biological subphenotypes of acute respiratory distress syndrome show prognostic enrichment in the general ICU population

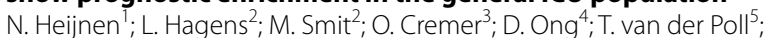
B. Scicluna ${ }^{6}$; R. Schnabel'; I. Van der Horst ; M. Schultzz; D. Bergmans"; L. Bos ${ }^{2}$

${ }^{1}$ Department of intensive care, Maastricht UMC + , Maastricht, Netherlands; ${ }^{2}$ Department of intensive care, Amsterdam UMC, locatie AMC, Amsterdam, Netherlands; ${ }^{3}$ Department of intensive care, University Medical Center Utrecht, Utrecht, Netherlands; ${ }^{4}$ Department of medical microbiology and infection control, Franciscus Gasthuis and Vlietland, Rotterdam, Netherlands; ${ }^{5}$ Center for experimental and molecular medicine, Amsterdam UMC, locatie AMC, Amsterdam, Netherlands; ${ }^{6}$ Department of clinical epidemiology, biostatistics and bioinformatics, infection and immunity, Amsterdam UMC, locatie AMC, Amsterdam, Netherlands

Correspondence: N. Heijnen

Intensive Care Medicine Experimental 2020, 8(2): 000555

Introduction: Biological subphenotypes have been identified in acute respiratory distress syndrome (ARDS) [1, 2], which have shown to provide prognostic- and possibly predictive enrichment [2-4]. Differentiation of biological subphenotypes in ARDS is mainly driven by inflammatory parameters measured in plasma. Considering that this inflammatory response can also be observed in other types of acute respiratory failure and patients admitted to the ICU for other reasons [5], the question arises whether these subphenotypes are limited to ARDS.

Objectives: To determine whether these biological subphenotypes and their prognostic- and predictive enrichment could be extended to a general ICU population, irrespective of fulfilling the definition of ARDS.

Methods: This was a secondary analysis of the Molecular Diagnosis and Risk Stratification of Sepsis (MARS) study (ClinicalTrials.gov identifier NCT01905033), a prospective observational study of adult patients admitted to the ICU. Two previously proven parsimonious models 
were used to determine the biological subphenotype of each patient. First, a cluster based model distinguishing an uninflamed and reactive subphenotype based on plasma levels of interleukin-6, interferon gamma, angiopoietin 1/2, and plasminogen activator inhibitor-1 [2]. Second, a latent class analysis (LCA) model revealing a hypo-inflammatory and hyper-inflammatory subphenotype using plasma levels of interleukin-8, protein C, and bicarbonate [4]. The prognostic enrichment was assessed with logistic regression analysis (30-day mortality) while correcting for the mediating effect of ARDS and with Cox regression analysis (ICU-mortality and ventilor-free days), for both biological phenotyping models. As part of predictive enrichment, blood leukocyte gene expression profiles in biological subphenotypes were analyzed by principal component analysis.

Results: We analyzed the data of 2500 patients (674 with ARDS and 1826 without ARDS). Here, we only report the results of patients without ARDS. The cluster-derived reactive subphenotype was, independently of ARDS, associated with (1) a higher 30-day mortality (OR 1.48, Cl 1.16-1.89), (2) a higher probability of ICU mortality (HR 2.43, Cl 1.90-3.11), and (3) a lower probability of successful extubation while alive (HR 0.66, Cl 0.59-0.74; Figure 1) compared to the uninflamed subphenotype. The blood leukocyte gene expression profiles of each individual subphenotype were similar for patients with and without ARDS (all $p>0.05$; Figure 2). LCA-derived subphenotypes showed similar profiles (data not shown).

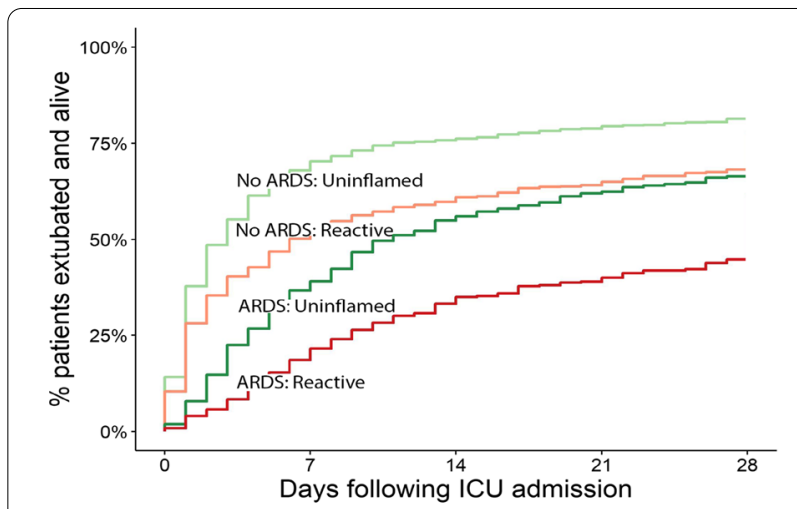

Figure 1: Percentage of patients extubated and alive over time by cluster subphenotype. The figure depicts the univariable cox proportional hazard modelling.

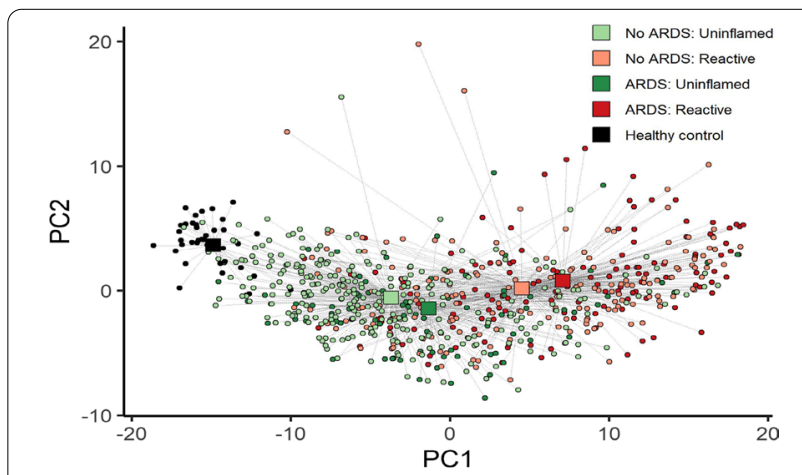

Figure 2: Principal component analysis by cluster subphenotype. On the $\mathrm{x}$-axis $\mathrm{PC} 1$ explaining approximately $56 \%$ of variance and on the y-axis PC2 explaining approximately $8 \%$ of variance
Conclusion: The prognostic- and predictive enrichment of biological ARDS subphenotypes can be extended to a general ICU population. Using the concept of biologicalsubphenotypes for splitting cohorts of critically ill patients could add to improving future precision-based trial strategies and lead to identifying treatable traits for all critically ill.

\section{Reference(s) and grant ackowledgment(s)}

1. [1] Calfee CS, Delucchi K, Parsons PE, et al (2014) Subphenotypes in acute respiratory distress syndrome: latent class analysis of data from two randomised controlled trials. Lancet Respir Med 2:611-20. https://doi. org/10.1016/S2213-2600(14)70097-9 2. Bos LD, Schouten LR, van Vught LA, et al (2017) Identification and validation of distinct biological phenotypes in patients with acute respiratory distress syndrome by cluster analysis. Thorax 72:876-883. https://doi.org/10.1136/thoraxjnl-2016-209719 3. Bos LDJ, Scicluna BP, Ong DSY, et al (2019) Understanding Heterogeneity in Biologic Phenotypes of Acute Respiratory Distress Syndrome by Leukocyte Expression Profiles. Am J Respir Crit Care Med 200:42-50. https ://doi.org/10.1164/rccm.201809-18080C 4. Sinha P, Delucchi KL, McAuley DF, et al (2020) Development and validation of parsimonious algorithms to classify acute respiratory distress syndrome phenotypes: a secondary analysis of randomised controlled trials. Lancet Respir Med 8:247-257. https ://doi.org/10.1016/S2213-2600(19)30369-8 5. Zador Z, Landry A, Balas M, et al (2020) Data Driven Analysis Reveals Shared Transcriptome Response, Immune Cell Composition, and Distinct Mortality Rates Across Differing Etiologies of Critical Illness. Crit Care Med 48:338-343. https://doi.org/10.1097/ CCM. 00000000000004128

2. [2] Bos $L D$, Schouten $L R$, van Vught $L A$, et al (2017) Identification and validation of distinct biological phenotypes in patients with acute respiratory distress syndrome by cluster analysis. Thorax $72: 876-883$. https://doi. org/10.1136/thoraxjnl-2016-209719

3. [3] Bos LDJ, Scicluna BP, Ong DSY, et al (2019) Understanding Heterogeneity in Biologic Phenotypes of Acute Respiratory Distress Syndrome by Leukocyte Expression Profiles. Am J Respir Crit Care Med 200:42-50. https://doi. org/10.1164/rccm.201809-18080C

4. [4] Sinha P, Delucchi KL, McAuley DF, et al (2020) Development and validation of parsimonious algorithms to classify acute respiratory distress syndrome phenotypes: a secondary analysis of randomised controlled trials. Lancet Respir Med 8:247-257. https://doi.org/10.1016/S2213 -2600(19)30369-8

5. [5] Zador Z, Landry A, Balas M, et al (2020) Data Driven Analysis Reveals Shared Transcriptome Response, Immune Cell Composition, and Distinct Mortality Rates Across Differing Etiologies of Critical IIIness. Crit Care Med 48:338-343. https://doi.org/10.1097/CCM.0000000000004128

6. This work was supported by the Center for Translational Molecular Medicine (http://www.ctmm.nl), project MARS (grant 04l-201).

\section{3}

Circulating bioactive adrenomedullin as a marker of sepsis, septic shock and critical illness

OHM. Lundberg'; M. Lengquist ${ }^{2}$; M. Spångfors ${ }^{3}$; M. Annborn ${ }^{4}$;

D. Bergmann ${ }^{5}$; J. Schulte ${ }^{5}$; H. Levin ${ }^{6}$; O. Melander ${ }^{7}$; A. Frigyesi ${ }^{2}$; H. Friberg ${ }^{1}$

${ }^{1}$ Department of intensive and perioperative care, Skåne University Hospital, SUS, Malmö, Malmö, Sweden; ${ }^{2}$ Departement of intensive and perioperative care, Skånes universitetssjukhus Lund, Lund, Sweden; ${ }^{3}$ Department of anaesthesia and intensive care, Kristianstad Hospital, Kristianstad, Sweden; ${ }^{4}$ Department of anaesthesia and intensive care, Helsingborgs hospital, Helsingborg, Sweden; ${ }^{5}$ Sphingotec gmbh, Sphingotec GmbH, Hennigsdorf, Germany; ${ }^{6}$ Department of clinical science, Lund University, Lund, Sweden; ${ }^{7}$ Department of internal medicine, Skåne University Hospital, SUS, Malmö, Malmö, Sweden

Correspondence: O.H.M. Lundberg

Intensive Care Medicine Experimental 2020, 8(2): 000663

Introduction: Biomarkers can be of help in understanding critical illness and to identifying and stratifying sepsis. Adrenomedullin is a peptide hormone with vasoactive properties, with reported prognostic and potentially therapeutic value in sepsis.

Objectives: The aim of this study was to investigate how levels of circulating bioactive adrenomedullin (bio-ADM) at admission to the 
intensive care unit (ICU) correspond to morbidity and mortality in sepsis patients as well as in a general ICU population.

Methods: Admission blood samples from all patients admitted to four ICUs during 2016 were batch-analysed. Patients fulfilling the sepsis-3 criteria were identified. Primary outcomes were the predictive capacity of bio-ADM to identify sepsis patients and the association of bioADM with 30-day mortality in the ICU. Secondary outcomes were the need for circulatory and renal support. A commonly used bio-ADM cutoff of $70 \mathrm{pg} / \mathrm{mL}$ was validated.

RESULTS. Bio-ADM in 1867 consecutive adult patients were analysed, and 632 patients fulfilled the sepsis criteria of whom 267 had septic shock. The median bio-ADM in the entire ICU population was $40 \mathrm{pg} /$ $\mathrm{mL}$, compared to $74 \mathrm{pg} / \mathrm{mL}$ in sepsis patients, $107 \mathrm{pg} / \mathrm{mL}$ in septic shock and $29 \mathrm{pg} / \mathrm{mL}$ in the non-septic patients. Sepsis was identified with an odds ratio (OR) of 1.64 for log2-transformed bio-ADM, after adjusting for severity of the disease. The association of elevated bio$\mathrm{ADM}$ and 30-day mortality was equally strong in sepsis patients and the ICU population with an adjusted OR of 1.23 and 1.22 , respectively. Higher levels of bio-ADM were associated with an increased need of renal replacement therapy with adjusted ORs of 2.28 and 1.97 for the ICU-population and the sepsis patients respectively, and with an increased need of vasopressors with adjusted OR of 1.33 for both populations. In the sepsis cohort, bio-ADM provided additional information to lactate on 30-day mortality. The cutoff of $70 \mathrm{pg} / \mathrm{mL}$ of bio-ADM was able to differentiate between survivors and non-survivors, but a threshold of $108 \mathrm{pg} / \mathrm{mL}$ performed better in the sepsis cohort.

Conclusion: Admission bio-ADM is an independent sepsis biomarker. In addition, bio-ADM levels are associated with 30-day mortality and organ failure in sepsis patients as well as in a general ICU-population.

\section{Reference(s) and grant ackowledgment(s)}

1. European Union Interreg programme IV A

2. Hans-Gabriel and Alice Trolle-Wachtmeister Foundation for Medical Research

3. Biobanking and BioMolecular resources Research Infrastructure (BBMRI, Sweden)

4. Government funding of clinical research within the Swedish National Health Services (ALF)

5. Regional research support, Region Skåne

\section{3}

Dysphagia and evolution during COVID-19 pandemic in critically ill patients. A multidisciplinary challenge for healthcare workers A. Glotta ; A. Galli²; M. Biggiogero ${ }^{3}$;. Tinessa'; C. Moro ${ }^{1}$; R. Mauri ${ }^{1}$;

S. Ceruti ${ }^{1}$

${ }^{1}$ Critical care department, Clinica Luganese Moncucco, Lugano, Switzerland; ${ }^{2}$ Physiotherapy service, Clinica Luganese Moncucco, Lugano, Switzerland; ${ }^{3}$ Clinical research unit, Clinica Luganese Moncucco, Lugano, Switzerland

\section{Correspondence: S. Ceruti}

Intensive Care Medicine Experimental 2020, 8(2): 000703

Introduction: Critically ill patients have an increased risk of dysphagia after prolonged oro-tracheal intubation (OTI) [1, 2]. COVID-19 is a multisystem disease caused by SARS-COV-2, characterized by acute respiratory failure; severe forms usually need mechanical ventilation (MV) support and required OTI; for prolonged courses tracheostomy is mandatory $[3,4]$.

Objectives: The purpose of this study was to evaluate dysphagia in critically ill COVID-19 patients, analyzing relations with clinical and biological data concerning MV during the ICU stay. Moreover, an analysis about recovery rate from dysphagia and its characteristics were reported.

Methods: This was a retrospective study on COVID-19 cohort patients admitted to intensive care unit (ICU); patients died before the first evaluation were excluded from the analysis. Dysphagia score has been reported in agreement with Gugging Swallowing Screen (GUSS) [5]. GUSS stratified the severity of dysphagia into severe (0-9), moderate (10-14), mild (15-19) or no dysphagia (20 points), according to clinical evaluation made by speech and language therapist (SLT). Data related to MV days, ICU length of stay (LOS) and other clinically relevant variables were analyzed.

Results: Forty-two consecutive patients were admitted to ICU, with an average age of 61 years (SD 12); according to exclusion criteria, 31 (74\%) were evaluated by the nurse and the SLT. Twenty-five of them were mechanically ventilated; 19 (76\%) patients were treated with OTI, and $6(24 \%)$ of them were tracheostomized; median MV days were 11 (7.5 16). At first SLT evaluation, 14 (45.2\%) patients showed no dysphagia, 5 (16.1\%) a mild degree of dysphagia, $6(19.4 \%)$ a moderate level of dysphagia and 6 (19.4\%) patients had a severe dysphagia. Mean first GUSS evaluation was 15.1 (SD 6.5), with a median of 19 (IQR = 12-20).

A correlation was found between distribution of GUSS score at first evaluation and ICU LOS $(p<0.001)$, MV days $(p<0.001)$, age $(p=0.03)$ and tracheostomy $(p=0.04)$. No correlation was found with BMI, SAPS, SOFA, NEMS at admission, sex, comorbidities (COPD, OSA, diabetes, Hypertension and IHD), pronation maneuvers and initial PEEP setting. After 7 days from the first SLT evaluation, GUSS stratification showed 2 (6.5\%) patients with severe form of dysphagia, 2 (6.5\%) with moderate dysphagia, $4(12.9 \%)$ with mild dysphagia and finally 23 (74.2\%) without dysphagia. This 7-days evolution resulted significantly different from baseline $(p<0.001)$. Severe, moderate, mild and none dysphagia significantly evolved on day $14(p=0.01)$ with a prevalence of $0 \%$, $3.2 \%, 9.7 \%, 87.1 \%$ respectively. Intriguingly, severe forms of dysphagia were no longer found at 12 days.

Conclusion: In COVID-19 patients, dysphagia disorders were related to the length of the MV, to ICU LOS and to tracheostomy placement. The GUSS temporal evolution could suggest that a multidisciplinary synergic approach to dysphagia management, which consist of a nurse and a SLT, would seems to improve the outcome of this symptom in critically ill COVID-19 patients. Futures analysis are necessary to confirm the hypothesis that the SLT treatment could have a positive predictive role in dysphagia recovery.

Reference(s) and grant ackowledgment(s)

1. No funding has been required

2. 1. Rassameehiran S, Klomjit S, Mankongpaisarnrung C, Rakvit A (2015) Postextubation Dysphagia. Baylor Univ Med Cent Proc 28:18-20

3. 2. Brodsky MB, Gellar JE, Dinglas VD, Colantuoni E, Mendez-Tellez PA, Shanholtz C, Palmer JB, Needham DM (2014) Duration of oral endotracheal intubation is associated with dysphagia symptoms in acute lung injury patients. J Crit Care 29:574-579

4. 3. McGrath BA, Brenner MJ, Warrillow SJ, et al (2020) Tracheostomy in the COVID-19 era: global and multidisciplinary guidance. Lancet Respir Med 2600:1-8

5. 4. Guan W, Ni Z, Hu Y, et al (2020) Clinical characteristics of coronavirus disease 2019 in China. N Engl J Med 382:1708-1720

6. 5. Trapl M, Enderle P, Nowotny M, Teuschl Y, Matz K, Dachenhausen A, Brainin M (2007) Dysphagia bedside screening for acute-stroke patients: The gugging swallowing screen. Stroke. https://doi.org/10.1161/STROK EAHA.107.483933

\section{0}

The use of high flow nasal cannula oxygen around end of life: indications, complications, outcomes

S. Jones'; L. Rose ${ }^{2}$; S. Bajwah ${ }^{3}$; N. Trenchard-Turner ${ }^{4}$; A. Rabbie'; A. Hassan ${ }^{5}$; V. Metaxa ${ }^{5}$

${ }^{1}$ Anaesthetics and critical care, Great Ormond Street Hospital, London, United Kingdom; ${ }^{2}$ Nursing \& midwifery, King's College London, London, United Kingdom; ${ }^{3}$ Palliative care, King's College Hospital, London, United Kingdom; ${ }^{4}$ Critical care outreach, St George's Hospital, London, United Kingdom; ${ }^{5}$ Critical care, King's College Hospital, London, United Kingdom Correspondence: $\mathrm{V}$. Metaxa

Intensive Care Medicine Experimental 2020, 8(2): 000750

Introduction: Over the past two decades, high-flow nasal cannula oxygenation (HFNCO) devices that deliver heated and humidified oxygen at flows up to $60 \mathrm{~L} / \mathrm{min}$, were developed as an alternative to low/ intermediate-flow devices. HFNCO has emerged as a safe and useful supportive therapy in many clinical situations and is considered a valuable tool in enhancing patients' comfort and oxygenation 1. 
The simplicity and potential benefit of HFNCO has led to its widespread use in and out of the Intensive Care Unit (ICU), even in situations where the evidence is sparse. Patients with established ceilings of treatment that prohibit intubation, who develop worsening respiratory failure is such an example. These patients are often treated with some form of non-invasive ventilation (NIV). However, discomfort and complications associated with NIV may outweigh potential benefits, and its use may extend the dying process without providing an acceptable quality of life in survivors or an acceptable quality of death in non survivors 2,3 . The use of HFNCO is seen as a potential alternative to NIV, however the impact of HFNCO around end of life (EoL) has not been extensively investigated.

Objectives: The aim of this study was to assess the number of patients receiving HFNCO initiated by an outreach team near the EoL, the indications for its use, as well as any beneficial and/or unintended effects. Methods: We conducted a retrospective observational study of all ward patients that were initiated on HFNCO within 10 days of death, over 2 years (January 2017 to January 2019), in a 950 bedded, tertiary London hospital, with a dedicated outreach service. All HFNCO initiation decisions and implementation are made by the outreach service. We reviewed electronic patient records and collected patient demographics, reasons for HFNCO initiation, initial HFNCO flow and oxygen settings, patients' response and adverse events, as well as EoL decision-making.

Results: During the study period, 736 patients received HFNCO with $95(12.9 \%)$ receiving it within 10 days prior to death. Sixty-five of them (68.4\%) were more than 70 years old and $44.2 \%$ were female. The commonest reason for hospital admission was respiratory failure $(51.6 \%)$ and the commonest reason for HFNCO initiation was hypoxia (67.4\%). Twenty-six patients (27.4\%) were recognised as EoL before initiation of HFNCO, and $6(6.3 \%)$ after the HFNCO failed to improve their condition. Details on EoL decision-making are shown in table 1. Palliative care referral was made in $82.1 \%$ of EoL patients. When HFNCO continued, 27/32 (87.1\%) diedwithin 7 days; of those in whom HFNCO was discontinued, $32 / 61$ (52.4\%) died within 7 days. In the majority of the cases $(61.1 \%)$, there were no adverse events associated with HFNCO. In the remaining patients, the most frequently documented adverse event was general discomfort, mainly due to nasal administration of high flow gas.

\begin{tabular}{|c|l|}
\hline HFNCO practices around EoL & n (\%) \\
\hline $\begin{array}{c}\text { Discussion of EoL preferences on HFNCO } \\
\text { initiation }\end{array}$ & \\
\hline $\begin{array}{c}\text { Discussed with patient } \\
\text { Discussed with family }\end{array}$ & $39(41.1)$ \\
\hline Not discussed & $32(33.7)$ \\
\hline Not documented & $20(21.1)$ \\
\hline Existing documentation of EoL preferences & $4(4.2)$ \\
\hline Documented & $73(76.8)$ \\
\hline Not documented & $22(23.2)$ \\
\hline HFNCO use around EoL & $53(55.8)$ \\
\hline Discontinued & $32(33.7)$ \\
\hline Continued & $8(8.4)$ \\
\hline Already discontinued prior to EoL & $2(2.1)$ \\
\hline determination & \\
\hline Not documented & \\
\hline
\end{tabular}

Conclusion: Approximately $13 \%$ of HFNCO use initiated by the outreach service in our hospital is for ward patients that are approaching EoL. This rescue therapy is primarily used in hypoxic respiratory failure, aiming to improve comfort. However, treatment related discomfort in almost $40 \%$ of patients highlights the importance of palliative care involvement and optimisation of traditional EoL comfort measures. Whether HFNCO actually extends the dying period in such patients requires further investigation.
Reference(s) and grant ackowledgment(s)

1. 3. Wilson ME, Majzoub MA, Dobler CC, et al. Noninvasive Ventilation in Patients With Do-Not-Intubate and Comfort-Measures-Only Orders: A Systematic Review and Meta-Analysis. Crit Care Med 2018; 46:1209-16

2. 2. Kacmarek RM. Should noninvasive ventilation be used with the do-notintubate patient? Respir Care 2009;54(2):223-9

3. 1. Papazian L, Corley A, Hess D,et al. Use of high-flow nasal cannula oxygenation in ICU adults: a narrative review. Intensive Care Med. 2016 Sep:42(9):1336-49

4. Nil

\section{0}

$(1,3)-\beta$-D-glucan-based diagnosis of invasive Candida infection versus culture- based diagnosis in patients with sepsis and with an increased risk of invasive Candida infection (CandiSep)

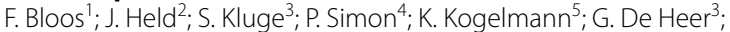

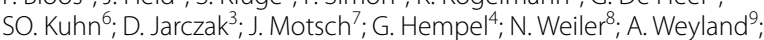
M. Drüner ${ }^{5} ;$ P. Meybohm ${ }^{10} ;$ U. Jaschinski ${ }^{11} ;$ O. Moerer ${ }^{12}$; R. Weiss ${ }^{13}$;

C. Putensen ${ }^{14}$; M. Bauer'; D. Thomas-Rüddel ${ }^{1}$

${ }^{1}$ Dept. of Anesthesiology and Intensive Care Medicine, Jena University Hospital, Jena, Germany; ${ }^{2}$ Institute of microbiology, University Hospital Erlangen, Erlangen, Germany; ${ }^{3}$ Department of intensive care medicine, University Medical Center Hamburg-Eppendorf, Hamburg, Germany; ${ }^{4}$ Dept. of anesthesiology and intensive care medicine, University Hospital Leipzig, Leipzig, Germany; ${ }^{5}$ Klinik für anästhesiologie und intensivmedizin, Klinikum Emden - Hans-Susemihl-Krankenhaus, Emden, Germany; ${ }^{6}$ Dept. of anesthesiology and intensive care medicine, Greifswald University Hospital, Greifswald, Germany; ${ }^{7}$ Dept. of anesthesiology, University Hospital Heidelberg, Heidelberg, Germany; ${ }^{8}$ Klinik für anästhesiologie und operative intensivmedizin, Universitätsklinikum Schleswig-Holstein Campus Kiel, Kiel, Germany; ${ }^{9}$ Research center neurosensory science, University of Oldenburg, Oldenburg, Germany; ${ }^{10}$ Department of anaesthesiology, University Hospital Würzburg, Würzburg, Germany; ${ }^{11}$ Anesthesiology and intensive care, University Hospital Augsburg, Augbsurg, France; ${ }^{12}$ Department of anesthesiology, emergency and intensive care medicine, University Hospital Göttingen - University Medical Center Göttingen, Göttingen, Germany; ${ }^{13}$ Dept. of anesthesiology and intensive care medicine, Universitätsklinikum Münster, Münster, Germany; ${ }^{14}$ Department of anaesthesiology and critical care medicine, University of Bonn, Bonn, Germany

Correspondence: F. Bloos

Intensive Care Medicine Experimental 2020, 8(2): 000790

Introduction: Invasive candida infection in sepsis patients is associated with a high mortality. Long time to positivity in culture-based pathogen detection further increases the risk of death by delaying antifungal therapy (1). Serum (1,3)- $\beta$-D-glucan (BDG) has a high diagnostic accuracy for detecting $\mathrm{ICI}(2)$ and is available within few hours. However, the clinical significance of BDG is unclear and current guidelines hesitate in recommending this diagnostic tool.

Objectives: To investigate whether guidance by serum-BDG concentrations results in an earlier antifungal therapy in sepsis patients with high risk of $\mathrm{ICl}$ and therefore reduces mortality.

Methods: CandiSep was a prospective multicenter randomized trial conducted in $18 \mathrm{ICU}$ in Germany (4). Patients within $24 \mathrm{hrs}$ after onset of severe sepsis or septic shock and high risk for ICI were included. Risk factors for $\mathrm{ICl}$ were total-parenteral nutrition, recent abdominal surgery, preexisting antimicrobial therapy, or renal replacement therapy. Exclusion criteria included a diagnosed $\mathrm{ICl}$ or anticipated antifungal therapy. Patients were randomized into a control or a BDG-group. Patients of the control group received targeted antifungal therapy according to current guidelines (3). In the BDG group, treating physicians received $B D G$-results taken at and 24 hours after enrollment. Antifungals were started when at least one BDG serum concentration was $\geq 80 \mathrm{pg} / \mathrm{ml}$. Primary endpoint was 28 day all-cause mortality.

Results: Of 2324 eligible patients, 339 patients were included in an intention-to-treat analysis comparing the BDG-group $(n=172)$ with standard of care $(n=167)$. Of those, 99 patients received antifungals in the BGD group and 46 patients in the control group. Time from 
randomization to antifungal therapy was 1.1 (IQR 1.0-2.2) days in the BDG group and 4.4 (IQR 2.0-9.1 days, $\mathrm{P}<0.01$ ) in the control group. The 28 -day mortality rate was $33.7 \%$ in the BDG group and $30.5 \%$ in the control group $(\mathrm{P}=0.53$; relative risk of death: $1.1,95 \% \mathrm{Cl} 0.8-1.5$, see figure for Kaplan-Meier-curve).

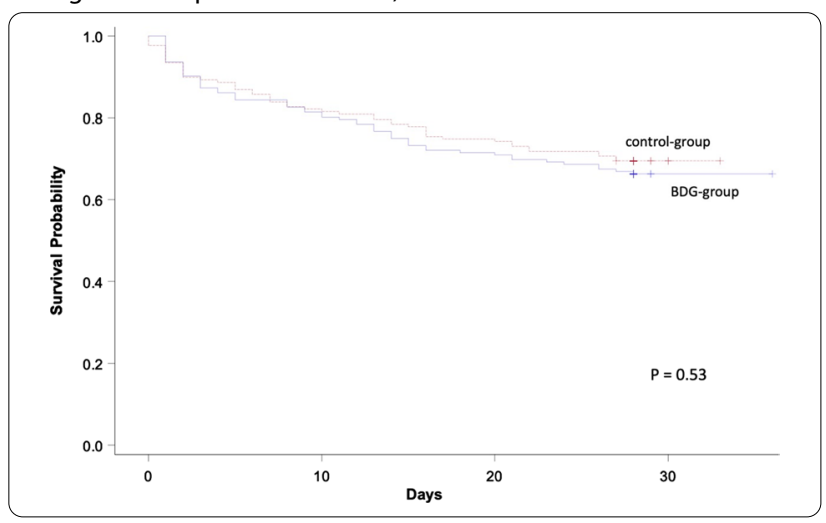

Conclusion: BDG guidance was associated with early initiation of antifungal therapy but did not reduce mortality. These results do not support BDG-guided preemptive antifungal therapy in sepsis patients.

\section{Reference(s) and grant ackowledgment(s)}

1. 1. Kollef M et al. Clin Infect Dis. 2012;54:1739; 2. Karageorgopoulos DE et al. Clin Infect Dis. 2011;52(6):750; 3. Cornely OA et al. Clinical Microbiology and Infection 18 Suppl 7:19; 4. Bloos F et al. Trials 2018:19:472

2. The study was funded by the Federal Ministry of Education and Research of Germany (grant 01EO1502).

\section{2}

Relationship between obesity and ventilator-associated pneumonia: a planned ancillary study of the NUTRIREA-2 randomized controlled trial

S. Nseir'; A. Le Gouge 2 ; O. Pouly ${ }^{3}$; J. Reignier ${ }^{4}$

${ }^{1}$ ICu, Lille Univ Hosp, Lille, France; ${ }^{2}$ Statistic department, Chru Hôpitaux De Tours, Hospital Bretonneau, Tours, France; ${ }^{3} \mathrm{cu}$, Hospital Saint Philibert, Lille, France; ${ }^{4}$ Médecine intensive réanimation, Nantes University Hospital Hotel-Dieu, Nantes, France

Correspondence: S. Nseir

Intensive Care Medicine Experimental 2020, 8(2): 000932

Introduction: Patients with obesity are at higher risk for community-acquired and ICU-acquired infections. However, no study has evaluated the relationship between obesity and ventilator-associated pneumonia (VAP).

Objectives: The aim of our study was to determine the impact of obesity on the incidence of VAP.

Methods: Planned ancillary study of the NUTRIREA-2 open-label RCT, performed in 44 French ICUs. Adults (18 years or older) receiving invasive mechanical ventilation and vasopressor support for shock were randomly assigned (1:1) to either parenteral nutrition or enteral nutrition, both targeting normocaloric goals ( $20-25 \mathrm{kcal} / \mathrm{kg}$ per day), within $24 \mathrm{~h}$ after intubation.

Obesity was defined as body mass index (BMI) $\geq 30 \mathrm{~kg} / \mathrm{m}^{2}$ at ICU admission. VAP diagnosis was adjudicated by an independent blinded committee, based on all available clinical, radiological, and bacteriological data. Only first VAP episodes were taken into account. Incidence of VAP was analyzed using a competing risk approach, with extubation, death and ICU discharge alive as competing risks. An adjusted analysis was performed including variables collected at ICU admission with $p<0.2$ in the univariate analysis. The choice of adjustment variables was based on scientific knowledge, and potential collinearity.

In a subgroup of patients $(n=151)$, we also evaluated the impact of obesity on abundant microaspiration of gastric contents and oropharyngeal secretions (defined as the presence of significant levels of pepsin (> $200 \mathrm{ng} / \mathrm{ml}$ ) and salivary amylase ( $>1685 \mathrm{Ul} / \mathrm{ml})$ in > 30\% of tracheal aspirates, collected during the $48 \mathrm{~h}$ following intubation). Results: Among the 2410 patients included in the NUTRIREA-2 trial, 85 (4\%) were excluded because of missing data on BMI. 699 of the 2325 (30\%) included patients had obesity. Female gender ( $36 \%$ vs $32 \%)$, age $(67 \pm 11$ vs $65 \pm 15$ years), Sofa score at ICU admission (med (IR) 11 $(8,13)$ vs $10(8,13)$, and comorbidities significantly $(p<0.05)$ differed between obese and non-obese patients. No significant difference was found in percentage of patients who received enteral nutrition between obese and non-obese patients ( $51 \%$ vs $50 \%$ ).

224 VAP were diagnosed (60 in the obese group and, 164 in the nonobese group). The incidence of VAP at day 28 was $8.6 \%$ vs $10.1 \%$ in obese and non-obese patients, respectively; HR: $0.845,95 \% \mathrm{Cl}$ : [0.63; $1.14], p=0.26$. After adjustment on gender, age and Knaus score, no significant difference was found in VAP incidence between obese and non-obese patients (ajusted HR: $0.914,95 \% \mathrm{Cl}$ : [0.675; 1.238], $\mathrm{p}=$ $0.56)$. Vomiting at day 7 was more frequent in obese than in non obese patients ( $24 \%$ vs $19 \%, p=0.008$ )

No significant difference was found in percentage of patients with abundant microaspiration of gastric contents ( $31 \%$ vs $19 \%, p=0.13$ ), or oropharyngeal secretions ( $63 \%$ vs $65 \%, p=0.86$ ) between obese and non-obese patients.

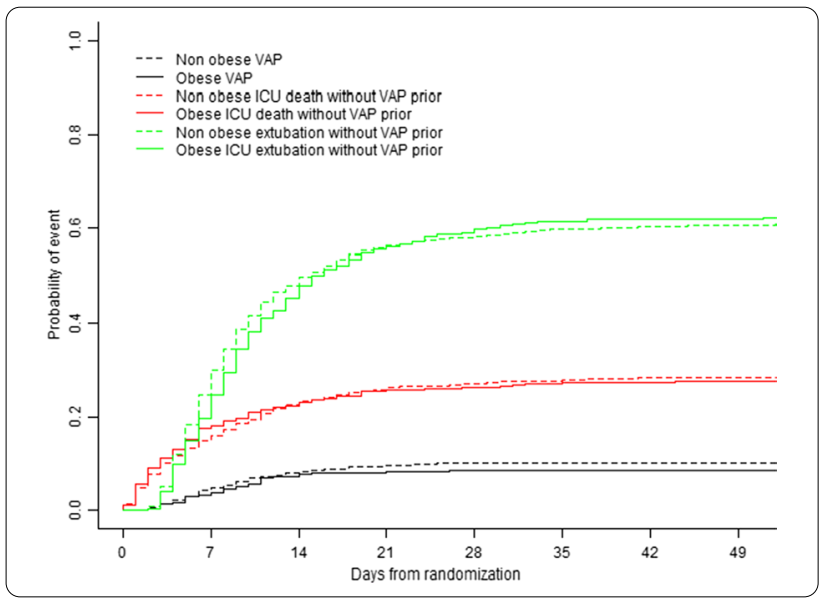

Conclusion: Our results suggest that obesity has no significant impact on the incidence of VAP.

Reference(s) and grant ackowledgment(s)

1. Reignier J, et al. Enteral versus parenteral early nutrition in ventilated adults with shock: a randomised, controlled, multicentre, open-label, parallelgroup study (NUTRIREA-2). Lancet 2018:13:133-143.

\section{8}

Incidence and risk factors of deep vein thrombosis after extracorporeal membrane oxygenation

O. van Minnen'; WM. van den Bergh ${ }^{1}$; J. Droogh ${ }^{1}$; L. Koehorst ${ }^{2}$; WK. Lagrand ${ }^{3}$; A. Oude Lansink-Hartgring ${ }^{1}$; A. Terpstra²; JM. Smit'; PR. Tuinman ${ }^{4}$; APJ. Vlaar ${ }^{3}$

${ }^{1}$ Department of critical care, University Medical Center Groningen, Groningen, Netherlands; ${ }^{2}$ Radiology, Amsterdam UMC, locatie AMC, Amsterdam, Netherlands; ${ }^{3}$ Intensive care, Amsterdam UMC, locatie AMC, Amsterdam, Netherlands: ${ }^{4}$ Intensive care, Amsterdam UMC, locatie VUmc, Amsterdam, Netherlands

Correspondence: $O$. van Minnen

Intensive Care Medicine Experimental 2020, 8(2): 000958

Introduction: Previous studies reported a various incidence of deep vein thrombosis (DVT) after decannulation from Extracorporeal membrane oxygenation (ECMO), ranging from 18 to $85 \%$ depending on screening method. The actual incidence of ECMO associated DVT is 
therefore unclear and the role of anticoagulation and potential risk factors is undetermined. Furthermore, it is unclear if cannula-associated DVT is more common in ECMO patients compared to critically ill patients without ECMO.

Objectives: To determine incidence and risk factors of DVT following decannulation from ECMO, to assess the relationship with level of anticoagulation and to compare the cannula-associated DVT rate in patients with and without ECMO.

Methods: We conducted a prospective cohort study in a 30-bed, closed format mixed medical-surgical ICU from a Dutch tertiary referral hospital. All consecutive adult patients who were successfully weaned from ECMO and underwent a routine venous duplex ultrasound of the large vessels following decannulation to assess the presence of DVT between January 2018 and March 2020 were included. We performed three multivariable logistic regression analysis to determine the association between anticoagulation with heparin and the occurrence of DVT after ECMO treatment: 1), mean aPTT per 10 seconds increase; 2 ), time weighted average aPTT under 50 seconds; and 3), time weighted average aPTT under 60 seconds, adjusted for sex, age, ECMO-mode and duration of ECMO treatment. Multivariable stepwise backward logistic regression was performed to assess risk factors for DVT. We compared cannula-associated DVT rate in ECMO patients with a prospective composed cohort of 53 critically ill adult patients without ECMO support receiving a central venous catheter (CVC) for at least 48 hours in which the inserted vein was screened for DVT after CVC removal with duplex ultrasound.

Results: During the study period 95 patients received ECMO of which 38 were successfully weaned from ECMO. Eight patients had no ultrasound of the large vessels after decannulation leaving 30 patients eligible for analyses. Of these 30 patients $14(47 \%)$ received veno-arterial ECMO. DVT was found in 15 patients $(50 \%)$ of which 10 patients (33\%) had a DVT in a cannulated vein (cannula-associated DVT). Overall, patients with DVT had a longer duration of ECMO treatment (4.7 days \pm 2.4 vs 6.9 days $\pm 3.4, p=0.047$ ) and were more often on veno-venous (VV) ECMO ( $33 \%$ vs $73 \%, p=0.07$ ). Multivariable logistic regression analysis showed no correlation between level of anticoagulation and the occurrence of DVT. With the stepwise backward logistic regression analysis, we found VV-ECMO mode to be the only independent risk factor for DVT after ECMO (OR 5.5; 95\%Cl 1.16-26.41). We found no difference between the ECMO and CVC cohort regarding cannula-associated DVT rate (33\% vs $32.1 \%$ ).

Conclusion: DVT following ECMO is frequent with half the patients showed DVT after decannulation, although pure cannula-associated DVT was not higher compared to CVC-associated DVT in a non-ECMO cohort of critically ill patients. We identified VV-ECMO as an independent risk-factor for DVT after ECMO. The level of anticoagulation based on aPTT values is not associated with the occurrence of DVT following ECMO. Administration of a higher dose of heparin seems therefore not justified to prevent DVT during ECMO treatment.

\section{Reference(s) and grant ackowledgment(s)}

1. Parzy G, Daviet F, Persico N, Rambaud R, Scemama U, Adda M, et al. Prevalence and Risk Factors for Thrombotic Complications Following Venovenous Extracorporeal Membrane Oxygenation: A CT Scan Study. Crit Care Med. 2020;48(2):192-9.

2. Fisser C, Reichenbächer C, Müller T, Schneckenpointner R, Malfertheiner MV., Philipp A, et al. Incidence and Risk Factors for Cannula-Related Venous Thrombosis After Venovenous Extracorporeal Membrane Oxygenation in Adult Patients With Acute Respiratory Failure. Crit Care Med. 2019:47(4)::332-9.

3. Menaker J, Tabatabai A, Rector R, Dolly K, Kufera J, Lee E, et al. Incidence of Cannula-Associated Deep Vein Thrombosis after Veno-Venous Extracorporeal Membrane Oxygenation. ASAIO J. 2017;63(5):588-91.

4. Trudzinski FC, Minko P, Rapp D, Fähndrich S, Haake H, Haab M, et al. Runtime and aPTT predict venous thrombosis and thromboembolism in patients on extracorporeal membrane oxygenation: a retrospective analysis. Ann Intensive Care. 2016Dec;6(1):66.

5. Cooper E, Burns J, Retter A, Salt G, Camporota L, Meadows CIS, et al. Prevalence of venous thrombosis following venovenous extracorporeal membrane oxygenation in patients with severe respiratory failure. Crit Care Med. 2015 Dec 1;43(12):e581-4.

\section{6}

Vasoactive effects of vanilloids in progressive peritoneal sepsis

J. Horak'; L. Nalos ${ }^{2}$; V. Tegl ${ }^{3}$; M. Peltanová; ; J. Beneš ${ }^{3} ;$ M. Matejovic ${ }^{4}$

${ }^{1} 1$ st medical dept., Faculty of Medicine in Pilsen, Charles University, Plzen, Czech Republic; ${ }^{2}$ Department of physiology, Faculty of Medicine in Pilsen, Charles University, Plzen, Czech Republic; ${ }^{3}$ Department of anaesthesiology, resuscitation and intensive care, University Hospital in Pilsen, Plzeň, Czechia, Czech Republic; ${ }^{4} 1$ st medical department, University Hospital in Pilsen, Pilsen, Czech Republic

Correspondence: J. Horak

Intensive Care Medicine Experimental 2020, 8(2): 001146

Introduction: Continuous administration of vasoactive drugs represents cornerstone of hemodynamic management in septic shock. Standard care of patients with vasodilatory shock includes infusions of catecholamines, vasopressin agonists and, newly, angiotensin II. Single dose of intravenous vanilloids - capsaicin and its analogue nonivamide - substances responsible for pungent taste of some eggplants, caused increase of blood pressure in anesthetized mice. We designed proof-of-concept study in a large porcine model of progressive peritoneal sepsis to analyze the hemodynamic effects of capsaicin and nonivamide.

Methods: 28 anesthetized, mechanically ventilated and instrumented pigs were assigned into six groups: control groups receiving either capsaicin (CC, $\mathrm{n}=3)$ or nonivamide $(\mathrm{CN}, \mathrm{n}=3)$; sepsis groups treated either with $(\mathrm{SNa}, \mathrm{n}=3)$ or without norepinephrine $(\mathrm{S}, \mathrm{n}=3)$, and sepsis groups treated with capsaicin $(\mathrm{SC}, \mathrm{n}=6)$ or nonivamide $(\mathrm{SN}, \mathrm{n}=$ 6). Sepsis was induced by autologous feces $(2 \mathrm{~g} / \mathrm{kg})$ inoculation into peritoneal cavity. Continuous infusion of vasopressors was administered if the mean arterial pressure (MAP) dropped below $65 \mathrm{mmHg}$ and no further positive hemodynamic response was elicited via fluid resuscitation. Vasopressors were titrated to maintain MAP between 65 and $70 \mathrm{mmHg}$. Before, $6,12,18$ and $24 \mathrm{~h}$ after the induction of peritonitis (fixed timepoints) we measured, in addition to systemic, regional (renal, portal and femoral) and microvascular (gut mucosal) hemodynamics, oxygen kinetics (DO2/VO2), organ function (lung, kidney, liver, heart), and energy metabolism (lactate kinetics, acid base balance).

Results: Administration of both vanilloids to non-septic animals did not induce significant changes in measured variables, although MAP and systemic vascular resistance tended to increase. Septic animals developed classical hemodynamic alterations with increased cardiac output and reduced systemic vascular resistance. Systemic hemodynamic effect of capsaicin and nonivamide administration was comparable to that observed in norepinephrine group. Nonivamide significantly increased blood flow through the portal vein at 6 and 9 hour after infusion initiation when compared to norepinephrine. Neither of tested vasopressors affected progressive sepsis-induced disturbances in energy metabolism and multiorgan functions.

Conclusion: To the best of our knowledge, this is the first report of intravenous administration of the vanilloids in the established porcine model of progressive pertioneal sepsis. We demonstrated their therapeutic potential in reversal of sepsis-induced vasodilatory shock. While promising, further investigation into the mechanisms of action is necessary.

Reference(s) and grant ackowledgment(s)

1. This work was supported by the Charles University Research Fund (Progres Q39) and by project No. CZ.02.1.01/0.0/0.0/16_019/0000787 "Fighting INfectious Diseases", awarded by the MEYS CR, financed from EFRR.

\section{0}

Validation of a novel non-invasive continuous method to monitor end expiratory lung volume based on $\mathrm{CO} 2$ kinetics

JA. Sanchez Giralt'; B. Abad Santamaría'; M. Wallin²; M. Hallbäck';

M. Trigueros Genao'; J. Lázaro Gonzalez'; B. Muñoz Molina'; G. Tusman"; F. Suarez Sipmann

${ }^{1}$ Intensive care medicine, Hospital de La Princesa, Madrid, Spain; ${ }^{2}$ Physiology and pharmacology, Karolinska Institute, Solna, Sweden; ${ }^{3}$ Critical care, MAQUET Critical Care, Solna, Sweden; ${ }^{4}$ Anesthesiology, Community Private Hospital, Mar del Plata, Argentina 
Correspondence: J.A. Sanchez Giralt Intensive Care Medicine Experimental 2020, 8(2): 001170

Introduction: The functionally respiratory monitoring is essential in the clinical management of patients under mechanical ventilation. Clinical monitoring is a field in constant evolution aiming at developing less-invasive, continuous, safer and inexpensive methods that provide accurate, and relevant data to aid in better clinical decision making.

Objectives: Validation of a novel, non-invasive and continuous method to monitor end expiratory lung volume based on expired $\mathrm{CO} 2$ kinetics (EELVCO2) by comparison with computer tomography measured volume in mechanically ventilated patients. Being a functional lung volume we specifically compared EELVCO2 with normally aerated end-expiratory lung volume measured by computed tomography (EELVCT-NA).

Methods: We included patients under passive mechanical ventilation. EELVCO2 was measured by the capnodynamic method, which combines the Fick principle for $\mathrm{CO} 2$, measured $\mathrm{CO} 2$ elimination obtained from volumetric capnography and the mole balance equation for $\mathrm{CO} 2$ across the lungs. Expired $\mathrm{CO} 2$ was synchronized with flow and volume measured by the ventilator's spirometer (Servo-I, Maquet Critical Care) to reconstruct a breath-by-breath volumetric capnogram. A modified repetitive breathing pattern adding brief expiratory holds in 3 consecutive breaths of the measuring cycle of 9 breaths, changed alveolar $\mathrm{CO} 2$ concentration to solve the capnodynamic equation. The resulting EELVCO2 values were continuously displayed on the computer screen. EELVCT-NA was measured during a 8-10 seconds expiratory hold. Lung EELVCT-NA was afterwards determined by manually segmenting all lung CT slices using Osirix ${ }^{\mathrm{TM}}$ (Pixmeo, Geneva, Switzerland) summing all CT compartments with a density between -900 to -500 Hounsfield Units. Pearson's correlation coefficient was used to assess correlation between methods and the Bland-Altman method was used to assess accuracy and precision.

Results: Presented data correspond to 19 patients. Median(minmax) $\mathrm{PaO}_{2} / \mathrm{FiO}_{2}$ was 201(95-430). In 3 patients $\mathrm{PaO}_{2} / \mathrm{FiO}_{2}$ was $<100$ and in 7 patients between 100-200. Median (min-max) lung volumes were $1445 \mathrm{ml}(564-2544 \mathrm{ml})$ for EELVCO2 and $1305 \mathrm{ml}(252-2361 \mathrm{ml})$ for EELVCT-NA. Correlation between EELVCO2 and EELVCT-NA was $r 2=0.78(p<0.001)$. Bias(limits of agreement) of EELVCO2 were $-139 \mathrm{ml}(-771 \mathrm{ml}$ to $493 \mathrm{ml})$.

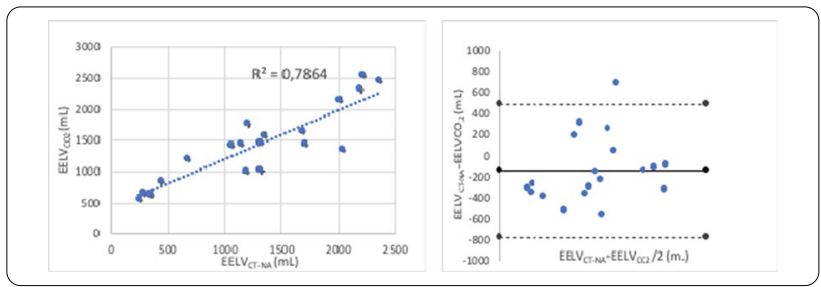

Conclusion: The new non-invasive and continuous EELVCO2 method presented a high correlation and good agreement with normally aerated CT lung volume performing with clinically relevant accuracy and precision.

\section{Reference(s) and grant ackowledgment(s)}

1. Albu G, Petak F, Zand T, Hallbäck M, Wallin M, Habre W. Lung volume assessments in normal and surfactant depleted lungs: agreement between bedside techniques and CT imaging. BMC Anesthesiol. 2014 Aug 5;14:64.

\section{5}

Where did all the women go? The underrepresentation of women in educational resources during COVID-19

T. Hall ${ }^{1}$; C. Dangoisse ${ }^{2}$; V. Metaxa

${ }^{1}$ Anaesthesia, Brighton and Sussex NHS Trust, Brighton, United Kingdom; ${ }^{2}$ Critical care, Ysbyty Gwynedd, Bangor, United Kingdom; ${ }^{3}$ Critical care, King's College Hospital, London, United Kingdom

Correspondence: T. Hall

Intensive Care Medicine Experimental 2020, 8(2):001215
Introduction: Intensive care medicine (ICM) is a growing specialty The Centre for workforce intelligence has predicted an expansion which would require anywhere between a 25 to $125 \%$ increase in the medical ICU workforce by 2033 [1]. This will require a substantial increase in recruitment to the specialty.

We know that women are currently underrepresented in ICM at consultant level and in leadership positions. According to General Medical Council (GMC) and Faculty of Intensive Care Medicine (FICM) data, $55 \%$ of UK medical graduates are women but only $20 \%$ of ICU consultants are women. These trends are similar in Europe and the rest of the world [2] and form part of a wider picture - less than 25\% of the most influential healthcare leadership positions worldwide are held by women [3].

This gender gap within ICM means that the specialty is excluding half of Medicine's talent pool and therefore not producing a workforce that is diverse and representative of the population it serves. The world economic forum showed in its' 2014 report that there is a correlation between a country's global competitiveness index and its' gender gap index [4]. If we extrapolate this to the field of Medicine, it suggests that a gender balanced ICU medical workforce could lead to improvements in the field of ICM and subsequently patient outcomes [5].

An important factor in career choice within medicine is the availability of role models and mentorship. Both male and female doctors are more likely to choose a specialty when they identify a positive role model [6]. For female trainees, having a female role seems to be particularly important [7].

Objectives: Covid-19 brought ICM into the spotlight. Whereas previously many trainees may not have had exposure to ICM, the pandemic changed this situation. Critical care societies from around the world produced excellent free online resources to educate and inform doctors who were now treating the surge of ICU patients in various settings.

We wanted to ascertain the percentage of female speakers at webinars, to see if intensive care societies are fostering female trainees' and consultants' careers by providing female role models. Most societies have issued statements regarding gender parity; we wanted to see whether they demonstrate inclusive leadership and are true advocates of gender equity [8].

Methods: We reviewed webinars produced in response to Covid-19 during a 3 month period (March, April and May 2020) by a few of the most prominent Intensive Care Medicine and Anaesthesia societies to which we were personally exposed. The choice was subjective but felt to represent webinars which were widely available and to which a significant proportion of doctors would have been exposed in Europe. The speakers were not approached individually and asked as to how they identify their gender. We therefore ascertained the gender of the speakers subjectively.

Results:

\begin{tabular}{|l|l|l|l|l|}
\hline \multicolumn{1}{|c|}{ Society } & M & F & & \multicolumn{2}{|c|}{$\begin{array}{c}\text { Percentage } \\
\text { Female }\end{array}$} \\
\hline European Society of Intensive Care Medicine & 57 & 14 & 19.7 \\
\hline Intensive Care Society & 31 & 13 & 29.5 \\
\hline $\begin{array}{l}\text { European Extracorporeal Life Support } \\
\text { Organization }\end{array}$ & 41 & 11 & 21.2 \\
\hline Association of Anaesthetists & 38 & 21 & 35.6 \\
\hline
\end{tabular}

These preliminary results show that women are grossly underrepresented in these webinars.

Conclusion: This lack of representation at a higher level within ICM will do little to encourage women into an already male dominated specialty. The ESICM equality goal which states "ESICM should aim for gender representation according to the gender proportion in the critical care medicine workforce" [9] does not go far enough to address this problem. To tackle the issue of gender inequality within ICM and build a diverse and sustainable workforce, we must provide role models and mentors to help attract female doctors to this specialty. It is time we start putting our theoretical aspiration for equal gender representation into practice. 
Reference(s) and grant ackowledgment(s)

1. 1. https://www.ficm.ac.uk/sites/default/files/cfwi_in-depth_review_of_ anaesthetics_and_icm_workforce_final.pdf (accessed 20/7/20)

2. 2. Venkatesh $B$, Mehta $\bar{S}$, Angus DC, et al. Women in Intensive Care study: a preliminary assessment of international data on female representation in the ICU physician workforce, leadership and academic positions. Crit Care. 2018;22(1):211. Published 2018 Sep 10. https://doi.org/10.1186/s1305 4-018-2139-1

3. 3. WHO (2019) Delivered by Women, Led by Men: A Gender and Equity Analysis of the Global Health Workforce, produced by the Global Health Workforce Network's Gender Equity Hub, 2019.

4. 4. http://www3.weforum.org/docs/GGGR14/GGGR_CompleteReport_2014. pdf (accessed 25/7/20)

5. 5. Lane-Fall MB, Miano TA, Aysola J, Augoustides JGT. Diversity in the Emerging Critical Care Workforce: Analysis of Demographic Trends in Critical Care Fellows From 2004 to 2014. Crit Care Med. 2017:45(5):822-827. https://doi. org/10.1097/CCM.0000000000002322

6. 6. Chadwick AJ, Baruah R. Gender disparity and implicit gender bias amongst doctors in intensive care medicine: A 'disease' we need to recognise and treat. J Intensive Care Soc. 2020;21(1):12-17. https://doi. org/10.1177/1751143719870469

7. 7. Yong et al. Sex Differences in the Pursuit of Interventional Cardiology as a Subspecialty Among Cardiovascular Fellows-in-Training. JACC February 2019

8. 8. Coe IR, Wiley R, Bekker LG. Organisational best practices towards gender equality in science and medicine. Lancet. 2019;393(10171):587-593

9. 9. Bjoern Weiss and The Task Force and Working Groups for Diversity and Equality of the ESICM. Statement paper on diversity for the European Society of Intensive Care Medicine (ESICM). Intensive Care Med (2019) 45:1002-1005

\section{4}

Temporary transvenous diaphragm neurostimulation in mechanically ventilated patients - preliminary results from a randomized controlled trial

M. Dres ${ }^{1}$; M. Gama De Abreu ${ }^{2}$; T. Similowski

${ }^{1}$ Respiratory and critical care, APHP. Sorbonne University, Paris, France; ${ }^{2}$ Klinik für anästhesie und intensivtherapie, Universitätsklinikum Carl Gustav Carus, Dresden, Germany

Correspondence: N. Mehta

Intensive Care Medicine Experimental 2020, 8(2):001224

Introduction: Mechanical ventilation (MV), while lifesaving, can lead to ventilator-induced diaphragm dysfunction (VIDD) and be associated difficulty in weaning and prolongation of MV. Mitigating VIDD may thus improve respiratory function and reduce duration of MV.

Objectives: We assessed the effects of temporary transvenous diaphragm neurostimulation (TTDN) using bilateral phrenic stimulation with a multi-electrode central venous catheter (Lungpacer Diaphragm Pacing Therapy System) on clinical outcomes and respiratory function in a multicenter, open label, randomized controlled trial. In contrast to inspiratory muscle strength training, this therapy does not depend on patients' cooperation.

Methods: Patients on invasive MV for $>96$ hours who failed at least two spontaneous breathing trials and satisfied readiness to wean criteria were randomized $(1: 1)$ to TTDN, consisting of up to 120 stimulations per day for up to 30 days, or standard of care (SoC). Clinical outcome measures included the proportion of successfully weaned subjects, mean days on MV and 30-day survival. The maximal inspiratory pressure (MIP) and rapid shallow breathing index (RSBI) were also determined. The diaphragm thickening fraction was assessed with M-mode ultrasound in a subset of patients and assessed by an ultrasound corelab blinded to group assignment.

Results: A total of 112 patients were randomly assigned to groups (57 TTDN and $55 \mathrm{SoC}$ ). The stimulation catheter was successfully placed in $43(75 \%)$ subjects and at least $50 \%$ of the protocol-required stimulations were successfully delivered in 34 (60\%) subjects. In this per-protocol subset, the incidence of successful weaning was $84 \%$ and $74 \%$ ( $p=0.436)$, duration of MV to successful weaning/study completion was $12.4 \pm 9.1$ and $14.1 \pm 10.8$ days $(p=0.438$ ); survival through
Day 30 was $91 \%$ and $85 \%(p=0.414)$ for the TTDN and SoC groups, respectively. Change in MIP from baseline was significantly different: $20 \pm 3$ (TTDN) and $5 \pm 2 \mathrm{cmH}_{2} \mathrm{O}$ (SoC) [difference $15 \pm 4 \mathrm{cmH}_{2} \mathrm{O}, \mathrm{p}=$ 0.0002 ]; change in RSBI from baseline was also significantly different: $-48 \pm 13$ (TTDN) and $-18 \pm 9$ (SoC) [difference $-30 \pm 15, \mathrm{p}=0.049$ ] with both variables showing a stimulation dose-response relationship in TTDN subjects. Compared to baseline, diaphragm thickening fraction changed by $60 \%$ (TTDN) vs $-39.5 \%$ (SoC) $(p=0.03)$ and $34.8 \%$ (TTDN) vs. $-34.2 \%(\mathrm{SoC})(\mathrm{p}=0.334)$ for the right and left hemidiaphragms, respectively. There were no unanticipated adverse device effects noted in the study.

Conclusion: TTDN increased the diaphragm thickening fraction and improved MIP and RSBI. While this study was not powered to show clinical efficacy, the results will contribute to defining the patient population and effect size to appropriately power future studies for clinical outcomes.

\section{1}

Identification of novel clinical subphenotypes in patients with community acquired pneumonia. A derivation study J. Aquino Esperanza ${ }^{1}$; P. Cardinal-Fernadéz ${ }^{2}$; C. Cillóniz ${ }^{3}$; C. Forne ${ }^{4}$; A. Gambarrus ${ }^{3}$; L. Sarlabous ${ }^{5}$; R. Magrans ${ }^{6}$; F. Ferreira ${ }^{6}$; L. Blanch ; R. Menendez; $z^{7}$ A. Torres ${ }^{3}$

${ }^{1}$ Isciii, CIBER Enfermedades Respiratorias. Institut d'linvestigació i Innovació Parc Taulí I3PT, Sabadell, Spain; ${ }^{2}$ Intensive care department, HM University Sanchinarro Hospital, Madrid, Spain; ${ }^{3}$ Pulmonology department, august pi i sunyer biomedical research institute - idibaps, Hospital Clinic de Barcelona, Madrid, Spain, Spain; ${ }^{4}$ Department of basic medical sciences, university of lleida, Heorfy Consulting, Lleida, Spain; ${ }^{5}$ Àrea de crítics, Institut d'Investigació i Innovació Parc Taulí I3PT, Sabadell, Spain; ${ }^{6}$ Bettercare, BetterCare, Sabadell, Spain; ${ }^{7}$ Pulmonology department, Hospital Universitario y Politécnico de La Fe, València, Spain

Correspondence: J. Aquino Esperanza

Intensive Care Medicine Experimental 2020, 8(2): 001241

Introduction: Community-acquired pneumonia (CAP) is the most common cause of sepsis in the developed world. Identifying factors associated with mortality is one of the main research issues with the aim to describe high-risk subsets (1). Different combinations of clinical data and host-response features may naturally cluster into previously undescribed subsets or subphenotypes (2) that may have different risks for a poor outcome and may respond to different etiologies. Similar studies have been made in sepsis (3)and ARDS (4) patients, but information in CAP is lacking.

Objectives: To derive CAP subphenotypes from clinical data and realationship with host-response inflammation, organ damage, etiology and clinical outcomes

Methods: Retrospective analysis of data sets using statistical, machine learning methods. Phenotypes were derived among 6963 patients (16 552 unique patients) who were evaluated in the emergency department (ED) from Spain and were diagnosed with pneumonia. Candidates variables were selected on clinical relevance and based on their association of CAP onset, availability during the first clinical examination, outcomes, missingness and correlations. The TRIPOD statement were followed (5). Missing data above $35 \%$ were excluded from analysis and the Little's test was used to assess the distribution and pattern of those included. Highly correlated variables were excluded ( $r$ $>0.85$ ). Multiple imputation with chained equations (MICE) was used to account for missing data. Ordering points to identify the clustering structure (OPTICS) plots were used to determine the optimal clustering strategy, and K-means clustering was the most appropriate method and therefore used. Robustness and internal validation were assessed trough Dunn's test and cluster silhouettes. We studied the frequency and clinical characteristics of the predicted phenotype groups. All analysis were performed with $\mathrm{R}$ version 3.6.1.

Results: Twenty-three variable were selected. These clusters were identified with different size, laboratory and clinical characteristics. Members of Cluster 1 (33.5\%) were the youngest [age: $45(\mathrm{SD}=13.8)$ ], less comorbid [Charlson Index: 0.7 (1)] $62.6 \%$ were male, $54.4 \%$ were current smoker and $4.9 \%$ used inhaled steroids. Individuals from 
Cluster 2 (39.5\%) were the eldest [age: $79.8(8.6)]$, the most comorbid [Charlson Index: 4.6 (1.6)], gender was equally distributed (male $49.8 \%)$, the lesser smokers (9.7\%), with a $15.4 \%$ of inhaled steroids usage. Patients from Cluster 3 (27\%) were old [age: 71.6 (13.1)], predominantly male (78.5\%), with comorbidities [Charlson Index: 3.8 (1.8)] and smokers $(58.9 \%)$, and were those ones who used inhaled steroids the most (33.2\%). Signs and symptoms in cluster 1, 2 and 3 respectively were: respiratory rate: $22,8(5.6), 24,8(5.9)$ and $31.7(7.8)$; heart rate: 98 (14.4), 92 (17) and 106 (21); SpO2: 96\% (IQR = 94-97), 94\% (92-96) and 90\% (85-93); systolic blood pressure: 121 (20), 139 (25), and 126 (29). Fever was more common in Cluster 1 (90.4\%). Inflammatory biomarkers as: C-reactive protein and white blood cells in Cluster 1,2 and 3 respectively were: $16.8(8.3-26.7), 14$ (6.4-23.1) and 20.4 (10.4-29); and: 11.405 (7.600-16.200), 11.520 (8.200-15.900) and 14.500 (10.200-19.800). Organ damage were more common in Cluster 3 with higher respiratory, hemodynamic and renal SOFA score system. Hospital admission occurred in $74.2 \%$ of Cluster 1,94.7\% of Cluster 2 and $99 \%$ of Cluster 3's patients. Critical care admission was more common in Cluster 3 (36.5\%) that Cluster 2 (8.8\%) and Cluster 1 (14.6) respectively. Thirty-day and 1-yr mortality was higher in Cluster $3(4.4 \%$ and $18.2 \%$, respectively) compared to Cluster $1(0.2 \%$ and $1.9 \%)$ and Cluster $2(0.3$ and $10.1 \%)$ respectively.

Pneumococcal pneumonia was the most frequent etiology [Cluster 1 (21.7\%), Cluster 2 (15.7\%) and Cluster 3 (25.4\%)]. Atypical pneumonia (Legionella, Mycoplasma and Chlamydia) was more frequent in Cluster 1 (3.6\%, $2.6 \%$ and $1.2 \%$ respectively).

Conclusion: In this retrospective analysis of data sets from patients evaluated in the ED with CAP, 3 clinical subphenotypes were identified that were related with host-response patterns, etiology and clinical outcomes. External validation and further research are needed to determine the utility of these subphenotypes in clinical care.

\section{Reference(s) and grant ackowledgment(s)}

1. 5. Collins GS, Reitsma JB, Altman DG, Moons KGM. Transparent reporting of a multivariable prediction model for individual prognosis or diagnosis (TRIPOD): The TRIPOD statement. Ann Intern Med 2015;162:55-63.

2. 4. Sinha P, Churpek MM, Calfee CS. Machine Learning Classifier Models Can Identify ARDS Phenotypes Using Readily Available Clinical Data. Am J Respir Crit Care Med 2020;1-46.

3. 3. Seymour CW, Kennedy JN, Wang S, Chang CCH, Elliott CF, Xu Z, Berry S, Clermont G, Cooper G, Gomez H, Huang DT, Kellum JA, Mi Q, Opal SM, Talisa V, Van Der Poll T, Visweswaran S, Vodovotz Y, Weiss JC, Yealy DM, Yende S, Angus DC. Derivation, Validation, and Potential Treatment Implications of Novel Clinical Phenotypes for Sepsis. JAMA 2019;321:2003-2017.

4. 2. Reddy K, Sinha P, O'Kane CM, Gordon AC, Calfee CS, McAuley DF. Subphenotypes in critical care: translation into clinical practice. Lancet Respir Med 2020;8:631-643

5. 1. Leoni D, Rello J. Severe community-acquired pneumonia: Optimal management. Curr Opin Infect Dis2017;30:240-247.

\section{7}

"Is this a good time to talk?" Improving telephone communication between junior doctors and relatives of patients in ICU during the COVID-19 pandemic

A. Rahim ${ }^{1}$; A. Shanmuganathan ${ }^{1}$; L. Vaccari'; G. Sartori' ; E. Leahy ${ }^{1}$; G. Erez ${ }^{2}$; V. Sathianathan ${ }^{1}$;. Husain ${ }^{1}$

${ }^{1}$ Intensive care unit, Northwick Park Hospital, London North West University Healthcare NHS Trust, Watford Road, London, HA1 3UJ, United Kingdom; ${ }^{2}$ Palliative medicine, Northwick Park Hospital, London North West University Healthcare NHS Trust, Watford Road, London, HA1 3UJ, United Kingdom

Correspondence: A. Rahim

Intensive Care Medicine Experimental 2020, 8(2): 001247

Introduction: During the COVID-19 pandemic, restrictions on hospital visitations created an unprecedented emotional stress on isolated patients and grieving families, leading to significant communication challenges [1-4]. Junior Doctors (JDs) have had to broach sensitive topics of life and death over the phone. Although there is a paucity of data on interventions to support effective communication, written and structured conversation guides are amongst the most validated [5-8].

Methods: We developed a framework in collaboration with our palliative care team to guide JDs through telephone communication with relatives of ICU patients; this outlined common events such as admission, daily updates, deterioration and bereavement.

We supported the roll out of a nurse-led video-calling service to facilitate sensitive communication, by creating a standard operating procedure (SOP) to outline instructions and define clinical governance limits $[3,6,7]$.

We distributed a questionnaire to JDs working in ICU during the pandemic, to evaluate whether our tools improved their confidence in effectively communicating with relatives (using a Likert scale 1 to $5=$ not at all confident to very confident) and their perception of whether the video-calling service improved communication with families.

Results: We received 21 responses from JDs, 57\% of whom had been redeployed from other departments. Pre COVID-19, only $10 \%$ of JDs had experienced difficult conversations with relatives about critically unwell patients once a week or more. During the pandemic, this increased to $100 \%$ of them having difficult conversations at least three times a week.

$75 \%$ of JDs scored their initial confidence in telephone communication with relatives as neutral/not confident/not at all confident; following the introduction of our framework, $76 \%$ felt confident/very confident. $71 \%$ of JDs found example conversations for different scenarios outlined in the framework helpful. Many commented that the framework was useful as point of reference and in providing phrases.

$81 \%$ of JDs were able to arrange a video-call post introduction of the SOP and felt that a nurse led video-calling service was helpful. All JDs felt that video-calls improved communication with patients' families. The main challenges identified with video-calling were concerns about confidentiality, time-burden and technical issues.

The difficulties faced by JDs in telephone discussions were the lack of non-verbal cues, technology issues, interruptions, and difficulty aligning perception of illness severity with reality. JDs commented on the emotional toll these conversations had taken on their mental health particularly when breaking bad news, describing these as "very sad", "stressful", and "traumatic".

Conclusion: The introduction of our framework for telephone communication has improved the confidence of JDs in their ability to interact with patient's relatives over the phone during the COVID-19 pandemic. The roll out of a nurse led video-calling service was felt to improve communication and was successfully facilitated by the introduction of a SOP.

Our work helps recognise the need to develop structured tools to support communication. It has been an opportunity to appreciate the emotional burden placed on JDs which is reflected in the experience of many filling this role throughout the country [9-10].

Future quality improvement interventions include assessing the families' experience of communication, providing relatives with written information about ICU and organising debriefing for JDs.

\section{Reference(s) and grant ackowledgment(s)}

1. The authors declare no conflicts of interest.

2. This research received no specific funding/grant from any funding agency in the public, commercial, or not-for-profit sectors.

3. 1. Back A, Tulsky JA, Arnold RM. Communication Skills in the Age of COVID-19. Annals of internal Medicine - Ideas and Opinions. Epub 2 June 2020. https://doi.org/10.7326/M20-1376.

4. 2. Goh KJ et. al. Preparing your intensive care unit for the COVID-19 pandemic: practical considerations and strategies. Critical Care 2020: $24,215$.

5. 3. Aziz S. et al. Managing ICU surge during the COVID-19 crisis: Rapid Guidelines. Intensive Care Medicine 2020. Epub 8 June 2020. https:// doi.org/10.1007/s00134-020-06092-5

6. 4. Akgun KM, Shamas TL, Feder SL, Schulman-Green, D. Communication strategies to mitigate fear and suffering among COVID-19 patients isolated in the ICU and their families. Heart and Lung 2020; 49(4): 344-345.

7. 5. Scheunemann LP, McDevitt M, Carson SS, Hanson LC. Randomized, Controlled Trials of Interventions to Improve Communication in 
Intensive Care: A Systematic Review. Chest 2011;139(3):543-554. Epub 24 November 2010. https://doi.org/10.1378/chest.10-0595.

8. 6. Negro A et. al. Introducing the Videocall to facilitate the communication between health care providers and families of patients in the intensive care unit during COVID-19 pandemia. Intensive Critical Care Nursing 2020: 102893. Epub 26 May 2020. May 26. https://doi. org/10.1016/j.iccn.2020.102893

9. 7. Hart JL, Turnbull AE, Oppenheim IM, Courtright KR. Family-Centered Care During the COVID-19 Era. Journal of Pain Symptom Management 2020. Epub 22 April 2020. https://doi.org/10.1016/j.jpainsymma n.2020.04.017

10. 8. Gray NA, Back AL. Covid-19 communication aids. British Medical Journal 2020; 369:m2255. Epub 11 June 2020. https://doi.org/10.1136/ bmj.m2255

11. 9. Wong H-Y. Communicating with relatives in a COVID ICU. Published 5th May 2020. https://rcoa.ac.uk/blog/communicating-relatives-covid -icu Accessed 01/07/2020

12. 10. Stuart JC. Making the call. New England Journal of Medicine 2020 . Epub 16 June 2020. https://doi.org/10.1056/NEJMp2014108

\section{4}

Epidemiology and risk factors of ventilator-associated pneumonia in COVID-19 patients: a propensity-score matched analysis

M. Martinez Martinez'; FJ. Ramos Gomez'; FX. Nuvials Casals'; O. Roca'; R. Ferrer Roca

'Intensive care department, Vall d'Hebron University Hospital, Barcelona, Spain

Correspondence: $M$. Martinez Martinez

Intensive Care Medicine Experimental 2020, 8(2): 001314

Introduction: Coronavirus disease 2019 (COVID-19) causes a respiratory failure that may require Intensive Care Unit (ICU) admission and mechanical ventilation (MV). Ventilator associated pneumonia (VAP) is a frequent complication in mechanically ventilated patients. However, there is no data about prevalence and risk factors for VAP in patients with COVID-19.

Objectives: To analyse the prevalence and risk factors for VAP in COVID-19 patients.

Methods: Retrospective observational study including all invasive mechanically ventilated COVID-19 patients admitted in the ICU in Vall d'Hebron University Hospital between March and April 2020. VAP was defined according to ATS/IDSA guidelines (1). Results were described using mean (standard deviation), median (interquartile range) or frequency (percentage). Differences between continuous variables were analysed using Student T-test or Mann-Withney U-test, as appropriate. Differences between categorical variables were assessed using Chi2 test or Fisher exact test.

To account for imbalances of baseline characteristics between both groups a propensity score matching was performed using the $R^{\circledR}$ package "matchit" with a nearest neighbour algorithm and a 1:1 matching ratio (2). Differences in variables in the matched cohort were assessed as previously described. To determine which variables were independently associated with VAP development, a logistic regression analysis was also performed in both non-matched and matched cohort using variables with $p<0.1$ in the univariate analysis. P-value $<$ 0.05 was considered statistically significant.

Results: A total of 353 patients were admitted on the study period and 250 required invasive MV. Median age was 59 (51-67), 69.2\% (173) were male. Median APACHE was 11 (8-13) and SOFA 4 (3-5). 100 patients $(40 \%)$ developed VAP. Patients who developed VAP were more frequently male, had a higher APACHE and SOFA score, were more frequently admitted to a newly open unit, and received more frequently treatment with corticosteroids and tocilizumab. These variables were included in the propensity score matching. The results of the multivariate logistic regression analysis for the risk of VAP are shown in table 1. Treatment with corticosteroids was independently associated with a higher risk of VAP in both non-matched (OR 3.364; $95 \% \mathrm{Cl} 1.829-6.185 ; \mathrm{p}<0.001$ ) and matched cohort (OR 2.071; 95\% Cl $1.141-3.758 ; p=0.017)$.

\begin{tabular}{|c|c|c|c|c|c|c|c|}
\hline \multicolumn{4}{|c|}{ Unmatched cohort } & \multicolumn{4}{|l|}{ Matched cohort } \\
\hline Variable & OR & $95 \% \mathrm{CI}$ & $p$-value & & OR & $95 \% \mathrm{CI}$ & $p$-value \\
\hline APACHE & 0.930 & $\begin{array}{l}0.849- \\
1.019\end{array}$ & 0.121 & APACHE & 0.960 & $\begin{array}{l}0.872- \\
1.056\end{array}$ & 0.400 \\
\hline SOFA & 1.539 & $\begin{array}{l}1.197- \\
1.979\end{array}$ & 0.001 & SOFA & 1.234 & $\begin{array}{l}0.960- \\
1.588\end{array}$ & 0.100 \\
\hline Corticosteroids & s 3.364 & $\begin{array}{l}1.829- \\
6.185\end{array}$ & $<0.001$ & Corticosteroids & 2.071 & $\begin{array}{l}1.141- \\
3.758\end{array}$ & 0.017 \\
\hline Tocilizumab & 1.918 & $\begin{array}{l}1.047- \\
3.512\end{array}$ & 0.035 & Age & 1.019 & $\begin{array}{l}0.993- \\
1.047\end{array}$ & 0.157 \\
\hline Gender (male) & 1.769 & $\begin{array}{l}0.944- \\
3.314\end{array}$ & 0.075 & & & & \\
\hline $\begin{array}{l}\text { Newly Open } \\
\text { ICU }\end{array}$ & 1.800 & $\begin{array}{l}1.002- \\
3.235\end{array}$ & 0.049 & & & & \\
\hline
\end{tabular}

OR: Odds ratio; CI: Confidence interval; APACHE: Acute Physiologic Assessment and Chronic Health Evaluation Score; SOFA: Sequential Organ Failure Assessment Score

Conclusion: VAP is a prevalent complication among COVID-19 patients. Those mechanically ventilated COVID-19 patients who received treatment with corticosteroids may present a higher risk of developing VAP.

\section{Reference(s) and grant ackowledgment(s)}

1. 2. Daniel Ho; Kosuke Imai; Gary King; and Elizabeth Stuart (2004) "Matchit: Matching as Nonparametric Preprocessing for Parametric Causal Inference," http://gking.harvard.edu/matchit/.

2. 1. Kalil, A, Metersky ML, Klompas M, Muscedere J, Sweeney DA, Palmer $\mathrm{LB}$, et al. Management of Adults With Hospital-acquired and Ventilatorassociated Pneumonia: 2016 Clinical Practice Guidelines by the Infectious Diseases Society of America and the American Thoracic Society. Clinical Infectious Diseases 2016.63 (5); e61-e111

\section{0}

$\mathrm{H}$ and L-subphenotype prevalence in ARDS patients with and without SARS-CoV-2

M. Chotalia'; JE. Alderman ; D. Parekh'; M. Bangash'; J. Patel ${ }^{1}$

${ }^{1}$ Birmingham acute care research group, University of Birmingham, Birmingham, United Kingdom

Correspondence: M. Chotalia

Intensive Care Medicine Experimental 2020, 8(2): 001350

Introduction: It is unclear whether patients with Severe Acute Respiratory Syndrome Coronavirus 2 (SARS-CoV-2) ARDS present with an atypical form of pulmonary illness, characterised by preserved lung compliance and low lung weight - termed L-subphenotype, or whether they reflect 'traditional' ARDS characterised by low lung compliance and high lung weight - $\mathrm{H}$-subphenotype [1].

Objectives: To calculate the prevalence of $\mathrm{H}$ and L-subphenotypes in ARDS patients with and without SARS-CoV-2 admitted to a single centre ICU.

Methods: This is a single-centre, retrospective study from the Queen Elizabeth Hospital Birmingham, UK. Two cohorts of ICU patients that received invasive mechanical ventilation were analysed: SARS-CoV-2 patients admitted between 11th March and 21st April 2020 and all patients with community acquired-pneumonia (CAP) that developed ARDS between 4th April 2016 and 5th July 2019. Patients were classified into putative $\mathrm{H}$ - and L-subphenotypes based on their admission dynamic compliance (Cdyn) and severity of opacification on chest radiography (graded by two independent reviewers using a validated, semi-quantitative opacification score ranging from 0 to 16 [2]). Cdyn is assumed to be $65 \%$ of static compliance in severe lung disease [3]. Patients with average semi-quantitative radiography scores $\leq 8$ and a Cdyn $>26 \mathrm{ml} / \mathrm{cmH}_{2} \mathrm{O}$ were classified as the L-subphenotype whilst patients with average semi-quantitative radiography scores $>8$ and a Cdyn $\leq 26 \mathrm{ml} / \mathrm{cmH}_{2} \mathrm{O}$ were classified as the $\mathrm{H}$-subphenotype. Patients who did not meet these criteria were placed in discordant 1 and 2 (D1, D2) groups. Statistical analysis was performed using GraphPad Prism v.8.0. Continuous data is presented as median (IQR) and analysed 
using a Mann-Whitney-U test. Categorical data is presented as number (\%) and analysed using a chi-squared test.

Results: 112 patients with SARS-CoV-2 ARDS and 121 patients with CAP ARDS met the inclusion criteria. The prevalence of $\mathrm{H}$ and $\mathrm{L}$-subphenotypes were similar between SARS-CoV-2 and CAP ARDS cohorts. ICU mortality was lower in patients classified as L-subphenotype and higher in patients classified as $\mathrm{H}$-subphenotype (Figure 1).

Table 1: Clinical characteristics of SARS-CoV-2 and CAP ARDS cohorts

\begin{tabular}{|l|l|l|l|}
\hline & SARS-CoV-2 & CAP & p value \\
\hline Age (years) & $56(46-63)$ & $64(49-74)$ & 0.0006 \\
\hline Gender (\%Male) & $85(76 \%)$ & $71(59 \%)$ & 0.0080 \\
\hline P/F ratio & $15(12-17)$ & $15(11-25)$ & 0.188 \\
\hline Dynamic compliance $(\mathrm{mls} / \mathrm{cmH} 2 \mathrm{O})$ & $28(23-34)$ & $27(22-32)$ & 0.156 \\
\hline CXR opacification score & $9(7-10)$ & $8(6-10)$ & 0.422 \\
\hline ICU mortality (\%) & $45(40 \%)$ & $42(35 \%)$ & 0.438 \\
\hline
\end{tabular}

Figure 1: Prevalence (A) and ICU mortality (B) of SARS-CoV-2 (CoV) and CAP ARDS cohorts
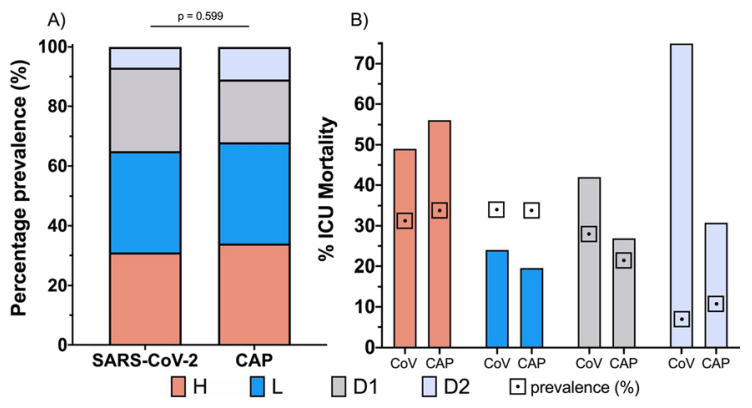

Conclusion: The prevalence of $\mathrm{H}$ and L-subphenotypes was similar in ARDS cohorts with and without and SARS-CoV-2, indicating that SARSCoV-2 respiratory failure is not an atypical form of ARDS requiring an alternative ventilation strategy. Given the lower mortality of patients classified as L-subphenotype, the heterogeneity in compliance and lung weight and the presence of these subphenotypes may simply reflect different patterns of disease severity.

\section{Reference(s) and grant ackowledgment(s)}

1. [1] Marini JJ, Gattinoni L. Management of COVID-19 Respiratory Distress. JAMA 2020.

2. [2] Mason SE, Dieffenbach PB, Englert JA, et al. Semi-quantitative visual assessment of chest radiography is associated with clinical outcomes in critically ill patients. Respir Res 2019; 20: 218.

3. [3] Stahl CA, Moller K, Schumann S et al. Dynamic versus static respiratory mechanics in acute lung injury and acute respiratory distress syndrome. Crit Care Med 2006; 34: 2090-2098

\section{2}

\section{Different renal trajectories and outcomes after Acute Kidney} Injury in Intensive Care Unit: a 5-years prospective cohort study

A. Orieux ${ }^{1}$; R. Prevel'; D. Gruson ' ; B. Clouzeau ' C. Rigothier ${ }^{2}$; C. Combe ${ }^{2}$; A. Boyer ${ }^{1} ;$ S. Rubin ${ }^{2}$

${ }^{1}$ Intensive care unit, Hospital Center University De Bordeaux, Bordeaux, France; ${ }^{2}$ Nephrology, Hospital Center University DeBordeaux, Bordeaux, France

Correspondence: A. Orieux

Intensive Care Medicine Experimental 2020, 8(2): 001362
Introduction: Acute kidney injury (AKI) is commonly observed in intensive care unit (ICU) and affects more than $50 \%$ of these patients. The consequences of AKI are well described (increased mortality, length of hospital stay, risk of chronic kidney disease (CKD)). Acute kidney disease (AKD) has been recently proposed to define the disease process after AKI and before CKD among patients in which renal pathophysiologic processes are in progress. We know that patients who suffered from AKI may have different clinical trajectories and outcomes (early, late or absence of recovery, early or late relapse, AKD or CKD), but to date, no cohort study has been able to accurately describe them

Objectives: The main objective of this study was to assess the different clinical trajectories and evaluate risk and timing of AKD and CKD after AKI in ICU. Secondary objectives were to evaluate the long-term incidence of CKD after AKI in critically ill patients and to propose the ideal timing for post-intensive care clinics.

Methods: We conducted a prospective 5-years follow-up cohort study in a medical ICU in Bordeaux University Hospital (France). Our center participated in the Artificial Kidney Initiation in Kidney Injury (AKIKI) study, during which all patients with AKI from stage 1 of the KDIGO classification were prospectively screened from September 2013 to May 2015. Patients were included if they were $\geq 18$ years of age, received invasive mechanical ventilation, catecholamine infusion, or both, and developed AKI. We excluded patients with estimated glomerular filtration rate (eGFR) $<90 \mathrm{~mL} / \mathrm{min} / 1.73 \mathrm{~m}^{2}$ (CKD-EPI) prior to ICU admission. AKI was classified according to the KDIGO classification. AKD was defined as a condition wherein criteria for AKI stage 1 or greater persists $\geq 7$ days after an exposure. CKD was defined by eGFR $<60 \mathrm{ml} / \mathrm{min} / 1.73 \mathrm{~m}^{2}$ (CKD-EPI) at least 90 days after AKI. Renal recovery was defined by the absence of KDIGO criteria. Early recovery was kidney recovery within 7 days and late recovery was renal recovery from 7 to 90 days after AKI.

Results: 304 patients suffered from AKI. Among them, 72/304 (24\%) had prior CKD and were excluded and 232 patients were enrolled: AKI stage 1, 62/232 (27\%), AKI stage 2, 50/232 (21\%) and AKI stage 3 , 120/232 (52\%). Age was $62 \pm 16$ years, 142/232 (63\%) were men. Basal serum creatinine $(\mathrm{SCr})$ was $78 \pm 18 \mu \mathrm{mol} / \mathrm{l}$ and no patients had missing baseline creatinine value. Mechanical ventilation was required for $192 / 232(83 \%)$ and $191 / 232(82 \%)$ required catecholamine use. Length of mechanical ventilation and catecholamine use were respectively 4 [2-8] days and 2.5 [1-5] days. SOFA score was $11.2 \pm 3.4$ and SAPS II was $62 \pm 19$. Among patients with AKI, 61/232 (26\%) died, 65/232 (28\%) recovered early and among them $3 / 65(5 \%)$ recovered early but secondary suffered from AKD resulting in 109/232 (47\%) who had been progressing to AKD (figure). Among AKD patients, 41/109 (38\%) died, 21/109 (19\%) had late recovery and 47/109 (43\%) developed CKD. CKD also developed in patients who recovered early and late, in respectively, $11 / 65(17 \%)$ and $5 / 21(24 \%)$ for a total of 63 out of 232 (27\%) patients included in the study. At the end of 5 years follow-up, CKD prevalence was 31\%. Among CKD patients, 16/65 (17\%) patients had already recovered and had developed a secondary relapse. A post-intensive care clinic from 30 to 90 days after ICU discharge could have failed to detect CKD in 16/63 (25\%) patients.

Conclusion: Because of many different clinical trajectories, a simple $\mathrm{SCr}$ dosage, within 3 months of ICU discharge in patients who had suffered from AKI was not sufficient to detect and prevent CKD. Assessment of progressive markers (hypertension, proteinuria) appears to be essential. 


\section{0}

Impact of sex and ICU treatment duration on creatinine and potassium excretion in critically ill patients

M. Van Bakelen-Knip ${ }^{1}$; L. Hessels ${ }^{2}$; C. Cordeiro ${ }^{3}$; S. Berger ${ }^{4}$; M. Nijsten ${ }^{2}$

${ }^{1}$ Critical care department, University Medical Center Groningen, Groningen, Netherlands; ${ }^{2}$ Critical care department, University Medical Center Groningen, Groningen, Netherlands; ${ }^{3}$ Research and development, Paramedir Bv, Groningen, Netherlands; ${ }^{4}$ Dt of nephrology, University Medical Center Groningen, Groningen, Netherlands

Correspondence: $M$. Nijsten

Intensive Care Medicine Experimental 2020, 8(2): 001370

Introduction: Critically ill patients may have considerable and sus tained loss of muscle mass. This loss may amount to more than $1 \%$ per day in ICU patients. It has been shown that urinary creatinine excretion (UCE) is a useful marker of muscle mass in non-ICU and ICU patients [1]. Recently we found that ICU patients display a negative potassium balance immediately after ICU admission, compatible with loss of intracellular volume, such as muscle mass.

Objectives: In the current study we compared UCE with urinary potassium excretion (UPE), where UCE served as marker of absolute muscle mass and UPE was hypothesized to indicate loss of muscle mass. We analyzed the time course of UCE with UPE in patients admitted for up to 30 days to our ICU and analyzed if there was a difference in UPE between males and females.

Methods: Patients for whom $24 \mathrm{~h}$ UCE and UPE were available between ICU day 1 and 30 were analyzed. Our computerized potassium regulation system regulated potassium administration in all patients. This system does not distinguish between males and females in their recommended potassium dose.

Results: 17,000 patients, aged $60 \pm 16$ year, $62 \%$ male, with a hospital mortality of $18 \%$ and 61,000 UCE and 45,000 UPE measurements were analyzed. UCE on ICU admission was $49 \%$ higher in males compared to females $(P<0.001)$ and decreased by $1.2 \% / d(P<0.001)$. Mean UPE showed a bimodal time course with a near stable excretion between ICU day 7 to 30 ( $88 \pm 59$ and $79 \pm 54 \mathrm{mmol} / \mathrm{d}$ in males and females respectively; $\mathrm{P}<0.001)$. This difference between males and females in UPE of $9 \mathrm{mmol} / \mathrm{d}$ corresponds to the expected difference on the basis of muscle intracellular potassium concentration and observed muscle loss as reflected by UCE.

Conclusion: During prolonged ICU stay, there is a sustained difference in potassium excretion between males and females that is consistent with the muscle mass loss derived from creatinine excretion. Future balance studies could establish if creatinine and potassium excretion are suitable markers for direct monitoring of muscle mass loss.

\section{9}

Effects of prone positioning on venous return in patients with acute respiratory distress syndrome

C. Lai ${ }^{1}$; I. Adda ${ }^{1}$; JL. Teboul ${ }^{1}$; R. Persichini ${ }^{1}$; F. Gavelli ${ }^{1}$; L. Guerin ${ }^{1}$; X. Monnet ${ }^{1}$ ${ }^{1}$ Médecine intensive réanimation, Bicetre Hospital AP-HP, Le Kremlin-Bicêtre, France

\section{Correspondence: $C$. La}

Intensive Care Medicine Experimental 2020, 8(2): 001429

Introduction: Prone positioning in patients with acute respiratory distress syndrome (ARDS) has variable effects on cardiac index. It has been reported that prone positioning increases cardiac preload and cardiac index in patients with preload responsiveness. We made the hypothesis that the increase in cardiac preload was related to an increased mean systemic filling pressure (Pms), the forward pressure of venous return.

Objectives: To examine the effects of prone positioning on venous return and its determinants such as Pms and venous return resistance (Rvr) in patients with ARDS.

Methods: We included 22 patients with moderate to severe ARDS in whom prone positioning was decided. Preload responsiveness was assessed at baseline using an end-expiratory occlusion test. Hemodynamic measurements, including cardiac index, central venous pressure (CVP), Pms and Rvr (the latter two estimated through the heart-lung interactions method) were measured in semi-recumbent, supine horizontal (in 15 patients) and after pronepositioning.

Results: Prone positioning significantly increased Pms (from 24 (1934) to $35(32-46) \mathrm{mmHg}$ ). This was partly due to the trunk lowering performed before prone positioning. In seven patients, prone positioning increased cardiac index $\geq 15 \%$. All were preload responsive. In these patients, prone positioning increased Pms by 82 (76-95)\%, CVP by 33 (21-59)\%, (Pms - CVP) by 144 (83-215)\%, while it increased Rvr by $71(60-154) \%$. In 15 patients, prone positioning did not increase cardiac index $\geq 15 \%$. In these patients, prone positioning increased Pms by $28(18-56) \%(\mathrm{p}<0.05 \mathrm{vs}$. patients with significant increase in cardiac index), CVP by 21 (7-54)\%, (Pms - CVP) by 28 (23-86)\%, and Rvr by 37 (17-77)\%. Eleven of these 15 patients were preload unresponsive.

Conclusion: Prone positioning increased Pms, CVP and Rvr. The resulting change in cardiac index depended on the extent of increase in (Pms-CVP), of preload responsiveness and of the increase in Rvr. Cardiac index increased only in preload responsive patients if the increase in Rvr was lower than the increase in the (Pms - CVP) difference.

\section{9}

The association between the Sequential Organ Failure Assessment scores in particular systems and intensive care unit mortality in patients $\geq \mathbf{8 0}$ years old - a prospective cohort study J. Fronczek ; H. Flaatten ${ }^{2}$; B. Guidet ${ }^{3}$; K. Polok'; D. De Lange ${ }^{4}$; J. Fjølner ${ }^{5}$; J. Górka ${ }^{1}$; C. Jung ${ }^{6}$; S. Leaver ${ }^{7}$; RP. Moreno ${ }^{8}$; A. Rhodes ${ }^{9}$; W. Szczeklik

${ }^{1}$ Intensive care and perioperative medicine, Jagiellonian University Medical College, Kraków, Poland; ${ }^{2}$ Department of anaesthesia and intensive care, Haukeland University Hospital, Bergen, Norway; ${ }^{3}$ Réanimation Médicale, Hôpital Saint-Antoine, Paris, France; ${ }^{4}$ Department of intensive care medicine, Utrecht University, Utrecht, Netherlands; ${ }^{5}$ Department of intensive care, Aarhus University Hospital, Aarhus, Denmark; ${ }^{6}$ Dep. of cardiology, pulmonology and angiology, University Hospital of Düsseldorf, Düsseldorf, Germany; 'Research lead critical care directorate, St George's Hospital, London, United Kingdom; ${ }^{8}$ Unidade de cuidados intensivos neurocríticos, Hospital de São José, Centro Hospitalar de Lisboa Central, Nova Médical School, Lisbon, Portugal; ${ }^{9}$ Anaesthesia and intensive care medicine, St George's University Hospitals NHS Foundation Trust, Londres, United Kingdom

Correspondence: J. Fronczek,

Intensive Care Medicine Experimental 2020, 8(2): 001619

Introduction: The Sequential Organ Failure Assessment (SOFA) score is being used to predict mortality in patients admitted to intensive care units (ICUs). The extent of organ failure in the SOFA is quantified using a point score with equal weights of abnormalities in different systems.

Objectives: To determine the relative contribution of alterations in different systems to the risk of ICU mortality in a population of very old intensive care unit patients (VIPs).

Methods: We used data from the Prognostic Score in the Very Old ICU Patients (VIP2) prospective, multicentre study (NCT03370692) which recruited consecutive patients $>80$ years old between May 2018 and May 2019. Worst SOFA scores within 24 hours from admission to the ICU was reported overall and in categories (i.e. respiratory, cardiovascular, hepatic, coagulation, renal and neurological systems). Logistic regression was used to study the association between the SOFA score and mortality.

Results: We included 3898/3919 (99.5\%) patients who had complete data on all components of the SOFA score in the analysis. The median age in the sample was equal to 84 years (IQR: 81-87), 2078 (53.3\%) patients were male. The median SOFA score was equal to 6 points (IQR: 4-9) and 1022/3898 (26.2\%) patients died in the ICU. Odds ratios for ICU mortality associated with the number of points in a given system $(1,2,3$, or 4 points respectively) with 0 points set as the reference category were as follows ( $95 \%$ confidence intervals shown in brackets):

respiratory: 1.21 (0.91-1.61), 1.67 (1.29-2.15), 1.63 (1.24-2.14) $3.51(2.53-4.86)$, 
- cardiovascular: 1.15 (0.90-1.45), 0.62 (0.36-1.07), 0.98 (0.77-1.25), $1.83(1.48-2.26)$,

- neurologic: 1.49 (1.20-1.85), 2.33 (1.76-3.09), 2.57 (1.95-3.39), $5.12(4.11-6.37)$

- renal: 1.57 (1.29-1.91), 1.78 (1.42-2.23), 2.59 (1.90-3.53), 2.21 (1.56-3.12),

- coagulation: 1.13 (0.91-1.39), 1.33 (0.99-1.79), 2.71 (1.54-4.76), $0.78(0.18-3.36)$,

- hepatic: 1.23 (0.98-1.55), 1.54 (1.14-2.06), 1.80 (0.85-3.81), 1.91 (0.68-5.30).

Conclusion: Our results suggest that the current SOFA score may be too simplistic to capture the relative contribution of individual organs' dysfunction to the risk of ICU mortality in a population of patients $\geq 80$ years old.

\section{Oral Presentations}

\section{1}

\section{Erythromycin as a prokinetic: what dose and duration are} optimal?

N. Shaikh'; M. Rahman²; C. Arshad²; M. Ranjan²

${ }^{1}$ Hamad Medical Corporation, Doha, Qatar; ${ }^{2}$ Sicu, HMC, Doha, Qatar

Correspondence: N. Shaikh

Intensive Care Medicine Experimental 2020, 8(2): 000011

Introduction: Erythromycin is a potent prokinetic agent and has a shorter onset of action time. The combination of prokinetic medications is increasingly used as they accelerate the prokinetic action and at the same time decrease the adverse effects.

Objectives: Aim of our study was to know the effective prokinetic dose, duration of usage, response and safety of erythromycin in patients already on metoclopramide.

Methods: All patients in surgical and trauma intensive care unit having enteral feed intolerance and already on metoclopramide for 24 hours were enrolled in the study prospectively. Patient's demographic data, diagnosis, surgical intervention, disease severity scores, erythromycin dose, duration, any adverse effects, factors affecting erythromycin response and outcome were recoded. All these patients received $125 \mathrm{mg}$ syrup erythromycin twice daily through nasogastric tube and nasogastric tube was clamped for 2 hours and half amount of previous enteral feeds were resumed. If patient did not tolerate the feeds, the erythromycin dose was increased every 24 hours in the increment of 250,500 and 1000 mgs.

Results: Total of 313 patients were included in the study. Males were higher in number $(77.6 \%)$ and majority $(48.2 \%)$ of patients with feed intolerance were post laparotomy. Ninety percent patients responded to prokinetic erythromycin therapy, $54 \%$ received lower dose $(125 \mathrm{mg}$ twice daily) and $14 \%$ had diarrhoea. Mean duration of erythromycin therapy was 4.98 days. The most effective dose of erythromycin was $125 \mathrm{mg}$ twice daily $(P=0.001)$. It was significantly effective in patients with multiple organ dysfunction and shock $(P=0.001$ and 0.003 respectively). Patients not responding to erythromycin therapy had a significant higher mortality $(p=0.001)$.

Conclusion: Post-laparotomy patients had higher enteral feed intolerance. Erythromycin was effective in $125 \mathrm{mgs}$ dose and minimal duration. Patients who did not tolerate feeds despite increasing dose of erythromycin had higher mortality.

\section{Reference(s) and grant ackowledgment(s)}

1. 8. Ritz MA, Chapman MJ, Fraser RJ et al. Erythromycin dose of $70 \mathrm{mg}$ accelerates gastric emptying as effective as 200mgs in critically ill patients. Intensive Care Med 2005; 31:949-54

2. 4. Catherine V. Hawkyard, Roland J. Koerner, The use of erythromycin as a gastrointestinal prokinetic agent in adult critical care: benefits versus risks, Journal of Antimicrobial Chemotherapy, Volume 59,Issue 3, March 2007, Pages 347-358

3. None
000015

Methotrexate carried in lipid core nanoparticles prevents cardiac dysfunction by increasing angiogenesis and availability of intracellular adenosine in the left ventricle of sepsis-induced rats

NM. Lopes'; MC. Guido'; Cl. Albuquerque'; L. Jensen²; RC. Maranhão' ${ }^{1}$ Lipids and Metabolism Laboratory, Heart Institute of the State of Sao Paulo (InCor), Sao Paulo, Brazil; ${ }^{2}$ Experimental biology laboratory, Heart Institute of the State of Sao Paulo (InCor), Sao Paulo, Brazil

Correspondence: N.M. Lopes

Intensive Care Medicine Experimental 2020, 8(2): 000015

Introduction: In sepsis, besides the dysregulation of the immune system and disturbances of several vital organs, life-threatening cardiac dysfunction often occurs. We showed in previous studies that $\mathrm{LDE}$, a non-protein lipid core nanoparticle, is able to concentrate in inflammatory sites. When carried in LDE, the cellular uptake of methotrexate (MTX) increased several-fold compared to the uptake of the commercial version. LDE-MTX formulation was shown to modulate the immune response in rats with myocardial infarction, reduce the inflammation and improve the post-infarction cardiac function by increasing angiogenesis, thereby improving hypoxia.

Objectives: To test whether LDE-MTX can improve the cardiac status in septic rats.

Methods: Sepsis was induced in Wistar rats by two I.P. injections of lipopolysaccharide (LPS, $10 \mathrm{mg} / \mathrm{kg}$ ) administered with a $24 \mathrm{~h}$ interval. Rats were allocated in a Control group (CT, without sepsis) and 3 groups with sepsis: LDE, treated with LDE only; MTX, with commercial MTX (1 mg/kg); and LDE-MTX, with LDE-MTX (1 mg/kg). Echocardiography was performed $72 \mathrm{~h}$ after sepsis induction. Animals were euthanized for morphometric analysis of the LV and protein expression analysis of inflammatory, apoptotic, angiogenesis, hypoxia and adenosine bioavailability markers in the LV.

Results: Septic rats treated with LDE developed diastolic dysfunction and presented decreased diastolic volume and diameter of the LV. LDE-MTX group had the appearance of diastolic dysfunction and alterations in LV dilation totally prevented. Also, LDE-MTX treatment elicited cardiac hypertrophy through the increase of the thickness of septum and of the LV posterior wall. LDE-MTX increased LV mass and relative heart weight, as compared to the other groups. Morphometric analysis showed increased myocyte diameter in LDE-MTX when compared to other groups. All groups showed no differences in the protein expression of inflammatory (lymphocytes, tumor necrosis factor, interleukins) and apoptotic (caspases and B-cell lymphoma family) markers, although LDE-MTX showed higher expression of CD68 (macrophage marker). In LDE, hypoxia was remarkably higher while LDE-MTX showed higher angiogenesis and lower cellular hypoxia, respectively represented by vascular endothelial growth factor (VEGF) and hypoxia-inducible factor 1 alpha. MTX showed no effects in angiogenesis or hypoxia. The intracellular adenosine bioavailability was increased in LDE-MTX-treated animals, promoted by higher expression of the A1 receptor, as compared to CT. Myocyte diameter was positively correlated with VEGF $(r 2=0.52 ; \mathrm{p}<0.0001)$.

Conclusion: The appearance of cardiac dysfunction associated to sepsis, was successfully avoided by the treatment with LDE-MTX. This outcome is conceivably associated to different effects observed in this group, such as the development of an adaptive hypertrophy as result of the modulation of different pathways, such as the activation of angiogenesis mechanisms, increase of adenosine bioavailability and immune recruitment to the LV.

Reference(s) and grant ackowledgment(s)

1. MOURA J.A. et al, Novel Formulation of a Methotrexate Derivative With a Lipid Nanoemulsion. Int J Nanomedicine, 6, 2285-95 2011

2. SINGER M. et al, The Third International Consensus Definitions for Sepsis and Septic Shock (Sepsis-3). JAMA, 315 (8), 801-10 2016 Feb 23

3. MARANHAO R.C. et al, Methotrexate Carried in Lipid Core Nanoparticles Reduces Myocardial Infarction Size and Improves Cardiac Function in Rats. Int J Nanomedicine, 12, 3767-3784 2017 May 17

4. BOWSER, J.L. et al, The hypoxia-adenosine link during inflammation. J Appl Physiol 123: 1303-1320, 2017. First published August 10, 2017 
5. ZHAO, T. et al, VEGF-CNEGFR-3 pathway promotes myocyte hypertrophy and survival in the infarcted myocardium. Am J Transl Res 2015:7(4):697-709

\section{9}

Stress ulcer prophylaxis plus enteral nutrition versus enteral nutrition alone in critically ill patients at risk for gastrointestinal bleeding: a propensity-matched analysis

H. Ohbe ; Y. Hideo

${ }^{1}$ Department of clinical epidemiology and health economics, The University of Tokyo, Bunkyo City, Japan

Correspondence: $\mathrm{H}$. Ohbe

Intensive Care Medicine Experimental 2020, 8(2): 000019

Introduction: As an alternative to stress ulcer prophylaxis (SUP) enteral nutrition (EN) alone may provide prophylaxis against stressrelated gastrointestinal bleeding. In animal studies, EN increased gastrointestinal blood flow and luminal $\mathrm{pH}$, contributing to the protection of the gastric mucosa [1]. Similarly, a previous prospective study of Intensive care unit (ICU) patients showed that both continuous EN and SUP independently increased gastric $\mathrm{pH}$ and gastric colonization [2]. However, the effects and safety of SUP in ICU patients receiving EN are poorly understood.

Objectives: To evaluate whether EN alone provides prophylaxis against stress-related gastrointestinal bleeding and reduces drug complications compared with concomitant pharmacological SUP and EN.

Methods: Using data for July 2010 to March 2018 from the Japanese Diagnosis Procedure Combination inpatient database, we identified adults with at least one risk factor for gastrointestinal bleeding and who had received EN within 2 days of intensive care unit admission. We allocated patients who had received intravenous or enteral SUP (proton-pump inhibitor and/or histamine-2 receptor antagonists) within 2 days of receiving EN as the SUP group, and patients who had received EN alone as the control group.

Results: We identified 50,663 eligible patients, and 4615 patients (9\%) constituted the SUP group. After propensity score matching, there was no significant difference in in-hospital mortality and endoscopic hemostasis for gastrointestinal bleeding between the two groups. The SUP group was more likely to have hospital-acquired pneumonia (odds ratio, 1.25; 95\% confidence interval, 1.06-1.46) than the control group. Patients who had received proton-pump inhibitors were more likely to receive percutaneous coronary intervention for acute myocardial infarction (odds ratio, 1.51; 95\% confidence interval, 1.04-2.18) than patients without SUP.

Conclusion: Our results suggest that among patients at risk for gastrointestinal bleeding who had received EN, SUP may not reduce in-hospital mortality or gastrointestinal bleeding but may increase complications of hospital-acquired pneumonia or acute myocardial infarction. Future randomized control trials are warranted to confirm our findings.

\section{Reference(s) and grant ackowledgment(s)}

1. Bonten MJM, Gaillard CA, Van Tiel FH et al (1994) Continuous enteral feeding counteracts preventive measures for gastric colonization in intensive care unit patients. Crit Care Med 22:939-944

2. Ephgrave KS, Kleiman-Wexler RL, Adair CG (1990) Enteral nutrients prevent stress ulceration and increase intragastric volume. Crit Care Med $18: 621-624$

\section{6}

Determining optimal mean arterial pressure after cardiac arrest: a Systematic Review

K. Rikhraj ${ }^{1}$; M. Wood ${ }^{2}$; R. Hoiland ${ }^{2}$; S. Thiara ${ }^{3}$; D. Griesdale ${ }^{3}$; M. Sekhon ${ }^{3}$

${ }^{1}$ Department of emergency medicine, faculty of medicine, The University of British Columbia, Vancouver, Canada; ${ }^{2}$ Department of anaesthesiology, pharmacology and therapeutics, faculty of medicine, The University of British Columbia, Vancouver, Canada; ${ }^{3}$ Division of critical care medicine, department of medicine, The University of British Columbia, Vancouver, Canada

Correspondence: K. Rikhraj

Intensive Care Medicine Experimental 2020, 8(2): 000066
Introduction: Hypoxic ischemic brain injury (HIBI) is the leading cause of morbidity and mortality in patients who survive cardiac arrest. The goal of HIBI management is to augment cerebral oxygen delivery by optimizing mean arterial pressure in these patients. Real-time cerebral autoregulation monitoring provides a method of identifying the patient specific optimal mean arterial pressure (MAPOPT) but while this method has been well established in traumatic brain injury patients, similar studies are lacking in HIBI patients.

Objectives: Our systematic review aims to determine (a) the average MAPOPT in post-cardiac arrest HIBI patients, (b) the feasibility of identifying MAPOPT, (c) the brain tissue oxygenation levels when within proximity to the MAPOPT, and (d) the relationship between neurological outcome and MAPOPT.

Methods: We carried out this review in accordance with the PRISMA guidelines. We searched MEDLINE (Ovid), Embase (Ovid), EBM Reviews (Ovid), CINAHL (EBSCO), the Cochrane Database of Systematic Reviews and the WHO Global Health Library from inception to October 31 2019. We also searched the Cochrane Central Register of Controlled Trials (CENTRAL), BioMed Central, ClinicalTrials.gov, WHO International Clinical Trials Registry Platform (ICTRP) and Thomson Center Watch for unpublished works and ongoing clinical trials.

We included all studies that used cerebral autoregulation to determine MAPOPT in adult patients ( $>16$ years old) who achieved return of spontaneous circulation (ROSC) post-cardiac arrest. Patients had to be intubated and ventilated while undergoing invasive cardiovascular and neurovascular monitoring in an Intensive Care Unit. We excluded studies where patients had any history of traumatic brain injury, ischemic stroke or intracranial hemorrhage.

Results: We identified six studies with 181 patients. There was wide variability in cerebral autoregulation monitoring methods, length of monitoring, calculation and reporting of MAPOPT. Amongst all studies, the median or mean MAPOPT was consistently above $65 \mathrm{mmHg}$ (range: 70-114 mmHg). Definitions of feasibility varied amongst studies and was difficult to summarize. Some studies defined feasibility as the ability to calculate one MAPOPT value during the monitoring period while others defined it as the duration of monitoring time for which MAPOPT could be calculated. Only one study noted that brain tissue oxygenation increased as patients' MAP approached MAPOPT. However, this effect disappeared when the MAP exceeded MAPOPT. Finally, there was no consistent association between MAPOPT and neurological outcome.

Conclusion: There is considerable heterogeneity in determining MAPOPT due to differences in autoregulation monitoring. Further standardized randomized controlled trials and prospective studies are needed to assess the clinical utility of MAPOPT guided strategies on improving neurological outcomes after ROSC.

Reference(s) and grant ackowledgment(s)

1. Griesdale D, Sekhon M, Wood M, et al. Near-infrared spectroscopy to assess cerebral autoregulation and optimal mean arterial pressure in patients with hypoxemic ischemic brain injury: A prospective multi-center pilot study. In Review

2. 5. Sekhon MS, Gooderham P, Menon DK, et al. The Burden of Brain Hypoxia and Optimal Mean Arterial Pressure in Patients With Hypoxic Ischemic Brain Injury After Cardiac Arrest. Crit Care Med 2019;47:960-9.

3. 4. Sekhon MS, Smielewski P, Bhate TD, et al. Using the relationship between brain tissue regional saturation of oxygen and mean arterial pressure to determine the optimal mean arterial pressure in patients following cardiac arrest: A pilot proof-of-concept study. Resuscitation 2016;106:120-5.

4. 3. Pham P, Bindra J, Chuan A, Jaeger $M$, Aneman A. Are changes in cerebrovascular autoregulation following cardiac arrest associated with neurological outcome? Results of a pilot study. Resuscitation 2015;96:192-8.

5. 2. Ameloot K, Genbrugge C, Meex I, et al. An observational near-infrared spectroscopy study on cerebral autoregulation in post-cardiac arrest patients: time to drop "one-size-fits-all" hemodynamic targets? Resuscitation 2015:90:121-6.

6. 1. Sundgreen C, Larsen FS, Herzog TM, Knudsen GM, Boesgaard S, Aldershvile J. Autoregulation of cerebral blood flow in patients resuscitated from cardiac arrest. Stroke 2001;32:128-32.

7. MS is funded through the Vancouver Coastal Health Research Institute Clinician Scientist Award. DG is funded through the Michael Smith HealthProfessional Investigator Award. 


\section{6}

Accuracy of Mortality Prediction by Machine Learning Models Compared to Clinician's Intuition in Critically III Patients

S. Patel ${ }^{1}$; Y. Wolfe ${ }^{1}$; N. Puri

${ }^{1}$ Critical care, Cooper University Health Care, Camden, United States of America

Correspondence: $Y$. Wolfe

Intensive Care Medicine Experimental 2020, 8(2): 000096

Introduction: Intensive Care Units (ICUs) treat patients with high mortality risk. Early and accurate mortality estimation is crucial for guiding care and resource allocation. Identification of patients with poor trajectories is difficult and often wrought with biases. Machine Learning (ML) with quality data is a means of accurate mortality prediction. Several studies compared machine learning models to severity illness scores in predicting mortality. To our knowledge, no study has compared ML and clinician feature importances.

Objectives: We compared important features for mortality chosen by ML models and clinicians.

Methods: We extracted data from the eICU Collaborative Research Database. The dataset included 27 variables (26 independent and 1 dependent) and 147,233 values. Mortality was the dependent variable for our prediction models. Exploratory data analysis performed in Python. Missing data imputed with Sklearn Iterative Imputer. Feature selection performed using Pearson correlation and Sklearn Select From Model library. Top ten features for the ML group chosen by the frequency in the top ten for Pearson Correlation and four ML model feature selection methods. ICU clinicians filled out a survey to choose the top ten features that predict mortality. Fifty-one surveys filled out, and the most common features compiled to create a top ten list. The mortality outcome of death was rare (136,451 alive versus 8,184 died). Given the imbalanced dataset, we employed an oversampling technique (SMOTE). The top features in both groups were passed through Logistic Regression (LR) and Naive Bayes (NB) algorithms. Area Under Receiver Operator Characteristic (AUROC) was chosen as our metric.

Results: White blood count (WBC), Prothrombin Time (PT), Lactate, Hypoglycemia, and $\mathrm{PaO}_{2}: \mathrm{FiO}_{2}$ ratio were the top features for the $\mathrm{ML}$ group. Clinicians chose hepatic failure, advanced age, immunosuppressed status, and history of metastatic cancer. The AUROC for the ML group in predicting mortality was 0.865 (LR); 0.840 (NB), as compared to 0.737 (LR); 0.744 (NB) for clinicians. As a benchmark, we compared our feature inputs to SAPS II and APACHE IV scores input into LR and NB. SAPS II and APACHE IV performance were 0.842 (LR);0.844 (NB) and 0.855 (LR);0.855 (NB) respectively.

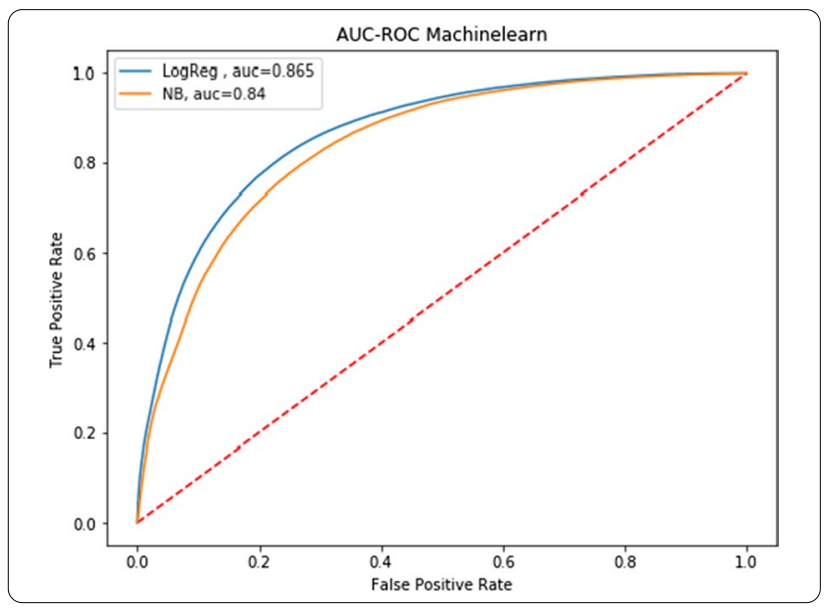

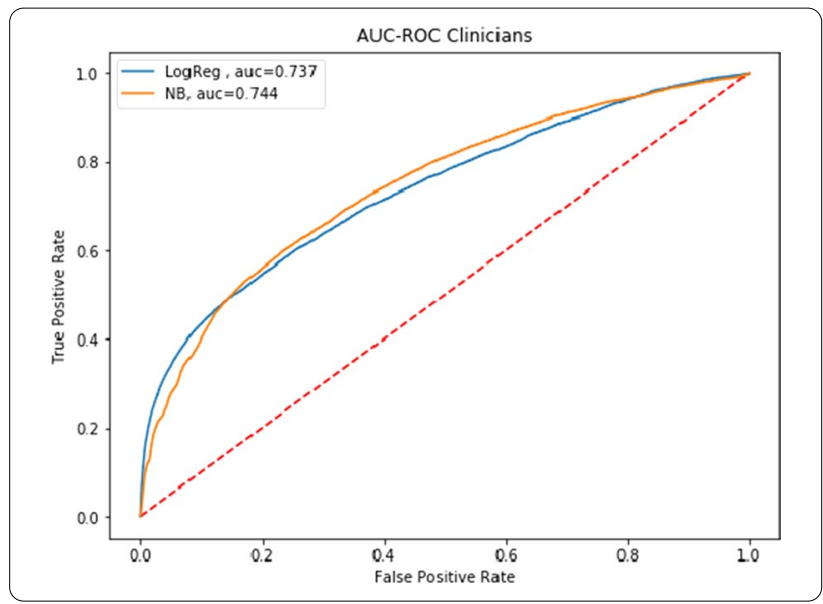

Conclusion: ML models can identify important features for accurate ICU mortality prediction. ML predictive models can be important adjuncts to augment clinical decision making.

Reference(s) and grant ackowledgment(s)

1. Lee J., Dubin J.A., Maslove D.M. (2016) Mortality Prediction in the ICU. In: Secondary Analysis of Electronic Health Records. Springer, Cham.

2. Johnson AEW, Mark RG, "Real-time mortality prediction in the Intensive Care Unit.", AMIA Annu Symp Proc. 2018 Apr 16; 2017:994.

3. Cosgriff, CV, Celi, LA, Ko, S, et al., "Developing well-calibrated illness severity scores for decision support in the critically ill.", NPJ Digit Med. 2019; 2:76.

4. The elCU Collaborative Research Database, a freely available multi-center database for critical care research. Pollard TJ, Johnson AEW, Raffa JD, Celi LA, Mark RG and Badawi O. Scientific Data (2018).

\section{8}

Lung-protective mechanical ventilation for $\mathbf{5 0}$ hours is associated with hippocampal apoptosis and shifts the behavior of hippocampal microglia from anti-inflammatory to pro-inflammatory

T. Bassi ${ }^{1}$; E. Rohrs ${ }^{2}$; K. Fernandez ${ }^{2}$; M. Ornowska ${ }^{2}$; M. Nicholas ${ }^{2}$; S. Reynolds ${ }^{1}$ ${ }^{1}$ Physiology, Simon Fraser University, Burnaby, Canada; ${ }^{2}$ Critical care, Fraser Health Authority, New Westminster, Canada

Correspondence: $T$. bassi

Intensive Care Medicine Experimental 2020, 8(2): 000128

Introduction: The hippocampus is part of the limbic system known as an emotional neuro-circuit, and its activity is coupled with the breathing cycle.1Ventilation-Induced Brain Injury (VIBI) was recently identified preclinically, 2 where VIBI was characterized by hippocampal neuroinflammation and apoptosis. However, these studies used high-tidal-volume mechanical ventilation for short durations (for up to $3 \frac{1}{2}$ hours), and the effect on the hippocampus of long-term lungprotective (i.e. gold standard) mechanical ventilation (MV) is not known. We hypothesize that lung-protective MV for 50 hours in a noninjured preclinical model shifts the behavior of hippocampal microglia towards inflammation, contributing to local apoptosis. The interaction between the apoptotic process and microglia cells has been described in other conditions, such as epilepsy and traumatic brain injury. 3-5 Characterizing changes in the behavior of hippocampal microglia might assist in a better understanding of the neuro-inflammatory process happening during $\mathrm{VIBI}$. This data might also contribute to the design of future studies into cognitive dysfunction after MV.

Objectives: To investigate whether lung-protective mechanical ventilation in a porcine model for 50 hours modifies microglia behavior, and to analyze the level of hippocampal apoptosis after 50 hours of lung-protective mechanical ventilation.

Methods: We ventilated eight human-size pigs (MV group) using lung-protective MV for 50 hours in a mock ICU, and compared them with six never-ventilated pigs (NV group). Ventilation settings were 
adjusted to achieve and maintain a tidal volume of $6-8 \mathrm{ml} / \mathrm{kg}$, with a PEEP of $5 \mathrm{~cm} \mathrm{H}_{2} \mathrm{O}$. The pigs' hippocampi were harvested, and machine-learning software was used to count and classify cells. Percentages of cells stained by the TUNEL and IBA1 markers were then determined. Microglia behavior was determined, and quantified following biometric cellular characteristics based on pre-established criteria in the literature.

Results: The hippocampal apoptotic percentage was $38 \%$ $(44,797 / 117,886)$ in the MV group and $1 \%(338 / 33,805)$ in the NV group $(p<0.01)$. Total microglia population as a percentage of total cell population, including those displaying pro- and anti-inflammatory characteristics, was $36 \%(74,212 / 205,006)$ in the MV group and $10 \%$ $(58,398 / 589,888)$ in the NV group $(p<0.01)$. Analysis of MV group microglia identified $81 \%(60,111 / 74,212)$ with anti-inflammatory characteristics and $19 \%(14,101 / 74,212)$ with pro-inflammatory characteristics $(p<0.01)$. Analysis of the NV group microglia identified $84 \%(49,288 / 58,398)$ with anti-inflammatory characteristics and $16 \%$ $(9,110 / 58,398)$ with pro-inflammatory characteristics $(p<0.01)$. The percentage of microglia with pro-inflammatory characteristics was slightly, but significantly higher in the MV group (19\%) than in the NV group $(16 \%, p<0.05)$. The percentage of microglia with anti-inflammatory characteristics was also significantly different; $81 \%$ in the MV group, compared to $84 \%$ in the NV group ( $p<0.05)$.

Conclusion: Lung-protective MV for 50 hours in a non-injured preclinical model is associated with VIBI, characterized by high hippocampal apoptotic percentage and also a shift in the behavior and number of hippocampal microglia from anti-inflammatory to pro-inflammatory.

\section{Reference(s) and grant ackowledgment(s)}

1. Kamuf J, Garcia-Bardon A, Ziebart A, et al. Lung injury does not aggravate mechanical ventilation-induced early cerebral inflammation or apoptosis in an animal model. PLoS One. 2018;13(8). https://doi.org/10.1371/journ al.pone.0202131

2. Ransohoff RM. A polarizing question: Do M1 and M2 microglia exist. Nat Neurosci. 2016;19(8):987-991. https://doi.org/10.1038/nn.4338

3. Lungpacer Medical Inc.

4. Cater HL, Sundstrom LE, Morrison B. Temporal development of hippocampal cell death is dependent on tissue strain but not strain rate. J Biomech. 2006;39(15):2810-2818. https://doi.org/10.1016/j.jbiomech.2005.09.023

5. González-López A, López-Alonso I, Aguirre A, et al. Mechanical ventilation triggers hippocampal apoptosis by vagal and dopaminergic pathways. Am J Respir Crit Care Med. 2013;188(6):693-702. https://doi.org/10.1164/ rccm.201304-06910C

6. Chen C, Zhang Z, Chen T, Peng M, Xu X, Wang Y. Prolonged mechanical ventilation-induced neuroinflammation affects postoperative memory dysfunction in surgical mice. Crit Care. 2015;19(1):1-12. https://doi.org/10.1186/ s13054-015-0882-0

\section{1}

Therapy limitation in octogenarians in German Intensive Care Units is associated with a longer length of stay and increased 30 days mortality: a prospective multicenter study

RR. Bruno ${ }^{1}$; B. Wernly'; M. Beil'; JM. Muessig ${ }^{1}$; T. Rahmel'; T. Graf5;

P. Meybohm ${ }^{6}$; S. Schaller ; M. Franz ${ }^{8}$; S. Dubler ${ }^{9}$; S. Schering ${ }^{10}$; G. Wolff';

S. Steiner ${ }^{11}$; C. Rabe ${ }^{12}$; M. Kelm ${ }^{1}$; D. De Lange ${ }^{13}$; B. Guidet ${ }^{14}$; H. Flaaten ${ }^{15}$;

C. Jung ${ }^{1}$; M. Schuster ${ }^{16}$

${ }^{1}$ Cardiology, Pulmonary Diseases, Vascular Medicine, Universität Düsseldorf, Düsseldorf, Germany; ${ }^{2}$ Cardiology, Paracelsus Medical University, Salzburg, Austria; ${ }^{3}$ Medical intensive care unit, Hadassah University Hospital Ein Kerem, Jerusalem, Israel; ${ }^{4}$ Department of anesthesiology, intensive care medicine and pain therapy, University Hospital Knappschaftskrankenhaus Bochum GmbH, Bochum, Germany; ${ }^{5}$ Department ofcardiology, angiology, and intensive care medicine, University Heart Center Luebeck, Lübeck, Germany; ${ }^{6}$ Department of anaesthesiology, University Hospital Würzburg, Würzburg, Germany; ${ }^{7}$ Department of anesthesiology and surgical intensive care, Charité - Universitätsmedizin Berlin, Berlin, Germany; ${ }^{8}$ Department of internal medicine i, Jena University Hospital, Jena, Germany; ${ }^{9}$ Department of anesthesiology and intensive care medicine, University Hospital Heidelberg, Heidelberg, Germany; ${ }^{10}$ Department of anesthesiology and intensive care, University Hospital Leipzig, Leipzig,
Germany; ${ }^{11}$ Department of cardiology, pneumology and intensive care medicine, St. Vincenz Hospital Limburg, Limburg an der Lahn, Germany; ${ }^{12}$ Department of clinical toxicology, Klinikum rechts der Isar, Technical University Munich, Munich, Germany; ${ }^{13}$ Department of intensive care medicine, Utrecht University, Utrecht, Netherlands; ${ }^{14}$ Réanimation Médicale, Hôpital Saint-Antoine, Paris, France; ${ }^{15}$ Department of anaestesia and intensive care, University of Bergen, Bergen, Norway; ${ }^{16}$ Department of anesthesiology, intensive care medicine and pain therapy, University Hospital Mainz, Mainz, Germany

Correspondence: R.R. Bruno

Intensive Care Medicine Experimental 2020, 8(2): 000131

Introduction: The approach to limit therapy in very old intensive care unit patients (VIPs) significantly differs between regions. The focus of this multicenter analysis is to illuminate, whether the Clinical Frailty Scale (CFS) is a suitable tool for risk stratification in VIPs admitted to intensive care units (ICUs) in Germany. Furthermore, this investigation elucidates the impact of therapeutic limitation on the length of stay and mortality in this setting.

Methods: German cohorts' data from two multinational studies (VIP-1, VIP-2) were combined. Univariate and multivariate logistic regression were used to evaluate associations with mortality.

Results: 415 acute VIPs were included. Frail VIPs (CFS $>4$ ) were older (85 [IQR 82-88] vs. 83 [IQR 81-86] years; $\mathrm{p}<0.001$ ) and suffered from an increased 30 -day-mortality $(43.4 \%$ versus $23.9 \%, \mathrm{p}<0.0001)$. CFS was an independent predictor of 30-day-mortality in a multivariate logistic regression model (aOR $1.2395 \% \mathrm{Cl} 1.04-1.46 ; p=0.02)$. Patients with any limitation of life-sustaining therapy had a significantly increased 30 -day mortality $(86 \%$ versus $16 \%, p<0.001)$ and length of stay (144 [IQR 72-293] versus 96 [IQR 47.25-231.5] hours, $p=0.026$ ).

Conclusion: In German ICUs, any limitation of life-sustaining therapy in VIPs is associated with a significantly increased ICU length of stay and mortality. CFS reliably predicts outcome.

\section{Reference(s) and grant ackowledgment(s)}

1. Guidet B, de Lange DW, Boumendil A, Leaver S, Watson X, Boulanger C, et al. The contribution of frailty, cognition, activity of daily life and comorbidities on outcome in acutely admitted patients over 80 years in European ICUs: the VIP2 study. Intensive Care Med 2019.

2. Flaatten H, De Lange DW, Morandi A, Andersen FH, Artigas A, Bertolini G, et al. The impact of frailty on ICU and 30-day mortality and the level of care in very elderly patients ( $>=80$ years). Intens Care Med 2017;43(12):1820-8.

3. The authors thank the steering committee of the VIP-1 and VIP-2 study for their support: Hans Flaatten, principal Investigator (Norway), Bertrand Guidet (France), Dylan de Lange (The Netherlands), Antonio Artigas (Spain), Finn Andersen (Norway), Carol Boulanger (UK), Ariane Boumendil (France), Maurizio Cecconi (Italy), Jesper FjøIner (Denmark), Brian Marsh (Ireland), Alessandro Morandi (Italy), Rui Moreno (Portugal), Sandra Oyen (Belgium), Joerg Schefold (Switzerland), Ivo Soliman (The Netherlands), Wojciech Szczeklik (Poland), Michael Joannidis (Austria), Sten Walther (Sweden), Ximena Watson (UK), Tilemachos Zafeiridis (Greece), Yuriy Nalapko (Ukranian).

\section{4}

\section{Management and outcomes in critically ill nonagenarian} versus octogenarian patients

RR. Bruno ; B. Wernly ${ }^{2}$; M. Kelm ; FH. Andersen ${ }^{3}$; A. Artigas ${ }^{4}$; M. Cecconi ${ }^{5}$; S. Christensen ${ }^{6}$ : M. Lichtenauer ${ }^{7}$; B. Marsh ${ }^{8}$ : RP. Moreno 9 : S. Oeyen ${ }^{10 .}$ B. Bollen Pinto ${ }^{11}$; W. Szczeklik ${ }^{12}$; S. Leaver ${ }^{13}$; M. Joannidis ${ }^{14}$; J. Fjølner ${ }^{15}$; D. De Lange ${ }^{16} ;$ B. Guidet ${ }^{17} ;$ H. Flaaten ${ }^{18} ;$ C. Jung ${ }^{1}$

${ }^{1}$ Cardiology, Pulmonary Diseases, Vascular Medicine, Universität Düsseldorf, Düsseldorf, Germany; ${ }^{2}$ Cardiology, Paracelsus Medical University, Salzburg, Austria; ${ }^{3}$ Dep of circulation and medical imaging, Alesund Hospital, Alesund, Norway; ${ }^{4}$ Department of intensive care medicine, Corporacion Sanitaria Universitaria Parc Tauli, Barcelona, Spain; ${ }^{5}$ Anesthesia and intensive care, Humanitas Research Hospital, Milan, Italy; ${ }^{6}$ Department of anaesthesia and intensive care medicine, Aarhus University Hospital, Aarhus, Denmark; ${ }^{7}$ Department of cardiology, Paracelsus Medical University, Salzburg, Austria; ${ }^{8}$ Intensive care unit, The Mater Misericordiae University Hospital, Dublin, Ireland; ${ }^{~}$ Unidade de cuidados intensivos neurocríticos, Hospital de São José, Centro Hospitalar de Lisboa Central, Nova 
Médical School, Lisbon, Portugal; ${ }^{10}$ Department of intensive care, Ghent University Hospital, Gent, Belgium; ${ }^{11}$ Anesthesiology, Hôpitaux Universitaires de Genève (HUG), Genève, Switzerland; ${ }^{12}$ Intensive care and perioperative medicine, Jagiellonian University Medical College, Kraków, Poland: ${ }^{13}$ Research lead critical care directorate, St George's Hospital, London, United Kingdom; ${ }^{14}$ Division of intensive care and emergency medicine, Medizinische Universität Innsbruck, Innsbruck, Austria; ${ }^{15}$ Department of intensive care, Aarhus University Hospital, Aarhus, Denmark; ${ }^{16}$ Department of intensive care medicine, Utrecht University, Utrecht, Netherlands; ${ }^{17}$ Réanimation Médicale, Hôpital Saint-Antoine, Paris, France; ${ }^{18}$ Department of anaestesia and intensive care, University of Bergen, Bergen, Norway

Correspondence: R.R. Bruno

Intensive Care Medicine Experimental 2020, 8(2): 000144

Introduction: The growing proportion of nonagenarian ICU patients challenges intensive care medicine. Due to the unclear benefit to patients and a high consumption of health care resources, these oldest-old patients represent both an ethical and political problem. This study compared outcome differences between nonagenarian and octogenarian ICU patients.

Methods: 7900 acutely admitted older critically ill patients from two large, multinational registers were included. The primary endpoint was ICU-, the secondary endpoint 30-day-mortality. Baseline characteristics including frailty assessed by the Clinical Frailty Scale (CFS), management and outcomes were compared between octogenarian (80-89.9 years) and nonagenarian ( $\geq 90$ years) patients. Logistic regression was used to calculate a propensity score on being nonagenarian and to evaluate univariable and multivariable associations with the endpoints.

Results: The nonagenarians accounted for $10 \%$ of the entire cohort. They evidenced higher rates of frailty ( $58 \%$ vs $42 \% ; p<0.001)$, but lower SOFA scores at admission ( $6 \pm 5$ vs. $7 \pm 6 ; p<0.001)$. Management strategies differed with octogenarians receiving higher rates of organ support while nonagenarians had higher rates of life-sustaining treatment limitation ( $40 \%$ vs. $33 \%$; $p<0.001)$. ICU mortality was similar $(27 \%$ vs. $27 \% ; p=0.973)$ in octogenarians and nonagenarians. However, being a nonagenarian was independently associated with increased 30-day-mortality after adjustment of the propensity score (OR 1.18 95\%Cl 1.02-1.38; $\mathrm{p}=0.03$ ).

Conclusion: Nonagenarians had higher 30-day mortality compared to octogenarian patients. Being a nonagenarianshould be considered an independent risk factor for mortality and - together with disease severity and pre-existing functional capacity - to effectively guide triage decisions.

\section{Reference(s) and grant ackowledgment(s)}

1. Guidet B, Flaatten H, Boumendil A, Morandi A, Andersen FH, Artigas A, et al. Withholding or withdrawing of life-sustaining therapy in older adults $(>/=80$ years $)$ admitted to the intensive care unit. Intensive Care Med. 2018;44(7):1027-38.

2. Guidet B, de Lange DW, Boumendil A, Leaver S, Watson X, Boulanger C, et al. The contribution of frailty, cognition, activity of daily life and comorbidities on outcome in acutely admitted patients over 80 years in European ICUs: the VIP2 study. Intensive Care Med. 2019.

3. Flaatten $\mathrm{H}$, De Lange DW, Morandi A, Andersen FH, Artigas A, Bertolini $\mathrm{G}$, et al. The impact of frailty on ICU and 30-day mortality and the level of care in very elderly patients ( $>/=80$ years). Intensive Care Med. 2017:43(12):1820-8.

4. Guidet B, de Lange DW, Flaatten H. Should this elderly patient be admitted to the ICU? Intensive Care Med. 2018.

\section{5}

Circadian rhythm of body temperature and prognosis of critically ill patients

R. Li ${ }^{1}$; J. Li' ${ }^{1}$;. Gao ${ }^{1}$; X. Jin ${ }^{1}$; J. Zhang ${ }^{1}$; J. Ren ${ }^{1}$; X. Wang ${ }^{1}$; G. Wang

${ }^{1}$ Department of critical care medicine, the Second Affiliated Hospital of Xi'an Jiaotong University, Xi'an, China

Correspondence: G. Wang

Intensive Care Medicine Experimental 2020, 8(2): 000155
Introduction: Most critically ill patients experience the disruption of circadian body temperature (BT) rhythm after intensive care unit (ICU) admission. However, there is limited evidence on the relationship between circadian BT variation and outcomes in patients during ICU stay.

Objectives: To assess the impact of circadian BT variation on prognosis in ICUs patients.

Methods: A multicenter retrospective cohort study was performed with elCU Collaborative Research Database, which comprises 139367 patients at 335 distinct ICUs across the United States from 2014 to 2015. Adult patients with complete records of continuous bedside core BT monitoring during the first 24 hours of ICU stay were included. According to circadian BT variation (mean night-time BT/mean daytime BT), participants were divided into two groups: ratio $\leq 1$ and ratio $>1$. Logistic and liner regression were performed to investigate the relationship between circadian BT variation and prognosis (ICU mortality, length of ICU stay [ICU-LOS], and length of hospital stay [hospital-LOS]). Sensitivity analysis was conducted according to Acute Physiology and Chronic Health Evaluation IV (APACHE IV) $\leq 64$ or $>64$. Furthermore, an external cohort was performed with Multiparameter Intelligent Monitoring in Intensive Care II database.

Results: A total of 6093 patients were analyzed. Patients with circadian BT variation $\leq 1(n=2473)$ had higher ICU mortality, longer ICU-LOS and hospital-LOS than those with circadian BT variation $>1$ $(\mathrm{n}=3620)$. After multivariable adjustments, circadian BT variation $\leq 1$ increased ICU mortality (odds ratio [OR], 1.231, 95\% confidence intervals $[\mathrm{Cl}], 1.009-1.502)$, prolonged ICU-LOS $(\beta,-0.420,95 \% \mathrm{Cl},-0.657$ to -0.182$)$, and hospital-LOS $(\beta,-0.836,95 \% \mathrm{Cl},-1.247$ to -0.426$)$ in ICU patients. The association remained significant in sensitivity analysis and external cohort.

Conclusion: Circadian BT variation may be associated with higher ICU mortality, longer ICU-LOS, and hospital-LOS in critically ill patients. Our findings will further develop our knowledge on the vital role of circadian rhythm in ICU and indicated that circadian BT variation may assist with early risk stratification.

Reference(s) and grant ackowledgment(s)

1. Pollard TJ, Johnson AEW, Raffa JD, et al. The elCU Collaborative Research Database, a freely available multi-center database for critical care research. Sci Data 2018;5:180178.

2. Oldham MA, Lee HB, Desan, PH. Circadian Rhythm Disruption in the Critically III: An Opportunity for Improving Outcomes. Crit Care Med 2016;44(1): 207-217.

3. Telias I, Wilcox ME. Sleep and Circadian Rhythm in Critical IIIness. Crit Care 2019;23(1):82

000162

Temporary transvenous diaphragm neurostimulation plus lung-protective mechanical ventilation is associated with greater heart rate variability after $\mathbf{5 0}$ hours compared to ventilation alone

T. Bassi'; M. Nicholas²; K. Fernandez ${ }^{2}$; M. Ornowska²; E. Rohrs²; S. Reynolds ${ }^{1}$ ${ }^{1}$ Physiology, Simon Fraser University, Burnaby, Canada; ${ }^{2}$ Critical care, Fraser Health Authority, New Westminster, Canada

Correspondence: $T$. bassi

Intensive Care Medicine Experimental 2020, 8(2): 000162

Introduction: Heart rate variability (HRV) is an accepted method for measuring sympathetic/parasympathetic balance and it is influenced by vagal afferent signals coming from the lungs.1,2 The effect of lung-protective mechanical ventilation (MV) on sympathetic/parasympathetic nervous system balance has not been demonstrated. Greater R-R interval variance reflects a normal physiological state with parasympathetic predominance; lower $\mathrm{R}-\mathrm{R}$ interval variance indicates sympathetic predominance, which correlates with high mortality and inflammation.3 Studying HRV during MV is important to better understand the inflammatory insult associated with MV. Temporary transvenous diaphragm neurostimulation (TTDN) is a novel technology that assists MV by creating negative pressure targeting the reduction of the pressure-time-product (PTP), and may influence the HRV. 
We hypothesize lung-protective MV progressively downregulates parasympathetic activity over the course of 50 hours and that MV + TTDN will upregulate parasympathetic activity due to more physiological alveolar stretch resulting in greater HRV after 50 hours of the experiment.

Objectives: To compare the sympathetic/parasympathetic nervous system balance after 50 hours of lung-protective MV alone and with TTDN assistance.

Methods: We used sixteen pigs between 51-64 kg, eight under lungprotective MV (MV-Only) and eight under lung-protective MV plus TTDN (MV + TTDN) for 50 hours. Ventilation parameters were set to achieve and maintain tidal volume $6-8 \mathrm{ml} / \mathrm{kg}$, and PEEP of $5 \mathrm{~cm} \mathrm{H}_{2} \mathrm{O}$. TTDN targeted between $15-20 \%$ reduction in PTP during MV. The MV + TTDN group received phrenic nerve stimulation every other breath in synchrony with MV. ECG data were analyzed over two periods after MV initiation; hours 0 to 6 (start) and hours 44 to hour 50 (end). Root mean square of the standard deviation of the R-R intervals (RMSSD) was chosen as the HRV time-domain method of analysis.

Results: In total, 815,658 R-R intervals were analyzed across the sixteen subjects; 407,762 in the MV-Only group and 407,896 in the MV + TTDN group $(p>0.05)$. In the MV-Only group the RMSSD was $0.90 \mathrm{~ms}$ at the start and $0.81 \mathrm{~ms}$ at the end of the study; and in the MV + TTDN group it was $0.81 \mathrm{~ms}$ at the start and $0.91 \mathrm{~ms}$ at the end $(p=0.007$, between groups).

Conclusion: MV resulted in a reduction of the HRV in the MV-Only group after 50 hours which might indicate a downregulation in parasympathetic activity. The MV + TTDN group showed higher HRV after 50 hours of MV which might point towards upregulation in parasympathetic activity.

\section{Reference(s) and grant ackowledgment(s)}

1. Guardiola J, Moffett B, Li H, et al. Airway mechanosensor behavior during application of positive end-expiratory pressure. Respiration. 2014;88(4):339344. https://doi.org/10.1159/000364947

2. Frasch MG, Szynkaruk M, Prout AP, et al. Decreased neuroinflammation correlates to higher vagus nerve activity fluctuations in near-term ovine fetuses: A case for the afferent cholinergic anti-inflammatory pathway? J Neuroinflammation. 2016;13(1):1-14. https://doi.org/10.1186/s12974-016-

3. Lungpacer Medical Inc.

4. Lotufo PA, Valiengo L, Benseñor IM, Brunoni AR. A systematic review and meta-analysis of heart rate variability in epilepsy and antiepileptic drugs. Epilepsia. 2012;53(2):272-282. https://doi.org/10.111 1/j.1528-1167.2011.03361.x

\section{6}

Intellivent-ASV ${ }^{\circledR}$ mode is superior to conventional ventilation modes after uncomplicated cardiac surgery during all phases of postoperative respiratory support

R. Komnov ${ }^{1}$; A. Eremenko ${ }^{2}$

${ }^{1}$ Cardiac Intencive Care Unit, Petrovsky National Research Center of Surgery, Moscow, Russia; ${ }^{2}$ Cardiac intensive care unit, Petrovsky National Research Centre of Surgery, Moskva, Russia

Correspondence: R. Komnov

Intensive Care Medicine Experimental 2020, 8(2): 000166

Introduction: Respiratory support plays an important role in patient's recovery after cardiovascular surgery. Intellivent-ASV ${ }^{\circledR}$ is a closed-loop ventilation mode that adjusts automatically ventilation and oxygenation settings according to patient weight, lung function (as assessed by the ventilator) and continuous input of end-tidal carbon dioxide and oxygen saturation in both passive and spontaneous breathing patients. There is an ability to improve patient's safety and reduce physician's workload with using intellectual modes of ventilation.

Objectives: To compare the effect(s) of Intellivent-ASV and conventional ventilation modes.

Methods: In this randomized controlled trial 40 adult patients were ventilated with conventional ventilation modes and 40 with Intellivent-ASV after uncomplicated cardiac surgery. Hamilton G5 ventilators were used and 8 physicians were involved into the study. All actions of physician's, ventilator settings and changes were monitored and recorded during respiratory support in ICU. Care of both groups was standardized, except modes of postoperative ventilation.

We compared:

the physician's workload, through accounting number of manual ventilator settings and time they spent near the ventilator in every group;

duration of tracheal intubation in ICU;

evaluation of ventilation safety by considering driving pressure, mechanical power, positive end expiratory pressure and tidal volume level.

Results: There were significant differences in the duration of respiratory support in ICU: $226 \pm 31 \mathrm{~min}$ (Intellivent group) vs $271 \pm 78 \mathrm{~min}$ (control) $(p=0,001267)$.

In Intellivent group the number of manual ventilator settings and physicians time spent near the ventilator before tracheal extubation were lower: 0 vs 4 (2-6), and 35 (25-53) sec vs $164 \pm 69$ sec respectively ( $p<$ 0,001 in both cases).

Intellivent-ASV provided a significant more protective ventilation through reduction in the driving pressure, tidal volume, $\mathrm{FiO}_{2}$ and $\mathrm{PEEP}$ levels, but without differences between $\mathrm{PaO}_{2} / \mathrm{FiO}_{2}$ ratio.

1. $\Delta \mathrm{P}$ and $\mathrm{Vt}$ were significantly lower in Intellivent group - $\triangle \mathrm{P}$ on mechanical ventilation was $6(5-7) \mathrm{cm} \mathrm{H}_{2} \mathrm{O}$ vs $7,25(6,5-9,5) \mathrm{cm} \mathrm{H}_{2} \mathrm{O}(\mathrm{p}<0,001)$ and pressure support level $-5(5-5) \mathrm{cm} \mathrm{H}_{2} \mathrm{O}$ vs $8(7-10) \mathrm{cm} \mathrm{H}_{2} \mathrm{O}$; Vt on mechanical ventilation was $6(5,2-7)$ vs $7(6-9,5) \mathrm{ml} / \mathrm{kg} / \mathrm{PBW}(\mathrm{p}=0,000003)$.

2. PEEP and $\mathrm{FiO}_{2}$ level were also significantly lower in Intellivent group during all phases of respiratory support in ICU. PEEP on mechanical ventilation was $5(5-7,5) \mathrm{cm} \mathrm{H}_{2} \mathrm{O}$ vs $7(5-11,5) \mathrm{cm} \mathrm{H}_{2} \mathrm{O}$ and $\mathrm{FiO}_{2}$ level was $26(22-30) \%$ vs 34 (30-40) \%. During spontaneous ventilation phase in Intellivent group PEEP was $5(5-5,5) \mathrm{cm} \mathrm{H}_{2} \mathrm{O}$ vs $7(5-10) \mathrm{cm} \mathrm{H}_{2} \mathrm{O}$ and $\mathrm{FiO}_{2} 26 \pm 4 \%$ vs 30 (2940) $\%$.

3. Mechanical power (simplified equation) was lower in Intellivent group 8,17 $(5,99-10)$ vs $8,58(6,34-13,7) \mathrm{J} / \mathrm{min}(p=0,0467)$.

There were no significant differences between the groups in Vt values during spontaneous ventilation: 8 (7-9) vs 8 (7-10) $\mathrm{ml} / \mathrm{kg} / \mathrm{PBW}$ in control group, as well as in undesirable events and duration of ICU and hospital stay.

Conclusion: Application of IntelliVent-ASV mode after uncomplicated cardiac surgery provides more protective mechanical ventilation and reduces the physician's workload without compromising the quality of respiratory support and safety of patients.

\section{0}

Modulation of the permeability-inducing factor Angiopoietin-2 through Bifonazole in systemic inflammation

T. Pape ${ }^{1}$;. Idowu' ;. Etzrodt ${ }^{1}$; H. Haller ${ }^{1}$; S. Parikh²; S. David ${ }^{1}$

${ }^{1}$ Nephrology, Hannover Medical School, Hannover, Germany; ${ }^{2}$ Center for vascular biology research, Beth Israel Deaconess Medical Center and Harvard Medical School, Boston, United States of America

Correspondence: $T$. Pape

Intensive Care Medicine Experimental 2020, 8(2): 000180

Introduction: Sepsis is a life-threatening organ dysfunction due to a pathological host response to an infection. Vascular barrier breakdown represents a key component of this maladaptive host response and the release of pre-stored Angiopoietin-2 (Angpt-2) from the endothelium is a direct driver of this phenomenon via deactivation of its target receptor "Tie2". Currently, an Angpt-2 modulating therapy does not exist. Screening an FDA-drug library, we identified the antifungal Bifonazole (BIFO) as a potential reducer of Angpt-2 in vitro. We hypothesize that BIFO might decrease circulating Angpt-2 and therefore, diminish capillary leakage.

Methods: Endothelial cells were isolated from human umbilical veins (HUVECs) and used for in vitro studies after stimulation with BIFO (RT$\mathrm{PCR}$, immunoblot, ELISA, immunocytochemistry, transendothelial electrical resistance (TER)). In vivo studies were performed on endotoxemic C57BI6 mice treated with BIFO (lung RT-PCR, immunoblot, ELISA). 
Results: We found that BIFO can reduce spontaneous Angpt-2 release in a time- and dose-dependent manner in HUVECs after $8(p<0.05)$, 12 and $24 \mathrm{~h}$ (veh: $15.58 \pm 0.7$ vs BIFO: $8.56 \pm 0.8 \mathrm{ng} / \mathrm{ml}, \mathrm{p}<0.001$ ). In addition, after TNF-a challenge, BIFO was still able to reduce this stimulated Angpt-2 release (veh: $30.3 \pm 3.8$ vs BIFO: $22.2 \pm 2.5 \mathrm{ng} / \mathrm{ml}, \mathrm{p}$ $<0.01$ ). Measuring the TER to quantify endothelial permeability, BIFO was sufficient to increase TER in two models of hyperpermeability (Thrombin and PMA) (veh: $0.82 \pm 0.10$ vs BIFO: $1.01 \pm 0.02, p<0.05$ ). As BIFO reduced not only Angpt- 2 release but also cellular content by $27 \%(p<0.05)$, an exocytosis-independent mechanism directly affecting Angpt-2 biosynthesis seems involved. Consistently, Angpt-2 transcription (mRNA) was reduced in lungs of BIFO-stimulated mice (veh: $1.00 \pm 0.38$ vs BIFO: $0.55 \pm 0.43, \mathrm{p}<0.05$ ).

Conclusion: The antifungal BIFO can reduce release and synthesis of the endothelial-destabilizing factor Angpt- 2 in vitro. Henceforth, our results suggest that BIFO might counterpart the pathophysiology of endothelial permeability in inflammation via a reduction of Angpt-2 transcription (mRNA). More in vitro studies are needed to further investigate mechanisms of thepharmacological effect of BIFO on the regulation of Angpt-2. In vivo studies that are currently ongoing are supposed to confirm the results in a murine sepsis-model.

Reference(s) and grant ackowledgment(s)

1. DZL

2. DFG 1209/4-3

\section{8}

Ultrasound can be used to ensure correct central venous catheter tip positioning during infraclavicular right subclavian vein catheterization; a prospective observational study

M. Adrian ${ }^{1} ;$ T. Kander $^{2} ;$ R. Lundén ${ }^{2} ;$ O. Borgquist ${ }^{2}$

${ }^{1}$ Department of cardiothoracic surgery, anaesthesia and intensive care, Skåne University Hospital Lund, Lund, Sweden; ${ }^{2}$ Department of intensive and peri-operative care, Skåne University Hospital Lund, Lund, Sweden

Correspondence: M. Adrian

Intensive Care Medicine Experimental 2020, 8(2): 000188

Introduction: Subclavian vein catheterization has many advantages compared to other sites for central venous access. Unfortunately, central venous catheter (CVC) misplacement is common (up to $9.3 \%$ after a right-sided catheterization). To avoid CVC misplacements, the right supraclavicular fossa ultrasound view can be used to confirm correct guidewire J-tip position in the lower part of the superior vena cava prior to CVC insertion.

Objectives: To demonstrate the usefulness of a real-time ultrasound method for guidewire positioning during infraclavicular CVC placement in the right subclavian vein, and to determine the number of CVC misplacements occurring when using this method. The hypothesis was that the incidence of misplaced CVCs could be reduced to $1 \%$.

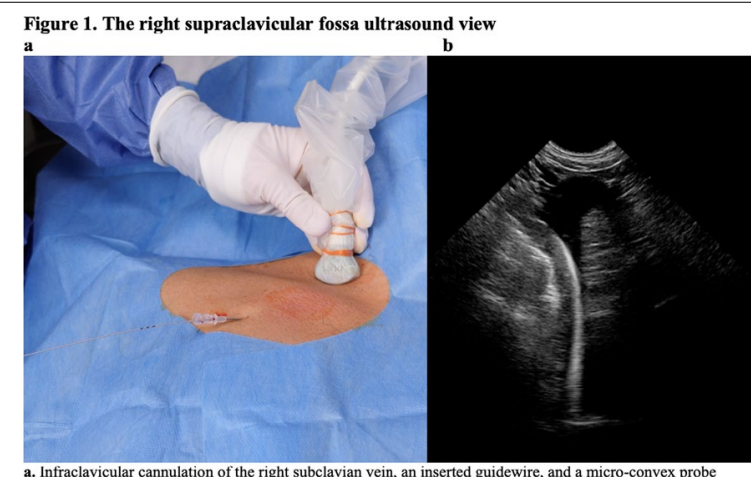

a. Infraclavicular cannulation of the right subclavian vein, an inserted guidewire, placed just above the medial part of the clavicle in the right supracla
Methods: The study was approved by the Swedish Ethical Review Authority and registered as a clinical trial at clinicaltrials.gov (NCT03812757). Sample size calculation showed that 93 patients should be included. All vein punctures were performed prospectively, under real-time ultrasound guidance with a micro-convex probe. The right supraclavicular fossa ultrasound view was used to confirm correct guidewire position (Figure 1) and misplaced guidewires were adjusted prior to CVC insertion. A post-procedural chest X-ray was obtained to determine definitive CVC tip position.

Results: One hundred patients were included. Successful catheterization of the right subclavian vein was achieved in all patients. In 14 cases the guidewire J-tip was initially misplaced in either the ipsilateral internal jugular vein $(n=8)$ or in the left brachiocephalic vein $(n=6)$. In 12 of these cases it was possible to adjust the guidewire J-tip to a correct position in the superior vena cava. In 2 cases it was impossible to adjust the guidewire J-tip from a position in the left brachiocephalic vein, and the suboptimal positions were finally accepted. Both CVC tips were confirmed with chest $\mathrm{X}$-rays in the left subclavian vein, as expected. The remaining 98 CVCs all had a tip position in the lower part of the superior vena cava, determined by chest X-ray.

Conclusion: Although the hypothesis could not be confirmed, this study demonstrates that the use of the right supraclavicular fossa ultrasound view to guide guidewire positioning reduced the incidence of CVC misplacements to $2 \%$ and thus is useful to avoid CVC misplacements in right infraclavicular subclavian vein catheterization.

\section{3}

Gradient-boosting machine learning models to predict creatinine clearance one day ahead

CY. Huang ${ }^{1}$; M. Schetz ${ }^{1}$; J. Gunst ${ }^{1}$; M. Casaer ${ }^{1}$; G. Van den Berghe ${ }^{1}$; F. Guiza Grandas'; G. Meyfroidt

${ }^{1}$ Laboratory of intensive care medicine, academic department of cellular and molecular medicine, KU Leuven, Leuven, Belgium

Correspondence: C.Y. Huang

Intensive Care Medicine Experimental 2020, 8(2):000193

Introduction: The glomerular filtration rate (GFR) is an index of the renal filtration function, and is estimated clinically by the creatinine clearance $(\mathrm{CrCl})$. Since the kidney is the primary excretory organ for most hydrophilic drugs such as beta-lactam antimicrobials, drug dosage is determined based on the $\mathrm{CrCl}$. It is well known that kidney function changes rapidly in critically ill patients. Predictions of $\mathrm{CrCl}$ could be useful to avoid subtherapeutic drug plasma levels in hyper-clearance patients and drug toxicity in hypo-clearance patients.

Objectives: The purpose of this study was to generate a daily prediction of kidney function on the next day in the ICU. For this purpose, we developed and internally validated prediction models for $\mathrm{CrCl}$ on the next day of ICU stay in adult patients.

Methods: This study was a retrospective preplanned secondary analysis of the EPaNIC multicenter randomized controlled trial database [1]. $\mathrm{CrCl}$ was calculated by daily urine output (UO), urine creatine $(\mathrm{UCr})$, and serum creatinine (SCr). The development cohort $(\mathrm{n}=1458$ patients, equivalent to 11726 patient-days) consisted of all ICU adult patients with available $\mathrm{CrCl}$ for the previous day. Clinical data were retrieved from the EPaNIC research database and from the clinical patient data management system database. The models were developed with gradient boosting method (GBM) machine-learning algorithm for both classification and regression. We first classified whether $\mathrm{CrCl}$ would be above $130 \mathrm{ml} / \mathrm{min} / 1.73 \mathrm{~m}^{2}$ and then regressed all the $\mathrm{CrCl}$ classified as below $130 \mathrm{ml} / \mathrm{min} / 1.73 \mathrm{~m}^{2}$. Model performance (Mean \pm SD) was internally validated with 10 -fold cross validation for discrimination, calibration, net benefit analysis, coefficient of determination (R2), mean absolute error (MAE), and root mean square error (RMSE).

RESULTS. A total of 479 patients (32.8\%), equivalent to 2077 patientdays $(17.7 \%)$, had $\mathrm{CrCl}$ above $130 \mathrm{ml} / \mathrm{min} / 1.73 \mathrm{~m}^{2}$ on the next day. The five most important variables for both the classification and regression models were mean $\mathrm{CrCl}, \mathrm{CrCl}$ of the previous day, age, $\mathrm{SCr}$ of the previous day, and UO of the previous day. As shown in Fig. 1, the classification model had an area under the receiver operating characteristic 
curve (AUROC) of $0.94 \pm 0.01$, calibration slope of $0.90 \pm 0.09$, and calibration in the large of $0.00 \pm 0.04$. At the classification threshold maximizing sensitivity and specificity, net benefit with respect to treat-none was $0.14 \pm 0.04$, and net benefit with respect to treat-all was $0.09 \pm 0.07$. For the remaining 979 patients having $\mathrm{CrCl}$ below 130 $\mathrm{ml} / \mathrm{min} / 1.73 \mathrm{~m}^{2}$ on the next day, the regression model had RMSE of $16.45 \pm 0.91$, MAE of $11.41 \pm 0.86$, and R2 of $0.80 \pm 0.04$.

Conclusion: Prediction models based on routinely collected clinical data were able to accurately predict $\mathrm{CrCl}$ of the next day in the ICU. These predictions could be used to more accurately determine hydrophilic drug doses in critically ill patients. These findings should be externally validated.
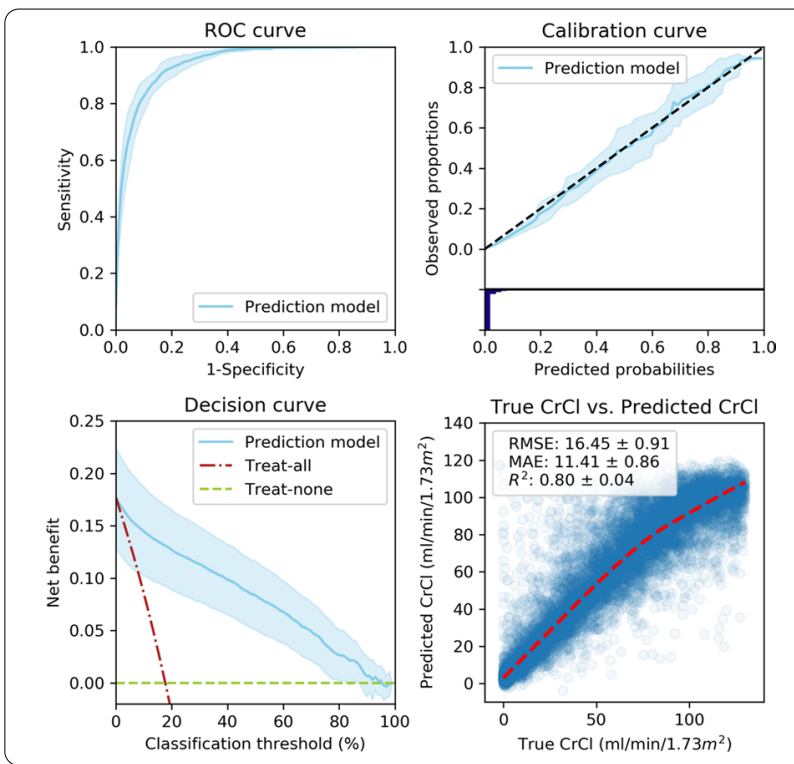

Reference(s) and grant ackowledgment(s)

1. 1. Casaer MP, et al (2011) N Engl J Med 365

2. Chao-Yuan Huang receives a Taiwan - KU Leuven scholarship.

\section{4}

Effects of colloids vs. crystalloids on microcirculation in ischemia-reperfusion injury: a randomized experimental study in pigs

C. Behem ${ }^{1}$; T. Friedheim ${ }^{1}$; H. Holthusen ${ }^{1}$; A. Rapp ${ }^{1}$; T. Suntrop'; H. Pinnschmidt'; M. Gräßler'; S. Wipper ${ }^{3}$; W. Schierling ${ }^{4}$; K. Pfister ${ }^{4}$;

C. Trepte

${ }^{1}$ Anesthesiology, University Medical Center Hamburg-Eppendorf, Hamburg, Germany; ${ }^{2}$ Medical biometry \& epidemiology, University Medical Center Hamburg-Eppendorf, Hamburg, Germany; ${ }^{3}$ Vascular medicine, University Heart Center Hamburg GmbH, Hamburg, Germany; ${ }^{4}$ Vascular surgery, University Medical Center Regensburg, Regensburg, Germany Correspondence: $\mathrm{C}$. Behem

Intensive Care Medicine Experimental 2020, 8(2):000194

Introduction: Introduction/Background: Ischemia/reperfusion (I/R) is frequently associated with microcirculatory deterioration (1). Microcirculatory disturbances are associated with reduced outcome (2). Both colloidal as well as crystalloid fluids are used for macrohemodynamic optimization in I/R. However, impact of colloids or crystalloids on microcirculation is unclear. Aim of this study was to compare the effects of colloids vs. crystalloids on microcirculation of vital organs in I/R injury.

Methods: Methods: This study was performed in 32 anesthetized and mechanically ventilated pigs. Animals were randomized in two equal groups receiving either colloids (Voluven ${ }^{\circledR} 6 \%$, Fresenius Kabi ${ }^{\circledR}$, Bad Homburg, Germany) or crystalloids (Sterofundin ${ }^{\circledR}$ ISO 1/1 E, B. Braun ${ }^{\circledR}$, Melsungen, Germany) for hemodynamic optimization. Volume optimization was performed before and after I/R using consecutive volume loading steps of $7 \mathrm{ml} / \mathrm{kg}$ of either colloids or crystalloids until increases of cardiac output $\geq 15 \%$ were absent. I/R was induced by 48 minutes of aortic cross clamping. Microcirculatory blood flow of kidney, liver and small intestine was assessed using Laser-Speckle-Contrast-Imaging providing mean microcirculatory blood flow (mFlux). Cardiac output was measured using pulmonary artery thermodilution and arterial pressure was assessed using invasive micro-tip catheter. Primary outcome measures were hepatic, renal and ileal microcirculatory blood flow $4,5 \mathrm{~h}$ after I/R. Secondary outcome measures were macrohemodynamic parameters and laboratory findings. Statistical analysis was performed using mixed-model analysis for comparison between groups. Values are given as mean ( $95 \%$ confidence interval), two-tailed $p$-values of $<0.05$ were considered significant.

Results: Results: Colloids significantly improved ileal mean microcirculatory blood flow (mFlux) compared to crystalloids $4,5 \mathrm{~h}$ after I/R (767.52 (676.47-858.57) vs. 594.24 (494.28-694.20), $\mathrm{p}=0.007)$. This was accompanied by a significant increase in cardiac output $(\mathrm{I} / \mathrm{min})(3.01$ (2.71-3.30) vs. 2.94 (2.09-2.70), $p=0.008)$. There were no significant differences of renal or hepatic microcirculatory blood flow between colloid and crystalloid group (mFlux kidney: 509.20 (426.87-591.52) vs. 443.41 (361.54-525.28), $p=0.304$; mFlux liver: 606.06 (509.44-702.67) vs. 546.70 (442.10-651.30), $\mathrm{p}=0.353$, respectively). Comparison of colloids vs. crystalloids revealed no significant difference in regard to arterial pressure. While there was no significant difference in regard to urine output $(\mathrm{ml})$ between groups (1144.82 (852.35-1537.63) vs. 780.55 (579.98-1.049.43), $p=0.071$ ), animals receiving colloids had significant lower values of serum creatinine $(\mathrm{mg} / \mathrm{dl})$ compared to crystalloids $(2.43(2.33-2.53)$ vs. $2.63(2.52-2.74), p=0.021)$.

Conclusion: Conclusions: Advantageous effects on intestinal microcirculation could be found for colloids. This was accompanied by improvements of macrocirculation. In conclusion, use of colloids may improve outcome in I/R injury.

Reference(s) and grant ackowledgment(s)

1. 2. Sakr, Y., et al., Persistent microcirculatory alterations are associated with organ failure and death in patients with septic shock. Crit Care Med, 2004. 32(9): p. 1825-31.

2. 1. Granger, D.N. and P.R. Kvietys, Reperfusion injury and reactive oxygen species: The evolution of a concept. Redox Biol, 2015. 6: p. 524-51

3. This study was supported by the ESICM Baxter Fluid Management Award 2016

\section{5}

Role of Transcranial Doppler Ultrasound as a predictor of outcome in severe traumatic brain injury and its correlation with Glascow Coma Scale and Full Outline Of Unresponsiveness Score T. Elsefi'; AM. Fayed ${ }^{2}$;. Zaytoun ${ }^{2}$

${ }^{1}$ Intensive Care, Health Education England, London, United Kingdom; ${ }^{2}$ Intensive care, Alexandria Faculty of Medicine, Alexandria, Egypt

Correspondence: $T$. Elsefi

Intensive Care Medicine Experimental 2020, 8(2):000195

Introduction: Traumatic brain injury (TBI) is a major public health problem. It is considered to be one of the leading causes of death and disability worldwide (1). After TBI cerebral blood flow (CBF) becomes extremely low approaching ischemic threshold (2). Concurrently, cerebral blood flow velocities become strongly correlated to CBF itself post injury. Identification of such haemodynamic disturbances can be used to predict outcome in severe TBI when measured immediately post-injury usingTranscranial Doppler (TCD). TCD permits non invasive assessment of different CBF velocities as well as pulsatility index (PI) (3). Abnormal measurement of such indices is believed to correlate to poor outcome.

Objectives: The aim of the work was to assess the predictive value of early TCD in patients with severe TBI in terms of mortality and GOSE at 3 months, and also to correlate different TCD measurements with GCS score and FOUR score

Methods: 120 patients with severe TBI, according to GCS, underwent TCD within 24 hours post trauma. Middle cerebral artery (MCA) velocities and pulsatility index, as well as other clinical and neuroimaging data, were recorded and accordingly patients were divided into 3 groups: patients with normal TCD measurements, patients 
with hypoperfusion and patients with vasospasm. Hypoperfusion was defined by meeting two out of three criteria: mean flow velocity (MFV) of MCA $<35 \mathrm{~cm} / \mathrm{sec}$, End diastolic velocity (EDV) of MCA $<20 \mathrm{~cm} / \mathrm{sec}$, $\mathrm{PI}>1.4$. Vasospasm was defined as MFV $>120 \mathrm{~cm} / \mathrm{sec}$. (4-9) Outcome was evaluated using the Glasgow Outcome Scale extended (GOSE) at 3 months, as well as in-hospital mortality. TCD measurements were also correlated to GCS and FOUR score.

Pearson's correlation coefficients $(r)$ were calculated to test the relation between TCD and other clinical variables. A binary logistic regression analyses were performed then variables that showed statistically significant result under univariate analysis were entered into a multivariate analysis to identify independent predictors of outcome measures. Discrimination of the logistic models was assessed by calculating the area under (ROC) curve (10). All hypotheses were constructed twotailed and $p \leq 0.05$ was considered significant

Results: There was a significant correlation between PI and GOSE 3 months. There was also significant correlation between PI and mortality. However, different MCA velocities did not show any correlation with GOSE or mortality. Strong negative correlation was recognized between PI and GCS and FOUR score.
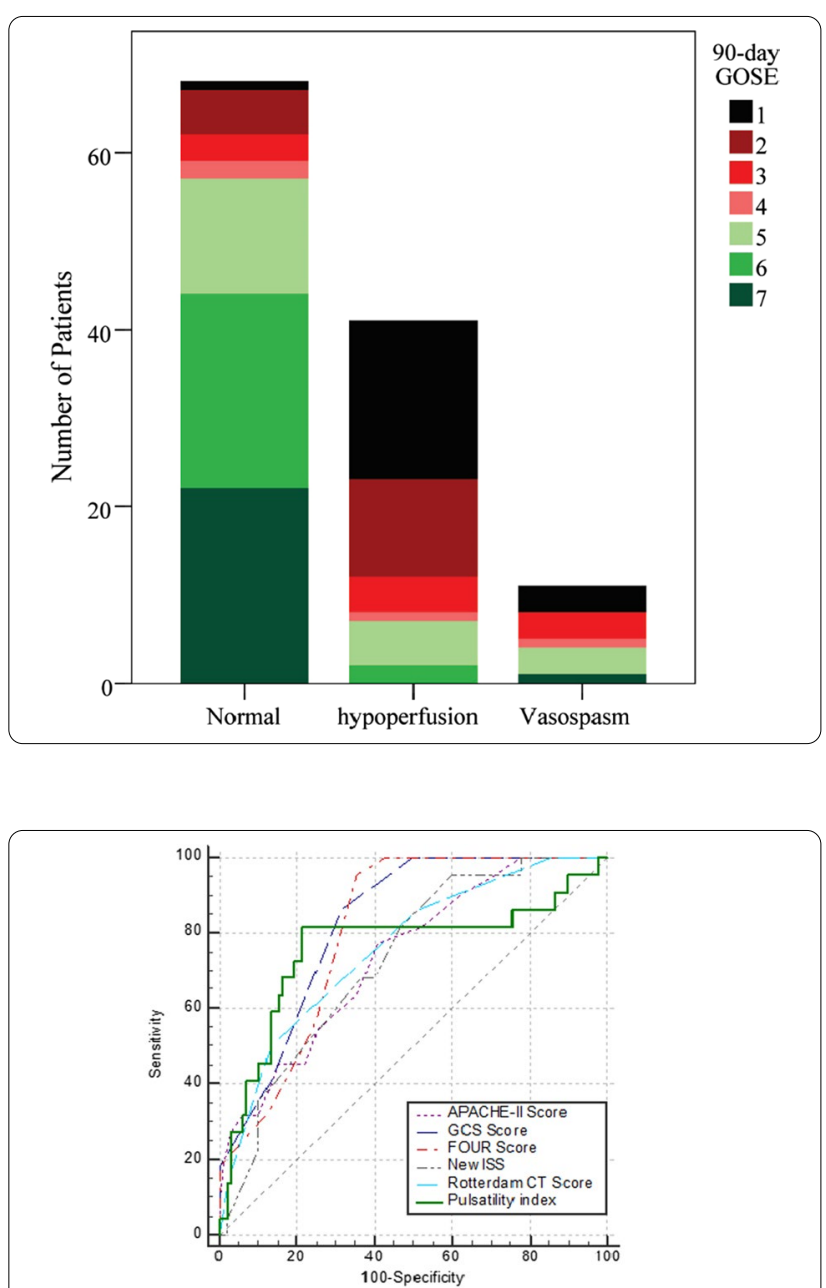

Fig: ROC curves comparing GCS Score, FOUR Score, APACHE-II Score, Rotterdam CT Score, New ISS, Pulsatility index in predicting in-hospital Mortality
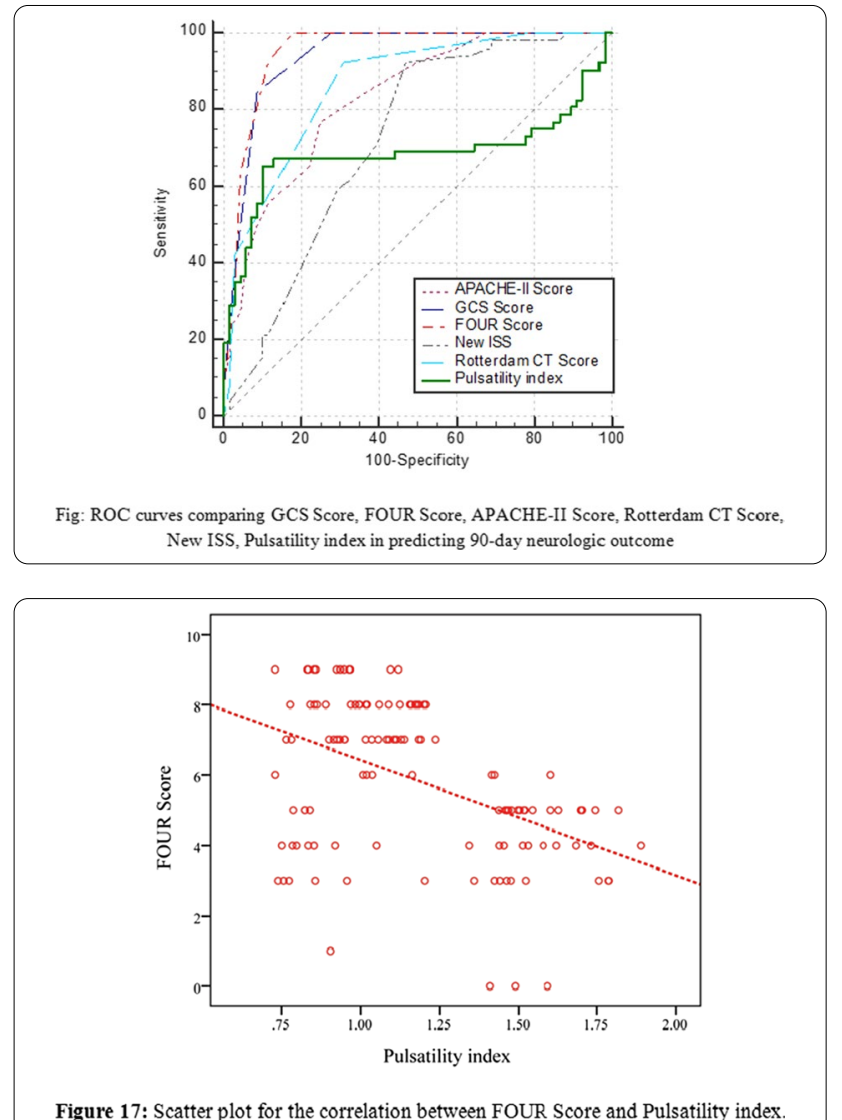

Figure 17: Scatter plot for the correlation between FOUR Score and Pulsatility index

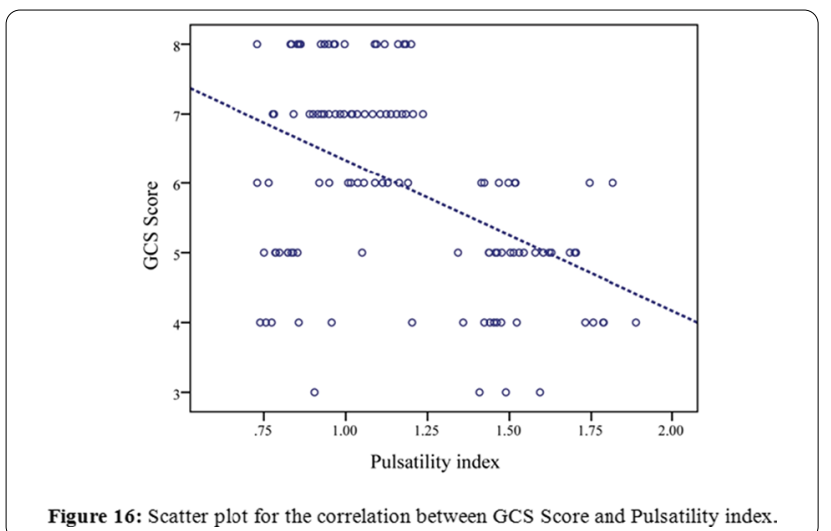

Conclusion: Pulsatility index, when measured within the first 24 hours post-trauma, is considered a good predictor of mortality as well as functional outcome at 3months. Abnormal pulsatility index values correlate with the severity of injury (in terms of GCS and FOUR score).

\section{Reference(s) and grant ackowledgment(s)}

1. 10. DeLong ER, DeLong DM, Clarke-Pearson DL. Comparing the areas under two or more correlated receiver operatingcharacteristic curves: a nonparametric approach. Biometrics 1988; 44: 837-45

2. 9. Zubkov AY, Lewis Al, Raila FA, Zhang J, Parent AD. Risk factors for the development of post-traumatic cerebral vasospasm. Surg Neurol 2000; 53: $126-30$ 
3. 8. Oertel M, Boscardin WJ, Obrist WD, Glenn TC, McArthur DL, Gravori T, et al. Posttraumatic vasospasm: the epidemiology, severity, and time course of an underestimated phenomenon: a prospective study performed in 299 patients. J Neurosurg 2005; 103: 812-24.

4. 7. Ract C, Le Moigno S, Bruder N, Vigué B. Transcranial Doppler ultrasound goal-directed therapy for the early management of severe traumatic brain injury. Intensive Care Med 2007; 33: 645-51.

5. 6. Van Santbrink H, Schouten JW, Steyerberg EW, Avezaat CJJ, Maas AIR. Serial transcranial Doppler measurements in traumatic brain injury with special focus on the early posttraumatic period. Acta Neurochir 2002; 144: 1141-9

6. 5. White H, Venkatesh B. "Applications of transcranial Doppler in the ICU: a review," Intensive Care Medicine 2006; (32)7: 981-94

7. 4. Gosling RG, King DH. "Arterial assessment by Doppler shift ultrasound," Proceedings of the Royal Society of Medicine1974; (67):6; 447-9.

8. 3. Aaslid R, Markwalder TM, Nornes H. Noninvasive transcranial Doppler ultrasound recording of flow velocity in basal cerebral arteries. J Neurosurg 1982; 57(6): 769-74

9. 2. Splavski B, Radanović B, Mužević D, Has B, Jančuljak D, Kristek J, et al. Assessment of intra-cranial pressure after severe traumatic brain injury by transcranial Doppler ultrasonography. Brain Inj. 2006 Jan 1:20(12):1265-70

10. 1. National centre for injury prevention and control. Report to congress on traumatic brain injury in the united states: Epidemiology and rehabilitation. Atlanta, GA: Division of Unintentional Injury Prevention. 2014. Available at: https://www.cdc.gov/traumaticbraininjury/pdf/tbi_repor t_to_congress_epi_and_rehab-a.pdf

11. None

\section{7}

Rapid Design and implementation of a data-driven forecast of iCU strain from COVID-19 for early surge planning in England

E. Rocheteau'; J. Deasy'; K. Kohler²; D. Stubbs²; P. Barbiero'; P. Liò';

A. Ercole

${ }^{1}$ Department of computer science, University of Cambridge, Cambridge, United Kingdom; '2Department of medicine, University of Cambridge, Cambridge, United Kingdom

\section{Correspondence: $\mathrm{E}$. Rocheteau}

Intensive Care Medicine Experimental 2020, 8(2):000267

Introduction: Creating ICU "surge capacity" was essential for the COVID-19 pandemic but takes time to implement and is costly and disruptive. A short-range forecast was needed in early March 2020 to guide costly and ethically challenging decisions in England and justify immediate service re-configuration from early epidemiological data. The design, construction, testing, implementation, documentation, dissemination and peer-review of a computer model is typically a lengthy process. Here we describe the use of agile techniques to develop a COVID-19 strain forecasting tool from ultra-early epidemiological data building a working tool within a week.

Objectives: To develop and implement a region-specific, short-range ICU demand forecast based on case data from the commissioning regions in England using agile software development techniques to build a public-facing tool as rapidly as possible.

Methods: We modelled the likely increases in regional ICU bed demand over a 14-day horizon under an exponential assumption. Daily COVID-19 cases were extrapolated from cumulative data using log-linear regression. Stochastic models for ICU admissions and length of stay linked expected case incidence to excess bed occupancy. Mortality and admission rates were regionally standardised. In parallel we developed a web application to publish updated predictions daily (Figure 1), with an explanatory manuscript on medRvix [1] and code on GitHub [2].

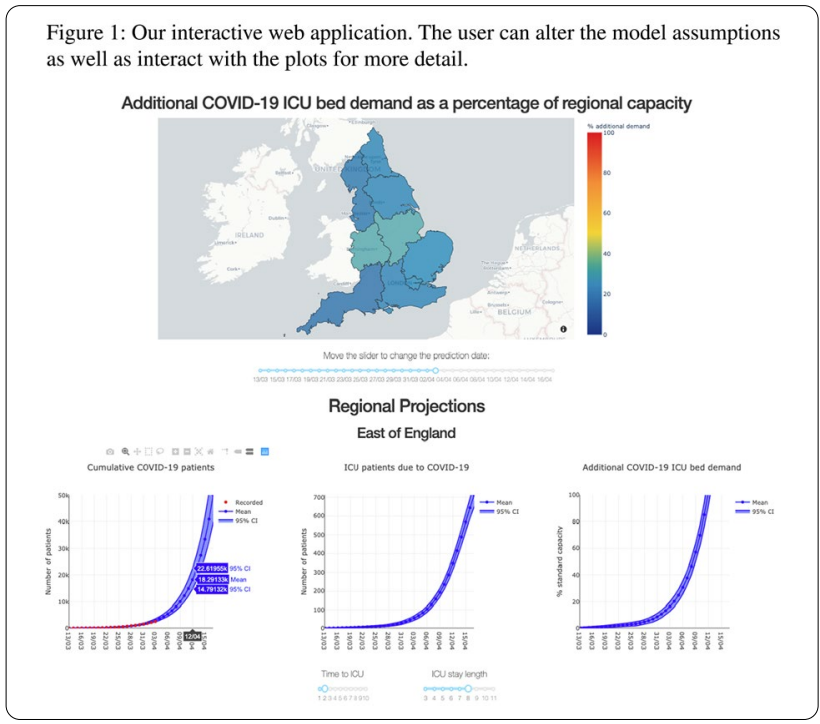

Results: We delivered an interactive web application along with explanatory notes and versioned code in 7 days. Community feedback allowed for a crowd-sourced review of manuscript and code. Users could explore the effect of changing various modelling assumptions interactively at a regional level. Over the preparatory time period, cumulative case data was well fitted by an exponential model. We predicted that ICU bed occupancy would grow very quickly with London and the Midlands predicted to be particularly hard-hit, motivating an immediate effort to create as much capacity as possible.

Conclusion: Uncertainties in modelling early epidemiological data notwithstanding, we accurately predicted that surge capacity would be required from the end of March. We were able to leverage software development techniques to deliver a working application on a timescale which was still actionable in the preparatory phase for COVID-19 in England. Our approach demonstrates that data-driven approaches to emergency healthcare planning can be highly agile.

\section{Reference(s) and grant ackowledgment(s)}

1. [2] J Deasy, E Rocheteau, K Kohler, DJ Stubbs, P Barbiero, P Liò, A Ercole. GitHub 2020 https://doi.org/10.5281/zenodo.3726134

2. [1] J Deasy, E Rocheteau, K Kohler, DJ Stubbs, P Barbiero, P Liò, A Ercole. Forecasting ultra-early intensive care strain from COVID-19 in England. MedRxiv 2020 https://doi.org/10.1101/2020.03.19.20039057

\section{8}

Post-operative near-infrared spectroscopy in pediatric patients after cardiac surgery: association with total IQ assessed at 2-years follow-up

G. Carra'; M. Flechet ${ }^{1}$; A. Jacobs ${ }^{1}$; S. Verstraete ${ }^{1}$; D. Vlasselaers ${ }^{1}$; L. Desmet ${ }^{1}$; H. Van Cleemput ${ }^{1}$; P. Wouters ${ }^{1}$; I. Vanhorebeek ${ }^{1}$; G. Van den Berghe'; F. Güiza'; G. Meyfroidt ${ }^{1}$

${ }^{1}$ Department and laboratory of intensive care medicine, KU Leuven, Leuven, Belgium

Correspondence: G. Carra

Intensive Care Medicine Experimental 2020, 8(2):000278

Introduction: During the early post-operative period, children with congenital heart disease can suffer from inadequate cerebral perfusion. Cerebral perfusion can be monitored non-invasively with the near-infrared spectroscopy (NIRS) cerebral oximeter, which measures local cerebral tissue oxygen saturation (SctO2) of the frontal lobe. In this study, we hypothesized that reduced SctO2 and increased dose of desaturation during the early post-operative period, may independently increase the probability of reduced total IQ score 2-years after Pediatric Intensive Care Unit (PICU) admission. 
Methods: The study included 90 children (median age (IQR) 4 (1 to 13) months) admitted to the PICU of the University Hospitals Leuven (Belgium) and included in the PEPaNIC trial [1] and NIRS study [2], after cardiac surgery for congenital heart disease. Inadequate post-operative cerebral perfusion was characterized by computing themean SctO2 -and dose of desaturation (defined as SctO2 $<65 \%$ ) from the first 12 hours of SctO2 monitoring after surgery. Trained psychologists assessed the total IQ at 2-years follow-up with the Wechsler Intelligence Quotient (IQ) tests. The independent association between mean SctO2, dose of desaturation and total IQ at 2-years follow-up was evaluated with a Bayesian linear regression model adjusted for age, syndrome, nutrition strategy (early vs late parenteral nutrition), cyanotic cardiopathy and severity of illness with Pediatric Index of Mortality 3 score.

Results: The results of the Bayesian model, summarized in Table 1, showed that reduced mean $\mathrm{SctO} 2$ and increased dose of SctO2 desaturation below $65 \%$ during the first 12 hours of SctO-2 monitoring, independently increased the probability of a lower total IQ at 2-years follow-up. For example, a difference of $10 \%$ in the mean SctO2 during the first 12 hours of SctO2 monitoring would result in a loss of 0.3 to $4 \mathrm{IQ}$ points, with an $80 \%$ probability. Similarly, a dose of desaturation of $300 \%$ minutes (resulting, for instance, from a SctO2 of $60 \%$ for 1 hour) would correspond to a loss of 0.3 to 4.8 IQ points with an $80 \%$ probability.

\begin{tabular}{|l|l|l|}
\hline & \multicolumn{2}{|c|}{ Table 1 } \\
\hline & $\begin{array}{l}\beta \text { estimates } \\
{[80 \% \text { credible }} \\
\text { interval }]\end{array}$ & $\begin{array}{l}\text { Probability that the } \beta \text { estimates for the } \\
\text { population is strictly negative(- } \\
/ \text { positive }(+)\end{array}$ \\
\hline $\begin{array}{l}\text { Desaturation } \\
\text { dose }\end{array}$ & $\begin{array}{l}-0.009[-0.016 \\
\text { to }-0.001]\end{array}$ & $90 \%(-)$ \\
\hline Mean SctO2 & $\begin{array}{l}0.227[0.037 \text { to } \\
0.412]\end{array}$ & $90 \%(+)$ \\
\hline
\end{tabular}

Table 1 . The $\beta$ estimates [ $80 \%$ credible interval] resulting from the Bayesian model are reported in the table together with the cumulative probability that the $\beta$ estimate of the population are strictly negative (-) or positive (+). The $80 \%$ credible interval indicates the range to which the $\beta$ estimates of the population belong with $80 \%$ probability. Results were obtained by using a noninformative prior.

Conclusion: Reduced mean $\mathrm{SctO} 2$ and increased dose of SctO2 below $65 \%$ during the first 12 hours of SctO2 monitoring, independently increase the probability of having a lower total IQ, 2 years after PICU admission. However, given the observational design of the study, no causal relations are proven. Whether intervention to improve SctO2 would result in better outcomes is still unclear.

\section{Reference(s) and grant ackowledgment(s)}

1. [1] S. Verstraete et al., Lancet Respir. Med., Sep. 2018.

2. [2] M. Flechet et al., Pediatr. Crit. Care Med., May 2018.

\section{3}

Mechanical chest compressions are associated with increased severity of post-cardiac arrest syndrome: a sub-study

\section{from the TTH48 trial}

G. Babini' ; H. Kirkegaard²; E. Søreide ${ }^{3}$; FS. Taccone ${ }^{4}$; S. Markus ${ }^{5}$

${ }^{1}$ Department of Pathophysiology and Transplantation, University of Milan, Milano, Italy; ${ }^{2}$ Research center for emergency medicine, department of emergency medicine and department of clini, Aarhus University Hospital and Aarhus University, Aarhus, Denmark; ${ }^{3}$ Critical care and anaesthesiology research group, Stavanger University Hospital, Stavanger, Norway; ${ }^{4}$ Soins intensif, ULB Erasme, Anderlecht, Belgium; ${ }^{5}$ Division of intensive care, department of anesthesiology, intensive care and pain medicine, University of Helsinki and Helsinki University Hospital, Helsinki, Finland

Correspondence: G. Babini

Intensive Care Medicine Experimental 2020, 8(2): 000283
Introduction: Mechanical chest compressions (CC) are frequently used during prolonged cardiopulmonary resuscitation in out-of-hospital cardiac arrest (OHCA), particularly in patients who do not have early return of spontaneous circulation (ROSC) on the scene. However, little is known about the characteristics of this population of OHCA patients, particularly after hospital admission.

Objectives: We hypothesized that the decision to initiate mechanical CC could identify, by itself, a specific subgroup of cardiac arrest patients with more severe clinical features.

Methods: This is a post hoc analysis of a sub-group of patients in the TTH48 trial (NCT01689077) who received or did not received mechanical CC during resuscitation manoeuvres. Baseline characteristics included age, sex, height, weight, neurological function before cardiac arrest and a prespecified set of comorbidities. Relevant data regarding OHCA were recorded following Utstein templates. Resuscitation outcomes included survival at ICU discharge, survival at hospital discharge, 6-months survival and 6-months survival with good neurological outcome (CPC 1-2). Continuous variables are presented as median [IQR] and nominal variables as percentage. Mann-Whitney u-test and chi-square test were used for groups comparison, accordingly. A p < 0.05 was considered as statistically significant.

Results: Out of 351 patients included in the analysis, $25.6 \%$ received mechanical CC. No major difference in baseline characteristics were observed between groups. Compared to patients that receive manual CC, mechanical CC patients showed significant prolonged time to ROSC $(26$ [20;38] vs $20[15 ; 25]$ min, $p<0.01)$. Accordingly, a greater percentage of patient received resuscitation drug (adrenaline: 80 vs $57 \%, \mathrm{p}<0.01$; amiodarone: 53 vs $37 \%, \mathrm{p}<0.01$ ) and percutaneous coronary intervention ( 93 vs $79 \%, p<0.01$ ). After hospital admission, mechanical CC patients demonstrate worse hemodynamic and acidbase status compared to patient who received manual CC; more specifically they showed lower systolic arterial pressure $(104[91 ; 120]$ vs 114 [97;132] $\mathrm{mmHg}, \mathrm{p}<0.01)$, higher blood lactate $(5.8[2.8 ; 10]$ vs 3.2 $[1.8 ; 5.1] \mathrm{mmol} / \mathrm{l}, \mathrm{p}<0.01)$ and lower base excess $(-8.4[-12.3 ;-4.0]$ vs $-5.8[-8.5 ;-3.5] \mathrm{mmol} / \mathrm{l}, \mathrm{p}<0.01)$. During hospital stay, mechanical CC patients develop adverse events more frequently, such as generalized seizure/myoclonus ( 27 vs $15 \%, p=0.03$ ), various grade of hypotension $(p=0.02)$ and arrhythmias $(p<0.01)$, greater incidence of acute renal failure needing continuous renal replacement therapy (17 vs $5 \%$, $p<0.01)$. Finally, mechanical CC patients showed increased mortality (ICU: 22 vs $14 \%, p=0.07$; in-hospital: 34 vs $20 \%, p=0.01 ; 6$-months 27 vs $15 \%, p<0.01)$ and worse neurological outcome after 6 months (CPC $1-252$ vs $71 \%, \mathrm{p}<0.01$ ).

Conclusion: This descriptive analysis identified an association between patient receiving mechanical CC and worse clinical status after hospital admission. Moreover, mechanical CC patients demonstrated poorer 6-months survival and neurological outcome. The longer duration of no-flow/low-flow time observed in the mechanical CC group could partially explain the increase severity in post-cardiac arrest syndrome observed in this subgroup of patients.

\section{Reference(s) and grant ackowledgment(s)}

1. Kirkegaard et al. JAMA 2017

\section{5}

Impact of a nurse-led family support intervention for families of critically ill persons: A mixed methods study

P. Massarotto ; S. Von Felten ${ }^{2}$; H. Petry ${ }^{3}$; R. Naef ${ }^{3}$

${ }^{1}$ Institute of intensive medicine, University Hospital of Zürich, Zürich, Switzerland; ${ }^{2}$ Department of biostatistics, institute of epidemiology, biostatistics, and prevention, University of Zurich, Zürich, Switzerland; ${ }^{3}$ Centre of clinical nursing science, University Hospital of Zürich, Zürich, Switzerland

Correspondence: P. Massarotto

Intensive Care Medicine Experimental 2020, 8(2): 000285

Introduction: Families of critically ill persons face uncertainty and psychological distress during and after their close other's stay on an intensive care unit (ICU). Poor communication, insufficient shared-decision making, and inadequate emotional support negatively affect their ICU 
experience and mental health. To increase quality of care and reduce adverse outcomes, guidelines recommend proactive family engagement and support. We thus implemented an advanced practice nurseled family support intervention in a 12-bed surgical-transplant ICU in Switzerland.

Objectives: To examine the impact of the intervention on family members' satisfaction with ICU care, sense of well-being, and post-ICU psychological distress.

Methods: We used a mixed methods approach with a quasi-experimental before and after design and an embedded qualitative study. Participants were family members of patients treated in ICU before and after introduction of the intervention, which consisted of relational family engagement, therapeutic family conversations, and facilitation of communication between the family and the ICU team. Data were collected post-ICU with the Family Satisfaction in ICU-24 Survey, Hospital Anxiety Depression Scale, Impact of Event Scale-Revised-6 and semi-structured interviews. Outcomevariables of the matched groups were analysed with linear mixed-effects models, and qualitative data using inductive content analysis.

Results: 139 family members in the control and 75 in the intervention group (51\% response rate) returned a questionnaire, and 19 participated in an interview. Families in the intervention group showed a trend for increased overall satisfaction (difference of $5.544,95 \% \mathrm{Cl}$ : -0.11 to 11.20 ), a statistically significant increase in satisfaction with decision-making (7.258, 95\% Cl: 0.89 to 13.63$)$, and a non-significant increase in satisfaction with care $(4.178,95 \% \mathrm{Cl}$ : 1.53 to 9.89$)$. Psychological distress was higher in the intervention group, with was more pronounced for depression (difference of 1.706, 95\% Cl: 0.16 to 3.25 ), which may be explained by considerably longer ICU stays and higher proportion of deaths in the intervention group. Qualitative findings indicated that the intervention was beneficial for family well-being, as families reported to feel acknowledged, cared for, well informed, and supported as an individual and a family living though critical illness. Families experienced the intervention as essential to ICU care. They appreciated to have a person to turn to who was easily accessible, knowledgeable, and part of the ICU team.

Conclusion: Our study suggests that family members appreciate a specific family nursing role that offers supportive interventions and facilitates interaction and communication with the ICU team, and experience it as beneficial for their well-being. It increased their satisfaction with ICU care, but was unable to demonstrate a beneficial impact on psychological distress.

\section{Reference(s) and grant ackowledgment(s)}

1. This work was supported by an innovation fund from the USZ Department of Nursing and Allied Health and the Health Services Research Prize awarded by the Ministry of Health, Canton of Zurich, Switzerland in 2018.

\section{3}

Photoacoustic mapping of tissue hypoxia after myocardial infarction in mice

H. DAVID'; A. Ughetto'; P. Gaudard'; N. Paiyabhroma²; S. Richard²; P. Colson ${ }^{3}$; P. Sicard ${ }^{2}$

'Phymedexp inserm u1046; department of anesthesiology and intensive care, CHU Arnaud de Villeneuve, Montpellier, France; ${ }^{2}$ Cardiovascular research, PhyMedExp, Montpellier University, INSERM, CNRS, IPAM, Montpellier, France; ${ }^{3}$ Department of anesthesiology and intensive care, $\mathrm{CHU}$ Arnaud de Villeneuve, Montpellier, France

Correspondence: $\mathrm{H}$. DAVID

Intensive Care Medicine Experimental 2020, 8(2): 000293

Introduction: Early alterations of microvascular blood flow after myocardial infarction (MI) may participate in the development of multiple organ failure.

Objectives: The aim of this study was to assess the contribution of photoacoustic imaging, a hybrid technology based on laser and ultrasound energy coupling, to detect early oxygen saturation $\left(\mathrm{sO}^{\mathrm{O}}\right)$ impairment in peripheral organs (kidney, liver) after Ml in mice.

Methods: An experimental preclinical study was carried out on two groups of six week BALB/c mice: Sham $(n=22)$ versus MI $(n=40)$. MI was obtained by permanent ligation of the left anterior descending artery within 7 days of follow-up. High resolution ultrasound was used to assess left ventricular (LV) function (ejection fraction (EF), Cardiac Output (CO) and longitudinal strain). Photoacoustic imaging (VevoLAZR, ViualSonics ${ }^{\circledR}$ ) was used to measure kinetics of the heart, kidney and liversO2after MI. Plasma biomarkers (ASAT, ALAT, creatinine, lactate) were assessed.

Results: LV function was altered $4 \mathrm{~h}$ post-MI (LVEF: Sham $61.7 \% \mathrm{n}=$ $16, \mathrm{Ml} 25.7 \% \mathrm{n}=32, \mathrm{p}<0.0001$; longitudinal strain: Sham $-19.6 \% \mathrm{n}=$ $13, \mathrm{Ml}-7.1 \% \mathrm{n}=31, \mathrm{p}<0.0001$; CO: Sham $11.6 \mathrm{~mL} / \mathrm{min} \mathrm{n}=13$, Ml 7.4 $\mathrm{mL} / \mathrm{min} \mathrm{n}=24, \mathrm{p}=0.003$ ). These alterations persisted after 7 days. A strong correlation was observed at $4 \mathrm{~h}$ and on day 1 between $\mathrm{LV}$ sO2 and longitudinal anterior myocardial strain $(r=-0.46, p<0.0001, n$ $=81$ ). Photoacoustic mapping showed a significant early decrease in sO2 level in both renal and hepatic tissues at $4 \mathrm{~h}$ and 1 day post-MI but this was normalized after 7 days (Figure 1).

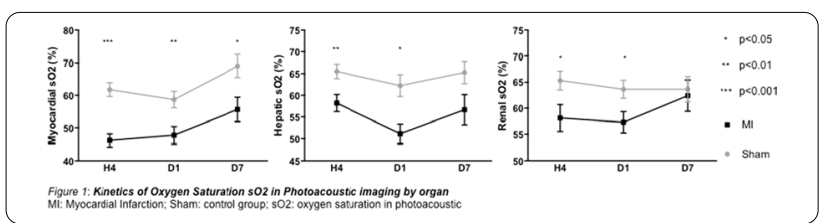

The kinetics of the biomarkers ASAT, ALAT and creatinine were correlated to a tissue alteration of $\mathrm{sO} 2$ at $4 \mathrm{~h}$. There was no correlation between alteration of $\mathrm{sO} 2$ and lactate (Figure 2).

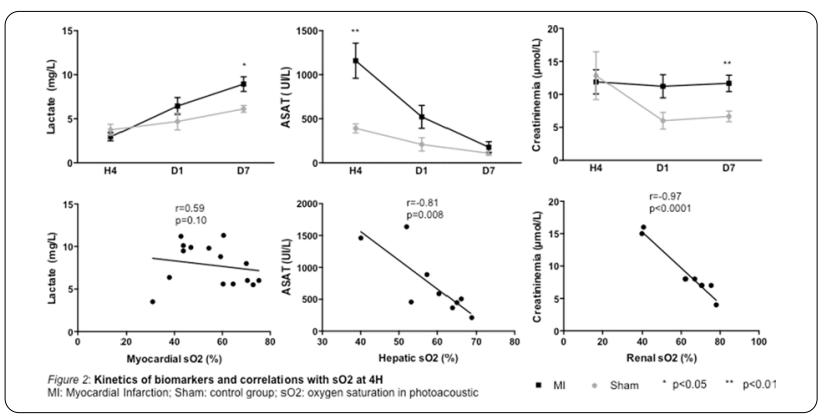

Conclusion: Post-MI heart failure generates moderate hypoxia of the peripheral organs in mice. Photoacoustic imaging enables early detection of $\mathrm{sO} 2$ multi-organ desaturation. The mapping of $\mathrm{sO} 2$ organs in real time seems to be an accurate marker to identify microcirculation alteration. This emerging imaging technology could be used to improve and evaluate early microvascular therapeutics.

Reference(s) and grant ackowledgment(s)

1. Al Mukaddim, Ultrasound in Medicine and Biology. 2018 Oct:44(10):2155-2164

2. Mebazaa, Eur J Heart Fail. 2017 Jul;19(7):821-836

3. Aissaoui, European Journal of heart failure (2017) 19, 192-200

\section{3}

Incidence and outcomes of acute kidney injury in intensive care units: Focus on high-risk surgical patients

TK. Henrique ; B. Cardoso Gomes ${ }^{2} ;$ JM. Silva ${ }^{3}$; M. Jacques ${ }^{4}$

${ }^{1}$ Anestesiologia, USP - University City, São Paulo, Brazil; ${ }^{2}$ Departamento de Medicina Integrada, Universidade Federal do Paraná, Curitiba, France; ${ }^{3} \mathrm{Icu}$, Hospital Israelita Albert Einstein, São Paulo, Brazil; ${ }^{4}$ Ssicologia, UniCesumar - Curitiba Campus, Curitiba, Brazil

Correspondence: B. Cardoso Gomes

Intensive Care Medicine Experimental 2020, 8(2): 000303

Introduction: The incidence of AKI in surgical patients varies according to the type and severity of surgery, with reported rates of $19 \%$ 
after cardiac surgery and approximately $12-13 \%$ after general and thoracic surgery. In a large epidemiological study of patients undergoing major noncardiac surgery in intensive care units (ICUs) in Brazil, 30\% of patients had postoperative complications, and AKI was the postoperative complication with the second-highest occurrence rate. Postoperative complications are common in high-risk patients after major surgery.

Therefore, our hypothesis is that perioperative AKI is common and is associated with a worse prognosis. Thus, evaluating the incidence and characteristics of patients with previously normal kidney function who underwent major noncardiac surgery and developed postoperative $\mathrm{AKI}$, in addition to determining whether perioperative volemic adjustments correlate with AKI, is relevant for adequately improving the therapeutic management of these patients.

Objectives: The main objective of this study was to evaluate the incidence, impact on outcomes and main risk factors for developing AKI in patients with previously normal kidney function who underwent noncardiac surgery and developed AKI after admission to the ICU. Because of the importance of fluid balance in surgical patients, we also evaluated the association between this variable and the development of AKI.

Methods: A multicenter, prospective cohort study with patients admitted to intensive care units (ICUs) after noncardiac surgery was conducted to assess whether they developed AKI. A total of 29 ICUs participated and provided data on 25,500 surgeries, of which 904 involved high-risk surgical patients who were included in the study. On the 28th day after surgery, the number of survivors and length of stay in the ICU and hospital were analyzed.

Results: The occurrence of AKI in the postoperative period was $15.8 \%$, and the mortality rate of postoperative AKI patients at 28days was $27.6 \%$, which represented $5.2 \%$ of the overall mortality of the study population. AKI was strongly associated with 28 -day mortality $(\mathrm{OR}=$ $2.91 ; 95 \% \mathrm{Cl} 1.51-5.62 ; \mathrm{P}=0.001$ ), and the assessment of ICU, hospital and 28-day postoperative survival identified significant differences between patients with and without postoperative AKI (log-rank test, P $<0.0001$ ). Independent factors for the risk of developing AKI were preoperative anemia ( $\mathrm{OR}=7.01 ; 95 \% \mathrm{Cl} 1.69$ - 29.07), elective surgery (OR $=0.45 ; 95 \% \mathrm{Cl} 0.21-0.97)$, SAPS $3(\mathrm{OR}=1.04 ; 95 \% \mathrm{Cl} 1.02-1.06)$, postoperative vasopressor use ( $\mathrm{OR}=2.47 ; 95 \% \mathrm{Cl} 1.34-4.55)$, postoperative infection (OR $=8.82 ; 95 \% \mathrm{Cl} 2.43-32.05)$ and need for reoperation (OR $=7.15 ; 95 \% \mathrm{Cl} 2.58-19.79$ ). Additionally, regarding fluid adjustment during the perioperative period, patients with higher fluid balance values presented an increased risk of developing AKI (OR $=1.001 ; 95 \%$ $\mathrm{Cl}, 1.000-1.002)$. It was noted that starting on the second day after surgery, the higher the fluid balance is, the more likely AKI is to develop.

$$
\text { Univariate Multivariate }
$$

\begin{tabular}{|c|c|c|c|c|c|}
\hline Variable (daily median & All patients & No AKI & AKI & P-value & $e_{\mathrm{CI})}^{\mathrm{OR}(95 \%}$ \\
\hline Total volume $(\mathrm{mL})$ & $\begin{array}{l}1900(1253- \\
2502)\end{array}$ & $\begin{array}{l}1820 \\
(1250- \\
2500)\end{array}$ & $\begin{array}{l}2000 \\
(1500- \\
2927)\end{array}$ & 0.007 & $\begin{array}{l}1.000 \\
(0.998- \\
1.001)\end{array}$ \\
\hline Fluid balance $(\mathrm{mL})$ & $\begin{array}{l}800(183- \\
1500)\end{array}$ & $\begin{array}{l}786(126- \\
1459)\end{array}$ & $\begin{array}{l}840(300- \\
1506)\end{array}$ & 0.04 & $\begin{array}{l}1.001(1.001- \\
1.002)\end{array}$ \\
\hline $\begin{array}{l}0.9 \% \text { saline solution } \\
(\mathrm{mL})\end{array}$ & $\begin{array}{l}1044(720- \\
2000)\end{array}$ & $\begin{array}{l}1000(642- \\
1750)\end{array}$ & $\begin{array}{l}1500 \\
(1000- \\
2527)\end{array}$ & 0.000 & $\begin{array}{l}0.999 \\
(0.999- \\
1.001)\end{array}$ \\
\hline $\begin{array}{l}\text { Lactated Ringer's } \\
\text { solution }(\mathrm{mL})\end{array}$ & $\begin{array}{l}1500(1000- \\
2500)\end{array}$ & $\begin{array}{l}1500 \\
(1000- \\
2500)\end{array}$ & $\begin{array}{l}1500(1000 \\
2500)\end{array}$ & 0.337 & $\begin{array}{l}1.000 \\
(0.999- \\
1.001)\end{array}$ \\
\hline Plasma-Lyte $(\mathrm{mL})$ & $\begin{array}{l}1500(1000- \\
2164)\end{array}$ & $\begin{array}{l}1500 \\
(1000- \\
2500)\end{array}$ & $\begin{array}{l}1150(500- \\
2000)\end{array}$ & 0.121 & $\begin{array}{l}0.999 \\
(0.998- \\
1.001)\end{array}$ \\
\hline $\begin{array}{l}\text { Hydroxyethylamide } \\
130 / 0.4(\mathrm{~mL})\end{array}$ & $\begin{array}{l}500(500- \\
1000)\end{array}$ & $\begin{array}{l}500(500- \\
500)\end{array}$ & $\begin{array}{l}1000(750- \\
1000\end{array}$ & 0.137 & ------- \\
\hline
\end{tabular}

median (interquartile range); adjusted OR in the multivariate analysis.
Conclusion: AKI is a major complication in high-risk surgical patients and is associated with risk of death. Patients with anemia prior to surgery who underwent elective surgery, had a higher SAPS 3, needed a vasopressor during the postoperative period or had postoperative infection or needed reoperation were more likely to develop AKI, as were those with an elevated perioperative fluid balance.

\section{Reference(s) and grant ackowledgment(s)}

1. Giglio M, Dalfino L, Puntillo F, Brienza N. Hemodynamic goal-directed therapy and postoperative kidney injury: an updated meta-analysis with trial sequential analysis. Crit Care. 2019;23(1):232.

2. Golden D, Corbett J, Forni LG. Peri-operative renal dysfunction: prevention and management. Anaesthesia. 2016;71 Suppl 1:51-7.

3. Gameiro J, Fonseca JA, Neves M, Jorge S, Lopes JA. Acute kidney injury in major abdominal surgery: incidence, risk factors, pathogenesis and outcomes. Annals of intensive care. 2018;8(1):22-.

4. Many thanks to the entire team that helped to recruit patients.

\section{2}

\section{Comparative effects of direct/indirect nitric oxide inhibition} on haemodynamics in a septic, hypotensive rat model

P. Arina ${ }^{1}$; A. Dyson ${ }^{1}$; J. Leiper ${ }^{2}$; M. Singer ${ }^{1}$

${ }^{1}$ University college london, Bloomsbury Institute of Intensive Care Medicine, London, United Kingdom; ${ }^{2}$ Institute of cardiovascular \& medical science, University of Glasgow, Glasgow, United Kingdom

\section{Correspondence: P. Arina}

Intensive Care Medicine Experimental 2020, 8(2): 000312

Introduction: Overproduction of nitric oxide (NO) plays an important role in sepsis-induced hypotension (1). However, a phase III study of L-NMMA, a non-selective NO synthase (NOS) inhibitor, was terminated early due to increased mortality (2). A new class of NOS inhibitor acts via inhibition of dimethylarginine dimethylaminohydrolase-1 (DDAH1). This increases levels of asymmetric dimethylarginine (ADMA), an endogenous methyl-arginine, which blocks NOS activity (3). Using a septic, hypotensive rat model, we hypothesized that indirect NOS inhibition would improve haemodynamics without detrimental effects on cardiac output that is associated with non-selective NOS inhibition.

Methods: Anaesthetized male Wistar rats underwent right jugular venous cannulation for fluid/drug administration. Sepsis was induced by intraperitoneal faecal slurry $(\mathrm{T}=0 \mathrm{~h})$. Animals were recovered and fluid $(1: 1$ mix of $5 \%$ glucose and Ringer's lactate [RL]) was infused at $10 \mathrm{ml} / \mathrm{kg} / \mathrm{h}$ from $2 \mathrm{~h}$. At $6 \mathrm{~h}$, animals were re-anaesthetized with cannulation of the carotid artery for BP monitoring and a tracheostomy to secure the airway. A further bolus of RL bolus $(20 \mathrm{ml} / \mathrm{kg})$ was given. At $8 \mathrm{~h}$, baseline measurements of BP, cardiac output (by echocardiography) and blood gas analysis were taken. Animals were then randomized to receive either direct (L-NMMA) or indirect (L-257) NOS inhibition, or to act as controls (equivalent volumes of RL). Treated animals received a bolus over 5 mins followed by infusion over 2 hours (to $\mathrm{T}=10 \mathrm{~h}$ ). Blood pressure was measured before and after the bolus. All other variables were measured hourly.

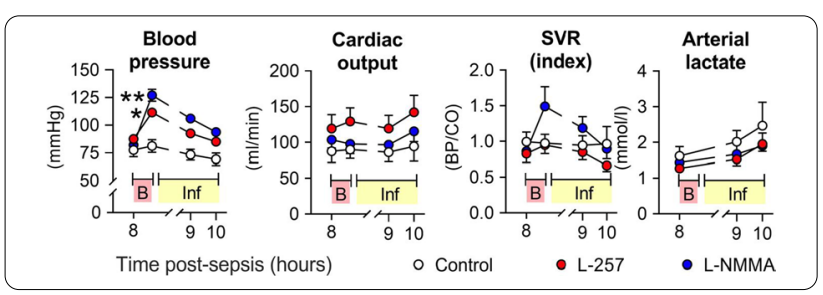

Results: Data shown as mean ( \pm SE). $B=$ bolus over 5 minutes. Inf $=$ infusion. Index of systemic vascular resistance (SVR) calculated as Blood pressure (BP)/Cardiac output (CO). Statistics: 2-way RM-ANOVA 
and post-hoc Tukey's test. ${ }^{*} p<0.05$ or ${ }^{* *} p<0.01$ between controls and drug-treated animals ( $\mathrm{n}=6 /$ group). $\mathrm{L}-257$ bolus $=30 \mathrm{mg} / \mathrm{kg}$, inf $=3.75 \mathrm{mg} / \mathrm{kg} / \mathrm{h}$. L-NMMA bolus $=3 \mathrm{mg} / \mathrm{kg}$, inf $=2.5 \mathrm{mg} / \mathrm{kg} / \mathrm{h}$. Conclusion: In hypotensive, septic rats, both direct and indirect NOS inhibition significantly increased blood pressure. While we did not use doses of either drug that significantly impacted on cardiac output, indirect NOS inhibition showed a lesser increase in SVR, in keeping with a more desirable safety profile.

Funding: Critical Pressure Ltd.

\section{Reference(s) and grant ackowledgment(s)}

1. References: 1. Pool R, et al. (2018) Crit Care Clin; 34:63-80. 2. López A, et al. (2004) Crit Care Med; 32:21-30. 3. Lambden S, et al. (2015) Arterioscler Thromb Vasc Biol; 35:1382-92.

\section{2}

Brain tissue oxygenation guided therapy in poor grade aneurysmatic subarachnoid haemorrhage patients E. Bogossian ${ }^{1}$; D. Diaferia'; N. Ndieugnou ${ }^{1}$; M. Menozzi ${ }^{1}$; JL. Vincent"; J. Creteur'. FS. Taccone

'Soins intensif, ULB Erasme, Brussels, Belgium

Correspondence: E. Bogossian

Intensive Care Medicine Experimental 2020, 8(2): 000342

Introduction: Aneurysmatic subarachnoid hemorrhage (aSAH) is responsible for severe disabilities and death (1). Tissue hypoxia can occur after $\mathrm{SAH}$, even with normal levels of intracranial pressure (ICP) (2). As such, brain tissue oxygen (PbtO2) monitoring has become widely used. Low PbtO2 has been associated with delayed cerebral ischemia and worse neurologic outcome(3-4).

Objectives: The aim of this study was to assess the impact of PbtO2guided therapy on patients' outcome.

Methods: Single center retrospective cohort study from January 2014 until March 2019 including adult patient admitted to the ICU with poor grade aSAH, that required invasive neurologic monitoring (ICP $+1-\mathrm{PbtO} 2$ catheter) within the first 48 hours of admission. Patients monitored with combined PbtO2 + ICP monitoring were compared with patients monitored with ICP alone. We considered as "PbtO2guided therapy", a bundle of interventions aiming to increase $\mathrm{PbtO} 2$ $>20 \mathrm{mmHg}$. Primary outcome was the presence of PbtO2 $<20 \mathrm{mmHg}$ and burden of tissue hypoxia.

Results: Of a total of 259 patients admitted to the ICU due to aSAH during the study period, 152 patients were included in the study: 96 were monitored with ICP alone and 56 with PbtO2 + ICP; of those $47(83.9)$ had at least one episode of PbtO2 $<20 \mathrm{mmHg}$ and 22 (39.3\%) received a PbtO2-guided therapy. PbtO2-guided therapy was associated with a reduction of the risk of unfavorable outcome (OR 0.03 $\mathrm{Cl} 95 \% 0.002-0.46, \mathrm{p}=0.01)$ and mortality (OR $0.01 \mathrm{Cl} 95 \% 0.16, \mathrm{p}=$ $0.002)$. $\mathrm{PbtO} 2<20 \mathrm{mmHg}$ was an independent predictor of poor neurological outcome(OR $24.48 \mathrm{Cl} 95 \%$ 1.49-470.77).

Conclusion: In this cohort of poor grade $\mathrm{SAH}, \mathrm{PbtO} 2$-guided therapy was an independent predictor of neurological recovery.

\section{Reference(s) and grant ackowledgment(s)}

1. 4. Maloney-Wilensky, E., et al., Brain tissue oxygen and outcome aftersevere traumatic brain injury: a systematic review. Crit Care Med, 2009. 37(6): p. 2057-63.

2. 3. Oddo, M., et al., Brain hypoxia is associated with short-term outcome after severe traumatic brain injury independently of intracranial hypertension and low cerebral perfusion pressure. Neurosurgery, 2011. 69(5): p. 1037-45; discussion 1045

3. 2. De Georgia, M.A., Brain Tissue Oxygen Monitoring in Neurocritical Care. J Intensive Care Med, 2015. 30(8): p. 473-83.

4. 1. Mozaffarian, D., et al., Heart disease and stroke statistics-2015 update: a report from the American Heart Association. Circulation, 2015. 131(4): p. e29-322.
000353

Therapeutic inhibition of the activated leukocyte integrin aMß2: A novel strategy in treating acute respiratory distress syndrome in mice

I. Bojti ${ }^{1}$; PM. Siegel ${ }^{1}$; AS. Przewosnik ; K. Peter ${ }^{2}$; T. Wengenmayer ${ }^{1}$; M. Moser ${ }^{1}$; C. Bode ${ }^{1}$; P. Diehl ${ }^{1}$

${ }^{1}$ Kardiologie und Angiologie I, Universitäts-Herzzentrum Freiburg- Bad Krozingen, Freiburg im Breisgau, Germany; ${ }^{2}$ Atherothrombosis and vascular biology laboratory, Baker Heart and Diabetes Institute, Melbourne, Australia

Correspondence: I. Bojti

Intensive Care Medicine Experimental 2020, 8(2): 000353

Introduction: Acute respiratory distress syndrome (ARDS) is a life threatening complication of various illnesses such as sepsis, pneumonia or cardiogenic shock. As there is no causal therapy available which is why the mortality of $>40 \%$ could not be significantly reduced within the last decades.

Activated polymorphonuclear (PMN) cells with systemic uncontrolled inflammatory responses and consecutive respiratory failure play a central role in the pathogenesis of acute respiratory distress syndrome. The leukocyte integrin aM $\beta 2$, which can be blocked activation specific by novel binding protein (Designed Ankyrin Repeat protein, DARPin) is a promising novel therapeutic target for acute respiratory distress syndrome as it has recently been shown to inhibit leukocyte transmigration and systemic inflammation.

Methods: ARDS was induced by intratracheal LPS instillation $(5 \mu \mathrm{g} / \mathrm{g})$. Recombinant anti-aM 22 DARPins were produced in E. coli. Purity of DARPins was assessed by SDS-Page electrophoresis. Activation specific binding of DARPins to activated mouse PMN cells was measured ex vivo using standard flow cytometry protocols. Disease severity was quantified through protein concentration and FACS analysis of bronchial lavage fluid. Animals were analysed on day 4 (acute phase) and on day 10 (chronic phase). Mice were weighed every day and eliminated if the weight loss was $20 \%$ over baseline. Animals were treated daily i.p. with anti-aM 2 DARPin $(n=11)$, PBS $(n=16)$ or control DARPin $(n=18)$. Statistical analysis was performed with one-way ANOVA and post hoc analysis, the survival curves were compared with Mantel-Cox test.

Results: 4 day survival was $100 \%$ (healthy controls) vs. $90 \%$ (anti-aM $\beta 2$ DARPin) vs. $38 \%$ (control DARPin) vs. $56 \%$ (PBS) $(p=0.0035)$. Absolute neutrophil number per $\mathrm{ml} \mathrm{BAL}$ fluid was $10 \pm 4.8$ (healthy controls) vs. $6,026 \pm 4,103$ (anti-aMß2 DARPin) vs. 19,582 $\pm 19,132$ (control DARPin) vs. $38,621 \pm 37,791$ (PBS) $(p=0.0031)$. The protein concentration in the BAL fluid was lower in the anti-aM $\beta 2$ DARPin group indicating a strong therapeutic effect in mice with acute respiratory distress syndrome (healthy controls vs. anti-aM $\beta 2$ DARPin vs. control DARPin vs. PBS: $0.16 \pm 0.08 \mathrm{mg} / \mathrm{ml}$ vs. $0.3 \pm 0.12 \mathrm{mg} / \mathrm{ml}$ vs. $0.62 \pm 0.38 \mathrm{mg} / \mathrm{ml}$ vs. $0.77 \pm 0.34 \mathrm{mg} / \mathrm{dl}, \mathrm{p}=0.0002)$.

Conclusion: Treating murine acute respiratory distress syndrome with the novel activation specific anti-aM 32 DARPin significantly reduces disease severity and improves survival.

\section{8}

The effect of short-chain fatty acid butyrate on metabolism, immune and mitochondrial function in sepsis

V. Peters ${ }^{1}$; N. Arulkumaran ${ }^{1}$; C. Gaupp ${ }^{1}$; T. Roger ${ }^{2}$; M. Shankar-Hari³ M. Singer

${ }^{1}$ Bloomsbury institute of intensive care medicine, University College London, London, United Kingdom; ${ }^{2}$ Infectious diseases service, Lausanne University Hospital, Lausanne, Switzerland; ${ }^{3}$ Peter gorer department of immunobiology, Kings College London, London, United Kingdom

Correspondence: $\vee$. Peters

Intensive Care Medicine Experimental 2020, 8(2): 000358

Introduction: Sepsis induces changes in cellular metabolic, immune and mitochondrial function [1]. The effect of nutrition on these changes is mostly overlooked. In addition to a caloric source, shortchain fatty acid butyrate may affect metabolism, immune and mitochondrial function. 
Objectives: To examine the therapeutic effect of butyrate in in vitro and in vivo models of sepsis.

Methods: Human peripheral blood mononuclear cells (PBMCs) from six healthy donors were incubated overnight with LPS (100 $\mathrm{ng} / \mathrm{ml}$ ), heat-killed Staphylococcus aureus or Escherichia coli 018 (PBMC:bacterium ratio 5:1) in the presence or absence of butyrate (1.8 $\mathrm{mM})$. Supernatants were collected and IL-6, IL-10 and TNF concentrations quantified by ELISA. Mitochondrial respiration was assessed using high-resolution respirometry.

A rat model of faecal peritonitis was developed to study the impact of butyrate in vivo. Rats were cannulated and received intraperitoneal injection of faecal slurry. Rats were housed in metabolic cages and, alongside fluid resuscitation, received intravenous butyrate infusion $(0.6 \mathrm{~g} / \mathrm{kg} / \mathrm{h})$ commencing 6 hours following sepsis induction. 24 hours following sepsis induction, splenocytes were isolated and oxygen consumption measured. Splenocyte membrane potential, reactive oxygen species (ROS), and intracellular cytokines (IL-10 and TNF) were assessed using flow cytometry. Non-parametric one-way ANOVA tests with Dunn's correction were used for statistical analyses.

Results: Butyrate significantly reduced IL-6, IL-10 and TNF secretion by PBMCs. Maximal respiration and spare-respiratory capacity were non-significantly increased. In vivo butyrate infusion stimulated fatty acid metabolism as shown by a fall in the respiratory exchange ratio. Ex vivo splenocytes from septic rats showed significantly increased TNF production, which was not affected by butyrate infusion. Butyrate infusion decreased mitochondrial respiration, increased mitochondrial ROS production and membrane potential.

Conclusion: Butyrate had anti-inflammatory and mitochondrial respiration stimulating effects in vitro. However, in vivo butyrate infusion caused mitochondrial stress in immune cells and did not impact immune function. Hence, beneficial effects of butyrate on immune cells in vitro could not be reproduced in vivo.

\section{9}

Inhibition of inflammasome-caspase-1 activation by tetracycline reduces direct acute respiratory distress syndrome

K. Peukert'; M. Fox'; S. Schulz'; C. Feuerborn ${ }^{1}$; S. Frede ${ }^{1}$; C. Putensen ${ }^{1}$; E. Latz ${ }^{2}$; H. Wrigge ${ }^{3}$; B. Kuemmerer ${ }^{4}$; S. David ${ }^{5}$; B. Seeliger ${ }^{6}$;. Welte ${ }^{6}$;

C. Wilhelm7 D. Klinman ${ }^{8}$; F. Steinhagen ${ }^{1}$; C. Bode

'Department of anaesthesiologyand intensive care medicine, University of Bonn, Bonn, Germany; ${ }^{2}$ Institute of innate immunology, University of Bonn, Bonn, Germany; ${ }^{3}$ Department of anesthesiology, intensive care and emergency medicine, pain therapy, Berufsgenossenschaftliche Kliniken Bergmannstrost Halle, Halle (Saale), Germany; ${ }^{4}$ Institute of virology, University of Bonn, Bonn, Germany; ${ }^{5}$ Nephrology, Hannover Medical School, Hannover, Germany; ${ }^{6}$ Department of Respiratory Medicine, Hannover Medical School, Hannover, Germany; ${ }^{7}$ Institute of clinical chemistry and clinical pharmacology, University of Bonn, Bonn, Germany; ${ }^{8}$ Cancer and inflammation program, National Cancer Institute - Frederick Campus, Frederick, United States of America

Correspondence: $\mathrm{C}$. Bode

Intensive Care Medicine Experimental 2020, 8(2): 000389

Introduction: Acute respiratory distress syndrome (ARDS) is a heterogeneous syndrome with high mortalty. Personalized medicine approaches targeting patients based on their molecular phenotypes of ARDS might help to identify effective pharmacotherapies. The inflammasome-caspase-1 pathway contributes to the development of ARDS via IL- $1 \beta$ and IL-18 production. Recent studies indicate that tetracycline (TET) can be used to treat inflammatory diseases mediated by IL-1 $\beta$ and IL-18 while the molecular mechanism by which TET inhibits inflammasome-caspase-1 signaling remains unknown.

Objectives: To identify patients with ARDS characterized by IL-1 $\beta$ and IL-18 production and investigate the ability of TET to inhibit inflammasome-caspase-1 signaling.

Methods: IL-1 $\beta$ and IL-18 levels were quantified in bronchoalveolar lavage fluid (BALF) from 62 patients from two ARDS referral centers with direct (viral, COVID-19 and bacterial pneumonia) or indirect (non-pulmonal) ARDS via multiplex assay. TET's effect on lung injury and inflammation were assessed in a LPS-induced mouse model of direct ARDS. Murine lung injury was determined by measurement of i) albumin concentration by ELISA, ii) neutrophil population by FACS (both in BALF) and iii) histopathology. Concentration of IL-1 $\beta$, IL-6, IL-18 and TNFa was measured by multiplex assay in BALF. The effect of TET on caspase- 1 was tested in BMDMs after stimulation with LPS plus nigericin. Concentration of IL-1 $\beta$ and TNFa was determined by ELISA and cleavage of caspase-1 via western blot. Alveolar leucocytes were obtained from BALF of patients with direct ARDS and the effect of TET on IL-1 $\beta$ and IL-18 production was measured ex vivo.

Results: BALF levels of IL-1 $\beta$ and IL-18 are significantly higher in patients with direct than those with indirect ARDS $(p<0.05)$. In experimental direct ARDS, TET treated mice showed significantly reduced weight loss and mortality $(43 \%$ vs. $7 \%: p<0.001)$ compared to PBS treated animals. TET treatment diminished lung injury in terms of lower albumin $(325 \pm 35$ vs $144 \pm 16 \mu \mathrm{g} / \mathrm{ml} ; \mathrm{p}<0.01)$ concentration and number of neutrophils. Histopathology showed reduced lung injury in TET-treated animals. TET decreased exclusively the concentration of IL-1 $\beta(152 \pm 16$ vs $86 \pm 8 \mathrm{pg} / \mathrm{ml} ; \mathrm{p}<0.01)$ and IL-18 $(92 \pm 7$ vs $31 \pm 2 \mathrm{pg} / \mathrm{ml} ; \mathrm{p}<0.05)$ in BALF. Immunoblot showed that TET reduced cleavage of the $\mathrm{p} 45$ caspase- 1 precursor into its p20 subunit. TET inhibited in a dose-dependent manner production of both IL-1 1 S and IL-18 from alveolar leucocytes obtained from patients with direct ARDS including COVID-19 by up to $85 \%(p<0.01)$.

Conclusion: This work demonstrates that i) patients with direct ARDS have significantly higher IL-1 $ß$ and IL-18 levels in their lungs than individuals with indirect ARDS, ii) TET may be effective in the treatment of direct ARDS including COVID-19 and iii) TET blocks the inflammasomecaspase-1 pathway by selective inhibition of caspase-1.

\section{1}

Assessment of Transient Hypaeremic Response as an index for Cerebral Autoregulation in patients with moderate and severe Traumatic Brain Injury using Transcranial Doppler Ultrasound M. Zahra'; T. Zaytoun²; A. Abdalla²; B. Beshay²; A. Elbeheiry²

${ }^{1}$ Intensive Care, Alexandria University Head office, Alexandria, Egypt; ${ }^{2}$ Intensive care, Alexandria Faculty of Medicine, Alexandria, Egypt

Correspondence: M. Zahra

Intensive Care Medicine Experimental 2020, 8(2): 000391

Introduction: Traumatic brain injury (TBI) has been increasing with greater incidence. It remains a leading cause of death (1). Cerebral pressure autoregulation impairment is a well-known pathology after TBI that worsens the prognosis and outcome (2). Transcranial Doppler (TCD) can be used to assess Transient Hyperaemic response ratio (THRR) after carotid compression which is a well demonstrated valid index for cerebral autoregulation $(3,4)$. A relative increase in mean flow velocity (MFV) of middle cerebral artery (MCA) above the baseline following the release of carotid compression denotes preserved autoregulation and sequentially predicts good outcome, whereas absence of such response indicates altered autoregulation with poor outcome (5).

Objectives: The aim of the work was to assess cerebral autoregulation and its relation to outcome in patients with moderate and severe TB using TCD. The primary end point is the Glasgow outcome score (GOS) (6) as a measure of outcome to assess THRR after carotid compression as an indicator of cerebral pressure autoregulation and as a predictor of GOS as well as a being prognostic tool. Other measures of TCD as MFV of MCA and Pulsatility Index (PI) were also used as predictors of GOS and correlated with THRR. The length of stay as well as mortality were recorded and correlated to THRR.

Methods: 120 patients with moderate or severe TBI according to Glasgow Coma Score (GCS) (7) underwent daily TCD, after intial hemodynamic and respiratory stabilization, for 5 days post trauma. All patients were managed according to guidelines for the management of head injury of the American Association of Neurologic Surgeons (8). A compression time of 10 seconds and a compression ratio of $40 \%$ or more, allow maximum expression of the hyperaemic response. Also carotid compression is accepted only when flow velocity is stable with no further decrease during the whole period of compression otherwise the compression will be terminated and repeated 60 seconds later (3). 
The Patients were divided into those with normal TCD measurements and those with poor cerebral circulation (MFV < 40, PI > 1.2). GOS scores are dichotomized into favorable or unfavorable outcomes. Patients in the upper two GOS outcomes groups (good recovery or moderate disability) are considered favorable outcome, and patients in other groups (severe disability, vegetative state or death) are considered unfavorable outcomes.

Pearson's correlation coefficiets were calculated to test the relation between TCD and other clinical variables. Discrimination of the logistic models was assessed by calculating the area under (ROC) curve (9). P value $<0.05$ was considered significant.

Results: There was a significant correlation between THRR and GOS (patients with THRR $\geq 1$ had favorable outcome). There was also significant correlation between THRR and mortality. Also significant correlation between THRR and MFV as well as PI was found. THRR $\geq 1$ showed sensitivity of $83.3 \%$, specificity of $72.9 \%$, postive predictive value (PPV) of $82.2 \%$ and negative predictive value (NPV) of $74.5 \%$ in predicting patients with favorable outcome.

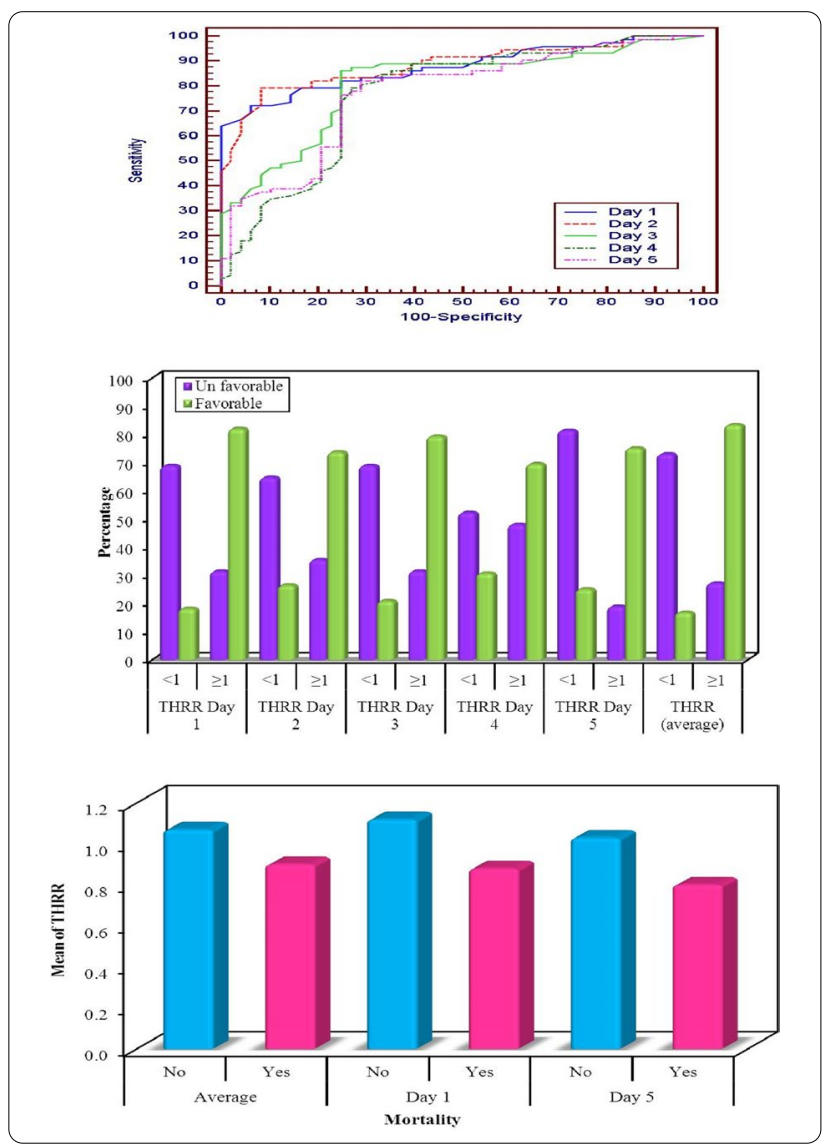

Conclusion: THRR provides a clinically useful index of cerebral autoregulation. THRR after carotid compression is a good predictor of GOS as well as being a prognostic tool in patients with moderate and severe TBI.

\section{Reference(s) and grant ackowledgment(s)}

1. 9. Delong ER, Delong DM, Clarke-Pearson DL. Comparing the areas under two or more correlated receiver operating Characteristic curves: a non parametric approach. Biometrics 1988; 44: 837-45

2. 8. Palmer S, Bader MK, Qureshi A, Palmer J, Shaver T, Borzatta M, et al. The impact on outcome in a community hospital setting of using AANS traumatic brain injury guidelines; American Association of Neurologic Surgeons. J Trauma 2001:50:657-64.

3. 7. Teasdale G, JennettB. Glassgow Coma Scale. Lancet1974;81-3.
4. 6. 36. Becker DP, MillerJD, Ward JD, et al. The outcome from severe head injury with early diagnosis and intensive management. J Neurosurgery 1977;47:491-4.

5. 5. Smielewski P, Czosnyka M, Kirkpatrick P, Pickard JD. Evaluation of the transient hyperaemic response test in head-injured patients. J Neurosurg 1997; 86: 773-8

6. 4. Moppett IK, Mahajan RP. Transcranial Doppler ultrasonographyin anaesthesia and intensive care. BJA 2004; 93(5): 710-24.

7. 3. Cavill G, Simpson EJ, Mahajan RP. Factors affecting assessment of cerebral autoregulation using the transient hyperemic response test. $\mathrm{Br} J$ Anaethe 1998; 81: 317-21.

8. 2. Puppo C, López L, Caragna E, Biestro A. Oneminute dynamic cerebral autoregulation in severe head injury patients and its comparison with static autoregulation. A transcranial Doppler study. Neurocritical Care 2008; 8(3):344-52.

9. 1. Roozenbeek B, Maas Al, Menon DK. Changing patterns in the epidemiology of traumatic brain injury. Nat Rev Neurol 2013;9(4):231-6.

\section{0}

What you give is what you get: fecal concentrations of piperacillin and tazobactam in ICU patients

I. De Ruyck'; S. Van Vooren²; A. Verstraeten ${ }^{2}$; J. De Waele ${ }^{1}$

${ }^{1}$ Intensive care medicine, Ghent University Hospital, Ghent, Belgium; ${ }^{2}$ Laboratory medicine, Ghent University Hospital, Gent, Belgium

Correspondence: I. De Ruyck

Intensive Care Medicine Experimental 2020, 8(2): 000400

Introduction: The widespread use of antibiotics poses a significant threat to human health because of the risk of antimicrobial resistance. While piperacillin/tazobactam (TZP) is commonly used in the empirical treatment of severe infections in the ICU, and the drug can be detected in plasma up to several days after discontinuation, it is unclear how long piperacillin is detectable in the feces of patients. Given the potential impact of antibiotics on the microbiome, we sought to investigate fecal concentrations of piperacillin in critically ill patients using a newly developed sensitive method.

Objectives: The objective of this study was to describe concentrations of TZP in the feces of critically ill patients during intravenous therapy and after treatment discontinuation.

Methods: Critical ill patients without chronic or acute abdominal disease and receiving intravenous piperacillin/tazobactam were included. The regimen consisted of a loading dose of $4,5 \mathrm{~g}$ of TZP injected over 30 minutes, followed by continuous infusion according to kidney function (standard therapy $16 \mathrm{~g} / 24 \mathrm{~h}$ ). Fresh stool specimens were collected after a minimum of 24 hours of antibiotic treatment, with up to ten samples per patient during therapy. Within ten minutes after collecting a stool sample, a serum sample was obtained for drug concentration analysis. Stool specimens collected up to one week after treatment discontinuation were analyzed, with a maximum of five samples. Concentrations of piperacillin and tazobactam were measured by a validated and sensitive ultra-high-performance liquid chromatography - high resolution mass spectrometric (UHPLCHRMS) method, with a lower limit of quantitation (LLOQ) of $1 \mu \mathrm{g} / \mathrm{g}$ and $0,5 \mu \mathrm{g} / \mathrm{g}$ lyophilized (lyo) feces and 1,5 mg/L and 0,5 mg/L plasma for piperacillin and tazobactam, respectively.

Results: Ten critically ill patients with a median age of 70,5 years (interquartile range (IQR) 50-74) were included. The specimens of one patient were excluded in further analyses due to pre-analytic errors. During therapy, piperacillin concentrations ranged from 18,65 to $289,18 \mathrm{mg} / \mathrm{L}$ in plasma and $1,63-23000,70 \mu \mathrm{g} / \mathrm{g}$ in lyo feces. Tazobactam concentrations ranged from $2,57-38,17 \mathrm{mg} / \mathrm{L}$ in plasma and could be detected and quantified in stool specimens of six patients $(67 \%)$ during therapy (range 0,08-576,18 $\mu \mathrm{g} / \mathrm{g}$ ). After discontinuation of therapy respectively $5(56 \%)$ and $3(33 \%)$ patients were found to have measurable fecal concentrations of piperacillin (range 5,48 $3477,27 \mu \mathrm{g} / \mathrm{g}$ ) and tazobactam (range 2,27 - 531,44 $\mu \mathrm{g} / \mathrm{g}$ ) for 1 to 2 days. In 2 patients, piperacillin and tazobactam were detectable in stool specimens for at least 4 to 6 days after discontinuation. Both of them suffered from acute kidney injury (AKI stage I and II). 
Conclusion: Both piperacillin and tazobactam are measurable in stool during therapy; after discontinuation of therapy, piperacillin can be detected in feces for up to 6 days. This may have important consequences for disturbances of the microbiome and the impact of antibiotic therapy.

\section{2}

\section{Impact of high doses of antibiotics and sequence}

on bacterial clearance in a rat model of $E$ coli peritonitis-induced septic shock

G. Vázquez-Grande ${ }^{1}$; E. Esfahani²; A. Kumar ${ }^{1}$

${ }^{1}$ Critical care, medical microbiology and infectious diseases, University of Manitoba, Winnipeg, Canada; ${ }^{2}$ Medical microbiology, University of Manitoba, Winnipeg, Canada

Correspondence: G. Vázquez-Grande

Intensive Care Medicine Experimental 2020, 8(2):000402

Introduction: Septic shock is one of the deadliest diseases worldwide, with a mortality rate of $30-50 \%$. Antibiotic delay and higher microbial loads are known to increase morbi-mortality in sepsis.

Loading doses achieve faster appropriate concentrations in plasma, allowing for a better bacterial kill. Bacterial kill and patient outcomes may be further improved by synergistic combination therapy, which is best established for betalactams and aminoglycosides. However, how the sequence of antibiotic administration impacts this synergy is unknown. While in vitro work suggests that giving the betalactam first improves bacterial clearance, the effect of antibiotic sequence has not been studied in vivo.

Objectives: Determine whether the sequence of antibiotic administration impacts bacterial kill and cytokine production in a monomicrobial rat model of septic shock.

Methods: Our monomicrobial peritonitis rat model of septic shock requires the surgical implant of a gelatin capsule with a fibrinogen clot, alpha cellulose and a known bacterial inoculum $\left(2^{*} 10^{\wedge} 6 \mathrm{cfu}\right.$ E. coli Bort) in the peritoneum of the rat. This capsule slowly dissolves and generates a progresive peritoneal infection that evolves into septic shock in 12 hours.

After septic shock is stablished, we fluid resuscitate the rats, connect them to mechanical ventilation, and start vasopressors if needed to achieve a MAP goal of $70 \mathrm{mmHg}$. We experiment with the order of antibiotics (cefotaxime [cef] and gentamicin [gent]) and compare them to monotherapy, untreated controls and sham controls, for a total of 7 treatment arms and 6 rats per arm. We used two doses of antibiotics: normal (cef $60 \mathrm{mg} / \mathrm{kg}$, gent $10 \mathrm{mg} / \mathrm{kg}$ ) and high (cef $120 \mathrm{mg} / \mathrm{kg}$, gent $20 \mathrm{mg} / \mathrm{kg}$ ).

We measure bacterial blood counts, cytokine profile, lactate and renal function at different time points. Following the ethics quidelines of University of Manitoba, we euthanize the rat and measure organ bacterial counts after homogenization.

Results: Rats treated with cefotaxime monotherapy had slow initial bacterial clearance compared to those treated with gentamicin alone. This improved with the higher dose of cefotaxime. Over time the rats treated with gentamicin showed bacterial regrowth (postantibiotic effect); this was delayed in the group treated with a higher gentamicin dose. All combination arms showed a rapid initial bacterial kill and maintained bacterial clearance over time, exhibiting the strengths of gentamicin and cefotaxime, respectively. No significant differences were observed between combination arms.

All treatment arms showed similar bacterial clearance from peritoneal fluid and organs.

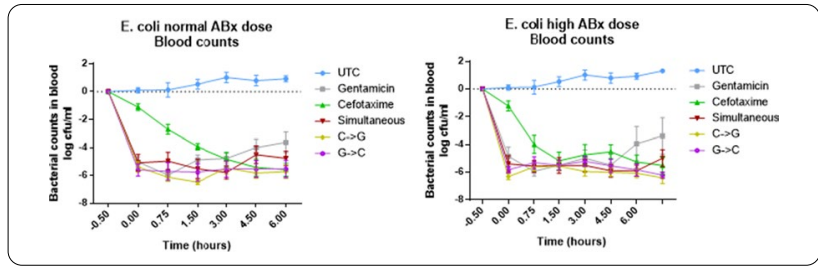

Conclusion: Combination therapy showed synergistic effects with a rapid and maintained bacterial clearance from the blood. Altering the antimicrobial sequence had nosignificant impact in our in vivo model of septic shock. Higher doses of antibiotics showed faster initial bacterial kill with cefotaxime monotherapy, and delayed the postantibiotic effect of gentamicin monotherapy.

\section{3}

Too old for the ICU? Long term prognosis of critically ill patients

A. Oliveira'; D. Rei'; M. Vera-Cruz'; J. Gonçalves-Pereira

${ }^{1}$ Intensive Care Unit, Hospital de Vila Franca de Xira, Estrada Carlos Lima Costa, Vila Franca de Xira, Portugal, Vila Franca de Xira, Portugal

Correspondence: A. Oliveira

Intensive Care Medicine Experimental 2020, 8(2): 000423

Introduction: In the recent years a rapid increase in the age of patients admitted to the Intensive Care Units (ICU) was noted. Fewer limitations are now imposed on performing invasive treatments or surgery in old or even frail patients, especially when complemented by optimal supportive intensive care. However, little is known about the long term follow up of these patients.

Objectives: To evaluate the age stratified long-term all-cause mortality, after admission to the ICU. Secondly, to compare a one-year mortality rate of this cohort of critically ill patients after hospital discharge, with that of a standard population with the same age distribution.

Methods: Retrospective cohort study of all patients admitted to a multipurpose ICU between 2015-2019. Patients $<18$ years or admitted for $<24 \mathrm{~h}$ were excluded. Only the first ICU admission was considered. Patients were followed until hospital discharge or death, whatever occurred first. One-year all-cause mortality was assessed for all patients. Standard Mortality Ratio (SMR) were determined at hospital discharge and at a 1-year follow-up.

We split the sample in 4 groups according to patients age at admission to the ICU: 18-50 (Adult); 51-65 (Senior); 66-80 (Old); > 80 (Veryold). Survival curves were plotted for all 4 groups according to the time of ICU admission. The ratio between observed and expected mortality was calculated, for patients discharged alive from the hospital and according to age-adjusted tables for the general Portuguese population.

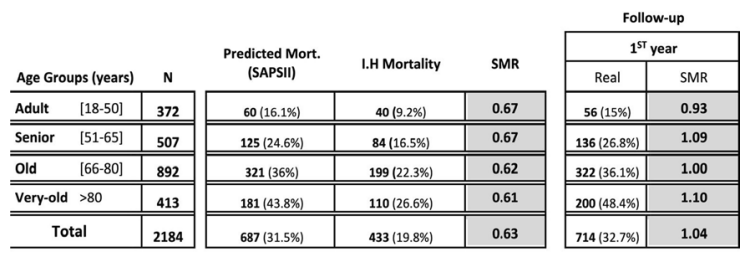

Table 1 -Mortality by Age Group. Predicted and observed In-hospital mortality (with SAPSII), and one-year follow-up SMR-Standard Mortality Ratio

Results: A total of 2184 patients were included. Male gender was predominant (57\%); mean age and SAPS II Score were $66 \pm 15.8$ and $40 \pm 18.9$. Mean ICU length of stay was $4.23 \pm 4.2$ days. ICU and Hospital mortality were $12.1 \%$ and $19.8 \%$ (SMR 0.63). SAPSII performance was accurate to predict one-year mortality, rather than in-hospital mortality, for all age groups (Table 1). 


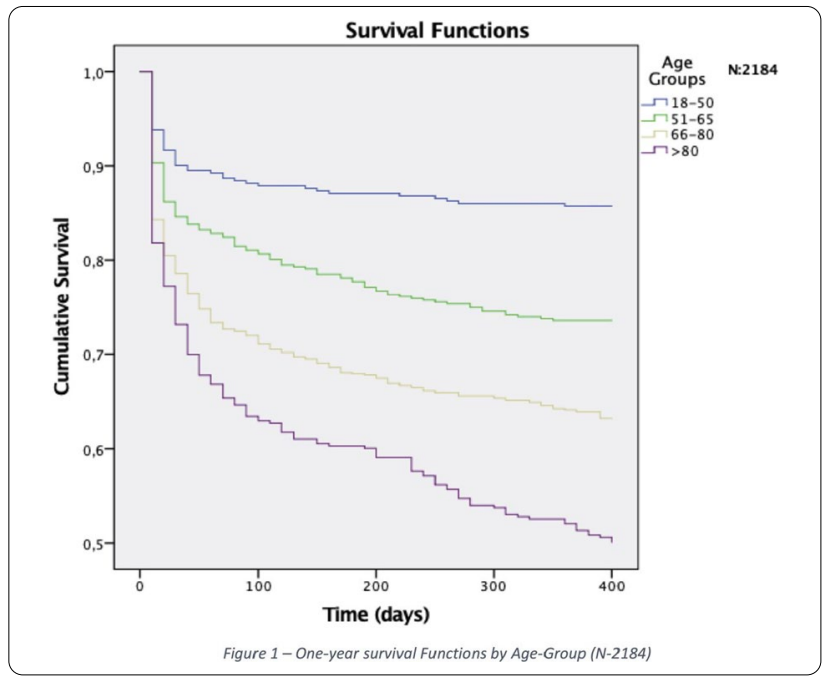

A Kaplan-Meyer survival curve (KM) was computed to assess the differences between long term prognosis for the different age groups (Fig 1) and unveiled a statistically significant difference in the survival probability (log rank test, $p<0.01$ ). A sharp drop in survival in the first days was noted, corresponding to the in-hospital mortality. A continuous decay in survival was observed in the Old and Very-old groups that was less evident in the younger population.

\begin{tabular}{|c|c|c|c|c|c|}
\hline \multirow{2}{*}{\multicolumn{2}{|c|}{ Age Groups (years) }} & \multirow[b]{2}{*}{$\mathrm{N}$} & \multicolumn{2}{|c|}{$1^{\text {st }}$ year } & \multirow[b]{2}{*}{ Ratio } \\
\hline & & & \multirow{2}{*}{$\begin{array}{c}\text { After discharge } \\
14(4.2 \%)\end{array}$} & \multirow{2}{*}{$\begin{array}{c}\begin{array}{c}\text { Population } \\
\text { Estimate }\end{array} \\
1(0.3 \%)\end{array}$} & \\
\hline Adult & [18-50] & 332 & & & 14 \\
\hline Senior & [51-65] & 423 & $51(12 \% \%)$ & $3(0.71 \%)$ & 16.9 \\
\hline Old & [66-80] & 693 & $125(18.4 \%)$ & $16(2.3 \%)$ & 8 \\
\hline \multicolumn{2}{|c|}{ Very-old $>80$} & 302 & 89 (29.5\%) & $32(10.6 \%)$ & 2.8 \\
\hline \multicolumn{2}{|c|}{ Total } & 1750 & $535(30.6 \%)$ & $69(3.9 \%)$ & 7.8 \\
\hline
\end{tabular}

Table 2 - One-year mortality after discharge and age adjusted control population estimate.

One-year mortality rate after hospital discharge was higher in the older patients. The ratio between the observed and expected mortality (for an age adjusted control population) was significantly high for all age groups (Table 2).

Conclusion: The one-year mortality risk of critically ill patients of all age groups is very high, even after hospital discharge, largely above of the general population. SAPSII showed good accuracy to predict oneyear mortality in this cohort. Although a continuous decline in survival is noted in the older patients, more than $50 \%$ of the Very-old patients are alive 1 year after ICU admission.

\section{Reference(s) and grant ackowledgment(s)}

1. Andersen, F.H., Flaatten, H., Klepstad, P. et al. Long-term survival and quality of life after intensive care for patients 80 years of age or older. Ann. Intensive Care 5, 13 (2015). https://doi.org/10.1186/s13613-015-0053-0

\section{6}

Prediction of Hypotension Events with Physiologic Vital Sign Signatures in the Intensive Care Unit

JH. Yoon ${ }^{1}$; V. Jeanselme'; A. Dubrawski²; M. Hravnak ${ }^{3}$; M. Pinsky ${ }^{4}$; G. Clermont ${ }^{4}$

${ }^{1}$ Pulmonary and Critical Care Medicine, University of Pittsburgh, Pittsburgh, United States of America; ${ }^{2}$ Auton lab. department of machine learning, Carnegie Mellon University, Pittsburgh, PA, United States of America;
${ }^{3}$ School of nursing, University of Pittsburgh, Pittsburgh, PA, United States of America; ${ }^{4}$ Critical care medicine, University of Pittsburgh, Pittsburgh, PA, United States of America

Correspondence: J.H. Yoon

Intensive Care Medicine Experimental 2020, 8(2): 000426

Introduction: Hypotension is known to be the most consistent manifestation of decompensated shock leading to major organ failure, and even brief hypotension is associated with increased morbidity and mortality. Several early warning scores have been introduced to identify patients at risk for decompensation and trigger escalation of care, but most of current scoring systems are unable to provide reliable, continuous feedback to clinicians who need to make time-sensitive decisions for rapidly fluctuating conditions in critically-ill patients.

Objectives: Using common vital sign data, we aim to develop a machine learning model to predict the initial hypotension event among intensive care unit (ICU) patients, and design an alert system for bedside implementation.

Methods: From the Medical Information Mart for Intensive Care III (MIMIC-3) dataset, minute-by-minute vital signs were extracted. An initial hypotension event was defined as at least 5 measurements within a 10-minute period of systolic blood pressure $\leq 90 \mathrm{mmHg}$ and mean arterial pressure $\leq 60 \mathrm{mmHg}$. The performance of various supervised machine learning models was measured with area under the receiver operating characteristic curve (AUROC) and area under the precision recall curve (AUPRC). A random forest (RF) classifier was selected, trained with 10 -fold cross validation method, then applied to an a priori separated out-of-sample validation cohort. Risk score trajectory was built from vital signs features on overlapping windows. For an alert system, hypotension alerts were generated using a two-step (stacked) approach. First, positive alerts were identified with risk score thresholds, then a second RF model was used to reduce false alerts, followed by imposing a lock-out time for alerts to decrease alarm fatigue.

Results: We identified 1532 subjects (1946 ICU stays) as the case group (experienced a hypotension event), and 1707 subjects (2585 ICU stays) as the control group. The RF model showed AUROC of 0.93 , 0.91 , and 0.88 at 15,30 , and 60 minutes before hypotension, AUPRC of 0.77 , and a low calibration score (Brier score 0.09 ). Mean risk score trajectories revealed a clear separation with $80 \%$ of cases predicted at 15 minutes before hypotension, and more than $60 \%$ at one hour before the hypotension. For the alert system, the stacked machine learning model with lock-out of 15 minutes produced on average 0.79 alerts/ subject/hour for future hypotension, with a positive predictive value (probability of developing hypotension) of $65 \%$ and sensitivity of 92.4\%.

Conclusion: Most clinically significant hypotension events in the ICU can be predicted from bedside physiologic monitoring at least 1 hour before the initial hypotension episode. Designing a practical alert system with high sensitivity and acceptable positive predictive value for hypotension prediction is feasible, with low rate of alerts.

\section{Reference(s) and grantackowledgment(s)}

1. Sendelbach S, Funk M. Alarm fatigue: a patient safety concern. AANC Adv Crit Care. 2013;24(4):378-386

2. Johnson AE, Pollard TJ, Shen L, Lehman LW, Feng M, Ghassemi M, Moody B, Szolovits P, Celi LA, Mark RG. MIMIC-III, a freely accessible critical care database. Sci Data 2016:3:160035

3. Vincent JL, Moreno R, Takala J, Willatts S, De Mendonca A, Bruining H, Reinhart CK, Suter PM, Thijs LG. The SOFA (Sepsis-related Organ Failure Assessment) score to describe organ dysfunction/failure. On behalf of the Working Group on Sepsis-Related Problems of the European Society of Intensive Care Medicine. Intensive Care Med 1996:22:707-710

4. Le Gall JR, Lemeshow S, Saulnier F. A new Simplified Acute Physiology Score (SAPS II) based on a European/North American multicenter study. JAMA 1993;270:2957-2963

5. Knaus WA, Draper EA, Wagner DP, Zimmerman JE. APACHE II: a severity of disease classification system. Crit Care Med 1985;13:818-829

6. Fitch W, MacKenzie ET, Harper AM. Effects of decreasing arterial blood pressure on cerebral blood flow in the baboon. Influence of the sympathetic nervous system. Circ Res 1975;37:550-557 
7. National Institute of Health award T32HL007820 (Yoon JH, Pinsky MR), T32HL007563 (Yoon JH, Morris A), R01HL126811 (Pinsky MR, Clermont G), R01GM117622 (Pinsky MR, Clermont G, Dubrawski A) 8. DARPA award FA8750-17-2-0130 (Dubrawski A)

\section{0}

\section{Dynamic MRI shows altered diaphragm movement} and configuration due to positive end-expiratory pressure M. Wennen ; AH. Jonkman²; D. Jansen ${ }^{3}$; HJ. De Vries ${ }^{4}$; C. Ottenheijm; JT. Marcus ${ }^{6} ;$ L. Heunks ${ }^{7}$

${ }^{1}$ Amsterdam UMC, locatie VUmc, Amsterdam, Netherlands; ${ }^{2}$ Intensive care, Amsterdam University Medical Center, location VUmc, Amsterdam, Netherlands; ${ }^{3}$ Anesthesiology, Radboud University Medical Center, Nijmegen, Netherlands; ${ }^{4}$ Intensive care medicine, Amsterdam UMC, locatie VUmc, Amsterdam, Netherlands; ${ }^{5}$ Physiology, Amsterdam UMC, locatie VUmc, Amsterdam, Netherlands; ${ }^{6}$ Radiology, Amsterdam University Medical Center, location VUmc, Amsterdam, Netherlands; ${ }^{7}$ Intensive care volwassenen, Amsterdam UMC, Amsterdam, Netherlands Correspondence: M. Wennen

Intensive Care Medicine Experimental 2020, 8(2):000460

Introduction: In mechanically ventilated patients, positive end-expiratory pressure (PEEP) is used to improve gas exchange and respiratory mechanics by preventing alveolar collapse. Recently, it has been demonstrated in rats, that PEEP induces diaphragm remodelling by altering the position and length of the muscle, causing longitudinal atrophy [1]. The effects of PEEP on the human diaphragm during tidal breathing are currently unknown.

Objectives: To study the effects of PEEP on diaphragm movement and configuration during tidal breathing, including velocity of muscle contraction.

Methods: Healthy subjects ( $n=17$ ) underwent dynamic magnetic resonance imaging (MRI) in five different planes during tidal breathing under non-invasive ventilation with PEEP. After $30 \mathrm{sec}$, PEEP was abruptly increased from 2 (baseline) to 5,10 or $15 \mathrm{cmH}_{2} \mathrm{O}$ (randomized) for 1 minute. The diaphragm dome and zone of apposition (ZOA) were tracked semi-automatically in every MRI frame using a software routine developed in Matlab (see Fig 1). Then, subjects were instrumented with nasogastric catheters to measure diaphragm neuromechanical efficiency (NME) during tidal breathing at different PEEP levels. Parameters were analysed using mixed models.

Results: Diaphragm tracking was successful (Fig 1). Increasing PEEP from 2 to $15 \mathrm{cmH}_{2} \mathrm{O}$ resulted 1) in decreased end-expiratory total diaphragm length (390 (234 - 445) to 351 (295 - 408) $\mathrm{mm}$ respectively), mostly due to decreased ZOA length (178 (166 - 190) to 140 (124 155) $\mathrm{mm}$ respectively), 2) flattening of the diaphragm with an increase of the radius of curvature ( $\mathrm{R}$ in Fig 1) of $1.4 \mathrm{~cm}$ and $1.9 \mathrm{~cm}$ for the right and left hemidiaphragm respectively and 3 ) increased peak contraction velocity $(14.2(13.3-15.1)$ to $15.4(14.0-16.7) \mathrm{mm} / \mathrm{s}$ respectively) but unchanged peak relaxation velocity and 4) decreased NME of $48 \%$ $(37.5-56.6 \%)$. All mentioned effects were significant $(p<0.03)$ and reported as estimated mean ( $95 \%$ confidence interval).

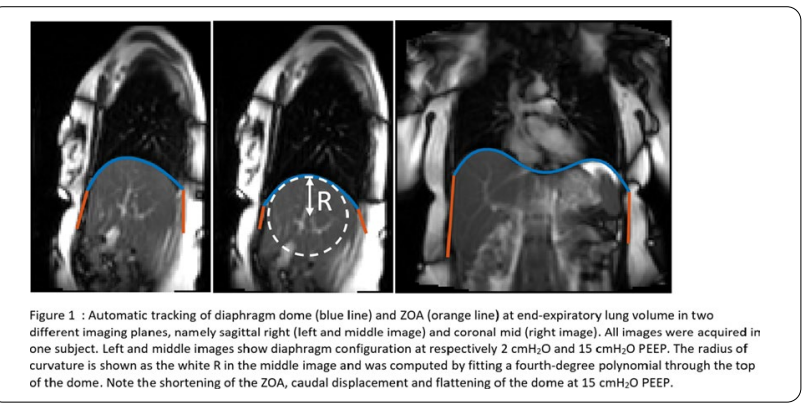

Conclusion: PEEP affects diaphragm geometry and function during tidal breathing. These findings suggest that conditions to develop longitudinal atrophy in the human diaphragm are present with the application of PEEP.

\section{Reference(s) and grant ackowledgment(s) \\ 1. Lindqvist J, van den Berg M, van der Pijl R, et al. Positive End-Expiratory Pressure Ventilation Induces Longitudinal Atrophy in Diaphragm Fibers. Am J Respir Crit Care Med. 2018;198(4):472-485. https://doi.org/10.1164/ rccm.201709-19170C \\ 2. This study was financially supported by ZonMW 09120011910004 \& Amsterdam UMC/ACS out of the Box grant.}

\section{3}

Can noble metal coating reduce the incidence of ventilator associated pneumonia (VAP): a multicenter double blind randomized pilot study

G. Kisoka'; S. Piret ${ }^{1}$; C. Legrain²; B. Lambermont ${ }^{1}$; L. Jadot ${ }^{2}$; J. Guntz ${ }^{2}$; Y. Kai-Larsen ${ }^{3}$; S. Grass ; H. Ammar ${ }^{4}$; A. Bertrand ${ }^{1}$; B. Misset ${ }^{1}$; P. DAMAS ${ }^{1}$ ${ }^{1}$ Intensive care, University Hospital Liege, Liège, Belgium; ${ }^{2}$ Intensive care, Clinical Chc Montlégia, Liège, Belgium; ${ }^{3}$ Clinical project manager, Bactiguard AB, TULLINGE, Sweden; ${ }^{4}$ Medical chief, Bactiguard AB, TULLINGE, Sweden

Correspondence: P. DAMAS

Intensive Care Medicine Experimental 2020, 8(2): 000483

Introduction: Ventilator associated pneumonia is still a problem in the intensive care unit (ICU). Noble metal coating of endotracheal tubes reduces the adhesion of bacteria and could reduce the incidence of VAP.

Methods: A controlled prospective randomized, double blind, multicenter study was launched in November 2018 in Liège, Belgium enrolling 324 patients from 8 ICUs from 4 sites of two hospital institutions using endotracheal tubes for ventilated patients from Bactiguard. These tubes with subglottic suctioning had, for half of them, a thin coating of noble metals (gold, silver and palladium) firmly attached to their surfaces, preventing bacteria from adhering and forming biofilm. Coated tubes were indistinguishable from control tubes. Coated and control tubes were coded and remained blinded for all the participants. Patients were followed during ventilation and ICU stay up to 28 days. Tracheal colonization, incidence of VAP, and antibiotic consumption were recorded.

Results: The study ended on 5 March 2020 before the Covid-19 pandemic occurrence in Liège. Data are given as a whole because the code is not yet broken. This will be done before the European convention. Among the 324 patients with a median age of 67.5 (IQR 56,574,6), there were 179 men (55.4\%). Their mean Charlson score was 2 (IQR 1-4) and the mean saps II score, calculated the day of intubation, was $50.5 \pm 17.5$. The median length of ventilation through both uncoated and coatedtubes was 5 days (IQR 3-9) totaling 2202 ventilatory days. No bacteria were found in tracheal sputum from 122 patients during ventilation. Persistence of bacteria already present in tracheal sputum the day of intubation was documented in 38 patients and new bacteria were found in 76 patients. No sampling was done in 88 patients, partially because of too short ventilation $(n=26)$. During ventilation and 2 days after extubation, there were 55 suspected episodes of VAP. The analysis of these episodes based on the presence of new infiltrate on X-ray exam, fever, leucocytosis, increase of the $\mathrm{FiO}_{2}$ or Peep level, quantitative bacteriological result and CRP change, leads to reject 25 suspicions and classifies the 30 other as confirmed $(n=$ $2)$, probable $(n=12)$ or possible $(n=16)$. The overall incidence of VAP was $13.7 / 1000$ ventilatory days or $9.3 / 100$ patients. Antibiotic from any kind was used during $68.7 \%$ of ventilatory days and $61.7 \%$ of ICU days. ICU mortality was $41.9 \%$ and hospital mortality was $50.8 \%$.

Conclusion: This is the first report of a randomized double blind study with Bactiguard tubes which will allow to define the number of patients for a confirmatory larger study. 
000521

Prognostic biomarkers for length of stay and mortality in patients admitted to critical care: a systematic review

YI. Wan ${ }^{1}$; A. Brayne ; RW. Haines ${ }^{1}$; JR. Prowle ${ }^{1}$

${ }^{1}$ William harvey research institute, Queen Mary University of London, London, United Kingdom

Correspondence: Y.I. Wan

Intensive Care Medicine Experimental 2020, 8(2): 000521

Introduction: Large numbers of observational studies have investigated the association of pre-specified laboratory tests within subgroups of critically ill patients. This systematic review aimed to determine evidence from pre-existing literature of associations between routine blood results and outcomes of patients admitted to critical care.

Methods: The study protocol was registered with the International Prospective Register of Systematic Reviews (PROSPERO; ID CRD42019122058). We followed the Preferred Reporting Items for Systematic Reviews and Meta-analyses (PRISMA) guidelines. [1] Searches were carried out using MEDLINE (PubMed) and Excerpta Medica dataBASE from inception through 29th January 2019. Outcome measures were 1) critical care or hospital length of stay, 2) mortality during critical care or in hospital or within 90 days of critical care admission. Identified studies were insufficiently homogenous to calculate pooled effect measures and qualitative summary assessments were undertaken. For biomarkers showing consistent effects, we reviewed proposed mechanisms and underlying hypotheses. Quality of evidence and risk of bias was assessed using the Newcastle-Ottawa Scale criteria. [2] We evaluated study methodology including diagnostic test accuracy, statistical approach and covariate selection.

Results: Of 441 records identified in our search, 98 met inclusion eligibility criteria. Studies analysed biomarkers individually or using a composite measure of two related biomarkers. The majority of studies were focused within mixed medical and surgical admissions $(n=62)$. All studies described retrospective data collection using cohort ( $\mathrm{n}=$ 84 ) and case-control $(n=14)$ designs. A total of 25 unique biomarkers were reported: 17 (one to four records), 5 (five to nine), and 3 (ten or more). RCDW was the biomarker most frequently assessed. Definitions varied in measurement of biomarkers and outcomes. $67.3 \%$ of studies measured admission values, $25.7 \%$ used repeated measures throughout the admission period. The biomarkers showing most consistent effects were RCDW, platelet count, NLR and albumin. A higher RCDW, a lower platelet count and a higher NLR were associated with both mortality and increased critical care length of stay. A lower level of albumin was associated with mortality. Of studies reporting statistical methods, the majority used logistic regression and Cox proportional hazards analysis. Studies which adjusted for confounders selected covariates using stepwise selection or bivariate screening.

Conclusion: Results from previous studies have limited applicability as methods of analyses tended to be restrictive and open to measured and unmeasured confounders. All identified studies focused on determining statistical significance prior to evaluating underlying pathophysiological mechanisms. Establishing a direct casual pathway among biomarkers and outcomes may be challenging and traditional statistical modelling may fail to adequately control for confounding.

Reference(s) and grant ackowledgment(s)

1. [1] Moher D, et al (2009). Preferred reporting items for systematic reviews and meta-analyses: the PRISMA statement. PLoS Med 6: e1000097.

2. [2] Wells G, et al (2013). The Newcastle-Ottawa Scale (NOS) for assessing the quality of nonrandomised studies in meta-analyses.

\section{7}

\section{Potential for lung recruitment in patients} with SARS-CoV2-associated acute respiratory distress syndrome

M. Combet ${ }^{1}$; X. Monnet ${ }^{1}$; A. Pavot ${ }^{1}$; S. Ayed ${ }^{1}$; A. Bertier ${ }^{1}$; T. Creutin ${ }^{1}$; D. Osman ${ }^{1}$; L. Guerin ${ }^{1}$; N. Anguel ${ }^{1}$; JL. Teboul ${ }^{1}$;T. Pham

${ }^{1}$ Médecine Intensive Réanimation, Bicetre Hospital AP-HP, Le Kremlin-Bicêtre, France

Correspondence: M. Combet

Intensive Care Medicine Experimental 2020, 8(2): 000527
Introduction: Many patients with SARS-CoV2 severe pneumonia received invasive mechanical ventilation and met the Berlin definition for ARDS. However, there is an ongoing debate regarding their respiratory mechanics. Several small studies found conflicting results: some showed a low potential for recruitability at an early stage of mechanical ventilation [1], others demonstrated a significant potential for recruitability, although with large variability between patients[2],[3].

Objectives: We aimed to assess lung recruitability in the first days of invasive mechanical ventilation in patients with SARS-CoV2 induced ARDS.

Methods: Single-center study in a French medical ICU.

We included patients undergoing invasive mechanical ventilation for SARS-CoV2 ARDS. Lung recruitability was assessed using recruitmentto-inflation ratio (R/I) measured within 72 hours after intubation. R/I ratio was obtained with a drop in PEEP over a single breath manoeuver, as previously described[4]. We also assessed airway opening pressure (AOP) by performing a low-flow inflation[5].

Results: Twenty-three patients were included in this study; 18 (78\%) were men with a mean \pm SD age, body mass index, SAPS II and SOFA of respectively $57 \pm 13,29 \pm 6 \mathrm{~kg} / \mathrm{m}^{2}, 45 \pm 15$ and $9 \pm 4$ at ICU admission. The majority of them $(65 \%, \mathrm{~N}=15)$ had at least one comorbidity, including hypertension $(48 \%, \mathrm{~N}=11)$, diabetes $(22 \%, \mathrm{~N}=5)$ or immunocompromised status $(22 \%, \mathrm{~N}=5)$. $\mathrm{R} / \mathrm{I}$ ratio was measured 1 [0;2.5] days after intubation and 14 patients $(60.9 \%)$ were considered to have recruitable lung $(\mathrm{R} / \mathrm{I}$ ratio $0.76[0.61 ; 1.01])$ with no difference in baseline characteristics as compared to the 9 patients with a R/I $<0.5$. Airway opening pressure was measured in eighteen patients and 4 patients $\left(22.2 \%\right.$ ) had an AOP $>5 \mathrm{cmH}_{2} \mathrm{O}$ (with a median of 6.50 $\mathrm{cmH}_{2} \mathrm{O}$ ), with no significant difference between recruiters and nonrecruiters. Overall survival rate was low (43.5\%), 57\% in the recruiter group ( $\mathrm{N}=8)$ and $22 \%$ in the non-recruiter group $(\mathrm{N}=2)$, not reaching statistical difference $(p=0.197)$.

Table 1. Respiratory parameters within 72 hours of mechanical ventilation.

\begin{tabular}{|c|c|c|c|c|}
\hline & $\begin{array}{l}\text { All patients, } \\
\mathbf{n}=\mathbf{2 3}\end{array}$ & $\begin{array}{l}\text { Non } \\
\text { recruiters, } \\
\mathbf{n}=9\end{array}$ & $\begin{array}{l}\text { Recruiters, } \\
\mathrm{n}=14\end{array}$ & p val \\
\hline $\begin{array}{l}\mathrm{VT} \mathrm{mL} / \mathrm{kg} \text { predicted } \\
\text { body weight }\end{array}$ & $5.95 \pm 0.43$ & $6.10 \pm 0.35$ & $5.85 \pm 0.46$ & 0.149 \\
\hline PEEP & $15.0[12.0 ; 15.0]$ & $\begin{array}{l}15.0 \\
{[12.0 ; 15.0]}\end{array}$ & $\begin{array}{l}14.5 \\
{[12.0 ; 15.0]}\end{array}$ & 0.972 \\
\hline Respiratory rate & $29 \pm 4$ & $27 \pm 4$ & $29 \pm 5$ & 0.275 \\
\hline $\mathrm{FiO} 2$ & $60.0[50.0 ; 80.0]$ & ] & $\begin{array}{l}65.0 \\
{[50.0 ; 80.0]}\end{array}$ & 0.974 \\
\hline $\mathrm{PaO} 2 / \mathrm{FiO} 2$ ratio & $135 \pm 39$ & $126 \pm 42$ & $141 \pm 37$ & 0.431 \\
\hline $\mathrm{R} / \mathrm{I}$ ratio & $0.59[0.39 ; 0.80]$ & ]$_{[0.29 ; 0.40]}^{0.38}$ & $\begin{array}{l}0.76 \\
{[0.61 ; 1.01]}\end{array}$ & $<0.001$ \\
\hline $\mathrm{AOP}$ & $4(22 \%)$ & $2(29 \%)$ & $2(18 \%)$ & 1.000 \\
\hline
\end{tabular}

Data are presented as median [interquartile range] or number (percentage) $V T$ tidal volume, $R / I$ ratio recruitment to inflation ratio, $A O P$ airway opening pressure.

$p$-values refer to the comparison between recruiters and non-recruiters.

Conclusion: Of the 23 patients with SARS-Cov2-associated acute respiratory distress syndrome included in this study, a majority (61\%) were considered as recruitable according to measured $\mathrm{R} / \mathrm{I}$ ratio and $22 \%$ presented an $\mathrm{AOP}>5 \mathrm{cmH}_{2} \mathrm{O}$. Given the large variability of potential for recruitment in our study, R/I ratio and AOP should be assessed to individualize PEEP settings for each patient.

Reference(s) and grant ackowledgment(s)

1. 1. Pan C, Chen L, Lu C, et al (2020) Lung Recruitability in SARS-CoV-2 Associated Acute Respiratory Distress Syndrome: A Single-center, Observational Study. Am J Respir Crit Care Med. https://doi.org/10.1164/rccm.20200 3-0527LE

2. 2. Mauri T, Spinelli E, Scotti E, et al (2020) Potential for Lung Recruitment and Ventilation-Perfusion Mismatch in Patients With the Acute Respiratory Distress Syndrome From Coronavirus Disease 2019. Crit Care Med. https:// doi.org/10.1097/CCM.0000000000004386 
3. 3. Beloncle F, Pavlovsky B, Desprez C, et al (2020) Recruitability and effect of PEEP in SARS-Cov-2-associated acute respiratory distress syndrome. Annals of Intensive Care. https://doi.org/10.1186/s13613-020-00675-7

4. 4. Chen L, Del Sorbo L, Grieco DL, et al (2020) Potential for Lung Recruitment Estimated by the Recruitment-to-Inflation Ratio in Acute Respiratory Distress Syndrome. A Clinical Trial. Am J Respir Crit Care Med. https://doi. org/10.1164/rccm.201902-03340C

5. 5. Chen L, Del Sorbo L, Grieco DL, et al (2018) Airway Closure in Acute Respiratory Distress Syndrome: An Underestimated and Misinterpreted Phenomenon. Am J Respir Crit Care Med. https://doi.org/10.1164/rccm.20170 2-0388LE

\section{7}

ICEBEARDS: Reliability of plateau pressure during assisted mechanical ventilation. A physiological analysis of ventilation traces from a multicentre prospective study

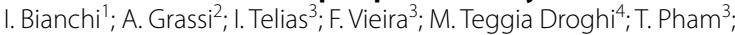
L. Brochard ${ }^{3}$; G. Bellani ${ }^{4}$

${ }^{1}$ Department of critical care and anesthesia, ASST Papa Giovanni XXIII, Bergamo, Italy; ${ }^{2}$ Department of anesthesiology and pain medicine, University of Toronto, Toronto, Canada; ${ }^{3}$ Interdepartmental division of critical care medicine, University of Toronto, Toronto, Canada; ${ }^{4}$ School of medicine and surgery, University of Milan-Bicocca, Monza, Italy

Correspondence: I. Bianchi

Intensive Care Medicine Experimental 2020, 8(2): 000537

Introduction: Measuring Plateau Pressure (PPlat) with an inspiratory hold during Pressure Support Ventilation (PSV) usually gives a value higher than the set pressure. This measurement can be used with two important clinical purposes. 1) The difference between Pplat and Peak Pressure can estimate patient's inspiratory effort (1) 2) Pplat, as in controlled mechanical ventilation (CMV), can be used to compute driving pressure and the respiratory system compliance (Crs) (2).

However, an inspiratory hold performed during PSV does not always reveal a reliable Pplat, due to ongoing muscular activity. The parameters defining Pplat as reliable during PSV are not established.

Objectives: To describe the features of a reliable inspiratory hold during PSV. To compare 1) the derived measure of inspiratory effort (Pressure Muscle Index, PMI) with the direct measure of muscular pressure (Pmusc) through esophageal pressure (Peso); 2) Crs measured during PSV and CMV when the two measurements are close enough in time.

Methods: This is an ancillary analysis of pressure and flow curves obtained during a multicenter observational study aimed to describe the incidence of patient ventilator asynchronies in moderate to severe ARDS (BEARDS, NCT03447288). We selected the tracings with an inspiratory hold in PSV and a reliable Peso. We analyzed the features of the readable Pplat and the correlation between 1) Crs measured during CMV and PSV 2) PMI and Pmusc. To be deemed reliable, Pplat had to be flat, flow had to be 0 , if inspiratory efforts were detected, Pplat had to be flat before and after the efforts. Univariate analysis was performed through t test for parametric data, Mann Whitney for nonparametric data. Data correlation was verified by Pearson's.

Results: 91 traces from BEARDS study (11centres, 35patients) met the inclusion criteria. 69/91 holds were deemed reliable (76\%), 30/35 $(86 \%)$ patients had at least one readable hold, according to visual inspection. The duration of the flat part of the hold was $1845 \mathrm{msec}$ [1207-2640]. The standard deviation of airway pressure (Paw) during the hold was significantly lower in readable versus non readable holds (1.6 [0.7-2.7] vs $2.1 \mathrm{cmH}_{2} \mathrm{O}$ [1.8-4.7], $\left.\mathrm{p}<0.005\right)$. The time to reach a plateau was lower in readable versus non readable holds (400 [229850] vs $1740 \mathrm{~ms}$ [905-3150], $\mathrm{p}<0.001)$. Peso swing was significantly lower in readable holds $\left(6.2 \pm 4.8\right.$ vs $\left.11.4 \pm 5.9 \mathrm{cmH}_{2} \mathrm{O}, \mathrm{p}<0.005\right)$. PMI showed a strong correlation with $\operatorname{Pmusc}(R=0.79, p<0.001$, Figure 1). In 21 patients, a measurement of Crs in CMV was available, with a strong correlation with $C r s$ measured during PSV $(R=0.71, p<$ 0.001 , Figure 2).

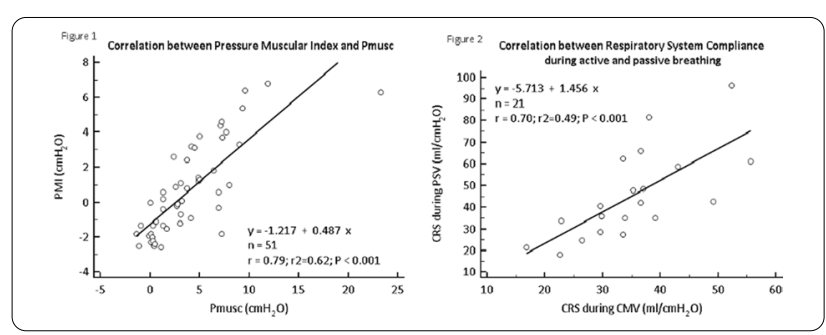

Conclusion: A readable measurement of Pplat could be obtained in most patients during PSV. Readable holds were characterized by low variability of Paw during the hold and by a short time to reach a stable plateau. When patient respiratory effort is vigorous, a reliable plateau might be more difficult to achieve. Pplat might be used to reliably compute Crs and Pmusc during PSV.

\section{Reference(s) and grant ackowledgment(s)}

1. 2. Driving Pressure Is Associated with Outcome during Assisted Ventilation in Acute Respiratory Distress Syndrome. G. Bellani, A. Grassi, S. Sosio, S. Gatti, B. P. Kavanagh, A. Pesenti, G. Foti. 2019, Anesthesiology, Vol. 131, pp. 594-604.

2. 1. End-Inspiratory Airway Occlusion A M ethod To Assess the Pressure Developed by Inspiratory M uscles in Patients with Acute Lung Injury Undergoing Pressure Support . G. Foti, M. Cereda, G. Banfi, P. Pelosi, R. Fumagalli, A. Pesenti. 1997, Am JRespir Crit Care Med, Vol. 156, pp. 1210-1216.

\section{4}

Correlation between abdominal ultrasound and intraoperative liver biopsy to evaluate organ viability in potential organ donors MD. Victoria Rodenas ; M. Royo-Villanova Reparaz ; AM. GÓMEZ DEL PULGAR VILLANUEVA ${ }^{1}$; A. López Domínguez ; M. Carrillo Cobarro ${ }^{1}$. AB. Pérez Pérez ${ }^{1}$; C. Manso Murcia’ ${ }^{1}$ M. Mateos Llosa ${ }^{1}$; M. Valer Rupérez ${ }^{1}$; M. Granados Madero ; P. Rivera Sánchez ; N. López Hernández ; E. Andreu Soler ${ }^{1}$; S. Sanchez Cámara ${ }^{\text {; }}$ DF. Pérez Martínez'; J. Moya Sánchez;

H. Vargas López ${ }^{1}$; M. Martinez Martinez ${ }^{1}$

${ }^{1}$ Servicio de Medicina Intensiva, Hospital Clínico Universitario Virgen de la Arrixaca, El Palmar, Spain

Correspondence: M.D. Victoria Rodenas

Intensive Care Medicine Experimental 2020, 8(2): 000554

Introduction: Liver biopsy remains the reference standard for diagnosing and quantifying steatosis. Ultrasonographyis a widely accesible, cheap, harmless imaging technique for the detection of fatty liver and other relevant chronic liver diseases, but the reported accuracy and reliability have been inconsistent across studies. We aimed to determine whether or not it is a suitable tool to evaluate organ viability in potential liver donors comparing with the gold standard technique.

Objectives: Determine the utility of abdominal ultrasound in organ donors to evaluate the level of hepatic steatosis and other relevant chronic liver diseases as any structural alteration in the organ.

Methods: We studied 226 consecutive donors during three years (january 2017 to december 2019) in an Intensive Care Unit from a tertiary referral hospital.

We correlated ultrasound findings with the results from the intraoperative biopsy, so we could establish the consistency between these two diagnostic tests calculated by an inter-rater agreement statistic (Kappa).

Results: Of 226 donors reviewed, 220 (97,3\%) had conducted an abdominal ultrasound and $164(72,6 \%)$ had a liver biopsy. $65 \%$ were male donors. The mean age of the donors was 60,6 years-old. $73 \%$ of evaluated organs were transplanted. $62,4 \%$ of ultrasonography operators were junior resident doctors. Agreement between ultrasonography and biopsy to evaluate hepatic steatosis is described in Table 1. We calculated a Cohen's Kappa coefficient (K) of 0,19 and a weighted 
Kappa coefficient of 0,32 . The ultrasonography was also able to detect other relevant, macroscopic findings, like space occupying liver lesions or ischemic lesions.

Table 1.

\begin{tabular}{|l|l|l|l|}
\hline & $\begin{array}{l}\text { MILD } \\
\text { STEATOSIS n }\end{array}$ & $\begin{array}{l}\text { MODERATE } \\
\text { STEATOSIS n }\end{array}$ & $\begin{array}{l}\text { SEVERE } \\
\text { STEATOSIS n }\end{array}$ \\
\hline ULTRASONOGRAPHY & 13 & 19 & 0 \\
\hline BIOPSY & 49 & 12 & 14 \\
\hline KAPPA & 0,02 & 0,02 & $<0,01$ \\
\hline
\end{tabular}

Conclusion: In our study, hepatic steatosis evaluated by abdominal ultrasound presented very low concordance rate with anatomopathological findings. Abdominal ultrasound results a very helpful tool to evaluate incidental structural injuries, though these could be uncommon findings. The use of newer quantitative ultrasound-based imaging techniques, which also are less operator dependent than traditional ultrasound, could improve the ability of ultrasound to detect and quantify hepatic steatosis and emerge as a solution to our concern.

\section{Reference(s) and grant ackowledgment(s)}

1. Castera L, Vilgrain V, Angulo P. Noninvasive evaluation of NAFLD. Nat Rev Gastroenterol Hepatol. 2013 Nov;10(11):666-75

2. Hernaez R, Lazo M, Bonekamp S, Kamel I, Brancati FL, Guallar E et al. Diagnostic accuracy and reliability of ultrasonography for the detection of fatty liver: a meta-analysis. Hepatology. 2011 Sep 2;54(3):1082-1090.

\section{4}

\section{Epidemiology and outcome of infective endocarditis in ICU} patients: preliminary results of a multi-centre prospective study (EPENDICUS)

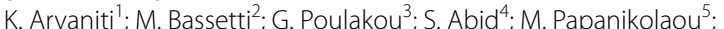

M. Peghin ; I. Karaiskos'; S. Dimopoulos ${ }^{8}$; P. Sotiriou'; M. Lukic ${ }^{9}$;

F. Ampatzidou' ${ }^{10} ;$ G. Papapathanakos ${ }^{11}$; K. Tsakalis ${ }^{12} ;$ N. Stamatiadis ${ }^{13}$.

EK. Dindar-Demiray ${ }^{14} ;$ MS. Sayar ${ }^{15}$; D. Lathyris ${ }^{16} ;$ G. Dimopoulos $^{17}$;

JF. Timsit ${ }^{4}$; M. Wolff ${ }^{4}$

${ }^{1} \mathrm{ICU}$, Papageorgiou General Hospital, Thessaloniki, Greece; ${ }^{2}$ Infectious diseases clinic, san martino univesrity hospital of genoa, Infectious diseases clinic, Genoa, Italy; ${ }^{3}$ Third department of medicine, national and kapodistrian university of athens, school of medicine, Athens, Greece; ${ }^{4}$ Medical and infectious diseases icu, Bichat-Claude Bernard Hospital, Paris, France;

${ }^{5} \mathrm{Icu}$, Hippocrateion General Hospital of Athens, Athens, Greece; ${ }^{6}$ Infectious diseases division, department of medicine, University of Udine and Azienda Sanitaria Universitaria Integrata di Udine, Udine, Italy; ${ }^{7}$ First department of internal medicine-infectious diseases, Hygeia General Hospital, Athens, Greece; ${ }^{8} \mathrm{Icu}$, Onassis Cardiac Surgery Center, Kallithea, Greece; ${ }^{9}$ Department of infectious diseases, University Medical Centre Ljubljana, Ljubljana, Slovenia; ${ }^{10}$ Cardiac surgery icu, General Hospital of Thessaloniki "George Papanikolaou", Thessaloniki, Greece; ${ }^{11} \mathrm{Icu}$, University Hospital of Ioannina, Neochoropoulo, Greece; ${ }^{12} \mathrm{lcu}$, Laikon General Hospital of Athens, Athens, Greece; ${ }^{13}$ Coronary icu, Papageorgiou General Hospital, Thessaloniki, Greece; ${ }^{14} \mathrm{I} \mathrm{Cu}$, Celal Bayar University Hafsa Sultan Hospital, Manisa, Turkey; ${ }^{15} \mathrm{Icu}$, Van training and education hospital, Van, Turkey; ${ }^{16} \mathrm{Icu}, \mathrm{G}$. Gennimatas Hospital, Thessaloniki, Greece; ${ }^{17} \mathrm{Icu}$, Attikon University Hospital, Chaidari, Greece

Correspondence: K. Arvaniti

Intensive Care Medicine Experimental 2020, 8(2): 000564

Introduction: Infective endocarditis (IE) is associated with considerable morbidity and mortality despite advances in diagnostic and therapeutic modalities.

Objectives: We sought to explore the epidemiology of IE in ICU patients with emphasis on severity, causes, management and outcome.

Methods: Preliminary analysis of EPENDICUS study (EPidemiology and outcome of ENDocarditis in ICU patientS: a multi-centre prospective epidemiological study on infectious endocarditis in ICU patients). Patients with a diagnosis of IE and ICU admission were included. Data were collected between December 2018 and April 2020. Of the collected data, demographics, comorbidities, community or healthcareonset, severity assessment on ICU admission, complications, surgical approach and ICU outcome were available for preliminary analysis. Continuous data are reported as median and interquartile range and categorical data as percentages.

Results: 73 patients were enrolled from 16 centers; median age 69 (IQR, 59-78), 69\% male, 74\% had at least one comorbidity, $49 \%$ presented valvular disease, $10 \%$ had a history of previous endocarditis, $7 \%$ were intravenous drug users. Median SAPS and SOFA on ICU admission were 52 (IQR, 45-64) and 9 (IQR, 5.25-11.75), respectively. Sepsis/septic shock and cardiac failure were recorded in $43 \%$ and $28 \%$ of the patients, respectively. Mechanical ventilation was performed in $67 \%$ of the patients. IE was healthcare-associated in $26 \%$ cases $(63 \%$ of them were vascular catheter- or prosthetic device-associated). A native valve was involved in $60 \%$ of the cases (aortic in $56 \%$, mitral in $28 \%$ of the cases). Embolic complications were observed in $18 \%$ of the patients (cerebrovascular in $46 \%$ of them). The predominant (52\%) pathogens were Staphylococci (Staphylococcus aureus in $61 \%$ of them), Enterococcus spp. in 16\%, Streptococcus spp. in $10 \%$. Surgery was performed in $56 \%$ patients (emergency/urgent in 33\% patients). ICU mortality was $29 \%$.

Conclusion: The preliminary descriptive analysis of EPENDICUS showed that IE cases present important severity on ICU admission including sepsis and septic or cardiogenic shock. IE in the ICU setting is associated with comorbidities and underlying valvular disease in the majority of the cases. A considerable number of the cases were healthcare-onset and device-associated while the predominant pathogens were Staphylococci. Surgery was performed in the majority of the patients. Enrollment and further analysis are actually ongoing.

\section{0}

Practice of ICP monitoring in acute brain-injured patients: results from the Synapse-ICU study

F. Elli' ; C. Bonetti ${ }^{1}$; F. Graziano '; G. Isernia'; G. Palio²; C. Robba ${ }^{3}$; P. Rebora'; S. Galimberti'; A. Vargiolu'; G. Citerio'

${ }^{1}$ School of medicine and surgery, University of Milano-Bicocca, Milan, Italy; 2 Department of intensive care, Clinica Santa Isabel, Universidad de Buenos Aires, Buenos Aires, Argentina; ${ }^{3}$ Department of anesthesia and intensive care, San Martino Policlinico Hospital, IRRCS for Oncology, Genova, Italy; ${ }^{4}$ Neurointensive care, department of emergency and intensive care, Ospedale San Gerardo di Monza, Monza, Italy

Correspondence: $C$. Bonetti

Intensive Care Medicine Experimental 2020, 8(2): 000600

Introduction: Increased intracranial pressure (ICP) is one of the major clinical complications of acute brain injuries (ABIs) and it is associated with poor outcome. ICP monitoring (ICPm) is considered the standard of care for the management of patients with intracranial hypertension, even if the procedure is not risk-free, and complications may occur. The indications for ICPm are mostly based on traumatic brain injury (TBI), whereas uncertainties remain for ICPm in non-TBI (subarachnoid haemorrhage (SAH) and intracerebral haemorrhage (ICH)).

Objectives: The main objective of the study is to describe the current practice of ICPm using a worldwide sample. Aim is to quantify practice variations in ICPm in TBI and non-TBI patients.

Methods: The SYNAPSE-ICU is an international, prospective, observational, cohort study (NCT03257904) including patients in coma after acute traumatic and non-traumatic brain damage admitted to the ICU. From March 2018 to June 2019, all patients fulfilling the following inclusion criteria were recruited: age $>18$ years; $A B I$ due to primary haemorrhagic stroke or TBl; Glasgow Coma Score (GCS) with Eyes score $(E)=1$ and Motor score $(M) \leq 5$ at ICU admission or within the first 48 hours. Data regarding the practice of ICPm insertion in both TBI and non-TBI patients was collected.

Results: 2504 patients were enrolled in the study (54.3\% TBI, 21.7\% $\mathrm{SAH}, 24 \% \mathrm{ICH})$, and 1358 (54\%) of them received an ICPm during ICU stay $(53.6 \%$ of $\mathrm{TBI}, 63.3 \%$ of $\mathrm{SAH}$ and $47.5 \%$ of ICH).

The most common reason for ICPm was clinical indication $(\mathrm{n}=948$, $70.1 \%)$, followed by radiology-anatomy of injury $(n=213,15.7 \%)$, 
neuroworsening ( $n=152,11.2 \%$ ), with no significant differences among primary diagnosis. Considering the type of device, in most TBI patients ( $n=144,72.3 \%)$ an intraparenchymal catheter was inserted, whereas in SAH and ICH patients DVE was the most frequently used (in $\mathrm{n}=179,53.8 \%$ and $\mathrm{n}=151,54.5 \%$ of patients, respectively).

In the majority of patients, the catheter was inserted in operating room (61.9\%). Alternatively, the insertion was performed in ICU (26.9\%) or in emergency department (5.5\%). The procedure was performed by neurosurgeons in more than $90 \%$ of cases, rarely by intensivists $(2.3 \%)$ or other medical specialists $(0.7 \%)$.

The mean length of ICPm was 8.7 days (standard deviation, $S D=16.9$ ) in $\mathrm{TBI}, 14.6$ days ( $\mathrm{SD}=10.7)$ in $\mathrm{SAH}$ and 10.4 days in $\mathrm{ICH}$ patients $(\mathrm{SD}=8.6)$. During ICU stay, the catheter was changed in 366 patients $(14.1 \%)$ due to fault or break $(25.5 \%)$, neurosurgery procedures $(22.9 \%)$, catheter displacement $(19.8 \%)$ or accidental removal $(15.1 \%)$, site infection (4.2\%) and other reasons (12.5\%) including insertion of another device, clinical worsening and hydrocephalus. The catheter has been changed, on average, after 5 days from the insertion.

Conclusion: A great variability regarding ICPm practice was observed. The results stress the need of evidence-based recommendation regarding ICPm worldwide.

\section{Reference(s) and grant ackowledgment(s)}

1. The study received an award from the ESICM, and it is inserted in the ESICM research portfolio.

\section{3}

Sepsis-induced Myopathy features a distinct proteomic

and metabolomic signature

B. Duceau'; M. Blatzer'; L. Duarte'; P. Rocheteau'; T. Lescot ${ }^{2}$; O. Langeron³ J. Bardon ${ }^{3} ;$ F. Chrétien'; A. Bouglé ${ }^{4}$

${ }^{1}$ Experimental neuropathology unit, Institut Pasteur, Paris, France; ${ }^{2}$ Surgical intensive care unit, Hospital Saint-Antoine, Paris, France; ${ }^{3}$ Anesthesiology and intensive care, Hôpital Henri-Mondor AP-HP, Avenue du Maréchal de Lattre de Tassigny, Créteil, France, Créteil, France; ${ }^{4}$ Paris, Pitié-Salpêtrière Hospital, ICAN, Paris, France

Correspondence: $\mathrm{B}$. Duceau

Intensive Care Medicine Experimental 2020, 8(2):000603

Introduction: Mechanistic studies of sepsis-induced myopathy are rare because of their complexity, both ethical and practical. Proteomics and metabolomics are high throughput technologies that aim to identify and quantify proteins and metabolites at a large scale. The analysis of protein and pathways that are involved at the cellular level, combined with metabolomic analysis of these pathway intermediates, could unravel innovative therapeutic strategies regarding muscle dysfunction in sepsis. Objectives: We aimed to investigate the sepsis-induced myopathy mechanisms with integrative and cutting-edge technologies.

Methods: We conducted a prospective study in three French Intensive Care Units. Muscle biopsies (vastus lateralis or rectus abdominis) were collected from 3 groups of patients: Septic Shock ("SS" group, $n=24$ ), Cardiogenic Shock ("CS" group, $\mathrm{n}=25$ ) and Brain Dead ("BD" group, $\mathrm{n}$ $=18$ ). The proteins/metabolites were extracted from muscle samples, then analyzed by High-Performance Liquid Chromatography-coupled to tandem Mass Spectrometry. We used enrichment analyses (differential analyses between two groups) to identify the impact of cellular pathways in muscle tissue during septic shock.

Results: Characteristics of the patients are detailed in Table 1. Proteomic and metabolomic analyses allowed the identification of 2426 proteins and 259 metabolites respectively. Despite the fact that biopsies were obtained from different muscle groups, proteomic analysis did not show any difference in the proportion of muscle fiber types. Auto-clustering methods (principal component analysis, hierarchical clustering) clearly differentiated the three groups, meaning that SS patients could be well discriminated using the proteomic and metabolomic datasets. Enrichment analysis was performed on the proteomic dataset (Figure 1). Each data point represents a biological pathway in the SS group, taking into account its significance (adjusted $p$-value, $y$-axis, logarithmic scale) and its magnitude of change ( $x$-axis) compared to the CS group (Figure 1A) or the BD group (Figure 1B). The dotted line represents the threshold for adjusted $p$-value significance. The most strongly negatively impacted pathways (adjusted p-value $<0.05$ ) were observed in the SS group: Oxidative phosphorylation; Valine, leucine and isoleucine degradation; Citrate cycle; Propanoate metabolism; Butanoate metabolism; and Beta-oxidation. Moreover, numerous key mitochondrial transporters, e.g. proteins that shuttle metabolites across the mitochondrial membranes, were no longer detected in the SS group. In the metabolomic dataset, fatty acid biosynthesis was the only up-regulated pathway in the SS group. Finally, consistent data in the proteomic and metabolomic datasets showed that anti-oxidant mechanisms have been surpassed: we observed increased polyamine and depletion of reduced glutathione (metabolomic dataset), decreased glutaredoxin, mitochondrial thioredoxin and peroxiredoxin ezymes (proteomic dataset).

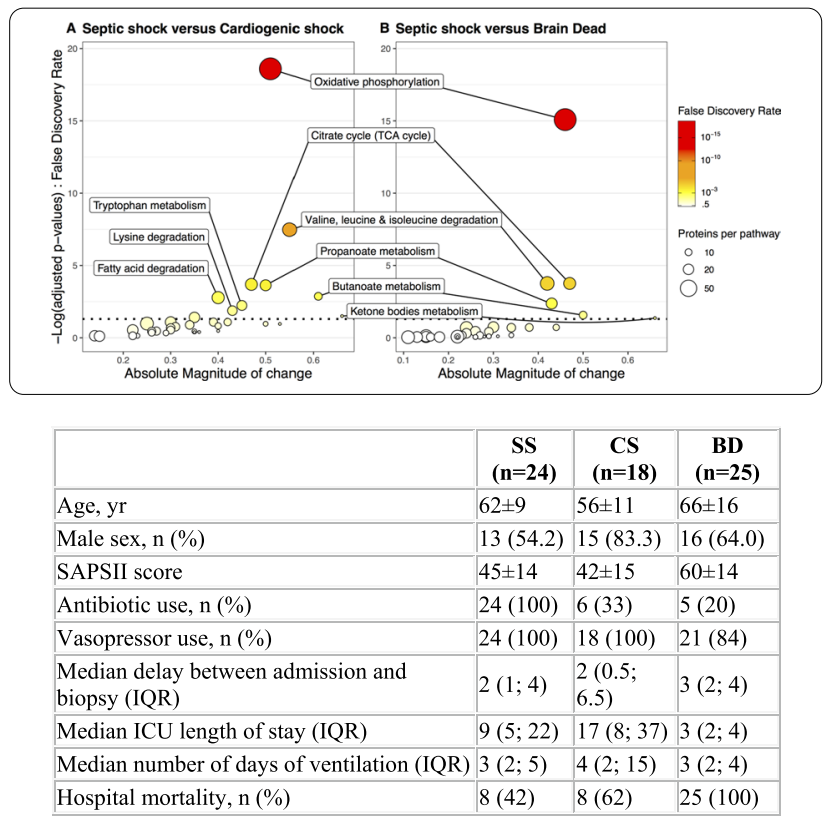

Conclusion: Our data provide a detailed and integrated in depth overview of proteins and metabolic analysis, of the pathogenesis of sepsisinduced myopathy in ICU patients. Interestingly, the mitochondrial response is not as impaired in major inflammatory processes without sepsis (brain death) or processes combining inflammation and hypoperfusion without sepsis (cardiogenic shock) as it is during sepsis.

Reference(s) and grant ackowledgment(s)

1. Institut Pasteur, Paris \& Direction de la Recherche Clinique et de I'Innovation, Assistance Publique - Hôpitaux de Paris

\section{7}

\section{Dysregulated expression of the b2-adrenergic receptor insepsis}

A. Kleyman ${ }^{1}$; A. Cesar ; G. Stanzami ${ }^{1}$; N. Arulkumaran ${ }^{1}$; W. Khaliq²

DT. Andreis ${ }^{1}$; B. Bollen Pinto ${ }^{3}$; M. Waugh ${ }^{1}$; M. Singer ${ }^{1}$

${ }^{1}$ University college london, Bloomsbury Institute of Intensive Care Medicine, London, United Kingdom; ${ }^{2}$ ntensive care unit, University Hospital Lewisham, London, United Kingdom; ${ }^{3}$ Anesthesiology, Hôpitaux Universitaires de Genève (HUG), Genève, Switzerland

Correspondence: A. Kleyman

Intensive Care Medicine Experimental 2020, 8(2): 000607

Introduction: Catecholamine hypo-responsiveness is a hallmark of sepsis, but its pathogenesis remains elusive. The adrenergic pathway, from catecholamine binding to the adrenergic receptor (AR) to the final physiological effect is a multistep process. Knowledge about protein expression and localisation of different constituents of the 
adrenergic signalling in sepsis, including adrenergic receptors themselves, is sparse.

Objectives: Using our well-characterised $72 \mathrm{~h}$ fluid-resuscitated rat model of faecal peritonitis (1), where accurate prognostication can be made as early as $6 \mathrm{~h}$, we analysed expression and membrane localisation of the b2-AR protein in heart tissue at early $(6 \mathrm{~h})$ and late $(24 \mathrm{~h})$ timepoints.

Methods: Sepsis was induced in male Wistar rats by i.p. injection of faecal slurry under general anaesthesia. The animals were then recovered and i.v. fluid resuscitation was started $2 \mathrm{~h}$ after sepsis induction. Sham-operated rats $(\mathrm{SH})$ were treated identically but did not receive the slurry. At $6 \mathrm{~h}$, an echo-measured heart rate cut-off of $460 \mathrm{bpm}$ was used to classify animals into predicted survivors (SR) and non-survivors (NSR). Rats were culled at either $6 \mathrm{~h}$ or $24 \mathrm{~h}$ with heart tissue collected and snap frozen in liquid nitrogen. To study b2-AR expression, either protein lysates of whole heart tissue or membrane fractions were prepared and analysed by Western Blot with antibodies against b2-AR and PFK or Caveolin 3. Specific signals were analysed in Image Studio Lite 5.2. Results are presented as mean $\pm \mathrm{SE}$ and considered significant at $\mathrm{p}<0.05$ (Student's t-test).

Results: The level of expression of b2-AR myocardial protein expression did not change in survivors but declined in non-survivors being significantly lower than survivors at $24 \mathrm{~h}$ (Table 1). Representative results of membrane localisation of b2-AR within different membrane fractions are presented in Figure 1. Lipid rafts were observed in only two of the 12 gradient fractions, namely fractions 4 and 5 . This was confirmed by cholesterol distribution and caveolin 3 staining. In sham operated animals and in predicted survivors at 24h, b2-AR appeared strongly in fraction 5 , associated with the lipid rafts. In predicted nonsurvivors, b2-AR was not detected in fractions 4 and 5 either at $6 \mathrm{~h}$ or $24 \mathrm{~h}$ post-sepsis.

\begin{tabular}{|c|c|c|c|}
\hline & Sham & Predicted survivor & $\begin{array}{l}\text { Predicted non- } \\
\text { survivor }\end{array}$ \\
\hline Early sepsis (6h) & $0.95 \pm 0.08$ & $0.91 \pm 0.04$ & $0.79 \pm 0.07$ \\
\hline Late sepsis $(24 \mathrm{~h})$ & & $1.10 \pm 0.15$ & $0.45 \pm 0.06 *$ \\
\hline
\end{tabular}

Figure 1. b2-AR localization within different membrane fractions

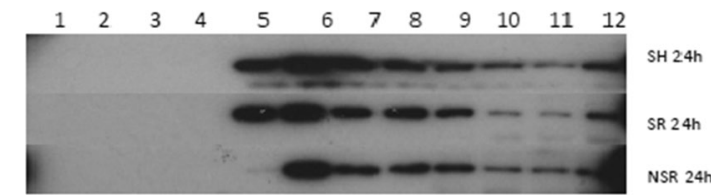

Conclusion: More severe sepsis, with corresponding poor prognosis, is accompanied by increasing catecholamine hyporesponsiveness. One cause could be a decrease in adrenergic receptor protein expression. Localisation of the b2-AR within lipid rafts is also a prerequisite for their normal functioning. In predicted non-survivors myocardial b2-AR was dissociated from the lipid rafts. Further studies are necessary to identify strategies that restore adrenergic signalling in sepsis

Reference(s) and grant ackowledgment(s)

1. Rudiger A. et al. Clin Sci 2013; 124:391-401

\section{4}

Premorbid functional status as an outcome predictor in intensive care patients aged over 85 years

L. Pietiläinen'; M. Bäcklund ${ }^{2}$; J. Hästbacka ${ }^{3}$; M. Reinikainen ${ }^{4}$

${ }^{1}$ Department of anesthesiology and intensive care, Kuopio University Hospital, Kuopio, Finland; ${ }^{2}$ Helsinki university and helsinki university central hospital, University of Helsinki, Helsinki, Finland; ${ }^{3}$ Intensive care units, department of anaesthesiology, intensive care and pain medicine, University of Helsinki and Helsinki University Hospital, Helsinki, Finland; ${ }^{4}$ Department of intensive care, Kuopio University Hospital, Kuopio, Finland
Correspondence: L. Pietiläinen

Intensive Care Medicine Experimental 2020, 8(2): 000684

Introduction: Poor premorbid functional status (PFS) is associated with a twofold increase in odds of death within 12 months after intensive care unit (ICU) admission in patients aged 80 years or over [1].

Objectives: We evaluated the one-year outcome of the oldest old ICU patients (aged 85 years or over) and the impact of PFS on outcome.

Methods: We analysed data from the nationwide Finnish Intensive Care Consortium database. We defined good PFS as independence in activities of daily living (ADL) and ability to climb stairs, whereas patients who were dependent on help in ADL or unable to climb stairs were defined as having poor PFS. Using multivariable logistic regression analysis to adjust for differences in admission types and severity of illness, we evaluated the independent impact of PFS on mortality.

To assess both the premorbid situation and functional outcome one year after ICU admission, we evaluated the patient's ability to live at home and to manage five physical activities (getting out of bed, moving indoors, dressing, climbing stairs and walking 400 metres) and created a functional status score (FSS) based on the number of manageable activities.

Results: During the years 2012-2015, 2224 (3.3\%) ICU patients were aged 85 years or older. PFS was available for $1431(64.3 \%)$ patients. PFS was good for $47.7 \%$ and poor for $52.3 \%$. Hospital mortality was $19.9 \%$ for patients with poor PFS, as compared to $13.3 \%$ for those with good PFS, $p=0.001$. One-year mortality was $47.4 \%$ for patients with poor PFS, as compared to $30.1 \%$ for those with good PFS, $p<0.001$.

Poor PFS was associated with increased mortality. For in-hospital death adjusted odds ratio (OR) was 1.35; 95\% confidence interval (Cl) 0.97-1.88; and for 12 months death OR was $1.85,95 \% \mathrm{Cl} 1.45-2.37$. For one-year survivors $(n=1244)$, data on the type of accommodation and the FSS one year after ICU admission were available for $49.8 \%$ and $47.2 \%$, respectively. Of those patients who had lived at home before ICU admission, $84.2 \%$ were still living at home one year afterwards. The FSS one year after ICU admission was the same or higher than the premorbid FSS for $69.5 \%$ of survivors. The premorbid and one-year FSS are presented in Fig. 1.

Fig. 1 Functional status scores (FSS) one year after ICU admission according to premorbid FSS

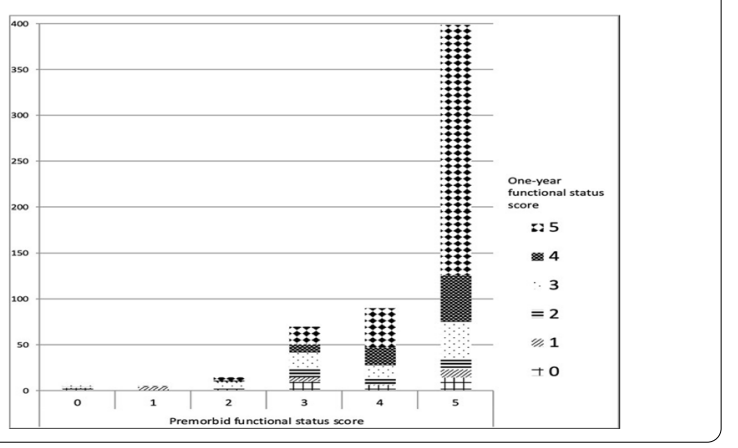

Conclusion: PFS is strongly associated with long-term outcome in ICU patients aged 85 years or older. For most survivors, functional recovery was favourable.

Reference(s) and grant ackowledgment(s)

1. 1. Pietiläinen L et al. Intensive Care Med 44: 1221-1229, 2018.

\section{5}

In which form is cholesterol present in blood?

A. Kleyman ${ }^{1}$; C. Gaupp ; M. Singer

${ }^{1}$ University College London, Bloomsbury Institute of Intensive Care Medi-

cine, London, United Kingdom

Correspondence: A. Kleyman

Intensive Care Medicine Experimental 2020, 8(2): 000695 
Introduction: Hypocholesterolaemia (HCE) is a well-known hallmark of sepsis, a reliable biomarker of disease severity and a predictor of poor outcome. The pathogenesis of HCE insepsis however remains elusive. The plasma cholesterol level reflects the balance between synthesis and ingestion versus metabolism. The rapid drop of cholesterol in sepsis onset suggests a leading role for metabolism in the development of HCE.

We postulate that sepsis-induced HCE is part of a big defensive reorganisation of the plasmalemma that includes activation of enzymes participating in membrane remodelling and production of bioactive lipids (phospholipases, sphingomyelinases and b-glucosidases). b-glucosidases transfer a sugar moiety, glucose or galactose, from glucosylceramide to cholesterol. The product is glycosylated cholesterol, which differs from cholesterol in its physical and chemical properties, including resistance to cleavage by cholesterol esterase, but is sensitive to alkaline hydrolysis.

Objectives: To study the chemical forms of cholesterol, we analysed cholesterol levels before and after alkaline hydrolysis in blood obtained from septic rats.

Methods: Seven awake instrumented male Wistar rats $(300 \pm 50 \mathrm{~g})$ received an i.p. injection of faecal slurry followed by a i.v. fluid resuscitation in $2 \mathrm{~h}$ (1). Blood was collected before sepsis initiation (base line), and at $24 \mathrm{~h}$ post-sepsis. Total and free cholesterol were measured by an enzymatic method and Amplex red assay with and without cholesterol esterase. Alkaline hydrolysis was performed with $2 \mathrm{~N} \mathrm{NaOH}$ at $45^{\circ} \mathrm{C}$ for $2 \mathrm{~h}$ and then neutralised by $10 \mathrm{~N} \mathrm{HCl}$. Statistical analysis was performed using Student's t test

Results: Plasma cholesterol dropped markedly $(p<0.05)$ after 24 hours of sepsis (Table 1). This was more prominent for cholesterol esters $(54 \%)$ compared to free cholesterol levels $(21 \%)$. Of note, total cholesterol concentration increased after alkaline hydrolysis and was unchanged after $24 \mathrm{~h}$ sepsis.

Table 1. Cholesterol concentration in blood (mg/ml)
\begin{tabular}{|l|l|l|l|}
\hline \multicolumn{5}{|l|}{ Before hydrolysis } & After hydrolysis \\
\hline & $\begin{array}{l}\text { Total Free } \\
\text { cholesterol }\end{array}$ cholesterol & $\begin{array}{l}\text { Cholesterol } \\
\text { Esters }\end{array}$ & Total cholesterol \\
\hline Baseline & $666 \pm 45 \quad 163 \pm 14$ & $503 \pm 31$ & $1081 \pm 128$ (c) \\
\hline Sepsis 24h & $348 \pm 18$ (a) $129 \pm 6$ & $219 \pm 13$ (a) & $905 \pm 90$ (c) \\
\hline
\end{tabular}

$\mathrm{a}=\mathrm{p}<0.01$ compared to corresponded baseline $\mathrm{c}=\mathrm{p}<0.01$ compared total cholesterol before an after hydrolysis

Conclusion: These results suggest that cholesterol is mainly present in blood as neither free cholesterol nor as a cholesterol ester, but rather as a substance that cannot be directly oxidized by cholesterol oxidase (ChO). However, after alkaline hydrolysis, cholesterol could be liberated from this substance and oxidised by ChO. Total cholesterol levels after hydrolysis at baseline and $24 \mathrm{~h}$ post-sepsis were similar, however the levels of any form of cholesterol without hydrolysis differed significantly. This suggests that during sepsis the proportion of cholesterol in this form had increased. Further analytical studies, MS analysis and digestion by b-glucosidases are required to identify this form of cholesterol and elucidate the pathogenesis of sepsis-induced hypocholesterolaemia.

\section{0}

Can physiology based mathematical models better represent arterial blood in the event of transient ventilatory changes? L. Shastri'; S. Kjærgaard²; PS. Thyrrestrup ${ }^{2}$; SE. Rees '; LP. Thomsen

${ }^{1}$ Respiratory and critical care group (rcare), department of health science and technology, Aalborg University, Aalborg, Denmark; ${ }^{2}$ Department of anesthesia and intensive care, Aalborg University Hospital North, Aalborg, Denmark

Correspondence: $L$. Shastri

Intensive Care Medicine Experimental 2020, 8(2):000700

Introduction: $\mathrm{ABG}$ are performed in acute conditions as the reference method for assessing the acid-base status of blood. Hyperventilation and breath-holding are common ventilatory changes that can occur around the time of sampling, rapidly altering the 'true' status of the blood.

Objectives: To determine whether mathematically calculated "arterialised" venous values (1) represent a more stable measure of arterial blood during rapid, transient, changes in ventilation.

Methods: 14 patients without cardiovascular and respiratory illnesses scheduled for elective surgery were studied. Following anaesthesia and before the start of the surgery, ventilator settings were altered to achieve $+100 \%$ or $-60 \%$ change in alveolar ventilation ('over-' or 'under-ventilation'), thus simulating a transient change in ventilation. Blood samples were drawn simultaneously from indwelling arterial and peripheral venous catheters at baseline and every $15 \mathrm{~s}$ for $1 \mathrm{~min}$ following the ventilatory change. Venous values were used to calculate arterial equivalents of acid-base status, using the mathematical arterialisation method (1).

Results: Figure 1 illustrates measured arterial and calculated arterialised values, shown as changes from baseline for $\mathrm{pH}$ and $\mathrm{PCO}$, for over- and underventilation. Arterial blood changes rapidly within the first 15 - 30s with arterialised values remaining relatively constant until $45 \mathrm{~s}$.

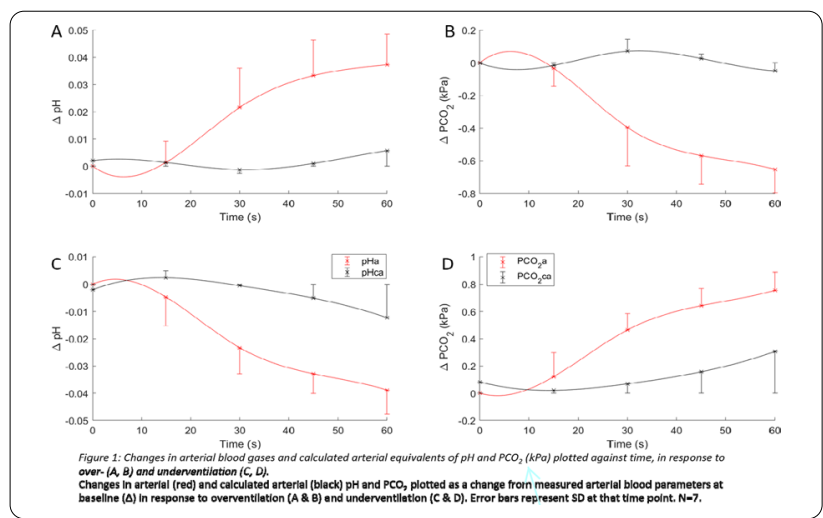

Conclusion: In situations prompting transient changes in ventilation, viz. anxiety in the emergency department or spontaneous breathing with assisted ventilation, mathematically arterialised venous values may provide a more stable value of arterial acid-base status closer to baseline values, prior to the transient change in ventilation.

Reference(s) and grant ackowledgment(s)

1. 1. Toftegaard M, Rees SE, Andreassen S. Evaluation of a method for converting venous values of acid-base and oxygenation status to arterial values. Emerg Med J. 2009;26:268-72.

000715

lonized Calcium levels predict adverse outcome in patients with acute upper gastrointestinal bleeding: a retrospective study of 1345 patients

D. Epstein ${ }^{1}$; A. Korytny ${ }^{2}$; N. Solomon ${ }^{3}$;. Freund ${ }^{4}$; E. Marcusohn ${ }^{5}$.

A. Neuberger ${ }^{6}$; A. Raz ; A. Miller ${ }^{8}$

${ }^{1}$ Medical ICU, Rambam Healthcare Campus, Haifa, Israel; ${ }^{2}$ Department of gastroenterology, Rambam Healthcare Campus, Haifa, Israel; ${ }^{3}$ Lis maternity and women's hospital, Ichilov Hospital, Tel Aviv-Yafo, Israel; ${ }^{4}$ Ruth and bruce rappaport faculty of medicine, The Ruth and Bruce Rappaport Faculty of Medicine, Haifa, Israel; ${ }^{5}$ Department of cardiology, Rambam Healthcare Campus, Haifa, Israel; ${ }^{6}$ Unit of infectious diseases, Rambam Healthcare Campus, Haifa, Israel; 'Department of anesthesiology, Rambam Healthcare Campus, Haifa, Israel; ${ }^{8}$ Medical intensive care unit, Rambam Healthcare Campus, Haifa, Israel

Correspondence: D. Epstein

Intensive Care Medicine Experimental 2020, 8(2):000715

Introduction: Acute upper gastrointestinal bleeding (UGIB) is a common condition associated with significant morbidity and mortality Previous studies in trauma patients have shown low ionized calcium 
$(\mathrm{Ca}++)$ levels to be associated with an increased need for transfusion of blood products and adverse outcome in this population.

Objectives: We hypothesized that admission $\mathrm{Ca}++$ levels could predict the need for blood transfusion and surgical/angiographic intervention in patients with UGIB.

Methods: We conducted a population-based retrospective cohort study, using the data of patients admitted to Rambam Medical center, Haifa, Israel due to UGIB for whom $\mathrm{Ca}++$ was measured within 24 hours before or after the endoscopy and before administration of blood transfusion. Patients on anticoagulants, with known varices or chronic liver disease were excluded as these cause complex alterations in coagulation and hemostasis. The primary outcome of this study was a composite of transfusion of two or more packed red blood cells (PC) or a need for urgent surgical or angiographic intervention during the index admission. Secondary outcomes included individual components of the composite outcome. Multivariable logistic regression was performed to determine whether $\mathrm{Ca}++$ was an independent predictor of these outcomes.

Results: A total of 1345 patients were included in the final analysis. Hypocalcemia $(\mathrm{Ca}++<1.16)$ was recorded in $604(45 \%)$ patients and $88(7 \%)$ had $\mathrm{Ca}++$ below $1 \mathrm{mmol} / \mathrm{L}$. The rates of composite adverse outcome were significantly higher in the low $\mathrm{Ca}++$ group, $14.4 \%$ vs. $5.1 \%, p<0.001$. In addition, multiple transfusions $(9.9 \%$ vs. $2.3 \%, p<0.001)$, need for surgical/angiographic interventions $(5.3 \%$ vs. $2.8 \%, p=0.03)$ and mortality $(33.3 \%$ vs. $24.7 \%, p<$ 0.001 ) were significantly increased in the hypocalcemia group. Multivariable logistic regression analysis identified $\mathrm{Ca}+<1.16$ as an independent predictor of the primary and secondary adverse outcomes.

$\mathrm{Ca}++$ performed better than modified Glasgow Blatchford score in predicting the need for multiple PC transfusion (AUC $0.7295 \% \mathrm{Cl}$ $0.69-0.74$ vs. AUC $0.5595 \% \mathrm{Cl} 0.53-0.58, \mathrm{p}=0.001)$. There was a trend towards a better performance of $\mathrm{Ca}++$ measurements on the composite adverse outcome (AUC $0.6695 \% \mathrm{Cl} 0.63-0.69$ vs. AUC $0.5895 \%$ $\mathrm{Cl} 0.56-0.61, \mathrm{p}=0.07$ )

Conclusion: Low calcium in patients with UGIB is common and associated with an increased requirement of PC transfusion and need for surgical or angiographic interventions. Admission calcium levels may facilitate the rapid identification of high-risk patients with UGIB. As $\mathrm{Ca}++$ plays a key role in the coagulation cascade, coagulopathy may occur as $\mathrm{Ca}++$ levels drop. In addition, low cardiac contractility and reduced systemic vascular resistance also occur when calcium levels drop. Correcting hypocalcemia may serve as an additional goal in the treatment of the bleeding patient. Further studies should evaluate the impact of correcting hypocalcemia on UGIB patients' outcome. Until definitive studies are available, clinicians should consider monitoring $\mathrm{Ca}++$ levels and maintaining it within the normal range in patients with UGIB.

\section{Reference(s) and grant ackowledgment(s)}

1. Ho KM, Yip CB. Concentration-dependent effect of hypocalcaemia on in vitro clot strength in patients at risk of bleeding: A retrospective cohort study. Transfus Med. 2016;26(1):57-62. https://doi.org/10.1111/tme.12272

2. Magnotti LJ, Bradburn EH, Webb DL, et al. Admission ionized calcium levels predict the need for multiple transfusions: A prospective study of 591 critically ill trauma patient. J Trauma - Inj Infect Crit Care. 2011;70(2):391-397. https://doi.org/10.1097/TA.0b013e31820b5d98

3. Stanley AJ, Laine L. Management of acute upper gastrointestinal bleeding. BMJ. 2019;364. https://doi.org/10.1136/bmj.I536

4. herry RA, Bradburn E, Carney DE, Shaffer ML, Gabbay RA, Cooney RN. Do early ionized calcium levels really matter in trauma patients? J Trauma - Inj Infect Crit Care. 2006;61 (4):774-779. https://doi.org/10.1097/01.ta.00002 39516.49799 .63

5. Ditzel RM, Anderson JL, Eisenhart WJ, et al. A review of transfusion- and trauma-induced hypocalcemia. Is it time to change the lethal triad to the lethal diamond? J Trauma Acute Care Surg. December 2019:1. https://doi. org/10.1097/ta.000000000000257
000724

Effects and complications of neostigmine for acute colonic ileus in critical care patients

SN. Jansen ${ }^{1}$; J. De Metz'; RJ. Bosman'; B. Van Den Bogaard

${ }^{1}$ Intensive care medicine, OLVG location East, Amsterdam, Netherlands

Correspondence: S.N. Jansen

Intensive Care Medicine Experimental 2020, 8(2): 000724

Introduction: Neostigmine is used to treat critical illness related colonic ileus in the intensive care unit (ICU). Data on the effectiveness and possible complications of intravenous administration of neostigmine in critically ill patients are scarce. Specifically data concerning patients with recent intestinal anastomosis are lacking.

Objectives: The primary objective of this study was to assess the effectiveness of neostigmine treatment on passing stool. The secondary objective was to assess the rate of hemodynamic and abdominal complications of neostigmine treatment in critically ill patients.

Methods: In this single-centre retrospective cohort study, all consecutive patients treated with neostigmine on the ICU of the OLVG hospital (Amsterdam, the Netherlands) between 2007 and 2019 were included. The primary outcome was the effectiveness of neostigmine treatment defined as the percentage of patients with $\geq 100 \mathrm{ml}$ stool, 6 and 24 hours after the start of neostigmine. The secondary outcome was the rate of complications, such as bradycardia and abdominal complications. Bradycardia was defined as heart rate $<50 / \mathrm{min}$ and/ or the use of atropine after administration of neostigmine. Abdominal complications such as bowel perforation and anastomotic leakage were assessed by automatic database extraction and consequently manually reviewing all relevant patient files. We studied the incidence of abdominal complications between patients with- and without a recent (within 10 days) intestinal anastomosis.

Results: Between 2007 and 2019 a total of 1008 patients were treated with neostigmine. $71 \%$ of these patients were male, mean age was 66 years (SD 13), mean SOFA score 10 (SD 3,8), mean APACHE IV 0,4 (SD 0,3 ). In 414 patients $(41 \%) \geq 100 \mathrm{ml}$ stool was passed after 6 hours, and in 784 patients (79\%) after 24 hours. Bradycardia was seen in 58 patients $(6 \%)$ and atropine was administered to 15 patients (1.5\%). A total of 148 patients (15\%) had abdominal surgery within 10 days prior to neostigmine treatment. Forty-nine (5\%) of them had an intestinal anastomosis. Twelve of these 49 patients $(24,5 \%)$ developed an abdominal complication requiring surgery during their treatment on the ICU. In eleven patients an anastomotic leakage was found, and one patient experienced a spontaneous perforation of an diverticula. In $1.65 \%$ of patients (13 out of 784 ) without recent abdominal surgery a spontaneous perforation or bowel ischemia requiring a surgical intervention was observed after neostigmine treatment. In the patients who underwent recent abdominal surgery without an intestinal anastomosis, 7\% developed an abdominal complication.

Conclusion: Neostigmine seems to be an effective treatment to resolve acute colonic ileus in critically ill patients. Bradycardia requiring atropine was observed rarely. However, in $24.5 \%$ of patients with recent abdominal surgery with an intestinal anastomosis treated with neostigmine anastomotic leakage or diverticular perforation was observed.

Reference(s) and grant ackowledgment(s)

1. De Giorgio et al. Aliment Pharmcol Ther 2001 Review article: the pharmacological treatment of acute colonic pseudo-obstruction

2. Ponec et al. NEJM 1999 Neostigmine fot the treatment of acute colonic pseudo obstruction

3. Van der Spoel et al ICM 2001 Neostigmine resolves critical illness related colonic ileus in intensive care patients with multiple organ failure - a prospective double-blind, placebo controlled trial. 
000725

External validation of a Gaussian Process model for the prediction of elevated intracranial pressure on the Center-TBI dataset

G. Carra' ; F. Güiza'1 ; B. Depreitere ${ }^{2}$; G. Meyfroidt ${ }^{1}$

${ }^{1}$ Department and laboratory of intensive care medicine, KU Leuven, Leuven, Belgium; '2Department of neurosurgery, KU Leuven, Leuven, Belgium Correspondence: $G$. Carra

Intensive Care Medicine Experimental 2020, 8(2): 000725

Introduction: Intracranial pressure (ICP) is continuously monitored in patients with severe Traumatic Brain Injury (TBI). Güiza et al. [1] developed a prediction model able to predict elevated ICP (defined as ICP $>30 \mathrm{mmHg}$ for more than 10 minutes) with useful forewarning. The model, which was developed on the Brain-IT database [2], presented good calibration (Cox calibration slope 1.02,calibration-in-the-large $=-0.02$ ) and good discrimination ( $A U C=0.87$ ).

It is good practice to periodically validate the performance of a prediction model on new and preferably external databases. First, it is important to assess whether the performance of the model have deteriorated as a result of the changes in the clinical practice that followed the new Brain Trauma Foundation guidelines for TBI of 2007 and 2016. Second, external validation allows to assess the model generalizability capacities. In this study, we validated the prediction model developed by Güiza et al. [1] on the Collaborative European NeuroTrauma Effectiveness Research in Traumatic Brain Injury (CENTER-TBI) dataset [3]. This validation follows a previous external validation performed on data prior to 2013 [4].

Methods: The external validation included 257 patients with continuous ICP and mean arterial blood pressure signals. Patients were prospectively recruited between January 2015 and December 2017 as part of the Center-TBI dataset. In accordance with the original publication [1], the performance of the model was quantified in terms of Area Under the ROC Curve (AUC) and calibration. Discrimination curve analysis was additionally performed to quantify the clinical utility of the model.

Results: On the external validation dataset, the model was still able to predict future episodes of elevated intracranial pressure with good calibration (Cox calibration slope 1.22, calibration-in-the-large = -0.04 , see panel A) of Fig. 1 ) and discrimination (AUC $=0.93)$. The discrimination curve showed clinical usefulness for almost all risk thresholds (respectively from $4 \%$ to $96 \%$ ), as shown in panel B) of Fig. 1.

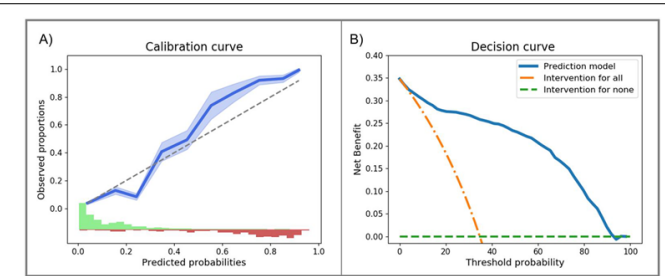

Figure 1 Panel A) The calibration curve of the prediction model is represented in blue, the shaded blue area represents the $95 \% \mathrm{CI}$. Below the curve, a histogram shows the distribution of the model predicted probabilities for episodes of elevated ICP, represented in red, and episodes of not elevated ICP, represented in green. Panel B) Decision curve of the prediction model, the blue line indicates the net benefit of the model, the dashed orange line indicates the net benefit in case all subjects are treated (intervention for all), while the dashed green line represents the net benefit in case no subjects are treated (intervention for none).
Reference(s) and grant ackowledgment(s)

1. [1] Güiza F, Piper I, Meyfroidt G, et al (2012) Novel Methods to Predict Increased Intracranial Pressure During Intensive Care and Long-Term Neurologic Outcome After Traumatic Brain Injury. Crit Care Med 41:554-564

2. [2] Brain-IT. http://www.brain-it.eu/

3. [3] Center TBI. https://www.center-tbi.eu/

4. [4] Güiza F, Depreitere B, Piper l, et al (2017) Early Detection of Increased Intracranial Pressure Episodes in Traumatic Brain Injury: External Validation in an Adult and in a Pediatric Cohort. Crit Care Med 45:e316-e320

\section{3}

Microcirculation alterations in severe COVID-19 pneumonia

VS. Kanoore Edul ${ }^{1}$; EFJ. Caminos ${ }^{2}$; G. Ferrara ${ }^{3}$; E. Estenssoro ${ }^{4}$; CE. Cesio ${ }^{3}$; DS. Páez Siles ${ }^{1}$; A. Dubin 5

${ }^{1}$ Servicio de terapia intensiva, Hospital Juan A Fernández, Buenos Aires, Argentina; ${ }^{2}$ Cátedra de farmacología aplicada, Universidad Nacional de La Plata, Facultad de Ciencias Médicas, La Plata, Argentina; ${ }^{3}$ Servicio de terapia intensiva, Sanatorio Anchorena, San Martín, Argentina; ${ }^{4}$ Servicio de terapia intensiva, Hospital San Martín, La Plata, Argentina; ${ }^{5}$ Servicio de terapia intensiva, Sanatorio Otamendi, Buenos Aires, Argentina

Correspondence: A. Dubin

Intensive Care Medicine Experimental 2020, 8(2): 000733

Introduction: Microvascular thrombosis has recently been diagnosed in patients with COVID-19, and it has been proposed to mediate the pathogenesis of organ injury in this disease. While widespread pulmonary microvascular thrombosis was demonstrated, reports about the compromise of extrapulmonary microvessels are controversial.

Objectives: To evaluate the characteristics of sublingual microcirculation and skin perfusion in patients with acute respiratory distress syndrome (ARDS) secondary to COVID-19.

Methods: We prospectively included 27 patients with diagnosis of ARDS secondary to COVID-19 (positive PCR testing of nasopharyngeal or tracheal samples), from 4 intensive care units in Argentina. All patients were intubated and mechanically ventilated, received infusions of midazolam, fentanyl and atracurium, and anticoagulant prophylaxis from their hospital admission. Blood pressure, heart rate and norepinephrine infusion remained unchanged for 3-h before the measurements. Sublingual microcirculation was assessed by means of hand-held videomicroscopy and software-assisted analysis (1).

The respective institutional review board approved this study and waived patient informed consent.

We present continuous variables as median [IQR]) or mean (SD), and categorical variables as numbers (\%).

Results: $\mathrm{PaO}_{2} / \mathrm{FiO}_{2}$ was $122 \pm 43$, and APACHE II and SOFA score were 12 [9-17] and 6 [3-8]. Norepinephrine (0.03 [0.01-0.17] $\mu \mathrm{g} / \mathrm{kg} / \mathrm{min})$ was required in 12 patients (44\%). D-dimer was elevated (1.30 [0.58-2.93] $\mu \mathrm{g} / \mathrm{mL}$. Median plasma lactate was normal (1.8 [1.6-2.5] mmol/L). Capillary refill time was prolonged (3.5 [3.0-5.0] s).

Compared to reported normal values (1), total and perfused vascular density and heterogeneity flow index were high, and the proportion of perfused vessels, microvascular flow index, and red blood cell velocity were reduced (Table 1 ). The proportion of perfused vessels was inversely correlated with total vascular density (Pearson $r=-0.41, P=$ 0.03).
Conclusion: These results demonstrate the robustness of the prediction model for elevated ICP developed by Güiza et al. [1]. The model shows good calibration and good discrimination even when evaluated on an external multi-center dataset collected 10 years later than the original development cohort. Future intervention studies are required to assess the impact of this model on patient outcome when used in clinical practice. 
Sublingual microcirculatory variables

\begin{tabular}{|l|l|}
\hline Total vascular density, $\mathrm{mm} / \mathrm{mm}^{2}$ & $21.9 \pm 3.9$ \\
\hline (normal values $=16.7 \pm 1.6)$ & $21.0 \pm 3.5$ \\
\hline Perfused vascular density, $\mathrm{mm} / \mathrm{mm}^{2}$ & \\
\hline $\begin{array}{l}\text { (normal values }=16.7 \pm 1.6) \\
\text { Proportion of perfused vessels } \\
\text { (normal values }=1.00 \pm 0.00)\end{array}$ & $0.96 \pm 0.03$ \\
\hline $\begin{array}{l}\text { Microvascular flow index } \\
\text { (normal values }=2.97 \pm 0.03 \text { ) }\end{array}$ & $2.79 \pm 0.10$ \\
\hline $\begin{array}{l}\text { Red blood cell velocity, } \mu \mathrm{m} / \mathrm{s} \\
\text { (normal values }=1331 \pm 190 \text { ) }\end{array}$ & $1124 \pm 161$ \\
\hline $\begin{array}{l}\text { Heterogeneity flow index } \\
\text { (normal values }=0.04 \pm 0.03 \text { ) }\end{array}$ & $0.91 \pm 0.24$ \\
\hline
\end{tabular}

Conclusion: Although most patients were hemodynamically stable, they had alterations in sublingual microvascular flow and capillary refill time. Microcirculatory abnormalities consisted in an increased number of unperfused vessels. Although these changes seem minor, compared to those described in septic shock, they represent a several time increase from normal values. In addition, heterogeneity was severely augmented and flow velociry was reduced. These abnormalities might contribute to the development of multiple organ failure. The most striking result was the increase in vascular densities, a finding that might be related to enhanced angiogenesis or hypoxiainduced capillary recruitment. The association of microthrombosis and increased angiogenesis has been found in COVID-19 and in other conditions.

In summary, patients with severe COVID-19 pneumonia showed subtle but consistent alterations in sublingual microcirculation and skin perfusion.

\section{Reference(s) and grant ackowledgment(s)}

1. Edul VS, Enrico C, Laviolle B, Vazquez AR, Ince C, Dubin A. Quantitative assessment of the microcirculation in healthy volunteers and in patients with septic shock. Crit Care Med 2012;40:1443-8

\section{5}

Development and validation of a sample entropy-based method to identify complex patient-ventilator interactions during mechanical ventilation

L. Sarlabous'; J. Aquino Esperanza'² R. Magrans ${ }^{3}$; C. De Haro ${ }^{4}$;

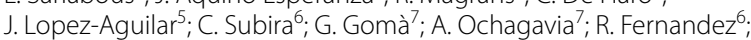

L. Blanch ${ }^{5}$

'Ârea de crítics, Institut d'Investigació i Innovació Parc Taulí I3PT, Sabadell, Spain; ${ }^{2}$ Isciii, CIBER Enfermedades Respiratorias. Institut d'Investigació i Innovació Parc Taulí I3PT., Sabadell, Spain; ${ }^{3}$ Bettercare, BetterCare, Sabadell, Spain; ${ }^{4}$ Àrea de crítics., Parc Tauli HospitalUniversitari. CIBER Enfermedades Respiratorias. Isciii, Sabadell, Spain; ${ }^{5}$ Isciii, CIBER Enfermedades Respiratorias. Institut d'linvestigació i Innovació Parc Taulí I3PT, Sabadell, Spain ${ }^{6}$ Department of intensive care, Fundació Althaia, Universitat Internacional de Catalunya, Manresa, Spain; ${ }^{7}$ Àrea de crítics, Parc Tauli Hospital Universitari. Institut d'Investigació i Innovació Parc Taulí I3PT, Sabadell, Spain

Correspondence: J. Aquino Esperanza

Intensive Care Medicine Experimental 2020, 8(2): 000755

Introduction: Patient-ventilator asynchronies occur when there is a mismatch between the ventilator's setting and patient's breathing pattern, and oudl trigger major consequences. Asynchronies are difficult to characterize when supported only by visual assessment carried out by inexperienced personnel, since different types may develop in a short time period or may even overlap with each other. Therefore, it would be extremely useful to have access to a method for assessing irregularity and complexity which could detect Complex PatientVentilator interactions (CP-VI), including not only asynchronies of any kind but also changes in the respiratory rate, in an automated, noninvasive and personalized fashion.

Sample Entropy (SE) is a non-linear method which measures complexity and regularity. Lower $S E$ value indicates more self-similarity in a time series of data. $S E$ has proved to be an effective tool for investigating different types of time series data derived from various biological conditions in the human body. We hypothesized that analyzing transient complexity of CP-VI may provide clinically relevant information about asynchronies and changes in respiratory rate during invasive mechanical ventilation.

Objectives: Develop and validate a non-invasive method based on SE measurement using the entire airway pressure (Paw) and airway flow (Flow) waveforms to detect CP-VI, defined as the occurrence of asynchronies and changes in the respiratory rate.

Methods:SE of airway flow (SE-Flow) and airway pressure (SE-Paw) waveforms from 92 15-minute-long segments obtained from 27 critically ill, mechanically-ventialted patients were used to develop and validate an automated algorithm for detecting CP-VI, defined as a $>50 \%$ change in the respiratory rate, and/or $>30 \%$ asynchronous breaths of any type (ineffective expiratory efforts, double cycling, premature cycling, prolonged cycling, or reverse triggering) over a 3-minute period.

The algorithm's performance was compared versus CP-VI scored visually by three experts, and measured through Fleiss's Kappa interrater agreement. A repeated holdout cross-validation procedure using the Matthews correlation coefficient (MCC) as a measure of effectiveness was used for optimization of different combinations of SE settings (embedding dimension, $m$, and tolerance value, $r$ ), derived $S E$ features (mean, [SE-Flowmean and SE-Pawmean] and maximum values, [SEFlowmax and SE-Pawmax]), and the thresholds of change (Th) from patient's own baseline SE value, applying different values of Th $(15 \%$, $20 \%, 25 \%, 30 \%, 35 \%, 40 \%, 45 \%, 50 \%)$. We hypothesized that $S E$ values would be higher in periods with CP-VI than in periods with regular patient-ventilator interactions

Results: Fleiss' kappa for inter-rater agreement was 0.90 (0.87-0.93), indicating almost perfect agreement. SE was highly sensitive to changes in the irregularity of the respiratory pattern occurring during ventilation. The most accurate results for the automated detection of $\mathrm{CP}-\mathrm{VI}$ were obtained using the maximum values of $S E$-Flow $(m=2, r=$ $0.2, T h=25 \%$, [SE-Flowmax25]) and SE-Paw $(m=4, r=0.2, T h=30 \%$, [SE-Pawmax30]) which report MCCs of $0.85(0.78-0.86)$ and $0.78(0.78-$ $0.85)$, and accuracies of $0.93(0.89-0.93)$ and $0.89(0.89-0.93)$, respectively. Once we had determined the settings that best detected CP-VI, we evaluated the performance of the algorithm in the 15 repetitions of the cross-validation procedure. Both SE-Flowmax 25 and SE-Pawmax30 yielded their highest MCC values in 13 of the 15 repetitions.

Conclusion: Our non-invasive method based on SE measurement of Paw and Flow is able to detect with high accuracy CP-VI, defined as the occurrence of clusters of asynchronies and changes in the respiratory rate, with high accuracy. Clinical relevance and usefulness of identifying Complex Patient-Ventilator Interactions in different clinical scenarios deserves to be explored.

Reference(s) and grant ackowledgment(s)

1. Suki, B., Bates, J. H. T. \& Frey, U. Complexity and Emergent Phenomena. Compr Physiol 1, 995-1029 (2011)

2. Keim-Malpass, J., Clark, M. T., Lake, D. E. \& Moorman, J. R. Towards development of alert thresholds for clinical deterioration using continuous predic tive analytics monitoring. J. Clin. Monit. Comput. (2019)

3. Brochard, L. Breathing: does regular mean normal? Crit Care Med 26, 1773-4 (1998)

4. Sarlabous, L. et al. Efficiency of mechanical activation of inspiratory muscles in COPD using sample entropy. Eur. Respir. J. 1808-11 (2015) 
5. Richman, J. S. \& Moorman, R. Physiological time-series analysis using approximate entropy and sample entropy. Am J Physiol Hear. Circ Physiol 278, 2039-2049 (2000)

6. Vaporidi, K. et al. Clusters of ineffective efforts during mechanical ventilation: impact on outcome. Intensive Care Med. 43, 184-191 (2017).

7. Thille, A. W., Rodriguez, P., Cabello, B., Lellouche, F. \& Brochard, L. Patientventilator asynchrony during assisted mechanical ventilation. Intensive Care Med. 32, 1515-22 (2006)

8. Blanch, L. et al. Asynchronies during mechanical ventilation are associated with mortality. Intensive Care Med. 41, 633-641 (2015).

9. This work was funded by projects PI16/01606, integrated in the Plan Nacional de R + D + I and co-funded by the ISCIII-Subdirección General de Evaluación y el Fondo Europeo de Desarrollo Regional (FEDER). RTC-20176193-1 (AEI/FEDER UE). CIBER Enfermedades Respiratorias, and Fundació Parc Taulí.

\section{5}

The mechanical power before and after initiation of VV-ECMO; the importance of correct calculations

V. Baartmans'; J. Goedegebuur'; A. Schoe ${ }^{1}$

${ }^{1}$ Intensive Care, Leiden University Medical Center (LUMC), Leiden, Netherlands

Correspondence: $\vee$. Baartmans

Intensive Care Medicine Experimental 2020, 8(2): 000775

Introduction: Ventilator induced lung injury (VILI) is associated with the amount of energy the ventilator transfers to the patient, which is called the mechanical power (MP) [1]. In a previous study, Schmidt et al. demonstrated that veno-venous extra corporal membrane oxygenation (VV-ECMO) can reduce the amount of energy transferred to the lungs to maintain adequate oxygenation and ventilation [2]. However, they used a formula developed for volume controlled ventilation (VCV) [1], whereas $40 \%$ of the population was ventilated with a pressure controlled mode (PCV) before start of VV-ECMO. This percentage was even a greater after the start of ECMO.

It is of utmost importance to use the right methods to calculate the MP. Using the formula for VCVon patients ventilated with PCV will lead to a systemic bias in which the MP will be underestimated.

Objectives: To describe the change of MP before and after initiation of ECMO using the correct calculations and to demonstrate the systemic bias when using the wrong calculations.

Methods: We performed a retrospective cohort study in all patients admitted to the adult ICU of the LUMC on VV-ECMO for respiratory indications between 2011 and 2020. We calculated the mean MP during 6 hours pre ECMO and 6 hours after initiation of ECMO with a pause of ca. 2 hours after placing the patient on ECMO. Mechanical power was calculated with the formula MP-PCV $=0.098 * \mathrm{RR} * \mathrm{Vt} *$ $\left[P E E P+\Delta\right.$ Pinsp * $\left(1-\mathrm{e}^{\wedge}\left(-\mathrm{Tinsp} /\left(\mathrm{R}^{*} \mathrm{C}\right)\right)\right]$ as described by van der Meijden et al. [3], which is suitable for PCV. We also calculated the MP in all patients using the formula described by Gattinoni et al. [1] MP-VCV = $0.098 * \mathrm{RR} * \mathrm{Vt}^{*}$ [Ppeak $\left.-0.5 * \Delta \mathrm{Pinsp}\right]$ which is suitable for VCV and used by Schmidt et al. on all patients.

All patients were ventilated with an Evita -XL (Dräger, Lubeck, Germany) or Hamilton 6 ventilator (Hamilton Medical, Bonaduz, Switzerland). Statistics were done with SPSS 25 (Armonk, NY: IBM Corp).

Results: See Table 1. for the population characteristics. The mechanical power before ECMO was $34.5 \mathrm{~J} / \mathrm{min}$ (sd 13.4), after start of ECMO the mean MP was $6.8 \mathrm{~J} / \mathrm{min}$ (sd 4.0). When calculated with the VCV method the MP was $27.2 \mathrm{~J} / \mathrm{min}$ (sd 11.1) before and $5.3 \mathrm{~J} / \mathrm{min}$ (sd 3.0) after ECMO. Ventilator settings and gas exchange parameters are shown in table 2. The mean difference of the MP before ECMO between the two calculations was $7.3 \mathrm{~J} / \mathrm{min}$ and after ECMO $1.5 \mathrm{~J} / \mathrm{min}$.
Table 1. population characteristics

\begin{tabular}{l} 
Table 1. population characteristics \\
\begin{tabular}{|l|l|}
\hline Number of patients & 40 \\
\hline Mean age (sd) & $51(15.9)$ \\
\hline Gender - Male / female & $77.5 \% / 22.5 \%$ \\
\hline ECMO indication - ARDS & $37.5 \%$ \\
\hline - Bacterial pneumonia & $30 \%$ \\
\hline - Asthma / COPD / other & $32.5 \%$ \\
\hline Mean RESP score (sd) & $1.3(3.2)$ \\
\hline ECMO survival & $75 \%$ \\
\hline ICU survival & $62.5 \%$ \\
\hline Hospital survival & $62.5 \%$ \\
\hline
\end{tabular} \\
\hline
\end{tabular}

Table 2. Results

\begin{tabular}{|c|l|l|}
\hline \multicolumn{2}{|c}{ Table 2. Results } \\
\hline & $\begin{array}{c}\text { Before } \\
\text { ECMO; } \\
\text { mean (sd) }\end{array}$ & $\begin{array}{c}\text { After } \\
\text { ECMO; } \\
\text { mean (sd) }\end{array}$ \\
\hline MP-PCV (J/min) & $34.5(13.4)$ & $6.8(4.0)$ \\
\hline Wrong calculation: MP-VCV (J/min) & $27.2(11.1)$ & $5.3(3.0)$ \\
\hline Mean difference MP-PCV and MP-VCV & $7.3(4.6)$ & $1.5(1.4)$ \\
\hline Percentage MP-VCV lower than MP- & $21 \%$ & $22 \%$ \\
\hline PCV & $7.20(0.15)$ & $7.30(0.10)$ \\
\hline pH & $9.1(3.2)$ & $6.5(1.6)$ \\
\hline pCO2 (kPa) & $10.8(6.7)$ & $9.8(3.3)$ \\
\hline pO2 (kPa) & $13.4(5.1)$ & $10.5(3.7)$ \\
\hline PEEP (cmH2O) & $\begin{array}{c}\text { Before } \\
\text { ECMO; }\end{array}$ & $\begin{array}{c}\text { After } \\
\text { ECMO; }\end{array}$ \\
\hline & mean (sd) & mean (sd) \\
\hline & $33.8(5.6)$ & $23.2(5.7)$ \\
\hline Ppeak (cmH2O) & $29.6(5.1)$ & $15.0(7.3)$ \\
\hline RR (/min) & $0.395(0.103)$ & $0.224(0.092)$ \\
\hline Vt (L) & $82.9(17.3)$ & $49.6(13.2)$ \\
\hline fiO2 (\%) & & \\
\hline & &
\end{tabular}

Conclusion: VV-ECMO lowers the mechanical power while maintaining adequate oxygenation and ventilation reducing the MP with $80 \%$. The MP calculation with the wrong formula underestimated the MP by more than $20 \%$ in this study. This study underlines that it is mandatory to use the correct methods to calculate the MP.

Reference(s) and grant ackowledgment(s)

1. 3. Van der Meijden S, Molenaar M, Somhorst P, Schoe A. Calculating mechanical power for pressure-controlled ventilation. Intensive Care Med. 2019;45(10):1495-1497. https://doi.org/10.1007/s00134-019-05698-8

2. 2. Schmidt M, Pham T, Arcadipane A, et al. Mechanical Ventilation Management during Extracorporeal Membrane Oxygenation for Acute Respiratory Distress Syndrome. An International Multicenter Prospective Cohort. Am J Respir Crit Care Med. 2019;200(8):1002-1012. https://doi.org/10.1164/ rccm.201806-10940C

3. 1. Gattinoni L, Tonetti T, Cressoni M, et al. Ventilator-related causes of lung injury: the mechanical power. Intensive Care Med. 2016;42(10):1567-1575. https://doi.org/10.1007/s00134-016-4505-2 


\section{8}

Tracheal and Enteral Aspirate pH Study: The begining of the end to $\mathrm{pH}$ testing for confirmation of enteral tube placement? JP. Mcnally-Reilly'; S. Giannaraki ${ }^{1}$; J. Wilcox ${ }^{1}$; NA. Barrett ${ }^{1}$; L. Wandrag ${ }^{1}$ ${ }^{1}$ Adult critical care, Guy's and St Thomas' NHS Foundation Trust, London, United Kingdom

Correspondence: J.P. McNally-Reilly

Intensive Care Medicine Experimental 2020, 8(2): 000788

Introduction: Delivering enteral feed or medications through an enteral feeding tube placed into the lung causes major morbidity. (1) To prevent this from occurring an accurate means of assessing enteral tube placement is essential. In the UK, the National guidelines for assessment the of enteral feeding tube position includes the measurement of gastric aspirate $\mathrm{pH}(<5.5)$ and assessment of tube placement using $x$-ray if indeterminate. (1) However there is little evidence to support this as a safe approach in critically ill patients. $(2,3)$

Objectives: - Establish the prevalence of acidic $\mathrm{pH}$ aspirates (defined as a $\mathrm{pH}<5.5$ ) within the critical care ventilated patient cohort. • Provide clarity on the use of $\mathrm{pH}$ strip testing as a method confirming nasogastric (NG) tube position in the critically ill patient cohort.

Methods: Simultaneous pH measurements were taken for aspirates obtained from the NG tube, subglottic port and tracheal samples in 106 ventilated patients from June - November 2019 at a universityaffiliated, tertiary hospital. The $\mathrm{pH}$ from the three sites was obtained and recorded once per nursing shift and electronically entered. Any sample taken as a non-directed bronchial lavage (NBL) also had the $\mathrm{pH}$ recorded. Baseline demographic data, relative time of NBL to sampling, and presence of medications used to suppress gastric acid production were recorded. Patient's notes were reviewed by the authors for history or suspicion of aspiration, and documented episodes of emesis. Institutional approval was given for this work. Statistical plan: The distribution of $\mathrm{pH}$ from each site was described using descriptive statistics. Specific note was made of any instances of $\mathrm{pH}<5.5$. Spearman's correlation was performed between subglottic and tracheal aspirate data.

Results: Data were included for 106 patients. Demographics include: mean age of 58.9 years, $78.3 \%$ referred from a medical speciality, average APACHE II score 14.6, average SOFA score 7.2, and 28.9\% mortality rate overall. Analysis of all aspirates collected demonstrated a significant proportion within both subglottic and sputum aspirates of a $\mathrm{pH}<5.5,5.3 \%$ and $6.3 \%$ respectively, see Table 1 . Fifteen patients accounted for the 27 acidic subglottic aspirates and four patients for the 7 sputum aspirates. Of note, there was a large proportion of NG aspirates with a $\mathrm{pH}>5.5,70.8 \%$.

Table 1. Summary of data points collected from subglottic, nasogastric and sputum aspirates

\begin{tabular}{|l|l|l|l|}
\hline \multicolumn{1}{|c|}{} & Subgottic & Nasogasrtic & Sputum \\
\hline Count & 513 & 510 & 111 \\
\hline Mean $\mathrm{pH}$ & 6.5 & 5.3 & 6.9 \\
\hline Standard Deviation & 1.4 & 1.8 & 1.6 \\
\hline Median $\mathrm{pH}$ & 6.5 & 6.0 & 7.0 \\
\hline Lower Range $\mathrm{pH}$ & 6.0 & 5.0 & 6.0 \\
\hline Upper Range $\mathrm{pH}$ & 7.0 & 6.5 & 8.0 \\
\hline No. $\mathrm{pH}<5.5$ & 27 & 149 & 7 \\
\hline Percentage $\mathrm{pH}<5.5$ & 5.3 & 29.2 & 6.3 \\
\hline
\end{tabular}

Analysis of simultaneous sputum and subglottic demonstrated a positive correlation, $r=0.52$ (Confidence Interval $0.31-0.69$ ), $p<0.0001$.

Conclusion: From this study we have demonstrated a large number of acidic samples outside of the gastrointestinal tract, $5.3 \%$ subglottic and $6.3 \%$ sputum, with a simultaneously high proportion of aspects from the gastrointestinal tract not being acidic (70.8\%). Given that the $\mathrm{pH}$ in tracheal and subglottic examples was strongly correlated, it is possible that the acidic $\mathrm{pH}$ in the airway was due to micro-aspiration past the cuff of the endotracheal tube. $(4,5)$ This raises the possibility that any patient with significant glottic dysfunction and either macroor micro-aspiration may have a an acidic tracheal $\mathrm{pH}$ and raises doubts about the validity of $\mathrm{pH}$ testing as a means of confirmation of correct placement of an enteral feeding tube in this population. The elevated $\mathrm{pH}$ in the gastric samples is to be likely due to the use of protein pump inhibitors as a medication for stress ulcer prophylaxis in the critically ill patient cohort.

The data presented here suggests that $\mathrm{pH}$ testing as a means of confirmation of enteral tube placement will not prevent feed installation into the lungs if the tube is misplaced and the patient has poor glottic function and a risk of aspiration.

\section{Reference(s) and grant ackowledgment(s)}

1. 1. NICE. (2006) Nutrition support for adults: oral nutrition support, enteral tube feeding and parenteral nutrition. Available from: https://nice.org.uk/ guidance/cg32 [Accessed 16th Mar 2020].

2. 3. NHS Improvement. (2016) Nasogastric tube misplacement: continuing risk of death and severe harm. Available from: https://improvement.nhs.uk/ news-alerts/nasogastric-tube-misplacement-continuing-risk-of-death-sever e-harm/ [Accessed 16th Mar 2020]

3. 4. Clayton J, Jack CIA, Ryall C, Tran J, Hilal E \& Gosney M (2006) Tracheal pH monitoring and aspiration in acute stroke. Age and Ageing, 35(1):47-53

4. 5. Hamilton VA, Grap MJ (2012) The role of the endotracheal tube cuff in micro-aspiration. Heart Lung 41(2):167-172.

5. This study was approved by Critical Care Clinical Governance of Guy's and St.Thomas' NHS Foundation Trust. No other external funding was provided.

6. 2. NHS Improvement. (2018) Never Events reported as occurring between 1 April 2016 and 31 March 2017 - final update. Available from: https://impro vement.nhs.uk/documents/2347/Never_Events_1_April_2016_-_31_March 2017_FINAL_v2.pdf. [Accessed 16th Mar 2020].

\section{3}

Ventilatory Ratio: a useful prognostic tool for ARDS due

\section{to SARS-CoV-2}

M. Monti ${ }^{1}$; P. Pugni'; M. Di Matteo ${ }^{2}$; L. Gandini ${ }^{1}$; FM. Russo ${ }^{1}$; L. Manesso ${ }^{1}$;

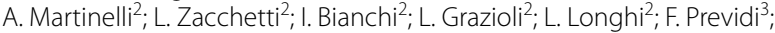

FL. Lorini ${ }^{2}$

${ }^{1}$ Anestesia, rianimazione e terapia intensiva e del dolore, University of Milan, Milano, Italy; ${ }^{2}$ Dipartimento di emergenza urgenza e area critica, ASST Papa Giovanni XXIII, Bergamo, Italy; ${ }^{3}$ Ingegneria, Università Degli Studi Di Bergamo Ingegneria Ed A, Dalmine, Italy

Correspondence: M. Monti

Intensive Care Medicine Experimental 2020, 8(2): 000813

Introduction: Increased dead space, as a reflection of global lung injury, is an independent predictor of mortality in both the early and intermediate stages of Adult Respiratory Distress Syndrome (ARDS) (1). Ventilatory ratio (VR) is a bedside index of impaired ventilatory efficiency with significant association with dead space and outcome in ARDS (2). Severe Adult Respiratory Syndrome Coronavirus-2 (SARS-CoV-2) disease partly differs from traditional ARDS; high dead space fraction may be a specific pathophysiological characteristic (3).

Objectives: To describe VR, its trend and association with mortality in a cohort of SARS-CoV-2 patients.

Methods: A cohort of consecutive patients affected by SARS CoV-2 admitted to Papa Giovanni XXIII Hospital ICU (Bergamo) from February 22th to March 22th and requiring invasive mechanical ventilation was retrospectively analysed. VR, defined as [minute ventilation $(\mathrm{ml} /$ $\mathrm{min}) * \mathrm{PaCO} 2(\mathrm{mmHg})] /[$ predicted body weight $* 100(\mathrm{ml} / \mathrm{min}) * 37.5$ $(\mathrm{mmHg})]$, was calculated within 48 hours since intubation. Patients were divided into two groups, low VR $(<2)$ and high VR $(\geq 2)(2)$ Demographics, ventilatory parameters (Mann Whitney test) and 28-day mortality (Fisher's exact test) were compared between the two groups. 


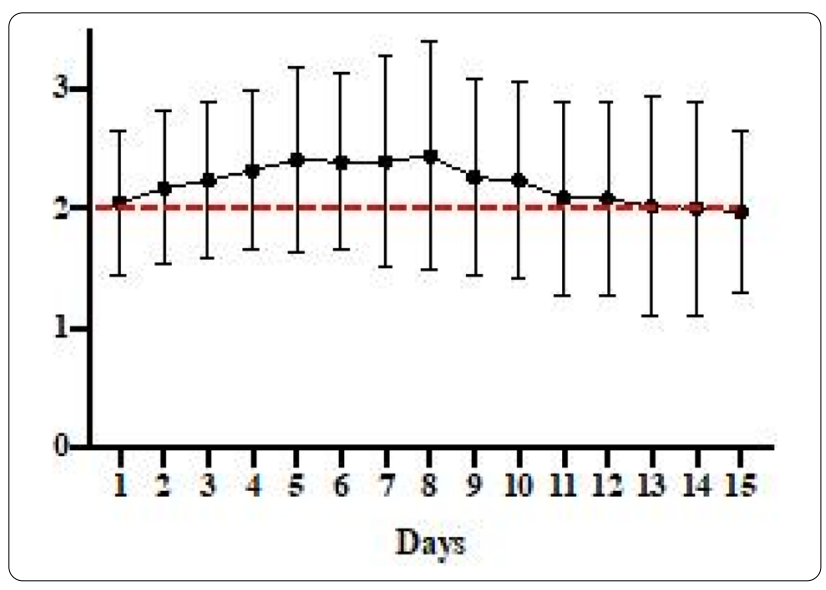

Results: 86 of the 115 patients enrolled had a baseline VR, with a mean value of $2.05( \pm 0.59)$. VR remained high over the first 15 day of ICU admission (mean value always $\geq 2$, Figure 1). Low VR group included 45 patients with mean VR $1.58( \pm 0.34)$; high VR group included 41 patients with mean VR $2.53( \pm 0.40)$. The groups were similar for age, SOFA-score, baselines ventilatory setting and gas exchange (Table 1). High VR was associated with significantly increased 28-day mortality (odds ratio $2.83,95 \% \mathrm{Cl} 1.08-7.39, \mathrm{p}=0.03$ ). Comparing survivors and non-survivors median VR was respectively $1.87(1.55-2.35)$ versus 2.22 $(1.72-2.71)(p<0.05)$.

\begin{tabular}{|l|l|l|l|}
\multicolumn{5}{c|}{ Table 1. Baseline characteristics in the two groups. } \\
\hline & VR<2 $\left(\mathrm{n}^{\circ} 45\right)$ & VR $\geq 2\left(\mathrm{n}^{\circ} 41\right)$ & $\mathrm{p}$-value \\
\hline Age & $57(51-66)$ & $64(55-69)$ & 0.07 \\
\hline SOFA score & $6(4-8)$ & $7(4-9)$ & 0.1 \\
\hline Driving Pressure & $11(10-13)$ & $11(10-14)$ & 0.5 \\
\hline PEEP & $15(13-16)$ & $15(14-17)$ & 0.2 \\
\hline Compliance & $41(35-46)$ & $41(32-46)$ & 0.4 \\
\hline PaO2/FiO2 & $117(98-151)$ & $98(76-135)$ & 0.09 \\
\hline
\end{tabular}

Conclusion: Patients with baseline VR $\geq 2$ had significantly higher 28-day mortality in ARDS due to SARS-CoV-2; VR could be a useful prognostic tool.

\section{Reference(s) and grant ackowledgment(s)}

1. 1. Kallet R. Measuring dead-space in acute lung injury. Minerva Anestesiol. 2012; 78(11): p. 1297-305.

2. 2. Sinha P, Calfee C, Beitler J, et al. Physiologic Analysis and Clinical Performance of the Ventilatory Ratio in Acute Respiratory Distress. Am J Respir Crit Care Med. 2019; 199(3): p. 333-341.

3. 3. Mauri T, Bellani G, Confalonieri A, et al. Topographic distribution of tidal ventilation in acute respiratory distress syndrome: effects of positive endexpiratory pressure and pressure support. Crit Care Med. 2013; 41(7): p. 1664-73.

\section{4}

SARS-CoV-2 is not a uniquely diabetogenic virus: insulin requirements in COVID-19 are similar to other viral pneumonias and resolve in convalescence

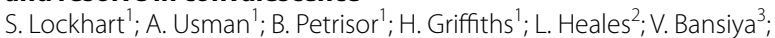

R. Mahroof'; A. Conway-Morris ${ }^{4}$

${ }^{1}$ John farman intensive care unit, Cambridge University Hospitals NHS Foundation Trust, Cambridge, United Kingdom; ${ }^{2}$ Department of anaesthetics \& intensive care medicine, Cambridge University Hospitals NHS Foundation Trust, Cambridge, United Kingdom; ${ }^{3}$ Department of diabetes \& endocrinology, Cambridge University Hospitals NHS Foundation Trust, Cambridge, United Kingdom; ${ }^{4}$ University division of anaesthesia, department of medicine, University of Cambridge, Cambridge, United Kingdom

Correspondence: $\mathrm{S}$. Lockhart

Intensive Care Medicine Experimental 2020, 8(2): 000814
Introduction: Anecdotal evidence suggests COVID-19 may be associated with severe insulin resistance in critical care. Indeed, a group of leading diabetologists have recently established a COVID-19-Diabetes registry to catalogue cases of new onset diabetes and severe insulin resistance associated with COVID-19, positing that COVID-19 may be a uniquely diabetogenic virus. However, to date there has been no longitudinal evaluation of insulin requirements in critically ill patients with COVID-19 and there is no evidence to suggest these observations represent anything other than stress hyperglycaemia.

Methods: To address this, we conducted a retrospective observational study of contemporaneous electronic medical records of insulin requirements and glycaemia in patients admitted to our intensive care unit (ICU) with COVID-19 in March and April 2020.

Results: 54 patients with COVID-19 were admitted to our unit for $>24$ hours during the study period and included in the analysis. We reasoned that the high insulin requirements observed in COVID-19 may represent the high prevalence of severe illness. To test this, we analysed insulin requirements according to interventions employed to treat respiratory failure, namely mechanical ventilation, neuromuscular blockade, nebulised epoprostenol and proning. Increased Insulin requirements (units/day) and worsening glycaemia were clearly associated with severity of illness as indicated by intensity of salvage therapies required for respiratory failure ( $P<0.01$ for all, ordinal regression). In a multivariate model adjusted for age and sex, being proned during ICU admission and premorbid Type 1 or Type 2 diabetes, but not steroid exposure or obesity, were independently associated with insulin requirements. To determine if COVID-19 had unique effects on glucose metabolism we compared insulin requirements and glycaemia in COVID-19 to a cohort of non-viral pneumonitis patients admitted to our ICU between 2018 and 2020, matched for age, sex, BMI and diabetesstatus. Insulin requirements (Mean Insulin Dose (Units/day), COVID-19: $19.8 \pm 8.3$, Non-COVID-19:24.8 $\pm 11.2, \mathrm{P}=\mathrm{NS}, \mathrm{N}=17$ per group) and glycaemic control (Time in range $4-10 \mathrm{mmol} / \mathrm{L}$ (\%), COVID19: $81.2 \pm 26.2$, Non-COVID-19: $82.1 \pm 20.9, \mathrm{P}=\mathrm{NS}, \mathrm{N}=17$ per group) were comparable between groups (Figure 1). Finally, we examined the short term metabolic sequalae of COVID-19 by looking at antihyperglycaemic use in 31 patients discharged from our hospital after their ICU admission for COVID-19. All patients who were commenced on insulin in ICU had insulin successfully withdrawn and there was no new initiation of oral anti-hyperglycaemic agents in this cohort.

Conclusion: Insulin requirements in severe COVID-19 are positively associated with severity of respiratory failure and pre-existing diabetes. They are comparable to those seen in non-COVID-19 viral pneumonitis and resolve in convalescence. We did not find evidence to support the emerging hypothesis that SARS-CoV-2 is a uniquely diabetogenic virus.

P N
Figure 1. Maximum insulin requirements in a single day (A), average insulin requirements across the whole ICU
stay (B) and mean blood glucose (C) are displayed for patients with non-COVID-19 viral pneumonitis and a
cohort with COVID-19, matched for age, sex and diabetes status.

Reference(s) and grant ackowledgment(s)

1. SL is supported by an Academic Clinical Fellowship from the National Institute of Health Research.

2. ACM is supported by a Wellcome Trust grant (WT 2055214/Z/16/Z) 
000860

A reduction in skin blood flow during vigorous diuretic therapy is associated to the development of acute kidney injury in patients with acute respiratory distress syndrome

W. Mongkolpun ${ }^{1}$; B. Péter ${ }^{1}$; QC. Armin ${ }^{1}$; V. Jean-Louis ${ }^{1}$; J. Creteur ${ }^{1}$

${ }^{1}$ Department of intensive care, Erasme Hospital, Brussels, Belgium

Correspondence: $\mathrm{W}$. Mongkolpun

Intensive Care Medicine Experimental 2020, 8(2): 000860

Introduction: Diuretic therapy to obtain a negative fluid balance is often used in patients with acute respiratory distress syndrome (ARDS) but its aggressive application can result in decreased renal perfusion and kidney injury (AKI). Since skin blood flow (SBF) is highly sensitive to a reduction in blood flow, we hypothesized that a reduction in SBF during tan attempt to decrease fluid balance could be associated with the development of AKI.

Methods: We included hemodynamically stable (mean arterial pressure (MAP) $\geq 65 \mathrm{mmHg}$ without an increase in norepinephrine (NE) dose in the least 6 hours at least) ARDS patients (Berlin criteria) in which intravenous furosemide administration was used in order to reach a negative fluid balance. Patients with AKI at baseline were excluded. SBF (Periflux 5000, Perimed; index finger; Perfusion unit: PU) together with hemodynamic variables, the ratio of arterial oxygen partial pressure to fractional inspired oxygen $\left(\mathrm{PaO}_{2} / \mathrm{FiO}_{2}\right)$, central venous oxygen saturation ( $\mathrm{ScvO} 2)$ and blood lactate concentrations were obtained at baseline (T0) and after 24 hours (T24). Relative changes in SBF during first 24 hours ( $\triangle \mathrm{SBF} 24)$ was recorded. Patients were separated by the occurrence of acute kidney injury (AKI) (AKIN criteria) within 7 days after T0. Receiver operating characteristic (ROC) curves were constructed and reported as areas under the curve (AUC) with $95 \%$ confidence intervals to predict AKI.

Results: Of the 36 ARDS patients (pneumonia (26), abdominal infection (8), pancreatitis (2)) we studied, 14 developed AKI (12 stage 3 and 2 stage 1). A strategy to achieve a negative fluid balance was initiated 73 (50-96) hours after ARDS diagnosis. At baseline, there were no significant difference between patients with and without AKI in SOFA score (8 (6-11) vs 11 (7-12), $p=0.2), \mathrm{PaO}_{2} / \mathrm{FiO}_{2}(\mathrm{p}=0.5), \mathrm{MAP}(\mathrm{p}=0.9)$, cardiac index $(p=0.4)$, lactate concentration $(p=0.1), \operatorname{ScvO} 2(p=0.3)$ and SBF $(p=0.1)$ (Table 1). At T24, lactate concentration increased and SBF decreased in both groups. However, $\triangle \mathrm{SBF} 24 \%$ was greater in patients who developed AKI than in those who did not (Table 1). $\triangle$ SBF24\% had the highest AUROC $(0.90 \pm 0.06[0.76-0.1])(p<0.01)$ to predict the occurrence of AKI, with a cut off value of $45 \%$ (sensitivity $74 \%$, specificity $89 \%$ ) (Figure 1)

\begin{tabular}{|c|c|c|c|c|c|c|c|c|c|}
\hline & \multicolumn{3}{|c|}{ All patients $(\mathrm{N}=36)$} & \multicolumn{3}{|c|}{ No AKI $(\mathrm{N}=22)$} & \multicolumn{3}{|c|}{ AKI $(\mathrm{N}=14)$} \\
\hline & Before & After & $p$ & Before & After & $\mathrm{p}$ & Before & After & $\mathrm{p}$ \\
\hline MAP (mmHg) & \begin{tabular}{|l|}
71 \\
$(69-$ \\
$72)$ \\
\end{tabular} & \begin{tabular}{|l|}
71 \\
$(69-$ \\
$73)$ \\
\end{tabular} & 1.0 & $\begin{array}{l}71(69- \\
72)\end{array}$ & $\begin{array}{l}71 \text { (69- } \\
73)\end{array}$ & 0.6 & $\begin{array}{l}72(69- \\
72)\end{array}$ & \begin{tabular}{|l|}
72 \\
$(69-$ \\
$75)$ \\
\end{tabular} & 0.8 \\
\hline $\begin{array}{l}\text { Cardiac index } \\
\left(\mathrm{L} / \mathrm{min} / \mathrm{m}^{2}\right)\end{array}$ & \begin{tabular}{|l|}
2.9 \\
$(2.5-$ \\
$3.3)$ \\
\end{tabular} & \begin{tabular}{|l}
3.0 \\
$(2.7-$ \\
$3.4)$ \\
\end{tabular} & 0.1 & $\begin{array}{l}2.8(2.5- \\
3.3)\end{array}$ & $\begin{array}{l}2.9 \\
(2.7- \\
3.4) \\
\end{array}$ & 0.2 & \begin{tabular}{|l|}
$3.0(2.5-$ \\
$3.3)$
\end{tabular} & \begin{tabular}{|l|}
3.0 \\
$(2.7-$ \\
$3.5)$ \\
\end{tabular} & 0.9 \\
\hline $\begin{array}{l}\text { Norepinephrine } \\
\text { dose } \\
(\mathrm{mcg} / \mathrm{kg} / \mathrm{min})\end{array}$ & \begin{tabular}{|l|}
0.2 \\
$(0.1-$ \\
$0.2)$ \\
\end{tabular} & $\begin{array}{l}0.1 \\
(0.05- \\
0.3) \\
\end{array}$ & 0.8 & \begin{tabular}{|l|}
0.1 \\
$(0.02-$ \\
$0.2)$ \\
\end{tabular} & $\begin{array}{l}0.1 \\
(0.02- \\
0.2) \\
\end{array}$ & 1.0 & \begin{tabular}{|l|}
$0.2(0.1-$ \\
$0.3)$
\end{tabular} & \begin{tabular}{|l|}
0.2 \\
$(0.1-$ \\
$0.4)$ \\
\end{tabular} & 0.3 \\
\hline $\begin{array}{l}\mathrm{PaO2} / \mathrm{FiO} 2 \\
(\mathrm{mmHg})\end{array}$ & \begin{tabular}{|l|}
163 \\
$(122-$ \\
$209)$
\end{tabular} & $\begin{array}{l}166 \\
(134- \\
224)\end{array}$ & 0.1 & $\begin{array}{l}151 \\
(126- \\
197)\end{array}$ & $\begin{array}{l}166 \\
(133- \\
204)\end{array}$ & 0.02 & \begin{tabular}{|l}
$180(123-$ \\
$220)$
\end{tabular} & $\begin{array}{l}166 \\
(137- \\
227)\end{array}$ & 0.2 \\
\hline $\begin{array}{l}\text { Lactate levels } \\
(\mathrm{mmol} / \mathrm{L})\end{array}$ & \begin{tabular}{|l|}
1.2 \\
$(0.8-$ \\
$1.5)$ \\
\end{tabular} & $\begin{array}{l}1.5 \\
(1.0- \\
1.8) \\
\end{array}$ & $<0.01$ & $\begin{array}{l}1.1(0.8- \\
1.2)\end{array}$ & $\begin{array}{l}1.3 \\
(1.0- \\
1.4)\end{array}$ & $<0.01$ & \begin{tabular}{|l|}
$1.4(0.9-$ \\
$1.8)$
\end{tabular} & \begin{tabular}{|l|}
1.7 \\
$(1.5-$ \\
$2.1)$ \\
\end{tabular} & 0.04 \\
\hline $\mathrm{ScvO2}(\%)$ & \begin{tabular}{|l|}
71 \\
$(70-$ \\
$74)$ \\
\end{tabular} & $\begin{array}{l}72 \\
(71- \\
73)\end{array}$ & 0.1 & $\begin{array}{l}70(70- \\
72)\end{array}$ & $\begin{array}{l}72(71- \\
73)\end{array}$ & 0.04 & $\begin{array}{l}73(70- \\
75)\end{array}$ & \begin{tabular}{l|}
72 \\
$(71-$ \\
$73)$
\end{tabular} & 0.7 \\
\hline SBF (PU) & \begin{tabular}{|l|}
120 \\
$(62-$ \\
$167)$ \\
\end{tabular} & $\begin{array}{l}64 \\
(20- \\
116) \\
\end{array}$ & \begin{tabular}{|l|}
0.01 \\
\end{tabular} & $\begin{array}{l}153(87- \\
210)\end{array}$ & $\begin{array}{l}105 \\
(56- \\
187)\end{array}$ & $<0.01$ & \begin{tabular}{|l|}
$91(51-$ \\
$134)$
\end{tabular} & $\begin{array}{l}45 \\
(14- \\
79) \\
\end{array}$ & $<0.01$ \\
\hline$\Delta \mathrm{SBF}_{24}(\%)$ & $36(19$ & & & $21(11-36$ & & & $51(34-64)$ & & $<0.01$ \\
\hline
\end{tabular}

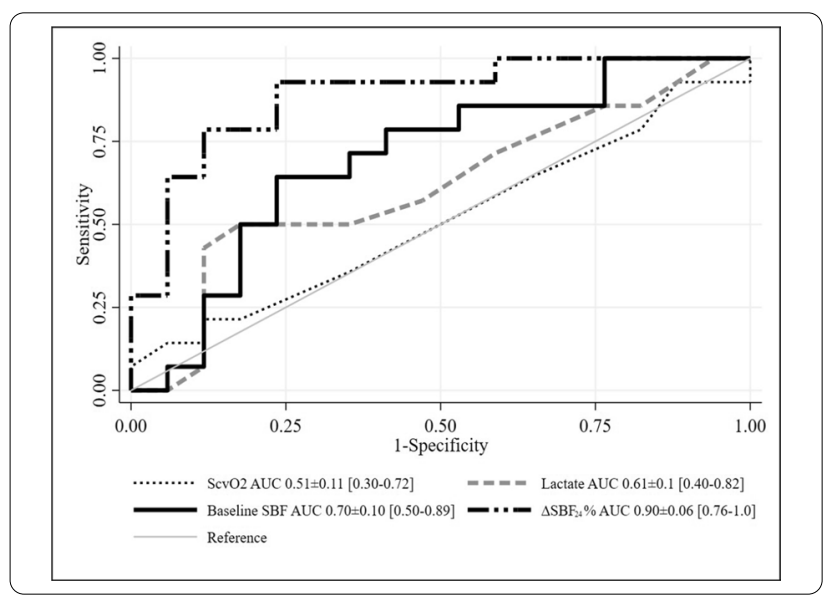

Conclusion: Monitoring of SBF can be valuable to decrease the risk of AKI during a negative fluid balance strategy for ARDS.

\section{3}

Compliance of critical care randomised controlled trials with the CONSORT statement: A Systematic Review

A. Fowler ${ }^{1}$;. Joseph ${ }^{2}$; D. Jafree ${ }^{3}$; R. Coe ${ }^{4}$; J. Garner ${ }^{5}$; K. Whitehurst ${ }^{6}$.

H. Sagoo ${ }^{7}$; R. Agha ${ }^{\top}$

${ }^{1}$ Department of surgery, Barts Health NHS Trust, London, United Kingdom:

2Department of medicine, North Middlesex University Hospital, London, France; ${ }^{3}$ Department of medical sciences, University College London, London, United Kingdom; ${ }^{4}$ Department of anaesthesia, Norfolk and Norwich University Hospital, Norwich, United Kingdom; ${ }^{5}$ Department of medicine, Frimley Park Hospital, Frimley, United Kingdom; ${ }^{6}$ Department of clinical pathology, Nottingham City Hospital, Nottingham, United Kingdom; ${ }^{7}$ Department of medicine, Luton and Dunstable University Hospital, Luton, United Kingdom

Correspondence: $T$. Joseph

Intensive Care Medicine Experimental 2020, 8(2): 000883

Introduction: Randomised controlled trials represent the cornerstone of modern evidence-based medicine [1]. There is increasing interest in the reporting of randomized controlled trials to ensure that research is transparently described [2]. Currently, the quality of reporting within the critical care literature is unclear.

Methods: A systematic search was undertaken to identify all randomized controlled trials published within the four highest impact factor critical care journals between 1st January 2013 and 31st December 2016. Randomised controlled trials involving children were excluded. Two researchers independently selected articles and marked them against the Jadad scale and the CONSORT reporting guideline to determine if any domains were lacking.

Results: 306 trials met the inclusion criteria over the four-year study period. Just under half were blinded randomized controlled trials (139 of 298; $46.6 \%)$, and the median number of participants was 95 (IQR: 175.25); nine trials enrolled in excess of 1000 participants. Median compliance with CONSORT was 75\% (IQR: 15.7\%). The three best reported items were background and rationale (298/298; 100\%), Interpretation consistent with results (99.3\%) and statistical methods comparing groups for primary/secondary outcomes (99.4\%). The three worst reported items were any changes to commencement with reasons (4.3\%), important changes to methods after trial recruitment, with reasons (11.2\%) and where the full trial protocol is if available $(17.6 \%)$. There was an improvement in reporting over time, blinded trials had better reporting than unblinded trials, and trials with a trial registration had higher compliance. 
Conclusion: Randomised controlled trials in critical care journals are typically well reported but some clear weaknesses should be addressed. These include location of a full protocol and a detailed description of any changes to the study after commencement.

\section{Reference(s) and grant ackowledgment(s)}

1. [1] Akobeng AK. Understanding randomised controlled trials. Arch Dis Child 2005: 90: 840-4

2. [2] Wallach JD, Boyack KW, loannidis JPA. Reproducible research practices, transparency, and open access data in the biomedical literature, 2015-2017. PLoS Biol PublicLibrary

\section{4}

Clinical characteristics and outcomes of COVID19 patients admitted in Catalan ICUs (UCIsCAT study group)

J. Mancebo'; R. Ferrer Roca; A. Mas ${ }^{3}$; JR. Masclans'; P.Vera'; R. Mañez ; J. Trenado ${ }^{6}$; R. Fernandez; JM. Sirvent Calvera ${ }^{8}$; M. Martínez $;$ M. Ibarz ${ }^{10}$; E. Sandoval ${ }^{11}$; P. Garro ${ }^{12}$; J. Lopera ${ }^{13}$; M. Bodi' ${ }^{14} ;$ JC. Yebenes-Reyes ${ }^{15}$; C. Triginer ${ }^{16}$; I. Vallverdú17 ${ }^{17}$; A. Baró Serra ${ }^{18} ;$ F. Bodi $^{19}$

${ }^{1}$ Intensive care dpt, Hospital de la Santa Creu i Sant Pau, Barcelona, Spain ${ }^{2}$ Intensive care dpt / sodir research group, Vall d'Hebron University Hospital, Barcelona, Spain; ${ }^{3}$ ntensive care dpt, Hospital de Sant Joan Despi Moisès Broggi, Sant Joan Despí, Spain; Intensive care dpt, Hospital del Mar, Barcelona, Spain: ${ }^{5}$ ntensive care dpt, Hospital Universitari de Bell-

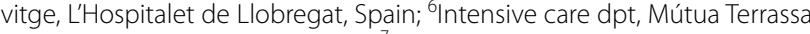
University Hospital, Terrassa, Spain; Intensive care dpt, Althaia, Xarxa Assistencial Universitària de Manresa, Manresa, Spain; ${ }^{8}$ Intensive care dpt, Hospital Universitari de Girona Dr Josep Trueta, Girona, Spain; ${ }^{9}$ Intensive care dpt, Hospital General De Cataluña, Sant Cugat del Vallès, Spain; ${ }^{10}$ Intensive care dpt, Hospital Universitari Sagrat Cor - Grup Quirónsalut, Barcelona, Spain; ${ }^{11}$ Cardiovascular surgery, Hospital Clínic de Barcelona, Barcelona, Spain; ${ }^{12}$ Intensive care dpt, Hospital General de Granollers, Granollers, Spain; ${ }^{13}$ Intensive care dpt, Hospital Universitari de Vic (Consorci Hospitalari de Vic), Vic, Spain; ${ }^{14}$ Intensive care dpt, Hospital Universitari de Tarragona Joan XXIII, Tarragona, Spain; ${ }^{15}$ Intensive care dpt, Hospital de Mataró, Mataró, Spain; ${ }^{16}$ Intensive care dpt, Igualada Hospital, Igualada, Spain; ${ }^{17}$ Intensive care dpt, Hospital Universitari Sant Joan de Reus, Reus, Spain; ${ }^{18}$ Intensive care dpt, Hospital de Santa Caterina, Salt, Spain; ${ }^{19}$ Intensive care dpt, Hospital de Sant Pau i Santa Tecla, Tarragona, Spain

Correspondence: J. Mancebo

Intensive Care Medicine Experimental 2020, 8(2): 000884

Introduction: COVID19 pandemic and the consequent surge of patients with acute hypoxemic respiratory failure has been challenging for intensive care departments around the globe.

Objectives: To describe the main clinical charateristics and ICU outcomes in a cohort of adult patients with severe acute hypoxemic respiratory failure due to COVID19 admitted between 15 March and 15 April in the ICU Catalan network.

Methods: Consecutive patients admitted between 15 March and 15 April in $26 \mathrm{ICUs}$, who tested positive for SARS-Cov-2 (RT-PCR in a nasopharingeal swab) and had severe acute hypoxemic respiratory failure (need of at least $10 \mathrm{~L} / \mathrm{min}$ oxygen flow to keep an oxygen arterial saturation -pulsioximeter- above $90 \%$, and presenting with bilateral infiltrates in the chest X-ray). Demographic and relevant clinical and physiological data were colected within the first $24 \mathrm{~h}$ of ICU admission. Follow-up time was til ICU discharge. Study was approved by the Ethics Committee of the the coordinating center (Hospital Universitari de la Santa Creu i Sant Pau).

Results: A total of 1704 patients (1187 men and 517 women) were admitted. Median age was $63 \mathrm{yr}$ (IQR 54-70) and median BMI was 28 $\mathrm{kg} / \mathrm{m}^{2}$ (IQR 26-32). Most frequent comorbidities were cardiovascular conditions $(n=480)$, diabetes $(n=90)$, cancer/immunosuppression ( $=74)$ and COPD $(n=31)$. ICU mortality was 505/1704 (29,6\%). Among patients who required invasive mechanical ventilation $(82 \%$ of the cohort), ICU mortality was 35\% (491/1388). ICU mortality very much differed between patients up to $60 \mathrm{yr}(\mathrm{n}=705)$ and older than $60 \mathrm{yr}$ $(\mathrm{n}=999): 12,8 \%(90 / 705)$ and $41,5 \%(415 / 999)$ respectively, $\mathrm{p}<0.001$. The median length of ICU stay was 13 days (IQR 7-25).

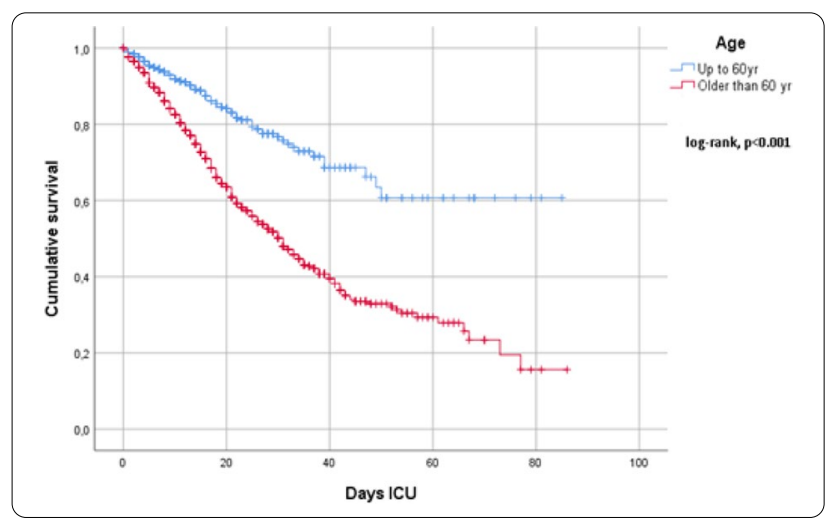

Conclusion: COVID19 severe hypoxemic respiratory failure mainly affects men, the vast majority will require invasive mechanical ventilation for a long period of time and the overall mortality is substantial. People older than $60 \mathrm{yr}$ have a dismal prognosis.

Reference(s) and grant ackowledgment(s)

1. Other contributors: J-A. Santos, J. Baldirà, A-J. Betbesé, M. Izura, I. Morán, J-C. Suárez, L. Zapata, N. Rodríguez, M. Torrens, A. Cordón, C. Gomila, M. Flores, A. Segarra, M. Morales, L. Mateo, M. Martos, P. Saludes, J-R. Cervelló, M. Valencia, F. Roche, G. Masdeu, B. Cancio, S. Hernández-Marín, C. Pedrós, P. Sebastian, X. Saiz, M. Morales, A. Huerta, E. Esteban, S. Benito, C. Barberà, J.

Echevarría, P. Santigosa, D. Tognetti, C. González

000887

Stress level assessment among healthcare workers involved in the management of critically ill COVID-19 patients, A cross sectional study. COVID SCREAM (Stress in Critical caRE health workers Assessment Model)

T. Aisa'; J. Thomas²; D. Diviney² ; H. Afify ${ }^{3}$; M. Abdelbaky ${ }^{3}$; N. Al Qadheeb ${ }^{4}$; M. Yasawy ${ }^{5}$ M. Mahmoud ${ }^{6}$; B. Elgammal $;$; A. Sherif ; A. Ahmad ${ }^{9}$

${ }^{1}$ Anesthesia and intensive care, Our Lady of Lourdes hospital, Drogheda, Ireland; ${ }^{2}$ Anesthesia and intensive care, Our Lady of Lourdes Hospital, Drogheda, Drogheda, Ireland; ${ }^{3}$ Intensive care department, King Abdullah Medical City Specialist Hospital, Makkah, Saudi Arabia; ${ }^{4}$ Intensive care department, King Fahad Specialist Hospital - Dammam., Dammam, Saudi Arabia; ${ }^{5}$ Intensive care department, King Fahad Medical City KFMC, Riyadh, Saudi Arabia; ${ }^{6}$ Anesthesia and intensive care, Manchester Royal Infirmary, Manchester, United Kingdom; ${ }^{7}$ Anesthesia and intensive care, The Mater Misericordiae University Hospital, Dublin, Ireland; ${ }^{8}$ Intensive care department, Hunter new England health district, Tamworth, Australia; ${ }^{9}$ Pharmaceutical sciences, Institute of pharmaceutical sciences, Jinnah Sindh Medical University, Karachi, Pakistan

Correspondence: T. Aisa

Intensive Care Medicine Experimental 2020, 8(2): 000887

Introduction: The declaration of the Novel Coronavirus (COVID-19) as a pandemic lead to the implementation of surge plans and an increase in the number of intensive care units beds across most healthcare facilities. Health care workers (HCWs) in the ICU are frontlines of this crisis as they deal with critically ill patients affected by COVID-19 which can potentially affect their mental wellbeing and causes different levels of stress and psychological disorders.

Objectives: We aimed to determine the prevalence of stress among HCWs involved in the management of critically ill COVID-19 patient identify the risk factors associated with stress, and highlight the availability of psychological support provided to HCWs and its effectiveness. Methods: A cross sectional multicenter, international study using a web-based questionnaire of 27 questions including the Perceived Stress Scale [PSS] for assessment of stress level. Questions to identify the risk factors for stress and the psychological support provided by each health care facility in addition to the socio-demographic characteristics were included. 
Results: We received a total 1501 response from 59 countries, 526 (35\%) were from Europe, 432 (28.7\%) from Asia, 283 (18.85\%) from Africa and 159 (10.59\%) from North America. A totalof 657 (43.95\%) were senior doctors, 455 (30.43\%) were nurses, 278 (18.60\%) were junior doctors and 105 (7.04\%) others (clinical pharmacists, physiotherapists and respiratory therapists). Most responders were male $844(56.22 \%)$. The overall average stress level was 22 points on the PSS denoting moderate stress, while 508 (33.36\%) respondents had a severe level of stress, $788(51.87 \%)$ respondents had moderate level of stress and only $223(14.61 \%)$ had mild stress.

Fear of infection was the major concern for more than $80 \%$ of all the respondents, where 717 (58.5\%) reported that fear of getting infected with COVID-19 increased their stress level while 290 (23.67\%) reported that the fear of transmitting the infection to their families was the main cause of their stress. Other contributing factors to stress were, the increased duty hours, the increased work load and shortage of staff as reported by $89(7.26 \%)$ while the lack of (personal protective equipments) PPEs accounted for stress in $68(5.63 \%)$ respondents. Social media and triaging COVID patients were the least causes of staff stress as quoted by $39(3.1 \%)$ and $38(3.1 \%)$ respectively.

Although 805 (53.67\%) of respondents reported receiving psychological support from their facility, $689(45.93 \%)$ reported not receiving any support. $302(26.24 \%)$ received psychiatric counselling, 613 $(53.26 \%)$ received phone calls and motivational emails/messages, 515 (44.74\%) received phone calls for psychological support from their facility, $428(37.19 \%)$ reported active listening to their concerns, 208 (18.07\%) received psychological support from the community in the form of positive words and applauses. 444 (38.61\%) reported the psychological support was somewhat effective, $371(32.26 \%)$ said that it was very effective while $335(29.19 \%)$ said that it was not effective at all. $829(55.45 \%)$ tried some ways to decrease their stress like religious services, anxyolytics, exercise and watching movies

Conclusion: Stress level was rated to be moderate to severe among intensive care HCWs during this pandemic, many risk factors were identified emphasizing the importance of psychological support during that unprecedented pandemic and adjusting different ways for mitigating such stress.

\section{3}

The Presence of Psychological Safety Within the Intensive Care Environment: A Qualitative Interview and Questionnaire Study K. Grailey ${ }^{1}$; E. Murray ${ }^{2}$; SJ. Brett ${ }^{3}$

${ }^{1}$ Imperial College London, London, United Kingdom; ${ }^{2}$ Said business school, University of Oxford, Oxford, United Kingdom; ${ }^{3}$ Critical care, Imperial College Healthcare NHS Trust, London, United Kingdom

Correspondence: K. Grailey

Intensive Care Medicine Experimental 2020, 8(2): 000893

Introduction: Psychological safety is defined as an environment "safe for interpersonal risk taking" (1). It allows staff to voice concerns without fear of negative repercussions. This can reduce clinical error, lead to workplace innovation and enhance staff wellbeing (2).

The importance of psychological safety has led to the study of its antecedents across many sectors (2-4). This understanding needs to be built upon, focusing on the specific needs of each workplace.

Objectives: Our study had 3 objectives:

1. How psychologically safe is our intensive care (ICU) workforce?

2. How does context influence psychological safety?

3. Are there any negative consequences of psychological safety?

Methods: Participants were recruited from the multidisciplinary teams of 3 ICU's within Imperial College NHS Trust. Semi-structured interviews were conducted, exploring attitudes towards psychological safety. Quantitative data were collected in the form of verbal agreement with 7 statements (1) and the completion of the Safety Attitudes Questionnaire (SAQ) (5). Qualitative audio files were transcribed and analysed within NVIVO software using a thematic analysis approach. Quantitative data were analysed using Microsoft Excel \& Graph Pad Prism.

Results: Interviews, verbal surveys and SAQ's were completed by 30 participants. 28 were in agreement that it was easy to ask for help, with
20 agreeing that it is safe to take a risk on the team. 6 reported feeling that a mistake would be "held against" them [Figure 1]. The SAQ data corroborated this - 28 felt encouraged to report safety concerns. However, 11 were unsure this would be acted upon by management. Our thematic analysis highlighted areas where the context influenced an individual's psychological safety [Figure 2]. These contextual factors included personality, culture \& leadership. Counterintuitively - psychologically safe teams in which individuals are encouraged to participate can lead to negative consequences for the team leader. This can take the form of distraction and fatigue. It was also reported that time taken for increased discussion and raising concerns could reduce the time available for other clinical work.

We demonstrated that psychological safety can be influenced by motivations other than patient safety - such as undermining others or self-promotion. [Figure 3]
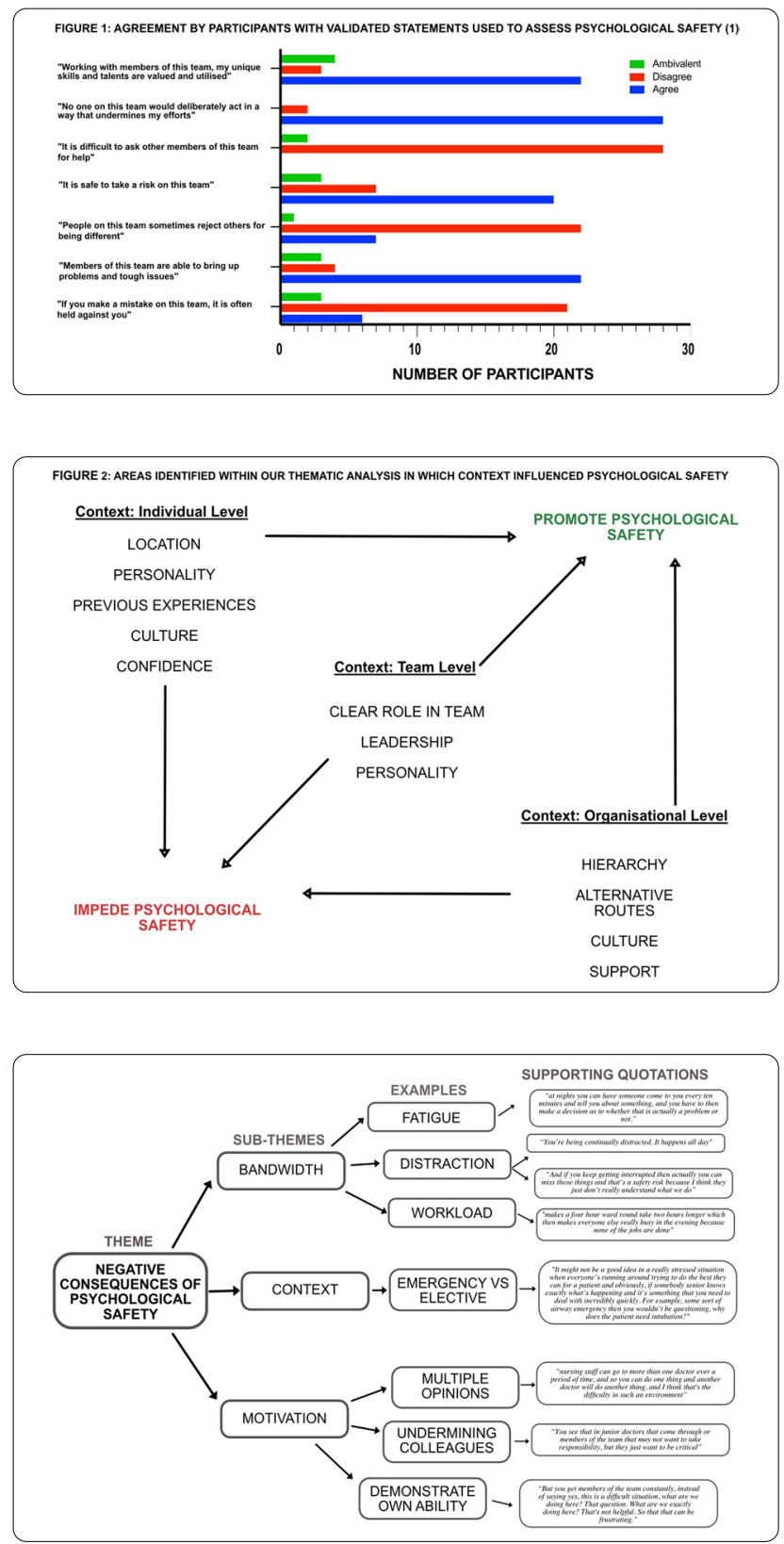
Conclusion: Our study demonstrates reassuring levels of psychological safety within our sample of ICU staff. It also highlighted room for improvement $-1 / 3$ felt at risk of repercussions after speaking up. We have created a model demonstrating how the context of each clinical scenario can influence psychological safety, and how this varies across different levels within the organisation. This allows leaders to identify contexts that can be modified in order to promote psychological safety.

Understanding the negative consequences of psychological safety and when they occur is important - if a clinician's bandwidth is overloaded this can have implications both for employee well-being and patient safety. Team leaders can use this data to help foster a culture of openness whilst minimising the risk of negative implications.

\section{Reference(s) and grant ackowledgment(s)}

1. Supported by an educational grant from Bupa Cromwell Hospital, U.K.

2. 1. Edmondson A. Psychological Safety and Learning Behavior in Work Teams. Administrative Science Quarterly. 1999;44(2):350-83.

3. 2. Edmondson A, Higgins M, Singer S, Weiner J. Understanding Psychological Safety in Health Care and Education Organizations: A Comparative Perspective. Research in Human Development. 2016;13:65-83

4. 3. Pattni N, Arzola C, Malavade A, Varmani S, Krimus L, Friedman Z. Challenging authority and speaking up in the operating room environment: a narrative synthesis. British Journal of Anaesthesia. 2019;122(2):233-44

5. 4. Newman A, Donohue R, Eva N. Psychological safety: A systematic review of the literature. Human Resource Management Review. 2017;27(3):521-35

6. 5. Sexton J, Helmreich R, Neilands T, Rowan K, Vella K, Boyden J, et al. The Safety Attitudes Questionnaire: Psychometric Properties, Benchmarking Data, and Emerging Research. BMC health services research. 2006;6:44.

\section{9}

Retrospective analysis of data collection of ventilator settings and ventilator-derived parameters with comparison of autonomous computer led data recording and nurse led paper flow sheet recording in Intensive Care

G. Murphy'; L. O'Gorman²; C. Power'; A. Fahy ${ }^{3}$

${ }^{1}$ Intensive Care Medicine, Tallaght University Hospital, Dublin, Ireland; ${ }^{2}$ Anaesthesiology, Tallaght University Hospital, Dublin, Ireland; ${ }^{3}$ Intensive care, Tallaght University Hospital, Dublin, Ireland

Correspondence: G. Murphy

Intensive Care Medicine Experimental 2020, 8(2): 000909

Introduction: Increased use of technology in Intensive Care Medicine has become integral to the specialty over the last 50 years (1). This, accompanied by advances in medical practice, has led to larger quantities of data amongst ventilated patients and a need to accurately record ventilator parameters to make clinical decisions (2). It is important to evaluate traditional paper-based data entry with new automated data capture in intensive care units to ensure continued best practice.

This audit aims to assess data collection failures in ventilator setting recordings amongst intubated and ventilated COVID-19 patients and directly contrast failures of hourly recording amongst the ICCA system and data entered in paper ICU flow sheets. We analysed 13,355 data entries over a one-week period in our ICU to compare recording failure of computer-based records and traditional flow sheet entry.

Objectives: The objective of this audit is to directly compare and analyse the performance of paper-based nurse led data entry against computer based patient recording. Primary end points were hourly recordings of the following values: $\mathrm{FiO}_{2}, \mathrm{SpO} 2, \mathrm{EtCO} 2$, Respiratory Rate, Ventilator Mode, Tidal Volumes, Minute Volume, Peak Airway Pressure and Positive End Expiratory Pressure over a one-week period. By analysing the results, we aim to gain insight into the benefits and potential failures of automated computing in data recording as its use is further incorporated into clinical practice.

Methods: Hourly ventilator data record entry was retrospectively analysed for intubated patients from the 13th -19th of April in a Dublin teaching hospital ICU. The IT system and patient flow sheet for each patient were analysed. All data was available for inclusion. Patients who were extubated during the week were included until the hour of extubation. Hours when a result was not available for $\mathrm{FiO}_{2}, \mathrm{SpO}$, EtcO2, Respiratory Rate, Ventilator Mode, Tidal Volumes, Minute Volume, Peak Airway Pressure and PEEP were recorded. These 13,355 data points were separated by patient hours when either the ICCA system or Nurse led patient flow sheet was used with 9270 patient hours in the ICCA arm and 4283 patient hours in the Paper recording arm, further analysis was based upon time of day, (day shift 0800-2000 and night shift 2000-0800). These data were then entered onto a database for analysis.

Results: 13,355 data entries were collated. Within the IT based ICCA system $4 \%$ (368 hours of 9270 hours) of data entries were unrecorded compared to paper flow sheet entries $7.6 \%$, (487 hours of 4283 hours data entries). Of ICCA recordings that were missed $62.5 \%$ were missed during day shifts, (0800-2000) and 37.5\% during night shifts, (20000800). This contrasts with paper flow sheet entry in which $39.5 \%$ of data entries missed during day shifts and $60.5 \%$ during night shifts.

Results were also separated by parameter missed as percentage of total recorded hours. The ICCA system resulted in: End Tidal (2\%), Peak Airway Pressure $(0.728 \%)$, Minute Volume $(0.65 \%)$, Tidal Volume $(0.287 \%), \mathrm{SpO} 2(0.12 \%)$, PEEP $(0.12 \%), \mathrm{FiO}_{2}(0.077 \%)$, Ventilator Mode $(0.066 \%)$ Respiratory Rate $(0 \%)$. Percentages refer to hours missed as a proportion of total hours.

Paper flow sheets recording resulted in: End Tidal $\mathrm{CO} 2(2.1 \%), \mathrm{SpO} 2$ (1.5\%), Ventilator Mode (1.42\%), $\mathrm{FiO}_{2}(1.12 \%)$, PEEP (1\%), Minute Volume $(0.21 \%)$ Peak Airway Pressure (0.163\%), Tidal Volume $(0.14 \%)$, Respiratory Rate $(0.02 \%)$.

Of note a range of data entry failures occurred across multiple parameters simultaneously. This was consistent with patient movement for diagnostic tests and procedures and was not removed from the data set.

EtCO2 performed poorly in both groups. It is hypothesised that air temperature and humidity change within the unit during the COVID19 epidemic contributed in part to these results.

Conclusion: Accurate data collection provides clinicians with the ability to make informed decisions on patient care. Our study concludes that ventilation parameters are recorded more accurately with the use of digital computer systems when total data failures are compared to total patient hours, (4\% and $7.6 \%$ ). The improved performance is even more clear if EtCO2 is removed as it results in $2.1 \%$ of $4 \%$ of the ICCA computing failures. While this confers more accurate data available for clinicians there were parameters where paper flow sheets had improved recording over automated data entry. These include peak airway pressure, tidal volume and minute ventilation while ICCA had improved performance in $\mathrm{FiO}_{2}, \mathrm{SpO} 2$, ventilator setting and PEEP. $\mathrm{EtCO} 2$ had the worst recording rate amongst both modalities. We can conclude that the use of automated computing provides superior overall recording rates, however, nurse led paper flow sheets had improved recording in some parameters and recorded less proportion of data misses during daylight hours.

\section{Reference(s) and grant ackowledgment(s)}

1. 2-Yongsheng Tan, Yibing Wang, \& Hongyan Li. (n.d.). A Management Strategy of Monitor Data in ICU Based on Data Stream Technology. IDEAS Workshop on Medical Information Systems: The Digital Hospital (IDEASDH'04). https://doi.org/10.1109/ideadh.2004.2

2. 1-Varon, J., \& Marik, P. E. (2002). Clinical information systems and the electronic medical record in the intensive care unit. Current Opinion in Critical Care, 8(6), 616-624. https://doi.org/10.1097/00075198-200212000-00022

\section{3}

Does the rate of administration of a fluid challenge have a haemodynamic impact?

V. Bennett ${ }^{1}$;H. Farrah'; A. Messina ${ }^{2}$; HD. Aya ${ }^{1}$; A. Rhodes ${ }^{1}$; N. Fletcher ${ }^{1}$; M. Cecconi ${ }^{2}$

${ }^{1}$ Intensive care medicine, St George's University Hospitals NHS Foundation Trust, London, United Kingdom; ${ }^{2}$ Anaesthesia and intensive care units, Humanitas Research Hospital, Rozzano, Italy

Correspondence: $\vee$. Bennett

Intensive Care Medicine Experimental 2020, 8(2): 000953 
Introduction: Peri-operative fluid management has long been identified as key to reducing post operative morbidity and mortality. Assessment of fluid responsiveness following administration of a fluid challenge can form part of a review of whether that patient requires additional fluid volume. (1) How the optimal fluid challenge should be delivered remains an area of ongoing research. Previous work has shown that $4 \mathrm{ml} / \mathrm{kg}$ is the minimum volume to reliably challenge the circulation but the rate at which the fluid should be given has not been fully investigated. (2)

Objectives: The primary objective was to determine if the rate of administration of a fluid challenge influences the percentage of responders and non-responders to fluid administration.

The secondary objective was to investigate whether the rate of administration influences the pharmacodynamic response to fluids in terms of maximum change and duration of change in haemodynamic variables including Pmsf and $\mathrm{CO}$.

Methods: A prospective study of 40 post-operative patients was conducted in the intensive care units at St George's Hospital, London. They were randomized to receive a post operative $4 \mathrm{ml} / \mathrm{kg}$ fluid challenge over either 5 or 20 minutes. Stability criteria were assessed and then baseline observations including heart rate (HR), blood pressure (BP), mean arterial pressure (MAP), stroke volume (SV), cardiac output (CO), mean systemic filling pressure (Pmsf) and central venous pressure (CVP), where available, were recorded. These were repeated on completion of the fluid challenge and after a 5 and 10 minute interval. Cardiac output measurements were recorded using a calibrated LiDCOplusTM monitor. Patients were considered fluid responsive if SV increased by more than $10 \%$ following a fluid challenge. Adequacy of fluid challenge was assessed as an increase in Pmsf of more than $14 \%$. The proportion of fluid and Pmsf responders were compared using Fisher's exact test. ANCOVA models adjusted by baseline values and fluid responsiveness, were used to assess the impact of rate on haemodynamics. P values less than 0.05 were considered significant.

Results: Of the 40 patients randomized7 were excluded from analyses, as they did not meet stability criteria. In the group that received a fluid challenge over 5 minutes, $40 \%$ were fluid responsive and $11.1 \%$ were fluid responsive in the 20 minute cohort. This difference was not statistically significant, $p=0.1$. In the 5 minute group $73.3 \%$ showed an increased in Pmsf of more than $14 \%$, and $72.2 \%$ in the 20 minute group, $p=0.8$. The rate of fluid administration does not affect the change in Pmsf, but has a significant effect on change in MAP ( $8.3 \mathrm{vs}-0.2 \mathrm{mmHg}, p=0.03$ ), CO $(0.6 \mathrm{vs}-0.3 \mathrm{~L} / \mathrm{min}, p=0.02)$ and SV ( $9.6 \mathrm{vs}-6.4 \mathrm{~mL}, p=0.02)$.

Conclusion: No statistically significant difference between the rate of responders and non responders could be demonstrated with varying the rate of administration of the fluid challenge. However, a potentially clinically significant difference was seen. The changes in Pmsf seen in both groups, suggests no difference in the adequacy of fluid challenge with varying rates. This may indicate a potential effect of the fluid challenge on venous resistance and highlights an area for future work.

\section{Reference(s) and grant ackowledgment(s)}

1. 2. Aya HD, Rhodes A, Ster IC, Fletcher N, Grounds RM, Cecconi M. Hemodynamic Effect of Different Doses of Fluids for a Fluid Challenge. Crit Care Med. 2017;45(2):e161-e168

2. This work received funding from the ESICM Baxter Fluid Management Award. Victoria Bennett was funded by a NIHR Academic Clinical Fellowship.

3. 1. Cecconi M, Hofer C, Teboul JL, Pettila V, Wilkman E, Molnar Z, et al. Fluid challenges in intensive care: the FENICE study: A global inception cohort study. Intensive Care Med. 2015;41(9):1529-37.

\section{9}

The potential magnitude of endogeneity bias in the evidence base of an international sepsis guideline

FS. van der Ven'; ARJ. Girbes²; AME. De Man²; HJ. de Grooth²

${ }^{1}$ Department of intensive care, Amsterdam UMC, locatie AMC, Amsterdam, Netherlands; ${ }^{2}$ Department of intensive care, Amsterdam UMC, locatie VUmc, Amsterdam, Netherlands

Correspondence: F.S. van der Ven

Intensive Care Medicine Experimental 2020, 8(2): 000979
Introduction: It has been shown that observational studies often lead to biased results, even when statistical methods such as logistic regression and propensity score matching are used to adjust for confounding. This has specifically been shown for intensive care-related inferential problems [1, 2].

In observational critical care studies, the initiation or dose of the investigated treatment is often associated with baseline severity of illness and mortality risk. This causes endogeneity in estimation models (a correlation between the investigated treatment and the error term), which biases the analysis results. Endogeneity is a separate entity from confounding by indication, which arises through omitted variables. It arises from measurement error (imperfect calibration of severityof-illness scoring systems) and simultaneity (the decision to initiate treatment or the dose is influenced by the severity of illness but also influences the severity of illness) $[2,3]$.

We aimed to investigate the potential magnitude of endogeneity bias in the evidence base of a major intensive care guideline.

\section{Objectives:}

1. To identify which observational studies in the Surviving Sepsis Campaign (SSC) Guidelines' evidence base are at risk of endogeneity and spurious causal inferences [4].

2. To identify which topics in the SSC Guidelines are most affected by these potentially biased studies.

Methods: We screened all references in the Surviving Sepsis Campaign (SSC) Guidelines. We considered studies to be at risk of endogeneity bias when five conditions were met:

1. Observational non-randomized study design, and;

2. Investigated an intervention whose initiation or dosing is positively or negatively associated with baseline severity of illness or mortality risk, and;

3. Aimed to analyse the relationship between the intervention and a clinical outcome, and;

4. Adjusted for baseline severity of illness by regression methods (such as linear, logistic or proportional hazards regression) or propensity matching, and;

5. Made implicit or explicit causal statements

Results: We identified 167 observational studies in the guidelines' evidence base. Sixty-five (39\%) of these studies were at risk of endogeneity bias (Figure 1). None of these studies used a validated causal inference method to reduce endogeneity (e.g. an instrumental variable approach.) Studies at risk of endogeneity bias were most prevalent among the guideline topics Fluid Therapy (5 studies) and Glucose Control (9 studies).

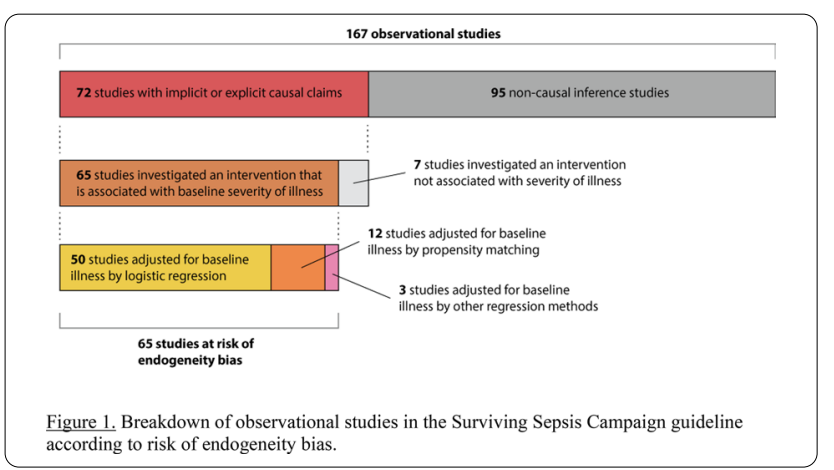

Conclusion: We found that $39 \%$ of the observational studies in the SSC Guidelines made causal statements while being at risk of endogeneity bias. The most affected topics were Fluid Therapy and Glucose Control. Their inclusion in a major guideline means these studies are not merely 'hypothesis-generating' and may influence treatment decisions. Biased results caused by endogeneity may thus lead to patient harm. Clinicians and authors of guidelines should be aware of this potential bias. 
Reference(s) and grant ackowledgment(s)

1. 4. Rhodes A, Evans LE, Alhazzani W, et al (2017) Surviving Sepsis Campaign. Intensive Care Med 43:304-377.

2. 1. Sjoding MW, Luo K, Miller MA, Iwashyna TJ (2015) When do confounding by indication and inadequate risk adjustment bias critical care studies? A simulation study. Crit Care 19:195.

3. 2. de Grooth H-J, Girbes ARJ, van der Ven FLIM, et al (2020) Observational research for therapies titrated to effect and associated with severity of illness: misleading results from commonly used statistical methods. Crit Care Med: in press.

4. 3. Leisman DE (2019) Ten Pearls and Pitfalls of Propensity Scores in Critical Care Research. Crit Care Med 47:176-185.

5. No grant acknowledgment.

\section{1}

Endothelial glycocalyx disruption and impaired microvascular flow following liver resection surgery

E. Potter ${ }^{1}$; B. Creagh-Brown'ㄹ L. Forni ${ }^{3}$; J. Mcvey

${ }^{1}$ Intensive Care Unit, Royal Surrey County Hospital, Guildford, France; ${ }^{2}$ Intensive care and perioperative medicine, Royal Surrey County Hospital, Guildford, United Kingdom; ${ }^{3}$ Intensive care, Royal Surrey County Hospital, Guildford, United Kingdom; ${ }^{4}$ Biochemical sciences, University Of Surrey, Guildford, United Kingdom

Correspondence: E. Potter

Intensive Care Medicine Experimental 2020, 8(2): 000991

Introduction: The endothelial glycocalyx is a bio-active structure which lines blood vessels. Its importance in critical and peri-operative care is increasingly recognised. 1 Shedding of key components of the endothelial glycocalyx such as syndecan-1, a proteoglycan, which bindsglycosaminoglycans, has been found to be associated with impaired microvascular blood flow following cardiac bypass surgery. 2 Glycocalyx degredation has also been found in ischaemia - reperfusion in trauma patients with shock.3 Animal models have found antithrombin to reduce endothelial dysfunction following ischaemia-reperfusion injury.4 In our centre we use a low central venous pressure (CVP) anaesthetic for liver resection surgery to decrease intraoperative blood loss, which is then followed by goal-directed fluid therapy.5 We investigated whether there was impairment of microvascular blood flow, evidence of endothelial glycocalyx shedding or changes in antithrombin concentrations in patients undergoing liver resection surgery.

Objectives: To establish:

- if there is evidence of endothelial glycocalyx shedding by measuring serum concentrations of: syndecan-1, thrombomodulin, vascular endothelial growth factor receptor (VEGF-R) or e-selectin in patients undergoing liver resection surgery

- if there were changes in antithrombin concentrations during liver resection surgery

- if endothelial glycocalyx shedding was related to microvascular blood flow.

Methods: This was a prospective observational study (REC no 14/ $\mathrm{NE} / 1150$ ) enrolling patients undergoing elective liver resection surgery. Microvascular blood flow, measured using the MicroScan device (MicroVision Medical, Amsterdam) and physiological measurements were taken at pre-specified time points throughout the peri-operative period (T1, awake on day of surgery pre-operatively, T2 following induction of anaesthesia, T3 on exposure of the liver, T4 completion of liver resection prior to fluid resuscitation, T5 arrival at ICU, T6 completion of goal directed fluid therapy (GDFT)). Serum samples were taken from a subset of 20 patients at T2, T4 and T6. ELISA's were carried out in duplicate for each sample at two dilutions. Results which fell outside of the standard curve were excluded from analyses.

Results: A total of 55 data sets were included in the analyses, 28 male and 27 female, mean age was 58 years (S.D. 13) and BMI $28.1 \mathrm{~kg} /$ $\mathrm{m}^{2}$ (S.D. 5.4 ), $18 \%$ were smokers, $13 \%$ were diabetic and $76 \%$ had neoadjuvant chemotherapy.

Microvascular flow measurements decreased post-operatively and this fall was sustained after completion of GDFT. There was a fall in microvascular flow index (MFI), proportion of perfused small blood vessels
(sPPV) and perfused small blood vessel density (sPVD) from time 1 to T6 of $2.78(2.42-2.98)$ vs $2.58(2.08-2.83) \mathrm{p}<0.0001,95.6(87.4-99.8)$ vs $65.1(61.3-66.8) \mathrm{p}<0.0001$ and $4.85(3.69-5.9)$ vs $5.77(4.91-7.2)$, $\mathrm{p}<0.0001$ respectively.

There was a significant increase in syndecan-1 shedding and fall in antithrombin concentrations on completion of goal directed fluid therapy. Other markers of endothelial glycocalyx disruption decreased.

Table 2 - Markers of endothelial disruption (results displayed as median (IQR), Wilcoxon signed rank test is used to establish difference between groups)

\begin{tabular}{|c|c|c|c|}
\hline Biomarker & Baseline (T2) & Post-operative (T6) & \\
\hline syndecan-1 $(\mathrm{pg} / \mathrm{mL})$ & $52.6(42.7-52.6)$ & $\begin{array}{l}18456(10047- \\
24301)\end{array}$ & $p<0.0001$ \\
\hline $\begin{array}{l}\text { Thrombomodulin } \\
\text { (ng/mL) }\end{array}$ & $6.73(5.6-7.6)$ & $3.3(2.5-3.7)$ & $\mathrm{p}<0.0001$ \\
\hline e-selectin $(\mathrm{ng} / \mathrm{mL})$ & $21.9(10.1-21.9)$ & $4.5(2.2-7.5)$ & $P=0.0001$ \\
\hline $\begin{array}{l}\text { vascular endothelial } \\
\text { growth factor receptor } \\
\text { (VEGF-R) }(\mathrm{ng} / \mathrm{mL})\end{array}$ & $10.4(9.7-10.5)$ & $1.7(1.3-2.5)$ & $\mathrm{p}=0.001$ \\
\hline Antithrombin & $110(106-115)$ & $96(92-114)$ & $P=0.04$ \\
\hline
\end{tabular}

Syndecan-1 and other biomarkers were not related to microvascular perfusion parameters, morbidity or length of stay.

Conclusion: Microvascular blood flow decreased post-operatively and this was sustained despite completion of GDFT. There was a significant increase in syndecan-1 concentration post-operatively, which may be due to disruption of the integrity of the endothelial glycocalyx membrane. However, there was a fall in other biomarkers, this could be dilutional. There was a fall in circulating antithrombin concentration which may be related to increased infiltration and binding to the endothelial glycocalyx following hypoperfusion. Further study of the glycocalyx in the peri-operative setting and its relation to microvascular blood flow is required.

\section{Reference(s) and grant ackowledgment(s)}

1. 1. Sakr Y, Dubois M-J, De Backer D, Creteur J, Vincent J-L: Persistent microcirculatory alterations are associated with organ failure and death in patients with septic shock*. Crit Care Med.32(9):1825-1831, 2004

2. 2. De Backer D, Donadello K, Sakr Y, Ospina-Tascon G, Salgado D, Scolletta S, Vincent JL: Microcirculatory alterations in patients with severe sepsis: impact of time of assessment and relationship with outcome. Crit Care Med.41(3):791-799, 2013.

3. 3. Arnemann P, Seidel L, Ertmer C. Haemodynamic coherence - The relevance of fluid therapy. Best Pract Res Clin Anaesthesiol 2016;30(4):419-427 (In eng). https://doi.org/10.1016/j.bpa.2016.11.003.

4. 4. Jones RM, Moulton CE, Hardy KJ. Central venous pressure and its effect on blood loss during liver resection. Br J Surg 1998;85(8):1058-60. (In eng). https://doi.org/10.1046/j.1365-2168.1998.00795.x.

\section{6}

Factors associated with ICU eligibility during the COVID 19 pandemic in a level-3 hospital in Madrid

H. Fernández-Hervás ${ }^{1}$; JA. Barea-Mendoza ${ }^{1}$; M. Chico-Fernández ${ }^{1}$; C. Mudarra-Reche1; C. García-Fuentes ' ; L. Orejón García'; S. Bermejo Aznárez'; M. Valiente Fernández'; JA. Cantalapiedra Santiago '; Y. Nieto Piñar $^{2}$; F. Delgado Moya ; M. González Fernández'; F. Eiras Abalde ${ }^{3}$ Y. Chicote Carasa ${ }^{1}$ : M. Sánchez-Bayton Griffith ${ }^{1}$

${ }^{1}$ Intensive care medicine, University Hospital 12 de Octubre, Madrid, Spain ${ }^{2}$ Intensive care medicine, Hospital Universitari Son Llàtzer, Palma, Spain; ${ }^{3}$ Intensive care medicine, Ourense University Hospital, Ourense, Spain

Correspondence: $H$. Fernández-Hervás

Intensive Care Medicine Experimental 2020, 8(2): 000996

Introduction: The COVID 19 pandemic outbreak in Madrid overwhelmed the capacities of our regional and local health system. Despite the local efforts to increase the critical care capacity we were faced with difficult decisions regarding allocation of a scarce resource. The optimal method for allocating critical care resources during a pandemic remains questionable [1]. 
Objectives: To describe the cohort of patients evaluated with COVID 19 disease and the associated factors with ICU eligibility. This study could provide relevant information to optimize benefits from critical care resources in future COVID 19 outbreaks.

Methods: This prospective observational study was conducted in a specialised trauma and emergency department ICU of a level-3 hospital in Madrid, Spain. Our ICU staff evaluated adult patients with respiratory failure due to suspected COVID 19 infection at the ED. The decision about eligibility for ICU admission was guided using an institutional protocol including age, comorbidities and disease severity. We also collected data regarding gender, severity scores at time of evaluation (SOFA, CURB65), decision about eligibility (with independence of ICU admission), ICU admission and in-hospital mortality. Data was analyzed using the software RStudio Version 1.2.5042 for windows. For the univariate analysis we used Chi square test for categorical variables and student T Test or Mann Whitney U Test for continuous variables. Multivariate analysis was performed with a logistic regression model.

Results: Between March 4th and April 20th 2020 we evaluated a total of 221 patients. In the univariate analysis, age, comorbid state and disease severity were inversely associated with ICU eligibility with statistical significance. The number of events in the non eligible group limited the number of explanatory variables in the multivariate model, we created an index of comorbid state simply adding the main comorbidities of each patient, SOFA score was excluded due to lack of linearity. The resultant ligistic regression model showed adequate goodness of fit and predictive accuracy (Likelihood ratio test $X^{2}(4)=94.945 p<$ 0.001 . Pseudo R McFadden: 0.3837 . Classification rate 0.85 . Area under the curve for the ROC curve 0.8875 (95\% Cl [0.8365; 0.9385]). In this model only age and comorbidities retained statistical significance (Table 1).

Table 1: Comparison between eligible and non eligible patients for ICU admission

\begin{tabular}{|c|c|c|c|c|}
\hline & $\begin{array}{c}\text { Non eligible } \\
(56)\end{array}$ & Eligible (165) & $\begin{array}{c}\text { p value } \\
\text { (Uni) }\end{array}$ & $\begin{array}{c}\text { OR }(95 \% \text { CI }) \\
\text { Multi }\end{array}$ \\
\hline Age (years) & 66.7 (SD 10.7) & $52.3(\mathrm{SD} 11.8)$ & $<0.001$ & $\begin{array}{l}0.91(0.86- \\
0.95, \mathrm{p}<0.001)\end{array}$ \\
\hline Female & $22(39.3 \%)$ & $44(26.7 \%)$ & 0.082 & $\begin{array}{l}0.60(0.25- \\
1.41, \mathrm{p}=0.233)\end{array}$ \\
\hline Hypertension & $29(51.8 \%)$ & $47(28.5 \%)$ & 0.002 & \\
\hline Diabetes & $20(35.7 \%)$ & $19(11.5 \%)$ & $<0.001$ & \\
\hline OSA & $12(21.4 \%)$ & $11(6.67 \%)$ & 0.004 & \\
\hline COPD & $10(17.9 \%)$ & $3(1.82 \%)$ & $<0.001$ & \\
\hline Obesity & $20(35.7 \%)$ & $41(24.8 \%)$ & 0.125 & \\
\hline Heart Disease & $17(30.4 \%)$ & $6(3.64 \%)$ & $<0.001$ & \\
\hline $\begin{array}{l}\text { Number of } \\
\text { comorbidities * T }\end{array}$ & $3.00[1.75 ; 4.00]$ & $1.00[0.00 ; 2.00]$ & $<0.001$ & $\begin{array}{l}0.46(0.33- \\
0.65, \mathrm{p}<0.001)\end{array}$ \\
\hline SOFA* & $3.00[2.00 ; 3.00]$ & $2.00[2.00 ; 3.00]$ & 0.012 & - \\
\hline CURB65* & $2.00[1.00 ; 3.00]$ & $1.00[0.00 ; 2.00]$ & $<0.001$ & $\begin{array}{l}0.71(0.42- \\
1.15, \mathrm{p}=0.170)\end{array}$ \\
\hline
\end{tabular}

Conclusion: The variables most associated with ICU eligibility were age and comorbid state proving that age was not the only factor accounted for. Female gender and CURB 65 was inversely associated with eligibility however this association was not significant in multivariate models.

\section{Reference(s) and grant ackowledgment(s)}

1. [1] Maves RC, Downar J, Dichter JR, et al. Triage of Scarce Critical Care Resources in COVID-19 An Implementation Guide for Regional Allocation: An Expert Panel Report of the Task Force for Mass Critical Care and the American College of Chest Physicians. Chest. 2020;158(1):212-225. https:// doi.org/10.1016/j.chest.2020.03.063

2. To the general population for their effort during confinement
001002

Paired nasopharyngeal and deep lung testing for SARS-CoV2 reveals a significant viral gradient in critically ill patients: a multi-centre study

I. Hamed'; N. Shaban²; M. Nassar²; D. Cayir²; S. Love²; MD. Curran³; S. Webb ${ }^{4} ;$ H. Yang ${ }^{4}$; K. Watson ${ }^{5}$; A. Rostron ${ }^{5}$; R. Mahroof'; VN. Vilas Navapurkar ${ }^{6}$; A. Conway-Morris ${ }^{1}$

${ }^{1}$ Department of anaesthetics \& intensive care medicine, Cambridge University Hospitals NHS Foundation Trust, Cambridge, United Kingdom; ${ }^{2}$ Intensive care, john farman intensive care unit, Addenbrooke's Hospital, Cambridge, United Kingdom; ${ }^{3}$ Clinical microbiology and public health laboratory, Public Health Laboratory, Cambridge, United Kingdom; ${ }^{4}$ Department of anaesthetics and intensive care medicine, Royal Papworth Hospital NHS Foundation Trust, Cambridge, United Kingdom; ${ }^{5}$ ntegrated critical care unit, Sunderland Royal Hospital, Sunderland, United Kingdom ${ }^{6}$ Intensive care, John Farman Intensive Care Unit, Addenbrooke's Hospital, Cambridge, UK, Cambridge, United Kingdom

\section{Correspondence: I. Hamed}

Intensive Care Medicine Experimental 2020, 8(2): 001002

Introduction: Samples for diagnostic tests for SARS-CoV-2 can be obtained from the upper (nasopharyngeal/oropharyngeal swabs) or lower respiratory tract (sputum or tracheal aspirate or broncho-alveolar lavage - BAL). Data from different testing sites indicates different rates of positivity1. Real-time polymerase chain reaction (RT-PCR) allows for semi-quantitative estimates of viral load as time to crossing threshold $(\mathrm{Ct})$ is inversely related to viral load.

Objectives: The objective of our study was to evaluate SARS-CoV2 RNA loads between paired nasopharyngeal (NP) and deep lung (endotracheal aspirate or BAL) samples from critically ill patients.

Methods: SARS-CoV-2 RT-PCR results were retrospectively reviewed for 51 critically ill patients from 5 intensive care units in 3 hospitals Addenbrookes Hospital Cambridge (3 units), Royal Papworth Cambridge (1 unit), and Royal Sunderland Hospital (1 unit). At the times when paired NP and deep lung samples were obtained, one patient had been on oxygen only, 6 patients on non-invasive ventilation, 18 patients on ECMO, and 26 patients mechanically ventilated.

Results: Results collected showed significant gradient between NP and deep lung viral loads (Figure 1). Median Ct value was 29 for NP samples and 24 for deep lung samples. Of 51 paired samples, 16 were negative (below limit of detection) on NP swabs but positive (above limit of detection) on deep lung sample, whilst 2 were negative on deep sample but positive on NP (both patients were on ECMO).

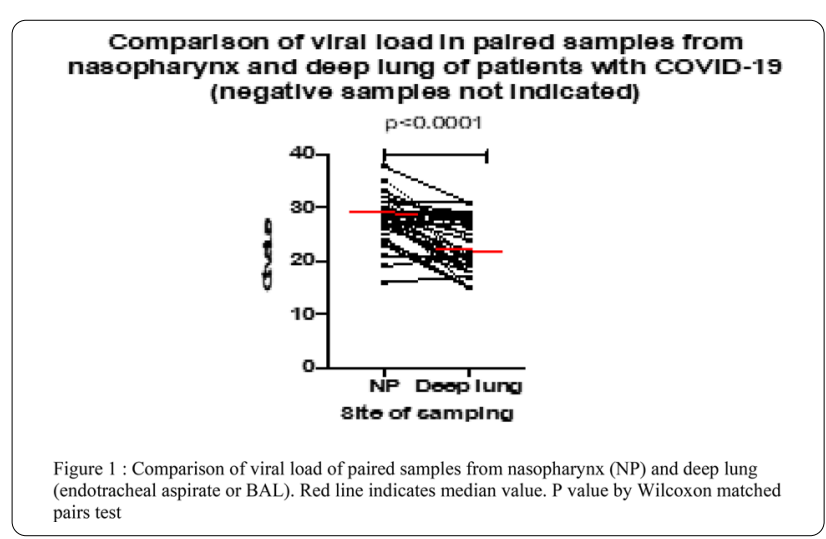

Conclusion: It has been suggested that whilst SARS-CoV1 tends to replicate in the lower respiratory tract, SARS-CoV2 replicates more vigorously in the upper respiratory tract2. These data challenge that assumption. These data suggest that viral migration to, and proliferation in, the lower respiratory tract may be a key factor in the progression to critical illness and the development of severe acute respiratory syndrome (SARS). Factors which promote this migration should be examined for association with severe COVID-19. From a practical point 
of view, patients with suspected severe COVID-19 should have virological samples obtained from the lower respiratory tract where-ever possible, as upper respiratory samples have a significant false-negative rate.

\section{Reference(s) and grant ackowledgment(s)}

1. 2- Gandhi M, et al. Asymptomatic Transmission, the Achilles' Heel of Current Strategies to Control Covid-19. N Engl J Med. 2020;382:2158-2160.

2. 1- Wang W, et al. Detection of SARS-CoV-2 in Different Types of Clinical Specimens. JAMA. March 2020:1-2.

\section{8}

Caregivers resilience during CoVID 19 pandemic, Return

from experience

YL. Nguyen ${ }^{1}$; C. Baillard'; M. Samama

${ }^{1}$ Anesthesiology and critical care department, Cochin Hospital, Paris, France

Correspondence: Y.L. Nguyen

Intensive Care Medicine Experimental 2020, 8(2): 001038

Introduction: The CoVID19 pandemic led to a complete reorganization of our anesthesiology and critical care medicine department (composed of 8 ICU beds, 12 intermediate care unit beds (IMCU) and 32 operating rooms $(\mathrm{OR}))(1)$. In order to face the increasing demand of ICU beds, our 12 IMCU beds were transformed into ICU beds, 40 ICU beds were created within the 3 post-anesthesia care units of our hospital, leading to a total of 60 ICU beds and only 13 OR remaining opened. The majority of caregivers working usually in the OR worked in these new ICUs outside the walls from March 23d to May 7th 2020.

Objectives: One month after the end of the pandemic, we conducted an anonymous e-survey among caregivers of our department, with the aim of identifying what happened, what went right, what went wrong and what to do in the case of a second wave of CoVID19 infections.

Methods: This project received the approval of the research ethics committee of the French Society of Anesthesiology and Intensive Care Medicine (SFAR). Participation was free. Our survey was composed of 10 questions with rating scales ( 0 to 10 ) to assess job satisfaction, quality of care delivered and quality of life at work, with tick box categories and free text to evaluate the main positive/negative points related from their experience and the main actions to be taken in case of a second wave, with Likert scales to evaluate the relationships between caregivers and on demographic data.

Results: 130 people answered our questionnaire, mainly women $(\mathrm{n}=$ $84 ; 65 \%)$, the majority younger than 40 years old $(n=94 ; 72 \%)$ and the majority with a past experience in critical care $(n=92 ; 71 \%)$. The participation rate varied across professions: $100 \%$ for head of nurses $(n$ $=3), 65 \%$ for nurses $(n=44), 45 \%$ for anesthetist nurses $(n=22), 47 \%$ for nurse assistants $(n=20), 100 \%$ for physiotherapists $(n=6) ; 100 \%$ dieticians $(n=2) ; 50 \%$ for residents $(n=16), 70 \%$ for physicians $(n=$ 14). The levels of job satisfaction, quality of care delivered and quality of life at work were respectively $6.9( \pm 2), 7.2( \pm 2)$ and $5.8( \pm 2)$. The main positive points reported were the collaboration and solidarity between colleagues $(n=76 ; 58 \%)$ and the feeling of being useful $(n=62 ; 48 \%)$. The main negative points reported were the working conditions difficulties (environment, logistics, material protection) (n $=95 ; 73 \%)$ and the lack of visibility of their schedule or hospital ICU organization $(n=36 ; 27 \%)$. The two main areas for improvement suggested were the optimization of logistics organization $(n=74 ; 57 \%)$ and working within the same unit $(n=59 ; 45 \%)$. The majority weresatisfied with their relationships with physicians $(n=123 ; 89 \%)$, nurses ( $n$ $=127 ; 98 \%)$ or other allied health workers $(n=95 ; 73 \%)$.

Conclusion: During the epidemic, the 3-fold increase of ICU beds and the closing of the majority of OR were associated with a big change in the work habits of our caregivers. Despite the difficulties related to working conditions, caregivers have been strongly resilient with the complete reorganization of our department during the CoVID19 pandemic. The existence of an association with this new organization and patient outcomes must be explored.
001048

Predictors of intubation in COVID-19 patients treated with noninvasive continuous positive airway pressure N. De Vita' ${ }^{1}$;. Scotti '; F. Barone-Adesi ${ }^{1}$; F. Racca ${ }^{2}$; C. Pissaia ${ }^{3}$; C. Maestrone ${ }^{4}$.

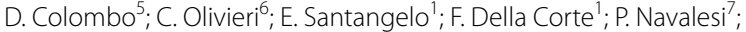

G. Cammarota ${ }^{8}$; R. Vaschetto ${ }^{1}$; F. Covid-19 Eastern Piedmont Network ${ }^{1}$

${ }^{1}$ Dipartimento di medicina traslazionale, Università Degli Studi Del Piemonte Orientale, Novara, Italy; ${ }^{2}$ Department of anesthesia and intensive care, Azienda ospedaliera SS Antonio e Biagio e Cesare Arrigo, Alessandria, Italy; ${ }^{3}$ Dipartimento di anestesia e terapia intensiva, Ospedale "Degli Infermi", Biella, Italy; ${ }^{4}$ Anestesia rianimazione asl vco, Presidio Ospedaliero Domodossola e Verbania, Verbania, Italy; ${ }^{5}$ Department of anesthesia and critical care, Ospedale Ss. Trinità Borgomanero, Borgomanero, Italy; ${ }^{6}$ Department of anesthesia and critical care, Azienda Ospedaliera Sant'Andrea, Vercelli, Italy; ${ }^{7}$ Istituto di anestesia e rianimazione, Azienda Ospedaliera Università Padova - Dipartimento di medicina DIMED - Università di Padova, Padova, Italy; ${ }^{8}$ Anestesia e terapia intensiva, Azienda Ospedaliero Universitaria Maggiore della Carità di Novara, Novara, Italy

Correspondence: $\mathrm{N}$. De Vita

Intensive Care Medicine Experimental 2020, 8(2): 001048

Introduction: Despite some new evidence, the use of noninvasive continuous positive airway pressure (nCPAP) during coronavirus disease 2019 (COVID-19) is still an issue. In a large population of COVID19 patients treated with nCPAP we aimed to identify early factors associated with intubation.

Methods: Post hoc analysis of an observational prospective-retrospective multicentre study performed in six Italian hospitals. We included in this analysis all patients treated with nCPAP outside ICU due to COVID-19 and candidate to intubation in case of nCPAP failure. We collected demographic and clinical data at hospital entrance and $\mathrm{PaO}_{2} / \mathrm{FiO}_{2}$ percentage increase between Venturi mask $\left(\mathrm{PaO}_{2} / \mathrm{FiO}\right.$ VENT) and nCPAP ( $\mathrm{PaO}_{2} / \mathrm{FiO}_{2}$ nCPAP) i.e., $\triangle \mathrm{PaO}_{2} / \mathrm{FiO}_{2}$, calculated as $\left(\mathrm{PaO}_{2} / \mathrm{FiO}_{2}\right.$ nCPAP $-\mathrm{PaO}_{2} / \mathrm{FiO}_{2}$ VENT $) / \mathrm{PaO}_{2} / \mathrm{FiO}_{2}$ VENT $x 100$.

Results: From March 1st to April 15th, a total of 397 patients with confirmed COVID-19 were treated with nCPAP with a full treatment therapeutic goal. Of these, $8 \%$ of the patients were excluded from the analysis as intubated on the same day nCPAP was started. Univariate analysis showed that among the demographic and clinical patients' characteristics, age $(65$ (IQR, 55-71) vs. 68 (IQR 58-73) years), lactate dehydrogenase (LDH) (518 (IQR, 360-676) U/L vs. 654 (IQR, 486-922) $\mathrm{U} / \mathrm{L})$, white blood cells $(6.75(\mathrm{IQR}, 5.11-8.91) \times 103 / \mu \mathrm{L}$ vs. $7.04(\mathrm{IQR}$ $5.17-10.00) \times 103 / \mu \mathrm{L}$ ) resulted significantly lower in patients succeeding nCPAP compared to those who failed nCPAP and were intubated. Percentage increase in $\mathrm{PaO}_{2} / \mathrm{FiO}_{2}(87(\mathrm{IQR}, 7-203)$ vs. 44(IQR,12-120)) was higher in patients who succeeded nCPAP compared to those who failed. Male gender was also significantly associated with intubation. A multivariate analysis adjusting for age, gender, Charlson comorbidity index, percentage increase in $\mathrm{PaO}_{2} / \mathrm{FiO}_{2}$, lactate, white blood cell count, $\mathrm{LDH}$ and $\mathrm{C}$-reactive protein levels led to an area under the curve of 0.77 and confirmed that age (relative risk, 1.023;95\% Cl, 1.005-1.043), LDH (relative risk, $1.001 ; 95 \% \mathrm{Cl}, 1.000-1.001$ ), and $\mathrm{PaO}_{2}$ / $\mathrm{FiO}_{2}$ (relative risk, $1.002 ; 95 \% \mathrm{Cl}, 1.001-1.003$ ), were strong predictors of intubation.

Conclusion: In patients with acute hypoxemic respiratory failure due to COVID-19 receiving $\mathrm{nCPAP}$ outside ICU $\mathrm{PaO}_{2} / \mathrm{FiO}_{2}, \mathrm{LDH}$ at hospital entrance and age were predictors of intubation.

Reference(s) and grant ackowledgment(s)

1. Covid-19 Eastern Piedmont Network: Gianluca Airoldi; Marta Baggiani; Sara Baino; Piero Balbo; Simona Bazzano; Valeria Bonato; Silvio Borrè; Luigi Castello; Tiziana Cena; Sara Carbonati; Federico Crimaldi; Veronica Daffara; Luca De Col; Luca Grillenzoni; Matteo Maestrone; Mario Malerba; Francesco Mojoli; Federica Moroni; Maria Adele Moschella; Raffaella Perucca; Mario Pirisi; Valentina Rondi; Daniela Rosalba; Martina Taverna; Letizia Vanni; Francesca Vigone 


\section{7}

Incidence of acute kidney injury and provision of renal replacement therapy in critically ill patients with COVID-19 R. Fisher ${ }^{1}$; J. Clarke ${ }^{1}$; K. Al Arfi'; R. Saha'; C. Sharpe ${ }^{2}$; K. Bramham²; SD. Hutchings ${ }^{1}$

${ }^{1}$ Critical care, King's College Hospital, London, United Kingdom; ${ }^{2}$ King's kidney care, King's College Hospital, London, United Kingdom

\section{Correspondence: R. Fisher,}

Intensive Care Medicine Experimental 2020, 8(2): 001077

Introduction: Amongst patients admitted to intensive care (ICU) with COVID-19 the reported incidence of acute kidney injury (AKI) ranges from $3 \%$ to $66 \%$.[1] In the United Kingdom (UK) $26 \%$ of patients admitted to ICU with COVID-19 required renal replacement therapy (RRT).[2] At our institution RRT in ICU is usually exclusively provided as Continuous Veno-Venous HaemoDiaFiltration (CVVHDF). During early stages of the pandemic it became apparent that demand for RRT - driven by a surge in ICU admissions, high rates of AKI and shorter than usual filter lifespan - threatened our ability to provide timely organ support to critically ill patients with COVID-19, a situation that was being replicated globally.[3] During the first 2 months of the pandemic in the UK we introduced a number of measures designed to extend our ability to provide RRT, including introduction of acute peritoneal dialysis (aPD) and intermittent haemodialysis (IHD).

Objectives: To describe the incidence of AKI in patients admitted to ICU with COVID-19 at a large teaching hospital in London, UK. To evaluate how the introduction of alternative modalities of RRT (aPD and IHD) - not usually offered in our ICU - were able to reduce dependence on CVVHDF. To report any complications associated with using aPD and IHD in these critically ill patients.

Methods: We carried out a retrospective review of patients' electronic medical records as part of a departmental service evaluation (ref: KCC06052020AIA). We recorded baseline demographics, incidence of AKI (defined using KDIGO serum creatinine criteria) [4] and details regarding RRT received and complications of RRT for all patients admitted to our ICU with a diagnosis of COVID-19 between 10/03/2020 (date of first patient admitted with COVID-19) and 10/05/2020.

Results: 177 patients with COVID-19 were admitted to the ICU. Excluding patients who were transferred from other institutions $(n=34)$ and patients with established End-Stage Kidney Disease (ESKD, $n=7)$ the incidence of AKI was 79\%. Most (86\%) developed severe (stage 3) AKI. $63 \%$ of patients required RRT, which was initiated a median of 4.5 (IQR 2-7) days after ICU admission. As of 16/06/2020, of patients who developed severe AKI, $49 \%$ had died, $8 \%$ were still admitted to the ICU, $22 \%$ had been discharged from the ICU but were still in hospital and $22 \%$ had been discharged home. Almost all ICU survivors hadrecovered renal function to the point of being free of RRT (95\%). Median duration of RRT in those who recovered was 21 (IQR 13-29) days. Peritoneal dialysis catheter insertion was attempted in 41 patients and successful in $34(83 \%) .15$ patients $(44 \%)$ were subsequently exclusively treated with aPD, whilst 19 required some supplemental CVVHDF. Common reasons for reverting to CVVHDF included: clinical deterioration not attributed to PD $(n=5)$; elevated urea $(n=5)$; fluid overload $(n=4)$; hyperkalaemia $(n=3)$. No patients were reported to have had deterioration in ventilatory parameters or peritonitis. A reverse osmosis (RO) plant was installed within the ICU on $18 / 04 / 20$, and IHD began on $20 / 04 / 20$. $82 \mathrm{HD}$ sessions were delivered to 15 patients. Transient hypotension was reported during 11 sessions (13.4\%). On one occasion the session was terminated due to marked hypotension. At the start of the pandemic $100 \%$ of RRT was delivered as CVVHDF. With the introduction of aPD and IHD this fell to a nadir of $39 \%$. During the study period a total of 463 patient days of CVVHDF were replaced with either aPD or IHD.

Conclusion: In our study most patients admitted to ICU with COVID19 developed severe AKI. It was only possible to provide RRT to these patients by expanding the range of RRT modalities offered to include aPD and IHD. Through close collaboration between the critical care and nephrology departments this was achieved rapidly, effectively and with no serious adverse events during pandemic conditions.

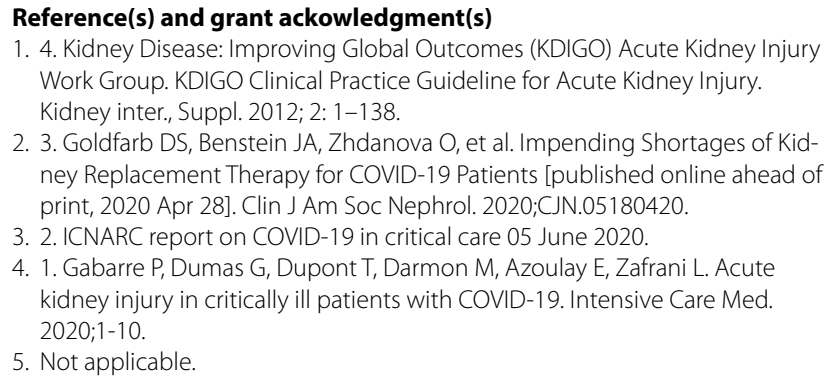

\section{7}

Comparison of mechanical power estimations in mechanically ventilated patients suffering from the acute respiratory distress syndrome

MS. Schaefer ${ }^{1}$; SH. Loring ${ }^{1}$; D. Talmor ${ }^{1}$; E. Baedorf-Kassis²

${ }^{1}$ Department of anesthesia, critical care \& pain medicine, Beth Israel Deaconess Medical Center (BIDMC), Boston, United States of America; ${ }^{2}$ Department of pulmonary, critical care \& sleep medicine, Beth Israel Deaconess Medical Center (BIDMC), Boston, United States of America

Correspondence: M.S. Schaefer

Intensive Care Medicine Experimental 2020, 8(2): 001097

Introduction: The energy delivered to the respiratory system during mechanical ventilation can be measured as mechanical power (i.e. work over time) and has been associated with increased in-hospital mortality. [1] A previously proposed concept of mechanical power (here referred to as Respiratory System-directed Power) [2] is easy to calculate but includes energy dissipated into the chest wall as well as static work from positive end-expiratory pressure (PEEP), both probably not causal to lung injury. These issues could be addressed by excluding PEEP as well as using transpulmonary pressure (PL) instead of airway pressure (Pao).

Objectives: To compare and assess the agreement between Respiratory System-directed Power and two alternative calculations in mechanically ventilated patients suffering from ARDS.

Methods: Using data from a previous, randomized trial (EPVent) [3], mechanical power was calculated from pressure-volume loops: (1) Respiratory System-directed Power based on Pao and including static work from PEEP; (2) Respiratory System-directed Driving Power based on driving pressure (Pao-PEEP); (3) Lung-directed Driving Power based on $\mathrm{PL}$ change from end-expiration. Estimates were compared at baseline using repeated measures ANOVA and Bland-Altman analysis. We further investigated how the study intervention - a PEEP titration to achieve positive $\mathrm{PL}$ - affected agreement among the estimations.

Results: In 4,803 breathing cycles from 53 patients, Respiratory Systemdirected Power yielded a median 37.2 [IQR 24.1;51.4] J/min, followed by Respiratory System-directed Driving Power (14.2 [11.0;19.8]) and Lungdirected Driving Power $(13.4[9.9 ; 18.8] \mathrm{J} / \mathrm{min}$, overall $\mathrm{p}<0.001)$. There was poor agreement between Respiratory System-directed Power and the other two estimates (bias $24.2[20.8 ; 27.7]$, limits $-0.2[-6.1 ; 5.7]$ to $48.7[20.8 ; 27.7] \mathrm{J} / \mathrm{min}$ against Respiratory System-directed Driving Power and 25.6 [21.9;29.2], limits -0.5 [-6.8;5.9] to 51.7 [45.3;58.0] $\mathrm{J} / \mathrm{min}$ against Lung-directed Driving Power) and good agreement between Respiratory System-directed Driving Power and Lung-directed Driving Power (bias 1.4 [95\% Cl 0.8;1.9], limits $-2.3[-3.2 ;-1.4]$ to $5.0[4.1 ; 5.9] \mathrm{J} /$ $\mathrm{min})$. In a subgroup of 21 patients, Respiratory System-directed Power increased after PL-guided PEEP titration by $5.8 \pm 12.4 \mathrm{~J} / \mathrm{min}(p=0.033)$ with no change in the other estimates, increasing bias and limits of agreement between Respiratory System-directed Power and the other two estimates.

Conclusion: In mechanically ventilated patients without spontaneous breathing activity, inclusion of PEEP into calculation of mechanical power may greatly increase the estimate. Calculations including PEEP show poor agreement with calculations excluding PEEP or made based on PL. Clinical data are required to elucidate how these different concepts relate to patient outcome. 
Reference(s) and grant ackowledgment(s)

1. [1] Intensive Care Med. 2018; 44:1914-22 [2] Intensive Care Med. 2016:42:1567-75 [3] NEJM 2008 13·359·2095-104

\section{3}

Blood Transfusion Effectiveness Evaluation: Looking for Better

\section{Transfusion Triggers}

A. Arynov ${ }^{1}$; Z. Chingissova 2 ; V. Chursin ${ }^{3} ;$ K. Lebedinskii

${ }^{1}$ Department of Anesthesiology and Intensive Care, Kazakh Institute of Oncology and Radiology, Almaty, Kazakhstan; ${ }^{2}$ Department of oncology, Kazakh Institute of Oncology and Radiology, Almaty, Kazakhstan; ${ }^{3}$ Department of anesthesiology and intensive care, Kazakh Medica University of Continuing Education, Almaty, Kazakhstan; ${ }^{4}$ Department of anesthesiology and intensive care, I.I. Mechnikov North-Western State Medical University, Saint Petersburg, Russia

Correspondence: A. Arynov

Intensive Care Medicine Experimental 2020, 8(2): 001103

Introduction: For many decades red blood cells (RBC) transfusion remains the main treatment for acute and even chronic anemia. However, numerous clinical trials estimatedthe risks of RBC transfusion ascomparable with those of anemia and associated with increased mortality [1]. Recognition of the fact promotes search for more rigorous and less formal transfusion triggers.

Objectives: To compare blood transfusion effectiveness assessment based on oxygen extraction ratio (ExRO2), arterial-venous oxygen difference (A-Vdiff) and central venous oxygen saturation (ScvO2) with those using only blood hemoglobin $(\mathrm{Hb})$ level.

Methods: Prospective observational study included 59 patients with mean age of $57 \pm 14,3$ years and initial $\mathrm{Hb} 72 \pm 12,0 \mathrm{~g} \times 1-1$. ScvO2, ExRO2 ((CtaO2-CtvO2)/CtaO2)'100\%), A-Vdiff (CtaO2-CtvO2) and serum lactate were measured andcalculated before and after RBC transfusion. According to the initial ExRO2 all the patients were divided between two groups $-\mathrm{I}(\geq 40,0 \%, \mathrm{n}=20)$ and $\mathrm{II}(<40 \%, \mathrm{n}=$ 39).

Results: After transfusion, in group IExRO2 significantly decreased by $9,1 \pm 2,5 \%$ ( $p<0,05)$, ScvO2 increased by 7,0 $\pm 2,6 \%(p<0,05)$, while in group Ilboth ExRO2 and ScvO2 changes were less $(5,9 \pm 1,0 \%$ and $5,9 \pm 1,1 \%$ respectively, both $p<0,05)$. A-Vdiff after transfusion did not drop significantly in both groups: $0,8 \pm 2,8$ and $0,7 \pm 1,2 \mathrm{ml} \times \mathrm{I}$ 1 respectively (both $p>0,05$ ). Serum lactate decrease was significant in groupl $(0,5 \pm 0,2 \mathrm{mmol} \times 1-1, \mathrm{p}<0,05)$ and negligible in group I $(0,3 \pm 0,1 \mathrm{mmol} \times 1-1, \mathrm{p}>0,05)$. Notably, $\mathrm{Hb}$ levels were similar among the two groups with different ExRO2 levelsbefore and after transfusion. These results are presented in Table 1.

\begin{tabular}{|l|l|l|l|l|}
\hline Parameters & \multicolumn{2}{l}{ Group I } & \multicolumn{3}{l|}{ Group II } \\
\cline { 2 - 5 } & $\begin{array}{l}\text { Before } \\
\text { transfusion }\end{array}$ & $\begin{array}{l}\text { After } \\
\text { transfusion }\end{array}$ & $\begin{array}{l}\text { Before } \\
\text { transfusion }\end{array}$ & $\begin{array}{l}\text { After } \\
\text { transfusion }\end{array}$ \\
\hline ExRO2, $\%$ & $43,7 \pm 5,6$ & $34,6 \pm 8,7$ & $34,0 \pm 5,6$ & $28,1 \pm 5,8$ \\
\hline ScvO2, $\%$ & $55 \pm 5,3$ & $62 \pm 8,6$ & $64,2 \pm 6,2$ & $70,1 \pm 5,8$ \\
\hline $\begin{array}{l}\text { A-Vdiff, } m l \times l- \\
1\end{array}$ & $40,7 \pm 8,4$ & $38,6 \pm 12,7$ & $28,6 \pm 7,2$ & $28,8 \pm 7,1$ \\
\hline Lac, $m$ mol $\times l-1$ & $1,3 \pm 1,0$ & $0,8 \pm 0,5$ & $1,1 \pm 0,7$ & $0,8 \pm 0,7$ \\
\hline Hb, $g \times l-1$ & $72,7 \pm 12,4$ & $81,2 \pm 13,0$ & $70,0 \pm 12$ & $82,0 \pm 12,5$ \\
\hline
\end{tabular}

Spearman correlation between ExRO2 and $\mathrm{ScvO} 2$ showed strong negative relationship in both groups (before 0,9 and 0,8; after 1,0 and 0,9 respectively); the same coefficient between ExRO2 and A-Vdiff was 0,5 before and 0,8 after transfusion. ExRO2 and $\mathrm{Hb}$ demonstrated weak negative connection: $-0,2$ before transfusion in both groups, 0,1 and-0,2 after transfusion in groups I and II respectively.

Conclusion: ExRO2 assesses anemia severity and compensation degree more correctly in comparison to $\mathrm{Hb}$ level only. This parameter reveals effectiveness of transfusion, and can be used as the alternative transfusion trigger. ScvO2 has also showed some value as blood transfusion trigger, while having certain restrictions as systemic oxygen balance criterion because of unreliable correlation with mixed venous oxygen saturation.
Reference(s) and grant ackowledgment(s)

1. 1. Vincent JL. Which carries the biggest risk: anaemia or blood transfusion?Transfus Clin Biol. 2015;22(3):148-150. https://doi.org/10.1016/j. tracli.2015.05.001

001121

Analysis of the STRIVE phase 2 trial of once-weekly rezafungin for treatment of candidemia and invasive candidiasis compared with caspofungin: outcomes by body mass index (BMI)

P. Honoré

${ }^{1}$ ICU department, CHU Brugmann, Brussels, Belgium

Correspondence: P. Honoré

Intensive Care Medicine Experimental 2020, 8(2): 001121

Introduction: Title

Analysis of the STRIVE Phase 2 Trial of Once-Weekly Rezafungin for Treatment of Candidemia and Invasive Candidiasis Compared with Caspofungin: Outcomes by Body Mass Index (BMI)

Authors

Jose A. Vazquez1; Shawn Flanagan2; Peter G. Pappas3; George R. Thompson III4; Taylor Sandison2; Patrick M. Honore5

1 Medical College of Georgia at Augusta University, Augusta, Georgia, USA; 2Cidara Therapeutics, Inc. San Diego, CA, USA; 3University of Alabama, Birmingham; 4Unversity of California-Davis, Davis, California, USA; 5CHU Brugmann University Hospital, Brussels, Belgium

Objectives: There is increasing evidence of antifungal underdosing in the treatment of invasive disease, particularly in special populations such as the obese. Body size is often an important variable affecting drug exposure, and pharmacokinetic (PK) models of antifungal dosing have suggested size-based dose adjustments to achieve target drug exposure.

Rezafungin (RZF) is a novel echinocandin in Phase 3 development for treatment of candidemia and invasive candidiasis (IC) and for prevention of invasive fungal disease caused by Candida, Aspergillus, and Pneumocystis in blood and marrow transplant recipients. Distinctive PK properties of RZF (e.g., long half-life, extensive tissue distribution, and front-loaded drug exposure) lend themselves to RZF once-weekly (QWk) dosing and antifungal efficacy.

Methods: To evaluate outcomes of the Phase 2 STRIVE trial of RZF QWk for the treatment of candidemia and/or IC compared with caspofungin (CAS) once-daily (NCT02734862)1, based on patient BMI.

Results: The STRIVE trial (NCT02734862) compared the safety and efficacy of RZF QWk compared with once-daily caspofungin. For this subanalysis, data were stratified by BMI categories of $<30 \mathrm{~kg} / \mathrm{m}^{2}$ and $\geq 30$ $\mathrm{kg} / \mathrm{m}^{2}$. Efficacy (overall response [resolution of clinical signs of infection + mycological eradication], mycological response, and investigator assessment of clinical response) and safety (treatment-emergent adverse events [TEAEs]) endpoints by treatment group were evaluated, as well as PK data (area under the curve [AUC]) from RZF-treated patients

Mean BMI values were similar across treatment arms $\left(26.9 \mathrm{~kg} / \mathrm{m}^{2}\right.$ in RZF Group 1 and $26.8 \mathrm{~kg} / \mathrm{m}^{2}$ in RZF Group 2 and CAS arms). Efficacy outcomes at Day 14 were similar between BMI categories (Table 1). Rates of TEAEs were generally similar between BMI categories as well (Table 2), with no concerning safety trends. Following one dose of RZF $400 \mathrm{mg}$ (Week 1), the ranges of AUCs by BMI category overlapped and there was a minor mean difference of $\sim 20 \%$ (lower for those with BMI $\geq 30 \mathrm{~kg} / \mathrm{m}^{2}$ ).

Conclusion: The safety, efficacy, and PK of RZF in the Phase 2 STRIVE trial was consistent across BMl categories. These results suggest that dose adjustments in obese patients are not necessary. These findings contribute to the evaluation of RZF in a range of patient populations and its ongoing development.

\section{Reference(s) and grant ackowledgment(s)}

1. 1. Thompson GR, Honore PM, Horcajada JP, Hites M, Bassetti M, Mena K, Navalta L, Viani R, Sandison T, Pappas P. Rezafungin Clinical Safety and Efficacy in the Treatment of Candidemia and/or Invasive Candidiasis: Combined Results from the STRIVE Phase 2 Trial Parts A and B. Trends in Medical Mycology; October 11-14, 2019; Nice, France.

2. None 
001127

Family support on intensive care units

during the COVID-19-imposed visit ban: a Delphi study in German speaking countries in Europe

MM. Jeitziner ${ }^{1}$; B. Jenni-Moser ${ }^{2}$; M. Brauchle ${ }^{3}$; SA. Moser ${ }^{4}$; JC. Schefolg1;

K. Amrein ${ }^{5}$; M. Hoffmann 6

${ }^{1}$ Department of intensive care medicine, University Hospital Bern, Bern, Switzerland; ${ }^{2}$ Department of intensive care medicine, University Hospital Bern, Bern, Switzerland; ${ }^{3}$ Department for Anesthesia and Intensive Care Medicine, Landeskrankenhaus Feldkirch, Feldkirch, Austria; ${ }^{4}$ Clinic for cardiovascular surgery, University Hospital Bern, Bern, Switzerland; ${ }^{5}$ Division of endocrinology and diabetology, Medical University of Graz, Graz, Austria; ${ }^{6}$ Division of endocrinology and diabetology/research unit for safety in health/ qm-rm, Medical University of Graz, University Hospital Graz, Graz, Austria

Correspondence: M.M. Jeitziner

Intensive Care Medicine Experimental 2020, 8(2): 001127

Introduction: A hospitalization due to COVID-19 in the intensive care units (ICU) is challenging for patients, their families, and ICU professionals. In addition to coping with the uncertainties of a widely unknown disease, visits is limited or even prohibited during a pandemic. These restrictions require special attention and trigger unconventional ways for personal contact among ICU professionals with families in order to provide required informations and support.

Objectives: This Delphi study aimed 1) to explore the opinions and experiences of experts during the COVID-19 pandemic in relation to supporting ICU patients and their families; and 2) to provide recommendations for supporting families during a pandemic.

Methods: A two-round modified Delphi processassessed the opinions and experiences of experts from Germany, Switzerland, Austria and Lichtenstein between May and June 2020. Experts included ICU professionals, patients, and family members. 120 experts answered anonymously the first online questionnaires with 33 questions based on a literature review and recommendations of ICU experts. Each question contained an additional comment section. The answers were analyzed and transformed into a second questionnaire with 16 questions and comment sections, which was answered by 131 experts. Data analysis will include descriptive statistics and content analysis of the answers in all comment sections.

Results: First results indicate that experts perceived family support on ICU as highly important during the COVID-19 pandemic. Enabling contact amongst patients, families and clinicians is regarded essential to build hope and confidence. The role of a designated contact person, who delivers frequent and consistent information and builds a supportive and professional relationship, may even be more important. The extraordinary situation leads to the implementation of new communication structures to support families and to document the patients' ICU stay for future processing. The use of video calls, or other forms of communication are mostly wanted under the condition that data security is guaranteed, and that enough personnel and technical resources are available. Some experts see a potential for application of such new resources beyond the current pandemic.

Conclusion: During a pandemic, virtual contacts are essential for COVID-19/ICU patients and respective families to gain and maintain faith confidence in ICU professionals and ICU treatment. Ethical considerations are crucial in deciding what forms of communication would be most appropriate, considering data security, resource distribution, and the information need of patients and their families.

\section{6}

Profiling the physical rehabilitation of COVID-19 patients

admitted to critical care

J. Dowds ${ }^{1}$; G. Sheill1 ; K. O Brien ${ }^{1}$; N. Murphy ${ }^{1}$; C. Bannan²;

I. Martin-Loeches ${ }^{3}$

'Department of physiotherapy, St James"s Hospital, Dublin, Ireland:

${ }^{2}$ Department of genito urinary medicine and infectious disease and school of medicine, St James"s Hospital and Trinity College Dublin, Dublin, Ireland; ${ }^{3}$ School of medicine, Trinity College Dublin, Dublin, Ireland Correspondence: $\mathrm{G}$. Sheill

Intensive Care Medicine Experimental 2020, 8(2): 001166
Introduction: The COVID-19 pandemic brings a large number of people experiencing critical care admissions. It is important to profile the physical rehabilitation required by patients admitted to hospital with COVID-19 in order to identify the physical side-effects of critical care admission and to determine the rehabilitation services required by this patient group.

Objectives: The aim of this study is to profile the acute rehabilitation pathway of people with COVID-19 admitted to critical care.

Methods: This study was a retrospective patient chart audit of all patients admitted to critical care with COVID-19 between March 16th and May 2nd, 2020. Ethical approval was granted for this study. Demographic information and information regarding critical care stay was collected for each patient. Physical rehabilitation was assessed against standardised rehabilitation milestones as per the ICU Mobility Scale.

Results: A total of 64 patients were admitted to critical care $(65 \%$ male, mean age 60 years SD 14.1). 56 had a confirmed diagnosis of COVID-19 on testing, while 8 patients were classified as 'highly suspicious' for COVID-19. A total of 14 patients died in critical care and 1 patient remains in critical care, leaving 49 patients for inclusion in the rehabilitation audit. $33(67 \%)$ of these patients were intubated, with an average critical care length of stay of 16 days (SD 15.1, range 2-69 days). All patients were referred for physical rehabilitation during their critical care stay (range: 0-49 sessions).

On average, patients began active rehabilitation sitting over the edge of the bed 11 days (range 3-46 days) after ICU admission. Patients progressed to step transfers from bed to chair a mean of 1.35 days later and mobilised away from the bedside a mean of 4.05 days later. In total 26 (53\%) participants experienced a documented oxygen desaturation during physical rehabilitation, the majority while transferring out of bed and mobilising ( $\mathrm{SaO} 290-95 \% \mathrm{n}=9$, SaO2 $85-90 \% \mathrm{n}=11$, $\mathrm{SaO} 280-85 \% \mathrm{n}=4$, $\mathrm{SaO} 275-80 \% \mathrm{n}=2$ ).

A total of $43(88 \%)$ patients had independent mobility before admission to critical care, while $6(12 \%)$ required mobility aid/assistance with mobility. On discharge from hospital 34 (69\%) patients had returned to baseline mobility levels, while 6 patients required increased aids/ assistance with mobility when compared to baseline levels. A further 9 patients (18\%) were still receiving inpatient rehabilitation at the time of this audit.

Conclusion: Findings of this study indicate that patients with COVID19 who survived a stay in critical required inpatient physical rehabilitation. Once rehabilitation began, a large proportion of patients progressed quickly through functional mobility classifications to become independently mobile. A sub-set of patients in this audit have required extensive inpatient physical rehabilitation following a critical care stay.

\section{3}

Thrombo-embolic events associated with Covid-19 ARDS Epidemiology and risk factors

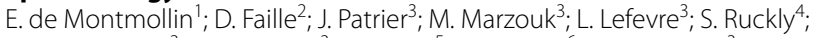
G. Franchineau $u^{3}$; L. Bouadma ${ }^{3}$; K. Peoch ${ }^{5}$; V. Andrieu ${ }^{6}$; R. Sonneville ${ }^{3}$;

N. Azjenberg ${ }^{2}$; JF. Timsit ${ }^{1}$

${ }^{1}$ Intensive care medicine department, Assistance Hopitaux Publique de Paris, Paris, France; ${ }^{2}$ Hematobiology, Assistance Hopitaux Publique de Paris Bichat, Paris, France; ${ }^{3}$ Paris, Medical and infectious Intensive Care Unit, Bichat-Claude Bernard Hospital, Paris, France; ${ }^{4}$ Umr 1137, INSERM, Paris, France; ${ }^{5}$ Biochemistery, Assistance Hopitaux Publique de Paris, Paris, France; ${ }^{6}$ Hematobiology, Assistance Hopitaux Publique de Paris, Paris, France

Correspondence: J.F. Timsit

Intensive Care Medicine Experimental 2020, 8(2): 001193

Introduction: Covid-19 severe ARDS is frequently associated with venous thrombosis and pulmonary emboli. The first events we diagnosed lead us to recommend a high heparin prophylaxis (4000 IU enoxaparin bid or $6000 \mathrm{IU}$ bid if weight $>120 \mathrm{~kg}$; targeted anti-Xa activity $0.3-0.5 \mathrm{UI} / \mathrm{mL}$ ) or an Immediate use of curative anticoagulant therapy (enoxaparin $100 \mathrm{lU} / \mathrm{kg}$ bid or unfractionated heparin $500 \mathrm{IU} /$ $\mathrm{kg} / \mathrm{d}$; targeted anti-Xa 0.5-0.7 Ul/mL). 
Objectives: To evaluate the risk factors and epidemiology of deep venous thrombosis (DVT) and pulmonary emboli (PE) when this strategy is applied to all the Covid-19 patients with ARDS.

Methods:All the consecutive patients with severe ARDS due to SARSCov2 were systematically followed until hospital discharge or day60. Doppler US of the vein was routinely performed to all patients. IV CT scan was performed as clinically indicated. We recorded daily clinical and biological covariates as well as antimicrobial, anticoagulant and anti-inflammatory therapies from ICU admission to ICU discharge. Patients were followed until hospital discharge or day 60 . No patient was lost to follow-up.

We use a competing cause-specific risk model to evaluate the risk of DVT/PE during the follow-up (SAS 9.X, SAS system NC, USA). We tested variables at ICU admission and variables collected daily as time-dependent covariates. Variables collected at time $\mathrm{t}-1$ were used to model even occurring at time t. A p value of 0.05 or less was considered significant. The impact of time-dependent DVT/PE on patients' survival was tested using a Cox model.

Results: Of the 134 patients with Sars-Cov2 ARDS admitted in our ICU (age 59.5 [51; 69]; SAPSII: SOFA 5 [4; 7]), 47 (35.1\%) died at day 28. 21 developed a DVT/PE (10/21 ultimately died), 45 died without DVT/PE and 68 were censored alive without DVT/PE. Main characteristics of the patients and anticoagulant therapy used at ICUadmission are on Table.

The cause specific model identified high SOFA score, use of central vein catheter, hypothermia less than $36^{\circ} \mathrm{C}$, high BUN, high ALAT, high $\mathrm{LDH}$, low hematocrit, high neutrophils count as risk factors of DVT/ PE. Neither anticoagulant therapy (curative vs prophylaxis) given the day before nor anti-Xa activity were related with DVT/PE risk. Platelet count, prothrombin time, fibrinogen level, fibrin monomers, D-dimer, troponin levels were not related to DVT/PE risk.

DVT/PE was not related to survival even after adjusting on comorbidities and SOFA score at ICU admission HR $=1.679 ; 95 \% \mathrm{Cl}[0.802 ; 3.512]$, $\mathrm{p}=0.17$

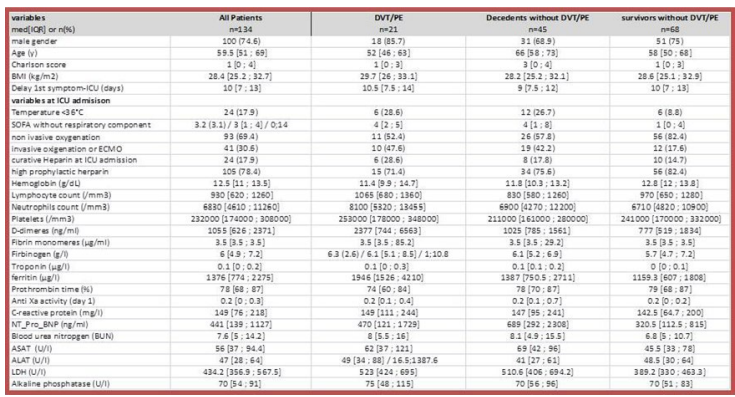

Conclusion: Even when using a high-risk prophylaxis, curative anticoagulant therapy, DVT/PE occurred in $16 \%$ of cases. The risk is higher in patients with high SOFA score, renal and hepatic failure, high neutrophil count suggesting that other mechanisms than coagulation (i.e. endothelial activation and/or hypofibrinolysis) may concur to thromboembolic events.

\section{7}

Acute kidney injury in critically ill patients with COVID - 19 disease: AKICOV multicenter study

A. E Mera'; A. Navas-Pérez²; FJ. González De Molina ${ }^{3}$; M. Rodríguez-López ${ }^{4}$ M. Torrens Sonet ${ }^{5}$; M. Alvarez Garcia-Pumarino ${ }^{6}$; TM. Tomasa-Irriguible J. Sabater-Riera ${ }^{8}$; M. Cerdá Martínez ${ }^{2}$; J. Prados Chica ${ }^{9}$; A. Baró Serra ${ }^{10}$; E. Casanova-Gongora ${ }^{11}$; M. Pérez_Carrasco'; Y. Díaz-Buendía ${ }^{12}$; P. Ortiz Ballujera ${ }^{13}$; A. Olmo-Isasmendii ${ }^{14}$; C. Rovira-Anglès ${ }^{15}$; I. Oliva-Zelaya ${ }^{16}$; RM. Catalán-Ibars ${ }^{17} ;$ M. Ibarz-Vilamayor ${ }^{18}$

${ }^{1}$ Intensive care, Vall d'Hebron University Hospital, Barcelona, Spain; ${ }^{2}$ Intensive care, Hospital Parc Taulí de Sabadell, Sabadell, Spain; ${ }^{3}$ Intensive care, Mútua Terrassa University Hospital, Terrassa, Spain; ${ }^{4}$ Intensive care, Hospital de Sant Joan Despí Moisès Broggi, Sant Joan Despí, Spain;
${ }^{5}$ Intensive care, Hospital Universitari Santa Creu i Sant Pau, Barcelona, Spain; ${ }^{6}$ Intensive care, Consorci Sanitari de Terrassa, Terrassa, Spain; ${ }^{7}$ Intensive Care Department, Hospital Germans Trias i Pujol, Badalona, Spain ${ }^{8}$ Intensive care, Bellvitge University Hospital, L'Hospitalet de Llobregat, Spain; ${ }^{9}$ Intensive care, University Hospital Arnau de Vilanova, Lleida, Spain: ${ }^{10}$ Intensive care, Hospital Santa Caterina en Salt, Salt, Spain; ${ }^{11}$ Intensive care, Hospital Asepeyo Barcelona, Sant Cugat del Valles, Spain; ${ }^{12}$ Intensive care, Hospital del Mar, Barcelona, Spain; ${ }^{13}$ Intensive care medicine, Hospital Universitari de Girona Dr Josep Trueta, Girona, Spain; ${ }^{14}$ Intensive care, Helipuerto Hospital General de Catalunya, Sant Cugat del Vallès, Spain; ${ }^{15}$ Intensive care, Hospital Universitari Sant Joan de Reus, Reus, Spain; ${ }^{16}$ Intensive care, Hospital Universitari de Tarragona Joan XXIII, Tarragona, Spain; ${ }^{17}$ Intensive care, Hospital Universitari de Vic (Consorci Hospitalari de Vic), Vic, Spain; ${ }^{18}$ Intensive care, Hospital Universitari Sagrat Cor - Grup Quirónsalut, Barcelona, Spain

Correspondence: $M$. Pérez_Carrasco

Intensive Care Medicine Experimental 2020, 8(2): 001197

Introduction: Severe Acute Respiratory Syndrome Coronavirus 2 has spread at great speed throughout the world. Between 15 and $25 \%$ of hospitalized patients infected with COVID-19 develop acute kidney injury (AKI).

Objectives: TTo describe the incidence, evolution and prognosis of Acute Kidney Injury (AKI) in critically ill patients with the COVID-19 disease.

Methods: A multicenter, descriptive, observational study including COVID-19 patients admitted to 19 Catalonian UCls. Usual demographic variables, drugs, AKI development, several therapies, mortality and ICU/hospital stay were collected.

Descriptive statistics and logistic regression analysis for AKI development and mortality were used.

Results: A total of 936 patients were enrolled, of whom $66 \%$ were men and mean age was 64(17) years. APACHE II and SOFA scores were $13(8)$ and 5(4), respectively. Viral pneumonia with ARDS was observed in $88 \%$ of the patients. MV was required by $76 \%$ of patients, and in addition, $56 \%$ required prone position, $66 \%$ received vasopressors and $51 \%$ treatment with glucocorticoids. AKI prevalence at ICU admission was $28.4 \%$ and increased during ICU stay up to $43.6 \%$ : $29.4 \%$ was developed in the first 72 hours and $36 \%$ in the following 7 days. Only $88(9.3 \%)$ required RRT.

AKI occurred more frequently in older patients (66 years vs 59 years), males (74\% vs $61 \%$ ), those with higher ICU scores (APACHE II: 15 vs 10 SOFA: 6.5 vs 5 ), hypertension ( $58 \%$ vs $42 \%)$, CPOD ( $9.7 \%$ vs $5.9 \%$ ), CKD ( $10 \%$ vs $1,3 \%)$, diabetes mellitus ( $29 \%$ vs $18 \%)$ and chronic ischemic heart disease ( $7 \%$ vs $4 \%$ ).

AKI was more frequent in ARDS severe patients (59\% vs $43 \%, p<$ $0.001)$, in MV patients $(91.5 \%$ vs $78 \%, p<0.001)$ and required more prone position $(69 \%$ vs $58 \%, p<0.001)$. The treatment included Remdesivir ( $2.3 \%$ vs $0.5 \%, p<0.039)$, interferon ( $32 \%$ vs $23 \%, p<0.009)$, glucocorticoids in patients with shock $(6 \%$ vs $1.1 \%, p<0,001)$ and vasopressors ( $86 \%$ vs $63 \%, p<0,001)$.

AKI patients developed more infections during ICU stay: ventilator associated pneumonia (VAP) $(35 \%$ vs $22 \%, p<0.001)$ and catheter related bloodstream infections $(17 \%$ vs $11 \%, p<0.021)$.

Risk factors associated with AKI development were age (IC 1.02-1.05), gender (male IC 1.4-2.9), APACHE II (1.02- 1.07), SOFA (1.1-1.2), CKD (3.13-21.6), Shock (2.03-4.4) and VAP (1.11-2.3).

Mortality in ICU stay was 30\% and overall in-hospital mortality $33 \%$. Mortality was increased in AKI patients in both groups: ICU stay (47\% vs $15 \%, p<0,001)$ and in-hospital $(51.7 \%$ vs $16.5 \%, p<0,001)$.

AKI was an independent factor associated with mortality (IC $1.5-3.45$ ). Mortality was higher in patients that required RRT both in the ICU (57 $\%$ vs $44 \%, p<0.04)$ and in-hospital $(62.5 \%$ vs $48 \%, p<0.03)$. The duration of ICU stay was longer in AKI patients (22 vs 16 days, $p<0.001$ ).

Conclusion: There is a high incidence of AKI in critically ill patients with COVID 19 disease and it is associated with higher mortality, increased organ failure, nosocomial infections and prolonged ICU stay. 
Reference(s) and grant ackowledgment(s)

1. Fanelli V, Fiorentino M, Cantaluppi V, Gesualdo L, Stallone G, Ronco C, Castellano G. Acute kidney injury in SARS-CoV-2 infected patients. Crit Care. 2020;24(1):155.

\section{3}

Delirium Prediction in COVID-19 Intensive Care Unit: A Bayesian Model with Informed Priors (DELIPIOUS)

A. Al-Hindawi ${ }^{1}$; J. Sokhi ; F. El-Hibri ${ }^{1}$; A. Hill' ${ }^{1}$; A. Sisson ${ }^{1}$; M. Vizcaychipi ${ }^{1}$ Intensive care unit, Chelsea and Westminster Hospital, London, United Kingdom

Correspondence: A. Al-Hindawi

Intensive Care Medicine Experimental 2020, 8(2): 001203

Introduction: Patients infected with COVID-19 are recognised to have a spectrum of neurological complications. In a systematic review, of those who survived, $65 \%$ developed delirium during their intensive care stay [1]. Current models assessing delirium in the Adult Intensive Care Unit (AICU), such as E-PRE-DELIRIC, lacks discriminant variables for the prediction of delirium in patients with COVID-19 [6]. Furthermore; the increased in length of stay (LOS) for those with COVID-19 (average 20 days) invalidates models such as E-PRE-DELIRIC which has been modelled on an average LOS of 6 days.

The pathogenesis of delirium in AICU is thought to be a combination of disease severity, hypoxia, highinflammatory response and thrombosis; all elements present in patients with COVID-19 [3-5]. Consequently, further research into mental health surrounding COVID-19 has been set as a priority [6].

\section{Objectives:}

1. To test the validity of E-PRE-DELIRIC in patients with COVID-19.

2. Develop an internally valid, COVID-19 specific, delirium prediction model

using Bayesian Hamiltonian Markov Chain Monte Carlo methods.

Methods: Anonymised data from SARS-CoV-2 positive patients admitted to AICU at Chelsea and Westminster Hospital was collected from the 9th of March to the 18th of May 2020. CAM-ICU or author consensus was used to identify patients who underwent delirium assessment. Observational, pathological, and ventilatory variables from patients were extracted from electronic patient records. Forward variable selection strategy was used in the patient's recovery phase; this phase was identified through dichotomisation of their AICU stay following an alignment procedure that centres the patients' stay, such that day 0 is the day with their lowest $\mathrm{PaO}_{2} / \mathrm{FiO}_{2}$ (PF) ratio. The resulting dataset was split into a training cohort (80\%) and a validation cohort (20\%).

Analysis was performed in $\mathrm{R}$ version 4.0. Candidate variables were standardised to a mean of 0 and a standard deviation of 1 prior to the use of BRMS as an interface to Stan. Receiver Operator Curves (ROC) and the Area Under the Curve (AUC) are used to demonstrate model performance.

Results: 66 patients were screened of which 31 were excluded resulting in the identification of 23 patients with delirium and 12 patients without delirium (Figure 1).

The alignment procedure successfully identified the recovery phase with U-shaped PF ratio pattern centred on the lowest ratio (Figure 2 ).

E-PRE-DELIRIC was found to be inaccurate in the prediction of delirium in patients with COVID-19 with an AUC of $48.6 \%$. DELIPIOUS, a Bayesian model with priors informed from both the delirium and COVID-19 literature, was developed demonstrating good performance with an AUC of $76.7 \%$ (Figure 3 ). The proposed model uses biologically plausible variables that reflect the pathogenesis of delirium in COVID-19. By defining the recovery period and using values within, temporal relationship between the timing of the variables' values and onset of delirium is maintained. This reduces the lag-time between variable value and outcome given the prolonged stay of COVID-19 patients resulting in higher accuracy.

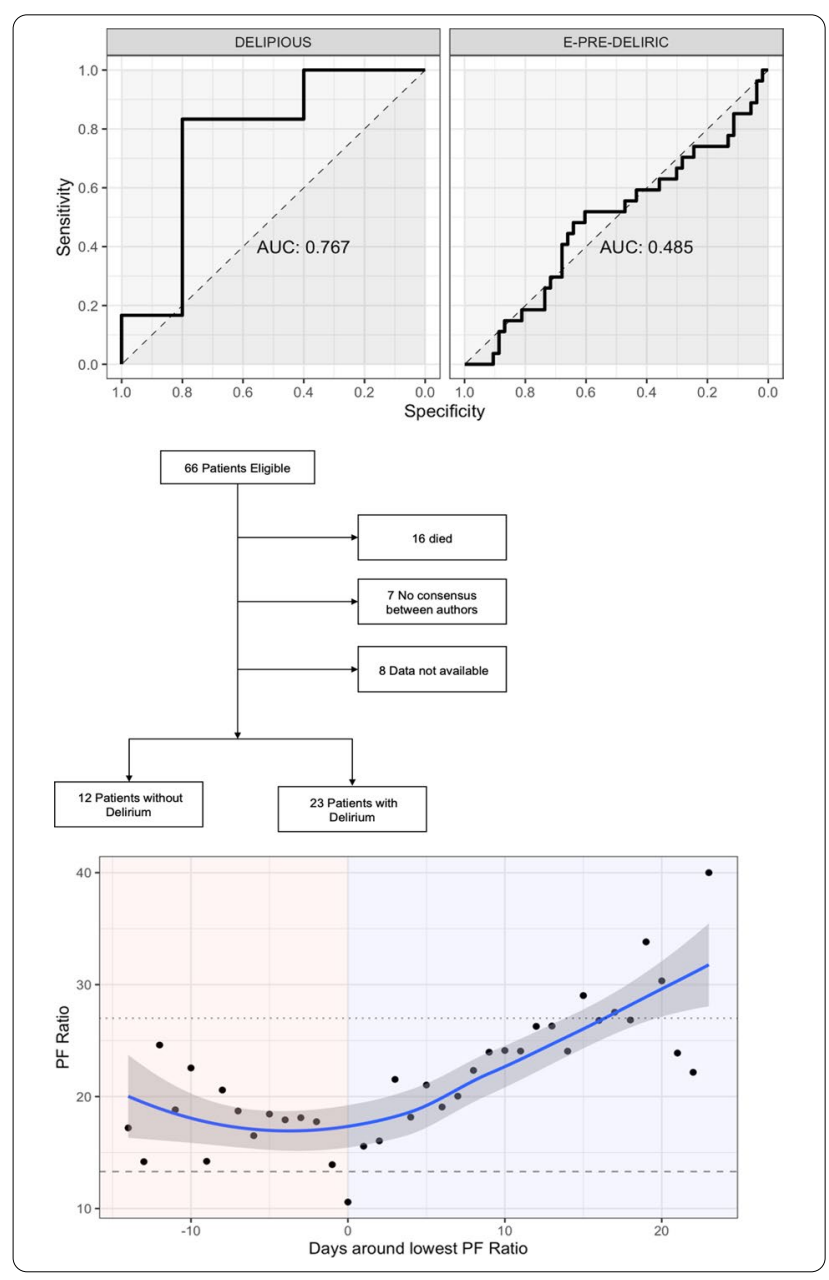

Conclusion: E-PRE-DELIRIC has a poor performance in those with COVID-19. DEPLIPIOUS uses biologically plausible variables that reflect the pathogenesis of delirium in COVID-19 resulting in an accuracy of $76.7 \%$. It requires external and prospective validation prior to its clinical use.

\section{Reference(s) and grant ackowledgment(s)}

1. None

2. [6] E. A. Holmes et al.,' Multidisciplinary research priorities for the COVID-19 pandemic: a call for action for mental health science', Lancet Psychiatry, vol. 7, no. 6, pp. 547-560, Jun. 2020, https://doi.org/10.1016/\$2215 $-0366(20) 30168-1$

3. [5] J. Cerejeira, H. Firmino, A. Vaz-Serra, and E. B. Mukaetova-Ladinska, 'The neuroinflammatory hypothesis of delirium', Acta Neuropathol. (Berl.), vol. 119, no. 6, pp. 737-754, Jun. 2010, https://doi.org/10.1007/s0040 1-010-0674-1

4. [4] W. A. C. Palmbergen, A. Van Sonderen, A. M. Keyhan-Falsafi, R. W. M. Keunen, and R. Wolterbeek, 'Improved perioperative neurological monitoring of coronary artery bypass graft patients reduces the incidence of postoperative delirium: the Haga Brain Care Strategy,' Interact. Cardiovasc. Thorac. Surg., vol. 15, no. 4, pp. 671-677, Sep. 2012, https://doi.org/10.1093/ icvts/ivs317

5. [3] J. Hirsch, G. DePalma, T. T. Tsai, L. P. Sands, and J. M. Leung, 'Impact of intraoperative hypotension and blood pressure fluctuations on early postoperative delirium after non-cardiac surgery', Br. J. Anaesth., vol. 115, no. 3 , pp. 418-426, Sep. 2015, https://doi.org/10.1093/bja/aeu458

6. [2] A. Wassenaar et al.,'Multinational development and validation of an early prediction model for delirium in ICU patients', Intensive Care Med., vol. 41, no. 6, pp. 1048-1056, Jun. 2015, https://doi.org/10.1007/s00134-015-3777-2. 
7. [1] J. P. Rogers et al.,'Psychiatric and neuropsychiatric presentations associated with severe coronavirus infections: a systematic review and metaanalysis with comparison to the COVID-19 pandemic', Lancet Psychiatry, May 2020, https://doi.org/10.1016/S2215-0366(20)30203-0.

\section{4}

\section{Intensive Care specific Virtual Reality (ICU-VR) improves} Post-Intensive Care Syndrome-related psychological sequelae in survivors of critical illness

JH. Vlake ; EJ. Wils ${ }^{1}$; J. Van Bommel ${ }^{2}$; T. Korevaar ${ }^{3}$; D. Gommers ${ }^{2}$; M. van Genderen $^{2}$

${ }^{1}$ Intensive care, Franciscus Gasthuis, Rotterdam, Netherlands; ${ }^{2}$ Intensive care, Erasmus MC, Rotterdam, Netherlands, Netherlands; ${ }^{3}$ Internal medicine,environmental epidemiology, Erasmus MC, Rotterdam, Netherlands, Netherlands

\section{Correspondence: J.H. Vlake}

Intensive Care Medicine Experimental 2020, 8(2): 001264

Introduction: A substantial proportion of Intensive Care unit (ICU) survivors develops psychological impairments in the aftermath of ICU treatment, such as post-traumatic stress disorder (PTSD), anxiety and depression (1). These impairments are the major determinant for decreased health-related quality of life (HRQoL) (2), and it is hypothesized that an unmet need for information regarding ICU-treatment might be an important contributing factor (3). An effective evidencebased treatment for these psychological impairments and this need for information is still lacking. Virtual reality (VR) exposure therapy for several psychological disorders is effective and safe and could provide an adequate method to deliver the desired information of the ICU period (3). To date, it is unknown whether Intensive Care specific VR exposure in survivors of critical illness decreases psychological sequelae.

Objectives: To examine the effect of ICU-specific Virtual Reality (ICUVR) on psychological sequelae and quality of life.

Methods: We conducted a multicenter, randomized pilot study in ICU patients admitted with sepsis or septic shock and mechanically ventilated for $\geq 24$ hours. A week after ICU discharge, patients were randomized into the control or intervention group. The intervention group received ICU-VR, providing both exposure to the environment traumatizing them in combination with information regarding several aspects of their ICU treatment. The control group received a static VR environment for the same duration of time. Safety of ICU-VR was assessed by monitoring vital signs and the simulator sickness questionnaire (SSQ). Psychological sequelae and HRQoL were assessed at baseline (T0) and 2 days (T1), 7 days (T2), 1 month (T3) and 6 months (T4) after initial exposure to VR. Depression was assessed using the Beck Depression Inventory (BDI), and PTSD using the Impact of Event Scale - Revised (IES-R). HRQoL was assessed using the Short-Form 12 (SF-12) and the EuroQol 5 dimensions (EQ-5D).

Results: We included 50 patients, median age 61 years (IQR: 51-68), median APACHE IV score of 77 (IQR: 57-106) and an median ICU length of stay of 16 days (IQR: 8-31). During VR exposure, no changes in vital signs were observed and none of the individual SSQ items were scored as severe. After 1 month (T3), patients in the intervention group scored significantly lower on PTSD (IES-R sum score, median (IQR): 8 (3-13) vs. $17(12-26), P=0.003)$ and depression (BDI sum score, median (IQR): 8 (6-12) vs. 12 (8-18), $P=0.04)$. This effect persisted at 6 months after ICU discharge (T4) for PTSD (IES-R sum score, median (IQR): 5 (1-13) vs. 23 $(10-27), P=0.01)$, but not for depression (BDI sum score, median (IQR): 4 (2-12) vs. 10 (6-17), $P=0.07$ ) (Figure). Subsequently, although we observed no difference in the physical quality of life between groups, patients in the intervention group reported a better mental quality of life 1 month after VR exposure (Mental Component Scale (MCS-12), median (IQR): 57 (53-61) vs. 47 (39-49), $P=0.002)$, but not at 6 months after ICU discharge (MCS-12, median (IQR): 50 (41-58) vs. 47 (39-56), $P=0.43$ ).

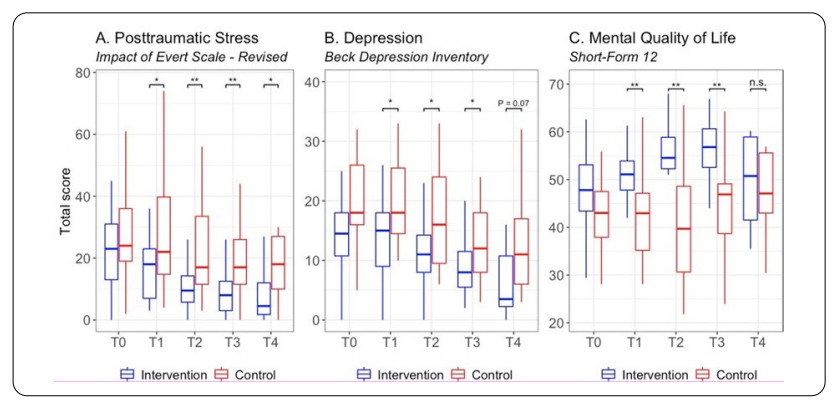

Conclusion: We demonstrate that ICU-VR decreases PTSD and depression up to 6 months after ICU discharge, is safe and improves mental quality of life. Further research is needed to confirm these effects on a larger scale.

Reference(s) and grant ackowledgment(s)

1. 1. Needham DM, Davidson J, Cohen H, Hopkins RO, Weinert C, Wunsch $\mathrm{H}$, et al. Improving long-term outcomes after discharge from intensive care unit: report from a stakeholders' conference. Crit Care Med. 2012:40(2):502-9.

2. 2. Kerckhoffs MC, Kosasi FFL, Soliman IW, van Delden JJM, Cremer OL, de Lange DW, et al. Determinants of self-reported unacceptable outcome of intensive care treatment 1 year after discharge. Intensive Care Med. 2019;45(6):806-14

3. 3. Vlake JH, van Genderen ME, Schut A, Verkade M, Wils E-J, Gommers D, et al. Patients suffering from psychological impairments following critical illness are in need of information. Journal of Intensive Care. 2020;8(1):6.

\section{8}

Relationship between SARS-CoV-2 infection and the incidence of ventilator-associated lower respiratory tract infections: a European multicenter cohort study

A. Rouzél; I. Martin-Loeches ${ }^{2}$; P. Povoa ${ }^{3}$; D. Makris ${ }^{4}$; A. Artigas ${ }^{5}$;

J. Labreuche ${ }^{6}$; S. Nseir ${ }^{1}$

${ }^{1}$ Icu, Chu De Lille, Lille, France; ${ }^{2}$ School of medicine, Trinity College Dublin, Dublin, Ireland; ${ }^{3}$ Polyvalent intensive care unit, São Francisco Xavier Hospital, CHLO, Lisbon, Portugal; ${ }^{4}$ Department of inttensive care medicine, University of Thessaly, Medical Schoos, Larissa, Greece; ${ }^{5}$ Department of intensive care medicine, Corporacion Sanitaria Universitaria Parc Tauli, Barcelona, Spain; ${ }^{6}$ Statistics, Chu De Lille, Lille, France

Correspondence: $S$. Nseir

Intensive Care Medicine Experimental 2020, 8(2): 001268

Introduction: Although patients with SARS-CoV-2 infection have several risk factors for ventilator-associated lower respiratory tract infections (VA-LRTI), the reported incidence of hospital-acquired infections is low.

Objectives: We aimed to determine the relationship between SARSCoV-2 pneumonia, as compared to influenza pneumonia or no viral infection, and the incidence of VA-LRTI. Secondary objectives were to determine the relationship between SARS-CoV-2 infection and the etiology and outcomes of VA-LRTI.

Methods: Multicenter retrospective European cohort performed in 36 ICUs. All adult patients receiving invasive mechanical ventilation for more than $48 \mathrm{~h}$ were eligible for this study if they had: SARS-CoV-2 pneumonia, influenza (A or B) pneumonia, or no viral infection at ICU admission. VA-LRTI, including ventilator-associated tracheobronchitis (VAT) and ventilator-associated pneumonia (VAP), were diagnosed using clinical, radiological and quantitative microbiological criteria. All VA-LRTI were prospectively identified, and chest- $X$ rays were analyzed by at least two physicians. Cumulative incidence of first episodes of VA-LRTI was estimated using Kalbfleisch and Prentice method, and 
compared using Fine-and Gray models. This study is registered on ClinicalTrials.gov, NCT04359693.

Results: 1576 patients were included (568 in SARS-CoV-2 group, 482 in influenza group, and 526 in no viral infection group). VA-LRTI incidence was significantly higher in SARS-CoV2 patients (287, 50.5\%), as compared to influenza patients $(146,30.3 \%$, adjusted sub hazard ratio (SHR) 1.60 (95\% confidence interval (Cl) 1.27 to 2.00$)$ ) or patients with no viral infection (133,25.3\%, adjusted SHR 1.65 (95\% CI 1.22 to 2.22$)$ ). VA-LRTI were mainly caused by Gram-negative bacilli (Pseudomona aeruginosa, Enterobacter spp., and Klebsiella spp.). VAP was associated with increased adjusted HR for 28-day mortality in SARS-CoV-2 and influenza groups, and for duration of ICU stay in all study groups.

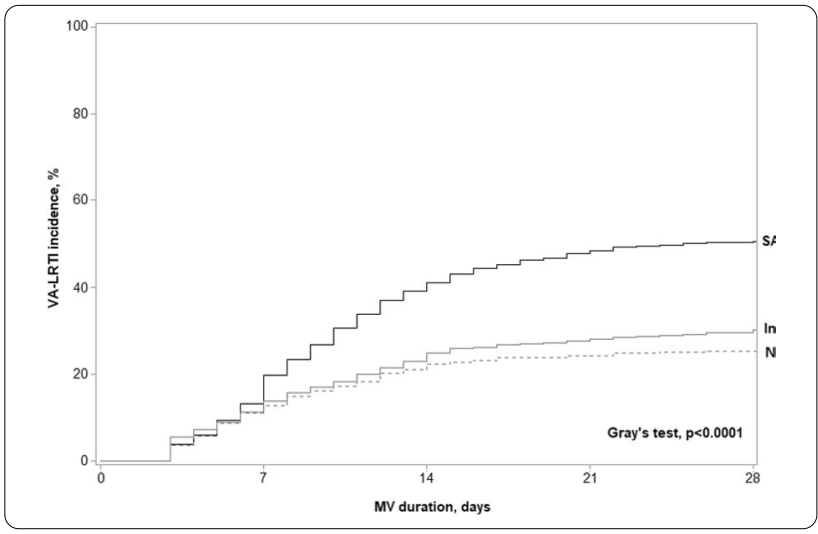

Conclusion: The incidence of VA-LRTI is significantly higher in patients with SARS-CoV-2 infection, as compared to patients with influenza pneumonia, or no viral infection.

\section{Reference(s) and grant ackowledgment(s)}

1. I-SITE Université Lille Nord-Europe

\section{9}

Zarit Burden Interview to evaluate main caregivers overload in Intensive Care Chronic patients

O. Moreno Romero ${ }^{1}$; A. Carranza Pinel' ${ }^{1}$ : M. Muñoz Garach

'Intensive care unit, Hospital Universitario Clínico San Cecilio, Granada, Spain

Correspondence: $\mathrm{O}$. Moreno Romero

Intensive Care Medicine Experimental 2020, 8(2): 001279

Introduction: Critically ill patients survival is increasing nowadays parallel to fragilty and morbidity in long therm surviving ICU patients, thus the role of caregivers in some cases has become essential.

Objectives: First, to evaluate the main caregiver overload of chronic patients roled in our Home Mechanical Ventilation Unit (driven by intensive care physicians) using the Zarit Burden Interview. Secondly, to describe the characteristics of our caregivers and patients.

Methods: The evaluation was perform with a presential interview to the main caregiver of all the patients included in our program during 12 months, from January 2018 to January 2019. We applied the Zarit Burden interview, which classifies the caregiver as a sum of various items, in 3 grades: absence of overload ( $<46$ points), mild overload (46-55), heavy overload ( $>56$ points) (Figure 1). Epidemiological data of caregivers (age, sex, number of carers, state economical help) and patients (age, sex, Barthel Index, diagnostic).
THE ZARIT BURDEN INTERVIEW

\begin{tabular}{|c|c|c|c|c|c|c|}
\hline & Never & Rarely & Sometimes & $\begin{array}{l}\text { Quite } \\
\text { Frevoently }\end{array}$ & $\begin{array}{l}\text { Neaty } \\
\text { Always }\end{array}$ & Score \\
\hline $\begin{array}{l}\text { 1. Do you feel that your relefitive askis } \\
\text { for more help than heishe needs? }\end{array}$ & 0 & 1 & 2 & 3 & 4 & \\
\hline $\begin{array}{l}\text { 2. Do you feel that because of the } \\
\text { fime you spend with your relative that } \\
\text { you don't have enought time for } \\
\text { hourself? }\end{array}$ & 0 & 1 & 2 & 3 & 4 & \\
\hline $\begin{array}{l}\text { 3. Do you feel stressed betwien } \\
\text { caning for your relative and trying to } \\
\text { meat other responsibilifias for your } \\
\text { family or wark? }\end{array}$ & 0 & 1 & 2 & 3 & 4 & \\
\hline $\begin{array}{l}\text { 4. Do you feet enbarrassed oner your } \\
\text { relative's behaviour? }\end{array}$ & 0 & 1 & 2 & 3 & 4 & \\
\hline $\begin{array}{l}\text { 5. Do you feel angry when you are } \\
\text { around your relatwe? }\end{array}$ & 0 & 1 & 2 & 3 & 4 & \\
\hline $\begin{array}{l}\text { 6. Do you feel that your relative } \\
\text { currently affects cur relationships with } \\
\text { other family members or triends in a } \\
\text { negative way? }\end{array}$ & 0 & 1 & 2 & 3 & 4 & \\
\hline $\begin{array}{l}\text { 7. Are you afraid what the future holds } \\
\text { for your relative? }\end{array}$ & 0 & 1 & 2 & 3 & 4 & \\
\hline $\begin{array}{l}\text { 8. Do you feel your relative is } \\
\text { dependent an you? }\end{array}$ & 0 & 1 & 2 & 3 & 4 & \\
\hline $\begin{array}{l}\text { 9. Do you feel statined whiten you are } \\
\text { around your relatave? }\end{array}$ & 0 & 1 & 2 & 3 & 4 & \\
\hline $\begin{array}{l}\text { 10. Do you feel your health has } \\
\text { suffered because of your involvement } \\
\text { with your realitive? }\end{array}$ & 0 & 1 & 2 & 3 & 4 & \\
\hline $\begin{array}{l}\text { 11. Do you feel that you don't have as } \\
\text { much privacy as you would like } \\
\text { becaune of your retathe? }\end{array}$ & 0 & 1 & 2 & 3 & 4 & \\
\hline $\begin{array}{l}12 \text { Do you feel ithat your sacial life } \\
\text { has suffered because you are caring } \\
\text { for your relative? }\end{array}$ & 0 & 1 & 2 & 3 & 4 & \\
\hline 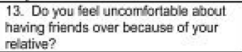 & 0 & 1 & 2 & 3 & 4 & \\
\hline
\end{tabular}

Results: In 12 months, 49 patients and caregivers were roled in our study. Zarit Burden Scale of caregivers: $6(12,2 \%)$ abscense of overload, $28(57,1 \%)$ mild overload and $15(30,6 \%)$ with heavy overload.

Caregiver Characteristics: mean age 53,58 $\pm 15,61$, women 38 (77,5\%); one single caregiver in $28(57,1 \%)$; state ecomomical significant help in 26 (53\%).

Patient data: mean age 43,73 $\pm 13,1$, man 33 (67,3\%), Barthel Index $45,42 \pm 33,2$ (IC95\% 36,1-54,7), diagnostic: Amyotrophic Lateral Sclerosis ALS in $22(45 \%)$, other myopathies and neuromuscular diseases $16(32,6 \%)$, dificulty in weaning $11(22,4 \%)$ due to mechanical ventilation dependency: COPD (6), critical patientpolineuromiopathy (4), others (1). From these, NIMV (6), IMV (5).

Conclusion: In our caregivers using the Zarit Burden Scale shows that more than $85 \%$ have moderate-severe overload. Women is the prevalent carer (77\%). Neumomuscular diseases is the most importante cause for roling our unit, becoming ALS the most prevalent disease $(45 \%)$. This unit has become a great alternative for patients with difficult in weaining in our in intensive care units, 11 patients $(22,4 \%)$ can be treated at home with their families, even so, bigger efforts needs to be performed to improve overload in caregivers.

\section{3}

Risk factors and outcomes for ICU-acquired infection: a genomics approach

TJ. McClelland ${ }^{1}$; C. Goh ${ }^{2}$; KL. Burnham ${ }^{3}$; P. Hutton ${ }^{4}$; EE. Davenport ${ }^{3}$;

CJ. Hinds ${ }^{5}$; JC. Knight ${ }^{2}$

${ }^{1}$ Oxford university clinical academic graduate school, University of Oxford, Oxford, United Kingdom; ${ }^{2}$ Wellcome centre for human genetics, University of Oxford, Oxford, United Kingdom; ${ }^{3}$ Wellcome sanger institute, Wellcome Genome Campus, Hinxton, United Kingdom; ${ }^{4}$ John radcliffe hospital, Oxford University Hospitals NHS Foundation Trust, Oxford United Kingdom; ${ }^{5}$ William harvey research institute, Barts and the London School of Medicine, London, United Kingdom

Correspondence: T.J. McClelland

Intensive Care Medicine Experimental 2020, 8(2): 001303

Introduction: ICU-acquired infections (ICU-AI) are associated with substantial morbidity and mortality, but there are few large-scale epidemiological studies and investigations of risk factors, including predictive biomarkers, are currently limited. Genomic-led approaches identified two distinct Sepsis Response Signatures (SRS1 and SRS2) using hierarchical clustering of leucocyte genome-wide gene 
expression in patients admitted to ICU with sepsis [1]. The SRS1 endotype is characterised by features of immunosuppression and increased mortality.

Objectives: To elucidate the risk factors for ICU-Al, compare outcomes between those with or without ICU-Al, and assess the influence of SRS endotype on ICU-AI.

Methods: Patients in whom ICU-AI was clinically diagnosed at any time during ICU admission were identified from a cohort of 2054 patients admitted with sepsis to 34 ICUs in the UK [2]. 1697 (82.6\%) patients with an ICU length of stay $>48$ hours were included in the analysis. Univariate analysis comparing between those with and without ICU-AI was calculated for continuous variables using linear model ANOVA and Kruskal-Wallis test (for non-normally distributed data), and Pearson's Chi-squared test (X2) (categorical variables). Logistic regression modelling used all potentially significant independent variables from univariate analysis $(p<0.2)$, with backward selection of significant variables for the final models. A weighted Cox-regression model was used to assess effect of ICU-AI on 6-month mortality.

Results: A total of 687 ICU-Als were recorded. 462 (27.2\%) patients developed one or more ICU-AI. The most common were ventilatorassociated pneumonia ( $n=208,12.3 \%$ of all patients), line-related infection $(n=115,6.8 \%)$, and wound infection $(n=111,6.5 \%)$. These accounted for $63.2 \%$ of all ICU-Als. Patient demographics (age, race, sex, Charlson score) did not differ between those with or without ICU-AI. Those with ICU-AI had higher severity scores on admission (APACHE II score [median 18 vs $16, \mathrm{p}<0.001$ ], SOFA score [median 8 vs $6, p<0.001])$. ICU-Al was significantly associated with the level of organ support and steroid therapy [p $<0.001]$. In those in whom SRS endotype had been assigned $(n=871)$, the last available SRS endotype was significantly associated with ICU-AI. In patients with the SRS1 (immunosuppressed endotype), 30.8\% $(n=89)$ developed an ICU-AI compared to $20.8 \%(n=121)$ of those with the SRS2 (immunocompetent endotype) $(p=0.002, X 2)$. Renal replacement therapy (RRT) (OR $1.85,95 \% \mathrm{Cl} 1.11-3.09, \mathrm{p}=0.019$ ) and inotropic support (OR $1.84,95 \%$ $\mathrm{Cl} 1.17-2.91, \mathrm{p}=0.009$ ) were independent risk factors for ICU-AI. ICUAl was associated with increased risk of death at six months (HR 1.31, $95 \% \mathrm{Cl} 1.08-1.61, \mathrm{p}=0.0056)$.

Conclusion: Risk factors for ICU-AI include illness severity, level of organ support, steroid therapy and the SRS1 transcriptomic endotype. RRT and inotropic support were independently associated with ICU-AI. ICU-AI is associated with increased mortality.

\section{Reference(s) and grant ackowledgment(s)}

1. 1. Davenport, Emma E et al. "Genomic landscape of the individual host response and outcomes in sepsis: a prospective cohort study." The Lancet. Respiratory medicine vol. 4,4 (2016): 259-71. https://doi.org/10.1016/S2213 -2600(16)00046-1

2. 2. Genomic Advances in Sepsis (GAinS) study (ukccggains.com)

3. The UK Genomic Advances in Sepsis (GAinS) study (original source of data used in this study) was supported by the European Union and 6th framework programme of RTD funding, Medical Research Council (UK), the National Institute for Health Research (NIHR) through the Comprehensive Clinical Research Network for patient recruitment, the NIHR Oxford Biomedical Research Centre, a Wellcome Trust Investigator Award (204969/Z/16/Z) (J.C.K.) and Wellcome Trust Grant (090532/Z/09/Z) to core facilities at the Wellcome Centre for Human Genetics.

\section{7}

A predictive model for seven-day mortality in Intensive Care Unit: findings from the SPIN-UTI project

M. Barchitta'; A. Maugeri'; G. Favara'; P. Riela ${ }^{2}$; G. Gallo ${ }^{2}$; I. Mura ${ }^{3}$; A. Agodi ${ }^{1}$ ${ }^{1}$ Department gf ingrassia, University of Catania, Catania, Italy; ${ }^{2}$ Department of mathematics and informatics, University of Catania, Catania, Italy; ${ }^{3}$ Department of biomedical sciences, University of Sassari, Sassari, Italy

Correspondence: A. Agodi

Intensive Care Medicine Experimental 2020, 8(2): 001307

Introduction: Patients admitted to intensive care units (ICUs) are at higher risk of death because of their underlying illness, often worsened by the occurrence of healthcare associated infections (HAls). The
Simplified Acute Physiology Score (SAPS) II is one of the most used scoring systems to evaluate the severity of illness of patients admitted to ICU and to predict their risk of dying.

Objectives: The main aim of this study was to evaluate the ability of SAPS II to predict the risk of 7-day mortality of patients admitted to ICU, and to compare its performance with a machine learning algorithm combining the SAPS II with additional patients' characteristics at ICU admission.

Methods: For this purpose, we used data from seven biennial editions (i.e. from 2006-2007 to 2018-2019) of the "Italian Nosocomial Infections Surveillance in Intensive Care Units" (SPIN-UTI) project. Overall, 3,782 patients staying in ICU for at least 7 days and those who died within the same timeframe constituted the test set. The training set, instead, was made of 4589 recovered and synthetic records. The accuracy of the SAPS II for predicting 7-day mortality was evaluated using the receiver operating characteristic (ROC) curve and computation of the Area Under the Curve (AUC). Next, the Support Vector Machine (SVM) algorithm was applied to improve the predicting performance of the model, by combining the SAPS II with the following variables collected at ICU admission: gender, origin of the patient, trauma patient, impaired immunity, antibiotic treatment, patient needed acute coronary care, surgery before ICU admission, presence of intubation, presence of urinary catheter, presence of central vascular catheter.

Results: The proportion of deaths was $23.1 \%$ in the test set and $43.4 \%$ in the training set, respectively. No differences in the SAPS II and other patient's characteristics at ICU admission were evident. The SAPS II was able to discriminate patients who died from those who did not with an accuracy of $69.3 \%$ and AUC of $0.678(95 \% \mathrm{Cl}=0.657-0.700)$. However, the SVM algorithm improved the predicting performance of the model, reaching anaccuracy of $83.5 \%$ and AUC of $0.896(95 \% \mathrm{Cl}=$ 0.881 - 0.910). Notably, the SAPS II was the predictor which weighted more on the model. Indeed, both accuracy and AUC dropped to $68.4 \%$ and $0.653(95 \% \mathrm{Cl}=0.632-0.675)$ by removing the SAPS II from the algorithm.

Conclusion: The SVM algorithm - which combined the SAPS II with additional patients' characteristics at ICU admission - exhibited higher predicting performance than the SAPS II alone. This model represented a simple tool to quickly predict patients at the highest risk of death at ICU admission, allowing them to be prioritized and potentially reducing the mortality rate.

Reference(s) and grant ackowledgment(s)

1. The Authors wish to thank all the SPIN-UTI network

\section{8}

Renal replacement therapy in critically ill patients with COVID 19: A multicenter study AKICOV

A. De La Vega Sánchez ; FJ. González De Molina²; BY. Díaz;

A. Navas-Pérez ${ }^{4}$; P. Ortiz Ballujera ${ }^{5}$; M. Pérez_Carrasco';

A. Olmo-Isasmendi ; C. Rovira-Anglès ${ }^{7}$; I. Oliva-Zelaya ${ }^{8}$; RM. Catalán-Ibars ${ }^{9}$; M. Ibarz-Vilamayor ${ }^{10}$; M. Rodríguez-López ${ }^{11}$; M. Torrens Sonet ${ }^{12} ;$ M. Alvarez Garcia-Pumarino $^{13}$; TM. Tomasa Irriguible ${ }^{14}$; J. Sabater-Riera ${ }^{15}$; J. Prados

Chica ${ }^{16} ;$ A. Baró Serra ${ }^{17}$; E. Casanova-Gongora ${ }^{18}$; C. Pedrós Mas ${ }^{19}$

${ }^{1}$ Intensive care, Vall d'Hebron University Hospital, Barcelona, Spain; ${ }^{2}$ Intensive care, Mútua Terrassa University Hospital, Terrassa, Spain; ${ }^{3}$ Intensive care, Hospital del Mar, Barcelona, Spain; ${ }^{4}$ Intensive care, Hospital Parc Taulí de Sabadell, Sabadell, Spain; ${ }^{5}$ Intensive care medicine, Hospital Universitari de Girona Dr Josep Trueta, Girona, Spain; Intensive care, Helipuerto Hospital General de Catalunya, Sant Cugat del Vallès, Spain; ${ }^{7}$ Intensive care, Hospital Universitari Sant Joan de Reus, Reus, Spain; ${ }^{8}$ ntensive care, Hospital Universitari de Tarragona Joan XXIII, Tarragona, Spain; ${ }^{9}$ Intensive care, Hospital Universitari de Vic (Consorci Hospitalari de Vic), Vic, Spain; ${ }^{10}$ Intensive care, Hospital Universitari Sagrat Cor - Grup Quirónsalut, Barcelona, Spain; ${ }^{11}$ Intensive care, Hospital de Sant Joan Despí Moisès Broggi, Sant Joan Despí, Spain; ${ }^{12}$ Intensive care, Hospital Universitari Santa Creu i Sant Pau, Barcelona, Spain; ${ }^{13}$ Intensive care, Consorci Sanitari de Terrassa, Terrassa, Spain; ${ }^{14}$ Intensive care unit, Hospital Germans Trias i Pujol, Badalona, Spain; ${ }^{15}$ Intensive care, Bellvitge University Hospital, L'Hospitalet de Llobregat, Spain; ${ }^{16}$ Intensive care, University Hospital Arnau de Vilanova, Lleida, 
Spain; ${ }^{17}$ Intensive care, Hospital Santa Caterina en Salt, Salt, Spain; ${ }^{18}$ Intensive care, Hospital Mutua de Terrassa, Terrassa, Spain; ${ }^{19}$ Intensive care, Hospital General de Granollers, Granollers, Spain

Correspondence: M. Pérez_Carrasco

Intensive Care Medicine Experimental 2020, 8(2): 001308

Introduction: Severe Acute Respiratory Syndrome Coronavirus 2 has spread at great speed throughout the world. Between 15 and $25 \%$ of hospitalized patients infected with COVID-19 develop acute kidney injury (AKI).

Objectives: To describe renal replacement therapy (RRT) in COVID-19 patients at the admission in intensive care units (ICU).

Methods: A multicenter, descriptive, observational study of COVID-19 patients admitted to 19 Catalonian ICUs. Patients with acute kidney injury (AKI) and RRT requirements were included. Data of descriptive variables including severity and evolution of the AKI, associated complication, circuit permeability, filter duration and need of RRT at discharge were analyzed. Frequency and percentage (categorical) and mean (SD) or median (RI) (quantitative) are presented. X2/T-Fischer or T-Student/U-Mann Whitney were applied. Data were presented as frequency (percentage) and mean (standard deviation) or median (95\%RI).

Results: A total of 934 patients were included, $66 \%$ men, mean age 64(16.8) years. APACHE II and SOFA at admission were 13(8) and 5(4) respectively. Information was collected on the following comorbidities: arterial hypertension (45\%), obesity (34\%), diabetes mellitus $(20 \%)$ and COPD (7\%). Chronic kidney disease was present in $4.5 \%$ of patients. AKI at the ICU admission was $28.4 \%$ and increased to $44 \%$ during ICU stay. NSAIDs were administrated to $25.9 \%$, contrast-media to $18,8 \%$ and ACE inhibitors to $10,5 \%$. AKI patients presented ventilator-associated pneumonia VAP (35.7\%), UTIs (16.6\%) and intravascular catheter-related infection (14\%).

A total of 88 patients $(9,3 \%)$ required RRT. At the initiation of RRT patients presented with KDIGO I: $2.5 \%$, II: $6.2 \%$, III: $91.4 \%$ (creatinine mean was $3.91(1.6) \mathrm{mg} / \mathrm{dL}$ ). Indications of RRT initiation were: anuria $(62 \%)$, fluid overload $(51.1 \%)$, hyperkalemia $(33 \%)$, sepsis $(20.5 \%)$ and uremic syndrome (14.8\%). RRT duration was 7 (RI 9) days (CVVHDF (62.4\%), CVVHD (35.3\%), CVH (2.,4\%)); and iHD 3 (RI 6.5) days.

Heparin was the most commonly prescribed anticoagulant for use during continuous RRT (61.4\%), followed by ARC $(45,5 \%)$ and a minor percentage of no anticoagulation (6.8\%). Filter/patient median was 3 (RI 4); filter life with ARC was 2 (RI 1.23), with heparin 1.71(1.53) and without anticoagulation 1(1.63) days. Complications: catheter dysfunction $(26.1 \%)$, early/non expected coagulation (23.9\%), thrombocytopenia (14.8\%), bleeding (5.7\%) and hypothermia (5.7\%). Only one patient required adsorption techniques. A quarter of patients $(24.7 \%)$ needed RRT at ICU discharge. Overall ICU mortality was $57.1 \%$ in RRT patients, $44.3 \%$ in AKI without RRT and $14.9 \%$ in patients without AKI.

Conclusion: $\mathrm{A}$ high percentage of patients required RRT for AKI at the admission or during ICU stay, with a mortality rate exceeding $50 \%$. Management and complications are not different from the usual clinical practice.

\section{Reference(s) and grant ackowledgment(s)}

1. Ronco C, Reis T, Husain-Syed F. Management of acute kidney injury in patients with COVID-19. Lancet Respir Medicine. 2020;8(7):738-42.

2. Fanelli V, Fiorentino M, Cantaluppi V, Gesualdo L, Stallone G, Ronco C, Castellano G. Acute kidney injury in SARS-CoV-2 infected patients. Crit Care. 2020;24(1):155.

\section{3}

\section{Dexamethasone for patients undergoing major noncardiac} surgery

K. Asehnoune'; C. Le Moal'² M. Lepenndu'; JN. Chatel'; M. Boissonn'; M. Faucher ${ }^{5}$; S. Jaber JT. Godet'; M. Leone $^{8}$; C. Motamed ${ }^{9}$; JS. David ${ }^{10}$; Y. El Amine $^{11} ;$ A. Roquilly ${ }^{1}$; M. Garrot ${ }^{12} ;$ E. Futier $^{13}$

'Service d'anesthésie-réanimation, Nantes University Hospital Hotel-Dieu, Nantes, France; ${ }^{2}$ Service d'anesthésie, C.H. - Le Mans, Le Mans, France; ${ }^{3}$ Service anesthésie, Hôpital Privé du Confluent, Nantes, France; ${ }^{4}$ Service d'anesthésie-réanimation, (5) CHU de Poitiers, Poitiers,
France; ${ }^{5}$ Service d'anesthésie, Institute Paoli-Calmettes, Marseille, France ${ }^{6}(8)$ anesthesia and critical care department b, Centre Hospitalier Universitaire Montpellier, Montpellier, France; ${ }^{7}$ Service d'anesthésie et réanimation, Chu Estaing, Clermont-Ferrand, France; ${ }^{8}$ Department of anesthesiology and critical care medicine, Hospital Nord, Marseille, France; ${ }^{9}$ Service d'anesthésie, Institut Gustave Roussy, Villejuif, France; ${ }^{10}$ Service d'anesthésie-réanimation, Hôpital Edouard Herriot, Hospices Civils de Lyon, Lyon, France; ${ }^{11}$ Serviced'anesthésie, Hospital Center De Valenciennes, Valenciennes, France; ${ }^{12}$ Pôle anesthésie réanimation, (3) Centre Hospitalier Universitaire (CHU) Lille, Lille, France; ${ }^{13}$ Department of anesthesiology and intensive care medicine, University Hospital of Clermont-Ferrand, Clermont-Ferrand, France

Correspondence: $\mathrm{K}$. Asehnoune

Intensive Care Medicine Experimental 2020, 8(2): 001313

Introduction: Surgery initiates inflammation which contributes to postoperative morbidity and mortality.

Objectives: We aimed to assess the effects of dexamethasone on postoperative complications in patients undergoing major surgery.

Methods: In a multicenter, randomized, double-blind trial, patients over 50 years of age undergoing non-cardiac surgery with expected duration of more than 90 minutes were recruited from 34 centers. We randomly assigned patients to dexamethasone $(0.2 \mathrm{mg} . \mathrm{kg}-1 \mathrm{immedi}-$ ately after the procedure, and on day +1 ) or to placebo. Randomization was stratified on two criteria: cancer and thoracic procedure. The primary outcome was a composite of major complications (including sepsis, pneumonia and the need for mechanical ventilation) and allcause mortality occurring within the first 14 days following surgery.

Results: Of the 1,222 patients, 613 patients were randomized to dexamethasone, and 609 to placebo. In intention-to-treat analysis after multiple imputation for missing data, 104 patients (17.1\%) in the dexamethasone group and 123 patients $(20.4 \%)$ in the placebo group had major complications or died within 14 days after surgery (adjusted odds ratio, 0.78; 95\% confidence interval [Cl], 0.59-1.04; $\mathrm{P}=0.09$, crude odds ratio $0.79 ; 95 \% \mathrm{Cl}, 0.60-1.07, \mathrm{P}=0.13)$. The hazard ratios with dexamethasone for sepsis (including pneumonia) and for mechanical ventilation were respectively $0.82(95 \% \mathrm{Cl} 0.61-1.12, \mathrm{P}$ $=0.21)$ and $0.68(95 \% \mathrm{Cl} 0.53-0.88, \mathrm{P}=0.003)$. In the a priori defined randomization stratum of patients with non-thoracic surgery, primary outcome occurred less frequently in the dexamethasone group than in the placebo group $(P=0.04)$. Adverse events were reported in $47 \%$ and $48.6 \%$ of patients who received dexamethasone or placebo (odds ratio: $0.92 ; 95 \% \mathrm{Cl} 0.74-1.15)$.

Conclusion: After major non-cardiac surgery, dexamethasone did not result in significantly less mortality or fewer major complications 14 days after surgery. (Funded by French Ministry of Health, PHRCN 2016, number clinicaltrials.gov NCT03218553).

\section{Reference(s) and grant ackowledgment(s)}

1. Source of funding: PHRC-N 2016.

\section{0}

Socially distanced rehabilitation: a potential new normal for post-critical care recovery?

A. Hunter ; L. Hodgson ; T. Leckie ; A. Richardson²; B. Hardy;

D. Fitzpatrick ${ }^{3} ;$ A. Goncalves ${ }^{4}$

${ }^{1}$ Intensive care, Worthing Hospital, Worthing, United Kingdom; ${ }^{2}$ Centre of stress, ageing and disease, University of Brighton, Brighton, United Kingdom; ${ }^{3}$ Sports and sports medicine, University of Brighton, London, United Kingdom; ${ }^{4}$ Physiotherapy, Worthing Hospital, Worthing, United Kingdom Correspondence: $L$. Hodgson

Intensive Care Medicine Experimental 2020, 8(2): 001320

Introduction: It is unclear when the effects of the coronavirus will subside and a surge in demand for critical care follow-up is anticipated. The requirement to provide a rehabilitation service within the context of social distancing and reconfigured outpatient infrastructure presents a set of new challenges to the delivery of optimal patient care. 
Objectives: 1 . To explore the feasibility of managing critical care recovery via remote coaching enabled by smartwatch technology.

2. Report physical measures at the point of hospital discharge and on follow-up

Methods: The use of smartwatch ambulatory health monitoring technology in COVID-19 critical care discharges was set up across eight trusts in Surrey, Sussex and Kent, UK. Worthing Hospital also implemented a digitally integrated multidisciplinary team (MDT) approach (physiotherapists, exercise physiologist, critical care, respiratory and general practice clinicians) using this smart watch data to support our critical care COVID-19 survivors. Smartwatch data was shared by the patient with the MDT via synchronisation with a smartphone app. The MDT met remotely every 4 weeks to review patients' progress and discuss goal setting. Baseline data was collected at discharge and follow up data points are at 3 months and 12 months respectively.

Results: From the first 13 patients reviewed (mean age 58, SD 10), mean heart rate per minute has slowed from 98 (SD 11) at hospital discharge to 75 (SD 5) at six weeks, whilst daily step count has increased from 1187 (SD 486) to 5407 (SD 3153). Shuttle walk test distance increased from 69 metres (SD 78) at hospital discharge to 364 (SD 221) on three-month follow-up, with air oxygen saturations increasing from $86 \%$ (SD 8) to $93 \%$ (SD 3). The MDT were able to provide a patient personalised follow up pathway, providing earlier intervention than would have occurred with a face-to-face patient follow up.

Patient engagement with the programme was high with over $90 \%$ of patients regularly wearing and uploading smartwatch data. Patients responded well to the technology and incorporated the feedback from the devices into their personalised rehabilitation programmes. As further patients return for follow up this dataset will increase substantially over time.

Conclusion: Remote rehabilitation is feasible and offers an alternative to traditional post critical care follow-up. Regular, proactive review of smartwatch data combined with a remote MDT faciliated a unique, personalised and sustainable model for post critical care follow up. Future work will aim to expand this model to a wider cohort of critical care survivors.

\section{Reference(s) and grant ackowledgment(s)}

1. Grant recieved from University of Brighton

\section{2}

Continuous positive airway pressure outside ICU for COVID-19 patients: a multicenter observational study

E. Santangelo ${ }^{1}$; N. De Vita ${ }^{1}$; L. Scotti ${ }^{1}$; F. Barone-Adesi ${ }^{1}$; F. Racca ${ }^{2}$; C. Pissaia ${ }^{3}$; C. Maestrone ${ }^{4}$; D. Colombo ${ }^{5}$; C. Olivieri ${ }^{6}$; F. Della Corte ${ }^{1}$; P. Navalesi ${ }^{7}$; G. Cammarota ${ }^{8}$; R. Vaschetto ; F. Covid-19 Eastern Piedmont Network ${ }^{1}$

${ }^{1}$ Dipartimento di medicina traslazionale, Università Degli Studi Del Piemonte Orientale, Novara, Italy; ${ }^{2}$ Department of anesthesia and intensive care, Azienda ospedaliera SS Antonio e Biagio e Cesare Arrigo, Alessandria, Italy; ${ }^{3}$ Dipartimento di anestesia e terapia intensiva, Ospedale "Degli Infermi", Biella, Italy; ${ }^{4}$ Anestesia rianimazione asI vco, Presidio Ospedaliero Domodossola e Verbania, Verbania, Italy; ${ }^{5}$ Department of anesthesia and critical care, Ospedale Ss. Trinità Borgomanero, Borgomanero, Italy; ${ }^{6}$ Department of anesthesia and critical care, Azienda Ospedaliera Sant'Andrea, Vercelli, Italy; ${ }^{7}$ Istituto di anestesia e rianimazione, Azienda Ospedaliera Università Padova - Dipartimento di medicina DIMED - Università di Padova, Padova, Italy: ${ }^{8}$ Anestesia e terapia intensiva, Azienda Ospedaliero Universitaria Maggiore della Carità di Novara, Novara, Italy

Correspondence: N. De Vita

Intensive Care Medicine Experimental 2020, 8(2): 001322

Introduction: During novel coronavirus disease (COVID-19) pandemic outbreak, a large number of patients required respiratory support due to severity of the respiratory involvement. Noninvasive ventilation delivered as continuous positive airway pressure (CPAP) for de novo acute hypoxemic respiratory failure (ARF) isstill debated. To face the overwhelming wave of patients, hospitals converted many general wards in respiratory intermediate units (RICU), where COVID-19 patients requiring respiratory support could be treated with CPAP outside intensive care unit (ICU).
Objectives: The study aims to investigate the role of CPAP outside ICU during COVID-19 pandemic, describing the clinical characteristics of patients and ascertaining whether the CPAP duration affects outcome in patients requiring endotracheal intubation.

Methods: We performed a multicenter, retrospective observational study in six hospitals of Eastern Piedmont Region in Northern Italy. All patients admitted to one of the participating hospitals from March 1st to April 15th 2020 with hypoxemic ARF secondary to COVID-19 infection were eligible. Inclusion criteria were: 1 ) age $\geq 18$ years, 2) respiratory distress and partial pressure of arterial oxygen to fraction of inspired oxygen ratio $<200 \mathrm{mmHg}$ during low flow oxygen therapy, 3) CPAP initiation outside ICU. Patients were classified according to predefined CPAP therapeutic goal in two subgroups: 1 ) full treatment, i.e. patients scheduled to receive intubation in case of CPAP failure; and 2) do-not-intubate (DNI), when CPAP was the ceiling of treatment.

Results: During the study period, 537 patients received CPAP outside ICU in the six Eastern Piedmont hospitals. The median age was 69 (IQR 60-76) years. Median CPAP duration was 4 (IQR, 1-8) days, while hospital LOS 16 (IQR, 9-27) days. According to predefined CPAP therapeutic goal, $397(74 \%)$ patients were included in full treatment subgroup, and $140(26 \%)$ in the do-not intubate (DNI) subgroup. Sixty-day in hospital mortality was overall $34 \%(95 \% \mathrm{Cl}, 0.304-0.384), 21 \%(95 \% \mathrm{Cl}$ $0.169-0.249)$ and $73 \%(95 \% \mathrm{Cl}, 0.648-0.787)$ for full treatment and DNI subgroup, respectively. In the full treatment subgroup, in-hospital mortality was $42 \%(95 \% \mathrm{Cl}, 0.345-0.488)$ for $180(45 \%)$ CPAP failures requiring intubation, while $2 \%(95 \% \mathrm{Cl}, 0.008-0.035)$ for the remaining $217(55 \%)$ patients who succeeded. Delaying intubation was associated with increased mortality [HR, $1.093(95 \% \mathrm{Cl}, 1.010-1.184)]$.

Conclusion: Our study suggests that CPAP is feasible outside ICU even during COVID-19 outbreak. In-hospital mortality is related to therapeutic goal and patients with pre-set therapeutic limitations showing a higher mortality rate. Intubation delay is an independent risk factor for mortality.

\section{Reference(s) and grant ackowledgment(s)}

1. Covid-19 Eastern Piedmont Network: Gianluca Airoldi; Marta Baggiani; Sara Baino; Piero Balbo; Simona Bazzano; Valeria Bonato; Silvio Borrè; Luigi Castello; Tiziana Cena; Sara Carbonati; Federico Crimaldi; Veronica Daffara; Luca De Col; Luca Grillenzoni; Matteo Maestrone; Mario Malerba; Francesco Mojoli; Federica Moroni; Maria Adele Moschella; Raffaella Perucca; Mario Pirisi; Valentina Rondi; Daniela Rosalba; Martina Taverna; Letizia Vanni; Francesca Vigone

\section{3}

Serum leptin is associated with hypercoagulation measured by thromboelastrometry (ROTEM) in COVID-19 disease

N. Van Veenendaal' ; T. Scheeren ${ }^{2}$; K. Meijer ${ }^{3} ;$ P. Van Der Voort ${ }^{4}$

${ }^{1}$ Department of critical care/department of anaesthesiology, University Medical Center Groningen, Groningen, Netherlands; ${ }^{2}$ Department of anaesthesiology/department of critical care, University Medical Center Groningen, Groningen, Netherlands; ${ }^{3}$ Department of hematology, University Medical Center Groningen, Groningen, Netherlands; ${ }^{4}$ Department of critical care, University Medical Center Groningen, Groningen, Netherlands

Correspondence: $\mathrm{N}$. Van Veenendaal

Intensive Care Medicine Experimental 2020, 8(2): 001333

Introduction: Coronavirus disease 2019 (COVID-19) is associated with coagulation abnormalities leading to numerous thromboembolic complications. Obesity is a risk factor for severe COVID-19 disease and patients with obesity have relatively high leptin serum levels. In addition, high serum leptin levels are associated with hypercoagulation. Rotational thromboelastrometry $\left(\right.$ ROTEM $\left.^{\circledR}\right)$ is a digitized modification of thromoboelastography reported to be useful in identifying hypercoagulable conditions.

Objectives: The aim of this study was to determine the correlation between serum leptin levels and ROTEM ${ }^{\circledast}$ profiles of COVID-19 patients.

Methods: In a cross-sectional design, we included patients who were treated in the Intensive Care Unit with COVID-19 at the same time. 
Blood samples were collected for serum leptin measurement (ELISA kit MD53001 IBL International, Hamburg, Germany, performed according to the manufacturer's instructions) and ROTEM ${ }^{\circledR}$ thomboelastometry. Coagulation patterns were analyzed using extrinsic and intrinsic rotational thromboelastometry activators (respectively EXTEM and INTEM). The following variables were recorded and analyzed: clotting time (CT), clotting formation time (CFT), clot firmness' amplitude after 5 minutes (A5) and after 10 minutes (A10), and maximum clot firmness (MCF). Spearman's correlation coefficients were determined for all variables.

Results: Thirty-one COVID-19 patients were included, of which 23 (74\%) were male. The mean age was 62.5 years (Standard Deviation (SD) 9.7), and the mean BMI was $31.2 \mathrm{~kg} / \mathrm{m}^{2}$ (SD 5.7). The median serum leptin level was 12.2 (range $1.0-85.2$ ) ug/L. BMI was significantly correlated to serum leptin level $r=0.49 ; p=0.001$. Table 1 shows the correlation of leptin and ROTEM variables.

\begin{tabular}{|l|l|l|}
\hline $\begin{array}{l}\text { Table 1. } \\
\begin{array}{l}\text { EXTEM clot } \\
\text { formation time } \\
\text { (CFT) }\end{array}\end{array}$ & Spearman's correlation & $\boldsymbol{P}$-value \\
\hline $\begin{array}{l}\text { EXTEM maximum } \\
\text { clot firmness (MCF) }\end{array}$ & -0.410 & 0.022 \\
\hline INTEM CFT & -0.422 & 0.258 \\
\hline INTEM MCF & 0.262 & 0.018 \\
\hline FIBTEM CFT & -0.108 & 0.154 \\
\hline FIBTEM MCF & 0.023 & 0.563 \\
\hline
\end{tabular}

Table 2 shows the correlation of leptin and conventional coagulation variables.

\begin{tabular}{|l|l|l|}
\hline Table 2. & Spearman's correlation & $\boldsymbol{P}$-value \\
\hline $\begin{array}{l}\text { Platelet function } \\
\text { Activated Partial } \\
\text { Thromboplastin } \\
\text { Time }\end{array}$ & 0.168 & 0.367 \\
\hline Prothrombin & -0.277 & 0.139 \\
\hline Fibrinogen & 0.143 & \\
\hline Antithrombin III & -0.010 & 0.350 \\
\hline
\end{tabular}

The only variable that was significantly correlated with serum leptin level was clot formation time, both for EXTEM and for INTEM tests. An inverse correlation was found, meaning that higher leptin levels were correlated with a shorter clot formation time. The conventional clotting tests were not significantly correlated with serum leptin level.

Conclusion: ROTEM ${ }^{\circledR}$ measurements of clot formation time were significantly inversely correlated with serum leptin levels, which may indicate that leptin plays a role in the hypercoagulable state that is present in COVID-19 patients treated in the ICU.

\section{4}

The amount of subpleural lung infiltrates in patients affected by SARS-CoV2 is proportional to the number of days of spontaneous breathing

M. Pellegrini' ; A. Larina' ; R. Frithiof'; M. Hultström'; E. Mourtos²;

T. Hansen'ㄹ M. Lipcsey ${ }^{1} ;$ G. Perchiazzi ${ }^{1}$

${ }^{1}$ Department of surgical sciences, anesthesiology and intensive care medicine, Uppsala University, Uppsala, Sweden; ${ }^{2}$ Department of surgical sciences, radiology, Uppsala University, Uppsala, Sweden

Correspondence: M. Pellegrini

Intensive Care Medicine Experimental 2020, 8(2): 001344

Introduction: Specific features differentiate SARS-CoV2-induced acute respiratory failure from the classical definition of acute respiratory distress syndrome (ARDS) [1]. By lung computed tomography (CT) it is possible to characterize extension and severity of lung damage and infer regional lungs mechanics during ARDS [2]. The role of lung CT for SARS-CoV2 related lung engagement remains to be confirmed. Objectives: A primarily subpleural distribution of lung infiltrates induced by SARS-CoV2 has been hypothesized. The correlation between the extent of lung infiltrated and days of spontaneous breathing has never been tested before.

Methods: This study has been performed on CT images collected from 20 patients affected by a severe form of SARS-CoV2 infection in need of intensive care. Chest $\mathrm{CT}$, acquired during static conditions and without contrast agent were selected. For each $\mathrm{CT}$, twenty equally spaced images between apex and diaphragmatic dome were analyzed. Each CT image was divided into three subpleural regions of interest (ROIs - between 0 and $1 \mathrm{~cm} ; 1$ and $2 \mathrm{~cm} ; 2$ and $3 \mathrm{~cm}$ ) concentric to the visceral pleura. Four quadrants (external non-dependent,internal nondependent, internal dependent, external dependent) were delineated for each lung, depending on figures centroids (Figure). Quadrants were used to differentiate between atelectasis, affecting dependent regions, and SARS-CoV2 specific lung infiltrates in the external and non-dependent regions. Four lung Hounsfield units (HU) compartments were defined [3]: hyper $(-1,000$ to $-800 \mathrm{HU})$, normally $(-800$ to $-500 \mathrm{HU})$, poorly $(-500$ to $-100 \mathrm{HU})$ and not inflated $(-100$ to $+100 \mathrm{HU}$ ). Friedman's test (a-value $<0.05$ ) was used for statistics. Days of spontaneous breathing with and without ventilatory support were defined for each patient. Correlations between HU compartments and days of spontaneous breathing were performed (Spearman correlation, rs, a-value $<0.05$ ).

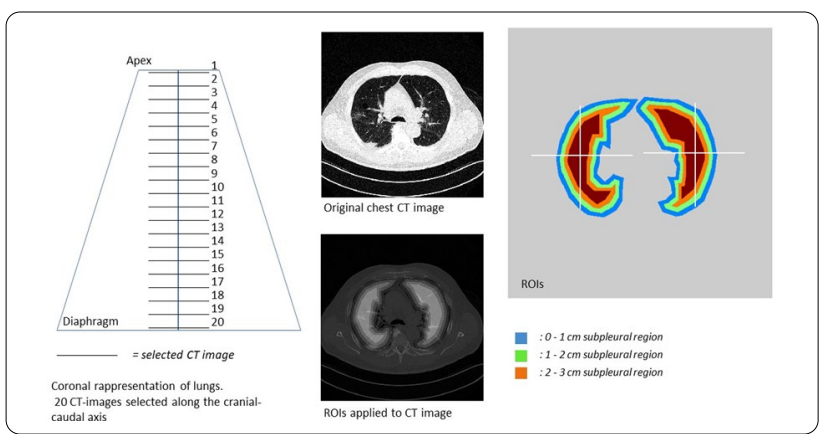

Results: Twenty-three chest CT were analyzed. Hypo-inflated and not-inflated compartments were significantly more represented in the subpleural regions of the lung $(0-1 \mathrm{~cm})$ compared to more central ones $(2-3 \mathrm{~cm})$, both in dependent and not dependent quadrants. Consistently, the amount of normo-inflated lung was more represented in deeper regions of the lung. The hyper-inflated compartment was significantly more represented in non-dependent subpleural lung regions $(0-1 \mathrm{~cm})$. A positive correlation was found between days of spontaneous breathing and hypo-/not- inflated lung compartments, in not-dependent ( $\mathrm{rs}=0.54, \mathrm{p}=0.017)$ and external regions ( $\mathrm{rs}=$ $0.49, p=0.032$ ).

Conclusion: The primarily subpleural distribution of SARS-CoV2 specific lung infiltrates has been confirmed using objective lung-CT analysis. Further, it subsequently extends towards more central regions of the lung. A direct correlation between extent of SARS-CoV2-induced lung infiltrated and days of spontaneous breathing was shown.

Reference(s) and grant ackowledgment(s)

1. 1. Gattinoni et al (2020) ICM. 46:1099-1102

2. 2. Ranieri et al (2012) JAMA 307:2526-33

3. 3. Gattinoni et al (2001) AJRCCM. 164:1701-1711

4. Swedish Heart-Lung Foundation (20170531) 


\section{7}

Corticosteroid therapy is associated with a decrease in mortality in a multicenter cohort of mechanically ventilated COVID-19 patients

B. Lambermont ${ }^{1}$; M. Ernst ${ }^{2}$; P. Demaret ${ }^{3}$; V. Fraipont ${ }^{4}$; C. Gurdebeke ${ }^{5}$;

T. Sottiaux $;$; M. Quinonez ; C. Dubois ${ }^{3}$;. Njambou' ${ }^{8}$; B. Akando';

T. Lemineur ${ }^{10}$; D. Wertz ${ }^{11}$; F. Forêt ${ }^{12}$; AF. Rousseau'; D. Ledoux ${ }^{1}$; N. Layios ${ }^{1}$;

S. Robinet ${ }^{1}$; P. Morimont ${ }^{1}$; B. Misset

${ }^{1}$ Department of intensive care, University Hospital Liege, Liège, Belgium; ${ }^{2}$ Biostatistics and medico-economic department, University Hospital Liege, Liège, Belgium; ${ }^{3}$ Department of anesthesia and intensive care., MontLegia Hospital - Groupe Santé CHC, Liège, Belgium; ${ }^{4}$ Department of intensive care, CHR de la Citadelle, Liège, Belgium; ${ }^{5}$ Department of intensive care, CHRVerviers, Verviers, Belgium; ${ }^{6}$ Department of intensive care, Clinique Notre-Dame de Grace, Gosselies, Belgium; ${ }^{7}$ Department of intensive care, Hospital Center Bois De L'abbaye, Seraing, Belgium; ${ }^{8}$ Department of intensive care, Hospital Center Reine Astrid, Malmedy, Belgium; ${ }^{9}$ Department of intensive care, Hospital Saint-Joseph Saint-Vith, Sankt Vith, Belgium; ${ }^{10}$ Department of intensive care, Clinical André Renard, Herstal, Belgium; ${ }^{11}$ Department of intensive care, Hospital Center Régional De Huy, Huy, Belgium; ${ }^{12}$ Department of intensive care, Chu Ucl Namur - Site De Dinant, Dinant, Belgium

Correspondence: B. Lambermont

Intensive Care Medicine Experimental 2020, 8(2): 001357

Introduction: In late 2019, the virus responsible for Covid-19 was identified and called SARS-CoV-2. In China, 5\% of Covid-19 patients were admitted in ICU, 2,3\% were ventilated and 1,4\% died. In early 2020, Covid-19 quickly spread in Europe and was responsible for high mortality. Since the start of the SARS-CoV-2 pandemic, the need for trials to assess the benefit of antiviral treatment, anti-cytokine drugs, convalescent plasma and hydroxychloroquine has been advocated by the world health organization. However, evidence of the efficacy of such strategies is still lacking.

Objectives: This observational multicentric study aimed to identify prognostic factors and therapies which could be valuable in mechanically ventilated Covid-19 patients for respiratory insufficiency.

Methods: The method consisted in a multicentric retrospective analysis in all consecutive Covid-19 patients admitted to intensive care unit (ICU) and mechanically ventilated for more than 24 hours from March 1 to April 25, 2020, in 12 hospitals. The study protocol was approved by our Ethics Committee and, due to the retrospective nature of the data collected, no consent from the patient was required.

Admission date, age, sex, body mass index, underlying conditions, treatments, physiological values, use of vasopressors, renal replacement therapy (RRT) and extracorporeal membrane oxygenation (ECMO), duration of mechanical ventilation, length of ICU stay, ICU and ventilator-free days at day 42 were collected during May 2020. A second call for missing data was done during June 2020 in each center. The primary outcome was survival during the hospital stay. Secondary outcomes included use of vasopressors, RRT or ECMO, ICU and ventilator-free days at day 42, and evolution of the main physiological values between days 0 and 7. Quantitative variables were reported as median and interquartile range (Q1-Q3). Categorical variables were expressed as number (\%). Simple and multiple time-dependent Cox regression models were used to assess the effects of factors on survival. All the variables which had a $p$-value lower than the critical level of 0.1 were selected for the multivariate model. A p-value $<0.05$ was considered significant.

Results: From March 1 to April 25, 2020, out of 2003 adult patients hospitalized for SARS-CoV-2 pneumonia, 361 were admitted to the 12 participating ICUs for acute respiratory failure. Of these, 257 patients were ventilated for more than 24 hours and 247 were included in the data base. The median age of the 247 patients was 65 (57-72), and 172 (70\%) were men. On admission, the median sequential organ failure assessment (SOFA) score was 6 (4-8), the median $\mathrm{PaO}_{2} / \mathrm{FiO}_{2}$ ratio was 103 (82-132) and $128(52 \%)$ patients were treated with norepinephrin. The median length of stay in ICU was 21 (12-32) days, the median survival time was 82 days, and the mortality rate was $45 \%$. Sixty-nine $(28 \%)$ patients needed RRT and 215 patients (87\%) were treated with norepinephrine during their ICU stay. Four patients (1,6\%) were on ECMO. Corticosteroid therapy was started in 58 (23\%) patients between days 0 and 7 of ICU admission and 225 (91\%) patients received hydroxychloroquine alone or in combination with corticosteroid and/or azythromycin. The mortality rate of the patients who received corticosteroid was $34 \%(20 / 58)$ while it was $48 \%$ $(91 / 189)$ for patients who did not $(p=0,01)$.

As opposed to survivors, non-survivors were older and suffered more often from chronic kidney disease. On admission in ICU, non-survivors had higher SOFA score and serum creatinine value, lower mean arterial pressure and diuresis. Non-survivors were also more often treated with norepinephrine during the first day in ICU. Survivors received more frequently corticosteroid and hydroxychloroquine.

Using multiple regression, the predictors of mortality were age, creatinine value, mean arterial pressure lower than $70 \mathrm{mmHg}$, lymphocytes count on day 0 and absence of corticosteroid use during the first week of mechanical ventilation. Survival probability was significantly higher in patients who received corticosteroid $(p=0,01)$. Survival probability was $75 \%$ by day 23 for patients who received corticosteroid versus by day 10 for those who did not.

Conclusion: Retrospectively analyzing the data of a multicenter cohort, we observed that mortality of patients with SARS-CoV-2 pneumoniatreated with mechanical ventilation was as high as $45 \%$ and median survival time was 82 days. In this series, the risk factors for mortality included age, renal and circulatory dysfunction, lymphopenia and the absence of corticosteroid use during the first week of mechanical ventilation. Corticosteroid therapy during the first week of mechanical ventilation was associated with a lower mortality ( $34 \%$ vs $48 \%)(p=0,01)$.

\section{3}

Artificial Intelligence to predict mortality in critically ill COVID-19 patients: a case study using Data from Lombardy outbreak

G. Angelotti'; PF. Caruso ; N. Stomeo ; C. Ciccone ${ }^{1}$; A. Zanella ${ }^{2}$; M. Greco ${ }^{1}$; G. Grasselli ${ }^{3} ; \mathrm{M} . \mathrm{Cecconi}^{4}$

${ }^{1}$ Anesthesia and intensive care, Humanitas Research Hospital, Milano, Italy; ${ }^{2}$ Department of anesthesiology, Fondazione IRCCS Ca'Granda Ospedale Maggiore Policlinico, Milano, Italy; ${ }^{3}$ Intensive care unit, Policlinico of Milan, Milano, Italy; ${ }^{4}$ Anesthesia and intensive care, Humanitas Research Hospital, Milan, Italy

Correspondence: P.F. Caruso

Intensive Care Medicine Experimental 2020, 8(2): 001363

Introduction: In mid-February 2020, an outbreak of atypical pneumonia caused by Severe Acute Respiratory Syndrome CoronaVirus 2 (SARS-CoV-2) represented the beginning of COVID-19 epidemic in Italy. Since then, the number of admissions in intensive care units (ICU) due to bilateral pneumonias progressing to Acute Respiratory Syndrome (ARDS) rose substantially. Lombardy, due to the high number of cases, created a network called 'COVID-19 Lombardy ICU Network' to manage the exponential surge of patients. With all the Data gathered in these patients, we hypothesized that machine learning models could predict the mortality risk in these patients.

Objectives: Predicting mortality at 7, 14 and 28 days from ICU admission in COVID-19 patients using machine learning models.

Methods: Data comprises medications, comorbidities and daily ventilation of 1503 ICU patients from Lombardy affected by COVID-19 during the early phase of the epidemic.

Two machine learning models where built: the first machine learning model was trained only with features available before ICU admission to investigate the predictive power of pre-existing conditions; the second one was integrated with all the data available during the first day of admission in ICU.

Results: The majority (51\%) of the cohort faced death; survivors were characterized by significantly lower age, higher length of stay and a minor number of comorbidities at admission. Kaplan-Meier curves reveal a survival percentage drop of $30 \%$ within the first 10 days, $45 \%$ after 20 days and reaches a plateau around day 30 where the population is halved. As age increases, the percentage of survivors decreases, with lowest survival in the age cohort between 80 and 90 .

Best model performance was reached when predicting mortality at 28 days, where average area under the curve (AUC) after multiple 
cross-validations were respectively 0.77 and 0.79 . There was no major difference in sensitivity and specificity between the two models (around $70 \%$ for both). Shortening the interval of time at 7 days led to a decrease in performance (AUC 0.68 and 0.72 ) with the model including data from the first day in ICU performing worse overall. Mortality predictions at 14 days performed in between with an AUC 0.72 and 0.73 .

Conclusion: Mortality prediction performances at 28 days showed promising results based merely on patient information that could be gathered at ICU admission. Further training on the model could improve more its ability to assess and give a score of severity at admission to patients for better resource management during the pandemic.

\section{Reference(s) and grant ackowledgment(s)}

1. Arentz M, Yim E, Klaff L, Lokhandwala S, Riedo FX, Chong M, et al. Characteristics and Outcomes of 21 Critically III Patients With COVID-19 in Washington State. JAMA. 28 aprile 2020;323(16):1612-4.

2. Grasselli G, Pesenti A, Cecconi M. Critical Care Utilization for the COVID19 Outbreak in Lombardy, Italy: Early Experience and Forecast During an Emergency Response. JAMA. 28 aprile 2020;323(16):1545.

3. Grasselli G, Zangrillo A, Zanella A, Antonelli M, Cabrini L, Castelli A, et al. Baseline Characteristics and Outcomes of 1591 Patients Infected With SARS-CoV-2 Admitted to ICUs of the Lombardy Region, Italy. JAMA. 28 aprile 2020:323(16):1574

4. World Health Organization. Novel coronavirus situation report -2. [Internet]. 2020 [citato 23 gennaio 2020]. Available at: https://www.who.int/docs/ default-source/coronaviruse/situation-reports/20200122-sitrep-2-2019ncov.pdf

5. European Centre for Disease Prevention and Control. Novel coronavirus in China. [Internet]. [citato 23 gennaio 2020]. Available at: https://www.ecdc. europa.eu/en/novel-coronavirus-china

6. Prevention Centers for Disease Control and. First travel-related case of 2019 novel coronavirus detected in United States [Internet]. 2020. Available at: https://www.cdc.gov/media/releases/2020/p0121-novel-coronavirus-trave l-case.html

\section{6}

Clinical Characteristics, Outcomes and Predictors of Survival in COVID-19 ICU patients with Tracheostomy

L. Skelly' ; K. Liatsikos'; S. Mcdougall2; G. Dempsey'; B. Borgatta

'Intensive Care, Aintree University Hospital, Liverpool, United Kingdom;

${ }^{2}$ Intensive care, The Walton Centre, Liverpool, United Kingdom

Correspondence: L. Skelly

Intensive Care Medicine Experimental 2020, 8(2): 001366

Introduction: The COVID-19 pandemic has led to unprecedented numbers of critically ill patients requiring prolonged mechanical ventilation [1]. Professional societies advocated the delay of tracheostomies or discouraged them altogether given the expected increase in risk of aerosol-generating procedure (AGP) [2]. We performed tracheostomy in an effort to deal with predicted pandemic related resource shortages and to reduce ICU weakness and hospital stay [3]. We developed a modified technique for the performance of percutaneous and surgical tracheostomies to reduce AGP potential, with a dedicated team performing them, presented as another abstract.

Methods: A prospective, single centre, observational study took place between March and July 2020. Patients underwent tracheostomy if expected to require prolonged mechanical ventilation and were either unready for trial extubation or unfit for extubation after failing a dedicated protocol for extubation within 7-15 days of intubation.

Results: Between March 2020 and July 2020, 74 patients were admitted to our ICU due to COVID-19 pneumonitis. Of these, 13 patients (17.6\%) required CPAP only and 61 patients (82.4\%) were intubated. No patient met the extubation criteria, so 34 underwent tracheostomy (15 percutaneous, 19 surgical). Patients were predominantly male (63.6\%), with a median age of 64 years (IQR: 58-68) (Table 1). Only $85.3 \%$ were confirmed cases of COVID-19. The rest could not be tested for antibodies, but had a strongly suggestive clinical picture. At the time of tracheostomy, $25 \%$ were receiving vasopressor support and $17.9 \%$ requiring renal replacement therapy (RRT) (Figure 1). Duration of oro-tracheal intubation prior to tracheostomy was 7.5 days (IQR: 5-12) and length of cannulation was then 12 days (IQR: 6-17). ICU-LOS was 23 days (median, IQR: 15-34.5) and ward-LOS was 9 days (median, IQR: 5-17). Mortality was $20.6 \%$ (7 patients); death occurred at a median of 9 days (IQR: 4-14).Twenty six patients (78.8\%) were discharged home and one remains in hospital. Three patients required a more protracted weaning phase with prolonged ICU and ward stay; all of them had developed fibrosis in HRCT. After treatment with high dose steroids, they were successfully decanulated. Predictors of survival were: age $\leq 67$ years $\mathrm{OR}=10.5(1.5-70.8 ; \mathrm{IQR} ; \mathrm{p}<0.05)$ and PEEP at day 4 post tracheostomy $<10 \mathrm{cmH} 20 \mathrm{OR}=13.8$ (1.9-97.2; IQR; $\mathrm{p}<$ 0.01 ). Comorbidity was a risk factor for death (Charlson's comorbidity index $\mathrm{OR}=0.43$ [0.18-0.99; IQR; $\mathrm{p}<0.05]$ ). See Figure 2 for KaplanMeier curves. Previous CPAP was not associated with worse outcomes. Conclusion: In a selected cohort of COVID-19 patients tracheostomy is associated with good outcomes. Predictors of ICU survival in these patients were age $\leq 67$ years and the ability to wean respiratory support 4 days after tracheostomy, whereas greater comorbidity is associated with worse outcomes. Patients with prolonged weaning might benefit from being investigated for development of pulmonary fibrosis.

\section{Reference(s) and grant ackowledgment(s)}

1. 1. Huang, Prof C et al. Clinical Features of patients with 2019 novel coronavirus in Wuhan, China. The Lancet 2020.395;497-506.

2. 2. The Faculty of Intensive Care Medicine. COVID-19: Very Rapid Updates and Safety (ViRUS). 2020

3. 3. National Tracheostomy Safety Project (NTSP). NTSP consideration for tracheostomy in the Covid-19 outbreak. 2020.

4. Nothing to declare.

\section{5}

Multistate modeling of COVID-19 patients in French Outcomerea ICU population: an observational study

U. Moreno ${ }^{1}$; C. DUPUIS ${ }^{2}$; E. de Montmollin ${ }^{3} ;$ L. Bouadma ${ }^{4}$

D. Goldgran-Toledano ${ }^{5}$; S. Ruckly ; M. Neuville' ; Y. Cohen ${ }^{8}$; B. Mourvillier';

B. Souweine ${ }^{10}$; M. Gainnier ${ }^{11}$; V. Laurent ${ }^{12}$; N. Terzi ${ }^{13}$; S. Siami ${ }^{14}$; C. Alberti ${ }^{15}$; JF. Timsit ${ }^{16}$

${ }^{1}$ Inserm umrs 1138 team 22, Paris-Sorbonne University, Paris, France; ${ }^{2}$ MEDECINE INTENSIVE REANIMATION, CHU Gabriel-Montpied, Clermont-Ferrand France; ${ }^{3}$ Medical and infectious Intensive Care Unit, Bichat-Claude Bernard Hospital, Paris, France: ${ }^{4}$ Paris, Medical and infectious Intensive Care Unit, Bichat-Claude Bernard Hospital, Paris, France, ${ }^{5}$ Médecine intensive et réanimation, Intercommunal Hospital Group Le Raincy Montfermeil, Montfermeil, France; ${ }^{6}$ Umr 1137, INSERM, Paris, France; ${ }^{7}$ Réanimation, Hospital Foch, Suresnes, France; ${ }^{8}$ Respiratory department, normadie univ, Avicenne Hospital (AP-HP), Bobigny, France; ${ }^{9}$ Médecine intensive et réanimation polyvalente, CHU Reims - Champagne Clinic, Reims, France; ${ }^{10}$ Médecine intensive et réanimation, CHU Gabriel-Montpied, Clermont-Ferrand, France; ${ }^{11}$ Intensive care unit, Hospital Timone, Marseille, France; ${ }^{12}$ Réanimation, C.H. de Versailles, Le Chesnay, France; ${ }^{13}$ Médecine intensive réanimation, C.H.U de Grenoble, La Tronche, France; ${ }^{14}$ Réanimation, Hospital Center Sud Essonne -, Étampes, France; ${ }^{15}$ Epidemiology, Assistance Publique - Hôpitaux de Paris, Hôpital Robert Debré, Paris, France; ${ }^{16}$ Réanimation médicale et infectieuse, Bichat-Claude Bernard Hospital, Paris, France Correspondence: C. DUPUIS

Intensive Care Medicine Experimental 2020, 8(2): 001375

Introduction: Covid-19 ARDS progress from hospital oxygen requirement to severe ARDS requiring ECMO, to final hospital discharge or death. The impact of antiviral therapy, corticosteroids (CS) and other immunomodulatory agents on the path between states is not known. 
The Recovery trial [1] found a better day 28 prognosis when using dexamethasone low dose (DXM-6mg) but results are only preliminary and CS adverse effects not followed.

Objectives: To model COVID-ARDS states transitions from ICU admission to day 60 prognosis and evaluate the CS impact on transitions and final outcome.

Methods: A prospective data collection of severe COVID-19 patients admitted in 10 ICUs in France. Patients were followed daily until hospital discharge for a maximum of 60 days. We considered a multistate model shown in Figure 1. The 17 possible transitions are modeled via a Cox proportional hazard model (Breslow method, robust variance). Continuous variables are discretized via nested coding using quartiles values and tested on transitions with more than 10 events. Covariates with a $p$-value $\leq 0.2$ in univariate analysis are retained and the final model is achieved via stepwise selection (BSC criterion). CS effect is tested using G-computation. Confidence intervals are obtained via a probabilistic sensitivity analysis (500 runs).

Results: From 401 patients in OutcomeRea, 6 are excluded since COVID-19 was acquired in the hospital. $77.5 \%$ are male, the median age is 61 years. Charlson score is greater than 0 in $61 \%$ of patients, the median BMI is 28.3 , median SAPS II is 33 . The median duration first symptom-ICU admission is 10 days, and the median hospital stay before ICU is 2 days. Median lymphocytes count is $0.9 \times \mathrm{G} / \mathrm{L}$, median C-reactive protein serum value is $157 \mathrm{mg} / \mathrm{L}$ and the median leucocytes is $9 \mathrm{G} / \mathrm{L}$. $28.6 \%$ have temperature $>39^{\circ} \mathrm{C}, 26.3 \%$ received $\mathrm{CS}$ at admission $(8.3 \%$ with dose $\leq 10 \mathrm{mg}$ of Dexamethasone or equivalent and $18 \%$ with DXM $20 \mathrm{mg}$ or equivalent), $34.7 \%$ lopinavir/ritonavir, $6.8 \%$ tocilizumab, $6.3 \%$ anakinera and $11.1 \%$ hydroxychloroquine. $20 \%$ received more than one therapy.

$64.3 \%$ were admitted in ICU with non-invasive oxygenation, 30.9\% with invasive mechanical ventilation and 5.8\% in ECMO. Overall day 60 mortality was $32.9 \%$ and $13.4 \%$ of patients were censored.

Transition selected variables are shown on Figure 1. Antiviral therapies did not result in significant impact. CS decrease the risk of invasive ventilation but decreased the risk of successful extubation. $\mathrm{G}$ computation suggests no significant effect on day 60 prognosis $(32.7 \% \mathrm{Cl}$ : $23.8-43.1 \%$ and $32.7 \% \mathrm{Cl}: 23.8-43.4 \%$ of being dead for CS and no CS, respectively). Low and high doses of CS provide similar effect.

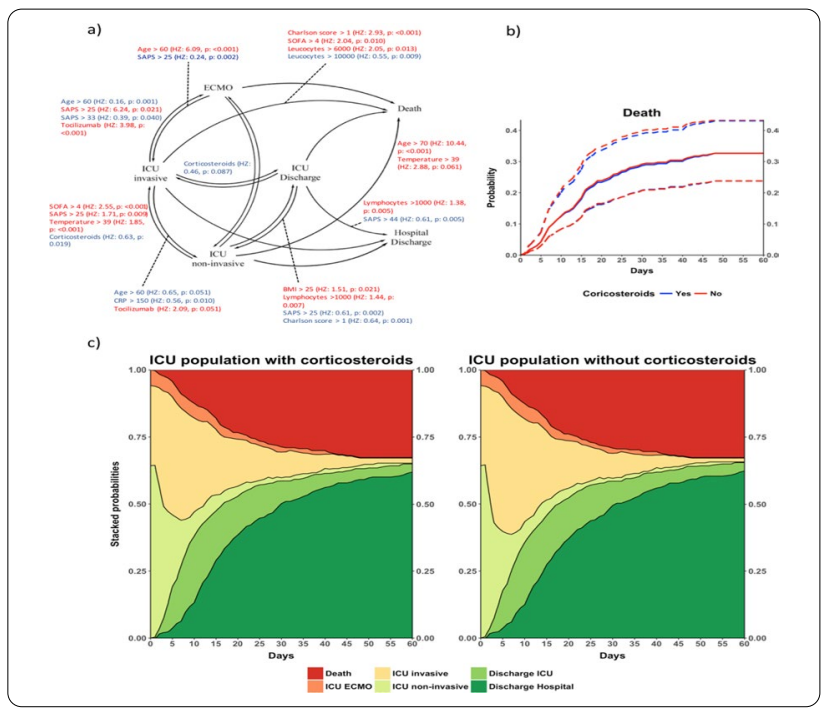

Conclusion: Using a multistate model, we found that CS decrease the risk of invasive ventilation need but is associated with a lower chance of being extubated. Impact on prognosis is not significant. Possible causes of this delayed adverse impact (infections or other adverse effects) require further analyses.

\section{6}

Impact of early corticosteroids on day-60 mortality in critically ill patients with COVID-19: a multicenter cohort study of the OUTCOMEREA network

C. DUPUIS ; E. de Montmollin²; D. Goldgran-Toledano ${ }^{3}$; J. Reignier ${ }^{4}$; C. Schwebel' ${ }^{5}$ M. Neuville ${ }^{6}$; U. Moreno ${ }^{7}$; S. Siami ${ }^{8}$; S. Ruckly ${ }^{9}$; C. Alberti ${ }^{10}$; B. Mourvillier ${ }^{11}$; S. Bailly ${ }^{12}$; V. Laurent ${ }^{13}$; M. Adda ${ }^{14}$; M. Gainnier ${ }^{15}$;

N. Buetti ${ }^{16} ;$ B. Souweine ${ }^{14} ;$ JF. Timsit $^{17}$

${ }^{1}$ MEDECINE INTENSIVE REANIMATION, CHU Gabriel-Montpied, Clermont-Ferrand, France; ${ }^{2}$ Medical and infectious Intensive Care Unit, Bichat-Claude Bernard Hospital, Paris, France; ${ }^{3}$ Médecine intensive et réanimation, Intercommunal Hospital Group Le Raincy Montfermeil, Montfermeil, France; ${ }^{4}$ Médecine intensive réanimation, Nantes University Hospital Hotel-Dieu, Nantes, France; ${ }^{5}$ Médecine intensive réanimation, C.H.U de Grenoble, La Tronche, France; ${ }^{6}$ Réanimation, Hospital Foch, Suresnes, France; ${ }^{7}$ Inserm umrs 1138 team 22, Paris-Sorbonne University, Paris, France, ${ }^{8}$ Réanimation, Hospital Center Sud Essonne -, Étampes, France; ${ }^{9}$ Umr 1137, INSERM, Paris, France; ${ }^{10}$ Epidemiology, Assistance Publique Hôpitaux de Paris, Hôpital Robert Debré, Paris, France; ${ }^{11}$ Médecine intensive et réanimation polyvalente, CHU Reims - Champagne Clinic, Reims, France; ${ }^{12}$ Laboratoire hp2, Chu Grenoble Universitaire, La Tronche, France; ${ }^{13}$ Réanimation, C.H. de Versailles, Le Chesnay, France; ${ }^{14}$ Médecine intensive et réanimation, CHU Gabriel-Montpied, Clermont-Ferrand, France; ${ }^{15}$ Intensive care unit, Hospital Timone, Marseille, France; ${ }^{16}$ Inserm umr 1137, Paris Diderot University, Paris, France; ${ }^{17}$ Réanimation médicale et infectieuse, Bichat-Claude Bernard Hospital, Paris, France

\section{Correspondence: C. DUPUIS}

Intensive Care Medicine Experimental 2020, 8(2): 001406

Introduction: The Recovery trial [1] found a better day 28 prognosis while using dexamethasone (DXM) low dose (DXM-6 mg) but results are only preliminary and not specific of the patients already admitted in ICU. Consequently, the benefit of Corticosteroids (CS) for the Covid19 pneumonia patients admitted in ICU on death is still under debate. Objectives: The objective of this study was to assess a non-biased effect of early CS in COVID-19 pneumonia patients admitted in ICU on the occurrence of death by the 60-day.

Methods: We selected patients from $11 \mathrm{ICUs}$ included in the French OutcomeReaTM group. We included patients over 18 years, with COVID proved by PCR, entered in database from January 2020 to May 2020, with an ICU stay longer than two days, not transferred from another ICU and without decision to forego life-sustaining therapies on admission. We excluded patients treated with CS before ICU admission. The primary outcome was death before day 60 . The intervention was an early administration of CS in ICU defined by the initiation of a new treatment by CS at day 1 or 2 after ICU admission. We used Cox proportional-hazard models with ponderation with inverse probability of treatment weighting (IPTW) to model the risk of death (IPTWHR $>1$ in favor of death).

Results: 302 patients were included. Their median age was 61.6 (5370) years; $78.8 \%$ were male; $58.6 \%$ had at least one comorbidity. On admission, SAPS II was 33(25-44); the $\mathrm{PaO}_{2} / \mathrm{FiO}_{2}$ ratio was under 200 $\mathrm{mmHg}$ in $74.2 \% ; 34.8 \%$ of the patients received invasive mechanical ventilation, $31.8 \%$ had a temperature over $39^{\circ} \mathrm{C}$.

$66(21.8 \%)$ patients were in the Early CS subgroups (11 (3.6\%) with dose less than $10 \mathrm{mg}$ of DXM or equivalent and $55(18.2 \%)$ with DXM $20 \mathrm{mg}$ or equivalent). In the No Early CS subgroup, 96 (40.7\%) received CS after day 2. Patients in the Early CS subgroup received more often anti-viral therapy and Anakinra $(p<0.01)$, had more hyperglycemia $(p<0.01)$ and needed more daily insulin dose $(p<0.01)$ during the ICU stay and tended to have more bacteriemia and HAP-VAP ( $p=0.06$ and 0.16 , see table).

The mortality at day 60 was $29.4 \%$ in the study population, $34.8 \%$ in the early CS and $28 \%$ in the non Early CS $(p=0.28)$. In the IPTW analysis, on day 60, early CS was not associated with an increase risk of death (IPTW-HR $=1.06, \mathrm{Cl} 95 \%, 0.67$ to $1.68, \mathrm{p}=0.79$ ). The results were similar limiting the analysis to high doses CS (IPTW-HR $=1.24, \mathrm{Cl} 95 \%$, 0.83 to $1.87, \mathrm{p}=0.29$ ). 


\begin{tabular}{|c|c|c|c|c|}
\hline & All $(n=302)$ & No Early CS (n=236) & Early CS ( $n=66)$ & pvalue \\
\hline Baseline caracteristics & & & & \\
\hline $\begin{array}{l}\text { Age } \\
\text { Sexe(Male) }\end{array}$ & $\begin{array}{l}61.6[153 ; 70] \\
238(78.8)\end{array}$ & $\begin{array}{l}6153 ; 70] \\
182(77,1)\end{array}$ & $\begin{array}{l}62.5(55 ; 71] \\
56(84.8)\end{array}$ & $\begin{array}{l}0.44 \\
0.17\end{array}$ \\
\hline Body mass Index & $28.4[25.6 ; 32.2]$ & $28.4[25.5 ; 32.2]$ & $\begin{array}{l}56(84.8) \\
27.6[25.1 ; 32.1]\end{array}$ & $\begin{array}{l}0.17 \\
0.35\end{array}$ \\
\hline At least one comorbidity & 177 (58.6) & $131(55.5)$ & $46(69.7)$ & \\
\hline Time from symptoms to ICU admission & $10[7 ; 12]$ & $10[7 ; 12]$ & $10[8 ; 13]$ & \\
\hline Characteristics on admis & & & & \\
\hline SAPS II & $33[25 ; 44]$ & $32[24 ; 43]$ & $37[29 ; 4$ & $<.01$ \\
\hline SOFA & $4[3 ; 7]$ & $4[2 ; 6]$ & $5[3 ; 8]$ & \\
\hline$T>39^{\circ} \mathrm{C}$ & $96(31.8)$ & $82(34.7)$ & $14(21.2)$ & 0.04 \\
\hline Neutrophils & $6760.8[4600 ; 9830]$ & $6700[4350 ; 9260]$ & $7415[5240 ; 11600]$ & 0.02 \\
\hline Lymphocytes & $800[500 ; 1100]$ & $800[500 ; 1065]$ & $775[500 ; 1200]$ & 0.59 \\
\hline & $157[83.6 ; 238]$ & $149.9[83.7 ; 238]$ & $162[82 ; 232.9]$ & 0.83 \\
\hline Ferritin & $1127[592 ; 1960.2]$ & $1107.4[581.7 ; 1941]$ & $1347.5[720 ; 2210]$ & \\
\hline & $1743.4[860 ; 5261.6]$ & $1800[901 ; 5347]$ & $1640.5[700 ; 4805.3]$ & 0.27 \\
\hline $\begin{array}{l}\text { Treatments on admission } \\
\text { Invasive Mechanical ventilation }\end{array}$ & & & & \\
\hline $\begin{array}{l}\text { Invasive Mechanical ventilation } \\
\text { Tocilizumab }\end{array}$ & $\begin{array}{l}105(3.84) \\
25(8.2)\end{array}$ & $\begin{array}{l}80(33.2) \\
17(7.2)\end{array}$ & $\begin{array}{l}25(37.9) \\
8(12.1)\end{array}$ & $\begin{array}{l}0.55 \\
0.20\end{array}$ \\
\hline Anakine & $22(7$ & & $22(33.3)$ & \\
\hline Anti-vir & 136 & $97(41.1)$ & 39159 & $<0.01$ \\
\hline Adverse events due to $C S$ & & & & \\
\hline & $15(3$ & 9 (25) & 46 (6 & $<.01$ \\
\hline $\begin{array}{l}\text { Mean daily dose of insulin } \\
\text { Outcomes }\end{array}$ & $8.8[0 ; 44.2]$ & $5.90 ; 36.3$ & $27.2[4.4 ; 58.4]$ & \\
\hline $\begin{array}{l}\text { Outcomes } \\
\text { Ventilatory free days }\end{array}$ & & & & \\
\hline Bacteriemia & & & & 0.06 \\
\hline & & & & 0.16 \\
\hline Nosocomial infections & & & 27 & 0.19 \\
\hline ICU LOS & $11.6[7 ; 20]$ & 20] & $11[7 ; 19]$ & 0.88 \\
\hline $\begin{array}{l}\text { ICUD Death } \\
\text { Death at day } 60\end{array}$ & $\begin{array}{l}85(28.2) \\
89(29.4)\end{array}$ & $\begin{array}{l}63(26.7) \\
66(28)\end{array}$ & $\begin{array}{l}22(333.3) \\
23(34.8)\end{array}$ & $\begin{array}{l}0.29 \\
0.28\end{array}$ \\
\hline
\end{tabular}

Conclusion: For the COVID-19 patients just admitted in ICU, in a prospective cohort with daily follow-up and a use a causal inference model, we were not able to unmask any positive impact of early CS therapy on patients' survival. Early CS was associated with hyperglycemia. The absence of benefit is not related to CS doses and may reflect difference in case-mix and/or standard of care used. Further studies are required to confirm the results of the RECOVERY trial.

\section{Reference(s) and grant ackowledgment(s)}

1. RECOVERY Collaborative Group. "Dexamethasone in Hospitalized Patients with Covid-19 -Preliminary Report." New England Journal of Medicine (2020).

\section{6}

Correlation between ventilatory parameters and gas exchange response to prone ventilation in SARS-CoV-2 ARDS

M. Chotalia'; JE. Alderman ${ }^{1}$; M. Bangash'; D. Parekh'; J. Patel ${ }^{1}$

${ }^{1}$ Birmingham acute care research group, University of Birmingham, Birmingham, United Kingdom

Correspondence: M. Chotalia

Intensive Care Medicine Experimental 2020, 8(2): 001416

Introduction: Prone ventilation has a demonstrated mortality benefit in moderate-severe ARDS [1]. It has been argued that the majority of patients with SARS-CoV-2 ARDS have preserved lung compliance and low lung weight and have minimal response to prone ventilation due to a low amount of recruitable lung tissue. [2]

Objectives: To determine the pre-prone parameters that correlate with $\mathrm{P} / \mathrm{F}$ ratio and $\mathrm{PaCO} 2$ response to prone ventilation in SARS-CoV-2 ARDS.

Methods: This is a single-centre, prospective study from the Queen Elizabeth Hospital Birmingham, UK. Patients undergoing invasive mechanical ventilation with SARS-CoV-2 pneumonia between the 11th March and 21st April that received at least one episode of prone ventilation were included. Prone ventilation was encouraged in patients who had an $\mathrm{FiO}_{2}$ concentration of greater than 0.6. Ventilatory parameters six hours before and during the first episode of prone ventilation were collected. Severity of opacification on chest radiography was graded by two independent reviewers using a validated, semiquantitative opacification score ranging from 0 to 16. [3] Deadspace fraction was estimated using the unadjusted Harris-benedict equation. [4] Statistical analysis was performed using GraphPad Prism v.8.0. Continuous data are presented as median (IQR). Correlation between variables was analysed using a nonparametric spearman correlation test. A p value $<0.05$ was used to determine significance.

Results: 143 patients were admitted to the ICU, of which 94 (66\%) received at least one episode of prone ventilation for a median time of $16(14-20)$ hours. $\mathrm{P} / \mathrm{F}$ ratio increased by $6.6(3.5-10.1)$ and $\mathrm{PaCO} 2$ decreased by $-0.03 \mathrm{kPa}(+0.9--1.0)$ in response to prone ventilation.
Figure 1: Correlation between ventilatory parameters and change in $\mathrm{P} / \mathrm{F}$ ratio and $\mathrm{PaCO} 2$ in response to prone ventilation

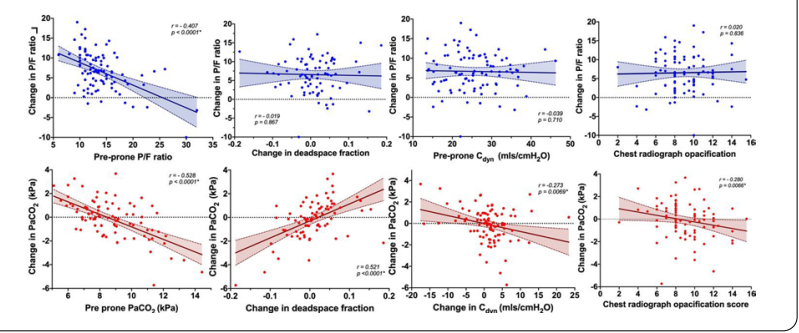

Conclusion: The $\mathrm{P} / \mathrm{F}$ ratio response to prone ventilation correlated with the degree of hypoxia prior to proning, indicating that stratifying the receipt of prone ventilation by the degree of hypoxia is entirely appropriate. The $\mathrm{P} / \mathrm{F}$ ratio response did not correlate with changes in deadspace fraction, pre-prone compliance or chest radiograph opacification, indicating that part of the response may be independent of lung recruitment, potentially due to improvements in cardiac output, distribution of blood flow and shunt fraction, as suggested by Gattinoni. [5]

The $\mathrm{PaCO} 2$ response to prone ventilation correlated with markers of lung recruitment, such as changes in dynamic compliance, deadspace fraction and pre-prone severity of chest radiograph opacification, similar to findings from previous ARDS cohorts. [5]

In summary, the majority of patients with SARS-CoV-2 ARDS had positive $P / F$ ratio responses with prone ventilation and receipt of this ventilation strategy should be stratified by the degree of hypoxia.

Reference(s) and grant ackowledgment(s)

1. • [1] Guérin C, Reignier J, Richard JC, et al. Prone positioning in severe acute respiratory distress syndrome. New England Journal of Medicine. 2013 Jun 6;368(23):2159-68.

2. • [2] Marini JJ, Gattinoni L. Management of COVID-19 respiratory distress. Jama. 2020 Apr 24

3. • [3] Mason SE, Dieffenbach PB, Englert JA, et al. Semi-quantitative visual assessment of chest radiography is associated with clinical outcomes in critically ill patients. Respiratory research. 2019Dec;20(1):1-9

4. - [4] Beitler JR, Thompson BT, Matthay MA, et al. Estimating dead-space fraction for secondary analyses of ARDS clinical trials. Critical care medicine. 2015 May;43(5):1026

5. • [5] Protti A, Chiumello D, Cressoni M, et al. Relationship between gas exchange response to prone position and lung recruitability during acute respiratory failure. Intensive care medicine. 2009 Jun 1;35(6):1011-7

\section{0}

Baseline ventilator settings, gas exchange and respiratory system mechanics in COVID19 patients with hypoxemic respiratory failure admitted in Catalan ICUs (UCIsCAT study group)

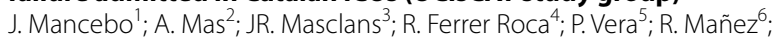

J. Trenado ; R. Fernandez ; JM. Sirvent Calvera9 ${ }^{9}$ M. Martínez ${ }^{10}$; M. Ibarz ${ }^{11}$; E. Sandoval12; P. Garro ${ }^{13}$; JL. Lopera ${ }^{14}$; M. Bodín ${ }^{15}$; JC. Yebenes-Reyes ${ }^{16}$; C. Triginer ${ }^{17}$; I. Vallverdú ${ }^{18}$; A. Baró Serra ${ }^{19}$; F. Bodi ${ }^{20}$

${ }^{1}$ Intensive care, Hospital de la Santa Creu i Sant Pau, Barcelona, Spain; ${ }^{2}$ Intensive care dpt, Hospital de Sant Joan Despí Moisès Broggi, Sant Joan Despí, Spain; Intensive care dpt, Hospital del Mar, Barcelona, Spain: ${ }^{4}$ Intensive care dpt / sodir research group, Vall d'Hebron University Hospital, Barcelona, Spain; Intensive care dpt, Hospital de la Santa Creu i Sant Pau, Barcelona, Spain; ${ }^{6}$ Intensive care dpt, Hospital Universitari de Bellvitge, L'Hospitalet de Llobregat, Spain; 'Intensive care dpt, Mútua Terrassa University Hospital, Terrassa, Spain; ${ }^{8}$ Intensive care dpt, Althaia, Xarxa Assistencial Universitària de Manresa, Manresa, Spain; ${ }^{9}$ Intensive care dpt, Hospital Universitari de Girona Dr Josep Trueta, Girona, Spain; ${ }^{10}$ Intensive care dpt, Hospital General De Cataluña, Sant Cugat del Vallès, Spain; ${ }^{11}$ Intensive care dpt, Hospital Universitari Sagrat Cor - Grup Quirónsalut, Barcelona, Spain; ${ }^{12}$ Cardiovascular surgery, Hospital Clínic de Barcelona, Barcelona, Spain; ${ }^{13}$ Intensive care dpt, Hospital General de Granollers, Granollers, Spain; ${ }^{14}$ Intensive care dpt, Hospital Universitari de Vic (Consorci Hospitalari de Vic), Vic, Spain; ${ }^{15}$ Intensive care dpt, Hospital Universitari de Tarragona Joan XXIII, Tarragona, Spain; ${ }^{16}$ Intensive care 
dpt, Hospital de Mataró, Mataró, Spain; ${ }^{17}$ Intensive care dpt, Igualada Hospital, Igualada, Spain; ${ }^{18}$ Intensive care dpt, Hospital Universitari Sant Joan de Reus, Reus, Spain; ${ }^{19}$ Intensive care dpt, Hospital de Santa Caterina, Salt, Spain; ${ }^{20}$ Intensive care dpt, Hospital de Sant Pau i Santa Tecla, Tarragona, Spain

\section{Correspondence: J. Mancebo}

Intensive Care Medicine Experimental 2020, 8(2): 001440

Introduction: COVID19 pandemic and the consequent surge of patients with acute hypoxemic respiratory failure has stretched the intensive care departments capacity to provide supportive treatment.

Objectives: To describe the baseline ventilator settings of adult patients with severe acute hypoxemic respiratory failure due to COVID19 admitted in the Catalan ICU network.

Methods: Consecutive patients admitted between 15 March and 15 April in $26 \mathrm{ICUs}$, who tested positive for SARS-Cov-2 (RT-PCR in a nasopharingeal swab) and had severe acute hypoxemic respiratory failure, presenting with bilateral infiltrates in the chest $\mathrm{X}$-ray and requiring invasive mechanical ventilation. Relevant data were colected in stable conditions shortly after intubation. Follow-up time was til ICU discharge. Study was approved by the Ethics Committee of the coordinating center (Hospital Universitari Sant Pau, Barcelona).

Results: A total of 1704 patients (1187 men and 517 women) were admitted, and their median age was $63 \mathrm{yr}$ (IQR 54-70). Of these, 1388 $(82 \%)$ required invasive mechanical ventilation. Median duration of mechanical ventilation was 14 days (IQR 7-24). Overall UCI mortality was 35\% (491/1388). Prone position was implemented in $77 \%$ (1070/1388). ARDS criteria was met by 1220 intubated patients (88\%, 1220/1338). Cumulative survival over ICU time is shown in the KaplanMeier plot.

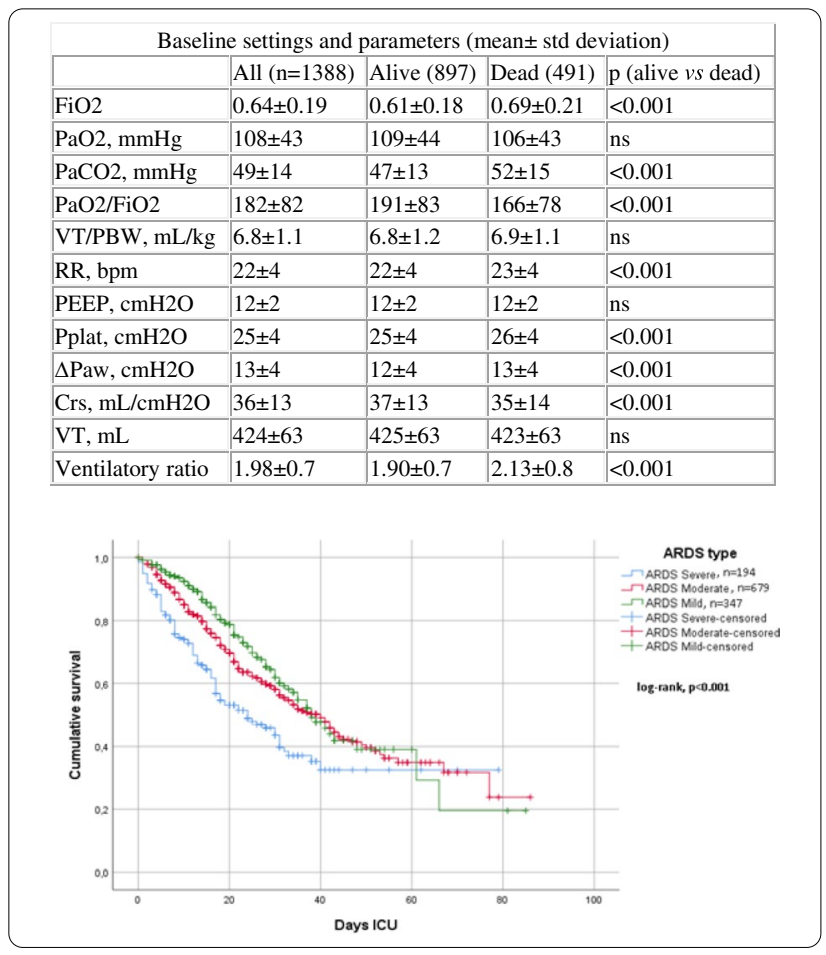

Conclusion: The vast majority of acutely hypoxemic covid 19 patients who required invasive mechanical ventilation met classical ARDS criteria.

\section{Reference(s) and grant ackowledgment(s)}

1. Other contributors: J-A. Santos, J. Baldirà, A-J. Betbesé, M. Izura, I. Morán, J-C. Suárez, L. Zapata, N. Rodríguez, M. Torrens, A. Cordón, C. Gomila, M. Flores, A. Segarra, M. Morales, L. Mateo, M. Martos, P. Saludes, J-R. Cervelló,
M. Valencia, F. Roche, G. Masdeu, B. Cancio, S. Hernández-Marín, C. Pedrós, P. Sebastian, X. Saiz, M. Morales, A. Huerta, E. Esteban, S. Benito, C. Barberà, J. Echevarría, P. Santigosa, D. Tognetti, C. González

\section{9}

The clinico-pathological spectrum of renal lesions in the course of COVID-19

M. Jamme ; S. Ferlicot ${ }^{2}$; F. Gaillard ${ }^{3}$; J. Oniszczuk ${ }^{4}$; A. Couturier ${ }^{5}$;

A. Grünenwald ${ }^{6}$; O. May ${ }^{7}$; A. Sannier ${ }^{8}$; A. Moktefi ${ }^{2}$; R. Arrestier ${ }^{9}$;

C. Petit-Hoang ${ }^{10}$; Z. Amoura ${ }^{11}$; M. Essig ${ }^{5}$; I. Brocheriou ${ }^{12}$; E. Daugas ${ }^{3}$;

V. Audard ${ }^{4}$; D. Buob ${ }^{13} ;$ Z. Massy $^{5} ;$ M. Zaidan ${ }^{14} ;$ G. Geri $^{15}$

${ }^{1}$ Médecine intensive Réanimation, Hospital Center Intercommunal Poissy/ Saint-Germain-En-Laye., Poissy, France; ${ }^{2}$ Anatomopathologie, Bicetre Hospital AP-HP, Le Kremlin-Bicêtre, France; ${ }^{3}$ Néphrologie, Bichat-Claude Bernard Hospital, Paris, France; ${ }^{4}$ Néphrologie, Hôpital Henri-Mondor Ap-Hp, Créteil, France; ${ }^{5}$ Néphrologie, Ambroise Paré Hospital (AP-HP), Boulogne-Billancourt, France; ${ }^{6}$ Médecine intensive réanimation, Bicetre Hospital AP-HP, Le Kremlin-Bicêtre, France; ${ }^{7}$ Néphrologie, Hospital André Grégoire, Montreuil, France; ${ }^{8}$ Anatomopathologie, Bichat-Claude Bernard Hospital, Paris, France; ${ }^{9}$ Médecine intensive réanimation, Hôpital Henri-Mondor Ap-Hp, Créteil, France; ${ }^{10}$ Urgences néphrologiques et transplantation rénale, Hospital Tenon Ap-Hp, Paris, France; ${ }^{11}$ Médecine interne, University Hospitals Pitié Salpêtrière - Charles Foix, Paris, France; ${ }^{12}$ Anatomopathologie, University Hospitals Pitié Salpêtrière - Charles Foix, Paris, France; ${ }^{13}$ Anatomopathologie, Hospital Tenon Ap-Hp, Paris, France; ${ }^{14}$ Néphrologie, Bicetre Hospital AP-HP, Le Kremlin-Bicêtre, France; ${ }^{15}$ Médecine intensive réanimation, Ambroise Paré Hospital (AP-HP), Boulogne-Billancourt, France

Correspondence: $M$. Jamme

Intensive Care Medicine Experimental 2020, 8(2): 001469

Introduction: The spectrum of kidney damage in COVID-19 has been mostly limited to autopsies and case reports. Here, we report the first case series of patients with COVID-19 who developed kidney injury and underwent a kidney biopsy in the Paris and its metropolitan area. The spectrum of kidney damage in COVID-19 has been mostly limited to autopsies and case reports. Here, we report the first case series of patientswith COVID-19 who developed kidney injury and underwent a kidney biopsy in the Paris and its metropolitan area.

Methods: We included all patients with COVID-19 who underwent a kidney biopsy between March 08 and May 19, 2020. Data were obtained through the review of the medical records.

Results: Forty-seven patients (80.9\% men) with COVID-19 were included in the present study. Median age was 63 years IQR [52-69]. Comorbidities included hypertension (66.0\%), diabetes mellitus (27.7\%), previous history of chronic kidney $(25.5 \%)$, cardiac $(38.6 \%)$ and respiratory $(27.3 \%)$ diseases. Initial symptoms were fever $(85.1 \%)$, cough (63.8\%), shortness of breath (55.3\%), and diarrhea (23.4\%). Almost all patients developed acute kidney injury (97.9\%) and $63.8 \%$ required renal replacement therapy. Kidney biopsy analysis showed two main histopathological patterns, including isolated acute tubular necrosis in $22(46.8 \%)$ patients, and dominant glomerular injury consisting of collapsing glomerulopathy or focal segmental glomerulosclerosis in $17(36.2 \%)$ patients. Eight (17\%) patients had alternative diagnosis, which were most likely unrelated to COVID-19. Acute tubular necrosis occurred almost invariably in the setting of severe forms of COVID-19, whereas patients with glomerular injury had various profiles of COVID-19 severity and collapsing glomerulopathy was only observed in patients harboring a combination of APOL 1 risk variants. At last follow-up, 16 of the 30 patients who initially required dialysis were still on dialysis, and 9 died from COVID-19 or related-adverse events.

Conclusion: The present study describes clinico-pathological spectrum of kidney lesions in yet-alive patients with COVID-19. While acute tubular necrosis is correlated with COVID-19 severity, the pattern of glomerular injury is intimately associated with the expression of $A P O L-$ 1risk variants. Whether SARS-CoV-2 directly underlies renal damage remains a major but unsolved issue. 
001472

Evaluation of oXiris in COVID-19 patients on VV-ECMO with AK

K. Aitchison ${ }^{1}$; R. Lewis ${ }^{1}$; R. Browning ${ }^{1}$; L. Thakuria ${ }^{1}$; J. Doyle ${ }^{1}$

'Intensive care, Royal Brompton \& Harefield NHS Foundation Trust, Lon-

don, United Kingdom

Correspondence: K. Aitchison

Intensive Care Medicine Experimental 2020, 8(2): 001472

Introduction: COVID-19 caused many deaths through a dysregulated immune response, with resultant "cytokine storms" contributing to multi-organ failure [1]. Patients with severe respiratory failure have required extra-corporeal membrane oxygenation (ECMO) as a rescue therapy [2]. The oXiris adsorptive haemofilter has the capacity to adsorb cytokines while delivering continuous renal replacement therapy (CRRT) [3]. Therefore, patients with multi-organ failure secondary to COVID-19 could be managed with ECMO and haemoadsorptive therapy as a novel approach [4]. Minimal evidence currently exists of its effectiveness or safety.

Objectives: To evaluate the safety and efficacy of oXiris in COVID-19 patients on VV-ECMO with AKI.

Methods: A single-site prospective observational case series was conducted from March to June 2020. Inclusion criteria were: COVID-19, age $>18, \mathrm{VV}$-ECMO, CRRT and severe septic shock. Data was collected at baseline and over three successive oXiris filters. Data included vasopressor requirements, biochemical markers and vital signs. Variables at baseline and completion of therapy were compared using Wilcoxon Signed Ranks Test, two-tailed $p$-values (significance $p<0.05$ ).

Results: Eight patients were included; 7 males and 1 female with a mean age of forty-seven. None had pre-existing renal disease and mean baseline creatinine on admission was 230 umol/L. Mean CRP was $252 \mathrm{mg} / \mathrm{dL}$ with a mean lactate of $3.12 \mathrm{mmol} / \mathrm{L}$. The median vasopressin dose fell significantly from 0.02 units $/ \mathrm{min}$ to 0.00 units $/ \mathrm{min}$ ( $p$ $=0.031$ ), median noradrenaline dose fell significantly from $0.23 \mathrm{mcg} /$ $\mathrm{kg} / \mathrm{min}$ to $0.06 \mathrm{mcg} / \mathrm{kg} / \mathrm{min}(\mathrm{p}=0.039)$ and median urine output significantly improved from $0 \mathrm{ml} /$ day to $151 \mathrm{ml} /$ day $(p=0.008)$. There were no adverse events reported.

Conclusion: The use of oXiris adsorbent membrane in patients requiring VV-ECMO for COVID-19 with clinical evidence of a "cytokine storm" was safe, with no adverse events. There was also significant improvement of haemodynamic stability and initial renal recovery. Further evaluation through a large randomised control trial is warranted.

\section{Reference(s) and grant ackowledgment(s)}

1. [1] Chen, G., et al., Clinical and immunological features of severe and moderate coronavirus disease 2019. J Clin Invest, 2020. 130(5): p. 2620-2629.

2. [2] World Health Organisation., Clinical management of severe acute respiratory infection (SARI) when COVID 19 disease is suspected. Interim report 2020.

3. [3] Malard, B., Lambert, C., Kellum, J.A. In vitro comparison of the adsorption of inflammatory mediators by blood purification devices. Intensive Care Med Exp, 2018. 6(1):12

4. [4] Kowalewski, M., et al., COVID-19 and ECMO: the interplay between coagulation and inflammation - a narrative review. Crit Care, 2020. 24(1): p. 205.

\section{8}

The use high levels of positive end-expiratory pressure in patients with massive non-traumatic subarachnoid hemorrhages and initially increased intracranial pressure

ED. Mekhia Mekhia'; A. Savenkov ; A. Solodov'; S. Petrikov'; V. Krylov

${ }^{1}$ Neurosurgical icu, Sklifosovsky Research Institute for Emergency Medicine, Moscow, Russia; ${ }^{2}$ Neurosurgery, Sklifosovsky Research Institute for Emergency Medicine, Moscow, Russia

Correspondence: A. Solodov

Intensive Care Medicine Experimental 2020, 8(2): 001478

Introduction: Positive end-expiratory pressure (PEEP) is one of the main parameters for preventing lung damage and ensuring gas exchange during mechanical ventilation. The use of high PEEP levels can lead to increase of intracranial pressure (ICP) in patients with massive non-traumatic subarachnoid hemorrhages (SAH). Therefore, the use of high levels of PEEP is limited in patients with initially high ICP.

Objectives: To determine the efficacy and safety of different levels of PEEP in patients with intracranial hemorrhage with initially increased ICP.

Methods: We analyzed the state of systemic and cerebral hemodynamics in different levels of PEEP in critically ill patients with SAH in situations with initially increased ICP $(n-31)$. The study was not performed in patients with intracranial pressure of $20 \mathrm{mmHg}$ and more. The examined patients had Glasgow coma scale below 9. Invasive ICP monitoring, transpulmonary thermodilution, respiratory monitoring were used in all patients. The studied parameters were determined with an increase in PEEP from $5 \mathrm{~cm} \mathrm{H}_{2} \mathrm{O}$ to 10,15 and $20 \mathrm{~cm} \mathrm{H}_{2} \mathrm{O}$. Minute ventilation volume and $\mathrm{PaCO} 2$ were stable at all stages of the study.

Results: The initial level of ICP was $17(16 ; 18) \mathrm{mmHg}$, cerebral perfusion pressure (CPP) - $93(86 ; 107) \mathrm{mmHg}$. A decrease of global end-diastolic index and compensatory increase of systemic vascular resistance index with normal mean arterial blood pressure indicated a state of latent hypovolemia. Increase of PEEP to 10 and $15 \mathrm{cmH}_{2} \mathrm{O}$ had no negative effects on ICP and CPP. We determined the tendency to decrease cardiac index, cardiac preload and to increase systemic vascular resistance index, stroke volume variation. High levels of PEEP did not lead to obstruction of extracranial venous outflow; the jugular bulb pressure were stable (Table 1). We identify increase ICP and decrease CPP, cardiac index, global end-diastolic index, systemic vascular resistance index when PEEP were $20 \mathrm{cmH} 20$. Hypovolemia limited the compensatory reserves of hemodynamics, which resulted a decrease in arterial and cerebral perfusion pressures.

Table 1. Parameters of systemic and cerebral hemodynamics during different levels of positive end-expiratory pressure in patients with high baseline intracranial pressure

\begin{tabular}{|c|c|c|c|c|}
\hline Parameters & $\begin{array}{l}\text { PEEP } 5 \\
\mathrm{cmH} 2 \mathrm{O}\end{array}$ & $\begin{array}{l}\text { PEEP } 10 \\
\mathrm{cmH} 2 \mathrm{O}\end{array}$ & $\begin{array}{l}\text { PEEP 15 } \\
\mathrm{cmH} 2 \mathrm{O}\end{array}$ & $\begin{array}{l}\text { PEEP 20 } \\
\mathrm{cmH} 2 \mathrm{O}\end{array}$ \\
\hline $\begin{array}{l}\text { Intracranial } \\
\text { pressure, } \\
\mathrm{mmHg}\end{array}$ & $17(16 ; 18)$ & $17,5(16 ; 18)$ & $18(16 ; 19,5)$ & $19(17 ; 20)$ *\# \\
\hline $\begin{array}{l}\text { Mean arterial } \\
\text { pressure, } \\
\mathrm{mmHg}\end{array}$ & $110(102 ; 122)$ & $109(100 ; 119)$ & $105(98 ; 114)$ & $\begin{array}{l}99(92 ; 110) \\
* \#\end{array}$ \\
\hline $\begin{array}{l}\text { Cerebral } \\
\text { perfusion } \\
\text { pressure, } \\
\text { mmHg }\end{array}$ & $93(86 ; 107)$ & $92(84 ; 102)$ & $89(80 ; 97)$ & $81(75 ; 90)$ *\# \\
\hline $\begin{array}{l}\text { Cardiac index, } \\
\text { litres } / \mathrm{min} / \mathrm{m} 2\end{array}$ & $3,5(3,0 ; 3,7)$ & $3,3(2,9 ; 3,4)$ & $3,1(3,0 ; 3,3)$ & $3,0(2,8 ; 3,3)$ \\
\hline $\begin{array}{l}\text { Global end } \\
\text { diastolic index, } \\
\mathrm{m} 1 / \mathrm{m} 2\end{array}$ & $608(598 ; 701)$ & $556(523 ; 666)$ & $564(527 ; 653)$ & $588(514 ; 662)$ \\
\hline $\begin{array}{l}\text { Systemic } \\
\text { vascular } \\
\text { resistance index, } \\
\text { dyn* }{ }^{*} \mathrm{~cm}-5^{*} \mathrm{~m} 2\end{array}$ & $\begin{array}{l}2450 \\
(1980 ; 2925)\end{array}$ & \begin{tabular}{l|l}
2645 & $2107 ; 3073)$ \\
$(213$
\end{tabular} & $\begin{array}{l}2590 \\
(2193 ; 3000)\end{array}$ & $\begin{array}{l}2725 \\
(2248 ; 3119)\end{array}$ \\
\hline $\begin{array}{l}\text { Stroke volume } \\
\text { variation, \% }\end{array}$ & $10,5(8 ; 14)$ & $13(9 ; 21,3)$ & $18(12 ; 22,8)$ & $\begin{array}{l}21,5(14 ; 22,8) \\
*\end{array}$ \\
\hline $\begin{array}{l}\text { Extravascular } \\
\text { lung water } \\
\text { index, } \mathrm{ml} / \mathrm{kg}\end{array}$ & $4,9(4,6 ; 7,7)$ & $5,3(5 ; 7,6)$ & $5,3(4,8 ; 7,8)$ & $6(5,2 ; 7,3)$ \\
\hline $\begin{array}{l}\text { Central venous } \\
\text { pressure, } \\
\text { mmHg }\end{array}$ & $5(4 ; 7,5)$ & $6(4 ; 7)$ & $6(3 ; 11)$ & $8(5,5 ; 12)$ \\
\hline $\begin{array}{l}\text { Jugular bulb } \\
\text { pressure, } \\
\text { mmHg }\end{array}$ & $14(13 ; 19)$ & $15(13 ; 18)$ & $14(13 ; 18)$ & $15(12,5 ; 16,8)$ \\
\hline $\mathrm{PaO} 2 / \mathrm{FiO} 2$ & $352(294 ; 390)$ & $364(276 ; 402)$ & $382(323 ; 429)$ & $397(313 ; 440)$ \\
\hline
\end{tabular}

(* - $\mathrm{p}<0,05$ compared with PEEP $5 \mathrm{cmH} 2 \mathrm{O}$, \# - $\mathrm{p}<0,05$ compared with PEEP $10 \mathrm{cmH} 2 \mathrm{O}$ ).

Conclusion: Increase PEEP up to $15 \mathrm{cmH}_{2} \mathrm{O}$ does not have a negative effect on ICP and CPP in patients with initially increased intracranial pressure. Further increase PEEP up to $20 \mathrm{cmH}_{2} \mathrm{O}$ can lead to increase ICP, to decrease mean arterial pressure and CPP. The increase ICP is on average 2-3 $\mathrm{mmHg}$, which may be acceptable for use high PEEP in 
conditions of stable blood pressure and significant improvement in pulmonary gas exchange.

\section{5}

A Good Death: A Complete Audit Cycle of End of Life care in the Intensive Care Unit at The Hammersmith Hospital

S. Bakare1; J. Irwin'²; R. Towning ${ }^{2}$; R. Stumpfle ${ }^{3}$

${ }^{1}$ Anaesthetics and intensive care medicine, Northwick Park Hospital, London, United Kingdom; ${ }^{2}$ Foundation programme, Imperial College Healthcare NHS Trust, London, United Kingdom; ${ }^{3}$ Intensive care, Hammersmith Hospital, London, United Kingdom

Correspondence: S. Bakare

Intensive Care Medicine Experimental 2020, 8(2): 001485

Introduction: Despite advances in medicine, death rates in Intensive Care Units (ICU) remain high (1). ICU patients are admitted for life saving organ support; however when this fails, it is vital to provide patients with dignified deaths. The ICU brings about unique circumstances that make palliative care challenging, highlighting the importance of auditing current practices. The aim of this project was to audit the provision of end of life care, identify areas for improvement and develop interventions to ensure we provide optimum care. This was particularly important during the COVID-19 Pandemic surge where we had higher than average ICU mortality rates and unique sets of challenges. Interventions included raising awareness through multidisciplinary education at various forums. We increased awareness of existing available tools such as electronic end of life care forms and prescription order sets. Trainees also had access to electronic copies of simple guides to facilitate discussions with patient and their families (2). Videoconferencing proved invaluable in allowing spiritual and psychological support for parents and their families.

Methods: A retrospective initial audit was carried out by reviewing the electronic medical records (ISCCA) of patients who died between April and September 2019, following the implementation of interventions, a re-audit was carried out between March and May 2020. Data was collected based on NICE and FICM guidelines for end of life care and summarised into 6 main criteria $(3,4,5)$. This included; Identification of dying patients, Treatment escalation and Resuscitation planning, Patient and Family Involvement, Symptom management, Comfort care, and Psychological and Spiritual needs. The data was analysed using simple statistical tests.

Results: There were 61 deaths during the initial audit period and 28 during the re-audit. The re-audit showed $93 \%$ (92\% initial audit) of patients were identified as dying in a timely manner with $86 \%$ having clearly documented treatment escalation and appropriate resuscitation planning. $93 \%$ (77\% initial audit) of patients had clearly documented Patient and Family Involvement with end of life discussions. Spiritual \& Psychological support and Comfort Care was documented in all patients. This was a significant improvement compared to $41 \%$ for Spiritual \& Psychological support and $26 \%$ for Comfort Care recorded in the initial audit. Symptom management also significantly improved from $13 \%$ to $100 \%$.

Conclusion: Despite the additional challenges posed by the COVID19 Pandemic these results show our ICU Team continued to provide high quality and compassionate End of Life Care 6 . We intend to continue carrying out multidisciplinary teaching sessions with input from Palliative care and Pharmacists. We will also encourage the use of the palliative care form on ISCCA to provide prompts and enable accurate documentation. A laminated aide memoire flow chart (4) will also be available in clinical areas. End of life care is an essential skill for all members of the critical care team, because a good death is as important as a good life (4).

\section{Reference(s) and grant ackowledgment(s)}

1. 6. Clinical guide for the management of palliative care in hospital during the coronavirus pandemic. April 2020 Accessed 25/07/20 https://www.engla nd.nhs.uk/coronavirus/wp-content/uploads/sites/52/2020/04/C0081-AMEND ED-Speciality-guide-Palliative-care-and-coronavirus-v2-2020-04-22.pdf

2. 5. Care at the end of life - Accessed 10/10/19 https://www.ficm.ac.uk/sites/ default/files/ficm_care_end_of_life_0.pdf
3. 4. NICE: Care of the dying in the last days of life. March 2017. Accessed 10/10/19 https://www.nice.org.uk/guidance/qs144

4. 3. NICE: End of life care for Adults. November 2011. Accessed 10/10/19 https //WWW.nice.org.uk/guidance/QS13

5. 2. Talking to relatives A guide to compassionate phone communication during COVID-19 Dr Antonia Field-Smith and Dr Louise Robinson Accessed 25/07/20 https://static1.squarespace.com/static/5e6613a1dc75b87df82b 78e1/t/5e8f2b2fc2a92a40be9e6b70/1586441009136/Talking+to+Relat ives+Poster.pdf

6. 1. Sebastiano Mercadante, Cesare Gregoretti and Andrea Cortegiani: Palliative care in intensive care units: why, where, what, who, when, how. BMC anaesthesiology: 2018, 106 Accessed 10/10/19 https://bmcanesthesiol. biomedcentral.com/articles/10.1186/s12871-018-0574-9

7. None

\section{9}

Predicting lactate levels after vasopressor administration

M. Mollura1'; F. Marian'; LH. Lehman²; RG. Mark²; R. Barbieri

${ }^{1}$ Dipartimento di elettronica, informazione e bioingegneria, Politecnico di Milano, Milan, Italy; ${ }^{2}$ Institute for medical engineering \& science (imes), Massachusetts Institute of Technology, Cambridge, United States of America

Correspondence: M. Mollura

Intensive Care Medicine Experimental 2020, 8(2): 001489

Introduction: Circulatory shock and infections are known to be among the major causes of mortality in the ICU. These critical states are often associated with vasodilation, which could cause a significant drop in blood pressure, reduced tissue oxygenation and eventually organ failure.

A common physiological index of metabolic dysfunction and tissue oxygenation is blood lactate level. In particular, vasopressor need and lactic acid level have been proven to provide an accurate severity of illness classification system [1] in predicting out-of-hospital mortality.

Objectives: The goal of this study is to identify effects of the initiated vasopressor therapy as related to Lactate levels. To this extent we developed a model to predict a change in blood lactate level 12 hours after the administration of vasopressors by combining baseline features extracted from the electronic health records with measures of vasopressor responses estimated from continuously recorded ECG and arterial blood pressure (ABP) waveforms.

Methods: The data used in this study comes from the MIMIC-IIIDatabase [2]. The study was carried out on a cohort of 123 patients resulting from selection of all patients undergoing vasopressor administration that passed waveform signal quality annotation and had matching clinical data.

Selected features were extracted from the waveform recordings considering 15-minute windows before and after vasopressor administration, as shown in Figure 1.

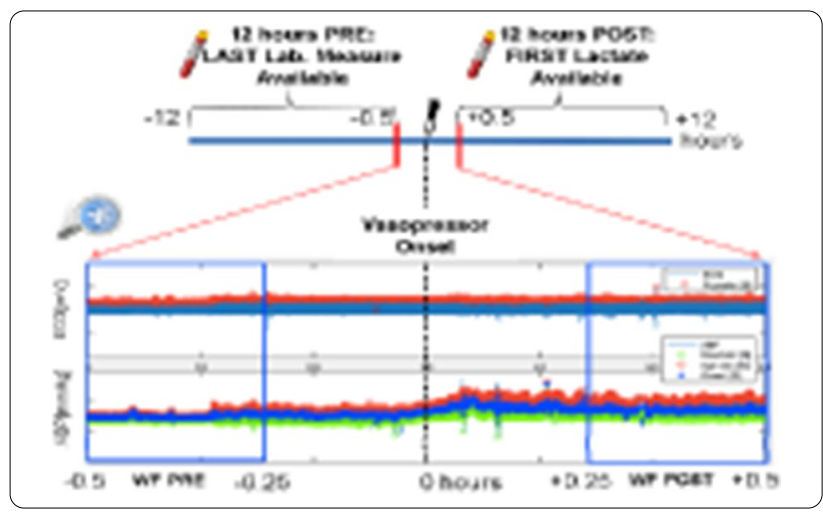

Clinical measurements from laboratory tests, blood gas analyses, pathologies, treatments and demographic information were also 
extracted for each patient within a 12 hour window before vasopressor onset.

We used all features to predict whether lactate level was normal ( < $2 \mathrm{mmol} / \mathrm{L}$ ) or abnormal ( $=>2 \mathrm{mmol} / \mathrm{L}) 12$ hours after the vasopressor onset.

The dataset was divided into a training set (80\%) and testing set (20\%). A Random Forest classifier was trained through a hyperparameter optimization with a 10 -fold cross validation and grid search procedure. Results:

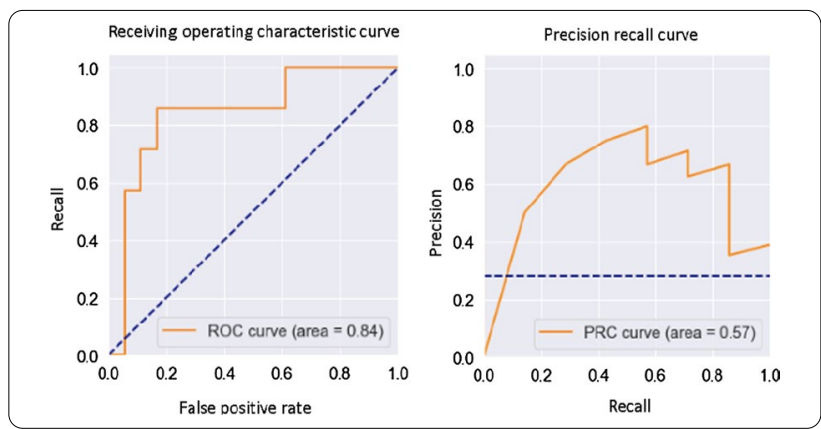

Results (Figure 2) showed an AUROC $=0.84$ and AUPRC $=0.57$. Feature importance analysis indicated that changes of the cardiovascular interaction between heartbeat and blood pressures after vasopressor administration are predictive of an abnormal lactate 12 hours after the start of vasopressor therapy.

Conclusion: Autonomic measures reveal an association between the response of the cardiovascular system to vasopressor therapy and changes in blood lactate level, suggesting that ECG- and ABP-derived indexes may be useful for predicting lactate level changes, i.e. tissue oxygenation level, after initiation of vasopressor therapy.

\section{Reference(s) and grant ackowledgment(s)}

1. [1]: Cocchi MN, Miller J, Hunziker S, et al. The association of lactate and vasopressor need for mortality prediction in survivors of cardiac arrest. Minerva Anestesiol. 2011;77(11):1063-107

2. [2]: Johnson, A., Pollard, T., Shen, L. et al. MIMIC-III, a freely accessible critical care database. Sci Data 3, 160035 (2016).

3. This work was partially supported by Progetto Roberto Rocca, MIT-Italy Program

\section{7}

An international survey about Above Cuff Vocalisation: what are the risks and benefits?

C. Mills ${ }^{1}$; E. Michou²; M. Bellamy ${ }^{3}$; H. Siddle ${ }^{4}$; C. Brennan ${ }^{1}$; C. Bojke ${ }^{1}$

${ }^{1}$ Leeds institute of health sciences, University of Leeds, Leeds, United Kingdom; ${ }^{2}$ Speech language therapy department, University Of Patras, Panepistimioupoli Patron, Greece; ${ }^{3}$ Leeds institute of medical research, University of Leeds, Leeds, United Kingdom; ${ }^{4}$ Leeds institute of rheumatic and musculoskeletal medicine, University of Leeds, Leeds, United Kingdom Correspondence: C. Mills

Intensive Care Medicine Experimental 2020, 8(2): 001507

Introduction: Tracheostomy is a life-saving intervention and facilitates prolonged ventilation in critical care. However, the negative consequences of tracheostomy include thirst and difficulty communicating which can be the most well recalled and distressing experiences for patients [1-3]. Above cuff vocalisation (ACV), also known as 'talking tracheostomy' and 'external subglottic airflow (ESAF)', has been used for $>50$ years [4]. A flow of oxygen or air is applied via the subglottic port of the tracheostomy to restore airflow through the vocal cords and enable vocalisation [5-7]. Some research has also reported improvements to swallowing and cough, hypothesised to be a result of improved laryngopharyngeal sensation $[5,8]$.

Objectives: To explore the extent of ACV use internationally, implementation approaches, risks, and benefits.
Methods: Ethical approval was obtained (MREC: 18-037). An international online survey was disseminated May-November 2019 via social media and professional networks. The survey included 73 questions covering topics such as: tracheostomy management, prevalence, practicalities, barriers, resource use, and personal experiences and opinions. Quantitative data was analysed using Microsoft Excel ${ }^{\circledR}$ (2016) and reported descriptively.

Results: The survey was completed by 244 healthcare professionals (HCPs), with one exclusion, from 9 different HCP groups and 25 countries. Respondents were primarily from critical care $(85 \% ; n=205)$. ACV was used by $94 \mathrm{HCPs}(39 \%)$. Most respondents had limited ACV experience; $54 \%(n=50)$ had used ACV with $<10$ people. There was a varied approach to ACV implementation (Figure 1). Most respondents wait $>72$ hours post-tracheostomy insertion prior to commencing ACV $(n=45 ; 48 \%)$, but $13(14 \%)$ commence ACV at $0-48$ hours. The most common complications or symptoms observed with ACV were patient reported discomfort $(n=54 ; 58 \%)$, strained vocal quality $(n=$ $39 ; 42 \%)$, and air escape via the stoma ( $n=32 ; 34 \%)$ (Figure 2). Various benefits were reported including: communication $(n=76 ; 82 \%)$, $\operatorname{mood}(n=62 ; 67 \%)$, laryngeal sensation $(n=49 ; 53 \%)$, swallowing frequency $(n=43 ; 46 \%)$, reduced volume of subglottic secretions ( $n$ $=39 ; 42 \%)$, expectorating secretions $(n=37 ; 40 \%)$, and earlier commencement of oral intake $(n=16 ; 17 \%)$. Incidences of more serious complications were observed less frequently, sub-cutaneous emphysema in 1-4 patients $(n=7 ; 8 \%)$, air trapping in $1-6$ patients $(n=10$; $11 \%)$, and bleeding in $1-30$ patients $(n=10 ; 11 \%)$. The most extreme barriers to ACV implementation were lack of access to knowledgeable staff $(n=92 ; 38 \%)$, lack of access to training $(n=73 ; 30 \%)$, and not using tubes with subglottic ports $(n=74 ; 31 \%)$.
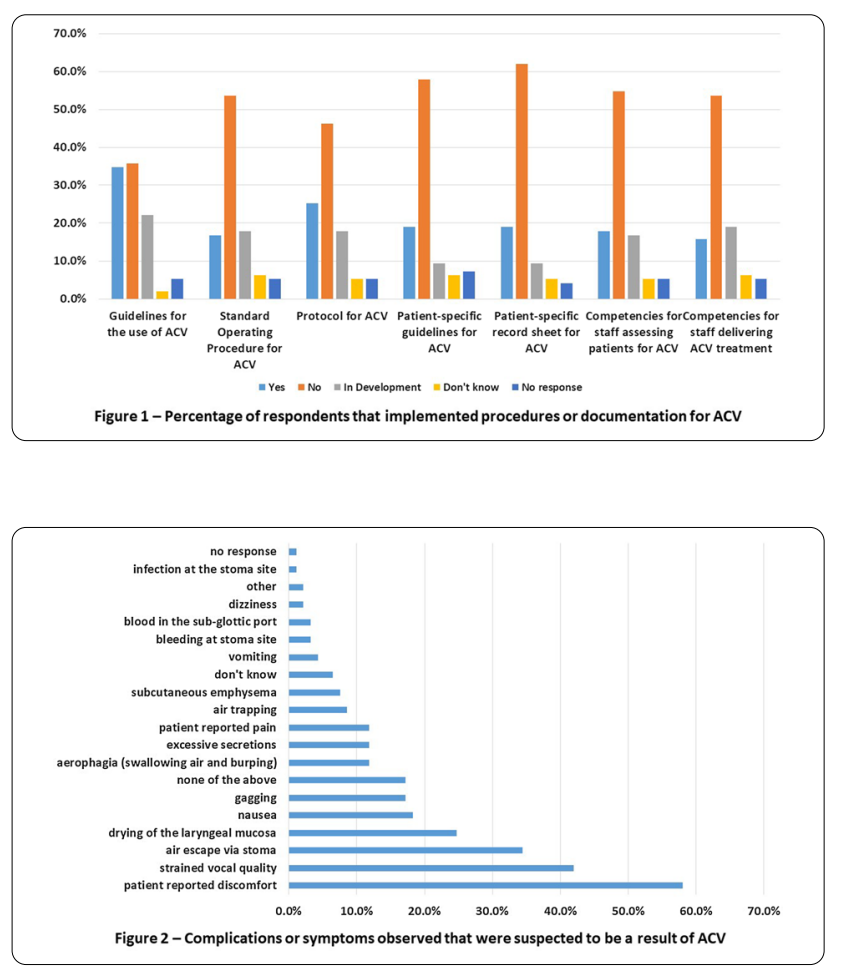

Conclusion: There is a varied approach to ACV implementation and delivery. The benefits are diverse, but minor complications are common. Future research should focus on establishing optimal implementation of ACV and developing a standardised approach to maximise benefits and minimise risks.

Reference(s) and grant ackowledgment(s)

1. Claire Mills is funded by a National Institute for Health Research (NIHR) Clinical Doctoral Research Fellowship for this research project. 
2. 8. Kothari M, Bjerrum K, Nielsen LH, Jensen J, Nielsen JF. Influence of External Subglottic Air Flow on Dysphagic Tracheotomized Patients With Severe Brain Injury: Preliminary Findings. Annals of Otology, Rhinology \& Laryngology. 2017 Mar;126(3):199-204.

3. 7. McGrath B, Lynch J, Wilson M, et al. Above cuff vocalisation: A novel technique for communication in the ventilator-dependent tracheostomy patient. J Intensive Care Soc 2016; 17: 19-26.

4. 6. Pandian V, Smith CP, Kling Cole T, et al. Optimizing Communication in Mechanically Ventilated Patients. J Med Speech Lang Pathol 2014; 21: 309-318.

5. 5. McGrath BA, Wallace S, Wilson M, et al. Safety and feasibility of above cuff vocalisation for ventilator-dependant patients with tracheostomies. J Intensive Care Soc 2019; 20: 59-65.

6. 4. Whitlock RML. A Means of Speaking for Patients with Cuffed Tracheostomy Tubes. Br Med J 1967; 3: 547.

7. 3. Freeman-Sanderson AL, Togher L, Elkins M, et al. Quality of life improves for tracheostomy patients with return of voice: A mixed methods evaluation of the patient experience across the care continuum. Intensive Crit Care Nurs 2018; 46: 10-16.

8. 2. Kjeldsen $C L$, Hansen MS, Jensen K, etal. Patients 'experience of thirst while being conscious and mechanically ventilated in the intensive care unit. 2017; 23: 75-81.

9. 1. Rose L, Nonoyama M, Rezaie S, et al. Psychological wellbeing, health related quality of life and memories of intensive care and a specialised weaning centre reported by survivors of prolonged mechanical ventilation. Intensive Crit Care Nurs 2014; 30: 145-151

\section{4}

\section{Delays in initiating the weaning process: Insights from the WEAN} SAFE Study

\section{T. Pham ${ }^{1}$}

'Service de médecine intensive-réanimation, Hôpital de Bicêtre, Le Kremlin-Bicêtre, France

\section{Correspondence: T. Pham}

Intensive Care Medicine Experimental 2020, 8(2): 001514

Introduction: Prolonged weaning from invasive mechanical ventilation (MV) is associated with poorer patient outcomes, including longer length of stay in the ICU and in the hospital and higher patient mortality [1]. It is recommended to start weaning based on general respiratory criteria (PEEP, $\mathrm{FiO}_{2} \ldots$ ) to avoid potential delays which may contribute to prolonged ventilation and poor outcomes.

Objectives: To evaluate the prevalence and types of delays in initiating the first separation attempt (SA) from invasive MV in patients enrolled in the WEAN SAFE study (WorldwidE AssessmeNt of Separation of pAtients From ventilatory assistancE; NCT03255109).

Methods: WEAN SAFE is a global, multi-centre prospective observational study that enrolled 6,167 patients in 476 centres receiving mechanical ventilation for at least 2 days. The time to initiation of the first SAs was examined in patients meeting general criteria. We considered the patients met criteria for initiating the weaning process when they were not receiving neuromuscular blocking agents, were on low dose or no vasopressors, had a PEEP $<10 \mathrm{cmH}_{2} \mathrm{O}$ and a $\mathrm{FiO}_{2}<50 \%$.

Patients were classified according to the WIND classification, which describes three different duration of weaning [2]. WIND groups were defined as the time from the first SA to successful weaning or death: group 1 within 24h; group 2: more than 1 day and less than a week; group 3: weaning not completed 7 days after the first SA.

Results: Of the 6167 patients enrolled, 4646 had at least 1 SA and could be grouped according to the WIND classification: $70 \%$ in group $1 ; 14 \%$ in group 2 and $16 \%$ in group 3 .

Of 4,212 patients that met criteria for weaning initiation, the median (interquartile range) time from these criteria to the first SA was 2 days $(0 ; 4)$. In the whole cohort, $30 \%$ of the patients had a SA the very day they met these criteria $(n=1257)$ whereas this delay was longer than 6 days in $16 \%(N=669)$. This delay statistically differed according to the WIND group (Table 1). We then assessed the time difference between the first day patients had spontaneous breathing activity and the first SA. In the whole population $(n=4646)$ this delay was 0 $(0 ; 3)$ days and was also significatively different across the WIND groups
(Table). Delay from the first SA to the weaning termination (successful weaning or death) was associated with the outcomes: hospital mortality was $15.6 \%$ in group $1,38.2 \%$ in group 2 and $35.4 \%$ in group $3(P<$ 0.001 between groups).

Delays in Initiating weaning attempts

\begin{tabular}{|c|c|c|c|c|}
\hline & $\begin{array}{l}\text { Group } 1 \\
(\mathrm{n}=\mathbf{3 2 3 1})\end{array}$ & $\begin{array}{l}\text { Group } 2 \\
(n=650)\end{array}$ & $\begin{array}{l}\text { Group } 3 \\
(n=765)\end{array}$ & $\begin{array}{l}P \\
\text { value }\end{array}$ \\
\hline $\begin{array}{l}\text { Time to 1st SA in patients } \\
\text { fulfilling weaning criteria, } \\
\text { days, median (IQR) }\end{array}$ & $\begin{array}{l}1.00 \\
{[0.00 ; 4.00]}\end{array}$ & $\begin{array}{l}2.00 \\
{[0.00 ; 5.00]}\end{array}$ & $\begin{array}{l}2.00 \\
{[0.00 ; 6.00]}\end{array}$ & $<0.001$ \\
\hline $\begin{array}{l}\text { Time to 1st SA since } \\
\text { spontaneous breathing, days, } \\
\text { median (IQR) }\end{array}$ & $\begin{array}{l}0.00 \\
{[0.00 ; 2.00]}\end{array}$ & $\begin{array}{l}1.00 \\
{[0.00 ; 3.00]}\end{array}$ & $\begin{array}{l}1.00 \\
{[0.00 ; 4.00]}\end{array}$ & 0.001 \\
\hline
\end{tabular}

Conclusion: Delays in initiating the weaning process in patients may contribute prolonged mechanical ventilation and subsequent higher mortality. The reasons underlying these delays in initiating weaning need to be explored.

\section{Reference(s) and grant ackowledgment(s)}

1. 1. Burns KEA, et al. International Practice Variation in Weaning Critically III Adults from Invasive Mechanical Ventilation. Ann Am Thorac Soc. 2018 Apr;15(4):494-502. https://doi.org/10.1513/AnnalsATS.201705-4100C. PMID: 29509509

2. 2. Béduneau $G$, et al; Epidemiology of Weaning Outcome according to a New Definition. The WIND Study. Am J Respir Crit Care Med. 2017 Mar 15;195(6):772-783. https://doi.org/10.1164/rccm.201602-03200C. PMID: 27626706

3. This abstract is submitted on behalf of the WEAN SAFE INVESTIGATORS

\section{1}

\section{Proinflammatory cytokines and endothelial cell activation} in human acute heart failure versus septic shock

M. Reina-Couto'; C. Silva-Pereira²; P. Terra²; J. Quelhas-Santos²; D. Pinho ${ }^{2}$.

S. Martins 3 ; P. Serrão 2 ; J. Afonso ${ }^{4}$; R. Roncon-Albuquerque ${ }^{5} ;$ JA. Paiva ${ }^{6}$;

A. Albino-Teixeira ${ }^{4}$;. Sousa ${ }^{4}$

${ }^{1}$ Intensive Care Medicine Department, Hospital São João, Porto, Portugal; ${ }^{2}$ Department of biomedicine - unit of pharmacology and therapeutics, Faculdade de Medicina da Universidade do Porto - FMUP, Porto, Portugal; ${ }^{3}$ Department of clinical pathology, São João Universitary Hospital Center, Porto, Portugal; ${ }^{4}$ Dep. biomedicina - unid. farmacologia e terapêutica, Faculdade de Medicina da Universidade do Porto, Porto, Portugal, Portugal; ${ }^{5}$ Intensive care department \& cirurgia e fisiologia department, Centro Hospitalar São João \& Faculdade de Medicina da Universidade do Porto, Porto, Portugal; ${ }^{6}$ Emergency and intensive care department, Centro Hospitalar de São João, Porto, Portugal

Correspondence: $M$. Reina-Couto

Intensive Care Medicine Experimental 2020, 8(2): 001521

Introduction: Inflammation-driven "endothelitis" appears to contribute to acute heart failure (AHF). We aimed at evaluating the proinflammatory status and endothelial activation, as well as their correlation, in human AHF, cardiogenic shock (CS) and to differentiate the same processes in patients with the diagnosis of non-cardiogenic shock (NCS), namely septic shock.

Methods: Patients with AHF $(n=23), C S(n=25)$ and NCS $(n=20)$ were included and blood and urine samples were collected at days $1-2$, days 3-4 and days 5-8. Blood donors were used as controls ( $n=$ 22) at a single time point collection. Serum IL-1 $\beta, I L-6$, tumoral necrosis factor-a (TNF-a), intercellular adhesion molecule-1 (ICAM-1), vascular cell adhesion molecule-1 (VCAM-1) and E-selectin were determined using Multiplex Immunoassays. Endocan was evaluated by ELISA kit.

Results: At admission, we found no significant differences in IL-1 $\beta$ concentration between groups. Admission IL-6 values $(\mathrm{pg} / \mathrm{mL})$ were higher in patients' groups (controls: $2.0 \pm 0.9$; AHF:40.0 \pm 22.3 ; CS: $95.9 \pm 26.4$, NCS: $598.1 \pm 311.5$; controls vs AHF, $p<0.01$; controls vs CS or NCS, $p<0.001)$. Admission TNF-a concentration $(\mathrm{pg} / \mathrm{mL})$ 
was higher in NCS (controls: $5.4 \pm 0.6$; AHF: $11.9 \pm 3.1$; CS: $9.4 \pm 1.5$ NCS:46.9 \pm 8.7 , controls vs NCS, p < 0.001; NCS vs CS, p < 0.001). Admission endocan $(\mathrm{ng} / \mathrm{mL})$ were higher in all patients' groups (controls: $1.4 \pm 0.2$; AHF: $6.1 \pm 1.8$; CS: $12.9 \pm 1.9$, NCS: $10.6 \pm 1.4$; controls vs AHF, $p<0.01$; controls vs CS or NCS, $p<0.001$, CS vs AHF, $p$ $<0.01)$. Admission ICAM-1 values $(\mathrm{ng} / \mathrm{mL})$ were increased in $\mathrm{CS}$ and NCS compared to controls and AHF (controls: $474.9 \pm 70.7$; AHF: $412.4 \pm 60.3$; CS: $665.5 \pm 72.6$, NCS: $726.6 \pm 55.3$, controls vs CS, $p<$ 0.05 ; controls vs NCS, $\mathrm{p}<0.01)$. Admission VCAM- $1(\mathrm{ng} / \mathrm{mL})$ was higher in all patients' groups (controls: $705.8 \pm 51.0$, AHF: $1840.0 \pm 290.0$, CS: $3425.0 \pm 645.6$, NCS: $4462.0 \pm 449.7$, controls vs AHF, $\mathrm{p}<0.01$ controls vs CS, $p<0.001$, controls vs NCS, $p<0.001$ ), while E-selectin presented higher values in NCS (controls: $28.7 \pm 1.6$, AHF: $33.1 \pm 2.3$, CS: $37.9 \pm 3.7$, NCS: $207.4 \pm 28.6$, controls vs NCS, $p<0.001$, CS vs $\mathrm{NCS}, \mathrm{p}<0.001$ ). During hospitalization, there was an overall decrease in TNF- $a$ values $(p=0.02)$ and also a decrease in all endothelial biomarkers (ICAM-1, VCAM- 1 and E-selectin concentration - $p=0.04$ and $p=0.001$ and $p<0.01$, respectively) in NCS. Withinpatients, we observed significant positive correlations between endocan and IL- 6 and VCAM-1, between ICAM-1 and IL-1 $\beta$, IL-6, VCAM- 1 and E-selectin, between VCAM- 1 and IL- 6 , TNF- $\alpha$ and all the endothelial biomarkers, and between E-selectin and IL-6, TNF- $a$, ICAM-1 and VCAM-1.

Conclusion: Proinflammatory status and endothelial cell activation are interrelated and significantly increased in all patient's groups. During hospitalization, there is significant decrease in all endothelial biomarkers only in NCS patients. This suggests that inflammation-driven "endothelitis" is constant in AHF and CS which might perpetuate and aggravate the prognosis heart failure.

\section{Reference(s) and grant ackowledgment(s)}

1. [Funded by FCT/FEDER-COMPETE,Portugal 2020-PTDC/

MEC-CAR/32188/2017]

\section{9}

Prediction tools developed in the early part of the COVID-19 pandemic lack validity

H. Al Hassan'; E. Cocks' ${ }^{2}$ L. Jesani²; S. Lewis ${ }^{3}$;. Szakmany ${ }^{4}$

${ }^{1}$ Unscheduled care directorate, Cardiff University, Cardiff, United Kingdom; ${ }^{2}$ Critical care directorate, Aneurin Bevan University Health Board, Newport, United Kingdom; ${ }^{3}$ Value based healthcare team, Aneurin Bevan University Health Board, Newport, United Kingdom; ${ }^{4}$ Department of anaesthesia, intensive care and pain medicine, division of population medicine, Cardiff University, Cardiff, United Kingdom

Correspondence: $\mathrm{H}$. Al Hassan

Intensive Care Medicine Experimental 2020, 8(2): 001579

Introduction: Since the outbreak of the SARS-CoV-2 pandemic, there has been a surge of clinical risk prediction scores developed, mostly at the first epicentre in China [1][2][3][4]. Predicting risk of deterioration or severe COVID-19 related illness is of significance, both as part of a clinical treatment guidance as well as to highlight the likely groups benefitting from novel disease modifying therapies. Although many of the risk prediction tools were developed in multi-centre studies, their external clinical utility and face validity has not been established in independent cohorts [5].

Objectives: We attempted to validate three previously published riskstratification tools in a multi-centre study.

Methods: Anonymised patient data was collected as part of a service evaluation and audit project by the Secondary Care Group Members of the Welsh Government COVID-19 response from patients admitted to the University Hospital of Wales, a tertiary academic centre and to the two district general hospitals in Aneurin Bevan UHB. Due to the anonymised nature of the data collection, formal written consent was waived.

Data was collected to enable to calculate the CALL-score, the COVIDGRAM risk score and a nomogram developed by Gong et al [1][2][3]. Primary outcome was a composite outcome of development of severe COVID-19 disease leading to ICU admission or death as described by the original publications. The outcome was censored at hospital discharge or 30-days.
For statistical analysis receiver operating characteristics (ROC) curves were used to establish predictive ability. Data is presented as $\mathrm{n}(\%)$, median (IQR) or ROC $(95 \% \mathrm{Cl})$ as appropriate.

Results: We collected data from 169 patients. Patients were 73 (5982) years old, 66 out of $169(39.1 \%)$ were female, 32 (18.9\%) had no significant co-morbidities, 57 (33.7\%) had one and 80 (47.3\%) had two or more comorbidities. Most prevalent comorbid conditions were diabetes in $42(24.9 \%)$, COPD in $32(19.0 \%)$ and ischaemic heart disease in $26(15.4 \%)$ of patients. $81(47.9 \%)$ of patients had reached the composite outcome of ICU admission or death, the hospital mortality was $33.7 \%$. Neither of the three risk-prediction tools were able to accurately predict outcome. ROC $(95 \% \mathrm{Cl})$ for the COVID-GRAM score was $0.636(0.550-0.722) p=0.003$, for the CALL-score $0.500(0.411-0.589)$ $p=0.997$ and for the nomogram $0.628(0.543-0.714) p=0.005$ (Fig 1).

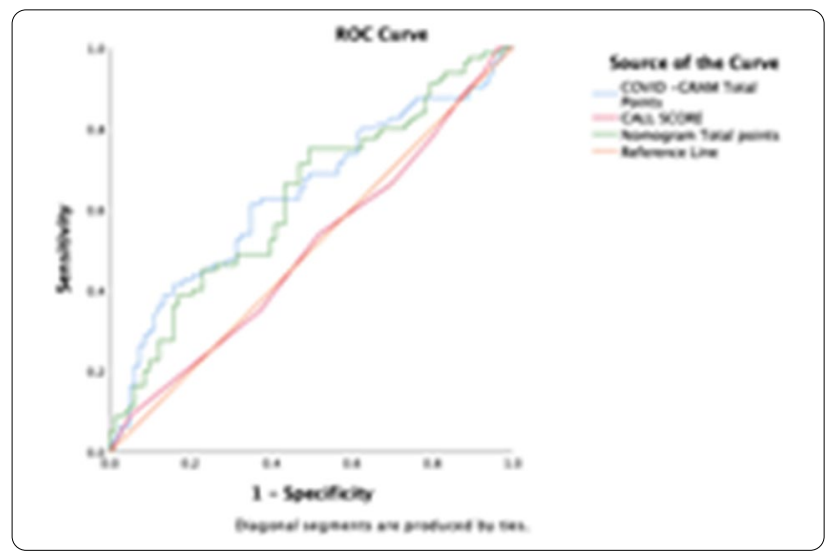

Conclusion: With respect to the composite outcome of ICU admission or death within our cohort, the discriminative value of all three predictive tools was poor. This is likely due to a variety of factors. The absence of calibration data to address the overfitting of the COVIDGRAM leaves the fine-tuning of regression coefficients as potentially overlooked [6]. Furthermore, each of the tools had relatively small and homogenous training and validation cohorts, despite being multi-centred in nature, in contrast to the international datasets now available. Moreover, our cohort differed as older, predominantly white and carrying a higher comorbidity burden.

\section{Reference(s) and grant ackowledgment(s)}

1. 1. Ji D, Zhang D, Xu J, et al. Prediction for Progression Risk in Patients with COVID-19 Pneumonia: the CALL Score. Clin Infect Dis Available at: https:// academic.oup.com/cid/advance-article/doi/10.1093/cid/ciaa414/5818317. Accessed 23 June 2020.

2. 2. Liang W, Liang $\mathrm{H}$, Ou L, et al. Development and Validation of a Clinical Risk Score to Predict the Occurrence of Critical IIIness in Hospitalized Patients With COVID-19. JAMA Intern Med 2020; Available at: https://jamanetwor k.com/journals/jamainternalmedicine/fullarticle/2766086. Accessed 23 June 2020.

3. 3. Gong J, Ou J, Qiu X, et al. A Tool for Early Prediction of Severe Coronavirus Disease 2019 (COVID-19): A Multicenter Study Using the Risk Nomogram in Wuhan and Guangdong, China. Clin Infect Dis Available at: https:// academic.oup.com/cid/advance-article/doi/10.1093/cid/ciaa443/5820684. Accessed 23 June 2020

4. 6. Steyerberg EW, Vickers AJ, Cook NR, et al. Assessing the performance of prediction models: a framework for traditional and novel measures. Epidemiology. 2010;21(1):128-138. https://doi.org/10.1097/EDE.0b013e3181c30fb 2.

5. 5. Wynants L, Calster BV, Collins GS, et al. Prediction models for diagnosis and prognosis of covid-19: systematic review and critical appraisal. BMJ 2020; 369. Available at: https://www.bmj.com/content/369/bmj.m1328. Accessed 23 June 2020.

6. 4. Zhou Y, He Y, Yang $H$, et al. Development and validation a nomogram for predicting the risk of severe COVID-19: A multi-center study in Sichuan, China. PLOS ONE 2020; 15:e0233328. 
001586

Ventilation strategy and outcomes of hospitalised COVID-19 patients

G. Sanson ${ }^{1}$; P. Giaccaglia ${ }^{2}$;. Montomoli3 ; M. Emily²; T. Venturi²;

F. Facondini ${ }^{3}$; E. Mosconi ${ }^{3}$; S. Guarino ${ }^{3}$; D. Guerra ${ }^{3}$; G. Giuliani ${ }^{3}$; M. Bitondo ${ }^{3}$; A. Potalivo ${ }^{3} ;$ G. Nardi ${ }^{3}$

${ }^{1}$ Dept. of medicine, surgery and health sciences, University of Trieste, Trieste, Italy; ${ }^{2}$ Anesthesia and intensive care unit, Marche Polytechnic University Faculty of Medicine, Ancona, Italy; ${ }^{3}$ Anaesthesia and intensive care, Infermi Hospital, AUSL della Romagna, Rimini, Italy

Correspondence: J. Montomoli

Intensive Care Medicine Experimental 2020, 8(2): 001586

Introduction: COVID-19 outbreak has represented a new challenge for intensivists worldwide in terms of patient and resources management. The decision of which ventilation strategy to adopt in each patient has been crucial and not guided by existing outcome evidence.

Objectives: We described the clinical characteristics and outcomes of hospitalized COVID-19 patients according to the adopted ventilatory strategy.

Methods: All COVID-19 patients hospitalized within the Rimini province (340,000 residents) from February 26 to April 18, 2020 were included in the present observational population-based study. Patients were classified according to the maximum level of ventilation support (oxygen, non-invasive ventilation (NIV), mechanical ventilation (MV)). Sixty-day mortality was computed and patient's risk of death was estimated with a Cox proportional hazard analysis adjusted by age, sex, and administration of steroids, canakinumab, and tocilizumab.

Results: 1,424 symptomatic patients were identified; 522 (36.7\%) were hospitalized, the remaining 902 (63.3\%) were treated at home with no deaths at 60 days. $408(78.5 \%)$ patients were treated only with standard care and oxygen supplementation during hospitalization (G1), 46 (8.8\%) patients underwent only NIV (G2), 25 (4.8\%) failed a NIV trial and received MV (G3), and 41 (7.9\%) only/or MV (G4). There was no significant difference in the P/F at MV inception among G3 and G4 (p-value 0.9 ). Overall 60 -day mortality was $24.2 \%$ and was $23.0 \%$ in $\mathrm{G} 1,19.6 \%$ in $\mathrm{G} 2,32.0 \%$ in $\mathrm{G} 3$, and $36.6 \%$ in G4 $(p=0.165)$. Compared with $\mathrm{G} 1$, corresponding adjusted HRs progressively increasing from $\mathrm{G} 2$ to G4 (HR 1.78, 95\% Cl 0.79-3.98; HR 2.78, 95\% Cl 1.14-6.73; HR 2.97, $95 \% \mathrm{Cl} 1 \cdot 55-5 \cdot 65$, Figure)

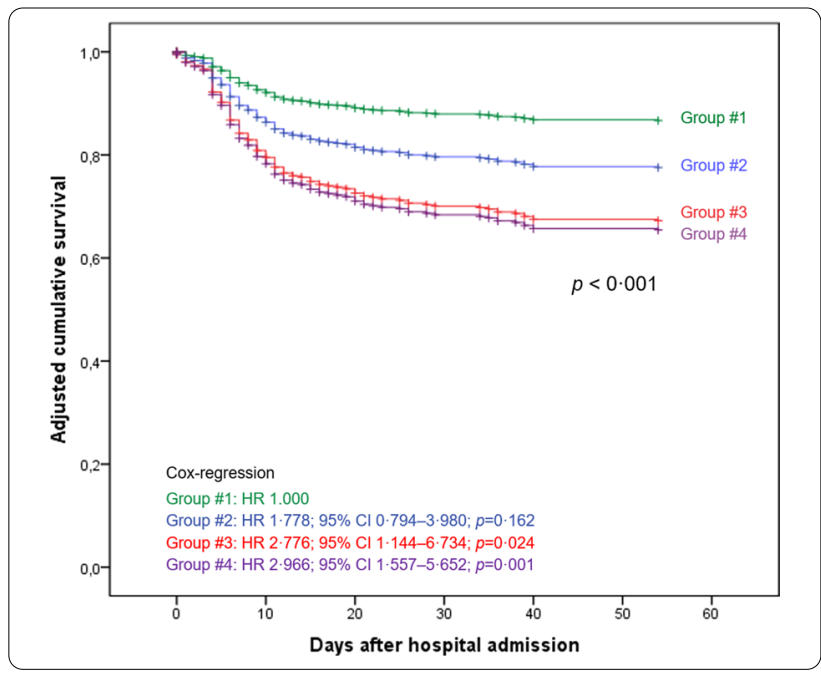

Conclusion: Our study suggested that patients who failed a NIV trial did not have a worse prognosis than patients treated directly with $\mathrm{MV}$, but the adopted strategy has helped with the optimization of the resources allocation.
Reference(s) and grant ackowledgment(s)

1. Zhou F, Yu T, Du R, et al. Clinical course and risk factors for mortality of adult inpatients with COVID-19 in Wuhan, China: a retrospective cohort study. The Lancet. 2020;395(10229):1054-1062. https://doi.org/10.1016/S0140 -6736(20)30566-3

2. Yang $X, Y u Y, X u J$, et al. Clinical course and outcomes of critically ill patients with SARS-CoV-2 pneumonia in Wuhan, China: a single-centered, retrospective, observational study. Lancet Respir Med. February 2020. https://doi. org/10.1016/S2213-2600(20)30079-5

3. ICNARC - Report on COVID-19 in critical care 22 May 2020. https://www. icnarc.org/Our-Audit/Audits/Cmp/Reports. Accessed May 27, 2020.

4. Grasselli G, Zangrillo A, Zanella A, et al. Baseline Characteristics and Outcomes of 1591 Patients Infected With SARS-CoV-2 Admitted to ICUs of the Lombardy Region, Italy. JAMA. April 2020. https://doi.org/10.1001/ jama.2020.5394

5. Richardson S, Hirsch JS, Narasimhan M, et al. Presenting Characteristics, Comorbidities, and Outcomes Among 5700 Patients Hospitalized With COVID-19 in the New York City Area. JAMA. April 2020. https://doi. org/10.1001/jama.2020.6775

\section{7}

The role of the physiotherapy team in a multidisciplinary management of pronation in critically ill COVID-19 patients

A. Glotta ${ }^{1}$; N. Faldarini²; M. Biggiogero ${ }^{3}$; D. Olivieri²; C. Molteni ${ }^{2}$; R. Mauri ${ }^{1}$. S. Ceruti ${ }^{1}$

${ }^{1}$ Critical care department, Clinica Luganese Moncucco, Lugano, Switzerland; ${ }^{2}$ Physiotherapy service, Clinica Luganese Moncucco, Lugano, Switzerland; ${ }^{3}$ Clinical research unit, Clinica Luganese Moncucco, Lugano, Switzerland

Correspondence: S. Ceruti

Intensive Care Medicine Experimental 2020, 8(2): 001597

Introduction: Critically ill COVID-19 patients often required mechanical ventilation (MV) support. For ICU nursing staff, COVID-19 patients' management presented a relevant increasing in workload, especially during pronation. In this emergency scenario Clinica Luganese Moncucco, identified as a COVID-19 Center by the Swiss Health Department, decided to introduce a specialized ICU Physiotherapy Team (IPT) to support nursing staff during pronations.

Objectives: Primary outcome was to analyze complications rate in critically ill COVID-19 patients managed by this specialized team during pronations. Secondary outcomes were to analyze all correlations between the incidence of minor complications, like pressure sores, and clinical data to find any potential protective factor.

Methods: A retrospective analysis on consecutive critically ill COVID19 patients from March 16th to April 16th, 2020. The purpose of this study was to analyze the rate of major and minor complications related to pronation performed by the IPT.

Results: Forty-two patients were treated by specialized IPT. Pronation maneuvers were performed on $81 \%$ of patients with 296 pronations, with an average of 3.52 cycles per patient (SD 2.47). The average BMI was $28.3 \mathrm{~kg} / \mathrm{m}^{2}$ (SD 5.1); $36(86 \%)$ patients were on MV (66\% ventilated by OTI and $19 \%$ by tracheotomy), with a mean duration of MV days of 10.5 days. The mean age was 65.61 (SD 10.95), 35 (83.3\%) were male. The nurse workload was increased, with a median NEMS score of 34.5, compared to previous year $(p<0.001)$

One major complication ( $0.3 \%$ ) identified as a dislocation of endotracheal tube was observed; 14 patients (33.3\%) were burdened by minor complications like pressure sores. The number of pressure sores was correlated with ICU LOS $(p=0.001)$ and MV days $(p=0.001)$; the presence of pressure sores was also related to ICU LOS $(p=0.029)$ and MV days $(p=0.015)$. No other significant correlations were found. To better estimate the effect of the observed data, the propensity score was applied, given the retrospective study design and the imbalance between treatment and pronation number. The propensity match score showed no protective pronation factor regarding the presence of ulcers $(1.0, p=0.448)$.

Conclusion: Specific pronation team resulted in a lower rate of major complications. The persistent high amount of minor complications appeared to be related rather to the disease severity than to pronation 
gesture, suggesting that minor complications were not protected by pronation.

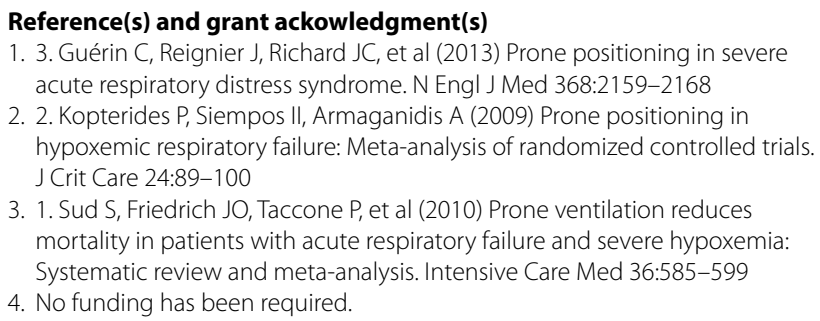

\section{8}

Machine Learning to tackle COVID-19: the collection of multicenter intensive care data from electronic health records during a crisis

L. Fleuren'; B. Vonk²; M. Fornasa²; DB. Aletta²; W. Herter²; H. Hovenkamp²; T. Guo ; L. Roggeveen ${ }^{1}$; P. Thoral ${ }^{1}$; E. Swart ${ }^{3} ;$ ARJ. Girbes ${ }^{1}$;

M. Hoogendoorn ${ }^{4}$; PWG. Elbers ${ }^{1}$

'Intensive care medicine, Amsterdam UMC, locatie VUmc, Amsterdam, Netherlands; ${ }^{2}$ Pacmed.ai, Pacmed, Amsterdam, Netherlands; ${ }^{3}$ Clinical pharmacology, Amsterdam UMC, locatie VUmc, Amsterdam, Netherlands; ${ }^{4}$ Computational intelligence, Vrije Universiteit Amsterdam, Amsterdam, Netherlands

Correspondence: $L$. Fleuren

Intensive Care Medicine Experimental 2020, 8(2): 001598

Introduction: The Corona virus disease 2019 (COVID-19) pandemic touches health care systems worldwide and surpasses intensive care capacity in many areas. Since COVID-19 is an emerging disease, high quality evidence about the course of the disease and optimal treatment is sparse. We envision that combining efforts of the intensive care community through knowledge and data is paramount to accumulate insight into this novel disease.

Objectives: We set out to gather data from COVID-19 patients for machine learning studies from all intensive care units in the Netherlands. We outline the process of data collection under European privacy law in times of crisis.

Methods: We devised a multidisciplinary report on the lawful collection of data from electronic health records during the COVID-19 pandemic in close collaboration with data protection officers (DPO), hospital lawyers, and intensivists. Similarly, a data sharing agreement was drafted based on a nation-wide template for sharing data between healthcare institutions. Documentation was reviewed by the institutional review board (IRB). All hospitals in the Netherlands with an intensive care unit were approached, and documentation was reviewed locally before permission was granted.

Results: Under European law, patient privacy is regulated in the General Data Protection Regulation (GDPR) (1). Both the GDPR and Dutch national law provide that data subjects have to give explicit consent for the processing of their data. We argue that during the COVID-19 crisis, asking consent cannot be reasonably expected from health care workers due to a) the large number of expected patients and associated time burden in an already overstrained health care system, b) the danger of spreading or contracting the virus upon contact with patients or their families, and c) the poor clinical condition of many patients in the intensive care. Consent is therefore not only impractical, but often impossible. In addition, alternative forms of data collection are unavailable and selection bias would lead to unreliable data. As under non-crisis circumstances, we may gather COVID-19 data necessary for scientific purposes when we "provide for suitable and specific measures to safeguard the fundamental rights and interests of the data subject" (GDPR, Article 9, paragraph j1). Therefore, we a) pseudonymized data in the providing hospital, b) informed patients through media and local hospital outlets about the possibility to opt out, and c) signed data sharing agreements regulating privacy of patients. We currently have data sharing agreements in place with 38 hospitals, and have received data from 32 hospitals. With this study we aspire to set an example for the lawful collection of data in times of a health crisis.

Conclusion: In times of the COVID-19 crisis, we consider large scale data collection pivotal to combat this novel disease and to improve patient outcomes. Data collection in times of crisis may be possible and lawful under European privacy laws.

\section{e-posters}

\section{5}

Hypothermia during CRRT, a comparative analysis

ME. Broman ${ }^{1}$; C. Ronco ${ }^{2}$; M. Bell ${ }^{3}$

${ }^{1}$ Perioperative and intensive care, Skåne University Hospital, Lund, Sweden; ${ }^{2}$ Department of medicine, San Bortolo Hospital Of Vicenza, Vicenza, Italy; ${ }^{3}$ Perioperative medicine and intensive care, Karolinska University Hospital, Stockholm, Sweden

Correspondence: M.E. Broman

Intensive Care Medicine Experimental 2020, 8(2): 000065

Introduction: One of the most common adverse events during continuous renal replacement therapy (CRRT) is hypothermia, $<35.0^{\circ} \mathrm{C}$, reported in $44 \%$ of all cases. Hypothermia may be less common over time, but available data is scarce. Hypothermia is associated with higher mortality rates; in a time series analysis of over 15000 admissions during 8.5 years, the incidence of hypothermia $\left(<36.0{ }^{\circ} \mathrm{C}\right)$ decreased from $29 \%$ to $21 \%$ during the study period and was associated with ICU mortality in both medical and surgical patients. The older Prismaflex CRRT system uses the Barkey blood warmer, also known as Prismacomfort. It operates by covering the blood return flow with a silicon tube heat exchanger and the heat is transferred by the contact of the resistance heating system. The Thermax blood warmer on the newer Prismax CRRT system responds to changing treatment parameters by repeatedly adjusting heating to fulfil the prescribed return blood temperature. We wanted to characterize the occurrence of hypothermia in CRRT patients and to compare wether the newer Thermax warmer was more effective in protecting against hypothermia compared to its predecessor Barkey.

Methods: The study was registered at Clinical Trials.gov (NCT03973814) and approved by the Swedish Ethical Review Authority (Dnr 2019-04388). A total of 9046 patients admitted to the Intensive Care Unit at Skåne University Hospital, Lund, Sweden during the years 2006-2019 were screened and 342 patients undergoing CRRT were identified and included. The old Prismaflex with the Barkey warmer was used until November 2018, thereafter the Prismax with the Thermax was used. Data was extracted from the electronic patient database management system ICCA (IntelliSpace Critical Care and Anesthesia, Philips, the Netherlands) which contains complete clinical datasets generated during the ICU stays. Inclusion criteria: > 18 years of age and admission to the adult intensive care unit at Skåne University Hospital, Lund during the period from November 2006 to August 2019 and treatment with CRRT during the ICU stay. Hypothermia was defined as $<36.0^{\circ} \mathrm{C}$. The temperature measurements (bladder, ear, blood) were carried out hourly. The fraction of hours in hypothermia were compared between the two warmers. A sensitivity analysis was performed where the first two hours of CRRT treatment were excluded in order to test if the phase of starting CRRT was the main driver of overall hypothermia. Means and standard deviations are presented. Significance was tested by using the chi squared test. For all calculations the SAS software version 9.4 (Statistical Analysis Software, Cary, USA) was used. Significance level was defined as $p<0.05$.

Results: In total, 57741 hours were available for evaluation in the cohort. Prismaflex patients spent $11.43 \%$ (6112 treatment hours below $36{ }^{\circ} \mathrm{C} / 53465$ treatment hours in total) of their time in hypothermia, as compared to the novel Prismax CRRT system, where $10.06 \%$ (430 treatment hours below $36^{\circ} \mathrm{C} / 4276$ treatment hours in total) were below $36.0^{\circ} \mathrm{C}$ (Chi-Square $\mathrm{p}=0.0063$ ). In the sensitivity analysis significant differences between the historic and novel systems prevailed; $12.7 \%$ as compared to $11.2 \%$ hypothermia hours $(p=0.0216)$. The Thermax blood warmer was associated with less heat loss compared to the Barkey warmer. This was highlighted by a mean patient temperature of 
$37^{\circ} \mathrm{C}$ vs $36.5^{\circ} \mathrm{C}$ correspondingly, but also by the fact that the mean set return temperature was $37.9^{\circ} \mathrm{C}$ vs $40.9^{\circ} \mathrm{C}$. This difference in mean set return temperature translates to a significant $(p<0.001)$ difference in the historic Barkey system compared to the new Thermax system. Seemingly, an overcorrection was needed in the Barkey system, probably because of its insufficiency to maintain the sought blood temperature in the patient. In addition, the use of adjacent Bairhugger bodywarmer was significantly less in the Thermax group compared to the Barkey group; 17/32 (53.1\%) patients had Bairhugger compared to $285 / 310(91.9 \%)(p<0.001)$ and for those patients with Bairhugger there were more Bairhugger free hours in the Thermax group compared to the Barkey group; $99.7 \pm 93.4$ hours without $(16.7 \pm 16.4$ $\mathrm{h}$ with) in the Thermax group compared to $73.2 \pm 107.3 \mathrm{~h}$ without $(40.3 \pm 45.7 \mathrm{~h}$ with) in the Barkey group $(\mathrm{p}<0.01)$.

Conclusion: In conclusion, every tenth hour during CRRT was spent in hypothermia. There was a signal that the Prismax CRRT system and the Thermax blood warmersignificantly decreased the risk of hypothermia as compared to historic controls with the older Prismaflex and Barkey warmer, in this pilot study. Furthermore, achieving target temperature was easier with the new system and use of auxilliary Bairhugger bodywarmer was signifcantly less.

\section{Reference(s) and grant ackowledgment(s)}

1. Yagi N, Leblanc M, Sakai K, Wright EJ, Paganini EP. Cooling effect of continuous renal replacement therapy in critically ill patients. American journal of kidney diseases : the official journal of the National Kidney Foundation 1998; 32: 1023-30.

2. Niven DJ, Stelfox HT, Laupland KB. Hypothermia in Adult ICUs: Changing Incidence But Persistent Risk Factor for Mortality. Journal of intensive care medicine 2016; 31: 529-36.

3. Sigwalt F, Bouteleux A, Dambricourt F, Asselborn T, Moriceau F, Rimmele T. Clinical Complications of Continuous Renal Replacement Therapy. Contributions to nephrology 2018; 194: 109-17.

4. Akhoundi A, Singh B, Vela M, Chaudhary S, Monaghan M, Wilson GA, Dillon $\mathrm{JJ}$, CartinCeba R, Lieske JC, Gajic O, Kashani K. Incidence of Adverse Events during Continuous Renal Replacement Therapy. Blood purification 2015; 39: 333-9.

\section{9}

Does haemoconcentration or passive leg raising predict cardiac index decrease during mechanical fluid removal with continuous renal replacement therapy?

M. Ruste ${ }^{1}$; M. Jacquet-Lagrèze ${ }^{1}$; W. Fornier ${ }^{1}$; E. Cancade ${ }^{1}$; R. Schweizer ${ }^{1}$; JL. Fellahi

'Departement of anaesthesiology and intensive care, Hôpital Louis Pradel - HCL, Bron, France

Correspondence: $M$. Ruste

Intensive Care Medicine Experimental 2020, 8(2): 000109

Introduction: After acute phase of circulatory failure, some authors suggest to have a derresuscitation strategy to avoid side effects of fluid overload, involving mechanical or diuretic's induced fluid removal (1). According to frank starling, hypovolemia should induce a decrease in cardiac index. We hypothesized that an absence of refilling detected by an hameoconcentration, or a preload responsiveness before fluid removal could predict cardiac index $(\mathrm{Cl})$ decrease during mechanical fluid removal induced by renal replacement therapy.

Methods: We conducted a single center prospective diagnostic accuracy study in an intensive care unit of an academic French hospital. Primary aim of the study was to evaluate diagnostic performance of haemoconcentration based on proteinemia variation to detect $\mathrm{Cl}$ decrease $\geq 12 \%$ during mechanical fluid removal. Secondary aims were to evaluate diagnostic performance of passive leg raising (PLR) or $\mathrm{Cl}$ trending by pulse contour analysis to detect $\mathrm{Cl}$ decrease, and to describe hemodynamic variations observed during mechanical fluid removal. Patients were adults treated with continuous renal replacement therapy, and fluid removal; and monitored by transpulmonary thermodilution technique. Non inclusion criteria were needs to sedation or vasoactive drug adjustement, compartment abdominal syndrome, pregnancy. Patients experienced a fluid removal challenge
(FRC), consisting in a mechanical fluid removal of $500 \mathrm{~mL}$ during one hour. Fluid removal was stopped if hypotension occurred. Biological samples with proteinemia and haemoglobin, PLR and transpulmonary thermodilution were carried out just before and just after the FRC. We constructed Receptor Operative Characteristics (ROC) curves with computation of area under the curve (AUC) to evaluate diagnostic performance of different variables. $95 \%$ confidence interval was determined by bootstrap technic with 2000 repetitions. Comparisons between groups were analysed with a Mann-Whitney $U$ test All tests were two-sided, and p-value below 0.05 defined statistical significance.

Results: We included sixty-nine patients between December 2016 and April 2020. Sixteen patients had a significant $\mathrm{Cl}$ decrease $(23 \%$ [Cl95\% 14-35]). Patient's age was 64 [52, 72], 46 (67\%) were cardiac surgical patients, $41(59 \%)$ benefited from mechanical ventilation, $29(42 \%)$ presented arrhythmia, cumulative fluid balance at baseline was $7 \%[-1,16]$, SOFA score was $12[8,14]$. To compare with patient without $\mathrm{Cl}$ decrease, patients with significant $\mathrm{Cl}$ decrease presented: no variation concerning arterial pressure or heart rate a preload decrease (global end diastolic volume index decrease $-8 \%$ $[\mathrm{Cl} 195 \%-20,-5]$ vs $0[\mathrm{Cl} 95 \%-4,6]) ;$ a systemic vascular resistance index increase $(29 \%$ [Cl95\% 14, 30] vs $2 \%[-7,14])$; a more important decrease in cardiac function index $(-9 \%[\mathrm{Cl} 95 \%-16,-2]$ vs $-4 \%$ $[\mathrm{Cl} 95 \%-8,3]$. Hemoconcentration evaluated by proteinemia or haemoglobin variations, PLR before or after $\mathrm{FRC}$, and $\mathrm{Cl}$ trending by pulse contour analysis failed to predict $\mathrm{Cl}$ decrease with AUC under ROC curve $>0.75$

Conclusion: Haemocontration variables, preload responsiveness status and $\mathrm{Cl}$ trending by pulse contour analysis failed to predict a $\mathrm{Cl}$ decrease during FRC. As we observed several events of $\mathrm{Cl}$ reduction, monitoring cardiac index with transpulmonary thermodilution technique might help to prevent deleterious effect of fluid removal.

\section{8}

The duration of acute kidney injury is an additional parameter to predict 1-year survival in very elderly patients

F. ZHOU ${ }^{1}$; L. Qinglin ${ }^{1}$; M. Zhi' ${ }^{1}$ T. Sheng

'Department of critical care medicine, Chinese PLA General Hospital, Beijing, China

Correspondence: $\mathrm{F}$. Z HOU

Intensive Care Medicine Experimental 2020, 8(2): 000178

Introduction: Acute kidney injury (AKI) severity is classified into three stages on the basis of either the increase in serum creatinine (Scr) level or the duration and extent of oliguria by KDIGO criteria. However, the diagnostic and staging criteria do not take into account the duration of Scr elevation, which reflects the time to renal function recovery and could be an important potential dimension of AKI along with severity. Objectives: we sought to explore the association between the duration of AKI based on Scr and 1-year mortality and to examine whether the duration of AKI offered any additional prognostic information over the magnitude of elevation in Scr.

Methods: This retrospective study was conducted from 2007 to 2018 with very elderly patients ( $>75$ years) from the Chinese PLA General Hospital. AKI was stratified by magnitude according to KDIGO stage and duration ( $1-2$ days, 3-4 days, $5-7$ days, and $>7$ days). The primary outcome was the 1-year mortality rate after AKI.

Results: During the study period, a total of 688 AKI patients were included in the analysis. The median age was 88 (84-91) years. According to the KDIGO criteria, 317 patients (46.1\%) had stage 1 AKI, 169 (24.6\%) had stage $2 \mathrm{AKI}$, and 202 (29.4\%) had stage 3 AKI. Of the 688 study subjects, we stratified patients by duration of AKI: $1-2$ days ( 61 ; $8.9 \%), 3-4$ days $(104 ; 15.1 \%), 5-7$ days $(140 ; 20.3 \%)$, and $>7$ days (383; $55.7 \%)$.

The Kaplan-Meier survival plot demonstrates that 1-year survival was significantly different according to both AKI stage and duration. As the duration of AKI increased within each strata of AKI severity as defined by the KDIGO criteria (magnitude), 1-year survival decreased, but no significant differences were found for patients with stage 1 and stage 2 AKI (stage 1, $P=0.526$; stage $2, P=0.818$; stage $3, P=0.016$ ). 
However, survival significantly changed within each strata of AKI classified by duration. Notably, the 1-year mortality rates were greater for those with a duration of 3-4 days, a duration of 5-7 days, and a duration of $>7$ days of AKI. These relationships were confirmed when we examined the stratified survival plots. When patients were stratified by the stage of AKI, patients with increasing duration of AKI had significantly worse 1-year survival in the group with AKI stage 3 (log-rank test: $P<0.001$ ), but no differences were found in the group with stage 1 or stage 2 (log-rank test: both $P>0.05$ ). In contrast, when patients were stratified by the duration of AKI, the magnitude of Scr increase as classified by KDIGO was significantly associated with 1-year mortality (log-rank test: $P<0.05$ for all four strata).

Accordingto the multivariate regression analysis, both AKI duration (3-4 days: $\mathrm{HR}=3.184 ; 95 \% \mathrm{Cl}: 1.733-5.853 ; P<0.001,5-7$ days: $\mathrm{HR}=1.915$ $95 \% \mathrm{Cl}: 1.073-3.416 ; P=0.028 ;>7$ days: $\mathrm{HR}=1.766 ; 95 \% \mathrm{Cl}: 1.017-$ $3.065 ; P=0.043$ ) and more advanced AKI stage (stage 2: $\mathrm{HR}=3.063 ; 95 \%$ Cl: 2.207-4.252; $P<0.001$; stage 3: HR $=7.333 ; 95 \%$ Cl: 5.274-10.197; $P<$ 0.001 ) were independently associated with 1-year mortality.

Conclusion: In conclusion, the duration of AKI in geriatric patients is independently associated with 1-year mortality and may adds prognostic value in addition to that provided by the magnitude of Scr alone. These data need to be validated in other settings of AKI, and if found to be valid, the duration of AKI should be incorporated into the consensus definitions of AKI and target AKI duration for clinical management.

\section{4}

Serum trace elements in critically iii patients on continuous renal replacement therapy

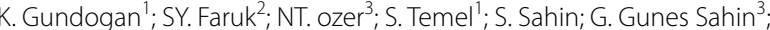
C. YazıcI'; A. Esmaoglu²; T. Talih'; M. Sungur

'Department of internal medicine, division of intensive care, Erciyes University, School of medicine, Kayseri, Turkey; ${ }^{2}$ Department of anesthesiology and reanimation, Erciyes University, School of medicine, Kayseri, Turkey; ${ }^{3}$ Deparment of health sciences institute, division of clinical nutrition, Erciyes University, Kayseri, Turkey; ${ }^{4}$ Department of clinical biochemistry, Erciyes University, School of medicine, Kayseri, Turkey; ${ }^{5}$ Department of general surgery, Erciyes University, School of medicine, Kayseri, Turkey

Correspondence: N.T. ozer

Intensive Care Medicine Experimental 2020, 8(2): 000334

Introduction: Acute kidney injury (AKI) is common and serious complication in critically. AKI treated with continuous renal replacement therapy (CRRT) may alter trace element levels in critically ill patients and at risk for disturbances in plasma levels of trace elements due to the underlying illness, $\mathrm{AKI}$, and dialysis 1.

Objectives: We aimed to determine trace element balances during CRRT in critically ill patients.

Methods: The study was conducted prospectively in intensive care unit (ICU). The study included patients aged $\geq 18$ years who were admitted in ICU with AKI and required CRRT support (continuous venovenous hemodiafiltration, CVVHDF). Serum trace elements (copper, zinc, selenium, chromium and cobalt) measured were measured using the high performance liquid chromatography on the first three days of CVVHDF and first two days after CVVHDF.

Results: We enrolled 50 patients. The median (min-max) age was 64(18-88) years. The most common reasons for ICU admission were respiratory failure (34\%) and metabolic disorders (20\%). The median (min-max) baseline APACHE II score and NUTRIC score of the patients was $24.0(6.0-33.0)$ and $5(0-8)$, respectively. The mean days on CRRT was $2.4 \pm 0.5$ days. Serum copper, zinc, selenium, chromium and cobalt levels at all times and referance range showed Table 1. Serum trace elements was not significantly different from baseline at all times $(p>0.05)$.
Table 1. Serum copper, zinc, selenium, chromium and cobalt levels

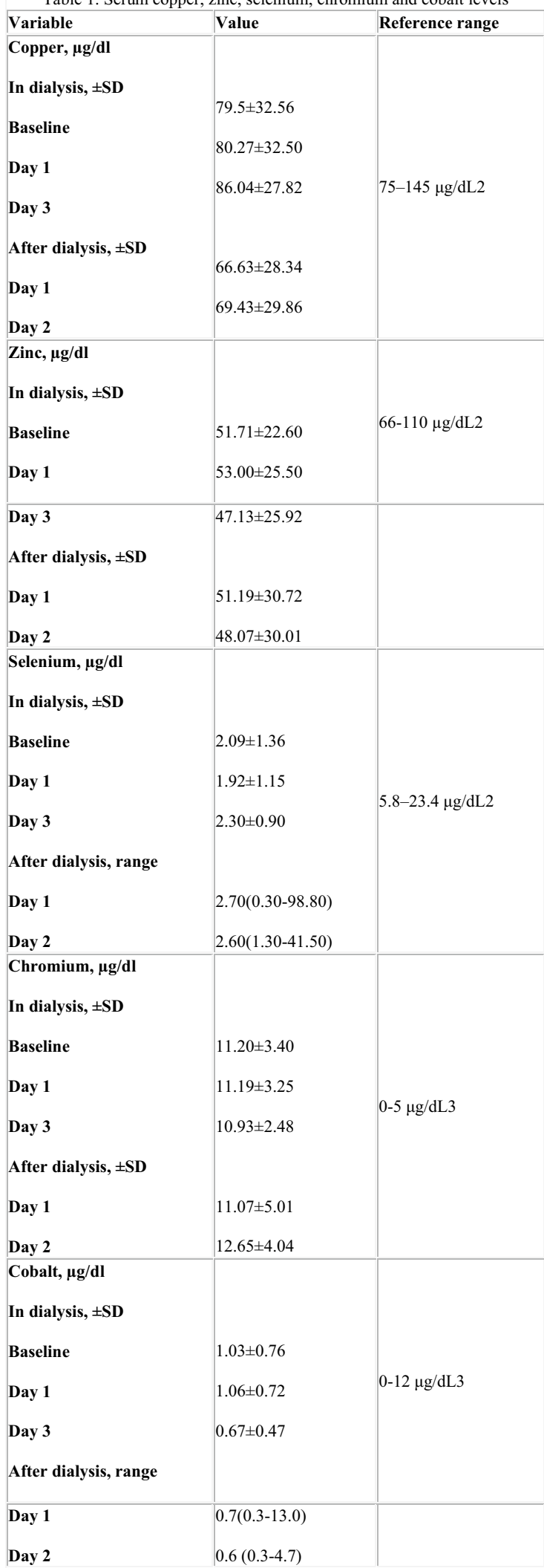


Conclusion: End of the study, serum chromium and selenium was tended to increase on follow-up while cobalt, copper and zinc was tended to decrease on follow-up. However, the difference in these parameters was not statistically significant.

\section{Reference(s) and grant ackowledgment(s)}

1. Editor: Kyle C. A Handbook for the interpretation of laboratory tests. 4th ed. Diagnostic Medlab;2008

2. URL: http://www.gclabs.co.kr/eng/information/search_test_item_view

3. Allegretti AS, Steele D, David-Kasdan JA, Bajwa E, Niles JL, Bhan I. Continuous renal replacement therapy outcomes in acute kidney injury and endstage renal disease: a cohort study. Crit Care. 2013;17(3):R109-R117.

4. Erciyes University Scientific Research Committee

\section{9}

Association of plasma and urine neutrophil gelatinase-associated lipocalin with acute kidney injury and long-term mortality in patients undergoing elective colorectal surgery per enhanced recovery after surgery protocols

N. Lumlertgul'; J. Van Dellen²; M. Ostermann ${ }^{1}$; S. Mccorkell'; A. Williams ${ }^{2}$

'Intensive care, Guy's and St Thomas' NHS Foundation Trust, London, United Kingdom; ${ }^{2}$ Colorectal surgery, Guy's and St Thomas' NHS Foundation Trust, London, United Kingdom; ' ${ }^{3}$ eppartment of anaesthetics, Guy's and St Thomas' NHS Foundation Trust, London, United Kingdom

Correspondence: $\mathrm{N}$. Lumlertgul

Intensive Care Medicine Experimental 2020, 8(2): 000369

Introduction: Acute kidney injury (AKI) is common in surgical patients. We aimed to investigate the validity of plasma and urine neutrophil gelatinase-associated lipocalin (NGAL) in the early detection of $\mathrm{AKI}$ and prediction of outcomes in patients undergoing major elective colorectal surgery.

Methods: This was a post-hoc analysis of a randomized controlled trial comparing oesophageal doppler and Lithium dilution cardiac output monitoring for intraoperative haemodynamic monitoring in high risk patients undergoing major colorectal surgery in a tertiary care hospital. Plasma and urine samples were collected before surgery ( $\mathrm{T} 1$ ), immediately after surgery (T2), and on postoperative day 1 (T3) and NGAL levels were measured. We collected baseline demographics, perioperative parameters, hospital and long-term mortality. We identified patients with AKI according to KDIGO criteria and compared them with patients without $A K I$

Results: A total of 89 patients were included, of whom 12 (13.5\%) developed AKI. Plasma NGAL significantly increased from T1 to T3 in both AKI $(P<0.001)$ and non-AKI $(P=0.048)$ patients, while urine NGAL did not change over time. There were no significant differences in plasma and urine NGAL in patients with and without AKI at all time points. Baseline plasma NGAL and post-operative urine NGAL were higher in 8-year non-survivors than survivors. AKI patients with raised plasma or urine NGAL had a higher long-term mortality than AKI patients without a raised NGAL.

Conclusion: Plasma and urine NGAL poorly predicted AKI post-colorectal surgery. Both plasma and urine NGAL were higher in non-survivors although further studies are needed to determine the association between NGAL and long-term outcomes.

\section{Reference(s) and grant ackowledgment(s)}

1. The authors acknowledged BioPorto Diagnostics A/S for assistance with urine and plasma NGAL measurement. Data collection was collated by the Colorectal Enhanced Recovery Team - Marie Morris, Lucy Morris, and Jane Varghese. Perioperative care was predominantly provided by the following consultant anesthetists and surgeons - Emin Carapeti, Amir Darakhshan, Vivek Datta, Mark George, David James, Stuart McCorkell, Veda Ponnaiah, Emma Taylor, Andy Williams, and Georgina Wilson - and staff in the Overnight Intensive Recovery Unit. Linda Tovey and the ICU technicians processed the NGAL samples.

2. The study was funded by a New Services and Innovation Grant awarded by the Guy's \& St Thomas' Charities. A full-time research assistant wasfunded jointly with contribution by the medical device manufacturer, Deltex Medical (Chichester, UK).
000509

Impact of a computerized decision support tool and care bundle on critical care costs of acute kidney injury (AKI) patients

L. Atallah'; E. Ghosh'; J. Rivers²; C. Bourdeaux ${ }^{2}$

${ }^{1}$ Connected care and personal health, Philips Research North America, Cambridge, United States of America; ${ }^{2}$ Department of intensive care, University Hospitals Bristol NHS Foundation Trust, Bristol, United Kingdom

Correspondence: E. Ghosh

Intensive Care Medicine Experimental 2020, 8(2): 000509

Introduction: Acute kidney injury is one of the most common complications in critical care. It is associated with high mortality, increased length of stay and adverse long-term outcomes. Clinical decision support (CDS) tools have provided means of alerting clinicians to deteriorating patients leading to better management and improved outcomes. A study was conducted in two ICU units in University Hospitals Bristol, UK. It involved integrating a CDS intervention based on Acute Kidney Injury Network (AKIN) guidelines and a care bundle consisting of alerts, dashboard and order sets.

Objectives: The study involved a before (control) and after (intervention) phases. It investigated the effect of the intervention on the number of patients deteriorating to higher AKI levels as well as measuring medication adherence. This work investigates the in-ICU costs relating to the length of stay of AKI patients before and after the intervention. Methods: AKI score was calculated anytime a new urine output or Serum Creatinine was measured. The costs for each stage of AKI (1-3, according to AKIN) were calculated as the number of patients per stage, multiplied by the average LOS and the average cost of an ICU day in intensive care based on hospital averages (£1981). This provides a minimal value, as the costs associated with higher AKI levels are higher.

Results: Each arm of the study was run for 1 year with 2523 ICU admissions in the control phase followed by 2521 ICU admissions in the intervention phase. The prevalence of patients developing any AKI decreased from $43.1 \%$ (control) to $37.5 \%$ (intervention). There was a reduction in the proportion of patients in each stage of AKI. This was accompanied with a reduction in LOS for the three levels. In control cohort, the mean LOS for AKI stage 1 was 6.4 days which reduced to 5.0 days in intervention. For stage 2 and stage 3, the reduction was ( 8.2 days; control to 7.3 days; intervention) and (10.8 days; control to 10.2 days; intervention) respectively. Overall, minimal ICU costs for all AKI patient days were $£ 14,459,874$ for 1018 AKI patients in the control phase and $£ 10,163,738$ for $870 \mathrm{AKI}$ patients in the intervention phase. Conclusion: In comparison to the control phase, the study showed that that the introduction of AKI guidelines and care bundle increased AKI vigilance, leading to proactive measures in managing AKI. This led to a reduction of patients with AKI in the intervention phase, accompanied by an overall shorter length of stay. ICU costs were reduced by $30 \%$ if only length of stay were to be considered as a contributing factor. This, however, is a rather simplified view of ICU costs. Further work should include the effect of treatments, long-term follow-up and readmission on costs.

\section{8}

Acute renal failure in COVID 19 patients on mechanical ventilation A. Ruiz García'; C. Serrano'; R. Araoz Illanes '; A. Acha '; DR. Beltran”;

M. Trascasa Muñoz' ; J. Higuera Lucas²; B. Llorente Ruiz'; P. Villa Díaz';

E. Serrano Yebenes ${ }^{3}$; F. Poveda Saenz ${ }^{4}$; C. Demartini Foj ${ }^{4}$; E. Nevado'

${ }^{1}$ Intensive care unit, Hospital Príncipe de Asturias, Alcalá de Henares, Spain;

${ }^{2}$ Medicina intensiva, Hospital Universitario Príncipe de Asturias, Meco, Spain; ${ }^{3}$ General surgery, Hospital Príncipe de Asturias, Alcalá de Henares, Spain; ${ }^{4}$ Anesthesiology, Hospital Príncipe de Asturias, Alcalá de Henares, Spain

Correspondence: A. Ruiz García

Intensive Care Medicine Experimental 2020, 8(2): 000548

Introduction: COVID 19 community acquired pneumonia is a recent and frequent cause of admission in European intensive care units. Currently, there are many open questions regarding the management 
and prognostic factors of these patients. Among them, its association with acute renal failure in patients on mechanical ventilation.

Objectives: Analysis of acute renal failure in COVID 19 patients on mechanical ventilation. Prognosis and factors related to its development.

Methods: Retrospective observational study carried out in the intensive care unit of a University Hospital during the COVID 19 pandemic. We analysed patients admitted to the ICU with the diagnosis of Respiratory Failure due to pneumonia COVID 19 between 6/3/2020 and 21/4/2020. Demographic data (age, sex), APACHE II, comorbidities, analytical data (ferritin, total bilirubin), length of stay, treatments administered (mechanical ventilation, muscle relaxant, vasoactive drugs, prone) and its association to acute renal failure were analysed.

Results: 67 patients received complete treatment in our hospital. $67.2 \%$ males with an average age 60 years, length of stay (days) $14.1 \pm 10$, APACHE II $14.3 \pm 5.2$. Maximum creatinine mean value $(\mathrm{mg} /$ dl) $1.48 \pm 1.26$, maximum ferritin mean value $(\mathrm{ng} / \mathrm{ml}) 2310 \pm 3322$, maximum total bilirubin mean value $(\mathrm{mg} / \mathrm{dl}) 2 \pm 2.2$. $100 \%$ of the patients required mechanical ventilation. $65.7 \%$ prone positioning, $74.6 \%$ neuromuscular blockers and $98.5 \%$ required vasoactive drugs. Establishing renal failure as creatinine values greater than $1.2 \mathrm{mg} / \mathrm{dl}$, the results shown in Table 1 were obtained.

\begin{tabular}{|l|l|l|l|}
\hline & $\begin{array}{l}\text { Creatinine }>\mathbf{1 , 2} \\
\mathbf{m g} / \mathbf{d l}\end{array}$ & $\begin{array}{l}\text { Creatinine }<\mathbf{1 , 2} \\
\mathbf{m g} / \mathbf{d l}\end{array}$ & $\mathbf{P}$ \\
\hline Age (years) & 61,3 & 59 & 0,37 \\
\hline APACHE II & 15,8 & 13,3 & 0,128 \\
\hline Ferritine (max) & 3421 & 1535 & $\mathbf{0 , 0 3 5}$ \\
\hline D-Dimer (max) & 23,5 & 15 & 0,19 \\
\hline Bilirubine (max) & 3 & 1,4 & $\mathbf{0 , 0 1 2}$ \\
\hline
\end{tabular}

Table 2 shows the mean creatinine values in patients, who require prone positioning, muscular blockers, have cardiovascular disease, dyslipidaemia, survivors, and non-survivors.

\begin{tabular}{|l|l|l|l|}
\hline & $\begin{array}{l}\text { Level of } \\
\text { creatinine (mg/dl) }\end{array}$ & P & \\
\hline & Yes & No & \\
\hline Prone & 1,51 & 1,43 & 0,8 \\
\hline Muscular bloq. & 1,49 & 1,46 & 0,9 \\
\hline Sex (male) & 1,81 & 0,8 & $\mathbf{0 , 0 0 2}$ \\
\hline CV desease & 1,69 & 1,3 & 0,22 \\
\hline Dyslipidemia & 1,92 & 1,22 & $\mathbf{0 , 0 2 6}$ \\
\hline Suoerinfection & 1,62 & 1,3 & 0,29 \\
\hline Survivors & $\mathbf{1 , 0 7}$ & $\mathbf{1 , 9 6}$ & $\mathbf{0 , 0 0 3}$ \\
\hline
\end{tabular}

Conclusion: In our sample, we have associated acute renal failure with higher ferritin values. Likewise, we have observed higher creatinine values in the group of non-survivors, those with dyslipidaemia and men, with statistical signification.

\section{7}

\section{The use of Polymyxin B Hemoperfusion for COVID-19 Patients} with endotoxic shock

S. De Rosa'; C. Ronco'; G. Golino'; M. De Cal'; G. Pierbellini'; V. Danzi'

${ }^{1}$ Department of anesthesia and intensive care unit, San Bortolo Hospital Of Vicenza, Vicenza, Italy; ${ }^{2}$ Department of nephrology, dialysis, and transplantation, San Bortolo Hospital Of Vicenza, Vicenza, Italy

Correspondence: S. De Rosa

Intensive Care Medicine Experimental 2020, 8(2): 000837

Introduction: Astrointestinal dysfunction may lead to increased mucosal permeability and leakage of endotoxin. Although coronaviruses are known mainly as respiratory pathogens, up to $60 \%$ of COVID-19 patients show gastrointestinal symptoms at admission or developed during hospitalization. Recent published data show how endotoxemia and bacterial DNA are frequently found in patients with COVID-19 pneumonia, indicating that loss of intestinal barrier function can contribute to the pathogenesis of COVID-19. In addition, patients who are hospitalized for extended periods in an ICU are more prone to superimposed infections.

Objectives: In this retrospective analysis we report our experienceof Polymyxin B hemoperfusion (PMX-HP) as complementary therapy for unresponsive endotoxic shock management in 5 patients with COVID19 hospitalized in our ICU ward between February and April 2020. To the best of our knowledge, there is no data published yet concerning PMX-HP use in COVID-19 patients.

Methods: In the present case series, we evaluated the impact of PMX-HP as adjunctive therapy in a population of patients affected by COVID-19 and confirmed endotoxic shock identified my measurement of endotoxin activity at enrollment. Hemodynamics and main clinically relevant outcome parameters were monitored. PMX-HP treatment consists of 2 hemoperfusion sessions, the second session performed 24 hours after the first one, at a blood flow rate of $100 \mathrm{ml} / \mathrm{min}$. Unfractionated heparin was used as standard anticoagulant.

Results: PMX-HP treatment was associated with rapid hemodynamic stabilization with reduction of Vasopressors Inotropic Score (VIS) - (from a mean value of $29 \pm 15,17$ to $2,60 \pm 2,88$ at 120 hours, $p<0,05$ ), reduction in blood lactate levels (from $2,84 \pm 1,43$ to $1,08 \mathrm{mmol} / \mathrm{l} \pm 0,54$ at 120 hours $-\mathrm{p}<0,05$ ), rapid decrease in Endotoxin Activity (EA) levels (from a mean EA value of $0,70 \pm 0,10$ to $0,49 \pm 0,13$ at 48 hours $-p<0,05)$ in a population affected by SARS CoV-2 and endotoxic shock. PMX-HP treatment resulted safe and well-tolerated with no device related adverse events during or after hemoperfusion sessions. All patients received 2 hemoperfusion sessions.

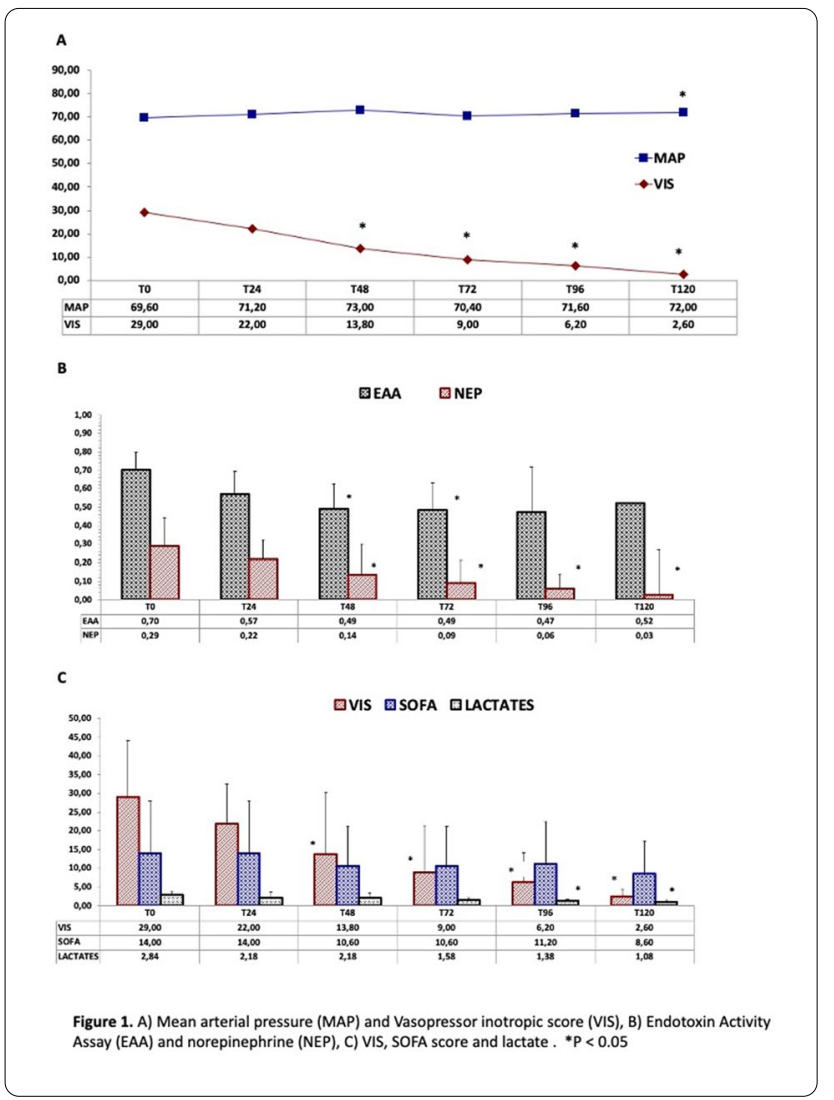

Conclusion. Endotoxic shock could be associated to SARS-CoV-2. PMX-HP can be considered for management of unresponsive endotoxic shock. In our cases, PMX-HP treatment was associated with rapid hemodynamic improvement associated with a rapid decrease in vasopressor use, blood lactates and EAA levels. PMX-HP treatment was safe with no device-related adverse events. 
Reference(s) and grant ackowledgment(s)

1. Ronco C. et al., Extracorporeal Blood Purification and Organ Support in the Critically III Patient During COVID-19 Pandemic: Expert Review and Recommendation. Blood Purif 2020 May 26;1-11.

2. Lin, L., et al., Gastrointestinal symptoms of 95 cases with SARS-CoV-2 infection. Gut, 2020.69(6): p. 997-1001.

3. Sirivongrangson, P., et al., Endotoxemia and circulating bacteriome in severe COVID-19 patients. medRxiv, 2020: p. 2020.05.29.20109785.

4. None

\section{9}

Acute Kidney Injury in critically ill trauma patients

G. Golino ; M. Greco ${ }^{2}$; A. Rigobello ${ }^{1}$; C. Ronco ${ }^{3}$; V. Danzi ${ }^{1}$; P. Navalesi ${ }^{4}$; S. De Rosa $^{1}$

${ }^{1}$ Department of anesthesia and intensive care unit, San Bortolo Hospital Of Vicenza, Vicenza, Italy; ${ }^{2}$ Anesthesia and intensive care, Humanitas Research Hospital, Milano, Italy; ${ }^{3}$ Department of nephrology, dialysis, and transplantation, San Bortolo Hospital Of Vicenza, Vicenza, Italy; ${ }^{4}$ Department of anesthesia and intensive care unit, University of Padua, Padova, Italy

Correspondence: S. De Rosa

Intensive Care Medicine Experimental 2020, 8(2): 000839

Introduction. Acute Kidney Injury (AKI) is a common complication in trauma with $24 \%$ of incidence in intensive care unit and it is independently associated with increased morbidity and mortality, but also prolonged length of stay. The high incidence of AKI in trauma patients should lead to early identification of those at risk of AKI to establish a resuscitation strategy that aims at preventing AKI.

Methods: Detailed data of trauma patients between May, 2017 and May, 2019 were retrieved from icu medical records. In particular, patients' demographics, hemodynamic parameters, biochemical parameter and fluid balance, urinary output, $\mathrm{sCr}$ values at baseline, $24 \mathrm{hrs}, 48 \mathrm{hrs}$, and $72 \mathrm{hrs}$. Shorts and long terms outcomes had been analysed.

Results. Based on KDIGO criteria, 45 patients developed AKI (29.4\%.) 29 patients (19.9\%) developed stage 1 AKI, 16 patients (10.5\%) stage 2-3 AKI. Patients with elevated urinary biomarkers alone undergoing therapeutics interventions had $8.5 \%$ stage $2-3 \mathrm{AKI}$ compared to $33 \%$ of patients with creatinine elevation only, and $50 \%$ of patients with both increased creatinine and AKI risk score. A number of 19 patients (23\%) with urinary markers driven interventions progressed to higher stages of $\mathrm{AKI}$, while $7(8.5 \%)$ improved $\mathrm{AKI}$ stage.

\begin{tabular}{|l|l|l|l|l|}
\hline \multicolumn{1}{|c|}{ Outcome } & $\begin{array}{c}\text { No AKI } \\
\text { (N=29) }\end{array}$ & $\begin{array}{l}\text { AKI stage 1 } \\
(\mathbf{N = 1 6 )}\end{array}$ & $\begin{array}{l}\text { AKI stage 2- } \\
\mathbf{3}(\mathbf{N}=\mathbf{2 9})\end{array}$ & $\begin{array}{c}\text { AKI (all } \\
\text { patients) } \\
\mathbf{N = 4 5}\end{array}$ \\
\hline RRT & 0 & 0 & 0 & $5(11 \%)$ \\
\hline $\begin{array}{l}\text { Ventilation free } \\
\text { days }\end{array}$ & $1.8(2.1)$ & $1.7(1.4)$ & $2.0(2.5)$ & $1.8(1.9)$ \\
\hline MV length & $5.9(9.3)$ & $4.7(6.6)$ & $7.4(10.1)$ & $5.9(8.6)$ \\
\hline ICU stay & $7.7(9.3)$ & $6.3(6.7)$ & $9.4(9.8)$ & $7.7(8.0)$ \\
\hline Hospital stay & $18.1(16.3)$ & $19.3(15.6)$ & $18.5(19.6)$ & $19.1(17.3)$ \\
\hline Renal recovery & NA & $26(89 \%)$ & $11(68.5 \%)$ & $37(82.2 \%)$ \\
\hline $\begin{array}{l}\text { Survival over 24 } \\
\text { hrs from ER } \\
\text { arrival }\end{array}$ & $108(100 \%)$ & $29(100 \%)$ & $16(100 \%)$ & $45(100 \%)$ \\
\hline
\end{tabular}

Conclusion: AKI is a frequent complication following trauma. Although the reduction of stage $2 / 3$ AKI based on therapeutic interventions driven by elevated urinary biomarkers was observed in $8 \%$ of patients, the use of biomarkers represent an important tool for the early detection of patients at high risk of AKI behind the local ICU experience based on multidisciplinary team.
Reference(s) and grant ackowledgment(s)

1. Perkins ZB, Haines RW, Prowle JR. Trauma-associated acute kidney injury. Curr Opin Crit Care. 2019;25(6):565-572.

2. Harrois A, Libert N, Duranteau J. Acute kidney injury in trauma patients. Curr Opin Crit Care. 2017;23(6):447-456

\section{6}

Can dexmedetomidine reduce the incidence of AKI?

M. Giovini ${ }^{1}$; M. Barbera ${ }^{1}$; E. Antonucci ${ }^{1}$

${ }^{1}$ Intermediate care unit, Ospedale "Guglielmo da Saliceto", Piacenza, Italy

Correspondence: $\mathrm{E}$. Antonucci

Intensive Care Medicine Experimental 2020, 8(2): 000886

Introduction: Dexmedetomidine (DEX) is a common sedative agent that may protect against organ dysfunction such as acute kidney injury (AKI) by reducing inflammatory response. A recent post-hoc analysis 1 has found that DEX treatment is associated with reduced $\mathrm{AKI}$ and mortality rate among septic patients. However, no human study has ever explored confounding factors that could increase AKIrisk in NO-DEX group (i.e. nephrotoxic drugs; chronic kidney disease, CKD). For these reasons, we analyzed a well stratified cohort of septic patients that need sedative agents and have been exposed to similar risk factors for AKI development.

Objectives: We hypothesized that DEX could reduce AKI incidence when compared to NO-DEX sedative agents in septic patients.

Methods: We performed a retrospective analysis, reviewing all patients admitted for sepsis to our intermediate care unit (IMCU) from January 1st 2018 to December 31st 2019. The inclusion criteria were: age $\geq 18$ years old; diagnosis of sepsis at admission; need of sedative agents for delirium. Exclusion criteria were: chronic need of hemodialysis; use of sedative agents beyond delirium. NO-DEX agents included benzodiazepines, quetiapine and haloperidol. AKI was identified by KDIGO criteria. We analyzed two groups (DEX and NO-DEX) matched for age, SAPS2 score, SOFA score and CKD. We investigated rates of $\mathrm{AKI}$ and mortality at 90 days in the two groups. We also analyzed some counfounding factors such as circulatory failure (CF), use of iodinated contrast media (ICM), aminoglycosides, glycopeptides, colistin, ACEi/ sartans and non steroidal anti-inflammatory drugs (NSAID).

Results: We reviewed 34 patients and 22/34 respected the inclusion criteria (11 in DEX group; 11 in NO-DEX group). The two groups were well matched (SAPS 2 score: $33 \pm 1$ (DEX), $33 \pm 3$ (NO-DEX) $p=0.5$; SOFA score: $4 \pm 1$ (DEX), $4 \pm 4$ (NO-DEX) $p=0.4$; age: $70 \pm 11$ (DEX), $70 \pm 3$ (NO-DEX) $p=0.5$; CKD: $3 / 11$ patients (DEX), 2/11 patients (NODEX). We found that DEX significantly decreases AKI rate (DEX $1 / 11$ patients; NO DEX 6/11 patients; OR 0.08, 95\% Cl 0.008-0.895, $\mathrm{p}=$ 0.04). We also found no significant difference in mortality at 90 days between the two groups(NO DEX 2/11 patients; DEX 5/11 patients; OR $3.75,95 \% \mathrm{Cl} 0.54-26.05, \mathrm{p}=0.2)$. In the Table 1 we have reported AKI characteristics for every single patient. About confounding factors, only a patient in NO DEX group received ICM, ACEi/sartans and NSAID all together. Remaining $5 \mathrm{AKI}$ patients in NO-DEX groups did not receive any nephrotoxic drug. Only a patient in DEX group showed AKI and received colistin and DEX treatment at the same time. In conclusion, the higher AKI rate in NO-DEX group was not significantly linked to confounding factors.

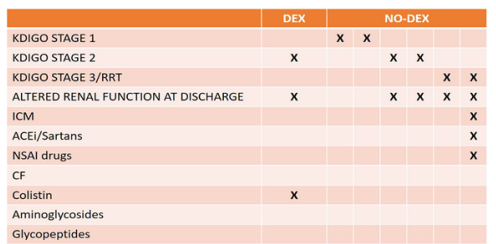

Table 1 - AKI characteristics and confounding factors. Every column represents a single patient RRT, renal replacement therapy. 
Conclusion: DEX could reduce the incidence of AKI in septic patients when compared with other sedative agents. Future large randomized studies are necessary to confirm these preliminary findings.

\section{Reference(s) and grant ackowledgment(s)}

1. 1. Nakashima T. Dexmedetomidine Improved Renal Function in Patients With Severe Sepsis: An Exploratory Analysis of a Randomized Controlled Trial. Journal of Critical Care 2020

\section{8}

Prevalence and risk factors of hemodynamic instability associated with preload-dependence during continuous renal replacement therapy in critically ill patients

L. Bitker'; G. Chazot ${ }^{1}$; M. Mehdi' ; N. Chebib'; P. Chabert'; L. Chauvelot ${ }^{1}$;

L. Folliet'; G. David'; J. Provoost ${ }^{1}$; H. Yonis ${ }^{1}$; JC. Richard'

${ }^{1}$ Médecine intensive - réanimation, Hospital La Croix-Rousse - Hcl, Lyon, France

Correspondence: J.C. Richard

Intensive Care Medicine Experimental 2020, 8(2): 000928

Introduction: Hemodynamic instability is a frequent complication of continuous renal replacement therapy (CRRT) in intensive care patients. Postural tests (i.e. passive leg raising in the supine position or Trendelenburg in the prone position) combined with continuous measurement of cardiac output are highly reliable to identify preload dependence and may provide new insights into the mechanisms involved in hemodynamic instability related to renal replacement therapy (HIRRT).

Objectives: We aimed to assess the prevalence and risk factors of HIRRT associated with preload-dependence in ICU patients.

Methods: We conducted a single-center prospective observational study in ICU patients with acute kidney injury KDIGO 3, started on CRRT in the last 24 hours, and monitored with a PiCCO ${ }^{\circledR}$ device. The primary endpoint was the rate of HIRRT episodes associated with preload dependence during the first 7 days of CRRT. HIRRT was defined as the occurrence of a mean arterial pressure below $65 \mathrm{~mm}$ $\mathrm{Hg}$ requiring any of the following therapeutic intervention (initiation or increase in vasopressor dose, discontinuation or decrease of fluid removal by CRRT, or fluid administration). Preload dependence was assessed every 4 hours, and during each hypotensive episode, and was deemed present if the continuous cardiac index increased by at least $10 \%$ during a passive leg raising test in supine patients, or $8 \%$ during Trendelenburg in prone patients. Data are expressed in median [1rst quartile-3rd quartile], unless stated otherwise.

Results: 32 patients (59\% male, age 67 [58-75] year, SAPS-2 71 [49-78]) were included 8 [1-14] hours after CRRT initiation and studied continuously for 99 [55-144] hours. 29 [18-40] hemodynamic evaluations per patient were performed for a total of 903 hemodynamic evaluations. Preload-dependence was present in $42 \%$ of the hemodynamic evaluations. RRT modality was CVVH during $91 \%$ of the hemodynamic evaluations and CVVHD in 9\%. Ultrafiltration rate was 27 [24-31] ml.kg-1. $\mathrm{hr}-1$ in CVVH-treated patients, dialysate rate was 26 [20-29] ml.kg-1. hr-1 in CVVHD-treated patients, and net UF was 100 [0-200] ml.hr-1. All patients experienced at least 1 episode of HIRRT. A median of 4 [3-7] HIRRT episodes occurred per patient, for a pooled total of 173 episodes. 94 episodes (54\% [C195\%: 47-62\%]) were associated with preload-dependence, 75 (43\%, [Cl95\%: 36-51\%]) without preloaddependence, and 2 were unclassified. Therapeutic management of HIRRT episodes is reported in the figure below. Multivariate analysis (using variables collected prior to HIRRT) identified the following variables as risk factors for the occurrence of HIRRT associated with preload-dependence: preload-dependence before HIRRT (odds ratio $=3.66, \mathrm{p}<0.001)$, delay since last HIRRT episode of at least 4 hours $(\mathrm{OR}=0.35, \mathrm{p}<0.01)$, lactate $(\mathrm{OR}=1.22$ per $1.9 \mathrm{mmol} / \mathrm{L}$ increase, $\mathrm{p}$ $<0.01)$, cardiac index $(0.58$ per 1 L.min-1.m-2 increase, $p<0.01)$ and SOFA at ICU admission $(0.90$ per 1 point increase, $\mathrm{p}<0.01$ ).

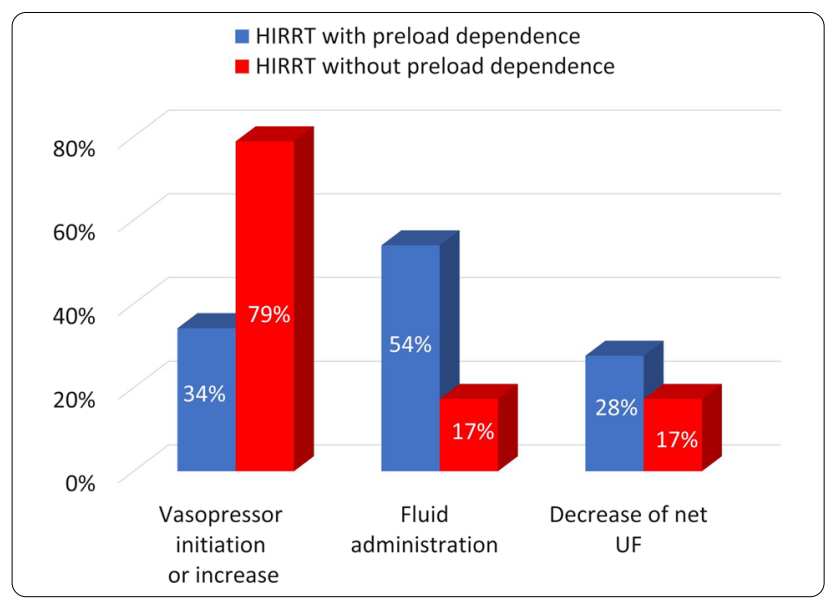

Conclusion: In this single center study, HIRRT associated with preload dependence was slightly more frequent than HIRRT without preload dependence in ICU patients undergoing CRRT. Testing for preload dependence to adjust fluid removal could help preventing HIRRT incidence during CRRT.

\section{1}

Prognostic value of [TIMP2] [IGFBP7] for mortality

J. Baldirà ; M. Flores Orella ${ }^{1}$; JA. Santos ${ }^{1}$; A. Cordon ${ }^{1}$; A. Betbesé-Roig ${ }^{1}$;

M. Pérez-Carrasco ${ }^{2}$;. Díaz-Buendía ${ }^{3}$; J. Sabater Riera ${ }^{4}$;

TM. Tomasa-Irriguible ${ }^{5}$; M. Cuartero ${ }^{6}$

${ }^{1}$ Intensive care, Hospital de la Santa Creu i Sant Pau, Barcelona, Spain ${ }^{2}$ Intensive care, Vall d'Hebron University Hospital, Barcelona, Spain; ${ }^{3}$ Intensive care, Hospital del Mar, Barcelona, Spain; ${ }^{4}$ Intensive care, Hospital Universitario de Bellvitge, Barcelona, Spain, Spain; ${ }^{5}$ Intensive care, Hospital Germans Trias i Pujol, Badalona, Spain; 'Intensive care, St Mary's Hospital, London, United Kingdom

Correspondence: M. Flores Orella

Intensive Care Medicine Experimental 2020, 8(2): 000971

Introduction: TIMP2 and IGFBP7 are specific biomarkers of structural renal injury in critically ill patients.The composite of [TIMP-2].[IGFBP7] in urine is suggested to better detect and stratify acutekidney injury (AKI). To date, there is not enough data in medical-surgical ICUs where renalcritical care is essentially led by primary intensivists. We designed an observational study todefine our population as a first step of an ongoing quality improvement project in AKI inCatalonia.

Objectives: To assess the prognostic value of a single [TIMP2]. [IGFBP7] determination for predicting mortality at ICU admission.

Methods: Prospective, observational study in five tertiary university intensive care units. We included medical and surgical adult patients with an expected ICU stay more than 24 hours. We excluded those pregnant, post cardiac surgery, with severe acute kidney injury (KDIGO3) at admission or requiring bladder washouts. Epidemiological, clinical and laboratory variables were collected during more than 7 days of stay. [TIMP2]. [IGFBP7] $((\mathrm{ng} / \mathrm{mL}) 2 / 1000)$ was analysed in an admission urine sample by Nephrocheck ${ }^{\circledR}$ Vitros 3600 platform (NC). Descriptive and survival analysis were made using Stata v.16. The descriptive results are shown in median and interquartile range (IQR). We stratified patients in three groups according acute kidney injury risk: low, NC1 < 0.3; high, NC2 0.3-2; very high, NC3 > 2 (ng/ $\mathrm{mL} 2 / 1000$ ).

Results: The sample included 476 patients (Table 1), with median age of 61 (IQR 48-71.5) years; 271 (57\%) were women. Median ICU length of stay was 7 (IQR 4-17) days. Patients in NC3 group had a hazard ratio for mortality of 2.8 compared to NC1 group (Cl 95\% 1.5-5.2; $p=0,001)$. According to the Kaplan-Meier curve, the probability of death at 28 
days of admission of patients in NC3 group was 52\% (Cl 95\% 24-68\%) (figure 1).

Table 1. Main characteristics of study population. Values expressed as either $\%$ or median with interquartile range (IQR)

VARIABLE

TOTAL $(n=476)$

\begin{tabular}{|l|l|}
\hline \multicolumn{1}{|c|}{ VARIABLE } & \multicolumn{1}{c|}{ TOTAL (n = 476) } \\
\hline Age (years) & $61(\mathrm{IQR} 48-71.5)$ \\
\hline Female & $57 \%(271 / 476)$ \\
\hline ICU length of stay (days) & $7(\mathrm{IQR} 4-17)$ \\
\hline Hospital length of stay (days) & $17(\mathrm{IQRR} 9-32)$ \\
\hline ICU mortality & $17 \%(81 / 476)$ \\
\hline Days on mechanical ventilation & $4(\mathrm{IQR} 2-10)$ \\
\hline SOFA day 1 & $5(\mathrm{IQR} 1-8)$ \\
\hline APACHE II & $20(\mathrm{IQR} 14-26)$ \\
\hline Baseline serum creatinine $(\mathrm{umol} / \mathrm{L})$ & $76(\mathrm{IQR} 63-98)$ \\
\hline [TIMP2]·[IGFBP7] in $(\mathrm{ng} / \mathrm{mL}) 2 / 1000$ & $0.07(\mathrm{IQR} 0.04-7.52)$ \\
\hline AKI $(\mathrm{KDIGO}>0)$ at $48 \mathrm{hours}$ & $30 \%(143 / 476)$ \\
\hline Renal replacement during ICU stay & $5.5 \%(26 / 476)$ \\
\hline
\end{tabular}

Figure 1. Kaplan-Meier graph depicting overall survival stratified by AKI groups risk NC1, NC2 and NC3 depending on [TIMP2]-[IGFBP7] cut-offs 0.3 and 2 ((ng/mL)2/1000).

Kaplan Meier Graph.

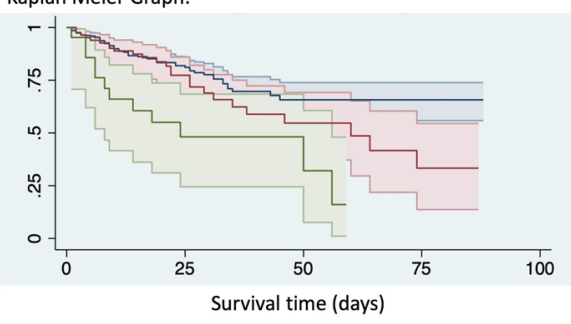

- NC1 - NC2 - NC3

$\square$
$\mathbf{I C 9 5}$
$\mathrm{IC95}$

Conclusion: In our study, patients with $\mathrm{NC}>2(\mathrm{ng} / \mathrm{mL}) 2 / 1000$ had a significant higher mortality rate compared to those with low NC values.

\section{Reference(s) and grant ackowledgment(s)}

1. Cuartero M, Ballus J, Sabater J et al. Ann. Intensive Care (2017).7:92

2. Kashani K, Al-Khafaji A, Ardiles T et al. Crit Care (2013) 17:R25

\section{8}

\section{Prognostic value of urinary [TIMP2].[IGFBP7] in acute kidney} injury

J. Baldirà'; M. Flores Orella'; JA. Santos'; A. Betbesé-Roig';

C. Gomila'; M. Pérez-Carrasco ${ }^{2}$;. Díaz-Buendía ${ }^{3}$; J. Sabater Riera ${ }^{4}$.

TM. Tomasa-Irriguible ${ }^{5}$; M. Cuartero ${ }^{6}$

'Intensive care, Hospital de la Santa Creu i Sant Pau, Barcelona, Spain: ${ }^{2}$ Intensive care, Vall d'Hebron University Hospital, Barcelona, Spain; Intensive care, Hospital del Mar, Barcelona, Spain; ${ }^{4}$ Intensive care, Hospital Universitario de Bellvitge, Barcelona, Spain, Spain; ${ }^{5}$ Intensive care, Hospital Germans Trias i Pujol, Badalona, Spain; 'Intensive care, St Mary's Hospital, London, United Kingdom

Correspondence: M. Flores Orella

Intensive Care Medicine Experimental 2020, 8(2): 000998

Introduction: TIMP2 and IGFBP7 are specific biomarkers of structural renal injury in critically ill patients. The composite of [TIMP-2].[IGFBP7] in urine is suggested to better detect and stratify acute kidney injury (AKI). To date, there is not enough data in medical-surgical ICUs where renal critical care is essentially led by primary intensivists. We designed an observational study to define our population as a first step of an ongoing quality improvement project in AKI in Catalonia.
Objectives: To analyse the predictive value of urinary [TIMP2].[IGFBP7] to predict acute kidney injury in critically ill patients.

Methods: Prospective, observational study in five tertiary university intensive care units. We included medical and surgical adult patients with an expected ICU stay more than 24 hours. We excluded those pregnant, post cardiac surgery, with severe acute kidney injury (KDIGO3) at admission or requiring bladder washouts. Epidemiological, clinical and laboratory variables were collected during the first 7 days of stay. [TIMP2].[IGFBP7] $((\mathrm{ng} / \mathrm{mL}) 2 / 1000)$ was analysed in an admission urine sample by Nephrocheck ${ }^{\circledR}$ Vitros 3600 platform (NC). Descriptive and predictive analysis were made using Stata v.16. The results are shown in median and interquartile range (IQR), sensibility (S) - specificity analysis (E), and predictive capability with ROC and area under the curve (AUC).

Results: Of 476 patients in our cohort, we selected 287 who did not present AKI (table 1). Median age was 59 (IQR 46-71) years; $54 \%$ were women. Patients with NC $>0.3$ had a higher ICU mortality rate ( $45 \%$ vs $20 \%, p=0.032$ ). The AUC ROC curve to predict AKI KDIGO 2-3 of NC during the first week of ICU stay was 0.68 (IC 95\% 0.48-0.89; $p<0.001$ ) (figure 1). The negative predictive value for $\mathrm{NC}<0.3(\mathrm{ng} / \mathrm{mL}) 2 / 1000$ was $98 \%$ (S 44.4\%, E $86.3 \%$ ). The positive predictive value for NC > 2 was $50 \%$ (S 11.1\%, E 99.6\%).

Table 1. Main characteristics of study population. Values expressed as either

\begin{tabular}{|l|l|}
\hline \multicolumn{2}{c}{$\%$ or median with interquartile range (IQR) } \\
\hline VARIABLE & TOTAL $(\mathrm{N}=287)$ \\
\hline Age (years) & $59(\mathrm{IQR} 46-71)$ \\
\hline Female & $54 \%(155 / 287)$ \\
\hline ICU length of stay (days) & $5(\mathrm{IQR} 3-10)$ \\
\hline Hospital length of stay (days) & $17(\mathrm{IQRR} 9-32)$ \\
\hline ICU mortality & $13.9 \%(40 / 287)$ \\
\hline Days on mechanical ventilation & $3(\mathrm{IQR} 2-7)$ \\
\hline SOFA day 1 & $4(\mathrm{IQR} 1-7)$ \\
\hline APACHE II & $18(\mathrm{IQR} 13-24)$ \\
\hline Baseline serum creatinine $(\mathrm{umol} / \mathrm{L})$ & $76(\mathrm{IQR} 62-94)$ \\
\hline [TIMP2]·[IGFBP7] in $(\mathrm{ng} / \mathrm{mL}) 2 / 1000$ & $0.07(\mathrm{IQR} 0.04-7.52)$ \\
\hline
\end{tabular}

Figure 1. ROC curve and area under the curve (AUC) for [TIMP2] [ [IGFBP7] to predict AKI KDIGO 2-3 during the first ICU week

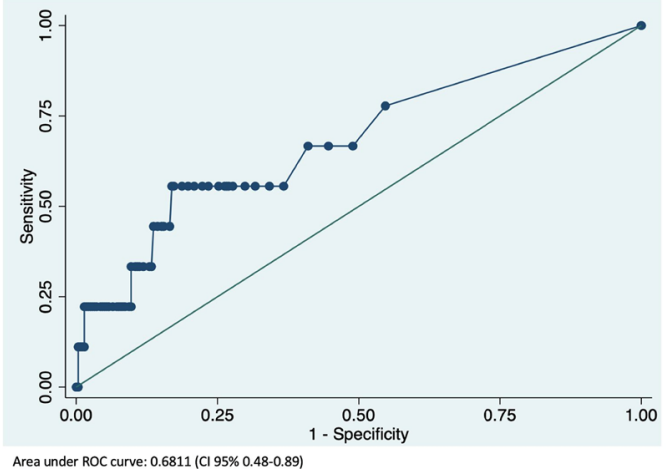

Conclusion: Although in our study [TIMP-2].[IGFBP7] had a low predictive capacity to diagnose severe AKI, it showed a very high negative predictive value.

Reference(s) and grant ackowledgment(s)

1. Cuartero M, Ballus J, Sabater J et al. Ann. Intensive Care (2017).7:92

2. Kashani K, Al-Khafaji A, Ardiles T et al. Crit Care (2013) 17:R25 


\section{9}

Prevalence and risk factors for development of acute kidney injury in patients after cardiac surgery

S. Dimopoulos ${ }^{1}$; G. Zagkotsis²; C. Kidi' ; I. Vasileiadis ${ }^{3}$; N. Rouvali'

M. Georgopoulou'; M. Mavraki'; E. Spiropoulou'; D. Markantonaki';

A. Tasouli'; 'K. Marathias'; S. Nanas ${ }^{2}$; A. Karabinis ${ }^{1}$

${ }^{1} \mathrm{Icu}$, Onassis Cardiac Surgery Center, Kallithea, Greece; ${ }^{2} \mathrm{lcu}$, Evaggelismos

General Hospital, Athina, Greece; ${ }^{3}{ }^{\mathrm{cu}}$, Sotiria Thoracic Diseases Hospital of Athens, Athina, Greece

Correspondence: S. Dimopoulos

Intensive Care Medicine Experimental 2020, 8(2): 000999

Introduction: Acute kidney injury (AKI) occurs frequently in patients after cardiac surgery and has been associated with poor outcome.

Objectives: To assess prevalence of AKI in patients admitted to intensive care unit (ICU) post-cardiac surgery during an immediate postoperative period of 24 hours, evaluate predisposing risk factors and its association with outcome.

Methods: 199 (60 women) consecutive patients admitted to ICU postcardiac surgery participated in this study. Kidney function evaluated pre-operatively and at 24 hours after procedure. An increase in serum creatinine by $0.3 \mathrm{mg} / \mathrm{dl}$ was considered as AKI, according to KDIGO classification.

Results: 46 patients (23.1\%) developed AKI 24 hours after ICU admittance. These patients received more vasoactive drugs (noradrenaline, vasopressin), (54\% vs $26 \%, p<0.001)$. They also had longer cardio-pulmonary bypass (CPB) time [AKI group: $119(104-164)$ min vs no-AKI group: $106(80-142) \mathrm{min}, p<0.027]$, longer administration of sedation [490(300 - 780) min vs 300(240 - 540) $\mathrm{min}), p<0.001]$ and longer duration of mechanical ventilation support [1084(732 - 1913) min vs $720(534-1073)$ min, $p \leq 0.0001]$. Finally, patients that developed AKI had significantly higher positive fluid balance during the first 24 hours [1038(363 - 1859) $\mathrm{ml}$ vs 501(23 - 1239) $\mathrm{ml}, p=0.005]$

Conclusion: There is a high prevalence of AKI in patients post cardiacsurgery admitted to ICU. AKI is associated with vasoactive treatment, positive fluid balance, duration of CPB and duration of administration of sedation and duration of mechanical ventilation support.

\section{2}

Early detection of serum biomarkers for sepsis-induced acute kidney injury through metabolomics

F. Ping ${ }^{1}$; Y. Guo ${ }^{1}$; Y. Cao ${ }^{1}$; J. Shang ${ }^{1}$; J. Zhang ${ }^{1}$; Y. Li ${ }^{1}$

${ }^{1}$ Deparment of critical care medicine, Shanghai Jiao Tong university affiliated sixth people's hospital, Shanghai, China

Correspondence: $Y$. GuO

Intensive Care Medicine Experimental 2020, 8(2): 000572

Introduction: Sepsis-induced acute kidney injury (AKI) causes high mortality in critical ill patients owing to elevated levels of endotoxin. This situation might be partially attributed to the delayed recognition of AKI because thecurrent diagnosis is based on either an elevation of serum creatinine levels or the detection of oliguria. Metabolomics including diverse detection platforms and multiple analysis methods is a potential tool for identifying small molecule biomarkers of renal diseases.

Objectives: This study intends to study the alterations of serum metabolomics in rats with sepsis-induced AKI and to find earlier biomarkers of sepsis-induced AKI for early clinical diagnosis and treatment through gas chromatography time-of-flight / mass spectrometry (GC-TOFMS).

Methods: A rat model of sepsis-induced AKI was established by intraperitoneal injection of Lipopolysaccharide. Thirty SD rats were randomly divided into control group, LPS $2 \mathrm{~h}$ group and LPS $6 \mathrm{~h}$ group with 10 rats in each group. Non-targeted metabolomics screening was performed on serum samples of control group and sepsis-induced AKI groups based on Gas chromatography time-of-flight / mass spectrometry (GC-TOFMS) techniques. Combining multivariate and univariate statistical analysis, the comparisons between every two groups were analyzed to screen out the serum metabolites that were statistically altered in the early stage of AKI in rats with sepsis. Finally differential metabolites obtained from each intergroup comparison are summarized.

Results: Orthogonal partial least square discriminant analysis (OPLSDA) showed the obvious separation between the control group and the LPS $2 \mathrm{~h}$ group, the control group and the LPS6h group, the LPS $2 \mathrm{~h}$ group and the LPS $6 \mathrm{~h}$ group. Each intergroup comparison can obtain a series of differential metabolites that meet the set threshold in the "potential biomarkers" section. The results of differential metabolites obtained from each intergroup comparison are intersected and summarized, which suggested that three metabolites were always significantly different in the development of sepsis-induced AKI. The potential three biomarkers for sepsis-induced AKI were Malic acid, Methionine sulfoxide and Petroselinic acid.

Conclusion: Our findings suggested that the levels of Malic acid Methionine sulfoxide and Petroselinic acid in the serum are expected to be candidates for early diagnosis of sepsis-induced AKI in rats.

\section{Reference(s) and grant ackowledgment(s)}

1. Biologic markers for the early detection of acute kidney injury. Curr Opin Crit Care 2004,10: 476-482.

2. Metabolomics Analysis of the Renal Cortex in Rats With Acute Kidney Injury Induced by Sepsis. Front Mol Biosci 2019, 6: 152.

3. This work was supported by grants from the Shanghai Municipal Commission of Health and Family Planning Foundation for Key Developing Disciplines (2015ZB0103), the Shanghai Jiao Tong University Biomedical Engineering Cross Research Foundation (YG2015MS15), and the Shanghai Pujiang Talent Plan with Class A in 2018 (18PJ1409200).

\section{3}

Cystatin C is a good predictor of acute kidney injury after elective aortic surgery

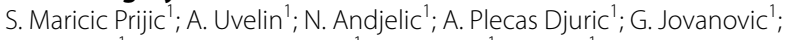

S. Vickovic'; M. Lukic Sarkanovic'; R. Popovic'; T. Tubic ${ }^{1}$

${ }^{1}$ Department of anesthesiology, intensive therapy and pain therapy, clinical centre of vojvodina, Faculty of Medicine, University of Novi Sad, Novi Sad, Serbia

Correspondence: S. Maricic Prijic

Intensive Care Medicine Experimental 2020, 8(2): 000113

Introduction: Acute kidney injury is frequent and serious complication after aortic surgery which increases length of hospital stay, costs, morbidity and mortality. Earlier recognition of patients at risk by predictive factors and identification of acute kidney injury could have important role on right timing of preventive and therapeutic measures and lower mortality of these patients.

Objectives: To investigate the most important preoperative and intraoperative predictive factors for acute kidney injury 72 hours after elective infrarenal aortic surgery.

Methods: This prospective observational study was performed at Clinic of Anesthesia, Intensive Care and Pain Therapy and Clinic of Vascular and Transplantation Surgery, Clinical Centre of Vojvodina, during the period of 18 months, from October 2017 till April 2019. It includes 140 adult patient who underwent elective infrarenal aortic surgery. Potential predictive factors were identified out of medical records such as: patient history, anesthesia lists, daily therapeutic lists, vital parameters and laboratory values lists. The occurrence of acute kidney injury was noted according to AKIN criteria. IBM SPSS version 21 (Chicago, Illinois) was used for statistical analysis. The results were presented in tables and graphs, statistical significance was set at $p$ value of less than 0,05 . Standard statistical tests were applied. Multivariate logistic regression model was used for potential predictive factors.

Results: The cut off value of cystatin $C$ serum concentration of 1,14 $\mathrm{mg} / \mathrm{l}$ has the highest sensitivity $(82,5 \%)$, and specificity $(76 \%)$ in the differentiation of patients who will develop acute kidney injury. The final model for predicting acute kidney injury in patients who underwent elective infrarenal aortic surgery contains the following variables: presence of chronic kidney disease, preoperative serum concentration of cystatin C > 1,14 mg/l, application of colloid solutions in volume > $500 \mathrm{ml}$ during the operation and total intravascular fluid replacement volume $>59 \mathrm{ml} / \mathrm{kg}$ in intraoperative period. The strongest predictor of 
AKI development was cystatin C concentration above $1,14 \mathrm{mg} / \mathrm{l}$ with a probability ratio of 17,811 .

Conclusion: Presurgical value of cystatin $\mathrm{C}$ is a good predictor of acute kidney injury after elective infrarenal aortic surgery.

\section{8}

\section{Free Hemoglobin Ratio as a Novel Predictive Biomarker of Acute} Kidney Injury after Cardiac Surgery: Secondary Analysis

\section{of a Randomized Controlled Trial}

J. Hu ; Z. Francesco ${ }^{2}$; AB. Edward ${ }^{3}$; L. Chong ${ }^{4}$; E. Rezoagli ${ }^{5}$; B. Lorenzo

${ }^{1}$ Department of critical care medicine, PLA general hospital, Beijing, China; ${ }^{2}$ Department of pathophysiology and transplantation, University of Milan, Via Della Commenda, Milan, Italy; ${ }^{3}$ Department of anesthesia, critical care and pain medicine, Mass General Hospital, Boston, United States of America; ${ }^{4}$ Department of anesthesiology and perioperative medicine, The Fourth Military Medical University Xijing Hospital, Xian Shi, China; ${ }^{5}$ School of medicine and surgery, University of Milano-Bicocca, Monza, Italy

Correspondence: J. Hu

Intensive Care Medicine Experimental 2020, 8(2): 000238

Introduction: Acute kidney injury (AKI) is a common complication among patients undergoing cardiopulmonary bypass (CPB) [1-3] leading to prolonged stay in the intensive care unit (ICU) and higher postoperative mortality [4]. Early identification paired with effective intervention has reduced the frequency and severity of postoperative AKI [2], indicating that early diagnosis is extremely warranted. Currently, AKI diagnosis is based on KDIGO criteria evaluating serum creatinine $(\mathrm{SCr})$ and urine output [5]. However, $\mathrm{SCr}$ has been challenged as it rises slowly after an initial insult (usually 48-72 h) and is influenced by numerous non-renal factors [6-8]. Urinary biomarkers have been extensively studied as promising strategy for early diagnosis. However, the following issues may limit urinary biomarkers from being widely used: (1) The detection needs specific testing facilities, which may not be available in all the hospitals; (2) The visible elevation can only be observed several hours after surgery [9], which still delayed the treatment to get maximum clinical benefit; (3) Most studies involved with urinary biomarkers [9-12] have been limited by the absence of statistical methods to account for overfitting due to small sample size [13] and non-linear correlation [14], leading to poor generalizability and flexibility; and (4) Urinary biomarkers prominently manifest tubular epithelium injury. New biomarkers for AKI induced by open-heart surgery are needed.

Hemolysis with subsequent free hemoglobin $(\mathrm{fHb})$ released by redblood cells is a significant contributor to AKI among patients undergoing CPB $[15,16]$. Levels of $\mathrm{fHb}$ peaked $15 \mathrm{~min}$ after weaning of CPB [17] and patients with AKI display significantly higher $\mathrm{fHb}$ levels compared to patients without AKI [16]. In addition, the difference between levels of $\mathrm{fHb}$ at the end of CPB and baseline $(\triangle \mathrm{fHb})$ was associated with AKI in pediatric patients [18]. Whether $\Delta \mathrm{fHb}$ or $\mathrm{fHb}$ ratio, defined as levels of $\mathrm{fHb}$ at the end of CPB divided by $\mathrm{fHb}$ at baseline, are independent risk factors of $A K I$ in adult patients requires further investigation. Moreover, whether $\Delta \mathrm{fHb}$ or $\mathrm{fHb}$ ratio could predict AKI remains unclear.

Therefore, we performed a secondary analysis on the control arm of a randomized controlled trial [3] to determine whether $\Delta \mathrm{fHb}$ or $\mathrm{fHb}$ ratio, an easily detected surrogate of hemolysis, could be identified as an early biomarker for postoperative AKI after open-heart surgery and whether incorporation of $\mathrm{fHb}$ ratio could improve predictive performance, compared with using urinary biomarkers alone.

Objectives: To determine whether free hemoglobin $(\mathrm{fHb})$ ratio could predict AKI immediately after open-heart surgery.

Methods: This is a secondary analysis of a randomized controlled trial comparing the effect of nitric oxide (intervention)versus nitrogen (control) on AKI after cardiac surgery (NCT01802619) [1]. 110 adult patients in the control arm were included. First, we determined whether $\mathrm{fHb}$ ratio (i.e., levels of $\mathrm{fHb}$ at the end of CPB divided by baseline $\mathrm{fHb}$ ) was associated with AKI via univariable and multivariable analyses.
Second, we verified whether $\mathrm{fHb}$ ratio could predict AKI and incorporation of $\mathrm{fHb}$ ratio could improve predictive performance of $\mathrm{AKI}$ at an early stage, compared with prediction using urinary biomarkers alone. We conducted restricted cubic spline in logistic regression for model development. We used bootstrap-based internal validation to account for overfitting. We also employed concordance (c) statistic, area under curve (AUC) test, resampling model calibration, and likelihood ratio test to compare the predictive performance (i.e., discrimination and calibration) between competing models.

Results: Data stratified by median $\mathrm{fHb}$ ratio showed that subjects with an $\mathrm{fHb}$ ratio $>2.23$ presented higher incidence of $\mathrm{AKI}(80.0 \%$ vs. $49.1 \%, p=0.001)$, more need of renal replacement therapy (10.9\% vs. $0 \%, p=0.036)$, and higher 28 -day mortality $(10.9 \%$ vs. $0 \%, p=0.036)$ than subjects with an $\mathrm{fHb}$ ratio $\leq 2.23$. $\mathrm{fHb}$ ratio was associated with AKI after adjustment for pre-established factors. Among the four biomarkers, $\mathrm{fHb}$ ratio outperformed other biomarkers with the highest AUC of 0.703 (0.600-0.806), best calibration, and minimaloptimism (bootstrap-adjusted AUC $0.704,95 \% \mathrm{Cl}$ 0.592-0.804). Incorporation of $\mathrm{fHb}$ ratio achieved better predictive performance in terms of better discrimination $(0.771$ versus $0.653, p=0.012)$ and calibration $(\mathrm{p}<$ 0.001 ) at an early stage, compared with prediction using urinary biomarkers alone.

Conclusion: $\mathrm{fHb}$ ratio at the end of CPB is a novel, widely applicable biomarker for AKI after open-heart surgery and incorporation of $\mathrm{fHb}$ ratio can achieve better predictive performance at an early stage, compared with prediction using urinary biomarkers alone.

\section{Reference(s) and grant ackowledgment(s)}

1. 1. Lei C, Berra L, Rezoagli E, Yu B, Dong H, Yu S, Hou L, Chen M, Chen W, Wang $\mathrm{H}$ et al: Nitric Oxide Decreases Acute Kidney Injury and Stage 3 Chronic Kidney Disease after Cardiac Surgery. Am J Respir Crit Care Med 2018, 198(10):1279-1287.

2. 2. Kidney Disease: Improving Global Outcomes (KDIGO) Acute Kidney Injury Work Group. KDIGO Clinical Practice Guideline for Acute Kidney Injury. Kidney international 2012, Suppl(2):1-138.

3. 3. Bosch JP: Renal reserve: a functional view of glomerular filtration rate. Seminars in nephrology 1995, 15(5):381-385.

4. 4. Han WK, Wagener G, Zhu Y, Wang S, Lee HT: Urinary biomarkers in the early detection of acute kidney injury after cardiac surgery. Clinical journal of the American Society of Nephrology: CJASN 2009, 4(5):873-882.

5. 5. Liu S, Che M, Xue S, Xie B, Zhu M, Lu R, Zhang W, Qian J, Yan Y: Urinary L-FABP and its combination with urinary NGAL in early diagnosis of acute kidney injury after cardiac surgery in adult patients. Biomarkers : biochemical indicators of exposure, response, and susceptibility to chemicals 2013, 18(1):95-101.

6. 6. Meersch M, Schmidt C, Van Aken H, Martens S, Rossaint J, Singbartl K, Gorlich D, Kellum JA, Zarbock A: Urinary TIMP-2 and IGFBP7 as early biomarkers of acute kidney injury and renal recovery following cardiac surgery. PloS one 2014, 9(3):e93460.

7. 7. Metzger J, Mullen W, Husi H, Stalmach A, Herget-Rosenthal S, Groesdonk HV, Mischak H, Klingele M: Acute kidney injury prediction in cardiac surgery patients by a urinary peptide pattern: a case-control validation study. Critical care (London, England) 2016, 20(1):157.

8. 8. Vermeulen Windsant IC, Hanssen SJ, Buurman WA, Jacobs MJ: Cardiovascular surgery and organ damage: time to reconsider the role of hemolysis. J Thorac Cardiovasc Surg 2011, 142(1):1-11.

9. 9. Rezoagli E, Ichinose F, Strelow S, Roy N, Shelton K, Matsumine R, Chen L, Bittner EA, Bloch DB, Zapol WM et al: Pulmonary and Systemic Vascular Resistances After Cardiopulmonary Bypass: Role of Hemolysis. J Cardiothorac Vasc Anesth 2017, 31 (2):505-515.

10. 10. Kim-Campbell N, Gretchen C, Callaway C, Felmet K, Kochanek PM, Maul T, Wearden P, Sharma M, Viegas M, Munoz R et al: Cell-Free Plasma Hemoglobin and Male Gender Are Risk Factors for Acute Kidney Injury in Low Risk Children Undergoing Cardiopulmonary Bypass. Critical care medicine 2017, 45(11):e1123-e1130.

11. National Natural Science Foundation of China (NSFC 81501642)

12. National Heart, Lung, and Blood Institute K23 HL128882-01 A1 
000331

\section{Comparison of AKI epidemiology using measured} versus calculated baseline creatinine in ICU patients D. Smagghe"; E. Hoste ${ }^{2}$

${ }^{1}$ AZ West, Veurne, Belgium; ${ }^{2}$ Icu, Ghent University Hospital, Gent, Belgium Correspondence: D. Smagghe

Intensive Care Medicine Experimental 2020, 8(2): 000331

Introduction: Acute kidney injury (AKI) occurs in over half of ICU patients and is associated with an increase in ICU and hospital mortality. The KDIGO consensus definition for AKI is based on an episode of oliguria or an increase of serum creatinine (Scr) above baseline Scr. In case no representative baseline $\mathrm{Scr}$ is available, an alternative based on the MDRD equation for estimated glomerular filtration rate (eGFR) may be used. In this study we evaluated the use of an MDRD estimated baseline Scr (MDRD-Scr) versus the use of a measured baseline Scr (BL$\mathrm{Scr}$ ) on the incidence of AKI in critically ill patients.

Methods: We analysed data collected during a four-year period in all consecutive admitted patients in the 5 different adult ICUs of the Ghent University Hospital. We excluded patients who did not have a $\mathrm{BL}-\mathrm{Scr}$ available. AKI was assessed using the KDIGO criteria for serum creatinine based on MDRD-Scr and BL-Scr during the first week of ICU admission. Precision was calculated as (BL-Scr - MDRD-Scr)/BL-Scr X $100 \%$, bias as BL-Scr - MDRD-Scr, and accuracy as the proportion of MDRD-Scr within 10\%, 20\%, 30\% or 50\% of BL-Scr (Acc10-20-30-50).

Results: Of 9,333 patients admitted, BL-Scr was available in 5,925 patients. Of these, $43.5 \%$ developed AKI based on Scr. AKI was correctly classified in $87.2 \%$ patients. No-AKI was correctly classified in $89 \%$ of no-AKI patients, and AKI stage 3 was correctly in $96.1 \%$ of AKI-3 patients. AKI-1 and AKI-2 was only correctly classified in 50.5 resp $54.7 \%$ (table 2 ). In $11.5 \%$ of patients MDRD-Scr led to classification of $\mathrm{AKI}$ in a higher stage, and in $7.5 \%$ to classification of $\mathrm{AKI}$ in a lower stage. MDRD-Scr had a sensitivity of $84.2 \%$ and specificity of $89.0 \%$ for diagnosis of AKI. Positive predictive value was $81.5 \%$, and negative predictive value $90.7 \%$. Precision was $27.5 \%$, bias -0.09 , and Acc1020-30-50 was 20.0\%, 38.9\%, 53.6\%, and 75.3\%. In Cardiac Surgery ICU patients precisionand bias were less and Acc was higher compared to Surgical ICU, Medical ICU and Burn Unit ICU patients.

Conclusion: The use of an MDRD estimated baseline Scr resulted in adequate classification of AKI in almost $90 \%$ of patients. There was an important bias and a limited accuracy when estimating baseline serum with the MDRD-Scr formula. For AKI stage 1 and 2 there was a high rate $(49.5 \%$ and $45.3 \%$ respectively) of over- or underclassification. In stage $3 \mathrm{AKI}$ the accuracy was $96.1 \%$ using MDRD-Scr. When available, a recorded baseline creatinine value should be used as a reference of baseline Scr.

\section{6}

\section{Accurate and Interpretable Prediction of ICU-acquired AKI}

E. Schwager ; E. Ghosh ${ }^{1}$; L. Eshelman ${ }^{1}$; K. Pasupathy²; E. Barreto ${ }^{3}$; K. Kashani ${ }^{4}$

${ }^{1}$ Connected care - personal health, Philips Research, Cambridge, United States of America; ${ }^{2}$ Healthcare and policy research, Mayo Clinic, Rochester, United States of America; ${ }^{3}$ Department of pharmacy, Mayo Clinic, Rochester, United States of America; ${ }^{4}$ Division of nephrology and hypertension, department of medicine, Mayo Clinic, Rochester, United States of America Correspondence: E. Schwager

Intensive Care Medicine Experimental 2020, 8(2): 000336

Introduction: Acute kidney injury ( $\mathrm{AKI})$ is a prevalent and detrimental condition in the intensive care unit (ICU) (Silver and Chertow 2017). Its impact can be prevented or mitigated by timely intervention. We hypothesized that boosting models could accurately predict the impending development of AKI and provide interpretable information to clinicians regarding AKI risk.

Methods: We used a large cohort of 98,051 ICU encounters admitted to Mayo Clinic ICUs between 2005 and 2017 to train abstainBoost (Schapire and Singer 1999) models to predict two classes of AKI patients: any-AKI or moderate/severe AKI (AKI stage $>1$ ). Models predicted any-AKI six hours in advance of its onset, and moderate/ severe AKI 12 hours in advance of its onset. We removed encounters with insufficient data prior to AKI onset, and divided the remaining cohort into 'clean' encounters (agreement between urine output [UO] and creatinine $[\mathrm{Cr}$ ] for maximum AKI stage) and 'noisy' encounters (disagreement between $\mathrm{UO}$ and $\mathrm{Cr}$ for maximum AKI stage). The clean encounters were further divided into training and testing cohorts. Encounters had a total of 388 predictive features measured during ICU stay, including laboratory results, vital signs, medications, and interventions. We compared the area under the ROCs (AUROCs) of this model with parsimonious models ( 30 features for any-AKI, 31 features for moderate/severe $\mathrm{AKI}$ ) selected by the Boruta method (Kursa and Rudnicki 2010; Homola, Daniel, n.d.). We evaluated the importance and contribution of the most predictive features.

Results: Features selected by Boruta yielded nearly the same performance as using the entire feature set. On the test set, the any-AKI model had an AUROC of 0.8301 vs. 0.8303 (all features vs. feature subset), while the moderate/severe model had an AUROC of 0.889 vs. 0.891 . On the noisy cohort, the models using the feature subset had AUROC of 0.74 (any-AKI) and 0.70 (moderate/severe AKI). Top features from the feature subset for predicting both any AKI and moderate/severe AKI included urine output and creatinine levels measured before AKI onset, as well as mechanical ventilation, lactate levels, and heart rate. The dosage of furosemide was predictive of any AKI, while $\mathrm{pH}$ and fluid balance were predictive of moderate/severe AKI.

Conclusion: This study demonstrated that parsimonious, interpretable models could accurately predict AKI in advance of its development or progression, allowing clinicians time to implement timely preventative measures. They provide a list of individualized risk factors for precision medicine.

\section{Reference(s) and grant ackowledgment(s)}

1. Silver, Samuel A., and Glenn M. Chertow. 2017. "The Economic Consequences of Acute Kidney Injury." Nephron 137 (4): 297-301. https://doi. org/10.1159/000475607.

2. Schapire, Robert E., and Yoram Singer. 1999. "Improved Boosting Algorithms Using Confidence-Rated Predictions." Machine Learning 37 (3): 297-336. https://doi.org/10.1023/A:1007614523901.

3. Kursa, Miron B., and Witold R. Rudnicki. 2010. "Feature Selection with the Boruta Package." Journal of Statistical Software 36 (1): 1-13. https://doi. org/10.18637/jss.v036.111.

4. Homola, Daniel. n.d. Boruta_py (version 0.3). Python. https://github.com/ scikit-learn-contrib/boruta_py/.

5. This project was supported in part by the National Institute of Allergy and Infectious Diseases of the National Institutes of Health under Award Number K23Al143882 (PI; EFB)

\section{4}

Discharge Documentation and Nephrology Follow-up of Critical Care Patients Requiring RRT

XY. Choon"; N. Lumlertgul'; 'L. Tovey²; A. Dixon²; A. Jones²; M. Ostermann² ${ }^{1}$ Nephrology, Guy's and St Thomas' NHS Foundation Trust, London, United Kingdom; ${ }^{2}$ Intensive care, Guy's and St Thomas' NHS Foundation Trust, London, United Kingdom

Correspondence: X.Y. Choon

Intensive Care Medicine Experimental 2020, 8(2): 000374

Introduction: An episode of acute kidney injury (AKI) with a requirement for renal replacement therapy (RRT) is associated with excess short- and long-term mortality, and chronic comorbidities, including chronic kidney disease (CKD). Follow-up after discharge from hospital is recommended. This requires detailed information transfer from Critical Care to the nephrology and primary care team.

Objectives: We set out to examine data from a single-centre population of critically ill patients who received RRT during a stay in Critical Care and were discharged from hospital alive. We sought to ascertain the following:

a) Comprehensiveness of discharge documentation;

b) Rates of renal follow-up;

and c) Survival outcomes at 1 year 
Methods: We performed a retrospective review of electronic medical notes and patient records of patients admitted to a 54-bedded critical care unit at a Tertiary Care Centre in the UK between June 2017 to May 2018 and who received RRT.

Exclusion criteria included: end-stage renal disease already receiving RRT; transfer from other catchment areas; and patients who died in hospital.

Results: A total of 433 patients received RRT, of whom 91 met the inclusion criteria.

Underlying co-morbidities included: hypertension (54\%), diabetes mellitus (25\%), pre-existing renal impairment (24\%) and coronary artery disease (21\%).

Review of discharge documentation from Critical Care showed: AKI was mentioned in $86 \%$ and need for RRT in $82 \%$ of discharge letters. Nephrology follow up was recommended in $47 \%$ of discharge letters. Only 22 of 91 patients (24\%) were followed-up by the renal team. 69 patients (76\%) received medical follow-up from other specialties. The overall survival rate was $82 \%$ at 1 year, and $70 \%$ at 2 years. In the group followed-up by nephrologists, survival rate at 1-year was $86 \%$, versus $81 \%$ in the group with no follow-up. There was no difference in survival in patients followed-up by other medical specialties, compared to those with no follow-up.

The average serum creatinine at point of hospital discharge was 160 $\mu \mathrm{mol} / \mathrm{L}$, and in survivors at 1 year it was $115 \mu \mathrm{mol} / \mathrm{L}$. 7 patients were dependent on RRT at point of discharge, with 3 recovering renal function sufficiently to be independent ofdialysis at 1 year. 2 out of these 7 patients were deceased at 1 year. 2 patients became newly dependent on RRT after 1 year.

Conclusion: Despite a large critical care capacity and a robust specialist renal service, our data points towards sub-optimal follow-up rates for this high risk patient cohort. Robust pathways at the point of discharge from Critical Care may help mitigate this and ensure consistent follow-up.

\section{Reference(s) and grant ackowledgment(s)}

1. Siew E et al. Outpatient nephrology referral rates after acute kidney injury. JASN 2012; 23(2):305-312.

2. Pannu, $\mathrm{N}$ et al. Renal Replacement Therapy in Patients With Acute Renal Failure: A Systematic Review. JAMA 2008; 299(7):793-805.

3. Bagshaw $S$ et al. Prognosis for long-term survival and renal recovery in critically ill patients with severe acute renal failure: a population-based study.

Crit Care 2005; 9(6):R700-R709

\section{7}

"Acute kidney injury in COVID-19 positive patients in the Mater Misericordiae University Hospital Critical Care"

R. Nolan'; B. Marsh'; R. Turner ${ }^{2}$

${ }^{1}$ Anaesthesia, The Mater Misericordiae University Hospital, Dublin, Ireland; ${ }^{2}$ Critical care, The Mater Misericordiae University Hospital, Dublin, Ireland Correspondence: R. Nolan

Intensive Care Medicine Experimental 2020, 8(2): 000827

Introduction: Renal injury has been reported to occur in up to $60 \%$ of high-risk COVID-19 positive patients admitted to intensive care [1]. Distinct mechanisms of renal injury in COVID-19, two of those being hypercytokinaemia and hypercoagulability, have been reported.

Objectives: To compare rates of AKI in COVID-19 positive patients in the Mater Misericordiae University Hospital Critical Care and record data on hypercoagulability and hypercytokinaemia.

Methods: Concurrent cohort study of COVID-19 positive patients admitted to the Mater Misericordiae University Hospital Critical Care between February and May 2020; exclusion criterion was ESRF preadmission. AKI was defined as per the AKIN criteria. Biochemical indicators of hypercytokinaemia (white cell count, neutrophils, leucocytes, ferritin) and hypercoagulability (d-dimer, platelets, fibrinogen) and data sets of individual patient's maximum vasopressor requirements, days pyrexial \& VTE prophylaxis were collected.
Results: 45 out of 47 COVID-19 positive patient were included (26 males, 19 females, median age 59 (range 37-77)). 8 patients had preexisting chronic renal disease. $64.4 \%$ of patients developed an AKI with patients with d-dimer $>5$ at higher risk $(R R=1.44)$. Thrombocytosis showed no correlation whereas thrombocytopaenia was weakly related $(R R=1.23$ ). Double dose VTE prophylaxis (enoxaparin $40 \mathrm{mg}$ BD) was not shown to be protective (RR 0.9). $97 \%$ of patients had hyperferritinaemia \& $78 \%$ of patients were pyrexic for at least 24 hours. Pyrexia was associated with AKI $(\mathrm{RR}=2.2)$, as was neutrophilia $(\mathrm{RR}=$ 2.24). Maximum dose vasopressor requirement was taken as an indicator of severity of cytokine storm-related hypotension. $79 \%$ of patients with a maximum dose of noradrenaline of $>5 \mathrm{mcg} / \mathrm{min}$ developed an AKI $(\mathrm{RR}=2.11)$.

Conclusion: Hypercytokinaemia was the most significant factor in development of AKI, with more vasoplegic patients likely to develop renal injury. Hypercoagulability was not closely related with AKI and higher dose VTE prophylaxis did not appear to impact the development of renal injury in our small cohort of patients.

\section{Reference(s) and grant ackowledgment(s)}

1. [1] Batlle, D. et al. (2020) "Acute Kidney Injury in COVID-19: Emerging

Evidence of a Distinct Pathophysiology", Journal of the American Society of Nephrology, p. ASN.2020040419. https://doi.org/10.1681/asn.2020040419.

\section{0}

Evaluation of urinary NGAL as a diagnostic tool for acute kidney injury in critically ill patients with infection

B. Cardoso Gomes ${ }^{1}$; F. Tuon'; JM. Silva ${ }^{3}$; M. Jacques ${ }^{4}$

1Departamento de Medicina Integrada, Universidade Federal do Paraná, Curitiba, Brazil; ${ }^{2}$ Infectologia, Complexo Hospital de Clínicas UFPR, Curitiba, Brazil; ${ }^{3}{ }^{2} \mathrm{Cu}$, Hospital Israelita Albert Einstein, São Paulo, Brazil; ${ }^{4}$ Psicologia, UniCesumar - Curitiba Campus, Curitiba, Brazil

Correspondence: B. Cardoso Gomes

Intensive Care Medicine Experimental 2020, 8(2): 000050

Introduction: Acute Kidney Injury (AKI) is a common complication in critical care patients. Several biomarkers, including urinary neutrophil gelatinase-associated lipocalin (UNGAL), have been used in the early detection of AKI.

Objectives: The aim of this study was to evaluate UNGAL for the diagnosis and prognosis of AKI in critical ill patients with infections.

Methods: This was a prospective observational study of critically ill patients with infections, sepsis, or septic shock in the intensive care unit. Clinical and laboratory data, including daily uNGAL levels, were assessed. The AKI stage using the KDIGO criteria was evaluated. Sensitivity, specificity, and the area under the curve-receiver operating characteristic (AUC-ROC) values were calculated to determine the optimal UNGAL level for predicting AKI. Ethics Approval: The study was approved by the Local Ethics Committee (\#58317216.2.0000.0096).

Results: We had 38 patients who completed the study during the screening period (November 2016 to May 2018). The incidence of AKI was $76.3 \%$. The hospitalization period was longer in the group that developed AKI, with 21 days of median, [IQR 13.5-25]; non-AKI group had a median of 13 days, [IQR 7-18], $(P=0.019)$. We found a direct relationship between UNGAL levels and the progression to AKI. Increased values of the biomarker were associated with the worsening of AKI $(P<0.05)$. The cut-off levels of uNGAL that identified patients who would progress to AKI were the following: (d1) $>116 \mathrm{ng} / \mathrm{mL}$; (d2) $>100 \mathrm{ng} / \mathrm{mL}$, and (d3) $284 \mathrm{ng} / \mathrm{mL}$. The value of the fourth and last measurement was not predictive of patients who would progress to AKI. The median urinary UNGAL were also associated with mortality on days ( 1,3 and 4$): \mathrm{d} 1, \mathrm{p}=0.039 ; \mathrm{d} 3, \mathrm{p}=0.005 ; \mathrm{d} 4, \mathrm{P}=0.005)$. The performance of UNGAL in detecting AKI patients (AUC-ROC $=0.881$ ) There were no risk factors other than AKI that could be correlated with increased UNGAL levels on day 1 . 
TABLE 1. Increased UNGAL values in the progression of the KDIGO score $(1,2,3)$ and without AKI.

\begin{tabular}{|c|c|c|c|c|c|c|c|c|}
\hline & & & NGAL & & NGAL & & NC & \\
\hline $\mathrm{N}$ & Median & $\begin{array}{l}\text { IQR [25 } \\
\mathrm{n}_{75 \%} \text { ] }\end{array}$ & Mediar & $\begin{array}{l}\mathrm{IQR}[2 \\
\mathrm{n} 75 \%]\end{array}$ & Median & $\begin{array}{l}\text { IQR [ } \\
\mathrm{n}_{75 \%}\end{array}$ & $\mathrm{Me}$ & IQR \\
\hline $\begin{array}{l}\text { o } \\
\text { KI }\end{array}$ & 78.2 & & 55.7 & & 45.3 & & 66.2 & \\
\hline $\begin{array}{l}\text { KI } \\
\text { tage } 1\end{array}$ & 213 & & 259.4 & 8 & 158.2 & & 63.3 & \\
\hline KI & 317.1 & $.0-$ & 318.8 & $\begin{array}{l}{[117.7-} \\
895.6]\end{array}$ & 513.2 & 133.8- & 560.1 & \\
\hline $\begin{array}{l}\text { KI } \\
\text { age } 3\end{array}$ & & $\begin{array}{l}{[504.4-} \\
1700.0]\end{array}$ & 1026 & $\begin{array}{l}{[368.9-} \\
2090.3]\end{array}$ & 961.6 & $\begin{array}{l}{[374.1-} \\
2377.5]\end{array}$ & 794.6 & $\begin{array}{l}{[403.9-} \\
4666.4]\end{array}$ \\
\hline
\end{tabular}

Conclusion: The uNGAL had an association in its values with the diagnosis and prognosis of patients with severe infections and AKI. We suggest that studies with a greater number of patients could better establish the cut-off values of UNGAL and/or serum NGAL in the identification of infected patients who are at a high risk of developing AKI.

\section{Reference(s) and grant ackowledgment(s)}

1. Hjortrup PB, Haase N, Wetterslev M, Perner A. Clinical review: Predictive value of neutrophil gelatinase-associated lipocalin for acute kidney injury in intensive care patients. Critical Care. 2013;17(2):211

2. This study was facilitated with great support from the Biochemistry Division of Hospital de Clínicas da Universidade Federal do Paraná, primarily by the Dr. Flávia Kazumi Shibata and Michelle Tomassini Jacques who revised the language.

\section{3}

Incidence of acute kidney injury in Covid-19 infected patients compared with non-Covid-19 patients admitted with respiratory

\section{failure}

A. Bhide'; HD. Aya ${ }^{1}$;JM. Low²; JK. Rane²; D. Padman³; M. Hamilton;

A. Rhodes ${ }^{4}$; S. Leaver ${ }^{5}$

${ }^{1}$ General Intensive Care Unit, St George's Hospital, London, United King dom; ${ }^{2}$ Intensive care unit, St George's Hospital, London, United Kingdom; ${ }^{3}$ Anaesthesia and Intensive Care, St George's Hospital, London, United Kingdom; ${ }^{4}$ Anaesthesia and intensive care medicine, St George's University Hospitals NHS Foundation Trust, Londres, United Kingdom; ${ }^{5}$ Research lead critical care directorate, St George's Hospital, London, United Kingdom

\section{Correspondence: A. Bhide}

Intensive Care Medicine Experimental 2020, 8(2): 001023

Introduction: A novel coronavirus (severe acute respiratory syndrome coronavirus 2, SARS-CoV-2) emerged in early December 2019 and has subsequently spread globally causing a pandemic. There remains clinical equipoise on whether the incidence of acute kidney injury (AKI) is higher in those patients with acute respiratory failure caused by SARSCoV-2 (Covid-19) than those without Covid-19 [1, 2, 3].

Objectives: To assess whether the incidence of AKI among patients admitted with acute respiratory failure caused by Covid-19 infection is higher than those with acute respiratory failure without Covid-19 infection. To describe the differences in AKI stages observed between the two groups.

Methods: This was a single centre, observational cohort study. Patients admitted with acute respiratory failure to the ICU at St George's Hospital were recruited to the study between December 2019 and May 2020. Covid-19 infection was detected by viral PCR of SARS-CoV-2 on nasopharyngeal or sputum samples. The presence and stage of AKI was defined as per AKIN criteria [4] and assessed on day 0,7 and 14 of ICU admission. Univariate analysis was conducted using the Chi squared test to compare the frequency of AKI between the two groups.

Results: 256 patients were included. 132 (51.6\%) were Covid-19 patients, whereas 124 (48.6\%) were non-Covid-19 ICU patients. Overall, $226(88.3 \%)$ patients had AKI over the study period; $112(84.8 \%)$ within the Covid-19 group and 114 (91.9\%) within the non-Covid-19 group (c2 (1) 3.1, p = 0.058).

On ICU admission, the distribution of AKI stages between Covid-19 and non-Covid-19 patients was similar (c2 (3) 2.0, $\mathrm{p}=0.58$ ).
At day 7 of admission, 127 patients were still in ICU - 88 in the Covid19 group and 39 in the non-Covid-19 group. There were $45(51.1 \%)$ in stage 0, $8(9.1 \%)$ in stage 1, $6(6.8 \%)$ in stage 2 and $29(33 \%)$ in stage 3 within the the Covid-19 group, compared to $32(82.1 \%)$ in stage 0 , $3(7.7 \%)$ in stage 1, $2(5.1 \%)$ in stage 2 and $2(5.1 \%)$ in stage 3 within the non-Covid-19 group (c2 (3) 13.0, $p=0.004$ ). At day 14, 71 patients remained in ICU, and the distribution of stages was similar between both groups (c2 (3) 4.9, p = 0.16).

Conclusion: The incidence and severity of AKI in Covid-19 infected patients are higher after a week of ICU admission compared with nonCovid-19 patients admitted with respiratory failure.

\section{Reference(s) and grant ackowledgment(s)}

1. 4. Mehta RL et al. Acute Kidney Injury Network: report of an initiative to improve outcomes in acute kidney injury. Crit Care. 2007;11:R31.

2. 3. Pei $\mathrm{G}$ et al. Renal involvement and early prognosis in patients with COVID19 pneumonia. J Am Soc Nephrol. 2020.

3. 2. Richardson $\mathrm{S}$ et al. Presenting characteristics, comorbidities, and outcomes among 5700 patients hospitalized with COVID-19 in the New York City area. JAMA. 2020

4. 1. Wang L et al. Coronavirus Disease 19 Infection Does Not Result in Acute Kidney Injury: An Analysis of 116 Hospitalized Patients from Wuhan, China. Am J Nephrol. 2020;51(5):343-348

\section{6}

A prospective study on blood citrate levels and clinical correlations in patients receiving regional citrate anticoagulation

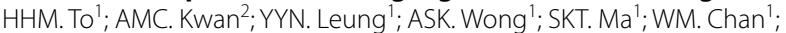
I. Yam ${ }^{3}$; P. Lee Le $^{3}$ : DYH. Yap ${ }^{3}$

${ }^{1}$ Adult intensive care unit, Queen Mary Hospital, Hong Kong, Hong Kong; ${ }^{2}$ Department of anaesthesia and intensive care, Tuen Mun Hospital, Hong Kong, Hong Kong; ${ }^{3}$ Division of nephrology, The University of Hong Kong, Hong Kong, Hong Kong

Correspondence: $\mathrm{H}$. TO

Intensive Care Medicine Experimental 2020, 8(2): 000036

Introduction: Regional citrate anticoagulation (RCA) is commonly used for continuous renal replacement therapy (CRRT). Citrate accumulation (CA) is a serious potential complication of RCA, and conventional ways to diagnose $C A$ such as increased total to ionized calcium ratio ( $\mathrm{T}$ : I Ca ratio) are confounded by various clinical conditions and thus remains suboptimal $[1,2]$. Blood citrate measurement emerges as a more direct way to determine CA but its clinical utility and optimal cut-off values for predicting CA remains undefined.

Objectives: To evaluate serum citrate kinetics of patients undergoing RCA CRRT and its correlations with clinical events and outcomes.

Methods: We prospectively recruited patients who received RCA CRRT and measured serial serum citrate levels at baseline, 2-, 6-, 12-, 24-, 36-, 48- and 72-hrs after initiation. The citrate levels were correlated with clinical parameters and patient outcomes.

Results: 91 patients were included, of which 15 patients developed CA defined as a combination of elevated $\mathrm{T}$ :I calcium ratio $>2.5$, high anion gap metabolic acidosis and systemic hypocalcaemia $<1 \mathrm{mmol} / \mathrm{L}$ or escalating calcium replacement requirement. The CA group showed higher APACHE IV score (136.5(99.5-167.0)vs 100.0(85.0$117.8), p=0.003)$. Serum citrate levels in the CA group at 2-, 6- and 12-hrs after initiation of RCA CRRT was significantly higher than the non-CA group ( $p<0.05$, for all) (Figure 1$)$. The peak citrate level was significantly higher in the CA group $0.78 \mathrm{mmol} / \mathrm{L}(0.39-0.96 \mathrm{mmol} / \mathrm{L})$ vs $0.38 \mathrm{mmol} / \mathrm{L}(0.23-0.67 \mathrm{mmol} / \mathrm{L})$ in the non-CA group, $\mathrm{p}=0.01)$. The serum citrate level correlated with the T:I Ca ratio at 6, 12, 24 and 36 hrs $(r=0.52,0.65,0.53$ and 0.59 respectively, $p$ all $<0.05)$. Using a $2-h r$ serum citrate cut-off of $0.33 \mathrm{mmol} / \mathrm{L}$, the sensitivity and specificity for predicting development of CA were 0.90 and 0.75 respectively (ROC AUC 0.83, $p=0.001$ ) (Figure 2). Peak serum lactate level was higher in the CA group [10.4mmol/L (7.1 - $17.7 \mathrm{mmol} / \mathrm{L})$ vs. $2.70 \mathrm{mmol} / \mathrm{L}(1.78-$ $5.25 \mathrm{mmol} / \mathrm{L})$, for CA vs. non-CA respectively, $\mathrm{p}<0.001$ ) and a cut-off value of $6.15 \mathrm{mmol} / \mathrm{L}$ also showed good predictive value for development of CA (sensitivity/specificity: 0.7/0.61, ROC AUC 0.82, p < 0.001). 


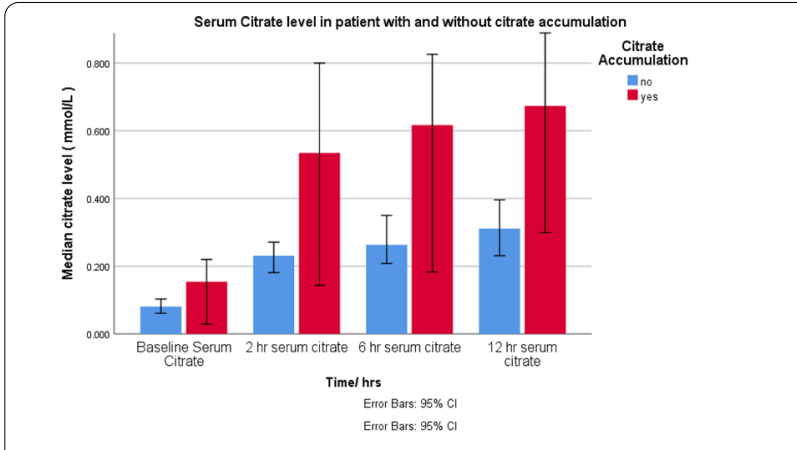

Figure 1. Serum citrate levels over time in patients who have or have not developed citrate accumulation (CA) during regional citrate anticoagulation

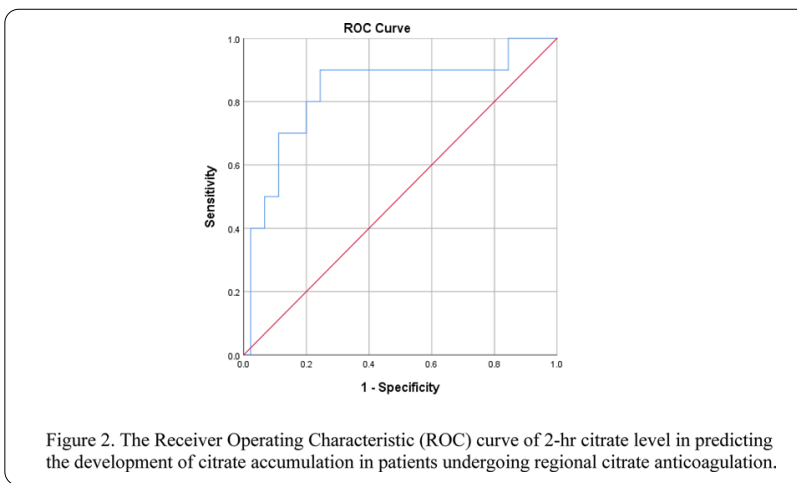

Conclusion: Patients who develop CA show higher serum citrate levels over time, and the 2 -hr citrate level shows good predictive value for subsequent development of CA in patients receiving RCA CRRT.

\section{Reference(s) and grant ackowledgment(s)}

1. 1. Hetzel, G.R., et al., Citrate plasma levels in patients under regional anticoagulation in continuous venovenous hemofiltration. Am J Kidney Dis, 2006. 48(5): p. 806-11. 2. Khadzhynov, D., et al., Incidence and outcome of metabolic disarrangements consistent with citrate accumulation in critically ill patients undergoing continuous venovenous hemodialysis with regional citrate anticoagulation. J Crit Care, 2014. 29(2): p. 265-71.

2. This study was supported by the Young Investigator Research Grant 2018 from Hong Kong College ofPhysicians.

\section{4}

Continuous Veno-Venous Haemodiafiltration - Are we getting it right?

VN. Gotz

${ }^{1}$ Critical Care Unit, University Hospitals of Morecambe Bay NHS Foundation Trust, Lancaster, United Kingdom

Correspondence: V.N. Gotz

Intensive Care Medicine Experimental 2020, 8(2): 000184

Introduction: Acute Kidney Injury presents one of the most common organ failures on the intensive care unit. We consider renal replacement therapy in all patients with Acute Kidney Injury level 3 and issues of potassium homeostasis, fluid balance or metabolic acidosis. At University Hospitals of Morecambe Bay NHS Foundation Trust (UHMBT) we use continuous veno-venous haemodiafiltration (CVVHDF) as modality with citrate anticoagulation (PRISMAFLEX dialysis machines) and aim for an effluent volume of $20-25 \mathrm{ml} / \mathrm{kg} / \mathrm{h}$ as per KDIGO Acute Kidney Injury Guidelines (2012); but, once we have decided on need for filtration and all is set up and ready to go, we often reflect on achieving prescribed target dose.

Objectives: To audit our perfomrance of achieving the target dose of CVVHDF

Methods: We prospectively audited all patients needing CVVHDF between July 2019 to October 2019 at our critical care unit at Royal Lancaster Infirmary, UHMBT, England, UK. We recorded demographics and indications, total length of, downtime of and reasons for discontinuing CVVHDF as much as effluent flow to be achieved and delivered, type of anticoagulation used, hourly fluid removal and type of vascular access.

Results: We sampled 23 data sets, 13 male and 10 female. The average age of patients was 62 years with an actual average body weight of 86 $\mathrm{kg}$. Length of stay on our critical care unit varied from 2 to 19 days with an average of 8.56 days. Indications for RRT included: 18 for acidosis, 9 for hyperkalaemia, 4 for anuria/fluid balance and 2 for uraemia. Average total length of CVVHDF was 100.39 hours (4.18 days) with actual CVVHDF delivered for an average of 72.78 hours (3.03 days). This means there was on average a $28 \%$ downtime of CVVHDF during dependancy. Reasons for stopping CVVHDF during dependancy included: access problems, recirculating e.g. for radiological examinations, blood flushed back, filter clotted and air in circuit. Effluent flow was prescribed as per actual body weight $(\mathrm{kg})$ according to a prepopulated prescription chart suplied by PRISMAFLEX and was $37.95 \mathrm{ml} / \mathrm{kg} / \mathrm{h}$ on average. The prepoluated chart accounted for a 15\% down time. 21 patients were anticoagulated using citrate and 2 with heparin. Fluid removal varied from $0-100 \mathrm{ml} / \mathrm{h}$ as per clinical need and was decided by consultants. 19 patients had internal jugular vein and 4 had femoral vein vascular access for CVVHDF, none had subclavian venous access due to risk of pneumothorax.

Conclusion: We prescribed effluent flow rates for all patients. However, we experienced a $28 \%$ downtime of CVVHDF during dependancy. The prepopulated prescription chart by PRISMAFLEX only accounted for $15 \%$ of downtime. The likelyhood of not achieving target effluent rates of at least $20-25 \mathrm{ml} / \mathrm{kg} / \mathrm{h}$ would have been high. We are, therefore, now recording our delivered effluent rate hourly on the critical care bedside chart and are calculating the average delivered rate per day, so adjustments to effluent rates can be made in order to achieve the recommended minimum of $20-25 \mathrm{ml} / \mathrm{kg} / \mathrm{h}$.

\section{Reference(s) and grant ackowledgment(s)}

1. Kidney Disease: Improving Global Outcomes (KDIGO), Acute Kidney Injury guidelines, 2012

\section{7}

The effects of regional citrate anticoagulation compared to non-citrate base techniques on the systemic ionised calcium levels accounting for the competing risks of treatment failures

M. Richardson ; J. Fotheringham² ; K. Bauchmuller'; A. Raithatha ${ }^{1}$

${ }^{1}$ Intensive care medicine, Sheffield Teaching Hospitals NHS Foundation Trust, Sheffield, United Kingdom; ${ }^{2}$ Nephrology, Sheffield Teaching Hospitals NHS Foundation Trust, Sheffield, United Kingdom

Correspondence: M. Richardson

Intensive Care Medicine Experimental 2020, 8(2): 000377

Introduction: Regional citrate anticoagulation in continuous renal replacement therapy (CRRT) offers longer filter life spans, lower bleeding complications, fewer blood transfusions and is recommended as the first line for anticoagulation despite associations with a range of metabolic complications including hypocalcaemia.1-6 Mild hypocalcaemia is common and appears protective in critically ill patients yet severe hypocalcaemia during CRRT is associated with increased mortality.7,8 How hypocalcaemia on CRRT competes with other endpoints according to citrate and non-citrate anticoagulation is unclear. Methods: Patients commencing CRRT in a single centre between Mar 2016 and Feb 2018 spanned a transition from non-citrate to citrate based anticoagulation in May 2017. Patient characteristics, biochemical data and endpoints of hypocalcaemia, filter/access dysfunction, death, elective discontinuation and recovery were analysed. 
Hypocalcaemia was defined as mild, moderate or severe by a systemic ionised calcium (iCa) of $<1.13,<1.0$ and $<0.9 \mathrm{mmol} / \mathrm{l}$. A competing risk analysis visualised time to events for the above endpoints and iCa $<1.0$ to reflect censoring events which terminate CRRT, with log-rank tests to statistically compare the groups.

Results: 220 patients were identified during the period. 113 patients were pre citrate; of the 107 after the transition 3 were excluded for not receiving citrate based therapy. Patient characteristics by anticoagulation regime were similar. The main precipitants for renal dysfunction were septic shock $43.3 \%$, cardiogenic shock $11.1 \%$ and hypovolaemia 9.2\% $(P=0.945)$. Non-Citrate patients received a mean cumulative total of $88.2 \mathrm{~h}$ CRRT vs $113.7 \mathrm{~h}$ for citrate $(\mathrm{P}=0.128) .28$ day mortality was not significantly different $(41.6 \%$ vs $37.5 \%, P=0.538)$.

Pre-treatment mean iCa was 1.12 in the non-citrate and 1.15 in the citrate group $(P=0.111)$. The proportion of patients experiencing an episode of hypocalcaemia $(<1.13,<1.0$ and $<0.9)$ was $67.3 \%, 12.4 \%$, and $2.7 \%$ for non-citrate and $93.3 \%, 26.0 \%$, and $5.8 \%$ for citrate $(P<$ 0.02 for $<1.13 \&<1.0$ ). The proportion of non-citrate and citrate patients stopping CRRT on first exposure for filter/access dysfunction was $19.5 \%$ vs $8.7 \%$, death $11.5 \%$ vs $22.1 \%$, recovery $15.9 \%$ vs $13.5 \%$ and elective discontinuation $53.1 \%$ vs $55.8 \%$ respectively (overall $\mathrm{P}=$ 0.038). At $24 \mathrm{~h} 18.6 \%$ of the non-citrate group had stopped CRRT due to filter/access dysfunction, compared to $5.7 \%$ in the citrate group ( $p$ $=0.009)$. Hypocalcaemia $(<1.0)$ had occurred in $5.3 \%$ vs $18.3 \%$ for the non-citrate vs citrate group $(p=0.02)$.

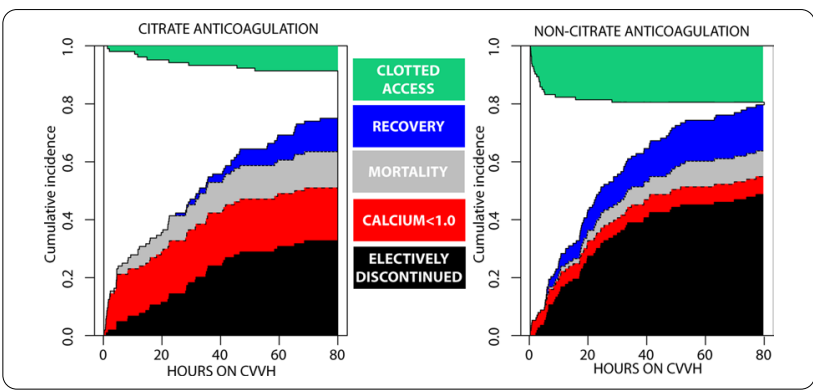

Conclusion: Although mild and moderate hypocalcaemia was more common in the citrate group no treatments were ceased or adverse events reported due to hypocalcaemia. The competing risk of filter/ access dysfunction was significantly higher in the non-citrate group. Differences in other endpoints during the first treatment reflected longer uninterrupted CRRT delivery in the citrate group. Existing recommendations on citrate-based anticoagulation appear justified.

\section{Reference(s) and grant ackowledgment(s)}

1. 8. Afshinnia F, Belanger K, Palevsky PM, Young EW. Effect of ionized serum calcium on outcomes in acute kidney injury needing renal replacement therapy: secondary analysis of the acute renal failure trial network study, Renal Failure, 2013; 35:10, 1310-1318

2. 7. Zhang Z., Xu X., Ni H., Deng H. Predictive Value of lonized Calcium in Critically III Patients: An Analysis of a Large Clinical Database MIMIC II. PLoS ONE. 2014;9:e95204

3. 6. Monchi M, Berghmans D, Ledoux D, et al. Citrate versus heparin for anticoagulation in continuous venovenous haemofiltration: a prospective randomized study. Intensive Care Med. 2004; 30:260-265

4. 5. Kutsogiannis DJ, Gibney RT, Stollery D, et al. Regional citrate versus systemic heparin anticoagulation for continuous renal replacement in criticallyill patients. Kidney Int. 2005; 67:2361-2367

5. 4. Monchi M, Berghmans D, Ledoux D, Canivet JL, Dubois B, Damas P. Citrate vs. heparin for anticoagulation in continuous venovenous hemofiltration: a prospective randomized study. Intensive Care Med. 2004 Feb; 30(2):260-265

6. 3. Morgera S, Scholle C, Voss G, Haase M, Vargas-Hein O, Krausch D et al. Metabolic complications during regional citrate anticoagulation in continuous venovenous hemodialysis: single-center experience. Nephron ClinPract. 2004; 97(4):C131-6
7. 2. Davenport A, Tolwani A. Citrate anticoagulation for continuous renal replacement therapy (CRRT) in patients with acute kidney injury admitted to the intensive care unit. NDT Plus. 2009;2: 439-447

8. 1. KDIGO Clinical Practice Guideline for Acute Kidney Injury OFFICIAL JOURNAL OF THE INTERNATIONAL SOCIETY OF NEPHROLOGY VOLUME 2 | ISSUE 1 | MARCH 2012 Section 5: Dialysis Interventions for Treatment of AKI 000500

Introduction:of Sustained Low-Efficiency Dialysis (SLED) to augment ICU RRT surge capacity during the COVID-19 crisis K. Allan ${ }^{1}$; K. Kohler ${ }^{2}$; A. Conway-Morris ${ }^{3}$; J. Varley ${ }^{1}$; C. Summers 3 ; A. Johnston ${ }^{1}$; D. Sapsford ${ }^{4}$; R. Mahroof ${ }^{1}$

${ }^{1}$ Department of anaesthetics \& intensive care medicine, Cambridge University Hospitals NHS Foundation Trust, Cambridge, United Kingdom; ${ }^{2}$ University division of anaesthesia, University of Cambridge, Cambridge, United Kingdom; ${ }^{3}$ Department of medicine \& intensive care, University of Cambridge, Cambridge, United Kingdom; ${ }^{4}$ Department of intensive care medicine, Cambridge University Hospitals NHS Foundation Trust, Cambridge, United Kingdom

Correspondence: K. Allan

Intensive Care Medicine Experimental 2020, 8(2): 000500

Introduction: Acute kidney injury (AKI) in critically ill COVID-19 patients is common and is associated with higher mortality1. The pandemic has placed ICUs under an unprecedented burden, and provision of RRT has been challenging.

Over 7 weeks we admitted 75 COVID-19 positive patients to our combined 59 bedded ICUs. Of those, $61 \%$ developed AKI and $40 \%$ required continuous renal replacement therapy (CRRT). In April, NHS England highlighted the difficulties with supply lines for RRT machines and disposables. Our hospital had 14 CRRT machines for the ICUs.

Objectives: An alternative strategy for RRT was needed, preferably effective and relatively unshackled by supply line issues of routine CRRT, usable with current infrastructure and affordable. The alternative needed to cover this crisis and provide increased future RRT capacity. Intermittent haemodialysis (IHD) and SLED were the 2 main options. SLED is modified IHD, where therapy occurs daily and has shown to be safe and efficacious2.

Methods: Surge capacity meetings between intensivists, nephrologists, pharmacists and nurse managers explored alternative RRT options for their relative risk/benefit, cost/benefit strategy and feasibility assessment for its physical provision and training, with potential problems identified via web-conferencing3. Plumbing and architecture were reviewed and assessed for capability to provide reverse osmosis (RO) water interface to ICU.

A strategy to provide clinical care alongside ongoing maintenance work was devised. With manufacturer's support, a focused training programme and protocols were developed. A business plan was submitted to hospital authorities for funding approval. The current demand for RRT and available supply of disposables was assessed. An appropriate manufacturer was identified by seeking multiple quotes.

Results: SLED was chosen as it combines the benefits of IHD (less time on RRT) with those of CRRT (haemodynamic stability) 4 .

Time to proceed to setup of a SLED service was 3 days followed by 8 days to commencement of works. These were completed in two of the ICUs within 2 days, during which the areas were isolated from clinical care for RO/water softener works. In total 5 RO machines and 10 SLED machines (SC + Quanta, QD Technologies) were set up in 4 separate ICU areas, thereby increasing our number of RRT machines by $70 \%$. The machines provide standard bicarbonate-based dialysis at highflow $(500 \mathrm{~mL} / \mathrm{min})$ and low-flow $(300 \mathrm{~mL} / \mathrm{min})$ rates, supporting treatment times up to $8 \mathrm{hrs}$. The machine cost for acquisition was $£ 25,000$ including set-up. Quanta staff trained 18 nurses in 10 days including practice development nurses who will become the future trainers. ICU developed protocols for the use of SLED and prescriptions were designed into our eMR platform (Epic, WI, USA). 


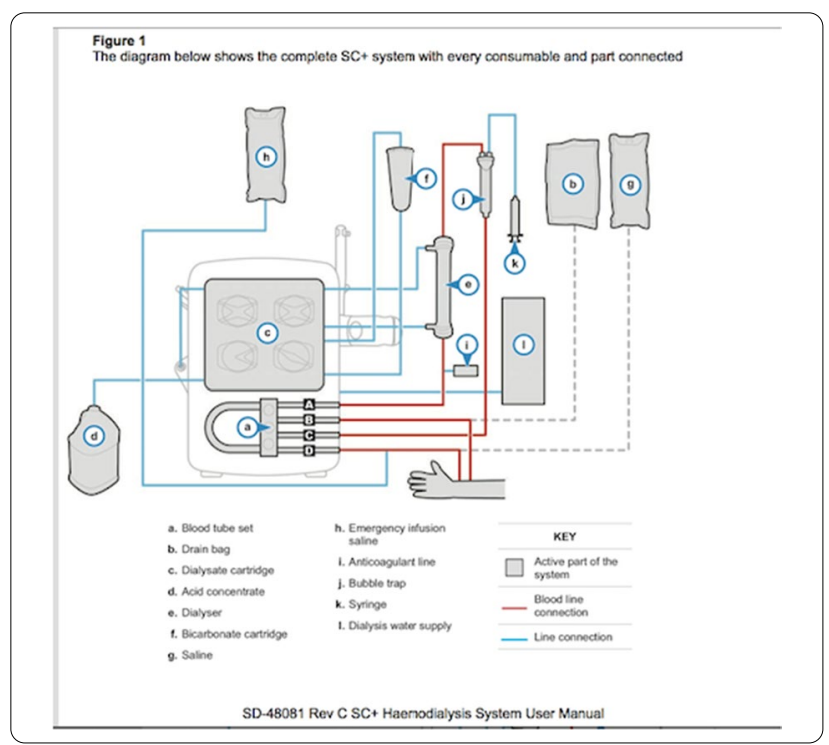

Conclusion: Surveillance of local and national demands on RRT alerted us to the need for surge provision of RRT. Rapid procurement and phased installation allowed introduction of a new RRT modality during a pandemic. As well as sparing stocks of RRT consumables in the short term, the longer-term improvement in ICU resilience will help manage potential future waves of COVID-19 and threats from other emerging infections. We are now disseminating this learning across the local, national and international ICU community.

\section{Reference(s) and grant ackowledgment(s)}

1. 1. "Renal Involvement and Early Prognosis in Patients with COVID-19 Pneumonia" Pei G et.al.; J Am Soc Nephrol 2020

2. 4."Comparison of sustained hemodiafiltration with continuous venovenous hemodiafiltration for the treatment of critically ill patients with acute kidney injury", Abe et. al.; Artificial Organs 2010; 34(4): 331-338

3. 2. "Systematic review and meta-analysis of renal replacement therapy modalities for acute kidney injury in the intensive care unit" Nash D et. al.; Journal of Critical Care 2017,41:138- 144

4. 3."Renal replacement therapy in the ICU: intermittent hemodialysis, sustained low-efficiency dialysis or continuous renal replacement therapy? Wang AY, Bellomo R; Curr Opin Crit Care 2018, 24:437-442

\section{2}

\section{Regional citrate anticoagulation in a continuous renal} replacement therapy protocol with dialysis offers superior filter life span to one using filtration

E. Taylor ' ; Z. Al-Faham²; S. lucena-amaro'; L. Jones' ; A. Chang 2 ;

J. Martinlazaro'2; S. Ilott ${ }^{3}$; J. Prowle'; CJ. Kirwan'1

${ }^{1}$ Intensive care, The Royal London Hospital, London, United Kingdom; ${ }^{2}$ Intensive care, Newham University Hospital, London, United Kingdom; ${ }^{3}$ Intensive care, Whipps Cross University Hospital, London, United Kingdom

Correspondence: E. Taylor

Intensive Care Medicine Experimental 2020, 8(2): 000882

Introduction: Regional citrate anticoagulation (RCA) is the gold standard anticoagulation for continuous renal replacement therapy (CRRT) as it reduces both clotting of the circuit and systemic bleeding events. There is no clear evidence that mode of therapy improves mortality, but dialysis may be superior to filtration in improving filter life span as it removes the filtration ratio and reduces the reliance on faultless venous access and high blood flows. We present data comparing protocols for post dilution continuous veno-venous haemofiltration $(\mathrm{CVVH})$ and continuous veno-venous haemodialysis (CVVHD) with or without RCA available for CRRT in critically ill patients.
Methods: 3 hospitals in the same NHS Trust (providing CRRT to general critical care patients (no cardiac surgery) for $\sim 350$ patients a year) followed the same CRRT protocols but the larger site offers a protocol with RCA as first line anticoagulation. In June 2019 all sitesswitched from the Nikkiso Aquarius ${ }^{\circledR}$ system (CVVH and RCA via post dilution method) to the BBraun OMN ${ }^{\circledR}$ system (CVVHD). Recent audit (September - November 2019) coupled with historical data (SeptemberNovember 2017) collected in the same way on each site, was used to compare filter life span across protocols. Heparin infusion into the filter or no anticoagulation was used when RCA was either not available or contraindicated. Only one site had data for CVVH with no RCA.

Results: In total 173 patients who used 524 filters were analysed (table 1).

When censored for reasons other than clotting, there was a significant advantage in filter survival of CVVHD over CVVH when RCA was available $(p<0.0001)$ (graph 1). There was no difference between protocols that used CVVH and CVVHD when RCA was not available $(p=0.64)$ but CVVH with RCA available, was significantly better than CVVHD when RCA is not available $(p=0.02)$.

\begin{tabular}{|c|c|c|c|c|}
\hline TABLE 1 & $\begin{array}{l}\text { CVVH } \\
\text { RCA }\end{array}$ & $\begin{array}{l}\text { CVVH no } \\
\text { RCA }\end{array}$ & $\begin{array}{l}\text { CVVHD } \\
\text { RCA }\end{array}$ & $\begin{array}{l}\text { CVVHD no } \\
\text { RCA }\end{array}$ \\
\hline \multirow{2}{*}{$\begin{array}{l}\text { Consecutive patients } \\
\text { Age }\end{array}$} & 41 & 24 & 50 & 58 \\
\hline & $56.5(27-75)$ & $61(27-85)$ & $64(86-23)$ & $63(29-81)$ \\
\hline \multicolumn{5}{|l|}{ Admission reason } \\
\hline \multirow{2}{*}{$\begin{array}{l}\text { Medical } \\
\text { Surgical }\end{array}$} & $29(70 \%)$ & $15(63 \%)$ & $38(76 \%)$ & $47(81 \%)$ \\
\hline & $11(30 \%)$ & $9(37 \%)$ & $12(24 \%)$ & $11(19 \%)$ \\
\hline \multirow[t]{4}{*}{ Filters } & 141 & 63 & 109 & 211 \\
\hline & $96(68 \%)$ & 0 & $69(63 \%)$ & 0 \\
\hline & $27(19 \%)$ & $27(43 \%)$ & $16(15 \%)$ & $124(59 \%)$ \\
\hline & $18(13 \%)$ & $36(57 \%)$ & $24(22 \%)$ & $87(41 \%)$ \\
\hline Filter life span (hrs) & 27 & 20 & 58 & 20 \\
\hline
\end{tabular}

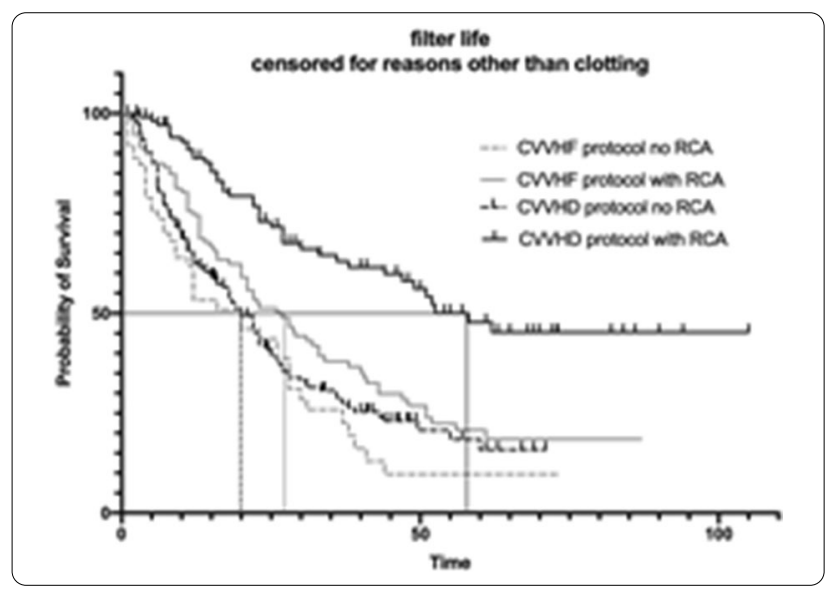

Significantly fewer filters per patient are used with a CVVHD protocol containing RCA compared to the other protocols $(2.2(1-10) ; \mathrm{p}=0.028)$ Conclusion: CRRT Protocols that contain RCA as first line anticoagulation significantly improve filter life span. There is a significant additional advantage in using dialysis alone over filtration. 


\section{5}

First use of NxStage portable intermittent haemodialysis in ICU patients with AKI requiring RRT during COVID-19 pandemic in UK S. Isralls ${ }^{1}$; G. Sartori ${ }^{1}$;. Edwards ${ }^{1}$; M. Griffith ${ }^{1}$; G. Saquing ${ }^{2}$; J. Gross ${ }^{3}$; T. Husain ${ }^{1}$; N. Duncan ${ }^{2}$

${ }^{1}$ Intensive care unit, Northwick Park Hospital, London, United Kingdom; ${ }^{2}$ Imperial college renal and transplant centre, Imperial College Healthcare NHS Trust, Hammersmith Hospital, DuCane Road, W12 OHS, London, United Kingdom; ${ }^{3}$ Intensive care, London North West University Healthcare NHS Trust, London, United Kingdom

Correspondence: G. Sartori

Intensive Care Medicine Experimental 2020, 8(2): 000895

Introduction: The Coronavirus disease (COVID-19) pandemic brought extraordinary demands on many Intensive Care Units (ICU) in the UK. Our ICU faced an unprecedent rate of admissions [ref1], with 32.7\% of patients requiring Renal Replacement Therapy (RRT) for Acute Kidney Injury (AKI) [ref2]. Pre-pandemic, our conventional RRT was Continuous Veno Venous Haemofiltration (CVVHF), favoured over other modalities in view of more stable haemodynamic profile [ref3]. With a shortage of RRT machines, consumables, and trained workforce, we explored NxStage Pure Flow System and Cycler (NxS) as an alternative to provide portable intermittent haemodialysis (iHD) as RRT in ICU.

Methods: This study was designed as a service evaluation to review NxS safety and efficacy, including all patients with COVID-19 and AKI Stage 3 by KDIGO criteria treated with NxS between 24/04 and $24 / 06 / 2020$. Exclusion criteria were liver failure or haemodynamic instability requiring vasopressors. Study approval SE20/015 (28/05/2020).

Results: Ten consecutive patients were included, 9/10 male, all of Black, Asian and Minority Ethnicity; mean age 54.9 years (SD 11.7). $7 / 10$ had diabetes, $6 / 10$ had hypertension, one had chronic kidney disease. All patients had radiological features typical for COVID-19 and 7 had positive nasopharyngeal swab. In the first 24 hours in ICU, mean APACHE II score was 19.8 (SD 5.7) and $\mathrm{PaO}_{2} / \mathrm{FiO}_{2}$ ratio $137 \mathrm{mmHg}$ (SD 69). All patients were intubated and ventilated during admission. $45 \mathrm{NxS}$ treatments were delivered over two months, with a median of 3.5 days of NxS per patient (IQR 1.5-8), versus 14 days of CVVHDF (IQR 8-22.5). 5 of 45 treatments were aborted: 3 secondary to vascular access mechanical issues, one for change in therapeutic priority, and one for hypotension requiring metaraminol. Adverse events per patient are detailed in the table below.

\begin{tabular}{|l|c|c|c|c|c|c|c|c|c|c|}
\hline PATIENT & $\mathbf{1}$ & $\mathbf{2}$ & $\mathbf{3}$ & $\mathbf{4}$ & $\mathbf{5}$ & $\mathbf{6}$ & $\mathbf{7}$ & $\mathbf{8}$ & $\mathbf{9}$ & $\mathbf{1 0}$ \\
\hline Hypotension & 0 & 0 & 0 & 0 & 0 & 0 & 0 & $\mathbf{1}$ & 0 & 0 \\
\hline Hypokalaemia & Yes & Yes & Yes & No & Yes & Yes & Yes & Yes & No & No \\
\hline Dialysis catheter site bleeding & 0 & 0 & 1 & 0 & 0 & 0 & 0 & 0 & 0 & 0 \\
\hline Bleeding - others & 0 & 1 & 0 & 0 & 0 & 1 & 3 & 0 & 0 & 0 \\
\hline Dialysis catheters sepsis & 2 & 0 & 0 & 1 & 0 & 0 & 0 & 0 & 0 & 0 \\
\hline Sepsis - others & 5 & 3 & 5 & 3 & 1 & 0 & 2 & 2 & 2 & 2 \\
\hline
\end{tabular}

$7 / 10$ patients developed hypokalaemia during NxS, requiring replacement in 24 of 45 treatments, without any arrhythmia. 36 treatments were delivered through standard vascath, and 9 through TesioCath. We report one bleeding episode from TesioCath site, but no episodes of catheter-associated thrombosis, nor any pneumothorax secondary to dialysis catheter insertion. We report 3 episodes of dialysis catheter associated sepsis, versus 26 septic episodes from other sources.

The $\mathrm{pH}$ variation over $\mathrm{NxS}$ treatment remained stable and no patient was acidotic at the end of NxS treatment. Serum lactate level increased during all treatments, by virtue of the lactate buffer used, and decreased to the normal reference range within five hours of $\mathrm{NxS}$ completion in 31 of 45 cases, without clinical sequelae in the remaining cases.
Efficacy was described by achieving the target fluid balance in $40 / 45$ treatments, and reducing blood urea nitrogen (BUN) post-NxS compared to pre-NxS in $37 / 45$ treatments (on average $5.1 \mathrm{mmol} / \mathrm{l}$ less than pre-NxS BUN samples, $\mathrm{p}<0.01$ on paired $t$ test).

$8 / 10$ patients survived to ICU discharge or last follow up; 3 have been discharged home, 2 remain in hospital and 3 in ICU. Mean ICU length of stay was 38.7 days (SD 16.8). At ICU discharge, median eGFR was 73 $\mathrm{mL} / \mathrm{min} / 1.73 \mathrm{~m}^{2}$ (IQR $14-84$ ) with no dialysis dependence.

Conclusion: This pilot project was developed and delivered through necessity, and it was not designed to compare or displace the use of CVVHF by iHD in ICU [ref4], but proved that NxS is safe and effective in selected patients in order to preserve the use of CVVHF for patients who are haemodynamically unstable or with liver failure.

\section{Reference(s) and grant ackowledgment(s)}

1. 1. Batchelor $G$. Revealed: The hospitals facing most pressure to meet coronavirus demand [Internet]. HSJ For Healthcare Leaders. 2020 [cited 2020 Jul 14]. Available from: https://www.hsj.co.uk/quality-and-performance/ revealed-the-hospitals-facing-most-pressure-to-meet-coronavirus-deman d/7027354.article

2. 2. Intensive care national audit \& research centre. ICNARC report on COVID19 in critical care - 26 June 2020. 2020;(June):1-16.

3. 3. Mehta RL, McDonald B, Gabbai FB, et al. A randomized clinical trial of continuous versus intermittent dialysis for acute renal failure. Kidney Int. 2001;60(3):1154-1163. https://doi.org/10.1046/j.1523-1755.2001.06000 31154.x

4. 4. Bell, M., Granath, F., Schön, S. et al. Continuous renal replacement therapy is associated with less chronic renal failure than intermittent haemodialysis after acute renal failure. Intensive Care Med 33, 773-780 (2007). https://doi. org/10.1007/s00134-007-0590-6

\section{2}

Wells and revised Geneva scores as predictors of pulmonary embolism in critically ill patients: a prospective study

A. Muradás Girardi ${ }^{1}$; M. Gazzana ${ }^{2}$; T. Rech

${ }^{1}$ Departamento de medicina intensiva, Hospital de Clínicas de Porto Alegre, Porto Alegre, Brazil; ${ }^{2}$ Departamento de pneumologia, Hospital de Clínicas de Porto Alegre, Porto Alegre, Brazil

Correspondence: A. Muradás Girardi

Intensive Care Medicine Experimental 2020, 8(2): 000112

Introduction: Critically ill patients are at high risk for pulmonary embolism (PE) (1). However, the diagnosis of this potentially fatal disease often goes unnoticed, as symptoms are nonspecific and other clinical conditions can manifest with similar features (2). Prediction rules, usually used in emergency departments, such as the Wells and Geneva scores, are not validated in this population. Therefore, the best diagnostic approach to investigate critically ill patients with suspected $P E$ remains uncertain.

Objectives: The aim of the present study was to assess the Wells and the revised Geneva scoring systems as predictors of PE in critically ill patients.

Methods: From July 2018 to November 2019, adult patients admitted to the intensive care unit (ICU) at the Hospital de Clínicas de Porto Alegre were considered eligible for the study if they had a pulmonary computed tomographic angiogram (CTA) as a confirmatory imaging study for suspected cases of PE. Clinical data was collected and the Wells and the revised Geneva scores were calculated. The researcher who calculated the scores was unaware of the CTA result. Two radiologists independently reviewed the scans to diagnose the presence of PE based on the presence of filling defects in the pulmonary artery or its branches. The discriminative power of the Wells and revised Geneva scores to predict risk of PE was determined by receiver operating characteristic curve (ROC) analysis. 
Results: Eighty critically ill patients were prospectively included in the study. The mean age of the patients was $59 \pm 14$ years, $52.5 \%$ were female, with a mean SAPS 3 score of $61 \pm 16$. The main cause for ICU admission was acute respiratory failure $(62 \%)$, followed by cardiac arrest (17\%) and shock (11\%). The incidence of PE was $39 \%(n=31)$. Segmental PE was the most common classification (58\%), followed by main artery PE (32\%), subsegmentary PE (6.5\%) and lobar PE (3\%). Inter-observer agreement for $\mathrm{PE}$ diagnosis was very good $(\mathrm{K}=0.89)$. The mean Wells score in patients without $P E$ was $3.0 \pm 1.9$ vs. $4.2 \pm 2.7$ in patients with PE $(p=0.027)$. The mean revised Geneva score was $5.0 \pm 3.0$ in patients without PE vs. $6.0 \pm 4.0$ in patients with PE ( $p$ $=0.08$ ). Figure 1 shows the ROC curves of the Wells and the revised Geneva scores, respectively. The area under the curve (AUC) for the Wells score was $0.64(\mathrm{Cl} 0.51-0.77)$ and for the revised Geneva score AUC was 0.60 (Cl 0.47-0.73).

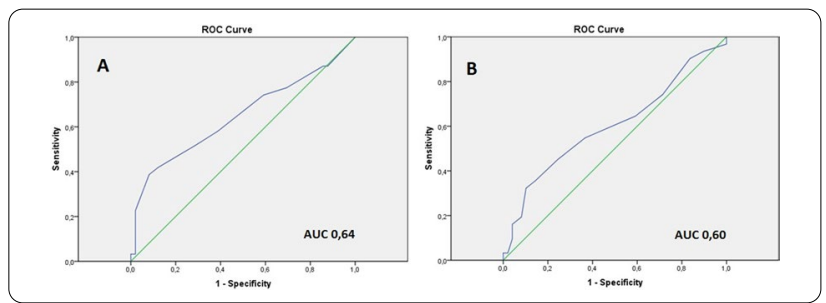

Conclusion: Wells and revised Geneva scores are not reliable predictors of PE in critically ill patients. These negative results, obtained prospectively, reinforce the importance to search for alternatives for a better diagnostic approach to PE in this population.

\section{Reference(s) and grant ackowledgment(s)}

1. 1 - Koch C, Schramm R, Roller FC, Hecker A, Henrich M, Schneck E, et al. Impact of unsuspected subsegmental pulmonary embolism in ICU patients. Anaesthesist. 2016;65(2):122-8.

2. 2 - Raja AS, Greenberg JO, Qaseem A, Denberg TD, Fitterman N, Schuur JD, et al. Evaluation of Patients With Suspected Acute Pulmonary Embolism: Best Practice Advice From the Clinical Guidelines Committee of the American College of Physicians. Ann Intern Med. 2015;163(9):701-11.

\section{3}

\section{Community acquired pneumonia in the intensive care unit}

C. Serrano '; A. Ruiz García ; J. Higuera Lucas ${ }^{1}$; E. Lopez Ramos ${ }^{1}$; B. Llorente Ruiz'; M. Trascasa Muñoz'; E. Nevado

${ }^{1}$ Intensive care unit, Hospital Príncipe de Asturias, Alcalá de Henares, Spain Correspondence: $C$. Serrano

Intensive Care Medicine Experimental 2020, 8(2): 000133

Introduction: The term severe community acquired pneumonia (CAP) identifies the group of patients with CAP who require admission to intensive care units (ICU). The incidence of severe acquired pneumonia has increased in the last decade and is one of the main infectious causes of admission in ICU.

Objectives: The aim of this study is to review and describe severe CAP patients in a level II hospital ICU, analyzing the patients' profile, microbiology isolations and treatment.

Methods: A retrospective observational study was performed. Consecutive critically ill CAP patients receiving treatment in level II hospital polivalent ICU were reviewed from january 1, 2018 to november 30, 2019. Categorical variables were analyzed using chi-square tests, and continuous variables were analyzed by T Student or ANOVA. Signification level was stablished as $p<0.05$.
Results: A total of 41 consecutive eligible individuals were reviewed. Mean age was $60.24( \pm 8)$, mean APACHE II was $16.77( \pm 8)$, mean hospital stay was 16 days ( \pm 23.24). $63.45 \%$ were men. $25 \%$ were inmunosupressed. $41.5 \%$ had COPD. The main reason for admission was respiratory failure in $87.8 \%$ of patients, followed by shock (need of vasoactive drugs). $14.6 \%$ required intubation as an initial respiratory support. NIMV was initiated in $48.8 . \%$, HFO in $26.8 \%$ and conventional oxygen in $9.8 \%$. Finally, $48.8 \%$ of pacients required mechanical ventilation (20/41). There was no statistically signifcant difference between NIMV and GNAF failure. The initial empiric treatment was a betalactamic (ceftriaxone) and a macrolid (claritromicina) or quinolone (levofloxacine) in $78 \%$ of cases. $17 \%$ received broad espectrum treatment (piperaciline-tazobactam or imipenem). $2.5 \%$ received only ceftriaxone. In 21 cases there was microbiology isolation: respiratory virus were isolated in 10 cases (Influenza 4, VMC 3, HVS 2 and adenovirus 1). In 6 patients S.pneumoniae was isolated. $H$. Influenzae, E Coli and $P$ Aeruginosa in one case each. Comparative analysis between bacterial and virical pnumonia is shown in the table below:

\begin{tabular}{|l|l|l|l|}
\hline & $\begin{array}{l}\text { Viral pneumonia } \\
\mathrm{n}=9\end{array}$ & $\begin{array}{l}\text { Bacterial pneumonia } \\
\mathrm{n}=9\end{array}$ & $\mathrm{p}$ \\
\hline Age & 57 & 64 & 0.33 \\
\hline Gender $\delta$ & $75 \%$ & $66.6 \%$ & 0.56 \\
\hline ICU stay (days) & 14.7 & 23.2 & 0.58 \\
\hline APACHE II & 11.7 & 19 & 0.045 \\
\hline Diabetes Mellitus & $37.5 \%$ & $22.2 \%$ & 0.437 \\
\hline CV illness previously & $37.5 \%$ & $44.4 \%$ & 0.58 \\
\hline Previous respiratory & $37.5 \%$ & $33.3 \%$ & 0.627 \\
\hline illnes & $0 \%$ & $11.1 \%$ & 0.5 \\
\hline Cirrosis & $37.5 \%$ & $77.7 \%$ & 0.12 \\
\hline Shock & $0 \%$ & $25 \%$ & 0.2 \\
\hline Inmunosupression & $62.5 \%$ & $66 \%$ & 0.63 \\
\hline Mechanical ventilation & $0 \%$ & $25 \%$ & 0.2 \\
\hline Exitus & & & \\
\hline
\end{tabular}

Mortality rate was $14.6 \%$. Hospital stay, inmunosuppression, vasoactive drugs requirement and mechanical ventilation requirement were found to be independent mortality risk factors.

Conclusion: In our ICU patient's profile, mortality risk factors and mortality rate are similar to other series of cases. A high percentage $(48.8 \%)$ required mechanical ventilation. It should be noted that the incidence of viral and bacterial pneumonia had the same number of cases. Bacterial pneumoniae seems to lead to a longer hospital stay and mortality but more studies would be required.

Reference(s) and grant ackowledgment(s)

1. Menendez R, Torres A, Reyes S, et al. Initial management of pneumonia and sepsis: factors associated with improved outcome. Eur Respir J 2012;39(1):156-62

2. Dellinger RP, Levy MM, Rhodes A, et al. Surviving sepsis campaign guidelines committee including the pediatric subgroup. Surviving Sepsis Campaign: international guidelines for management of severe sepsis and septic shock, 2012. Intensive Care Med 2013;39(2):165-228

3. Restrepo MI, Mortensen EM, Rello J, et al. Late admission to the ICU in patients with community-acquired pneumonia is associated with higher mortality. Chest 2010;137(3):552-7

4. Woodhead M, Welch CA, Harrison DA, et al. Community-acquired pneumonia on the intensive care unit: secondary analysis of 17,869 cases in the ICNARC Case Mix Programme Database. Crit Care 2006; 10(Suppl 2):S1

5. Adamantia Liapikou, Edmundo Rosales Mayor and Antoni Torres. The management of severe community acquired pneumonia in the intensive care unit. Expert Rev. Respir. Med. 8(3), 293-303 (2014) 
000280

Comparison of anticoagulation strategies for veno-venous ECMO support in acute respiratory failure: a retrospective comparative cohort study

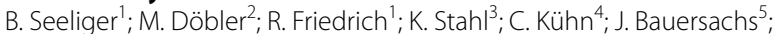

F. Steinhagen ${ }^{2}$; S. Ehrentraut ${ }^{2}$; JC. Schewe ${ }^{2}$; C. Putensen ${ }^{2}$;. T. Welte'; MM. Hoeper'; S. David ${ }^{6}$; C. Bode ${ }^{2}$

'Department of Respiratory Medicine, Hannover Medical School, Hannover, Germany; ${ }^{2}$ Department of anaesthesiology and critical care medicine, University of Bonn, Bonn, Germany; ${ }^{3}$ Department of gastroenterology, hepatology and endocrinology, Hannover Medical School, Hannover, Germany; ${ }^{4}$ Department of cardiothoracic, transplantation and vascular surgery, Hannover Medical School, Hannover, Germany; ${ }^{5}$ Department of cardiollogy and angiology, Hannover Medical School, Hannover, Germany; ${ }^{6}$ Nephrology, Hannover Medical School, Hannover, Germany

Correspondence: B. Seeliger

Intensive Care Medicine Experimental 2020, 8(2): 000280

Introduction: Extracorporeal membrane oxygenation (ECMO) support in acute respiratory failure may be lifesaving, but bleeding and thromboembolic complications are common. The optimal anticoagulation strategy balancing these factors remains to be determined.

Objectives: This study was carried out to determine whether a lowdose prophylactic heparinization strategy is associated with similar rates of oxygenator changes while reducing bleeding events when compared to a high-dose therapeutic heparinization strategy.

Methods: We conducted a retrospective unblinded observational cohort study between 04/2015 - 02/2020 in two ECMO referral centres in Germany in patients receiving veno-venous (VV)-ECMO support for acute respiratory failure for $>24 \mathrm{~h}$ and unfractionated heparin (UFH) for anticoagulation. One centre routinely applied low-dose heparinization aiming for a partial thromboplastin time (PTT) of 35-40s and the other routinely used a high-dose therapeutic heparinization strategy aiming for an activated clotting time (ACT) of 140-180s. We assessed number of and time to ECMO oxygenator changes, 15-day freedom from oxygenator change, major bleeding events, thromboembolic events, 30-day ICU mortality, activated clotting time and partial thromboplastin time, red blood cell transfusions and platelet transfusions. Primary outcome was the occurrence of oxygenator changes depending on heparinization strategy; main secondary outcomes were the occurrence of severe bleeding events and occurrence of thromboembolic events.

Results: Of 375 screened patients receiving VV-ECMO support, 218 were included in the analysis (117 high-dose group; 101 low-dose group). Disease severity measured by SAPS II score was 46 (IQR 36-57) vs 47 (IQR 37-55) and ECMO runtime was 8 (IQR 5-12) vs 11 (IQR 7-17) days $(P=0.003)$. There were 14 oxygenator changes in the high-dose group vs 48 in the low-dose group. Freedom from oxygenator change at 15 days was 73 vs $55 \%$ (adjusted HR 2.74 [95\% confidence interval 1.2-6.5]; $P=0.023$ ) (Figure). Severe bleeding events occurred in $23(19.7 \%)$ vs 14 (13.9\%) patients $(P=0.256)$ and thromboembolic events occurred in 8 (6.8\%) vs. $19(19 \%)$ patients $(P=0.007)$. Mortality at 30 days was $33.3 \%$ vs $30.7 \%(P=0.11)$. Mean ACT in the high-dose group (158s [IQR 151165]) and PTTs (48s [IQR 41-57] vs 38s [IQR 34-42]) corresponded well with predefined target ranges. Red blood cell and platelet transfusions were less frequent in the high-dose group (6 [IQR 2-10] vs 8 [IQR 6-19] $P$ $<0.001$ and 0 [IQR $0-1]$ vs $4[0-10], P<.001$, respectively).

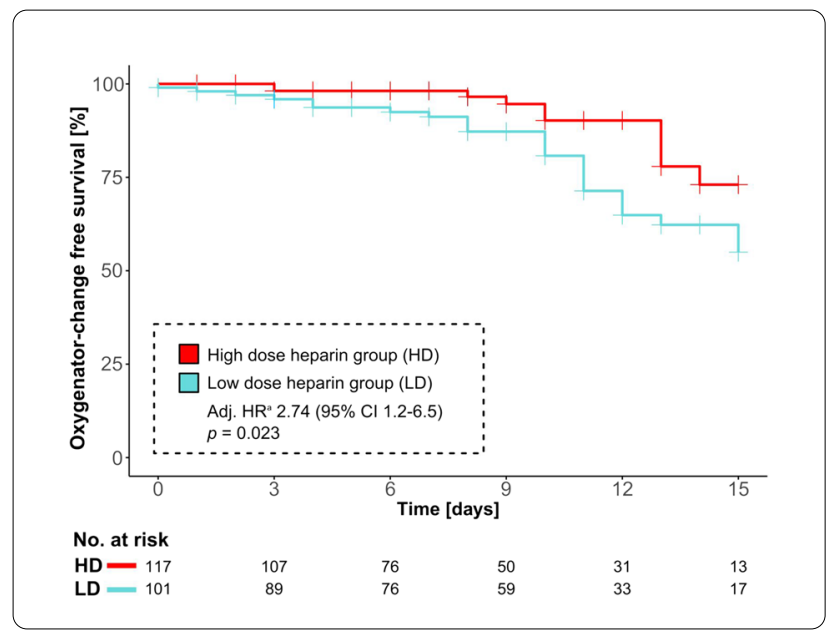

Conclusion: Compared with low-dose heparinization, high-dose heparinization during ECMO support was associated with lower rates of oxygenator changes and thromboembolic events, but with a numerically higher number of bleeding events.

\section{5}

Systematic review of minimal invasive methods for diagnosis of AcuteRespiratory Distress Syndrome

L. Hagens'; N. Heijnen²; M. Smit ; M. Schultz'; D. Bergmans ${ }^{2}$; R. Schnabel²; L. Bos ${ }^{1}$

${ }^{1}$ Department of intensive care, Amsterdam UMC, locatie AMC, Amsterdam, Netherlands; ${ }^{2}$ Department of intensive care, Maastricht UMC + , Maastricht, Netherlands

Correspondence: L. Hagens

Intensive Care Medicine Experimental 2020, 8(2): 000315

Introduction: Acute Respiratory Distress Syndrome (ARDS) is currently diagnosed by the Berlin definition, which does not include a direct measure of pulmonary oedema, endothelial permeability, pulmonary inflammation, coagulation or oxidative stress. A diagnostic test that could capture these processes might improve the diagnosis of ARDS. We hypothesized that biomarkers of these processes have good diagnostic accuracy for ARDS.

Methods: Medline and Scopus were searched with broad keyword criteria and selection was performed by two independent researchers. Inclusion criteria were: (1) original research, (2) that reported diagnostic accuracy, (3) used a minimal invasive test for (4) pathophysiological mechanisms of ARDS and (5) compared the results to a relevant control group. The primary outcome was the diagnostic accuracy per test, categorized by pathophysiological mechanism, control group and analyzed biomaterial. Secondary, the methodological quality was assessed with Quadas-2 tool. Biomarkers that had an area under the Receiver Operating Characteristic curve (AUROCC) of more than 0.75 and were studied with minimal bias against an unselected control group were considered to be promising. 
Results: The search led to 929 independent articles of which 42 were included. The median AUROCC for all evaluated tests was 0.80 (25th to 75th percentile: $0.72-0.89$ ). The type of control group influenced the diagnostic accuracy (Figure 1, $p=0.01$ ). Higher risk of bias was associated with higher diagnostic accuracy (AUROCC 0.74 for low bias, 0.78 for medium bias and 0.84 for high bias studies; $p=0.0021$ ). Club Cell protein 16 (CC16) and soluble receptor for advanced glycation end-products (sRAGE) in plasma and three biomarkers of oxidative stress in breath showed good diagnostic accuracy in low bias studies that compared ARDS patients to an unselected intensive care unit (ICU) population.

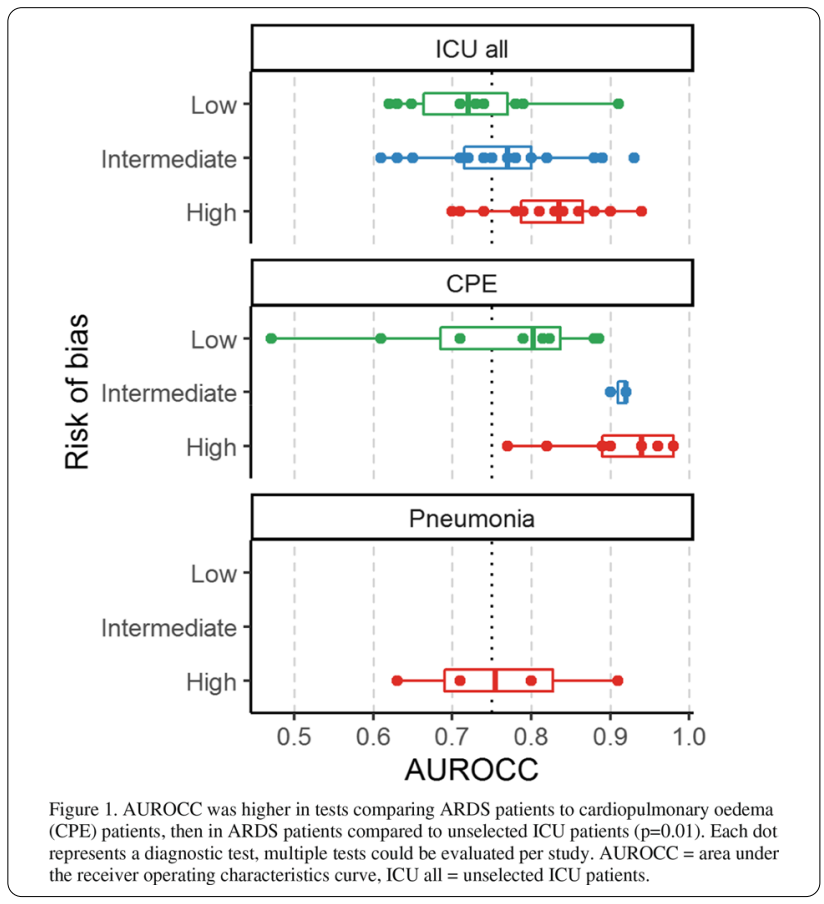

Conclusion: This systematic review revealed only three diagnostic tests that fulfilled stringent criteria for a promising biomarker in a low bias setting. For implementation into the clinical setting, prospective studies in a general unselected ICU population with good methodological quality are needed.

\section{6}

\section{Development and validation of a point of care breath test}

\section{for octane detection in the intensive care unit}

L. Hagens ${ }^{1}$; A. Verschueren²; M. Lammers ${ }^{3}$; H. Knobel'; N. Heijnen ${ }^{5}$ M. Smit'; M. Schultz'; D. Bergmans ${ }^{5}$; R. Schnabel ${ }^{5}$;. Nijsen ${ }^{2}$; L. Bos 'Department of intensive care, Amsterdam UMC, locatie AMC, Amsterdam, Netherlands; ${ }^{2}$ Research, Philips Research, Global Headquarters, Eindhoven, Netherlands: ${ }^{3}$ Department of respiratory medicine, Amsterdam UMC, locatie AMC, Amsterdam, Netherlands; ${ }^{4}$ Analytics, Eurofins Materials Science Netherlands B.V., Eindhoven, Netherlands; ${ }^{5}$ Department of intensive care, Maastricht UMC + , Maastricht, Netherlands

Correspondence: L. Hagens

Intensive Care Medicine Experimental 2020, 8(2): 000316

Introduction: Acute Respiratory Distress Syndrome (ARDS) is currently diagnosed by the Berlin definition, which involves subjective criteria and leads to late diagnosis. There is a demand for a non-invasive bedside method to diagnose ARDS. Three metabolites in breath, of which octane was the most important, showed good diagnostic accuracy when analysed with gas chromatography and mass spectrometry (GCMS). However, mass-spectrometry is unsuitable for a point-of-care (POC) test. Therefore, we aimed to develop a POC test that measures octane in exhaled breath of invasively ventilated intensive care unit (ICU) patients at trace amounts (parts-per-billion). We hypothesized that the breath analyser (BA) can reliably detect octane in exhaled breath of mechanically ventilated ICU patients.

Methods: Two steps were taken in development of the BA. A laboratory BA was validated in healthy subjects with calibration measurements. Comparison to gold standard GCMS was performed in healthy subjects and in patients receiving mechanical ventilation. Subsequently, the BA was further developed into a POC BA, which was also calibrated and then used for assessment of exhaled breath of ventilated ICU patients. Accuracy of the BA was reflected by repeatability between duplicate samples and reproducibility for detecting a known amount of octane. Octane concentration was based on the area under the curve (AUC), which was extracted from the chromatogram and compared to known values from calibration measurements.

Results: Five healthy subjects and 40 patients were included for assessment with the laboratory BA, 12 patients were included for validation of the POC BA. A linear association with an R2 of 0.99 was reached with the laboratory BA with breath samples spiked with octane. In contrast, reproducibility with GCMS was moderate (R2 = 0.68). In patients 40 duplicate measurements were performed with the laboratory BA and 25 duplicate measurements with the POC BA In 5 out of 25 measurements with the POC BA, one of the octane peaks could not be fitted due to co-elution or a noisy signal. The repeatability was strong for the laboratory- and POC-BA, with an intraclass correlation coefficient (ICC) of 0.87 and 0.95 and a coefficient of variation of $17 \%$ and $8 \%$, respectively (Figure 1 and 2 ).
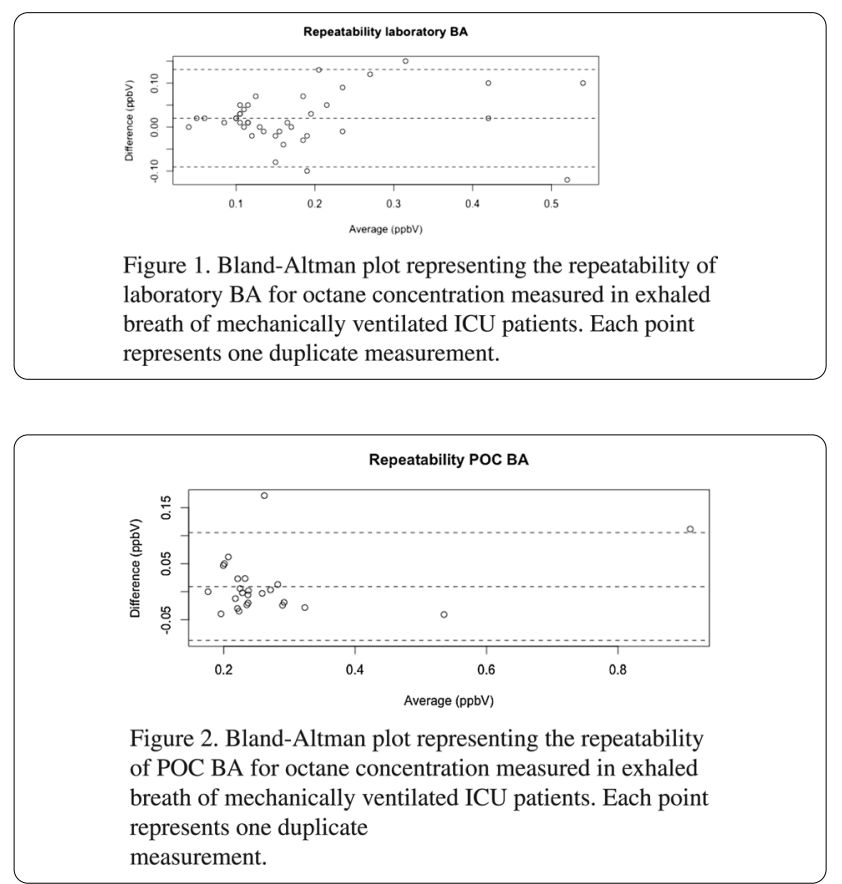

Conclusion: The laboratory- and POC-BA were both able to accurately detect trace amounts of octane in exhaled breath of ventilated ICU patients. Reproducibility was acceptable and repeatability was good. Further validation and implementation steps are needed to progress to a POC breath test for diagnosing ARDS in ventilated ICU patients.

Reference(s) and grant ackowledgment(s)

1. The authors received a grant from Health Holland and the Dutch Lung Foundation for the DARTS study. 


\section{7}

Pulmonary consolidation alters the estimate of pleural fluid volume before considering chest drainage in patients on ECMO M. Maly ${ }^{1}$; C. Mokotedi ; M. Porizka ${ }^{1}$; M. Otahal ${ }^{1}$; J. Rulisek ; M. Balik ${ }^{1}$ ${ }^{1}$ Dept of anaesthesia and intensive care, General University Hospital in Prague, Prague, Czech Republic

Correspondence: $M$. Balik

Intensive Care Medicine Experimental 2020, 8(2): 000347

Introduction: The estimate of pleural fluid volume on chest ultrasound (CUS) (1) helps to decide on pleural drainage in relation to weaning of the ECMO and IPPV. The method of multiplying maximum transverse pleural separation at the lung base in $\mathrm{mm}$ by 20 has been constructed on medical mechanically ventilated patients (1). The method may fail in severely consolidated lungs encircled by pleural fluid.

Objectives: To test the established method of pleural fluid estimation in haevily consolidated lungs and to find optimal way of the volume assessment.

Methods: 35 pleural effusions were drained in 28 mechanically ventilated patients (APACHE II $26.3 \pm 5$, SOFA $10.9 \pm 2$ ) with severe lung consolidation of 4th degree on CUS at least in basal lung regions and no better than 3rd degree in the apical regions. All were prospectively observed while treated on VV, VAV or VA ECMO. All drainages were performed by intensivists by blunt forceps technique, most of them with $12 \mathrm{~F}$ chest drain (CD).

Results: The maximum pleural separation was $23 \pm 6 \mathrm{~mm}$, correlating with separation at the lateral chest wall $(19 \pm 8 \mathrm{~mm}, \mathrm{r}=0.79)$. The dorsal and frontal separations were $16 \pm 7 \mathrm{~mm}$ and $16 \pm 7 \mathrm{~mm}$, respectively. The classic (1) method of pleural fluid estimate correlated weakly with pleural fluid volume $(r=0.32)$ and produced a mean underestimation error of $-371 \pm 207 \mathrm{ml}$ contrasting with drained $830 \pm 209 \mathrm{ml}$. Better correlation was found for the frontal pleural separation ( $r=0.44, p<0.05$ ). The volume of pleural fluid may be estimated with equation $\mathrm{V}[\mathrm{ml}]=620+13^{*}$ frontal separation $[\mathrm{mm}]$ which results in a mean prediction error of $156 \pm 101 \mathrm{ml}$. Similarly, the sum of basal, lateral and ventral pleural separations $(51 \pm 15 \mathrm{~mm})$ correlated with drained volume $(r=0.42, p<0.05)$ showing mean prediction error of $159 \pm 100 \mathrm{ml}$.

Conclusion: The known CUS assessment of volume of pleural fluid largely underestimates reality in severely consolidated lungs surrounded by potentially misleadingly thin layer of circumferential effusion. The multiple measurements are feasible paying attention to a degree of lung consolidation and separation of pleural layers under the frontal chest wall.

\section{Reference(s) and grant ackowledgment(s)}

1. 1. Balik M, Plasil P, Pazout J, et al: Ultrasound guided thoracentesis in mechanically ventilated patients. Intensive Care Med 2006,32:318-321

\section{9}

\section{Administration of large volume of Hydroxyethyl starch (HES)} in the first $\mathbf{4 8}$ hours of resuscitation is associated with early ARDS development in patients with blunt thoracic trauma

D. Mihajlovic ; A. Uvelin²; M. Armus²

${ }^{1}$ Department of anesthesiology, intensive therapy and pain therapy, clinical centre of vojvodina, Medical Faculty in Novi Sad, Novi Sad, Serbia; ${ }^{2}$ Department of anesthesiology, intensive therapy and pain therapy, clinical centre of vojvodina, Faculty of Medicine, University of Novi Sad, Novi Sad, Serbia

\section{Correspondence: $D$. Mihajlovic}

Intensive Care Medicine Experimental 2020, 8(2): 000399

Introduction: Blunt thoracic trauma is a major risk factor for development of Acute Respiratory Distress Syndrome (ARDS). Although blunt thoracic trauma is recognized as a specific risk factor for ARDS development, other predictors of early ARDS have not been precisely defined in this group patients.
Objectives: To define specific predictors that can identify patients who are at greater risk of ARDS development in an early resuscitation phase (first 48 hours) after blunt thoracic trauma.

Methods: During one-year study period, 50 patients with blunt thoracic trauma who were treated in intensive care unit after the initial resuscitation in Emergency center- Clinical center of Vojvodina were included in our study. Data were analyzed using SPSS 20.0 software. Differences between groups of patients were assessed by Student's t-test. Categorical variables were compared using chi-square test. Statistical significance ( $p)$ was set at a value of 0.05 .

Results: 16 patients (32\%) developed early ARDS during the first 48 hours after blunt thoracic trauma. Patients who developed ARDS received higher volumes of hydroxyethyl starch (HES) $(1607 \pm 594.15$ vs. $678.57 \pm 530.79 . \mathrm{p} \leq 0.05)$ and fresh frozen plasma (FFP) $(662.85 \pm 616.51$ vs. $365.35 \pm 894.64 . p \leq 0.05)$ during the first 48 hours after the trauma. Thoracic Trauma Severity Score (TTS) and Injury Severity Score (ISS) were significantly higher in patients with ARDS in comparison to patients who did not develop ARDS (TTS: $13.29 \pm 3.12$ vs. $9.25 \pm 4.30$; ISS: $51.92 \pm 20.94$ vs. $34.5 \pm 17.23$. $\mathrm{p} \leq 0.05)$. Patients with ARDS had significantly lower values of Glasgow coma score (GCS): $(9.69 \pm 4.88$ vs. $13.48 \pm 3.05 . p \leq 0.05)$

Conclusion: Significant predictors of ARDS development in patients with blunt thoracic trauma are multiple traumatic injuries defined with higher values of ISS and TTS score, presence of traumatic brain injury with lower values of GCS and administration of higher volumes of colloids and FFP.

\section{Reference(s) and grant ackowledgment(s)}

1. 5. Daurat A, Millet I, Roustan JP, Maury C, Taourel P, Jaber S, et al. Thoracic trauma severity score on admission allows to determine the risk of delayed ARDS in trauma patients with pulmo-nary contusion. Injury 2016;47(1):147-53.

2. 4. Watkins TR, Nathens AB, Cooke CR, Psaty BM, Maier RV, Cuschieri J, et al. Acute respira-tory distress syndrome after trauma: development and validation of a predictive model. Critical care medicine 2012;40(8):2295-303.

3. 3.O'Leary MP, Keeley JA, Yule A, Suruki C, Plurad DS, Moazzez A, et al. Clinical predictors of early acute respiratory distress syndrome in trauma patients. The American Journal of Surgery 2016;212(6):1096-100.

4. 2. Ranieri VM, Rubenfeld GD, Thompson BT, Antonelli M, Anzueto A, Beale $R$, et al. Acute respiratorydistress syndrome: the Berlin definition. JAMA 2012:307:2526-33.

5. 1.Silva PL, Pelosi P, Rocco PR. Fluids in acute respiratory distress syndrome: pros and cons. Current opinion in critical care 2014;20(1):104-12.

\section{9}

High dead space and dissociation between severe hypoxia and relatively low shunt might be distinguishing features of C-ARDS vs. ARDS

I. Marongiu'; E. Spinelli²; T. Mauri²; G. Colussi²; MC. Basile²; P. Tagliabue²; G. Grasselli²;A. Pesenti ${ }^{2}$

${ }^{1}$ Department of pathophysiology and transplantation, University of Milan, Milano, Italy; ${ }^{2}$ Department of anesthesia, critical care and emergency, Fondazione IRCCS Ca'Granda Ospedale Maggiore Policlinico, Milano, Italy

Correspondence: I. Marongiu

Intensive Care Medicine Experimental 2020, 8(2): 000429

Introduction: The pathophysiology of Coronavirus disease 2019 acute respiratory distress syndrome (C-ARDS) might have some peculiarities distinguishing it from ARDS of other etiology. Preliminary data suggest a unique contribution of vascular dysfunction induced by inflammation and microthrombosis to the imbalances in ventilation and perfusion within the lung [1].

Objectives: To characterize with electrical impedance tomography (EIT) the distribution of ventilation and perfusion in a cohort of patients affected by C-ARDS and to compare these findings with data collected in ARDS patients. 
Methods: We included 19 patients with C-ARDS between March 2020 and April 2020 and 50 patients with ARDS between March 2019 and December 2019 admitted to the Intensive Care Unit of the Ospedale Maggiore Policlinico (Milan, Italy). Gas exchanges and the distribution of ventilation and perfusion measured by EIT were assessed at positive end expiratory pressure (PEEP) of $5 \mathrm{cmH}_{2} \mathrm{O}$ for C-ARDS and at clinical PEEP for ARDS patients. Perfusion was obtained through injection of $10 \mathrm{~mL}$ of a $5 \%$ saline bolus during end-inspiratory occlusion. Dead space fraction was calculated as the percentage of pixels ventilated but not perfused, while shunt fraction as those perfused but not ventilated. Data are presented as median [IQR], differences between groups were assessed by Mann-Whitney on ranks test.

Results: Gas exchange and respiratory data are summarized in Table 1.

\begin{tabular}{|c|c|c|c|}
\hline & $C-A R D S(n=19)$ & $\operatorname{ARDS}(n=50)$ & P value \\
\hline PEEPtot (cmH2O) & $6[5 ; 6]$ & $11[8 ; 15]$ & $<0.0001$ \\
\hline $\mathrm{Vt} / \mathrm{PBW}(\mathrm{mL} / \mathrm{kg})$ & $7.4[6.7 ; 8.1]$ & $7.3[6.1 ; 8.0]$ & 0.53 \\
\hline Compliance $(\mathrm{mL} / \mathrm{cmH} 2 \mathrm{O})$ & $49[30 ; 52]$ & $42[35 ; 52]$ & 0.61 \\
\hline Driving Pressure $(\mathrm{cmH} 2 \mathrm{O})$ & $10[9 ; 14]$ & $11[8 ; 14]$ & 0.67 \\
\hline Respiratory rate (breaths/min) & $22[15 ; 26]$ & $16[14 ; 22]$ & 0.02 \\
\hline $\mathrm{PaCO} 2$ (mmHg) & $51[44 ; 60]$ & $44[37 ; 47]$ & 0.0009 \\
\hline $\mathrm{pH}$ & $7.39[7.33 ; 7.43]$ & $7.39[7.34 ; 7.43]$ & 0.90 \\
\hline Ventilatory ratio & $2.0[1.6 ; 2.6]$ & $1.4[0.9 ; 1.9]$ & 0.001 \\
\hline $\mathrm{PaO2} / \mathrm{FiO} 2$ & $81[69 ; 137]$ & $168[122 ; 241]$ & 0.0001 \\
\hline
\end{tabular}

*Mann-Whitney test, C-ARDS vsARDS. PBW: predicted body weight Compared to patients with ARDS, patients with C-ARDS showed no differences in tidal volume (Vt) and respiratory mechanics. Efficiency of ventilation was more impaired in C-ARDS, as indicated by higher respiratory rate, $\mathrm{PaCO} 2$ and ventilatory ratio. Accordingly, EIT documented a higher dead space fraction $(23 \%[13 ; 29]$ vs $12 \%[5 ; 19]$, C-ARDS vs ARDS, $p<0.01$ ). (figure $1 \mathrm{~A}$ ). Hypoxemia was more severe in C-ARDS, but shunt fraction didn't differ (figure 1B).

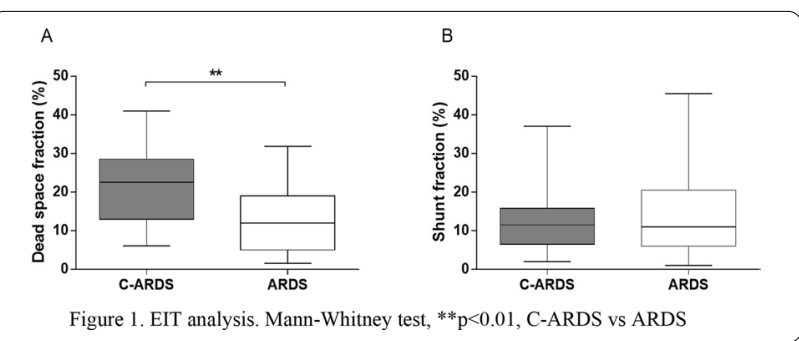

Conclusion: Compared to ARDS of other etiology, C-ARDS is characterized by more severe hypoxia and less efficient $\mathrm{CO} 2$ clearance, even in the presence of preserved mechanics. The mechanisms underlying derangements of gas exchange during C-ARDS may differ from ARDS, with increased dead space likely playing a key role.

\section{Reference(s) and grant ackowledgment(s)}

1. [1] Gattinoni L, Coppola S, Cressoni M, Busana M, Rossi S, Chiumello D. Covid-19 Does Not Lead to a "Typical" Acute Respiratory Distress Syndrome [published online ahead of print, 2020 Mar 30]. Am J Respir Crit Care Med. 2020;https://doi.org/10.1164/rccm.202003-0817le. https://doi.org/10.1164/ rccm.202003-0817LE
000435

COVID-19 in Critically III Patients in North Brabant,

the Netherlands

M. Linssen'; F. Aleva²; L. Van Mourik²; M. Broeders²; CPC. De Jager';

AJ. Paling

${ }^{1}$ Intensive Care, Jeroen Bosch Ziekenhuis, 's-Hertogenbosch, Netherlands;

${ }^{2}$ Respiratory medicine, Jeroen Bosch Ziekenhuis, 's-Hertogenbosch, Neth-

erlands

Correspondence: M. Linssen

Intensive Care Medicine Experimental 2020, 8(2): 000435

Introduction: Since the SARS-CoV-2 pandemic, countries are overwhelmed by critically ill Coronavirus disease 2019 (COVID-19) patients. As ICU capacity becomes limited we characterized critically ill COVID19 patients in the Netherlands.

Methods: In this case series, COVID-19 patients admitted to the ICU of the Jeroen Bosch Hospital were included from March 9, 2020. COVID19 was confirmed by a positive result by a RT-PCR of a specimen collected by nasopharyngeal swab. Clinical data were extracted from medical records.

Results: All patients were admitted to the ICU due to acute hypoxemic respiratory failure in absence of hemodynamic instability. The mean age was $64 \pm 10$ years, the mean BMI was $30.1 \pm 7$ and $73 \%$ were men. Thirty percent of patients had no comorbidities. With $33 \%$, hypertension was the most common comorbidity.

Of the 68 admitted patients, 62 were in need of invasive respiratory support (91\%). Six patients were successfully treated with high flow oxygen therapy. Six patients initially treated with a high flow oxygen cannula deteriorated requiring invasive mechanical ventilation.

Among the mechanically ventilated patients, $77 \%$ required ventilation in prone position during their stay in ICU according to our local protocol. The average length of invasive ventilation was 22.5 days (range 3-53 days). Renal replacement therapy was required in $14(21 \%)$ patients.

Venous thromboembolism was recognized in $40 \%$ of patients. In 20 patients pulmonary embolism was diagnosed, 1 patient developed a deep venous thrombosis of the forearm and 6 patients developed a catheter related thrombus. These complications occurred despite regular thrombosis prophylaxis.

The overall mortality of our patients was $20.5 \%$.

Conclusion: The survival rate of COVID-19 critically ill patients in our population is lower than previously reported. Dedicated selection and screening of patients before potential admission to the ICU could be a possible explanation for this low mortality. Thrombotic complications were commonly found and merit additional clinical attention.

\section{3}

A Retrospective Analysis of Influenza H1N1 ARDS

C. Joya Montosa'; JF. Martínez Carmona²; MP. Benitez Moreno33; M. Delgado-Amaya ${ }^{2}$

${ }^{1}$ Unidad de Cuidados Intensivos, Regional Hospital of Malaga, Málaga, Spain; ${ }^{2}$ ntensive care unit, Hospital Carlos Haya, Málaga, Spain; ${ }^{3}$ Intensive care, HOSPITAL CARLOS HAYA, MALAGA, Spain

Correspondence: J.F. Martínez Carmona

Intensive Care Medicine Experimental 2020, 8(2): 000453

Introduction: Influenza A (Influenza H1N1) is one of the great epidemics of our time. It had a great repercussion due to the associated morbidity and mortality, been moderate-severe ARDS one of the most common forms of presentation that requires prolonged admission to the ICU.

Methods: Retrospective, descriptive study of patients admitted in ICU from 2009 to 2019, with H1N1 influenza diagnosis. Clinical, epidemiological and result-related variables were analyzed. Quantitative variables are expressed as mean and standard deviation, while qualitative variables are expressed as ratios and absolute value. 
Results: A total of 78 patients were collected. Mean age was 47 years $+/-16.77 .53 .8 \%$ were women. APACHE II average upon admission: $14+/-7.23$ and average SOFA at admission: $5.5+/-3.83$

Between the comorbidities: $41 \%$ of the patients presented COPD, $33.3 \% \mathrm{BPH}, 32.1 \%$ Obesity and $21.3 \%$ had Heart disease and Dyslipidemia.

The $66.7 \%$ of the patients received previous NIMV; $74.4 \%$ required MV. Mean duration of MV was 15 days $+/-14.68$. Most of the patients required pulmonary recruitment maneuvers, and Prone Position was needed in $35.9 \%$. Renal failure was presented in $50 \%$ of the patients, and $21.8 \%$ of them required RRT. Tracheostomy, due to weaning, was performed in $39.7 \%$ of the patients.

Between risk factors Alcoholism (OR 9,025 p 0.042 IC 1,079- 75.51) Malignancy (OR 41.45 p 0.001 IC 4.47 - 384,437) and Autoimmune Disease (OR 7.3 p 0.042 IC 1,075 - 49.65) were the most frequent.

When the sample was divided into two groups, survivors and non-survivors, it was observed that the deceased patients were older (Median 46 years vs. 53 years), and presented greater renal failure (38.18\% vs. $78.26 \%)$ with a greater need for RRT $(28.57 \%$ vs. $61.11 \%)$ as well as greater severity in the Berlin Criteria for distress (severe ARDS $38.18 \%$ vs. $78.26 \%)$

The length of stay in the ICU was 13 days $+/-18.47$. The ICU observed mortality was $29.5 \%$ and the hospital mortality $33.33 \%$.

Conclusion: Severe ARDS caused by H1N1 Influenza associates great morbidity and mortality, being a very relevant pathology today in Intensive Care Units.

\section{6}

Impact of NMBAs on ventilation homogeneity and lung volume in ARDS patients

Y. Ichikawa'; A. Shono ${ }^{2}$; M. Somei ${ }^{2}$;. Kotani ${ }^{3}$

IIntensive Care Medicine, Showa University, Hatanodai Campus, 1 Chome-5-8 Hatanodai, Shinagawa City, Tokyo, Japan, Shinagawa City, Japan; ${ }^{2}$ Department of Intensive Care Medicine, Showa University, School of Medicine, Tokyo, Japan; ${ }^{3}$ Intensive care medicine, Showa University Hospital, Shinagawa City, Japan

Correspondence: Y. Ichikawa

Intensive Care Medicine Experimental 2020, 8(2): 000486

Introduction: Excessive inspiratory effort in mechanically ventilated patients with acute respiratory distress syndrome (ARDS) increases stress and strain in the lungs leading to ventilator-induced lung injury (VILI). The use of neuromuscular blocking agents (NMBAs) can however, synchronize with ventilator, but the impact of use of NMBAs on ventilation distribution and lung volume has not been fullyinvestigated.

Objectives: The objective of this study was to examine the impact of NMBAs on ventilation homogeneity and end-expiratory lung volume (EELV) evaluated by electric impedance tomography (EIT) in ARDS patients with strong inspiratory effort.

Methods: In this retrospective study, we performed off-line analysis of EIT data previously recorded before and after NMBA. The ventilation homogeneity was evaluated using EIT measures, the proportion of ventilation distribution (ventral and dorsal lung regions, expressed in percentage to a tidal volume $=100 \%$ ), center of ventilation, spatial dispersion in regional distribution of tidal breath (global inhomogeneity index: GI index). End-expiratory lung impedance (EELI), which is corresponding to EELV was measured and changes in EELI from at before NMBA administration were calculated and expressed as a percentage of tidal impedance change at before NMBA administration. These EIT measures and oxygenation after NMBA administration were compared to the data measured before administration of NMBA. For the comparison, paired t-test or Wilcoxon rank sum test were used for statistical analysis as appropriate.

Results: The EIT data of 6 patients (mean age 54) were analyzed. In all the patients rocuronium was administrated because of the strong inspiratory effort and asynchrony with ventilator. The mean proportions of ventilation distribution in ventral and dorsal region before NMBA were $39 \pm 14 \%$ (mean \pm SD) and $61 \pm 14 \%$ respectively, indicating that the active movement of diaphragm induced the predominant distribution of tidal volume to the dorsal region. After NMBA, the inspired gas distributed evenly ( $48 \pm 11 \%$ to ventral and $52 \pm 11 \%$ to dorsal region). $\mathrm{Gl}$ index were decreased after administration of NMBA in 5 cases (before NMBA: $53 \pm 4 \%$ after NMBA: $44 \pm 7 \%$ ). The EELI showed increase in 4 cases and decrease in 2 cases, the mean difference in EELI between before and after administration of NMBA was $26 \pm 59 \%$ (95\% confidence interval, -35 to $88 \%$ ). Mean difference in $\mathrm{PaO}_{2} / \mathrm{FiO}_{2}$ ratio increased after NMBA (before NMBA: $127 \pm 34 \mathrm{mmHg}$, after NMBA: $177 \pm 77 \mathrm{mmHg}$ ), however no significant difference was detected.

Conclusion: After administration of NMBA, the ventilation inhomogeneity was decreased by suppressing strong inspiratory effort, resulting in better oxygenation. In addition, NMBAs might maintain EELV by controlling not only inspiratory effort but also active expiration.

\section{Reference(s) and grant ackowledgment(s)}

1. The role of neuromuscular blockers in ARDS: benefits and risks/Sami Hraiech, Jean-Marie Forel, Laurent Papazian/ Current Opinion Critical Care 2012,18:495-502

2. Balancing neuromuscular blockade versus preserved muscle activity / Sami Hraiech, Takeshi Yoshida, Laurent Papazian/ Current Opinion Critical Care 2015,21:26-33

\section{0}

Mechanical power and intensive care mortality in ARDS patients A. Caccioppola ${ }^{1}$; T. Pozzi ${ }^{1}$; V. Camponetti ${ }^{2}$; F. Bichi ${ }^{2}$; C. Bianchi ${ }^{2}$; S. Cenci ${ }^{2}$; L. Meloni ${ }^{3}$; F. Bianchi'; S. Coppola ; D. Chiumello ${ }^{5}$

${ }^{1}$ Anesthesia and intensive care, University of Milan, Milano, Italy; ${ }^{2}$ Department of anesthesia and intensive care, san paolo hospital, University of Milan, Milano, Italy; ${ }^{3}$ Emergenza \& urgenza, University of Milan, Milano, Italy: ${ }^{4}$ Emergenza e urgenza, University of Milan, Milano, Italy: ${ }^{5}$ Anesthesia and intensiva care, San Paolo, Milano, Italy

Correspondence: A. Caccioppola

Intensive Care Medicine Experimental 2020, 8(2): 000490

Introduction: In ARDS patients, mechanical ventilation should minimize ventilator induced lung injury. The mechanical power which is the energy per unit time released to the respiratory system according to the applied tidal volume, PEEP, respiratory rate and flow should reflect the ventilator induced lung injury. However similar levels of mechanical power applied in different lung size could be associated to different effects.

Objectives: Aim of this study was to assess the role both of the mechanical power and of the transpulmonary mechanical power normalized to predicted body weight, respiratory system compliance, lung volume and amount of aerated tissue on intensive care mortality. Methods: Retrospective analysis of ARDS patients previously enrolled in seven published studies. All patients were sedated, paralyzed and mechanically ventilated. After 20 minutes from a recruitment maneuver, partitioned respiratory mechanics measurements and blood gas analyses were performed with a PEEP of $5 \mathrm{cmH}_{2} \mathrm{O}$ while the remaining setting was maintained unchanged from the baseline. A whole lung $\mathrm{CT}$ scan at $5 \mathrm{cmH}_{2} \mathrm{O}$ of PEEP was performed to estimate the lung gas volume and the amount of well inflated tissue. Univariate and multivariable Poisson regression models with robust standard error were used to calculate risk ratios and $95 \%$ confidence intervals of ICU mortality.

Results: 222 ARDS patients were included, 88 (40\%) died in ICU [Table 1]. Mechanical power was not different between survivors and non-survivors 14.97 [11.51-18.44] vs. 15.46 [12.33-21.45] J/min and did not affect intensive care mortality. 


\begin{tabular}{|c|c|c|c|}
\hline & $\begin{array}{l}\text { Survivors } \\
N=134\end{array}$ & $\begin{array}{l}\text { Non- } \\
\text { survivors }\end{array}$ & $p$ \\
\hline & & $N=88$ & \\
\hline Age (years) & $60[43-70]$ & 64 [54-75] & 0.005 \\
\hline Female N (\%) & $38(31 \%)$ & $31(35 \%)$ & 0.48 \\
\hline BMI (kg/m2) & $\begin{array}{l}24.8[22.6- \\
28.2]\end{array}$ & $\begin{array}{l}24.2[22.2- \\
29.0]\end{array}$ & 0.97 \\
\hline $\mathrm{PaO} 2 / \mathrm{FiO} 2$ & $195[146-231]$ & 153 [115-189] & 0.001 \\
\hline $\mathrm{PaCO} 2(\mathrm{mmHg})$ & $40[35-40]$ & 44 [39-52] & 0.001 \\
\hline Respiratory Rate (bpm) & $16[13-20]$ & 17 [14-20] & 0.07 \\
\hline Tidal Volume $(\mathrm{mL})$ & 510 [450-600] & $480[420-550]$ & 0.004 \\
\hline Clinical PEEP (cmH2O) & $10[10-13]$ & $10[10-12]$ & 0.68 \\
\hline Driving Pressure $(\mathrm{cmH} 2 \mathrm{O})$ & $12.5 \pm 3.3$ & $14.0 \pm 3.5$ & 0.004 \\
\hline $\begin{array}{l}\text { Respiratory system elastance } \\
(\mathrm{cmH} 2 \mathrm{O} / \mathrm{mL})\end{array}$ & $\begin{array}{l}23.9[18.4- \\
29.2]\end{array}$ & $\begin{array}{l}28.5[22.9- \\
34.8]\end{array}$ & 0.001 \\
\hline Intensive Care Unit stay (days) & 19 [11-30] & $15[8-24]$ & 0.009 \\
\hline
\end{tabular}

The multivariable robust regression models showed that the mechanical power normalized to well inflated tissue (RR: 2.69 [95\% Cl: 1.10$6.56] p=0.029$ ) and the mechanical power normalized to respiratory system compliance (RR 1.79 [95\% Cl: 1.16-2.76], $\mathrm{p}=0.008$ ) were independently associated with intensive care mortality after adjusting for age, SAPS II and ARDS severity. Also, transpulmonary mechanical power normalized to respiratory system compliance and to well inflated tissue significantly increased intensive care mortality (RR 1.74 $[1.11-2.70] p=0.015 ; \operatorname{RR} 3.01$ [1.15-7.91] $p=0.025)$. [Figure 2].

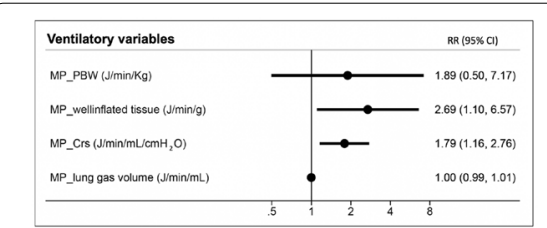

Figure 2. Predictive performance of ventilatory variables for Intensive care unit mortality

Conclusion: In our ARDS population there is not a causal relationship between the mechanical power itself and mortality, while mechanical power normalized to the compliance or to the amount of well aerated tissue is independently associated to the intensive care mortality. Further studies are needed to confirm this data.

\section{Reference(s) and grant ackowledgment(s)}

1. Gattinoni L, Tonetti T, Cressoni M, Cadringher P, Herrmann P, Moerer O, et al. Ventilator-related causes of lung injury: the mechanical power. Intensive Care Med. 2016;42(10):1567-1575.

2. Serpa Neto A, Deliberato RO, Johnson AEW, Bos LD, Amorim P, Pereira $\mathrm{SM}$, et al. Mechanical power of ventilation is associated with mortality in critically ill patients: an analysis of patients in two observational cohorts. Intensive Care Med. 2018;44(11):1914-1922.

\section{9}

Glucocorticoids as Rescue Therapy in Severe COVID-19 Respiratory Failure

T. Woodhead ${ }^{1}$; A. Joseph ${ }^{1}$; R. Kumar ${ }^{1}$

'Department of Anaesthetics and Intensive Care, Kingston Hospital, London, United Kingdom

Correspondence: $T$. Woodhead

Intensive Care Medicine Experimental 2020, 8(2): 000499

Introduction: SARS-CoV-2, the causative organism of COVID-19, is known to trigger a hyperinflammatory acute respiratory distress syndrome (ARDS) in a subset of patients leading to rapid deterioration and respiratory failure. This is associated with raised inflammatory markers and carries a poor prognosis. At present there is a paucity of interventions available to clinicians to treat this. Glucocorticoids are a plausible treatment option for this presentation given their established application in reducinginflammatory burden and association with improved outcomes in ARDS. Targeted use of methylprednisolone in patients with COVID-19-induced ARDS has been anecdotally reported to improve outcomes but limited data to support this is available.

Objectives: To examine outcomes in patients with severe ARDS secondary to COVID-19 treated with methylprednisolone, identify predictors of favourable response and any associated risk factors with poor outcomes.

Methods: We conducted a single centre case-control study in a London district general hospital intensive care unit. 27 participants with confirmed SARS-CoV-2 infection, severe ARDS and biochemical evidence of hyperinflammation receiving invasive mechanical ventilatory support between 01/02/2020 and 26/05/2020 were included. 21 patients treated with minimum of one dose of methylprednisolone ( $1 \mathrm{~g} /$ day or $1 \mathrm{mg} / \mathrm{kg} /$ day) were identified as the case group, and compared with a control group of 6 patients requiring an $\mathrm{FiO}_{2} \geq 0.6$ for $>48$ hours who received no corticosteroids during ICU admission. Outcomes were measured from first dose of methylprednisolone in the case group and after requiring a fraction of inspired oxygen $\left(\mathrm{FiO}_{2}\right)$ $\geq 0.6$ for $48 \mathrm{~h}$ in the control group. Primary outcome was 28 -day mortality. Secondary outcomes included $\mathrm{FiO}_{2}, \mathrm{C}$-reactive protein (CRP) and ferritin at $48 \mathrm{~h}$ and day 7 . Data on timing of methylprednisolone initiation, demographics and comorbidities were also collected.

Results: 28 -day mortality was $38.1 \%(8)$ in the methylprednisolone group vs $66.7 \%$ in the control group (4), odds ratio 0.31 (95\% Cl 0.045 to $2.08, \mathrm{p}=0.358$ ). $52.3 \%$ (11) required an $\mathrm{FiO}_{2} \leq 0.5$ on day 7 in the methylprednisolone group vs $0 \%$ in the control group $(p=0.0003)$. As a predictor of 28-day survival, an $\mathrm{FiO}_{2} \leq 0.5$ at day 7 had a sensitivity of $100 \%$ ( $95 \% \mathrm{Cl}: 71.5$ to $100 \%$ ) and a specificity of $75.0 \%$ (95\% Cl: 41.3 to $92.7 \%)(p=0.0002)$. In participants receiving $>48 \mathrm{~h}$ methylprednisolone, an absolute reduction in $\mathrm{FiO}_{2}$ requirement at day 7 of $0.25(0.15$ to $0.35, \mathrm{p}=0.0002$ ) and an $85.1 \%$ relative reduction in CRP at day 7 ( 69.6 to $93.1 \%, p=0.0002$ ) was observed. Higher age was associated with mortality in the methylprednisolone group $(p=0.003)$.

Conclusion: Methylprednisolone may reduce early mortality in COVID-19 patients with severe ARDS and is associated with lower oxygen requirements and CRP at day 7.

\section{5}

Physiological effects and safety of bed verticalization in COVID-19 patients with acute respiratory distress syndrome (CARDS):

A Preliminary Report

J. Audard'; L. Bouchant '; G. Arpajou'; L. Aupetitgendre'; S. Cayot ';

R. Guerin'; M. Jabaudon'; C. Verlhac'; R. Blondonnet'; L. Borao' ; B. Pereira ${ }^{2}$. JM. Constantin ${ }^{3}$; JE. Bazin ${ }^{1}$; E. Futier ${ }^{1}$; T. Godet ${ }^{1}$

${ }^{1}$ Department of anesthesiology and intensive care medicine, University Hospital of Clermont-Ferrand, Clermont-Ferrand, France; ${ }^{2}$ Biostatistics unit, direction de la recherche clinique (drci), University Hospital of Clermont-Ferrand, Clermont-Ferrand, France; ${ }^{3}$ Department of anesthesiology and intensive care medicine, University Hospitals Pitié Salpêtrière - Charles Foix, Paris, France

Correspondence: L. Bouchant

Intensive Care Medicine Experimental 2020, 8(2): 000525

Introduction: Prone positioning has been proposed as a key aspect of care in ARDS (1), more than ever with emergence of COVID-19 pandemic (2), but sometimes at the cost of burdensome procedure and serious adverse events. Bed verticalization (standing upright) could be an efficient alternative to prone position. Semi-seated position (45-degree head-up, 45-degree legs-down) have already been studied and shew promising results $(3,4)$, but to our knowledge no study has evaluated the mechanical and physiological impacts of complete patient verticalization (standing upright) during ARDS.

Objectives: To evaluate the safety and physiological effects of bed verticalization of sedated and ventilated COVID-19 patients with ARDS (CARDS).

Methods: Patients were gradually verticalized, using a dedicated bed, at $0^{\circ}, 30^{\circ}, 60^{\circ}$ and $90^{\circ}$ by steps of 30 minutes. At each position step, multiparametric measurements were performed, including 
hemodynamic data with pulmonary artery catheter, ventilatory parameters, end-expiratory lung volume (EELV), esophageal pressures and gazometric parameters.

Results: Ten out of 30 were included for this interim analysis. No serious side effects or adverse event occurred during the verticalization procedures. Mean arterial pressure, and cardiac output remained stable in different patient position. EELV of predicted body weight (PBW) increased significantly from $23(15 ; 29)$ to $35(28 ; 39) \mathrm{mL} . \mathrm{kg}-1$, between baseline to $90^{\circ}$. Alveolar strain was significantly lower $(0.28[0.19 ; 0.46]$ to $0.18[0.15 ; 0.24])$. Although static compliance decreased (36 [27; 43] to $25[19 ; 32] \mathrm{mL} . \mathrm{cmH}_{2} \mathrm{O}-1 . \mathrm{kg}-1$, baseline and $90^{\circ}$ position, respectively, $\mathrm{P}=0.01)$, transpulmonary driving pressure did not increased significantly between these two steps $\left(7[5 ; 11]\right.$ to $9[5 ; 11] \mathrm{cmH}_{2} \mathrm{O}, \mathrm{P}=$ $0.9)$, as well as total mechanical power $(10[8 ; 13]$ to $10[9 ; 16]$ J.min-1, $\mathrm{P}=0.5$ ).

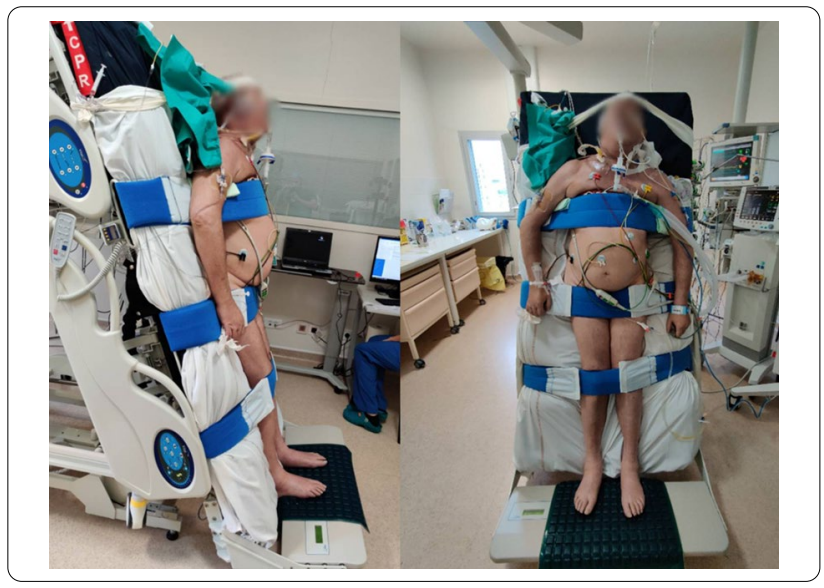

Conclusion: Bed verticalization in ARDS is feasible, safe, and shows promising results on respiratory mechanic with minimal impact on hemodynamics. The rest of inclusions and data are needed to conclude on verticalization efficiency and eventually to identify some responders patients.

\section{Reference(s) and grant ackowledgment(s)}

1. VitalGo Systems Inc., Arjo AB, Illinois, USA, for providing us verticalization beds

2. 1. Guérin $C$, Reignier J, Richard J-C, Beuret $P$, Gacouin A, Boulain T, Mercier E, Badet M, Mercat A, Baudin O, Clavel M, Chatellier D, Jaber S, Rosselli S, Mancebo J, Sirodot M, Hilbert G, Bengler C, Richecoeur J, Gainnier M, Bayle F, Bourdin G, Leray V, Girard R, Baboi L, Ayzac L, PROSEVA Study Group. Prone positioning in severe acute respiratory distress syndrome. N Engl I Med 2013;368:2159-2168.

3. 2. Marini JJ, Gattinoni L. Management of COVID-19 Respiratory Distress. JAMA 2020;https://doi.org/10.1001/jama.2020.6825.

4. 3. Dellamonica J, Lerolle N, Sargentini C, Hubert S, Beduneau G, Di Marco F, Mercat A, Diehl JL, Richard JCM, Bernardin G, Brochard L. Effect of different seated positions on lung volume and oxygenation in acute respiratory distress syndrome. Intensive Care Med 2013;39:1121-1127.

5. 4. Richard J-CM, Maggiore SM, Mancebo J, Lemaire F, Jonson B, Brochard L. Effects of vertical positioning on gas exchange and lung volumes in acute respiratory distress syndrome. Intensive Care Med 2006;32:1623-1626.

\section{2}

\section{Respiratory pattern of critically ill patients with SARS COV 2} Infection at ICU admission

M. Perez Ruiz'; S. Moreno Cano'; A. Breval Flores'; B. Gimenez Beltrán'; B. Diez Del Corral' ${ }^{1}$; A. Estella ${ }^{1}$

${ }^{1}$ University hospital of jerez., Intensive care unit, Jerez, Spain

Correspondence: M. Perez Ruiz

Intensive Care Medicine Experimental 2020, 8(2): 000562
Introduction: Respiratory failure is the most severe form of clinical presentation of SARS COV 2 pandemic infection. 10\% of hospital admissions required to be treated in Intensive Care Units (ICU).

Objectives: To describe clinical characteristics and respiratory patterns in critically ill patientsadmitted in ICU with SARS COV 2 infection. Methods: Retrospective study in a community hospital with a 17 bed ICU. We included patients with confirmed SARS COV 2 respiratory infection. Variables analyzed were clinical characteristics, respiratory support requirements, respiratory rate and inspiratory fraction of oxygen in Emergency room, $\mathrm{PaO}_{2} / \mathrm{FiO}_{2}$ ratio at admission, 24, 48 and 72 hours, peep, $\mathrm{FiO}_{2}$ requirements and mortality.

Statistical analysis: Data were analyzed by SPSS 18 and quantitative variables were expressed as a mean \pm standard deviation.

Results: Twenty consecutive patients were included. Mean age was $61,7 \pm 8,25$. $65 \%$ male and $35 \%$ female. Apache II at admission was $10,3 \pm 4,21$ and SOFA score $3,45 \pm 1,87$. Chest CT was performed in $20 \%$ of the patients. Predominant chest radiograph findings were bilateral lung involvement in $90 \%$ of cases. Mean PEEP at admission was $11,88 \pm 1,85$ and inspiratory fraction of oxygen was $65,94 \pm 18,9$. Non-invasive ventilation BIPAP mode was used in $5 \%$ and high flow oxygen therapy in $45 \%$ of the admitted patients. Mechanical ventilation was prescribed in $80 \%$ of patients, in $56,3 \%$ of them high flow oxygen therapy was used before intubation. Prone ventilation was indicated in most of the patients, $93,8 \%$. Mortality was $30 \%$, figure 1 shows $\mathrm{PaO}_{2} / \mathrm{FiO}_{2}$ ratio based on mortality.

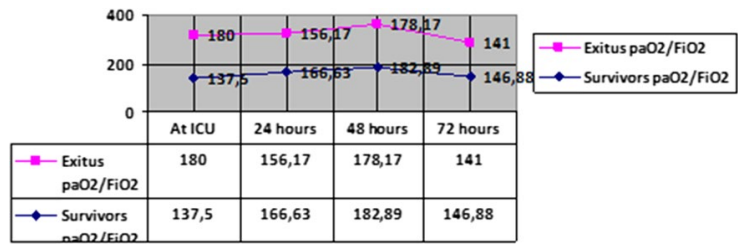

Conclusion: Mechanical ventilation requirement and prone ventilation at ICU admission was common in SARS COV 2 critically ill patients. Improvement of $\mathrm{PaO}_{2} / \mathrm{FiO}_{2}$ in first 48 hours of ICU admission was related with better prognostic.

\section{Reference(s) and grant ackowledgment(s) \\ 1. No}

000577

Therapeutic effects of high-frequency oscillatory ventilation as the rescue management for premature infants with severe refractory respiratory failure

MH. Tsai'; SM. Chu²; JF. Hsu²

${ }^{1}$ Pediatrics, Chang Gung Memorial Hospital, Yunlin, Taiwan; ${ }^{2}$ Pediatrics, Chang Gung Memorial Hospital, Linkou, Taiwan

Correspondence: M.H. Tsai

Intensive Care Medicine Experimental 2020, 8(2): 000577

Introduction: Despite wide application of high frequency oscillatory ventilation (HFOV) in neonates with respiratory distress, little has been reported about its rescue use in preterm infants

Objectives: We aimed to evaluate the therapeutic effects of HFOV in preterm neonates with refractory respiratory failure, investigate the parameters during HFOV and the independent risk factor for 120-day outcomes.

Methods: We retrospectively analyzed data collected prospectively (January 2014-December 2018) in multiple neonatal intensive care units of tertiary-level medical centers in Taiwan. All premature infants (gestational age $<34$ weeks) receiving HFOV as rescue management for refractory respiratory failure were included.

Results: We analyzed the demographics, pre- and daily post-HFOV mechanical ventilation settings and use of adjunctive therapies, therapeutic responses and 120-day outcome data for 668 preterm neonates with refractory respiratory failure. The median (IQR) gestational age 
and birth weight were 27.3 (25.3-31.0) weeks and 915.0 (710.0-1380.0) $\mathrm{g}$, respectively. Pre-HFOV use of cardiac inotropic agents and inhaled nitric oxide were $70.5 \%$ and $23.4 \%$, respectively. The oxygenation index $(\mathrm{OI}), \mathrm{FiO}_{2}$, and $\mathrm{AaDO} 2$ were markedly increased after HFOV initiation (all $p<0.001$ ), and can be decreased within 24-48 hours (all $p$ $<0.001)$ after use of HFOV. $547(81.9 \%)$ patients had a good response to HFOV within 3 days. The final in-hospital mortality rate was $34.7 \%$. No association was found between specific primary pulmonary disease and survival in multivariate analysis. Initial severity of respiratory failure, response to HFOV treatment, occurrence of sepsis, severe hypotension and patients with underlying perinatal asphyxia were independently associated with the 120-day mortality.

Conclusion: Nearly two-thirds of preterm infants with severe respiratory failure had well response to HFOV, mostly within the first 48 hours. For preterm infants with sepsis, severe hypotension and perinatal asphyxia, alternative strategies are warranted and urgently needed to investigate, in order to optimize outcomes.

\section{5}

\section{Differences in evolution during ICU admission between patients} with acute respiratory failure by SARS-COV 2 who received non-invasive respiratory support prior to admission versus those who did not receive it

B. Llorente Ruiz ; A. Ruiz García ; C. Serrano ${ }^{1}$; R. Araoz Illanes ${ }^{1}$; A. Acha ${ }^{1}$; J. Hiquera Lucas'; M. Trascasa Muñoz'; R. Molina Montero'; E. Serrano Yebenes ${ }^{3}$; F. Poveda Saenz ${ }^{4}$; C. Demartini Foj'; E. Nevado

'Intensive care unit, Hospital Príncipe de Asturias, Alcalá de Henares, Spain; ${ }^{2}$ Medicina intensiva, Hospital Universitario Príncipe de Asturias, Alcala de Henares, Spain; ${ }^{3}$ General surgery, Hospital Príncipe de Asturias, Alcalá de Henares, Spain; ${ }^{4}$ Anesthesiology, Hospital Príncipe de Asturias, Alcalá de Henares, Spain

Correspondence: A. Ruiz García

Intensive Care Medicine Experimental 2020, 8(2): 000635

Introduction: Spain has been one of the most affected countries by the SARS-COV2 pandemic with thousands of cases in Madrid. The healthcare overload and excessive number of critical patients increased the capacity of the ICU. Due to assitance pressure, many of the patients with acute respiratory failure de novo had to be treated at the beginning with conventional respiratory support, CPAP systems and NIMV before their admission in ICU.

Objectives: To analyse the evolution, average hospital stay and mortality of our patients who received respiratory support with CPAP, HFOT or BiPAP before their admission in the ICU. Comparing with the critical patients admitted after conventional oxygen therapy failed.

Methods: Retrospective observational study completed in an ICU of a second degree hospital. During the pandemic, ICU beds went from 14 to 39 . We analysed the patients with diagnosis of acute respiratory failure and severe atypical pneumonia by SARS-COV2 admitted from $6 / 3 / 20$ to $21 / 4 / 20$. Patients who are still admitted at the time of data collection are excluded. Demographic variables, medical history, APACHE II, days elapsed from hospital admission to ICU admission, presence of associated organic failure, need for rescue treatment in refractory hypoxemia (prone position, neuromuscular relaxation) were collected. We also gathered analytical parameters of bad prognosis (creatinine, bilirubin, ferritin, D-dimer), days of ICU stay and mortality. Statistical analysis is performed using the IBM ${ }^{\circledR}$ SPSS Statistics 23 tool. Results: We analyse 90 patients. Only 67 received full treatment at our centre due to the lack of beds. All requiring invasive respiratory support, of whom $46.3 \%$ of these patients (31) received respiratory support with CPAP or NIMV systems prior to admission in the ICU. In the subgroup analysis, we did not find statistical significant differences in terms of age, gender, personal history, APACHE II or development of organ failure. No differences were found in the number of prones, tracheostomies or need of neuromuscular blockers. There were not differences in the analytical parameters nor in the number of superinfections, neither in the ICU stay nor in mortality. The only difference was in the days elapsed from hospital admission and ICU admission, being $5.6 \pm 6.15$ days in patients who received non-invasive respiratory support prior to ICU admission versus $3.03 \pm 2.48$ days in patients who did not $(p<0.05)$

Table 1.

\begin{tabular}{|c|c|c|c|}
\hline & Device $(n=32)$ & $\begin{array}{l}\text { No device } \\
(n=35)\end{array}$ & $\mathbf{p}$ \\
\hline Age(y) & $59.32 \pm 9.26$ & $60.66 \pm 10.5$ & $>0.05$ \\
\hline $\operatorname{Sex} \delta / q(\%)$ & $71 / 29$ & $65.71 / 34,29$ & $>0.05$ \\
\hline CV $(\%)$ & 48.39 & 42.86 & $>0.05$ \\
\hline \multirow{2}{*}{$\begin{array}{l}\text { Medical Respiratory } \\
\text { history }(\%) \\
\text { Others(\%) }\end{array}$} & 22.58 & 17.14 & $>0.05$ \\
\hline & 64.52 & 60 & $>0.05$ \\
\hline $\begin{array}{l}\text { Days before ICU } \\
\text { admission }\end{array}$ & $5.64 \pm 6.15$ & $3.03 \pm 2.48$ & $<0.05$ \\
\hline APACHE II & $15.08 \pm 5.8$ & $13.16 \pm 4.56$ & $>0.05$ \\
\hline Vasoactive support & 100 & 97.14 & $>0.05$ \\
\hline Creatinine(mg/dl) & $1.55 \pm 1.55$ & $1.42 \pm 0.99$ & $>0.05$ \\
\hline Bilirubine (mg/dl) & $1.98 \pm 2.03$ & $2.09 \pm 2.41$ & $>0.05$ \\
\hline Ferritine (ng/ml) & $1743,27 \pm 1726,38$ & $2627.73 \pm 4216.26$ & $>0.05$ \\
\hline D-dimer (mg/l) & $17.13 \pm 22,15$ & $18.85 \pm 26.59$ & $>0.05$ \\
\hline Prone (\%) & 67.74 & 62.86 & $>0.05$ \\
\hline $\begin{array}{l}\text { Neuromuscular } \\
\text { blockers(\%) }\end{array}$ & 74.19 & 74.28 & $>0.05$ \\
\hline Tracheostomy (\%) & 45.16 & 43.75 & $>0.05$ \\
\hline Mortality (\%) & 45.16 & 48.57 & $>0.05$ \\
\hline Days at UCI & $13.97 \pm 8.37$ & $14.14 \pm 11.65$ & $>0.05$ \\
\hline
\end{tabular}

Conclusion: In our sample, the patients diagnosed with refractory hypoxemia who were treated with non invasive respiratory devices had not a worse evolution, longer ICU stay or increased mortality.

We did find a difference in the time elapsed between hospital admission and ICU admission. Patients who were treated with non invase respiratory devices were admitted later than patients who did not require them.

\section{8}

Derivation of "specific population who could benefit from Rosuvastatin": A secondary analysis on randomised controlled trial to uncover novel value of Rosuvastatin for precise treatment of ARDS

S. Zhang

'zhongda hospital, Nanjing, China

Correspondence: $\mathrm{S}$. Zhang

Intensive Care Medicine Experimental 2020, 8(2): 000648

Introduction: The high heterogeneity of ARDS contributes to paradoxical conclusions from previous investigations of rosuvastatin for ARDS. Identification of the population (phenotype) who could benefit from rosuvastatin is a novel exploration for precise treatment of ARDS. Methods: The patient population for this analysis consisted of unique patients with ARDS enrolled in the SAILS trial (Rosuvastatin vs. Placebo). Phenotypes were derived using consensus $\mathrm{k}$ means clustering applied to routinely available clinical variables within 6 hours of hospital presentation before receiving placebo or rosuvastatin. KaplanMeier statistic was used to estimate the 90 day cumulative mortality for screening specific population who could benefit from rosuvastatin, with cut-off value as $\mathrm{P}<0.05$.

Results: The derivation cohort included 585 patients with ARDS. Of the 4 derived phenotypes, phenotype 3 was identified as "specific population who could benefit from rosuvastatin" since rosuvastatin resulted in a significant reduction in 90 day cumulative mortality for ARDS (hazard ratio [HR] $0.29[95 \% \mathrm{Cl} 0.09,0.93] ; \mathrm{P}=0.027$ ). Meanwhile, there were no significant differences in baseline characteristics between those assigned to rosuvastatin and those assigned to placebo. Additionally, rosuvastatin markedly improved the free of cardiovascular failure $(10.08 \pm 3.79$ in Rosuvastatin group vs $7.31 \pm 4.94$ in Placebo group, $\mathrm{P}=0.01)$ and coagulation abnormality $(13.65 \pm 1.33$ vs $12.15 \pm 3.77, \mathrm{P}=0.02$ ) to day 14 in phenotype 3 . Patients classified as phenotype 3 exhibited but not limited to the relative higher platelet 
count $\left(390.05 \pm 79.43 \times 10^{\wedge} 9 / \mathrm{L}\right)$, lower CRP $(20.23 \pm 11.99 \mu \mathrm{g} / \mathrm{L})$ and Creat $(1.42 \pm 1.08 \mathrm{mg} / \mathrm{dl})$, compared with patients classified as other phenotypes. Besides that, rosuvastatin seemed to increase 90 day mortality for patients in phenotype $4(\mathrm{HR} 2.76[95 \% \mathrm{Cl} 0.09,9.93], \mathrm{P}=$ $0.076)$, with its adverse effect on the reduction of free of renal failure to day $14(4.70 \pm 4.99$ vs $10.17 \pm 4.69, P=0.01)$. Patients in phenotype 4 showed a relative severe illness baseline features particularly renal failure.

Conclusion: This secondary analysis of SAILS trial identified the specific population who can benefit from rosuvastatin using machine learning applied to clinical variables at the time of hospital presentation, which uncovered a novel value of rosuvastatin for the treatment of ARDS, with validation clinical trials to be warranted to assess these further.

\section{Reference(s) and grant ackowledgment(s)}

1. Matthay, M., Zemans, R. The Acute Respiratory Distress Syndrome: Pathogenesis and Treatment. Annual Review of Pathology 2011;6(1): 147-163.

2. Bellani G, Laffey JG, Pham T, et al. Epidemiology, patterns of care, and mortality for patients with acute respiratory distress syndrome in intensive care units in 50 countries. JAMA 2016;315(8):788-800

3. Thompson BT, Chambers RC, Liu KD. Acute respiratory distress syndrome. N Engl J Med 2017;377(19):1904-1905.

\section{0}

Improved survival outcome in SARS-COV-2(COVID-19) acute respiratory distress syndrome patients with Tocilizumab administration

N. Wadud'; N. Ahmed ${ }^{1}$; M. Shergill'; M. Khan ${ }^{1}$; M. Krishna ${ }^{2}$; A. Gilani²;

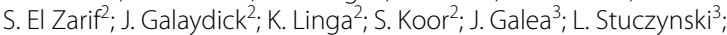

M. Osundele

${ }^{1}$ Internal Medicine, Orange Regional Medical Center, Middletown, United States of America; ${ }^{2}$ Pulmonary, critical care and sleep medicine, Orange Regional Medical Center, Middletown, United States of America; ${ }^{3}$ Pharmacy, Orange Regional Medical Center, Middletown, United States of America

Correspondence: N. Wadud

Intensive Care Medicine Experimental 2020, 8(2): 000710

Introduction: The novel human coronavirus, severe acute respiratory syndrome coronavirus-2 (SARs-CoV-2), was declared a global pandemic by the World Health Organization on March 11, 2020. Hence, there is an urgency to find effective treatment. Of those patients afflicted in the United States, many have required treatment with ventilator secondary to acute respiratory distress syndrome (ARDS). Data are needed regarding the benefit of treatment and prevention of the cytokine storms in COVID-19 patients with Tocilizumab.

Objectives: There are over 5.8 million confirmed cases and 360,412 deaths have been identified worldwide as of May 28, 2020 due to SARs-CoV-2 (COVID-19). Our study showed improved survival outcome in COVID-19 ARDS patients treated with Tocilizumab.

Methods: Clinical outcomes data for patients admitted to Orange Regional Medical Center with confirmed COVID-19 from Mar 15, 2020 to Apr 20, 2020 were identified through electronic health record chart review. We conducted a retrospective case-control study in confirmed COVID 19 positive patients with ARDS requiring mechanical ventilation and compared outcome in terms of mortality and length of stay amongst those who received Tocilizumab as treatment modality opposed to those that did not.

Results: A total of 94 patients with COVID-19 ARDS were analyzed. 44 were in the study group and 50 in the control group. We tried to match both group as close as possible in terms of age, sex, BMI and HS score- calculated using inflammatory markers- ferritin, triglycerides, AST and fibrinogen. The median age was 55.5 years in the study group and 66 in the control group, difference was not statistically significant. Average HS score was 114 in the Tocilizumab group and 92 in the control group, difference was statistically significant with $P<0.0001$. Also, the patients in the study group had elevated levels of IL-6, triglycerides, AST, ferritin which were statistically significant with $p<0.0001$ when compared to the control group. Length of stay was longer, average 17.9 days in the Tocilizumab. Survival rate was much lower at $48 \%$ in the control group and $61.36 \%$ in patients who received Tocilizumab with significant $P$ value of $<0.00001$. The number needed to treat (NNT) was 7.48, if we treat 8 patients with Tocilizumab, 1 will not die.

Conclusion: Cytokine Release Syndrome (CRS) occurs in a large number of patients with severe COVID-19, which is also an important cause of death. IL- 6 is the key molecule of CRS, so IL-6R antagonist Tocilizumab may be of value in improving outcomes. In our study Tocilizumab group seemed to have improved survival outcome.

Reference(s) and grant ackowledgment(s)

1. Ortiz-Martínez Y. Tocilizumab: A new opportunity in the possible therapeutic arsenal against COVID-19.Travel Med Infect Dis. 2020 Apr

2. Puja Mehta, Daniel McAuley, Michael Brown, Emilie Sanchez, Rachel Tattersall, Jessica Mason. COVID-19: Consider cytokine storm syndromes and immunosuppression. The Lancet, March 2020

3. Zhang $W$, Zhao Y, Zhang F, et al. The use of anti-inflammatory drugs in the treatment of people with severe coronavirus disease 2019 (COVID-19): The Perspectives of clinical immunologists from China. Clin Immunol. 2020

4. Xiaoling Xu, MingfengHan, Tiantian Li, Wei Sun, Dongsheng Wang, Binqing Fu, Yonggang Zhou, Xiaohu Zheng, Yun Yang, Xiuyong Li, Xiaohua Zhang, Aijun Pan, Haiming Wei. Effective Treatment of Severe COVID-19 Patients with Tocilizumab.

5. Zhang C, Wu Z, Li JW, Zhao H, Wang GQ. The cytokine release syndrome (CRS) of severe COVID-19 and Interleukin-6 receptor (IL-6R) antagonist Tocilizumab may be the key to reduce the mortality. Int J Antimicrob Agents. Mar 2020

6. Guan W, Ni Z, Hu Y, et al. Clinical Characteristics of Coronavirus Disease 2019 in China. N Engl J Med 2020

7. Chen C, Zhang XR, Ju ZY, He WF. Advances in the research of cytokine storm mechanism induced by CoronaVirus Disease 2019 and the corresponding immunotherapies Chinese Journal of Burns. March 2020

8. Grasselli G, Zangrillo A, Zanella A, et al. Baseline Characteristics and Outcomes of 1591 Patients Infected With SARS-CoV-2 Admitted to ICUs of the Lombardy Region, Italy. JAMA 2020

9. Bingwen Liu, Min Li, Zhiguang Zhou, Xuan Guan, Yufei Xiang. Can we use interleukin-6 (IL-6) blockade for coronavirus disease 2019 (COVID19)- induced cytokine release syndrome (CRS)? Journal of Autoimmunity, April 2020

10. Michot JM, Albiges L, Chaput N, Saada V, Pommeret F, Griscelli F,Balleyguier C, Besse B, Marabelle A, Netzer F, Merad M, Robert C, Barlesi F, Gachot B, Stoclin A.Tocilizumab, an anti-IL6 receptor antibody, to treat Covid-19-related respiratory failure: a case report. Annals of Oncology, 2020

11. Funding statement: No external funding was received

\section{7}

\section{Injury to the Endothelial Glycocalyx in critically ill Covid-19} patients

PA. Gronski'; S. Klaus²; Y. Kiyan ${ }^{1}$; B. Seeliger ${ }^{3}$; A. Bertram ${ }^{1}$; T. Pape ${ }^{1}$; T. Welte ${ }^{3}$. MM. Hoeper'; M. Busch' O. Wiesner'; H. Haller'; S. David

${ }^{1}$ Department of nephrology and hypertension, Medizinische Hochschule Hannover MHH, Hannover, Germany; ${ }^{2}$ Department of gastroenterology, hepatology and endocrinology, Medizinische Hochschule Hannover $\mathrm{MHH}$, Hannover, Germany; ${ }^{3}$ Department of Respiratory Medicine, Hannover Medical School, Hannover, Germany

Correspondence: P.A. Gronski

Intensive Care Medicine Experimental 2020, 8(2): 000727

Introduction: The severe acute respiratory syndrome coronavirus 2 (SARS-CoV-2) can infect the endothelium leading to microvascular endothelialitis. The endothelial glycocalyx (eGC) plays an essential role in maintaining endothelial function.

Objectives: To investigate if injury to the eGC can be found in critical ill Covid-19 patients

Methods: Sublingual assessment of the eGC via sublingual dark field imaging was performed in 19 critically ill Covid-19 patients within $24 \mathrm{~h}$ after intubation and in 10 controls. Blood samples 
were analyzed for markers of endothelial and eGC injury. A 3D microfluidic flow assay was performed to examine eGC ex vivo in naïve and Heparanase-2 overexpressing endothelial cells (ECs).

Results: In Covid-19 patients, transmembrane proteins such as Syndecan-1 and sTie2 were increased in the circulation indicting pathological shedding. Hpa-1, a key regulator of eGC, and its enzymatic activity were unchanged. In contrast, its protective counterpart $\mathrm{Hpa}-2$ was profoundly reduced (median (IQR) 18.7 (10.6-31.1) U/ml vs. 4.7 (2.6-5.6) $\mathrm{U} / \mathrm{ml}, \mathrm{p}=0.014$ ). Ex vivo stimulation of $\mathrm{ECs}$ with serum from Covid-19 patients was sufficient to lower $\mathrm{Hpa}-2$ transcription and to induce glycocalyx damage that could be rescued by lentiviral $\mathrm{Hpa-2}$ overexpression. Consistently, in vivo imaging of the eGC revealed a reduced thickness (increased perfused boundary region (PBR) 1.9 (1.8-1.9) $\mu \mathrm{m}$ vs. 2.1 (1.8-2.2) $\mu \mathrm{m}, \mathrm{p}=0.009)$ in Covid-19.

Conclusion: In critically ill patients with Covid-19, early endothelial injury involves the integrity of the glycocalyx. Acquired Hpa-2 deficiency might be a potentially causative factor. These observations may trigger novel therapeutic interventions aiming at protecting endothelial function.

\section{4}

COVID-19 Patients treated in the Intensive Care Unit: a case series study from Cyprus

A. Kastoris ' ; C. Efseviou' ; A. Papastylianou'; E. Soteriades ${ }^{2}$; L. Palazis

${ }^{1}$ Intensive care unit, Nicosia General Hospital, Nicosia, Cyprus; ${ }^{2}$ College of medicine and health sciences, Institute of Public Health, Al Ain, United Arab Emirates

Correspondence: A. Kastoris

Intensive Care Medicine Experimental 2020, 8(2): 000734

Introduction: Understanding the progression and optimum management of the COVID-19 as a "new disease" is of vital importance for healthcare workers worldwide to improve severe disease outcomes.

Objectives: The main purpose of the study is to investigate the progression of critically ill patients with confirmed COVID-19 infection in the ICU.

Methods: All consecutive patients who were admitted in the ICU of the Nicosia General Hospital, Cyprus due to severe COVID-19, between March 10th and May 1st, 2020 were reviewed. A confirmed case of COVID-19 was defined as a positive result on a reverse-transcriptase-polymerase-chain-reaction (RT-PCR) assay of a specimen collected either from nasopharyngeal swab or bronchial secretions. Data was collected regarding respiratory, cardiovascular, infectious, and other parameters. Results: A total of 19 patients with confirmed COVID-19 were identified. Their mean age was 64 , while $74 \%$ were men. Obesity $(63 \%)$ and hypertension (53\%) were the most common risk factors for ICU admission. Most patients were admitted with severe respiratory failure $(68 \%)$ and all required mechanical ventilation. Patients were categorized in four groups of ventilation, based on $\mathrm{H}$ or $\mathrm{L}$ ventilation phenotype in association with co-morbidities. The first category were $L$ phenotype patients that seemed to benefit from a few days of mechanical ventilation. These patients suffered from no of minor comorbidity. The second category $L$ phenotype patients that seemed to suffer from ICU complications and had a prolonged weaning process. These patients had several comorbidities and/or older age. The third category were $\mathrm{H}$ phenotype patients that eventually changed to L phenotype. Among this group the patients exhibited radiological and ultrasonographic signs of atelectasis. Prone positioning seemed of great benefit in these patients. It may be argued that the transition from $\mathrm{H}$ to $\mathrm{L}$ phenotype may be due to the improvement of atelectasis rather than a change or improvement of lung inflammation. This group exhibited obesity with a high waist circumference partially explicatory of the high atelectasis rate found. Lastly the category "classic" ARDS patients or pure $\mathrm{H}$ phenotype. They had the worst outcome since they suffered from severe ARDS. $68 \%$ of patients required vasopressor support, and $42 \%$ developed AKI during their stay. Diarrhea was common with a median day of onset of 8 days. Lactate levels above $2 \mathrm{mmol} / \mathrm{L}$ in the first four days of admission were correlated with a negative outcome. Eight patients $(42 \%)$ were successfully discharged from the ICU, 7 (39\%) died and 4 patients remain in the ICU.
Conclusion: Obesity and male gender are significant risk factors for ICU admission. Ventilation phenotype on admission and during hospitalization may be predictive of outcome. Mechanical ventilation, lower PEEP values and early prone position may provide more favorable outcomes especially in $L$ phenotype patients.

\section{1}

Acute pancreatitis complicated with refractory ARDS supported by ECMO: a French multicentric retrospective study (preliminary results)

S. Rozencwajg ${ }^{1}$; A. Tran Dinh' ${ }^{1}$; A. Foubert ${ }^{2}$; P. Seguin²;G. Haye ${ }^{3}$; H. Dupont ${ }^{3}$; PG. Guitard ${ }^{4}$; B. Veber ${ }^{4}$; T. Godet ${ }^{5}$; JE. Bazin ${ }^{5}$; P. Montravers ${ }^{1}$

${ }^{1}$ Anaesthesiology and surgical intensive care unit, Bichat-Claude Bernard Hospital, Paris, France; ${ }^{2}$ Anaesthesiology and surgical intensive care unit, Pontchaillou University Hospital Center, Rennes, France; ${ }^{3}$ Anaesthesiology and surgical intensive care unit, Amiens-Picardie University Hospital Center, Amiens, France; ${ }^{4}$ Anaesthesiology, pre-hospital and surgical intensive care unit, Charles Nicolle University Hospital, Rouen, France; ${ }^{5}$ Department of anesthesiology and intensive care medicine, University Hospital of Clermont-Ferrand, Clermont-Ferrand, France

Correspondence: S. Rozencwajg

Intensive Care Medicine Experimental 2020, 8(2): 000751

Introduction: Acute pancreatitis (AP) is described as a common cause of acute respiratory distress syndrome (ARDS). Paradoxically, despite exponential increase in ECMO (ExtraCorporeal Membrane Oxygenation) use over the past decade1, literature remains very poor concerning its use in these patients2.

Objectives: Our objective was to describe the use of ECMO in this specific population.

Methods: French multicentric retrospective study. All adult patients on ECMO for an ARDS secondary to AP between 2010 and 2020 were included in the analysis. Demographic data, AP severity, respiratory complications and outcomes were collected. Results are expressed in median [interquartile range] and proportions.

Results: Twenty-five patients with severe ARDS following PA requiring ECMO were identified, of which 5 were secondarily excluded (4 ECMO post-cardiac arrest, 1 for aspiration pneumonia unrelated to PA).

Patients were young (47 [38-60] years) and obese (body mass index $30[26-34] \mathrm{kg} / \mathrm{m}^{2}$ ) men (90\%) admitted for a severe AP (BISAP 2 score [1-2]), mainly of alcoholic (45\%) and biliary (20\%) origin. They presented a median sequential organ failure assessment (SOFA) score of 8 [4-13] at admission and a simplified acute physiology score (SAPS 2) of 50 [34-61]

Prior to ECMO initiation, ventilatory characteristics were as follows: tidal volume of 6.9 [5.7-8.6] mL/kg of ideal body weight, positive endof-expiration pressure of 10 [8-12] $\mathrm{cmH}_{2} \mathrm{O}$ and a plateau pressure of 30 [30-32] $\mathrm{cmH}_{2} \mathrm{O}$. Associated adjuvant therapies were neuromuscular blockades (100\%), prone positioning (30\%) and nitric oxide (30\%). Before ECMO initiation, patients had a median pH of 7.26 [7.20-7.38] and $\mathrm{P} / \mathrm{F}$ ratio of 58 [52-82].

ECMO support was mainly venovenous $(80 \%)$ with details presented in the Table. Thirteen patients $(65 \%)$ underwent at least one surgical intervention under ECMO, mainly for digestive ischemia or abdominal compartment syndrome. Outcomes are listed in the Figure.

\begin{tabular}{|l|l|}
\hline \multicolumn{2}{|c|}{ Main ECMO characteristics } \\
\hline \begin{tabular}{l|l|}
\hline \multicolumn{2}{|c|}{ Variables } \\
Time from mechanical ventilation to ECMO, median \\
{$[$ IQR], days }
\end{tabular} & $2[0-10]$ \\
\hline VV-ECMO / VA-ECMO / VAV-ECMO & $\begin{array}{l}16(80) / 3(15) / 1 \\
(5)\end{array}$ \\
\hline Refractory hypoxaemia & $14(70)$ \\
\hline Unable to achieve protective ventilation & $3(15)$ \\
\hline Uncontrolled hypercapnia & $2(10)$ \\
\hline Cardiogenic shock & $1(5)$ \\
\hline
\end{tabular}




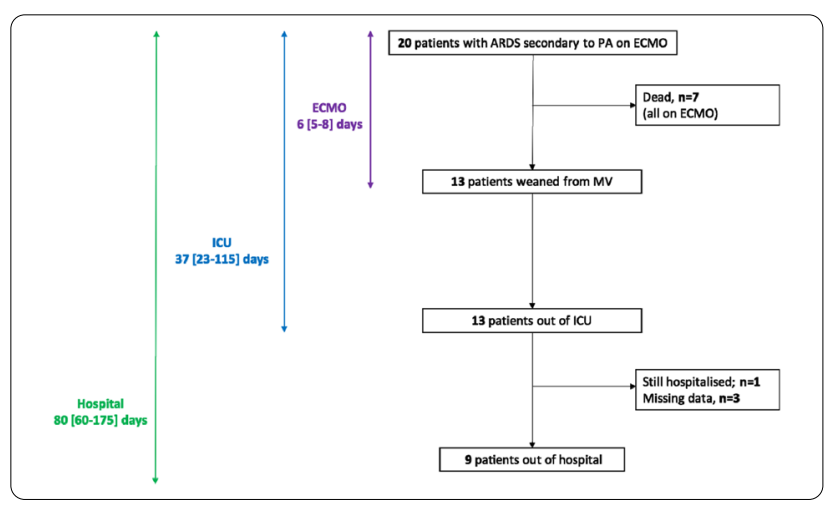

Conclusion: ECMO appears to be an acceptable therapy for ARDS secondary to AP due to the young age and limited comorbidities of these patients. In particular, the need for abdominal surgery does not seem to change the risk-benefit balance and should not contraindicate ECMO in this population. A study comparing this cohort to AP in ARDS without ECMO will provide more answers and is currently underway.

\section{Reference(s) and grant ackowledgment(s)}

1. 1 https://www.elso.org/Registry/Statistics/InternationalSummary.aspx 2. 2 Bryner BS, Smith C, Cooley E, Bartlett RH, Mychaliska GB. Extracorporeal life support for pancreatitis-induced acute respiratory distress syndrome. Ann Surg. 2012;256(6):1073-1077. https://doi.org/10.1097/SLA.0b013e3182 $5 \mathrm{~d} 33 \mathrm{c} 1$

\section{3}

\section{High-Flow Nasal Cannula in patients with COVID-19; a prospective} cohort study

JP. van Oosten ${ }^{1}$; Y. Türk ${ }^{2}$; M. van Bochove-Waardenburg ${ }^{1}$; Y. Aga ${ }^{2}$;

WEJJ. Hanselaar ${ }^{2}$; HE. Endeman ${ }^{1}$; EJ. Wils ${ }^{3}$

${ }^{1}$ Intensive care, Erasmus University Medical Center, Rotterdam, Netherlands; ${ }^{2}$ Pulmonology, Franciscus Gasthuis, Rotterdam, Netherlands; ${ }^{3}$ Intensive care, Franciscus Gasthuis, Rotterdam, Netherlands

Correspondence: J.P. van Oosten

Intensive Care Medicine Experimental 2020, 8(2): 000763

Introduction: Since the end of 2019 the world is enthralled by the novel COVID-19 pandemic, putting extreme pressure on Intensive Care Units. The general advice for patients with progressive respiratory failure due to COVID-19 disease was to start invasive mechanical ventilation (MV) early in the clinical course. This was based on the assumption that COVID-19 led to a moderate to severe ARDS requiring MV with PEEP and to prevent patient self-inflicted lung injury (P-SILI) [1]. In addition, non-invasive ventilation and High-Flow Nasal Cannula (HFNC) were discouraged because of the perceived risk of aerosol formation leading to disease transmission. However, it has become increasingly clear that patients with COVID-19 do not necessarily develop typical ARDS and the transmission risk of HFNC is considered comparable to conventional oxygen therapy [1-3]. HFNC is a validated therapy that can prevent intubation in patients with progressive hypoxemic respiratory failure and can also be used outside the ICU [4]. We hypothesize that implementation of HFNC in the pre-ICU setting can successfully reduce the need for MV and ICU-admission of COVID19 patients.

Objectives: To investigate to what extent HFNC can prevent MV in patients with progressive hypoxemic respiratory failure due to COVID-19.

Methods: We performed a multicenter prospective cohort study between March 2020 and June 2020 on the regular pulmonary wards of an academic and teaching hospital in Rotterdam, the Netherlands. Patients on the regular wards with progressive hypoxemic respiratory failure due to COVID-19 while being treated with maximum oxygen (O2) therapy (peripheral 02 saturation $<92 \%$ or high work of breathing), started with HFNC. Maximum $\mathrm{O} 2$ therapy before HFNC consisted of $15 \mathrm{~L} \mathrm{O2/min} \mathrm{flow} \mathrm{via} \mathrm{a} \mathrm{non-rebreathing} \mathrm{mask} \mathrm{(NRM)} \mathrm{in} \mathrm{one} \mathrm{center}$ and $6 \mathrm{~L} \mathrm{O2/min} \mathrm{flow} \mathrm{via} \mathrm{a} \mathrm{nasal} \mathrm{cannula} \mathrm{in} \mathrm{the} \mathrm{other.} \mathrm{Patients} \mathrm{with} \mathrm{con-}$ traindications for HFNC (such as hypercapnia, severe hypoxia) were excluded and intubated directly. Patients vital parameters (peripheral $\mathrm{O} 2$ saturation, respiratory rate (RR)) and ROX-scores were monitored after one hour, followed by every two hours during HFNC. In case of an increasing oxygen demand or exhaustion, assessed by the corresponding ROX-scores, MV was initiated [5].

Results: HFNC was initiated in a total of 30 patients for progressive respiratory failure on conventional $\mathrm{O} 2$ therapy ( $\mathrm{n}=25$ nasal cannula; $\mathrm{n}=5$ NRM). Patients started HFNC after a median of 3 days (IQR: 1-4) after hospital admission, while having symptoms for a median of 11 days (IQR: 9-15). The median peripheral O2 saturation and RR at start of HFNC was 94\% (IQR: 93-96) and 28/min (IQR: 24-32) respectively. 14 out of $30(47 \%)$ were eventually intubated after a median of 12 hours (IQR: 8-24) after starting HFNC. 2 out of 5 on an NRM (40\%) were intubated versus 12 out of 25 (48\%) on a nasal cannula. Median ROX-score at time of intubation was 4.82 (IQR: 4.60-4.98). Reasons for intubation were hypoxia $(n=1)$ and somnolence $(n=1)$ but mainly exhaustion $(n=12)$ defined as a $R R \geq 30 / m i n$ with corresponding low ROX-scores. Patients stabilizing on HFNC continued therapy for a median of 5 days (IQR: 2-8) before switching to a nasal cannula.

Conclusion: Our study suggests that HFNC may prevent intubation in a relevant proportion of COVID-19 patients with isolated hypoxemic respiratory failure.

\section{Reference(s) and grant ackowledgment(s)}

1. 5. Roca O, Caralt B, Messika J, Samper M, Sztrymf B, Hernández G, et al. An Index Combining Respiratory Rate and Oxygenation to Predict Outcome of Nasal High-Flow Therapy. American journal of respiratory and critical care medicine 2019 Jun 1;199(11):1368-1376.

2. 4. Rochwerg B, Granton D, Wang DX, et al. High flow nasal cannula compared with conventional oxygen therapy for acute hypoxemic respiratory failure: a systematic review and meta-analysis. Intensive Care Med. 2019;45(5):563-572. https://doi.org/10.1007/s00134-019-05590-5

3. 3. Kotoda M, Hishiyama S, Mitsui K, Tanikawa T, Morikawa S, Takamino A, et al. Assessment of the potential for pathogen dispersal during high-flow nasal therapy. Journal of Hospital Infection 2020 Apr;104(4):534-537.

4. 2. Loh, N.W., Tan, Y., Taculod, J. et al. The impact of high-flow nasal cannula (HFNC) on coughing distance: implications on its use during the novel coronavirus disease outbreak. Can J Anesth/J Can Anesth 67, 893-894 (2020). https://doi.org/10.1007/s12630-020-01634-3

5. 1. Gattinoni L. et al. COVID-19 pneumonia: different respiratory treatment for different phenotypes? (2020) Intensive Care Medicine; https://doi. org/10.1007/s00134-020-06033-2

\section{6}

Lung and chest wall viscoelastic properties of patients with acute respiratory distress syndrome. The role of expiratory flow limitation

C. Guérin ${ }^{1}$; N. Terzi'; M. Cour ${ }^{1}$; L. Argaud ${ }^{1}$; B. Louis ${ }^{3}$

${ }^{1}$ Service de médecine intensive-réanimation, Hospital Édouard Herriot, Lyon, France; ${ }^{2}$ Médecine intensive réanimation, C.H.U de Grenoble, La Tronche, France; ${ }^{3}$ Inserm 955, IMRB, Creteil, France

Correspondence: $C$. Guérin

Intensive Care Medicine Experimental 2020, 8(2): 000796

Introduction: Lung and chest wall tissues absorb the dissipation of the energy during lung inflation through their viscoelastic properties. These can be represented by the Mount rheological model that consists of a Maxwell body (lung and chest wall viscoelastic elastance (E2) and resistance (R2) arranged in series) in parallel with the standard lung and chest wall elastance and resistance, according to the following equation: Pvisc(ti) = R2 V' (1- exp(-ti/t2)) (1)

where Pvisc(ti) represents the viscoelastic elastic pressure dissipation into lung/chest wall during inflation time (ti), $V^{\prime}$ the inflation flow and $\tau 2$ the lung/chest wall viscoelastic time constant $(\tau 2=R 2 / E 2)$. 
Lung and chest wall viscoelastic properties have not previously been described during acute respiratory distress syndrome (ARDS) according to presence or absence of expiratory flow limitation (EFL).

Objectives: We aimed at assessing the relation-ship between EFL and viscoelastic dissipation characteristics of lung/chest wall in ARDS patients.

Methods: This is a secondary analysis of a previously reported study on ARDS patients meeting the Berlin definition criteria (1). Patients were intubated, sedated and paralyzed. Airway and esophageal pressures and flow signals were recorded in semi-recumbent position at PEEP 5, 10 and $15 \mathrm{cmH}_{2} \mathrm{O}$. At PEEP 5, EFL was assessed by the atmospheric method as previously reported (2). Patients were classified as flow-limited (FL) or non-flow-limited (NFL). At each PEEP a 5-s endinspiratory occlusion was performed at baseline ventilator settings. We used the single breath method (2) to estimate the viscoelastic elastance $(E 2, L$ and $E 2, \mathrm{CW})$ and resistance $(\mathrm{R} 2, \mathrm{~L}$ and $\mathrm{R} 2, \mathrm{cW})$ of lung and chest wall, respectively. Values are shown as median (1st-3rd quartiles) and compared between FL and NFL at PEEP 5 by using Wilcoxon rank test. The effect of EFL, PEEP and their interaction was further tested by linear mixed-effects model.

Results: Twenty-five ARDS patients were included. Eight were FL and 17 NFL. At PEEP 5, E2,L and R2,L were significantly higher in FL than in NFL patients, amounting to $12(10-4)$ vs. $6(5-8) \mathrm{cmH}_{2} \mathrm{O} / \mathrm{L}(\mathrm{P}=0.03)$ and $7(5-10)$ vs. $4(2-6) \mathrm{cmH}_{2} \mathrm{O} / \mathrm{L} / \mathrm{s}(\mathrm{P}=0.04)$, respectively. Chest wall $\mathrm{E} 2, \mathrm{CW}$ and $\mathrm{R} 2, \mathrm{CW}$ were not different between $\mathrm{FL}$ and NFL patients. PEEP had no effect on viscoelastic constants but EFL had a significant effect on E2,L and E2,cw without interaction between PEEP and EFL. Over all PEEP levels, E2, L was 11 (7-14) in $\mathrm{FL}$ and 5 (4-7) $\mathrm{cmH}_{2} \mathrm{O} / \mathrm{L}$ in $\mathrm{NFL}(\mathrm{P}=0.02)$ and $\mathrm{E} 2, \mathrm{cw}$ was $2(1-4)$ in $\mathrm{FL}$ and $3(1-3) \mathrm{cmH}_{2} \mathrm{O} / \mathrm{L}$ in NFL $(P=0.003)$.

Conclusion: In ARDS, lung viscoelastic constants are significantly higher in FL than in NFL patients. The mechanisms behind this finding should be explored.

\section{Reference(s) and grant ackowledgment(s)}

1. 1. Journal of Applied Physiology 2020.

\section{1}

\section{Steroid immunomodulation in SARS-CoV-2 patients}

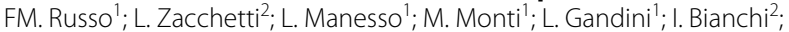
M. Di Matteo ${ }^{2}$; A. Martinelli²; G. Polii' L. Grazioli ${ }^{2}$; F. Previdi ${ }^{3}$; FL. Lorini ${ }^{2}$

${ }^{1}$ Anestesia, rianimazione e terapia intensiva e del dolore, University of Milan, Milano, Italy; ${ }^{2}$ Dipartimento di emergenza urgenza e area critica, ASST Papa Giovanni XXIII, Bergamo, Italy; ${ }^{3}$ Dipartimento di ingegneria e scienze applicate, Università degli studi di Bergamo, Dalmine, Italy Correspondence: F.M. Russo

Intensive Care Medicine Experimental 2020, 8(2): 000801

Introduction: Severe Acute Respiratory Syndrome Coronavirus 2 (SARS-CoV-2) is an atypical Acute Respiratory Distress Syndrome (ARDS), characterized by an initial direct viral insult that could lead to a disproportionate inflammatory response that worsens lung function [1]. To date it is unknown if steroids may be a strategy in viral ARDS [2]. We tested the hypothesis that methylprednisolone reduces mortality at 28 days.

Objectives: Determine the effect of methylprednisolone on mortality at 28 days, on mechanical ventilation (MV) free days and major complications.

Methods: From February 22nd to March 22nd, 130 consecutive patients admitted in 6 ICUs of Ospedale Papa Giovanni XXIII (Bergamo) were retrospectively enrolled. Age, Charlson comorbidity index, SOFA score and $\mathrm{PaO}_{2} / \mathrm{FiO}_{2}$ ratio (P/F) at admission were collected. We compared 67 control patients (control group) with 63 receiving methylprednisolone (steroid group) at either low dose $(1 \mathrm{mg} / \mathrm{kg} / \mathrm{day}, \mathrm{n}=58)$ for 10 days or up to three high dose boluses $(1000 \mathrm{mg}, \mathrm{n}=5)$. Primary outcome was mortality at 28 days. Secondary outcomes were MV-free days and secondary infections. Baseline characteristics and MV-free days were compared in two groups using Mann Whitney test. The association with mortality and infection was evaluated with Fisher exact test.
Results: In our population median age was 62 (IQR 14), 107 (82\%) were male, $115(88 \%)$ required intubation.

In the steroid group all patients received methylprednisolone within the first 14 days after ICU admission, median therapy duration was 10 days (IQR 10). Steroid and control groups were similar in terms of age, Charlson comorbidity index, SOFA score and P/F. Methylprednisolone was associated with reduced mortality at 28 days (OR $0.35,95 \%$ $\mathrm{Cl}$ 0.17-0.77, p 0,009). No differences were observed in MV-free days, secondary infections and septic shock. The table summarizes baseline characteristics and main findings of the study.

$\begin{array}{llll} & \begin{array}{l}\text { Steroid Group } \\ (63)\end{array} & \begin{array}{l}\text { Control Group } \\ (67)\end{array} & p \\ \begin{array}{l}\text { Age, median (IQR) } \\ \text { Charlson comorbidity index, median3 (2) }\end{array} & 60(11) & \mathrm{ns} \\ \text { (IQR) } & & 2(2) & \mathrm{ns} \\ \text { SOFA score, median (IQR) } & 6(2) & 6(2) & \mathrm{ns} \\ \text { PaO2/FiO2, median (IQR) } & 110(59) & 109(69) & \mathrm{ns} \\ \text { Secondary infections, n (\%) } & 30(52) & 25(40) & \mathrm{ns} \\ \text { Septic shock, n (\%) } & 20(32) & 15(26) & \mathrm{ns} \\ \text { MV-free days, median (IQR) } & 0(15) & 0(8.5) & \mathrm{ns} \\ \text { Mortality at 28 days, n (\%) } & 14(22) & 30(45) & *\end{array}$

Conclusion: In our cohort of SARS CoV-2 patients, immunomodulation therapy with methylprednisolone was associated with reduced mortality at 28 days.

\section{Reference(s) and grant ackowledgment(s)}

1. [1] Ingraham NE, Lotfi-Emran S, Thielen BK, et al. Immunomodulation in COVID-19. Lancet Respir Med 2020;2019(20):2019-21.

2. [2] Arabi YM, Fowler R, Hayden FG. Critical care management of adults with community-acquired severe respiratory viral infection. Intensive Care Med 2020:46(2):315-28

\section{1}

Radiological findings in patients with COPD exacerbation as determinants of morbidity and mortality: A cohort study F. Jaimes ${ }^{1}$; S. Lopera²; B. Noreña²; J. Ascuntar ${ }^{3}$; D. Mendoza'; V. Garcia ${ }^{2}$; C. Vallejo ${ }^{3}$

${ }^{1}$ Internal medicine, Universidad de Antioquia, Medellin, Colombia; ${ }^{2}$ Radiology, Universidad de Antioquia, Medellín, Colombia; ${ }^{3}$ Internal medicine, Universidad de Antioquia, Medellín, Colombia

Correspondence: F. Jaimes

Intensive Care Medicine Experimental 2020, 8(2): 000821

Introduction: Patients with chronic obstructive pulmonary disease (COPD) have recurrent exacerbations that contribute to their clinical impairment (1). Computed tomography (CT) scanning can distinguish the underlying changes driven the airflow limitation, and many studies have showed the main pathological characteristics of emphysema, airway wall thickening and reduced lumen caliber (1-3).

CT has been used to quantify emphysema by detecting low attenuation areas (LAA\%), and quantitative parameters of the parenchyma on inspiration and expiration allow indirect evaluation of small airway obstruction. While the quantification of emphysema by CT imaging has been well established and validated, the quantification of airway disease has proven to be more difficult to stablish. The segmental and subsegmental bronchial wall area percent (WA\%) have been found to be correlated with the forced expiratory volume in $1 \mathrm{~s}$ (FEV 1) (1-3).

Objectives: To estimate the association between radiological findings and the need for intensive care unit (ICU) or mortality in patients hospitalized by the emergency room with COPD exacerbation.

Methods: Prospective cohort study with adults attended in the emergency room of three reference hospitals in Medellín (Colombia), who consulted for acute exacerbation of COPD and required hospitalization between 2017 and 2020. Inspiratory and expiratory scans were performed. Morphometric assessment of the airways ([WA\%] and airway inner luminal area [Ai]), as well as extent of emphysema (LAA\%), were performed using 3D Slicer software. Data were collected from the segmental and subsegmental generations of the apical segment 
of the right upper lobe (RB1) and the posterior-basal segmental and subsegmental bronchi of the right lower lobe (RB10). LAA thought to reflect emphysematous destruction of the lung parenchyma were defined using a Hounsfield Unit threshold of - 50 (\%LAA-950). The association between radiological measurements and inpatient death or admission to ICU was explored by locally weighted regression (LOWESS) and simple univariate logistic regression models.

Results: Among 1411 eligible patients, 326 (23\%) were included. The main exclusions were pneumonia (30\%), limiting to perform the CT (24\%) and terminal illness (11\%). The median age was 74 years (IQR $66-82)$ and $56 \%$ were women. Smoking history $(86 \%)$ and exposure to biomass $(61 \%)$ were the main risk factors. Hospital mortality rate was $2.5 \%$ and 40 (12.3\%) patients required admission to ICU, with 43 (13.2\%) patients with the outcome of interest. The means of WA\% were: RB1 segmental 64.6 (58.8-70.7), subsegmental 70.3 (64.5-75.5), and RB10 segmental 63.3 (57.5-70.6), subsegmental 69.4 (62.8 -76.1). The mean for extent of emphysema (\%LAA-950) was 14.7\% (4.2-29.3). In univariate logistic regression analyzes, all radiological measurements showed no association with the outcome of death or need for ICU (Table 1).

\begin{tabular}{|l|l|}
\multicolumn{2}{c}{ Table 1. CT variables } \\
Morphometric variable & Odds Ratio $(95 \% \mathrm{CI})$ \\
\hline Mean airway wall area percent $(\%)[\mathrm{WA}]$ & \\
\hline B1 Segmental & $0.99(0.96-1.02)$ \\
\hline B10 Segmental & $1.01(0.98-1.04)$ \\
\hline Mean airway inner luminal area $\left(\mathrm{mm}^{\wedge}\right.$ 2) $[\mathrm{Ai}]$ & \\
\hline B1 Segmental & $0.99(0.98-1.02)$ \\
\hline B10 Segmental & $0.99(0.98-1.01)$ \\
\hline Volumetry & \\
\hline \%LAA-950 & $0.99(0.97-1.02)$ \\
\hline 15th percentile & $1.00(0.97-1.01)$ \\
\hline
\end{tabular}

Conclusion: Morphometric assessment of the airways and the extent of emphysema did not differ between patients with and without the outcome of death or need for ICU.

\section{Reference(s) and grant ackowledgment(s)}

1. 1. Cheng, T. et al. Computed tomography manifestation of acute exacerbation of chronic obstructive pulmonary disease: A pilot study. Exp. Ther. Med. 11,519-529 (2016). 2. Diaz, A. A. et al. Effect of Emphysema on CT Scan Measures of Airway Dimensions in Smokers. Chest 143, 687-693 (2013).

3. Matsuoka, S., et al. Airway Dimensions at Inspiratory and Expiratory

Multisection CT in Chronic Obstructive Pulmonary Disease: Correlation with Airflow Limitation. Radiology 248, 1042-1049 (2008).

2. COLCIENCIAS (Grant 111577757025, Convocatoria 777-2017) and Universidad de Antioquia

\section{2}

Covid-19 thromboembolic complications and risk factors for poor outcomes: experience from a single ICU

L. Bullman ${ }^{1}$; N. Boyer ${ }^{1}$; BL. Alvarez ${ }^{1}$; R. Pierce ${ }^{1}$; S. Navaneetham ${ }^{1}$;

K. Redington ${ }^{1}$; M. Richardson ${ }^{1}$; Z. Campbell'; K. Papadopoulou' ${ }^{1}$; E. Potter ${ }^{1}$; L. Forni ${ }^{1}$

${ }^{1}$ Intensive care unit, Royal Surrey County Hospital, Guildford, United Kingdom

Correspondence: $\mathrm{K}$. Papadopoulou

Intensive Care Medicine Experimental 2020, 8(2): 000822

Introduction: COVID-19 may be associated with severe respiratory failure that, for patients requiring invasive ventilation carries an estimated mortality of $49 \%$ in the UK (1). A prominent feature of the disease is the development of thromboembolic complications that despite thromboprophylaxis, occurs with an incidence of approximately $50 \%(2,3)$. We evaluated the presenting characteristics and outcomes of the patients admitted with COVID-19 in our ICU, particularly as regards to the development of thromboembolic complications.
Methods: Baseline characteristics, respiratory variables, coagulation and inflammatory parameters and ICU mortality were collected retrospectively on all patients admitted with either PCR-confirmed or clinically diagnosed COVID-19.

Results: Between March and May 202059 admissions with COVID19 were recorded. Thirty-two patients $(58 \%)$ required invasive ventilation and 27 patients (48\%) received therapeutic anticoagulation. Thromboembolic complications occurred in 8 patients (14\%) and $50 \%$ of those died. Of the patients that had ischaemic complications $5(63 \%)$ were on prophylactic and $3(38 \%)$ on therapeutic anticoagulation. Six patients developed haemorrhagic complications and 2 of those required blood transfusion. Mortality in the intubated patients was $47 \%$. There was no difference in the mortality of patients that received therapeutic anticoagulation versus those that did not. Factors associated with mortality included CRP and respiratory variables, whereas D-dimer was associated with the development of thromboembolic complications. Maximum CRP in the first 72 hours of admission was significantly higher in the patients that died (mean (SD) 311 (80) vs 207 (86) $\mathrm{mg} / \mathrm{L}, \mathrm{p}=0.0002$ ); > $300 \mathrm{mg} / \mathrm{L}$ was associated with $>70 \%$ mortality. Minimum S/F ratio (median [IQR]) within the first 72 hours of admission in non-survivors vs survivors was 149 [114-165] vs 192 [157-269], $\mathrm{p}=0.0004$. Patients with lowest $\mathrm{S} / \mathrm{F}<150$ and $\leq 100$ had $60 \%$ and $>80 \%$ mortality respectively. Both D-dimer on admission and mean D-dimer during ICU stay were higher in the patients that experienced thromboembolic complications. However, there was no association between APTTR, anti-Xa, or Fibrinogen values with either mortality or the of thromboembolic complications (Figure).

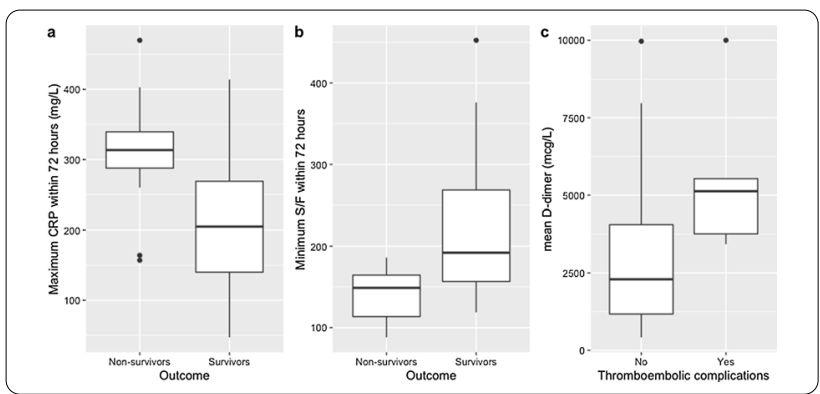

Conclusion: Our analysis shows a lower incidence of thrombotic complications compared to other centres, likely because of our low threshold for anticoagulation. Therapeutic anticoagulation was not associated with improvement in outcomes and that was likely a result of patient selection, as those with clinically severe disease received therapeutic anticoagulation. Similar to other studies, D-dimer and CRP levels were associated with poor outcomes and may be used for prognostication and to guide anticoagulation treatment $(4,5)$.

Reference(s) and grant ackowledgment(s)

1. 1. Intensive care national audit \& research centre. ICNARC report on COVID19 in critical care - 04 April 2020. 2020;(April):1-16. 2. Klok FA, Kruip MJHA, van der Meer NJM, Arbous MS, Gommers D, Kant KM, et al. Confirmation of the high cumulative incidence of thrombotic complications in critically ill ICU patients with COVID-19: An updated analysis. Thromb Res. 2020;191:148. 3. Middeldorp S, Coppens M, van Haaps TF, Foppen M, Vlaar AP, Müller MCA, et al. Incidence of venous thromboembolism in hospitalized patients with COVID-19. J Thromb Haemost [Internet]. 2020 May 5 [cited 2020 Jun 28];jth.14888. Available from: https://onlinelibrary.wiley. com/doi/abs/10.1111/jth.14888 4. Zhou F, Yu T, Du R, Fan G, Liu Y, Liu Z, et al. Clinical course and risk factors for mortality of adult inpatients with COVID-19 in Wuhan, China: a retrospective cohort study. Lancet. 2020 Mar 28;395(10229):1054-62. 5. Wang G, Wu C, Zhang Q, Wu F, Yu B, Lv J, et al. $\mathrm{C}$-reactive protein level may predict the risk of COVID-19 aggravation. Open Forum Infect Dis. 2020;7(5):1-5. 


\section{5}

Characteristics and outcomes of ECMO implementation by a multidisciplinary team in two hospitals in Madrid during 2020 SARS-CoV-2 pandemic

A. Santos ${ }^{1}$; V. Hortiguela'; R. Hernandez-Estefania ${ }^{2}$; M. Perez-Marquez ${ }^{3}$; AM. Ioan ${ }^{1}$; E. Rosas-Carvajal ${ }^{1}$; J. Martinez-Milla ${ }^{4}$; J. Marquez ${ }^{3}$; G. Aldámiz ${ }^{2}$. C. Perez-Calvo

${ }^{1}$ Intensive care medicine department, Hospital Universitario Fundación Jiménez Díaz, Madrid, Spain; ${ }^{2}$ Cardiac surgery, Hospital Universitario Fundación Jiménez Díaz, Madrid, Spain; Intensive care medicine department, Hospital Universitario Rey Juan Carlos, Móstoles, Spain; ${ }^{4}$ Cardiology, Hospital Universitario Fundación Jiménez Díaz, Madrid, Spain

Correspondence: A. Santos

Intensive Care Medicine Experimental 2020, 8(2): 000825

Introduction: SARS-CoV-2 overwhelmed intensive care units capacity around the world. In this circumstances providing high complexity and resources consuming techniques as ECMO was a challenge.

Objectives: To describe characteristics and outcomes of patients receiving ECMO to support complications related with COVID-19 infection.

Methods: ECMO decision was based on ELSO recommendations for SARS-CoV-2 pandemic after discussion between ECMO team members (which consisted of cardiac surgeons, cardiologists and intensive care medicine specialists). Clinical records were analyzed to obtain relevant demographic, gas exchange and mechanical ventilation characteristics and outcomes. Descriptive statistics were performed.

Results: From February to May 2020 around 230 patients ( > 95\% invasive mechanical ventilation application) with SARS-CoV-2 diagnosis were admitted to the intensive care units of the two study centers and ECMO was used in 6 patients. Two H1N1 patients (with $0 \%$ mortality) were also subjected to ECMO during this time period but were excluded of this analysis. Five patients had ARDS as main diagnosis and reason for ECMO (veno-venous) while one had a diagnosis of miopericarditis and cardiogenic shock secondary to COVID-19 and veno-arterial ECMO was used. All patients were male, mean APACHE II was $15 \pm 7$, age $51 \pm 4$ years, post intubation $\mathrm{PaO}_{2} / \mathrm{FiO}_{2}$ ratio was $152 \pm 57 \mathrm{mmHg}(131 \pm 28 \mathrm{mmHg}$ when miopericarditis patient was excluded), $\mathrm{PaCO} 255 \pm 12 \mathrm{mmHg}, \mathrm{pH} 7.32 \pm 0.1$, tidal volume $442 \pm 38$ $\mathrm{ml}$ (always below $8 \mathrm{ml} / \mathrm{kg}$ PBW) and PEEP $13.8 \pm 1.6 \mathrm{cmH}_{2} \mathrm{O}$ and none of them had evidence of right ventricle failure. All veno-venous patients were in protective mechanical ventilation settings including prone position at the time of ECMO decision which occurred at day $13 \pm 8$ of admission and after $10 \pm 7.16$ mechanical ventilation days. In two patients ECMO was started after extubation failure and restart of mechanical ventilation. Variables at the time of ECMO start were $\mathrm{PaO}_{2} / \mathrm{FiO}_{2}$ ratio $89 \pm 14 \mathrm{mmHg}, \mathrm{PaCO} 274 \pm 15 \mathrm{mmHg}, \mathrm{pH} 7.35 \pm 0.07$, tidal volume $412 \pm 30$ and PEEP $12 \pm 2.4 \mathrm{cmH}_{2} \mathrm{O}$. In the veno-arterial patient ECMO was started in the admission day without data of respiratory failure. ECMO was used during $13 \pm 7$ days, survival was $66 \%$ and intensive care unit length of stay $56 \pm 25$ days (one patient remained in the ICU at the time of analysis). No differences in the analyzed variables were found between survivors and not survivors.

Conclusion: In this cohort of patients with severe COVID-19 infection ECMO showed encouraging results. Mortality was below the usual reported data. However length of stay was high. As long as material and human resources were available and well-coordinated, ECMO could be delivered in a safe way in times of SARS-CoV-2 pandemic.

\section{6}

Enhanced thromboprophylaxis dosing or therapeutic anticoagulation are not associated with a decrease in thrombo-embolic events in patients with COVID19: A single centre experience

AM. Karimi i ; PJ. Masters ${ }^{2}$; E. Riddell1; J. Ramzi ${ }^{1}$; K. Patel ${ }^{2}$; M. Trivedi';

R. Mahroof ${ }^{1}$; A. Conway-Morris ${ }^{1}$

'Intensive care, Addenbrooke's Hospital, Cambridge, United Kingdom; ${ }^{2}$ Acute medicine, Addenbrooke's Hospital, Cambridge, United Kingdom

Correspondence: A.M. Karimi

Intensive Care Medicine Experimental 2020, 8(2): 000876
Introduction: Multiple studies report that patients with COVID-19 have a high incidence of arterial and venous thromboses despite standard prophylaxis 1,2,3. Although this has led to recommendations for higher anticoagulant dosing 4 in patients on ICU, it is unclear if this will reduce thrombotic complications.

Objectives: 1. To quantify the difference in thrombotic events between ICU and ward level patients.

2. To ascertain whether a higher prophylactic low molecular weight heparin (pLMWH) dose regime for ICU patients conferred protection from developing thromboses.

Methods: We collected data retrospectively on patients admitted to medical wards between 8th Apr 2020 to 22nd Apr 2020, who were PCR positive for SARS-CoV-2. Data collected included the use of prophylactic or therapeutic anticoagulation, clinically detected thrombotic complications or bleeding.

We also collected data on patients admitted to ICU from 15th Mar 2020 to 14th Jun 2020 with PCR confirmed COVID-19. These patients received either standard $\mathrm{pLMWH}$, or, following introduction of new guidance, 'enhanced' pLMWH.

Results: 83 ward admissions were identified, of which 53 had standard pLMWH, 18 were already on therapeutic anticoagulation, and 12 received neither. 1 patient developed a venous thromboembolism (VTE) (1.2\%). 2 patients, both on pre-admission therapeutic anticoagulation, had bleeding complications (2\%).

73 ICU patients were identified; 13 VTE events (12 PE and 1 DVT) (18\%). A further 20 filter-related line thromboses occurred, amongst 22 receiving $\mathrm{CVVH}$. In total 29 patients (40\%) experienced intra or extracorporeal thrombotic events.

34 patients received standard pLMWH, whilst 39 received enhanced thromboprophylaxis or therapeutic anticoagulation initiated prior to development of any thrombotic event. Peak D-dimer was higher in patients developing thrombotic events or bleeding, (median 1410 vs $5374, p<0.0001$ ); none of the other measures were significantly different in either group (Table 1).

\begin{tabular}{|c|c|c|}
\hline & $\begin{array}{c}\text { Standard } \\
\text { thrombophylaxis } \\
(34)\end{array}$ & $\begin{array}{c}\text { Enhanced } \\
\text { thrombophylaxis (39) }\end{array}$ \\
\hline Median age (range) & $62(55)$ & $61(60)$ \\
\hline$\%(95 \%$ CI $)$ female & $10[29 \%(17-46 \%)]$ & $10[26 \%(15-41 \%)]$ \\
\hline $\begin{array}{c}\text { Median (range) APACHE } \\
\text { II score }\end{array}$ & $12(18)$ & $14(24)$ \\
\hline $\begin{array}{c}\text { Median (range) admission } \\
\text { D-Dimer }\end{array}$ & $384(13033)$ & $423(36213)$ \\
\hline $\begin{array}{c}\text { Median (range) Peak D- } \\
\text { Dimer }\end{array}$ & $2566(12944)$ & $1491(58383)$ \\
\hline$\%(95 \%$ CI $)$ ventilated & $25[74 \%(57-85 \%)]$ & $33[85 \%(70-93 \%)]$ \\
\hline $\begin{array}{l}\%(95 \% \text { CI }) \text { developing } \\
\text { pulmonary or deep vein } \\
\text { thrombosis }\end{array}$ & $6[18 \%(8-34 \%)]$ & $7[18 \%(9-33 \%)]$ \\
\hline $\begin{array}{c}\%(95 \% \text { CI }) \text { Bleeding } \\
\text { complications }\end{array}$ & $2[6 \%(2-19 \%)]$ & $5[13 \%(6-27 \%)]$ \\
\hline
\end{tabular}

Conclusion: Thrombotic events were significantly more common amongst patients admitted to ICU than those who had ward level management only. Although the rate of these events was higher, we found no signal that increasing pLMWH doses was effective, and may have been associated with an increased risk of bleeding.

Baseline inequalities between our groups and relatively small numbers mean that these conclusions should be tested in larger data sets and trials of enhanced thromboprophylaxis are warranted.

\section{Reference(s) and grant ackowledgment(s)}

1. Clinical guide for the prevention, detection and management of thromboembolic disease in patients with COVID-19. 19 June 2020. https://icman aesthesiacovid-19.org/clinical-guide-prevention-detection-and-manag ement-of-vte-in-patients-with-covid-19 
2. Nahum J, Morichau-Beauchant T, Daviaud F, et al. Venous Thrombosis Among Critically III Patients with Coronavirus Disease 2019 (COVID-19). JAMA Netw Open. 2020;3(5):e2010478. https://doi.org/10.1001/jamanetwor kopen.2020.10478

3. Helms J, Tacquard C, Severac F, et al. High risk of thrombosis in patients in severe SARS-CoV-2 infection: a multicenter prospective cohort study. Intensive Care Med. 2020:

4. Thomas W, Varley J, Johnston A, et al. Thrombotic complications of patients admitted to intensive care with COVID-19 at a teaching hospital in the United Kingdom. Thromb Res. 2020;191:76-77. https://doi.org/10.1016/j. thromres.2020.04.028

\section{2}

\section{The Veronese Paradigm Facing COVID-19}

K. Donadello ; L. Gottin ${ }^{2}$; P. Zanatta ${ }^{3}$; V. Schweiger ${ }^{1}$; M. Taiana ${ }^{4}$; R. Boetti

Pettiti'; D. Michelon ${ }^{1}$; G. Pavan ${ }^{1}$; E. Polati ${ }^{1}$

${ }^{1}$ Anesthesia and intensive care b unit, University of Verona, AOUI-University Hospital Integrated Trust of Verona, Verona, Italy; ${ }^{2}$ Cardio-thoracic anesthesia and intensive care unit, University of Verona, AOUI-University Hospital Integrated Trust of Verona, Verona, Italy; ${ }^{3}$ Anesthesia and intensive care a unit, AOUI-University Hospital Integrated Trust of Verona, Verona, Italy; ${ }^{4}$ Anesthesia and intensive care b unit, AOUI-University Hospital Integrated Trust of Verona, Verona, Italy

Correspondence: K. Donadello

Intensive Care Medicine Experimental 2020, 8(2): 000892

Introduction: On Feb 20,2020, the first patient with COVID-19 in Italy developed respiratory failure and was admitted to the ICU and the number of recorded cases increased rapidly throughout the country.

Objectives: Strong from Chinese experience and with 2 week-gap from Lombardy, we organized our hospital (AOUI of Verona, Italy) from 60 to 114 ICU beds.

We aimed to describe our COVID-19 critically ill patients.

Methods: Prospective observational study of all COVID19-confirmed critically ill patients,treated at our ICUs between 6 March to 23 April, 2020.

Date of final follow-up was June 25, 2020.

Demographic and daily clinical data were collected, including data on clinical management, respiratory and other organ failure, management and outcome.

Results: Of the 122 pts included, the median age was 65 (56-71) yrs and $96(78,7 \%)$ were male.

66 (54\%) had cardiovascular disorders, 24 (20\%) were obese, 17 (14\%) had dyslipidemia, 20 (16\%) had diabetes, 20 (16\%) were current smokers, $16(13 \%)$ had COPD.

$34(28 \%)$ patients were directly admitted from the ED, 60 (49\%) from a medical ward and $27(22 \%)$ were transferred from another hospital. The mean duration of symptoms before hospital admission was 7 days and 9 days before ICU admission.

The most common symptoms on admission to the hospital were shortness of breath (dyspnoea, 91 [74.2\%]) and cough (60 [49.5\%]).

At ICU admission, APACHE II and SOFA scores were 20 (13-28) and 8 (5-11), respectively.

Lymphocytopenia was present (0.5 [0.1-0.8] 109/L); CRP was 137 (61194) $\mathrm{mg} / \mathrm{L}, \mathrm{PCT}$ was $0.3(0.2-0.8) \mathrm{ng} / \mathrm{mL}$. Total CPK, LDH and D-Dimer were 131 (77-339) U/L, 421 (311-505) U/L and 1265 (570-2032), respectively; arterial lactate was $1.2(0.9-1.6) \mathrm{mmol} / \mathrm{L}$.

$\mathrm{P} / \mathrm{F}$ at baseline was 81 (65-118); 117 pts (96\%) received invasive mechanical ventilation and after tracheal intubation P/F was 115 (98149], TV was 495 (450-500) mL, RR was 17 (15-20) apm, PEEP was 11 (10-12) $\mathrm{cmH}_{2} \mathrm{O}$, PPlat was $20(18-22) \mathrm{cmH}_{2} \mathrm{O}$, DP was $9(8-10) \mathrm{cmH}_{2} \mathrm{O}$ and compliance was $47.25 \mathrm{~mL} / \mathrm{cmH}_{2} \mathrm{O}$.

2 pts (1.6\%) were treated with Sildenafil, 5 (4\%) received iNO and 3 (2.5\%) needed ECMO.

All received hydroxychloroquine, 112 (92\%) lopinavir-ritonavir, $12(9.8 \%)$ remdesivir, $18(15 \%)$ tocilizumab. On D1 $104(86 \%)$ pts were under antibiotic therapy. 28 day ICU mortality was $18.9 \%$. ICU LOS was 13 (2-40) for survs and 7 (1-35) for non survs.
Conclusion: Almost all our COVID-19 patients received a homogeneous management schema and invasive mechanical ventilation and ICU mortality was less than $20 \%$.

\section{2}

The effect of prone positioning in SARS-CoV-2: a case series

L. Manesso ; I. Bianchi ${ }^{2}$; L. Gandini ${ }^{1}$; M. Monti ${ }^{1}$; FM. Russo ${ }^{1}$; L. Zacchetti ${ }^{2}$; P. Pugni ; M. Di Matteo ${ }^{2}$; L. Longhi²; L. Grazioli²; F. Previdi ${ }^{3}$; FL. Lorini ${ }^{2}$

${ }^{1}$ Anestesia, rianimazione e terapia intensiva e del dolore, University of Milan, Milano, Italy; ${ }^{2}$ Dipartimento di emergenza urgenza e area critica, ASST Papa Giovanni XXIII, Bergamo, Italy; ${ }^{3}$ Dipartimento di ingegneria gestionale, dell'informazione e della produzione, Università Degli Studi Di Bergamo, Bergamo, Italy

Correspondence: $L$. Manesso

Intensive Care Medicine Experimental 2020, 8(2): 000902

Introduction: Patients suffering from Severe Acute Respiratory Syndrome Coronavirus 2 (SARS-CoV-2) disease develop an atypical form of ARDS characterized by severe hypoxemia and preserved lung mechanics1. Prone positioning (PP) is successfully used as a rescue therapy for severe hypoxemia in classic ARDS. Its effects are not yet clear in SARS-CoV-2.

Objectives: To describe the effects of PP on gas exchange and respiratory mechanics in a group of severely hypoxic patients with SARS-CoV-2.

Methods: From February 22th to March 22th, consecutive SARS-CoV-2 patients admitted to the ICUs of Papa Giovanni XXIII Hospital in Bergamo, were studied. PROSEVA study group criteria were used to identify modalities and clinical indications to PP2. Data about ventilation (positive end-expiratory pressure (PEEP), driving pressure (DP), compliance of respiratory system $(\mathrm{Crs}))$ and gas exchange $\left(\mathrm{PaO}_{2} / \mathrm{FiO}_{2}(\mathrm{P} / \mathrm{F}\right.$ ratio) and PCO2) were collected at three time points: before (supine_ pre), at the end (prone) and after 6 hours (supine_post) of each session. Data were analyzed using repeated measurements ANOVA followed by Dunnet post-hoc analysis.

Results: Among 115 SARS-CoV-2 patients admitted to the ICU receiving mechanical ventilation, 71 were pronated. $80 \%$ patients were male, mean age and BMI were $60 \pm 10$ and $30 \pm 5$, similarly to the rest of the population. At the beginning of mechanical ventilation $\mathrm{P} / \mathrm{F}$ ratio and Crs were $123 \pm 63$ and $40 \pm 11 \mathrm{ml} / \mathrm{cmH}_{2} \mathrm{O}$. Median time to the first prone session was $1(0 ; 3)$ day. 25 patients (35\%) were pronated once while the remaining 46 needed more than a session. Table shows the results of the PP.

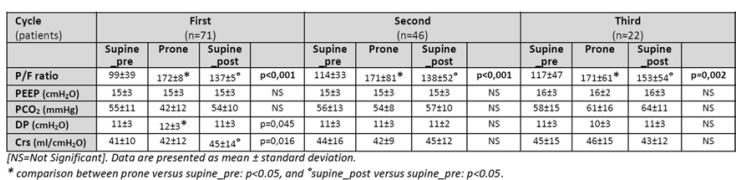

Major complications occurred in 16 cycles (11\%): severe hypoxemia in 7 , pneumothorax in 1, airway-related complications in 4, pulmonary embolism in 2 and cardiovascular instability in 2 cycles.

Conclusion: $\mathrm{PP}$ resulted in a significant increase in $\mathrm{P} / \mathrm{F}$ ratio despite no effect on respiratory mechanics. These data suggest that PP could be used as rescue therapy for severe hypoxemia in SARS-CoV-2.

\section{Reference(s) and grant ackowledgment(s)}

1. 1. Gattinoni L, Coppola S, Cressoni M et al. Covid-19 Does Not Lead to a "Typical" Acute Respiratory Distress Syndrome. Am J Respir Crit Care Med. $2020 \mathrm{Mar}$

2. 2. Guérin C1, Reignier J, Richard JC et al. Prone positioning in severe acute respiratory distress syndrome. N Engl J Med. 2013 Jun; 368:2159-2168. 


\section{5}

Early risk factors associated to mortality in critical COVID-19 patients in Bergamo

A. Galli'; D. Corbella²; A. Marino²; D. Adelmann ${ }^{3}$; F. Restuccia²; I. Riva ${ }^{2}$

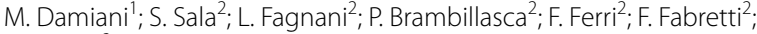

FL. Lorini ${ }^{2}$

${ }^{1}$ Department of pathophysiology and transplantation, University of Milan, Milano, Italy; ${ }^{2}$ Department of emergency and critical care, ASST Papa Giovanni XXIII, Bergamo, Italy; ${ }^{3}$ Department of anesthesia \& perioperative care, University of California San Francisco Parnassus Campus, San Francisco, United States of America

Correspondence: A. Galli

Intensive Care Medicine Experimental 2020, 8(2): 000925

Introduction: Identification of risk factors associated with mortality in coronavirus disease 2019 (COVID-19), could promote rapid and proper allocation of resources. (1)

Objectives: We sought to identify early detectable risk factors associated with 120-day mortality in subjects with COVID-19 admitted to the Intensive Care Unit (ICU).

Methods: We included all consecutive adult patients with COVID-19 admitted to the ICU at our institution between February 22 and March $22,2020$.

COVID-19 was defined according to at least one between clinical, radiological and/or laboratory criteria.

Variables were considered if clinically relevant, promptly available and were excluded if their accuracy was not verifiable (e.g. viral exposure and date of start symptoms, which were self-reported).

First, we used univariable logistic regressions to explore risk factors associated with 120-day mortality.

Secondly, variables with a p value at least inferior to 0.05 at univariable analyses were included in a multivariable logistic regression model; to avoid overfitting, we limited the number of regressors to 1 variable every 15 outcomes.

Finally, we computed a ROC curve of the multivariate analysis and its relative area under the curve (AUC) in order to assess the discriminative power of the model.

Results: In our cohort of 130 patients, 51 patients (39.2 \%) died within 120 days after ICU admission.

\begin{tabular}{|c|c|c|c|c|}
\hline \multirow{2}{*}{$\begin{array}{l}\text { TABLE } 1 \\
\text { Variable }\end{array}$} & \multicolumn{2}{|c|}{ Univariable analyses } & \multicolumn{2}{|c|}{$\begin{array}{l}\text { Multivariable } \\
\text { analysis }\end{array}$} \\
\hline & OR (95\% CI) & $\begin{array}{l}p \\
\text { value }\end{array}$ & $\begin{array}{l}\text { OR }(95 \% \\
\text { CI) }\end{array}$ & p value \\
\hline Age (year) * & $1.14(1.09-1.21$ & $<0.001$ & $\begin{array}{l}1.13(1.07 \text { - } \\
1.19)\end{array}$ & $<0.001$ \\
\hline BMI * & $\int^{1.01(0.94-1.08}$ & 0.77 & - & - \\
\hline Male sex ^ & $1.01(0.40-2.61$ & 0.99 & - & - \\
\hline $\mathrm{COPD}^{\wedge}$ & )$^{10.3(1.68-197}$ & $<0.05$ & $\begin{array}{l}5.54(0.74- \\
115)\end{array}$ & 0.14 \\
\hline $\mathrm{RR}$ admission $>24 \mathrm{bpm}$ & $0.91(0.38-2.10$ & 0.83 & - & - \\
\hline CPAP/NIV to TI ( days & $0.96(0.80-1.15$ & 50.68 & - & - \\
\hline$)^{*}$ & ) & & & \\
\hline ACE-I therapy ${ }^{\wedge}$ & $2.32(0.8-7)$ & 0.12 & - & - \\
\hline Blood group $0^{\wedge}$ & $1.66(0.69-3.94$ & 0.25 & - & - \\
\hline Blood group A & $0.54(0.22-1.25$ & 0.15 & - & - \\
\hline SOFA * & $\begin{array}{l}1.29(1.10- \\
1.54)\end{array}$ & $<0.05$ & $\begin{array}{l}1.28(1.06- \\
1.57)\end{array}$ & $<0.05$ \\
\hline
\end{tabular}

TABLE 1: ACE-I = Angiotensin Converting Enzyme Inhibitors, BMI = Body Mass Index, bpm $=$ breath per minute, $C O P D=$ Chronic Obstructive Pulmonary Disease, CPAP $=$ Continuous Positive Airway Pressure, NIV = Non Invasive Ventilation, RR = Respiratory Rate, SOFA = Sequential Organ Failure Assessment, $\mathrm{TI}=$ Tracheal Intubation ${ }^{*}=$ per unit increase, $\stackrel{\wedge}{=}$ true
We identified age, COPD and SOFA- score as independent predictors of mortality at 120 days (see Table 1 ).

In the multivariable model, both age and SOFA- score were associated with mortality and the AUC of the relative ROC curve was 0.84 .

Conclusion: Facing the unpredicted trajectory of the still ongoing outbreak of COVID-19, we identified a "three early-detectable variables" model associated with 120-day mortality in critically ill COVID19 patients.

If confirmed in other cohorts, our findings could promote the development of a mortality-predicting score, improving resource allocation in COVID-19 pandemic.

Reference(s) and grant ackowledgment(s)

1. 1. Zhou F, Yu T, Du R, et al. Clinical course and risk factors for mortality of adult inpatients with COVID-19 in Wuhan, China: a retrospective cohort study. The Lancet. 2020;395(10229):1054-1062. https://doi.org/10.1016/ S0140-6736(20)30566-3

\section{5}

Dosing of Thromboprophylaxis and Mortality in Critically ill COVID-19 patients

S. Jonmarker'; J. Hollenberg ${ }^{2}$; M. Dahlberg ${ }^{1}$; O. Stackelberg'; J. Litorell³;

Å. H. Everhov ${ }^{1}$; H. Järnberg-Petterson ${ }^{1}$; M. Söderberg ${ }^{1}$; J. Grip ${ }^{4}$; A. Schandl ${ }^{5}$; M. Gunther ${ }^{1}$; M. Cronhjort ${ }^{1}$

${ }^{1}$ Department of clinical science and education, Karolinska Institutet, Södersjukhuset, Stockholm, Sweden; ${ }^{2}$ Department of medicine, center for resuscitation science, Karolinska Institutet, Stockholm, Sweden; ${ }^{3}$ Department of anaesthesia and intensive care, Södersjukhuset, Stockholm, Sweden; ${ }^{4}$ Department of perioperative medicine and intensive care, Huddinge Hospital, Huddinge, Sweden; ${ }^{5}$ Department of molecular medicine and surgery, Karolinska Institutet, Stockholm, Sweden

Correspondence: S. Jonmarker

Intensive Care Medicine Experimental 2020, 8(2): 000965

Introduction: A substantial proportion of critically ill COVID-19 patients develop thromboembolic complications (ref 1-3). It is unclear what the optimal dose of thromboprophylaxis is for critically ill COVID19 patients and whether higher doses are associated with lower mortality rates.

Objectives: To evaluate the association of initial dosing strategy of thromboprophylaxis in critically ill COVID-19 patients and the risk of death, thromboembolism, and bleeding.

Methods: This was an observational cohort study in a 600-bed emergency hospital in Stockholm, Sweden. All critically ill COVID-19 patients admitted to two ICUs in March and April 2020 were eligible. Exclusion criteria were short length of stay, ongoing anticoagulation therapy due to diagnosed thromboembolic disease, or no initial thromboprophylaxis in the ICU. Patients were categorized into three groups according to initial daily dose; low (2500-4500 IU tinzaparin or 2500-5000 IU dalteparin), medium ( $>4500 \mathrm{IU}$ but $<175 \mathrm{IU} / \mathrm{kg}$ of body weight tinzaparin or $>5000 \mathrm{IU}$ but $<200 \mathrm{IU} / \mathrm{kg}$ of body weight dalteparin), and high dose thromboprophylaxis ( $\geq 175 \mathrm{lU} / \mathrm{kg}$ of body weight tinzaparin or $\geq 200 \mathrm{IU} / \mathrm{kg}$ of body weight dalteparin). The three study groups reflected a gradual increase in dose of thromboprophylaxis during the study period.

Results: A total of 152 patients were included, out of which 67 received low dose thromboprophylaxis, 48 medium dose thromboprophylaxis, and 37 high dose thromboprophylaxis. Demographic andclinical characteristics were similar in the three groups at baseline. Mortality was lower in patients treated with high $(13.5 \%)$ vs medium $(25.0 \%)$ and low dose thromboprophylaxis $(38.8 \%), p=0.02$. The multivariable hazard ratio of death was 0.33 ( $95 \%$ confidence interval $0.13-0.87$ ) with high vs low dose thromboprophylaxis when adjusted for sex, age, body mass index, Simplified Acute Physiology Score III and invasive respiratory support. There were fewer thromboembolic events in patients treated with high (2.7\%) vs medium (18.8\%) and low dose thromboprophylaxis $(17.9 \%), p=0.04$, but no difference in the proportion of bleeding events, $\mathrm{p}=0.16$. 


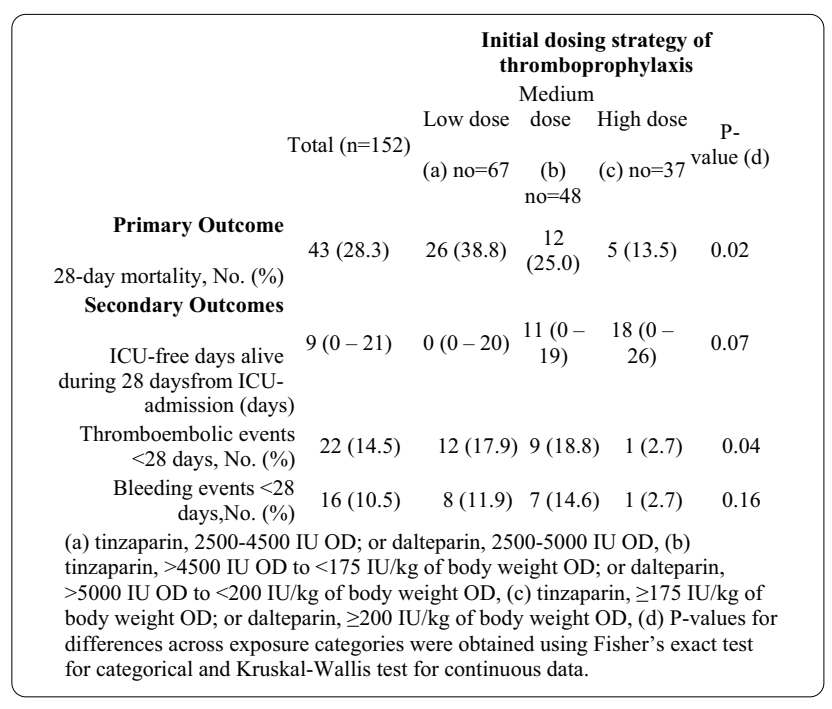

Conclusion: Among critically ill Covid-19 patients, high dose thromboprophylaxis was associated with a lower risk of death and a lower cumulative incidence of thromboembolic events compared with lower doses. There was no difference in the proportions of bleeding events between the groups.

\section{Reference(s) and grant ackowledgment(s)}

1. 1. Klok FA, Kruip M, van der Meer NJM, et al. Incidence of thrombotic complications in critically ill ICU patients with COVID-19. Thromb Res. 2020;191:145-147. 2. Helms J, Tacquard C, Severac F, et al. High risk of thrombosis in patients with severe SARS-CoV-2 infection: a multicenter prospective cohort study. Intensive Care Med. 2020. 3. Llitjos JF, Leclerc M, Chochois C, et al. High incidence of venous thromboembolic events in anticoagulated severe COVID-19 patients. J Thromb Haemost. 2020.

\section{3}

\section{National questionnaire of ECMO in Spanish Intensive Care Units}

A. Delgado ; P. Millán²; A. García De Lorenzo ${ }^{2}$; JM. Añon Elizalde²

${ }^{1}$ Intensive Care, Ramón y Cajal University Hospital, Madrid, Spain; ${ }^{2}$ Intensive care medicine, La Paz University Hospital, Madrid, Spain

\section{Correspondence: A. Delgado}

Intensive Care Medicine Experimental 2020, 8(2): 000983

Introduction: The main objetive is to analyse the organisation of ECMO in our country

Methods: A national questionnaire was performed(January 20192020) consisting in questions about pre-ECMO management, accesibility of the technique, characteristics of the ECMO team, quality, safety, training and membership of the EuroELSO.

Results: $86(29 \%)$ ICU units answered. In 57\% ( $n=49)$ ECMO was established. Only 8 regions of 17 have an ECMO retrieval team. In the management before ECMO: in $95 \%(n=81)$ prone position was trialled prior to ECMO. In $43 \%(n=36)$ ECMO was considered if prone position was not sucessfull in 12-24h. In $65 \%(n=55)$ ECMO was considered if $\mathrm{Pa} / \mathrm{FiO}_{2} \leq 100$. In the hospitals with established ECMO, $63 \%(n=41)$ do not have a specialised team. The Intensivist is the one that most frequently does the cannulation $(40 \%(n=15)$ and management $(95 \%(n=37))$. V-V ECMO is the type most frequently used and ARDS is the most frequent indication. There is a large heterogeneity in the number of cases done per centre ranging from centres that have $>$ 70 cases per year $(n=2)$ to centres that do $>10$ cases $(n=23)$. Hypovolaemia and recirculation are the most frequent complications. 93\% $(n=39)$ of the ICUs recognise that they do not do a monthly ECMO educational or simulation session. $81 \%(\mathrm{n}=35)$ do not belong to EuroELSO and $74 \%(n=31)$ do not participate in the ECMO registry.
Conclusion: There is a wide heterogeneity of the accesibility of ECMO in Spain. There is a high rate of trial of prone position prior to ECMO, as per current recommendations. A significant number of ICUs do not reach the minimal number of cases recommended by ELSO Criteria. There is ample heterogeneity in the teams and mangement. There is room from improvement in education, simulation, and membership of EuroELSO.

\section{0}

Validation of clinically applicable techniques for measurement of end-inspiratory lung volume

L. Roesthuis ${ }^{1}$; H. Van Der Hoeven ${ }^{1} ;$ J. Doorduin ${ }^{2}$; L. Heunks ${ }^{3}$

${ }^{1}$ Intensive care, Radboud University Medical Center, Nijmegen, Netherlands; ${ }^{2}$ Donders institute for brain, cognition and behaviour, department of neurology, Radboud University Medical Center, Nijmegen, Netherlands; ${ }^{3}$ Intensive care volwassenen, Amsterdam UMC, Amsterdam, Netherlands Correspondence: L. Roesthuis

Intensive Care Medicine Experimental 2020, 8(2): 001040

Introduction: Dynamic hyperinflation may develop in patients with chronic obstructive pulmonary disease (COPD) and asthma due to dynamic airway collapse or due to airway narrowing by bronchospasm or bronchial wall edema. Dynamic hyperinflation during controlled mechanical ventilation may result in barotrauma and hemodynamic instability. To limit these risks, it is important to quantify pulmonary hyperinflation. Tuxen et al. $(1,2)$ demonstrated that maintaining endinspiratory lung volume (Vei) below $1.4 \mathrm{~L}$ (or below $20 \mathrm{~mL} / \mathrm{kg}$ when corrected for ideal body weight) prevents development of adverse effects related to pulmonary hyperinflation.

Objectives: To validate two clinically applicable bedside techniques for the measurement of Vei.

Methods: Vei was assessed using three techniques: i) Gold standard: the patient exhaled from end-inspiration in a calibrated glass tube with liquid level indicator. ii) Formula based on physiology: (Vt * Pplateau)/(Pplateau - PEEPtotal)), with Vt the tidal volume, Pplateau the plateau pressure obtained after an inspiratory occlusion maneuver and PEEPtotal the positive end-expiratory pressure obtained after an expiratory occlusion maneuver. iii) Ventilator maneuver: measuring the expired volume using the flow sensor of the ventilator during passive exhalation from end-inspiration during pressure support ventilation. Vei was determined using these three techniques in each patient with 5 minute interval. The gold standard was repeated after the three measurements to exclude changes in respiratory mechanics due to the study protocol.

Results: Seventeen patients were evaluated. Vei according to the gold standard was $16.08 \pm 1.17 \mathrm{~mL} / \mathrm{kg}$. Vei according to the formula and ventilator maneuver had a low bias but large limits of agreement $(0.64 \pm 6.70 \mathrm{~mL} / \mathrm{kg}$ and $0.46 \pm 6.48 \mathrm{~mL} / \mathrm{kg}$, respectively) and were moderately correlated with the gold standard $(r \wedge 2=0.47$ with $\mathrm{P}=0.0035$ and $\mathrm{r}^{\wedge} 2=0.56$ with $\mathrm{P}=0.0009$, respectively). Vei according to the gold standard did not change due to the study protocol: $0.21 \pm 1.73 \mathrm{~mL} / \mathrm{kg}$ as compared to the initial Vei measurement.

Conclusion: In patients with airflow obstruction it is important to quantify pulmonary hyperinflation to limit risks associated with hyperinflation. We showed that using a formula to estimate Vei and a ventilator maneuver to measure Vei are too cumbersome to use at the bedside, due to low agreement and moderate correlations compared to the gold standard to measure Vei.

\section{Reference(s) and grant ackowledgment(s)}

1. 2. Williams TJ, Tuxen DV, Scheinkestel CD, Czarny D, Bowes G. Risk factors for morbidity in mechanically ventilated patients with acute severe asthma. Am Rev Respir Dis 1992; 146: 607-615.

2. 1. Tuxen DV, Lane $S$. The effects of ventilatory pattern on hyperinflation, airway pressures, and circulation in mechanical ventilation of patients with severe air-flow obstruction. Am Rev Respir Dis 1987; 136: 872-879. 
001043

ECMO for CoVid19 patients with severe ARDS - is the glass half

full or half empty?

JKM. Fichte ${ }^{1} ; \mathrm{HB}$. Hopf ${ }^{2}$

${ }^{1}$ Anesthesia and intensive care medicine, Asklepios Klinik Langen, Langen (Hessen), Germany; ${ }^{2}$ Anestesia and perioperative medicine, Asklepios Klinik Langen, Langen (Hessen), Germany

Correspondence: J.K.M. Fichte

Intensive Care Medicine Experimental 2020, 8(2):001043

Introduction: The humanopathogen coronavirus CoVid-19 may cause life threatening pneumonia. $20 \%$ of those tested positive show severe signs of hypoxic respiratory failure, with $5 \%$ needing intensive care unit treatment, with a reported mortality between 30-60\% (Grasselli,JAMA) While knowledge about the disease is increasing, no firm recommendations concerning treatment strategies have been made.

Objectives: The humanopathogen coronavirus CoVid-19 may cause life threatening pneumonia. $20 \%$ of those tested positive show severe signs of hypoxic respiratory failure, with $5 \%$ needing intensive care unit treatment, with a reported mortality between 30-60\% (Grasselli,JAMA) While knowledge about the disease is increasing, no firm recommendations concerning treatment strategies have been made.

Methods: From January 2020 to June 2020 data of all patients with respiratory failure who tested positively for SARS-COV-2, treated in the ICU of the Asklepios Klinik Langen, Germany, were sampled. We differentiated between those patients who required extracorporeal membrane oxygenation therapy (ECMO) and those who did not. Comorbidities, clinical examination and laboratory values were recorded. In case ECMO therapy had been necessary, we noted which type we used as well as the patients' outcome.

Results:

\begin{tabular}{|c|c|c|}
\hline $\begin{array}{l}\text { All patients } \mathbf{n}=\mathbf{2 7} \\
\text { Asklepios Klinik Langen, Germany }\end{array}$ & $\begin{array}{l}\text { ECMO } \\
\mathbf{n}=13\end{array}$ & $\begin{array}{l}\text { without } \\
n=14\end{array}$ \\
\hline \multicolumn{3}{|l|}{ Demographics } \\
\hline male & 10 & 10 \\
\hline female & 3 & 4 \\
\hline BMI & 28,7 & 26,2 \\
\hline \multicolumn{3}{|l|}{ Co-morbidities } \\
\hline Hypertension, adiposity, pulmonary diseases, diabetes & yes & yes \\
\hline \multicolumn{3}{|l|}{ Presenting symptoms } \\
\hline Median time to invasive ventilation (d) & 4,2 & 3,2 \\
\hline Median time of invasive ventilation (h) & 776 & 363,8 \\
\hline Tracheostomy & 12 & 4 \\
\hline Median time to tracheostomy (d) & 1 & 1 \\
\hline Thrombotic event & 6 & 3 \\
\hline Pneumothorax & 6 & 2 \\
\hline \multicolumn{3}{|l|}{ Presenting Laboratory Values } \\
\hline White blood cell count $(\mathrm{G} / \mathrm{l})$ & 10,7 & 9,7 \\
\hline Lymphocyte count (G/1) & 9,7 & 13,9 \\
\hline C-reactive protein $(\mathrm{mg} / \mathrm{dl})$ & 255 & 148,6 \\
\hline $\operatorname{PCT}(\mu \mathrm{g} / \mathrm{l})$ & 10,3 & 4,18 \\
\hline Ferritin $(\mu / 1)$ & 1932 & 1903 \\
\hline D-dimer (mg/l) & 29,1 & 11,94 \\
\hline Troponin $(\mu \mathrm{g} / \mathrm{l})$ & 277 & 26,3 \\
\hline Lactate $(\mathrm{mmol} / \mathrm{l})$ & 2,5 & 1,1 \\
\hline Creatine kinase (U/1) & 1195 & 217,78 \\
\hline SOFA score & 5,1 & 1,2 \\
\hline SAPS II & 35,6 & 28,1 \\
\hline SARS-Cov IgA & 10 & 5,8 \\
\hline SARS- Cov IgG & 12 & 6 \\
\hline \multicolumn{3}{|l|}{ Respiratory Paramters on intubation } \\
\hline Bilateral infilttrates on chest $\mathrm{x}$-ray/typical CT-scan & 13 & 14 \\
\hline $\mathrm{PaO} 2: \mathrm{FiO} 2$ & 83 & 205,2 \\
\hline Median symptom onset to intubation (d) & 4,2 & 3,2 \\
\hline Median time to admission & 3,2 & 8,3 \\
\hline \multicolumn{3}{|l|}{ Outcome } \\
\hline Median ICU stay (d) & 32,5 & 11,9 \\
\hline \multirow[t]{2}{*}{ Death } & 9 & 2 \\
\hline & 9 & 2 \\
\hline
\end{tabular}

Conclusion: Patients with ARDS caused by SARS-COV-2 treated with ECMO had a two times higher death rate compared to ARDS caused by other diseases (Combes, EOLIA). Especially patients of hospitals with lower care levels who underwent prolonged prone position with controlled ventilation and higher ventilation pressures had a more serious course of the disease and suffered from more complications compared to the patients who had immediate access to ECMO therapy if required.

\section{Reference(s) and grant ackowledgment(s)}

1. Grasselli G. et. al., Patients Infected With SARS-CoV-2 Admitted to ICUs of the Lombardy Region, Italy. JAMA 2020; Combes A et. al. Extracorporeal Membrane Oxygenation for Severe Acute Respiratory Distress Syndrome. NEJM 2018

\section{5}

The Impact of Standard Transthoracic Echocardiography (TTE) on the Management of COVID- 19 patients in Critical Care

M. Carton ${ }^{1}$; J. Hastings ${ }^{2}$; R. Ryan ${ }^{3}$; J. O'neill ${ }^{4}$; F. Colreavy

${ }^{1}$ Anaesthesia, Mater Misericordiae University Hospital, Dublin, France; ${ }^{2}$ Intensive care, The Mater Misericordiae University Hospital, Dublin, Ireland; ${ }^{3}$ Cardiology, The Mater Misericordiae University Hospital, Dublin, Ireland; ${ }^{4}$ Cardiology, The Mater Misericordiae University Hospital, Dublin, Ireland; ${ }^{5}$ Intensive care, The Mater Misericordiae University Hospital, Dublin, Ireland

\section{Correspondence: M. Carton}

Intensive Care Medicine Experimental 2020, 8(2): 001055

Introduction: Among patients with COVID-19, there is a high prevalence of cardiovascular disease, and it has been reported that $22 \%$ of critically ill patients experience myocardial injury from the infection. 1 The British Society of Echocardiography and The European Association of Cardiovascular Imaging advise performing a focused TTE, on patients during the COVID -19 pandemic.2,3

In our critical care unit we performed a full standard TTE on COVID$19+$ patients and we report the impact on the management of these patients.

Methods: This prospective study took place in the 36 bed critical care unit of the Mater Hospital. Critically ill COVID-19 + patients who had a $\Pi T E(s)$ were included. The studies were performed by an intensivist, or cardiac physiologist.

Results: Fifty-three COVID-19 + patients were admitted to critical care between 31/3/20 -17/6/20. Thirty-six TTE studies were performed on 22 patients. Indications; haemodynamic shock (11), assess RV (7), assess LV (12), ? endocarditis (6). Mean age; $60 \pm 11$ years, male ( $\mathrm{n}=$ 12) median critical care length of stay; $17(12,32)$ days, mean SOFA score $5 \pm 2$. Non- invasive ventilation $(n=2)$ and mechanical ventilation $(\mathrm{n}=17)$. Thirteen patients received vasoactive medication, 4 patients required Extra Corporeal Membranous Oxygenation. Seven patients died.

\begin{tabular}{|c|c|}
\hline & Total $n=22$ \\
\hline CAD & $\mathrm{n}=4 \quad(18 \%)$ \\
\hline Valvular heart disease & $\mathrm{n}=3 \quad(13 \%)$ \\
\hline Congestive heart disease & $\mathrm{n}=1 \quad(4 \%)$ \\
\hline Hypertension & $\mathrm{n}=9 \quad(40 \%)$ \\
\hline Atrial fibrillation & $\mathrm{n}=1 \quad(4 \%)$ \\
\hline COPD & $\mathrm{n}=1 \quad(4 \%)$ \\
\hline Asthma & $\mathrm{n}=4 \quad(18 \%)$ \\
\hline Diabetes & $\mathrm{n}=6 \quad(27 \%)$ \\
\hline Chronic kidney disease & $\mathrm{n}=4 \quad(18 \%)$ \\
\hline ACEi /ARB use & $\mathrm{n}=7 \quad(31 \%)$ \\
\hline
\end{tabular}

CAD: Coronary Artery Disease, COPD: Chronic Obstructive Pulmonary Disease, ACEi: angiotensin converting enzyme inhibitor, ARB angiotensin receptor blocker.

Seven patients had 1 normal TTE. Seven patients had 19 TTE 's with echocardiographic abnormalities that did not immediately change management; including reduced LV EF, left ventricular hypertrophy, 
regional wall motion abnormalities, diastolic dysfunction, cor pulmonale, tricuspid regurgitation and pericardial effusion. Eight patients had 10 TTE's which led directly to a change in management. These are summarized in Table 2.

\begin{tabular}{|l|l|l|l|}
\multicolumn{5}{|c|}{ Table 2. TTE studies that led to change in management } \\
\hline Indication & TTE Findings & Management change & Outcome \\
\hline Assess RV & $\begin{array}{l}\text { RV severe dilatation, } \\
\text { pressure overload LV }\end{array}$ & $\begin{array}{l}\text { Cancellation } \\
\text { discharge critical care }\end{array}$ & S \\
\hline $\begin{array}{l}\text { Haemodynamic } \\
\text { shock }\end{array}$ & $\begin{array}{l}\text { Severe LV hypertrophy } \\
\text { normal LVEF }\end{array}$ & Noradrenaline & D \\
\hline $\begin{array}{l}\text { Haemodynamic } \\
\text { shock }\end{array}$ & $\begin{array}{l}\text { Large hemothorax } \\
\text { tamponade effect }\end{array}$ & IR guided drainage & S \\
\hline Assess LV & $\begin{array}{l}\text { Normal LVEF Grade } 2 \\
\text { diastolic dysfunction }\end{array}$ & ACEi + diuretic & S \\
\hline Assess LV & $\begin{array}{l}\text { Normal LVEF Grade 1 } \\
\text { diastolic dysfunction }\end{array}$ & ACEi & S \\
\hline Assess LV & $\begin{array}{l}\text { Normal LVEF left } \\
\text { ventricular hypertrophy }\end{array}$ & $\begin{array}{l}\text { BP control GTN } \\
\text { before extubation }\end{array}$ & S \\
\hline $\begin{array}{l}\text { Haemodyanmic } \\
\text { shock }\end{array}$ & $\begin{array}{l}\text { Pericardial effusion, RV } \\
\text { collapse }\end{array}$ & IR guided drainage & D \\
\hline $\begin{array}{l}\text { Haemodynamic } \\
\text { shock }\end{array}$ & $\begin{array}{l}\text { Pericardial effusion, RV } \\
\text { collapse }\end{array}$ & IR guided drainage & D \\
\hline $\begin{array}{l}\text { Query } \\
\text { endocarditis }\end{array}$ & $\begin{array}{l}\text { No vegetations, evidence } \\
\text { hypovolaemia }\end{array}$ & Fluid therapy & S \\
\hline Assess RV & RV severe dilatation & $\begin{array}{l}\text { Nitric oxide }+ \\
\text { sildenafil }\end{array}$ & S \\
\hline
\end{tabular}

RV: Right ventricle, LV: Left Ventricle, LVEF Left ventricular ejection fraction, IR: interventional radiology, BP: Blood pressure, GTN: Glyceryl trinitrate, D: Dead, S: Survived.

No Intensivist or cardiac physiologist involved in the study became infected with COVID-19.

Conclusion: Critically ill COVID-19 positive patients may develop cardiovascular pathologies in addition to myocardial injury. We recommend a standard TTE in such patients in order to diagnose and effect management changes as appropriate.

\section{Reference(s) and grant ackowledgment(s)}

1. 3. Skulstad H, et al. COVID-19 pandemic and cardiac imaging: EACVI recommendations on precautions, indications, prioritization, and protection

for patients and healthcare personnel. Eur Heart J Cardiovasc Imaging. 2020;(6):592-598.

2. 2. D. Augustine, et al. British Society of Echocardiography Clinical Guidance regarding providing echocardiography during the COVID . pandemic.https ://www.bsecho.org/Public/Education/COVID-19-clinical-guidance.aspx

3. 1. K. Clerkin, et al. Covid-19 and Cardiovascular Disease. Circulation.2020;(141):1648-1655.

4. Nil

\section{0}

Characteristics of patients with COVID-19 admitted to a tertiary referral Intensive Care Unit

S. Shinnors ${ }^{1}$; J. Kelliher'기. Conrick-Martin ${ }^{2}$

${ }^{1}$ Intensive Care Medicine, Mater Misericordiae University Hospital, Dublin, Ireland; ${ }^{2}$ Intensive care medicine, Mater Misericordiae University Hospital, Dublin, Ireland

Correspondence: S. Shinnors

Intensive Care Medicine Experimental 2020, 8(2): 001070

Introduction: COVID-19 was declared a global pandemic by the World Health Organization (WHO) on March 11th 2020. The first case of COVID-19 identified in the Republic of Ireland was on February 29th 2020.

Objectives: Our aim was to collect data in order to examine clinical characteristics of COVID-19 patients admitted to Critical Care (ICU/
HDU) in an inner-city, university-affiliated, 570-bed, tertiary referral centre in Dublin, Ireland.

Methods: Prospective data collection took place from the arrival of the first COVID-19 positive patient to our ICU on March 8th 2020 until June 1 st 2020

Results: During the study period, 46 patients were admitted to Critical Care with symptoms of COVID-19. 45/46 (98\%) were identified as COVID-19 positive by oro/nasopharyngeal specimens. 1 patient out of $46(2 \%)$ was confirmed by bronchoalveolar lavage (BAL).

The median age was 59. 28/46 (61\%) were male and 18/46 (39\%) were female. In total, $28 / 46(61 \%)$ patients required invasive mechanical ventilation, which lasted greater than 2 days forall.

The most common comorbidity was hypertension in 16/46 (35\%) of patients, followed by both obstructive pulmonary disease and diabetes mellitus in 11/46 (24\%) patients, chronic kidney disease (CKD) in $8 / 46(17 \%)$ and ischemic heart disease in $7 / 46(15 \%) .7 / 46(15 \%)$ patients were healthcare workers and 4/46 (9\%) were identified as positive contacts

The most predominant symptom was dyspnoea in $37 / 46(80 \%)$ patients, followed by cough in $19 / 46(41 \%)$, fever in $16 / 46(35 \%)$, myalgia in $8 / 46(17 \%)$, diarrhoea in $8 / 46(17 \%)$ and altered consciousness in $1 / 46(2 \%) .41 / 46(89 \%)$ patients were lymphopaenic. $22 / 46(47 \%)$ patients were proned for $>16$ hours per day. $4 / 46(9 \%)$ patients were placed on extracorporeal membrane oxygenation (ECMO). At the end of the study period, 5/46 (11\%) patients remain in Critical Care.

Conclusion: The first patient tested positive for COVID-19 in Ireland in February 2020. It was essential to collect data on this novel virus as it presented to our Critical Care Unit. We identified the most prominent clinical characteristic in order to deliver effective treatment. In total, two thirds of patients presenting to Critical Care required invasive mechanical ventilation. Almost $70 \%$ survived their Critical Care admission, while $15 \%$ died. The clinical symptoms and co-morbidities of patients admitted to our ICU were comparable to that seen internationally at this time [1, 2].

\section{Reference(s) and grant ackowledgment(s)}

1. 2. Wang, D., Clinical Characteristics of 138 Hospitalized Patients With 2019 Novel Coronavirus-Infected Pneumonia in Wuhan, China, B. Hu, et al., Editors. 2020: JAMA

2. 1. Guan, W.J., Clinical Characteristics of Coronavirus Disease 2019 in China, Z.Y. Ni, et al., Editors. 2020: N Engl J Med. p. 1708-1720.

3. No funding received.

\section{4}

Pandemic ICU Research as a Newly Qualified Doctor: An Experience from a District General Hospital

J. Caterson ${ }^{1}$; MC. Frise

${ }^{1}$ Intensive care unit, Royal Berkshire Hospital NHS Foundation Trust, Reading, United Kingdom

Correspondence: J. Caterson

Intensive Care Medicine Experimental 2020, 8(2): 001074

Introduction: Prior to the World Health Organisation declaring a pandemic in March 2020, research efforts into coronavirus disease 2019 (COVID-19) were rapidly gathering pace.[1] Simultaneously in the UK, final year medical students were graduating early,[2] having completed their core studies, to join the workforce as junior doctors. Locally, research training including a Good Clinical Practice course and close support from research clinicians was provided to some new doctors, offering a unique opportunity for involvement in pandemic research studies at the outset of their medical careers.

Objectives: To describe the challenges associated with recruiting to a pandemic study at a UK District General Hospital serving a population 
of over $500,000,[3]$ and inform preparations for research during a possible 'second-wave' or future pandemics. A core focus was obstacles to recruitment.

Methods: Patient screening and recruitment was conducted in accordance with the GenOMICC study protocol.[4] Demographic data were anonymously collected for patients screened and deemed eligible. The Chi-Squared or Two-Tailed Fisher's Exact Test were used to compare enrolment into the study according to demographic characteristics. Statistical significance was taken as $p<0.05$.

Results: Approval for recruitment was given on 19th April 2020 and the first patient recruited on 21st April. Over 6 weeks, 39 eligible patients were identified. 6 were approached directly and in 33 an opinion sought from relatives as the patients lacked capacity due to illness severity. 26 patients were enrolled, 5 declined, and 8 died during the process. 23 were recruited by the newly qualified doctor and 3 by research nurses.

There was no significant difference in enrolment according to sex ( $p$ $>0.99)$, age $(p=0.15), B M I(p=0.21)$, or 60 -day mortality $(p=0.48)$. There was a trend towards lower enrolment in Black, Asian and Minority Ethnic (BAME) groups compared with white patients $(p=0.05)$. The predominant reason for enrolment was a desire to improve understanding of COVID-19. Reasons for declining included fears of sharing genetic data and lack of acute benefit to the patient.

Conclusion: Newly qualified doctors can play a valuable role in the recruitment of patients to priority pandemic studies when other staff have been redeployed. Our analysis identifies several challenges to conducting observational pandemic research; notably timely set up to capture the population affected when the condition has a high early mortality, and communication when constrained by infection control requirements for a highly contagious airborne pathogen. The suggestion of lower recruitment from the BAME population - if real - would be of particular concern given the urgent need to determine the extent to which genetic factors contribute to apparently worse outcomes in these patients.[5]

\section{Reference(s) and grant ackowledgment(s)}

1. [1] https://www.who.int/emergencies/diseases/novel-coronavirus-2019/ global-research-on-novel-coronavirus-2019-ncov

2. [2] BMJ 2020;368:m1227

3. [3] Royal Berkshire NHS Foundation Trust. Vision 2025, Our Strategy. 2018.

4. [4] https://genomicc.org/uk/

5. [5] Price-Haywood EG, Burton J, Fort D, Seoane L. Hospitalization and Mortality among Black Patients and White Patients with Covid-19. N Engl J Med. 2020;382(26):2534-2543

6. No external funding received

\section{0}

\section{Extracorporeal CO2 Removal in acute exacerbation of COPD} not responding to noninvasive ventilation : a single center experience

D. Silva'; M. Azzi' ; D. Ushmorova'; 'L. Lainé ${ }^{1}$; V. loos $^{1}$; M. Lermuzeaux'; N. Mémain ${ }^{1}$; S. Alviset' ; L. Ferreira'; J. Aboab ${ }^{1}$

'Intensive care unit, Hospital Center De Saint-Denis, Saint-Denis, France

Correspondence: D. Silva

Intensive Care Medicine Experimental 2020, 8(2): 001090

Introduction: Acute exacerbation of chronic obstructive pulmonary disease (eCOPD) can lead to hypercapnia and respiratory acidosis. It is a major determinant in health status. Non-invasive ventilation (NIV) is the gold standard therapy as it reduces work of breathing and improves gas exchange. However, for some patients NIV fails and intubation is needed which exposes them to mechanical invasive ventilation (MIV) complications and weaning difficulties. For those patients extracorporeal carbon dioxide removal (ECCO2R) may be an option.
Methods: We conducted a single center retrospective analysis on successive eCOPD patients for whom NIV failed and didn't have limitation for MIV. For the first step (from January 2010 to February 2015) we observed blood gas measurements, respiratory rates and complication rates on 26 eCOPD patients treated with MIV after NIV failure. Then, for the second period (from February 2015 to February 2019) we observed the same parameters for 28eCOPD patients failing NIV for whom ECCO2R was performed with the iLA activve, iLA kit (Xenios Novalung, Heilbronn, Germany) device at a targeted blood flow of $1 \mathrm{~L} /$ $\min$.

Variables are reported as mean $+/-$ sd. Quantitative values are compared with $\mathrm{t}$ test ( $95 \%$ confidence interval) and qualitative values with Pearson's Chi-squared test with Yates' continuity correction. Population distribution was tested with the Shapiro-Wilk normality test.

Results: The two groups were comparable at baseline in demographics : gender, BMI, SAPS and age : respectively in the ECCO2R and MIV group SAPS $48+/-15$ and $50+/-14$, age $66,1+/-11,4$ years and $71,4+/-11$ years. $\mathrm{pH}$ and $\mathrm{PaCO} 2$ are showed in Figure 1 . The respiratory rate 6 hours before ECCO2R was $27,00+/-8,37$ and 22,18 $+/-3,79$ at decannulation $(p=0,22)$. In the MIV group, respiratory rate 6 hours before intubation was $25,88+/-3,83$ and $21,25+/-5,44$ at extubation $(p=0,30)$

We were able to avoid MIV for 25 patients $(89,3 \%)$ in the ECCO2R group.

Complications for patients affected are listed in Table 1. The ECCO2R duration was $5,7+/-4,2$ days and in the MIV group ventilation lasted $28,2+/-42,5$ days. In the MIV group, 5 patients had an extubation failure and 6 patients had autoextubated. ECCO2R was stopped because of recovery for 20 patients, complication for 6 patients (with a mean duration of ECCO2R of 5,7 days) and death for 2 patients. MIV was stopped because of recovery for 17 patients, tracheotomy for 2 patients and death for 7 patients.

Length of stay in the Intensive Care Unit for the ECCO2R and the MIV group was $19+/-15,5$ days and $31+/-42,2$ days respectively ( $p=$ 0.18 ). Length of stay in the hospital for the ECCO2R and the MIV group was $29.46+/-21.1$ days and $50.58+/-52.4$ days respectively ( $p=$ $0.06)$. Mortality at 28 days and 90 days was $8 \%$ and $14 \%$ respectively in the ECCO2R group and $16 \%$ and $30 \%$ in the MIV group.

Conclusion: Our results show that ECCO2R is feasible and brings significant improvement on $\mathrm{pH}$ and $\mathrm{PaCO} 2$ with relatively low complication rate.

Evidence for a mortality benefice of ECCO2R in eCOPD patients failing NIV therapy has yet to be proven in a larger randomized study.

\section{4}

Effect of different corticosteroid regimens on the outcome of patients with severe COVI19-related acute respiratory failure E. Pisano ${ }^{1}$; C. Bonino ; A. Arena ${ }^{1}$; G. Montanari ${ }^{1}$; F. Cerbone ${ }^{1}$; M. Umbrello'; S. Muttini

${ }^{1}$ Uo anestesia e rianimazione, Ospedale San Carlo Borromeo, ASST Santi Paolo e Carlo, MILANO, Italy

Correspondence: M. Umbrello

Intensive Care Medicine Experimental 2020, 8(2): 001094

Introduction: The risk/benefit ratio of corticosteroids for patients with COVID19-related acute respiratory failure has not been yet established.

Objectives: We compared the outcomes of patients receiving dexamethasone (DEXA) vs. methylprednisolone (METHYL), and the effects of rescue, short-term/high-dose pulses of glucocorticoids (BOLUS).

Methods: Consecutive patients admitted from March 1st to April 30th 2020 to the general ICU of a tertiary care hospital for COVID19 were enrolled. As per Institutional protocol, from March 1st severe cases were given DEXA; as of April 10th the protocol was modified and 
METHYL was suggested. On top of that, physicians were allowed to administer a 3-day course of $1000 \mathrm{mg} /$ day of methylprednisolone in case of refractory hypoxemia within the first week of treatment. Results: We enrolled 81 consecutive patients, 69 (85\%) males, age $60 \pm 10$ years. At ICU admission SAPS II was $27 \pm 7$ and SOFA 4 [3 6] points. 46 patients $(56.8 \%)$ received DEXA, whereas $30(37 \%)$ had METHYL; 5 patients did not receive corticosteroids. The groups were well-matched for age, comorbidities and severity at admission. No differences were found in the duration of mechanical ventilation (11 [7; $17]$ vs. $11[8 ; 18]$ days, $p=0.7021)$, ICU mortality ( 37.0 vs $43.3 \%, p=$ 0.578 ), incidence of VAP (47.8 vs $53.3 \%, p=0.639$ ) or bacteremia $(39.0$ vs. $36.7 \%, p=0.829$ ). A significantly lower number of patients who had DEXA received a rescue BOLUS ( 34.8 vs. $66.7 \%, p=0.007$ ).

36 patients $(44.4 \%)$ received a rescue BOLUS: they were more likely to be female ( 25.0 vs. $7.0 \%, p=0.021$ ) and obese ( 38.9 vs. $8.9 \%, p=$ 0.002 ). Table 1 shows the outcomes of patients who did vs. those who did not receive BOLUS.

\begin{tabular}{|l|l|l|l|}
\hline & No BOLUS & BOLUS & p \\
\hline $\begin{array}{l}\text { (N=45) } \\
\text { Duration of mechanical } \\
\text { ventilation (days) }\end{array}$ & $9[4 ; 16]$ & $13[8 ; 21]$ & 0.0074 \\
\hline $\begin{array}{l}\text { Duration of pressure support } \\
\text { ventilation (days) }\end{array}$ & $2[1 ; 5]$ & $4[2 ; 9]$ & 0.0349 \\
\hline ICU length of stay (days) & $13[9 ; 19]$ & $18[11 ; 26]$ & 0.0287 \\
\hline Ventilator-free days (days) & $12[0 ; 22]$ & $6[0 ; 19]$ & 0.0580 \\
\hline ICU mortality $-\mathbf{n}(\%)$ & $15(33.3)$ & $17(47.2)$ & 0.204 \\
\hline $\begin{array}{l}\text { Patients who developed VAP }-\mathbf{n} \\
\text { (\%) }\end{array}$ & $17(37.8)$ & $23(63.9)$ & 0.020 \\
\hline $\begin{array}{l}\text { Number of VAP per patient } \\
\text { Patients who developed } \\
\text { bacteremia }-\mathbf{n}(\%)\end{array}$ & $2[1 ; 2]$ & $2[1 ; 2]$ & 0.6898 \\
\hline $\begin{array}{l}\text { Number of bacteremias per } \\
\text { patient }\end{array}$ & $14(31.1)$ & $17(47.2)$ & 0.138 \\
\hline & $1 ; 2]$ & $2[1 ; 2]$ & 0.2748 \\
\hline
\end{tabular}

Conclusion: we were unable to find any difference between DEXA or METHYL on the explored outcomes; high-dose BOLUS of glucocorticoids seemed to be associated with a higher duration of mechanical ventilation and a higher incidence of complications. Further, prospective investigations are needed to confirm these results.

\section{Reference(s) and grant ackowledgment(s)}

1. Veronese et al. Use of Corticosteroids in Coronavirus Disease 2019 Pneumonia: A Systematic Review of the Literature. Front Med 2020; 7: 170

\section{8}

Incidence of barotrauma in patients with severe COVID-19 acute respiratory failure: a descriptive study

S. Nespolii'; G. Babini ${ }^{1}$; E. Piacentino ${ }^{1}$; E. Tognacci'; M. Umbrello²;

S. Muttini

${ }^{1}$ Uo anestesia e rianimazione, Ospedale San Carlo Borromeo, ASST Santi Paolo e Carlo, MILANO, Italy; ${ }^{2}$ UO Anestesia e Rianimazione, Ospedale San Paolo - Polo Universitario, ASST Santi Paolo e Carlo, MILANO, Italy

\section{Correspondence: M. Umbrello}

Intensive Care Medicine Experimental 2020, 8(2): 001098

Introduction: Barotrauma is a well-known complication of invasive mechanical ventilation and it has been anecdotally reported in critically ill patients with COVID-19.

Objectives: To analyse the incidence and factors associated with development of barotrauma in a cohort of COVID-19 patients.

Methods: Retrospective case series of consecutive patients with laboratory-confirmed COVID-19 admitted from March 1st to April 30th 2020 to the general ICU of a tertiary care hospital. Data regarding the use of CPAP before ICU admission, as well as the parameters of mechanical ventilation during the ICU stay were collected. Barotrauma was defined as pneumothorax, pneumomediastinum or subcutaneous emphysema by means of clinical examination, lung ultrasound, chest XR or CT scan.

Results: 81 patients were enrolled in the study; age was $60 \pm 10$ years, $69(85 \%)$ were male. 16 patients (20\%) developed barotrauma. Table 1 shows the comparison of patients who did vs. those who did not develop barotrauma.

\begin{tabular}{|c|c|c|c|}
\hline \multirow[b]{2}{*}{ Variable } & \multirow{2}{*}{$\begin{array}{l}\text { No } \\
\text { barotrauma } \\
(n=65)\end{array}$} & \multicolumn{2}{|l|}{ Barotrauma } \\
\hline & & $(n=16)$ & $\mathbf{p}$ \\
\hline Age (years) & $59[53 ; 68]$ & $68[58 ; 73]$ & 0.0496 \\
\hline COPD - n (\%) & $3(4.7)$ & $1(6.3)$ & 0.798 \\
\hline SAPS II score (points) & $26.5 \pm 6.2$ & $27.8 \pm 7.3$ & 0.5024 \\
\hline SOFA score at ICU admission (points) & $4 \pm 2$ & $5 \pm 2$ & 0.6070 \\
\hline Awake prone position - n (\%) & $32(57.1)$ & $5(38.6)$ & 0.224 \\
\hline $\begin{array}{l}\text { Onset of symptoms to hospital } \\
\text { admission (days) }\end{array}$ & $7[5 ; 10]$ & $7[5 ; 10]$ & 0.8519 \\
\hline Hospital admission to CPAP (h) & $5[1 ; 45]$ & $8[2 ; 15]$ & 0.4400 \\
\hline $\begin{array}{l}\text { Beginning of CPAP to ICU admission } \\
\text { (h) }\end{array}$ & $53[19 ; 96]$ & $47[28 ; 132]$ & 0.7519 \\
\hline $\begin{array}{l}\text { Duration of CPAP before ICU } \\
\text { admission (days) }\end{array}$ & $2[1 ; 4]$ & $3[1 ; 5]$ & 0.7046 \\
\hline $\begin{array}{l}\text { Respiratory system compliance at ICL } \\
\text { admission }(\mathrm{ml} / \mathrm{cmH} 2 \mathrm{O})\end{array}$ & $\mathrm{J}_{51.9 \pm 13.3}$ & $49.4 \pm 13.3$ & 0.5286 \\
\hline $\begin{array}{l}\text { Plateau pressure at ICU admission } \\
\text { (cmH2O) }\end{array}$ & $24.3 \pm 3.1$ & $24.8 \pm 3.4$ & 0.5797 \\
\hline Number of prone-positioning cycles & $3[1 ; 6]$ & $5[4 ; 7]$ & 0.0289 \\
\hline $\begin{array}{l}\text { Duration of controlled mechanical } \\
\text { ventilation (days) }\end{array}$ & $6[3 ; 10]$ & $11[9 ; 14]$ & 0.0011 \\
\hline $\begin{array}{l}\text { Duration of assisted mechanical } \\
\text { ventilation (days) }\end{array}$ & $3[1 ; 5]$ & $5[1 ; 13]$ & 0.0790 \\
\hline $\begin{array}{l}\text { Incidence of VAP from } S \text { aureus - n } \\
(\%)\end{array}$ & $20(31.3)$ & $6(37.5)$ & 0.633 \\
\hline ICU & & & 0.9766 \\
\hline ICU mortality - n (\%) & $23(35.9)$ & $10(66.7)$ & 0.030 \\
\hline
\end{tabular}

3 patients already had barotrauma at ICU admission; in the remaining cases, barotrauma was diagnosed after 14 [10; 22] days of mechanical ventilation. At the diagnosis, 3 patients (19\%) were spontaneously breathing, 5 (31\%) were receiving pressure support ventilation and 8 $(50 \%)$ controlled ventilation; in patients undergoing mechanical ventilation PEEP was $11.6 \pm 2.6 \mathrm{cmH}_{2} \mathrm{O}$ and Plateau airway pressure was $28 \pm 5 \mathrm{cmH}_{2} \mathrm{O}$

Conclusion: Barotrauma is a frequent, severe, late complication in mechanically ventilated COVID-19 patients. An older age and a longer course of controlled mechanical ventilation seem associated with the development of barotrauma.

Reference(s) and grant ackowledgment(s)

1. Grasselli et al, Baseline characteristics and outcomes of 1591 patients infected with SARS-CoV-2 admitted to ICUs of the Lombardy Region, Italy. JAMA 2020; 323(16):1574-1581

\section{2}

Neutrophil-to-Lymphocyte Ratio as a prognostic marker in Covid 19 M. Portilla-Botelho' ;A. Bueno-Gonzalez' ; M. Sanchez Casado'; J. Vejo ${ }^{3}$; J. Monserrat ${ }^{4}$; M. Alvarez-Mon ${ }^{5}$

${ }^{1}$ Intensive care unit, Hospital General Universitario de Ciudad Real, Ciudad Real, Spain; ${ }^{2} \mathrm{cu}$, Virgin Health Hospital, Toledo, Spain; ${ }^{3}$ Intensive care unit, Hospital Universitario La Paz, Madrid, Spain, Spain; ${ }^{4}$ Inmunology department, University of Medicine of Alcalá de Hemares, Alcala de Henares, Spain; ${ }^{5}$ nnmunology department, Hospital Príncipe de Asturias, Alcalá de Henares, Spain

Correspondence: $\mathrm{M}$. Portilla-Botelho

Intensive Care Medicine Experimental 2020, 8(2): 001102 
Introduction: The Neutrophil-to-Lymphocyte Ratio (NLR) is an expression of systemic inflammation in multiple immunologic, cardiovascular, and cancer diseases. An excesive inflammatory answer to SARS Cov2 can lead to acute respiratory distress that increases mortality

Objectives: We considered 1/- studying characteristics of patients with Covid19 admitted to Intensive Care Unit (ICU) due to SARS Cov2 severe pneumonia; and 2/- analysing if NLR and other inflammatory markers are associated with poor prognosis

Methods: We included Covid patients admitted to our ICU, with acute respiratory failure that required invasive mechanical ventilation (IMV). We analysed different inflammatory markers at the momento of ICU admission: NLR, plasmatic lymphocites (L), fibrinogen, D dimer (DD), and C-Reactive Protein (CPR). Statistical analysis with STATA version 13.

Results: We analysed 63 patients, mean age of 62.3 years (SD 11.3), $63 \%$ were males. The most frequent risk factor was high blood pressure. Mean stay in ICU was 26.4 days (SD 19.4), and mean hospital stay was 59.4 days (SD 19.3). $71 \%$ of patients needen prone position, 13 patients $(20 \%)$ suffered pulmonary barotrauma, and 29 patients (46\%) died in ICU. All patients that survived in ICU were discharged to home.

In the study of prognostic factors, we found that patients who died, had a higher NLR (33.40 vs $15.75, p<0.001)$, and more lymphopenia (772 vs $1540, p=0.004$ ). We did not find significant differences in DD or PCR values. Patients with barotrauma had a higher NLR than patients without it (43.51 vs $18.76, p<0.001$ ) and there were not differences in the other inflammatory markers studied. Patients wth longer mechanical ventilation and longer ICU stay, did not present differences in NLR or L, but had higher DD (7509 vs $2364, p=0.002$ ). In multiple regression analysis, RNL and $L$ at ICU admission, were independent risk factors of mortality (AUC ROC curve $0.75 \mathrm{IC}: 0.62-0.87$ ) and pulmonary barotrauma (AUC ROC curve 0.75 IC: 0.59-0.90). However these parameters did not correlate with ICU stay nor days of IMV.

Conclusion: Neutrophyl-to-lymphocyte ratio at ICU admission, is a simple low cost index that has important prognostic value in patients with severe SARS-Cov2 pneumonia.

\section{Reference(s) and grant ackowledgment(s)}

1. Ethers $\mathrm{J}$, et al. Prognostic role of Neutrophil-to-Lymphocyte Ratio in breast cancer: a systematic review and meta-analysis. Breast Cancer Res.2017 Jan $5 ; 19(1)$

\section{7}

Utility of Bronchoalveolar Lavage in the diagnosis of Acute Respiratory Failure Diagnosis in the criticaly ill hematological patient

M. Ana'; M. Bento 2 ; R. Lídia'; F. Pedro 3 ; M. Catarina ${ }^{3}$; C. Inês ${ }^{3}$; E. Fragoso ${ }^{3}$; L. Carlos ${ }^{3}$; A. Pilar ${ }^{3}$

${ }^{1}$ Hematology and transplantation department, Centro Hospitalar Universitário Lisboa Norte- Hospital de Santa Maria, Lisboa, Portugal; ${ }^{2}$ Hematology and Transplantation Department, Centro Hospitalar Universitário Lisboa Norte- Hospital de Santa Maria, Lisbon, Portugal, Lisboa, Portugal; ${ }^{3}$ Respiratory intensive care unit, Centro Hospitalar Universitário Lisboa NorteHospital de Santa Maria, Lisboa, Portugal

Correspondence: M. Bento

Intensive Care Medicine Experimental 2020, 8(2): 001107

Introduction: Bronchoalveolar lavage (BAL) is the current gold-standard for the differential diagnosis of alveolar infiltrates in immunosuppressed patients. Its reported diagnostic yield has been between 30 to $67 \%$, but its results have marginal repercussions on treatment options and prognosis. Performing BAL is not risk free and with the development of non-invasive techniques, the clinical relevance of BAL inestablishing the etiology of acute respiratory failure (ARF) has recently been questioned.
Objectives: 1) To assess the diagnostic yield of BAL in establishing the etiology of ARF; 2) To compare the mortality rate between patients subjected to BAL or not; and 3) To evaluate the impact of BAL on time until diagnosis in hematologic patients admitted to the intensive care unit (ICU) with ARF.

Methods: Retrospective cohort study. Inclusion criteria: hematooncological patients admitted to a tertiary hospital's ICU due to ARF between January 2012 and June 2020 (8.5 years). Exclusion criteria: patients without infiltrates on chest radiography at admission. Primary endpoint was diagnostic yield of BAL, defined as the identification of microbiological agent or exclusion of other causes. Secondary endpoints were intra-ICU mortality rate, 30-day mortality rate, survival time and time until etiological diagnosis.

Results: A total of 79 hematological patients were included in the study, of which $34(42.5 \%)$ were female, with a mean age of $59.01 \pm 16.59$ years. Twenty-one $(26.3 \%)$ of the patients were subjected to BAL. Age, gender and need for mechanical ventilation were comparable between groups. Mean SOFA at admission, mean APACHE II and the number of neutropenic patients were significantly higher in the non-BAL group $(p<0.05)$

BAL contributed to the etiological diagnosis in $47.37 \%$ of the clinical cases. Mortality rate was lower in the BAL group either evaluated as Intra-ICU mortality rate $(38.1 \%$ for BAL vs. $71.9 \%$ for non-BAL, $p=$ $0.006)$ or 30 -day mortality $(42.9 \%$ vs. $75.4 \%, p=0.013)$. Survival time was longer in the BAL group (19.00 vs. 5.00 days, $p=0.002)$. On a multivariate analysis including APACHE II or OFA at admission and neutropenia, APACHE II was a significant confounding factor in the mortality rate difference between the 2 groups; yet, when analyzing survival time controlling for these same factors, BAL remained significantly associated with longer survival times $(p=0.003)$. We found no differences between the groups on the frequency of etiological diagnosis, time to diagnosis or duration of mechanical ventilation.

Conclusion: BAL is a useful diagnostic tool for acute respiratory failure in selected hematological patients in which diagnosis is not possible by non-invasive techniques.

\section{Reference(s) and grant ackowledgment(s)}

1. 4 - Oren I et al., Does molecular analysis increase the efficacy of bronchoalveolar lavage in the diagnosis and management of respiratory infections in hemato-oncological patients? International Journal of Infectious Diseases 50 (2016) 48-53.

2. 3 - Svensson T, Lundström K, Höglund M, Cherif H, Utility of bronchoalveolar lavage in diagnosing respiratory tract infections in patients with hematological malignancies: are invasive diagnostics still needed? Upsala Journal of Medical Sciences, 2017 VOL. 122, NO. 1, 56-60.

3. 2 - Katsumata Y, Terada J, Abe M, Suzuki K, Ishiwata T et al. An Analysis of the Clinical Benefit of 37 Bronchoalveolar Lavage Procedures in Patients with Hematologic Disease and Pulmonary Complications, Intern Med 58: $1073-$ 1080, 2019. https://doi.org/10.2169/internalmedicine.1606-18.

4. 1 - Azoulay E, Mokart D, Rabbat A, Pene F, Kouatchet A, Bruneel F, et al. Diagnostic bronchoscopy in hematologyand oncology patients with acute respiratory failure: prospective multicenter data. Crit Care Med. 2008;36:100-7.6.

001134

A review of proning in non-intubated patients with Covid 19 who presented to a tertiary referral ICU

J. Kelliher

${ }^{1}$ ICU, HSE Ireland, Dublin, Ireland

Correspondence: J. Kelliher

Intensive Care Medicine Experimental 2020, 8(2): 001134

Introduction: A review of proning in non-intubated patients with Covid 19 who presented to a tertiary referral ICU.

Objectives: This small retrospective study investigates 16 patients admitted to our ICU who were treated with awake proning. 
The primary outcome studied was the difference in oxygen saturation before and 1 hour after self proning.

Secondary Outcomes include;decrease in the respiratory rate, increase in PF ratio, rates of subsequent intubation and the length of time proning was tolerated.

Methods: We conducted a retrospective chart review of consecutive patients admitted to our ICU between 31/03/20 to 25/04/20. Covid 19 diagnosis was confirmed by oropharyngeal and nasopharyngeal PCR. 52 patients with Covid 19 were admitted to our ICU during this time, 16 of whom self proned.

We document the changes in oxygen saturations, PF ratio and respiratory rate before and one hour after proning.

We also note the duration proning was tolerated in each session and how many proning sessions patients received.

Results: RESULTS

Oxygen saturations improved on average $3.6 \%$ on proning (range 1 to 6.8\%; median 3.7\%).

The average pre-proning 02 saturation was $94 \%$ (on supplemental oxygen delivered by nasal prongs or Venturi mask).

Patients who required intubation had a poorer response to proning from the outset with an mean improvement in oxygen Saturations of only $2.2 \%$ (range $1-3.7 \%$ median $2 \%$ ) compared with $4.6 \%$ (range 2 to $7 \%$ median $4.5 \%$ ) in the patients who did not require intubation.

The day of illness when proning was initiated was similar in both groups (8.8 $\mathrm{v} 8.0)$.

Overall, the PF ratio post proning improved by an average of $6.9 \mathrm{kPa}$ (ranging from $2.3 \mathrm{kPa}$ to $23 \mathrm{kPa}$ : median $4.5 \mathrm{kpa}$ ). The PF ratio improved significantly more on self proning in those patients that did not require intubation $(9.14 \mathrm{kPa}$ compared to an average improvement of only $5.4 \mathrm{kPa}$ in those who required intubation)

$\mathrm{PF}$ ratios were lower from the outset in the patients who were subsequently intubated after trial self proning-average PF ratio 17.1 (range PF 14.6- 21.4: median 17) compared to PF ratio of 25.9 in patients who did not require intubation.

Proning was tolerated well with only one patient unable to continue for more than an hour.

The average hours proned per session was 2.7 hours; ranging from 1 hour to 8hours per session.

The number of sessions ranged from 1 to 13 sessions.

The Respiratory Rates after proning did not significantly change with proning 25.4 to 24.4 average. 7 patients out of the 16 patients were ultimately intubated (43\%).

All patients survived to ICU discharge.

Conclusion: CONCLUSIONS

This study adds to a body of observational evidence that awake prone position improves oxygenation in the non-intubated patient with respiratory failure and that it is well tolerated in short sessions.[i]Limitations include small sample size and its retrospective nature.

\section{Reference(s) and grant ackowledgment(s)}

1. Scaravilli V, Grasselli G, Castagna L, et al. Prone positioning improves oxygenation in spontaneously breathing nonintubated patients with hypoxemic acute respiratory failure: A retrospective study. J Crit Care. 2015;30(6):1390-1394. https://doi.org/10.1016/j.jcrc.2015.07.008

2. Guérin C, Reignier J, Richard J-C, et al. Prone positioning in severe acute respiratory distress syndrome. N Engl J Med 2013; 368: 2159-2168. 3. nil

\section{9}

Response to prone positioning in patients with severe Covid19-induced severe Acute Respiratory Distress Syndrome

M. Gabenesch'; F. Sinnah ${ }^{1}$; Q. Staiquly $;$ E. de Montmollin ${ }^{1}$; C. Dupuis' ; PH. Wicky'; L. Lefevre'; R. Sonneville ${ }^{1}$; P. Jaquet ${ }^{1}$; J. Patrier ${ }^{1}$; G. Franchineau'; M. Marzouk'; A. Grinea'; L. Bouadma'; JF. Timsit

${ }^{1}$ Paris, Medical and infectious Intensive Care Unit, Bichat-Claude Bernard Hospital, Paris, France
Correspondence: M. Gabenesch

Intensive Care Medicine Experimental 2020, 8(2): 001149

Introduction: The coronavirus disease 2019 (Covid-19) pandemic mainly affects the respiratory system and the evolution into acute respiratory distress syndrome (ARDS) is the main cause of admission in ICU. It is known now that the Covid-19-induced ARDS presents some unusual characteristics compared to common ARDS. Although the practice of prone positioning (PP) is part of the actual recommendations in Covid-19 severe ARDS management (based on common ARDS trials), there is, to our knowledge, no studies which assessed the efficacy of prone positioning on blood oxygenation and mortality in this context.

Methods: We performed a monocentric analysis of prospectively collected data in the medical ICU of Bichat-Claude-Bernard Hospital (France) between January and June 2020. Covid-19 confirmed patients, admitted for ARDS, who required intubation and prone positioning (practiced for a $\mathrm{PaO}_{2} / \mathrm{FiO}_{2}(\mathrm{P} / \mathrm{F})$ ratio $<150 \mathrm{mmHg}$ after positive end-expiratory pressure (PEEP) optimization) were included. P/F ratio before and after the first PP were collected, and response was defined as $[(\mathrm{P} / \mathrm{F}$ after $\mathrm{PP}-\mathrm{P} / \mathrm{F}$ before $\mathrm{PP}) / \mathrm{P} / \mathrm{F}$ before $\mathrm{PP}] \mathrm{X} 100$. A response was considered significant if $\geq 20 \%$. We analyzed predictors of significant response and day 60 (d60) mortality.

Results: 42 patients were included. 31 patients were dead at d60 (mortality rate: $64.5 \%$ ). No difference in baseline characteristics were found between survivors and decedents (Table 1). Compliance (mean $28.9 \mathrm{vs} 29 \mathrm{ml} / \mathrm{cmH}_{2} \mathrm{O}$ ) at the time of prone position did not differentiate survivors from decedents (Table 1).

In the overall population, the response was high, with a mean rate of $94.4 \%$ and was correlated with a better outcome at d60 (mean $145 \%$ vs $76 \%, p=0.038$, Wilcoxon test, Figure 1 ). Significant response to first PP was observed in 73.8\%. 10/11 (90.9\%) in d60 survivors responded to first PP vs $21 / 31(67.7 \%)$ in d60 decedents $(p=0.23$, Fischer test, Table 1).

Higher response remained associated with a lower d60 mortality after adjustment on age, extra-respiratory components of SOFA at day 1 and first Covid-19 viral load recovered from the respiratory tract (adjusted OR (per \% of response) $[\mathrm{Cl}]=0.992[0.98 ; 1], \mathrm{p}=0.0618$ ) Significant response was not associated to age, body mass index, severity at admission, first Covid-19 viral load, time from intubation to PP or compliance.

\begin{tabular}{|c|c|c|c|}
\hline Characteristics & $\begin{array}{l}\text { Alive at day-60 } \\
\quad(n=11)\end{array}$ & $\begin{array}{l}\text { Dead at day-60 } \\
\quad(n=31)\end{array}$ & Pvalue \\
\hline Age, year, median [IQR] & $56[51 ; 69]$ & $63[51 ; 69]$ & 0.5351 \\
\hline Simplified Acute Physiology Score II, median [IQR] & $37[27 ; 46]$ & $32[25 ; 50]$ & 0.7967 \\
\hline $\begin{array}{l}\text { Sepsis-related Organ Failure Assessment score at day } 1 \text {, } \\
\text { median [IOR] }\end{array}$ & $1[0 ; 4]$ & $2[1 ; 5]$ & 0.1735 \\
\hline Body Mass Index, median [IQR] & $33[26 ; 34]$ & $28[24 ; 32]$ & 0.1752 \\
\hline Delay from intubation to PP, hour, median [IQR] & $64.5[25 ; 136]$ & $46[18 ; 100]$ & 0.6783 \\
\hline $\mathrm{PaO}_{2} / \mathrm{FiO}_{2}$ ratio before $\mathrm{PP}$, median [IQR] & $102[78 ; 110.1]$ & $101.3[83 ; 121]$ & 0.5576 \\
\hline Admission nCov19 viral load', log (10) copy, median [IQR] & $6[5 ; 9]$ & $8[6 ; 8]$ & 0.1430 \\
\hline $\begin{array}{l}\text { Static compliance of the respiratory system, before PP, } \\
\mathrm{mL} / \mathrm{cmH} \mathrm{H}_{2} \mathrm{O} \text {, median [IQR]] }\end{array}$ & $25[23.1 ; 34]$ & $28.6[21.4 ; 34.2]$ & 0.9668 \\
\hline Response to $\mathrm{PP}^{2}$, percentage, median [IQR] & $149[52 ; 232]$ & $62[11 ; 119]$ & 0.0381 \\
\hline Significant response to $\mathrm{PP}^{3}, \mathrm{n}(\%)$ & $10(90.9)$ & $21(67.7)$ & 0.2342 \\
\hline \multicolumn{4}{|c|}{ 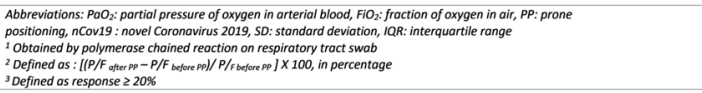 } \\
\hline
\end{tabular}


Figure 1: Response to first prone positioning (PP) according to mortality at day 60 .

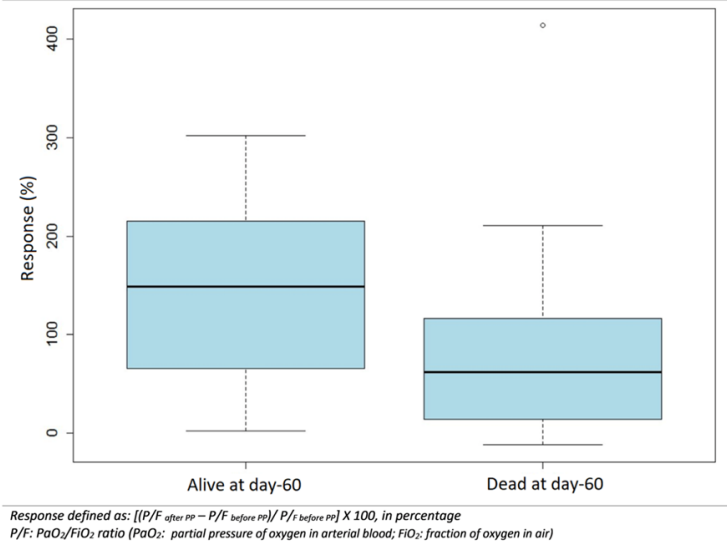

Conclusion: In our study, patients with severe Covid-19 induced ARDS mainly responded to the first PP, although mortality rate was high. Using a 20\% increase threshold, no difference was found regarding response rate to first PP between patients alive and dead at day 60 . However, the response was significantly better in the $d 60$ survivors. This suggests that the threshold used to define our endpoint may be too low to use it as a predictive factor. These results tendto highlight that response to first PP is correlated with survival.

Reference(s) and grant ackowledgment(s)

1. Li, X., Ma, X. Acute respiratory failure in COVID-19: is it "typical" ARDS?. Crit Care 24, 198 (2020). https://doi.org/10.1186/s13054-020-02911-

2. Alhazzani W, Møller MH, Arabi YM, Loeb M, Gong MN, Fan E, et al. Surviving Sepsis campaign: guidelines on the management of critically ill adults with coronavirus disease 2019 (COVID-19) Intensive Care Med. 2020;46(5):854887. https://doi.org/10.1007/s00134-020-06022-5

\section{0}

\section{Rotational thromboelastometry and thromoboembolic} complications in COVID-19 patients

N. Van Veenendaal ${ }^{1}$; T. Scheeren ${ }^{2}$; K. Meijer ${ }^{3}$; P. Van Der Voort ${ }^{4}$

${ }^{1}$ Department of critical care/department of anaesthesiology, University Medical Center Groningen, Groningen, Netherlands; ${ }^{2}$ Department of anaesthesiology/department of critical care, University Medical Center Groningen, Groningen, Netherlands; ${ }^{3}$ Department of hematology, University Medical Center Groningen, Groningen, Netherlands; ${ }^{4}$ Department of critical care, University Medical Center Groningen, Groningen, Netherlands

Correspondence: N. Van Veenendaal

Intensive Care Medicine Experimental 2020, 8(2): 001160

Introduction: Coronavirus disease 2019 (COVID-19) is associated with hypercoagulable states and high incidences of thromboembolic complications. Conventional coagulation tests show trends of coagulopathy, but currently no test reliably identifies COVID-19 patients at increased risk of developing thromboembolic complications. Rotational thromboelastrometry $\left(\right.$ ROTEM $\left.^{\circledR}\right)$ is a point-of-care device that evaluates viscoelastic changes during coagulation and may provide valuable information to identify patients at risk of developing thromboembolic complications.

Objectives: The aim of this study was to evaluate ROTEM ${ }^{\circledR}$ profiles of COVID-19 patients in identifying thromboembolic complications.

Methods: All patients diagnosed with COVID-19 admitted to the ICU in April 2020 were enrolled in this single-center, retrospective observational study. Blood samples were collected for ROTEM ${ }^{\circledR}$ thromboelastometry. Coagulation patterns were analyzed using extrinsic, intrinsic and fibrinogen rotational thromboelastometry activators (respectively EXTEM, INTEM and FIBTEM). ROTEM ${ }^{\circledR}$ patterns were analyzed for all COVID-19 patients and were compared between patients with and without thromboembolic complications. Hypercoagulability was defined as a reduction in clot formation time (CFT) and a maximum clot firmness (MCF) above the upper limit of the normal reference values.

Patients who were clinically suspected to have a thromboembolic complication underwent additional imaging. The decision for imaging was based on clinical features and was at the discretion of the attending intensivist.

Additionally, ROTEM ${ }^{\circledR}$ parameters were compared with conventional coagulation parameters including, partial thromboplastin time (PT), activated partial thromboplastin time (APTT), fibrinogen, and platelet count.

Results: ROTEM ${ }^{\circledR}$ patterns of forty-seven COVID-19 patients were analyzed. Ten patients (21.3\%) developed thromboembolic complications during ICU admission, all being pulmonary embolisms confirmed by CT-scan. MCF in EXTEM was lower in patients with and without thromboembolic complications (75.0 vs. $77.9 \mathrm{~mm}, \mathrm{p}=0.047$ ). COVID-19 patients with thromboembolic complications had an increased CFT compared to patients without complications in EXTEM (54.1 vs. $42.9 \mathrm{~s}$, $\mathrm{p}=0.001)$ and INTEM (53.3 vs. $43.5 \mathrm{~s}, \mathrm{p}=0.03$ ). EXTEM clot firmness at 5 minutes (A5) was significantly decreased in patients with thromboembolic complications compared to patients without complications (58.5 vs. $64.3 \mathrm{~mm}, \mathrm{p}=0.009$ ).

The conventional coagulation tests showed no significant differences between patients with and without thromboembolic complications. Univariate linear analysis identified nine variables for multiple regression analysis. None of the ROTEM ${ }^{\circledR}$ variables were associated with thromboembolic complications in the multiple linear regression model. ROTEM ${ }^{\circledR}$ variables could not predict the risk of developing thromboembolic complications $(R 2=0.266 ; F(9,37)=1,489 ; \mathrm{p} 0.188)$. Conclusion: ROTEM ${ }^{\circledast}$ patterns of COVID-19 patients showed no clinical significant differences between patients with and without thromboembolic complications. Hence, our study does not support the use of ROTEM ${ }^{\circledR}$ in identifying COVID-19 patients at risk for developing thromboembolic complications.

\section{5}

The mobile ECMO team to retrieve refractory ADRS patients justify the results the efforts ?

JKM. Fichte ${ }^{1}$; M. Schoeffner' ${ }^{1}$; S. Ullrich ${ }^{1}$; A. Starl ${ }^{1}$; A. Ernst ${ }^{1}$; HB. Hopf ${ }^{1}$

${ }^{1}$ Anesthesia and perioperative medicine, Asklepios Klinik Langen, Langen

(Hessen), Germany

Correspondence: J.K.M. Fichte

Intensive Care Medicine Experimental 2020, 8(2): 001165

Introduction: Mortality rates of patients with ARDS still are around $45 \%$ (Combes, NEJM). Since the resources for delivering an ECMO therapy are available only in a minority of hospitals, mobile ECMO teams have been established by ECMO centres to retrieve ARDS patients from primary or secondary care hospitals after implanting an ECMO system on side and consecutively transporting the patient in an ECMO centre. However, data on survival rates of those patients compared to patients who were implanted in the ECMO centre are scarce. Accordingly we retrospectively examined the data of our patients in which the ECMO system has been implanted in the submitting hospital with emphasize on survival rates compared to those primarily admitted to our ECMO centre.

Methods: We examined the data of all patients retrieved by our ECMO team in primary and secondary hospitals who had called our ECMOsupport hotline. All patients had been initially admitted in hospitals in the Rhine-Main region from January 2017 till June 2020. We restricted our evaluation to patients with ARDS

We divided the patients in two groups - ECMO implantation in the requesting hospital or in our hospital. We retrospectively noted the following variables: type of cannula, CPR before or during ECMO implantation, the frequency of palliative treatment, mortality rate. We compared the data of the ECMO patients implanted at the requesting hospital with those of the patients who were implanted at our hospital. Primary outcome variable was hospital mortality rate. Secondary 
outcome variables: SAPS II score, sofa-score, incidence of acute renal failure, time to weaning off the ventilator, duration of ICU stay.

Results: From 2017-2020 we treated 234 patients with ECMO on our intensive care unit. 170 patients have been admitted directly to our hospital. 66 patients have been retrieved from external clinics with a majority of patients who suffered from severe acute respiratory distress syndrome (ARDS) of different origins.

Remarkably is the extremely higher survival rate with ECMO treatment in patients initiated already at an external clinic. $36 \%$ compared to $55 \%$ of deceased patients in the in hospital patients group.

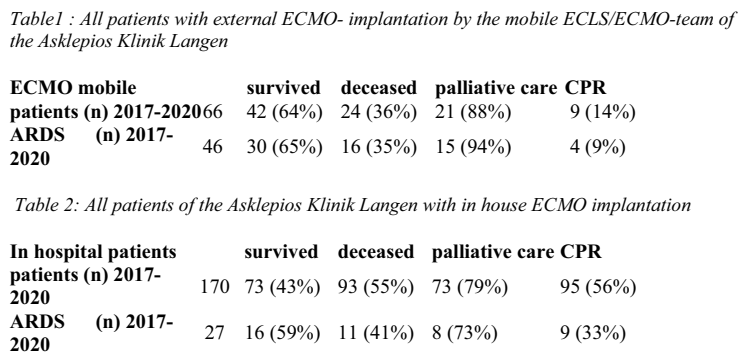

Conclusion: Our results suggest, that ECMO treatment, if required is more helpful to improve mortality the sooner the better. Especially patients with ARDS obviously seem to benefit from immediate treatment changes to ECMO therapy which gets along with shorter ventilation times, lower ventilation pressures, less sedation of the patients and spontaneously breathing patients. Patients initially admitted to external hospitals have been presented for ECMO therapy only after having exploited all other possible treatment options. Presumably ECMO should become more than only a rescue treatment, but a more and earlier considered option to improve mortality in serious medicalconditions.

\section{5}

Shuttlescope ${ }^{\circledR}$, a novel laryngoscope. One-handed endotracheal intubation makes a difference by allowing continuous suction JM. Alonso Babarro'; HN. Bui ${ }^{2}$; P. Boyer ${ }^{2}$; A. Boyer ${ }^{2}$; J. Medrano ${ }^{3}$

${ }^{1}$ Intensive care unit, The Prince Charles Hospital, Brisbane, Australia; ${ }^{2}$ Service de médecine intensive réanimation, CHU Pellegrin, Bordeaux, France; ${ }^{3}$ Bioaraba, Basque Country University, UPV/EHU, Vitoria-Gasteiz, Spain

Correspondence: J. Medrano

Intensive Care Medicine Experimental 2020, 8(2): 000035

Introduction: Despite the fact that videolaryngoscopy has largely improved laryngoscopy, endotracheal intubation (ETI) is still a twohanded procedure. While the first hand holds the videolayngoscope, the second hand is required to introduce the endotracheal tube (ETT). Shuttlescope ${ }^{\circledR}$ is a novel laryngoscope that allows performing both laryngoscopy and delivering of the ETT with the same hand, leaving an extra hand free. This may improve safety by allowing continuous oral suction in difficult scenarios.

Objectives: Our hypothesis was that Shuttlescope ${ }^{\circledR}$ videolaryngoscope allows similar efficiency in ETI than conventional laryngoscope while improving safety by allowing simultaneous suction. Main objective was to compare efficacy and safety of endotracheal intubation with Shuttlescope ${ }^{\circledR}$ and Macintosh laryngoscope by intensivists in a simulated difficult scenario.

Methods: We conduct a randomized cross-over trial to assess efficacy and safety of Shuttlescope ${ }^{\circledR}$ videolaryngoscope compaired to Macintosh laryngoscope in a manikin model (AirSim, TrueCorp ${ }^{\circledR}$ ). A difficult scenario which required continuous suction was designed, with a modified yaunkauer suction tip capable to determine suction time. All operators were intensivists from the service de Médecine Intensive Réanimation (Bordeaux, France) who received a rigorous $15 \mathrm{~min}$ standardized training on the new device and technique, which they didn't experience before. Three attempts with each device were compaired for each operator. The primary endpoint was a combination of time to intubation and success rate. Secondary endpoints were cumulative suction time and capability to aspirate continuously. Mean times and proportions were compaired with the T-Student and chi-square test, respectivelly.

Results: In total, 20 operators participate to the study. After randomisation, 10 intensivists started ETI with the Shuttlescope ${ }^{\circledR}$ and 10 started with Macintosh, providing a total of 60 intubations with each device. Mean time for ETI with Macintosh laryngoscope compaired to Shuttlescope ${ }^{\circledR}$ was $14 \mathrm{~s}$ [6-37] and $11 \mathrm{~s}$ [6-28], $\mathrm{p}<0.001$, respectively. Success rate was $100 \%$ of ETT in the trachea with both devices ( $p=$ N.S). Mean suction time was $2 \mathrm{sg}$ [1-10] with Macintosh and $3.6 \mathrm{sg}$ [2-10], with Shuttlescope ${ }^{\circledR}(p<0.001)$. Finally, only $5 \%$ of ETI done with Macintosh could be performed with a continuous suction compared to $90 \%$ with Shuttlescope ${ }^{\circledR}(p<0.001)$.

Conclusion: Shuttlescope ${ }^{\circledR}$ is a novel device that allows safe and easy ETI while leaving the right hand of the operator free during the procedure. It allows effective suction which may confer a relevant advantage in situations with a high risk of aspiration.

\section{Reference(s) and grant ackowledgment(s)}

1. Shuttlescope ${ }^{\circledR}$, a novel laryngoscope. Is one-handed endotracheal intubation feasible? European Journal of Anaesthesiology (Volume 37, e-Supplement 58, June 2020)

2. SHUTTLESCOPE ${ }^{\circledR}$, a novel laryngoscope for one-handed endotracheal intubation: one helping hand can make a difference. 2nd World Airway Management Meeting 2019.13 - 16 Nov, 2019| Amsterdam, NL.

3. 'Building Better Future for Health Challenge' in Australia

4. MIT-Harvard Medical School Healthcare Bootcamp

5. 'Ignite Ideas Grant' by the Queensland Government in Australia

\section{3}

Combined cell therapy and anticoagulants as a treatment for acute respiratory distress syndrome

M. Camprubí-Rimblas ${ }^{1}$; A. Areny²; L. Morales-Quinteros ${ }^{3}$.

R. Guillamat-Prats ${ }^{4}$; N. Tantinyà ${ }^{4}$; L. Blanch ${ }^{5} ;$ A. Artigas $^{6}$

${ }^{1}$ Centro de investigación biomédica en red enfermedades respiratorias (ciberes i i3pt), Universitat Autònoma de Barcelona, Cerdanyola del Vallès, Spain; ${ }^{2}$ Institut d'investigació i innovació parc tauli (i3pt), Universitat Autònoma de Barcelona, Cerdanyola del Vallès, Spain; ${ }^{3}$ Intensive care, Hospital Universitari Sagrat Cor - Grup Quirónsalut, Barcelona, Spain; ${ }^{4}$ Institut d'investigació i innovació parc taulí (i3pt), Centro de Investigación Biomédica en Red de Enfermedades Respiratorias, Sabadell, Spain; ${ }^{5}$ nnstitut d'investigació i innovació parc taulí i3pt, Parc Taulí Hospital Universitari, Sabadell, Spain; ${ }^{6}$ Department of intensive care medicine, Corporacion Sanitaria Universitaria Parc Tauli, Barcelona, Spain

Correspondence: M. Camprubí-Rimblas

Intensive Care Medicine Experimental 2020, 8(2): 000243

Introduction: The lungs of patients with acute respiratory distress syndrome (ARDS) are characterized by an acute inflammation and an increased procoagulant activity. Recent evidence suggests the beneficial effects of cell therapies and nebulized anticoagulants for ARDS $(1,2)$. Considering the different mechanism through which cell therapies and antithrombin act in ARDS, we propose a combined administration of these therapies to potentiate their therapeutic properties and face the different pathways and processes involved in the pathophysiology of ARDS.

Objectives: Determine the therapeutic benefit of a combined cell therapy (alveolar type II cells (ATII), mesenchymal stem cells (MSC), supernatant (SN) ATII, SN MSC) with antithrombin in vitro in a coculture of ATII and alveolar macrophages injured with lipopolysaccharide (LPS).

Methods: Cocultured ATII and alveolar macrophages isolated from rat lungs were injured with LPS $(10 \mathrm{ng} / \mathrm{ml})$. Two hours after the injury a combined cell therapy (ATII, MSC, SN ATII, SN MSC) with antithrombin $(0.1 \mathrm{IU} / \mathrm{ml})$ was administered. The effect of the administered treatments was assessed by the analysis of proinflammatory mediators and coagulants factors via qRT-PCR at $18 \mathrm{~h}$. Data are expressed as 
mean \pm SEM. Statistical analysis was performed using One-Way-ANOVA and Newman-Keuls post-hoc test (statistical significance $p \leq 0.05$ ).

Results: Proinflammatory mediators and coagulant factors were significantly increased after LPS administration (Figure). After administering the different treatments the expression of IL1 $\beta$, IL6, TF and PAI-1 was not as high as in the LPS group.
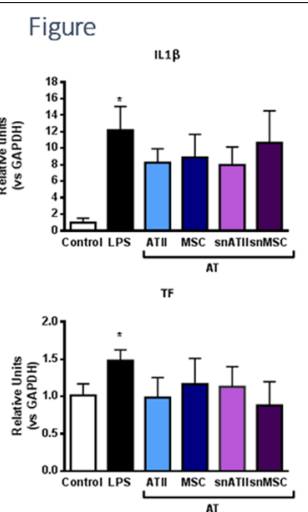

Fig. 1: Gene expression of proinflammatory mediators; IL $1 \beta$, IL6 and coagulant factors; TF, PAl1, evaluated by qRT-PCR at $18 \mathrm{~h}$. Data are expressed mean \pm SEM in relation to GAPDH expression ( $n=6)$. ${ }^{*} p \leq 0.05$. vs control group. One-way ANOVA, Bonferroni post-hoc test

Conclusion: The results indicate that combined cell therapy with antithrombin are able to attenuate inflammation and coagulation in vitro in a coculture of ATII and macrophages. Further studies need to be performed to elucidate the effect of both treatments in the different processes involved in lung injury.

\section{Reference(s) and grant ackowledgment(s)}

1. 2. Guillamat-Prats $R$, Camprubí-Rimblas $M$, Bringué J, Tantinyà N, Artigas A. Cell therapy for the treatment of sepsis and acute respiratory distress syndrome. Ann Transl Med. 2017 Nov;5(22):446. https://doi.org/10.21037/ atm.2017.08.28

2. 1. Camprubí-Rimblas M, Tantinyà N, Bringué J, Guillamat-Prats R, Artigas A. Anticoagulant therapy in acute respiratory distress syndrome. Ann Transl Med. 2018 Jan;6(2):36. https://doi.org/10.21037/atm.2018.01.08.

3. 2. Fundació d'Investigació i Innovació Parc Taulí

4. 1. Instituto de Salud Carlos III (PI18/00677)

\section{1}

Creating Organizing and Managing a National Randomized controlled study in Corona Pandemic:The use of Tocilizumab in the management of patients who have severe COVID-19 with suspected pulmonary hyperinflammation

J. Benbenishty ${ }^{1}$;. Zhitomirsky ${ }^{2}$; R. Pizov' ${ }^{3}$ EG. Prof ${ }^{4}$

${ }^{1}$ \{street_address\}, Jerusalem, Israel; ${ }^{2}$ Nursing administration, Hadassah University Hospital - Ein Kerem, Jerusalem, Israel; ${ }^{3}$ Anesthesia and critical care, Hadassah University Hospital - Ein Kerem, Jerusalem, Israel; ${ }^{4}$ Gene theary, Hadassah University Hospital - Ein Kerem, Jerusalem, Israel

Correspondence: J. Benbenishty

Intensive Care Medicine Experimental 2020, 8(2): 000311

Introduction: This talk will share trials and tribulations of creating National randomized controlled study during Corona pandemic.This is a study designed to assess the therapeutic value of intravenous tocilizumab administered as single $8 \mathrm{mg} / \mathrm{Kg}$ dose compared to placebo in patients affected by SARS-CoV2 infection with a pulmonary manifestation causing hypoxia

Objectives: Aim of the study is to test the hypothesis that an antiIL6 treatment can be effective in calming the virus-induced cytokine storm, blocking deterioration of lung function or even promoting a rapid improvement of clinical conditions, preventing naso-tracheal intubation and/or death. In additon, lessons learned from institutional and national ethics committee submissions, safety board review and revisions, creating digital data capture, and data monitoring will be shared.

Methods: When COVID 19 pandemic started in China we approached government offices for support and stategy. A protocal was developed and implementation plans were established. When ethical approval was granted in one medical institution digital electronic data capture platform was created, tested and launched. We recruited all Intenisve care and infectious disease units, to join the national study. After national Ministry of health approval was granted each hospital needed institutional approval. Within 1 week, 6 other centers recieved approval, recieved study drug and started recruting patients. Nurses trained medical centers on data capture and protocol adherence. Zoom and whatsapp platforms were utilized for training. Close Data monitoring was preformed at base hospital.

Results: At present 20 patients have been recruited and by time of presentation many more will be added. We will demonstrate change from baseline in cytokines IL-1 $\beta$, IL-6, IL-8 and TNF- $\alpha$ [ Outcome data -mortality, length of Hosptial and ICU stay, ventilation days,and ventilation methods. In addition, technologies used to organzie and manage multi center- multi disciplanary communications in an randomized placebo controlled drug study during Corona epidemic will be shared and discussed.

Conclusion: Sharing lessons learned in creating, organizing and managing a multi center mulit-disciplinary national randomized controlled trial is vital for applying in order to duplicate during the next pandemic.

\section{Reference(s) and grant ackowledgment(s)}

1. Behrens EM, Koretzky GA. Review: Cytokine Storm Syndrome: Looking Toward the Precision Medicine Era [Internet]. Vol. 69, Arthritis and Rheumatology. John Wiley and Sons Inc.; 2017 [cited 2020 Mar 17]. p. 1135-43. Available from: http://www.ncbi.nlm.nih.gov/pubmed/28217930

\section{7}

Lung and intestinal microbiome changes in a murine model of acute lung injury treated with Anti-IL33

R. Muñoz-Bermúdez'; J. Marin-Corral'; I. Dot-Jordana';

A. Salazar-Degracia'; B. Carbonetto²; P. Gonzalez-Torres²; T. Gabaldón².

G. Tagmouti ${ }^{3}$ 'L. Pijuan ${ }^{3}$; JR. Masclans-Enviz ${ }^{4}$

${ }^{1}$ Critical care department, Hospital del Mar, IMIM-GREPAC, Barcelona, Spain; ${ }^{2}$ Bioinformatics and genomics program, Centre for Genomic Regulation (CRG), Barcelona, Spain; ${ }^{3}$ Anatomic pathology department, Hospital del Mar, Barcelona, Spain; ${ }^{4}$ Critical care department, Hospital del Mar, IMIM-GREPAC, UAB, Barcelona, Spain

Correspondence: R. Muñoz-Bermúdez

Intensive Care Medicine Experimental 2020, 8(2): 000497

Introduction: Alterations of the microbiota can cause a dysregulation of the immune system locally and systemically, facilitating the clinical progression in different pathologies with inflammation as ALI.

Objectives: To assess changes in lung and intestinal microbiome in a murine model of acute lung injury (ALI) and subsequent treatment with an anti-IL-33 antibody.

Methods: Experimental model in which 36 male BALB/c mice were included (CEEA: JME-16-0025). ALI was induced at Day 1 by the intranasal administration of lipopolysaccharide (LPS) at a dose of $8 \mathrm{mg} / \mathrm{kg}$.

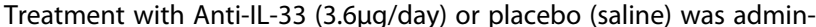
istered, intraperitoneally, at days 2, 3 and 4 . On day 5, body weight gain was registered and bronchoalveolar lavage (BAL), lung, feces and blood samples were collected. The studied groups were: 1) Control group without $\mathrm{ALI}$ including those treated with placebo or anti-IL-33 (CTL-PCB, $n=6$ and CTL-Anti-IL33, $n=6$ ), 2) Experimental group with $A L I$ and placebo (ALI-PCB, $n=12$ ) and 3) Experimental group with ALI and anti-IL-33 (ALI-Anti-IL-33, $\mathrm{n}=12$ ). Microbiome was assessed by the analysis of 16SrRNA in BAL and feces and bioinformatic analysis was performed. Cell count, total protein and IL-33 levels were measured in BAL fluid. IL-33 levels were also measured in blood samples. ALI was assessed by histology and wet-to-dry lung weight ratio (W/D). The data was expressed as mean (standard deviation) or as median (interquartile range). Significance was set to $p<0.05$. The comparison 
of the microbiome between samples was carried out by correspondence analysis with the QIIME and UNIFRAC programs.

Results: Intestinal microbiota showed a greater richness but not eveness (a diversity) in comparison with lung microbiota $(p<0.000)$ in all groups. Differences between the microbial communities (betadiversity) in gut CTL, ALI-PCB and ALI-Anti-IL-33 groups were observed (Bray Curtis, Permanova 0.027, Permdisp > 0.05, ANOSIM 0.0797, p = 0.062). Clear differences in microbial communities were observed in the three groups when comparing gut vs lung (Unweighted Unifrac, Permanova $=0.001$, Permdisp $>0.05$, ANOSIM 0.5025, $p=0.001$ ). ALIPCB group compared with the CTL group presented a decreased in the body weight gain [-11.5 (6.2) vs $2.8(1.8), \mathrm{p}<0.001]$, an increase in the W/D [0.16 (0.02) vs $0.10(0.00), \mathrm{p}<0.001]$ and higher neutrophil percentage [69.4 (67.4-71.1) vs $2.7(2.0-3.1), \mathrm{p}<0.001]$, protein levels [596.2 (462.8-760.8) vs $112.0(81.5-194.9), \mathrm{p}<0.001]$ and IL-33 levels [26.1 (25.2-29.6) vs 15.1(11.1-20.1), $\mathrm{p}=0.004$ ] in BAL. It also showed higher rates of proteinaceous debris [1.1 (0.7) vs $0.0(0.0), p=0.01]$ and polymorphonuclear (PMN) cells in alveolar and interstitial space [1.7 (0.4) vs $0.0(0.0), p<0.001 ; 1.1(0.4)$ vs $0.0(0.0), p=0.002$ ]. Differences between gut and lung communities were lower in ALI groups than in CTL groups, showing a greater similarity in the lung-gut microbial communities in this group $(p<0.001)$.

Treatment with Anti-IL-33 compared with placebo reduced the W/D $[0.13(0.02)$ vs $0.16(0.02), p=0.02]$, the BALF neutrophil percentage [63.1(52.6-68.1) vs 69.4(67.4-71.1); $p=0.03$ ], the interstitial PMN $[0.4(0.5)$ vs $1.1(0.4), p=0.01]$ and proteinaceous debris $[0.3(0.4)$ vs $1.1(0.7), p=0.02]$. Anti-IL-33 treatment did not reverse the observed changes in the microbiome of both echosystems.

Conclusion: ALI modifies the respiratory microbiome, resembling lung microbiota to intestinal. Anti-IL-33 could partially restore the ALIinduced histopathological and biochemical but no the microbiome changes. Treatment directed to restore microbiomein ALI could be a new therapeutic target independent to anti-inflammatory approach.

\section{6}

Regional Lung Stress and Strain during spontaneous breathing in an animal model of Acute Respiratory Distress Syndrome E. Chiodaroli ${ }^{1}$; F. Liggieri ${ }^{1}$; PS. Mariotti ${ }^{1}$; M. Pellegrini ${ }^{2}$; G. Hedenstierna ${ }^{3}$; G. Perchiazzi ${ }^{2}$

${ }^{1}$ Hedenstierna laboratory, department of surgical sciences, Uppsala University, Uppsala, Sweden; ${ }^{2}$ Hedenstierna laboratory, department of surgical sciences and, Department of Anesthesia and Intensive Care Medicine of Uppsala University Hospital, Uppsala, Sweden; ${ }^{3}$ Department of medical sciences, uppsala university, Hedenstierna Laboratory, Uppsala, Sweden Correspondence: E. Chiodaroli

Intensive Care Medicine Experimental 2020, 8(2): 000516

Introduction: It is questioned whether spontaneous breathing (SB) should be used in early Acute Respiratory Distress Syndrome (ARDS). Recent papers have reported concerns regarding SB, for the potential of triggering the so-called patient self-induced lung injury (1). Despite a clear improvement in ventilatory strategies, we still lack knowledge that could make SB fully protective. Assessment of potential parenchymal injury must take into account stress and strain as the real physical quantities acting on the lung structures. Moreover, the lack of information regarding regional lung mechanics and deformation at the acinar level is likely the main determinant of these unresolved issues.

Objectives: Aim of the present study was to investigate regional lung Strain and Stress during continuous positive airway pressure (CPAP) ventilation, in an animal model of mild ARDS during SB.

Methods: Mild experimental ARDS $\left(\mathrm{PaO}_{2} / \mathrm{FiO}_{2}\right.$ ratio of $\left.250 \mathrm{mmHg}\right)$ was induced in anesthetized pigs $(n=5)$ breathing at three positive end-expiratory pressure (PEEP) levels during CPAP: 12,6 and $0 \mathrm{cmH}_{2} \mathrm{O}$. Computed tomography (CT) of the lung was performed at Functional Residual Capacity (FRC) and then during uninterrupted CPAP breathing at the three PEEP levels. Airway pressure and flow together with gastric and esophageal pressures were acquired simultaneously to $C T$ execution. One lung slice above the diaphragm dome was continuously exposed and successively analysed. By analysing representative breaths at each PEEP level, regional Strain and Stress (transpulmonary pressure, PTP) were computed in three regions of interest (ROI) of the lung: nondependent, middle and dependent.

The obtained data were successively analysed for statistical significance by applying ANOVA test for repeated measurements with $a=$ 0.05 (SigmaPlot, Systat Soft.,USA).

Results: When decreasing PEEP from 12 to $0 \mathrm{cmH}_{2} \mathrm{O}$, regional lung Strain decreased from $0.78 \pm 0.17$ to $0.37 \pm 0.10(p<0.001)$ respectively. Regional lung Stress was significantly higher at PEEP 12 $\left(4.30 \pm 0.90 \mathrm{cmH}_{2} \mathrm{O}\right)$ than PEEP $6\left(3.83 \pm 0.70 \mathrm{cmH}_{2} \mathrm{O}\right)$, but the highest PTP values were recorded at PEEP $0\left(5.00 \pm 1.1 \mathrm{cmH}_{2} \mathrm{O}\right)(\mathrm{p}<0.001)$ Stress significantly increased in all the studied ROls passing from PEEP 0 to 6 and from 0 to $12 \mathrm{cmH}_{2} \mathrm{O}$. Both stress and strain were significantly higher in dependent ROls than in middle and non-dependent.

Conclusion: This study shows that high regional Stress and Strain occur during spontaneous breathing in dependent ROls and at the two extremes of the PEEP spectrum: high regional Stress is present during CPAP at the lowest extreme (PEEP 0) whereas high regional strain at the highest one (PEEP 12). Titrating PEEP to reach the best compromise between preventing both lung regional strain and stress can enable a fully protective CPAP ventilation during SB.

\section{Reference(s) and grant ackowledgment(s)}

1. 1. Brochard L et al. Mechanical Ventilation to Minimize Progression of Lung

Injury in Acute Respiratory Failure. Am J Respir Crit Care Med 2017;195:1-16. 2. The Swedish Heart and Lung Foundation

\section{8}

\section{Lung viscoelastic properties in supine and prone position} in a porcine model of acute respiratory distress syndrome C. Guérin ${ }^{1}$; N. Terzi ${ }^{2}$; N. Noury ${ }^{3}$; M. Cour ${ }^{4}$; L. Argaud ${ }^{4}$; B. Louis ${ }^{5}$; S. Bayat ${ }^{6}$ ${ }^{1}$ Medecine intensive réanimation, Hospital Édouard Herriot, Lyon, France; ${ }^{2}$ Médecine intensive réanimation, C.H.U de Grenoble, La Tronche, France: ${ }^{3}$ Laboratoire de physique appliquée, Claude Bernard University Lyon 1, Villeurbanne, France; ${ }^{4}$ Service de médecine intensive-réanimation, Hospital Édouard Herriot, Lyon, France; ${ }^{5}$ Inserm 955, IMRB, Creteil, France; ${ }^{6}$ Labo des efr, C.H.U de Grenoble, La Tronche, France

Correspondence: $C$. Guérin

Intensive Care Medicine Experimental 2020, 8(2): 000798

Introduction: Lung tissues absorb the dissipation of the energy during lung inflation through their viscoelastic properties. These can be represented by the Mount rheological model that consists of a Maxwell body (lung viscoelastic elastance (E2) and resistance (R2) arranged in series) in parallel with the standard lung elastance and resistance, according to the following equation: Pvisc(ti) = R2 V' (1- exp(-ti//2)) (1) where Pvisc(ti) represents the viscoelastic elastic pressure dissipation into the lungs during inflation time (ti), $\mathrm{V}^{\prime}$ the inflation flow and $\tau 2$ the lung viscoelastic time constant ( $\tau 2=\mathrm{R} 2 / \mathrm{E} 2)$.

Objectives: In present study we aimed at assessing the effect of supine versus prone position on global and regional $E 2, L$ and $R 2, L$ at different positive end-expiratory pressure (PEEP). We also analyzed the relationship of regional $E 2, L$ and $R 2, L$ to lung collapse and overdistension.

Methods: This is a secondary analysis of a previous study in 6 pigs in whom ARDS was induced by surfactant depletion (1). Overall TransPulmonary pressure (PL) was measured as Paw minus esophageal pressure. Regional dorsal and ventral PL were obtained by subtracting Paw from dorsal and ventral pleural pressure, respectively. Pleural pressure was measured directly into the anterior and posterior 6th intercostal space. Lung ventilation was measured with electrical impedance tomography (EIT). Animals were ventilated in volume controlled mode at constant flow inflation, $\mathrm{FiO}_{2} 100 \%$ and tidal volume (VT) $6 \mathrm{ml} / \mathrm{kg}$. After ARDS induction the lungs were recruited and then PEEP was reduced from 20 to $5 \mathrm{cmH}_{2} \mathrm{O}$ by steps of $5 \mathrm{cmH}_{2} \mathrm{O}$. E2,L and $R 2, L$ were obtained by fitting the global and regional PL during a 5 s end-inspiratory hold to equation 1 as initially proposed (2). The effect of PEEP and position was analyzed by a linear mixed-effects model. The lung collapse and overdistension was measured by EIT as the change in impedance pixel-by-pixel during the decremental PEEP 
trial. Correlation was assessed by Spearman Rho coefficient. Values are median (1st-3rd quartiles).

Results: The fitting of data to equation 1 was successful in every instance.

\begin{tabular}{|c|c|c|c|c|c|c|c|c|}
\hline Position & \multicolumn{4}{|c|}{ Supine } & \multicolumn{4}{|c|}{ Prone } \\
\hline PEEP & 20 & 15 & 10 & 5 & 20 & 15 & 10 & 5 \\
\hline$E_{2}, L(\mathrm{cmH} H \mathrm{O} / \mathrm{L})$ & $8(6-9)$ & $4(3-6)$ & $5(4-7)$ & $10(9-12)$ & $12(8-18)$ & $6(4 \cdot 6)$ & $6(3-10)$ & $6(5-12)$ \\
\hline Dorsal $E_{2}, L$ (cmH & $8(6.8)$ & 5(4-6) & $5(5.6)$ & $9(9-15)$ & $12(9-15)$ & $5(4-8)$ & $6(5-10)$ & $10(6-21)$ \\
\hline Ventral $\mathrm{E}_{2} \mathrm{~L}\left(\mathrm{cmH} \mathrm{H}_{2} \mathrm{OL} \mathrm{L}\right)$ & $8(6.9)$ & $5(3-6)$ & $6(6-7)$ & $10(9-14)$ & $13(11-16)$ & $5(3-7)$ & $9(4+13)$ & $9(5-18)$ \\
\hline $\mathrm{R}_{2}, \mathrm{~L}\left(\mathrm{cmH} \mathrm{H}_{2} \mathrm{OL} / \mathrm{s} / \mathrm{s}\right)$ & $37(11-65)$ & $5(4-6)$ & $12(8.20)$ & $41(27.54)$ & $15(0-50)$ & $8(6-11)$ & $8(5-12)$ & $13(6-19)$ \\
\hline corsal R,L $\mathrm{L}(\mathrm{cmH}: \mathrm{O} / \mathrm{T} / \mathrm{s})$ & $18(16-20)$ & $9(6-11)$ & $8(6.9)$ & $29(24-37)$ & $29(15-63)$ & $9(4-13)$ & $24(5-50)$ & $19(13-23)$ \\
\hline entral $R_{2} L\left(\mathrm{cmH} H_{2} \mathrm{OIL} / \mathrm{s}\right)$ & $20(9.25)$ & $14(9-16)$ & $12(7.30)$ & $28(17-33)$ & $16(11-32)$ & $7(6.12)$ & $12(11-98)$ & $18 \mathrm{C}$ \\
\hline
\end{tabular}

Conclusion: Prone position does not significantly change the lung viscoelastic properties in an ARDS porcine model of surfactant depletion.

Reference(s) and grant ackowledgment(s)

1. 1. JAP 2020

2. 2. ERJ 1998

\section{6}

Hemodynamic profile of ARDS caused by SARS-CoV2

AM. Ioan ; J. Martínez Milla²; A. Durante López²; A. Kallmeyer Mayor²;

M. López Castillo²; O. GonzálezLorenzo ${ }^{2}$; C. Pérez Calvo ${ }^{1}$; A. Santos ${ }^{1}$

${ }^{1}$ Intensive care medicine department, Hospital Universitario Fundación Jiménez Díaz, Madrid, Spain; ${ }^{2}$ Cardiology department, Hospital Universitario Fundación Jiménez Díaz, Madrid, Spain

Correspondence: A.M. loan

Intensive Care Medicine Experimental 2020, 8(2): 000226

Introduction: It has been proposed that SARS CoV2 patients requiring invasive mechanical ventilation represent a particular type of ARDS with a different ventilatory profile, being managed with lower pressures, as well as having a distinguished physiopathology element involving blunted hypoxic pulmonary vasoconstriction. Therefore, we might expect a specific hemodynamic profile of these patients, with less prevalence of right ventricular (RV) failure.

Objectives: To describe the hemodynamic profile, measured by conventional monitoring as well as echocardiography, of the patients with ARDS caused by SARS CoV2 admitted to the ICU and treated with protective mechanical ventilation.

Methods: From April to May 2020, we performed a single-centre, prospective, observational study, which included consecutive patients with a diagnosis of ARDS caused by SARS CoV2 who required treatment with invasive mechanical ventilation and who had no previous cardiac nor respiratory chronic condition. The patients were managed with a protective ventilatory strategy. Hemodynamic parameters, as well as echocardiographic biventricular function and respiratory mechanics were measured.

Two transthoracic echocardiograms, separated by an interval of 30 minutes, were performed during the first 72 hours after initiation of mechanical ventilation. The first one with parameters set by the attending physician following our approach of reaching a $\mathrm{SaO} 2>90 \%$ with a low PEEP high $\mathrm{FiO}_{2}$ strategy. The second echocardiogram was performed after either increasing the PEEP 2-4 $\mathrm{cmH}_{2} \mathrm{O}$ if a $\mathrm{FiO}_{2}>60 \%$ were necessary to reach the $\mathrm{SaO} 2$ goal or increasing the $\mathrm{FiO}_{2}$ to $100 \%$ if the $\mathrm{SaO} 2$ goal were reached with a lower $\mathrm{FiO}_{2}$. A third echocardiogram was performed $72 \mathrm{~h}$ hours later with the ventilatory adjustments considered by the physician in charge of the patient. Arterial blood gas analysis was performed previously to each echocardiogram.

Results: We included 8 patients. Respiratory and hemodynamic variables are shown in Table 1. All patients showed hemodynamic stability with a median of MAP $>75 \mathrm{mmHg}$, both at baseline and $72 \mathrm{~h}$ later and low dose norepinephrine was used in one of them. We observed that in both groups, and particularly in the PEEP group, ventilatory changes did not have a negative effect on hemodynamics except by a trend to a decrease in the pulmonary acceleration time. All patients had preserved both left and right ventricular ejection fraction. None of the patients developed RV failure. A slight increase in PEEP tended to worsen pulmonary compliance but this was not followed by a hemodynamic worsening.

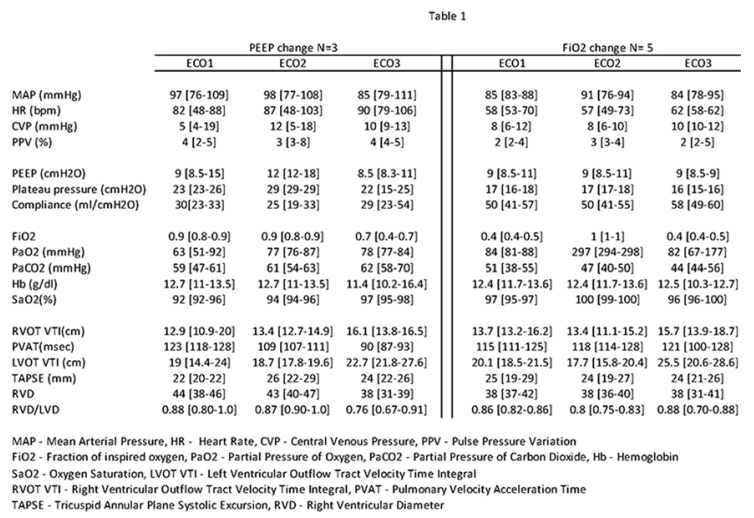

Conclusion: Despite the low oxygenation levels, this cohort of patients with SARS CoV2 shows hemodynamic stability and presents normal RV function. An increase in PEEP tends to worsen pulmonary mechanics but without an enormous impact in hemodynamics. This hemodynamic profile could be related with a blunted hypoxic pulmonary vasoconstriction and could be relevant at the time of choosing the best management strategy for this kind of patients.

\section{5}

\section{Lung ultrasound patterns in patients with COVID-19 pneumonia} at admission

S. Mongodi'; M. Cavagnino ${ }^{1}$; A. Orlando ${ }^{1}$; M. Pozzi ${ }^{1}$; G. Tavazzi ${ }^{2}$; E. Arisi ${ }^{1}$; L. Caneva'; G. Maggio'; A. Stella'; A. Colombo'; S. Bonaiti' ; S. Pregnolato'; G. Salve ; F. Daverio ; E. Pariani ${ }^{1}$; G. Bettini'; GM. Siano ${ }^{1}$; CL. Villa' ; F. Mojoli ${ }^{1}$ ${ }^{1}$ Department of anesthesia and intensive care, Fondazione I.R.C.C.S. Policlinico San Matteo, Pavia, Province of Pavia, Italy, Italy; ${ }^{2}$ Department of anesthesia and intensive care, Fondazione IRCCS Policlinico S Matteo, Pavie, Italy

Correspondence: M. Cavagnino

Intensive Care Medicine Experimental 2020, 8(2): 000395

Introduction: In February 2020, Italy reported the first cases of novel coronavirus disease (COVID-19) requiring admission to ICU for acute respiratory failure (ARF)[1]. Lung ultrasound (LUS) is useful in ARF for lung assessment and monitoring[2]: performed bedside avoiding transport to radiology, it is easily repeatable and it doesn't involve further healthcare providers, thus limiting potential exposure to COVID19 contamination[3].

Objectives: To describe LUS patterns at admission to ICU in patients with COVID-19.

Methods: Retrospective observational study; analysis of the LUS performed within the first 24 hours from admission to ICU. We computed the LUS score, from 0 (normal aeration) to 3 (complete loss of aeration), in 6 standard regions per hemithorax and registered lung features (subpleural/lobar consolidations, pleural irregularities, sliding, artefacts distribution, pleural effusion)[2].

Results: We present the first 13 patients admitted to our ICU $(77 \%$ males, median age 64 [56-73] year-old, median BMI 28 [26-29] kg/ $\mathrm{m}^{2}$ ). They were all examined under pressure-controlled ventilation (median PEEP 14 [11-15] $\mathrm{cmH}_{2} \mathrm{O}, \mathrm{FiO}_{2}>50 \%$ in $85 \%$ of patients). In all patients, we observed bilateral distribution of pathological findings, with at least one area with B-pattern (score $>1-100 \%$ ) and pleural 
irregularities (100\%). We frequently observed subpleural consolidation (85\%) and lobar consolidation (77\%); lung sliding was frequently reduced in anterior fields (92\%). The median LUS score was 18 [14-19]. Small pleural effusion was visualized in 3 patients $(23.1 \%$ median interpleural distance $0.8 \mathrm{~cm}$ ). $23.1 \%$ of patient had a bacterial overinfections, confirmed with positive BAL; they all had lobar consolidations.

Conclusion: LUS features of COVID-19 include bilateral loss of aeration with diffuse B-pattern, pleural irregularities and frequent subpleural consolidations. Lobar consolidations may suggest bacterial overinfection.

\section{Reference(s) and grant ackowledgment(s)}

1. Mongodi, Ultrasound Med Biol 2020; doi.org/https://doi.org/10.1016/j.ultra smedbio.2020.04.033

2. Mojoli, Am J Respir Crit Care Med. 2019;199:701-714

3. Grasselli, JAMA 2020; https://doi.org/10.1001/jama.2020.5394.

\section{7}

Defining the roles of CPAP and HFOT in the management

\section{of COVID-19 hypoxaemic respiratory failure}

K. El-Shakankery'; G. Sismey ${ }^{1}$; J. Cai ${ }^{2}$; V. Whitford'; D. Fink ${ }^{3}$; N. Goldman';

C. Tai $^{4}$

${ }^{1}$ Respiratory medicine, Whipps Cross University Hospital, London, United Kingdom; ${ }^{2}$ Ucl medical school, University College London, London, United Kingdom; Infectious diseases, Whipps Cross University Hospital, London, United Kingdom; ${ }^{4}$ Anaesthetics and critical care, Whipps Cross University Hospital, London, United Kingdom

\section{Correspondence: $C$. Tai}

Intensive Care MedicineExperimental 2020, 8(2): 000457

Introduction: Coronavirus disease 2019 (COVID-19) is a potentially severe acute respiratory infection which presents most commonly as a pneumonia, complicated by hypoxaemic respiratory failure in up to $41.8 \%$ of patients(1). Pandemic COVID-19 has placed unprecedented pressures on critical care services. Safe delivery of respiratory support on a large scale has proved a major challenge. High flow oxygen therapy (HFOT) and continuous positive airway pressure (CPAP) devices are recommended by interim guidance but significant controversy remains over their use for COVID-19-associated hypoxaemic respiratory failure $(2,3)$.

Objectives: We describe the real-world use of HFOT and CPAP in a single centre during the COVID-19 epidemic. We ask: 1 . What respiratory parameters predict failure of respiratory devices in COVID-19? 2. Can the ROX index be used to monitor HFOT for COVID-19? 3. Does HFOT compare to CPAP for management of hypoxaemic respiratory failure in COVID-19?

Methods: Data were collected retrospectively for all patients admitted to a single hospital with confirmed or probable COVID-19 between 27/03-06/04/20. Confirmed COVID-19 was defined by laboratory PCR testing of respiratory samples. Probable COVID-19 was defined as high clinical suspicion with negative COVID-19 testing. Outcomes were defined as severe (death or intubation) and non-severe (no intubation) at 30 days. Digital notes review captured demographic, clinical and respiratory parameters. Qualitative data were compared with the Fisher exact test. Quantitative data were compared with the Student's t-test.

Results: Of 239 adults with confirmed $(221,92.4 \%)$ or probable COVID$19(18,7.6 \%), 149$ patients received HFOT and/or CPAP. 45 (30.3\%) and $104(69.7 \%)$ patients had severe and non-severe outcomes respectively. 60 patients received HFOT and/or CPAP in 78 episodes of device use (Table 1). Mean $\mathrm{PaO}_{2} / \mathrm{FiO}_{2}$ (PF) ratios for patients initiating CPAP were lower than for those initiating HFOT (Table 1). In patients receiving HFOT, ROX index at all time points was significantly lower in those with severe versus non-severe outcomes (Figure 1). The mean ROX index at 0 to 12 hours was less than 4.88 in the severe outcome group, and above 4.88 in the non-severe group. For all patients receiving respiratory support devices, PF ratio was significantly lower in those with severe versus non-severe outcomes (Figure 2).
Table 1. Summary data for patients receiving HFOT and/or CPAP

\begin{tabular}{|c|c|c|c|c|}
\hline & HFOT & & CPAP & \\
\hline & Severe & Non-severe & Severe & Non-severe \\
\hline $\begin{array}{l}\text { Total (n, \% all } \\
\text { device use) }\end{array}$ & $30(38 \%)$ & $17(22 \%)$ & $24(31 \%)$ & $7(9 \%)$ \\
\hline $\begin{array}{l}\text { PF ratio at } \\
\text { admission(mean, } \\
\text { range) }\end{array}$ & $\begin{array}{l}154.5(45.5- \\
367.9)\end{array}$ & $\begin{array}{l}206.5(71.3- \\
341.5)\end{array}$ & $\begin{array}{l}125.5(45.5- \\
283.6)\end{array}$ & $\begin{array}{l}140.7(72.7- \\
317.9)\end{array}$ \\
\hline $\begin{array}{l}\text { PF ratio before } \\
\text { device(mean, range) }\end{array}$ & $\begin{array}{l}89.5(45.5- \\
269)\end{array}$ & $\begin{array}{l}122.3(59.2- \\
250)\end{array}$ & $\begin{array}{l}76.5(45.5- \\
195.0)\end{array}$ & $\begin{array}{l}105.2(74.9- \\
150.0)\end{array}$ \\
\hline $\begin{array}{l}\text { ROX index at } 12 \\
\text { hours HFOT (mean, } \\
\text { range) }\end{array}$ & $\begin{array}{l}4.83 \\
(2.2-7.6)\end{array}$ & $\begin{array}{l}7.69 \\
(4.7-10.8)\end{array}$ & & d \\
\hline $\begin{array}{l}\text { First device (n, \% all } \\
\text { device use) }\end{array}$ & $19(24 \%)$ & $16(21 \%)$ & $16(21 \%)$ & $4(5 \%)$ \\
\hline $\begin{array}{l}\text { Only device (n, \% all } \\
\text { device use) }\end{array}$ & $16(21 \%)$ & $13(17 \%)$ & $10(22 \%)$ & $3(4 \%)$ \\
\hline $\begin{array}{l}\text { Time to } \\
\text { intubation(days, } \\
\text { range) }\end{array}$ & $3.2(0-9)$ & - & $4.5(0-12)$ & - \\
\hline
\end{tabular}

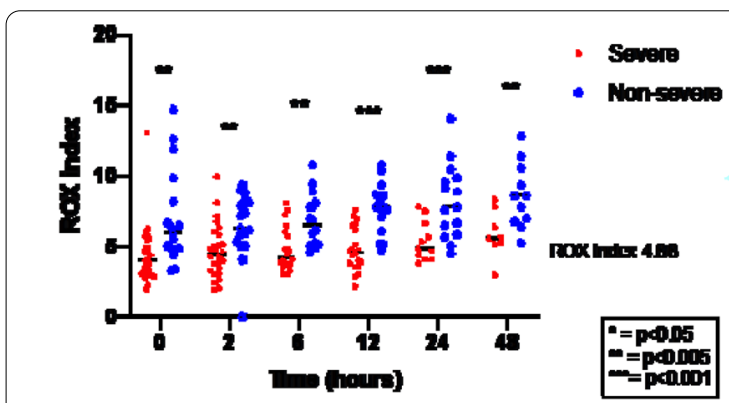

Figure 1. ROX indices for patients receiving HFOT

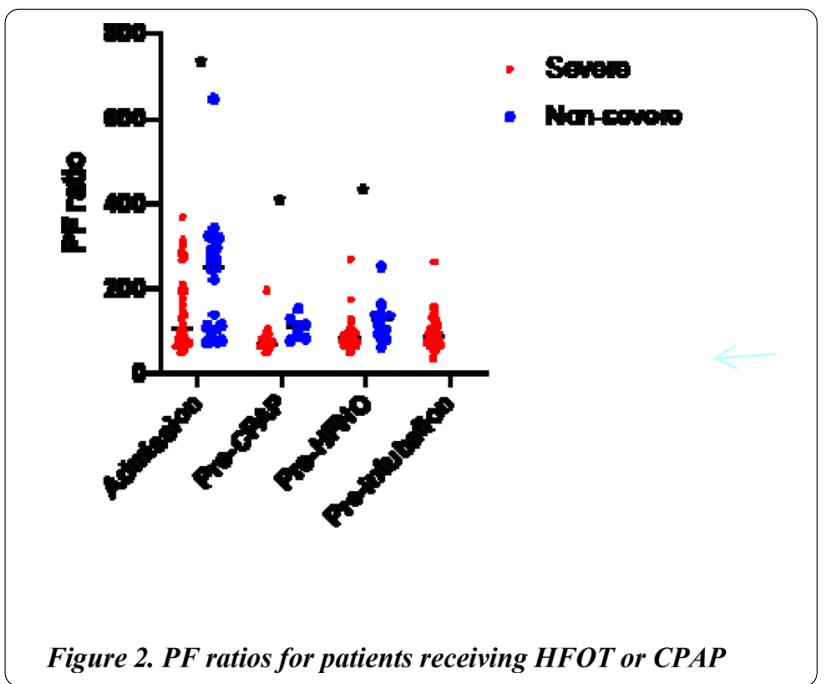

Conclusion: Data from our single site retrospective cohort study suggest ROX index parameters may predict failure of HFOT, and PF ratio may predict failure of HFOT and CPAP in COVID-19 consistent with non-COVID-19 literature(4). In our study, clinicians favoured CPAP for individuals presenting with more severe hypoxaemic respiratory 
failure making comparisons between HFOT and CPAP challenging. However HFOT was associated with successful outcomes in certain patients. ROX index may be useful in selecting this sub-group and warrants prospective study.

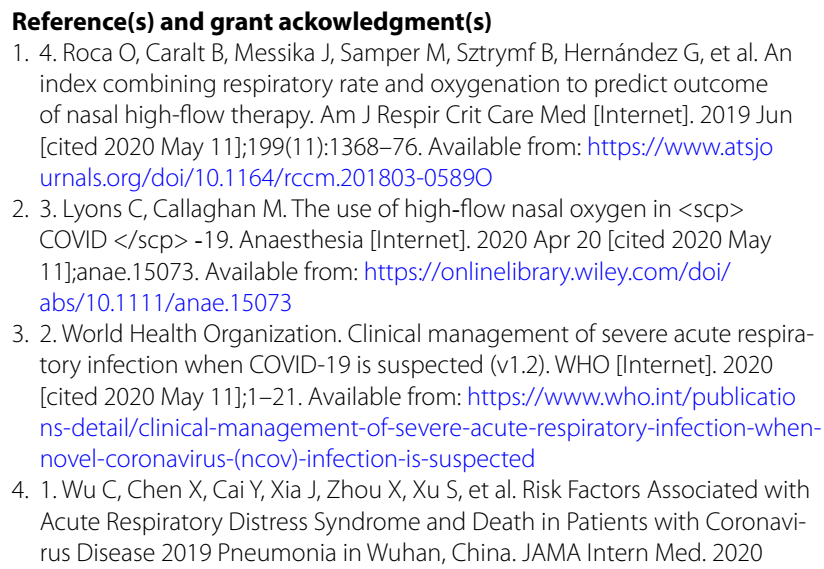

4. 1. Wu C, Chen X, Cai Y, Xia J, Zhou X, Xu S, et al. Risk Factors Associated with Acute Respiratory Distress Syndrome and Death in Patients with Coronavirus Disease 2019 Pneumonia in Wuhan, China. JAMA Intern Med. 2020

\section{0}

Estimation of respiratory work and mechanical power transmitted to ARDS patients

G. Ferrigno'; N. Rodrigo Castroviejo²; A. Tejero Pedregosa 2 ;V. Losada Martínez²; R. Beltrán Bernáldez²; C. Sanmartino Gonzalez²; D. Monge Donaire'; LL. Beltran Martínez²; C. Tarancón Maján²; A. Marcos Gutiérrez² ${ }^{1}$ Intensive Care Unit, Hospital Virgen De La Concha, Zamora, Spain; ${ }^{2}$ Intensive care, Hospital Virgen De La Concha, Zamora, Spain

Correspondence: $G$. Ferrigno

Intensive Care Medicine Experimental 2020, 8(2): 000470

Introduction: Mechanical ventilation (MV) is vital for the management of patients with acute respiratory distress syndrome (ARDS). In recent years there has been important technological development. Along with technological advances and a better understanding of respiratory pathophysiology, it has been determined that MV can have deleterious effects on these kinds of patients.

Objectives: to estimate respiratory work by means of the pressuretime product (PTP) and the Campbell's diagram (WOB), to estimate different parameters related to ventilator induced lung injury (VILI) according to classical and rheological theories: driving pressure (DP), stress index (SI) and mechanical power (PM)

Methods: Data were collected from adult patients connected to MV with ARDS criteria as proposed in the Berlin definition. The Fluxmed ${ }^{\circledR}$ respiratory mechanics monitor was used after insertion of an esophageal pressure probe and placement of a flow sensor (Image 1). Parameters related to VILI were collected: DP, SI and MP was calculated according to the formula proposed by Gattinoni. PTP and WOB were also calculated with the FluxReview ${ }^{\circledR}$ software (Picture 2). Data were collected for 5 minutes after esophageal probe insertion, in volume controlled mode and under the effects of a neuromuscular blocking agent. The variables are presented as an average $(95 \% \mathrm{Cl})$. Hypothesis contrast for mean difference was performed with Mann-Whitney test. Results: 8 patients with the following baseline characteristics were included. Age 69.4 (61.6-77.2) years, sex 87.5\% men, weight 71.6 (60.383) $\mathrm{kg}$, height $166.8(158.1-175.4) \mathrm{cm}$, APACHE 26 (9.2-42.8), SAPSII 53.3 (42-64.5), SOFA 6.9 (3.4-10.4). Patients were ventilated with Vt 7.6 (6.6-8.7) $\mathrm{ml} / \mathrm{kg}$, PEEP 9.8 (7.9-11.6) $\mathrm{cmH}_{2} \mathrm{O}, \mathrm{FiO}_{2}$ 0.63, FR 22 (18.7-25.3) rpm. $\mathrm{PaO}_{2} / \mathrm{FiO}_{2}$ was $140.5(88-193) \mathrm{mmHg}$. PTP of the respiratory system was $9.5(7.8-11.2) \mathrm{cmH}_{2} \mathrm{O} \times$ sec., with an elastic component of 49.6 $\%(37.6-61.5) \%$ and a resistive component of $43 \%$ (33.4-52.5) \%. The elastic WOB was $4.3(3.2-5.5) \mathrm{cmH} 20 \mathrm{xl}$, expiratory resistive WOB was $2.8(1.8-3.8) \mathrm{cmH} 20 \times \mathrm{I}$, inspiratory resistive WOB was $-3.4(-4.5-2.2)$ $\mathrm{CmH}_{2} \mathrm{O} \times$ I. DP was $13.6(10-17.2) \mathrm{cmH}_{2} \mathrm{O}, \mathrm{MP} 10.2$ (7-13.4) $\mathrm{J} / \mathrm{min}$ and SI 0.95 (0.8-1.1). Mortality was $62.5 \%$ and duration of MV was 16.6 (5.527.8) days. A statistically significant difference was observed in the level of PEEP among survivors and non-survivors: $3 \mathrm{cmH}_{2} \mathrm{O}(0.2-6) \mathrm{p}<$ 0.05. Differenceswere also observed in MP $5.9 \mathrm{~J} / \mathrm{min}$ (1.5-10.3) and PTP $2.7 \mathrm{cmH}_{2} \mathrm{O} \times$ sec. (0.5-5.4) but without reaching statistical significance $(\mathrm{p}=0.053)$.

Conclusion: Advanced monitoring and knowledge of the different theories that explain VILI may allow us to optimize MV programming and reduce the damage associated with it.

\section{1}

Impact of prone ventilation in mechanically ventilated \& rescue self positioned awake ARDS patients with Covid-19

S. Hartigan ${ }^{1}$; C. Joyce ; R. Turner'; B. Marsh

${ }^{1}$ Intensive care unit, The Mater Misericordiae University Hospital, Dublin, Ireland

Correspondence: S. Hartigan

Intensive Care Medicine Experimental 2020, 8(2): 001001

Introduction: SARS-CoV2 is a novel pathogen which, in humans, can lead to respiratory failure and acute respiratory distress syndrome (ARDS)(1). Prone ventilation has been shown to improve mortality in patients with severe ARDS(2). From early case reports in SARS-CoV2 infected patients and previous literature on ARDS, we hypothesised that prone ventilation may play a role improving oxygenation(2)(3). Prone positioning for patients receiving non-invasive ventilation has been demonstrated to improve oxygenation in a case series(4). Given potential issues with resource allocation and simplicity of this measure, patients requiring NIV were encouraged to self prone(5). We used SpO2:Fi02 (SPF) ratio as a surrogate for Pa02/Fi02 (PF) ratios these have been shown to have a linear relationship with PF ratios previously(6).

Objectives: 1 . To assess the impact of prone ventilation on $\mathrm{P}: \mathrm{F}$ ratios for invasively ventilated patients with SARS-CoV2 infection with severe ARDS.

2. To assess impact on $\mathrm{SpO2:Fi02} \mathrm{ratio,} \mathrm{intubation} \mathrm{rates} \mathrm{and} \mathrm{patient}$ compliance with prone positioning for conscious patients with SARSCoV2 infection receiving NIV

Methods: Retrospective observational cohort study of patients with severe ARDS secondary to SARS-CoV2, admitted to the ICU receiving mechanical ventilation in the prone position for at least twelve consecutive hours. Prone Ventilation occurred as per local protocol, aiming for 18 hours prone per day. All patients receiving mandatory mechanical ventilation received lung protective ventilation as per ARDSNET protocol $6 \mathrm{ml} / \mathrm{kg}$ PBW, with titrated PEEP. For proning patients received a bolus of non-depolarising muscle relaxant and a weight based atracurium infusion for the duration of prone ventilation.

In the self prone group we assessed patients who had non invasive ventilatory support who had any proning event.

Results: 17 patients with SARS-CoV2 infection at MMUH received prone position invasive mechanical ventilation (3 during venoveno extra corporeal life support). This was associated with a mean improvement in P:F ratio of $16 \mathrm{kPa}$ during the first episode of prone ventilation. This improvement declined with subsequent episodes. Patients received a mean of 4.2 episodes of prone ventilation. The rate of improvement in PF ratio was greatest in the first 6 hours of proning and declined from hour 6-17.

11 patients undertook awake prone positioning whilst receiving NIV. The average number of awake proning events was 5.4 events per patient. The mean Sp02 / Fi02 ratio prior to the first self prone event was 179 There was an average best increase in SpFi02 ratio of 33.6. In the awake proning group only $18 \%$ of patients required mechanical ventilation.

Conclusion: In our cohort of patients who received prone ventilation for severe ARDS secondary to SARS-CoV2 there was an improvement in their PF ratios, the greatest benefit was seen in the first 6 hours of the prone period. This is consistent with data previously seen in other causes of ARDS. Although not all patients demonstrated a significant improvement in SPF ratio on self proning, we note that this intervention may have avoided intubation for some patients where patient adherence to the protocol was high. 
Reference(s) and grant ackowledgment(s)

1. Bilan, $\mathrm{N}$ et al. Comparison of the spo $2 / \mathrm{FiO}_{2}$ Ratio and the $\mathrm{PaO}_{2} / \mathrm{FiO}_{2}$ Ratio in Patients With Acute Lung Injury or Acute Respiratory Distress Syndrome. Journal of Cardiovascular and Thoracic Research. 2015; 7 (1): 28-31.

2. Caputo, $\mathrm{N}$ et al. Early self-proning in awake, non-intubated patients in the emergency department: A single ED's experience during the COVID-19 pandemic. Academic Emergency Medicine. 2020;27 (5):375-378.

3. Elharrar $X$, et al. Use of Prone Positioning in Nonintubated Patients With COVID-19 and Hypoxemic Acute Respiratory Failure. JAMA. 2020;323 (22): 2336-2338.

4. Pan C, et al. Lung Recruitability in COVID-19-associated Acute Respiratory Distress Syndrome: A Single-Center Observational Study. American Journal of Respiratory and Critical Care Medicine. Mar 2020;201 (10):1294-1297.

5. Guerin, C et al. Prone Positioning in Severe Acute Respiratory Distress Syndrome. New England Journal of Medicine. 2013:368:2159-2168

6. Huang, C et al. Clinical features of patients infected with 2019 novel coronavirus in Wuhan, China. The Lancet. Feb 2020;395(10223):497-506.

7. None

\section{0}

Ventilatory support in COVID-19 patients: shift in clinical practice L. Rodrigues dos Santos' ; A. Mendes De Castro²; F. Leal'; A. Dias ${ }^{3}$; R. Pinto Silva ${ }^{4}$; G. Cabral Campello ${ }^{4}$

'Internal medicine, Centro Hospitalar do Tâmega e Sousa, Avenida do Hospital Padre Américo, Portugal, Porto, Portugal; ${ }^{2}$ Anesthesiology, São João Universitary Hospital Center, Porto, Portugal; ${ }^{3}$ Anesthesiology, Centro Hospitalar do Tâmega e Sousa, Avenida do Hospital Padre Américo, Portugal, Porto, Portugal; ${ }^{4}$ Intensive care, Centro Hospitalar do Tâmega e Sousa, Avenida do Hospital Padre Américo, Portugal, Porto, Portugal

Correspondence: $L$. Rodrigues dos Santos

Intensive Care Medicine Experimental 2020, 8(2): 001030

Introduction: Coronavirus Disease 2019 (COVID-19) presented as a challenge to Intensive Care Units (ICU). An effort is still being made to clarify the main features and the impact of invasive mechanical ventilation (IMV) in outcomes.

Objectives: To describe the characteristics of an ICU COVID-19 population and to analyze the evolution of the decision on the ideal ventilation moment and the impact on mortality between invasively ventilated and non-invasively ventilated patients.

Methods: Retrospective cohort study of Polymerase Chain Reaction confirmed SARS-COV-2 adult patients admitted to an ICU in the interior north of Portugal between March 23 and May 23, 2020. Data collection on clinical characteristics and management decisions was performed. Patients were grouped according to the oxygen or ventilation support mode: group 1 - IMV; group 2 - non-invasive ventilation, high flow nasal canula and facial mask oxygen. Variables were analyzed using the SPSS statistical software and the significance level used was 0.05 .

Results: During the study period were admitted 20 patients, $60 \%$ were women, the median age was 67.5 years $[44 ; 87]$.

Seventeen patients (85\%) were admitted for respiratory failure, $35 \%$ of whom with $\mathrm{PaO}_{2} / \mathrm{FiO}_{2}$ ratio below $100 \mathrm{mmHg}, 2$ patients $(10 \%)$ for septic shock in context of gastroenteritis and 1 patient with acute coronary syndrome and mild respiratory symptoms.

The median SAPS II at admission was 34 [15;50], the mortality rates in the ICU and at 30 days were $25 \%$ and $35 \%$, respectively.

When analyzing the ventilation support, $60 \%$ required IVM and $55 \%$ were proned. When comparing groups, group 1 mortality was clinically significative higher $(p=0.055)$ and had a significative longer ICU stay $(p=0.006)$. In terms of laboratorial features on admission there wereno differences between groups. Regarding COVID 19 and ICU complication, group 1 had more superinfections $(p=0.019)$, but without impact in mortality. Otherwise, we found no differences in renal, cardiac or thromboembolic complications.

Within group 1, the median $\mathrm{PaO}_{2} / \mathrm{FiO}_{2}$ ratio was initially higher, mainly in the first month. As time went by and experience accumulated, a tendency to tolerate lower ratios before invasive ventilation and an inverted ratio between the number of ventilated and non-ventilated patients can be seen.
Conclusion: Patients who received IMV had a higher rate of infection and a significantly longer stay in ICU with impact on mortality.

Initially patients were invasively ventilated earlier in accordance with studies and expert opinion, however with experience, non-invasive strategies were preferred without impact in the outcomes. The decision not to ventilate was well tolerated and allowed patients to overcome infection without the need to be ventilate, even under traditional "mandatory" ventilation ratios. Outcomes like neurological dysfunction were not evaluated but we raise the hypothesis that non-ventilation probably spares that dysfunction, as well as decreases secondary lung infection.

The main limitation of this study is the small sample. Further largescale studies would be interesting analyzing the mortality and prevalence of post-ICU impairment in ventilated and non-ventilated patients.

\section{Reference(s) and grant ackowledgment(s)}

1. Gattinoni L. et al. COVID-19 pneumonia: different respiratory treatment for different phenotypes? (2020) Intensive Care Medicine

2. Phelan A. et al. The Novel Coronavirus Originating in Wuhan, China - Challenges for Global Health Governance (2020) JAMA

\section{0}

Pandemic Pedagogy: Upskilling and educating a District General Hospital workforce for COVID-19

E. Jenkins ${ }^{1}$; D. Turton ${ }^{1}$; D. Hobden ${ }^{2}$;. T. Sayani ${ }^{3}$; S. Bill ${ }^{3}$; C. Walters ${ }^{4}$; C. Tai

${ }^{1}$ Anaesthetics and intensive care, Whipps Cross University Hospital, London, United Kingdom; ${ }^{2}$ Respiratory medicine, Whipps Cross University Hospital, London, United Kingdom; ${ }^{3}$ Division of medicine, Whipps Cross University Hospital, London, United Kingdom; ${ }^{4}$ Department of physiotherapy, Whipps Cross University Hospital, London, United Kingdom

Correspondence: $C$. Tai

Intensive Care Medicine Experimental 2020, 8(2): 001110

Introduction: On 11 March 2020, the WHO characterised COVID-19 as a pandemic. At that point, in the UK, there were only 364 patients with proven coronavirus infection and only seven deaths. We could, however, glimpse the possible future from the horrors that struck Italy and China. We therefore began plans to upskill and re-organise our workforce, both medical and nursing staff.

Objectives: Due to resource limitations, non-respiratory doctors and nurses would have to learn how to manage patients with COVID-19 related respiratory failure. Consequently, we wanted to ensure that staff were as well prepared and confident in their new roles as possible.

Methods: We developed 'Respiratory Failure Escalation Guidelines' and a twice daily, two-hour teaching session delivered by a team of experienced doctors and nurses from Acute and Respiratory Medicine and Intensive Care (ITU). These started with a lecture on the basics of respiratory failure and its management - including the use of High Flow Oxygen Therapy (HFOT), Continuous Positive Airway Pressure (CPAP) and Non-Invasive Ventilation (NIV) - followed by a demonstration of our hospital's respiratory support devices with time for hands-on experience. We advertised the training to all members of the multidisciplinary team, from all specialties, working within the hospital.

Results: Between 17 March and 15 May 2020, we had 435 confirmed attendees (as per registers collected during teaching times). The below pie charts demonstrate the broad professional backgrounds (Fig. 1) and specialties (Fig. 2) of the attendees.

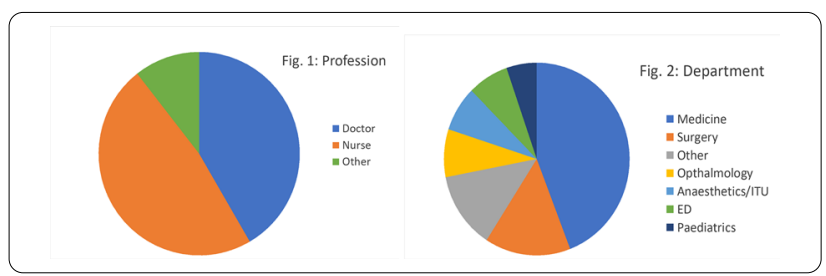


We received post-course feedback from 64 attendees, across different departments and professions, which was overwhelmingly positive (Fig. 3). Many requested further teaching on the subject, even suggesting annual training, and around $72 \%$ of respondents attended our associated courses, suggesting there was a genuine desire for this type of education.

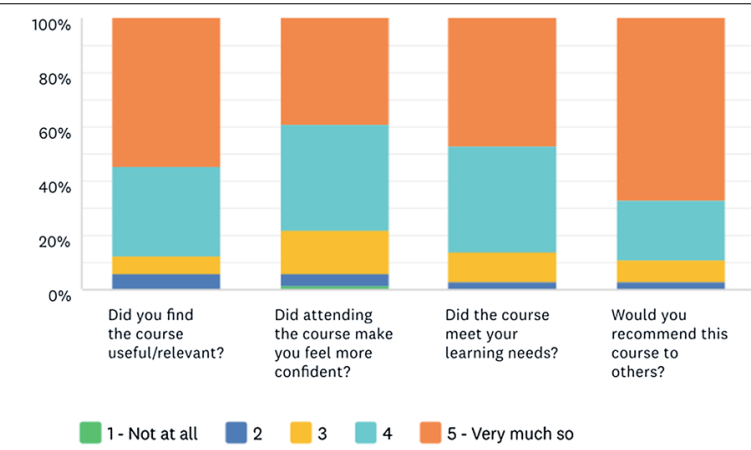

Fig. 3: Feedback

Conclusion: During the first wave of the pandemic, 78 patients with COVID-19 requiring enhanced respiratory support were managed on wards previously unfamiliar with this treatment modality. The educational sessions were essential in building the confidence of our staff in managing COVID-19 related respiratory failure throughout the hospital and were extremely well received. In response to their requests for further teaching, and in order to facilitate ongoing learning and revision, we developed complementary material including respiratory support device set-up videos, online presentations and e-assessment modules accessible to all staff through the Trust's intranet.

The key lessons we learned from the development and delivery of these training sessions were as follows:

Truly multidisciplinary training is both possible and effective. The skill mix and varied backgrounds of the participants appeared to have no negative effects on the sessions.

We did not have the time available to deliver the longer sessions normally used in nurse-training. Instead, we adopted an approach more typical of medical education. This style was, nevertheless, warmly received by all participants and showed that shorter sessions may be effective for future nurse-training in our Trust.

As the programme developed, the importance of providing resources in a variety of formats became increasingly clear. Our complimentary resources, developed by the same team of educators, was easily accessible to all staff to review whenever they wished.

\section{0}

\section{Prognostic Capacity of Oxygenation parameters in the evolution}

\section{of patients with Severe ARDS by H1N1 Influenza}

JF. Martínez Carmona ${ }^{1}$; C. Joya Montosa²; MP. Benitez Moreno ${ }^{3}$;

M. Delgado-Amaya

${ }^{1}$ Intensive care unit, Hospital Carlos Haya, Málaga, Spain; ${ }^{2}$ Unidad de Cuidados Intensivos, Regional Hospital of Malaga, Málaga, Spain; ${ }^{3}$ Intensive care, HOSPITAL CARLOS HAYA, MALAGA, Spain

Correspondence: J.F. Martínez Carmona

Intensive Care Medicine Experimental 2020, 8(2): 001150

Introduction: ARDS ventilator management is one of the most difficult tasks in the ICU. Monitoring lung mechanics and oxygenation parameters is one of the keys in this entity, and provide useful information about the evolution as well as prognosis.

Methods: Retrospective, descriptive study of patients admitted in ICU from 2017 to 2019, with H1N1 influenza diagnosis, ARDS and MV. Clinical, epidemiological and result-related variables were analyzed, between them: MV and sedative timing, needs of vasoactives, renal failure, prone position, $\mathrm{PaO}_{2}-\mathrm{FiO}_{2}, \mathrm{~A}-\mathrm{aO} 2$ gradient, $\mathrm{OI}, \mathrm{OSI}, \mathrm{PaO}_{2}$, $\mathrm{PaCO} 2, \mathrm{ICU}$ mortality and UCI length of stay, as well as SOFA, APACHE II. Quantitative variables are expressed as mean and standard deviation, while qualitative variables are expressed as ratios and absolute value.

Results: A total number of 17 patients were included. $52.9 \%$ were men, with a mean age of $53.93 \pm$ years. APACHE and SOFA scores at the ICU admission were $5.65 \pm 3.06$ and $13.88 \pm 3.53$ respectively. Prone position was needed in $58.8 \%$ of the patients. Length of mechanical ventilation was $17.7 \pm 9.47$ days; sedatives were needed an average of $17.47 \pm 9.47$ days. $58.8 \%$ of the patients developed renal injury, 29.4\% among them needed RRT.

When the sample was divided into two groups, survivors and nonsurvivors, statistically significant differences $(p<0.05)$ were observed in oxygenation parameters: $\mathrm{PaO}_{2}-\mathrm{FiO}_{2}, \mathrm{~A}-\mathrm{aO} 2$ gradient, OI, and OSI. There were no significant differences ( $\mathrm{p} 0.07)$ in $\mathrm{PaO}_{2}$ and $\mathrm{pCO} 2$. The best cut-points were: $\mathrm{PaO}_{2}-\mathrm{FiO}_{2} 202.5$ (S 83.3\%; E 72.7\%); A-aO2 gradient was 193.18 (S 66.7\%; E 72.7\%), Ol 9.21 (S 66.7\%; E 72.7\%), and OSI 8.66 (S 83.3\%, E 72.7\%).

The length of stay in the ICU was $21.71 \pm 11.53$ days. ICU observed mortality was $35.29 \%$.

Conclusion: An increase in mortality risk was observed in those patients who kept $\mathrm{PaO}_{2}-\mathrm{FiO}_{2}$ values $<202$ and $\mathrm{OI}>9.21$.

Oxygenation parameters monitoring in ARSD patients provide prognosis value and allow for the assessment in the day-to-day patient situation.

\section{7}

Protecting Prone Patients: Collateral Complications of COVID-19 R. Carrington ${ }^{1}$; V. Jenkins'; M. Phull ${ }^{1}$

'Intensive care, Queen's Hospital, London, United Kingdom

Correspondence: $\mathrm{R}$. Carrington

Intensive Care Medicine Experimental 2020, 8(2): 000447

Introduction: Covid-19 has resulted in a proning pandemic. Intensive care units (ICUs) across the world have proned more patients in March and April 2020 than they would expect to in a year. The prone position is thought to improve the condition of patients with COVID-19 by increasing recruitment in dorsocaudal regions of the lung, reducing alveolar shunt and increasing tidal volume(1). Putting patients in the prone position, along with the necessary frequent head-turns, has well-recognised risks and complications(2). The increased need for this labour-intensive procedure, combined with overstretched staff with varied levels of ICU experience, has led to a surge in the number of proning-related complications.

\section{Objectives:}

1. To identify the number of patients with COVID-19 admitted in ICU at Queen's Hospital on 23rd April 2020 that had been proned during their admission

2. To determine the number of complications that occurred secondary to proning and head-turns

3. To design and implement a simple, efficient and effective method of reducing common complications

Methods: Retrospective data collection from all patients admitted in Queen's Hospital COVID-19 ICU on 23rd April 2020. The number of patients that had been proned during their admission, the duration of each prone period and any complications that occurred were recorded. Directed by the most common complications, and by the Faculty of Intensive Care Medicine guidelines(3), we designed a graphic highlighting five simple checks to be carried out before each proning/head-turn, and five simple checks to be carried out afterwards (Fig. 1). 


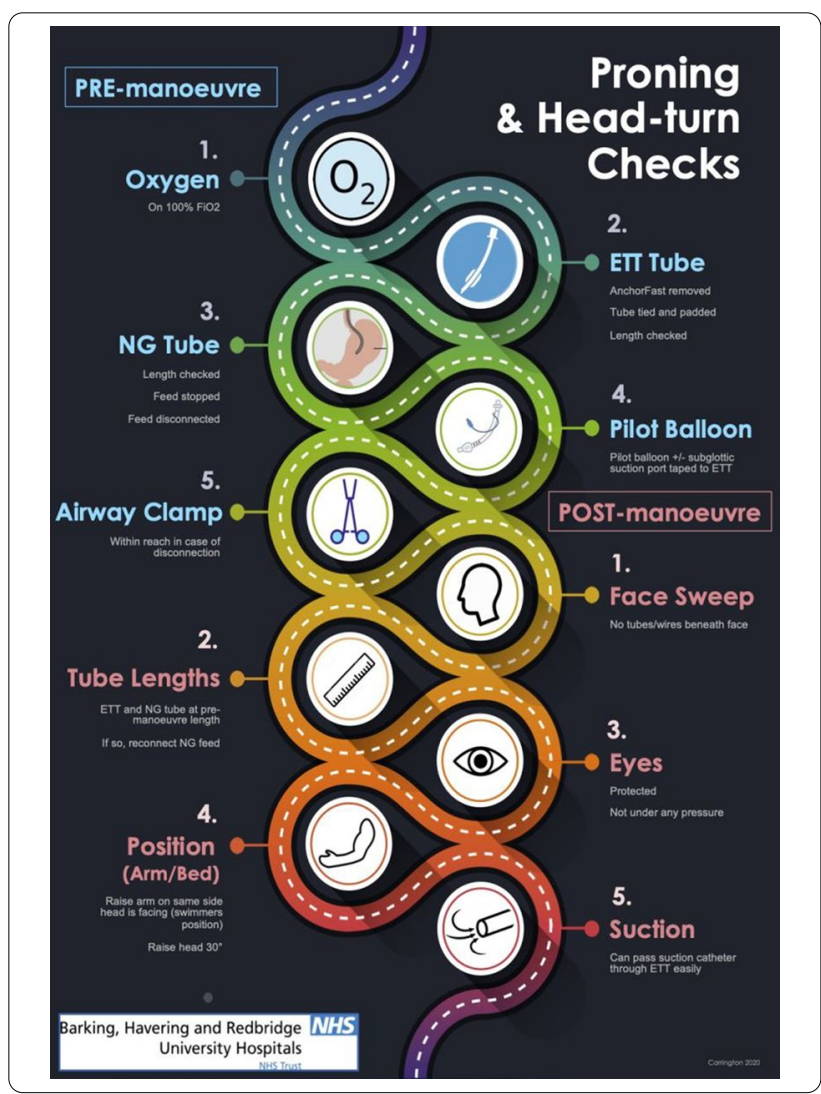

Results: 31 patients with COVID-19 were admitted in ICU at Queen's Hospital on 23rd April 2020. 22 (71\%) were proned during their admission. 17 (77\%) of these patients experienced at least one complication secondary to being proned. There was a total of 52 prone periods, with an average duration of 27.8 hours. The occurrence of different types of complications are shown in Table 1.

\begin{tabular}{|l|l|l|}
\hline \multicolumn{2}{|c|}{ Table 1. Complications secondary to proning and head-turns } \\
\hline \multicolumn{1}{|c|}{ Complications } & $\begin{array}{c}\text { Number of } \\
\text { patients }\end{array}$ & $\begin{array}{c}\text { Percentage of proned } \\
\text { patents }\end{array}$ \\
\hline Nasogastric tube removal & 12 & $55 \%$ \\
\hline Facial pressure sores & 8 & $36 \%$ \\
\hline $\begin{array}{l}\text { Endotracheal tube removal to level } \\
\text { needing replacing }\end{array}$ & 2 & $9 \%$ \\
\hline Central venous access removal & 1 & $5 \%$ \\
\hline Retro-orbital haematoma & 1 & $5 \%$ \\
\hline
\end{tabular}

Conclusion: There has been a high rate of complications secondary to proning during this period of the COVID-19 pandemic. Our results show that $77 \%$ of patients who were proned experienced at least one complication. The consequences of these ranged from the need to replace an NG tube to potentially permanent loss of sight. Going forward, we have implemented our proning checks graphic by displaying it across the ICU, attaching it to dedicated proning equipment packs and explaining it to members of the proning team. We continue to monitor its effect and strive to improve ways of protecting our patients from these complications.

\section{Reference(s) and grant ackowledgment(s)}

1. 3. Bamford P, et al. Guidance For: Prone Positioning in Adult Critical Care.

The Faculty of Intensive Care Medicine. Nov 2019
2. 2. Bloomfield R, Noble DW, Sudlow A. Prone position for acute respiratory failure in adults. Cochrane Database of Systematic Reviews 2015, Issue 11. Art. No.: CD008095. https://doi.org/10.1002/14651858.CD008095.pub2.

3. 1. Ghelichkhani P, Esmaeili M. Prone Position in Management of COVID-19 Patients; a Commentary. Arch Acad Emerg Med. 2020 Apr 11;8(1):e48. eCollection 2020.

\section{7}

Bronchoscopy assisted percutaneous dilational tracheostomy in critically ill patients with SARS COV 2 Infection

A. Estella ${ }^{1}$; M. Gracia Romero ; V. Perez Madueño ${ }^{1}$; R. Monterroso. ;

R. Bohollo De Austria'; I. Blanco Saez ; J. Flores'; JM. Martín Cano';

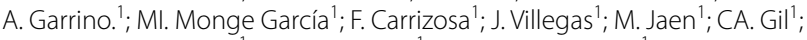

F. Valenzuela Sanchez ; JM. Sampedro ${ }^{1}$; MD. Sandar Nuñez ${ }^{1}$

${ }^{1}$ University hospital of jerez., Intensive care unit, Jerez, Spain

Correspondence: A. Estella

Intensive Care Medicine Experimental 2020, 8(2): 000597

Introduction: Percutaneous dilational tracheostomy is usually performed blindly, entry into the trachea can be visualized using bronchoscopy. With SARS COV 2 pandemic, measures to perform the procedure safely for protecting healthcare personnel from undue risk of exposure and infection must be taken. To delay the procedure has been recommended due to a significant risk of viral transmission because it is an aerosol-generating procedure.

Objectives: The aim of the present study are: to describe the clinical experience in the realization of tracheostomy guided by bronchoscopy in critically ill patients with SARS COV 2 infection and to analyze the complications related to the procedure.

Methods: Retrospective study in a 17 bed medical-surgical ICU during two months: march and april 2020. We included patients with confirmed SARS COV 2 respiratory infection. Variables analyzed were clinical characteristics and outcome, day of tracheostomy during ICU admission, duration of procedure, complications related with the tracheostomy. Statistical analysis: Data were analyzed by SPSS 18 and quantitative variables were expressed as a mean \pm standard deviation. Results: Twenty consecutive patients with confirmed SARS COV 2 infection were admitted in ICU. Mean age was $61,7 \pm 8,25$. $65 \%$ male and $35 \%$ female. Apache II at admission was $10,3 \pm 4,21$ and SOFA score $3,45 \pm 1,87$. Mechanical ventilation was required in $80 \%$ of patients. In nine patients, $45 \%$, tracheostomy was performed, all the procedure were performed guided by videobronchoscopy to avoid the use of laringoscopy and to distance itself from the oral cavity and airways during the procedure during withdrawing orotraqueal tube. Mean duration of procedure was 5 minutes and 19 seconds.

No major complications related with the procedure were described, in two patients first attempt of needleinsertion failed and with the bronchoscope the positioning of the needle were directly visualized before entry into the trachea and a guidewire was introduced (figure 1). Four patients developed hypoxemia during the procedure, they had no clinical significance and were transient.

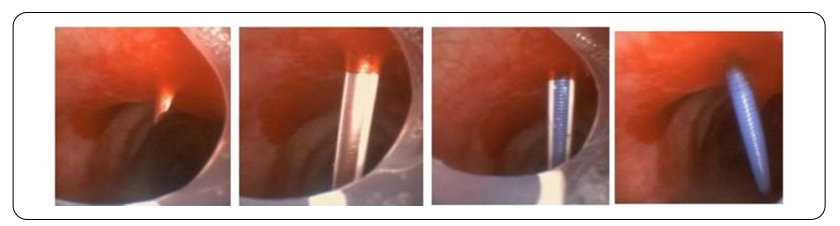

Conclusion:

- Video bronchoscopy visualization during tracheostomy minimizing risk for healthcare practitioners in SARS COV 2 infected patients.

- Bronchoscopy facilitates optimal visualization of the wall of the trachea, to locate the correct needle entrance during percutaneous dilational tracheostomy improving safety of the procedure. 
000630

Patient and staff safety of bedside percutaneous tracheostomy in COVID-19 patients in a medical ICU

E. Sancho ${ }^{1}$; M. Diaz ${ }^{1}$; V. Carlos ${ }^{1}$; P. Ramirez ${ }^{1}$;. Calleja ${ }^{1}$; C. Verdeguer ${ }^{1}$ MD. Gomez ${ }^{2}$; C. Lloret ${ }^{2}$; I. Sigona ${ }^{2}$; A. Castellanos ${ }^{1}$

${ }^{1}$ Intensive care unit, Hospital Universitario y Politécnico de La Fe, València, Spain; ${ }^{2}$ Microbiology, Hospital Universitario y Politécnico de La Fe, València, Spain

Correspondence: E. Sancho

Intensive Care Medicine Experimental 2020, 8(2): 000630

Introduction: During COVID 19 pandemic, many considerations have been discussed about tracheostomy in ICU patients, in terms of efficacy, timing, and health care professionals safety. Tracheostomy is an aerosol generating procedure and therefore some recommendations have been proposed: skilled intervening staff, surgical technique, full personal protective equipment (PPE) use and negative pressure environment if possible. However in our medical ICU we were not able to apply some of these recommendations.

Objectives: To describe and analyse patient and staff safety of bedside percutaneous tracheostomy in COVID-19 patients in a medical ICU

Methods: We recorded data of consecutive patients diagnosed with ARDS secondary to COVID 19 who required extended mechanical ventilation and tracheostomy during their ICU stay. We registered demographic data, mechanical ventilation parameters, information about the procedure and we finally prospectively evaluated SARS-CoV-2 transmission to health care professionals.

Results: We included 35 COVID-19 mechanically ventilated patients tracheostomy was performed in 11 patients $(31 \%)$. Negative pressure environment was unavailable. Percutaneous technique with single dilator was applied at bedside in ICU. Previous ultrasound assessment was implemented and bronchoscopy was used as a standard of care during the procedure. The mean duration of the procedure was 38 minutes [35.1-40.9]. Nine out of 11 tracheostomies $(81.8 \%)$ were performed by critical care resident doctors (4th-5th year of training). Only 4 patients (36.36\%) had negative SARS-CoV-2 PCR at the moment of the procedure. PPE including fluid-resistant gown, ocular protection (goggles or face shield), gloves and FFP3 mask, was used in all cases. Median of mechanical ventilation days up to tracheostomy was 21 [2024]. Any technique complication was detected. SARS-CoV-2 PCR and serology $(\mathrm{lgM} / \mathrm{lgG})$ were negative in all staff participants after a proper window period.

Conclusion: Despite not following all the expert opinion recommendations regarding tracheostomy in COVID-19 patients, no safety events occurred to our patients and no SARS-CoV-2 transmission to healthcare providers was detected.

\section{Reference(s) and grant ackowledgment(s)}

1. Thamboo A. et al. Clinical evidence based review and recommendations of aerosol generating medical procedures in otolaryngology - head and neck surgery during the COVID-19 pandemic.J Otolaryngol Head Neck Surg. 2020 May 6;49(1):28. https://doi.org/10.1186/s40463-020-00425-6

2. Michetti $C P$ et al. Performing tracheostomy during the Covid-19 pandemic: guidance and recommendations from the Critical Care and Acute Care Surgery Committees of the American Association for the Surgery of Trauma. Trauma Surg Acute Care Open. 2020 Apr 15;5(1):e000482. https://doi. org/10.1136/tsaco-2020-000482. eCollection 2020.

3. Mattioli F et al. Tracheostomy in the COVID-19 pandemic. Eur Arch Otorhinolaryngol. 2020 Apr 22

\section{2}

The Impact of Surgical vs. Percutaneous Tracheostomy - An Audit of Tracheostomy Insertion in Intensive Care

T. Ross ${ }^{1}$; M. Komorowski ${ }^{1}$

${ }^{1}$ Intensive Care, Charing Cross Hospital, London, United Kingdom

Correspondence: $T$. Ross

Intensive Care Medicine Experimental 2020, 8(2): 000632
Introduction: Up to a third of patients in intensive care requiring prolonged mechanical ventilation receive a tracheostomy. In the United Kingdom, this equates to around 15,000 tracheostomies annually. Tracheostomy insertion aims to facilitate ventilator wean through reducing dead space and improving secretion clearance. They can be performed either surgically or percutaneously. Tracheostomy timing has been an area of controversy in the literature, with some groups advocating that early insertion can lead to reduced sedative use, faster ventilator wean, shorter hospitalisation and reduced mortality.

Objectives: In 2013, a large randomised controlled trial, TracMan, was carried out assessing the impact of early versus late tracheostomy insertion on outcomes. This audit aimed to compare outcomes following tracheostomy insertion in our tertiary intensive care to those of the TracMan study.

Methods: All ventilated patients undergoing tracheostomy (either surgically or percutaneously) in 2019 were included $(n=53)$. Patients undergoing emergency tracheostomy, intra-operative tracheostomy during elective head and neck surgery and with a pre-existing tracheostomy were excluded. Data was collected from electronic health records retrospectively.

Results: In contrast to the TracMan study where $89 \%$ of tracheostomies were performed percutaneously, we performed only $17 \%$ this way. This introduced quite a significant time delay between decision to insertion of tracheostomy. Often patients were clinically deteriorating prior to going to theatre for a surgical tracheostomy, resulting in even further delays. "Short neck" was cited as the most common reason for referral for a surgical tracheostomy. More complications were seen in the cohort receiving surgical tracheostomies, however this group contained higher risk patients. Early tracheostomy also reduced the risk of complications. Contrary to the TracMan population, our patients achieved ventilator wean faster when tracheostomy was performed earlier, which we felt was related to differing definitions of this term. Similar, early tracheostomy reduced length of stay on intensive care and shortened time on sedation. Mortality was also higher in the group receiving late tracheostomy.

Conclusion: Overall, duration from decision to insertion of tracheostomy was shorter when a percutaneous approach was chosen. If a percutaneous approach is feasible, this should therefore be preferable over a surgical approach, as it expedited tracheostomy insertion, reduced length of stay and appeared to be associated with fewer complications. Similarly, identifying patients for early tracheostomy insertion should be advocated, given the improved outcomes seen in our unit.

\section{Reference(s) and grant ackowledgment(s)}

1. Young D, Harrison DA, Cuthbertson BH, Rowan K; TracMan Collaborators. Effect of early vs late tracheostomy placement on survival in patients receiving mechanical ventilation: the TracMan randomized trial. JAMA.2013;309(20):2121-2129.

\section{6}

Regional Experiences of Endotracheal Intubation During The COVID-19 Pandemic in The United Kingdom

B. Shuker ; E. Smith²; P. Checketts ${ }^{3}$; Q. Khan

${ }^{1}$ Department of Anaesthesia, Warwick Hospital, Warwick, United Kingdom; ${ }^{2}$ Department of anaesthesia, University Hospital Coventry \& Warwickshire, Coventry, United Kingdom; ${ }^{3}$ Department of anaesthesia, The Shrewsbury and Telford Hospital NHS Trust, Shrewsbury, United Kingdom; ${ }^{4}$ Department of anaesthesia, University Hospitals Birmingham (Heartlands Hospital), Birmingham, United Kingdom

Correspondence: $B$. Shuker

Intensive Care Medicine Experimental 2020, 8(2): 000686

Introduction: In the United Kingdom (UK), consensus guidelines for airway management were published early in the COVID-19 pandemic making recommendations to support clinicians during this potentially challenging intervention (1). Adaptions to existing guidance for airway management in critically ill adults from the Difficult Airway Society (2) included: use of personal protective equipment (PPE), preferential use of the best skilled airway manager to maximise chance of first-pass 
success, avoidance of aerosol-generating procedures (such as noninvasive ventilation, high flow nasal oxygenation), and use of reliable well practiced techniques (including videolaryngoscopy where appropriate).

Objectives: Areas of the West Midlands were some of the worst affected by the COVID-19 pandemic in the UK (3). We aimed to gain insight into the experiences of clinicians involved with airway management during the COVID-19 pandemic in this region.

Methods: An online survey was distributed to multiple centres within the West Midlands region of the UK. Clinicians who had experience of endotracheal intubation in patients with confirmed, suspected, or unknown COVID-19 status were asked to reflect upon their experience of one patient intubation.

Results: 127 clinicians from 16 hospitals including 3 large university hospitals responded to the online survey, most were consultant grade (56.7\%). Clinicians self-reported an average approximate number of pandemic intubations of 7.35 (range 1-30).

When asked to reflect on a single intubation, clinicians reflected on intubations in ICU (42.5\%), emergency departments (20.5\%), wards $(8.7 \%)$, and theatre (28.3\%). Appropriate PPE was available in $96.1 \%$. The most senior clinician available intubated in $65.4 \%$.

Clinicians reported first pass success in $93.7 \%$ of responses. Most intubators reported use of videolaryngoscopy (74.8\%), however $26 \%$ reported not using this equipment regularly and $5.5 \%$ did not feel confident with their equipment. Despite a high success rate, difficulties were reported in $15.1 \%$. The most common was desaturation. Other common difficulties included equipment or environment unfamiliarity, lack of skilled support.

When asked what advice they would give to colleagues, frequently occurring themes included: ensuring familiarity with equipment, use of a checklist, use of videolaryngoscopy, and availability of a second intubator. Desire for simulation and equipment familiarisation was highlighted in multiple responses, and in one example a clinician attributed their success to a simulation session performed in the week prior.

Conclusion: Experiences from clinicians in this region highlight the specific challenges encountered involved in airway management of patients with COVID-19, in particular highlighting the importance of advance preparation for intubation when faced with unfamiliar circumstances. Simulation sessions, use of checklists and standard operating procedures for emergency intubation may contribute to maintaining preparedness for intubation in this challenging patient group.

\section{Reference(s) and grant ackowledgment(s)}

1. 2) Higgs A, McGrath BA, Goddard C, et al. Guidelines for the management of tracheal intubation in critically ill adults. Br J Anaesth. 2018;120(2):323-352.

2. 1) CookTM, El-Boghdadly K, McGuire B, McNarry AF, Patel A, Higgs $A$. Consensus guidelines for managing the airway in patients with COVID-19: Guidelines from the Difficult Airway Society, the Association of Anaesthetists the Intensive Care Society, the Faculty of Intensive Care Medicine and the Royal College of Anaesthetists. Anaesthesia. 2020;75(6):785-799.

3. 3) Public Health England. Disparities In The Risk And Outcomes From COVID-19. London: PHE Publications; 2020:20-31. 4. Nil.

\section{6}

Performance of tracheostomy in COVID-19 critically ill patients A. Ruiz García'; C. Serrano²; R. Araoz Illanes²; A. Acha²; J. Higuera Lucas $^{3}$; M. Trascasa Muñoz ${ }^{2}$; J. Tato ${ }^{4}$; C. Vaduva ${ }^{4}$; J. Arteaga ${ }^{4}$; RR. Teresa ${ }^{4}$; E. Nevado ${ }^{2}$

${ }^{1}$ Medicina intensiva, Hospital Universitario Príncipe de Asturias, Meco, Spain; ${ }^{2}$ Intensive care unit, Hospital Príncipe de Asturias, Alcalá de Henares, Spain; ${ }^{3}$ Medicina intensiva, Hospital Universitario Príncipe de Asturias, Alcala de Henares, Spain; ${ }^{4}$ Otorhinolaryngology, Hospital Príncipe de Asturias, Alcalá de Henares, Spain

Correspondence: A. Ruiz García

Intensive Care Medicine Experimental 2020, 8(2): 000776
Introduction: Community-acquired pneumonia COVID 19 has been a recent and frequent cause of admission to intensive care units worldwide. Its rapid expansion and high number of cases mean that there are many open questions regarding its management, treatment and prognostic. The performance of a tracheostomy in the affected patients is one of these questions.

Methods: A retrospective, observational study was carried out with all the patients admitted to the Intensive Care Unit at a University Hospital with the diagnosis of COVID-19 pneumonia. All patients who require mechanical ventilation support and tracheostomy during the pandemic were included.

Results: 37 patients were analysed. $70.3 \%$ of the sample were men (26/37). Average age $59.4 \pm 9.4$. APACHE II $14.8 \pm 4.67$. The average days of mechanical ventilation prior to performing a tracheostomy was $11 \pm 2.66$. Three of them were completed during the first week Percutaneous tracheostomy was performed in $86.5 \%$ (32/37) of the cases; $31 / 37$ in the course of the second week and 3 of them after the first two weeks. The average days from tracheostomy to decannulation was $17.7 \pm 10.6 .17$ patients have been decannulated (46\%). 16 of these 17 patients have been discharged from the hospital. The characteristics of surviving and non-surviving patients are shown in the Table 1.

\begin{tabular}{|l|l|l|l|}
\hline & Survivors & Non survivors & p \\
\hline N & 26 & 11 & \\
\hline Age & 59 & 60,5 & 0,68 \\
\hline Sex (M) & $65 \%$ & $81,8 \%$ & 0,28 \\
\hline APACHE II & 14,2 & 16 & 0,4 \\
\hline $\begin{array}{l}\text { Cardiovascular } \\
\text { history }\end{array}$ & $57,7 \%$ & $45 \%$ & 0,57 \\
\hline $\begin{array}{l}\text { Respiratory } \\
\text { history }\end{array}$ & $7,6 \%$ & $18,1 \%$ & 0,34 \\
\hline $\begin{array}{l}\text { Other medical } \\
\text { history }\end{array}$ & $34,6 \%$ & $18,1 \%$ & 0,28 \\
\hline $\begin{array}{l}\text { Prone } \\
\text { Neuromuscular } \\
\text { blockers }\end{array}$ & $77 \%$ & $100 \%$ & 0.09 \\
\hline $\begin{array}{l}\text { Days to } \\
\text { traqueostomy }\end{array}$ & 11 & $100 \%$ & 0,33 \\
\hline $\begin{array}{l}\text { Percutaneous } \\
\text { procedure }\end{array}$ & $88,4 \%$ & 11 & 1 \\
\hline
\end{tabular}

Conclusion: We present the results of 37 patients who required a tracheostomy as a part of the management of COVID 19 pneumonia. We describe the performance and the prognostic of the technique

\section{4}

Comparison of complication rates of surgical vs. percutaneous approach to tracheostomy insertion in a specialist burns critical care unit

A. Gandhi ${ }^{1}$. L Christie ${ }^{1}$

${ }^{1}$ Magill department of anaesthesia, Chelsea and Westminster Hospital, London, United Kingdom

Correspondence: A. Gandhi

Intensive Care Medicine Experimental 2020, 8(2): 000834

Introduction: Critically ill burns patients frequently require a tracheostomy, but pose particular challenges including airway and/or neck swelling, face and neck grafting and high oxygen and ventilation requirements. Grade 2Devidence exists for percutaneous dilation technique (PDT) over surgical technique (ST) in the burns setting[1]. $\mathrm{ST}$ is associated with lower costs when performed at the bedside or in association with another surgical procedure [2].

Objectives: We sought to compare the rate and severity of complications between PDT and ST in critically ill burns patients, within a regional burns critical care unit.

Methods: Retrospective data was collected over 33 months (May 2017 to February 2020) from all admissions to a regional burns critical care unit. Basic demographic data was collected in addition to data regarding technique, staff performing tracheostomy and tracheostomy 
complications during hospital stay. Complications were classed as severe or not severe based on the necessity for significant ventilator changes, transfusion or tracheostomy change/bronchoscopy. Averages were expressed as medians with interquartile ranges. Mann Whitney- $U$ analysis was used for non-parametric continuous data and $X 2$ Fisher's exact test for categorical data using SPSS (IBM corp). A p-value $<0.05$ was considered significant.

Results: From 71 admissions, 49 required ventilation and 13 patients required a tracheostomy. Of these 13 tracheostomy patients, the median burn surface area was $23 \%$ (IQR $37 \%), 46 \%(n=6 / 13)$ had inhalational injuries of grade 2 or worse and $92 \%(n=12 / 13)$ had burns involving their neck. ST was employed in $70 \%$ of cases. Demographic data showed overall homogeneity between the PDT and ST groups. However the ST group had a greater proportion of males, severe inhalational injuries and burns involving the neck was although not significant. The average time between intubation and tracheostomy formation was comparable between groups. Decannulation time also did not differ significantly. The majority of tracheostomies were performed in the theatre setting $(n=12 / 13)$ to coincide with debridement or skin grafting. PDT was performed predominantly by senior trainee anaesthetists (75\%) and in the theatre setting with surgical presence. All STs were performed by consultant surgeons, using an open approach.

At least one pre-procedural concerns was highlighted in $69 \%$ of all cases, including ventilatory concerns (3) (e.g. pneumonia, frequent desaturation), oedema at desired tracheostomy site (2), neck burns (2), distorted anatomy (1) and presence of graft at tracheostomy site (1). Similar rates of pre-procedural concerns were highlighted for ST and PDT cases (6/9 vs $3 / 4$ respectively)

Early complication rate was greater with ST, with the majority being considered severe such as dislodgement, stomal leak, significant bleeding, surgical emphysema and occlusion due to malposition. PDT demonstrated a lower complication rate but all were severe.

\begin{tabular}{|c|c|c|c|}
\hline & PDT & ST & \\
\hline & $(n=4)$ & $(n=9)$ & \\
\hline Age (years) & $38(23)$ & $55.5(16.75)$ & 0.266 \\
\hline Weight $(\mathrm{kg})$ & $74.5(28.3)$ & $89(16.4)$ & 0.559 \\
\hline Male Sex & $1(25 \%)$ & $6(67 \%)$ & 0.266 \\
\hline Inhalational injuries grade $\geq 2$ & $1(25 \%)$ & $5(56 \%)$ & 0.559 \\
\hline Burns to neck & $3(75 \%)$ & $9(100 \%)$ & 0.308 \\
\hline Burns Surface Area (\%) & $26(17.5)$ & $23(47)$ & 1.00 \\
\hline $\begin{array}{l}\text { Time to tracheostomy } \\
\text { formation (days) }\end{array}$ & $9.5(6.75)$ & $8(3.0)$ & 1.00 \\
\hline Time to de-cannulation (days) & $20(5)$ & $18.5(13.75)$ & 1.00 \\
\hline Complications & $2(50 \%)$ & $8(88.9 \%)$ & 0.41 \\
\hline Severe & $2(50 \%)$ & $6(66.7 \%)$ & 0.203 \\
\hline Not severe & $0(0 \%)$ & $2(22.2 \%)$ & 0.510 \\
\hline
\end{tabular}

Conclusion: Tracheostomies in critically ill burns patients are associated with a high risk of severe early complications[3]. We observed a higher rate of early complications (loss of airway and bleeding) in ST vs PDT. Although this data appears to favour PDT, the ST group had more pre-procedural concerns (e.g. more neck burns, distorted neck anatomy, oedema and inhalational injury) and therefore represent a higher risk group. We believe a national database is required to capture tracheostomy technique and complications to enable recommendations in this high risk specialised cohort.
Reference(s) and grant ackowledgment(s)

1. 1. Raimondi N, Vial MR, Calleja J, et al. Evidence-based guidelines for the use of tracheostomy in critically ill patients. J Crit Care 2017;

2. 2. Caruso DM, Al-Kasspooles MF, Matthews MR, Weiland DE, Schiller WR. Rationale for 'early' percutaneous dilatational tracheostomy in patients with burn injuries. J Burn Care Rehabil 1997; 18: 424-8

3. 3. Depetris $N$, Stella M, Mininanni $P$, et al. TRACHEOSTOMY IN BURN PATIENTS-WHEN AND HOW? THE CLINICAL EXPERIENCE OF A BURN CENTRE. (V001). Ann. Burns Fire Disasters. 2015

4. No funding acquired from external sources.

\section{7}

A new emergency tracheostomy algorithm for the icu

S. paulich ${ }^{1}$; F. Kelly ${ }^{1}$; T. Cook ${ }^{1} ;$ H. Hall ${ }^{1} ; H_{\text {. Churchill }}{ }^{1}$

${ }^{1}$ Intensive Care, Royal United Hospital Bath, Bath, United Kingdom

Correspondence: $S$. paulich

Intensive Care Medicine Experimental 2020, 8(2): 000047

Introduction: The National Tracheostomy Safety Project (NTSP) produces resources to improve multidisciplinary management of tracheostomies [1]. However, tracheostomy tube emergencies on the Intensive Care Unit (ICU) differ significantly to the rest of the hospital. We therefore created new algorithms specific to ICU with emphasis on the use of capnography.

Methods: The algorithms were designed using 'Plan, Do, Study, Act' (PDSA) cycles to sit alongside existing NTSP bedhead tracheostomy or laryngectomy signs and use a stepwise approach suitable for any member of the multidisciplinary team to use in an emergency. We use the headings: 'Tracheostomy: with patent upper airway' and 'Laryngectomy: Neck-ONLY breather' to emphasise that while both patients are 'neck breathers' only the laryngectomy patient is an obligate neck breather [2]. Using a multidisciplinary bedside training programme, using the 'tea trolley' method to provide 10-15 minute teaching sessions for each staff member [3], we familiarised staff with the algorithms and enabled them to employ them in a low fidelity simulation tool.

Results: We trained 67 staff members including all grades of nurses and doctors. $100 \%$ of participants preferred our algorithms to the NTSP algorithm, judging them to be clearer and easier to follow in an emergency. $100 \%$ of participants recommended this training programme to other ICUs.

Conclusion: In ICU the patient with a tracheostomy is often ventilatordependent, capnography-monitored and displacement is more common than obstruction. This necessitates a different pathway to that on the wards (where obstruction is more common). Our ICU-specific algorithms were preferred and easier to follow. Conversely the NTSP algorithms, while an excellent teaching resource, can become overwhelming in an emergency due to excessive detail.

Reference(s) and grant ackowledgment(s)

1. 1. National Tracheostomy Safety Project. http://www.tracheostomy.org.uk (accessed 02/10/2019)

2. 2. Paulich et al. Anaesthesia74:947, 2019

3. 3. O'Farrell et al. Anaesthesia 2014; 70(1): 104 
001067

Percutaneous tracheostomy in COVID-19 patients: assessing patient benefits and clinician risks

A. Williamson' ${ }^{1}$; M. Roberts ${ }^{1}$; J. Phillips ${ }^{1}$; R. Saha

${ }^{1}$ Critical care, Princess Alexandra Hospital, Harlow, United Kingdom

Correspondence: A. Williamson

Intensive Care Medicine Experimental 2020, 8(2): 001067

Introduction: Many patients with COVID-19 require long-term mechanical ventilation. Early tracheostomy may offer a route to weaning ventilation, decreased sedation, and reduced cardiovascular support for intensive care patients. However, at the peak of the COVID-19 pandemic, advice in the UK was to wait for 14 days and/or a negative COVID-19 test due to perceived risks to the operator $[1,2]$.

Objectives: To determine the impact of early percutaneous tracheostomies ( $<10$ days after intubation) on sedation andvasopressor requirements. In addition, we examine whether modifications to reduce viral exposure avoided COVID-19 transmission to the treating team.

Methods: We conducted a retrospective analysis of all mechanically ventilated COVID-19 patients receiving tracheostomies at a singlecentre general intensive care unit in the UK from 19 March to 29 June $2020(n=31)$. This involved a modified percutaneous technique to minimise aerosol generation. We compare patient sedation and cardiovascular support over a five-day follow-up period, as well as 30-day discharge and mortality, with an indicative control group of five clinically similar patients who did not receive a tracheostomy as they were transferred to different hospitals for exogenous reasons of ICU bed capacity. The treating team were also screened for COVID-19 antibodies $>2$ weeks after the procedures.

Results: Compared to baseline preceding tracheostomy, patients showed a decrease in both sedative and cardiovascular support requirements with no similar improvements in the control group. Median sedative requirements decreased from an average of over 10 $\mathrm{mg} / \mathrm{hr}$ of both morphine and midazolam or equivalent to less than 5 $\mathrm{mg} / \mathrm{hr}$ of both five days after tracheostomy, whilst median sedation remained above $10 \mathrm{mg} / \mathrm{hr}$ of both for the controls. Median noradrenaline decreased from 5 to $0 \mathrm{mcg} / \mathrm{min}$ over the same period, whilst the control group increased from 2.66 to $8 \mathrm{mcg} / \mathrm{min}$. After 30 days, 15 tracheostomy patients (48.3\%) had been discharged from hospital, two $(6.5 \%)$ were receiving ongoing care on step-down wards, and 14 were deceased (45.1\%). Of the indicative control group, after 30 days since meeting the criteria for tracheostomy two $(40 \%)$ had been discharged and three $(60 \%)$ were deceased. Of the 31 procedures only two involved complications (6.4\%); both featured transient desaturation and hypoxia but neither resulted in lasting sequelae. In addition, we find that all clinicians involved with tracheostomies received negative COVID-19 antibody tests.

Conclusion: In a small sample size, we demonstrate the potential for early tracheostomy as a strategy for reducing sedative and vasopressor requirements. This may improve outcomes for mechanically ventilated patients in a pandemic setting without compromising the safety of the treating team.

The authors acknowledge Aisling Corcoran, Becca Parrott, and Cheryl Pittuck for helpful data assistance.

\section{Reference(s) and grant ackowledgment(s)}

1. 2. National Tracheostomy Safety Project, 2020. NTSP Considerations For Tracheostomy In The COVID-19 Outbreak. http://www.tracheostomy.org. uk/storage/files/NTSP\%20COVID_19\%20tracheostomy\%20guidance \%2031_3_20.pdf

2. 1. Jacob T, 2020. Framework for open tracheostomy in COVID-19 patients. ENT UK. https://www.entuk.org/sites/default/files/COVID\%20tracheos tomy\%20guidance\%20-\%206\%20Apri|\%202020\%20update.pdf
001085

Early vs late tracheostomy in SARS-COV-2 patients. Does it really make a difference?

J. Gonzalez Londoño ${ }^{1}$; C. Vera Ching ${ }^{2}$; M. Buxó3 ${ }^{3}$ P. Ortiz Ballujera²;

JM. Sirvent Calvera ${ }^{2}$

${ }^{1}$ Intensive care medicine, Hospital de Santa Caterina, Salt, Spain; ${ }^{2}$ Intensive care medicine, Hospital Universitari de Girona Dr Josep Trueta, Girona, Spain; ${ }^{3}$ Statistical and metodological support unit, Fundació Privada Institut d'Investigació Biomèdica de Girona Doctor Josep Trueta, Salt, Spain

Correspondence: J. Gonzalez Londoño

Intensive Care Medicine Experimental 2020, 8(2): 001085

Introduction: The most serious form of COVID 19 infection manifested itself with a high number of patients with severe and longstanding ARDS, many of whom required a tracheostomy during the course of their illness. Currently, there is no consensus on the best time to perform tracheostomies. According to current recommendations, in the SARS-COV-2 pandemic situation, it is recommended to perform a tracheostomy after 10 to 14 days of mechanical ventilation (MV) to minimize exposure to the viral load that exists in earlier stages and for a better selection of patients.

Objectives: The objective of this study is to compare the results of patients with ARDS secondary to SARS-COV-2 that required a tracheostomy because of prolongued mechanical ventilation in two Intensive Care Units (ICU) in Spain

Methods: We carried out a retrospective observational study in the period between March and June 2020, in which SARS-COV-2 patients that developed ARDS and required a tracheostomy were divided in two groups. We considered early-tracheostomy patients as the ones who had a tracheostomy during the first 14 days of MV and late-tracheostomy after the 14th day. Data are presented as the mean \pm standard deviation (SD), the median with the $25-75$ percentiles and the frequencies with percentages, as appropriate. Continuous variables were analyzed with Student's t-test or the Mann-Whitney test, and categorical variables were analyzed with the chi-square test or Fisher's exact test. A level of statistical significance was set for values of $\mathrm{p}<0.05$

Results: 57 patients were included, of whom $73.7 \%$ were men, the mean age was 60.4 years old $( \pm 13.2)$. APACHE II at admission was $21.5( \pm 8.1)$ and SOFA $8.9( \pm 8.1) .29 .6 \%$ of the patients were obese, $43.9 \%$ had a history of HT and $22.8 \%$ were diagnosed with diabetes. At admission $29.8 \%$ had severe ARDS and $56.1 \%$ moderate ARDS. The CURB-65 and PORT-PSI mean were $2.07( \pm 0.8)$ and $4.09( \pm 0.8)$ respectively. In $42 \%$ of patients, an early tracheostomy was performed (12.1 ( \pm 1.7 ) days) and $58 \%$ late (17.4 ( \pm 2.7 ) days).

The duration of MV in the early TQT group was 23.5 days (20.2-33)) and in the late 30 days (25-41) ( $p$ 0.049); no significant differences were found in the days of ICU stay or in mortality.

Conclusion: In our series, patients with early-tracheostomy required fewer days of MV; however, we did not find significant differences in the days of ICU stay or mortality. These findings are similar to other studies in non SARS-COV-2 patients.

001172

Suction assisted containment of respiratory droplets during airway management with the SALAD technique

C. Della Vella ; R. Barrix²; C. Root ${ }^{3}$; J. Horowitz ${ }^{4}$; J. Ducanto ${ }^{5}$

${ }^{1}$ Catholic University of the Sacred Heart, Agostino Gemelli Hospital, Rome, Italy; ${ }^{2}$ Ems, Emergency Medical Services, Madison, United States of America; ${ }^{3}$ Emergency medicine, Department of Emergency Medicine, Albuquerque, United States of America; ${ }^{4}$ Emergency medicine, NYU Langone Health, New York, United States of America; ${ }^{5}$ Anesthesia, Medical College of Wisconsin, Milwaukee, United States of America

Correspondence: $C$. Della Vella

Intensive Care Medicine Experimental 2020, 8(2): 001172

Introduction: The COVID-19 pandemic has generated significant discussion of different means to reduce the risk of transmission to the clinician tasked with intubating an infected patient. Appropriate personal protective provides a barrier and video laryngoscopy increase 
the distance between the clinician and the respiratory tract, however additional proposed methods such as translucent plastic boxes have been show to be less than helpful. We present a simulation to examine the implications of the deliberate use of suction during the airway management of a patient with a respiratory infectious disease, and to examine a technique that may serve to reduce respiratory droplet emissions and potentially increase the safety of the procedure for the clinician.

Methods: The right mainstem bronchus of the 7-Sigma mannequin was coupled with two medication nebulizer systems filled with diet tonic water, which fluoresces on contact with ultraviolet (UV) light. The nebulizers are then connected to two separate medical flowmeters set to $15 \mathrm{~L}$ per minute flow. The left mainstem bronchus and esophagus of the mannequin are occluded to enhance thenebulizer gas flow through the mannequin to the oropharynx. The fresh gas flow through the nebulizer units produces a fine mist/aerosol, which is visible in the room air when illuminated by UV light. Two separate 60-watt UV lights, which are placed immediately to the sides and behind the endoscopist - to minimize the exposure of UV light to the endoscopist's eyes-are utilized to illuminate the aerosol mist.

When the clinician introduces the suction catheter into the oropharynx, the stream of aerosolized ceases to be visible as it is fully diverted into the stream of the suction. Utilizing the Suction Assisted Laryngoscopy and Airway Decontamination (SALAD) technique, the intubation can continue while leaving the suction catheter in place in the hypopharynx.

Results: This simulation shows the potential for a medical suction system to decontaminate the air space directly above the mouth of a mannequin emitting a nebulizer aerosol that is visible on UV light; and furthermore, it demonstrates the "intraoral" and "hypopharyngeal" decontamination of the nebulizer aerosol during simulated tracheal intubation. Battery-powered suction systems, such as those used on code carts and by prehospital providers, typically draw approximately 25 to $30 \mathrm{~L}$ per minute of gas through the suctionsystem. This airflow is equivalent to 1 cubic foot per minute. Though this volume is small, and it is dubious at best to predict whether this procedure would improve clinical safety of airway management in the presence of a respiratory infectious disease especially if the patient were to forcefully cough. Indeed, this implies that proper PPE, adequate paralysis and formal protocols are mandatory to maintain caregiver safety during an airway management procedure.

\section{Conclusion:}

The concept here is to reduce the potential inoculum that the caregiver's PPE is exposed to during the procedure, such that the filtering capability of their respiratory protection mask is not overwhelmed. Proactive use of the suction should prove to reduce the exposure of the physician PPE to a large inoculum of infectious respiratory particles if it accompanies the intubation attempt. Keeping the suction alongside the laryngoscope but above the esophagus and larynx could further help to reduce the airborne infectious respiratory particles in a patient under full induction and paralysis during intubation. Further focused studies are warranted.

\section{5}

Real time assessment of diaphragmatic muscle motion with Tissue Doppler Imaging; data from a single-center observational study in ICU patients

A. Kefalidou'; E. Doumaki'; S. Savvidou'; I. Iordanidou'; E. Soilemezi';

M. Tsagourias'; D. Matamis ${ }^{1}$

${ }^{1}$ Icu, Papageorgiou General Hospital, Thessaloniki, Greece

Correspondence: E. Soilemez

Intensive Care Medicine Experimental 2020, 8(2): 000205

Introduction: Diaphragmatic Tissue Doppler Imaging (TDI) allows real time assessment of the diaphragmatic tissue motion velocity. TDI of diaphragmatic tissue motion has been studied very little so far in adults, and no information exists regarding its referrence values nor its clinical significance in ICU patients.
Objectives: To determine whether TDI-derived parameters can differentiate patients who succeed from patients who fail a spontaneous breathing trial.

Methods: In consecutive ICU patients undergoing a T-tube trial, the following TDI-derived parameters were measured: Peak Contraction Velocity (PCV), defined as the maximal diaphragmatic velocity during contraction, measured in $\mathrm{cm} / \mathrm{sec}$; Peak Relaxation Velocity (PRV), defined as the maximal diaphragmatic velocity during relaxation, measured in $\mathrm{cm} / \mathrm{sec}$; TDI-derived Maximal Relaxation Rate (TDI-MRR), defined as the slope of the initial steepest part of the diaphragmatic motion velocity curve during relaxation, measured in $\mathrm{cm} / \mathrm{sec}^{\wedge} 2$. Measurements are presented as medians with 25th-75th interquartile ranges (IQR). Areas under the receiver operating characteristic (AUROC) with $95 \%$ Confidence Intervals $(95 \% \mathrm{Cl})$ have been calculated. Results: A total of 116 patients were included $(67$ succeed and 49 failed weaning). Baseline patient characteristics: 87 (75.2\%) males, aged $66.2 \pm 12.5$ years old, median 6 days under mechanical ventilation, reason for ICU admission: respiratory failure $18.9 \%$, multiple trauma $13.8 \%$, sepsis $13.8 \%$, coma $5.2 \%$. Groups did not differ in their baseline characteristics, but in TDI-derived measurements: PCV was 1.36 (IQR 1.06-1.79) vs. 2.02 (IQR 1.28-2.89) $\mathrm{cm} / \mathrm{sec}, \mathrm{p}=0.001$; PRV 1.36 (IQR 0.96-1.78) vs. 2.70 (IQR $1.41-4.86) \mathrm{cm} / \mathrm{sec}, \mathrm{p}<0,001$; and TDI-MRR 10.31 (IQR 5.66-13.74) vs. 21.75 (IQR 11.83-38) cm/sec^2, p < 0.001, for weaning success and weaning failure patients respectively. AUROC for PCV, PRV and TDI-MRR were $0.712(95 \% \mathrm{Cl} 0.607-0.816, \mathrm{p}<0.001)$, $0.772(95 \% \mathrm{Cl} 0.677-0.867, \mathrm{p}<0.001)$ and $0.804(95 \% \mathrm{Cl} 0.716-0.893, \mathrm{p}<$ $0.001)$, respectively.

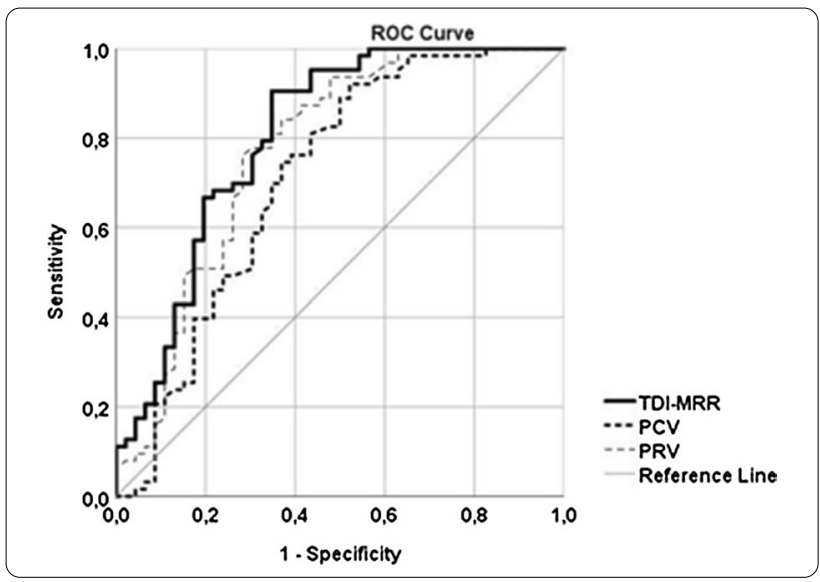

Conclusion: The results of this study suggest that TDI-derived measurements may serve as good markers for predicting success in a spontaneous breathing trial in ICU patients.

000287

Effects of End-Expiratory Lung Volume (EELV) versus $\mathrm{PaO}_{2}$ Guided PEEP Determination on Respiratory Mechanics and Oxygenation in Moderate to Severe ARDS: A Randomised Controlled Trial

K. Rollas ${ }^{1}$; P. Hanci'; A. Topeli ${ }^{1}$

${ }^{1}$ Department of internal medicine division of intensive care medicine, Hacettepe University Faculty of Medicine, Ankara, Turkey

Correspondence: $\mathrm{K}$. Rollas

Intensive Care Medicine Experimental 2020, 8(2): 000287

Introduction: There is no ideal method for determination of PEEP in ARDS patients. We compared the effects of an EELV-guided versus $\mathrm{PaO}_{2}$ guided PEEP determination on respiratory mechanics and oxygenation during the first 48 hours in moderate to severe ARDS.

Methods: Twenty-two patients with moderate to severe ARDS admitted to an academic MICU were randomly assigned to $\mathrm{PaO}_{2}$-guided $(n=11)$ or to an EELV-guided PEEP determination $(n=11)$ group. Incremental PEEP trial was performed by increasing PEEP by $3 \mathrm{cmH}_{2} \mathrm{O}$ 
steps from 8 to $20 \mathrm{cmH}_{2} \mathrm{O}$ and in each step EELV and lung mechanics were measured in both groups. EELV was measured with a modified nitrogen multiple breath wash-out/wash-in technique. In the $\mathrm{PaO}_{2}$-guided group, optimal PEEP was accepted as the minimum PEEP level associated with $\mathrm{PaO}_{2} \geq 60 \mathrm{mmHg}$ and $\mathrm{FiO}_{2} \leq 0.60$. In the EELVguided group, optimal PEEP was accepted as the PEEP that caused a $>10 \%$ increase in EELV. Oxygenation and respiratory mechanics were measured under the determined PEEP at 4, 12, 24, and 48th hours. Results are presented as median [25-75th percentile]. Friedman and Spearman's rho tests were used where appropriate. $P$ value $<0.05$ is accepted as statistically significant.

Results: As seen in the Table after the incremental PEEP trial, over the 48 hours of the study, $\mathrm{PaO}_{2}$ and $\mathrm{PaO}_{2} / \mathrm{FiO}_{2}$ increased in the EELVguided group as compared to $\mathrm{PaO}_{2}$-guided group. EELV, static compliance (Cs), driving pressure, and strain remained similar in the two groups. In all patients, the median value of EELV change ( $\triangle E E L V$ ) during incremental PEEP trial was $25 \%$. As seen in the Table in patients with $\triangle E E L V>25 \%(n=11) \mathrm{PaO}_{2}, \mathrm{PaO}_{2} / \mathrm{FiO}_{2}$ and $\mathrm{Cs}$ increased over time in 48 hours, whereas they did not change in those with $\triangle E E L V \leq 25 \%$ $(n=11)$. There was a negative correlation between EELV and driving pressure measured at 48 th hour of the study period $(r=-0.77, p<$ $0.001)$.

\begin{tabular}{|c|c|c|c|c|c|}
\hline & 4th hour & 12th hour & 24th hour & 48th hour & p value \\
\hline \multicolumn{6}{|l|}{$\mathrm{PaO}, \mathrm{mmHg}$} \\
\hline $\mathrm{PaO} 2$ guided & $63[59-71]$ & 60 [53-69] & $61[55-90]$ & 68 [62-84] & 0.09 \\
\hline EELV guided & $66[61-74]$ & $68[62-81]$ & $66[61-75]$ & $70[62-88]$ & 0.04 \\
\hline$\Delta \mathrm{EELV}>25 \%$ & $67[59-73]$ & 68 [53-80] & 62 [60-90] & 76 [68-91] & 0.03 \\
\hline$\Delta \mathrm{EELV} \leq 25 \%$ & $64[61-71]$ & $62[60-69]$ & $63[59-75]$ & $62[58-81]$ & 0.73 \\
\hline $\mathrm{PaO2} / \mathrm{Fi}$ & $\begin{array}{l}108[90- \\
130]\end{array}$ & $\begin{array}{l}103[88- \\
137]\end{array}$ & $92[91-168$ & ${ }_{162]}^{124[100-}$ & \\
\hline $\begin{array}{l}\mathrm{PaO} 2 \text { guided } \\
\mathrm{EELV} \text { guided }\end{array}$ & $\begin{array}{l}111[100- \\
142]\end{array}$ & $\begin{array}{l}130[108- \\
150]\end{array}$ & $\begin{array}{l}127[107- \\
151]\end{array}$ & $\begin{array}{l}145[95- \\
176]\end{array}$ & 0.02 \\
\hline$\triangle \mathrm{EELV}>25 \%$ & $\begin{array}{l}111[95- \\
142]\end{array}$ & $\begin{array}{l}133[88- \\
138]\end{array}$ & $\begin{array}{l}127[91- \\
168]\end{array}$ & $\begin{array}{l}140[108- \\
200]\end{array}$ & $<0.01$ \\
\hline$\Delta \mathrm{EELV} \leq 25 \%$ & $\begin{array}{l}101[90- \\
132]\end{array}$ & $\begin{array}{l}124[100- \\
138]\end{array}$ & $\begin{array}{l}107[101- \\
146]\end{array}$ & $\begin{array}{l}111[90- \\
155]\end{array}$ & 0.51 \\
\hline \multicolumn{6}{|l|}{$\mathrm{Cs}, \mathrm{mL} / \mathrm{cmH} 2 \mathrm{O}$} \\
\hline $\mathrm{PaO} 2$ guided & 26 [23-29] & 25 [19-33] & $25[22-44]$ & 29 [21-36] & 0.21 \\
\hline EELV guided & 27 [22-38] & $29[25-31]$ & 28 [26-32] & 30 [23-33] & 0.84 \\
\hline$\Delta \mathrm{EELV}>25 \%$ & $28[22-37]$ & $28[22-33]$ & $30[17-37]$ & $30[23-42]$ & 0.04 \\
\hline$\Delta \mathrm{EELV} \leq 25 \%$ & $25[23-29]$ & 27 [24-31] & 26 [25-29] & $27[22-35]$ & 0.73 \\
\hline $\begin{array}{l}\text { Driving pressure } \\
\mathrm{PaO} 2 \text { guided }\end{array}$ & 15 [12-17] & 15 [13-17] & $14[10-17]$ & 12 [10-19] & 0.41 \\
\hline EELV guided & 14 [10-18] & 14 [11-16] & 14 [13-18] & 14 [13-18] & 0.45 \\
\hline$\Delta \mathrm{EELV}>25 \%$ & $12[11-15]$ & $13[12-15]$ & $14[10-17]$ & $12[10-18]$ & 0.25 \\
\hline$\Delta \mathrm{EELV} \leq 25 \%$ & $17[12-18]$ & $16[12-17]$ & $15[13-18]$ & $14[12-22]$ & 0.41 \\
\hline Strain (VT/EELV & $\begin{array}{l}0.22[0.12- \\
0.44]\end{array}$ & $\begin{array}{l}0.24[0.11- \\
0.39]\end{array}$ & $\begin{array}{l}0.28[0.11- \\
0.39]\end{array}$ & $\begin{array}{l}0.12[0.10- \\
0.40]\end{array}$ & \\
\hline $\begin{array}{l}\mathrm{PaO} 2 \text { guided } \\
\text { EELV guided }\end{array}$ & $\begin{array}{l}0.24[0.21- \\
0.32]\end{array}$ & $\begin{array}{l}0.24[0.18- \\
0.36]\end{array}$ & $\begin{array}{l}0.22[0.17- \\
0.31]\end{array}$ & $\begin{array}{l}0.24[0.15- \\
0.31]\end{array}$ & 0.84 \\
\hline$\Delta \mathrm{EELV}>25 \%$ & $\begin{array}{l}0.22[0.12- \\
0.42]\end{array}$ & $\begin{array}{l}0.21[0.11- \\
0.30]\end{array}$ & $\begin{array}{l}0.22[0.13- \\
0.32]\end{array}$ & $\begin{array}{l}0.21[0.11- \\
0.33]\end{array}$ & 0.31 \\
\hline$\Delta \mathrm{EELV} \leq 25 \%$ & $\begin{array}{l}0.23[0.13- \\
0.37]\end{array}$ & $\begin{array}{l}0.31[0.16- \\
0.40]\end{array}$ & $\begin{array}{l}0.25[0.15- \\
0.34]\end{array}$ & $\begin{array}{l}0.24[0.13- \\
0.40]\end{array}$ & \\
\hline
\end{tabular}

* Friedman tests were conducted to test whether there is a significant change in the variables within each group.

Conclusion: Compared to $\mathrm{PaO}_{2}$-guided PEEP determination, EELVguided PEEP determination resulted in greater improvement in oxygenation over time. Patients who had $>25 \%$ improvement inEELV during a PEEP trial had greater improvement in oxygenation and compliance over 48 hours. Measurement of EELV might not only help to determine optimal PEEP but also imply lung recruitability in patients with moderate to severe ARDS.

\section{Reference(s) and grant ackowledgment(s)}

1. Dellamonica J., Lerolle N., Sargentini C., et al. PEEP-induced changes in lung volume in acute respiratory distress syndrome.Two methods to estimate alveolar recruitment. Intensive Care Med 2011; 37:1595-1604.

2. Olegard C., Sondergaard S., Houltz E., et al. Estimation of functional residual capacity at the bedside using standard monitoring equipment: a modified nitrogen washout/washin technique requiring a small change of the inspired oxygen fraction. Anesth Analg 2005; 101(1):206-212.

3. Study supported by Coordination Unit For Scientific Research Projects, Hacettepe University, Ankara.

4. We are thankful to Franco Laghi, MD for his critical review.

\section{8}

Neuromuscular blocking agents in acute respiratory distress syndrome: updated systematic review and meta-analysis of randomised trials

N. Tarazan'; M. Alshehri' ; S. Sharif ${ }^{2}$; Z. Al Duhailib ${ }^{1}$; M. Hylander Møller ${ }^{3}$. E. Belley-Côté ${ }^{1} ;$ M. Alshahrani ${ }^{4}$; J. Centofanti'; L. Mcintyre ${ }^{5}$; B. Baw' M. Meade ${ }^{1}$; W. Alhazzani ${ }^{6}$

${ }^{1}$ Medicine, McMaster University, Hamilton, Canada; ${ }^{2}$ Medicine, division of emergency medicine \& critical care, McMaster University, Hamilton, France; ${ }^{3}$ Department of intensive care, Copenhagen University Hospital, Rigshospitalet, Copenhagen, Denmark; ${ }^{4}$ Emergency and critical care, Imam Abdulrahman Bin Faisal University Hospital, Dammam, Saudi Arabia; ${ }^{5}$ Medicine, University of Ottawa, Ottawa, Canada; ${ }^{6}$ Department of medicine/department of health research methods, evidence and impact, McMaster University, Hamilton, Canada

Correspondence: $\mathrm{S}$. Sharif

Intensive Care Medicine Experimental 2020, 8(2): 000318

Introduction: Existing clinical practice guidelines support the use of neuromuscular blocking agents (NMBA) in acute respiratory distress syndrome (ARDS). However a recent large randomized clinical trial (RCT) has questioned this practice. Therefore, we updated a previous systematic review to determine the efficacy and safety of NMBAs in ARDS.

Methods: We searched MEDLINE, EMBASE (October 2012 to July 2019), the Cochrane (Central) database, and clinical trial registries (clinicaltrials.gov, ISRCTN Register, and WHO ICTRP) for RCTs comparing the effects of NMBA as a continuous infusion versus placebo or no NMBA infusion on patient-important outcomes for adults with ARDS. Two independent reviewers assessed methodologic quality of the primary studies and abstracted data.

Results: Seven RCTs, including four new RCTs, met eligibility criteria for this review. These trials enrolled 1,598 patients with moderate to severe ARDS at centres in the United States, France, and China. All trials assessed short-term continuous infusions of cisatracurium or vecuronium. The pooled estimate for mortality outcomes showed significant statistical heterogeneity, which was only explained by a subgroup analysis by depth of sedation in the control arm. A continuous NMBA infusion did not improve mortality when compared to a light sedation strategy with no NMBA infusion (relative risk [RR] $0.99 ; 95 \% \mathrm{Cl}$ $0.86-1.15$; moderate certainty; $P=0.93$ ). On the other hand, continuous NMBA infusion reduced mortality when compared to deep sedation with as needed NMBA boluses (RR $0.71 ; 95 \% \mathrm{Cl} 0.57-0.89$; low certainty; $P=0.003$ ). Continuous NMBA infusion reduced the rate of barotrauma (RR $0.55 ; 95 \% \mathrm{Cl} 0.35-0.85$, moderate certainty; $P=0.008$ ) 
across eligible trials, but the effect on ventilator-free days, duration of mechanical ventilation, and ICU-acquired weakness was uncertain.

Conclusion: Inconsistency in study methods and findings precluded the pooling of all trials for mortality. Earliest trials suggested a mortality reduction with a continuous NMBA infusion; however, when compared to the current practice of ARDS management with lighter sedation, use of continuous NMBA infusion did not reduce the risk of death. In both situations, a continuous NMBA infusion may reduce the risk of barotrauma, but the effects on other patient-important outcomes remain unclear.

\section{9}

Comparison of survival outcomes between patients ventilated on anaesthetic machines and conventional ventilators during the Coronavirus pandemic: A retrospective case series P. Jain ' ${ }^{\prime}$ J. Dixon ${ }^{\prime}$; V. Shukla'

'Intensive care unit st helier hospital, Epsom and St Helier University Hospitals, London, United Kingdom

Correspondence: P. Jain

Intensive Care Medicine Experimental 2020, 8(2): 000329

Introduction: The coronavirus disease 2019 (COVID-19) pandemic has led to rapid exhaustion of intensive care facilities across the UK and the world. The use of anaesthetic machines for long term ventilation as an off label use received regulatory clearance in this setting. We compare the survival outcomes of COVID-19 patients between 2 groups - those ventilated on anaesthetic machines and on those on standard ventilators. This was a retrospective observational case series conducted at Epsom and St Helier university hospitals in London.

Methods: 63 patients admitted to our intensive care unit with confirmed SARS-CoV2 (severe acute respiratory syndrome coronavirus-2) infection between 07 March and 27 April were included in this case series. These patients were either ventilated on anaesthetic machines or conventional ventilators depending on the availability of these machines. All the patients were treated with similar management protocols. The mortality rates, APACHE 2 scores and the length of ventilator days of the deceased patients were compared between the two groups.

Results: The mean \pm standard deviation (SD) APACHE2 score in the anaesthetic machine group was $14.21 \pm 4.05$ and in the ventilator group was $19.72 \pm 5.66$; $\mathrm{p}$-value 0.0002 . The mean $\pm S D$ number of ventilator days was $6.87 \pm 4.3$ and $8.36 \pm 6.68$ in the anaesthetic machine and ventilator groups respectively ( $p$-value 0.41 ). The mortality rate in the anaesthetic machine group was $80 \%$ and in the ventilator group was $66.66 \%$; $p$ value 0.82 .

Conclusion: There was no difference in outcomes between the patients ventilated on conventional ventilators versus those with anaesthetic machines. However, the results could be different if the APACHE scores were similar.

Anaesthetic machines were not intended for long term ventilation and their use in the times of such pandemics seem justified and essential when the availability of the conventional ventilators is limited. This is important because having a back up stock / inventory of conventional ventilators anticipating the occasional pandemic may not be viable financially for most healthcare organisations.

\section{Reference(s) and grant ackowledgment(s)}

1. Orser, B.A., Byrick, R., Cooper, R. et al. Locating and repurposing anesthetic machines as intensive care unit ventilators during the COVID-19 pandemic. Can J Anesth. [Internet]. 2020 [Cited 2020 May 05]. Available from: https:// doi.org/10.1007/s12630-020-01657-w.

2. Medicines and healthcare products regulatory agency. Anaesthetic machines: off-label use during the COVID-19 pandemic, MDA/2020/012 [Internet]. 2020 [Cited 2020 May 05]. Available from: https://www.gov.uk/ drug-device-alerts/anaesthetic-machines-off-label-use-during-the-covid -19-pandemic-mda-2020-012.
000410

Appraisal of different inspiratory flow curves during volume-controlled ventilation in ARDS patients

A. Meli ${ }^{1}$; V. Galanti ${ }^{1}$; A. Caccioppola ${ }^{1}$; V. Camponetti ${ }^{1}$; C. Bianchi ${ }^{1}$;

G. Catzula ${ }^{1}$; S. Cenci'; F. Bichi' ; V. Castagna ${ }^{1}$; S. Coppola ${ }^{2}$; D. Chiumello ${ }^{2}$

${ }^{1}$ Department of anesthesia and intensive care, san paolo hospital, University of Milan, Milano, Italy; ${ }^{2}$ Anesthesia and intensiva care, San Paolo, Milano, Italy

Correspondence: A. Meli

Intensive Care Medicine Experimental 2020, 8(2): 000410

Introduction: In ARDS patients, controlled mechanical ventilation - either volumetric or pressometric - is commonly employed. Several studies investigated the effects of inspiratory flow on clinical parameters. 1 Yet, a systematic approach to this issue is still lacking, and so are its clinical consequences.

Objectives: To appraise the effects of four different inspiratory flow waveforms on gas exchange, pulmonary mechanics and hemodynamics during volume-controlled mechanical ventilation.

Methods: Within 72 hours from the diagnosis of ARDS, patients were enrolled. Supine position, myorelaxation and esophageal pressure monitoring were inclusion criteria. Volume-controlled ventilation with Hamilton S1 (Hamilton Medical, Bonaduz, CH) was instituted as it follows: Tidal Volume 6-8 $\mathrm{ml} / \mathrm{kglBW}$, Plateau Pressure $\leq 25 \mathrm{~cm} \mathrm{H}_{2} \mathrm{O}$, PEEP as described by the high-PEEP/low- $\mathrm{FiO}_{2}$ branch of the LOV study. 2 Patients were randomized to receive: square (SQ), decelerated (DE), sinusoidal (SIN), and trunk decelerated (TDE) waveform. After $20 \mathrm{~min}$ of clinical stabilization following each waveform change, data were collected and analyzed.

Results: Twenty-six patients were enrolled and randomized. Gas exchange, pulmonary mechanics and hemodynamic data are clustered on the basis of inspiratory waveform (Table 1). Significant difference was observed in peak inspiratory flow (Figure 1) between DE and TDE $(p<0.001)$, SQ $(p<0.001)$ and SIN $(p=0.001)$; among TDE and SIN $(p<0.001)$; between SQ and SIN $(p<0.001)$. Nevertheless, variations in terms of gas exchange, pulmonary mechanics and hemodynamics resulted shy of statistical relevance.

Table 1. Grouped gas exchange, pulmonary mechanics and hemodynamic

\begin{tabular}{|c|c|c|c|c|c|}
\hline & DE & TED & SQ & SIN & p \\
\hline $\begin{array}{c}\text { Peak } \\
\text { Inspiratory } \\
\text { Flow (ml/sec) }\end{array}$ & $707.9 \pm 121.1$ & $475.5 \pm 74.1$ & $423.1 \pm 88.7$ & $598.1 \pm 105.1$ & $<0.01$ \\
\hline $\begin{array}{l}\text { Ppeak (cm } \\
\text { H2O) }\end{array}$ & $28.3 \pm 4.8$ & $27.1 \pm 4.5$ & $28.4 \pm 4.9$ & $28.4 \pm 4.8$ & 0.719 \\
\hline $\begin{array}{l}\text { MAPaw (cm } \\
\text { H2O) }\end{array}$ & $16.6 \pm 2.6$ & $15.9 \pm 2.5$ & $15.5 \pm 2.6$ & $15.6 \pm 2.7$ & 0.428 \\
\hline $\begin{array}{c}\text { ERS (cm } \\
\text { H2O/l) }\end{array}$ & $20.6 \pm 6.9$ & $20.1 \pm 6.7$ & $19.6 \pm 6.2$ & $20.4 \pm 6.6$ & 0.964 \\
\hline EL(cm H2O/l) & $13.4 \pm 6.3$ & $3.7 \pm 4.2$ & $12.6=$ & $13.5 \pm 6.1$ & 0.918 \\
\hline $\begin{array}{c}\text { ECW (cm } \\
\text { H2O/l) }\end{array}$ & $7.1 \pm 2.2$ & $6.4 \pm 2.8$ & $7.1 \pm 2.1$ & $6.9 \pm 2.3$ & 0.746 \\
\hline$\Delta \mathrm{PL}(\mathrm{cm} \mathrm{H2O})$ & $13.1 \pm 4.2$ & $13.7 \pm 4.2$ & $12.6 \pm 3.8$ & $13.3 \pm 3.9$ & 0.813 \\
\hline EELV (ml) & $1321.5 \pm 584.7$ & $1271.2 \pm 556.2$ & $1304.8 \pm 645.9$ & $1314.9 \pm 585.9$ & 0.990 \\
\hline MP (J/min) & $16.9 \pm 4.4$ & $16.4 \pm 4.8$ & $16.9 \pm 4.1$ & $17.4 \pm 4.5$ & 0.883 \\
\hline MAP (mmHg) & $76.4 \pm 9.1$ & $76.1 \pm 10.8$ & $77.7 \pm 14.5$ & $77.5 \pm 10.6$ & 0.939 \\
\hline & DE & TED & SQ & SIN & p \\
\hline HR (bpm) & $82.1 \pm 15.7$ & $80.4 \pm 14.3$ & $84.0 \pm 16.9$ & $83.5 \pm 16.7$ & 0.852 \\
\hline $\mathrm{PaCO2}(\mathrm{mmHg})$ & $49.70 \pm 9.92$ & $51.74 \pm 11.55$ & $52.76 \pm 11.78$ & $52.18 \pm 11.34$ & 0.776 \\
\hline
\end{tabular}

Data are displayed as mean \pm SD. Ppeak, Peak Pressure; Pplat, Plateau Pressure; MAPaw, Mean Airway Pressure; ERS, Respiratory System Elastance; EL, Lung Elastance; ECW, Chest Wall Elastance; $\triangle \mathrm{PL}$, Transpulmonary pressure; EELV, End-Expiratory Lung Volume; MP, Mechanical Power; MAP, Mean Arterial Pressure; HR, Heart Rate; $\mathrm{PaCO} 2$, Arterial partial pressure of $\mathrm{CO} 2 ; \mathrm{PaO}_{2} / \mathrm{FiO}_{2}$, arterial partial pressure of $\mathrm{O} 2$ to inspired fraction of $\mathrm{O} 2$ ratio. 


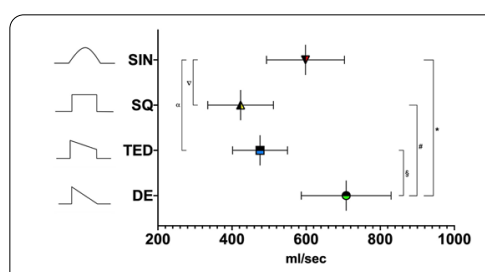

Figure 1. Peak inspiratory flow. Data are displayed as mean $\pm \mathrm{SD}$. ${ }^{*}, \mathrm{p}=0.001 ; \#, \mathrm{p}<0.001 ; \S$, $\mathrm{p}<0.001 ; \nabla, \mathrm{p}<0.001 ; \mathrm{a}, \mathrm{p}<0.001$

Conclusion: In our study, the application of different inspiratory waveforms resulted in sensible variations of peak inspiratory flow. Nevertheless, this did not translate in relevant shifts of clinical parameters.

\section{Reference(s) and grant ackowledgment(s)}

1. 2 Meade M, Cook D, Guyatt G et al. Ventilation strategy using low tidal volumes, recruitment maneuvers, and high positive end-expiratory pressure for acute lung injury and acute respiratory distress syndrome: A randomized controlled trial. JAMA 2008;299;637-645

2. 1 Rittayamai N, Katsios CM, Beloncle F, Friedrich JO, Mancebo J, Brochard L. Pressure-controlled vs volume-controlled ventilation in acute respiratory failure: A physiology-based narrative and systematic review. Chest 2015;148:340-355

\section{6}

Impact of a stepwise increase in PEEP (PEEP-trial)

on haemodynamics, static compliance, functional residual capacity and tidal volume: a study in patients

\section{with PiCCO-monitoring}

J. Wettstein'; U. Mayr ${ }^{2}$; S. Rasch²; T. Lahmer²; G. Batres-Baires²; R. Schmid ${ }^{2}$.

W. Huber ${ }^{2}$

${ }^{1}$ Klinikum rechts der Isar der Technischen Universität München, München, Germany; ${ }^{2}$ Medizinische klinik und poliklinik ii, Klinikum rechts der Isar; Technische Universität München, Munich, Germany

Correspondence: $J$. Wettstein

Intensive Care Medicine Experimental 2020, 8(2): 000476

Introduction: Advanced respirator technologies provide automated stepwise PEEP-increases in parallel with the measurement of functional residual capacity (FRC) and static pulmonary compliance (C_stat). While this PEEP-trial (PT) may help facilitate optimal PEEPsetting, it also carries the risk of haemodynamic instability due to the reduced venous return. We hypothesized that a standard PT with stepwise increases in PEEP of a total of $8 \mathrm{cmH}_{2} \mathrm{O}$ might result in substantial haemodynamic changes. Since heart lung interactions have been suggested to diagnose volume responsiveness, the PT could be used as a combined approach to optimize ventilation and haemodynamics.

Methods: In 50 mechanically ventilated patients (Carescape R860; GE; pressure-controlled ventilation; PF-ratio of $249+-83$ ) and PiCCO monitoring, an automated five step PEEP-trial was performed. PEEP was increased from values of $4 \mathrm{cmH} 20$ below, to $4 \mathrm{cmH}_{2} \mathrm{O}$ above the present PEEP-level. PiCCO-data, ventilator settings, FRC and C_stat were measured at baseline and after each increase.

Results: Mean values for most haemodynamic parameters including heart rate, MAP, CVP, global end-diastolic volume index GEDVI, stroke volume index SVI, stroke volume variation SW, extravascular lung water index EVLWI and cardiac power index CPI were not significantly different at the end compared to the start of the PT. Mean cardiac index $\mathrm{Cl}$ decreased significantly as the heart rate decreased with increasing PEEP. $\operatorname{PEEP}\left(5,1 \pm 1,7\right.$ vs. $\left.12,8 \pm 2,4 \mathrm{cmH}_{2} \mathrm{O} ; \mathrm{p}<0.001\right)$ and FRC $(1493 \pm 588$ vs. $1940 \pm 778 \mathrm{ml} ; \mathrm{p}<0.001)$ were significantly higher after the PT, while all other respiratory data including tidal volume and C_stat were not different after the PT. Individual maximum values during the PT were significantly higher for tidal volume $(461 \pm 151$ vs. $526 \pm 126 \mathrm{~mL} ; \mathrm{p}<0.001)$ and C_stat ( $38 \pm 21$ vs. $48 \pm 23 \mathrm{~mL} / \mathrm{cmH}_{2} \mathrm{O} ; \mathrm{p}<0.001$ ) compared to baseline. Conclusion: 1.) The PT did not impair haemodynamics. While the PT is haemodynamically safe, its use to detect volume deficiency appears to be low. 2.) Adjustment of PEEP in response to PT data substantially improves FRC, C_stat and tidal volume.
000493

Establishing a COVID-19 Tracheostomy Pathway: a Tertiary Centre Experience

AA. Singh ${ }^{1} ;$ H. Griffiths ${ }^{2}$; J. Ashcroft ${ }^{1}$; J. Tysome ${ }^{3}$; A. Conway-Morris ${ }^{4}$;

R. Mahroof ${ }^{4}$

${ }^{1}$ Department of surgery, Cambridge University Hospitals NHS Foundation Trust, Cambridge, United Kingdom; ${ }^{2}$ Department of anaesthesia, Cambridge University Hospitals NHS Foundation Trust, Cambridge, United Kingdom; ${ }^{3}$ Department of ear, nose and throat surgery, Cambridge University Hospitals NHS Foundation Trust, Cambridge, United Kingdom; ${ }^{4}$ Department of anaesthetics \& intensive care medicine, Cambridge University Hospitals NHS Foundation Trust, Cambridge, United Kingdom

Correspondence: A. Singh

Intensive Care Medicine Experimental 2020,8(2): 000493

Introduction: A tracheostomy (TSY) is a well-recognised means to provide a safe interface for weaning off prolonged mechanical ventilation, especially in those with severe respiratory failure. Aerosol generating procedures (AGPs) are associated with significant cross infection risks to healthcare workers with deaths reported, including those of ENT surgeons. No national consensus exists on the role or timing of TSY in COVID-19 pts. Initially TSYs were requested ad-hoc based on clinical need. However, with the dynamic and rapidly evolving pandemic it became clear an agreed pathway was required with clarity on indications and timing of the procedure. We present a tertiary centre experience of creating a novel TSY pathway for long stay COVID-19 patients.

Methods: Regular multidisciplinary team (MDT) discussions between intensivists, ENT surgeons and operating room staff provided a forum to address and discuss all relevant issues, concerns and risks (both to patients as well as staff) of undertaking a TSY in COVID-19 pts. An agreed step-wise approach was instituted with potential for review. Consecutive COVID-19 positive pts admitted to ICU at a tertiary referral centre over 8 weeks were included. Contemporaneous electronic case notes and results were retrospectively reviewed.

Results: The step-wise plan agreed was as follows

1. Avoid TSY before 14 days

2. TSY only in patients with favourable prognosis

3. Need for TSY agreed by 2 ICU Consultants

4. Aim percutaneous TSY as first option if possible

5. Surgical TSY following MDT discussion

6. All procedures to be undertaken under full PPE

MDT meetings took place at set times, 3 times a week. The order of patients for surgical TSY was also agreed.

During this time 72 COVID pts were admitted to ICU with 59/72 (82\%) intubated and mechanically ventilated; their characteristics are shown in Table 1.

The median time from admission to intubation was 1 (range 0-34) day and from intubation to TSY 16 (2-34) days. TSY was performed in 27/59 $(46 \%)$ of patients; with a surgical approach $24 / 27$ (89\%) being commonest. Median time from decision for TSY and the procedure was 4 $(0-26)$ days. Median time from TSY to spontaneous breathing trial was 4 (2-19) days and to decannulation was 12 (6-24) days. There were no TSY related complications. 13 patients were successfully primarily extubated at a median time of 10 (3-17) days after endotracheal intubation. Importantly, no healthcare worker cross infection from TSY has been recorded.

\begin{tabular}{|l|l|}
\hline Characteristics & Total $\mathbf{n = 7 2}$ \\
\hline Patient characteristics & \\
\hline Age (years) - mean (standard deviation) & $57.9( \pm 12.7)$ \\
\hline Male sex $-\mathrm{n}(\%)$ & $53(74 \%)$ \\
\hline BMI - mean (standard deviation) & $29.1( \pm 6.2)$ \\
\hline Comorbidity $-n(\%)$ & \\
\hline Cardiac & $20(28 \%)$ \\
\hline Respiratory & $12(17 \%)$ \\
\hline Renal & $5(7 \%)$ \\
\hline Neurological & $6(8 \%)$ \\
\hline Diabetes & $10(14 \%)$ \\
\hline
\end{tabular}


Conclusion: Engagement of key stakeholders is essential to create a streamlined TSY pathway for COVID-19 pts. Clarity and agreement between multiple specialties has helped provide an effective service without an increased infection risk to staff.

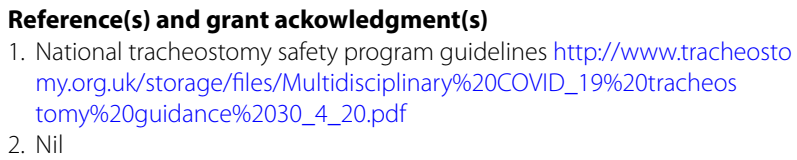

\section{3}

Clinical features, ventilatory management, and outcome of ARDS caused by COVID-19 does not differ from other causes of ARDS GS. Angeles ${ }^{1}$; L. Zattera ${ }^{2}$; C. Sole ; A. Fernandez ; C. De Peray ${ }^{1}$; P. Benet ${ }^{1}$; T. Ramis ${ }^{1}$; O. Comino'; J. Aliaga ; A. Fervienza'; D. Forne'; M. Arias'; R. Mellado Artigas ${ }^{3} ;$ C. Ferrando ${ }^{3}$

${ }^{1}$ Anaesthesiology, Hospital Clínic de Barcelona, Barcelona, Spain; ${ }^{2}$ Anaesthesiology, Hospital del Mar, Barcelona, Spain; ${ }^{3}$ Anaesthesiology, critical care unit, Hospital Clínic de Barcelona, Barcelona, Spain

Correspondence: G.S. Angeles

Intensive Care Medicine Experimental 2020, 8(2): 000513

Introduction: Specific respiratory features, ventilatory management, and outcomes of patients with acute respiratory distress syndrome (ARDS) caused by coronavirus disease 2019 (COVID-19) have not been well established yet.

Objectives: The aim of this observational study was to describe characteristics of confirmed ARDS COVID-19 patients that received invasive mechanical ventilation (MV) and other adjunctive therapies.

Methods: From March 12th to April 8th 2020, this prospective, multicenter, observational, cohort study enrolled patients with COVID-19 ARDS admitted to the ICU, in 28 hospitals, with a 28-day follow up. Patient medical data were collected into an anonymized online data acquisition system (CoVid19.ubikare.io). Inclusion criteria: $>18$ y/o, intubated and mechanically ventilated, confirmed SARS-CoV-2 infection using PCR-based tests, and ARDS, as defined by the Berlin criteria. Recorded data included demographics, ventilator parameters, adjunctive therapies, disease chronology (initiation of MV, ventilatorfree-days, ICU-length-of-stay), discharge from ICU and 28-day mortality. Patients were stratified by severity of ARDS. Descriptive variables were expressed as percentage, mean and standard deviation (SD), or median and interquartile range (IQR), as appropriate. To compare variables, Student t-test or Mann-Whitney test and one-way ANOVA or Kruskal-Wallis test for numerical variables, and Chi squared test or Fisher exact test for categorical variables were used. Time to event curves were plotted using the Kaplan-Meier method and analyzed with log-rank test and univariable Cox regression analysis.

Results: A total of 504 patients were included: 96 (19.0\%) with mild, $235(46.6 \%)$ with moderate, and $173(34.3 \%)$ with severe ARDS. Median (IQR) values were: $\mathrm{PaO}_{2} / \mathrm{FiO}_{2} 127(88-182) \mathrm{mmHg}$, VT 6.9 (6.3-7.8) ml/ kg PBW, PEEP 12 (10-14) $\mathrm{cmH}_{2} \mathrm{O}, \mathrm{Crs} 35(28-45) \mathrm{ml} / \mathrm{cmH}_{2} \mathrm{O}$, Pplat 24 (20-28) $\mathrm{cmH}_{2} \mathrm{O}$, and driving pressure 12 (9-14) $\mathrm{cmH}_{2} \mathrm{O}$. Recruitment maneuvers (RM) were used in $414(82 \%)$ without differences between groups. Prone position was used in 385 (76\%) patients, and neuromuscular blocking agents (NMBA) were used in $340(66 \%)$ patients. Degree of ARDS severity was associated with significant differences in the use of prone position $(p=0.001)$ and NMBA $(p=0.008)$. There was a higher likelihood of discontinuation of MV $(p=0.006)$, ICU discharge $(p=0.010)$ and 28-day survival $(p=0.004)$ with decreasing severity of ARDS. All-cause 28-day mortality was $18 \%$ (94 patients). The risk of 28-day mortality was higher in severe ARDS compared to moderate ARDS [hazard ratio (RR) 0.47 (95\%Cl: 0.30-0.76), $p=0.002$ ].

Conclusion: On average, in this large series, COVID-19 ARDS patients were managed with standard practice of lung-protective mechanical ventilation frequently associated to RM and prone position. General clinical features, ventilatory management, and 28-day outcome did not differ from other causes of ARDS. The degree of ARDS severity was a significant prognostic factor.

\section{Reference(s) and grant ackowledgment(s)}

1. Bellani G, Laffey JG, Pham T, et al. Epidemiology, patterns of care, and mortality for patients with acute respiratory distress syndrome in intensive care units in 50 countries. JAMA 2016; 315:788-800.

2. Ranieri VM, Rubenfeld GD, Thompson BT, et al; ARDS Definition Task Force. Acute respiratory distress syndrome: the Berlin Definition. JAMA. 2012;307(23):2526-2533.

\section{4}

Experience with COVID-19 related severe acute respiratory distress syndrome (ARDS) patients admitted to a medical Intensive Care Unit in a second-level university Hospital

I. Gallego Barbachano'; A. Ortega Montes'; E. Moreno Lopez ${ }^{2}$; JL. Martinez Melgar'; E. Sanmartin Mantiñan'; T. Sanchez De Dios' ; A. De La Campa Gestido'; J. Cenoz Osinaga'; JV. Bravo Doviso'; P. Posada Gonzalez

${ }^{1}$ Intensive care unit, Complexo Hospitalario Universitario De Pontevedra, Pontevedra, Spain; ${ }^{2}$ Anaesthesia and postoperative care, Complexo Hospitalario Universitario De Ferrol, Ferrol, Spain

Correspondence: E. Moreno Lopez

Intensive Care Medicine Experimental 2020, 8(2): 000534

Introduction: A new coronavirus (SARS-CoV-2) emerged from China in December 2019, causing an severe acute respiratory distress syndrome (ARDS) that rapidly spread to become a pandemic.

Objectives: To describe patients admitted to a medical Intensive Care Unit (ICU) in a second-level university Hospital due to severe ARDS related to COVID-19 infection.

Methods: Retrospective and descriptive analysis between March and April 2020 of critically ill patients with COVID-19-related severe acute respiratory distress syndrome admitted to a medical ICU. The following parameters were analyzed: demographic values (gender, age, severity scores, comorbidities), hemodinamic instability, renal failure, respiratory failure (ventilatory mode, $\mathrm{FiO}_{2}$ level, $\mathrm{PEEP}$, prone ventilation, continuous neuromuscular blockade (NMB), endotracheal tube exchange, tracheostomy, days spent under mechanical ventilation), laboratory parameters (lactate dehydrogenase (LDH), low white blood cell (WBC) count, C-reactive protein (CRP), D-dimer, serum ferritine), length of ICU stay and outcomes.

Results: 22 patients ( 19 male, 3 female) were admitted, mean age: $67 \pm 6,1$ ( $<60$ years old:4, 60-70 years old: $13(59 \%)$, > 70 years old 7). APACHE II score: $26 \pm 7$, SOFA : 7,5 $\pm 1,9$. Comorbidities: chronic airflow limitation (CAL): 4 , obesity (BMI > 30): $5(22,7 \%)$, hypertension: 13 (59\%), diabetes: 4. Inotropic support: $6(27,3 \%)$, renal failure (continuous renal replacement therapy(CRRT)): $3(13,7 \%)$, respiratory support: $22(100 \%)$, ventilatory mode: pressure $14(64 \%)$, volumen $8, \mathrm{FiO}_{2}$ (max): < 0.6: 5, between 0.6-0.8: $12(54,5 \%),>0.8$ : 5. PEEP (max): $<9$ $\mathrm{CmH}_{2} \mathrm{O}: 3$, between 9-12: $13(60 \%),>12: 6$. Prone ventiltation: $18(81 \%)$ : within the first 24 hours:8, after 24 hours: 10. Continuous NMB: 14 (64\%). Reintubation: $4(18 \%)$, endotracheal tube exchange (obstruction):7 (32\%), tracheostomy:5 (22,7\%), 4 surgical tracheostomy (3 difficult airway, 1 coagulopathy), 1 percutaneous. Time spent under mechanical ventilation: $18 \pm 8,5$ days. Laboratory parameters: $14 \mathrm{LDH}$, 10 low WBC count, 19 (86\%) CPR, 18 (81\%) D-dimer, 19 (86\%) serum ferritine. Length of ICU stay: $19,8 \pm 8$ days. Outcome: $18(81,8 \%)$ discharged (alive), 3 deaths, 1 still in ICU.

Conclusion: According to our series, the type of patient admitted with COVID-19 related severe acute respiratory distress syndrome (ARDS) was a male between 60-70 years old, with hypertension and/or obesity, under mechanical ventilation (pressure mode:64\%) and $\mathrm{FiO}_{2}$ (within the first 24 hours): $0.6-0.8(54,5 \%)$, PEEP $9-12 \mathrm{cmH}_{2} \mathrm{O}(60 \%)$, in prone positioning $(81 \%)$ and continuous NMB infusion $(64 \%)$, requiring tracheostomy $(22,7 \%)$ and a $81,8 \%$ of survival rate. 


\section{4}

\section{Ventilator-Associated Pneumonia in COVID19: a single centre} experience

S. Hajela' ; B. Ravenhill' ${ }^{1}$ S. Ubhi ${ }^{1}$; VN. Vilas Navapurkar'; A. Conway-Morris²; R. Mahroof'; M. Mailis ${ }^{3} ;$ G. Dougann 3 ; M. Martin ${ }^{4}$

'John farman intensive care unit, addenbrooke's hospital, cambridge, uk, Cambridge University Hospitals NHS Foundation Trust, Cambridge, United Kingdom, Cambridge, United Kingdom; ${ }^{2}$ Department of anaesthetics \& intensive care medicine, Cambridge University Hospitals NHS Foundation Trust, Cambridge, United Kingdom; ${ }^{3}$ Centre for therapeutic immunology and infectious diseases, university of cambridge, uk, University of Cambridge, UK, Cambridge, United Kingdom; ${ }^{4}$ Public health england microbiology laboratory, cambridge, uk, Public health england microbiology laboratory, addenbrooke's hospital, cambridge, uk, Cambridge, United Kingdom

Correspondence: S. Hajela

Intensive Care Medicine Experimental 2020, 8(2): 000594

Introduction: Ventilator associated pneumonia (VAP) is the commonest complication of mechanical ventilation and is a major cause of morbidity and mortality. VAP incidence varies based on definitions, but has been estimated to affect between $8-28 \%$ of patients(1). The SARS-CoV-2 pandemic has resulted in a large increase in the numbers of patients being mechanically ventilated. We examine the incidence and cause of VAP in COVID positive and negative individuals during the first wave of SARS-CoV-2 in a UK tertiary care intensivist unit.

Objectives: To determine the incidence of suspected VAP in patients with COVID-19.

Methods: We performed a retrospective chart review of all patients admitted to Addenbrooke's hospital requiring mechanical ventilation with confirmed SARS-CoV-2 for 7 weeks up to and including the $1 / 5 / 2020$. Patients were defined as having a suspected VAP based on worsening oxygenation or increased sputum combined with systemic inflammation (pyrexia and/or raised neutrophil count) and radiological features (new or progressive chest $x$-ray infiltrates), confirmed VAP required positive quantitative microbiological culture or molecular detection of a pathogenic organism.

Results: 64 COVID-19 positive patients required invasive ventilation, receiving a median 15 (IQR 10-22) days of ventilation and a total of 1017 ventilator days. 53 (83\%) developed a suspected VAP, with 26 cases confirmed by microbiology. The incidence density of suspected VAP amongst all COVID-19 positive intubated patients was 52 cases per 1000 ventilator-days. ICU Mortality was $56 \%$ in this cohort, amongst those who did not develop suspected or confirmed VAP it was $29 \%$. The organisms detected are shown in table 1 below.

\begin{tabular}{|l|l|}
\hline \multicolumn{1}{|c|}{ Organism } & Frequency \\
\hline Gram-Positive & \\
\hline Staphylococcus aureus & 3 \\
\hline Gram-Negative & \\
\hline Escherichia Coli & 3 \\
\hline Enterobater sp. & 4 \\
\hline Hemophilus influenzae & 3 \\
\hline Klesiella sp. & 9 \\
\hline Proteus sp. & 1 \\
\hline Pseudomonas aeruginosa & 7 \\
\hline Serratia liquifaciens & 1 \\
\hline Fungi & \\
\hline Aspergillus fumigatus & 1 \\
\hline
\end{tabular}

Conclusion: VAP rates appear to be high amongst patients with COVID19, reflecting both their prolonged need for ventilation(1) and critical-illness induced immunosuppression(2). The rate reported here may reflect the diagnostic modalities used, as early on endotracheal aspirate (which over-estimates true incidence) (3) was used until we developed a safe system for bronchoscopic sampling. Gram-negative organisms predominate. The extent to which VAP mediates the high mortality seen remains uncertain but calls for antibiotic stewardship, vigilance, and detailed work in this area.

Reference(s) and grant ackowledgment(s)

1. 1) Chastre J, Fagon J-Y. Ventilator-associated pneumonia. Am J Respir Crit Care Med. 2002;165(7):867-903. 2) Morris AC, Datta D, Shankar-Hari M et al. Cell-surface signatures of immune dysfunction risk-stratify critically ill patients: INFECT study. Intensive Care Med. 2018:44(5):627-635. 3) Morris AC, Kefala K, Simpson AJ, et al. Evaluation of the effect of diagnostic methodology on the reported incidence of ventilator-associated pneumonia. Thorax. 2009;64(6):516-522.

2. NA

\section{5}

COVID-19 ARDS. Does compliance phenotype impact on ventilatory management and outcomes?

A. Fernandez"; GS. Angeles ${ }^{1}$; C. Sole ${ }^{1}$; L. Zattera ${ }^{2}$; C. De Peray ${ }^{1}$; P. Benet ${ }^{1}$; T. Ramis ${ }^{1}$; O. Comino ${ }^{1}$; J. Aliaga ${ }^{1}$; A. Fervienza ${ }^{1}$;D. Forne ${ }^{1}$; M. Arias ${ }^{1}$;

R. Mellado Artigas ${ }^{3}$; C. Ferrando ${ }^{3}$

${ }^{1}$ Anaesthesiology, Hospital Clínic de Barcelona, Barcelona, Spain; ${ }^{2}$ Anaesthesiology, Hospital del Mar, Barcelona, Spain; ${ }^{3}$ Anaesthesiology, critical care unit, Hospital Clínic de Barcelona, Barcelona, Spain

Correspondence: G.S. Angeles

Intensive Care Medicine Experimental 2020, 8(2): 000605

Introduction: An atypical behavior of ARDS caused by COVID-19 has been suggested, since several patients with profound hypoxemia had close to normal respiratory system compliance (Crs). However, data confirming this assumption are scarce, and there is still controversy on the most appropriate oxygenation and ventilation strategies for these patients.

Objectives: We performed a secondary analysis of an observational study to compare clinical features, ventilatory management and clinical outcomes in COVID-19 ARDS patients on mechanical ventilation (MV) with normal and low Crs.

Methods: From March 12th to April 8th 2020, a prospective, multicenter, observational, cohort study that enrolled patients with COVID19 ARDS admitted to the ICU in 28 hospitals was performed, using a specifically designed online anonymized data acquisition system (CoVid19.ubikare.io). Inclusion criteria: > $18 \mathrm{y} / \mathrm{o}$, intubated and MV confirmed SARS-CoV-2 infection from a respiratory tract sample using PCR-based tests, and ARDS, as defined by the Berlin criteria. Recorded data: demographics, ventilator parameters, adjunctive therapies, disease chronology (initiation of MV, ventilator-free-days, ICU-length-ofstay), discharge from ICU and 28-days in-hospital mortality. Patients were stratified as having normal $\mathrm{Crs}\left(\geq 50 \mathrm{ml} / \mathrm{cmH}_{2} \mathrm{O}\right)$ or low $\mathrm{Crs}$ (< $50 \mathrm{ml} / \mathrm{cmH}_{2} \mathrm{O}$ ) according to baseline values. Descriptive variables were expressed as percentage, mean and standard deviation (SD), or median and interquartile range (IQR), as appropriate for each variable. To compare variables across groups, Student t-test or Mann-Whitney test and one-way ANOVA or Kruskal-Wallis test for numerical variables, and Chi squared test or Fisher exact test for categorical variables were used. Time to event curves were plotted using the Kaplan-Meier method and analyzed with log-rank test and univariable Cox regression analysis.

Results: A total number of 504 patients were included. From the 286 patients (56\%) with Crs data, median baseline was 35 [28-48], 216 (75.5\%) were classified as having low $\mathrm{Crs}$ and $70(24,5 \%)$ as having normal Crs. Time from symptoms onset to MV showed no differences between groups [11 (ICR: 8-15)]. There were no significant differences in $\mathrm{PaO}_{2} / \mathrm{FiO}_{2}$ between patients with normal or low $\mathrm{Crs}$ and the ventilation strategy (VT and PEEP) did not vary. Although the distribution of patients with normal or low Crs showed significant differences in driving pressure, both at baseline [9 (IQR: 6-10) vs 12 (IQR: 10-16) $\mathrm{cmH}_{2} \mathrm{O}, \mathrm{p}<0.001$ ] and at maximum values [12 (IQR: 9-15) vs 16 (IQR: 12- 18) $\left.\mathrm{cmH}_{2} \mathrm{O}, \mathrm{p}<0.001\right]$, these differences were not associated with ARDS severity. No differences were observed in the use of recruitment maneuvers and prone positioning. The probability of discontinuation from MV and the probability of ICU discharge did not increase with normal Crs. The risk of 28-day mortality was not impacted by $\mathrm{Crs}$. 
Conclusion: We found a median baseline Crs similar to non-COVID-19 ARDS patients, and $75 \%$ of patients had low Crs right after initiation of MV. No differences were found in ventilation management and outcomes.

\section{Reference(s) and grant ackowledgment(s) \\ 1. Ranieri VM, Rubenfeld GD, Thompson BT, et al; ARDS Definition Task Force. Acute respiratory distress syndrome: the Berlin Definition. JAMA. 2012;307(23):2526-2533 \\ 2. Gattinoni L. Chiumello E, Caironi P, et al. COVID-19 pneumonia: different respiratory treatment for different phenotypes? Intensive Care Medicine 2020; https://doi.org/10.1007/s0013 4-020-06033-2.}

\section{1}

Ventilating the COVID19 patient: Experience from a tertiary hospital

R. Neto'; D. Adrião'; AL. Rios'1; P. Fernandes'; P. Castelões

${ }^{1}$ Intensive care department, Centro Hospitalar de Vila Nova Gaia/ Espinho, Vila Nova de Gaia, Portugal

\section{Correspondence: R. Neto}

Intensive Care Medicine Experimental 2020, 8(2): 000621

Introduction: Corona Virus Disease 2019 (COVID-19) is a life-threatening viral infection caused by a new strand of the coronaviridae family[1], that was declared a pandemic by WHO on March 11th. The most severe form of the disease is characterized by severe hypoxemic respiratory failure with a need for ventilatory support. Despite fulfilling the Berlin ARDS Criteria, these patients have severe hypoxemia with a near-normal lung compliance, differing from traditional ARDS patients[2]. This presented a new challenge for Intensivists, who had to develop new ventilatory strategies.

Objectives: Study the ventilatory dynamics of COVID-19 patients and their impact on critical care outcomes.

Methods: Retrospective observational cohort study of all patients with a laboratory confirmation of SARS-CoV-2 infection, admitted to the Intensive Care Unit (ICU) of a tertiary Hospital in Northern Portugal, from March 15th to May 10th.

Results: During the study period, 264 COVID-19 patients were admitted to our hospital and 36 required ICU admission. Median age was 62 years $[58,25-65,75]$ and the majority were male (63.9\%). Median ICU stay was 16 days [7.0-23.5]. 86.1\% of patients required invasive mechanical ventilation, with a median duration of 17 days [8-24]. One patient required veno-arterial extracorporeal oxygenation. Mean pO2/ $\mathrm{FiO}_{2}$ ratio at admission was $174.1 \pm 67.4$. $\mathrm{pO} 2 / \mathrm{FiO}_{2}$ ratio at admission and variation during the first $48 \mathrm{~h}$ of stay were not associated with ICU length of stay ( $p=0.063$ and $p=0.820$, respectively) or duration of invasive mechanical ventilation $(p=0.103$ and $p=0.916$, respectively).

At admission, median Positive End-Expiratory Pressure (PEEP) levels were $9 \mathrm{cmH} 20$ [8-10] and patients were ventilated with an average $7.6 \pm 1.1 \mathrm{ml} / \mathrm{kg}$ of tidal volume. During the total length of ICU stay, median PEEP levels were $8.5 \mathrm{~cm} \mathrm{H} 20$ [7.7 -9.7] and patients were ventilated with $7.6 \pm 0.9 \mathrm{ml} / \mathrm{kg}$ of tidal volume. Average driving Pressure levels at admission and during ICU stay were $14.6 \pm 4.8$ and $12.8 \pm 3.9$, respectively.

Neuromuscular block perfusion was used in $64.5 \%$ of patients and $54.8 \%$ of patients required at least one period of prone position (mean duration of $3.6 \pm 2.7$ days). Due to prolonged weaning, 33.3\% of patients were tracheostomized.

During the ICU stay 9 (25\%) patients died. Non-survivors had significantly lower $\mathrm{pO} 2 / \mathrm{FiO}_{2}$ ratio (168.3 VS 176.0, $\mathrm{p}=0.001$ ), higher driving pressures (15.40 vs $11.7, p=0.018)$ and lower tidal volumes $(6.95$ vs $7.85 ; p=0.022$ ) during ICU stay. No other ventilatory variable was associated with ICU mortality. At the time of analysis, 1 patient remained in the ICU and only 9 (25\%) patients were discharged from the hospital.

Conclusion: COVID-19 patients require lengthy periods of invasive mechanical ventilation, with higher tidal volume strategies than normal ARDS patients and high rates of tracheostomy as part of the ventilatory weaning strategy. The retrospective nature and low sample size are the study main limitations.

\section{Reference(s) and grant ackowledgment(s)}

1. Gattinoni, L., et al., COVID-19 pneumonia: different respiratory treatments for different phenotypes? Intensive Care Medicine, 2020

2. Zhu, N., et al., A Novel Coronavirus from Patients with Pneumonia in China, 2019. New England Journal of Medicine, 2020. 382(8): p. 727-733.

\section{0}

Pneumoniae in patient with Covid-19: Strategies to Decrease Patient's Self-Inflicted Lung injury

JA. Benitez Lozano'; JM. Serrano Simón ${ }^{2}$

${ }^{1}$ Intensive care medicine, Hospital Quirónsalud Málaga, Málaga, Spain ${ }^{2}$ Intensive care medicine, Reina Sofia University Hospital, Córdoba, Spain

Correspondence: J.M. Serrano Simón

Intensive Care Medicine Experimental 2020, 8(2): 000640

Introduction: Spontaneous breathing in hypoxemic respiratory failure can lead to Patient self-inflicted lung injury (P-SILI) due to various factors related to intense inspiratory effort with high transpulmonary pressures $(1,2)$

Objectives: To evaluate respiratory mechanics, Effort (PTP/min), Work of breathing (WOB), and delta muscle pressure (Dpmus) in patients with COVID-19 pneumonia during mechanical ventilation (MV), with three ventilatory strategies during spontaneous ventilation, as well as in passive mode.

Methods: We have studied eleven patients with COVID-19 pneumonia mechanically ventilated during volume-controlled ventilation (CMV), pressure support ventilation (PSV) with inspiratory pressure 10/PEEP $10 \mathrm{cmH}_{2} \mathrm{O}$, airway pressure release ventilation (APRV) $20 / 0 \mathrm{cmH}_{2} \mathrm{O}$ with expiratory time for $75 \%$ peak flow expiratory (Pef) and double CPAP (CPAPd) $20 / 10 \mathrm{cmH}_{2} \mathrm{O}$. Respiratory signals: Flow, airway pressure and volume were recording from the Medibus protocol of the Evita Dräger ventilator, at $125 \mathrm{htz}$, using personal software. The three components of the equation of motion for the respiratory system: elastance (Ers), Resistances (Rrs) and total PEEP (PEEPt) were obtained in the passive mode using multiple linear regression. In support pressure, we use the inspiratory, expiratory occlusion manoeuvres for Ers, and the measurement of the time constant $(t)$, as the time required to empty the lung $2 / 3$ of its tidal volume to obtain Rrs. In this way, the signals of Pressure Muscular, alveolar and total pressure were obtained. Parameters related to the respiratory drive, effort, work of breathing, and breathing pattern were calculated in each ventilatory mode, cycle by cycle. The clinical and demographic characteristics were collected. Data were analyzed for descriptive methods and expressed as mean $\pm S D$, coefficient of variation (CV) and medians (IRQ).

Results: $\mathrm{N} 11$ cases. Sex M 7 (63\%). Age (y): $63 \pm 9.81$. Died 4. Apachell Score 18.92 (17.25-20.75). BMI $28.58 \pm 2.43$. MV, days 12 (5.25-21.7). $\mathrm{P} / \mathrm{F} 194.8 \pm 49.64 \mathrm{mmHg} ; \mathrm{FiO}_{2} 0.48 \pm 0.08 \mathrm{mmHg}$. PCO2 $53.09 \pm 15.59$ mmHg. ECMO-vv 1.

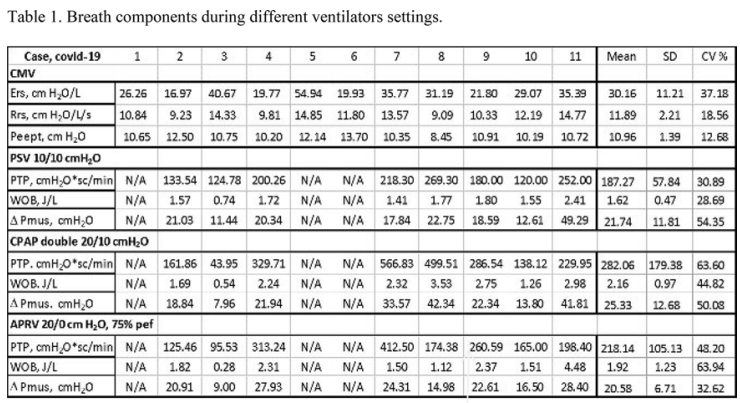

Conclusion: We have found an elevated respiratory driver in almost all patients COVID-19, associated with high inspiratory effort, although with wide dispersion. That is not controlled with any spontaneous 
mode tested that resemble VNI; and this could be a cause of potential P-SILI.

\section{Reference(s) and grant ackowledgment(s)}

1. 1) Grieco DL, Menga LS, Eleuteri D, Antonelli M. Minerva Anestesiologica 2019 September;85(9):1014-23.

2. 2) Brochard L. Slutsky A. Pesenti A. Mechanical Ventilation to Minimize Progression of Lung Injury in Acute Respiratory Failure. Am J Respir Crit Care Med 2017;195:438-42

\section{1}

Low-PEEP strategy effect on mortality and ICU stay in SARS-COV-2 patients

G. Luvini'; M. Biggiogero' P. Maida'; R. Mauri'; C. Garzoni'; S. Ceruti ${ }^{3}$

${ }^{1}$ Internal medicine, Clinica Luganese Moncucco, Lugano, Switzerland;

${ }^{2}$ Clinical research unit, Clinica Luganese Moncucco, Lugano, Switzerland; ${ }^{3}$ Critical care department, Clinica Luganese Moncucco, Lugano, Switzerland; ${ }^{4}$ Internal medicine department, Clinica Luganese Moncucco, Lugano, Switzerland

Correspondence: S. Ceruti

Intensive Care Medicine Experimental 2020, 8(2): 000691

Introduction: COVID-19 is a pandemic associated with acute respiratory failure [1]. Management of critically ill COVID-19 patients with severe hypoxemia is associated with high mortality [2].

Objectives: To reduce ICU length-of-stay (LOS), mechanical ventilation (MV) and mortality implementing a standardized "care map".

Methods: A retrospective analysis was conducted on consecutive COVID-19 patients admitted in ICU for severe pneumonia in our Center in Lugano. A standardized multidisciplinary approach was implemented to improve patients monitoring using a) uniform patient selection for ICU admission, b) a low-PEEP strategy and c) an enlarged pharmacologic venous thromboembolism (VTE) management.

1. Dyspnea, mental confusion or SpO2 less than $85 \%$ were criteria for ICU admission.

2. Ventilation approach employed low PEEP values (about $10 \mathrm{cmH}_{2} \mathrm{O}$ in presence of lung compliance $>40 \mathrm{~mL} / \mathrm{cmH}_{2} \mathrm{O}$ ) and $\mathrm{FiO}_{2}$ as needed. In presence of lower lung compliance ( $<40 \mathrm{~mL} / \mathrm{cmH}_{2} \mathrm{O}$ ) PEEP value was increased to about $14 \mathrm{cmH}_{2} \mathrm{O}$.

3. Enlarged prophylactic anticoagulation (Enoxaparine $60 \mathrm{mg}$ bid SC if weight $>80 \mathrm{Kg}$, Enoxaparine $40 \mathrm{mg}$ bid SC if weight $<80 \mathrm{Kg}$, Unfractioned Heparin in case of AKI) with daily ultrasound Color-Doppler lower limbs and plasmatic D-dimer level monitoring was performed; in case of suspected or confirmed thromboembolism, treatment was switched to therapeutic anticoagulation (Enoxaparine $1 \mathrm{mg} / \mathrm{Kg}$ bid SC - Unfractioned Heparin in case of AKl at $14 \mathrm{UI} / \mathrm{Kg} /$ day in perfusion, adapted according to anti-Xa values).

Results: From March 16th to April 12th 2020, 48 patients were presented to be evaluated for ICU admission. Twenty-two of them were not admitted, they have been carefully monitored and presented a $100 \%$ of overall survival at 30 days.

Forty-one patients were admitted to ICU: 26 from the Internal Medicine Department, 10 from Emergency Department and 5 transferred from other hospitals. Mean SAPS was 45 (SD 18.4), mean NEMS was 30 (SD 10.4). Thirty-four (82.9\%) patients needed MV; low PEEP values based on BMI (PEEP $11 \pm 3.8(10-12) \mathrm{cmH}_{2} \mathrm{O}$ if $\mathrm{BMl}<30 \mathrm{~kg} / \mathrm{m}^{2}$; PEEP $15 \pm 3.26(12-18) \mathrm{cmH}_{2} \mathrm{O}$ if $\left.\mathrm{BMI}>30 \mathrm{~kg} / \mathrm{m}^{2}\right)$ was employed. Six patients (14.6\%) presented major VTE phenomena. According to VTE prophylaxis strategy, $15(36.5 \%)$ patients were treated through prophylaxis, $26(63.4 \%)$ were managed by full therapeutic anticoagulation. No patient presented bleeding complication, nor clinical sign requiring anticoagulation removal.

Median duration of MV was 7 days; the median LOS was 9 days. After 4 weeks, 25 patients $(61 \%)$ were discharged from ICU, $4(9.7 \%)$ were still under MV, 2 were transferred to another hospital; ICU mortality rate was $24.3 \%$. P/F ratio had daily improvement during first 72 hours $(p<$ $0.0001)$.

Conclusion: Our multidisciplinary program allowed to reduce MV days, ICU LOS and mortality in COVID-19 critical care patients; a standardized "multimodal approach" could be the base for further specific patients' management in this emergency clinical scenario.
Reference(s) and grant ackowledgment(s)

1. Bouadma I, Lescure FX, Lucet JC, Yazdanpanah Y, Timsit JF. Severe SARSCoV-2 infections: practical considerations and management strategy for intensivists. Intensive Care Med 2020;46(4):579-82

2. Center for Systems Science and Engineering. Coronavirus COVID-19 global cases. 2019 (https://gisanddata.maps.arcgis.com/apps/opsdashboard/index /html\#/bda7594740fd40299423467b48e9ecf6).

3. No funding has beenrequired.

\section{9}

Predictive models of postextubation respiratory failure and reintubation

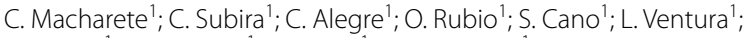

I. Catalan ${ }^{1}$; G. Rognoni ${ }^{1}$; M. Batlle ${ }^{1}$; R. Fernandez ${ }^{1}$

${ }^{1}$ Intensive care unit, Althaia, Xarxa Assistencial Universitària de Manresa, Manresa, Spain

Correspondence: C. Macharete

Intensive Care Medicine Experimental 2020, 8(2): 000759

Introduction: Postextubation respiratory failure is associated with bad prognosis, leading to reintubation within 72 hours in up to $50 \%$ of cases. A model to identify which patients are at high risk of failure can be useful to improve outcome.

Objectives: To design a model to identify those patients with high risk of postextubation failure

Methods: Post-hoc analysis of a randomized clinical trial (reference). Variables associated with postextubation respiratory failure and reintubation were tested by multiple logistic analyses. Then, using logit methodology, we designed models to predict postextubation respiratory failure and reintubation. We calculated the area under the curve (AUC) for each model.

Results: We analyzed 950 patients extubated after a successful SBT, 199 (21\%) of them developed respiratory failure, and 104 (11\%) needed reintubation. The variables related with postextubation respiratory failure were respiratory rate (RR) $>25 x^{\prime}$ at the end of SBT (OR $1.73, \mathrm{p}=0.02$ ), duration of MV $>4$ days (OR 1.75, $\mathrm{p}=0.001$ ), SatO2/ $\mathrm{FiO}_{2}<240$ (OR 1.89, $\mathrm{p}=0.002$ ), and neoplastic disease (OR 1.62, $\mathrm{p}=$ 0.02 ). Reintubation was only associated with duration of $M V>4$ days (OR 1.86, $p=0.005$ ) and $R R>25$ at the end of SBT (OR 1.99, $p=0.002$ ). The AUCs were low in both models $(0.63$ for respiratory failure and 0.60 for reintubation).

The model classified patients in 3 groups of risk for respiratory failure. The actual rate of respiratory failure in patients classified as low risk was $13 \%, 22 \%$ at middle risk and $34 \%$ at high risk of failure patients.

The model also classified patients in 3 groups of risk for reintubation. The reintubation rate was $7 \%$ in patients in low risk, $14 \%$ in patients in middle risk, and $24 \%$ in patients in high risk of reintubation.

Conclusion: $\mathrm{RR}>25 \mathrm{bpm}$, length of $\mathrm{MV}>4$ days, SatO2/FiO $2<240$ and neoplastic disease were related with higher postextubation respiratory failure. Only the length of $M V>4$ days and $R R>25$ were related with reintubation. Despite low AUC, both models for respiratory failure and reintubation identified 3 groups of patients with very different risks.

Reference(s) and grant ackowledgment(s)

1. Subirà C, Hernández G, Vázquez A, et al. Effect of Pressure Support vs T-Piece Ventilation Strategies During Spontaneous Breathing Trials on Successful Extubation Among Patients Receiving Mechanical Ventilation: A Randomized Clinical Trial JAMA. 2019;321(22):2175-2182

\section{3}

Factors associated to mortality in COVID 19 patients on mechanical ventilation

C. Serrano1; A. Ruiz García'; J. Higuera Lucas'; J. Tato²; C. Vaduva²;

J. Arteaga²; E. Nevado ${ }^{1}$

${ }^{1}$ Intensive care unit, Hospital Príncipe de Asturias, Alcalá de Henares, Spain; ${ }^{2}$ Otorhinolaryngology, Hospital Príncipe de Asturias, Alcalá de Henares, Spain

Correspondence: $C$. Serrano

Intensive Care Medicine Experimental 2020, 8(2): 000773 
Introduction: Respiratory failure requiring mechanical ventilation due to COVID 19 pneumonia has been the principal cause of admission to Intensive Care during the months of March, April, May and June in Europe.

Objectives: We present a sample with the factors that we have associated with mortality in patients on mechanical ventilation.

Methods: A retrospective, observational study was performed with all the patients admitted to the Intensive Medicine Service at a University Hospital with the diagnosis of Community-acquired pneumonia due to COVID 19.

Variables analysed age, sex, APACHE II, mechanical ventilation, length of stay, laboratory values, prone, neuromuscular blocking drugs, tracheostomy and mortality during their stay in intensive care.

Results: 90 patients were analysed. 40\% (36/90) have been discharged. The sample has a mortality rate of $34.4 \%$ (31/90) and $25.1 \%$ (23/90) have been transferred.

Excluding the transferred patients, analysing the 67 patients who received complete treatment in our hospital: Length of stay $14.1 \pm 10$, average age 60 years, APACHE II $14.3 \pm 5.2$. 67.2\% males. Average worst creatinine result $1.48 \pm 1.26 \mathrm{mg} / \mathrm{dl}$, Average worst ferritin value $2310 \pm 3322 \mathrm{mg} / \mathrm{l}$, Mean value of worst total bilirubin value $2 \pm 2.2$ $\mathrm{mg} / \mathrm{dl}$. $100 \%$ of the patients required mechanical ventilation. $65.7 \%$ prone position, $74.6 \%$ neuromuscular blockers and $98.5 \%$ vasoactive drugs. Tracheostomy was performed in $45.3 \%$ of the patients. The table shows the values in the group of survivors and non-survivors.

\begin{tabular}{|l|l|l|l|}
\hline & Survivors & Non survivors & P \\
\hline Age & 56,9 & 63,4 & 0,006 \\
\hline Sex (Male) & $55 \%$ & $80 \%$ & 0,026 \\
\hline APACHE II & 12,6 & 16 & 0,03 \\
\hline Length of stay & 16,5 & 11,5 & 0,042 \\
\hline Creatinine Max. & 1 & 1,96 & 0,003 \\
\hline Bilirrubine Max. & 1,71 & 2,4 & 0,2 \\
\hline Ferritine Max. & 1836 & 2990 & 0,2 \\
\hline D Dimer & 17,2 & 19,9 & 0,67 \\
\hline CV desease & $38,8 \%$ & $51 \%$ & 0,21 \\
\hline Pulmonar desease & $19,4 \%$ & $19,3 \%$ & 0,6 \\
\hline Dyslipidemia & $27,7 \%$ & $48 \%$ & 0,069 \\
\hline Traqueostomy & $51 \%$ & $37,4 \%$ & 0,2 \\
\hline Prone & $52,7 \%$ & $80 \%$ & 0,015 \\
\hline $\begin{array}{l}\text { Neuromuscular } \\
\text { Bloq. }\end{array}$ & $58 \%$ & $93 \%$ & 0,001 \\
\hline
\end{tabular}

Conclusion: In our sample the need of prone position, neuromuscular blocking drugs in relation to distress severity have been related to increased mortality. Furthermore, acute renal failure, age and sex (male) are statistically significantly related factors.

\section{6}

Evaluation of a 6-hour trial of inverse-ratio Airway Pressure Release Ventilation (APRV) in Covid-19 pneumonia

B. Pequignot' ; M. Koszutski'; P. Guerci²; B. Levy ${ }^{1}$

${ }^{1}$ Service de Medecine Intensive et Réanimation Brabois, CHRU de NancyHôpitaux de Brabois, Vandœuvre-lès-Nancy, France; ${ }^{2}$ Department of anesthesiology and critical care medicine, surgical icu, university hospital of na, CHRU Nancy, Nancy, France

Correspondence: $B$. Pequignot

Intensive Care Medicine Experimental 2020, 8(2): 000806

Introduction: Covid-19 pneumonia can lead to a severe respiratory failure requiring invasive ventilation. Inverse-ratio APRV is a ventilation mode in which Tidal Volume (VT) is delivered during a short decrease in pressure. The expiration phase is interrupted before the expiratory flow reaches zero (1). This refers to the concept of Time Controlled Adaptive Ventilation (TCAV) (2).

Inverse-ratio Airway Pressure Release Ventilation (APRV) was proposed as a ventilation mode for ARDS in a monocentric randomized study showing a reduction in ventilation time in the APRV group (3). We report our experience of switching patients from conventional ventilation (volume control or pressure support) to APRV in two French ICUs during the Covid-19 pandemic.

Methods: Seventeen consecutive patients who have completed a 6-hour APRV trial in April 2020 were studied (we excluded 3 patients on VV-ECMO and 3 who were unable to be maintained on APRV). Initial APRV settings were: P-high $28 \mathrm{cmH} 20$, T-high $3.5 \mathrm{sec}-$ onds, P-low $5 \mathrm{cmH}_{2} \mathrm{O}$ and T-low 0.5 seconds. T-low was then immediately adjusted to obtain a sufficient tidal volume and the expiratory flow curve was observed to ensure an interruption of the release phase at around 50 to $75 \%$ of the peak expiratory flow. P-low was seldom set to $0 \mathrm{cmH}_{2} \mathrm{O}$ due to concerns of alveolar pressure reaching the set P-low (in case of inappropriately long T-low) and was frequently set to $5 \mathrm{cmH}_{2} \mathrm{O}$ as it was by Zhou et al (3). Within the first hour of APRV, blood gases were measured to detect an eventual increase in $\mathrm{PaCO} 2$ and ifnecessary, increase the respiratory rate (by decreasing T-high).

Results: The mean $\left( \pm\right.$ SD) $\mathrm{PaO}_{2}: \mathrm{FiO}_{2}$ ratio increased from $126( \pm 28)$ to $178( \pm 53)$ after 6 hours of APRV $(p<0.001)$. In $12 / 17$ patients, the PF ratio increase was of more than $20 \%$. Two patients presented a decrease of the $\mathrm{PaO}_{2}: \mathrm{FiO}_{2}$ ratio after 6 hours of APRV. Median (interquartile range) ventilation time before the APRV trial was of 14 (8-19) days. At baseline, 12 of the 17 patients were on Volume Assist Control and 5 patients were on Pressure Support. After 6 hours of APRV, the mean Tidal Volume was of $6.5( \pm 1.7) \mathrm{ml}$. There was no significant hemodynamic impairment appearing during APRV and an eventual increase in $\mathrm{PaCO} 2$ during the first hour of APRV was managed by increasing respiratory rate (i.e. shortening Thigh) and/or increasing tidal volume (i.e. increasing T-low).

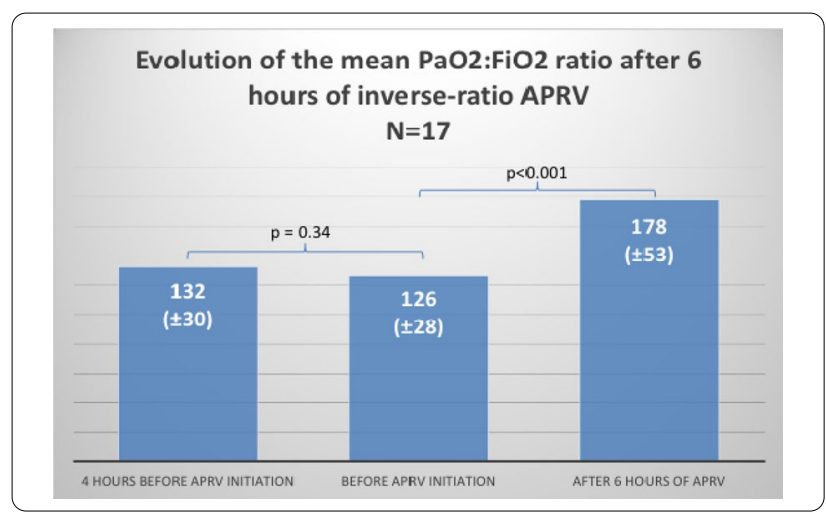

Conclusion: To conclude, in our severe ARDS covid19 patients, the use of inverse ratio APRV was well tolerated and associated with a marked improvement in oxygenation parameters. This could be explained by the high recruitability potential in some cases of Covid-19 associated $\operatorname{ARDS}(4,5)$.

\section{Reference(s) and grant ackowledgment(s)}

1. (1) Kollisch-Singule M, Satalin J, Blair SJ, Andrews PL, Gatto LA, Nieman GF, et al. Mechanical Ventilation Lessons Learned From Alveolar Micromechanics. Front Physiol. 24 mars 2020;11:233

2. (2) Jain SV, Kollisch-Singule M, Sadowitz B, Dombert L, Satalin J, Andrews P, et al. The 30-year evolution of airway pressure release ventilation (APRV). ICMx. déc 2016:4(1):11.

3. (3) Zhou Y, Jin X, LvY, Wang P, Yang Y, Liang G, et al. Early application of airway pressure release ventilation may reduce the duration of mechanical ventilation in acute respiratory distress syndrome. Intensive Care Med. nov 2017;43(11):1648-59.

4. (4) Gattinoni L, Chiumello D, Caironi P, Busana M, Romitti F, Brazzi L, et al. COVID-19 pneumonia: different respiratory treatments for different phenotypes? Intensive Care Med. 14 avr 2020;1-4.

5. (5) Beloncle FM, Pavlovsky B, Desprez C, Fage N, Olivier P-Y, Asfar P, et al. Recruitability and effect of PEEP in SARS-Cov-2-associated acute respiratory distress syndrome. Ann Intensive Care. 12 mai 2020;10(1):55. 


\section{8}

Automatic Tube Compensation for spontaneous breathing trial-A systematic review and a network meta-analysis

P. Cardinal-Fernández'; J. Bougnaud ${ }^{2}$; L. Argaud²; M. Cour²; C. Guérin²

${ }^{1}$ Intensive care unit, HM University Sanchinarro Hospital, Madrid, Spain;

${ }^{2}$ Intensive réanimation, groupement hospitalier centre, Hôpital Edouard Herriot, Lyon, Université de Lyon, Lyon, France

Correspondence: $\mathrm{P}$. Cardinal-Fernández

Intensive Care Medicine Experimental 2020, 8(2): 000818

Introduction: Spontaneous Breathing Trial (SBT) is an established test for evaluating the capability of the patient to breathe without ventilator assistance before a scheduled extubation. Among the different techniques used to perform SBT, Automatic Tube Compensation (ATC) calculates the pressure drop across the endotracheal tube and provides with a breathing assistance that counteracts it. In that way ATC acts as a within-cycle closed loop.

Objectives: To conduct a system review (SR) with a network metaanalysis (NMA) that compares the effect of ATC with other techniques for SBT in terms of test tolerance and extubation success.

Methods: This NMA of published studies was conducted following the Preferred Reporting Items for Systematic Reviews and Meta-Analyses (PRISMA) guidelines. Studies were identified using MEDLINE, Web of Science, Cochrane Register of Controlled Trials, LILACS and citation review. The inclusion criteria used the PICO approach. Patients are adults, intubated in the ICU; Intervention is ATC; Control is either the patient (before/after) or another non-ATC intervention; Outcome is either physiologic or patient-centered depending on the category the study falls into. Two authors, independently and blinded each to otherselected studies for inclusion (JB and PCF). Were excluded animal studies, other languages than English, Spanish or French, not in full text format (eg. abstract or letter were excluded) and reviews.

Data analysis. Publication bias was analyzed with a funnel plot for the endpoint extubation success. Relative risk (RR) with random-effects models were used to pool the effect of each SBT technique. Statistical significance was set at $\mathrm{P}<0.05$ with a two-tailed test. Data were analyzed using "netmeta" library from R package.

Results: Four of the 108 studies included the ATC as a SBT representing 769 patients and 4 interventions (ATC100\% + Continuous Positive Airway Pressure (CPAP) $<7.5 \mathrm{cmH}_{2} \mathrm{O}[\mathrm{n}=319], \mathrm{CPAP}<7.5 \mathrm{cmH}_{2} \mathrm{O}[\mathrm{n}=120]$, Pressure Support (PS) $5 \mathrm{cmH}_{2} \mathrm{O}+\mathrm{CPAP}<7.5 \mathrm{cmH} 20[\mathrm{n}=30]$ and T-tube [ $n=300$ ]. In terms of successful SBT, the probability of being the best (in decremental order) were: ATC100\% + CPAP $<7.5 \mathrm{cmH}_{2} \mathrm{O}$ (reference group), $\mathrm{CPAP}<7.5 \mathrm{cmH}_{2} \mathrm{O}, \mathrm{PS} 5 \mathrm{cmH}_{2} \mathrm{O}+\mathrm{CPAP}<7.5 \mathrm{cmH} 20$ and T-tube. For extubation success the decremental order from the best to the worst was: ATC $100 \%+$ CPAP $<7.5 \mathrm{cmH}_{2} \mathrm{O}$, PS $5 \mathrm{cmH}_{2} \mathrm{O}+$ CPAP $<7.5 \mathrm{cmH} 20$, T-tube and CPAP $<7.5 \mathrm{cmH} 20$. No publication bias was identified. Conclusion: ATC $100 \%$ associated to CPAP $<7.5 \mathrm{cmH}_{2} \mathrm{O}$ may be the best technique in terms of SBT and extubation success.

\section{Reference(s) and grant ackowledgment(s)}

1. Low-pressure Support vs Automatic Tube Compensation During Spontaneous Breathing Trial for Weaning. Guérin C, Terzi N, Mezidi M, Baboi L, Chebib N, Yonis H, Argaud L, Heunks L, Louis B. Ann Intensive Care. 2019 Dec 13:9(1):137

2. What have we learned from network meta-analyses applied to critical care? Baez-Pravia OV, Montes-Andujar L, Menéndez J, Cardinal-Fernández P. Minerva Anestesiol. 2019 Apr;85(4):433-442. https://doi.org/10.23736/S0375 $-9393.19 .13267-$

\section{7}

Association of peak inspiratory muscle pressure and work of breathing estimated by proportional assist ventilation during spontaneous breathing trial with duration of mechanical ventilation in patients who failed SBT: An interim report A. Ishizuka ; Y. Norisue

${ }^{1}$ Intensive care medicine, Tokyo Bay Urayasu Ichikawa Medical Center, Urayasu, Japan

Correspondence: A. Ishizuka

Intensive Care Medicine Experimental 2020, 8(2): 000927
Introduction: Ventilator-induced diaphragmatic dysfunction is known to be one of the main causes of prolonged mechanical ventilation. We hypothesized that patients who failed spontaneous breathing (SBT) due to respiratory muscle weakness are more likely to need longer duration of mechanical ventilator support than patients who failed SBT not because of inspiratory muscle weakness but because of abnormal respiratory mechanics which potentially improves rapidly. Proportional assist ventilation with load adjustable gain factors $(\mathrm{PAV}+)$ is the ventilator mode that calculates the pressure generated by patient (Pmus) and patient's work of breathing (WOBpt) during spontaneous breathing.

Objectives: We investigated whether Pmus and WOBpt estimated by $\mathrm{PAV}+$ during SBT can predict duration of mechanical ventilation in patients who failed SBT.

Methods: This study was a prospective observational study in a single center in Japan.All patients over 18 years old who were mechanically ventilated and failed SBT using a mode of PAV+ with a support rate $20 \%$ were included. We recorded the average of WOBpt, peak inspiratory pressure (Ppeak), compliance and resistance measured by $\mathrm{PAV}+$ during SBT. We also calculated the peak Pmus from Ppeak. The primary outcome of this study was duration of mechanical ventilation. We defined prolonged mechanical ventilation group as patients who failed at least three weaning attempts or require $>7$ days of weaning after the first SBT.

Results: A total of 20 mechanically ventilated patients who failed SBT were included. All patients weaned from mechanical ventilator eventually. 3 patients were categorized into prolonged mechanical ventilation group. The medians of WOB, Pmus, compliance and resistance measured by PAV+ were $0.600(0.580-0.780), 24.00(20.60-24.60)$, $48.00(41.00-56.00), 5.900(5.000-8.200)$ in prolonged mechanical ventilation group and $0.700(0.560-0.800), 21.60(11.72-25.60), 50.00(35.00-$ $64.00), 5.900(5.000-8.200)$ in non-prolonged mechanical ventilation group respectively without significant differences in all variables.

Conclusion: There are no significant differences in Peak Pmus and WOBpt estimated by PAV+ during failed SBT between prolonged mechanical ventilation group and non-mechanical ventilation group in this interim analysis with the small sample size. Larger sample size is needed to further investigate our hypothesis.

\section{7}

Linear approach of respiratory mechanics during VCMV and PCMV modes of mechanical ventilation

N. Karampela'; C. Bostantzoglou'; G. Marinakis ${ }^{1}$; T. Tsoutsouras ${ }^{1}$;

M. Patrani'; M. Paraschos'; M. Vassiliou

${ }^{1} \mathrm{Icu}$, Korgialeneio-Mpenakeio Red Cross Hospital, Athens, Greece

Correspondence: M. Paraschos

Intensive Care Medicine Experimental 2020, 8(2): 001087

Introduction: Volume control (VCMV) and Pressure control mechanical ventilation (PCMV) are the main modes of respiratory mechanical support. Linear regression analysis (LRA) and Fourier analysis (FA) are methods used for the linear evaluation of Respiratory system (RS) mechanics, independently of the mode of ventilation and without intervening with the ventilators settings.

Objectives: The present study attempts a comparison of RS mechanics on linear basis between two different modes of mechanical ventilation (MV): VCMV and PCMV.

Methods: The Airway Pressure (Paw) and Flow (V') signals were digitally acquired from 25 intubated and mechanically ventilated patients in the Red Cross Hospital ICU. Data were recorded under VCMV and PCMV with stable ventilatory settings (frequency, VT and PEEP). The off-line analysis, included the tidal volume (VT) calculation by numerical integration of $\mathrm{V}^{\prime}$ and the application of LRA and FA with the aid of a specifically developed software. The calculated mechanical parameters were: RS Elastance (Ers), RS Resistance (Rrs), End Expiratory Pressure (EEP). The Error of the applied models (RMSD) according to both techniques were also evaluated. Comparisons for every index between the four applied instances were evaluated with the aid of Analysis of Variance $(p=0.05)$. 
Results: All calculated indices are presented in the following table as mean values \pm standard deviations.

\begin{tabular}{|c|c|c|c|c|c|c|c|c|}
\hline Ventilation & $\begin{array}{l}\text { Ers } \\
\text { (LRA) }\end{array}$ & $\begin{array}{l}\text { Ers } \\
\text { (FA) }\end{array}$ & $\begin{array}{l}\text { Rrs } \\
\text { (LRA) }\end{array}$ & $\begin{array}{l}\text { Rrs } \\
\text { (FA) }\end{array}$ & EEP & EEP & RMSD & RMSD \\
\hline Mode & $(\mathrm{hPa} / \mathrm{L})$ & $(\mathrm{hPa} / \mathrm{L})$ & $(\mathrm{hPa} / 1 / \mathrm{s}$ & $(\mathrm{hPa} / 1 / \mathrm{s})$ & (hPa) & $(\mathrm{hPa})$ & $(\mathrm{hPa})$ & \\
\hline CMV & $\begin{array}{l}31.6 \pm \\
13.68\end{array}$ & $\begin{array}{l}31.4 \pm \\
14.16\end{array}$ & $\begin{array}{l}12.9 \pm \\
4.67\end{array}$ & $\begin{array}{l}15.4 \pm \\
7.00\end{array}$ & $6.4 \pm$ & $6.4 \pm$ & $\begin{array}{l}1.25 \pm \\
0.97\end{array}$ & $\begin{array}{l}1.64 \pm \\
1.63\end{array}$ \\
\hline C) & $\begin{array}{l}31.4 \pm \\
12.82\end{array}$ & $\begin{array}{l}31.7 \pm \\
14.16\end{array}$ & $\begin{array}{l}13.0 \pm \\
4.04\end{array}$ & $\begin{array}{l}15.0 \pm \\
5.89\end{array}$ & $\begin{array}{l}6.4 \pm \\
3.78\end{array}$ & $6.2 \pm$ & $\begin{array}{l}1.22 \pm \\
0.95\end{array}$ & $\begin{array}{l}1.56 \pm \\
1.53\end{array}$ \\
\hline
\end{tabular}

Applied comparisons revealed not significant differences for every mechanical index in all studied cases $(p>0.05)$. Nevertheless, FA showed a tendency for higher Rrs values especially in patients with underlying obstructive disorder, which is in accordance with previous findings of ours. The same issue is true for the Error of the applied linear modeling.

Conclusion: Respiratory mechanics are equivalently evaluated with the aid of two different linear methods (LRA and FA) under two different modes of MV (VCMV and PCMV). Our results reinforce the applicability of both calculating techniques and also suggest that both modes of MV have almost identical mechanical effect on respiration. Further research in homogenous conditions concerning the underlying respiratory disorder (COPD, ALI, ARDS e.t.c.) would further clarify the issue.

\section{Reference(s) and grant ackowledgment(s)}

1. 1. Chacko B., Peter J.V., Tharyan P., John G. \& Jeyaseelan L. and Cochrane Emergency and Critical Care Group. Pressure-controlled versus volumecontrolled ventilation for acute respiratory failure due to acute lung injury (ALI) or acute respiratory distress syndrome (ARDS) Cochrane Database of Systematic Reviews, Jan; 2015(1): CD008807

2. 2. M.P.Vassiliou, L.Petri, A.Amygdalou, M.Patrani, C.H.Psarakis, D.Nikolaki, G.Georgiadis, P.K.Behrakis. Linear and Nonlinear analysis of pressure and flow during mechanical ventilation. Intensive Care Medicine 26,1057-1064 (2000)

3. 3. A.Amygdalou, C.Psarakis, P.Vassiliou, Y.A.Dalavanga, C.Mandragos, S.H. Constantopoulos, P.K.Behrakis, M.P.Vassiliou. Evaluation of the end-expiratory pressure by multiple linear regression and Fourier analysis in humans. Respiratory Medicine, 96(7), 499-505 (2002)

\section{2}

Magnetic fields generated by a magnetic coil used for stimulation of the phrenic nerve for diaphragm stimulation - an ICU simulation study

KF. Kuhn'; J. Grunow'; M. Lorenz'; JC. Schefold²; D. Berger²;

S. Weber-Carstens' ${ }^{1}$ ' S. Schaller ${ }^{1}$

${ }^{1}$ Department of anaesthesiology and operative intensive care medicine, Charité - Universitätsmedizin Berlin, Berlin, Germany; ${ }^{2}$ Department of intensive care medicine, Inselspital, Bern University Hospital, University of Bern, Bern, Switzerland

Correspondence: K.F. Kuhn

Intensive Care Medicine Experimental 2020, 8(2): 000262

Introduction: Approximately $60 \%$ of ventilated patients are affected by weakness of the diaphragm at the time of the first spontaneous breathing trial [1, 2]. A possible non-invasive intervention to preserve diaphragmatic function is magnetic stimulation of the phrenic nerve [3]. Since safety instructions state a minimum distance to magnetically sensitive objects of at least $1 \mathrm{~m}$ and prohibit the use near life-sustaining equipment, it is currently unknown if such stimulation would be safe in an intensive care unit (ICU) environment.

Objectives: This investigation aimed to (1) measure distances between patients and life-sustaining medical equipment in a typical ICU surrounding and (2) measure the magnetic fields generated by stimulation coils (located at the patient's neck) at the patient and surrounding medical equipment with regard to safety in a pilot study.
Methods: A typical ICU patient room was simulated and equipped at the Berliner Simulations- und Trainingszentrum at Charité - Universitätsmedizin Berlin (full data set) and at the Inselspital Bern (distances to devices measured only). A resuscitation manikin was used to simulate the patient position in bed. Stimulations were performed using the PowerMag Research 100 Stimulator $^{\circledR}$ (MAG \& More GmbH, Munich, Germany) with a butterfly coil (PMD70-pCool) generating a single, biphasic stimulus at maximum intensity. Magnetic field intensity $B$ in Tesla [T] was measured at various distances vertical to the center of the coil (table 1) and raw data analyzed. Furthermore, possible heating of the metal bed rails and ECG electrodes was investigated using $85 \%$ intensity, a frequency of $20 \mathrm{Hertz}[\mathrm{Hz}]$ and 2-3 sequences with a length of 5-10 $\mathrm{s}$ and $0 \mathrm{~mm}$ safety distance.

Table 1. Measurements of the magnetic field intensity at different distances with a single
stimulus at 100\% power. Direction means position of the sensor to the stimulation coil.
\begin{tabular}{|l|l|l|l|l|}
\hline$\#$ & Power [\%] & Stimulus & Distance [mm] & Direction \\
\hline 1 & 100 & Single & 0 & $\begin{array}{l}\text { center of the stimulation } \\
\text { coil }\end{array}$ \\
\hline 2 & 100 & Single & 20 & $\begin{array}{l}\text { vertical to center of the } \\
\text { stimulation coil }\end{array}$ \\
\hline 3 & 100 & Single & 30 & $\begin{array}{l}\text { vertical to center of the } \\
\text { stimulation coil }\end{array}$ \\
\hline 4 & 100 & Single & 70 & $\begin{array}{l}\text { vertical to center of the } \\
\text { stimulation coil }\end{array}$ \\
\hline 5 & 100 & Single & 300 & $\begin{array}{l}\text { vertical to center of the } \\
\text { stimulation coil }\end{array}$ \\
\hline 6 & 100 & Single & 400 & $\begin{array}{l}\text { vertical to center of the } \\
\text { stimulation coil }\end{array}$ \\
\hline 7 & 100 & Single & 150 & $\begin{array}{l}\text { horizontal to stimulation } \\
\text { coil }\end{array}$ \\
\hline
\end{tabular}

Results: Distances were $1.2 \mathrm{~m}$ and $0.8 \mathrm{~m}$ to the ventilator, $1 \mathrm{~m}$ and 1 $\mathrm{m}$ to the perfusors and $0.7 \mathrm{~m}$ and $1 \mathrm{~m}$ to the touch panel or vital sign monitor in Berlin and Bern, respectively.

The magnetic field intensity declined with rising distance to the coil (figure 1). It illustrates the results of our measurements at different distances tothe coil during stimulation. At $40 \mathrm{~cm}$ distance, only general signal noise could be observed.

The heating test resulted in heating of the bed rail to $70^{\circ} \mathrm{C}$ and a safety stop of the coil at the temperature of $40^{\circ} \mathrm{C}$. The ECG electrodes did not heat up. The ECG signal at the monitor showed artefacts during stimulation as known from surgical equipment. Direct stimulation of the perfusor did not lead to any obvious malfunction.

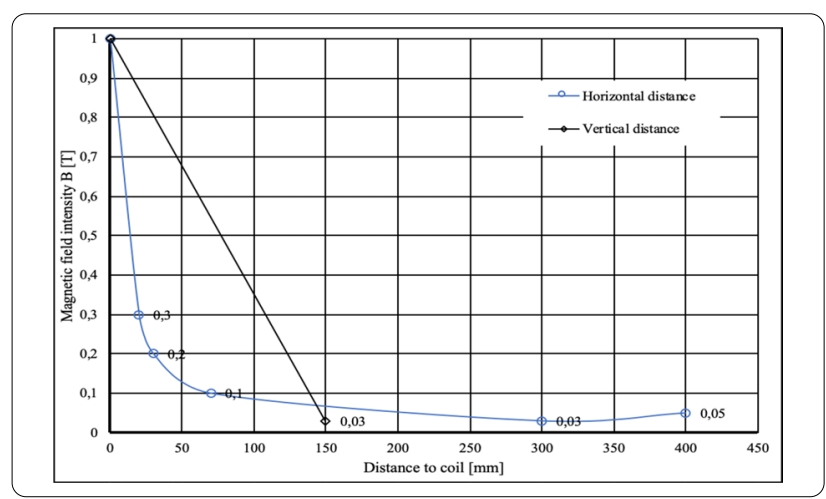

Magnetic field intensity was measured at six points vertical to the center of the stimulation coil and once horizontal to the center of the stimulation coil. Stimulation was done with $100 \%$ power. Magnetic field intensity $B$ is measured in Tesla [T]. 
Conclusion: In standard ICU environment, the distance of magnetically-sensitive objects to the coil can be lower than $1 \mathrm{~m}$. The magnetic fields in this experiment declined with distance from the stimulator. The measurements are providing relevant information for the riskbenefit assessment of magnetic coils to stimulate the phrenic nerve in an ICU environment. With only $40 \%$ intensity necessary to generate sufficient tidal volume [3], field intensity should be even lower in clinical practice.

\section{Reference(s) and grant ackowledgment(s)}

1. 3. Sander BH, DieckT, Homrighausen F, Tschan CA, Steffens J, Raymondos K: Electromagnetic ventilation: first evaluation of a new method for artificial ventilation in humans. Muscle \& nerve 2010, 42(3):305-310.

2. 2. Berger D, Bloechlinger S, von Haehling S, Doehner W, Takala J, Z'Graggen WJ, Schefold JC: Dysfunction of respiratory muscles in critically ill patients on the intensive care unit. Journal of cachexia, sarcopenia and muscle 2016, 7(4):403-412.

3. 1. Dres M, Dubé B-P, Mayaux J, Delemazure J, Reuter D, Brochard L, Similowski T, Demoule A: Coexistence and impact of limb muscle and diaphragm weakness at time of liberation from mechanical ventilation in medical intensive care unit patients. American journal of respiratory and critical care medicine 2017, 195(1):57-66

4. Charité - Universitätsmedizin Berlin received a grant from STIMIT AG, Nidau, Switzerland to support this investigation. STIMIT AG was allowed to give feedback to the study design, and be present at the data collection. STIMIT AG had no role in analysis, decision to publish, or preparation of the manuscript.

\section{9}

Individualization of tidal volume based on lung compliance in flow controlled ventilation - a step towards personalized medicine in artificial ventilation

P. Spraider ${ }^{1}$; J. Abram ${ }^{1}$; G. Putzer ${ }^{1}$; J. Martini

${ }^{1}$ Department of anaesthesiology and intensive care medicine, Medizinische Universität Innsbruck, Innsbruck, Austria

Correspondence: P. Spraider

Intensive Care Medicine Experimental 2020, 8(2): 000279

Introduction: Flow-controlled ventilation (FCV) guarantees a constant gas flow during in- and expiration. Coupled with direct intratracheal pressure measurement, it allows for a precise determination of dynamic lung compliance and ventilation can be adjusted accordingly. Objectives: Aim of this study was to investigate correlation of observed tidal volume and lung compliance in individualized FCV based on dynamic compliance measurements, compared to state-ofthe-art pressure-controlled ventilation (PCV) in lung healthy- and lung injured animals.

Methods: Anaesthetized pigs were ventilated with either FCV or PCV over a period of 10 hours in lung healthy animals (FCV $n=6, P C V n$ $=6$ ), and 2 hours after oelic acid induced acute respiratory distress syndrome (ARDS) ( $F C V n=8, P C V n=8)$. FCV was individualized with compliance guided positive end-expiratory (PEEP) and peak pressure settings. PCV was performed with a PEEP of $5 \mathrm{cmH}_{2} \mathrm{O}$ and a peak pressure set to achieve a tidal volume of $6-8 \mathrm{ml} / \mathrm{kg}$ in lung healthy animals and a tidal volume of $6 \mathrm{ml} / \mathrm{kg}$ and compliance guided PEEP titration in lung injured animals as a lung protective low tidal volume strategy. Tidal volume and pressure measurement were recorded directly from the respirator; compliance was calculated subsequently. As a surrogate parameter of lung stiffness compliance was then correlated to applied tidal volume.

Results: Overall, applied tidal volume showed a significant correlation to lung compliance $(r=0.704, p<0.001)$ in individualized FCV. Lung healthy animals showed a slightly lower correlation $(r=0.375, p=$ $0.0014)$ compared to ARDS animals $(r=0.734, p<0.001)$. Obviously, in PCV there was no correlation since tidal volume was set to $6-8 \mathrm{ml} / \mathrm{kg}$ in lung healthy and $6 \mathrm{ml} / \mathrm{kg}$ in lung injured animals.

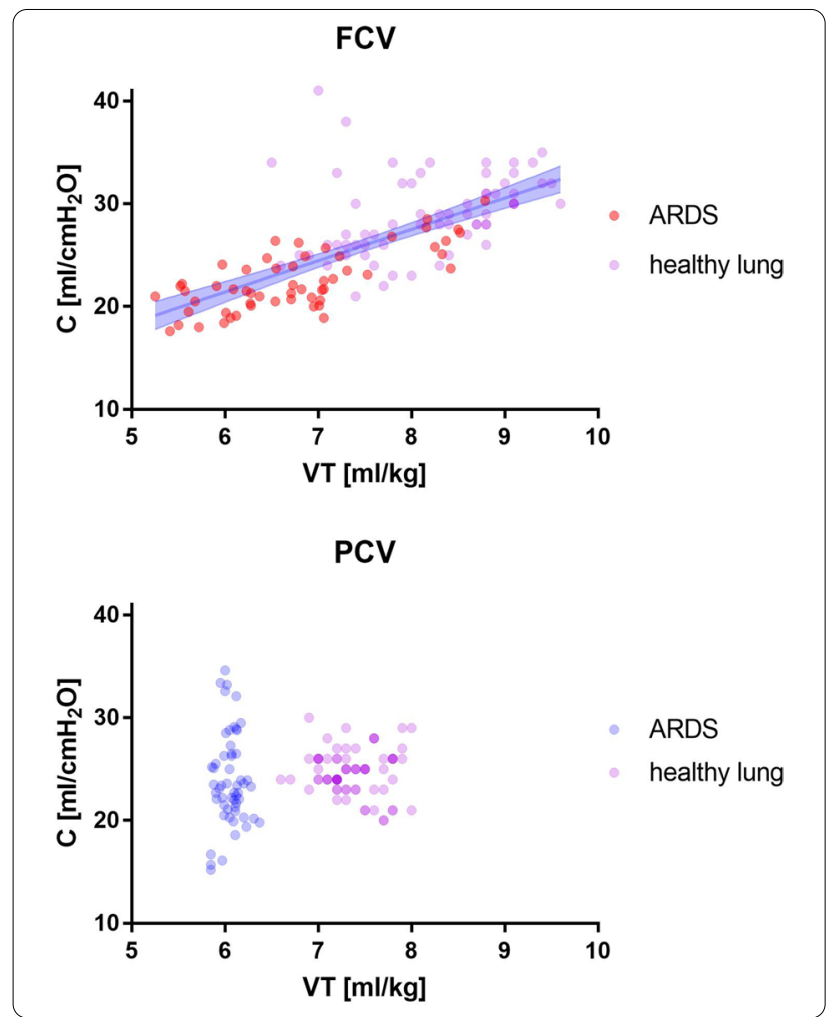

Conclusion: Compliance guided pressure settings in FCV showed a significant correlation of applied tidal volume to lung compliance as a surrogate parameter of lung stiffness. Thus, we hypothesize individualized FCV to be a more lung protective ventilation strategy as applied tidal volumes are based on individualized lung mechanics measurements. "One size does not fit all", even if best standard of care low tidal volume ventilation is performed.

000450

Increased non-aerated lung in healthy swines undergoing progressive decrease of respiratory rate with $\mathrm{ECCO}_{2} \mathrm{R}$

G. Colussi'; ;. Marongiu' ${ }^{2}$; A. Mazzucco ${ }^{3}$; E. Scotti ${ }^{1}$; R. Maia' ${ }^{2}$; G. Dal Santo ${ }^{2}$; E. Garbelli2; D. Dondossola ${ }^{4} ;$ G. Lopez $^{5}$; G. Fumagalli'; A. Damia'; M. Battistin ${ }^{1}$; O. Biancolilli'; S. Gatti ${ }^{6}$; A. Zanella ${ }^{1}$; E. Spinelli ${ }^{1}$; T. Mauri ${ }^{1}$; A. Pesenti ${ }^{1}$

${ }^{1}$ Department of anesthesia, critical care and emergency, Fondazione IRCCS Ca'Granda Ospedale Maggiore Policlinico, Milano, Italy; ${ }^{2}$ Department of pathophysiology and transplantation, University of Milan, Milano, Italy; ${ }^{3}$ Thoracic surgery and lung transplantation unit, Fondazione IRCCS Ca'Granda Ospedale Maggiore Policlinico, Milano, Italy; ${ }^{4}$ Liver transplant and general surgery unit, Fondazione IRCCS Ca'Granda Ospedale Maggiore Policlinico, Milano, Italy; ${ }^{5}$ Department of pathology, Fondazione IRCCS Ca'Granda Ospedale Maggiore Policlinico, Milano, Italy; ${ }^{6}$ Center for preclinical research, Fondazione IRCCS Ca'Granda Ospedale Maggiore Policlinico, Milano, Italy

Correspondence: $G$. Colussi

Intensive Care Medicine Experimental 2020, 8(2): 000450

Introduction: Extracorporeal $\mathrm{CO}_{2}$ Removal $\left(\mathrm{ECCO}_{2} \mathrm{R}\right)$ allows ultraprotective ventilation avoiding hypercapnia and thus can be used in ARDS patients with the aim of reducing the ventilation load to the lungs [1], but only a few studies investigated the effects of a decrease in respiratory rate during $\mathrm{ECCO}_{2} \mathrm{R}$. 
Objectives: The aims of our study were: to investigate the effects of decreased respiratory rate during $\mathrm{ECCO}_{2} \mathrm{R}$ on respiratory mechanics, oxygenation, hemodynamics and the development of atelectasis. Methods: Six female pigs were sedated and paralyzed; volume controlled ventilation was set with TV $10 \mathrm{ml} / \mathrm{Kg}$, PEEP $5 \mathrm{cmH}_{2} \mathrm{O}$ and respiratory rate (RR) $24 \mathrm{bpm}$. Animals underwent surgical cannulation of the right iliac and jugular veins, then were placed on v-v ECLS (iliacjugular) with extracorporeal blood flow maintained at $1.5 \mathrm{~L} / \mathrm{min}$. RR was reduced in three 8-hours steps to 18,12 and 6 breaths/min while keeping same TV and PEEP, inspiratory time was adapted to maintain $\mathrm{I}: \mathrm{E}=1: 2$. Gas flow was adjusted to maintain $\mathrm{PaCO}_{2} 30-40 \mathrm{mmHg}$. At Baseline and at the end of each step respiratory, hemodynamic, ECLS and gas exchange parameterswere collected, and end-expiratory chest CT scan was performed.

Results: All animals completed the study protocol. During progressive decrease of $\mathrm{RR}$ the $\mathrm{VCO}_{2}$ of the membrane lung progressively increased to maintain a stable $\mathrm{PaCO}_{2}$. The mechanical power progressively diminished, while plateau pressure, compliance and $\mathrm{PaO}_{2} / \mathrm{FiO}_{2}$ ratio worsened. Mean pulmonary artery pressure rose. Quantitative analysis of CT scan showed increase in the non-aerated lung compartment but no change in total lung weight. (See Table).

\begin{tabular}{|c|c|c|c|c|c|}
\hline & $\begin{array}{l}\text { RR24 } \\
\text { NO } \\
\text { ECCO } \square \mathrm{R}\end{array}$ & $\begin{array}{l}\text { RR18 } \\
+ \\
\text { ECCO } \square \mathrm{R}\end{array}$ & $\begin{array}{l}\mathrm{RR} 12 \\
+ \\
\mathrm{ECCO} \square \mathrm{R}\end{array}$ & $\begin{array}{l}\text { RR6 } \\
+ \\
\text { ECCO } \square \mathrm{R}\end{array}$ & $\begin{array}{l}\text { RM One } \\
\text { Way } \\
\text { Anova }\end{array}$ \\
\hline $\begin{array}{l}\text { Membrane Lung } \\
\text { VCO } \square / \text { tot, \% }\end{array}$ & 0 & $18 \pm 7^{*}$ & $33 \pm 14 *$ & $64 \pm 8^{*}$ & $\mathrm{p}<0.001$ \\
\hline $\mathrm{PaCO} \square, \mathrm{mmHg}$ & $34.6 \pm 3.8$ & $30.7 \pm 2.8$ & $34.0 \pm 3.0$ & $32.5 \pm 2.3$ & $\mathrm{p}=0.060$ \\
\hline $\begin{array}{l}\text { Mechanical Power, } \\
\mathrm{J} / \mathrm{min}\end{array}$ & $9.7 \pm 1.0$ & $8.8 \pm 1.7$ & $5.4 \pm 0.8^{*}$ & $3.4 \pm 0.7^{*}$ & $\mathrm{p}<0.001$ \\
\hline $\begin{array}{l}\text { Mean Airway } \\
\text { Pressure, cmH } \square \mathrm{O}\end{array}$ & $9.2 \pm 0.7$ & $9.8 \pm 0.7$ & $8.7 \pm 2.1$ & $8.3 \pm 0.5$ & $\mathrm{p}=0.276$ \\
\hline $\begin{array}{l}\text { Plateau Pressure, } \\
\text { cmH } \square \mathrm{O}\end{array}$ & $14.0 \pm 0.9$ & $16.8 \pm 1.6^{*}$ & $16.8 \pm 1.5 *$ & $16.8 \pm 1.2 *$ & $\mathrm{p}<0.001$ \\
\hline $\begin{array}{l}\text { Respiratory System } \\
\text { Compliance, } \\
\mathrm{mL} / \mathrm{cmH} \square \mathrm{O}\end{array}$ & $44.4 \pm 5.9$ & $33.7 \pm 2.8^{*}$ & $33.8 \pm 4.4 *$ & $33.6 \pm 2.9 *$ & $\mathrm{p}<0.001$ \\
\hline $\mathrm{PaO} \_/ \mathrm{FiO} \square$ & $526 \pm 46$ & $501 \pm 49$ & $514 \pm 32$ & $436 \pm 46^{*}$ & $\mathrm{p}<0.001$ \\
\hline $\begin{array}{l}\text { Mean Pulmonary } \\
\text { Artery Pressure, } \\
\mathrm{mmHg}\end{array}$ & $15 \pm 2$ & $18 \pm 1$ & $19 \pm 4$ & $20 \pm 2 *$ & $\mathrm{p}<0.050$ \\
\hline $\begin{array}{l}\text { Total Lung Weight, } \\
\text { g }\end{array}$ & $661 \pm 119$ & $647 \pm 125$ & $631 \pm 96$ & $695 \pm 107$ & $\mathrm{p}=0.260$ \\
\hline $\begin{array}{l}\text { Non-Aerated Lung, } \\
\%\end{array}$ & $\begin{array}{l}1.7[1.1- \\
5.2]\end{array}$ & $\begin{array}{l}2.4[1.5- \\
4.2]\end{array}$ & $\begin{array}{l}4.5[2.5- \\
9.3]\end{array}$ & $\begin{array}{l}9.5[7.4- \\
16.5] \#\end{array}$ & $\mathrm{p}<0.050$ \\
\hline
\end{tabular}

*Post-Hoc Holm-Sidak p $<0.05$ vs RR24 \#Post-Hoc Dunnett's on Ranks p $<0.05$ vs RR24

When dependent and non-dependent lung regions are analyzed separately, it can be noted that the increase in non-aerated compartment is larger in the dependent regions of the lung (see Figure below : Twoway RM Anova:region $p=0.022$, RR $p=0.007$, interaction $p<0.001$; Post-Hoc: \# $p<0.05$ NDEP vs DEP § DEP $p<0.05$ vs RR24).

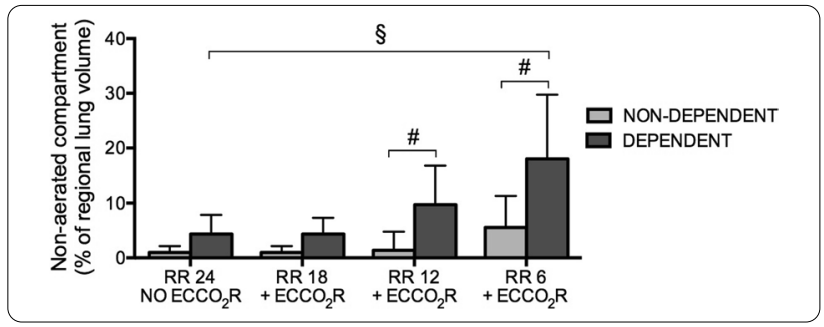

Conclusion: In healthy lungs decreased respiratory rate during $\mathrm{ECCO}_{2} \mathrm{R}$ allows a reduction of the ventilatory load to the lung at the expense of worsening oxygenation and compliance. Atelectasis progressively develop mainly in the dependent lung.
Reference(s) and grant ackowledgment(s)

1. [1] Combes A, Fanelli V, Pham T, Ranieri VM; European Society of Intensive Care Medicine Trials Group and the "Strategy of Ultra-Protective lung ventilation with Extracorporeal CO2 Removal for New-Onset moderate to severe ARDS" (SUPERNOVA) investigators. Feasibility and safety of extracorporeal $\mathrm{CO} 2$ removal to enhance protective ventilation in acute respiratory distress syndrome: the SUPERNOVA study. Intensive Care Med. 2019:45(5):592-600. https://doi.org/10.1007/s00134-019-05567-4

\section{0}

An investigation into patient-derived bioaerosol escape to the local environment when using a Vibrating Mesh Nebuliser during mechanical ventilation

M. Joyce; PJ. McKiernan²; JA. Mcgrath ${ }^{3}$; R. Macloughlin'; E. Fernandez Fernandez ${ }^{2}$

${ }^{1}$ Science, Aerogen, Galway, Ireland; ${ }^{2}$ Medical affairs, Aerogen, Galway, Ireland; ${ }^{3}$ School of physics \& ryan institute's centre for climate \& pollution studies, National University of Ireland Galway, Galway, Ireland

Correspondence: E. Fernandez Fernandez

Intensive Care Medicine Experimental 2020, 8(2): 000590

Introduction: Transmission of infectious diseases through patientderived aerosols (bioaerosols) may increase the prevalence of a disease in the general population[1]. In the critical care setting, aerosol therapy is often used concurrently with mechanical ventilation in the treatment of patients with respiratory issues[2]. If these patients are infectious, the breaking of the circuit, and subsequent potential release of patient-derived bioaerosols during nebuliser refill has the potential to impact the health of caregivers near the patient. Unlike conventional nebulisers, the Aerogen Solo nebuliser is designed to not require breaking of the ventilator circuit when refilling.

Objectives: To assess the potential for release of patient-derived bioaerosols into the local environment during drug refill of a vibrating mesh nebuliser (VMN) during mechanical ventilation.

Methods: A mechanical ventilator (Bellavista, IMT Medical, Switzerland), with a dual limb circuit (RT200, Fisher \& Paykel, New Zealand) incorporating a heat moisture exchange filter (HMEF) (Intersurgical, UK) at the patient side of the wye, was set to simulate an adult ventilation pattern (Vt $500 \mathrm{~mL}$, 15BPM, \& I:E Ratio 1:1). A VMN (Aerogen Solo, Aerogen, Ireland) was placed between the HMEF and the endotracheal tube (ETT) $(8.0 \mathrm{~mm}$, Flexicare, UK). An experimental breath actuated aerosol generator was placed at the end of the ETT to generate simulated exhaled patient-derived bioaerosol, using saline $(0.9 \%$, Braun, Germany). Aerosol concentrations of particles sized between 0.5 and $20 \mu \mathrm{m}$ per $\mathrm{cm} 3$ were recorded using two Aerodynamic Particle Sizers (APS, Model 3321, TSI Inc., US) positioned at $30 \mathrm{~cm}$ and $90 \mathrm{~cm}$ from the nebuliser. Test runs were 10 minutes in duration. An initial two-minute period without simulated bioaerosol being generated established ambient aerosol concentrations. Testing (in triplicate) was completed with the VMN in the circuit with the silicon medication cup cap opened or closed for the 10-minute test period.

Results: Results are illustrated in Figure 1.

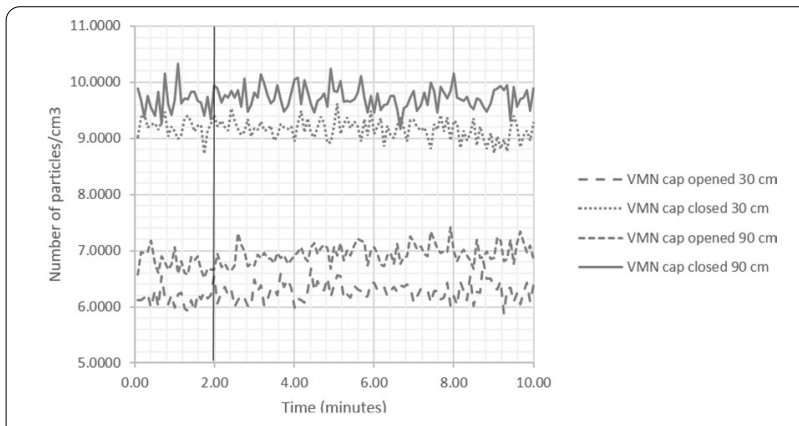

Figure 1: Particle concentration across all tests 
Conclusion: There was no increase in particle concentration noted over the 10-minute test period. Results on average ranged from 6-10 particles per $\mathrm{cm} 3$ across all tests at both distances and were in line with ambient levels, also recorded at 6-10 particles per $\mathrm{cm}^{3}$. Variations in ambient levels are due to localised conditions during testing. The results of this study confirm that there is no release of patient-derived bioaerosol through the VMN with the silicon plug opened or closed during mechanical ventilation.

\section{Reference(s) and grant ackowledgment(s)}

1. 2. Dhand R. Basic Techniques for aerosol delivery during mechanical ventilation. Respir. Care. 2004; 49: 611-622.

2. This work was funded by Aerogen Ltd, Ireland

3. 1. World Health Organization. Infection prevention and control of epidemicand pandemic-prone acute respiratory infections in health care. Geneva: World Health Organization; 2014; https://www.who.int/csr/bioriskreductio n/infection_control/publication/en/

\section{4}

Transvenous-stimulated diaphragm contractions combined with mechanical ventilation reduce systemic inflammation in healthy pigs

E. Rohrs ${ }^{1}$; T. Bassi ${ }^{1}$; K. Fernandez ${ }^{1}$; M. Ornowska ${ }^{1}$; M. Nicholas ${ }^{1}$; S. Reynolds ${ }^{1}$ ${ }^{1}$ Physiology, Simon Fraser University, Burnaby, Canada

Correspondence: $\mathrm{E}$. Rohrs

Intensive Care Medicine Experimental 2020, 8(2): 000644

Introduction: Mechanical ventilation damages the alveolar epithelium through increased and cyclical stretch due to non-physiological distribution of tidal volume.1 This, combined with shear stress from repeated opening and closing of alveoli in atelectatic regions, triggers the release of proinflammatory cytokines such as IL-1 $\beta$, IL- 6 and IL-8.2Excessive cyclic stretch induces cell membrane leakage, causing a spillover of intracellular contents. 3 In addition, injured cells release alarmins that elicit a local pro-inflammatory response. 4 If the injury to lung epithelium progresses such that the compartmentalization of the alveoli is lost, these cytokines translocate into the circulation and have the potential to cause secondary organ injury that leads to multi-system organ failure, which contributes to morbidity and mortality. 5 This is known as biotrauma. Contracting the diaphragm during mechanical ventilation may reduce atelectrauma that leads to biotrauma.

Objectives: We studied diaphragm contractions elicited by transvenous phrenic nerve stimulation (TTDN) delivered in synchrony with the inspiratory phase of ventilation as a method to reduce lung injury and biotrauma in a sedated, preclinical, ICU model with non-injured lungs.

Methods: Human-sized pigs were sedated and ventilated under mock ICU conditions for 50 hours. They were ventilated with protective volume control, and one group received temporary transvenous diaphragmaticneurostimulation during inspiration on every second breath (MV + TTDN group) while the other did not (MV group). IL-1 $\beta$, IL- 6 and IL- 8 were measured in BAL fluid and serum.

Results: BAL fluid concentrations were not significantly different for IL-1 $\beta$ (14324 pg/ml MV group vs. $9287 \mathrm{pg} / \mathrm{ml} \mathrm{MV} \mathrm{+} \mathrm{TTDN} \mathrm{group,} \mathrm{p}=$ 0.27), IL-6 (210.7 pg/ml MV group vs. $186.2 \mathrm{pg} / \mathrm{ml} \mathrm{MV} \mathrm{+} \mathrm{TTDN} \mathrm{group,}$ $\mathrm{p}=0.67)$, or IL-8 (15814 pg/ml MV group vs. $15401 \mathrm{pg} / \mathrm{ml} \mathrm{MV} \mathrm{+} \mathrm{TTDN}$ group, $p=0.99)$. Serum concentrations were significantly lower in the MV + TTDN group for IL-1 $(434.6 \mathrm{pg} / \mathrm{ml} \mathrm{MV} \mathrm{group} \mathrm{vs.} 104.1 \mathrm{pg} / \mathrm{ml}$ $\mathrm{MV}+$ TTDN group, $\mathrm{p}=0.02), \mathrm{IL}-6(182.1 \mathrm{pg} / \mathrm{ml} \mathrm{MV}$ group vs. $30.7 \mathrm{pg} /$ $\mathrm{ml} \mathrm{MV}+$ TTDN group, $\mathrm{p}=0.03)$, and IL-8 $(35.0 \mathrm{pg} / \mathrm{ml} \mathrm{MV}$ group vs. $13.2 \mathrm{pg} / \mathrm{ml} \mathrm{MV}+$ TTDN group, $\mathrm{p}=0.04)$.

Conclusion: BAL washings were taken both at the start and the end of the experiment and the initial wash may have initiated an inflammatory process that was independent of ventilation, confounding the BAL cytokine results. Transvenous phrenic-nerve-stimulated diaphragm contraction combined with protective mechanical ventilation on every second breath reduced biotrauma in healthy porcine lungs that were ventilated for 50 hours.
Reference(s) and grant ackowledgment(s)

1. 5. Vlahakis NE, Hubmayr RD. Invited review: Plasma membrane stress failure in alveolar epithelial cells. J Appl Physiol (1985). 2000;89(6):2490-6;discussion 2497.

2. 4. McDonald B, Pittman K, Menezes GB, et al. Intravascular danger signals guide neutrophils to sites of sterile inflammation. Science. 2010;330(6002):362-366

3. 3. Stroetz RW, Vlahakis NE, Walters BJ, Schroeder MA, Hubmayr RD. Validation of a new live cell strain system: Characterization of plasma membrane stress failure. J Appl Physiol (1985). 2001;90(6):2361-2370.

4. 2. Birukov KG, Birukova AA, Dudek SM, et al. Shear stress-mediated cytoskeletal remodeling and cortactin translocation in pulmonary endothelial cells. Am J Respir Cell Mol Biol. 2002;26(4):453-464

5. 1. Curley GF, Laffey JG, Zhang H, Slutsky AS. Biotrauma and ventilatorinduced lung injury: Clinical implications. Chest. 2016;150(5):1109-1117.

6. Mitacs

7. Royal Columbian Hospital Foundation

8. Lungpacer Medical Inc.

\section{1}

In vivo performance of the OxVent rapidly manufactured

\section{ventilator}

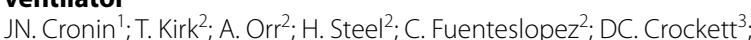
KM. Ahlgren ; J. Beddoe Rosendo ${ }^{5} ;$ C. Bergeles 5 ; S. East ${ }^{2}$; C. Edwardes J. Fisk ${ }^{2}$; M. Garstka ${ }^{2}$; C. Heaysman ${ }^{5}$; A. Hussain ${ }^{2}$; I. Kempf ${ }^{2}$; D. Salisbury²; C. Seneci ${ }^{5}$; R. Staruch'²; V. Vitiello ${ }^{5}$; M. Xochicale ${ }^{5}$; A. Castrejon-Pita ${ }^{2}$;

T. Denison'; P. Goulart'; P. Jha ; S. Ourselin'; M. Thompson²; R. Beale; L. Camporota ${ }^{8} ;$ AD. Farmery ${ }^{3} ;$ F. Formenti $^{1}$

${ }^{1}$ Centre for human and applied physiological sciences, King's College London, London, United Kingdom; ${ }^{2}$ Department of engineering science, University of Oxford, Oxford, United Kingdom; ${ }^{3}$ Nuffield division of anaesthetics, nuffield department of clinical neurosciences, University of Oxford, Oxford, United Kingdom; ${ }^{4}$ Hedenstierna laboratoriet, department of surgical sciences, Uppsala University, Uppsala, Sweden; ${ }^{5}$ School of biomedical engineering and imaging sciences, King's College London, London, United Kingdom; ${ }^{6}$ Worcester college, University of Oxford, Oxford, United Kingdom; ${ }^{7}$ Pulmonary, adult critical care and sleep, Guy's and St Thomas' NHS Foundation Trust, London, United Kingdom; ${ }^{8}$ Critical care, St Thomas' Hospital, London, United Kingdom

Correspondence: J.N. Cronin

Intensive Care Medicine Experimental 2020, 8(2): 000721

Introduction: The OxVent [1] is a low-cost volume control mechanical ventilator designed to be rapidly produced at scale to meet a large and sudden increase in ventilators' need, like during the COVID19 pandemic. It is based on a pneumatic core component driven by compressed air flow controlled by a solenoid valve. Near-patient spirometry measures delivered tidal volume and solenoid actuation is adjusted by a closed feedback loop. Expiration occurs through a commodity mechanical PEEP valve. The device has been characterised on the bench to meet the UK rapidly manufactured ventilator system requirements [2].

Objectives: Determine the performance of the OxVent in uninjured and lung-lavaged pigs.

Methods: Variations in PEEP and tidal volume were set on the OxVent in a protocolised manner both before and after surfactant depletion with saline lung lavage in 4 anaesthetised and paralysed pigs (30 (1) $\mathrm{kg}$; mean (SD)). Set tidal volumes (VTset) ranged from 170 to $340 \mathrm{~mL}$ (6 to $11 \mathrm{~mL} / \mathrm{kg}$ ). Respiratory rate (RRset) was set at either 16 or $28 \mathrm{~min}-1$ to maintain minute ventilation around $170 \mathrm{~mL} / \mathrm{min} / \mathrm{kg}$. PEEP was varied between 5, 10 and $20 \mathrm{cmH}_{2} \mathrm{O}$ (PEEPset). Airway flow and pressure waveforms were measured independently (Datex-Ohmeda Capnomac Ultima) and analysed to determine actual tidal volume (VTact) and PEEP (PEEPact). End-expiratory lung volume (EELV) was measured using a sulphur hexafluoride washout technique.

Results: Lung lavage decreased respiratory system compliance from 22 (5) to $15(5) \mathrm{mL} / \mathrm{cmH}_{2} \mathrm{O}(\mathrm{P}<0.001)$, EELV from 570 (158) to 272 (97) $\mathrm{mL}(\mathrm{P}=0.04)$, and $\mathrm{PaO}_{2} / \mathrm{FiO}_{2}$ ratio from 438 (39) $\mathrm{mmHg}$ to 298 (133) $\mathrm{mmHg}(\mathrm{P}=0.11)$, all measured at PEEP $5 \mathrm{cmH}_{2} \mathrm{O}$. Airway resistance was $7.8(2.8) \mathrm{cmH}_{2} \mathrm{O} / \mathrm{L} / \mathrm{s}$. 
VTact correlated with VTset $(\mathrm{R} 2=0.71$, gradient of regression line 1.02), mean bias was -1 [-11 to 9 ] $\mathrm{mL}$ (mean [95\% confidence interval]) with $95 \%$ limits of agreement -90 [-107 to -73$] \mathrm{mL}$ and 88 [71 to 105] $\mathrm{mL}$. PEEPact was 3.9 (1.2), 8.1 (1.9) and 13.7 (14.8) $\mathrm{cmH}_{2} \mathrm{O}$ for PEEPset of 5,10 and $20 \mathrm{cmH}_{2} \mathrm{O}$ respectively. Airway pressure did not drop to PEEPact immediately during expiration, but remained 2.7 [2.5 to 2.9] and 1.1 [1.0 to 1.2] $\mathrm{cmH}_{2} \mathrm{O}$ above PEEPset respectively at $50 \%$ and $75 \%$ of the expiratory period. End-expiratory flow was 50 (17) and 88 (15) $\mathrm{mL} / \mathrm{s}$ respectively at PEEPset 5 and $20 \mathrm{cmH}_{2} \mathrm{O}$.

The presence of lung injury did not alter any outcome measure.

Conclusion: The OxVent delivered tidal volumes and PEEP similar to those set over a wide range in uninjured pigs and those with a salinelavage lung injury model. The commodity PEEP valve was unable to maintain PEEP at the set value and our tests identified an expiratory flow limitation, which was not apparent on bench-top testing. This flow limitation was PEEP-dependent, suggesting that the valve was the culprit. The system has since been redesigned following profiling of the valve. Rapidly developed safety critical devices for pandemic use should still be thoroughly tested in vivo over a range of typical usage scenarios to mitigate all possible risks to patients.

\section{Reference(s) and grant ackowledgment(s)}

1. [1] OxVent - A collaboration for COVID 19 ventilation (2020). OxVent Team. https://oxvent.org Accessed 30 June 2020

2. [2] Rapidly Manufactured Ventilator System, v4 (2020). Medicines \& Healthcare products Regulatory Agency.https://assets.publishing.service.gov. uk/government/uploads/system/uploads/attachment_data/file/879382/ RMVS001_v4.pdf Accessed 30 June 2020

3. This work was supported by the King's Together Multi and Interdisciplinary Research Scheme \{Wellcome Trust Institutional Strategic Support Fund\}.

\section{5}

The Effect of Closed Loop Ventilation on Mechanical Power results from a pilot study of Adaptive Support Ventilation

LA. Buiteman-Kruizinga'; HE. Mkadmi ${ }^{2}$; MJ. Schultz ${ }^{3}$; PL. Tangkau'; PLJ. Van

Der Heiden

'Intensive care, Reinier de Graaf Gasthuis, Delft, Netherlands; ${ }^{2}$ Research, Reinier de Graaf Gasthuis, Delft, Netherlands; ${ }^{3}$ Intensive care, Academic Medical Centre, Amsterdam, Netherlands

Correspondence: L.A. Buiteman-Kruizinga

Intensive Care Medicine Experimental 2020, 8(2): 000785

Introduction: 'Mechanical Power of ventilation' (MP) is a summary variable that includes tidal volume (VT), respiratory rate (RR), flow and driving pressure $(\Delta \mathrm{P})$, all the components that play a role in VILI [1]. MP has been shown to be associated with important patient-centered outcomes [2]. The complex interplay between its components makes it difficult to set a ventilator so that the least amount of energy per time (MP) is transferred from the ventilator to the respiratory system in an individual patient. Adaptive Support Ventilation (ASV) is a closedloop mode that provides pressure-controlled or pressure-support ventilation depending on patient's activity. With ASV, all components that can cause VILI are under control of the ventilator.

Objectives: The aim of this study is to compare MP under ASV and under conventional ventilation.

Methods: We calculated and compared MP in 12 patients under standard ventilation in which VT, peak- and driving pressure, and RR were set by experienced caregivers, with MP in 12 patients under ASV. Patients were sedated and without spontaneous breathing activity. MP was calculated three times per day over the first 4 days of ventilation, using the following equation [3]: $0.098 * V T * R R *($ Ppeak- $1 / 2 * \Delta P)$ A linear mixed model regression was used to assess the effect of time (as random factor) on MP. The regression was adjusted for ventilation mode and gender as fixed factors, and the APACHE IV score as a covariate. A p-value $<0.05$ was considered significant. This pilot study was performed on the ICU in the Reinier de Graaf hospital in Delft, the Netherlands, from march till august 2019.

Results: Median MP was 15.1 [6.1-32.0] J/min with ASV versus 22.9 [4.9-49.1] J/min with conventional ventilation (mean difference $6.2 \mathrm{~J} /$ min). MP with ASV was lower at all time points data were collected.
Linear mixed model regression analysis showed that the factor ventilation mode was statistically significant (95\%-confidence interval 1.45 to $11.62, P=0.013$ ). With ASV, VT was not different form that with pressure controlled ventilation, but Ppeak and RR were significantly lower $(P=0.012)$ (Figure 1).

Conclusion: Findings of this pilot study suggest that concerning the MP transferred from the ventilator to the respiratory system, ASV is superior to standard ventilation. This effect is not achieved by simple limitation of VT alone, but by a reduction in applied pressure and RR.

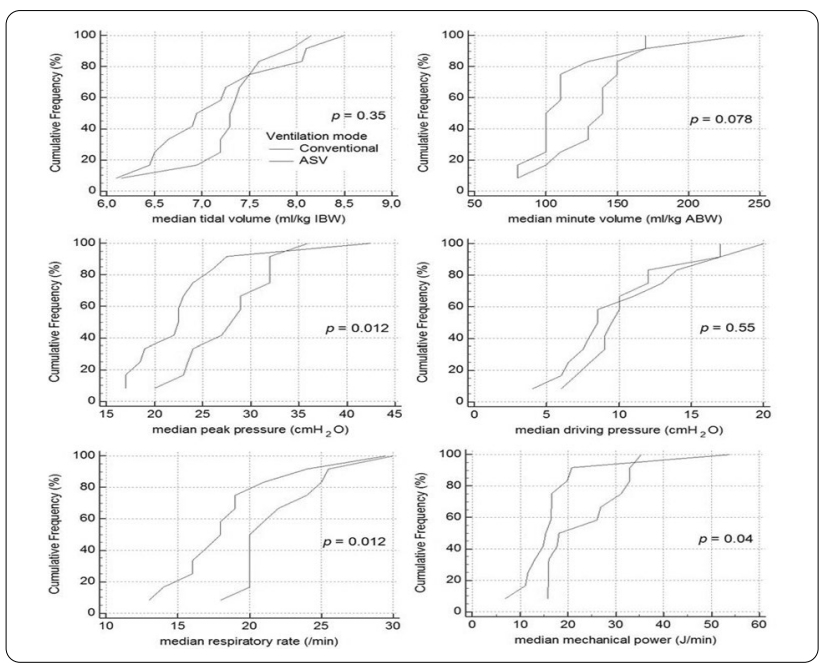

\section{Reference(s) and grant ackowledgment(s)}

1. 1. Giosa, L., Busana, M., Pasticci, I., Bonifazi, M., Macrì, M. M., Romitti, F., Vassalli, F., Chiumello, D., Quintel, M., Marini, J. J., \& Gattinoni, L. (2019). Mechanical power at a glance: a simple surrogate for volume-controlled ventilation. Intensive Care Medicine Experimental, 7(1)

2. 2. Serpa Neto, A., Deliberato, R. O., Johnson, A. E. W., Bos, L. D., Amorim, P., Pereira, S. M., Cazati, D. C., Cordioli, R. L., Correa, T. D., Pollard, T. J., Schettino, G. P. P., Timenetsky, K. T., Celi, L. A., Pelosi, P., Gama de Abreu, M., \& Schultz, M. J. (2018). Mechanical power of ventilation is associated with mortality in critically ill patients: an analysis of patients in two observational cohorts. Intensive Care Medicine, 44(11), 1914-1922.

3. 3. Gattinoni, L., Tonetti, T., Cressoni, M., Cadringher, P., Herrmann, P., Moerer, O., Protti, A., Gotti, M., Chiurazzi, C., Carlesso, E., Chiumello, D., \& Quintel, M. (2016). Ventilator-related causes of lung injury: the mechanical power. Intensive Care Medicine, 42(10), 1567-1575.

\section{1}

Comparison of Extubation Outcome after Spontaneous Breathing Trials with T-piece and Pressure Support Ventilation in Patients with Expiratory Flow Limitation

Y. Kluanwan ${ }^{1}$; N. Kongpolprom²

${ }^{1}$ Department of internal medicine, faculty of medicine, King Chulalongkorn Memorial Hospital, Bangkok, Thailand; ${ }^{2}$ Department of internal medicine, Faculty of Medicine, Chulalongkorn University, King Chulalongkorn Memorial Hospital, Bangkok, Thailand

Correspondence: $Y$. Kluanwan

Intensive Care Medicine Experimental 2020, 8(2): 000881

Introduction: Expiratory Flow Limitation (EFL) is an inability to exceed a certain flow regardless of the pressure exerted, which accounts for $60 \%$ of extubation failure. However, a spontaneous breathing trial (SBT) method that suits for this specific condition is uncertain.

Objectives: We aimed to evaluate an effect of 30-minute T-piece and 30-minute pressure support ventilation (PSV) on a success rate of extubation in patients with EFL. 
Methods: We conducted a non-inferiority randomized controlled trial comparing 30-minute T-piece SBT and 30-minute SBT with PSV (PS 6 $\mathrm{CmH}_{2} \mathrm{O}$ and PEEP $5 \mathrm{cmH}_{2} \mathrm{O}$ ) in mechanically ventilated patients with $\mathrm{EFL}$ who were considered as readiness to wean. The primary outcome was successful extubation (72-hour ventilator free after extubation). Secondary outcomes were a reintubation rate, time to reintubation, and a success rate of SBT.

Results: A total of 40 ventilated patients with EFL were recruited. The majority of them were male (62.5\%) with the median age of 72[IQR 21] years. The most common cause of acute respiratory failure was the intrapulmonary cause (87\%). The eligible patients were randomized into 2 groups: 21 patients in the T-piece group and 19 patients in the PSV group. The success rates of extubation were not significantly different between 2 groups: $85.71 \%$ in the T-piece group and $84.21 \%$ in the PSV group, $\mathrm{p}=0.62$. There were no significant differences in the reintubation rate $(9.52 \%$ in the T-piece group VS $5.26 \%$ in the PSV group, $p=0.54$ ), time to reintubation (41 hours in the T-piece group VS 6.5 hours in the PSV group, $p=0.221$ ) and the SBT success rate (95.24\% in the T-piece group VS $89.47 \%$ in the PSV group, $p=0.462$ ).

Conclusion: Among the patients with EFL, the 30-minute T-piece SBT was non-inferior to the 30-minute SBT with PSV in terms of the successful extubation, successful SBT, reintubation rate and time to reintubation

\section{Reference(s) and grant ackowledgment(s)}

1. Volta CA, Dalla Corte F, Ragazzi R, Marangoni E, Fogagnolo A, Scaramuzzo G, et al. Expiratory flow limitation in intensive care: prevalence and risk factors. Crit Care. 2019;23(1):395.

2. Subira C, Hernandez G, Vazquez A, Rodriguez-Garcia R, Gonzalez-Castro A, Garcia C, et al. Effect of Pressure Support vs T-Piece Ventilation Strategies During Spontaneous Breathing Trials on Successful Extubation Among Patients Receiving Mechanical Ventilation: A Randomized Clinical Trial. Jama.2019;321(22):2175-82.

3. Esteban A, Alia I, Gordo F, Fernandez R, Solsona JF, Vallverdu I, et al. Extubation outcome after spontaneous breathing trials with $\mathrm{T}$-tube or pressure support ventilation. The Spanish Lung Failure Collaborative Group. Am J Respir Crit Care Med. 1997;156(2 Pt 1):459-65

4. Expiratory Flow Limitation During Mechanical Ventilation Junhasavasdikul, Detajin et al. CHEST, Volume 154, Issue 4, 948-962

5. Koutsoukou A, Armaganidis A, Stavrakaki-Kallergi C, et al. Expiratory flow limitation and intrinsic positive end-expiratory pressure at zero positive end-expiratory pressure in patients with adult respiratory distress syndrome. Am J Respir Crit Care Med. 2000;161(5):1590-1596.

\section{0}

Effect of Fully-automated versus Non-automated Ventilation on Mechanical Power of Ventilation in COVID-19 an observational crossover study

LA. Buiteman-Kruizinga ${ }^{1}$; HE. Mkadmi ${ }^{2}$; MJ. Schultz ${ }^{3}$; PLJ. Van Der Heiden ${ }^{1}$ 'Intensive care, Reinier de Graaf Gasthuis, Delft, Netherlands; ${ }^{2}$ Research, Reinier de Graaf Gasthuis, Delft, Netherlands; ${ }^{3}$ Intensive care, Academic Medical Centre, Amsterdam, Netherlands

Correspondence: L.A. Buiteman-Kruizinga

Intensive Care Medicine Experimental 2020, 8(2): 001100

Introduction: Patients with severe coronavirus disease 2019 (COVID19) often need admission to an intensive care unit (ICU) for invasive ventilation [1]. This new disease often challenged the caregiver to initiate ventilation in a lung protective manner. The 'mechanical power of ventilation' (MP), a summary variable that includes all components that play a role in worsening of lung injury, is associated with outcome in patients with ARDS from another cause. Fully-automated ventilation has been shown to reduce MP in patients not having COVID-19 [2]. Whether fully-automated ventilation affects MP in COVID-19 patients is uncertain.

Objectives: The aim of this study is to compare MP under INTELLiVENT-ASV, a fully-automated mode of ventilation, versus conventional ventilation in COVID-19 patients.

Methods: Observational crossover study performed in the ICU in the Reinier de Graaf Hospital in Delft, The Netherlands, during the first months of the outbreak from march till May 2020. Ventilation variables and parameters, necessary to calculate MP, were collected in 31 invasively ventilated COVID-19 patients -2 hours during pressure controlled ventilation (conventional ventilation) and 2 hours during INTELLiVENT-ASV (fully-automated ventilation). During conventional ventilation, tidal volume (VT) peak and driving pressure (Ppeak and $\Delta \mathrm{P})$, and respiratory rate (RR) were set by the caregivers strictly following the local guideline for invasive ventilation. During fully-automated ventilation, these settings were under control of the ventilator, and a target etCO2 and $\mathrm{SpO} 2$ was set as targeted with conventional ventilation. MP was calculated using the following equation [3] $0.098 * V T * R R *($ Ppeak $-1 / 2 * \Delta P)$

Mean MP during conventional ventilation and fully-automated were compared using paired T-test; a p-value $<0.05$ was considered statistically significant.

Results: Mean MP under conventional ventilation was $31.5 \mathrm{~J} / \mathrm{min}$ (SD 13.9) and $22.4 \mathrm{~J} / \mathrm{min}$ (SD 9.2) under fully-automated ventilation (mean difference 9.1 J/min; 95\%-confidence interval 6.1 to $12.2, P=<$ $0.0001)$. Individual data are shown in the figure.

Conclusion: INTELLiVENT-ASV is superior to conventional ventilation with respect to the amount of energy transferred from the ventilator to a patient's respiratory system in invasively ventilated COVID-19 patients.

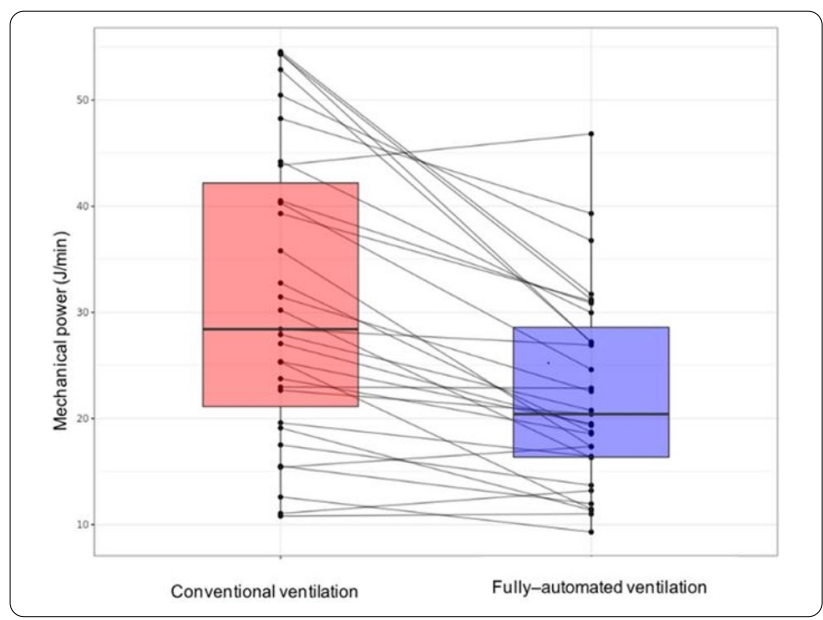

Reference(s) and grant ackowledgment(s)

1. 1. Guan, W.J., et al., Clinical Characteristics of Coronavirus Disease 2019 in China. N Engl J Med, 2020.

2. 2. Arnal, J.M., Saoli, M., Garnero, A. Airway and transpulmonary driving pressures and mechanical powers selected by INTELLiVENT-ASV in passive, mechanically ventilated ICU patients (2019) Heart Lung. S0147-9563(19)30533-3.

3. 3. Gattinoni, L., Tonetti, T., Cressoni, M., Cadringher, P., Herrmann, P., Moerer, O., Protti, A., Gotti, M., Chiurazzi, C., Carlesso, E., Chiumello, D., \& Quintel, M. (2016). Ventilator-related causes of lung injury: the mechanical power. Intensive Care Medicine, 42(10), 1567-1575.

\section{2}

\section{Why recording ideal body weight matters}

S. Nasser

${ }^{1}$ Intensive Care Department, Surrey and Sussex Healthcare NHS Trust, Redhill, United Kingdom

Correspondence: S. Nasser

Intensive Care Medicine Experimental 2020, 8(2): 000032

Introduction: ARDSNet demonstrated $22 \%$ increased mortality in patients ventilated at $11.8 \mathrm{ml} / \mathrm{kg}$ rather than $6.2 \mathrm{ml} / \mathrm{kg}$, with the promotion of lung inflammation as a possible mechanism for its deleterious effects [1]. Lung protective ventilation however, depends on the 
correct assessment of the patient's Ideal Body Weight (IBW). Measurement and accurate recording of IBW is, therefore, of fundamental importance in the Intensive Care Unit (ICU), to enable implementation of lung protection strategies.

Objectives: Assessment of recording of patient weight in a UK District General Hospital ICU, with analysis of implications in discrepancies between recorded weight and IBW as measured by ulna-length, on lung-protective ventilation strategies.

Methods: Collection of recorded weights for 49 patients admitted to ICU between 06/11/2019-27/11/2019 and comparison to IBW using ulna-length measurement to calculate IBW.

Results: IBW was not the recorded weight in any patients ( 22 female; 27 male), with only actual body weight (ABW) recorded, derived from visual estimation, measured weight, or from pre-admission notes. The recorded weight was, on average, 1.3 times higher than IBW as derived from ulna-length measurement. Had $8 \mathrm{ml} / \mathrm{kg}$ tidal volumes been used on patients at the recorded weight, the mean equivalent tidal volume delivered would have been $10.5 \mathrm{ml} / \mathrm{kg}$ of IBW (median $9.92 \mathrm{ml} / \mathrm{kg}$ ), giving an average higher tidal volume of $147 \mathrm{ml}$. For the 16 obese patients in the sample (BMI > 30) the corresponding values were $13.04 \mathrm{ml} / \mathrm{kg}$ of IBW and $296 \mathrm{ml}$.

Conclusion: In the UK, $28.7 \%$ of the population are obese (BMI > 30) with $3.6 \%$ morbidly obese (BMI $>40$ ) [2], constituting around $20 \%$ of ICU admissions [3]. Obese individuals are more prone to Acute Respiratory Distress Syndrome [4] due to de-recruitment and atelectasis as well as a decreased Functional Residual Capacity [5], and thus have been posited as a population requiring more aggressive implementation of ventilation protection strategies. Yet this is precisely the population most prone to erroneous recording of their IBW, and thus, overventilation, due to failure to proactively record IBW rather than $A B W$. Whether through ulna-length derived values or through direct measurement of patient height, recording of IBW with a standardised method should be a high priority for any patients admitted to ICU.

\section{Reference(s) and grant acknowledgment(s)}

1. 1. Acute Respiratory Distress Syndrome Network. Ventilation with lower tidal volumes as compared with traditional tidal volumes for acute lung injury and the acute respiratory distress syndrome. New England Journal of Medicine. 2000 May 4;342(18):1301-8.

2. 2. House of Commons, Obesity Statistics, Briefing Paper Number 3336, 6 August 2019

3. 3. Lewandowski K, Lewandowski M. Intensive care in the obese. Best Pract Res Clin Anaesthesiol. 2011;25:95-108.

4. 4. Gong MN, Bajwa EK, Thompson BT, Christiani DC. Body mass index is associated with the development of acute respiratory distress syndrome. Thorax. 2010 Jan 1;65(1):44-50

5. 5. De Jong A, Verzilli D, Jaber S. ARDS in obese patients: specificities and management. Critical Care. 2019 Dec 1;23(1):74.

\section{5}

Change in lung impedance monitored by electrical impedance tomography during bronchoalveolar lavage in patients with acute respiratory distress syndrome

A. Shono ${ }^{1}$; M. Somei ${ }^{1}$; T. Watanabe ${ }^{1}$; Y. Ichikawa ${ }^{1}$; M. Mori ${ }^{1}$; R. Miyashita'; T. Kotani ${ }^{i}$

'Department of Intensive Care Medicine, Showa University, School of Medicine, Tokyo, Japan

Correspondence: A. Shono

Intensive Care Medicine Experimental 2020, 8(2): 000105

Introduction: Bronchoalveolar lavage (BAL) is a useful tool for the diagnostic evaluation and often performed under mechanical ventilation. The concerns about performing BAL in hypoxemic patients are shunt caused by administrated fluid and/or loss of lung aeration during procedure, resulting in impairment of oxygenation. In mechanically ventilated patients positive end-expiratory pressure (PEEP) could maintain lung volume during $B A L$, however, the impact of $B A L$ on regional ventilation and aeration has not been fully investigated. Electrical impedance tomography (EIT) can evaluate lung volume, regional ventilation non-invasively in a real-time at the bedside. Fluid instillation in the lung decreases impedance value.

Objectives: The objective of this study was to examine the change in lung impedance throughout the BAL procedure in acute respiratory distress syndrome (ARDS) patients.

Methods: The moderate ARDS patients with bilateral ground-glass opacity were included. All patients were ventilated with pressurecontrolled ventilation with PEEP of 5 to $10 \mathrm{cmH}_{2} \mathrm{O}$. We monitored end-expiratory lung impedance (EELI), which is impedance value at the end of expiration and corresponds to end-expiratory lung volume. The measurements were performed at four time points, (1) before BAL, (2) during fiberoptic examination, (3) after instillation of fluid for lavage, (4) 15 min after completion of BAL. The value of EELI at before BAL was defined as baseline, and changes in EELI from at before BAL were calculated at each time point and expressed as a percentage of tidal impedance change at before BAL. The change in EELI was analyzed by one-way ANOVA with repeated measures and Turkey test as post-hoc analysis. $\mathrm{PaO}_{2} / \mathrm{FIO}_{2}$ ratio before and after $\mathrm{BAL}$ was compared by paired t test.

Results: Five patients were included. The entire procedure was finished within $20 \mathrm{~min}$ in all cases. Mean collected volume of fluid was $45 \%$ of administered fluid. EELI significantly changed during the entire course of the procedure $(P=0.024)$. EELI at after instillation of fluid for lavage was significantly decreased from at before BAL (mean values: $-53 \pm 16 \%, P=0.030$ ). However, EELI was recovered gradually after removal of bronchoscopy towards the level of at baseline, showing no significant difference at 15 min after completion of BAL (mean values: $-43 \pm 16 \%, P=0.103$ ) compared to at before BAL. Mean difference in $\mathrm{PaO}_{2} / \mathrm{FIO}_{2}$ ratio between before and after BAL was $-4 \pm 20$ ( $95 \%$ confidence interval, -60 to 52 ) and no significant difference was detected.

Conclusion: Lung impedance value significantly decreased during $\mathrm{BAL}$, probably due to saline instillation, collapse in the lung during the procedure etc. However, it recovered promptly resulting in maintenance of oxygenation.

\section{5}

Early ventilator-induced diaphragmatic dysfunction

in polytraumatized patients

C. Cobilinschi ${ }^{1}$; A. Baetu' ; M. Tiglis' ${ }^{1}$; AM. Cotae ${ }^{1}$; C. Minoiu²; E. Nestianu ${ }^{2}$; OM. Melente; ${ }^{1}$ M. Costache'; IM. Grintescu

${ }^{1}$ Anesthesiology and Intensive Care, Clinical Emergency Hospital, Bucharest, Romania; ${ }^{2}$ Radiology Department, Clinical Emergency Hospital, Bucharest, Romania

Correspondence: $C$. Cobilinschi

Intensive Care Medicine Experimental 2020, 8(2): 000325

Introduction: Pulmonary dysfunction in trauma patients may be induced by a variety of causes. In addition to the trauma-related etiologies, mechanical ventilation itself may contribute to the deterioration of respiratory function through ventilator induced lung injury or the impairment of respiratory muscle function. Ventilator-induced diaphragmatic dysfunction (VIDD) is an entity induced by the inactivity during invasive mechanical ventilation, associated with a profound loss of diaphragm muscle mass.

Methods: In order to assess the incidence of VIDD in polytrauma patients we performed an observational, retrospective, longitudinal study which included 24 polytraumatized patients, who were mechanically ventilated for at least $48 \mathrm{~h}$ and underwent two chest CT scans for clinical reasons during their ICU stay. Diaphragmatic thickness was measured by two independent radiologists on coronal and axial images, at the level of celiac plexus. Statistical analysis was performed using MedCalc 14.1, applying T-test and correlation tests.

Results: Mean age in the study group was 59 years old, APACHE II score had a mean value of 19 and the Injury severity score 35 . The mean duration between chest CT scans was 7 days. The thickness of the diaphragm was significantly decreased on both the left and right sides (left side: $-0.82 \mathrm{~mm}$ axial $p=0.034 ;-0.79 \mathrm{~mm}$ coronal $p=0.05$; right side: $-0.94 \mathrm{~mm}$ axial $p=0.016$; -0.91 coronal $p=0.013$ ). In addition, we obtained a positive correlation between 
the number of days of mechanical ventilation and the difference between the two measurements of the diaphragm thickness on both sides $(r=0.5 ; p=0.02)$. There was no statistically significant correlation between the body mass indexes on admission, use of vitamin $C$ or $\mathrm{N}$-acetyl cysteine and the differences of diaphragmatic thickness. Conclusion: Considering prioritization of life-threatening injuries in the trauma care, secondary dysfunctions such as VIDD are often overlooked. This study provides evidence of early impairment of diaphragm after mechanical ventilation in polytraumatized patients. Moreover, we obtained that a prolonged period of mechanical ventilation is proportional with the loss of diaphragmatic thickness.

\section{0}

A bench assessment of the resistance of expiratory valve of current ICU ventilators in dynamic conditions

A. Pinède ; ${ }^{1}$ M. Cour ${ }^{1}$; F. Degivry'; C. Guérin ${ }^{1}$; B. Louis²; L. Argaud ${ }^{1}$

${ }^{1}$ Service de médecine intensive-réanimation, Hospital Édouard Herriot, Lyon, France; ${ }^{2}$ Inserm 955, IMRB, Creteil, France

Correspondence: C. Guérin

Intensive Care Medicine Experimental 2020, 8(2): 000360

Introduction: We hypothesized that the lack of benefit of low vs. high positive end-expiratory pressure (PEEP) in patients with acute respiratory distress syndrome may partly come from a difference in the dynamic behavior of the active expiratory valve in ICU ventilators.

Objectives: Our goal was to compare the resistance of the expiratory valve across current ICU ventilators on the bench.

Methods: Seven current ICU ventilators (C5, C6, Carescape, PB980, ServoU, V500 and V680) were attached to an ASL 5000 lung model set in passive condition with compliance $20 \mathrm{ml} / \mathrm{cmH} 2 \mathrm{O}$ and resistance 5 $\mathrm{cmH} 2 \mathrm{O} / \mathrm{L} / \mathrm{s}$. Ventilators were set in volume-controlled mode with a tidal volume of $0.8 \mathrm{~L}$ and respiratory rate of 10 breaths $/ \mathrm{min}$. Flow and pressure were measured just before the exhalation valve. PEEP was set at 5, 10 and $15 \mathrm{cmH} 2 \mathrm{O}$. Flow and pressure signals were acquired at $200 \mathrm{~Hz}$ (Biopac 150). On three consecutive breaths the instantaneous expiratory resistance was computed as the pressure drop to atmosphere divided by the flow. The valve opening delay was measured as the maximal slope of pressure-flow relationship from zero flow to peak flow. Finally, the time spent with PEEP above or below the set value, ie the pressure-time product (PTP) was computed. The median value of instantaneous expiratory resistance was taken as the primary end-point. Median (1st-3rd quartiles) were compared by two-factor ANOVA. P-value was set to $P<0.0007$ to take into account the number of comparisons done.

Results: The median instantaneous expiratory resistance was significantly different between ventilators and PEEP with a significant interaction between them. Therefore, ventilators were compared at each PEEP. Between C5, C6, Carescape, PB980, ServoU, V500 and V680, the median expiratory resistance was 3.9 (3.5-4.7), 3.0 (3.0-3.1), 20.9 (15.8-24.9), $27.4(26.5-43.2), 13.8$ (13.6-13.9), $4.4(4.0-4.6)$ and 34.3 $(33.7-33.8) \mathrm{cmH} 2 \mathrm{O} / \mathrm{L} / \mathrm{s}$, respectively, at PEEP5 $(\mathrm{P}<0.0007$ with all pairwise differences $P>0.0007)$. Over all PEEP, the corresponding opening delay was $0.080(0.077-0.082), 0.082(0.080-0.085)$, Carescape 0.110 (0.105-0.110), $0.100(0.085-01.05), 0.072(0.062-0.072), 0.145(0.115-$ $0.150)$ and $0.075(0.070-0.080)$, respectively. Over all PEEP PTP was $2.8(2.1-7.4), 6.8(6.7-7.3), 2.4(2.1-2.4), 3.5$ (2.7-3.6), $1.8(1.8-2.1), 2.8$ (2.7-2.9), and $5.7(5.4-5.9) \mathrm{cmH} 2 \mathrm{O}$.

Conclusion: We found on the bench that the resistance of active expiratory valve significantly differ between seven current ICU ventilators.

\section{1}

Sharing ventilators in the Covid-19 pandemics. A bench study C. Guérin'; M. Cour ${ }^{1}$; N. Stevic ' F. Degivry'; E. L'Her' ${ }^{2}$; B. Louis ${ }^{3}$; L. Argaud ${ }^{1}$ ${ }^{1}$ Service de médecine intensive-réanimation, Hospital Édouard Herriot, Lyon, France; ${ }^{2}$ Service de médecine intensive-réanimation, Hospital Center Regional University Morvan De Brest, Brest, France; ${ }^{3}$ Inserm 955, IMRB, Creteil, France

Correspondence: C. Guérin

Intensive Care Medicine Experimental 2020, 8(2): 000361
Introduction: COVID-19 pandemics has set the healthcare system to a shortage of ventilators. Among the strategies to overcome this burden one is to divide the same ventilator to more than one patient.

Objectives: We aimed at assessing tidal volume (VT) delivery and air recirculation during expiration when one ventilator is divided to 2 patients. We designed the study to mimic nonsymmetrical impairment in respiratory mechanics.

Methods: We performed a bench study in a research lab taking part of a University medical ICU. Four ventilators (V500, Servo-I, MonnalT60 and Elisee350) were attached to $A$ and $B$ test-lungs (QuickLung) through a dedicated flow-splitter (Michelin, France). A $50 \mathrm{~mL} / \mathrm{cmH} 2 \mathrm{O}$ Compliance (C) and $5 \mathrm{cmH} 2 \mathrm{O} / \mathrm{L} / \mathrm{s}$ Resistance (Rp) were set in both (C1 condition), C50Rp20 in A/C20Rp20 in B (C2), C20Rp20 in A/C10Rp20 in $B(C 3)$, and C50Rp20 in A/C20Rp5 in B (C4). Each ventilator was set in volume-controlled (VCV) and pressure-controlled (PCV) mode targeting $0.8 \mathrm{~L}$ as delivered global volume. VT was measured from a pneumotachograph placed immediately before each lung. Values are median (1st-3rd quartiles) and compared between ventilators by Kruskal-Wallis test in each condition.

Results: In VCV VT was greater in A than in B by a factor of 1.83 (1.22-2.07), 2.14 (1.68-2.15), and 1.65 (1.27-1.67) for C2, C3 and C4 conditions, setting $A$ to risk of overdistension. In PCV targeted VT was maintained in each lung for A in C2 (0.388L (0.321-0.540)) and C4 $(0.445 \mathrm{~L}(0.416-0.454))$, but not in C3 $(0.180 \mathrm{~L}(0.169-0.368))$. The corresponding VT in B were $0.230 \mathrm{~L}(0.147-0.342), 0.320 \mathrm{~L}(0.185-0.338)$, and $0.09 \mathrm{~L}(0.08-0.18)$, respectively. Recirculated air was substantial in VCV, $53 \mathrm{~mL}$ in C4, setting risk to cross-infection, but negligible in PCV. Conclusion: Dedicating one ventilator to two or three patients is feasible. For safety reasons, due to dependence of VT to $C$ and risk of crosscontamination, the ventilator should be set in PCV in order to maintain adequate in VT at least in one patient when $\mathrm{C}$ and/or Rp changes abruptly and monitoring of VT should be done carefully.

\section{3}

The assessment of esophageal pressure using different devices: a validation study

A. Ciabattoni ${ }^{1}$; A. Caccioppola ${ }^{1}$; T. Pozzi ${ }^{1}$; AC. Lusardi' ;. De Giorgis';

V. Galanti' ; E. Ferrari'; G. Catzula'; S. Coppola ${ }^{2}$; D. Chiumello ${ }^{2}$

${ }^{1}$ Anesthesia and intensive care, University of Milan, Milano, Italy; ${ }^{2}$ Department of anesthesia and intensive care, asst santi paolo e carlo, San Paolo University Hospital, Milano, Italy

Correspondence: $T$. Pozzi

Intensive Care Medicine Experimental 2020, 8(2): 000543

Introduction: Esophageal pressure monitoring could help clinicians to better tailor ARDS-patients ventilatory setting, to reduce the risk of VILI; however, it has been mainly used in clinical research and not in daily clinical practice, mostly because of technical difficulties and the inability to obtain easy and accurate measurements. Recently, an automatic monitoring system (Optivent, Sidam Srl, Modena, Italy), able to directly measure both airway pressure and esophageal pressure, has been released.

Objectives: Aim of this study was to compare measurements of respiratory mechanics in ARDS patients by using a dedicated manual device, assumed as gold standard, with those obtained by two other systems: Optivent and a bedside equipment. We focused on endexpiratory esophageal pressure, end-expiratory transpulmonary pressure and lung stress.

Methods: Forty mechanically ventilated sedated and paralyzed ARDS patients admitted in the intensive care unit (ICU) from February to September 2019, were enrolled. Patients were ventilated with a tidal volume between $6-8 \mathrm{ml} / \mathrm{kg}$ of predicted body weight and a respiratory rate to maintain an arterial pH between 7.30 and 7.45. Esophageal pressure was measured by using a radiopaque catheter equipped with a balloon (Nutrivent, Sidam Srl., Modena, Italy). Measurements of the airway pressure and esophageal pressure during end-inspiratory and end-expiratory pauses were performed at three levels of PEEP, randomly applied: clinical level, $50 \%$ higher and $50 \%$ lower. 
- Optivent system: Optivent is an automatic self-calibrating system composed by a touchscreen monitor directly connected to the patient with a real time airway and esophageal pressure measurements;

- Bedside system: This hybrid system is composed by the ventilator and the patient's hemodynamic monitor. Airway pressure was measured by the mechanical ventilator and esophageal pressure by a pressure transducer (TruWave/VAMP Plus, Edwards Lifesciences, Unterschleissheim) connected to a hemodynamic monitor.

- Dedicated system: The airway and esophageal pressure were measured by pressure transducers and recorded on a personal computer using a specific software for subsequent analysis (Colligo, Elekton, Milan, Italy).

Results: End-expiratory esophageal pressure measured by the dedicated system, the bedside system and Optivent were $10.0 \pm 4.2,10 \pm$ 4 and $9.9 \pm 4.0 \mathrm{cmH} 2 \mathrm{O}$, respectively. End-expiratory transpulmonary pressures, respectively computed with data acquired by the dedicated system, bedside system and Optivent, were $-1.6 \pm 4.6,-1.4 \pm 4.5$ and $-1.2 \pm 4.3 \mathrm{cmH} 2 \mathrm{O}$. Lung Stress values, respectively computed with data acquired by dedicated system, bedside system and Optivent, were $14.0 \pm 5.8,15.0 \pm 6.6$, and $15.0 \pm 6.1 \mathrm{cmH} 2 \mathrm{O}$. Both Optivent and the bedside system exhibited a high correlation with the dedicated system for all the three investigated variables. The respective bias and limits of agreement between the dedicated system and Optivent and between the dedicated system and the bedside system were as follows: end-expiratory esophageal pressure, $0.25 \mathrm{cmH} 2 \mathrm{O},(-0.4$ to 0.9$)$ and $-0.1 \mathrm{cmH} 2 \mathrm{O}$ ( -1.9 to 1.7$)$; end-expiratory transpulmonary pressure, $-0.6 \mathrm{cmH} 2 \mathrm{O}(-1.7$ to 0.4$)$ and $-0.4 \mathrm{cmH} 2 \mathrm{O}$, (-2.2 to 1.5$)$; lung stress $-0.9 \mathrm{cmH} 2 \mathrm{O}(-3.00$ to 1.10$)$ and $-1.50 \mathrm{cmH} 2 \mathrm{O}(-4.4$ to 1.4$)$.

Conclusion: Both Optivent and the bedside system present clinical acceptability in measuring esophageal pressure and its derived variables. The possibility to apply one of these systems could make the measurement of esophageal pressure easier, with a consequent wider application in clinical practice.

\section{Reference(s) and grant acknowledgment(s)}

1. 2. Mauri T, Yoshida T, Bellani G, Goligher EC, Carteaux G, Rittayamai N, et al. Esophageal and transpulmonary pressure in the clinical setting: meaning, usefulness and perspectives. Intensive Care Med. 2016;42(9):1360-73

2. 1. Akoumianaki E, Maggiore SM, Valenza F, Bellani G, Jubran A, Loring SH, et al. The application of esophageal pressure measurement in patients with respiratory failure. Am J Respir Crit Care Med. 2014;189(5):520-31

\section{5}

Assessing the health and cost benefits of a temporary IMCU with advanced telemonitoring for the treatment of Covid-19 patients

B. Truschel ${ }^{1} ;$ M. Patout ${ }^{2}$; E. Fresnel ${ }^{3} ;$ K. Adrien $^{3} ;$ A. Sabil $^{4}$; L. Sese ${ }^{2} ;$ T. Gille $^{2}$; Y. Cohen ${ }^{2}$; N. Hilario ${ }^{2}$;. Tandjaoui-Lambiotte ${ }^{2}$; L. Milojevic ${ }^{1}$

${ }^{1}$ Research, Philips Respironics, Inc., Murrysville, United States ofAmerica; ${ }^{2}$ Respiratory department, normadie univ, Avicenne Hospital (AP-HP), Bobigny, France; ${ }^{3}$ Institute for research and innovation in biomedicine (irib), KerNel Biomedical, Bois-Guillaume, France; ${ }^{4}$ Research, Philips, Paris, France

Correspondence: $\mathrm{B}$. Truschel

Intensive Care Medicine Experimental 2020, 8(2): 000655

Introduction: In northern France during March of 2020, a surge in patients presenting withinfection and acute respiratory failure (ARF) from the novel 2019 coronavirus prompted urgent action. The surge created a heavy burden on French hospital facilities and finances. This paper contains an assessment of the health and cost benefits obtained by the construction of a temporary intermediate care unit(IMCU) with an advanced remote monitoring system. Facilities that include anIMCU in combination with ICUs have demonstrated significantly lower mortality rates in large multinational studies. [1] Improved health outcomes were an expected result. Furthermore, the addition of real time patient remote monitoring in the form of telemedicine in ICUs has been proven to reduce both mortality and length of stay (LOS) within critical care. [2] Over a three-day period, a team built a temporary IMCU with an advanced monitoring system in Avicenne Hospital,Bobigny France.

Methods: Mimicking modern ICU and emergency department telemonitoring systems, the network in the IMCU consisted of a 10-bed facility connected to a central workstation. The central workstation allowed a staff-worker who remained isolated from the infected patients to monitor respiratory data and pulse oximetry.

The system would display real time data along with patient alarms through aconnection to a dedicated USB port on a portable life-support ventilator, (TrilogyEvo, Philips Respironics, USA). The workstation had dedicated software to reviewall patient data and it included a mathematical algorithm determining the degree ofpatient-ventilator asynchrony.[3] This station contained an early warning system(EWS) to alert the clinical staff in the IMCU when it was appropriate to intervene.

Results: As a result, 40 patients were treated during the month of April in the AvicennelMCU. Twenty-four patients (60\%) treated at the IMCU had ARF but wereconsidered ineligible for ICU due to frailties such as age $(\geq 80)$ or high-riskcomorbidities. The expected mortality of this critical population with ARF is near49\% $[4,5] .4$ patients in this cohort $(17 \%)$ died, thus exceeding survival expectations. 12 patients (30\%) were admitted into the IMCU due to a progression from mild to severe Covid 19 related hypoxemia. Within this cohort, 4 patients(33\%) progressed to the ICU. 4 patients (10\%) were admitted into the IMCU following discharge from the ICU, and 1 patient (25\%) was readmitted to the ICU.

The incidence of alerts prompting intervention were $\mathrm{HR}(<50$ or $>120)$ $=3.1 \pm 7.5$ alarm/hour, SpO2 $(<90)=8.1 \pm 10.0$ alarm $/ \mathrm{hr}$ and leak $(>40 \mathrm{~L} / \mathrm{min})=6.7 \pm 12.9 \mathrm{alarm} / \mathrm{hr}$. (Total $=17.9 \mathrm{alerts} / \mathrm{hour}) .280 \mathrm{ICU}$ bed days were spared in the month of April 2020 due to the construction of this ICU. According to the cost data collected from 7 European countries, the average daily cost in ICU is approximately $1850 €$, resulting in a computed savings of $518 \mathrm{k} €[6]$.

Conclusion: The construction of a temporary IMCU, using advanced telemonitoring with anEWS, successfully improved patient outcomes and reduced the financial burden on the hospital system. The frequency of interventive care recorded within this unit was once per 3.5 minutes on average. Without alerts, this level of care would require continuous, rotating bedside contact by staff. Consequently, the directed interventions from the central workstation may have reduced exposure times.

\section{Reference(s) and grant acknowledgment(s)}

1. [1] M. Capuzzo, C. A. Volta, T. Tassinati et al., Hospital mortality of adults admitted to intensive care units in hospitals $w$ ith and $w$ ithout intermediate care units: a multicentre European cohort study, Critical Care, vol. 18, no. 5, article no. 18, 2014.

2. [2] Sajeesh Kumar, Shezana Merchant and Rebecca Reynolds, Tele-ICU: Efficacy and Cost-Effectiveness Approach of Remotely Managing the Critical Care, The Open Medical Informatics Journal, 2013, 6, 24-29

3. [3] Emeline Fresnel, Adrien Kerfourn, Antoine Cuvelier, Maxime Patout, Automated detection of patient-ventilator asynchronies during noninvasive ventilation (NIV) European Respiratory Journal 2019 54: OA3828; https://doi. org/10.1183/13993003.congress- 2019.0A3828

4. [4] Emanuel EJ, Persad G, Upshur R, et al. Fair allocation of scarce medical resources in the time of Covid-19. N Engl J Med. 2020; (published online March 23.)

5. [5] Wu Z, McGoogan JM. Characteristics of and important lessons from the coronavirus disease 2019 (COVID-19) outbreak in China: summary of a report of 72314 cases from the Chinese Center for Disease Control and Prevention. JAMA 2020 February 24 (Epub ahead of print).

6. [6] Bittner et al. How is intensive care reimbursed? A review of eight European countries, Annals of Intensive Care 2013, 3:37 
000687

A tidal volume calculator to improve lung protective ventilation in COVID-19 related Acute Respiratory Distress Syndrome (ARDS) J. Blair ${ }^{1}$; A. Baldwin ${ }^{2}$; S. Hester ${ }^{1}$; T. Ali ${ }^{2}$

${ }^{1}$ Intensive care, The Shrewsbury and Telford Hospital NHS Trust, Shrewsbury, United Kingdom; 'Intensive care, Stoke Mandeville Hospital, Stoke Mandeville, United Kingdom

Correspondence: J. Blair

Intensive Care Medicine Experimental 2020, 8(2): 000687

Introduction: Routine use of lower tidal volumes (TVs) for the mechanical ventilation of patients with ARDS results in decreased mortality and increases the number of days without ventilator use [1]. Severe COVID-19 pneumonia has been associated with the development of ARDS as characterised by the Berlin definition [2]. A multi-centre preliminary audit was undertaken to identify whether ventilated COVID-19 related ARDS patients were receiving optimal TVs, as recommended by the Faculty of Intensive Care Medicine (FICM) and Intensive Care Society (ICS) ARDS management guidelines [3].

Objectives: As a result of the audit, three main areas for improvement were identified.

1. To achieve accurate calculations for ideal body weight (IBW) and target TV

2. To improve documentation of IBW and target TV

3. To achieve TVs no greater than $6 \mathrm{ml} / \mathrm{kg}$

Methods: A 'tidal volume calculator' tool was developed using Microsoft Excel, which was simple, colour coded and kept on all Intensive Care Unit (ICU) computer desktops. This tool was designed to use height to calculate IBW and, if the patient's height was unavailable, could also be utilised to calculate height from ulna length. IBW was subsequently used to calculate a target TV. Staff received training on how to apply the tool.

Two snapshot audits were carried out in April and May 2020 at two ICUs. The first was conducted prior to the tool's introduction with the second two weeks after its implementation. All patients receiving mechanical ventilation, except those spontaneously breathing, were included. Data was extracted from patient notes, charts and ventilator settings.

Results: The initial audit included 14 patients. Six patients did not have an IBW documented. Three patients had documented IBWs that were $12 \mathrm{~kg}, 15 \mathrm{~kg}$ and $23 \mathrm{~kg}$ greater than the weight calculated using the tool, leading to increased tidal volume targets. Only three patients were achieving TVs of 4-6 ml/kg. Eleven patients were achieving a TV greater than $6 \mathrm{ml} / \mathrm{kg}$, with two of these achieving a TV of greater than $8 \mathrm{ml} / \mathrm{kg}$.

The follow-up audit included ten patients. This revealed that all patients had an IBW clearly documented. Moreover, nine patients were achieving TVs within 4-6 ml/kg, with only one patient found to be achieving a TV greater than $6 \mathrm{ml} / \mathrm{kg}$.

Conclusion: This audit cycle revealed that initially adherence to lung protective ventilation and documentation of IBW was poor. In some instances, documented IBW was vastly different to the calculated IBW, suggesting that in these situations actual body weight may have been used.

In a time when clinicians were being redeployed to support ICU, this simple tool was shown to support staff by clearly calculating and displaying IBW and target TV for reference. This directly led to improved adherence to lung protective ventilation and optimisation of patient care.

Limitations include that no consideration was made for overall patient outcome, and only a snapshot of achieved TVs from ventilators were recorded; daily/weekly trends were not studied.

\section{Reference(s) and grant acknowledgment(s)}

1. 2. ARDS Definition Task Force, Ranieri VM, Rubenfeld GD, et al (2012) Acute respiratory distress syndrome: the Berlin Definition. JAMA 307:2526-2533

2. 3. The Faculty of Intensive Care Medicine, Intensive Care Society (2018) Guidelines on the management of Acute Respiratory Distress Syndrome. https://www.ficm.ac.uk/sites/default/files/ficm_ics_ards_guideline_-_ july_2018.pdf. Accessed 11 June 2020

3. 1. Acute Respiratory Distress Syndrome Network, Brower RG, Matthay MA, et al (2000) Ventilation with lower tidal volumes as compared with traditional tidal volumes for acute lung injury and the acute respiratory distress syndrome. N Engl J Med 342:1301-1308

\section{6}

End tidal $\mathrm{CO} 2$ in out of hospital intubated critically ill patients: Is it a reliable surrogate for plasma $\mathrm{CO} 2$ concentration?

S. Naeem ${ }^{1}$; S. Bloomfield ${ }^{2}$; J. Leung ${ }^{3}$; R. Lyon ${ }^{4}$

${ }^{1}$ Accident and Emergency, East Kent Hospitals University Foundation Trust, Maidstone, United Kingdom; ${ }^{2}$ Accident \& emergency, East Kent Hospitals University Foundation Trust, Maidstone, United Kingdom; ${ }^{3}$ Accident \& emergency, East Kent Hospitals University Foundation Trust, Ashford, United Kingdom; ${ }^{4}$ Hems, Air Ambulance Kent Surrey Sussex, Redhill, United Kingdom

Correspondence: S. Naeem

Intensive Care Medicine Experimental 2020, 8(2): 000896

Introduction: End tidal $\mathrm{CO} 2$ (ETCO2) monitoring is a standard for all intubated patients in the pre-hospital environment. ETCO2 is used as surrogate marker for determining plasma $\mathrm{CO} 2$ concentration (pCO2) in intubated patients as pCO2 measurement is not readily available in the resource limited pre-hospital environment. Maintenance of normocapnia $(4.4-5 \mathrm{kPa}$ ) is crucial for neuroprotection in critically ill patients.

Objectives: The objective was to determine whether ETCO2 can serve as a reliable surrogate marker for pCO2.

Methods: Data was retrospectively collected from the Air Ambulance Kent, Surrey and Sussex hospital database for critically ill non-trauma patients conveyed to the William Harvey Hospital between September 2017 and March 2019. The latest pre-hospital ETCO2 value was paired with pCO2 values from initial blood gas results. Patient demographics, pre-hospital haemodynamic instability (use of vasopressor), other blood gas parameters and mortality within the same admission were also recorded. The paired data sets were analysed using the MannWhitney test.

Results: 18 data sets that met the inclusion criteria were generated Median ETCO2 and pCO2 was $4.4 \mathrm{kPa}$ (IQR 3.9-4.8 kPa) and 7.3 kPa (6.5$9.4 \mathrm{kPa}$ ) respectively. Median difference between ETCO2 \& pCO2 was significant $3.0 \mathrm{kPa}(\mathrm{p}<0.001, \mathrm{Cl} 2.6-3.6 \mathrm{kPa})$. Median ETCO2,pCO2 and difference was $4.4 \mathrm{kPa}$ (IQR 4.0- $4.5 \mathrm{kPa}$ ), 7.2 kPa (IQR 6.5-8.0 kPa) and $3.3 \mathrm{kPa}$ (IQR 2.1-3.6 kPa) respectively in patients who survived. Median ETCO2, pCO2 and difference was $4.3 \mathrm{kPa}$ (IQR 4.0-4.5 kPa), 8.3 kPa (IQR 7.0-11.3 kPa) and 3.0 $\mathrm{kPa}(\mathrm{IQR} 2.9-4.5 \mathrm{kPa})$ respectively in patients who died. Median difference was not significantly associated with mortality $(p=0.69)$. The median difference between the pCO2 and ETCO2 in cardiovascularly stable vs unstable patients was $3.0 \mathrm{kPa}$ and $3.4 \mathrm{kPa}$ and was not statistically significant $(p=0.59)$.

Table 1: Comparison of ETCO2 and PCO2 values

\begin{tabular}{|l|l|l|c|l|}
\hline Measurement & n & Median [IQR] & Difference $\left(^{*}\right)$ & P-value \\
\hline & & & & \\
\hline ETCO2 & 18 & $4.4[3.9,4.8]$ & 0 & \\
\hline PCO2 & 18 & $7.3[6.5,9.4]$ & $3.0(2.6,3.6)$ & $<\mathbf{0 . 0 0 1}$ \\
\hline & & & & \\
\hline
\end{tabular}

(*) Differences calculated as PCO2 - ETCO2

\begin{tabular}{|l|l|l|l|l|l|}
\hline Measurement & Stable & \multicolumn{3}{|l|}{ Unstable } & P-value \\
\hline & $\mathrm{n}$ & Median [IQR] & Median [IQR] & \\
\hline & & & & & \\
\hline ETCO2 & 7 & $4.5[4.0,5.5]$ & 11 & $4.3[3.5,4.4]$ & 0.13 \\
\hline PCO2 & 7 & $7.3[6.5,8.8]$ & 12 & $7.6[6.6,10.7]$ & 0.93 \\
\hline CO2 difference $(*)$ & 7 & $3.0[2.5,3.3]$ & 11 & $3.4[2.1,4.5]$ & 0.59 \\
\hline & & & & & \\
\hline
\end{tabular}

(*) Differences calculated as PCO2 - ETCO2 
Conclusion: $\mathrm{ETCO} 2$ is an unreliable surrogate marker of $\mathrm{pCO} 2$ and consistently underestimates the true pCO2 value by $3.0 \mathrm{kPa}$ [C.I 2.6$3.6 \mathrm{kPa}$. The use of vasopressor does not appear to significantly impact the ETCO2 and pCO2 difference. Hence ETCO2 should not be used to determine normocapnia in the pre-hospital environment.

\section{Reference(s) and grant acknowledgment(s)}

1. Lewis L.M. Stothert J. Standeven J. Chandel B. Kurtz M. Fortney J. Correlation of end-tidal CO2 to cerebral perfusion during CPR. Ann Emerg Med. 1992; 21:1131-1134

2. Tang W. Weil M.H. Gazmuri R.J. Sun S. Duggal C. Bisera J. Pulmonary ventilation/perfusion defects induced by epinephrine during cardiopulmonary resuscitation. Circulation. 1991; 84: 2101-2107

3. T. M. Cook, N. Woodall, C. Frerk, on behalf of the Fourth National Audit Project, Major complications of airway management in the UK: results of the Fourth National Audit Project of the Royal College of Anaesthetists and the Difficult Airway Society. Part 1: Anaesthesia, BJA: British Journal of Anaesthesia, Volume 106, Issue 5, May 2011, Pages 617-631, https://doi.org/10.1093/ bja/aer058

\section{2}

Does the introduction of a ventilation team improve compliance with lung protective ventilation for patients with COVID-19?

A. Harriman'; P. Isherwood ${ }^{2}$; D. McWilliams ${ }^{3}$

${ }^{1}$ Therapy services, Queen Elizabeth Hospital Birmingham, Birmingham, United Kingdom; ${ }^{2}$ Critical care, Queen Elizabeth Hospital Birmingham, Birmingham, United Kingdom; ${ }^{3}$ Critical care rehab team, Queen Elizabeth Hospital Birmingham, Birmingham, United Kingdom

Correspondence: D. McWilliams

Intensive Care Medicine Experimental 2020, 8(2): 000942

Introduction: Lung protective ventilation (LPV) is an essential strategy in the management of patients mechanically ventilated due to severe respiratory failure, including those with COVID-19. When utilised, LPV has been proven to reduce absolute mortality by $8.8 \%$ and significantly increase ventilator free days (1). Despite this, a recent study of 459 ICUS's from across 50 countries found that only $65 \%$ of patients received adequate LPV and $35 \%$ actually received potentially harmful volumes [2]. To manage the increased numbers of mechanically ventilated patients during the COVID-19 pandemic, a ventilation team was created to support the management and adherence to LPV targets.

Objectives: To evaluate the impact of a multi-faceted approach to ventilatory management on adherence to LPV targets.

Methods: A new supportive ventilation team was created, utilising existing members of the physiotherapy service with training taking place in March 2020, with a focus on promoting compliance with LPV. After an initial training period, this team implemented a multi-faceted approach to ventilatory management. This included measurement of patient height on admission and calculation of ideal body weight, documented LPV targets in each bed space on whiteboards and twice daily physiotherapy led ventilation ward rounds to review and amend ventilator settings as required. Hourly tidal volume data was collected for all patients mechanically ventilated over a 2 month period during the COVID-19 pandemic (April-May 2020) and compared to a 2 month baseline period (January-February 2020). Primary outcome was compliance with target tidal volumes of $<8 \mathrm{ml} / \mathrm{kg}$, with secondary outcome was PEEP/FiO2 compliance. Data was analysed using Chi Square test.

Results: A total of 62,656 hours of mechanical ventilation were included for analysis, with the results presented in table. 1. The introduction of the ventilation team was associated with a significant increase in compliance with target tidal volumes of $<8 \mathrm{ml} / \mathrm{kg}$ (76\% vs $69 \%, \mathrm{p}<0.0001)$ and a significant reduction in occurrence of volumes $>10 \mathrm{ml} / \mathrm{kg}(4 \%$ vs $10 \%, \mathrm{p}<0.0001)$. No significant differences were observed with regards to PEEP/Fi02 compliance.

\begin{tabular}{|l|l|l|l|}
\multicolumn{5}{c|}{ Table 1 - Results } \\
\hline & $\begin{array}{l}\text { Baseline (Pre } \\
\text { COVID) }\end{array}$ & $\begin{array}{l}\text { Ventilation Team } \\
\text { (COVID) }\end{array}$ & $\mathrm{p}$ \\
\hline $\mathrm{n}$ & 25237 & 38529 & \\
\hline$<8 \mathrm{mls} / \mathrm{kg}$ (hours) & $17290(69 \%)$ & $28086(76 \%)$ & $<0.0001$ \\
\hline $\begin{array}{l}>10 \mathrm{mls} / \mathrm{kg} \\
\text { (hours) }\end{array}$ & $2590(10 \%)$ & $1424(4 \%)$ & $<0.0001$ \\
\hline
\end{tabular}

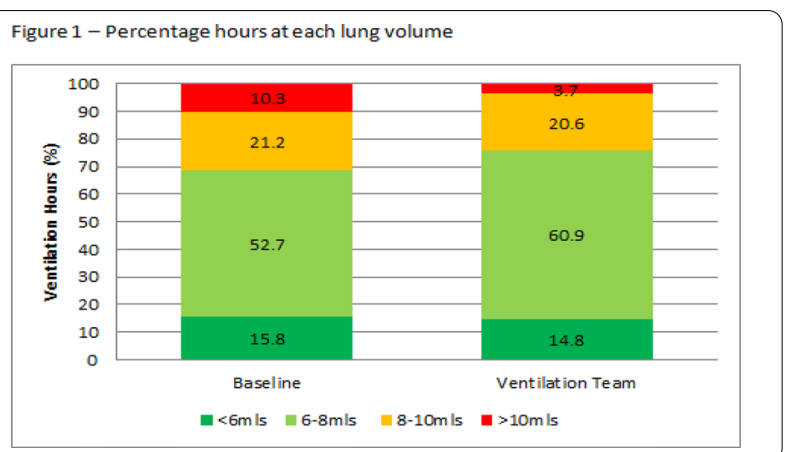

Conclusion: The implementation of a specialist ventilation team was associated with a significant improvement in compliance with LPV targets for tidal volumes. This also led to a significant reduction in the percentage of ventilation hours in which patients may receive potentially harmful tidal volumes.

\section{Reference(s) and grant acknowledgment(s)}

1. 2. Bellani G, Laffey JG, Pham T, et al. Epidemiology, Patterns of Care, and Mortality for Patients With Acute Respiratory Distress Syndrome in Intensive Care Units in 50 Countries. JAMA. 2016;315(8):788-800. https://doi. org/10.1001/jama.2016.0291

2. 1. The Acute Respiratory Distress Syndrome Network. Ventilation with lower tidal volumes as compared with traditional tidal volumes for acute lung injury and the acute respiratory distress syndrome. The New England journal of medicine. 2000; 342(18):1301-8. [PubMed: 10793162] 3. N/A

000968

Central venous pressure swing outperforms diaphragm ultrasound as a measure of inspiratory effort during pressure support ventilation in covid-19 patients

S. Lassola'; S. Miori'; A. Sanna ${ }^{1}$; A. Cucino ${ }^{1}$; S. Magnoni'; M. Umbrello ${ }^{2}$

${ }^{1}$ Anesthesia and intensive care, OSPEDALE S. CHIARA APSS, Trento, Italy; ${ }^{2}$ Anestesia e rianimazione, Ospedale San Paolo - Polo Universitario, ASST Santi Paolo e Carlo, Milano, Italy

Correspondence: S. Magnoni

Intensive Care Medicine Experimental 2020, 8(2): 000968

Introduction: An increasing number of patients required prolonged mechanical ventilation during the recent COVID-19 pandemic. The associated shortage of ICU beds and monitoring devices magnified the need of simple tools to properly titrate the level of ventilator assistances. Observation of breathing pattern with utilization of accessory muscles, rapid or shallow breathing, or the inspection of ventilator waveforms are not useful to asses breathing efforts in a quantitative way. Thus, appropriate bedside monitoring of inspiratory effort is strongly required.

Objectives: We investigated whether bedside-available indices such as the ultrasonographic changes in diaphragm thickening ratio (TR) and the tidal swing in central venous pressure $(\Delta C V P)$ are reliable 
estimates of respiratory effort, as assessed by the tidal swing in esophageal pressure ( $\triangle \mathrm{Pes})$.

Methods: Fourteen critically-ill patients were enrolled (age $64 \pm 7$ years, BMI $29 \pm 4 \mathrm{~kg} / \mathrm{m} 2$ ), after 6 [3; 9] days from onset of assisted ventilation. A three-level pressure support trial was performed, at 10 (PS10), 5 (PS5) and $0 \mathrm{cmH} 2 \mathrm{O}$ (PS0). In each step, the esophageal and central venous pressure tidal swing were recorded, as well as diaphragm ultrasound.

Results: The reduction of pressure suppor was associated with an increased respiratory rate and a reduced tidal volume, while minute ventilation was unchanged. $\triangle$ Pes significantly increased with reducing support $(5[3 ; 8]$ vs. $8[14 ; 13]$ vs. $12[6 ; 16] \mathrm{cmH} 2 \mathrm{O}, \mathrm{p}<0.0001)$, as did the diaphragm TR $(9.2 \pm 6.1$ vs. $17.6 \pm 7.2$ vs. $28.0 \pm 10.0 \%, \mathrm{p}<0.0001)$ and the $\Delta$ CVP $(4[3 ; 7]$ vs. $8[5 ; 9]$ vs. $10[7 ; 11] \mathrm{cmH} 2 \mathrm{O}, \mathrm{p}<0.0001) . \Delta \mathrm{CVP}$ was significantly associated with $\triangle \mathrm{Pes}(\mathrm{R} 2=0.810, \mathrm{p}<0.001)$, as was diaphragm TR, albeit with a lower coefficient of determination $(R 2=0.399$, $\mathrm{p}<0.001$ ). In a multivariable, linear, fixed-effects regression, only $\Delta$ CVP was significantly associated with $\Delta$ Pes (coefficient: $1.45 \pm 0.16$, $\mathrm{p}<0.001$ ), while diaphragm TR was not (coefficient: $-0.07 \pm 0.05, \mathrm{p}=$ $0.184)$.

Conclusion: In patients with COVID-19-associated respiratory failure undergoing assisted mechanical ventilation, $\triangle \mathrm{CVP}$ is a better estimate of inspiratory effort than diaphragm ultrasound.

\section{Reference(s) and grant acknowledgment(s)}

1. Grasselli G, Pesenti A, Cecconi M. Critical Care Utilization for the COVID19 Outbreak in Lombardy, Italy: Early Experience and Forecast During an Emergency Response. JAMA 2020

2. Roger C, Muller L, Riou B, et al. Comparison of different techniques of central venous pressure measurement in mechanically ventilated critically ill patients. Br J Anaesth 2017; 118: 223-31

3. Umbrello M, Chiumello D. Interpretation of the transpulmonary pressure in the critically ill patient. Ann Transl Med 2018; 6: 383

4. Bellani G, Mauri T, Coppadoro A, et al. Estimation of patient's inspiratory effort from the electrical activity of the diaphragm. Crit Care Med 2013; 41: 1483-91

5. This study was supported by internal departmental funding.

\section{9}

Noninvasive ventilation (NIV) in an intermediate care unit

H. van Nieuw Amerongen'; JJ. Spijkstra'; L. Heunks

'Intensive care volwassenen, Amsterdam UMC, Amsterdam, Netherlands

Correspondence: $\mathrm{H}$. van Nieuw Amerongen

Intensive Care Medicine Experimental 2020, 8(2): 000089

Introduction: In the past decades noninvasive ventilation (NIV) has obtained an important role in the treatment of patients with acute respiratory failure (ARF). Most NIV studies have been conducted in an intensive care unit (ICU) setting. There are studies which have shown that NIV can be performed safely and effectively outside the ICU. The outcome of patients failing NIV in an intermediate care unit remains to be established. The aim of the current study was to investigate the safety and feasibility of providing acute NIV in a heterogeneous group of patients with ARF in an intermediate care unit.

Methods: This study was a retrospective analysis of patients admitted to the intermediate care unit of Amsterdam UMC, location VUmc between January 2010 and January 2016. Patients with acute respiratory failure were included as well as those whose respiratory status slowly deteriorated, whatever underlying disease. Patients with CPAP at home or obstructive sleep apnea syndrome (OSAS) were excluded. Patients were subdivided into two categories: Hypercapnic failure and non-hypercapnic failure before the start of NIV. In both groups we analyzed admission source and their outcome (discharge destination).
Results:

\begin{tabular}{|c|c|c|c|}
\hline \multicolumn{4}{|c|}{ RESULTS. } \\
\hline & Total group of & f Hypercapnia & $\begin{array}{l}\text { Non } \\
\text { hypercapnia }\end{array}$ \\
\hline & patients & group & Group \\
\hline n (\%) & $241(100)$ & $75(31)$ & $152(63)$ \\
\hline \multicolumn{4}{|l|}{$\begin{array}{l}\text { Demographic } \\
\text { characteristics }\end{array}$} \\
\hline Male sex $(\%)$ & $150(62)$ & $43(57)$ & $100(66)$ \\
\hline Age (SD) & $66(16)$ & $69(14)$ & $64(16)$ \\
\hline \multicolumn{4}{|l|}{ Location prior to admission } \\
\hline General ward (\%) & $53(22)$ & $28(37)$ & $28(18)$ \\
\hline Intensive Care Unit (\%) & $110(46)$ & $22(29)$ & $81(53)$ \\
\hline $\begin{array}{l}\text { Post Anaesthesia Care Unit } \\
(\%)\end{array}$ & $32(13)$ & $9(12)$ & $19(13)$ \\
\hline Emergency Department (\%) & $39(16)$ & $14(19)$ & $20(13)$ \\
\hline Other $(\%)$ & $7(3)$ & $2(3)$ & $4(3)$ \\
\hline \multicolumn{4}{|l|}{ Discharge location } \\
\hline General ward (\%) & $109(45)$ & $25(33)$ & $76(50)$ \\
\hline Intensive Care Unit (\%) & $85(35)$ & $28(37)$ & $54(36)$ \\
\hline Died $(\%)$ & $33(14)$ & $19(25)$ & $13(9)$ \\
\hline Other destinations (\%) & $14(6)$ & $3(4)$ & $9(6)$ \\
\hline
\end{tabular}

NIV at intermediate care $(n=241)$ :

No arterial blood gas before start NIV $(\mathrm{n}=14)$

Hypercapnia $(n=75)$ :

Endotracheal intubation $(n=23): 4$ patients died and 19 survived

No endotracheal intubation ( $n=52): 23$ patients died and 29 survived

Non hypercapnia $(\mathrm{n}=152)$ :

Endotracheal intubation ( $\mathrm{n}=43): 10$ patients died and 33 survived

No endotracheal intubation $(n=109)$ : 19 patients died and 90 survived

The admission diagnosis was heterogeneous: $36 \%$ of all the patients who received NIV was admitted to the intermediate care unit primarily for respiratory failure. The duration of NIV treatment ranged from 1 hour to more than 40 hours. Overall 61 of the 241 patients treated with NIV died within 28 days of the start of NIV on the intermediate care. They died either in de intermediate care unit (33 patients), or in the ICU after the transfer from intermediate care unit (12 patients), or elsewhere (16 patients). Patients dying in the intermediate care unit had do-not-intubate orders.

Conclusion: We found that it is feasible to treat patients with NIV in an intermediate care unit, both for hypercapnic and non-hypercapnic indications. There is a need for more studies to confirm the safety of providing NIV in an intermediate care setting. 
000375

Can a peak C-Reactive Protein in viral pneumonias patients during COVID-19 pandemic predict CPAP failure leading to invasive ventilation in Intensive Care? (Retrospective analysis)i T. Wray ; M. Mazalkov²

${ }^{1}$ Intensive Care, Peterborough City Hospital, Peterborough, United Kingdom; ${ }^{2}$ Anaesthetics, Peterborough City Hospital, Peterborough, United Kingdom

Correspondence: T. Wray

Intensive Care Medicine Experimental 2020, 8(2): 000375

Introduction: C-reactive protein (CRP) is an acute inflammatory protein known to increase in those with COVID-19 pneumonia caused by the novel SARS-CoV-2 virus. Several studies have correlated an increased in pneumonia severity in those with higher CRP levels. Patients with severe/critical COVID-19 infections often require additional support with oxygenation and ventilation in the intensive care unit (ICU) in the form of Continuous Positive Airway Pressure (CPAP) or invasive mechanical ventilation (IMV).

Some critical care societies had advised during the initial phases of the 2020 pandemic for an "earlier" intubation in those with hypoxia resistant to standard oxygen therapy. Intubation and mechanical ventilation comes with its own risks, from the difficulty of intubating the critically ill patient to other ventilator associated complications in those who may have improved on CPAP alone. With CPAP there are concerns over viral aerosolization transmission to healthcare staff and insufficient hospital oxygen supply if it is extensively used during pandemic. A proportion of those started on CPAP will deteriorate and require intubation, but as yet there seems to be little evidence if CRP can help predict those who might deteriorate.

Objectives: To see if the initial peak CRP in patients started on CPAP can predict those who will go on to need invasive mechanical ventilation.

Methods: We included patients accepted to the Intensive Care Unit (ICU) during February to May 2020 who presented with viral pneumonia (either a laboratory confirmed SARS-CoV-2 viral PCR test or strong clinical suspicions of a COVID-19 infection) causing hypoxia refractory to standard ward oxygen supplementation. Patients in ICU were either immediately intubated and mechanically ventilated (primary IMV), or started on CPAP. No high flow nasal oxygenation was used at this hospital. Some of these patients improved on CPAP only and were successfully stepped down from ICU to the wards. Others on CPAP deteriorated and were subsequently intubated (CPAP failure). Excluded from the study were; patients on CPAP who died in $I C U$, patients who had a limit on respiratory therapeutic level of care to CPAP only, or were on still on CPAP in ICU at time of data collection. All patients included had their CRP $(\mathrm{mg} / \mathrm{L})$ measured at hospital admission and 1 st peak level.

The results are presented as full sets of numbers for respective groups. Statistical analysis was performed using Kruskal-Wallis test.

Results: A total of 40 patients with viral pneumonia were identified. Of those in the CPAP group, 3 were excluded from analysis. This meant there were $70.3 \%(n=26 / 37)$ in the CPAP arm and $29.7 \%(n=11 / 37$ mean age 67) who underwent primary IMV. Of those who started on CPAP $62.0 \%$ ( $n=16 / 26$, mean age 56.4 ) were successfully discharged with only CPAP, while $38.5 \%$ ( $n=10 / 26$, mean age 55$)$ failed CPAP and underwent secondary intubation.

Patients for whom CPAP was successful had a mean peak CRP of $\underline{240}$ $(110,145,153,162,173,228,230,244,245,254,268,287,304,324,350$ and 359). Those who failed CPAP and underwent secondary intubation had a mean peak of $353(204,283,298,323,329,338,345,346$ 526 and 538). For the primary intubation group the mean peak CRP was $342(192,212,237,314,345,345,349,349,377,493$ and 547).

The difference in the peak CRPs was found to be statistically significant between the CPAP only group when compared to the CPAP failure group ( $p=0.0098)$. The difference between the CPAP only group and primary IMV was also significant $(p=0.023)$.
Time difference between the date of peak CRP and date of intubation from failure of CPAP ranged between 4 days prior to 6 days post intubation. Only in half of the cases $(n=5 / 10)$ did the CRP peak before intubation.

Time taken from starting CPAP to CPAP failure on ICU was between 1-8 days (median 3.5).

The 1 month mortality was higher in the primary intubation group at $45.5 \%(n=5 / 11)$. There were $2 / 16$ deaths $(12.5 \%)$ in the CPAP only group and $3 / 10$ (33.3\%) who died in the failed CPAP group.

Conclusion: Whilst a small study, these results seem to indicate that patients with a higher peak CRP on CPAP with COVID-19 pneumonia are more likely to fail CPAP support and require intubation than those with lower values. However at present we cannot advocate the use of peak CRP as a predictor for CPAP failure with current data showing that CRP peaked in half cases after intubation. The authors feel that a larger population is needed to see if the peak CRP (or CRP at time of ICU admission) could be used to prognosticate CPAP failure in COVID19 pneumonias.

\section{Reference(s) and grant acknowledgment(s)}

1. None

2. Cook, T., El-Boghdadly, K., McGuire, B., McNarry, A., Patel, A., \& Higgs, A. (2020, Mar 27). Consensus guidelines for managing the airway in patients with COVID-19. Guidelines from the Difficult Airway Society, the Association of Anaesthetists the Intensive Care Society, the Faculty of Intensive Care Medicine and the Royal College of Anaesthetists.

3. Griesdale, D., Bosma, T., Kurth, T., Isac, G., \& Chittock, D. (2008, Oct). Complications of endotracheal intubation in the critically ill. Intensive Care Medicine, 34 (10), 1835-42.

4. NHS England and NHS Improvement Estates and Facilities. (31 Mar 2020). Use of high flow Oxygen therapy devices (including wall CPAP and high flow face mask or. NHS England and NHS Improvement Estates and Facilities.

5. Young, B., ONG, S., Kalimuddin, S., \& et al. (2020). Epidemiologic Features and Clinical Course of Patients Infected With SARS-CoV-2 in Singapore. JAMA, 323(15), 1488-1494.

6. Arulkumaran, N., Brealey, D., Howell, D., \& Singer, M. (2020, Apr 20). Use of non-invasive ventilation for patients with COVID-19: a cause for concern? The Lancet.

7. Chen, Y., \& Lanjuan, L. (2020, May 01). SARS-CoV-2: virus dynamics and host response. The Lancet: Infectious Diseases, 20 (5), 515-516.

8. National Emergency Critical Care Committee, Intensive Care Society, UCL Partners, NIHR. (3 Apr 2020). COVID-19: a synthesis of clinical experience in UK intensive care settings. The Intensive Care Society. Retrieved May 6, 2020, from https://www.ics.ac.uk/ICS/Pdfs/COVID-19/NECCC_Thema tic_Analysis_Summary.aspx

9. Ruan, Q., Yang, K., Wang, W., Jiang, L., \& Song, J. (2020, March 3). Clinical predictors of mortality due to COVID-19 based on an analysis of data of 150 patients from Wuhan, China. Intensive Care Medicine .

\section{9}

CPAP versus standard oxygen in acute respiratory failure sustained by SARS-CoV-2

M. Giovini ${ }^{1}$; C. San Nicola ${ }^{1}$; M. Bergonzi ${ }^{1}$; M. Barbera ${ }^{1}$; E. Antonucci ${ }^{1}$ ${ }^{1}$ Intermediate care unit, Ospedale "Guglielmo da Saliceto", Piacenza, Italy Correspondence: $\mathrm{E}$. Antonucci

Intensive Care Medicine Experimental 2020, 8(2): 000419

Introduction: Severe acute respiratory syndrome coronavirus 2 (SARS-CoV-2) outbreak was declared pandemic on March 2020, due to increasing number of cases around the world. Patients with SARSCoV-2 can develop coronavirus disease 2019 (COVID-19), which has resulted in high rates of intensive care unit (ICU) admission 1 . Alternatives to endotracheal intubation (ETI) such as continuous Positive Airvway Pressure (C-PAP) were adopted to supply the lack of ICU beds during this extraordinary pandemic. C-PAP can be used such as a "bridge" to ETI in intermediate care units (IMCU) or in general wards. 
Objectives: We hypothesized that C-PAP can reduce ETI and mortality rates when compared to standard oxygen (SO) in COVID-19 patients with moderate-to-severe acute respiratory failure (ARF).

Methods: We performed a retrospective analysis, reviewing all COVID19 patients with moderate-to-severe ARF and admitted to our IMCU from March 1st to March 31st 2020. The inclusion criteria were age $\geq$ 18 years old; diagnosis of COVID-19 by a nasopharyngeal swab; moderate-to-severe ARF at IMCU admission $(\mathrm{PaO} / \mathrm{FiO} 2$ ratio $<200 \mathrm{mmHg}$ during oxygen therapy at a flow rate $>10 \mathrm{~L} / \mathrm{min}$ for at least $15 \mathrm{~min}-$ utes); oxygenation with SO or helmet CPAP (stand-alone system) for 2 consecutive hours at least. Exclusion criteria were: not confirmed diagnosis of COVID-19 infection; do-not-intubate order. We analyzed two groups (C-PAP and SO) matched for age, SAPS2 score, SOFA score and $\mathrm{PaO} 2 / \mathrm{FiO} 2$ at IMCU admission. We also collected demographic characteristics and we investigated rates of ETI and hospital mortality.

Results: We reviewed 93 patients and $42 / 93$ respected the inclusion criteria (21 in SO group; 21 in C-PAP group). The two groups were well matched (PaO2/FiO2 ratio at admission: $86 \pm 20$ (C-PAP), $92 \pm 17$ (SO) $\mathrm{p}=0.3$; SAPS 2 score: $29 \pm 2$ (C-PAP), $35 \pm 7$ (SO) $\mathrm{p}=0.2$; SOFA score: $3 \pm 2$ (C-PAP), $3 \pm 2$ (SO) $p=0.4$; age: $62 \pm 3$ (C-PAP), $64 \pm 10$ (SO) $p$ $=0.2$. Demographic characteristics are shown in Table 1. Patients in C-PAP group were more frequently intubated (14/21) than patients in SO group (8/21) although in not statistically significant way (OR 3.25 , $95 \% \mathrm{Cl} 0.92$ to $11.51, \mathrm{p}=0.07)$. We also found no significant difference in rate of hospital mortality [C-PAP $8 / 21, \mathrm{SO} 6 / 21, \mathrm{OR}=1.54,95 \% \mathrm{Cl}$ 0.42 to $5.61, p=0.51$ ] between the two groups (see Figure 1).

\begin{tabular}{|l|c|c|}
\hline & $\begin{array}{c}\text { C-PAP } \\
\text { n. of patients (\%) }\end{array}$ & $\begin{array}{c}\text { SO } \\
\text { n. of patients (\%) }\end{array}$ \\
\hline Obesity & $3 / 21(14 \%)$ & $6 / 21(29 \%)$ \\
\hline Malignancy & $2 / 21(9 \%)$ & $2 / 21(9 \%)$ \\
\hline COPD/asthma & $2 / 21(9 \%)$ & $1 / 21(5 \%)$ \\
\hline Diabetes & $5 / 21(24 \%)$ & $3 / 21(14 \%)$ \\
\hline Cardiovascular disease & $3 / 21(14 \%)$ & $5 / 21(24 \%)$ \\
\hline Hypertension & $8 / 21(28 \%)$ & $11 / 21(52 \%)$ \\
\hline
\end{tabular}

Table 1.Demographic characteristic

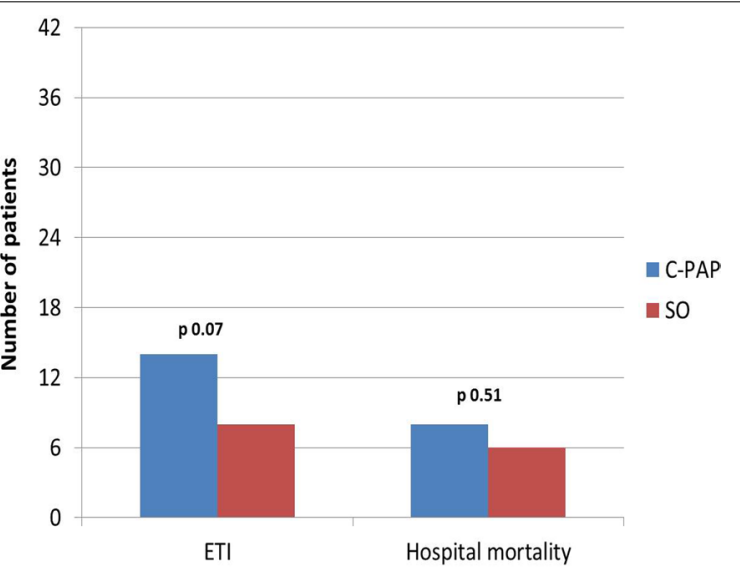

Figure 1. ETI and mortality rates
Conclusion: C-PAP did not reduce ETI and mortality rates when compared to SO in COVID-19 patients with ARF.

Reference(s) and grant acknowledgment(s)

1. 1. Grasselli G et al. Early experience and forecast during an emergency response. JAMA 2020 Mar 13. https://doi.org/10.1001/jama.2020.4031.

\section{3}

A trial of non invasive ventilation in severe COVID-19

T. Grundy ${ }^{1}$; R. Bandopadhyay ${ }^{1}$; S. Yoon ${ }^{1}$; R. Breeze ${ }^{1}$

${ }^{1}$ Intensive care unit, University Hospital Lewisham, London, United Kingdom

Correspondence: $S$. Yoon

Intensive Care Medicine Experimental 2020, 8(2): 000503

Introduction: While the optimal mode of respiratory support in severe COVID-19 disease has been the subject of ongoing debate, non-invasive ventilation (NIV) has been included in management guidelines from WHO (1), NHS England (2) and the Surviving Sepsis Campaign (3). NIV is an effective treatment for mild-moderate acute respiratory failure with evidence of mortality reduction when compared to mechanically ventilated or spontaneously breathing patients (4). There is the risk of nosocomial transmission via viral aerosolisation (5-6), however NIV may offer benefits over an early invasive ventilation strategy in terms of resource allocation and preventing morbidity from intubation and mechanical ventilation (IMV), especially in healthcare settings with limited invasive ventilation capacity. There are a number of evolving safety considerations alongside this.

Objectives: To characterise and describe initial outcomes of patients receiving a trial of NIV in a small, single centre intensive care unit in London. We aim to assess whether NIV is a safe therapy in pandemic surge planning, and understand its impact on the need for IMV in anticipation of future waves of COVID-19 infection.

Methods: Data was collected retrospectively on 44 patients admitted with severe respiratory failure during the first wave of the COVID-19 pandemic between March and April 2020, all of whom received NIV as part of their initial critical care management. Data was collected on age, gender, BMI, requirement for IMV, and survival. We collected data on critical care staff infection rates during the same period as part of hospital wide audit.

Results: Of the 44 patients, 35 (79.5\%) were male and 9 (20.5\%) were female. Median age of the group was 56 (IQR 52-64), and median BMI was 29 (IQR 27-34). Overall $40.9 \%$ of patients died. 23 (52.3\%) of patients went on to require IMV, on average after 56 hours of NIV. $60.9 \%$ of those who required IMV subsequently died, whereas $19 \%$ of those who only received NIV for respiratory support died. Key characteristics for both sub-groups were similar. Median APACHE II score and Clinical Frailty Score (CFS) for those who required IMV was 16 (IQR 13-17) and 2 respectively and 15 (IQR 12-19) and 2 for patients who remained on NIV only. Prior to commencing NIV, baseline median $\mathrm{SpO} 2$ was $91 \%$ and $\mathrm{FiO} 2$ requirement was $80 \%$ in both groups. Importantly, there was marked improvement in $\mathrm{PaO} 2, \mathrm{FiO} 2$, and $\mathrm{SpO} 2$ 2-4 hours after starting NIV, with $\mathrm{PaO} 2$ increased to $9.67 \mathrm{kPa}$, $\mathrm{FiO} 2$ requirement reduced to $60 \%$ and $\mathrm{SpO} 2$ increased to $95 \%$. There was greater improvement in $\mathrm{PaO} 2$ 2-4 hours after starting NIV in the NIV only subgroup when compared to those who required IMV (10.45 kPa NIV, $8.98 \mathrm{kPA}$ IMV). Average length of time to discharge home from critical care admission was reduced from 17.2 days in the IMV sub-group to 15.9 days in the NIV only sub-group. We did not note any increase in staff sickness rates in comparison with other departments, including emergency medicine, general medicine and anaesthetics. 
Conclusion: Our data suggests there are benefits of administering a trial of NIV for respiratory failure in COVID-19. We demonstrate that in a significant proportion of cases it prevented or delayed IMV despite similar clinical severity at time of admission, and we also observed rapid improvements in work of breathing and oxygen requirements after initiation of NIV. This could ensure that invasive ventilators are kept free for those requiring emergent IMV. Survival was similar to national outcomes (7). NIV is not without its complications and therefore frequent review of these patients for escalation of their care is important. Particular consideration needs to be given to staff safety, including adequate supply of appropriate protective equipment, hospital ventilation and airflow dynamics, and an adequate supply of oxygen and consumables needs to be ensured.

\section{Reference(s) and grant acknowledgment(s)}

1. None

2. 1. Clinical management of severe acute respiratory infection when nove coronavirus (2019-nCoV) infection is suspected: interim guidance, 28 January 2020

3. 2. NHS England Guidance for the role and use of non-invasive respiratory support in adult patients with COVID19 (confirmed or suspected) 6 April 2020, Version 3

4. 3. Alhazzani, W., Møller, M.H., Arabi, Y.M. et al. Surviving Sepsis Campaign: guidelines on the management of critically ill adults with Coronavirus Disease 2019 (COVID-19). Intensive Care Med 46, 854-887 (2020). https:// doi.org/10.1007/s00134-020-06022-5

5. 4. Cabrini, Luca MD; Landoni, Giovanni MD; Oriani, Alessandro MD; Plumari, Valentina P. MD; Nobile, Leda MD; Greco, Massimiliano MD; Pasin, Laura MD; Beretta, Luigi MD; Zangrillo, Alberto MD Noninvasive Ventilation and Survival in Acute Care Settings, Critical Care Medicine: April 2015 - Volume 43 - Issue 4 - p 880-888 https://doi.org/10.1097/ccm.0000000000000819

6. 5. Hui DS, Hall SD, Chan MT, et al. Noninvasive positive-pressure ventilation: An experimental model to assess air and particle dispersion. Chest. 2006;130(3):730-740. https://doi.org/10.1378/chest.130.3.730

7. 6. Hui DS, Chow BK, Ng SS, et al. Exhaled air dispersion distances during noninvasive ventilation via different Respironics face masks. Chest 2009;136(4):998-1005. https://doi.org/10.1378/chest.09-0434

8. 7. Intensive Care National Audit and Research Centre. ICNARC report on COVID-19 in critical care 08 May 2020 [Internet]. 2020. Available from: https ://www.icnarc.org/Our-Audit/Audits/Cmp/Reports

\section{0}

\section{Comparative study of two modalities of oxygen therapy} during flexible bronchoscopy in critically ill patients: High-flow nasal oxygen therapy vs conventional oxygen therapy

J. Cobos'; G. Ledesma1; H. Tirape1; B. Jaramillo1; J. Ruiz'; L. Pacheco1;

J. Martinez ${ }^{1}$; R. Maldonado ${ }^{1}$; H. Aguirre-Bermeo ${ }^{1}$

${ }^{1}$ Intensive care unit, Hospital Vicente Corral Moscoso, Cuenca, Ecuador

Correspondence: $\mathrm{H}$. Aguirre-Bermeo

Intensive Care Medicine Experimental 2020, 8(2): 000610

Introduction: Performing diagnostic fiberoptic bronchoscopy in critically ill patients may produce arterial blood gas and hemodynamic changes. The use of High-flow nasal oxygen therapy (HFOT) could decrease these complications.

Objectives: To compare the incidence of complications in the two studied oxygen therapy modalities during fiberoptic bronchoscopy.

Methods: Forty critically ill patients without invasive mechanical ventilation were consecutively collected. Their responsible physician decided to perform diagnostic fiberoptic bronchoscopy suspecting lung infection. Twenty patients received HFOT (Flow 60Lt /min and $\mathrm{FiO} 240 \%$ ) and 20 received conventional oxygen therapy (nasal cannula at $5 \mathrm{Lt} / \mathrm{min}$ ). The fiberoptic bronchoscopy was performed at bed side. Variables were collected before, during and 2 hours after performing the bronchoscopy. All the patients were sedated with Dexmedetomidine Hydrochloride. Delta of oxygen saturation was defined as the difference between the initial saturation and the lowest saturation during bronchial fibroscopy measured by pulse oximetry. The data was compared with the Mann-Whitney test. Data are presented in median and interquartile range.
RESULTS. The main results are shown in the table below.

\begin{tabular}{|l|l|l|l|}
\hline VARIABLES & $\begin{array}{l}\text { HIGH FLOW OXYGEN } \\
\text { THERAPY }(\mathrm{n}=20)\end{array}$ & $\begin{array}{l}\text { CONVENTIONAL } \\
\text { OXYGEN }(\mathrm{n}=20)\end{array}$ & $\mathrm{P}$ \\
\hline Age (years) & $51[28,72]$ & $44[30,70]$ & 0.86 \\
\hline $\begin{array}{l}\text { Days of } \\
\text { hospitalization }\end{array}$ & $5[2,10]$ & $4[2,5]$ & 0.11 \\
\hline $\begin{array}{l}\text { SAPS III } \\
\text { (admission) }\end{array}$ & $44[36,60]$ & $49[40,59]$ & 0.68 \\
\hline PAM (before study, & $83[76,94]$ & $94[84,104]$ & 0.4 \\
\hline mmHg) & $95[92,99]$ & 0.85 \\
\hline $\begin{array}{l}\text { Sat O2 (before } \\
\text { study, \%) }\end{array}$ & $96[92,98]$ & $18[14,26]$ & 0.59 \\
\hline FR (before study) & $18[15,20]$ & $89[71,97]$ & 0.5 \\
\hline $\begin{array}{l}\text { PAM (during study, } \\
\text { mmHg) }\end{array}$ & $76[70,97]$ & $90[85,94]$ & 0.43 \\
\hline $\begin{array}{l}\text { Sat O2 (during } \\
\text { study, \%) }\end{array}$ & $94[89,96]$ & $19[17,26]$ & 0.28 \\
\hline FR (during study) & $19[17,22]$ & $5[2,10]$ & 0.01 \\
\hline $\begin{array}{l}\text { Delta Sat O2 } \\
\text { (during study, \%) }\end{array}$ & $2[1,3]$ & $81[76,91]$ & 0.6 \\
\hline $\begin{array}{l}\text { PAM (two hours } \\
\text { after study, mmHg) }\end{array}$ & $82[72,87]$ & $94[91,96]$ & 0.38 \\
\hline $\begin{array}{l}\text { Sat O2 (two hours } \\
\text { after, } \%\end{array}$ & $92[87,96]$ & $19[16,22]$ & 0.37 \\
\hline $\begin{array}{l}\text { FR (two hours } \\
\text { after) }\end{array}$ & $18[15,20]$ & & \\
\hline
\end{tabular}

Conclusion: The use of high-flow oxygen therapy is related to a lower decrease in saturation compared to conventional oxygen therapy.

\section{1}

Incidence of pneumothorax and pneumomediastinum in Covid-19 critically ill patients

L. Gandini ${ }^{1}$; M. Di Matteo ${ }^{2}$; M. Monti ${ }^{1}$; L. Manesso ${ }^{1}$; FM. Russo ;

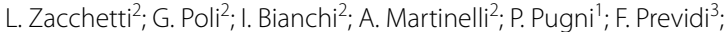

FL. Lorini ${ }^{2}$

${ }^{1}$ Anestesia, rianimazione e terapia intensiva e del dolore, University of Milan, Milano, Italy; ${ }^{2}$ Dipartimento di emergenza urgenza e area critica, ASST Papa Giovanni XXIII, Bergamo, Italy; ${ }^{3}$ Dipartimento di ingegneria e scienze applicate, Università Degli Studi Di Bergamo, Dalmine, Italy

Correspondence: L. Gandini

Intensive Care Medicine Experimental 2020, 8(2): 000651

Introduction: Barotrauma, including both pneumothorax and pneumomediastinum, is a well-known complication of prolonged non-invasive ventilation (NIV) [1]. Vigorous respiratory efforts lead to increased transpulmonary pressure and to patient self induced lung injury [2]. In Coronavirus Disease 2019 (Covid-19), correct timing to declare failure of NIV and proceed to intubation and invasive ventilation is unknown. We tested the hypothesis that in Covid-19 prolonged NIV is associated with barotrauma.

Objectives: To describe incidence of barotrauma in Covid-19 and its onset time from hospital admission.

To evaluate association between barotrauma and duration of NIV in Covid-19.

Methods: We retrospectively enrolled 104 consecutive Covid-19 patients, admitted to our ICU from February 22nd to March 22nd, requiring intubation after failure of NIV. Age, sex and SOFA score were collected at ICU admission. Diagnosis of barotrauma was confirmed by radiological evidence (ultrasound/chest $x$-ray, chest $C T$ ) during the first 2 weeks from ICU admission. Different time intervals (from hospital admission to intubation (T1) and from beginning of NIV to intubation (T2)) were compared between patients with barotrauma and controls, using the Mann-Withney test. We then identified a subgroup of barotrauma patients, defined "early", characterized by occurrence of barotrauma before or within the first 24 hours after intubation. We compared this group with controls with the Mann-Whitney test.

Results: Mean age was $61 \pm 10,84(80.7 \%)$ were male and median SOFA score was 6 (range 4-8). Barotrauma occurred in 15 patients (14\%), at a median time of 8 days (IQR 6-12) from hospital admission. No differences were found either in T1 (median days 3, IQR 2-5.5 vs 5, IQR 2-9, p ns), or in T2 (median days 2, IQR 1-4 vs 3, IQR 2-6, p ns) 
between controls and patients with barotrauma. However 6 patients had an early barotrauma; in this subgroup, $\mathrm{T} 2$ was longer in patients who developed early barotrauma compared to controls (Table).

\begin{tabular}{|c|l|l|}
\hline Time intervals & Controls $(\mathbf{n}=\mathbf{8 9})$ & \multicolumn{1}{|c|}{ Early Barotrauma $(\mathbf{n = 6})$} \\
\hline T1, days (median, IQR) & $3.0(2.0-5.5)$ & $6.5(2.5-13.2)$ \\
\hline T2, days (median, IQR) & $2.0(1.0-4.0)$ & $6.0(1.7-9.2)^{*}$ \\
\hline
\end{tabular}

${ }^{*} \mathrm{p}<0.05$ between early barotrauma and controls

Conclusion: In Covid-19 barotrauma had an incidence similar to other forms of acute respiratory failure. However, early barotrauma was associated with prolonged use of NIV.

\section{Reference(s) and grant acknowledgment(s)}

1. 1. Rochwerg et al, Official ERS/ATS clinical practice guidelines: noninvasive ventilation for acute respiratory failure. Respir J. 2017 31:50(2)

2. 2. Yoshida $T$ et al, Patient self-inflicted lung injury and positive end-expiratory pressure for safe spontaneous breathing. Curr Opin Crit Care. 2020 26(1):59-65

\section{8}

\section{Retrospective study of non-invasive ventilation use in a district} hospital in Portugal

A. Paula ${ }^{1}$; F. Lage ${ }^{2}$; F. Morais ${ }^{2}$. P. Cesar²; D. Pinto ${ }^{1} ;$ J. Vaz $^{1}$

${ }^{1}$ Intensive care unit, Hospital José Joaquim Fernandes, Beja, Portugal; ${ }^{2}$ Internal medicine, Hospital José Joaquim Fernandes, Beja, Portugal

Correspondence: A. Paula

Intensive Care Medicine Experimental 2020, 8(2): 000678

Introduction: The decision to use non-invasive ventilation (NIV) to treat acute respiratory diseases in the ICU avoids intubation and reduces invasive mechanical ventilation risks [1].

Objectives: To investigate the patients who started NIV in our ICU and study their failure and mortality rates.

Methods: Retrospective single-centre study of all patients admitted during a five-year period who received NIV, except for chronic therapeutic purposes. Data of demographics, mortality scores, cause for NIV and NIV failure (defined as intubation need) of 167 patients were recorded and processed with SPSS 23 . NIV failure was studied, excluding patients on a weaning strategy.

Results: The average age was 70 years, with a mean SOFA score on admission of 8.7 compared with 6.6 at discharge. The mean ICU stay was 10 days with NIV duration of 53 hours. The patients admitted were mainly medical, with only $10.8 \%$ admitted due to emergency surgery. Half the patients had previous respiratory diseases, accounting COPD with $44.3 \%, 46.7 \%$ had cardiovascular pathology and $36.5 \%$ had respiratory illnesses. The number of patients who started NIV due to COPD and acute cardiogenic pulmonary oedema was similar (16.8\%), while $11.4 \%$ had pneumonia. $13.6 \%$ were on mechanical ventilation weaning. Considering all patients, $36.5 \%$ had NIV failure rate and $32.3 \%$ of mortality rate.

Patients who survived were younger (69.2 vs 72.7 years), had better scores on admission (SAPS II 51 vs 64; APACHE II 26.9 vs 31.6; SOFA 7.7 vs 10.7), had less need for haemodynamic support ( 27 vs $54 \%$ ) and less time gap between admission and NIV start (45 vs 70 hours). These patients had more respiratory and cardiovascular diseases than those who died and there were fewer patients in need for NIV due to pneumonia ( 9.7 vs $14.8 \%$ ). About $28 \%$ of patients that lived had NIV failure compared with those that did not (54\%).

A significant difference was observed in those who failed NIV trial with those who did not with in between admission and discharged SOFA score (on admission 7.27 vs 10.34; on discharge 5.56 vs 8.34 ), aminergic support ( 42 vs $58 \%$ ), ICU stay ( 6 vs 15 days) and mortality rate ( 21 vs $48 \%$ ). Average SAPS II score of those who survived and did not fail NIV trial was 47 compared with 59 in intubated patients. Failed NIV survivors had higher $\mathrm{pH}$ levels (7.28 vs 6.7), while the opposite happened within those who succeeded NIV trial (7.07 vs 7.28).
Conclusion: Patients who had the best outcomes had more comorbidities, opposing to the higher percentage of pneumonia in between those who died. Despite studies showing a SAPS II score $>34$ to be independently related to NIV failure [2], our patients who succeeded had an average score of 47. Although indications for NIV trial in patients with a pH $>7.25[3,4]$, there was no significant $\mathrm{pH}$ difference. NIV is a useful treatment and more studies are needed to identify early factors associated with higher mortality.

Reference(s) and grant acknowledgment(s)

1. 1. Lindenauer PK et al. Outcomes Associated With Invasive and Noninvasive Ventilation Among Patients Hospitalized With Exacerbations of Chronic Obstructive Pulmonary Disease. JAMA internal medicine. 2014;174(12).

2. 2. Yamauchi LY et al. Noninvasive positive-pressure ventilation in clinical practice at a large university-affiliated Brazilian hospital. Clinics (Sao Paulo). 2012;67(7):767-72.

3. 3. Osadnik CR et al. Non-invasive ventilation for the management of acute hypercapnic respiratory failure due to exacerbation of chronic obstructive pulmonary disease. Cochrane Database Syst Rev. 2017;7:Cd004104.

4. 4. Rochwerg B et al. Official ERS/ATS clinical practice guidelines: noninvasive ventilation for acute respiratory failure. 2017.

\section{6}

Withdrawal of noninvasive ventilation in chronic obstructive pulmonary disease exacerbation. a spanish national survey on clinical practice

M.R. Koborzan'; A. Mas'; M. Lujan²; G. Rialp ${ }^{3}$

${ }^{1}$ Intensive Care, Hospital de Sant Joan Despí Moisès Broggi, Sant Joan Despí, Spain; ${ }^{2}$ Pneumology, Hospital Parc Taulí de Sabadell, Sabadell, Spain; ${ }^{3}$ Intensive care, Hospital Universitari Son Llàtzer, Palma, Spain

Correspondence: M.R. Koborzan

Intensive Care Medicine Experimental 2020, 8(2): 000846

Introduction: Noninvasive ventilation (NIV) is considered as the standard treatment for moderate to severe exacerbations of Chronic Obstructive Pulmonary Disease (COPD). However, the way to withdraw NIV after stabilization is not well established.

Objectives: We performed a National Survey to disclose this issue in clinical practice.

Methods: A national survey was launched to Spanish hospitals having general or respiratory ICU through the intensive (SEMICYUC) and pulmonology (SEPAR) national societies. The survey was carried out between January and March 2019.

One response per Unit was analyzed (that of the NIV expert or the head of the service in his absence). Characteristics of the center, specialty and withdrawal strategies are collected: time of beginning of disconnection, direct or progressive withdrawal, type of progression, with or without analytic control (arterial or venous), among others.

Data analysis was conducted using SPSS Version 21.0. The data are presented as $n(\%) ; p$ value $<0.05$ were considered statistically significant doing contingency tables and using the Chi-Square or the Fisher test.

Results: We received a total of 179 responses, leaving us with 141 after the elimination of double responses. The final analysis was performed on 129 answers after eliminating 12 answers from third level hospitals that do not admit this pathology (AECOPD).

Responses from 129 units were analyzed, 84(60\%) by intensivists and $57(40 \%)$ by pulmonologists. The $54.6 \%$ were tertiary hospitals.

$96.6 \%$ of physicians (n 128) perform blood gases to decide to initiate disconnections. The use of an arterial sample is more common among pulmonologists than among intensivists $(94.7 \%$ vs $66.2 \%, p<0.05)$.

Only in 26 units (20.3\%) is direct withdrawal attempted and it is more frequent among intensivists $(28.2 \%$ vs $10.5 \% \mathrm{p}<0.05)$.

When they make a progressive withdrawal, $42.2 \%$ extend the disconnections, $6.9 \%$ decrease the support and $51 \%$ combine both. Nighttime NIV is indicated in $88.4 \%$ of cases.

The majority of the respondents required hemodynamic stability $(82 \%)$, respiratory rate $<30 \mathrm{~b} / \mathrm{min}(98 \%)$ and blood gas analysis $(97 \%)$ for making decisions. Venous blood gas samples were used in $21 \%$ 
of the cases. Pulmonologists conducted progressive withdrawal and used arterial blood gases significantly more often than intensivists.

Conclusion: To our knowledge, this is the first descriptive study of NIV withdrawal in patients with AECOPD.

The criteria for initiating withdrawal are fairly homogeneous, although some different strategies may be evident depending on the specialty. Pulmonologist usually do withdrawal progressively and use arterial blood gases more often than intensivists.

\section{Reference(s) and grant acknowledgment(s)}

1. Jacobo Sellares, Miquel Ferrer, Antonio Anton et al. Discontinuing noninvasive ventilation in severe chronic obstructive pulmonary disease exacerbations: a randomised controlled trial. European Respiratory Journal 2017 50: 1601448

2. Lun, Chung-Tat; Chan, Veronica L.;Leung, Wah-Shing et al. A pilot randomized study comparing two methods of non-invasive ventilation withdrawal after acute respiratory failure in chronic obstructive pulmonary disease. Respirology 2013; 18(5):814-819

3. Duan, Jun; Tang, Xiaokui;Huang, Shicong et al. Protocol-directed versus physician-directed weaning from noninvasive ventilation: The impact in chronic obstructive pulmonary disease patients. Journal of Trauma and Acute Care Surgery 2012; 72(5):1271-1275

4. Damas, C. Andrade, C. Araújo, J.P et al Weaning from non-invasive positive pressure ventilation: Experience with progressive periods of withdraw. Revista Portuguesa de Pneumologia 2008, 14 (1): 49-53

\section{5}

Unilateral diaphragm paralysis: the evolving role of diaphragm pacing (DP)

R. Onders; ${ }^{1}$ H. R. ${ }^{2} ;$ M. Elmo ${ }^{1}$

${ }^{1}$ Surgery, University Hospitals Cleveland Medical Center, Cleveland, United States of America; ${ }^{2}$ Pulmonary, University Hospitals Cleveland Medical Center, Cleveland, United States of America

Correspondence: R. Onders

Intensive Care Medicine Experimental 2020, 8(2): 001005

Introduction: Unilateral diaphragm dysfunction (UDD) is an underdiagnosed condition. Of the benign causes, the most common is the idiopathic type. Other causes include complications of cardiac surgery, trauma, metabolic derangements, as well as some infections. It has been postulated that the functional diaphragm increases in strength and compensates for the dysfunctional side thus the asymptomatic state in the majority of patients. However, in a smaller group this dysfunction may lead to dyspnea, sleep-disordered breathing, exercise intolerance, fatigue and even respiratory failure.

Objectives: We report the outcome of symptomatic UDD patients implanted with DP to treat the isolated dysfunction

Methods: This is an IRB approved retrospective analysis of a prospective, non-randomized interventional experience at a single institution. Patients underwent laparoscopic diaphragm mapping and electrode implantation. Post implantation patients were evaluated radiographically with either chest radiography or fluoroscopic sniff test, reported symptoms and diaphragm electromyography (dEMG) using the implanted DP electrodes. Success was considered when there was improvement or recovery in 2 of the 3 data points

Results: In the diaphragm dysfunction database, 62 patients with UDD were identified. Etiologies of the paralysis codified into 7 groups: idiopathic $(n=27)$, chest/thoracic procedure $(n=13)$, neck/shoulder surgery/injection $(n=8)$, trauma $(n=4)$, infection $(n=3)$, chemo/ radiation $(n=3)$, Parsonage Turner's syndrome $(\mathrm{N}=3)$, and post liver transplant $(n=1)$. The average age was $60 \pm 10$ years. All patients had significant respiratory symptoms and abnormal chest radiographs. Symptom duration was greater than 6 months in 49 patients (79\%). Fifty-nine (95\%) had fluoroscopic evaluation of the diaphragm with $51 \%$ showing paradoxical motion; the others showed no movement. Post implantation, $44(70.9 \%)$ of patients reported improvement in symptom, 46 (74\%) had improvement in dEMG and $23(37 \%)$ had improved radiologic testing. Thirty nine patients (62.9\%) had recovery of 2 data points. Recovery rate was at $63.2 \%$ and $40 \%$ in patients with symptom duration greater than 6 and 18 months respectively. Additionally, there was a significant improvement in those with paradoxical motion $78 \%$ compared to those without paradoxical movement (59\%). Conclusion: DP can play a significant role in patients with symptomatic UDD. It could be offered as a treatment modality in UDD where the diaphragm can be electrically stimulated prior to proceeding with plication procedures

\section{Reference(s) and grant acknowledgment(s)}

1. Onders RP, Elmo MJ, Kaplan C, Schilz R, Katirji B, Tinkoff G. Long-term experience with diaphragm pacing for traumatic spinal cord injury: Early implantation should be considered. Surgery 2018. 164(4):705-711

2. Onders RP, Markowitz A, Ho VP, Hardacre J, Novitsky Y, Towe C, Elmo M, Kaplan C, Schilz R. Completed FDA feasibility trial of surgically placed temporary diaphragm pacing electrodes: A promising option to prevent and treat respiratory failure.Am J Surg. 2018 Mar;215(3):518-521

3. Onders R, Elmo MJ, Kaplan C, Katirji B, Schilz R. Extended Use of Diaphragm Pacing in Patients with Unilateral of Bilateral Diaphragm Dysfunction: A New Therapeutic Option. Surgery 2014;156:772-86

\section{1}

Outcomes of CPAP usage for COVID-19 pneumonitis

T. Disney'; G. Dempsey'; E. O'callaghan

${ }^{1}$ Critical care unit, Aintree University Hospital, Liverpool, United Kingdom Correspondence: $T$. Disney

Intensive Care Medicine Experimental 2020, 8(2): 001021

Introduction: The outcome for COVID-19 patients undergoing a trial of continuous positive airway pressure (CPAP) prior to invasive ventilation is unclear. There may be a role for avoiding invasive ventilation at the risk of worsening outcomes for those failing such a trial, however there is a lack of definitive evidence.

Objectives: To identify prognostic factors which may indicate a successful CPAP trial for COVID-19 patients and determine the effect of CPAP on mortality for those invasively ventilated after a CPAP trial.

Methods: A prospective cohort study of adult patients admitted to the ICU with COVID-19 pneumonitis in respiratory failure. Primary outcome: failure of CPAP trial resulting in invasive ventilation. Secondary outcome: mortality in patients requiring invasive ventilation after a CPAP trial.

Results: 45 patients underwent a trial of CPAP during the study period. $33(73 \%)$ patients failed the trial and required invasive ventilation. There was no significant difference in age, Clinical Frailty Score, Extended Medical Research Council Dyspnoea Score (eMRCD), APACHE and SOFA scores (see Table 1). All patients over 70 years failed their CPAP trial. Patients undergoing a successful trial had shorter duration of ICU and hospital stay (see Table 2). 14 (42\%) patients died after they failed a CPAP trial. 12/22 (55\%) patients undergoing invasive ventilation without CPAP died.

\begin{tabular}{|l|l|l|l|}
\hline & & CPAP & $\begin{array}{l}\text { Failed CPAP \& } \\
\text { subsequently intubated }\end{array}$ \\
\hline \multirow{2}{*}{ Age (Years) } & Mean & 54.6 & 56.4 \\
\hline \multirow{2}{*}{ Clinical Frailty Score } & Median & 53.0 & 58.5 \\
\hline \multirow{2}{*}{ MRRCD } & Mean & 1.5 & 1.5 \\
\cline { 2 - 4 } & Median & 1.0 & 1.0 \\
\hline \multirow{2}{*}{ APACHE } & Mean & 1.4 & 1.3 \\
\hline \multirow{2}{*}{ SOFA } & Median & 1.0 & 1.0 \\
\hline & Mean & 11.4 & 12.6 \\
\hline & Median & 10.0 & 12.0 \\
\hline
\end{tabular}

Table 1. Comparison of attributes on admission between patients receiving successful CPAP and those failing CPAP and requiring invasive ventilation. 


\begin{tabular}{|l|l|l|l|}
\hline & & CPAP & $\begin{array}{l}\text { Failed CPAP \& } \\
\text { subsequently intubated }\end{array}$ \\
\hline \multirow{2}{*}{ ICU Length of Stay (Days) } & Mean & 2.8 & 21.9 \\
\cline { 2 - 4 } $\begin{array}{l}\text { Hospital Length of Stay } \\
\text { (Days) }\end{array}$ & Mean & 15.1 & 22.5 \\
\hline & Median & 13.0 & 34.1 \\
\hline
\end{tabular}

Table 2. Comparison of ICU and hospital length of stay between patients receiving successfu $\mathrm{CPAP}$ and those failing CPAP and requiring invasive ventilation.

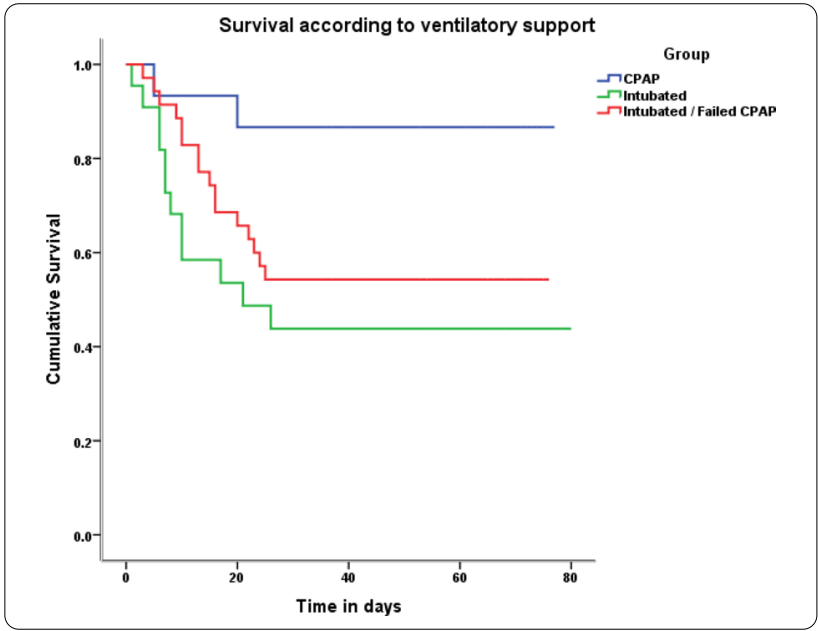

Conclusion: Mortality for patients undergoing a CPAP trial does not appear to be increased. All patients over 70 years failed CPAP. Successful CPAP trial results in reduced ICU and hospital stay.

\section{4}

Effects of awake prone positioning in non-intubated spontaneously breathing COVID-19 patients requiring high flow oxygen therapy in high dependency unit (HDU): an observational study

R. Najeeb ${ }^{1}$; T. masoodi' ${ }^{2}$; K. Muneer ${ }^{1}$; M. Ommid ${ }^{1}$; I. Hussain ${ }^{1}$

${ }^{1}$ Anesthesiology and critical care, Government Medical College (GMC), Srinagar, India; ${ }^{2}$ Anesthesiology and critical care., Government Medical College (GMC), Srinagar, India

Correspondence: T. masoodi

Intensive Care Medicine Experimental 2020, 8(2): 001174

Introduction: Prone positioning increases oxygenation by recruiting dorsal lung regions and drain airway secretions, improves gas exchange and survival in ARDS. We described efficacy of prone positioning in awake non-intubated spontaneously breathing COVID-19 positive patients with hypoxemic acute respiratory failure.

Methods: We studied 26 awake non-intubated spontaneously breathing patients with hypoxemic respiratory failure treated with prone positioning. Patients were kept in prone positioning for two hours in each session and four such sessions were given to patients in 24 hours. $\mathrm{SPO} 2, \mathrm{PaO} 2, \mathrm{RR}$ and hemodynamics were measured before prone positioning (PRE), 60 minutes of prone positioning (PRONE) and after one hour of the completion of session (POST).

Results: 26 patients ( 12 males and 14 females) non-intubated spontaneously breathing with $\mathrm{SPO} 2<94 \%$ on $0.4 \mathrm{FiO} 2$ were treated with prone positioning. One patient required intubation and was shifted to ICU, rest 25 patients were discharged from HDU. Mean hours of prone positioning was $19.4 \pm 2.06 \mathrm{hr}$. There was significant improvement in oxygenation (increase in $\mathrm{PaO} 2$ from $53.15 \pm 6.0 \mathrm{mmHg}$ to $64.23 \pm$ $6.96 \mathrm{mmHg}$ in PRE and POST sessions respectively, likewise there was increase in SPO2). No complications were noted with various sessions. Conclusion: Prone positioning was feasible and improved oxygenation in awake non-intubated, spontaneously breathing COVID-19 patients with hypoxemic acute respiratory failure.

\section{Reference(s) and grant acknowledgment(s)}

1. Grasselli G, Pesenti A, Cecconi M. Critical care utilization for the COVID19 outbreak in Lombardy, Italy: early experience and forecast during an emergency response. JAMA. Published online March 13, 2020. https:// doi.org/10.1001/jama.2020.4031 2. Grasselli G, Zangrillo A, Zanella A, et al; COVID-19 Lombardy ICU Network.Baseline characteristics and outcomes of 1591 patients infected with SARS-CoV-2 admitted to ICUs of the Lombardy region, Italy. JAMA. Published online April 6, 2020. https://doi.org/10.1001/ jama.2020.5394 3. Guérin C, Reignier J, Richard J-C, et al; PROSEVA Study Group. Prone positioning in severe acute respiratory distress syndrome. N Engl J Med. 2013;368(23):2159-2168. https://doi.org/10.1056/nejmo a1214103. 4. Gattinoni L, Carlesso E, Taccone P, Polli F, Guérin C, Mancebo J. Prone positioning improves survival in severe ARDS: a pathophysiologic review and individual patient meta-analysis. Minerva Anestesiol. 2010; 76: 448-454. 5. Slutsky AS, Ranieri VM. Ventilator-Induced Lung Injury. N. Engl. J. Med. 2013; 369: 2126-2136. 6. Hess DR. Patient Positioning and VentilatorAssociated Pneumonia. Respir. Care 2005; 50: 892 LP - 899. 7. Scaravilli V, Grasselli G, Castagna L, et al. Prone positioning improves oxygenation in spontaneously breathing nonintubated patients with hypoxemic acute respiratory failure: a retrospective study. J Crit Care. 2015;30(6):1390-1394. https://doi.org/10.1016/j.jcrc.2015.07.008 8. Nyrén S, Radell P, Lindahl SGE, Mure M, Petersson J, Larsson SA, et al. Lung ventilation and perfusion in prone and supine postures with reference to anesthetized and mechanically ventilated healthy volunteers. Anesthesiology 2010;112:682-7. 9. Protti A, Chiumello D, CressoniM, Carlesso E,Mietto C, Berto V, et al. Relationship between gas exchange response to prone position and lung recruitability during acuterespiratory failure. Intensive Care Med 2009;35:1011-7. 10. Galiatsou E, Kostanti E, Svarna E, Kitsakos A, Koulouras V, Efremidis SC, et al. Prone position augments recruitment and prevents alveolar overinflation in acute lung injury. Am J Respir Crit Care Med 2006;174:187-97. 11. Gattinoni L, Coppola S, Cressoni M, Busana M, Rossi S, Chiumello D.Covid-19 Does Not Lead to a "Typical" Acute Respiratory Distress Syndrome.Am J RespirCrit Care Med. 2020 Mar 30. https://doi.org/10.1164/rccm.202003-0817le. 12. Chatte G, Sab JM, Dubois JM, Sirodot M, Gaussorgues P, Robert D.Prone position in mechanically ventilated patients with severe acute respiratory failure.Am J RespirCrit Care Med. 1997 Feb;155(2):473-8. 13. Feltracco P, Serra E, Barbieri S, Milevoj M,Michieletto E, Carollo C, et al. Noninvasive high-frequency percussive ventilation in the prone position after lung transplantation. Transplant Proc 2012;44:2016-21. 14. Feltracco P, Serra E, Barbieri S, Persona P, Rea F, LoyM, et al. Non-invasive ventilation in prone position for refractory hypoxemia after bilateral lung transplantation. Clin Transplant 2009;23:74850. 15. Valter C, Christensen AM, Tollund C, Schønemann NK. Response to the prone position in spontaneously breathing patients with hypoxemic respiratory failure. Acta Anaesthesiol Scand 2003;47:416-8.

03. AIDS, hematologic-oncologic issues in the ICU > AIDS, hematologic-oncologic issues in the ICU

\section{0}

Prognostic factors in critically ill patients with hematologic malignancies admitted to Intensive Care Units

T. Isidoro Duarte ${ }^{1}$; P. Faria ${ }^{1}$; A. Martins ${ }^{1}$; N. Germano ${ }^{1}$

${ }^{1}$ Intensive Care Medicine Department, Centro Hospitalar Universitário de

Lisboa Central, Lisboa, Portugal

Correspondence: $T$. Isidoro Duarte

Intensive Care Medicine Experimental 2020, 8(2): 000340

Introduction: Survival of patients with hematologic malignancies (HM) admitted to Intensive Care Units (ICU) have been changing over the years. Nevertheless, still exists some reluctance in intensive care providers to admit and treat patients with these diseases. The ability to identify key prognostic variables of outcome within the first days may help to recognize those who are most likely to benefit from ICU therapy.

Methods: We studied a retrospective cohort of 56 adult patients with HM admitted to a non-hematologic ICU between January 2015 and September 2019. Patient's baseline characteristics, organ failure scores and outcome were analyzed in order to identify clinically useful prognostic factors.

Results: Median age was 56 years. The most common hematologic diseases were acute myelogenous leukemia (17; 30\%), malignant 
lymphoma (16; 29\%), acute lymphoblastic leukemia $(6 ; 11,4 \%)$ and multiple myeloma $(5 ; 8,6 \%)$. Primary reason for ICU admission were septic shock (30; $54 \%)$, respiratory failure $(11 ; 20,3 \%)$, acute kidney injury AKIN $>=2(5 ; 9 \%)$ and gastrointestinal complications mainly due to upper gastrointestinal bleeding (3; 5,7\%). Median time in-hospital before ICU admission was 10 days and the median duration of ICU stay was 6 days. Median ICU and in-hospital mortality were 57 and 68,5\%, respectively. Median SAPS II score was 56 with an estimated mortality of 59,6\%. Median SOFA score 48 and 24 hours before ICU admission was higher in non-survivors group ( 6 vs 4 and 9 vs 7, respectively). Mortality was higher in patients with acute leukemia, septic shock and need for mechanical ventilation and vasopressor support during ICU admission.

Conclusion: Higher mortality in patients with HM admitted to the ICU was associated with more severe illness and respiratory or hemodynamic instability. Organ failure scores done before admission may suggest delay on ICU admission and worst outcome.

\section{Reference(s) and grant acknowledgment(s)}

1. Hampshire PA et al. Admission factors associated with hospital mortality in patients with haematological malignancy admitted to UK adult, general critical care units: a secondary analysis of the ICNARC Case Mix Programme Databse. Critical Care. 2009. 13:R157

2. Bird GT et al. Outcomes and prognostic factors in patients with haematological malignancy admitted to a specialist cancer intensive care unit: a $5 \mathrm{yr}$ study. British Journal of Anaesthesia. 2012. 108 (3): 452-9

03. AIDS, hematologic-oncologic issues in the ICU > AIDS, hematologic-oncologic issues in the ICU

\section{2}

Predictors of mortality of patients with hematologic malignancies admitted to the Intensive Care Unit

A. Herraiz'; P. Marcos ${ }^{1}$; R. Roig'; A. Torrent ${ }^{2}$; M. Moreno²; S. Vives²;

MJ. Jiménez $z^{2}$

${ }^{1}$ Intensive care unit, Hospital Germans Trias i Pujol, Badalona, Spain; ${ }^{2} \mathrm{Hae}-$ matology, Hospital Germans Trias i Pujol, Badalona, Spain

Correspondence: A. Herraiz,

Intensive Care Medicine Experimental 2020, 8(2): 000432

Introduction: Patients affected by hematologic malignancies admitted to an Intensive Care Unit (ICU) have been a subject of debate for many years. Reported survival rates are low, especially in those patients who develop several organ failures.

Objectives: To determine the factors associated with ICU mortality in patients with hematologic malignancies.

Methods: Study period: January 2015 to December 2019. It has been collected demographic variables, severity scores, hematological characteristics, organ dysfunctions, microbiological data, length of stay and ICU and hospital mortality. Statistical analysis includes qualitative variables, presented as proportions and $95 \%$ confidence interval (Cl); quantitative variables have been presented as medians and $95 \% \mathrm{Cl}$. Shapiro-Wilk test has been used for the assessment of normality. The univariate analysis was performed using the simple linear regression test. ICU mortality was determined in the multivariate analysis using the logistic regression test.

Results: $\mathrm{n}=84$. Males $0.61(95 \% \mathrm{Cl}: 0.5$ to 0.7$)$. Age: 57.5 years $(95 \% \mathrm{Cl}$ : 52.5 to 61.5$)$. Hematologic therapies: hematopoietic stem cell transplant 0.38 ( $95 \% \mathrm{Cl}$ : 0.28 to 0.48$)$; intra-ICU chemotherapy $0.14(95 \% \mathrm{Cl}$ : 0.08 to 0.23 ). Severity scores and biomarkers on admission: APACHE II 27 (95\%Cl: 25 to 28$)$; SOFA 8 ( $95 \% \mathrm{Cl}: 7$ to 9); procalcitonin 4 ( $95 \% \mathrm{Cl}$ : 0.9 to 19.5). Acute respiratory failure (ARF) was the main reason for admission 0.5 (95\% Cl: 0.4 to 0.6$)$, followed by sepsis $0.34(95 \% \mathrm{Cl}$ : 0.24 to 0.44 ). The proportion of patients with positive blood cultures on admission was 0.21 ( $95 \% \mathrm{Cl}: 0.13$ to 0.31 ), all of them of unknown focus. Pathogen most frequently isolated: Escherichia coli 0.33 ( $95 \% \mathrm{Cl}$ : 0.14 to 0.59$)$. Organ dysfunctions developed in ICU: ARF $0.85(95 \% \mathrm{Cl}$ : 0.76 to 0.91$)$; shock 0.64 ( $95 \% \mathrm{Cl}: 0.54$ to 0.74$)$; renal failure $0.64(95 \% \mathrm{Cl}$ : 0.54 to 0.74 ); coma 0.14 ( $95 \% \mathrm{Cl}: 0.08$ to 0.23 ). Life support measures: high flow nasal cannula 0.3 ( $95 \% \mathrm{Cl}$ : 0.21 to 0.41 ); non-invasive ventilation 0.47 ( $95 \% \mathrm{Cl}$ : 0.36 to 0.57 ); invasive ventilation 0.7 (95\% Cl: 0.65 to
0.83 ); continuous renal replacement therapy (CRRT) 0.34 (95\%Cl: 0.24 to 0.44$)$. The ICU stay was 9 days ( $95 \% \mathrm{Cl}: 6.9$ to 14$)$ and the hospital stay was 18 days ( $95 \% \mathrm{Cl}: 15$ to 23$)$. The ICU mortality was $0.46(95 \% \mathrm{Cl}$ : 0.36 to 0.57 ) and the hospital mortality was 0.59 ( $95 \% \mathrm{Cl}: 0.48$ to 0.69 ). Variables significantly associated with mortality: APACHE II (OR 1.1; 95\%Cl: 1.04 to 1.19 ); SOFA (OR $1.21 ; 95 \% \mathrm{Cl}: 1.04$ to 1.4 ); shock (OR 3.7 $95 \% \mathrm{Cl}: 1.4$ to 9.8$)$ ) ARF (OR $12 ; 95 \% \mathrm{Cl}: 1.5$ to 100$)$; invasive ventilation (OR 30.4; 95\%Cl: 3.8 to 241.1 ); CRRT (OR 3; 95\%Cl: 1.16 to 7.7). Regarding multivariate analysis, the variable which independently explains mortality is invasive ventilation (OR $22.7 ; 95 \% \mathrm{Cl}$ : 2.8 to 184.9 ).

Conclusion: The main determinant of mortality in patients admitted to the ICU due to hematologic malignancies is the acute respiratory failure and the need of invasive ventilation.

\section{Reference(s) and grant acknowledgment(s)}

1. Riedijk M, van den Bergh WM, van Vliet M, et al. Characteristics and outcomes of patients with a haematological malignancy admitted to the intensive care unit for a neurological event. Crit Care Resusc. 2015;17(4):268-273.

2. van Beers EJ, Müller MC, Vlaar AP, Spanjaard L, van den Bergh WM; HEMAICU Study Group. Haematological malignancy in the intensive care unit: microbiology results and mortality. Eur J Haematol. 2016;97(3):271-277.

3. Kawada T. Risk factors of infection in patients with hematological malignancy. Ann Hematol. 2019;98(9):2251.

03. AIDS, hematologic-oncologic issues in the ICU > AIDS, hematologic-oncologic issues in the ICU

\section{4}

Short- and long-term survival and functional assessment of critically ill patients with hematologic malignancies A. Mata'; PD. Vasconcelos M'; S. Fernandes'; D. Silva²; J. Raposo'; J. Ribeiro ${ }^{2}$

${ }^{1}$ Hematology and bone marrow transplant department, Centro Hospitalar Universitário Lisboa Norte, Hospital Santa Maria, Lisbon, Portugal; ${ }^{2}$ Intensive care service, Centro Hospitalar Universitário Lisboa Norte, Hospital Santa Maria, Lisbon, Portugal

Correspondence: A. Mata

Intensive Care Medicine Experimental 2020, 8(2): 000474

Introduction: ICU admission and organ support in patients with hematologic malignancies with systemic organ failure is still debatable, given the high associated mortality and absence of reliable outcome predictors $(1,2)$.

Objectives: Identification of prognostic factors in patients with hematologic malignancy and multisystemic organ failure admitted to an ICU. Mortality and functional outcome 3-months and 1-year after ICU discharge were the primary and secondary outcomes, respectively.

Methods: Retrospective, single centre study from a tertiary care, university hospital. Clinical and laboratory data from patients with hematologic malignancies admitted to ICU, from June 2014 to June 2019, were extracted from electronic registries. Functional assessment was performed through clinical registries plus phone contact and scored according to Eastern Cooperative Oncology Group (ECOG) scale. Statistical analysis was performed through IBM ${ }^{\circledR}$ SPSS $^{\circledR}$ Statistics 25 .

Results: We included 100 patients (112 ICU admissions), mostly male $(60 \%)$ with a median age of 60 years old [inter-quartile range (IQR): 45-65], median SAPS II of 56 (IQR: 19-129) and median admission SOFA of 9 (IQR: 2-22). Acute leukemia (34.8\%), non-Hodgkin lymphoma (25.9\%) and multiple myeloma (13.4\%) were the most prevalent diagnosis. Most of the patients had active disease (70\%) and were under treatment, half of them on first line. $25 \%$ had been submitted to allogeneic stem cell transplant. Septic shock (40\%) and respiratory failure (38.4\%) were the most frequent causes for ICU admission. During their ICU stay, $80 \%$ had respiratory failure $(50 \%$ received invasive mechanical ventilation) and $68.8 \%$ had cardiovascular failure; half of the patients had 3 or more organ failures. We observed a $54 \%$ ICU, $38 \%$ hospital, $28 \% 3$ months and $26 \% 1$ year survival rates. Main cause of death was refractory shock (37\%). Each additional organ failure was associated with lower survival probability by univariate analysis, except for neurologic failure; age was not a predictor of early death; by multivariate analysis, only respiratory failure and mechanical 
ventilation were associated with lower survival ( $\mathrm{HR} 11.59,95 \% \mathrm{Cl} 1.56$ $-86.45, p=0.02$ and $\mathrm{HR} 3.05,95 \% \mathrm{Cl} 1.41-6.57, p=0.01$, respectively). Type of hematologic malignancy and disease status at ICU admission were not predictors of ICU survival (Fig. 1 and 2, respectively). One month after ICU discharge, lower ECOG scores were associated with higher survival $(p<0.001)$. One year after ICU discharge, $74 \%$ of the survivors had ECOG score 0 or 1 and $68.4 \%$ were in complete remission.

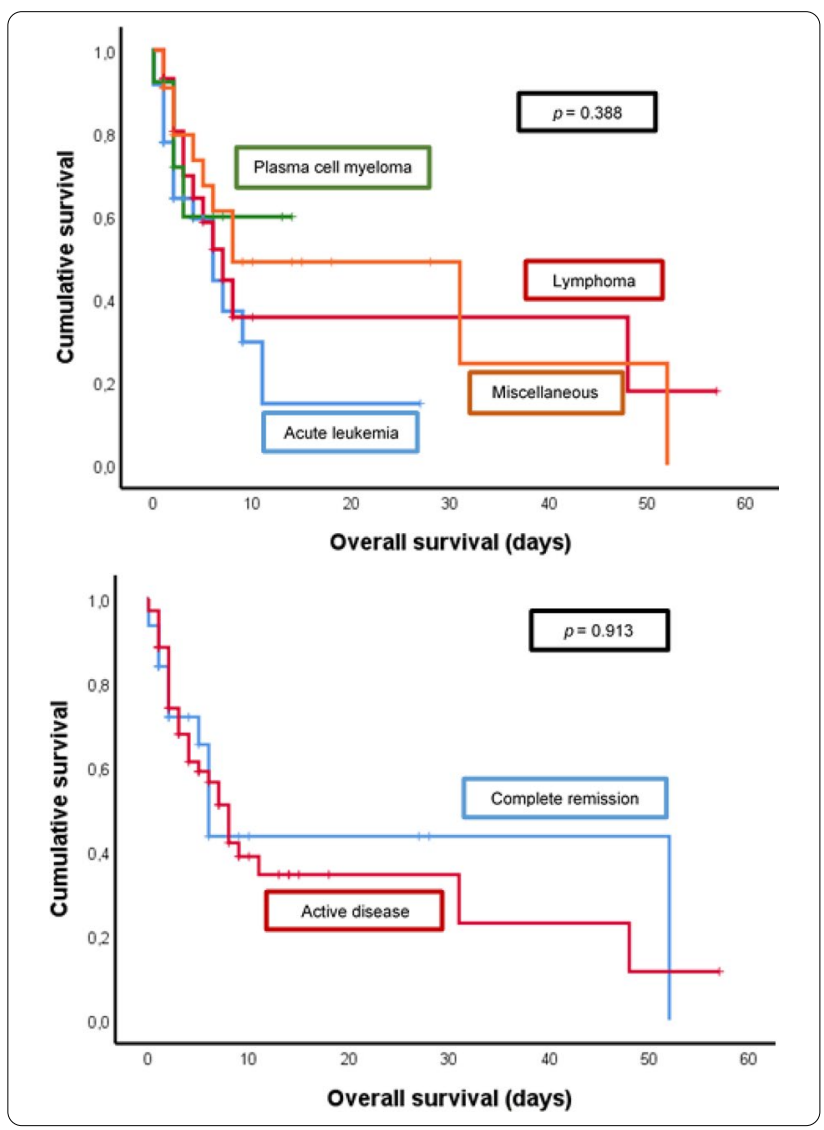

Conclusion: Patients with hematologic malignancy and multiorgan failure are at increased risk of death, however timely ICU care can still be associated with $40 \%$ survival. Mechanical ventilation, but not hematologic diagnosis nor age, was associated with lower survival probability. Importantly, patients surviving ICU admission attain good functional state at 1-year.

Reference(s) and grant acknowledgment(s)

1. Azoulay E, et al, J Clin Oncol, 2013, 31:2810-2818

2. De Vries, et al, "Long Term Outcome of Patients With Hematologic Malignancy and Multiple Organ Failure Admitted ant the Intensive Care"; Crit Care Med Journal, Feb 2019, Volume 47, num. 2

03. AIDS, hematologic-oncologic issues in the ICU > AIDS, hematologic-oncologic issues in the ICU

\section{8}

Acute kidney injury in critically ill oncohaematological patients: single centre experience

S. Judickas'? R. Stasiūnaitis² ; J. Sipylaite

${ }^{1}$ Institute of Clinical Medicine, Clinic of Anaesthesiology and Intensive Care, Faculty of Medicine of Vilnius University, Vilnius, Lithuania; ${ }^{2}$ Faculty of medicine, Vilnius University, Vilnius, Lithuania

Correspondence: $\mathrm{S}$. Judickas

Intensive Care Medicine Experimental 2020, 8(2): 000518
Introduction: Organ dysfunction is one of the main reasons for admission to intensive care unit (ICU) and it is associated with higher mortality. Acute renal injury (AKI) might occur not only because of general reasons but it is also associated with haematological malignancy itself, treatment and complications.

Objectives: The aim of our study was to analyse AKI in critically ill oncohaematological patients.

Methods: We retrospectively analysed data of oncohaemathological patients who were admitted to the 3rd ICU in Vilnius university hospital Santaros clinics from 13072017 to 2712 2019. Inclusion criteria were: oncohaematological malignancy, age $>18$ years, arterial or central line inserted, signed informed consent form. AKI was diagnosed according to KDIGO and RIFLE scoring systems. Baseline creatinine level was calculated at least 6 months before admission to ICU. Levels of creatinine were taken and recorded on admission to the hospital and ICU. Decision to admit patient to ICU and/or start renal replacement therapy was made by ICU doctor in charge. All of the continuous renal replacement therapies were performed with Prismaflex machine using citrate anticoagulation and CVVHDF mode. The effluent dose was prescribed by attending ICU doctor. SOFA score was calculated on admission to ICU. Statistical analysis was performed with $\mathrm{R}$ statistical package. A p-value of less than 0,05 was considered to be statistically significant.

Results: 117 patients $(67$ males and 50 females with the average age of $59,86 \pm 15,26)$ were included into the study. The baseline creatinine was $91,07 \mu \mathrm{mol} / \mathrm{L} \pm 59,34$, creatinine on admission to hospital was $118,96 \pm 91,86$, on admission to ICU $130,37 \pm 94,61$. Incidence of acute renal injury on admission to hospital according to KDIGO and RIFLE score were $35(29,91 \%)$ and $15(12,82 \%)$ on the admission to ICU $48(41,03 \%), 19(16,24 \%)$. Patients with KDIGO stage ${ }^{3} 1$ had lower arterial $\mathrm{pH}(7,41$ vs $7,46, \mathrm{p}$ value $<0.05)$, lower $\mathrm{BE}(-4,0$ vs $-0,2$, $p$ value $<0,05)$, higher lactate $(1,3$ vs $2,0, p$-value $<0,001)$ on admission to ICU. Patients with AKI according to RIFLE on admission to ICU had higher creatinine on admission to hospital ( 151 vs 75 , p-value < $0,001)$. CVVHDF was used for $30(25,42 \%)$ patients. Higher proportion of patients who received CVVHDF had renal injury according to RIFLE not only in ICU $(52,63 \%$ vs $20,20 \%$, p value $<0,05)$, but also on a ward $(46,66 \%$ vs $22,33 \%$, $p$ value $<0,05)$. Patients who received CVVHDF had lower arterial $\mathrm{pH}(7,37$ vs $7,45, \mathrm{p}$ value $<0,05)$, lower $\mathrm{BE}(-5,9$ vs $-0,3, \mathrm{p}$ value $<0,001)$, higher lactate $(2,1$ vs $1,4 p$ value $<0,05)$, higher creatinine on admission to hospital ( 108 vs $82,5 p$ value $<0,05$ ), higher creatinine on admission to ICU $(174,5$ vs $86, p$ value $<0,05)$. Patients who received CVVHDF had higher ICU mortality and worse overall survival $(83,33 \%$ vs $38,64 \%$, p value $<0,0001,96,67$ vs 64,77 , $p$ value $<0,001)$.

Conclusion: Acute renal injury is common in critically ill oncohamatological patients. Higher incidence is seen if diagnosed with KDIGO scoring system. Patients with AKI and those who received renal replacement therapy had more derangements on arterial blood gases. Patients who received CVVHDF had signs of acute renal injury already on a ward they also had higher ICU mortality and lower overall survival.

03. AIDS, hematologic-oncologic issues in the ICU > AIDS, hematologic-oncologic issues in the ICU

\section{9}

High dose vitamin C therapy for oncohaemathological patients with septic shock

S. Judickas'; R. Pelanis²; J. Sipylaite

${ }^{1}$ Institute of Clinical Medicine, Clinic of Anaesthesiology and Intensive Care, Faculty of Medicine of Vilnius University, Vilnius, Lithuania; ${ }^{2}$ Faculty of medicine, Vilnius University, Vilnius, Lithuania

Correspondence: $\mathrm{S}$. Judickas

Intensive Care Medicine Experimental 2020, 8(2): 000519

Introduction: Patients with septic shock have a very high mortality rate despite of aggressive treatment in intensive care unit (ICU). Prognosis of oncohaematological patients is even worse. High dose vitamin C therapy received a lot of attention over the last years as a supplementary therapy which could lead to improvement during septic shock. 
Objectives: The aim of our study was to evaluate the impact of high dose vitamin $C$ therapy during septic shock in oncohaematological patients.

Methods: We retrospectively analysed data of oncohaematological patients with septic shock who were admitted to the 3rd Intensive care unit from 13072017 to 19022019 in Vilnius university hospital Santaros clinics. Inclusion criteria were: oncohaematological malignancy, age $>18$ years, septic shock, arterial or central line inserted, signed informed consent form. Sepsis and septic shock were defined as per SEPSIS-3 definition. When noradrenaline dose reached $0,1 \mathrm{mcg} / \mathrm{kg} /$ min. patients received vitamin $C 1500 \mathrm{mg}$ every six hours $\mathrm{i} / \mathrm{v}$ together with hydrocortisone $50 \mathrm{mg}$ every six hours $\mathrm{i} / \mathrm{v}$, thiamine $200 \mathrm{mg} \mathrm{i} / \mathrm{v}$ once per day supplementary to the standard treatment of septic shock according to local practice. Control group consisted of oncohaematological patients with septic shock who didn't receive high dose vitamin C therapy. High dose vitamin C therapy was stopped when patients became haemodynamically stable. Statistical analysis was performed with R statistical package. A p-value less than 0.05 was considered to be statistically significant.

Results: 71 patients with haematological malignancies were admitted to ICU. 36 patients were diagnosed with sepsis and 33 of them had septic shock. 23 patients received high dose Vitamin C therapy. 9 patients were excluded from the analysis due to the violation of dosage of vitamin C. Final analysis included 14 patients who received high dose vitamin $C$ therapy. Control group consisted of 10 patients.

Patients in high dose vitamin C group were older $(65,57$ vs 53,95, p $<0.0027$ ), they had a higher SOFA score on day1, day2 and day 3 in intensive care unit (accordingly 8,64 vs $6,2^{*}, 10,62$ vs $7,12^{* *}, 10,09$ vs $5,92^{* * *}, \mathrm{p}$ value $\left.{ }^{*} 0.0534, * *-0.0051, * * *-0.002597\right)$. Arterial $\mathrm{pH}, \mathrm{BE}$, lactate did not differ between groups on arrival to intensive care unit (accordingly 7.46 vs $7,43(p=0,44), B E-2,99$ vs $0,53(p=0,48)$, lactate 1,9 vs $1,2(p=0.09)$ ). Intensive care unit survival of patients treated with high dose vitamin C therapy was $21,43 \%$ (3/14) compared with $47,37 \%(9 / 19)$ in standard treatment group ( $p$ value 0.1258 ). There was no difference in terms of intravenous fluids (per hour), time spent in intensive care unit, length of vasopressor therapy and renal replacement therapy between the groups $(149.15 \mathrm{ml}$ vs $131,1 \mathrm{ml}, 69,5 \mathrm{~h}$ vs $71 \mathrm{~h}, 62 \mathrm{~h}$ vs $10 \mathrm{~h}$ and $50 \%(7 / 7)$ vs $26,32 \%(5 / 19))$.

Conclusion: High dose vitamin C therapy didn't improve survival of oncohaematological patients with septic shock. Patients with high dose vitamin C had higher SOFA scores on the first, second and third days in intensive care unit.

03. AIDS, hematologic-oncologic issues in the ICU > AIDS, hematologic-oncologic issues in the ICU

\section{3}

Lymphocyte subpopulations in critical SARS-CoV2 infection

L. P. Moulaert' ${ }^{1}$;. Henin ${ }^{1}$; V. Huberlant ${ }^{1}$; J. B. Mesland

'Icu, Jolimont Hospital, La Louvière, Belgium

Correspondence: J.B. Mesland

Intensive Care Medicine Experimental 2020, 8(2): 000553

Introduction: Since the beginning of the new SARS-CoV2 pandemic, numerous biological and clinical findings have been described through the literature. Lymphopenia is one of them and is found in nearly all severe cases (1). Little is known about the lymphocyte subset in this pathology, despite interesting data that could help to better understand SARS-CoV2 infection.

Objectives: To review the lymphocyte subpopulations in SARS-CoV2 infected patients admitted in our ICU.

Methods: Observational, retrospective and descriptive study. We reviewed all our SARS-CoV2 patients admitted in our ICU between march 2020 and may 2020 and selected patients who had lymphocytes subset requested at admission. Epidemiological data, hemoglobin, leucocyte count, leucocyte population, and lymphocyte subpopulation (studied by flow cytometry) were collected.

Results: Of 46 patients admitted in our ICU (22 beds), 10 had lymphocyte subset requested. Mean age (+/-SD) was 56,18 years (+/- 11). All of these 10 patients were critical SARS-CoV2 pneumonia, requiring mechanical ventilation within their stay. $5 / 10$ patients required VVECMO. All patients were HIV negative.

$8 / 10$ patients had low CD4+ T-lymphocyte count $(317 / \mathrm{mm} 3+/-94)$, with proportionally more profound alteration in CD8+ T-lymphocyte count $(82 / \mathrm{mm} 3+/-30)$. The T-Lymphocyte Helper/Suppressor ratio was elevated in most of the patients $(7 / 10)$, with mean value of 4,3 $(+/-2)$. No patient had a ratio less than 1 . There was an increase in proportion of circulating B lymphocytes $(24 \%+/-10 \%)$, with normal absolute value $(148 / \mathrm{mm} 3+/-53)$. All patients had normal NK cells value $\left(75 / \mathrm{mm}^{3}+/-31\right)$.

Opportunistic infections are classically described in patients having specific risk factor (malignancies, solid organ transplant, specific drugs) or HIV patients having CD4+ count lower than $200 / \mathrm{mm} 3$ (2) which was the case in only $2 / 10$ patients (lowest value of $126 / \mathrm{mm} 3$ ).

Conclusion: Lymphocytes are playing an important role in the main tenance of immune system function. Subsets are uniformly affected with low CD4+ and CD8+ T-lymphocyte, higher ratio, and increased proportion of circulating B lymphocytes. These findings are correlated to the severity of the disease (2), but underlying consequence of it is unknown. Potential treatment aimed to restore a better immune function as used in cancer immunotherapy or tested in sepsis might be a promising tool to correct those alterations(3).

\section{Reference(s) and grant acknowledgment(s)}

1. (3) Kenneth E Remy, Scott C Brakenridge, Bruno Francois, et al. Immunotherapies for COVID-19: lessons learned from sepsis. Lancet Respir med, https:// doi.org/10.1016/s2213-2600(20)30217-4

2. (2) Chen G, Wu D, Guo W, et al. Clinical and immunological features of severe and moderate coronavirus disease 2019. J Clin Invest. 2020;130(5):2620-2629. https://doi.org/10.1172/jci137244

3. (1) Wang D, Hu B, Hu C, et al. Clinical Characteristics of 138 Hospitalized Patients With 2019 Novel Coronavirus-Infected Pneumonia in Wuhan, China. JAMA. 2020;323(11):1061-1069. https://doi.org/10.1001/ jama.2020.1585

03. AIDS, hematologic-oncologic issues in the ICU > AIDS, hematologic-oncologic issues in the ICU

\section{6}

Cytokine release syndrome after CAR T cell therapy. A multidisciplinary approach. The Advanced Therapies in Hematology Unit-Vall d'Hebron Hospital (Barcelona) experience

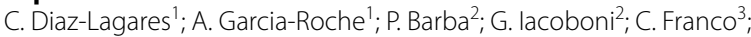
M. Linares ${ }^{4}$; M. Tintore ${ }^{5}$; A. Farriols ${ }^{6}$; I. Losarcos ${ }^{7}$; O. Roca ${ }^{1}$; R. Ferrer Roca ${ }^{1}$

${ }^{1}$ Intensive care department, Vall d'Hebron University Hospital, Vall d'Hebron Research Institute, Barcelona, Spain; ${ }^{2}$ Hematology department. vhio., Vall d'Hebron University Hospital., Barcelona, Spain; ${ }^{3}$ mmunology department. vhir., Vall d'Hebron University Hospital., Barcelona, Spain; ${ }^{4}$ Blood bank., Vall d'Hebron University Hospital, Barcelona, Spain; ${ }^{5}$ Neurology department. cem-cat, Vall d'Hebron University Hospital, Barcelona, Spain; ${ }^{6}$ Pharmacy department, Vall d'Hebron University Hospital, BarceIona, Spain; ${ }^{7}$ Infectious diseases department, Vall d'Hebron University Hospital, Barcelona, Spain

Correspondence: C. Díaz

Intensive Care Medicine Experimental 2020, 8(2): 000716

Introduction: Cytokine release syndrome (CRS) is a clinical syndrome derived from the massive release of cytokines after immunotherapy, which can range in severity from a flu-like illness to multiorgan dysfunction syndrome.

Objectives: To describe the clinical profile of CRS and to identify risk factors for developing CRS and for CRS severity after Chimeric Antigen Receptor (CAR) T-cell therapy.

Methods: Retrospective study that included those patients treated with CAR T-cell therapy in the Advanced Therapies in Hematology Unit (UTAH) at Vall d'Hebron University Hospital (Barcelona) from August 2018 to December 2019. The UTAH is a 12-bed, monitored unit, staffed by Hematologists, with the support of a multidisciplinary team including ICU, Neurology, Pediatrics, Infectious Diseases, Microbiology, Immunology and Pharmacy specialists. Patients were admitted before 
CART-cell infusion for lymphodepletive chemotherapy and were followed up during at least 10 days after CAR T-cell infusion, per protocol. CRS and immune effector cell-associated neurotoxicity syndrome (ICANs) were defined and graded according to ASTCT Consensus (Biol Blood Marrow Transplant 2019; 25: 625-38). We considered CRS onset in the first 72 hours after CAR T-cell infusion as early CRS (eCRS), and severe CRS (sCRS) as CRS grade $>1$. Chi-Square, Fisher's test, T test, $U$ Mann-Whitney and logistic regression were employed as appropriate. Quantitative variables are reported as median (IQR) and categorical as frequency (\%).

Results: Forty-one patients, 31 (76\%) male with a median age of 57 (47-66) years, were treated with anti-CD19 CAR T-cells for refractory non Hodgkin lymphoma $(38,93 \%)$ and acute lymphoblastic leukemia $(3,7 \%)$. Costimulatory domains for CAR T-cells were 4-1BB and CD28 in $39(95 \%)$ and $2(5 \%)$ patients, respectively. Thirty $(73 \%)$ developed CRS:13 (32\%) grade 1, $16(39 \%)$ grade 2, and $1(2 \%)$ grade 3. All of them had fever, with a median peak temperature of $38.4^{\circ} \mathrm{C}$ (38-39), $13(32 \%)$ developed hypotension that required fluid bolus and 11 (27\%) hypoxemia requiring supplemental oxygen (6 liters per minute or less in 10 and $30 \mathrm{lpm}$ administered with high-flow nasal cannula in 1 patient). Time from CAR T-cell infusion to CRS was 4 (2-6) days. Ten (33\%) patients developed eCRS. Duration of CRS was 5 (3-8) days. Tocilizumab was used in $8(20 \%)$ patients and was associated with resolution of CRS in all treated cases; one patient $(2 \%)$ needed a second dose to control CRS. Moreover, 8 (20\%) patients developed ICANs, at a median of 7 (5-9) days after CAR T cell infusion; 1 (2\%) grade 1, 6 (15\%) grade 2 and $1(2 \%)$ grade 3 . Seven of these 8 patients developed ICANs after CRS onset. Patients with ICANs grade 2 and 3 were treated with corticosteroids with complete resolution in all patients. Baseline biomarkers were not predictors of CRS (Table 1). Patients with sCRS had earlier CRS onset, higher temperature, higher CRP at day 1 of of CRS, higher CRP peak, LDH peak and interleukin 6 peak levels (Table 2). After multivariate analysis, peak temperature (OR 44.6, 95\% IC 1.6$1243, p=0.025$ ) and early CRS (OR $39.7,95 \%$ IC $1.2-1279, p=0.038$ ) were associated with severity. Most patients were successfully managed in the UTAH, with only 2 requiring admission to the ICU. No fatal events were observed due to CAR T-cell related toxicity.

Table 1. Baseline biomarkers in patients with and without CRS

\begin{tabular}{|l|l|l|l|l|}
\hline Variable & All $(\mathrm{n}=41)$ & No CRS $(\mathrm{n}=11)$ & CRS $(\mathrm{n}=30)$ & $\mathrm{p}$ value \\
\hline CRP $(\mathrm{mg} / \mathrm{dL})$ & $3(1.1-8.7)$ & $1.6(1.1-7.8)$ & $3.1(1.5-11.4)$ & 0.311 \\
\hline LDH $(\mathrm{UI} / \mathrm{L})$ & $594(399-1084$ & $441(332-659)$ & $650(416-1405)$ & 0.499 \\
\hline Ferritin $(\mathrm{ng} / \mathrm{mL})$ & $741(396-1578)$ & $1426(387-1542)$ & $702(406-1695)$ & 0.547 \\
\hline
\end{tabular}

Table 2. Characteristics of CRS depending on severity

\begin{tabular}{|l|l|l|l|l|}
\hline Variable & CRS $(\mathrm{n}=30)$ & $\begin{array}{l}\text { Grade 1 CRS } \\
(\mathrm{n}=13)\end{array}$ & $\begin{array}{l}\text { Severe CRS } \\
(\mathrm{n}=17)\end{array}$ & $\begin{array}{l}\mathrm{p} \\
\text { value }\end{array}$ \\
\hline Day 1 of CRS & & & & \\
\hline $\begin{array}{l}\text { CRP } \\
(\mathrm{mg} / \mathrm{dL})\end{array}$ & $3(1.7-8.7)$ & $1.8(1.7-3)$ & $5.4(3-18.4)$ & 0.029 \\
\hline Peak value & & & & \\
\hline $\begin{array}{l}\text { CRP } \\
(\mathrm{mg} / \mathrm{dL})\end{array}$ & $12.3(6.7-25)$ & $8.9(6.6-9.8)$ & $19.8(13.6-42.4)$ & 0.016 \\
\hline LDH (UI/L) & $809(474-2003$ & $553(474-1092)$ & $1485(613-3095)$ & 0.035 \\
\hline $\begin{array}{l}\text { IL-6 } \\
(\mathrm{pg} / \mathrm{mL})\end{array}$ & $\begin{array}{l}68.7(31.6- \\
269.3)\end{array}$ & $46.5(20-63.7)$ & $\begin{array}{l}214.5(72.3- \\
317.8)\end{array}$ & 0.001 \\
\hline Temp $\left({ }^{\circ} \mathrm{C}\right)$ & $\begin{array}{l}38.8(38.2- \\
39.1)\end{array}$ & $38.4(38.1-38.5)$ & $39.1(38.8-39.3)$ & $<0.001$ \\
\hline Early CRS & $10(33)$ & $1(8)$ & $9(53)$ & 0.017 \\
\hline
\end{tabular}

Conclusion: Most patients treated with CAR T-cell therapy presented some adverse event, including CRS and/or ICANs. Resolution was fast in most cases, with no fatal events in our cohort. These results were achieved with a proactive multidisciplinary approach, leading to an early detection and appropriate therapy. Peak temperature and early onset of CRS may be useful to identify those patients with higher CRS severity.

03. AIDS, hematologic-oncologic issues in the ICU > AIDS, hematologic-oncologic issues in the ICU

\section{1}

Prognostic factors in critically ill oncological patients admitted in a tertiary ICU: The Vall d'Hebron Intensive Care Department/ Vall d'Hebron Institute of Oncology cohort (VHIO)

A. Pacheco ; C. Diaz-Lagares ${ }^{1}$; A. Garcia-Roche1; I. Romera1'; O. Roca1; E. Elez ${ }^{2} ;$ R. Ferrer Roca'

${ }^{1}$ Intensive care department, Vall d'Hebron University Hospital, Vall d'Hebron Research Institute, Barcelona, Spain; ${ }^{2}$ Oncology department, Vall d'Hebron University Hospital. VHIO, Barcelona, Spain

Correspondence: $C$. Díaz

Intensive Care Medicine Experimental 2020, 8(2): 000731

Introduction: Over the last two decades, the number of cancer patients requiring ICU care has dramatically increased with $15 \%$ of ICU beds occupied by cancer patients. Thus, in the following years intensive care medicine will become a key component in the management of these patients.

Objectives: Our aim was to describe the cohort of patients with solid organ cancer admitted to a tertiary hospital ICU and analyze factors associated with survival.

Methods: Seven-year (2010-2017) retrospective study, including adult patients with solid organ cancer who required ICU admission. Chi-Square, Fisher's test, T test, U Mann-Whitney and Cox's regression (including all significant variables in the univariate analysis) were employed as appropriate. Quantitative variables are reported as median (IQR) and categorical as frequency (\%).

Results: Two hundred patients were included, 107 (54\%) were male with a median age of 60 (50-68) years. Lung cancer was present in $53(27 \%)$, followed by colon $27(14 \%)$, breast $16(8 \%)$ and ovary 15 (7\%). One hundred and six (53\%) had metastatic disease. Most of the patients $(181 ; 91 \%)$ were on ECOG $0-2$ and Charlson Index was 9 (5-11). Respiratory failure was the reason for ICU admission in 72 (36\%) patients, shock in 41 (21\%), respiratory failure and shock in $33(17 \%)$, urgent surgery in $36(18 \%)$ and central nervous system involvement in $12(6 \%)$. Neutropenia was present in 27 (14\%). SOFA score at day 1 was 6 (4-9). Eighty-four (42\%) had a lower SOFA score at day 3 and 60 (30\%) at day 5 . One hundred and twenty-five $(62 \%)$ required vasopressors (VP), 89 (45\%) invasive mechanical ventilation (IMV), 72 (36\%) nasal high flow (NHF) and $18(9 \%)$ renal replacement therapy (RRT). Of those patients treated with NHF, 24 (33\%) were intubated, while 35 (49\%) were not intubated after improvement in their condition and $7(10 \%)$ because of a do not intubate order. ICU mortality affected to 50 (25\%) and in-hospital mortality to 86 (43\%). Differences between hospital survivors and non-survivors are detailed in Table 1. Patients requiring RRT (HR 2.7, 95\% IC (1.1-6.8) $p=0.036)$ and those who did not decrease their SOFA score at day 5 (HR 2.3 IC 95\% (1.1-4.9) had higher in-hospital mortality after multivariate analysis. 
Table 1. Characteristics of hospital survivors and non-survivors

\begin{tabular}{|l|l|l|l|}
\hline Variable & Survivors $(\mathrm{n}=114)$ & Non-survivors $(\mathrm{n}=86)$ & $\mathrm{p}$ value \\
\hline Age (years) & $59(50-68)$ & $61(48-69)$ & 0.420 \\
\hline Male & $53(49 \%)$ & $38(44 \%)$ & 0.537 \\
\hline Lung cancer & $22(20 \%)$ & $30(35 \%)$ & 0.021 \\
\hline Charlson & $9(5-10)$ & $7(5-10)$ & 0.478 \\
\hline Metastasis & $57(56 \%)$ & $49(60 \%)$ & 0.767 \\
\hline Neutropenia & $14(13 \%)$ & $13(15 \%)$ & 0.648 \\
\hline NHF & $35(32 \%)$ & $37(43 \%)$ & 0.117 \\
\hline IMV & $31(29 \%)$ & $57(67 \%)$ & $<0.001$ \\
\hline VP & $61(57 \%)$ & $61(73 \%)$ & 0.021 \\
\hline RRT & $5(4 \%)$ & $13(15 \%)$ & 0.012 \\
\hline SOFA day 1 & $5(2-7)$ & $7(3-11)$ & 0.001 \\
\hline No decrease in SOFA & & & \\
\hline \multicolumn{1}{|c|}{ Day 3 } & $21(27 \%)$ & $25(49 \%)$ & 0.01 \\
\hline \multicolumn{1}{|c|}{ Day 5 } & $10(18 \%)$ & $23(64 \%)$ & $<0.001$ \\
\hline
\end{tabular}

Conclusion: Hospital mortality of oncologic patients that require ICU admission remains high. Mortality may be more dependant on organ failure development and need for organ support suggesting that these patients may benefit of an earlier ICU admission. Persistent organ failure is associated with worse outcomes and, therefore, this information could be useful in the bedside clinical decision making process during an ICU trial, although clinical global assessment must prevail.

03. AIDS, hematologic-oncologic issues in the ICU > AIDS, hematologic-oncologic issues in the ICU

\section{4}

ICU-outcomes in CAR-T patients-A single centre experience

T. Materski ${ }^{1}$; M. john ${ }^{1}$; T. Pirani ${ }^{1}$; R. Benjamin ${ }^{2}$; A. Kuhnl ${ }^{2}$; V. Potter ${ }^{2}$; R. Sanderson ${ }^{2}$; V. Metaxa ${ }^{1}$

${ }^{1}$ Critical care, Kings college hospital, LONDON, United Kingdom; ${ }^{2}$ Hematology, Kings college hospital, LONDON, United Kingdom

Correspondence: $M$. john

Intensive Care Medicine Experimental 2020, 8(2): 000954

Introduction: Chimeric antigen receptor-modified T (CAR-T) cells are a revolutionary treatment for patients with advanced blood cancers. A proportion of these patients will develop significant toxicities, requiring critical care management. Our hospital was one of 7 in the UK approved for the treatment of relapsed/refractory high-grade lymphoma and refractory acute lymphoblastic leukaemia (ALL).

Objectives: To describe our experience with lymphoma and ALL patients, who received CAR-T CD19 cells and were admitted to the intensive care unit (ICU).

Methods: From January 2019 until May 2020, patients with diffuse large $B$ cell lymphoma (DLBCL), transformed follicular lymphoma, primary mediastinal $B$-cell lymphoma (PMBCL) or ALL, who were infused with CAR-T CD19 cells and developed $\geq$ grade 2 Cytokine Release Syndrome (CRS) and Immune Effector Cell Associated Neurotoxicity Syndrome (ICANS) were analysed.

Results: Sixty-one patients underwent CAR-T therapy during the study period, with 21 (34\%) requiring admission to ICU . The mean age at presentation to ICU was 57 years and $76 \%$ of the admissions were males. All patients were receiving broad spectrum antibiotics and 19\% of treatments were broadened in ICU and an antifungal was added. CRS/sepsis ( $n=12,57 \%$ ) was the most common documented reason for admission to ICU, with a proven infection documented in only 2 patients. The reasons for admission are shown in figure 1. Median duration of ICU stay was 4 (range 1-21) days. Grade 2 CRS was documented in 12 patients (57\%) for a median duration of 2 (range 0-6) days. Grade 3 CRS was seen in 6 patients (29\%) for 1-3 days. No patient progressed to grade 4 CRS during their ICU stay. Six patients had grade 1 ICANS each for a median duration of 4 (2-6) days, whereas another 6 had grade 3 ICANS for 2 days (1-4).
During their ICU stay, 9 patients (48\%) required vasopressors for a median duration of 2 (1-3) days. Three patients (14\%) needed mechanical ventilation with a median duration of 5 (4-9) days and 2 patients needed renal replacement therapy for a median duration of 4 (1-7) days. IL-1, IL-6 antagonists and steroid administration are shown in figure 2 . Three patients $(14 \%)$ died in ICU, all in multi-organ failure. Two deaths were attributed to invasive pulmonary aspergillosis and the third to disease progression. Further 7 patients that were discharged alive from ICU, died before hospital discharge from disease progression.

Conclusion: A significant number of patients receiving CAR-T therapy will need admission to ICU, requiring various level of organ support. Differentiation between CRS and neutropenic sepsis was difficult, and all patients stayed on antibiotics for the duration of their ICU stay. The observed ICU mortality (14\%) was in accordance to the one reported in international literature and no deaths were attributed to treatment toxicity. Close collaboration between haematology and ICU is warranted for the optimal management of CAR-T patients.

\section{Reference(s) and grant acknowledgment(s)}

1. Lee DW, Santomasso BD, Locke FL, et al. ASTCT Consensus Grading for Cytokine Release Syndrome and Neurologic Toxicity Associated with Immune Effector Cells. Biol Blood Marrow Transplant. 2019 Apr;25(4):625-638

2. No grants

03. AIDS, hematologic-oncologic issues in the ICU > AIDS, hematologic-oncologic issues in the ICU

\section{5}

Is there a possible benefit in therapeutic anticoagulation for all COVID-19 patients?

H. Aly ; M. Parris ${ }^{2}$

${ }^{1}$ Anaesthetics and Intensive Care, Ashford and St Peters NHS Foundation Trust, CHERTSEY, SURREY, United Kingdom; ${ }^{2}$ Anaesthetics, ASHFORD AND ST PETERS NHS FOUNDATION TRUST, Chertsey, United Kingdom

Correspondence: $\mathrm{H}$. Aly

Intensive Care Medicine Experimental 2020, 8(2): 001105

Introduction: Since the beginning of the COVID-19 pandemic, rapid evidence was suggesting a pro-coagulant state (1).

There are currently insufficient data to recommend for or against the use of thrombolytics or increasing anticoagulant doses for VTE prophylaxis in hospitalised COVID-19 patients outside the setting of a clinical trial (2). Currently, the guidance adopted by the national institute of health recommends anticoagulation treatment risk to be assessed on individual patient basis and therapeutic anticoagulation to commence in the event when it's clinically indicated (2), namely when patients develop thrombo-embolic manifestations.

Objectives: We have undertaken a retrospective data analysis of the patients admitted to our hospital between Mid February to mid April 2020 with COVID-19 illness to ITU and to the general wards. We have established the number need to treat for both patients groups based on a single outcome (death), in an effort to establish a possible benefit from starting anticoagulation earlier to ward patients as ITU patients.

Methods: Drug history was identified through hospital records for confirmed patients with COVID-19 disease to identify patients who have been on anticoagulation prior to hospital admission for an independent reason. For both groups (ward patients and ITU patients) the outcome was established as Death or improvement at the time of data collection. Total number of cases explored for ITU is 51 patients, compared to 156 patients on the ward.

Results: For ITU patients the NNT(number needed to treat) to prevent adverse outcome (death) $=2$, with an absolute risk reduction of $52.17 \%$ and $\mathrm{a} \mathrm{Cl}$ (confidence interval) for NNT =1.5-2.6.

For ward patients the NNT $=4$, with an absolute risk reduction of 23.4\% and $\mathrm{a} \mathrm{Cl}$ of 2.2-162.0.

Conclusion: The above mentioned results does not support changing the current practice to starting therapeutic anticoagulation on ward patients "empirically". The risk assessment therefore is to be conducted on individual basis and assessed for each patient individually. 
Reference(s) and grant acknowledgment(s)

1. COVID-19-Related Severe Hypercoagulability in Patients Admitted to Intensive Care Unit for Acute Respiratory Failure, Thromb Haemost 2020; 120(06): 998-1000.

2. COVID-19 Treatment Guidelines Panel. Coronavirus Disease 2019 (COVID19) Treatment Guidelines. National Institutes of Health.

3. This work did not receive any grants or funding.

\section{5}

Lean body mass as a prognostic factor in post-cardiac arrest patients treated by targeted temperature management: a retrospective cohort study

H. H. Kim¹; E. L. Sung

${ }^{1}$ Emergency Medicine, Ajou University Hospital, Suwon, Republic of Korea Correspondence: $H$. H. Kim

Intensive Care Medicine Experimental 2020, 8(2): 000005

Introduction: Post-cardiac arrest patients with return of spontaneous circulation have a very low survival rate and poor prognosis despite aggressive treatments. Prediction these patients' prognosis is important for planning the treatment of the patients and providing information to their families. Several studies have reported body mass index (BMI) as a prognostic factor in post-cardiac arrest patients, which is called "obesity paradox". In this study, researchers aimed to investigate the relationship of LBM with the prognosis of post-cardiac arrest patients.

Methods: This retrospective cohort study included adult patients of out-of-hospital cardiac arrest between January 2015 and August 2018. The enrolled patients were divided into 2 groups based on clinical outcomes (cerebral performance category score, 1 to 2 and 3 to 5) and compare the characteristics of these two groups. Association of LBM with good neurologic outcomes (CPC score 1 to 2) was analyzed by dividing patients into quartile segment by their LBM. Predictive value of optimal cutoff points of LBM in post-cardiac arrest patients was evaluated.

Results: A total 169 patients were analyzed (CPC score 1 to $2, n=55$ CPC score 3 to $5, n=114$ ). Patients' age, frequency of witnessed cardiac arrest, initial shock rhythm, post-hospital flow time, estimated cardiac arrest, and LBM were different in the two groups ( $p<0.05)$. LBM was associated with the prognosis of post cardiac arrest patients in the 4th quartile segment. Cutoff point of lean body mass for prediction of cardiac arrest outcomes was 48.34 (sensitivity $=0.600$, specificity $=$ 0.763 , accuracy $=0.710$ ) and the predictive value was 0.68 (95\% confidence interval, 0.60 to 0.75 ).

Conclusion: High LBM is related to good neurological prognosis of patients after cardiac arrest. The increase in muscle mass due to exercise is interpreted as showing positive effects on hypoxic injury in cardiac arrest and reperfusion injury after return of spontaneous circulation.

\section{Reference(s) and grant acknowledgment(s)}

1. Ma, Y., et al., Association between body mass index and clinical outcomes of patients after cardiac arrest and resuscitation: A meta-analysis. Am J Emerg Med, 2018. 36(7): p. 1270-1279.

2. Geri, G., et al., Influence of body mass index on the prognosis of patients successfully resuscitated from out-of-hospital cardiac arrest treated by therapeutic hypothermia. Resuscitation, 2016. 109: p. 49-55

3. Leary, M., et al., The association of body mass index with time to target temperature and outcomes following post-arrest targeted temperature management. Resuscitation, 2014. 85(2): p. 244-7.

4. Jain, R., et al., Body mass index and survival after in-hospital cardiac arrest. Circ Cardiovasc Qual Outcomes, 2010. 3(5): p. 490-7.

5. Fugate, J.E., et al., Predictors of neurologic outcome in hypothermia after cardiac arrest. Ann Neurol, 2010. 68(6): p. 907-14

6. Sandroni, C., et al., Predictors of poor neurological outcome in adult comatose survivors of cardiac arrest: a systematic review and metaanalysis. Part 2: Patients treated with therapeutic hypothermia. Resuscitation, 2013. 84(10): p. 1324-38.
7. Herlitz, J., et al., Characteristics and outcome among patients having out of hospital cardiac arrest at home compared with elsewhere. Heart, 2002. 88(6): p. 579-82.

8. Marwick, T.H., et al., Prediction of survival from resuscitation: a prognostic index derived from multivariate logistic model analysis. Resuscitation, 1991. 22(2): p. 129-37.

9. Weaver, W.D., et al., Factors influencing survival after out-of-hospital cardiac arrest. J Am Coll Cardiol, 1986. 7(4): p. 752-7.

10. Kamps, M.J., et al., Prognostication of neurologic outcome in cardiac arrest patients after mild therapeutic hypothermia: a meta-analysis of the current literature. Intensive Care Med, 2013. 39(10): p. 1671-82.

11. This research did not receive any specific grant from funding agencies in the public, commercial, or not-for-profit sectors.

\section{4}

Probability of receiving mechanical chest compressions is associated with cardiac arrest mortality: a post-hoc, propensity score analysis from the TTH48 trial

G. Babini ; H. Kirkegaard ${ }^{2}$; E. Søreide ${ }^{3}$; FS. Taccone ${ }^{4}$; M. Skrifvars ${ }^{5}$

${ }^{1}$ Department of Pathophysiology and Transplantation, University of Milan, Milano, Italy; ${ }^{2}$ Research center for emergency medicine, department of emergency medicine and department of clini, Aarhus University Hospital and Aarhus University, Aarhus, Denmark; ${ }^{3}$ Critical care and anaesthesiology research group, Stavanger University Hospital, Stavanger, Norway; ${ }^{4}$ Department of intensive care, ULB Erasme, Brussels, Belgium; ${ }^{5}$ University of helsinki and helsinki university hospital, Perioperative, intensive care and pain medicine, Helsinki, Finland

\section{Correspondence: $\mathrm{G}$. Babin}

Intensive Care Medicine Experimental 2020, 8(2): 000284

Introduction: Various randomized clinical trials reported no differences in outcome when mechanical chest compressions (CC) were used in cardiac arrest patients [1-3]; however, in these trials mechanical CC were systematically applied soon after ALS arrival to evaluate its effectiveness compared to manual CC. The evidence regarding the impact of mechanical CC on mortality in patients with refractory outof-hospital cardiac arrest (OHCA) and prolonged resuscitation efforts, when mechanical CC are initiated later, is scarce.

Methods: This is a post hoc, propensity score (PS) analysis of OHCA patients who received or did not received mechanical CC during resuscitation manoeuvres in the TTH48 trial. A predefined set of covariates (recruiting centre, age, height, weight, gender, location of OHCA, witnessed OHCA, initial cardiac rhythm, bystander cardiopulmonary resuscitation, automatic external defibrillator used, time from OHCA to basic life support, time from OHCA to advanced life support, resuscitation drugs used, intubation on scene) were explored and used for developing a PS for the receipt of mechanical CC using multiple binary logistic regression. The PS was then applied as an adjustment factor, together with the receipt of mechanical CC, in a Cox proportional hazard model to estimate the impact on 6-months mortality.

Results: Out of 351 patients included in the analysis, $25.6 \%$ received mechanical CC. No major differences in baseline characteristics were observed between groups, except for prolonged time to ROSC ( 26 [20;38] vs $20[15 ; 25] \mathrm{min}, \mathrm{p}<0.01)$ and more frequent use of resuscitation drug (adrenaline: 80 vs $57 \%, p<0.01$; amiodarone: 53 vs $37 \%$, $\mathrm{p}<0.01)$ in the mechanical CC group. The Cox proportional hazard models did not show a significant impact of the probability to receive mechanical CC and the receipt of mechanical CC on 6-months mortality (1.676 [0.580-4.842], $p=0.34$ and 1.683 [0.925-3.061], $p=0.09$, respectively). We computed another PS including time from OHCA to ROSC to the same covariates used previously; in this case, the predicted probability to receive mechanical CC was significantly associated with 6 months mortality (3.713 [1.437-9.599], $\mathrm{p}<0.01)$.

Conclusion: When time to ROSC was included in the PS, the likelihood to receive mechanical CC during cardiac arrest significantly correlated with 6-months mortality while receipt of mechanical chest compressions did not. The same association was not observed when time to ROSC wasn't accounted in the PS. The longer duration of resuscitation 
efforts observed in patients that received mechanical CC could explain the results. Notably, mechanical CC, per se, was never associated with mortality.

\section{Reference(s) and grant acknowledgment(s) \\ 1. Perkins et al. Lancet 2015 \\ 2. Wik et al. Resuscitation 2014 \\ 3. Rubertsson et al. JAMA 2014}

\section{0}

In-hospital cardiorespiratory arrest at NSTEMI: is it possible to identify at-risk patients early?

H. Miranda'; S. Marianaa'; H. Santos'; I. Almeida'; C. Sousa'; L. Almeida ${ }^{1}$ Cardiology, Centro Hospitalar Barreiro Montijo, Barreiro, Portugal; ${ }^{2}$ Cardiology, Centro Hospitalar Barreiro Montijo, E.P.E, Barreiro, Portugal

Correspondence: $\mathrm{H}$. Miranda

Intensive Care Medicine Experimental 2020, 8(2): 000380

Introduction: The occurrence of in-hospital cardiopulmonary arrest (CPA) is associated with worse outcomes. Identifying the most at-risk patients is crucial to ensure greater vigilance and a more "offensive" posture in these cases.

Objectives: Characterization of patients admitted by NSTEMI with progression in Cardiac Arrest (CPA). Creation of intra-hospital CPA predictor score in this population and comparison with 2 recently published SAFER and CHADS2 scores in international publications.

Methods: Multicenter study conducted over a period of 7 years and 9 months. Patients without diagnosis of NSTEMI, with CPA as a symptom of hospital admission or without information on the occurrence of in-hospital CRP were excluded from the study.

Results: A total of 10430 patients were admitted, 95 of whom had in-hospital CPA. Patients with CPA were older, with more hypotensive profile and Killip class $>1$. Electrocardiographic changes (LBB (left bundle block), RBB (right bundle block) and ST depression) were also more frequent in this group, as well as the use of IAB, Invasive Mechanical Ventilation and temporary PM.

By logistic regression 6 CPA predictor variables were identified: 1) admission SAP $\leq 125 \mathrm{mmHg}$ (1 point), 2) Killip class $>1$ at admission (1 point), 3) LBB at admission EkG (2 points), 4) RBB on admission EkG (1 point), 5) ST depression on admission EkG (1 point), 6) CHB (complete heart block) / VT / cardiogenic shock within the first 24 hours after admission ( 2 points). This allowed the creation of 3 risk groups according to the score obtained: low risk (0-2 points), medium risk (3-4 points) and high risk (5-7 points).

ROC curves were created comparing the new score with the SAFER and CHADS2 score. The new score was found to have a higher predictive power than the 2 previously reported scores (AUC: $0.723 ; 95 \% \mathrm{Cl}$ 0.714-0.731; p-value <0.05).

Conclusion: Our study allowed us to create a new score that is easy to apply on a daily basis. Internal and external validation is crucial to assessing its true impact.

\section{4}

The relation between cerebral tissue oxygen saturation and end tidal carbon dioxide during cardiopulmonary resuscitation K. Sakaguchi': S. Saito ${ }^{1}$ : M. Takada ${ }^{1}$ : K. Takahashi ${ }^{1}$;. Onodera ${ }^{2}$;

T. Kobayashi'; K. Kawamae²; M. Nakane

'Department of emergency and critical care medicine, Yamagata University Faculty of Medicine, Yamagata, Japan; ${ }^{2}$ Department of anesthesiology, Yamagata University Faculty of Medicine, Yamagata, Japan

Correspondence: K. Sakaguchi

Intensive Care Medicine Experimental 2020, 8(2): 000414

Introduction: The quality of chest compressions is very important in improving prognosis in patients following cardiac arrest. High quality chest compressions leads to increased cardiac output and maintains perfusion of systemic organs. Thus, resuscitation team members should evaluate the degree of systemic organ perfusion to improve the quality of chest compressions during cardiopulmonary resuscitation (CPR).
End tidal carbon dioxide (EtCO2) is one of the most useful physiological markers to evaluate the degree of systemic organ perfusion during CPR. EtCO2 is an indicator of cardiac output and reflects pulmonary perfusion, but $\mathrm{EtCO} 2$ measurement requires intubation, which is not easily and rapidly performed.

In contrast, cerebral tissue oxygen saturation can be rapidly assessed during CPR, and serves as a possible indicator of return of spontaneous circulation (ROSC). Cerebral tissue oxygen saturation can be easily assessed by placing oximeter probes on the patients' forehead. Increase in cardiac output and pulmonary perfusion improves systemic oxygenation and elevates regional cerebral tissue oxygen saturation. Studies that elucidate the relationship between cerebral tissue oxygen saturation and EtCO2 during CPR are warranted.

Objectives: We investigated the relationship between cerebral tissue oxygen saturation and $\mathrm{EtCO} 2$ to determine if cerebral tissue oxygen saturation can evaluate the quality of chest compression during CPR.

Methods: This is an observational study conducted from April to September 2019. Patients arrived in a state of cardiac arrest at the emergency department of Yamagata University Hospital in Japan. Cerebral tissue oxygen saturation was measured by near infrared spectroscopy (NIRO-200NX, Hamamatsu photonics, Japan) during CPR.

We assessed cerebral tissue oxygen saturation using two approaches. The first is TOI, which represents the ratio of oxygenated haemoglobin to total haemoglobin and the second is $\mathrm{SnO} 2$, which is the ratio between the change in oxygenated haemoglobin and change in total haemoglobin. We also measured EtCO2 following intubation. We compared cerebral tissue oxygen saturation with $\mathrm{EtCO} 2$ every minute in cases in which both parameters were measured.

Results: Seven cases were included, and the median age was 81 years old. Three of the seven cases achieved ROSC, but none survived for one month. The median values for $\mathrm{EtCO} 2, \mathrm{TOl}$, and $\mathrm{SnO} 2$ were $14.0 \mathrm{mmHg}$ (inter quartile range (IQR): 10.0-18.0), 37.7\% (IQR: 32.4 42.7) and 38.2\% (IQR: 17.8-59.5), respectively. The correlation coefficient between TOI and EtCO2 was 0.681 (95\% confidence interval $(95 \% \mathrm{Cl}) 0.559-0.774, \mathrm{p}<0.001)$, and between $\mathrm{SnO} 2$ and $\mathrm{EtCO} 2$ was $0.459(95 \% \mathrm{Cl} 0.288-0.601, \mathrm{p}<0.001)$, respectively.

Conclusion: This study showed a strong correlation between tissue oxygen saturation and EtCO2. This suggests that cerebral tissue oxygen saturation may reflect the degree of cardiac output that results from chest compressions during CPR.

\section{Reference(s) and grant acknowledgment(s)}

1. None

\section{2}

Myeloperoxidase and Resistin as inflammation biomarkers in cardiac arrest patients

H. Vuopio ; F. Carbone ${ }^{2}$; S. Minetti ${ }^{2}$; D. Ramoni ${ }^{2}$; G. Ristagno ${ }^{3}$; R. Latini ${ }^{4}$.

P. Jakkula ${ }^{5} ;$ M. Reinikainen ${ }^{6}$; M. Skrifvars ${ }^{1}$; F. Montecucco ${ }^{2}$; PT. Pekkarinen

${ }^{1}$ Division of emergency care and services, University of Helsinki, Helsinki University Hospital, Helsinki, Finland; ${ }^{2}$ Irccs ospedale policlinico san martino genoa-italian cardiovascular network, 10 largo benzi, Dept. of internal medicine, First Clinic of Internal Medicine, University of Genoa, Genoa, Italy; ${ }^{3}$ Cardiovascular research, Mario Negri Institute for Pharmacological Research, Milano, Italy; ${ }^{4}$ Cardiovascular research, Istituto di Ricerche Farmacologiche Mario Negri IRCCS, Milano, Italy; ${ }^{5}$ Perioperative, intensive care and pain medicine, HUS Helsinki University Hospital, Helsinki, Finland: ${ }^{6}$ Department of intensive care, Kuopio University Hospital, Kuopio, Finland; ${ }^{7}$ Division of intensive care medicine, department of anaesthesiology, intensive care and pain medi, Helsinki University Hospital, Helsinki, Finland Correspondence: $\mathrm{H}$. Vuopio

Intensive Care Medicine Experimental 2020, 8(2): 000482

Introduction: Activation of a systemic inflammatory response after cardiac arrest (CA) due to whole-body ischemia/reperfusion injury complicates recovery and may lead to organ dysfunction. Neutrophilic granulocytes are rapidly reacting inflammatory cells that play a role in ischemia/reperfusion injury. Myeloperoxidase (MPO) and resistin are released from activated neutrophils. MPO is involved in the generation of reactive oxygen species upon neutrophil activation. Resistin has a 
dual role in inflammation, it increases the production of several proinflammatory cytokines but inhibits neutrophil migration.

Objectives: We tested the association of MPO and resistin levels with 6-month neurological outcome in ICU-treated out-of hospital cardiac arrest (OHCA) patients.

Methods: We analyzed blood samples taken upon ICU admission and at 48 hours from 108 OHCA patients with shockable rhythm included in the COMACARE study. We defined 6-month cerebral performance category (CPC) 1-2 as good and CPC 3-5 as poor outcome. We calculated area under receiver operating characteristic curve (AUC) to determine association of biomarker levels with poor outcome. We tested the relationship between return of spontaneous circulation (ROSC) delay and admission biomarker levels with linear regression.

Results: At ICU admission, median (Interquartile range, IQR) concentration of MPO was 97 (46-170) ng/mL in good and 98 (52-197) ng/ $\mathrm{mL}$ in poor outcome groups, and of resistin $39(25-57) \mathrm{ng} / \mathrm{mL}$ in good and 43 (29-64) $\mathrm{ng} / \mathrm{mL}$ in poor outcome groups. At admission the AUC for the association of MPO levels with poor outcome was $0.52(95 \%$ $\mathrm{Cl} 0.41-0.64)$ and for resistin $0.57(95 \% \mathrm{Cl} 0.46-0.69)$. At $48 \mathrm{~h}$ the median (IQR) concentration of MPO was 92 (50-163) ng/mL in good and $104(50-138) \mathrm{ng} / \mathrm{mL}$ in poor outcome groups, and of resistin $83(57-146) \mathrm{ng} / \mathrm{mL}$ in good and $86(53-172) \mathrm{ng} / \mathrm{mL}$ in poor outcome groups. At $48 \mathrm{~h}$ the AUC values were $0.47(95 \% \mathrm{Cl} 0.36-0.58)(\mathrm{MPO})$ and $0.52(95 \% \mathrm{Cl} 0.40-0.64)$ (resistin). The linear regression analysis showed that MPO levels at the time of admission were associated with ROSC delay (B 4.8, 95\% Cl 0.75 - 8.9, p-value 0.02). The same was observed for admission levels of resistin (B $0.77,95 \% \mathrm{Cl} 0.09-1.45$, p-value 0.03)

Conclusion: Duration of ischemia is associated with ICU admission levels of MPO and resistin, suggesting neutrophil involvement in the systemic inflammatory response after CA. The markers showed no ability to predict long term neurological outcome.

\section{5}

Osteopontin and Proprotein convertase subtilisin/kexin type 9 as biomarkers for evaluating prognosis after out-of-hospital cardiac arrest

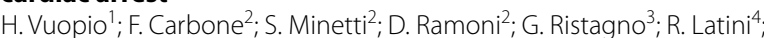
P. Jakkula ${ }^{5}$; M. Reinikainen ${ }^{6}$; M. Skrifvars ${ }^{1}$; F. Montecucco ${ }^{2}$; PT. Pekkarinen

'Division of emergency care and services, University of Helsinki, Helsinki University Hospital, Helsinki, Finland; ${ }^{2}$ Irccs ospedale policlinico san martino genoa-italian cardiovascular network, 10 largo benzi, Dept. of internal medicine, First Clinic of Internal Medicine, University of Genoa, Genoa, Italy; ${ }^{3}$ Cardiovascular research, Mario Negri Institute for Pharmacological Research, Milano, Italy; ${ }^{4}$ Cardiovascular research, Istituto di Ricerche Farmacologiche Mario Negri IRCCS, Milano, Italy; ${ }^{5}$ Perioperative, intensive care and pain medicine, HUS Helsinki University Hospital, Helsinki, Finland ${ }^{6}$ Department of intensive care, Kuopio University Hospital, Kuopio, Finland: ${ }^{7}$ Division of intensive care medicine, department of anaesthesiology, intensive care and pain medi, Helsinki University Hospital, Helsinki, Finland Correspondence: $\mathrm{H}$. Vuopio

Intensive Care Medicine Experimental 2020, 8(2): 000485

Introduction: Cardiac arrest (CA) leads to activation of the inflammatory system due to whole body ischemia-reperfusion injury. Osteopontin (OPN), an extracellular matrix protein produced by many tissues and Proprotein convertase subtilisin/kexin 9 (PCSK9), a regulator of cholesterol homeostasis, play a role in the low-grade inflammation leading to coronary artery disease (CAD).

Objectives: Since CAD is the most important cause for $C A$, we tested if the release of OPN and PCSK9 is increased in the acute inflammation after out-of-hospital cardiac arrest (OHCA) and hypothesized, that they have prognostic value.

Methods: We studied blood samples of 112 patients with OHCA and shockable rhythm included in the COMACARE study. Biomarker levels were measured at the time of ICU admission and at 24, 48 and 72 hours. We used 6-month cerebral performance category (CPC) as the main outcome and defined CPC 1-2 as good and CPC 3-5 as poor outcome. We evaluated the association of admission biomarker levels with outcome using area under receiver operating characteristic curve
(AUC) analysis. We assessed temporal differences in biomarker levels with linear modeling with restricted maximum likelihood estimation (REML).

Results: Median (interquartile range IQR) concentration of OPN was $53(41-79) \mathrm{ng} / \mathrm{mL}$ at admission, $160(100-190) \mathrm{ng} / \mathrm{mL}$ at $24 \mathrm{~h}, 240$ $(170-360) \mathrm{ng} / \mathrm{mL}$ at $48 \mathrm{~h}$ and $240(190-320) \mathrm{ng} / \mathrm{mL}$ at $72 \mathrm{~h}$. For PCSK9 the concentrations were $160(100-210) \mathrm{ng} / \mathrm{mL}$ at admission, 110 (74$160) \mathrm{ng} / \mathrm{mL}$ at $24 \mathrm{~h}, 160(92-220) \mathrm{ng} / \mathrm{mL}$ at $48 \mathrm{~h}$ and $240(170-330) \mathrm{ng} /$ $\mathrm{mL}$ at $72 \mathrm{~h}$. The predictive value of OPN at admission was weak (AUC $0.548,95 \%$ confidence interval, $\mathrm{Cl} 0.43-0.66$ ) and similar results were observed for PCSK9 (AUC $0.551,95 \% \mathrm{Cl} 0.44-0.67$ ). With linear modeling we observed an effect of sample collection timepoint ( $F 98, P<$ $0.01)$ and poor outcome ( $F 6.8, P=0.01)$ on OPN concentration. Sample collection timepoint had an effect $(F 78, P<0.01)$ on PCSK9 concentration but poor outcome did not show a statistically significant effect. The interaction of sample collection timepoint and poor outcome did not show a statistically significant effect on the concentrations of OPN or PCSK9.

Conclusion: Cardiac arrest is followed by changes in OPN and PCSK9 concentrations and the temporal course of OPN response differs between 6-month neurological outcome groups. The biomarkers did not have clinically relevant predictive value at ICU admission.

\section{0}

Non-traumatic out-of-hospital cardiac arrest-review of pre-hospital critical care team attendances

V. Rehnberg ${ }^{1}$; C. Louisa ${ }^{2}$

${ }^{1}$ Anaesthesia / Intensive Care, Poole Hospital, Bournemouth, United Kingdom; ${ }^{2}$ Prehospital emergency medicine, University of Southampton Faculty of Medicine, SGH Campus, Southampton, United Kingdom

Correspondence: $V$. Rehnberg

Intensive Care Medicine Experimental 2020, 8(2): 000510

Introduction: The Hampshire \& Isle of Wight Air Ambulance (HIOWAA) works alongside the ambulance service to provide a Prehospital Critical Care (PHCC) team to the south central region of England. It responds between the hours of $0700-0200$ to both medical emergencies and major trauma, either by road or by helicopter. In November 2018, HIOWAA adopted a new database (ARCnet) to support its commitment to a robust clinical governance frame

Objectives: This report reviews cases of medical (i.e. non-traumatic) outof-hospital cardiac arrests (OOHCA) attended by the HIOWAA team from the inception of the database to the end of December 2019 (13 months). Methods: The ARCnet database was interrogated for all cardiac arrests from the beginning of November 2018 to the end of December 2019. Cases were included where HIOWAA attended the scene and the patient had received chest compressions at any point. Likely or confirmed traumatic cardiac arrests were excluded. Retrospective review of the patient record forms (PRFs) were then carried out and key data collected.

Results: A total of 192 cases of non-traumatic cardiac arrest were identified over the study period. The median age was 61 (IQR 50-71; range). Over two thirds of patients were male (133 [69.3\%] vs 58 [30.2\%]). Most missions were in the more populated urban and suburban mainland areas in the south of the region (Southampton, Portsmouth and the M27 corridor), with another cluster around Basingstoke. Overall, 106 patients (55.2\%) were conveyed to hospital with HIOWAA.

The majority of patients conveyed were taken to hospital by road (99 [94.3\%]). Six patients (5.7\%) were taken to their destination hospital by air, of which 5 were from the Isle of Wight (IOW) and one from Winchester to University Hospital Southampton (UHS). All patients transported by air had established return of spontaneous circulation (ROSC) prior to transfer and were either fully conscious and self-ventilating ( 2 patients) or sedated and ventilated (4 patients). In one case the mode of transport was not recorded and could not be established from the patient record. A doctor and paramedic crew attended 107 missions (55.7\%) and $85(44.3 \%)$ were attended by a paramedic only crew. Initial 999 calls occurred between the hours of 20:00-08:00 for 22 missions (11.5\%). The initial rhythm in most patients was non-shockable (i.e. asystole or pulseless electrical activity [PEA]). A quarter of patients had ROSC prior 
to the arrival of HIOWAA. Of the remainder, a quarter subsequently achieved ROSC while treated by HIOWAA.

Cardiac arrest was witnessed in 126 patients (65.6\%), unwitnessed in $58(30.2 \%)$ and not recorded or unknown for 8 patients (4.2\%). Of witnessed arrests, 102 patients (81\%) received documented bystander CPR of which 67 (53.2\%) had ROSC on arrival in ED. Unwitnessed arrests received bystander CPR in 41 patients $(70.7 \%)$, did not receive bystander CPR in $10(17.2 \%)$ and was not recorded in $7(12.1 \%)$. Of the 10 patients that were neither witnessed nor received bystander CPR, two $(20 \%)$ had ROSC on arrival in the ED.

A number of interventions were performed in both groups. In survivors, prehospital emergency anaesthesia (PHEA) was carried out in 24 patients (29.3\%), vasopressors post-ROSC in $34(41.5 \%)$ and ongoing intravenous sedation in 31 (37.8\%). In non-survivors, life was declared extinct on scene in 81 patients (73.6\%)

Conclusion: Our data is consistent with known determinants of outcome following cardiac arrest (witnessed cardiac arrest, bystander CPR and shockable rhythm all associated with higher chance of ROSC). Increasing age was associated with a lower chance of survival. In successful resuscitation, key interventions performed by the PHCC team not available to standard ambulance crews related primarily to postresuscitation care following ROSC. In unsuccessful resuscitation, the PHCC team was also able to support decision-making and aid prognostication with the aid of ultrasound, which allowed termination of futile treatment without admission to hospital in the majority of cases without ROSC

\section{8}

Early Diffusion Tensor Imaging predicts lack of cerebral recovery after cardiac arrest

H. Keijzer'; ${ }^{1}$ M. Duering ${ }^{2}$; FJA. Meijer ${ }^{3}$; BAR. Tonino ${ }^{4}$; MJ. Blans ${ }^{5}$; CJM. Klijn ${ }^{6}$; CWE. Hoedemaekers; J. Hofmeijer

${ }^{1}$ Neurology, Rijnstate, Arnhem, Netherlands; ${ }^{2}$ Institute for stroke and dementia research, Ludwig Maximilian University of Munich, München, Germany; ${ }^{3}$ Radiology and nuclear medicine, Radboud University Medical Center, Nijmegen, Netherlands; ${ }^{4}$ Radiology, Rijnstate, Arnhem, Netherlands; ${ }^{5}$ Intensive care medicine, Rijnstate, Arnhem, Netherlands; ${ }^{6} \mathrm{Neu}$ rology, Radboud University Medical Center, Nijmegen, Netherlands; ${ }^{7}$ Intensive care medicine, Radboud University Medical Center, Nijmegen, Netherlands

Correspondence: $\mathrm{H}$. Keijzer

Intensive Care Medicine Experimental 2020, 8(2): 000528

Introduction: Prolonged depletion of oxygen and glucose during cardiac arrest may result in cytotoxic cerebral oedema. In cytotoxic oedema, water diffusion within the brain becomes restricted. Diffusion Tensor Imaging (DTI) allows quantification of diffusion restriction through Fractional Anisotropy (FA, the directionality of diffusion) and Mean Diffusivity (MD, the amount of diffusion). White matter FA appeared a promising outcome predictor after prolonged postanoxic coma $[1,2]$. We now investigate predictive values of FA and MD in both grey and white matter, 2-4 days after cardiac arrest.

Objectives: To study differences in diffusion restriction of the brain using FA and MD, between comatose patients after cardiac arrest with good and poor neurological recovery, and estimate predictive values.

Methods: In an ongoing prospective cohort study, 33 comatose patients after cardiac arrest underwent $3 \mathrm{~T}$ brain MRI $3 \pm 1$ days after return of spontaneous circulation. From the DTI sequence, we calculated FA and MD of the whole brain, grey matter (GM) and skeletonised white matter (WM). MD and FA are likely influenced by free water, e.g. cerebrospinal fluid and vasogenic oedema. Other than in previous studies, we corrected MD and FA for the influence of free water [3]. As predictive marker, we calculated the percentage of brain volume with severe oedema, defined as $M D<500^{*} 10-6$. We compared FA and MD between patients with good (Cerebral Performance Category (CPC) $1-2$ ) and poor (CPC3-5) outcome at 6 months and estimated predictive value based on a preliminary logistic regression model.

Results: Patients with poor outcome $(n=16)$ showed significantly lower whole brain mean FA and MD (FA: 0.31, MD: 544*10-6) than patients with good outcome (FA: $0.34, \mathrm{MD}: 589^{*} 10-6, \mathrm{p}=0.01, \mathrm{p}=$
$0.01)$. $M D$ within the $G M$ showed the highest between-group difference (poor: $532 * 10-6$, good: $585^{*} 10-6, p=0.03$ ). Patients with poor outcome had a significantly larger proportion of the brain with severe cytotoxic oedema (poor: $20 \%$, good: $5 \%, p=0.01$ ). In a preliminary prediction model, we could predict poor outcome at $100 \%$ specificity with $75 \%$ sensitivity ( $95 \% \mathrm{Cl}$ : 46-93\%, figure 1)

Conclusion: MD and FA of grey and white matter differ between patients with good and poor outcome, and hold potential to add to prediction of neurological recovery of comatose patients after cardiac arrest. The study is ongoing and updated results will be presented.

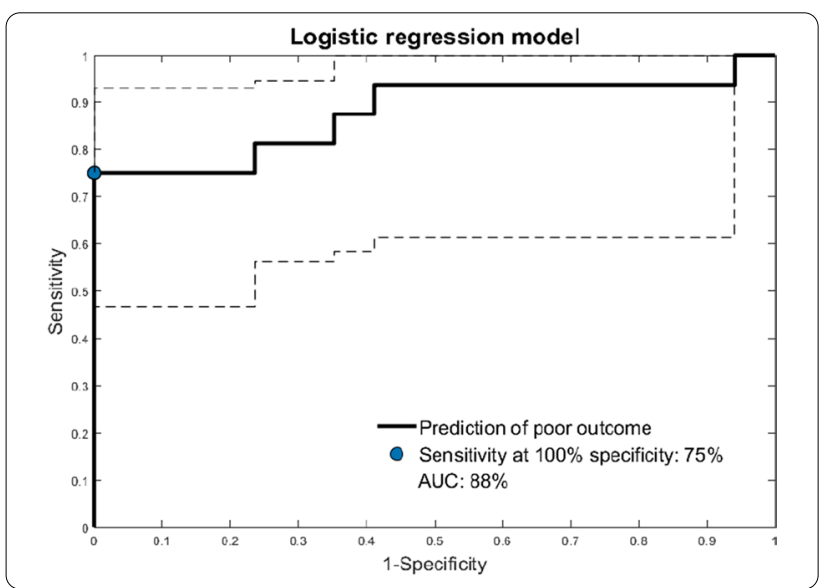

Reference(s) and grant acknowledgment(s)

1. Pasternak, O., et al., Free water elimination and mapping from diffusion MRI. Magnetic Resonance in Medicine: An Official Journal of the International Society for Magnetic Resonance in Medicine, 2009. 62(3): p. 717-730.

2. Velly, L., et al., Use of brain diffusion tensor imaging for the prediction of long-term neurological outcomes in patients after cardiac arrest: a multicentre, international, prospective, observational, cohort study. The Lancet Neurology, 2018. 17(4): p. 317-326

3. luyt, C., et al., Diffusion Tensor Imaging to Predict Long-term Outcome after Cardiac Arrest - A Bicentric Pilot Study. Anesthesiology, 2012. 117(6)

4. HK is funded by the Rijnstate-Radboud promotion fund

\section{2}

The use of neuron specific enolase to predict long term neurological outcome in OOHCA survivors

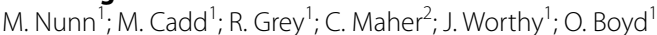

${ }^{1}$ Intensive care unit, Brighton \& Sussex University Hospitals NHS Trust, Brighton, United Kingdom; ${ }^{2}$ Brighton \& sussex medical school, Brighton \& Sussex Medical School, Brighton, United Kingdom

Correspondence: $M$. Cadd

Intensive Care Medicine Experimental 2020, 8(2): 000552

Introduction: There are approximately 60,000 out-of-hospital cardiac arrests $(\mathrm{OOHCA})$ in the UK each year. Less than $10 \%$ of these patients survive to discharge (1) and hypoxic-ischaemic brain injury (HIBI) is common (2). HIBI can cause a range of sequelae from mild cognitive impairment to brain death.

Prognostication for HIBI post OOHCA remains difficult. The European Resuscitation Council (ERC) suggests a multimodal approach to prognostication including electroencephalogram (EEG), brain imaging and Neuron Specific Enolase (NSE) (2).

Objectives: To ascertain if there is a relationship between NSE, longterm neurological outcome and quality of life in OOHCA survivors.

Methods: This study was carried out at a tertiary centre with 31 critical care beds and was approved by the local research committee. The critical care electronic database was retrospectively analysed for OOHCA patients who survived to ICU discharge between May 2017 and November 2018. Patient demographics, NSE values, EEG and imaging reports were obtained for each patient. 
Follow up telephone contact was made with each patient at least 14 months following ICU discharge. Verbal consent was obtained to collect anonymised information. A single investigator, who was blinded to NSE values, conducted all patient interviews. Neurological outcome was stratified using in-house standardised questions and the validated Cerebral Performance Category (CPC) (3).

Patients were asked to subjectively assess their QoL on a locally devised numerical scale (Figure 1).

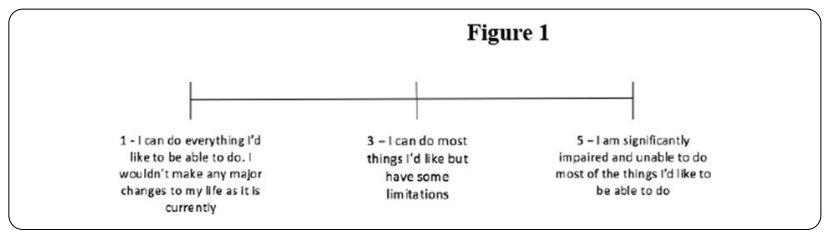

Results: Thirty-two patients (36.0\%) survived to hospital discharge, four patients had subsequently died and two were not contactable; these patients were excluded from analysis. Eighty four percent of patients were male, the median age was 65 and $66 \%$ had known cardiovascular disease. Twenty patients (77\%) had an NSE level. Data were analysed using Mann Whitney $U$ test.

There were 3 patients with a poor neurological outcome (defined as $C P C \geq 3)$ therefore it is a small patient group for analysis. However, there was no significant relationship between NSE and neurological outcome (good outcome defined as CPC 1 or 2, poor outcome as $C P C \geq 3) p=0.0963$.

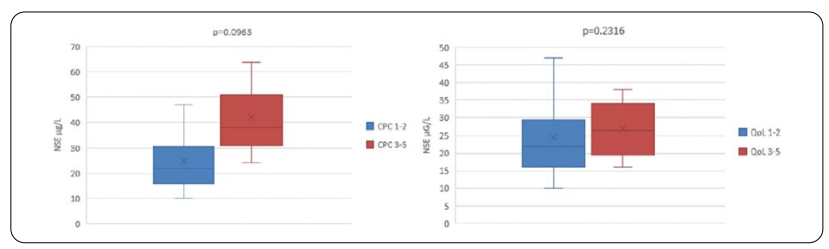

Conclusion: The 72-hour NSE result in survivors showed no relationship with neurological outcome post-OOHCA at 14 months. It is noteworthy that the patient with the highest NSE had the worst outcome. Previous in-house research has shown an NSE of $>64.5 \mu \mathrm{g} / \mathrm{L}(100 \%$ specificity, $78 \%$ sensitivity) correlates with poor neurological outcome (4). One study suggests the NSE ratio at 48 and 24 hours can be used as a reliable prediction of outcome, which should be further investigated in our patient group (8). Larger scale follow-up studies should therefore be conducted to further assess the use of NSE in prognostication of long-term neurological outcomes for OOHCA patients.

\section{Reference(s) and grant acknowledgment(s)}

1. 1. Bækgaard JS, Viereck S, Møller TP, Ersbø\|l AK, Lippert F, Folke F. The effects of public access defibrillation on survival after out-of-hospital cardiac arrest: a systematic review of observational studies. Circulation. $2017 \mathrm{Sep}$ 5;136(10):954-65.

2. 2. Monsieurs KG, Nolan JP, Bossaert LL, Greif R, Maconochie IK, Nikolaou NI, Perkins GD, Soar J, Truhlar A, Wyllie J, Zideman DA. European resuscitation council guidelines for resuscitation 2015 section 1. Executive summary. Resuscitation.-Limerick, 1972, currens. 2015;95:1-80.

3. 3. Jennett $B$, Bond M. Assessment of outcome after severe brain damage: a practical scale. The Lancet. 1975 Mar 1;305(7905):480-4.

4. 4. C. Maher, J. Worthy, R. Gray, O. Boyd. The clinical application of Neurone Specific Enolase to prognosticate neurological recovery in cardiac arrests patients. Intensive Care Medicine Experimental 2019 7(Suppl 3):55 P110

5. 5. Luescher T, Mueller J, Isenschmid C, Kalt J, Rasiah R, Tondorf T, Gamp M, Becker C, Sutter R, Tisljar K, Schuetz P. Neuron-specific enolase (NSE) improves clinical risk scores for prediction of neurological outcome and death in cardiac arrest patients: Results from a prospective trial. Resuscitation. 2019 Sep 1;142:50-60.

6. 6. Streitberger KJ, Leithner C, Wattenberg M, Tonner P, Hasslacher J, Joannidis M, Pellis T, Di Luca E, Födisch M, Krannich A, Ploner CJ. Neuronspecific enolase predicts poor outcome after cardiac arrest and targeted temperature management: a multicenter study on 1,053 patients. Critical care medicine. 2017 Jul 1;45(7):1145-51.

7. 7. Stammet P, Collignon O, Hassager C, Wise MP, Hovdenes J, Åneman A, Horn J, Devaux Y, Erlinge D, Kjaergaard J, Gasche Y. Neuron-specific enolase as a predictor of death or poor neurological outcome after out-of-hospital cardiac arrest and targeted temperature management at $33 \mathrm{C}$ and $36 \mathrm{C}$. Journal of the American College of Cardiology. 2015 May 19;65(19):2104-14.

8. 8. Chung-Esaki HM, Mui G, Mlynash M, Eyngorn I, Catabay K, Hirsch KG. The neuron specific enolase (NSE) ratio offers benefits over absolute value thresholds in post-cardiac arrest coma prognosis. Journal of Clinical Neuroscience. 2018 Nov 1;57:99-104.

\section{3}

Differential patterns of $\mathbf{2 0 - 3 0 m s e c ~ S o m a t o s e n s o r y ~ E v o k e d ~}$ Potential [SSEP] complex and neurological prognosis in comatose survivors of Out of Hospital Cardiac Arrest [OHCA]

L. Lankester'; C. Bigham'; C. Wells'; N. Broomfield²; L. Nye²; E. Dunn²;

A. Maccormick'; N. Hudson ${ }^{2}$; N. Nikitas ${ }^{1}$

${ }^{1}$ Department of Intensive Care Medicine, Derriford Hospital, Plymouth, United Kingdom; '2Department of neurophysiology, Derriford Hospital, Plymouth, United Kingdom

Correspondence: $L$. Lankester

Intensive Care Medicine Experimental 2020, 8(2): 000593

Introduction: Recent studies [1] suggested a potential role of P25/30 SSEP in prediction of neurological outcome after OHCA. However it is not clear whether any particular patterns of 20-30msec SSEP complex could potentially improve the already established predictive value of N20.

Objectives: The main objective of this study is the integrated analysis of the 20-30 msec SSEP complex in order to identify all possible patterns and their potential prognostic value.

Methods: Single-centre, prospective, observational study. Adult comatose survivors of OHCA with Targeted Temperature Management [TTM] [normothermia], neuroprotection and organ support on ICU. SSEP recorded 24-36 h post return of spontaneous circulation. Analysis of the $20-30$ msec SSEP complex was performed by 2 independent interpreters: 1. The amplitude of both N20 and P25/30 was measured bilaterally from an agreed baseline. An amplitude threshold of 0.5 microvolt [ $\mathrm{mcV}$ ] determined the presence or absence of each SSEP component. 2. The Peak To Peak Amplitude (PTPA) of the $20-30 \mathrm{msec}$ complex (peak of N20 to peak of P25) was also measured bilaterally. The largest PTPA was used to test potential correlations with patients' neurological outcome. Short term neurological outcome assessed at hospital discharge used the cerebral performance category [CPC] score: favourable outcome 1-2, unfavourable outcome 3-5.

Results: Data from the first 52 patients: 37 male, 15 female, median age 59.5 years. OHCA rhythm: 31 VF, 21 asystole. Cause of OHCA: 38 cardiac, 13 non-cardiac, 1 unknown. Median anoxic time $25 \mathrm{~min}$. Median Temperature [T] in the first $24 \mathrm{~h} 360 \mathrm{C}$. Median time of SSEP recording $25 \mathrm{~h}$. Median T during SSEP recording $36.1 \mathrm{oC}$. The preliminary clinical and neurophysiological analysis has done for the first 48 patients. 23 patients had favourable and 25 had unfavourable neurological outcomes. Among the 23 patients with a favourable outcome, both $\mathrm{N} 20$ and P25/30 SSEP were present in all. Among patients with an unfavourable outcome, both $\mathrm{N} 20$ and P25/30 were absent in 22, and N20 was absent but $\mathrm{P} 25 / 30$ was present in 3 .

Use of $0.5 \mathrm{mcV}$ as a threshold for the N20-P25/30 presence provided an objective method to minimise inter-interpreter variability and limit the effect of background noise in the analysis of the SSEP.

Median N20 amplitudes were consistently lower than P25/30 median amplitudes in patients with both favourable [N20: $1.325 \mathrm{mcV}, \mathrm{P} 25 / 30$ : 
2.6mcV] and unfavourable outcomes [N20: $0.275 \mathrm{mcV}, \mathrm{P} 25 / 30$ : $0.35 \mathrm{mcV}]$.

The highest PTPA was $\geq 1.20 \mathrm{mcV}$ in all patients with a favourable outcome [range $1.2-15.7 \mathrm{mcV}$, median $3.825 \mathrm{mcV}$ ], whereas in patients with an unfavourable outcome the highest PTPA was $\leq 1.3 \mathrm{microV}$ [range $0.1-1.3 \mathrm{mcV}$, median $0.5 \mathrm{mcV}$ ]. In the latter group, the PTPA was $\leq 1.0 \mathrm{mcV}$ in all except 1 patient [1.3mcV]. PTPA values between 1.1 and $1.3 \mathrm{mcV}$ could be correlated with both outcomes. We are further investigating whether separate measurement of N20 or P25/30 amplitude could be used in this patient cohort as a distinctive feature between outcomes.

Conclusion: Addition of P25/30 in the analysis of 20-30msec SSEP complex may potentially increase $\mathrm{N} 20^{\prime}$ 's positive predictive value for the short term favourable neurological outcome after OHCA. Analysis of P25/30 does not affect N20's already established negative predictive value.

Direct PTPA measurements of the 20-30 msec SSEP complex provides an easy to measure value for future objective quantitative assessments. Preliminary results suggest PTPA of $<1.1 \mathrm{mcV}$ is associated with an unfavourable neurological outcome whereas a PTPA of $>1.3 \mathrm{mcV}$ appears to be associated with a favourable neurological outcome. As the number of patients increases, it will become clearer whether the above suggestions will be consistently reproduced and are statistically significant.

\section{Reference(s) and grant acknowledgment(s)}

1. 1. Short latency positive peak following N20 somatosensory evoked potential is superior to N2O in predicting neurologic outcome after out of hospital cardiac arrest. Kim SW,et al. Crit Care Med 46(6):545-551,2018

2. Study is funded by local charitable funds

\section{0}

\section{Prognostic significance of time intervals after sudden coronary}

\section{death in non-city areas}

J. Higny' ${ }^{1}$; F. Forêt ${ }^{2}$; A. Guédès ${ }^{1}$; J. Flament ${ }^{3}$

${ }^{1}$ Cardiovascular Disease, CHU UCL Namur - Site Godinne, Yvoir, Belgium; Intensive care medicine, Chu Ucl Namur - Site De Dinant, Dinant, Belgium: ${ }^{3}$ Emergency department, CHU UCL Namur - Site Godinne, Yvoir, Belgium Correspondence: J. Higny

Intensive Care Medicine Experimental 2020, 8(2): 000620

Introduction: Out-of-hospital cardiac arrest (OHCA) carries a low survival rate, especially in rural areas. Coronary artery disease (CAD) represents the most common cause of collapse. Predictors of survival have been described in the literature. However, most studies have been conducted in city areas. Therefore, little is known about the resuscitation performances of emergency medical services (EMS) in non-city areas.

Objectives: We sought to perform a quality control assessment on the management of acute coronary syndrome (ACS) complicated with OHCA in non-urban areas. We explored standard parameters, resuscitation settings, pre- and in-hospital response times, lactate level, cath lab findings and clinical outcomes at 30 days.

Methods: The location of our institution consists of non-urban areas with a low population density. Built at the top of a hill, the hospital was initially founded in 1903 to treat patients suffering from tuberculosis. In this context, we performed a retrospective mono-center study including 40 patients resuscitated from OHCA with ACS.

Results: Parameters associated with 30-day mortality included age, no-bystander CPR, no use of AED, non-convertible rhythm, duration of CPR, recurrence of cardiac arrest, epinephrine dose, cardiac dysfunction, LAD culprit lesion and delivery time intervals (cath lab/ICU admission). Particularly, lactate level, distance from hospital, duration of pre-hospital management (no-flow, low-flow) and delayed response times (EMS arrival, ER admission) were predictive of early mortality.
Table : 30-day mortality related to patient characteristics, resuscitation settings, time intervals and demographic parameters.

\begin{tabular}{|c|c|}
\hline $\begin{array}{l}\text { Age: }<50 \text { yrs }=7(17 \%), \text { D30: } 1 \\
(14 \%) ; 50-60 \text { yrs }=10(25 \%) \\
\text { D30: } 3(30 \%) ; 60-70 \text { yrs }=14\end{array}$ & $\begin{array}{l}\text { No bystander CPR: } 12(30 \%) \text {, D30: } 11 \\
(92 \%) \text {, D6: } 7(64 \%) \text {; bystander CPR: } 28 \\
(70 \%) \text {, D30: } 4 \text { (14\%); No use of AED: } 12\end{array}$ \\
\hline $\begin{array}{l}(35 \%), \text { D } 30: 6(43 \%) ;>70 \mathrm{yrs} \\
=9(23 \%), \text { D30: } 5(56 \%), \text { D4: } 4 \\
(80 \%)\end{array}$ & $\begin{array}{l}(30 \%), \text { D30: } 6(50 \%) \text {; Use of AED: } 28 \\
(70 \%), \text { D30: } 9(32 \%) \text {; Non } \\
\text { convertible rhythm: } 6(15 \%) \text {, D30: } 5 \\
(83 \%), \text { D5: } 4(80 \%) \text {; Convertible rhythm: } \\
34(85 \%), \text { D30: } 10(29 \%) \text { Recurrent } \\
\text { cardiac arrest: } 7(17 \%), \text { D30: } 6(86 \%), \text { D0: } \\
4(67 \%)\end{array}$ \\
\hline 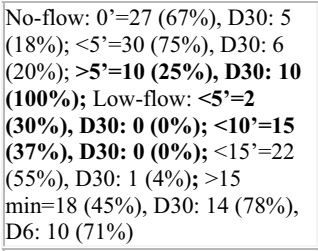 & $\begin{array}{l}\text { Epinephrine: } \leq 1 \mathrm{mg}=15(38 \%), \mathrm{D} 30: 1 \\
(7 \%) ; 2-5 \mathrm{mg}=16(40 \%), \mathrm{D} 30: 7(44 \%) ;>5 \\
\mathrm{mg}=9(22 \%), \mathrm{D} 30: 7(78 \%) ; \text { Lactate: }<5 \\
\mathrm{mmol} / \mathrm{l}=25(63 \%), \mathrm{D} 30: 2(8 \%) ; 5-10 \\
\mathrm{mmol} / \mathrm{l}=11(27 \%), \mathrm{D} 30: 9(81 \%), \text { D6: } 6 \\
(66 \%) ;>\mathbf{1 0} \mathbf{~ m m o l} / \mathrm{l}=\mathbf{4}(\mathbf{1 0} \%), \text { D30: } 3 \\
(\mathbf{7 5 \%}), \text { D6: } 4(\mathbf{1 0 0} \%)\end{array}$ \\
\hline $\begin{array}{l}\text { LVEF: }<30 \%=16(40 \%), \text { D30: } \\
12(75 \%) ; 30-50 \%=9(22 \%), \\
\text { D30: } 9(22 \%) ;>50 \%=15(38 \%), \\
\text { D30: } 1(6 \%) ; \text { Cardiogenic shock: } \\
11(27 \%), \text { D30: } 9(82 \%), \text { D6: } 6 \\
(67 \%) ; \text { Culprit lesion: LAD=24 } \\
(60 \%), \text { D30: } 12(50 \%), \text { D6: } 9 \\
(75 \%) \text {, RCA }=13(32 \%), \text { D30: } 3 \\
(23 \%) ; \text { LCX }=3(8 \%), \text { D30: } 0 \\
(0 \%)\end{array}$ & $\begin{array}{l}\text { Distance from hospital: }<10 \mathrm{~km}=23(57 \%) \text {, } \\
\text { D30: } 5(22 \%), \text { D6: } 2(40 \%) ;<20 \mathrm{~km}=34 \\
(85 \%), \mathrm{D} 30: 9(26 \%), \mathrm{D} 6: 5(55 \%) ;>10 \\
\mathrm{~km}=17(43 \%), \mathrm{D} 30: 11(65 \%), \mathrm{D} 6: 8 \\
(73 \%) ;>\mathbf{2 0} \mathbf{k m}=\mathbf{6}(\mathbf{1 5 \%}), \mathrm{D30}: \mathbf{6}(\mathbf{1 0 0 \%}) \text {, } \\
\text { D6: } 5(\mathbf{8 3} \%)\end{array}$ \\
\hline 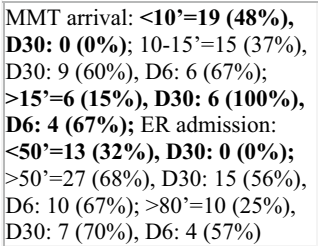 & $\begin{array}{l}\text { Cath lab admission: }<300^{\prime}=10(25 \%), \text { D30: } \\
1(10 \%) ; 30-60^{\prime}=14(35 \%), \text { D } 30: 5(36 \%), \\
\text { D6: } 3(60 \%) ;>60^{\prime}=16(40 \%), \text { D30: } 9 \\
(56 \%), \text { D6: 6 } 67 \%) \text {, ICU admission: } \\
<60^{\prime}=27(67 \%), \text { D30: } 5(18 \%), \text { D6: } 2 \\
\left(40^{\circ}\right) ;>60^{\prime}=12(30 \%), \text { D30: } 8(67 \%), \text { D6: } \\
5(63 \%)\end{array}$ \\
\hline
\end{tabular}

Conclusion: Pre-hospital emergency care of patients with cardiac arrest remains challenging, particularly in non-city areas. Beyond the usual prognostic factors, it would appear that demographic parameters and pre-hospital time intervals represent major determinants of mortality. Therefore, shortening response times and prompt delivery to hospital facilities may help to improve the prognosis, rather than prolonged pre-hospital management. Further RCTs are needed to assess the effect of the 'scoop and run' approach versus the 'stay and play' approach in this setting.

\section{Reference(s) and grant acknowledgment(s)}

1. Mathiesen WT, Bjarshol CA, Kvalay JT, Sareide E. Effects of modifiable prehospital factors on survival after out-of-hospital cardiac arrest in rural versus urban areas.(Report). Critical Care. 2018;22(1). https://doi.org/10.1186/s1305 4-018-1946-8

2. Dumas F, Cariou A, Manzo-Silberman S, et al. Immediate percutaneous coronary intervention is associated with better survival after out-of-hospital cardiac arrest: insights from the PROCAT (Parisian Region Out of hospital Cardiac ArresT) registry. Circ Cardiovasc Interv. 2010;3(3):200-207. https:// doi.org/10.1161/circinterventions.109.913665

3. Spaulding CM, Joly LM, Rosenberg A, et al. Immediate coronary angiography in survivors of out-of-hospital cardiac arrest. N Engl J Med. 1997;336(23):1629-1633. https://doi.org/10.1056/nejm199706053362302

4. Sasson C, Rogers MA, Dahl J, Kellermann AL. Predictors of survival from out-of-hospital cardiac arrest: a systematic review and meta-analysis. Circ Cardiovasc Qual Outcomes. 2010;3(1):63-81. https://doi.org/10.1161/circo utcomes.109.889576. 


\section{4}

Association of dispatcher-assisted bystander cardiopulmonary resuscitation with the likelihood of an initial shockable rhythm after out-of-hospital cardiac arrest

Y. Goto ${ }^{1}$; A. Funada 2 ;. Maeda'; Y. Goto ${ }^{3}$

${ }^{1}$ Department of emergency and critical care medicine, Kanazawa University Hospital, Kanazawa, Japan; ${ }^{2}$ Department of cardiology, Saiseikai Senri Hospital, Suita, Japan; ${ }^{3}$ Department of cardiology, Yawata Medical Centre, Komatsu, Japan

Correspondence: $Y$. Goto

Intensive Care Medicine Experimental 2020, 8(2): 000624

Introduction: Data are insufficient to address the association of dispatcher-assisted bystander cardiopulmonary resuscitation (DA-BCPR) before emergency medical services (EMS) arrival with the likelihood of an initial shockable rhythm after out-of-hospital cardiac arrest (OHCA). We hypothesised that more patients who received DA-BCPR would have initial shockable rhythm than those without bystander CPR (NoBCPR) and that DA-BCPR has an equivalent effect on the likelihood of initial shockable rhythm to that of bystander CPR without dispatcher assistance (Non-DA-BCPR).

Objectives: We aimed to examine the incidence rate of initial shockable rhythm according to the type of bystander CPR performed after $\mathrm{OHCA}$ in relation to bystander CPR duration.

Methods: The study included 114,473 patients (age, $\geq 18$ years) who had an OHCA with a presumed cardiac origin witnessed by a layperson in a prospectively recorded Japanese nationwide Utstein-style database from 2013 to 2017 . Patients who received public-access defibrillation before arrival of the EMS were excluded. The patients were divided into 3 groups as follows: DA-BCPR $(n=42,709)$, Non-DA-BCPR $(n=16,979)$, and No-BCPR $(n=54,785)$. The primary end point was initial shockable rhythm recorded by the EMS after arrival. A Cox proportional hazards model was applied to reflect the different bystander CPR durations before/after propensity score (PS) matching.

Results: The crude rate of the initial shockable rhythm in DA-BCPR $(21.7 \%, 9254 / 42,709)$ was significantly higher than those in Non-DABCPR $(18.5 \%, 3133 / 16,979)$ and No-BCPR $(17.5 \%, 9569 / 54,785$; both $\mathrm{p}<0.0001$ ). Before PS matching, age (adjusted hazards ratio [aHR] for 1 year decrement, $1.01 ; 95 \%$ confidence interval $[\mathrm{Cl}], 1.01-1.01)$, rural area (aHR, 1.27; 95\% Cl, 1.25-1.29, p<0.0001), male sex (aHR, 1.12; 95\% $\mathrm{Cl}, 1.11-1.14, \mathrm{p}<0.0001)$, arrest witnessed by family members (aHR, $1.06 ; 95 \% \mathrm{Cl}, 1.05-1.08, \mathrm{p}<0.0001)$, and presence of bystander CPR $(p<0.0001)$ were associated with substantially higher initial shockable rhythm rate at EMS arrival. As for the type of bystander CPR, we found no significant differences between DA-BCPR and Non-DA-BCPR in the association with the likelihood of initial shockable rhythm as follows: before PS matching, aHR of DA-BCPR for initial shockable rhythm compared with Non-DA-BCPR, 1.00; 95\% Cl, 0.99-1.03 ( $p=0.52)$; after PS matching, aHR, 0.99; $95 \% \mathrm{Cl}, 0.97-1.02$ ( $p=0.56$ ). As compared with No-BCPR, DA-BCPR was positively associated with initial shockable rhythm before $(\mathrm{aHR}, 1.17 ; 95 \% \mathrm{Cl}, 1.16-1.19 ; \mathrm{p}<0.0001)$ and after PS matching (aHR, 1.07; 95\% Cl, 1.05-1.08; $\mathrm{p}<0.0001)$. Non-DA-BCPR was also positively associated with initial shockable rhythm before (aHR, 1.16; 95\% Cl, 1.14-1.19; $\mathrm{p}<0.0001)$ and after PS matching (aHR, 1.03; $95 \% \mathrm{Cl}, 1.00-1.05 ; \mathrm{p}=0.04$ ) as compared with No-BCPR

Conclusion: The patients who received DA-BCPR after witnessed OHCA due to presumed cardiac causes were more likely to have an initial shockable rhythm than those who did not receive bystander CPR. Moreover, DA-BCPR had the same multivariable-adjusted association with the likelihood of initial shockable rhythm as Non-DA-BCPR.

\section{Reference(s) and grant acknowledgment(s)}

1. This work was supported by the Japan Society for the Promotion of Science (KAKENHI Grant No. 18K09999).
000633

Relationship between dispatcher-assisted bystander cardiopulmonary resuscitation and initial shockable rhythm in children with out-of-hospital cardiac arrest

Y. Goto'; A. Funada ${ }^{2}$;. T. Maeda ${ }^{3}$; Y. Goto ${ }^{4}$

${ }^{1}$ Emergency and Critical Care Medicine, Kanazawa University Hospital, Kanazawa, Japan; ${ }^{2}$ Department of cardiology, Saiseikai Senri Hospital, Suita, Japan; ${ }^{3}$ Department of emergency and critical care medicine, Kanazawa University Hospital, Kanazawa, Japan; ${ }^{4}$ Department of cardiology, Yawata Medical Centre, Komatsu, Japan

Correspondence: Y. Goto

Intensive Care Medicine Experimental 2020, 8(2): 000633

Introduction: The association of dispatcher-assisted bystander cardiopulmonary resuscitation (DA-BCPR) before emergency medical services arrival with the likelihood of initial shockable rhythm in children with out-of-hospital cardiac arrest (OHCA) is unclear. We hypothesised that more children with OHCA who received DA-BCPR would have initial shockable rhythm than those without bystander CPR (No-BCPR) and that DA-CPR has an equivalent effect on the likelihood of initial shockable rhythm to that of bystander CPR without dispatcher assistance (Non-DA-BCPR).

Objectives: We aimed to examine the incidence rate of initial shockable rhythm according to the type of BCPR after OHCA in children. We aimed to examine the incidence rate of initial shockable rhythm according to the type of BCPR after OHCA in children.

Methods: We included 2198 paediatric patients aged 8 to 17 years with a witnessed OHCA who were identified from a prospectively recorded Japanese registry from January 2005 to December 2017. Patients who received public-access defibrillation before arrival of the emergency medical services were excluded. The patients were divided into 3 groups according to the type of BCPR as follows: DA-BCPR $(n=564)$, Non-DA-BCPR $(n=466)$ and No-BCPR $(n=1168)$. The primary end point was initial shockable rhythm recorded by the emergency medical service. A Cox proportional hazards model was applied to reflect the different bystander CPR durations before/after propensity score (PS) matching.

Results: The crude rate of the initial shockable rhythm in DA-BCPR $(24.3 \%, 137 / 564)$ was significantly higher than that in No-BCPR $(11.0 \%$, $129 / 1168 ; p<0.0001)$. No significant difference was found between DA-BCPR and Non-DA-BCPR $(27.0 \%, 126 / 466)$ in terms of the crude rate of the initial shockable rhythm $(p=0.31)$. Before PS matching, rural area (adjusted hazards ratio [aHR], 1.20; 95\% confidence interval $[\mathrm{Cl}], 1.07-1.35, \mathrm{p}<0.01)$, male sex $(\mathrm{aHR}, 1.18 ; 95 \% \mathrm{Cl}, 1.07-1.31$, $\mathrm{p}<0.01$ ), and Non-DA-BCPR compared with No-BCPR (aHR, 1.14; 95\% $\mathrm{Cl}, 1.00-1.29, \mathrm{p}<0.05)$ were associated with substantially higher initial shockable rhythm rate at EMS arrival. As for the type of bystander CPR, DA-BCPR was positively associated with the initial shockable rhythm after PS matching (aHR, 1.27; 95\% Cl, 1.11-1.45; $\mathrm{p}<0.0001)$ when compared with No-BCPR. Non-DA-BCPR was positively associated with the initial shockable rhythm after PS matching (aHR, $1.41 ; 95 \% \mathrm{Cl}, 1.22-$ 1.63; $\mathrm{p}<0.0001)$ when compared with No-BCPR. However, we found no significant difference between DA-BCPR and Non-DA-BCPR in their association with the likelihood of initial shockable rhythm after PS matching (aHR, 0.98; 95\% Cl, 0.83-1.16; $\mathrm{p}=0.81$ ).

Conclusion: Paediatric patients aged 8 to 17 years who received DABCPR after a witnessed OHCA were more likely to have an initial shockable rhythm than those who did not receive bystander CPR. Moreover, DA-BCPR had the same multivariable-adjusted association with the likelihood of initial shockable rhythm as Non-DA-BCPR.

Reference(s) and grant acknowledgment(s)

1. This work was supported by the Japan Society for the Promotion of Science (KAKENHI Grant Number 18K09999). 


\section{8}

\section{Prediction criteria for poor outcomes in children}

with out-of-hospital cardiac arrest in the field

Y. Goto' ${ }^{1}$; A. Funada²; T. Maeda ${ }^{3}$; Y. Goto

${ }^{1}$ Emergency and Critical Care Medicine, Kanazawa University Hospital, Kanazawa, Japan; ${ }^{2}$ Department of cardiology, Saiseikai Senri Hospital, Suita, Japan; ${ }^{3}$ Department of emergency and critical care medicine, Kanazawa University Hospital, Kanazawa, Japan; ${ }^{4}$ Department of cardiology, Yawata Medical Centre, Komatsu, Japan

Correspondence: Y. Goto

Intensive Care Medicine Experimental 2020, 8(2): 000638

Introduction: The current guidelines for cardiopulmonary resuscitation (CPR) after out-of-hospital cardiac arrest (OHCA) recommend the use of a universal basic life support termination-of-resuscitation (BLS-TOR) rule for emergency medical service (EMS) providers that was developed to identify adult patients with refractory OHCA eligible for field termination of CPR. However, the BLS-TOR rule is not recommended for children with refractory OHCA due to lack of evidence. We hypothesized that the establishment of outcome prediction criteria in the field for children with OHCA may be useful for better utilization of hospital healthcare resources and reducing attendant hazards for the EMS personnel.

Objectives: We aimed to develop and validate outcome prediction criteria children with OHCA that may aid EMS personnel in deciding to which hospital should they transport the patients.

Methods: We analysed the records of 20,872 children (age $<18$ years) who experienced OHCA treated by the EMS providers. Data were obtained from a prospectively recorded Japanese nationwide Utsteinstyle database for a 13-year period (2005-2017). Outcome measures were specificity and positive predictive value (PPV) of the newly developed criteria in predicting 1-month poor outcomes, namely, unfavourable neurological outcomes, defined as Cerebral Performance Categories score 3 to 5 (CPC 3-5), and mortality. Patients were divided into the development $(2005-2014, n=16,660)$ and validation $(2015-$ 2017, $\mathrm{n}=4212$ ) groups.

Results: The overall 1-month mortality and 1-month CPC 3-5 rates were $88.5 \%(18,471 / 20,872)$ and $95.6 \%(19,944 / 20,872)$, respectively. Multivariate logistic regression analyses for the development group showed that the EMS personnel could predict poor outcomes in patients if the patients met all of the following three prehospital criteria before performing EMS-initiated interventions in the field: asystole as the initial rhythm recorded by EMS personnel, OHCA unwitnessed by laypersons, and no bystander-administered intervention. For patients meeting all three criteria, the specificity and PPV for predicting 1-month CPC 3-5 were 97.8\% (95\% confidence interval [Cl], $96.4 \%-98.7 \%)$ and $99.6 \%(95 \% \mathrm{Cl}, 99.4 \%-99.8 \%)$, respectively, for the development group and $97.1 \%(95 \% \mathrm{Cl}, 94.1 \%-99.8 \%)$ and $99.6 \%$ (95\% Cl, 99.1\%-99.8\%), respectively, for the validation group. The specificity and PPV of predicting 1-month mortality were 91.9\% (95\% $\mathrm{Cl}, 90.6 \%-93.1 \%)$ and $96.5 \%(95 \% \mathrm{Cl}, 95.9 \%-97.0 \%)$, respectively, for the development group and $91.8 \%(95 \% \mathrm{Cl}, 89.3 \%-93.7 \%)$ and $94.4 \%$ (95\% Cl, 92.7\%-95.8\%), respectively, for the validation group.

Conclusion: With high specificity and PPV for predicting the 1-month unfavourable outcome, the EMS personnel could predict poor outcomes if children with OHCA met all three criteria, namely, asystole as the initial recorded rhythm, OHCA unwitnessed by laypersons, and no bystander CPR, soon after arrival of the EMS personnel at the patients' site.

\section{Reference(s) and grant acknowledgment(s)}

1. This work was supported by the Japan Society for the Promotion of Science (KAKENHI Grant Number 18K09999), which had no role in the design and implementation of the study, analysis and interpretation of the data, or approval of the manuscript.
000689

Predictors of mortality and neurological dysfunction in patients successfully resuscitated from in-hospital and out-of-hospital cardiac arrest: observational retrospective single centre study M. Cabral ${ }^{1}$; D. Castro ${ }^{2}$; MJ. Palavras ${ }^{3}$; F. Santos ${ }^{3}$; F. Sequeira ${ }^{2}$; L. Linhares ${ }^{2}$; L. Pereira ${ }^{2}$

${ }^{1}$ Department of cardiology, Hospital Santo Andre - Hospital Distrital De Leiria, Leiria, Portugal; ${ }^{2}$ Intensive care unit, Hospital Santo Andre - Hospital Distrital De Leiria, Leiria, Portugal; ${ }^{3}$ Department of internal medicine, Hospital Santo Andre - Hospital Distrital De Leiria, Leiria, Portugal

Correspondence: M. Cabral

Intensive Care Medicine Experimental 2020, 8(2): 000689

Introduction: Recent international out-of-hospital and in-hospital cardiac arrest statistics show that despite a decrease in heart disease and stroke mortality, the burden of disease remains on top of society's health problems. There is a high variation in survival to hospital discharge and survival with functional recovery between different regions and different countries. Early identification of predictors for mortality and poor long-term neurological outcome in patients who survive the initial phase of cardiac arrest may provide critical information for physicians and facilitate clinical approach.

Objectives: The aim of this study was to identify the mortality rate of out-of-hospital and in-hospital cardiac arrest in our institution and to determinate the association between variables available from the patient's history and status at intensive care unit admission with mortality rate and neurological outcome. In subgroup analysis, we intend to study the impact on mortality of performing emergent coronary angiography on non-ST-segment elevation myocardial infarction (NSTEMI) patients.

Methods: We performed a retrospective review of all patients with the first diagnosis of "cardiac arrest" (CA) admitted to our intensive care unit (ICU) from 31 May 2015 to 31 May 2020. Patient selection and information collection were obtained through medical records. Outcomes were early mortality, including in-hospital and 1 month after discharge mortality, and neurological function as defined by the Cerebral Performance Category (CPC) scale after CA.

Group comparisons between survivals and non-survivals were performed using the Student-T test or Mann-Whitney U-test for continuous variables and Pearson chi-square test or Fisher test for categorical variables. For mortality outcome, multivariate logistic regression analysis with forward stepwise selection was performed to assess the association between the variables with statistically significant differences between the two groups. Correct adjustment of the model was analysed by the Hosmer and Lemeshow Test, and the ROC curve. A p-value less than 0.05 is statistically significant. Statistical analysis was performed using SPSS software version 25.0 (IBM, Armonk, NY, USA).

Results: Of the 114 patients included, 42 (36.8\%) admitted to our ICU with the diagnosis of cardiac arrest were non-survivors and 72 $(63.2 \%)$ were discharged alive from the hospital and survive at least one month after. We observe no significant difference between mortality rates of in-hospital CA (64.6\%) versus out-of-hospital CA (59.4\%) $(p=0.60)$. Univariate logistic regression analysis found the following factors associated with mortality: age greater than 75 years old ( $p$-value $<0.01)$, asystole or pulseless electrical activity (PEA) as cardiac rhythm at presentation ( $p$-value $=0.03$ ), total cardiopulmonary resuscitation $(C P R)$ time greater than 5 minutes ( $p$-value $=0.02$ ), Glasgow coma scale (GCS) after the return of spontaneous circulation (ROSC) less than $5(p$-value $<0.01)$ and another cause for CA besides acute ST-elevation myocardial infarction (STEMI) $(p<0.01)$. In multivariate logistic regression, only GCS after ROSC less than 5 (odds ratio = $3.63,95 \% \mathrm{Cl}=[1.25 ; 10.56], \mathrm{p}$-value $=0.02)$ and another cause for $\mathrm{CA}$ besides STEMI (odds ratio $=4.88,95 \% \mathrm{Cl}=[0.98 ; 24.34], \mathrm{p}$-value $=0.05$ ) proved to be predictive factors of mortality. Regarding the predictors of poor neurological prognosis, we identified the following variables as significant: CPR time greater than 5 minutes $(p$-value $=0.03$, 
$95 \% \mathrm{Cl}=[-8.82 ;-0.37])$ and GCS after ROSC less than $5(\mathrm{p}<0.01,95 \% \mathrm{Cl}$ $=[2.26 ; 5.34])$. Analytic parameters at admission $(\mathrm{pH}$, lactate, bicarbonate, calcium, magnesium, potassium, phosphate and haemoglobin) and comorbidities (hypertension, diabetes mellitus, prior myocardial infarction and heart failure) show no significant difference on mortality or neurological prognostic. In subgroups analysis of acute coronary syndromes $(n=35)$, we observed 21 patients with STEMI and 14 patients with NSTEMI. Between NSTEMI patients, 12 were submitted to coronary angiography, which 7 required percutaneous coronary intervention (PCI). We show no significant difference between mortality on NSTEMI patients submitted to emergent coronary angiography versus conservative approach $(p=1.00)$

Conclusion: Our risk prediction model identified GCS after ROSC and cause of CA as important factors determining survival outcomes. Others factors like patient age, CPR time and cardiac rhythm at presentation showed less importance. We identified CPR time and GCS after ROSC as major predictors of a poor neurological prognosis. We showed no difference in early mortality for NSTEMI patients submitted to immediate invasive strategy.

\section{Reference(s) and grant acknowledgment(s)}

1. Hirlekar G, Karlsson T, Aune S, et al. Survival and neurological outcome in the elderly after in-hospital cardiac arrest. Resuscitation. 2017;118:101-106. https://doi.org/10.1016/j.resuscitation.2017.07.013

2. Cheng A, Nadkarni VM, Mancini MB, et al. Resuscitation Education Science: Educational Strategies to Improve Outcomes From Cardiac Arrest: A Scientific Statement From the American Heart Association. Circulation 2018;138(6):e82-e122. https://doi.org/10.1161/cir.0000000000000583

3. Welbourn C, Efstathiou N. How does the length of cardiopulmonary resuscitation affect brain damage in patients surviving cardiac arrest? A systematic review. Scand J Trauma Resusc Emerg Med. 2018;26(1):77. Published 2018 Sep 10. https://doi.org/10.1186/s13049-018-0476-3

4. Madder RD, Reynolds JC. Multidisciplinary Management of the Post-Cardiac Arrest Patient. Cardiol Clin. 2018;36(1):85-101. https://doi.org/10.1016/j. cCl.2017.08.005

5. Andersen LW, Holmberg MJ, Berg KM, Donnino MW, Granfeldt A. In-Hospital Cardiac Arrest: A Review. JAMA. 2019;321(12):1200-1210. https://doi. org/10.1001/jama.2019.1696

\section{9}

Influence of the time of admission to a Heart Attack Centre on patient journey following an out of hospital cardiac arrest

J. Stephens' ; S. Cuddy ${ }^{2}$; S. Hutchison ${ }^{3}$; P. Patel2 ${ }^{2}$; R. Stümpfle

${ }^{1}$ Imperial College Healthcare NHS Trust, London, United Kingdom; ${ }^{2}$ Anaesthetics and critical care, Imperial College Healthcare NHS Trust, London, United Kingdom; ${ }^{3}$ Anaesthetics and critical care, Hammersmith Hospital, London, United Kingdom

Correspondence: J. Stephens

Intensive Care Medicine Experimental 2020, 8(2): 000729

Introduction: Within the UK, health care outcomes following admission to hospital either 'in-hours' or 'out-of-hours' has recently gained significant interest. Variation in service availability and staffing levels across the week may have an impact on this. However, specialist NHS services, such as Heart Attack Centres (HAC) and ICU are mandated to run a fully operational service 24-hours a day, 7-days a week.

Objectives: We have undertaken a service evaluation of all patients admitted to ICU at a Central London HAC, who suffered an OOHCA within the last five-years.

Methods: All patients who suffered an OOHCA and admitted to the ICU at a Central London HAC were identified and clinical notes were reviewed. We classified 'in-hours' attendance to HAC as Monday- Friday from 08:00 - 17:00 (excluding public holidays). All hours outside of these periods were considered 'out-of-hours'. This service evaluation was registered locally.

Results: Of the 279 patients admitted to ICU following an OOHCA 102 (36.6\%) patients attended in-hours with 177 (63.4\%) out-of-hours. Despite the fact that the patient cohort attending in-hours was significantly older than those attending out-of-hours, timing of HAC admission showed no significant impact upon whether patients had a primary percutaneous intervention (PPCl), time taken for admission to ICU, ICU length of stay or survival to ICU and hospital discharge or at 1-year post cardiac arrest (Table 1).

\begin{tabular}{|c|c|c|c|c|}
\hline & $\begin{array}{l}\text { All } \\
\text { patients }\end{array}$ & In-Hours & s Out-of-Hours & p-value \\
\hline Number of patients & 279 & 102 & 177 & N/A \\
\hline Median age (years) & 63.0 & 67.5 & 61.0 & 0.010 \\
\hline Survival to ICU discharge & $58.8 \%$ & $55.9 \%$ & $60.5 \%$ & 0.455 \\
\hline $\begin{array}{l}\text { Survival to hospital } \\
\text { discharge }\end{array}$ & $52 \%$ & $52.0 \%$ & $52.0 \%$ & 0.998 \\
\hline One-year survival & $42.2 \%$ & $50.0 \%$ & $49.7 \%$ & 0.964 \\
\hline MRS of survivors & 1.89 & 2.13 & 1.75 & 0.219 \\
\hline Median Temperature & 35.82 & 35.77 & 35.84 & 0.194 \\
\hline $\begin{array}{l}\text { Frequency of PPCI before } \\
\text { GICU admission }\end{array}$ & $78.9 \%$ & $83.3 \%$ & 76.3 & 0.164 \\
\hline $\begin{array}{l}\text { Median time taken for GICU } \\
\text { admission (mins) }\end{array}$ & $\mathrm{J}_{185}$ & 187 & 184 & 0.267 \\
\hline $\begin{array}{l}\text { Median length of stay on } \\
\text { GICU (days) }\end{array}$ & 6.81 & 6.50 & 6.83 & 0.230 \\
\hline
\end{tabular}

Irrespective of time of day, attending either during the week $(67.4 \%)$ or at the weekend (32.6\%) had no impact on frequency of $\mathrm{PPCl}$ or hospital survival rate (Table 2 ).

$\begin{array}{llll}\text { Table } 2 & \text { Week Day } & \text { Weekend } & \text { p-value } \\ \begin{array}{l}\text { Frequency of PPCI before GICU } \\ \text { admission }\end{array} & 153(81.4 \%) & 67(73.6 \%) & 0.137 \\ \text { Survival to hospital discharge } & 98(52.1 \%) & 47(51.6 \%) & 0.940\end{array}$

Conclusion: In our hospital experience, timing of admission had no significant impact on patient outcome. There was no greater length of time taken to be admitted to the ICU or length of stay on the unit. However, although not statistically significant, there was a greater tendency to perform a PPCI before admission to ICU when attending in hours or during a week day. This may be due to greater availability of staff or laboratory space.

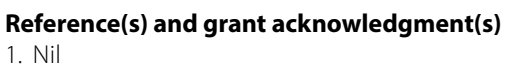

000730

Intensive care outcome predictors for out of hospital cardiac arrest following admission to a UK Heart Attack Centre. A 5-year retrospective analysis

S. Cuddy ${ }^{1}$; J. Stephens ${ }^{2}$; S. Hutchison ${ }^{3}$; P. Patel ${ }^{1}$; R. Stümpfle

${ }^{1}$ Anaesthetics and critical care, Imperial College Healthcare NHS Trust, London, United Kingdom; ${ }^{2}$ Imperial College Healthcare NHS Trust, London, United Kingdom; ${ }^{3}$ Anaesthetics and critical care, Hammersmith Hospital, London, United Kingdom

Correspondence: J. Stephens

Intensive Care Medicine Experimental 2020, 8(2): 000730

Introduction: Survival following ROSC from OOHCA is poor. Data from the London Ambulance Service suggests admission to a Heart Attack Centre (HAC) may improve outcome with $45.1 \%$ of patients surviving to hospital discharge, independent of initial heart rhythm1.

Objectives: We investigate the effect of patient demographics, coronary angiography, $\mathrm{PPCl}$ and temperature on survival and neurological outcomes in all patients admitted to our ICU in the last 5-years.

Methods: A single centre, retrospective analysis of all OOHCA patients admitted to ICU from 2015-2019. We used hospital data on patient demographics, coronary angiography reports, temperatures during the first 24 hours and patient survival. Neurological outcomes of survivors were determined by modified rankin scale (MRS).

Results: 279 patients were admitted to ICU, of which 58.8\%, 52.0\% and $42.2 \%$ survived to ICU discharge, hospital discharge and 1 year 
post-cardiac arrest respectively. Although males were more likely to be admitted post-OOHCA compared to females ( $76.4 \%$ vs $23.3 \%$ ), males had a significantly better 1 year survival despite equivalent survival at ICU discharge (males $45.5 \%$, females $30.1 \%, p=0.033$ ).

Table 1: Outcome divided by gender.

\begin{tabular}{llll}
\multicolumn{1}{c}{ Survival } & Male & Female & p-value \\
ICU (\%) & 58.68 & 58.46 & 0.97 \\
Hospital (\%) & 53.99 & 44.62 & 0.19 \\
1-year (\%) & 45.54 & 30.77 & $\mathbf{0 . 0 3}$
\end{tabular}

Age did not affect survival at unit discharge (age of survivors 62.0 vs non survivors 65.0, $p=0.215$ ) but there was a significant difference at hospital discharge (survivors vs non-survivors 60.0 vs 66.0 respectively, $p=0.007)$. Gender did not affect age of survivors at hospital discharge (median male age $=60$, female $=63, p=0.190$ ) or by 1 year (median male age $=60$, female $=58, p=0.38$ ) nor did BMI (median BMI of survivors $26.78 v$ non-survivors $27.68(p=0.254)$ ). The median MRS for survivors at hospital discharge was 1.89. Univariate analysis showed that gender was not a predictor of neurological outcome (Median MRS for males $=2$, females $=1, p=0.26)$ nor was age $(p=0.25)$.

Table 2: Effect of coronary angiography on patient survival, MRS scores and temperature.

\begin{tabular}{|c|c|c|c|}
\hline & Angiography & No angiography & p-value \\
\hline Total & 220 & 59 & - \\
\hline $\begin{array}{l}\text { \% Hospital } \\
\text { survival }\end{array}$ & 53.6 & 45.8 & 0.448 \\
\hline$\%$ 1-year survival & 52.3 & 40.7 & 0.114 \\
\hline MRS & 1.94 & 1.59 & 0.271 \\
\hline $\begin{array}{l}\text { Median Temp } \\
\left({ }^{\circ} \mathrm{C}\right)\end{array}$ & 35.86 & 35.64 & 0.478 \\
\hline
\end{tabular}

Coronary stenting was not shown to be a predictor of survival, quality of survival or average temperature (Table 3 ). Temperature had a significant effect on survival but there was no correlation between mean temperatures and MRS in survivors $(p=0.24)$.

Table 3: Effect of coronary stent insertion on outcome.

\begin{tabular}{|c|c|c|c|}
\hline & Stent inserted & No stent inserted & p-value \\
\hline Total & 127 & 84 & - \\
\hline $\begin{array}{l}\text { \% Hospital } \\
\text { survival }\end{array}$ & 56.6 & 51.0 & 0.527 \\
\hline $\begin{array}{l}\text { \% 1-year } \\
\text { survival }\end{array}$ & 55.0 & 50.0 & 0.471 \\
\hline MRS & 1.74 & 2.14 & 0.258 \\
\hline $\begin{array}{l}\text { Median Temp } \\
(\circ \mathrm{C})\end{array}$ & 35.86 & 35.85 & 0.889 \\
\hline
\end{tabular}

Table 4: Effect of temperature on outcome.

\begin{tabular}{lll} 
& $\begin{array}{l}\text { Temperature on } \\
\text { Admission }\end{array}$ & Mean \\
Total & 35.1 & 35.8 \\
$\begin{array}{l}\text { Died before hospital } \\
\text { discharge }\end{array}$ & 34.7 & 35.5 \\
$\begin{array}{l}\text { Hospital Survival } \\
\text { p-value }\end{array}$ & 35.4 & 36.0 \\
\hline
\end{tabular}

Conclusion: Our data suggests our hospital had favourable outcomes compared with London. Negative outcomes after OOHCA may be associated with a colder initial and first 24-hour average temperature and a poorer 1-year outcome for those of female gender. Further work is required to understand these associations.

\section{Reference(s) and grant acknowledgment(s)}

1. London Ambulance Service NHS Trust. Cardiac Arrest Annual Report: 2018/19. December 2019. Accessed on 02/06/20.
000933

Intensive care clinician's identification of poor neurologic prognosis for post-cardiac arrest patients from EEG patterns identified in neurophysiologist reports: a questionnaire survey L. Lilja'; S. Joelsson²; S. Lindgren ${ }^{1}$; P. Redfors ${ }^{3}$; P. Lundgren ${ }^{4}$; C. Rylander ${ }^{1}$ ${ }^{1}$ Anesthesiology and intensive care medicine, Sahlgrenska University Hospital, Gothenburg, Sweden; ${ }^{2}$ Clinical neurophysiology, Sahlgrenska University Hospital, Gothenburg, Sweden; ${ }^{3}$ Neurology, Sahlgrenska University Hospital, Gothenburg, Sweden; ${ }^{4}$ Cardiology, Sahlgrenska University Hospital, Gothenburg, Sweden

Correspondence: L. Lilja

Intensive Care Medicine Experimental 2020, 8(2): 000933

Introduction: "Highly malignant" EEG patterns are predictive of poor neurologic outcome in comatose survivors from cardiac arrest. To identify such patterns, the referring intensive care clinician depends on the neurophysiologist report. An alternative to plain text EEG descriptions could be a short report confirming or denying the presence of EEG patterns compliant with the standardized EEG pattern categories "highly malignant", "malignant" and "benign" as proposed by Westhall et al. We hypothesised that identification of EEG patterns known to have a specific prognostic association may be difficult in plain text EEG neurophysiologist reports.

Objectives: To test whether the plain text EEG reports, or the EEG short statements granted higher degree of correct identification of prognosis.

Methods: Volunteering ICU clinicians in two university clinics were introduced to 17 authentic plain text neurophysiologist reports and three reports limited to a short statement declaring that "highly malignant", "malignant" or "benign" EEG patterns were present. They were asked to classify how the described EEG patterns would impact the neurologic prognosis of a comatose patient. The standardised scenario included a high neuron specific enolase (NSE), but other neurologic tests and neuroimaging were inconclusive. In consequence, the clinician's perception of the prognostic value of the EEG neurophysiologist report would affect the neurological prognostication according to international guidelines. The primary outcome was the proportion of clinicians who correctly identified the poor neurologic prognosis inferred by the "highly malignant" EEG patterns. Secondary outcomes were how "malignant" and "benign" EEG patterns were classified.

Results: Fifty-seven intensive care clinicians, 36 of whom were specialists, participated in the study. "Highly malignant" patterns consistent with poor neurologic prognosis were correctly identified by $73 \%$ in the plain text reports and by $93 \%$ in the short statements. "Malignant" patterns consistent with no impact on prognosis were correctly identified by $27 \%$. A "benign" pattern consistent with a positive impact on prognosis were correctly identified by $58 \%$ in plain text reports.

\begin{tabular}{|c|c|c|c|}
\hline EEG short statement & "Highly malignant" & "Malignant" & "Benign" \\
\hline Prognosis poor & $53(93 \%)$ & $34(60 \%)$ & 0 \\
\hline Prognosis not affected & $4(7 \%)$ & $23(40 \%)$ & $4(7 \%)$ \\
\hline Prognosis positive & 0 & 0 & $53(93 \%)$ \\
\hline
\end{tabular}

Conclusion: ICU clinicians exposed to standardized EEG short statements defining the presence or absence of "highly malignant" EEG patterns, compared to plain text EEG neurophysiologist reports, are to a higher degree able to correctly identify a poor neurological prognosis in comatose survivors of cardiac arrest.

\section{Reference(s) and grant acknowledgment(s)}

1. Backman S, Cronberg T, Friberg H, Ullen S, Horn J, Kjaergaard J, et al. Highly malignant routine EEG predicts poor prognosis after cardiac arrest in the Target Temperature Management trial. Resuscitation. 2018;131:24-8.

2. Westhall E, Rossetti AO, van Rootselaar AF, Wesenberg Kjaer T, Horn J, Ullen $\mathrm{S}$, et al. Standardized EEG interpretation accurately predicts prognosis after cardiac arrest. Neurology. 2016;86:1482-90. 
3. Westhall E, Rosen I, Rossetti AO, van Rootselaar AF, Kjaer TW, Horn J, et al Electroencephalography (EEG) for neurological prognostication after cardiac arrest and targeted temperature management; rationale and study design. BMC Neurol. 2014;14:159.

4. Nolan JP, Soar J, Cariou A, Cronberg T, Moulaert VR, Deakin CD, et al. European Resuscitation Council and European Society of Intensive Care Medicine Guidelines for Post-resuscitation Care 2015: Section 5 of the European Resuscitation Council Guidelines for Resuscitation 2015. Resuscitation. 2015;95:202-22

5. We would like to acknowledge the work done by our colleges dr Nilsson, dr Krysl and dr Hedstrom at the clinical neurophysiology department of the Sahlgrenska University Hospital.

\section{0}

Evaluation of healthcare providers results after cardiopulmonary resuscitation training programme

C. Palau Marti'; S. Moliner Velázquez ; I. Girbes Ruiz ; JM. Blanco

Rodríguez ; ; J. De Andrés Ibáñez ${ }^{1}$

${ }^{1}$ Anaesthesiology, Critical Care and Pain Therapy Service, Consorci Hospital General Universitari de València, València, Spain

Correspondence: C. Palau Marti

Intensive Care Medicine Experimental 2020, 8(2): 000950

Introduction: Evidence shows that the mortality rate and the effects derived from a CPRlower if care response is improved and the survival chain amongst hospital is optimised. In this sense, medical staff's life support techniques training is essential, as it goes without saying that this improves the survival rates. Having said this, we proceed to examine the assessment results from the CPR training programme which started in 2017 and 2018 in a university tertiary hospital.

Objectives: The main objective of this project is to provide training for basic and advanced life support based on clinical simulations to a certain number of healthcare workers (nurses and medical assistants). Furthermore, didactic methods will be assessed using quality surveys.

Methods: Theory and practice classes were conducted by qualified basic and advanced life support teachers in groups of 12-15 healthcare workers. Advanced CPR mannequins as well as airway material were used. The participants were also provided with online access to extra teaching material. Finally, the participants had to fill in the satisfaction survey.

Results: There were 38 sessions in which 695 healthcare workers took part. Only $60 \%$ of the nurses and $38 \%$ of the medical assistants who participated were familiar with CPR, and $22 \%$ of the participants had never been present during a CPR. The programme received positive feedback. $90 \%$ of the participants were satisfied with aspects such as the teachers' teaching skills, the possibility to participate and the understanding of the theory.

Conclusion: The CPR training conducted in 2017 and 2018 has allowed healthcare workers to update the CPR knowledge they need. The use of clinical simulations has been regarded as effective in order to acquire skills to deal with risky situations. The professionals considered the training they received was excellent.

\section{Reference(s) and grant acknowledgment(s)}

1. Girotra S, Nallamothu BK, Spertus JA, et al. Trends in survival after inhospital cardiac arrest. N Engl J Med. 2012;367(20):1912-1920. https://doi. org/10.1056/nejmoa1109148

\section{6}

Impact of catecholamines dose on metabolism and brain injury after out-of-hospital cardiac arrest

K. Czerwinska-Jelonkiewicz'; A. Wood ${ }^{2}$; A. Bohm³ ; P. Kwasiborski;

J. Grand ${ }^{5}$; A. Oleksiak ; G. Tavazzi7; A. Sionis ${ }^{8}$; J. Stępińska ${ }^{6}$

${ }^{1}$ Intensive Therapy Unit, Harefield Hospital, Harefield, United Kingdom; ${ }^{2}$ Department of cardiology, University of Leicester, Leicester, United Kingdom; ${ }^{3}$ Department of acute cardiology, National Institute of Cardiovascular Diseases, Bratislava, Slovakia; ${ }^{4}$ Third department of internal diseases and cardiology, Medical University of Warsaw, Warszawa, Poland; ${ }^{5}$ Department of cardiology, BørneRiget - Rigshospitalet, København, Denmark; ${ }^{6}$ Department of intensive cardiac therapy, National Institute of Cardiology, Warsaw, Poland; ' Department of anesthesia and intensive care, Fon- dazione IRCCS Policlinico S Matteo, Pavie, Italy; ${ }^{8}$ Cardiology department, Hospital de la Santa Creu i Sant Pau, Barcelona, Spain

Correspondence: K. Czerwinska-Jelonkiewicz

Intensive Care Medicine Experimental 2020, 8(2): 001006

Introduction: Catecholamines are recommended as the first-line drugs to treat hemodynamic instability after out-of-hospital cardiac arrest (OHCA) [1,2]. However, the benefit-to-risk ratio of catecholamines in the critical setting is dose dependent and their effect on metabolism and organ function early after OHCA has not been investigated.

Objectives: The aim of the analysis was to investigate the association between catecholamine and metabolism and organ function parameters during the first 72 hours after OHCA as well as to assess the importance of catecholamines dose for short-term survival.

Methods: The Post-Cardiac Arrest Syndrome (PCAS) pilot study was a prospective, observational, multicenter study. The details of the project were described elsewhere [3]. The endpoints of the prespecified analysis presented here were: 1 . Lactate concentration as an indicator of oxygen delivery/anaerobic metabolism and neuron-specific enolase (NSE) as a brain injury marker, both assessed every 24 hours, over the first 72 hours after OHCA, 2. in-hospital mortality. Risk factors: 1. Norepinephrine dose ( $\mu \mathrm{g} / \mathrm{kg} / \mathrm{min}), 2$. Cumulative dose of catecholamines ( $\mu \mathrm{g} / \mathrm{kg} / \mathrm{min}), 3$. Vasoactive-inotropic score (VIS)

Results: Simple and multiple linear regression analysis showed that norepinephrine dose and cumulative catecholamines dose are both independent predictors of lactate concentration on admission, at 24 and 48 hours after OHCA (Table 1). Furthermore, norepinephrine dose and cumulative catecholamine dose were independent predictors of NSE concentration on admission $(r=0.466, p<0.0001 ; r=0.158, p=0.01)$ and at 24 hours after OHCA $(r=0.327, p=0.04 ; r=0.345, p=0.003)$. The VIS was independently correlated with NSE concentration on admission $(r=0.61, p<0.0001)$. Cumulative catecholamine dose as well as VIS on admission were significantly associated with in-hospital mortality in univariate and multivariate analysis. Norepinephrine dose on admission was associated with in-hospital mortality in univariate analysis (Table 1).

Table 1. Impact of catecholamine dose on lactate concentration within the first 48 hours after OHCA and on in-hospital mortality.

\begin{tabular}{|c|c|c|c|c|}
\hline $\begin{array}{l}\text { Multiple regression } \\
\text { model* }\end{array}$ & B coefficient & $r$ coefficient & t Stat & p-value \\
\hline $\begin{array}{l}\text { Norepinephrine dose on } \\
\text { admission }\end{array}$ & 2.264 & 0.238 & 2,743 & 0.007 \\
\hline $\begin{array}{l}\text { Norepinephrine dose } 24 \mathrm{~h} \\
\text { after OHCA }\end{array}$ & 3.283 & 0.36 & 4.138 & $<0.0001$ \\
\hline $\begin{array}{l}\text { Norepinephrine dose } 48 \mathrm{~h} \\
\text { after OHCA }\end{array}$ & 1.856 & 0.314 & 3.21 & 0.001 \\
\hline $\begin{array}{l}\text { Cumulative catecholamine } \\
\text { dose } \\
\text { on admission }\end{array}$ & 0.073 & 0.184 & 2.091 & 0.03 \\
\hline $\begin{array}{l}\text { Cumulative catecholamine } \\
\text { dose } 24 \mathrm{~h} \text { after OHCA }\end{array}$ & 0.099 & 0.4291 & 4.959 & $<0.0001$ \\
\hline $\begin{array}{l}\text { Cumulative catecholamine } \\
\text { dose } 48 \mathrm{~h} \text { after OHCA }\end{array}$ & 0.02 & 0.229 & 2.291 & 0.02 \\
\hline In-hospital mortality & $\begin{array}{l}\text { Univariate } \\
\text { OR }(95 \% \mathrm{CI}) ; \mathrm{p}- \\
\text { vaule }\end{array}$ & \multicolumn{3}{|c|}{ OR $(95 \% \mathrm{CI}) ; \mathrm{p}$-vaule } \\
\hline $\begin{array}{l}\text { Norepinephrine dose on } \\
\text { admission }\end{array}$ & $\begin{array}{l}2.330(1.106- \\
4.909) ; 0.04\end{array}$ & \multicolumn{3}{|c|}{$\begin{array}{l}11.95(0.802-178.00) \\
0.07\end{array}$} \\
\hline $\begin{array}{l}\text { Cumulative catecholamine } \\
\text { dose } \\
\text { on admission }\end{array}$ & $\begin{array}{l}2.14(1.876- \\
2.654) ; 0.02\end{array}$ & \multicolumn{3}{|c|}{$1.09(1.003-1.189) ; 0.04$} \\
\hline VIS on admission & $\begin{array}{l}3.54(3.104- \\
3.967) ; 0.001\end{array}$ & \multicolumn{3}{|c|}{$1.04(1.011-1.073) ; 0.006$} \\
\hline
\end{tabular}


Conclusion: Dose of catecholamines administered early after OHCA is associated with lactate and NSE concentration, which may suggest their importance for impaired tissue oxygen delivery, enhanced anaerobic metabolism and organ injury. Dose of catecholamines on admission is related with in-hospital mortality after OHCA.

\section{Reference(s) and grant acknowledgment(s)}

1. 1. Nolan JP, Soar J, Cariou A, et al. European Resuscitation Council and European Society of Intensive Care Medicine 2015 guidelines for post-resuscitation care. 2015. Intensive Care Med 2015; 41: 2039-2056. 2. Dellinger RP, Levy MM, Carlet JM, et al. Surviving Sepsis Campaign: international guidelines for management of severe sepsis and septic shock: 2008. Crit Care Med.2008;36(1):296-327. 3. CzerwińskaJelonkiewicz K, Grand J, Tavazzi G, et al. Acute respiratory failure and inflammatory response after out-of-hospital cardiac arrest: results of the Post-Cardiac Arrest Syndrome (PCAS) pilot study. Eur Heart J Acute Cardiovasc Care 2020. https://doi.org/10.1177/2048872619895126. 4. Oh YT, Oh J, Park SM, et al. Vasoactive inotropic score as a predictor of in-hospital mortality in out-of-hospital cardiac arrest. SIGNA VITAE. 2019;15(2):40-44.

\section{2}

A 2-year retrospective review of out of hospital cardiac arrests presenting to a Scottish Major Trauma Centre to identify candidates for a proposed extracorporeal cardiopulmonary resuscitation service

C. Edmunds'; G. Baillie'2; I. Scott ${ }^{2}$

${ }^{1}$ Intensive Care Unit, I. M. \& T., Aberdeen, United Kingdom; ${ }^{2}$ Intensive care department, NHS Grampian, Aberdeen, United Kingdom

Correspondence: $C$. Edmunds

Intensive Care Medicine Experimental 2020, 8(2): 001022

Introduction: Extracorporeal Cardiopulmonary Resuscitation (ECPR) is internationally recognised as an intervention to enable physiological support of patients in refractory cardiac arrest. The evidence base for this type of support comes from a mixture of both cohort and case series and is applied to specific group of patients. To assess the potential benefit of an ECPR service a review of 2 years of patients presenting to the Emergency Department with out of hospital cardiac arrest was undertaken.

Methods: Inclusion criteria were defined based on evidence available and the practice undertaken in other parts of the UK. These criteria were; age $<60$ years, witnessed arrest, immediate bystander CPR, $>10$ minutes of resuscitation but $<45$ minutes of CPR on arrival in the Emergency Department, the presence of electrical activity. Postcodes were also looked at to assess the distances from the department within a $10 \mathrm{~km}$ and $20 \mathrm{~km}$ radius. Timings of cases were assessed to give an overview of in hours (08:00 to 17:00) and out of hours (17:00 to 08:00) workloads. Data was taken from Emergency Department presentations recorded as cardiac arrest, with a presentation review from electronic systems.

Results: 109 patients were identified over a two-year period between January 2018 to December 2019. This was split into 46 patients in hours and 63 patients out of hours. Not accounting for age or CPR extending beyond 45 minutes, 17 patients $(36.9 \%)$ met the criteria in hours and 19 patients (30.1\%) out of hours. However, when excluding patients with CPR ongoing longer than 45 minutes on arrival there were 11 patients $(23.9 \%)$ in hours and 11 patients $(17.4 \%)$ out of hours. Further considering patients that were 60 years or younger found that only 6 patients $(13.0 \%)$ in hours and 3 patients $(4.7 \%)$ out of hours met criteria for extracorporeal resuscitation. Considering in hours cases there were 35 within $20 \mathrm{~km}$ and 30 within $10 \mathrm{~km}$. Considering distances for cases out of hours there were 43 within $20 \mathrm{~km}$ and 40 within $10 \mathrm{~km}$ of Aberdeen Royal Infirmary. All the patients that met criteria died in the Emergency department.

Conclusion: Over two years the case load for patients that meet the criteria for ECPR in the local areas is low, and even when considering a 24-hour period it remains a small number of patients. However, when considering service implementation, it is important to note that all the patients meeting criteria died in the Emergency Department and that there is a significant number of cardiac arrest cases within close traveling distance of Aberdeen Royal Infirmary. Due to this there may be an reasaonable argument for the development of an ECPR service. Any service development would need to consider the pathway of care that is delivered from the pre-hospital setting as well as the instigation of ECPR in the hospital as timeframes for establishing a patient on VAECMO are crucial to overall outcome. More work is needed to further develop local cardiac arrest care in the North-East of Scotland.

Reference(s) and grant acknowledgment(s)

1. 4. Soar J, Deakin C, Lockey A, et al. Adult Advanced Life support. Resuscitation Council (UK) guidelines 2015, www.resus.org.uk/resuscitation-guide lines/adult-advanced-life-support/ (Accessed 23/06/20).

2. 3. Kim, SJ, Kim, HJ, Lee, HY, et al. Comparing extracorporeal cardiopulmonary resuscitation with conventional cardiopulmonary resuscitation: a meta-analysis. Resuscitation 2016; 103: 106-116.

3. 2. Kagawa, E, Inoue, I, Kawagoe, T, et al. Assessment of outcomes and differences between in- and out-of-hospital cardiac arrest patients treated with cardiopulmonary resuscitation using extracorporeal life support. Resuscitation 2010; 81: 968-973.

4. Harrogate S, Stretch B, Seatter R, Finney S, Singer B. A retrospective analysis of inpatient cardiac arrests over one year at a tertiary heart attack and cardiothoracic centre identifying potential candidates for an inpatient extracorporeal cardiopulmonary resuscitation service. J Intensive Care Soc. 2020;21(2):105-110. https://doi.org/10.1177/1751143719848660

\section{5}

An estimate of the need for somatosensory evoked potential examination for assessing the neurological prognosis in comatose survivors of cardiac arrest

M. Thuccani' ' S. Joelsson²; L. Liljä3; C. Rylander ${ }^{4}$

${ }^{1}$ Department of anaesthesiology and intensive care medicine, institute of clinical sciences, Sahlgrenska Academy, University of Gothenburg, Gothenburg, Sweden; ${ }^{2}$ Department of clinical neurophysiology, institute of neuroscience and physiology, Sahlgrenska Academy, University of Gothenburg, Gothenburg, Sweden; ${ }^{3}$ Department of anesthesiology and intensive care medicine, institute of clinical sciences, Sahlgrenska Academy, University of Gothenburg, Gothenburg, Sweden; ${ }^{4}$ Department of anaesthesiology and intensive care medicine, Institute of Clinical Sciences, Sahlgrenska Academy, University of Gothenburg, Gothenburg, Sweden

Correspondence: M. Thuccani

Intensive Care Medicine Experimental 2020, 8(2): 001075

Introduction: The neurological prognosis of comatose survivors of cardiac arrest is recommended to be multimodal (1). Somatosensory Evoked Potentials (SSEP) is one of the methods used. Bilateral absence of cortical SSEP response is associated with poor prognosis. As a tool for hospitals lacking the SSEP modality, Daubin et al. constructed a scoring system based on clinical signs and electroencephalography (EEG) aiming to predict the absence of the cortical N20-signal (2).

Objectives: We applied this scoring system to assess the need for the SSEP modality as a potential addition to the current multimodal prognostic examinations of comatose survivors of cardiac arrest in the Sahlgrenska University Hospital, Gothenburg, Sweden.

Methods: Survivors of out-of-hospital cardiac arrests (OHCA) who remained unconscious $>72$ hours after return of spontaneous circulation (ROSC) were classified according to the scoring system. We had to assume that EEG patterns classified according to Westhall et al. scored similar points as EEG classified according to Synek which was used by Daubin $(3,4)$. Using the cut-off values defined by its authors, the proportion of patients with predicted absent N20-signal on SSEP was calculated. Results: 37 patients with OHCA were included and 13 (35\%) of these were predicted to have bilaterally absent N20-signals based on the total score on day 3, 48-72 hours after ROSC. 
Conclusion: Using the scoring system constructed by Daubin et al. (2) with minor adaptations, we calculated that one third of patients with OHCA, who remained unconscious $>72$ hours after ROSC, would have absent cortical signals on SSEP. Hence, the use of SSEP would be meaningful in at least the other two thirds of OHCA patients. These results are essential for the dimensioning of a future SSEP service for the intensive care units in the Sahlgrenska University Hospital.

\section{Reference(s) and grant acknowledgment(s)}

1. Westhall E, Rossetti AO, van Rootselaar A-F, Wesenberg Kjaer T, Horn J, Ullén $\mathrm{S}$, et al. Standardized EEG interpretation accurately predicts prognosis after cardiac arrest. Neurology. 2016:86(16):1482-90.

2. Synek VM. EEG abnormality grades and subdivisions of prognostic importance in traumatic and anoxic coma in adults. Clin Electroencephalogr. 1988;19(3):160-6.

3. Daubin C, Guillotin D, Etard O, Gaillard C, du Cheyron D, Ramakers M, et al. A clinical and EEG scoring system that predicts early cortical response (N20) to somatosensory evoked potentials and outcome after cardiac arrest. BMC Cardiovasc Disord. 2008:8:35

4. Nolan JP, Soar J, Cariou A, Cronberg T, Moulaert VRM, Deakin CD, et al. European Resuscitation Council and European Society of Intensive Care Medicine Guidelines for Post-resuscitation Care 2015: Section 5 of the European Resuscitation Council Guidelines for Resuscitation 2015. Resuscitation. 2015;95:202-22

5. This work was supported by institutional funding only.

\section{5}

Initial base deficit and return of spontaneous circulation in refractory out-of-hospital cardiac arrest predict successful extracorporeal cardiopulmonary resuscitation

P. Massion ${ }^{1}$; S. Joachim²; P. Morimont ${ }^{1}$; GL. Dulière ${ }^{2}$; R. Betz ${ }^{3}$; A. Benoit ${ }^{1}$.

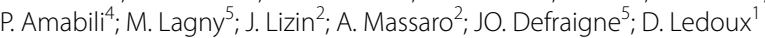
${ }^{1}$ Department of intensive care, Chu De Liège, Liège, Belgium; ${ }^{2}$ Department of intensive care, CHR de la Citadelle, Liège, Belgium; ${ }^{3}$ Department of emergency, Chu De Liège, Liège, Belgium; ${ }^{4}$ Department of anaesthesiology, Chu De Liège, Liège, Belgium; ${ }^{5}$ Department of cardiothoracic surgery, Chu De Liège, Liège, Belgium

Correspondence: $\mathrm{P}$. Massion

Intensive Care Medicine Experimental 2020, 8(2): 001115

Introduction: Extracorporeal cardiopulmonary resuscitation (ECPR) for refractory out-of-hospital cardiac arrest (OHCA) recently developed with disappointing limited survival (1) and still requires more selective inclusion criteria.

Objectives: To evaluate clinical benefit of an ECPR protocol in OHCA and to investigate potential predictive variables on admission.

Methods: Between 2011 and 2020, we analysed 80 ECPR candidates admitted in 2 Belgian reference centres. From 2016 an ECPR protocol based on 6 prehospital criteria was implemented (no flow $<3$ minutes, low flow $<60$ minutes, initial shockable rhythm, end-tidal $\mathrm{CO} 2>$ $15 \mathrm{mmHg}$, age $<65$ years and absence of comorbidities). Scoop-andrun strategy and admission metabolic criteria for resuscitation discontinuation were also evaluated.

Results: Twenty-six (32\%) OHCA patients benefited from ECPR, their hospital survival rate was $43 \%(n=9 / 21)$ after protocol implementation. Prehospital inclusion criteria did not discriminate survivors and nonsurvivors $(p=0.92)$. By contrast, any transient return of spontaneous circulation (ROSC) before ECPR ( 89 vs. $17 \%, \mathrm{p}=0.002$ ), higher serum bicarbonate (14.0 (10.6 -15.2) vs. $7.5(3.7-10.5) \mathrm{mml} / \mathrm{l}, \mathrm{p}=0.019)$, lower base deficit (14.9 (11.9- 18.2) vs. $21.6(17.9-28.9) \mathrm{mmol} / \mathrm{l}, \mathrm{p}=0.039)$ and initial lactate $<900 \mathrm{mg} / \mathrm{dl}(5 / 9 \mathrm{vs} .1 / 12, \mathrm{p}=0.046)$ were associated with a more favourable outcome. Best cut-offs were bicarbonate $>10 \mathrm{mmol} / \mathrm{L}$, base deficit $<18 \mathrm{mmol} / \mathrm{L}$, lactate $<900 \mathrm{mg} / \mathrm{L}$ and fibrinogen $>1,8 \mathrm{~g} / \mathrm{l}$ (figure). Four patients became organ donors after brain death.

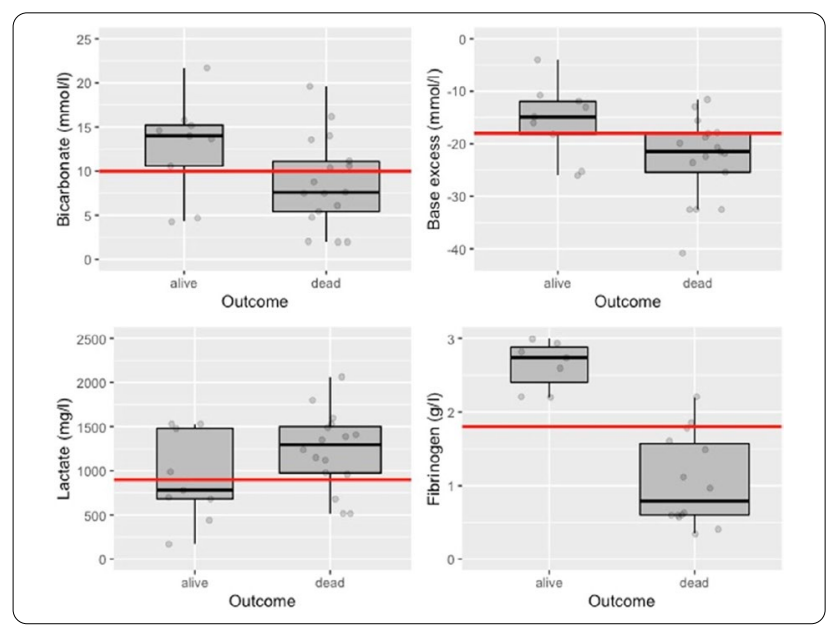

Conclusion: This observational study shows that, in ECPR candidates, base deficit on admission and any transient ROSC before cannulation were significantly associated with better survival. Integration of ROSC and base deficit into inclusion criteria for ECPR in refractory OHCA could improve resources allocation.

Reference(s) and grant acknowledgment(s)

1. (1) Bougouin W, Dumas F, Lamhaut L, et al. Extracorporeal cardiopulmonary resuscitation in out-of-hospital cardiac arrest: a registry study. Eur Heart J 2019; 41: 1961-71.

2. None

\section{2}

The risk factors analysis in patients after In-Hospital Cardiac

\section{Arrest: a nationwide study}

M. T. Wang ; W. C. Huang ${ }^{2}$; E. H. Yeh ${ }^{3}$; H. H. Liao ${ }^{3}$; Y. S. Lin ${ }^{4}$

${ }^{1}$ Department of critical care medicine, Kaohsiung Veterans Genera Hospital, Kaohsiung, Taiwan; ${ }^{2}$ Critical care medicine and cardiovascular center, Kaohsiung Veterans General Hospital, Taipei, Taiwan; ${ }^{3}$ The joint commission of taiwan, The Joint Commission of Taiwan, New Taipe City, Taiwan; ${ }^{4}$ Critical care medicine, Kaohsiung Veterans General Hospital, Kaohsiung, Taiwan

Correspondence: W.C. Huang

Intensive Care Medicine Experimental 2020, 8(2): 001152

Introduction: In-hospital cardiac arrest (IHCA) is common and associated with a high mortality rate. However, it has received little attention compared with other critical cardiovascular conditions.

Objectives: This study is aimed to analyze the association between IHCA patients and healthcare related risk factors.

Methods: Data for this study enrolled IHCA patients from 14 regional hospitals, 2 district hospitals, and 5 medical centers between 2013 June to 2018 December. Primarily, the registry enrolled patients once "collapse" event was disclosed regardless of the witness, and a total of 7,731 cases was included initially. Patients with index hospitalization for Out-of-Hospital Cardiac Arrest (OHCA), IHCA patients without acute life support (ALS) attempt, undetermined sex, Age $£ 18$ or ${ }^{3} 120$ years old, and patients with "do not resuscitate (DNR)" consent were excluded. Finally, a total of 5,306 patients were included in the analysis Results: As for overall IHCA patients, HR for mortality was higher in older patients $(H R=1.11)$, and those underwent vasoactive agents in $\mathrm{ALS}(\mathrm{HR}=1.20)$. While, initial rhythm as ventricular fibrillation (HR $=0.62$ ) reduced the risk of mortality in IHCA patients. With regard to 
subgrouping IHCA patients in ICU and ER, patients underwent vasoactive agents in ALS (HR = 1.19) had poor survival. Whereas, better survival was reported in patients with initial ventricular tachycardia (HR $=0.63)$ and ventricular fibrillation $(H R=0.59)$. Regarding patients in general wards, old age $(H R=1.21)$ and shift between 24:00 to 08:00 (compared to 08:00 17:00) (HR=1.26) lead to poor outcome.

Conclusion: The majority of initial detected rhythm in IHCA patients was non-shockable. Factors for poor survival included old age, overnight shift, and vasoactive agents. While, better survival was noted in patients with witness, those underwent targeted temperature management, and those with initial detected ventricular fibrillation. With regard to conditions at discharge, patients with regained conscious and those with good neurologic performance had better survival.

\section{4}

\section{Hemodynamic effect of hypertonic lactate infusion}

after resuscitation in an experimental model of cardiac arrest

F. Annoni

${ }^{1}$ Intensive Care, Université Libre De Bruxelles / Campus Érasme, Anderlecht, Belgium

Correspondence: $\mathrm{F}$. Annoni

Intensive Care Medicine Experimental 2020, 8(2): 000484

Introduction: Among cardiac arrest (CA) survivors, post CA syndrome is responsible for poor outcome and is one of the main causes of deaths in these patients1,2. Lactate enriched solutions could exerts positive effects on the heart, where the myocardial reperfusion injury is responsible for a consistent fraction of the organ damage3-5. Several protocols have demonstrated how an ischemic post-conditioning strategy based on lactate-enriched solutions could reduce the magnitude of the reperfusion injury6,7, but no study has investigated the impact of hypertonic sodium lactate (HSL) infusion on macro-hemodynamic variables after CA.

Objectives: To investigate the hemodynamic impact of the infusion of HSL after resuscitation in an experimental model of cardiac arrest Methods: 27 adult domestic pigs were enrolled in the study. After proper sedation and preparation, including endotracheal intubation, invasive arterial pressure monitoring and pulmonary artery catheter, a ventricular fibrillation was induced via a pace-maker wire and left untreated for 10 minutes, when cardio-pulmonary resuscitation (CPR) were started at the rate of 100 /minute with Lucas III device and continued for 5 more minutes. After the first minute of CPR and eventually after 7 minutes, an injection of Adrenaline was provided and at the end of the 5 minutes an electric shock (200 J) was delivered and repeated every minute to achieve Retour of Spontaneous Circulation (ROSC). The animals were divided as follow: 9 as control group received a bolus of crystalloids during CPR and infusion during $12 \mathrm{~h}$ after ROSC (G0); 9 received HSL both during CPR and after ROSC (G1) and 9 received a bolus of crystalloids during CPR and HSL infusion after ROSC (G2). HSL solution was prepared by diluting into injectable sterile water a $2 \mathrm{mEq} / \mathrm{mL}$ solution (Monico spa, Mestre, Italy) to achieve a $1 \mathrm{M}$ solution with calculated osmolarity of $2000 \mathrm{mOsm} / \mathrm{L}$.

Results: All the animals in the study achieved ROSC. No differences were found between groups in the distribution of sex and weight, as well as in number of shocks needed to achieve ROSC, adrenaline dose, first end tidal $\mathrm{CO} 2$ measurement or baseline $\mathrm{pH}$. To investigate differences on dependent variables over time between groups, a general linear mixed model was used. Arterial lactate levels were higher in the group of animals which received HSL (G1-G2) compared to G0 during the study course $(p<0.001)$. To achieve the same mean arterial pressure level of $65 \mathrm{mmHg}$, there was a significantly lower requirements for vasopressors between groups over the study period $(p<0.01)$ and in the post-hoc analysis with Bonferroni correction, there was a significant difference between G0 compared to G1 $(p<0.001)$ and G2 $(p<0.001)$, but not between $\mathrm{G} 1$ and $\mathrm{G} 2(\mathrm{p}=0.55)$.

Conclusion: In animals treated with continuous HSL infusion there is a significantly lower requirements of vasopressors in the first hours following resuscitation after cardiac arrest.

\author{
Reference(s) and grant acknowledgment(s) \\ 1. 1. Favaretto E, Tarantini G. Cardiology 2013; 125: 94-95. \\ 2. 6. Yellon DM, Hausenloy DJ. NEJM 2007; 357: 1121-1235. \\ 3. 5. Jentzer JC, Chonde MD, Dezfulian C. Biomed Res Int 2015, ID 314796 \\ 4. 4. Knott, EM Ryou MG, Sun J et al. Am J Physiol Heart Circ Physiol 2005; 289; \\ $1123-1130$ \\ 5. 3. Heart IJC, Koyama T. IJC Hear Vasc 2017; 15: 1-8. \\ 6. 1. Nolan, JP et al. Resuscitation 2015; 95: e1-e31. \\ 7. 2. Lemiale V, Dumas F, Mongardon N et al. Intensive Care Med 2013; \\ 39:1972-1980
}

\section{7}

Family member presence during simulated cardiopulmonary resuscitation-frustrating but not impairing

M. Willmes- Pflüger ${ }^{1}$; T. Sellmann²; N. Semmer ${ }^{3} ;$ F. Tschan $^{4}$;

D. Wetzchewald ${ }^{1} ;$ H. Schwager ${ }^{1}$; S. Russo ${ }^{5}$; S. Marsch ${ }^{6}$

${ }^{1}$ Institut für notfallmedizin, AIM Institut für Notfallmedizin gGmbH, Arnsberg, Germany; ${ }^{2}$ Klinik für anästhesiologie und intensivmedizin, Bethesda Lutheran Hospital to Duisburg, Duisburg, Germany; ${ }^{3}$ Institut für psychologie, University of Bern Institute of Psychology, Bern, Switzerland; ${ }^{4}$ Institut de psychologie du travail et des organisations, University of Neuchâtel, Neuchâtel, Switzerland; ${ }^{5}$ Lehrstuhl für anästhesiologie i, Zentrale der Universität Witten/Herdecke, Witten, Germany, Germany; ${ }^{6}$ Department of intensive care, University Hospital of Basel, Basel, Switzerland

Correspondence: M. Willmes- Pflüger

Intensive Care Medicine Experimental 2020, 8(2): 000037

Introduction: Current international guidelines recommend the presence of a family member during cardiopulmonary resuscitation (CPR) as this has beneficial psychological effects for relatives witnessing the event $[1 ; 2]$. Contrary, there is only sparse data on the quality of team performance and the psychological well-being of health-care workers during relative-witnessed CPR.

Objectives: This prospective randomized trial investigates the effects of a family witness on team performance and perceived stress levels in simulated cardiac arrests.

Methods: 325 teams of 3 to 4 physicians each were randomized to perform CPR in a simulated cardiac arrest in the presence or absence of a family witness (trained actor). Additionally, teams were randomized to designated or emergent leadership. Video-recordings obtained during CPR simulations were used for data analysis. The perceived task load was assessed by the NASA task load index (TLX), Primary endpoint was percentage of "hands-on" time. Secondary outcomes included adherence to treatment algorithms, the perceived task load and the effects of designated leadership on primary and secondary outcomes.

Results: In groups with a present relative, verbal interaction occurred cumulatively during $105 \pm 66 \mathrm{sec}$, representing $27 \pm 16 \%$ of the study time. Teams without a leader had significantly more verbal interaction with the relative $(123 \pm 75 \mathrm{sec} v \mathrm{~s} .96 \pm 58 \mathrm{sec}, \mathrm{p}=0.014)$ corresponding to $33 \pm 20 \%$ vs $24 \pm 14 \%$ ( $p=0.001)$ of the study time. Primary outcome: percentage "hands-on" time was $86.6 \pm 6.3 \%$ in the relative present groups vs. $87.3 \pm 5.6 \%$ in the control groups $(p=0.25)$. Secondary outcomes: time to first defibrillation was $81 \pm 38 \mathrm{sec}$ in groups with a relative present and $77 \pm 41 \mathrm{sec}$ in control groups ( $p=0.49$ ). Designated leadership had no effect on medical performance markers. The presence of a relative was associated with significantly higher NASA-TLX ratings for the domains "temporal demands" $(p<0.001)$ and "frustration" ( $p<0.001)$ while there was a borderline difference ( $p=0.057$ ) for the domain "mental demand" and no significant differences for "effort" ( $p=0.62$ ) and "performance" (0.96). Designated leadership had no significant effect on NASA-TLX ratings.

Conclusion: The presence of a relative during simulated CPR significantly increased the perceived task load for resuscitation teams without impairing their medical team performance measures. Having designated team leaders was associated with decreased verbal interaction with the relative but had no effect on the perceived work load or the team performance. Thus, resuscitation teams seem capable to cope with the additional psychological burden imposed by a family witness. 
000003

Analyze the clinical characteristics of critically ill Adult Congenital Heart Disease (ACHD) Patients and its impact on outcomes

S. Kadir ${ }^{1}$

${ }^{1}$ Cardiology, University of Nebraska Omaha, Omaha, United States of America

Correspondence: S. KADIR

Intensive Care Medicine Experimental 2020, 8(2): 000003

Introduction: The population of ACHD patients continues to grow in the USA $1,2,3$. There exists a paucity of data in the realm of evidencebased critical care medicine management of ACHD patients.

Objectives: To analyze our ACHD population with respect to clinical, anatomical, and physiological characteristics in order to understand associations to Intensive Care Unity (ICU) morbidity and outcomes. This information will serve to improve clinicians' ability to understand the various factors that influence outcomes in critically ill ACHD patients with unplanned and post-operative ICU admissions.

Methods: Retrospective chart review was completed on all ACHD patients with ICU admissions between 01/2015-12/2018. Demographic, anatomic, and physiologic characteristics were recorded. We examined associations between these characteristics and patient outcomes.

Results: There were 134 ICU admissions ages 18 to $79.71 \%(n=95)$ were admitted after elective cardiac surgery and $29 \%(n=39)$ were non-surgical, unplanned admissions. Factors associated with ICU Length of Stay (LOS) in the elective, surgical cohort are shown in the table. Right Ventricle (RV) dysfunction was associated with more ventilator days (median 2.0 vs $1.0, p=0.009$ ) and a higher rate of Acute Kidney Injury (AKI) after cardiac surgery ( $57 \%$ vs $19 \% ; p=0.001)$. NYHA class 3 or 4 vs 1 or 2 was associated with increased post-operative complications ( $56 \%$ vs $22 \% ; p=0.001$ ) and AKI ( $44 \%$ vs $17 \% ; p=0.004$ ). Post-operative arrhythmia was associated with increased ventilator days $(p=0.0002)$. For unplanned admissions, higher lactic acid was associated with AKI $(p=0.01)$, increased ICU LOS $(p=0.02)$ and ventilator days $(p=0.016)$.

\begin{tabular}{|l|l|l|l|}
\hline $\begin{array}{l}\text { Planned Surgical Cohort } \\
(\mathrm{n}=95)\end{array}$ & $\begin{array}{l}\text { Percentage } \\
(\mathrm{n}=\mathrm{number})\end{array}$ & $\begin{array}{l}\text { ICU LOS Median } \\
\text { days }\end{array}$ & $\begin{array}{l}\text { P } \\
\text { Value }\end{array}$ \\
\hline Physiology class 3,4 vs 1,2 & $\begin{array}{l}80(\mathrm{n}=76) \text { vs } \\
20(\mathrm{n}=19)\end{array}$ & 2.0 vs 1.0 & 0.03 \\
\hline NYHA 3,4 vs 1,2 & $\begin{array}{l}43(\mathrm{n}=41) \text { vs } \\
57(\mathrm{n}=54)\end{array}$ & 3.0 vs 1.0 & 0.01 \\
\hline Tobacco use vs none & $\begin{array}{l}33(\mathrm{n}=31) \text { vs } \\
67(\mathrm{n}=64)\end{array}$ & 3.0 vs 1.0 & 0.03 \\
\hline LV Dysfunction vs none & $\begin{array}{l}13(\mathrm{n}=12) \text { vs } \\
87(\mathrm{n}=83)\end{array}$ & 5.0 vs 1.0 & 0.01 \\
\hline RV Dysfunction vs none & $\begin{array}{l}24(\mathrm{n}=23) \text { vs } \\
76(\mathrm{n}=72)\end{array}$ & 5.0 vs 1.0 & 0.0001 \\
\hline Shock vs no shock & $\begin{array}{l}47(\mathrm{n}=45) \text { vs } \\
53(\mathrm{n}=50)\end{array}$ & 3.0 vs 1.0 & 0.0001 \\
\hline Redo sternotomy vs none & $\begin{array}{l}48(\mathrm{n}=46) \text { vs } \\
49(\mathrm{n}=47)\end{array}$ & 2.5 vs 1.0 & 0.04 \\
\hline prior & $\begin{array}{l}29(\mathrm{n}=28) \text { vs } \\
71(\mathrm{n}=67)\end{array}$ & 4.0 vs 1.0 & 0.003 \\
\hline Arrhythmia & & & 0.04 \\
\hline
\end{tabular}

Conclusion: Clinical characteristics of ACHD patients are associated with ICU morbidity and outcome. By better understanding these associations we may improve critical care management in the ACHD population.

\section{Reference(s) and grant acknowledgment(s)}

1. 1. Dearani JA, Connolly HM, Martinez R, Fontanet H, Webb GD. Caring for adults with congenital cardiac disease: successes and challenges for 2007 and beyond. Cardiol Young. 2007 Sep; 17(Suppl 2):8796

2. 3. J.I. Hoffman, S. Kaplan et al. The incidence of congenital heart disease. J Am Coll Cardiol, 39 (2002), pp. 1890-1900

3. 2. Dearani JA, Mavroudis C, Quintessenza JA, Deal BJ, Backer CL, Fitzgerald $\mathrm{P}$, et al. Surgical advances in the treatment of adults with congenital heart disease. CurrOpinPediatr. 2009 Sep 9
000071

Right ventricle dysfunction correlation with short term outcome in cardiogenic shock post ST elevation myocardial infarction H. Lashin ${ }^{1}$;OO. Olusanya ${ }^{1}$; S. Bhattacharyya ${ }^{2}$

${ }^{1}$ Adult Critical Care Unit, St Bartholomew's Hospital, London, United Kingdom; ${ }^{2}$ Cardiology department, St Bartholomew's Hospital, London, United Kingdom Correspondence: $\mathrm{H}$. Lashin

Intensive Care Medicine Experimental 2020, 8(2): 000071

Introduction: Right ventricular (RV) function is increasing recognised as a contributing factor to outcome in cardiogenic shock (CS) post ST elevation myocardial infarction (STEMI). This was previously demonstrated with regards to long term outcome1, but not short term.

Objectives: In this study we sought to determine the correlation between RV dysfunction and 28-day mortality in CS post STEMI with impaired left ventricle (LV).

Methods: Retrospective analysis of 100 patients admitted to intensive care unit (ICU) with CS post STEMI. Medical history, culprit lesion, clinical data, echocardiography data including multiple parameters of RV and LV. Clinical and Echocardiographic parameters were correlated with 28-days mortality.

Results: 100 patients admitted over 3 years period were included. Mean age $62.6 \pm 12.7 y, 78 \%$ were male, $38 \%$ suffered out of hospital cardiac arrest and 28 -day morality was $37 \%$. Non-survivors had no statistically significant difference in age, gender, medical history or culprit lesion. Non-survivors had significantly higher acute physiology and chronic health score II (APACHE II, 20 \pm 6 v 15 \pm 4 p .0001), creatinine (133 (IQR 114 - 210) v $103(96-150) \mathrm{mmol} / \mathrm{L}, \mathrm{p} .005)$, lactate $(5.2($ IQR $2.5-8)$ v $3(2.2$ $5.2) \mathrm{mmol} / \mathrm{Lp} \mathrm{p.03)}$ and lower mean arterial pressure $(50 \pm 1 \vee 55 \pm 7 \mathrm{mmHg}$, $\mathrm{p}$.02). Tricuspid annulus peak systolic velocity by tissue doppler (RV $\left.\mathrm{S}^{\prime}\right)$ was significantly lower in non-survivors $(10 \pm 3.5 \vee 12 \pm 3.3 \mathrm{~cm} / \mathrm{s}, \mathrm{p} .03)$. Otherwise all parameters were comparable. In multivariate analysis of significantly significant clinical and echocardiographic parameters, RV S was found to be an independent predictor of 28-days mortality. In univariate analysis RV $\mathrm{S}^{\prime}$ significantly predicted outcome (OR $1.2,95 \% \mathrm{Cl}$ $1.1-1.4$ p .04) (Figure 1A). RV S' of $10.5 \mathrm{~cm} / \mathrm{s}$ exhibited best sensitivity and specificity in receiver operator curve analysis $(64 \%$ and $65 \%$ respectively, area under the curve $0.6695 \% \mathrm{Cl} 0.52-0.80, \mathrm{p} .02$ ) for prediction of 28 -days outcome. Kaplan-Meier survival curves revealed $85 \%$ survival for RV $\mathrm{S}^{\prime}>10.5 \mathrm{~cm} / \mathrm{s}$ and $53 \%$ for RV $\mathrm{S}^{\prime}<10.5 \mathrm{~cm} / \mathrm{s}$ and the curves were significantly different (log rank p .01) (Figure 1B).

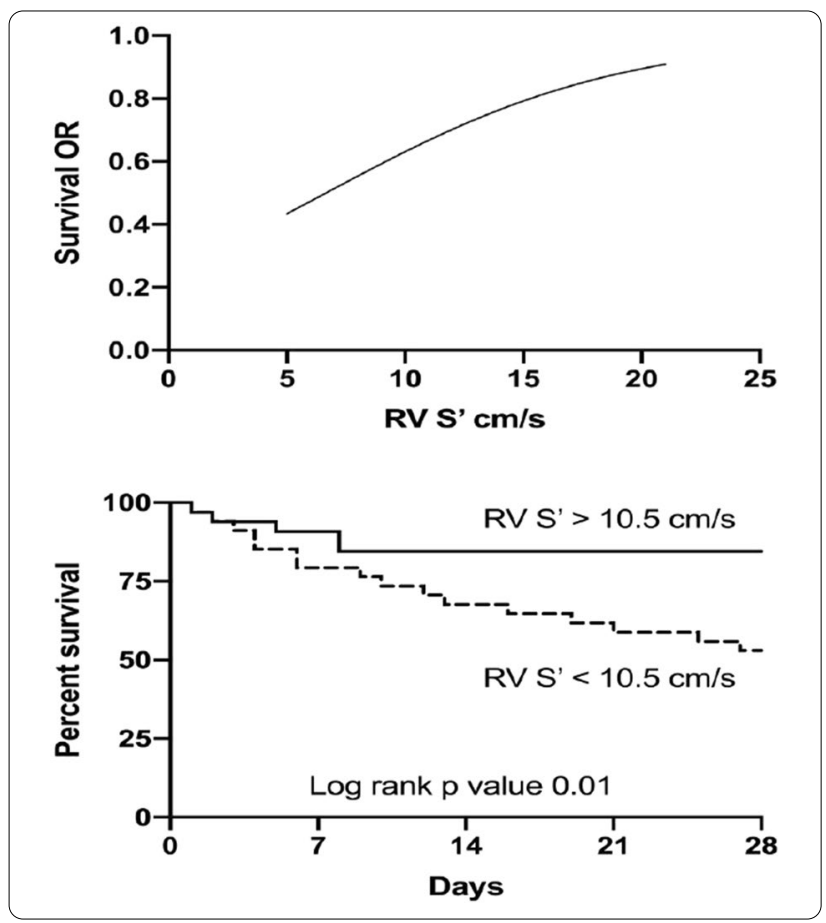


Figure 1. RV $\mathrm{S}^{\prime}$ prediction of survival. A. Univariate analysis of RV $\mathrm{S}^{\prime}$ association with 28-days outcome. Kaplan-Meier survival curves of 28 days mortality using RV S' cut-off value of $10.5 \mathrm{~cm} / \mathrm{s}$.

Conclusion: RV dysfunction influences short term outcome in patients admitted with CS post STEMI and impaired LV. RV S' was an independent predictor of 28-days mortality and a cut-off of $10.5 \mathrm{~cm} / \mathrm{s}$ was able to predict outcome with reasonable sensitivity and specificity.

\section{Reference(s) and grant acknowledgment(s)}

1. 1 Engström AE, Vis MM, Bouma BJ, van den Brink RBA, Baan J, Claessen $B E P M$, et al. Right ventricular dysfunction is an independent predictor for mortality in ST-elevation myocardial infarction patients presenting with cardiogenic shock on admission. Eur J Heart Fail 2010;12:276-82.

\section{0}

Efficacy of a novel angiotensin II receptor agonist (Giapreza) in septic shock

M. Krishna'; I. Pak'; M. Chaudry²; H. Rasool'; E. Lucks²; L. Stuczynski

${ }^{1}$ Director, department of pulmonary, critical care and sleep medicine, Orange Regional Medical Center, Middletown, United States of America; ${ }^{2}$ Pulmonary, critical care and sleep medicine, Orange Regional Medical Center, Middletown, United States of America

Correspondence: M. Krishna

Intensive Care Medicine Experimental 2020, 8(2): 000080

Introduction: Septic shock, a form of distributive shock, is characterized by decreased organ perfusion due to severe peripheral vasodilation in the setting of sepsis and dysregulated host inflammatory response to infection that is unresponsive to adequate fluid resuscitation. It is unclear what the ideal third line vasopressor is after norepinephrine and vasopressin.

Objectives: We conducted a retrospective cohort study, examining patients with septic shock who were not responsive to maximal doses of two vasopressors and required a third agent; we compared patients who received angiotensin II versus any other vasopressor as their third agent.

Methods: Patients included in the study were admitted to the ICU between July 2017 and March 2019. Eligible patients were at least 18-years-old, with catecholamine-resistant hypotension, defined as those who require a total sum catecholamine dose of $>0.2 \mu \mathrm{g} / \mathrm{kg} / \mathrm{min}$ to maintain MAP between 55 and $70 \mathrm{mmHg}$, typically a combination of norepinephrine and vasopressin. After screening patients who did not meet inclusion criteria and those with incomplete data points, a total of 50 patients were studied, 29 in the angiotensin II group, 21 in the control group. Primary endpoint was the change in mean arterial pressure- MAP. Secondary endpoints included total length of ICU stay. Results: In our 50 patients, there was a statistically significant increase in MAP at three hours in the angiotensin II group compared to the control group (16.3 $\mathrm{mmHg}$ vs. $4.3 \mathrm{mmHg} ; \mathrm{p}=0.0304$ ). Overall, calculated lactic acid clearance at 3 hours in the angiotensin II group was better than in the control group (13.39\% vs $-57.2 \%)$, but this was not statistically significant $(p=0.1338)$. There was no significant difference in ICU length of stay.

Conclusion: Initiation of an angiotensin II agonist as a third-line agent showed a statistically significant improvement in mean arterial pressire at 3 hours compared to a traditional third-line agent. Further prospective studies are required to evaluate clinical outcomes in the intensive care unit.

\section{Reference(s) and grant acknowledgment(s)}

1. Busse LW, Wang XS, Chalikonda DM, et al. Clinical experience with IV angiotensin II administration: a systematic review of safety. Crit Care Med. 2017 Aug;45(8):1285-94
2. Tumlin JA, Murugan R, Deane AM, et al. Outcomes in patients with vasodilatory shock and renal replacement therapy treated with intravenous angiotensin II. Crit Care Med. 2018 Aug;46(6):949-57

3. Khanna A, English SW, Wang XS, et al. Angiotensin II for the treatment of vasodilatory shock. N Engl J Med. 2017 Aug 33;377(5):419-30

\section{3}

Mechanical thrombectomy in acute pulmonary embolism: clinical and echocardiographic evolution

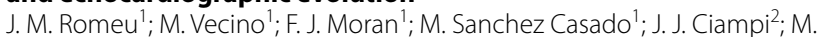

L. Rodriguez ${ }^{1}$; M. Buj ${ }^{1}$; M. Márquez ${ }^{1}$; M. J. Sánchez ${ }^{1}$; C. Lanciego ${ }^{2}$

${ }^{1}$ Intensive care unit, Virgin Health Hospital, Toledo, Spain; ${ }^{2}$ Interventional radiology, Virgin Health Hospital, Toledo, Spain

Correspondence: J.M. ROMEU

Intensive Care Medicine Experimental 2020, 8(2): 000083

Introduction: Pulmonary thromboembolism continues to be a high incidence pathology, with a high mortality rate, whose treatment depends on the risk of mortality at 30 days. In low and intermediate risk thromboembolisms the indicated treatment is anticoagulation, and in high risk thromboembolism fibrinolysis if it is not contraindicated. Patients with intermediate-high risk do not have a clearly defined treatment since fibrinolysis has shown an increase in the incidence of bleeding without an improvement in survival, with thrombectomy being a therapeutic possibility in this group of patients.

Objectives: Evaluate clinical and echocardiographic evolution in patients with acute intermediate-high and high risk pulmonary thromboembolism (PTE).

Methods: Patients collected between January 2016 and August 2019 with diagnosis of PTE of intermediate-high risk, defined by the guidelines of the European Society of Cardiology as positive markers of myocardial damage, right ventricular dysfunction measured by echocardiogram and stable hemodynamically; and high risk, hemodynamically unstable; who underwent mechanical thrombectomy in the first 24 hours of admission in ICU with aspiration of approximately 70 to $80 \%$ of the thrombus. Thrombectomy was performed in 42 patients, transthoracic echocardiogram (ETT) was performed at admission and 24 hours post-procedure, collecting parameters of right ventricle dysfunction (TAPSE, Wave S, right ventricle diameter [RV], pulmonary hypertension [HTP]); arterial blood gas at admission and after 24 hours to calculate $\mathrm{PaFi}$; and clinical assessment of the patient at admission and 24 hours after thrombectomy. The analysis of the data obtained comparing the situation before and after the procedure was carried out.

Results: Echocardiographically: significant improvement was obtained in the diameter of the RV $\left(46^{\prime} 34 \pm 4^{\prime} 71 \mathrm{~mm}\right.$ at $42^{\prime} 13 \pm$ $\left.6^{\prime} 37 \mathrm{~mm}, \mathrm{p}<0.05\right)$, TAPSE $\left(15^{\prime} 29 \pm 3^{\prime} 63 \mathrm{~mm}\right.$ at $18^{\prime} 49 \pm 3^{\prime} 98 \mathrm{~mm}, \mathrm{p}$ $\left.<0^{\prime} 05\right)$, S wave $\left(9^{\prime} 45 \pm 1^{\prime} 93 \mathrm{~cm} / \mathrm{sg}\right.$ at 11'9 $\left.\pm 3^{\prime} 26 \mathrm{~cm} / \mathrm{sg}, \mathrm{p}<0^{\prime} 05\right)$ and not significant of the HTP $\left(50^{\prime} 63 \pm 13^{\prime} 80 \mathrm{mmHg}\right.$ at $46^{\prime} 81 \pm$ $\left.19^{\prime} 14 \mathrm{mmHg}, \mathrm{p0} 256\right)$. Analytically: significant improvement of the PaFi $\left(186^{\prime} 87 \pm 127^{\prime} 9\right.$ to $\left.287^{\prime} 58 \pm 97^{\prime} 348, p<0.05\right)$. Clinically there was no significant improvement in chest pain, dyspnea or hemodynamic instability.

Conclusion: Mechanical thrombectomy in patients with acute pulmonary thromboembolism of intermediate-high and high risk is an effective technique that significantly improves the parameters of right ventricular dysfunction and the oxygenation of patients. There was no significant improvement in clinical aspects such as dyspnea, chest pain or hemodynamic stability.

\section{Reference(s) and grant acknowledgment(s)}

1. Chatterjee S, Chakraborty A, Weinberg I, et al. Thrombolysis for Pulmonary Embolism and Risk of All-Cause Mortality, Major Bleeding, and Intracranial Hemorrhage. A Meta-analysis JAMA. 2014; 311(23):2414-2421. https://doi. org/10.1001/jama.2014.5990 
2. Ciampi-Dopazo JJ, Romeu-Prieto JM, Sánchez-Casado M, et al. Aspiration thrombectomy for treatment of acute massive and submassive pulmonary embolism: Initial single-center prospective experience. J Vasc Interv Radiol 2018;29:101-106

3. Konstantinides SV, Meyer G, Becattini C. 2019 ESC Guidelines for the diagnosis and management of acute pulmonaryembolism developed in collaboration with the European Respiratory Society (ERS). European Heart Journal 2020; 41: 543-603

4. Keller K, Hobohm L, Ebner M, Kresoja KP, Munzel T, Konstantinides SV, Lankeit M. Trends in thrombolytic treatment and outcomes of acute pulmonary embolism in Germany. Eur Heart J 2020;41:522 529

5. Wendelboe AM, Raskob GE. Global burden of thrombosis: epidemiologic aspects. Circ Res 2016;118:1340-1347

\section{9}

Bilirubin-possible prognostic mortality marker in patients with VA-ECMO

S. Roth ${ }^{1}$; S. Bunte ${ }^{1}$; G. Lurati Buse ; H. Aubin²; R. Walz ; R. Huhn ${ }^{1}$

${ }^{1}$ Department of anesthesiology, University Hospital Duesseldorf, Duesseldorf, Germany; ${ }^{2}$ Department of cardiovascular surgery, University Hospital Duesseldorf, Duesseldorf, Germany

Correspondence: $S$. Roth

Intensive Care Medicine Experimental 2020, 8(2): 000149

Introduction: Veno-arterial membrane oxygenation (VA-ECMO) is a promising therapeutic option for patients with refractory cardiogenic shock or circulatory failure. However, as mortality rate of VA-ECMOtreated patients still remains high, there is a need for early outcome parameters reflecting on therapy success or futility.

Objectives: In the present study we investigated whether the liver enzyme levels in patients that survived the first four days of VA-ECMO therapy could serve as prognostic marker, hypothesizing that Bilirubin has the potential to be a mortality marker in this cohort.

Methods: The present study is a retrospective single center cohort study approved by the Ethics Committee of the Heinrich Heine University Duesseldorf (Reference Number 5141R). Adult patients over 18 years of age who received VA-ECMO therapy for refractory cardiogenic shock or circulatory failure between 2011 and 2018 were included. VA-ECMO was deployed either at the home center or by the local team on-site in external hospitals. Only patients who survived the first four days of VA-ECMO therapy were included into the analysis. For quantification of liver function Bilirubin was analysed at predefined time points (day $0,5,10,15$ ). The primary endpoint was all-cause in-hospital mortality. The association between Bilirubin and mortality was examined by receiver operating characteristic curve (ROC) and the area under the curve (AUC) as well as univariate and multivariable cox regression. In a sensitivity analysis, patients with shock-liver (defined as GOT > 10 x cutoff $(=35 \mathrm{U} / \mathrm{l})$ )) were excluded.

Results: A total of 438 patients received VA-ECMO therapy during the observation period. Based on the inclusion criteria, 298 patients (mean age $59 \pm 14$ years) remained for statistical analysis. Overall mortality rate was $42.6 \%(n=127)$. The AUC in the ROC for Bilirubin on day five was 0.72 [95\% confidence interval $(\mathrm{Cl})$ : 0.66-0.78]. Youden-Index showed a cutoff for Bilirubin on day five of $2.23 \mathrm{mg} / \mathrm{dl}$ with a sensitivity of 0.70 . Cox regression with multivariable adjustment revealed a significant association between Bilirubin on day five and mortality with a hazard ratio (HR) of 2.24 [95\% Cl: 1.53- 3.30]. After excluding patients with shock liver from the sensitivity analysis, this association was still significant (HR 2.08 [95\% Cl: 1.33-3.26]. ROC for patients without shock liver revealed an AUC of 0.67 [95\% confidence interval $(\mathrm{Cl})$ : 0.59-0.75].
Conclusion: Based on the results of the current study, an increase in serum Bilirubin on day 5 in patients receiving VA-ECMO therapy correlates independently with mortality regardless of the presence of shock liver. Thus, Bilirubin might serve as a prognostic marker in these patients and may be used as an indicator for early recognition of problems as well as optimization of VA-ECMO therapy.

000174

TIMI risk score: a valuable tool in the prognostic stratification of non-st-segment-elevation acute coronary syndrome

A. M. Garcia Bellon 1; A. M. González González; D. Gaitan-Roman 1 ; M. De Mora-Martin ${ }^{1}$

${ }^{1}$ Cardiology, Regional Hospital of Malaga, Málaga, Spain

Correspondence: A.M. González González

Intensive Care Medicine Experimental 2020, 8(2): 000174

Introduction: Prognostic stratification of acute coronary syndrome is of vital importance, determining the strategy in these patients.

Objectives: The aim of this study was to analyze the importance of TIMI Risk score (TRS) as a prognostic tool of non-ST-segment elevation acute coronary syndrome (NSTEACS).

Methods: Retrospective analysis of all patients consecutively admitted with NSTEACS, from January 2016 to December 2018. Clinical and epidemiological characteristics and prognostic variables were studied, completing follow-up for a median of 24 months.

Results: We included 824 patients, $33.9 \%$ women. In $76.4 \%$ of cases the acute coronary event corresponded to an unstable angina and the other $23.6 \%$ to a non-Q wave myocardial infarction. $65.3 \%$ of the patients had a TRS moderate to high risk (C3), corresponding to patients with higher comorbidity (Charlson Index $2.9 \pm 2.2$ vs. $1.4 \pm$ $0.6, p=0.0001$ ).

Highest scores in the TRS associated with greater development of heart failure $(3.6 \pm 1.2$ vs. $2.6 \pm 1.3, \mathrm{p}=0.0001)$ and mortality (3.9 \pm 1.1 vs. $2.7 \pm 1.3, p=0.0001)$ during in-hospital period. This relationship remained after completing the long-term follow-up (3.5 \pm 1.2 vs. $2.6 \pm 1.3, p=0.0001$ for heart failure and $3.6 \pm 0.9$ vs. $2.6 \pm 1.3, p=$ 0.0001 for mortality).

After adjustment, the TRS predicted higher risk of presenting complicated forms of acute

Coronary events both during in-hospital and long-term follow-up (OR 1.6, 95\% Cl, 1.3-2.0 and OR1.3, 95\% Cl, 1.1-1.4, respectively).

Conclusion: The TIMI Risk score is a valuable tool in the prognostic stratification of Patients admitted for non-ST-elevation acute coronary syndrome.

\section{7}

Predictors of mortality in the first year after ACS in patients admitted to the ICU of a regional hospital

I. Fernández ; R. Torcuato ; A. Alvarez; M. Salgado1; P. Rojas; A. Ubeda

${ }^{1}$ Intensive care unit, Hospital Point Europe, Algeciras, Spain

Correspondence: A. Ubeda

Intensive Care Medicine Experimental 2020, 8(2): 000197

Introduction: Acute coronary syndrome (ACS) continues to be one of the most frequent admission pathologies in an intensive care unit (ICU). There are some predictors and risk factors that could indicate a higher probability of mortality during the first year.

Objectives: To analyze factors associated with one-year mortality in patients with ACS admitted to the ICU.

Methods: Retrospective descriptive study conducted in a 12-bed ICU from January 2017 to September 2019. Demographic variables, 
comorbidities, type of ACS and location, left ventricular ejection fraction (LVEF), Killip, severity scales, responsible artery, complications and mortality. Statistical analysis: categorical (frequencies and percentages) and numerical variables (mean and standard deviation or median and interquartile range). Comparisons: X2 test (percentages) and Student test (means). Multivariate logistic regression. Statistical significance with $\mathrm{p}<0.05$.

Results: 278 patients were included. Deceased in the first year after ACS $56(20.14 \%)$ vs. 222 survivors (79.86\%): male (58.9\% vs. 80.2, $\mathrm{p}=.001)$, age $(73.88[ \pm 10.99], \mathrm{p}=.001)$, smoking $(21.4 \%$ vs. $43.7 \%$, $\mathrm{p}=.002)$, high blood pressure (64.3\% vs. $38.7 \%, \mathrm{p}=.001)$, diabetes mellitus (DM) $(51.8 \%$ vs. $33.8 \%, p=.013)$, previous coronary lesions $(30.4 \%$ vs. $16.2 \%, \mathrm{p}=.016)$, chronic heart failure $(26.8 \%$ vs. $3.2 \%, \mathrm{p}<.001)$, chronic kidney disease (CKD) $(19.6 \%$ vs. $3.6 \%$, $p<.001)$, COPD $(14.4 \%$ vs. $3.6 \%, p=.006)$, cerebrovascular disease $(14.3 \%$ vs. $4.1 \%, p=.009)$, heart rate $(97.32[ \pm 27.18]$ vs. $79.70[ \pm 17.19], p<.001)$, systolic blood pressure $(100.04[ \pm 25.49]$ vs. $119.54[ \pm 20.99], p<.001)$, TIMI $(5.91$ $[ \pm 3.42]$ vs. $2.84[ \pm 2.12], p<.001), \operatorname{GRACE}(216.73[ \pm 50.56]$ vs. 149.01 $[ \pm 38.53], p<.001)$, CRUSADE (59.17 [ \pm 15.79$]$ vs. $29.45[ \pm 17.0], p<.001)$, previous acute myocardial infarction $(51.8 \%$ vs. $29.3 \%) \mathrm{p}<.001$, LVEF (38.03 $[ \pm 15.48]$ vs. $52.84[ \pm 11.51], p<.001)$, norepinephrine usage (38.5\% vs. $1.8 \%, \mathrm{p}<.001)$, dobutamine usage $(25 \%$ vs. $1.4 \%, \mathrm{p}<.001)$, shock (61.1\% vs. $2.3 \%$. p <.001), hypotension ( $18.5 \%$ vs. $5.9 \%, p=.006)$, cardiac arrest $(18.5 \%$ vs. $4.1 \%, \mathrm{p}=.001)$, atrial fibrillation / flutter $(11.1 \%$ vs. $2.7 \%, p=.016)$, kidney complication $(9.3 \%$ vs. $2.3 \%, p=.028)$, invasive mechanical ventilation $(25.5 \%$ vs. $3.2 \%, p<.001)$. Multivariate analysis: age (OR 1.22, 95\% Cl [1.09-1.37], p<.001), Killip IV (OR 5871.59, $95 \% \mathrm{Cl}$ [1.06-32626571.9], $\mathrm{p}=.049$ ), shock (OR 251.56, Cl 95\% [10.026317.5], $\mathrm{p}=.001$ ), COPD (OR 12.5, 95\% Cl [1.10-141.72], $\mathrm{p}=.041$ ), CKD (OR 88.1, 95\% Cl [7.87- 987.8], $\mathrm{p}=.041$ ), LVEF (OR 0.92, 95\% Cl [0.880.97], $\mathrm{p}=.010$ ). AUROC: $0.978,95 \% \mathrm{Cl}$ [0.958-0.998].

Conclusion: Age, COPD, CKD, Killip IV on admission and shock during ICU stay behaved as independent predictors of mortality after 1 year from ACS. Higher LVEF and DM were associated with greater survival.

\section{9}

28-day mortality-related factors in patients with an acute coronary syndrome

I. Fernández'; R. Torcuato'; A. Alvarez'; M. Salgado ${ }^{1}$; P. Rojas ' ; A. Ubeda

'Intensive care unit, Hospital Point Europe, Algeciras, Spain

Correspondence: A. Ubeda

Intensive Care Medicine Experimental 2020, 8(2): 000199

Introduction: Acute coronary syndrome (ACS) is one of the main causes of death worldwide. The improvement in the control of cardiovascular risk factors, diagnosis and treatment, has increased the diseases' prevalence. Therefore, its mortality has diminished. However, the proportion of sudden death remains the same. Studying mortality related factors has proven crucial for the prevention of the disease.

Objectives: To analyze factors associated with 28-day mortality in patients with ACS admitted to the ICU.

Methods: A retrospective descriptive analysis on a prospective cohort, performed in a 12-bed ICU from january 2017 to september 2019. Variables: demographic, comorbidities, ACS type and location, left ventricular ejection fraction (LVEF), Killip, severity's scales, responsible artery, complications and mortality. Statistical analysis: categorical (frequencies and percentages) and numerical variables (mean and standard deviation). Comparisons: test of X2 (percentages), Student's t (means). Multivariate logistical regression. Statistical significance with $p<0.05$.

Results: 389 patients were included, 292 male $(75.1 \%)$ and 97 female (24.9\%). 28 day mortality: 46 (11.8\%). Deceased vs. survivors: male $(56.5 \%$ vs. $77.6 \%, p=.002)$, age $(73.30[ \pm 11.17]$ vs. $62.72[ \pm 12.16]$, $\mathrm{p}<.001)$, DM ( $56.5 \%$ vs. $32.9 \%, \mathrm{p}=.007)$, hypertension ( $63 \%$ vs. $43.4 \%$, $\mathrm{p}=.042)$, smoking ( $23.9 \%$ vs. $42.9 \%, \mathrm{p}=.044)$, previous coronary lesions ( $30.4 \%$ vs. $15.7 \%, p=.014)$, previous acute myocardial infarction $(26.1 \%$ vs. $12.8 \%, p=.016)$, COPD ( $15.2 \%$ vs. $4.1 \%, p=.007)$, chronic kidney disease $(15.2 \%$ vs. $5.5 \%, p=.023)$, chronic heart failure $(30.4 \%$ vs. $4.1 \%, \mathrm{p}<.001)$, NSTEMI ( $43.5 \%$ vs. $54.8 \%, \mathrm{p}=.148)$, systolic blood pressure $(94.67[ \pm 20.89]$ vs. $117.61[ \pm 20.97], p<.001), \operatorname{HR}(100.22[ \pm 26.29]$ vs. $78.30[ \pm 17.50], p<.001), \operatorname{LVEF}(32.62[ \pm 12.73]$ vs. $53.36[ \pm 11.49]$, $\mathrm{p}<.001)$, TIMI $(6.30[ \pm 3.55]$ vs. $3.07[ \pm 2.28], \mathrm{p}<.001)$, GRACE $(226.06$ $[ \pm 53.11]$ vs. $150.30[ \pm 38.84], p<.001), C R U S A D E(59.74[ \pm 17.56]$ vs. $29.58[ \pm 16.97], p<.001)$, dobutamine usage ( $31 \%$ vs. $1.7 \%, p<.001)$, noradrenaline usage $(45.2 \%$ vs. $2.3 \%, \mathrm{p}<.001)$. Invasive mechanical ventilation $(28.9 \%$ vs. $2.9 \%, p<.001)$, shock $(72.7 \%$ vs. $2.6 \%, p<.001)$, cardiac arrest $(20.5 \%$ vs. $4.4 \%, \mathrm{p}<.001)$, hypotension $(12.8 \%$ vs. $5.9 \%$, $\mathrm{p}=.008)$, kidney-related complications $(11.4 \%$ vs. $1.8 \%, \mathrm{p}=.004)$. Multivariate analysis: age (OR 1.18, 95\% Cl [1.04-1.32], $\mathrm{p}=.010)$, LVEF (OR $0.85,95 \% \mathrm{Cl}$ [0.77-0.93], $\mathrm{p}=.001)$, COPD (OR 7.66, 95\% Cl [1.01-57.84], $\mathrm{p}=.048$ ), Killip IV (OR 628.42, 95\% Cl [7.28-54261.48], $\mathrm{p}=.005)$, shock during ICU stay (OR $95.35,95 \% \mathrm{Cl}$ [6.85-1327.48], $\mathrm{p}=.001)$. AUROC: $0.989,95 \% \mathrm{Cl}$ [0.979-0.998].

Conclusion: Age, COPD, Killip IV on ICU admission and shock during the patient's ICU stay, behaved as independent 28-day mortality predictors after an ACS. LVEF was associated with higher survival rate.

\section{7}

In-hospital mortality and major complications in acute coronary syndrome patients over 75 years. Russian single center study findings

Y. Kalashnikova'; D. Pek'; N. Glushkov'; ${ }^{1}$ Y. Schneider ${ }^{1}$

${ }^{1} \mathrm{Icu}$, Kaliningrad Federal High Medical Technologies Center, Kaliningrad, Russia

Correspondence: J. Kalashnikova

Intensive Care Medicine Experimental 2020, 8(2): 000217

Introduction: Patients older than 75 years are often underrepresented in published acute coronary syndrome (ACS) studies, although they make up a significant and ever-increasing portion of this population. Mortality from ACS progressively increases with age and remains high among elderly and very elderly patients. It was shown that elderlies receive optimal treatment and myocardial revascularization less often than young patients [1], however, if revascularization is performed, hospital mortality decreases, 6-month survival improves, and the risk of stroke does not increase [2]. Currently, further studies are needed on the ACS course features in patients of older age groups to develop optimal treatment tactics for this category of patients in order to reduce in-hospital mortality and the incidence of long-term complications.

Objectives: To assess the clinical status, comorbidities, complications, in-hospital mortality and it structure in depend of age and acute coronary syndrome (ACS) type in case of early invasive strategy.

Methods: Retrospective all-over study was carried out in ICU of High Medical Technologies Center, Kaliningrad, Russia. 1353 patients with ACS and emergency and urgent myocardial revascularization in the 2014-2016 years period were included in the study. The first group (gr.1) consisted of 984 patients younger than 75 years old, and the second group (gr.2) was compound of 369 patients aged ${ }^{3} 75$. We were performed the analysis of anamnestic, clinical and laboratory data from medical documentation.

Results: The average age in the gr. 1 was $60 \pm 8,6$ years old, in the second $-80,1 \pm 4,2$. There was significantly myocardial infarction and stroke anamnesis burden, and reduced ejection fraction (EF), congestive heart failure, valve disorders, atrial fibrillation prevalence in very elderly patients. 
There was no significant difference in ACS structure in studied groups $(47,8 \%$ in gr. 1 vs $45,5 \%$ in gr. $2, p=0,512$ for ST elevation myocardial infarction). There were more frequent severe manifestations of acute heart failure (AHF) and cardiogenic shock cases (Killip III-5,9\% vs $11,4 \%(p=0,002)$; Killip IU $2,1 \%$ vs $9,1 \%(p<0,0001)$ in the first and second group respectively), higher score of GRACE ischemic risk and higher bleeding risk in very elderly patients in compare with persons younger than 75 years old. "First Medical Contact - balloon" time did not significantly differ in the studied groups $(86 \pm 19,1$ vs $94 \pm 21,3 \mathrm{~min}-$ utes, $\mathrm{p}=0,072$ for emergency $\mathrm{PCl}$ or $48,6(95 \% \mathrm{Cl} 24-136)$ vs $34(95 \% \mathrm{Cl}$ $25-45$ ) minutes, $p=0,084$ for urgent $\mathrm{PCl}$ in group 1 and group 2 . Allcases in-hospital mortality was $3,1 \%$ in the group 1 , and $10 \%$ in very elderly patients, $p<0,0001$. ST elevation myocardial infarction mortality was $5 \%$ among patients younger than 75 years old and it was $20 \%$ among very elderly patients, $p<0,0001$. Non-ST elevation myocardial infarction+unstable angina mortality didn't significantly differ in studied groups and composed $0,97 \%$ and $0,99 \%, p=0,52$. In-hospital mortality from AHF ( $2 \%$ in the group 1 vs $6,5 \%$ in the group $2, p<0,0001)$ and percutaneous coronary intervention complications (stent thrombosis and no-reflow) $(0,5 \%$ vs $2,4 \%, p=0,01)$ was significantly different depending on age. AHF mortality didn't differ among different age patients with preserved and mid-range $E F$, but it had significant difference in patients with reduced EF: $7,3 \%$ in the gr. 1 и $18,6 \%$ in the gr.2 $(p<0,0001)$. Studied group didn't differ from the rate of gastrointestinal bleedings and major bleedings $(0,7 \%$ in gr.1 vs $1,4 \%$ in gr.2, $\mathrm{p}=0,225$ ).

Conclusion: ACS has more severe clinical course in very elderly patients in comparison with persons younger than 75 years old. Early invasive strategy is effective in spite of age and ACS type. Fatal outcomes from percutaneous coronary intervention complications and AHF are more frequent in very elderly patients. ACS in-hospital mortality with the AHF as main reason is the prevalent mortality reason in very elderly patients with reduced EF. Otherwise, the structure of complications and mortality is similar in patients of different ages.

Reference(s) and grant acknowledgment(s)

1. [2] Vandermolen S, Abbott J, Silva K. What's Age Got to do with it? A Review of Contemporary Revascularization in the Elderly. Current Cardiology Reviews. 2015;11(3):199-208. https://doi.org/10.2174/1573403×1066614 1020110122

2. [1] Shanmugam VB, Harper R, Meredith I, Malaiapan Y, Psaltis PJ. An overview of $\mathrm{PCl}$ in the very elderly. Journal of geriatric cardiology: JGC 2015;12(2):174-84. https://doi.org/10.11909/j.issn.1671-5411.2015.02.012

\section{5}

Acute coronary syndrome in ICU: predictive factors for 90-day mortality

I. Fernández ${ }^{1}$; R. Torcuato ${ }^{1}$; A. Alvarez ${ }^{1}$; M. Salgado ${ }^{1}$; P. Rojas ${ }^{1}$; A. Ubeda ${ }^{1}$

${ }^{1}$ Intensive care unit, Hospital Point Europe, Algeciras, Spain

Correspondence: A. Ubeda

Intensive Care Medicine Experimental 2020, 8(2): 000225

Introduction: Coronary heart disease (CHD) is a leading cause of death. Accurate prediction of risk of death after acute coronary syndromes (ACS) is important for prognostication and decision making about treatment and management of these patients.

Objectives: To analyze predictive factors associated to 90-day mortality in patients admitted to intensive care unit (ICU) with an ACS.

Methods: Retrospective descriptive analysis of patients admitted in a 12-bed ICU performed from January 2017 to September 2019. Demographic variables, comorbidities, type of ACS, left ventricular ejection fraction (LVEF), initial Killip, extension, coronary arteries affected, risk scores (TIMI, GRACE, CRUSADE), complications and mortality have been registered.Statistical analysis: categorical (frequencies and percentages) and numerical variables (mean and standard deviation). Comparisons: test of X2 (percentages), Student's t (means). Multivariate logistic regression. Statistical significance with $p<0.05$.

Results: 380 patients were included, $287(75.52 \%)$ males and 93 (24.47\%) females. 90-day mortality: 51 (13.4\%). Non survivors vs. survivors: male $(56.9 \%$ vs. $78.4 \%, p=.001)$, age $(73.94[ \pm 10.94]$ vs. 62.44 $[ \pm 12.05], p<.001)$, diabetes mellitus $(54.9 \%$ vs. $32.5 \%, p=.008)$, high blood pressure $(64.7 \%$ vs. $43.2 \%, p=.016)$, smoking $(21.6 \%$ vs. $43.2 \%$, $\mathrm{p}=.012)$, previous coronary lesions $(29.4 \%$ vs. $15.8 \%, \mathrm{p}=.018)$, previous ACS ( $27.5 \%$ vs. $12.5 \%, p=.005)$, COPD ( $13.7 \%$ vs. $4.3 \%, p=.014)$, chronic kidney disease $(15.7 \%$ vs. $5.2 \%, p=.011)$, congestive heart failure $(27.5 \%$ vs $4.3 \%, p>.001)$, STEMI $(39.2 \%$ vs. $55.6 \%, p=.029)$, systolic blood pressure (97.63 $[ \pm 24.13]$ vs. $117.75[ \pm 20.63], p<.001)$, heart rate $(100.18[ \pm 25.68]$ vs. $78.06[ \pm 17.15], \mathrm{p}<.001), \operatorname{LVEF}(34.84[ \pm 13.47]$ vs. $53.46[ \pm 11.42], p<.001)$, TIMI $(5.91[ \pm 3.43]$ vs. $3.04[ \pm 2.27], p<.001)$, GRACE $(220.86[ \pm 48.89]$ vs. $149.09[ \pm 38.08], \mathrm{p}<.001)$, CRUSADE $(59.81$ [ \pm 16.75$]$ vs. $29.10[ \pm 16.80], p<.001)$, number of implanted stents $(2.48$ $[ \pm 1.25]$ vs. $1.91[ \pm 1.25], p=.045)$, dobutamine usage $(27.7 \%$ vs. $1.8 \%$, $\mathrm{p}<.001)$, norepinephrine usage $(42.6 \%$ vs. $2.1 \%, \mathrm{p}<.001)$. Invasive mechanical ventilation $(28 \%$ vs. $2.5 \%, p<.001)$, shock $(67.3 \%$ vs. $2.4 \%$, $\mathrm{p}<.001)$, cardiac arrest $(20.4 \%$ vs. $4.3 \%, \mathrm{p}<.001)$, infectious complication $(6.1 \%$ vs. $1.2 \%, p=.05)$, kidney complication $(10.2 \%$ vs. $1.8 \%$, $\mathrm{p}=.008)$. ICU length of stay (LOS) $(4.65[ \pm 7.05]$ vs. $2.43[ \pm 3.32]$, $\mathrm{p}=.031$ ).

Multivariate analysis: age (OR 1.14 IC $95 \%$ [1.05-1.24], $\mathrm{p}=.001$ ), LVEF (OR 0.91, IC95\% [0.86-0.96], $\mathrm{p}=.002$ ), Killip III (OR 80.40, IC 95\% [2.45-2633.07], $\mathrm{p}=.014$ ), shock (OR 27.65, IC95\% [4.34-175.95], $\mathrm{p}=.004)$. AUROC: 0.976 , IC 95\% [0.958-0.993].

Conclusion: Age, Killip III at ICU admission and shock during ICU stay were found as independent predictive factors for 90-day-mortality in patients diagnosed of ACS. LVEF was an independent predictor of survival.

\section{4}

Acute pulmonary thromboembolism treated by mechanical thrombectomy: clinical, radiological, echocardiographic and analytical differences between massive and submassive thromboembolism

J. M. Romeu' ; F. J. Moran²; M. Vecino²; M. Sanchez Casado²; J. J. Ciampi i ; M. L. Rodriguez ; M. Márquez ${ }^{2}$; M. Buj ${ }^{2}$; MJ. Sánchez ${ }^{2}$; C. Lanciego ${ }^{3}$

${ }^{1}$ INTENSIVE CARE UNIT, HOSPITAL VIRGEN DE LA SALUD, TOLEDO, Spain; ${ }^{2}$ Intensive care unit, Virgin Health Hospital, Toledo, Spain; ${ }^{3}$ nterventional radiology, Virgin Health Hospital, Toledo, Spain

Correspondence: J.M. ROMEU

Intensive Care Medicine Experimental 2020, 8(2): 000244

Introduction: Pulmonary thromboembolism it's still a high incidence pathology with a high mortality risk. Depending on the hemodynamic situation of the patient, it is classified as massive, if the patient is hemodynamically unstable; or submassive if the patient does not have hemodynamic instability. But do the clinical, ecocardiographic and blood tests improve depending on the type of pulmonary thromboembolism?

Objectives: Analyze the clinical, echocardiographic and analytical differences between patients with acute massive (hemodynamically unstable) and submassive (hemodynamically stable) pulmonary thromboembolism (APE).

Methods: Recruited patients between January 2016 and August 2019 with a diagnosis of submassive pulmonary embolism (hemodynamically stable); and massive pulmonary embolism (hemodynamically unstable); who underwent mechanical thrombectomy in the first 24 hours of admission with aspiration of between 70 and $80 \%$ of the 
total thrombus. 42 thrombectomies were collected, performing transthoracic echocardiogram (TTE) at admission and at 24 hours postprocedure analyzing parameters of right ventricle dysfunction (TAPSE, Wave S, right ventricle diameter [RV], pulmonary hypertension [HTP]); arterial blood gas at admission and at 24 hours to calculate PaFi; and clinical assessment of the patient at admission and at 24 hours. We compare between patients with massive and submassive pulmonary thromboembolism.

Results: A significant relationship was found between diabetes mellitus and massive thromboembolism ( $30^{\prime} 8 \%$ patients, $p<0.05$ ); syncope being the significant manifestation among patients with massive PE $(57.7 \%, p<0.05)$. Radiologically, the involvement of the main pulmonary arteries was significantly associated with massive PE $(100 \%, p$ $<0.05$ ). Echocardiographically no significant differences were found in the parameters of right ventricular dysfunction (diameter, TAPSE, S wave or pulmonary hypertension) between massive or submassive PE. The echocardiographic improvement by TAPSE was significant among patients with submassive $P E$, improving the figures from $15^{\prime} 83 \pm 2^{\prime} 23$ to $20^{\prime} 17 \pm 2^{\prime} 85(\mathrm{p}<0.05) 24$ hours after procedure.

Conclusion: Massive pulmonary embolism is significantly related to DM, syncope and involvement of major pulmonary arteries. Echocardiographic improvement of the right ventricle is significantly earlier in submassive PET after mechanical thrombectomy.

\section{Reference(s) and grant acknowledgment(s)}

1. Raskob GE, Angchaisuksiri P, Blanco AN, Buller H, Gallus A, Hunt BJ, Hylek EM, Kakkar A, Konstantinides SV, McCumber M, Ozaki Y, Wendelboe A, Weitz Jl. Thrombosis: a major contributor to global disease burden. Arterioscler Thromb Vasc Biol 2014;34:2363_2371.

2. Lario S, Bello C, Figueredo AL, Guirola JA. Massive and submassive pulmonary embolism treated by thromboaspiration (INDIGO) and low-dose fibrinolysis. Single center experience. Intervencionismo 2017;17(4):111-9

3. Dutta, Tanya MD; Frishman, William H. MD; Aronow, Wilbert S. MD Echocardiography in the Evaluation of Pulmonary Embolism. Cardiology in Review. 25(6):309-314, November/December 2017.

4. Pieraccini M, Guerrini S, Laiolo E. Acute massive and submassive pulmonary embolism: preliminary validation of aspiration mechanical thrombectomy in patients with contraindications to thrombolysis. Cardiovasc Intervent Radiol 2018; 41:1840-1848

5. Tu T, Toma C, Tapson V, et al. A prospective, sincle-arm, multicenter trial catheter-directed mechanical thrombectomy for intermediate-risk acute pulmonary embolism. The FLARE study. J Am Coll Cardiol Intv 2019; 12(9):859-69.

\section{3}

Tricuspid valve replacement: mechanical or biological prostheses? A Systematic review and Meta-Analysis

Z. Cheng ${ }^{1}$

${ }^{1}$ cardiovascular surgery, West china hospital Sichuan University, Chengdu, China

Correspondence: $Z$. Cheng

Intensive Care Medicine Experimental 2020, 8(2): 000343

Introduction: Tricuspid valve replacement (TVR) is seldom performed in cardiac valve surgery, and there are currently no clinical guidelines suggest which type of prostheses is better in tricuspid valve position. This meta-analysis was performed to compare the results of mechanical and biological prostheses for TVR.

Methods: We searched Pubmed, Cochrane, Embase, Clinical trial databases to collect all the related studies published during recent 10 years (from January 1, 2000 to October 31, 2019). A random-effects model was used to evaluate the OR (odds ratios) and its $95 \% \mathrm{Cl}$ (confidence intervals) of time-to-event related effects of the surgical procedures, every study's quality evaluated by the Newcastle-Ottawa Scale (NOS).
Results: A total of 13 retrospective studies including 1407 patients were analyzed. There were no statistically differences between mechanical and biological prostheses with respect to prosthetic valve failure $[O R=1.35,95 \% \mathrm{Cl}(0.21,2.96), \mathrm{P}=0.46]$, bleeding $[\mathrm{OR}=0.68$, $95 \% \mathrm{Cl}(0.33,1.40), \mathrm{P}=0.29]$, reoperation[ $\mathrm{OR}=1.00,95 \% \mathrm{Cl}(0.59,1.72)$, $\mathrm{P}=0.99]$, early mortality $[\mathrm{OR}=1.13,95 \% \mathrm{Cl}(0.58,2.18), \mathrm{P}=0.73]$ and long-time survival $[\mathrm{OR}=1.06,95 \% \mathrm{Cl}(0.68,1.66), \mathrm{P}=0.80]$. $\mathrm{A}$ trend toward greater risk of thrombosis can be seen in mechanical prostheses $[\mathrm{OR}=0.19,95 \% \mathrm{Cl}(0.06,0.65), \mathrm{P}=0.008, \mathrm{I}=0 \%]$.

Conclusion: In tricuspid valve position, mechanical valve prostheses has a higher risk of thrombosis than biological prostheses, but no statistically differences between mechanical and biological prostheses with respect to prosthetic valve failure, bleeding, reoperation, early mortality and long-time survival. The more specific conclusion need to be further proved by large-sample, multi-center, randomized, doubleblind and control trials.

\section{5}

\section{Vasopressin in patients with septic shock and dynamic left} ventricular outflow tract obstruction

M. Balik'; A. Novotny'; D. Suk'; V. Matousek'; M. Maly'; T. Brozek;

G. Tavazzi ${ }^{2}$

${ }^{1}$ Dept of anaesthesia and intensive care, General University Hospital in Prague, Prague, Czech Republic; ${ }^{2}$ Department of anesthesia and intensive care, Fondazione IRCCS Policlinico S Matteo, Pavia, Italy

Correspondence: $M$. Balik

Intensive Care Medicine Experimental 2020, 8(2): 000345

Introduction: Left ventricular outflow tract obstruction (LVOTO) is a relatively uncommon but severe condition that may lead to hemodynamic impairment. It can be elicited by morphological (left ventricular hypertrophy, sigmoid septum, prominent papillary muscle, prolonged anterior mitral valve leaflet) and functional (hypovolemia, low afterload, hypercontractility, catecholamines) factors.

Objectives: We sought to determine the incidence of the most severe form of LVOTO in septic shock patients and describe the therapeutic effects of vasopressin.

Methods: 527 patients in septic shock were screened for LVOTO over a period of 29 months. All were mechanically ventilated and treated according to sepsis bundles, including pre-load optimization and norepinephrine infusion. Vasopressin was added in addition to norepinephrine to reduce the adrenergic burden and decrease LVOTO.

Results: 10 patients were diagnosed with the most severe form of LVOTO including SAM (systolic anterior mitral valve motion) and severe MR (mitral regurgitation) with pulmonary oedema. The median norepinephrine dosage to obtain a mean arterial pressure of $>/=70 \mathrm{mmHg}$ was $0.58 \mathrm{mcg} / \mathrm{Kg} / \mathrm{min}$ (IQR 0.40-0.78). All patients had a hyper-contractile left ventricle, septal hypertrophy, significant LVOTO (peak gradient 78 [56-123] $\mathrm{mmHg}$ ) associated with SAM and severe MR with pulmonary oedema. Vasopressin (median $4 \mathrm{IU} / \mathrm{h}$ ) allowed a significant reduction of norepinephrine $(0.18[0.14-0.30] \mathrm{mcg} / \mathrm{kg} / \mathrm{min}$; $\mathrm{p}=0.01$ ), LVOT gradient (35 [24-60] $\mathrm{mmHg} ; \mathrm{p}=0.01$ ) and MR with a significant paO2/FiO2 increase (174 [125-213] mmHg; $\mathrm{p}=0.01)$.

Conclusion: Vasopressin allowed a reduction of norepinephrine with subsequent LVOTO reduction and hemodynamic improvement of the most severe form of LVOTO which represented $1.9 \%$ of all septic shock patients.

\section{Reference(s) and grant acknowledgment(s)}

1. 1. Martin Balik, Adam Novotny, Daniel Suk, Vojtech Matousek, Michal Maly, Tomas Brozek, Guido Tavazzi: Vasopressin in patients with septic shock and dynamic left ventricular outflow tract obstruction. Cardiovasc Drugs Ther 2020, accepted, in press 
2. Supported from the grant project NV 18-06-00417 of the Czech Ministry of Health.

\section{1}

Antithrombotic therapy in cardiogenic shock: Could glycoprotein Ilb/Illa inhibitors be useful?

H. Miranda ${ }^{1}$; H. Santos ${ }^{1}$; S. Mariana ${ }^{2}$; C. Sousa ${ }^{1}$ I. Almeida ${ }^{1}$; L. Almeida ${ }^{1}$

${ }^{1}$ Cardiology, Centro Hospitalar Barreiro Montijo, Barreiro, Portugal; ${ }^{2}$ Cardi-

ology, Centro Hospitalar Barreiro Montijo, E.P.E, Barreiro, Portugal

Correspondence: $\mathrm{H}$. Miranda

Intensive Care Medicine Experimental 2020, 8(2): 000381

Introduction: Impaired enteral perfusion and mechanical ventilation, with inability to swallow, play a major role in the bioavailability of antiplatelets. Data on the use of glycoprotein Ilb/Illa receptor inhibitors (GPI) in acute coronary syndrome patients presenting with cardiogenic shock is sparse.

Objectives: Evaluate the impact of the use of GPI on the outcome (MACE/death) of patients (P) admitted with CS

Methods: Retrospective study from $01 / 10 / 2010$ to $08 / 01 / 2019$. All $P$ admitted for CS in the context of ACS were selected. Exclusion criteria: Killip class I-III, lack of information about APT during hospital length. We created 2 groups based in the use/absence of GPI during hospital length.

Results: 329 P were admitted, hypertension and dyslipidaemia were the most frequent comorbidities. Mean Systolic and diastolic pressure at admission of $96 \pm 33$ and $59 \pm 21 \mathrm{mmHg}$, respectively. Haemoglobin at admission of $13,1 \pm 2 \mathrm{~g} / \mathrm{dl}$. platelets on admission of $221 \pm 89 \times 103$ / $\mathrm{mm} 3$.

Comparing the 2 groups we found out GPI was preferred in younger $P(p<0,001)$, with higher levels of haemoglobin and platelets $(p=0,04$ and P00,046, respectively) and was associated with higher use of intraaortic balloon $(p=0,003)$. Also, $100 \%$ of patients with GPI performed coronary angiography.

Comparing the occurrence of MACE/death during hospital length we only found differences at cardiac arrest (higher in the group with GPI, $p=0,003)$. There weren't any differences regarding other MACE and death between the 2 groups. Furthermore, we haven't seen any differences regarding bleedings complications in patients using GPI.

Conclusion: With a lack of specific randomized trials in CS the same recommendations apply as for other types of acute coronary syndrome. Our study suggests that GPI may be beneficial in this high-risk group of patients in whom a delayed onset of action of oral antiplatelet therapy would be expected. More selected trials are needed to clarify which is the best pharmacological option for these patients.

\section{4}

\section{Medical Doctor}

M. Aitavaara-Anttila ; H. Pernu ${ }^{2}$; T. Rumpunen ${ }^{3}$; J. Similä4 ; J. Liisanantti ${ }^{5}$;

T. Kaakinen ${ }^{6}$; L. Raatiniemi ${ }^{7}$

${ }^{1}$ Medical research center, research group of surgery, anesthesiology and intensive care, University of Oulu, Oulu, Finland; ${ }^{2}$ Emergency medical services, Jokilaaksojen pelastuslaitos, Ylivieskan paloasema, Ylivieska, Finland; ${ }^{3}$ Emergency medical services, Oulu-Koillismaan pelastuslaitos, Oulu, Finland: ${ }^{4}$ Centre for pre-hospital emergency care, Pohjois-Pohjanmaan sairaanhoitopiirin kuntayhtymä, Oulu, Finland; ${ }^{5}$ Study group of surgery, anesthesiology and intensive care, Oulu University, Medical Research Center, Oulu, Finland, Finland; ${ }^{6}$ Department of anesthesiology, University of Oulu, Oulu, Finland; ${ }^{7}$ Centre for pre-hospital emergency care, Oulu University Hospital, Oulu, Finland

Correspondence: M. Aitavaara-Anttila

Intensive Care Medicine Experimental 2020, 8(2): 000424

Introduction: Factors associated with time delay to angiography in acute st-elevation myocardial infarction - a retrospective cohort study in Northern Finland

Objectives: Chest pain is a common mission (16.4\%) for emergency medical service (EMS) but only in $10,5 \%$ there is a acute myocardial infarction (AMI) behind the symptom. (1) Delay in percutaneous coronary interversion $(\mathrm{PCl})$ with $\mathrm{AMI}$ is associated with increased heart failure and mortality (2). Aim of this study was to examine time intervals of EMS and factors associated

Methods: Study was conducted in hospital district of Northern Ostrobothnia, Finland between 2014- 2016. Study included all the patients diagnosed with STEMI that underwent angiography within 24 hours from arrival to hospital and had been transported to hospital by EMS. We defined angiography delayed if it was performed over 120 minutes from first medical contact.

Results: 330 patients met the inclusion criteria in the study period. Time from first medical contact to angiography (TFMCA) was <120 minutes with 231 patients (70.0\%). In multivariant analysis, OR for TFMCA over 120 minutes was higher if there was absence of chest pain (OR $2.4695 \% \mathrm{Cl}(1.18-5.13), \mathrm{P}=0.016)$, dyspnea (OR $3.1195 \% \mathrm{Cl}$ (1.54-6.28), $\mathrm{P}=0.002)$, treatment protocol not followed (OR $2.4195 \% \mathrm{Cl}$ $(0.99-5.80), P=0.050)$, treatment started at health care center (OR 3.64 $95 \% \mathrm{Cl}(1.39-9.48), \mathrm{P}=0.008)$ and distance to hospital over $100 \mathrm{~km}$ (OR $11.8795 \% \mathrm{Cl}(6.14-22.93), \mathrm{P}<0.001)$.

Conclusion: We demonstrated patient and ems related factors, that were associated with TFMCA time over 120 minutes.

\section{Reference(s) and grant acknowledgment(s)}

1. 3) Nallamothu BK et al. Relation between door-to-balloon times and mortality after primary percutaneous coronary intervention over time: a retrospective study. Lancet.2015 Mar 21;385(9973):1114-22.https://doi.org/10.1016/ s0140-6736(14)61932-2.epub 2014 Nov 19

2. 2) Zurowska-Wolak M et al. The effects of prehospital system delays on the treatment efficacy of STEMI patients. Scand J Trauma Resusc Emerg Med.2019 Apr 8;27(1):39.https://doi.org/10.1186/s13049-019-0616-4.

3. 1) Pedersen et al. Chest pain in ambulance; prevalence, causes and outcome - a retrospective cohort study. Scand J Trauma Resusc Emerg Med.2019 Aug 29;27(1):84.https://doi.org/10.1186/s13049-019-0659-6.

4. Grant application for Finnish Medical Foundation has been made.

\section{6}

GRACE score vs ACTIVE ICU score: predicting MACE and intra-hospital death in NSTEMI

M. Santos ${ }^{1}$; H. Miranda ${ }^{1}$; I. Almeida ${ }^{1} ;$ H. Santos ${ }^{1}$; C. Sá ${ }^{1}$; J. Chin ${ }^{1}$;

S. Almeida'; C. Sousa ${ }^{1}$; J. Tavares ${ }^{1}$; L. Almeida

${ }^{1}$ Cardiology, Centro Hospitalar Barreiro Montijo, E.P.E, Barreiro, Portugal

Correspondence: $M$. Santos

Intensive Care Medicine Experimental 2020, 8(2): 000466

Introduction: Regarding prognosis, patients with a non-ST elevation myocardial infarction (NSTEMI) are a heterogeneous population. Therefore, early risk stratification at admission is essential. GRACE score estimates risk of death, including in-hospital death. ACTION ICU score predicts the risk of NSTEMI complications requiring ICU care.

Objectives: To evaluate and compare GRACE and ACTION ICU scores as predictors of in-hospital death (IHD) and in-hospital major adverse cardiovascular events (IHMACE) in our center population using reallife data.

Methods: Based on a single-center retrospective study, data collected from admissions between 1/01/2016 and 11/12/2019. Patients with cardiac arrest or hemodynamically unstable patients at admission were excluded. GRACE and ACTION ICU scores were calculated, using Killip-Kimball classification as a surrogate of heart failure at admission. Logistic regression analysis and ROC curve analysis were performed to assess their performance as predictors of IHD and IHMACE.

Results: We identified 389 patients with NSTEMI, 63.5\% were males with an average age of $68.7 \pm 12.6$ years old. Regarding past medical history, $75.1 \%$ were hypertensive, $38.8 \%$ had diabetes, $54.8 \%$ had dyslipidaemia, $6.2 \%$ had chronic kidney disease, $3.1 \%$ had chronic obstructive pulmonary disease, $29.0 \%$ had a previous acute coronary syndrome (ACS) and $28.5 \%$ had been submitted to coronary revascularization.

Mean GRACE score was $132.4 \pm 1.8$ and median ACTIVE ICU score was $4 \pm 4$. Median heart rate was $77 \pm 23 \mathrm{bpm}$, median arterial pressure was $146 \pm 38 \mathrm{mmHg}, 88.7 \%$ had Killip-Kimball classification II-IV, 25.7\% had ST-depression at admission. Logistic regression analysis confirmed that GRACE score was a good predictor of IHMACE $(p<0.001)$ and 
IHD $(p=0.005)$ in our population. ACTIVE ICU score was also a predictor of both IHMACE $(p<0.001)$ and IHD $(p=0.005)$. ROC curve analysis revealed that GRACE and ACTIVE ICU scores are accurate at predicting risk of IHMACE (area under curve (AUC) 0.726 and 0.737 , respectively) and IHD (AUC 0.886 and 0.868 , respectively).

Conclusion: This study confirms that, in our population, both scores are accurate at predicting the outcomes. As expected, GRACE score was slightly more accurate at predicting IHD while ACTIVE ICU score was more accurate at predicting IHMACE.

\section{1}

Does the rhythm at admission influence times to medical care in acute coronary syndrome?

M. Santos ${ }^{1}$; H. Miranda $:$ H. Santos ${ }^{1}$ I. Almeida ${ }^{1}$; C. Sá ${ }^{1}$ J. Chin ${ }^{1}$.

S. Almeida'; C. Sousa ${ }^{1}$; J. Tavares ${ }^{1}$; L. Almeida

${ }^{1}$ Cardiology, Centro Hospitalar Barreiro Montijo, E.P.E, Barreiro, Portugal

Correspondence: $M$. Santos

Intensive Care Medicine Experimental 2020, 8(2): 000471

Introduction: Acute coronary syndrome (ACS) and atrial fibrillation (AF) are frequent disorders and it is not uncommon for AF to be present at admission of ACS. However, the presence of an arrhythmia can make the diagnosis of ACS more challenging and, therefore, it may increase in pre-hospital and admission delay in diagnosis.

Objectives: To evaluate if the presence of AF or other rhythms at admission had influence in the period from symptom to first medical care, time from first medical care and admission and overall time from symptoms to admission.

Methods: Based on a multicenter retrospective study, data collected from admission between $1 / 10 / 2010$ and $8 / 01 / 2019$. Patients were divided into 3 groups: A - sinus rhythm, B - AF, and C - other rhythms at admission. Patients without data on previous cardiovascular history or uncompleted clinical data were excluded. Chi-square test, T-student test and Mann-Whitney $\mathrm{U}$ test were used in the analysis.

Results: 15927 patients were included, 14637 in group A (91.9\%), 1049 in group B (6.6\%) and 241 in group C (1.5\%).

Group B (GB) patients were older ( $65 \pm 13$ vs $75 \pm 10$ vs $73 \pm 13, p<0.001$ ) and had higher rates of comorbidities such as arterial hypertension (67.1 vs 83.2 vs $79.2 \%, p<0.001$ ), diabetes ( 30.4 vs 36.0 vs $36.9 \%$, $\mathrm{p}<0.001)$, previous history of stroke (6.7 vs 14.9 vs $11.7 \%, p<0.001)$, peripheral artery disease ( 4.8 vs 8.9 vs $4.6 \%, p<0.001)$, chronic kidney disease (4.7 vs 10.5 vs $10.4 \%, p<0.001)$ and dementia ( 1.5 vs 4.2 vs $3.1 \%, \mathrm{p}<0.001$ ) but fewer smokers ( 31.0 vs 8.7 vs $20.2 \%, p<0.001$ ).

At presentation GB had lower rates of STEMI ( 42.6 vs 33.6 vs $50.6 \%$, $\mathrm{p}<0.001$ ), were more frequently admitted by the emergency room $(53.8 \%$ vs $62.6 \%$ vs $61.3 \%, p<0.001)$ and had higher heart rates at admission ( $77 \pm 18$ vs $91 \pm 29$ vs $68 \pm 30, p<0.001)$.

The three groups were similar regarding times from symptoms to first medical care, times from first medical care and admission and overall times from symptoms to admission, rates of multivessel disease and culprit lesion in ST-segment elevation myocardial infarction (STEMI). Regarding in-hospital adverse events, group $B$ and group $C$ had higher rates of de novo heart failure ( 13.0 vs 29.7 vs $26.6 \%, p<0.001$ ), sustained ventricular tachycardia ( 1.5 vs 3.1 vs $3.7 \%, p<0.001)$, cardiac arrest $(2.8$ vs 4.9 vs $7.1 \%, p<0.001)$, stroke ( 0.6 vs 1.7 vs $0.4 \%, p<0.001)$ and death ( 2.7 vs 9.0 vs $10.4 \%, \mathrm{p}<0.001$ ).

Conclusion: The rhythm at admission does not seem to have an effect in the time until medical care. Nevertheless, it was associated to worse prognosis and complications.

\section{2}

Acute coronary syndrome and atrial fibrillation onset

M. Santos ${ }^{1}$; H. Santos ; I. Almeida ; H. Miranda ${ }^{1}$; C. Sá1 ; J. Chin ${ }^{1}$; S. Almeida'; C. Sousa ${ }^{1}$; J. Tavares ${ }^{1}$; L. Almeida

${ }^{1}$ Cardiology, Centro Hospitalar Barreiro Montijo, E.P.E, Barreiro, Portugal Correspondence: M. Santos

Intensive Care Medicine Experimental 2020, 8(2): 000472

Introduction: Atrial Fibrillation (AF) complicates approximately $10 \%$ of acute coronary syndromes (ACS) and it is, therefore, important to access its impact on prognosis and identify patients with higher risk of AF.

Objectives: To evaluate predictors of early onset $(<48 \mathrm{~h})$ and late onset ( $\geq 48 \mathrm{~h}$ ) de novo atrial fibrillation (AF).

Methods: Based on a multicenter retrospective study, data collected from admissions between $1 / 10 / 2010$ and $8 / 01 / 2019$. Patients were divided in two groups: A - patients that presented early onset de novo $\mathrm{AF}$ (EOAF), and $\mathrm{B}$ - patients that presented late onset de novo AF (LOAF). Patients without data on previous cardiovascular history or uncompleted clinical data were excluded. Logistic regression was performed to assess predictors of de novo AF in ACS. Survival analysis was evaluated through Kaplan Meier curve.

Results: Population - 257272 patients (pts) with ACS. Group A (GA) 584 pts (2.3\%); group B (GB) - 360 pts (1.4\%).

GA were younger ( $73 \pm 13$ vs $77 \pm 10, p<0.001)$ with a higher prevalence of smokers ( $21.3 \%$ vs $12.1 \%, p<0.001)$. GB had higher rates of diabetes mellitus $(40.1 \%$ vs $30.2 \%, p<0.001)$, angina $(30.8 \%$ vs $21.4 \%, p<0.001)$ previous ACS ( $22.5 \%$ vs $15.4 \%, p=0.006)$, previous revascularization (percutaneous coronary intervention $14 \%$ vs $9.5 \%, p=0.032$; coronary artery bypass surgery $8.4 \%$ vs $3.9 \%, \mathrm{p}=0.004)$.

GA had more chest pain $(76.1 \%$ vs $67.3 \%, p=0.001)$, more ST-segment elevation myocardial infarction $(56.8 \%$ vs $46.9 \%, \mathrm{p}=0.003)$ and were admitted directly to the cath lab more often $(21.7 \%$ vs $13.4 \%$, $\mathrm{p}=0.001)$. Normal $\mathrm{QRS}$ at admission was more common in $\mathrm{GA}(80 \%$ vs $70.2 \%, p<0.001)$ and complete right branch block (CRBB) was more frequent in GB $(13.5 \%$ vs $6.7 \%$, $\mathrm{p}<0.001)$.

$\mathrm{GB}$ had lower hemoglobin $(\mathrm{Hb})$ levels at admission $(12.9 \pm 2$ vs $13.4 \pm 1.9, p<.001)$, first medical evaluation $>120$ minutes after symptoms onset $(\mathrm{FME}>120 \mathrm{~m})$ more often $(71.6 \%$ vs $57.8 \%, \mathrm{p}<0.001)$ and more in-hospital diuretics usage $(72.8 \%$ vs $54.3 \%, \mathrm{p}<0.001)$. Logistic regression confirmed that $\mathrm{FME}>120 \mathrm{~m}(\mathrm{OR} 1.6, \mathrm{p}=0.005, \mathrm{Cl} 1.15-2.22)$, $\mathrm{Hb} \leq 12 \mathrm{~g} / \mathrm{dL}$ (OR 1.53, $\mathrm{p}=0.018, \mathrm{Cl} 1.02-2.18$ ), CRBB (OR 1.72, $\mathrm{p}=0.043$, $\mathrm{Cl} 11.02-2.92)$ and diuretics usage (OR 2.18, $\mathrm{p}<0.001, \mathrm{Cl} 1.57-3.01$ ) were predictors of late onset AF.

140 LOAF patients and 236 EOAF patients had 1 year of follow up. Event-free survival was higher in EOAF than LOAF $(80,3 \%$ vs $5,9 \%$; $\mathrm{p}=0.002$, OR 2.072).

Conclusion: LOAF patients seem to have a poorer prognosis compared to EOAF.

\section{7}

Cardiac biomarkers and electrocardiogram for detection of heart failure in the critically ill

O. Cavefors ${ }^{1}$; F. Einarsson ${ }^{2}$; B. Redfors ${ }^{3}$; J. Oras

${ }^{1}$ Anesthesiology and intensive care medicine, Sahlgrenska University Hospital, Gothenburg, Sweden; ${ }^{2}$ Medical student, Sahlgrenska University Hospital, Gothenburg, Sweden; ${ }^{3}$ Department of cardiology, Sahlgrenska University Hospital, Gothenburg, Sweden

Correspondence: J. Oras

Intensive Care Medicine Experimental 2020, 8(2): 000477

Introduction: Left ventricular (LV) dysfunction is potentially harmful in the critically ill patient as it might compromise circulatory status. LV dysfunction can be triggered by many conditions in the critically ill e.g. myocardial infarction, toxic cardiomyopathy, hypoxia or severe stress (Takotsubo syndrome). The gold standard for diagnosing left ventricular function is echocardiography, but it might not be feasible to screen all ICU patients without discretion.

Objectives: This study evaluated troponin T, NTproBNP and ECG as more readily available surrogate markers for heart failure in critically ill patients.

Methods: This is a secondary analysis of a single-center prospective observational study which consecutive patients were examined with echocardiography (ClinicalTrialsID: NCT03787810). Included patients were examined with echocardiography according to the recommendations from ESC [1] within 24 hours from admission to the general ICU in our hospital. All echoes were recorded and blindly reviewed by an independent examiner. Blood samples were analyzed for high sensitive troponin T (hsTnT) and NTproBNP and an ECG was obtained in 
conjunction with the echocardiography of the patients. Heart failure was defined as having an LV ejection fraction $<50 \%$.

Results: A total of 411 patients were included in the final analysis of whom $64(16 \%)$ had an $\mathrm{EF}<50 \%$. Measurement of hsTnT and NTproBNP was available in $314(76 \%)$ and $277(67 \%)$ patients, respectively. An ECG was obtained in 374 (91\%) patients. A sensitivity analysis showed that the population having measurement of cardiac biomarkers or an ECG-recording was a representative sample of the whole study population.

Levels of hsTnT were higher in patients with an $\mathrm{EF}<50 \%$ (median, IQR 27 [12-64] vs 176 [62-503], $\mathrm{p}<0.001$ ), as was also the case with NTproBNP (median, IQR 683 [212-2810] vs 4175 [1570-12900], $\mathrm{p}<0.001)$. An ECG with q-waves, ST-elevation, ST segment depression, negative T-waves or bundle block branch were all significantly associated with an $\mathrm{EF}<50 \%$.

Using ROC curves, the area under the curve (AUC) for hsTnT to detect an $\mathrm{EF}<50 \%$ was 0.81 . The best-cut off value was $68 \mathrm{ng} / \mathrm{L}$ with a sensitivity of $75 \%$, a specificity of $75 \%$, a positive predictive value (PPV) of $40 \%$ and a negative predictive value (NPV) of $93 \%$ in the detection of an $\mathrm{EF}<50 \%$. The AUC for NTproBNP to detect an $\mathrm{EF}<50 \%$ was 0.79 . The best cut-off value was $1230 \mathrm{ng} / \mathrm{l}$ with a sensitivity of $84 \%$, a specificity of $60 \%$, a PPV of $60 \%$ and a NPV of $95 \%$. The ECG finding with the highest predictive value was an ECG with any pathology which had a sensitivity of $92 \%$, a specificity of $38 \%$, a PPV of $23 \%$ and a NPV of $95 \%$ for detection of an $\mathrm{EF}<50 \%$ (Table).

A lower ejection fraction was associated with an increased risk of 30 days mortality $(<0.001)$. Levels of hsTnT and NTproBNP were both associated with an increased risk of 30 -days mortality $(p<0.001)$. Both biomarkers were also significantly associated with an increased risk of death when adjusting for SAPS 3 score, age and EF in $\%(p=0.024$ and $p=0.025$, respectively). An ECG with any pathology was associated with an increased risk of 30-days mortality $(p=0.005)$ but not when adjusting for SAPS 3 score, age and EF in $\%(p=0.813)$.

\begin{tabular}{ll|l} 
hsTnT $>68 n g / 1$ & NTproBNP $>1230 n g / l$ & ECG with any pathology
\end{tabular}

\begin{tabular}{|l|l|l|l|}
\hline Sensitivity & $75 \%$ & $84 \%$ & $92 \%$ \\
\hline Specificity & $75 \%$ & $60 \%$ & $38 \%$ \\
\hline PPV & $40 \%$ & $60 \%$ & $38 \%$ \\
\hline NPV & $93 \%$ & $95 \%$ & $95 \%$ \\
\hline
\end{tabular}

Conclusion: The cardiac biomarkers hsTnT and NTproBNP and ECG are only moderate in detection of heart failure in a mixed population of critically ill patients. However, the high negative predicitive value of these biomarkers, as well as a normal ECG, could be used to rule out heart failure in the critically ill.

Reference(s) and grant acknowledgment(s)

1. The Swedish Heart-Lung foundation, The Swedish state support for clinical research (ALF-agreement)

2. 1. Evangelista, A et al, Eur J Echocardiogr 9(4): 438-448 (2008).

\section{1}

Influence of pre-transplant assistance with venoarterial ECMO in transplanted cardiac patients in the ICU

AM. GÓMEZ DEL PULGAR VILLANUEVA'; R. Jara Rubio'; AB. Pérez Pérez ${ }^{1}$. CM. Carrillo'; A. López Domínguez'; RMD. Victoria²; DGJ. Higinio'; FA. Moreno'; S. Sanchez Cámara'; C. Manso Murcia'; LM. Mateos'; M. Valer Rupérez

${ }^{1}$ Intensive care unit, Hospital clinico universitario Virgen de la Arrixaca, Murcia, Spain; ${ }^{2}$ ntensive care unit, Hospital Clínico Universitario Virgen de la Arrixaca, Murcia, Spain

Correspondence: A.M. GÓMEZ DEL PULGAR VILLANUEVA

Intensive Care Medicine Experimental 2020, 8(2): 000541

Introduction: Cardiac assistance with venoarterial ECMO (ECMO-VA) is increasingly being used in patients with heart failure as a bridge to cardiac transplantation in code 0. In our study, we study how this fact influences the evolution of transplanted cardiac patients in ICU.
Methods: Review of cardiac transplants between January 2013-March 2020 that required ECMO-VA pre-transplantation in a retrospective, descriptive study. Age, Euroscorell value, vasoactive drugs requirements and stay times, as well as troponin concentration values and mortality rates were analyzed. A basic descriptive analysis and comparisons were performed with the appropriate statistical tests.

Results: 74 patients were included in the study. Pre-transplant ECMO was implanted in 9 patients $(12,2 \%), 2$ of them in INTERMASC 1 . No statistically significant differences in ECMO-VA group age were reported compared to elective transplant group[E1] . No significant differences were reported between the groups compared to Euroscore II, Apache II score or lactate and bilirrubine concentration levels. However, patients with pre-transplant ECMO-VA had higher average of ICU stay than the elective[E2] $(40,4 \pm 21,5$ vs $10,5 \pm 9,6$, respectively) $p<0,001$. Ultrasensitive $T$ troponin levels were significantly higher in ECMO $(10568,8 \pm 16351,3$ vs $2582,8 \pm 1975,3, p<0,001)$. Hours of treatment with vasoactive drugs as dobutamine $(569,6 \pm 595,5$ vs $133,1 \pm 73,4$, $\mathrm{p}<0.001)$ and norepinephrine $(560 \pm 370,9$ vs $55,9 \pm 59,5, \mathrm{p}<0.001)$ were significantly higher in the ECMO group compared to elective group, respectively. Mortality was significantly higher in the pre-transplant ECMO group (44.4\%) compared to a elective transplants (10.8\%). Conclusion: Transplanted cardiac patients in urgency 0 with pretransplant ECMO-VA had significantly higher ICU stay than elective patients, with higher troponin numbers, longer periods of vasoactive drugs treatment, and higher mortality rates than those reported in elective transplants.

\section{Reference(s) and grant acknowledgment(s)}

1. Hirsch Mehta, MD, FACC; Howard J. Eisen, MD, FACC; Joseph C. Cleveland, Jr., MD, FACC. Indications and Complications for VA-ECMO for Cardiac Failure. ACC. Jul 14, 2015. https://www.acc.org/latest-in-cardi ology/articles/2015/07/14/09/27/indications-and-complications-forva-ecmo-for-cardiac-failure

2. Thomas C. Hanff, MD, MPH1; Michael O. Harhay, PhD, MPH2; Stephen E. Kimmel, MD, MSCE1; et al. Trends in Mechanical Support Use as a Bridge to Adult Heart Transplant Under New Allocation Rules JAMA Cardiol. Published online April 15, 2020. https://doi.org/10.1001/ jamacardio.2020.0667

3. Francisco González-Vílchez, Luis Almenar-Bonetb, María G. CrespoLeiroc, Javier Segovia-Cubero et al. Spanish Heart Transplant Registry. 30th Official Report of the Spanish Society of Cardiology Working Group on Heart Failure (1984-2018)

\section{3}

Copeptin and hepatocyte growth factor at hospital arrival as a prognostic tool in acute myocardial infarction

MC. Pintado'; L. Maceda ${ }^{2}$; M. Trascasa' ; I. Arribas ${ }^{3}$; E. Nevado ${ }^{1}$; R. De Pablo ${ }^{4}$ ${ }^{1}$ Unidad de Cuidados Intensivos, Hospital Universitario Príncipe de Asturias, Alcala de Henares, Spain; ${ }^{2}$ Servicio de bioquímica, Hospital Universitario Príncipe de Asturias, Alcala de Henares, Spain; ${ }^{3}$ Servicio de bioquimica, Hospital Ramón y Cajal, Madrid, Spain; ${ }^{4}$ Unidad de cuidados intensivos, Hospital Ramón y Cajal, Madrid, Spain

Correspondence: M.C. Pintado

Intensive Care Medicine Experimental 2020, 8(2): 000563

Introduction: Actually, copeptin is an additional biomarker for the early rule-out of myocardial infarction in patients in emergency department or with early-onset chest pain. It also, have been associated with severity of the post cardiac arrest syndrome, 1-year mortality in ST-segment elevation acute myocardial infarction patients or the identification of major trauma, hospital admission and blood transfusion in adult trauma patients.

Objectives: To explore the association between early measurements of copeptin and hepatocyte growth factor with hospital and 1-year follow-up mortality, in patients with acute myocardial infarction.

Methods: We prospectively measured hepatocyte growth factor and copeptin in blood samples collected at hospital arrival in consecutive patients diagnoses of type 1 acute myocardial infarction during 8 months, and prospectively we follow-up them to measure hospital and 1-year follow-up mortality. 
Results: 84 patients were included in the study, 55 patients $(65 \%)$ were male with a mean age of 70.3 ( \pm 13.56 ) years. 25 patients $(29.8 \%)$ had an ST-segment elevation myocardial infarction. Hospital mortality was $11.9 \%, 1$-year follow-up mortality was $21.4 \%$. Mean copeptin levels were higher in patients who died during hospital admission (145.60 [52.21-588.50] vs. 24.79 [10.90-84.82] pmol/L, $\mathrm{p}=0.01)$ or during follow-up (112.28 [25.10-418.27] vs. 23.82 [10.9677.30] $\mathrm{pmol} / \mathrm{L}, \mathrm{p}=0.02)$. There were no differences on hepatocyte growth factor levels between patients who died or no during hospital admission (381.05 [189.95-736.65] vs. 355.24 [175.55-521.76] pg/ $\mathrm{ml}, \mathrm{p}=0.73)$ nor during follow-up (350.00 [175.05-555.08] vs. 345.53 [183.68-561.15] pg/ml, $\mathrm{p}=0.679$ )

Copeptin measured at hospital arrival was found to be independently associated with hospital mortality, hazard ratio 1.004 [1.001-1.007], $\mathrm{p}=0.011$; and 1-year follow-up mortality, hazard ratio 1.004 [1.0011.007], $p=0.010$. Conversely, hepatocyte growth factor measured at hospital arrival was not found to be independently associated with hospital mortality (hazard ratio 1.000 [0.999-1.001], $\mathrm{p}=0.794$ ) nor 1 -year follow-up mortality (hazard ratio 1.000 [0.999-1.001], $p=0.396$ ). Conclusion: Copeptin measured at hospital arrival, but not the hepatocyte growth factor, is an independent marker of mortality in patients with acute myocardial infarction.

\section{Reference(s) and grant acknowledgment(s)}

1. Int J Cardiol 2019 Jan 1:274:337-341.

2. BMC Emerg Med. 2020 Feb 24;20(1):14

3. Ann Lab Med 2020;40:7-14

4. European Heart Journal. https://doi.org/10.1093/eurheartj/ehv3202015

5. This study is no granted.

\section{6}

\section{Cardiac function and cor pulmonale on mechanical ventilated} Covid-19 patients. A multicenter observational study

ED. Valenzuela Espinoza'; P. Mercado ${ }^{2}$; R. Pairumani ${ }^{3}$; N. Medel ${ }^{4}$;

E. Petruska ${ }^{3}$; D. Ugalde ${ }^{4}$; F. Morales ${ }^{3}$; D. Eisen ${ }^{4}$; C. Araya ${ }^{3}$; J. Ramirez ;

J. Montoya ${ }^{4}$; M. Rovegno ; A. Bruhnn ; J. Bakker ; M. Slama ${ }^{6}$

'Departamento de medicina intensiva, Pontificia Universidad Católica de Chile, Santiago, Chile; ${ }^{2}$ Departamento de paciente crítico., Clínica Alemana, Santiago, Chile; ${ }^{3}$ Hospital barros luco trudeau, Unidad de Cuidados Intensivos, Santiago, Chile; ${ }^{4}$ Unidad de pacientes críticos, University of Chile Clinical Hospital, Santiago, Chile; ${ }^{5}$ Department of intensive Care Adults, Erasmus University Medical Center, Rotterdam, Netherlands; ${ }^{6}$ Medical intensive care unit, Chu D'amiens-Picardie Site Sud, Amiens, France

Correspondence: E.D. Valenzuela Espinoza

Intensive Care Medicine Experimental 2020, 8(2): 000576

Introduction: Cardiac injury has been reported to be a major complication associated to COVID-19, with reported incidences ranging from 7 to $28 \%$ patients $(1,2)$. It has even been associated with increased mortality(1). Circulatory failure due to cardiogenic shock or fulminant myocarditis has been described in some case reports (3). It has been reported that $95 \%$ of COVID-19 patients on mechanical ventilation require norepinephrine (NE)(4), nonetheless, no systematic data have been reported about the hemodynamic profiles, cardiac function, or incidence of acute cor pulmonale (ACP), in critically ill COVID-19 patients.

Objectives: To perform a systematic characterization of cardiac function in COVID-19 mechanically ventilated patients using critical care echocardiography.

Methods: The echocardiographic group of Sociedad Chilena de Medicina Intensiva (SOCHIMI) prospectively included COVID-19 patients admitted to ICU and connected to mechanical ventilation. A comprehensive echocardiographic assessment has been done within the first 12 hours of mechanical ventilation in four University Hospitals of Santiago.

Results: 60 patients were consecutively included. The mean age was $58 \pm 12$ y.o., 42 (70\%) were male. APACHE II was $13 \pm 4$ and SOFA $6 \pm 2$.
Forty seven (78\%) patients required low doses of NE $(0.05 \pm 0.06 \mathrm{mcg} /$ $\mathrm{kg} / \mathrm{min}$ ), median cardiac output was 4.9 [IQR 4.4-6.1] L min-1 and Lactate was 1.4 [1.0-1.9] $\mathrm{mmol} / \mathrm{L}$.

Forty three $(72 \%)$ patients exhibited a left ventricular ejection fraction (LVEF) $>60 \%$ (hyperkinetic), 16 patients had LVEF between 45 and $60 \%$ (normokinetic) and one patients had hypokinetic heart function (LVEF $<45 \%)(6)$. Right ventricle function was preserved in 55 (92\%) patients $($ TAPSE $>17 \mathrm{~m})(7)$. In eighteen patients (30\%) mild right ventricle dilation (right to left ventricular area ratio between 0.6-0.8) was observed and four $(7 \%)$ patients exhibited moderate acute cor pulmonale (right to left ventricular area ratio between $>0.8$ ). In addition, no correlation was observed between LVEF and troponin levels. Echocardiography parameters are shown in the Table.

\begin{tabular}{|c|c|}
\hline Echocardiography variables & $N=60$ \\
\hline \multicolumn{2}{|l|}{ Left Ventricular Function } \\
\hline Left ventricular outflow tract diameter, $\mathrm{cm}$ & $2.13[2.0-2.2]$ \\
\hline LVEDV, ml & $116[76-137]$ \\
\hline LVESV, $\mathrm{ml}$ & $37[28-52]$ \\
\hline Left Ventricular Ejection Fraction, \% & $65[60-70]$ \\
\hline Stroke Volume, $\mathrm{ml}$ & $68[57-88]$ \\
\hline MAPSE, $\mathrm{mm}$ & $15[14-17]$ \\
\hline Mitral Tissue Doppler Image s' wave, $\mathrm{cm} / \mathrm{sec}$ & $11[9.4-13]$ \\
\hline LVOT VTI, cm & $22[18-24]$ \\
\hline Cardiac Output, L.min ${ }^{-1}$ & $4.9[4.4-6.1]$ \\
\hline \multicolumn{2}{|l|}{ Right Ventricular Function } \\
\hline TAPSE, $\mathrm{mm}$ & $22[20,24]$ \\
\hline Tricuspid Tissue Doppler Image s' wave, $\mathrm{cm} / \mathrm{sec}$ & $13[11-16]$ \\
\hline Right end diastolic area, $\mathrm{cm}^{2}$ & 17 [13-19] \\
\hline Left end diastolic area, $\mathrm{cm}^{2}$ & 30 [24-35] \\
\hline $\mathrm{RV} / \mathrm{LV}$ ratio & $0.57[0.48-0.67]$ \\
\hline Mild Cor Pulmonale RV/LV ratio (0.6-0.8), n (\%) & $18(30)$ \\
\hline Moderate Cor Pulmonale, RV/LV ratio (>0.8), n (\%) & $4(7)$ \\
\hline Severe Cor Pulmonale, RV/LV ratio (>1), n (\%) & $0(0)$ \\
\hline Left ventricular D Shape, n (\%) & $0(0)$ \\
\hline \multicolumn{2}{|l|}{ Diastolic Function } \\
\hline Doppler Trans-mitral E wave, $\mathrm{cm} / \mathrm{sec}$ & $71[56-81]$ \\
\hline Doppler Trans-mitral A wave, $\mathrm{cm} / \mathrm{sec}$ & $62[46-76]$ \\
\hline E/A ratio & $1.1[0.8-1.5]$ \\
\hline Mitral Tissue Doppler Image e' wave, $\mathrm{cm} / \mathrm{sec}$ & $10[8-12]$ \\
\hline E/e' ratio & $6.7[5.3-9.1]$ \\
\hline \multicolumn{2}{|l|}{ Fluid responsiveness predictors } \\
\hline Maximum IVC diameter, $\mathrm{mm}$ & 20 [14-24] \\
\hline Minimum IVC diameter, $\mathrm{mm}$ & $18[11-22]$ \\
\hline IVC distensibility index, \% & $8[5-12]$ \\
\hline Stroke Volume Variation, \% & $5[3-7]$ \\
\hline Pulse Pressure Variation, \% & $4[3-5]$ \\
\hline
\end{tabular}

Conclusion: The most frequent hemodynamic profile of mechanically ventilated patients with severe COVID-19 pneumonia was a normodynamic circulatory state with hyperkinetic cardiac performance, associated with normal intravascular volume and normal tissue perfusion. In contrast with usual ARDS, severe acute cor pulmonale was not observed in this COVID-19 patient series. Although some cases of circulatory failure have been described, our data suggest that cardiac dysfunction is not a frequent feature of COVID-19. Further studies are necessary to assess the dynamic changes in cardiac performance over time.

Reference(s) and grant acknowledgment(s)

1. 3. Inciardi RM, Lupi L, Zaccone G, Italia L, Raffo M, Tomasoni D, et al. Cardiac Involvement in a Patient With Coronavirus Disease 2019 (COVID-19). JAMA Cardiol. 2020

2. 2. Deng Q, Hu B, Zhang Y, Wang H, Zhou X, Hu W, et al. Suspected myocardial injury in patients with COVID-19: Evidence from front-line clinical observation in Wuhan, China. Int J Cardiol. 2020.

3. 1. Shi S, Qin M, Shen B, Cai Y, Liu T, Yang F, et al. Association of Cardiac Injury With Mortality in Hospitalized Patients With COVID-19 in Wuhan, China. JAMA Cardiol. 2020 
000602

A Shunt Demonstration: intracardiac gradients following left heart unloading as a cause of hypoxaemia

M. Hoy ${ }^{1}$; N. Palomo-Lopez ; S. Escalona-Rodriguez ${ }^{2}$; A. Simon³.

M. Balakrishnan ${ }^{3}$; H. Soliman Aboumarie ${ }^{1}$; N. Lees ${ }^{1}$

${ }^{1}$ Critical care, Harefield Hospital, Harefield, United Kingdom; ${ }^{2}$ Critical care unit, Virgen del Rocio University Hospital, Seville, Spain; ${ }^{3}$ Cardiothoracic transplant surgery, Harefield Hospital, Harefield, United Kingdom

Correspondence: M. Hoy

Intensive Care Medicine Experimental 2020, 8(2): 000602

Introduction: Atrial septal defects (ASD) are common but rarely symptomatic. Alteration of right (Rt) and left ( $\mathrm{Lt}$ ) sided pressures may create a gradient across the interatrial septum causing shunt. If rightto-left shunt (RLSh) occurs, systemic hypoxaemia may ensue.

Objectives: To use 3 cardiac anatomical variants and physiological manipulation thereof to demonstrate how intracardiac gradients can be manipulated to both unmask and improve RLSh.

Methods: Case 1: 77 year old male with good biventricular function underwent tissue aortic valve replacement for aortic stenosis with epicardial pacing postoperatively. He failed extubation due to hypoxaemia. CXR, bronchoscopy and CTPA were normal. With ongoing deterioration (P/F ratio 10, PEEP 14) transoesophageal echocardiography (TOE) showed a large secundum ASD with continuous RLSh. Reducing pacing from $100 \mathrm{bpm}$ to $60 \mathrm{bpm}$ reduced shunt and increased pO2 from $10.1 \mathrm{kPa}$ to $32.3 \mathrm{kPa}$ on the same ventilator settings. Unfortunately, oxygenation could not be managed by manipulation of cardiac filling pressures non-invasively and he recovered after surgical correction of his ASD.

Case 2: 51 year old female with a background of Arrhythmogenic Right Ventricular Cardiomyopathy was admitted in cardiogenic shock on inotropes and underwent LVAD implantation. On day 7 postoperatively, she developed severe hypoxaemia. CXR was clear but oxygenation poor: P/F ratio 14 with PEEP 10 . TOE demonstrated a previously undetected large PFO with continuous right to left shunt. Oxygenation could not be improved despite reducing PEEP or LVAD RPM. She underwent Amplatzer closure of the PFO and recovered before being listed for transplantation.

Case 3: 40 year old male presented with fulminant lymphocytic myocarditis requiring pVAECMO and subsequent Total Artificial Heart (TAH) placement. He was readmitted a year later with bacterial pneumonia and fluid overload requiring intubation. TAH rate was increased to optimise pulmonary offloading and Lt vacuums manipulated to maximise this. Despite improved radiographic appearances and pulmonary compliance, oxygenation failed to resolve with a $P / F$ ratio of 11, PEEP 10. TOE was undertaken which showed a small PFO with continuous RLSh. Increasing Rt vacuum + iNO improved systemic oxygenation. After a few days, oxygenation worsened due to pulmonary congestion. Lt vacuum was increased coupled with diuresis. As chamber fills fell, both vacuums were increased. This maximised both systemic and pulmonary haemodynamics without provoking further shunt. He subsequently weaned from the ventilator before being discharged home.

Results: All 3 cases demonstrate a variety of techniques capable of managing RLSh across the IAS. Understanding of the physiology involved, particularly with mechanical devices is important.

Conclusion: RLSh can be a consequence of iatrogenic unloading of the left side of the heart. Management may be physiological, interventional or surgical. In the setting of unexplained hypoxaemia and in the right clinical setting, TOE is an essential part of investigation whose utility should not be overlooked. A high index of suspicion is needed and this diagnosis should not be forgotten.

\section{Reference(s) and grant acknowledgment(s)}

1. Bancal A, Arnoult F,Krapf L, Bonay M. Patent foramen ovale and hypoxaemia with or without elevated right heart pressures. Revue des Maladies Respiratoires (2011) 28, 967-977
000623

Individual and clinical characteristics associated with the presence of atherosclerotic coronary artery disease in patients with acute coronary syndrome in an intensive care unit in the city of Bogota

F. Morales'; D. Barrios"; A. Buitrago ${ }^{2}$

${ }^{1}$ Intensive Care Unit, Clinica Nueva el Lago, Bogotá, Colombia; ${ }^{2}$ Cardiology department, Fundacion Santa Fe de Bogota University Hospital, Bogotá, Colombia

Correspondence: F. Morales

Intensive Care Medicine Experimental 2020, 8(2): 000623

Introduction: Acute coronary syndrome (ACS) is a group of clinical diseases which occur as a result of acute myocardial ischemia, whether it be secondary to obstruction of coronary flow (atherosclerotic coronary artery disease) or to an imbalance in myocardial oxygen supply and demand, based on the fourth definition of acute myocardial infarction. Classic risk factors for coronary artery disease include older age, arterial hypertension, smoking, diabetes mellitus and family history of ischemic heart disease. In Colombia, the current scale of the disease in terms of risk factor prevalence is unknown.

We determine individual and clinical factors associated with acute coronary syndrome and their association with the presence of coronary atherosclerosis, documented using cardiac catheterization in patients in an intensive care unit during the first half of 2018, in the city of Bogota.

Objectives: To determine individual and clinical factors associated with the presence of atherosclerotic coronary artery disease in patients with acute coronary syndrome in an intensive care unit, during the first half of 2018 in the city of Bogota.

Methods: It is a cross-sectional study which included 223 patients diagnosed with coronary syndrome, and who received cardiac catheterization during the first half of 2018. We performed a bivariate analysis of the individual and clinical characteristics, using the Chi-squared test to determine their level of association with the dependent variable, i.e., the presence of atherosclerotic coronary artery disease. We performed a multivariate analysis through logistic regression to determine the independent risk factors. A p-value $\leq 0.05$ was considered significant.

Results: We found that 1 in 5 patients diagnosed with acute coronary syndrome does not show significant atherosclerotic coronary artery disease in their catheterization. $25 \%$ of patients with acute coronary syndrome are under 50 years old. Men show a greater prevalence, at $65 \%$. Contrary to what we expected, there was no statistically significant association in the variables that are widely described in literature, such as hypertension, smoking, or family history and personal history of ACS. Out of the individual and clinical characteristics assessed in this study, a history of Diabetes Mellitus (OR. 4.9, Cl 1.680-14.393, $p$ 0.004) and the presence of changes in the admission electrocardiogram (OR. 2.96, IC 1.285-6.854, p 0.011) had the greatest statistical significance.

Conclusion: This study shows that the variables that better account for the probability of evidence of atherosclerotic coronary artery disease in the catheterization of patients with acute coronary syndrome are a history of diabetes mellitus and the presence of electrocardiographic changes at the moment of diagnosis. These findings can give the clinician additional tools when deciding on the risk stratification strategy, thus avoiding unnecessary invasive procedures, and reducing the risk of complications and other considerations that derive from this.

Reference(s) and grant acknowledgment(s)

1. Lee DW, Stouffer GA. Diagnostic Coronary Angiography. En: Stouffer GA MD, Runge MS MD, PhD, Patterson C MD, MBA, Rossi JS MD, editores. Netter's Cardiology [Internet]. 2019. p. 81-91. Disponible en: https://www.clinicalke y.es/\#!/content/3-s2.0-B9780323547260000121

2. Silva SY, Rincón MY, Dueñas RE, Chaves ÁM, Camacho PA, Arenas MA, et al. El sobrepeso es el factor determinante en la presentación de síndrome coronario agudo en adultos jóvenes colombianos. Rev Colomb Cardiol. 2008;15(6):8. 
3. Yusuf S, Hawken S, Ôunpuu S, Dans T, Avezum A, Lanas F, et al. Effect of potentially modifiable risk factors associated with myocardial infarction in 52 countries (the INTERHEART study): case-control study. 2004; 364 : 16. 4. Consenso ESC 2018 sobre la cuarta definición universal del infarto. Rev Esp Cardiol. January 2019;72(1):72.e1-72.e27.

\section{9}

The impact of end stage renal disease on long term outcome in patients after acute myocardial infarction: a nationwide observational study

TH. TAl ${ }^{1}$; WC. Huang ${ }^{2}$; KC. Lin ${ }^{1}$; FY. Kuo ${ }^{1}$; SH. Kuo ${ }^{3}$; HL. Liang ${ }^{4}$

${ }^{1}$ Critical care medicine and cardiovascular center, Kaohsiung Veterans General Hospital, Kaohsiung, Taiwan; ${ }^{2}$ Critical care medicine and cardiovascular center, Kaohsiung Veterans General Hospital, Taipei, Taiwan; ${ }^{3}$ Department of Critical Care Medicine, Kaohsiung Veterans General Hospital, Kaohsiung City, Taiwan; ${ }^{4}$ Ritical care medicine and cardiovascular center, Kaohsiung Veterans General Hospital, Kaohsiung, Taiwan

Correspondence: T.H. TAl

Intensive Care Medicine Experimental 2020, 8(2): 000639

Introduction: Although a possible association between chronic kidney disease and coronary artery disease or acute myocardial infarction has been identified, the impact of end-stage-renal-disease (ESRD) under dialysis on long-term prognosis after an acute myocardial infarction (AMI) is uncertain. Therefore, this study was to evaluate the specific impact of the patient with ESRD under dialysis on survival after a first AMI through a retrospective analysis of data from the Taiwan National Health Insurance Research Database.

Methods: This was a nationwide, propensity score-matched casecontrol study of patients admitted to hospitals between January 2000 and December 2012 with a primary diagnosis of a first AMI. Among the 186,326 prospective patients, 8056 patients with a confirmed diagnosis of ESRD under regular dialysis were identified. A propensity score, one-to-one matching technique was used to match 8056 controls to the AMI group for analysis. Controls were matched on the following variables: sex, age, hypertension, dyslipidemia, diabetes, peripheral vascular disease, heart failure, cerebrovascular accidents, end stage renal disease, chronic obstructive pulmonary disease, and percutaneous coronary intervention.

Results: The 12-year survival rate was significant lower in AMI with ESRD group than control group (log rank $p<0.01$ ). No matter male or female, the survival rate was significant lower in AMI with ESRD group (log rank $\mathrm{p}<0.0001$ ). In both younger (Age<65 years) and older category, the survival rate was also lower in AMI with ESRD group (log rank $\mathrm{p}<0.0001)$. The survival rate of patients who had received $\mathrm{PCI}$ management was comparable among patients in the ESRD and control groups (log rank $\mathrm{p}<0.0001)$.Similarly, the rate of survival among patients who did not receive $\mathrm{PCI}$ management was comparable for both the ESRD and control groups (log rank $\mathrm{p}<0.001$ ).

Conclusion: In this nationwide study, using matched-case control we provide evidence of a possible effect of end-stage-renal-disease on the survival after a first AMI. Independent of sex and age, and revascularization management, ESRD plays an important role in the long-term outcome of AMI patients.

\section{4}

\section{Control of arterial hypertension in critical patient} with clevidipino: safe and effective therapy. descriptive study M. T. Cruces Moreno ; C. V. Ines²; J. Tejero Aranguren ${ }^{1}$; P. A. Carranza ${ }^{2}$ ${ }^{1}$ Intensive care unit, Hospital Universitario San Cecilio, Granada, Spain; ${ }^{2}$ Intensive care unit, Hospital PTS, Granada, Spain

Correspondence: M.T. Cruces Moreno

Intensive Care Medicine Experimental 2020, 8(2): 000654

Introduction: It is common to find ourselves in intensive care units with patients with difficult blood pressure control. In many occasions, the impossibility of administering oral antihypertensive therapy is added.
Objectives: to describe the utility of the use of clevidipine as a safe and effective antihypertensive in different medical and surgical pathologies, in an Intensive Care unit.

Methods: Single-center, descriptive and retrospective study of a series of cases. We included patients admitted to our unit for any pathology between January 2018 and December 2019, treated with clevidipine for blood pressure control. The software used was IBM SPSS v.20.

Results: A total of 20 patients were included, 17 males (85\%) and 3 females (15\%) with a mean age in the range of $58 \pm 14.9$ years. The pathologies that led to admission were: 7 (35\%) coronary, $5(25 \%)$ neurological, 3 (15\%) vascular, $2(10 \%)$ hypertensive crisis, $2(10 \%)$ heart failure and 1 (5\%) postoperative period of general surgery. The mean APACHEll score was $14.4 \pm 6.5$. It was recorded as a history of known arterial hypertension on admission in 15 patients (75\%).

Different combinations of antihypertensive drugs were started prior to the use of clevidipine in 12 patients (60\%), the most frequent being: a combination of three oral antihypertensive drugs plus IV nitroglycerin in 4 of them (20\%); ACE inhibitors plus BB in $2(10 \%)$ and IV nitroglycerin monotherapy in $2(10 \%)$. In 8 patients (40\%) clevidipine was chosen as the first option due to contraindication for the use of other antihypertensive drugs and / or the impossibility of oral intake.

The maximum average dose used was $6.24 \mathrm{ml} / \mathrm{h}(3.12 \mathrm{mg} / \mathrm{h})$ for about 2.35 days until optimization and / or onset of action of the different oral antihypertensives. Very good tolerance to the drug was observed without registering any side effect for which the therapy had to be suspended.

Conclusion: The main indication for clevidipine is the reduction of blood pressure in the perioperative environment. We consider that it is also a useful alternative for a rapid control of blood pressure in other clinical settings, also reducing the length of stay in the Intensive Care Unit, with the consequent improvement in the therapeutic efficiency of this type of patient. However, more randomized prospective studies would be necessary to corroborate these conclusions.

\section{Reference(s) and grant acknowledgment(s)}

1. Deeks ED, Keating GM, Keam SJ. Clevidipine: a review of its use in the management of acute hypertension. Am J cardiovasc Drugs. 2009; 9 (2): 117-34 2. Yu Lin, MM and Lina Ma, MM. Blood pressure lowering efecct of calcium channel blockers on perioperative hypertension. A systematic review and meta-analysis. Medicine (Baltimore) 2018 Nov; 97 (48): e13152

3. Avad AS, Goldberg ME. Role of clevidipine butyrate in the treatmen of acute hipertension in the critical care setting: a rewiew. Vasc Health Risk Manag 2010; 6: 457-64

4. I assign all copyrights

\section{6}

Pilot study: implementation of a protocol for the management of intermediate-risk pulmonary embolism

I. Cruz Valero'; A. Carranza Pinel²; MT. Cruces Moreno1; J. Tejero Aranguren ${ }^{1}$; AME. Poyatos

${ }^{1}$ Intensive care unit, Hospital Universitario San Cecilio, Granada, Spain; ${ }^{2}$ Intensive care unit, Hospital Universitario Clínico., Granada, Spain

Correspondence: I. Cruz Valero

Intensive Care Medicine Experimental 2020, 8(2): 000656

Introduction: High risk pulmonary embolism (PE) should be monitored in an Intensive Care Unit (ICU) because of the severity and high mortality it has. However, there is controversy regarding the monitoring and treatment of patients with intermediate-risk PE, since they are initially hemodynamically stable patients, but with a high risk of deterioration.

Objectives: In order to optimize its management, the ICU of the San Cecilio University Hospital has created a protocol in collaboration with the Emergency, Pneumology and Internal Medicine units.

Methods: Prospective, descriptive study over a period of four months. Patients with diagnosis of acute PE in the Emergency Department or in the hospital ward are included, with the following criteria:

- PE diagnosed by CT-scan.

- Hemodynamic stability. 
- Suspected right ventricular dysfunction on CT-scan and elevation of cardiac markers (troponin and NT-proBNP).

A transthoracic echocardiogram was performed in the first 24 hours to evaluate right ventricular function.

Results: 29 patients met the inclusion criteria. The mean age was 69 years, being $41 \%$ women and 59\% men. APACHE-II average of 9 and dyspnea as the main symptomatology (79\% of patients). $100 \%$ presented some echocardiographic alteration consisting of RV dilatation, systolic dysfunction, or pulmonary hypertension. Three of the patients presented a torpid evolution towards high-risk PE, being able to be diagnosed and treated early with systemic fibrinolysis, which conditioned a favourable subsequent evolution normalizing RV systolic function.

The rest of the patients has a standard anticoagulant regimen and evolved properly, although all of them have persistent echocardiographic alterations 5-7 days after the treatment begin.

Conclusion: Patients with diagnosis of intermediate-risk PE benefited from closer monitoring thanks to the existence of a consensus protocol for the management of these patients, since the initial unsatisfactory evolution of three of them towards high-risk PE was diagnosed early, allowing an early approach.

Reference(s) and grant acknowledgment(s)

1. 2. Desai H, Natt B, Bime C, Dill J, Dalen JE, Alpert JS. Pulmonary em-bolism with right ventricular dysfunction: who should receive throm-bolytic agents? Am J Med. 2017;130:e29-e32.

2. 1. Meyer G, Sánchez $O$, Jiménez D. Risk Assessment and Management of High and Intermediate Risk Pulmonary Embolism. Presse Med. 2015 Dec;44(12 Pt 2):e401-8.https://doi.org/10.1016/j.lpm.2015.10.009. Epub 2015 Nov 14

\section{1}

Evaluated benefical effects of inhaled nitric oxide using the ventilatory ratio in adult cardiac surgery patients

T. Kamo' ${ }^{1}$ K. Misumi ${ }^{1}$;. Koinuma'; Y. Hagiwara'; T. Ogura ${ }^{1}$

${ }^{1}$ Critical care medicine, Saiseikai Utsunomiya Hospital, Utsunomiya, Japan Correspondence: T. Kamo

Intensive Care Medicine Experimental 2020, 8(2): 000681

Introduction: Cardiac surgery produces a systemic inflammatory response syndrome (SIRS) that has been highly related with lung injury. This reaction is associated with abnormalities in gas exchange, such as increased pulmonary shut fraction. Inhaled nitric oxide (iNO) plays a roll of potent dilator of pulmonary vessels and improves such a ventilator/perfusion mismatche. Indirect indices for assessing impaired ventilation, such as dead space fraction and ventilatory ratio, have been used in acute respiratory distress syndrome (ARDS) and other respiratory failure. However, no studies have evaluated how much iNO improves shunt rate in cardiac surgery patients with postoperative pulmonary dysfunction using these formulas.

Objectives: We conducted to eavluate the effectiveness of iNO therapy for cardiac surgey patients with respiratory failure or right herat failure using indirect indicies.

Methods: We retrospectively reviewed the medical records of 19 adult cardiac patients treated with iNO postoperatively between July 2018 and March 2020. iNO was used to pulmonary hypertension and respiratory failure in patients who had undergone total arch replacement $(n=9)$, valve replacement $(n=8)$ and coronary artery bypass graft $(C A B G)(n=2)$. Hemodynamic measurements, dead space fraction and ventilatory ratio before iNO and again 6 and 12 hours after administration were examined. The effect of iNO infusion on arterial oxygenation was quantified using the $\mathrm{PaO} 2 / \mathrm{FiO}_{2}(\mathrm{P} / \mathrm{F})$ ratio. The data were analyzed by using student's $t$ test. And we assessed the correlation between these indices and P/F ratio using Pearson correlation coefficient.

Results: Average administration duration of iNO (20 ppm) was 54.4 hours. Eight patients underwent emergency or urgent operation and all had type $A$ aortic dissection. There was no significant difference in mean pulmonary artery pressure, however $\mathrm{P} / \mathrm{F}$ ratio increased significantly from 182 to $265(p<0.05)$ at 12 hours after initiation. Mean arterial pressure and cardiac index did not change before and after used iNO. Although P/F ratio at 6 hours after initiation increased, it did not reached statistical significance. Improving oxygenation was associated with decreasing impaired ventilation measured by dead space fraction and ventilatory ratio at 12 hours after initiation. There was a significant negative correlation between VR and P/F ratio (Pearson, $\mathrm{r}=-0.5 ; \mathrm{Cl}$, -0.78 to -0.05 ). The improvement of oxygenation due to the vasodilatory effect of iNO was proved.

Conclusion: In cardiac surgery patients, calculating dead space fraction and the ventilatory ratio during using iNO proved indirectly how iNO improve oxygenation without deterioration of the circulation indices. From these results, the vasodilatory effects of nitric oxide improved shunt fraction and oxygenation

\section{5}

Thromboembolic stroke risk of postoperative atrial fibrillation following cardiac surgery

R. Pierik'; M. Nijsten'; ME. Erasmus²; GJR. Luijckx³ ; M. Rienstra4;

T. Scheeren ${ }^{5}$; M. Uyttenboogaart ${ }^{3}$; M. Zeillemaker-Hoekstra ${ }^{5}$; WM. van den Bergh $^{1}$

${ }^{1}$ Department of critical care, University Medical Center Groningen, Groningen, Netherlands; ${ }^{2}$ Department of cardiac surgery, University Medical Center Groningen, Groningen, Netherlands; ${ }^{3}$ Department of neurology, University Medical Center Groningen, Groningen, Netherlands; ${ }^{4}$ Department of cardiology, University Medical Center Groningen, Groningen, Netherlands; ${ }^{5}$ Department of anesthesiology, University Medical Center Groningen, Groningen, Netherlands

Correspondence: R. Pierik

Intensive Care Medicine Experimental 2020, 8(2): 000685

Introduction: Atrial fibrillation and atrial flutter are the most common cardiac arrhythmias in patients admitted to the intensive care unit (ICU) after cardiac surgery. Even in the ICU environment short episodes can be missed and left untreated.[1,2] If a clear increase of stroke risk in patients with postoperative AF/AFI (POAF) is established this might implicate that POAF should be detected meticulously and treated expeditiously.

Objectives: The primary aim of the current study was to establish thromboembolic stroke risk during hospital admission in patients with POAF in the first week after cardiac surgery in our institution. The secondary aim was to study the association of clinical characteristics including the occurrence of POAF with occurrence of thromboembolic stroke during hospital admission in patients after cardiac surgery.

Methods: A single-center retrospective observational study was performed with all consecutive cardiac surgery patients admitted postoperatively to the ICU of our institution between 2009 and 2010. $[3,4]$ Brain imaging was studied for the occurrence of thromboembolic stroke (other subtypes of stroke were censored) during hospital admission and POAF was monitored meticulously for seven days after cardiac surgery. The proportion of patients with POAF among patients with thromboembolic stroke was compared to those without thromboembolic stroke and odds ratios (ORs) were calculated with corresponding $95 \% \mathrm{Cl}$ using logistic regression analyses. To assess which characteristics were associated with occurrence of thromboembolic stroke, a stepwise backward regression analysis was performed.

Results: Of the 910 patients included in this study POAF occurred in 360 patients (40\%) during the first seven days after cardiac surgery, and 26 patients (2.9\%) had a thromboembolic stroke during hospital admission. The proportion of patients with POAF among patients with thromboembolic stroke $(65 \%)$ was higher compared to those without thromboembolic stroke (39\%): adjusted OR 3.01 (95\% Cl; 1.13 - 8.00). POAF, a history of peripheral vascular disease, a higher EuroSCORE and a longer duration of surgery were associated with thromboembolic stroke.

\begin{tabular}{|lcccc|}
\hline \multicolumn{5}{|c|}{ Table 1. Odds ratio for it -hospital thromboembolic stroke with $P O A F<7 d$} \\
\hline Variable & $\begin{array}{c}\text { Total (n=910) } \\
\text { N (\%) }\end{array}$ & $\begin{array}{c}\text { TE stroke } \\
(\mathrm{n}-26,2.9 \%)\end{array}$ & $\begin{array}{c}\text { No TE stroke } \\
(\mathrm{n}=884,97.1 \%)\end{array}$ & OR (95\%CT) \\
& & $\mathrm{N}(\%)$ & $\mathrm{N}(\%)$ & \\
\hline POAF $<7 \mathrm{~d}$ & $360(40 \%)$ & $17(05 \%)$ & $343(39 \%)$ & $2.98(1.31-6.70)$ \\
& & & $3.01(1.13-8.00)^{*}$ \\
\hline
\end{tabular}


Figure 1. Thromboembolic stroke free interval after cardiac surgery dependent on POAF. Straight line shows $10 P Q 4 F$, and dotted line shons $P O A F$.

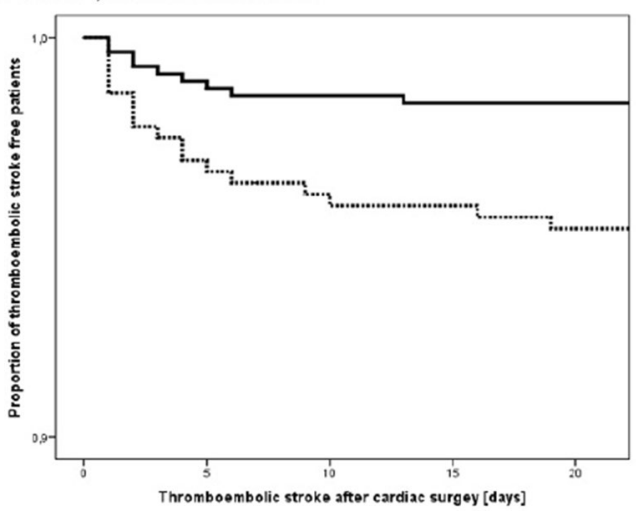

Conclusion: POAF within seven days after cardiac surgery was associated with a threefold increased risk for a thromboembolic stroke during hospital admission. POAF, a history of peripheral vascular disease, a higher EuroSCORE, and a longer duration of surgery were independent risk factors for thromboembolic stroke directly post-cardiac surgery of which POAF was the strongest independent predictor. Perioperative prophylaxis or expeditious treatment of POAF may therefore prevent early stroke after cardiac surgery.

\section{Reference(s) and grant acknowledgment(s)}

1. 4. Pierik R, Uyttenboogaart M, Erasmus ME, Scheeren TW, van den Bergh WM. Distribution of perioperative stroke in cardiac surgery. European Journal of Neurology. 2019; 26(1): 184-90.

2. 3. Hoekstra M, Hessels L, Rienstra M, et al. Computer-guided normal-low versus normal-high potassium control after cardiac surgery: no impact on atrial fibrillation or atrial flutter. American Heart Journal. 2016; 172, p. 45-52.

3. 2. Healey JS, Connolly SJ, Gold MR, et al. Subclinical atrial fibrillation and the risk of stroke. New England Journal of Medicine. 2012; 366: 120-129.

4. 1. Testai FD. The relevance of atrial fibrillation in stroke prevention. The Lancet Neurology. 2017; 16(4): 253-5.

\section{4}

Oral tap water ingestion versus intravenous fluid in shock patients: a prospective randomized study

M. Nguyen ; V. Berthoud ; L. Bartamian 1 ; A. Martin ; V. Duclos ; O. Ellouze ; R. Mohamed '; C. Tiberiu'; S. Grosjean'; E. Rafrafi'; B. Bouhemad';

PG. Guinot

${ }^{1}$ Anesthesiology and critical care medicine, Hospital Center University François Mitterand, Dijon, France

Correspondence: $M$. Nguyen

Intensive Care Medicine Experimental 2020, 8(2): 000694

Introduction: In patient with shock, fluid therapy is tailored to optimize cardiac preload and tissue perfusion. Oral tap water administration is known to be associated with an improvement of blood pressure and cardiac output in non-critically-ill patients (1). Whether water digestive absorption is impaired in critically ill and whether tap water administration increase stroke volume in preload dependent patients remains uncertain.

Objectives: to determine the safety and effectiveness of tap water administration (via naso-gastric tube) in preload dependent patient with shock.

Methods: We performed a monocentric open label, randomize clinical trial in Dijon teaching hospital. Randomization was stratified on stroke volume (SV) change induce by passive leg raising. The study was approved by an independent ethics committee (Ethics Committee No. 2018-A03368-47, NCT03951519). Inclusion criteria were: aged 18 years or older, suffering from acute circulatory failure (systolic blood pressure $<90 \mathrm{mmHg}$, and/or mean blood pressure $<65 \mathrm{mmHg}$, and/or need for infusion of vasopressor amines, and/or skin mottling, and/or diuresis < $0.5 \mathrm{ml} / \mathrm{kg} / \mathrm{h}$ for 2 hours $\geq$, and/or arterial lactate level $>2 \mathrm{mmol} / \mathrm{l}$ ), with stroke volume change following passive leg raising over $10 \%$, and with no contraindication to a nasogastric tube. Exclusion criteria were: permanent atrial fibrillation, hypothermia, concurrent participation in another study, and pregnant women. Patients were assigned to receive either $500 \mathrm{~m}$ of nasogastric tap water (at room temperature) or $500 \mathrm{ml}$ of intravenous (IV) saline solution over a 15 minutes period. The primary endpoint was the change in stroke volume at the end of fluid administration expressed as percentage. Secondary endpoints were mean arterial pressure, cardiac output and oxygen delivery changes following fluid administration. Safety was assessed by the incidence of adverse events (arrythmia, vomiting, abdominal distension, worsening arterial lactate and death).

Results: Fifty patients were randomized. Most of patients (70\%) had septic shock. Baseline SV did not differ between the two groups. The median change in SV with fluid expansion did not differ between the two groups $(22 \%[16 ; 51]$ vs $21 \%[16 ; 35], p=0.578)$. The number of fluid responsive patients (i.e. SV > 15\% between baseline and end of fluid expansion) did not differ between the two groups $(n=19(76 \%)$ vs $n=18(72 \%)$ ). In the two groups, blood pressure and oxygen delivery increased, with no differences between groups (table 1). The incidence of adverse event did not differ between groups.

\begin{tabular}{|c|c|c|c|c|}
\hline & & $\begin{array}{l}\text { Saline Group } \\
(\mathrm{n}=25)\end{array}$ & $\begin{array}{l}\text { Water Group } \\
(\mathbf{n}=\mathbf{2 5})\end{array}$ & $\begin{array}{l}\text { p-value } \\
\text { intergroup }\end{array}$ \\
\hline \multirow[b]{2}{*}{ Heart rate (BPM) } & Before & $94[80 ; 107]$ & $90[80 ; 109]$ & 0.580 \\
\hline & After & $86[77 ; 104]^{*}$ & $89[79 ; 104]$ * & \\
\hline \multirow[t]{2}{*}{ MAP (mmHg) } & Before & $66[62 ; 72]$ & $68[62 ; 73]$ & 0.449 \\
\hline & After & $74[67 ; 81]$ * & $77[72 ; 84]$ * & 0.105 \\
\hline \multirow[t]{2}{*}{ CVP (mmHg) } & Before & $9[6 ; 11]$ & $10[8 ; 12]$ & 0.234 \\
\hline & After & $11[8 ; 15]$ * & $11[9 ; 12]$ * & 0.967 \\
\hline \multirow[t]{2}{*}{ SV (ml) } & Before & $36[28 ; 51]$ & $38[30 ; 51]$ & 0.900 \\
\hline & After & $44[36 ; 60]$ * & $44[37 ; 59]$ * & 0.977 \\
\hline \multirow{2}{*}{$\begin{array}{l}\text { Cardiac output (ml } \\
\text { min-1) }\end{array}$} & Before & $3.40[2.62 ; 4.92]$ & $3.89[2.34 ; 4.58]$ & 0.839 \\
\hline & After & $\underset{*}{4.41[3.47 ; 5.13]}$ & $\underset{*}{4.46}[2.55 ; 5.28]$ & 0.808 \\
\hline \multirow{2}{*}{$\begin{array}{l}\text { O2 delivery (ml } \\
\text { min-1 m-2) }\end{array}$} & Before & $298[188 ; 364]$ & $242[209 ; 327]$ & 0.455 \\
\hline & After & $299[255 ; 404] *$ & $271[181 ; 369]$ * & * 0.607 \\
\hline
\end{tabular}

Table 1. Change in the hemodynamic parameters induced by Tap water and IV saline administration.

Conclusion: There was no difference between SV change induce by the administration of $500 \mathrm{ml}$ of enteral tap water and SV change induced by the administration of $500 \mathrm{ml}$ of IV saline solution. The incidence of adverse event did not differ between groups. The administration of enteral tap water for fluid expansion appear to be safe and effective. Further studies are needed to confirm our results.

Reference(s) and grant acknowledgment(s)

1. Callegaro CC, Moraes RS, Negrão CE, Trombetta IC, Rondon MU, Teixeira MS, Silva SC, Ferlin EL, Krieger EM, Ribeiro JP. Acute water ingestion increases arterial blood pressure in hypertensive and normotensive subjects. J Hum Hypertens 2007;21:564-570.

\section{8}

Complications in venoarterial extracorporeal membrane oxygenation: the key to survival

A. Marcos-Morales ${ }^{1}$; P. Pagliarani Gill'; JL. PerezVela'; I. Martín Badía ${ }^{1}$; N. Quílez-Trasobares ${ }^{1}$; M. Corres Peiretti ${ }^{1}$; E. Renes Carreño ${ }^{1}$;

JC. Montejo-Gonzalez

${ }^{1}$ Intensive care department, Hospital Doce de Octubre, Madrid, Spain

Correspondence: A. Marcos-Morales

Intensive Care Medicine Experimental 2020, 8(2): 000868

Introduction: Extracorporeal membrane oxygenation (ECMO) is a fundamental tool in refractory cardiorespiratory failure, however, complications are common and can be severe (1) 
Objectives: The purpose of this study was to evaluate the different complications occurring in patients with refractory cardiogenic shock (CS) supported with veno-arterial ECMO.

Methods: Data was collected prospectively from 2010 to 2020, patients with CS having received VA-ECMO were reviewed retrospectively. Baseline demographic variables, cause of CS, ECMO characteristics and complications were analyzed using SPSS software, version 20. Results: We included 92 patients who received 101 VA-ECMO. Median age was 54 (interquartile range IQR 42-63), 64\% were male. Causes of CS were mostly decompensated advanced heart failure (32\%), acute myocardial infarction (26\%), post-cardiotomy (21\%), post-surgical pulmonary thromboendarterectomy (13\%) and acute myocarditis (7\%). A peripherally VA-ECMO was established in $91 \%$ of cases. The median length of ECMO was 186 hours (IQR 5.4 - 13 days), median ICU stay 18 days (IQR 12-33 days). In our series, device related complications were as follows: bleeding from cannula site occurred in $35 \%$ of patients, requiring surgical intervenction in $9 \%$ of them. One patient suffered an unintended decannulation. Lower limb ischemia after femoral cannulation occurred in $22 \%$, which is comparable to the literature (2). Limb amputation was required in 3\% of patients. Thrombi formation in the oxygenator membrane was found in $19 \%$ of cases, $3 \%$ required urgent membrane replacement.

Patient related complications may be due to profound shock at the time of ECMO cannulation, or to the ECMO itself. Acute renal failure occurred in $68 \%$ of patients, half of which received renal replacement therapy (RRT). Major neurological complications were recorded: $9 \%$ of patients suffered from anoxic brain injury frequently related to previous cardiac arrest, 2 patients had a central nervous system (CNS) hemorrhage and CNS infarction was observed in $4 \%$ of patients. Only three of these patients diagnosed with stroke, either hemorrhagic or ischemic, were weaned from ECMO and discharged with good neurological recovery. Infectious complications occurred on $48 \%$ patients, mostly catheterrelated bacteremia (27\%) and ventilator-associated pneumonia (19\%). Global survival rate during ECMO support was $68 \%$, with $43 \%$ of patients being discharged home. Severe complications had an enormous impact in outcome, regardless of the initial CS cause. After hemorrhagic stroke or anoxic brain injury survival was as low as $21 \%$. However, some complications as the need of RRT were met with $34 \%$ survival, maybe due to the removal of inflammatory cytokines (3).

Conclusion: Patients needing VA-ECMO for CS have an already slim chance of survival. Complications may further increase their morbidity and mortality. A better understanding of their incidence and characteristics is paramount to a better management of these patients and to achieving better outcomes in the future.

\section{Reference(s) and grant acknowledgment(s)}

1. 1. Lo Coco V, Lorusso R, Raffa GM, et al. Clinical complications during venoarterial extracorporeal membrane oxigenation in post-cardiotomy and non post-cardiotomy shock: still the achille's heel. J Thorac Dis. 2018;10(12):69937004. https://doi.org/10.21037/jtd.2018.11.103

2. 2. Bonicolini, E., Martucci, G., Simons, J. et al. Limb ischemia in peripheral veno-arterial extracorporeal membrane oxygenation: a narrative review of incidence, prevention, monitoring, and treatment. Crit Care 23, 266 (2019). https://doi.org/10.1186/s13054-019-2541-3

3. 3. Mu TS, Palmer EG, Batts SG, Lentz-Kapua SL, Uyehara-Lock JH, Uyehara CF. Continuous renal replacement therapy to reduce inflammation in a piglet hemorrhage-reperfusion extracorporeal membrane oxygenation model. Pediatr Res. 2012;72(3):249-255. https://doi.org/10.1038/pr.2012.69

\section{1}

Comparison of epidemiological features and outcomes

between patients with Acute Coronary Syndrome (ACS)

with and without coronary obstruction in the Intensive Care Unit (ICU) from Hospital Virgen de las Nieves (HUVN)

I. Guzmán Adum; MD. Paez Sánchez; A. López Fernández; R. De La

Chica Ruano

${ }^{1}$ Intensive care unit, Hospital Universitario Virgen de las Nieves, Granada, Spain

Correspondence: I. Guzman

Intensive Care Medicine Experimental 2020, 8(2): 000901
Introduction: MINOCA is defined by the AMI criteria, absence of obstructive coronary artery disease $(\geq 50 \%)$, and no other cause for the clinical presentation. Research studies of MINOCA reported a 12-month mortality of $4.7 \%(95 \% \mathrm{Cl} 2.6-6.9)$, consistently demonstrating a better prognosis than for those with AMI and obstructive coronary disease. Therefore we can no longer dismiss patients with MINOCA as having false-positive AMIs but need to consider the potential benefit of statins and ACE inhibitors/ blockers.

Objectives: To describe the characteristics of patients admitted in the ICU with acute coronary syndrome, differentiating between the groups of acute myocardial infarction with and without coronary obstruction (MINOCA)

Methods: An observational study that includes all patients $(\mathrm{N}=$ 498) admitted for ACS in HUVN- ICU's Coronary Unit from January to December 2015. The ARIAM registry was used and computerized medical records were reviewed to collect data not included in the registry. Patients who did not undergo cardiac catheterization, echocardiography, or did not have the study variables recorded in their history were excluded. The data obtained was collected in an SPSS sheet and was subsequently evaluated in terms of descriptive statistics with mean, standard deviation (SD) and frequencies, and comparisons were made between the groups with and without coronary artery disease, using Student's $T$ test for quantitative variables and Chi square test for qualitative variables.

Results: The study includes 391 patients, 270 (69.2\%) men and 120 (30.8\%) women. The mean age was 62.9 (SD 12.6). The most common reason for admission was STEMI (49\%) and $83.4 \%$ were in a Killip I clinical situation. The most prevalent cardiovascular risk factors were: hypertension $(59.5 \%)$, dyslipidemia $(50.5 \%)$, smoking (34.6\%), type 2 Diabetes Mellitus (33.8\%). LVEF at discharge was 53.2 (DS 10). $83.6 \%$ of the patients had coronary lesions, but only $62.4 \%$ were discharged with dual antiplatelet therapy. $16.4 \%$ had no coronary lesions; however, only 19 (3\%) were considered MINOCA and received treatment. After 3 years, $7.7 \%$ of patients had another myocardial infarction, $16.4 \%$ were hospitalized due to cardiovascular causes, $0.8 \%$ had a recovered cardiorespiratory arrest, and mortality was 3.3\% (17). Patients without coronary obstruction were more frequently women ( 56.5 vs. $44.5 \% \mathrm{p}<0.01)$, younger ( $61 \pm 10.8$ vs. $65.7 \pm 12.9$ years, $p$ $=0.06$ ) and with a better cardiovascular risk profile (DM 15 vs $30.8 \%$ $\mathrm{p}<0.01$, dyslipidemia 23.7 vs $55.8 \%, \mathrm{p}<0.01$, hypertension 33.4 vs $56.6 \%, p=0.06$ and tobacco 28.8 vs $54.6 \%, p=0.02$ ).

Conclusion: - Admissions to the ICU's Coronary Unit with significant coronary lesion are much more frequent, which makes a reliable comparison between both groups difficult.

- MINOCA patients were more frequently women, younger and with a better cardiovascular risk profile. They are underdiagnosed and undertreated at hospital discharge.

\section{Reference(s) and grant acknowledgment(s)}

1. Agewall $S$ et al. Myocardial infarction with angiographically normal coronary arteries.Atherosclerosis. 2011; 219:10-14.

2. McCabe James, et al. Prevalence and factors associated with false-positive ST-segment elevation myocardial infarction diagnoses at primary percutaneous coronary intervention-capable centers: a report from the Activate-SF registry.Arch Intern Med. 2012; 172:864-871.

3. Sivabaskari Pasupathy, BSc(Hons). Myocardial Infarction With Nonobstructive Coronary Arteries (MINOCA). Volume 135, Issue 16, 18 April 2017, Pages 1490-1493 
000908

The use of carotid doppler to determine volume responsiveness following passive leg raising in hemodynamically unstable patients

M. Boraie ${ }^{1}$; C. Ibtissam ${ }^{1}$

${ }^{1}$ Icu, Burjeel Royal Hospital, Al Ain, United Arab Emirates

Correspondence: $M$. Boraie

Intensive Care Medicine Experimental 2020, 8(2): 000908

Introduction: Critically ill patients have a mixture of intravascular volume depletion, low systemic vascular resistance, and decreased cardiac output, which makes responses to attempts at volume resuscitation difficult to predict. The objectiveness of this study was to determine the value of passive leg raising (PLR)-induced changes in the carotid volumetric blood flow, as assessed by carotid Doppler, which can predict volume responsiveness in a heterogeneous group in the ICU.

Methods: The following patients were enrolled in the study: 1. Patients who present with clinical and laboratory evidence of sepsis/septic shock. 2. Patients who present with evidence of fluid loss due to hyperosmolar hyperglycemia syndrome (HHS). 3. Patients who present with a picture of acute renal failure with no obvious etiology. Common carotid artery Doppler is performed before and after PLR to determine the carotid volumetric blood flow using the formula: Carotid Volume Flow = Cross sectional Area (A) X Time Average Velocity (TAV) $\mathrm{ml} / \mathrm{s}$. A is obtained as $\pi \times$ radius $(D 2), A=D 2 \times 0.785$. A patient whose carotid volume flow increased by $>20 \%$ following PLR was considered a fluid responder. We based our estimate on the study done by Paul Mark et al 1 .

Results: 36 patients were enrolled in the study, $67 \%$ were male; with an average age of $48 \pm 18$ years. The increase in carotid volume flow by $>20 \%$ following PLR was significant in fluid responders $(p<0.05)$. Eighteen patients (50\%) had sepsis/septic shock, five of them were fluid unresponsive and required a vasopressor. In the other thirteen patients of the same group, hypotension and tachycardia resolved with limited fluid resuscitation. We also admitted twelve patients with $\mathrm{HHS}$, ten of those patients were fluid responsive and required limited fluid resuscitation. The other two patients were fluid unresponsive and required maintenance fluid. We also admitted six patients with acute renal failure with no apparent etiology, three of those patients were fluid responsive and required controlled fluid resuscitation. The other three patients were fluid unresponsive and required inotropic support for heart failure.

Conclusion: Our study supports the concept of a hemodynamicallyguided, restricted fluid resuscitation strategy in the hemodynamically unstable ICU patients. Initial fluid resuscitation in those patients should be limited and guided by an assessment of fluid responsiveness through the estimation of carotid volume flow following PLR.

\section{Reference(s) and grant acknowledgment(s)}

1. The author has no conflict of interest to declare

2. 1. The Use of Bioreactance and Carotid Doppler to determine Volume

Responsiveness and Blood Flow Redistribution Following Passive Leg Rais-

ing in Hemodynamically Unstable Patients. Paul E Mark, Alex Levitov, Alisha

Young, Lois Andrews. Chest. 2013; 143(2):364-370.

\section{8}

\section{Safety and effectiveness of temporary cardiac pacing} with the new kronosafe system

R. Vicente-Miralles ${ }^{1}$; DA. Martín-Langerwerf ${ }^{1}$; JM. Nuñez ${ }^{1}$; A. Marco ${ }^{1}$;

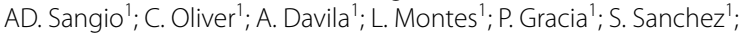

MC. Prieto

${ }^{1}$ Intensive care unit - cardiac stimulation, Vinalopo University Hospital, Elche, Spain

Correspondence: $R$. Vicente-Miralles

Intensive Care Medicine Experimental 2020, 8(2): 000048

Introduction: Temporary Cardiac Stimulation with active-fixation leads has demonstrated to be more effective and safer than the other done without fixation $(1,2,3)$. Nowadays doesn't exist a pacemaker to do it and physicians usually use definitive cardiac stimulation devices (leads and generator) off label. We have developed a new subjection system called KronoSafe ${ }^{\circledR}$, that allows us to hold all the external part of the temporary pacemaker. With our new subjection system, we provide the security that the procedure needs against device detachment or intravascular infection.

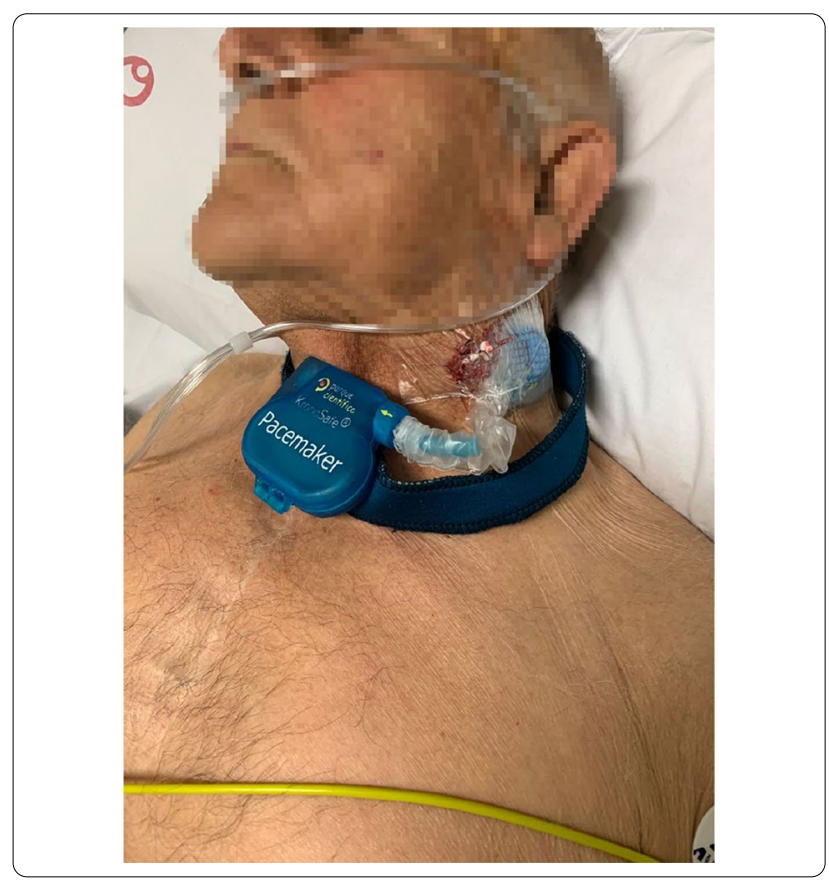

Objectives: The aim of this study was to demonstrate the effectiveness and safety of temporary cardiac stimulation with active-fixation leads subjected with the new KronoSafe System ${ }^{\circledR}$.

Methods: The study was approved by the Clinical Research Ethics Committee of our hospital. We include all consecutive patients underwent temporary cardiac stimulation over 6 months at our centre. A $65 \mathrm{~cm}$ active-fixation lead was implanted throw the left jugular vein to right ventricle apex and connected to a reusable generator We measure $\mathrm{R}$ wave sensed, lead impedance and threshold every 48 hours. Complications related with cardiac stimulation were collected: pace/sense failure, lead or generator detachment and right ventricle perforation. We also describe how much time the patient was in the intensive care unit and standard hospitalization floor under temporary pacing.

Results: 12 patients were included. Every 48 hours medium of R wave sensed was 10-9,56-9,98-9,71 mV; lead impedance was 673-584-573$569 \mathrm{Ohm}$ and the threshold was 0,63-0,42-0,47-0,46 V No complication was registered. The average duration of temporary cardiac stimulation was 7,6 days (min 44 h- max 600 h), 24 hours in ICU and 157 hours in standard hospitalization floor.

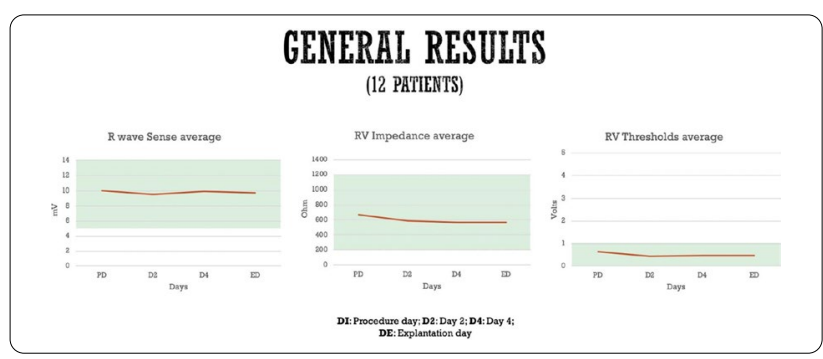


Conclusion: 1- Temporary Cardiac Stimulation with active-fixation leads is safer than conventional temporary stimulation and a safe procedure outside ICU.

2- This kind of temporary cardiac pacing allows an early discharge from ICU.

3- KronoSafe ${ }^{\circledR}$ can provide the security that this procedure needs.

\section{Reference(s) and grant acknowledgment(s)}

1. 1 - Betts TR. Regional survey of temporary transvenous pacing procedures and complications. Postgrad Med J. 2003;79(934):463-5.

2. 2 - Zei PC, Eckart RE, Epstein LM. Modified Temporary Cardiac Pacing Using Transvenous Active Fixation Leads andExternal Re-Sterilized Pulse Generators. J Am Coll Cardiol. 2006;47(7):1487-9

3. 3 - Kawata H, Petrorius V, Phan H, Mulpuru S, Gadiyaram V, Patel J. Utility and safety of temporary pacing using active fixation leads and externalized re-usable permanent pacemakers after lead extraction. Europace. 2013 Sep;15(9):1287-91

4. - We thank the Miguel Hernandez University Scientific Park, for providing the support to develope the project

\section{9}

Prehospital care administration of unfractionated heparin in STEMI as protective factor against no-reflow phenomenon L. Hidalgo Manchado ; RF. Rivera López; M. Morales García; J. Sánchez Gila²; E. Molina Navarro²; R. De La Chica Ruano

${ }^{1}$ Intensive care unit, University Hospital Complex of Granada, Granada, Spain; ${ }^{2}$ Cardiology, University Hospital Complex of Granada, Granada, Spain

\section{Correspondence: L. Hidalgo}

Intensive Care Medicine Experimental 2020, 8(2): 001099

Introduction: The presence of No-reflow (NR) during primary coronary intervention (PCl) in STEMI is associated with worse outcomes and higher mortality. Different strategies have been studied to decrease NR's incidence without success.

Objectives: To asset if there is a reduction in NR's incidence depending on the time of administration of unfractionated heparin (UFH) in patients with STEMI, before or during PCI.

Methods: We perform a retrospective cohort study that includes patients diagnosed of STEMI treated with PCl during 2017 and 2018 in our hospital. Patients who had out-of-hospital cardiac arrest, those who were treated with fibrinolysis and those withouth reperfusion treatment or without obstructive coronary artery disease in $\mathrm{PCl}$ were excluded. We compare 2 groups, in the first one UFH was administered during $\mathrm{PCl}$, and in the second one UFH was administered previously. Arterial flow was analyzed at the beggining and at the end of $\mathrm{PCl}$, using TIMI scale. We also analyzed NR's incidence, mortality and complications.

Results: 389 patients were included, with ages between $61,38 \pm 12,6$ in group 1 vs $64,4 \pm 12$ in group $2(p=0.02)$. The studied variables are shown in table 1, there weren't statistically significant differences in cardiovascular risk factors or previous pharmacological treatment between the groups. Arterial flow at the begging of $\mathrm{PCl}$ and the incidence of in-hospital major bleeding were similar in both groups (TIMI $>1->48.9 \%$ in group 1 vs $52 \%$ in group $2, p=0.56)(1.4 \%(n=2)$ vs $1.6 \%(n=4) ; p=0.9)$ respectively. Mortality rate was $5,9 \%(N=8)$ vs $4,4 \%$ $(\mathrm{N}=11)(\mathrm{p}=0,52)$.

Statistically significant difference was found in NR's incidence, $12,9 \%$ $(\mathrm{N}=18)$ vs $6,4 \%(\mathrm{~N}=16)(\mathrm{p}=0,0028)$, with an $\mathrm{OR}$ of $2,1 \quad(\mathrm{IC} 1,07-4,41)$ $(p=0,036)$.

The multivariable analysis shows that the lack of previous administration of UFH is associated with an increase in NR (Or: 2.48 [1.20-5.33] $\mathrm{p}=0.014)$ and major age too (OR $1.04(1.009-1.07), \mathrm{p}=0.008)$, getting renal insufficiency out of the analysis.
Table 1. Sample's baseline characteristics:

\begin{tabular}{|c|c|c|c|c|}
\hline & $\begin{array}{c}\text { Total } \\
\mathrm{N}=\mathbf{3 9 8}\end{array}$ & $\begin{array}{c}\text { Group } 1 \text { (UFH } \\
\text { during PCI) } N= \\
139\end{array}$ & $\begin{array}{c}\text { Group } 2 \text { (previous } \\
\text { UFH) } N=250\end{array}$ & $\mathbf{P}$ \\
\hline Age & $\begin{array}{l}63.4 \%+/- \\
12.4\end{array}$ & $61.38+/-12.6$ & $64.4+/-12.0$ & 0.021 \\
\hline Male & $79,7 \%$ & $77,7 \%$ & $80,8 \%$ & 0.46 \\
\hline $\begin{array}{l}\text { Arterial } \\
\text { hypertension }\end{array}$ & $29 \%$ & $27,3 \%$ & $30 \%$ & 0.6 \\
\hline Smoker & $15,4 \%$ & $17,3 \%$ & $14,4 \%$ & 0.45 \\
\hline Diabetes & $20,3 \%$ & $22,3 \%$ & $19,2 \%$ & 0.5 \\
\hline Dislipemia & $64,2 \%$ & $51,8 \%$ & $51,6 \%$ & 0.7 \\
\hline $\begin{array}{l}\text { Previous renal } \\
\text { insufficiency }\end{array}$ & $6,2 \%$ & $7,2 \%$ & $5,6 \%$ & 0.7 \\
\hline $\begin{array}{l}\text { TIMI > } 1 \text { before } \\
\text { angioplasty }\end{array}$ & $50,9 \%$ & $48,9 \%$ & $52 \%$ & 0.56 \\
\hline Anti IIB/IIIA & $36,8 \%$ & $38,8 \%$ & $35,6 \%$ & 0.52 \\
\hline NR & $8,7 \%$ & $12,9 \%$ & $6,4 \%$ & 28 \\
\hline In-hospital & $1,5 \%$ & $1,4 \%$ & $1,6 \%$ & 0.9 \\
\hline \multicolumn{5}{|l|}{ bleeding } \\
\hline Stent thrombosis & $2 \%$ & $0 \%$ & $0,5 \%$ & 0.54 \\
\hline Mortality & $4,9 \%$ & $5,9 \%$ & $4,4 \%$ & 0.52 \\
\hline
\end{tabular}

Table 2. Multivariable analysis with logistic regression; dependent variable:

\begin{tabular}{|l|l|l|}
\hline \multicolumn{1}{|c|}{ Variable } & \multicolumn{1}{c|}{ OR } & \multicolumn{1}{c|}{ P } \\
\hline Previous UFH & $2.48(1.20-5.12)$ & 0,014 \\
\hline Age & $1.04(1.01-1.07)$ & 0,008 \\
\hline
\end{tabular}

Conclusion: Early UFH administration is a protective factor against NR and is associated with a better outcome after PCI in STEMI.

001155

The impact of dipyridamle added on dual antiplatelet therapy on stroke prevention and long-term outcome in patients after first acute myocardial infarction

M. T. Wang ; W. C. Huang ${ }^{2}$; C. K. Tsai ${ }^{3}$; S. H. Kuo ${ }^{1}$; K. C. Lin ${ }^{4}$; W. T. Hung ${ }^{4}$; C. C. Cheng ${ }^{5}$; P. L. Tang ${ }^{4}$; C. C. Hung ${ }^{5}$; H. L. Liang $;$; G. Y. Mar ${ }^{4}$

${ }^{1}$ Department of critical care medicine, Kaohsiung Veterans General Hospital, Kaohsiung, Taiwan; ${ }^{2}$ Critical care medicine and cardiovascular center, Kaohsiung Veterans General Hospital, Taipei, Taiwan; ${ }^{3}$ Cardiovascular surgery, Zuoying Branch of Kaohsiung Armed Forces General Hospital, Kaohsiung, Taiwan; ${ }^{4}$ Critical care medicine, Kaohsiung Veterans General Hospital, Kaohsiung, Taiwan; ${ }^{5}$ Critical care medicine and cardiovascular center, Kaohsiung Veterans General Hospital, Kaohsiung, Taiwan

Correspondence: W.C. Huang

Intensive Care Medicine Experimental 2020, 8(2): 001155

Introduction: Dipyridamole based triple antiplatelet therapy (TAPT) on stroke prevention and long-term outcome was unknown. Our study is aimed to evaluate long-term efficacy and safety of dipyridamole based TAPTafter acute myocardial infarction (AMI).

Methods: This is a nationwide, case-control study involving 186,112 first AMI patients. We adopted a ratio of 1:4 propensity score matching based on multiple variables. Finally, we enrolled4,540 patients in the dual antiplatelet therapy (DAPT) group and 1,135 patients in the TAPT group in our final analysis.

Results: Overall survival rate was comparable between two groups of patients (log-rank $P=.0569$ ), regardless ofsex. In age subgroup, TAPT group showed poor survival in younger patients (log-rank $\mathrm{P}=$ .0072), but no survival difference compared with DAPT group in older patients.There was no extra benefit of TAPT for patients after AMI regardless of types of myocardial infarction (MI).DAPT was superior 
to TAPT for patients underwent percutaneous coronary intervention $(\mathrm{PCl})$ (log-rank $\mathrm{P}=.0022)$. TAPT did not reduce cumulative event rate of recurrent $\mathrm{MI}$ or recurrent stroke in patients after AMI. TAPT increased cumulative intracerebral hemorrhage $(\mathrm{ICH})$ rate (log-rank $P=.0026)$ but did not affect cumulative event rate of gastrointestinal (GI) bleeding free survival. Dipyridamole did not contribute to AMI survival.

Conclusion: TAPTdid not contribute to overall AMI survival, and did not reduce recurrent rate of MI or stroke. TAPT increased ICH ratewithout effect onthe incidence of Gl bleeding compared with DAPT.

\section{6}

The impact of Ambulance prehospital dual-antiplatetet medication system in patients with acute myocardial infarciton:a 8-year city-based multicenter study

C. C. Hung' ; W. C. Huang ${ }^{2}$;. J. Liou ${ }^{3}$; C. K. Lin ${ }^{3}$; C. H. Chiang ${ }^{1}$; C. C. Cheng'; F. Y. Kuo ; ' L. S. Yan ${ }^{4}$; P. L. Kang ${ }^{5}$; G. Y. Mar ${ }^{6}$

${ }^{1}$ Critical care medicine and cardiovascular center, Kaohsiung Veterans General Hospital, Kaohsiung, Taiwan; ${ }^{2}$ Critical care medicine and cardiovascular center, Kaohsiung Veterans General Hospital, Taipei, Taiwan; ${ }^{3}$ Fire bureau, Fire Bureau of Kaohsiung City, Kaohsiung, Taiwan; ${ }^{4}$ Health, Kaohsiung City of Government, Kaohsiung, Taiwan; ${ }^{5}$ Department of critical care medicine, Kaohsiung Veterans General Hospital, Kaohsiung, Taiwan; ${ }^{6}$ Critical care medicine, Kaohsiung Veterans General Hospital, Kaohsiung, Taiwan

\section{Correspondence: W.C. Huang}

Intensive Care Medicine Experimental 2020, 8(2): 001156

Introduction: Early use of dual anti-platelet therapy (DAPT) in ST elevation myocardialinfarction (STEMI) can reduce stent thrombosis and further save the patients. However, it is still difficult to set up ambulance paramedics DAPTsystem for patients after STEMI. Therefore, the aim of this studyis to investigate improving use of DAPTin ambulance for acute myocardial infarction patients in a city-based program.

Methods: A multidisciplinary team among 15 hospitals, fire bureau and departmentof health, Kaohsiung city government was organized since 2016. The key interventionsinclude Asian first innovativecity-based prehospital ambulance paramedic STEMI dual anti-platelet medicationonline instruction system, direct chewing DAPT at ambulance, training programfor ambulance paramedics, 24 hours STEMI interpretation and on-line intrusctionteam. The consecutativeSTEMIdiagnosed by ambulance electrocardiogram system were enrolled from Jan. 2011 toDec. 2018 in Kaohsiung city. The patients weredivided into three group: preinterventional group from 2011-2015, Interventionalgroup from Jan to Dec 2016 and post-interventional group from 2016 to 2018.

Results: Thepercentage of ambulance DAPT use in adequate STEMI patients increased from $0 \%$ in pre-interventional group, to $2.8 \%$ ininterventional group and to $57.8 \%$ in post-interventional group $(p<0.001)$. Inthese STEMI patients received DAPT at ambulance, there was not any bleeding complications.Furthore, the mortality was $0 \%$ in these patients. Conclusion: This study showed, from 2011 to 2018, innovative citybased pre-hospitalambulance paramedic STEMI dual anti-platelet medication on-line instruction system can improve percentage of ambulance DAPT use and also outcomes inSTEMI patients.

\section{9}

Application of sequential organ failure assessment (SOFA) score in patients who underwent cardiac surgery: a prospective study

A. M. Noble ${ }^{1}$; C. Permejo

'Department of Adult Cardiology, Philippine Heart Center, Quezon City, Philippines

\section{Correspondence: A.M. Noble}

Intensive Care Medicine Experimental 2020, 8(2): 000119

Introduction: After cardiovascular (CV) surgery, clinicians must identify patients at high risk to develop mortality/morbidity from multi-organ failure, and should quantify it through time to intensify treatment. The SOFA score is an objective tool in predicting outcomes in patients with severe sepsis1.
Objectives: This study identified the association of SOFA score with organ dysfunction/failure, death or clinical improvement in patients who underwent CV surgery.

Methods: This is a single-center prospective cohort study that included 63 patients, $\geq 19$ years old, admitted at the Surgical Intensive Care Unit (SICU) $\leq 3$ days, who underwent any CVS (cardiopulmary bypass, valve, congenital and vascular surgeries). The Institutional Ethics Review Board (IERB) of the institution approved the study in accordance with the ethical principles set forth in the Declaration of Helsinki and the National Ethical Guidelines for Health and Health-related Research of 2017. Informed consent was obtained prior to the conduct of the study.

The SOFA variables were obtained pre-operatively and post-operative at day 0 and day 3 . The score was computed using the $O x$ calculator $^{\circledR}$. Total maximum SOFA (TMS SOFA) and the delta SOFA ( $\triangle$ SOFA) were also calculated.

Results:SOFA score analysis. The SOFA score was compared pre-operatively, on Day 0 post-operative and day 3 post-operative. In addition, the SOFA variables were also compared on these three periods. Table 4 compared the SOFA score and the SOFA variables on these periods. $\triangle$ SOFA score and the individual SOFA parameters (except for serum creatinine and bilirubin levels) were significantly different in the three periods $(p<0.001)$.

\begin{tabular}{|c|c|c|c|c|}
\hline & Pre-operative & $\begin{array}{l}\text { Day 0 Post- } \\
\text { operative }\end{array}$ & $\begin{array}{l}\text { Day } 3 \text { Post- } \\
\text { operative }\end{array}$ & \multirow[t]{2}{*}{ P-valu } \\
\hline & \multicolumn{3}{|l|}{ Median (IQR) } & \\
\hline $\mathrm{PaO2}$ & $\begin{array}{l}95(85.6 \text { to } \\
112)\end{array}$ & 203 (147 to 241$)$ & $130(98$ to 165$)$ & $<0.001$ \\
\hline $\mathrm{FiO2}$ & 21 (21 to 80$)$ & $60(60$ to 100$)$ & 21 (21 to 100$)$ & $<0.001$ \\
\hline Platelet count & $\begin{array}{l}254(217 \text { to } \\
288)\end{array}$ & 169 (116 to 195$)$ & 177 (125 to 261$)$ & ) $<0.001$ \\
\hline MAP & 83 (72 to 93 ) & 93 (85 to 98 ) & $84(75$ to 90$)$ & $<0.001$ \\
\hline $\begin{array}{l}\text { Use of } \\
\text { inotropes }\end{array}$ & 1 ( 1 to 1$)$ & 3 (1 to 4$)$ & 1 (1 to 4$)$ & $<0.001$ \\
\hline GCS & $15(15$ to 15$)$ & $10(10$ to 19$)$ & $15(10$ to 15$)$ & $<0.001$ \\
\hline Creatinine & $\begin{array}{l}0.9(0.79 \text { to } \\
1.13)\end{array}$ & $\begin{array}{l}0.9(0.68 \text { to } \\
1.13)\end{array}$ & $\begin{array}{l}0.85(0.68 \text { to } \\
1.03)\end{array}$ & 0.203 \\
\hline Urine output & $\begin{array}{l}1500(1150 \text { to } \\
1900)\end{array}$ & $\begin{array}{l}2450(1790 \text { to } \\
3100)\end{array}$ & $\begin{array}{l}1800(1350 \text { to } \\
2120)\end{array}$ & $<0.001$ \\
\hline eGFR & $\begin{array}{l}93.2(68.8 \text { to } \\
106.5)\end{array}$ & $\begin{array}{l}95.4(68 \text { to } \\
112.9)\end{array}$ & $\begin{array}{l}99(72.2 \text { to } \\
115.8)\end{array}$ & $<0.001$ \\
\hline Bilirubin & $\begin{array}{l}1.04(0.81 \text { to } \\
1.58)\end{array}$ & $\begin{array}{l}1.7(1.23 \text { to } \\
2.68)\end{array}$ & $\begin{array}{l}1.52(0.92 \text { to } \\
2.14)\end{array}$ & 0.391 \\
\hline SOFA score & 1 (0 to 2$)$ & 7 (6 to 9$)$ & 2 (1 to 3 ) & $<0.001$ \\
\hline
\end{tabular}

$\mathrm{PaO} 2$ partial pressure of oxygen $\mathrm{FiO} 2$ fraction of inspired oxygen in the air $\mathrm{MAP}$ mean arterial pressure GCS Glasgow coma scale $e G F R$ estimated glomerular filtration rate $S O F A$ sequential organ failure assessment score

Predictors of organ dysfunction, failure and mortality. Valvular surgeries, platelet count and bilirubin levels, as well as the MAP, were shown to be predictors of outcome ( $P$ valve $0.032,0.006,0.023$ and 0.041 respectively). In patients undergoing valvular surgeries, they have more than 3 times risks to develop organ failure/dysfunction or mortality as compared to those who underwent other cardiovascular surgeries (OR 3.1429, 1.1068 to $8.924595 \% \mathrm{Cl}, 0.032$ p-value). Postoperatively, for every unit increase in platelet count, the odds of developing organ failure/dysfunction or mortality decreased by $0.94 \%$ (OR $0.9906,0.9839-0.997495 \% \mathrm{Cl}, 0.006$ p-value). Post-operatively, for every unit increase in MAP, the odds of developing organ failure/dysfunction or mortality decreases by $5.31 \%$ (OR $0.9469,0.8987-0.9977$ $95 \% \mathrm{Cl}, 0.041 \mathrm{p}$-value). Post-operatively, for every unit increase in bilirubin, the odds of developing organ failure/dysfunction or mortality increased by $103 \%$ (OR 2.0303, $1.1013-3.742995 \% \mathrm{Cl}, 0.023 \mathrm{p}$-value). Conclusion: Exposure of patients undergoing CV surgery to the bypass machine may lead to deleterious sequelae explaining the high TMS SOFA and $\triangle$ SOFA score in the immediate post-operative period. As organ dysfunction improves or deteriorates in the succeeding days after intervention, it is coupled to a corresponding decrease or increase of the said scores. Our study showed that the SOFA score is a simple, reproducible, and readily available tool that could assist the clinician in intensifying treatment in this subset of patient; thereby, decreasing the risk for morbidity or death. 
Reference(s) and grant acknowledgment(s)

1. 1. Ferreira F. Serial Evaluation of the SOFA Score to Predict Outcome in Critically III Patients. JAMA. 2001;286(14):1754

2. 2. Ceriani R, Maurizio M, Bortone F, Gandini S, Solina C, Susini G, Parodi O. Application of the Organ Failure Assessment Score to Cardiac Surgical Patients. Chest Journal. 2003;123(4):1229-1239

3. 3. Janssens U, Dujardin R, Graf J, Lepper W, Ortlepp J, Merx M et al. Value of SOFA (Sequential Organ Failure Assessment) score and total maximum SOFA score in 812 patients with acute cardiovascular disorders. Critical Care. 2001;5(Suppl 1):P225

4. 4. Vincent J, de Mendonca A, Cantraine F, Moreno R, Takala J, Suter P et al. Use of the SOFA score to assess the incidence of organ dysfunction/failure in intensive care units. Critical Care Medicine. 1998;26(11):1793-1800

5. 5. Bingold T, Lefering R, Zacharowski K, Meybohm P, Waydhas C, Rosenberger $\mathrm{P}$ et al. Individual Organ Failure and Concomitant Risk of Mortality Differs According to the Type of Admission to ICU - A Retrospective Study of SOFA Score of 23,795 Patients. PLOS ONE. 2015;10(8):e0134329

6. 6. Vincent J, de Mendonca A, Cantraine F, Moreno R, Takala J, Suter P et al. Use of the SOFA score to assess the incidence of organ dysfunction/failure in intensive care units. Critical Care Medicine. 1998;26(11):1793-1800

7. 7. Definition of Death. www.medicinenet.com

8. 8. Stephens RS, Whitman GJR. Postoperative Critical Care of the Adult Cardiac Surgical Patient. Part 1: Routine Postoperative Care. Critical Care Medicine 2015;43(7)1477-1479

9. 9. Argyriou G, Vrettou C, Filippatos G, Sainis G, Nanas S, Routsi C. Comparative evaluation of Acute Physiology and Chronic Health Evaluation II and Sequential Organ Failure Assessment scoring systems in patients admitted to the cardiac intensive care unit. Journal of Critical Care. 2015;30(4):752-757

\section{9}

Analysis of right ventricular failure after cessation of nitric oxide inhalation in left ventricular assist device implantation

R. Sakaguchi ${ }^{1}$; A. Tanaka ${ }^{1}$;.. Horiguchi ; R. Higeno ${ }^{1}$; A. Uchiyama”;

Y. Fujino

${ }^{1}$ Anesthesiology and intensive care medicine, Osaka University, Suita, Japan

Correspondence: R. Sakaguchi

Intensive Care Medicine Experimental 2020, 8(2): 000139

Introduction: Right ventricular failure (RVF) after left ventricular assist device (LVAD) implantation increases morbidity and mortality1. Nitric oxide inhalation (NOi) is frequently used to prevent and treat RVF. Previous studies reported a few preoperative hemodynamic predictors of RVF such as CVP/PCWP (pulmonary wedge pressure) ratio, RV stroke work index1,2. However, the criteria to wean of and stop NOi are not fully standardized.

Objectives: The aim of our study was to investigate the risk factors and the outcome of restart of NOi after cessation in LVAD implantation.

Methods: A retrospective medical review was performed on 79 patients who underwent continuous flow type of LVAD (CF-LVAD) implantation and received NOi at the arrival of ICU (intensive care unit) rom April 2014 to March 2019 at Osaka University Hospital. Patients under 18 years of age and those who underwent biventricular assist device implantation were excluded. Univariate and ROC analysis were performed to clarify the risk factors and cut-off value. This retrospective study was approved by the clinical research ethics committee of Osaka University Hospital (Ethical Committee number 19244).

Results: One patient without PA catheter was excluded. Of the entire 78 patients, $17(22 \%)$ restarted NOi within 48 hours after cessation. In hemodynamic parameters just before cessation of NOi, CVP and CVP/ dPAP (diastolic pulmonary pressure) ratio was higher in patients with restart of NOi $(p<0.01)$. ROC analysis showed Area Under the curve (AUC) of CVP was larger than that of CVP/dPAP $(p<0.05)$. Cut-off value were $11 \mathrm{mmHg}$ in CVP (sensitivity 0.88 , specificity 0.75 ), and 1.3 (sensitivity 0.77 , specificity 0.79 ) in CVP/dPAP ratio. Compared with patients without restart of NOi, patients with restart of NOi had shorter ventilator-free days within 28 days [13 days (interquartile range, $0-20$ days) vs. 23 days (interquartile range, 19-26 days), $p<0.01$ ] and longer ICU length of stay [22 days (interquartile range, 12-44 days) vs. 10 days (interquartile range, $7-16$ days), $\mathrm{p}<0.01]$.

Conclusion: CVP and CVP/dPAP ratio are useful criteria to discontinue $\mathrm{NO}$ inhalation. Also, restart of NO inhalation decreases ventilator-free days within 28 days and increases ICU length of stay.

\section{Reference(s) and grant acknowledgment(s)}

1. 1. Kormos RL, Teuteberg JJ, Pagani FD, et al. J Thorac Cardiovasc Surg 2010; 139(5):1316-24.

2. 2. Fitzpatrick JR 3rd, Frederick JR, Hsu VM, et al. J Heart Lung Transpl 2008;27(12): 1286-92.

\section{0}

24 hours ABPM, vascular risk profile and ventricular function deterioration in patients type 2 diabetes mellitus with acute heart failure

A. M. González González; A. M. Garcia Bellon; J. A. Cano-Nieto1; M. De Mora-Martin

${ }^{1}$ Cardiology, Regional Hospital of Malaga, Málaga, Spain

Correspondence: A.M. González González

Intensive Care Medicine Experimental 2020, 8(2): 000160

Introduction: Ambulatory blood pressure monitoring (ABPM) permites the evaluation of 24 hours blood pressure pattern.

Objectives: Our aim was to evaluate a possible negative influence of the presence of Type 2 Diabetes mellitus (T2DM) on $24 \mathrm{~h}$. ABPM pattern, vascular risk profile and severity of left ventricular (LV) function in patients with Acute Heart Failure (AHF).

Methods: In 122 patients with AHF we compare clinical features of 42 (65 \pm 9 years / 87,5\% males) with T2DM versus 80 (63 \pm 11 years / 68,3\% males) non-T2DM.

In addition to clinical examination and analytical parameters, all patients underwent a $24 \mathrm{~h}, \mathrm{ABPM}$ and echocardiogram and evaluation of degree of severity LV Dysfunction.

Results: Patients with T2DM had higher $(p<0.001)$ prevalence $(\%)$ of Hypertension (75 vs 46.7), dyslipidemia (65.6 vs 28.3 ), obesity (47 vs 43), and worse renal function (fGe: 64.7 vs $72.3 \mathrm{ml} / \mathrm{min} / 1.75 \mathrm{~m} 2$ ). Etiology IC: Hypertensive and/or ischemic heart disease: $75 \%$ vs $60 \%$. Mean Values of $24 \mathrm{~h}$ ABPM measurements are in table 1.

\begin{tabular}{|c|c|c|c|}
\hline $\begin{array}{l}\text { Mean Values } \\
(\mathrm{mmHg})\end{array}$ & T2DM & $\mathbf{p}$ & NonT2DM \\
\hline 24. SBP & $116 \pm 16$ & $<0.05$ & $111 \pm 15$ \\
\hline Daytime SBP & $117 \pm 15$ & $<0.05$ & $112 \pm 15$ \\
\hline Nightime SBP & $114 \pm 18$ & $<0.04$ & $108 \pm 16$ \\
\hline 24h DBP & $65 \pm 7$ & ns & $65 \pm 8$ \\
\hline Daytime DBP & $67 \pm 8$ & ns & $67 \pm 9$ \\
\hline Nightime DBP & $64 \pm 7$ & ns & $69 \pm 9$ \\
\hline Pulse Pressure & $50 \pm 13$ & 0.05 & $46 \pm 11$ \\
\hline Nocturnal PP & $51 \pm 15$ & 0.05 & $47 \pm 12$ \\
\hline
\end{tabular}

No significant differences in DBP between groups, but patient with T2DM present higher average values $(\mathrm{p}<0.05)$ of SBP $24 \mathrm{~h}(\mathrm{mmHg})$ : 116/111); daytime (117/112) and nightime (114/108); higher $24 \mathrm{~h}$ Pulse Pressure (50 vs $46 \mathrm{mmHg}$ ) and non-dipper pattern frequency (84.7 vs 79.35), as well as a greater proportion of patients with moderate/severe LV dysfunction ( 87.6 vs $81.7 \%)(p<0,05)$.

We also observed worse NYHA functional class in T2DM patients: NYHA II-III 84,4 vs 58,3\%, p<0,001.

Conclusion: In patients with $\mathrm{AHF}$, the presence of T2DM contributes to show greater hypertensive and/or ischaemic ethiology, further deterioration of $24 \mathrm{~h}$ ABPM pattern and worse left ventricular myocardial function.

T2DM can be considered as a risk factor and worsening of heart failure. $24 \mathrm{~h}$ ABPM may contibute to a better prognostic evaluation in these patients. 
000211

Dynamic arterial elastance after thoracoadominal aortic repair M. Gräßler'; C. Behem ${ }^{1}$; S. Wipper ${ }^{2}$; A. Dupree ${ }^{3}$; DA. Reuter ${ }^{4}$; E. Debus ${ }^{2}$; C. Trepte ${ }^{1}$

${ }^{1}$ Department of anaesthesiology, University Medical Center Hamburg-Eppendorf, Hamburg, Germany; ${ }^{2}$ Department of vascular medicine, University Heart \& Vascular Center Hamburg, Hamburg, Germany; ${ }^{3}$ Department of general, visceral and thoracic surgery, University Medical Center Hamburg-Eppendorf, Hamburg, Germany; ${ }^{4}$ Department of anaesthesiology and intensive care medicine, University Hospital of Rostock, Rostock, Germany

Correspondence: M. Gräßler

Intensive Care Medicine Experimental 2020, 8(2): 000211

Introduction: Dynamic arterial elastance (Eadyn) has been shown to be a dynamic parameter for arterial load assessment and to predict arterial pressure response to volume administration 1 . Since volume administration is a key part of hemodynamic resuscitation, arterial load assessment and knowledge of arterial pressure response to volume administration have lately become of particular interest. This is especially relevant in aortic repair, since maintenance of adequate spinal cord, renal and visceral perfusion pressure is a key element of peri-operative management. Although several studies could show that changes in cardiac preload and afterload influence Eadyn, the impact of changes in vascular properties, like aortic repair, have not been assessed so far2.

Objectives: To date the effect of changes in vascular properties, like aortic repair, on dynamic arterial elastane have not been assessed systematically. Therefore the aim of this study was to test whether aortic repair affects dynamic arterial elastance and its ability to predict arterial pressure response to volume administration.

Methods: The study was designed as prospective observational trial in 18 domestic pigs. Animals were anaesthetized and mechanically ventilated. Hemodynamic data were obtained using transcardiopulmonary thermodilution and pulse contour analysis. 4 consecutive volume loading steps each consisting of $6 \mathrm{ml} / \mathrm{kg}$ bodyweight hydroxyethyl starch were performed in baseline conditions and repeated 6 hours after hybrid aortic repair. Aortic repair was performed using a hybrid-graft technique consisting of a thoracic stent graft and open re-insertion of the abdominal and iliacal branches. An increase in mean arterial pressure (MAP) $>15 \%$ was considered as a positive pressure response. Values are given as mean ( $95 \%$ confidence interval), two-tailed $p$-values of $<0.05$ were considered significant.

Results: In baseline conditions Eadyn decreased (1.20 vs. 0.94, $\mathrm{p}<0.001)$ during volume loading and presented with an AUC of 0.84 $(0,73-0.95)$. After aortic repair Eadyn also decreased (0.97 vs. 0.85 , $\mathrm{p}=0.08$ ) during volume loading while the ability to predict pressure response decreased to an AUC of $0.56(0.38-0.74)$. Baseline values were significantly changed after aortic repair $(1.20$ vs $0.97, p<0.05)$

Conclusion: Eadyn values were significantly changed after aortic repair. Further Eadyn was not able to predict arterial pressure response to volume administration when vascular properties changed after thoracoabdominal aortic repair

\section{Reference(s) and grant acknowledgment(s)}

1. 2. Monge García MI, Guijo González P, Gracia Romero M, Gil Cano A, Rhodes A, Grounds RM, Cecconi M .Effects of arterial load variations on dynamic arterial elastance: an experimental study Br J Anaesth. 2017 Jun 1;118(6):938-946

2. 1. Monge García MI, Gil Cano A, Gracia Romero M. Dynamic arterial elastance to predict arterial pressure response to volume loading in preload-dependent patients Crit Care. 2011;15(1):R15

3. The study was supported by departmental funds of the Department of Anaesthesiology, University Medical Centre Hamburg-Eppendorf
000378

Volume status assessment with cardiac variation of internal jagular vein

H. Nakano'; M. Masaki ${ }^{1}$; N. Hiromu' ${ }^{1}$;. Yuji'; S. Tomohiro ${ }^{1}$; H. Hideki';

N. Kensuke

${ }^{1}$ Department of emergency and critical care medicine, Hitachi Genaral Hospital, Hitachi, Japan

Correspondence: $\mathrm{H}$. Nakano

Intensive Care Medicine Experimental 2020, 8(2): 000378

Introduction: Minimally invasive volume status assessments are highly required in emergency and critical care medicine, such as the respiratory variation of inferior vena cava (IVC) [1]. Previously, we focused on cardiac variation of major veins as a new index of it $[2,3]$, and reported the association between cardiac variation of internal jugular vein (IJV) and stroke volume (SV) via venous return [3]. This is the first study which evaluated the cardiac variation of IJV in the emergency department.

Objectives: The purpose of this study was to confirm the efficacy of cardiac variation of IJV as an indicator for hemodynamics related to fluid volume.

Methods: This is a single-center prospective observational study. Patients over the age of 20 with need of fluid resuscitation who came in by ambulance were included from August 2019 to March 2020. Those who were severely ill and needed immediate treatments were excluded.

Cardiac variation of right IJV cross section area and respiratory variation of IVC diameter were evaluated by echography, SV, stroke volume variance (SVV) were evaluated by ClearSight system (Edwards Lifesciences, Irvine, CA, USA), and vital signs were recorded before infusion. Then, after infusion of $500 \mathrm{~mL}$ crystalloid, SV, SVV and vital signs were recorded again. To evaluate cardiac variation, we applied speckle tracking technique with ACUSON S2000 HELX EVOLUTION (Siemens, Munich, Germany). Collapse index was calculated by following formula; collapse index = (maximum - minimum) / maximum. Fluid responsiveness was defined as SV increase after infusion of $15 \%$ or more. The setting of age, gender, height, and weight were fixed in ClearSight measurement to shorten the evaluation time before treatment and provisional SV were recorded. Since the provisional SV is higher with age, we analyzed the provisional SV divided by age.

The primary outcome was fluid responsiveness. We evaluated the correlation with IJV cardiac variation, IVC respiratory variation or SVV. The secondary outcome was SV before infusion. We assessed correlation with IJV cardiac variation.

Results: Among 12,009 patients visiting emergency department during the study period, 103 patients were included in analysis. The mean age of patients was 74.0 years old, $55.3 \%$ were male, and $37.9 \%$ responded to fluid infusion. The mean collapse index of IJV cardiac variation was $15.6 \pm 13.6 \%$. Collapse index of IJV cardiac variation $(16.8 \pm 14.9 \%$ vs $13.6 \pm 10.9 \%, p=0.24)$ and collapse index of IVC respiratory variation $(41.8 \pm 27.5 \%$ vs $45.6 \pm 27.6 \%, p=0.53)$ were not different between those with and without fluid responsiveness, and SVV $(p=0.02)$ before infusion showed significant difference. On the other hand, correlation was found between collapse index and SV/age $(r=0.25, p=0.01)$.

Conclusion: Not IJV cardiac variation and IVC respiratory variation, but only SVV was correlated to fluid responsiveness. IJV cardiac index was associated with SV in emergency clinical setting similarly with the previous study.

\section{Reference(s) and grant acknowledgment(s)}

1. [3] Nakamura K, Qian K, Ando T, et al. Cardiac Variation of Internal Jugular Vein for the Evaluation of Hemodynamics. Ultrasound Med Biol. 2016;42(8):1764-1770.

2. [2] Nakamura K, Tomida M, Ando T, et al. Cardiac variation of inferior vena cava: new concept in the evaluation of intravascular blood volume. J Med Ultrasonics. 2013:40(3):205-209.

3. [1] Nagdev AD, Merchant RC, Tirado-Gonzalez A, Sisson CA, Murphy MC. Emergency department bedside ultrasonographic measurement of the 
caval index for noninvasive determination of low central venous pressure. Ann Emerg Med. 2010;55(3):290-295

4. This work was supported by Grant-in-Aid for Young Scientists (19K18314)

\section{5}

Decrease of haemoconcentration in dyspnoeic patients reliably detects hydrostatic pulmonary oedema in the Emergency Department

F. Gavelli' ; I. Nerici'; X. Monnet ${ }^{2}$; D. Azzolina ${ }^{3}$; S. Priora ${ }^{1}$; V. Giai Via';

M. Bertoli ${ }^{1}$; C. Foieni ${ }^{1}$; N. De Vita ${ }^{4}$; F. Patrucco ${ }^{5}$; PP. Sainaghi' ${ }^{1}$ GC. Avanzi ${ }^{1}$;

JL. Teboul ${ }^{2}$; LM. Castello'

${ }^{1}$ Emergency medicine department, Department of Translational Medicine - Università degli Studi del Piemonte Orientale, Novara, Italy; ${ }^{2}$ Service de médecine intensive-réanimation, Hôpital de Bicêtre, Hôpitaux universitaires Paris-Sud, AP-HP, Le Kremlin-Bicêtre, France; ${ }^{3}$ Research support unit, Department of Translational Medicine - Università degli Studi del Piemonte Orientale, Novara, Italy; ${ }^{4}$ Intensive care unit, Department of Translational Medicine - Università degli Studi del Piemonte Orientale, Novara, Italy; ${ }^{5}$ Division of respiratory diseases, Department of Translational Medicine - Università degli Studi del Piemonte Orientale, Novara, Italy

Correspondence: F. Gavelli

Intensive Care Medicine Experimental 2020, 8(2): 000405

Introduction: To diagnose the cause of acute respiratory failure (ARF) in the emergency department (ED) is difficult. In particular, the differential diagnosis between hydrostatic pulmonary oedema (HPO) and other lung diseases is often challenging. Today, the diagnosis of HPO is mainly based on clinical criteria and the dosage of B-type natriuretic peptide, which is costly and suffers from some false positives, especially in case of renal failure. Since HPO is due to transfer of a significant volume of plasma through the pulmonary capillary barrier toward the interstitium and alveoli, it has been demonstrated that this volume is large enough for inducing haemoconcentration, whose level reflects the volume of lung oedema. However, the diagnostic accuracy of haemoconcentration in a population of dyspnoeic patients in the ED has never been investigated.

Objectives: To evaluate whether the variation of haemoglobin concentration $(\Delta \mathrm{Hb})$ over the first hours of treatment in dyspnoeic patients allows discriminating HPO from other causes of dyspnoea and ARF.

Methods: We retrospectively collected data of patients admitted to a medium-size ED for dyspnoea and ARF as leading problem, and for whom at least two $\mathrm{Hb}$ measurements (one at ED admission and one within the next 6 hours) were available. Patients were excluded if data regarding therapeutic management, radiological and biochemical investigations were not retrievable and if they were not subsequently admitted to an hospital ward. Two expert investigators, unaware of $\Delta \mathrm{Hb}$, defined a posteriori the cause of dyspnoea as HPO and non-HPO, according to clinical, instrumental and biochemical findings. The ability of $\Delta \mathrm{Hb}$ to detect HPO as the main cause of dyspnoea was evaluated through the area under the receiver operating characteristic (AUROC) curve.

Results: Between November 1st, 2018 and December 31st, 2019, 1391 dyspnoeic patients were admitted to our ED. 572 patients were included in the analysis, among which 174 (30.4\%) were diagnosed with $\mathrm{HPO}$. HPO patients showed a significantly higher value of $\mathrm{Hb}$ at ED presentation compared to non-HPO (12.8[11.5-14.1] g/dL vs. 12.2[10.8$13.7 \mathrm{gg} / \mathrm{dL}$, respectively; $\mathrm{p}=0.002$ ). At 6 hours from admission and the beginning of therapeutic management, relative $\Delta \mathrm{Hb}$ was significantly higher in HPO than in non-HPO patients $(-8.4[-11.4$ to -5.8$] \%$ vs. 0.7[$1.9-3.6] \%$, respectively; $\mathrm{p}<0.0001)$. The AUROC for $\triangle \mathrm{Hb}$ to detect HPO was $0.896[0.889-0.903]$ (sensitivity and specificity $0.81[0.80-0.84]$ and $0.90[0.87-0.92]$, respectively) with a cut-off of $-4.3 \%$. When absolute $\Delta \mathrm{Hb}$ was considered, similar results were observed $(-1.0[-1.5$ to -0.8$]$ $\mathrm{g} / \mathrm{dL}$ vs. $0.1[-0.2-0.4] \mathrm{g} / \mathrm{dL}$, respectively; $\mathrm{p}<0.0001)$, with an AUROC of $0.892[0.885-0.899]$ (sensitivity and specificity $0.82[0.80-0.83]$ and $0.88[0.87-0.90]$, respectively) and a cut-off value of $-0.6 \mathrm{~g} / \mathrm{dL}$.

Conclusion: $\mathrm{A}$ reduction of $\mathrm{Hb}$ concentration $\geq 4.3 \%$ over the first hours of treatment reliably identifies HPO as the main cause of dyspnoea and ARF in patients admitted to the ED.

\section{2}

Echocardiographic assessment of systolic and diastolic function in critical care unit. Prognostic influence

G. Ferrigno'; V. Losada Martínez ${ }^{2}$; A. Tejero Pedregosa²; N. Rodrigo

Castroviejo ${ }^{2}$; R. Beltrán Bernáldez²; C. Sanmartino Gonzalez²; C. Tarancón

Maján²; A. Marcos Gutiérrez²; P. Cañizares Ortiz²; S. Cortés Díaz

${ }^{1}$ Intensive Care Unit, Hospital Virgen De La Concha, Zamora, Spain; ${ }^{2}$ Inten-

sive care, Hospital Virgen De La Concha, Zamora, Spain

Correspondence: $\mathrm{G}$. Ferrigno

Intensive Care Medicine Experimental 2020, 8(2): 000452

Introduction: Critical-care echocardiography (CCE) has been recently recognize by the Echocardiography Working Group of the Cardio- vascular Dynamics section of the European Society of Intensive Care Medicine (ESICM) and several studies have shown its growing importance in the critical patient.

Objectives: To evaluate biventricular systolic and diastolic function in ICU patients. To estimate the delay in performing the echocardiogram, as well as its prognostic influence in patients with different pathologies.

Methods: Echocardiographic studies conducted during the period 2018-2019 were reviewed and the parameters of systolic (LVEF by Simpson and Teicholz method, TAPSE) and diastolic (E/A and E/e' ratio) function as well as preload parameters (diastolic LV volume, inferior vena cava diameter) were collected. The delay in carrying out the study from the time of admission and the distribution by shifts (morning, afternoon, night) of these wereanalyzed. The influence of systolic and diastolic dysfunction on mortality and ICU stay was evaluated. The variables are expressed as a mean $(95 \% \mathrm{Cl})$.

Results: 197 patients with the following baseline characteristics were included. Age 68.8 (66.9-70.7) years, sex $64.6 \%$ men, weight 75.4 (72.6-78.2) kg, height $165.3(163.9-166.7) \mathrm{cm}$, APACHE 17.8 (16.5-19.1), SAPS II 41.8 (39.2-44.3), SOFA 4.8 (4.2-5.4). The reason for admission was: sepsis/septic shock $14.8 \%$, heart failure $12.3 \%$, acute coronary syndrome $12.3 \%$, pulmonary embolism $10.3 \%$, acute respiratory failure $8.7 \%$, cardiorespiratory arrest $7.2 \%$, neurocritical $5.6 \%$, acute on chronic respiratory failure $5.1 \%$, trauma $2.6 \%$, myopericarditis $2.6 \%$, other $17.9 \%$. The average stay was $7.8(6.1-9.5)$ days, with a mortality rate of $20 \%$. Systolic function was $41.7 \%$ (39.6-43.9) \% Teicholz, $48.8 \%$ (39.7-57.8) \% Simpson, 20.3 (19-21.6) mmTAPSE. Diastolic function was: E/A 1.8 (1.2-2.4), E/e' 12.5 (11-13.9). The LVEDV was 106.4 (99.9$112.9) \mathrm{ml}$ and the IVC was $17.8(16.5-19) \mathrm{mm}$. 4.1 (3.6-4.6) images and 2.9 (2.4-3.3) video clips were archived per study. The study was performed at 45.1 (31.9-58.3) hours after admission, the distribution by shifts was: morning $63.1 \%$, afternoon $25.6 \%$ and night $11.3 \%$. Statistically significant differences (ANOVA test) were observed in the Simpson LVEF $(p=0.006)$ and the E/A ratio $(p=0.007)$ between the different diagnostic groups, with the heart failure and cardiac arrest groups showing the highest degree of dysfunction. The presence of systolic or diastolic dysfunction did not influence ICU stay or mortality.

Conclusion: Conclusions: Routine echocardiographic evaluation of critical patients provides information on systolic and diastolic function. It can guide treatment and advise on prognosis.

\section{Reference(s) and grant acknowledgment(s)}

1. Huang et al. Ann. Intensive Care (2020) 10:49 https://doi.org/10.1186/s1361 3-020-00662-y

\section{8}

Interaction of stroke volume variance and pulse pressure variance with parameters of mechanical ventilation

F. Wagner ; K. Putko ; D. Busch ${ }^{1}$; H. Schuster ${ }^{1}$; W. Huber ${ }^{1}$; T. Lahmer

${ }^{1}$ Medizinische klinik und poliklinik ii, Klinikum rechts der Isar; Technische

Universität München, Munich, Germany

Correspondence: F. Wagner

Intensive Care Medicine Experimental 2020, 8(2): 000498 
Introduction: Haemodynamic parameters play an essential role in intensive care units. Stroke volume variance (SVV) and pulse pressure variance (PPV) are used for predicting fluid responsiveness in mechanically ventilated patients.

Objectives: However, their applicability is limited as they require sinus rhythm and controlled ventilation modes which both is only available in $43 \%$ of ICU patients (Huber et al., 2018). This study's goal was to investigate the impact of change in ventilation pressure (Pinsp) on change in SVV and PPV in pressure-controlled ventilated patients. Due to former studies (Sakka, Becher, Kozieras, \& van Hout, 2009; VieillardBaron et al., 2016), we hypothesized that there's a significant correlation between tidal volume(VT), Ppeak, Pmean, lung compliance and SVV or PPV. It was aimed to generate a formula using multiple regression to predict deltaSVV and deltaPPV in dependence of ventilation parameters.

Methods: In total 43 pressure-controlled ventilated patients from ICU were investigated. Transpulmonary thermodilution (TPTD; PiCCO, Pulsion Medical Systems SE, Germany) was performed before and after a change in inspiratory pressure (Pinsp) on the ventilator machine (EvitaXL,Drägerwerk ${ }^{\circledR}$ AG \& Co. KGaA, Germany and Carescape ${ }^{\mathrm{TM}} R 860$, GE Healthcare, Germany). Magnitude of change in Pinsp was based on medical indication due to arterial blood gas analysis or weaning. Patients were measured at least once and not more than eight times, resulting in 90 total measurements. We compared deltaSVV and deltaPPV to deltatVT, deltaPpeak, deltaPmean and lung compliance. Due to multiple measurements in some individuals we analysed the withinsubject correlation using ANCOVA and between-subject correlation using subjects' means(Bland \& Altman, 1994).

Results: No significant $(\mathrm{p}<0.05)$ within-subject or between-subject correlation could be shown between deltaSVV/ deltaPPV and deltaVT, deltaPpeak, deltaPmean, lung compliance. No formula using ventilation parameters as predictors for deltaSVV and deltaPPV in multiple regression could be generated.

Conclusion: Former findings of correlation between VT and SVV or PPV could not be confirmed in our study. Further investigations need to be performed to find out more about these discrepancies in results.

\section{Reference(s) and grant acknowledgment(s)}

1. Vieillard-Baron, A., Matthay, M., Teboul, J. L., Bein, T., Schultz, M., Magder, S., \& Marini, J. J. (2016). Experts' opinion on management of hemodynamics in ARDS patients: focus on the effects of mechanical ventilation. Intensive Care Medicine, 42(5), 739-749.

2. Sakka, S. G., Becher, L., Kozieras, J., \& van Hout, N. (2009). Effects of changes in blood pressure and airway pressures on parameters of fluid responsiveness. European Journal of Anaesthesiology, 26(4), 322-327.

3. Huber, W., Mayr, U., Umgelter, A., Franzen, M., Reindl, W., Schmid, R. M., \& Eckel, F. (2018). Mandatory criteria for the application of variability-based parameters of fluid responsiveness: a prospective study in different groups of ICU patients. Journal of Zhejiang University-Science B, 19(7), 515-524.

4. Bland, J. M., \& Altman, D. G. (1994). Correlation, regression, and repeated data. BMJ, 308(6933), 896.

\section{5}

Evaluating the implementation of a remote patient monitoring platform in the ICU: a qualitative study

L. Mosch ${ }^{1}$; AS. Poncette ; S. Weber-Carstens ${ }^{1}$; M. Schieler ${ }^{1}$; H. Krampe ; F. Balzer ${ }^{1}$

${ }^{1}$ Department of Anesthesiology and Operative Intensive Care Medicine, Charité - Universitätsmedizin Berlin, Berlin, Germany

Correspondence: F. Balzer

Intensive Care Medicine Experimental 2020, 8(2): 000515

Introduction: In intensive care medicine, digital health technologies promise to improve outcomes [1], yet their implementation is lagging behind, challenged by various factors [2].

We aimed to explore influencing factors to the implementation of a remote patient monitoring platform in an intensive care unit (ICU) and suggestions for improving the implementation performance of novel digital health technology in ICU.

Methods: The study was conceived June-November 2019 in an ICU of a German university hospital during the implementation of Vital Sync 2.4 (Medtronic plc) as an additional tablet computer based remote patient monitoring to the primary patient monitoring. After IRB approval (EA1/031/18), seven semistructured interviews with key stakeholders-closely involved in the implementation process-were conducted. The interview guideline was developed on the basis of findings of a previous study [3] and the Consolidated Framework for Implementation Research [4]. Transcripts were analyzed following an inductive approach [5].

Results: Main obstacles identified by interviewees were lack of staff commitment due to little and non-persistent leadership engagement, unequal distribution of workload for the project among professions, technical instructions not reaching all staff members, limitations of the intervention in its current version, and low perceived benefit of remote monitoring for ICU staff. High stress levels in the ICU and an increased workload due to a complex set-up of the system that was additional to the primary monitoring system obstructed regular usage.

Suggestions to improve implementation of novel digital applications in the ICU aimed to enhance staff engagement through the nomination of specific responsible staff members for the implementation process, continuous technical instructions and feedback workshops as well as transparent and early communication of the project aims. The intervention should provide a high intuitivity, interoperability with other devices, and a clear advantage for its usage. Regarding the implementation site, a ward with a lower staffing ratio per patient, less fluctuation of staff members, and less patient turnover was suggested. For example, patients with a relatively weak indication for admission to the ICU could be remotely monitored on a normal ward.

Conclusion: A technologized setting with many different devices and a high stress level of staff make the ICU a particular environment for the implementation of new technology. Staff engagement and identification with the intervention are essential for effective implementation and could be enhanced through persistent leadership engagement, regular instructions, and early, transparent communication of the intervention. Intuitivity and interoperability are indispensable intervention features for implementation success. However, the additional benefit of remote monitoring in the ICU was regarded as low, other settings were perceived as more suitable for this technology.

\section{Reference(s) and grant acknowledgment(s)}

1. 1. Hawkins HA, Lilly CM, Kaster DA, Groves RH, Khurana H. ICU Telemedicine Comanagement Methods and Length of Stay. Chest 2016 Aug;150(2):314319. [https://doi.org/10.1016/j.chest.2016.03.030]

2. 2. Reynolds HN. The Tele-ICU: Formative or Out-of-Date or Both? Practice Models and Furture Directions. In: Koenig MA, editor. Telemed ICU [Internet] Cham: Springer International Publishing; 2019 [cited 2019 Jun 23]. [https:// doi.org/10.1007/978-3-030-11569-2]

3. 3. Poncette A-S, Spies C, Mosch L, Schieler M, Weber-Carstens S, Krampe H, Balzer F. Clinical Requirements of Future Patient Monitoring in the Intensive Care Unit: Qualitative Study. JMIR Med Inform 2019 Apr 30;7(2):e13064. [https://doi.org/10.2196/13064]

4. 4. Damschroder LJ, Aron DC, Keith RE, Kirsh SR, Alexander JA, Lowery JC. Fostering implementation of health services research findings into practice: a consolidated framework for advancing implementation science. Implement Sci 2009 Aug 7:4(1):50. [https://doi.org/10.1186/1748-5908-4-50]

5. 5. Boyatzis RE. Transforming Qualitative Information: Thematic Analysis and Code Development. London and New Delhi: SAGE; 1998. ISBN:978-0-7619-0961-3 
000530

Real-time changes in pulse pressure detect preload responsiveness through the end-expiratory occlusion test F. Gavelli ${ }^{1}$; N. De Vita ${ }^{1}$; JL. Teboul ${ }^{1}$; D. Azzolina ${ }^{2}$; C. Lai ${ }^{1}$; A. Beurton ${ }^{1}$; A. Pavot ${ }^{1}$; R. Shi ${ }^{1}$;I. Adda'; X. Monnet

'Service de médecine intensive-réanimation, Hôpital de Bicêtre, Hôpitaux universitaires Paris-Saclay, AP-HP, Le Kremlin-Bicêtre, France; ${ }^{2}$ Research support unit, Department of Translational Medicine - Università degli Studi del Piemonte Orientale, Novara, Italy

Correspondence: F. Gavelli

Intensive Care Medicine Experimental 2020, 8(2): 000530

Introduction: The end-expiratory (EEXPO) test detects preload responsiveness by observing changes in cardiac index (Change $\mathrm{Cl}$ ) during a 15-sec interruption of ventilation at end-expiration. First, it is not clearly established whether a simple measurement of the changes in arterial pulse pressure (ChangePP) during EEXPO could reliably reflect ChangeCl and detect preload responsiveness. Second, if it was the case, this should allow the shortage of the EEXPO test. Indeed, many devices measuring $\mathrm{Cl}$ do not display the beat-to-beat value of $\mathrm{Cl}$ but average it over several seconds, so that the EEXPO test must be long enough for allowing significant changes to appear. ChangePP, which are instantaneously measured, might require a shorter test to be evidenced, but this has to be proven.

Objectives: First, to test whether beat-to-beat ChangePP measured during a 15-sec EEXPO detect preload responsiveness. Second, to evaluate whether it is still the case when test's duration is shortened.

Methods: In 30 mechanically ventilated patients we performed a 15-sec EEXPO. PP was continuously and digitally recorded. $\mathrm{Cl}$ was measured by pulse contour analysis (PiCCO2 device). Patients were defined as "preload responders" if $\mathrm{Cl}$ increased $\geq 10 \%$ during a passive leg raising.

ChangePP was calculated as the difference between the mean of PP values over the last 5 seconds of tests (both for 15-sec and 10-sec EEXPO) and the baseline value. The ability of ChangePP to detect preload responsiveness through the EEXPO test was evaluated through the area under the receiver operating characteristic curve (AUROC). The same analysis was repeated on a validation cohort of 126 patients enrolled from previous studies, in which real-time ChangePP during EEXPO had been already recorded.

Results: In the prospective cohort, 15 patients were preload responders and 15 preload non-responders. ChangePP during 15-sec EEXPO was significantly higher in responders than in non-responders (11 [8$15] \%$ vs. 2[1-4]\%, respectively, $\mathrm{p}<0.0001)$. ChangePP during $15-\mathrm{sec}$ EEXPO detected preload responsiveness with an AUROC of $0.90 \pm 0.05$ (sensitivity 0.85 , specificity 0.84 , cut-off $5 \%$ ). When the duration of EEXPO was shortened to 10 seconds, ChangePP during EEXPO was still larger in responders than non-responders (11 [8-14]\% vs. 3[2-3]\%; $\mathrm{p}<0.0001)$. ChangePP during a 10-sec EEXPO detected preload responsiveness with an AUROC of $0.88 \pm 0.07$ (sensitivity 0.84 , specificity 0.85 , cut-off $5 \%$ ). The analysis on the validation cohort showed similar results for ChangePP, with AUROCs of $0.89 \pm 0.07$ for 15 -sec EEXPO and $0.91 \pm 0.05$ for 10 -sec EEXPO.

Conclusion: Real-time measurement of PP during EEXPO detects preload responsiveness, even when the duration of the test is shortened to 10 seconds. This opens the possibility of assessing preload responsiveness without any need for cardiac output monitoring in ventilated patients.

\section{0}

\section{Alarm fatigue reported by ICU staff: a survey study}

A. S. Poncette ; M. M. Wunderlich²; M. Schmieding ${ }^{1}$; F. Schiefenhoevel ${ }^{1}$;

E. Salgado ${ }^{1}$; M. Feufel ${ }^{2}$; F. Balzer

${ }^{1}$ Department of anesthesiology and intensive care medicine ccm / cvk, Charité - Universitätsmedizin Berlin, Berlin, Germany; ${ }^{2} \mathrm{Fg}$ arbeitswissenschaft, Technische Universität Berlin, Berlin, Germany

Correspondence: F. Balzer

Intensive Care Medicine Experimental 2020, 8(2): 000550
Introduction: Alarm fatigue on intensive care units (ICU) is defined as a sensory overload due to an excessive number of alarms from medical devices, which can result in desensitization to alarms and missed alarms [1].

Objectives: We assessed to what extent ICU staff at a university hospital experiences alarm fatigue from patient monitoring alerts, whether false alarms are perceived to disrupt patient care, and what strategies staff developed to handle alarms, such as adjusting or communicating thresholds or pausing alarms when examining patients. Because patient monitoring is a multidisciplinary team effort, we also aimed to uncover differences between health professions in the ICU.

Methods: A survey study was conducted with ICU staff from four ICUs of a German university hospital between November 2019 and January 2020 after IRB approval (EA1/031/18). We designed a webbased 12-item questionnaire based on literature analyses and focus groups [2-6], and distributed it by email to all 270 ICU staff members, which included both physicians and nurses. The response format was a 5-point Likert-type scale (e.g., never - always). We cleaned and analysed the data with $\mathrm{R}$ in combination with the packages tidyverse and infer [7]. Statistical analyses included median values with bootstrapped $95 \%$ confidence intervals and permutation tests to compare the distributions of responses of nurses and physicians. An item median was considered statistically significant $\left({ }^{*}\right)$ when the $95 \%$ bootstrap confidence intervals of the median did not include 3 , which indicates the response sometimes.

Results: Eighty-nine of the 270 ICU staff members (33\%) completed the questionnaire. $63 \%$ * of the respondents had always or often so much to do that they could not respond to alarms quickly enough. $45 \%$ always or often ignored alarms that keep repeating, $24 \%$ stated to overhear critical alarms often, and $22 \%$ were always or often overwhelmed by too many alarms. The majority strongly agreed or agreed that false alarms disrupt patient care $\left(76 \% \%^{*}\right)$, and reduce trust in alarms $\left(63 \%^{*}\right) .82 \% *$ of participants reported to always or often adjust alarm settings (e.g., alarm thresholds) individually for each patient, but documentation of adjustments of alarm settings in the patient data management system was always or often omitted by $76 \% *$. $29 \%$ of the respondents stated to always or often set the alarms of patient monitors on Pause when examining the patient. Between nurses and physicians, no significant differences of the median values of the items were revealed.

Conclusion: Our results suggest that both ICU physicians and nurses experience noticeable alarm fatigue due to false alarms which could harm patients. To remedy this situation, alarm management in the ICU should be optimized such as by more targeted and regular use of the alarm pause function, and by introducing a hospital-wide standard operating procedure for alarm management.

\section{Reference(s) and grant acknowledgment(s)}

1. 1. Sendelbach S, Funk M. Alarm Fatigue: A Patient Safety Concern. AACN Adv Crit Care 2013;24(4):378-386. [https://doi.org/10.1097/nci.0b013e3182 a903f9]

2. 2. Torabizadeh C, Yousefinya A, Zand F, Rakhshan M, Fararooei M. A nurses' alarm fatigue questionnaire: development and psychometric properties. J Clin Monit Comput 2017 Dec;31(6):1305-1312. [https://doi.org/10.1007/ s10877-016-9958-x]

3. 3.Sowan AK, Gomez TM, Tarriela AF, Reed CC, Paper BM. Changes in Default Alarm Settings and Standard In-Service are Insufficient to Improve Alarm Fatigue in an Intensive Care Unit: A Pilot Project. JMIR Hum Factors [Internet] 2016 Jan 11;3(1). PMID:27036170

4. 4. Ruppel H, Vaux LD, Cooper D, Kunz S, Duller B, Funk M. Testing physiologic monitor alarm customization software to reduce alarm rates and improve nurses' experience of alarms in a medical intensive care unit. PLOS ONE 2018 Oct 18;13(10):e0205901. [https://doi.org/10.1371/journal.pone.02059 $01]$

5. 5. Van de Pol I, Wirds JW. St. Antonius Hospital reduces non-actionable ICU alarms by $40 \%$ to improve patient care and staff satisfaction. Philips Clinical Services; 2017.

6. 6. Li A, Gretzinger D. Applying an Evidence-based Approach to Managing Alarm Safety: A University Health Network Case Study. In: Jaffray DA, editor. 
World Congr Med Phys Biomed Eng June 7-12 2015 Tor Can [Internet] Cham: Springer International Publishing; 2015 [cited 2019 Apr 8]. p. 1538-1541. [https://doi.org/10.1007/978-3-319-19387-8_374]

7. 7. R Core Team. R: A language and environment for statistical computing. $R$ Foundation for Statistical Computing [Internet]. Vienna, Austria; 2018. Available from: https://www.R-project.org/

8. 8. This study was funded by a grant from the Einstein Center Digital Future, Berlin, Germany.

\section{9}

Non-invasive monitoring of pulmonary blood flow and shunt fraction in a porcine model

M. Edlinger-Stanger ${ }^{1} ;$ H. Mcgregor ${ }^{2} ;$ T. Neugebauer $^{1} ;$ J. Müller ${ }^{3}$;

S. Khünl-Brady ${ }^{4}$; S. Bustin²; N. Ayoubi ; N. Fleming ${ }^{5} ;$ A. Bukaty ${ }^{3}$; S. Böhme ${ }^{3}$;

K. Markstaller ${ }^{3}$; A. Lassnigg ${ }^{1}$; M. Hiesmayr ${ }^{1}$

${ }^{1}$ Division of cardiac thoracic vascular anaesthesia and intensive care medicine, Medical University of Vienna, Wien, Austria; ${ }^{2}$ Rostrum medical innovations inc., Rostrum Medical Innovations Inc., Vancouver, Canada; ${ }^{3}$ Department of anesthesia, intensive care medicine and pain medicine, Medical University of Vienna, Wien, Austria; ${ }^{4}$ Medical university of vienna, Medical University of Vienna, Wien, Austria; ${ }^{5}$ Department of anesthesiology and pain medicine, University of California, Davis, Davis, United States of America

Correspondence: $M$. Edlinger-Stanger

Intensive Care Medicine Experimental 2020, 8(2): 000569

Introduction: Knowing trends in pulmonary blood flow (PBF) and shunt fraction (Qs/Qt) could guide hemodynamic and respiratory management and improve outcomes in critically ill patients. PBF and shunt fraction are typically determined invasively using systemic and pulmonary arterial catheters and repetitive blood gas analyses. We present a porcine study utilizing novel techniques to obtain PBF and shunt measurements via the breathing tube.

Objectives: We tested whether PBF and Qs/Qt can be measured via the breathing tube by means of a new monitoring system (VQm $\mathrm{PHM}^{\mathrm{TM}}$, Rostrum Medical Innovations Inc., Canada).

Methods: 9 pigs (70-88 kgs) were anesthetized, intubated and instrumented with a systemic and pulmonary artery catheter and a pumpdriven extracorporeal circuit connecting the right and left atrium to produce shunt. Qs/Qt was measured at baseline and pump flows of $1 \mathrm{~L} / \mathrm{min}$ and $3 \mathrm{~L} / \mathrm{min}$. Additionally, in four animals, cardiac output (CO) was increased to $130 \%$ of baseline using dobutamine. Measurements of PBF and shunt fraction from the VQm PHM and the reference were conducted at baseline and during interventional periods (increased CO or shunt, respectively).

VQm PHM estimated PBF using the differential Fick equation and sequential gas delivery (SGD). VQm PHM assessed Qs/Qt by measuring the decay of $\mathrm{N} 2 \mathrm{O}$ after breathing FiN2O of 30\% for 25 breaths (N2O challenge). The number of breaths required to reach an end-tidal value of $2.5 \%$ of the end-tidal after cessation of the $\mathrm{N} 2 \mathrm{O}$ challenge was calculated and expressed as shunt index (SI). These values were compared to PBF and Qs/Qt calculated by means of bolus thermodilution and the Berggren equation, respectively1. PBF was estimated by subtracting shunt fraction from thermodilution-derived $\mathrm{CO}$. Agreement between the two methods was assessed by Bland-Altman analysis.

Results: For PBF, 17 paired measurements of the VQm PHM and reference were obtained. The mean difference between paired values was $0.21 \mathrm{~L} / \mathrm{min}$, and the $95 \%$ limits of agreement were -2.15 and $2.57 \mathrm{~L} /$ min. For Qs/Qt, 64 paired measurements of the VQm PHM SI and reference were obtained. The mean difference between paired values was -0.22 , and the $95 \%$ limits of agreement were -1.34 and 0.89 .

Conclusion: These results suggest that the VQm PHM measurements of PBF and Shunt Index were able to track changes in PBF and shunt fraction in anesthetized pigs.

\section{Reference(s) and grant acknowledgment(s)}

1. Berggren SM:. Acta Physiol Scand Suppl 4:1-92, 1942

2. Laboratory and experimental costs were donated by Rostrum Medical Innovations Inc.
000606

Comparison of hemodynamic parameters derived by PiCCO and GE E-PiCCO

K. Putko ; F. Wagner ${ }^{1}$; D. Busch ${ }^{1}$; H. Schuster ${ }^{1}$; R. Schmid ${ }^{1}$; W. Huber ${ }^{1}$; T. Lahmer ${ }^{1}$

${ }^{1}$ Medizinische klinik und poliklinik ii, Klinikum rechts der Isar; Technische Universität München, Munich, Germany

Correspondence: K. Putko,

Intensive Care Medicine Experimental 2020, 8(2): 000606

Introduction: The monitoring of hemodynamic parameters to assess the cardiovascular status and the efficiency of therapy is of high importance in critically ill patients. Due to increased interest in advanced hemodynamic monitoring (AHM) several new devices were introduced within the last years. In this context, the new E-PiCCO device (GE Healthcare, Helsinki, Finnland) recently became available.

Objectives: To compare the E-PiCCO device with the established PiCCO $^{\circledR}$ device (PULSION Medical Systems, Munich, Germany) and to quantify the impact of proper and improper settings on AHM.

Methods: Both devices are based on pulse contour (PC) analysis and transpulmonary thermodilution (TPTD). Sixteen adult intensive care patients ( 8 female, 8 male, age 35-88 yr) undergoing clinically indicated $\mathrm{AHM}$ with $\mathrm{PiCCO}{ }^{\circledR}$ were enrolled in this study. All patients had femoral (FEM) as well as jugular (JUG) central venous catheter (CVC). In these patients, 30 measurement sequences were completed, with one sequence consisting of 5 TPTDs (divided into 3 measurements by $\mathrm{PiCCO}^{\circledR}$ and 2 by E-PiCCO). Each of the 5 TPTDs included 3 cold saline injections of $15 \mathrm{ml}$. Usually, the measurements are performed in JUG site, whereas the $\mathrm{PiCCO}^{\circledR}$ device also allows the measurement in FEM CVC site by applying a built-in correction formula. The E-PiCCO device only allows correct JUG CVC site measurements. For comparison both devices were used in the proper and improper way. Proper use: JUG $\left(\mathrm{PiCCO}{ }^{\circledR}\right.$ and $\mathrm{E}-\mathrm{PiCCO}$ ) and FEM with correction formula (only $\mathrm{PiCCO}^{\circledR}$ ); improper use: FEM (E-PiCCO and $\mathrm{PiCCO}^{\circledR}$ without correction formula). The following hemodynamic parameters were estimated by both devices: cardiac index $(\mathrm{Cl})$ by $P C(\mathrm{PCCl})$ and by TPTD $(\mathrm{TDCl})$, indexed global end-diastolic volume (GEDVI) and extravascular lung water (EVLWI). These were compared by Bland-Altman analysis. IBM SPSS 26. Results: Mean $\mathrm{PCCl}$ in $\left[\mathrm{L} / \mathrm{min} / \mathrm{m}^{2}\right]$ were $3.75,3.64,3.96,3.92,3.99$ for PiCCO ${ }^{\circledR}$ JUG, E-PiCCO JUG, E-PiCCO FEM, PiCCO ${ }^{\circledR}$ FEM corrected, $\mathrm{PiCCO}^{\circledR}$ FEM uncorrected, respectively. In the same order, mean values for TDCl in $\left[\mathrm{L} / \mathrm{min} / \mathrm{m}^{2}\right]$ were $3.73,3.59,3.90,3.94,4.01$; for GEDVI in $\left[\mathrm{ml} / \mathrm{m}^{2}\right]$ 855, 810, 1067, 815, 1116; for EVLWI in $[\mathrm{mL} / \mathrm{kg}]$ 9.33, 9.27, $10.3,10.3,10.4$, respectively. The mean bias between E-PiCCO and PiCCO ${ }^{\circledR}$ in JUG site were: $-0.11(\mathrm{PCCl}),-0.14(\mathrm{TDCl}),-47.3$ (GEDVI), -0.07 (EVLWI), with limits of agreement (LOA, $1.96 \mathrm{SD}, \pm 95 \% \mathrm{Cl}$ ) of \pm 0.55 , $\pm 0.45, \pm 117, \pm 1.48$, respectively, and the percentage error of 15,12 , $14,16 \%$, respectively. The bias results for the comparison of E-PiCCO in FEM and PiCCO ${ }^{\circledR}$ in JUG were: 0.21 (PCCI), 0.17 (TDCI), 212 (GEDVI), 0.97 (EVLWI), with LOA of $\pm 0.71, \pm 0.74, \pm 286, \pm 3.83$, respectively, and the percentage error of $18,18,26,37 \%$, respectively.

Conclusion: The E-PiCCO measurements showed only small differences to the $\mathrm{PiCCO}{ }^{\circledR}$ device when used in JUG site. As in this study, $\mathrm{PiCCO}^{\circledR}$ JUG is considered as the gold-standard, the improper measurement with the E-PiCCO in FEM site shows a considerably higher error but except for EVLWI would still meet the "Critchley-Criterion" (1). In conclusion, the E-PiCCO device is a reasonable alternative to the $\mathrm{PiCCO}^{\circledR}$ device. When using the devices improperly, especially EVLWI and GEDVI showed higher discrepancies than TDCI and PCCI measurements.

\section{Reference(s) and grant acknowledgment(s)}

1. Critchley, L. A., \& Critchley, J. A. (1999). A meta-analysis of studies using bias and precision statistics to compare cardiac output measurement techniques. J Clin Monit Comput, 15(2) 
000611

Comparative study of two central venous catheter placement methods: conventional method vs a modified method guided by the intracavitary electrocardiogram

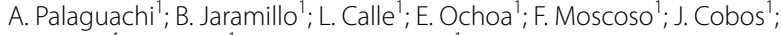
S. Alvarez ${ }^{1}$; H. Tirape ${ }^{1}$; H. Aguirre-Bermeo

${ }^{1}$ Intensive care unit, Hospital Vicente Corral Moscoso, Cuenca, Ecuador

Correspondence: $\mathrm{H}$. Aguirre-Bermeo

Intensive Care Medicine Experimental 2020, 8(2): 000611

Introduction: The correct placement of the central venous catheter (CVC) has to be verified by chest radiography. The technique guided by intracavitary electrocardiogram (EKG-IC), allows a correct placement of the catheter avoiding radiological control. The classic method of CVC placement by EKG-IC places the test electrode at the end of the procedure (when passing the catheter through the guidewire). We have made a modification of this method by performing the check by connecting the electrode to the guidewire when it is placed through the dilator. This modification allows the guide to be repositioned through the dilator and avoids the new puncture and restart of the procedure if the guide is in an extrathoracic location.

Objectives: Compare the correct placement of the CVC in the two methods studied and validate the modified CVC placement method by EKG-IC.

Methods: Fifty patients in whom CVC was placed were prospectively collected. Two methods were evaluated, conventional method (seldinger technique) and a modified method guided by EKG-IC. The method to be used was indicated by the doctor responsible of the patient. The location of the catheter was evaluated by chest radiography. The radiologist who evaluated the radiography was unaware of the method used. Correct CVC placement was defined with radiologic landmark of the lower third of the SVC (zone2): under the carina but within the first 3 distal $\mathrm{cm}(1)$. The data are presented in median, interquartile range and number and percentage as appropriate. Results:

\begin{tabular}{|l|l|l|l|}
\hline VARIABLE & $\begin{array}{l}\text { CONVENTIONAL } \\
\mathrm{N}=25\end{array}$ & $\begin{array}{l}\text { EKG_IC } \\
\text { MODIFICATED N=25 }\end{array}$ & $\mathrm{P}=$ \\
\hline $\begin{array}{l}\text { Procedure Time, } \\
\text { Minuts }\end{array}$ & $12(7,19)$ & $13(9,19)$ & 0,66 \\
\hline Zone 1 & $9(36)$ & $3(12)$ & 0.05 \\
\hline Zone 2 & $3(12)$ & $18(72)$ & $<0,01$ \\
\hline Zone 3 & $12(48)$ & $4(16)$ & 0,02 \\
\hline Zone out & $1(4)$ & $0(0)$ & 0,31 \\
\hline
\end{tabular}

Conclusion: In this patient sample, the modified CVC placement method by EKG - IC significantly allowed correct placement. This modified method, in addition to avoiding control radiology, would avoid puncturing again if the catheter location is extrathoracic.

\section{Reference(s) and grant acknowledgment(s)}

1. 1. Pittiruti M, La Greca A, Scoppettuolo G The electrocardiographic method for positioning the tip of central venous catheters. J Vasc Acces 2011; 12(4):280-91

\section{6}

\section{Heparin resistance during ECMO-alternative anticoagulation} with Argatroban

C. Efseviou ${ }^{1}$; A. Kastoris ${ }^{1}$; L. Palazis ${ }^{1}$

${ }^{1}$ Intensive care unit, Nicosia General Hospital, Nicosia, Cyprus

Correspondence: A. Kastoris

Intensive Care Medicine Experimental 2020, 8(2): 000736

Introduction: Prompt anticoagulation and coagulation monitoring is an essential aspect of patients during extracorporeal membrane oxygenation (ECMO) therapy. Heparin infusion is the gold standard regarding anticoagulation during ECMO. Nevertheless, resistance to heparin may arise that may be difficult to diagnose and manage.

Methods: A search of the literature on PubMed, Google Scholar and Scopus with the keywords "ECMO or ECLS" and "heparin resistance" was conducted. Published case reports and series written in English were included with adult patients. Abstracts were not included in the review. A case of heparin resistance during ECMO from our ICU is also presented.

Results: The mechanism and pathophysiological process that leads to the development of heparin resistance is not fully understood, however acquired antithrombin (AT) deficiency is a recognized causative factor. The Extracorporeal Life Support Organization (ELSO) recommends that in established heparin resistance AT-III levels are maintained within the normal range in patients on ECMO by administration of fresh frozen plasma (FFP) or AT-III concentrate. Nevertheless, Ciolek et al (2017) mentioned in pediatric ECMO patients that AT-III repletion was associated with high cost, significant increases in anticoagulation parameters, and no significant change in the heparin infusion rate. In total 6 studies in the international literature were identified regarding heparin resistance during ECMO therapy. The studies identified are summarized in Table 1. Three of the 6 studies studied the use of Argatroban during heparin resistance in a total of 13 patients with good results regarding safety, cost, and ease of administration. The other three studies suggested the use of Bivalirudin, FFP and ATIII administration, respectively.

A 29-year-old woman with a history of obesity, hypothyroidism and polycystic ovary disease was admitted to the ICU of the Nicosia General Hospital with type I respiratory failure due to COVID-19 infection. On the 4th day of mechanical ventilation, she presented severe respiratory deterioration. A decision was made to proceed with VV ECMO support. Heparin infusion was initiated with a target to maintain aPTT 1.5 to 2 times initial patient values. During the first 48 hours of ECMO support the patient was unable to maintain anticoagulation targets despite progressively increased heparin dose, furthermore she presented signs of thrombosis on her left index finger. ATIII levels were in the normal range and the anti-PF4 test was negative. FFP administration was unable to reduce the heparin dose. Heparin infusion was stopped and anticoagulation with Argatroban was initiated. During Argatroban administration the patient presented a non-clinically significant bleeding from the tracheostomy and the ECMO intravenous cannulas. There were no apparent thrombotic events and the thrombosis on the index finger resolved without intervention. The patient was successfully weaned off the ECMO after 10 days and made a full recovery.

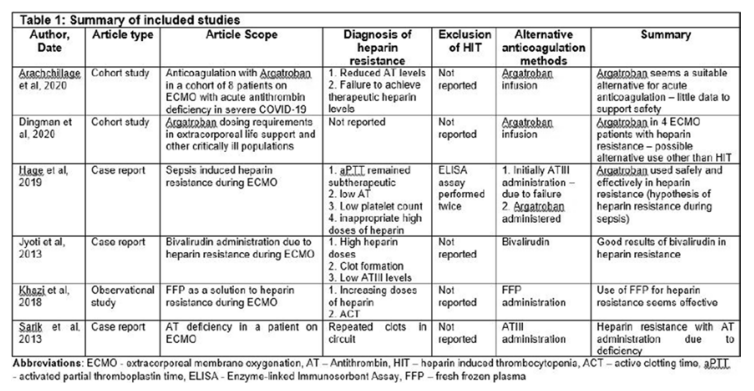

Conclusion: It seems that patients with heparin resistance have higher mortality rates during ECMO highlighting the need for developing alternative anticoagulation strategies. The recommended ELSO guidelines of ATIII or FFP administration in heparin resistance during ECMO may be costly, and ineffective. The role of Argatroban, as alternative anticoagulation, in this group of patients should be further researched to establish both safety and efficacy. 
Reference(s) and grant acknowledgment(s)

1. Dirnberger, D., Fiser, R., Harvey, C., Lunz, D., Bacchetta, M., Frenckner, B., .. \& \& Fan, E. (2015). Extracorporeal Life Support Organization (ELSO). Guidelines for ECMO Transport

2. Khazi, F. M., Elhoufi, A. M., AbdelAziz, T. A., Siddiqui, N. R., Al-Zamkan, B., Robert, S. B., .. \& Aljassim, O. (2018). Fresh frozen plasma: A solution to heparin resistance during extracorporeal membrane oxygenation (ECMO). The Egyptian Journal of Critical Care Medicine, 6(3), 79-86.

3. Jyoti, A., Maheshwari, A., Daniel, E., Motihar, A., Bhathiwal, R. S., \& Sharma, D. (2014). Bivalirudin in venovenous extracorporeal membrane oxygenation. The journal of extra-corporeal technology, 46(1), 94.

4. Sarik, J. R., Pitcher, H. T., Hirose, H., \& Cavarocchi, N. C. (2014). Antithrombin III deficiency in patients requiring mechanical circulatory support. J Cardiol, 2(1), 1017

5. Hage, A., Louzada, M., \& Kiaii, B. (2019). Sepsis-induced heparin resistance during extracorporeal membrane oxygenation. CMAJ, 191(10), E283-E285.

6. Dingman, J. S., Smith, Z. R., Coba, V. E., Peters, M. A., \& To, L. (2020). Argatroban dosing requirements in extracorporeal life support and other critically ill populations. Thrombosis research.

7. Arachchillage, D. J., Remmington, C., Rosenberg, A., Xu, T., Passariello, M., Hall, D., .. \& Patel, B. V. (2020). Anticoagulation with Argatroban in patients with acute antithrombin deficiency in severe COVID-19. British Journal of Haematology

8. Ciolek, A., Lindsley, J., Crow, J., Nelson-McMillan, K., \& Procaccini, D. (2018). Identification of cost-saving opportunities for the use of antithrombin III in adult and pediatric patients. Clinical and Applied Thrombosis/Hemostasis, 24(1), 186-191.

\section{9}

\section{An analysis of transthoracic echocardiography and its impact} on patient outcomes in the Intensive Care Unit

M. Carton ; F. O'riordan²; A. Fahy 3 ; J. Coughlan²; D. Moore ${ }^{2}$

${ }^{1}$ Anaesthesia, Mater Misericordiae University Hospital, Dublin, France; ${ }^{2} \mathrm{Car}$ diology, Tallaght University Hospital, Dublin, Ireland; ${ }^{3}$ Intensive care, Tallaght University Hospital, Dublin, Ireland

\section{Correspondence: M. Carton}

Intensive Care Medicine Experimental 2020, 8(2): 000809

Introduction: Transthoracic echocardiography (TTE) is regularly requested in critically ill patients. However to date there has been limited data published on the association between TTE results and patient outcomes.

Objectives: The purpose of this study was to assess the prognostic function of transthoracic echocardiography in the intensive care unit.

Methods: A retrospective, cross sectional, single centre study, was performed. All patients admitted to the Intensive care unit (ICU) over the 13-month period (January 2018- February 2019) were included. Baseline characteristics were obtained from the Clinical Information System. All TTE's were performed by a cardiac physiologist with final approval of reports from a consultant cardiologist. Echocardiographic reports were extracted from the Cardiovascular Information System. ICU and hospital outcomes were extracted from the 'Acubase Ltd' system.

Results: Three hundred and fifty eight patients were admitted to the ICU over the 13 month period (55\% Male, Mean age $59 \pm 17$ years). Just under one-third of all patients received a TTE during their ICU admission $(\mathrm{N}=115 / 358)$.

Results included; normal left ventricular (LV) function; $n=56$, left ventricular hypertrophy $(\mathrm{LVH}) ; \mathrm{n}=19$, LV dysfunction; $n=19$, moderate to severe valvular pathology $+/-$ LV dysfunction; $n=9$, right ventricular (RV) dysfunction; $n=7$. Five $T$ TE's were not reported therefore excluded from our study.

Each TTE subgroup was then tested against the study population as a whole to assess for any significant impact on patient outcome such as ICU and hospital length of stay and mortality.

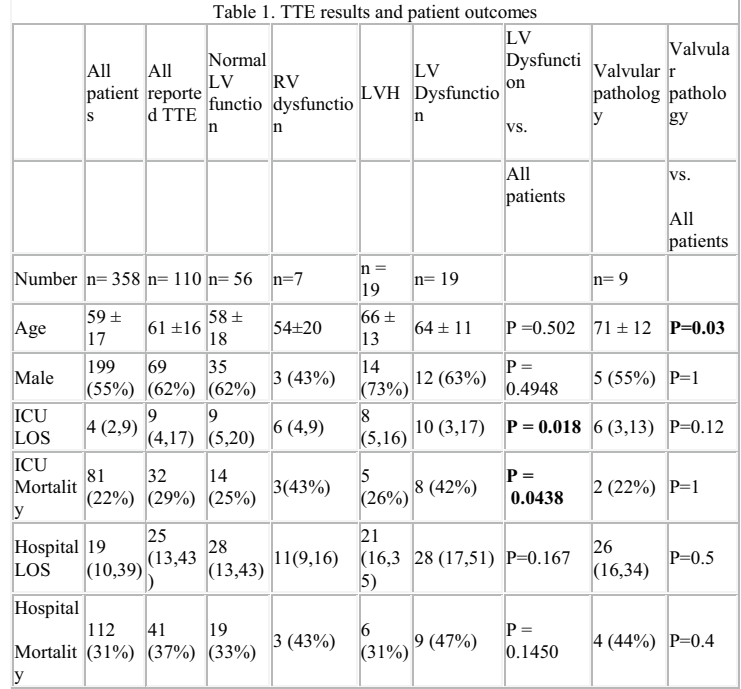

Patients with reduced LV function were found to have a significant increase in ICU mortality when compared to the study population $(42 \% n=8 / 19$ vs $22 \% n=81 / 358: p=0.04)$. This patient cohort was also found to have a longer ICU length of stay ( 10 vs. 4 days $p=0.018$ ). Hospital mortality and length of stay did not differ significantly between groups.

Of note the patients with moderate to severe valvular pathology (+/- impaired LV function) were found to be older (71 $\pm 11 \mathrm{vs.59} \pm 17$ $\mathrm{P}=0.03$ ) yet outcomes did not differ significantly when compared to the study population as a whole.

Patients found to have right ventricular dysfunction or left ventricular hypertrophy were not found to have a significant increase in mortality rate or length of stay.

Conclusion: A finding of moderate to severe LV dysfunction on Transthoracic Echocardiography in ICU setting, is associated with a significant increase in ICU mortality and length of stay.

\section{Reference(s) and grant acknowledgment(s) \\ 1. Nil}

\section{2}

Milrinone concentration monitoring during continuous renal replacement therapy

H. T. Sørensen ${ }^{1}$; S. Christensen ${ }^{1}$; CU. Andersen²; DV. Nielsen ${ }^{1}$

${ }^{1}$ Department of anaesthesia and intensive care medicine, Aarhus University Hospital, Aarhus, Denmark; ${ }^{2}$ Department of clinical pharmacology, Aarhus University Hospital, Aarhus, Denmark

Correspondence: $\mathrm{H}$.T. Sørensen

Intensive Care Medicine Experimental 2020, 8(2): 000842

Introduction: Milrinone is an inodilator drug widely used in the treatment of both surgical and non-surgical patients with severe heart failure. These patients often present with acute renal failure (ARF), and a substantial proportion will require continuous renal replacement therapy (CRRT).

Several studies indicate an increased risk of supratherapeutic plasma Milrinone concentrations in patients with ARF not treated with CRRT. This has led to guidelines advising Milrinone dosage reduction for these patients, as supratherapeutic plasma concentrations may increase the risk of serious adverse effects such as arrhythmias and hypotension.

Data on Milrinone pharmacokinetics in patients receiving CRRT is sparse and based on one study of 6 patients [1]. Accordingly, no prescribing guidance exists for Milrinone dosing during CRRT. 
Objectives: The objective of the present study is to describe Milrinone plasma concentrations in relation to the therapeutic index, in patients receiving intravenous Milrinone infusion while on CRRT.

Methods: A prospective observational descriptive study including intensive care patients $>18$ years receiving intravenous Milrinone infusion while on CRRT. Both patients with low cardiac output syndrome following cardiac surgery, and patients with non-surgical acute decompensated heart failure are included.

Milrinone prescribing including dosage, and settings and duration of CRRT are at the discretion of the attending intensive care doctors. We expect to include approximately 15 patients.

Guided by results from a pilot study, arterial plasma samples are collected at 8, 16, 24, 36 and 48 hours after initiation of Milrinone infusion and/or CRRT, and once every 24 hours thereafter. Plasma sampling is continued until Milrinone infusion is discontinued. An additional plasma sample is collected prior to initiation of CRRT, in case Milrinone infusion is initiated before CRRT.

The arterial whole blood samples are centrifuged on site. Plasma is obtained and stored at $-80{ }^{\circ} \mathrm{C}$ until analysis is performed. Milrinone plasma concentrations will be quantified using liquid chromatography tandem mass spectrometry (LC-MS/MS).

Milrinone plasma concentrations versus time after initiation of CRRT will be described and stratified according to different dosages of Milrinone infusion. Milrinone concentrations will be described according to therapeutic index. The study will not have power to evaluate relationship between Milrinone concentration and outcomes.

Results: The study is still recruiting. Inclusion is expected to finish October 1 2020. Results on the Milrinone plasma concentration analysis will be presented.

Conclusion: This study reflects common practice of the use of Milrinone while on CRRT. The results from this study will clarify further whether specific dosing recommendations should be considered for patients receiving Milrinone infusion while on CRRT.

\section{Reference(s) and grant acknowledgment(s)}

1. Taniguchi, T., et al., Pharmacokinetics of milrinone in patients with congestive heart failure during continuous venovenous hemofiltration. Intensive Care Med, 2000. 26(8): p. 1089-93.

2. This study was supported by grants from the Gunnar Steen Jacobsen and Kirsten Hjortholm Foundation and the Health Fund of Central Denmark Region

\section{6}

The shock index and the shock index delta as predictors of anti-shock garment removal in patients with obstetric hemorrhage from an ICU in Ecuador

S. Cruz Montesinos ${ }^{1}$;TM. Nares ${ }^{2}$; O. Perez ${ }^{3}$; F. Morales ${ }^{4}$; C. Sánchez ${ }^{5}$;

C. Cevallos ${ }^{6}$; MG. Caicedo ${ }^{7}$; D. Muñoz ; A. Teran ${ }^{1}$; M. Barrionuevo ${ }^{1}$;

D. Vaca ; JO. Montoya Rojo ${ }^{9}$; G. Cruz ${ }^{10}$; J. Jimenez ; N. Laica ${ }^{11}$; P. Gutierrez'; N. Benavides ${ }^{1}$; R. Lasluisa

${ }^{1}$ Intensive Care, Provincial General Hospital, Latacunga, Ecuador; ${ }^{2}$ Intensive care, Centro Medico Nacional La Raza, Ciudad de México, Mexico; Intensive care, General Hospital San Juan River, San Juan del Río, Mexico; ${ }^{4}$ Intensive care, Hospital Oncologico Dr. Julio Villacreses Colmont, Portoviejo, Ecuador; ${ }^{5}$ ntensive care, Hospital General de Quevedo (IESS), Quevedo, Ecuador; ${ }^{6}$ Intensive care, Hospital Carlos Andrade Marín, Quito, Ecuador; ${ }^{7}$ General surgery, Provincial General Hospital, Latacunga, Ecuador; ${ }^{8}$ Gynecology and obstetrician, Provincial General Hospital, Latacunga, Ecuador: ${ }^{9}$ Intensive care, Hospital Hispano Americano, Mexicali, Mexico; ${ }^{10} \mathrm{Critical}$ care, Clinica Novasalud, Latacunga Canton, Ecuador; ${ }^{11}$ Emergency medicine, Hospital del I.E.S.S, Ambato, Ecuador

Correspondence: S. Cruz Montesinos

Intensive Care Medicine Experimental 2020, 8(2): 000916

Introduction: Obstetric hemorrhage is the main cause of maternal morbidity and mortality in developing countries. 1 Despite the fact that the anti-shock garment (AG) is an alternative for the management of obstetric hemorrhage, there is no evidence about when is the optimal time of its withdrawal.2.
Objectives: To determine the predictive capacity of the shock index, and the delta of the shock index, to determine hemodynamic instability, after the removal of the antishock garment in obstetric hemorrhage.

Methods: This is a prospective diagnostic test validation study. It evaluates the shock index (SI) and the shock index delta $(\Delta \mathrm{SI})$ prior to removal of the AG as a predictor of hemodynamic instability after removal of the AG. This study focuses on patients with obstetric hemorrhage admitted to a second level ICU of a hospital in Ecuador between the months of March and December of 2019. Sensitivity (Se), Specificity (Sp), positive and negative predictive values were studied, and the positive and negative Likelihood Ratio (LR) calculated.

Results: Thirteen patients were included, with a mean age of 25.6 years (17-34), with an Apache II mean of 6.4 (2-17) and estimated mean bleeding volume of $1875 \mathrm{ml}$. (500-2500) The main etiology of bleeding was uterine atony (69\%), $15 \%$ had placental retention, $7 \%$ had placenta previa, and $7 \%$ of women experienced a grade III vaginal tear at the time of childbirth, with the average shock index at ICU admission of $1.04(0.8-1.6)$. The mean of the SI before the removal of the AG was $0.88(0.71-1.03)$ in the patients who remained stable after their removal, and the mean of the SI was $0.89(0.83-1.0)$ in those who showed instability, with an Se of $50 \%$ and Sp $66 \%$, a positive LR 1.47 and a negative LR 0.75 . Additionally a $\Delta$ SI of $0.21(0.05-0.58)$ was found for those who remained stable, and $0.07(0.01-0.22)$ for those who presented hemodynamic instability, with Se $75 \%$, Sp $85 \%$, positive LR of 3 and LR. negative of 0.33 .

Conclusion: The Shock Index and shock index delta, are not good predictors of hemodynamic instability after removal of the shock garment in patients with obstetric hemorrhage. However, more studies are required with major samples.

\section{Reference(s) and grant acknowledgment(s)}

1. 2. El Ayadi A, Butrick E, Geissler J, Miller S. Combined analysis of the nonneumatic anti-shock garment to on mortality from hypovolemic shock secondary to obstetric hemorrhage. BMC Pregnancy Childbird 2013;13:208. 2. 1. Escobar M, Fuchtner C, Carvajal J, Nieto A. et al. Experience in the use of non-pneumatic anti-shock garment (NASG) in the management of postpartum haemorrhage with hypovolemic shock in the Fundacion Valle del Lili, Cali, Colombia. Reproductive Health (2017) 14:58 3. None

000945

The reliability of duplex ultrasound in the diagnosis of catheter-related thrombosis performed by general intensive care nurses in the critically ill patients

B. Pakostová'; R. Skulec'i' L. Mikšová'; V. Cerny

${ }^{1}$ Department of anesthesiology, perioperative medicine and intensive care, Masaryk Hospital, Usti nad Labem, Czech Republic

Correspondence: B. Pakostová

Intensive Care Medicine Experimental 2020, 8(2): 000945

Introduction: It has been previously shown that general intensive care nurses are able to perform bedside examination of deep vein thrombosis by compression ultrasound test with a high degree of reliability.1 Another challenge in critically ill patients is the diagnosis of central venous catheter-related thrombosis (CRT). Therefore, we conducted prospective observational study to compare the diagnostic reliability of vascular Point-of-Care duplex ultrasound (compression ultrasound test combined with colour doppler imaging) in diagnostics of CRT performed by general intensive care nurses in the setting of intensive care unit with the examination performed by intensive care physicians, the experts in vascular ultrasound.

Methods: Prior to the study, each nurse participating in the study completed two-hour training in duplex ultrasound and examined 5 patients under supervision. Then, intensive care unit (ICU) patients with central venous catheter placed more than 48 hours in the catchment area of the inferior or superior caval vein underwent duplex ultrasound performed by the nurse. On the same day, the examination was repeated by an ICU physician. In the case of catheter placement in the internal jugular vein or subclavian vein, jugular, subclavian 
and axillar vein were examined bilaterally by a nurse and a physician. When a catheter was placed in the femoral vein, patients underwent duplex ultrasound of the femoral and popliteal region of both lower extremities. The results of the examinations of each patient were blinded until both tests were performed. The calculations evaluating the reliability of the test were applied.

Results: A total of 160 patients of age $62.9 \pm 12.3$ years were included. The prevalence of CRT of $41 \%$ has been found in our sample. The overall sensitivity, specificity, positive predictive value, negative predictive value, and accuracy of the examination performed by general intensive care nurses were $71.2 \%, 100.0 \%, 100.0 \%, 83.3 \%$ and $88.2 \%$, respectively.

Conclusion: The results of our study indicate that general ICU nurses are able to perform bedside examination of CRT by duplex ultrasound with an excellent specificity but only with a moderate sensitivity after a brief predefined training.

\section{Reference(s) and grant acknowledgment(s)}

1. 1. Skulec $R$ et al. The reliability of ultrasound compression test performed

by general ICU nurses in the critically ill patients: A preliminary prospective clinical study. Eur J Intern Med 2020;76:130-131.

\section{3}

High central venous pressure and high ventilatory pressures are associated with acute kidney injury in COVID19 critically ill patients

G. Colombo ${ }^{1}$; M. Brivio ${ }^{1}$; D. Bonacina' ; D. Ferrari ${ }^{1}$; E. Hila ${ }^{1}$; L. Scarpa'

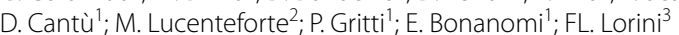

${ }^{1}$ Anaesthesia emergency and intensive care department, ASST Papa Giovanni XXIII, Bergamo, Italy; ${ }^{2}$ Anesthesia and critical care medicine, University of Milan, Milano, Italy; ${ }^{3}$ Dipartimento di emergenza urgenza e area critica, ASST Papa Giovanni XXIII, Bergamo, Italy

Correspondence: $\mathrm{G}$. Colombo

Intensive Care Medicine Experimental 2020, 8(2): 001013

Introduction: Acute Kidney Injury (AKI) is a frequent complication for COVID-19 patients admitted to Intensive Care Unit (ICU) and is independently associated with higher mortality. The patophysiology of COVID-19 related AKI is supposed to be multifactorial: haemodynamic alteration due to high levels of PEEP may play a role.

Objectives: We investigate if higher central venous pressure (CVP) and higher positive end expiratory pressure (Peep) were associated to higher incidence of AKI and if AKI was related to 28 days mortality.

Methods: 130 consecutive COVID-19 patients admitted to the Intensive Care Unit (ICU) at Bergamo General Hospital for acute respiratory failure from 22th February to 22th March were enrolled. AKI was defined by KDIGO criteria. Age, sex and SOFA score were collected at ICU admission. Mean values of PVC and Peep during the first 14 days in ICU were compared between AKI group and controls, using Mann Whitney test. The association between AKI and mortality was evaluated with Fisher exact test.

Results: In our population median age was 62 (IQR 14), 107 (82\%) were male and median SOFA score was 6 (range 4-8). 74 (57\%) patients developed AKI; $17(13 \%)$ in stage 1, $16(12 \%)$ in stage 2, and $41(31 \%)$ in stage $3 ; 22$ patients $(17 \%)$ required continuous renal replacement therapy (CRRT).

28 days mortality among AKI group was significantly higher (46\% vs $18 \%$ p-value 0.0008 ).

Furthermore mean values of PVC and Peep were higher in AKI group compared to controls; relevant data are represented in the Table 1.
Table 1.

$\begin{array}{llll} & \text { controls }(\mathrm{n}=56) & \text { AKI }(\mathrm{n}=74) & \mathrm{p} \\ \text { Age (y) } & 59(51-66) & 63(56-70) & \mathrm{ns} \\ \text { Gender M/F (\%) } & 43 / 13(77 / 23) & 64 / 10(86 / 14) & \mathrm{ns} \\ \text { SOFA (\%) } & 5(4-6) & 6(4-8) & 0.011 \\ \text { Median Peep (cmH2O) } & 13(10-14) & 14(13-15) & 0.0028 \\ \text { PVC (mmHg) } & 11(9-13) & 13(10-15) & 0.0067 \\ \text { 28 days mortality, n (\%) } & 10(18) & 34(46) & 0.0008\end{array}$

Data are presented as median and interquartile range. Two groups are compared using Mann Whitney test for continuous variables and using Fisher's exact test for categorical variables

Conclusion: Our data confirm that AKI represent a risk factor for mortality in COVID patients (odds ratio 3.9, 95\% IC 1.8-8.8). Both higher PEEP and PVC are significantly associated with $\mathrm{AKI}$, suggesting that the haemodynamic impact of high intrathoracic pressure may be involved in its pathogenesis.

Reference(s) and grant acknowledgment(s)

1. 2. Cooke CR et al. Predictors of hospital mortality in a population-based cohort of patients with acute lung injury. Crit Care Med. 2008;36(5)

2. 1.S J. S. Hirsch et Al. Acute kidney injury in patients hospitalized with COVID19. Kidney International (2020)

001036

Cardiogenic shock and 28-days mortality in acute heart failure: possible role for 24-hour lactate clearance

Y. F. Borquez Lopez'; M. G. Gómez García'; A. Palacios Chavarria'; J. Franco Granillo'; J. Aguirre Sánchez'; R. Chaires Gutierrez

${ }^{1}$ Intensive care unit, The American British Cowdray Medical Center, Mexico City, Mexico

Correspondence: Y.F. Borquez Lopez

Intensive Care Medicine Experimental 2020, 8(2): 001036

Introduction: Implications of lactate clearance in patients with acute heart failure (AHF) in intensive care unit (ICU) setting is limited. A recent report suggests that a 24-hour lactate clearance ( $\Delta 24$ lac) of less than $19 \%$ could have a negative prognostic role in septic patients in $\mathrm{ICU}$, therefore we conducted a study to assess the prognostic value of $\Delta 24$ lac for mortality in AHF.

Objectives: To compare the $\Delta 24$ lac with clinical variables and mortality in AHF.

Methods: We conducted an observational retrospective cohort with critical patients from a single center in an intensive care unit (ICU) in Mexico City, Mexico. Demographic, comorbidities and laboratory data was collected for all patients. We estimated the acute physiology and chronic health evaluation (APACHE) II score, and $\triangle 24$ lac. Additionally, treatment with vasoactive agents, diuresis, fluid balance was assessed during first 24 hours and length of stay (LOS). Primary outcome was 28-days mortality.

Results: Data from 100 patients was included, $63 \%$ male, with mean age 63.1 (SD \pm 10.6 ) years and mean BMI $28.8(S D \pm 2.7) \mathrm{Kg} / \mathrm{m} 2$. Most frequent comorbidities were COPD, Hypertension and chronic kidney disease and diabetes in 34,32,19\% and 13\%, respectively. Cardiogenic shock was observed in 54\%; Systolic and diastolic dysfunction was documented in 23 and $27 \%$, respectively. APACHE II score with median of 25 (RIQ 22-27.5) and $\triangle 24$ lac < $19 \%$ in $42 \%$; Overall mortality $35 \%$, median LOS 8 (7-9) days. Variables on admission were similar for mortality and $\Delta 24$ lac groups were similar. Treatment with levosimendan was higher in mortality group. 94.3 vs. $70.8 \%, p=0.006$; when stratified according to $\Delta 24$ lac, we found that patients with $<19 \%$, patients with requirement of inotropic support with levosimendan was a strong predictor for mortality [HR $=10.9$ (IC95\% 1.4-84.7, $\mathrm{p}=0.02)]$, in contrast to $\Delta 24 \mathrm{lac}>19 \%[(\mathrm{HR}=5.6$ (IC95\% 0.74-42.6, $\mathrm{p}=0.09)]$. Further multivariate analysis and stratifications found no association with other $\Delta 24 \mathrm{lac}$ cutoff values, comorbidities, fluid balances and vasopressor support. 
Conclusion: Cardiogenic shock with requirement for inotropic support in subjects with AHF was associated with mortality and inadequate tissue perfusion. Failure to stablish associations with AHF clinical variables with mortality is likely explained by methodological limitations (e.g. lack of a control group). We present additional evidence supporting the use of $\Delta 24 \mathrm{lac}$ as prognostic marker of global tissue hypoxia in circulatory shock, however further controlled studies and a prospective design may be needed to explain the role of this marker in AHF.

\section{Reference(s) and grant acknowledgment(s)}

1. Hugo Bitterncour et all, Non-Invasive Ventilation in Patients with Heart Failure: A Systematic Review and Meta-Analysis, Arq Bras Cardiol. 2017 feb; 108 (2): 161-168.

2. Tara $L$ Jones, Cardiogenic shock: evolving definitions and future directions in management Open Heart 2019; 6

3. Masyuk M, Prognostic relevance of serum lactate kinetics in critically ill patients. Intensive Care Med 2019 Jan;45(1):55-61

3. The ABC Medical Center, and the hospital for all the support provided

\section{2}

Simplified approach in limited resources settings

to estimate cardiac output with Pulse pressure variation and plethysmography in critical care patients

M. G. Gómez García'; Y. F. Borquez Lopez'; E. Monares Zepeda'; J. Franco Granillo'; J. Aguirre Sánchez'; R. Chaires Gutierrez ${ }^{1}$

${ }^{1}$ Intensive care unit, The American British Cowdray Medical Center, Mexico City, Mexico

Correspondence: M.G. Gómez García

Intensive Care Medicine Experimental 2020, 8(2): 001042

Introduction: Measurement of cardiac output (CO) is an important diagnostic maneuver and adds valuable information for the management of critically ill patients. However, estimation of $\mathrm{CO}$ in limited resource settings may be limited in terms of accessibility to noninvasive, continuous, reliable, reproducible methods. Pulse pressure variation (PPV) and plethysmographyc variability (PV) are dynamic and widely extended indicators that in certain conditions may be useful to estimate $\mathrm{CO}$, however, information regarding its reliability is scarce. Therefore, we propose a study with information obtained from PP and PV with other clinical variables and a proposed simplified equation to estimate $\mathrm{CO}$ with echocardiography as gold standard.

Objectives: To develop a simplified method to estimate CO with PPV and PV.

Methods: We conducted a transversal study in critical patients with sepsis recruited from a single center in an intensive care unit (ICU) in Mexico City, Mexico during 2019 through 2020. We calculated the real $\mathrm{CO}$ as stroke volume (SV) per minute measured by echocardiogram; PP was defined as the difference between systolic (DP) and diastolic pressure (DP); The proposed Estimated CO formula was calculated as follows: estimated $\mathrm{CO}=\mathrm{PP}^{*}$ heart rate $(\mathrm{HR}){ }^{*} \mathrm{k}$; where $\mathrm{k}$ was defined as the estimated difference in SV in $\mathrm{mm} \mathrm{Hg}$ and was adjusted according to variability of the PP and plethysmography patterns. We determined correlation with Pearson coefficient ( $r$ ) and absolute agreement with intraclass correlation coefficient $(\mathrm{CCl})$ of estimated $\mathrm{CO}$ compared to the gold standard.

Results: Data from 53 patients was analyzed. We included 6 PV wave patterns and $\mathrm{k}$ values: type 1 and 2 (group 1), $\mathrm{k}=0.9$; type 3 and $4 \mathrm{k}=1.2$ (group 2) and type 5 and $6, \mathrm{k}=1.4$ (group 3). Additionally, if patients showed clinical signs of hypoperfusion we adjusted the $k$ value for groups 1,2 and 3 to 1.2, 1.6 and 2, respectively. Estimated CO correlated with echocardiogram $\mathrm{r}=0.76,(\mathrm{p}<0.01)$ and $\mathrm{CCl}=849$ (IC $95 \%=0.739-0.912, p<0.01)$.

Conclusion: We present a simplified and accessible approach for CO estimation with PPV and PV in critical care patients. This method may be cost effective and easily performed in low-income and limited resources settings, however, additional studies should be considered for further external validation and the possible role and clinical implications of PPV and PV for clinical outcome and mortality is yet to be defined in prospective clinical trials.

\section{Reference(s) and grant acknowledgment(s)}

1. 1. Liljestrand G, Zander E. Vergleichen die Bestimmungen des Minutenvolumens des Herzens beim Menschen mittels der Stichoxydulmethode und durch Blutdruckmessung. Ztschr ges exper med. 1928;59:105-122

2. 2. Hill LK, et al. Evaluation of a simple estimation method for the derivation of cardiac output from arterial blood pressure and heart rate. Biomed Sciences Instrumentation. 2011:48:165-170.

3. 3. Sun JX, et al. Estimating cardiac output from arterial blood pressure waveforms: a critical evaluation using the mimic ii database. Computers in Cardiology. 2005:295-298.

4. 4. Frank O. Evaluation of the shock volume of the human heat on the basis of waves and Windkessel theory. Zeitschrift fur Biologie. 1930;90:405-409.

5. 5. Westerhof, N., Lankhaar, J. \& Westerhof, B.E. The arterial Windkessel. Med Biol Eng Comput 2009;47, 131-141

6. 6. Herd J, Leclair N, Simon W. Arterial pressure pulse contours during hemorrhage in anesthetized dogs. Journal of Applied Physiology 1966;21.

7. 7. Erlanger, J, Hooker B. Johns Hopkins Hospital Report 1904; 12:147-378.

8. 8. Tusman G, Acosta CM, Pulletz S, Böhm SH, Scandurra A, Arca JM, Madorno M, Sipmann FS. Photoplethysmographic characterization of vascular tone mediated changes in arterial pressure: an observational study. J Clin Monit Comput. 2019 Oct;33(5):815-824

9. 9. Lee QY, Chan GS, Redmond SJ, Middleton PM, Steel E, Malouf P, Critoph C, Flynn G, O'Lone E, Lovell NH. Multivariate classification of systemic vascular resistance using photoplethysmography. Physiol Meas 2011;32:111732.

10. 10. Awad AA, Haddadin AS, Tantawy H, Badr TM, Stout RG, Silverman DG, 10. Shelley $\mathrm{KH}$. The relationship between the photoplethysmographic waveform and systemic vascular resistance. J Clin Monit Comput 2007;21:36572.

11. 11. The American British Cowdray Medical Center

\section{3}

The ability of superior vena cava collapsibility and left ventricular surface area to predict volume responsiveness in cardiac surgical patients

A. Babuk'; K. Parhar²; D. Swedlo ${ }^{3} ;$ P. Boucher ${ }^{2}$

${ }^{1}$ Department of Critical Care Medicine, Alberta Health Services, Calgary, Canada; ${ }^{2}$ Department of Critical Care Medicine, University of Calgary, Calgary, Canada; ${ }^{3}$ Department of anesthesiology, Alberta Health Services, Calgary, Canada

\section{Correspondence: $A$. Babuk}

Intensive Care Medicine Experimental 2020, 8(2): 001053

Introduction: Evaluation of fluid responsiveness in critically ill patients is challenging. Echocardiographically obtained variation in inferior vena cava (IVC) and superior vena cava (SVC) have shown promise in their ability to address this challenge. However, they have been predominantly used to assess fluid responsiveness in patients with sepsis, and it is less clear whether they apply to patients undergoing cardiac surgery. Additionally, left ventricular surface area (LVSA) has been shown to be correlated with stroke volume and in a study by Cannesson et. al. respiratory variation in LVSA ( $\triangle$ LVSA) obtained by transesophageal echo (TEE) automatic border detection was shown to predict fluid responsiveness (Cannesson et al., 2006).

Objectives: The primary purpose of this study was to assess whether variation in SVC diameter, during positive pressure ventilation, is predictive of volume responsiveness in cardiac surgical patients. Additionally, $\triangle$ LVSA was assessed for ability to predict fluid responsiveness. Methods: This prospective study enrolled patients undergoing elective or semi-elective cardiac surgery and was conducted in operating theaters at a tertiary care academic hospital. Volume challenges were administered pre-sternotomy and cardiopulmonary bypass (pre-CPB) and after sternal closure (post-CPB) to patients under general anesthesia with standardized mechanical ventilation settings. Hemodynamic measurements and TEE images were collected before and after each volume challenge. Cardiac output (CO) was obtained from TEE images as previously described (Vieillard-Baron et. al., 2004) and fluid responders were defined by an increase in CO of $11 \%$ or more after a volume challenge. The SVC diameters (Dmax and Dmin) were measured over a respiratory cycle and the SVC collapsibility index (SVC-CI) was calculated as (Dmax - Dmin)/Dmax and expressed as a percentage. SVC-CI 
was compared between fluid responders (FR) and non-responders (NR) to determine if there is a significant difference and the optimal SVC-CI threshold was determined for optimizing the sensitivity and specificity. Additionally, LVSA was measured over the respiratory cycle and $\triangle$ LVSA was calculated as (SA max-SA min)/SA max, expressed as a percentage. $\triangle$ LVSA underwent the same analysis as SVC-Cl.

Results: 11 out of 20 enrolled patients (55\%) were fluid responders. There was no difference in heart rate, mean arterial pressure or CVP between responders and non-responders. Pre-CPB SVC-Cl was greater in $R$ vs. NR but not statistically significant $(0.18 \pm 0.15$ vs. $0.11 \pm 0.05$; $\mathrm{p}=0.456$ ). Pre-CPB SVC-Cl threshold of $15 \%$ was found to be most predictive of fluid responsiveness with an area under the receiver operating curve (AU-ROC) of $0.62(0.41-0.84,95 \% \mathrm{Cl})$, NPV of $50 \%$ and PPV of $75 \%$. Post-CPB SVC-Cl was also greater in R vs NR but not statistically significant $(0.18 \pm 0.12$ vs. $0.11 \pm 0.05 ; p=0.241)$. Post-CPB SVC$\mathrm{Cl}>11 \%$ was most predictive of fluid responsiveness with an AU-ROC of $0.66(0.41-0.84,95 \% \mathrm{Cl}), 58 \% \mathrm{NPV}$ and $75 \%$ PPV. Pre-CPB $\triangle \mathrm{LVSA}$ was greater in $R$ vs NR $(0.08 \pm 0.13$ vs $0.01 \pm 0.08)$ but did not reach statistical significance $(p=0.151)$ and Post-CPB $\triangle$ LVSA was minimally greater in R vs NR $(0.08 \pm 0.06$ vs $0.05 \pm 0.11)$ and not statistically significant $(p=0.456)$. Pre-CPB $\triangle L V S A>10 \%$ was predictive of fluid responsiveness with an AU-ROC of $0.79(0.65-0.94,95 \% \mathrm{Cl}), \mathrm{NPV}$ of $62 \%$ and PPV of $100 \%$. Post-CPB $\triangle L V S A>2 \%$ was predictive of fluid responsiveness with AU-ROC of $0.63(0.42-0.84,95 \% \mathrm{Cl})$, NPV of $68 \%$ and PPV of $64 \%$.

Conclusion: Our study shows that SVC-Cl is not a useful measure for predicting fluid responsiveness in cardiac surgical patients either pre or post-operatively. Post-sternotomy there are multiple changes to the cardiopulmonary physiology (to PVR, SVR, venous capacitance, $\mathrm{RV}$, pericardium, etc.) which may be some of the reasons why SVC-CI is not predictive of $\mathrm{FR}$ in this population. SVC-CI not being predictive of $F R$ pre-CPB may be potentially due to the fact that these were elective or semi-elective surgical patients (and not ICU patients in septic shock, like in most other studies). These findings suggest that perhaps the lack of venous return is not the only mechanism for tidal changes in stroke volume with respiration. It is also possible that SVC-Cl is not as good of a predictor of FR as was demonstrated in early studies since many later studies had different optimal thresholds and less impressive predictive values.

Although the differences in $\triangle \mathrm{LVSA}$ pre-CPB did not reach statistical significance, $\triangle \mathrm{LVSA}>10 \%$ was helpful in predicting fluid responsiveness pre-sternotomy with a PPV of $100 \%$ and NPV of $62 \%$. These findings suggest that $\triangle$ LVSA is likely a marker of preload status with higher $\triangle$ LVSA indicating lower preload and therefore more potential for enhancing the CO with a preload challenge. $\triangle \mathrm{LVSA}$ was not helpful in predicting fluid responsiveness post-sternotomy. It is possible that losing the integrity of the pericardium makes $\triangle$ LVSA a less reliable predictor of preload and therefore fluid responsiveness.

\section{Reference(s) and grant acknowledgment(s) \\ 1. Cannesson, M., Slieker, J., Desebbe, O. et al. Prediction of fluid responsive- ness using respiratory variations in left ventricular stroke area by transoe- sophageal echocardiographic automated border detection in mechanically ventilated patients. Crit Care 10, R171 (2006). \\ 2. Vieillard-Baron, A., Chergui, K., Rabiller, A., Peyrouset, O., Page, B., Beauchet, A., \& Jardin, F. (2004). Superior vena caval collapsibility as a gauge of volume status in ventilated septic patients. Intensive Care Medicine, 30(9), 1734-1739.}

\section{0}

Short-term coronary surgery outcomes in patients with variable levels of left venrtricular function

M. Kahloot' ; A. Omar' P. Sivadasan²; S. Sudarsanan²; S. Hanoura²; R. Singh ${ }^{3}$ ${ }^{1}$ Cardiothoracic surgery, Hamad medical corporation, Doha, Qatar; ${ }^{2}$ Department of cardiothoracic surgery/cardiac anaesthesia \& icu, Hamad Medical Corporation, Doha, Qatar; ${ }^{3}$ Medical research center, Hamad Medical Corporation, Doha, Qatar

Correspondence: A. Omar

Intensive Care Medicine Experimental 2020, 8(2): 001140
Introduction: Survival after coronary revascularization surgery is influenced by many variables. Poor left ventricular function (LVF) is believed to be associated with worse outcomes (1).

Objectives: We aim to study the outcome measures at varying levels of LVF.

Methods: A single center retrospective descriptive study. According to the simplified American Society of Echocardiography classification (2), patients were divided into three groups: Group I - severe impairment of LVF; EF < 30\% (38 patients), Group II - mild-moderate impairment of LVF; EF $30 \%$ to $51 \%$ (182 patients), and Group III - normal LVF; $\mathrm{EF}>52 \%$ (186 patients). The outcome variables were compared, and mortality was used as the primary outcome.

Results: The mean age of patients was $55.34 \pm 9.9$ years. The mortality rate was significantly higher in Group I compared to the other groups (13.2\% in Group I vs. $2.2 \%$ and $1.6 \%$ in Groups II and III, respectively; $p=0.007)$. Patients in Group I had a significantly higher incidence of postoperative acute kidney injury (AKI) $(47 \%$ in Group I vs. $28.6 \%$ and $22.6 \%$ in Groups II and Group III, respectively; $p=0.007$ ). No significant differences were found between the studied groups for postoperative atrial fibrillation (POAF), length of ventilation, length of stay (LOS) in intensive care, and length of (LOS) stay in the hospital.

Conclusion: Among patients undergoing coronary surgery, preoperative left ventricular function appears to influence short-term outcomes. Patients with severely impaired left ventricular systolic function had higher mortality, more complications, particularly AKI, and needed more pharmacological and mechanical circulatory support.

\section{Reference(s) and grant acknowledgment(s)}

1. 1. Topkara VK, Cheema FH, Kesavaramanujam S, et al.,. Coronary artery bypass grafting in patients with low ejection fraction. Circulation. 2005 Aug 30;112(9_supplement):I-344. 2) Lang RM, Badano LP, Mor-Avi V, et al., Recommendations for cardiac chamber quantification by echocardiography in adults: an update from the American Society of Echocardiography and the European Association of Cardiovascular Imaging. European Heart JournalCardiovascular Imaging. 2015 Mar 1;16(3):233-71.

2. This work would not have been possible without the kind support and help of many individuals and our organization.

\section{7}

The intensity of rapid response team operation and general ward cardiopulmonary resuscitation incidence

D. Lee'; JE. Kim²; S. Kim³; T. Kim

${ }^{1}$ Intensive care medicine, Dong-a University Hospital, Busan, Republic of Korea; ${ }^{2}$ Emergency medicine, Dong-a University Hospital, Busan, Republic of Korea; ${ }^{3}$ Rapid response team, Dong-a University Hospital, Busan, Republic of Korea; ${ }^{4}$ Internal medicine, Gyeongsang National University, College of Medicine, Changwon, Republic of Korea

Correspondence: $\mathrm{D}$. Lee

Intensive Care Medicine Experimental 2020, 8(2): 000027

Introduction: Rapid response team (RRT) was initially regarded as fulltime working team. However, because of several reasons such as lack of professional manpower, cost effectiveness and size and requirement of each hospital, part-time RRT was introduced. Outcome of part-time RRT recently suggested similary efficacy compared to fulltime RRT. However it is difficult to make a head-to-had comparison and there are no available data comparing the efficacy of part-time and full-time RRT. Authors sought to investigate the intensity of RRT and incidence of in-hospital cardiopulmonary resuscitation (CPR).

Methods: Dong-A University Hospital - a tertiary referral center with 1,000 beds - started to operate RRT in April 2017 as a part-time RRS operating 8 hours on weekdays. In March 2018, RRT operation time was extended to 15 hours on weekdays and finally, extended to 24 hours a day, 7 days a week on February 2019.

We retrospectively reviewed the incidence of in-hospital cardiopulmonary resuscitation (CPR) according to stepwise extension of RRT operation.

Results: For the study period ( 36 months before and 33 months after RRT implementation), 113,394 patients admitted and 127 cases of CPR occurred. For the 36 months before RRT implementation, the next 
initial RRT period (11 months), the second RRT period (11 months), and the third RRT period (11 months), CPR per 1000 admission was 1.27, $1.08,0.90$ and $0.86(p=0.381)$.

In logistic regression analysis, Age, Hospital length of stay, Charlsoncomorbidity score, and incremental intensity was independently associated with CPR development (Table 1).

\begin{tabular}{|c|c|c|c|c|c|c|}
\hline \multirow[b]{2}{*}{ variables } & \multicolumn{3}{|c|}{ Univariate } & \multicolumn{3}{|c|}{ Multivariate } \\
\hline & OR & $95 \% \mathrm{CI}$ & $p$ & OR & $95 \%$ CI & $p$ \\
\hline Age & 1.040 & $1.026-1.055$ & $5<0.001$ & 1.038 & $1.023-1.053$ & $3<0.001$ \\
\hline $\begin{array}{l}\text { Hospital length of } \\
\text { stay }\end{array}$ & 1.010 & $1.006-1.013$ & $3<0.001$ & 1.009 & $1.006-1.013$ & $<0.001$ \\
\hline $\begin{array}{l}\text { Charlson- } \\
\text { comorbidity score }\end{array}$ & 1.038 & $1.020-1.055$ & $5<0.001$ & 1.044 & $1.025-1.063$ & $<0.001$ \\
\hline RRT intensity & 0.849 & $0.719-1.002$ & 20.053 & 0.787 & $0.663-0.934$ & 40.006 \\
\hline Sex & 0.646 & $0.452-0.924$ & 40.017 & 0.715 & $0.499-1.025$ & 0.068 \\
\hline
\end{tabular}

Conclusion: The incremental intensity of RRT operation is associated with decremental tendency of in-hospital CPR rate suggesting the more CPR prevention efficacy with the more operation time of RRT.

Reference(s) and grant acknowledgment(s)

1. Critical Care Medicine. 45(6):e592-e599, JUNE 2017

2. No grant

\section{8}

Intensive care unit without barriers: impact on the forecast of patients with septic shock, after the implementation of an intensive care reference program in the colombian amazonia

A. Ortiz'; D. Molano'; M. Gómez'; E. Beltran'; M. Villabon²; L. Diaz

${ }^{1}$ Medicina crítica y cuidado intensivo, Foundation University of Health Sciences, Bogotá, Colombia; ${ }^{2}$ Medicina crítica y cuidado intensivo, fucs, bogo, Colombia

Correspondence: A. Ortiz

Intensive Care Medicine Experimental 2020, 8(2): 000028

Introduction: Mortality due to septic shock is around $40 \%$ globally (1), these reach figures close to $70 \%$ in peripheral areas devoid of health systems with capacity to manage patientswith intensive care requirements. Given the impossibility of opening intensive careunits in these regions, several options have been raised, such as telemedicine, referral systems, training of non-intensivist health personnel, among others.

Objectives: We propose in this study, to establish the impact on mortality in patients with septicshock, of the establishment of an early identification program and transfer to a reference ICU, with 24-hour availability of acceptance of these patients.

Methods: Prospective observational descriptive study, between January 2016 and October2019, in the intensive care unit of the Hospital de San José in Bogotá Colombia, of patients diagnosed with septic shock, who were treated by transfer from the regionof Guainía-Amazonas, within the MIAS program (Integral model of health care) (2).

Results: Twenty-three patients with septic shock or multi-organ dysfunction secondary to sepsis were attended, mean age 44.1 years, APACHE II 16.68 with expectedmortality of $24 \%$. $25 \%$ of infections correspond to tropical infections, the mostcommon being tuberculosis and secondly malaria. Within the non-tropical etiology, $40 \%$ sepsis of lung origin and $12 \%$ soft tissue sepsis. $60 \%$ of the patients requiredinvasive mechanical ventilation and $80 \%$ vasopressor support. In $90 \%$ of patients the empirical antibiotic management was beta-lactam antipseudomonas + inhibitor. Only in $36 \%$ of the patients, the etiological germ was isolated, with $45 \%$ grampositive cocci, $30 \%$ gram negative bacilli and the remaining 30\% atypical germs, viruses, fungi and mycobacteria. Gross mortality was $12 \%$.

Conclusion: The implementation of rapid care models with immediate transfer services to intensive care units, reduces mortality by up to $50 \%$ in patients with septic shock in the region of the Colombian
Amazon. Tropical, respiratory and skin and soft tissue infections constitute the main and most serious diseases.

\section{Reference(s) and grant acknowledgment(s)}

1. Moreno Gomez Germán Alberto. El nuevo modelo de Atención Integral en Salud -MIAS- para Colombia. ¿La solución a los problemas del sistema. Revista médica Risaralda [Internet]. 2016 July [cited 2020 Jan 23]; 22(2): 73-74. Available from: http://www.scielo.org.co/scielo.php?script=sci_artte xt\&pid=S0122-06672016000200001\&lng=en.

2. Singer M, Deutschman CS, Seymour CW, Shankar-Hari M, Annane D, Bauer $M$, et al. The Third International Consensus Definitions for Sepsis and Septic Shock (Sepsis-3). JAMA. 2016;315(8):801-10.

\section{7}

A cross-sectional survey of the research environment in critical care in Japan, South Korea, and Singapore

Y. Kotani ${ }^{1}$; S. Na ${ }^{2}$; J. Phua ${ }^{3}$; N. Shime ${ }^{4}$;. K. Kawasaki ${ }^{5}$; H. Yasuda ${ }^{1}$;

A. Kawaguchi ${ }^{6}$

${ }^{1}$ Department of intensive care medicine, Kameda Medical Center, Kamogawa, Japan; ${ }^{2}$ Anesthesiology and pain medicine, Severance Hospital, Seoul, Republic of Korea; ${ }^{3}$ Division of respiratory and critical care medicine, National University Hospital, Singapore, Singapore; ${ }^{4}$ Department of emergency and critical care medicine, Graduate School of Biomedical and Health Sciences, Hiroshima University, Hiroshima, Japan; ${ }^{5}$ Department of pediatric critical care, Shizuoka Children's Hospital, Shizuoka, Japan; ${ }^{6}$ Department of pediatrics, University of Montreal, $\mathrm{CHU}$ Sainte Justine Hospital, Montreal, Canada

Correspondence: $Y$. Kotani

Intensive Care Medicine Experimental 2020, 8(2): 000077

Introduction: The strengthening of research infrastructure is key for the advancement of academic medicine in critical care. However, while there have been dramatic clinical developments in Asian critical care in the last few decades, research has lagged behind.

Objectives: The aim of this study was to describe the current status of academia in critical care in Japan, South Korea, and Singapore, particularly from the research environment perspective.

Methods: This was a cross-sectional questionnaire survey conducted between June 2019 and January 2020. We included all physician members of the Japanese Society of Intensive Care Medicine (JSICM) and the Korean Society of Critical Care Medicine (KSCCM) as of June 2019, and intensivists in Singapore as of November 2019. The questionnaire was drafted by two content experts and validated through pre-determined testing with a clinical sensitivity test and a specificity table. We collected data on the characteristics of the participants and their affiliated institutions, the research environment such as funding and support for personnel, and ethics boards and their role. We investigated the association between the presence of time dedicated to research and the number of research publications in English (1-10 versus $>10$ ) among the participants with at least one English original article.

Results: In total, 490 responded (response rate: 5.6\%) to the survey and all responses were included for analysis. The clinical experience in critical care was 20 years or more in $41 \%, 10-19$ years in $42 \%, 6-9$ years in $14 \%$, and $2-5$ years in $3 \%$. Forty-eight percent of the respondents held a university faculty appointment. Fifty-five percent worked for a university-affiliated hospital while $45 \%$ worked for a community hospital. Fifty-one percent reported that they used $50 \%$ or more of their work hours for bedside patient management in the intensive care unit. Thirty-four percent replied that there was at least one dedicated staff member for research work (epidemiologist or biostatistician) available in their hospitals. Twenty-four percent had dedicated time for research within their full-time work hours. When looking at the respondents with at least one original article in English language as a first or corresponding author, the odds to have more than 10 of the English publications for whom with protected time for research was 3.3 (95\% confidence intervals: 1.8-6.1) compared to those without.

Conclusion: We found that many critical care physicians in the three countries had limited support for their research work and that dedicated time for research was positively associated with the number of research publications. The research environment and infrastructure in 
these countries require substantial enhancement for better research productivity.

\section{Reference(s) and grant acknowledgment(s)}

1. Burns KE, Zubrinich C, Tan W, Raptis S, Xiong W, Smith O, et al. Research recruitment practices and critically ill patients. A multicenter, cross-sectional study (the Consent Study). American journal of respiratory and critical care medicine. 2013;187(11):1212-8.

\section{7}

The cost-effectiveness of blood-based brain biomarkers for screening adults with mild traumatic brain injury

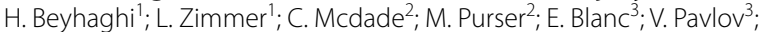
S. Earnshaw ${ }^{2}$

${ }^{1}$ Global medical affairs, bioMerieux Inc, Durham, United States of America; ${ }^{2}$ Health economics, RTI-Health Solutions, Research Triangle Park, United States of America; ${ }^{3} \mathrm{Global}$ medical affairs, bioMerieux SA, Marcy-l'Étoile, France

\section{Correspondence: M. Purser}

Intensive Care Medicine Experimental 2020, 8(2): 000107

Introduction: Mild traumatic brain injury (mTBI) is a frequent cause of emergency department (ED) visits in France, with a substantial impact on healthcare utilization and ED costs. Blood-based, brain biomarker tests such as $\mathrm{S100B}$ and a combination of glial fibrillary acidic protein (GFAP) and ubiquitin C-terminal hydrolase-L1 (UCH-L1) have been shown to accurately identify mTBI patients who do not have intracranial lesions (ICL), potentially reducing the need for computed tomography $(\mathrm{CT})$ scanning.

Objectives: To assess the cost-effectiveness of the GFAP + UCH-L1 compared with S100B for screening adults with suspected mTBI presenting to an ED.

Methods: We developed a decision tree model to estimate the costs and health outcomes of using two biomarker tests in patients presenting to the ED with suspected mTBI (Glasgow Coma Scale of 13-15). Model parameters were extracted from peer-reviewed papers, clinical guidelines, and expert opinion. The diagnostic test accuracy measures were extracted from the pivotal trial (GFAP + UCH-L1) and a meta-analysis (S100B). Lifelong costs $(2020 €)$ and health outcomes were calculated from a French healthcare system perspective and discounted over time at $2.5 \%$. Cost for the GFAP + UCH-L1 test was assumed to be the same as the S100B test. In this model, patients with a positive biomarker receive a CT scan to confirm the presence of ICLs. Depending on the biomarker test outcome and the CT results (where relevant), patients may be discharged immediately from the ED, kept for <24-hour observation, admitted to a short stay general ward, or admitted to a neurosurgery ward. Patients with ICLs not identified by the biomarker (false negatives) could suffer from delayed treatment and poorer health outcomes. Clinical outcomes, including Glasgow Outcome Score and radiation-induced cancer were used to calculate quality-adjusted life years (QALYs). One-way and probabilistic sensitivity analyses were conducted to manage uncertainty.

Results: In a hypothetical cohort of 1,000 patients, the GFAP + UCHL1 biomarker was associated with a decrease of $48 \mathrm{CT}$ scans compared to S100B (769 versus 817 , respectively). In the same cohort, the GFAP $+\mathrm{UCH}-\mathrm{L} 1$ biomarker resulted in $€ 4,785$ overall savings and a minor improvement of 0.08 QALYs. In probabilistic sensitivity analysis, at the willingness to pay threshold of $€ 50,000$ per QALY, there is $63 \%$ probability of the GFAP + UCH-L1 test to be cost-saving and a 73\% probability to be cost-effective when compared to S100B.

Conclusion: The findings of this cost-effectiveness model show that for patients with suspected $\mathrm{mTBI}$ who present to an ED, compared to $\mathrm{S} 100 \mathrm{~B}$, screening with the GFAP + UCH-L1 test is associated with cost savings and a reduction in CT scans, while resulting in similar health outcomes.

\section{Reference(s) and grant acknowledgment(s)}

1. Bazarian JJ, et al. Serum GFAP and UCH-L1 for prediction of absence of intracranial injuries on head CT (ALERT-TBI): a multicentre observational study. Lancet Neurol. 2018 Sep;17(9):782-9.
2. Gerlach R, et al. Traumatic epidural hematomas in children and adolescents: outcome analysis in 39 consecutive unselected cases. Pediatr Emerg Care. 2009 Mar;25(3):164-9.

3. Mondello S, et al. Blood-Based Protein Biomarkers for the Management of Traumatic Brain Injuries in Adults Presenting to Emergency Departments with Mild Brain Injury: A Living Systematic Review and Meta-Analysis. J Neurotrauma. 2018 Jul 2.

4. Pandor A, Goodacre S, Harnan S, Holmes M, Pickering A, Fitzgerald P, et al. Diagnostic management strategies for adults and children with minor head injury: a systematic review and an economic evaluation. Health Technol Assess. 2011 Aug;15(27):1-202.

5. The study was funded by bioMérieux. HB, LZ, EB and VP are employees of bioMerieux, which has a licensing agreement with Banyan Biomarkers, the company that produces the GFAP + UCH-L1 combination biomarker. CM, $\mathrm{MP}$ and SE are employees of RTI, which received consulting fees for this project.

6. Cheung PS, et al. Outcome of traumatic extradural haematoma in Hong Kong. Injury. 2007 Jan;38(1):76-80.

7. Deverill J, Aitken LM. Treatment of extradural haemorrhage in Queensland: interhospital transfer, preoperative delay and clinical outcome. Emerg Med Australas. 2007 Aug;19(4):325-32.

8. Fabbri A, et al. Observational approach to subjects with mild-to-moderate head injury and initial non-neurosurgical lesions. J Neurol Neurosurg Psychiatry. 2008 Oct;79(10):1180-5.

9. Stein SC, Hurst RW, Sonnad SS. Meta-analysis of cranial CT scans in children. A mathematical model to predict radiation-induced tumors. Pediatr Neurosurg. 2008;44(6):448-57.

\section{1}

Minimising extravasation risk with central venous catheters

\section{in critical care}

E. McKemey'; N. Manning ${ }^{1}$; R. Chauhan ${ }^{1}$

${ }^{1}$ Itu, Queen Elizabeth Hospital Birmingham, Birmingham, United Kingdom Correspondence: E. McKemey

Intensive Care Medicine Experimental 2020, 8(2): 000111

Introduction: Extravasation is the accidental injection or leakage of fluid into the subcutaneous or perivascular tissues. Risk of extravasation injury related to intra venous therapy is between $10-30 \%$ resulting in varying complications (1). Extravasation associated with Central Venous Catheters (CVCs) is rarer but consequences can be severe, potentially requiring skin grafts or even leading to death $(2,3)$. This is minimised by ensuring the line is secured at appropriate depth and by potency (toxicity) of drugs in the line. Currently there is no guidance on selection of drugs to ports on CVC. On a Quad lumen CVC, there is $6 \mathrm{~cm}$ from the distal to the proximal port, increasing risk if toxic drugs are placed on the proximal port. Distal ports are traditionally utalised for Central Venous Pressure (CVP) however evidence suggests there is no difference in accuracy when CVP is on distal or proximal port (4).

Objectives: Minimising risks of extravasation with CVCs in Critical care.

Methods: Data was collected from a tertiary centre based in the West Midlands, UK. It is a large Critical care unit (CCU) with over 100 beds with specialised cardiac, neurology, trauma, burns, surgical and liver patients, over 4 units. Data was collected by 2 auditors in 2019 over 2 days across all patients within CCU who had a CVC. The following was assessed: were CVCs sutured to the hilt, what length/type of CVC was used, which port was transducing CVP and which "Toxic drugs" were on the proximal port. We defined "Toxic drugs" as inotropes, neat potassium and $50 \%$ magnesium.

Results: Of 61 Critical Care patients, 51 patients had an internal jugular, 4 femoral and 6 subclavian CVC. $21 \%$ of the lines were not sutured at the hub. $77 \%$ of lines used the distal port to transduce the CVP which is current practice and $6 \%$ on Proximal. $41 \%$ of the lines had a Toxic drug on the proximal line.

Conclusion: This quality improvement project demonstrated that extravasation risks for CCU patients could be further minimised by ensuring CVCs are sutured at the hub, and careful selection of toxic drugs to the most distal ports. Changing the location of CVP monitoring from the distal port may help facilitate this. This is a cost neutral 
way or minimising extravasation risk and promoting patient safety in Critical care.

\section{Reference(s) and grant acknowledgment(s)}

1. (4) Alsafi, T., Pahamin, I., Selvaraj, I., and Wollard, C. (2010) Monitoring of central venous pressure via the proximal lumen of central venous catheter: a safety issue. Anaesthesia. Abstract presented at AAGBI Conference Harrogate 2010.65. Pp 1241

2. (3) Davies AG, Russell WC, Thompson JP. Extravasation and tissue necrosis secondary to central line infusions. Anaesthesia 2003; 58: 820-1.

3. (2) Bolton DT. Extravasation associated with a multilumen central catheter. Anaesthesia 1997: 52: 1119.

4. (1) Lake, C. and Beecroft, C.L. (2010) Extravasation injuries and accidental intra-arterial injection. Continuing Education in Anaesthesia, Critical Care and Pain. Vol. 10, No. 4, pp 109-113

\section{8}

\section{Delayed discharges from critical care unit and its impact}

R. AGRAWAL'; I. Dsouza'; J. Reeves ${ }^{2}$

${ }^{1}$ Critical Care Department, University hospitals of North Midlands, STOKE ON TRENT, United Kingdom; ${ }^{2} \mathrm{Pdm}$ s systems administrator, University hospitals of North Midlands, STOKE ON TRENT, United Kingdom

Correspondence: R. AGRAWAL

Intensive Care Medicine Experimental 2020, 8(2): 000168

\section{Introduction:}

Delayed Discharge : Defined as 'the period of continued hospital stay after a patient is deemed medically fit to leave hospital but is unable to do so for non-medical reasons'.(1)

Costs of NHS in England associated with delayed discharge are approximately 100 million GBP per year and resulted in 1.2 million bed days lost in 2013-14.(2)

\section{Objectives:}

FICM Edition 2 June 2019: Guidelines for the provision of intensive care services.

Discharge from critical care to a general ward must occur within four hours of the decision and must occur between 07:00 and 21:59. QIPP Reference 16-17 S4-Trauma

National standard that all discharges from adult critical care should be made within 4 hours of a clinical decision to discharge.

Compliance of UHNM to national guidance and FICM guidance

\section{Methods:}

Retrospective observational study

12 month period from Jan-Dec 2019

All admissions to adult critical care

Extract CCMDS information from 4D auditing software

Wardable date input by nurses after consultant ward round and counter checked by validator

$>4$ hours after ready date

Convert hours delayed to days

HRG code * Days Delayed = Cost of delay

Results:

$921(86.4 \%)$ delayed discharges

1,096 days

Average 1.2 days delay per patient

Cost over the year $£ 1,919,252$

Average 3 beds/day

70 band 5 salaries

\section{Conclusion:}

Delayed discharge is recognised to be a system level problem requiring effective team working within hospitals and coordination between health and social care .

Adverse outcomes of delayed discharge include :

For patients:

an association with increased risk of mortality,Hospital-acquired infections, mental ill health

a reduction in patient's mobility and activities of daily living

For health care staff
Stress, diversion from a primary focus on patient care and deleterious interprofessional relationships,all with potentially harmful

implications for patient's health and well being

As well as impact on inpatient costs, there are additional economic repercussions for other services.

\section{Reference(s) and grant acknowledgment(s)}

1. 2. Hospital discharges delays cost NHS 100 m'Health serv J. 2014 3.https

2. 1. Bates A. Delayed transfers of care in the NHS. In: common H, ed. 2015.

3. No grants required

\section{4}

National Early Warning Score (NEWS) and the outcomes

of a district general hospital wards' patients referred to Critical

Care Outreach service, but not admitted to Intensive Care Unit due to their co-morbidities

M. Mazalkov'; E. Kazantzidis²; R. Samanta ${ }^{3}$; M. Camilleri ${ }^{1}$

${ }^{1}$ Anaesthetics, Peterborough City Hospital, Peterborough, United Kingdom; ${ }^{2}$ Emergency department, Peterborough City Hospital, Peterborough, United Kingdom; ${ }^{3}$ Anaesthetics, Addenbrooke's Hospital, Cambridge, United Kingdom

Correspondence: M. Mazalkov

Intensive Care Medicine Experimental 2020, 8(2): 000224

Introduction: Critical Care consultant led outreach services (Outreach) are set to assist the hospital wards in the prompt detection and care of the acutely unwell and deteriorating patients. The patients on the general wards are referred to the Outreach if their National Early Warning Score (NEWS 2), which in the UK may range from 0 up to 20 maximal, reach certain thresholds or if there appear any other significant concerns. According to the algorithm printed on NEWS-2 charts: values 5 or more (or 3 in one parameter) trigger an urgent patient's review by the parental team; values 7 or more trigger the urgent automatic referral to Outreach team. After a prompt review by an outreach consultant and a senior nurse, the decision is made whether the patient needs an urgent Critical Care admission. In more reassuring cases, patients continue to be managed on the wards under a close supervision by the Outreach. Unfortunately, some of the patients have multiple co morbidities and their physiological reserve is very limited. Therefore, any potential Level 2 or Level 3 technological organ support would be futile. In those cases a difficult (but necessary) decision is made that the ward based care should be the ceiling of treatment for the patient. This group of patients is very diverse in the setting of a district general hospital. Nevertheless, there are certain similarities within this cohort. Typically, these patients are frail and old with concurrent chronic illnesses and a poor excercize tolerance. The vast majority are medical patients with sepsis of various origins. Their increased NEWS reflects patients' acute deterioration and impending organ failure. Therefore, it is paramount that such decisions are absolutely correct. Thus, we have conducted a retrospective, observational, single centre study of the relevance of NEWS magnitude to the outcomes of the hospital wards' patients referred to the outreach service, but deemed not to be appropriate for critical care admission secondary to their comorbidities and a limited physiological reserve. The project has been approved by the Health Research Authority, reference number is 268879 .

Methods: We followed up 152 patients admitted to our hospital between 1/12/2017 and 24/12/2018 who at any point were referred to Outreach, but deemed not appropriate for any critical care organ support as above. The ceiling of care would be at Level 1 (ward-based care only).

The analysed data included NEWS and a fraction of inspired oxygen (FiO2) (delivered through an appropriate device) as documented at the time of Outreach referral; mortality in-hospital and for up to 3 months after the hospital discharge, if happened; length of hospital stay (LOS), age, gender and diagnosis on admission. The patients' groups were defined as: "hospital non survivors or HNS"; if died within three months after the date of discharge - " 3 months non survivors or $3 \mathrm{mNS}$ " and, if survived for more than 3 months - " 3 months survivors or $3 \mathrm{mS}^{\prime \prime}$. 
The statistics are presented as medians with interquatile ranges or means with standard deviation. Comparisons between groups were made using Wilcoxon rank sum test for non-normally distributed data. We used logistical regression for associations and reported results as odds ratios with $95 \%$ confidence interval. Time dependent outcomes were assessed with Kaplan Meier analysis as hazard ratios. Length of stay data were modelled using Poisson regression.

Results: The hospital mortality (HNS) was $46.7 \%$ (71 patients). $3 \mathrm{mNS}$ comprised $15.8 \%$ (24 patients). $3 \mathrm{mS}$ constituted $37.5 \%$ (57 patients). Median (quartiles 1 and 3) NEWSs were: HNS - 7 (5.5-9), 3mNS - 7 (5-8.25), 3mS - 6 (5-8). There was no statistically significant difference between the groups.

Median FiO2 levels were: HNS - 35\% (28-60), 3mNS - 28\% (23.25-60) $3 \mathrm{mS}-28 \%(24-40)$. There was a trend for a better survival in patients on lower $\mathrm{FiO} 2$.

Median patients' age was: HNS $-75,3 \mathrm{mNS}-74.5$ and $3 \mathrm{mS}-73$ years. Median lengths of hospital stay (LOS) were: HNS $-8(4-13)$ days, $3 \mathrm{mNS}$ - 19 (9.5-31) days, 3mS - 20 (11-30) days.

Subsequently, the sample was split in: patients with NEWS " 5 and above" (123/152) median NEWS 7 (6-10) and with NEWS "4 and below" (29/152) median NEWS $3(2-4)$

Their respective: hospital mortality was $47.2 \%$ versus $44.8 \%$, 3 months mortality was $15.4 \%$ versus $17.2 \%$ and 3 months survival was $37.4 \%$ versus $38 \%$.

Conclusion: High NEWS scores are not associated with worse 30 days or 3 months mortality in this patients' cohort.

High NEWS scores are associated with shorter LOS within the survivors group.

Chronological age does not appear to be a risk factor.

No particular diagnosis strongly predicts in-hospital mortality.

\section{Reference(s) and grant acknowledgment(s)}

1. 1. Sparkes, D., Smith, G. (2004) Intensive care requirements for an ageing population - a microcosm of problems facing the NHS? Clin Med May/June 2004 vol. 4 no. 3263-266 https://doi.org/10.7861/clinmedicine.4-3-263 2. Malycha J, Farajidavar N, Pimentel MAF, et al. The effect of fractional inspired oxygen concentration on early warning score performance: A database analysis. Resuscitation 2019; 139:192-199. https://doi.org/10.1016/j.resus citation.2019.04.002 3. Chelluri L, Pinsky MR, Donahoe M P, Grenvik A. Long-term Outcome of Critically III Elderly Patients Requiring Intensive Care. JAMA. 1993; 269 (24):3119-3123. https://doi.org/10.1001/jama.1993.03500 240063027 4. Lingsma H F, Bottle A, Middleton S, Kievit J, Steyerberg EW, Marang-van de Mheen P J Evaluation of hospital outcomes: the relation between length-of-stay, readmission, and mortality in a large international administrative database BMC Health Services Research 18, article 116 (2018) 5. Jayasundera R, Neilly M, Smith TO, Myint PK. Are Early Warning Scores Useful Predictors for Mortality and Morbidity in Hospitalised Acutely Unwell Older Patients? A Systematic Review. J Clin Med. 2018; 7 (10):309. Published 2018 Sep 28, https://doi.org/10.3390/jcm7100309 6.Jo S, Yoon J, Lee JB, Jin Y, Jeong T, Park B. Predictive value of the National Early Warning Score-Lactate for mortality and the need for critical care among general emergency department patients. J Crit Care. 2016 Dec; 36:60-68, https://doi. org/10.1016/j.jcrc.2016.06.016 2. None

\section{4}

\section{ICU follow-up: a national survey in Belgium}

D. Prevedello'; FS. Taccone ${ }^{1}$; JC. Preiser ${ }^{1}$

1 Intensive Care, Hospital Erasme, Bruxelles, Belgium

Correspondence: D. Prevedello

Intensive Care Medicine Experimental 2020, 8(2): 000264

Introduction: The concept of ICU follow-up clinics arose in 1993 in the United Kingdom to identify and rehabilitate patients with postintensive care syndrome (PICS)(1). Recently, an increasing number of ICUs had organized the same program to assess patient's conditions after ICU discharge. In Belgium, we do not have a national guideline or recommendation for the ICU follow-up program as in the UK and Netherlands for instance $(2,3)$. We therefore performed a national survey to assess Belgian ICU follow-up practice.
Methods: This Survey was designed based on the International Handbook of Survey Methodology sponsored by the European Association of Methodology (EAM) and the Revised WAPOR Code of Ethics, 2011 (WAPOR - World Association for Public Opinion Research). The Survey has been endorsed by the Belgian Society of Intensive Care (SIZ). We developed a closed and self-enumerated questionnaire that had initiated its content validated by four experts and a pilot phase. After the validation process, in March 2019, the questionnaire and the cover letter were addressed to directors of ICUs in Belgian hospitals electronically (one per ICU). The questionnaire was anonymous, and no electronic tracker was used to identify the responders. Two weeks following the distribution, a reminder letter and the link to access the questionnaire were resent by email. After eight months, a new reminder was sent by the Belgium Society of Critical Care, trying to increase the response rate.

Results: Thirty-six out of 82 potential respondents (43\%) completed the Survey. Specifically, $35 \%(16 / 46), 46 \%(12 / 26)$, and $80 \%(8 / 10)$ of respondents were considered from Flanders, Wallonia, and BrusselsCapital, respectively. Six centers (17\%) have an ICU follow-up program currently running. When we analyzed the profile of patients involved, the ICU length of stay, and the duration of mechanical ventilation were the two mean criteria to select eligible patients. Only one of the six centers received external funding to support this program; two of the five remaining centers used internal financial support to afford the ICU follow-up activities. Thirty centers did not run an ICU followup clinic. The main reasons were the lack of human resources $(20 / 30$ $67 \%)$ and financial constraints (12/30 40\%). Seven centers (23\%) also justified the lack of ICU follow-up program with the absence of clinical need and of current evidence for benefits. Only 4 (13\%) centers were not interested in starting an ICU follow-up clinic if possible.

Conclusion: The use of an ICU follow-up program is implemented in a minority of Belgian ICUs. Funding and human resources are critical issues to solve and promote this activity in more centers.

\section{Reference(s) and grant acknowledgment(s)}

1. 1. Griffiths JA, Gager M, Waldmann C. Follow-up after intensive care. Contin Educ Anaesthesia, Crit Care Pain. 2004;4(6):202-5.

2. 2. Rehabilitation after critical illness in Rehabilitation after critical illness in adults adults Clinical guideline. 2009;(March 2009).

3. 3. Van Schaaf M Der, Bakhshi-Raiez F, Van Der Steen M, Dongelmans DA, De Keizer NF. Recommendations for intensive care follow-up clinics; Report from a survey and conference of Dutch intensive cares. Minerva Anestesiol. 2015;81(2):135-44.

\section{7}

Role of Levosimendan in heart failure patient undergoin cardiac surgery

F. Marchese ${ }^{1} ;$ M. L. ${ }^{2} ;$ S. G. ${ }^{3} ;$ R. M. ${ }^{2}$; C. G. ${ }^{2}$

${ }^{1}$ Ospedali Riuniti, Foggia, Italy; ${ }^{2}$ Anestesia e rianimazione, Hospital Oo Rr Di Foggia, Foggia, Italy; ${ }^{3}$ Cardiochirurgia, Città di Lecce Hospital, Lecce, Italy Correspondence: $F$. Marchese

Intensive Care Medicine Experimental 2020, 8(2): 000307

Introduction: Levosimendan is a calcium sensitizer developed for intravenous use in hospitalized patients with acute heart failure (AHF) (1).

Aim of this study is to observe the cardiac function's index, Mean Arterial Pressure (MAP) and diuresis after the administration of Levosimendan (G1) rather than control group (G2) started 12 hours before cardiac surgery (2).

Objectives: In this prospective observational study 47 patients consecutive admitted in ICU of "Città di Lecce Hospital- GVM Care and Research" with AHF (Ejection Fraction $<40 \%$ ) were included.

Methods: Patients admitted in ICU were randomized to receive either Levosimendan infusion duration up to 24 hours $(0,1 \mathrm{mcg} / \mathrm{Kg} / \mathrm{min})(\mathrm{G} 1)$ (18 patients).

Patients undergoing urgent cardiac surgery, severe ipotensive syndrome or left ventricular ejection stenosis were excluded (29 patients). Measurement were collected at TO (before to start Levosimendan infusion), $\mathrm{T} 1$ (in operation room before to start cardiac surgery), $\mathrm{T} 2$ (when 
was interrupted extra corporeal circulation), T3 (in Intensive Care Unit) and T4 ( $24 \mathrm{~h}$ after Levosimendan administration). Were recordered major functional cardiac index such as Ejection Fraction, Stroke Volume, Cardiac Output and TAPSE with echocardiography transesofageal (T0, T1, T2) and transthoracic (T3, T4) and were collected Mean Arterial Pressure, ScvO2, lactate in blood gas analysis, diuresis. Pvalue of $0,05 \%$ was considered statistically significant.

Results: Echocardiografy showed no statistically significant difference in Stroke Volume $(p=0.8)$ and Cardiac Output $(p=0.99)$ between two groups. In both groups SV and CO parametres increased progressively rather than baseline, expecially in $\mathrm{G} 1$.

Ejection Fraction increased significantly at any time vs baseline in $\mathrm{G} 1$ $(p<0,049)$, whilst they remained stable over time in $\mathrm{G} 2$.

TAPSE was stable during the study period in G2, while the trend increased progressively only in G1. Howewer no statistically difference between groups.

Comparisons between groups showed similar ScvO2 levels at baseline whilst they significanlty increased in G1 vs G2 at T2, T3 and T4 ( $p>0.05)$ as anaerobic metabolism' index.

In both groups lactate levels increased during the study period especially at $T 2$, but non statistically difference between $G 1$ vs $G 2$. In postoperative times blood-gas assessment showed impaired lactate values in $\mathrm{G} 1$ vs $\mathrm{G} 2$.

The analysis found that diuresis was lower in patients treated with Levosimendan (G1) vs placebo (G2) in 24 hour after surgery. Perhaps no statistical difference between two groups.

No patient had adverse reactions or oversensitiveness to Levosimendan infusion.

Conclusion: The preliminary data of the study support these conclusions: (i) the optimal dosing is $0.1 \mathrm{mcg} / \mathrm{kg} / \mathrm{min}$ for 24 hours of Levosimendan did not increased the SV and CO value, whilst increased EF statistically significant (ii)lactate concentration in gas analysis decrease in patients treated with Levosimendan, whilst also suggest that Levoimendan improved ScvO2 (iii) levosimendan did not enhance the diuresis in G1 vs $\mathrm{G} 2$ and (iv) Levosimendan infusion should be started before surgery to exert its preconditioning effect.

Levosimendan infusion sustained haemodynamic improvement in the acute heart failure patients.

\section{Reference(s) and grant acknowledgment(s)}

1. 1) McMurray JJV, Adamopoulos S, Anker SD, Auricchio A, Böhm M, Dickstein

$\mathrm{K}$, et al. ESC Guidelines for the diagnosis and treatment of acute and chronic heart failure 2012. European journal of heart failure. 2012; 14(8): 803-69.

2) De Lissovoy G, Fraeman K, Teerlink JR, Mullahy J, Salon J, Sterz R, et al.

Hospital costs for treatment of acute heart failure: economic analysis ofthe

REVIVE II study. The European journal of health economics : HEPAC : health economics in prevention and care. 2010; 11(2): 185-93.

2. 2) No grants were given

\section{7}

Developing a Family Liaison Team during the COVID-19 pandemic: the experience of a tertiary London teaching hospital

C. Lopez Soto'; L. Flood'; E. Bates ${ }^{1}$; C. Anderson'; V. Metaxa

'Critical care medicine, King's College Hospital, London, United Kingdom Correspondence: $\mathrm{C}$. Lopez Soto

Intensive Care Medicine Experimental 2020, 8(2): 000357

Introduction: Communication with and participation of families and patients during their stay in an intensive care unit (ICU) has evolved over the last decades, by means of different approaches, such as: family-centred rounds, daily updates, electronic patient portals and interdisciplinary family meetings. Family-centred care is desired by patients and families, and evidence suggests that it may improve user outcomes, and reduce burnout and moral distress among clinicians. This patient/family-centred approach is challenged during large-scale disasters, where movement restrictions are implemented to preserve public safety/health.

In March 2019, our hospital following Public Health England guidance, restricted patients' family and friends (PFFs) visitation rights in response to the COVID-19 pandemic
Objectives: We describe the setting up of a service to communicate with patients' families.

Methods: A service improvement project in a tertiary London hospital, during the 2020 novel COVID-19 pandemic. The Trust increased its beds from 81 to 140 to cope with the surge in patients and a total of 10 critical care (CC) areas were developed (increased from 5). As quarantine and national guidance prevented relatives from visiting the hospital, we explored the possibility of creating a dedicated team of professionals that would focus on communications with patients' families and friends (PFFs)

Results: A dedicated team of redeployed healthcare professionals was assembled on 25th March (family liaison team, FLT), with a total of 40 people volunteering. The vast majority $(75 \%)$ were medical consultant grade. Their background specialty is shown in figure 1 and their main responsibilities in figure 2 . The teams were allocated to each CC area, with a maximum ratio of 1:10 and underwent 2 1-hourly webinars around basic family communication skills. The following routine was followed: at 8am the FLT attended the doctors' handover and identified any patients who had deteriorated overnight in order to update their families. They then communicated with the medical team after the ward round and transferred the information to the next of kin of each patient. Video calls were facilitated during the day, depending on the availability of the family and the bedside nurse. Until May 10th, the FLT has facilitated over 12,000 (video-) calls and communicated with $>90 \%$ of PFFs daily.

We defined the FLT responsibilities as follow: daily medical updates, facilitation of video calls, information, provision and suppor for families visiting (visiting was allowed for deteriorating patients or approaching end of life), obtaining social history if not completed at time of admission, communicate information to medical and nursing team and identification of families/friends with specific needs (young children, impending bereavement, social and psychological support) and refer them to the appropiate services.

We also developed a document to serve as a guide for the FLT during the communications with PFFs, which included: introduction to the team, how to give daily updates and how to manage difficult conversations if patients were deteriorating.

Figure 1. Specialties taking part in the FLT

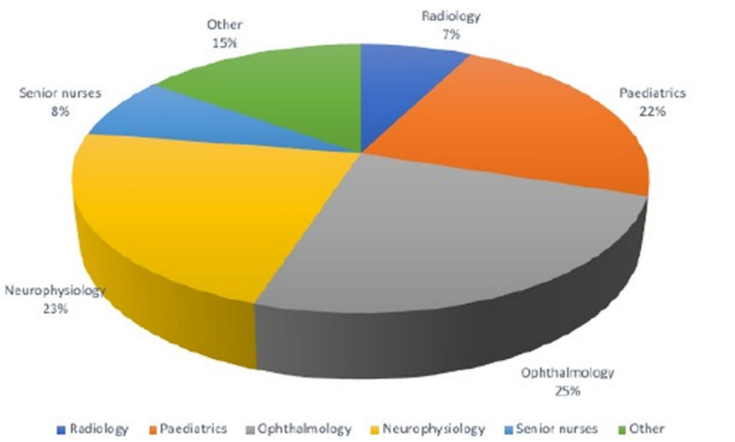

Conclusion: Non-CC professionals have been pivotal in continuing the patient-family connection in our institution during the pandemic. The model is successful, requiring moderate training for the FLT. Further evaluation both for the team and around PFF satisfaction is currently underway.

\section{Reference(s) and grant acknowledgment(s)}

1. No grants received

2. White DB, Angus DC, Shields AM, et al. A Randomized Trial of a Family-Support Intervention in Intensive Care Units. N Engl J Med 2018;378:2365

3. Hart JL, Turnbull AE, Oppenheim IM, Courtright KR. Family-Centered Care

During the COVID-19 Era. J Pain Symptom Manage 2020 
000383

\section{Dependency and vulnerability of intensive care medicine} in times of COVID-19 crisis

M. T. Lingitz'; G. Roth'; C. G. Krenn ${ }^{2}$

${ }^{1}$ Department of anesthesia and general intensive care medicine, Franziskus Spital Margareten, Wien, Austria; ${ }^{2}$ Department of anesthesia, general intensive care and pain medicine, Vienna General Hospital, Vienna Austria, Austria

Correspondence: M.T. Lingitz

Intensive Care Medicine Experimental 2020, 8(2): 000383

Introduction: Since December 2019, Coronavirus Disease-2019 (COVID-19) spread rapidly all over the world, resulting in pandemic $[1,2,3]$. Through high hospitalization rates and via potentially exponential expansion of the disease, the COVID-19 crisis places a tremendous strain on healthcare systems, especially on limited numbers of hospital and Intensive Care Unit (ICU) - beds and equipment $[4,5]$.

Objectives: The aim of this study was to elucidate the possible dependency of ICUs in European Countries with Austria as a pars pro toto in particular on long transglobal supply chains and its potential vulnerability by reported shortages of essential medicines and limited capacities in times of globalization and asserted economic advantages versus the pandemic threat of COVID-19 $[4,5,6]$.

Methods: From 3 April to 8 April 2020 we performed a short survey with heads of Austria's ICU-wards in different institutions following a structured questionnaire to enquire their personal experience and assessment.

At that time containment measures with exit restrictions, starting on 16 March 2020, has lasted for a time span of three weeks. Due to the dynamic situation during pandemic with constantly changing export bans and travel restrictions especially represents a current snapshot of the situation in that time.

Results: We surveyed nearly half of the head physicians of ICUs $(n=40)$ in Austria [7]. 55 percent $(n=22)$ are already treating COVID19 affected patients at their ICU department. Only 45 percent $(n=18)$ of them had received the amount of personal protective equipment they deemed enough, and 90 percent $(n=36)$ of surveyed ICUs were already informed about shortage supplies concerning personal protective equipment. (Fig. 1) 45 percent $(n=18)$ already reported infections within their medical personnel. According to the estimation of heads of ICUs the duration of pandemic should not last more than 6 weeks averagely without facing difficulties in storage and availability of supply. 35 percent $(n=14)$ already reported supply difficulties in several pharmaceuticals, whereas 73 percent $(n=29)$ were informed or already warned by their economic or purchase departments about further potential or real shortages concerning essential pharmaceuticals in near future. Detailed results are illustrated in Fig. 2.

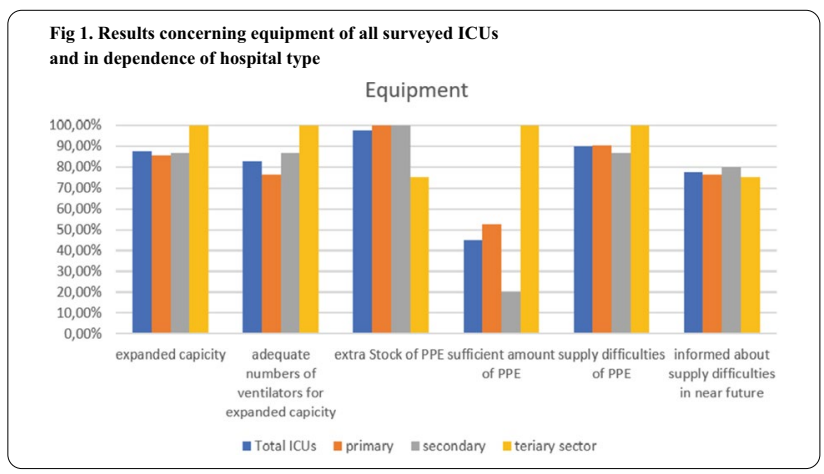

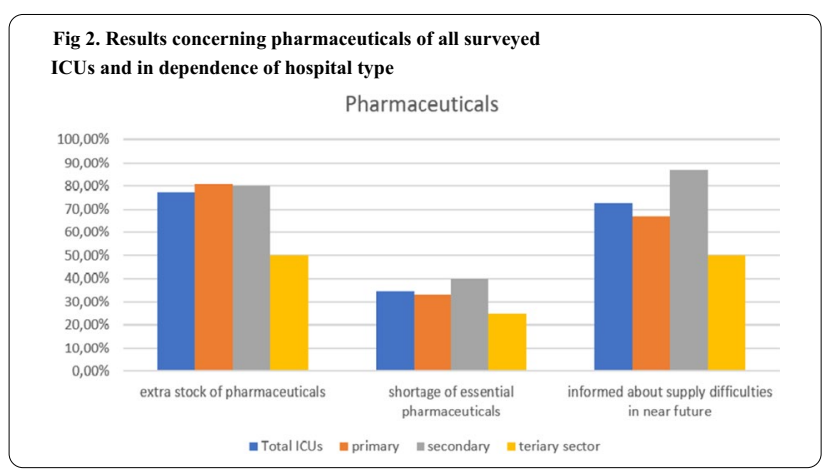

Conclusion: Summarizing the results of our survey we would like to draw attention to the lessons we can learn from the COVID-19 crisis in the EU: European Countries with Austria as a pars pro toto should establish a national production of key equipment and disposables, at least a minor part of PPE as well as key pharmaceuticals. European countries need to improve far distance supplies and invest in maintaining critical infrastructure especially in those with limited capacities such as health care in general and ICUs in particular.

Reference(s) and grant acknowledgment(s)

1. 1. Huang C, Wang Y, Li X, Ren L, Zhao J, Hu Y, et al. Clinical features of patients infected with 2019 novel coronavirus in Wuhan, China. The Lancet. 2020 Feb 15;395(10223):497-506.

2. 2. Guan W-J, Ni Z-Y, Hu Y, Liang W-H, Ou C-Q, He J-X, et al. Clinical Characteristics of Coronavirus Disease 2019 in China. The New England journal of medicine [Internet]. 2020 Feb 28 [cited 2020 Apr 5]

3. 3. WHO/Europe | Coronavirus disease (COVID-19) outbreak - About the virus [Internet]. [cited 2020 Apr 5]. Available from: http://www.euro.who.int/en/ health-topics/health-emergencies/coronavirus-covid-19/novel-coronaviru s-2019-ncov

4. 4. Shortage of personal protective equipment endangering health workers worldwide [Internet]. [cited $2020 \mathrm{Apr} 11]$. Available from: https://www.who. int/news-room/detail/03-03-2020-shortage-of-personal-protective-equip ment-endangering-health-workers-worldwide

5. 5. Addressing the potential impact of novel coronavirus disease (COVID-19) on medicines supply in the EU | European Medicines Agency [Internet]. [cited 2020 Apr 11]. Available from: https://www.ema.europa.eu/en/news/ addressing-potential-impact-novel-coronavirus-disease-covid-19-medic ines-supply-eu

6. 6. The Pharmaceutical Industry in Figures [Internet]. [cited 2020 Apr 12]. Available from: www.efpia.eu

7. 7. Federal Ministry Republic Austria [Internet]. [cited 2020 Apr 23]. Available from: http://www.kaz.bmg.gv.at/fileadmin/user_upload/Krankenanstalte n/5_T_KH.pdf

000416

Experience of repurposing the non-invasive V60 Ventilator for invasive ventilation to produce surge capacity during covid19

S. Petty ${ }^{1}$; M. Jeffrey ${ }^{1}$; Y. Yang ${ }^{1}$; A. Conway-Morris ${ }^{1}$; R. Mahroof ${ }^{1}$

${ }^{1}$ Department of anaesthetics \& intensive care medicine, Cambridge University Hospitals NHS Foundation Trust, Cambridge, United Kingdom

Correspondence: M. Jeffrey

Intensive Care Medicine Experimental 2020, 8(2): 000416

Introduction: We present an early analysis and account of our experiences using our primary non-invasive ventilation (NIV machine), Respironics V60 ventilator ${ }^{\circledR}$, as a first line expansion for invasive ventilation.

Objectives: Review how the V60 ventilator may be used for invasive ventilation

Methods: We obtained 25 V60 units for critical care. Our surge plan was shaped by staff familiarity with the V60 versus staff unfamiliarity/ limited supply of consumables for Anaesthetic Machines 
We maximised staff protection from aerosolised SARS CoV-2 during V60 use by adding a Clear-Therm 3 HMEF $(1541)^{\circledR}$ to allow circuit breaks. We used Pressure Control Ventilation (PCV) as our mandatory mode, which was not used prior to COVID19. Spontaneous/Timed $(\mathrm{S} / \mathrm{T})$ mode was used for support ventilation.

Laminated setup guides for circuits and initial settings were attached to all machines supported by comprehensive face-to-face and videoconferencing training delivered to all relevant staff.

Results: The V60 ventilator has formed a significant part of our surge response, used in 30 out of 81 (37\%) patients. These patients received 572 ventilator days, with 239 on a V60 (42\%).

Key issues arising from use were filter saturation, oxygen consumption in $P C V$ vs $S / T$ mode, requirement for increased pressure support for CPAP equivalence and the lack of an inspiratory hold for recruitment manoeuvres.

The proximity of the HMEF to nebuliser caused frequent filter saturation and high resistance. Filter issues were noted in 11 (37\%) patients. This was worse in cases using nebulised epoprostenol (epo). Using a trial of epo $15 \mathrm{ng} / \mathrm{kg} / \mathrm{min}$ for $30 \mathrm{~min}$ we could identify patients better suited to ventilation with a full service (Servo-i) ICU ventilator.

Oxygen usage of $\mathrm{V} 60$ was much higher than the anticipated 10-15LPM. In PCV on $100 \%$ O2 the V60 used 46LPM, at 60\% 21LPM, making patient transfer difficult.

We created a recruitment manoeuvre which was used successfully in 9 (30\%) patients. Settings of RR to 10, i-time 3 s, IPAP 40, EPAP 25 were used for this.

Though V60 ventilators were unable to invert ratios $>1: 1,2$ patients were proned on V60 ventilation, 1 died but the other has been discharged from intensive care.

Lastly, we noted the standby function would continue producing aerosol by blowing when disconnected. However the V60 loads its previous settings when switched on, and does this in $<5 \mathrm{~s}$, so on/off was used in place of standby.

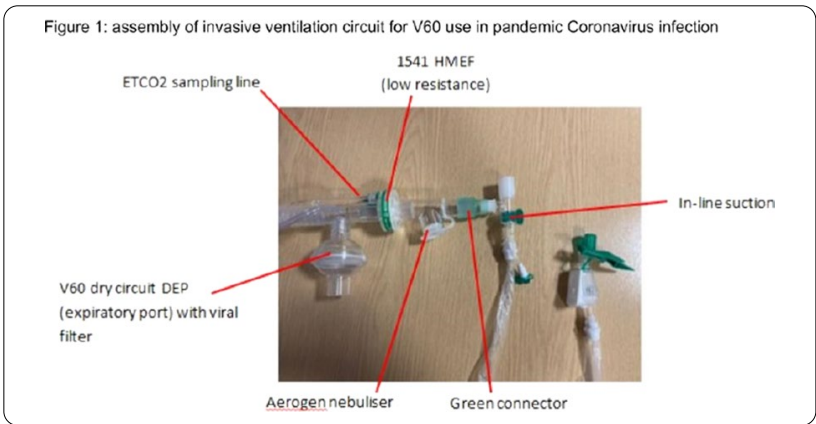

Conclusion: The V60 ventilator is a viable invasive ventilator if problems are addressed. With appropriate patient selection it can spare full-service ventilator capacity, and at $25 \%$ of cost of our full-service ICU ventilator it allowed a cost-effective expansion of our ventilator fleet.

Reference(s) and grant acknowledgment(s)

1. https://www.medonegroup.com/pdf/manuals/userManuals/Respi ronics-V60-Users-Manual.pdf

\section{3}

\section{Family members' satisfaction in a Tunisian medical ICU}

M. zghidi'; I. Ben Saida ${ }^{2}$; A. Elhadhri'; W. Zarrougui'; M. Boussarsar ${ }^{2}$

${ }^{1}$ Medical intensive care unit, Farhat hached university hospital, Sousse, Tunisia; ${ }^{2}$ Farhat hached university hospital, medical intensive care unit, Université de Sousse, Faculté de Médecine de Sousse, UR Nº LR12SP09. Heart Failure, Sousse, Tunisia

Correspondence: M. Zghidi

Intensive Care Medicine Experimental 2020, 8(2): 000433
Introduction: Family satisfaction is an important indicator for quality improvement of intensive care units. Compliance with implemented protocols are often poor.

Objectives: The purpose of this study was to describe levels of family satisfaction with the care provided in a Tunisian intensive care unit (ICU).

Methods: It was a prospective observational and descriptive study involving family members of patients admitted in a Tunisian medical ICU between November 2018 and Mai 2019. Family members of adult patients, staying at an ICU for $\geq 48$ hours, were included. A questionnaire to measure family satisfaction with care provided in the ICU was developed. Family members were asked to complete the questionnaire and to provide information about their own demographics. Data on patient's baseline characteristics were abstracted from charts retrospectively.

Results: Ninety-three family members completed the questionnaire. Demographic characteristics of study related patients were: male, 76(81.7\%) mean age, 56.6 \pm 15.4 years; respiratory distress at admission, $79(84.9 \%)$; mean length of stay, $14.4 \pm 18.5$ days. Respondents characteristics were: mean age, $39 \pm 13$ years; female, 51(54.8\%); firstdegree parents $48(51.6 \%)$; mean waiting delay, $42.4 \pm 28.1 \mathrm{mn}$.

Twenty-eight (30.1\%) of respondents were satisfied with overall care. Families reported good satisfaction with the waiting room atmosphere, visitor's number and coordination and teamwork staff respectively in $56(60.2 \%), 33(35.5 \%)$ and $28(30.1 \%)$. Families reported the least satisfaction for the availability and communication by nurses. Responses to family satisfaction questionnaire are displayed in Table 1. Table 1 : Responses to questionnaire : Family satisfaction with intensive care unit (ICU)
experience $(\mathbf{N}=93)$

\begin{tabular}{|l|l|l|l|}
\hline & Good n(\%) & $\begin{array}{l}\text { Fair } \\
\mathbf{n}(\%)\end{array}$ & $\begin{array}{l}\text { Poor } \\
\mathbf{n}(\%)\end{array}$ \\
\hline -Waiting delay before visits & & & \\
\hline -Visit duration & $21(22.6)$ & $58(62.4)$ & $14(15.1)$ \\
\hline -Visitors' number & $26(28)$ & $46(49.5)$ & $21(22.6)$ \\
\hline -Completeness of information about & $33(35.5)$ & $43(46.2)$ & $17(18.3)$ \\
patient's condition & $25(26.9)$ & $43(46.6)$ & $25(26.9)$ \\
\hline -Atmosphere of the waiting room & $56(60.2)$ & $24(25.8)$ & $13(14)$ \\
\hline -Psychological support of family members & $10(10.8)$ & $59(63.4)$ & $24(25.8)$ \\
\hline -Availability of nurses & $20(21.5)$ & $42(45.2)$ & $31(33.3)$ \\
\hline -Availability of physicians & $27(29)$ & $50(53.8)$ & $16(17.2)$ \\
\hline -Management of anxiety and depression of $24(25.8)$ & $49(52.7)$ & $20(21.5)$ \\
their patients & $25(22.6)$ & $56(60.2)$ & $12(12.9)$ \\
\hline -Medical secret respect & $21(22.6)$ & $65(69.9)$ & $7(7.5)$ \\
\hline -Courtesy, respect, and compassion & $17(18.3)$ & $64(68.8)$ & $12(12.9)$ \\
toward patient & $28(30.1)$ & $57(61.3)$ & $8(8.6)$ \\
\hline -Pain's patient management & $19(20.4)$ & $40(43)$ & $34(36.6)$ \\
\hline $\begin{array}{l}\text {-Coordination and teamwork by staff } \\
\text {-Communication by nurses } \\
\text {-Psychological support of their patients } \\
\text {-Global satisfaction of cares given to their }\end{array}$ & $28(30.1)$ & $54(58.1)$ & $11(11.8)$ \\
\hline & $21(22.6)$ & $60(64.5)$ & $12(12.9)$ \\
\hline
\end{tabular}

Conclusion: Most family members were satisfied with the care provided in the intensive care unit. Communication and availability of nurses were areas with potential for improvement.

000434

Self-perceived discomforts in critically ill patients

M. Zghidi ; I. Ben Saida ${ }^{2}$ AT. Ben ${ }^{1}$; E. Ennouri'; M. Boussarsar ${ }^{2}$

${ }^{1}$ Medical intensive care unit, Farhat hached university hospital, Sousse,

Tunisia; ${ }^{2}$ Farhat hached university hospital, medical intensive care unit, Uni- 
versité de Sousse, Faculté de Médecine de Sousse, UR N LR12SP09. Heart Failure, Sousse, Tunisia

Correspondence: M. Zghid

Intensive Care Medicine Experimental 2020, 8(2): 000434

Introduction: Critically ill patients are exposed to stressful conditions and may experience discomforts from multiple sources. Modification of the physical and psychological stressors would contribute positively to the short-term and long-term outcomes during the ICU stay and after the discharge.

Objectives: To explore self-perceived discomforts in critically ill patients.

Methods: A prospective cohort study was performed in medical ICU and anesthesiology department between September 2018 and Mars 2019. All patients admitted during the study period were included. Patients with diminished mental capacity, unable to communicate or refusing to participate in the study were excluded. Patient discomforts were assessed using the 16 items self-reported discomfort questionnaire on discomforts in ICU patients (IPREA). Patients were asked to rate the severity of each discomfort experienced during their ICU stay using a 10-point numerical rating scale (example: $0=$ no pain and $10=$ worst pain possible). Patients characteristics were recorded from medical patients 'charts.

Results: One hundred twenty patients completed the questionnaire. $51(42 \%)$ patients were admitted in the medical ICU. Clinical characteristics were: female, $60(50 \%)$; mean age, $47.9 \pm 18.5$ years; mean length of stay, $10 \pm 2$ days; acute respiratory failure, 39 (32.5\%); invasive mechanical ventilation, $48(40 \%)$; noninvasive mechanical ventilation, $72(60 \%)$; tracheostomy, $18(15 \%)$.

The four highest discomforts were the absence of a phone, pain, sleep deprivation and limited visiting hours and the 4 lowest discomforts were feeling of cold, hunger, bed-related discomfort and feeling of heat. Self-perceived discomforts of the 16-item IPREA are displayed in Table 1.

\begin{tabular}{|c|c|}
\hline Items & Mean score \pm SD $/ 10$ \\
\hline Noise & $5.4 \pm 3.27$ \\
\hline Excess of light & $5.1 \pm 3.28$ \\
\hline Bed related discomfort & $5.0 \pm 3.25$ \\
\hline Sleep deprivation & $6.1 \pm 3.11$ \\
\hline Thirst & $5.7 \pm 3.07$ \\
\hline Cold & $4.6 \pm 2.99$ \\
\hline Heat & $5.0 \pm 3.07$ \\
\hline Hunger & $4.7 \pm 3.42$ \\
\hline Pain & $6.2 \pm 3.46$ \\
\hline Discomfort du to tubes & $5.6 \pm 3.53$ \\
\hline Lack of privation & $5.9 \pm 3.58$ \\
\hline Anxiety & $5.7 \pm 3.38$ \\
\hline Isolation & $5.3 \pm 3.54$ \\
\hline Limited visits hours & $6.1 \pm 3.12$ \\
\hline Absence of phone & $6.6 \pm 3.42$ \\
\hline Lack of information & $5.8 \pm 3.44$ \\
\hline
\end{tabular}

Conclusion: The findings of this study shed light on the difficulties that Tunisian ICU patients may experience and that could adversely affect their outcomes.

\section{0}

ARDS treated in a super-obese COVID-19 patient admitted

\section{to an OR converted into ICU}

G. Salve ${ }^{1}$; E. Francesco ${ }^{2}$; S. Pregnolato ; T. Giulia 3 ; F. Riccardi ${ }^{3}$; B. Mascia ${ }^{3}$; S. Bonaiti ${ }^{1}$; M. Pizzullii ; L. Fontanelli ${ }^{1}$; S. Mongodii ${ }^{3}$;. Mojoli ${ }^{1}$; R. Veronesi ${ }^{2}$

${ }^{1}$ Department of clinical-surgical, diagnostic and pediatric sciences, unit of anaesthesia and inte, The University of Pavia, Pavia, Italy; ${ }^{2}$ Department of intensive medicine,anaesthesia and intensive care 2, Fondazione IRCCS Policlinico San Matteo, Pavia, Italy; ${ }^{3}$ Department of intensive medicine,anaesthesia and intensive care 1, Fondazione IRCCS Policlinico San Matteo, Pavia, Italy
Correspondence: G. Salve

Intensive Care Medicine Experimental 2020, 8(2): 000440

Introduction: On February 18, 2020 the first Italian case of coronavirus disease 19 (COVID-19) was identified in Lombardy. It was centralized in our hospital Fondazione IRCCS Policlinico S. Matteo, in Pavia. [1] Because of the rapid outbreak, the number of dedicated ICU beds was increased up to 72 , even adapting operating rooms (OR) to a 6-beds intensive care unit (ICU). As described in this case report, we treated there an obese COVID-19 patient with acute respiratory distress syndrome.

Methods: On ICU admission, a 33-years-old super-obese patient (body mass index, BMl $52 \mathrm{~kg} / \mathrm{m} 2$ ) presented respiratory distress and critical hypoxemia, despite CPAP helmet. We promptly proceeded to videolaryngoscopy intubation for invasive mechanical ventilation (MV). Past medical history revealed diabetes mellitus and psoriasis. Respiratory symptoms and fever began a week before. COVID-19 was confirmed by the reverse-transcription polymerase chain reaction analysis of the bronco-alveolar lavage. We faced several problems during admission: the limited respiratory mechanics data of an anaesthesia workstation (Flow-i ${ }^{\circledR}$, Getinge, Göteborg, Sweden), the unfeasibility of oesophageal pressure measurement [2] and the difficulties to obtain a computed tomography scan due to the high BMI. However, we used available lung ultrasound (LUS) scan to quantify lung de-aeration [3] and set the positive end-expiratory pressure (PEEP) level up to $20 \mathrm{cmH} 2 \mathrm{O}$. [4] (Figure 1) According to the LUS posterior consolidations and the anterior-lateral interstitial pattern [5] we carried out 12-16 hours prone positioning cycles $[6,7]$ on neuromuscular blocking agents continuous infusion and proper sedation. Prophylactic antimicrobial therapy was carried out with azithromycin, ceftriaxone and trimethoprim/sulfamethoxazole. Prophylactic heparin dosage (6000 IU q12h) was adjusted according to antiFXa monitoring, without major bleedings. [8] On day 1 the patient was enrolled in the convalescent plasma experimental treatment protocol. [9] On day 15, Klebsiella Pneumuoniae ESBL ventilator-associated pneumonia occurred, with clinical status worsening. Antimicrobial therapy was then adjusted with meropenem. On day 19, percutaneous tracheostomy was performed to facilitate MV weaning. Intravenous sedation was de-escalated implementing oral sedatives, [10] without delirium or agitation.

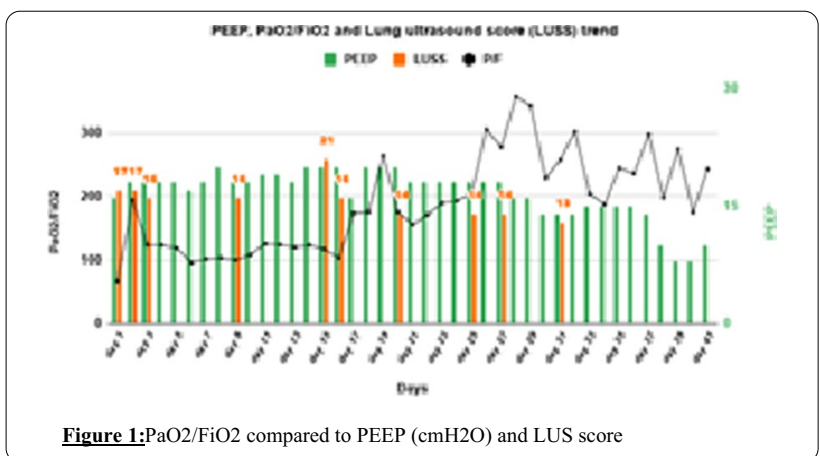

Results: On day 41 the patient was transferred to another ICU on assisted MV. Discharging was mandatory: our ICU was closed after the COVID-19 cases reduction in Italy, to allow return to surgical activity. Conclusion: According to our experience, despite the limited resources it is possible to treat successfully a super-obese patient with severe COVID-19 infection in an OR converted into ICU.

Reference(s) and grant acknowledgment(s)

1. 1. Mojoli F, Mongodi S, Grugnetti G, Muzzi A, Baldanti F, Bruno R, et al. Setup of a Dedicated Coronavirus Intensive Care Unit. Anesthesiology [Internet]. 2020;1. Available from: http://anesthesiology.pubs.asahq.org/artic le.aspx?doi=10.1097/ALN.0000000000003325

2. 2. Akoumianaki E, Maggiore $S M$, Valenza F, Bellani G, Jubran A, Loring SH, et al. The Application of Esophageal Pressure Measurement in Patients with Respiratory Failure. Am J Respir Crit Care Med [Internet]. 2014;189:520-31. 
Available from: http://www.atsjournals.org/doi/abs/10.1164/rccm.20131 2-2193Cl

3. 3. Chiumello D, Umbrello M, Sferrazza Papa GF, Angileri A, Gurgitano M, Formenti P, et al. Global and Regional Diagnostic Accuracy of Lung Ultrasound Compared to CT in Patients With Acute Respiratory Distress Syndrome. Crit Care Med. 2019;

4. 4. Mojoli F, Bouhemad B, Mongodi S, Lichtenstein D. Lung Ultrasound for Critically III Patients. Am J Respir Crit Care Med [Internet]. 2019;199:701-14. Available from: https://www.atsjournals.org/doi/10.1164/rccm.20180 2-0236Cl

5. 5. Gattinoni L, Chiumello D, Caironi P, Busana M, Romitti F, Brazzi L, et al. COVID-19 pneumonia: different respiratory treatments for different phenotypes? Intensive Care Med. 2020.

6. 6. Prat G, Guinard S, Bizien N, Nowak E, Tonnelier J-M, Alavi Z, et al. Can lung ultrasonography predict prone positioning response in acute respiratory distress syndrome patients? J Crit Care. 2016;32:36-41.

7. 7. Bouhemad B, Mongodi S, Via G, Rouquette I. Ultrasound for "Lung Monitoring" of Ventilated Patients. Anesthesiology [Internet]. 2015;122:437-47. Available from: http://content.wkhealth.com/linkback/openurl?sid=WKPTL P:landingpage\&an $=00000542-201502000-00034$

8. 8. Egan G, Ensom MHH. Measuring antifactor xa activity to monitor lowmolecular-weight heparin in obesity: A critical review. Can. J. Hosp. Pharm. 2015.

9. 9. Alhazzani W, Møller MH, Arabi YM, Loeb M, Gong MN, Fan E, et al. Surviving Sepsis Campaign: guidelines on the management of critically ill adults with Coronavirus Disease 2019 (COVID-19). Intensive Care Med [Internet]. 2020;46:854-87. Available from: http://link.springer.com/10.1007/s0013 4-020-06022-5

10. 10. Mistraletti G, Umbrello M, Salini S, Cadringher P, Formenti P, Chiumello $D$, et al. Enteral versus intravenous approach for the sedation of critically ill patients: a randomized and controlled trial. Crit Care [Internet]. 2019;23:3. Available from: https://ccforum.biomedcentral.com/articles/10.1186/s1305 $4-018-2280-x$

\section{8}

Coping with COVID-19 in a low resource country: ethical, social, and economic dilemmas

T. Jreis ${ }^{1} ;$ H. Ashhab ${ }^{2}$

${ }^{1}$ Anesthesiology \& CCM, Hadassah Medical Center, Bethlehem, Occupied Palestinian territories; ${ }^{2}$ Internal medicine, Ahli Hospital, Hebron, Occupied Palestinian territories

Correspondence: $T$. Jreis

Intensive Care Medicine Experimental 2020, 8(2): 000488

Introduction: Palestinian authority, population 2.8 million, is a low resource country facing COVID-19 pandemic, neighboring high resource country fighting the epidemic. In preparing ourselves, we creating a disaster committee, included private and governmental hospitals CEOs, infectious disease specialists and Intensivists.

Objectives: To share lessons learned, barriers overcome, discuss ethical and social implications of decisions made in the low resource country.

Methods: Epidemiological and national statistics will be demonstrated, dealing with unestablished infrastructures and utilizing the existing resources. Enforced early total lockdown while saving lives had many ethical and social implications.

Results: 547 confirmed infected, 4 COVID-19 related deaths all in provisional, urgently created ICUs. 40513 COVID-19 tests were performed. High risk population testing positive COVID-19 were workers transiting daily into neighboring countries (75\%), medical staff (3.7\%), tourist industry related personnel (20.3\%).

Conclusion: Early enforced lockdown the most effective governmental decision preventing population from getting sick. However, the burden of ethical, social and economic consequences is still being evaluated and will be discussed in this abstract.
000491

Goals of care bundle via a critical care outreach team for critical care provision during the COVID-19 outbreak in Lombardy: baseline characteristics and outcomes

A. Messina ${ }^{1}$; P. Andrea ${ }^{1}$; A. Valeria ${ }^{1}$; N. Maxim ${ }^{1}$; M. Cecconi ${ }^{1}$

${ }^{1}$ Anesthesia and intensive care, Humanitas Research Hospital, Rozzano, Italy

Correspondence: A. Messina

Intensive Care Medicine Experimental 2020, 8(2): 000491

Introduction: During the Coronavirus disease 2019 (COVID-19) outbreak (1), the mission of our intensive care unit (ICU) team was to provide intensive care to whoever needed intensive care. Upon reaching the plateau of the outbreak in Italy, 7 wards were dedicated to high dependency units, counting approximately 100 beds [including 50 level-2 beds delivering forms of non-invasive ventilatory assistance such as continuous positive airways pressure (CPAP), non-invasive ventilation (NIV) and high flow nasal cannula (HFNC)] The outreach team provided senior ICU decision support using a Goals Of Care Bundle (Figure 1).

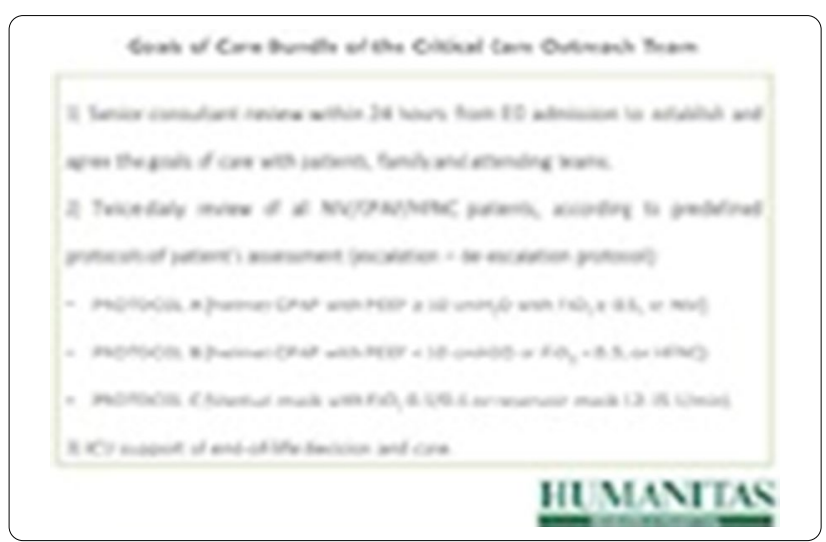

Methods: We retrospectively evaluated data from 125 patients referred to the outreach team from March 22- April 22, 2020 and outcomes after a minimum of 15-days follow-up.The data analysis refers to three subgroups of COVID-19 patients:patients admitted to the wards with respiratory symptom and receiving a ceiling of care decision (PCEI); patients requiring more detailed observation or intervention including non-invasive forms of ventilatory support and considered eligible level-3 care, if needed (PWARD); patients admitted to the ICU within $24 \mathrm{~h}$ after the after the evaluation of the outreach team (PICU).

Results: From March 22 to April 22, 2020, 125 consecutive adult patients with a confirmed diagnosis of COVID-19 [a median (IQR) of $25 \%(21 \%-27 \%)$ of the overall number of COVID-19 positive patients admitted to the Humanitas Research Hospital] have been referred to the outreach team (Figure 2). Overall, 61 patients $(48.8 \%)$ were included in the PCEI group, 30 in the PWARD and, finally, 34 in the PICU. The median (IQR) number of patients receiving non-invasive respiratory support in the wards (including either helmet CPAP or NIV delivered by facemask or HFNC) was 9 (4-16), with a maximum of 19 patients on March 28.

The overall in-hospital mortality of COVID-19 patients (including the emergency department, wards and ICU) in the considered period was $26.8 \%$, while the overall ICU mortality was $21.9 \%$. At the end of the follow-up, 19 patients in the PCEI group (31.1\%), 29 in the PWARDgroup $(96.7 \%)$ and 26 in the PICUgroup (76.2\%) were discharged from hospital, to either home or rehabilitation facilities. Moreover, 41/65 patients in the PCEI group (67.2\%) died in hospital as compared to none in the PWARD and PICUgroups ( $p<0.0001)$. 


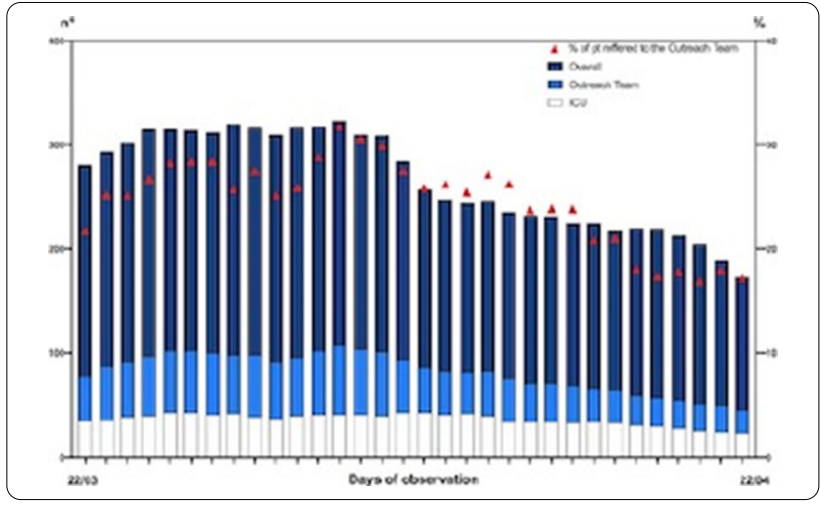

Conclusion: In a context of hospital and ICU surge, implementation of a dedicated Critical Care Outreach was associated with proper allocation of ICU resources, a low rate of cardiac arrest calls and no emergency intubations in the ward.

\section{Reference(s) and grant acknowledgment(s)}

1. 1. Grasselli G, Pesenti A, Cecconi M (2020) Critical Care Utilization for the COVID-19 Outbreak in Lombardy, Italy: Early Experience and Forecast During an Emergency Response. JAMA. https://doi.org/10.1001/jama.2020.4031 2. 2. None.

\section{5}

Outcomes from patients invasively ventilated with repurposed non-invasive V60 Ventilator during covid19

M. Jeffrey ${ }^{1}$;. Y. Yang ${ }^{2}$; S. Petty ${ }^{2}$; R. Mahroof ${ }^{2}$; A. Conway-Morris ${ }^{2}$

1JVF Intensive Care Unit, Addenbrooke's Hospital, Cambridge, United Kingdom; ${ }^{2}$ Department of anaesthetics \& intensive care medicine, Cambridge University Hospitals NHS Foundation Trust, Cambridge, United Kingdom

Correspondence: $M$. Jeffrey

Intensive Care Medicine Experimental 2020, 8(2): 000495

Introduction: The COVID-19 pandemic created many challenges for ventilator surge capacity. Solutions included use of anaesthetic machines and the repurposing of Non-Invasive Ventilators. Use of Phillips Respironics V60 Non-Invasive Ventilator ${ }^{\circledR}$ in invasive, mandatory ventilation is previously unreported. Here, we describe our outcomes repurposing the V60 ventilator for COVID19 critical care admissions in a single UK tertiary centre.

Objectives: To describe the use of V60 Ventilators during a pandemic emergency. We focus on their utility, problems and place in our response going forward.

Methods: We performed retrospective analysis of all swab or BAL confirmed COVID19 positive cases admitted to intensive care in a University Teaching Hospital for 7 weeks upto 1/5/20. Descriptive statistics were performed using Microsoft Excel.

Results: 82 patients were admitted to intensive care with confirmed COVID19. Of these, 30 were ventilated with a V60. These 30 patients recieved 572 ventilator days with 239 days $(42 \%)$ delivered by a $V 60$ The mean ventilator days were 19.1 with 8 V60 ventilator days. A V60 was used for Mandatory ventilation in 19 patients, of these 12 were ventilated on a V60 immediately following intu. 11 used a V60 for spontaneous ventilation/weaning only.

In total 14 patients failed on a V60 and were changed to a full-service ICU ventilator (Servo-i): 2 for non-clinical reasons, 2 for bronchoscopy, 1 for ECMO, 2 for humidified circuits, 2 for proning and 2 for dyssynchrony. $93 \%$ of these failures occurred in the patients requiring mandatory ventilation and those with more severe ARDS, only 1 occured in the spontaneous ventilation only group. Proning was performed in 2 patients whilst ventilated on a V60, of which 1 survived to ICU discharge.
A total of 64/82 COVID19 patients admitted to critical care have either been discharged from ICU or died. 39 (61\%) were alive at ICU discharge and 25 (39\%) died. For the patients ventilated on a V60 at any point $18 / 26(69 \%)$ where discharged from ICU alive and $8 / 26(31 \%)$ died. For those not ventilated on a V60 at any point $21 / 38$ (55\%) left ICU alive and 17/38 (45\%) died.

Figure 1: Demographics of patients using V60 ( $n=30)$

\begin{tabular}{|c|c|c|}
\hline & Mean & Range \\
\hline Age, years & 59 & $33-82$ \\
\hline Male, $n(\%)$ & $21(70)$ & $9(30)$ \\
\hline Minimum P/F ratio & Number & Percentage \\
\hline Mild(<13.3) & 2 & $7 \%$ \\
\hline Mod (13.3 - 23.2) & 12 & $41 \%$ \\
\hline Severe & 15 & $52 \%$ \\
\hline
\end{tabular}

" 1 patient excluded due to being a transfer with no clear way of finding minimum PF ratio

Figure 2: V60 use data across 83 ventilated patients patients

\begin{tabular}{|l|c|c|}
\cline { 2 - 3 } \multicolumn{1}{c|}{} & Absolute number, $\mathbf{n}$ & Percentage, (fraction) \\
\hline Total patient number using v60s & 30 & $38 \%(30 / 83)$ \\
\hline $\begin{array}{l}\text { Primary ventilation (placed within } 24 \text { hours of } \\
\text { admission) }\end{array}$ & 12 & $14 \%(12 / 30)$ \\
\hline Mandatory & 7 & $8 \%(7 / 30)$ \\
\hline Socondary & 11 & $13 \%(11 / 30)$ \\
\hline
\end{tabular}

\begin{tabular}{|l|c|}
\hline Total days spent on a ventilator, days & 572 \\
\hline Average total ventilator deys, days & 19.1 \\
\hline
\end{tabular}

\begin{tabular}{|l|c|}
\hline Total ventilatory days on V60, days & 239 \\
\hline Average ventilator days on V60, days & 8 \\
\hline
\end{tabular}

\begin{tabular}{|l|l}
\hline Average proportion of total ventilator days on v60 & $46 \%$
\end{tabular}

Figure 3A: Disease severity and outcomes among patients on V60 in those with an ICU outcome

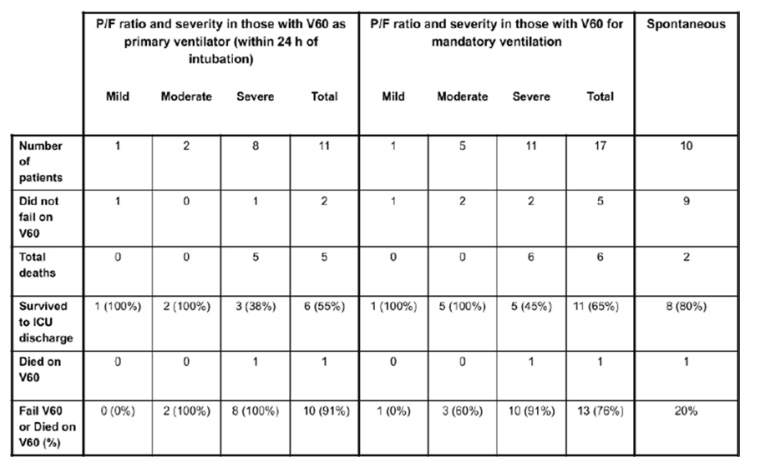

Conclusion: When a V60 was used, it was able to provide 239/572 $(42 \%)$ of ventilator days. However a universally high failure rate, 13/16 patients $(81 \%)$, in those with moderate-severe ARDS when used for mandatory ventilation makes it clear that it cannot be used as a direct replacement for an ICU ventilator. If used in carefully selected patients, with experienced staff, it can contribute significantly to surge capacity by sparing ICU ventilators for those requiring advanced respiratory support. At $25 \%$ cost of a full-service ICU ventilator it can, when used 
appropriately, allow for cost-effective surge expansion of ventilator capacity.

Reference(s) and grant acknowledgment(s)

1. https://www.medonegroup.com/pdf/manuals/userManuals/Respironic s-V60-Users-Manual.pdf

\section{6}

Experience of managing essential critical care medication shortages during COVID19 Pandemic

L. Radford'; M. Jeffrey ${ }^{2}$;Y. Yang ${ }^{3}$; S. Dabasia ${ }^{1}$; S. Pacey ${ }^{1}$; J. Kent ${ }^{4}$;

R. Mahroof ${ }^{2}$; A. Conway-Morris²; D. Sapsford ${ }^{1}$

${ }^{1}$ Pharmacy department, Cambridge University Hospitals NHS Foundation Trust, Cambridge, United Kingdom; ${ }^{2}$ Department of anaesthetics \& intensive care medicine, Cambridge University Hospitals NHS Foundation Trust, Cambridge, United Kingdom; ${ }^{3}$ Department of intensive care medicine, Cambridge University Hospitals NHS Foundation Trust, Cambridge, United Kingdom; ${ }^{4}$ Pharmacy department, Southend University Hospital NHS Foundation Trust, Southend-on-Sea, United Kingdom

Correspondence: M. Jeffrey

Intensive Care Medicine Experimental 2020, 8(2): 000496

Introduction: We present the experience of a UK single tertiary centre in managing supplies of essential critical care medications during the COVID-19 pandemic.

Objectives: To identify challenges and solutions in managing supplies of essential critical care medications during the COVID-19 pandemic. Methods: During the surge of COVID-19 patients, shortages of propofol $2 \%$, fentanyl, norepinephrine and atracurium were experienced. To facilitate safe sedation, a policy was developed to prioritise short-acting agents for light sedation and patients likely to accumulate other agents. Combinations of long-acting agents including morphine and midazolam were used for deep sedation and a nurse-led bolus dosing strategy was used in selected patients.

A coordinated switch to $1 \%$ propofol was made, requiring staff training and dietetic input for calorie differences. Shortages of atracurium were managed with bolus regimes of pancuronium.

To maintain supplies of noradrenaline, an alternative unlicensed formulation was received. We coordinated the local setup of a Centralised Intravenous Additive Service for preparation of noradrenaline pre-filled syringes. This mitigated risks of incorrect concentrations or diluents and reduced nursing workload, particularly given use of noncritical care nursing staff.

Availability of Continuous Veno-Venous Haemodiafiltration (CVVHDF) sets and fluids has been challenging, requiring supply management on a regional level. Locally, risk of shortage was mitigated through elective filter breaks, requirement for Consultant approval for restart following clotting and judicious use of exchange rates. A protocol for the addition of potassium to potassium-free fluids was also developed. Figure 1 demonstrates the baseline and peak demand across the region for each of these therapies.

Throughout the surge, real-time administration data was able to be extracted from the local electronic prescribing system for all critical drugs, facilitating timely and more accurate planning of management strategies.

Results: There were challenges related to timely availability of drugs in areas where Aerosol Generating Procedures were performed. We installed additional controlled drug cupboards and fridges in isolation areas and emergency drugs in each bed space drug locker, so drugs could be available quickly whilst maintaining storage and governance requirements.

To manage the shortage of CVVHDF supplies, work to plumb reverse osmosis water supplies to our ICUs has been completed. This has allowed the use of Sustained Low Efficiency Dialysis (SLED) in selected patients.

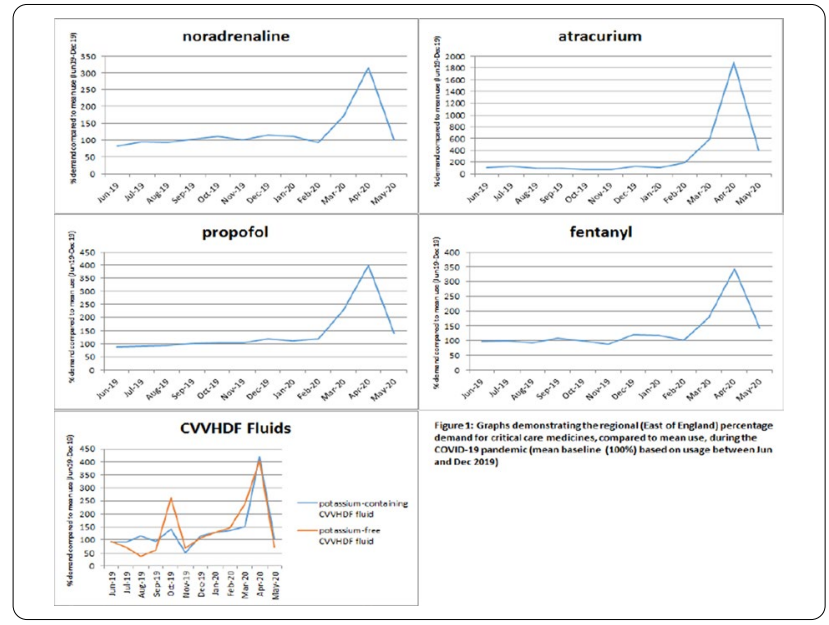

Conclusion: Our response locally has focused on rapid-response amendments to formulary with new policies and adaptations to usual practice to support use of alternative agents. A prospective, pragmatic and flexible approach has been required with actions to mitigate, rather than eliminate, risk. This has minimised the need for emergency, unplanned changes in policy.

Reference(s) and grant acknowledgment(s)

1. Liu, S Int J Clin Pharm 42, 299-304 (2020)

2. The Pharmaceutical Journal, April 2020, Vol 304, No 7936

\section{5}

Establishing a rapid response system in an arctic hospital

S. Christiansen ${ }^{1}$; P. Lennert ${ }^{2}$

${ }^{1}$ Department of medicine, Queen Ingrids Hospital, Nuuk, Greenland; 2Department of anaesthesiology, Queen Ingrid's Hospital, Nuuk, Greenland

Correspondence: S. Christiansen

Intensive Care Medicine Experimental 2020, 8(2): 000545

Introduction: The 1st of May 2019 a rapid response system (RRS) was established at Queen Ingrid's Hospital, Nuuk, Greenland to ensure immediate assistance to patients suffering from critical illness, trauma or are in cardiac arrest. A rapid response team (RRT) composed of physicians, nurses, patient transporters and laboratory technicians is triggered by an early warning system (EWS). The EWS is defined by certain thresholds, such as deviating vital parameters or mechanism of trauma. Queen Ingrid's Hospital have a capacity of approximately 100 beds, including 4 in the intensive care unit. The emergency personnel are on call from their homes.

Objectives: The objective of the study is to describe the incidence, typical location, type of event (i.e. critical illness, trauma or cardiac arrest) and triggers activating the RRT. Furthermore, we want to measure certain quality indicators and describe the outcome of the patients managed by the RRT.

Methods: From 1st of May 2019 until the 1st of May 2020, we plan to examine and collect data from the electronic health record each time the RRT is deployed. Here we obtain information on the type of event, vital parameters, mechanism of trauma and treatment given (e.g. intubation, medicine). Furthermore, we obtain information on the time of death.

Results: During the first 11 months the RRT have treated 88 patients corresponding to 1,83 per week. The patients had a median age of 55 (interquartile range (IQR) 31-67) and 53 (60.2\%) being male. 25 patients $(28.4 \%)$ have been in cardiac arrest, $25(28.4 \%)$ suffering from traumas and 38 (43.2\%) presented with critical illness. $71(80.7 \%)$ were received as out-of-hospital patients while $17(19.3 \%)$ were hospitalized. The 30-day survival for patients in cardiac arrest, critically 
ill patients and patients suffering from trauma was $13.7 \%, 84.2 \%$ and $88.0 \%$, respectively.

Conclusion: In 2019 a RRS was established at Queen Ingrid's Hospital, Nuuk, Greenland. To monitor and evaluate the system, we plan to collect data from the first year after implementation. Preliminary results from the first 11 months indicate that the system is frequently being activated and especially from the emergency room.

\section{8}

\section{Converting a surgical unit into a COVID-19 intensive care unit} during Northern Italy outbreak

S. Pregnolato'; F. Epis²; G. Salve'; G. Ticozzelli ${ }^{3}$; M. Fuardo ${ }^{3}$; G. Negri' ${ }^{4}$;

N. Peroni'; A. Carletti' ; F. Mojoli'; R. Veronesi ${ }^{2}$

${ }^{1}$ Department of clinical-surgical, diagnostic and paediatric sciences, The University of Pavia, Pavia, Italy; ${ }^{2}$ Anaesthesia and intensive care 2 , department of intensive medicine, IRCCS Policlinico San Matteo Foundation, Pavia, Italy; ${ }^{3}$ Anaesthesia and intensive care 1 , department of intensive medicine, IRCCS Policlinico San Matteo Foundation, Pavia, Italy; ${ }^{4}$ Anaesthesia service, IRCCS Maugeri Clinical Scientific Institutes, Pavia, Italy Correspondence: $\mathrm{S}$. Pregnolato

Intensive Care Medicine Experimental 2020, 8(2): 000658

Introduction: After severe acute respiratory syndrome coronavirus 2 outbreak in Wuhan, China, coronavirus disease 2019 (COVID-19) spread out in a pandemic. [1] On February 20, 2020 the first Italian case of secondary transmission was centralized in our hospital Fondazione IRCCS Policlinico San Matteo, in Pavia. The rapidly growing numbers in Northern Italy [2] imposed our hospital to increase greatly the already available 31 intensive care unite (ICU) beds [3].

Methods: On March 14, 2020, after the surgical activities suspension, we converted in a short time (8 hours) a surgical unit (SU) into a 6-beds COVID-19 ICU, reaching the hospital total capacity of 72 ICU beds.

Results: This cardiothoracic SU was square-shaped with four operating rooms (OR) on the corners, connected by an interior passage. We isolated this area with a double filter system in the pre-operative holding areas: a "clean filter", at the entrance, was reserved for disposable personal protective equipment [4] wearing, while a "contaminated" one, at the exit, was for undressing procedures. In the interior corridor we allocated the drugs storage, while a blood gas analyser and a point-of-care coagulation monitoring system were already in place. An ultrasound machine, a fibre optic bronchoscope and a defibrillator were also available (Figure 1).

Outside this space we set up a "control unit" area, equipped with a centralized parameters monitoring system and a video surveillance system, with a camera in each OR.

Communications were possible thanks to an internal telephone line and professional walkie-talkies.

In a situation of ICU ventilators shortage, three beds were equipped with Servo $900 C^{\circledR}$ ventilators (Siemens Elema, Solna, Sweden) kept as back up machines till then, while the others had the Flow- ${ }^{\circledR}{ }^{\circledR}$ anaesthesia workstations (Getinge, Göteborg, Sweden) routinely used in the SU. The wall gas aspiration system was connected to the ventilators expiratory valve, preventing airdrops dispersion. Essential devices, such as monitoring tools and aspiration systems, were already available in each OR. In a limited resources context this was an undoubted advantage, compared with the rearrangement of a traditional ward. Many of the nurses and health workers employed were ordinarily working in this SU. Medical staff was composed of anaesthetist-intensivist physicians and residents, most of them with an anesthesiological background.

We performed daily bedside lung ultrasound (LUS) monitoring to minimize the use of traditional imaging, reducing transports to the radiology department. Moreover, with limited lung mechanic measurements availability, we used LUS to titrate ventilation settings. [5,6]

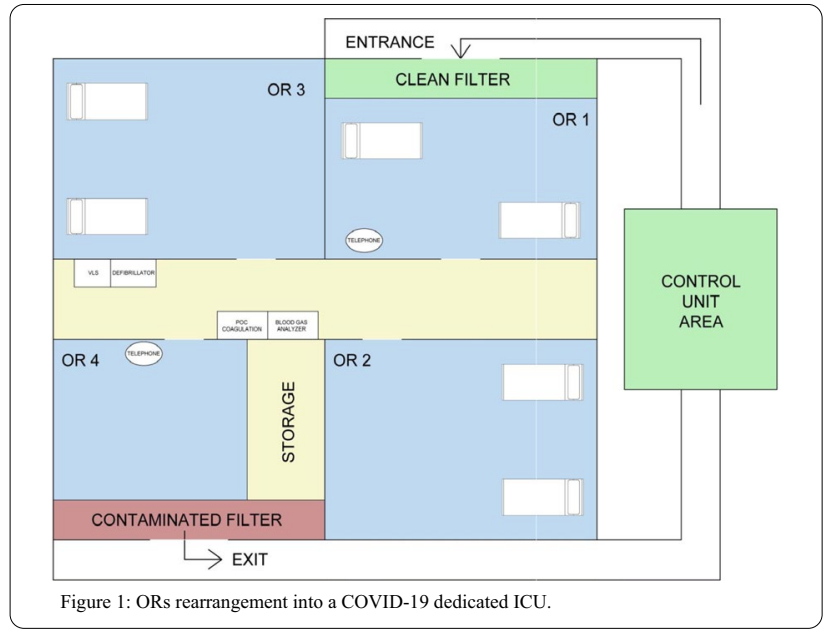

Conclusion: According to our positive experience, during a health emergency OR represents a real and feasible source of ICU beds, quickly available and easy to set-up.

\section{Reference(s) and grant acknowledgment(s)}

1. 1. Zhu N, Zhang D, Wang W, Li X, Yang B, Song J, et al. A novel coronavirus from patients with pneumonia in China, 2019. N Engl J Med. 2020

2. 2. Grasselli G, Zangrillo A, Zanella A, Antonelli M, Cabrini L, Castelli A, et al. Baseline Characteristics and Outcomes of 1591 Patients Infected With SARSCoV-2 Admitted to ICUs of the Lombardy Region, Italy. JAMA [Internet]. 2020;323:1574. Available from: https://jamanetwork.com/journals/jama/ fullarticle/2764365

3. 3. Mojoli F, Mongodi S, Grugnetti G, Muzzi A, Baldanti F, Bruno R, et al. Setup of a Dedicated Coronavirus Intensive Care Unit. Anesthesiology [Internet]. 2020;1. Available from: http://anesthesiology.pubs.asahq.org/artic le.aspx?doi=10.1097/ALN.0000000000003325

4. 4. World Health Organization (WHO). Rational use of personal protective equipment for coronavirus disease 2019 (COVID-19). Who. 2020

5. 5. Mongodi S, Orlando A, Arisi E, Tavazzi G, Santangelo E, Caneva L, et al. Lung Ultrasound in Patients with Acute Respiratory Failure Reduces Conventional Imaging and Health Care Provider Exposure to COVID-19. Ultrasound Med Biol [Internet]. 2020; Available from: http://www.sciencedir ect.com/science/article/pii/S0301562920302052

6. 6. Mojoli F, Bouhemad B, Mongodi S, Lichtenstein D. Lung Ultrasound for Critically III Patients. Am J Respir Crit Care Med [Internet]. 2019;199:701-14. Available from: https://www.atsjournals.org/doi/10.1164/rccm.20180 $2-0236 \mathrm{Cl}$

\section{9}

Shared decision making impact on hospice palliative care over terminal critical patient

HC. Chen'; SY. Lin ${ }^{2}$; SH. Kuo ${ }^{3}$; JM. Chung ${ }^{4}$; SH. Tzeng ${ }^{4}$; MY. Lin ${ }^{4}$; RY. Chen ${ }^{4}$; HL. Liang ${ }^{5}$; WC. Huang

${ }^{1}$ Department of critical care medicine, Kaohsiung Veterans General Hospital, Kaohsiung, Taiwan; ${ }^{2}$ Master program of school of nursing, Kaohsiung Medical University, Kaohsiung, Taiwan; ${ }^{3}$ Graduate institute of clinical medicine, Kaohsiung Medical University, Kaohsiung, Taiwan; ${ }^{4}$ Department of family medicine, Kaohsiung Veterans General Hospital, Kaohsiung, Taiwan; ${ }^{5}$ Department of nursing, Shu-Zen Junior College of Medicine and Management, Kaohsiung, Taiwan

Correspondence: H.C. Chen

Intensive Care Medicine Experimental 2020, 8(2): 000659

Introduction: The idea of shared decision making (SDM) was first proposed in 1972, with gradually increasing its importance as the upsurging interest in patient centredness and autonomy in health care interactions since the $1970 \mathrm{~s}$. It has became the major care model 
enbodying patient centered care and physician-patient equality interaction. Hospice palliative care among terminal critical illed patient, determined by two separate specialist based on the Hospice Palliative Care Act definition, has long been poorly accepted owing to cultural mis-understanding, especially among Chinese. By retrospective cohort data analysis, we try to unvealed the impact of introducing SDM on hospice palliative care in terminal critical adult ICU patients

Methods: Medical record of medical center adult ICU admitted terminal critical illed patient from Jan. 1st, 2017 to Dec. 31th, 2019 was retrospectively reviewed, and further subdivided into pre-SDM and post-SDM (after Oct. 1st, 2018) intervention period for analysis. Terminal critical illed was defined as ICU admitted patient fit following criteria including advanced malignancy, end-stage motor neuron disease, decompensated heart failure with NYHA Fc IV, oxygenation or ventilator dependent pulmonary and airway disease, liver cirrhosis Child-Pugh C with episodes of hepatic encephalopathy, hemodialysis dependent acute or chronic renal failure, extremely elderly associated organic psychosis or central nervous system degeneration, and fulminant critical condition, which post tolerated available treatment without clinically improvement determined by two separate specialist. Decision about withdrawal, discontinuing, or refusing any add-on life supporting management was considered as palliative care. Data including SDM impact on palliative decision associated withdraw or withhold rate, ventilator dependent days, length of ICU stay, length of hospital stay, means of withdraw or withhold, and average medical cost were collected. SPSS was applied for statistically analysis, and a $p$ value less than 0.05 was considered significant difference.

Results: Medical records from total 9198 patients from Jan. 1st, 2017 to Dec. 31th, 2019 were reviewed based on tertiary medical center adult ICU record. According to inclusion criteria, 56 patients out of 5209 patients fit terminal critical illed patient during the pre-SDM (before Oct. 1st, 2018) in compared to 153 patients from 3989 patients in post-SDM (after Oct. 1st, 2018), and 173\% improvement over identifying terminal critical illed patient impressed. In compared to pre-SDM, post-SDM terminal critical illed patient had less ventilator dependent days $(23.9+/-18.5$ v.s $21.2+/-16.7, \mathrm{p}<0.05)$, shorter length of ICU stay $(24.9+/-19.2$ v.s $21.8+/-15.3, p<0.05)$, and shorter length of hospital stay $(31.2+/-25.9$ v.s $27.6+/-23.3, p<0.05)$. The most often chosen withdrawal is hospice endotracheal tube extubation, and the most frequent chosen withhold is vasopressor pump prescription. After SDM was introduced, ventilator days, length of ICU stay, and length of hospital stay of terminal critical illed patient was found reduced 2.7 days, 3.1 days, and 3.6 days individually in compared to each other. The average medical cost was also decreased after SDM introduced.

Conclusion: Our retrospective cohort study from a single medical center adult ICU revealed introducing shared decision making on hospice palliative care over terminal critical illed patient might improve palliative decision associated withdrawal or withhold rate, reduce ventilator days, length of ICU stay, and length of hospital stay. Introducing shared decision making on hospice palliative care over terminal critical illed patient reduce futile medical treatment and associated excessive medical cost.

Keywords: shared decision making, hospice palliative care, terminal critical illed patient.

\section{2}

\section{Reducing breathing system transmission of Covid-19: The} Addenbrooke's experience

J. Gutsell ${ }^{1}$;. Y. Yang ${ }^{1}$; M. Jeffrey ${ }^{1}$; A. Conway-Morris ${ }^{1}$; R. Mahroof ${ }^{1}$ J. Martin ${ }^{1}$ 'Department of anaesthetics \& intensive care medicine, Addenbrooke's Hospital, Cambridge University Hospitals NHS Foundation Trust, Cambridge, United Kingdom

Correspondence: $Y$. Yang

Intensive Care Medicine Experimental 2020, 8(2): 000712

Introduction: The SARS-Cov-2 coronavirus partly spread via aerosolisation. To reduce nosocomial infection within our ICU it was necessary to effect equipment and behavioural changes. We provide an experiential narrative of a single UK tertiary centre of the adaptations to reduce viral particulate load, and steps to disseminate this information. Subsequent issues and how these were resolved, are then described.

Objectives: To identify aerosol generating procedures (AGP) within ICU and to create a breathing circuit aiming to minimise viral transmission.

Methods: We adopted a pragmatic approach, intending a log 5-6 reduction in viral exposure. Equipment and procedural changes were implemented to effect this change. We replaced current heat and moisture exchangers (HME) with high efficiency filters (HEF) after comparing manufacturer values for bacterial vs viral efficiency. We adopted a modified RSI technique using apnoeic oxygenation was encouraged, with bagging avoided. An 'optimal' breathing circuit setup was proposed (see Fig 1). Setup then focused on ensuring expired gas always passed via a HEF, and that circuit breaks occurred distal to this. Specific high risk situations were considered; if circuit breaks were necessary, these occurred during apnoea with ventilators on standby and portable ventilators and bag valve masks ventilate to air, though not via expiratory limb - we focused on education to address this issue.

Results: Filter saturation was the most serious difficulty we encountered. The combination of Aerogen nebulisers, and in particular epoprostenol nebulisers, caused abrupt occlusions of HEF risking airway emergencies. We considered both modifiable and non-modifiable factors to manage occult respiratory gas loss:

Modifiable consideration included positioning of capnography sampling, which exhaust to air, post-filter. Aerogen nebulisers required distal placement relative to the HME. We received reassurance from the manufacturer that these nebulisers had not produced detectable leak during normal use.

Non-modifiable concerns included that 'suction above cuff' endotracheal tubes were not easily clamped, thus this was discouraged owing to ineffectiveness and risk of damage. We adopted a practice of paralysis with ventilator standby during planned disconnection. Similarly, portable suction devices lack viral filters and any use had to be treated as an AGP.

As a number of modifications to usual practice were required, multidisciplinary education was necessary. Regular tutorials were available, with time for discussion regarding airway management. Teaching was available within ICU, dynamically changing as the situation developed.

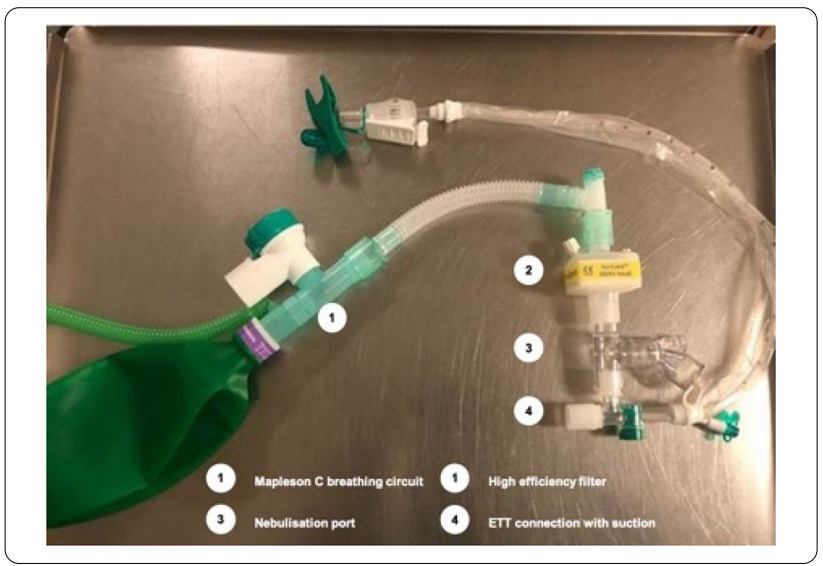

Conclusion: Through consideration of changes to usual equipment and behaviours, we were able to achieve effective reduction in viral transmission in the context of an emergent pandemic.

Reference(s) and grant acknowledgment(s)

1. Intersurgical Ltd. (2020) Filtration and humidification. Available from: https ://uk.intersurgical.com/info/filtrationandhumidification [Accessed 2nd June 2020] 
000756

Debriefing of critical situations: experiences and expectations of ICM residents

A. Trifi ${ }^{1}$; M. Tobich ${ }^{1}$; C. Abdennebi ${ }^{1}$; Y. Touil ${ }^{1}$; F. Daly ${ }^{1}$; S. Abdellatif ${ }^{1}$; S. Ben Lakhal ${ }^{1}$

'Intensive care unit, Hospital la Rabta, Tunis, Tunisia

Correspondence: A. Trif

Intensive Care Medicine Experimental 2020, 8(2): 000756

Introduction: At any moment of their exercise, residents of intensive care medicine (ICM) confront insurmountable stress. This is mainly due to the critical situations they encounter, especially as it is associated with lack of experience. Debriefing is a tool designed to relieve this stress and transform the error in learning.

Objectives: to assess the application of debriefing in clinical situations in residents of ICM and to inquire about their expectations.

Methods: Descriptive survey, focusing on residents of ICM from all over Tunisia after their consent. Data were collected using a 30-questions computerized questionnaire developed on the "Google Forms" platform which was sent to their personal email addresses. The topics investigated their experiences of debriefing after critical situations, the interest and expectations brought to debriefing in real practice.

Results: 100 responses were collected from 220 questionnaires sent representing a participation rate of $45,5 \% .70 \%$ of participants were of female gender. The mean of cumulated semesters at the study time was $4 \pm 2$. In $90 \%$ of cases, the senior doctor on call being at home with displacement and in $10 \%$ of cases the senior being on site. More than 10 calls per guard were recorded, in a quarter of interrogated residents, in order to manage a critical situation. 57 residents participated in a debriefing as part of simulation training whereas 86 have experienced it in clinical practice. In $40 \%$ of the cases, the Debriefing took place with a staff of several teaching doctors. 74 of the participants gave a satisfaction score between 3 and 5 out of 5 . In this case, debriefing concerned the diagnostic discussion and the taken attitude. $95 \%$ preferred a collective debriefing than the individual one. $44 \%$ of the participants experienced at least one emotional shock during their course and they affirm the necessity of defusing even outside of a debriefing in $40 \%$ of cases. $52 \%$ of residents have claimed the ideal time for debriefing would be on day 1 of the managed critical situation and day 0 was considered in $32 \%$ of residents. Considering those who rated more than 6 out of 10 , the expectations from debriefing in the studied population were: improvement of theoretical skills $(92 / 100)$, improvement of technical skills $(82 / 100)$ coordination within the team $(89 / 100)$ and psychological interest (appeasement, guiltfree...) (87/100).

Conclusion: Our survey confirms the value of debriefing in clinical practice among ICM residents, both in the theoretical as technical skills and in psychological management.

\section{4}

ICU resources during COVID-19 pandemia: Optimising the timing of tracheostomy with a modified "Failure-free days" outcome G. Hernandez'; O. Roca'; JM. Añón ${ }^{3}$; L. Colinas'; R. Ortiz'; JR. Masclans ${ }^{5}$; C. Vaquero ${ }^{6}$; A. Canabal Berlanga $;$; O. Peñuelas ${ }^{8}$; R. Corrales ${ }^{9}$; J. Prada ${ }^{10}$; R. Cuena ${ }^{11}$; F. J. Ramos'2 ; J. C. Figueira ${ }^{3}$; F. Muñoz ; F. A. Castellví; ; R. De Pablo ${ }^{6}$; M. Martinez ${ }^{2}$; C. Diaz ${ }^{3}$

${ }^{1}$ Critical care department, Virgen de la Salud University Hospital, Toledo, Spain; ${ }^{2}$ Critical care department, Vall d'Hebron University Hospita, Barcelona, Spain; ${ }^{3}$ Department of intensive care medicine, Hospital La Paz-madrid, Madrid, Spain; ${ }^{4}$ Critical care department, Ciudad Real University Hospital, Ciudad Real, Spain; ${ }^{5}$ Critical care department, Hospital del Mar, Barcelona, Spain; ${ }^{6}$ Critical care department, Hospital Ramón y Cajal, Madrid, Spain; ${ }^{7}$ Critical care department, Hospital de La Princesa, Madrid, Spain; ${ }^{8}$ Intensive care medicine, Getafe University Hospital, Getafe, Spain; ${ }^{9}$ Ent surgical team, Virgen de la Salud University Hospital, Toledo, Spain; ${ }^{10}$ Ent surgical team, Hospital de La Princesa, Madrid, Spain; ${ }^{11}$ Medical research council, Virgen de la Salud University Hospital, Toledo, Spain

Correspondence: G. Hernandez

Intensive Care Medicine Experimental 2020, 8(2): 000764
Introduction: Shortage of both ventilators and ICU beds overwhelmed further health care systems during COVID-19 pandemia1,2. Early tracheostomy (TRQ) can possibly reduce the duration of mechanical ventilation (MV) and ICU lenght of stay but counterbalanced with an increased risk for health-care workers3. COVID-19 is a clinical condition with easy-to-predict prolonged MV and a high mortality rate1. Objectives: Modified "failure-free days" combined outcome focused on resources (respirators and ICU beds) could contribute to decide the optimal timing of TRQ in consumed health care systems during future viral pandemia periods 1,4 .

Methods: Multicenter retrospective cohort study including consecutive TRQ patients diagnosed with COVID-19 in 8 spanish high-volume centers. The case selection, timing and technique of TRQ based on weekly burden degree in ICUs: the greater the burden the earlier and the higher the TRQ and surgical procedure rates. Modified ventilator and ICU bed-free days (VFD and BFD respectively) within 28 and 60-day time frame were defined as follows, respectively: VFD/BFD= $28 / 60-x$ if successfully liberated from MV/discharged to ward $x$ days after initiation/admission and VFD/BFD $=28 / 60-\mathrm{x}$ if subject dies within $28 / 60$ days of MV/admission. Modified VFD and BFD were calculated for different timings (10, 14 and 20 days) and compared with multivariate ANOVA test. Matching bias was exluded with a propensity score. Results: TRQ was performed in $48 \%$ of intubated patients; 504 tracheostomized patients were finally included. Table 1 shows the preliminary results of 143 patients. The median (IQR) time under MV was $23 \mathrm{~d}$ (19-32); mortality rate was $49.2 \%$ and surgical TRQ was performed in $38.1 \%$ of patients. COVID-19 PCR was positive at any timing in all monitored patients $(58.8 \%)$.

\begin{tabular}{|l|l|l|}
\hline \multicolumn{1}{|c|}{ Table1 } \\
& $\begin{array}{l}\text { Early TRQ } \\
(\leq 10 d / 14 d / 20 d)\end{array}$ & $\begin{array}{l}\text { Delayed TRQ } \\
(>10 d / 14 d / 20 d)\end{array}$ \\
\hline Time under MV, d (median) & $25.3 / 24.7 / 25.9$ & $29.7 / 35 / 46.6$ \\
\hline Mortality rate, $\%$ & $54 / 45 / 50$ & $50 / 60 / 57$ \\
\hline VFD at 28d, d & $6.6 / 6.1 / 5.2$ & $4.1 / 2.4 / 1.4$ \\
\hline BFD at 28d, d & $3.9 / 3.1 / 2.7$ & $1.9 / 1.2 / 1.4$ \\
\hline VFD at 60d, d & $34.7 / 35.7 / 34.4$ & $31.3 / 26.4 / 17.3$ \\
\hline BFD at 60d, d & $30.5 / 32.1 / 30.6$ & $27.9 / 22.9 / 14.8$ \\
\hline
\end{tabular}

Conclusion: Pending definitive results, we conclude that ICU resources could be optimized with early tracheostomy at 10d according to the 28-d modified VFD and BFD outcome under the conditions of the COVID-19 pandemia.

\section{Reference(s) and grant acknowledgment(s)}

1. Yehya N, Harhay MO, Curley MAQ, et al. Reappraisal of ventilator-free days in critical care research. Am J Respir Crit Care Med 2019; 200(7):828-836.

2. Chao TN, Braslow BM, Martin ND, et al. Tracheostomy in ventilated patients with COVID-19. Annals of Surgery https://doi.org/10.1097/sla.0000000000 003956

3. McGrath BA, Brenner M, Warrillow S, et al. Tracheostomy in the COVID-19 era: global and multidisciplinary guidance. Lancet Respir 2020; https://doi. org/10.1016/S2213-2600(20)30230-7.

4. Wang R, Pan C, Wang X, et al. The impact of tracheostomy in critically ill patients undergoing mechanical ventilation: A meta-analysis of randomized controlled clinical trials with trial sequential analysis. Heart \& Lung 2019; 48:46-54.

000792

Intensive care communication with families during COVID: a national UK survey

A. Boulton ${ }^{1}$; H. Jordan'; C. Adams 3 ; A. Conway Morris ${ }^{4}$; N. Arora ${ }^{5}$

${ }^{1}$ University of Warwick, Coventry, United Kingdom; ${ }^{2}$ Division of anaesthesia, department of medicine, University of Cambridge, Cambridge, United Kingdom; ${ }^{3}$ John $v$ farman intensive care unit, Addenbrooke's Hospital, Cambridge, United Kingdom; ${ }^{4}$ Division of anaesthesia, department of medicine, University of Cambridge, Cambridge, UK, Cambridge, United Kingdom; ${ }^{5}$ Academic department of anaesthesia, critical care, pain 
and resuscitation, Birmingham Heartlands Hospital, Birmingham, United Kingdom

Correspondence: A. Boulton

Intensive Care Medicine Experimental 2020, 8(2): 000792

Introduction: Frequent communication with a patient's relatives is a part of normal ICU care, however has been challenged by intensified clinical care demands of the COVID-19 pandemic.1,2 Regular visiting and communication with the ICU team is a priority for many families and crucial to coping. 3 Visiting loved ones at the end-of-life is fundamental within many cultures and is a crucial part of the grieving process for the bereaved.4 Restrictions to hospital visiting policies during the COVID-19 pandemic have left relatives unable to visit their loved ones in ICU and receive face-to-face updates from clinicians and nurses.5-7 It is unclear how UK ICUs are dealing with this challenging situation.

Objectives: We aimed to investigate how UK ICUs are approaching relative communications and visiting during the COVID-19 pandemic.

Methods: This web-based snapshot survey was delivered between 16th April and 24th May 2020 and was open to UK ICUs. It was completed by individual clinicians regarding practices of the ICU they were currently working at during the COVID-19 pandemic.

Results: There were replies from 134 individual NHS ICUs with COVID patients for analysis. All units reported that visiting was more restricted than in normal times. Twenty-nine (22\%) units reported that no relative visiting was allowed, $71(53 \%)$ reported visiting was allowed at end of life only and $30(23 \%)$ reported visiting was allowed for vulnerable or end of life patients.

Nearly all ICUs ( $n=130,97 \%)$ were updating families daily. Most ICUs were initiating the family update $(n=120,92 \%)$. Daily phone calls were routinely initiated by the medical team in 75 (55\%) ICUs and by the nursing team in 50 (37\%) ICUs. Thirty-nine (29\%) units used a dedicated team for regular family updates. Video calling was being used by $63(47 \%)$ ICUs.

Do not attempt resuscitation and end of life family discussions were commonly done by consultants ( $n=132,99 \%)$, followed by PGY4-7 doctors $(n=60,45 \%)$. Six $(4 \%)$ units reported that PGY1-3 doctors may have these discussions. These discussions were most frequently phone calls initiated by the ICU medical team ( $N=129,96 \%)$. Video calling was utilised by $24(18 \%)$, and $15(11 \%)$ reported that discussion may occur in person.

Conclusion: Relative visiting to UK ICUs during the COVID-19 pandemic has been severely restricted compared to normal times. Despite visiting restrictions, many units are adapting to support family visiting for end of life patients. ICUs are actively contacting families daily to provide updates, most commonly by ICU medical teams and many units have developed bespoke family communication teams. Modern technology has been utilised to deliver video calling by many ICUs.

\section{Reference(s) and grant acknowledgment(s)}

1. 7. NHS England and NHS Improvement. Clinical guide for the management of surge during the coronavirus pandemic: rapid learning. 12 April 2020, Version 2, 2020.

2. 6. Phua J, Weng $L$, Ling $L$, et al. Intensive care management of coronavirus disease 2019 (COVID-19): challenges and recommendations. Lancet Respir Med 2020.

3. 5. Liao X, Wang B, Kang Y. Novel coronavirus infection during the 2019-2020 epidemic: preparing intensive care units-the experience in Sichuan Province, China. Intensive care medicine 2020; 46(2): 357-60.

4. 4. Heyland DK, Rocker GM, O'Callaghan CJ, Dodek PM, Cook DJ. Dying in the ICU: perspectives of family members. Chest 2003; 124(1): 392-7.

5. 3. Quinio P, Savry C, Deghelt A, Guilloux M, Catineau J, de Tinteniac A. A multicenter survey of visiting policies in French intensive care units. Intensive Care Med 2002; 28(10): 1389-94.

6. 2. Intensive Care National Audit and Research Centre. ICNARC report on COVID-19 in critical care. 19 June 2020, 2020.

7. 1. Paul F, Rattray J. Short- and long-term impact of critical illness on relatives: literature review. Journal of advanced nursing 2008; 62(3): 276-92.

8. ACM is supported by the Wellcome Trust (WT 2055214/Z/16/Z).
000847

Unindicated daily blood testing on ICU: an avoidable expense?

J. Sugarman'; J. Malik'; J. Hill'; P. Morgan ${ }^{1}$

${ }^{1}$ Intensive care unit, East Surrey Hospital, Redhill, United Kingdom

Correspondence: J. Sugarman

Intensive Care Medicine Experimental 2020, 8(2): 000847

Introduction: ICU care often generates daily blood tests, which can provide essential diagnostic information. However, protracted blood sampling may contribute to anemia in ICU patients, with knock-on negative impacts on patient outcome. In addition, testing without clinical indication contributes to healthcare spending, creating an avoidable cost burden. We therefore set out to investigate the proportion of blood sampling that could have been avoided, as well as its total cost.

Objectives: We audited adherence to a departmental standard that no greater than $25 \%$ of blood sampling should be without clear clinical indication. This follows a 2015 audit of the same department, in which $46 \%$ of blood tests $(n=339)$ were deemed unnecessary over 70 patient days, reducing to $41 \%$ with intervention.

Methods: We limited eligible patient data to blood samples collected after the first 48 hours of admission, to exclude stable post-surgical admissions. Patient data was collected between 21 January 2020 and 17 February 2020. Indications were listed for each of the seven most common blood tests: full blood count, urea \& electrolytes, liver function tests, CRP, magnesium, phosphate, and clotting. These were stratified according to patient level of care in a 16-bed unit, with 10 Level 3 (ICU) beds and 6 Level 2 (HDU) beds. Daily blood tests were deemed indicated if the patient required advanced end-organ support (such as mechanical ventilation, vasopressors, or continuous veno-venous hemofiltration), if previous tests were acutely deranged, or if requested by a senior practitioner.

Results: 191 patient days were audited, with 1096 blood tests ordered total (Level 3: $n=715$, Level 2: $n=388$ ). Of all tests performed, $31.5 \%$ $(n=345)$ were unindicated (Level 3: 22.4\%, Level 2: 48.2\%). The rate of blood sampling without clear clinical indication was within the departmental standard of $25 \%$ for CRP (13.1\%), full blood count (15.4\%), and urea \& electrolytes (18.1\%). Liver function tests $(51 \%)$, magnesium (42.2\%), phosphate (42.7\%), and clotting (40.4\%) failed to meet departmental guidelines, as did all blood tests requested for Level 2 patients. A potential cost of $£ 2101$ was estimated for all blood tests, with $£ 505$ unindicated.

Conclusion: We found that common blood tests in ICU are ordered regularly without clear clinical indication. We further determine that liver function tests, magnesium, phosphate, and clotting are ordered regularly without considering their diagnostic value.

This data also demonstrates that a regimen of testing without indication can significantly impact the running cost of an intensive care unit at a district general hospital. This may be a consequence of a critical care blood test set available on our electronic requesting system. Following the COVID-19 pandemic, we aim to introduce clearer guidelines on appropriate daily testing, to reduce the burden on patients and our healthcare system.

Our department will look to re-audit our practice following changes, to determine the degree of impact.

\section{Reference(s) and grant acknowledgment(s)}

1. Cioc A, Fodor R, Benedek O, Moldovan A, Copotoiu SM. Blood sampling as a cause of anemia in a general ICU - a pilot study. Rom J Anaesth Intensive Care 2015, 22(Suppl 1):P13-16.

2. Gray R, Baldwin F. Targeting blood tests in the ICU may lead to a significant cost reduction. Critical Care 2014, 18(Suppl 1):P15 https://doi.org/10.1186/ cC13205

3. Hall T, Ngu WC, Jack JM, Morgan P. Are daily blood tests on the intensive care unit necessary? Critical Care 2016, 20(Suppl 2):P422 
000850

'Surviving an overwhelming COVID-19 ICU Surge': a case study and timeline from Northwick Park Hospital, UK

D. Ball' ; M. O'connor ${ }^{1}$; O. Elsaka' ; J. Borkowski ${ }^{1}$; V. Sathianathan

${ }^{1}$ Intensive Care Department, London North West University Healthcare NHS Trust, London, United Kingdom

Correspondence: $\mathrm{D}$. Ball

Intensive Care Medicine Experimental 2020, 8(2): 000850

Introduction: In December 2019, a pneumonia of unknown cause was detected in Wuhan, China. A novel strain of coronavirus (SARS-CoV 2) was identified as the cause. The virus spread rapidly and the World Health Organization declared a Public Health Emergency of International Concern on 30 January. Worldwide, cases of new coronavirus disease (COVID-19) began to increase exponentially with Italy, Spain, France and the UK reporting the highest rates of new infections within Europe. Northwick Park Hospital, a large district general hospital in North West London was one of the first UK centres to experience a surge in COVID-19 admissions requiring ICU level care. In relation to its baseline critical care capacity, it was one of the worst hit centres in the UK and a substantial increase in transfers to neighbouring hospitals was necessary to ensure safe, quality care was maintained.

Objectives: We provide an overview of the ICU surge experience due to COVID-19 admissions from Northwick Park Hospital. We establish a timeline, focusing on several distinct themes including capacity, staffing, operational issues and training. Data on admissions, transfers and patient demographics is included.

Methods: All available data from the period 16 February to 12 May 2020 (coinciding with the peak ICU surge) was collected via audit of in-house electronic reporting systems (PathPoint, ePRO, ICE) and informal channels (NHSMail).

Results: We present a timeline detailing the challenges faced during the surge around establishing and maintenance of bed capacity; adoption of new staffing ratios, rotas and redeployment; equipment and medication supply; establishing new surgical pathways and 'returning to normal'.

A total of 257 patients with COVID-19 were admitted to the ICU. Length of stay on the unit ranged from 1 to 41 days. Maintenance of spare capacity was facilitated by transfers to other sites within the North West London Critical Care Network. 124 patients were transferred to satellite sites with 11 patients repatriated. Of the 22 admissions to the London Nightingale Hospital, 19 were from Northwick Park. The highest number of transfers off site in one day was 16 . With the expanded ICU capacity, the peak number of inpatients in a 24-hour period was 57 and the highest admissions for one day was 15 .

The ICU population had several risk factors which have since been shown to be associated with worse outcomes in COVID-19, namely age, ethnicity and obesity. Of the total admissions, $75 \%$ fell within the 51 to 80 years of age group, $84 \%$ were from a Black, Asian or Minority Ethnic background, and 58\% were classified as either overweight or obese by BMI.

Conclusion: We report our experience of preparing for, receiving and management of the initial ICU surge of COVID-19 patients. We present a timeline, our patient population and report on several of the challenges we faced.

\section{3}

\section{A rapid response to the COVID-19 pandemic: the North Central} London Critical Care Transfer Hub

H. Rowley'; S. Amdekar'; H. Hayakawa ${ }^{3}$; S. Mallory 3 ; R. May ${ }^{4}$; A. Menon ${ }^{4}$ M. Abbink ${ }^{5}$; G. Emery ${ }^{5}$; T. North Central London Transfer Team ${ }^{6}$; D. Howell

${ }^{1}$ Anaesthetics, Royal Free and Barnet Hospital Trust, London, United Kingdom; ${ }^{2}$ Anaesthetics, University College Hospital, London, United Kingdom;

${ }^{3}$ Anaesthetics, Great Ormond Street Hospital, London, United Kingdom; ${ }^{4}$ Anaesthetics, National Hospital for Neurology and Neurosurgery, London, United Kingdom; Inforcehub, Inforcehub, London, United Kingdom; ${ }^{6} \mathrm{Ncl}$ transfer hub, NHS England, London, United Kingdom; ${ }^{7}$ Critical care and anaesthetics, University College Hospital, London, United Kingdom Correspondence: $\mathrm{H}$. Rowley

Intensive Care Medicine Experimental 2020, 8(2): 000853
Introduction: The COVID-19 pandemic led to an unprecedented demand for critical care beds across the world(1). North Central London (NCL) Sustainable Training Partnership (STP) critical care capacity expanded by over $100 \%(2)$, presenting extreme pressures on staff and consumables, with significant logistical challenges.

Objectives: The NCL transfer team was established on 3rd April 2020, with the primary aim to manage the safe movement of critically unwell patients to decompress overwhelmed critical care units and optimise patient care. The secondary aim was to alleviate the workload of stretched referring units by organising the logistics of transfer, relaying clinical patient information and mobilising a dedicated transfer team to retrieve the patient, whilst protecting the resources at the referring site. (Diag. 1).

Methods: A central point of contact was established to streamline referrals with real-time updates of bed capacity across the sector. Three dedicated consultant-led teams were created and responsible for the movement of patients both in and out of the sector.

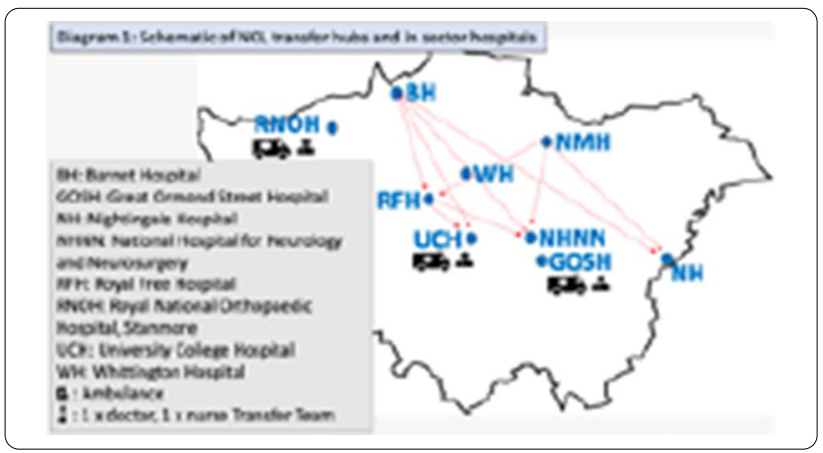

The Hub provided operational support to the transfer teams and sector hospitals. Ambulances provided by London Ambulance Service (LAS) were matched with teams at their base hospitals (Diagram 1). The Hub created a central gateway through which activity and outcomes could be recorded for quality improvement. A COVID-19 transfer app was designed in partnership with InforceHub (data analytics company), ensuring NHS data security governance.

Results: A total of 81 transfers were performed during April 2020, with a peak of 7 transfers per day. No critical incidences were reported. 60 patients were transferred in sector, follow up showed a $48 \% 30$-day mortality, with men aged between 60-69 most affected, comparable to ICNARC data (3). $68 \%$ represented BAME groups, with a proportional mortality of $48 \%$, comparable to Caucasian group at $50 \%$ in this data set.

The most prevalent complications within 30 days of admission were: PE/DVT (40\%), renal failure requiring haemofiltration (36\%), and ongoing ventilatory failure requiring proning, $(42 \%)$. This is a small data set with inherent limiting factors.

Conclusion: NHS England (NHSE) project that the demand for critical care across London will continue to increase with plans to double the critical care capacity (2).

Critical transfers for specialist care and bed capacity will continue to require a co-ordinated response for the benefit of patients and workforce. The NCL transfer hub provided a valuable service during this novel pandemic. An NHSE advisory group is producing a National business case for a new Pan-London Critical Transfer Service.

As board advisors, our collective experiences and expertise will help to shape a broader and cohesive vision for critical care transfers in London.

\section{Reference(s) and grant acknowledgment(s)}

1. Ma, X\& Vervoort,D., (2020). Critical care capacity during the COVID-19 pandemic: Global availability of intensive care beds Journal of Critical Care 58: $96-972$.

2. North Central London Intensive Care Unit Expansion plan. May 2020 V2.0 060520. NHS England NHS Improvement 
3. https://www.statista.com/chart/21360/uk-intensive-care-covid-19-survi val-rate/

\section{5}

Bath Tea Trolley as educational tool in Intensive Care. Analysis of theoretical framework

A. Serrano ; F. Kelly ${ }^{2}$

${ }^{1}$ Accp intensive care, Royal United Hospital, Bath, United Kingdom; ${ }^{2}$ Consultant intensive care, Royal United Hospital Bath, Bath, United Kingdom Correspondence: A. Serrano

Intensive Care Medicine Experimental 2020, 8(2): 000865

Introduction: Bath Tea trolley is a teaching initiative that has shown excellent results improving knowledge, skills and satisfaction of health professionals. It is based on a bedside discussion between the instructor and the trainee about a topic always related to the current care of the patient. The teaching session does not last more than 5 minutes and always includes tea and cake as a refreshment. The combination of peer-to-peer bedside learning, relevant topic, and tea and cake as an element to facilitate the learning break, make the Tea Trolley a powerful learning tool. Vygotsky's, Wenger's, Bateson's levels of learning, Malinowski's, Akerlof strategy and Rich's class and gender construction concept support this educational tool.

Objectives: Discuss the theoretical framework behind Bath Tea Trolley as educational tool.

Methods: The interventions and results of the innitiatives that included Bath Tea Trolley and elements of the intervention were reviewed and the theoretical framework behind them analysed.

Results: Bath Tea trolley has shown excellent results on either learning progress and trainee satisfaction. Bath Tea Trolley has been utilised as teaching tool with different learning topics related to critical care.

It has been tried in other clinical settings. From teaching sessions on airway rescue techniques and maternal sepsis, to acute kidney injury and hypoglycaemia, it has proved very successful-analysis feedback forms indicates that after receiving tea trolley training $98 \%$ of staff have felt more confident in one or more of the aspects that were covered.

This teaching technique has potential in many settings including anaesthetic rooms, wards, emergency departments, outpatient departments, GP surgeries, and administration areas. It can be adapted to suit different learning and teaching styles (visual, auditory, verbal, physical, one-on-one, group), grades of participants, and previous levels of experience.

So far, it has been included in 54 projects in Bath, with 2977 staff 'educational encounters' to date. It has been utilised in 39 other UK hospitals and Internationally in countries as Australia, Canada, USA, France and Germany.

The elements of the intervention were analysed from the theoretical point of view. The combination of peer-to-peer bedside learning relevant topic and tea and cake as an element to facilitate the learning break make the Tea Trolley a powerful learning tool. Vygotsky's theory of "Zone of Proximal Development" and "Scaffolding", Wenger's hypothesis of "Situated learning", Bateson's levels of learning, Malinowski's concept of "Reciprocity", Akerlof's "Tit-for-tat" strategy and Rich's class and gender construction concept support this powerful educational tool.

Conclusion: Tea trolley is based on a peer-to-peer bedside learning in which the instructor offers tea and cake to facilitate a short break that allows the intervention. It has shown to be an excellent teaching tool and has been utilised in different clinical settings nationally and internationally.

Every element of this intervention is supported on a solid theoretical framework. Peer-to-peer interaction is supported on Vygotsky's theory of "Zone of Proximal Development" and "Scaffolding", bedside offers the adequate learning context as per Wenger's hypothesis of "Situated learning" and Bateson's levels of learning, tea and cake leans on
Malinowski's concept of "Reciprocity", Akerlof's "Tit-for-tat" strategy and Rich's class and gender construction concept.

\section{Reference(s) and grant acknowledgment(s)}

1. * Corbett L, Davies A, McDonald M, Kelly FE, Jordan L. Bath Tea Trolley'training part one. RCoA bulletin 2020; 119: 28-29. In press.

2. * Kerton MCS, Gough C, Jones A, Hardy R, Kelly FE. Paediatric Anaphylaxis Management: training staff to actually draw up the correct dose of adrenaline. British Journal of Anaesthesia 2018; 120(4): 881-882

3. Penketh J, McDonald M, Kelly FE. EZ-IO ${ }^{\circledR}$ intraosseous access teaching in the workplace using a mobile 'tea trolley' training method. Resuscitation 2015; 99: e17-8

4. O'Farrell G, McDonald M, Kelly FE. 'Tea trolley' difficult airway training. Anaesthesia 2014; 70(1): 104.

\section{9}

Evaluation of the impact of sideskilling workshops during Covid 19 redployment

H. O'mahony'; R. Saleh ${ }^{2}$

${ }^{1}$ Education and anaesthesia, St Bartholomew's Hospital, London, United Kingdom; ' $E d u c a t i o n$ and intensive care, St Bartholomew's Hospital, London, United Kingdom

Correspondence: $\mathrm{R}$. Saleh

Intensive Care Medicine Experimental 2020, 8(2): 000879

Introduction: The Covid19 pandemic saw a large-scale redeployment of doctors in the UK. In expanding Intensive Treatment Unit (ITU) capacity, doctors at St Bartholomew's Hospital, often with little or no prior ITU experience, were asked to staff this area at short notice.

To support the transition we created a half day face-to-face Sideskilling Workshop (SW) prior to redeployment, aimed at non-ITU physicians. We surveyed them after two months of working in ITU to assess the impact of the SW on their self-assessed performance and morale.

Objectives: To assess the impact of the SW for non-ICU doctors being redeployed to ICU.

To assess perceived change in knowledge, confidence, and anxiety as a result of SW.

Methods: Senior ITU clinicians and the education team developed a syllabus of key topics which we felt would be most useful to doctors being redeployed.

The SW consisted of 4 stations:

1. Assessment and monitoring of the ITU patient

2. ITU observation chart, drug chart, and infusion pumps

3. Introduction to Invasive Ventilation

4. Proning a ventilated patient

Evaluation was performed as an anonymous, online post hoc survey.

Candidates were asked questions regarding:

their perceived knowledge pre-SW, immediately post-SW, and after working on ITU

their agreement with statements regarding the impact of the SW on their anxiety and confidence surrounding redeployment

their experiences, indicating the most beneficial element of the SW and suggested improvements

Answers were indicated on a 5-point Likert scale, and others given as comments.

Results: 34 of 104 candidates responded. $58 \%$ had no previous ITU experience. Candidates reported a mean of 1.6 point increase on a 5-point Likert scale ( $1=$ no knowledge, $5=$ expert knowledge) across all knowledge domains investigated. Ventilation knowledge had the lowest pre-SW score (1.4/5), and saw the largest mean increase: $1.94 .44 \%$ indicated the 'crisis atmosphere' increased or strongly 
increased their ability to take in information. $71 \%$ agreed or strongly agreed that knowledge gained from the SW was useful for working in ITU and $79 \%$ agreed or strongly agreed that the SW helped them deliver good patient care during redeployment.

$82 \%$ reported decreased anxiety and $88 \%$ reported increased confidence regarding the prospect of working in ITU following the SW; 84\% agreed or strongly agreed that the SW reassured them they would be well supported in ITU.

The structured patient assessment, ventilator knowledge, and reassurance/confidence featured prominently in the comments.

Suggested improvements included more interactivity and simulation. Conclusion: The results demonstrate improved self-perception of knowledge in all aspects of ITU care explored by the workshops. Candidates also reported allayed anxiety, improved confidence, and increased camaraderie. Our workshops serve as a good model for future induction of doctors that are redeployed during further Covid19 surges, both for improving knowledge and maintaining wellbeing, and could serve as a foundation for further ITU education.

\section{5}

Patient and family centered actionable processes of care for persistent or chronic critical illness: systematic review, interview and Delphi study

L. Rose ; I. Perform²

${ }^{1}$ Florence Nightingale Faculty of Nursing, Midwifery and Palliative Care, King's College London, London, United Kingdom; ${ }^{2} \mathrm{Na}$, NA, NA, Canada

Correspondence: $L$. Rose

Intensive Care Medicine Experimental 2020, 8(2): 000885

Introduction: Patients with persistent or chronic critical illness (P/CCI) require adaption of their clinical management plan and goals of care from an acute to a rehabilitative focus. Development and implementation of quality improvement $(\mathrm{QI})$ tools such as daily or rounding checklists focused on actionable processes of care are needed as existing QI tools were not developed with $\mathrm{P} / \mathrm{CCl}$ patients in mind. Actionable processes are those over which clinicians have direct control and are able to action.

Objectives: To develop end-user (patients, family, all professions of the ICU interprofessional team) consensus on the most important actionable processes of care for inclusion in tools designed to improve quality and consistency of patient/family-centred care for $\mathrm{P} / \mathrm{CCl}$ patients and family.

Methods: Systematic review, end-user qualitative interviews with touch-point video, and 3 round (R) Delphi consensus of actionable processes. Participants rated actionable processes on 9-point Likert scales. Actionable processes considered critical for inclusion in future development of $\mathrm{QI}$ tools were those sored between 7-9 on the Likert scale by $\geq 70 \%$ of participants and between $1-3$ by $<15 \%$ of participants.

Results: During item generation phase, we screened 13,130 references, included 114 primary studies (24,251 participants), 102 reviews and 94 abstracts and identified 40 distinct actionable processes of care. Interviews with $\mathrm{P} / \mathrm{CCl}$ survivors/family $(\mathrm{N}=17)$ identified 15 actionable processes with $3 / 15(20 \%)$ distinct from the systematic review. Interviews with clinicians $(\mathrm{N}=31)$ identified 35 actionable processes with $4 / 35(11 \%)$ distinct from other item generation procedures (Total=47). Following iterative discussion to combine similar processes removing redundancy and to word as actionable processes in lay terms, 31 actionable processes were included in Delphi R1. Nine additional actionable processes suggested by Delphi participants were added to R2 (Total=40). Response rates were 83\% (138/166) R1, 79\% (109/138) R2 and 74\% (81/109) R3. Of the 40 actionable processes, 34 (85\%) were considered critical for inclusion in future QI tools. The top 3 actionable processes with the highest proportion of clinician, ICU survivor, and family participants considering them critical for inclusion were (1) establish and track rehabilitation/physiotherapy goals including early mobilization while still in the ICU (99\%); (2) assess and manage symptoms (i.e., pain, breathlessness, tiredness, thirst) (98\%); and (3) review ongoing need for drugs used for sedation and decrease their use as able $(98 \%)$. Actionable processes not considered critical for inclusion were (1) limit physiologic monitoring and routine blood tests (54\%); (2) offer chaplaincy/spiritual support to the patient and family (57\%); (3) provide activities to promote relaxation (e.g. music, pet visitation) based on patient preferences (62\%); (4) de-escalate (including change to oral instead of IV drugs) or stop ICU pharmacotherapy and restart previous comorbidity pharmacotherapy (62\%); (5) enable appropriate and timely discharge planning (67\%); and (6) conduct multi-disciplinary rounds that include non-ICU subspecialty teams (68\%).

Conclusion: The large number of items considered critical for inclusion in QI tools reflects the complex and multi-factorial needs of PCCI patients and families. Further consensus building work is planned to enable development and testing of QI tools.

\section{6}

\section{Characteristics of the Non-COVID patients seen in our Intensive} Care Unit during the pandemic in contrast to those seen

\section{in the same period of time the year before}

N. Cruza Leganés ${ }^{1}$; F. Arbol'; C. Sena'; V. Losada'; A. Simon ${ }^{1}$; A. Escudero';

JL. Martin ${ }^{1}$; B. Segovia'; JR. Herrera'; LJ. Serrano'; MA. Taberna ${ }^{1}$

${ }^{1}$ Intensive Care Unit, General Hospital Nuestra Señora del Prado, Talavera de la Reina, Spain

Correspondence: N. Cruza Leganés

Intensive Care Medicine Experimental 2020, 8(2): 000956

Introduction: Given the intense healthcare demand of COVID patients (COVp) during the pandemic, ICU beds had to be tripled. Non-COVID patients (nCOVp) were reduced to a lower number than they used to be. Ordinary hospital activity was interrupted due to the high COVID patients demand.

Objectives: To analyze the nCOVp admitted in our ICU during the pandemic in contrast to those admitted in the same period of time the previous year.

Methods: A restrospective descriptive observational study of $n C O V p$ admitted in our ICU during the pandemic, from March 14th to June 1st 2020 , versus the patients admitted in the same period of time the previous year. The number of COVp-nCOVp patients were analyzed, also: sex, age, provenance, diagnosis, severity, hospital stay and nCOVp mortality, as well as the admissions in the same period of time in 2019. Results: From March 14th (admission of the first COVp) to May 4th 2020, 48 COVp were admitted in our ICU (last COVp the 1st June). In the meantime, $32 \mathrm{nCOVp}$ were admitted, whereas 101 patients were admitted in 2019. It's a polyvalent ICU with 10 beds, which were expanded during the pandemic to 36 beds. High occupation period (HOP) was from March 14th to April 13th 2020, in which 40/48 (83,3\%) COVp and $4 / 32(12,5 \%)$ nCOVp were admitted. Over this period in 2019, 37/101 (36,7\%) patients were admitted. 2020 nCOVp: 23 (71,9\%) were males, with a mean age of 54,8 y.o. (Cl 95\% 49,5-61,2). 18 patients $(56,2 \%)$ came from emergency department. Ten patients $(31,2 \%)$ were admitted because of different etiologies of shock, which was the most frequent reason for admission, followed by: acute coronary syndrome (ACS) $6(18,7 \%)$, cardiac arrhythmias (CA) $3(9,3 \%)$, cardiorespiratory arrest (CRA) 3 (9,3\%), neurological disorders (ND) $3(9,3 \%)$, acute respiratory failure (ARF) $3(9,3 \%)$, polytrauma (PT) $2(6,2 \%)$, and $2(6,2 \%)$ oncological surgery postoperative (OSP). The average APACHE II was 20,8 (Cl 95\% 16, 2-25,4) and $27(\mathrm{Cl} 95 \% 19,6-34,4)$ during HOP, with an average hospital stay of 5,5 days $(\mathrm{Cl} 95 \% 2,3-8,7)$ and $9(28,12 \%)$ patients died, two of them during HOP. As for the patients admitted in 2019: 61 (60,4\%) were males, with an average age of 63,8 y.o.(IC $95 \%$ $60,5-67,1) .80$ patients $(79,2 \%)$ came from the emergency department The most frequent reason for admission was ACS with 23 patients $(22,8 \%)$ followed by: ARF 15 patients $(14,9 \%)$, shock 14 patients 
(13,9\%), CA 13 patients (12,9\%), ND 7 patients (6,9\%), OSP 7 patients $(6,9 \%)$, CRA 5 patients $(4,9 \%)$. The average APACHE II score was $15(\mathrm{Cl}$ $95 \% 13,6-16,4)$, and the average hospital stay of 3,8 days (CI 95\% 2,84,7). 12 patients died $(11,9 \%)$.

Conclusion: During the pandemic, there has been a significant reduction of non-COVID admissions in our ICU. 2020 patients had a higher APACHE II score, a longer hospital stay and an increase in mortality regarding 2019 patients.

\section{3}

A scoping review into the rationale and behaviours underpinning routine blood tests in critically ill patients, effect on patient outcomes and possible strategies to reduce this

F. Davies ${ }^{1}$

'Respiratory Medicine, Glan Clwyd Hospital, Rhuddlan Road, Bodelwyddan, Rhyl, UK, Bodelwyddan, United Kingdom

Correspondence: F. Davies

Intensive Care Medicine Experimental 2020, 8(2): 000963

Introduction: Unnecessary blood tests in the critically ill lead to adverse patient outcomes and significant financial cost. It has been shown that $90 \%$ of patients admitted to critical care will be anaemic by day 3 of their stay (1). Several physiological mechanisms that protect against anaemia are impaired in the critically ill (2), and repeated blood tests make a significant contribution towards iatrogenic anaemia. Unnecessary blood tests can also lead to organ dysfunction, prolonged length of stay, and increased mortality. Unnecessary blood tests also incur significant expense, with one study estimating blood tests cost $£ 362,587.80 /$ year was spent per year on blood tests (3).

Objectives: This review aims to identify the outcomes of routine blood tests, rationale and behaviours underpinning routine blood tests in critically ill patients, and possible strategies to reduce this in the future.

Methods: A scoping review was conducted in accordance with Arksey and O'Malley's principles (4). As there is not a large amount of quality data in this area, a scoping review was chosen over a literature review to allow greater flexibility. This review did not attempt to evaluate the quality of the literature as there is not enough quality data published yet. A PEO format was used to formulate the research question. OVID, EMbase and Medline were searched with the key terms 'intensive care' and 'routine blood tests'. 432 articles were identified and reduced to 17 articles which were included in the final review. Thematic analysis was conducted on the texts included in the final review in accordance with Braun and Clarke's key steps (5).

Results: Three key themes were identified, with associated subthemes - outcomes of routine blood tests, the rationale underpinning this, and stategies to reduce this. Patient centred outcomes featured heavily in the analysis, as this is clinically most important, and widely undisputed. The literature demonstrated that unnecessary blood tests can lead to iatrogenic anaemia, organ dysfunction, increased length of stay and mortality. The relationship between iatrogenic anaemia and an increased need for transfusion was less concrete, with some studies demonstrating a reduction in RBC transfusion and others failing to demonstrate a difference. Significant financial costs were associated with unnecessary blood tests and the follow up incurred, as well as the cognitive demand on clinicians of reviewing blood results. Rationale behind routine and unnecessary blood tests was discussed in several papers. Rationale was influenced by clinicians knowledge, time constraints on the decision maker, and reflex ordering. It is often easier to adopt a 'one-size-fitsall' strategy when it comes to blood tests in order to minimize decision making. Additionally, less experienced clinicians have a tendency to order more blood tests and a lack of guidelines in this area means there is significant variation between clinicians. Environmental factors influencing routine blood tests were the presence of central/arterial lines, increased frequency of blood tests in intensive care and increased use of point of care testing. Several strategies were employed by the literature, with bundled interventions demonstrating the best outcomes. Staff education for nurses and clinicians was a common theme. There were no local/national/international guidelines identified which needs addressing to underpin practice in this area. This can be further supported by equipment such as low-volume blood tubes, paediatric blood tubes and blood conservation sampling devices (BCSDs). Modification of checklists and electronic ordering systems also play an important part in reducing the amount of unnecessary blood tests.

Conclusion: This scoping review has identified several themes in the literature which provide evidence that routine blood tests can lead to adverse outcomes for patients, and unnecessary costs for healthcare organizations. Some key behaviours and influencing factors have been identified as important reasons for routine blood tests, that can be targeted in order to change practice. This review has identified several promising strategies to reduce the amount of unnecessary blood tests in ICU, which when combined can lead to better patient care.

The results of this scoping review will inform ongoing work in this area and may help to develop consensus led national guidelines and a gold standard model for blood tests in ICU. The authors hope to develop consensus around new guidelines and implementation of a package of change incorporating several of the strategies identified in this review. It is anticipated that this scoping review will help to inform core outcomes to measure future progress in this area.

\section{Reference(s) and grant acknowledgment(s)}

1. 1. Society for the Advancement of Blood Management. (2018). 5 Things Physicians and Patients Should Question. Retrieved from Choosing Wisely: https://www.choosingwisely.org/wp-content/uploads/2018/07/SABMChoosing-Wisely-List.pdf

2. 2. Page, C., Retter, A., \& Wyncoll, D. (2013). Blood conservation devices in critical care: a narrative review. Annals of Intensive Care, 14.

3. 3. Cumber, E., Channon, L., \& Wong, A. (2017). Blood tests in the intensive care unit: a necessary cost? 37th International Symposium on Intensive Care and Emergency Medicine.

4. 4. Arksey, H., \& O'Malley, L. (2003). Scoping Studies: Towards a Methodological Framework. International Journal of Social Methodology, 19-32.

5. 5. Braun, V., \& Clarke, V. (2006). Using thematic analysis in psychology. Qualitative Research in Psychology, 77-101.

\section{6}

Collaboration in Covid-19 crisis: an international survey on supervision, quality and safety, and collaboration amongst (redeployed) ICU personnel

M. Hennus '; JQ. Young ${ }^{2}$; M. Hennessy ${ }^{3}$; K. Friedman ${ }^{4}$; B. De Vries ${ }^{5}$; R. Hoff E. O'connor ${ }^{7}$; A. Patterson ${ }^{8}$; G. Curley $;$; K. Thakker ${ }^{10}$; M. van Dam ${ }^{11}$; D. van Dijk ${ }^{11}$ :W. Van Klei ${ }^{6}$ : O. Ten Cate ${ }^{12}$

${ }^{1}$ Pediatric Intensive Care, University Medical Center Utrecht, Utrecht, France; ${ }^{2}$ Psychiatry, Donald and Barbara Zucker School of Medicine at Hofstra/Northwell, Hempstead, United States of America; ${ }^{3}$ School of medicine, Trinity College, Dublin, Ireland; ${ }^{4}$ Medicine, Donald and Barbara Zucker School of Medicine at Hofstra/Northwell, Hempstead, United States of America; ${ }^{5}$ Quality and patient safety, UMC Utrecht, Utrecht, Netherlands; ${ }^{6}$ Anesthesiology, UMC Utrecht, Utrecht, Netherlands; ${ }^{7}$ Anaesthesia and intensive care, St James hospital, Dublin, Ireland; ${ }^{8} \mathrm{School}$ of medicine, Trinity College, Dublin, United Kingdom: ${ }^{9}$ Department of anaesthesia and critical care medicine, Royal College of Surgeons in Ireland, Beaumont Hospital, Dublin, Ireland; ${ }^{10}$ Research, Zucker Hillside Hospital, Hempstead, United States of America; ${ }^{11}$ Intensive care center, UMC Utrecht, Utrecht, Netherlands; ${ }^{12}$ Center for research and development of education, UMC Utrecht, Utrecht, Netherlands

Correspondence: M. Hennus

Intensive Care Medicine Experimental 2020, 8(2): 000976 
Introduction: To meet Covid-19 demands, intensive care units (ICU) had to rapidly expand capacities with help of health care workers redeployed outside their regular scope of practice. During the height of the pandemic, (re-)deployed ICU personnel was surveyed regarding level and quality of supervision, quality and safety of care provided, and interprofessional collaboration.

Methods: In an international, prospective, observational cohort survey study, physicians, nurses and allied health personnel, redeployed at ICUs in Utrecht, New York and Dublin completed the survey. Data analysis focused on: Supervision; Quality and safety of care; Collaboration, communication and atmosphere; Recruitment, scheduling and team composition; Organization and facilities.

Results: Quality and safety of care were perceived as lower than usual but acceptable by the majority respondents $(n=368)$. Supervision, was rated less than optimal but overall quality was acceptable to good. Where IC-trained nurse-to-patient ratios decreased most critically (Utrecht), nurses were most critical about supervision and quality of care. The working atmosphere was overwhelmingly felt as collaborative and supportive. Continuity of working environment and team composition, informal ('curbside') consultations and availability of protocols were critical mediators.

Conclusion: A surge of critically ill patients can be accommodated by IC trained personnel on expanded or new ICUs when assisted by redeployed non-ICU trained personnel. Although quality of care, supervisory capacity, training background and experience were compromised to meet COVID-19 demands, key features to maintain acceptable quality of care included stable teams matching less with more experienced personnel allowing for adequate supervision, better than normal interprofessional collaboration and a robust culture of informal consultation.

\section{0}

Intensive care unit (ICU) referrals and admissions at a district general hospital (DGH) in light of the COVID-19 pandemic

J. Blair ; E. Naughton ${ }^{1}$; N. Pradhan ${ }^{1}$

'Intensive Care, The Shrewsbury and Telford Hospital NHS Trust, Shrewsbury, United Kingdom

\section{Correspondence: J. Blair}

Intensive Care Medicine Experimental 2020, 8(2): 001010

Introduction: The COVID-19 pandemic has led to an increase in ICU referrals and admissions across the UK during 2020 [1]. Intensive care beds are a limited and expensive resource and decisions on patient admission are often very challenging [2]. Proformas help to standardise documentation and decision logging during patient referrals [3]. They provide easily accessible evidence in case of a future referral and allow audit of decision-making processes. A preliminary survey of doctors working in a DGH ICU was undertaken to assess the current referral and admission process in expectation of an increased volume of work.

Objectives: As a result of the survey, three main areas for improvement were identified:

1. To maintain a record of all ICU referrals and decision-making processes

2. To reduce the time taken for documentation of referrals and admissions

3. To improve the quality and appropriateness of referrals from parent specialities

Methods: A proforma was designed for dual use as a referral and admission document. All referrals were recorded on paper and staff received training on how to apply the proforma. After assessment of each referral, irrespective of admission outcome, a completed copy of the proforma was placed in both the patient's notes and a dedicated referrals folder.
After one month, a further survey was designed to assess the response post-implementation of the proforma. All referrals made over a threemonth period between April and June 2020 were audited.

Results: The initial survey received 12 responses. Prior to the COVID19 pandemic, documentation of any referral or admission took on average 10-15 minutes. All survey participants felt that referring teams did not have a good understanding of the role of ICU care and estimated that up to $40 \%$ of all referrals received were inappropriate.

The follow-up survey received 14 responses. Implementation of the proforma reduced the time taken to document a referral or admission on average by 5-10 minutes. Twelve participants found the proforma a useful aid, helping to provide clear documentation and ease communication between ICU team members.

Less than $9 \%$ of the referrals made between April and June 2020 were admitted to ICU with over $32 \%$ of referrals deemed unsuitable for further escalation. Approximately $50 \%$ of referrals were made by registrars, with $13 \%$ discussed by consultants. The median age of patients referred was 67.5 and the most common reason was for respiratory deterioration.

Conclusion: This quality improvement project successfully reduced the time taken to document ICU referrals and admissions. Use of a proforma has provided many benefits, including standardisation of documentation, decision logging and improvement of intra- and inter-team communication.

Only a small proportion of patients referred to ICU have been suitable for admission. A teaching session is being designed so that referral information can be fed-back to parent specialties. Referrals will be reaudited after this.

Data analysis of this project has been limited by incomplete proforma documentation from participating users.

Reference(s) and grant acknowledgment(s)

1. 1. Public Health England (2020) Public Health England (2020) National COVID-19 surveillance report: 28 May 2020 (week 22). https://www.gov.uk/ government/publications/national-covid-19-surveillance-reports. Accessed 11 June 2020

2. 2. Fullerton JN, Perkins GD (2011) Who to admit to intensive care? Clin Med (Lond) 11:601-604

3. 3. Power N, Plummer NR, Baldwin J, James FR, Laha S (2018) Intensive care decision-making: Identifying the challenges and generating solutions to improve inter-specialty referrals to critical care. J Intensive Care Soc 19:287-298

001027

The implementation of a structured daily assessment proforma to critical care areas during the COVID-19 pandemic

M. Watanabe ${ }^{1}$; S. $\mathrm{Ng}^{1}$; A. Manoras ${ }^{1}$; J. Mandeville ${ }^{1}$; P. Shanmugasundaram'; P. Duggleby ${ }^{1} ;$ T. Ali $^{\top}$

${ }^{1}$ Intensive care department, Stoke Mandeville Hospital, Aylesbury, United Kingdom

Correspondence: M. Watanabe

Intensive Care Medicine Experimental 2020, 8(2): 001027

Introduction: With early reports from other countries of the enormous strain COVID-19 had placed on healthcare systems, a decision was made at Buckinghamshire Healthcare NHS Trust to increase the capacity of Critical Care beds and redeploy junior doctors from other specialities to prepare for a rapid surge in patient numbers.

To facilitate ongoing daily review of patients, a structured assessment proforma was produced to be used in all Critical Care areas for COVID19 patients.

Principles by which the proforma were designed included: easy and rapid to complete (by doctors with limited ICU experience), enables rapid decision-making by senior ICU doctors, ensures thorough and appropriate reviews. 
The benefits of protocolisation in ensuring patient safety, standardising assessments, and minimising error have previously been detailed in the literature $(1,2)$

This project aimed to determine whether the implementation of a structured assessment proforma had enabled appropriate daily ICU reviews to be conducted despite the challenges posed by the pandemic.

Methods: A structured assessment proforma was produced by members of the Consultant body, agreed by the department, and subsequently disseminated to Critical Care areas in early April.

Clinical records of all patients admitted to ICU within a random oneweek period were reviewed.

An online survey was sent out to all junior doctors working on ICU. The survey asked two key questions:

How confident they felt in conducting an ICU review prior to using and with the proforma.

How likely they felt they were to omit important information prior to using and with the proforma.

Answers were recorded as a score between 0 and 10 and paired-samples t-tests conducted.

Results: All proformas completed within a random one-week period $(30 / 05 / 2020$ to $05 / 06 / 2020)$ were reviewed $(n=47)$, from a total of 8 patients admitted on ICU with COVID-19.

The date was written on $100 \%$ and time on $89.4 \%$ of proformas. $97.9 \%$ of the proformas contained an appropriate A to E assessment (as defined by completion of the proforma), $100 \%$ contained a summary of main clinical issues, and a documented plan. $100 \%$ of the proformas had a named doctor completing it and $85.1 \%$ had a clearly documented Consultant.

17 doctors completed the survey (sent out on 29/05/2020).

There was a significant difference in confidence prior to using (mean score $=6, S D=3.41$ ) and with the proforma (mean score $=8.76, \mathrm{SD}=$ 1.09); $\mathrm{t}(16)=-3.62, \mathbf{p}=\mathbf{0 . 0 0 3}$.

There was a significant difference in how likely doctors felt they were to omit important information prior to using (mean score $=4.76$, SD $=2.54$ ) and with the proforma (mean score $=7.12, \mathrm{SD}=2.37$ ); $\mathrm{t}(16)=$ $-2.59, \mathbf{p}=\mathbf{0 . 0 2}$.

Conclusion: Implementation of a structured assessment proforma has enabled appropriate daily ICU reviews to be conducted despite the surge in patient numbers during the pandemic.

With a possible second wave on the horizon, it may be prudent for other hospitals to implement a similar proforma to facilitate daily reviews of COVID-19 patients on ICU.

\section{Reference(s) and grant acknowledgment(s)}

1. 1. Chang SY, Sevransky J, Martin GS. Protocols in the management of critical illness. Critical Care. 2012;16(2):306.

2. 2. Weiss $\mathrm{CH}$, Moazed F, McEvoy CA, et al. Prompting physicians to address a daily checklist and process of care and clinical outcomes: a single-site study. Am J Respir Crit Care Med. 2011;184(6):680-686.
001072

Emergency planned Hub \& Spoke system in support to COVID-19 ICUs in Lombardy: the experience of Niguarda Hospital

F. Fossi ${ }^{1}$; F. Pozzi ${ }^{1}$; F. Zumbo' ${ }^{1}$ C. Scattolini' ${ }^{1}$ L. Pressato ${ }^{1}$; G. Chevallard'; M. Zaniboni' ${ }^{1}$ F. Curto ${ }^{1}$; A. Chieregato ${ }^{1}$

${ }^{1}$ Neurointensive care unit, Grande Ospedale Metropolitano di Niguarda, Milan, Italy

Correspondence: F. Pozzi

Intensive Care Medicine Experimental 2020, 8(2): 001072

Introduction: On March 8th a regional law planned a new Hub and Spoke system for time-dependent pathology in order to allocate resources for an expected increase in COVID patients [1] and to divide hospital pathways to prevent further diffusion of the pandemia. By Regional Decree Niguarda Hospital (Milan) become Hub, for direct admission of major traumas, strokes and neurosurgical emergencies as well as Hub secondary referral for 16 Spoke hospitals. The serving area was at least of 6376970 inhabitants $(63.4 \%$ of the total Lombardy population). Duration of Hub function was from March 8th to May 8th $.3,4$ Therefore the Hospital augmented our NICU beds from 11 to 20 beds.

Objectives: The aim of the study was to report the main findings of the Hub activity.

Methods: All patients admitted since the 8th of March to the 8 of May to the Neurointensive care unit of the Niguarda Hospital were selected. The patients admitted in the same period of the previous year (2019) was also selected as control group. All the data were retrospectively obtained from the institutional clinical reports. The medical staff remained the same, increasing work commitments by $25 \%$. The nursing staff was increased by 23 untrained nurses. Access to our ICU was allowed only after negative nasal swab and bronchoalveolar lavage for COVID-19 (patients were held in a dedicated room for 6 hours while waiting for the results). Family visiting and interviews were unchanged. The activity of the Hub center lasted 2 months, infact once the COVID epidemic the Regional Government declared since the 8th of May to revert the system [2].

Results: 151 consecutively patients were admitted to our ICU in 2020 compared to 60 in 2019. Table 1 shows patient characteristics. Twelve of them became utilized organ donor accounting for $2 / 3$ of all the organ donors in Lombardy. In the same period of the 2019 utilized organ donors from Niguarda were 3 (7.8\% of the 38 organ donors in our region)The absolute number of patients was 2.5 times compared to 2019 , for all the quota of patients arriving from Spoke areas (Figure 1).Figure 2 shows the occupation rate trend showing a progressive increase in the occupation rate along the days even once the CODIV patients in the Lombardy ICU was in a progressive and constant reduction. An increase of withdrawal-withholding was observed. 
Tabie 1
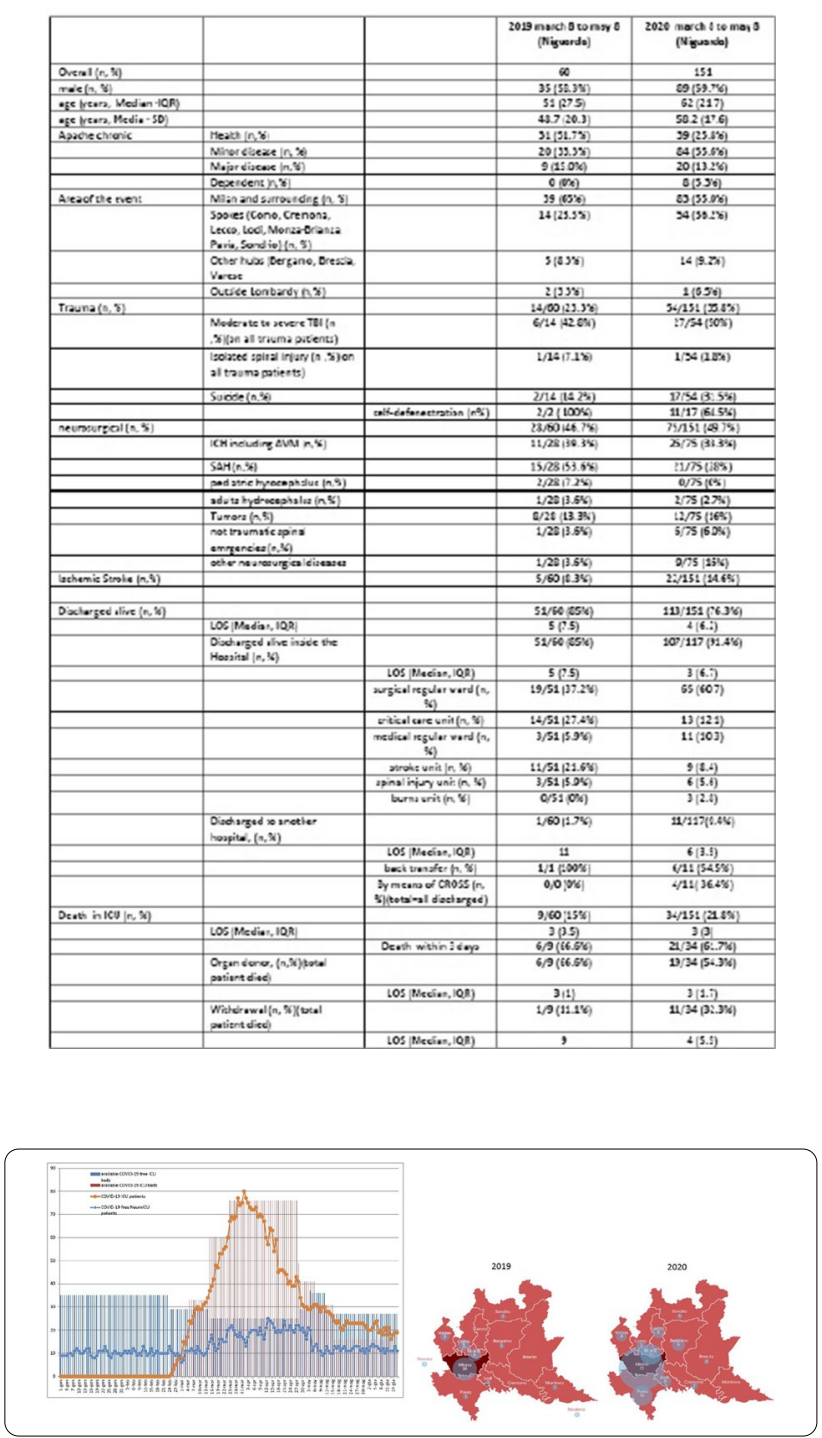

Conclusion: The comparison between the trends of the amount of COVID patients in Lombardy and of the amount of patients entering our Hub shows the support given to the system. In Lombardy a lack of available ICU beds was evident [3]. In such extraordinary condition the creation of an emergency Hub and spoke system seemed to have indirect benefits.

\section{Reference(s) and grant acknowledgment(s)}

1. 1. Remuzzi A, Remuzzi G (2020) COVID-19 and Italy: what next? Lancet 395:1225-1228. https://doi.org/10.1016/S0140-6736(20)30627-9

2. 2. https://www.regione.lombardia.it/wps/portal/istituzionale/HP/DettaglioR edazionale/servizi-e-informazioni/cittadini/salute-e-prevenzione/preve nzione-e-benessere/red-coronavirusnuoviaggiornamenti. Accessed 26 Jun 2020

3. 3. Grasselli G, Pesenti A, Cecconi M (2020) Critical Care Utilization for the COVID-19 Outbreak in Lombardy, Italy: Early Experience and Forecast During an Emergency Response. JAMA. https://doi.org/10.1001/jama.2020.4031
001080

Shared decision making impact on early tracheostomy in prolonged intubated critical patient-post implementation 2 year follow up data

S. H. Kuo ; Z. X. Lin²; H. C. Chen ${ }^{3}$; Y. C. Jiang ${ }^{1}$; T. H. Tai ${ }^{1}$; H. L. Liang ${ }^{4}$; W.

C. Huang

${ }^{1}$ Department of critical care medicine, Kaohsiung Veterans General Hospital, Kaohsiung, Taiwan; ${ }^{2}$ Graduate institute of clinical medicine, Kaohsiung Medical University, Kaohsiung, Taiwan; ${ }^{3}$ Master program of school of nursing, Kaohsiung Medical University, Kaohsiung, Taiwan; ${ }^{4}$ Department of nursing, Shu-Zen Junior College of Medicine and Management, Kaohsiung, Taiwan

Correspondence: S.H. Kuo

Intensive Care Medicine Experimental 2020, 8(2): 001080

Introduction: By previous collected retrospective cohort data analysis, our team revealed introducing shared decision making on early Tracheostomy(less than 14 days) in prolonged intubated critical patient improve total Tracheostomy rate, reduce ventilator days and length of hospital stay, and shorten the Tracheostomy decision time delay.

Methods: Medical record of tertiary medical center adult ICU admitted respiratory failure patient from Jan. 1st, 2018 to Dec. 31th, 2019 was retrospectively reviewed as post-SDM(after Jan. 1st, 2017) intervention follow up period for analysis. Data including total Tracheostomy rate, early and late Tracheostomy rate, ventilator weaning rate and ventilator days, in-hospital mortality, and length of hospital stay of prolonged intubated patient were collected. SPSS was applied for statistically analysis, and a p-value less than 0.05 was considered significant difference.

Results: By inclusion and exclusion criteria, medical records from 366 patients from Jan. 1st, 2018 to Dec. 31th, 2019, who were considered prolonged intubated s/p Tracheostomy SDM, were applied for further analysis. Among them the early and late Tracehostomy rate was $41 \%$ and $69 \%$ individually. In compared to previous reported pre-SDM data, ventilator weaning rate(289(79.2\%) vs. $89(69.3 \%)$, $\mathrm{p}<0.05)$, ventilator days(39.3+/-20.5 vs. $42.3+/-17.3, p<0.05)$, in hospital mortality((10.3\%) vs. $7(3.3 \%)$, p: 0.737$)$, and length of hospital stay $(61.2+/-23.4$ vs. $64.3+/-29.2$, p: 0.04$)$ of prolonged intubated patient. The continued impact on introducing SDM was impressed, with improving ventilator weaning rate, reduced ventilator days, and shortening the length of hospital stay of prolonged intubated patient by 3 days for each in compared in between groups. The Tracheostomy decision making time delay was found 6 days less after SDM introduced was also consistently noted.

Conclusion: Our retrospective cohort study follow-up data revealed introducing shared decision making on early Tracheostomy in prolonged intubated critical patient persistently improve total Tracheostomy rate, improve ventilator weaning rate, reduce ventilator days and length of hospital stay, and shorten the Tracheostomy decision time delay.

\section{3}

\section{Quantifying the impact of informed code status discussions} in critically ill elderly patients in the ICU

J. Kent ${ }^{1}$; E. Mulroney ${ }^{2}$; R. Ghosh ${ }^{3}$

${ }^{1}$ Emergency medicine, University of Toronto, Toronto, Canada; ${ }^{2}$ Research, Sault Ste Marie Academic Medical Association, Sault Ste. Marie, Canada; ${ }^{3}$ Critical care and cardiology, Sault Area Hospital, Sault Ste. Marie, Canada

Correspondence: J. Kent

Intensive Care Medicine Experimental 2020, 8(2): 001083

Introduction: Resuscitation Code Status is a controversial topic that often fails to obtain truly informed consent and is highly contextual depending on the nature and severity of illness. While studies have identified DNR status in ICU patients as a risk factor for mortality and 
adverse events, no literature has targeted elderly, critically ill patients for whom these conversations were intended.

Objectives: Our study aimed to assess the relationship between code status and mortality in critically ill, elderly patients admitted to the ICU. As a secondary outcome, we sought to analyze the impact of an informed code status discussion on resource utilization. Specifically, our study focused on ascertaining if critically ill, elderly patients with DNR code status have different hospital outcomes relative to similarly ill patients who are full code.

Methods: Data for this retrospective study was obtained through a review of medical records at Sault Area Hospital, an accredited 289 bed large community hospital in Northern Ontario spanning a 5-year period. During this time, 613 patients were admitted to the ICU, 163 of whom met the inclusion criteria of being $\geq 70$ years of age and APACHE II score $\geq 20$. A Kaplan-Meier Survival Curve examined survival to hospital discharge comparing DNR and full code groups. DNR code status was defined as omission of cardiopulmonary resuscitation in the event of a cardiac arrest.

Results: 64 patients (39.3\%) had a DNR order based on a documented conversation within 72 hours of admission. The remaining $99(60.7 \%)$ patients were deemed to be full code.There was a significant association between DNR status and increased mortality $(p=0.004)$. Patients with DNR code status stayed fewer days in ICU (7.7 days ( \pm 3.6$)$ vs. 9.9 days $( \pm 8.3), p=0.03)$ and used less resources (4.5 diagnostic images/pt ( \pm 3.4$)$ vs. 6.2 diagnostic images/pt $( \pm 6.4), p=0.02 ; 0.9$ consultations $( \pm 1.1)$ vs. 1.1 consultations $( \pm 0.9), p=0.48)$ than similarily ill patients who were full code with no benefit in long term survival at 21 days. Patients with full code status had a higher average cost of hospital and ICU admission in comparison to patients with DNR status $(59,704.70 \mathrm{CND} /$ pt. vs $49,589.10 \mathrm{CND} /$ pt. respectively).

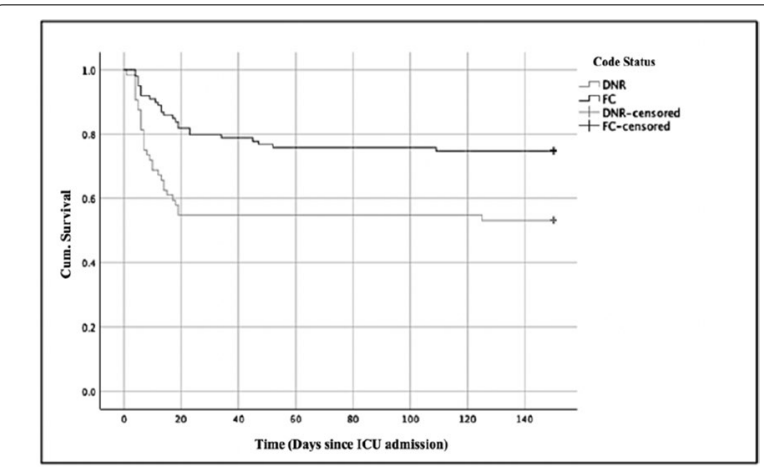

Figure 1. Kaplan-Meier Survival Curve to Hospital Discharge in between DNR and Full-Code patients.

Conclusion: Our study has demonstrated the benefit of having early, informed code status discussions in the ICU. In our study population, DNR code status is associated with significantly reduced resource utilization. Furthermore, no mortality benefit was seen in DNR vs full code patients after 21 days suggesting that resuscitation limits may be objectively placed in long stay, elderly ICU patients.

\section{Reference(s) and grant acknowledgment(s)}

1. 4. Walkey AJ, Weinberg J, Wiener RS, Crooke CR, Lindenauer PK (2016) Association of do-not-resuscitate order and hospital mortality rate among patients with pneumonia. JAMA Intern Med 176:97-104

2. 3. Fuchs L, Anstey M, Feng M et al (2017) Quantifying the mortality impact of do-not-resuscitate orders in the ICU. Crit Care Med 45:1019-1027

3. 2. Chen YY, Chen YS, Chu TS, Lin KH, Wu CC (2016) Further deliberating the relationship between do-not-resuscitate and the increased risk of death. Sci Rep 6:23182

4. 1. Becker C, Lecheler L, Hochstrasser S et al (2019) Association of communication interventions to discuss code status with patient decisions for do-not-resuscitate orders: A systematic review and meta-analysis. JAMA Netw Open 2:1-17
5. Sault Ste. Marie Academic Medical Association (SSM AMA) XL Research Grant $<30,000$

\section{8}

LocSSIPs in an intensive care setting: generating solutions to improve compliance

C. Chaplin ${ }^{1}$; N. Shah; N. Anderson²; R. Saleh²

${ }^{1}$ Medical school, Queen Mary University of London, London, United Kingdom; ${ }^{2}$ Intensive care, St Bartholomew's Hospital, London, United Kingdom Correspondence: $\mathrm{R}$. Saleh

Intensive Care Medicine Experimental 2020, 8(2): 001088

Introduction: In 2015, NHS England published the National Safety Standards for Invasive Procedures (NatSSIPs), based on analysis of Never Events, Serious Incidents and near misses. These standards provided the basis for the creation of Local Safety Standards for Invasive procedures (LocSSIPS). To meet our ITU standards, at least $90 \%$ of invasive procedures performed should have a LocSSIP completed: this takes the form of a specific checklist, conducted by a team pre-, during, and post-procedure.

Objectives: To assess LocSSIP compliance in our Intensive Treatment Unit (ITU) with a view to generating solutions for improved compliance

Methods: We consulted the paper notes for all patients resident on two ITU wards to take a 'snapshot view' of compliance at a single point in time.

In situ invasive devices and corresponding LocSSIPs were identified from the patients' notes and recorded. Devices to be recorded included: Dialysis Catheters, Arterial Catheters, Peripherally Inserted Central Catheter (PICC) line, Central Venous Catheters, Tracheostomy, Bronchoscopy, Chest Drain, Extracorporeal Membranous Oxygenation (ECMO) Catheters, and Endotracheal Tubes.

\section{Results:}

There were a total of 27 devices in situ across both ICU wards Only devices that were in situ at the time of data collection were audited for an accompanying LocSSIP

Of 27 invasive devices, LocSSIPs were only identified for 3, a compliance rate of $11.1 \%$

The only devices for which LocSSIPs had been recorded were tracheostomies and CVCs

\begin{tabular}{|l|l|l|l|}
\hline Device & $\begin{array}{l}\text { Number in } \\
\text { situ }\end{array}$ & $\begin{array}{l}\text { Completed } \\
\text { LocSSIPs }\end{array}$ & $\begin{array}{l}\text { Compliance } \\
(\%)\end{array}$ \\
\hline Dialysis Catheter & 1 & 0 & 0 \\
\hline Intercostal Drain & 2 & 0 & 0 \\
\hline PICC Line & 2 & 0 & 0 \\
\hline Tracheostomy & 4 & 2 & 50 \\
\hline $\begin{array}{l}\text { Central Venous } \\
\text { Catheter }\end{array}$ & 9 & 1 & 11 \\
\hline Arterial Catheter & 9 & 0 & 0 \\
\hline
\end{tabular}

Conclusion: The barriers to implementing LocSSIPs in the 2018 NatSSIP implementation survey include: lack of time, staff culture, limited expertise, and poor clinical engagement.

Education on LocSSIPs is already part of doctors' induction and nurse education. The time taken to conduct a LocSSIP checklist is not significant. Staff culture of LocSSIPs being considered 'someone else's problem', and not in the forefront of the team's mind may play a part. However, given that details of the procedure are often recorded electronically, it seems that the systemic culture of not having the LocSSIP easily available is a significant factor in its omission. The team is usually at least a doctor and a nurse, so educational reinforcement must target both groups. We are contacting our device manufacturers to try and attach our LocSSIP forms to the kit used for the procedures - this 
would ensure a visual reminder and access to the form is easy for each procedure.

\section{1}

Communicating with relatives of patients admitted to critical care during the Covid-19 pandemic

D. El-Dalil'; C. Morris'²; G. Gibbon ${ }^{1}$

${ }^{1}$ Critical Care, Queens Medical Centre, Nottingham, United Kingdom; ${ }^{2}$ Critical care, Nottingham City Hospital, Nottingham, United Kingdom

Correspondence: D. El-Dali

Intensive Care Medicine Experimental 2020, 8(2): 001101

Introduction: Relatives of patients in critical care experience high levels of stress and anxiety [1,2,3]. Prioritising good communication and facilitating visiting are the most important factors in meeting the needs of families [2]. During the COVID-19 pandemic, infection control procedures have prevented family and friends from visiting their loved ones, potentially having a negative psychological impact on patients' relatives [3].

Objectives: To develop a system to ensure comprehensive and effective communication with the relatives of patients admitted to critical care at Nottingham University Hospitals Trust during the Covid-19 pandemic.

Methods: A multidisciplinary forum developed a communication strategy whereby the next of kin was updated daily and then asked to relay information to other friends and family. This was an iterative process guided by online feedback from junior doctors and nursing interviews. Feedback from medical and nursing staff was explored using thematic analysis and utilised to refine the programme.

Results: Informal comments from patients' relatives have been largely positive and feedback from doctors and nurses suggest the process was achievable. Data collected from 55 online feedback forms and 16 nursing interviews were analysed and 7 main themes and 4 sub themes were identified. Overarching themes included 'Updates are Important' and that there is a 'Role for Doctor and Nurse Updates'. The theme 'Be Flexible' identified that daily updates were sometimes overwhelming and may contribute to relative anxiety. Other themes included 'Utilise Video Calling' and creating a 'Universal Communication Document'.

Conclusion: Feedback from Doctors and Nurses has shown that the process is feasible and acceptable to both groups of staff. Continuing to pro-actively contact a named relative daily is important and ensures relatives are well informed in the absence of visiting times. We aim to collect formal feedback from patients' relatives to evaluate how well we are meeting their needs. A communication booklet is in development to help aid clarity of documentation and to provide a single area for recording communications. We will be exploring simulation based teaching in managing difficult conversations over the phone.

\section{Reference(s) and grant acknowledgment(s)}

1. 1. Alvarez, G. F., \& Kirby, A. S. (2006). The perspective of families of the critically ill patient: their needs. Current opinion in critical care, 12(6), 614-618.

2. 2. Alsharari, A. F. (2019). The needs of family members of patients admitted to the intensive care unit. Patient preference and adherence, 13, 465.

3. 3. Paul, F., \& Rattray, J. (2008). Short-and long-term impact of critical illness on relatives: literature review. Journal of advanced nursing 62(3), 276-292.

4. 4. No grants have been issued in relation to this quality improvement project.

\section{9}

Finding resilience against burn-out syndrome and moral distress in an academic intensive care unit

T. Witter'; J. Hancock'; S. Comber²; O. Kits ${ }^{3}$

${ }^{1}$ Critical care, QEIl HSC, Halifax, Canada; ${ }^{2}$ Economics, Dalhousie University, Halifax, Canada; ${ }^{3}$ Department of community health and epidemiology, Dalhousie University, Halifax, Canada

Correspondence: $T$. Witter

Intensive Care Medicine Experimental 2020, 8(2): 001139

Introduction: Burnout Syndrome (BOS) is the result of chronic exposure to stress in the workplace and is characterized by emotional exhaustion, depersonalization and reduced personal accomplishment (1). Moral Distress (MD) occurs when individuals are placed in situations that are at odds with their core values and have little power to make changes (2). BOS and MD is a rising problem in Medicine and in Intensive Care in particular, not only affecting healthcare providers (HCPs)and their families but also affecting the quality of care provided by the HCPs $(3,4)$. In a recent survey done in our ICUs, we found a high prevalence of BOS and MD amongst all groups of health care professionals using the MBI. While the MBI is well validated to detect BOS and MD, it lacks the depth to identify causes of BOS and MD.

Objectives: To perform an in-depth analysis of causes of BOS and Moral distress amongst HCPs in an academic intensive care unit using focus group methodology.

Methods: Focus groups were conducted with HCP groups and were cohorted based on professional designation to facilitate psychological saftey. The session were open to all members of the health care team and participation was voluntary. The focus group interview guide used in the sessions underwent iterative review and explored the daily work environment at both the organizational and individual level. It probed around burnout, moral distress, team dynamics, currently used coping strategies and interventions that were thought to be helpful in building resilience.

All sessions were audiotaped and transcribed verbatim. A qualitative software (Quirkos - www.quirkos.com) was used to store, sort, index and code the information provided in the session. All transcripts were read individually and codes to relevant passages were collected under themes, disagreements were resolved by consensus.

Results: We were able to recruit 35 HCPs for 6 focus groups: 1 group with MDs, 1 group with RTs and 4 groups with RNs, capturing 19\% of the RNs, $20 \%$ of the RTs and $43 \%$ of the physicians.

There was a great degree of overlap between themes associated with $\mathrm{BOS}$ and MD. We were able to identify three overarching themes causing BOS and MD: organizational issues, exposure to high intensity situations and poor team experience. These themes were further broken down into multiple subthemes, again with a high degree of overlap between BOS and MD.

When asked about coping strategies for dealing with workplace stressors participants in all groups named self-constructive (e.g. performing a workout) and self-destructive examples (e.g. excessive alcohol intake). Particpants suggested multiple strategies around interventions that they felt would help build resilience. These interventions can be categorized according to the three major themes.

1. Organizational: Improved staffing and infrastructure, improved education

2. Exposure to high intensity situations: implementation of regular debriefing sessions, de-stigmatizing the need for support, mental health wellness days, long-term follow up of ICU survivors, and addressing workplace violence.

3. Poor team dynamics: improved relationship builiding (inter and intra-professional), teaching respectful communication and behaviour, acknowledgement of hard work and job well done, support for just-culture, hospital leadership engagement and management support.

Conclusion: We found three main themes when looking at associations with BOS and MD in our ICU HCPs: Organizational, exposure to high intensity situations and poor team dynamics. Some of the suggetions provided by the participants for interventions to reduce BOS and MD are challenging to execute while others are doable (e.g. regular debriefing sessions, improved communication) and should be considered for implementation.

\section{Reference(s) and grant acknowledgment(s)}

1. 4. Henrich N, Dodek P, Gladstone E, Alden L, Keenan S, Reynolds S, et al. Consequences of Moral Distress in the Intensive Care Unit: A Qualitative Study. Am J Crit Care 2017:26:e48-e57.

2. 3. Moss M, Good V, Gozal D, Kleinpell R, Sessler C. An Official Criti-

cal Care Scoieties Collaborative Statement. Critical Care Medicine 2016;44(7):1414-1421

3. 3. Jameton A. Nursing practice. Englewood Cliffs, NJ: Prentice-Hall 1984 
4. 1. Maslach C, Jackson S. MBI: Maslach burnout inventory; manual research edition Palo Alto, CA: Consulting Psychologist Press; 1986

5. Departmental Research Grant

\section{1}

Long term survival effect of chronic obstructive pulmonary disease and inhaled bronchodilators in acute myocardial infarction

Y. C. Jiang ${ }^{1}$; S. H. Kuo ${ }^{2}$; K. C. Lin ${ }^{1}$; T. H. TAl ${ }^{3}$; H. L. Liang ${ }^{1}$; W. C. Huang ${ }^{4}$ ${ }^{1}$ Critical care medicine, Kaohsiung Veterans General Hospital, Kaohsiung, Taiwan; ${ }^{2}$ Department of critical care medicine, Kaohsiung Veterans general hospital, Kaohsiung, Taiwan; ${ }^{3}$ Critical care medicine, Kaohsiung Veterans General Hospital, Kaohsiung, Taiwan; ${ }^{4}$ Critical care medicine and cardiovascular center, Kaohsiung Veterans General Hospital, Taipei, Taiwan

Correspondence: W.C. Huang

Intensive Care Medicine Experimental 2020, 8(2): 001151

Introduction: Chronic obstructive pulmonary disease (COPD) in Asia was different from Europe in higher cigarette smoking rate and more severe air pollution. Varies inhaled COPD medications had safety concern in acute myocardial infarction (AMI). The aim of this study was to investigate the effect of COPD in Asia AMI patients and real world safety of COPD medications in AMI patients.

Methods: We conducted a nationwide cohort study extracted data from the Taiwan National Health Insurance Research Database. Patients who hospitalized between 2000 and 2012 with a primary diagnosis of first AMI were included. Among the 186,112 prospective AMI patients, COPD was diagnosed in 13,065 (7.0\%) patients. AMI patients without COPD were 1:1 matched by propensity score matching. AMI cohort was further divided to STEMI and NSTEMI cohort. STEMI without COPD was matched by propensity score. Cox proportional hazards regression model was used to estimate adjusted hazard ratios (HR) with $95 \%$ confidence intervals $(95 \% \mathrm{Cl})$.

Results: During 12 years follow up, there were 18405 (77.65\%) and 16093 (67.89\%) deaths in AMI with COPD and without COPD group, respectively. The adjusted hazard ratio (aHR) of mortality in AMI with COPD group was 1.12 (95\% Cl 1.09 to 1.14). In STEMI with COPD group, aHR was 1.20 (95\% Cl 1.14 to 1.25$)$. NSTEMI with COPD, aHR was 1.07 (95\% Cl 1.04 to 1.10). Using short-acting inhaled bronchodilators in AMI patients increased mortality (short acting beta-agonist: aHR 1.2, $95 \% \mathrm{Cl} 1.16$ to 1.23 , short acting muscarinic antagonist: aHR 1.3, 95\% Cl 1.26 to 1.34). Corticosteroids using also increased $10 \%$ mortality (aHR $1.10,95 \% \mathrm{Cl} 1.07$ to 1.14). However, long acting inhaled bronchodilators reduced mortality (long acting beta-agonist: aHR $0.87,95 \% \mathrm{Cl}$ 0.81 to 0.94 , long acting muscarinic antagonist: aHR $0.82,95 \% \mathrm{Cl} 0.69$ to 0.96$)$.

Conclusion: In Asia, AMI patient with COPD was associated with higher mortality compared with those without COPD. Guideline recommended AMI medications decreased mortality in patient with or without COPD, but they were underused in Taiwan. Using inhaled short-acting bronchodilators and corticosteroids in AMI patient reduced survival. On the contrary, long acting inhaled bronchodilators were associated with survival benefit. Appropriate COPD medications and adequate standard AMI medications in AMI patient were equal crucial in improving long term survival.

\section{4}

The role of neuropathic pain in critical illness survivors. A prospective 12 month follow-up study

A. Calvo Barrera'; A. Ojeda²; M. Chanza ${ }^{3}$; D. Christian ${ }^{4}$; C. Ferrando';

V. Zafra ${ }^{5}$

'ANAESTHESIOLOGY, CRITICAL CARE UNIT, Hospital Clínic de BarceIona, Barcelona, Spain; ${ }^{2}$ Aanesthesiology, ojeda@clinic.cat, BARCELONA, Spain; ${ }^{3}$ Anaesthesiology, Hospital del Mar, Barcelona, Spain; ${ }^{4}$ Anaesthesiology, pain unit, Hospital Clínic de Barcelona, Barcelona, Spain; ${ }^{5}$ Nurse of critical care unit, Hospital Clínic de Barcelona, Barcelona, Spain

Correspondence: A. Calvo Barrera

Intensive Care Medicine Experimental 2020, 8(2): 000014
Introduction: Post-intensive Care Syndrome (PICS) is a major health problem that describes impairment in their physical, cognitive, or mental health status. Neurophatic pain (NP) is related with worst painrelated interference and poor quality of life $(\mathrm{QoL})$. Despite of that, the role of NP in Critical IIIness survivors (CIS) and its relationship with PICS has been not establish.

Objectives: The goal of this study was to evaluate the prevalence and impact of NP in CIS, and its relationship with anxiety, depression and post-traumatic stress disorder (PTSD), majors PICS components

Methods: We conducted a prospective 12-month follow-up study. Patients admitted in ICU were consecutively recruited and were evaluated before ICU discharge, and at 3 and 12 months after. Evaluations included: NP (DN4: Douleur Neuropathique en 4 Questions); pain intensity and pain related-interference (BPI-LV: Brief Pain Inventory Long Version); anxiety and depression (HAD Test: Hospital Anxiety and Depression Scale); QoL (EuroQoL Test 5 dimension, 3 levels); PTSD (PTSD Checklist-Civilian Version).

\begin{tabular}{|l|l|l|l|}
\hline \multicolumn{4}{|c|}{ Table 1. Evolution of pain, NP and PICS variable. } \\
\begin{tabular}{|l|l|l|l|}
\hline QUESTIONNAIRE & \multicolumn{1}{|c|}{ ICU } \\
DISCHARGE(\%)
\end{tabular} & $\begin{array}{c}\text { 3 } \\
\text { MONTHS(\%) }\end{array}$ & $\begin{array}{c}\text { 12 MONTHS } \\
\mathbf{( \% )}\end{array}$ \\
\hline BPI INTENSITY >3 & 78,6 & 37 & 37,5 \\
\hline BPI & 84,1 & 42,6 & 46,2 \\
\hline INTERFERENCE $>3$ & & 37 & 24,4 \\
\hline DN4>4 & 25,7 & 39,6 & 31,7 \\
\hline HAD ANXIETY & & 28,3 & 31,7 \\
\hline HAD DEPRESSION & & 15,7 & 24,3 \\
\hline PTSD POSITIVE & & & \\
\hline
\end{tabular}

Results: A DN4 test positive was related with a major pain intensity (BPI intensity score $>=3$ ), worse pain-related interference (BPI interference score $>=3$ ), worse value of EuroQoL visual analogue scale and more depression in the 3 and 12-month follow-up, and more anxiety and PTSD in the 12-month $(p<0,05)$.

Conclusion: Neuropathic pain is common in Critical Illness survivors, affects their QoL and interferes with their daily live activities. Moreover, its related with anxiety, depression, and PTSD, key components of PICS.

\section{Reference(s) and grant acknowledgment(s)}

1. Herrero MJ, Blanch J, Peri JM, De Pablo J, Pintor L, Bulbena A. A validation study of the hospital anxiety and depression scale (HADS) in a Spanish population. Gen Hosp Psychiatry. 2003 Aug;25(4):277-83.

2. Torres J, Carvalho D, Molinos E, Vales C, Ferreira A, Dias CC, et al. The impact of the patient post-intensive care syndrome components upon caregiver burden. Med Intensiva. 2017 Nov;41 (8):454-60

3. Badia X, Muriel C, Gracia A, Manuel Núñez-Olarte J, Perulero N, Gálvez R, et al. Validación española del cuestionario Brief Pain Inventory en pacientes con dolor de causa neoplásica. Med Clínica. 2003 Jan;120(2):52-9.

4. Kyranou M, Puntillo K. The transition from acute to chronic pain: might intensive care unit patients be at risk? Ann Intensive Care. 2012;2(1):36.

5. Kemp HI, Laycock H, Costello A, Brett SJ. Chronic pain in critical care survivors: a narrative review. BJA Br J Anaesth. 2019 Aug;123(2):e372-84.

\section{1}

Mineralocorticoid receptor expression in critically-ill patients A. G. Vassiliou'; A. Kotanidou²; D. Vassiliadi ${ }^{3}$; C. Keskinidou'; E. Jahaj²; S. Orfanos ${ }^{2}$; S. Tsagarakis ${ }^{3} ;$ I. Dimopoulou ${ }^{2}$

${ }^{1} 1$ st department of critical care medicine \& pulmonary services, gp livanos and $m$ simou laboratori, National and Kapodistrian University of Athens, Athina, Greece; ${ }^{2} 1$ st department of critical care medicine \& pulmonary services, Evangelismos Hospital, Medical School, National and Kapodistrian University of Athens, Athens, Greece; ${ }^{3}$ Department of endocrinology, diabetes and metabolism, Evaggelismos General Hospital, Athina, Greece; ${ }^{4}$ Medical school, Democritus University of Thrace, Alexandroupoli, Greece Correspondence: A.G. Vassiliou Intensive Care Medicine Experimental 2020, 8(2): 000021 
Introduction: The mineralocorticoid receptor (MR) belongs to the steroid receptor superfamily. Discoveries from the past two decades reveal that MRs are expressed in non-epithelial cells and exert numerous additional functions beyond electrolyte handling in the distal tubule of the kidney. The recent identification of MRs in immune cells has led to novel functions of MR, such as modulation of immunity and inflammation. The MR is unique in this family in that it has two ligands, aldosterone and cortisol. Aldosterone and cortisol have a similar affinity for the MR.

Objectives: To evaluate the expression of $M R$ in peripheral polymorphonuclear cells in critical illness for a 13-day period.

Methods: Forty-two mechanically ventilated, steroid-free patients were studied. Blood samples were collected on admission in the ICU and on days 4,8 , and 13 , to measure $M R$ expression. Twenty-five ageand sex-matched subjects were used as controls.

Results: Compared to healthy controls, severely ill patients on admission in the ICU exhibited a 12-fold lower MR mRNA expression [0.08 (0.04-0.92); $p<0.0001]$. During ICU stay, MR expression levels remained reduced up to day 13 . More specifically, on day 4 patients exhibited 8 -fold reduced levels [0.13 (0.04-0.41); $\mathrm{p}<0.001]$, on day 8 , 5-fold $[0.20(0.05-0.50) ; \mathrm{p}<0.01]$ and on day 13,14 -fold $[0.07(0.05-0.25) ; \mathrm{p}<$ $0.001]$. This was corroborated by the fact that compared to admission MR expression levels during the stay period remained unaltered. The MR-downstream target gene galectin-3 (GAL3) also exhibited reduced expression on ICU admission, compared to healthy controls [0.51 (0.37-0.68); $\mathrm{p}<0.0001]$.

Conclusion:MR exhibits a very low expression in critically ill patients, and remains low during the 13-day observation period. The downstream target of MR, GAL3, as expected is also low on ICU admission.

\section{0}

Healthcare utilization, costs and survival of a Canadian cohort of patients discharged from a specialized weaning centre with matched ICU controls: a retrospective longitudinal health administrative database study

L. Rose ${ }^{1}$; E. Dvorani² ${ }^{2}$ E. Homehauth ${ }^{2}$; L. Istanboulian ${ }^{3}$; I. Fraser $^{3}$

${ }^{1}$ Florence Nightingale Faculty of Nursing, Midwifery and Palliative Care, King's College London, London, United Kingdom; ${ }^{2}$ Ahrq, Institute of Clinical Evaluative Science, Toronto, Canada; ${ }^{3}$ Prolonged-ventilation weaning centre, Michael Garron Hospital, Toronto, Canada

Correspondence: $L$. Rose

Intensive Care Medicine Experimental 2020, 8(2): 000100

Introduction: Specialized weaning centres (SWCs) provide an alternate model of care to the ICU for patients requiring prolonged mechanical ventilation. Many studies representing numerous countries report short term outcomes of SWCs; limited data describe mortality beyond 1 year. Furthermore, comparative data on long term outcomes with a control group representing PMV patients who remain in an ICU setting are lacking. Quantification of long-term survival, healthcare utilization and costs of patients experiencing PMV informs patient/family decision making, healthcare spending policy, and understanding of specialized weaning centres (SWC) as an alternate care model.

Objectives: To compare long-term healthcare utilization, costs, and survival trajectory of Canadian SWC patients with a matched cohort of $\geq 21$-day stay ICU patients.

Methods: Retrospective longitudinal (2004-2016, 12-year) study. We linked patients discharged alive identified from the clinical/quality database of the Prolonged-ventilation Weaning Centre (PWC) (Toronto, Canada) to Canadian health administrative databases. We used 1:1 matching (age, sex, Charlson Comorbidity Index, income quintiles) to patients experiencing prolonged ICU stay only. We created multivariable Cox proportional hazard models of survival and time to home discharge.

Results: We matched 228 SWC to 228 prolonged ICU stay survivors (456 patient cohort); $42 \%$ had Charlson score of $>4$. Mean (SD) duration of SWC admission was 62.2 (85.7) days with mean (SD) 95 (87.7) ICU days before SWC admission. Mean (SD) ICU LOS of the prolonged ICU cohort was 43.4 (32.7) days. SWC survivors required more ICU admissions, hospital and ICU days 12 months before index admission. Six months and 12 months following unit discharge, more SWC patients required ICU admission ( $30.3 \%$ vs $16.7 \%, P<0.001$ ), with more frequent (and longer) hospital and ICU admissions, specialist visits, and billable procedures. Median costs were $\$ 10,853$ (6-months) $(P=0.01)$ and $\$ 13,138$ (12-months) $(P=0.004)$ higher for SWC survivors. At 12-years, hospitalization/ICU admissions and hospital days were similar. SWC survivors consumed more ICU bed days (25 (106.3) vs $7.8(21.9), \mathrm{P}=0.02)$ and cost more healthcare dollars $(\$ 135,975$ vs $\$ 119,763, \mathrm{P}=0.02)$. There was no difference in home discharge (hazard ratio (HR) $1.30,95 \%$ confidence interval $(\mathrm{Cl}) 0.95-1.80)$. Survival was not different ( $72 \%$ vs $61 \%$ dead at 12 years; HR $1.18,95 \% \mathrm{Cl} 0.93-1.50$ ). Longer length of index hospital admission (HR 1.02, 95\% Cl 1.00-1.04 per month increase) was associated with increased survival whereas increasing numbers of care location transfers was associated with decreased survival (HR 0.88, 95\% Cl 0.79-0.98).

Conclusion: SWC patients used more acute healthcare services before index admission suggesting greater medical fragility and after unit discharge with higher public healthcare costs. Repatriation of non-weaned patients to acute settings due to limited long-term care resources may have influenced this finding. Survival trajectory was similar.

\section{Reference(s) and grant acknowledgment(s)}

1. This study was supported by the Institute for Clinical Evaluative Sciences (ICES), which is funded by an annual grant from the Ontario Ministry of Health and Long-Term Care (MOHLTC).

\section{3}

The change in in-hospital mortality after discharge from intensive care over a period of $\mathbf{2 0}$ years

A. Coppola'; S. Pararajasingam'; P. Morgan

${ }^{1}$ Critical care, East Surrey Hospital, Redhill, United Kingdom

Correspondence: A. Coppola

Intensive Care Medicine Experimental 2020, 8(2): 000123

Introduction: The Intensive care setting has undergone major paradigm shifts over the years with the ability to expand beyond the physical ICU setting and the transition to continuous monitoring and appropriately minimising invasive procedures. We performed a large retrospective cohort study to analyse the changes to in-hospital mortality after ICU discharge over time.

Methods: A retrospective cohort study on patients discharged from ICU in a District General Hospital between 2000-2020. Palliative patients and missing patient data were excluded. Patient search was obtained via ward watcher software. Total patient number was 12762 and following exclusion 9081 were included. The patients were grouped into in-hours (08:00-17:59) and out of-hours (18:0007:59) depending on the hour of discharge. The mortality data was collected and then compared over 5-year increments to evaluate progression over time. The results underwent statistical Chi Square analysis.

Results: A total of 9081 patients discharged alive from ICU were studied. Mean age 62.7 years (range, 16-98), 4861 (53.5\%) were male, $4220(46.5 \%)$ were female. The overall in-hospital mortality was 744 (8.2\%). The mortality rate in 5 year increments from 2000 to 2020 are $9.53 \%, 10.33 \%, 10.37 \%$ and $5.42 \%$ respectfully. The most recent 5 years showed a statistically significant drop in mortality of $4.95 \%$ (p-value < 0.00001 ) to the $2010-2015$ group. The results show a consistent higher mortality for those discharge out of hours.

Conclusion: Measure taken place over the years have shown improvement in in-hospital mortality after ICU discharge. Several factors have potential to influence the result such as demographics, improvements in ward care and ICU paradigm shifts. It is rational to note the significant changes have occurred over the latter 10 years, with little differences from 2000 to 2015 . Essential work is require to highlight the key factors influencing the result.

\section{Reference(s) and grant acknowledgment(s)}

1. 1. Vincent, J. L. \& Creteur, J. Paradigm shifts in critical care medicine: The progress we have made. Critical Care (2015) https://doi.org/10.1186/cc14728. 


\section{4}

Serum ammonia level is associated with mortality in critically ill patients: a multicenter retrospective cohort study

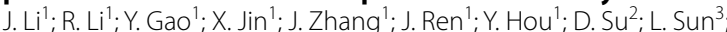

X. Wang ${ }^{1}$; G. Wang

${ }^{1}$ Department of critical care medicine, the Second Affiliated Hospital of Xi'an Jiaotong University, Xi'an, China; ${ }^{2}$ Department of cardiology, the Second Affiliated Hospital of Xi'an Jiaotong University, Xi'an, China; ${ }^{3}$ Department of ultrasound, the Second Affiliated Hospital of Xi'an Jiaotong University, Xi'an, China

Correspondence: $G$. Wang

Intensive Care Medicine Experimental 2020, 8(2): 000164

Introduction: Ammonia, generated from the deamination of amino acids, is a constituent of all human body fluids. Elevated serum ammonia is commonly observed in patients with hepatic diseases, but large and multicenter studies on the association of serum ammonia levels with clinical outcomes in a broad population of adult patients are scant.

Objectives: The study was aimed to assess the association of serum ammonia upon admission during initial intensive care unit (ICU) stay with ICU and in-hospital mortalities.

Methods: This retrospective observational cohort study included 2,703 adult patients with available admission serum ammonia measurements from 335 ICUs within 208 hospitals in elCU Collaborative Research Database (eICU-CRD). Ammonia levels were divided into deciles, and the ICU mortalities within ammonia deciles were assessed. Univariable and multivariable logistic regression analyses were performed to analyze the relationship between serum ammonia and mortalities. The covariates included demographics, Acute Physiology and Chronic Health Evaluation (APACHE) IV score, treatments, and diseases diagnosed during the first $24 \mathrm{~h}$ of ICU stay. Model discrimination was assessed by the calculation of the receiver operating characteristic curve and reporting the area under the curve (AUC). Interaction and subgroup analyses were conducted to determine whether the effect of ammonia on mortalities varied in patients with or without hepatic diseases.

Results: The median of serum ammonia was 39 (23-65) $\mu \mathrm{g} / \mathrm{dL}$. Totally, we defined three ammonia categories: < $47 \mu \mathrm{g} / \mathrm{dL}, 47 \mu \mathrm{g} / \mathrm{dL}$ to $111 \mu \mathrm{g} / \mathrm{dL}$, and $\geq 111 \mu \mathrm{g} / \mathrm{dL}$, corresponding to low, intermediate, and high ICU mortality. ICU and in-hospital mortalities in the three ammonia categories were $7.59 \%, 16.52 \%$, and $32.72 \%$ and $14.05 \%, 22.96 \%$, and $42.28 \%$, respectively. Increased ammonia was significantly associated with increasing ICU mortality (per $10 \mu \mathrm{g} / \mathrm{dL}$ increase: odds ratio, 1.069 [ 95\% confidence intervals, 1.048-1.091]; Intermediate vs. Low: 1.935 [1.445-2.592]; High vs. Low: 4.239 [2.916-6.162]), and in-hospital mortality (per $10 \mu \mathrm{g} / \mathrm{dL}$ increase: 1.060 [1.040-1.081]; Intermediate vs. Low: 1.440 [1.127-1.839]; High vs. Low: 3.249 [2.330-4.529]). Adding ammonia to APACHE IV score improved the AUC from 0.826 to $0.839(P$ $<0.001)$ for ICU mortality and 0.806 to $0.813(P=0.001)$ for in-hospital mortality. Interaction and subgroup analyses demonstrated consistent results in patients with or without hepatic diseases. In patients with hepatic disease, serum ammonia levels were significantly associated with the ICU and in-hospital mortalities (1.053 [1.025-1.081], 1.040 [1.015-1.067], per $10 \mu \mathrm{g} / \mathrm{dL}$ increase), the results were similar in the non-hepatic disease group (1.078 [1.051-1.106], 1.071 [1.046-1.096], per $10 \mu \mathrm{g} / \mathrm{dL}$ increase).

Conclusion: Serum ammonia levels upon admission might be an early risk factor for higher ICU and in-hospital mortalities in ICU patients.

\section{2}

\section{Doctor}

T. Ylimartimo'; M. Koskela²; J. Liisanantti ${ }^{3}$

${ }^{1}$ University hospital of Oulu, Oulu, Finland, Finland; ${ }^{2}$ Surgery, University hospital of Oulu, Oulu, Finland, Finland; ${ }^{3}$ Study group of surgery, anesthesiology and intensive care, Oulu University, Medical Research Center, Oulu, Finland, Finland

Correspondence: T. Ylimartimo

Intensive Care Medicine Experimental 2020, 8(2): 000172
Introduction: Emergency laparotomy (EL) is among one of the most common surgical operations. Previous studies have shown that emergency surgery is associated with higher morbidity and mortality compared to elective surgery 1-4. It has also been reported that patients with direct intensive care unit (ICU) admission after EL have significantly high mortality5. However, there are no studies comparing the impact of direct and delayed admission from recovery room (RR) to ICU on the outcome.

Objectives: Aim of the study was to compare outcomes between direct and delayed ICU admission after EL.

Methods: The study was a retrospective single center registry study. Data were collected from operation registry and ICU patient data management system's database of Oulu University Hospital. All patients undergoing $E L$ and admitted to ICU directly from the operation room (OR) or from the RR between the January 2005 and May 2015 were included. Appendectomies, cholecystectomies, laparotomies due traumas or gynecological reasons and patients less than 16 years of age were excluded.

Results: There were a total of 450 patients in the study. A total of $323(71.8 \%)$ patients were admitted directly from OR to ICU and 127 (28.2\%)patients were admitted to ICU from the RR. The patients admittet directly to ICU were younger, had higher SOFA-scores and lower first portoperative day (POD) CRP-levels. There were no differences in short-term mortality between the groups, but 5-y mortality was higher in indirect ICU admission group (Table 1).

\begin{tabular}{|l|l|l|l|}
\hline & $\begin{array}{l}\text { Direct ICU } \\
\text { admission }\end{array}$ & $\begin{array}{l}\text { Indirect ICU } \\
\text { admission }\end{array}$ & P-Value \\
\hline N323 & N127 & \\
\hline Age (y) & $65[54-76]$ & 71[] & $<0.001$ \\
\hline Male Gender & $183(56.7)$ & $69(54.3)$ & 0.655 \\
\hline SOFA adm & $6[4-8]$ & $5[3-7]$ & 0.02 \\
\hline 1pod CRP & $169[100-253]$ & $238[159-302]$ & $<0.001$ \\
\hline Hospital mortality & $47(14.6)$ & $16(12.6)$ & 0.591 \\
\hline 30-d mortality & $61(18.9)$ & $22(17.3)$ & 0.700 \\
\hline 90-d mortality & $78(24.1)$ & $32(25.2)$ & 0.816 \\
\hline 5y- mortality & $54.3 \%$ & $67.7 \%$ & 0.015 \\
\hline
\end{tabular}

Conclusion: Younger patients were more often admitted directly to ICU from OR. Patients admitted to ICU from RR had higher CRP at first postoperative day. Short-term prognosis was not dependent on timing of the ICU admission in this patient cohort. Higher long-term mortality can be explained by the age distribution of the study population.

Reference(s) and grant acknowledgment(s)

1. 1. Peponis T, Bohnen JD, Sangji NF, et al. Does the emergency surgery score accurately predict outcomes in emergent laparotomies? Surgery. 2017;162(2):445-452. Accessed Feb 18, 2020. https://doi.org/10.1016/j. surg.2017.03.016.

2. 2. al, Havens JM, et. The excess morbidity and mortality of emergency general surgery. - PubMed - NCBI. . . https://www-ncbi-nlm-nih-gov.pc124152. oulu.fi:9443/pubmed/25757115. Accessed Feb 16, 2020.

3. 3. Scott JW, Olufajo OA, Brat GA, et al. Use of national burden to define operative emergency general surgery. JAMA Surg. 2016;151(6):e160480. Accessed Mar 10, 2020. https://doi.org/10.1001/jamasurg.2016.0480.

4. 4. al, Sharoky CE, et. Outcomes of hospitalized patients undergoing emergency general surgery remote from admission. - PubMed - NCBI. https://www-ncbi-nlm-nih-gov.pc124152.oulu.fi:9443/pubmed/28689604. Accessed Feb 16, 2020

5. 5. Vester-Andersen M, Lundstrøm LH, Møller MH, Waldau T, Rosenberg J, Møller AM. Mortality and postoperative care pathways after emergency gastrointestinal surgery in 2904 patients: A population-based cohort study. Br J Anaesth. 2014;112(5):860-870. Accessed Mar 13, 2020. https://doi. org/10.1093/bja/aet487. 


\section{7}

Trained ICU doulas can provide early psychological support to the critically ill patients

L. Karnatovskaia'; K. Varga ${ }^{2}$; P. Schulte ${ }^{3}$; G. Ognjen ${ }^{1}$; K. Philbrick

${ }^{1}$ Division of pulmonary and critical care, Mayo Clinic, Rochester, United States of America; ${ }^{2}$ Affective psychology department, Eötvös Loránd University, Budapest, Hungary; ${ }^{3}$ Division of biomedical statistics and informatics, Mayo Clinic, Rochester, United States of America; ${ }^{4}$ Department of psychiatry and psychology, Mayo Clinic, Rochester, United States of America Correspondence: L. Karnatovskaia

Intensive Care Medicine Experimental 2020, 8(2): 000177

Introduction: Over a third of critical illness survivors suffer from mental health problems following hospital discharge. Memories of delusional experiences are a major risk factor. Research on the formation of fear demonstrates that if mitigating information about a traumatic event is introduced during the time of memory formation or upon its subsequent recall, the emotional experience of the memory may be modified. Given that semantic processing continues during altered states of consciousness, and that the thinking process of the critically ill is characterized by heightened suggestibility, we adapted an approach based on positive suggestions to provide early psychological support for the critically ill in parallel with medical treatment.

Objectives: We used trained ICU doulas to provide an intervention targeting the vulnerable time period when memories are being formed.

Methods: Adult patients on mechanical ventilation and/or vasopressors were recruited for this single-arm prospective study. Intervention was administered daily during the ICU stay in parallel with medical treatment. Approach consisted of three phases: informing the patient about details of their ICU treatment while providing positive reframing and pre-framing of the events in their clinical course; actively involving the patient in the treatment process once they were able to communicate; and debriefing prior to ICU dismissal to clarify potential memory distortions. As a secondary analysis, subjects were compared to a historic cohort of patients receiving standard care in the ICU using adjusted linear regression models. Interactions with mechanical ventilation assessed whether the relationship depended on ventilation status.

Results: Forty-three patients received the intervention provided by two trained ICU doulas for an average of 5 days with each session lasting an average of 20 minutes. Questionnaires were administered following ICU transfer. Compared to historical controls $(N=299)$, intervention was associated with reduced Hospital Anxiety and Depression Scale (HADS) - Depression subscale (adjusted mean difference estimate $=-1.3,95 \% \mathrm{Cl}=(-2.6,0.0), \mathrm{p}=0.049)$. In a model with ventilation interaction, intervention was associated with reduced HADS-Anxiety subscale among ventilated patients (estimate $=-2.0,95 \% \mathrm{Cl}=(-3.6$, $-0.3), p=0.019)$, but not among those without mechanical ventilation $(p=0.73)$. There was insufficient evidence to suggest an association with MoCA-Blind or IES-R subscores. These initial results suggest the intervention may be beneficial.

Conclusion: Positive therapeutic suggestion is a unique approach aimed to reduce formation of traumatic memories and to humanize critical care. It was feasible to successfully intervene on 43 critically ill patients. Testing the intervention on a wider scale with randomized treatment arms is warranted.

\section{Reference(s) and grant acknowledgment(s)}

1. Research reported in this abstract was supported by NHLBI, of the National Institutes of Health under award number $\mathrm{K} 23 \mathrm{HL} 146741-1$

\section{3}

Improving CDC definition of ventilator associated condition (VAC) A. K. I. Wong ; H. Kim²; M. Charpignon ${ }^{3}$; E. Mireles-Cabodevila ${ }^{4}$; M. Lough ${ }^{5}$; L. Carvalho6; E. Monares-Zepeda7; A. Madushani ${ }^{8}$; L. Adhikari'; L. Celi ${ }^{10}$; R. Kindle Kin $^{10}$

${ }^{1}$ Division of Pulmonary, Allergy, Critical Care, and Sleep Medicine, Emory University, Atlanta, United States of America; ${ }^{2}$ Department of biomedical engineering, Johns Hopkins University, Baltimore, United States of Amer- ica; ${ }^{3}$ Mit institute for data, systems and society, Massachusetts Institute of Technology, Massachusetts Avenue, Cambridge, MA, USA, Cambridge, United States of America; ${ }^{4}$ Respiratory institute, Cleveland Clinic - Main Campus, Cleveland, United States of America; ${ }^{5}$ Medicine - primary care and population health, Stanford Health Care, Palo Alto, United States of America; ${ }^{6}$ Cursos de imersão, Somiti - Sociedade Mineira de Terapia Intensiva, Belo Horizonte, Brazil; ${ }^{7}$ Medicina crítica por la universidad, Centro Médico ABC, Mexico City, Mexico; ${ }^{8}$ Department of infectious diseases, Boston Medical Center, Boston, United States of America; ${ }^{9}$ Philips research north america, Philips Research, Cambridge, United States of America; ${ }^{10}$ Laboratory for computational physiology, Massachusetts Institute of Technology, Cambridge, United States of America

Correspondence: A.K.I. Wong

Intensive Care Medicine Experimental 2020, 8(2): 000213

Introduction: Patients on mechanical ventilation (MV) can develop a ventilator associated event (VAE), which is associated with higher morbidity and mortality. The new Centers for Disease Control (CDC) VAE definition, updated early 2020, replaces prior surveillance criteria. [1] The most inclusive VAE definition is the ventilator associated condition (VAC), requiring at least two consecutive calendar days of MV stability followed by two consecutive calendar days of increased MV support (defined as lowest change $[\Delta]$ PEEP $\geq 3$ or $\mathrm{FiO} 2 \geq 20 \%$ maintained $>=1$ hour, hereafter $\triangle \mathrm{PEEP}$ or $\triangle \mathrm{FiO} 2)$. Although $\mathrm{VAC}$ is designed as a population-level surveillance definition, it was developed by expert consensus and has not been validated on population data.

Objectives: The aims are to evaluate VAC definition performance by clinical outcomes and to optimize the definition in prediction of clinical outcomes when applying different $\triangle \mathrm{PEEP}, \triangle \mathrm{FiO} 2$, and time-window combinations.

Methods: We utilized the multi-center eICU Collaborative Research Database [2-4][5] Inclusion criteria: ICU stay $\geq 4$ days, MV $\geq 4$ days, with $\mathrm{PEEP}$ and $\mathrm{FiO} 2$ data availability. Exclusion criteria: age $<18$ years. $\triangle$ PEEP was examined in a range of 1 to 7 and $\Delta \mathrm{FiO} 2$ was examined from $10 \%$ to $60 \%$ above stability baseline. The time-windows for stability and deterioration were varied by calendar days [CDC definition] and rolling 24-hour time windows from the start of MV. Within these time-windows, patients with VAC and without VAC were compared by in-hospital mortality log odds ratios.

Results: From a total of 200,859 patients in the elCU database, we identified 8,230 patients who met our inclusion criteria (Table 1).

For patients with VAC, the mortality log odds ratio heatmap by calendar day (Figure $1 \mathrm{a}$ ) stratifies at $\triangle \mathrm{PEEP} \geq 4$ and $\triangle \mathrm{PEEP} \geq 6$. Increasing the $\triangle \mathrm{FiO} 2$ criteria increases mortality log odds at the expense of sensitivity. Heatmap color gradations suggest greater increases of $\triangle P E E P$ and $\triangle \mathrm{FiO} 2$ clusters mortality into mild, moderate, and severe VAC. Similar trends are seen in 24-hour time-windows from the start of MV to determine stability and deterioration periods in Figure $1 \mathrm{~b}$. Unlike calendar day, the 24-hour time-window allows equitable comparison of MV periods between all patients.

Conclusion: This data-driven approach validates CDC VAC surveillance criteria in a publicly available ICU dataset. Furthermore, data analysis shows that the VAC definition can be improved to differentiate mild moderate, and severe $\mathrm{VAC}$ by redefining time-windows, $\triangle \mathrm{PEEP}$, and $\Delta \mathrm{FiO} 2$.

\section{Reference(s) and grant acknowledgment(s)}

1. Goldberger AL, Amaral LAN, Glass L, et al (2000) PhysioBank, PhysioToolkit, and PhysioNet. Circulation 101

2. Pollard TJ, Johnson AEW, Raffa JD, et al (2018) The elCU Collaborative

Research Database, a freely available multi-center database for critical care research. Sci Data 5:180178

3. (2019) elCU Collaborative Research Database v2.0. https://physionet.org/ content/eicu-crd/2.0/. Accessed 15 Feb 2020

4. Rello J, Ramírez-Estrada S, Romero A, et al (2019) Factors associated with ventilator-associated events: an international multicenter prospective cohort study. Eur J Clin Microbiol Infect Dis 38:1693-1699

5. LAC is funded by the NIH through the grant R01 EB017205.

6. AIW is supported by NIGMS 2T32GM095442. 


\section{6}

Vancomycin trough concentration was associated with mortality in critically ill patients

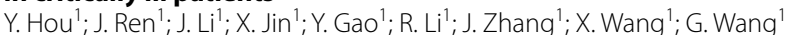

${ }^{1}$ Department of critical care medicine, the Second Affiliated Hospital of Xi'an Jiaotong University, Xi'an, China

Correspondence: G. Wang

Intensive Care Medicine Experimental 2020, 8(2): 000216

Introduction: A series of previous guidelines recommended vancomycin trough concentration (VTC) should be maintained at $10-20 \mathrm{mg} / \mathrm{L}$ to avoid drug resistance and $15-20 \mathrm{mg} / \mathrm{L}$ to improve the clinical outcomes of patients with severe infections. However, the latest consensus suggested that VTCs with a target of $15-20 \mathrm{mg} / \mathrm{L}$ should no longer be recommended based on existing data of efficacy and nephrotoxicity in patients with serious MRSA infections. To date, the optimal target of VTC for critically ill patients who receive vancomycin remains unclear considering limited evidence.

Objectives: The aim of this study was to explore the association between serum VTC and mortality in intensive care unit (ICU) settings. Methods: This multicenter, retrospective cohort study included 7,975 patients from 335 different ICUs at 208 hospitals in the elCU Collaborative Research Database. Serum VTC was continuously monitored, and mean serum VTC, calculated by dividing the sum of all collected VTCs by the frequency of VTC monitoring, was investigated as a continuous and categorical variable. Patents with different mean serum VTCs were classified into four groups: $<10 \mathrm{mg} / \mathrm{L}, 10-15 \mathrm{mg} / \mathrm{L}, 15-20 \mathrm{mg} / \mathrm{L}$, and $>20 \mathrm{mg} / \mathrm{L}$. Multivariable logistic regression, interaction, and subgroup analyses were performed to investigate the relationship of mean VTC with hospital and ICU mortalities.

Results: Hospital mortality rates were $13.7 \%, 15.1 \%, 19.4 \%$, and $25.8 \%$ and ICU mortality rates were $7.8 \%, 8.8 \%, 12.1 \%$, and $17.1 \%$, respectively, in the four groups. After adjusting for age, sex, ethnicity, body mass index, Acute Physiology and Chronic Health Evaluation IV (APACHE IV) score, the use of ventilation, dialysis, and vasopressor, and diagnoses at ICU admission, mean VTC, as a continuous variable, was positively correlated with hospital (odds ratio [OR], 1.032, 95\% confidence interval [Cl], 1.024-1.041) and ICU (OR, 1.037, 95\% $\mathrm{Cl}, 1.028-1.047)$ mortalities. Moreover, as a categorical variable, a mean VTC of $10-15 \mathrm{mg} / \mathrm{L}$ failed to reduce hospital (OR, $0.973,95 \% \mathrm{Cl}$, 0.802-1.180) and ICU (OR, 1.002, 95\% Cl, 0.785-1.279) mortalities, and mean VTCs of $15-20 \mathrm{mg} / \mathrm{L}$ and $>20 \mathrm{mg} / \mathrm{L}$ were significantly associated with higher hospital (OR, 1.232, 95\% Cl, 1.015-1.496; OR, 1.747, 95\% Cl, 1.437-2.123) and ICU (OR, 1.301, 95\% Cl, 1.021-1.658; OR, 1.858, 95\% $\mathrm{Cl}, 1.459-2.368)$ mortalities than mean VTC $<10 \mathrm{mg} / \mathrm{L}$. The interaction analysis had no statistical significance, and similar results persisted in the patients with different APACHE IV scores.

Conclusion: An increasing serum VTC was associated with incremental hospital and ICU mortalities in critically ill patients. Therefore, our results do not support the use of serum VTC as a monitoring indicator for vancomycin regimens in critically ill patients, and the necessity of vancomycin therapeutic drug monitoring based on VTC needs to be investigated in future prospective studies.

\section{Reference(s) and grant acknowledgment(s)}

1. Pollard TJ, Johnson AEW. The elCU Collaborative Research Database, a freely available multi-center database for critical care research. 2018; 5: 180178.

2. Rybak MJ, Le J, Lodise TP, et al. Therapeutic monitoring of vancomycin for serious methicillin-resistant Staphylococcus aureus infections: A revised consensus guideline and review by the American Society of Health-System Pharmacists, the Infectious Diseases Society of America, the Pediatric Infectious Diseases Society, and the Society of Infectious Diseases Pharmacists. American journal of health-system pharmacy : AJHP : official journal of the American Society of Health-System Pharmacists 2020.

3. Rybak M, Lomaestro B, Rotschafer JC, et al. Therapeutic monitoring of vancomycin in adult patients: a consensus review of the American Society of Health-System Pharmacists, the Infectious Diseases Society of America, and the Society of Infectious Diseases Pharmacists. American journal of health-system pharmacy: AJHP : official journal of the American Society of Health-System Pharmacists 2009; 66(1): 82-98.
4. We appreciate the researchers at the MIT Laboratory for Computational Physiology and collaborating research groups to keep eICU-CRD databases available.

\section{8}

Impact of spontaneous respiratory rate variability on clinical outcomes in critically ill patients

Y. Gao ${ }^{1}$; X. Jin ${ }^{1}$; R. Li ${ }^{1}$; J. Li ' ; J. Zhang ${ }^{1}$; X. Wang ${ }^{1}$; G. Wang ${ }^{1}$

${ }^{1}$ Department of critical care medicine, the Second Affiliated Hospital of Xi'an Jiaotong University, Xi'an, China

Correspondence: $G$. Wang

Intensive Care Medicine Experimental 2020, 8(2): 000218

Introduction: Previous studies have explored several different approaches to assess breathing changes and to evaluate their prognostic value. However, the relationship between spontaneous respiratory rate variability (RRV) using the coefficient of variation (CV) and mortality in intensive care units (ICU) patients remains to be unclear.

Objectives: To investigate the association between spontaneous RRV and clinical outcomes in ICU patients.

Methods: Adult patients having spontaneous RR records during the first 24 hours after ICU admission with an ICU length of stay (LOS) $\geq 1$ day were enrolled from elCU Collaborative Research Database. The CV of RR was calculated as standard deviation/mean $\times 100 \%$. Then patients were divided into low RRV group (CV of RR $\leq 20 \%$ ) and high RRV group (CV of RR $>20 \%$ ). The outcomes included ICU mortality, hospital mortality, and LOS in ICU. Logistic regression was performed to investigate the relationship between spontaneous RRV and outcomes. External validation was also conducted in patients from Multiparameter Intelligent Monitoring in Intensive Care II database.

Results: Based on the CV of RR results, 36,880 patients were divided into two groups: low RRV group $(n=24490)$ and high RRV group $(n=12390)$. The rate of ICU and in-hospital mortality, and long ICU LOS were found significantly higher in low RRV group than in high RRV group $(2.7 \%$ vs. $2.3 \%, P=0.034 ; 5.7 \%$ vs. $5.1 \%, P=0.018 ; 5.6 \%$ vs. $4.8 \%$, $P=0.001$; respectively). Multivariate regression analysis showed that compared with high RRV, patients with a low RRV had a higher ICU mortality (odds ratio [OR]: 1.225, 95\% confidence interval [Cl]: $1.056-$ 1.422), higher in-hospital mortality (OR: 1.155, 95\% Cl: 1.041-1.282), and a longer ICU LOS (OR: 1.199, 95\% Cl: 1.084-1.327) after adjusting for covariates. Furthermore, external validation conducted in 13,506 patients revealed that low RRV showed an OR of $1.329(P<0.001)$ for ICU mortality, $1.267(P<0.001)$ for in-hospital mortality, and 1.357 $(P<0.001)$ for long ICU LOS. In addition, the Cox proportional hazards models also confirmed the significantly association between spontaneous RRV and 90-day (hazard ratio [HR]: 1.295, 95\% confidence interval [Cl]: 1.165-1.439), 180-day (HR: 1.316, 95\% Cl: 1.195-1.450), and 1-year mortality (HR: 1.300, 95\% Cl: 1.191-1.418).

Conclusion: The low RRV may serve as an independent risk factor for ICU mortality, hospital mortality and LOS in ICU in critically ill patients. To our knowledge, this is the first attempt to measure the spontaneous RRV by CV based on continuously monitored records and confirm its prognostic value in two large multicenter ICU database.

\section{1}

Effect of admission serum chloride level on the prognosis of critically ill patients: a multicenter retrospective cohort study

X. Jin ${ }^{1}$; R. Li ${ }^{1}$; J. Li $\mathrm{Li}^{1}$; J. Zhang ${ }^{1}$; Y. Gao ${ }^{1}$; J. Ren ${ }^{1}$; X. Wang ${ }^{1}$; G. Wang ${ }^{1}$

${ }^{1}$ Department of critical care medicine, the Second Affiliated Hospital of Xi'an Jiaotong University, Xi'an, China

Correspondence: $G$. Wang

Intensive Care Medicine Experimental 2020, 8(2): 000231

Introduction: Critically ill patients are vulnerable to the alteration of acid-base homeostasis and plasma electroneutrality, which is profoundly influenced by the serum chloride level. Abnormally low or high serum chloride levels were associated with adverse outcomes in intensive care unit (ICU) settings. However, the serum chloride levels of most patients in ICU are within a relatively normal range, and 
among those patients, the prognostic implication of serum chloride levels has scarcely been assessed.

Objectives: To analyze the relation of admission serum chloride levels with ICU mortality.

Methods: The multicenter retrospective cohort study recruited 59,807 patients from the elCU Collaborative Research Database (eICU-CRD), which includes data from 335 distinct ICUs throughout the continental United States between 2014 and 2015. The clinical records of adult patients with a single hospital admission and serum chloride level, measured during the initial 24 hours of first ICU stay, between $96 \mathrm{mEq} / \mathrm{L}$ and $111 \mathrm{mEq} / \mathrm{L}$ were analyzed in the present study. Restricted cubic splines were used to visualize the relationship between serum chloride levels and ICU mortality. Patients were categorized into low $(<104 \mathrm{mEq} / \mathrm{L})$ or high $(\geq 104 \mathrm{mEq} / \mathrm{L})$ chloride groups. And multivariable logistic regression models were further used to explore the association between serum chloride levels and ICU mortality. We also performed an external validation in the Multiparameter Intelligent Monitoring in Intensive Care II Database.

Results: The multivariable logistic regression models revealed that for every unit increase in serum chloride level, there was an $8 \%$ decline in mortality [odds ratio (OR) $0.92,95 \%$ confidence interval (CI) 0.90 0.93]. And restricted cubic splines revealed that the risk of ICU mortality increased rapidly with serum chloride level decreased to lower than $104 \mathrm{mEq} / \mathrm{L}$. In comparison to those with serum chloride levels $\geq 104 \mathrm{mEq} / \mathrm{L}$, patients with serum chloride level $<104 \mathrm{mEq} / \mathrm{L}$ had a higher risk of mortality (OR $1.64,95 \% \mathrm{Cl} 1.49-1.81)$. In external validation cohort with 16,543 patients, we found a consistent result that patients in the low chloride group also had an increased risk of ICU mortality (OR 2.16, 95\% Cl 1.82-2.56).

Conclusion: Our study revealed that, within a relatively normal range, the admission serum chloride level is still independently and inversely associated with ICU mortality. And a serum chloride level below $104 \mathrm{mEq} / \mathrm{L}$ may increase the mortality risk in critically ill patients, which may facilitate early risk stratification and improve fluid management for critically ill patients in the future.

\section{2}

Serum lactate dehydrogenase may serve as a risk factor for prognosis of critically ill patients

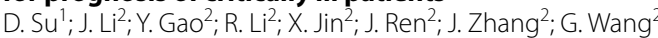

${ }^{1}$ Department of cardiology, the Second Affiliated Hospital of Xi'an Jiaotong University, Xi'an, China; ${ }^{2}$ Department of critical care medicine, the Second Affiliated Hospital of Xi'an Jiaotong University, Xi'an, China

Correspondence: G. Wang

Intensive Care Medicine Experimental 2020, 8(2): 000232

Introduction: Lactate dehydrogenase (LDH), widely synthesized in muscle, liver and red blood cells, is one of the key enzymes in the glycolytic pathway. Although serum LDH is correlated with the outcomes of septic patients, whether serum LDH impacts on mortality in unselected ICU patients remains unclear.

Objectives: To investigate the potential relationship between serum LDH levels and ICU and hospital mortalities.

Methods: Patients with serum LDH measurements during the initial $24 \mathrm{~h}$ of first ICU stay from 335 ICUs within 208 hospitals in elCU Collaborative Research Database (eICU-CRD) were included in this retrospective observational cohort study. Patients were divided into four groups according to quartiles of the serum LDH levels. The associations of serum LDH with ICU and hospital mortalities were assessed using univariable and multivariable logistic regression analyses. The covariates included Acute Physiology and Chronic Health Evaluation IV (APACHE IV) score, demographics, treatments performed between admission and LDH tested, and diseases diagnosed during the first $24 \mathrm{~h}$ of ICU stay. Interaction and subgroup analyses were performed for those patients who had different levels of APACHE IV score.

Results: The overall cohort comprised 1,981 patients. ICU and hospital mortalities in the four groups were $7.33 \%, 10.10 \%, 14.86 \%$ and $23.34 \%, P<0.001 ; 11.0 \%, 15.56 \%, 22.09 \%$, and $30.99 \%, P<0.001$; respectively. Univariable logistic regression analysis showed that serum LDH level was significantly associated with mortalities. Using the Q1 (lowest quartile) as a reference, the odds ratios (95\% confidence intervals) with ICU and hospital mortalities of Q2, Q3 and Q4 were 1.420 (0.907-2.222), 2.206 (1.450-3.356), and 3.848 (2.585-5.728) and 1.491 (1.027-2.164), 2.294 (1.612-3.266), and 3.633 (2.584-5.108), respectively. After further adjustment for all covariates, the influence of serum LDH on ICU and hospital mortalities were similar (ICU mortality, Q2 vs. Q1: 1.019 [0.606-1.713]; Q3 vs. Q1: 1.676 [1.037-2.710]; Q4 vs. Q1: 1.747 [1.086-2.809]; hospital mortality, Q2 vs. Q1: 1.193 [0.777-1.832]; Q3 vs. Q1: 1.931 [1.290-2.889]; Q4 vs. Q1: 2.066 [1.3783.098]). In addition, a linear association between serum LDH quartiles and ICU and hospital mortalities (ICU: 1.243 [1.075-1.438]; hospital: 1.300 [1.148-1.473]) were found. When stratified by APACHE IV score < 61 (975 patients) and $\geq 61$ (1006 patients), no significant interaction was observed and similar results persisted in the patients with different APACHE IV scores.

Conclusion: The level of serum LDH may aid in the early identification of critically ill patients for higher risks of ICU and hospital mortalities.

\section{4}

\section{Serum albumin level may serve as a predictor of all-cause} mortality in critically ill patients

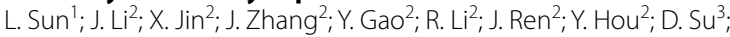

X. Wang ${ }^{2} ;$ G. Wang ${ }^{2}$

'Department of ultrasound, the Second Affiliated Hospital of Xi'an Jiaotong University, Xi'an, China; ${ }^{2}$ Department of critical care medicine, the Second Affiliated Hospital of Xi'an Jiaotong University, Xi'an, China; ${ }^{3}$ Department of cardiology, the Second Affiliated Hospital of Xi'an Jiaotong University, Xi'an, China

Correspondence: G. Wang

Intensive Care Medicine Experimental 2020, 8(2): 000234

Introduction: Albumin plays an essential role in the maintenance of colloid osmotic pressure and is involved in several physiological and pathological processes, such as keeping vascular permeability, binding and transporting numerous endogenous and exogenous compounds, regulating of acid-base balance, scavenging free radical, and inhibiting platelet function as well as antithrombotic effect. An abnormally low serum albumin level (SAL) was common in intensive care unit (ICU) and was related to adverse outcomes. However, the optimal SAL was inconsistent across different studies.Albumin plays an essential role in the maintenance of colloid osmotic pressure and is involved in several physiological and pathological processes, such as keeping vascular permeability, binding and transporting numerous endogenous and exogenous compounds, regulating of acid-base balance, scavenging free radical, and inhibiting platelet function as well as antithrombotic effect. An abnormally low serum albumin level (SAL) was common in intensive care unit (ICU) and was related to adverse outcomes. However, the optimal SAL was inconsistent across different studies.

Objectives: The study was aimed to assess the association of SAL with hospital and ICU mortalities in critically ill patients.

Methods: This retrospective multicenter cohort study includes 36,448 patients extracted from the elCU Collaborative Research Database (elCU-CRD) from 335 distinct ICUs throughout the continental United States. Patients with single hospital admission and having albumin records in the initial $24 \mathrm{~h}$ of the first ICU admission in elCU-CRD database were included. The outcomes of this study were ICU and hospital mortalities. Restricted cubic splines and logistic regression were performed to investigate the relationship between SAL and mortalities.

Results: The restrictive cubic splines revealed a rapid increase of ICU mortality when SAL declined to around $3.5 \mathrm{~g} / \mathrm{dl}$. Thus, 36,448 ICU patients were divided into two groups according to the $S A L \leq$ and $>3.5 \mathrm{~g} / \mathrm{dl}$. Patients with SAL $\leq 3.5 \mathrm{~g} / \mathrm{dl}(\mathrm{n}=28,972)$ had higher ICU and hospital mortalities than those with SAL $>3.5 \mathrm{~g} / \mathrm{dl}(\mathrm{n}=7476$; $9.8 \%$ vs $4.2 \%, P<0.001$ and $14.7 \%$ vs $5.9 \%, P<0.001$, respectively). After adjusted for demographic factors, Acute Physiology and Chronic Health Evaluation IV score, treatments before admission SAL examination and primary diagnoses, logistic regression models showed that $\mathrm{SAL} \leq 3.5 \mathrm{~g} / \mathrm{dl}$ was independently correlated with higher risks of ICU and hospital mortalities [odds ratio (OR): $1.161,95 \%$ confidence interval $(\mathrm{Cl}): 1.012-1.331, P=0.033$ and OR: $1.415,95 \% \mathrm{Cl}: 1.259-1.590, P$ 
$<0.001$, respectively]. Furthermore, the association between $S A L$, as a continuous variable, and mortalities in patients with $\mathrm{SAL} \leq$ or $>$ $3.5 \mathrm{~g} / \mathrm{dl}$ were assessed, respectively. The results showed that SAL was inversely associated with ICU and hospital mortalities (OR: 0.976, 95\% Cl: 0.968-0.984, $P<0.001$; OR: 0.958, 95\% Cl: 0.952-0.965,P<0.001, respectively) in the $S A L \leq 3.5 \mathrm{~g} / \mathrm{dl}$ group, but the associations were not found in the $S A L>3.5 \mathrm{~g} / \mathrm{dl}$ group.

Conclusion: Patients with SAL $\leq 3.5 \mathrm{~g} / \mathrm{dl}$ had higher risks of ICU and hospital mortalities than those with $\mathrm{SAL}>3.5 \mathrm{~g} / \mathrm{dl}$. Among patients with $\mathrm{SAL} \leq 3.5 \mathrm{~g} / \mathrm{dl}$, increasing SAL was associated with an incremental improvement of survival in critically ill patients. Therefore, a SAL $\leq 3.5 \mathrm{~g} / \mathrm{dl}$ may serve as an optimal therapeutic target for exogenous albumin infusion in ICU.

\section{6}

No impact of weather conditions on the outcome of intensive care unit patients

R. R. Bruno ; B. Wernly ${ }^{2}$; M. Masyuk'; J. M. Muessig ${ }^{1}$; R. Schiffner ${ }^{3}$; L. Baez ${ }^{4}$; P. C. Schulze; M. Franz ; M. Kelm ${ }^{1}$; C. Jung

${ }^{1}$ Cardiology, Pulmonary Diseases, Vascular Medicine, Universität Düsseldorf, Düsseldorf, Germany; ${ }^{2}$ Cardiology, Paracelsus Medical University, Salzburg, Austria; ${ }^{3}$ Orthopaedic department, Jena University Hospital, Jena, Germany; ${ }^{4}$ Department of internal medicine i, Jena University Hospital, Jena, Germany

Correspondence: R.R. Bruno

Intensive Care Medicine Experimental 2020, 8(2): 000246

Introduction: Global warming leads to increased exposure of humankind to meteorological variation, including short-term weather changes. Weather conditions involve changes in temperature, heat and cold, in air pressure and in air humidity. Every single condition influences the incidence and mortality of different diseases such as myocardial infarction and stroke.

Objectives: This study investigated the impact of weather conditions on short- and long-term mortality of critically ill patients admitted to an Intensive Care Unit (ICU).

Methods: A total of 4321 medical patients (66 14 years, 2638 male) admitted to a German ICU over five years were retrospectively investigated. Meteorological information (mean, maximum and minimum air temperature, air pressure and humidity) for the same period was retrieved. The influence of absolute weather parameters, different seasons, sudden weather changes (delta $24 \mathrm{~h}$ ) including "warm" and "cold" spells on ICU- and long-term mortality was analyzed. Significant results $(p<0.05)$ were adjusted for SAPS-2 scores. Additionally, subgroups of patients older than 75 years, patients with myocardial infarction and with pneumonia were investigated.

Results: After correction for SAPS-2, no impact of meteorological conditions on mortality was found. Different seasons, sudden weather changes, "warm spells" or "cold spells" did not affect the outcome. In a subgroup analysis, none of the subgroups demonstrated increased mortality during the studied weather conditions. Additionally, no difference between males and females could be found.

Conclusion: Sudden weather changes and seasonal variations have no impact on short- and long-term mortality in critically ill patients.

\section{3}

Influence of serum anion gap on mortality in critically ill patients: a multicenter cohort study

R. Li'; J. Li'; Y. Gao ${ }^{1}$; X. Jin¹; J. Ren ${ }^{1}$; J. Zhang ${ }^{1}$; X. Wang ${ }^{1}$; G. Wang

${ }^{1}$ Department of critical care medicine, the Second Affiliated Hospital

of Xi'an Jiaotong University, Xi'an, China

Correspondence: G. Wang

Intensive Care Medicine Experimental 2020, 8(2): 000263

Introduction: Anion gap (AG), which is the difference between unmeasured anions and unmeasured cations, has been used to evaluate acid-base statues and guide the management of intensive care units (ICUs) patients. However, there were controversies in the relationship between AG and mortality.
Objectives: To evaluate the association between AG and ICU mortality in a large sample of ICU patients.

Methods: We retrospectively enrolled adult patients with complete serum sodium, potassium, chloride, and bicarbonate in the first 24 hours of initial ICU stay from 335 ICUs at 208 hospitals across the United States in elCU Collaborative Research Database. AG and corrected AG by serum albumin (CAG) were calculated as [sodium]+[potassium]-[chloride]-[bicarbonate] and AG+2.5(4.4-albumin). The ICU mortalities in patients with different deciles of admission AG or CAG were assessed, respectively. Multivariable logistic regression models were applied to investigate the association between $A G$ or CAG, as continuous and dichotomous variable, and ICU mortality. Interaction analysis between AG or CAG and acute physiology and chronic health evaluation (APACHE) IV score was assessed for the association with ICU mortality.

Results: A total of 15,927 patients with AG $(10,442$ patients with CAG) entered into the final cohort. After adjustment for demographics, APACHE IV score, lactate, and diseases diagnosed during the first $24 \mathrm{~h}$ of ICU stay, elevated admission serum AG or CAG, as continuous variable, were related with increased ICU mortality (odds ratios [95\% confidence intervals]: AG, 1.024 [1.011-1.037); CAG, 1.017 [1.0021.032]). Furthermore, the ICU mortality was higher in patients with the highest three deciles of admission $A G(>16.90 \mathrm{mEq} / \mathrm{L}, 18.9 \%$ vs. $9.8 \%, P<0.001)$ or $\mathrm{CAG}(>21.45 \mathrm{mEq} / \mathrm{L}, 22.7 \%$ vs. $11.0 \%, P<0.001)$ than those with the lowest seven deciles of admission $A G(\leq 16.90 \mathrm{mEq} / \mathrm{L})$ or cAG ( $\leq 21.45 \mathrm{mEq} / \mathrm{L})$. In multivariable models, $A G>16.90 \mathrm{mEq} / \mathrm{L}$ or CAG $>21.45 \mathrm{mEq} / \mathrm{L}$ were associated with higher risks of ICU mortality (AG: 1.027 [1.077-1.354]; cAG: 1.171 [1.023-1.341]) compared with AG $\leq 16.90 \mathrm{mEq} / \mathrm{L}$ or $\mathrm{CAG} \leq 21.45 \mathrm{mEq} / \mathrm{L}$. Interaction analyses showed that the association between AG $(P$ Interaction $=0.226)$ or CAG ( $P$ Interaction $=0.067$ ) and ICU mortality could not be modified by APACHE IV score.

Conclusion: Elevated admission serum AG or CAG might be an early risk factor for ICU mortality.

\section{5}

Association between circadian variation of heart rate and mortality among critically ill patients

J. Zhang ${ }^{1}$; J. Li' ${ }^{\text {; R. R. Li }}{ }^{1}$; X. Jin ${ }^{1}$; Y. Gao ${ }^{1}$; G. Wang ${ }^{1}$

${ }^{1}$ Department of critical care medicine, the Second Affiliated Hospital of Xi'an Jiaotong University, Xi'an, China

Correspondence: G. Wang

Intensive Care Medicine Experimental 2020, 8(2): 000275

Introduction: Heart rate (HR) variations, such as HR variability, HR turbulence, resting HR, and nighttime mean $H R$ have been recognized as independent predictors of mortality. However, it is unclear whether the circadian changes in HR influence the risk of death in the intensive care unit (ICU).

Objectives: To evaluate the relationship between the circadian variation of HR and the mortality among critically ill patients.

Methods: The records of 4760 patients were extracted from the Multiparameter Intelligent Monitoring in Intensive Care II (MIMIC II) database. The circadian variation in HR was evaluated as nighttime mean HR/daytime mean HR. Participants were divided into two groups according to the median score of the circadian variation in HR: group $A$ $(\leq 0.98)$ and group B (>0.98). The endpoints were ICU, hospital, 30-day, and 1-year mortality. Multivariable logistic regression models and Cox proportional hazards models were used to investigate the relationship between circadian HR variation and mortality in ICU. Sensitivity analyses were also conducted in different Sequential Organ Failure Assessment (SOFA) scores subgroups.

Results: Patients in group $B(n=2471)$ had higher mortality than those in group $A(n=2289)$. After multivariable adjustments, the associations of circadian HR variation with ICU mortality (odds ratio [OR], 1.419; $95 \%$ confidence interval $[\mathrm{Cl}], 1.097-1.835 ; P=0.008)$, hospital mortality $(\mathrm{OR}, 1.394 ; 95 \% \mathrm{Cl}, 1.122-1.747 ; P=0.004), 30$-day mortality (hazard ratio $[\mathrm{HR}], 1.283 ; 95 \% \mathrm{Cl}, 1.085-1.518 ; P=0.004)$, and 1 -year mortality $(\mathrm{HR}, 1.215 ; 95 \% \mathrm{Cl}, 1.064-1.387 ; P=0.004)$ were statistically significant Furthermore, the association became more statistically remarkable 
in patients with higher SOFA scores ( $\geq 6$ subgroup) in the sensitivity analysis.

Conclusion: The circadian HR variation may predict short-term and long-term mortality in critically ill patients, especially in patients with higher SOFA scores.

\section{6}

The search for optimal range of platelets count in critically ill patients: an observational study from eICU databases

J. Zhang ${ }^{1}$; X. Jin ${ }^{1}$; J. Li ${ }^{1}$; R. Li ; J. Ren ${ }^{1}$; X. Wang ${ }^{1}$; G. Wang

'Department of critical care medicine, the Second Affiliated Hospital of Xi'an Jiaotong University, Xi'an, China

Correspondence: G. Wang

Intensive Care Medicine Experimental 2020, 8(2): 000276

Introduction: Platelets are involved in coagulation system, immune system, inflammation and stress responses. Abnormal platelet counts have been linked to increased mortality in critically ill patients. However, the optimal platelets count target remains unclear.

Objectives: The study is designed to explore the relatively optimal range of platelets count in critically ill patients with real world data; Furthermore, the relationship between the platelets count and mortality in ICU was also studied.

Methods: This is a multicenter and retrospective cohort study using the elCU Collaborative Research Database (elCU-CRD) with authorized access. The restricted cubic splines were used to explore the crude relationship between platelets count and mortality. We derived an optimal range of platelet counts and analyzed the association between the optimal range of platelet counts and mortality. Multivariable logistic regression models were used to further explore the association between the level of platelet counts and mortality in intensive care unit (ICU). The sensitivity analyses were performed in patients with sepsis or not.

Results: In total, 49,875 adult patients with platelet counts within 24 hours of admission to the ICU were included. According to the restricted cubic splines, the optimal range of platelets count was $125-185 \times 109 / L$ (reference group). Patients were divided into lower platelet group $(<125 \times 109 / \mathrm{L})$, higher group $(>185 \times 109 / \mathrm{L})$ and reference group (125-185 $\times 109 / \mathrm{L})$. Compared with those in the reference group, patients with lower platelet counts and higher platelet counts are associated with a higher hospital mortality ([odds ratio (OR) 1.226, 95\% confidence interval (Cl) $1.116-1.347]$; OR $1.276,95 \%$ Cl $1.278-$ 1.381; respectively) and ICU mortality (OR $1.260,95 \% \mathrm{Cl} 1.127-1.409$; OR 1.284, 95\% Cl 1.166-1.413; respectively) after adjustment for a series of variables. The results of subgroup analyses are consistent with overall population.

Conclusion: The optimal range of platelet counts was $125-185 \times 109 / \mathrm{L}$ in critically ill patients. platelet counts lower than $125 \times 109 / \mathrm{L}$ or higher than $185 \times 109 / \mathrm{L}$ may increase the mortality in ICU.

\section{2}

A 16 year review of patients admitted to an adult Intensive Care Unit (ICU) following drug overdose at a district general hospital in Surrey, England

A. Nechowska' ${ }^{1}$ T. Samuels ${ }^{1}$; S. Ranjan ${ }^{1}$

${ }^{1}$ Critical care, East Surrey Hospital, Redhill, United Kingdom

Correspondence: A. Nechowska

Intensive Care Medicine Experimental 2020, 8(2): 000322

Introduction: An annual review of suicides in the UK (Office for National Statistics) for 2018 showed a significantly higher rate of deaths by suicide than the previous year [1]. This was the first reported increase since 2013. Deliberate and accidental drug overdose cases that result in critical care admission often require a period of artificial ventilation and may also require renal replacement therapy. Identifying trends in admission could improve the ability for ICU to plan with regards to budget and resources.

Objectives: To review whether there has been a change in the number of patients admitted to a 16 -bed adult ICU during a 16 year period and whether it is possible to identify seasonal variations.
Methods: The WardWatcher database for a single 16-bed adult critical care unit was interrogated for the period January 1993 to September 2019. To account for different classification and coding, search terms containing the following were deemed suitable: overdose, selfharm, poison, suicide. Hanging and asphyxia were not included in the search. Duplicated information was removed manually. Data was analysed according to year, season and outcome (ie. death or survival to ICU discharge). There was insufficient information available to investigate method of overdose. Statistical analysis was performed using $\mathrm{R}$ version 3.6.3 (Austria-Vienna).

Results: A database of 12,671 patients was reviewed and 331 cases involving drug overdose were identified. The dataset showed a minimum of 2 and maximum of 33 overdoses per year, with a median number of 10 (IQR 5-16). The raw time series for the data demonstrates an increasing trend. Using a bias-corrected and accelerated bootstrap percentile method, we estimated the $95 \%$ confidence interval for the $\mathrm{r} 2$ value of 0.41 to be between 0.17 and 0.64 . This suggested there was a small positive association between the number of total overdoses over time.

Analysis by season suggested that the highest percentage of overdoses were admitted in Spring (annually 20th March to 20th June), accounting for $28.01 \%$ (93) of total overdose admissions. The numbers were, however, closely clustered for all four seasons.

Out of the 331 patients, 315 (95\%) survived to discharge from ICU. There was not enough data to study their course following this, such as their neurological outcomes. No trends by year or season were observed due to the small number of deaths in this period.

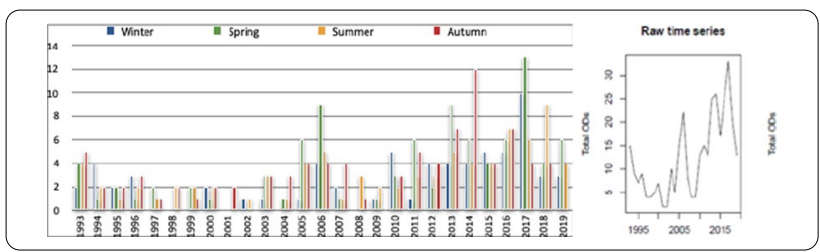

Conclusion: By reviewing a large dataset over a prolonged period of time we were able to show that an increase in suicide death rates at a national level, as reported by ONS, was not accompanied by an increase in ICU admissions at a local level. This study showed that there has been a relatively poor increase in the annual number of overdoses admitted to this particular ICU since 1993 with a median of 10 per annum.

\section{Reference(s) and grant acknowledgment(s)}

1. [1] Office for National Statistics - Suicides in the UK: 2018 registrations. 3 Sept 2019. Available at: https://www.ons.gov.uk/peoplepopulationandc ommunity/birthsdeathsandmarriages/deaths/bulletins/suicidesintheun itedkingdom/2018registrations

\section{1}

Ventricular-arterial coupling: a non-invasive parameter

for prognostic evaluation in critical cardiological patients

P. Trambaiolo ${ }^{1}$ I. Figliuzzi ${ }^{1}$; M. Salvati ${ }^{1}$; P. Bertini ${ }^{2}$; G. Ferraiuolo';

F. Guarracino ${ }^{2}$

${ }^{1} \mathrm{ICCU}$, Hospital Sandro Pertini, Roma, Italy; ${ }^{2}$ Cardiothoracic and vascular anesthesia and intensive care, Azienda Ospedaliero Universitaria Pisana, Pisa, Italy

Correspondence: P. Trambaiolo

Intensive Care Medicine Experimental 2020, 8(2): 000351

Introduction: Ventriculo-arterial coupling (VAC), is a major determinant of cardiovascular efficiency in terms of the ratio between work required for the stroke volume relative to the total work for the contraction.

Objectives: Aim of this study was to investigate the relationship between ventricular-arterial coupling (VAC) and in-hospital outcomes and to evaluate its prognostic value in critical ill patients. 
Methods: We evaluated 333 (age 66,8 $\pm 15,4$ years, male 67,3\%) consecutive patients admitted in ICCU of our hospital. Blood pressure and trans-thoracic cardiac ultrasound were collected for assessment of left ventricular elastance (Ees), arterial elastance (Ea) and VAC calculated through Chen's method. In-hospital we recorded events related with acute heart failure and study population was stratified according to events development (need for invasive and NIV, amine, IABP, CVVHD, anuria, MOF, and death).

Results: In our population 190 pts (57\%) presented in-hospital complications, and 143 (43\%) didn't. Ea and VAC were found to be significantly higher in the first group than in the latter, and a trend toward decrease for Ees was observed in the first group (Figure). VAC resulted a strong and independent predictor of in-hospital clinical outcomes [odds ratio ( $95 \%$ confidence interval): 2,016 (1.287-3,159); $\mathrm{P}=0.002$ ] even after univariate and multivariate analysis adjusted for age, dyslipidaemia, diabetes, obesity, coronary artery disease and chronic kidney disease.

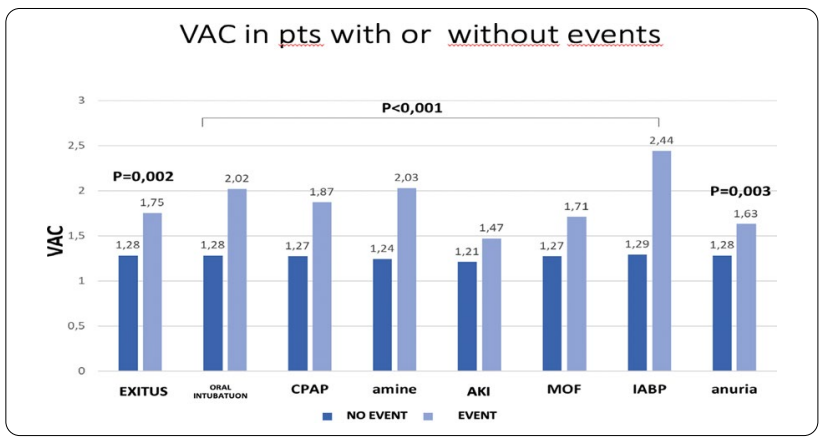

Conclusion: Assessment of VA coupling might be an additional noninvasive parameter that can be used to determine prognosis in critically ill patients. Although those encouraging results, the benefit of such an approach for understanding the pathophysiology of altered hemodynamic profiles and to guide the therapeutic strategies, has to be confirmed in larger clinical trials.

\section{6}

Post-intensive care syndrome in patients and their caregivers

J. Tejero Aranguren ${ }^{1}$; C. M. Mt. ; I. Cruz Valero' ; F. M. Purificación';

A. Carranza Pinel

'Intensive care unit, Hospital Universitario Clínico San Cecilio, Granada, Spain

Correspondence: J. Tejero Aranguren

Intensive Care Medicine Experimental 2020, 8(2): 000376

Introduction: During the first year, ICU survivors show a greater use of health services and a high percentage become chronic patients in addition to presenting high rates of hospital readmission (up to $47 \%$ ) and mortality (17\%). The most common consequences, after admission in ICU, are included in what has recently been called Post-Intensive Care Syndrome (PICS).

Objectives: To determine the frequency of appearance of the PostIntensive Care Syndrome (PICS) in patients and their main caregiver, treated in an Intensive Care Unit (ICU), 3 months after discharge.

Methods: Prospective observational study in a 14-bed ICU for 12 months. Inclusion criteria were admission $>7$ days and at least one of the following: mechanical ventilation (invasive or non-invasive)> 3 days, sepsis or shock. Patients with cognitive impairment or severe dependency prior to admission were excluded. The variables analyzed (physical, cognitive and psychoemotional), were assessed using validated scales and physical examination in the follow-up consultation The presence of overload from the main caregiver was assessed using two validated scales. Prevalence was measured as the percentage of cases of patients with PICS identified of the total number of patients discharged with risk criteria.
Results: Of the 52 patients who presented risk factors for developing PICS, 36 were discharged from the unit. Of the 25 patients who attended the follow-up consultation at 3 months, 18 had PICS. The fundamentally affected dimension was physical $55.5 \%$, followed by psycho-emotional $27.7 \%$ and cognitive $16.6 \%$. In all the cases of patients with PICS, the main relative had caregiver overload.

Conclusion: The frequency of PICS at three months after ICU discharge is at least $50 \%$ in patients with risk factors. In all cases presented by the patient, their primary caregiver is affected too. The high frequency of this syndrome would require adopting measures for it to be foreward, and follow-up as well as therapeutic approach, both for patients and for their main caregivers.

Reference(s) and grant acknowledgment(s)

1. Hill AD, Fowler RA, Pinto R, Herridge MS, Cuthbertson BH, Scales DC. Long-term out comes and healthcare utilization following critical illness-a population-based study. Crit Care 2016;20:76

2. Herridge MS, Chu LM, Matte A, Tomlinson G, Chan L, Thomas C, et al. The RECOVER program: disability risk groups and 1-year outcome after 7 or more days of mechanical ventilation. Am J Respir Crit Care Med 2016:194(7):831-44

3. The authors declare no conflict of interest

\section{8}

Venoarterial extracorporeal membrane oxygenation in refractory cardiogenic shock: a look into long-term survival

A. Marcos-Morales '; I. Martín Badía'; JL. PerezVela ${ }^{1}$; P. Pagliarani

Gil $^{1}$; R. Ashbaugh-Lavesiera'; M. Corres Peiretti ${ }^{1}$; E. Renes Carreño'.

JC. Montejo-Gonzalez

${ }^{1}$ Intensive care department, Hospital Doce de Octubre, Madrid, Spain

Correspondence: A. Marcos-Morales

Intensive Care Medicine Experimental 2020, 8(2): 000388

Introduction: Cardiogenic shock (CS) is the fatal common path of some of the most prevalent diseases in the world (1), yet its management is not straightforward. Veno-arterial extracorporeal membrane oxygenation (VA-ECMO) use as a rescue therapy for refractory cases is growing in popularity (2).

Objectives: The purpose of this study was to evaluate the different outcomes of patients with refractory CS supported with VA-ECMO, stratified by etiology, in the short-term and after 1-year follow-up.

Methods: Data was collected prospectively from 2010 to 2020, patients with CS having received VA-ECMO were reviewed retrospectively. We analysed baseline demographic variables, cause of CS, ECMO characteristics, complications and global survival (at ECMO weaning, hospital discharge, and after 1 year), using SPSS software, version 20.

Results: We included 92 patients who received 101 VA-ECMO. The median age was 54 (interquartile range IQR 42-63), 64\% were male. Causes of CS were: decompensated advanced heart failure (32\%), acute myocardial infarction (AMI) $(26 \%)$, post-surgical pulmonary thromboendarterectomy (13\%), post-cardiotomy, (10\%), acute myocarditis from infectious, toxic or metabolic causes (7\%).

Before VA-ECMO cannulation $61 \%$ of patients were supported by an intraaortic balloon pump.

A peripherally VA-ECMO was established in $91 \%$ of cases. The median length of ECMO was 186 hours (8 days, IQR 5.4 - 13 days), median ICU stay 18 days (IQR $12-33$ days).

The global survival rate during ECMO support was $68 \%$, with $43 \%$ of patients being discharged home, and all of them were alive one year later. Patients with reversible causes of cardiogenic shock (acute myocarditis, right ventricular failure following pulmonary embolism) fared better overall, with a $100 \%$ survival rate.

In $50 \%$ of patients with CS secondary to AMI (with previous normal or unknown cardiac function) a "bridge to decision" strategy was implemented. Sixty-four percent were weaned from ECMO, but only $37 \%$ of weaned patients survived. A heart transplantation was made in $83 \%$ of the survivors of this subgroup.

Patients with CS secondary to acute decompensation of previous cardiopathy were usually placed in the "bridge to transplant" category. 
Out of 32 patients, half of them died, but in those succeeding into getting a heart transplantation (59\%), global survival was of $83 \%$.

Postcardiotomy CS patients requiring VA-ECMO had a global survival of $43 \%$, the most common surgeries needing this kind of support being pulmonary thromboendarterectomy and valvular surgery with previous systolic dysfunction.

One year after hospital discharge, 39 out of the 40 surviving patients remained alive and out of the hospital.

Conclusion: Refractory CS patients are a very heterogeneous cohort. Outcomes were far better in potentially reversible causes of CS like acute myocarditis. VA-ECMO offers an effective support tool for these patients, achieving favorable results at hospital discharge and after 1 -year follow-up.

Reference(s) and grant acknowledgment(s)

1. 1. Vahdatpour C, Collins D, Goldberg S. Cardiogenic Shock. J Am Heart Assoc. 2019;8(8):e011991. https://doi.org/10.1161/jaha.119.011991

2. 2. Guglin M, Zucker MJ, Bazan VM, et al. Venoarterial ECMO for Adults: JACC Scientific Expert Panel. J Am Coll Cardiol. 2019;73(6):698-716. https://doi. org/10.1016/j.jacc.2018.11.038

\section{6}

Mechanical ventilation in octogenarians, intensive care and subsequent hospital survival in a medium sized district general hospital in the UK

M. Allsop ${ }^{1}$; M. Mazalkov²

${ }^{1}$ Peterborough City Hospital, Peterborough, United Kingdom; ${ }^{2}$ Anaesthetics, Peterborough City Hospital, Peterborough, United Kingdom

Correspondence: M. Allsop

Intensive Care Medicine Experimental 2020, 8(2): 000406

Introduction: With an increasingly aging and co-morbid population coupled with the increasing demand on NHS intensive care beds it is more important than ever to have a clear understanding of the benefit added by admission to intensive care for mechanical ventilation. In addition there is an increasing drive to provide patients with the information allowing them to make an informed decision regarding their escalation plan. Currently despite making up only $4 \%$ of the population, octogenarians account for around $10 \%$ of ICU admissions in the developed world [1]

We set out to perform a retrospective study looking at the previous 4 years of intensive care admissions for mechanical ventilation at Peterborough City Hospital. We then compared the survival of all patients $<80$ years old with the survival of patients 80 years and over. For the 80 and over age group we then compared the medical admission vs surgical admission survival outcome.

It should be noted that the intensive care department already puts a strong emphasis on requiring a good pre-morbid state prior to admission to the ICU, and all admissions are approved by an intensive care consultant. Therefore, the patients with a high frailty score deemed unlikely to benefit from mechanical ventilation, are not reported in these figures.

Objectives: To compare intensive care survival between patients younger than 80 years and those patients 80 years and over. To compare the above patient's hospital survival once they have been stepped down from intensive care.

To compare whether medical vs surgical admission in 80 and over affects survival.

Methods: Data was extracted from Intensive Care National Audit \& Research Centre (ICNARC) and is based on data exclusively from PCH.

Results: Over a 4-year period (2015-2019) 1025 patients received ventilatory support in PCH's ICU. Of these 99 (9.7\%) were $\geq 80$.

$52.5 \%$ of $>80$ survived ICU and $41.4 \%$ were discharged home $(11.1 \%$ dying in hospital post ICU). Compared with $74.4 \%$ of $<80$ 's surviving ICU and $68.3 \%$ being discharged home $(6.1 \%$ dying in hospital post $I C U)$.

Even though the vast majority of octogenarians admitted to PCH's ICU for mechanical ventilation had a very good pre-morbid status, compared to all those $<80$, they had a $21.9 \%$ lower chance of ICU survival and $27.1 \%$ lower chance of being discharged home.
Overall $91.7 \%$ of all patients $<80$ discharged from ICU were subsequently discharged from hospital. This is to be compared with only $78.8 \%$ of the $\geq 80$ cohort who are stepped down, showing they are $12.9 \%$ less likely to be discharged after an ICU admission.The data also shows a year on year increase in numbers of patients admitted for mechanical ventilation 232 patients in 2015-2016, 244 in 2016-2017, 261 in 2017-2018 and 288 patients in 2018-2019. There is also a general trend of increasing numbers of octogenarians being ventilated ie: 18 in 2015-16 and 31 in 2018-2019.

\begin{tabular}{|l|lll|}
\hline Age & Total patients & Survived ICU & Survived Hospital \\
\hline$<\mathbf{8 0}$ years & 926 & 689 & 632 \\
\hline $\mathbf{8 0}$ years & 99 & 52 & 41 \\
\hline
\end{tabular}

Results:part 2, medical Vs surgical admission for $\geq \mathbf{8 0}$ year olds between 2015-2018.

Medical patients represented $63.2 \%$ of $\geq 80$ ICU admissions for mechanical ventilation with $35 \%$ of these patients surviving to be stepped down to the ward. Of those stepped down $100 \%$ were discharged home.

Of the surgical patients a higher percentage of $77 \%$ survived to be stepped down to the ward with $67 \%$ of these being discharged home. Therefor hospital survival was $35 \%$ for medical patients $\geq 80$ and $52.4 \%$ for surgical admissions $\geq 80$.

(Patients were excluded from part 2 of this study if it wasn't clear if they were medical or surgical (16 patients). 2019 data for part 2 was not readily available at time of this project (31 patients)).

Conclusion: The data from this study, shows that overall an octogenarian with a good pre-morbid status stands a $41.4 \%$ chance of surviving the acute hospital admission. This percentage varies from some of the reported data, however in our study, we only include those who have received mechanical ventilation.

It can be speculated that should all octogenarians requiring mechanical ventilation be admitted to ICU, rather than those with a good pre-morbid status, mortality among this group of $\geq 80 \mathrm{~s}$ would be significantly increased.

\section{Reference(s) and grant acknowledgment(s)}

1. [1] Caring for the critically ill patients over 80: a narrative review. 2018. Bertrand Guidet et al. Annals of Intensive Care. Nov 2018114.

2. [2] Are we every too old? Characteristcs and outcome of octogenarians admitted to a medical intensive care unit. 2017. Johanna Maria Muessig et al. Medicine (Baltimore) 2017 Sept 96(37)

\section{1}

Is lactate variation important? Different cut-off analysis for prognostication in critically ill patients

R. Martins Fernandes ${ }^{1}$; I. Jesus Pereira'; D. Nuñez ${ }^{2}$; CC. Dias ${ }^{3}$; C. Granja ${ }^{4}$ ${ }^{1}$ Internal medicine department, University Hospital Center of Algarve, Faro, Faro, Portugal; ${ }^{2}$ Emergency and intensive care unit, University Hospital Center of Algarve, Faro, Faro, Portugal; ${ }^{3}$ Department of community medicine, information and health decision, Faculty of Medicine of Oporto, Porto, Portugal; ${ }^{4}$ Anesthesiology department, São João Universitary Hospital Center, Porto, Portugal

Correspondence: $\mathrm{R}$. Martins Fernandes

Intensive Care Medicine Experimental 2020, 8(2): 000411

Introduction: Hyperlactacidemia is a marker of organ dysfunction widely used in critically ill patients with a direct relation with tissue hypoxia, but it is difficult to draw conclusions with isolated lactate values.

The topic of lactate kinetics has been gathering increased interest and decreasing blood lactate concentrations have been associated with better outcomes.

However, controversy remains over what is the better cut-off value to use when considering prognosis. In studies that were conducted in a 24 -hour period a $10 \%$ cut-off has been proposed. A new study from 2019 proposed $19 \%$ as the ideal cut-off. 
In the present study we aimed to investigate the prognostic impact of two different values of lactate variation in the first 24 hours of critically ill patients admited to a polyvalent intensive care unit (ICU).

Methods: We conducted a retrospective investigation of a prospectively gathered database collecting subjects admitted to a single ICU from January 2014 to December 2018. Inclusion criteria stated a lactate value above $2,0 \mathrm{mmol} / \mathrm{L}$ on admission.

The maximum value of lactate concentration in the first 2 days was collected in order to calculate its variation in the first 24 hours after admission. Data on ICU mortality, ICU length of stay, days of invasive mechanical ventilation (IMV), need for vasopressors and renal replacement therapy (RRT) were also collected.

We performed the same statistical analysis for the two cut-off values: $10 \%$ and $19 \%$. The primary end-point was the association between lactate variation in the first 24 hours and ICU mortality. The secondary end-points were association between lactate variation and ICU lenght of stay, days of IMV, need of vasopressors and need of RRT.

Results: $A$ total of 674 patients (male $=409$, age (mean \pm SD) $=$ $63.4 \pm 16,3$ years) were included. The global mortality rate was $39,9 \%$. Analysis of mortality showed a statistically significant association with both cut-offs $(p<0.001)$, higher with a lower lactate variation.

A decreased lactate variation was associated with the need for vasopressors $(p=0.001$ for $10 \%$ and $19 \%)$ and RRT $(<=10 \%$ with $p=0.003$; $<=19 \%$ with $p=0.010$ ). The ICU length of stay had a statistically significant association with a lactate variation $<=10 \%(p=0.015)$ but not with $<=19 \%(p=0.057)$. We did not find a significant association between IMV days and lactate variation $(<=10 \%$ with $p=0.647$ $<=19 \%$ with $\mathrm{p}=0.312$ ).

The $10 \%$ cut-off showed a sensitivity of $71 \%(95 \% \mathrm{Cl} 67-76)$ and specificity of $54 \%(95 \% \mathrm{Cl} 48-60)$ for mortality compared to a $64 \%$ sensitivity (95\% Cl 59-68) and $62 \%$ specificity $(95 \% \mathrm{Cl} 56-67)$ for the $19 \%$ cut-off.

Conclusion: Both cut-offs were associated with ICU patients' mortality. Only the $10 \%$ cut-off was significantly associated with the ICU length of stay.

When analyzing sensitivity and specificity, we could not find a statistically significant difference between cut-offs. However, given that the $10 \%$ cut-off has a greater sensitivity we would select this value for our clinical practice.

\section{Reference(s) and grant acknowledgment(s)}

1. Masyuk M, Wernly B, Lichtenauer M, Franz M, Kabisch B, Muessig JM, et al.

Prognostic relevance of serum lactate kinetics in critically ill patients.

Intensive Care Med [Internet]. 2019;45(1):55-61. Available from: https://doi. org/10.1007/s00134-018-5475-3

2. Lee SM, Kim SE, Kim E Bin, Jeong HJ, Son YK, An WS. Lactate Clearance and Vasopressor Seem to Be Predictors for Mortality in Severe Sepsis Patients with Lactic Acidosis Supplementing Sodium Bicarbonate: A Retrospective Analysis. PLoS One. 2015;10(12):1-14

3. Vincent $\mathrm{L}$, e Silva AQ, Couto L, Taccone FS. The value of blood lactate kinetics in critically ill patients: A systematic review. Crit Care [Internet]. 2016;20(1):1-14. Available from: http://dx.doi.org/10.1186/s1305 4-016-1403-5

\section{1}

Patient and family feedback to introducing ICU doulas to provide psychological support for the critically ill

K. Johnson ; K. Philbrick'; K. Varga ${ }^{3}$; M. Mujic ${ }^{4}$; L. Karnatovskaia

${ }^{1}$ Division of pulmonary and critical care, Mayo Clinic, Rochester, United States of America; ${ }^{2}$ Psychiatry, Mayo Clinic, Rochester, United States of America; ${ }^{3}$ nstitute of psychology, Eötvös Loránd University, Budapest, Hungary; ${ }^{4}$ Anesthesia information management and analytics, Mayo Clinic, Rochester, United States of America

Correspondence: $\mathrm{K}$. Johnson

Intensive Care Medicine Experimental 2020, 8(2): 000421

Introduction: Negative psychological sequelae following an ICU admission remain a prevalent issue for critical illness survivors. Delusional memories are the biggest modifiable risk factor. Mitigating the negative character of formed memories may be possible during a critical window of initial memory consolidation when the memories are malleable. Previously, psychological support based on positive suggestions (PSBPS) was identified as a viable psychotherapeutic intervention in the ICU setting. Given that the spectrum of emotional support doulas provide is very similar to PSBPS, we trained doulas to work in the ICU setting. Introducing themselves as "members of the team", doulas provided daily PSBPS sessions throughout the patients' ICU stays.

Objectives: Acceptance of the intervention is a vital part of the intervention's success. We have therefore evaluated feedback from patients and their families regarding the intervention.

Methods: Following ICU discharge, patients and their family members who were present during the intervention were asked to provide their impressions of the intervention including open-ended and multiple choice questions.

Results: Of the 48 patients who received the intervention, all provided feedback. 40 patients recalled a team member explaining what was happening to them and 37 found this comforting. 29 recalled a team member holding their hand and 28 found this comforting. When asked what made them feel better while in the ICU, 34 recalled specific aspects with themes summarized in the table; others had no factual memories. Nine family members who had witnessed an interventional session also provided feedback. When asked what they thought about the intervention, all provided positive feedback. Family members were also asked if the doula interaction was helpful to them, and again all also responded positively. Representative themes are provided in the table.

\begin{tabular}{|l|l|}
\hline \multicolumn{1}{|l|}{ Patient Perspective } & (number of responses) \\
\hline $\begin{array}{l}\text { Things that made } \\
\text { me feel better in } \\
\text { the ICU }\end{array}$ & -Positive Interaction with team/feeling cared for (15) \\
& -Reassurance/explanations of medical course (5) \\
\hline Family Perspective (number of responses) \\
\hline $\begin{array}{l}\text { Things I liked } \\
\text { about the } \\
\text { intervention }\end{array}$ & -Relaxing/soothing/calming (3) \\
\hline & -All aspects (3) \\
\hline & -Spending time with family (1) \\
\hline $\begin{array}{l}\text { How the } \\
\text { intervention was } \\
\text { helpful to me }\end{array}$ & -Providing compassion (1) \\
\hline
\end{tabular}

Conclusion: Patients and their families welcomed ICU doulas providing psychological support during the ICU stay. Patient responses highlight the importance of personal interaction with care team members during the ICU stay. Family member responses are uniformly positive regarding the intervention.

\section{1}

The relationship between post-traumatic stress disorder, anxiety and depression among icu survivors

M. Zghidi' ; I. Ben Saida'; R. Toumi²; T. Amal'; M. Boussarsar ${ }^{2}$

${ }^{1}$ Medical intensive care unit, Farhat hached university hospital, Sousse,

Tunisia; ${ }^{2}$ Farhat hached university hospital, medical intensive care unit, Université de Sousse, Faculté de Médecine de Sousse, UR Nº LR12SP09. Heart Failure, Sousse, Tunisia

Correspondence: $M$. Zghidi

Intensive Care Medicine Experimental 2020, 8(2): 000431 
Introduction: Many mental disorders can arise after critical illness, including anxiety, depression and post traumatic stress disorder (PTSD). Those psychiatric disorders can impair the quality of life of ICU survivors.

Objectives: The study aimed to estimate the frequency PTSD, anxiety and depression among ICU survivors and to investigate the relationship between those psychiatric disorders.

Methods: It is a mixed method study conducted in a 9-bed medical ICU of Farhat teaching hospital from January 2017 to January, 31 2018 including all ICU survivors beyond 3 months after discharge. Information regarding demographic and clinical characteristics were obtained from medical records. At 3 months post-ICU discharge, the patients were contacted by phone to complete the Hospital Anxiety and Depression Scale (HADS) and Impact Event Revised Scale (IES-R). Spearman's correlation test was done to find the association between PTSD, anxiety and depression among ICU survivors.

Results: Three hundred ninety-three patients were admitted during the study period. One hundred ninety-one $(48.6 \%)$ were discharged alive, 56(29.3\%) were never successfully contacted and $21(11 \%)$ died within the 3 months' period. One hundred fourteen patients fulfilled the inclusion criteria and were enrolled in the study.

Clinical characteristics were: mean age, $56 \pm 17.8$ years. The majority of patients were males, 66(57.9\%); current alcohol consumers, 13(11.4); median Charlson comorbidity index, 1[1-2]; Respiratory distress, 79(69.3\%); median SAPS II at admission, 25[17-31]; invasive mechanical ventilation, 47(41\%); vasopressors, $13(26 \%)$; median of duration of IMV, 0[0-4]; length of stay, 6[4-10]; Median IES-R, 19[14-28]; median HADS A, 4[ 3-10]; median HADS D, 5[ 4-6]. Twenty-five (21.9\%) patients met diagnostic criteria for PTSD (IESR $\geq 37$ ). Twenty-four (21\%) patients had anxiety (HADS A>11) and only eleven (9.6\%) had depression (HADS D>11).

There was significant correlation between PTSD and anxiety $(r=0.662$; $p=0.000)$ and PTSD and depression $(r=0.473 ; p=0.000)$. There was also a statistically significant correlation between anxiety and depression $(r=0.196 ; p=0.037)$.

Conclusion: Psychiatric disorders after clinical illness are common. There was a strong and significant statistical correlation between anxiety and PTSD, PTSD and depression, and anxiety and depression in ICU survivors at 3 months post ICU discharge.

\section{3}

\section{The post-intensive care syndrome in general practice; barriers} and opportunities

JH. Vlake'; J. Van Bommel'; EJ. Wils'; M. Hobers²; P. Vlake ${ }^{3}$; D. Gommers'; M. van Genderen

${ }^{1}$ Intensive care, Franciscus Gasthuis, Rotterdam, Netherlands; ${ }^{2}$ Intensive care, Erasmus MC, Rotterdam, Netherlands, Netherlands; ${ }^{3}$ General practice, Hoed Pax Et Bonum, Vught, Netherlands

\section{Correspondence: J.H. Vlake}

Intensive Care Medicine Experimental 2020, 8(2): 000443

Introduction: A substantial number of patients treated in ICUs suffer from a loss of health-related quality of life (HRQoL) in the aftermath of their critical illness. This is due to new or worsened physical, cognitive or psychological impairments, collectively referred to as the Post-Intensive Care Syndrome (PICS). As a result ICU survivors have more consultations with their general practitioner (GP) after their ICU treatment (1). Relatives of ICU patients are also at risk of developing psychological impairments, referred to as PICS Family (PICS-F). The General Practitioner (GP) may have a pivotal role in the aftercare of ICU survivors and their relatives. However data on the perceived boundaries for GPs to become involved in PICS (-F) care is largely lacking.

Objectives: To identify perceived boundaries for GPs to become more involved in post-ICU care and identify possible targets for improvement.

Methods: 769 Dutch GPs were approached to fill out a self-composed questionnaire to assess the following questions: 1) What is the current state of knowledge regarding PICS and PICS-F 2) How often is the GP confronted with PICS-related symptoms in his practice? 3) Who is responsible for recognition and treatment of PICS in the opinion of the
GP? and 4) What is the current state of information sharing between the hospital/ICU and the GP concerning ICU treatment and is the GP satisfied with this information?

Results: $258 \mathrm{GPs}$ responded to the questionnaire. As much as $58 \%$ of these GPs was not familiar with PICS and $44 \%$ not with PICS-F prior to receiving the questionnaire, although annually 2 (median, IQR: 1-3) patients and 3 (median, IQR: 1-3) relatives of ICU patients visit their GP with impairments compatible with PICS(-F). Patients most frequently present themselves to GPs with fatigue, psychological or cognitive impairments. GPs refer these patients to a psychologist or to a general practice assistant, while only $15 \%$ of the GPs were familiar with the concept of dedicated ICU follow-up clinics. GPs report that they feel responsible for both recognition and treatment of PICS-related sequelae in their patients, but they have insufficient knowledge. Up to $87 \%$ of GPs expressed a wish to obtain more knowledge about PICS(-F) via an e-learning or by providing education for GPs. Furthermore, information sharing between the hospital/ICU and the GP in its present state is suboptimal and could therefore be improved, for instance by including information regarding the long-term consequences of ICU treatment in the discharge letter of patients.

Conclusion: Although PICS(-F) is a widely recognized problem within the ICU community, the majority of Dutch GPs is not familiar with PICS(-F)-related sequelae, but nevertheless feel a responsibility to recognize and treat them. GPs want to be educated preferably via an e-learning or educational programs, and informed about the longterm consequences of ICU treatment and existing ICU follow-clinics in the discharge letter, to improve GP's involvement in post-ICU care.

\section{Reference(s) and grant acknowledgment(s)}

1. 1. van Beusekom I, Bakhshi-Raiez F, de Keizer NF, van der Schaaf M, Termorshuizen F, Dongelmans DA. Dutch ICU survivors have more consultations with general practitioners before and after ICU admission compared to a matched control group from the general population. PLoS One. 2019;14(5):e0217225.

\section{7}

Epidemiology and outcome of elderly patients after renal replacement therapy in the intensive care unit: an observational study

C. Salathé ${ }^{1}$; M. Altarelli²; E. Poli²; P. Eckert ${ }^{2}$; A. Schneider ${ }^{2}$

${ }^{1}$ Hospital Neuchâtel - Pourtalès, Neuchâtel, Switzerland; ${ }^{2}$ Service of adult inensive care \& burns, Lausanne University Hospital, Lausanne, Switzerland Correspondence: C. Salathé

Intensive Care Medicine Experimental 2020, 8(2): 000487

Introduction: Renal replacement therapy (RRT) is commonly performed in patients admitted to the intensive care unit (ICU). However, it is associated with high morbidity and mortality and its relevance is sometimes questioned in elderly patients in particular when they suffer from other premorbid illnesses.

Objectives: We sought to evaluate the long-term outcomes and quality of life (QOL) of elderly patients who survived critical illness requiring renal replacement therapy (RRT).

Methods: We retrospectively identified all patients aged over 55 admitted to our ICU between January 2015 and April 2018 who received RRT for acute kidney injury (AKI). We assessed their vital status by cross-referencing our hospital database and the Swiss national death registry. Mortality and time to death were recorded. Patients still alive at the time of the study underwent a phone interview. They were asked about their dialytic status and their quality of life was evaluated using an EQ-5D survey with visual analog scale (VAS). Results were stratified according to patients' age group (group 1, G1: "55-64 yo"; group 2, G2: "65-74 yo"; group 3, G3: ">75 yo") and comparisons between these groups and European standards were made.

Results: During the study period, 354 eligible patients were admitted to our ICU. Of those, 173 (48.9\%) died during the index hospital admission (150 in ICU and 23 on the ward). After a median follow-up of 2.7 years (IQR 1.6), a further 62 had died with a mean time to death for hospital survivors of 149.9 days (IQR 450.4). Hence, 119 patients were still alive at the time of the study. We successfully contacted 96 
(80.7\%) of them and $83(69.7 \%)$ were included in the study (G1:24, G2: 44, G3: 15). Among the survivors, only 6 (7.2\%) were still RRT dependant at time of the study. Median EQ-5D and VAS scores were respectively 6 (IQR 2) and 75 (IQR 30). Pain and mobility issues were the most frequent items quoted in the survey. Group comparisons are shown in figure 1. Both score were lower in patients in G3 compared to the other two groups (both $p$ values $<0.01$ ). However, they were comparable with EQ-5D index population norms of aged matched patients (1).

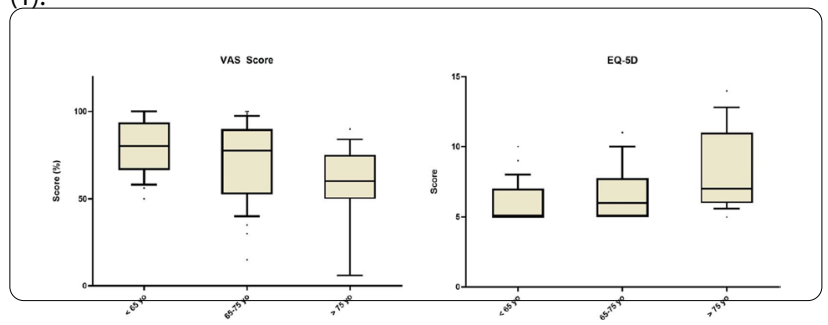

Conclusion: The need for renal replacement therapy identifies a population at very high risk of mortality with a survival of $33 \%$ at 2.5 years. However, quality of life for survivors was acceptable and comparable to age matched control population.

\section{Reference(s) and grant acknowledgment(s)}

1. Agota Szende, Bas Janssen, Juan Cabases. Self Reported Population Health: An international Perspective based on EQ-5D. Springer Open, 2014

\section{6}

Evaluation of an implementation process of post-crisis debriefing: a tool for improving quality in the medical emergency team system

E. Conoscenti ${ }^{1}$; G. Martucci ${ }^{2}$; M. Piazza ${ }^{3}$; F. Tuzzolino ${ }^{4}$; B. Ragonese ${ }^{5}$; G. Burgio ${ }^{6}$;. Arena 7 ; S. Blot ${ }^{8}$; A. Luca ${ }^{9}$; A. Arcadipane ${ }^{10}$; G. Chiaramonte ${ }^{10}$ 'Infectious disease and infection control service ismett, ISMETT, Palermo, Italy; ${ }^{2}$ Department of anesthesia and intensive care, ISMETT, Palermo, Italy; ${ }^{3}$ Department of anesthesia and intensive care, ismett, palermo, italy, ISMETT, Palermo, Italy; ${ }^{4}$ Research officer, ISMETT, Palermo, Italy; ${ }^{5}$ Quality and patient safety department, ISMETT, Palermo, Italy; ${ }^{6}$ Anesthesia and intensive care unit, IRCCS-ISMETT, Palermo, Province of Palermo, Italy, Italy; ${ }^{7}$ Chief of nursing, ISMETT, Palermo, Italy; Internal medicine department, Ghent University Hospital, Gent, Belgium; ${ }^{9}$ Director, ISMETT, Palermo, Italy; ${ }^{10}$ Anesthesia and intensive care unit, IRCCS-ISMETT, Palermo, Italy Correspondence: $\mathrm{E}$. Conoscenti

Intensive Care Medicine Experimental 2020, 8(2): 000556

Introduction: The ability of first responders to deliver appropriate care after medical emergencies determines positive outcomes for patients. Clinicians' skills, best practice, and knowledge are gained from basic life-support courses, unfortunately often lost due to a lack of hands-on practice.The purpose of debriefing is to facilitate the identification of errors, and eventually develop plans to improve future performances specifically to both individual and team achievement. It also refers to a valid approach for correcting clinical behavior, and allows clinicians to reflect on knowledge, skills, attitude or teamwork revealed during a clinical event that if modified would benefit other patients in similar situations.At our institute a well-structured Rapid Response System (RRS) has been implemented;when a patient deteriorates a Medical Emergency Team (MET) is called to the bedside of the patient in crisis. The MET is summoned to the bedside between 80 and 100 times a year, and is composed of health care responders: an anesthesiologist, 3 nurses, an aide, and a clinical clerk who is involved in alerting and initiating the RRS.Anesthesiologists and nurses are trained in basic life support (BSL)/advanced cardiac life support/pediatric advanced life support, and aides are trained in BLS regularly (once every two years). So far, at the end of a crisis, the debriefing has been left as optional for the team despite the fact that it has always been taught and performed regularly during ACLS/PALS training sessions.
Furthermore,anesthesiologists and senior nurses have been receiving training in how to conduct a structured debriefing after clinical crisis. The questionnaire was submitted as a part of a larger and multifaceted quality improvement project aimed at implementing regular post crisis debriefing, and to detect areas of improvement .

Methods: This is a descriptive, structured and voluntary, anonymous, non-funded, self-administered cross-sectional survey conducted at a 78-bed medical institute accredited with Joint Commission International standards and focused on all solid organ transplantation medicine. The Quality and Patient Safety Department approved the conduction of the survey. The need for ethics committee approval was waived given the voluntary nature of the survey.The aim of the survey was to generate hypotheses and identify critical issues among responders, to guide educational activities, and to detect the attitudes of the personnel toward debriefing implementation as routine. The survey was developed by three investigators based on a review of the theoretical basis of survey elaboration, reappraisal of the relevant literature in the field of communication, team dynamics, and debriefing, which later was piloted by the quality department for clarity of the questions.We conducted a Web-based survey sent by e-mail with a link to a total of 593 potential responders involved in clinical activity:118 physicians, 342 clinical nurses, 97 aides, and 36 unit clerks.The survey was anonymously self-administered on a voluntary basis by the respondents, and submitted online using a Survey Monkey system.The survey was conducted using 17 multiple-choice questions organized in four domains:Respondent characteristics;adherence to resuscitation guidelines and assessment of value of training; teamwork and leadership and awareness of mutual respect;professional development and debriefing .We recorded respondents' characteristics inherent to work activity: years of service at the institute, clinical role, ward of principal activity, and number of emergency conditions experienced in previous years. The survey was checked for content validity by members of the quality coordinating team at the hospital.Survey results were analyzed using descriptive statistics (numbers and percentage). Chi-square test or Fisher's exact tests were applied where appropriate. Exploratory data analyses with contingency tables were prepared to illustrate different responses among groups according to roles, years of service at the hospital and number of yearly-managed conditions experienced. Data management and statistical analysis were done with SAS 9.4 by SAS Institute Inc., Cary, NC, USA.

Results: Response rate to the survey was 25\% (148 healthcare workers). Of all respondents, $86 \%$ were employed $>10$ years, $75 \%$ were involved in $\leq 5$ emergencies over the previous year. Resuscitation guidelines were considered fully applied by $55 \% ; 64 \%$ of respondents considered the teaching program as sufficient. Of all participants, $97 \%$ were aware of the importance of teamwork dynamics, $79 \%$ were aware of the importance of the personal performance, and $52 \%$ considered emergencies as valid opportunities for professional growth.Leadership was considered important by $45 \%$ of respondents; debriefing implemented by $41 \%$, and considered a potentially useful tool by $85 \%$.

Conclusion: We hypothesize that quality improvement programs based on debriefing implementation and communication may improve the outcome during emergency conditions, and increase satisfaction for the operators. Future prospective studies are needed to substantiate this hypothesis.

\section{Reference(s) and grant acknowledgment(s)}

1. Hunziker S, Tschan F, Semmer NK, Zobrist R, Spychiger M, Breuer M, et al. Hands-on time during cardiopulmonary resuscitation is affected by the process of teambuilding: a prospective randomised simulator-based trial. BMC emergency medicine. 2009;9:3.

2. Herbers MD, Heaser JA. Implementing an in Situ Mock Code Quality Improvement Program. American journal of critical care : an official publication, American Association of Critical-Care Nurses. 2016;25:393-9.

3. Healy S, Tyrrell M. Importance of debriefing following critical incidents, Emergency nurse : the journal of the RCN Accident and Emergency Nursing Association. 2013:20:32-7.

4. Hamilton R. Nurses' knowledge and skill retention following cardiopulmonary resuscitation training: a review of the literature. Journal of advanced nursing. 2005;51:288-97. 
5. Fanning RM, Gaba DM. The role of debriefing in simulation-based learning. Simulation in healthcare : journal of the Society for Simulation in Healthcare. 2007:2:115-25.

6. Coppens I, Verhaeghe S, Van Hecke A, Beeckman D. The effectiveness of crisis resource management and team debriefing in resuscitation education of nursing students: A randomised controlled trial. Journal of clinical nursing. 2018;27:77-85.

7. Clark R, McLean C. The professional and personal debriefing needs of ward based nurses after involvement in a cardiac arrest: An explorative qualitative pilot study. Intensive \& critical care nursing. 2018;47:78-84

8. Chesham B, Dawber C. The "All of Us" study - Non-clinical staff members' experience of performing cardiopulmonary resuscitation in acute care settings. Australasian emergency care. 2019;22:243-8.

9. Cheng A, Hunt EA, Donoghue A, Nelson-McMillan K, Nishisaki A, Leflore $J$, et al. Examining pediatric resuscitation education using simulation and scripted debriefing: a multicenter randomized trial. JAMA pediatrics. 2013;167:528-36.

10. Andersen PO, Jensen MK, Lippert A, Østergaard D. Identifying non-technical skills and barriers for improvement of teamwork in cardiac arrest teams. Resuscitation. 2010;81:695-702.

\section{7}

Analysis of the effect of fragility as a risk factor for hospital mortality in ICU patients

R. Molina Lobo'; L. López de la Oliva Calvo ; ; I. Torrejón Pérez ; ; B. Lobo Valbuena ${ }^{1}$; A. Naharro Abellan ${ }^{1}$; D. Janeiro Lumbreras'; F. Gordo Vidal ${ }^{2}$

${ }^{1}$ Intensive care medicine, Hospital Universitario del Henares, Madrid, Spain; ${ }^{2}$ Intensive care medicine, Universidad Francisco de Vitoria, Madrid, Spain

Correspondence: L. López de la Oliva Calvo

Intensive Care Medicine Experimental 2020, 8(2): 000557

Introduction: Fragility assessment in very elderly patients ( $\geq 80$ years) is inversely associated with short-term survival. In this regard, we believe that the implementation of this scale to all ICU patients on admission, regardless of age, can provide us with additional objective information.

Objectives: To assess the impact of fragility, defined by the Clinical Frailty Scale (CFS), on hospital mortality of ICU admitted patients regardless of their age.

Methods: Prospective observational study evaluating the role of frailty on patients admitted to a medical-surgical ICU from October 2016 to April 2019. Patients who required transfer to another centre during their admission were excluded. Analysed variables were age, sex, comorbidities, SAPS 3, SOFA, type of patient, hospital stay before ICU admission, length of stay and outcome at discharge. We also included other variables of clinical interest during ICU admission. Fragility was estimated using the CFS scale, using a cut-off value of $\geq 3$. Continuous variables are presented as median and interquartile range. To analyse the association with hospital mortality, a univariate and multivariate analysis was performed using backward logistic regression and recursive partitioning using CHAID tree to estimate special risk populations. Results: 1462 patients were included. Mean age of 66 years (55-74), $42 \%$ women. Medical reason for admission in 54.5\%. SAPS 346 (38-56), SOFA on admission 2 (0-10). Average ICU length-of-stay of 2 days (1-4), and time in-hospital after ICU discharge of 6 days (3-12). ICU survival was $96.8 \%$, and hospital survival was $93.8 \%$, with a rate of ICU readmissions of $3.2 \%$. The CFS was 3 (2-3). The significant results regarding hospital mortality obtained from the multivariate analysis are shown in Table 1. Recursive partitioning model established that the greatest risk factor for mortality in the population was the development of neurological failure during ICU admission, with a mortality rate of 31\% (51\% in frail patients vs $17 \%$ in non-fragile patients: $p<0.0001)$. Regarding patients without neurological failure, the main risk factor for mortality was presenting a CFS $>3$ before ICU admission, with a mortality rate of $3.2 \%$ (11.2\% in frail patients vs 1.2 in non-fragile patients: $p<0.0001)$

Conclusion: The fragility index is an independent factor of hospital mortality in patients requiring ICU admission. The other factors identified are the need for mechanical ventilation, the need for preventive isolation or the development of organ failure.

\section{5}

Mapping patient, family and healthcare professional outcomes of studies reporting on ICU design: a scoping review

H. Noble ${ }^{1}$; S. Saha1; D. Hadfield ${ }^{1}$; T. Best ${ }^{1}$; A. Xyrichis 2 ; PA. Hopkins ${ }^{1}$; L. Rose ${ }^{2}$ ${ }^{1}$ Critical care, King's College Hospital, London, United Kingdom; ${ }^{2}$ Florence nightingale faculty of nursing, midwifery and palliative care, King's College London, London, United Kingdom

Correspondence: $\mathrm{H}$. Noble

Intensive Care Medicine Experimental 2020, 8(2): 000565

Introduction: The ideal intensive care unit (ICU) design creates a 'healing environment' humanizing the medical setting, minimizing environmental stressors for healthcare professionals (HCP) and families while supporting patient recovery 1,2 . Due to the multifactorial nature of ICU design, understanding the effect on patient, family and staff outcomes is complex and remains limited.

Objectives: To conduct a scoping review of studies of ICU design features. Specifically, to map outcomes and measures evaluating the effect of these features on patients, family and HCPs. Data will inform future studies evaluating new or existing ICU designs.

Methods: In consultation with an information specialist, we iteratively designed and executed a search strategy across seven databases from January 2007 to May 2020. All research designs were eligible as long as studies explored the effect of ICU design features on patients, family and/or HCPs. Reviewers independently confirmed study eligibility, one reviewer extracted data with accuracy confirmed by a second.

Results: Of the 18,577 citations screened; 49 studies met inclusion criteria; most (41/49, 84\%) were published after 2012. 28 (57\%) studies evaluated a newly built or renovated ICU/ICU room, the remainder evaluated existing ICU designs or features. We grouped studies into 12 design feature categories, the most common being light $(25 / 49,51 \%)$, single vs multi-occupancy rooms/pods (19/49, 39\%), and family-centred $(13 / 49,27 \%)$.

\begin{tabular}{|l|l|}
\hline Types of design features & $\mathrm{n}(\% *)$ \\
\hline Light & $25(51)$ \\
\hline Occupancy & $19(39)$ \\
\hline Family centered & $14(29)$ \\
\hline Workspace Layout & $13(26)$ \\
\hline Equipment related & $11(22)$ \\
\hline Noise & $10(20)$ \\
\hline View & $9(18)$ \\
\hline Outside Space & $9(18)$ \\
\hline Aesthetics & $8(16)$ \\
\hline Privacy & $7(14)$ \\
\hline Unit Layout & $7(14)$ \\
\hline Visibility & $5(10)$ \\
\hline
\end{tabular}

${ }^{*}$ All categories were categorised for multi-design feature studies and therefore the $\%$ do not sum to 100 .

We identified 67 distinct outcomes. Patient clinical outcomes were included in most studies with the most commonly reported being length of stay $(13 / 49,26 \%)$, mortality $(10 / 49,20 \%)$ and psychological outcomes such as delirium occurrence $(9 / 49,18 \%)$ and duration (5/49, $10 \%)$. Objective ICU/ICU workspace physical environment outcomes were reported in only 8/49 (16\%) studies and included physical/visual accessibility $(3 / 49,6 \%)$, sound levels $(3 / 49,6 \%)$, light levels $(2 / 49,4 \%)$, and corridor utility/traffic patterns $(1 / 49,2 \%)$. Most commonly measured HCP reported outcomes were staff experience/perception (12/49, $24 \%)$, staff stress $(4 / 49,8 \%)$, team interaction $(3 / 49,6 \%)$ and team collaboration $(3 / 49,6 \%)$. Only $5 / 49(10 \%)$ studies measured patient reported outcomes; $7 / 49$ (14\%) studies measured family reported outcomes.

Conclusion: The last decade has seen an increase in studies evaluating the effect of ICU design. However, few studies characterise objective environmental characteristics and fewer use patient or family reported outcome or experience measures. Our findings can inform design of future studies evaluating new or existing ICU designs. 
Reference(s) and grant acknowledgment(s)

1. Lorenz, S.G. (2007) The Potential of the Patient Room to Promote Healing and Well-being in Patients and Nurses. Holistic Nurse Practitioner; 21(5) pp 263-277.

2. Rubert, R. Long, L.D \& Hutchinson, M.L (2007) 'Creating a Healing Environment in the ICU' in Kaplow, R. \& Harding S,R. (ed.) Critical Care Nursing: Synergy for Optimal Outcomes. Massachusetts, USA. Jones \& Bartlett Publishers. Pp 27.

3. Faculty of Nursing, Midwifery and Palliative Care, King's College London

\section{1}

Sex and gender differences in the intensity of intensive care

E. Zettersten ${ }^{1}$; G. Jäderling ${ }^{1}$; M. Bell ${ }^{1}$; E. Larsson

${ }^{1}$ Departement of physiology and pharmacology, Karolinska Institute, Stockholm, Sweden

Correspondence: E. Zettersten

Intensive Care Medicine Experimental 2020, 8(2): 000581

Introduction: It has been previously reported that there are differences in the care given within the ICU between men and women 1,2, and that men have a lower probability of being discharged from the ICU given equal disease burden as women3. To date there are no satisfactory explanation as to why that is. The aim of this study is to investigate if any differences exist between men and women regarding the level of intensive care provided, given equal disease burden.

Objectives: To investigate differences in mechanical ventilation, invasive monitoring, vasoactive treatment, placement of dialysis catheters and central venous catheters between men and women in the ICU.

Methods: This is a retrospective cohort study of 9067 ICU patients admitted to the Karolinska University Hospital Stockholm, Sweden, between 2006-2016. Data was extracted from the ICU medical record (PDMS, Clinisoft ${ }^{\circledR}$, GE Healthcare). Differences in use of mechanical ventilation, invasive monitoring, vasoactive treatment, echocardiograms, placement of dialysis catheters and central venous catheters based on the sex of the patient were analysed using univariate and multivariable logistic regressions, adjusted for Estimated Mortality Rate (calculated using SAPS3 and Apache II), Charlsons comorbidity index and age. Subanalyses were performed on patients diagnosed with sepsis, cardiac arrest and respiratory disease.

Results: Approximately one third (36.4\%) of the patients were women. Overall, men received more mechanical ventilation $(57.7 \%$ vs $51.9 \%$, OR $1.2895 \% \mathrm{Cl} 1.17-1.40)$, more central dialysis catheters (11.8\% vs $9.9 \%$, OR $1.2195 \% \mathrm{Cl} 1.05-1.40)$ and vasoactive treatment $(49.3 \%$ vs $46.6 \%$, OR $1.6195 \% \mathrm{Cl} 1.06-1.28)$. Of the 1799 patients admitted with a respiratory disease, men were more likely to receive mechanical ventilation (55.5\% vs 50.0\%, OR 1.22 95\% Cl $1.01-1.49$ ). Among patient diagnosed with cardiac arrest $(n=536)$, men were more likely to receive a central venous line $(80.3 \%$ vs $71.9 \%$, OR $1.6095 \% \mathrm{Cl} 1.04$ - 2.45). Women did not receive more of any of the items investigated. Conclusion: We conclude that differences in the level of intensive care provided to men and women exists. Knowing that baseline level of disease has been adjusted for, it is clear that this finding warrants further investigation.

\section{Reference(s) and grant acknowledgment(s)}

1. 1. Valentin A, Jordan B, Lang T, Hiesmayr M, Metnitz PG. Gender-related differences in intensive care: a multiple-center cohort study of therapeutic interventions and outcome in critically ill patients. Crit Care Med. 2003;31(7):1901-1907.

2. 2. Fowler RA, Sabur N, Li P, et al. Sex-and age-based differences in the delivery and outcomes of critical care. CMAJ. 2007;177(12):1513-1519.

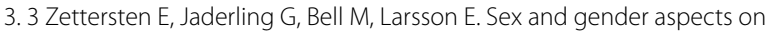
intensive care. A cohort study. J Crit Care. 2019;55:22-27.

4. This study is supported by Stockholm County Council and The Swedish Society of Medicine
000583

Patient centred care and early rehabilitation for enhanced ICU outcome and recovery

A. Ersson ${ }^{1} ;$ L. Orvelius ${ }^{2}$

${ }^{1}$ Anaesthesia and Intensive Care, Region Sormland, Nykoping, Sweden;

${ }^{2}$ Anaesthesia and intensive care, Region Ostergotland, Linkoping, Sweden

Correspondence: $A$. Ersson

Intensive Care Medicine Experimental 2020, 8(2): 000583

Introduction: The development in critical care during the past decades have improved ICU outcome and long-time survival (1), even in highly co-morbid patients. This, in combination with increasing frailty in the ICU case mix, have increased the number of ICU survivors with a poor physical and psychical capacity at the time of discharge (2) and thus, a rising demand for individualised rehabilitation. Furthermore, increasing awareness of post intensive care syndrome (PICS) related sequels, further increases the demand for an adapted and individualised aftercare to preserve function and improve health-related quality of life (HRQoL) (3).

Current data suggests that rehabilitation actions should be initiated early and that programs for PICS prevention and family/ patient centred care (PCC) have favourable effects on ICU outcome and potentially reduce anxiety and PTSD in patients and relatives (4). Early initiation of rehabilitation actions has been shown both feasible and safe to perform (5).

Objectives: To investigate the feasibility and safety of a PCC and early rehabilitation program in a general ICU.

Methods: Setting: A feasibility and safety study in a 12 bed ICU in a university hospital in Sweden.

Population: 21 consecutive adult ICU patients with an ICU LOS $>48 \mathrm{~h}$ and on mechanical ventilation were subjected to a PCC program containing early initiated rehabilitation. A multi-professional team performed daily physiotherapeutic interventions and psychosocial activities parallel with normal ICU care procedures. During the study period the mean age of the ICU population was 58 years ( $73 \%$ male). Mean length of stay was 3 days and mean time on ventilator was 59 hours.

Endpoints:

Protocol adherence

Achievement of sedation (RASS)/ pain (BPS, NRS), physiotherapeutic targets (MMS) and Psychosocial asssessmet (MoCA)

Ability to perform PCC/ family support

Patient/ family participation

Frequency of ICU Diaries

Risk assessment for physiotherapy

Adverse events

Results: The full program was completed in $88 \%$ of the patients. The physiotherapy program was fully applicable in all patients while patient cognitive limitations prevented cognitive assessment during the study period.

Sedation (RASS $</=-2$ ) targets were met within $4 \mathrm{~d}$. BPS /NRS were completed in $80 \%$ of the patients during the first week

Interactive rounds were feasible in $>80 \%$ after two days and diaries were kept in $80 \%$ of all patients. Integrity issues at rounds and inability for relatives to participate were common ( $>70 \%)$.

Low risk for rehabilitation procedures were scored in $66 \%$ of the patients, $27 \%$ at intermediate and $7 \%$ at high risk

No adverse events were recorded

Conclusion: The suggested program was highly feasibly, could be safely performed and could be executed with a high level of participation. Dropouts or late initiation were commonly due to the level of sedation and cognitive impairment which also limited the progression 
rate of the physical rehabilitation and made cognitive assessment impossible during the ICU stay. Co-morbidity or severity of organ dysfunction did not prevent execution of the program but required minor adaptions. Interactive rounds were well received by all staff and patients but required procedural and timely adaption for ensuring patient integrity and to encourage family participation. To enhance and facilitate paramedical participation and integration of relatives, ICU work schedules have to be adapted accordingly.

\section{Reference(s) and grant acknowledgment(s)}

1. 1. Adhikari NK, Fowler RA, Bhagwanjee S, Rubenfeld GD. Critical care and the global burden of critical illness in adults. Lancet. 2010;376(9749):1339-46

2. 2. Azoulay E, Vincent J-L, Angus D et al: Recovery after critical illness: putting the puzzle together - a consensus of 29. Critical Care 2017: 21:296

3. 3. Fuke R, et al. BMJ Open 2018;8:e019998. https://doi.org/10.1136/bmjop en-2017-019998

4. 4. Lee. H, Park Y, Jang E, Lee Y. Intensive Care Med (2019) 45:1072-1081 https ://doi.org/10.1007/s00134-019-05681-3

5. 5. Hodgson et al. Critical Care (2018) 22:77

\section{4}

\section{Influence of medical history on the prognosis of patients}

\section{on mechanical ventilation due to COVID 19}

C. Serrano ${ }^{1}$ : A. Acha'; A. Ruiz García²; R. Araoz Illanes ${ }^{1}$ : M. Trascasa Muñoz J. Higuera Lucas ${ }^{3}$; B. Llorente Ruiz'; MC. Pintado ${ }^{4}$; E. Serrano Yebenes ${ }^{5}$; F. Poveda Saenz ${ }^{6}$; C. Demartini Foj ; $;$ A. Revuelta Rebollo6; E. Nevado ${ }^{6}$

'Intensive care unit, Hospital Príncipe de Asturias, Alcalá de Henares, Spain; ${ }^{2}$ Medicina intensiva, Hospital Universitario Príncipe de Asturias, Meco, Spain; ${ }^{3}$ Medicina intensiva, Hospital Universitario Príncipe de Asturias, Alcala de Henares, Spain; ${ }^{4}$ Unidad de Cuidados Intensivos, Hospital Universitario Príncipe de Asturias, Alcala de Henares, Spain; ${ }^{5}$ General surgery, Hospital Príncipe de Asturias, Alcalá de Henares, Spain; ${ }^{6}$ Anesthesiology, Hospital Príncipe de Asturias, Alcalá de Henares, Spain

Correspondence: A. Ruiz García

Intensive Care Medicine Experimental 2020, 8(2): 000604

Introduction: COVID 19 pandemic affected thousands of people in Spain. Younger and older people have been infected, even some people without any previous medical history were admitted in a critical care units.

Objectives: Determine if there are any comorbilities related to the increased lenght of stay in ICU and the prognosis of the patients with COVID 19 pneumonia.

Methods: Retrospective observational study conducted in an intensive care unit of a university Hospital. During the pandemic, ICU beds went from 14 to 39 . We analysed the patients admitted to the ICU with the diagnosis of respiratory failure due to COVID 19 pneumonia between $6 / 3$ and 21/4 2020. Patients who are still admitted at the time of data collection and those who moved to another centre due to lack of beds are excluded. Demographic data (age, sex), APACHE II, comorbidities (arterial hypertension, previous pulmonary pathology, dyslipidemia, diabetes mellitus and obesity) are analyzed. We describe the relationship between comorbidities, length of stay and mortality rate. Statistical analysis is performed using the IBM ${ }^{\circledR}$ SPSS Statistics 23 tool.

Results: 67 patients are analysed. Male: $65.6 \%$ (44/67). Average age 60 years, APACHE II $14.3 \pm 5.2$, average stay $14.1 \pm 10$ days. $80.5 \%$ (54/67) had previous pathology: cardiovascular 44.8\% (30/67) (HT $41.7 \%(28 / 67)$ ). Pulmonary $19.4 \%$ (13/67) (asthma 7, 4\% (5), SAHS 5.9\% (4), COPD $2.9 \%$ (2)). $71.6 \%$ had other comorbidities (48/67) (dyslipidaemia $40.2 \%$ (27), diabetes $22.3 \%$ (15). $19.4 \%$ (13/67) did not present any disease prior to their admission to the ICU. $100 \%$ of patients received invasive mechanical ventilation since admission. Characteristics and prognosis are shown in Table 1.

\begin{tabular}{|c|c|c|c|c|c|c|c|c|c|c|c|c|}
\hline \multirow[t]{2}{*}{$\begin{array}{l}\text { Medical } \\
\text { history }\end{array}$} & \multirow{2}{*}{$\begin{array}{l}\text { CV } \\
\text { Yes }\end{array}$} & \multirow[b]{2}{*}{ No } & \multirow[b]{2}{*}{$\mathbf{P}$} & \multirow{2}{*}{$\begin{array}{l}\text { Pulmona } \\
\text { r } \\
\text { Yes }\end{array}$} & \multirow[b]{2}{*}{ No } & & No & \multirow[b]{2}{*}{ No } & \multirow[b]{2}{*}{$\mathbf{P}$} & \multirow{2}{*}{$\begin{array}{l}\text { DL } \\
\text { Yes }\end{array}$} & \multirow[b]{2}{*}{ No } & \multirow[b]{2}{*}{$\mathbf{P}$} \\
\hline & & & & & & P & Yes & & & & & \\
\hline $\begin{array}{l}\text { Characteristic } \\
\text { s }\end{array}$ & 30 & 37 & & 13 & 54 & & 13 & 54 & & 25 & 42 & \\
\hline Sex (male) & $\begin{array}{l}76,6 \\
\%\end{array}$ & $\begin{array}{l}59,4 \\
\%\end{array}$ & 0,1 & $76,9 \%$ & $\begin{array}{l}64,7 \\
\%\end{array}$ & 0,3 & $\begin{array}{l}76,9 \\
\%\end{array}$ & $\begin{array}{l}64,8 \\
\%\end{array}$ & 0,3 & $64 \%$ & $69 \%$ & 0,43 \\
\hline Apache II & 14,7 & 14 & $\begin{array}{l}0,6 \\
8\end{array}$ & 14,3 & 14,3 & 0,9 & 11,89 & 14,9 & $\begin{array}{l}0,12 \\
5\end{array}$ & 17,1 & 12,35 & $\begin{array}{l}0,00 \\
2\end{array}$ \\
\hline Ferritine & 1526 & 2856 & $\begin{array}{l}0,1 \\
4\end{array}$ & 2440 & 2285 & 0,9 & 3000 & 2100 & 0,4 & 2156 & 2389 & 0,8 \\
\hline Creatininie & 1,69 & 1,31 & 0,2 & 1,2 & 1,55 & $\begin{array}{l}0,3 \\
7\end{array}$ & 1,3 & 1,5 & 0,6 & 1,9 & 1,22 & 0,02 \\
\hline Bilirrubin & 2,27 & 1,8 & 0,4 & 1,6 & 2,1 & $\begin{array}{l}0,4 \\
8\end{array}$ & 2,5 & 1,9 & 0,47 & 2,65 & 1,6 & 0,07 \\
\hline Prone & $\begin{array}{l}66,6 \\
\%\end{array}$ & $64 \%$ & $\begin{array}{l}0,3 \\
4\end{array}$ & $53,8 \%$ & $68 \%$ & $\begin{array}{l}0,2 \\
4\end{array}$ & $60 \%$ & $66 \%$ & 0,48 & $80 \%$ & $57 \%$ & 0,04 \\
\hline Traqueostomy & $50 \%$ & $\begin{array}{l}41,6 \\
\%\end{array}$ & $\begin{array}{l}0,3 \\
4\end{array}$ & $50 \%$ & $44 \%$ & $\begin{array}{l}0,4 \\
8\end{array}$ & $30 \%$ & $50 \%$ & 0,19 & $\begin{array}{l}43,4 \\
\%\end{array}$ & $\begin{array}{l}46,6 \\
\%\end{array}$ & 0,5 \\
\hline Mortality rate & $\begin{array}{l}46,6 \\
\%\end{array}$ & $59 \%$ & 0,2 & $53,8 \%$ & $\begin{array}{l}53,7 \\
\%\end{array}$ & 0,6 & $30 \%$ & $50 \%$ & $\begin{array}{l}0,17 \\
4\end{array}$ & $40 \%$ & $61 \%$ & 0,06 \\
\hline Lenght of stay & 13,6 & 14,6 & $\begin{array}{l}0,6 \\
7\end{array}$ & 11,2 & 14,9 & $\begin{array}{l}0,2 \\
4\end{array}$ & 13 & 14,4 & 0,6 & 15 & 13,5 & 0,6 \\
\hline
\end{tabular}

Conclusion: In our sample, we did not find statistically significant differences between the medical personal history of cardiovascular pathology $(\mathrm{AHT})$, pulmonary disease or dyslipidemia and length of stay or mortality rate of critically ill patients on mechanical ventilation for COVID 19 pneumonia. Medical history of dyslipidaemia is related with higher creatinine values in our sample.

000616

Evaluation of the relationship between body mass index with sepsis and intensive care outcomes in critically ill patients

Z. çınar'; B. ÖZtürk²; M. Aydoğdu ${ }^{3}$

${ }^{1}$ Department of anaesthesiology, division of critical care, Gazi University School of Medicine, Anaesthesiologist, fellow of critical care, Ankara, Turkey; ${ }^{2}$ Department of pulmonary critical care medicine, Gazi University School of Medicine, Resident of Pulmonary Diseases, Ankara, Turkey; ${ }^{3}$ Pulmoner diseases department intensive care unit, Gazi University Faculty of Medicine,Professor of Respiratory Medicine, Ankara, Turkey

Correspondence: $Z$. çınar

Intensive Care Medicine Experimental 2020, 8(2): 000616

Introduction: Conflicting results have been reported regarding the potential impact of obesity on intensive care unit mortality and sepsis. Sepsis and obesity independently have high incidence and mortality rates. Physiological consequences of obesity increase the risk of complications and the possibility of slow recovery from sepsis. However, obese patients with sepsis had a lower mortality rate than non-obese patients for the first time in 1999 and it was described as obesity survival paradox.

Objectives: To evaluate the relationship between body mass index (BMI) and intensive care outcomes and development of sepsis in critical patients.

Methods: A retrospective cohort study involving $>18$-year-old patients who were followed up in a tertiary care medical intensive care unit from January 2015 to December 2019. Patients' age, gender, arrival diagnosis, APACHE II score, SOFA score, BMIs, comorbid diseases, presence of infection during ICU admission and follow-up, invasive (IMV) and noninvasive mechanical ventilation (NIMV) states, CRP and Procalcitonin levels were recorded. According to their BM patients were classified as, "underweight" $<20 \mathrm{~kg} / \mathrm{m} 2$, "normal" 20-25 kg / m2, "overweight" 25-30 kg / m2, "obese" 30-40 kg / m2 and "morbid / super obese "> $40 \mathrm{~kg} / \mathrm{m} 2$ and compared for intensive care outcomes and sepsis development. IBM SPSS version 23 program was used for data analysis.

Results: A total of 410 patients, $54 \%$ of whom were male and the mean age was $71 \pm 15$, were included in the study. When categorized according to body mass indexes, $6 \%$ of patients were underweight, $36 \%$ were normal, $32 \%$ were overweight, $20 \%$ were obese, $6 \%$ were morbidly obese. Overweight, obese and morbid obesity were significantly higher in women than in men $(p=0.001)$. Hospitalization 
APACHE II score was significantly higher in the underweight and normal patient groups than in the other patient groups $(p=0.013)$.Sepsis was detected in 113 (28\%) cases; $4 \%$ of them were underweight, 35\% normal, $32 \%$ overweight, $21 \%$ obese and $7 \%$ morbid obese. There was no statistical difference in terms of sepsis and SOFA scores between the groups $(p=0.232$ and $p=0.111)$. Besides no difference was identified in terms of hospital acquired and ventilator associated pneumonia development and the causative pathogens. Ninety-three patients (23\%) resulted in mortality; $8 \%$ was in underweight, $43 \%$ in normal, $\% 33$ in overweight, $\% 13$ in obese and $\% 3$ in morbid obese groups $(p=$ $0.031)$. BMI was evaluated as one of the significant risk factors for mortality ( $26 \pm 5$ in exitus group and $28 \pm 7$ in discharge group; $p=0.007$ ). One hundred and twenty-eight (31\%) patients received IMV and 187 $(46 \%)$ patients received NIMV. There was no statistical significance in the distribution of patients who underwent invasive MV $(49 \%$ in normal group, $51 \%$ in overweight and obese group, $p=0.285$ ). NIMV administration was higher in overweight and obese patients group $(61 \%)(p=0.008)$. While no difference was observed between the groups in terms of invasive MV duration, NIMV duration was found to be longer in obese and morbidly obese patients $(p=0.018)$. There was no statistically significant difference between the groups in terms of length of ICU and hospital stay ( $p>0.05$ ).

Conclusion: With this study, it has been shown that disease severity and mortality were lower in overweight and obese critical patients; the rate and duration of NIMV usage is higher. But there was no relationship between BMI and sepsis and length of ICU and hospital stay. Studies with more patient number are needed to demonstrate the exact effect of BMI on sepsis development and ICU results.

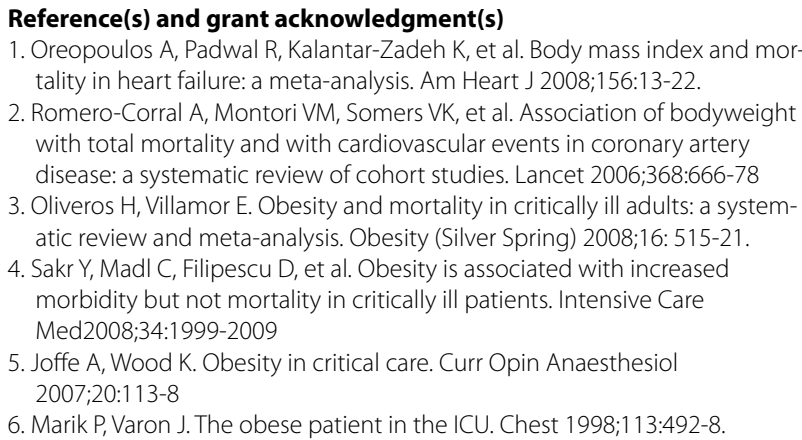

5. Joffe A, Wood K. Obesity in critical care. Curr Opin Anaesthesiol 2007;20:113-8

6. Marik P, Varon J. The obese patient in the ICU. Chest 1998:113:492-8.

\section{7}

Impact of neurological problems on icu outcome in pulmonary icu patients

Z. Çınar'; ;. Macit Aydın'; N. Navruzvai²; A. Mammadova²; S. Kodalak²;

A. Abbasova²; G. Gursel ${ }^{3}$

${ }^{1}$ Department of anaesthesiology, division of critical care, Gazi University School of Medicine, Anaesthesiologist, fellow of critical care, Ankara, Turkey; ${ }^{2}$ Department of pulmonary critical care medicine, Gazi University School of Medicine,Resident of Pulmonary Diseases, Ankara, Turkey;

${ }^{3}$ Department of pulmonary critical care medicine, Gazi University School of Medicine,Professor of Respiratory Medicine, Ankara, Turkey

Correspondence: $Z$. çınar

Intensive Care Medicine Experimental 2020, 8(2): 000617

Introduction: There are many risk factors for impaired cognitive function and neurologic damage in intensive care patients including pre-existing cognitive impairment, metabolic factors, mechanical ventilation, prolonged exposure to sedative drugs, sepsis and systemic inflammation. Neurologic problems are frequently associated with other critical illnesses in ICU patients and they may have an influence on ICU outcome.

Objectives: Aim of this study was to investigate whether neurologic problems have any impact on ICU outcome and to determine what are the risk factors for the development of neurologic problems after ICU admission in pulmonary ICU patients.

Methods: This retrospective observational single center study was performed an University hospital ICU and patients admitted between 2015 and 2019 years were included in the study. Frequency of neurologic problems at admission, their impact on mechanical ventilation (MV), infections, ICU length of stay and mortality, development rate of neurologic problems during ICU stay and risk factors for them were investigated.

Results: 360 patients were included in the study and there were neurologic problems in the 130 of them (36\%) (Group 1). They were stroke, parkinson's disease, Alzheimer, neuromuscular diseases, delirium and muscle weakness acquired in intensive care unit. NIV requirement rate was less than the patients without neurologic problems (group 2 ) and requirement of mechanical ventilation was significantly more frequent in this group $(37 \% \& 19 \%, \mathrm{p}<0.05)$. Duration of MV was significantly longer in the Group 1 also( $19 \pm 27 \& 8 \pm 6$, days p:0.003). Lastly neurologic problems was independent risk factor for extubation failure(OR:3). In the presence of neurologic problems length of ICU stay was significantly longer and they were independent risk factor(OR:3) for the increased length of ICU stay more than 10 days. When infectious findings were analyzed sepsis rate was significantly higher before and after ICU admission. Urinary and pulmonary infection rates were also significantly higher in these patients.

Neurologic complications developed median 7 days after ICU admission in the $19 \%$ of the patients. Most frequent ones were delirium and muscle weakness acquired in intensive care unit. Neurologic problems developed after ICU admission increased mechanical ventilation requirement 3 times as an independent risk factor. Risk factors for ICU acquired neurological problems were existance of sepsis during admission(OR:2.01, 95\% Cl:1.02-4, p:0.045) and longer MV durations before ICU admission(OR:1.05, 95\%Cl:1.004-41.103, p:0.033). Neurological problems were not independent risk factor for mortality.

Conclusion: Neurologic problems did not increase mortality but caused more frequently mechanical ventilation requirement, more extubation failure and longer ICU stay in this study population. Additionally our data suggest that having sepsis during admission and longer length of MV prior to admission may increase neurological complication rate.

\section{Reference(s) and grant acknowledgment(s)}

1. Holland MC, Mackersie RC, Morabito D, Campbell AR, Kivett VA, Patel R, et al. The development of acute lung injury is associated with worse neurologic outcome in patients with severe traumatic brain injury. J Trauma. 2003;55(1):106-11.

2. Oddo M, Citerio G. ARDS in the brain-injured patient: what's different? Intensive Care Med. 2016;42(5):790-3.

\section{2}

Liver injury in critically ill ECMO patients with COVID-19: a case series

C. mccarthy ${ }^{1} ; H_{.} J^{1} ; H . J^{1}$

${ }^{1}$ Icu, Barts Health NHS Trust, London, United Kingdom

Correspondence: $C$. mccarthy

Intensive Care Medicine Experimental 2020, 8(2): 000622

Introduction: Infection with severe acute respiratory syndrome coronavirus 2 (SARS-CoV-2) has become a rapidly spreading pandemic Extracorporeal Membrane Oxygenation (ECMO) has become a rescue therapy for some refractory critically ill patients. COVID-19 studies to date showed that patients with abnormal liver tests are at higher risk of progressing to severe disease1,2,3. ECMO patients being the most critically unwell patients, we have used our case series of all patients with COVID-19 on ECMO admitted to the intensive care unit to describe the dysfunction of blood liver tests and some potential causes.

Methods: We included into our case series a total of 18 SARS-CoV-2 positive patients undergoing VA/VV/VAV ECMO as rescue therapy at a university-affiliated tertiary care centre from March 1st to May 28th 2020. Liver test abnormalities were defined as the elevation of the following liver enzymes in serum: ALT >40 U/L, AST > 40 U/L, gamma-glutamyltransferase (GGT) $>49 \mathrm{U} / \mathrm{L}$, alkaline phosphatase (ALP) $>135 \mathrm{U} / \mathrm{L}$, and total bilirubin (TBIL) $>17.1 \mathrm{Imol} / \mathrm{L}$. Patients who had raised ALT and/or AST more than $3 \times$ the upper limit unit of normal (ULN) were classified as hepatocyte type; patients who had raised ALP or GGT twice the ULN were classified as cholangiocyte type; and patients who had a combination of both ALT/AST elevated more than $3 \times$ the ULN and ALP/ GGT twice the ULN were classified as mixed type. 
Results: Of the 18 patients mean age was 47.5(27-59)years with $94 \%$ being male. $100 \%$ had abnormal liver results. No patient had documented liver disease prior to hospitalization. Average BMI was $30.4,64 \%$ having pre-existing T2DM and 52\% having essential hypertension. Average APACHE2 Scores was 19[6-32]. 88\% commenced VVECMO, $12 \%$ VA-ECMO with average duration of 30.4 days. $23 \%$ had a hepatocellular picture, $13 \%$ cholangiocyte, $11 \%$ mildly raised LFTs and $43 \%$ a mixed type picture. $83 \%$ had preserved synthetic function. On examination of causes, $70 \%$ had normal echocardiography, $11 \%$ global biventricular failure and 19\% mild- moderately impaired RV function. $70.5 \%$ patients were on pipercillin/tazobactum, $47 \%$ clarithromycin $41 \%$ co-amoxiclav $23 \%$ levofloxacin, $11.7 \%$ had oseltamivir at the time of the liver injury. $5 \%$ (1 patient) was on parenteral nutrition. $11.7 \%$ had documented fatty liver on US during their ICU stay. Mortality was $11.7 \%$. Ferritin levels were wide ranging $[390-38,000]$ with mean of 5800 and median of 3100 . Average platelets count $150 \times 109 / \mathrm{L}$.

Conclusion: From previous studies, we know those who had abnormal liver test results, especially in hepatocyte type or mixed type, had significantly higher risks of developing severe disease secondary to SARS-CoV-2 3. $100 \%$ of our patients had abnormal liver tests while requiring ECMO representing the most critically unwell SARS-CoV-2 population.Mixed hepatocellular/cholangiocyte picture was the most prevalent at $43 \%$, as seen in previous studies 4 with no correlation between type of liver dysfunction and mortality.The liver dysfunction in our study was higher than previously reported 3,4 and with no previous history of underlying liver disease, suggesting that liver damage in patients with coronavirus infection might be directly caused by the viral infection of liver cells as suggested by previous studies due to SARS-CoV-2 might directly binding to ACE2-positive cholangiocytes to dysregulate liver function5. It is also possible that the liver impairment is due to drug hepatotoxicity, which might explain the large variation observed across the different cohorts. There was no direct correlation appreciated between antimicrobials and liver dysfunction in our population.In addition, immune-mediated inflammation, such as cytokine storm and pneumonia-associated hypoxia, might also contribute to liver injury or even develop into liver failure in patients with COVID-19 who are critically ill3. Ferritin levels were wide ranging $[390-38,000]$ with mean of 5800 suggesting systemic inflammatory process. High positive end expiratory pressure and aggressive ventilator strategies can cause RV strain/ pressure overload. While our patients had reduced ventilation on ECMO $33 \%$ had RV dysfunction. This right ventricular dysfunction can be associated with severe hepatic congestion. The primary pathophysiology involved in hepatic dysfunction is either passive congestion from increased filling pressures or low cardiac output and the consequences of impaired perfusion.Cardiogenic ischemic hepatitis may ensue compounding the insult6. It is important to identify RV strain on echo and avoid precipitating factors such as fluid overload and aggressive ventilation. Early consideration for extracorporeal support is important in these patients to avoid RV failure from medical management of respiratory failure. Our case series suggests that there might be concomitant liver injury as a consequence of the critical illness or than direct viral damage on the hepatocytes C19. Attention to modifiable factors and further research into liver injury in SARS-CoV-2 is key with the emphasis on clinical management of these severely ill patients that may consequently impact on liver function.

\section{Reference(s) and grant acknowledgment(s)}

1. 4. Bangash MN, Patel J, Parekh D. COVID-19 and the liver: little cause for concern. Lancet Gastroenterol Hepatol. 2020. https://doi.org/10.1016/S2468 $-1253(20) 30084-4$

2. 3. Cai O, COVID-19: Abnormal liver function tests. J. Hepatol. 2020, https:// doi.org/10.1016/j.jhep.2020.04.006

3. 2. Roth C, Liver function predicts survival in patients undergoing extracorporeal membrane oxygenation following cardiovascular surgery, Crit Care. 2016 Mar 11;20:57. https://doi.org/10.1186/s13054-016-1242-4

4. 1. Guan W-J, Clinical characteristics of 2019 novel coronavirus infection in China.N Engl J Med. 2020; https://doi.org/10.1056/nejmoa2002032

5. N/a

6. 6. Chai X, Hu L Zhang, Y Specific ACE2 expression in cholangiocytes may cause liver damage after 2019-nCoV infection. bioRxiv. 2020 https://doi. org/10.1101/2020.02.03.931766
7. 5. Alvarez A, Mukherjee, D, Liver Abnormalities in Cardiac Diseases and Heart Failure Int J Angiol. 2011 Sep; 20(3): 135-142. https://doi. org/10.1055/s-0031-1284434

\section{8}

Survival characteristics of COVID-19 patients requiring invasive ventilation in a district general hospital in East London

S. B. Tay ${ }^{1}$; L. Fletcher' ${ }^{1}$; C. Tai

${ }^{1}$ Anaesthetics and intensive care, Whipps Cross University Hospital, London, United Kingdom

Correspondence: C. Tai

Intensive Care Medicine Experimental 2020, 8(2): 000628

Introduction: There is limited evidence to guide UK based critical care management in patients with novel coronavirus disease 2019 (COVID19) despite high demand for this service. Throughout the pandemic, emerging trends and initial outcome data have been important to modifying practice. Risk factors for severe disease in the general population have been described [1] but their impact on intensive care outcome is unclear.

Objectives: This is a detailed assessment of local management and outcomes for an east London district general hospital, following the first peak of infection and aims to inform future patient care.

Methods: Adult patients ( $>18$ years) who tested positive on PCR for COVID-19 or those with strong clinical suspicion for COVID-19, admitted to critical care for intubation and ventilation between 14/03-01/05/2020 were identified. Patients transferred to other hospitals pre-extubation, were excluded, as were four patients who remained on invasive ventilation. Epidemiological, clinical, biochemical, temporal and outcome data was collected. Continuous data was analysed with Student's t-test /Mann Whitney's $U$ test and Fisher's exact test was used for categorical data.

Results:

\begin{tabular}{|c|c|c|c|c|}
\hline & & Survivors $(n=15)$ & Non-survivors $(n=24)$ & $p$ \\
\hline Age & (years) & $62.0(36.0-76.8)$ & $67.5(38.5-77.2)$ & 0.095 \\
\hline Gender & (female) & $6(40.056)$ & $4(16.7 \%)$ & 0.141 \\
\hline BMI & $(\mathrm{kgm}-2)$ & $26.2(20.3-40.0)$ & $30.5(18.7 .56 .0)$ & 0.382 \\
\hline \multirow[t]{2}{*}{ Ethnicity } & White & $8(53.3 \%)$ & $9(37.5 \%)$ & 0.508 \\
\hline & BAME & $7(46.7 \%)$ & $15(62.5 \%)$ & 0.507 \\
\hline \multirow{6}{*}{ Co-morbidities } & Diabetes & $3(20.05)$ & $10(41.7 \%)$ & 0.295 \\
\hline & Hypertension & $8(53.3 \%)$ & $16(66.7 \%)$ & 0.505 \\
\hline & Obstructive lung disease & $4(26.7 \%)$ & $2(8.3 \%)$ & 0.180 \\
\hline & Cardiac disease & $4(26.7 \%)$ & $4(16.766)$ & 0.686 \\
\hline & Chronic kidney disease & $1(6.7 \%)$ & $2(8.3 \%)$ & $>0.99$ \\
\hline & Smoking history & $6(40.0 \%)$ & $9(37.5 \%)$ & $>0.99$ \\
\hline \multirow{6}{*}{$\begin{array}{l}\text { Laboratory } \\
\text { parameters }\end{array}$} & Hospital admission CRP & $107.0(57.2)$ & $178.2(103.0)$ & 0.032 \\
\hline & Hospital admission NLR & $5.3(3.1-10.5)$ & $8.8(2.5-38.0)$ & 0.020 \\
\hline & ICU admission CRP & $155.4(64.8)$ & 242.9 (118.1) & 0.013 \\
\hline & ICU admission NLR & $6.8(3.1-23.5)$ & $12.7(4.6-36.8)$ & 0.0003 \\
\hline & ICU admission ferritin & 1329 (239-2800) & 1389 (478-4042) & 0.680 \\
\hline & ICU admission D-dimer & $1.5(0.4-47.1)$ & $3.5(0.5-80)$ & 0.062 \\
\hline \multirow{6}{*}{$\begin{array}{l}\text { ICU admission } \\
\text { features }\end{array}$} & APACHE score & $10.3(5.3)$ & $16.5(5.7)$ & 0.003 \\
\hline & Atrocurlum & $10(66.78 \%)$ & 23 (95.8\%) & 0.024 \\
\hline & Noradrenaline & $10(66.7 \%)$ & 23 (95.8\%) & 0.024 \\
\hline & Renal replacement & $5(33.3 \%)$ & $7(29.2 \%)$ & $>0.99$ \\
\hline & Corticosteroid & $3(20.056)$ & $10(41.796)$ & 0.295 \\
\hline & Proning & $5(33.3 \%)$ & $16(66.7 \%)$ & 0.055 \\
\hline
\end{tabular}

Thirty-nine patients met the inclusion criteria; overall there were 15 survivors. There was no significant difference in co-morbidity prevalence, however Asian ethnicity was more highly represented in nonsurvivors. Illness duration prior to hospital or critical care admission did not differ significantly, nor did time from hospital to ICU admission, during which the majority received advanced respiratory support. Preintubation $\mathrm{PaO} 2 / \mathrm{FiO} 2$ ratio was not statistically different between survivors and non-survivors (median 78.3 vs 82.5 respectively; $p=0.584$ ). Median ICU stay in survivors was 9.0 days (range 3.0-24.0) and hospital admission 17 days (range 11.0-35.0). CRP values were significantly higher in those who died at the initial hospital presentation $(p=0.032)$ and ICU admission $(p=0.013)$. Neutrophil to lymphocyte ratio (NLR) was significantly higher in non-survivors at both hospital admission $(p=0.020)$ and ICU admission $(p=0.0003)$. ICU admission D-dimer showed a trend towards higher levels in the non-survivors.

Conclusion: The APACHE II score appears helpful in predicting COVID19 mortality and may aid clinical decision making. Higher ICU admission CRP and neutrophil count in non-survivors may suggest a bacterial 
superinfection or increased pro-inflammatory response. NLR $\geq 3.13$ plus age $\geq 50$ has been proposed as a useful early indicator of severe disease [2]. Higher NLR in our non-survivors could suggest extension to its use as an early indicator of ICU mortality. Lack of significant difference in comorbidities, age, $\mathrm{PaO} 2 / \mathrm{FiO} 2$ ratio, ferritin and $\mathrm{D}$ dimer may suggest these indicators of more severe disease do not impact so greatly on critical care survival, though noting our sample size is small. Significantly higher noradrenaline and paralysis use, plus trend towards proning in non-survivors is likely reflective of disease severity. Patients who are from Black, Asian or minority ethnic (BAME) backgrounds are over-represented in our study, consistent with emerging national evidence [3].

\section{Reference(s) and grant acknowledgment(s)}

1. 1. Zhou F, Yu T, Du R et al (2020) Clinical course and risk factors for mortality of adult inpatients with COVID-19 in Wuhan, China: a retrospective cohort study. Lancet. 395(10229):1054-1062. https://doi.org/10.1016/S0140 $-6736(20) 30566-3$

2. 2. Liu J, Liu Y, Xiang P et al (2020) Neutrophil-to-Lymphocyte Ratio Predicts Severe Illness Patients with 2019 Novel Coronavirus in the Early Stage. medRxiv. https://doi.org/10.1101/2020.02.10.20021584

3. 3. Platt L, Warwick R (2020) Are some ethnic groups more vulnerable to COVID-19 than others? The Institute for Fiscal Studies. https://www.ifs.org. uk/inequality/chapter/are-some-ethnic-groups-more-vulnerable-to-covid -19-than-others/. Accessed 13 May 2020

\section{7}

The ICU Impact: results from a short-term follow-up programme R. Neto ${ }^{1}$; M. Carvalho'; Al. Paixão'; AL. Rios'; D. Adrião' ; P. Fernandes ${ }^{1}$; P. Castelões

'Intensive care department, Centro Hospitalar de Vila Nova Gaia/ Espinho, Vila Nova de Gaia, Portugal

Correspondence: R. Neto

Intensive Care Medicine Experimental 2020, 8(2): 000637

Introduction: Transition from the Intensive Care Unit (ICU) to the general ward represents a challenge for the critical care patient. Some patients will have an unfavourable evolution, which may be potentially avoidable. A short-term follow-up programme (FU-P) may identify patients at risk of deterioration, with a potential to improve quality of care [1].

Objectives: Evaluate the impact of a post-discharge short-term FU-P on patients from a Critical Care Department (CCD).

Methods: Prospective interventional study performed in a tertiary hospital in Northern Portugal. All patients with an ICU stay $>48$ hours or intermediate care stay $>72$ hours or tracheostomized patients were included in the follow-up programme. Patients were excluded if not eligible to be readmitted in the ICU. Follow-up programme consisted of a medical evaluation by an intensivist at 24,48 or 72 hours after discharge to the ward and a review of the clinical case with the attending physician. Patients were discharged from the follow-up programme if they presented no organ dysfunction after the visit.

Results: Between September 15th 2019 and March 15th 2020, 29.6\% of the patients admitted to the CCD $(n=153)$ were included in the FU-P. The majority were male (65.4\%) with a median age of 66 years [52.5 - 76]. Median ICU length of stay was 6 days [4-12.5], 56\% of patients required invasive mechanical ventilation (IMV), for a median of 6 days [3-13], and $10.1 \%$ were tracheostomized. Most patients were evaluated at 48 hours post-discharge. 20 patients were discharged from the hospital and 1 died before the first evaluation. Median Glasgow coma scale (GCS) and median Barthel Scale at follow-up were 15 and 60, respectively. At followup, $16.4 \%$ of patients presented some level of pain according to visual analogue scale (VAS). ICU-acquired weakness (ICUAW) was diagnosed in $23.1 \%$ of patients. Length of ICU stay $(p<0.001)$ and duration of IMV $(p<0.001)$ were significantly associated with ICUAW. Intensivist's visit resulted in a medical action in $15.0 \%$ of patients. 2 patients were readmitted. Admission due to urgent surgery was significantly associated with the need for medical intervention (OR 3.38, $\mathrm{p}=0.01$ ), as well as longer IMV duration $(p=0.03)$ and higher VAS pain levels $(p=0.03)$.

Ten patients died and the median time to hospital discharge was 7 days [3.0-16.5]. Longer duration of IMV $(p=0.015)$ and lower GCS scores $(p=0.05)$ were significantly associated with hospital death. No other variables were associated with hospital mortality.
Conclusion: This short-term follow-up programme was essential to improve the quality of care provided to our patients, while maximizing communication between Intensivists and attending physicians. This is a feasible programme that helps to identify deteriorating patients and further analysis will allow to improve eligibility criteria to better allocate resources.

\section{Reference(s) and grant acknowledgment(s)}

1. Ball, C., M. Kirkby, and S. Williams, Effect of the critical care outreach team on patient survival to discharge from hospital and readmission to critical care: non-randomised population based study. Bmj, 2003. 327(7422): p. 1014.

\section{5}

COVID 19 - experience from a 400-bedded District General

Hospital in the North West of England, UK

V. N. Gotz

${ }^{1}$ Critical Care Unit, University Hospitals of Morecambe Bay NHS Foundation Trust, Lancaster, United Kingdom

Correspondence: V.N. Gotz

Intensive Care Medicine Experimental 2020, 8(2): 000675

Introduction: To date, there are about 280,000 confirmed COVID 19 cases in the UK with about 40,000 deaths. Reviewing Public Health England data, the first wave of the COVID 19 pandemic seems to have passed. Our national peak was at the end of March and the beginning of April 2020. The same trend we observed at our local critical care unit and in view of preparedness for a second wave, we analysed our own data from COVID 19 positive patients.

Objectives: We are reviewing our perfomrance during the COVID 19 pandemic and are using the data and reflection to prepare for a second wave.

Methods: We collected demographic data and other variables of all adult male and female patients confirmed positive with COVID 19 by nasal/oropharyngeal swab. All patients were admitted to critical care at Royal Lancaster Infirmary, University Hospitals of Morecambe Bay, Lancashire, UK, in March and April 2020.

Results: We had 21 adimission in total, 16 males (76.2\%) and 5 females (23.8\%), with an average age of 62.14 years. The clinical frailty score was on average 2.05 , characterising our patients as 'generally well' with 'no active disease symtpoms'. The APACHE score on average was 15.43 and length of symptoms (defined as shortness of breath, cough, pyrexia) pre admission to hospital was 8.05 days on average. The predominant past medical histories were hypertension, diabetes mellitus and hypercholesterolaemia and $57.1 \%$ presented with fulminant four quadrant consolidation on admission chest $x$-ray (due to ARDS). Patients admitted to critical care were either in the emergency department $(47.6 \%)$ or on the respiratory ward $(42.9 \%)$. Patients that came to us from the emergency department were on a $15 \mathrm{l} / \mathrm{min}$ non-rebreather mask and patients from the respiratory ward were treated with eith High Flow Nasal Cannulae, CPAP or Venturi. Average length of hospital to critical care admission was 17 hours, but when patients were admitted directly from the emergency department the time reduced to 2-5 hours. We observed markers for multi-organ involvement: positive D-dimer (on average $611.5 \mathrm{ng} /$ $\mathrm{ml})$, elevated procalcitonin $(6.43 \mathrm{ng} / \mathrm{ml})$, elevated highly sensitive cardiac troponin HsCTNI (on average $1035.92 \mathrm{ng} / \mathrm{l}$ ). Average length of stay on ITU was 8.33 days with intubations of 7.85 days. Our patients were all ventilated by Draeger Evita XL ventilators and modes included CPAP/ ASB, BiPAP and APRV. 6 patients were proned, 3 patients were referred for ECMO (all declined) and 1 patient received a tracheostomy to support weaning from the ventilator. Cardiovascular support was delivered for 8.5 days on average and 3 patients also received renal replacement therapy (CVHDF). Patients were extubated to High Flow Nasal Cannulae based on better patient tolerance than CPAP mask and ability to deliver higher concentrations of oxygen when compared to usual face mask. $52 \%$ of our patients died ( 11 in total) and $43 \%$ survived ( 9 in total). One patient is still on critical care past day 60 of the admission. All patients that survived to ITU discharge also survived to hospital discharge and are still alive as per our Lorenzo electronic record system (3 months post admission). Stay on the repsiratory ward pre discharge home lasted for an average of 3.6 days. One out of the 21 patients developed a pulmonary embolism after discharge from ITU. The patient was subsequently 
anticoagulated by direct oral anticoagulants. All patients will be followed up in 6 months time in our local respiratory out-patient clinic.

Conclusion: Reviewing our local data, outcomes are very much in line with the national COVID 19 ICNARC data. Our predominat patient on ITU would be a male diabetic hypertensive patient, over 60 , well \& independant in activities of daily living with primarily two organ failures, respiratory and cardio-vascular. Mortality was $52 \%$. Those that also progressed to multi-organ failure involving the renal system displayed a $100 \%$ mortality. The most hypoxic patients were proned and ventilator modes were changed to APRV, but numbers are too small to deduct correlations with survival benefit. The review of data has helped us to strategically plan for a potential second wave of COVID 19.

\section{Reference(s) and grant acknowledgment(s)}

1. Fei Zhou (2020) 'Clinical course and risk factors for mortality of adult inpatients with COVID-19 in Wuhan, China: a retrospective cohort study', The Lancet, 395(10229), pp. 1054-1062.

\section{0}

\section{Outcomes of frail critically ill patients}

L. Wilson" ; A. Waite'; N. Main

${ }^{1}$ Intensive care unit, Royal Liverpool University Hospital, Liverpool, United Kingdom

Correspondence: A. Waite

Intensive Care Medicine Experimental 2020, 8(2): 000680

Introduction: Clinical frailty describes a syndrome of reduced physical, physiological and cognitive reserves. Frail patients who are admitted to critical care have a higher mortality rate and prolonged hospital stays $[1,2]$. However, the Clinical Frailty Score (CFS) [3] has only been validated for use in patients aged 65 years and older.

Objectives: We aimed to assess the prevalence of clinical frailty and the associated mortality and morbidity in all admissions to a tertiary mixed medical and surgical intensive care unit (ICU) during a 6 month period. Methods: We retrospectively reviewed electronic records of adult patients admitted to the Royal Liverpool University Hospital ICU between July and December 2018. Clinical Frailty Scores (CFS) were calculated using information documented in patient notes. Patients with a CFS of $\geq 5$ were categorised as frail. Demographic data, mortality and length of stay (LOS) were also collected.

Results: During the period reviewed 424 patients were admitted to ICU, of which $62 \%$ were medical and $38 \%$ surgical patients. The prevalence of frailty was $32.5 \%$ (138 patients). Both ICU and hospital LOS were longer in frail patients ( 7.3 and 30.8 days, respectively) compared to non-frail patients ( 6.5 and 23.4 days, respectively).

The table below outlines the ICU, hospital and one year mortality rates of frail and non-frail patients aged $<65$ years compared to those $\geq 65$ years. Frail patients in both age categories were less likely to survive ICU and hospital admission and less likely to survive to one year.

\begin{tabular}{|l|l|l|l|l|l|l|}
\hline \multicolumn{3}{|l}{$<\mathbf{6 5}$ years } & \multicolumn{6}{l|}{$\geq \mathbf{6 5}$ years } \\
\hline & Non-frail & Frail & Total & $\begin{array}{l}\text { Non- } \\
\text { frail }\end{array}$ & Frail & Total \\
\hline $\begin{array}{l}\text { Number of } \\
\text { patients }\end{array}$ & $199 / 267$ & $68 / 267$ & $267 / 424$ & $87 / 157$ & $70 / 157$ & $157 / 424$ \\
\hline & $(74.5 \%)$ & $(25.5 \%)$ & $(63.0 \%)$ & $(55.4 \%)$ & $(44.6 \%)$ & $(37.0 \%)$ \\
\hline ICU mortality & $23 / 199$ & $14 / 68$ & $37 / 267$ & $20 / 87$ & $24 / 70$ & $44 / 157$ \\
\hline $\begin{array}{l}\text { Hospital } \\
\text { mortality }\end{array}$ & $31 / 199$ & $18 / 68$ & $49 / 267$ & $23 / 87$ & $30 / 70$ & $53 / 157$ \\
\hline $\begin{array}{l}\text { 1 year } \\
\text { mortality }\end{array}$ & $(15.6 \%)$ & $(26.5 \%)$ & $(18.4 \%)$ & $(26.4 \%)$ & $(42.9 \%)$ & $(33.8 \%)$ \\
\hline
\end{tabular}

Conclusion: Frail adult patients admitted to ICU are less likely to survive their admission and less likely to survive to one year compared to non-frail patients, irrespective of age. Further research should be conducted to validate the Clinical Frailty Score in patients $<65$ years of age, in order to facilitate evidence-based discussions regarding admission to critical care.

\section{Reference(s) and grant acknowledgment(s)}

1. 1. Muscedere J, Waters B, Varambally A, et al. The impact of frailty on intensive care unit outcomes: a systematic review and meta-analysis. Intensive Care Med. 2017:43(8):1105-1122.

2. 2. De Geer L, Fredrikson M, Tibblin AO. Frailty predicts 30 -day mortality in intensive care patients: A prospective prediction study Eur J Anaesthesiol. 2020; 37:1-8

3. 3. Rockwood K, Song X, MacKnight C, et al. A global clinical measure of fitness and frailty in elderly people. CMAJ. 2005;173(5):489-495.

\section{3}

Indications and outcomes of maternal peripartum intensive care unit admissions: a retrospective study

H. Taube'; I. Matot' ${ }^{2}$; N. Levy ${ }^{3}$; O. Goren ${ }^{2}$; R. Marom ${ }^{4}$; CF. Weiniger ${ }^{2}$

${ }^{1}$ General intensive care unit, Sheba Medical Center, Ramat Gan, Israel; ${ }^{2}$ Anesthesia, intensive care and pain medicine, Ichilov Hospital, Tel Aviv-Yafo, Israel; ${ }^{3}$ Anesthesia, critical care and pain medicine, Beth Israel Deaconess Medical Center (BIDMC), Boston, United States of America; ${ }^{4}$ Newborn care, Lis Maternity Hospital, Tel Aviv-Yafo, Israel

Correspondence: $\mathrm{H}$. Taube

Intensive Care Medicine Experimental 2020, 8(2): 000753

Introduction: The most common indications for peripartum ICU admissions are hypertensive diseases of pregnancy and obstetric hemorrhage. Admission indications are well-reported, $(1,2)$ but hemodynamic and laboratory details rarely are. We characterize laboratory values and vital signs according to admission diagnosis in peripartum ICU admissions.

Methods: Retrospective study, IRB approval, 2011-15, an annual birth rate $>11000$. General maternal characteristics, obstetric characteristics, management and ICU admission details including hemodynamics and laboratory test results were assessed. P-value of 0.05 or less was considered statistically significant.

Results: 96 out of 56,865 women had a peripartum ICU admission. The most common admission diagnosis was PPH (post-partum hemorrhage) $(n=35,38.5 \%)$, followed by hypertensive diseases of pregnancy $(n=19,20.9 \%)$, and respiratory complications ( $n=13,14.3 \%) .98 .9 \%$ were admitted post-partum. Mean (SD) ICU LOS was 4.5 (9.2) days. $62.6 \%$ required mechanical ventilation. Demographic and obstetric data according to ICU admission diagnosis are shown in Table 1. We compared women with each diagnosis to the rest of the ICU cohort. Women with PPH had a lower platelet count and temperature, table 2 . Women with hypertensive diseases of pregnancy had higher blood pressure and different laboratory results including higher creatinine, urea and liver enzymes, table 3 . Women with respiratory complications had lower oxygen saturations, and a higher temperature, table 4.

\begin{tabular}{|c|c|c|c|c|}
\hline Parameter & $\begin{array}{l}\text { PPH } \\
\mathrm{N}=\mathbf{3 5}\end{array}$ & $\begin{array}{l}\text { Hypertensive } \\
\text { diseases of } \\
\text { pregnancy } \\
N=19\end{array}$ & $\begin{array}{l}\text { Respiratory } \\
\mathrm{N}=13\end{array}$ & $\begin{array}{l}\text { Other } \\
N=24\end{array}$ \\
\hline Age, years, mean $=\mathrm{SD}$ & $34.52 \pm 5.30$ & $32.38 \pm 4.48$ & $31.31 \pm 5.50$ & $32.96 \pm 5.69$ \\
\hline $\mathrm{BMI}, \mathrm{kg} / \mathrm{m}^{2}$, mean $\pm \mathrm{SD}$ & $27.57 \pm 4.62$ & $27.59 \pm 8.82$ & $27.25 \pm 5.12$ & $26.08 \pm 4.53$ \\
\hline $\begin{array}{l}\text { Gravidity, } \mathrm{n} \text {, median }[\mathrm{TQR}] \text {; } \\
\text { range }\end{array}$ & $3[2-4] ; 1-7$ & $2[1-3] ; 1-7$ & $2[1-3] ; 1-7$ & $2[1-3] ; 1-6$ \\
\hline Parity, n, median [IQR]; range & $2[1-2] ; 0-6$ & $0[0-1] ; 0-5$ & $1[0-2] ; 0-5$ & $1[0-1] ; 0-4$ \\
\hline $\begin{array}{l}\text { Previous abortion, n, median } \\
\text { [IQR]; range }\end{array}$ & $0[0-1] ; 0-3$ & $0[0-1] ; 0-3$ & $0[0-0] ; 0.2$ & $0[0-0.75] ; 0-2$ \\
\hline $\begin{array}{l}\text { Previous cesarean delivery, } \mathbf{n} \text {, } \\
\text { median [IQR]; range }\end{array}$ & $0[0-2] ; 0-6$ & $0[0-0] ; 0-1$ & $0[0-0] ; 0.0$ & $0[0-0.75] ; 0-4$ \\
\hline $\begin{array}{l}\text { Gestational age, weeks, mean } \\
\pm \mathrm{SD}\end{array}$ & $36.90+3.92$ & $33.43+3.89$ & $35.38+3.25$ & $36.54+4.08$ \\
\hline ICU LOS, days, mean $\neq$ SD & $2.63+1.43$ & $2.78+1.51$ & $7.45+13.61$ & $7.08+14.61$ \\
\hline $\begin{array}{l}\text { PC transfusion, } \mathrm{n} \text {, median } \\
{[\mathrm{IQR}] \text {; range }}\end{array}$ & $8[5-13] ; 0-28$ & $0[0-3] ; 0-9$ & $1[0-3] ; 0-9$ & $0[0-2.75] ; 0-14$ \\
\hline
\end{tabular}


Table 2. Admission laboratory parameters and vital sign measurements for women with PPH and the rest of the ICU cohort

\begin{tabular}{|c|c|c|c|}
\hline \multirow[t]{2}{*}{ Parameter } & PPH & Rest of the ICU cohort & \multirow[t]{2}{*}{ p-value } \\
\hline & $\mathrm{N}=35$ & $N=56$ & \\
\hline $\mathrm{Hb}, \mathrm{g} / \mathrm{dL}, \mathrm{mean} \pm \mathrm{SD}$ & $9.43 \pm 1.54$ & $9.92 \pm 2.43$ & 0.289 \\
\hline PLT, $x 1000 / \mu \mathrm{L}$, mean $\pm S D$ & $120.23 \pm 45.84$ & $181.23 \pm 109.88$ & 0.003 \\
\hline SNIBP, $m m H g$, mean \pm SD & $132.5 \pm 29.63$ & $131.42 \pm 22.69$ & 0.903 \\
\hline $\mathrm{DNIBP}, \mathrm{mmHg}$, mean $\neq \mathrm{SD}$ & $76.40 \pm 21.11$ & $81.85 \pm 18.38$ & 0.432 \\
\hline Temperature, ${ }^{\circ} \mathrm{C}$, mean $\pm \mathrm{SD}$ & $35.68 \pm 1.10$ & $36.47 \pm 1.16$ & 0.002 \\
\hline
\end{tabular}

Table 3. Admission laboratory parameters and vital sign measurements for women with hypertensive diseases of pregnancy and the rest of the ICU cohort

\begin{tabular}{llll}
\hline Parameter & $\begin{array}{l}\text { Hypertensive diseases of } \\
\text { pregnancy } \\
\mathrm{N}=\mathbf{1 9}\end{array}$ & $\begin{array}{l}\text { Rest of the study } \\
\text { eohort } \\
\mathrm{N}=72\end{array}$ & p-valuc \\
\hline Creatinine, mg/dL, mean \pm SD & $1.05 \pm 0.57$ & $0.75 \pm 0.30$ & 0.003 \\
\hline UREA, mg/dL, mean \pm SD & $14.63 \pm 7.74$ & $10.52 \pm 4.71$ & 0.005 \\
\hline AST, U/L, mean \pm SD & $258.35 \pm 296.99$ & $41.38 \pm 42.87$ & 0.000 \\
\hline ALT, U/L, mean \pm SD & $184.41 \pm 199.22$ & $35.12 \pm 75.94$ & 0.000 \\
\hline ALP, U/L, mean \pm SD & $166.85 \pm 112.64$ & $95.97 \pm 59.97$ & 0.006 \\
\hline SNIBP, mmHg, mean \pm SD & $150.38 \pm 29.06$ & $127.40 \pm 21.03$ & 0.013 \\
\hline DNIBP, mmHg, mean \pm SD & $100.25 \pm 18.67$ & $76.09=16.10$ & 0.001 \\
\hline Key: SD=standard deriation; ICU=intensive care unit; AST= aspartate transaminase; ALT= \\
alaninc transaminasc; ALP= alkalinc phosphatasc: SNIBP= systolic noninvasivc blood
\end{tabular}

pressure; DNIBP $=$ diastolic noninvasive blood pressur

Table 4. Admission laboratory parameters and vital sign measurements for women with respiratory complications and the rest of the ICU cohort

\begin{tabular}{llll}
\hline Parameter & $\begin{array}{l}\text { Respiratory } \\
\text { complications }\end{array}$ & $\begin{array}{l}\text { Rest of the study } \\
\text { cohort }\end{array}$ & p-value \\
& $\mathbf{N}=13$ & $\mathbf{N}=78$ & \\
\hline WBC, $x 1000 / \mu \mathrm{L}$, mean \pm SD & $12.30 \pm 4.63$ & $16.95 \pm 8.74$ & 0.088 \\
\hline SNIBP, mmHg, mean \pm SD & $127.88 \pm 22.20$ & $132.54 \pm 24.73$ & 0.627 \\
\hline DNIBP, mmHg, mean \pm SD & $79.38 \pm 15.23$ & $80.86 \pm 19.86$ & 0.844 \\
\hline O2 Saturation, mean \pm SD & $93.69 \pm 6.06$ & $98.03 \pm 2.62$ & 0.000 \\
Temperature, ${ }^{\circ} \mathrm{C}$, mean \pm SD & $37.12 \pm 0.77$ & $36.01 \pm 1.18$ & 0.001
\end{tabular}

Key: $\mathrm{SD}=$ standard deviation; $\mathrm{ICU}=$-intensive care unit; $\mathrm{WBC}=$ white blood cell count:

$\mathrm{SNIBP}=$ systolic noninvasive blood pressure $\mathrm{DNIBP}=$ diastolic noninvasive blood pressure

Conclusion: In characterizing demographic, obstetric, hemodynamic and laboratory data according to the admission diagnosis, we report unique findings. Notable findings included a lower admission temperature among women with $\mathrm{PPH}$, possibly associated with a higher rate of packed cells administration, and a lower gestational age and higher incidence of liver and renal dysfunction among women with hypertensive diseases of pregnancy. Considering our findings, the maternal ICU population might be more heterogeneous than previously reported and patient assessment and care should be individualized accordingly.

\section{Reference(s) and grant acknowledgment(s)}

1. 2. Martin SR, Foley MR. Intensive care in obstetrics: An evidence-based review.American Journal of Obstetrics and Gynecology. 2006;195(3):673689. https://doi.org/10.1016/j.ajog.2006.05.042.

2. 1. Pollock W, Rose L, Dennis C-L. Pregnant and postpartum admissions to the intensive care unit: a systematic review.Intensive Care Medicine. 2010;36(9):1465-1474. https://doi.org/10.1007/s00134-010-1951-0.
000791

Short-term psychological outcomes in covid-19 patients

following admission to intensive care

V. Pattni ${ }^{1}$; S. Dewan ${ }^{1}$; G. Collins ${ }^{1}$; I. Rodriguez ${ }^{1}$; L. Kankowski².

C. Mclaughlin²; A. Asardag ${ }^{2}$; J. Phillips'; R. Saha

${ }^{1}$ Critical Care, Princess Alexandra Hospital, Harlow, United Kingdom; ${ }^{2}$ Medical school, Queen Mary University of London, London, United Kingdom

Correspondence: $V$. Pattni

Intensive Care Medicine Experimental 2020, 8(2): 000791

Introduction: Studies indicate high rates of psychological morbidity amongst patients following admission in an intensive care unit (ICU), including acute stress disorder which is predictive of post traumatic stress disorder (PTSD).[1-3] The recent emergence of covid-19 has had a profound impact on patients, with additional psychological burdens implicated with ICU admission.

Objectives: We aimed to establish the prevalence of acute stress disorder in a single cohort of covid-19 patients discharged from ICU, and to assess which variables could be correlated to their psychological outcomes.

Methods: Data was prospectively collated following local audit approval in a UK district general ICU over a 3 month period between March and May 2020. Patients diagnosed with covid-19, who survived ICU care and were discharged to the ward, were eligible for inclusion. A validated screening questionnaire called the "Acute Stress Disorder Scale" was used to assess the acute stress disorder score (ASDS), the primary outcome.[4] Variables including APACHE II score; CAM-ICU score; duration of stay and sedation in ICU; insertion of tracheos tomy; and receipt of invasive ventilation were recorded. A "covid-19 score" was formed to observe the impact of covid-19 specifically on a patient's psychological experience of social isolation.

Results: 75 patients were admitted to ICU, with 22 patients discharged to the ward. 9 patients were included for screening; the other 13 patients eligible for inclusion had been discharged to the community on commencing screening so were unable to participate. The median ASDS score was 39 (IQR 33-54). 3 patients recorded ASDS scores of $\geq 52$, which as an independent cut-off score can predict PTSD with a sensitivity and specificity of $91 \%$ and $86 \%$ respectively. There was a strong positive correlation between ASDS and duration of stay in ICU and sedation (Table 1). There was a weaker correlation with "covid-19 scores" and APACHE II scores. 8 patients had documented CAM-ICU scores, with 6 patients screened as positive.

Table 1: ASDS Scores by tertiles for recorded patient variables

\begin{tabular}{|c|c|c|c|c|c|c|c|}
\hline $\begin{array}{l}\text { Total } \\
\text { Patient } \\
(N=9)\end{array}$ & $\begin{array}{l}\text { sASDS } \\
\text { Score } / 95\end{array}$ & $\begin{array}{l}\text { Median } \\
\text { "COVID- } \\
19 \text { " } \\
\text { Score } / 10\end{array}$ & $\begin{array}{l}\text { Median } \\
\text { APACHE } \\
\text { Score }\end{array}$ & $\begin{array}{l}\text { Median } \\
\text { Number } \\
\text { Eof Days in } \\
\text { Critical } \\
\text { Care }\end{array}$ & $\begin{array}{l}\text { Median } \\
\text { Number } \\
\text { of Days } \\
\text { Sedated }\end{array}$ & $\begin{array}{l}\text { Invasively } \\
\text { Ventilated }\end{array}$ & $\begin{array}{l}\text { Number } \\
\text { Undergoing } \\
\text { Tracheostomy }\end{array}$ \\
\hline$n=3$ & $\begin{array}{l}50-<65 \\
(52,56,60)\end{array}$ & $\begin{array}{l}8 \\
(6,8,8)\end{array}$ & $\begin{array}{l}14 \\
(7,14,19)\end{array}$ & $\begin{array}{l}37 \\
(26,37,43)\end{array}$ & $\begin{array}{l}26 \\
(18,26,28)\end{array}$ & 3 of 3 & 3 of 3 \\
\hline$n=3$ & $\begin{array}{l}35-<50 \\
(37,39,40)\end{array}$ & $\begin{array}{l}9 \\
(6,9,10)\end{array}$ & $\begin{array}{l}16 \\
(8,16,17)\end{array}$ & $\begin{array}{l}30 \\
(21,30,33)\end{array}$ & $\begin{array}{l}16 \\
(10,16,20)\end{array}$ & 3 of 3 & 1 of 3 \\
\hline$n=3$ & $\begin{array}{l}20-<35 \\
(27,32,34)\end{array}$ & $\begin{array}{l}7 \\
(4,7,8)\end{array}$ & $\begin{array}{l}14 \\
(8,14,15)\end{array}$ & $\begin{array}{l}21 \\
(4,21,31)\end{array}$ & $\begin{array}{l}14 \\
(0,14,15)\end{array}$ & 2 of 3 & 2 of 3 \\
\hline
\end{tabular}

Conclusion: In this cohort of covid-19 patients, there is a high incidence of acute stress disorder following admission into ICU. There appears to be a strong correlation between ASDS and duration of ICU stay and sedation. No statistical significance can be derived from these data. Further large studies with long-term follow-up are required to assess if acute stress disorder in this cohort of covid-19 patients is statistically linked to PTSD. 
Reference(s) and grant acknowledgment(s)

1. 1. Jackson, J. C., Jutte, J. E., Hunter, C. H., Ciccolella, N., Warrington, H., Sevin, C., \& Bienvenu, O. J. (2016). Posttraumatic Stress Disorder (PTSD) After Critical IIIness: A Conceptual Review of Distinct Clinical Issues and Their Implications. Rehabilitation Psychology, 61(2), 132-140. https://doi.org/10.1037/ rep0000085

2. 2. Parker, A. M., Sricharoenchai, T., Raparla, S., Schneck, K. W., Bienvenu, O. J., \& Needham, D. M. (2015). Posttraumatic stress disorder in critical illness survivors: A metaanalysis. Critical Care Medicine, 43(5), 1121-1129. https:// doi.org/10.1097/CCM.0000000000000882

3. 3. Davydow, D. S., Zatzick, D., Hough, C. L., \& Katon, W. J. (2013). In-hospital acute stress symptoms are associated with impairment in cognition 1 year after intensive care unit admission. Annals of the American Thoracic Society, 10(5), 450-457. https://doi.org/10.1513/AnnalsATS.201303-0600C

4. 4. Bryant, R. (1999). The acute stress disorder scale: a tool for predicting post-traumatic stress disorder. Australian Journal of Emergency Management,13-15

5. Not applicable

\section{3}

The effect of ICU diaries on the occurrence of impaired mental health and quality of life of ICU SARS-CoV-2 patients and their families: a protocol for an observational cohort study

L. Rutjes-Weurding ${ }^{1}$; S. Pouwels ${ }^{1}$; M. Oomen ${ }^{1}$; S. Op 'T Hoog ${ }^{1}$;

M. Prins-Smulders ${ }^{1}$; C. Van Slobbe ${ }^{1}$; R. Marijnissen-De Jong ${ }^{1}$;

D. Ramnarain

${ }^{1}$ Department of intensive care medicine, St. Elisabeth Hospital, Tilburg, Netherlands

\section{Correspondence: L. Rutjes-Weurding}

Intensive Care Medicine Experimental 2020, 8(2): 000803

Introduction: Patients admitted at the ICU and their family members experience many different stressors, which make them prone for developing psychological disorders like Post Traumatic Stress Disorder (PTSD), anxiety, depression and cognitive dysfunction, also called the post-intensive care syndrome (PICS and PICS-Family). Patients with SARS- CoV-2 infection with acute respiratory failure requiring ICU care and mechanical ventilation are isolated from their loved ones and face an uncertain course of the disease. Most patients have long ICU length of stay with prolonged sedation, analgesia and even muscular blocking agents. Due to longer sedation and mechanical ventilation post-ICU COVID-19 patients will have impaired recall of the ICU stay accompanied by hallucinations or delusions with increased risk of PTSD. The use of an ICU diary for the patient could help fill in memory gaps, gain a sense of reality and complete their fragmented illness narrative.

Objectives: The aim of this study is to evaluate the effect of a doctor and nurse driven ICU diary on the occurrence of impaired mental health in patients admitted with a SARS-CoV-2 infection and their family members.

Methods: An observational cohort study of SARS-CoV-2 patients treated in the ICU of Elizabeth-Tweesteden Hospital in Tilburg, The Netherlands will be conducted. Adult patients admitted at the ICU because of respiratory insufficiency due to SARS-CoV-2, who are mechanically ventilated, are included. During treatment in the ICU the ICU staff initiates a written diary with additional photographs of the patient during treatment. Family members will also be stimulated to make a contribution to this diary. After discharge to a ward, the ICU diary will be continued by nurses of the ward and an ICU nurse practitioner, who is guiding the transfer and treatment on the ward.

After hospital discharge patients' mental health will be monitored on our post-ICU aftercare clinic at 3,6 and 12 months after discharge. Anxiety, depression and PTSD, cognitive dysfunction and quality of life will be screened in patients and their relatives. The Psychosocial Screening Instrument for adult trauma patients (PSIT), a validated tool, will be used for screening for anxiety, depression and PTSD. Cognitive dysfunction will be screened using the Cognitive Failure Questionnaire (CFQ) and quality of life will be assessed by the Short Form Health Survey 36 (SF-36). The recollection of patients' memory of the ICU stay will be evaluated by the ICU memory tool.
Results: The quantitative data of the PSIT, CFQ, SF-36 will be compared over time and between patients and their relatives. Participants' characteristics including the recollection of memory will be reported and if necessary multivariate analysis will be computed.

Conclusion: This study will contribute to the research on ICU diaries and pioneers on the psychological problems of PICS of SARS-CoV-2 patients and the use of ICU diaries in this group.

\section{4}

The effect of ICU diaries on the recollection of memories and the occurrence of impaired mental health in post-ICU patients: a prospective cohort study

L. Rutjes-Weurding ${ }^{1}$; S. Pouwels ${ }^{1}$; M. Oomen ${ }^{1}$; D. Ramnarain ${ }^{1}$

${ }^{1}$ Department of intensive care medicine, St. Elisabeth Hospital, Tilburg, Netherlands

Correspondence: $L$. Rutjes-Weurding

Intensive Care Medicine Experimental 2020, 8(2): 000804

Introduction: Post-intensive care syndrome (PICS) affects $50-70 \%$ of ICU survivors. ICU patients as well as their close relatives are at risk of developing PICS symptoms comprising Post Traumatic Stress Disorder (PTSD), anxiety or depression. Post-ICU patients having delusional memories may have a higher risk for developing PTSD symptoms. (Jones, Griffiths, Humphris, \& Skirrow, 2001). Little is known about the mental health of patients who have complete loss of memory without fragmented or delusional memories. ICU diaries may contribute to the recollection of memories or completion of a fragmented illness narrative. We hypothesize that the use of ICU diaries will increase memory recollection of patients' ICU stay.

Objectives: The aim of this study is to evaluate the effect of ICU diaries on the recollection of ICU memories. Secondary, we investigate the occurrence of impaired mental health in patients with and without memories of their ICU stay.

Methods: Post-ICU patients, admitted to the ICU from January 2015 to January 2017, were included. During ICU treatment the diary was written by either ICU staff and/or patients' relatives. During follow-up at the outpatient ICU aftercare clinic, 12 weeks after hospital discharge, patients were screened for complete loss of memory of the ICU period. PTSD symptoms were screened by the Impact of Event Scale-Revised (IES-R). The Hospital Anxiety and Depression scale (HADs) was used to assess anxiety or depression symptoms.

Results: A total of 174 patients were included, of which 83 men and 91 females, with respectively a mean ICU stay of eleven and nine days. Of the males 48 had an ICU diary and 35 had memories of their ICU stay. Of the females 33 had an ICU diary and 49 had memories of their ICU stay. A multivariate analysis was conducted on the factors that may influence having memories of the ICU. Patients with a diary had three times higher odds of having memories (OR 3.37 (95\%Cl: $1.51-7.51, p=0.003)$. Of the patients who had an ICU diary, there were significantly more men than women who had no ICU-recall $(p=0.013)$. Patients were divided into two groups; 1 . Complete loss of ICU memory. 2. Complete recall, fragmented or delusional memories. Ten patients without any memory of their ICU stay had an IES-R score of $\geq 33$, considered a probable case of PTSD, compared to twenty-two patients with any memory of their ICU stay $(p=0.04)$. HADs scores were not significantly different between both groups.

Conclusion: ICU diaries may contribute to the recollection of memories of an ICU stay. Having any kind of memory of the ICU admission seems to result in a higher risk for developing PTSD symptoms. This study suggests a difference of the effect of ICU diaries between males and females, however further research is needed to elucidate whether this matter is clinically relevant.

\section{Reference(s) and grant acknowledgment(s)}

1. Jones, C., Griffiths, R. D., Humphris, G., \& Skirrow, P. M. (2001). Memory, delusions, and the development of acute posttraumatic stress disorder-related symptoms after intensive care. Critical Care Medicine, 29(3), 573- 580. 


\section{5}

Post Intensive Care Syndrome in patients suffering from Acute Subarachnoid Haemorrhage: results of an outpatient post-ICU aftercare clinic

L. Rutjes-Weurding '; D. Ramnarain '; B. Den Oudsten²; D. Van Rijswijk'; AW. Oldenbeuving ${ }^{1}$; S. Pouwels ${ }^{1}$; J. De Vries ${ }^{3}$

'Department of intensive care medicine, St. Elisabeth Hospital, Tilburg, Netherlands; ${ }^{2}$ Dep. of medical and clinical psychology, center of research on psychological and somatic disease, Tilburg University, Tilburg, Netherlands; ${ }^{3}$ Department of medical psychology, St. Elisabeth Hospital, Tilburg, Netherlands

Correspondence: L. Rutjes-Weurding

Intensive Care Medicine Experimental 2020, 8(2): 000805

Introduction: Survivors of acute subarachnoid haemorrhage (aSAH) may suffer from long-term neurological disability but also from cognitive impairment, anxiety and depression. These symptoms can also be related to the Post Intensive Care Syndrome (PICS) defined as new or worsened impairment in physical, cognitive, psychological domains after ICU treatment. The prevalence of PICS after aSAH is not exactly known and the syndrome is poorly understood.

Objectives: The aim of the study was to investigate the prevalence of PICS and examine ICU- and patient related risk factors.

Methods: We conducted an observational cohort study of post- ICU patients after aSAH, who were evaluated in our post-ICU aftercare clinic three months after hospital discharge. Patients were included between January 2012 and December 2016. Patients were screened for physical complaints, anxiety, depression and PTSD symptoms using respectively an in-hospital developed questionnaire, the Hospital Anxiety and Depression scale (HADS) and the Impact of Event-Revised questionnaire (IES-R). Subjective cognitive complaints were assessed by asking three questions about short-term memory deficits, concentration problems and speed of thinking.

Results: In total, 110 patients visited the post-ICU aftercare clinic after aSAH. When looking at the separate domains of PICS the prevalence of anxiety and depression was $24.3 \%$ and $19.6 \%$, respectively. PTSD was seen in $28.6 \%$ of the patients. There was a high prevalence of cognitive complaints; lack of concentration (63.6\%), short-term memory problems $(45.8 \%)$ and reduced speed of thinking $(60.9 \%)$. Fatigue $(75.0 \%)$ and limitations in daily activity (74.1\%), muscle weakness (42.6\%), pain (37.4\%) and weight loss (32.1\%) were the top five most reported physical complaints. Thirty per cent of patients had PICS symptoms related to all three domains of physical, cognitive and psychological functioning. Only $6(5.5 \%)$ patients had no complaints. High prevalence of PICS symptoms was reported even in good outcome aSAH patients.

Conclusion: In post-ICU patients suffering from aSAH there is a high prevalence of PICS. Even in patients with good outcome after aSAH high prevalence of PICS symptoms was reported. Future studies concerning PICS should focus on the full domain of complaints comprising PICS.

\section{2}

\section{A retrospective study of obstetric admissions in an intensive care} unit

D. Castro ${ }^{1}$; F. Sequeira ${ }^{1}$; C. Roriz ${ }^{1}$; L. Linhares ${ }^{1}$; N. Ferreira ${ }^{1}$; T. Carlos ${ }^{2}$;

L. Pereira ${ }^{1}$

${ }^{1}$ Serviço de medicina intensiva, Centro Hospitalar de Leiria, Leiria, Portugal; ${ }^{2}$ Serviço de anestesiologia, Centro Hospitalar de Leiria, Leiria, Portugal

Correspondence: $D$. Castro

Intensive Care Medicine Experimental 2020, 8(2): 000852

Introduction: Obstetric admissions to an Intensive Care Unit (ICU) may be considered a marker of morbidity but are a rare event because pregnant women are usually young and healthy. The incidence varies between 0.4 to $16 \%$ of critical care admissions or 0.7 to 13.5 per 1000 deliveries. Reasons for admissions are conditions related to pregnancy (haemorrhagic events, preeclampsia, eclampsia or puerperal infections) or conditions unrelated to pregnancy (congenital and valvular heart disease, pulmonary hypertension, etc). These patients represent a challenge to intensivists due to the physiological adaptations and pathological concerns during puerperium.

Objectives: The aim of present study is determining the incidence, epidemiological characteristics and outcomes of postpartum women who required ICU admission.

Methods: A retrospective cohort study of all obstetric admissions in a Portuguese 10-bed ICU between January 2015 to January 2020.

Results: In the period from January 2015 to January 2020, 17 obstetric patients were admitted to our ICU, representing $1.3 \%$ of all ICU admissions and 1,7 per 1000 deliveries. The mean patient's age was 32.5 years (21-45 years old) and the mean of gestational age was 36.9 weeks (28.1-40.7 weeks). Out of all women, $55.6 \%$ were primiparous, all of them were single pregnancies and all were admitted in postpartum care, most patients $(76,5 \%)$ were admitted in immediate postpartum period.

Most common delivery type was caesarean in $88.2 \%$ women, instrumented delivery in 5.9\% and eutocic delivery in 5.9\%. The three most common causes of admissions were obstetric haemorrhage in 6 cases (33.3\%); HELLP Syndrome in 4 cases $(22.2 \%)$ and pneumonia in 3 cases $(16.7 \%)$. Both instrumented and eutocic deliveries had obstetric haemorrhage as cause of admission.

The median length of ICU stay was 4 days. The most common interventions during ICU admission were transfusion of blood products in 8 patients $(47.1 \%)$, mechanical ventilation in 9 patients $(52.9 \%)$, with 3 patients for a period $<24$ hours. Only 2 patients needed vasopressors and 1 patient needed Renal Replacement Therapy. The mean of APACHE II score was 10 and SAPS II was 23. One patient died, with a maternal mortality rate of 0,1 per 1000 deliveries. This casualty was a 45-years-old woman who was admitted with a pulmonary thromboembolism.

Conclusion: Although obstetric admissions to ICU may be considered a marker of morbidity, there are few national studies to investigate these admissions. In our study, these patients accounted for $1.3 \%$ of total admissions or 1,7 per 1000 deliveries. These rates are very heterogeneous between ICUs and it may be explained by several factors, including the number of ICU beds or the existence of maternal and child centres where women are followed when there is a potential problem in pregnancy or prepartum.

The length of ICU stay is 4 days and the most common reasons for ICU admission are hypertensive disorders of pregnancy and obstetric haemorrhage and it is comparable with other studies. These patients typically have a short length of ICU stay and low hospital mortality. The only fatality case reported is the oldest pregnant woman. Increased maternal age is related to many complications during pregnancy and delivery, thus we need to be considered as a risk factor Obstetric patients in ICU are a challenge that needs a multidisciplinary approach.

\section{Reference(s) and grant acknowledgment(s)}

1. Seppänen PM, Sund RT, Uotila JT, Helminen MT, Suominen TM. Maternal and neonatal characteristics in obstetric intensive care unit admissions. Int J Obstet Anesth. 2020;41:65-70. https://doi.org/10.1016/j.ijoa.2019.07.002

2. Oliveira S, Filipe C, Husson N, et al. Obstetric Admissions to the Intensive Care Unit: A 18-Year Review in a Portuguese Tertiary Care Centre. Acta Med Port. 2019;32(11):693-696. https://doi.org/10.20344/amp.11410

3. Barry Y, Deneux-Tharaux C, Saucedo M, et al. Maternal admissions to intensive care units in France: Trends in rates, causes and severity from 2010 to 2014. Anaesth Crit Care Pain Med. 2019;38(4):363-369. https://doi. org/10.1016/j.accpm.2018.12.007

4. Fadiloglu E, Bulut Yuksel ND, Unal C, et al. Characteristics of obstetric admissions to intensive care unit: APACHE II, SOFA and the Glasgow Coma Scale. J Perinat Med. 2019;47(9):947-957. https://doi.org/10.1515/jpm-2019-0125

5. Maiden MJ, Finnis ME, Duke GJ, et al. Obstetric admissions to intensive care units in Australia and New Zealand: a registry-based cohort study [published online ahead of print, 2020 May 2]. BJOG. 2020;https://doi. org/10.1111/1471-0528.16285. https://doi.org/10.1111/1471-0528.16285 
000873

\section{Monitoring outcomes in intensive care unit: the transfusion} traceability quality indicator

A. ARGYROU ${ }^{1}$; A. Pouliakis²; A. Gafou ${ }^{3} ;$ K. Tsoumakas ${ }^{4}$;

T. Mariolis-Sapsakos ${ }^{4}$; G. Fildisis ${ }^{4}$

${ }^{1}$ Blood transfusion service/ faculty of nursing, Agioi Anargyroi General \& Oncology Hospital/ National and Kapodistrian University of Athens, Athens, Greece; ${ }^{2}$ 2nd department of pathology, Attikon University Hospital, Chaidari, Greece; ${ }^{3}$ Blood transfusion service, Agioi Anargyroi General \& Oncology Hospital, Athens, Greece; ${ }^{4}$ Faculty of nursing, National and Kapodistrian University of Athens, Athens, Greece

Correspondence: A. Argyrou

Intensive Care Medicine Experimental 2020, 8(2): 000873

Introduction: According to European Directorate for the Quality of Medicines \& HealthCare "traceability is the ability to trace each blood component unit from donor to its final destination (patient, medicinal products manufacturer, disposal) \& vice versa." It is mandatory procedure \& prerequisite for Hemovigilance Networks. Ideally, Blood Transfusion Services (BTS) should be able to trace every blood unit they issue.

Traceability is achieved by recording transfusion data (component's unique identification number, patient's data) in electronic files of computer systems and/or in paper-forms, both in BTS \& clinical ward.

Nurses contribute to implementing traceability since their actions mark the beginning \& the end of a transfusion. Therefore, it is vital to train them on traceability-issues.

Objectives: To measure effectiveness of educational interventions regarding traceability, which were implemented in 2018 in the 8-bedICU of an oncology hospital, by recording the change in traceability levels, the years before (2017) \& after (2019) implementing relevant educational activities.

Methods: During 2017 \& 2019, 84 \& 91 patients respectively were hospitalized in the ICU. All 20 ICU nurses were trained on traceability issues during 2018 (lectures, distribution of printed material in the ward, training in small groups or individually) by a BTS doctor. Training effectiveness was checked in two ways:

1.Nurses answered a traceability-relevant questionnaire in 2017 \& 2019 (before \& after implementing educational activities). Change in percentage of correct answers was recorded.

2.All blood products transfused before (2017) \& after (2019) training interventions were traced (both as a whole \& per blood component category). In our hospital, transfusion-data are recorded in paper files (ICU) \& in electronic files (BTS). A BTS Health Visitor checked \& collated all data recorded in both files. Transfusions were described as "confirmed" only when the patient's \& unit's data were identical in both files- otherwise, they were described as "probable".

Results were statistically evaluated (t-test).

Results: 1.A significant improvement in the percentage of questionnaire correct answers before \& after training interventions was recorded $(81.66 \%$ and $95.83 \%$ respectively).

2.Results regarding traceability are depicted in Table 1. The lack of statistical significance in platelet/plasma results is attributed to low transfusion numbers.
Table 1: Traceability of blood transfusion (years 2017 and 2019)

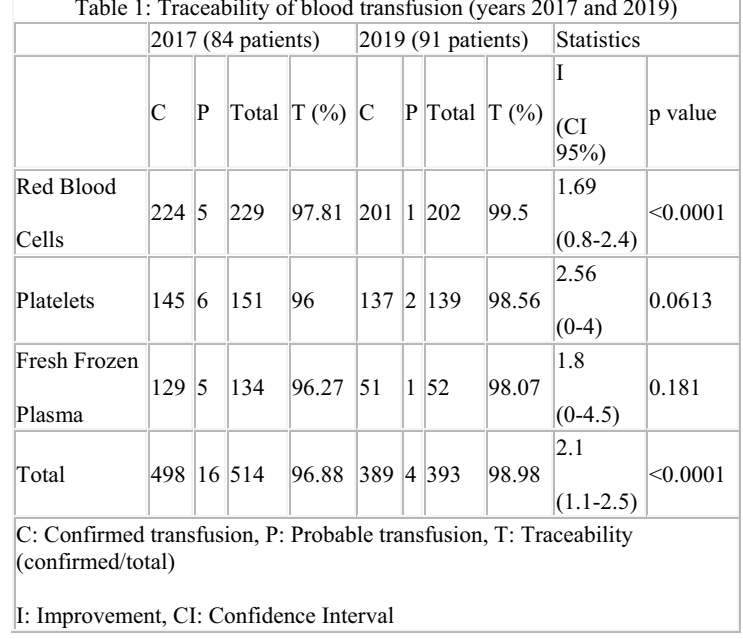

Conclusion: 1. Implemented educational interventions contributed to the improvement of ICU nurses' knowledge \& practices on traceability issues.

2. Auditing cases of non-traceability per nurse, could help in planning targeted training to nurses with frequent "probable transfusion" results.

3. Repeated training can strengthen \& consolidate proper traceability practices, enhance safety of all involved in transfusion chain (blood donor, patient, staff) \& upgrade quality of health services provided.

\section{Reference(s) and grant acknowledgment(s)}

1. Commission Directive 2005/61/EC of 30 September 2005 implementing Directive 2002/98/EC of the European Parliament and of the Council as regards traceability requirements and notification of serious adverse reactions and events

2. Guide to the preparation use and quality assurance of Blood Components. 20th ed. Strasbourg. EDQM 2020. Chapter 10, Haemovigilance, p 324-6 3. N/A

\section{1}

Clustering of ARDS-covid 19 patients based on admission characterististics and 3 days follow-up

C. DUPUIS ${ }^{1}$; PH. Wicky²; M. Neuville ${ }^{3} ;$ J. Oziel ${ }^{4} ;$ J. Reignier ${ }^{5}$; T. Nicolas'; B. Mourvillier ; D. Goldgran-Toledano ${ }^{8}$; S. Siami ${ }^{9}$; L. Le Fèvre ${ }^{2}$; B. Souweine ${ }^{1}$; JF. Timsit ${ }^{2}$

${ }^{1}$ Médecine intensive et réanimation, CHU Gabriel-Montpied, Clermont-Ferrand, France; ${ }^{2}$ Réanimation médicale et infectieuse, Bichat-Claude Bernard Hospital, Paris, France; ${ }^{3}$ Réanimation, Hospital Foch, Suresnes, France ${ }^{4}$ Réanimation médico-chirurgical, Avicenne Hospital (AP-HP), Bobigny, France; ${ }^{5}$ Médecine intensive réanimation, Nantes University Hospital Hotel-Dieu, Nantes, France; ${ }^{6}$ Médecine intensive et réanimation, C.H.U de Grenoble, La Tronche, France; ${ }^{7}$ Médecine intensive et réanimation polyvalente, CHU Reims - Champagne Clinic, Reims, France; ${ }^{8}$ Médecine intensive et réanimation, Intercommunal Hospital Group Le Raincy Montfermeil, Montfermeil, France; ${ }^{9}$ Réanimation, Hospital Center Sud Essonne -, Étampes, France 
Correspondence: $C$. DUPUIS

Intensive Care Medicine Experimental 2020, 8(2): 000891

Introduction: Outbreaks of the coronavirus disease 2019 (COVID-19) epidemic have been causing worldwide health concerns since December 2019. Most of the patients admitted in ICU fulfill acute respiratory distress syndrome. Little is known about the heterogeneity of those patients and their outcomes.

Objectives: Identification of more homogeneous subgroups of COVID-19 patients admitted in ICU with similar characteristics, independently of any outcome measures.

Methods: We included patients admitted for COVID-19 disease to one of the French ICU participating in the French Outcomerea $\odot$ prospective cohort between March 1st and April 31th 2020 and having a ICU length of stay longer than 72 hours.We recorded baseline characteristics including age, comorbidities, BMI and severity and main laboratory features at day 3 including blood cell counts, Ferritin, CRP, DDimer and the evolution of all those indices between admission to day 3 .

Heterogeneity was explored using a Hierarchical clustering in a principal component approach. Random forest algorithm allowed the identification of the most important predictors in each sub group.

Results: 357 patients still alive at day 3 could be included. The median age was $61[52 ; 70]$ years, 277 (77.6) were male, the median body mass index was 28.4 [25.4; 32], the median charlson score was 1 [0; 3]. At day 3 , the median SOFA without respiratory was $3[1 ; 4]$ and the median SOFA respiratory was 3 [2; 3]. At day 28, $147(41.2)$ were still under mechanical ventilation or died including 104 (29.2) dead patients.

Finally, 5 sub-groups could be identified.

Cluster A ( $\mathrm{N}=76,21 \%, 28 \%$ mortality) was characterized by quite old patients $(63[55 ; 72.5]$ years old), with multiorgan failure at day 3 , and low lymphocytes, monocytes and neutrophils levels which tended to decrease from day 1 to day 3 with a moderate increase of CRP and ferritin levels.

Cluster $\mathrm{B}(\mathrm{N}=79,22 \%, 23 \%$ mortality) had younger patients $(53[48 ; 61]$ years old), mainly with respiratory failure and the lowest inflammatory syndrome at day 3

Cluster C ( $\mathrm{N}=95,26.7 \%, 31 \%$ mortality) gathered quite old patients ( 64 $[55 ; 73]$ years), with a less severe respiratory failure, but others organ failures, and with a moderate inflammatory syndrome.

Cluster $\mathrm{D}(\mathrm{N}=100,28 \%$, 34\% mortality), was remarkable for the severity of their patients at day 3 with multi organ failures and for their inflammatory profile with high level of ferritin, CRP and neutrophils at day 3.

Cluster $\mathrm{E}(\mathrm{N}=7,2 \%, 29 \%$ mortality) gathered young, obese patients with multi organ failure and high level of inflammation.

Conclusion: This clustering approach revealed several profiles of COVID-19 patients, with several levels of organ failures and variations in inflammation markers, highlighting for some immunosuppression and others hyper-inflammatory conditions.

\section{4}

\section{Effect of smoking on COVID-19 severity: a systematic review} and meta-analysis

W. N. Charles ; R. K. Reddy²; A. Sklavounos ${ }^{3}$; A. Dutt ${ }^{1}$; P. T. Seed ${ }^{4}$;

A. Khajuria

${ }^{1}$ Department of surgery and cancer, Imperial College London, London, United Kingdom; ${ }^{2}$ Department of medicine, Hull York Medical School, York, United Kingdom; ${ }^{3}$ Department of medicine, King's College London, London, United Kingdom; ${ }^{4}$ Department of women and children's health, King's College London, London, United Kingdom; ${ }^{5}$ Nuffield department of surgical sciences, University of Oxford, Oxford, United Kingdom

Correspondence: W.N. Charles

Intensive Care Medicine Experimental 2020, 8(2): 000914

Introduction: Various comorbidities have been described as risk factors for severe COVID-19. The impact of smoking on COVID-19 severity has been previously reported in several meta-analyses limited by small sample sizes and poor methodology.

Objectives: To rigorously quantify the effects of smoking on COVID19 severity, including hospital and critical care outcomes.
Methods: This review was registered on PROSPERO a priori, CRD42020180920, and adhered to PRISMA guidelines. Two authors independently searched Medline, Embase, CENTRAL and Web of Science for studies published between December 1, 2019 and April 24, 2020 , with an updated search on June 2, 2020. The COVID-19 resource centres of major journals were also screened for relevant studies. All clinical studies reporting the smoking status of hospitalised patients presenting with different severities of disease and/or at least one clinical endpoint of interest (disease progression, ICU admission, need for mechanical ventilation and mortality) were included. Smoking history included current and former tobacco smokers or e-cigarette users. Studies with insufficient information to distinguish outcomes based on smoking status were excluded. Random effects meta-analyses of pooled raw data were employed for each outcome with sufficient data. Results are presented as risk ratios (RR) with corresponding $95 \%$ Confidence Intervals $(\mathrm{Cl})$. Study quality was independently assessed using the Newcastle-Ottawa Scale.

Results: After screening 1050 papers, we analysed the findings of 47 eligible studies reporting on 32849 hospitalised COVID-19 patients. Of these, 8417 (25.6\%) reported a smoking history, comprising 1501 current smokers, 5676 former smokers and 1240 unspecified smokers. Current smokers had an increased risk of presenting with severe COVID-19 (RR 1.80, 95\% Cl 1.14-2.85; $p=0.012$ ), as well as severe or critical COVID-19 (RR 1.98, 95\% Cl 1.16-3.38; $\mathrm{p}=0.012$ ), compared to former and never-smokers. However, the increased risk of in-hospital mortality was not statistically significant (RR $1.46,95 \% \mathrm{Cl} 0.83-2.60$ $\mathrm{p}=0.192$ ). Compared to never-smokers, patients with a smoking history had a significantly increased risk of severe COVID-19 (RR 1.31, 95\% Cl 1.12-1.54; $\mathrm{p}=0.001$ ), severe or critical COVID-19 (RR 1.35, 95\% Cl 1.19-1.53; $p<0.001$ ), in-hospital mortality (RR 1.26, 95\% Cl 1.20-1.32 $\mathrm{p}<0.001$ ), disease progression ( $R R 2.18,95 \% \mathrm{Cl} 1.06-4.49 ; \mathrm{p}=0.035$ ), and need for mechanical ventilation (RR $1.20,95 \% \mathrm{Cl} 1.01-1.42$ $\mathrm{p}=0.043$ ).

Conclusion: Smokers are vulnerable to increased severity of COVID-19 and worse in-hospital outcomes. In the absence of current targeted therapies against COVID-19, governments, policymakers and other key stakeholders must focus on preventative and supportive strategies to reduce morbidity and mortality in this patient population.

\section{8}

Clinical parameters that affect the outcome in ICU patients

with severe influenza infection

A. Vakalos'; N. Tsikliras' ${ }^{1}$ : S. Patsatzakis

${ }^{1}$ Icu, Xanthi General Hospital, Xanthi, Greece

Correspondence: A. Vakalos

Intensive Care Medicine Experimental 2020, 8(2): 000038

Introduction: Influenza virus infection is an important cause of mortality worldwide, leading to 250000 to 500000 deaths each year in the developed world. Mortality is caused by the primary viral infection, but also by influenza-associated pulmonary co-infections.

Objectives: Our retrospective observational study aimed to test the hypothesis that there is a difference in mean values of clinical data on ICU admission day, among patients with confirmed influenza infection who died and patients who survived in both Medical and Surgical ICU served to a community hospital.

Methods: From January 2006 to October 2019, 1332 patients admitted to our ICU. From these, 25 (1.87\%), 14 males and 11 females, were indicated with the diagnosis of severe influenza infection. Mean age 61.08 years, length of stay (LOS) 17.68 days, days under mechanical ventilation (MVD) 13.6 days. The patients separated into two groups. Group A involved all patients who survived ICU and group B all patients who died in ICU. We looked for statistically significant differences ( $p$-value two-tailed) between the medians values of two groups, using the unpaired Mann - Whitney test or the unpaired t-test Welch corrected according to the normality test, according to clinical data on the ICU admission day.

Results: We detected no statistical difference between the two groups according: Temperature: $(p=0.3022)$, MAP: $(p=0.1750), H R:(p=0.1410)$, RR: (0.2800), Na: $(p=0.4029), k:(p=0.1539), B U N:(p=0.1384), C r:$ 
$(p=0.0675), W B C:(p=0.1340), H t:(p=0.4889), P a O 2:(p=0.3680)$, $p H:(p=0.1096)$, albumin: $(p=0.0576)$, glucose: $(p=0.1139)$, Bilirubin: $(p=0.9359), P a C O 2:(p=0.2966)$, glucose: $(p=0.1139), P a O 2 / F i O 2$ ratio: (0.2364). We only detected statistical significant higher group $B$ value according HCO3: (0.040).

Conclusion: According to our data, there was no statistical difference detected between the two groups according to all clinical parameters we measured, except $\mathrm{HCO} 3$ values, which were lower in patients who died. Our data suggest that even though pH didn't achieve to make a difference, acid-base disorders may be so important to make as give a priority to these patients, as more likely to have a more severe illness.

\section{6}

Changes in high-risk surgical practices in patients in Brazilian intensive care units from 2008 to 2018

J. M. Silva' ; R. C. D. F. Chaves ${ }^{1}$; T. Corrêa' ; M. S. C. Assuncao ${ }^{2}$; C. Amendola ${ }^{3}$. A. Serpa Neto ${ }^{2}$;. K. Henrique ${ }^{4}$; L. M. S. Malbouisson ${ }^{5}$; B. Cardoso Gomes ${ }^{6}$; M. Jacques ${ }^{7}$; S. M. Lobo ${ }^{8}$

${ }^{1} \mathrm{Icu}$, Hospital Israelita Albert Einstein, São Paulo, Brazil; ${ }^{2}$ Intensive care unit, Hospital Israelita Albert Einstein, São Paulo, Brazil; ${ }^{3}$ Fundação pio xii, Hospital de Câncer de Barretos, Barretos, Brazil; ${ }^{4}$ Anestesiologia, USP University City, São Paulo, Brazil; ${ }^{5}$ Trauma and emergency surgery intensive care unit, Hospital das Clínicas Faculdade de Medicina USP, São Paulo, Brazil; ${ }^{6}$ Departamento de Medicina Integrada, Universidade Federal do Paraná, Curitiba, Brazil; ${ }^{7}$ Psicologia, UniCesumar - Curitiba Campus, Curitiba, Brazil; ${ }^{8}$ Intensivist, Hospital de Base, São José do Rio Preto, Brazil

Correspondence: $\mathrm{B}$. Cardoso Gomes

Intensive Care Medicine Experimental 2020, 8(2): 000046

Introduction: Many advances in the care of high-risk surgical patients have been made. Nevertheless, temporal trends in outcomes in this population of patients in developing countries are lacking.

Objectives: This study aimed to investigate trends in mortality and complications in high-risk surgical patients over a ten-year period.

Methods: Ethical approval was obtained N. 1.570.327-2016. Data from two prospective multicenter cohort studies (SCORIS AND BRASIS studies) conducted by AMiB Network from 2008 to 2018 to address the outcomes of high-risk surgical patients in Brazil were compared. All non-cardiac surgical patients admitted to intensive care units (ICU) were daily followed up to hospital discharge to determine complications and mortality.

Results: Individual patient data from 1,491 high-risk surgical patients (904 patients from BRASIS study and 587 from SCORIS study) in 50 ICUs were enrolled in this analysis. table 1.

\begin{tabular}{|c|c|c|c|c|c|c|}
\hline \multicolumn{7}{|c|}{ Baseline characteristics } \\
\hline Variable & Original $\mathrm{C}$ & Cohort & & tched Cohort & & \\
\hline & $\begin{array}{l}\text { Scoris } \\
(n=587)\end{array}$ & $\begin{array}{l}\text { Brasis } \\
(n=904)\end{array}$ & $\mathrm{P}$ & $\begin{array}{l}\text { Scoris }(\mathrm{n}= \\
509)\end{array}$ & $\begin{array}{l}\text { Brasis } \\
(n=509)\end{array}$ & $\mathrm{P}$ \\
\hline Male, n (\%) & $\begin{array}{l}363 \\
(61.9 \%)\end{array}$ & $\begin{array}{l}605 \\
(67 \%)\end{array}$ & 0.40 & $277(54.4 \%)$ & ) $265(53.3 \%$ & 0.56 \\
\hline $\begin{array}{l}\text { Age }(y) \\
\text { mean } \pm \text { SD }\end{array}$ & $71.1 \pm 7.4$ & $69.5 \pm 7.0$ & 0.07 & $62.2 \pm 16.5$ & $61.9 \pm 16.5$ & 0.83 \\
\hline ASA Score & & & 0.16 & & & 0.76 \\
\hline $\begin{array}{l}\text { P 2 score } \\
\text { n (\%) }\end{array}$ & $\begin{array}{l}301 \\
(51.3 \%)\end{array}$ & $\begin{array}{l}522 \\
(57.8 \%)\end{array}$ & & $272(53.6 \%)$ & $278(54.8 \%)$ & \\
\hline $\begin{array}{l}\text { P } 3 \text { score } \\
\text { n }(\%)\end{array}$ & $\begin{array}{l}275 \\
(46.9 \%)\end{array}$ & $\begin{array}{l}381 \\
(42.2 \%)\end{array}$ & & $230(45.2 \%)$ & $230(45.2 \%)$ & \\
\hline $\begin{array}{l}\text { SOFA scor } \\
\text { median (mi } \\
\max )\end{array}$ & $5.0(0-20)$ & $4(0-19)$ & 0.04 & $4.0(0-19)$ & $3.0(0-19)$ & 0.06 \\
\hline
\end{tabular}

The crude hospital mortality rate decreased from $20.6 \%(95 \% \mathrm{Cl}, 17.1$ to $24.6 \%$ ) in 2008 to $8.9 \%(95 \% \mathrm{Cl}, 7.0$ to $11.2 \%)$ in 2018 . After adjusting for baseline Sequential Organ Failure Assessment (SOFA) Score, age and type of ICU, patients enrolled in $2018(10.1 \%$; $95 \% \mathrm{Cl}, 7.5$ to 13.4) compared those enrolled from $2008(16.1 \% ; 95 \% \mathrm{Cl}, 12.8$ to 20.0$)$ exhibited a lower risk of hospital death $(\mathrm{OR}, 0.58 ; 95 \% \mathrm{Cl}, 0.40$ to 0.85 ; $\mathrm{P}=0.005$ ) and ICU mortality rate, $5.5 \%$ in 2018 and $11.2 \%$ in 2008 (OR, $0.46 ; 95 \% \mathrm{Cl}, 0.29$ to $0.75 ; \mathrm{P}=0.001)$.
The frequency of infection decreased significantly from $19.8 \%$ in 2008 to $2.9 \%$ in $2018(\mathrm{OR}, 0.12 ; 95 \% \mathrm{Cl}, 0.07$ to $0.21 ; \mathrm{P}<0.001)$ while general rate of complications did not differ between the two periods, $34.8 \%$ (95\% Cl, 29.8 to $40.3 \%$ ) in 2008 and $33.8 \%$ (95\% Cl, 28.9 to $39.2 \%$ ) in 2018.

Conclusion: Over the 10-year period between 2008 and 2018, mortality and incidence of infection improved in high-risk surgical patients admitted to Brazilian intensive care units, however general complications rate remained unchanged.

\section{Reference(s) and grant acknowledgment(s)}

1. Lobo SM, Rezende E, Knibel MF, Silva NB, Paramo JA, Nacul F, et al. Epidemiology and outcomes of non-cardiac surgical patients in Brazilian intensive care units. Rev Bras Ter Intensiva. 2008;20(4):376-84.

2. Acknowledgements: To the data collection BRASIS study group, Israelita Albert Einstein Hospital and Barretos Cancer Hospital for the support to the study. The study was endorsed by the Brazilian Network of Research in Intensive Care (AMIBnet).

000931

"A tidal wave of patients": effects of COVID-19 on a busy district general hospital serving a large BAME population

S. Bakare ${ }^{1}$; M. Wilson ${ }^{2}$; J. Borkowski ${ }^{3}$

${ }^{1}$ Anaesthetics and Intensive Care Medicine, London North West University Healthcare NHS Trust, London, United Kingdom; ${ }^{2}$ Anaesthetic, London North West University Healthcare NHS Trust, London, United Kingdom; ${ }^{3}$ Intensive care, London North West University Healthcare NHS Trust, London, United Kingdom

Correspondence: S. Bakare

Intensive Care Medicine Experimental 2020, 8(2): 000931

Introduction: Northwick Park Hospital (NPH) is a busy District General Hospital in Northwest London serving the Harrow, Brent and Ealing Councils which are very multi-ethnic areas with a dense BAME population. NPH has a tertiary Infectious Diseases Unit, is one of the busiest A\&E Departments in the UK and is in close proximity to London Heathrow, one of the busiest airports in the world.(1) NPH became the epicentre of the COVID-19 Pandemic in the UK in Mid-March as the country transitioned from the containment to the delay phase of pandemic management. A "Tidal Wave"(1) of COVID-19 patients hit NPH early with cases doubling every two days before moving across the country. NPH was reconfigured and its services upscaled (one of the first in the country) in anticipation and duly surpassed its baseline 22 Intensive Care Unit (ICU) beds during the transition. (2) Over 4 weeks, ICU Beds increased to 70, and CPAP capabilities also quadrupled seperately. Despite this increase, half of the over 260 COVID patients admitted to our ICU were transferred to other hospitals within our North West London critical care network (NWCCN) to manage the significant increase in demand and alleviate pressure

Objectives: We aim to illustrate the increased burden the COVID-19 pandemic put on our critical care services and the impact of the pandemic on the largely BAME population served by our hospital by comparing our admissions between March and May 2020 to the same time period in 2019.

Methods: Data was collected from our ICNARC Database and analysed using Simple Statistical Method.

Results:

- 87\% higher admissions between March and May 2020 compared to the same period in 2019. A total of 379 Patients admitted to the ICU. 70\% of which were diagnosed with COVID-19.

- Mortality Rate in 2020 was $26 \%$ vs. 22\% in 2019. COVID-19 Subgroup (CS) analysis showed a mortality rate of $44 \%$.

- Average APACHE Scores were lower in $2020,17 \%$ vs. $23 \%$ in 2019. CS was also 17.

- Organ Support

- Increased Ventilatory Support 74\% in 2020 vs. 54\% in 2019. $85 \%$ in CS.

- Decreased RRT $21 \%$ in 2020 vs. $37 \%$ 2019. $22 \%$ in CS. 
- Significantly Increased CVS Support $95 \%$ in 2020 vs. $38 \%$ in 2019. $100 \%$ in CS

Ethnicity

- Increased BAME admissions $73 \%$ in 2020 vs. $49 \%$ in 2019. 83\% in CS

Gender

- Increased Male admissions $74 \%$ in 2020 vs. $60 \%$ in 2019. $80 \%$ in CS

Conclusion: Our Data shows there was both an increase in the number of patients and the proportion of patients requiring organ support, with BAME and Male patients disproportionally affected in line with recent publications (3). Despite the 4 -fold increase in our critical care capacity (the generally accepted protocol that left other centres with excess capacity), our systems were stretched during these unprecedented times to the extent we had to transfer half our patients out (4). The mutual aid response by NWCCN and the dedication of the hospital multidisciplinary teams at all levels whose lives were also severely disrupted helped maintain patient care and safety throughout this period.

\section{Reference(s) and grant acknowledgment(s)}

1. None to declare

2. 5. NHS England. Specialty guides for patient management during the coronavirus pandemic. Clinical guide for the management of surge during the coronavirus pandemic: rapid learning, 12 April 2020, Version 2. https:// www.england.nhs.uk/coronavirus/wp-content/uploads/sites/52/2020/03/ C0167-specialty-guide-surge-based-on-current-hospital-experience-v2.pdf Accessed 24/6/2020

3. 4. HospitalsurgecapacityinatertiaryemergencyreferralcentreduringtheCOVID-19 outbreak in Italy L.Carenzo et al Anaesthesia 2020, 75, 928-934

4. 3. ICNARC report on COVID-19 in critical care 19 June 2020 https://www. icnarc.org/Our-Audit/Audits/Cmp/Reports Accessed 24/6/2020

5. 2. ICU surge capacity in a busy London district general hospital during the COVID-19 pandemic Martin Shao Foong Chong, Tun Win Hla, Giulia Sartori Anaesthesia News https://anaesthetists.org/Home/Resources-publications/ COVID-19-guidance/ICU-surge-capacity-in-a-busy-London-district-gener al-hospital-during-the-COVID-19-pandemic Accessed 24/6/2020

6. 1. Coronavirus: The London hospital hit by a 'tidal wave' of patients Thomas Mackintosh June 2nd 2020 https://www.bbc.co.uk/news/uk-england-londo n-52812457 Accessed 24/6/2020

\section{0}

\section{Neutrophil-to-lymphocyte ratio as a risk marker of bad outcome} in patients hospitalized with COVID-19

R. Thouny ${ }^{1}$; P. Goffin ${ }^{1}$; J. Guntz ${ }^{1}$; L. Jadot ' ; C. Legrain ${ }^{1}$; D. Noirot ${ }^{1}$; I. Noirot'; S. Verscheure'; P. Demaret ${ }^{1}$

${ }^{1}$ Department of anesthesia and intensive care., MontLegia Hospital Groupe Santé CHC, Liège, Belgium

Correspondence: P. Goffin

Intensive Care Medicine Experimental 2020, 8(2): 000980

Introduction: The fast worldwide spread of Coronavirus disease 2019 (COVID-19) and the resulting dramatic pandemic have stimulated clinical research to describe patients risks and evolution. Neutrophilto-lymphocyte ratio (NLR) has shown its prognostic value in cardiovascular diseases, cancer, infections or inflammatory diseases, probably because it is correlated to the level of dysregulation of the immune response. We therefore studied the association between NLR and inhospital death or intensive care unit (ICU) admission in patients hospitalized with COVID-19.

Methods: We retrospectively analyzed a cohort of patients with confirmed COVID-19 admitted to the MontLegia Hospital (Liège, Belgium) between $15 / 03 / 20$ to $04 / 05 / 20$. We recorded baseline data including biological and clinical features, as well as ICU admission and hospital mortality.

The independent association between NLR and the composite outcome of ICU admission and/or hospital mortality was assessed with multivariable logistic regression.
Results: Two hundred and thirteen patients were admitted to our hospital with a confirmed diagnosis of COVID-19 (polymerase chain reaction positive for SARS-CoV-2) during the study period, among which $92(43.2 \%)$ were admitted to the ICU and/or died during the hospital stay (composite primary outcome).

The median (interquartile range (IQR)) NLR was significantly higher for patients with the composite primary outcome (7 (4.8-11.6) versus $4.6(3.1-6.6), p<0.001)$. At hospital admission, patients with the composite primary outcome had a worse oxygenation (SpO2 / FiO2 $3.8(223-399)$ versus 323 (304-424), $\mathrm{p}<0.001)$, a higher creatinine level (1.14 (0.84-1.66) versus $0.92(0.79-1.21) \mathrm{mg} / \mathrm{dL}, \mathrm{p}=0.006)$ and a higher C-reactive protein (CRP) level (108 (64-183) versus 67 (32-125) mg/L, $\mathrm{p}<0.001$ )

The area under the receiver operating characteristic curve describing NLR as a predictor of ICU admission and/or death was 0.71 (95\% confidence interval $(\mathrm{Cl})$ 0.63-0.77), which was significantly different from the non-discrimination line $(p<0.001)$. The threshold with the best global predictive value for ICU admission and/or death, according to the Youden index, was 6.1 (sensitivity $66.3 \%$, specificity $74.4 \%$ ).

After adjustment for potential confounders, NLR remained associated with ICU admission or death: adjusted odds ratio (OR) 1.1, 95\% Cl 1.04$1.17, \mathrm{p}=0.002$. Patients with an NLR $\geq 6.1$ had a 5.72 -fold increased risk of ICU admission or death (adjusted OR 5.72, Cl95\% 2.80-11.71, $\mathrm{p}<0.001)$.

Conclusion: The NLR is independently associated with ICU admission or death during hospital stay for the patients hospitalized with COVID-19. In association with other clinical and biological variables, this risk marker should be useful in helping the clinicians to identify the patients needing a close monitoring.

\section{4}

Evaluation of hepatic injury definitions in pulmonary ICU patients E. Macit Aydın'; A. Mammadova²; Z. çınar'; N. Navruzvai²; S. Kodalak²; G. Gursel ${ }^{3}$

${ }^{1}$ Department of anaesthesiology and reanimation, division of critical care, Gazi University Faculty of Medicine, Anaesthesiologist, fellow of critical care, Ankara, Turkey; ${ }^{2}$ Department of pulmonary critical care medicine, Gazi University Faculty of Medicine, Resident of Pulmonary Diseases, Ankara, Turkey; ${ }^{3}$ Department of pulmonary critical care medicine, Gazi University Faculty of Medicine, Professor of Respiratory Medicine, Ankara, Turkey

Correspondence: E. Macit Aydın

Intensive Care Medicine Experimental 2020, 8(2): 001034

Introduction: Hepatic injury( $\mathrm{HI})$ and failure are observed in up to $20 \%$ of patients in intensive care units and are associated with increased morbidity and mortality. Secondary or acquired liver injury and failure such as hypoxic liver injury, cholestatic dysfunction in critical illness, sclerosing cholangitis in critical illness, and drug-induced liver injury (DILI) represent the most frequent forms of hepatic injury in critical illness. More importantly, retrospective population studies showed that liver injury was not mentioned in the diagnosis or in the physician's discharge letter in $52-68 \%$ of cases. On the other hand definition of $\mathrm{HI}$ influences the incidence, prognosis, mortality, and treatment management of the diseases.

There is no uniform definition of hepatic injury in critically ill patients. According to some studies, total bilirubin levels greater than $2 \mathrm{mg} / \mathrm{dL}$ represent a cutoff in daily practice. Others use 2-fold to 3 -fold elevation of ALP/AST, alkaline phosphatase (AP) and/or gamma-glutamyl transpeptidase (GGT) to define it.

Objectives: Aim of this study is to compare $\mathrm{HI}$ definitions in pulmonary ICU patients with secondary hepatic injury.

Methods: This retrospective cohort study was performed in an University Hospital Pulmonary ICU. Patients with hepatic injury included in the study. 478 patients were included in the study. We compared 3 definition of hepatic injury 1 . hepatic criteria of the SOFA score (HISOFA: biluribin>2 mg/dl), 2. 2017 definition of American Collage of Gastroenterology (HI-ACG1), 2017 definition of American Collage of Gastroenterology without borderline patients (HI-ACG2) and 3. 2019 
EASL DILI definition (HI-EAS).To define if $\mathrm{HI}$ is cholestatic, hepatocellular or mixed we calculated $\mathrm{R}$ ratio. We also calculated severity of $\mathrm{HI}$ in all patients.

Incidence, consistency (level of agreement-Kappa statistics), correlations, sensitivity and specificity of the definitions were also calculated for each definition taking one of them as gold standart. Lastly mortality rates according to 3 different definition were also calculated.

Results: As can be seen in table 1 and $2 \mathrm{HI}$ incidence and mortality rates are highly variable across the definitions and there is no good aggrement and correlation between them. Sensitivity and/or specificity numbers were also low in general also.

\begin{tabular}{|c|c|c|c|c|}
\hline & \multicolumn{2}{|c|}{ Incidence } & \multicolumn{2}{|c|}{ Mortality, } \\
\hline HI-ACG2, n (\%) & \multicolumn{2}{|c|}{$121(25.3 \%)$} & \multirow{2}{*}{\multicolumn{2}{|c|}{$\frac{49 / 121(41 \%)}{50 / 88(57 \%)}$}} \\
\hline HI-EASL, $\mathbf{n}(\%)$ & \multirow{2}{*}{\multicolumn{2}{|c|}{$\frac{88(18.4 \%)}{210(44 \%)}$}} & & \\
\hline HI-ACGl, n (\%) & & & \multicolumn{2}{|c|}{$79 / 210(38 \%)$} \\
\hline HI-SOFA, n (\%) & \multicolumn{2}{|c|}{$\begin{array}{l}210(44 \%) \\
49(10.3 \%)\end{array}$} & \multicolumn{2}{|c|}{$27 / 49(55 \%)$} \\
\hline \multicolumn{5}{|c|}{ 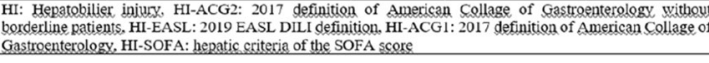 } \\
\hline \multirow{2}{*}{\multicolumn{3}{|c|}{$\frac{\text { Kappa test }}{\text { Kappa }}$}} & \multicolumn{2}{|c|}{ Correlations } \\
\hline & & & $\mathrm{x}$ & p-value \\
\hline HI-SOFA-HI-ACG2 & 0.325 & 0.0001 & 0.374 & 0.0001 \\
\hline HI-SOFA-HI-ACG1 & 0.227 & 0.0001 & 0.340 & 0.0001 \\
\hline
\end{tabular}

Conclusion: These results showed that definition of $\mathrm{HI}$ according to biluribin or ALT/AST or together changes $\mathrm{HI}$ diagnosis. To standardize epidemiologic data, diagnosis, severity assessment, treatment of $\mathrm{HI}$ we need a generally accepted definition for ICU patients.

\section{Reference(s) and grant acknowledgment(s)}

1. Paul Y. Kwo, Stanley M. Cohen, Joseph K. Lim. ACG Clinical Guideline: Evaluation of Abnormal Liver Chemistries. Am J Gastroenterol 2017; 112:18-35

2. EASL Clinical Practice Guidelines: Drug-induced liver injury. J Hepatol 2019 Jun;70(6):1222-1261

\section{6}

The link between risk factors and mortality rate in coma patients

D. Adukauskiene'; V. Jasinskas ${ }^{1}$

'Intensive care unit, Lithuanian University of Health Sciences, Kaunas, Lithuania

Correspondence: $\vee$. Jasinskas

Intensive Care Medicine Experimental 2020, 8(2): 001096

Introduction: Coma - a formidable disorder of consciousness caused by a various etiological factors. According to some authors, the mortality rate of patients in coma may be higher than $87.0 \%$ [1]. For this reason more and more factors, increasing the mortality of coma patients rate, are being sought and investigated.

Methods: 82 Intensive Care Unit patients retrospective analysis was performed. Chosen patients who had a score of 8 or less, according to the GCS and were not medically suppressed. Mortality rate and influence of the risk factors of the patients in coma were observed. $95.0 \%$ significance level $p \leq 0.05$ was chosen for significance evaluation.

Results: Male patients made $53.7 \%(n=44)$. The average age of patients was $67.6 \pm 15.2$ years. The outcomes of coma patients' mortality makes $65.9 \%(n=54)$.

In $74.0 \%$ of the dead patients had metabolic etiology coma, $\mathrm{p}=0.004$. According to the ROC test, metabolic etiology coma increased the odds ratio for mortality by 3,8 times, the area under the curve $71.8 \%$, the sensitivity $61.5 \%$ and the specificity $80.0 \%$. In $80.6 \%$ of the dead patients had 5 or less score by Glasgow coma scale, $p<0.001$. According to the ROC test, 5 and less points in the GCS increased the odds ratio for mortality by 16.7 times, the area under the curve $81.7 \%$, the sensitivity $92.6 \%$ and the specificity $57.1 \%$.

In $83.3 \%$ of the dead patients experienced tachycardia which occurred more than 100 beats per minute, $p<0.001$. According to the ROC test, tachycardia more than 100 beats per minute increased the odds ratio for mortality by 7.1 times, the area under the curve $78.0 \%$, the sensitivity $74.1 \%$, and the specificity $71.4 \%$.

In $88.9 \%$ of the dead patients creatinine concentration was higher than $170 \mu \mathrm{mol} / \mathrm{l}, \mathrm{p}=0.038$. According to the ROC test, creatinine value more than $170 \mu \mathrm{mol} / \mathrm{l}$, increased the odds ratio for mortality by 5.5 times, the sensitivity $30.0 \%$, and the specificity $92.6 \%$.

In $76.9 \%$ of the dead patients had lactate value more than $3.5 \mathrm{mmol} / \mathrm{l}$, $\mathrm{p}=0.05$. According to the ROC test, lactate concentration more than $3.5 \mathrm{mmol} / \mathrm{l}$, increased the odds ratio for mortality by 5.9 times, the sensitivity $82.0 \%$, and the specificity $57.0 \%$.

Conclusion: 1. Mortality rate of coma patients was $65.9 \%$.

2. Mortality rate of coma patients increases due to coma of metabolic origin, GCS $\leq 5$, tachycardia $>100$ beats per minute, creatinine concentration $>170 \mu \mathrm{mol} / \mathrm{l}$, lactate concentration $>3.5 \mathrm{mmol} / \mathrm{l}$.

Reference(s) and grant acknowledgment(s)

1. Kraus JF., McArthur DL. Epidemiologic aspects of brain injury. 1996; 14(2):435-50.

001157

How "door-to-icu" time influences outcome

A. L. Rios ${ }^{1}$; N. R. ${ }^{1}$; A. D. ${ }^{1}$; A. Marques ${ }^{1}$; P. Castelões

${ }^{1}$ Medicina intensiva, Centro Hospitalar de Vila Nova Gaia/Espinho, Vila

Nova de Gaia, Portugal

Correspondence: A. L. Rios

Intensive Care Medicine Experimental 2020, 8(2): 001157

Introduction: The pandemic of COVID-19 has overcrowded our ICUs. In Italy, the country besides China with the most patients with COVID19 until March 29, 2020, up to $12 \%$ of all positive cases required ICU admission. The median time from symptom onset to the development of pneumonia was approximately 5 days, and the median time from symptom onset to severe hypoxaemia and ICU admission was approximately 7-12 days.(1)

Data from Wuhan, China, concluded that older patients ( $>60$ years) were more likely to exhibit severe and critical COVID-19 conditions; patients with chronic disease were more prone to severe forms of COVID-19 and regarding the symptoms, dyspnea, chest tightness, diarrhea, fatigue, dizziness, muscle ache, and unconsciousness were significantly linked to severe COVID-19 cases.(2) Prediction of trajectory of illness from symptom onset is difficult, and prognostic tools and biomarkers are urgently needed. (3)

Objectives: etermination of factors that predict this rapid respiratory deterioration may help to anticipate rapid need for intubation and respiratory support. This work relates patient status at ICU admission and the time to invasive ventilation, complications and outcome.

Methods: This is a retrospective study of all patients admitted to a tertiary hospital ICU in Portugal. Primary outcomes were mortality, time of mechanical ventilation; Secondary outcomes were association with higher APACHEII, SAPSII and greater need for invasive ventilatory maneuvers (like pronning).

Results: Between March 17th and May 10th 2020 were admitted 36 SARS-CoV-2 patients to our ICU, 10 of those were direct admissions from the emergency department. The other 26 ICU admissions were transfers from wards and the majority (35\%) only after $72 \mathrm{~h}$ of hospital stay. $31 \%$ during the first $24 \mathrm{~h}$ after admission.

There was no difference in APACHE II and SAPS II scores between the two groups at ICU admission: direct admissions from the emergency department or from the wards. There was a positive association 
between the need for pronning maneuvers and longer time-to-ICU $(p=0,008)$, although there was no difference in admission PO2/FiO2 ratio.

Conclusion: There was no identified correlation between mortality and time-to-ICU admission.

We should look into other parameters as predictors of prognosis.

\section{Reference(s) and grant acknowledgment(s)}

1. Xie J. et al. Critical care crisis and some recommendations during the COVID19 epidemic in China. Intensive Care Med 2020; published online March 2. https://doi.org/10.1007/s00134-020-05979-7.

2. Phua J. et al., for the Asian Critical Care Clinical Trials Group; Intensive care management of coronavirus disease 2019 (COVID-19): challenges and recommendations; Lancet Respir Med 2020, Published Online April 6, 2020 https://doi.org/10.1016/S2213-2600(20)30161-2

3. Zhang J et al., Risk factors for disease severity, unimprovement, and mortality in COVID-19 patients in Wuhan, China, Clinical Microbiology and Infection, https://doi.org/10.1016/j.cmi.2020.04.012

\section{8}

\section{Impact of clinical frailty score and the national early warning} score 2 on ICU and 30-day mortality

G. Azzopardi'; J. Hill' ; P. Morgan ${ }^{1}$

'Intensive Care, East Surrey Hospital, Redhill, United Kingdom

Correspondence: G. Azzopardi

Intensive Care Medicine Experimental 2020, 8(2): 001158

Introduction: ICU outcome can be predicted using multiple methods, these tend to be retrospective, time consuming and require a large number of data points.

The National Early Warning Score 2 (NEWS2) is a widely used severity of illness scale to detect the acutely unwell and deteriorating patient from the end of the bed (1). A Rockwood Clinical Frailty Score (CFS) (2) can be easily calculated following discussion with the patient, their family or review of clinical notes. It has been demonstrated that a higher CFS correlates with a worse ICU outcome (3), studies looking at NEWS2 and ICU outcome are not widely published.

We hypothesise that a higher CFS and NEWS2 will correlate with a higher ICU and 30-day mortality. Combined, both provide a snapshot assessment of the patient and may help inform future decision making with regards to ICU admission and predicted outcome.

Objectives: To determine the effect of CFS and NEWS2 on ICU mortality and 30-day mortality.

Methods: Observational data was collected on 106 patients admitted in ICU between $9 / 01 / 2020$ and $12 / 03 / 2020$. CFS was calculated by ICU clinicians from reviewing the patient, nursing and therapy notes, as well as speaking to the patient or family members where possible. This score was based on the patients status two weeks prior to admission to ICU. NEWS2 was calculated from the last set of observations performed prior to admission to ICU. The primary outcome was ICU mortality, secondary outcomes were 7-day and 30-day mortality. The data was collected in Microsoft Excel and analysed.

Results: Data was collected on 106 patients. There were 62 patients admitted under medicine, 32 emergency surgical cases and 12 elective surgical cases. The average age of patients was 64 . Overall ICU mortality was $20.8 \%$.

A CFS of 5 or more was used as a cut off to indicate frailty. $24.5 \%$ of patients had a CFS of 5 or more. Patients were further stratified into a low (0-4), medium (5-6) or high ( $>7)$ NEWS2 category. Patients with a CFS $\geq 5$ (38.5\%) had a higher ICU mortality than those with a CFS $<5$ (15\%). Patients with a high NEWS2 had a higher ICU mortality (32\%) than those with a low NEWS2 (11\%) (Figure 1).

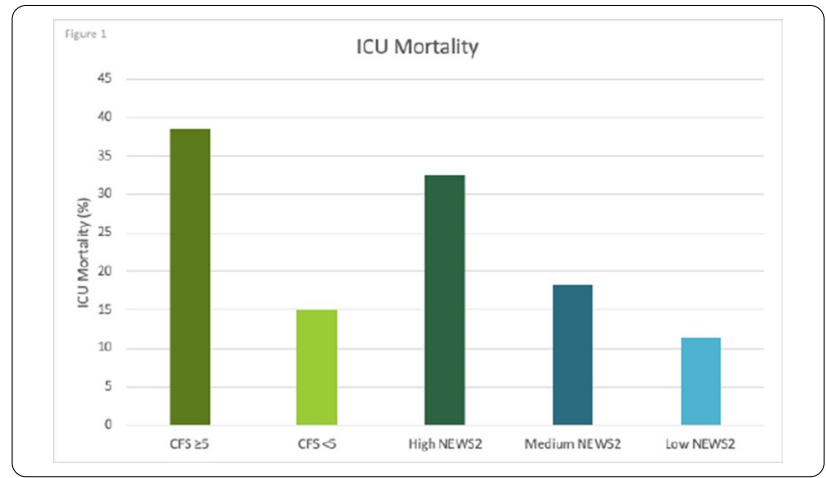

Figure 2 shows survival at 7 and 30 days in each of the NEWS2 categories, divided into those with a CFS $<5$ and $\geq 5$.

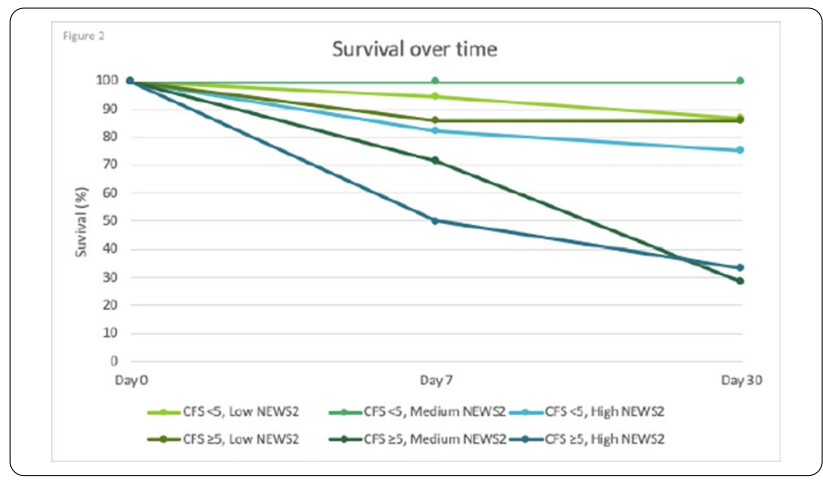

Conclusion: Our data shows that frail patients, with a high NEWS2 have a higher ICU and 30-day mortality rate than patients without frailty and a low NEWS2. Larger scale studies should be performed to further investigate this, as well as correlating this data with existing ICU outcome predictor tools such as APACHE II. Future use of the CFS and NEWS2 as an 'end of the bed' assessment tool may help to augment decision making with regards to ICU admissions and predicted outcomes.

\section{Reference(s) and grant acknowledgment(s)}

1. 3. Guidet $B$, et al. The contribution of frailty, cognition, activity of daily life and comorbidities on outcome in acutely admitted patients over 80 years in European ICUs: the VIP2 study. Intensive Care Med. 2020;46(1):57-69. https://doi.org/10.1007/s00134-019-05853-1

2. 2. Rockwood $\mathrm{K}$, et al. A global clinical measure of fitness and frailty in elderly people. CMAJ. 2005;173(5):489-495. https://doi.org/10.1503/cmaj.050051

3. 1. Royal College of Physicians. National Early Warning Score (NEWS) 2: Standardising the assessment of acute-illness severity in the NHS. 2017

\section{4}

Does age always matter for the outcome in the Intensive Care Unit ?

G. Koukoulitsios ${ }^{1}$; K. Tsikritsaki'; M. Anifanti ${ }^{1}$ I. Tsoni ${ }^{1}$; S. Bakouli ${ }^{1}$;. Ionna ${ }^{1}$;

G. Maglaras'; A. Koutivas'; C. Mandila'; S. Antonopoulou'; V. Koutsoukou'; V. Salma'; A. Kalogeromitros

"ICU, General Hospital of Athens "G. Gennimatas", Athina, Greece

Correspondence: $\mathrm{G}$. Koukoulitsios

Intensive Care Medicine Experimental 2020, 8(2): 001184 
Introduction: Aged patients represent a significant proportion of patients who require admission to the Intensive Care unit (ICU). Advanced age is associated with higher mortality and adverse outcomes in ICU, however according to some studies is not only the age that determines the prognosis, co morbidities and previous health status could affect the outcome as well.

Objectives: The aim of this study was to evaluate the possible effect of age, co morbidities, reason for admission to the ICU, length of stay in ICU and the final outcome.

Methods: Our study included 157 patients aged 68 and older that were admitted to the ICU from January 2018 till September 2019. Demographic parameters, admission diagnosis, underling diseases, ICU length of stay and finally outcome were recorded.

Results: We enrolled 157 patients, 85 males, 72 females with mean age 74,6 years. They main reasons for admission were 1 st.post operative (after elective operation) 64 patients 2 nd. trauma 40 patients, 3 rd. acute respiratory failure 30 patents, 4th. sepsis 23 patients. Out of 157 patients the 112 had co morbid medical illness (e.g. ischemic heart disease, arterial hypertension,diabetes mellitus, COPD, chronic renal failure). The mean length of stay was 23 days but it ranged from 1 to 65 days. Out of 157 patient 95 were discharged from the ICU and 62 patient died. Post operative patients independently from the age and co morbidities had the best outcome and discharged earlier from ICU than other etiological groups. There were not differences in the outcome as far as the gender was concerned,

Conclusion: Reasons for mortality appear to be multifactorial in elderly patients. High age alone is not a factor to predict morbidity and mortality in ICU patients. Previous health state-co morbidities and the reason for admission in the ICU play a very important role for the final outcome.

\section{7}

Association of Sequential Organ Failure Assessment (SOFA) components with outcome in contemporary critical care

A. Pölkki' ; PT. Pekkarinen²; M. Reinikainen ${ }^{3}$

'Department of anesthesiology and intensive care, Kuopio University Hospital, Kuopio, Finland; ${ }^{2}$ Division of intensive care medicine, department of anaesthesiology, intensive care and pain medi, Helsinki University Hospital, Helsinki, Finland; ${ }^{3}$ Department of intensive care, Kuopio University Hospital, Kuopio, Finland

Correspondence: A. Pölkki

Intensive Care Medicine Experimental 2020, 8(2): 000327

Introduction: Sequential Organ Failure Assessment (SOFA) score is a practical method to describe the severity of multi-organ failure. It has been suggested that SOFA score could be employed as a tool to predict outcome and as an endpoint in interventional trials (1). To justify these assumptions all SOFA components should carry comparable weights as outcome predictors. SOFA score was introduced in 1996 and has remained unchanged to date, although there have been changes in clinical practice (2).

Objectives: The aim of this study was to investigate if SOFA score components are comparable in association with in-hospital mortality. Methods: We performed a national observational cohort study on adult (18 years of age or older) patients admitted to 26 Finnish intensive care units (ICUs) between 2012-2015. SOFA score was determined as the most severe SOFA score component value within the first 24 hours after admission to ICU. We performed statistical analysis employing age- and sex-adjusted multivariable logistic regression. Results: We included 67095 admissions after excluding 4289 readmissions and 112 admissions with missing in-hospital mortality data. Total in-hospital mortality was $11.4 \%$. Multivarible logistic regression revealed that increase in SOFA points was associated with higher risk of in-hospital death in respiratory (age-adjusted odds ratio [OR] 1.40, $95 \%$ confidence interval $[\mathrm{Cl}] 1.36-1.44, \mathrm{p}<0.001)$ per point; in coagulation (OR 1.22, 95\% Cl 1.18-1.26, $\mathrm{p}<0.001$ ); in hepatic (OR 1.36, 95\% Cl 1.31-1.42, $\mathrm{p}<0.001$ ); in cardiovascular (OR 1.16, 95\% Cl 1.14-1.19, $\mathrm{p}<0.001)$; in neurologic (OR 1.72, 95\% Cl 1.69-1.75, $\mathrm{p}<0.001)$, and in renal component (OR 1.63, 95\% Cl 1.59-1.66, $\mathrm{p}<0.001)$.

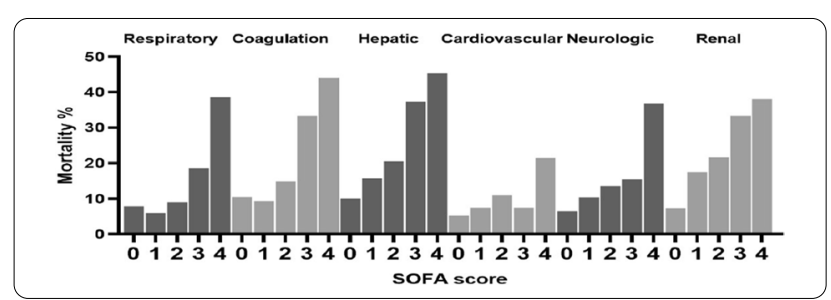

Conclusion: We found that all SOFA score components have independent predictive value. However increased risk of in-hospital death per SOFA score point was not comparable between components. Cardiovascular component has the weakest and neurologic component has the strongest association with increased in-hospital mortality (Figure 1).

\section{Reference(s) and grant acknowledgment(s)}

1. 2. Vincent, J.L., Moreno, R., Takala, J. et al. The SOFA (Sepsis-related Organ Failure Assessment) score to describe organ dysfunction/failure. Intensive Care Med 22, 707-710 (1996). https://doi.org/10.1007/BF01709751

2. 1. Lambden, S., Laterre, P.F., Levy, M.M. et al. The SOFA score-development, utility and challenges of accurate assessment in clinical trials. Crit Care 23, 374 (2019). https://doi.org/10.1186/s13054-019-2663-7

\section{2}

GRACE versus CHADS2: a paradigm change in ACS stratification?

H. Miranda'; H. Santos ${ }^{2}$; S. Mariana ${ }^{3}$; I. Almeida ${ }^{2}$;. Sousa ${ }^{2}$;. Almeida ${ }^{2}$

${ }^{1}$ Intensive Medicine Care, Hospital São Pedro de Vila Real (Centro Hospitalar de Trás-os-Montes e Alto Douro, EPE), Vila Real, Portugal; ${ }^{2}$ Cardiology, Centro Hospitalar Barreiro Montijo, Barreiro, Portugal; ${ }^{3}$ Cardiology, Centro Hospitalar Barreiro Montijo, E.P.E, Barreiro, Portugal

Correspondence: $\mathrm{H}$. Miranda

Intensive Care Medicine Experimental 2020, 8(2): 000382

Introduction: The GRACE Score is a prospectively studied scoring system which stratify patients with diagnosed ACS to estimate in-hospital and 6-month to 3-year mortality. Knowing patient's risk earlier may help with management and prognostication. Recent studies refer that CHADS2 could be as useful as GRACE score in this scenario.

Objectives: To compare GRACE and CHADS2 scores regarding MACE, in-hospital mortality and 1-year mortality in Portuguese population. Methods: Retrospective study during a period of 8 years +8 months. Only NSTEMI patients were admitted in the study. GRACE and CHADS2 groups, based on mortality risk, were created and compared regarding MACE and mortality.

Results: 7537 patients were admitted. Male predominance $(70,9 \%)$ and mean age of $67 \pm 13$ years. Hypertension $(73,2 \%)$, diabetes $(34,4 \%)$ and dyslipidaemia $(61,4 \%)$ were the most frequent comorbidities. $85 \%$ were submitted to angiography with DA being the most common culprit vessel $(33,2 \%)$. Multivessel disease in $55,1 \%$ of the patients. MACE occurred in $5,1 \%$ of the cases with 1,9\% of the patients dying in hospital.

$60,8 \%$ of the patients presented a high risk (GRACE score) compared with CHADS2 score $(7,6 \%)$. We verified a predominance of low risk patients in this last score. ROC curves were created and the results were compared. Comparing the occurrence of MACE during hospital length we found that GRACE was a better predictor than CHADS2 (AUC(grace) 0,708 versus AUC(chads2) 0,598, $\mathrm{p}<0,001$ ). We also point out the best performance of GRACE score when we compare in-hospital (AUC (grace) 0,834 versus AUC(chads2) $0,669, p<0,001$ ) and 1 -year death (AUC(grace) 0,667 versus AUC(chads2) $0,645, p=0,045$ ).

Conclusion: Our study has reinforced the idea that the GRACE score remains an extremely reliable score today. 
000446

"Patients' quality of life with critical ill and the support of family's members after discharge from the intensive care unit"

K. Augeri ${ }^{1}$

${ }^{1}$ Medical, UNIVERSITY OF THESSALY, Larisa, Greece

Correspondence: K. Augerl

Intensive Care Medicine Experimental 2020, 8(2): 000446

Introduction: Patients following intensive care unit hospitalization may present disability and dysfunction in their physically and cognitive function.(1)(2)

Objectives: We investigated the quality of life of patients following discharge from the intensive care unit and we assessed the role of support by the family members.

Methods: This retrospective study conducted at the University Hospital of Thessaly and included patients who stayed for at least 48 hours in ICU and were alive at discharge between 2014 and 2019. The quality of life was assessed by the questionnaire SF-36.

Results: A total of 671 were included. The SF score overall was 51.32. There were correlations between SF score and daily duration of support by spouses $(r=0.2046, p=<0.0001)$., friends $(r=-$ $0.2536, p=<0.0001)$ and duration of hospitalization $(r=-0.2324$, $p=<0.0001)$. Patients who were supported by their spouses for more than 8 hours daily or patients who were supported by their friends for more than 3 times/week or were hospitalized for less than 10 days had increased SF compared to the rest of the cohort [mean(SD) $54.49(28.07)$ vs 50.14, (28.69), $\mathrm{p}=0.05$ and 58.07 (28.54) vs 45.20 (27.93), $\mathrm{p}=<0.0001$, and $68.45,27.57)$ vs 49.07 (27.53) $\mathrm{p}=<0.0001$, respectively].

Conclusion: The present study shows that the quality of life of patients following ICU discharge may be positively affected by the support from spouses or friends.

\section{Reference(s) and grant acknowledgment(s)}

1. 2. Sarah E. Jolley, MD, Aaron E. Bunnell, and Catherine L. Hough, MD. Chest 2016 Nov; 150(5): 1129- 1140. Icu- Acquired weakness

2. 1. Amy B. Petrinec, Bradley R. Martin. Palliat support care. 2018 Dec; 16(6): 719- 724. Post- intensive care syndrome symptoms and health- related quality of life in family decision- makers of critically ill patients.

\section{8}

Evaluation of demographic and clinical characteristics in intensive care unit with critical end stage renal disease patients

E. N. Karaoglu ${ }^{1}$; M. Caliskan ${ }^{1}$; M. Yıldırım ${ }^{1}$; A. Merve ${ }^{1}$; S. Temel ${ }^{1}$; M. Sungur ${ }^{2}$; K. Gundogan ${ }^{3}$

${ }^{1}$ Internal medicine, Erciyes University, Kayseri, Turkey; ${ }^{2}$ Division of critical care medicine, department of internal medicine, Erciyes University School of Medicine, Kayseri, Turkey, Kayseri, Turkey; ${ }^{3}$ Department of internal medicine, division of intensive care, Erciyes University, School of medicine, Kayseri, Turkey

Correspondence: E.N. Karaoglu

Intensive Care Medicine Experimental 2020, 8(2): 000508

Introduction: Patients diagnosed with end stage renal failure (ESRD) who are likely to be hospitalized due to comorbidites, advanced age, immuncompression caused by uremia and phagocyte disfunction.

Objectives: The aim of this study is to procpectively monitor and evaluate the clinical and laboratory features of the patients with ESRD in the intensive care unit(ICU).

Methods: This study was performed prospectively in the Medical ICU. Patients in need of ICU admission, over 18 years old and diagnosed with ESRD were included in the study.

Results: A total of 100 patients were included in the study. The mean age of patients was $68 \pm 14$ years.The most common reason for ICU admission were sepsis/septic shock (26\%) and respiratory failure (21\%).The most common causes of ESRD were diabetes mellitus (DM) (49\%) and hypertension (HT) (21\%). Mean APACHE II score was $22 \pm 6$. Median SOFA score was 8 (min:2-max:19). A total of $52 \%$ of patients received dialysis treatment $(96 \%$ of hemodialysis, $4 \%$ peritoneal dialysis) before admission to ICU. The length of ICU stay was 7 (min:2 max:36) days. Cox regression analysis was performed to determine the factors affecting mortality. APACHE II score was identified as an independent risk factor that determined mortality. (OR:1.059, $95 \mathrm{Cl}, 1.01$ $1.09, \mathrm{p}=0.015)$. ICU mortality rate was $45 \%$.

Conclusion: As a result of this study, DM was the most common cause for ESRD. High APACHE II score was identified as an independent risk factor for mortality in ICU with end stage critically ill renal failure patients.

\section{Reference(s) and grant acknowledgment(s)}

1. Manhes G, Heng AE, Aublet-Cuvelier B, Gazuy N, Deteix P, Souweine B. Clinical features and outcome of chronic dialysis patients admitted to an intensive care unit. Nephrology Dialysis Transplantation. 2005;20(6):1127-33. 2. Goswami J, Balwani MR, Kute V, Gumber M, Patel M, Godhani U. Scoring systems and outcome of chronic kidney disease patients admitted in intensive care units. Saudi Journal of Kidney Diseases and Transplantation. 2018;29(2):310

\section{3}

A modified SOFA score: testing a new prognostic score for critically ill patients

ALN. Gobatto ${ }^{1}$; GL. Silva'; MS. Manciola ${ }^{1}$; MSM. Reis ${ }^{1}$; CC. Starteri'

LS. Sancho ; VOS. Junior ${ }^{1}$; MCC. Bispo ${ }^{1}$; JC. Ribeiro ${ }^{1}$; SF. Da Guarda1;

JGR. Ramos ${ }^{1}$; RDH. Passos ${ }^{1}$

${ }^{1}$ Critical care medicine, Hospital São Rafael, Salvador, Brazil

Correspondence: M.S. Manciola

Intensive Care Medicine Experimental 2020, 8(2): 000573

Introduction: The Sequential Organ Failure Assessment (SOFA) score was created in 1994, in an effort to describe organ dysfunction/failure over time in the critically ill, and has been widely used in medical practice ever since. However, the need for future alterations was highlighted by the original paper, as better parameters could be suggested by future re-evaluation and updated data. Thus, additional studies are appropriate for re-evaluating current parameters of the SOFA score, as well as possibly providing a modified version with higher accuracy for outcome prediction.

Objectives: This study aimed to test the accuracy of a modified SOFA score as a mortality predictor by using different parameters for neurological and gastrointestinal evalutation.

Methods: A prospective cohort was performed in a Brazilian private tertiary hospital between June and November 2018. Inclusion criteria were adult patients in the intensive care unit (ICU) under nutrology follow-up. Demographic and clinical characteristics, nutritional status, laboratory results, physiological parameters, use of medical devices and outcomes were collected from electronic and physical medical records.

Glasgow Coma Scale (GCS) and Richmond Agitation-Sedation Score (RASS) were used to determine the neurological component in SOFA and RSOFA, respectively. Total bilirubin and the Acute Gastrointestinal Injury (AGI) score were used to determine the gastrointestinal component in SOFA and ASOFA, respectively. AGI score and RASS were used to determine the gastrointestinal and neurological components of ARSOFA, respectively. To test our hypothesis, accuracy of the SOFA, ASOFA, RSOFA and ARSOFA scores to predict mortality was determined by the area under the curve (AUC) using the Receiver Operating Characteristic (ROC) curve.

Results: During the study period, 52 patients met the inclusion criteria and were included. Patients' demographic characteristics were: median age 73.9 [60.47-84.01]; male, 25 (48.1\%); on admission: 26 patients were on mechanical ventilation (50\%), mean SAPS III was 52.3 $( \pm 15.5)$, mean APACHE II was $23.2( \pm 11.4)$, mean SOFA score was 3.7 $( \pm 2.5)$ and $33(63,5 \%)$ individuals had sepsis. The AUC of the SOFA, RSOFA, ASOFA and ARSOFA scores were 0.813, 0.831, 0.793 and 0.807, respectively. See the attached figures. 


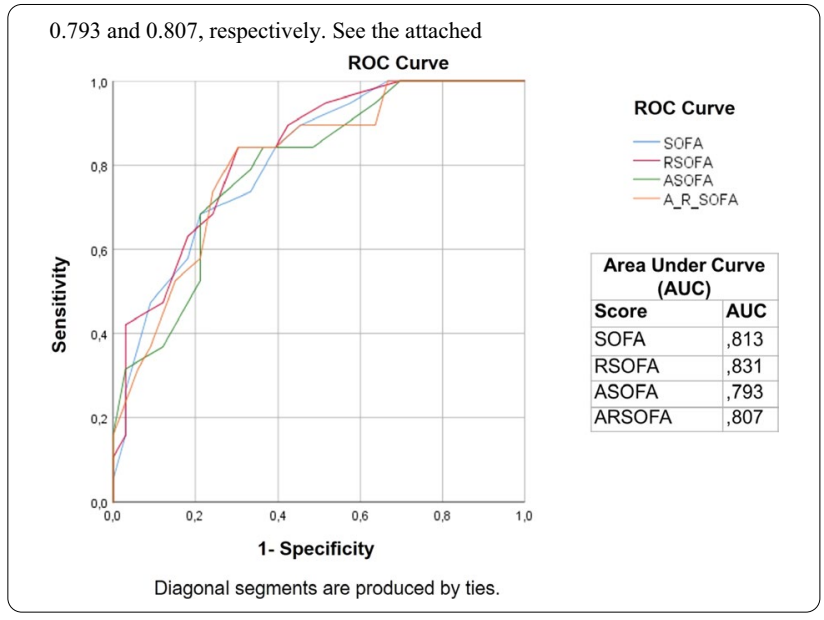

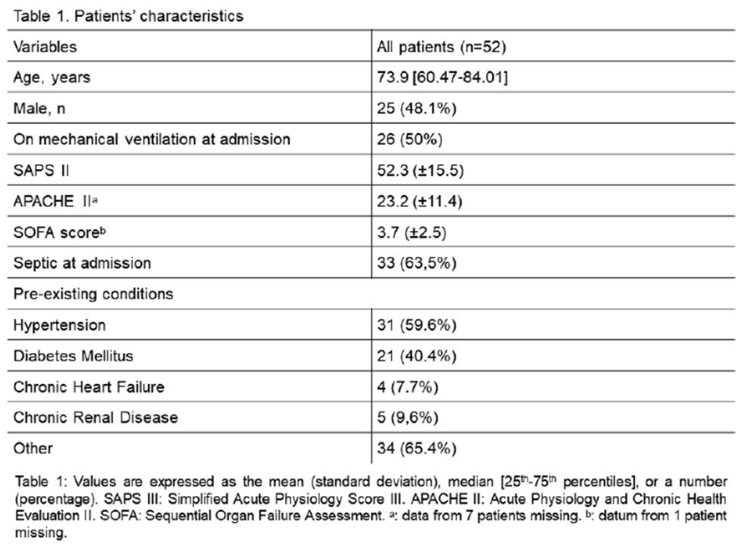

\begin{tabular}{|c|c|}
\hline Variables & Frequency \\
\hline Hospital length of stay, days ${ }^{c}$ & 24,9 days $( \pm 22,3)$ \\
\hline ICU length of stay, days $d$ & 11.6 days $( \pm 13.4)$ \\
\hline Hospital mortality, n & $19(36.5 \%)$ \\
\hline ICU mortality, n & $15(28.8 \%)$ \\
\hline
\end{tabular}

Conclusion: The RASS scale and bilirubin level as neurological and gastrintestinal components, respectively, had higher accuracy for mortality prediction than the GCS and the AGI score. Therefore, the use of the RSOFA score should be encouraged over traditional SOFA score. The ASOFA and ARSOFA scores, which used the AGI scale, must not be used as it had lower accuracy than currently used bilirubin levels.

\section{Reference(s) and grant acknowledgment(s)}

1. Knaus WA, Draper EA, Wagner DP, Zimmerman JE. APACHE II: A severity of disease classification system. Crit Care Med. 1985; 13(10):818-29.

2. Metnitz PGH, Moreno RP, Almeida E, Jordan B, Bauer P, Campos RA, et al. SAPS 3-From evaluation of the patient to evaluation of the intensive care unit. Part 1: Objectives, methods and cohort description. Intensive Care Med. 2005; 31(10):1336-44.

3. Blaser AR, Malbrain MLNG, Starkopf J, Fruhwald S, Jakob SM, De Waele J, et al. Gastrointestinal function in intensive care patients: Terminology, definitions and management. Recommendations of the ESICM Working Group on Abdominal Problems. In: Intensive Care Medicine. 2012; 38(3):384-94.

4. Sessler CN, Gosnell MS, Grap MJ, Brophy GM, O'Neal PV., Keane KA, et al. The Richmond Agitation-Sedation Scale: Validity and reliability in adult intensive care unit patients. Am J Respir Crit Care Med. 2002; 166(10):1338-44

5. Teasdale G, Jennett B. ASSESSMENT OF COMA AND IMPAIRED CONSCIOUSNESS. A Practical Scale. Lancet. 1974; 2(7872):81-4.

6. Vincent JL, Moreno R, Takala J, Willatts S, De Mendonça A, Bruining H, et al. The SOFA (Sepsis-related Organ Failure Assessment) score to describe organ dysfunction/failure. Intensive Care Med. 1996; 22(7):707-10.

7. The authors declare that they have no competing interests.

\section{9}

\section{Predictors of lethality in ICU patients with COVID-2019}

\section{pneumonia}

O. Dymova'; M. Petrushin²; M. Babaev³; A. Eremenko ${ }^{3}$

${ }^{1}$ Clinical diagnostic laboratory, Petrovsky National Research Centre of Surgery, Moskva, Russia; ${ }^{2}$ Intensive care unit, Tver Regional Hospital, Tver, Russia; ${ }^{3}$ Intensive care unit, Petrovsky National Research Centre of Surgery, Moskva, Russia

Correspondence: M. Babaev

Intensive Care Medicine Experimental 2020, 8(2): 000779

Introduction: Understanding the risk factors and early predictors of the severe course of the disease allows us to choose the right treatment tactics.

Objectives: To identify risk factors and predictors of poor outcom in patients with COVID-19 pneumonia.

Methods: We performed a retrospective analysis of the treatment results of 118 patients admitted to ICU of Tver Regional Hospital in April-May 2020. The mortality rate was 61\% (72 from 118 patients). Patients were assessed according to 21 clinical and laboratory indicators using a multivariate analysis of the obtained data.

Results: Using the comparative and ROC analysis, we selected 4 most informative predictors of the development of the lethal outcome from 17 initial indicators. These 4 predictors were used to build a multidimensional regression equation. In the obtained regression equation, each of the 4 qualitative predictors had a certain weight: age over 65.5 years (31 points), SOFA score $>3.5$ (29 points), serum creatinine $>149.5 \mu \mathrm{mol} / \mathrm{I}$ ( 25 points), the ratio of CRP $(\mathrm{mg} / \mathrm{l})$ to the relative number of lymphocytes (\%) > 8.18 (16 points). The obtained regression equation allows us to assess the risk of death (AUC 0.859, $95 \% \mathrm{Cl} 0.792-0.927$, see Figure 1). The risk of death increased to $93 \%$ (Cl 89-97\%), OR $=12.85,(95 \% \mathrm{Cl} 4.8-34.2, \mathrm{p}=0.0000)$ when a sum of values approached to 50.5 points. It is also possible to use a simplified regression equation, where each of 4 predictors means 1 point (AUC $0.840,95 \% \mathrm{Cl} 0.767-0.913)$. In patients without the risk factors the probability of death was $7.7 \%$. If any 1 predictor was noted, the probability of death was $30 \%$, two $-61.9 \%$, three $-96 \%$. None of the patients with 4 risk factors at the admission survived.

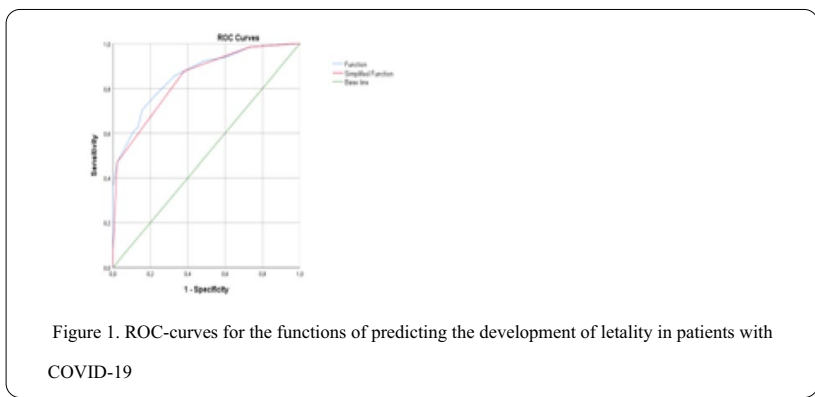

Conclusion: The group of the highest risk for adverse outcomes in patients with COVID-19 is represented by patients over 65 years of age who have signs of impaired renal function and/or a pronounced inflammatory reaction upon admission. 


\section{5}

Decreased serial scores of severe organ failure assessments are associated with survival in mechanically ventilated patients; the prospective Maastricht Intensive Care COVID cohort

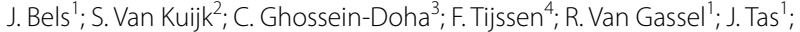
R. Schnabel ${ }^{1}$; M. Aries ${ }^{1}$; MC. Van De Poll ${ }^{1}$; D. Bergmans ${ }^{1}$; S. Meex ${ }^{5}$; W. Mook Van $^{1}$; I. Van der Horst ${ }^{1}$; B. Van Bussel ${ }^{1}$

${ }^{1}$ Department of intensive care, Maastricht UMC+, Maastricht, Netherlands; ${ }^{2}$ Department of clinical epidemiology and medical technology assessment, Maastricht UMC+, Maastricht, Netherlands; ${ }^{3}$ Department of cardiology, Maastricht UMC+, Maastricht, Netherlands; ${ }^{4}$ Department of anaesthesiology and pain medicine, Maastricht UMC+, Maastricht, Netherlands; ${ }^{5}$ Department of clinical chemistry, Maastricht UMC+, Maastricht, Netherlands

\section{Correspondence: J. Bels}

Intensive Care Medicine Experimental 2020, 8(2): 000845

Introduction: The vast majority of patients with severe acute respiratory syndrome coronavirus 2 (SARS-CoV-2) infection are primarily admitted to the Intensive Care Unit (ICU) for mechanical ventilation. The role of multi-organ failure during ICU admission as driver for outcome remains to be investigated yet.

Objectives: The aim of the study was to determine whether patients who survive the ICU develop a lower (i.e. decreasing) Sequential Organ Failure Assessment (SOFA) score over time compared to patients that die in the ICU.

Methods: Prospective cohort of mechanically ventilated critically ill with SARS-CoV-2 infection. The study includes 81 participants (23\% women) of the MaastrICCht cohort of whom 54 reached primary outcome at follow-up. The median length of stay was nine days, with a maximum of 30 . The study includes a total of 1101 serial SOFA scores, an established score to evaluate multi-organ function in the ICU on a daily basis. The cohort was divided into survivors $(n=28$, discharged from the ICU) and non-survivors $(n=26$, died during their ICU stay). Serial data were analysed using linear mixed-effects models. Trial registration: Netherlands Trial Register number NL8613.

Results: On admission the mean SOFA score was $7.8 \pm 2.8$. Survivors improved on average one SOFA score point more per four days (95\% Cl: 2.5-9) than non-survivors. Adjustment for age, sex, the presence of chronic lung, renal and liver disease, body-mass index, diabetes mellitus and cardiovascular risk factors, and Acute Physiology and Chronic Health Evaluation II score did not change this result. This association, however, was stronger for women than men (P-interaction 0.006).

Conclusion: The decrease in SOFA score associated with survival strongly suggests involvement of multi-organ failure during the clinical course of mechanically ventilated patients with SARS-CoV-2 infection. Surviving women appeared to improve faster than surviving men. Serial SOFA scores may unravel an unfavourable trajectory and guide decisions in mechanically ventilated patients with SARS-CoV-2.

\section{Reference(s) and grant acknowledgment(s)}

1. Zhang J, Wang X, Jia X, et al. Risk factors for disease severity, unimprovement, and mortality of COVID-19 patients in Wuhan, China. Clin Microbiol Infect. 2020

2. Arabi YM, Murthy S, Webb S. COVID-19: a novel coronavirus and a novel challenge for critical care. Intensive Care Med. 2020

3. Gattinoni L, Coppola S, Cressoni M, Busana M, Rossi S, Chiumello D. Covid-19 Does Not Lead to a "Typical" Acute Respiratory Distress Syndrome. Am J Respir Crit Care Med. 2020.

4. Organisation WH. Epidemiology: Q\&A: similarities and differences - COVID19 and influenza. 2020;viewed 16.04.2020:https://www.who.int/news-room/qa-detail/q-a-similarities-and-differences-covid-19-and-influenza.
000920

Hospital clinical alert capacity for critical events. Descriptive analysis. Capacicritic study

L. Sánchez-Alés ; J. Ruiz²; L. Chiscano ${ }^{3}$; R. Algarte ${ }^{2}$; L. Almorín ${ }^{4}$; P. Velasco ${ }^{5}$; JC. Ruiz-Rodríguez ; B. Sánchez González²

${ }^{1}$ Critical care department, Consorci Sanitari de Terrassa, Rubí, Spain; ${ }^{2}$ Intensive care department, Mútua Terrassa University Hospital, Terrassa, Spain; ${ }^{3}$ Intensive care department, Hospital Vall d'Hebron, Barcelona, Spain; ${ }^{4}$ Intensive care department, Hospital Plató, Barcelona, Spain; ${ }^{5}$ Intensive care department, Hospital General de Granollers, Granollers, Spain; ${ }^{6}$ Intensive Care Department, Hospital Vall d'Hebron, Barcelona, France

Correspondence: $B$. Sánchez González

Intensive Care Medicine Experimental 2020, 8(2): 000920

Introduction: It has been observed that some physiological parameters are altered in the hours prior to a critical hospital event. With the application of early warning systems by evaluating the physiological parameters of patients, they can be classified as low, intermediate or high clinical risk of suffering a critical event, reducing or anticipating the risk ofcritical events.

Objectives: Analyze the capacity of clinical alert and describe the characteristics of patients who suffer a critical event in a conventional ward.

Methods: Prospective, observational and multicenter study. The patients admitted to the conventional plant who suffered a critical event from February to May 2019 were analyzed.The NEWS-2 (NHS Early Warning Score-2) was calculated to stratify the patient's clinical risk prior to the event: low $(<5)$, medium $(5-6)$ and high $(>6)$ or some parameter of the NEWS- $2=3$.

Qualitative variables are expressed as percentages and quantitative variables are expressed as means and standard deviations (SD).

Results: 231 patients from 5 hospitals in Catalonia were included Table 1: describe the main characteristics of the population.

\begin{tabular}{|l|l|}
\hline Table 1 & $\mathrm{N}=231$ \\
\hline Age mean (SD) & $70.9(15.6)$ \\
\hline Male (\%) & 56 \\
\hline Medical pacient (\%) & 83 \\
\hline Hospital (\%) & 48 \\
\hline $\begin{array}{l}\text { Event occurs at night or on a holiday (\%) } \\
\text { Cause of the event related to the admission (\%) }\end{array}$ & 53 \\
\hline $\begin{array}{l}\text { Active infection at the time of the event (\%) } \\
\text { Patient monitored prior to the event (\%) }\end{array}$ & 46 \\
\hline NEWS-2 at the time of the event . mean (SD) & 44.6 \\
\hline $\begin{array}{l}\text { Some parameter of the NEWS-2 =3, 48 h before the event. } \\
\text { mean (SD) }\end{array}$ & $8,2(3.8)$ \\
\hline $\begin{array}{l}\text { Some parameter of the NEWS-2 =3, at the moment of the } \\
\text { event (\%) }\end{array}$ & $7.1(3.7)$ \\
\hline \begin{tabular}{l} 
Some parameter of the NEWS-2 =3, 48h before the event (\%) \\
\hline Hospital long of stay, mean (SD)
\end{tabular} & 53.7 \\
\hline \begin{tabular}{l} 
Hospital mortality (\%) \\
\hline
\end{tabular} & 69.7 \\
\hline
\end{tabular}

Critical event: unexpected admission to the ICU 27\%, ICU consultation without admission $22 \%$, non-resuscitable death on the ward $37 \%$ PCRIH 9\%. Medical Service: Internal Medicine (44.6\%), Onco-Hematology: $22.6 \%$. Frequency in taking constants: $<6$ h $(35.1 \%), 6$ h (21\%), 8 h (34\%), > 8 h (9.2\%). Nursing: patient ratio: 1: 8 (40\%), 1:14 (23\%), $1: 10(18 \%)$. Most frequent pre-event infection: respiratory $56 \%$. Cause of the event: Hypoxemia (39\%). $72 \%$ of patients received supplemental pre-event 02 . In $42 \%$ of the cases some of the NEWS-2 parameters were missing, $60 \%$ the Respiratory F. and $10 \%$ the Sat. O2.Figure 1 describes the clinical risk calculated using the NEWS- 2 at the time of the event and $48 \mathrm{~h}$ before. 


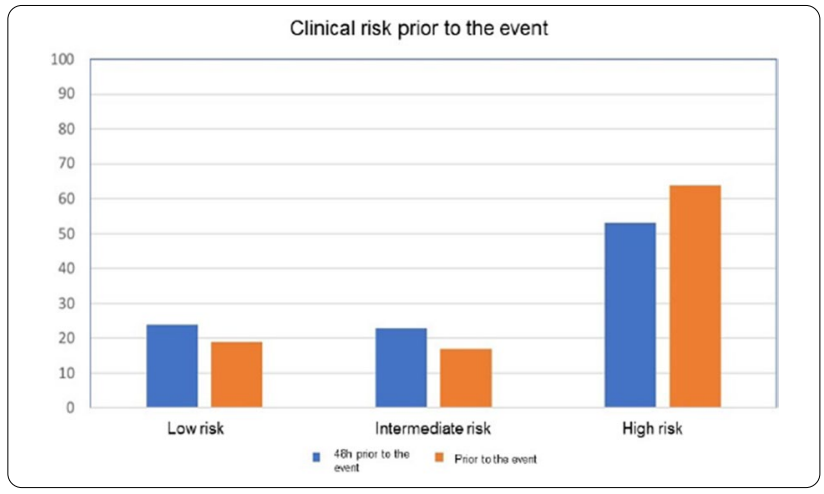

Conclusion: A medium and high clinical risk up to $48 \mathrm{~h}$ prior to a critical event in a conventional plant is frequent. Respiratory function is the most affected and its clinical record is the most incomplete.

Reference(s) and grant acknowledgment(s)

1. Not funded.

\section{1}

\section{Hospital clinical alert capacity. Analysis of factors related} to mortality. Capacicritic study

J. Ruiz ; L. Chiscano²; R. Algarte'; L. Sánchez-Alés ${ }^{3}$; L. Almorín ${ }^{4}$; P. Velasco ${ }^{5}$; JC. Ruiz-Rodríguez ; ; B. Sánchez González

'Intensive care department, Mútua Terrassa University Hospital, Terrassa, Spain; ${ }^{2}$ Intensive care department, Hospital Vall d'Hebron, Barcelona, Spain; ${ }^{3}$ Critical care department, Consorci Sanitari de Terrassa, Rubí, Spain; ${ }^{4}$ Intensive care department, Hospital Plató, Barcelona, Spain; ${ }^{5}$ Intensive care department, Hospital General de Granollers, Granollers, Spain; 6 Intensive Care Department, Hospital Vall d'Hebron, Barcelona, France

Correspondence: B. Sánchez González

Intensive Care Medicine Experimental 2020, 8(2): 000921

Introduction: It has been observed that some physiological parameters are altered in the hours prior to a critical hospital event. With the application of early warning systems by evaluating the physiological parameters of patients, they can be classified as low, intermediate or high clinical risk of suffering a critical event, reducing or anticipating the risk of critical events.

Objectives: To analyze the clinical alert capacity and the factors related to the mortality of patients who suffer a critical event in a conventional ward.

Methods: Prospective, observational and multicenter study. The patients admitted to the conventional plant who suffered a critical event from February to May 2019 were analyzed.Demographic and clinical data were collected. The NEWS-2 (NHS Early Warning Score) was calculated to stratify the patient's clinical risk prior to the event: low $(<5)$, medium $(5-6)$ and high $(>6)$ or some parameter of the NEWS-2 $=3$. Qualitative variables are expressed as percentages and were compared using the X2-test, quantitative variables are expressed as means and standard deviations (SD) and were compared using Student's t-test. Multivariate logistic regression was performed for hospital mortality.

Results: 231 patients from 5 ICU hospitals in Catalonia were included. Prior to the nursing event, between 1 and 4 medical alerts per patient were performed in $75 \%$ of the events, and between 1 and 2 medical consultations per patient were performed in $58 \%$ of the events. Hospital mortality was $60 \%$. Table 1 shows the factors related to mortality.

\begin{tabular}{|l|l|l|l|}
\hline \multicolumn{1}{|c}{ Vable 1 } & ALIVE & DEAD & $\mathrm{p}<0,05$ \\
\hline Age mean (SD) & $62,56(17,1)$ & $76,8(12,2)$ & 0,001 \\
\hline Hospital discharge $<30$ days (\%) & 24,6 & 75,4 & 0,001 \\
\hline $\begin{array}{l}\text { Event occurs at night or on holiday (\%) } \\
\text { Pacient comes from chronic center (\%) }\end{array}$ & 13,2 & 62,4 & 0,001 \\
\hline $\begin{array}{l}\text { Medical pacient (\%) } \\
\text { Cause of admission related to critical } \\
\text { event (\%) }\end{array}$ & 62,7 & 36,8 & 0,001 \\
\hline $\begin{array}{l}\text { Some parameter of the NEWS-2 =3, } \\
\text { Pre- Event (\%) }\end{array}$ & 26,7 & 65,7 & 0,001 \\
\hline $\begin{array}{l}\text { Some parameter of the NEWS-2=3, } \\
\text { 48 h before the event (\%) }\end{array}$ & 26,9 & 73,3 & 0,001 \\
\hline
\end{tabular}

Figure 1 shows the relationship between hospital mortality and PreEvent risk and 48 before the event.

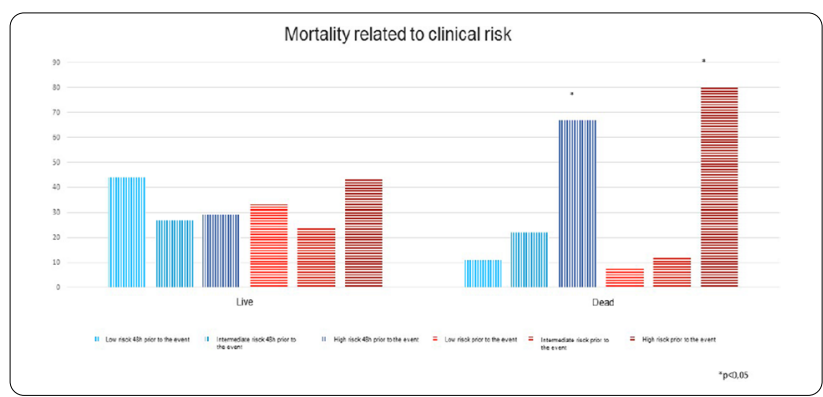

Figure 2 shows the multivariate analysis of the factors related to mortality.

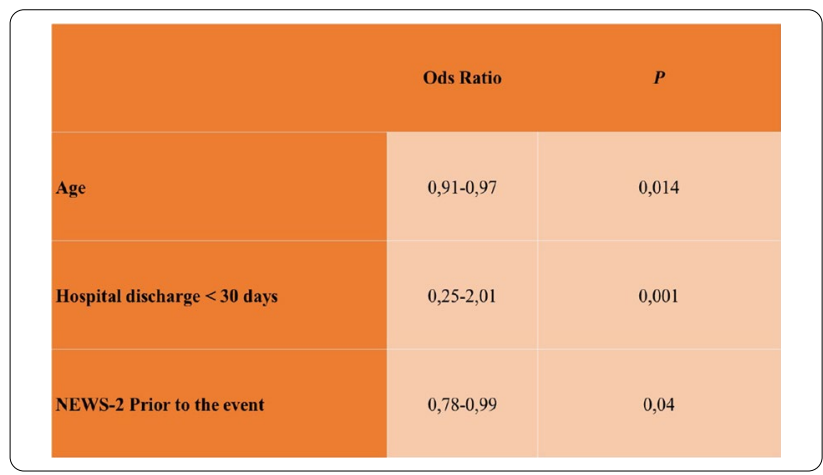

Conclusion: The mortality of patients admitted to the conventional ward who present a critical event is very high and is related to the level of clinical risk of the patient prior to the event.

Reference(s) and grant acknowledgment(s)

1. Not funded

000923

Hospital clinical alert capacity. Prediction of evolution to sepsis/ septic shock. Capacicritic study

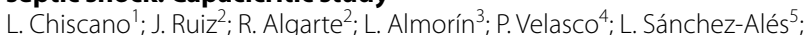
B. Sánchez González; ; JC. Ruiz-Rodríguez ${ }^{6}$

${ }^{1}$ Intensive care department, Hospital Vall d'Hebron, Barcelona, Spain; ${ }^{2}$ Intensive care department, Mútua Terrassa University Hospital, Terrassa, Spain; Intensive care department, Hospital Plató, Barcelona, Spain; ${ }^{4}$ Intensive care department, Hospital General de Granollers, Granollers, Spain; 
${ }^{5}$ Critical care department, Consorci Sanitari de Terrassa, Rubí, Spain; ${ }^{6}$ Intensive Care Department, Hospital Vall d'Hebron, Barcelona, France

Correspondence: B. Sánchez González

Intensive Care Medicine Experimental 2020, 8(2): 000923

Introduction: It has been observed that some physiological parameters are altered in the hours prior to a critical hospital event. With the application of early warning systems by evaluating the physiological parameters of patients, they can be classified as low, intermediate or high clinical risk of suffering a critical event, reducing or anticipating the risk of critical events.

Objectives: Objectives: to analyze the clinical alert capacity and describe the characteristics of the patients who suffer a critical event in the conventional ward.

Methods: Prospective, observational and multicenter study. The patients admitted to the conventional plant who suffered a critical event from February to May 2019 were analyzed.The NEWS-2 and qSOFA of the patients who had active infection at the time of the event were calculated, and the risk of progressing to sepsis / septic shock was assessed: NEWS-2 low $(<5)$ and high $(\geq 5)$ and qSOFA: low (0-1) and high (2-3). Qualitative variables are expressed as percentages and were compared using the X2-test, quantitative variables are expressed as means and standard deviations (SD) and were compared using Student's t-test. Multivariate logistic regression was performed for hospital mortality.

Results: 231 patients from 5 ICU hospitals in Catalonia were included. $46 \%$ of patients had an active infection at the time of the event, the most frequent type of infection was respiratory (56\%). 44\% evolved towards sepsis / septic shock, Figure 1 shows the prognostic capacity of the NEWS- 2 and qSOFA score.

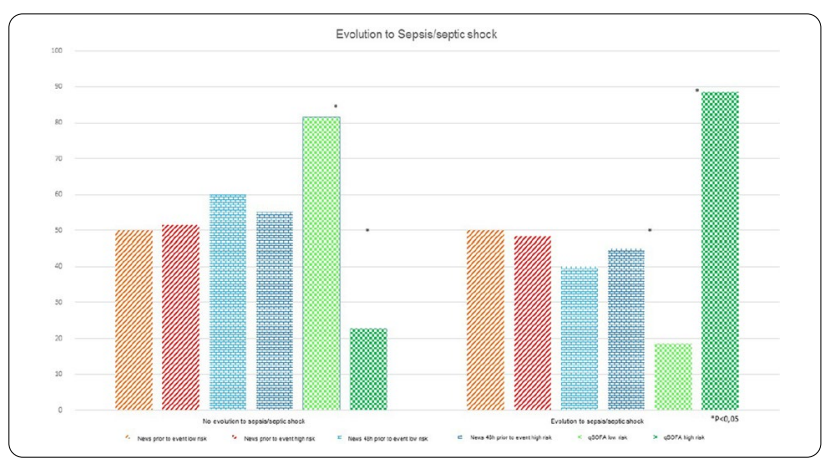

Hospital mortality was $62 \%$. Table 1 shows the relationship between hospital mortality and factors related to infection.

\begin{tabular}{|l|l|l|l|}
\hline Table 1 & ALIVE & DEAD & $\mathrm{p}<0,05$ \\
\hline Age mean (SD) & $62,9(16,4)$ & $74,5(12,7)$ & 0,001 \\
\hline Patient comes from chronic center (\%) & 11,8 & 76,5 & 0,014 \\
\hline qSOFA=2- 3 (\%) & 22,2 & 77,8 & 0,004 \\
\hline NEWS-2 Pre- Event $\geq 5(\%)$ & 30,7 & 69,3 & 0,001 \\
\hline NEWS-2 48 h before the event $\geq 5(\%)$ & 26,7 & 73,3 & 0,001 \\
\hline $\begin{array}{l}\text { Some parameter of the NEWS-2 =3, } \\
\text { Pre- Event (\%) }\end{array}$ & 25 & 74 & 0,001 \\
\hline $\begin{array}{l}\text { Some parameter of the NEWS-2 =3, } \\
\text { 48 h before the event }(\%)\end{array}$ & 26,9 & 73,1 & 0,001 \\
\hline
\end{tabular}

Figure 2 shows the multivariate analysis of the factors related to mortality.

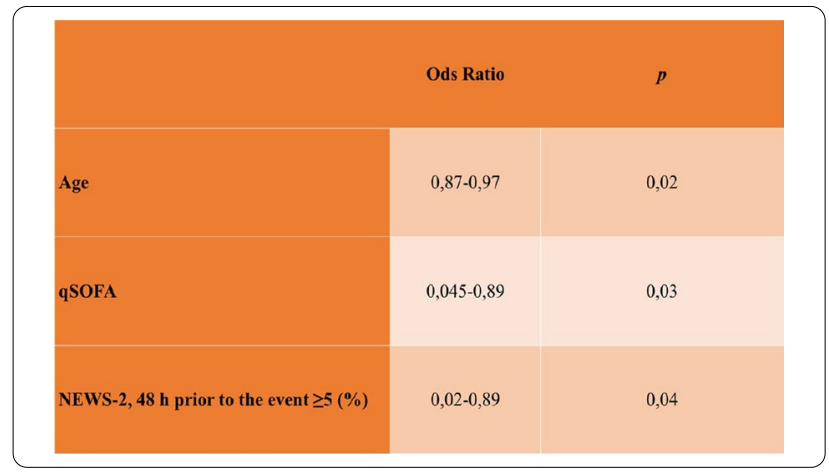

Conclusion: The infection is present in a very high percentage of patients at the time they present a critical event in the conventional plant, presenting a high mortality that can be predicted by qSOFA and NEWS-2 up to 48 hours prior to the event. In our population, qSOFA better predicts evolution to sepsis / septic shock than NEWS-2 in these patients.

\section{Reference(s) and grant acknowledgment(s)}

1. Not funded

\section{4}

Assessment prognostic value of gravity scores in cirrhotic patients admitted in intensive care units

H. Pérez Chomón ${ }^{1}$; M. Pinilla-De-Torre ${ }^{1}$; M. Blázquez Romero';

J. Garnacho-Montero ${ }^{1}$

${ }^{1}$ Servicio medicina intensiva, hospital universitario virgen macarena, Sevilla, Spain

Correspondence: $\mathrm{H}$. Pérez Chomón

Intensive Care Medicine Experimental 2020, 8(2): 001104

Introduction: Cirrhotic patients often develop severe complications requiring ICU admission. The evolution in treatments and liver transplant programs have improved the prognosis of critical cirrhotic patients, a fact that has facilitated their admission to the intensive care units (ICU). However, knowledge on the pathogenesis, diagnosis and treatment of these complications and the prognostic assessment of critical cirrhotic patients is still limited.

Objectives: To know survival prognosis of cirrhotic patients admitted to the ICU and the prognostic value of general scores for critically ill patients and those specific for cirrhosis.

Methods: Prospective, descriptive and analytical study. All patients admitted to our ICU from April 2017 to May 2020 with a history of liver cirrhosis were evaluated and were followed after 90 days of admission. Different variables such as age, sex, cirrhosis etiology, reason for admission, laboratory data, APACHE II and SOFA scales at baseline and specific scales score (Child-Pugh, CLIF-SOFA, Life Score and MELD) were registered upon admission, 7 days and discharge or death. The need for invasive mechanical ventilation, continuous renal replacement therapy or vasoactive support, as well as the development of infectious complications, were also noted.

Statistical analysis: qualitative variables are expressed as percentages. For quantitative variables, measures of central tendency and dispersion were used. The comparison of quantitative variables was performed using the Student T test. Chi-square test was used to compare qualitative variables. ROC curves were built to evaluate the ability of prognostic scores to predicte mortality at 90 days of admission.

Results: One hundred and one patients (77 men) were included; age $56.61 \pm 11.41$ years. APACHE II mean at admission was $22.38 \pm 10.08$ SOFA average $9.06 \pm 4.89$. Average MELD $20.73 \pm 9.91 .43 .6 \%(n=44)$ presented Child-Pugh C. $43.6 \%(n=44)$ had CLIF-SOFA grade 3 and $35.6 \%(n=36)$ had an intermediate risk Life- Score. Mortality in the 
ICU and at 90 days was $41.6 \%$ and $53 \%$ respectively. Statistical analysis showed worse APACHE II, SOFA and MELD values at admission ( $p$ 0.000 ) in patients who died in the ICU and at 90 days. There were no significantly differences regarding age. Analysis of ROC curves showed that the SOFA (0.738) and APACHE II (0.762) calculated in the first $24 \mathrm{~h}$ of admission predicted mortality at 90 days whereas the AUC of specific scores were 0.783 for Child-Pugh and 0.780 for MELD.

Conclusion: Cirrhotic patients who died during their stay in the ICU or at 90 days of follow-up showed significantly higher values in all prognostic scores at admission compared to those who survived. ChildPugh and MELD seem to be the better predictors of 90-day mortality.

\section{9}

A prognostic prediction model for critically ill patients with covid-19

J. Cedeño Mora'; P. García Olivares ${ }^{1}$; N. Cango ; A. Garrido ${ }^{1}$; P. Oliver ${ }^{1}$; J. Cuil; C. Mata'; G. Castañeda'; C. Alvarez'; C. Sotillo

${ }^{1}$ Intensive care unit, H.G.U Gregorio Marañón, Madrid, Spain

Correspondence: P. García Olivares

Intensive Care Medicine Experimental 2020, 8(2): 001119

Introduction: Covid-19 pandemic has conditioned a high demand for critical care beds. The develop of a prognostic score could help to better management of health resources.

Objectives: Our aim was to obtain a prognostic prediction model for critically ill patients with severe SARS-COV-2 coronavirus infection (Covid-19).

Methods: Prospective observational study of patients with diagnosis of Covid-19 admitted to critical care units of the H.G.U Gregorio Marañón. Epidemiological data, previous comorbidities, clinical and analytical data, specific treatments administered, organic support used, complications during stay and final outcome, were collected.

Descriptive data were described as medians with interquartile range (IOR) and as percentages for categorical data. The continuous variables were categorised according to maximum discrimination point by the area under the receiver operating characteristic curve (AUROC). Univariate analysis was performed using a simple logistic regression (OR). The predictive model was obtained by means of multiple logistic regression analysis. For model validation, its discrimination capacity (AUROC), its calibration capacity (Hosmer-Lemeshow test) and its quality diagnostic indices (sensitivity, specificity, positive and negative predictive value), were analysed.

Results: One hundred and fifty patients were included, $71 \%$ male. Age was 61 years (49-71) and Charlson Comorbidity Index 1 pts (0-3). Severity scores were: APACHE II 16 pts (12-20), SOFA 6 pts (4-8). $88 \%$ of patients required mechanical ventilation, $77 \%$ prone-position and $63 \%$ vasopressor therapy. Acute renal failure was present in 33\% $(10 \%$ needing renal replacement therapy). $77 \%$ presented any complication. Some specific treatment was administered in $98 \%$. Hospital mortality was $40.5 \%$.

In the univariate analysis the variables related to mortality were: Age (decades) OR 1.59; 95\% Cl 1.21-2.09, Charlson I. OR 1.40; 95\% Cl 1.141.72 , SOFA > 3 pts $O R 3.03 ; 95 \% \mathrm{Cl} 1.06-8.63$, Mechanical ventilation OR 13.1; $95 \% \mathrm{Cl}$ 1.69-101.78, Prone-positioning OR 2.93; 95\% Cl 1.276.75 , Vasopressors OR 4.45; $95 \% \mathrm{Cl} 2.05-9.68$, Atrial fibrillation $\mathrm{OR}$ 3.58; $95 \% \mathrm{Cl} 1.63-7.87$, C-reactive protein OR 1.03; $95 \% \mathrm{Cl} 1.01-1.06$ Procalcitonin OR 1.05; 95\% Cl 1.01-1.10, IL-6 400 pg/ml OR 6.31; 95\% Cl 2.55-15-61, D-dimer 4000 ng/ml OR 7.00; 95\% CI 3.36-14.57, Lactate $2 \mathrm{mmol} / \mathrm{I}$ OR $7.26 ; 95 \% \mathrm{Cl} 3.47-15.21$

The prognostic predictive model included the following variables: age (decades) (OR 1.72; 95\% 95\% Cl 0.98-2.99), Charlson I. (OR 1.44; $95 \% 95 \% \mathrm{Cl} 0.92-2.26)$, SOFA > 3 pts (OR 5.59; $95 \% \mathrm{Cl} 0.89-34.79$ ), IL-6 > $\mathbf{4 0 0} \mathbf{~ p g} / \mathbf{m l}$ (OR 4.05; 95\% Cl 1.15-14.18), D-Dimer> $\mathbf{4 0 0 0} \mathbf{~ n g} /$ ml (OR 11.85; 95\% Cl 2.95-57.66), Lactate > 2 mmol/l (OR 5.41; 95\% $\mathrm{Cl}$ 1.56-18.68).

The model obtained (figure 1) had a good discriminatory capacity AUROC 0.90; $\mathrm{Cl}$ 0.84-0.96, a proper calibration (Hosmer-Lemeshow test): Chi-square 3.38, $p=0.9$, and good diagnostic quality indices: $S$ $81 \%$, E $81 \%$, PPV 0.73, NPV 0.87, VPR 4.26, VNR 0.23

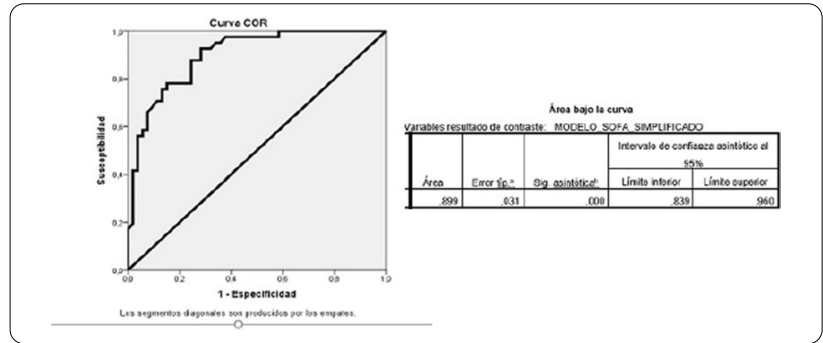

Conclusion: In our experience, the predictive model created using clinical and analytical data obtained early on ICU admission, was able to discriminate the prognosis of Covid-19 patients.

001142

Emergency department visits, Emergency Severity Index and Intensive Care Unit admissions in the era of COVID19; A single center experience

G. Karlis ${ }^{1}$; S. Orfanos ${ }^{1}$; P. Zafeiriadou ${ }^{1}$; S. Kolokytha'; S. Christakopoulos'; K. Georgiopoulos ${ }^{1}$; A. Sofianou ${ }^{1}$; K. Tsekouras ${ }^{1}$; P. Batiani ${ }^{1}$; E. Ntikoudi'; V. Kaldis ${ }^{1}$

${ }^{1}$ Emergency department, Sismanogleio General Hospital, Marousi, Greece Correspondence: $G$. Karlis

Intensive Care Medicine Experimental 2020, 8(2): 001142

Introduction: The purpose of triage in the Emergency Department $(E D)$ is to prioritize incoming patients and to identify those who cannot wait to be seen (1). One of the most widely used triage tools is the Emergency Severity Index (ESI), which includes a five-level scale with ESI 1 describing a patient that requires immediate life-saving intervention/resuscitation and ESI 5 a non-urgent patient (2). COVID19 has set new challenges in the organization and practice of emergency and critical care medicine, prompting for re-evaluation of existing tools.

Objectives: To assess the effects of the "lockdown" on ED visits and the relationship between ESI 1 patients and short-term outcomes in the era of COVID19, in a tertiary Greek hospital.

Methods: ED data from Sismanoglio General Hospital, a tertiary Greek hospital in Athens metropolitan area, was retrospectively studied from $1 / 1 / 2020$ to $30 / 6 / 2020$. In Greece, a full "lockdown" was imposed between $13 / 3 / 2020$ and 4/5/2020.

Results: The number of ED visits per month was significantly affected by "lockdown". We noted $42.2 \%$ reduction in total ED visits during March-April compared to January-February and $41.7 \%$ compared to March-April 2019. After the "lockdown" period a $24.1 \%$ increase in the number of ED visits was seen (Fig.1). Interestingly, the peak of epidemic in Greece did not influence ED attendances for immediate lifethreatening conditions (Fig.2). In our analysis patients assessed as ESI 1 constituted $0.46 \%$ of all ED patients for the 6 -month period. Furthermore, in the group of patients triaged to ESI 1, 20.8\% died, $65.3 \%$ were admitted to the ICU and $13.9 \%$ were admitted to the ward. None was discharged home from the ED (Fig. 3).

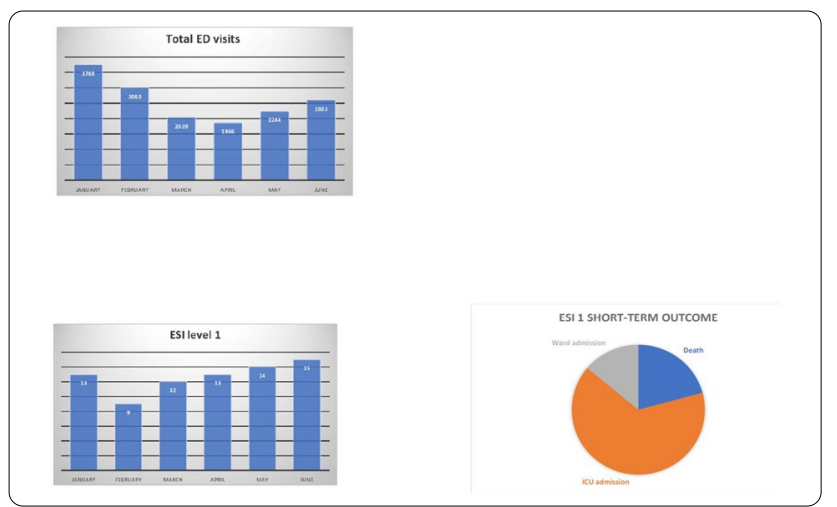


Conclusion: Our data is consistent with other reports of dramatic falls in ED attendances amid the outbreak of SARS-CoV-2, due to fear of contracting the disease $(3,4)$. However, we noticed that individuals with critical illness (ESI 1) found their way to the ED at any given time during the coronavirus crisis. From previous research we know that most ESI 1 patients are admitted to ICU, while some die in the $\operatorname{ED}(5,6)$. Our results show that during COVID19 crisis, ESI 1 was associated with high likelihood of ICU admission and death in the ED, suggesting that it remains a useful tool for predicting short-term outcomes of critically ill ED patients.

\section{Reference(s) and grant acknowledgment(s)}

1. 6. Eitel DR, Travers DA, Rosenau AM, Gilboy N, Wuerz RC. The emergency severity index triage algorithm version 2 is reliable and valid. Acad Emerg Med. 2003;10(10):1070-1080. https://doi.org/10.1111/j.1553-2712.2003. tb00577.x

2. 5. Kwak H, Suh GJ, Kim T, et al. Prognostic performance of Emergency Severity Index (ESI) combined with qSOFA score. Am J Emerg Med. 2018;36(10):1784-1788. https://doi.org/10.1016/.ajem.2018.01.088

3. 4. Bodington R, Bhandari S. Falling usage of hospital-based emergency care during the COVID-19 pandemic in the UK. J R Coll Physicians Edinb. 2020;50(2):207-214. https://doi.org/10.4997/jrcpe.2020.234

4. 3. Anteby R, Zager Y, Barash Y, et al. The Impact of the Coronavirus Disease 2019 Outbreak on the Attendance of Patients with Surgical Complaints at a Tertiary Hospital Emergency Department [published online ahead of print, 2020 Jun 23]. J Laparoendosc Adv Surg Tech A. 2020;https://doi. org/10.1089/lap.2020.0465. https://doi.org/10.1089/lap.2020.0465

5. 2. Ganjali R, Golmakani R, Ebrahimi M, Eslami S, Bolvardi E. Accuracy of the Emergency Department Triage System using the Emergency Severity Index for Predicting Patient Outcome; A Single Center Experience. Bull Emerg Trauma. 2020;8(2):115-120. https://doi.org/10.30476/beat.2020.46452

6. 1. Tanabe P, Gimbel R, Yarnold PR, Kyriacou DN, Adams JG. Reliability and validity of scores on The Emergency Severity Index version 3. Acad Emerg Med. 2004;11(1):59-65. https://doi.org/10.1197/j.aem.2003.06.013 7. None

\section{8}

Early-stage errors after the implementation of a clinical information system in intensive care unit

Y. Seino ${ }^{1}$; N. Sato ${ }^{1}$; M. Idei ${ }^{1}$; Y. Saishu'; M. Iwabuchi ${ }^{1}$; K. Fukui ${ }^{1}$;

M. Namekawa'; K. Ota ; J. Ishikawa'; M. Nakagawa' ${ }^{1}$ T. Nomura

${ }^{1}$ Department of Intensive Care Medicine, Tokyo Women's Medical University, Tokyo, Japan

Correspondence: $Y$. Seino

Intensive Care Medicine Experimental 2020, 8(2): 000248

Introduction: Clinical information systems (CIS) have been developed in intensive care units (ICU) to aggregate information, promote operational efficiency, and obtain accurate records. However, in settings wherein hospital electric medical record (hEMR) systems have operated, the differences in the operability and performance between CIS in ICUs and hEMR systems and the constraints of cooperation of both the systems can lead to new errors when using both the systems in ICUs. However, the incidence rate and the type of errors recorded at an early-stage after implementation of CIS in ICUs are unknown.

Objectives: To investigate the incidence rate and the type of errors in ICUs for 9 months after implementation of CIS.

Methods: CIS (PrimeGaia, Nihon Kohden, Tokyo, Japan) was introduced into our medical and surgical ICU (18 beds, nursing-to-patient ratio 1:2) and intermediate medical care unit (IMU, 15 beds, nursingto-patient ratio 1:4) at a university hospital (1,335 beds). After implementation and a 3-month training period, CIS was used in all the patients starting in April 2019.The data of incidents and accidents in the ICU and IMU in the 9-month period after the launch of the CIS (from January to September 2019) were extracted from the hospital's incident reporting system, and the incidence rate was determined. In our incident reporting system, errors have been divided into two categories: "incident" (indicating no error or error that caused no harm such as near-misses) and "accident" (indicating error resulting in harm and error resulting in patient death). Poisson distribution was used to calculate the $95 \%$ confidence interval $(\mathrm{Cl})$ of the incidence rate of events.

Results: From January to September 2019, CIS was used in 942 (90\%) of the 1,046 patients admitted to ICU and in 1,278 (89\%) of the 1,439 patients in IMU. The total length of stay in ICU and IMU was 3,854 and 2,728 patient-days, respectively, and the duration of using CIS was 3,200 and 2,311 patient-days, respectively. The number of incidents and accidents was 123 in ICU and 140 in IMU. The incidence rates of events were $31.9(95 \% \mathrm{Cl} 25.5-38.0)$ and $51.3(43.2-60.6)$ events per 1000 patient-days, respectively. The number of incidents and accidents related to CIS were $18(15 \%)$ in ICU and $15(11 \%)$ in IMU. The incidence rates of events were $5.6(3.3-8.9)$ and 6.5(3.6-10.7) events per $1000 \mathrm{CIS}$ operation patient-days, respectively.

The major background factors for the incidents and accidents related to CIS were unfamiliarity with its operations $(11 / 33)$, inadequate coordination between the CIS and hEMR (11/33), specifications of the CIS (7/33), and others (4/33).

Conclusion: Our study has revealed that $12 \%$ of the incidents and accidents in ICU and IMU were related to CIS and that the incidence did not vary with the management intensity of care units. The errors during the early stages after implementation of the CIS occurred because of unfamiliarity with its operation, inadequate coordination between CIS and hEMR, or the specifications of CIS.

Reference(s) and grant acknowledgment(s)

1. Friedman LN, Halpern NA, et.al. Implementing an electronic medical record. Critical Care Clinics. 2007:23:347-81.

2. Mason C, Leong T. Clinical information systems in the intensive care unit. Anaesthesia and Intensive Care Medicine 2016;17:13-16.

000354

Can anyone talk to me? Communication with families during the days of corona in intensive care unit

HY. Y. ; N. V. ; SH. Tal'; ML. R.

${ }^{1}$ Icu. sheba medical center, Emek Haela 9, Ramat Gan, Israel

Correspondence: R. Mor levy

Intensive Care Medicine Experimental 2020, 8(2): 000354

Introduction: With the outbreak of the Corona virus SARS-CoV-2, communication with family members became even more challenging and critical than it had been in the first place. Group meetings for families which we usually convened every week were no longer possible due to the prohibition on gathering, and therefore we were in need of a solution that would meet the needs of patients and their families. On April 10, all family visits were stopped in order to prevent infection. This situation created a new reality, where family members could not visit the unit at all and could not see their loved ones. This reality required us to make a quick assessments and response to the situation, understanding that the emotional burden would weigh heavily on the families.

Objectives: Our objective was to find a way to preserve the critical connection and communication lines between the families in the ICU to their loved ones and the staff in a way that met their emotional needs, while operating within the existing limitations that were necessary to prevent the spread of the virus.

Methods: The program was implemented on four levels: (1) Initiated conversations of physicians with the primary caregiver: From April 10th until May 5th the doctors made phone calls to family members of 42 patient. (2) Video calls of the single UI with the families via tablet: During video calls that were connected and accompanied by the social worker, the families could see the patient and speak to them (3) Providing telephone information by the nursing staff: Since the family members did not enter the unit at all, it was decided that the information they provided would be slightly more detailed than usually acceptable and tailored to their needs. (4) A video that provides general information to families, in order to familiarize them with the unit, the management team, and the nature of the outpatient care: The video was created as a replacement for family gatherings where information is usually transmitted. The video will be used in the future as it was found to be helpful as a day to day tool as well. 
Results: As a result of the program, we were able to positively influence intensive care patients' families, include them in the treatment process without being physically present in the unit, and maintain quality communication that influences their motivation and commitment to the care plan and hospitalization provided by their loved ones. We received immediate positive feedback from the families that took part, verbally, via text messages and thank you letters. In addition, there was a decrease in the amount of pressure and phone calls to the nursing station from families who were trying to get information, once they started getting the information in an organized way initiated by the multi-professional staff.

Conclusion: The coronavirus, which has spread rapidly around the world, has tested the readiness of the health system to cope with pandemic conditions and the entire system has had to reformulate coping methods in many areas including communication between patients and their families in isolation and risk of infection. The program was implemented on four levels that combined the knowledge and practice we accumulated and is the basis upon which we added more knowledge during each shift. We hope to continue developing as we proceed.

\section{8}

\section{Seeking for interoperability standards on e-health medication} records in intensive care medicine

E. Salgado ${ }^{1}$; R. Heidepriem ${ }^{1}$; J. Sass² ; F. Schiefenhoevel'; AS. Poncette'; S. Thun' F. Balzer

'Department of anesthesiology and intensive care medicine, Charité Universitätsmedizin Berlin, Berlin, Germany; ${ }^{2}$ Core unit e-health and interoperability, Berlin Institute of Health, Berlin, Germany

Correspondence: $\mathrm{E}$. Salgado

Intensive Care Medicine Experimental 2020, 8(2): 000398

Introduction: Medication errors have been a frequent and safety-relevant problem in medical care for years[1]. In this context, the lack of standardized, automatic, reliable, consistent documentation and information systems represents a major obstacle. The high dependency on manual input in many steps of the process is a particular challenge[2].

Objectives: This work aimed to analyze the medical records of intensive care unit (ICU) patients admitted to a large university hospital in Germany, as well as to explore and propose an information technology (IT) standard to build an interoperable medication interface.

Methods: We accessed a series of databases to collect the information about 81 medications that were frequently used in our ICUs from the hospital's IT department, pharmacy, as well as the ICU's Patient Data Management System (PDMS). We further obtained data drawn from the most comprehensive German medication register, the ABDA/ ABDAMED database[3]. To gather data according to European standards, we used information available on the European Directorate for the Quality of Medicines and HealthCare (EDQM)[4].

We oriented our search with the Health Level Seven International (HL7) standards and definitions[5] with the IT architecture and guidelines of our institution. To ensure fulfillment of the HL7 v2.3.1 requirements, we selected the following data components: medication name, Pharmacy Central Number (PZN), institution-specific material number used in the electronic medical record (EMR), Pharmacy Product Number (PPN), Global Trade Item Number (GTIN), National Trade Item Number (NTIN), Anatomical Therapeutic Chemical (ATC), Arzneistoffkatalo $\mathrm{g} /$ German drug substance catalogue (ASK), and EDQM standards for administration method, intended site, and routes of administration. Finally, we compiled the data into a single data set.

Results: Most medications documented data was not digitalized and therefore not interoperable (e.g. pharmacy and PDMS databases). Moreover, we found that it is difficult to access the data mostly due to a deep fragmentation throughout the stakeholders. We observed that the interoperability problems were not a specific difficulty of our hospital but a nationwide challenge, due to the lack of standardization. Nonetheless, we could build a unified and standardized database out of the information of each mentioned database according to the HL7 guidelines of our institution, which led us to generate examples of codes for communication between different IT systems. We present an example below (Figure 1).
MSH $\left.\right|^{\wedge} \sim|\&|$ COPRA|| SAP-ISH | 20191017175721||RDE || 2.3.1| PID | | 90501847 || MUSTERFRAU^MAXIMILIANE^| | 19670525|F| PV1 $\mid$ I | AL1 $|1|{ }^{\wedge}$ No Known Drug Allergies^| $\mathrm{ORC}|\mathrm{NW}|$ RXE $\mid 1^{\wedge} T G L \wedge \wedge 201910171200^{\wedge \wedge \wedge \wedge}$ TGL 1 TBL MITTAGS^^|51055^PLAVIX 75MG FILMTABLETTEN^PZN|1||0069^TABLET^EDQM| RXR $\mid 20053000^{\wedge} O R A L$ USE^EDQM

Figure 1: Example order message with instructions for administration of a medication to a patien (MSH: Mossage header, RDE: Pharmacyareatment encoded order messago; PID: Patient identfication. encocied order, RXA: Pharmacyltreatment routes

Conclusion: Our results show that it is a challenge to achieve standardization and therefore interoperability of medical records within the different IT platforms used in intensive care medicine. However, we proposed a solution based on international standards that may be applied to a broader spectrum of processes, such as the digitalization of the discharge medication from the ICU to the normal ward.

\section{Reference(s) and grant acknowledgment(s)}

1. 5. HL7-Definition V2, "Caristix HL7-Definition V2 reference site." Retrieved May 4, 2020 (https://hl7-definition.caristix.com/v2/HL7v2.3.1)

2. 4. European Directorate for the Quality of Medicines and HealthCare (EDQM), "Standard Terms." Retrieved April 29, 2020 (https://standardterms. edqm.eu)

3. 3. ABDATA Pharma-Daten-Service, "Pharmazeutische Stoffliste." Retrieved February 17, 2020 (https://abdata.de)

4. 2. Balzer F, Wickboldt N, Spies C, et al. Standardised drug labelling in intensive care: results of an international survey among ESICM members. Intensive Care Med. 2012;38(8):1298-305

5. 1. Valentin A, Capuzzo M, Guidet B, et al. Errors in administration of parenteral drugs in intensive care units: multinational prospective study. BMJ. 2009;338:b814

6. The project was financed with special funds from the Berlin Institute of Health $(\mathrm{BIH})$ in 2019.

\section{9}

The adaptation and utilisation of a best-of-breed informatics system to support safe operational expansion during the COVID19 epidemic in a London University hospital at the UK outbreak epicentre

T. Lalmahomed ${ }^{1}$; I. Salako ${ }^{1}$; E. Cenizo ${ }^{1}$; O. Wong ${ }^{1}$; C. Bell ${ }^{1}$; R. Sloss ${ }^{1}$;

H. Lewis ${ }^{1}$; R. West ${ }^{1}$; F. Anwar ${ }^{2}$; L. Mccaulsky²; C. Chan-Graschitz ; K. Child ${ }^{1}$; R. Mehta ${ }^{1}$; T. Best ${ }^{1}$; P. Hopkins ${ }^{3}$

${ }^{1}$ King's critical care, King's College Hospital, London, United Kingdom; ${ }^{2}$ King's ict, King's College Hospital, London, United Kingdom; ${ }^{3}$ King's critical care, King's College London, London, United Kingdom

Correspondence: $P$. Hopkins

Intensive Care Medicine Experimental 2020, 8(2): 000899

Introduction: Best of breed informatics systems have an unclear evidence base for delivering clinically efffective support over paperbased systems or large institutional electronic health records (Rubenfeld, 2004; Morrison, 2008). During the COVID 19 pandemic, King's College Hospital admitted over 2000 patients with COVID 19, 190 of whom required an admission for critical care. Severity in our population was higher than seen in other UK centres, in part due to the high incidence of renal failure (50\%). We assessed the use and effectiveness of a best-of-breed critical care informatics system in this unprecedented period of operational and clinical strain.

Objectives: To use SQL reports and general database to assess the scope and effectiveness of a rapid electronic/informatics systems expansion during the COVID 19 pandemic in a central London critical care service.

Methods: The configuration of the ICCA system was compared post COVID 19 to that pre-COVID 19. In addition, pre-existing and new ad hoc SQL queries were constructed from the Intellivue, Critical Care \& Anaesthesia Informatics System (ICCA, Philips HealthCare) database 
using Sequel Server Management Studio 17. These were used to assess changes to configuration and workflow support; activity; electronic workflow alerts and adverse event capture.

Results: The ICCA informatics support system was rapidly expanded from 51 to 196 licensed support beds, including Census Page and Dashboard expansions. Over the pandemic period 1022 healthcare professionals were added to the system users database. 506 additional client computers were also added. Remote service review was set up for anaesthesia, renal, cardiology, diabetes, haematology and nutrition teams. The system and systems team provided a bespoke pathophysiological flowsheet and designed three clinical reports to support strategic mangement of overall clinical operations, renal replacement and airway management. 29 major urgent prescription changes were delivered to reflect supply chain breakdown and the need to deliver new COVID 19 trials. This included changes to sedation regimens, muscle relaxants, fluids and electrolyte replacement. There were also several hundred individual text changes to prescription/administration of medications to support the major category updates. System screening was used to issue 1288 workflow support alerts during the pandemic period. Commonest alerts were around sedation levels; ventilator driving pressure; patient allergies and patient identity. Importantly, several operationally important system updates were developed. A visual basic solution was written to provide a physical paper medication chart to go with the patient at discharge and the servers received improved power redundancy to decrease downtime. Finally, the system captured 159 adverse incidents that were not reported in the institutional 'Datix' system.

Conclusion: We have described the rapid and effective expansion of a critical care informatics system from 51 beds to 196 beds during the COVID 19 pandemic response of a large critical care service. The level of rapid adaptation may not have been possible on paper or across an institutional EHR.

\section{Reference(s) and grant acknowledgment(s)}

1. Morrison C et al. 2008. Electronic patient record use during ward rounds: a qualitative study of interaction between medical staff. Crit Care 12: R148

2. Rubenfeld GD. 2004. Using computerised medical databases to measure and to improve the quality of intensive care. J Crit Care 19: 248-56 3. NHS Digital Support Grant

\section{8}

\section{Future FICE faculty: ensuring the standard of future faculty} with a mentorship scheme

S. Morton' ; R. Fisher'; J. Powell-Tuck'; G. Zilahi' ; J. Aron ${ }^{5}$

${ }^{1}$ Intensive care, Chelsea and Westminster Hospital, London, United Kingdom; ${ }^{2}$ Intensive care, King's College Hospital, London, United Kingdom; ${ }^{3}$ Intensive care, St Thomas' Hospital, London, United Kingdom; ${ }^{4}$ Intensive care, St George's Hospital, London, United Kingdom; ${ }^{5}$ General intensive care unit, St. George's Hospital, London, United Kingdom

Correspondence: $\mathrm{S}$. Morton

Intensive Care Medicine Experimental 2020, 8(2): 000008

Introduction: Focused Intensive Care Echo (FICE) is a training scheme developed by the UK Intensive Care Society to train clinical practitioners in the use of echocardiography in intensive care. [1] Learning a new physical skill normally requires one-to-one tuition. With an increasing demand for FICE courses there is therefore increasing demand for faculty; ensuring the quality of the faculty is maintained can become difficult. For many faculty intensive courses (such as Advanced Life Support), instructor courses and mentorship schemes exist to ensure the future faculty meet the expected standard.[2] Currently there is no such scheme for FICE faculty.

Objectives: Our aim was to develop a mentorship scheme for prospective faculty to ensure new faculty were supported through the course and to ensure standards were met to guarantee the quality of teaching desired on our FICE course.

Methods: Nine prospective faculty candidates, who were all FICE (or equivalent) qualified, were recruited to teach on two FICE courses held on consecutive days in October 2019. Prior to the course an anonymous online survey was conducted to gain background information and their pre-course teaching confidence. Throughout the course the candidates were then paired with experienced FICE faculty to support their teaching; at the end of the course candidates received written feedback, including an overall assessment as to whether it was felt they could be recommended as full faculty in the future. Following this another anonymous online survey was conducted to review the experience of the faculty candidates and to assess any changes in confidence. Results: Table 1 shows the demographics of the faculty candidates:

\begin{tabular}{|l|l|}
\hline \multicolumn{2}{|c|}{ Table 1: Demographics of faculty candidates } \\
\hline Qualified $\leq 5$ years & Number \\
\hline Qualified $>5$ years & 2 \\
\hline Echo experience $\leq 2$ years & 7 \\
\hline Echo experience $>$ 2 years & 7 \\
\hline $\begin{array}{l}\text { Had previously recieved formal teaching instruction e.g. GIC } \\
\text { course? }\end{array}$ & 2 \\
\hline Had previously taught on echo courses? & 5 \\
\hline
\end{tabular}

Seven of the faculty candidates completed the post course questionnaire. Confidence improved in all aspects assessed (Figure 1) with every candidate at least feeling "OK/assistance only needed occasionally" following the course. Comments relating to the mentorship included "well supported", "constructive" and "good opportunity". 86\% of candidates agreed absolutely that all people who want to mentor on a FICE course should do something similar and that it will improve the quality of mentorship. $71 \%$ found the paper feedback useful. All faculty candidates apart from one were recommended as full faculty; the one not recommended has been invited to attend again as a faculty candidate to increase their confidence.

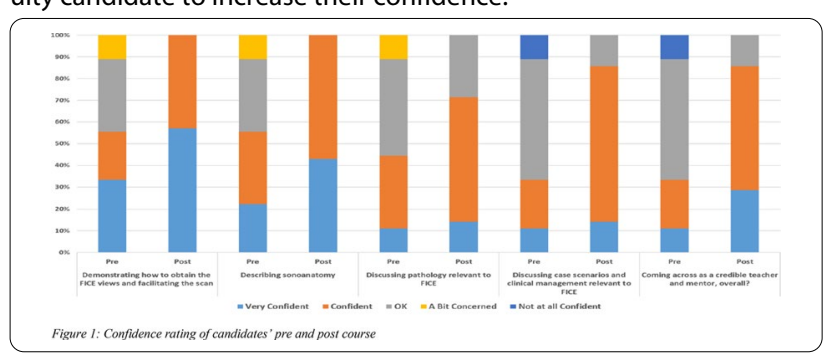

Conclusion: With the increasing need for FICE faculty we have shown that a faculty mentorship scheme is practical and well received by the faculty candidates. This is a low cost method of providing structured mentorship to prospective faculty, without the need for specialised instructor courses. We believe that by rolling this mentorship scheme out nationwide it will ensure that we continue to develop a pool of quality faculty to meet the increasing demand for FICE courses.

\section{Reference(s) and grant acknowledgment(s)}

1. Intensive care society. FICE. 2018. https://www.ics.ac.uk/ICS/fice.aspx (Accessed 20th December 2019).

2. Resuscitation Council UK. Generic instructor course. 2019. https://www. resus.org.uk/information-on-courses/generic-instructor-course/ (Accessed 20th December 2019)

\section{1}

Point of care ultrasound in medical ICU patients with intra-abdominal hypertension

Z. Bitar ${ }^{1}$; O. Maadrani ${ }^{1}$; M. Alsamar ${ }^{1}$; T. Zaalouk ${ }^{1}$

${ }^{1}$ Critical care unit, New Ahmadi hospital KOC

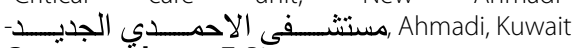

Correspondence: Z. Bitar

Intensive Care Medicine Experimental 2020, 8(2): 000031

Introduction: Intraabdominal hypertension (IAH) is well-documented causes of morbidity and mortality in the critically ill and may affect almost every organ system (1). The Abdominal Compartment Society (WSACS) developed a medical management algorithm with a stepwise approach according to the evolution of the intra-abdominal pressure 
and aiming to keep IAP $\leq 15 \mathrm{~mm} \mathrm{Hg}$ (2). Point of care ultrasound as a bedside modality in the critical care patients, could be used as an adjuvant point-of-care tool during IAH management.

Objectives: To test the role of Point of Care Ultrasound (POCUS) in the medical management of patients with IAH.

Methods: We conducted a Prospective observational study and enrolled fifty-three consecutive adult critically ill patients admitted to the medical critical care unit (CCU) of Ahamdi hospital with risk factors for IAH. Those who met the inclusion criteria were allocated to undergo POCUS and small bowel ultrasound as an adjuvant tool in their IAH management

Results: A total of 22 patients met the inclusion criteria and were included in the study (Table 2) Following step 1 of the WSACS IAH medical management algorithm, ultrasound was used for NGT placement, confirmation of correct positioning, and evaluation of stomach contents. Ultrasound was comparable to abdominal X-ray but shown to be superior in determining the gastric content (fluid vs. solid) and diagnoses gastric paresis in 2 cases. Ultrasound also proved useful in (table 3 ): (1) evaluating for intestinal obstruction. ((2) identification of large bowel contents; (3) identification of patients that would benefit from bowel evacuation (enema) as an adjuvant to lower IAP; (4) and in the diagnosis of moderate to large amounts of free intra-abdominal fluid. Small bowel obstruction and were present on four patients and confirmed with CT abdomen, and two of the patients underwent surgical intervention for mesenteric vessel occlusion and transmenteric internal hernia. Six patients were identified to have ileus in septic patients and responded to prokinetic drugs. Enema treatment was found to empty the bowel incompletely in $72 \%, 56 \%$ and $42 \%$ of the times on days 1,2 and 3 respectively. colonoscopy decompression was not needed in any of our patients. Four patients with cirrhosis that were admitted with upper gastrointestinal bleeding and hepatic encephalopathy (out of a total of 8) were found to have large amounts of ascites and the US-guided paracentesis was carried out. The average amount of ascites removed was $3600 \pm 1.6 \mathrm{~mL}$ and resulted in a significant drop in the IAP average from $21 \pm 4.1 \mathrm{~mm} \mathrm{Hg}$ to $13 \pm 2.0 \mathrm{~mm} \mathrm{Hg}$ in all four patients.

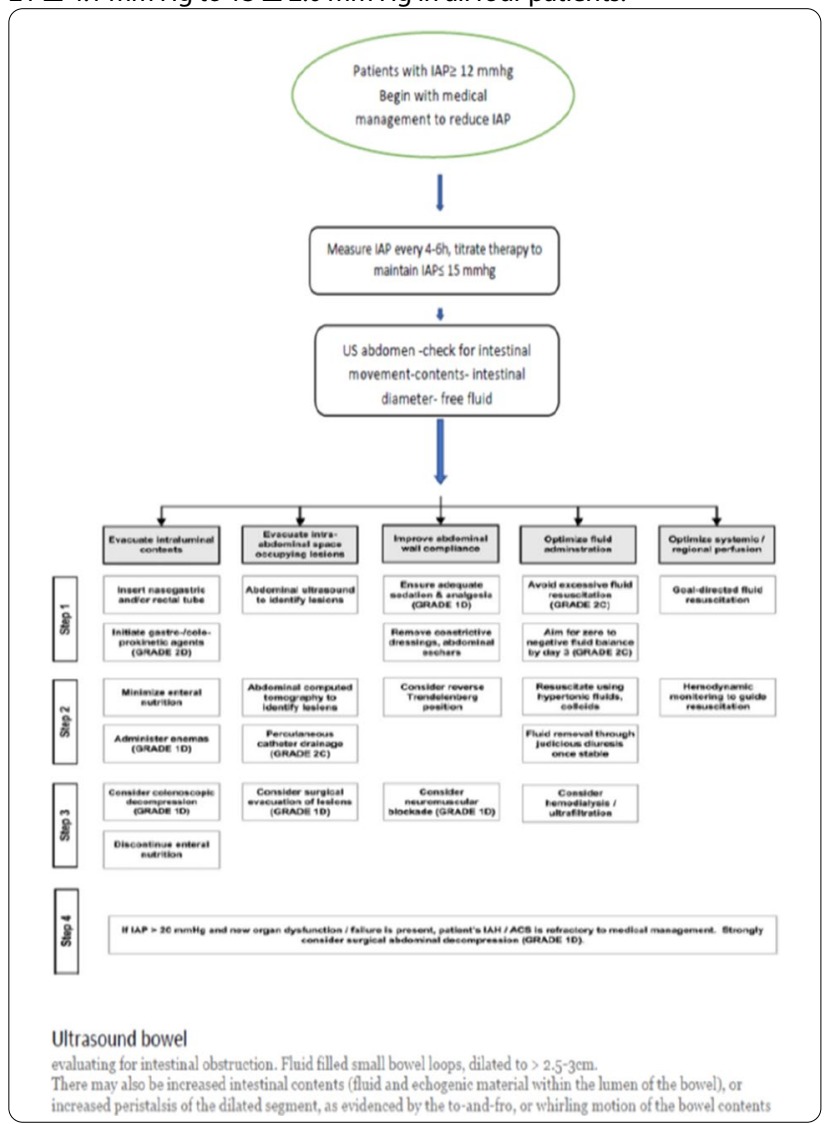

Conclusion: POCUS can be used in nonoperative management of $\mathrm{IAH}$ and ACS. It is an important tool in the diagnosis and treatment in patients with IAH.

\section{Reference(s) and grant acknowledgment(s)}

1. 2. Andrew W. Kirkpatrick, Derek J. Roberts, Jan De Waele...etal. Intra-abdominal hypertension and the abdominal compartment syndrome: updated consensus definitions and clinical practice guidelines from the World

Society of the Abdominal Compartment Syndrome. Intensive Care Med (2013) 39:1190-1206

2. 1. Cheatham ML, Malbrain ML, Kirkpatrick A, et al: Definitions and pathophysiological implications of intra-abdominal hypertension and abdominal compartment syndrome, Am Surg 77(Suppl 1): S6-S11 2011. 3. None.

\section{5}

Comparison of linear and sector array probe for handheld lung ultrasound in invasively ventilated ICU patients

M. Smit' ; J. De Vos' ; L. Pisani' ; L. Hagens'; C. Almondo'; N. Heijnen²; R. Schnabel'2 I. Van der Horst'; D. Bergmans²; M. Schultz'; L. Bos'

${ }^{1}$ Department of intensive care, Amsterdam University Medical Centers, University of Amsterdam, Amsterdam, Netherlands; ${ }^{2}$ Department of intensive care, Maastricht UMC+, Maastricht, Netherlands

Correspondence: M. Smit

Intensive Care Medicine Experimental 2020, 8(2): 000175

Introduction: International guidelines do not recommend a specific probe for assessment of lung aeration using Lung Ultrasound (LUS). The aim of this study was to assess the concordance between linear and sector array probes of a handheld ultrasound device in assessment of lung aeration in invasively ventilated Intensive Care Unit (ICU) patients.

Methods: This study included invasively ventilated patients admitted to the ICU expected to be ventilated $>24$ hours. A trained examiner performed a 12-region LUS exam with a linear and a sector array probe. In each image the LUS aeration score and the 'number of Blines' was determined. The LUS aeration score was defined as; ' 0 ', Apattern; '1', B1-pattern; '2', B2-pattern; or ' 3 ', C-pattern. Adding LUS aeration scores of all regions resulted in a global LUS aeration score [Crit Care. 2007;11(1):205]. Agreement between the two probes was calculated using intraclass correlation coefficients (ICC).

Results: 30 LUS exams were performed in 19 mechanically ventilated ICU patients resulting in a total of 328 pairs of images. 29 pairs of images were excluded for analysis because the images from the linear probe were unable to score (UTS) (Table 1). ICC's calculated for the remaining images revealed a good concordance for the LUS aeration score for individual images, ICC: 0.73 (Cl: $0.67-0.78$ ), 'number of Blines', ICC: 0.79 (Cl: $0.72-0.83$ ) and global LUS aeration score, ICC: 0.74 (Cl: 0.52-0.87)

\begin{tabular}{|c|c|c|c|c|c|c|c|}
\hline \multirow{8}{*}{$\begin{array}{l}\text { Sector } \\
\text { array } \\
\text { probe }\end{array}$} & \multicolumn{7}{|c|}{ Linear probe } \\
\hline & & $\mathbf{A}$ & B1 & B2 & $\mathrm{C}$ & UTS & Total \\
\hline & $\mathbf{A}$ & 185 & 7 & 2 & 4 & 14 & 212 \\
\hline & B1 & 23 & 10 & 1 & 2 & 8 & 44 \\
\hline & B2 & 6 & 7 & 23 & 1 & 3 & 40 \\
\hline & $\mathrm{C}$ & 5 & 1 & 1 & 21 & 4 & 32 \\
\hline & UTS & 0 & 0 & 0 & 0 & 0 & 0 \\
\hline & Total & 219 & 25 & 27 & 28 & 29 & 328 \\
\hline
\end{tabular}

Conclusion: There is a good concordance between linear and sector array probes of a handheld ultrasound device in assessment of lung aeration patterns in mechanically ventilated ICU patients. However, in roughly $10 \%$ of the images acquired using the linear probe the aeration pattern could not be scored.

\section{Reference(s) and grant acknowledgment(s)}

1. The authors received a grant from Health Holland and the Dutch Lung Foundation for the DARTS study. 
2. Bouhemad B, Zhang M, Lu Q, Rouby JJ. Clinical review: Bedside lung ultrasound in critical care practice. Crit Care. 2007;11(1):205.

\section{5}

A prospective study of effect of Short End Expiratory Hold (SEEH) on Echocardiography image quality and hemodynamic parameters in patients on mechanical ventilation

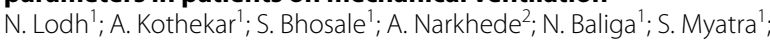
A. Kulkarni ${ }^{1}$; J. Divatia ${ }^{1}$; V. Patil ${ }^{1}$

${ }^{1}$ Department of Anesthesia, Critical Care and Pain, Tata Memorial Hospital, Mumbai, India; ${ }^{2}$ Department of critical care medicine, Jupiter Hospital, Thane, India

Correspondence: $\mathrm{N}$. Lodh

Intensive Care Medicine Experimental 2020, 8(2): 000415

Introduction: The lung interference due to mechanical ventilation is known to cause poor acoustic window making transthoracic echocardiography difficult 1 .Interference of the lungs is least during expiration however, this period is too short for interpretation. End Expiratory hold has potential to improve acoustic window by reducing lung interference.End Expiratory hold of 15 seconds acts like a fluid bolus and changes cardiovascular physiology especially during last five seconds limiting it's application solely for improving acoustic window2.We hypothesized that a Short End Expiratory Hold (SEEH) of eight seconds improves acoustic window leading to superior echocardiographic image quality without significant hemodynamic changes.

Objectives: Primary objective of the study was to determine effect of SEEH on image quality score. Secondary objectives of the study were to determine the change in IVC diameter and Carotid Doppler peak velocity $(\triangle C D P V)$ with SEEH. Tertiary objective was to look for presence of any Auto-PEEP revealed during SEEH.

Methods: Twenty patients on mechanical ventilation with poor acoustic window during point of care echocardiography examination were assessed for both apical four chamber(A4C) and parasternal short axis(PSAX) view, video clips of six second duration were recorded both with tidal ventilation and with eight second SEEH. Assessors blinded to the intervention scored the video clips based on the quality of the image using a previously validated scoring criteria3.Clips were scored from 0 (no image visualization), 1 (visualization of less than $70 \%$ of the left ventricle(LV) walls), 2 (Visualization of $70 \%$ to $95 \%$ of the LV walls) to 3 (visualization of $95 \%$ to $100 \%$ of the LV walls). Increase in one or more point was considered significant. To determine hemodynamic effects of SEEH on change in IVC diameter and Carotid Doppler peak velocity ( $\triangle$ CDPV) were noted at the baseline and just before end of SEEH. Any change $(\Delta)$ more than $14 \%$ was considered as significant4. Auto-PEEP displayed on the ventilator at the end of SEEH was noted.

Results: In A4C view baseline worst median image quality score during tidal ventilation was 1 (Interquartile range(IQR) 1-1) and in PSAX view was 1 (IQR 0.25-2) which with SEEH improved to 2 (IQR 2-3) for A4C view and 3 (IQR 1-3) for PSAX. 18 out of 20 (90\%) patients in A4C view and 11 out of 20(55\%) patients for PSA had at least one point increment in worst image quality score with SEEH. The median $\triangle$ CDPV was $1.95 \%$ (IQR 1-3.175) and median change in IVC diameter was $4.45 \%$ (IQR 0-7.925) indicating no significant hemodynamic change due to SEEH. Median auto-PEEP was found to be 0.55 (IQR 0-1.075) $\mathrm{cm} \mathrm{H} 2 \mathrm{O}$ indicating absence of auto-PEEP.

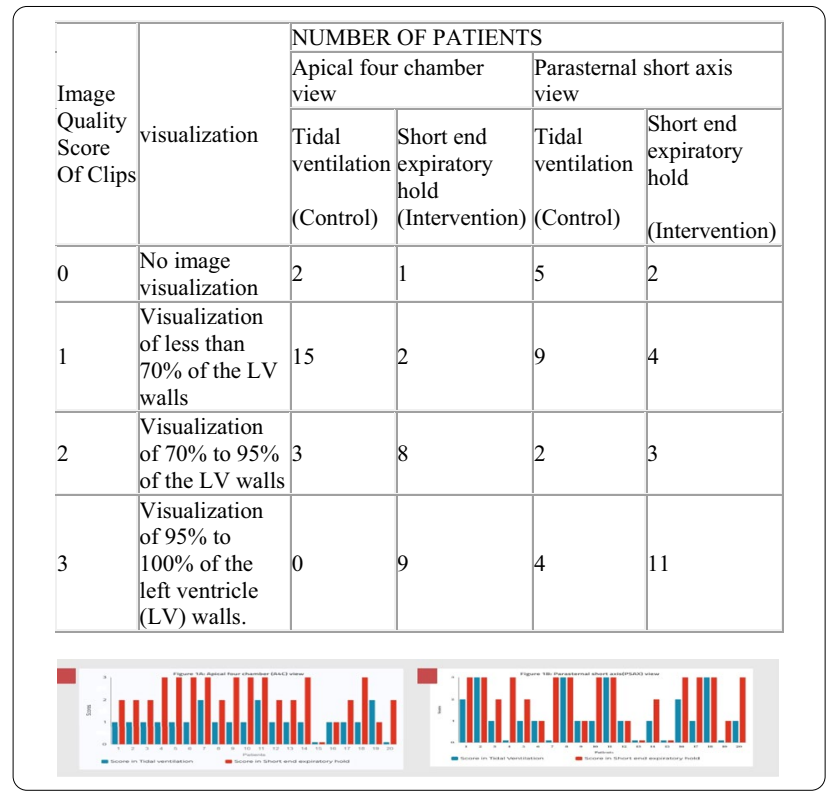

Conclusion: In mechanically ventilated critically ill patients with poor acoustic window, SEEH causes significant improvement in echocardiography image quality without significant change in hemodynamic parameters. This improvement occurs in the absence of auto-PEEP.

\section{Reference(s) and grant acknowledgment(s)}

1. Nil

2. 1. Lancellotti P Price S Edvardsen T Cosyns B Neskovic AN Dulgheru R Flachskampf FA Hassager C Pasquet A Gargani L Galderisi M Cardim N Haugaa KH Ancion A Zamorano JL Donal E Bueno H Habib G. The use of echocardiography in acute cardiovascular care: recommendations of the European Association of Cardiovascular Imaging and the Acute Cardiovascular Care Association. Eur Heart J Acute Cardiovasc Care2015;4:3-5

3. 2. Monnet $X$, Osman D, Ridel $C$, et al: Predicting volume responsiveness by using the end- expiratory occlusion in mechanically ventilated intensive care unit patients. Crit Care Med 2009; 37:951-956

4. 3. Ibarra-Estrada MÁ, López-Pulgarín JA, Mijangos-Méndez JC, Díaz-Gómez $J$, Aguirre- Avalos $G$. Respiratory variation in carotid peak systolic velocity predicts volume responsiveness in mechanically ventilated patients with septic shock: a prospective cohort study. Critical Ultrasound Journal. 2015;7(1).

5. 4. Johri AM, Chitty DW, Hua L, Marincheva G, Picard MH. Assessment of Image Quality in Real Time Three-Dimensional Dobutamine Stress Echocardiography: An Integrated 2D/3D Approach. Echocardiography. 2014;32(3):496-507 
000567

The impact of thoracic ultrasound on ICU management: a prospective observational study

M. Heldeweg ${ }^{1}$; P. R. Tuinman ${ }^{2}$

${ }^{1}$ Intensive Care Adults, Amsterdam UMC, locatie VUmc, Amsterdam, Netherlands; ${ }^{2}$ Intensive care, Vrije Universiteit Amsterdam, Amsterdam, Netherlands

Correspondence: $M$. Heldeweg

Intensive Care Medicine Experimental 2020, 8(2): 000567

Introduction: Point-of-care-ultrasound is a useful diagnostic instrument on critical care units. Its diagnostic accuracy supersedes chest $x$-ray and is similar to chest CT(1). However, the effect it has on management strategies is less clear.

Objectives: Our objective is to quantify the impact of thoracic ultrasound on ICU management.

Methods: This prospective observational study was conducted at a tertiary ICU. The study was approved by the local medical ethics committee and the need for consent was waived.A treating intensivist recorded a diagnosis and management plan before and after a thoracic ultrasound. Changes in management and diagnoses were recorded. Patient characteristics, fluid balance and whether the changes were carried out within 8 hours were extracted from the electronic health records.

Results: Thirty-seven thoracic ultrasound examinations were included. Management changes occurred in 22 (59.5\%). Seventeen (77.3\%) out of these 22 had their management changes carried out within 8 hours. The thoracic ultrasound yielded a new diagnosis in 13 (35.1\%) examinations. No significant effect was observed for management changes, patient characteristics, or fluid balance within 8 hours.

Conclusion: This single-arm observational study shows that a majority of thoracic ultrasounds alter patient management plans with feasible changes. More research is needed to give conclusive evidence regarding patient centric outcomes like mortality, duration of mechanical ventilation and time to discharge.

Reference(s) and grant acknowledgment(s)

1. Mayo PH, Copetti R, Feller-Kopman D, Mathis G, Maury E, Mongodi S, et al.

Thoracic ultrasonography: a narrative review. Vol. 45, Intensive Care Medi-

cine. Springer Verlag; 2019. p. 1200-11.

\section{8}

Serial lung ultrasounds for detection of pulmonary complications in critically ill obstetric patients: preliminary findings from a prospective observational study in a low-resource setting L. Pisani ${ }^{1}$; A. De Nicolo ${ }^{2}$; M. Schiavone ${ }^{3}$; AO. Adeniji ${ }^{4}$; EE. Emuveyan ${ }^{5}$.

S. Grasso ; ${ }^{6}$. Henciles ${ }^{7}$; MM. Koroma ${ }^{8}$; M. Schultz

'Department of intensive care, Amsterdam UMC, locatie AMC, Amsterdam, Netherlands; ${ }^{2}$ Intensive care unit, department of emergency and organ transplantation (deto), University of Bari, Bari, Italy; ${ }^{3}$ Thoracic surgery unit, department of emergency and organ transplantation (deto), University of Bari, Bari, Italy; ${ }^{4}$ Department of obstetrics and gynecology, Princess Christian Maternity Hospital, Freetown, Sierra Leone; ${ }^{5}$ Department of obstetrics and gynaecology, Princess Christian Maternity Hospital, Freetown, Sierra Leone; ${ }^{6}$ Department of emergency and organ transplants (deto), anesthesiology and intensive care, University of Bari Aldo Moro, Bari, Italy; ${ }^{7}$ Department of anesthesia and intensive care, Connaught Hospital, University of Sierra Leone, Freetown, Sierra Leone; ${ }^{8}$ Department of anesthesia, Princess Christian Maternity Hospital, Freetown, Sierra Leone; ${ }^{9}$ Intensive care, Amsterdam University Medical Centers, University of Amsterdam, Amsterdam, Netherlands

Correspondence: L. Pisani

Intensive Care Medicine Experimental 2020, 8(2): 000578

Introduction: Critically ill parturients have an increased risk of developing pulmonary complications. Lung ultrasound could be effective in addressing the cause of respiratory distress in limited-resource settings[1] with high maternal mortality.
Objectives: To determine the frequency, timing of appearance and type of pulmonary complications in critically ill parturients in an obstetric unit in Sierra Leone.

Methods: Prospective observational study conducted between July 2018 and February 2019 in critically ill parturients admitted to the high dependency unit (HDU) of Princess Christian Maternity Hospital in Freetown, Sierra Leone. Twelve-regions lung ultrasound examinations were performed on admission, after 24 and 48 hours, and in case of respiratory deterioration, using a low frequency $(2.5-5 \mathrm{MHz})$ convex probe.

Primary endpoint was the proportion of parturients with one or more pulmonary complications, stratified for presence of respiratory distress. Secondary endpoints included timing and types of complications, and their association with 'poor outcome', defined as a composite of transfer for escalation of care or death. The study was approved by the Sierra Leone Ethical Research Committee on June 5, 2018 and registered at clinicaltrials.gov (NCT 03828630).

Results:Measurements and main results. 166 patients were enrolled (median age 25 years [IQR 22-30]). A total of 383 LUS examinations, median 2 [2-3] LUS per patient, were performed. Of all patients, 34\% presented with or developed respiratory distress during HDU stay; 35 patients (21\% [95\%-confidence interval 15 to $28 \%$ ]) had one or more pulmonary complications, the majority diagnosed on admission. Acute respiratory distress syndrome (period prevalence $4 \%$ ) and hydrostatic pulmonary edema (4\%) were only observed in patients with respiratory distress. Pneumonia (2\%), atelectasis (10\%) and pleural effusion (7\%) were present irrespective of respiratory distress. In patients in whom ultrasound excluded pulmonary complications, respiratory distress was related to anemia or metabolic acidosis. In total, 18 out of 166 patients met the composite endpoint of a poor outcome (11\%). Pulmonary complications were associated with an increased risk of poor outcome (odds ratio, 5.0 [95\%-confidence interval 1.7 to 14.6]; $P=0.003$ ).

Conclusion: One out of five critically ill parturients admitted to the HDU of a resource-limited obstetric unit developed one or more pulmonary complications. Lung ultrasound contributed to address the cause of respiratory distress by identifying or excluding ARDS, pulmonary edema, pneumonia, atelectasis and pleural effusion. These complications were associated with a poor outcome.

\section{Reference(s) and grant acknowledgment(s)}

11 Leopold SJ, Ghose A, Plewes KA, Mazumder S, Pisani L, Kingston HWF, Paul S, Barua A, Sattar MA, Huson MAM, Walden AP, Henwood PC, Riviello ED, Schultz MJ, Day NPJ, Kumar Dutta A, White NJ, Dondorp AM. Point-of-care lung ultrasound for the detection of pulmonary manifestations of malaria and sepsis: An observational study. PLoS One 2018;13:e0204832

2. This study was funded by the Amsterdam University Medical Centers, location AMC and Doctors with Africa - CUAMM, Padova, Italy.

\section{9}

Diagnostic accuracy of early sonographic signs in COVID-19 pneumonia

Z. Bitar'; M. Shamsah²; H. Al-Foudari ${ }^{3}$

${ }^{1}$ Critical care unit, New Ahmadi hospital KOC مستشــــــ Ahmadi, Kuwait; ${ }^{2}$ Intensive care depärtmenẗ, Adan Hospital, Hadiya, Kuwait; ${ }^{3}$ Head of intensive care department and anesthesia, Adan Hospital, Hadiya, Kuwait

Correspondence: Z. Bitar

Intensive Care Medicine Experimental 2020, 8(2): 000619

Introduction: Chest radiography (CXR) and computerized tomography $(\mathrm{CT})$ scan are the standard methods for lung imaging in diagnosing COVID-19 pneumonia in the intensive care unit, despite their limitations. This study aimed to assess the performance of bedside lung ultrasound examination by a critical care physician, in the diagnosis of COVID-19 pneumonia in acute admission to the ICU.

Methods: The study is an observational, prospective, single-center study conducted in the intensive care unit of Adan General Hospital 
from Apr 10, 2020, till May 10, 2020. Adolescent or adult with suspicion of COVId19 infection transferred to ICU with fever or suspected respiratory infection, plus one of the following: respiratory rate $>30$ breaths/min; severe respiratory distress; or $\mathrm{SpO} 2<93 \%$ on room air (1). Patients admitted directly from ER to ICU after performing the reverse transcriptase-polymerase chain reaction (RT-PCR) and send to the central virology in Kuwait. A certified intensivist in critical care ultrasound performed the lung ultrasound within 12 hours of admission to ICU and blinded to the result of the RT-PCR, if available at the time of examination. The four signs at lung ultrasound COVID-19 pneumonia are (2):

1. B-lines as Bilateral patchy distribution of multiform clusters, both in separate and coalescent forms, sometimes giving the appearance of a shining white lung. They can arise from one point of the pleural line and small peripheral consolidations and spread down like rays maintaining their brightness until the edge of the screen without fading. 2. Bilateral diffuse irregularities of the pleural line.

3. Absence of significant pleural effusion.

4. Presence of multiple subpleural consolidations of various size. The treating physician confirmed the diagnosis of COVID-19 pneumonia by a set of clinical features, inflammatory markers, biochemical profile studies, RT-PCR test, and CXR.

Results: Out of 57 patients with suspected COVID-19 pneumonia, 46 $(80.7 \%)$ were confirmed. Mean age 51 with a range of 39 to 69 years and $32(76 \%)$ men.

In the group of patients with confirmed COVID-19 pneumonia (table 1), 44 (98.6\%) LUSs revealed the four signs suggestive of COVID19 pneumonia (sensitivity $95.6 \%, \mathrm{Cl} 85 \%-99.5 \%$ ).Two patients presented with unilateral lobar pneumonia and without other ultrasonic signs of COVID-19 pneumonia but positive RT-PCR. The group without COVID-19 pneumonia and negative RT-PCR were 10 (90\%) had LUS negative for COVID-19 pneumonia (specificity $90.91 \%, 95 \% \mathrm{Cl}$ $58.72 \%-99.77 \%)$. One case declared positive by LUS, and with negative RT-PCR, it was a case of non-Hodgkin lymphoma post-radiation pulmonary fibrosis and presented with hypertensive pulmonary edema. The other cases of negative LUS and negative RT-PCR where three cases on chronic dialysis and pulmonary edema, a 25-year-old woman with miliary tuberculosis and severe myocarditis, three cases of NSTEMI and pulmonary edema, a 26-year-old man with uncontrolled hypertension, renal failure and possible vasculitis, one case of diabetic ketoacidosis and right basal pneumonia and another case of hypertensive pulmonary edema with UTI.

Two cases where considered COVID-19 pneumonia by the treating team despite negative RT-PCR because the clinical picture, lab findings, and CT chest finding keeping with COVID-19 pneumonia in concur with the ultrasound chest. Pleural effusion was not detected in any patient with confirmed COVID-19 pneumonia.

Table 1. Lung ultrasound and RT-PCR profile in patients with respiratory distress and suspicionof COVID-19 in ICU $(N=57)$

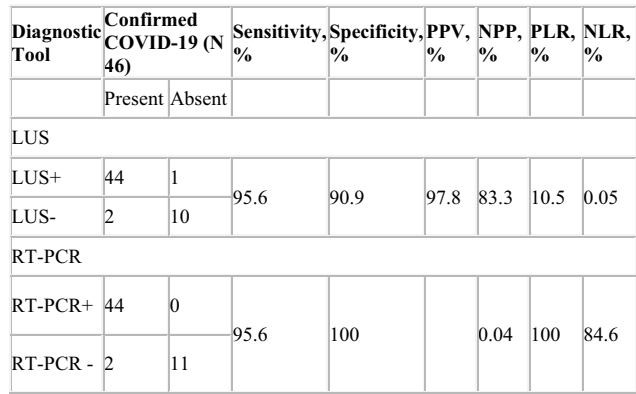

LUs, lung ultrasound; positive (+) or negative (-) for the abnormality; COVID-19 pneumonia present or absent based on final diagnosis;

Conclusion: LUS pattern is sensitive and specific for diagnosing COVID-19 pneumonia in acutely admitted patients to ICU with respiratory involvement.

\section{Reference(s) and grant acknowledgment(s)}

1. 2. Peng QY, Wang XT, Zhang LN; Chinese Critical Care Ultrasound Study Group (CCUSG). Findings of lung ultrasonography of novel coronavirus pneumonia during the 2019-2020 epidemic. Intensive Care Med. 2020 May;46(5):849-850. https://doi.org/10.1007/s00134-020-05996-6. Epub 2020 Mar 12.

2. 1. WHO. Volume 2 IMAI District Clinician Manual. Hospital care for adolescents and adults. Geneva: World Health Organization; 2011 (https://apps. who.int/iris/bitstream/handle/10665/77751/9789241548290_Vol2_eng. pdf? sequence $=3$, accessed Mar 4, 2020)

3. The research was performed as part of the employment of the authors in Ministry of health and Kuwait oil Company

\section{7}

Deep vein thrombosis screening of COVID-19 patients in intensive care

C. McGovern ${ }^{1}$; C. Mccue ${ }^{1}$; G. Bainbridge ${ }^{1}$; J. Selfridge ${ }^{1}$

${ }^{1}$ Intensive care unit, glasgow royal infirmary, NHS Greater Glasgow and Clyde, Glasgow, United Kingdom

Correspondence: C. McGovern

Intensive Care Medicine Experimental 2020, 8(2): 000717

Introduction: Patients infected with COVID-19 are at increased risk of thromboembolic complications as demonstrated in autopsy findings(Wichmann et al) and observational clinical studies(Helms et al).

Recognising this increased risk, our intensive care unit started a screening programme using compression ultrasound to detect venous thrombosis.

Methods: From 1st April 2020, any patient admitted to our adult intensive care unit with proven or suspected COVID-19 underwent compression ultrasound of the external iliac veins to popliteal trifurcation and the internal jugular veins. This scan was carried out shortly after admission to ICU and repeated weekly until ICU discharge.

Scans were performed by medical staff trained in compression ultrasound. Any scans felt to demonstrate thrombus meriting anticoagulation were reviewed by a senior clinician with experience in compression ultrasound. Any doubt or disagreement prompted further assessment by a radiologist or sonographer.

Results: Twenty-two consecutive patients with a diagnosis of COVID19 pneumonia were cared for in ICU during this project. The demographics are summarised below.

\begin{tabular}{|l|l|}
\hline Age - median (IQR) & $\mathbf{n}=\mathbf{2 2}$ \\
& $60.5(55-66)$ \\
\hline Male - n. (\%) & $17(77.3)$ \\
\hline BMI - median (IQR) & $31.5(25.5-36.7)$ \\
\hline Co-morbidities - n. (\%) & \\
\hline Cardiovascular disease & $9(40.9)$ \\
\hline Previous cancer & $2(9.1)$ \\
\hline Previous VTE & $1(4.5)$ \\
\hline Diabetes & $3(13.6)$ \\
\hline Hypothyroid & $3(13.6)$ \\
\hline Asthma/COPD & $5(22.7)$ \\
\hline CKD & $2(9.1)$ \\
\hline Disease Severity & \\
\hline APACHE Score - median (IQR) & $16(14-19)$ \\
\hline APACHE Mortality - median (IQR) & $23.4(19.2-28.6)$ \\
\hline Interventions $-\mathbf{n}$. (\%) & \\
\hline Invasive mechanical ventilation & $22(100)$ \\
\hline Muscle relaxant infusion & $21(95.5)$ \\
\hline Prone position & $19(86.4)$ \\
\hline Renal replacement therapy & $8(36.4)$ \\
\hline
\end{tabular}

Fifteen patients $(68.2 \%)$ were found to have thrombus of varying severity on compression ultrasound. Eight were judged to merit treatment with anticoagulation.

Of the eight patients that were anticoagulated, 7 were diagnosed on compression ultrasound screening. Five were found to have lower 
limb DVTs, 2 of which were bilateral. Two patients had occlusive thrombus in an internal jugular vein. One patient was anticoagulated following thrombolysis for a clinical diagnosis of massive pulmonary embolus.

Patients with proximal femoral vein DVTs were treated in the prone position more times (median 5 [4-6]) than those without (median 2 [1-5]). They were also more likely to have a higher Body Mass Index (median 32 [31-36] vs 27.5 [25-36]).

Eight patients had non-occlusive thrombus in an internal jugular vein, not considered large enough to merit systemic anticoagulation and all were associated with central venous catheters sites.

Five patients underwent computed tomography pulmonary angiograms (CTPA), all of which were negative.

Two patients suffered bleeding complications during anticoagulation therapy, one developed minor haematuria and one life threatening spontaneous retroperitoneal haemorrhage requiring embolisation. Conclusion: Given the recognised risk of thromboembolic complications in critically unwell COVID-19 patients, the higher prevalence of severe disease in obese patients(Simonnet et al, Caussy et al) and the frequent use of prone position ventilation, we feel that DVT screening merits consideration as a routine part of the care of these patients. Compression ultrasonography is a simple, non-invasive bedside investigation that uses existing technology that is familiar to critical care staff.

\section{Reference(s) and grant acknowledgment(s)}

1. Caussy C, Pattou F, Wallet F, et al. Prevalence of obesity among adult inpatients with COVID-19 in France. Lancet Diabetes Endocrinol. 2020. https:// doi.org/10.1016/s2213-8587(20)30160-1

2. Simonnet A, Chetboun M, Poissy J, et al. High prevalence of obesity in severe acute respiratory syndrome coronavirus-2 (SARS-CoV-2) requiring invasive mechanical ventilation. Obesity. 2020. https://doi.org/10.1002/ oby.22831

3. Helms J, Tacquard C, et al. High risk of thrombosis in patients with severe SARS-CoV-2 infection: a multicenter prospective cohort study. Intensive Care Med. 2020. https://doi.org/10.1007/s00134-020-06062-x

4. Wichmann D, Sperhake J-P, Lütgehetmann M, et al. Autopsy Findings and Venous Thromboembolism in Patients With COVID-19. Ann Intern Med. 2020. https://doi.org/10.7326/m20-2003

\section{7}

\section{Critically ill COVID-19 patients with acute kidney injury have} reduced renal blood flow and perfusion despite preserved cardiac function; a case-control study using contrast enhanced ultrasound

J. Watchorn'; D. Huang ${ }^{2}$; J. Joslin ' ; K. Bramham³ ; SD. Hutchings

${ }^{1}$ Critical care, King's College Hospital, Denmark Hill, London, UK, London, UK, United Kingdom; ${ }^{2}$ Radiology, King's College Hospital, London, United Kingdom; ${ }^{3}$ Nephrology, King's College Hospital, London, United Kingdom Correspondence: $J$. Watchorn

Intensive Care Medicine Experimental 2020, 8(2): 000787

Introduction: Acute Kidney Injury (AKI) is a common complication of COVID-19 critical illness but the pathophysiology is uncertain. Much of the limited current evidence comes from autopsies demonstrating the presence of viral inclusion bodies within tubular epithelial cells alongside endothelial injury and erythrocyte aggregation within peritubular capillaries. These findings contribute to the hypothesis that COVID-19 disease induces widespread changes in vascular endothelial cells and that resultant changes in regional blood flow may be important in the pathogenesis of COVID-19 related critical illness. We used contrast enhanced ultrasound (CEUS) and echocardiography to study renal perfusion and global blood flow and compared our findings with measurements taken in a group of septic shock patients and healthy volunteers.

Methods: Prospective case-control study. Renal perfusion variables were assessed with contrast enhanced ultrasound (CEUS); macrovascular blood flow was assessed using Doppler analysis of large renal vessels; echocardiography was used to assess right and left heart function and cardiac output.
Results: CEUS derived parameters were reduced in in COVID-19 associated AKI compared to healthy controls (perfusion index 3415 v 548 a.u., $p=0.001$; renal blood volume 7794 v 3338 a.u., $p=0.04$ ). Renal arterial flow quantified using time averaged peak velocity (TAPV) was also reduced compared to healthy controls $(36.6 \mathrm{v} 20.9 \mathrm{~cm} / \mathrm{s}$, $\mathrm{p}=0.004)$ despite cardiac index being similar between groups $(2.8 \mathrm{v}$ 3.7 L/min $/ \mathrm{m} 2, p=0.07$ ) and right heart function being normal. There were no differences in CEUS derived or cardiac parameters between COVID-19 and septic shock patients but patients with septic shock had more heterogeneous perfusion variables.

Conclusion: Both large and small vessel blood flow is reduced in patients with COVID-19 associated AKI compared to healthy controls, which does not appear to be a consequence of right or left heart dysfunction. A reno-vascular pathogenesis of COVID-19 AKI seems likely

\section{Reference(s) and grant acknowledgment(s)}

1. Ronco C, Reis T, Husain-Syed F. Management of acute kidney injury in patients with COVID-19. Lancet Respir Med 2020; published online May 14. https://doi.org/10.1016/s2213-2600(20)30229-0

2. Su H, Yang M, Wan C, et al. Renal histopathological analysis of 26 postmortem findings of patients with COVID-19 in China. Kidney International 2020; published online April 9. https://doi.org/10.1016/j.kint.2020.04.003.

3. Zhou F, Yu T, Du R, et al. Clinical course and risk factors for mortality of adult inpatients with COVID-19 in Wuhan, China: a retrospective cohort study. Lancet 2020; 395: 1054-62.

4. Richardson S, Hirsch JS, Narasimhan M, et al. Presenting Characteristics, Comorbidities, and Outcomes Among 5700 Patients Hospitalized With COVID-19 in the New York City Area. JAMA 2020; 323: 2052

5. Royal Centre for Defence Medicine (UK Ministry of Defence), 2019; European Society of Intensive Care Medicine Point of Care Ultrasound award, 2019

\section{9}

Association of baseline diaphragm, rectus femoris and vastus intermedius muscle thickness with weaning in critically-ill patients

B. Er ${ }^{1}$; M. Simsek'; M. Yıldırım ; B. Halacli ; S. ÖCal ; E. Ortac Ersoy';

AU. Demir'; A. Topeli

${ }^{1}$ Department of internal medicine, division of intensive care medicine, Hacettepe University Faculty of Medicine, Ankara, Turkey; ${ }^{2}$ Department of chest diseases, Hacettepe University Faculty of Medicine, Ankara, Turkey

Correspondence: $\mathrm{B}$. $\mathrm{Er}$

Intensive Care Medicine Experimental 2020, 8(2): 000929

Introduction: Weaning from mechanical ventilation in critically-ill patients is associated with many factors, baseline muscle thickness is one of them $[1,2]$.

Objectives: To determine whether baseline diaphragm (Tdi), rectus femoris (RF) and vastus intermedius (VI) muscle thickness (TRF and $\mathrm{TRF}+\mathrm{VI}$ ) are associated with weaning.

Methods: The study was performed between 1st April-30th June 2019 and 1st October 2019-31st March 2020. Right Tdi, TRF and TRF+VI were measured by ultrasonography within 36 hours of intubation and diaphragmatic excursion (DE) was evaluated at the first spontaneous breathing trial in adult critically-ill patients. Successful weaning was defined as extubation without reintubation within 7 days after extubation. Reintubation or death within 7 days after extubation were grouped as weaning failure. Two groups were compared in terms of ultrasonographic measurements and clinical features.

Results: A total of 62 patients were enrolled in the study. The patients $(n=24)$ who had no attempt for weaning were excluded from the analysis. Clinical features and ultrasonographic measurement results of weaning groups are shown in the Table. In the weaning failure group $(n=15)$, the median body mass index (BMI), DE, TRF+VI were lower while the median clinical frailty scale (CFS), duration of mechanical ventilation, ICU and hospital mortality rate were higher. The median baseline diaphragm thickness was similar in two groups. In ROC analysis, AUC for TRF+VI was $0.71(95 \% \mathrm{Cl}: 0.51-0.90 ; \mathrm{p}=0.035)$, with $21 \mathrm{~mm}$ cut-off having sensitivity of $82 \%$ and specificity of $57 \%$. Logistic regression analysis revealed $\mathrm{TRF}+\mathrm{Vl}<21 \mathrm{~mm}$ as the only predictor 
of weaning failure with an odds ratio of $7.6(95 \% \mathrm{Cl}: 1.1-52.8, \mathrm{p}=0.04)$ after adjusting for BMI and CFS.

Table: Clinical features and ultrasonographic measurements of patient groups

\begin{tabular}{|c|c|c|c|}
\hline & $\begin{array}{c}\begin{array}{c}\text { Weaning } \\
\text { success }\end{array} \\
\mathbf{n}=\mathbf{2 3}\end{array}$ & $\begin{array}{c}\text { Weaning failure } \\
\mathrm{n}=15\end{array}$ & p value \\
\hline Age, years & $61[50-70]$ & $71[55-80]$ & 0.15 \\
\hline Female sex* & $8(34.7)$ & $4(26.6)$ & 0.72 \\
\hline Body mass index, $\mathrm{kg} / \mathrm{m} 2$ & $\begin{array}{l}26.1[24.1- \\
31.3]\end{array}$ & $23.5[21.1-25.6]$ & 0.04 \\
\hline Charlson co-morbidity score & $3[1-5]$ & $4[3-6]$ & 0.22 \\
\hline $\begin{array}{l}\text { Nutrition risk in the critically- } \\
\text { ill (NUTRIC) score }\end{array}$ & $5[4-6]$ & $5[4-6]$ & 0.26 \\
\hline Clinical fraility scale & $4[3-6]$ & $6[5-7]$ & 0.02 \\
\hline APACHE II & $24[13-26]$ & $16[14-21]$ & 0.06 \\
\hline SOFA & $7[5-9]$ & $8[6-10]$ & 0.40 \\
\hline Prealbumin, mg/dl & $\begin{array}{l}12.2[8.8-20.0] \\
(n=14)\end{array}$ & $\begin{array}{l}11.8[6.5-13.9] \\
(\mathrm{n}=10)\end{array}$ & 0.34 \\
\hline $\mathrm{PaO2} / \mathrm{FiO} 2$ & $171[90-208]$ & $106[88-179]$ & 0.15 \\
\hline
\end{tabular}

Table: Clinical features and ultrasonographic measurements of patient groups

\begin{tabular}{|c|c|c|c|}
\hline & $\begin{array}{c}\text { Weaning } \\
\text { success } \\
\mathbf{n}=\mathbf{2 3}\end{array}$ & $\begin{array}{c}\text { Weaning failure } \\
n=15\end{array}$ & p value \\
\hline pH & $\begin{array}{l}7.37[7.20- \\
7.45]\end{array}$ & $7.37[7.31-7.44]$ & 0.34 \\
\hline $\mathrm{PaO2}, \mathrm{mmHg}$ & $\begin{array}{l}69.0[60.0- \\
89.0]\end{array}$ & $62.2[55.4-83.1]$ & 0.15 \\
\hline $\mathrm{PaCO} 2, \mathrm{mmHg}$ & $\begin{array}{l}37.0[28.5- \\
52.0]\end{array}$ & $37.8[32.1-55.4]$ & 0.28 \\
\hline ICU length of stay, days & $18[11-34]$ & $26[21-52]$ & 0.12 \\
\hline Hospital length of stay, days & $\begin{array}{l}31[17-54] \\
(n=21)\end{array}$ & 37 [26-64] & 0.42 \\
\hline $\begin{array}{l}\text { Mechanical ventilation } \\
\text { duration, days }\end{array}$ & $8[4-14]$ & $18[8-23]$ & 0.004 \\
\hline ICU mortality* & $2(8.6)$ & $14(93.3)$ & $<0.001$ \\
\hline Hospital mortality* & $\begin{array}{l}2(9.5) \\
(n=21)\end{array}$ & $14(93.3)$ & $<0.001$ \\
\hline $\begin{array}{l}\text { Time to first spontaneous } \\
\text { breathing trial, days }\end{array}$ & $7[4-11]$ & $8[4-15]$ & 0.47 \\
\hline Tdi, $\mathbf{m m}$ & $2.0[1.7-2.4]$ & $1.9[1.5-2.3]$ & 0.26 \\
\hline DE, mm & $\begin{array}{l}25.9[19.3- \\
38.5] \\
(n=19)\end{array}$ & $\begin{array}{l}19.4[14.6-24.0] \\
(\mathrm{n}=8)\end{array}$ & 0.045 \\
\hline TRF, mm & $\begin{array}{l}12.7[11.7- \\
16.4]\end{array}$ & $\begin{array}{l}7.2[5.9-15.3] \\
(n=14)\end{array}$ & 0.06 \\
\hline TRF+VI, mm & $\begin{array}{l}23.6[21.3- \\
27.1]\end{array}$ & $\begin{array}{l}14.0[12.3-26.2] \\
(\mathrm{n}=14)\end{array}$ & 0.03 \\
\hline \multicolumn{4}{|c|}{$\begin{array}{l}\text { *number (\%), others median [IQR]; Tdi: diaphragm thickness, DE: } \\
\text { diaphragmatic excursion, TRF: rectus femoris thickness, VI: vastus } \\
\text { intermedius }\end{array}$} \\
\hline
\end{tabular}

Conclusion: TRF+VI lower than $21 \mathrm{~mm}$, measured by ultrasonography within 36 hours of intubation, was associated with weaning failure among critically-ill patients.

\section{Reference(s) and grant acknowledgment(s)}

1. Formenti P, UMbrello M, Coppola S, Froio S, Chiumello D. Clinical review: peripheral muscular ultrasound in the ICU. Annals of Intensive Care 2019; 9:57.
2. Boles JM, Bion J, Connors A, Herridge M, Marsh B, et al. Weaning from mechanical ventilation. European Respiratory Journal 2007; 29(5): p. 1033-56.

000974

Point of care lung ultrasound and focused echocardiography findings in a cohort of patients with severe COVID-19

B. Shuker ${ }^{1}$

${ }^{1}$ Department of Anaesthesia, Warwick Hospital, Warwick, United Kingdom Correspondence: $\mathrm{B}$. Shuker

Intensive Care Medicine Experimental 2020, 8(2): 000974

Introduction: Point of care ultrasound (POCUS) is a valuable tool in intensive care medicine. During the COVID-19 pandemic there has been significant interest in the utility of POCUS as an aid to management of critical illness.

Preliminary work indicated characteristic lung ultrasound findings in COVID-19: pleural thickening, pleural irregularity, presence of $B$ lines, consolidations, absence of pleural effusions, and resolution to $A$ lines during recovery (1). Echocardiographic features associated with COVID-19 include: hyperdynamic cardiac function, acute cardiomyopathy, right ventricular dysfunction, and diffuse myocardial inhibition (2).

Objectives: We aimed to validate these preliminary findings in a UK cohort of ICU patients with severe COVID-19.

Methods: All invasively ventilated patients admitted to a single ICU in a single centre in the UK with severe COVID-19 illness confirmed by RTPCR who underwent routine POCUS examination during their admission were included. Lung ultrasound and focused echocardiography were performed using standardised protocols (3).

Results: Five patients with a mean age of 58.4 years were included. Except for a previously well slim female, all were overweight or obese male patients with pre-existing hypertensive disease. The patients were severely ill with a mean APACHE II score of 27 (range 17-34) and mean P:F of $14.2 \mathrm{kPa}(7-27)$.

Patients were scanned between day 5 and 10 of their ICU admission (mean day 7.8). None had been treated prone. All patients had evidence of pleural thickening, irregularity of the pleural lines, and presence of B lines. Only 2 patients had evidence of subpleural or overt consolidations. One patient had a small pleural effusion.

Three patients were receiving vasopressor therapy. One demonstrated hyperdynamic heart function associated with left ventricular systolic impairment. Three patients exhibited signs of right ventricular dysfunction.

Conclusion: In a cohort of severely ill mechanically ventilated patients with COVID-19 in the UK, we identified similar POCUS features to the preliminary findings reported by clinicians working in China notably a universal presence of pleural irregularity and a high burden of right ventricular dysfunction. An important finding in our cohort was the infrequency of lung consolidations, a finding thought to be present in the most severe loss of lung aeration (4).

\section{Reference(s) and grant acknowledgment(s)}

1. Nil.

2. 1. Peng QY, Wang XT, Zhang LN; Chinese Critical Care Ultrasound Study Group (CCUSG). Findings of lung ultrasonography of novel corona virus pneumonia during the 2019-2020 epidemic. Intensive Care Med. 2020;46(5):849-850

3. 2. Peng QY, Wang XT, Zhang LN; Chinese Critical Care Ultrasound Study Group (CCUSG). Using echocardiography to guide the treatment of novel coronavirus pneumonia. Crit Care. 2020;24(1):143.

4. 3. Intensive Care Society. Focused Ultrasound for Intensive Care. https:// www.ics.ac.uk/ICS/FUSIC/ICS/FUSIC/FUSIC_Accreditation.aspx. Published 2020

5. 4. Smith MJ, Hayward SA, Innes SM, Miller ASC. Point-of-care lung ultrasound in patients with COVID-19 - a narrative review [published online ahead of print, 2020 Apr 10]. Anaesthesia. 2020;https://doi.org/10.1111/anae.15082. https://doi.org/10.1111/anae.15082 


\section{3}

Introduction:of lung ultrasonography (LUS) in ICU: a single center experience

X. Vanderspikken ${ }^{1}$; T. Ecker ${ }^{1}$;T. Fivez ${ }^{1}$; M. Vander Laenen ${ }^{1}$; W. Boer

${ }^{1}$ Department of Anesthesiology, Intensive Care Medicine, Emergency

Medicine and Pain Medicine, Ziekenhuis Oost-Limburg, Genk, Belgium

Correspondence: X. Vanderspikken

Intensive Care Medicine Experimental 2020, 8(2): 000993

Introduction: Since 1989 when Jardin (1) introduced bedside ultrasonography in his ICU, lung US has become increasingly popular in the ICU community. In our center, the value of LUS is becoming apparent and fellows in training are interested in learning this important technique. We initiated a simple learning program and followed 2 fellows in their learning trajectory.

Objectives: To determine the time needed to develop basic LUS skills in the ICU.

Methods: After a literature review the modified Bedside Lung Ultrasound in Emergency (BLUE)- protocol (2) was selected for implementation, (excluding the venous vascular part of the protocol for deep vein thrombosis). An introductory session was organized, lead by an experienced lung US specialist, entailing a 30-minute instruction for knobology and a protocol trial run in 2 patients. Patients selected were admitted to ICU and in need of O2-therapy. Patients below 18 year of age, patients after thoracic and cardiac surgery and morbidly obese in whom limited LUS resolution was expected, were excluded. Patients were selected, in whom objectifiable lung lesions were most expected. Patients could only be included once. Length of time for the examination was noted and findings describing the different LUS study points were noted and retrospectively compared to classic radiological findings (chest X-Ray (CXR) and/or CT) and medical history. These data were subsequently analyzed.

Results: A total of 63 patients were included in the study over a period of 1 month divided over 2 ICU fellows. One fellow performed 45 examinations (71\%), the other 18 (29\%).Time of study. Mean study time of all examinations $(n=63)$ was 254 seconds $(s d=104)$. In the first 15 examinations mean time was $380 \mathrm{~s}(\mathrm{sd}=106)$, the second was $261 \mathrm{~s}(\mathrm{sd}=33)$, the third $246 \mathrm{~s}(\mathrm{sd}=52)$, dropping to a mean of $148 \mathrm{~s}(\mathrm{sd}=31)$, in the last 18 examinations ( $p<0.001$ compared to all previous periods). The maximum time of examination in the last period was $195 \mathrm{~s}$. Findings according to BLUE protocol. In a total of 63 patients: PLAPS profile 15(23.8\%); A profile: 29 (46\%); A' profile 2 (3.2\%); B profile: $8(12.7 \%)$; B' profile $2(3.2 \%)$; $A B$ profile 2 (3.2\%); undetermined 5 (7.9\%). Comparison of LUS to CXR and medical history. In 50 (79.4\%) patients findings on LUS confirmed CXR and medical history. In 7 (11.1\%) patients LUS likely fell short. In 6 (9.5\%) patients LUS had added value to previous findings on CXR and from medical history.

Conclusion: The learning curve for LUS in the ICU is steep. After a short introduction, within about 50 examinations fellows could perform lung US with confidence within just over 2 minutes. LUS findings proved robust or had added value compared to CXR and medical history in $89 \%$ of examinations, comparable to results in the literature.

\section{Reference(s) and grant acknowledgment(s)}

1. 2. BLUE protocol and FALLS protocol. Two applications of lung ultrasound in the critically ill. DA Lichtenstein. Chest 2015: 147(6):1659-1670

2. Influence of positive end-expiratory pressure on left ventricular performance. Jardin, F, Farcot, JC, Boisante, L, Curien, N, Margairaz, A, and Bourdarias, JP. N Engl J Med. 1981; 304: 387-392
001133

Distribution and main characteristics of patients admitted for pneumonia by COVID-19 in ICU during the pandemic period A. Falcon Marchena'; M. De La Maza Segovia²; E. Lucas Romero ${ }^{3}$;

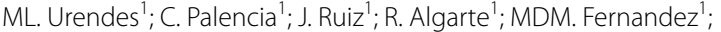
B. Sánchez González'; J. Trenado ${ }^{1}$

${ }^{1}$ Intensive care department, Mútua Terrassa University Hospital, Terrassa, Spain; ${ }^{2}$ Anesthesiology and resuscitation department., Mútua Terrassa University Hospital, Terrassa, Spain; ${ }^{3}$ Internal medicine department, Mútua Terrassa University Hospital, Terrassa, Spain

Correspondence: $\mathrm{B}$. Sánchez González

Intensive Care Medicine Experimental 2020, 8(2): 001133

Introduction: During the months of March to May we have experienced in Spain the consequences of the COVID 19 pandemic. Different factors can be related to the evolution of a pathology, both those of the pathology and the patient, as well as those derived from the high number of cases in a short period of time. Below we intend to analyze the different factors that are related to the evolution of COVID-19 pneumonia in our area.

Objectives: Describe the distribution and main characteristics of the patients. admitted to the ICU for COVID-19 pneumonia during the pandemic period.

Methods: Descriptive study carried out in a multipurpose ICU with 12 beds of critical care and 21 semi-critical care. All the patients admitted for COVID-19 related pneumonia admitted during the pandemic period were included.

Statistics: Qualitative variables are expressed as percentages and quantitative variables they are expressed as means and standard deviations (SD).

Results: 95 patients were admitted from March 14 to April 24. Were enabled 50 ventilators in 5 critical areas, reaching 40 intubated patients simultaneously. Figure 1: shows the distribution of income by week. $60 \%$ of admissions occurred in the first 2 weeks. Table1: describes the maincharacteristics of the patients.

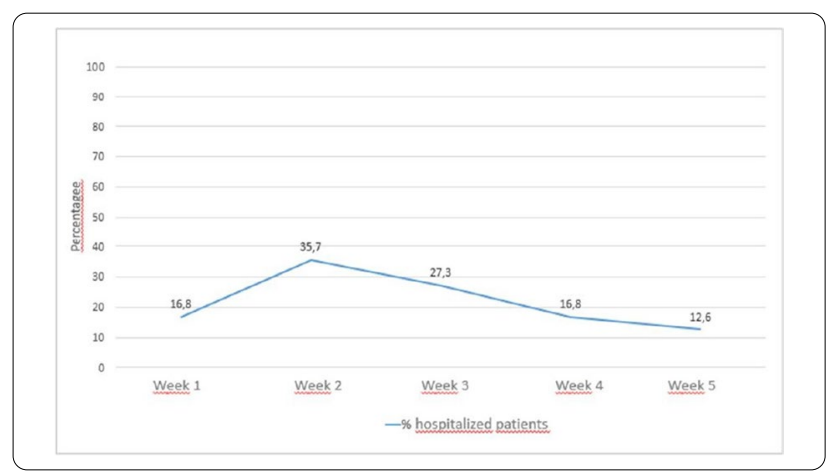

$100 \%$ of patients received Kaletra, Hydroxychloroquine and Azithromycin, $40 \%$ also received Tozilucimab, 34\% Interferon and 58\% corticosteroids. Table 2: describes other clinical characteristics and aspects related to mechanical ventilation. 


\begin{tabular}{|l|c|}
\multicolumn{1}{|c|}{ Variables } & 84,2 \\
\hline Lymphopenia on admission (\%) & 73,7 \\
\hline Lymphopenia recovered at discharge ICU (\%) & 24,1 \\
\hline Nosocomial pneumonia (\%) & 9,2 \\
\hline Non-catheter-related bacteremia(\%) & 9,2 \\
\hline Catheter-related bacteremia(\%) & 20,7 \\
\hline Urinary tract infection(\%) & 2,3 \\
\hline Endocarditis (\%) & 3,3 \\
\hline Clostridium infection(\%) & 4,6 \\
\hline Fungal infection(\%) & 22,3 \\
\hline CMV (\%) & 56,6 \\
\hline Antibiotic treatment for Nosocomial infection(\%) & 42,5 \\
\hline norepinephrine (\%) & $3,9(5,3)$ \\
\hline Midazolam infusion days (days, mean (SD)) & $7,8(8,9)$ \\
\hline Propofol infusion days(days, mean(SD)) & $5,2(6,5)$ \\
\hline Remifentanil infusion days(days, mean (SD)) & $3,9(5,6)$ \\
\hline Perfusion days Morphic Chloride(days, mean(SD)) & 56 \\
\hline Patients with muscle relaxation in continuous infusion(\%) & $3,9(5,8)$ \\
\hline Dexmedetomidine infusion days(days, mean(SD)) & 47 \\
\hline Delirim (\%) & 14 \\
\hline Refeeding (\%) & 23 \\
\hline Discharge to plant with antipsychotics(\%) & 1,1 \\
\hline Adverse events(\%) & \\
\hline
\end{tabular}

ICU mortality was $19 \%$ and hospital mortality $20 \%$.

\begin{tabular}{|c|c|}
\hline Variables & \\
\hline Age (years, mean (SD) & $58,6(10)$ \\
\hline Sex (male\%) & 71 \\
\hline SOFA entry $\leq 5(\%)$ & 76 \\
\hline Rockwood frailty scale upon admission $\leq 3(\%)$ & 100 \\
\hline ICU stay (days, mean (SD)) & $13.1(12.9)$ \\
\hline Hospital stay (days, mean (SD)) & $25.4(16.7)$ \\
\hline Cardiovascular history (\%) & 52.6 \\
\hline Immunosuppression history (\%) & 4.2 \\
\hline Background taking corticosteroids (\%) & 0 \\
\hline FA Background (\%) & 3.4 \\
\hline Thrombosis history (\%) & 2.1 \\
\hline Background taking blood thinners (\%) & 1.1 \\
\hline Psychiatric history (\%) & 11.4 \\
\hline Antecedent taking antipsychotics (\%) & 1.1 \\
\hline History take drugs or alcohol (\%) & 0 \\
\hline Smoker Background (\%) & 8.4 \\
\hline ICU ventilator only (\%) & 57.7 \\
\hline Multi-organic failure (\%) & 16 \\
\hline Shock (\%) & 6 \\
\hline Renal insufficiency (\%) & 11.6 \\
\hline Adverse events (\%) & \\
\hline
\end{tabular}

Conclusion: The distribution of ICU admission for COVID-19 related pneumonia required quadrupling the capacity of ventilated patients in the first 2 weeks of the pandemic. The characteristics of the admitted patients, their location and resources used for treatment were conditioned by this aspect.

Reference(s) and grant acknowledgment(s)

1. Acknowledgments to all the nursing and auxiliary staff of the ICU of HUMT. 2. Not funded
000013

Community acquired urosepsis: a surgical intensive care experience

N. Shaikh'; S. Ahmed ${ }^{2}$;. Kazi ${ }^{3}$; M. Muna ${ }^{4}$

${ }^{1}$ Hamad Medical Corporation, Doha, Qatar; ${ }^{2}$ Clinical pharmacy, HMC, doha, Qatar; ${ }^{3}$ Micu, HMC, Doha, Qatar; ${ }^{4}$ Cdc, CDC, Doha, Qatar

Correspondence: $\mathrm{N}$. Shaikh

Intensive Care Medicine Experimental 2020, 8(2): 000013

Introduction: Urosepsis contributes significantly to the epidemiology of sepsis. Urosepsis is divided into community-acquired or hospitalacquired, depending upon the origin of infection acquired either from the community or from the health care setup. The literature about community-acquired urosepsis (CAUs) is limited and studies are small powered.

Objectives: Aim of our study was to know the epidemiology, bacteriology, severity and outcome of CAUs patients.

Methods: All patients admitted from the emergency department to the surgical intensive care unit (SICU) with urosepsis over a period of 10 years were identified and included retrospectively from the SICU register.

Results: During the study period 302 patients admitted with CAUs. The common etiology was obstructive uropathy (60\%). Local Arabic population outnumbered the non-Arabic population (164/54.3\%) and there were equal number of patients from both genders, $75 \%$ had acute kidney injury (AKI). Thirty eight percent patients had percutaneous nephrostomy and $24.8 \%$ patients underwent endoscopic stent insertion to relieve the obstruction. Ninety three percent patients were admitted with septic shock and $71.5 \%$ had bacteraemia.

The common bacteria (36.1\%) was extended-spectrum beta-lactamase $(\mathrm{ESBL})$ producing bacteria, with a predominance of $\mathrm{E}$. coli (ESBL) (31.5\%) and 54\% required change of antibiotics to carbapenem. Eighty two percent patients had ARDS (acute respiratory distress syndrome). Patients with bacteraemia had a statistically significant AKI, ARDS and septic shock $(P<0.001)$. Male patients had significantly higher incidence of oliguria, intubation and ARDS $(P<0.05)$. Eight patients died of urosepsis giving mortality of $2.6 \%$.

Conclusion: Patients obstruction to the flow of urine was the common cause for the CAUs. Urosepsis patients had a higher bacteraemia rate, corresponding to higher incidence of organ dysfunction and septic shock. ESBL bacteria were frequently causing urosepsis, requiring initial antibiotic to be changed to carbapenem. Mortality of our urosepsis patients were lower than mentioned in the literature.

\section{Reference(s) and grant acknowledgment(s)}

1. 3. Nissar Shaikh, Umm-E-Amara, Jazib Hassan, Zeeshan Qazi, Arshad Chanda, Zia Mahemood, Mahommad Zubair, AR Raju Vegensa, Abdul Gafoor M Tharayil, Adel Ganaw, Ranjan Matthias and Muna Al Musalmani (November 22nd 2018). Urosepsis: Flow is Life, IntechOpen, https://doi.org/10.5772/ intechopen.82262.

2. 4. Xia Liang, Jiangju Huang, Manyu Xing, Liqiong He, Xiaoyan Zhu, Yingqi Weng, Qulian Guo and Wangyuan Zou. Risk factors and outcomes of urosepsis in patients with calculous pyonephrosis receiving surgical intervention: a single center retrospective study. BMC Anesthesiology 2019; 61:2-8 3. None.

\section{8}

"Find It, quick!": the challenge of finding the source of CPE bacterium in ICU

RY. G. ; HY. Y. ; PZ. N. ${ }^{1}$; N. V. ${ }^{1}$; SY.T. ; ML. R. ${ }^{1}$

${ }^{1}$ Icu. sheba medical center, Emek Haela 9, Ramat Gan, Israel

Correspondence: R. Mor levy

Intensive Care Medicine Experimental 2020, 8(2): 000068

Introduction: The Respiratory Intensive Care Unit at the Sheba Medical Center consists of 14 beds with $~ 470$ admissions per year. The patient are exposed to infectious risk factors, suppressed immune status and are monitored via many central line catheters. Therefore, they are unable to cope with further infections. The data from the hospital's infection prevention unit were shown to be alarming in 
terms of Carbapenemase-producing Enterobacteriaceae (CPE). CPE can produce a broad spectrum of infections; it is associated with high mortality and very limited therapeutic options. CPE cases outbreaks are mostly attributed to patient-to-patient transmission via healthcare workers. The challenge we faced with was to locate the source of the infection and solve it.

Methods: Active CPE surveillance included twice-weekly rectal screening of all patients. A case was defined as a patient detected with OXA$48 \mathrm{CPE}>72$ hours after admission. Sevral meetings were scheduled between hospital's infection prevention unit and the department staff, in order to define the source of the outbreak. Over the course of the several months samples were calculated from patients and their units. A root-cause analysis "Fish Bone" method was used to investigate the results. The clone was detected in 16 sink traps.

Results: From January 2016 to May 2017, 32 OXA-48 CPE cases were detected, $81 \%$ of these were $\mathrm{S}$. marcescens. The common factor in all cases was the use of relatively large amounts of tap water. Educational intervention that engaged the ICU team led to high adherence to 'sink-contamination prevention guidelines'. No additional infections detected for 12 months.

Conclusion: Finding the source of CPE outbreak was necessary for the educational intervention and collaboration with the team of ICU to reduce the contagion of OXA-48 producing Serratia marcescens. Due to the fact that it is not possible to predict where the next infection will stem from, it is important to sample all sites of the patient environment, to conduct a multidisciplinary brainstorming session to increase the chances of finding the source.

\section{Reference(s) and grant acknowledgment(s)}

1. Gili Regev-Yochay MD, MS, Gill Smollan MD, llana Tal RN et al, 2018, Sink

traps as the source of transmission of OXA-48 producing Serratia marcesens in an intensive care unit, Infection Control \& Hospital Epidemiology,

30,1307-1315

\section{2}

\section{The gut microbiota in the clinical care setting: a study}

\section{of the microbiome of the critically ill patient}

S. Cuenca Barrero'; C. Manichanh²; J. Willamil Dos Santos²; E. Valera

Castro $^{2}$; J. Gonzalez Londoño'; JM. Sirvent Calvera

${ }^{1}$ Intensive care medicine, Hospital Universitari de Girona Dr Josep Trueta, Girona, Spain; ${ }^{2}$ Microbiology department, Vall d'Hebron University Hospital, Barcelona, Spain

Correspondence: J. Gonzalez Londoño

Intensive Care Medicine Experimental 2020, 8(2): 000092

Introduction: Patients admitted to the ICU are exposed to risk factors such as: use of antibiotics, prolonged fasting, enteral and / or parenteral nutrition and proton pump inhibitors (PPI). These factors influence and promote an imbalance in the intestinal microbiota (Dysbiosis). As a result, there are multiple alterations that can develop locally and systemically, such as the increase in antibiotic resistance, decrease in immunoregulation and increase in pathogenic bacteria.

Objectives: Our objective was to study with molecular (metagenomic) techniques the variation suffered by the intestinal microbiota of the previously healthy patient who enters the ICU and the possible dysbiosis generated during the time of admission.

Methods: Patients admitted to the ICU are exposed to risk factors such as: use of antibiotics, prolonged fasting, enteral and / or parenteral nutrition and proton pump inhibitors (PPI). These factors influence and promote an imbalance in the intestinal microbiota (Dysbiosis). As a result, there are multiple alterations that can develop locally and systemically, such as the increase in antibiotic resistance, decrease in immunoregulation and increase in pathogenic bacteria.

Our objective was to study with molecular (metagenomic) techniques the variation suffered by the intestinal microbiota of the previously healthy patient who enters the ICU and the possible dysbiosis generated during the time of admission.

Results: The fecal microbiome of 30 patients was analyzed, 21 of them with three samples (M1, M2) and the remaining 9 with two samples (M1, M2 and M3). A total analysis of 81 rectal swabs was done. According to the UniFrac (unweighted) beta diversity analysis, samples from previously healthy ICU patients show a different overall composition of the intestinal microbiota than they have one week after admission. Alpha diversity decreased significantly in the M3 samples compared to the admission samples. Taxonomic alterations in the samples (M3) included the enrichment of intestinal pathogens such as Enterococcus and the disappearance of several usual pathogens such as Prevotella and Peptoniphilus.

Conclusion: Our study showed that critical patients undergo rapid dysbiosis after admission to the ICU, promoting the development of possible infectious complications. Such alterations could be a target to study and develop therapies aimed at restoring a healthy microbiome in critical patients.

\section{2}

Management of infections in the intensive care units: an international survey

A. Roshdy'; AS. Elsayed ${ }^{2} ;$ A. Sabry ${ }^{3}$

${ }^{1} \mathrm{Icu}$, Whipps Cross University Hospital, London, United Kingdom; ${ }^{2} \mathrm{Icu}$, King Fahd Military Medcial Complex, Dhahran, Saudi Arabia; ${ }^{3} \mathrm{Cu}$, Aboukir General Hospital, Alexandria, Egypt

Correspondence: A. Roshdy

Intensive Care Medicine Experimental 2020, 8(2): 000122

Introduction: Infections affect $54 \%$ while antimicrobials (AM) are prescribed to $70 \%$ of ICU patients [1]. The heterogeneity managing infectious diseases (ID) in different ICUs remains a main obstacle for stewardship programs. Easy, fast transmissibility and AM resistance are of global concern. Altogether, they make the assessment of management, knowledge and training for intensivists an essential step toward improving outcome from ID in the ICUs.

Objectives: To explore the practice of Infectious diseases management in different ICUs.

Methods: An International cross sectional descriptive and analytical internet based open survey of Intensivists. The survey consisted of 25 closed and open-ended questions divided into 4 sections including free text annotations. Likert and semantic scales were used in 4 questions. After external peer-review, it gained endorsement by the ESICM. It was hosted on SurveyMonkey (July 30,2019) and was available for 82 days. Invitations were shared on social media and by emails sent to the ID section members of the ESICM.

Results: The survey was initiated by 561 respondents from 82 countries. The $470(83.8 \%)$ respondents who completed the survey were analysed. Respondents had a median ICU experience of 10 years (IQR 5-19) and were trained in Intensive Care Medicine as a sole specialty $(30 \%)$, or dual along with anaesthesia (35.3\%) or medicine (30.2\%). $76.2 \%$ of the respondents were practicing as full-time Intensivists and $63.4 \%$ in University hospitals. $34 \%$ of the participants were from lowand middle-income countries (LMICs). The latter were more frequently working in smaller hospitals in the private sector compared to those from high-income countries (HICs) $(23.1 \%$ versus $9 \%, p<0.001$ and $32.5 \%$ versus $8.1 \%, p<0.001$ respectively).

Only $2 / 3$ of participants had adequate knowledge of the ID diagnostic capabilities in their hospitals. Despite expert opinion is available within 1 hour for $82.5 \%$ of them, the initiation of AM was protocolised in $68.5 \%$ of cases, and half of Intensivists seldom request non-ICU opinion. Most frequently, Intensivists sought non-ICU expert opinion in immunocompromised patients and in case of Extended or Panresistant microorganisms as well as fungal infections $(63 \%, 43 \%, 55 \%$, and $32 \%$ respectively). At the time of the survey, only $27.2 \%$ of Intensivists felt a need for expert opinion in case of viral infections.

Stewardship program was in place in just $66 \%$ of ICUs, and a regular microbiology round is lacking in about $40 \%$ of them. The implementation of Stewardship programs differed significantly between LMICs and $\mathrm{HICs}(61.9 \%$ versus $68.1 \%, p=0.013)$.

Satisfaction with ID training and knowledge among Intensivists was low at $43.2 \%$; even less in those working in LMICs $(28.8 \%, p<0.001)$. $95.5 \%$ of Intensivists think addressing such gap can improve patients' outcome and decrease cost and AM resistance. 
Conclusion: From Intensivists' prospect, there is a need to improve training and knowledge in the field of ID. Stewardship programs are not universally implemented and ID management follow mostly a protocolised approach.

\section{Reference(s) and grant acknowledgment(s) \\ 1. The survey had been sponsored by the ESICM. The authors would like to thank Pedro Povoa and Andrew Conway Morris from the Infection Section of the ESICM for their support and guidance; and Sherihane Bensemmane at ESICM research office for her valuable support in creating and promoting the online survey. \\ 2. 1. Vincent J, Sakr Y, Singer M, et al. Prevalence and Outcomes of Infec- tion Among Patients in Intensive Care Units in 2017. JAMA. https://doi. org/10.1001/jama.2020.2717}

\section{9}

Effect of bundle approach on preventing hospital-acquisition of drug-resistant pathogens in intensive care units

A. Ota ${ }^{1}$; M. Kawanami ${ }^{1}$; J. Yosimura ${ }^{2}$; Y. Umemura ${ }^{2}$; K. Yamakawa ${ }^{2}$.

S. Fujimi ${ }^{2}$

${ }^{1}$ Nursing Department, Osaka General Medical Center, Osaka, Japan ${ }^{2}$ Division of trauma and surgical critical care, Osaka General Medical Center, Osaka, Japan

Correspondence: A. Ota

Intensive Care Medicine Experimental 2020, 8(2): 000129

Introduction: The increasing emergence and spread of drug-resistant pathogens all over the world has become catastrophic world health problems. In fact, we experienced an uncontrollable outbreak of multidrug-resistant pathogens and ICU closure for 3 weeks in January 2013. Based on the experience, we built an infection control bundle including contact precautions for every patient in the ICU, carrying personal alcohol-based hand rubs, cohorting patients who acquired drug-resistant pathogens, active surveillance cultures once a week, and enhanced environmental disinfection in January 2013.

Objectives: The purpose of this study is to evaluate the effectiveness of the systemic infection control bundle approach against acquisition of drug-resistant pathogens.

Methods: This was a retrospective historical control study conducted from April 2008 to March 2019 in 18-bed ICU of a tertiary care hospital in Japan. Every patient admitted to the ICU during the study period was eligible. Patients were divided into 2 groups: Conventional (from April 2008 to December 2012) or Intervention (from January 2013 to March 2019).The primary outcome was the number of hospitalacquired MRSA. We defined hospital-acquisition as the isolation of MRSA after 48 hours of hospitalisation.

Results: A total of 13,047 patients were included during the study period. There were 5,128 patients in the control group (39.3\%) from 2008 to 2012 and 7,919 patients in the intervention group (61.7\%) from 2013 to 2019. The hospital-acquisition of MRSA was observed 284 patients $(5.5 \%)$ in the control group and 150 patients $(1.9 \%)$ in the intervention group. The interrupted time-series analysis suggested that the intervention was significantly associated with reduced hospital-acquisition of MRSA [risk ratio, $0.342(95 \%$ confidence interval, 0.282-0.416); <0.01].

Conclusion: The Bundle approach could be an effective strategy to prevent hospital-acquisition of drug-resistant pathogens in ICUs.

\section{0}

Impact of team-based approach on preventing catheter-related

bloodstream infection in critically ill setting

A. Hamana'; A. Sawada'; J. Yosimura²; Y. Umemura²; K. Yamakawa²,

S. Fujimi²; Y. Sakajo

${ }^{1}$ Department of Pharmacy, Osaka General Medical Center, Osaka, Japan; ${ }^{2}$ Division of trauma and surgical critical care, Osaka General Medical Center, Osaka, Japan

Correspondence: A. Hamana

Intensive Care Medicine Experimental 2020, 8(2): 000130
Introduction: Catheter-related blood stream infection (CRBSI) is a common serious infection and associated with increased mortality in intensive care units (ICUs). One of the most important strategies to prevent CRBSI is to minimize the duration of central venous catheterization. So We built a medical team consisting of doctors, nurses and pharmacists in ICU to discuss whether patients needed central venous catheter (CVC) in terms of monitoring hemodynamics and administering drugs, and recommend catheter removal to attending physicians every day in April 2019.

Objectives: The purpose of this study is to evaluate whether our team-based approach could shorten the total duration of catheterization and reduce CRBSI.

Methods: This was a retrospective historical control study conducted from April 2018 to January 2020 in the ICU of a tertiary care hospital in Japan. Every patient admitted to the ICU during the study period was eligible if they were inserted CVC. Patients were divided into 2 groups: Conventional group (from April 2018 to March 2019) or Interventional group (from April 2019 to January 2020). We set the primary endpoint as onset of CRBSI. The secondary endpoints included the duration of central venous catheterization, the length of ICU stay and hospital mortality. CRBSI was defined as bloodstream infection in patients with CVC, not related to another site.

Results: We included 490 patients: 259 in the Conventional group and 231 in the Interventional group. The reduced tendency of CRBSI was observed in the Interventional group, though nonsignificant [hazard ratio, 0.249 (95\% confidence interval, 0.054-1.141; $p=0.067)$ ]. The Interventional group was significantly associated with reduced duration of central venous catheterization ( 5 days vs 7 days; $p<0.001$ ). No difference was observed in the length of ICU stay and in-hospital mortality between groups.

Conclusion: The team-based approach to assess CVC necessity could shorten the duration of central venous catheterization and might reduce CRBSI.

\section{3}

Risk factors and clinical characteristics of Stenotrophomonas maltophilia bacteremia acquired in Intensive care unit : A Propensity Score-Matched Analysis

T. Kamo ${ }^{1}$; K. Misumi ${ }^{1}$;. Koinuma ${ }^{1}$; Y. Hagiwara ${ }^{1}$; T. Ogura

${ }^{1}$ Critical care medicine, Saiseikai Utsunomiya Hospital, Utsunomiya, Japan Correspondence: $T$. Kamo

Intensive Care Medicine Experimental 2020, 8(2): 000163

Introduction: Bacteremia is an important problem in intensive care units (ICUs) due to high rates of antimicrobial resistance and mortality. Especially Stenotrophomonas maltophilia (SM) is an important pathogen that induced nosocomial infections. However, the risk factors for SM bacteremia in critically ill patients admitted to the ICU who are not immunocompromised, such as hematological tumor patients and malignant tumor patients, are unknown.

Objectives: We conducted a propensity score matching analysis using control patients with the other Gram negative bacteremia in ICU.

Methods: This study was conducted at ICU in the Saiseikai Utsunomiya Hospital, a critical care center in Tochigi, Japan. The medical charts of ICU- acquired bacteremia were retrospectively reviewed between January 2013 and March 2020. ICU acquired bacteremia was defined as at least one positive blood culture together with clinical features compatible systemic inflammatory response syndrome and occurring $\geq 72$ hours after ICU admission. Gram-positive bacteremia and fungemia were excluded. Because, these bacteremia are likely to have different initial treatment options. Because of the relatively few SM group, for multivariable modeling we performed a propensity score analysis with 1:1 matching with age, sex, neutropenia, and APACHEll score.

Results: Of 345 bacteremia, 92 (27\%) were included, and 15 (4\%) of which were SM bacteremia.

Propensity score matching identified 14 patients in each cohort. With propensity score matching, there were no significant difference in 30 days mortality and 90 days mortality between SM bacteremia and the other Gram negative bacteremia. 
However, the length of hospital stay was significantly longer in $\mathrm{SM}$ group ( 66 days vs 106 days, $\mathrm{P}<0.005$ ). Days to positive blood culture from ICU admission were longer in SM group(15 days vs 24 days, $\mathrm{p}<0.005)$. The ratio of carbapenems and anti-MRSA drugs used in combination was significantly higher in the SM bacteremia group( $28 \%$ vs $64 \%, p<0.05)$, and the administration duration was also significantly longer than the other bacteremia group ( 0.8 days vs 6.0 days, $p<0.05$ ). Use rate of the extracorporeal membrane oxygenation (ECMO), there was no significant difference but higher in SM bacteremia than the other bacteremia at $70 \%$ and $0 \%$, respectively.

Conclusion: Our study showed that SM should be considered as the causative bacteria that developed after prolonged ICU stay. And, in addition to the previously known risk factor for immunosuppression, longer combination use of broad spectrum antibiotics use was could be risk factor of SM bacteremia in ICU settings. Although there was no significant difference in mortality, it was found that the acquisition of SM bacteremia in ICU prolongs the hospitalization period.

\section{2}

\section{Previous infections as a risk factor for multidrug-resistant} Acinetobacter baumannii in ventilator-associated pneumonia

\section{S. J. kwon ${ }^{1}$; I. Jung ${ }^{1}$; J. Son ${ }^{1}$; J. Kim²; D. Kang ${ }^{1}$; S. Park ${ }^{1}$}

${ }^{1}$ Respiratory and critical care medicine, Konyang University Hospital, Daejeon, Republic of Korea; ${ }^{2}$ Respiratory and allergic medicine, Konyang University Hospital, Daejeon, Republic of Korea

Correspondence: S.J. kwon

Intensive Care Medicine Experimental 2020, 8(2): 000242

Introduction:Acinetobacter baumannii $(\mathrm{AB})$ has high resistant to carbapenems and other antibiotics and has become an increasingly important cause of intensive care units (ICU) nosocomial infections. Factors increasing significantly the risk of ventilator-associated pneumonia (VAP) due to Acinetobacter spp. include long duration of hospital stay, longer time on mechanical ventilation (MV), prior episode of sepsis, and prior antibiotic use. A preexistent acute-phase response can reduce the proinflammatory responses to $A B$, which is associated with an impaired bacterial clearance.

Objectives: The purpose of this study is whether the risk factors for VAP by multidrug-resistant $A B$ (MDR-AB) are the previous infections, the prior antibiotic uses, and a longer duration of mechanical ventilation, or not.

Methods: Retrospectively analysis of medical records of 209 patients receiving bronchoscopy due to suspected VAP during mechanical ventilation in ICU from January 2011 to December 2019 in Konyang University hospital, South Korea. Pathogens were isolated by a quantitative bacterial culture and susceptibility test of the lower respiratory specimens using bronchoscopy.

Results: 116 patients excluding 93 patients that met exclusion criteria were available for analysis.

54 patients (46.5\%) of VAP patients had MDR-AB. 48 patients $(41.4 \%)$ of patients was received mechanical ventilation due to infections, including 44 pneumonia patients (91.6\%). Although the 28 days mortality was $29.6 \%$ of VAP by MDR-AB, there was no significant difference between MDR-AB and other pathogens. There was no significant difference in late onset VAP between MDR-AB and other pathogens (85.2\% vs. $80.6 \%, p=0.518)$.

The previous infections including pneumonia were more frequent in those with VAP by MDR-AB than in those with other pathogens $(51.9 \%$ vs. $27.4 \%, p=0.007)$. The risk factor for VAP by MDR-AB was not associated to the prior antibiotic uses (OR $=6.737,95 \% \mathrm{Cl} ; 0.800-56.765, p$ $=0.079$ ), but was associated with the previous infections in a logistic regression analysis (OR $=2.361,95 \% \mathrm{Cl} ; 1.061-5.250, p=0.035)$.

Conclusion: The risk factor for VAP patients by MDR-AB are associated with the previous infections rather than the prior antibiotic uses, irrespective of the duration of mechanical ventilation.

\section{Reference(s) and grant acknowledgment(s)}

1. American Thoracic Society (ATS) and Infectious Diseases Society of America (IDSA). Management of Adults With Hospital-acquired and Ventilatorassociated Pneumonia: 2016 Clinical Practice Guidelines by the Infectious
Diseases Society of America and the American Thoracic Society. Clinical infectious diseases : an official publication of the Infectious Diseases Society of America 2016;63:e61-e111

2. Renckens R, Roelofs JJ, Knapp S, de Vos AF, Florquin S, van der Poll T. The acute-phase response and serum amyloid $A$ inhibit the inflammatory response to Acinetobacter baumannii pneumonia. J Infect Dis 2006;193:187-195

3. Hotchkiss RS, Monneret G, Payen D. Sepsis-induced immunosuppression: from cellular dysfunctions to immunotherapy. Nat Rev Immunol 2013;13:862-874

4. Luna CM, Aruj P. Nosocomial Acinetobacter pneumonia. Respirology 2007; 12:787-791

5. Restrepo MI, Peterson J, Fernandez JF, Qin Z, Fisher AC, Nicholson SC. Comparison of the bacterial etiology of early-onset and late-onset ventilatorassociated pneumonia in subjects enrolled in 2 large clinical studies. Respiratory care 2013;58:1220-1225

6. Koulenti D, Tsigou E, Rello J. Nosocomial pneumonia in 27 ICUs in Europe: perspectives from the EU-VAP/CAP study. European journal of clinical microbiology \& infectious diseases : official publication of the European Society of Clinical Microbiology 2017;36:1999-2006

7. Weber DJ, Rutala WA, Sickbert-Bennett EE, Samsa GP, Brown V, Niederman MS. Microbiology of ventilator-associated pneumonia compared with that of hospital-acquired pneumonia. Infection control and hospital epidemiology 2007;28:825-831

8. Chung DR, Song JH, Kim SH, et al. High Prevalence of Multidrug-Resistant Nonfermenters in Hospital-acquired Pneumonia in Asia. Am J Respir Crit Care Med 2011;184:1409-1417

\section{5}

Effectiveness and safety of intravenous fosfomycin in multi-drug resistant organism (MDRO) infections in critically ill patients an Indian experience

K. Zirpe';Y. Mehta ${ }^{2}$; P. Rajesh ${ }^{3}$; P. Rahul ${ }^{4}$; D. Abhijit' ; S. Patil ${ }^{5}$; B. Sagar ${ }^{5}$; B. Hanmant ${ }^{5}$

${ }^{1}$ Neuro critical care, Ruby Hall Clinic, Pune, India; ${ }^{2}$ Critical care, Medanta -The Medicity, Gurugram, India; ${ }^{3}$ Critical care, BLK Hospital, New Delhi, India; ${ }^{4}$ Critical care, Fortis Hospital Mulund, Mumbai, India; ${ }^{5} \mathrm{Global}$ medical affairs, Glenmark Pharmaceuticals Limited, Mumbai, India

Correspondence: S. Patil

Intensive Care Medicine Experimental 2020, 8(2): 000255

Introduction: IV fosfomycin is used particularly in difficult-to-treat or complex infections, caused by both Gram-positive and Gram-negative pathogens including multidrug-resistant strains. Despite its clinical use for almost five decades, data on the use of Intravenous (IV) fosfomycin in clinical routine is still limited. We intent to observe the effectiveness and safety of intravenous fosfomycin in real-life setting.

Objectives: The primary objective is to observe the current clinical use of IV fosfomycin. Secondary objectives are clinical outcome, impact of variables on mortality and adverse events.

Methods: Multicentric retrospective study conducted among recipients of IV fosfomycin for the treatment of multi-drug resistant organism (MDRO) infections from 4 tertiary care centers from India. Medical records were analyzed for clinical diagnosis, pathogen, fosfomycin posology, adverse events with $\mathrm{CCl}$, Pitts, qSOFA score.

Results: Overall, 309 patients received IV fosfomycin of which 17\% were immunocompromised. Main indications for fosfomycin administration were bacteremia (45.3\%), pneumonia (15.8\%), septic shock (14.3\%), UTI (13.9\%). Common pathogens were K. pneumoniae $(43.3 \%)$, E. coli $(21.7 \%)$, polybacterial $(20.8 \%)$, Pseudomonas spp. (11.7\%), Staphylococcus spp. (2.9\%). Average dose of fosfomycin was $9.8 \pm 4.06 \mathrm{~g} / \mathrm{d}$ (Range: $1 \mathrm{~g}$ to $24 \mathrm{~g} / \mathrm{d}$ ) administered for average duration of $4.66 \pm 3.68$ days (Range: 1-16 days). Hypokalemia was the most 
common (62.1\%) adverse event observed followed by hypernatremia (24.2\%). Overall clinical improvement was observed in 55\% patients. Immunocompromised status, Pitts score, CCl, qSOFA score and hypernatremia were associated with significant impact on survival (Table 1).

Table 1: Comparison of variable among survivor and non-survivor $(n=309)$

\begin{tabular}{|l|l|l|l|}
\hline Variables & $\begin{array}{l}\text { Survivor } \\
(\mathbf{n}=170)\end{array}$ & $\begin{array}{l}\text { Non survivor } \\
(\mathbf{n}=139)\end{array}$ & P value \\
\hline Age & $61.91 \pm 15.24$ & $58.99 \pm 16.58$ & 0.14 \\
\hline Sex $(\mathrm{M})$ & $98(57.64 \%)$ & $95(68.34 \%)$ & 0.05 \\
\hline $\begin{array}{l}\text { Charlsons comorbidity } \\
\text { index }(\mathrm{CCI})\end{array}$ & $1.11 \pm 1.11$ & $3.22 \pm 1.96$ & $<0.0001$ \\
\hline Immunocompromised & $22(12.94 \%)$ & $30(21.58 \%)$ & 0.04 \\
\hline Septic shock & $19(11.17 \%)$ & $25(17.98 \%)$ & 0.10 \\
\hline Pitts score $($ Overall) & $2.32 \pm 2.52$ & $3.51 \pm 2.22$ & $<0.0001$ \\
\hline qSOFA score & $1.44 \pm 0.86$ & $1.85 \pm 0.95$ & 0.0003 \\
\hline Enterobacteriaceae & $101(59.41 \%)$ & $87(62.58 \%)$ & 0.63 \\
\hline Complicated UTI & $42(24.7 \%)$ & $11(7.9 \%)$ & 0.0001 \\
\hline $\begin{array}{l}\text { Average dose }(\mathrm{g} / \mathrm{d}) \\
\text { (Excluding } \geq 24 \text { hrs } \\
\text { dosing) }\end{array}$ & $\begin{array}{l}11.88 \pm 3.83 \\
(\mathrm{n}=134)\end{array}$ & $\begin{array}{l}11.62 \pm 4.32 \\
(\mathrm{n}=117)\end{array}$ & 0.92 \\
\hline Average duration $(\mathrm{d})$ & $4.71 \pm 3.81$ & $4.50 \pm 3.21$ & 0.77 \\
\hline Hypokalaemia & $110(64.7 \%)$ & $82(58.9 \%)$ & 0.34 \\
\hline Hypernatremia & $31(18.2 \%)$ & $44(31.6 \%)$ & 0.007 \\
\hline
\end{tabular}

Conclusion: IV fosfomycin was used across different indications and sites of infection in critically ill patients with clinical improvement in $55 \%$ patients. Hypokalemia is very common adverse event in practice; however, hypernatremia is associated with increased association with mortality in our study.

\section{5}

Prevalence of intestinal colonization with Carbapenem resistant Gram-negative bacteria (CRB) on admission to intensive care unit and incidence of subsequent CRB infections

A. Sakagianni' ${ }^{1}$; O. Kampouropoulou' ${ }^{1}$ E. Kourtelesi ${ }^{1}$; N. Skarmoutsou ${ }^{2}$;

G. Feretzakis ${ }^{3} ;$ K. Valakis

${ }^{1}$ Intensive care unit, SISMANOGLIO GENERAL HOSPITAL, ATHENS, Greece; ${ }^{2}$ Clinical microbiology department, SISMANOGLIO GENERAL HOSPI-

TAL, ATHENS, Greece; ${ }^{3}$ Department of quality control, research and continuing education, SISMANOGLIO GENERAL HOSPITAL, Athens, Greece

Correspondence: A. Sakagianni

Intensive Care Medicine Experimental 2020, 8(2): 000305

Introduction: Carbapenem resistant Gram-negative bacterial (CRB) infections constitute a significant menace for healthcare systems, especially in high endemicity countries, such as Greece. Limited therapeutic options and high mortality rates call for prompt recognition and isolation of CRB carriers, preventing patient-to patient transmission.

Objectives: To evaluate the prevalence of intestinal colonization with CRB on admission to Intensive Care Unit (ICU), assess the rate of CRBrelated infections and identify risk factors.

Methods: This is a retrospective observational study that included all patients admitted to the ICU of a tertiary hospital in Greece, between September 2019 and March 2020. Rectal swabs for CRB detection were collected upon ICU admission in the first 24 hours. Medical records of the patients were reviewed for evaluation of age, sex, source of ICU admission, previous antibiotic therapy, comorbidities, CRB infections and length of ICU and hospital stay. Rectal swabs were cultured for screening at Brilliance CRE agar; in all positive results resistance to imipenem and meropenem was confirmed with susceptibility testing and phenotypical methods of carbapenemases production. We used chi-square with Yates' correction to compare categorical attributes, Kruskal-Wallis Test for the medians and t-test for the means of numerical variables, with a $p$ value $<0.05$ statistically significant.
Results: A total of 158 patients were admitted to the ICU during a 6 -month period. All of them were screened for intestinal colonization with CR-GnB. Among them, 27 patients (17\%) were colonized with one or more Carbapanemase producing (CP)-CRB strains, with Klebsiella pneumoniae being the most prevalent (74\%), followed by Acinetobacter baumanii (37\%) and Pseudomonas aeruginosa (7.4\%). Predisposing factors for CRB colonization were the presence of comorbidities, the source of ICU admission with over $85 \%$ transferred from another acute care setting (ICU, non-ICU ward), recent antibiotic exposure (100\%), and hospital admission in the previous 6 months (52\%) (table1). The rate of CRB infections among carriers was 52\% ( $n=14 / 27)$. In all but three patients the colonizing and infecting strains were the same. Median time from rectal screening to infection was $10 \mathrm{~d}$. The most common site of infection was the lung in ten patients (37\%), followed by primary bloodstream infection in seven (26\%). Baseline characteristics among colonized patients with CRB infections and non-CRB infections were not significantly different, except for the length of ICU and hospital stay (table1).

\begin{tabular}{|c|c|c|c|c|}
\hline Variables & $\begin{array}{l}\text { colonized } \\
\text { patients } \\
(\mathrm{n}=27)\end{array}$ & $\begin{array}{l}\text { non-CRB } \\
\text { infection } \\
(\mathrm{n}=13)\end{array}$ & $\begin{array}{l}\text { CRB } \\
\text { infection } \\
(n=14)\end{array}$ & $\begin{array}{l}\text { P- } \\
\text { value* }\end{array}$ \\
\hline Median age (IQR), years ${ }^{2}$ & $71(29-89)$ & $72(39-89)$ & $69(29-86)$ & 0.13 \\
\hline Male gender $\square$ & $21(77 \%)$ & $9(69 \%)$ & $12(86 \%)$ & 0.32 \\
\hline Hospital stay pre-ICU ${ }^{3}$ & $17.4(14.6)$ & $12.2(13)$ & $21.9(14.4)$ & 0.1 \\
\hline $\begin{array}{l}\text { Hospital admission in the } \\
\text { previous } 6 \mathrm{mo}\end{array}$ & $14(52 \%)$ & $8(62 \%)$ & $6(43 \%)$ & 0.34 \\
\hline Heart disease $\square$ & $18(85 \%)$ & $9(69 \%)$ & $9(64 \%)$ & 0.89 \\
\hline \multirow{2}{*}{ Diabetes mellitus II $\square$} & $7(26 \%)$ & $4(31 \%)$ & $3(21 \%)$ & 0.91 \\
\hline & $7(26 \%)$ & $4(31 \%)$ & $3(21 \%)$ & 0.91 \\
\hline \multirow{2}{*}{$\begin{array}{l}\text { Chronic pulmonary } \\
\text { disease } \square\end{array}$} & $13(48 \%)$ & $7(54 \%)$ & $6(43 \%)$ & 0.85 \\
\hline & $3(11 \%)$ & $2(15 \%)$ & $1(7 \%)$ & $\mathrm{NA}$ \\
\hline Immunosuppression $\square$ & & & & \\
\hline $\begin{array}{l}\text { Chronic Kidney } \\
\text { Disease } \square\end{array}$ & $3(11 \%)$ & $1(7 \%)$ & $2(14 \%)$ & NA \\
\hline \multicolumn{5}{|l|}{ Malignancy history $\square$} \\
\hline Length of ICU stay ${ }^{2}$ & $8(5-26)$ & $5(4-8)$ & $\begin{array}{l}25.5(12.25- \\
50.25)\end{array}$ & $0.0014^{*}$ \\
\hline Length of hospital stay ${ }^{2}$ & $\begin{array}{l}32.5(12.75- \\
53)\end{array}$ & $14(10.5-27)$ & $39(34-54.5)$ & $0.008^{*}$ \\
\hline ICU mortality rate $\square$ & $12(44 \%)$ & $5(38 \%)$ & $7(50 \%)$ & 0.83 \\
\hline
\end{tabular}

$\square$ Chi-square Test, ${ }^{2}$ Kruskal-Wallis (days, median, IQR), ${ }^{3}$ t-test (days, mean, SD) ${ }^{*} \mathrm{P}<0.05$

Conclusion: Our findings suggest that CRB carriers admitted to the ICU suffer from an increased risk of developing CRB infections with high mortality rate, and significantly prolonged ICU and hospital stay. Surveillance cultures on admission and early patient and staff cohorting may limit CRB transmission and guide empirical treatment in case of subsequent infection.

\section{Reference(s) and grant acknowledgment(s)}

1. Centers for Disease Control and Prevention. Facility guidance for control of carbapenem-resistant Enterobacteriaceae (CRE), November 2015 update CRE toolkit. Atlanta (GA): United States Department of Health and Human Services

2. European Centre for Disease Prevention and Control. Carbapenem-resistant Enterobacteriaceae. Second update - 26 September 2019. ECDC: Stockholm; 2019

3. None. 
000332

Association of thyroid status with risk of bloodstream infections: results from the prospective population-based HUNT Study in Norway

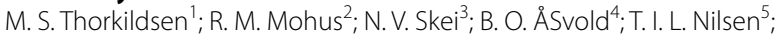
A. Mehl'; E. Solligård'; J. K. Damås ${ }^{8}$; L. T. Gustad ${ }^{9}$

${ }^{1}$ Gemini center for sepsis research at institute of circulation and medical imaging, St. olavs hospital, Trondheim, Norway, Norway; ${ }^{2}$ Clinic of anaesthesia and intensive care, St. Olavs Hospital, Trondheim, Norway; ${ }^{3}$ Department of anesthesia and intensive care, Levanger Hospital, Trondheim, Norway; ${ }^{4}$ K.g. jebsen center for genetic epidemiology, St Olavs Hospital, Trondheim, Norway; ${ }^{5}$ Department of public health and nursing, St. olavs hospital, Trondheim, Norway, Norway; ${ }^{6}$ Department of medicine, Levanger Hospital, Levanger, Norway; ${ }^{7}$ Clinic of anaesthesia and intensive care, St. Olavs Hospital, Trondheim, Norway; ${ }^{8}$ Department of clinical and molecular medicine, St. Olavs Hospital, Trondheim, Norway; ${ }^{9}$ Department of medicine and rehabilitation, levanger Hospital, Levanger, Norway

\section{Correspondence: M.S. Thorkildsen}

Intensive Care Medicine Experimental 2020, 8(2): 000332

Introduction: Studies of patients admitted to intensive care units (ICU) with sepsis have shown that thyroid function abnormalities are associated with adverse outcomes. It has also been suggested that patients' thyroid levels prior to critical illness could affect sepsis mortality, even at subclinical levels(1). As thyroid dysfunction is common in the general population, this may represent a modifiable risk factor for sepsis $(2,3)$. To our knowledge, the association between thyroid status and risk of bloodstream infections (BSI) has not previously been investigated in a large population-based study.

Objectives: To determine if thyroid hormone levels in the general population is associated with future risk of BSI and mortality.

Methods: Thyroid function was measured in 35,263 Norwegian men and women participating in the second HUNT Study(HUNT2, 199597). Thyroid function was defined as Thyroid Stimulating Hormone (TSH) levels combined with free thyroxine (FT4) and total triiodthyronine (T3). HUNT2 data was linked to prospective information from the Mid-Norway Sepsis Register (MNSR), on clinically relevant BSI from local and regional hospitals until 2011. TSH levels where classified into six categories. Most of the BSI patients had sepsis. We evaluated the risk of first-time BSI, of all causative agents and by the most common infecting bacteria E. coli, S. aureus and S. pneumoniae. Risk of mortality was assessed within 30 days of BSI and and all cause-mortality during the whole follow-up period. We used Cox regression to estimate hazard ratios with $95 \%$ confidence interval, using TSH 0,5-1,4 $\mathrm{mU} / \mathrm{L}$ as reference.

Results: After excluding participants with known thyroid disease, 31,707 HUNT2 participants where eligible for follow-up of BSI. During a median of 14,8 follow-up years, 1,138 experienced at least one episode of BSI and 198 died within 30 days. Table 1 show the hazard ratios adjusted for age, sex, socioeconomical status, BMI and smoking. The higher TSH levels showed some association with development of BSI, but the results have a low precision and the confidence interval is also in agreement with no association. The results where robust for further adjustments of comorbidities (data not shown). The observed pattern displayed in Table 1 did not change across follow-up of sepsis due to E. coli, S. aureus and S. pneumoniae or for 30-day mortality after BSI. Conclusion: We found an association between higher TSH levels and a lower risk of hospitalizations and mortality due to BSI. However, the width of the confidence intervals also coincides with no risk difference. All participants with abnormal levels of TSH where advised for further investigations, leaving a possibility that our associations could have been stronger in an untreated population.

\section{Reference(s) and grant acknowledgment(s)}

1. (1) Ling XW, Howe TS, Koh JS, Wong MK and Ng AC. Preoperative thyroid dysfunction predicts 30-day postoperative complications in elderly patients with hip fracture. Geriatr Orthop Surg Rehabil. 2013; 4: 43-9

2. (2) Luo B, Yu Z and LiY.Thyroid hormone disorders and sepsis. Biomed Mater Eng. 2017; 28: S237-S41.
3. (3)Tasci HI, Erikoglu M, Toy H and Karaibrahimoglu A. Course of sepsis in rats with thyroid dysfunction. Turk J Surg. 2017; 33: 175-9

4. MST has received Grants from Clinic of Anaesthesia and Intensive Care at St. Olavs Hospital, Trondheim, Norway.

000356

Body Mass Index and ARDS severity in patients with and without SARS-CoV-2 infection

T. Pozzi ${ }^{1}$; A. Caccioppola ${ }^{1}$; A. Ciabattoni ${ }^{1}$; V. Castagna ${ }^{1}$; L. Meloni ${ }^{2}$;

F. Bianchi ${ }^{2} ;$ S. Coppola ${ }^{3}$; D. Chiumello ${ }^{4}$

${ }^{1}$ Anesthesia and intensive care, University of Milan, Milano, Italy; ${ }^{2}$ Emergenza e urgenza, University of Milan, Milano, Italy; ${ }^{3}$ Anesthesia and intensiva care, San Paolo, Milano, Italy; ${ }^{4}$ Anesthesia and intensive care, San Paolo, Milano, Italy

Correspondence: $T$. Pozzi

Intensive Care Medicine Experimental 2020, 8(2): 000356

Introduction: Since December 2019 a pandemia of new acute respiratory distress syndrome due to coronavirus disease (C-ARDS) has been reported worldwide, with high need of intensive care admission and mechanical ventilation. A high frequency of obesity among patients admitted to ICUs for C-ARDS has been demonstrated; in previous studies, Body Mass Index (BMI) has been described as a major risk factor for ICU admission and ARDS severity.

Objectives: The aim of the study was to investigate if C-ARDS patients had a higher BMI compared to a previous population of ARDS patients. Methods: We conducted a retrospective analysis of 140 consecutive C-ARDS patients admitted to the intensive care units of San Paolo Hospital in Milan and of Maggiore Hospital in Lodi from February 25th 2020 to April 19th 2020. All patients had a laboratory-confirmed SARS-CoV-2 infection diagnosis by RT-PCR on nasopharyngeal swabs. We compared C-ARDS patients with 247 consecutive ARDS patients enrolled in previous studies and ongoing studies.

Results: BMI was significantly higher in C-ARDS compared to ARDS (28 [25-31] kg/m2 vs 25 [22-29] kg/m2, p<0.0001). We found a significantly higher percentage of overweight and obese patients in C-ARDS than ARDS patients admitted to the ICU (49\% vs $33 \%$ and $29 \%$ vs $16 \%, p<0.0001)$. At the time of ICU admission, mechanically ventilated C-ARDS patients had $\mathrm{PaO} 2 / \mathrm{FiO} 2$ ratio significantly lower (120 [90-165] vs 169 [127-216] with positive end-expiratory pressure (PEEP) level significantly higher (12 [10-14] cmH2O vs 10 [10-12] $\mathrm{cmH} 2 \mathrm{O}, \mathrm{p}<0.0001)$. According to the ARDS Berlin definition, we found that the percentages of severe ARDS patients was significantly higher in C-ARDS patients ( $36 \%$ vs $13 \%, \mathrm{p}<0.0001$ ).

Conclusion: We found that, among patients admitted in ICU and requiring mechanical ventilation, C-ARDS population had both a higher percentage of patients with $\mathrm{BMI} \geq 25$ and higher percentage of patients with severe ARDS compared to "other ARDS". Our results suggest that a possible relationship between higher BMI and development of severe ARDS patients could exist among C-ARDS patients.

\section{Reference(s) and grant acknowledgment(s)}

1. 2. Simonnet A, Chetboun M, Poissy J, Raverdy V Lille Intensive Care COVID19 and Obesity study group. High prevalence of obesity in severe acute respiratory syndrome coronavirus-2 (SARS-CoV-2) requiring invasive mechanical ventilation. Obesity (Silver Spring) 2020;https://doi.org/10.1002/ oby.22831. https://doi.org/10.1002/oby.22831.

2. 1. Gong MN, Bajwa EK, Thompson BT, Christiani DC. Body mass index is associated with the development of acute respiratory distress syndrome. Thorax 2010; 65(1):44-50.

\section{9}

Anti-influenza vaccination among healthcare personnel of the intensive care units of Catalonia. Preliminary results R. Muñoz-Bermúdez ; A. Alabart-Llinas ; ; A. Salazar-Degracia';

D. Ruiz-Sanchez ${ }^{1}$; C. Serra-Pujadas²; F. Alvarez-Lerma³; J.

R. Masclans-Enviz ${ }^{3}$;. Marin-Corral

${ }^{1}$ Critical care department, Hospital del Mar, IMIM-GREPAC, Barcelona, Spain; ${ }^{2}$ Occupational health department, Hospital del Mar, Barcelona, 
Spain; ${ }^{3}$ Critical care department, Hospital del Mar, IMIM-GREPAC, UAB, Barcelona, Spain

Correspondence: R. Muñoz-Bermúdez

Intensive Care Medicine Experimental 2020, 8(2): 000359

Introduction: Healthcare personnel working in contact with severe patients are a risk group for which annual vaccination against influenza $A$ is recommended.

Objectives: To identify the adherence rate to the influenza A vaccine among healthcare personnel in the Intensive Care Units (ICUs) of Catalonia during 2018-2019 season. Describe and analyze the reasons for non-vaccination in different worker groups.

Methods: Multicentre, observational and cross-sectional study based on an anonymous paper survey sent to healthcare personnel from 19 ICUs in Catalonia. Healthcare workers were classified as physicians (including residents and fellows) and non-physicians [nurses and Auxiliary Nursing Care Technician (TCNA)]. The survey included sociodemographic and basic clinical information related to knowledge regarding anti-influenza vaccination guidelines as well as attitudes regarding campaigns and recommendations made by the competent authorities. Reasons for non-vaccination were classified as either personal, professional or scientific. Chi-square and t-test were used for the statistical analysis and $p<0.05$ was considered statistically significant. The study was approved by the PSMar Clinical Research Ethics Committee (No. 2019/8495/I).

Results: Among 917 answered surveys, 730 (69\%) were properly completed and included in the study. 79\% of the participants were women and the mean age of the total sample was 39 (12) years old. 170 surveys $(23.3 \%)$ corresponded to physicians and $560(76.7 \%)$ to non-physicians (421 nursing and 139 TCNA). Total vaccination rate was $43.4 \%$ and it was higher in physicians compared to non-physicians $(66.5 \%$ vs. $36.4 \% ; p<0.000)$. Vaccination was more frequent in men than in women $(57.1 \%$ vs. $39.8 \%, \mathrm{p}<0.000)$. Factors related to the decision of vaccination were self-protection (86\%) and patient protection (63\%). Reasons for non-vaccination in physicians $(n=57 ; 33.5 \%)$ and non-physicians group $(n=356 ; 63.6 \%)$ were scientific [no protection of the vaccine $(8.7 \%$ vs. $23.7 \%, p=0.011)$, financial motivations related to vaccination campaigns (5.3\% vs. $19.5 \%, p=0.008)$, the possibility of contracting the flu with the vaccine $(1.8 \%$ vs $17.3 \%, p=0.002)$ and low effectiveness of the vaccine $(28.1 \%$ vs. $36.5 \%, p=0.217)$, personals [forgetfulness $(24.6 \%$ vs. $8.1 \%, p=0.000)$, lack of time $(24.6 \%$ vs. $6.7 \%$, $\mathrm{p}=0.000)$ and fear of side effects $(21.1 \%$ vs. $25.9 \%, \mathrm{p}=0.433)]$ and professional as inconvenience on the vaccination site ( $7 \%$ vs. $0.5 \%$, $\mathrm{p}=0.004)$. Overall $57 \%$ of healthcare personnel included in this study (31.6\% of physicians and $5.9 \%$ of non-physicians; $\mathrm{p}<0.001)$ has either no intention or doubts about being vaccinated in the 2019-2020 campaign. The most effective reported factor influencing the decision to vaccination were hospital teaching sessions (60\%) and social media (40\%).

Conclusion: Vaccination rate among ICU healthcare personnel is low however it is higher in physicians than in non-physicians. Reasons for non-vaccination differ between both worker groups. A low percentage of ICU workers are decided to be vaccinated next season. To stress in the evaluated factors related to vaccination and non-vaccination could be helpful in design and establish measures to promote vaccination among ICU healthcare personnel

Reference(s) and grant acknowledgment(s)

1. This team obtained the SOCMIC 2019 grant to conduct this study.

\section{0}

\section{Management of covid 19 critically ill patients in a local hospital} hit by pandemic. What can we learn?

JP. Avilés Parra ; OF. Fong Ruíz; ; EM. Sánchez Sánchez ; M. Fuentes Ponte ; F. Muñoyerro González'; A. Orejas Gallego'; D. Roa Alonso; LA. Matesanz Canencia”; I. Jiménez Del Río; M. Vicente Orgaz²; S. Gholamian Ovejero²; u. Flordelís Lasierra

${ }^{1}$ Intensive Care Medicine, Hospital Universitario Severo Ochoa, Leganés, Spain; ${ }^{2}$ Anaesthesia and pain medicine, Hospital Universitario Severo Ochoa, Leganés, Spain
Correspondence: J.P. Avilés Parra

Intensive Care Medicine Experimental 2020, 8(2): 000390

Introduction: Our 350 bed center (10 ICU beds) assisted 2200 COVID19 patients in the period March to June 2020, of whom 90 were admitted to ICU. A great healthcare challenge.

Objectives: To describe the characteristics of COVID-19 critically ill patients admitted to the ICU.

Methods: RRetrospective descriptive study including critically ill COVID-19 patients admitted to the ICU from March 2020 to June 2020. Variables: sociodemographic, comorbidities, COVID-19 treatment, adverse drug reactions, severity scores at ICU admission, organ supports, complications, ICU and hospital stay/mortality. A descriptive analysis was performed. Continuous variables have been expressed as median/ interquartile range and categorical as percentage.

Results: 90 COVID-19 patients confirmed by PCR were included. 10 patients were excluded due to transfer to another hospitals. Mean age 65 years (56-72); 68\% male; symptoms began 9 (7-15) days before admission. Main comorbidities were obesity $43 \%$ and hypertension 41\%. Hospital stay before ICU admission was 3 (2-6) days. APACHE II 15 (10-18), SAPS 35 (48-65), SOFA at ICU admission 5 (3-8); 94\% were admitted for severe respiratory failure, $\mathrm{PaO} 2 / \mathrm{FiO} 285$ (66-125), 84\% required invasive mechanical ventilation $(\mathrm{MV}), 4 \%$ non-invasive $M V$, and $11 \%$ high flow nasal cannula. Videolaryngoscopy device was used to intubate in 95\%; 74\% needed prone position, with a median of 2 cycles (1-3) per patient, MV 9 (2-22) days, 23\% needed tracheostomy, $56 \%$ required vasopressors, $4 \%$ continuous renal replacement therapy. At ICU admission the mean white blood cell count was $9300 \mu \mathrm{L}$ (6222-13330), absolute lymphoctyte count 700 $\mu \mathrm{L}$, LDH 479U/L (350582), D-dimer $1.97 \mu \mathrm{g} / \mathrm{ml}(0.76-7.6)$, At the first $48 \mathrm{~h}$ ferritin levels was $1713 \mathrm{ng} / \mathrm{ml}$ (1020-2481) and IL-6 $130 \mathrm{pg} / \mathrm{ml}$ (38-358). COVID-19 treatments: hydroxychloroquine $90 \%$, lopinavir/ritonavir $91 \%$, interferon beta-1b $41 \%$, azithromycin $75 \%$, tocilizumab $49 \%$, remdesivir $1 \%$, systemic glucocorticoids $75 \%$, anticoagulation $40 \%$ prophylactic, $14 \%$ intermediate dose, $45 \%$ therapeutic dose. Adverse drug reactions were $26 \% ; 12 \%$ suffered thromboembolic events and $16 \%$ haemorrhagic events, $46 \%$ required change of anticoagulant drug, 5\% developed antiheparin antibodies. Nosocomial infection was diagnosed in $51 \%$, with an outbreak of Stenotrophomonas maltophilia affecting $21 \%$ of patients. ICU stay: 12 (5-25) days, ICU mortality 53\%, Hospital mortality $56 \%$.

Conclusion: COVID-19 patients have shown a high mortality, conditioned by their respiratory evolution, with an infrequent use of non-invasive MV and high flow nasal cannula; associated with a high incidence of nosocomial infection and a delayed ICU admission.

Reference(s) and grant acknowledgment(s)

1. Cummings MJ, Baldwin MR, Abrams D, et al. Epidemiology, clinical course, and outcomes of critically ill adults with COVID-19 in New York City: a prospective cohort study. Lancet. 2020;395(10239):1763-1770. https://doi. org/10.1016/s0140-6736(20)31189-2

2. Grasselli G, Zangrillo A, Zanella A, et al. Baseline Characteristics and Outcomes of 1591 Patients Infected With SARS-CoV-2 Admitted to ICUs of the Lombardy Region, Italy. JAMA. 2020;323(16):1574-1581. https://doi. org/10.1001/jama.2020.5394

\section{7}

Eight years application of Selective digestive decontamination in a mixed ICU: impact on colonization, nosocomial multi-resistant infection and antibiotic consumption C. Sánchez Ramírez ; S. Hipola Escalada'; RE. Morales Sirgado ${ }^{1}$ MA. Hernandez Viera'; M. Cabrera Santana ${ }^{1}$; L. Caipe Balcázar ${ }^{1}$; SM. Marrero Penichet ${ }^{2}$; CF. Lübbe Vázquez ${ }^{1}$; A. Padrón Mujica ${ }^{1}$; P. Saavedra-Santana ${ }^{3}$; S. Ruiz-Santana

${ }^{1}$ Intensive care medicine, University Hospital of Gran Canaria Dr. Negrin, Las Palmas de Gran Canaria, Spain; ${ }^{2}$ Pharmacy department, University Hospital of Gran Canaria Dr. Negrin, Las Palmas de Gran Canaria, Spain; ${ }^{3}$ Mathematics and informatics deparment, University of Las Palmas:, Las Palmas de Gran Canaria, Spain

Correspondence: C. Sánchez Ramírez 
Intensive Care Medicine Experimental 2020, 8(2): 000397

Introduction: Selective digestive decontamination (SDD) have been associated with reduced mortality and lower ICU-acquired rates However, the effect SDD in areas where multidrug-resistant Gram-negative bacteria are endemic is of great interest.

Methods: This study was conducted in a 30-bed-medical-surgical ICU. All consecutive patients admitted to the ICU from October 1, 2011 to September 30, 2019 expected to require tracheal intubation $>48$ hours were given SDD (SDD study group) with a 4-day course of intravenous cefotaxime, plus enteral colistin, tobramycin, nystatin in an oropharyngeal paste and in a digestive solution. Oropharyngeal and rectal swabs were obtained on admission and once weekly. We used ENVIN nosocomial infections (NI) criteria. We compared all patients admitted to ICU with ICU NI from October 1, 2010 to September 30, 2011 (non-SDD group) to the SDD study group. A univariate and a multivariate logistic regression analysis were performed. For each one of the infections the incidences per 1000 days of exposure in each cohort and the corresponding relative risks were obtained using the Poisson regression. Statistical significance was $p \leq 0.05$. We analyzed colistin- and tobramycin-resistant colonization and also antibiotic consumption as Defined antibiotics Daily Doses (DDD).

Results: Results are shown in Tables 1, 2 and 3.

\begin{tabular}{|c|c|c|c|}
\hline & \multicolumn{2}{|c|}{ Selective decontamination of the digestive tract } & \multirow[b]{2}{*}{$\mathrm{P}$} \\
\hline & $\begin{array}{c}N o \\
N=110\end{array}$ & $\begin{array}{c}\text { Yes } \\
N=599\end{array}$ & \\
\hline Age, years & $59.5 \pm 15.8$ & $61.4 \pm 15.0$ & 0.241 \\
\hline APACHE II socre & $21.2 \pm 7.7$ & $21.7 \pm 7.7$ & 0.516 \\
\hline Sex male & $0(0.0)$ & $559(100.0)$ & $<0.001$ \\
\hline Trauma petients & $74(67.3)$ & $364(652)$ & 0.025 \\
\hline Corchary atery disease patients & $17(15.4)$ & $\varpi(11.3)$ & 0.219 \\
\hline Emergency surgery & $19(17.3)$ & $125(224)$ & 0.378 \\
\hline Immunosuppression & $34(30.9)$ & $131(23.4)$ & 0.006 \\
\hline Neutropenia & $8(7.3)$ & $\wp(10.7)$ & 0.272 \\
\hline Immunodepression & $3(27)$ & $23(4.1)$ & 0.726 \\
\hline Parenteral nutuition & $3(27)$ & $2(0.4)$ & 0.034 \\
\hline RRT & $34(30.9)$ & $214(38.3)$ & 0.209 \\
\hline Melnutrition & $12(10.9)$ & $55(9.8)$ & 0.165 \\
\hline Diabetes mellitus & $34(30.9)$ & $175(31.3)$ & 0.935 \\
\hline COPD & 9(8.2) & $94(16.8)$ & 0.022 \\
\hline Renal failure & $40(36.4)$ & $107(19.1)$ & $<0.001$ \\
\hline Oimhosis & $6(5.5)$ & $28(5.0)$ & 0.178 \\
\hline Necolasm & $10(9.1)$ & $57(10.2)$ & 0.724 \\
\hline VAP & $59(53.6)$ & $186(33.3)$ & $<0.001$ \\
\hline$C R B$ & $26(23.6)$ & $211(37.8)$ & 0.014 \\
\hline Seccondary badterenia & $31(282)$ & $146(26.1)$ & 0.807 \\
\hline Uninary infection & $29(26.4)$ & $149(26.6)$ & 0.95 \\
\hline ATB 48 hours before admission & $28(25.4)$ & $153(27.4)$ & 0.05 \\
\hline Death & $36(327)$ & $202(36.1)$ & 0.495 \\
\hline 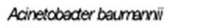 & $13(11.8)$ & $6(1.1)$ & $<0.001$ \\
\hline MRSA & $4(36)$ & $9(1.6)$ & 0.245 \\
\hline ESQ & $38(34.5)$ & $115(20.6)$ & 0.001 \\
\hline MRPseudomonas & $10(9.1)$ & $41(7.3)$ & 0.628 \\
\hline MRGNB & $12(10.9)$ & $23(4.1)$ & 0.003 \\
\hline Admission & & & 0.244 \\
\hline Medical & $79(71.8)$ & $398(71.3)$ & \\
\hline Scheduled surgery & $10(9.1)$ & $78(14.0)$ & \\
\hline Emergency surgery & $21(19.1)$ & $82(14.7)$ & \\
\hline Infarmatory response: & & & 0.228 \\
\hline Nen sepsis & $2(1.8)$ & $23(4.1)$ & \\
\hline Sepsis & $23(20.9)$ & $151(27.0)$ & \\
\hline Septic shock & $85(77.3)$ & $385(68.8)$ & \\
\hline IOUdays & $28(16-44.8)$ & $33(20-50)$ & 0.025 \\
\hline
\end{tabular}

edended spectum bedaxtamese; MR mutresistent, GNB gamnegative bach

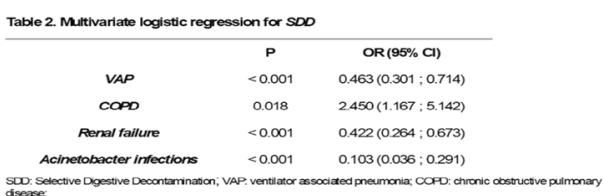

Table 3. Incidences per 1,000 days of exposures (Poisson regression)

\begin{tabular}{|c|c|c|c|c|}
\hline & \multicolumn{2}{|c|}{$S D O$} & \multirow[b]{2}{*}{$\mathbf{P}$} & \multirow{2}{*}{$\begin{array}{c}R R \\
(95 \% \text { a) }\end{array}$} \\
\hline & No & Yes & & \\
\hline VAP 1000 days of MV & 10.31 & 3.86 & $<0.001$ & $\begin{array}{c}0.374 \\
(0.282-0.496)\end{array}$ \\
\hline $\begin{array}{l}\text { lurinary nfections } / 1000 \text { days of } \\
\text { urinary catheter }\end{array}$ & 3.79 & 234 & 0.011 & $\begin{array}{c}0.618 \\
(0.426-0.897\end{array}$ \\
\hline CRB'1000 days of CVC & 3.59 & 3.62 & 0.962 & $\begin{array}{c}1.010 \\
(0.674-1.514)\end{array}$ \\
\hline $\begin{array}{l}\text { Secondary bacteremias. } 1000 \text { ICU } \\
\text { days }\end{array}$ & 4.69 & 205 & $<0.001$ & $\begin{array}{c}0.438 \\
(0.313-0.613)\end{array}$ \\
\hline $\begin{array}{l}\text { Multiresistant germs/1000 ICU } \\
\text { days }\end{array}$ & 9.59 & 253 & $<0.001$ & $\begin{array}{c}0.263 \\
(0.205-0.339)\end{array}$ \\
\hline
\end{tabular}

VAP? ventlator associded pneumonia; $\mathrm{M}$ : mechanical ventlation; $\mathrm{QRB}$ Catheter related bacteremia

There were no statistical significant differences between both groups in type of admission or demographic data. Patients with SDD had significantly less Extended Spectrum Betalactamase (ESBL), Gram Negative Bacteria Multirresistant (GNB-MR) and Acinetobacter spp infections. We had also a significant reduction in ventilator associated pneumonias (VAP), urinary infections and other secondary bacteremias and antibiotic resistant bacteria infection (ARB) rates, in SDD group versus non SDD. There was no infection by Clostridium difficile. Colistin-resistant colonization was $16,5 \%$ and tobramycin-resistant colonization was $25,7 \%$ of samples. There was a decrease on the DDD/100 ICU stays after SDD.

Conclusion: After 8 years applying SDD a significant reduction of infections by ESBL, GNB-MR and Acinetobacter, was observed. A significant decrease of VAP, secondary bacteremias, urinary and ARB infections rates was also shown. An antibiotic consumption reduction was found after SDD. Low rates of colistin- and tobramycin-resistant colonization bacteria were observed

\section{9}

What influences duration of antibiotic therapy?

Results:from a systematic literature review

R. Janssen ${ }^{1}$; A. Oerlemans²; H. Van Der Hoeven ${ }^{1}$; M. Hulscher ${ }^{2}$.

J. Schouten ${ }^{1}$

'Intensive care, Radboud University Medical Center, Nijmegen, Netherlands; ${ }^{2}$ lq healthcare, Radboud University Medical Center, Nijmegen, Netherlands

Correspondence: R. Janssen

Intensive Care Medicine Experimental 2020, 8(2): 000409

Introduction: Antibiotic use in hospitals, also in intensive care units (ICUs), is often inappropriate or unnecessary. Overuse of antibiotics drives antibiotic resistance. There are merely two ways to reduce the quantity of antibiotic use: not to start or early withdrawal. In ICUs the latter is far more acceptable. Even though emerging evidence suggests that reducing the duration of antibiotic therapy is safe for various types of infections, antibiotic therapy is commonly prescribed longer than recommended in daily practice. Professional performance of recommended care may be influenced by a large variety of factors. It is unclear, however, which factors influence the chosen duration of antibiotic therapy.

Objectives: The objective of this systematic literature review was to identify factors of antibiotic therapy duration in hospitals, with a special focus on ICUs.

Methods: A systematic literature search was conducted using PubMed, Embase, PsyclNFO and Web of Science from 2000 onwards. All qualitative, quantitative and mixed-method studies in any language, concerning adults, and performed in an in-hospital setting were 
eligible for inclusion. Studies were included if they reported original data on determinants of antibiotic therapy duration. The identified factors were categorized using the comprehensive, integrated checklist of determinants of practice as develop by Flottorp et al. (2013).

Results: Seventeen articles were included in this review, of which one specifically focussed on the ICU. Several determinants influencing duration of antibiotic therapy were found (see Table 1 for some examples). Most of these were factors related to individual health professionals (e.g. knowledge and skills, cognitions and attitudes, professional behaviour), followed by professional interactions (e.g. team processes, communications and peer influences), patient factors (e.g. patients' perceived needs), and guideline factors (e.g. (in)consistency with other guidelines, feasibility of the recommendations, quality of the recommendation).

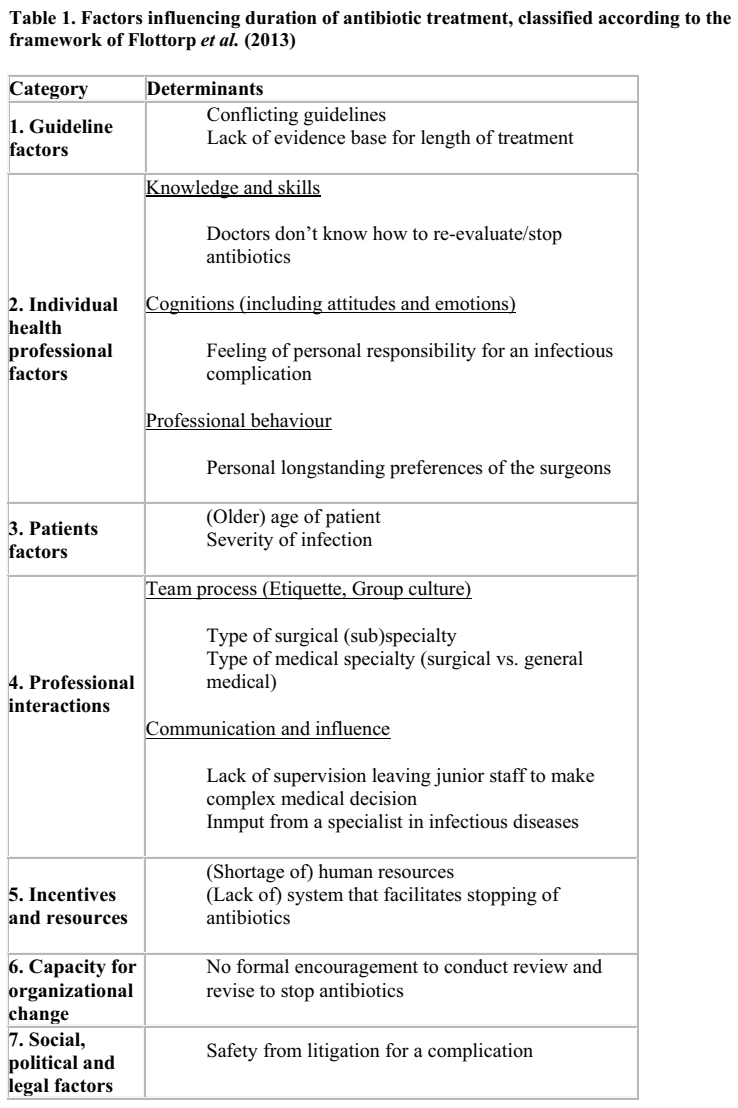

Conclusion: Only a few original studies have been performed investigating determinants of antibiotic therapy duration, with a remarkable lack of ICU studies. To design the most appropriate and effective improvement intervention, interventions need to be informed by clear information on determinants of behaviour. This review provides the first step for the development of effective behavioural interventions to optimize duration of antibiotic therapy. Further studies need to be conducted, specifically at ICUs, to gain more insight into factors that influence professional behaviour regarding the duration of antibiotic therapy.

\section{Reference(s) and grant acknowledgment(s)}

1. Flottorp SA, Oxman AD, Krause J, Musila NR, Wensing M, Godycki-Cwirko

$M$, et al. A checklist for identifying determinants of practice: a systematic review and synthesis of frameworks and taxonomies of factors that prevent or enable improvements in healthcare professional practice. Implementation Science. 2013:8(1):35.
2. This work was supported in part by a research grant from the Investigator Initiated Studies Program of Merck Sharp \& Dohme Corp (grant number 58288). The opinions expressed in this paper are those of the authors and do not necessarily represent those of Merck Sharp \& Dohme Corp.

000413

Risk factors associated with poor prognosis in patients with secondary peritonitis admitted to the Intensive Care Unit (ICU)

A. Abella Alvarez ; S. Garcia Manzanedo ${ }^{1}$; V. Enciso Calderon ${ }^{1}$; I. Conejo Márquez ; B. Lobo Valbuena ${ }^{1}$; C. San Miguel Méndez²; A. Cruz Cidoncha²; F. Gordo Vidal ${ }^{3}$

${ }^{1}$ Intensive care medicine, Hospital Universitario del Henares, Madrid, Spain; ${ }^{2}$ General surgery, Hospital Universitario del Henares, Madrid, Spain; ${ }^{3}$ Intensive care medicine, Universidad Francisco de Vitoria, Madrid, Spain

Correspondence: S. Garcia Manzanedo

Intensive Care Medicine Experimental 2020, 8(2): 000413

Introduction: Faecal peritonitis is a common cause of sepsis and ICU admission leading to up to $29 \%$ hospital mortality, prolonged ICU and hospital length-of-stays and increased costs. Risk factors associated with prognosis vary in different published studies.

Objectives: To analyse clinical, analytical, surgical, microbiological and physiological factors associated with poor prognosis (defined by ICU and hospital mortality, need for ICU readmission and prolonged ICU and hospital length-of-stay), within a cohort of ICU patients with the diagnosis of secondary peritonitis.

Methods: Retrospective single-centre observational cohort study from January 2015 to June 2018. Patients included suffered secondary peritonitis due to hollow viscera perforation, both as a reason for admission and/or during ICU stay. Quantitative variables are shown as mean and standard deviation, or as median with the interquartile range in case of failure to comply with normality. Qualitative variables are expressed as percentages. Multivariate analysis with binary backward stepwise logistic regression was performed. Odds ratio of the predictor variables of ICU mortality and bad prognosis are shown.

Results: 130 patients were recruited. Median age of 65 years (55-74), $45 \%$ were women. SAPS 3 median of 53.5 (43 - 65), ICU median stay 7 days (4 - 12) and ICU hospital stay 14 days (8 - 21). Readmission rate of $6.2 \%$. ICU mortality $5.4 \%$ and hospital mortality $9.2 \%$. In the multivariate analysis, endocrine comorbidity showed an OR $3.611(\mathrm{Cl} 95 \%$ : 1.217-10.715; $\mathrm{p}=0.021)$, need for mechanical ventilation OR 12.275 (Cl 95\%: 1.974-76.327; $\mathrm{p}=0.007$ ), need for parenteral nutrition OR 5.747 (Cl 95\%: 1. 565-21,101; $\mathrm{p}=0.008)$, faecal peritonitis OR 2,908 (Cl $95 \%: 1,176-7,192 ; p=0.021)$, need for new surgery after peritonitis OR 79,746 (Cl 95\%: 6,718-946,643; $\mathrm{p}=0.001)$ and SOFA at day 1 above 7 OR 6,939 (Cl 95\%: 2,208-21,814; $\mathrm{p}=0.001$ ). Microbiological variables showed no correlation with bad prognosis.

Conclusion: Clinical, physiological and surgical characteristics showed a relationship with poor prognosis of patients with secondary peritonitis. Microbiological variables did not.

000494

Penetration of anidulafungin in ascitic liquid in patients admitted to the ICU

R. Muñoz-Bermúdez ; F. Alvarez-Lerma²; MP. Gracia-Arnillas ${ }^{1}$;

M. Acer-Puig'; S. Luque-Pardos ${ }^{3}$; N. Campillo-Ambros ${ }^{3}$; S. Grau-Cerrato ${ }^{4}$;

JR. Masclans-Enviz²

${ }^{1}$ Critical care department, Hospital del Mar, IMIM-GREPAC, Barcelona, Spain; ${ }^{2}$ Critical care department, Hospital del Mar, IMIM-GREPAC, UAB, Barcelona, Spain; ${ }^{3}$ Pharmacy department, Hospital del Mar, Barcelona, Spain; ${ }^{4}$ Pharmacy department, Hospital del Mar, UAB, Barcelona, Spain

Correspondence: R. Muñoz-Bermúdez

Intensive Care Medicine Experimental 2020, 8(2): 000494

Introduction: Anidulafungin is commonly used for the treatment of abdominal diseases caused by Candida spp. in critically ill patients. 
Objectives: To analyze the variability in the pharmacokinetics (PK) of anidulafungin and its diffusion in ascitic fluid in patients admitted to an Intensive Care Unit (ICU).

Methods: A prospective, observational, and descriptive pharmacokinetic study in a single ICU. Included were all patients, who received intravenous anidulafungin from October 2014 to June 2019 with a loading dose of $200 \mathrm{mg}$ followed by $100 \mathrm{mg} /$ day as maintenance dose and for whom the extraction was possible. Extraction of plasma samples and ascites fluid was performed at the second day of treatment [valley or predose (Cmin) and/ or peak (Cmax) at the end of the infusion]simultaneously whenever possible. Demographic variables, pathological, analytical history and PK data were included. Quantitative variables are described as means (standard deviation) or medians (interquartile range) and qualitative variables as relative frequencies. This project was approved by the PSMar Committee for Clinical Research Ethics (No. 2016/6987/l)

Results: A total of 10 patients aged 65 (10.6) years, $6(60 \%)$ men, Body Mass Index (BMI) $26.2(3.6) \mathrm{kg} / \mathrm{m} 2.6(60 \%)$ of them hadsurgical pathology. A total of 14 plasma and ascites fluid extractions were performed. The analytical values corresponding to the day of extraction were: mean serum creatinine of $1.09(0.53) \mathrm{mg} / \mathrm{dL}$, albumin $3.17(0.96) \mathrm{mg} /$ $\mathrm{dL}$ and a median bilirubin 4.9 [2, 3-17.8] mg/dL. PK data: Mean Cmin 3.4 (1.5) $\mathrm{mg} / \mathrm{dL}$ and $\mathrm{Cmax} 5.4$ (3.2) $\mathrm{mg} / \mathrm{dL}$ in plasma; mean concentration in ascitic fluid $1.11(0.8) \mathrm{mg} / \mathrm{dL}$. Ratio Cascitic/Cmin plasma was calculated in 5 patients with a median of 0.23 [0.17-0.29]. In one case, ascites fluid levels could be extracted at $\mathrm{Cmin}, \mathrm{Cmax}, 4 \mathrm{~h}$ and $6 \mathrm{~h}$ after one administration. Anidulafungin concentrations were $0.52 \mathrm{mg} / \mathrm{dL}$, $0.51 \mathrm{mg} / \mathrm{dL}, 0.59 \mathrm{mg} / \mathrm{dL}$ and $0.68 \mathrm{mg} / \mathrm{dL}$, respectively. The concentrations of anidulafungin observed in ascites fluid were higher than the epidemiological cut-off points (ECOFF value) of this echinocandin for the different Candida spp species, which ranged from $0.015 \mathrm{mg} / \mathrm{L}$ for C. albicans to $0.25 \mathrm{mg} / \mathrm{L}$ for C. krusei.

Conclusion: The mean anidulafungin penetration in ascitic fluid was low, $23 \%$. However, the concentrations reached in ascites fluid could be sufficient to treat Candida spp since they exceed the epidemiological cut-off points.

\section{4}

Selective digestive decontamination in critically ill burn patients: does it modify the incidence of infections, type of microorganisms and use of antimicrobial agents?

D. Pérez-Torres'; C. Cuenca-Rubio ${ }^{1}$; C. Díaz-Rodríguez' I. Canas-Pérez'; GJ. Posadas-Pita'; JÁ. Berezo-García'; A. Martín-Luengo

${ }^{1}$ Servicio de medicina intensiva, Hospital Universitario Río Hortega, Valladolid, Spain

Correspondence: D. Pérez-Torres

Intensive Care Medicine Experimental 2020, 8(2): 000504

Introduction: Infections represent at least half of the clinically relevant complications in critically ill burn patients, and are one of the most frequent causes of death in this population. Selective Digestive Decontamination (SDD) is an infection prevention and control strategy conceived to minimize infections and, thus, it may have potential benefits in burn patients.

Objectives: We aim to assess the trend in the incidence of infections, type of microorganisms and use of antimicrobial agents before and after the implementation of SDD in a Burns Unit (BU).

Methods: We conducted a retrospective observational study in a 5 -bed BU of a University Hospital. We collected data from all the patients admitted to the BU within a baseline period without SDD (2 years) and an SDD period (8 months). Chi-square, Student's T and Mann-Whitney $\mathrm{U}$ tests were applied for comparisons, as appropriate.

Results: A total of 48 patients were included, of which $35 \%$ received SDD $(n=17)$. We did not find any statistically significant differences between baseline and SDD periods in terms of age (62 vs 61 years-old, $p=0.86)$, gender ( $71 \%$ vs $65 \%$ male, $p=0.65)$, BSA ( 23 vs $20 \%, p=0.52$ ), smoke inhalation injury ( $32 \%$ vs $24 \%, p=0.53)$, APACHE-II (19 vs 16 , $p=0.08), A B S I$ ( 7 vs $6, p=0.50$ ) or mortality ( $26 \%$ vs $12 \%, p=0.25$ ). Our findings are depicted in the Tables.

\begin{tabular}{|c|c|c|c|}
\hline & $\begin{array}{l}\text { Baseline } \\
\text { period }\end{array}$ & SDD period & p-value \\
\hline \multicolumn{4}{|l|}{ All infections } \\
\hline Incidence & $58 \%$ & $47 \%$ & 0.46 \\
\hline $\mathrm{N}^{\mathrm{o}}$ episodes/patient & 1.94 & 0.88 & 0.20 \\
\hline Days to event & $11(5-15)$ & $21(6-30)$ & 0.05 \\
\hline \multicolumn{4}{|l|}{ Bacteraemia } \\
\hline Incidence & $16 \%$ & $6 \%$ & 0.31 \\
\hline $\mathrm{N}^{\circ}$ episodes/patient & 0.23 & 0.06 & 0.25 \\
\hline Days to event & $22(20-27)$ & $54(54-54)$ & 0.38 \\
\hline \multicolumn{4}{|l|}{ Respiratory infections } \\
\hline Incidence & $36 \%$ & $29 \%$ & 0.67 \\
\hline $\mathrm{N}^{\mathrm{o}}$ episodes/patient & 0.81 & 0.35 & 0.25 \\
\hline Days to event & $6(5-11)$ & $7(5-17)$ & 0.69 \\
\hline \multicolumn{4}{|c|}{ Skin and soft tissue infections } \\
\hline Incidence & $32 \%$ & $24 \%$ & 0.53 \\
\hline $\mathrm{N}^{0}$ episodes/patient & 0.90 & 0.47 & 0.38 \\
\hline Days to event & $16(12-31)$ & $30(27-32)$ & 0.26 \\
\hline
\end{tabular}

Table 2. Type of microorganisms before and after the implementation of

\begin{tabular}{|c|c|c|c|c|c|c|c|}
\hline \multicolumn{8}{|c|}{ SDD. } \\
\hline & & & $\begin{array}{l}\text { Baseline } \\
\text { period }\end{array}$ & \multicolumn{2}{|c|}{ SDD period } & \multicolumn{2}{|c|}{ p-value } \\
\hline \multicolumn{3}{|c|}{ Staphylococcus aureus infections } & $3 \%$ & \multicolumn{2}{|l|}{$12 \%$} & \multicolumn{2}{|c|}{0.24} \\
\hline \multicolumn{3}{|c|}{$\begin{array}{l}\text { Streptococcus pneumoniae } \\
\text { infections }\end{array}$} & $10 \%$ & \multicolumn{2}{|l|}{$0 \%$} & \multicolumn{2}{|c|}{0.19} \\
\hline \multicolumn{3}{|c|}{ Other Gram-Positive infections } & $32 \%$ & \multicolumn{2}{|l|}{$6 \%$} & \multicolumn{2}{|c|}{0.04} \\
\hline \multicolumn{3}{|c|}{ NF Gram-Negative bacilli } & $23 \%$ & \multicolumn{2}{|l|}{$18 \%$} & \multicolumn{2}{|c|}{0.69} \\
\hline \multicolumn{8}{|l|}{ infections } \\
\hline Enterobacteriacea & ae infectio & & $29 \%$ & \multicolumn{2}{|l|}{$6 \%$} & \multicolumn{2}{|c|}{0.05} \\
\hline \multicolumn{8}{|c|}{ infections } \\
\hline \multicolumn{3}{|c|}{ Enterobacteriaceae infections } & $29 \%$ & \multicolumn{2}{|l|}{$6 \%$} & \multicolumn{2}{|c|}{0.05} \\
\hline \multicolumn{8}{|c|}{$\begin{array}{l}\text { Table 3. Use of antimicrobial agents before and after the implementation of } \\
\text { SDD. }\end{array}$} \\
\hline & \multicolumn{3}{|c|}{ Exposed patients, $\%$} & \multicolumn{4}{|c|}{$\begin{array}{l}\text { Days of treatment, P50 (P25- } \\
\text { P75) }\end{array}$} \\
\hline & $\begin{array}{l}\text { Baseline } \\
\text { period }\end{array}$ & $\begin{array}{l}\text { SDD } \\
\text { period }\end{array}$ & $\mathrm{p}$ & $\begin{array}{l}\text { Baseline } \\
\text { period }\end{array}$ & $\begin{array}{l}\mathrm{SDD} \\
\text { peric }\end{array}$ & & $\mathrm{p}$ \\
\hline NAP penicillins & 40 & 29 & 0.48 & $4(2-8)$ & $8(6$ & & 0.29 \\
\hline AP penicillins & 36 & 21 & 0.34 & $6(4-10)$ & $\begin{array}{l}13(4 \\
14)\end{array}$ & & 0.35 \\
\hline Cephalosporins & 48 & 93 & 0.01 & $\begin{array}{l}7(17- \\
21)\end{array}$ & $4(4$ & & 0.27 \\
\hline Carbapenems & 44 & 50 & 0.72 & $\begin{array}{l}11(3- \\
15)\end{array}$ & $\begin{array}{l}18(3 \\
23)\end{array}$ & & 0.52 \\
\hline Glycopeptides & 16 & 0 & 0.11 & $\begin{array}{l}12(5- \\
19)\end{array}$ & & & \\
\hline Quinolones & 28 & 14 & 0.33 & $9(4-7)$ & $5(3$ & & 0.24 \\
\hline Aminoglycosides & 8 & 0 & 0.28 & $\begin{array}{l}10(9- \\
11)\end{array}$ & & & \\
\hline Nitroimidazoles & 8 & 0 & 0.28 & $7(5-8)$ & & & \\
\hline Oxazolidinones & 48 & 43 & 0.76 & $8(4-12)$ & $5(3$ & $-10)$ & 0.51 \\
\hline Macrolides & 8 & 0 & 0.28 & $8(7-9)$ & & & \\
\hline
\end{tabular}

Conclusion: SDD was significantly associated with a later onset of all types of infection, a lower incidence of Enterobacteriaceae and other Gram-Positive infections, and a higher exposure to cephalosporins. A nonsignificant downtrend was observed in the incidence of all types of infection, use of antimicrobial agents (except cephalosporins) and mortality. 


\section{5}

Implementation of an Antimicrobial Stewardship Program reduces antimicrobial agents use and exhibits a broad acceptance in the Intensive Care Unit

D. Pérez-Torres ${ }^{1}$; M. Domínguez-Gil González²; C. Díaz-Rodríguez ; I. Canas-Pérez ; R. Almendros-Muñoz ${ }^{3}$; M. A. Sacristán-Salgado4; M E. González-González; M. C. Ramos-Sánchez²; J. M. Eiros-Bouza²; J. Á. Berezo-García'; L. M. Tamayo-Lomas

${ }^{1}$ Servicio de medicina intensiva, Hospital Universitario Río Hortega, Valladolid, Spain; ${ }^{2}$ Servicio de microbiología, Hospital Universitario Río Hortega, Valladolid, Spain; ${ }^{3}$ Servicio de farmacia hospitalaria, Hospital Universitario Río Hortega, Valladolid, Spain; ${ }^{4}$ Servicio de medicina preventiva y salud pública, Hospital Universitario Río Hortega, Valladolid, Spain

Correspondence: D. Pérez-Torres

Intensive Care Medicine Experimental 2020, 8(2): 000505

Introduction: Use of antimicrobial agents is a common intervention in the management of the critically-ill patient admitted to the intensive care unit (ICU). Optimizing antimicrobial use is imperative to reduce infection-related morbimortality and to limit the emergence of multidrug-resistant microorganisms (MDRMs).

Objectives: To describe the results of the implementation of an antimicrobial stewardship program (ASP) in the ICU of a University Hospital in Spain, after a year of operation (from mid-2018 to mid-2019).

Methods: A multidisciplinary ASP working group was created (intensivist, microbiologist, pharmacist, preventivist and nurse). Theoretical need for antimicrobial agents use was assesed in daily meetings of the group, whenever a result of a microbiological test was available, taking into account the clinical setting, infectious syndrome, isolated organism and antimicrobial pharmacology. Non-mandatory recommendations concerning optimization of antimicrobial treatment were proposed to the attending physician after each meeting, including deescalation (switch to narrower spectrum or discontinuation of treatment), adequacy (change to same spectrum or start a new treatment), optimization (duration, dose or route of administration) and escalation (switch to broader spectrum). We report antimicrobial utilization expressed as daily defined dose (DDD) per 100 bed-days (BD) in the ASP period and in previous periods.

Results: We held 260 meetings during the study period, where 230 recommendations were proposed over 110 patients $(64 \%$ male, aged $63 \pm 15)$, with a $96 \%$ of adherence. Intervention proposal was more frequently triggered by blood cultures (47\%) and respiratory samples (30\%), with Gram-negative bacteria being the most frequent isolated type of microorganism (41\%). Most interventions were advised over respiratory infections (46\%), followed by abdominal organs $(17 \%)$ and urinary tract infections (15\%).

Antimicrobial stewardship use was fundamentally proposed as deescalation (67\%), but also as adequacy (15\%), optimization (13\%) and escalation (5\%). Most interventions were advised over linezolid (22\%) and the groups of carbapenems (21\%) and penicillins (13\%). Antibiotic utilization, expressed as DDD/100 BD, decreased from 175 in 2017 (no ASP), to 154 in 2018 (ASP implemented) and to 136 in 2019 (ASP fully operational).

Conclusion: We found a broad adherence rate to the ASP-group proposed recommendations. De-escalation was the most common intervention, specially in clinically relevant groups of antibiotics. We observed a downtrend in antibiotic use after the implementation of the ASP.

\section{6}

Retrospective comparison of admission versus critical

care acquired methicillin-resistant staphylococcus aureus, vancomycin-resistant Enterococci and Clostridium difficile on patient mortality and critical care length of stay at one London hospital

S. HELYAR ${ }^{1}$; C. Bell²; E. Cole ${ }^{2}$; I. Salako ${ }^{3}$; P. Hopkins ${ }^{4}$

${ }^{1}$ ACET Research, King's College Hospital, London, United Kingdom; ${ }^{2}$ Audit critical care, King's College Hospital, Denmark Hill, London, UK, London, United Kingdom; ${ }^{3}$ Icca critical care, Kingston Hospital, London, United Kingdom; ${ }^{4}$ Critical care, King's College Hospital, London, United Kingdom
Correspondence: $\mathrm{S}$. Helyar

Intensive Care Medicine Experimental 2020, 8(2): 000506

Introduction: Critical care patients are at particular risk of contracting a nosocomial infection within the critical care unit1. Nosocomial infection is a major cause of mortality and morbidity for critical care patients and a leading factor in increased treatment costs2. However, many patients are at risk of hospital acquired infection pre admission to the critical care unit which adversely impacts on patient outcomes3. Methicillin-Resistant Staphylococcus Aureus (MRSA) Bactereamia4, Vancomysin-Resistant Enterococci (VRE)5 and Clostridium Deficile (CDiff)6 are leading hospital and critical care aquired nosocomial infections, Limited data exist on the impact of hospital versus critical care unit acquired infection on patients critical care length of stay (LOS) and critical care mortality.

Objectives: To compare impact of admission versus critical care unit acquired MRSA, VRE and C. Diff on critical care patient mortality and critical care LOS.

Methods: A retrospective analysis of all patients (critical care stay 48 hours or more) admitted to one of four critical care units (54 beds in total) at one central London hospital between March 2011 and December 2018. Patients with MRSA, VRE or C. Diff status reported to United Kingdom's Intensive Care National Audit and Research Centre (ICNARC) were included in the analysis. Admission NI was defined as an infection diagnosed within 48 hours of admission. Critical care $\mathrm{NI}$ was defined as any new infection diagnosed 48 hours or more post admission.

Results: There were 22,320 critical care patient admissions during the study period with an average age of 56.3 years and mortality rate of 12.7\%. 21,909 (98.1\%), 3041 (15.2\%) and 4791 (21.4\%) of patients were screened for MRSA, VRE and C. Diff respectively. 411 patients tested positive for MRSA (40\% female, mean 55.9 years), with $40(9.73 \%)$ unit acquired. Unit versus admission MRSA mortality rate was $12.1 \%(\mathrm{~N}=5)$ versus $13.5 \%(\mathrm{~N}=5)$ respectively $(\mathrm{p}=0.81) .632$ patients tested positive for VRE (35.1\% female, average age 55.5 years), with 90 (9.1\%) unit acquired. Unit versus admission VRE mortality rate was $39.2 \%(\mathrm{~N}=44)$ and $23.9 \%(\mathrm{~N}=126)$ respectively $(\mathrm{p}=0.008) .310$ patients tested positive for C. Diff (44.5\% female, average age 58.9 years), with 147 (47\%) unit acquired. Unit versus admission C. Diff mortality rate was $13.2 \%$ $(\mathrm{N}=20)$ and $20.7 \%(\mathrm{~N}=126)$ respectively $(\mathrm{p}=0.078)$. Mean and median LOS for admission MRSA, VRE and C. Diff were 9.5/5 days, 11.2/6 days and 10.9/6days respectively. Mean and median LOS for unit MRSA bacteraemia, VRE and C. Diff were 29.5/22 days, 43.8/25 days and 25.5/6 days respectively.

Conclusion: This study highlights that there is no significant impact on mortality rates if patients contract admission versus unit MRSA bacteraemia. Patients who contract unit VRE bacteraemia are at a significantly higher risk of mortality compared to patients infected pre admission. In contrast, patients who are infected with C. Diff pre admission are at a significantly higher risk of mortality as compared to unit patients. Despite variance in mortality, patients with unit MRSA, VRE and C. Diff had increased LOS. Sub-categorisation of C. Diff and VRE mortality risk should include admission/unit infection and differences in LOS and patient mortality rates highlight the need for heightened surveillance of VRE and C. Diff within the critical care, parallel with MRSA surveillance levels.

Reference(s) and grant acknowledgment(s)

1. 6. Khanna, S., Pardi, D. S., Aronson, S. L., Kammer, P. P., Baddour, L, M. (2012). Outcomes in Community-Acquired Clostridium Difficile Infection. Alimentary Pharmacology and Theraputics. Mar;35(5):613-8.

2. 5. Papadimitriou-Olivgeris, M.; Fligou, F.; Drougka, E.; Spiliopoulou, I.; Marangos, M.; Filos, K. S. (2013). Mortality rate of ICU patients is influenced by VRE colonization at ICU admission but not by VRE acquired infections during the ICU stay. PLOS 8(9) e75658.

3. 4. Mehta, Y., Hegde, A., Pande, R., Zirpe, K. Gupta V., Ahdal, J., Qamra, A., Motlekar, S., Jain R., (2020) Methicillin-resistant Staphylococcus aureus in Intensive Care Unit Setting of India: A Review of Clinical Burden, Patterns of Prevalence, Preventive Measures, and Future Strategies. Indian Journal of Critical Care Medicine Jan;24(1):55-62. 
4. 3. Borg MA, Kraker M, Scicluna EA, van de Sande-Bruinsma N, Tiemersma E, Monen J, et al. (2007). Prevalence of methicillin-resistant Staphylococcus aureus (MRSA) in invasive isolates from southern and eastern Mediterranean countries. Journal of Antimicrobial Chemotherapy. 60(6):1310-1315

5. 2. Dasgupta, S., Das,S., Chawan, N., Hazra, A. (2015). Nosocomial infections in the intensive care unit: Incidence, risk factors, outcome and associated pathogens in a public tertiary teaching hospital of Eastern India. Indian Journal of Critical Care Medicine. 19(1)14-20.

6. 1. Ylipalosaari, P., Ala-Kokko, T., Laurila, J., Ohtonen, P., Syriälä H. (2006) Intensive care acquired infection is an independent risk factor for hospital mortality: a prospective cohort study. Critical Care. Volume 10, R66

\section{3}

Concordance between clinical and epidemiological dignosis of tracheobronchitis and ventilator associated pneumonia in Complexo Hospitalario Universitario de Pontevedra (Spain)

J. I. Cenoz Osinaga ${ }^{1}$; J. L. Martinez Melgar ${ }^{1}$; E. Moreno Lopez ${ }^{2}$; L. Lores Aguin $^{3}$; I. Gallego Barbachano ${ }^{1}$; E. Sanmartin Mantiñan ${ }^{1}$; A. Ortega Montes ; J. V. Bravo Doviso'; A. De La Campa Gestido ; P. Posada

Gonzalez

${ }^{1}$ Intensive care unit, Complexo Hospitalario Universitario De Pontevedra, Pontevedra, Spain; ${ }^{2}$ Anesthesia and perioperative care, Complexo Hospitalario Universitario de Ferrol, Ferrol, Spain; ${ }^{3}$ Radiology department, Complexo Hospitalario Universitario De Pontevedra, Pontevedra, Spain

Correspondence: E. Moreno Lopez

Intensive Care Medicine Experimental 2020, 8(2): 000533

Introduction: Although trachebronchitis (VAT) and ventilator-associated pneumonia (VAP) are two different clinical entities, they have some factors in common in terms of clinical and microbiological diagnosis, treatment and prognosis. The result of the evaluation of patients with these type of ventilator-associated respiratory infections can be interpreted differently if clinical and epidemiological factors are considered.

Objectives: To describe the concordance between clinical and epidemiological diagnosis, and, as a secondary objective, to know the clinical an epidemiological characteristics as well as the rates of VAT and VAP.

Methods: Retrospective and descriptive study carried out between 1st May 2015 and 31st October 2017 of patients admitted to our Intensive Care Unit (ICU). Sources: ENVIN-UCI study, Department of Preventive Medicine, electronic medical record and retrospective review of imaging studies by radiology specialist. Parameters analized: age, gender, date of admission, intubation and discharge, comorbidities, microorganisms, antibiotics, associated infections and mortality. Statistical analysis: qualitative values (presented as absolute and percentages), quantitative values (presented as mean and standard deviation). Concordance between clinical diagnosis (data from ENVIN-UCI) and epidemiological diagnosis (data from Department of Preventive Medicine) was measured using kappa coefficient.

Results: 290 patients needed mechanical ventilation (MV) with 4523 stays (patients $x$ mean days under MV). VAP rate: 3,98 and VAT rate: 10,39. ICU infections: 65 (18 VAP, 47 VAT) and according to Preventive Medicine: 43 (19 VAP and 24 VAT). In imaging studies reviewed by radiology specialist, coincidence with ICU diagnosis was found in $70 \%$ of the infections and in $60 \%$ of diagnosis made by Preventive Medicine. The concordance observed between clinical and epidemiological diagnosis in VAT and VAP according to Landis and Koch was: moderate in VAP (kappa value: 0,56; Cl 95\%: 0.35-0,78) and fair in VAT (kappa value: 0,$32 ; \mathrm{Cl} 95 \%: 0,13-0,51)$. In the bivariate analysis, relationship was only found between concordance and length of stay.

Conclusion: Moderate agreement was observed in VAP and fair in VAT. None of the parameters studied were associated with no concordance, except for the length of stay
000546

Infectious complications in ICU patients with SARS COV 2 respiratory infection

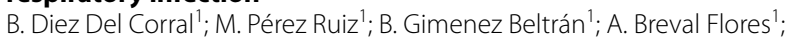

S. Moreno Cano'; A. Estella

${ }^{1}$ University Hospital of Jerez., Intensive care unit, Jerez, Spain

Correspondence: $\mathrm{B}$. Diez Del Corral

Intensive Care Medicine Experimental 2020, 8(2): 000546

Introduction: Many critically ill patients infected with SARS COV 2 have worse clinical course or die in ICU due to secondary infections.

Objectives: The aim of the present study is to describe the infectious complications in patients admitted in ICU with confirmed respiratory infection by Sars-Cov-2, and to assess factors related with these complications

Methods: Retrospective case series of consecutive critically ill patients admitted in ICU of a community hospital with severe pneumonia caused by SARS COV 2. Microbiological analysis of bronchoscopic bronchoalveolar lavage (BAL), urine antigen, PCR for Influenza and blood cultures were performed at ICU admission. We categorized infections as coinfections, within the first 72 hours in ICU, and those with a later onset. We analyzed if the use of corticoid therapy was related with developing infectious complications. Medical history, microbiological cultures, ICU length of stay and mortality were collected. Statistical analysis: Data was analyzed by SPSS 18 and quantitative variables were expressed as a mean \pm standard deviation.

Results: Twenty patients were included. Mean age was $61,7 \pm 8$ years old. $80 \%$ required mechanical ventilation. Mortality was $30 \%$. Positive PCR SARS COV 2 resulted in $100 \%$ of BAL samples. Pneumococcal urine antigen test and Influenza resulted negative in all cases.

ICU length of stay was significatively longer in patients who developed bacterial infections; $20,11 \pm 11.4$ days vs $10.5 \pm 7.29$ days in the noninfected group $(p<0,05)$. Regarding fungal infections, ICU length of stay was longer in infected $23.5 \pm 10.36$ days vs $11.27 \pm 8.3(p<0,05)$. In seven patients, 35\%, BAL performed at ICU admission resulted positive: 2 patients with Staphylococcus, 3 with Candida spp. and 2 cases with bacterial/fungal co-infection. Candida isolation was associated with previous corticosteroid treatment.

During the ICU stay, 56,25\%, developed ventilator associated pneumonia caused by Candida spp, Pseudomona aeruginosa, Klebsiella aerogenes. 30\% developed urinary tract infection, half of these cases due to Candida spp. In $30 \%$ it was described as bacteremia and $10 \%$ presented catheter-related bloodstream infection.

\begin{tabular}{|l|l|l|}
\hline & $\begin{array}{l}\text { Previous } \\
\text { corticosteroid }\end{array}$ & $\begin{array}{l}\text { No } \\
\text { corticosteroid }\end{array}$ \\
\hline Fungal infection & n: 10 & n:10 \\
\hline Fungal + Bacterial infection & 5 & 1 \\
\hline Bacterial infection & 1 & 3 \\
\hline No infection & 3 & 2 \\
\hline
\end{tabular}

Dividing the described infections by previous corticosteroids use: The most frequent isolation were Candida spp 10 (C albicans $7+C$ parapsilosis $2+C$ guillermondii 1), Pseudomona aeruginosa 7, Enterococcus faecalis 5, Klebsiella spp 4 and Staphylococcus aureus 4.

Conclusion: Fungal infections were frequent in critically ill patients withs SARS COV2 infection, probably related with corticosteroid therapy.

ICU length of stay was associated with more incidence of infections. Gram positive predominate first days of ICU admission and gram-negative infections in the later phase of ICU stay.

Health care associated infections were more frequent than co-infection.

Reference(s) and grant acknowledgment(s)

No 


\section{7}

Using VAC-therapy in skin plastic in patients with necrotic form of erysipelas

J. Fuss'; A. Voloboyeva²

${ }^{1}$ Regional hospital of Pustomyty, Lviv, Ukraine; ${ }^{2} \mathrm{Cu}$, Regional hospital of Pustomyty, Lviv, Ukraine

Correspondence: J. Fuss

Intensive Care Medicine Experimental 2020, 8(2): 000547

Introduction: Extensive purulent-necrotic wounds are one of the urgent and unresolved problems in purulent surgery [1,2,3]. However along with this, adequate surgical treatment often leads to the formation of extensive post-necrectomy wounds, the independent healing of which is impossible due to their significant size $[4,5]$. One of the effective treatment methods in this category of patients is the use of local vacuum aspiration [6,7].Extensive purulent-necrotic wounds are one of the urgent and unresolved problems in purulent surgery $[1,2,3]$. However, along with this, adequate surgical treatment often leads to the formation of extensive post-necrectomy wounds, the independent healing of which is impossible due to their significant size [4,5]. One of the effective treatment methods in this category of patients is the use of local vacuum aspiration $[6,7]$.

Objectives: Improve the treatment results of patients with extensive purulent-necrotic wounds caused by erysipelas.

Methods: In this work, we present data on the results of treatment of 89 patients with necrotic complications of erysipelas with the use of vacuum therapy in combination with autodermoplasty. The defect sizes varied from 20 to $152 \mathrm{~cm} 2$. The effectiveness of vacuum therapy was evaluated based on the dynamics of clinical manifestations, determining the degree of microbial contamination in the wound, analysis of the cellular composition of wound prints, phagocytic number, phagocytic activity of neutrophils, determination of the index of completion of phagocytosis.

Results: Patient wound cleansing during preparation of a wound for transplantation in the control and main groups occurred after $5.1 \pm$ 0.5 days from the start of treatment, the appearance of granulation tissue after $8.1 \pm 0.6$ days, and granulation tissue covered the entire surface of the wound after $11.0 \pm 0.4$ days. Upon admission to the clinic, patients underwent surgical treatment, consisting in opening a purulent focus, necrectomy. Purulent discharge was observed from the wound, there was redness of the skin around the wound, edema, local temperature increase, fibrinous-purulent plaque and pain on palpation of the wound edges. After installing a vacuum aspirator, the general condition and general well-being of patients improved markedly, by the 5th day, wound cleansing and the appearance of granulation were noted. In parallel with cleansing the wound surface from necrosis and filling them with granulation tissue, local regional signs of inflammation disappeared. The degree of microbial contamination ranged from $1.9 \pm 0.7 \times 104$ to $2.6 \pm 0.5 \times 104 / \mathrm{g}$ of tissue

It should be noted that patients of both groups needed autodermoplasty to close skin defects.

Skin transplantation was performed in the second phase of the wound process with bacterial contamination of the wound less than 102 CFU per $1 \mathrm{~g}$ of tissue and upon receipt of a regenerative type of wound exudate cytograms.

The use of a vacuum apparatus contributed to the metered pressing of the skin flap to the wound surface, reliable fixation of the skin flap and, to a certain extent, improved local microcirculation. Carried out autodermoplasty was effective in all cases. When using vacuum in the postoperative period in the main group, the engraftment of the skin flap accounted for $82 \%$ of the wound surface, and in patients of the control group, without vacuum therapy in the postoperative period, the engraftment amounted to $75 \%$ of the surface.

Conclusion: The use of vacuum therapy for 4-5 days in a continuous aspiration mode $(-125 \mathrm{~mm} \mathrm{Hg})$ helps to purify a purulent wound, shortens the first phase of the wound process and potentiates the filling of the wound surface with mature granulation tissue $(p<0.005)$. It is advisable to use an isolated vacuum in skin plasty in clinical practice in patients with purulent-necrotic wounds and with a necrotic form of erysipelas to heal extensive skin defects.

\section{Reference(s) and grant acknowledgment(s)}

1. 1. Palm HG, Hauer T, Simon C, Willy C. [Vacuum-assisted closure of head and neck wounds]. HNO. 2011;59(8):819-30. 2. Baisch A, Hörmann K, Goessler UR, Sauter A, Riedel F. [Vacuum-assisted closure of nonhealing wounds in head and neck reconstructive surgery]. HNO. 2007;55(5):392-8. 3. Hsia JC, Moe KS. Vacuum-assisted closure therapy for reconstruction of soft-tissue forehead defects. Arch Facial Plas Surg. 2011;13(4):278-82. 4. Powers AK, Neal MT, Argenta LC, Wilson JA, DeFranzo AJ, Tatter SB. Vacuum-assisted closure for complex cranial wounds involving the loss of dura mater. J Neurosurg. 2013;118(2):302-8. 5. Bovill E, Banwell PE, Teot L, Eriksson E, Song C, Mahoney J, et al. Topical negative pressure wound therapy: a review of its role and guidelines for its use in the management of acute wounds. Int Wound J. 2008;5(4):511-29. 6. Kairinos N, Hudson D, Solomons M. Depth of penetration of negative pressure wound therapy into underlying tissues. Wound repair and regeneration : official publication of the Wound Healing Society [and] the European Tissue Repair Society. 2009;17(3):456. 7. Kairinos N, Solomons M, Hudson DA. Negative-pressure wound therapy I: the paradox of negative-pressure wound therapy. Plast Reconstr Surg. 2009;123(2):589-98; discussion 99-600.

2. No acknowledgment.

\section{9}

Using ointments Argosulfan in treatment of purulent wounds

J. Fuss ${ }^{1}$; A. Voloboyeva ${ }^{2}$; V. Polovyj ${ }^{3}$

${ }^{1}$ Regional hospital of Pustomyty, Lviv, Ukraine; ${ }^{2} \mathrm{cu}$, Regional hospital of Pustomyty, Lviv, Ukraine; ${ }^{3}$ General surgery, Bukovinian State Medical University, Chernivtsi, Ukraine

Correspondence: J. Fuss

Intensive Care Medicine Experimental 2020, 8(2): 000549

Introduction: In the structure of surgical pathology, the leading place is occupied by purulent-inflammatory diseases of soft tissues [1] which are associated with the infectious process in the skin and cellular spaces caused by pathogenic or opportunistic flora [2]. Patients with this pathology make up $20-45 \%$ of all surgical patients $[3,4]$. The rapid development of the resistance of microorganisms to the antibacterial drugs used requires the selection of the most effective method for the local treatment of wound infections. To solve this problem, antibacterial preparations containing silver are actively used in modern practice $[5,6]$.

Objectives: Evaluation of the effectiveness of the treatment of purulent wounds with Argosulfan ointment in a surgical hospital.

Methods: 264 patients who were admitted to the hospital in 20182020 with purulent wounds of various etiologies were treated. All patients were divided into 2 groups: group I $(n=156)$ included patients in whom, in the postoperative period, ointment dressings with silver sulfathiazole were used for local treatment of wounds group II $(n=108)$ included patients who in the postoperative period for topical wound treatment used ointment dressings with antibacterial ointments - bactroban.

Upon admission to everyone, a wound was opened and a purulent cavity was drained, or primary surgical treatment of an infected wound in the first day after admission. Wounds were washed with an antiseptic solution, an aseptic dressing was applied. Patients in both study groups received the same broad-spectrum antibacterial therapy. Local treatment of purulent wounds began the next day after surgery. The following indicators were studied in dynamics: the nature of the wound discharge, the presence of edema and hyperemia of the tissues around the wound, the timing of the cleansing of the wound defect, the results of microflora culture and sensitivity to antibacterial therapy on the 1st, 5th, 10th day after the operation.

Results: Absence of hyperemia of the skin around the wound and soft tissue edema in group I averaged $4.3 \pm 1.7$ days, in group II $-5.5 \pm$ 2.5 days $(\mathrm{t}=0.379 ; \mathrm{p}<0.05)$.

Sterile inoculation from the wound on the 5 th day was observed in $42.3 \%$ of patients in group I, and only $5.9 \%$ in group II. Statistically significant differences between the groups were obtained: $x 2=33.68 ; p$ $<0.05$. 
In group I, a decrease in bacterial contamination by $50 \%$ was noted in $57.3 \%$ of patients, in group II - in $47.4 \%$. There were no statistically significant differences between the groups: $x 2=2.47 ; p<0.05$.

In group I, sterile discharge on the 10th day was observed in $100 \%$ of patients, in group II - only in $88.5 \%$. Statistically significant differences between the groups were found: $x 2=12.2 ; p<0.05$.

In group I, wound cleansing from pus and fibrin and the appearance of granulations on the 5 th day were noted in $87.8 \%$ of patients, in group II - only in $35.9 \%$. Statistically significant differences between the groups were revealed: $x 2=57.07 ; p<0.05$.

In group I, wound cleansing from pus and fibrin and the appearance of granulations on the 10th day were noted in $100 \%$ of patients, in group II - only in $88.5 \%$. Statistically significant differences between the groups were obtained: $\mathrm{X} 2=12.2 ; \mathrm{p}<0.05$.

Conclusion: Use of Argosulfan ointment can improve the results of treatment of patients with purulent pathology of soft tissues and reduce the risk of secondary infection in comparison with the use of traditional antibacterial ointments - by reducing bacterial contamination, faster cleaning of the postoperative wound from pus and fibrin.

\section{Reference(s) and grant acknowledgment(s)}

1. 1. Zieliński M, Kucharzewski M, Szewczyk MT et al. Opatrunki lipidokoloidowe- nowatorska koncepcja leczenia szerokiego spektrum ran ostrych i przewlekłych. Leczenie Ran 2016;13(3):77-83. 2. Sopata M, Tomaszewska E, Kotlińska-Lemieszek A. Nowoczesne zasady zachowawczego leczenia odleżyn. Leczenie Ran 2012;9(3):93-97. 3. Stożkowska W, WroczyńskaPałka M. Badania przeciwwirusowej aktywności soli srebrowej sulfatiazolu. Med Dośw 1999;51(1-2):167-174. 4. Stożkowska W, Grubska-Suchanek E. Ocena właściwości alergizujących preparatów z solą srebrową sulfatiazolu (krem, areozol). Prz Dermatol 1988;75(4):320-322. 5. Harding K, Gottrup F, Jawień A et al. A prospective, multi-centre, randomised, open label, parallel, comparative study to evaluate effects of Aquace ${ }^{\circledR} \mathrm{Ag}$ and

Urgotul $^{\circledR}$ Silver dressing on healing of chronic venous leg ulcers. Int Wound J 2012;9(3):285-294. 6. Leaper D, Münter C, Meaume S et al. The use of

Biatain ${ }^{\circledR} \mathrm{Ag}$ in hard-to-heal venous leg ulcers: meta-analysis of randomised controlled trials. PLoS One 2013:8(7):e67083.

2. No acknowledgment.

\section{0}

A Case series on Macrophage activation syndrome

with infections. More common than we think??

J. Nikhilesh'; V. Bohara ${ }^{2}$; A. Gupta ${ }^{3} ;$ V. Joshi ${ }^{4}$

${ }^{1}$ Dept of critical care medicine, CHL Hospital, Indore, India; ${ }^{2}$ Dept of hematology, CHL CBCC hospital, Indore, Madhya Pradesh, India, India; ${ }^{3}$ Dept of internal medicine, CHL hospital, Indore, Madhya Pradesh, India, India; ${ }^{4}$ Dept of critical care services, Shalby Hospitals, Indore, Madhya Pradesh, India, India

\section{Correspondence: J. Nikhilesh}

Intensive Care Medicine Experimental 2020, 8(2): 000560

Introduction: Association of Macrophage activation syndrome (MAS) and infections has been documented earlier in literature however, there have been case reports and isolated cases and we did not come across a big series on above in association with infections. This communication delineates a case cohort of macrophage activation syndrome secondary to infectious diseases.

Objectives: To study the epidemiology and pattern of common variables in a cohort of Macrophage activation syndrome associated with an infectious etiology..

Methods: Setting: Sixty bedded multidisciplinary ICUs of tertiary care units.

Methodology: Consecutive patients suspected of macrophage activation syndrome fulfilling diagnostic criteria as per HLH 2004 trial were included for a duration dated first January 2017-till date with an infectious disease etiology were included. They were evaluated with a focus on demographics, SOFA scores, ALT levels, AST levels, Hemoglobin, platelet counts, serum creatinine levels, bone marrow findings, incidence of AKI, LDH levels, splenomegaly, length of stay and mortality. Death/discharge from ICU were considered as end points. Statistical analysis was done using SPSS version 23.
Results: Total of thirty-seven patients with high grade fever were included ( $n=37, M$ : F-24:13) with age distribution of $44.8 \pm 14.3$ years (Range-21-70). SOFA scores were 14.8 \pm 2.2 (Range-10-18) and length of stay (LOS) was $10.4 \pm 3.31$ days (Range-5-17). Hemoglobin levels were $11.7 \pm 2.8$ (Range 7.9-17.5) $\mathrm{gm} / \mathrm{dl}$ and platelet counts were $60324.32 \pm 25075.50$ (Range12,000 -90,000). AST and ALT levels were $164 \pm 54$ (Range-61-263) and $164.9 \pm 48.7$ (Range-82-247) respectively [Ref <30]. Serum triglycerides, Serum ferritin levels, D-Dimer and LDH levels were $326 \pm 34.6 \mathrm{mg} / \mathrm{dl}$ (Range-290-410), 1530.4 \pm 909.7 (Range $740-4500) \mathrm{ngm} / \mathrm{ml}, 4000 \pm 1640 \mathrm{ng} / \mathrm{ml}$ (Range 3260-5900) and 295 \pm 15.7 (Range- 190-384) units/I respectively. The incidence of AKI for the entire cohort was $n=17(45.9 \%)$ with serum creatinine levels $1.44 \pm 1.08$ (Range-0.5-5.8) mg/dl. Bone marrow was consistent with hemophagocytosis in $n=22(59.5 \%)$. In hospital mortality was $n=6$ (16.2\%) and splenomegaly was noted in $n=19(51.4 \%)$. The etiology included Dengue $n=7$ (18.9\%), Typhoid $n=10(27 \%)$, HIV $n=4(14.8 \%)$, Malaria $n=5(18.5 \%)$, COVID $19 n=3(8.1 \%)$ and gram-negative infections $n=8(29 \%)$. On statistical analysis only Platelet levels and Ferritin levels had an association with mortality ( $p$ value $0.0003 \mathrm{~S}$ and 0.00001 S).

Conclusion: MAS remains a suspect cause in any new onset fever in ICU. Its associations with infectious diseases and ability to alter outcomes should prompt an early recognition for the same. This communication documents a bad prognosis with thrombocytopenia and elevated ferritin levels in these cohorts.

Reference(s) and grant acknowledgment(s)

1. 1. Br J Haematol 2004;124:4-14

2. 2. Crit Rev Oncol Hematol $2004 ; 50: 157-174$

000561

Can donor multidrug-resistant bacteria cause infection in organ recipients?

AM. Gómez del pulgar villanueva'; M. Royo-Villanova Reparaz';

RMD. Victoria'; AB. Pérez Pérez'; CM. Carrillo'; A. López Domínguez';

R. Jara Rubio ${ }^{1}$; SJ. Moya ${ }^{1}$; C. Manso Murcia'; LM. Mateos ${ }^{1}$; E. Andreu Soler ${ }^{1}$; A. Del Rey Carrión'; D. Pérez Fernández'; M. Valer Rupérez

${ }^{1}$ Intensive care unit, Hospital clinico universitario Virgen de la Arrixaca, Murcia, Spain; ${ }^{2}$ ntensive care unit, Hospital Clínico Universitario Virgen de la Arrixaca, Murcia, Spain

Correspondence: A.M. Gómez del pulgar villanueva

Intensive Care Medicine Experimental 2020, 8(2): 000561

Introduction: Colonizations and infections by multi-drug-resistant (MDR) bacteria are a common occurrence in patients treated in the Intensive Care Units. Most donors have previously been treated in these intensive care units and may therefore have colonizations or infections from these pathogens. In this study, we analyze whether the organ recipients of donors who were colonized or infected with an MDR bacteria develop an infection with the treatment challenges that this would entail.

Methods: Consecutive deceased organs donors in a critical care unit from a tertiary hospital with high donation activity were registered for 72 months (January-14 to December-19). We included in the study organ donors with MDR bacteria isolates and traced the transplanted organs and the MDR isolates in their corresponding recipients. Methods consisted in an active detection of any colonization by MDR bacteria through screening with swabs twice a week. As for the diagnosis of infection is made according to clinical, analytical and imaging criteria (as in the case of pneumonia) . For each case of nosocomial infection, further variables were documented, such as confirmed pathogens, date of infection, and temporal association of the infection with devices (tracheal tube, central venous catheter, urinary catheter).

Results: From 385 deceased organ donors, we detected 31 patients colonized or infected by MDR bacteria. In average, they were 67 years old with an ICU stay of 9 days. From these 31 organ donors, we followed the 59 patients who received a graft from a donor with a positive culture for MDR bacteria. Transmission of the MDR pathogen was detected in two receptors: a Methicillin- resistant Staphylococcus aureus (MRSA)bacteremia whose donor was treated 36 hours before 
the donation and a urinary tract infection for Klebsiella. pneumoniae BLEE from an untreated donor due to the fact that isolates identification results were obtained after donor death and transplantation. Conclusion: The transmission of MDR bacteria from organ donors to organ recipients is a rare occurrence. In our series, the use of organs from MDR bacteria-colonized donors is safe for recipients of these organs in terms of MDR pathogen transmission.

\section{6}

\section{Descriptive analysis of COVID 19 patients in the ICU}

during the pandemic

A. Ruiz García'; C. Serrano². A. Acha². R. Araoz Illanes². M. Trascasa Muñoz ${ }^{2}$. J. Higuera Lucas ${ }^{1}$; B. Llorente Ruiz'; E. Serrano Yebenes ${ }^{3}$; F. Poveda Saenz ${ }^{4}$; C. Demartini Foj ${ }^{4}$; E. Lopez Ramos ${ }^{2}$; L. Alcazar Sanchez-Elvira²; E. Nevado² ${ }^{1}$ Medicina intensiva, Hospital Universitario Príncipe de Asturias, Alcala de Henares, Spain; ${ }^{2}$ Intensive care unit, Hospital Príncipe de Asturias, Alcalá de Henares, Spain; ${ }^{3}$ General surgery, Hospital Príncipe de Asturias, Alcalá de Henares, Spain; ${ }^{4}$ Anesthesiology, Hospital Príncipe de Asturias, Alcalá de Henares, Spain

Correspondence: A. Ruiz García

Intensive Care Medicine Experimental 2020, 8(2): 000566

Introduction: COVID 19 pneumonia is exceeding the intensive care units capacity worldwide. It is important to analyse the results to tackle the disease successfully.

Objectives: To analyse the characteristics of the patients admitted to the ICU during the COVID 19 pandemic.

Methods: Retrospective observational study conducted in an intensive care unit of a university Hospital. During the pandemic, ICU beds went from 14 to 39 . We analysed patients admitted to the ICU with the diagnosis of respiratory failure due to COVID 19 pneumonia between $6 / 3$ and 21/4 2020. Patients who are still in the ICU at the time of data collection were excluded. Demographic data (age, sex), APACHE II, comorbidities (hypertension, previous pulmonary pathology, dyslipidaemia, diabetes mellitus, obesity), laboratory data (creatinine, ferritin, total bilirubin), length of stay and treatments administered (mechanical ventilation, muscular blockers, vasoactive drugs, prone positioning) were collected.

Results: 90 patients were analysed. Male 67.8\% (61/90). Average age $60.6 \pm 9.6$ years. APACHE II $14.3 \pm 5.1 .48 .9 \%$ (44/90) had cardiovascular history of which AHT $46.6 \%$ (42/90). Chronic lung disease $20 \%$ (18/90) (asthma 5.5\% (5), OSAHS 5.5\% (5), COPD 4.4\% (4), OSAHS + asthma 3.3\% (3)). 65.5\% (59/90) had other comorbidities (Diabetes mellitus $23.3 \%$ (21), dyslipidaemia $35.5 \%$ (32). $26.6 \%$ (24/90) had no previous pathology. Length of stay (days): $12 \pm 10.1$. The average of the maximum creatinine, ferritin and total bilirubin values were: creatinine $1.45 \pm 1.23 \mathrm{mg} / \mathrm{dl}$, ferritin $2244 \pm 3193 \mathrm{ng} / \mathrm{ml}$ and total bilirubin $1.8 \pm 2 \mathrm{mg} / \mathrm{dl}$. $100 \%$ of the patients required mechanical ventilation. Prone positioning was performed in $57.8 \%$ (52/90), infusion of muscular blocker $77.7 \%$ (70/90) and vasoactive drugs $96.7 \%$ $(87 / 90)$. Microbiological isolation was observed in $45.6 \%$ (41/90) of the patients. 40\% (36/90) were discharged and $25.1 \%(23 / 90)$ were transferred to another centre due to lack of beds. Mortality rate was $34.4 \%$ (31/90).

Conclusion: As in other series, males predominate in the group of critically ill patients. Among the most frequent comorbidities correlated to COVID 19 pneumonia, we find arterial hypertension, dyslipidaemia and diabetes mellitus. Mortality is high despite the low average age and the absence of severe comorbidities. $25 \%$ of hospitalized patients were transferred so their evolution is unknown, mortality and length of stay may be underestimated.
000580

Characterizing secondary infections in COVID 19 critically ill patients

C. Serrano ; A. Ruiz García ; A. Acha ${ }^{1}$; R. Araoz Illanes ${ }^{1}$; M. Trascasa Muñoz'; Y. Ortiz De Zarate'; J. Higuera Lucas'; B. Llorente Ruiz' ; F. Poveda Saenz²;

C. Demartini Foj ${ }^{2}$; E. Serrano Yebenes ${ }^{3}$; E. Nevado' ${ }^{1}$

${ }^{1}$ Intensive care unit, Hospital Príncipe de Asturias, Alcalá de Henares, Spain

${ }^{2}$ Anesthesiology, Hospital Príncipe de Asturias, Alcalá de Henares, Spain ${ }^{3}$ General surgery, Hospital Príncipe de Asturias, Alcalá de Henares, Spain

Correspondence: $C$. Serrano

Intensive Care Medicine Experimental 2020, 8(2): 000580

Introduction: Bacterial or fungal coinfection and secondary infections have an important role in comorbidity and mortality in COVID 19 critical patients. The typical COVID-19 clinical manifestations and laboratory findings make it quite challenging for the clinician to suspect and actively look for a different microbiological agent.

Objectives: To describe and analyse the microbiological isolations in the COVID-19 patients treated in our ICU (Príncipe de Asturias hospital).

Methods: A retrospective observational study was performed. All the critically ill COVID-19 positive patients who received treatment in our ICU (a polivalent, level II hospital ICU) were reviewed (from march 6, 2020 to april 21, 2020). Demographical, main laboratory findings, type of microbiological samples and isolated agents were collected. Chisquare tests, T Student or ANOVA were used. Signification level was stablished as $\mathrm{p}<0.05$.

Results: A total of 67 patients were enrolled in our study. Microbiological isolations were positive in $45.6 \%$ of patients, all of them were secondary infections. In 18 patients $>1$ isolation was found. The microorganisms isolated were: S. Aureus 11 cases, Coagulase-Negative Staphylococcus 12 (8 Epidermidis, 1 Hominis), E. Faecium(8), E. Faecalis(7), Pseudomonas(9), E. coli(4), Klebsiella(2), Serratia(3), Haemophillus (1). Strenotrophomonas (2). Acinetobacter(1), Corynebacterium(1). Candida was the fungus most frequently isolated (12); it is remarkable that it was isolated in endotraqueal aspirates (ETA) in 6 cases. There was one case of aspergillosis. The most frequently positive sample was ETA (28); followed by blood cultures (19). The most frequently isolated agent in ETA was Pseudomonas (10) and S. Aureus (10), 2 were SAMR. In blood cultures, the most frequently isolated agents were $S$. Epidermidis, E. Faecalis and E. Faecium ( 6 each), followed by S. Aureus (4). We found 5 catheter related bacteriemias (S. Epidermidis 3, SAMR 1, Pseudomonas 1) and 7 bacteriemic pneumonia (SAMS 3, Pseudomonas 2, E. Coli 1 and SAMR 1).

We didn't find statistically significant the assotiation between microbiological isolation and any of the patients' underlying diseases $(p=0.528)$, nor with vasoactive support $(p=0.43)$, nor with need of neuromuscular blockers $(p=0.25)$, nor with mortality $(p=0.5)$.

Need for prone position, traqueostomy and media hospital stay were associated with microbiological isolations with statistical significance ( $p$ value in table).

Comparative analysis between positive microbiological isolations' group and negative is shown in the table below. 


\begin{tabular}{|c|c|c|c|}
\hline \multicolumn{4}{|c|}{ Microbiological isolation } \\
\hline & Yes & No & $\mathbf{P}$ \\
\hline Sex (masculine) & $55 \%$ & $59 \%$ & 0,49 \\
\hline APACHE II & 14 & $\begin{array}{l}14.6 \\
5\end{array}$ & 0.73 \\
\hline Ferritine (ng/ml) & $\begin{array}{l}218 \\
4\end{array}$ & 2479 & 0.7 \\
\hline Creatinine (mg/dl) & 1.6 & 1.3 & 0.29 \\
\hline Bilirrubin (mg/dl) & 2.1 & 1.9 & 0.7 \\
\hline Prone & $65 \%$ & $39 \%$ & $\begin{array}{l}0.03 \\
3\end{array}$ \\
\hline Traqueostomy & $79 \%$ & $37 \%$ & $\begin{array}{l}0.00 \\
1\end{array}$ \\
\hline \multicolumn{4}{|c|}{ Microbiological isolation } \\
\hline & Yes & No & $\mathbf{P}$ \\
\hline Mortality rate & $55 \%$ & $58 \%$ & 0.5 \\
\hline Leght of stay (days) & 18.6 & 8.3 & $\begin{array}{l}0.00 \\
5\end{array}$ \\
\hline
\end{tabular}

Conclusion: Secondary infections in COVID-19 patients are a frequent finding in our group (48\%), being more frequent than in other published series of cases. In our study microbiological isolations were associated to longer hospital stay, prone position and traqueostomy with with statistical significance.

\section{6}

\section{Design of a study to evaluate the safety and efficacy} of convalescent plasma to treat COVID-19 in critically ill patients

R. Ferrer Roca”; P. Llamas²; R. Mañez ; C. Galbán ${ }^{4} ;$ M. Quintana ;

M. Sanchez-García ${ }^{6}$; M. Torres ${ }^{7}$; F. Mota ${ }^{8}$; N. Campins ; E. Mondou 9

${ }^{1}$ Intensive care department / sodir research group, Vall d'Hebron University Hospital, Barcelona, Spain; ${ }^{2}$ Department of haematology, Hospital Universitario Fundación Jiménez Díaz, Madrid, Spain; ${ }^{3}$ Intensive care department, Hospital Universitari de Bellvitge, L'Hospitalet de Llobregat, Spain; ${ }^{4}$ Intensive care division, Complejo Hospitalario Universitario de Santiago, Santiago de Compostela, Spain; ${ }^{5}$ Intensive care department, Hospital Universitario La Paz, Madrid, Spain; ${ }^{6}$ Intensive care department, Hospital Clinico Universitario San Carlos, Madrid, Spain; ${ }^{7}$ Bioscience research group, Grifols, Barcelona, Spain; ${ }^{8}$ Scientific and medical affairs group, Grifols, Barcelona, Spain; ${ }^{9}$ Bioscience research group, Grifols, NC, United States of America

Correspondence: E. Rosado

Intensive Care Medicine Experimental 2020, 8(2): 000586

Introduction: Coronavirus disease 2019 (COVID-19) is a respiratory infection caused by a newly emergent coronavirus, SARS-CoV-2, that was first recognized in Wuhan, China, in December 2019. There is currently no approved treatment for COVID-19. Convalescent plasma has been used as a treatment in other recent epidemics, including SARSCoV-1, Ebola, and MERS.

Objectives: We have designed a study with the main objectives to evaluate the safety and efficacy of the administration of anti-SARSCoV-2 plasma from recovered COVID-19 donors to critically ill COVID19 patients.

Methods: The study design is a prospective, multi-center, randomized, open-label trial comparing convalescent, ABO-compatible, anti-SARS-CoV-2 methylene blue-treated (MBT) plasma plus standard medical treatment (SMT) to SMT alone in a 2:1 ratio. Eligible patients are those hospitalized for COVID-19 (tested positive for SARS-CoV-2) and admitted to the ICU for respiratory support due to severe pneumonia (lung infiltrates). Patients will be eligible for inclusion within 48 hours of ICU admission. Exclusion criteria are prior anaphylactic reactions to blood products or methylene blue, severe acute renal or heart failure, and refractory shock. The study has been approved by the applicable regulatory authorities (https://clinicaltrials.gov/ct2/show/ NCT04280705).

Results: Enrollment target is approximately 100 symptomatic COVID19 adults. Interim analyses will be performed every 30 patients. Study variables will be collected daily during hospitalization from day 2 through $29 \pm 1$. All patients (independent of hospitalization status) will be monitored daily until day 10 . Study visits for discharged patients have been scheduled on days $15 \pm 1$ and $29 \pm 1$. The primary assessment is all-cause mortality through day 29. Secondary assessments are: change in National Early Warning Score [NEWS] from baseline, time to clinical response [NEWS $\leq 2$ for $24 \mathrm{~h}$ ], time to ICU discharge and hospital discharge, duration of mechanical ventilation and oxygen therapy and absolute value and change from baseline in the Ordinal Scale. Change from baseline in quantitative SARS-CoV-2 viral load and in quantitative anti- SARS-CoV-2 lgM and lgG antibodies will be measured. In addition, patients will be monitored for treatment-emergent adverse events for the entire study period.

Conclusion: The contribution of the study results to COVID-19 therapy would be the validation of the efficacy and safety of convalescent antiSARS-CoV-2 MBT plasma in critically ill patients.

\section{Reference(s) and grant acknowledgment(s)}

1. The study is funded by Grifols

\section{9}

Retrospective comparative study with historical control between 2009 pandemic influenza H1N1 viral infections, 2010-2011 influenza season and SARS COV 2 infection in an ICU of a community hospital

A. Estella'; JM. Martín Cano ${ }^{1}$; A. Garrino. ; J. Flores' ${ }^{1}$; R. Bohollo De

Austria' ; F. Carrizosa'; MI. Monge García'; I. Blanco Saez' ; R. Monterroso.'; M. Gracia Romero'; V. Perez Madueño ${ }^{1}$; M. Jaen ${ }^{1}$; J. Villegas' ${ }^{1}$; CA. Gill';

JM. Sampedro'; MD. Sandar Nuñez ; F. Valenzuela Sanchez ${ }^{1}$

${ }^{1}$ University hospital of jerez., Intensive care unit, Jerez, Spain

Correspondence: A. Estella

Intensive Care Medicine Experimental 2020, 8(2): 000609

Introduction: The current SARS COV 2 pandemic has led to a global crisis in the health system. In ICUs we have the recent past of a decade ago with the H1N1v Influenza pandemic, since this time annually admissions in ICU of several cases of Influenza A infections have been described.

Objectives: The aim of the study were to describe clinical features of patients admitted in ICU with SARS COV 2 infections occurred during this epidemic time and to compare clinical characteristics and outcome with severe 2009 pandemic Influenza A (H1N1)v infections and 2010-2011 Influenza season infections.

Methods: Comparative study that included a prospective cohort of patients with SARS COV 2 severe infections and an historical group of patients with 2009 pandemic and 2010-2011 seasonal Influenza A H1N1v pneumonia. Setting: ICU of a community hospital. We included patients with confirmed SARS COV 2 respiratory infection. Variables analyzed were clinical characteristics, APACHE II at admission, respiratory support requirements, ICU lenght of stay, radiological pattern, serum reactive $C$ protein (PCR) and procalcitonin (PCT) levels, leukocite count (WBC) and mortality. Statistical analysis: Data were analyzed by SPSS 18 and quantitative variables were expressed as a mean \pm standard deviation.

Results: Forty five patients were included, 18 with pandemic 2009 Influenza A H1N1v pneumonia, 7 with Seasonal Influenza A 201011 pneumonia and twenty consecutive patients with SARS COV2 pneumonia. At present, 14th may 2020, three patients are currently admitted to ICU. Comparative table shows clinical characteristics and outcome. 


\begin{tabular}{|c|c|c|c|}
\hline & $\begin{array}{l}\text { Pandemic } 2009 \text { Influenza A } \\
\text { n:18 }\end{array}$ & $\begin{array}{l}\text { Seasonal } \\
\text { Influenza A } \\
\text { 2010-11 n:7 }\end{array}$ & $\begin{array}{c}\text { SARS COV } 2 \\
\mathrm{n}: 20\end{array}$ \\
\hline Age & $42,6 \pm 12,7$ & $38,4 \pm 19,3$ & $61,7 \pm 8,2$ \\
\hline $\begin{array}{l}\text { Apache II at } \\
\text { admission }\end{array}$ & $12,8 \pm 6,5$ & $10 \pm 2,9$ & $10,30 \pm 4,2$ \\
\hline $\begin{array}{l}\text { Mechanical } \\
\text { ventilation (\%) }\end{array}$ & $66,7 \%$ & $57 \%$ & $80 \%$ \\
\hline $\begin{array}{l}\text { Time of } \\
\text { mechanical } \\
\text { ventilation } \\
\text { (days) }\end{array}$ & $8,7 \pm 8,8$ & $15,2 \pm 9,8$ & $12,25 \pm 10,3$ \\
\hline $\begin{array}{l}\text { ICU lenght of } \\
\text { stay (days) }\end{array}$ & $13,9 \pm 11$ & $11,9 \pm 10,2$ & $16,13 \pm 10,7$ \\
\hline $\begin{array}{l}\text { Prone } \\
\text { ventilation } \\
\text { (MV patients) }\end{array}$ & $54,54 \%$ & $25 \%$ & $93,75 \%$ \\
\hline $\begin{array}{l}\text { Non invasive } \\
\text { ventilation }\end{array}$ & $27,8 \%$ & $57,1 \%$ & $5 \%$ \\
\hline $\begin{array}{l}\text { High flow } \\
\text { oygen therapy }\end{array}$ & N/A & N/A & $45 \%$ \\
\hline $\begin{array}{l}\text { Bilateral } \\
\text { radiological } \\
\text { infiltrate }\end{array}$ & $77,8 \%$ & $42,9 \%$ & $80 \%$ \\
\hline $\begin{array}{l}\text { Reactive C } \\
\text { protein PCR } \\
\mathrm{mg} / 1\end{array}$ & $200 \pm 150,3$ & $139 \pm 170,6$ & $163,21 \pm 177,15$ \\
\hline $\begin{array}{l}\text { Procalcitonin } \\
\text { PCT ng/ml }\end{array}$ & $4,94 \pm 15,8$ & $1,17 \pm 2,1$ & $0,47 \pm 0,55$ \\
\hline PCT $>0,8$ & $46,4 \%$ & $28,6 \%$ & $15 \%$ \\
\hline $\begin{array}{l}\text { Leukocite } \\
\text { count (WBC) }\end{array}$ & $8588 \pm 6852$ & $\begin{array}{l}8702 \pm \\
6256\end{array}$ & $9595 \pm 3924$ \\
\hline $\begin{array}{l}\text { Confirmed } \\
\text { coinfection }\end{array}$ & $22,2 \%$ & $14,3 \%$ & $10 \%$ \\
\hline $\begin{array}{l}\text { Confirmed } \\
\text { NAVM }\end{array}$ & $16,7 \%$ & $14,3 \%$ & $30 \%$ \\
\hline Mortality & $16,7 \%$ & $28,6 \%$ & $30 \%$ \\
\hline
\end{tabular}

Conclusion: The clinical profile and outcome of SARS COV2 pneumonia differs considerably from those caused by Influenza in critically ill patients.

Despite no differences in the scales of severity at ICU admission were observed, severity of respiratory failure and the need for mechanical ventilation is greater in SARS COV2 infections.

Increased hospital length of stay and more healthcare-related infections could justify the worst patient prognosis in SARS COV 2 pneumonia.

\section{Reference(s) and grant acknowledgment(s)}

1. Martin-Loeches I, Díaz E, Vidaur L, et al. Pandemic and post-pandemic influenza A (H1N1) infection in critically ill patients. Crit Care. 2011;15(6):R286. https://doi.org/10.1186/cc10573

2. Bhatraju PK, Ghassemieh BJ, Nichols M, et al. Covid-19 in Critically III Patients in the Seattle Region - Case Series [published online ahead of print, 2020 Mar 30]. N Engl J Med. 2020;NEJMoa2004500. https://doi.org/10.1056/ nejmoa2004500.

\section{6}

\section{Characteristics of ICU patients with COVID-19 in a Spanish} second-level hospital

B. Lobo Valbuena'; S. Garcia Manzanedo ${ }^{2}$; L. López de la Oliva Calvo'; A. Abella Alvarez'; I. Conejo Márquez²; A. Naharro Abellan²; V. Enciso Calderon²; DD. Varillas ${ }^{3}$; F. Gordo'

${ }^{1}$ Intensive care unit, $\mathrm{H}$. U del Henares, Madrid, Spain; ${ }^{2}$ Intensive care unit, H.U del Henares, Madrid, Spain; ${ }^{3}$ Unidad de apoyo a la investigación, facultad de medicina, Universidad Francisco de Vitoria, Madrid, Spain

Correspondence: B. Lobo Valbuena

Intensive Care Medicine Experimental 2020, 8(2): 000646
Introduction: According to published epidemiological data, critical care is needed by $5 \%$ of COVID-19 patients [1]. An impressive effort from ICU teams allowed to multiply their capacity by two or three-fold, trying to cover the continuous need $[2,3]$.

Objectives: To report ICU admissions related to COVID-19 at a polyvalent ICU of a second-level hospital, with eight beds available in summer, and 10 in winter (0.47-0,59 ICU beds/10,000 inhabitants).

Methods: Retrospective study of ICU patients ( $>18$ years old) with nasal/throat swabs or bronchial aspirate positive for SARS-CoV-2 by PCR, between March 5th and May 7th, 2020. Patients transferred to other centres and those still in ICU were excluded. The local IRB approved the study. Patients were treated according to current national protocols. After analysis of clinical characteristics, we performed a multivariate analysis to check the causes and associated factors of ICU mortality. Statistical analyses performed using SPSS 20.0.

Results: 75 patients presented a positive PCR; 49 finally included. Median age 65 years (IQR: $59-72$ ); $65.3 \%$ men. $61.2 \%$ came from hospital ward. $93.9 \%$ presented on admission respiratory failure due to pneumonia. $73.5 \%$ suffered from some comorbidity $(44.9 \%$ cardiovascular, $42.9 \%$ endocrine, $12.2 \%$ malignancy, $10.2 \%$ respiratory, $6.1 \%$ chronic liver disease, $6.1 \%$ neurologic and $4.1 \%$ chronic renal disease). Table 1 shows relevant clinical data. Median ICU length-of-stay 12 days (IQR 9-19.5). Survival upon ICU discharge was $77.1 \%$.

Regarding multivariate analysis, no statistically significant association was noted between mortality and comorbidities $(p=0.126)$ except cardiovascular comorbidity (OR 4.8, Cl95\%: 1.2-26.4; $\mathrm{p}=0.028$ ) when performing a multivariate backward stepwise regression model. Albeit no organic failure (OF) presented individual association with mortality, we did find an association with the number: a threshold value of $4 \mathrm{OF}$ was associated with ICU mortality $(p<0.001)$. We also observed a statistical trend between mortality and number of days on MV: 19.88 days in non-survivors vs 14 among survivors $(p=0.056)$. Finally, we did not find any relationship between severity scores (SAPS3, Clinical Frailty Scale, SOFA on admission) and mortality.

Table 1. Relevant clinical data

\begin{tabular}{|c|c|c|}
\hline \multicolumn{2}{|l|}{ Variable } & All adults $(\mathrm{N}=49)$ \\
\hline \multirow{6}{*}{$\begin{array}{l}\text { Organ failure (number, } \\
\text { percentage) }\end{array}$} & Cardiovascular & $40(81.6)$ \\
\hline & Respiratory failure & $45(91.8)$ \\
\hline & Kidney failure & $22(44.9)$ \\
\hline & Hepatic failure & $8(16.3)$ \\
\hline & Hematologic & $10(20.4)$ \\
\hline & Neurological & $12(24.5)$ \\
\hline \multicolumn{2}{|c|}{ Number of organ failures per patient (median, IQR) } & $3(2-4)$ \\
\hline \multirow{7}{*}{$\begin{array}{l}\text { Respiratory failure } \\
\text { management (number, } \\
\text { percentage) }\end{array}$} & Invasive MV & $44(89.8)$ \\
\hline & ARDS diagnosis & $43(87.8)$ \\
\hline & Need for reintubation & $4(8.2)$ \\
\hline & Days on MV (median, IQR) & $11(9.3-19.8)$ \\
\hline & Percutaneous tracheostomy & $2(4.1)$ \\
\hline & Prone positioning & $25(51)$ \\
\hline & Neuromuscular blockade & $36(73.5)$ \\
\hline
\end{tabular}

Conclusion: In our cohort, mortality was significantly associated with cardiovascular comorbidities (OR 4.8) and a higher number of associated organ failures. The present study tries to highlight the importance of inclusive strategy based on multidisciplinary collaboration and maintenance of well-established protocols despite adversities [4].

\section{Reference(s) and grant acknowledgment(s)}

1. 4. Abella Alvarez A, Torrejon Perez I, Enciso Calderon V, Hermosa Gelbard C, Sicilia Urban JJ, Ruiz Grinspan M, et al (2013). ICU without walls project. Effect of the early detection of patients at risk. Med Intensiva;37(1):12-8.

2. 3. Ballesteros Sanz MA, Hernández-Tejedor A, Estella Á, Jiménez Rivera JJ, González de Molina Ortiz FJ, Sandiumenge Camps A et al. Recommendations of the Working Groups from the Spanish Society of Intensive and Critical Care Medicine and Coronary Units (SEMICYUC) for the management of adult critically ill patients in the coronavirus disease (COVID-19). Med Intensiva 2020; https://doi.org/10.1016/j.medin.2020.04.001 
3. 2. Ma X, Vervoort D (2020). Critical care capacity during the COVID-19 pandemic: Global availability of intensive care beds. Journal of Critical Care. https://doi.org/10.1016/j.jcrc.2020.04.012.

4. 1. Dong E, Du H, Gardner L (2020). An interactive web-based dashboard to track COVID-19 in real time [Epub 2020 Feb 19]. Lancet Infect Dis;20(5):533-534.

\section{9}

Investigating hospital acquired pneumonia in critical care

P. Beddoes ${ }^{1}$; D. Melia ${ }^{2}$

${ }^{1}$ Anaesthetics Department, Whipps Cross University Hospital, London, United Kingdom; ${ }^{2}$ Critical care, Whipps Cross University Hospital, London, United Kingdom

Correspondence: $P$. Beddoes

Intensive Care Medicine Experimental 2020, 8(2): 000649

Introduction: Responsible for $5.9 \%$ of all UK critical care admissions (M Woodhead, 2006), community acquired pneumonia (CAP) is a common cause of critical care admission. Antibiotics are vital in the treatment of CAP but their use is associated with morbidity such as colitis, infection with resistant organisms and kidney injury. The use of investigations to identify a causative organism may facilitate better antibiotic stewardship (I Johnson, 2017).

The 2009 British Thoracic Society (BTS) guideline recommends that patients admitted to critical care with severe CAP should have blood cultures, sputum or other respiratory samples for microscopy, culture and sensitivity (MC\&S), urine pneumococcal and legionella antigen testing performed(Lim WS, 2009).

In this audit cycle, on finding adherence to BTS guidelines at our institution to be low, we used a simple aide-memoire for nursing staff and doctors requesting investigations for patients with CAP and demonstrated improved adherence with an increase in identification of causative pathogens in severe CAP. Our intervention was simple, inexpensive and resulted in significant improvement in the investigation of severe CAP, adherence to guidelines and more precise microbiological diagnosis in patients with severe pneumonia.

Methods: 20 consecutive adult patients with a diagnosis of CAP admitted to a London ICU December 2018 - February 2019 were identified. We excluded patients $<18$ years and those with hospital acquired pneumonia. Using the electronic patient record (EPR), we noted investigations performed and results.

On discussion with nurses and junior doctors responsible for ordering investigations, it became clear that while an 'atypical screen' was commonly requested, there was poor understanding of what tests were then required. We made a laminated card listing required investigations for patients with severe CAP and attached this at eye line to computer monitors where tests are requested on the unit to serve as a visual prompt to staff requesting investigations. We listed investigations as they appear on our electronic requesting system to ensure that the correct search terms were used.

The following winter, the audit was repeated to re-assess compliance with guidelines.

Results: There was improvement in testing across all investigations. There was significant increase in legionella and pneumococcal antigen testing, respiratory virus PCR, blood cultures and mycoplasma DNA. The proportion fully investigated as per BTS guidelines rose by $40 \%$ (11.5930\% to $61.3271 \% \mathrm{P}=0.006$ ). The commonest missing test was sputum culture. The percentage of patients with a causative organism identified for their CAP rose by $30 \%(-0.1759 \%$ to $53.6369 \% p=0.05)$

Conclusion: CAP is a common cause of admission to intensive care. If investigated thoroughly, most patients will have an underlying organism identified, allowing for a narrower spectrum of antibiotic, which should not be forgotten in the context of COVID19.

Simple aide memoirs can bring clarity to poorly understood terms such as 'Atypical screen'.

\section{Reference(s) and grant acknowledgment(s)}

1. British Thoracic Society Standards of Care Committee. BTS Guidelines for the Management of Community Acquired Pneumonia in Adults. Thorax. 2001;56 Suppl 4(Suppl 4):IV1-IV64. https://doi.org/10.1136/thorax.56.suppl $-4 . i v 1$
2. Johnson I, Banks V, Antibiotic stewardship in critical care, BJA Ed. 2017;17(4), 111-116, https://doi.org/10.1093/bjaed/mkw059

3. Woodhead M, Welch CA, Harrison DA, Bellingan G, Ayres JG. Communityacquired pneumonia on the intensive care unit: secondary analysis of 17,869 cases in the ICNARC Case Mix Programme Database. Crit Care. 2006;10 Suppl 2(Suppl 2):S1. https://doi.org/10.1186/cc492

\section{8}

Assessment of antibody-in-lymphocyte assay for the etiological diagnosis of RSV pneumonia

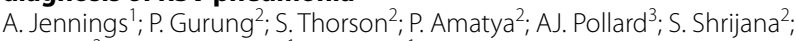
DF. Kelly ${ }^{3}$; M. Shankar-Hari ${ }^{1}$; M. Carter ${ }^{1}$

${ }^{1}$ School of immunology and microbial sciences, King's College London, London, United Kingdom; ${ }^{2}$ Paediatric research group, Patan Academy of Health Sciences, School of Public Health, Patan, Nepal; ${ }^{3}$ Oxford vaccine group, University of Oxford, Oxford, United Kingdom

Correspondence: A. Jennings

Intensive Care Medicine Experimental 2020, 8(2): 000688

Introduction: Circulating antibody-producing B cells secrete antibody following context-dependent stimulation by pathogen antigens. Secreted antibody from lymphocytes harvested and ex vivo cultured from patients with infection (antibodies-in-lymphocyte supernatant, ALS, assay) could be used as a diagnostic test that, unlike conventional serology, removes the requirement for convalescent sampling.

Objectives: We compared the diagnostic accuracy of ALS assay with standard molecular tests for respiratory syncytial virus (RSV), globally the most prevalent cause of childhood pneumonia.

Methods: We enrolled children admitted to Patan Hospital, Nepal, with a clinical diagnosis of pneumonia from October 2016 to June 2017. Peripheral blood mononuclear cells (PBMCs) were separated from whole blood and cultured for $48 \mathrm{~h}$ using standard methods. We used a IgG ELISA to RSV F protein (Fitzgerald) to calculate antibody concentrations in ALS. We compared ALS to RSV $F$ protein to the results of the NxTAG Luminex Respiratory Pathogen Multiplex PCR panel (Luminex Corp, USA) on nasopharyngeal (NP) swab specimens from the same patients.

Results: Of 280 children admitted to hospital, 83 (30\%) had primary endpoint pneumonia on chest radiographs (PEP). RSV was detected from NP specimens by PCR in 29/280 (10\%) children, of whom 5/29 (17\%) had PEP. Blood samples were taken a median of 4 days following onset of symptoms. There were significantly decreased concentrations of lgG to RSV in ALS samples from patients in whom RSV was detected in comparison with others (Figure 1).

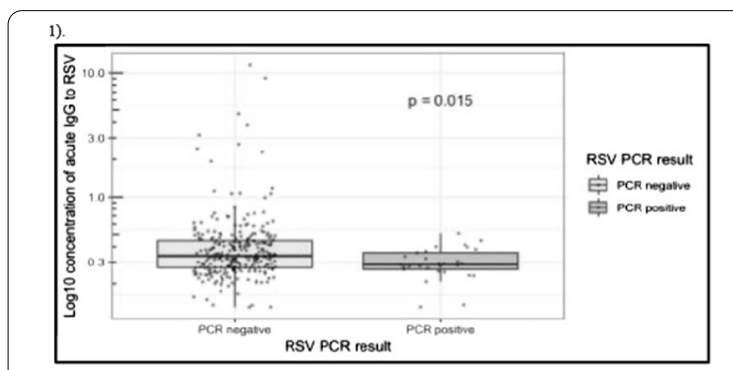

Figure 1: Comparison between ALS and PCR for RSV diagnosis for the study cohort. $]$ $\log 10$ ) versus RSV PCR result from nasopharyngeal swabs ( $\mathrm{x}$-axis; $\mathrm{p}=\mathbf{0 . 0 1 5}$, Wilcoxon

Conclusion: Children with RSV pneumonia (by PCR of NP specimens) had lower ALS to RSV F protein than children with other pneumonia etiologies. This result supports the lack of protective humoral immunity induced by either RSV infection, or previous RSV vaccines.

Reference(s) and grant acknowledgment(s)

1. Funded by Wellcome (grant 104439/Z/14/Z) and by Gavi to the PneumoNepal project. 
000705

Covid-19: clinical outcome of younger people admitted to ICU. A retrospective single-center cohort study

L. Oyaert ${ }^{1}$; A. Vandebroek ${ }^{1}$; D. Helsloot ${ }^{1}$; C. Missant ${ }^{1}$; W. De Corte ${ }^{1}$; S. Lamote ${ }^{1}$

${ }^{1}$ Anesthesia, intensive care \& emergency medicine, AZ Groeninge, Kortrijk, Belgium

\section{Correspondence: S. Lamote}

Intensive Care Medicine Experimental 2020, 8(2): 000705

Introduction: The Coronavirus disease 2019 (COVID-19) pandemic, caused by the Severe Acute Respiratory Syndrome Coronavirus 2 (SARS-CoV-2), led in spring 2020 to a worldwide crisis. In the majority there is a mild course, however in up to respectively $20 \%$ and $5 \%$ there is a severe and even critical presentation. Initial reports from China and Italy showed poor outcome for patients admitted to Intensive Care Unit (ICU), particularly for the elderly. We focused in our analysis on the outcome of younger patients admitted to ICU.

Methods: In this retrospective, single-center cohort study, we assessed clinical outcome of younger patients by comparing those aged below 66 years with those aged 66 years and above. We further divided the former group in 2 cohorts, namely patients with (young $+R F$ (risk factor)) and without (young-RF) any comorbidity including obesity (BMI > 30). Between March 13 and May 15, patients admitted with confirmed COVID-19 to ICU were consecutively screened. Exclusion criteria were COVID-19 cases without respiratory failure and Do Not Resuscitate (DNR) order at admission. Primary outcome was mortality, secondary outcome were need for mechanical ventilation (MV), duration of MV, proportion successfully weaned off ventilator, length of ICU stay (ICULOS), incidence of associated organ failure and rate of recovery. The latter was assessed at day 10, 14, 21 and 28 by using an ordinal clinical scale: 1. no hospitalization; 2 . at hospital; 3. at ICU; 4. ICU with high flow oxygen therapy (HFOT) or non-invasive ventilation (NIV); $5 . \mathrm{MV} ; 6$. dead. Mann-Whitney $U, X^{2}$ or Fisher's exact tests were used as appropriate and analyses were performed using SPSS.

Results: Of the 61 patients screened, 55 were eligible (6 patients excluded: 5 with DNR-code, 1 patient who didn't suffer from respiratory failure). Median age overall was 66 years (IQR 57-78) and males (M) were predominant, especially in the younger group (overall $65 \% M$, young $80 \% M$ vs old $53 \% M, p=0.038$ ). The younger cohort accounted for $45 \%(n=25)$, one-third of them had no comorbidities at all ( $n=9 / 55,16 \%)$. Most frequently reported concomitant pathology was obesity (overall 27\%, median BMI 27.5 ; young $32 \%$ vs old $23 \%, \mathrm{p}=0.303$ ), hypertension (overall $49 \%$, young $40 \%$ vs old $57 \%$, $\mathrm{p}=0.218$ ), cardiovascular disease (overall $33 \%$, young $16 \%$ vs old $47 \%, p=0.0 .016$ ) and diabetes (overall $29 \%$, young $12 \%$ vs old $43 \%$ $p=0.11)$. There was a significantly higher incidence of obesity and hypertension in the young $+\mathrm{RF}$ cohort $(50 \%, \mathrm{p}=0.0088$ and $63 \%$, $\mathrm{p}=0.003$ ) compared to the young-RF cohort.

Overall ICU mortality was $32 \%$. Even though the fatality rate for younger people was lower (young $22 \%$ vs old $40 \%, p=0.158$ ), this was not statistically significant due to high mortality in the young+RF group ( $36 \%$ for young $+R F$ vs $40 \%$ old, $p=0.786$ ), while none of the young-RF group died (vs $36 \%$ for young+RF, $p=0.0465$ ). More than half of the patients $(n=31 / 55,56 \%)$ required MV, without significant differences between young and older patients (52\% vs $60 \%$, $p=0.551)$, although only $11 \%(n=1 / 9)$ of the young-RF group ended up with MV versus three-quarters of the young+RF group $(p=0.002)$. Prone ventilation was applicated in 16 cases $(n=16 / 31,52 \%)$. Fortyfive percent of the ventilated patients were successfully weaned off the ventilator ( $46 \%$ young vs $44 \%$ old, p NS; $100 \%$ young-RF vs $42 \%$ young $+R F, p=0.154)$. Duration of MV for survivors was shorter for the younger vs the elderly ( 11.73 vs 18.76 days, $p=0.66$ ) and for youngerRF vs younger+RF (8.38 vs 15.07 days), but none of these differences were significant. ICU-LOS didn't differ among the different cohorts with a median duration of 10 days overall (young 11.5 vs old 9, $\mathrm{p}=0.59$; young-RF 9 vs young $+\mathrm{RF} 13, \mathrm{p} 0.26)$. Incidence of severe associated organ failure was slightly lower in the younger population ( $24 \%$ vs old $33 \%, p=0.448$ ), with kidneys as most vulnerable organ. None of the young-RF patients experienced any severe associated organ failure while young + RF patients had even higher incidence of such an extrapulmonary manifestation than the elderly (38\% vs 33\%, $p=0.777)$. Younger people tended to recover faster than the elderly, particularly the young-RF group outperformed the other cohorts with significantly higher proportions of patients clinically improving on the ordinal clinical scale by 1 point as evaluated at day $10(89 \%$, $\mathrm{p}=0.06), 14(89 \%, \mathrm{p}=0.013)$ and $21(100 \%, \mathrm{p}=0.005)$ as well as by 2 points at day $28(100 \%, p=0.01)$.

Conclusion: This study demonstrates that younger people can be severely hit by COVID-19 as well. Only those without any comorbidities at all have better clinical outcome in terms of mortality, MV requirement, associated organ failure and rate of recovery. As functional outcome after surviving remains unclear, caution and prevention remain mandatory for patients in all age groups, irrespective of their comorbidity.

\section{Reference(s) and grant acknowledgment(s)}

1. Grasselli G, Zangrillo A, Zanella A, Antonelli M, Cabrini L, Castelli A, et al. Baseline Characteristics and Outcomes of 1591 Patients Infected with SARS-CoV-2 Admitted to ICUs of the Lombardy Region, Italy. JAMA. 2020;323(16):1574-1581

2. Wu Z, McGoogan JM. Characteristics of and Important Lessons From the Coronavirus Disease 2019 (COVID-19) Outbreak in China: Summary of a Report of 72314 Cases From the Chinese Center for Disease Control and Prevention. [published online ahead of print, 2020 Feb 24]

3. Guan WJ, Ni ZY, Hu Y, Liang WH, Ou CQ, He JX, et al. Clinical Characteristics of Coronavirus Disease 2019 in China. N Engl J Med. 2020;382 (18):1708-20.

4. Zhu N, Zhang D, Wang W, Li X, Yang B, Song J, et al. A novel coronavirus from patients with pneumonia in China, 2019. N Engl J Med. 2020;382(8):727-33.

\section{8}

The association between d-dimer, mortality and ITU admission in COVID-19-is there a connection? A retrospective study L. Fakes ${ }^{1}$; H. Said ${ }^{2}$

${ }^{1}$ West Middlesex University Hospital, Isleworth, United Kingdom; ${ }^{2}$ Intensive care, West Middlesex University Hospital, Isleworth, United Kingdom Correspondence: $L$. Fakes

Intensive Care Medicine Experimental 2020, 8(2): 000728

Introduction: Coronavirus disease 2019 (COVID-19) was first reported in Wuhan, China in December 2019. Many studies have since focused on identifying parameters by which to risk stratify patients. Elevated d-dimer levels have been reported in up to $46 \%$ of patients (1), with higher levels noted in patients requiring intensive care unit (ICU) admission (2). A recent systematic review including six retrospective cohort studies conducted in China, found raised admission d-dimer levels are significantly associated with increased mortality in COVID19 (3). Given patients present at different time points in their illness, measuring d-dimer at admission may not accurately represent the potential severity of infection. We therefore sought to evaluate whether this association was maintained in a United Kingdom patient cohort when maximal d-dimer was measured.

Objectives: The aim of this study was to investigate the association between maximum d-dimer and both ITU admission and mortality in patients with COVID-19.

Methods: This retrospective single-centre study included patients presenting to West Middlesex University Hospital (London, United Kingdom) with a laboratory confirmed diagnosis of COVID-19. We selected a sample of 150 consecutive survivor and non-survivors. Patients in which no d-dimer sample was taken were excluded, giving a total 
sample analysed of 99 survivors and 118 non-survivors. In all cases, electronic medical records were used to obtain the highest d-dimer value during admission, survivor status, and whether ITU admission occurred. Median and mean d-dimer values were calculated, and confidence intervals $(\mathrm{Cl})$ used to assess statistical significance.

Results: D-dimers were elevated in $74.7 \%$ of survivors and $94.9 \%$ of non-survivors with mean d-dimers of $2683 \mu \mathrm{g} / \mathrm{mL}$ (Standard deviation [SD] 4350, Cl 857) and $3588 \mu \mathrm{g} / \mathrm{mL}$ (SD 3790, Cl 683) respectively, and median d-dimers $1070 \mu \mathrm{g} / \mathrm{mL}$ and $2200 \mu \mathrm{g} / \mathrm{mL}$ respectively. In patient admitted to ITU the mean and median d-dimer was $5597 \mu \mathrm{g} /$ $\mathrm{mL}$ (SD 5272, Cl 1654) and $3377 \mu \mathrm{g} / \mathrm{mL}$ respectively. In patients with ward-based care the mean d-dimer was $2635 \mu \mathrm{g} / \mathrm{mL}$ and median d-dimer $1600 \mu \mathrm{g} / \mathrm{mL}$ (SD 3184, Cl 456). No differences were statistically significant $(p<0.05)$.

Conclusion: This study supports previous studies in China showing d-dimer levels are commonly raised in COVID-19 positive patients (1, $4,5)$. However, in contrast, we found no significant difference between d-dimers in survivors and non-survivors. Similarly, we found patients admitted to ITU had higher d-dimers than ward-based patients supporting the findings of Wang et al. (2) but this was not statistically significant. This may reflect differences in the studied patient populations, or more likely could be attributable to the different approach adopted in this study of measuring maximal rather than admission d-dimer. Overall, these findings suggest parameters other than $\mathrm{d}$-dimer may be more useful clinically in risk stratifying COVID-19 patients and predicting need for ITU admission or mortality.

\section{Reference(s) and grant acknowledgment(s)}

1. 1. Guan WJ, Ni ZY, Hu Y, Liang WH, Ou CQ, He JX et al. Clinical Characteristics of Coronavirus Disease 2019 in China. N Engl J Med. 2020;382: 1708-1720

2. 2. Wang D, Hu B, Hu C, et al. Clinical Characteristics of 138 Hospitalized Patients With 2019 Novel Coronavirus-Infected Pneumonia in Wuhan, China. JAMA. 2020;323(11):1061-1069. https://doi.org/10.1001/ jama.2020.1585

3. 3. Sakka M, Connors JM, Heikimian G, Martin-Toutain I, Crichi B, Colmegna l et al. Association between d-dimer levels and mortality in patients with coronavirus disease 2019 (COVID-19): a systematic review and pooled analysis. Journal de Medicine Vasculaire. 2020;https://doi.org/10.1016/j. jdmv.2020.05.003

4. 4. Tang N, Bai H, Chen X, Gong J, Li D and Sun Z. Anticoagulant treatment is associated with decreased mortality in severe coronavirus disease 2019 patients with coagulopathy. J Thromb Haemost. 2020;18(5):1094-1099

5. 5. Zhou F, Yu T, Du R, Fan G, Liu Y, Liu Z et al. Clinical course and risk factors for mortality of adult inpatients with COVID-19 in Wuhan, China: a retrospective cohort study. Lancet. 2020;395(10229):1054-1062

The authors have no funding to declare.

\section{7}

Acute kidney injury in patients with COVID-19 is associated with severity of illness: A single centre experience

N. Shaban'; S. Love'; I. Hamed'; D. Cayir' ; R. Mahroof ${ }^{2}$; A. Conway-Morris ${ }^{2}$ 'John farman intensive care unit, Cambridge University Hospitals, Cambridge, United Kingdom; ${ }^{2}$ Department of anaesthetics \& intensive care medicine, Cambridge University Hospitals, Cambridge, United Kingdom Correspondence: N. Shaban

Intensive Care Medicine Experimental 2020, 8(2): 000747

Introduction: Acute kidney injury (AKI) is a frequent complication seen in patients with COVID-19 infection admitted to intensive care. National UK data indicates $26 \%$ of patients admitted to critical care with COVID-19 infection require renal replacement therapy (RRT), $61 \%$ of whom died (1). This has significant implications for resource utilisation. Better understanding of this disease and requirement for renal support will not only help plan local services but may also give insights into improved treatment and preventative strategies.

Objectives: We aimed to assess the incidence of AKI and need for RRT in critically ill patients with COVID-19 in a single large UK tertiary centre. The association between need for RRT and the presence of several co-morbidities as well as overall mortality was also examined. These local findings were also compared with national data.
Methods: Contemporaneous electronic medical records were retrospectively reviewed for all patients admitted to ICU for 10 weeks from March 2020 in a single large UK tertiary centre. Data extracted included demographics, co-morbidities, fluid balance, creatinine, eGFR, RRT, APACHE II score and outcome. AKI was defined as per the KDIGO criteria (2) and renal recovery defined as free from RRT for over $48 \mathrm{hrs}$.

Results: A total of 93 patients with COVID-19 were admitted to ICU during this period. The median age was 62 and $28(30 \%)$ were female. $51(56 \%)$ developed AKI and RRT was provided in $33(36 \%)$. The overall ICU mortality was $35 \%$, excluding 2 patients who remained in ICU at the time of reporting. Factors associated with the development of AKI are shown in Table 1. Multiple regression analysis retained APACHE II and requirement for mechanical ventilation as significant associations with the development of AKI (Table 2), with similar results for requirement for RRT.

\begin{tabular}{|c|c|c|c|}
\hline & AKI (51) & No AKI (42) & P value \\
\hline $\begin{array}{l}\text { Median age } \\
\text { (range) }\end{array}$ & $64(17-82)$ & $57(28-77)$ & 0.05 \\
\hline Female sex (n/\%) & $14(27 \%)$ & $14(33 \%)$ & 0.53 \\
\hline Hypertension & $19(37 \%)$ & $14(33 \%)$ & 0.69 \\
\hline Diabetes & $10(20 \%)$ & $10(24 \%)$ & 0.62 \\
\hline Obesity $($ BMI $>30)$ & $21(41 \%)$ & $15(36 \%)$ & 0.59 \\
\hline $\begin{array}{l}\text { Chronic Kidney } \\
\text { disease }\end{array}$ & $10(20 \%)$ & $2(5 \%)$ & 0.034 \\
\hline $\begin{array}{l}\text { Pre-existing } \\
\text { nephrotoxic } \\
\text { therapy } \\
\text { Admission }\end{array}$ & $20(39 \%)$ & $14(33 \%)$ & 0.558 \\
\hline $\begin{array}{l}\text { APACHE II } \\
\text { (median, IQR) }\end{array}$ & $17(14-19)$ & $12(9-16)$ & $<0.0001$ \\
\hline $\begin{array}{l}\text { Diuretic use in } \\
\text { ICU }\end{array}$ & $36(72 \%)$ & $29(69 \%)$ & 0.76 \\
\hline $\begin{array}{l}\text { Total Furosemide } \\
\text { dose (mg) (median, } \\
\text { IQR) }\end{array}$ &, $165(60-260)$ & $260(89-510)$ & 0.65 \\
\hline $\begin{array}{l}\text { Mechanical } \\
\text { ventilation }\end{array}$ & $48(94 \%)$ & $30(73 \%)$ & 0.005 \\
\hline $\begin{array}{l}\text { Median (IQR) ICU } \\
\text { Length of stay }\end{array}$ & $J_{19}(9-29)$ & $15(7-24)$ & 0.03 \\
\hline ICU Survival & $25(50 \%)$ & $34(83 \%)$ & 0.001 \\
\hline
\end{tabular}

Table 1 : Clinical and demographic factors associated with AKI

\begin{tabular}{|c|c|c|}
\hline & Odds ratio ( $95 \%$ CI) & $P$ value \\
\hline Age (per year increase) & $1.0(0.9-1.03)$ & 0.45 \\
\hline CKD (presence) & $2.7(0.4-19.2)$ & 0.32 \\
\hline $\begin{array}{l}\text { APACHE II (per } 1 \\
\text { point increase) }\end{array}$ & $1.2(1.1-1.4)$ & 0.002 \\
\hline Mechanical ventilation & $11.2(1.6-80.7)$ & 0.016 \\
\hline
\end{tabular}

Table 2 : Binary logistic regression for Independent association with the development of AKI

Conclusion: COVID-19 patients in our cohort experienced a high rate of AKI and need for RRT, although rates were in keeping with those seen in other causes of acute respiratory distress syndrome (3). Although AKI was associated with a higher risk of death, this is mostly explained by the severity of presenting illness. We found no evidence that diuretic therapy was independently associated with the development of AKI or need for RRT.

\section{Reference(s) and grant acknowledgment(s)}

1. 1. ICNARC report on COVID-19 in critical care 12 June 2020

2. 2. Kellum JA, et al; Crit Care. 2013:17:204.

3. 3. Panitchote, A et al. Ann Intensive Care. 2019;9:74

4. None. 
000794

Evolution of infections acquired intra-ICU in our Intensive Care unit during the period 2015-2019. Descriptive study

C. M. Rodríguez Mejías; A. López Fernández¹ ; J. P. Valencia Quintero²; A. Sánchez González ; J. Machado Casas ${ }^{1}$

${ }^{1}$ Medicina Intensiva, Hospital Universitario Virgen de las Nieves, Granada, Spain; ${ }^{2}$ Medicina intensiva, Regional Hospital Santa Ana de Motril, Motril, Spain

Correspondence: A. López Fernández

Intensive Care Medicine Experimental 2020, 8(2): 000794

Introduction: Studying the characteristics of infections acquired within the ICU during the last 5 years.

Methods: Descriptive study, based on the data collected in the ENVIN - ICU base of our medical-surgical unit, collected during the months of the official participation period. To do this, we have selected infections acquired within the ICU from 2015 to 2019. We will describe the location of the infection, the most frequently isolated microorganisms, the number of infections that received antibiotic treatment, appropriate antibiotic treatment

Results: During the official ENVIN-ICU registration periods from 2015 to 2019 , there are 132 records of infections acquired intra ICU. Of these 132 , the most frequent locations in decreasing order are: pneumonia related to mechanical ventilation or intubation (27), tracheobronchitis associated with mechanical ventilation (21), bacteremia of unknown focus (18), non-surgical infection of the digestive system (10), bacteremia secondary to abdominal infection (9), bacteremia secondary to catheter infection (9), bacteremia secondary to respiratory infection (8), urinary tract-related urinary infection (6), other infection (6). The most frequently isolated microorganisms in these records of intra-ICU infection are: pseudomona aeruginosa $13.74 \%(n=18)$, enterococcus faecium $12.21 \%(n=16)$, klebsiella pneumoniae $9.16 \%(n=12)$, staphylococcus epidermidis $8.40 \%(n=11)$, stenotrophomonas maltophilia $5.34 \%(n=7)$, escherichia coli 5.34\% $(n=7)$. Of the 132 registered intra-ICU infections, $87.88 \%(n=116)$ received antibiotic treatment. Of those treated with antibiotherapy $(n=116), 79.17 \%(n=24)$ of pneumonias associated with mechanical ventilation received the appropriate antibiotic treatment, $100 \%(n=5)$ of urinary tract-related urinary tract infections, $71.43 \%(n=21)$ of bacteremia primary and secondary to catheter infection, $75 \%(n=20)$ of bacteremia secondary to infection of another focus, and $78.26 \%(n=46)$ of those classified as other intra-ICU infections.

Conclusion: In the period 2015-2019 of ENVIN-ICU registration in our unit, the most frequent intra-ICU infections acquired were pneumonia and tracheobronchitis associated with mechanical ventilation and bacteremia of unknown focus. The most frequently isolated microorganisms are pseudomonas aeruginosa, enterococcus faecium, and klebsiella pneumoniae. Receiving antibiotic treatment in $87.88 \%$ of these infections, being correct in a very high percentage.

\section{5}

Attitudes and beliefs of Spanish healthcare professionals during the COVID-19 pandemic

B. N. Santana-Lopez' Y. G. Santana-Padilla; J. M. González-Martín;

L. Santana-Cabrera'

${ }^{1}$ Intensive care unit, Maternal and child Hospital, Las Palmas de Gran Canaria, Spain

Correspondence: B.N. Santana-Lopez

Intensive Care Medicine Experimental 2020, 8(2): 000795

Introduction: During the CoVID-19 pandemic, healthcare professionals are taking the risk of becoming infected or infecting their families. Spain is the country with the highest number of infected healthcare professionals worldwide.

Objectives: Our aim was to study the attitudes and beliefs of these professionals during the current pandemic.

Methods: Descriptive study conducted by using an online questionnaire - based on an earlier one - which was sent to healthcare professionals at the national level, during the week March 20-27, 2020.
Results: Healthcare professionals returned 971 completed questionnaires. A total of $803(82.7 \%)$ participants thought that they did not have suitable PPEs to protect them from infection with CoVID-19. In spite of this, 606 (62.4\%) of them were ready to work, even with a higher-than-usual risk of becoming infected at work and getting ill. Furthermore, 815 (83.93\%) participants were ready to work more hours than usual and 697 (71.78\%) were ready to work in a different workplace. Finally, $669(68.9 \%)$ of them put their duties towards their families before their responsibility at work.

Conclusion: Remarkably high professional commitment has been observed among Spanish healthcare workers in the current pandemic They were ready to work even when many of them considered that they did not have suitable PPEs, and were thus taking a higher than usual infection risk. However, they put the health of their relatives before their duties at work.

\section{Reference(s) and grant acknowledgment(s)}

1. Santana-López BN, Santana-Padilla YG, Martin-Santana JD, Santana-Cabrera L, Escot-Rodríguez C. Creencias y actitudes de trabajadores sanitarios y estudiantes de enfermería de una región de España ante una pandemia de gripe. Rev Peru Med Exp Salud Publica. 2019;36(3):481-6. Available from:

https://doi.org/10.17843/rpmesp.2019.363.4371

\section{1}

Bacteraemia and VV-ECMO Cannulation: A single centre audit J. Medhora'; S. Williams' ${ }^{1}$ I. Scott ${ }^{1}$

${ }^{1}$ Intensive Care Department, NHS Grampian, Aberdeen, United Kingdom Correspondence: J. Medhora

Intensive Care Medicine Experimental 2020, 8(2): 000811

Introduction: Veno-Venous Extra Corporeal Membrane Oxygenation (VV-ECMO) requires central access with either bifemoral cannulae (FF), a bicaval dual lumen cannula in the internal jugular vein $(\mathrm{J})$ or femoral access with jugular return (FJ) (1). Bacteraemia secondary to cannula site infection in patients supported by VV-ECMO poses an increased risk in this group. Currently there is little published work on ECMO cannula site infections however, evidence based on central venous catheter infections is considered to be, in part, transferable.

The relevance of the site of central venous catheters to incidence of bacteraemia in critically unwell patients is controversial. Some studies suggest that the femoral site poses a higher risk of catheter infection (2) whilst others suggest there is no significantly increased risk in using the femoral vein compared to the jugular vein (3). Work on central lines for dialysis may be more valid than other types of central access as dialysis lines are not used for drug delivery, whilst also being exposed to a continuous flow (4).

Objectives: To assess whether the incidence of bacteraemia in patients cannulated for VV-ECMO varies based on cannulation site.

Methods: Data was collected for patients admitted to the Intensive Care Unit at Aberdeen Royal Infirmary, a centre for VV-ECMO, between February 2014-February 2020. We carried out a retrospective audit of the cannulation site in each patient and the number that developed a bacteraemia. Bacteraemia was defined as a positive blood culture result in the time period between cannulation date up until 48 hours after decannulation $(2,4)$. Data on duration of cannulation, cannulation to bacteraemia time and location of patient at time of cannulation was also collected.

Results: Sixty patients were identified. Of these, 35 had a bicaval dual lumen cannula in the internal jugular vein, 24 had bifemoral cannulation and 1 patient had femoral access with jugular return. Ten bacteraemias were identified overall. A larger proportion of patients with bifemoral cannulation developed bacteraemias and mean time to infection in these patients was shorter. Sixty percent of the bacteraemias identified were due to a staphylococcal organism.

Conclusion: Our results suggest that VV-ECMO patients with femoral cannulae placed are more at risk of bacteraemia. This is in keeping with previous work on central venous catheter infections showing that femoral lines increased infection risk despite a shorter duration of insertion2. 
One patient developed two separate bacteraemias, one of which was caused by Streptococcus mitis, an organism found in the oropharynx and therefore a bacteraemia which is unlikely to have originated from the ECMO cannulae. This highlights the difficulty in discerning if a bacteraemia is truly secondary to ECMO cannulation, especially in the absence of line tip culture.

The impact of ECMO cannula related bacteraemia on mortality cannot be accurately assessed. Whilst it is important to consider infection risk when selecting cannulation insertion site, other factors will affect decision making such as ease of transfer, risk of thrombosis, risk of mechanical complications at time of insertion, ease of proning and the patient's Body Mass Index.

\section{Reference(s) and grant acknowledgment(s)}

1. (1) Hartley and Gillon Extracorporeal support of the respiratory system Anaesthesia and Intensive Care Medicine 2019:20;11

2. (2) Merrer et al Complications of Femoral and Subclavian Venous Catheterization in Critically III Patients Journal of the American Medical Association 2001:286;700-707

3. (3) Deshpande et al The incidence of infectious complications of central venous catheters at the subclavian, internal jugular, and femoral sites in an intensive care unit population Critical Care Medicine 2005: 33;13-20

4. (4) Parienti et al Femoral vs jugular venous catheterization and risk of nosocomial events in adults requiring acute renal replacement therapy: A randomized controlled trial Journal of the American Medical Association 2008: 299;2413-2422

5. There is no funding attached to this project

\section{7}

Bacterial and/or fungal infection in patients with respiratory virus pneumonia

JJ. Pineda-Capitán ' ; J. Rodriguez-Gomez²; M. Rojas-Amezcua ${ }^{3}$; R. León ${ }^{1}$; A. Mula'; JC. Pozo Laderas ${ }^{4}$

${ }^{1}$ Intensive care unit, Reina sofia universtiy hospital-imibic-university of cordoba, Córdoba, Spain; ${ }^{2}$ Intensive care unit, Reina Sofia Universtiy Hospital-IMIBIC-University of Cordoba, Cordoba, Spain; ${ }^{3}$ Intensive care unit, Hospital Cabra, Cabra, Spain; ${ }^{4}$ Intensive care medicine, Hospital Universitario Reina Sofía, Córdoba, Spain, Spain

Correspondence: J. Rodriguez-Gomez

Intensive Care Medicine Experimental 2020, 8(2): 000817

Introduction: There is scare information of bacterial and/or fungal infections (coinfections or superinfections) in critical ill patients with COVID-19 pneumonia.

Objectives: The objetive of the study is evaluate bacterial and/or fungal infections in respiratory viral infections and discuss antibiotic empirical management.

Methods: This is a multicentre, observational and retrospective study. Patients with confirmed COVID-19 and other respiratory virus infection which required ICU admission were recruited from October 2018 to April 30th 2020. We described the population and distribution of infections.

Results: We evaluated 112 critical patients with respiratory virus infections. 58 critical care patients with COVI-19 and a further 54 patients with other respiratory infections. COVID-19 patients had significantly less respiratory co-infection ( 3 vs $29 ; p^{<} 0.001$ ). $96 \%$ of patients were treated with antibiotics at admission. In comparison with those with other respiratory infection, superinfection was not significantly higher (46 vs $31 ; p=0.1$ ). Probable/possible ventilator associated pneumonia was the most frequent source of infection (29\%) in COVID-19 patients while in other respiratory virus infection were bacteriamia and urintary tract infection (25\%). In COVID-19 patients the most frequent bacteria involved was Pseudomonas aeruginosa (24\%) while the most frequent in other respiratory virus were Coagulase-negative Staphilococcus (29\%)

Conclusion: In COVID-19 patients the incidence of coinfection is low (3\%). Because of this fact, empirical antibiotic at admission should be closely evaluated. In this group, the incidence of superifection was
$46 \%$; ventilator associated pneumonia and pseudomonas aureginosa were the most frecuent source and etiology.

\section{Reference(s) and grant ackowledgment(s)}

1. Arentz M, Yim E, Klaff L, et al. Characteristics and Outcomes of 21 Critically III Patients With COVID-19 in Washington State. JAMA. 2020;323(16):1612-1614

2. Maclntyre CR, Chughtai AA, Barnes $M$, et al. The role of pneumonia and secondary bacterial infection in fatal and serious outcomes of pandemic influenza a(H1N1). BMC Infect Dis. 2018;18(1):637.

3. Horan TC, Andrus M, Dudeck MA. CDC/NHSN Surveillance Definition of Health Care-Associated Infection and Criteria for Specific Types of Infections in the Acute Care Setting. Am J Infect Control. 2008; Jun;36(5):309-32

4. Miniserio de Sanidad, Gobierno de España. Procedimiento ade actuación frenta a casos por infección por el nuevo coronavirus (SARS-CoV-2). Actualización 10.04.2020.https://www.mscbs.gob.es/profesionales/saludPublica/ ccayes/alertasActual/nCov-China/documentos.htm. Last visit 10th June 2020

5. Zhou F, Yu T, Du R, et al. Clinical course and risk factors for mortality of adult inpatients with COVID-19 in Wuhan, China: a retrospective cohort study. Lancet. 2020; Mar 28;395(10229):1054-1062

6. Clancy CJ, Nguyen MH. COVID-19, superinfections and antimicrobial development: What can we expect?. Clin Infect Dis. 2020;ciaa524. doi:10.1093/ cid/ciaa524. Online ahead of print.

7. Collaboration of authors who are members of the Spanish Network for Research in Infectious Diseases (REIPI RD16/0016/0008/ REIPI RD16/0016/0001). No other finantial support.

8. We gratefully acknowledge the collaboration of Maimonides Biomedical Research Institute of Cordoba (IMIBIC), Reina Sofía University Hospital and the University of Córdoba.

\section{9}

The impact of dexamethasone on clinical outcome of patients with COVID-19 admitted to ICU. A retrospective, single-center cohort study

E. De Greef ${ }^{1}$; A. Vandebroek ${ }^{1}$; L. Vanfleteren ${ }^{1}$; W. De Corte ${ }^{1}$; S. Lamote

${ }^{1}$ Anesthesia \& intensive care, AZ Groeninge, Kortrijk, Belgium

Correspondence: S. Lamote

Intensive Care Medicine Experimental 2020, 8(2): 000819

Introduction: When the Coronavirus Disease 19 (COVID-19) pandemic emerged in spring 2020, there was no effective treatment available. In a recent randomized clinical trial dexamethasone (DXM), a pure glucocorticoid agonist, was shown to have a beneficial impact on outcome of patients with Acute Respiratory Distress Syndrome (ARDS) As severe COVID-19 is characterized by an excessive systemic inflammation, we gradually implemented DXM in our therapeutic strategy, although initial guidelines didn't support systematic use of steroids, based on former data with viral infections and ARDS. This study evaluates the impact of DXM on the outcome of patients with COVID-19 admitted to our Intensive Care Unit (ICU).

Methods: In this retrospective, single-center cohort study, we consecutively screened all confirmed COVID-19 patients admitted to ICU between March 13 and May 15 and compared patients who received DXM (DXM group) to those who didn't (no-DXM group). Exclusion criteria were length of ICU stay less than or equal to 5 days, COVID19 positive case without respiratory failure and Do Not Resuscitate (DNR) order at admission. Subgroups we analyzed were mechanically ventilated patients and patients getting DXM prior to initiation of mechanical ventilation (MV). Primary outcome was mortality, secondary outcomes were need for MV, duration of MV, proportion successfully weaned off ventilator, ICU-length of stay (ICU-LOS), rate of recovery, incidence of associated organ failure and incidence of infections. Rate of recovery was assessed at day 10, 14, 21 and 28 by using an ordinal clinical scale: 1 . nohospitalization; 2 . at hospital; 3. at ICU; 4. ICU with high flow oxygen therapy (HFOT) or non-invasive ventilation (NIV); 5. MV; 6. dead. Mann-Whitney $U, x^{2}$ or Fisher's exact tests were used as appropriate and analyses were performed using SPSS.

Results: Of the 61 patients screened, 47 were eligible (14 patients excluded: 8 with ICU-LOS $\leq 5$ days, 5 with DNR-code, 1 patient who 
didn't suffer from respiratory failure). Eighteen cases (38\%) received dexamethasone, 6 of them before intubation. Baseline characteristics and severity of disease scores (APACHE II, SAPS II and worst SOFA score day 1 to 5 ) were similar in both groups. Median age was 67 years (IQR 57-78) overall, patients in the DXM group tended to be younger $(63$, IQR 54-71) than those in the no-DXM group $(70,62-78)$, although statistically not significant $(p=0.203)$. Up to two-thirds were male (DXM 72\% vs no-DXM 62\%, $p=0.475$ ). Nearly all our patients received hydroxychloroquine, frequently in combination with azithromycin. Rarely tocilizumab (DXM 17\%, no-DXM $3 \%, p=0.54$ ) or remdesivir (only one patient, in the DXM group) was associated.

Overall ICU mortality was 31\% (DXM $33 \%$ vs no-DXM $28 \%$, p=0.67). Up to almost three quarters of the DXM group were ventilated, compared to $55 \%$ of the no-DXM group $(p=0.96)$. This higher proportion is rather due to selection bias than caused by DXM, as the drug was frequently started after initiation of MV. In both groups, a similar proportion of patients has been successfully weaned off the ventilator (DXM 54\% vs no-DXM 56\%, $\mathrm{p}=0.87$ ). MV duration (26.2 (IQR 18.9-32.1) vs 10.0 days (IQR 7.5-18.6, $\mathrm{p}=0.05)$ ) and ICU-LOS (21 (IQR 14-40) vs 9 days (IQR 8-14), $\mathrm{p}=0.019$ ) were both significantly longer in the DXM group. Proportion of patients clinically improving as assessed by an ordinal scale at several points in time was lower in the DXM group compared to those of the no-DXM group, although not statistically significant. Moreover, incidence of severe associated organ failure in the DXM group was twice as high as in the no-DXM group ( $44 \%$ vs $21 \%$, $\mathrm{p}=0.75$ ) with kidneys as the most affected extrapulmonary organs, with respectively up to $40 \%$ and $10 \%$ requiring Renal Replacement Therapy $(p=0.75)$. There were more nosocomial infections reported in the DXM-group than in the no-DXM group ( $56 \%$ vs $17 \%, \mathrm{p}=0.04$ ). Similar findings were observed in the MV-subgroup (total of 29 patients with 13 getting DXM) with comparable mortality (46\% vs $44 \%, p=0.581)$ for both groups but longer duration of MV ( 26.2 vs 10.0 days, $p=0.05$ ), trend to longer ICU-LOS ( 40 vs 26 days, $p=0.11$ ) and slower recovery ( $8 \%$ clinically improved with 2 points at day 28 for DXM vs $56 \%$ for no-DXM patients, $\mathrm{p}=0.006$ ) in the DXM group. Incidence of severe associated organ failure $(62 \%$ vs $38 \%, p=0.198)$ and nosocomial infections ( $69 \%$ vs $25 \%, p=0.017$ ) was higher as well in this group.

Finally, 5 out of 6 patients (83\%) getting DXM prior to intubation responded well, while only one patient further deteriorated ending up on the ventilator and deceased eventually.

Conclusion: On the one hand, we couldn't demonstrate a beneficial impact of DXM on the outcome of patients for COVID-19 with even a higher incidence of ICU-acquired infections in the DXM group. On the other hand, the vast majority of patients getting the drug prior to intubation didn't further deteriorate suggesting a possible positive effect of prompter administration. Due to the small size and the study design with a presumably sicker DXM cohort, it is difficult to draw any firm conclusions. Further research is warranted to establish the role and the perfect timing of administration of DXM to treat COVID-19.

\section{Reference(s) and grant ackowledgment(s)}

1. Ye Q, Wang B, Mao J. The pathogenesis and treatment of the 'Cytokine Storm' in COVID-19. J of infect. 2020;80(6):607-613.

2. Villar J, Ferrando C, Martínez D, Ambrós A, Muñoz T, Soler JA, et al. Dexamethasone treatment for the acute respiratory distress syndrome : a multicentre, randomised controlled trial. Lancet Respir Med. 2020;8(3):267-276.

3. Bhimraj A, Morgan RL, Shumaker AH, et al. Infectious Diseases Society of America Guidelines on the Treatment and Management of Patients with COVID-19 [published online ahead of print, 2020 Apr 27]. Clin Infect Dis. 2020. doi:10.1093/cid/ciaa478.

4. Li L, Li R, Wu Z, Yang X, Zhao M, Liu J, Chen D. Therapeutic strategies for critically ill patients with COVID-19. Ann Intensive Care. 2020;10(45).

5. Ingraham NE, Lotfi-emran S, Thielen BK, Techar K, Morris RS, Holtan SG, et al. Immunomodulation in COVID-19. Lancet 2020;10(1)45.

6. Sanders JM, Monogue ML, Jodlowski TZ, Cutrell JB. Pharmacologic Treatments for Coronavirus Disease 2019 (COVID-19): A Review. JAMA. 2020;323(18):1824-1836.

7. Alhazzani W, Møller MH, Arabi YM, et al. Surviving Sepsis Campaign: quidelines on the management of critically ill adults with Coronavirus Disease 2019 (COVID-19). Intensive Care Med. 2020;46(5):854-887.

8. Bouadma L, Lescure FX, Lucet JC, Yazdanpanah Y, Timsit JF. Severe SARS CoV-2 infections: practical considerations and management strategy for intensivists. Intensive Care Med. 2020:46(4):579-82.

9. Zhu N, Zhang D, Wang W, Li X, Yang B, Song J, et al. A novel coronavirus from patients with pneumonia in China, 2019. N Engl J Med. 2020;382(8):727-33.

\section{8}

Interactive online tutorials for knowledge-sharing during a pandemic: Experience of the OxPal Partnership

R. Conway-Jones ${ }^{1}$; R. Jurdon ${ }^{1} ;$ T. Ali $^{2}$

${ }^{1}$ Medical sciences division, University of Oxford, Oxford, United Kingdom: ${ }^{2}$ Intensive care medicine, Buckinghamshire Healthcare NHS Trust, Aylesbury, United Kingdom

Correspondence: R. Conway-Jones

Intensive Care Medicine Experimental 2020, 8(2): 000828

Introduction: Web-based distance-learning is increasingly used during the COVID-19 pandemic. OxPal is a pre-established medical education partnership between University of Oxford medical students and their counterparts in Palestine. Our work has shown online learning to be feasible and effective for knowledge sharing to the occupied Palestinian territories (oPt) 1. In response to the COVID-19 pandemic, OxPal rapidly organised and conducted a lecture on the virology and epidemiology of COVID-19. In feedback, the most common request was for further teaching on COVID-19 management (13/36 requests), which was supported by focus group discussion. Conducting teaching internationally raises unique challenges in understanding and responding rapidly to local needs.

Objectives: We seek to evaluate the feasibility and efficacy of interactive, real-time online tutorials on the management of COVID-19 as a method for knowledge sharing to the oPT during the pandemic.

Methods: Our established network was used to advertise tutorials to medical students and clinicians in the oPt ( $>800$ unique emails and 2 social media platforms). Tutorials were conducted by a critical care consultant via Zoom. A systematic approach to management of severe COVID-19 was provided. The lecturer's local guidance was shared and its evidence base discussed. Feedback was elicited via an online survey. The primary outcomes were attendance and learner-reported change in confidence in COVID-19 management. As of June 2020, 3 such sessions have been conducted and placed on Youtube2.

Results: Primary outcome data is shown in Table 1. Feedback came from students at all medical schools in the oPT, from levels spanning Year 4 Medical Student to Resident doctor.

Table 1.

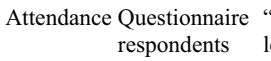

(n)

$\begin{array}{lll}\text { Lecture 1: } & 24 & 8 \\ \begin{array}{l}\text { Assessment } \\ \text { \& Imaging }\end{array} & & \\ \begin{array}{l}\text { Lecture 2: } \\ \text { Sepsis \& }\end{array} & & \\ \text { ARDS } & & \\ \text { Lecture 3: } & 5 & \text { N/A } \\ \text { Ventilation } & & \\ \text { \& Proning } & \end{array}$

When asked to rate tutorial quality on a 1-5 scale, $7 / 8$ respondents for Lecture 1 rated tutorial quality as 4 (Good); for Lecture $24 / 9$ respondents rated the tutorial quality as 4 (Good) and 2 rated it as 5 (Excellent). 
$7 / 8$ and $7 / 9$ described the difficulty of Lectures 1 and 2 respectively as '3- About right' on a 1-5 scale.

Conclusion: Online distance-learning is feasible and effective for knowledge sharing to the oPt during the COVID-19 pandemic. We saw a drop in attendance over 3 sessions. This could be due to a reduction in new cases in the oPt in that time (May to mid-June 2020)3, reducing perceived need. There were also medical school examinations in June. Therefore, lecture plans must be made in response to immediate need and be systematically adaptable as the target population's situation changes.

\section{Reference(s) and grant ackowledgment(s)}

1. This research received no specific grant from any funding agency in the public, commercial, or not-for-profit sectors.

2. 1. Penfold RS, Ali MA, Ali AM, Patel I, MacGregor T, Shankar S, et al. Evaluation of the first year of the Oxpal Medlink: A web-based partnership designed to address specific challenges facing medical education in the occupied Palestinian territories. JRSM Open. 2014;5(2):204253331351769.

3. 2. OxPal Medlink. Ox Pal Medlink - YouTube [Internet]. [cited 2020

Jun 27]. Available from: https://www.youtube.com/channel/ UCImkl_oB5igJjU2-zpjjCjg

4. 3. Nazzal S. Corona Virus (COVID-19) in Palestine [Internet]. 2020 [cited 2020 Apr 28]. Available from: https://corona.ps/

\section{0}

Infectious diseases prevention: Two years after enrolling on a national programme

A. Paula'; D. Mestre ; J. Oliveira ${ }^{1}$; D. Pinto ${ }^{1}$; J. Vaz ; M. Galado ${ }^{1}$; J. Espinho ${ }^{2}$

${ }^{1}$ Intensive care unit, Hospital José Joaquim Fernandes, Beja, Portugal;

${ }^{2}$ Infection control committee, Hospital José Joaquim Fernandes, Beja, Portugal

Correspondence: A. Paula

Intensive Care Medicine Experimental 2020, 8(2): 000830

Introduction: Throughout 2016 to 2018 our centre enrolled in a national programme that aimed to study the good practices in infectious diseases prevention and to implement a national bundle. This project was conducted due to Portugal's high rates of nosocomial infections, reaching almost twice the European numbers in 2013, with substantially high expenses on health [1].

Methods: Two different types of infections were studied in our intensive care unit (ICU): central venous-catheter (CVC) related bacteremia and intubation-associated pneumonia. Collaborative Breakthrough was implemented and results were analysed.

Results: Our ICU represents $2,2 \%$ of the national patients studied (in 19 hospitals), with an average age difference of 3 years older than the national mean. Despite a high variability, the mean duration period in the ICU was similar to the other hospitals studied, with an average 10,6 days stay. Our mortality was slightly higher than the studied ( $18 \%$ mortality), but with no high variability throughout the years analysed, in oppose to our higher SAPS II scores values, that averaged 49, in comparison with a national SAPS II score mean of 44 . Our patients mostly diverged on the necessity for antibiotics, with only $16 \%$ need, compared with $51 \%$ nationally. Also showing smaller values were the need for intubation ( 58 vs $75 \%$ ) and the average intubation period (5,7 vs 6,6 days).

The results of the infectious outcomes were consistent and improving throughout the years [2]. Both CVC placement and intubation numbers per patient were lower in our ICU than expected. In 2015 there were 0,7 blood infections per 1000 days of CVC, with a reduction to 0,6 in 2019, one year after the national program (representing a $14 \%$ reduction). Nationally there was a significant reduction of $23 \%$, but rarely reaching values lower than one blood infections per 1000 days of central venous access. On the other hand, the number of intubation-associated pneumonia reported was reduced by $68 \%$ in our ICU for the past 10 years, in comparison to a $30 \%$ national reduction, with only $1 \%$ of patients admitted to our ICU in 2019 suffering intubationassociated pneumonia.
Conclusion: Hospital-acquired infections are a significant health issue [3]. Several guidelines and protocols have been implemented worldwide, aiming for its reductions. A lot has been accomplished the past decade, but there is still a lot that can be done, as this national program has come to show us.

Reference(s) and grant ackowledgment(s)

1. 3. Report on the burden of endemic health care-associated infection worldwide. World Health Organization. 2011

2. 1. Xavier LL. Stop infecção hospitalar - Um desafio Gulbenkian. Fundação Calouste Gulbenkian. 2015.

3. 2. Paiva JA, Sousa P, Fonseca R. Stop infecção hospitalar - Um desafio Gulbenkian: Resultados. Fundação Calouste Gulbenkian. 2018.

000844

Immunoglobulins as anti-inflammatory treatment for SARS CoV19

J. Vilches Mira'; C. López Martin ${ }^{1}$; M. De Alba Aparicio ${ }^{1}$; J. Rodríguez.

Gómez'; R. León López'; C. De La Fuente Martos

${ }^{1}$ Intensive Care Unit, Hospital Universitario Reina Sofia, Córdoba, Spain

Correspondence: J. Vilches Mira

Intensive Care Medicine Experimental 2020, 8(2): 000844

Introduction: The pandemic caused by SARS-CoV-2 is an epidemiological emergency that has led to the rapid search for effective treatments to reduce the complications and improve the survival rate of the population affected by this virus.

One of the main complications that can result from SARS-CoV- 2 is the cytokine storm or hypercytokinemia: a syndrome characterised by the elevation of inflammatory markers, such as D-dimer, interleukin-6, ferritin and C-reactive protein, which, in addition, leads to clinical and radiological deterioration. In light of this inflammatory response, some therapies have been considered, such as corticosteroids in high doses or monoclonal antibodies like Tocilizumab (anti-IL-6) or Bevacizumab (anti-IL-2).

Although scientific associations have not endorsed the use of immunoglobulins as immunomodulators, this therapy could be considered for those patients with refractory acute respiratory distress syndrome (ARDS) and contraindications to previous treatments (due to suspected superinfection, among other causes).

Objectives: To describe our experience in the use of immunoglobulins in CoVid-19 patients with refractory ARDS with inflammatory response criteria and contraindications to the immunomodulatory therapy.

To describe radiological evolution, changes in the inflammatory markers, mortality and complications after the administration of immunoglobulins.

Methods: Retrospective, descriptive, observational study conducted in the Intensive Care Unit of the Hospital Universitario Reina Sofía in Córdoba, Spain.

Epidemiological variables, complications, mortality, radiological controls and inflammatory markers (interleukin-6, D-dimer, ferritin, C-reactive protein, lymphocytes) were collected in three different stages: immediately before, during and after administering a course of immunoglobulins in doses of $2 \mathrm{~g}$ per $\mathrm{kg}$ of the patient's body weight over a period of 3 to 6 days.

At the time of writing, some of the patients involved are still in the Intensive Care Unit.

Results: Of the total number of patients admitted to our ICU due to CoVid-19 $(n=41), 8$ received treatment with immunoglobulins. 3 of the patients were women; one of them died. One of the patients is still in ICU and the rest were discharged. The average age was 59.8 years old. The time elapsed between the admission to the ICU and the initial treatment with immunoglobulins ranges from 3 to 8 days.

After analysing the data, we noticed the average of all inflammation reactants had decreased, while lymphocytes had increased after the immunoglobulins treatment: 


\begin{tabular}{|llll|}
\hline & Before & During & After \\
C-reactive protein & 257 & 151 & 85 \\
D-dimer & 13,631 & 13,604 & 6,293 \\
Interleukin-6 & 1,263 & 296 & 295 \\
Ferritin & 5,248 & 5,614 & 1,520 \\
Lymphocytosis & 0.78 & 1.16 & 1.39 \\
(Mean values) & & & \\
\hline
\end{tabular}

There was a significant response in the decrease of the interleukin- 6 $(n=7)$. D-dimer dropped over $50 \%$ in 3 patients. Ferritin decreased over $50 \%$ in 3 of the cases. One of the patients showed elevation of theinterleukin-6, ferritin and D-dimer, probably due to superinfection. The CRP also fell by half in other 3 cases. Only one patient suffered from thrombotic events (thrombophlebitis in inferior vena cava). While monitoring the affected pulmonary fields in the serial chest $x$-rays, we noticed a drop in the average of affected fields: 3.12 before the treatment, 3.8 during and 2.5 after the treatment.

We also observed an improvement in the lymphopenia of our patients. However, it should be noted that in 3 of the 5 patients who developed an increase of absolute lymphocytes and of their white blood cell percentage, this was due to a general increase of the leukocytosis.

Conclusion: In cases of refractory ARDS and increase of the inflammatory response in CoVid-19 patients admitted to ICU with contraindications to monoclonal antibody therapy or to corticosteroid therapy in high doses, we could consider administering $1 \mathrm{~g}$ of immunoglobulin per $\mathrm{kg}$ of the patient's body weight for a time period between 3 and 6 days.

Studies with a larger sample size are required to confirm the effectiveness of immunoglobulins in this group of CoVid-19 positive patients.

\section{Reference(s) and grant ackowledgment(s)}

1. Channappanavar et al. 2017. Pathogenic human coronavirus infections: causes and consequences of cytokine storm and immunopathology. Semin. Immunopathol. https://doi.org/10.1007/s00281- 017-0629-x

2. Guo et al. 2018. Adverse Effects of Immunoglobulin Therapy. Front Immunol. https://doi.org/10.3389/fimmu.2018.01299

3. Shen et al. 2020. Treatment of 5 Critically III Patients With COVID-19 With Convalescent Plasma. JAMA. https://doi.org/doi:10.1001/jama.2020.4783

4. Hartung HP, Mouthon L, Ahmed R, Jordan S, Laupland KB, Jolles S. Clinical applications of intravenous immunoglobulins (IVIg)-beyond immunodeficiencies and neurology. Clin Exp Immunol. 2009;158 Suppl 1(Suppl 1):23-33. doi:10.1111/j.1365-2249.2009.04024.x

5. Wei Cao, Xiaosheng Liu, Tao Bai, Hongwei Fan, Ke Hong, Hui Song, Yang Han, Ling Lin, Lianguo Ruan, Taisheng Li, High-Dose Intravenous Immunoglobulin as a Therapeutic Option for Deteriorating Patients With Coronavirus Disease 2019, Open Forum Infectious Diseases, Volume 7, Issue 3, March 2020

6. Not

\section{9}

Nosocomial infections during COVID-19 surge: increased incidence and very common use of broad-spectrum antibiotics

C. Simon' ; L. Allemann²; M. Thalmann²; Y. Fleury ${ }^{1}$; H. Ksouri ${ }^{1}$; C. Chuard ${ }^{3}$; G. Sridharan ${ }^{1}$

${ }^{1}$ Intensive care unit, Fribourg hospital, Villars-sur-Glâne, Switzerland; ${ }^{2}$ Medical faculty, University of Fribourg, Fribourg, Switzerland; ${ }^{3}$ Infectious disease, Fribourg hospital, Villars-sur-Glâne, Switzerland

Correspondence: $C$. Simon

Intensive Care Medicine Experimental 2020, 8(2): 000889

Introduction: COVID-19 has challenged our health system and our standard practices. Mechanically ventilated (MV) COVID-19 patients have long intensive care unit (ICU) stays [1] increasing their exposure to nosocomial infections (NI).
Objectives: In a perspective of quality improvement, we studied the incidence of $\mathrm{NI}$ and use of broad-spectrum antibiotics (BSA) in MV COVID-19 patients.

Methods: We collected data retrospectively for every MV COVID-19 patient admitted to our 18- to 38-bed expanded ICU between 01.03. and 30.04.2020. ICU days, ventilation days (VD), catheter days (CD), use of $\mathrm{BSA}$, fever days $\left(>38^{\circ} \mathrm{C}\right)$ and c-reactive protein (CRP) were recorded. Each suspected $\mathrm{NI}$, defined by the introduction of $\mathrm{BSA} \geq 48 \mathrm{~h}$ after admission or repeated administration of BSA after an antibiotic free period $\geq 48 \mathrm{~h}$, was reviewed by an infectiologist. NI were confirmed using CDC criteria [2] for ventilator associated pneumonia (VAP), central line associated blood stream infections (CLABSI), urinary tract infections (UTI), or clinical evidence for septic shock (SS) and bacteremia (BE) from another origin.

Results: Of 54 COVID-19 patients, $37 \mathrm{MV}$ patients (69\%) spent a total of 672 ICU days, with 504 VD and 545 CD. They had fever during $26.5 \%$ of ICU days. Median peak CRP was 309 mg/l (IQR, 264-365) after a median stay of 3 days (IQR, 2-5). Among 31 suspected NI, 21 (68\%) NI were confirmed: 4 VAP, 6 CLABSI, 1 UTI, 8 SS and 2 BE from another origin. The incidence of VAP and CLABSI was respectively 7.9/1000 VD and 11/1000 CD. Our 2019 annual incidence for VAP and CLABSI was $3.9 / 1000 \mathrm{VD}$ and 1.7/1000 CD. Global incidence of confirmed $\mathrm{NI}$ was $31.3 / 1000$ ICU days.

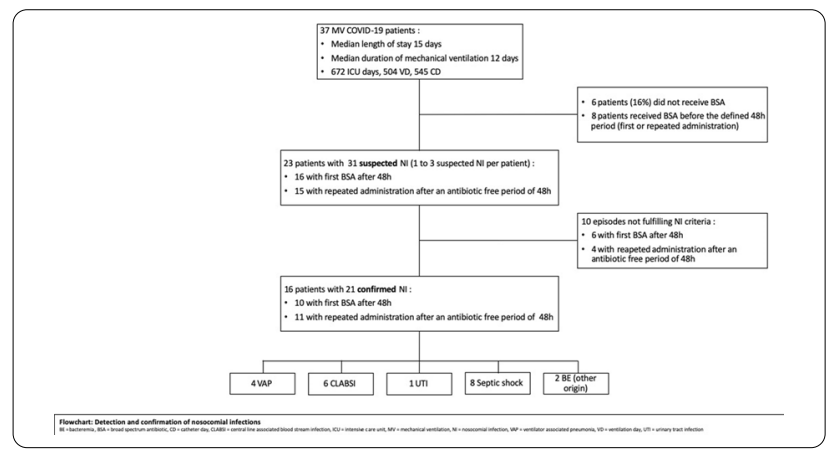

Conclusion: The incidence of confirmed VAP and CLABSI was higher in MV COVID-19 patients than in our standard ICU population of 2019. COVID-19 patients might have a greater risk of $\mathrm{NI}$ due to anatomical barrier disruption and maladaptive immune response, but other factors such as cohorting of several patients in the same room, using gloves for self-protection with insufficient replacement and temporary deviation from standardized procedures could have contributed to the incidence of NI. BSA were often prescribed early during ICU stay, reflecting physician's anxiety facing this new disease and the difficulty to diagnose NI. Severe COVID-19 pneumonia induces a prolonged fever, high inflammatory state, diffuse lung infiltrates and protracted respiratory failure, which makes classical criteria for VAP less specific. In that setting, NI definitions should be refined to find an optimized set point for BSA introduction, avoiding under- and overuse.

Reference(s) and grant ackowledgment(s)

1. [2]T. C. Horan et al. Am. J. Infect. Control, 2008;36(5):309.

2. [1]G. Grasselli et al. JAMA, 2020;323(16):1574.

000897

Therapeutic management in COVID-19 patients admitted in our Intensive Care Unit during the pandemic

N. Cruza Leganés ${ }^{1}$; C. Sena ${ }^{1}$; F. Arbol' ${ }^{1}$; A. Simon ${ }^{1}$; V. Losada'; A. Escudero'; B. Segovia'; JL. Martin ${ }^{1}$; JR. Herrera'; 'L. Serrano'; MA. Taberna ${ }^{1}$

${ }^{1}$ Intensive Care Unit, General Hospital Nuestra Señora del Prado, Talavera de la Reina, Spain

Correspondence: N. Cruza Leganés

Intensive Care Medicine Experimental 2020, 8(2): 000897 
Introduction: COVID-19 pandemic has been a challenge for healthcare professionals. This is a virus that seemed to follow a clinical pattern with an intense inflammatory response and thrombogenic load, for which we don't have a specific treatment.

Objectives: Different therapeutic strategies for COVID-19 have been used to target the viral replication, to limit the inflammatory response and to avoid thrombotic complications. We describe the prescribed treatment of COVID-19 patients (COVp) admitted in our ICU during the pandemic. This treatment was based on the protocol of our center, the availability of drugs and the published literature at the time.

Methods: A retrospective descriptive observational analysis about treatment of COVp admitted in our ICU during the pandemic, from March 13 to May 4, 2020, along with the ICU clinical evolution of the same patients under these treatment guidelines.

Results: From March 13th to May 4th 2020, 48 COVp were admitted in our ICU: 31 males $(64,6 \%)$, with an average age of 57,7 y.o. (CI 95\% $54,6-60,9)$ and APACHE II score 17,25 (CI 95\% 15,6-18,7). The time lapse from the onset of symptoms until admission in ICU was 8,8 days $(\mathrm{Cl}$ $95 \% 7,6-10,1)$. The average white bloodcells when entering was 9,8 x 109/L (Cl 95\% 8,3-11,3), PCR 169 mg/L (Cl 95\%141-197), D dimer $5841 \mathrm{ng} / \mathrm{ml}$ (Cl 95\% 1737-9945), ferritin $1807 \mathrm{ng} / \mathrm{ml}$ (Cl 95\% 17261888 ) and 37 patients $(77,1 \%)$ had a lymphocyte count $<1.0 \times 109 / \mathrm{L}$. Upon admission to the ICU, all patients were treated with hydroxychloroquine, 33 patients $(68,8 \%)$ with azithromycin associated and 47 patients $(97,9 \%)$ with lopinavir/ritonavir. Methylprednisolone was administrated to 44 patients $(91,7 \%)$, of which $13 / 44$ (29,5\%) $40 \mathrm{mg} / 8 \mathrm{~h}$ with variable duration (discretion of the responsible physician), and $31 / 44(70,4 \%) 500 \mathrm{mg} / 24 \mathrm{~h}$ for five days. Interferon beta- $1 \mathrm{~b}$ was administered to 38 patients $(79,2 \%)$, tocilizumab to 10 patients $(20,8 \%)$, and anakinra to 4 patients $(8,3 \%)$. Enoxaparin was given to 44 patients (91,7\%): 18/44 (37,5\%) with antithrombotic prophylaxis dose, 20/44 $(45,4 \%)$ with $1 \mathrm{mg} / \mathrm{kg} / 24 \mathrm{~h}$ and $6 / 44(13,6 \%)$ with $1 \mathrm{mg} / \mathrm{kg} / 12 \mathrm{~h}$. Forty patients $(83,3 \%)$ needed invasive mechanical ventilation with an average duration of 22,2 days (IC $95 \%$ 17,3-27); 35 patients (72,9\%) needed vasopressors and 6 patients $(12,5 \%)$ renal replacement therapy. Our patients showed: tetraparesis that increased the mechanical ventilation and the $\mathrm{UCl}$ stay days, 22 patients $(45,8 \%)$; delirium, 21 patients $(43,8 \%)$; nosocomial infection, 17 patients $(35,4 \%)$; arrhythmias, 11 patients $(22,9 \%)(9 / 11$ atrial fibrilation and supraventricular tachycardia); and vascular thrombosis, 2 patients $(4,2 \%)$. The average ICU stay was 23,1 days ( $\mathrm{Cl} 95 \%$ 18,4-27,8). 13 patients died (27\%).

Conclusion: All our patients were treated with hydroxychloroquine and most of them with lopinavir/ritonavir, methylprednisolone and interferon beta-b1. Half of them received higher heparin doses than those used for antithrombotic prophylaxis.

\section{8}

Combination Therapy with Meropenem and Ciprofloxacin shows a Synergistic Benefit against Intermediate and Resistant Pseudomonas aeruginosa Isolates from Critically III Patients in the Hollow-Fiber Infection Model

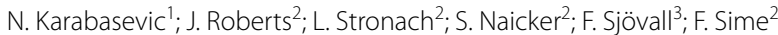

${ }^{1}$ Medical faculty, Lund University, Lund, Sweden, Lund, Sweden; ${ }^{2}$ Centre for translational anti-infective pharmacodynamics, school of pharmacy, The University of Queensland, Brisbane, Australia; ${ }^{3}$ Department of intensive care and perioperative medicine, Skåne University Hospital, Malmö, Sweden

Correspondence: N. Karabasevic

Intensive Care Medicine Experimental 2020, 8(2): 000898

Introduction: Patients with sepsis and septic shock have altered antimicrobial pharmacokinetics, which often results in suboptimal drug concentrations. Septic patients are also at risk for infections with resistant organisms such as Pseudomonas aeruginosa. Combination therapy against $P$. aeruginosa infections is still debatable.

Objectives: This study aims to investigate whether there is any synergistic benefit, in terms of bacterial killing and suppression of emergence of resistance, of a meropenem-ciprofloxacin combination therapy in comparison to monotherapy with the same antibiotics against $P$. aeruginosa in septic patients with altered pharmacokinetics, using a dynamic Hollow-Fiber Infection Model (HFIM).

Methods: Two intermediate to resistant $P$. aeruginosa isolates code numbered 27 (MICmeropenem of $4 \mathrm{mg} / \mathrm{L}$ and MICciprofloxacin of 1 $\mathrm{mg} / \mathrm{L}$ ) and 40 (MICmeropenem of $16 \mathrm{mg} / \mathrm{L}$ and MICciprofloxacin of 1 $\mathrm{mg} / \mathrm{L}$ ) were evaluated in a 5-day HFIM investigation. Changes of the total and resistant bacterial populations were evaluated using quantitative cultures. The studied regimens were equivalent to $2 \mathrm{~g}$ of meropenem every $8 \mathrm{~h}$ (30 min infusion), $600 \mathrm{mg}$ of ciprofloxacin every 8 $\mathrm{h}$ (45 min infusion) and the combination of the same antibiotics. The doses and pharmacokinetic simulations were based on recommendations and data from previous studies on critically ill patients $(1,2)$.

Results: In both HFIM investigations, combination therapy achieved considerably greater bacterial killing and resistance suppression compared to the monotherapies. The monotherapies started to fail after $24 \mathrm{~h}$, with increased bacterial concentrations of 3-6 $\log 10 \mathrm{CFU} / \mathrm{ml}$. From there, strain 40 plateaued at $8-9 \log 10 \mathrm{CFU} / \mathrm{ml}$ after $48 \mathrm{~h}$. Strain 27 continued increasing from 3-6 $\log 10 \mathrm{CFU} / \mathrm{ml}$ at $48 \mathrm{~h}$ until reaching 9-10 $\log 10 \mathrm{CFU} / \mathrm{ml}$ at $120 \mathrm{~h}$. In contrast, the combination therapies suppressed bacterial growth from $6 \mathrm{~h}$ and onward. Additionally, the monotherapies had accelerated growth of resistant subpopulations reaching 8-9 $\log 10 \mathrm{CFU} / \mathrm{ml}$, while no resistance was detected in the combination therapies.

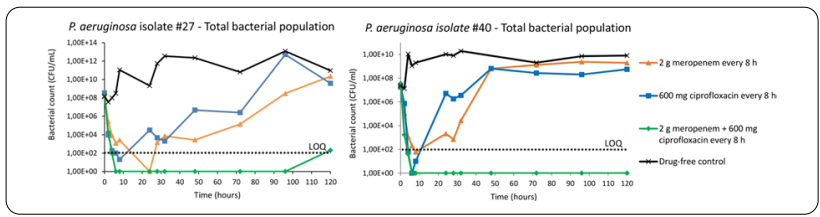

Conclusion: This study suggests a synergistic benefit of combination therapy with meropenem and ciprofloxacin against $P$. aeruginosa in critically ill patients with altered pharmacokinetics. Our findings support empirical combination therapy against $P$. aeruginosa, especially for resistant isolates.

Reference(s) and grant ackowledgment(s)

1. This study was funded by the Australian National Health and Medical Research Council for a Centre of Research Excellence (APP1099452)

2. 1. Roberts JA, Alobaid AS, Wallis SC, Perner A, Lipman J, Sjövall F. Defining optimal dosing of ciprofloxacin in patients with septic shock. J Antimicrob Chemother. 2019 Jun 1;74(6):1662-9.

3. 2. Sjövall F, Alobaid AS, Wallis SC, Perner A, Lipman J, Roberts JA. Maximally effective dosing regimens of meropenem in patients with septic shock. J Antimicrob Chemother. 2018 Jan 1;73(1):191-8

\section{1}

Antimicrobial consumption during SARS-CoV2 outbreak

A. Knapen'; LP. Moulaert'; R. Courcelle'; P. Henin'; JB. Mesland ${ }^{1}$

${ }^{1} \mathrm{ICu}, \mathrm{CH}$ Jolimont, La Louvière, Belgium

Correspondence: J.B. Mesland

Intensive Care Medicine Experimental 2020, 8(2): 000911

Introduction: Since the beginning of SARS-CoV2 outbreak, paucity of data has been published regarding rate of co-infection and super infection. Most guidelines have recommended empirical antimicrobial for mechanically ventilated patients admitted in ICU (1). In the meantime, the differentiation between bacterial super infection and bacterial colonization of the respiratory tract has been challenging especially with the hyperinflammatory state of critical cases admitted in ICU.

Consequently, high prescription rate of antibiotics has been described in the literature for SARS-CoV2 patients but no data has been published on antibiotic consumption specifically in ICU patients.

Objectives: Our main objective is to evaluate the antibiotic consumption (Days Of Therapy, antibiotic prescribed, timing within ICU 
hospitalization) in our critical SARS-Cov2 patients and evaluate the potential effect on antimicrobial resistance.

Methods: In an observational, retrospective and monocentric study, we reviewed antibiotic prescription (name, duration, indication) of all SARS-Cov2 patients admitted in our ICU between march 2020 and may 2020. $\beta$-Lactams were then stratified by their tendency to drive antimicrobial resistance based on classification developed by Weiss and al. (class 1 to 6 ) (2).

Results: Between march 2020 and may 2020, 44 critical SARS-CoV2 have been admitted to our ICU ( 22 beds). We have been able to collect data from 41/44 patients. Mean age at admission was 63 years. $83 \%$ received mechanical ventilation (34/41 patients), for a total of 505 days, and 24\% (10/41 patients) received ECMO therapy, for a total of 172 days. Mortality in our unit was $50 \%$. All but one patient received hydroxychloroquine and 38/41 patients received azythromycine as it was recommended by our national Belgian guidelines. Both medications were given during 5 days.

$92 \%$ received an antibiotic at some point during their ICU stay. Based on recommendations, $46 \%$ (19/41 patients) received empirical therapy at admission despite the fact that none of our patients were in shock. Considering co-infectionas positive microbiology within the first 48 hours after admission, then $17 \%$ were in that case. First antibiotic prescription for the rest of the cohort occurred for $9 / 41$ patients within the first week and for $6 / 41$ patients within the second week.

From 682 patient ICU days, 396 Days Of Therapy (DOT) of antibiotics were documented, representing 581 DOT/1000 patient-days. Prescription was distributed as follows: $26 \%$ for amoxicilline-clavulanate, $23 \%$ for piperacilline/tazobactam, $12 \%$ for cefuroxime, $7 \%$ for ceftriaxone, ceftazidime, and vancomycine, $6 \%$ for meropenem, $5 \%$ for amikacine, $2 \%$ for amoxicilline, flucloxacilline, temocilline, ciprofloxacine, and $1 \%$ for cefepime.

$37 \%$ of all antibiotics were at least classified as class 4 (2), and $44 \%$ of prescription had an anti-pseudomonal coverage. Non effective prescription (pathogen with documented resistance to the antibiotic prescribed) in $8 \%$ of cases (33/396 DOT).

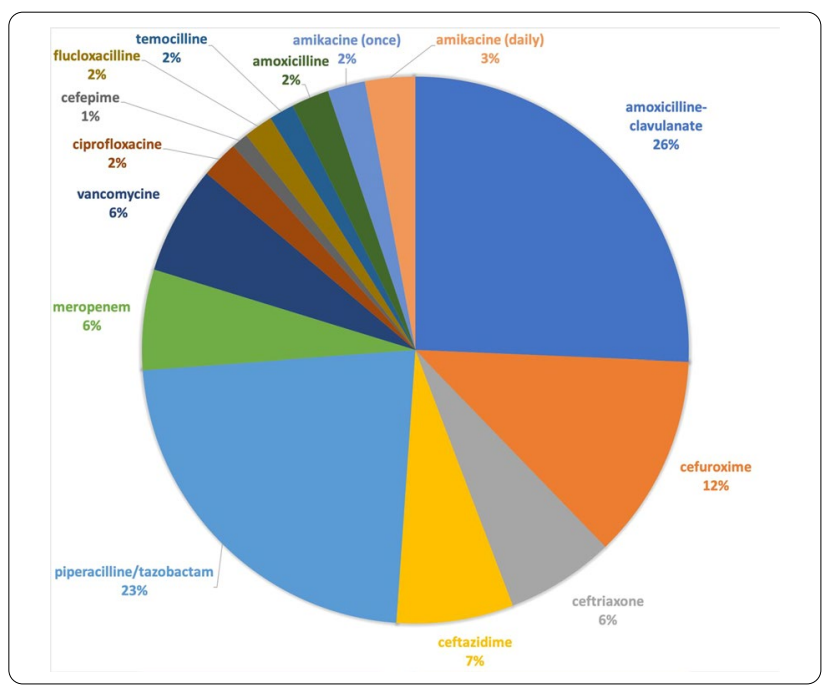

Conclusion: Antibiotic consumption was in the range of previously described consumption from general ICU outside of SARS-CoV2 pandemic : De Bus and al. reported 1232 DOT/1000 patient-days (3). Consumption decrease would have been possible by avoiding empirical administration at admission (absence of shock in all patients), and use of newly available technique like RT-PCR.

Concern about antimicrobial resistance need to be emphasized, resulting from increased patient exposure to antimicrobials, often suboptimally or inappropriately used (4). Indeed, on the one hand hyperinflammatory state might have increased the likelihood of antibiotic prescription in case of bacterial superinfection. And on the other hand long duration of ventilation and previous antimicrobial exposure might have prompted the clinician to prescribe a broad spectrum antibiotherapy.

\section{Reference(s) and grant ackowledgment(s)}

1. (1) Alhazzani W, Møller MH, Arabi YM, et al. Surviving Sepsis Campaign: guidelines on the management of critically ill adults with Coronavirus Disease 2019 (COVID-19). Intensive Care Med. 2020;46(5):854-887. doi:10.1007/ s00134-020-06022-5

2. (2) Weiss E, Zahar JR, Lesprit P, et al. Elaboration of a consensual definition of de-escalation allowing a ranking of $\beta$-lactams [published correction appears in Clin Microbiol Infect. 2015 Nov;21(11):e81. Whoerter, P-L [corrected to Woerther, P L]]. Clin Microbiol Infect. 2015;21(7):. doi:10.1016/j. cmi.2015.03.013

3. (3) De Bus L, Gadeyne B, Steen J, et al. A complete and multifaceted overview of antibiotic use and infection diagnosis in the intensive care unit: results from a prospective four-year registration. Crit Care. 2018;22(1):241. Published 2018 Sep 29. doi:10.1186/s13054-018-2178-7

4. (4) Timothy M Rawson, Luke S P Moore, Nina Zhu, Nishanthy Ranganathan, Keira Skolimowska, Mark Gilchrist, Giovanni Satta, Graham Cooke, Alison Holmes, Bacterial and fungal co-infection in individuals with coronavirus: A rapid review to support COVID-19 antimicrobial prescribing, Clinical Infectious Diseases, ciaa530, https://doi.org/10.1093/cid/ciaa530

\section{2}

Tigecycline reduces blood glucose in the critically ill patients with severe infection

W. Huang ${ }^{1}$; B. Li ${ }^{2} ; Y$. Lu' ${ }^{2} ; Y$. Li ${ }^{1}$

${ }^{1}$ Department of intensive care medicine, The Sixth People's Hospital, Shanghai Jiao Tong University, Shanghai, China; ${ }^{2}$ Department of epidemiology, Fudan University School of Public Health, Shanghai, China

Correspondence: W. Huang

Intensive Care Medicine Experimental 2020, 8(2): 000922

Introduction: Tigecycline is a last-resort antibiotic to treat severe infection caused by multidrug-resistant bacteria. It may induce decreased blood glucose, as described elsewhere [1-3]. In the drug inserts, incidence of decrease in blood glucose is labeled as $<2 \%$. In the critically ill patients, severe infection is common; however, the impact of Tigecycline reducing blood glucose remains to be assessed. Objectives: This study is to determine if Tigecycline reduces blood glucose in critically ill patients with severe infection and further identify the factors associated with decreased blood glucose.

Methods: We retrospectively studied critically ill patients who received Tigecycline in the ICU department of the Sixth's People Hospital in Shanghai, China from January, 2018, through July, 2019. Mixedmodel repeated measure was utilized to compare the mean blood glucose before and after Tigecycline administration. The two phases (before and after Tigecycline administration) and daily blood glucose (measured at $6 \mathrm{am}, 2 \mathrm{pm}$ and $10 \mathrm{pm}$ ) were presented as two repeated factors. Other factors were considered covariates or between-subjects factors in the model, including sex, age, comorbidity, prognosis, duration and dosage of Tigecycline administration, and intravenous glucose infusion. Multivariate linear regression model was employed to determine the association of above-mentioned factors with decrease in blood glucose. Moreover, binary logistic regression was utilized to determine the factor associated with hypoglycemia that was defined as a decrease $\geq 2.8 \mathrm{mmol} / \mathrm{L}$ in the study. Receiver operating characteristic (ROC) and its area under curve (AUC) were then employed to evaluate the factor in predicting hypoglycemia. All analyses were performed using SPSS Statistics, version 23.0 (IBM Corporation, Armonk, NY). A $P$ value $<0.05$ was considered statistically significant.

Results: A total of 168 patients were studied, including 105 male and 63 female, with an average age of $64.2 \pm 15.8$ yrs. After Tigecycline administration, daily blood glucose at three points of time significantly decreased $(P<0.001)$, regardless of other factors. The decrease in blood glucose, defined as the difference between the minimum blood glucose before and after Tigecycline administration, was positively correlated with death $(\beta=0.78, P=0.016)$, maximum decrease 
in blood glucose within $24 \mathrm{hrs}(\beta=0.67, P=0.032)$, and duration of administration (hrs) $(\beta=0.004, P<0.001)$. Hypoglycemia was identified in 19 patients $(11.3 \%)$, which was determined to be associated with death $(\mathrm{OR}=3.71, P=0.014)$ and maximum decrease in blood glucose within $24 \mathrm{hrs}(\mathrm{OR}=1.18, P=0.002)$. The AUC for the maximum decrease in blood glucose within $24 \mathrm{hrs}$ was determined to be $0.76(95 \% \mathrm{Cl}$, $0.66-0.86$ ) and best cutoff value was $4.35 \mathrm{mmol} / \mathrm{L}$ (sensitivity, 0.90 ; specificity, 0.53).

Conclusion: Tigecycline significantly reduces blood glucose in the critically ill patients with severe infection. In addition, decrease in blood glucose is associated with risk of death, duration of Tigecycline administration, and maximum decrease in blood glucose within 24 hrs, further suggesting usage with extreme caution. A decrease $\geq 4.35$ $\mathrm{mmol} / \mathrm{L}$ in blood glucose may predict hypoglycemia, which would facilitate early warning in the critically ill patients.

\section{Reference(s) and grant ackowledgment(s)}

1. Chen Y, Li L, Zhang N, Li H. Tigecycline-induced Sustained Severe Hypoglycemia: A Case Report. BMC Pharmacol Toxicol, 2019, 20(1): 50.

2. Wallace Jr RJ, Dukart G, Brown-Elliott BA, et al. Clinical Experience in 52 Patients With Tigecycline-Containing Regimens for Salvage Treatment of Mycobacterium Abscessus and Mycobacterium Chelonae Infections. J Antimicrob Chemother, 2014, 69(7): 1945-53.

3. Kadoyama K, Sakaeda T, Tamon A, Okuno Y. Adverse Event Profile of Tigecycline: Data Mining of the Public Version of the U.S. Food and Drug Administration Adverse Event Reporting System. Biol Pharm Bull, 2012, 35(6): $967-70$.

4. This study did not receive any specific grant from funding agencies in the public, commercial, or not-for-profit sectors.

\section{9}

Differences between survivors and no survivors in ICU patients with severe influenza infection, according to nursing and severity indexes

A. Vakalos ; N. Tsikliras ${ }^{1}$; S. Patsatzakis

${ }^{1}$ Icu, Xanthi General Hospital, Xanthi, Greece

Correspondence: A. Vakalos

Intensive Care Medicine Experimental 2020, 8(2): 000039

Introduction: Influenza virus infection is an important cause of mortality worldwide, leading to 250000 to 500000 deaths each year in the developed world. Mortality is caused by the primary viral infection, but also by influenza-associated pulmonary co-infections.

Objectives: Our retrospective observational study aimed to evaluate the difference in the outcome of patients with influenza infection requiring admission to both Medical and Surgical Intensive Care Unit (ICU) served to a community hospital, according to nursing and severity indexes.

Methods: From January 2006 to October 2019, 1332 patients admitted to our ICU. From these, 25 (1.87\%), were indicated with the diagnosis of severe influenza infection. The patients separated into two groups. Group A involved all patients who survived ICU and group B all patients who died in ICU. We looked for statistically significant difference ( $p$-value two-tailed) between the medians values of two groups, using the unpaired Mann - Whitney test or the unpaired t-test Welch corrected according to the normality test, according to nursing (Age, sex, LOS, MVD) and severity (Simplified Acute Physiology Score: SAPS II, predicted mortality by SAPS II, Acute Physiology and Chronic Health Evaluation: APACHE II, predicted mortality by APACHE II, Glasgow Coma Scale: GCS) indexes.

Results: We detected no statistical difference between the two groups according: age $(p=0.6314)$, sex $(p=1.000), G C S(p=0.3830)$, LOS $(p=0.9979), \operatorname{MVD}(p=0.4342)$, predicted mortality by SAPS II score $(\mathrm{p}=0.2001)$

We detected statistical significant higher group $B$ value according to APACHE II score $(p=0.0281)$, predicted mortality by APACHE II score $(p=0.0276)$, SAPS II score $(p=0.0356)$

Conclusion: According to our data, demographic data and nursing indexes were about the same among the two groups. On the other hand, severity scores like APACHE II score and SAPS II score were both statistically significantly higher in group $B$, assuming that patients who died in ICU admitted to ICU in more severe illness. But, only predicted mortality by APACHE II score achieved to be statistically significantly higher in group B. Our data suggest that we can use the APACHE II severity score as an accurate severity score in these patients.

\section{0}

Implementation of an Antibiotic Stewarship Program in an ICU

S. Carvalho Brugger ${ }^{1}$; B. Balsera Garrido'; M. Miralbés Torner ${ }^{1}$; A. Bellés Bellés'; J. Caballero López ${ }^{1}$; M. Vallverdú Vidal ${ }^{1}$

${ }^{1}$ Intensive care unit, University Hospital Arnau de Vilanova, Lleida, Spain: ${ }^{2}$ Microbiology department, University Hospital Arnau de Vilanova, Lleida, Spain

Correspondence: S. Carvalho Brugger

Intensive Care Medicine Experimental 2020, 8(2): 000930

Introduction: Antibiotic Stewarship Programs, in Spain known as PROA, are becoming increasingly important in both hospital and primary care. These programs work on optimizing antibiotic (ATB) prescribing to improve patient prognosis, minimize adverse effects, monitor the onset of resistance, and ensure the use of cost-effective treatments.

Objectives: In this work we review the performance of a PROA team in an ICU.

Methods: The authors present a descriptive analysis of the daily performance of a team of intensivists dedicated to infections, with the application of PROA in a polyvalent $\mathrm{UCl}$, during the year 2019, comparing to the previous year. The interventions carried out by the PROA team were: general recommendations, initiate, de-escale, shift or stop ATB, sampling for cultures, pk/pd optimization, and consults directly made by other doctors to the PROA team.

Results: During 2019, 632 interventions have been made. The recommendation was initiating ATB treatment in $38.1 \%$ of them, and discontinue it in $63,45 \%$, with an adherence of $91.1 \%$, higher than the previous year $(87.9 \%)$. Non-adherence was observed in interventions about days of ATB treatment $(35.71 \%)$, discontinuation of antibiotic therapy by negative cultures (17.85\%) and general recommendations made to the responsible physician (16\%). Adherence was observed mainly in interventions about days of treatment, general recommendations, de-escalation of ATB and culture- directed ATB treatment. The interventions that most often motivated the onset of ATB were: culture - directing ATB treatment (28.63\%), de-escaling (22.82\%), expanding spectrum (15.76\%) and initiate empirical treatment in cases of suspected infection (12.45\%). The most frequently used ATBs on interventions were 3rd generation cephalosporins (cefotaxime 15.35\%), ureidopenicillines (piperacillin-tazobactam 9.5\%), and aminoglycosides and linezolid (8.2\%). $63.44 \%$ of interventions recommended the suspension of ATB treatment, the most common reasons were: days of ATB treatment $(37.65 \%)$, de-escalate $(21 \%)$, negative cultures $(10.22 \%)$ and a few times the suspension because a limitation of therapeutic effort. The most frequently discontinued ATBs were ureidopenicillines (piperacillin-tazobactam 21.7\%), aminoglycosides (amikacin 9.23\%), 3rd generation cephalosporins (cefotaxime $9 \%$ ), linezolid (6.5\%) and azoles (6.23\%). Regarding inquiries made to the PROA team by the UCI team, the advices given were not initiate ATB, not changing antimicrobial therapy and extending the spectrum, with an adhesion of the doctors of $100 \%$. In addition, a total of 100 recommendations have been made (15.8\%) with $91 \%$ adhesion relative to contact isolations (22\%), microbiological sampling (20\%), initiation of ATB therapy (13\%) and not starting ATB or non-indicated ATB treatment (9\%). Overall mortality in 2018 was $10.95 \%$, and $10.67 \%$ in 2019 (not significant). It has been observed a trend for the reduction of multi-resistance since the implementation of the PROA. The rates of BMR infection during ICU stay have been $1.55 / 100$ patients and 2.9/1000 days of stay in 2018, and $1.22 / 100$ patients and $2.47 / 1000$ days of stay in 2019 .

Conclusion: The interventions carried out have had a high adhesion, which reflects the trust placed by the doctors responsible towards the PROA UCI team. These interventions have resulted in a decrease 
in multidrug-resistant bacteria in the ICU, antimicrobial consumption and a better ATB policy, with no implications for mortality. It is important to give out the results obtained to positively strengthen its good work and to be able to identify areas of improvement.

\section{4}

\section{Post-mortem lung biopsies of COVID-19 patients in an Intensive} Care Unit

Á. Vidal González'; JJ. Paez Vargas'; ; J. Fortes²; D. Robaglia'; E. Rosas Carvajal'; VA. Hortigüela Martín ${ }^{1}$; Al. Tejero Redondo'; A. Robles' ${ }^{1}$.

JL. Franqueza'; 'L. Colino Gomez ; M. Piris²; M. Górgolas ${ }^{3}$; L. Prieto-Pérez ${ }^{3}$

${ }^{1}$ Intensive care medicine, Hospital Universitario Fundación Jiménez Díaz, Madrid, Spain; ${ }^{2}$ Pathological anatomy, Hospital Universitario Fundación Jiménez Díaz, Madrid, Spain; ${ }^{3}$ Internal medicine, Hospital Universitario Fundación Jiménez Díaz, Madrid, Spain

Correspondence: Á. Vidal González

Intensive Care Medicine Experimental 2020, 8(2): 000944

Introduction: The full spectrum of COVID-19 infection ranges from an asymptomatic disease, through mild respiratory tract illness to a severe pneumonia and acute respiratory distress syndrome (ARDS), including multiorgan failure and death. This novel coronavirus induces the release of inflammatory cytokines, and it is the disruption of the immune response that can lead to multiorgan dysfunction and ARDS (1). Coagulopathy in COVID-19 infection is correlated with mortality, with $D$ - dimer levels being of particular importance as biomarker (2).

Objectives: To describe the pathological findings of post-mortem lung biopsies in patients who died of SARS-CoV-2 infection.

Methods: A prospective observational study of post-mortem lungbiopsies in patients who died of COVID-19 was conducted. Biopsies were obtained by $14 \mathrm{G}$ tru-cut from March 31 to May 25, 2020 in the ICU of the Fundación Jiménez Díaz in Madrid. Morphological studies were performed using immunohistochemical techniques (IHQ). On the last day of admission, data regarding the epidemiology, respiratory mechanics and severity of disease was collected.

Results: In total, 16 patients were studied $(100 \% \mathrm{H})$. The median age was 63 years (IQI 56-66 years). The most frequent previous medical history included: ETS $31 \%$, LD 31\%, DM $18 \%$ and OM $31 \%$. Median of previous admission day was 28 (IQI14-39). Median APACHE II 14. Days of admission to ICU were 27 (range 1-44). The median number of days on MV was 24 . Tracheotomy was performed in $63 \%$. The ventilatory mode was volume control in $100 \%$, with an average tidal volume of $6.21 \mathrm{ml} /$ $\mathrm{kg}$ of predicted body weight. This resulted in a plateau pressure of 31 $\mathrm{cmH} 20$, a driving pressure of $21 \mathrm{cmH} 20$ and a static compliance of 17.5 $\mathrm{ml} / \mathrm{cmH} 2 \mathrm{O}$. Regarding arterial gases: $\mathrm{pH}$ of 7.29 (IQ of 7.17-7.38), $\mathrm{pCO} 2$ of 74(IQ 53-87) and a PaO2/FiO2 ratio of 64 (IQ 58-108). The average laboratory values were: D-dimer (DD) $3860 \mu \mathrm{g} / \mathrm{L}$, lymphocytes 850 (abs), platelets $138000 \mu \mathrm{ll}, \mathrm{Hb} 8.5 \mathrm{~g} / \mathrm{L}$, creatinine $1 \mathrm{mg} / \mathrm{dl}, \mathrm{PCR} 13.6 \mathrm{mg} /$ dl, ferritin $892 \mathrm{ng} / \mathrm{mL}$. All patients received prophylaxis with $\mathrm{LMWH}$. If the DD was higher than $3000 \mu \mathrm{g} / \mathrm{l}$, the LMWH was administered at therapeutic dose $(1 \mathrm{mg} / \mathrm{kg} / 24 \mathrm{~h})$. Diffuse alveolar damage and frequent formation of hyaline membranes were observed in $100 \%$ (Fig. b), $44 \%$ presented pneumonia in cryptogenic organization. It should be noted that $78 \%$ of the patients had frequent capillary microthrombosis(Fig. a). In addition, the IHQ showed intense expression of CD163+ macrophages as well as CD8.

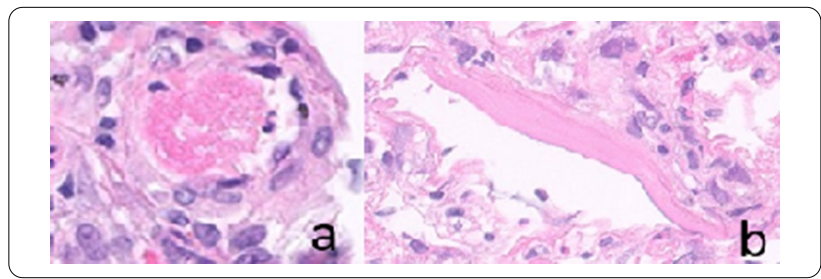

The results showed diffuse alveolar damage and hyaline membrane formation in all patients, and capillary microthrombosis in more than
$75 \%$ of them. In the pre-mortem analysis the DD was higher than $3,000 \mu \mathrm{g} / \mathrm{l}$ in more than half of cases. The frequent finding of CD163+ macrophages represents the histopathological substrate for the macrophage activation syndrome seen in these patients.

Conclusion: Our results confirm the hypothesis that SARS-Cov-2 may trigger a macrophage activation syndrome, as well as an state of hypercoagulability, which would support the implementation of immunosuppressive and anticoagulant treatment, beside supportive therapy.

\section{Reference(s) and grant ackowledgment(s)}

1. 1. Channappanavar R, Perlman S. Pathogenic human coronavirus infections: causes and consequences of cytokine storm and immunopathology. Semin Immunopathol. 2017;39(5):529-39

2. 2. Tang N, Li D, Wang X, Sun Z. Abnormal coagulation parameters are associated with poor prognosis in patients with novel coronavirus pneumonia. $J$ Thromb Haemost. 2020:18(4):844-7

\section{5}

Empirical antibiotic treatment in our ICU from the year 2002 to 2019

A. López Fernández¹; CM. Rodríguez Mejías'; JP. Valencia Quintero².

MDM. Jiménez Quintana'; J. Machado Casas

${ }^{1}$ Medicina intensiva, Hospital Universitario Virgen de las Nieves, Granada, Spain; ${ }^{2}$ Medicina intensiva, Regional Hospital Santa Ana de Motril, Motril, Spain

Correspondence: A. López Fernández

Intensive Care Medicine Experimental 2020, 8(2): 000985

Introduction: Analyzing which antibiotics have been used more frequently as empirical treatment in our unit over almost two decades and the duration of their use

Methods: Descriptive study based on the data collected in the ENVIN - ICU base of our medical-surgical unit, collected during the months of the official participation period. We describe the most frequently used antibiotics (meropenem, piperacillin-tazobactam, levofloxacin, azithromycin and linezolid), their percentage of use and average duration of treatment, analyzing two time periods, 2002-2009 and 2010-2019

Results: During the period 2002-2009, there were 916 records of antibiotics, the most used was levofloxacin $13.32 \%$, with an average duration of $6.71 \pm 4.99$ days, piperacillin-tazobactam $12.77 \%$ and an average of $7.37 \pm 4.41$ days, meropenem $4.91 \%$ and an average of 8.40 \pm 6.07 days, linezolid $2.07 \%$ and $7.74 \pm 68.48$ days on average, azithromycin $0.22 \%$ with an average of $4^{\prime} 00 \pm 0^{\prime} 00$ days.

During the period 2010-2019 there were 1570 records of antibiotics, the most used was meropenem $17.26 \%$ with an average duration of $6.22 \pm 4.92$ days, piperacillin-tazobactam $15.16 \%$ and $5.81 \pm 4.17$ days, linezolid $12.23 \%$ and $5.38 \pm 3.60$ days, levofloxacin $7.45 \%$ and $4.84 \pm 4.02$ days, azithromycin $3.444 \%$ with an average of $3.96 \pm 1.38$ days. Extracting only the data for the year 2019, there are 204 records, the most used antibiotic is piperazillin tazobactam $20.59 \%$ and $5.52 \pm$ 3.41 days, meropenem $19.12 \%$ and $5.87 \pm 3.11$ days, linezolid $14^{\prime} 71 \%$ and $5^{\prime} 17 \pm 3$ ' 27 days, azithromycin $4^{\prime} 90 \%$ and $4^{\prime} 40 \pm 1^{\prime} 78$ days, levofloxacin $1^{\prime} 47 \%$ and $5^{\prime} 67 \pm 2^{\prime} 31$ days.

Conclusion: We observe that the most used antibiotics have been modified, shortening their time of use, during the study period. Highlight the decrease in the use of levofloxacin and the increase in azithromycin, in addition to the frequent use of piperacillin-tazobactam maintained over time for almost two decades.

\section{6}

General characteristics of the patients admitted to our ICU in the last 20 years

A. López Fernández ; CM. Rodríguez Mejías'; JP. Valencia Quintero²;

E. Castillo Lorente' ${ }^{1}$ J. Machado Casas $^{1}$

${ }^{1}$ Medicina intensiva, Hospital Universitario Virgen de las Nieves, Granada, Spain; ${ }^{2}$ Medicina intensiva, Regional Hospital Santa Ana de Motril, Motril, Spain 
Correspondence: A. López Fernández

Intensive Care Medicine Experimental 2020, 8(2): 000986

Introduction: Descriptive analysis of the population admitted to our medical-surgical ICU in the last two decades.

Methods: Descriptive study based on the data collected in the ENVIN - ICU base of our medical-surgical unit, collected during the months of the official participation period. We divided the total number of patients into two periods: 2002-2009 and 2010-2019, and studied characteristics such as: age, sex, APACHE, SAPS II, underlying disease (medical, surgical, trauma, coronary), deaths, days of ICU stay, origin of the patients (hospitalization unit, another ICU, their home, long-stay center).

Results: During the period 2002-2009, 1114 patients were registered. The average age was $61,44 \pm 14,67$ years old, $62,48 \%$ male. The average score on the SAPS II scale was $29,77 \pm 12,63$ points, and APACHE was $16,61 \pm 8,53$ points. The base disease registered was medical $79,44 \%$, surgical $11,22 \%$, trauma $1,53 \%$, coronary $7,81 \%$. The origin of the patients $(n=466)$ prior to ICU admission was a $25.99 \%$ hospitalization unit, another ICU 2,07\%, home addres 12,95\%, long-stay center $0,90 \%$. Regarding mortality $(n=1109) 176$ deaths occurred $(15,87 \%)$.

During the period 2010-2019, 2533 patients were registered. The average age was $63,12 \pm 14,80$ years, with $67,11 \%$ males. The average SAPS II score was $30,64 \pm 16,35$ points and in APACHE 15,19 $\pm 8,70$ points. The registered base disease was medical $48,72 \%$, coronary $40,43 \%$, surgical $9,87 \%$ trauma $0,99 \%$. Its origin prior to income $(n=2531)$ was his home $61,39 \%$, one hospitalization unit $31,35 \%$, another ICU $6,20 \%$, long stay center $0,99 \%$. Regarding mortality $(n=2513) 322$ were recorded death $(12,81 \%)$.

Conclusion: Throughout these two decades, there are an increasing number of registered patients, whose most frequent origin was their home, with the medical reason persisting as the most frequent, and finding a decrease in mortality. Coronary patients go from 7 to $40 \%$ in the second decade, wich could partially explain the decrease in mortality, without affecting the severity of the patients.

\section{8}

Histiocytic hyperplasia with haemophagocytosis in COVID-19 patients admitted to ICU

Á. Vidal González ; JJ. Paez Vargas' ;D. Robaglia'; C. Soto²; J. Fortes³;

L. Prieto-Pérez ; N. Arias Martínez'; JM. Milicua'; LM. Polanco Mahecha'; P. Turrión ${ }^{1}$; A. Del Pino ${ }^{1}$; M. Górgolas ${ }^{4}$; M. Piris

${ }^{1}$ Intensive care medicine, Hospital Universitario Fundación Jiménez Díaz, Madrid, Spain; ${ }^{2}$ Hematology, Hospital Universitario Fundación Jiménez Díaz, Madrid, Spain; ${ }^{3}$ Pathological anatomy, Hospital Universitario Fundación Jiménez Díaz, Madrid, Spain; ${ }^{4}$ Internal medicine, Hospital Universitario Fundación Jiménez Díaz, Madrid, Spain

Correspondence: Á. Vidal González

Intensive Care Medicine Experimental 2020, 8(2): 000988

Introduction: The full spectrum of COVID-19 infection ranges from an asymptomatic disease, through mild respiratory tract illness to a severe pneumonia and acute respiratory distress syndrome (ARDS), including multiorgan failure and death. This novel coronavirus induces the release of inflammatory cytokines, and it is the disruption of the immune response that can lead to multiorgan dysfunction and ARDS (1). Growing evidence suggests that asubgroup of patients with severe disease might experience a deregulated systemicinflammation in conjunction with cytokine storm syndrome, leading to death $(1,2)$. There is an increasing interest on describing the histopathology of COVID-19 infection in different organs, through the study of samples obtained by means of core needle biopsies or full necropsies. Extending our knowledge will allow a better understanding of the disease and the development of new therapeutic targets.

Objectives: To describe the histopathological changes of the bone marrow in patients who died in ICU due to SARS-CoV-2 infection Methods: A prospective observational study of bone marrow biopsy (BMB) in patients who died of COVID19 was carried out in the ICU of the Fundación Jiménez Díaz in Madrid from March 31 to May 25, 2020. Morphological and immunohistochemical (IHQ) studies were performed on the samples. Epidemiological, severity and analytical data were also collected.

Results: 4 BMBs were performed. $100 \%$ of patients were male. The median age was 60 years (IQI 56-65). The most frequent past medical history were: LD and morbid obesity in $50 \%$, ATH in $25 \%$. The APACHE II at $24 \mathrm{~h}$ of admission was 13.5. The mean number of days of admission to the ICU was 14.5 days (IQI 3.5-25) with 23 days of MV. Prone ventilation was performed in $75 \%$ and tracheostomy in $50 \%$. The median of values in the pre-death analysis was: D-dimer $4209 \mu \mathrm{g} / \mathrm{L}$, fibrinogen $816 \mathrm{~g} / \mathrm{dl}$, lymphocytes $400 \mathrm{abs}$, platelets $83,000 / \mu \mathrm{l}, \mathrm{Hb} 10.35 \mathrm{~g} / \mathrm{L}$, creatinine $1.15 \mathrm{mg} / \mathrm{dl}$, PCR $37.8 \mathrm{mg} / \mathrm{dl}$, ferritin $892 \mathrm{ng} / \mathrm{mL}$, LDH $332 \mathrm{u} / \mathrm{L}$, total bilirubin $1.3 \mathrm{mg} / \mathrm{dl}$ and lactic $2.4 \mathrm{mmol} / \mathrm{L}$. They were treated in $75 \%$ of cases with LPV/RTV, hydroxychloroquine and $250 \mathrm{mg}$ methylprednisolone pulses, in $50 \%$ of them with interferon alpha- $2 \mathrm{~b}$ and only one patient received tocilizumab. Histiocytic hyperplasia with hemophagocytosis (Fig. a) was observed in $75 \%$ of patients and nonspecific inflammatory myelopathy in one patient.

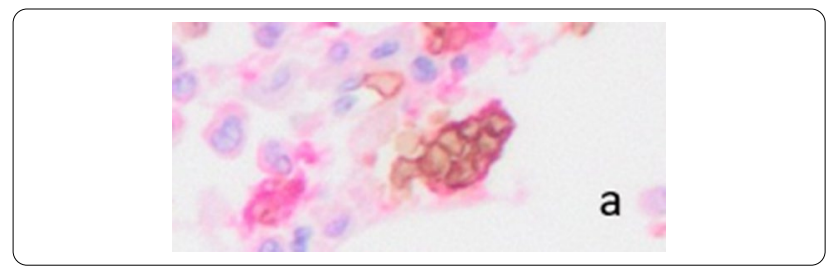

The $\mathrm{BMB}$ results show findings consistent with the diagnosis of hemophagocytic histiocytosis $(\mathrm{HH})$. $\mathrm{HH}$ is a common finding associated with severe viral problems and represents the histological counterpart of the macrophage activation (MAS) suffered by these patients. Macrophage activation syndrome (MAS), also known as hemophagocytic lymphohistiocytosis (sHLH), is characterized by the overactivation of cytotoxic T cells with hemophagocytosis by macrophages and a massive release of inflammatory cytokines. For these reason it is classified within the cytokine storm syndromes.

Conclusion: In our series, not all cases met the diagnostic criteria of $\mathrm{HLH}$, but we do consider that it represents the histological substrate for MAS in these patients, supporting the indication of immunomodulatory treatment.

\section{Reference(s) and grant ackowledgment(s)}

1. 1. Channappanavar R, Perlman S. Pathogenic human coronavirus infections: causes and consequences of cytokine storm and immunopathology. Semin Immunopathol. 2017;39(5):529-39

2. 2. Mehta P, McAuley DF, Brown M, Sanchez E, Tattersall RS, Manson JJ, et al. COVID-19: consider cytokine storm syndromes and immunosuppression. Lancet. 2020;395(10229):1033-4.

000989

Use of antibiotics in a medical surgical ICU. Descriptive study of the last 20 years

CM. Rodríguez Mejías'; A. López Fernández' ; JP. Valencia Quintero²;

JC. Luque Moscoso ${ }^{1}$; J. Machado Casas ${ }^{1}$

${ }^{1}$ Medicina Intensiva, Hospital Universitario Virgen de las Nieves, Granada, Spain; ${ }^{2}$ Medicina intensiva, Regional Hospital Santa Ana de Motril, Motril, Spain

Correspondence: A. López Fernández

Intensive Care Medicine Experimental 2020, 8(2): 000989

Introduction: To know how the general use of antibiotics in an ICU has evolved over two decades.

Methods: Descriptive study, based on the data collected in the ENVIN - ICU base of our medical-surgical unit, collected during the months of the official participation period. To do this, we have divided the total number of patients collected into two periods: 2002-2009 and 20102019. To study the evolution, we are going to describe the number of 
antibiotics per patient with antibiotics, relationship days without antibiotics / patient stays with antibiotics, most frequent causes of antibiotic indication, description of the use of antibiotics used (empirical or specific, change of treatment, Reason for change). The qualitative variables will be expressed in percentages.

Results: During the first period, the number of antibiotics per patient with antibiotics was 2.21, while in the second period it was 2.53 . The ratio of days without antibiotics / patient stays with antibiotics was 0.19 in the first period and 0.21 in the second period. In the first period, the use of 1760 antibiotics was recorded, the most frequent indication was other prophylaxis with $34.20 \%$, hospital infection extra ICU $25.06 \%$, community infection $21.59 \%$, hospital infection intra ICU $18.01 \%$. In the second period, the use of 2624 antibiotics was recorded, the most frequent indication was community infection $33.16 \%$, extra-ICU hospital infection $25.76 \%$, intra-ICU hospital infection $19.36 \%$, another prophylaxis $10.75 \%$. Regarding the reason for antibiotherapy, in the first period the empirical treatment was $82.12 \%$ and in the second $76.74 \%$. Antibiotic treatment (empirical or specific) produced a change of $25.73 \%$ in the first period, and $21.79 \%$ in the second period. The reasons for the change in antibiotherapy during the first period were a poor clinical evolution $38.26 \%$, unknown $33.56 \%$, microorganisms not covered $21.48 \%$, others $4.03 \%$, toxicity (Adverse effects) $2.01 \%$, spectrum reduction $0.67 \%$, resistance during treatment $0 \%$. The reasons for the change in antibiotherapy during the second period were the reduction of the spectrum $41.91 \%$, poor clinical evolution $28.68 \%$, microorganisms not covered $13.73 \%$, others $7.60 \%$, toxicity (adverse effects) $6.37 \%$, resistance during treatment $1.47 \%$, unknown $0.25 \%$.

Conclusion: In this descriptive study, it is observed how over two decades (coinciding with the implementation of the Zero projects), more antibiotics have been used, having more days free of antibiotherapy, changing the most frequent cause of antibiotic indication, and highlighting the largest use of de-escalated antibiotics today.

\section{4}

Severe SARS-CoV-2 infections in the Intensive Care Unit: Is it possible to predict mortality from routine clinical data on admission?

D. Pérez-Torres ${ }^{1}$; C. Díaz-Rodríguez ; I. Canas-Pérez ${ }^{1}$;

JÁ. Berezo-García'; P. Blanco-Schweizer'; V. Fraile-Gutiérrez';

AM. Olmos-Linares ${ }^{1}$; ML. Fernández-Rodríguez'; JJ. Sanz-Hernán;

JM. Martínez-García ';A. Martín-Luengo ${ }^{1}$; J. Sánchez-Ballesteros”;

PA. Merino-García ; R. Herrán-Monge ${ }^{1}$; C. Cuenca-Rubio ${ }^{1}$;

GJ. Posadas-Pita'; AM. Prieto-Lamo'; MM. García-García';

LM. Tamayo-Lomas'; PM. Enríquez-Giraudo ${ }^{1}$

${ }^{1}$ Servicio de medicina intensiva, Hospital Universitario Río Hortega, Valladolid, Spain

Correspondence: D. Pérez-Torres

Intensive Care Medicine Experimental 2020, 8(2): 001024

Introduction: Rapid spread of SARS-CoV-2 pandemic (COVID-19) has taken traditional ICU capacity to the limit worldwide. A demand overcoming availability has forced intensivists to select the patients who can benefit the most from admission. Predictive models based on routine clinical data may be valuable in the decision-making process.

Objectives: We aim to assess whether chronic comorbidities and laboratory tests drawn at presentation are good predictors of mortality in patients with severe SARS-CoV-2 infection (COVID-19) admitted to the Intensive Care Unit (ICU).

Methods: We conducted a retrospective observational single-centre study in the ICU of a University Hospital in Spain, over 3 months. All the patients who were admitted to the ICU with PCR-confirmed severe SARS-CoV-2 infection were included. Past medical history, laboratory tests drawn at presentation and clinical outcomes were obtained from the electronic medical record. Association among each variable and mortality was evaluated by bivariate analysis (Chi-square, Student's T and Mann-Whitney $\mathrm{U}$ tests, as appropriate). Potential explanatory variables from bivariate analysis were used to build a multivariate logistic regression model to predict mortality.

Results: We included 87 patients, $77 \%$ male, age $60 \pm 12$ years-old [survivors $56 \pm 12$ vs non-survivors $67 \pm 10, p<0.01]$, BMI $27(25-32) \mathrm{kg} / \mathrm{m}^{2}$ [survivors 27 (26-32) vs non-survivors 27 (24-32), $p=0.94$ ], APACHE-II $17 \pm 6$ [survivors 14 vs non-survivors $20, p<0.01$ ], mechanical ventilation $93 \%$ [survivors 59 vs non-survivors $41, p=0.73$ ], hospital mortality $38 \%$. Our findings are depicted in Tables 1 and 2. Our model (Table 3) showed $76.2 \%$ sensitivity and $89.7 \%$ specificity to predict mortality (area under the ROC curve 0.91).

Table 1. Association of chronic comorbidities with mortality.

\begin{tabular}{|c|c|c|c|c|}
\hline & $\begin{array}{l}\text { Overall } \\
\text { frequency, } \%\end{array}$ & Survivors, $\%$ & $\begin{array}{l}\text { Non- } \\
\text { survivors, \% }\end{array}$ & $\begin{array}{l}\mathrm{p}- \\
\text { value }\end{array}$ \\
\hline Hypertension & 30 & 20 & 45 & 0.01 \\
\hline Asthma & 8 & 9 & 6 & 0.59 \\
\hline COPD & 3 & 0 & 9 & 0.02 \\
\hline Hypothyroidism & 11 & 9 & 15 & 0.40 \\
\hline $\begin{array}{l}\text { Ischemic heart } \\
\text { disease }\end{array}$ & 5 & 0 & 12 & 0.01 \\
\hline Diabetes & 14 & 7 & 24 & 0.03 \\
\hline Neoplasia & 13 & 9 & 18 & 0.22 \\
\hline
\end{tabular}

\begin{tabular}{|l|l|l|l|}
\hline \multicolumn{4}{|c|}{ Table 2. Association of laboratory values at presentation with mortality. } \\
\hline & $\begin{array}{l}\text { Survivors, median } \\
\text { (IQR) }\end{array}$ & $\begin{array}{l}\text { Non-survivors, } \\
\text { median (IQR) }\end{array}$ & p-value \\
\hline Leukocytes, $\times 10^{3} / \mu 1$ & $7.1(5.4-9.5)$ & $7.7(5.8-14.7)$ & 0.69 \\
\hline Lymphocytes, $\times 10^{3} / \mu 1$ & $0.8(0.5-1.1)$ & $0.6(0.3-0.8)$ & 0.01 \\
\hline $\begin{array}{l}\text { D-dimer, } \mathrm{ng} / \mathrm{ml} \\
\text { High sensitivity troponin I, } \\
\text { ng/1 }\end{array}$ & $685(399-1041)$ & $641(531-2070)$ & 0.65 \\
\hline Creatinine, $\mathrm{mg} / \mathrm{dl}$ & $9(5-17)$ & $35(13-225)$ & $<0.01$ \\
\hline C-reactive protein, $\mathrm{mg} / 1$ & $0.9(0.7-1.1)$ & $1(0.7-1.3)$ & 0.15 \\
\hline Procalcitonin, $\mathrm{ng} / \mathrm{ml}$ & $123(61-216)$ & $108(59-179)$ & 0.39 \\
\hline Lactate, $\mathrm{mmol} / \mathrm{l}$ & $0.2(0.1-0.3)$ & $0.3(0.1-0.7)$ & 0.11 \\
\hline & $1.6(1.3-2.2)$ & $1.8(1.4-2.3)$ & 0.43 \\
\hline
\end{tabular}

\begin{tabular}{|l|l|l|l|l|}
\hline \multicolumn{5}{|c|}{ Table 3. Multivariate logistic regression model to predict mortality. } \\
\hline & Coefficient & $\begin{array}{l}\text { Standard } \\
\text { error }\end{array}$ & \multicolumn{1}{l|}{ Odds ratio $(95 \%$} & p-value \\
\hline Age, per year & 0.112 & 0.05 & $1.12(1.01-1.23)$ & 0.02 \\
\hline Gender, female & 0.915 & 1.01 & $\begin{array}{l}2.45(0.35- \\
17.99)\end{array}$ & 0.36 \\
\hline Hypertension, yes & 0.996 & 0.87 & $\begin{array}{l}2.71(0.49- \\
14.88)\end{array}$ & 0.25 \\
\hline Diabetes, yes & 0.586 & 1.24 & $1.80(0.16-$ & 0.64 \\
\hline $\begin{array}{l}\text { Lymphocytes, per } \\
1 \times 10^{3} / \mu 1\end{array}$ & -3.944 & 1.53 & $0.02(0.01-0.39)$ & 0.01 \\
\hline Creatinine, per mg/dl & -0.045 & 0.13 & $0.96(0.74-1.23)$ & 0.72 \\
\hline $\begin{array}{l}\text { Troponin, per ng/L } \\
\text { Intercept }\end{array}$ & 0.006 & 0.01 & $1.01(0.01-1.02)$ & 0.18 \\
\hline
\end{tabular}

Conclusion: In our series, mortality in severe SARS-CoV-2 infections requiring admission to the ICU was significantly associated with age and APACHE-II.

A prediction model based upon basic demographic data (age and gender), chronic comorbidities (hypertension and diabetes) and 
laboratory values at presentation (lymphocytes, creatinine and troponin) is able to reliably predict mortality in this patient population.

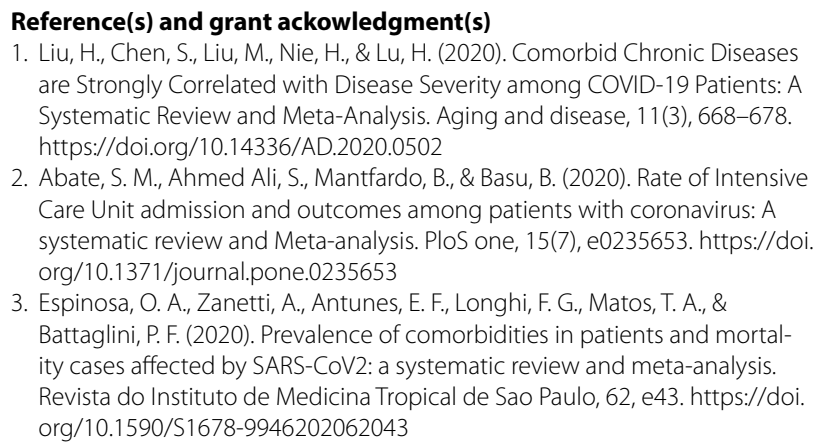

2. Abate, S. M., Ahmed Ali, S., Mantfardo, B., \& Basu, B. (2020). Rate of Intensive Care Unit admission and outcomes among patients with coronavirus: A systematic review and Meta-analysis. PloS one, 15(7), e0235653. https://doi. org/10.1371/journal.pone.0235653

3. Espinosa, O. A., Zanetti, A., Antunes, E. F., Longhi, F. G., Matos, T. A., \& Battaglini, P. F. (2020). Prevalence of comorbidities in patients and mortality cases affected by SARS-CoV2: a systematic review and meta-analysis. Revista do Instituto de Medicina Tropical de Sao Paulo, 62, e43. https://doi. org/10.1590/S1678-9946202062043

\section{3}

Infections caused by pandrug-resistant Acinetobacter baumannii in critically ill patients: clinical characteristics, treatment and outcome

F. Frantzeskaki'; MP. Almyroudi'; S. Patsilinakou'; E. Chrysanthopoulou'; M. Theodorakopoulou' ${ }^{1}$;. Diakakil; A. Armaganidis ${ }^{1}$;. Tsangaris ${ }^{1}$

'2nd department of critical care, Attikon University Hospital, National and Kapodistrian University of Athens, Medical school, Rimini 1, Chaidari, Greece

Correspondence: F. Frantzeskaki

Intensive Care Medicine Experimental 2020, 8(2): 001073

Introduction: Infections caused by A. baumannii are associated with high mortality and healthcare costs. In critical care settings, the incidence of acquired resistance of $A$. baumannii to commonly prescribed antibiotics is increasing, rendering the treatment of the relevant infections rather challenging and compromising patients' outcomes.

Objectives: To prospectively record the incidence, clinical characteristics, treatment and outcome of infections caused by pandrug - resistant (PDR) A.baumannii in critically ill patients.

Methods: The study was performed at a 19-bed polyvalent intensive care unit (ICU). All the mechanically ventilated patients with documented infection caused by PDR A. baumannii, from November 2018 until February 2020 were enrolled in the study. Concentrations (MICs) were determined by the broth microdilution method, as indicated by the CLSI. Disease severity was evaluated by APACHE-II and SOFA score on ICU admission. Patients' demographics, clinical characteristics, treatment and outcomes in 7 and 30 days were recorded. Clinical success was defined by a lessening of the signs and symptoms of infection, while microbiologic success was considered, as the eradication of the pathogen from cultures.

Results: Ten patients (mean age $\pm \mathrm{SD}=58 \pm 17 \mathrm{yrs}$, 8 males/2 females) with documented infection due to $A$. baumannii were enrolled in the study. Regarding comorbidities, diabetes mellitus was present in $3(30 \%)$ pts, arterial hypertension in $5(50 \%)$ pts, and coronary artery disease in $3(30 \%)$ pts. APACHE-II score in ICU admission was $18 \pm 7$, while SOFA score was $8 \pm 3$. Bloodstream infection was present in $6(60 \%)$ pt. Among them, central line associated bloodstream infection (CLABSI) was observed in 5 (83\%) pts, and primary bacteremia in $1(17 \%)$ pt. Ventilator associated pneumonia (VAP) was diagnosed in $4(40 \%)$ pts. Mean length of stay of enrolled patients in the ICU, prior to infection, was $18.5 \pm 10$ days. Regarding prior colonization, PDR-A.baumannii was isolated in stool and bronchial secretion surveillance cultures of $8(80 \%)$ pts. $7(70 \%)$ pts had received broad spectrum antibiotics prior to infection due to A.baumannii, while 5 among them had also received intravenous and nebulised colistin. All patients were empirically treated with combinations of colistin, high dose ampicillin/ sulbactam (A/S), rifampicin or tigecycline with broad spectrum antibiotics (meropenem or ceftazidim/avibactam), for $14 \pm 4$ days. Clinical and microbiological success was observed in 7 (70\%) of patients. In two patients (20\%), blood cultures remained positive, while in another patient, A.baumannii infection was complicated with meningitis. ICU mortality in 10 days of infection was $30 \%$. ICU mortality was significantly associated with SOFA score $(p<0.05)$. Mean length of stay in ICU after infection was $39 \pm 17$ days.

Conclusion: Broad use of antibiotics has increased the isolation rate of PDR-A.baumannii in critical care settings. Antibiotic combinations might offer a therapeutic benefit, as a result of possible synergistic effect. Further studies are required in order to elucidate the therapeutic options and clinical outcomes of infections caused by PDR pathogens.

\section{Reference(s) and grant ackowledgment(s)}

1. Basseti M et al. Curr Opin Crit Care. 2018, Oct (24)5, 385-393

\section{2}

Nosocomial Gram-Negative Rod Monobacteremia in ICU: A 10-Year Study

D. Adukauskiene'; A. Dambrauskiene²; D. Valanciene ${ }^{3}$;V. Micpovilyte

${ }^{1}$ Intensive care unit, Hospital of Lithuanian University of Health Sciences Kaunas Clinics, Kaunas, Lithuania; ${ }^{2}$ Infection control service, Hospital of Lithuanian University of Health Sciences Kaunas Clinics, Kaunas, Lithuania; ${ }^{3}$ Anesthesiology and intensive care department, Klaipeda Seamen's Hospital, Klaipèda, Lithuania

Correspondence: $\mathrm{V}$. Micpovilyte

Intensive Care Medicine Experimental 2020, 8(2): 001092

Introduction: Antibiotics resistant Gram-negative rod (GNR) bacteremia is increasing worldwide every year. Diagnosis of nosocomial Gram-negative rod monobacteremia (N-GNRM) in critically ill patients has been associated with prolonged hospitalization in intensive care units (ICUs) and a higher rate of mortality. The identification of pathogens, the pervasiveness of multiple antibiotic resistance, and risk factors for resistant strains are helpful in selecting appropriate empirical antibiotic therapy to reduce hospitalization time and mortality. The aim of this study was to identify the risk factors for the development of nosocomial multiple antibiotic resistance strains and mortality.

Methods: A retrospective cohort study was performed, analyzing the data of patients treated in ICU after 72 hours of hospitalization, with positive gram-negative rod monobacteremia over a period of 10 years. Results: Over the year 2009-2018, 261 cases of N-GNRM were identified. The detected pathogens include: $31.8 \%(n=83)$ Acinetobacter spp., 19.9\% ( $n=52)$ Klebsiella spp., 18\% ( $n=47)$ Escherichia coli and etc. Monobacteremia caused by $P$. aeruginosa was detected only in the last three years of the researched period, from 4 (8.5\%) cases in 2016 to 7 $(28 \%)$ cases in $2018(p<0.05)$. The sensitivity to Imipenem was $88.8 \%$ $(n=206)$, to Meropenem $85.4 \%(n=199)$, to Amikacin $65.1 \%(n=$ $142)$, to Cefoperazone / Sulbactam 61.6\% $(n=141)$ and to Piperacillin / Tazobactam $48 \%(n=120) .61 .6 \%(n=161)$ of all the cases studied, had multiple antibiotic resistance, out of which $39.8 \%(n=104)$ was extensively drug-resistant (XDR) $(p<0.001)$. Of all Acinetobacter spp., XDR made up $69.9 \%(n=58)(p<0.001)$. The identified risk factors for bacteremia in resistant strains include male gender $(p=0.012)$, age $>=65$ year old $(p=0.010)$, hospitalization in ICU $>=16$ days $(p=$ 0.012 ), and the duration of mechanical lung ventilation (IMV) $>=14$ days $(p<0.001)$. In a multivariate analysis, $69.3 \%(n=181)$ of mortality was associated with male gender $(p=0.001)$, acute kidney injury with the need for hemodialysis (AKI-HD) $(p=0.001)$ and shock $(p<0.001)$.

Conclusion: Over a ten-year period, the most common cause of $\mathrm{N}-$ GNRM in ICU was Acinetobacter spp., dominated by XDR strains. Male gender, older age, prolonged stay in ICU and longer duration of IMV were identified as risk factors for multiple antibiotic resistance strains. Furthermore, male gender, acute kidney injury with the need for hemodialysis and shock, were related to almost $70 \%$ rate of mortality. 
001122

Analysis of the STRIVE Phase 2 Trial of Once-Weekly Rezafungin for Treatment of Candidemia and Invasive Candidiasis Compared with Caspofungin: Outcomes by ICU Status and APACHE II Score P. Honoré

${ }^{1}$ Icu department, CHU Brugmann, Brussels, Belgium

Correspondence: $P$. Honoré

Intensive Care Medicine Experimental 2020, 8(2): 001122

Introduction: Analysis of the STRIVE Phase 2 Trial of Once-Weekly Rezafungin for Treatment of Candidemia and Invasive Candidiasis Compared with Caspofungin: Outcomes by ICU Status and APACHE II Score

AuthorsPatrick M. Honore1, Anastasia Kotanidou2, Massimo Girardis3, Shawn Flanagan4, Taylor Sandison4

1CHU Brugmann University Hospital, Brussels, Belgium; 2General Hospital of Athens "Evangelismos", Athens, Greece; 3University Polyclinic Hospital of Modena, Modena, Italy; 4Cidara Therapeutics, Inc. San Diego, CA, USA

\section{Objectives: INTRODUCTION}

Invasive fungal disease (IFD) caused by Candida spp. is associated with longer hospital duration and higher mortality rates for the critically ill.1-3 For such patients, treatment with an echinocandin is recommended first-line therapy.4 Rezafungin (RZF) is a novel, nextgeneration echinocandin in Phase 3 development for treatment of candidemia and invasive candidiasis (IC) and for prevention of IFD caused by Candida, Aspergillus, and Pneumocystis in blood and marrow transplant recipients.

Objectives: To evaluate outcomes of the Phase 2 STRIVE trial of RZF QWk for the treatment of candidemia and/or IC compared with caspofungin (CAS) once-daily (NCT02734862)5, based on ICU status and APACHE II scores.

Methods: Patients in STRIVE were randomized to 1 of 2 doses of RZF (400 mg QWk or $400 \mathrm{mg}$ in Week 1, then $200 \mathrm{mg} \mathrm{QWk}$ [400 mg/200 $\mathrm{mg}$ ], the latter being studied in Phase 3) or CAS (70 mg on Day 1, then $50 \mathrm{mg}$ QD) for $\geq 14$ days. For this ad-hoc subanalysis, data were stratified by ICU status (no ICU or ICU admission within Days 1-4) and by APACHE II score category $\left(<10,10-19\right.$, and $\left.{ }^{3} 20\right)$. Efficacy (overall success [resolution of clinical signs of infection + mycological eradication], mycological success, and investigator assessment of clinical cure at Day 14; and 30-day all-cause mortality) and safety (treatment-emergent adverse events [TEAEs]) endpoints were evaluated.

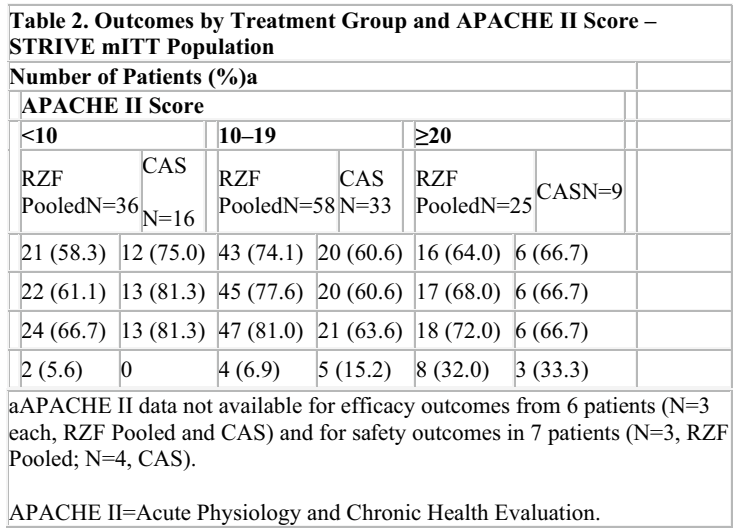

Results: Efficacy rates among RZF-treated patients admitted to the ICU were similar to or higher than those with no ICU admission (Table 1). On the primary efficacy endpoint of overall response, $75 \%$ of the RZF-treated ICU cohort and $60.7 \%$ of the non-ICU cohort achieved overall success. In CAS-treated patients, efficacy rates were lower among the ICU cohort than in the non-ICU cohort (Table 1). In general, response rates with RZF were comparable across APACHE II scores and response rates with CAS were lower in patients with higher APACHE II scores (Table 2). Analysis of TEAEs by ICU status and by APACHE II scores showed no concerning trends or differences between RZF and CAS; not surprisingly, higher numbers of SAEs were seen in patients with ICU admission (vs no admission) and with APACHE II scores $\geq 20$ (vs scores <20) (data not shown).

Conclusion: This Phase 2 subanalysis demonstrated RZF efficacy in patients admitted to the ICU and across APACHE II scores, and the safety of rezafungin in these subpopulations was comparable to that of caspofungin. While RZF outcomes appeared worse in the lower acuity patients, this was primarily due to the high numbers of indeterminate outcomes (i.e., missing data and lost to follow-up) among these patients. These findings contribute to the evidence of RZF safety and efficacy in a broad range of patients and support the ongoing Phase 3 development of RZF for the treatment of candidemia and IC (ReSTORE; NCT03667690).

\section{Reference(s) and grant ackowledgment(s)}

1. Thompson GR, Honore PM, Horcajada JP, et al. Rezafungin Clinical Safety and Efficacy in the Treatment of Candidemia and/or Invasive Candidiasis: Combined Results from the STRIVE Phase 2 Trial Parts A and B. Trends in Medical Mycology; October 11-14, 2019; Nice, France.

2. Pappas PG, Kauffman CA, Andes DR, et al. Clinical Practice Guideline for the Management of Candidiasis: 2016 Update by the Infectious Diseases Society of America. Clinical infectious diseases : an official publication of the Infectious Diseases Society of America. 2016;62(4):e1-50.

3. Bassetti M, Giacobbe DR, Vena A, et al. Incidence and outcome of invasive candidiasis in intensive care units (ICUs) in Europe: results of the EUCANDICU project. Critical care (London, England). 2019;23(1):219.

4. 2. KettDH, Azoulay E, Echeverria PM, Vincent JL. Candida bloodstream infections in intensive care units: analysis of the extended prevalence of infection in intensive care unit study. Critical care medicine. 2011;39(4):665-670.

5. 1. Prowle JR, Echeverri JE, Ligabo EV, et al. Acquired bloodstream infection in the intensive care unit: incidence and attributable mortality. Critical care (London, England). 2011;15(2):R100-R100.

6. None

001123

Analysis of Outcomes by Geographic Region of Enrollment

in STRIVE, the Phase 2 of Rezafungin for the Treatment of

Candidemia and Invasive Candidiasis (IC)

P. Honoré

${ }^{1}$ Icu department, CHU Brugmann, Brussels, Belgium

Correspondence: P. Honoré

Intensive Care Medicine Experimental 2020, 8(2): 001123

Introduction: Analysis of Outcomes by Geographic Region of Enrollment in STRIVE, the Phase 2 of Rezafungin for the Treatment of Candidemia and Invasive Candidiasis (IC)

\section{AUTHORS}

Patrick M. Honore (CHU Brugmann University Hospital, Brussels, Belgium)

Jesus Fortun (University Hospital Ramon y Cajal, Madrid, Spain) Athanasios Skoutelis (General Hospital of Athens Evangelismos) Rolando Viani (Cidara Therapeutics, Inc., San Diego, CA, USA)

Taylor Sandison (Cidara Therapeutics, Inc., San Diego, CA, USA)

Objectives: Rezafungin is a novel echinocandin in Phase 3 development. STRIVE (NCT02734862) 1 is the global, Phase 2 trial of rezafungin once-weekly for treatment of candidemia and/or IC versus standard of care. Results of STRIVE were evaluated by geographic region to assess for differences in the complete trial population

Methods: Data were stratified by region of enrollment (EU vs North America [NA]) and analysed for differences in patient demographics and baseline characteristics, treatment patterns, and efficacy outcomes.

Results: Patients enrolled in EU $(\mathrm{N}=131)$ were older than in $N A$ $(\mathrm{N}=76)$ (mean, 64 vs 52 years, respectively) and were predominantly male $(61.1 \%)$ and White $(93.1 \%)$. The NA population was $50 \%$ male 
and comprised $23.7 \%$ Black or African-American patients. On average, NA patients weighed more and had higher BMI $(+2.3 \mathrm{~kg} / \mathrm{m} 2)$.

The same leading Candida species were isolated at baseline in both regions but with differing distribution. Candida albicans comprised $44 \%$ of EU isolates, and proportions of Candida parapsilosis, Candida glabrata, and Candida tropicalis were similar (13-18\%). In NA, C. albicans accounted for $52 \%$ of isolates, followed by $25 \%$ C. glabrata and $7 \%$ each for C. parapsilosis and C. tropicalis.

In both regions, $55 \%$ of patients received 8-14 days of IV treatment; $27 \%$ of EU patients received $>14$ days versus $18 \%$ in NA. Fewer patients in EU were switched to oral step-down ( $24.4 \%$ vs $42.7 \% \mathrm{NA})$. The NA population had a higher proportion of patients with IC $(26.3 \%$ vs $17.6 \% \mathrm{EU})$.

Outcomes were comparable between regions except for higher rates of overall success in EU patients treated with rezafungin $400 \mathrm{mg}$ in Week 1 followed by $200 \mathrm{mg}$ once weekly, compared with the NA cohort.

Conclusion: The Phase 2 STRIVE trial demonstrated few differences by region in demographic and baseline characteristics. The EU population was slightly older, and NA patients were generally heavier. Nonalbicans species were predominant in the $\mathrm{EU}$ and comprised almost half of the NA isolates. Efficacy by region showed no consistent trends, although interpretation of efficacy-related differences are limited by group size. Results of this analysis may inform future evaluation of data from the rezafungin clinical trial program.

\section{Reference(s) and grant ackowledgment(s)}

1. 1. Thompson GR, Honore PM, Horcajada JP, Hites M, Bassetti M, Mena K, Navalta L, Viani R, Sandison T, Pappas P. Rezafungin Clinical Safety and Efficacy in the Treatment of Candidemia and/or Invasive Candidiasis: Combined Results from the STRIVE Phase 2 Trial Parts A and B. Trends in Medical Mycology; October 11-14, 2019; Nice, France.

2. None

\section{9}

\section{Related factors to mortality in patients admitted to the ICU due to} COVID-19 pneumonia

J. Ruiz'; T. Moreno López²; J. Llabata' ; E. Lucas Romero²; M. De La Maza Segovia $^{3}$; R. Algarte ${ }^{1}$; F. Meza Samaniego ${ }^{2}$; E. Piacentini ${ }^{1}$; B. Sánchez González ${ }^{1}$. J. Trenado

${ }^{1}$ Intensive care department, Mútua Terrassa University Hospital, Terrassa, Spain; ${ }^{2}$ Internal medicine department, Mútua Terrassa University Hospital, Terrassa, Spain; ${ }^{3}$ Anesthesiology and resuscitation department., Mútua Terrassa University Hospital, Terrassa, Spain

Correspondence: B. Sánchez González

Intensive Care Medicine Experimental 2020, 8(2): 001129

Introduction: During the months of March to May we have experienced in Spain the consequences of the COVID 19 pandemic. Different factors can be related to the evolution of a pathology, both those of the pathology and the patient, as well as those derived from the high number of cases in a short period of time. Below we intend to analyze the different factors that are related to the evolution of COVID-19 pneumonia in our area.

Objectives: Describe the related factors to mortality in patients admitted to the ICU due to COVID-19 pneumonia during the pandemic period.

Methods: Descriptive study conducted in a polyvalent ICU with 12 critical care beds and 21 semi-critical beds. All patients admitted for COVID-19 pneumonia during the pandemic period were included.

The qualitative variables are expressed as percentages and were compared using the $\mathrm{X} 2$-test, the quantitative variables are expressed as means and standard deviations (SD) and were compared using the student's t test. A logistic regression model was constructed with ICU mortality as a dependent variable.

Results: 95 patients were admitted, Table 1 describes the main characteristics of the patients.

RESULTS. 95 patients were admitted, Table 1 describes the main characteristics of the patients.
\begin{tabular}{|l|l|} 
Table 1 & \\
\hline Variables & $58.6(10)$ \\
\hline Age (years, mean (SD)) & 71 \\
\hline Sex (male\%) & 76 \\
\hline SOFA at admission $\leq 5(\%)$ & 100 \\
\hline Rockwood frailty scale at income $\leq 3(\%)$ & 16 \\
\hline Multi-organ failure (\%) & 6 \\
\hline Shock (\%) & 11.6 \\
\hline Acute kidney failure (\%) & $13.1(12.9)$ \\
\hline ICU long of stay (days, mean (SD)) & $25.4(16.7)$ \\
\hline Hospital stay (days, mean (SD)) & 19 \\
\hline ICU mortality (\%) & 20 \\
\hline Hospital mortality (\%)
\end{tabular}

$100 \%$ of the patients received Kaletra, hydroxychloroquine and Azithromycin as treatment, $40 \%$ in addition, Tozilucimab, 34\% interferon. On March 31th, the corticosteroid treatment protocol was implemented in the institution, $62 \%$ of patients were admitted before this date (first 2 weeks) and $58 \%$ of patients were treated with corticosterioids. Table 2 show the factors related to ICU mortality.

\begin{tabular}{|c|c|c|c|c|}
\hline & Dead & & \\
\hline & & $64.83(6.492)$ & $57.19(10.159)$ & 0.007 \\
\hline \multicolumn{2}{|l|}{$\begin{array}{l}\text { Age (years, median (DE)) } \\
\text { Patients before corticosteroid protocol }\end{array}$} & & 76.3 & \\
\hline $\begin{array}{l}\text { Age (Years.median (DE)) } \\
\text { Patients before corticosteroid protocol }\end{array}$ & $\begin{array}{l}\text { Si } \\
\text { No }\end{array}$ & $\begin{array}{l}11.1 \\
10.0\end{array}$ & $\begin{array}{l}89.9 \\
90.0\end{array}$ & 0.019 \\
\hline Cardiovascular comorbiditites (50) & & & & \\
\hline \multirow{2}{*}{ Tracheal intubation(\%) } & No & 0.0 & 100.0 & \\
\hline & & 28.6 & 71.4 & \\
\hline Pronations on intubated pacients (\%) & No & 7.7 & 92.3 & 0.002 \\
\hline \multirow{2}{*}{ Tracheostamy(\%) } & $\begin{array}{l}\text { i } \\
\text { No }\end{array}$ & $\begin{array}{l}31.7 \\
35.9\end{array}$ & 68.3 & 0.049 \\
\hline & & 14.3 & 85.7 & \\
\hline \multirow[t]{2}{*}{ Acute kidney failure(\%) } & No & 14.3 & 85.7 & 0.001 \\
\hline & si & & 45.5 & \\
\hline $\begin{array}{l}\text { Midazolam infusion's days (days, median (DE)) } \\
\text { Remifentanil infusion's days (days, median (DEE)) }\end{array}$ & & $\begin{array}{l}1126.5(1708) \\
129.2(175.3)\end{array}$ & $\begin{array}{c}482.6(702.2) \\
65.9(104.5)\end{array}$ & $\begin{array}{c}<0.001 \\
0.005\end{array}$ \\
\hline \multirow{3}{*}{ 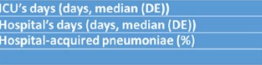 } & & $15.06(17.3)$ & $12.68(11.7)$ & 0.1 \\
\hline & No & $\begin{array}{c}32.83(17.20) \\
10.6\end{array}$ & $\begin{array}{l}23.5(16.2) \\
89.4\end{array}$ & $\begin{array}{l}0.39 \\
<0.001\end{array}$ \\
\hline & $\mathrm{si}$ & 52.4 & 47.6 & \\
\hline $\begin{array}{l}\text { Antibiotic treatment for hospital-acquired } \\
\text { infections }(\%)\end{array}$ & No & 5.6 & $94.4 \%$ & 0.002 \\
\hline \multirow{2}{*}{ Tireatment with ${ }^{\prime}$ ozilucimab(\%) } & $\begin{array}{l}\text { Si } \\
\text { No }\end{array}$ & $\begin{array}{c}34 \\
19.3\end{array}$ & $\begin{array}{c}66 \\
80.7\end{array}$ & 0.9 \\
\hline & & 18.4 & 81.6 & \\
\hline $\begin{array}{l}\text { Treatment with muscle relaxer'scontinuous } \\
\text { infusion (\%) }\end{array}$ & No & 8.3 & 91.7 & 0.007 \\
\hline \multirow{2}{*}{ Treatment with corticoesteroids (\$) } & $\begin{array}{l}\text { Si } \\
\text { jNo }\end{array}$ & $\begin{array}{l}35.8 \\
17.5\end{array}$ & $\begin{array}{l}64.1 \\
82.5\end{array}$ & 0.75 \\
\hline & & & & \\
\hline Corticoesteroids and muscle relaxers\% & No & 12.7 & 87.3 & 0.007 \\
\hline \multirow[t]{2}{*}{ SOFA<5 } & No & 16.7 & 83.3 & 0.042 \\
\hline & $\begin{array}{l}\text { Si } \\
\text { No }\end{array}$ & $\begin{array}{c}40 \\
14.3\end{array}$ & $\begin{array}{l}60 \\
857\end{array}$ & 0.047 \\
\hline \multirow{2}{*}{ Hospital floor's discharge with delirium(\%) } & S & 32.3 & 677 & \\
\hline & No & 14.7 & 85.3 & 0.043 \\
\hline
\end{tabular}

Table 3 shows the logistic regression analysis for mortality in the ICU after adjusting for possible confounding factors.

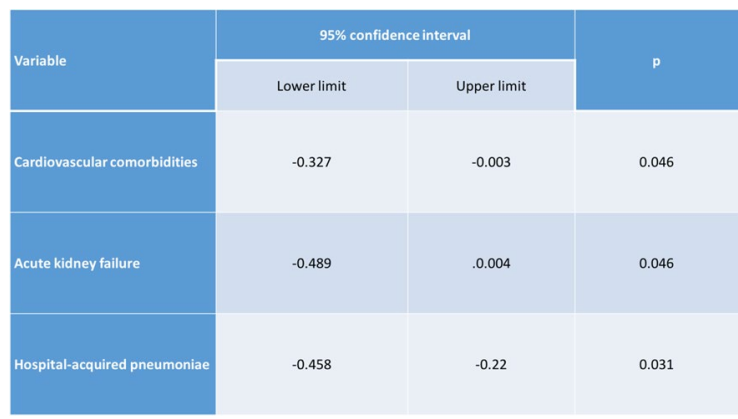

Conclusion: Only a relationship between mortality in the ICU and previous cardiovascular comorbidity is observed, as well as with the development of acute renal failure and nosocomial pneumonia. However, other aspects that should be considered in the face of a possible new outbreak are the use of corticosteroids, the doses of sedation and muscle relaxants and the appearance of delirium.

Reference(s) and grantackowledgment(s) 
1. Acknowledgments to all the nursing and auxiliary staff of the ICU at HUMT 2. Not funded

\section{0}

Corticosteroid use related factors in the treatment of admitted patients in ICU with covid-19 pneumonia

ML. Urendes'; F. Meza Samaniego ${ }^{2}$; C. Palencia ${ }^{1}$; T. Moreno López²;

E. Lucas Romero²; M. De La Maza Segovia ${ }^{3}$; E. Saavedra Sanjuan';

R. Algarte'; B. Sánchez González'; J. Trenado'

'Intensive care department, Mútua Terrassa University Hospital, Terrassa, Spain; ${ }^{2}$ Internal medicine department, Mútua Terrassa University Hospital, Terrassa, Spain: ${ }^{3}$ Anesthesiology and resuscitation department., Mútua Terrassa University Hospital, Terrassa, Spain

Correspondence: B. Sánchez González

Intensive Care Medicine Experimental 2020, 8(2): 001130

Introduction: During the months of March to May we have experienced in Spain the consequences of the COVID 19 pandemic. Different factors can be related to the evolution of a pathology, both those of the pathology and the patient, as well as those derived from the high number of cases in a short period of time. Below we intend to analyze the different factors that are related to the evolution of COVID19 pneumonia in our area.

Objectives: Describe the corticosteroids use related factors in the treatment of covid-19 pneumonia in patients admitted in ICU during the pandemic period.

Methods: Descriptive study carried out in a polyvalent ICU. All patients admitted because of COVID-19 pneumonia during the coronavirus outbreak were included.

A corticosteroid treatment protocol was established for patients with severe covid-19 pneumonia whose institutional implementation was on $03 / 31 / 2020$

Statistics: Qualitative variables are expressed as percentages and were compared using the X2-test.

The quantitative ones are expressed as means and standard deviations (SD) and were compared using the t-Student test.

A logistic regression model was constructed with treatment with corticosteroids as a dependent variable.

Results: Table 1 describes their main features.

\begin{tabular}{|l|l|}
\hline \multicolumn{1}{|c|}{ Vable 1} & \\
\hline Age (years, mean (SD)) & $58.6(10)$ \\
\hline Sex (male\%) & 71 \\
\hline SOFA at admission $\leq 5(\%)$ & 76 \\
\hline $\begin{array}{l}\text { Rockwood frailty scale at income } \leq \\
3(\%)\end{array}$ & 100 \\
\hline Multi-organ failure (\%) & 16 \\
\hline Shock (\%) & 6 \\
\hline Acute kidney failure (\%) & 11.6 \\
\hline ICU long of stay (days, mean (SD)) & $13.1(12.9)$ \\
\hline Hospital stay (days, mean (SD) & $25.4(16.7)$ \\
\hline ICU mortality (\%) & 19 \\
\hline Hospital mortality (\%) & 20 \\
\hline
\end{tabular}

$100 \%$ of patients received all Kaletra, Hydroxychloroquine and Azithromycin treatment.

$40 \%$ of them, in addition, received Tozilucimab and $34 \%$ of them received interferon too.

55 patients (58\%) were treated with corticosteroids and 52 of them followed the institutional protocol started on March 31.
Table 2 shows corticosteroids use related factors.

\begin{tabular}{|c|c|c|c|c|}
\hline \multirow{2}{*}{\multicolumn{2}{|c|}{$\begin{array}{l}\text { Variable } \\
\text { Ventilation's days (days. median (DEE)) }\end{array}$}} & & & \\
\hline & & $10.7(8.2)$ & $13.4(10.3)$ & 0.037 \\
\hline & No & 57.1 & 42.9 & 0.72 \\
\hline & & & 38.9 & \\
\hline \multirow{2}{*}{ Hospital's mortality (\%) } & & 00.2 & & 0.057 \\
\hline & No & 656 & 34.9 & 0.27 \\
\hline \multirow{2}{*}{\multicolumn{2}{|c|}{ Propofol Infusion's days (days, median (DE) }} & & & \\
\hline & & $8.6(9.7)$ & $6.5(6.7)$ & 0.001 \\
\hline \multicolumn{2}{|l|}{ Remifentanil infusion's days (days, median (DE)) } & $6.9(6.5)$ & $2.2(4.3)$ & 0.003 \\
\hline \multicolumn{2}{|c|}{ Dexmedetomidine infusion's days (days. median (DE)) } & $4.7(6.6)$ & $2.8(4.3)$ & 0.009 \\
\hline \multicolumn{2}{|l|}{ Maximum value of D-dimer. median (DE) } & $8481.8(9214.5)$ & 16220.8 (32114.) & 0.02 \\
\hline \multirow{2}{*}{ Hospital-acquired pneumoniae (\%) } & No & 60.6 & 39.4 & 0.9 \\
\hline & $\mathrm{Si}$ & 61.94 & 38.1 & \\
\hline \multirow{2}{*}{$\begin{array}{l}\text { Antibiotic treatment for hospital-acquired } \\
\text { infections (\%) }\end{array}$} & No & 50 & 50 & 0.1 \\
\hline & si & 66 & 34 & \\
\hline $0 \pi 1 \%$ & $\begin{array}{l}\text { No } \\
\text { Si }\end{array}$ & 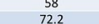 & $\begin{array}{l}42 \\
27.8\end{array}$ & 0.27 \\
\hline \multirow{3}{*}{$\begin{array}{l}\text { SOFA } 5 \\
\text { Delirium \% } \\
\end{array}$} & No & $16.7 \%$ & $83.3 \%$ & 0.042 \\
\hline & No & 62.5 & 37.5 & 0.6 \\
\hline & & 58.1 & 41.9 & \\
\hline
\end{tabular}

Table 3 shows the logistic regression analysis with the use of corticosteroids as a dependent variable.

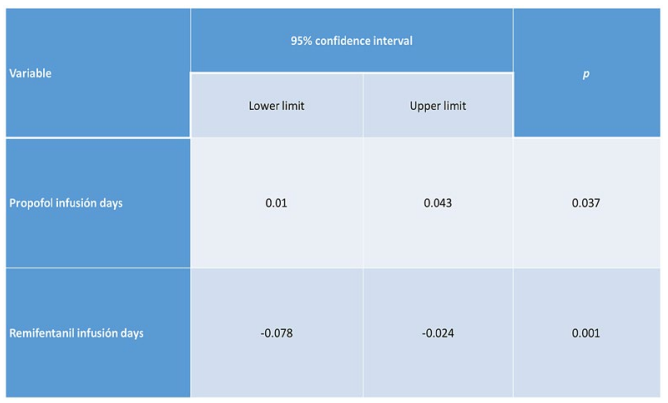

Conclusion: After adjusting for different confounding variables, a statistically significant relation is maintained between the use of corticosteroids for treatment of patients with COVID-19 pneumonia admitted in ICU and infusion days of propofol and remifentanil. However, its relation with other aspects (tendency to fewer days in mechanical ventilation and less ICU and hospital mortality) should be taken into account for future outbreaks and studies.

Reference(s) and grant ackowledgment(s)

1. Acknowledgments to all the nursing and auxiliary staff of the ICU at HUMT.

2. Not funded.

001131

D-dimer and Ferritin utility in patients admitted to the ICU due to COVID-19 pneumonia

J. Llabata'; E. Saavedra Sanjuan ${ }^{1}$; A. Falcon Marchena ${ }^{1}$; ML. Urendes ${ }^{1}$;

C. Palencia'; J. Ruiz'; M. López De La Fuente²; T. Villalobos Prego²;

B. Sánchez González'; J. Trenado ${ }^{1}$

${ }^{1}$ Intensive care department, Mútua Terrassa University Hospital, Terrassa, Spain; ${ }^{2}$ Hematology department, Mútua Terrassa University Hospital, Terrassa, Spain

Correspondence: B. Sánchez González

Intensive Care Medicine Experimental 2020, 8(2): 001131

Introduction: During the months of March to May we have experienced in Spain theconsequences of the COVID 19 pandemic. Different factors can be related to theevolution of a pathology, both those of the pathology and the patient, as well asthose derived from the high number of cases in a short period of time. Below we intend to analyze the different factors that are related to the evolution of COVID-19 pneumonia in our area. 
Objectives: To analyze the clinical utility of measuring D-dimer and Ferritin in patients admitted to the ICU with COVID-19 pneumonia during the SARS-CoV-2 outbreak.

Methods: Descriptive study carried out in a polyvalent ICU. All the patients admitted for pneumonia-COVID-19 during the pandemic period. Analytical measurements of $d$-dimer and ferritin were made from admission to hospital discharge. All patients had minimum 2-3 weekly measurements of these values.

Statistics:ROC curve analysis was performed for the maximum value of D-dimer and Ferritin and the difference between the minimum and maximum value (delta) of these parameters regarding different clinical variables.

Results: 95 patients were admitted. Table 1: describes the clinical variables analyzed with ROC curve with respect to the value of D-dimer and Ferritin.

\begin{tabular}{|l|l|}
\hline Table 1 & \\
\hline Age (years, mean (SD)) & $58.6(10)$ \\
\hline Sex (male\%) & 71 \\
\hline SOFA at admission $\leq 5(\%)$ & 76 \\
\hline Pulmonary embolism (\%) & 13 \\
\hline Patient receives anticoagulation (\%) & 26 \\
\hline Patient receives corticosteroids (\%) & 58 \\
\hline ICU long of stay (days, mean (SD)) & $13.1(12.9)$ \\
\hline Hospital stay (days, mean (SD) & $25.4(16.7)$ \\
\hline ICU mortality (\%) & 19 \\
\hline Hospital mortality (\%) & 20 \\
\hline
\end{tabular}

13 cases of pulmonary embolism (PE) were diagnosed, 3 of them were from the main pulmonary artery, 5 of them from segmental pulmonary artery, 1 of them from small glass and 5 of them from subsegmental arteries. In the group of 22 descoagulated patients, PE was only confirmed in 5 cases. Figure 1: represents the behavior of the ROC curves for ICU mortality and corticosteroid treatment.

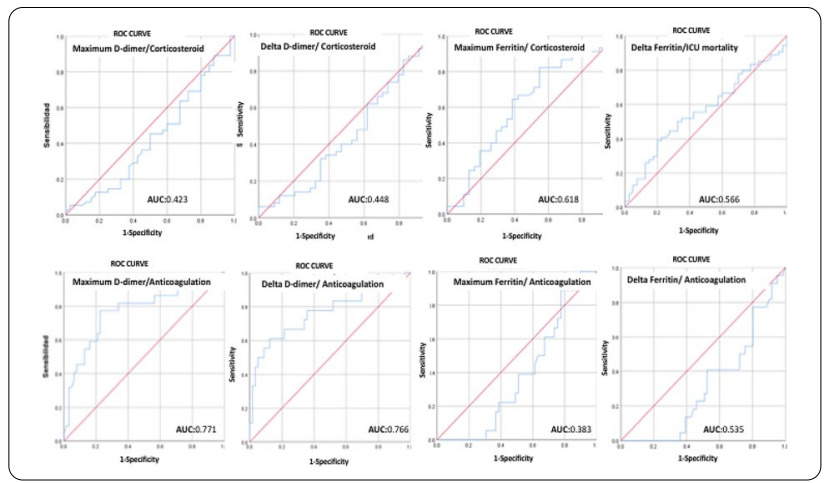

Figure 2: represents the behavior of ROC curves for diagnosis of PE and anticoagulated pacient.

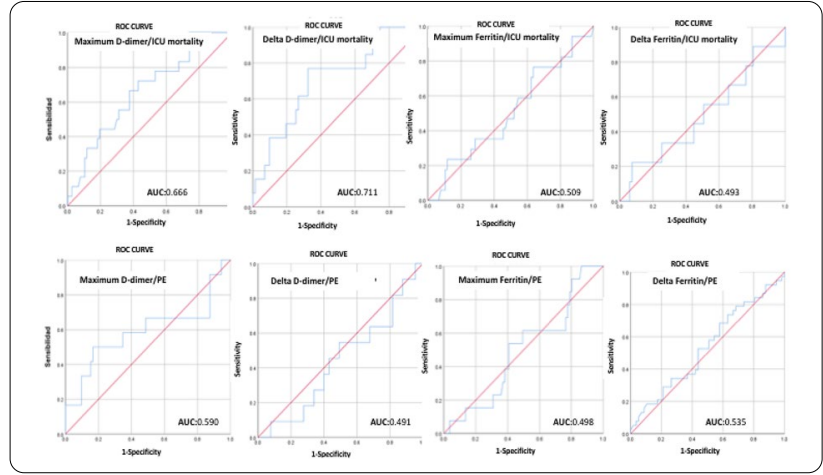

As the best result, we look at the area under the curve (AUC) for ICU mortality with respect to the delta of the D-dimer that shows a value of 0.711 with a $95 \% \mathrm{Cl}(0.561-0.861)$ and a significance asymptotic: 0.016 . An increase in D-dimer between 5500 and $6000 \mathrm{ng} / \mathrm{mL}$ is related to ICU mortality with a sensitivity between $0.77-0.61$ and specificity between $0.68 / 0.69$. The AUC for anticoagulated patient and PE shows the relationship of the D-dimer value and its delta to empirically anticoagulate a patient but not to diagnose PE.

Conclusion: The behavior of the maximum values of D-Dimer and Ferritin, as well as its delta, showed no prognostic neither diagnostic utility for PE and no utility in therapeutic decision-making in ICU hospitalized patients with COVID-19 relatedpneumonia. The need for routine measurement of these parameters in this group of patients should be assessed.

Reference(s) and grant ackowledgment(s)

1. Acknowledgments to all the nursing and auxiliary staff of the ICU at HUMT. 2. Not funded.

001132

Pharmacological treatment and ventilatory management of patients admitted to the ICU for COVID-19 pneumonia C. Palencia'; E. Lucas Romero²; J. Ruiz'; J. Llabata'; T. Moreno López²; F. Meza Samaniego ${ }^{2}$; M. De La Maza Segovia ${ }^{3}$; R. Algarte'; B. Sánchez González'; J. Trenado'

'Intensive care department, Mútua Terrassa University Hospital, Terrassa, Spain; ${ }^{2}$ Internal medicine department, Mútua Terrassa University Hospital, Terrassa, Spain; ${ }^{3}$ Anesthesiology and resuscitation department., Mútua Terrassa University Hospital, Terrassa, Spain

Correspondence: $B$. Sánchez González

Intensive Care Medicine Experimental 2020, 8(2): 001132

Introduction: During the months of March to May we have experienced in Spain the consequences of the COVID 19 pandemic. Different factors can be related to theevolution of a pathology, both those of the pathology and the patient, as well asthose derived from the high number of cases in a short period of time. Below we intend to analyze the different factors that are related to the evolution of COVID-19 pneumonia in our area.

Objectives: Describe the main characteristics of the patients admitted in ICU due to covid-19 related pneumonia during the coronavirus outbreak.

Methods: Descriptive study carried out in a polyvalent ICU with 12 beds for critical care and 21 for semi-critical care. All patients with covid-19 related pneumonia admitted in ICU in the outbreak were included in the study.

Statistics: Qualitative variables are expressed as percentages and quantitative variables are expressed as means and standard deviations (SD).

Results: 95 patients were entered,Table 1 described the main clinical characteristics of the patients. 


\begin{tabular}{|l|l|}
\hline \multicolumn{1}{|c|}{ Vable 1 } & \\
\hline Age (years, mean (SD)) & $58.6(10)$ \\
\hline Sex (male\%) & 71 \\
\hline SOFA at admission $\leq 5(\%)$ & 76 \\
\hline $\begin{array}{l}\text { Rockwood frailty scale at income } \leq \\
3(\%)\end{array}$ & 100 \\
\hline Multi-organ failure (\%) & 16 \\
\hline Patient receives anticoagulation (\%) & 26 \\
\hline Acute kidney failure (\%) & 11.6 \\
\hline ICU long of stay (days, mean (SD)) & $13.1(12.9)$ \\
\hline Hospital stay (days, mean (SD) & $25.4(16.7)$ \\
\hline ICU mortality (\%) & 19 \\
\hline Hospital mortality (\%) & 20 \\
\hline
\end{tabular}

Table 2: describes other clinic characteristics and complications.

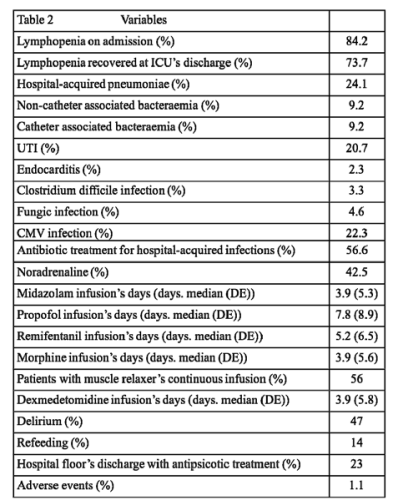

Table 3 describes respiratory failure and mechanical ventilation characteristics

\begin{tabular}{|c|c|}
\hline $\begin{array}{lll}\text { Variables } \\
\text { s. }\end{array}$ & \\
\hline Non-invasive ventilation (NIV) (\%) & 33 \\
\hline NIV beforct tasahcal inutuation (\%) & 25.3 \\
\hline $\begin{array}{l}\begin{array}{l}\text { High flow nasal cannula oxygen therapy (HFNC) } \\
\text { before tracheal intubation }(\%)\end{array} \\
\end{array}$ & 16.8 \\
\hline & 66.3 \\
\hline 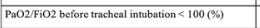 & 41 \\
\hline \begin{tabular}{|l} 
PEEP the first 24 hours of intubation (cmH2O. \\
median (DE))
\end{tabular} & $12.3(2.4)$ \\
\hline Fio2 the first 24 hours of intubation (median (DE)) & $0.5(0.2)$ \\
\hline $\begin{array}{l}\text { P.Platcau the first } 24 \text { hours of intubation (cmH2O. } \\
\text { mectian (DE) }\end{array}$ & $25.2(2.7)$ \\
\hline 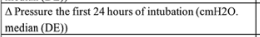 & $12.6(2.4)$ \\
\hline Compliance the first 2 thours of intubation $(\%)$ & $35.5(11.2)$ \\
\hline 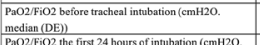 & $117.5(56.8)$ \\
\hline \begin{tabular}{|l|} 
PaO22fio2 the first 24 hours of intubation (cmH20. \\
median (DE))
\end{tabular} & $211(84.5)$ \\
\hline $\begin{array}{l}\text { Veidical weight the first } 24 \text { hours of fintubation (m/kg. } \\
\text { median (DE)) }\end{array}$ & $6.9(1.1)$ \\
\hline Pronations on intubated pacicnts (\%) & 45.3 \\
\hline ECMO (\%) & 3.2 \\
\hline \begin{tabular}{|l|} 
Tracheostomy $(\%)$ \\
\end{tabular} & 41.8 \\
\hline \begin{tabular}{|l|l|} 
ICU's ventilator $(\%)$ \\
\end{tabular} & 72 \\
\hline \begin{tabular}{|l|l} 
Only ICU's veatilator $(\%)$ \\
\end{tabular} & 57.7 \\
\hline
\end{tabular}

ICU mortality was $19 \%$ and $20 \%$ at hospital discharge.

Conclusion: Patients admitted in ICU for COVID-19 related pneumonia presented severe hypoxemia as the main organ affectation upon admission, requiring protective ventilation. Prone ventilation and tracheostomy were performed in a high percentage of patients. Infectious complications, PE and delirium were frequent.

Reference(s) and grant ackowledgment(s)

1. Acknowledgments to all the nursing and auxiliary staff of the ICU of HUMT.

2. Not funded.
001145

Factors related with delirium in patients admitted in ICU because of COVID-19 pneumonia

E. Saavedra Sanjuan'; A. Falcon Marchena ${ }^{1}$; ML. Urendes ${ }^{1}$; J. Llabata';

J. Ruiz'; T. Moreno López²; F. Meza Samaniego²; R. Algarte'; B. Sánchez

González'; J. Trenado

${ }^{1}$ Intensive care department, Mútua Terrassa University Hospital, Terrassa, Spain; ${ }^{2}$ Internal medicine department, Mútua Terrassa University Hospital, Terrassa, Spain

Correspondence: B. Sánchez González

Intensive Care Medicine Experimental 2020, 8(2): 001145

Introduction: During the months of March to May we have experienced in Spain theconsequences of the COVID 19 pandemic. Different factors can be related to theevolution of a pathology, both those of the pathology and the patient, as well asthose derived from the high number of cases in a short period of time. Below we intend to analyze the different factors that are related to the evolution of COVID-19 pneumonia in our area.

Objectives: Describe the factors related to delirium in patients admitted in ICU due to COVID-19 pneumonia.

Methods: Descriptive study carried out in a polivalent ICU. All patients admitted because of pneumonia by COVID-19 during the pandemia period were included. It was considered that a patient presented delirium by the ICU medical teams' clinical diagnosis. Delirium treatment was confirmed and adequated together with a psychiatry team destinated to ICU.

Statistics: Qualitative variables were expressed as percentages and were compared using the $\mathrm{X} 2$-test, quantitative were expressed as means and standard deviations (SD) and were compared using T-Student test. A logistic regression model with delirium as dependent variable was constructed.

Results: 95 patients were admitted, 35.6\% presented delirium during ICU admission and $16 \%$ at discharge to hospitalization plant. 66 ' $3 \%$ of all patients were intubated. $100 \%$ received Kaletra, Hydroxicloroquine and Azithromycin as treatment, additionally, 40\% received Tocilizumab, 34\% Interferon and 58\% corticoids. Table 1 describes clinical characteristics.

\begin{tabular}{|l|l|}
\hline Variables & Table 1 \\
\hline Age (years, mean (SD)) & $58.6(10)$ \\
\hline Sex (men, \%) & 71 \\
\hline Psychiatric history \% & 11.4 \\
\hline Antidepressant treatment \% & 7.4 \\
\hline Antipsychotic treatment \% & 1.1 \\
\hline Narcotic abuse \% & 0 \\
\hline Smoker \% & 9.5 \\
\hline Enolism \% & 0 \\
\hline Dexmetomedine \% & 42.1 \\
\hline Clonidine \% & 22.1 \\
\hline Olanzapine \% & 19.5 \\
\hline Quetiapine \% & 23 \\
\hline Other antipsychotic \% & 21.8 \\
\hline Other anxiolytic \% & 33.7 \\
\hline $\begin{array}{l}\text { Antipsychotic treatment at discharge to hospitalization } \\
\text { plant \% }\end{array}$ & 26.8 \\
\hline
\end{tabular}


Table 2 shows factors related to delirium.

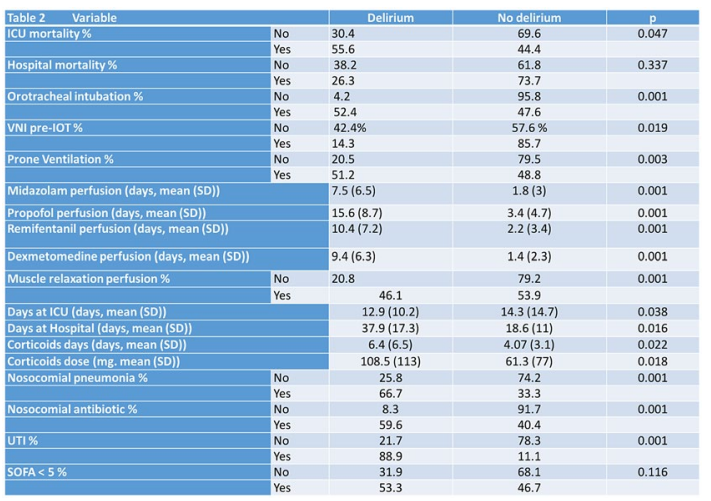

Table 3 shows logistic regression analysis of delirium as dependent variable.

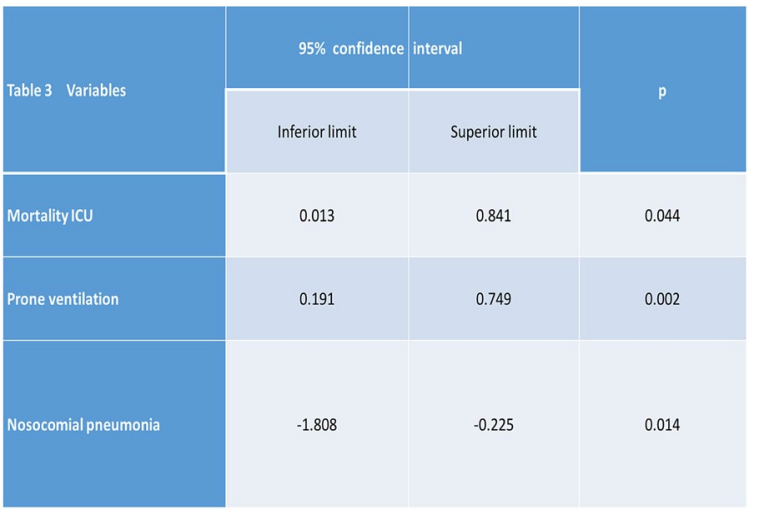

Conclusion: The patients admitted at our ICU due to COVID-19 pneumonia presented a high delirium incidence. Different factors as intubation and mechanical ventilation were associated with its development, aswell as sedation and muscle relaxants. However, it should be noted the statistically significant association after adjusting by different confusion factors between delirium with mortality in ICU, nosocomial infection appearance and prone ventilation.

\section{Reference(s) and grant ackowledgment(s)}

1. Not funded

2. Acknowledgments to all the nursing and auxiliary staff of the ICU at HUMT.

\section{3}

\section{Co/Super infection in critical SARS-CoV2 pneumonia}

B. Rodrigues De Castro ; A. Knapen ${ }^{1}$; LP. Moulaert ${ }^{1}$; P. Henin ${ }^{1}$; JB. Mesland ${ }^{1}$ ${ }^{1}$ Icu, CH Jolimont, La Louvière, Belgium

Correspondence: J.B. Mesland

Intensive Care Medicine Experimental 2020, 8(2): 001173

Introduction: Knowledge and understanding about SARS-CoV2 pathology is still poor. Viral pneumonia are known to promote coinfection or secondary bacterial infection. Diagnosis might be difficult between colonization of the respiratory tract and real infection. We need to be aware of specific patterns of infection as it is crucial for empiric prescription when needed.
Objectives: To review microbiological data regarding our critical SARS-Cov2 patients and evaluate if there is specific patterns of bacterial co-infection and super infection.

Methods: In an observational, retrospective and monocentric study, we reviewed microbiological data (blood culture, endotracheal aspirate, bronchoalveolar lavage) of all SARS-Cov2 patients admitted in our ICU between march 2020 and may 2020. If the same bacteria grew in different sample, it was counted as a unique episode.

Results: Between march 2020 and may 2020, 44 patients have been admitted to our 22 beds ICU for critical SARS-CoV2 pneumonia. Mean age at admission was 62,44 years (range 38 years -83 years). $37 / 44$ patients received mechanical ventilation, for a total of 638 days, and 13/44 patients received ECMO therapy, for a total of 249 days. ICU Mortality of our cohort is $50 \%$.

From our routine microbiology testing from tracheal aspirate, 34/44 patients had documented positive culture. 80 specimens were identified, distributed in order of prevalence as : Klebsiella species for $20 \%$ (Klebsiella pneumoniae for $8,75 \%$, Klebsiella oxytoca for 6,25\%, Klebsiella aerogenes for $3,75 \%$, Klebsiella varicola for $1,25 \%$ ), Pseudomonas aeruginosa for $13,75 \%$, E. Coli for $8,75 \%$, Proteus species for $7,5 \%$ (Proteus mirabilis for $3,75 \%$, Proteus vulgaris for $2,5 \%$, Proteus hauseri for $1,25 \%$ ), Staphylococcus aureus for $7,5 \%$ (MSSA for $6,25 \%$, MRSA for 1,25\%), Enterobacter species for 6,5\% (Enterobacter cloacae for 3,75\%, Enterobacter asburiae and kobei for 1,25\%), Citrobacter koseri for $3,75 \%$, Hafnia alvei for $2,5 \%$, Streptococcus pneumoniae for $2,5 \%$, Serratia species for $2,5 \%$, Haemophilus influenzae for $1,25 \%$. We noted a high number of Candida albicans positive specimens $(13,75 \%)$, which is not considered to be able to be an agent of pneumonia.

We performed 16 bronchoalveolar lavage (BAL). 87,5\% were predominantly neutrophilic. Regarding Pneumocystis jirovecii, indirect immunofluorescence using monoclonal antibodies were negative in all patients. All but one patient had BAL galactomannan negative, and none of our patients had positive respiratory culture for Aspergillus fumigatus. Multiplex RT-PCR was requested in 6 patients. 2/6 were strongly positive for HSV, and all were negative for Pneumocystis jirovecii.

12/44 patients developed at one time during their ICU stay an episode of bacteremia. $9 / 12$ were secondary to ventilatory associated pneumonia. From our cohort, $17 \%$ had co-infection at admission (considering co-infection as positive microbiology within the first 48 hours in our ICU).

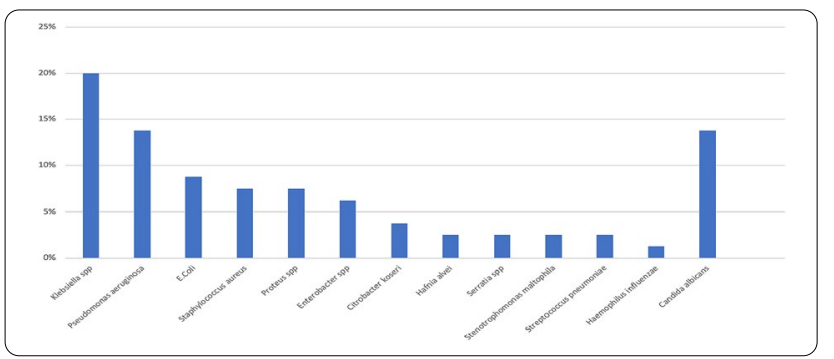

Conclusion: To conclude, we did not find typical organism described in community acquired pneumonia as being prevalent in SARS-CoV2 pneumopathy. Most of our positive microbiology were Gram negative bacteria, typical of ventilatory associated pneumonia. Indeed patients had to be mechanically ventilated during prolonged period of time. None of our patient had documented PCP or invasive pulmonary aspergillosis (IPA), as opposed to case series who found until one-third of patients with putative IPA(1)(2).

Reference(s) and grant ackowledgment(s)

1. (1) Menon AA, Berg DD, Brea EJ, et al. A Case of COVID-19 and Pneumocystis jirovecii Co-infection [published online ahead of print, 2020 May 15]. Am J Respir Crit Care Med. 2020;10.1164/rccm.202003-0766LE. doi:10.1164/ rccm.202003-0766LE

2. (2) Alanio A, Dellière S, Fodil S, Bretagne S, Mégarbane B. Prevalence of putative invasive pulmonary aspergillosis in critically ill patients with 
COVID-19 [published online ahead of print, 2020 May 20]. Lancet Respir Med. 2020;S2213-2600(20)30237-X. doi:10.1016/S2213-2600(20)30237-X

\section{0}

Effects of intravenous immunoglobulin (IVIG) on the course of severe COVID-19: results from a retrospective data analysis of a patient cohort in Turkey treated with or without Octagam ${ }^{\circledR}$ F. Esen ${ }^{1}$; P. Ergin Ozcan ; G. Orhun ${ }^{1}$; Ö. Polat ${ }^{1}$; I. Anakli'; G. Alay ${ }^{1}$; V. Tuna1; E. Celiksoy ${ }^{1}$; M. Kilic ${ }^{1}$; M. Mercan ${ }^{1}$; T. Tukek ${ }^{2}$

${ }^{1}$ Department of anesthesiology and intensive care, Istanbul University, Faculty of Medicine, İstanbul, Turkey; ${ }^{2}$ Department of internal medicine, Istanbul University Faculty of Medicine, İstanbul, Turkey

Correspondence: E. Clodi

Intensive Care Medicine Experimental 2020, 8(2): 001180

Introduction: The incidence of infection with severe acute respiratory syndrome coronavirus 2 (SARS-CoV 2) worldwide is still on the rise and the prevention and treatment of coronavirus disease - 2019 (COVID19) still a major challenge. Among those existing medications used for COVID-19 are intravenous immunoglobulins (IVIG) which are known for their anti-inflammatory effects and approved in several immune mediated disorders, including Kawasaki syndrome. Several case reports showing beneficial effects of IVIG administration in COVID-19 patients have been reported recently (1-5)

Objectives: To compare clinical outcomes and biomarkers in patients with severe COVID-19 treated with standard of care (SoC) alone or with SoC and IVIG.

Methods: Data from a cohort of severely ill COVID-19 patients requiring intensive care at a center of excellence in Turkey was analysed retrospectively. A subgroup of patients received high-dose IVIG (Octagam 5\%) treatment at $0.4 \mathrm{~g} /$ day for 5 consecutive days in addition to SoC according to a site-specific treatment algorithm and was compared to those patients receiving $\mathrm{SoC}$ alone. Clinical outcome measures included duration of specific treatment modalities, time to start of mechanical ventilation, change in ventilation mode, ICU and hospital discharge, and overall survival. Plasma biomarkers of inflammation (CRP, ferritin, PCT, IL-6, D-dimer, leukocytes) were measured at baseline and 6 days after ICU admission and treatment start. Imbalance in APACHE II scores was addressed by propensity-score-matching; otherwise Kaplan-Meier and multiple regression models were used.

Results: Data from 93 patients were included into analysis. Of these, 51 had received IVIG plus SoC and 42 were treated with SoC alone. In both groups, about $75 \%$ of patients were male and patients had comparable BMI and blood group distribution as well as comparable vital signs. The IVIG group was slightly younger (mean: 65 vs. 71 years) and had slightly lower baseline disease scores (mean APACHE II: 20.6 vs. 22.4; mean SOFA: 5.0 vs. 7.0). Apart from IVIG, patients in both groups were treated with hydroxychloroquine, favipiravir, azithromycin, oseltamivir, tocilizumab/anakinra, methylprednisolone, and/or ascorbate. Analysis showed that overall survival (OS) was $61 \%$ in the IVIG and 38\% in the control group. After controlling for imbalances at baseline, there remained a trend for better OS (OR: 2.2, 95\%Cl: 0.9-5.4, $\mathrm{p}=0.091$ ) and a significantly longer median survival (68 vs. 18 days, $\mathrm{p}=0.014$ ). Moreover, IVIG treatment significantly reduced CRP levels by $46 \%$ within 6 days compared to SoC alone $(p=0.049)$, but had no relevant effect on any other inflammation marker measured.

Conclusion: Adjunct treatment with IVIG might add to the currently limited armamentarium against COVID-19, especially due to its wellknown anti-inflammatory properties. However, results need to be confirmed in a randomized, controlled trial.

\section{Reference(s) and grant ackowledgment(s)}

1. 1) Mohtadi N, Ghaysouri A, Shirazi S, Sara A, Shafiee E, Bastani E, et al. Recovery of severely ill COVID-19 patients by intravenous immunoglobulin (IVIG) treatment: A case series. Virology. 2020;548:1-5. Epub 2020/06/13.

2. 2) Shi $H$, Zhou $C$, He $P$, Huang $S$, Duan $Y$, Wang $X$, et al. Successful treatment with plasma exchange followed by intravenous immunoglobulin in a critically ill patient with COVID-19.International journal of antimicrobial agents. 2020:105974. Epub 2020/04/17.
3. 3) Cao W, Liu X, Bai T, Fan H, Hong K, Song H, et al. High-Dose Intravenous Immunoglobulin as a Therapeutic Option for Deteriorating Patients With Coronavirus Disease 2019. Open forum infectious diseases. 2020;7(3):ofaa102. Epub 2020/04/08.

4. 4) Lanza M, Polistina GE, Imitazione P, Annunziata A, Di Spirito V, Novella C, et al. Successful intravenous immunoglobulin treatment in severe COVID19 pneumonia. IDCases. 2020;21:e00794-e.

5. 5) Chiotos K, Bassiri H, Behrens EM, Blatz AM, Chang J, Diorio C, et al. Multisystem Inflammatory Syndrome in Children During the Coronavirus 2019 Pandemic: A Case Series. J Pediatric Infect Dis Soc. 2020;9(3):393-8.

6. This retrospective study was sponsored by Octapharma AG, Lachen, Switzerland

\section{3}

Microbiological profile of infections in patients admitted by COVID-19 to the intensive care unit (ICU)

S. Ramiro González ; MDM. Molina Morales ${ }^{1}$; CM. Rodríguez Mejías ${ }^{1}$;

J. Machado Casas $^{1}$

${ }^{1}$ Medicina intensiva, Hospital Universitario Virgen de las Nieves, Granada, Spain

Correspondence: S. Ramiro González

Intensive Care Medicine Experimental 2020, 8(2): 001183

Introduction: Coronavirus infection (COVID-19) produces a severe acute respiratory syndrome that in up to $5-10 \%$ of cases requires admission to the ICU, with overinfection being one of the most frequent complications during a stay in this Unit.

Objectives: To describe the main intra-ICU infections according to location and microbiological germ isolated in patients admitted for COVID-19.

Methods: Retrospective descriptive study conducted with the data collected for the ENVIN registry in the period from March 13, 2020 to April 30, 2020 in the ICU of the Virgen de las Nieves Hospital in Granada.

Patients over 18 years old with a stay of more than 24 hours were included. Baseline characteristics: age, sex, degree of severity (APACHE II), mean stay, ICU mortality and extrinsic risk factors. The type of intraICU infections are exposed according to the isolated microorganism and the location of the infection.

Results: A total of 60 patients admitted to the ICU of our hospital are included, $80 \%$ of which are male, with a mean age of $60.03 \pm 11.25$ years and with APACHE II at admission of $13.76 \pm 5.65$ years. The average stay in the ICU was $21.11 \pm 13.45$ and the mortality was $38.18 \%$. Among the extrinsic risk factors, more than $80 \%$ of these patients required an artificial airway, $98.33 \%$ carried a central venous catheter and $98 \%$ a urinary catheter and $60 \%$ had received antibiotherapy prior to admission to the ICU.

The rate of intra-ICU infections was 90 infections per 100 patients admitted, the most frequent location being bacteremias of unknown focus and secondary to catheter infection (50\%), followed by pneumonias associated with mechanical ventilation (27.59\%) and urinary tract infections (13.79\%). In the case of bacteremias, up to $67.74 \%$ of large positive microorganisms were isolated, most frequently E. Faecalis and S. epidermidis, and up to $16.13 \%$ candida spp. Faecalis (50\%), followed by Candida spp.; on the other hand, in pneumonias associated with mechanical ventilation, $57.14 \%$ of gram-negative bacilli stand out, among them Pseudomona aeruginosa and klebsiella pneumoniae, and up to $19 \%$ of fungal isolation.

Conclusion: $\mathrm{A}$ high rate of intra-ICU infections is observed in patients admitted for COVID-19, the most frequent being labacteremia of unknown focus and secondary to catheter infection. This high rate of infections may be justified by the high percentage of invasive devices used as well as by the degree of complexity and severity of these patients. 


\section{0}

Serum coenzyme Q10 levels decrease during progression from critical illness to sepsis/septic shock

AG. Vassiliou'; A. Kotanidou²; Z. Mastora²; E. Jahaj²; C. Keskinidou³

M. Pratikaki'; E. Kampisioulii' S. Orfanos ${ }^{2}$; I. Dimopoulou ${ }^{2}$

${ }^{1} 1$ st department of critical care medicine \& pulmonary services, gp livanos and $m$ simou laboratori, National and Kapodistrian University of Athens, Athina, Greece; ${ }^{2} 1$ st department of critical care medicine \& pulmonary services, Evangelismos Hospital, Medical School, National and Kapodistrian University of Athens, Athens, Greece; ${ }^{3}$ Medical school, Democritus University of Thrace, Alexandroupoli, Greece; ${ }^{4}$ Biochemical department, Evaggelismos General Hospital, Athina, Greece

Correspondence: A.G. Vassiliou

Intensive Care Medicine Experimental 2020, 8(2): 000020

Introduction: Coenzyme $\mathrm{Q} 10(\mathrm{CoQ} 10)$ is a lipid-soluble vitaminlike compound present in the inner membrane of the mitochondria, where it transports electrons from complexes I and II to complex III of the mitochondrial respiratory chain (MRC). CoQ10 removes free radicals, inhibits the initiation and propagation of lipid peroxidation in cellular membranes, and aids in the regeneration of other antioxidants, such as vitamin E and C. CoQ10 levels in septic ICU patients have been investigated by a limited number of studies. The possibility that CoQ10 plasma concentrations exhibit time course changes from the initial period of critical illness to sepsis and septic shock, remains currently unexplored.

Objectives: We sought to investigate whether in a group of initially non-septic, critically ill patients, coenzyme Q10 (CoQ10) changes during progression from critical illness to sepsis and septic shock and assess its associations with inflammatory biomarkers, and intensive care unit (ICU) outcome.

Methods: This was an observational analysis on 63 initially non-septic ICU patients. CoQ10 was sequentially measured on admission in the ICU (baseline) and at sepsis and septic shock onset. Additionally, CoQ10 was measured in 25 age and sex-matched healthy controls. Inflammatory biomarkers were determined at baseline.

Results: On ICU admission and prior to sepsis development, patients had lower CoQ10 levels compared to healthy controls ( 0.89 vs. 1.04 $\mu \mathrm{g} / \mathrm{ml}, \mathrm{p}<0.01)$. At sepsis onset, CoQ10 levels decreased to $0.63 \mu \mathrm{g} / \mathrm{ml}$ $\mathrm{p}<0.001$, and at septic shock $(\mathrm{N}=14)$ to $0.42 \mu \mathrm{g} / \mathrm{ml}, \mathrm{p}<0.0001$. Baseline CoQ10 correlated with low density lipoprotein $(r 2=0.409, p=0.002)$, and tended to negatively correlate with IL-8 and TNF-a. CoQ10 at sepsis was not associated with ICU mortality.

Conclusion: Initially non-septic, critically ill patients have lower CoQ10 on ICU admission compared to healthy controls, with a further decrease in sepsis and septic shock, suggesting that CoQ10 depletion is related to sepsis severity. CoQ10 is a component of the mitochondria, and supplementation might improve mitochondrial function in septic shock.

\section{2}

Prevalence of relevant Augmented Renal Clearance in critically ill patients with sepsis. An Epidemiological Multicenter Study

T. Tomasa'; J. Sabater-Riera²; M. Pérez-Carrasco ${ }^{3}$; A. Navas-Pérez ;

A. Betbesé-Roig ${ }^{5}$; M. Rodríguez-López ${ }^{6}$; M. Ibarz-Vilamayor ${ }^{7}$;

Y. Díaz-Buendía; ; A. Olmo-Isasmendi'; I. Oliva-Zelaya ${ }^{10}$; C. Rovira-Anglès ${ }^{11}$; P. Ortz-Ballujera ${ }^{12}$; S. Cano-Hernández ${ }^{13}$. E. Vendrell-Torra ${ }^{14}$.

RM. Catalán-Ibars ${ }^{15}$; M. Miralbés Torner ${ }^{16}$; FJ. Gonzalez-Molina ${ }^{17}$;

J. Xirgu-Cortacans ${ }^{18}$; MP. Marcos-Neira ${ }^{19^{\prime}}$

'Intensive care unit, Hospital Germans Trias i Pujol, Badalona, Spain; Intensive care, Bellvitge University Hospital, L'Hospitalet de Llobregat, Spain; ${ }^{3}$ Intensive care, Vall d'Hebron University Hospital, BarceIona, Spain; ${ }^{4}$ ntensive care, Hospital Parc Taulí de Sabadell, Sabadell, Spain; ${ }^{5}$ ntensive care, Hospital de la Santa Creu i Sant Pau, Barcelona, Spain; ${ }^{6}$ ntensive care, Hospital de Sant Joan Despí Moisès Broggi, Sant Joan Despí, Spain; Intensive care, Hospital Universitari Sagrat Cor - Grup Quirónsalut, Barcelona, Spain; ${ }^{8}$ Intensive care, Hospital del Mar, Barcelona, Spain; ${ }^{9}$ Intensive care, Helipuerto Hospital General de Catalunya, Sant Cugat del Vallès, Spain; ${ }^{10}$ Intensive care, Hospital Universitari de Tarragona
Joan XXIII, Tarragona, Spain; ${ }^{11}$ Intensive care, Hospital Universitari Sant Joan de Reus, Reus, Spain; ${ }^{12}$ Intensive care, Hospital Universitari de Girona Doctor Josep Trueta, Girona, Spain; ${ }^{13}$ Intensive care, Althaia, Xarxa Assistencial Universitària de Manresa, Manresa, Spain; ${ }^{14}$ Intensive care, Hospital de Mataró, Mataró, Spain; ${ }^{15}$ Intensive care, Hospital Universitari de Vic (Consorci Hospitalari de Vic), Vic, Spain; ${ }^{16}$ Intensive care unit, University Hospital Arnau de Vilanova, Lleida, Spain; ${ }^{17}$ Intensive care, Mútua Terrassa University Hospital, Terrassa, Spain; ${ }^{18}$ Intensive care, Hospital General de Granollers, Granollers, Spain; ${ }^{19}$ Intensive care, Hospital Universitari Germans Trias i Pujol, Badalona, Spain

Correspondence: T. Tomasa,

Intensive Care Medicine Experimental 2020, 8(2): 000022

Introduction: Acute renal failure has been studied extensively, mainly to try to minimize pharmacological toxicity. Early administration of appropriate and correctly dosed antibiotics is one of the cornerstones of sepsis treatment. At present, the opposite situation is also disturbing, in which there is an augmented renal clearance (ARC), because it could affect the outcome of these patients.

Objectives: The purpose was to determine the prevalence of the phenomenon of relevant augmented renal clearance $(>170 \mathrm{~mL} / \mathrm{min} / 1.73$ $\mathrm{m} 2, \mathrm{rARC}$ ) and analyze the characteristics of these patients.

Methods: Multicenter, observational, prospective, epidemiological study in the critically ill patient with sepsis. 18 hospitals were recruited where 3 prevalence points were made, calculating the glomerular filtration rate (GFR) in 4-hour urine collection sample. The characteristics of these patients were compared with those with non-rARC group (GFR $90-170 \mathrm{~mL} / \mathrm{min} / 1.73 \mathrm{~m} 2$ ). A descriptive analysis and a univariate analysis were performed. A type I error $<0.05$ was considered statistically significant. The results are given in medium and $95 \% \mathrm{Cl}$.

Results: 561 patients of 65.5 (63.5-66.5) years were included. We focus on those 116 patients with TFG $>90 \mathrm{ml} / \mathrm{min} / \mathrm{m} 2$. Of these 116 patients, $34(29.31 \%)$ were classified in the rARC group and $82(70.7 \%)$ in the non-rARC group. The rARC patients were younger $59.5(51-64)$ years vs. 64.5 (61-70) years, $\mathrm{P}=0.03$. However, the sex ratio was also: $55.9 \%$ in rARC vs. $68.3 \%$ in non-rARC, $P=0.56$. Mortality was also similar in both groups: ICU mortality was $11.8 \%$ in rARC vs. $14.6 \%$ in non-rARC, $P=0.94$. In-hospital mortality was the same: $17.6 \%$ in rARC vs. $17.1 \%$ in non-rARC, $P=0.56$. And length of stay (LOS) was also similar: ICU-LOS was 15.5 (13-36) days in rARC group, vs. 20.5 (15-27) days in non-rARC $\mathrm{P}=0.56$. And HOSP-LOS was 34 (15-53) days in rARC group, vs. 34 (2743) days in non-rARC, $P=0.3$.

Conclusion: Relevant-ARC is found in almost $30 \%$ of ICU patients. rARC patients are younger. Conversely, sex is similar, and so is mortality and length of stay.

\section{Reference(s) and grant ackowledgment(s)}

1. Carrié Carrié C, Chadefaux G, Sauvage N, Courson H, Petit L, Nouette-Gaulain $\mathrm{K}$, et al. Increased $\beta$-Lactams dosing regimens improve clinical outcome in critically ill patients with augmented renal clearance treated for a first episode of hospital or ventilator-acquired pneumonia: a before and after study. Critical Care (2019) 23:379 https://doi.org/10.1186/s13054-019-2621-4 2. None

\section{9}

Assessment of lipolysis activity by the composition of plasma fatty acids

S. Tachyla ${ }^{1}$; A. Osipenko ${ }^{2}$; O. Tupitsyna ${ }^{3}$

${ }^{1}$ Department of Anesthesiology and Intensive Care, Mogilev Regional Hospital, Mogilev, Belarus; ${ }^{2}$ Central research laboratory, Mogilev State University named after A. A. Kuleshov, Mogilev, Belarus; ${ }^{3}$ Department of anesthesiology and intensive care, Mogilev Emergency City Hospital, Mogilev, Belarus

Correspondence: S. Tachyla

Intensive Care Medicine Experimental 2020, 8(2): 000079

Introduction: Sepsis and multiple organ dysfunction syndrome (MODS) accompanied by an increase of catabolic reactions, which 
include activation of lipolysis. Assessment of lipolysis activity allows us to judge the severity of the patient's condition and its changes during treatment. It may also be necessary to determine the amount of nutritional support.

Objectives: To develop a method for assessing lipolysis activity by the composition of blood plasma fatty acids in patients in abdominal surgery.

Methods: A prospective case-control study of the composition of blood plasma fatty acids was performed in 31 patients after abdominal operations during the first $24 \mathrm{~h}$ after admission in the intensive care unit (ICU). The patients were divided into two groups: group 1 (n $=15)$ - without sepsis and MODS, group $2(n=16)$ - the presence of sepsis and MODS. Sepsis and MODS were established by the criteria of the 2016 SCCM / ACCP conference.

Measurements of the composition of fatty acids were carried out on gas chromatographs $\Gamma X-1000$, ЦВЕТ-800 (Russian Federation). Final identification was carried out using the method of chromatographymass spectrometry using Thermo Scientific DSQ II (USA). The determination of the proportion of individual fatty acids was carried out by the internal normalization method using the Unichrom-97 spectrometric information recording system.

Statistical processing of the results was performed using the SPSS and Statistica 7.0 programs. Data are presented as medians and quartiles. Differences between groups were determined by the Mann-Whitney $U$ test.

Results: Of the 31 patients, there were 19 men and 12 women, age 40 (35-46) years, height $172(168-175) \mathrm{cm}$, body weight 80 (64-92) kg Patients in groups with no statistical differences in sex, age, weight, height. The Apache III score was significantly higher in the group 2 of $53.0(39.2-74.7)$ points vs $33.3(9.9-53.2)$ points $(p=0.01)$. The duration of treatment in the ICU was significantly higher in the group 2 of 11 $(5-15)$ days vs $2(2-3)$ days $(p<0.001)$. Mortality in the group $1-0 \%$, in the group $2-25 \%$.

Patients had the following pathology: acute pancreatitis -7 , chronic pancreatitis - 11, cholelithiasis - 5, acute intestinal obstruction - 3, abdominal trauma - 4, peptic ulcer with perforation - 1 . They performed laparotomy - 26, laparoscopy - 5 relaparotomy - 3 .

It was found that in the composition of total plasma lipids, the total proportion of palmitoleic and oleic fatty acids increases. If in patients of the group 1 this proportion is $18.2(16.2-19.6) \%$, then in patients of the group 2 it is $27.9(24.5-31.3) \%(p<0.001)$.

The construction and analysis of the receiver operating characteristic curve for the method is performed. It was established that the area under the curve is 0.929 with a $95 \%$ confidence interval from 0.837 to 0.978 ( $p<0.0001)$, sensitivity $87.5 \%$, specificity $93.3 \%$, threshold $21.7 \%$. Conclusion: The increase in the proportion of palmitoleic and oleic fatty acids in the composition of total blood plasma lipids reflects an increase in lipolysis activity.

Assessing the activity of lipolysis allows us to judge the intensity of catabolic processes in the body in patients.

\section{Reference(s) and grant ackowledgment(s)}

1. Novak F, Borovska J, Vecka M et al. Plasma phospholipid fatty acid profile is altered in both septic and non-septic critically ill: a correlation with inflammatory markers and albumin. Lipids 2017;52(3):245-254.

2. Osipenko AN, Marochkov AV. Blood plasma plasmalogens and fatty acids in multiple organ dysfunction syndrome. Critical Care and Shock 2017:20(2):40-45

3. Rival T, Cinq-Frais C, Silva-Sifontes $S$ et al. Alteration of plasma phospholipid fatty acid profile in patients with septic shock. Biochimie 2013;95(11):2177-81.

\section{5}

Predictive utility cannot be inferred from associations - a systematic review of the sepsis literature to quantify the confusion in reporting

T. Varga'; AS. Andreasen ${ }^{2}$; P. Moseley ${ }^{3}$

${ }^{1}$ Department of public health, Copenhagen University, København, Denmark; ${ }^{2}$ Anesthesia and critical care, Herlev Hospital, Herlev, Denmark;
${ }^{3}$ Novo nordisk foundation center for protein research, Copenhagen University, København, Denmark

Correspondence: $P$. Moseley

Intensive Care Medicine Experimental 2020, 8(2): 000115

Introduction: Precision medicine is considered to be the means to improve outcome of sepsis patients in the future. Statistical analyses applied to results may uncover high degrees of associations between markers or interventions and outcomes; however, even high degrees of association do not necessarily imply a predictive value of an intervention. Precision medicine requires prediction; thus attributing predictive value to associations diverges from this path and may lead to erroneous conclusions that can ultimately harm patients.

Objectives: To determine whether and to what extent published literature in sepsis with claims of prediction in their titles in fact do provide relevant measures of prediction in their abstracts. Our core premise was that abstracts, as the most informative summaries of published papers, should always confirm the claims of the title by reported statistical methodology that pertains to predictive utility.

Methods: A systematic search was performed using NCBI PubMed on Jan 27, 2020, aiming to identify articles that contain the terms sepsis and prediction in the titles using the following search term: sepsis[Title] AND (prediction[Title] OR predict[Title] OR predicts[Title] OR predictive[Title] OR predicting[Title]) AND (hasabstract[text] AND "humans"[MeSH Terms]). General terms and defined measures of individualized prediction, as well as other reported statistical methodologies related to associations, were extracted from the abstracts of these papers. Prediction metrics were defined as measures derived from individualized predicted outcomes or probabilities, while association metrics were defined as measures pertaining to group results.

Results: In total, 518 articles, published between 1978 and 2019, were identified. Of these, 34 were excluded due to non-relevance to this systematic assessment. The remaining 484 articles, published in 227 unique journals, comprised our study sample. Of these articles, $36 \%$ $(n=173)$ did not report metrics of predictive utility, while the remaining $64 \%(n=311)$ included at least one metric of predictive utility in their abstracts. Of the abstracts that reported measures of predictive utility, the most common reported metrics were ROC AUC (70\%), sensitivity (40\%) and specificity (36\%). Of the abstracts without measures of predictive utility, $54 \%$ did not define their methodology, while $28 \%$ performed logistic regression and $15 \%$ performed Cox models. Full review of the 173 undefined manuscripts revealed that more than $75 \%$ $(n=117)$ were actually describing a relationship based upon associations only, while $17 \%(n=31)$ contained some measure of prediction. Conclusion: We demonstrate evidence that more than one-third of abstracts from papers that claim to provide insights on prediction in the sepsis literature actually provide no methodology to evaluate prediction in their abstracts. In most cases, these manuscripts offer a measure of association or no defined methodology at all. When no information was available in the abstract, more than $75 \%$ of these manuscripts did not provide measures of prediction and often claimed prediction while using only tests of association. Such confusion may ultimately harm patients, for instance by undergoing unnecessary and inappropriate treatment of abnormalities that proves not to be predictive despite statistically significant associations.

Reference(s) and grant ackowledgment(s)

1. Novo Nordisk Foundation (NNF16OC0020698, NNF14CC0001,

NNF170C0027594), Swedish Research Council (Dnr 2009-1039), Swedish

Foundation for Strategic Research (Dnr IRC15-0067).

\section{6}

Endothelial Microparticles Is Novel Markers of Endothelial Injury in Patients with Sepsis

Y. Takei'; M. Yamada ${ }^{2}$; K. Saito ${ }^{3}$; S. Kazutomo ${ }^{1}$; T. Shiga ${ }^{3}$; Y. Kameyama ${ }^{3}$;

M. Yamauchi'

${ }^{1}$ Anesthesiology and Perioperative medicine, Tohoku University Graduate School of Medicine, Sendai, Japan; ${ }^{2}$ Respiratory medicine, Tohoku Univer- 
sity Graduate School of Medicine, Sendai, Japan; ${ }^{3}$ Intensive care of medicine, Tohoku University Hospital, Sendai, Japan

Correspondence: Y. TAKEI

Intensive Care Medicine Experimental 2020, 8(2): 000116

Introduction: Endothelial cells are involved in the pathogenesis of sepsis and organ dysfunction caused by sepsis. There is no wellestablished method in clinical practice for evaluating endothelial injury in sepsis. Endothelial microparticles (MPs) (EMPs) are extracellular vesicles derived from the cellular membranes of injured or apoptotic endothelial cells, which suggests that EMPs may be a marker for endothelial injury in sepsis.

Objectives: To examine whether the number of EMPs is increased in patients with sepsis and reflects the severity of sepsis.

Methods: This prospective observational study was performed to examine the numbers of circulating EMPs in patients with sepsis using flow cytometry (ethics committee approval: 2018-1-353). We collected platelet-poor plasma (PPP) from patients diagnosed with Sepsis-3 on admission and on days 2, 3, 5, and 7 of hospitalisation. We also collected PPP for a non-sepsis control group from patients who were admitted to the intensive care unit after undergoing scheduled head and neck surgery on admission and on days 2, 3, and 5 of hospitalisation. EMPs were defined as CD31+/CD41- MPs [platelet endothelial cell adhesion molecule (PECAM)+ EMPs], CD144+ MPs [vascular endothelial (VE)-cadherin + EMPs], CD62E+ MPs (E-selectin+ MPs), CD54+ MPs [intercellular adhesion molecule (ICAM)-1+ EMPs], and CD106+ MPs [vascular cell adhesion molecule (VCAM)-1+ EMPs] MPs. Results: We examined the numbers of circulating EMPs in 72 patients with sepsis (sepsis, 29; septic shock, 43) and 30 non-sepsis patients. The numbers of all types of EMPs were significantly higher in patients with sepsis than in non-sepsis patients during the study period, except for VCAM-1 + EMPs on admission. The numbers of PECAM+ and VEcadherin+ EMPs, the expressed endothelial antigens of which are rich in endothelial cell intercellular junctions and regulate endothelial junctional integrity and permeability, were significantly higher in patients with septic shock than in those with sepsis until day 5 . On the other hand, the number of E-selectin+ EMPs, which are endothelial adhesion molecules induced by inflammatory stimuli, such as E-selectin, ICAM-1, and VCAM-1, on admission was significantly higher in patients with septic shock than in patients with sepsis. The numbers of PECAM+, VE-cadherin+, and E-selectin + EMPs on admission, as well as PECAM+ EMPs on day 2, were significantly higher in patients who died in the hospital than those in survivors.

Conclusion: The number of EMPs significantly increased in patients with sepsis, which may suggest that an increase in the number of EMPs may reflect endothelial injury in sepsis. Furthermore, the numbers of PECAM+ and VE-cadherin + EMPs are higher in patients with septic shock than in patients with sepsis and might be novel markers of the severity of septic endothelial injury.

Reference(s)and grant ackowledgment(s)

1. Grant-in-Aid for Scientific Research (B:18-7 to Y.Takei) from the Japanese Society of Anesthesiologists (JSA)

2. Grants-in-Aid for Scientific Research (18K08907 to K. Saito) from the Japan Society for the Promotion of Science (JSPS)

\section{2}

Secondary sepsis in the ICU. A retrospective study

M. Karvouniaris ${ }^{1}$; M. Chatzimihail'; P. Papamichalis ${ }^{1}$;. T. Zafeiridis';

A. Palioura'; A. Papadogoulas ${ }^{1}$; P. Katsiafylloudis'; A. Komnos

${ }^{1} \mathrm{ICU}$, Koutlimbaneio \& Triantafylleio General Hospital of Larissa, Larisa, Greece

Correspondence: M. Karvouniaris

Intensive Care Medicine Experimental 2020, 8(2): 000142
Introduction: The presence of sepsis on patients' admission to the intensive care unit (ICU) is associated with adverse outcomes [1]. It has been demonstrated that many patients suffer additional infectious and septic episodes, which might increase mortality [2].

Objectives: Our aim is to delineate the incidence and clinical characteristics of the secondary sepsis event in comparison with sepsis on admission in a setting of endemic difficult-to-treat Gram-negative microbial infections.

Methods: Secondary ICU-associated sepsis was defined as a second septic event, $>48$ hours after admission. Data were retrieved from the records of all patients admitted in our 16-bed mixed ICU between July 2015 and December 2017. We excluded patients with an ICU stay of $<48 \mathrm{hs}$. We described continuous variables as median (IQR) and categorical variables as number (percentage) and we performed comparisons with Mann-Whitney, Wilcoxon test and chi-square test, where appropriate. We used survival analysis to evaluate ICU mortality.

Results: Sixty-five septic patients (65 episodes), 42 (64.6\%) male, and 35(53.4\%) medical, were analyzed. Their age, Charlson comorbidity index, APACHE II, and SOFA on admission were 73 (65-79) years, 4 (3-5), 24 (18-27.5), and 10 (8-12), respectively. The most commonly encountered primary septic focus was intraabdominal (46.2\%), and 42(64.6\%) patients were in septic shock. A pertinent culture displayed a pathogen in $35(53.8 \%)$ episodes, and Gram-negative isolates prevailed $(26 / 35,74.3 \%)$.

Sixteen (24.6\%) patients presented secondary sepsis on day 9(6-15.5), an isolate was identified in $93.7 \%$ of events, and Acinetobacter baumannii was isolated in $8 / 15$ events (53.3\%). The presence of hypothermia on the day of both episodes was the same (20\%); temperature and white blood cells did not differ between the events.

Regarding the outcome, overall ICU mortality was $46.2 \%$. The KaplanMaier curve analysis of 30-day mortality did not show any difference between patients with and without secondary sepsis. However, secondary sepsis was associated with prolonged length of stay in the ICU (21 vs. ten days, $p=0.001$ ). Eventually, more patients died after their first month in the critical care department (ICU mortality of $75 \%$ vs. $37 \%, p=0.02$ ).

Conclusion: In a setting of endemic difficult-to-treat Gram-negative bacteria, secondary sepsis, on top of primary sepsis, can lead to both prolonged hospital stay and late mortality.

\section{Reference(s) and grant ackowledgment(s)}

1. Van Vught LA, Klein Klouwenberg P, Spitoni K, et al. Incidence, Risk Factors, and Attributable Mortality of Secondary Infections in the Intensive Care Unit After Admission for Sepsis. 2016; 315(14): 1469-79

2. Rhodes A, Evans LE, Alhazzani W., et al. Surviving Sepsis Campaign: International Guidelines for Management of Sepsis and Septic Shock: 2016. Intensive Care Med; 43(3): 304-377

3. None

\section{9}

Characteristics and outcomes of frail patients with suspected infection in intensive care units

A. Komori ${ }^{1}$;. Abe ${ }^{2}$; K. Yamakawa ${ }^{3}$; H. Ogura ${ }^{4}$; S. Kushimoto ${ }^{5}$; D. Saitoh ${ }^{6}$; S. Fujishima ${ }^{7}$;. Otomo ${ }^{8}$; J. Kotani ${ }^{9}$; Y. Sakamoto ${ }^{10}$; J. Sasaki ${ }^{11}$; Y. Shiino ${ }^{12}$; N. Takeyama ${ }^{13}$;. Tarui ${ }^{14}$; R. Tsuruta ${ }^{15}$;A. Nakada ${ }^{16}$;. T. Hifumi ${ }^{17}$; H. Iriyama'; T. Naito'; S. Gando ${ }^{18}$

1Department of general medicine, Juntendo University, Bunkyo City, Japan; ${ }^{2}$ Department of emergency and critical care medicine, Tsukuba Memorial Hospital, Tsukuba, Japan; ${ }^{3}$ Division of trauma and surgical critical care, Osaka General Medical Center, Osaka, Japan; ${ }^{4}$ Department of traumatology and acute critical medicine, Osaka University Graduate School of Medicine, Suita, Japan; ${ }^{5}$ Division of emergency and critical care medicine, Tohoku University Graduate School of Medicine, Sendai, Japan; ${ }^{6}$ Division of traumatology, research institute, National Defense 
Medical College, Tokorozawa, Japan; ${ }^{7}$ Center for general medicine education, Keio University School of Medicine, Tokyo, Japan; ${ }^{8}$ Trauma and acute critical care center, Tokyo Medical and Dental University, Bunkyo City, Japan; ${ }^{9}$ Division of disaster and emergency medicine, Kobe University Graduate School of Medicine, Kobe, Japan; ${ }^{10}$ Emergency and critical care medicine, University Hospital attached to the Faculty of Medicine, Saga University, Saga, Japan; ${ }^{11}$ Department of emergency and critical care medicine, Keio University School of Medicine, Tokyo, Japan; ${ }^{12}$ Department of acute medicine, Kawasaki Medical School Hospital, Kurashiki, Japan; ${ }^{13}$ Emergency and critical care medicine, Aichi Medical University, Nagakute, Japan; ${ }^{14}$ Department of trauma and critical care medicine, Kyorin University, Mitaka, Japan; ${ }^{15}$ Acute and general medicine, Yamaguchi University Graduate School of Medicine, Ube, Japan; ${ }^{16}$ Department of emergency and critical care medicine, Chiba University Graduate School of Medicine, Chiba, Japan, Japan; ${ }^{17}$ Department of emergency and critical care medicine, St. Luke's International Hospital, Chuo City, Japan; ${ }^{18}$ Division of acute and critical care medicine, Hokkaido University Graduate School of Medicine, Sapporo, Japan

Correspondence: A. Komori

Intensive Care Medicine Experimental 2020, 8(2): 000159

Introduction: Frailty increases the risk of morbidity and mortality in patients with critical illnesses. However, most previous studies evaluated a heterogeneous population. The specific characteristics of patients with sepsis according to frailty in the intensive care units (ICUs) remains unclear.

Objectives: We aimed to investigate the association between frailty and characteristics, clinical features, and outcomes among patients with suspected infection in ICUs.

Methods: This is a secondary analysis of the Japanese Association for Acute Medicine Sepsis Prognostication in Intensive Care unit and Emergency room (JAMA SPICE)-ICU study, which was a multicenter study of patients with suspected infection. We included adult patients ( $\geq 18$ years) with suspected infection who were admitted to ICUs. Frailty was evaluated using the Canadian Study on Health and Aging Clinical Frailty Scale (CFS)[1]. As previous reported [2], baseline patient characteristics and outcomes were compared between three frailty groups: fit (scale 1-3), vulnerable (scale 4) and frail (scale 5-9). We also performed a subgroup analysis of patients with sepsis, which was defined based on sepsis- 3 criteria. The Kaplan-Meier survival curve for 90 days was also drawn.

Results: We enrolled 650patients with suspected infection, including 599 (92.2\%) patients with sepsis, for this study from the SPICE-ICU database. Patients with a median rating of $3(3-5)$ according to the CFS, were included: 337 (51.8\%) were fit, 109 (16.8\%) vulnerable, and $204(31.4 \%)$ frail. Comorbidities were more prevalent in frail and vulnerable patients than in fit patients. The APACHE II scores for fit, vulnerable, and frail patients were 18 (12-25), 22 (17-28), and 21 (15-27), respectively $(p<0.01)$. The body temperatures of patients were as follows: fit $37.5(36.5-38.5)^{\circ} \mathrm{C}$; vulnerable $37.5(36.4-38.6)^{\circ} \mathrm{C}$; frail 37.0 $(36.3-38.1)^{\circ} \mathrm{C}(p<0.01)$. Regarding the laboratory data, the CRP level in fit, vulnerable and frail patients were $13.6(4.6-24.5) \mathrm{mg} / \mathrm{dl}, 12.1$ $(3.9-24.9) \mathrm{mg} / \mathrm{dl}, 10.5(3.03-21.0) \mathrm{mg} / \mathrm{dl}$, respectively $(p<0.01)$. There was no statistically significant difference regarding organ dysfunction among the groups. In-hospital mortality did not differ statistically according to frailty: fit 55/335 (16.4\%); vulnerable 23/107 (21.5\%); frail $45 / 203(22.2 \%), p=0.19$. Kaplan-Meier survival curves showed that there was little difference in the mortality rate among groups during the acute phase. However, vulnerable and frail patients tended to die after the acute phase while the fit did not, although the difference was not statistically significant ( $p=0.25$, Figure). Compared to fit or vulnerable patients, fewer frail patients were discharged to home: fit 125/280 (44.6\%); vulnerable 36/84 (42.9\%); frail 40/158 (25.3\%).

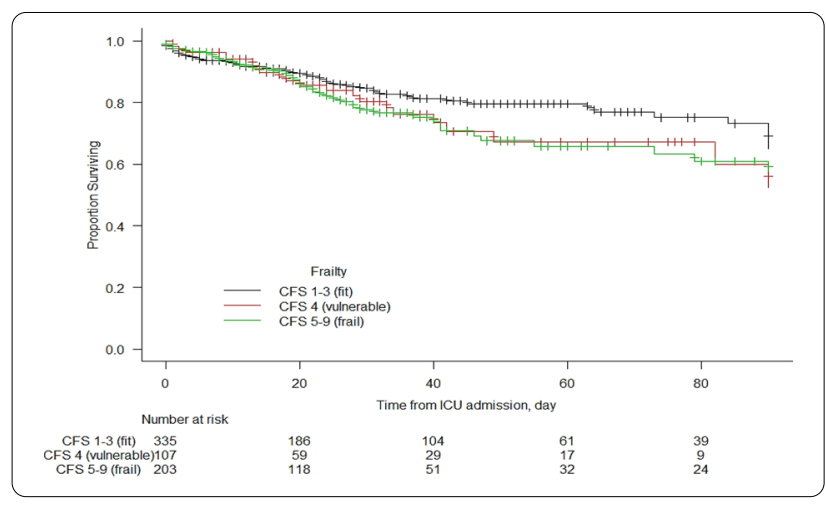

Conclusion: Frailty was associated with a poor response to inflammation of infection.Frail and vulnerable patients with suspected infection including sepsis tended to have poor outcomes after the acute phase in their clinical courses.

\section{Reference(s) and grant ackowledgment(s)}

1. [2] Hans Flaatten, et al. Intensive Care Med. 2017;43:1820-28

2. [1] Rockwood K, et al. CMAJ. 2005;173(5):489-95

000235

The temporal relationship between mitochondrial- and monocyte cell functionality during sepsis

MJ. Melis'; E. Karakike2; N. Koliakos³ EJ. Giamarellos-Bourboulis²; M. Shankar-Hari ${ }^{4} ;$ M. Singer ${ }^{1}$

${ }^{1}$ Bloomsbury institute of intensive care medicine, University College London, London, United Kingdom; ${ }^{2} 4$ th department of internal medicine, National and Kapodistrian University of Athens, Medical School, Athens, Greece; ${ }^{3} 3$ rd department of surgery, National and Kapodistrian University of Athens, Medical School, Athens, Greece; ${ }^{4}$ School of immunobiology and microbial sciences, King's College School, London, United Kingdom

Correspondence: M.J. Melis

Intensive Care Medicine Experimental 2020, 8(2): 000235

Introduction: Immune responses in sepsis involve simultaneous activation and suppression of circulating leukocytes. These immune responses are often associated with mortality [1]. Alterations in mitochondrial functionality of monocytes and lymphocytes are associated with immune suppression, severity of sepsis, and mortality [2-4]. Monocyte HLA-DR expression is an important marker for monitoring immune alterations during sepsis [5]. We postulated that immune dysfunction with decreased monocyte HLA-DR expression during sepsis may be induced by a bioenergetic shutdown.

Objectives: To assess the correlation between mitochondrial function (as measured by mitochondrial membrane potential $\left(\Delta \Psi_{\mathrm{m}}\right)$ and ROS production), and immune cell functionality (measured by monocyte HLA-DR expression) during the time-course of sepsis.

Methods: Septic and pre-operative control patients were recruited at the ATTIKON University Hospital in Athens, Greece. Whole blood was collected at four different timepoints (days 1, 2, 4 and 7 after admission) for septic patients and at one timepoint for control patients. Whole blood was lysed and stained with anti-CD14 to identify monocytes, TMRM and MitoSox as measures of $\Delta \Psi \mathrm{m}$ and ROS production, anti-HLA-DR for HLA-DR receptor expression, and Annexin-V and 7AAD as markers for apoptosis and cell death. Appropriate positive-, negative-, and isotype controls were used. Samples were measured 
using a BD FC500 flow cytometer and analysed using FlowJo. Prism was used for statistical analysis with Kruskal-Wallis non-parametric tests comparing different groups at day 1 , and a repeated measures mixed ANOVA model to analyse trends over time within groups.

Results: 16 septic patients (10 survivors), and 13 pre-operative control patients were enrolled. There were no differences in $\Delta \psi_{\mathrm{m}}$ between the three groups on day 1 . A change over time was found for all septic patients $(P=0.02)$, without any differences between survivors and non-survivors. Higher levels of mitochondrial ROS were found in nonsurvivors on day 1 compared to controls $(P=0.02)$. No differences were however found over time for either survivors or non-survivors. Compared to controls, HLA-DR expression was lower in septic patients on day 1 ( $P=0.05$ for survivors). HLA-DR expression recovered over time in survivors ( $P=0.003$ versus non-survivors).
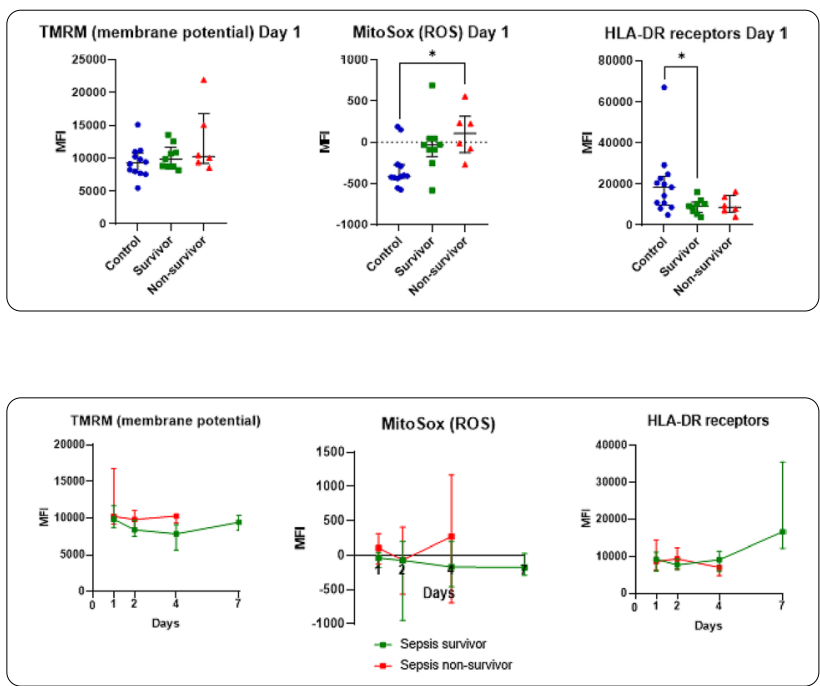

Conclusion: Bioenergetic alterations (mitochondrial ROS production and $\Delta \Psi_{\mathrm{m}}$ ) do not explain the reduction in HLA-DR levels in sepsis patients.

\section{Reference(s) and grant ackowledgment(s)}

1. 1. Hotchkiss RS, Karl, IE. N Engl J Med 2003; 348: 138-50.

2. 2. Adrie C et al. Am J Resp Crit Care Med 2001: 164:389-95.

3. 3. Belikova I et al. Crit Care Med 2007; 35:2702-8.

4. 4. Sjövall F et al., Crit Care 2013; 17:R152.

5. 5. Venet $F$ et al. Curr Opin Immunol 2013; 25:477-83

6. MJM was supported by the Boehringer Ingelheim Fonds Travel Grant.

7. MJM and EK are supported by the European Union's Horizon 2020 research and innovation programme under the Marie Sklodowska-Curie grant agreement No 676129.

\section{6}

\section{Characteristics and in-hospital mortality according} to whether patients who met sepsis-2 and sepsis-3 definitions among patients with suspected infection in intensive care units: Sepsis Prognostication in Intensive Care Unit and Emergency

\section{Room (SPICE-ICU)}

T. Abe'; K. Yamakawa²; H. Ogura ${ }^{3}$; S. Kushimoto ${ }^{4}$; D. Saitoh ${ }^{5}$; S. Fujishima ${ }^{6}$.

Y. Otomo ; J. Kotani ${ }^{8}$; Y. Umemura ${ }^{3}$; J. Sasaki ${ }^{9}$; Y. Shiino ${ }^{10}$; T. Tarui ${ }^{11}$

SI. Shiraishi ${ }^{12}$;TA. Nakada ${ }^{13}$; A. Hagiwara ${ }^{14}$; U. Masashi ${ }^{15}$; N. Yamashita ${ }^{16}$;

T. Masuno ${ }^{17} ;$ H. Ikeda ${ }^{18}$; S. Gando ${ }^{19}$

'Department of emergency and critical care medicine, department of health services research, Tsukuba Memorial Hospital, University of Tsukuba, Tsukuba, Japan; ${ }^{2}$ Division of trauma and surgical critical care, Osaka General Medical Center, Osaka, Japan; ${ }^{3}$ Department of traumatology and acute critical medicine, Osaka University Graduate School of Medicine, Suita, Japan; ${ }^{4}$ Division of emergency and critical caremedicine, Tohoku University Graduate School of Medicine, Sendai, Japan;
${ }^{5}$ Division of traumatology, research institute, National Defense Medical College, Tokorozawa, Japan; ${ }^{6}$ Center for general medicine education, Keio University School of Medicine, Tokyo, Japan; ${ }^{7}$ Trauma and acute critical care center, Tokyo Medical and Dental University, Bunkyo City, Japan; ${ }^{8}$ Division of disaster and emergency medicine, Kobe University Graduate School of Medicine, Kobe, Japan; ${ }^{9}$ Department of emergency and critical care medicine, Keio University School of Medicine, Tokyo, Japan; ${ }^{10}$ Department of acute medicine, Kawasaki Medical School Hospital, 577 Matsushima, Kurashiki, Okayama, Japan, Kurashiki, Japan; ${ }^{11}$ Department of trauma and critical care medicine, Kyorin University, 5 Chome-4 Shimorenjaku, Mitaka, Tokyo, Japan, Mitaka, Japan; ${ }^{12}$ Department of emergency and critical care medicine, Aizu Chuo Hospital, Aizuwakamatsu, Japan; ${ }^{13}$ Department of emergency and critical care medicine, Chiba University Graduate School of Medicine, Chiba, Japan, Japan; ${ }^{14}$ Department of emergency medicine, Niiza Shiki Chūō General Hospital, Niiza, Japan; ${ }^{15}$ Department of trauma, critical care medicine, and burn center, Chūkyō Hospital, Nagoya, Japan; ${ }^{16}$ Advanced emergency medical service center, Kurume University, Kurume, Japan; ${ }^{17}$ Department of emergency and critical care medicine, Nippon Medical School, Bunkyo City, Japan,

${ }^{18}$ Department of emergency medicine, Teikyo University, Hachioji, Japan;

${ }^{19}$ Division of acute and critical care medicine, Sapporo Higashi Tokushukai Hospital, Sapporo, Japan

Correspondence: T. Abe

Intensive Care Medicine Experimental 2020, 8(2): 000236

Introduction: Diagnosing sepsis remains difficult because it is not a single disease but a syndrome with various pathogen- and host factor-associated symptoms. Sepsis-3 was established to improve risk stratification among patients suspected with infection based on organ failures but it has been still controversial compared with previous definitions.

Objectives: We aimed to investigate characteristics according to whether patients who met sepsis-2 (severe sepsis) 1 and sepsis-3 definitions 2 among patients with suspected infection in ICU.

Methods: This was a multicenter, prospective cohort study. It conducted by 22 intensive care units (ICUs) in Japan. Adult patients ( $\geq 16$ years) with newly suspected infection from December 2017 to May 2018 were included. All patients were admitted to the ICUs in study hospitals. Patients were divided into four groups according to whether they met each definition or not: (both) sepsis-2 and sepsis-3; sepsis-2 and no sepsis-3; no sepsis-2 and sepsis-3; and no sepsis-2 and no sepsis-3. Characteristics and in-hospital mortality were compared according to the groups.

Results: In total, 652 patients with suspected infection were admitted to 22 ICUs during the study, of whom 558 (85.6\%) met the sepsis-2 definition and 600 (92.0\%) met the sepsis-3 definition. The sepsis-2 and sepsis-3 definitions comprised different individuals, and 527 (80.8\%) patients met both definitions (sepsis-2 and sepsis-3). Thirty one exclusively met the sepsis-2 (sepsis-2 and no sepsis-3) and 73 exclusively met the sepsis-3 (no sepsis-2 and sepsis-3) definition. A total of 21 patients did not meet either definitions (no sepsis-2 and no sepsis-3). Systemic inflammatory response syndrome (SIRS) positive ( $\geq 2$ ) among patients with sepsis-2 and sepsis-3; sepsis-2 and no sepsis-3; no sepsis-2 and sepsis-3; and no sepsis-2 and no sepsis-3 were $100 \%$; $100 \%$; $68.5 \%$; and $66.7 \%$, respectively. Quick sequential organ failure assessment (qSOFA) positive $(\geq 2)$ among patients with sepsis- 2 and sepsis-3; sepsis-2 and no sepsis-3; no sepsis-2 and sepsis-3; and no sepsis-2 and no sepsis-3 were $66.8 \% ; 35.5 \% ; 27.4 \%$; and $14.3 \%$, respectively. Inhospital mortality among suspected infection, sepsis-2, and sepsis-3 patients was comparable $(19.2 \%, 21.7 \%$, and $20.0 \%$, respectively). Inhospital mortality among patients with sepsis-2 and sepsis-3; sepsis-2 and no sepsis-3; no sepsis-2 and sepsis-3; and no sepsis-2 and no sepsis-3 were $22.0 \% ; 16.1 \% ; 5.5 \%$; and $0 \%$, respectively. Patients who met sepsis-3 shock definition had higher in-hospital mortality than those who met sepsis-2 shock definition ( $27.4 \%$ vs $34.3 \%)$.

Conclusion: Most patients with suspected infection admitted to ICU meet sepsis-2 and sepsis-3 definitions. They were less suitable for risk stratification in this setting. However, in-hospital mortality did not occur if patients did not meet any criteria. Better criteria might 
be developed by better selection and combining elements in both definitions.

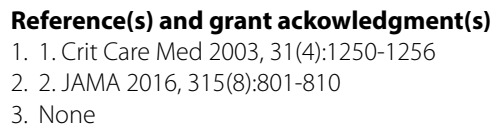

\section{2}

\section{Role of transesophageal echocardiography in non-cardiac} medical intensive care patients with sepsis of an unknown origin without preknown heart disease

C. Ditges ${ }^{1}$; R. Schmid ${ }^{1}$; W. Huber ${ }^{1}$; T. Lahmer ${ }^{1}$

${ }^{1}$ Medizinische klinik und poliklinik ii, Klinikum rechts der Isar; Technische Universität München, Munich, Germany

Correspondence: $C$. Ditges

Intensive Care Medicine Experimental 2020, 8(2): 000282

Introduction: In septic patients - besides a rapidly beginning therapy the effective search for the inflammatory focus is imperative to reduce morbidity and mortality. Therefore the prior diagnostic steps entail laboratory and imaging investigations (i.e. computer tomography). If these do not lead to any concrete result, transesophageal echocardiography (TEE) is recommended to exclude an infective endocarditis (IE). As the lethality of infective endocarditis can be up to $30 \%$, TEE is an important diagnostical tool in intensive care medicine. In this study we investigated the role of TEE to detect a septic focus in patients on medical intensive care units especially without underlying cardiac disease as until now it is still unclear.

Methods: We conducted a retrospective 10 years analysis of non-cardiac medical intensive care unit patients with sepsis of unknown origin but without preknown heart disease, who underwent a TEE to detect IE as the focus of the inflammation. We collected data about laboratory findings, risk-scores, TEE-results, DUKE-criteria and mortality.

Results: From 1925 septic patients 108 (6\%) underwent a TEE and were included in the study (average age 60 years, $61 \%$ male). Among these patients $15(14 \%)$ were diagnosed an IE and six (42\%) of them died. APACHE II- as well as SOFA-score were lower in patients with IE compared to patients with another septic focus ( 23 vs 27 and 9 vs $11)$, but showed statistical significance. Neither leucocytes (18.1 vs $20.6 \mathrm{G} / \mathrm{l}), \mathrm{CRP}$ (20.8 vs $13.4 \mathrm{mg} / \mathrm{dl}$ ), procalcitonin ( $32.9 \mathrm{vs} 17.9 \mathrm{ng} / \mathrm{ml}$ ) nor body temperature $\left(36.9\right.$ vs $37.2^{\circ} \mathrm{C}$ ) showed significant differences between both groups. Positive blood cultures were found in $80 \%$ of patients with IE and in $42 \%$ of the others. Comparing the results of the modified DUKE-criteria the patient with IE met in average 1.5 major criteria and 2.1 minor criteria. Patients without IE ( 0.2 major and 1.4 minor criteria) met significantly less criteria $(p<0.001)$. Those who left the intensive care unit alive received their TEE significantly earlier $(p=0.001)$.

Conclusion: This study demonstrates the relevance of TEE in sepsis of unknown focus and without underlying cardiac disease. Due to the high incidence of IE in septic patients in combination with an increased morbidity and mortality a transesophageal echocardiography should be carried out as soon as possible when having such patients on a medical intensive care unit. Clinical findings in patients with infective endocarditis show a high heterogeneity, so that the diagnosis is hard to make - especially in patients without preknown heart disease. Neither laboratory findings nor scoring systems were unequivocal or groundbreaking.

\section{Reference(s) and grant ackowledgment(s)}

1. Habib, G., Lancellotti, P., Antunes, M. J., Bongiorni, M. G., Casalta, J. P., Del Zotti, F., .. Group, E. S. C. S. D. (2015). 2015 ESC Guidelines for the management of infective endocarditis: The Task Force for the Management of InfectiveEndocarditis of the European Society of Cardiology (ESC). Endorsed by: European Association for Cardio-Thoracic Surgery (EACTS), the European Association of Nuclear Medicine (EANM). Eur Heart J, 36(44), 3075-3128. doi:10.1093/eurheart//ehv319
2. Singer, M., Deutschman, C. S., Seymour, C. W., Shankar-Hari, M., Annane, D., Bauer, M., ... Angus, D. C. (2016). The Third International Consensus Definitions for Sepsis and Septic Shock (Sepsis-3). JAMA, 315(8), 801-810. doi:10.1001/jama.2016.0287

3. Rhodes, A., Evans, L. E., Alhazzani, W., Levy, M. M., Antonelli, M., Ferrer, R., Dellinger, R. P. (2017). Surviving Sepsis Campaign: International Guidelines for Management of Sepsis and Septic Shock: 2016. Intensive Care Med, 43(3), 304-377. doi:10.1007/s00134-017-4683-6

\section{8}

Different associations between the body temperature and mortality in elderly and nonelderly patients with severe sepsis

T. Shimazui ${ }^{1}$;TA. Nakada ${ }^{1}$; K. Walley²; T. Oshima ${ }^{1}$;T. Abe ${ }^{3}$; H. Ogura ${ }^{4}$.

A. Shiraishi ${ }^{5}$; S. Kushimoto ${ }^{6}$; Y. Otomo ${ }^{7}$; S. Gando ${ }^{8}$

${ }^{1}$ Emergency and Critical Care Medicine, Chiba University Graduate School of Medicine, Chiba, Japan; ${ }^{2}$ Centre for heart lung innovation, University of British Columbia, Vancouver, Canada; ${ }^{3}$ Department of general medicine, Juntendo University, Tokyo, Japan; ${ }^{4}$ Department of traumatology and acute critical medicine, Osaka University Graduate School of Medicine, Osaka, Japan; ${ }^{5}$ Emergency and trauma center, Kameda Medical Center, Chiba, Japan; ${ }^{6}$ Division of emergency and critical care medicine, Tohoku University Graduate School of Medicine, Miyagi, Japan; ${ }^{7}$ Trauma and acute critical care center, Medical hospital, Tokyo Medical and Dental University, Tokyo, Japan; ${ }^{8}$ Acute and critical care center, department of acute and critical care medicine, Sapporo Higashi Tokushukai Hospital, Hokkaido, Japan

Correspondence: $T$. Shimazui

Intensive Care Medicine Experimental 2020, 8(2): 000298

Introduction: A dysregulated host response, which is a cornerstone of the pathophysiology of sepsis, induces alteration in vital signs. Elderly patients have a blunted host response, which may influence vital sign alterations and clinical outcomes of sepsis. We investigated the different associations between the vital signs and mortality in elderly and nonelderly patients with sepsis.

Methods: The current observational study was retrospectively conducted. A prospective multicenter severe sepsis cohort acquired from 59 Japanese ICUs (FORECAST, $n=1148$ ) was used for the derivation analyses. Significant results were tested for replication using two validation cohorts of severe sepsis (JAAMSR, prospective multicenter cohort from Japan, $\mathrm{n}=624 ; \mathrm{SPH}$, a sigle center cohort including different ancestry [Canada], $n=1004$ ). Patients were categorized into elderly and nonelderly groups (age $\geq 75$ or $<75$ years). The associations of the vital signs (body temperature [BT], heart rate, mean arterial pressure, systolic blood pressure, respiratory rate) within 24 hours of severe sepsis diagnosis and 90-day in-hospital mortality (primary outcome) were studied using Cox regression analysis.

Results: Nonelderly patients $(\mathrm{n}=628)$ with $\mathrm{BT}<36.0^{\circ} \mathrm{C}$ showed significantly increased 90 -day mortality $(P=0.025$, adjusted hazard ratio 1.70 , $95 \% \mathrm{Cl} 1.07-2.71)$ in the derivation cohort. While those with BT>38.3 ${ }^{\circ} \mathrm{C}$ showed decreased trend for mortality $(\mathrm{P}=0.11$, adjusted hazard ratio $0.71,95 \% \mathrm{Cl} 0.47-1.08)$. In the validation cohorts, nonelderly patients with $\mathrm{BT}<36.0{ }^{\circ} \mathrm{C}$ showed significantly increased mortality (JAAMSR, $\mathrm{P}=0.0024$, adjusted hazard ratio $2.05,95 \% \mathrm{Cl} 1.29-3.26$; $\mathrm{SPH}, \mathrm{P}=0.029$, adjusted hazard ratio $1.36,95 \% \mathrm{Cl} 1.03-1.80)$, and those with BT $>38.3^{\circ} \mathrm{C}$ displayed significantly decreased mortality (JAAMSR, $\mathrm{P}=0.0043$, adjusted hazard ratio $0.51,95 \% \mathrm{Cl} 0.32-0.81 ; \mathrm{SPH}, \mathrm{P}=0.018$, adjusted hazard ratio $0.76,95 \% \mathrm{Cl} 0.61-0.95)$. These differences were not observed in elderly patients in all the three cohorts. Associations between the other four vital signs and mortality were not different in elderly and nonelderly patients.

Conclusion: Among the vital signs, body temperature showed significantly different effects on mortality between nonelderly and elderly patients. Nonelderly severe sepsis patients with hypothermia and fever showed decreased and increased mortality, respectively. 
However, mortality in elderly patients was not associated with body temperature.

Reference(s) and grant ackowledgment(s)

1. This work was supported by the Japanese Association for Acute Medicine (JAAM) and Kashiwado Memorial Foundation for Medical Research.

\section{2}

Biomarkers of alveolar capillary membrane disruption in mechanically ventilated patients with sepsis - a pilot study A. Andrijevic'; S. Gavrilovic'; J. Matijasevic ${ }^{1}$; D. Obradovic ${ }^{2}$; U. Batranovic ${ }^{1}$ Intensive Care Unit, Institute for Pulmonary Diseases of Vojvodina, Sremska Kamenica, Serbia; ${ }^{2}$ High dependency unit, Institute for Pulmonary Diseases of Vojvodina, Sremska Kamenica, Serbia

Correspondence: A. Andrijevic

Intensive Care Medicine Experimental 2020, 8(2): 000302

Introduction: Pulmonary vascular endothelium is a key modulator in acute respiratory distress syndrome (ARDS) development (1). Recent investigations suggest that greater systemic endothelial activation might be associated with poor outcome of mechanically ventilated patients with sepsis (2).

Objectives: The aim of this study was to investigate serum biomarkers of alveolar capillary damage (sRAGE, endocan, angiopoetin-2) as outcome predictors in mechanically ventilated patients with sepsis.

Methods: A prospective, observational pilot study was conducted on mechanically ventilated patients with sepsis admitted in the ICU of the Institute for pulmonary diseases of Vojvodina (IPDV) from January until March 2018. Diagnosis of sepsis was based on Sepsis-3 criteria (3) and ARDS was defined by using Berlin criteria (4). Blood samples for the assessment of serum biomarkers (sRAGE, endocan, angiopoetin-2) were taken within the first 24 hours from starting the mechanical ventilation.

Baseline demographic data, Charlson comorbidity index, arterial blood gas analysis, Tidal volume, PEEP value, complete blood count and biochemistry findings (procalcitonin, CRP, lactate, creatinine, bilirubin) were collected. Severity of illness was defined by Acute Physiology and Chronic Health Evaluation II (APACHE II) and Sequential Organ Failure Assessment (SOFA) score.

Results: A total of 24 mechanically ventilated patients treated in the ICU of IPDV were included. Average age was 54,8 and $33,3 \%$ of patients were males. The Berlin criteria for ARDS met 14 patients (58,3\%). Average APACHE II score at ICU admission was 22,71 $\pm 6,9$ and average SOFA score $7,13 \pm 2,74$. ICU mortality was $37,5 \%$.

There were no differences observed in levels of biomarkers with respect to 28-day mortality for SRAGE, angiopoetin-2 and endocan, as shown in Table 1 . Subset of ARDS patients had significantly lower values of angiopoetin- $2(9,93 \pm 7,04$ vs $16,31 \pm 7,7, p=0,047)$ comparing to patients without ARDS. Regrading hospital mortality and ventilator free days, there was no statistically significant difference between SRAGE, angiopoetin-2 and endocan. There was no correlation between values of specific biomarkers.

\begin{tabular}{|l|l|l|l|}
\hline Biomarker & $\begin{array}{l}28 \text { day } \\
\text { mortalitydied }\end{array}$ & $\begin{array}{l}28 \text { day } \\
\text { mortalitysurvived }\end{array}$ & p value \\
\hline sRAGE & $3031 \pm 1818$ & $3052 \pm 1983$ & 0,979 \\
\hline angiopoetin-2 & $15.6 \pm 8,8$ & $10,4 \pm 6,6$ & 0,118 \\
\hline endocan & $5,62 \pm 3,94$ & $5,34 \pm 3,56$ & 0,855 \\
\hline
\end{tabular}

Table 1. Correlation between 28-day mortality and biomarkers of alveolar capillary damage.
Conclusion: Our results suggest that mechanically ventilated patients with ARDS have different expression of alveolar capillary damage biomarkers comparing to patients without ARDS. Biomarker changes are less useful for prediction of other hospital outcomes in our study.

Reference(s) and grantackowledgment(s)

1. Ranieri VM, Rubenfeld GD, Thompson BT, Ferguson ND, Caldwell E, Fan E, et al. Acute respiratory distress syndrome: The Berlin definition. JAMA - J Am Med Assoc. 2012;307(23):2526-33.

2. Singer M, Deutschman CS, Seymour C, Shankar-Hari M, Annane D, Bauer $M$, et al. The third international consensus definitions for sepsis and septic shock (sepsis-3). JAMA - Journal of the American Medical Association. 2016;315(8):801-10.

3. Uchimido R, Schmidt EP, Shapiro NI. The glycocalyx: A novel diagnostic and therapeutic target in sepsis. Critical Care. 2019;23(1):16.

4. Millar FR, Summers C, Griffiths MJ, Toshner MR, Proudfoot AG. The pulmonary endothelium in acute respiratory distress syndrome: Insights and therapeutic opportunities. Thorax. 2016;71: 462-473.

000364

A multi-site retrospective analysis of angiotensin II utilization and clinical outcomes in septic shock

S. Choi ${ }^{1}$; A. Nagy ${ }^{1}$; R. Shata ${ }^{2}$; C. Adams ${ }^{2}$

${ }^{1}$ Pharmacy, Saint Barnabas Medical Center, Livingston, United States of America; ${ }^{2}$ Pharmacy, RWJ University Hospital Somerset, Somerville, United States of America

Correspondence: A. Nagy

Intensive Care Medicine Experimental 2020, 8(2): 000364

Introduction: Septic shock, a form of distributive shock, is the most common cause of death in patients admitted into the intensive care unit (ICU).1 Persistent hypotension is a hallmark sign of shock and its resulting end-organ hypoperfusion is directly related to mortality. 2 Initial management of septic shock focuses on the maintenance of mean arterial pressure (MAP), which includes early and adequate fluid resuscitation and the initiation of vasopressors.3 Angiotensin II is a novel treatment modality for patients unresponsive to the current standard of care recommended by consensus guidelines. 4 Its administration was associated with a $660 \%$ increased chance of obtaining MAP response when compared to placebo.5 We investigated the utility of angiotensin II for the treatment of patients experiencing septic shock in the ICU.

Objectives: To assess the clinical outcomes of patients that were administered angiotensin II in the ICU of two academic medical centers.

Methods: In this retrospective chart review, we evaluated patients who received angiotensin II in the ICU of two academic medical centers from July 1, 2018, to January 31, 2020. Eligible patients were at least 18 years of age, had vasodilatory shock, and received angiotensin II. Baseline characteristics included age, sex, body mass index, clinical markers, severity of illness scores, and prior exposure to an angiotensin-converting enzyme inhibitor and/or to an angiotensin receptor blocker. Data for evaluation included changes in MAP, vasopressor use prior to and during angiotensin II use, Sequential Organ Failure Assessment (SOFA) score change at hour 48, adverse events, and mortality. All the data were analyzed using descriptive statistics.

Results: A total of 28 patients received angiotensin II during the studied period. The average baseline MAP was $60 \pm 13 \mathrm{mmHg}$. The mean MAP increase from baseline at hour 3 was $12 \pm 18 \mathrm{mmHg}$ (24 patients) and at hour 48 was $17 \pm 15 \mathrm{mmHg}$ (13 patients). When examining vasopressor use, the specific agent(s) used varied on the patient and site, 
however, the two most common vasopressors used before angiotensin II administration were norepinephrine (NE) and vasopressin. The baseline total NE-equivalent dose was $0.435 \pm 0.189 \mathrm{mcg} / \mathrm{kg} / \mathrm{min}$, at hour 3 was $0.406 \pm 0.184 \mathrm{mcg} / \mathrm{kg} / \mathrm{min}$, and at hour 48 was $0.385 \pm 0.226$ $\mathrm{mcg} / \mathrm{kg} / \mathrm{min}$. The mean baseline SOFA score was $10 \pm 4$. At hour 48 , SOFA increased by $1 \pm 6$ points. Side effects that arose included fungal infections (18\%) and new-onset thromboembolism (4\%). Mortality occurred in $46 \%$ of patients at hour $24,68 \%$ of patients at day 7 , and $71 \%$ of patients at day 28 .

Conclusion: Angiotensin II effectively increased MAP in patients with vasodilatory shock that did not respond to high doses of conventional vasopressors. Administration allowed the requirements of other vasopressors to be reduced over time. However, the patients still showed a rise in their SOFA scores and had a high mortality rate. More investigation is warranted to prove utility of angiotensin II at our clinical sites.

\section{Reference(s) and grant ackowledgment(s)}

1. 3. Pollard S, Edwin SB, Alaniz C. Vasopressor and inotropic management of patients with septic shock. P \& T. 2015;40(7):438-50.

2. 4. Rhodes A, Evans LE, Alhazzani W, et al. Surviving Sepsis Campaign: international guidelines for management of sepsis and septic shock. Crit Care Med. 2017;45(3):486-552.

3. 5. Khanna A, English SW, Wang XS, et al. Angiotensin II for the treatment of vasodilatory shock. N Engl J Med. 2017;377:419-30.

4. 2. Hotchkiss RS, Moldawer LL, Opal SM, et al. Sepsis and septic shock. Nat Rev Dis Primers. 2016;2:16045.

5. 1. Parrillo JE. Pathogenic mechanisms of septic shock. N Engl J Med. 1993;328:1471-7.

\section{3}

Mortality risk factors in patients with nosocomial infection, in an ICU after 8 years of Selective Digestive Decontamination C. Sánchez Ramírez'; RE. Morales Sirgado'; S. Hipola Escalada';

MA. Hernandez Viera'; M. Cabrera Santana ${ }^{1}$; L. Caipe Balcázarr'; CF. Lübbe Vázquez ${ }^{1}$; P. Saavedra-Santana²; S. Ruiz-Santana ${ }^{1}$

${ }^{1}$ Intensive care medicine, University Hospital of Gran Canaria Dr. Negrin, Las Palmas de Gran Canaria, Spain; ${ }^{2}$ Mathematics and informatics deparment, University of Las Palmas:, Las Palmas de Gran Canaria, Spain

Correspondence: $C$. Sánchez Ramírez

Intensive Care Medicine Experimental 2020, 8(2): 000403

Introduction: Recognition of mortality risk factors and early intervention of appropriate broad-spectrum antimicrobial administration may significantly improve the outcome. Selective Digestive Decontamination (SDD) has been associated with reduced ICU mortality and acquired infection rates.

Objectives: To analyze mortality risk factors in patients with nosocomial infection (NI) in an ICU after 8 years of SDD.

Methods: Prospective study. Patients with $\mathrm{NI}$ were included from October 1, 2010 to September 30, 2019 in a polyvalent ICU of 30 beds. SDD was applied for 8 years, from October 1, 2011 to September 30 2019. Patients requiring mechanical ventilation for more than 48 hours, an enteral solution and a paste with colistin, tobramycin and nystatin every 8 hours until discharge were applied. Also intravenous cefotaxime were administered during the first 4 days. Rectal and pharyngeal exudates were collected on admission and weekly. The categorical variables were summarized in frequencies and percentages and the numerical variables as means and standard deviations or in medians and interquartile ranges. The percentages were compared with the $\mathrm{X} 2$ test or the Fisher exact test, the means with the $\mathrm{t}$-test and the medians with the Wilcoxon test for independent data. A multidimensional logistic analysis was carried out. It was considered significant if $\mathrm{p} \leq .05$

Results: Of 8202 patients admitted, $238(35,57 \%)$ out of 669 patients with Nls died. In an univariate analysis we did no find statistically significant difference in ICU stay. Multiresistant (MR) Pseudomonas and MR Gram negative bacteria (GNB) were significantly higher in patients who died (Table 1)

\begin{tabular}{|c|c|c|c|}
\hline & $\begin{array}{c}\text { Alive } \\
\mathrm{N}=431\end{array}$ & $\begin{array}{c}\text { Death } \\
\mathrm{N}=238\end{array}$ & $\mathbf{P}$ \\
\hline Age, years & $59.6 \pm 15.6$ & $63.8 \pm 13.7$ & $<0.001$ \\
\hline APACHEII & $20.2 \pm 7.4$ & $24.3 \pm 7.6$ & $<0.001$ \\
\hline Sexmale & $283(65.7)$ & $155(65.4)$ & 0.453 \\
\hline Trauma patients & $69(16.1)$ & $11(4.6)$ & $<0.001$ \\
\hline Coronary artery disease patient & $91(21.1)$ & $53(22.3)$ & 0.427 \\
\hline Emergency surgery & $109(25.3)$ & $56(23.5)$ & 0.613 \\
\hline Immunosuppression & $25(5.8)$ & $43(18.1)$ & $<0.001$ \\
\hline Neutropenia & $8(1.9)$ & $18(7.6)$ & $<0.001$ \\
\hline Parenteral nutrition & $79(18.3)$ & $92(38.7)$ & $<0.001$ \\
\hline Ventricular device & $53(12.3)$ & $7(2.9)$ & $<0.001$ \\
\hline RRT & $96(22.3)$ & $152(63.9)$ & $<0.001$ \\
\hline Malnutrition & $31(7.2)$ & $36(15.1)$ & 0.002 \\
\hline Diabetes mellitus & $110(25.5)$ & $99(41.6)$ & $<0.001$ \\
\hline COPD & $53(12.3)$ & $50(21.0)$ & 0.003 \\
\hline Renal failure & $69(16.0)$ & $78(32.8)$ & $<0.001$ \\
\hline Cimhosis & $14(3.2)$ & $20(8.4)$ & 0.007 \\
\hline Neoplasm & $26(6.0)$ & $41(17.2)$ & $<0.001$ \\
\hline VAP & $139(32.2)$ & $106(44.5)$ & 0.002 \\
\hline CRB & $161(37.4)$ & $76(31.9)$ & 0.351 \\
\hline Seconctary bacteremia & $107(24.8)$ & $70(29.4)$ & 0.463 \\
\hline Uninary infection & $118(27.4)$ & $60(25.2)$ & 0.543 \\
\hline ATB 48 hours before admission & $101(23.4)$ & $80(33.6)$ & 0.009 \\
\hline Acinetobacter baumarnii & $12(28)$ & $7(2.9)$ & 0.907 \\
\hline MRSA & $10(23)$ & $3(1.3)$ & 0.399 \\
\hline ESE & $90(20.9)$ & $63(26.5)$ & 0.099 \\
\hline MRPseudomonas & $22(5.1)$ & $29(12.2)$ & 0.002 \\
\hline MRGNB & $16(3.7)$ & $19(8.0)$ & 0.018 \\
\hline SDD & $357(82.8)$ & $202(84.9)$ & 0.495 \\
\hline Admission: & & & $<0.001$ \\
\hline Medical & $279(64.7)$ & $198(83.5)$ & \\
\hline Scheduled surgery & $75(17.4)$ & $13(5.5)$ & \\
\hline Emergency surgery & $\pi(17.9)$ & $26(11.0)$ & \\
\hline Inflammatory response: & & & $<0.001$ \\
\hline Nan sepsis & $17(3.9)$ & $8(3.4)$ & \\
\hline Sepsis & $137(31.8)$ & $37(15.5)$ & \\
\hline Septic shock & $277(64.2)$ & $193(81.1)$ & \\
\hline ICU days & $31(18.2-48)$ & $35(20-54)$ & 0.138 \\
\hline
\end{tabular}

- Independent mortality risk factors were: APACHE ॥ Odd ratio (OR) 1.060 (1.033; 1.087), renal replacement therapy OR: 4.306(2.966; $6.251)$, septic shock OR: $2.636(1.818 ; 3.824)$, parenteral nutrition OR $1.970(1.303 ; 2.979)$ and neoplasm OR 3.334 (1.843; 6.030). (Table 2).

Table 2. Multivariate logistic regression for exitus

\begin{tabular}{ccc}
\hline & $\mathbf{P}$ & $\mathbf{O R}(95 \% \mathrm{Cl})$ \\
\hline APACHEII & $<.001$ & $1.060(1.033 ; 1.087)$ \\
Septic shock & $<.001$ & $2.636(1.818 ; 3.824)$ \\
Parenteral nutrition & 0.001 & $1.970(1.303 ; 2.979)$ \\
RRT & $<.001$ & $4.306(2.966 ; 6.251)$ \\
Neoplasm & $<.001$ & $3.334(1.843 ; 6.030)$
\end{tabular}

RRT: Renal replacement therapy,

Conclusion: In an ICU with SDD, factors that were independently associated with motality were: APACHE II, renal replacement therapy, septic shock, parenteral nutrition and neoplasm. MR Pseudomonas and MR GNB also had significantly higher mortality.

000420

Multiple organ dysfunction in COVID-19 patients

E. Antonucci ${ }^{1}$; M. Giovini ${ }^{1}$; C. San Nicola ; M. Bergonzi ${ }^{1}$; M. Barbera

IIntermediate care unit, Ospedale "Guglielmo da Saliceto", Piacenza, Italy Correspondence: $\mathrm{E}$. Antonucci

Intensive Care Medicine Experimental 2020, 8(2): 000420

Introduction: The most common reason for intensive care unit (ICU) admission for patients with severe coronavirus disease 2019 (COVID19 ) is acute respiratory failure (ARF). However, data on organ dysfunctions (OD) other than lungs remains poorly defined in this field. The aim of this study was to analyze the rate of various OD (acute kidney injury, AKl; liver failure, LF; circulatory failure, CF; coagulopathy; 
pancytopenia) in a cohort of COVID-19 patients admitted in a Intermediate Care Unit (IMCU) for ARF.

Methods: We performed a retrospective analysis, reviewing all COVID19 patients admitted for ARF in our IMCU from March 1st to March 31 st 2020 . The inclusion criteria were age $\geq 18$ years old; diagnosis of COVID-19 by a nasopharyngeal swab. AKI was defined by KDIGO criteria; LF was defined by high levels of bilirubin (> $2 \mathrm{mg} / \mathrm{dL}$ ), prolonged prothrombin time (PT) $>18 \mathrm{sec}$; circulatory failure was defined by mean arterial pressure (MAP) $<65 \mathrm{mmHg}$ and requiring the use of vasopressors; coagulopathy was defined by prolonged PT > $18 \mathrm{sec}$, activated partial thromboplastin time (aPTT) $>60 \mathrm{sec}$ and low platelet count $(<150,000 /$ microL); pancytopenia was defined by neutrophil count $<1800 /$ microL; $\mathrm{Hb}<12 \mathrm{~g} / \mathrm{dL}$ for women and $<13 \mathrm{~g} / \mathrm{dL}$ for men; platelet count $<150,000 /$ microL. We collected the demographic characteristics and the rate of the OD associated to ARF. We also reported the rate of COVID-19 patients affected by 2, 3 o more than 3 simultaneous OD.

Results: We reviewed 134 patients and 93/134 respected the inclusion criteria. Demographic characteristics are shown in the Table 1. All patients showed ARF with a $\mathrm{PaO} 2 / \mathrm{FiO} 2$ ratio $115 \pm 78$. SAPS2 score was $33 \pm 9$, SOFA score was $3 \pm 1$. The rate of endotracheal intubation (ETI) was $42 / 93$ patients $(45,2 \%)$. We found AKI in $19 / 93$ patients $(20,4 \%)$, LF in $23 / 93$ patients $(24,7 \%)$, CF in $7 / 93(7,5 \%)$, coagulopathy in $22 / 93$ patients $(23,7 \%)$, pancytopenia $3 / 93(3,2 \%)$. 45/93 (48,4\%) showed multiple OD. In particular 22/93 patients had 2 simultaneous OD; $14 / 93$ showed 3 simultaneous OD and 9 patients $>3$ OD (see figure 1)

\begin{tabular}{|c|c|}
\hline Demographic characteristics & Values \\
\hline Sex & Male: $70 / 93(75 \%)$ \\
\hline Age (mean \pm SD) & $64 \pm 12$ \\
\hline Obesity & $18 / 93(19,4 \%)$ \\
\hline Malignancy & $6 / 93(6,5 \%)$ \\
\hline COPD/Asthma & $8 / 93(8,6 \%)$ \\
\hline Diabetes & $16 / 93(17,2 \%)$ \\
\hline Cardiovascular disease & $26 / 93(28 \%)$ \\
\hline Hypertension & $42 / 93(45,2 \%)$ \\
\hline
\end{tabular}

\section{Table 1 - Demographic characteristics}

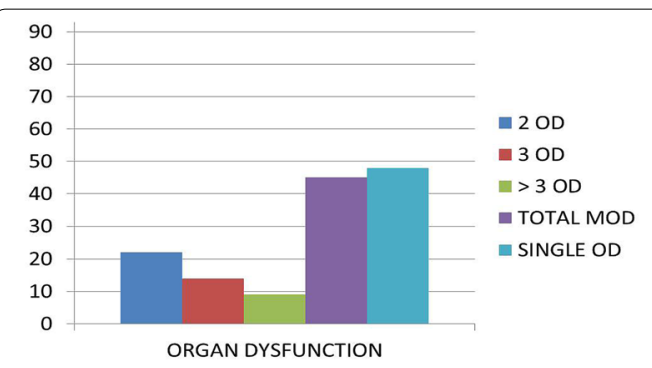

Figure 1 - Organ dysfunction - MOD (multiple organ dysfunction)

Conclusion: In our cohort of COVID-19 patients, multiple organ dysfuntion was a common finding with a rate close to $50 \%$ of cases.
000438

A comparison of sub-therapeutic Vancomycin levels

between critically ill surgical and medical patients in a mixed ICU setting

A. Kassab ${ }^{1}$; A. Hallal ${ }^{1}$; M. Homsi ${ }^{1}$; R. Jaafar'; D. Ghaziri² ; H. Farhat ${ }^{1}$;

R. Farsakoury ${ }^{1}$

${ }^{1}$ Department of surgery, AUB Medical Center, Beirut, Lebanon; ${ }^{2}$ Department of pharmacy, AUB Medical Center, Beirut, Lebanon

Correspondence: A. Kassab

Intensive Care Medicine Experimental 2020, 8(2): 000438

Introduction: Adequate utilization of vancomycin is essential for achieving therapeutic targets while avoiding clinical failure and development of antimicrobial resistance, especially in critically ill patients.

Objectives: We aim to determine whether there is a difference in the incidence of sub-therapeutic vancomycin level between surgical and medical patients in a mixed intensive care unit (ICU) and identify the possible factors that lead to this difference.

Methods: A retrospective electronic medical chart review was done for all adult ICU patients who received intravenous Vancomycin between 2012 and 2017 at our center. Patients with renal failure were excluded. The Vancomycin dose used in our population was $15-20 \mathrm{mg} / \mathrm{kg}$ every 8 hours, adjusted according to each patient's GFR. Blood Vancomycin trough levels below $15 \mathrm{mg} / \mathrm{l}$ before the 4 th dose were reported as subtherapeutic. The lab studies and illness severity scores of our patients during their ICU stay were also collected. A descriptive data analysis was done followed by a multivariate analysis to study the subtherapeutic Vancomycin levels between medical and surgical patients.

Results: A total of 85 patients were included in the study, divided into 44 surgical ICU (SICU) patients and 41 medical ICU (MICU) patients. No difference was found in age, gender, BMI, and eGFR between the two groups. Subtherapeutic vancomycin levels were significantly higher in SICU patients as compared to MICU patients $(29(66 \%)$ out of 44 vs. $14(34 \%)$ out of $41, P=0.003$ ). SICU patients were found to have a higher total volume intake, higher fluid balance, and lower $\mathrm{Hb}$ levels as compared to MICU patients. SICU patients also had a longer stay in the ICU before the initiation of Vancomycin treatment and lower APACHE and SAPS mortality scores as compared to MICU patients. Multivariate logistic regression including all the above factors associated with subtherapeutic vancomycin showed that admission to the ICU from a surgical service is an independent risk factor for subtherapeutic vancomycin levels $(P=0.001, O R=6.48, C l(2.14-19.59))$. Further subgroup analysis could not be done due to the relatively small sample size.

Conclusion: Critically ill surgical patients receiving intravenous Vancomycin in the ICU are inherently at a higher risk for subtherapeutic vancomycin levels than medical patients. We recommend further studies with larger sample sizes to further investigate the factors leading to the higher incidence of subtherapeutic vancomycin levels in surgical patients.

Reference(s) and grant ackowledgment(s)

1. Medellín-Garibay, Susanna E., et al. "Pharmacokinetics of vancomycin and dosing recommendations for trauma patients." Journal of Antimicrobial Chemotherapy 71.2 (2016): 471-479.

2. Radke, Christian, et al. "Development of a physiologically based pharmacokinetic modelling approach to predict the pharmacokinetics of vancomycin in critically ill septic patients." Clinical pharmacokinetics 56.7 (2017): 759-779.

3. Abraham, Jacob, et al. "Plasma and interstitial fluid population pharmacokinetics of vancomycin in critically ill patients with sepsis." International journal of antimicrobial agents 53.2 (2019): 137-142.

4. Kumar, Anand, et al. "Duration of hypotension before initiation of effective antimicrobial therapy is the critical determinant of survival in human septic shock." Critical care medicine 34.6 (2006): 1589-1596.

5. None 


\section{8}

Preload Functional Status and Stroke Volume in Sepsis

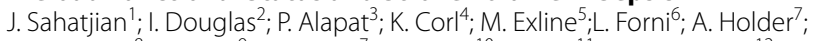
D. Kaufman ${ }^{8}$; M. Levy ${ }^{9}$; G. Martin ${ }^{7}$; E. Seeley ${ }^{10}$; W. Self ${ }^{11}$; J. Weingarten ${ }^{12}$; M. Williams ${ }^{13}$ : D. Hansell ${ }^{14}$

${ }^{1}$ Clinical affairs, Cheetah Medical, Newton Center, United States of America; ${ }^{2}$ Critical care, Denver Health, Denver, United States of America; ${ }^{3} \mathrm{lcu}$, Ben Taub General Hospital, Houston, United States of America; ${ }^{4}$ Critical care, Rhode Island Hospital, Providence, United States of America; ${ }^{5} \mathrm{Icu}$, Ohio State University Hospital, Columbus, United States of America; ${ }^{6}$ Renal, Royal Surrey County Hospital, Guildford, United Kingdom; ${ }^{7} \mathrm{Icu}$, Emory University Hospital Midtown, Atlanta, United States of America; ${ }^{8} \mathrm{ICu}$, NYU School of Medicine, New York, United States of America; ${ }^{9}$ Division of pulmonary, critical care and sleep medicine, Warren Alpert School of Medicine at Brown University, Providence, United States of America; ${ }^{10} \mathrm{Icu}$, University of California San Francisco Parnassus Campus, San Francisco, United States of America; ${ }^{11} \mathrm{Icu}$, Vanderbilt University, Nashville, United States of America; ${ }^{12} \mathrm{ICu}$, NewYork-Presbyterian Brooklyn Methodist Hospital, New York, United States of America; ${ }^{13} \mathrm{lcu}$, Methodist Hospital, Indianapolis, United States of America; ${ }^{14}$ Anesethesiology, Massachusetts general hospital, Boston, United States of America

Correspondence: J. Sahatjian

Intensive Care Medicine Experimental 2020, 8(2): 000458

Introduction: Cardiac function is known to be negatively impacted by sepsis. Monitoring Cardiac Output (CO) and Stroke volume (SV) trends over the course of treatment may provide insight into cardiac function and may be used to predict patient outcome.

Objectives: The goal of this study was to explore the relationship between the change in cardiac output over time in septic shock.

Methods: FRESH is randomized controlled study, evaluating hemodynamics in critically ill patients with sepsis or septic shock (NCT02837731). Patients randomized to PLR guided resuscitation received hemodynamic monitoring for 72 or until ICU discharge, whichever occurred first (Starling SV, Cheetah Medical). Patients that exhibited an improvement in stroke volume at 12, 24, 36 and 48 hours were compared to those who did not exhibit improvement. Overall improvement in stroke volume (first SV measurement compared to last SV measurement) was also compared between groups.

Results: 90 patients with septic shock received hemodynamic monitoring over a 72 hour monitoring period. $60 \%$ were female, and the average age was 61 years. Overall, $44 \%$ of assessments demonstrated a fluid responsive positive response after receiving initial resuscitation fluid of 2.3 L. Patients who exhibited improved SV at 24 and 36 hours exhibited a decreased fluid balance over the course of their ICU stay at both 24 hours $(1.06 \pm 3.40$ vs $3.15 \pm 4.38, p=0.029)$ and 36 hours $(1.18 \pm 2.58$ vs $3.41 \pm 4.52, p=0.032)$. Notably, patients who exhibited an overall improvement in SV also required less hours on vasopressors ( $34.50 \pm 41.03$ vs $64.28 \pm 65.07, p=0.05)$ and decreased ICU length of stay ( $3.93 \pm 3$ vs $5.59 \pm 5.24$ days, $p=0.08$ ) (Figure 1$)$.

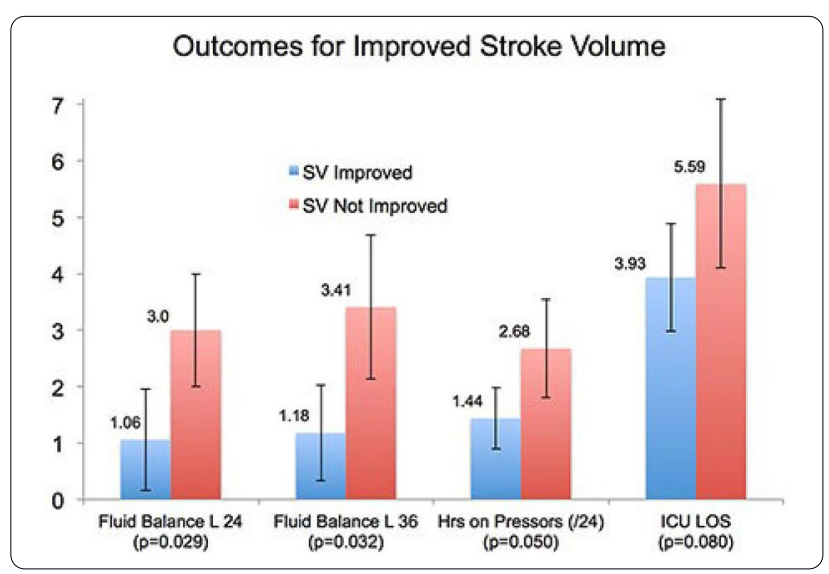

Conclusion: We have previously shown that patients who improve $\mathrm{SV}$ in response to the resuscitation exhibited improved outcome. Trending cardiac output over the 72 hour monitoring period revealed additional usefulness in predicting patients with improved outcome. The results highlight the importance of trending hemodynamics in therapy.

\section{8}

Global assessment of cardiac function in septic shock patients: effect of Norepinephrine on cardiac performance

ED. Valenzuela Espinoza'; D. Carpio '; P. Mercado²; G. Hernández'; A. Bruhn'; J. Bakker ${ }^{3}$

${ }^{1}$ Departamento de medicina intensiva, Pontificia Universidad Católica de Chile, Santiago, Chile; ${ }^{2}$ Departamento de paciente crítico., Clínica Alemana, Santiago, Chile; ${ }^{3}$ Department of intensive Care Adults, Erasmus University Medical Center, Rotterdam, Netherlands

Correspondence: E.D. Valenzuela Espinoza

Intensive Care Medicine Experimental 2020, 8(2): 000468

Introduction: Current guidelines recommend to increase mean arterial pressure (MAP) in previously hypertensive septic shock patients to higher levels $(80-85 \mathrm{mmHg})$ when tissue hypoperfusion has not been corrected with the initial fluid resuscitation and restoration of MAP to $65 \mathrm{mmHg}$. The impact of incremental NE titration on cardiac function depend on the balance between the effects on preload, contractibility and afterload. As NE may both increase venous return and afterload, a particular concern is a potential decrease in stroke volume (SV) in fluid-unresponsive patients.

Objectives: Determine whether septic shock patients requiring a high MAP exhibit afterload dependence, defined as a decrease in stroke volume $>10 \%$.

Methods: We evaluated a cohort of previously hypertensive patients with septic shock in whom the attending physician decided to increase MAP from 65 to $85 \mathrm{mmHg}$ because of persistent hypoperfusion after initial resuscitation. A comprehensive echocardiographic assessment was performed at baseline 1 (MAP $65 \mathrm{mmHg}$ ), after reaching the MAP target (MAP $85 \mathrm{mmHg}$ ), and after return to baseline 2 (MAP $65 \mathrm{mmHg}$ ). Preload, contractility and afterload variables were assessed. Statistical analysis was performed with Wilcoxon signed-rank test, $\mathrm{P}<0.05$ was considered as significant.

Results: Twelve mechanically ventilated patients (age $72 \pm 9 \mathrm{yr}$, APACHE II $23 \pm 5$, SOFA on admission $10 \pm 3$ ) were included in this preliminary report. No significant decrease in stroke volume was shown; in contrast, $60 \%$ of the studied patients increased their stroke volume $>10 \%$ despite being fluid unresponsive at baseline and with a significant increase in afterload. Hemodynamic and echocardiography variables are shown in Table 1. 


\begin{tabular}{|c|c|c|c|}
\hline \multicolumn{4}{|c|}{ Table 1. Hemodynamics variables and echocardiography variables } \\
\hline & Baseline 1 & MAP 85 & Baseline 2 \\
\hline MAP, $\mathrm{mmHg}$ & $65[63,67]$ & $84[83,88]^{*}$ & $66[62,66] \dagger$ \\
\hline $\mathrm{NE}, \mathrm{mcg} / \mathrm{kg} / \mathrm{min}$ & $0.17[0.08,0.37]$ & $0.41[0.13,0.49]^{*}$ & $0.28[0.06,0.37] \dagger$ \\
\hline HR, beats/min & $86[74,101]$ & $88[71,100]$ & $93[69,106]$ \\
\hline $\mathrm{CVP}, \mathrm{mmHg}$ & $10.0[6.5,12.5]$ & $10.0[7.0,13.0]$ & $9.0[5.5,10.5] \dagger$ \\
\hline Pmsf, mmHg & $18.1[15.0,20.2]$ & $18.9[18.2,22.2]^{*}$ & $15.9[15.3,19.5] \dagger$ \\
\hline Pvr, $\mathrm{mmHg}$ & $7.8[6.6,9.6]$ & $8.8[7.7,11.1]^{*}$ & $8.5[6.9,10.2]^{*} \dagger$ \\
\hline $\mathrm{CO}, \mathrm{L} \cdot \mathrm{min}-1$ & $4.9[3.4,6.2]$ & $5.0[3.9,6.7]^{*}$ & $5.3[3.9,6.7] \dagger$ \\
\hline $\begin{array}{l}\text { RVR, } \\
\mathrm{mmHg} \cdot \mathrm{min} \cdot \mathrm{mL}-1\end{array}$ & $1.60[1.49,1.99]$ & $1.80[1.55,2.03]^{*}$ & $1.61[1.44,1.86] \dagger$ \\
\hline $\begin{array}{l}\text { SVR, } \\
\mathrm{mmHg} \cdot \mathrm{min} \cdot \mathrm{mL}-1\end{array}$ & $880[679,1391]$ & $1246[858,1552]^{*}$ & $890[615,1277] \dagger$ \\
\hline Aortic Elastance & $1.52[1.33,2.21]$ & $1.92[1.65,2.69]^{*}$ & $1.42[1.28,1.77] \dagger *$ \\
\hline \multicolumn{4}{|l|}{ Left Ventricle } \\
\hline GLS, $\%$ & $-15.0[-18.9,-12.1$ & ] $-16.5[-17.4,-13.7]$ & $-13.4[-18.1,-11.8]$ \\
\hline S' wave, $\mathrm{cm} / \mathrm{sec}$ & $12.6[6.7,16.2]$ & $14.2[6.9,18.0]$ & $13.3[6.5,18.0]$ \\
\hline MPI & $0.63[0.55,1.08]$ & $0.68[0.45,10.93]$ & $0.75[0.54,1.06]$ \\
\hline IVA, $\mathrm{cm} / \mathrm{sec} 2$ & $318[172,522]$ & $322[243,556]$ & $394[139,776]$ \\
\hline VTI, cm & $18.9[14.5,26.7]$ & $20.7[15.6,27.7]^{*}$ & $20.1[15.8,25.4]$ \\
\hline $\begin{array}{l}\text { VTI Descendent } \\
\text { Aorta, cm }\end{array}$ & $11.3[8.5,13.53]$ & $11.9[8.9,14.0]$ & $11.6[8.5,13.9]$ \\
\hline LV EF, \% & $66[45,75]$ & $67[49,71]$ & $65[52,69]$ \\
\hline $\begin{array}{l}\text { LV EndSyst- } \\
\text { Elastance }\end{array}$ & $1.84[0.80,2.33]$ & $2.53[1.15,3.12]^{*}$ & $1.77[0.85,1.94] \dagger$ \\
\hline VA coupling & $1.19[063,1.79]$ & $1.01[0.59,1.79]$ & $0.96[0.65,1.83]$ \\
\hline \multicolumn{4}{|l|}{ Preload } \\
\hline E wave, $\mathrm{cm} / \mathrm{sec}$ & $67[55,96]$ & $80[67,113]^{*}$ & $68[51,108] \dagger$ \\
\hline $\begin{array}{l}\text { DT E wave, } \\
\mathrm{cm} / \mathrm{sec} 2\end{array}$ & $223[143,289]$ & $229[139,281]$ & $223[142,302]$ \\
\hline A wave, $\mathrm{cm} / \mathrm{sec}$ & $70[50,106]$ & $74[54,122]^{*}$ & $84[59,101]$ \\
\hline E/e'ratio & $5.7[4.3,8.2]$ & $6.7[4.9,9.1]^{*}$ & $5.4[4.3,10.0]$ \\
\hline \multicolumn{4}{|l|}{ Right Ventricle } \\
\hline TAPSE & $20.3[17.9,23.6]$ & $23.2[20.5,27.8]^{*}$ & $21.0[19.6,27.5]$ \\
\hline S'wave, $\mathrm{cm} / \mathrm{sec}$ & $13.7[12.1,18.6]$ & $16.4[11.9,20.9]$ & $15.6[13.4,18.6]$ \\
\hline MPI & $0.60[0.38,0.69]$ & $0.63[0.49,0.86]$ & $0.64[0.49,0.77]$ \\
\hline PASP, $\mathrm{mmHg}$ & $38.5[36.2,43.7]$ & $42.9[38.4,53.63]$ & $38.8[31.0,46.0]$ \\
\hline
\end{tabular}

Conclusion: In previously hypertensive and currently fluid unresponsive septic shock patients in whom MAP was increased from 65 to $85 \mathrm{mmHg}$ stroke volume did not decrease despite the increased afterload due to an increase in left ventricular contractility.

Reference(s) and grant ackowledgment(s)

1. Cecconi M, De Backer D, Antonelli M, Beale R, Bakker J, Hofer C, et al. Consensus on circulatory shock and hemodynamic monitoring. Task force of the European Society of Intensive Care Medicine. Intensive Care Med. 2014;40(12):1795-815.

2. European Society of Intensive Care Medicine, GE Challenge Awards, Point of Care (POC) 2019.

\section{5}

Effects of remote ischaemic conditioning on the sublingual microcirculation in septic shock patients

I. Kiudulaite ${ }^{1}$; A. Pranskunas

${ }^{1}$ Intensive care medicine, Lithuanian University of Health Sciences, Kaunas, Lithuania

Correspondence: I. Kiudulaite

Intensive Care Medicine Experimental 2020, 8(2): 000475

Introduction: Introduction: The severity of microcirculatory alterations is related to mortality in septic shock patients (1). Remote ischaemic conditioning (RIC) is a promising technique that may protect organs and tissues from the effects of further ischaemic episodes. Studies have shown that remote ishaemic conditioning may improve endothelial function,red blood cell deformability, reduce leukocyte adhesion and etc. These mechanisms may lead to improved microcirculation. RIC preserved microcirculation and prolonged survival in septic shock animals (2). However, RIC's impact on microcirculation in septic shock patients has not been evaluated.

We hypothesize that RIC improves sublingual microcirculation in septic shock patients.

Objectives: This study aims to evaluate RIC effects on sublingual microcirculation in septic shock patients.

Methods: This single-center open-label trial was performed in a mixed ICU in a tertiary teaching hospital. We included patients with septic shock within the first $24 \mathrm{~h}$ after ICU admission. RIC procedure involved three cycles of $5 \mathrm{~min}$ brachial cuff inflation to $200 \mathrm{mmHg}$ and then deflation to $0 \mathrm{mmHg}$ for another $5 \mathrm{~min}$. RIC was performed at inclusion and repeated $12 \mathrm{~h}$ and $24 \mathrm{~h}$ later. Sublingual microcirculatory measurements were obtained before and after each RIC procedure, using a sidestream dark-field imaging device. Data are reported as medians with 25th and 75th percentiles.

Results: Twenty-six septic shock patients with a median age of 65 (5781) were enrolled in this study. The median APACHE II and SOFA scores at admission were 20 (13-23) and 10 (9-12), respectively. We found a significant increase in microvascular flow index compared before and after the first RIC procedure $(p=0.003)$. We found no change in microcirculatory flow and density parameters during repeated RIC after 12 $\mathrm{h}$ and $24 \mathrm{~h}$.

Conclusion: The first remote ischaemic conditioning procedure improved microcirculatory flow, while subsequent procedures did not affect sublingual microcirculation in septic shock patients.

\section{Reference(s) and grant ackowledgment(s)}

1. 2. Orbegozo Cortés D et al. Ischemic conditioning protects the microcirculation, preserves organ function, and prolongs survival in sepsis. Shock. 2016:45:419-427.

2. 1. De Backer $D$ et al. Microcirculatory alterations in patients with severe sepsis: impact of time of assessment and relationship with outcome. Crit Care Med. 2013;41:791-9.

\section{9}

Evolution and prognosis in acute pancreatitis and abdominal compartment syndrome accordind to different treatments G. Ferrigno ${ }^{1}$; R. Beltrán Bernáldez²; A. Tejero Pedregosa ${ }^{2}$; V. Losada Martínez²; N. Rodrigo Castroviejo ${ }^{2}$; C. Sanmartino Gonzalez²; P. Cañizares Ortiz²; S. Cortés Díaz'; D. Monge Donaire²; LL. Beltran Martínez²

${ }^{1}$ Intensive Care Unit, Hospital Virgen De La Concha, Zamora, Spain; ${ }^{2}$ Intensive care, Hospital Virgen De La Concha, Zamora, Spain

Correspondence: $\mathrm{G}$. Ferrigno

Intensive Care Medicine Experimental 2020, 8(2): 000479

Introduction: Severe acute pancreatitis (SAP) is associated with multiple organ failure and systemic inflammatory response syndrome what leads to high mortality rate. Patients often require different interventional measures including surgical procedures to improve outcomes.

Objectives: to describe the evolution of patients with SAP and its associated complications. To analyze the medical and surgical management of secondary abdominal compartment syndrome (ACS).

Methods: retrospective study including patients admitted to ICU with a diagnosis of SAP during the period 2013-2019. Demographic data, cause, severity scales, and occurrence of associated complications are recorded: acute renal failure (ARF), infections, ACS, need for ventilatory support. Treatment with continuous renal replacement techniques (CRRT), the need for a surgical approach and nutritional support are recorded. Results are expressed as mean $(95 \% \mathrm{Cl})$.

Results: 43 patients were studied, $58.1 \%$ male, age 72.1 (63.3-76.9) years, APACHE 16.3 (12.9-19.8), SAPS II 38.1 (31.8-44.3), SOFA 4.3 (2.95.7). The cause of SAP was: lithiasic $30.2 \%$, alcoholic $9.3 \%$, post-ERCP $16.3 \%$, idiopathic $44.2 \%$. Up to $41.9 \%$ required mechanical ventilation (MV) for 17.1 (6.1-28.2) days, of which 7\% underwent tracheostomy. High-flow oxygen therapy was administered to $9.3 \%$. The prevalence of ARF was $55.8 \%, 18.6 \%$ of patients were treated with CRRT. ACS was present in $18.6 \%$ of patients, and laparotomy was performed in $20.9 \%$ (11.6\% decompressive, $4.7 \%$ necrosectomy). After decompressive laparotomy, the duration of the vacuum-assisted closure system (VAC) 
was 15.2 (0.3-30.1) days with 2.3 (0.6-4) mesh replacements. Abdominal infection rate $21 \%$, respiratory infection $23.3 \%$, multi-resistant colonization $2.3 \%$. $74.4 \%$ received nutritional support (39.5\% parenteral, $7 \%$ enteral, $27.9 \%$ mixed). ICU stay was 13.7 (7.4-20) days, hospital stay 24.9 (22.4-27.4) days and mortality $34.9 \%$. The absence of ARF was associated with less need for surgery (39\% in the ARF group VS $0 \%$ in the group without ARF) $p<0.05$ (Fisher's exact test). Surgical treatment did not influence stay or mortality in our sample.

Conclusion: SAP is an entity with high morbimortality, multidisciplinary management may improve mortality and reduce sequels.

\section{2}

Association between symptoms of early onset and mortality or ICU admission in patients with suspected infection or sepsis

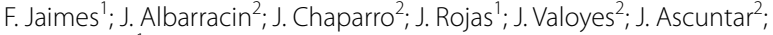
D. Mendoza ${ }^{1}$

${ }^{1}$ Internal medicine, Universidad de Antioquia, Medellin, Colombia; ${ }^{2}$ nternal medicine, Universidad de Antioquia, Medellín, Colombia

Correspondence: $\mathrm{F}$. Jaimes

Intensive Care Medicine Experimental 2020, 8(2): 000532

Introduction: The early recognition of sepsis is one of the pillars to improve its prognosis. It is necessary to explore if there are symptoms or early clinical characteristics associated with the worse outcomes such as the need for Intensive Care Unit (ICU) admission or in-hospital mortality. This information could lead to the optimization of timely care protocols.

Objectives: To identify the earliest symptoms of patients with suspected infection or sepsis that may be associated with the need for ICU admission or in-hospital mortality.

Methods: Prospective cohort study conducted between June/2019 and March/2020 in a university hospital of Medellín, Colombia. We included patients older than 18 years with diagnosis of any type of infection or sepsis (suspected or confirmed), requiring hospitalization by the emergency department (ED). Previously trained researchers collected symptoms and signs, by reviewing the clinical history and interviewing the patient and their relatives, using standardized equipment and formats. The primary outcome was ICU admission or mortality. We did univariate logistic regression models for each one of the independent variables. Then a multivariable adjusted logistic regression model was fitted to estimate the independent effect of each symptom on the outcome. The results are shown as OR with $95 \% \mathrm{Cl}$.

Results: Among 965 eligible patients, 245 (25.4\%) were included. The main causes of exclusion were use of antibiotics in the last 15 days $(44 \%)$ and immunodeficiency (21\%). The median age was 60 years (IQR $=45-71)$ and $51 \%$ were male. The main comorbidities were kidney disease $(23 \%)$, congestive heart failure $(15.1 \%)$ and chronic respiratory disease $(15.1 \%)$. The most common symptoms were general malaise $(80.6 \%)$, subjective fever $(76 \%)$ and chills $(70.5 \%)$. The median SOFA score was $2(\mathrm{IQR}=0-5)$ and the main foci of infection were the respiratory tract $(29.4 \%)$, followed by skin and soft tissues $(21.2 \%)$ and the gastrointestinal tract (19.2\%). The in-hospital mortality rate was $12.7 \%$ and 119 (48.6\%) patients required admission to the ICU. In the univariate analysis, the symptoms significantly associated with hospital mortality or ICU admission were chills, subjective fever, productive cough, dyspnea and altered mental status. However, after multivariate adjustment of symptoms for age, SOFA and Charlson score, only dyspnea and altered mental status remained associated, but they were not longer statistically significant $(\mathrm{OR}=2.16,95 \% \mathrm{Cl}=0.9 ; 5.2$ and $\mathrm{OR}=$ $1.51,95 \% \mathrm{Cl}=0.7 ; 3.2$, respectively)
Conclusion: A few symptoms, easily identified in the ED by a simple interrogatory, may be early indicators of ominous prognosis in patients with sepsis. A prospective multicenter cohort study is warranted for confirmation and external validation of our findings.

\section{Reference(s) and grant ackowledgment(s)}

1. Latten GHP, Claassen L, Jonk M, Cals JWL, Muris JWM, Stassen PM. Characteristics of the prehospital phase of adult emergency department patients with an infection: A prospective pilot study. PLoS One. 2019 Feb 7;14(2):e0212181

2. Filbin MR, Lynch J, Gillingham TD, Thorsen JE, Pasakarnis CL, Nepal S, et al. Presenting Symptoms Independently Predict Mortality in Septic Shock. Crit Care Med 2018 Oct; 46(10):1592-9

3. School of Medicine, Office of undergraduate students, Universidad de Antioquia

\section{2}

Improving the diagnosis of sepsis according to the sources of infection

A. Jaimes' ${ }^{1}$; D. Mendoza'; J. Ascuntar ${ }^{2}$; O. Rosero ${ }^{2}$

${ }^{1}$ Internal medicine, Universidad de Antioquia, Medellin, Colombia; ${ }^{2}$ nternal medicine, Universidad de Antioquia, Medellín, Colombia

Correspondence: F. Jaimes

Intensive Care Medicine Experimental 2020, 8(2): 000542

Introduction: The initial clinical presentation of sepsis is heterogeneous and largely depends on the primary site of infection. This situation makes the early diagnosis of this condition a clinical challenge. Tools such as qSOFA help to assess the risk of complications in patients with suspected infections; however, this approach is indifferent to the site of infection itself. A more individualized identification would allow focusing on earlier targeted therapy

Objectives: To identify simple variables associated with risk of inhospital mortality or Intensive Care Unit (UCI) admission, according to infection sites

Methods: Secondary analysis of a multicenter prospective cohort. We included patients older than 18 years hospitalized between 2014 and 2016 with suspected sepsis, who were admitted to Emergency Departments (ED) of three tertiary care university hospitals in Medellín, Colombia. Bivariate and multivariate logistic regression models were performed to estimate the association of widely available and clinically relevant variables with in-hospital mortality or ICU admission according to 5 infection sites: urinary tract infection (UTI), community acquired pneumonia (CAP), intra-abdominal infection (IAI), sepsis without evident source (primary) and others (soft tissue and bone infections, catheter-associated bacteremia, meningitis, surgical site infections, others). The independent effect of each variable on the outcome was estimated using Odds Ratio (OR) with its respective $95 \%$ confidence interval $(\mathrm{Cl})$.

Results: A total of 5,022 patients were screened, of which 2,454 entered the primary study. In this secondary analysis, we included 1,947 patients with confirmed diagnosis of infection. The most common sites of infection were UTI $(n=586,30 \%)$, CAP $(n=585,30 \%)$, IAI $(n=213,11 \%)$, primary $(n=22411.5 \%)$ and others $(n=339,17.4 \%)$ patients, respectively. After multivariable adjustment, the most significant signs varied widely according to the site of infection (Table 1): Respiratory rate (RR), Systolic Blood Pressure (SBP) and lactate for ITU; Heart Rate (HR), RR and temperature $<38^{\circ} \mathrm{C}$ for CAP; Glasgow coma scale (GCS), lactate and age $<65$ for IAl; SBP, GCS, lactate and temperature $<38^{\circ} \mathrm{C}$ for primary and RR, GCS and temperature $<38^{\circ} \mathrm{C}$ for others. 
Table 1. Relevant clinical variables according to infection site

\begin{tabular}{|c|c|c|c|c|c|}
\hline $\begin{array}{l}\text { Variable (OR } \\
95 \% \mathrm{CI})\end{array}$ & UTI & CAP & IAI & Primary & Others \\
\hline $\mathrm{HR}>120$ & $\begin{array}{l}1.4(0.8- \\
2.2)\end{array}$ & $\begin{array}{l}1.6(1.0- \\
2.5)\end{array}$ & $\begin{array}{l}1.6(0.6- \\
3.9)\end{array}$ & $\begin{array}{l}1.2(0.6- \\
2.4)\end{array}$ & $\begin{array}{l}1.6(0.8- \\
3.0)\end{array}$ \\
\hline $\mathrm{RR}>22$ & $\begin{array}{l}3.0(1.8- \\
5.0)\end{array}$ & $\begin{array}{l}1.9(1.3- \\
2.8)\end{array}$ & $\begin{array}{l}1.8(0.7- \\
4.5)\end{array}$ & $\begin{array}{l}2.0(0.9- \\
3.9)\end{array}$ & $\begin{array}{l}3.4(1.7- \\
6.5)\end{array}$ \\
\hline $\mathrm{SBP}<100$ & $\begin{array}{l}3.0(2.0- \\
4.4)\end{array}$ & $\begin{array}{l}1.4(0.9- \\
2.1)\end{array}$ & $\begin{array}{l}1.3(0.7- \\
2.6)\end{array}$ & $\begin{array}{l}2.6(1.4- \\
4.8)\end{array}$ & $\begin{array}{l}1.6(0.9- \\
2.7)\end{array}$ \\
\hline $\mathrm{GCS}<15$ & $\begin{array}{l}1.7(0.9- \\
3.0)\end{array}$ & $\begin{array}{l}1.3(0.8- \\
2.2)\end{array}$ & $\begin{array}{l}9.6(1.2- \\
80.5)\end{array}$ & $\begin{array}{l}4.5(1.7- \\
11.4)\end{array}$ & $\begin{array}{l}3.0(1.6- \\
5.8)\end{array}$ \\
\hline Lactate $>2$ & $\begin{array}{l}1.6(1.1- \\
2.4)\end{array}$ & $\begin{array}{l}1.2(0.8- \\
1.7)\end{array}$ & $\begin{array}{l}3.1(1.6- \\
6.0)\end{array}$ & $\begin{array}{l}2.0(1.1- \\
3.9)\end{array}$ & $\begin{array}{l}0.6(0.3- \\
1.1)\end{array}$ \\
\hline$\geq 38^{\circ} \mathrm{C}$ & Ref & Ref & Ref & Ref & Ref \\
\hline$<38^{\circ} \mathrm{C}$ & $\begin{array}{l}0.7(0.5- \\
1.0)\end{array}$ & $\begin{array}{l}2.1(1.4- \\
3.0)\end{array}$ & $\begin{array}{l}1.0(0.5- \\
2.2)\end{array}$ & $\begin{array}{l}2.1(1.1- \\
3.9)\end{array}$ & $\begin{array}{l}2.9(1.6- \\
5.1)\end{array}$ \\
\hline Age $>65$ & $\begin{array}{l}1.0(0.7- \\
1.5)\end{array}$ & $\begin{array}{l}0.5(0.4- \\
0.7)\end{array}$ & $\begin{array}{l}2.3(1.2- \\
4.5)\end{array}$ & $\begin{array}{l}0.8(0.4- \\
1.6\end{array}$ & $\begin{array}{l}1.2(0.7- \\
2.1)\end{array}$ \\
\hline
\end{tabular}

Conclusion: Our results suggest that the best approach for sepsis diagnosis in clinical practice, should consider different criteria depending on the site of infection. More research efforts in the area are required to integrate these variables into a qSOFA-type tool, with the aim of achieving a more individualized treatment according to the suspicion of the infection site.

\section{Reference(s) and grant ackowledgment(s)}

1. Londono J, Nino C, Archila A, Valencia M, Cardenas D, Perdomo M, et al. Antibiotics has more impact on mortality than other early goal-directed therapy components in patients with sepsis: an instrumental variable analysis. J Crit Care. 2018;48:191-7.

2. Funded by the Colombian Agency of Science, Technology and Innovation (COLCIENCIAS) (code 111556933362) and the University of Antioquia (Code 2582)

\section{7}

Inflammatory mediator differences between immunological phenotypes in a prospective clinical intensive care cohort study A. Person"; M. Jonasson ${ }^{1}$; A. Larsson²; M. Lipscey ${ }^{3}$; J. Johansson ${ }^{4}$; J. Tyden ${ }^{4}$; M. Castegren ${ }^{2}$

${ }^{1}$ Centre for clinical research, Uppsala University, Uppsala, Sweden; ${ }^{2}$ Medical sciences, Uppsala University, Uppsala, Sweden; ${ }^{3}$ Surgical sciences, Uppsala University, Uppsala, Sweden; ${ }^{4}$ Suregery and perioperative sciences, Umeå University, Östersund, Sweden

Correspondence: $M$. Castegren

Intensive Care Medicine Experimental 2020, 8(2): 000587

Introduction: Sepsis is a heterogenous syndrome caused by a dysregulated immune response to infection. Numerous studies on immunomodulatory therapies have failed to improve outcome. Identification of distinct immunological phenotypes in septic patients may lead to more precise clinical immunomodulatory studies. Clinical distinct septic patient phenotypes have different clinical presentations, microbial findings and outcome1.

Objectives: To study whether four different clinical phenotypes, based on the presence or not of preexisting inflammatory insults, differed in inflammatory mediators in intensive care treated septic patients.

Methods: For 12 months, 276 adult patients admitted to a single ICU and provided with an arterial catheter were consecutively included in a prospective study after informed consent. Plasma was obtained for the first 3 days for the analyses of heparin binding protein, NGAL, tumor necrosis factor receptor 1 and 2, endostatin, procalcitonin, calprotectin and C-reactive protein. Patients were retrospectively grouped as either septic or non-septic according to the Sepsis-3 classification. Further, the septic patients were classified based on the presence of preexisting inflammatory insults into either primary (no prior insults), secondary (one recent insult) or tertiary sepsis (several recent insults). Independent of prior inflammatory insults, patients with immunosuppressive treatments or immunosuppressive diseases were classified as immunosuppressive sepsis.

Results: Septic patients had significantly higher plasma levels in all measured inflammatory mediators at admission to the ICU compared to non-septic patients, with the exception of procalcitonin where no significant difference could be noted $(p=0.29)$. During the first 3 days, patients with primary sepsis had higher levels of heparin binding protein $(p<0.02)$ than secondary, tertiary or immunosuppressive sepsis. Patients with primary and immunosuppressive sepsis had lower levels of calprotectin $(p<0.01)$, than those with secondary or tertiary sepsis. No differences in plasma levels of endostatin, NGAL, procalcitonin, tumor necrosis factor receptor 1 or 2 , were noted between the immunological phenotype groups.

Conclusion: Classification of patients into inflammatory phenotypes based on preexisting inflammatory insults prior to sepsis, is supported by early differences in plasma levels of heparin binding protein and calprotectin. Procalcitonin performed poorly as a biomarker to distinguish septic patients from non-septic patients in the ICU.

Reference(s) and grant ackowledgment(s)

1. 1 Castegren M etal. Crit Care Resusc 2015;17, 174-181.

2. N/A

\section{9}

Clinical utility of procalcitonin in critically ill medical patients

in Croatia

A. Vujaklija Brajković ${ }^{\text {; I. Košuta }}{ }^{1}$; M. Rora ${ }^{1}$; D. Tomek ${ }^{2}$; J. Babel ${ }^{1}$; R. Radonić1

${ }^{1}$ Department of Internal Medicine, University Hospital Centre Zagreb, Zagreb, Croatia; ${ }^{2}$ Department of Oncology and Radiotherapy, University Hospital Centre Zagreb, Zagreb, Croatia

Correspondence: A. Vujaklija Brajković

Intensive Care Medicine Experimental 2020, 8(2): 000629

Introduction: Procalcitonin (PCT) is one of the most studied biomarkers regarding antibiotic stewardship1. In the critical care setting, published studies differ greatly considering patient case-mix, the source of infection, the severity of the disease, exclusion criteria as well as cutoffs and laboratory methods for PCT measurements2-4.

Objectives: PCT testing was implemented in the emergency lab of the Department of Laboratory Diagnostics in October 2016. As an initial demand management strategy, only the emergency department and intensive care units gained unlimited access to direct test ordering. The study aimed to evaluate the applicability of PCT testing in severely ill patients with suspected infection in a tertiary referral hospital in Croatia.

Methods: Patients with suspected infection were included in a prospective, observational study in the medical intensive care unit (ICU) at the University Hospital Centre Zagreb, Croatia. Analysis of disease severity (APACHE II, SOFA score), microbiological cultures (blood culture (BC), urine culture (UC), endotracheal aspirate culture (ETA)), C-reactive protein (CRP), and PCT was performed.

Results: 129 patients were included, 83 males $(63.6 \%)$ of median age 64 (39-89). Median APACHE II was 21 (7-35) and SOFA 7 (1-13). Sepsis $(50,38.8 \%)$ and pneumonia $(37,28.7 \%)$ were the most common diagnoses. Overall PCT was $1.66 \pm 11.43 \mu \mathrm{g} / \mathrm{L}$ and CRP $102 \pm 166 \mathrm{mg} / \mathrm{L}$. Most patients $(83,64.3 \%)$ received antibiotics before admission. No difference in PCT $(1.56 \pm 8$ vs $1.44 \pm 13, \mathrm{p}=0.6)$ nor in CRP $(98 \pm 137$ vs $127 \pm 171, p=0.5$ ) was observed regardless of previous antibiotic therapy.

Infection was clinically confirmed in 106 (82.2\%) patients. Microbiological cultures were positive in $86(66.7 \%)$ and $81.1 \%$ of patients with clinically confirmed infections. PCT and CRP were significantly increased in patients with positive $B C$, the infection caused by Gramnegative microorganism regardless of disease severity (no difference in SOFA and APACHE score) and pneumonia with complications (Table 1). PCT did not differ among patients with positive vs negative UC $(4.6 \pm 16$ vs $1.76 \pm 11.9)$ nor positive vs negative ETA (1.93 \pm 11.4 
vs $1.76 \pm 1.11)$. The change of antimicrobial treatment based on PCT value was done in 36 patients ( $28 \%$ ).

\begin{tabular}{|c|c|c|c|c|c|c|c|c|}
\hline $\begin{array}{l}\text { Variabl } \\
\text { e }\end{array}$ & $\mathrm{BC}+$ & $\mathrm{BC}-$ & $\begin{array}{l}\mathrm{BC}+ \\
\mathrm{G}+\end{array}$ & $\begin{array}{l}\mathrm{BC}+ \\
\mathrm{G}-\end{array}$ & $\begin{array}{l}\text { PNM, not } \\
\text { complicate } \\
\text { d }\end{array}$ & $\begin{array}{l}\text { PNM, } \\
\text { with } \\
\text { shock }\end{array}$ & $\begin{array}{l}\text { PNM, } \\
\text { BC - }\end{array}$ & $\begin{array}{l}\text { PNM, } \\
\mathrm{BC}+\end{array}$ \\
\hline $\mathrm{N}$ & 54 & 61 & 24 & 30 & 46 & 29 & 35 & 40 \\
\hline $\begin{array}{l}\mathrm{PCT}, \\
\mu \mathrm{g} / \mathrm{L}\end{array}$ & $\begin{array}{l}8.2 \pm 24.1 \\
*\end{array}$ & $\begin{array}{l}0.6 \pm 3.7 \\
*\end{array}$ & $1.99 \pm 12 *$ & $15.9 \pm 50^{*}$ & $1.1 \pm 3.6^{*}$ & $\begin{array}{l}16.5 \pm 44.2 \\
*\end{array}$ & $0.9 \pm 4.0 \mathrm{a}$ & $7.2 \pm 20.2 \mathrm{a}$ \\
\hline $\begin{array}{l}\mathrm{CRP}, \\
\mathrm{mg} / \mathrm{L}\end{array}$ & $\begin{array}{l}167 \pm 212 \\
*\end{array}$ & $\begin{array}{l}86 \pm 116 \\
*\end{array}$ & $\begin{array}{l}139 \pm 202 * \\
*\end{array}$ & $\begin{array}{l}181.5 \pm 222 * \\
*\end{array}$ & $80 \pm 115^{*}$ & $194 \pm 204^{*}$ & $\begin{array}{l}108 \pm 140 \mathrm{~N} \\
\mathrm{~S}\end{array}$ & $\begin{array}{l}143.5 \pm 19 \\
1 \mathrm{NS}\end{array}$ \\
\hline
\end{tabular}

Conclusion: PCT level was not influenced by previous antibiotic exposure. Patients with infection caused by gram-negative strains had a higher level of both CRP and PCT. The incomplete application of PCT guided stewardship in our patient series could be at least partially explained by a lack of experience in the routine clinical application of the studied biomarker.

\section{Reference(s) and grant ackowledgment(s)}

1. 4. Andriolo BN, Andriolo RB, Salomao R, Atallah AN. Effectiveness and safety of procalcitonin evaluation for reducing mortality in adults with sepsis, severe sepsis or septic shock. Cochrane Database Syst Rev.

2017;1:Cd010959

2. 3. Wirz Y, Meier MA, Bouadma L, Luyt CE, Wolff M, Chastre J, et al. Effect of procalcitonin-guided antibiotic treatment on clinical outcomes in intensive care unit patients with infection and sepsis patients: a patient-level metaanalysis of randomized trials. 2018;22(1):191.

3. 2. Huang HB, Peng JM, Weng L, Wang CY, Jiang W, Du B. Procalcitoninguided antibiotic therapy in intensive care unit patients: a systematic review and meta-analysis. Ann Intensive Care. 2017;7(1):114.

4. 1. Schuetz P, Beishuizen A, Broyles M, Ferrer R, Gavazzi G, Gluck EH, et al. Procalcitonin (PCT)-guided antibiotic stewardship: an international experts consensus on optimized clinical use. 2019:57(9):1308-18.

5. The study did not receive any additional funding.

\section{7}

\section{Monocyte distribution width: new biomarker of sepsis}

Herraiz' ; P. Marcos ${ }^{1}$; C. Morales ${ }^{2}$; F. Arméstar ${ }^{1}$; R. Roig ${ }^{1}$; B. Catalán ${ }^{1}$; L. Tello ${ }^{1}$; A. García

${ }^{1}$ Intensive Care Unit, Hospital Germans Trias i Pujol, Badalona, Spain; ${ }^{2} \mathrm{Clini}$ cal Chemistry, Hospital Germans Trias i Pujol, Badalona, Spain

Correspondence: A. Herraiz,

Intensive Care Medicine Experimental 2020, 8(2): 000647

Introduction: Sepsis is a major healthcare problem which affects millions of people around the world each year. Despite significant advances in diagnostic tools and the increasing use of biomarkers, the early recognition of sepsis in critically ill patients could be challenging. Recently, it has been described that morphological changes in leukocyte populations, especially the morphology and size of monocytes, could be used as a new biomarker for the early detection of sepsis.

Objectives: The main purpose is to determine if the monocyte distribution width (MDW) could be used as a new biomarker of sepsis. The secondary objective is to assess if MDW could be a prognostic factor of mortality in septic patients.

Methods: Prospective, observational study carried out during 2019 in the Intensive Care Unit (ICU). Critically ill patients with all kind of pathologies were included. It has been collected demographic variables, severity scores and ICU mortality. According to Sepsis-3 criteria, patients were divided into 3 groups on admission: non-infected (NI), sepsis (S) and septic shock (SS). MDW and traditional biomarkers were analyzed on admission. Statistical analysis includes qualitative variables, presented as proportions, and quantitative variables, presented as medians and minimum-maximum (Min-Max). Kolmogorov-Smirnov test was used for the assessment of normality. Kruskal-Wallis test was used to compare biomarkers among groups. The association between
ICU mortality and each biomarker was performed using Mann-Whitney $\mathrm{U}$ test. The area under the receiver operating characteristics (ROC) curve (AUC) was used to assess the prediction of ICU mortality. The study was previously approved by the Hospital Ethics Committee.

Results: A total of 188 patients were included: NI 127 (0.676), S 35 (0.186), SS 26 (0.138). Age 56.7 years; males 0.59 . Severity scores: APACHE II NI 15 (Min-Max: 2-58), S 16 (Min-Max: 9-41), SS 23.5 (MinMax: 8-45; $p<0.001$ ); SOFA NI 6 (Min-Max: 0-17), S 8 (Min-Max: 0-17), SS 10.5 (Min-Max: 3-17; p < 0.001). Biomarkers on admission: MDW NI 20.5 (Min-Max: 15,3-40.6), S 24 (Min-Max: 16.4-39.8), SS 35.5 (Min-Max: 21-58.9; $\mathrm{p}<0.001$ ); CRP NI $19.8 \mathrm{mg} / \mathrm{L}$ (Min-Max: 0.8-343.7), S 106.5 mg/L (Min-Max: 3.6-478.2), SS 249 mg/L (Min-Max: 86.7-437.6; $\mathrm{p}<$ 0.001 ); PCT NI $0.15 \mathrm{ng} / \mathrm{mL}$ (Min-Max: 0.2-46.7), S $1.2 \mathrm{ng} / \mathrm{mL}$ (Min-Max: $0.15-75)$, SS $24.5 \mathrm{ng} / \mathrm{mL}$ (Min-Max: 1.27-100; $\mathrm{p}<0.001$ ); lactate and total leukocytes were not statistically significant (pns). The ICU mortality was 0.138 . Association of biomarkers and mortality: MDW alive patients (AP) 21.1 (Min-Max: 15.3-57.7), dead patients (DP) 28.3 (MinMax: 17.3-58.9; $p$ <.001); CRP AP $34.8 \mathrm{mg} / \mathrm{L}$ (Min-Max: 0.8-478.2), DP $112.6 \mathrm{mg} / \mathrm{L}$ (Min-Max: 9.6-355; $\mathrm{p}=0.007$ ); PCT AP $0.31 \mathrm{ng} / \mathrm{mL}$ (MinMax: 0.02-100), DP $2.8 \mathrm{ng} / \mathrm{mL}$ (Min-Max 0.07-100; $\mathrm{p}=0.005$ ); lactate AP $1.16 \mathrm{mmol} / \mathrm{L}$ (Min-Max: 0-4.9), DP $2 \mathrm{mmol} / \mathrm{L}$ (Min-Max: 0-16.6; $\mathrm{p}=$ 0.001 ); total leukocytes were not statistically significant (pns). Mortality prediction according to each biomarker: AUC MDW 0.74(Min-Max: 0.63-0.85; $\mathrm{p}<0.001$ ); AUC CRP 0.67 (Min-Max: 0.57-0.77; $\mathrm{p}=0.007$ ); AUC lactate 0.71 (Min-Max: 0.59-0.83; $p=0.001$ ); AUC PCT 0.69 (MinMax: 0.58-0.8; $\mathrm{p}=0.005)$.

Conclusion: MDW could be considered as a new biomarker for the diagnosis of sepsis. Additionally, MDW could have prognostic performance in septic patients.

\section{Reference(s) and grant ackowledgment(s)}

1. Rhodes A, Evans LE, Alhazzani W, et al. Surviving Sepsis Campaign: International Guidelines for Management of Sepsis and Septic Shock: 2016. Intensive Care Med. 2017:43(3):304-377.

2. Polilli E, Sozio F, Frattari A, et al. Comparison of Monocyte Distribution Width (MDW) and Procalcitonin for early recognition of sepsis. PLoS One. 2020;15(1):e0227300.

3. Crouser ED, Parrillo JE, Seymour CW, et al. Monocyte Distribution Width: A Novel Indicator of Sepsis-2 and Sepsis-3 in High-Risk Emergency Department Patients. Crit Care Med. 2019;47(8):1018-1025.

\section{6}

Cytokine adsorbtion in severe therapy-refractory septic shock: an ambispective matched cohort study

PD. Wendel Garcia ${ }^{1}$; MP. Hiltyy ${ }^{1}$ U. Held ${ }^{2}$; EM. Kleinert ${ }^{1}$; M. Maggiorini ${ }^{1}$ ${ }^{1}$ Institute of Intensive Care Medicine, University Hospital of Zurich, Zürich, Switzerland; ${ }^{2}$ Department of Biostatistics and Epidemiology, University of Zürich, Zürich, Switzerland

Correspondence: P.D. Wendel Garcia

Intensive Care Medicine Experimental 2020, 8(2): 000706

Introduction: Septic shock and the underlying cytokine storm remain a major contributor to mortality in critically ill patients. The introduction of cytokine haemoadsorption techniques offer a novel approach to treatment of septic shock [1-3], nevertheless the efficacy of these devices in cytokine removal, as well as their effect on the course of disease remain to be further assessed.

Objectives: To study the effect of the Cytosorb (I) directly on the levels of Interleukin-6 (IL-6), (II) on the need for vasopressors assessed through the Vasopressor Dependency Index (VPI) [4], and (III) on intensive care unit (ICU) mortality.

Methods: We analysed 48 patients prospectively treated with a proinflammatory (IL-6 $\geq 1000 \mathrm{ng} / \mathrm{l}$ ), therapy refractory (VPI $\geq 3$ ) septic shock, admitted to the medical ICU of the University Hospital of Zurich between 2014 and 2018, with a Cytosorb coupled in series to a CRRT for $3 \times 24$ hours after septic shock diagnosis, in an "intention to treat" approach. A historical cohort of 437 patients admitted with septic shock to the same ICU between 2011 and 2018 was further retrospectively constituted. To reduce biased estimation of the treatment 
effect of Cytosorb, six matching algorithms based on the variables age, sex, BMI, SAPS II, SOFA, IL-6, lactate, PCT and VPI at septic shock diagnosis were employed and compared to determine the most balanced population of control patients to the Cytosorb cohort. Finally, a genetic matching algorithm demonstrated its superiority regarding the achievement of a standardized mean difference of under 0.1 and a variation ratio of between 0.5 and 2.0 for all matching variables, selecting 48 control patients [5].

Results: After matching, all matching variables and other baseline characteristics were balanced between the matched cohort (MC) and the Cytosorb cohort (CC). Cytokine adsorption did not result in a greater relative decrease in IL-6 over 72 hours as compared to the matched control (MC: $85 \%$ [0-95] vs. CC: $54 \%$ [0-73], $\mathrm{p}=0.09$ ). Further, there was no evidence that Cytosorb use lead to an increased reduction in the VPI (MC: $88 \%$ [54-95] vs. CC: $51 \%$ [6-83], $p=0.56$ ). Finally, 30-day ICU mortality was more pronounced in the CC (Hazard Ratio $1.79, \mathrm{Cl}[1.01-3.18], \mathrm{p}=0.04)$.

Conclusion: Our ambispective study suggested that broad adsorption of cytokines as provided by the Cytosorb may have, as compared to the matched control of septic shock patients, an uncertain effect on IL- 6 kinetics and the need for vasopressors over an observation period of 72 hours, this with a potential deleterious effect on 30-day ICU survival. A large randomized controlled trial is ugently needed to prove the efficacy of the Cytosorb treatment in patients with septic shock. Awating these results its use in septic shock should be carefully considered.

\section{Reference(s) and grant ackowledgment(s)}

1. This study is supported by an unrestricted grant by CytoSorbents Europe $\mathrm{GmbH}$.

2. 1. Kogelmann, K., Jarczak, D., Scheller, M., \& Drüner, M. (2017). Hemoadsorption by CytoSorb in septic patients: a case series. Critical Care, 21(1), 74

3. 2. Friesecke, S., Stecher, S. S., Gross, S., Felix, S. B., \& Nierhaus, A. (2017). Extracorporeal cytokine elimination as rescue therapy in refractory septic shock: a prospective single-center study. Journal of Artificial Organs, 20(3), 252-259.

4. 3. Hawchar, F., László, I., Öveges, N., Trásy, D., Ondrik, Z., \& Molnar, Z. (2019). Extracorporeal cytokine adsorption in septic shock: A proof of concept randomized, controlled pilot study. Journal of critical care, 49, 172-178.

5. 4. Cruz, Dinna N., et al. "Early use of polymyxin B hemoperfusion in abdominal septic shock: the EUPHAS randomized controlled trial." Jama 301.23 (2009): 2445-2452

6. 5. Linden, A., \& Samuels, S. J. (2013). Using balance statistics to determine the optimal number of controls in matching studies. Journal of evaluation in clinical practice, 19(5), 968-975.

\section{2}

A case of Lemierre Syndrome presenting with Type 1 respiratory failure and septic shock following a recent diagnosis with Covid 19

C. Costello; A. Gillece ${ }^{2}$; C. Cody ${ }^{1}$; C. Murphy

'Department of Anaesthesia and Critical Care Medicine, Connolly Hospital Blanchardstown, Dublin, Ireland; ${ }^{2}$ Department of clinical microbiology, Connolly Hospital Blanchardstown, Dublin, Ireland

Correspondence: $C$. Costello

Intensive Care Medicine Experimental 2020, 8(2): 000742

Introduction: Lemierre Syndrome is a a rare and potentially life threatening condition characterized by septic thrombophlebitis of the internal jugular vein (IJV). First identified in 1900, it was described by André Lemierre in 1936 and had a rapidly fatal course in the preantibiotic era. [1] The incidence appears to be rising with a mortality of $5-18 \%$ [2]

Methods: We present a case of a Lemierre Syndrome,which was admitted to our Intensive Care Unit following a recent history of Covid19.

Results: A 39 year old male presented with a 7 day history of pharyngitis, pyrexia and vomiting. His medical history was significant for seizures associated with a traumatic brain injury. The patient tested positive for SARS-CoV 2 infection 12 weeks previously. SARS-CoV 2 was not detected on multiple investigations during this presentation.

The patient developed type 1 respiratory failure. Chest X Ray and CT Thorax revealed a bilateral cavitating pneumonia and pleural effusions. Blood cultures were positive for Fusobacterium necrophorum. He was also severely thrombocytopenic.

Increasing oxygen requirements necessitated mechanical ventilation. A subsequent CT neck revealed a left sided $3.5 \mathrm{~cm}$ peritonsillar abscess with an associated internal jugular vein thrombosis.

The patient was transferred to another Intensive Care Unit with on site ENT support. Spontaneous rupture of the peritonsillar abscess precipitated septic shock requiring debridement and washouts in the Intensive Care Unit and in Theatre. Bilateral chest drains were inserted to facilitate oxygenation and ventilation.

The patient was weaned from vasopressors and mechanical ventilation and subsequently dischargedhome on long term intravenous antibiotics
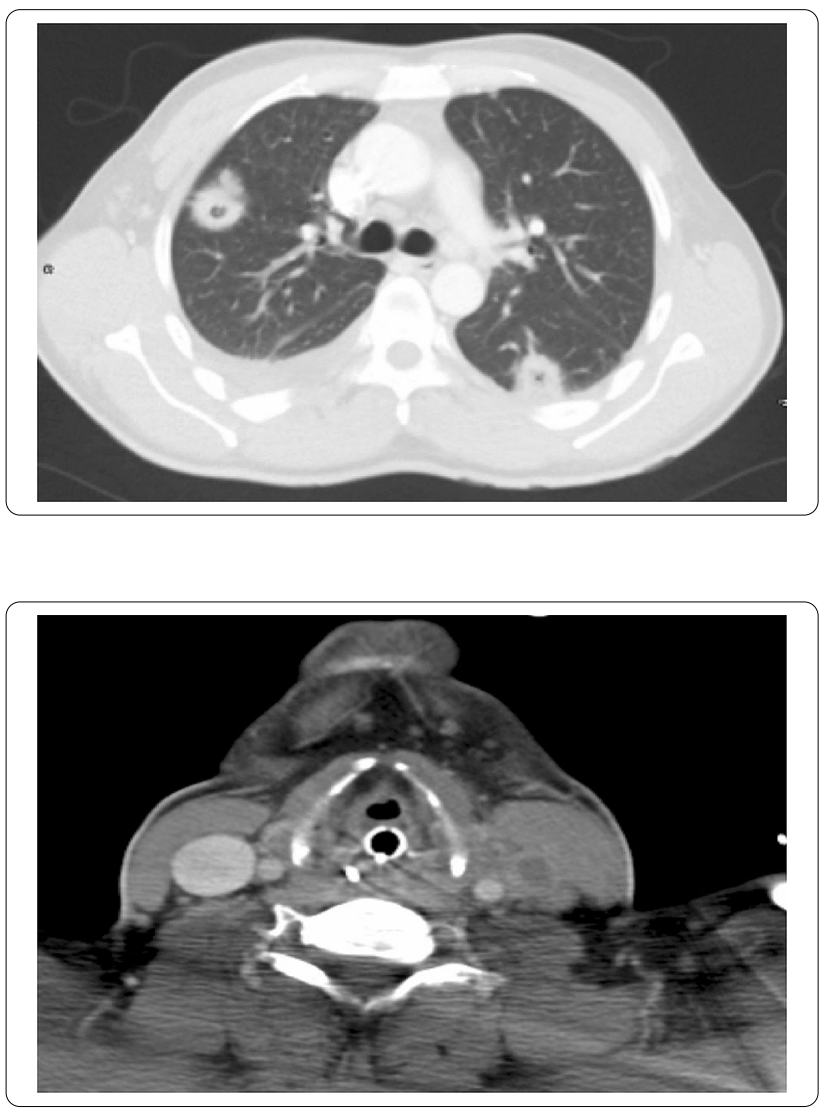

Conclusion: Lemierre syndrome is a rare condition precipitated by a bacteral pharyngitis typically caused by anaerobic organisms including Fusobacterium necrophorum. [1] It has been previously linked with Epstein-Barr Virus infection. However, given the recent pandemic we question the impact of SARS-CoV-2 on the pathogenesis of the disease and psychological factors at play leading to this critical illness?

Reference(s) and grant ackowledgment(s)

1. Kuppalli K, Livorsi D, Talati NJ, Osborn M. Lemierre's syndrome due to Fusobacterium necrophorum. Lancet Infect Dis. 2012 Oct: 12(10):808-15.

2. Osowicki J, Kapur S, Phuong LK, Dobson S. The long shadow of lemierre's syndrome. J Infect. 2017;74 Suppl 1:S47-S53. doi:10.1016/ S0163-4453(17)30191-3 
000772

Validation of sepsis-induced coagulopathy score in critically ill septic shock patients: a post hoc analysis of nationwide multicenter registry in Japan

C. Tanaka ${ }^{1}$; T. Tagami'; K. Unemoto ${ }^{1}$; M. Kuno ; S. Kudo'; F. Nakayama';

J. Kaneko ${ }^{1}$; A. Kitahashi'; S. Sato ${ }^{1}$; Y. Ishiki ${ }^{1}$; R. Fukuda ${ }^{1}$

${ }^{1}$ Emergency and critical care medicine, Nippon Medical School Tama Nagayama Hospital, Tama, Japan; ${ }^{2}$ Department of emergency and critical care medicine, Nippon Medical School Musashi Kosugi Hospital, Kawasaki, Japan

Correspondence: $C$. Tanaka

Intensive Care Medicine Experimental 2020, 8(2): 000772

Introduction: Coagulopathy is one of the major causes of organ dysfunction and death in sepsis; however, there are no standard criteria for diagnosis of it. Recently, sepsis-induced coagulopathy (SIC) was developed as a new criterion for coagulation disorder of sepsis patients. It includes the sequential organ failure assessment (SOFA), similarly the new definition of sepsis revised in 2016 (sepsis-3 definition). Previous studies suggested that SIC score might be a prognostic value for patients with sepsis and coagulopathy, however, there are few studies about SIC of septic shock patients who are defined as a subset of critically ill sepsis patients.

Objectives: To evaluate the accuracy of SIC score as a prognostic value for both sepsis patients and septic shock patients.

Methods: Coagulation [JSEPTIC-DIC] study), which investigated patients with sepsis in 42 intensive care units (ICUs) from 2011 to 2013, were analyzed. This contained information about patients' backgrounds, examination results, treatments, and mortality. Primary outcome measure was in-hospital mortality. Firstly, we grouped subjects into two (patients with vasopressor and patients without vasopressor). This database didn't contain information about vital signs, therefore, we analyzed the patients needed vasopressor instead of septic shock patients. Seconodly, we assessed patient characteristics and mortality of vasopressor groups between SIC and non-SIC, using $X 2$ test or Fisher's test. Then, we compared the same things between SIC and non-SIC in no vasopressor group. Furthermore, we analyzed in-hospital mortality of vasopressor group using a logistic regression analysis adjusted for age, gender, SIC and treatment for coagulation abnormality.

Results: This study included 1894 patient: 1453 in vasopressor group, and 441 in no vasopressor groups. In vasopressor group, 965 (66.4\%) were diagnosed with SIC. In-hospital mortality was significantly higher among SIC patients than non-SIC patients $(35.8 \%$ vs $27.9 \%, p<0.01$ ). Besides, ventilator free days and ICU free days were significantly shorter in SIC than non-SIC (mean days \pm standard deviations, $14.7 \pm 11.4$ vs $15.5 \pm 11.0 \mathrm{p}<0.01,12.0 \pm 10.2$ vs $13.2 \pm 10.1 \mathrm{p}$ $<0.01$, respectively). On the other hand, 186 (42.2\%) were diagnosed as SIC in no vasopressor group. There were no statistically significant differences about in-hospital mortality, ventilator free days, and ICU free days $(15.6 \%$ vs $12.2 \% p=0.33,21.9 \pm 9.8$ vs $22.0 \pm 9.3 p=0.22$, $16.9 \pm 9.7$ vs $17.8 \pm 8.6 p=0.29$, respectively). Moreover, the diagnosis of SIC in vasopressor group had significant association with worse outcome via a logistic regression analysis adjusted for factors independently associated with mortality (odds ratio: $1.52,95 \%$ confidence interval: 1.23-1.86).

Conclusion: SIC might be a good diagnostic value of fatal coagulation abnormally among sepsis patients who needed vasopressor.

\section{4}

The Apelinergic; Renin-Angiotensin \& Kallikrein Systems in Early Human Septic Shock

W. Salvail ${ }^{1}$; F. Chagnon ${ }^{2}$; D. Coquerel ${ }^{1} ;$ K. Tran $^{3}$; E. Marsault ${ }^{3} ;$ O. Lesur ${ }^{1}$ ${ }^{1}$ Médecine/icu, Université de Sherbrooke, Sherbrooke, Canada; ${ }^{2}$ Icu, CRCHUS, Sherbrooke, Canada; ${ }^{3}$ Institut de pharmacologie de sherbrooke, Université de Sherbrooke, Sherbrooke, Canada

Correspondence: $O$. Lesur

Intensive Care Medicine Experimental 2020, 8(2): 000774
Introduction: While catecholamine reactivity and response are welldocumented in septic shock, those related to the apelin and related renin-angiotensin and kallikrein-kinin systems are mostly unknown. From both experimental and human standpoints, the apelin system (i.e., apelinergics: apelins -APLNs- and Elabela -Ela-) offers an alternative pathway for hemodynamic support with evidence of efficiency and protection.

Objectives: Determine the endogenous blood contents of apelinergics; the related breakdown enzymatic profile; and the link with outcomes for septic patients.

Methods: An observational cohort study in 52 subjects: 33 patients admitted to the intensive care unit (ICU) for sepsis/septic shock (S) and 19 healthy volunteers $(\mathrm{H})$ with early blood sampling and data recording up to the outcome.

Results: The endogenous apelinergic system is reactive to $S$ (1.5-to3-fold increase of APLNs and Ela blood contents, respectively, $p<$ 0.01). Concomitantly, lower angiotensin converting enzyme-ACE2- but higher angiotensin converting enzyme -ACE1-, Neprilysin -NEP-, Kallikrein -KLK1-, and ACE1 enzymatic activities are found in S compared to $H$ ( $p<0.01$-to- 0.0001$)$. Such a huge elevation of systemic ACE1:2 ratio (16.5 [2.3-60.8] in S vs. 1.2 [0.9-1.3] in $H, p=0.0004)$ and of related enzymatic breakdown activities can jeopardize endogenous apelinergics with potential impacts on the outcome. Indeed: 1) S non survivors exhibit much higher ACE1:2 ratio than survivors (61.2 [6.3-77.9] vs. 8.8 [0.7-27], $p=0.0352), 2$ ) exogenously added APLN-13 and Ela to plasma in vitro display lower stability in S, suggesting an enhanced breakdown environment (half-life (min): APLN-13; H: $194 \pm 15$, S: $143 \pm 20 \mathrm{p}=$ 0.045; Ela, H: $258 \pm 45$, sepsis: $69 \pm 12 \mathrm{p}=0.003$ ). Decreased APLN13 half-life is significantly associated with higher NEP and KLK1 enzymatic activities ( $p=0.0116$ and 0.0003 , respectively). Also, increased ACE1/2ratio and NEP activities are significantly linked with higher circulating Ang1-7 levels in S ( $p<0.0001)$. PCA analysis showed a strong association between the following variables: arterial lactates, creatinine and cortisol circulating levels, APACHE II and SOFA scores, which altogether defined a $\mathrm{PC} 1$ axis. A second relationship gathering circulating APLNs, Ela, and Ang1-7 levels as well as ACE1, KLK1, and NEP activities, defined a PC2 axis. In this illustration, sepsis non-survivors were widely distributed in the positive area of the PC1 and PC2 axes, suggesting that worse sepsis outcomes could have been influenced by the profile of the apelinergic release-degradation system in the critically ill patients.

Conclusion: Sepsis-induced renin-angiotensin and kallikrein-kinin system disturbances potentiallyimpair the apelin system-driven hemodynamic support ability and affect the outcome in human patients with septic shock.

\section{9}

Microcirculatory, endothelial and inflammatory responses in critically ill patients with COVID-19 are distinct from those seen in septic shock: a case control study

J. Watchorn'; SD. Hutchings'; F. Trovato ${ }^{2}$; S. Napoli ${ }^{3}$; S. Mujib ${ }^{4}$;

PA. Hopkins ${ }^{5}$; M. Mcphail ${ }^{5}$

${ }^{1}$ Critical Care, King's College Hospital, Denmark Hill, London, UK, London, UK, United Kingdom; ${ }^{2}$ Hepatology, King's College London, London, United Kingdom; ${ }^{3}$ Hepatology, King's College Hospital, London, United Kingdom; ${ }^{4}$ Hepatology, King's College Hospital, Denmark Hill, London, UK, London, United Kingdom; ${ }^{5}$ Critical care, King's College Hospital, London, United Kingdom

Correspondence: J. Watchorn

Intensive Care Medicine Experimental 2020, 8(2): 000789

Introduction: Critically ill patients with COVID-19 infection frequently exhibit a hyperinflammatory response and develop organ failures, however the underlying mechanisms are unclear. We investigated the microcirculatory, endothelial and inflammatory responses in critically ill COVID-19 patients and compared them to a group of patients with septic shock. 
Methods: Prospective observational case control study. 30 critically ill patients with COVID-19 were compared to 33 patients with septic shock. Measurements of sublingual microcirculatory flow using Incident Dark Field (IDF) video-microscopy and serial measurements of IL- 6 and Syndecan- 1 levels were performed.

Results: COVID-19 patients had significantly less vasoactive drug requirement and lower plasma lactate than those with septic shock. Microcirculatory flow was significantly worse in septic patients than those with COVID-19 (MFI 2.6 v 2.9 p 0.02, PPV 88 v 97\% p <0.001). IL-6 was higher in patients with septic shock than COVID-19 (1653 v 253 $\mathrm{pg} / \mathrm{ml}, \mathrm{p}$ 0.03). IL-6 levels in COVID 19 patients were not elevated compared to healthy controls except on the day of ICU admission. Syndecan-1 levels were not different between the 2 pathological groups.

Conclusion: Compared to patients with bacterial sepsis an overt shock state with tissue hypoperfusion does not appear typical of COVID-19 infection. There was no evidence of significant sublingual microcirculatory impairment, widespread endothelial injury or marked inflammatory cytokine release in this group of critically ill COVID-19 patients. COVID-19 may have a different pathogenesis to bacterial induced septic shock

\section{Reference(s) and grant ackowledgment(s)}

1. Royal Centre for Defence Medicine and European Society of Intensive Care Medicine

\section{2}

Impact of hypothermia on the course of sepsis: a single-center observational study

M. Kirov' ; S. Makoveev²; A. Hussain ; E. Lochehina'; A. Lychakov'

R. Vinogradova'; T. Semenkova'

'Department of Anesthesiology and Intensive Care Medicine, Northern State Medical University, Arkhangelsk, Russia; ${ }^{2}$ Department of Anesthesiology and Intensive Care Medicine, City Hospital\#1, Arkhangelsk, Russia

\section{Correspondence: M. Kirov}

Intensive Care Medicine Experimental 2020, 8(2): 000042

Introduction: Body temperature abnormalities including hypothermia occur frequently in sepsis patients and can influence the inflammatory response to infection [1]. However, the relationship between hypothermia and clinical parameters of sepsis diagnosed with modern definitions is still unsettled.

Objectives: To estimate the impact of hypothermia upon admission to the intensive care unit (ICU) on the course and outcomes of sepsis. Methods: We performed a retrospective analysis of the ICU charts of all patients admitted to the 44-bed adult ICU of the City hospital \#1 of Arkhangelsk in 2018. We included for analysis all patients who was diagnosed with sepsis upon admission to ICU according to Sepsis-3 definitions [2] and recorded diagnosis, body temperature, demographic, clinical and laboratory data, duration of ICU stay and mortality. We used the Student's t-test, Mann-Whitney's test or chi-square test to compare the data according to their type and distribution. The correlation analysis was performed using Spearman's rho coefficient. The results are presented as $\mathrm{n}(\%), \mathrm{M} \pm \mathrm{SD}$ or median (25-75 percentiles). $p$ values $<0.05$ were regarded as statistically significant.

Results: Totally, 3103 ICU charts were examined. Upon admission to ICU, sepsis was observed in 121 patients (3.9\% from all admissions, $51.2 \%$ males and $48.8 \%$ females). The mean age was $66.5 \pm 14.7$ years, the duration of ICU stay was 4 (1.0-7.2) days. The most common origins of sepsis were pulmonary and abdominal. The organ dysfunctions included septic shock (57.9\% of cases), kidney injury (57.9\%), delirium $(53.7 \%)$, acute respiratory distress syndrome $(50.4 \%)$, disseminated intravascular coagulation (28.9\%), metabolic disorders (26.4\%), and hepatic failure (23.1\%). The mean SOFA score during the first $24 \mathrm{hrs}$ of ICU stay was 9 points; the hospital mortality was $51 \%$. The mean body temperature on ICU admission was 36.2 (35.9-36.7) ${ }^{\circ} \mathrm{C}$. Hypothermia $<36^{\circ} \mathrm{C}$ was diagnosed in $24 \%$ of cases; $67 \%$ of patients had body temperature within $36-38^{\circ} \mathrm{C}$, and $9 \%$ were febrile $\left(>38^{\circ} \mathrm{C}\right)$. Among hypothermic patients with sepsis, 59\% were postoperative. The patients with sepsis and hypothermia had more severe lactic acidosis as compared to normal or febrile body temperature: arterial $\mathrm{pH} 7.22$ (7.14-7.33) vs. $7.30(7.20-7.41)$ and $7.38(7.34-7.49)(p<0,002)$, lactate $5.0(2.5-9.5)$ vs. $2.6(1.5-4.9)$ and $4.1(2.1-5.3) \mathrm{mmol} / \mathrm{L}$, respectively $(\mathrm{p}=$ $0,04)$. Body temperature on ICU admission correlated with mean arterial pressure (rho $=-0,24, p=0,015)$ and platelet count (rho $=-0,34$, $p=0,001)$. Hypothermia in septic patients on ICU admission was not related with increased mortality or duration of ICU stay.

Conclusion: In sepsis, hypothermia $<36^{\circ} \mathrm{C}$ on admission to ICU occurs in $24 \%$ of patients and is accompanied by worsening lactic acidosis. The relationship between hypothermia and clinical outcome of sepsis requires further analysis.

Reference(s) and grant ackowledgment(s)

1. 1. Kushimoto S. et al. Crit Care Med 2019;47:691-699.

2. 2. Singer M. et al. JAMA 2016:315:801-810.

\section{7}

Variability of piperacillin-tazobactam pharmacokinetics in ICU patients: interim analysis of the OPTTAZ study

S. Sarfati ${ }^{1}$;T. Leroux ${ }^{2}$; C. Niles ${ }^{3}$; D. Carpentier ${ }^{1}$; C. Brault ${ }^{4}$; P. Gouin ${ }^{5}$;

L. Imbert ${ }^{2}$; B. Misset ${ }^{6}$; F. Lamoureux ${ }^{2}$

${ }^{1}$ Medical Intensive Care Unit, Rouen University Hospital, Rouen, France; ${ }^{2}$ Clinical Pharmacology Unit, Rouen University Hospital, Rouen, France; ${ }^{3}$ Medical Intensive Care Unit, Lille University Hospital, Lille, France; ${ }^{4}$ Medical Intensive Care Unit, Amiens University Hospital, Amiens, France; ${ }^{5}$ Surgical Intensive Care Unit, Rouen University Hospital, Rouen, France; ${ }^{6}$ Department of Intensive Care, CHU de Liège, Avenue de L'Hòpital, Liège, Belgium, Liège, Belgium

Correspondence: S. Sarfati

Intensive Care Medicine Experimental 2020, 8(2): 000977

Introduction: In septic patients, the piperacillin-tazobactam (PipTaz) association is one of the most used antibiotics. Pharmacokinetics of Pip-Taz depend mainly on distribution volume, renal clearance, patient's weight and serum albuminlevel. These parameters are highly variable in sepsis. Predictive algorithms based on clinical and biological parameters may help optimise dosing and improve outcome.

Objectives: To build a Bayesian model predicting optimal dosing regimen of Pip-Taz in septic patient. We designed the OPTAZ study with a 60-patient construction cohort and a 30-patient validation cohort. We present here the pharmacokinetics in the first patients recruited.

Methods: Patients were included prospectively in $4 \mathrm{ICUs}$ in France. Pip $4 \mathrm{~g}$ and Taz $0.5 \mathrm{~g}$ were infused every 6 hours (group 1 ) or 8 hours (group 2) according to clinician choice. Infusion time was of 3 hours. For patients receiving Pip-Taz every 6 hours, plasma level of Pip and Taz was assessed before infusion $(\mathrm{H} 0)$ and $1.5,3,4,6,12,24$ and 48 hours after the beginning of the first infusion. For patient receiving Pip-Taz every 8 hours, plasma level of Pip and Taz was assessed at $\mathrm{H} 0, \mathrm{H} 1.5$, $\mathrm{H} 4, \mathrm{H6}, \mathrm{H} 8, \mathrm{H} 16, \mathrm{H} 24$ and H48. Serum level of Pip-Taz was assessed by mass spectrometry. Values expressed as med [Q1-Q3]. We assessed the proportion of residual points over 4 times the EUCAST non-speciesrelated breakpoint for Pip-Taz (4mg/L). 


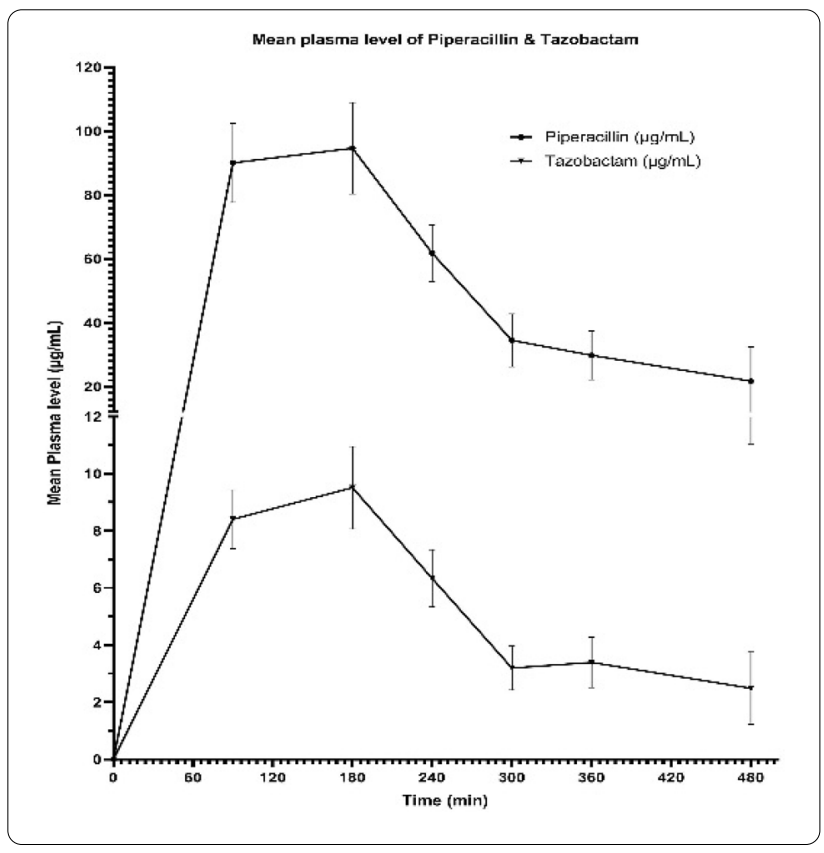

Results: Nineteen patients included. 14/19 (73\%) men, age 54 [4762] years, weight 88 [76-92] kg, BMl 27.47 [23.59-33.18] kg/m². On day $1,5(26 \%)$ received norepinephrine, $4(21 \%)$ lactate $\geq 2 \mathrm{mmol} / \mathrm{L}$ and urine output was 1580 [1350-2180] mL/24h. Patients received 44 [39-52] mg/kg of Pip and 5.65 [5.28-6.54] mg/kg of Taz. Seven patients received the infusion every 8 hours and 12 patients every 6 hours. After the first infusion, the AUC of Pip was 280 [218-367] mg.h/L in group 1, and 437 [313-566] mg.h/L in group 2. The AUC of Taz was 22.9 [19.4-36.8] mg.h/L in group 1, and 40.0 [27.1-46.2] mg.h/L in group 2. After the first infusion, the residual concentration was 13.77 [5.75$31.95] \mathrm{mg} / \mathrm{L}$, 33\% being under $16 \mathrm{mg} / \mathrm{L}$ in group $1,71 \%$ in group 2 . At $\mathrm{H} 48$, these values were 37.99 [15.00-48.19] and 9.68 [6.37-100.15], 33 $\%$ and $71 \%$.

Conclusion: In this prospective pilot series of patients admitted with sepsis and treated empirically with Pip-Taz, the blood exposure to the drug was consistent with the dosing interval but was highly variable, including values below the inferior (efficacy) target and over the superior (toxicity) target.

\section{8}

\section{Initial Ferritin levels predict poor evolution of patients infected} with Covid-19

L. Fernandez Ruiz'; B. Valenzuela-Mendez ${ }^{2}$; F. Valenzuela-Sánchez ${ }^{3}$;

JF. Rodriguez-Gutierrez ; MA. Gonzalez-Garcia ; L. Puget Martinez ; B. Diez Del Corral'; R. Bohollo De Austria'; J. Rello ${ }^{8}$

${ }^{1}$ Unidad de cuidados intensivos, Hospital San Juan de La Cruz, Úbeda, Spain; ${ }^{2}$ Ginecologia, Hospital Municipal de Badalona, Badalona, Spain; ${ }^{3}$ Ciberes. unidad de cuidados intensivos, Hospital Universitario de Jerez, Jerez de la Frontera, Spain; ${ }^{4}$ Hematología, Hospital Universitario de Jerez, Jerez de la Frontera, Spain; ${ }^{5}$ Analisis clinicos. bioquimica, Hospital Universitario de Jerez, Jerez de la Frontera, Spain; ${ }^{6}$ Servicio de medicina intensiva, Hospital Santa María del Puerto, El Puerto de Santa María, Spain ${ }^{7}$ Unidad de cuidados intensivos, Hospital Universitario de Jerez, Jerez de la Frontera, Spain; ${ }^{8}$ Ciberes. vall d'hebron research institute, Vall d'Hebron Barcelona Hospital Campus, Barcelona, Spain

Correspondence: F. Valenzuela-Sánchez

Intensive Care Medicine Experimental 2020, 8(2): 001078

Introduction: Biomarkers are essential to adequate triage and risk stratification of patients at hospital admission in order to provide an appropriate treatment.
Objectives: To compare the value as predictive biomarkers of different analytical parameters routinely performed in these patients.

Methods: Multicenter, prospective, observational study, carried out in the emergency department from 7 Spanish hospitals. Epidemiological and analytical data including demographics, therapy, inflammatory and immunity parameters were collected. Patients with the diagnosis of COVID-19 infection were included from two groups: patients admitted to the ICU and another group admitted to the hospital with less severity. The levels are expressed in median (IRQ).

Results: 203 patients admitted to the hospital emergency department with clinical symptoms and positive PCR for Covid-19 were recruited: 59 were admitted to the ICU during its evolution, 121 patients were admitted to the hospital for treatment and 22 were discharged and treated at home. The mortality of the patients admitted to the ICU was $42.37 \%$ and in the hospital $17 \%$.

All Biomarkers were significantly higher $(p<0.05)$ in the most severe group. Ferritin levels were significantly elevated in patients admitted to the ICU $1319 \mathrm{ng} / \mathrm{ml}$ compared to those admitted to the hospital $468 \mathrm{ng} / \mathrm{ml}$ and $85 \mathrm{ng} / \mathrm{ml}$ in those discharged; the CRP levels were $14.12 \mathrm{mgs} /$ I vs 8.65 vs $0.84 \mathrm{mgs} / \mathrm{L}$, respectively; PCT levels 0.245 vs. 0.07 vs. $0.03 \mathrm{ng} / \mathrm{ml}$ respectively; DD 1590 vs. 806.5 vs. $632 \mathrm{ng} / \mathrm{ml}$; LDH 437 vs 280.5 vs $195 \mathrm{U} / \mathrm{L}$; IL6 146 vs 14; Lactate 1.52 vs. 0.87 . mOsm / I respectively. The lymphocyte count showed a significant decrease in the severity group 765 vs 1070 vs 1540 cel / $\mu$ l respectively.

Regarding the relationship with gender, men predominate with $66.6 \%$ of those admitted to the ICU, with a lower percentage of those admitted to the hospital (42.97\%). Moreover, ferritin levels in men admitted to the hospital were significantly higher than in women at admission $(625.5 \mathrm{vs} 354 \mathrm{ng} / \mathrm{ml} ; \mathrm{p}=0.0033)$ and in the highest during evolution (777 vs $393 \mathrm{ng} / \mathrm{ml} ; \mathrm{p}=0.0007$ ).

The ROC-AUC to predict ICU admission and the need for MV were: Ferritin 0.821; PCT 0.767; DD 0.728; LDH 0.720; Lymphocyte count 0.663 . To predict mortality, the ROC-AUC were: Ferritin at admission 0.703; higher value of Ferritin 0.776; PCT 0.786; LDH 0.753; DD 0.680; PCR 0.613 ; Lymphocyte count 0.649 . In the multivariate analysis, Ferritin and LDH levels at admission were predictors of mortality in patients with severe Covid-19 pneumonia admitted to the ICU.

Conclusion: Ferritin levels are the best marker of severity and mortality in patients infected with Covid -19 virus, with a component linked to male sex.

\section{1}

Iron admission values predict respiratory failure in patients with SARS-Cov-2

F. Valenzuela-Sánchez'; B. Valenzuela-Mendez ${ }^{2}$; L. Fernandez Ruiz ${ }^{3}$.

JF. Rodriguez-Gutierrez ; MA. Gonzalez-Garcia ${ }^{4}$; P. Martínez-Garcia ${ }^{6}$;

S. Moreno Cano ; J. Rello ${ }^{8} ;$ A. Estella ${ }^{7}$

${ }^{1}$ Ciberes. unidad de cuidados intensivos, Hospital Universitario de Jerez, Jerez de la Frontera, Spain; ${ }^{2}$ Ginecologia, Hospital Municipal de Badalona, Badalona, Spain; ${ }^{3}$ Unidad de cuidados intensivos, Hospital San Juan de La Cruz, Úbeda, Spain; ${ }^{4}$ Hematología, Hospital Universitario de Jerez, Jerez de la Frontera, Spain; ${ }^{5}$ Analisis clinicos. bioquimica, Hospital Universitario de Jerez, Jerez de la Frontera, Spain; ${ }^{6}$ Servicio medicina intensiva, University Hospital of Puerto Real, Puerto Real, Spain; ${ }^{7}$ University hospital of jerez., Intensive care unit, Jerez, Spain; ${ }^{8}$ Ciberes. vall d'hebron research institute, Vall d'Hebron Barcelona Hospital Campus, Barcelona, Spain

Correspondence: F. Valenzuela-Sánchez

Intensive Care Medicine Experimental 2020, 8(2): 001081

Introduction: Although iron ( $\mathrm{Fe}$ ) is essential for biological processes, excessive iron levels can cause tissue damage and increased infection susceptibility. Also the immune response to pathogens could modify the iron concentration.

Objectives: To relate parameters of Fe metabolism with respiratory failure, oxygen requirements, and the need for invasive mechanical ventilation in patients with Covid-19.

Methods: Multicenter,prospective, observational study, carried out in 7 Spanish hospitals. Epidemiological and analytical data including 
demographics, therapy, inflammatory and immunity parameters were collected, including iron metabolism, and compared from two groups: patients admitted to the ICU and another group admitted to the hospital with less severity. The levels are expressed in median (IRQ).

Results: 180 patients with clinical symptoms attended in the Emergency Department, including respiratory failure, and a positive PCR for Covid-19 were recruited: 59 were admitted to the ICU during their evolution and 121 patients were admitted to the hospital. The global mortality rate was $17 \%$. Mortality rate for patients admitted to the ICU (59) was $42.3 \%$ and $55 \%$ for patients that required mechanical ventilation (47). Ferritin levels were significantly elevated $(p<0.05)$ in patients admitted to the ICU $1319 \mathrm{ng} / \mathrm{ml}$ compared to those admitted to the plant $468 \mathrm{ng} / \mathrm{ml}$. Iron levels were $24 \mu \mathrm{g} / \mathrm{dl}$ (16-44.75) in ICU patients $\mu \mathrm{g} / \mathrm{dl}$ vs. 56 (28.5-82.5) $\mu \mathrm{g} / \mathrm{dl}$ in the control group. The ROC curve for predicting ICU admission showed an AUC of 0.820 for Ferritin and 0.711 for Fe levels. The ROC curve for mechanical ventilation showed an AUC of 0.777 for Ferritin and 0.775 for Fe levels.

In the multiple linear regression, only Fe levels were directly and significantly associated with the worst $\mathrm{PaO} 2 / \mathrm{FiO} 2$ ratio (PAFI) with a r coefficient $=0.6688(\mathrm{p}<0.0001)$.

Conclusion: a) Ferritin and Iron levels are related to the severity of respiratory failure and the need for mechanical ventilation in Covid-19 patients

b) Iron levels are associated with oxygenation disorders in these patients.

\section{4}

Immunophenotypes predict need of invasive mechanical ventilation and death among Covid-19 adults: A prospective, multicenter, study

B. Valenzuela-Mendez ${ }^{1}$; F. Valenzuela-Sánchez²; L. Fernandez Ruiz³;

JF. Rodriguez-Gutierrez ; C. Martin González ; A. Sainz De Baranda

JC. García Ortiz ; A. Breval Flores ${ }^{8}$; J. Rello ${ }^{9}$

${ }^{1}$ Ginecologia, Hospital Municipal de Badalona, Badalona, Spain; ${ }^{2}$ Ciberes. unidad de cuidados intensivos, Hospital Universitario de Jerez, Jerez de la Frontera, Spain; ${ }^{3}$ Unidad de cuidados intensivos, Hospital San Juan de La Cruz, Úbeda, Spain; ${ }^{4}$ Hematología, Hospital Universitario de Jerez, Jerez de la Frontera, Spain; ${ }^{5}$ Servicio de analisis clinicos. bioquimica, Hospital Universitario de Jerez, Jerez de la Frontera, Spain; ${ }^{6}$ Servicio de medicina intensiva, Hospital HLA Jerez Puerta del Sur, Jerez de la Frontera, Spain; ${ }^{7}$ Servicio de urgencias, Hospital Universitario de Jerez, Jerez de la Frontera, Spain; ${ }^{8}$ University hospital of jerez., Intensive care unit, Jerez, Spain; ${ }^{9}$ Ciberes. vall d'hebron research institute, Vall d'Hebron Barcelona Hospital Campus, Barcelona, Spain

Correspondence: F. Valenzuela-Sánchez

Intensive Care Medicine Experimental 2020, 8(2): 001084

Introduction: Multiorgan dysfunction and death in sepsis is modulated by a balance between exaggerated hyperinflammation state and persistent immune dysfunction leading to anergy.

Objectives: To compare the state of immune dysfunction present in a cohort of adults with COVID-19 compared with a control group of influenza and predict immunophenotypes identifying need for invasive mechanical ventilation (MV) and death.

Methods: Multicenter, prospective, observational cohort study, carried out in the emergency department from 7 Spanish hospitals. A cohort of adults with confirmed PCR diagnosis of COVID-19 was compared with a historical control group with confirmed H1N1pdm2009 influenza virus admitted to the ICU. Epidemiological and analytical data including demographics, therapy, inflammatory and immunity parameters were collected. Patients were stratified depending on a) no need of oxygen therapy b) Oxygen requirements and c) Invasive mechanical ventilation. Findings were adjusted by age ( 65 and $80 \mathrm{yr}$ ). Mortality and progression to invasive MV were the main end-points.

Results: 203 COVID19 symptomatic adults were assessed in the emergency department by positive PCR for Covid-19. The global mortality rate was $17 \%$. Mortality rate for patients admitted to the ICU (59) was $42.3 \%$ and $55 \%$ for patients that required mechanical ventilation (47), whereas in the 75 ICU admission adults with influenza $A$ H1N1pdm2009 was $29.33 \%$.

In comparison to immunity parameters the results were the following: the number of lymphocytes per $\mu \mathrm{l}$ were significantly lower in patients admitted to the ICU 730 (530-1150) vs. 1070 (790-1450) at the hospital and 1470 (1030-1965) in discharged patients. This difference is at expense of the TCD4+ (584.17 vs. 921.42$) ; B C D 19+(66.12$ vs. 264.5$)$ and NKCD56+ (69.46 vs.138.18) the number of monocytes was similar but HLA-DR expression in monocytes was significantly lower in those admitted to ICU 1323 (854.25-1739.5) vs. 4618.5 (4537- 4700) MFI.

Ferritin levels were significantly elevated in patients admitted to the ICU $1319 \mathrm{ng} / \mathrm{ml}$ compared to those admitted to the hospital $468 \mathrm{ng} /$ $\mathrm{ml}$ and $85 \mathrm{ng} / \mathrm{ml}$ in those discharged. CRP levels $14.16 \mathrm{mg} / \mathrm{dl}(5.23-$ 24.37) in ICU admitted vs. $8.58 \mathrm{mg} / \mathrm{dl}$ (3.07-15.9) in hospital admitted $(p=0.01)$ vs. $0.86 \mathrm{mg}(0.26-4)(p<0.0001)$ in those discharged. PCT levels $0.25 \mu \mathrm{g} / \mathrm{l}(0.1-0.62)$ in ICU admitted vs. $0.07 \mu \mathrm{g} / \mathrm{l}(0.04-0.08)$ in hospital admitted vs $0.03 \mu \mathrm{g} / \mathrm{l}(0.02-0.05)(\mathrm{p}<0.0001)$ in those discharged. IL6 levels 146 pg/ml (32.9-1201) in ICU admitted vs. $14.56 \mathrm{pg} / \mathrm{ml}(7.24-$ 29.53) $(p<0.0014)$ in hospital admitted.

In survivors, the numbers of cells TCD4+ were significantly higher (921 vs. 263$)$ as NKCD56+ (138.18 vs. 59.91$)$, IgM (112 vs 60$)$ and HLA-DR expression in monocytes (1600 vs. 847 IMF); IL6 levels were higher in the non-survivors: $641.55 \mathrm{pg} / \mathrm{ml}(114.3-1156)$ vs. $81.9 \mathrm{pg} / \mathrm{ml}$ (26-1624) as well as Ferritin 1172 vs $500 \mathrm{ng} / \mathrm{ml}$.

Similar findings were reported in the control group of H1N1pdm influenza admitted to the ICU, which differed in a significantly $(p=0.0017)$ lower BCD19+ (9.750 vs 15.65) rates and IgM levels (72 vs. $160 \mathrm{mgs} / \mathrm{dl})$ but significantly higher Ferritin median plasma levels were $1345 \mathrm{ng} / \mathrm{ml}$ (819.25-2124.25) vs. $558.1 \mathrm{ng} / \mathrm{ml}(180-1880)$.

The ROC-AUC analysis to predict ICU admission and the need for MV were: Ferritin 0.821; NKCD56\% 0.818; CD19\% 0,773; for IL6 levels 0.731; IgM 0.682; lymphocyte count 0.663 . The ROC-AUC analysis to predict mortality, were: Ferritin at admission 0.703; highest value of Ferritin 0.776; IL6 levels: 0.921; HLA-DR: 0.857; IgM: 0.755; lymphocyte count: 0.649 .

Stratification by age groups showed higher mortality in the 65-79 age group: $21.9 \%$ compared to those under $65,10.3 \%$. Likewise, they required a greater need for MV $36.1 \%$ vs. $20.8 \%$ and treatment with corticosteroids. There were no differences in immunity cells levels, but significantly higher levels of inflammatory parameters: Ferritin, CPR, PCT and IL6.

Conclusion: A) Covid-19 patients showed a patent alteration in immunity that worsen with disease severity, similar to what happened in Influenza A N1H1 pneumonia, with a marked dysfunction of lymphocytes TCD4+ lymphocytes BCD19+, NKCD56+ and monocytes manifested by the low HLA-DR expression.

B) Covid-19 ICU admitted patients showed an important proinflammatory state expressed by high levels of IL6 and Ferritin, higher than those showed by severe A H1N1pdm2009 Influenza patients.

C) Both components influence the severity and poor evolution of the patients.

D) After age stratification, there is a greater hyperinflammation, need for respiratory support and mortality in the 65-79 age group.

\section{8}

Anakinra for treatment of critically ill patients with COVID-19: a retrospective study

N. Lumlertgul'; W. Kok'; B. Ficial'; 'L. Camporota ${ }^{1}$; NA. Barrett ${ }^{1}$; S. Agarwal'; B. Lams ${ }^{3}$; F. Hanks'; M. Ostermann ${ }^{1}$

${ }^{1}$ Critical care, Guy's and St Thomas' NHS Foundation Trust, London, United Kingdom; ${ }^{2}$ Interstitial lung disease unit, department of rheumatology, Guy's and St Thomas' NHS Foundation Trust, London, United Kingdom; 'Interstitial lung disease unit, Guy's and St Thomas' NHS Foundation Trust, London, United Kingdom

Correspondence: N. Lumlertgul

Intensive Care Medicine Experimental 2020, 8(2): 001118 
Introduction: Coronavirus disease 2019 (COVID-19) may be associated with systemic hyperinflammation, with consequent increase in pro-inflammatory cytokines (e.g. interleukin (IL)-1, IL-6, tumor necrosis factors) which leads to multiple organ dysfunction and increased mortality. Anakinra, an interleukin-1 receptor antagonist, is a potential therapy in patients with severe COVID-19 and may provide clinical benefits.

Objectives: We aimed to investigate the effects of anakinra on inflammatory markers and clinical outcomes in critically ill patients with COVID-19.

Methods: This was a retrospective observational study at a universityaffiliated tertiary hospital during March 18th and May 9th, 2020. We included adult patients ( $>18$ years) with confirmed COVID-19 admitted in intensive care units (ICU) and received at least one dose of anakinra. We compared them to a group of patients matched for age $( \pm 2$ years), date of ICU admission ( \pm 2 days), type of ventilatory support. The primary outcome was the change in c-reactive protein (CRP) 72 hours post-treatment. The secondary outcomes were changes in white blood cells (WBC), D-dimer, ferritin over 72 hours, and ICU mortality. (NCT04408326)

Results: 21 patients (male 90.5\%) received anakinra. Time from ICU admission to the first dose of anakinra was 8 days (interquartile range [IQR] 4-16). The duration of anakinra administration was 11 days (IQR 8-12), and the median total received dose was $2600 \mathrm{mg} \mathrm{(2100-3400}$ $\mathrm{mg}$ ). Compared with the control group, patients who were treated with anakinra had significantly higher pre-treatment CRP (32.7 (IQR 21.1,37.3) vs. 16.9 (IQR 7.7,30.1) $\mathrm{mg} / \mathrm{dL} ; \mathrm{p}=0.03)$ and $\mathrm{D}$-dimer $(9975$ (IQR 6390,72830) vs. 1580 (IQR 1000,7080) $\mathrm{ng} / \mathrm{mL} ; \mathrm{p}=0.001)$. Over 72 hours, there were significant differences in post-pre CRP $(-13.3$ (95\% confidence interval $[\mathrm{Cl}]-17.5,-9.2)$ vs. $0.2(95 \% \mathrm{Cl}-3.8,4.3) \mathrm{mg} / \mathrm{dL}$; $\mathrm{p}=0.006), \mathrm{WBC}(1266(95 \% \mathrm{Cl}-1171,3703)$ vs. $2717(95 \% \mathrm{Cl} 280,5154)$ cells $/ \mathrm{mm3} ; \mathrm{p}=0.002)$, and $\mathrm{D}-\operatorname{dimer}(-10729(95 \% \mathrm{Cl}-19089,-2369)$ vs. $-2758(95 \% \mathrm{Cl}-10441,4924) \mathrm{ng} / \mathrm{mL} ; \mathrm{p}=0.004)$, but similar changes in ferritin $(-864(95 \% \mathrm{Cl}-2903,1175)$ vs. $-2005(95 \% \mathrm{Cl}-3948,-62) ; \mathrm{p}=0.25)$. ICU mortality was not different between the groups.

Table 1 Baseline characteristics between patients who received anakinra and control $(\mathrm{n}=42)$

\begin{tabular}{|l|l|l|l|}
\hline Parameters & Anakinra group $(\mathrm{n}=21)$ & Control $(\mathrm{n}=21)$ & P-value \\
\hline Age (years) & $49.2 \pm 11.4$ & $49.6 \pm 11.1$ & 0.90 \\
\hline Male sex (\%) & $19(90.5)$ & $18(85.7)$ & 1.00 \\
\hline SOFA score & $6.5 \pm 2.9$ & $6.8 \pm 3.2$ & 0.73 \\
\hline Mechanical ventilation (\%) & $20(95.2)$ & $20(95.2)$ & 1.00 \\
\hline Vasopressor (\%) & $10(47.6)$ & $8(38.1)$ & 0.53 \\
\hline Venovenous ECMO (\%) & $10(47.6)$ & $6(28.6)$ & 0.20 \\
\hline
\end{tabular}

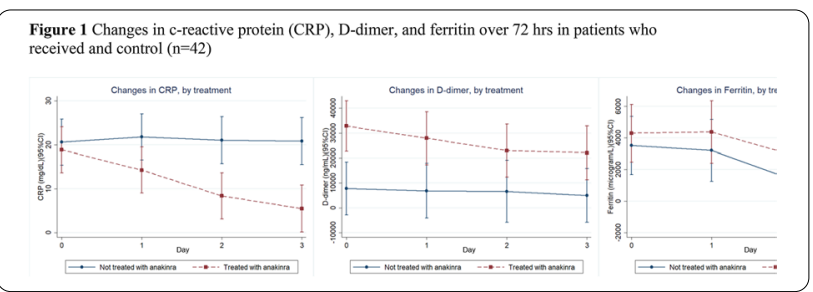

Conclusion: Administration of anakinra in critically ill patients with COVID-19 was associated with significant decrease in inflammatory markers. Further clinical trials are required to confirm the effects of anakinra on clinical outcomes.
001182

Does the Timing of Inter-Hospital Transfer for Critically III Patients With Sepsis Affect Outcomes?

C. Schorr'; C. Johnson'2; K. Hunter ${ }^{3}$; K. Mchugh²; E. Marone ${ }^{2}$; K. Gruber ${ }^{4}$. N. Loperfido ${ }^{5}$ P. Modelewski ${ }^{6}$

${ }^{1}$ Cooper research institute, critical care, Cooper University Hospital, Camden, United States of America; ${ }^{2}$ Student, Cooper Medical School of Rowan University, Camden, United States of America; ${ }^{3}$ Cooper research institute, Cooper University Hospital, Camden, United States of America; ${ }^{4}$ Nursing administration, Cooper University Hospital, Camden, United States of America; ${ }^{5}$ Critical care administration, Cooper University Hospital, Camden, United States of America; ${ }^{6}$ Critical care, Cooper University Hospital, Camden, United States of America

Correspondence: C. Schorr

Intensive Care Medicine Experimental 2020, 8(2): 001182

Introduction: Inter-hospital transfers (IHTs) to tertiary care centers are commonly due to limited availability of time-critical interventions and resources. Transfer patients are complex often requiring organ support with treatment delays resulting in worse outcomes. In one study, IHT patients with severe sepsis or septic shock had significant delays in appropriate antibiotic and resuscitation therapy compared to nontransferred patients, with no difference in ICU LOS or mortality. In a surgical population, increased complications were observed in IHTs for acute appendicitis versus patients that presenting directly. Weekend neurosurgical IHTs had worse functional outcomes with daytime IHTs experiencing longer length of stay (LOS). The "weekend effect" has been associated with higher mortality in the emergency department.

Objectives: The objective of this study was to evaluate whether the timing of IHTs affects outcomes in critically ill patients with sepsis.

Methods: A retrospective cohort study of IHT patients $\geq 18$ years old, admitted to the critical care service with a primary sepsis diagnosis during $1 / 1 / 2016-12 / 31 / 2018$. Data sources were merged from the transfer center, administrative databases, and the electronic health record chart review. Timing was defined as weekday (Monday 7:00 to Friday 17:59) vs. weekend (Friday 18:00 - Monday 06:59), and daytime (7:00 - 17:59) vs. nighttime (18:00 - 06:59). Evaluation included patient characteristics, sepsis diagnosis, therapies (days on mechanical ventilation (MV), dialysis, and vasopressors, and surgical procedures), and outcomes. Descriptive analysis included $95 \%$ confidence intervals to describe the data using means (SD) or median (IQR).

Results: A total of 521 patients were included of which 199 were daytime admissions, 322 nighttime, 319 weekday and 202 weekend. Comparing weekday vs. weekend, the groups were similar for age (60 vs. $61, \mathrm{p}=0.61)$, sex, race, BMI and APACHE II score (25.4 (8.71) vs. 26.92 (7.92), $p=0.08)$. More patients received MV on the weekend $(67.3 \%$ vs $58 \%$ weekday, $\mathrm{p}=0.33$ ), with weekend patients on MV longer (6 (3-12) days vs $5(3-9), p=0.05)$. Vasopressors were administered in $62 \%$ with weekend admissions receiving more days on vasopressors (3 (2-6) vs. weekday $2(1-4), p=0.03)$. Shorter LOS was observed in weekday admissions $(3(1-8)$ vs $5(2-10.25)$ weekend, $p=0.03)$. No difference in hospital LOS, mortality, or discharge disposition. Comparing daytime vs. nighttime, no significant differences were observed for patient characteristics, treatments, or outcomes. Most IHTs had sepsis with an unspecified organism. Mortality was $\geq 27 \%$, and discharge to hospice was $>30 \%$ in all groups.

Conclusion: In this cohort, IHTs were severely ill requiring life-sustaining therapies with increased resource use for weekend transfers. Contrary to previous studies, the timing of transfer did not have a significant effect on patient survival. Clinician awareness of IHT on the weekend with attention on weaning vasopressors and mechanical ventilation as appropriate, may contribute to decreased resource utilization and improved patient outcomes.

\section{Reference(s) and grant ackowledgment(s)}

1. Bell CM, Redelmeier DA. Mortality among patients admitted to hospitals on weekends as compared with weekdays. N Eng J Med. (2001); 345(9):663-668. 
2. Holland, M et al. Interhospital transfer of neurosurgical patients: Implications of timing on hospital course and clinical outcomes. Neurosurgery. (2017) 81(3), 450-457.

3. Leberer, D et al. Patient characteristics, outcomes and costs following interhospital transfer to a tertiary facility for appendectomy versus patients who present directly. American Journal of Surgery. (2017) 215(5). 825 - 830.

4. Faine et al: Interhospital Transfer Delays Appropriate Treatment for Patients With Severe Sepsis and Septic Shock: A Retrospective Cohort Study. Critical Care Medicine. (2015), 43(12) 2589-2596

5. Bernard, A et al: Comparing the hospitalizations of transfer and non-transfer patients in an academic medical center. Academic Medicine. (1996), 71(3): 262-266.

6. Flabouris, A; Hart, G; George, C: Outcomes of patients admitted to tertiary intensive care units after interhospital transfer: comparison with patients admitted from emergency departments. Critical Care and Resuscitation. (2008), 10(2) 97-105

7. Duke, G; Green, J: Outcome of Critically III Patients Undergoing Interhospital Transfer. Medical Journal of Australia: (2001); 174: 122-125

8. Gerber D; Schorr, C, et al:Location of patients before transfer to a tertiary care intensive care unit: Impact on outcome. Journal of Critical Care: (2009) $24,108-113$

\section{0}

Evolution of rheology and red blood cell complement regulatory proteins in septic patients

J. Vanderelst ${ }^{1}$; A. Rousseau ${ }^{2}$; BK. Zouaoui ${ }^{2}$; P. Biston ${ }^{1}$; M. Piagnerelli ${ }^{1}$

${ }^{1}$ Intensive Care, Civil Hospital Marie Curie, Charleroi, Belgium; ${ }^{2}$ Experimental Medicine Laboratory, Hospital André Vésale, Montigny-le-Tilleul, Belgium

Correspondence: J. Vanderelst

Intensive Care Medicine Experimental 2020, 8(2): 000310

Introduction: During sepsis, red blood cells (RBCs) rheology are altered (more spherical shape, decreased deformability and increased aggregation). Persistence of these alterations were associated with poor outcome [1,2]. Moreover, RBCs are physiologically protected by membrane surface proteins (CD35, CD55 and CD59) against the complement system activation [3]. In malaria, Waitumbi et al. reported links between the modification of the expression of these RBCs membrane proteins and the erythrophagocytosis, a cause of anaemia in this disease. [4]

Objectives: We studied the time course of RBCs deformability and the surface $\lg G$ and regulatory proteins content in RBCs from septic patients (S) compared to healthy volunteers (V).

Methods: By flow cytometry technics, we measure at ICU admission, at day 3-5 and day 7-10, the RBC membrane expression of IgG and complement proteins (CD35, 55, 59 and CD95) from $S$ patients compared to RBCs from V. We assessed the RBC shape by the second moment of dissymetry of Pearson as already reported [1]. RBCs deformability was estimated by an ektacytometry technique (laser-assisted optical rotational cell analyzer) for shear stress from 0.3 to $50 \mathrm{~Pa}$.

Results: RBCs from $11 \mathrm{~S}$ patients and $21 \mathrm{~V}$ were included. At ICU admission, RBCs from $\mathrm{S}$ patients were more spherical than $\mathrm{V}$ (moment $\mathrm{S}:-0.193(-0.561 ;-0.033)$ vs $\mathrm{V}:-0.538(-0.657 ;-0.491) ; p=0.026)$ and deformability was significantly altered for all shear stress $\geq 3.07 \mathrm{~Pa}$ However, these alterations of shape and deformability persists at Day 3.

We observed only a significant decrease at ICU admission in CD35 expression in S patients compared to $\mathrm{V}(9.2(5.02-13.18)$ vs 23.04 (11.39-32.57); $p=0.011)$. No significant changes were observed over time.

Conclusion: Our study suggests that a decrease in CD35 expression at the surface of the RBCs as well, as we have already observed (an increase in RBC sphericity and decrease membrane sialic acid content) [1], could be at the root of the accelerated aging of RBCs. This could contribute to the anaemia observed in septic patients.

\section{Reference(s) and grant ackowledgment(s)}

1. [1] Piagnerelli M et al. Crit Care Med; 2003, 31, 2156-62

2. [2] Donadello K et al. Microvascuc. Res $2015 ; 101: 8-14$
3. [3] Ugur Kurtogllu B et al. Central European Journal of Immunology 2017; 42(1)

4. [4] Waitumbi J-N et al.. Blood 2000 95: 1481-1486.

5. [5] Reggiori G et al. Crit Care Med 2009; 37 : 3041-3046

000473

Machine learning technique for severity classification in sepsis patients

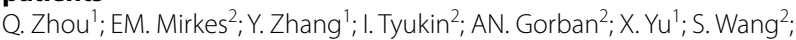
M. Charlton ${ }^{3} ;$ T. Coats ${ }^{4}$; MR. Sims ${ }^{5}$; JP. Thompson ${ }^{4}$

${ }^{1}$ School of informatics, University of Leicester, Leicester, United Kingdom; ${ }^{2}$ School of mathematics and actuarial science, University of Leicester, Leicester, United Kingdom; ${ }^{3}$ Anaesthesia, critical care and pain management, department of cardiovascular sciences, University of Leicester, Leicester, United Kingdom; ${ }^{4}$ Emergency medicine academic group, department of cardiovascular sciences, University of Leicester, Leicester, United Kingdom; ${ }^{5}$ Space research centre, department of physics and astronomy, University of Leicester, Leicester, United Kingdom

Correspondence: $\mathrm{Q}$. Zhou

Intensive Care Medicine Experimental 2020, 8(2): 000473

Introduction: Sepsis describes the condition of life-threatening organ dysfunction due to a dysregulated host response to infection. Early diagnosis and treatment can significantly reduce mortality. Microcirculatory dysfunction is an important pathophysiological feature of sepsis and may correlate with disease severity [1,2]. Infrared thermal imaging is a novel approach for monitoring the microcirculation via cutaneous temperature gradients [3]. Machine Learning shows great promise as a tool for medical image analysis, approaching the accuracy of clinicians in a growing range of tasks [4]. The combination of infrared thermal imaging and machine learning classification may be a promising approach for the automating the diagnosis of sepsis severity.

Objectives: This study is aimed at the preliminary development of a machine learning technique for identification of sepsis severity utilising infrared thermal images as a means of interrogating the microcirculation.

Methods: Participants were recruited in to two groups based on the 2009 sepsis definitions; early sepsis patients without organ dysfunction $(n=33)$, and severe sepsis patients with evidence of organ dysfunction $(n=34)$. Standardised infrared images were acquired of the anterior knee for each participant. A sliding window approach was used to extract image patches from the images. The patches were grouped by category and clustered with K-Means clustering. The Elbow method was applied to find the optimal number of clusters for each category. The patches were then projected onto the cluster centroids. With a large number of patches for each image, the image-wise features were reconstructed as mean values of patch-wise features. A number of classifiers were trained for the binary classification task in the space of the extracted features. The model performance was evaluated by 5 -fold cross-validation, where the samples were the samples were separated 5 folds, and for each fold (testing set) four other folds were used as training set. We accumulate the validation predictions from each fold and compare them to their actual grouping for evaluation.

Results: The highest performing classifier found is a support vector machine with gridsearch optimized hyper-parameters. This classifier produced an accuracy of $83.58 \%$, or error of $16.42 \%$, for the binary classification of early and severe sepsis in the cross-validation protocol.

Conclusion: The preliminary results show that the presented technique, in this h dimensional yet low-sample setting, may be a feasible approach to the binary discrimination of severity for infrared thermal imaging monitoring of microcirculation in sepsis patients. Further research is required to test and develop this preliminary technique, while more data is required to assure that the model does not overfit.

Reference(s) and grant ackowledgment(s)

1. [1] C. Ince, "The microcirculation is the motor of sepsis," Critical Care, vol. 9, no. 4, p. $\$ 13,2005$ 
2. [2] P. E. Spronk, D. F. Zandstra, and C. Ince, "Bench-to-bedside review: sepsis is a disease of the microcirculation," Critical Care, vol. 8, no. 6, p. 462, 2004

3. [3] M. Charlton, M. Sims, T. Coats, and J. P. Thompson, "The microcirculation and its measurement in sepsis," (in eng), Journal of the Intensive Care Society, vol. 18, no. 3, pp. 221-227, 2017.

4. [4] S. M. McKinney et al.,"International evaluation of an Al system forbreast cancer screening," Nature, vol. 577, no. 7788, pp. 89-94, 2020.

5. Royal Society International Exchanges Cost Share Award, UK (RP202G0230), Medical Research Council Confidence in Concept (MRC-CIC) Award, UK, Hope Foundation for Cancer Research, UK (RM60G0680)

\section{1}

MicroRNA-125a specific functional network increases endothelial permeability in acute inflammatory conditions

MB. Müller ${ }^{1}$; M. Hübner ${ }^{1}$; L. Li ${ }^{2}$; V. Ließke²; S. Kreth

${ }^{1}$ Department of anesthesiology, University Hospital, LMU Munich, Munich, Germany; ${ }^{2}$ Walter Brendel Centre of Experimental Medicine, Faculty of Medicine, LMU Munich, Munich, Germany

Correspondence: M.B. Müller

Intensive Care Medicine Experimental 2020, 8(2): 000511

Introduction: Acute inflammatory processes like sepsis are often accompanied by a dysfunctional endothelial barrier causing hyperpermeability and organ failure. While impaired expression of cell adhesion molecules is a typical feature of barrier disruption, it is known that microRNAs (miRs) play an essential role in regulating these adhesion molecules [1]. Recently, miR-125a was found to be upregulated in the blood of patients with sepsis [2]. Therefore, we wanted to investigate whether there is a miR-125a-specific functional network controlling endothelial barrier in acute inflammatory conditions.

Methods: MiR-125a was transfected into primary human umbilical vein endothelial cells (HUVECs). After stimulation with TNF transcriptome was analyzed using Next Generation Sequencing (NGS) followed by bioinformatics pathway analysis. NGS-results were validated via immunoblot and RT-PCR. To verify a direct miR-125a-3'-UTR interaction, we analyzed luciferase reporter assays. We tested endothelial barrier function with permeability assays and with electric cell substrate impedance sensing (ECIS) in HUVECs transfected with miR-125a, specific target siRNAs and blocking antisense miRNA (Antimir). For statistical evaluation we used student's t-test.

Results: In response to various inflammatory stimuli (cytokines, hypoxia, septic serum) HUVECs significantly upregulated miR-125a (Fig. 1A). NGS identified the pathways, GO_regulation of cell adhesion and KEGG_adherens junctions, as significantly regulated by miR-125a. We focused on five potential targets with an in-silico predicted miR125a binding site: The adhesion molecules VE-Cadherin (CDH5) and Claudin-5 (CLDN5) as well as the protein phosphatases PTPN1, PPP1CA and the transcription factor ETS-1. Validation of the NGS confirmed a significant downregulation of RNA and protein expression (Fig. 1B-D). Via reporter construct analysis, we newly identified $\mathrm{CDH} 5$ and PTPN1 as direct targets of miR-125a. Whereas expression of CLDN5 was dependent on CDH5 expression [3] and PPP1CA and ETS-1 are published targets of miR-125a [4, 5]. MiR-125a transfected HUVECs show a significantly increased permeability in permeability assays and decreased barrier function in ECIS. Specific knock-down of each of the five targets in the miR-125a functional network created a comparable phenotype. In contrast, hyperpermeable phenotype in the acute inflammation could be partially rescued by specific inhibition of miR125a (Fig. 1E, F).

Conclusion: MiR-125a induced by inflammatory stimulation directly targets the five target molecules CDH5, CLD5, PTPN1, PPP1CA and ETS-1 leading to increased permeability of the endothelial monolayer. In acute inflammatory diseases like sepsis this newly identified miR-125a-specific functional network could be a novel mechanism involved in the pathologic loss of intravascular fluids and thus blockade of miR-125a may be of therapeutic interest.

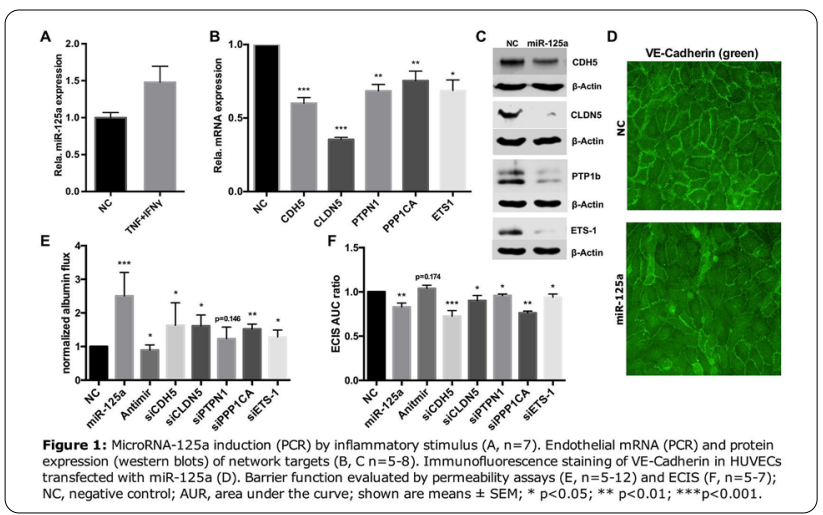

Reference(s) and grant ackowledgment(s)

1. 1. Young JA, Ting KK, Li J, Moller T, Dunn L, Lu Y, et al: Regulation of vascular leak and recovery from ischemic injury by general and VE-cadherinrestricted miRNA antagonists of miR-27. Blood 2013;122:2911-9

2. This work was supported by the Clinician Scientist PRogram In Vascular MEdicine (PRIME) funded by the Deutsche Forschungsgesellschaft (DFG, German Research Foundation) - project number MA 2186/14-1

3. 5. Ge Y, Sun M, Wu W, Ma C, Zhang C, He C, et al: MicroRNA-125a suppresses intestinal mucosal inflammation through targeting ETS-1 in patients with inflammatory bowel diseases. J Autoimmun 2019;101:109-20

4. 4. Guo S, Bai H, Megyola CM, Halene S, Krause DS, Scadden DT, et al: Complex oncogene dependence in microRNA-125a-induced myeloproliferative neoplasms. Proc Natl Acad Sci U S A 2012;109:16636-41

5. 3. Taddei A, Giampietro C, Conti A, Orsenigo F, Breviario F, Pirazzoli V, et al: Endothelial adherens junctions control tight junctions by VE-cadherinmediated upregulation of claudin-5. Nat Cell Biol 2008;10:923-34

6. 2. Zhao D, Li S, Cui J, Wang L, Ma X, Li Y: Plasma miR-125a and miR-125b in sepsis: Correlation with disease risk, inflammation, severity, and prognosis. J Clin Lab Anal 2020;34:e23036

\section{8}

Methotrexate carried in lipid core nanoparticles reduces the inflammatory process in septic rats with acute lung injury by increasing the bioavailability of intracellular adenosine

MC. Guido ; NM. Lopes ${ }^{1}$; Cl. Albuquerque ; R. Kalil-Filho'; RC. Maranhão ${ }^{1}$ ${ }^{1}$ Laboratory of Metabolism and Lipids, Heart Institute, University of São Paulo, São Paulo, Brazil

Correspondence: M.C. Guido

Intensive Care Medicine Experimental 2020, 8(2): 000618

Introduction: Acute lung injury (ALI) is the leading cause of mortality in critically ill patients. Exacerbated inflammatory response is a key mechanism of sepsis-induced ALI, so that attenuating inflammation is crucial1. Previously, we showed that methotrexate (MTX) carried in lipid core nanoparticles (LDE) had powerful anti-inflammatory and anti-proliferative effects on rheumatoid arthritis 2 and atherosclerotic lesions 3 and improved left cardiac dysfunction in myocardial infarction4. When MTX is associated to LDE, the cellular uptake of the drug is increased several-fold, which conceivably endows MTX with increased therapeutic action and diminished toxicity.

Objectives: To investigate whether treatment with LDE-MTX can attenuate the development of inflammatory process in septic rats with ALI.

Methods: Sepsis was induced in Wistar rats by two I.P lipopolysaccharide (LPS, $10 \mathrm{mg} / \mathrm{kg}$ ) injections administered at $24 \mathrm{~h}$ interval. Rats were allocated to Control group (CT, without sepsis) and 2 groups with sepsis: LDE, treated with LDE only; and LDE-MTX, with LDE-MTX at a dose of $1 \mathrm{mg} / \mathrm{kg}$ I.P. $72 \mathrm{~h}$ after sepsis induction, the animals were euthanized for protein expression analysis by Western blot of inflammatory, 
apoptotic, antioxidant enzymes, angiogenesis, cell hypoxia, fibrosis and adenosine bioavailability markers in lungs.

Results: Compared to $\mathrm{CT}$, the antioxidant enzymes, (catalase and superoxide dismutase-1) and fibrosis (collagen types 1 and 3) were lower in LDE and LDE-MTX groups. Although the expression of leucocytes and interleukins levels were similar in all 3 groups, septic rats treated with LDE had higher expression of macrophage recruitment marker (CD68) and tumor necrosis factor alpha, while LDE-MTX was able to decrease these levels, both compared to CT. Apoptotic markers, such as caspase 3 and b-cell associated X protein/b-cell lymphoma- 2 ratio, were higher in LDE group. LDE-MTX treatment was able to lower these pro-apoptotic factors to CT levels. Regarding angiogenesis and cell hipoxia, protein expression of CD31 and angiostatin, pro and anti-angiogenesis markers, respectively, and hypoxia-inducible factor 2-alpha were normalized to $\mathrm{CT}$ levels by the treatment with LDE-MTX, when compared to LDE group. The increase in thebioavailability of intracellular adenosine of septic animals treated with LDEMTX was suggested by the higher expression of $A 1$ and $A 2 b$ receptors in the lungs.

Conclusion: Possibly by increasing the bioavailability of intracellular adenosine, LDE-MTX treatment was effective in reducing the inflammatory process, decreasing cell death by apoptosis and normalizing angiogenesis and cell hypoxia factors in septic rats with ALI. The marked improvement of inflammatory response in ALI by LDE-MTX may pave the way for a novel strategy to treat this condition.

\section{Reference(s) and grant ackowledgment(s)}

1. 1. Chalmers S, Khawaja A, Wieruszewski PM, Gajic O, Odeyemi Y. Diagnosis and treatment of acute pulmonary inflammation in critically ill patients: The role of inflammatory biomarkers. World J Crit Care Med. $2019 \mathrm{Sep}$ 11;8(5):59-71. doi: 10.5492/wjccm.v8.15.59. 2. Mello SB, Tavares ER, Bulgarelli A, Bonfá E, Maranhão RC. Intra-articular methotrexate associated to lipid nanoemulsions: anti-inflammatory effect upon antigen-induced arthritis. Int J Nanomedicine. 2013;8:443-9. doi: 10.2147/IJN.S29392. 3. Bulgarelli A, Leite AC Jr, Dias AA, Maranhão RC. Anti-atherogenic effects of methotrexate carried by a lipid nanoemulsion that binds to $L D L$ receptors in cholesterol-fed rabbits. Cardiovasc Drugs Ther. 2013 Dec;27(6):531-9. doi: 10.1007/s10557013-6488-3. 4. Maranhão RC, Guido MC, de Lima AD, Tavares ER, Marques AF, Tavares de Melo MD, Nicolau JC, Salemi VM, Kali--Filho R. Methotrexate carried in lipid core nanoparticles reduces myocardial infarction size and improves cardiac function in rats. Int J Nanomedicine. 2017 May 17;12:37673784. doi: 10.2147/IJN.S129324.

\section{0}

\section{The role of Regulatory $\mathrm{T}$ cell subsets in nosocomial infection} of critically ill patients: a post-hoc analysis of the INFECT study A. Jennings'; M. Fish ${ }^{1}$; J. Simpson ${ }^{2}$; T. Walsh ${ }^{3}$; M. Shankar-Hari ${ }^{1}$;

A. Conway-Morris ${ }^{4}$

${ }^{1}$ School of Immunology and Infectious Diseases, King's College London, London, United Kingdom; ' ${ }^{2}$ Faculty of Medical Sciences, Newcastle University, Newcastle upon Tyne, United Kingdom; ${ }^{3}$ Department of Anaesthesia, Critical Care and Pain Medicine, The University of Edinburgh, Edinburgh, United Kingdom; ${ }^{4}$ Department of Anaesthetics \& Intensive Care Medicine, Cambridge University Hospitals NHS Foundation Trust, Cambridge, United Kingdom

Correspondence: A. Jennings

Intensive Care Medicine Experimental 2020, 8(2): 000670

Introduction: Regulatory T cells (Tregs) are a subset of CD4+ T cells which have an immunosuppressive function and essential for maintaining peripheral tolerance. In critically ill patients, the risk of adverse outcomes (including nosocomial infections), increase with increasing proportion of total Tregs. We hypothesised that this increase in risk is secondary to increases in proportions of effector Tregs and effector Tregs will be higher in sepsis compared to non-sepsis critical illness. Our aims were to compare the proportion of total Tregs and Treg subsets in critically ill patients with sepsis and without sepsis (non-sepsis) over the first 3-days following critical care admission.
Methods: INFECT was an observational study of critically ill adults admitted to ICU ( $n=148$ ), involving measurement of percentage Tregs to predict the risk of nosocomial infection. Data from 140 critically ill adult patients were included in this post-hoc analysis. Patients were categorised into those admitted with sepsis (57/140 and those who developed nosocomial infection during their ICU stay $(n=53)$. We measured total Treg proportions by CD25+ CD127- expression on CD4+ T cells. Treg subsets studied included naïve (CD4+, CD25+, CD127-, CD45RA+, CD15s-), effector (CD4+, CD25+, CD127-, CD45RA-, CD15s+), CD161+ cytokine-secreting Tregs (CD45RA-, CD15s-, CD161+) and CD161- non-regulatory cells (CD45RA+, CD15s+, CD161-).

Results: We found no significant difference in proportions of total Tregs (Fig. 1A), naïve Tregs (Fig.1B), CD161+ Tregs (Fig. 1D) and CD161cells (Fig. 1E) in septic and non-septic patients at admission (T1) ( $p$ $>0.05$ ). However, we found significantly less effector Tregs in septic patients versus non-septic patients at T1 (Fig. 1C, p $<0.05$, MannWhitney). We further categorised septic/non-septic patients according to the development of nosocomial infection. We found no significant changes in proportions of total Tregs and Treg subsets in infected and non-infected patients at T1 and T3 ( $p>0.05$, Two-way ANOVA).

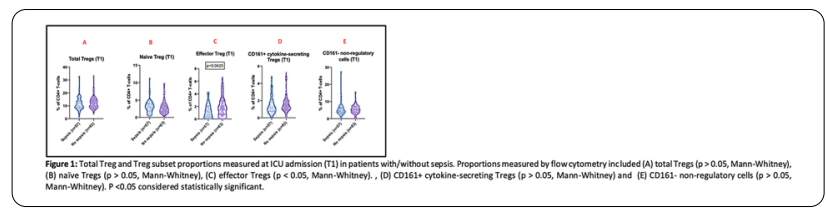

Conclusion: We found lower proportions of effector Tregs in patients admitted to ICU with sepsis compared to other admissions. Our data does not suggest that total Treg and Treg subsets proportions can be used to predict nosocomial infection in critically ill adults admitted to ICU.

\section{Reference(s) and grant ackowledgment(s)}

1. The study was funded by Innovate UK (Sepsis 2: 101193), BD Biosciences and the National Institute for Academic Anaesthesia. Dr Conway Morris is supported by a Clinical Research Career Development Fellowship from the Welcome Trust (WT 2055214/Z/16/Z). Dr Shankar-Hari is supported by the National Institute for Health Research Clinician Scientist Award (CS-2016-16011). The views expressed in this publication are those of the author(s) and not necessarily those of the NHS, the National Institute for Health Research or the Department of Health and Social Care.

\section{4}

Survivin and caspases upregulation in sepsis

M. Miliaraki'; P. Briassoulis'; 'S. Ilia'; A. Polonifi2; M. Mantzourani²;

E. Briassouli2 ; S. Nanas ${ }^{3}$; A. Pistiki ${ }^{4}$; M. Theodorakopoulou ${ }^{4}$; G. Daikos ${ }^{2}$;

G. Briassoulis

${ }^{1}$ Pediatric Intensive Care Unit, Medical School, University of Crete, Heraklion, Greece; ${ }^{2}$ First department of internal medicine, laiko hospital, National and Kapodistrian University of Athens, Athens, Greece; ${ }^{3}$ First critical care department, National and Kapodistrian University of Athens, Athens, Greece; ${ }^{4}$ 2nd department of critical care, Attikon General University Hospital, Haidari, Greece

Correspondence: M. Miliaraki

Intensive Care Medicine Experimental 2020, 8(2): 000994

Introduction: Sepsis is a dysregulated host response to infection, related to devastating outcomes. Recently, interest has been shifted towards apoptotic and antiapoptotic pathobiology (1). Apoptosis is executed through the activation of caspases (2) and regulated through the activation of a number of anti-apoptotic proteins, such as survivin, with different biological functions (pro-apoptotic or antiapoptotic), depending on the expression of its different splice variants (wild type-WT, $-\Delta \mathrm{E} \times 3,-2 \mathrm{~B},-3 \mathrm{~B}$ ) (3). The apoptotic and antiapoptotic responses have not yet been elucidated in sepsis. 
Objectives: The present study seeks to ascertain whether sepsis is associated with an upregulation of certain apoptotic (caspases) or anti-apoptotic biomolecules (survivin), and also seeks to assess the prognostic value of survivin transcript variant expression (survivinWT, $-\Delta \mathrm{E} \times 3,-2 \mathrm{~B}$ and $-3 \mathrm{~B}$ ), in ICU patients with sepsis and non-infectious (trauma-related) SIRS.

Methods: This prospective observational study was performed in a sample of critically ill adult patients with sepsis $(n=107)$ or traumatic SIRS $(n=75)$, compared to healthy controls $(n=89)$. The expression of survivin transcript variants was analyzed by real-time quantitative PCR in peripheral blood leukocytes, whereas the apoptotic or antiapoptotic equilibrium was specified by measuring caspases- 3 and -9 , along with survivin-WT serum protein concentrations, through enzymelinked immunosorbent assay (ELISA).

Results: The survivin-WT, $-2 \mathrm{~B},-\triangle \mathrm{Ex} 3 \mathrm{mRNA}$, survivin protein and caspases-3 and -9 showed an escalated increase in sepsis (Figure 1), compared to traumatic SIRS and controls, whereas survivin-3B was repressed in sepsis $(p<0.05)$. For discriminating sepsis, caspase9achieved the best receiver operating characteristic curve (AUROC) of 0.95 . In predicting mortality, caspase- 9 and survivin protein achieved an $A \cup R O C=0.70$

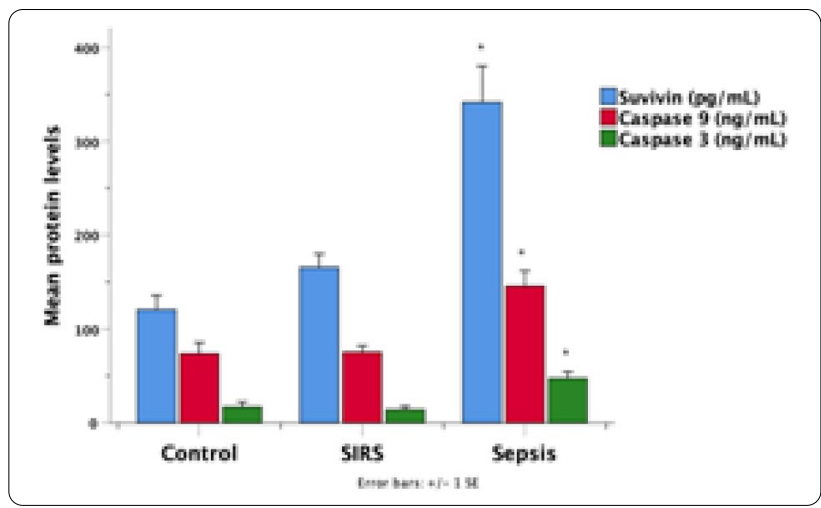

Conclusion: The upregulated apoptotic and anti-apoptotic pathways might represent attractive targets for future research in the clarification of sepsis. The mRNA expression of survivin variants and protein activity quantification of survivin and caspases could predict outcome and provide additional information about clinical management of patients suffering from sepsis.

\section{Reference(s) and grant ackowledgment(s)}

1. 1. Vaki, I. et al. An early circulating factor in severe sepsis modulates apoptosis of monocytes and lymphocytes. J. Leukoc. Biol.89, 343-349 (2011).

2. 2. Aziz, M., Jacob, A. \& Wang, P. Revisiting caspases in sepsis. Cell Death Dis.5, e1526 (2014)

3. 3. Wheatley, S. P. \& Altieri, D. C. Survivin at a glance. J. Cell Sci.132, (2019).

\section{9}

\section{Changes in monocyte subpopulations are associated}

\section{with a fulminant course of sepsis}

M. Helan'; M. Hortová-Kohoutková'; V. Tomášková'; P. Lázníčková2

K. Bendíčková2; M. De Zuani'; I. Andrejčinová²; M. ŠíTina'; J. Hruda'; P. Suk' V. Sramek' ; J. Frič²

'Department of Anaesthesiology and Intensive Care, St. Anne's University Hospital, Brno, Czech Republic; ${ }^{2}$ International Clinical Research Centre, (ICRC), Brno, Czech Republic

Correspondence: M. Helan

Intensive Care Medicine Experimental 2020, 8(2): 001109

Introduction: Sepsis is characterized by dynamic changes of the immune system resulting in deregulated inflammation. In a certain group of patients, the robust hyperactivation of inflammation occurs very early. This so-called "cytokine storm" leads frequently to patient's death and could be considered as a potential target group for future early immunotherapy. Circulating monocytes are among the first ones to recognize and clear pathogens and thus have an important role in early stages of infection and sepsis (1). Three distinct subpopulations of monocytes have been described: classical (CD14+ CD16-), intermediate (CD14+ CD16+) and non-classical (CD14lo CD16+) (2-3).

Objectives: The aim of the study was to evaluate monocyte populations in the early stages of septic shock in relation to patient mortality. Specifically, we focus on the group of patients with very early mortality (up to $72 \mathrm{hr}$. after ICU admission). We aim to determine whether specific changes in monocyte populations could be used as biomarker in prediction of early mortality and whether it correlates with severity of septic shock.

Methods: Patients admitted to the ICU with early septic shock were prospectively enrolled into the study. Whole blood samples and clinical data were collected at within $12 \mathrm{hr}$. after admission. Peripheral blood monocytic cells were isolated and labelled with specific antibodies. Presence of particular populations were determined by flow cytometry. With the use of Spearman correlation and Mann-Whitney test, the obtained data were related to 3-days mortality and the relationships with patient's age, sex, number of comorbidities, SOFA, CRP and the need of catecholamines were estimated. The predictive potential was assessed using receiver-operating curve. Data are provided as median (IQR).

Results: Recruited septic shock patients $(n=41)$ were predominantly male (57.1\%) with median age 73.0 (66.5-77.0) years. All patients were mechanically ventilated and the SOFA score at admission were 11 (1014). There was no statistically significant difference in the patients' comorbidities, chronic medication or age between patients who died in first 72 hours (early deaths) and the others. 3-day and 28-day mortality rate were $14 \%$ and $51 \%$, respectively. Group of early deaths had a lower percentage of classical monocytes (57.6 (42.5-62.0) \% vs. 81.6 (65.5-90.1) \%; $p=0.0098)$ and conversely had a higher proportion of intermediate (29.1 (26.2-36.1) \% vs. $8.9(4.2-19.6) \% ; p=0.0005)$ and non-classical (5.4 (4.5-6.5) \% vs. $3.1(1.9-6.4) \% ; p=0.1485)$ monocytes than other patients. AUC of receiver-operating curve for prediction of 3-day mortality was 0.8357 for classical, 0.9095 for intermediate and 0.6905 for non-classical monocytes. The ratio classical/intermediate shows even higher predictive potential $(A U C=0.9238)$. Furthermore, there were no statistically significant correlation between monocyte subsets and other characteristics (SOFA, lactate, CRP, catecholamines, etc.).

Conclusion: The determination of monocyte subsets frequency (especially intermediate monocytes) or its ratio can be used to predict a fulminant course of septic shock and to identify patients with excessive inflammatory response who might profit from personalized immunomodulatory therapy.

Reference(s) and grant ackowledgment(s)

1. 3. Cormican $\mathrm{S}$ et al.: Human monocyte subset distinctions and function: Insights from gene expression analysis. Front Immunol. 2020.

2. 2. Gainaru G et al.: Increases in inflammatory and CD14dim/CD16pos/ CD45pos patrolling monocytes in sepsis: Correlation with final outcome. Crit Care. 2018

3. 1. Hortová-Kohoutková M et al.: Phagocytosis-inflammation Crosstalk in Sepsis: New Avenues for Therapeutic Intervention. Shock. 2020.

4. This work has been supported by the Ministry of Education, Youth and Sports (LQ1605) and the Ministry of Health (NV18-06-00529) of the Czech Republic.

\section{2}

Procalcitonin in Management of COVID19 infection

K. Apostolidou'; N. Rapti ' J. Ramzi'; R. Mahroof'; A. Conway-Morris ${ }^{1}$;

A. Conway-Morris²

${ }^{1}$ Department of Anaesthetics \& Intensive Care Medicine, Cambridge University Hospitals NHS Foundation Trust, Cambridge, United Kingdom, ${ }^{2}$ Division of Anaesthesia, Department of Medicine, University of Cambridge, Cambridge, United Kingdom

Correspondence: $\mathrm{K}$. Apostolidou

Intensive Care Medicine Experimental 2020, 8(2): 000582 
Introduction: Procalcitonin (PCT) has emerged as a tool to guide antibiotic (ABx) therapy in bacterial pneumonia, but PCT's role in distinguishing between bacterial \& viral pneumonia is uncertain(1). With the novel SARS-CoV-2 pandemic there's emerging interest in the role of this biomarker for assessing COVID-19 disease severity \& progress(2). In COVID-19 pts, bacterial or fungal coinfection incidence is $8 \%$, whilst $72 \%$ of the pts have been reported to receive antimicrobial therapy(3). In the UK a recent guideline from NICE suggests early review of need for antibiotic treatment in COVID-19 on basis other than PCT(4). We have reviewed our institutional data in order to assess current practice \& usefulness of using PCT as a tool for assessing disease severity, progress \& for guiding $A B x$ therapy.

Objectives: 1) Compare PCT between COVID-19 pts admitted to ICU and not

2) Describe how concurrent bacterial infection in ICU pts influenced PCT

3) Describe how PCT was associated with duration of ABx therapy.

Methods: Contemporaneous eMR records were reviewed retrospectively for 70 COVID-19 pts admitted to ICU vs 70 who were not. Evidence of concurrent bacterial infection was sought; defined as bacteraemia, positive sputum/tracheal aspirate culture or molecular testing. Duration of ABx therapy was also collected.

Student's t-test was used to compare mean PCT values, whilst diagnostic performance of PCT $>0.5 \mu \mathrm{g} / \mathrm{mL}$ in bacterial co-infection was assessed. Duration of $A B x$ was assessed in pts with $P C T>\&<0.5 \mu \mathrm{g} / \mathrm{mL}$ threshold.

Results: Data was available from completed 70 ICU \& 70 ward admissions 35/70 ward pts had PCT measured. Similarly, 17/70 ICU pts had PCT measured prior to ICU. PCT levels were not clinically \& statistically significantly different (mean; ward $0.69 \mathrm{mg} / \mathrm{mL}, \mathrm{ICU} 0.57 \mathrm{mg} / \mathrm{mL}$, $\mathrm{p}=0.87$ ).

Of the 70 ICU pts, PCT was tested in $6 / 9$ pts with evidence of bacterial co-infection \& in 39/61 pts without bacterial infection. PPV of PCT $>0.5$ to indicate bacterial co-infection was found to be $21 \%$, but on the contrary the NPV of PCT $<0.5$ to exclude was found to be $95 \%$. There was no difference in median no. of ABx-days amongst pts with PCT $>0.5$ \& $<0.5$ (9.5 vs 10 days) but amongst pts with PCT $>$ of $2 \mathrm{mg} / \mathrm{ml}$ there was a trend towards longer therapy ( 9 vs 11 days).

Conclusion: It's not clear from this data that PCT on presentation discriminates between pts with severe \& non-severe COVID-19. Although the usual threshold PCT $<0.5 \mathrm{mg} / \mathrm{mL}$ was able to effectively rule out bacterial co-infection, its PPV was modest. This reflects limited impact that PCT had on duration \& intensity of ABx therapy. In pts with severe respiratory failure clinicians may be reluctant to stop $A B x$, given the non-trivial rate of bacterial co-infection. The combination of PCT \& a rapid, sensitive molecular test may improve antimicrobial stewardship.

\footnotetext{
Reference(s) and grant ackowledgment(s)

1. Kamat Clin Infect Dis 2020;70(3):538-42

2. Lippi Clin Chim Acta 2020;505:190-1

3. Rawson Clin Infect Dis 2020;ciaa530

4. https://www.nice.org.uk/guidance/ng173
}

\section{7}

\section{Continuous non-Invasive temperature monitoring as a means}

\section{of early detection of sepsis}

M. Albarade ${ }^{1}$; T. Alekberli²; P. Vernon Van Heerden ${ }^{2}$

${ }^{1}$ Department of Anesthesiology, Critical Care and Pain Medicine, Sandwell and West Birmingham Hospitals NHS Trust, Birmingham, United Kingdom; ${ }^{2}$ Department of Anesthesiology, Critical Care and Pain Medicine, Hadassah Medical Center, The Hebrew University of Jerusalem, Jerusalem, Israel Correspondence: M. Albarade

Intensive Care Medicine Experimental 2020, 8(2): 000767

Introduction: Sepsis is a host response to infection, which when exaggerated can lead to multiple organ dysfunction and death. Early treatment of sepsis decreases mortality and early diagnosis of sepsis, especially in critically ill patients, is essential.
Objectives: This study aimed to analyze abnormal body temperature patterns in intensive care patients using the PreEmpsis ${ }^{\text {TM }}$ proprietary algorithm developed by Medisim Ltd. and visual inspection to see if they could predict the subsequent diagnosis of sepsis.

Methods: This retrospective study was from January 2016 to December 2016. A total of 50 patients ( 35 male and 15 female) out of 1365 patients admitted to the ICU were included in the study when they developed a temperature above $38.3^{\circ} \mathrm{C}$ at least 72 hours after admission to the ICU. 24 of them were subsequebtly diagnosed as septic and 26 were non-septic patients. Patients were individuals aged 18 years and above (average age 58.34 years). Sepsis was diagnosed according to the Third International Consensus Definitions for Sepsis and Septic Shock (Sepsis-3, 2016) if these findings occurred 72 hours after admission to the ICU.

The temperature was measured non-invasively from the axillary, femoral, or rectal sites with a standard continuous temperature probe connected to our monitoring system. The investigators retrospectively screened all the temperature recordings retrieved from the electronic patient record of the patients admitted to the ICU during the study period. Patients with a temperature recorded at least once of more than $38.3^{\circ} \mathrm{C}$ were then screened for the additional criteria to determine the diagnosis of sepsis or not. The control group comprised those patients who developed temperatures above $38.3^{\circ} \mathrm{C}$ after 72 hours of admission to ICU but were not subsequently diagnosed as septic. Temperature curves for the 72 hours before the clinical suspicion of sepsis (for septic patients) and for the 72 hours before developing a temperature above $38.3^{\circ} \mathrm{C}$ (for non-septic patients) were quantitatively analyzed using visual inspection and the PreEmpsis ${ }^{\mathrm{TM}}$ proprietary, predictive temperature trend analysis algorithm.

Results: The mortality rate was $26 \%$ ( 13 out of 50 ). As expected, the mortality rate for septic patients was higher $-50 \%$ ( 12 out of 24 ) compared to the control group $-3.8 \%$ ( 1 out of 26 ). We did not find any difference between the temperatures of the two groups by visual inspection alone, but the PreEmpsis ${ }^{\mathrm{TM}}$ proprietary algorithm detected a difference by monitoring the maximum temperature changes within 24 hours. The maximum temperature change within 24 hours for septic patients was $1.8 \pm 0.54$, and for non-septic patients was $2.0 \pm 0.39$. In addition the algorhithm also detected differences in the patterns of temperature recording between the two groups - frequency and amplitude changes, as well as change from baseline. The accuracy of the temperature changes detected by thealgorithm as compared to clinical diagnosis of sepsis was 0.71 , with a sensitivity of 0.78 and a specificity of 0.65 .

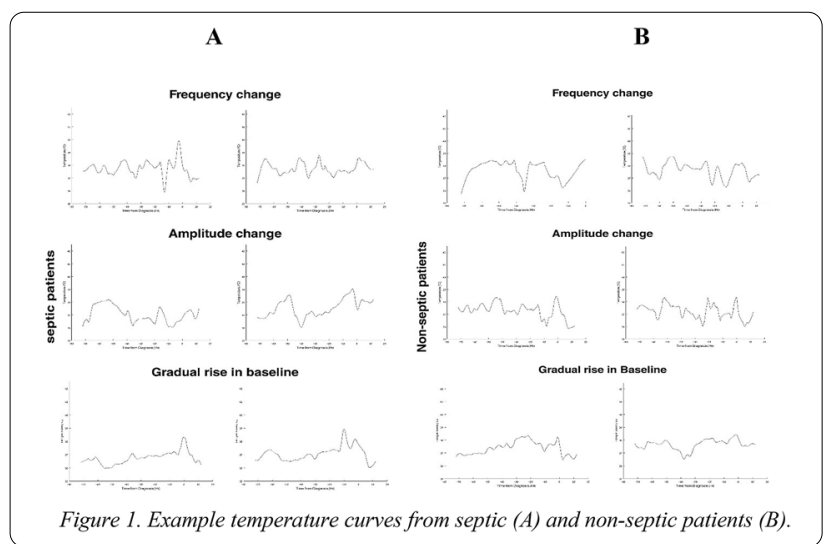

Conclusion: The visual inspection method of temperature changes alone is not accurate, nor specific enough to differentiate between septic and non-septic patients with high temperatures. However, PreEmpsis ${ }^{\mathrm{TM}}$ computerised predictive trends analysis was superior to visual inspection. Nevertheless, we should not use the temperature changes alone as a sole diagnostic method for predicting the onset of sepsis until validated by larger studies. 
Reference(s) and grant ackowledgment(s)

1. (7) Papaioannou VE, Chouvarda IG, Maglaveras NK, Pneumatikos IA: Temperature variability analysis using wavelets and multiscale entropy in patients with systemic inflammatory response syndrome, sepsis, and septic shock. Crit Care 2012, 16:R51

2. (6) Mohr NM, Hotchkiss RS, Micek ST, Durrani S, Fuller BM: Change in temperature profile may precede fever and be an early indicator of sepsis: a case report. Shock 2011, 36:318-321

3. (5) Circiumaru B, Baldock G, Cohen J: A prospective study of fever in the intensive care unit. Intensive Care Med 1999, 25:668-673

4. (4) Peres Bota D, Lopes Ferreira F, Mélot C, Vincent JL: Body temperature alterations in the critically ill. Intensive Care Med 2004, 30:811-816

5. (3) Apaioannou VE, Chouvarda IG, Maglaveras NK, Pneumatikos IA (2012) Temperature variability analysis using wavelets and multiscale entropy in patients with systemic inflammatory response syndrome, sepsis, and septic shock. Crit Care 16

6. (2) Drewry AM, Fuller BM, Bailey TC, Hotchkiss RS. Body temperature patterns as a predictor of hospital-acquired sepsis in afebrile adult intensive care unit patients: a case-control study. Crit Care. 2013;17(5):

7. (1) Levy MM, Fink MP, Marshall JC, Abraham E, Angus D, Cook D, Cohen J, Opal SM, Vincent JL, Ramsay G, International Sepsis Definitions Conference: 2001 SCCM/ESICM/ACCP/ATS/SIS International Sepsis Definitions Conference. Intensive Care Med 2003, 29:530-538

8. We would like to thank the Medisim LTD company (http://www.medisim. co.il)for their assistance

9. The author(s) received no financial support for the research, authorship, and/or publication of this article

\section{0}

Prescribing Practices of Vitamin C in Patients Admitted with Sepsis to an Intensive Care Unit at a District General Hospital R. Phillips ${ }^{1}$; S. Sahota ${ }^{1}$; R. Creamer ${ }^{1}$; M. Spivey

${ }^{1}$ Critical Care Unit, Royal Cornwall Hospital, Treliske, United Kingdom

Correspondence: R. Phillips

Intensive Care Medicine Experimental 2020, 8(2): 000970

Introduction: Vitamin C's role in the management of sepsis was first popularised in 2017 with the work of Marik et al1. Vitamin C is considered a safe therapy in other contexts such as management of malnutrition, itself shown to adversely affect outcome2. As such, we audited our current use of Vitamin C in sepsis.

Objectives: To investigate prescribing practice of Pabrinex (Vitamin B and $C$ ) in sepsis admissions to a district general hospital intensive care unit, and investigate its impact on mortality or requirement for organ support.

Methods: A retrospective analysis of prescribing practice was conducted studying all admissions to critical care who met the physiological parameters for Sepsis 3 during a 1 year time period. Outcomes including mortality and requirement for invasive organ support were monitored. The total dose of vitamin $C$ prescribed during admission was calculated.

Results: A total of 253 patients met sepsis criteria between April 2018April 2019. Pabrinex was prescribed in 91 cases (36\% of admissions). There was no uniformity in prescribing of Vitamin C. Regimes ranged from 1 pair a day to 3 pairs, three times a day. There was no correlation between severity of illness and dose of Pabrinex prescribed, nor association between higher doses of Vitamin $C$ and outcomes measured. Total dose received during admission ranged from $0 \mathrm{mg}$ to $32 \mathrm{~g}$.

The mean age of those receiving Pabrinex was 61, with ICNARC mortality predicted at $30.8 \%$. Actual mortality was 23\% (SMR 0.77 ). $23 \%$ required vasopressors, $58 \%$ required invasive ventilation and $28 \%$ required renal replacement therapy.

The non-Pabrinex group ( $64 \%$ of total admissions) had a mean age of 55 , with ICNARC mortality $23 \%$. Actual mortality was $12 \%$ (SMR 0.5 ). $59 \%$ required vasopressors, $34 \%$ required invasive ventilation and $8 \%$ required renal replacement therapy.

Conclusion: The survival rates in both groups were better than predicted using ICNARC Mortality calculators, indicating our centre performs above expectations in the management of sepsis admissions.
Due to the heterogeneity of the study groups we were unable to accurately ascertain the impact of vitamin $C$ in modifying patient outcomes. Vitamin $\mathrm{C}$ was often prescribed to older, more unwell patients, possibly reflecting the severity of their illness. Concurrent use of Pabrinex in the management of malnutrition also acts as confounding factor. Due to the disparities in prescribing practice we have initiated a standard prescription bundle for all admissions with sepsis.

\section{Reference(s) and grant ackowledgment(s)}

1. 2. Lew C, Wong G, Cheung K et al. 2017. Association between Malnutrition and 28-Day Mortality and Intensive Care Length-of-Stay in the Critically ill: A Prospective Cohort Study. Nutrients. 10;1: 10

2. 1. Marik P, Khangoora V, Rivera R et al. 2017. Hydrocortisone, Vitamin C, and Thiamine for the Treatment of Severe Sepsis and Septic Shock. Chest; $151 ; 6: 1229-123$

\section{9}

$\triangle$ PCO2 ALTERATIONS DURING THE COURSE OF SEPSIS -

\section{Pathophysiological Conclusion:}

E. Kaniaris ${ }^{1}$; I. Vasileiadis ${ }^{1}$; M. Kompoti ${ }^{1}$; N. Rovina ${ }^{2}$; ES. Tripodaki ${ }^{3}$;

D. Toumpanakis 2 ; I. Ioannidou²; D. Theodoulou ${ }^{2}$; E. Potamianou ${ }^{2}$;

I. Voulgareli²; A. Kyriakoudi²; E. Kostakou²; E. Filiou'²; M. Kyriakopoulou²;

V. Derveni'; A. Koutsoukou ${ }^{2}$; DD. Papadakis ${ }^{2}$

${ }^{1}$ Intensive Care Unit, Thriasio General Hospital of Elefsina, Athens, Greece; ${ }^{2}$ Intensive Care Unit, 1st Department of Respiratory Medicine, Sotiria Thoracic Diseases Hospital of Athens, Athina, Greece; ${ }^{3} 1$ st Depatment of Internal Medicine, "Agios Savvas" - Anticancer Hospital, Athina, Greece

Correspondence: E. Kaniaris

Intensive Care Medicine Experimental 2020, 8(2): 001059

Introduction: Patients in acute circulatory failure are characterized by inequality between tissue oxygen demand and supply. The difference of carbon dioxide tension between central venous and arterial blood $(\triangle \mathrm{PCO} 2)$ reflects the veno-arterial $\mathrm{CO} 2$ concentration difference and is inversely related to cardiac output, tissue oxygen delivery and perfusion [1]. High $\triangle \mathrm{PCO} 2$ in sepsis and septic shock is associated with poor outcome [2]. However, there has not been any study yet to evaluate $\triangle \mathrm{PCO} 2$ during the course of a septic episode.

Objectives: To follow and evaluate the difference of PCO2 between central venous and arterial blood sample ( $\triangle \mathrm{PCO} 2)$, during the progress of a septic episode

Methods: Seventy nine (79) patients were included and were separated into two groups. Group A included 52 patients who had sepsis remission while group $B$ included 27 patients who had sepsis deterioration. $\triangle P C O 2$ was calculated in septic patients on admission and at either deterioration or remission of the septic episode.

Results: $\triangle \mathrm{PCO} 2$ was higher in the sepsis deterioration group on admission $(6.47 \pm 3.03$ vs. $7.71 \pm 3.07 \mathrm{mmHg}$, for group $A$ and group $B$ respectively, $p=0.08$ ), although the difference did not reach a statistically significant level. In group A $\triangle \mathrm{PCO} 2$ increased at sepsis remission (6.47 \pm 3.03 vs. $7.41 \pm 3.24 \mathrm{mmHg}, \mathrm{p}=0.05)$. In contrast, in group B $\triangle \mathrm{PCO} 2$ decreased at sepsis deterioration (7.71 \pm 3.07 vs. $6.30 \pm 3.23$ $\mathrm{mmHg}, \mathrm{p}=0.04)$. Group $B$ patients were also characterized by increased use of vasopressors $(0.532 \pm 0.315$ vs. $0.258 \pm 0.166 \mu \mathrm{g} / \mathrm{kg} /$ min, $\mathrm{p}<0.001$ ), increased lactate concentration (5.2 \pm 3.8 vs. $2.4 \pm 1.3$ $\mathrm{mmol} / \mathrm{l}, \mathrm{p}=0.001)$ and higher SOFA score (13.4 \pm 3.0 vs. $10.4 \pm 3.0, \mathrm{p}<$ 0.001 ) at deterioration compared to admission, as expected.

Conclusion: The higher $\triangle \mathrm{PCO} 2$ on admission, in the group of patients who deteriorated compared to those who improved, might indicate tissue hypoperfusion occurring early in sepsis. However, at sepsis remission/deterioration, the results of our study probably reflect the different basal metabolic rate at the different stages of sepsis. Thus, at deterioration basal metabolic rate/mitochondrial metabolism decreases, leading to decreased $\mathrm{CO} 2$ production and low $\triangle \mathrm{PCO} 2$. On the contrary, during sepsis remission, mitochondrial metabolism recovers and, consequently, $\triangle \mathrm{PCO} 2$ increases[3]. 
Reference(s) and grant ackowledgment(s)

1. 3. Kreymann, G.; Grosser, S.; Buggisch, P.; Gottschall, C.; Matthaei, S.; Greten, $\mathrm{H}$. Oxygen consumption and resting metabolic rate in sepsis, sepsis syndrome, and septic shock. Crit. Care Med. 1993, 21, 1012-1019

2. 2. Kao, C.C.; Guntupalli, K.K.; Bandi, V.; Jahoor, F. Whole-body $\mathrm{CO} 2$ production as an index of the metabolic response to sepsis. Shock. 2009, 32, 23-28.

3. 1. Bakker, J.; Vincent, J.L.; Gris, P.; Leon, M.; Coffernils, M.; Kahn, R.J. Venoarterial carbon dioxide gradient in human septic shock. Chest. 1992, 101, 509-515.

4. No Ackonowledgmet

\section{1}

SEPSIS SAVER 2.0. New preliminary results after improvements in this automatic electronic alarm tool for early detection of sepsis based on Classic Sepsis Definitions (Sepsis 1 and 2) and Sepsis 3 Definition

A.O. Badallo Arévalo; MD. Valle1; R. Vara Arlanzón ${ }^{1}$; S. Ossa1; R. Zaragoza². J. Santos Gonzalez ${ }^{3}$; D. Iglesias Posadilla'; S. Sancho²; JA. Fernández Ratero ; L. Sanchez Santos ${ }^{4}$; V. Ramirez ${ }^{2}$; M. Parejo ${ }^{2}$;M. Borges ${ }^{5}$

${ }^{1}$ Intensive care unit, University Hospital of Burgos, Burgos, Spain; ${ }^{2}$ Intensive care unit, Hospital Universitario Doctor Peset, Valencia, Spain; ${ }^{3}$ General surgery, University Hospital of Burgos, Burgos, Spain; ${ }^{4}$ Emergency department, University Hospital of Burgos, Burgos, Spain; ${ }^{5}$ Intensive care unit, Multidisciplinary Sepsis Unit, Palma, Spain

Correspondence: A.O. Badallo

Intensive Care Medicine Experimental 2020, 8(2): 001091

Introduction: It is known that automatic alert systems are helpful in early detection of sepsis, considering how important early diagnosis as a prognostic factor. So far, automated monitoring electronic programs have been used with this objective, but not based on Sepsis-3.

Objectives: The main aim of this study is to compare the 3 different algorithms that could define sepsis including the classic sepsis definitions (Defs.) and Sepsis-3.

As secondary goals, it is described the automatic alert system itself, representing its sensibility $(S)$, specificity $(E)$, negative predictive value (NPV) and positive predictive value (PPV).

Besides, some of the clinical information that is provided by the program, such as focus and source of infection and mortality rate are analyzed.

Methods: SEPSIS SAVER is a new electronic software counselled by expert clinicians of 3 different Intensive Care Units of University Hospitals in Spain.

After improvements in the software (integration of basal SOFA scale estimation for each patient before testing any of the algorithm) and a friendly \& useful designed tool in pursuit of clinicians, during 2 months (February and March 2020) has been implemented as a pilot testing in our 1063-bed University Hospital of Burgos (UHB). The prospective evaluation was compared to the the Standard Sepsis Codification System (SSCS) of our UHB.

Any patient admitted in the hospital, through Emergency Room or during whole stay in ward, is monitored and after evaluating the clinical and lab tests results, the tool observes if patient matches any of the algorithms:

1. Systemic Inflammatory Response Sindrome $[S I R S]^{3} 2+1$ Organ Failure [OF].

2. Sequential Organ Failure scale $[S O F A]^{3} 2$

3. quickSOFA [qSOFA] ${ }^{3}$ 2. The validated clinical score in Sepsis-3 Definition. There can be matches to any of them or to more than one. Instantly, the tool will inform to the clinician(by email, cell phone or checking the tool)=patient at increased risk of sepsis.

Results: During February-March 2020, SEPSIS SAVER 2.0 monitored $13.584(100 \%)$ patients. Among them, $844(6.21 \%)$ were warned at risk of sepsis and 191 (prevalence[P] 1.40\%) were finally confirmed as sepsis by members of Sepsis Team. It means a rate of 703 sepsis episodes/100.000 inhabitants per month. Instead, the Standard Sepsis Codification System (SSCS) of our UHB, confirmed only 81 sepsis cases (P $0.60 \%$ ) and a rate of 22,8 ep./100.000 inh./month the preliminary software used in 531-bed Doctor Peset University Hospital (SEPSIS SAVER 1.01).

Only $9.42 \%$ of patients of the confirmed cases were admitted to ICU due to Septic Shock. Global mortality attributable to sepsis was $11 \%$.

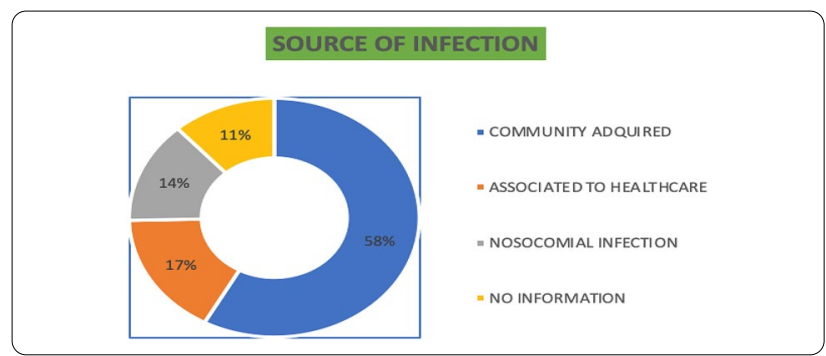

SOFA was the algorithm that more sepsis cases detected with $67,53 \%$ of cases. SIRS +1 OF $31,46 \%$ and qSOFA only 16 cases (assumption $8,40 \%$ ) but these episodes were also detected at the same time by the other 2 algorithms.
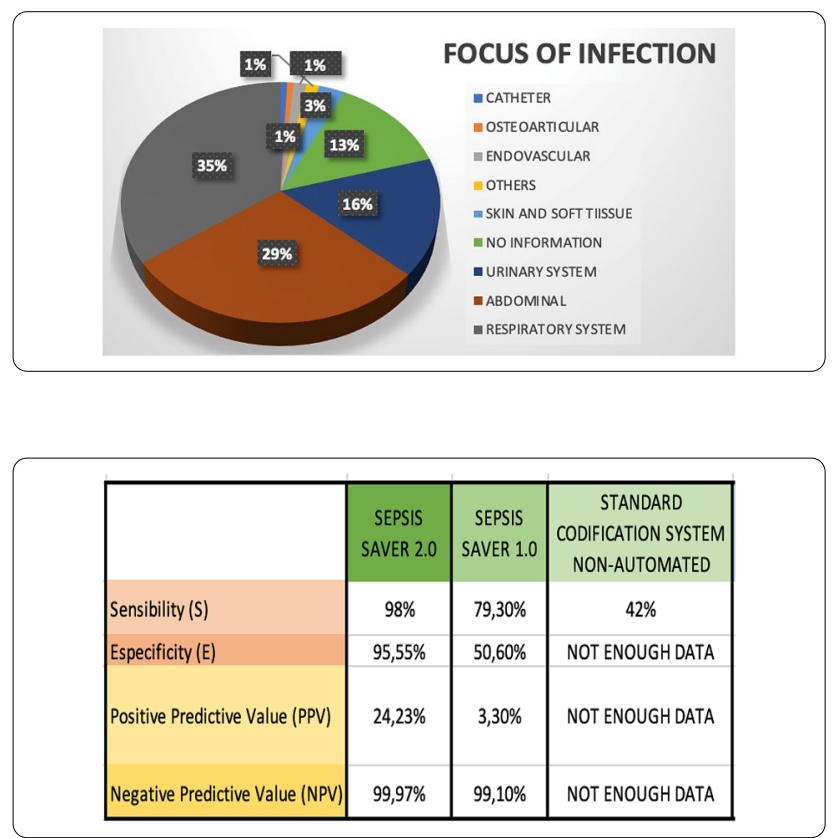

Conclusion: SOFA is the most sensitive tool, although $\mathrm{S}$ improves implementing SIRS+OF at the same time, therefore, Sepsis-2 Defs. Instead, Sepsis-3 Defs where qSOFA is used, is not valuable as sepsis detector, launching debate about validity of the "New Sepsis Defs 2016".

The SSCS of our UHB vs the use of automatic alarm tool and the enhancements of Sepsis Saver 2.0, verifies the importance \& usefulness of automatic alarm guided electronic tools. The upgrade of $E, S$, NPV and PPV, encourage us to continue in the design of the "future perfect automatic tool" in sepsis detection.

\section{Reference(s) and grant ackowledgment(s)}

1. 1. R. Zaragoza, S. Sancho, V. Ramirez, O. Badallo Arévalo, et al. SEPSIS SAVER. Preliminary results of a pilot implementation of a new automatic electronic alert for early detection of sepsis based on Sepsis 3 definition. Intensive Care Medicine Experimental 2019, 7(Suppl 3):000703

2. 2. A. Rhodes 1, L. E Evans, et al. Surviving Sepsis Campaign: International Guidelines for Management of Sepsis and Septic Shock: 2016. Intensive 
Care Med. 2017 Mar;43(3):304-377. doi: 10.1007/s00134-017-4683-6. Epub 2017 Jan 18

3. 3. Warttig S, Alderson P, Evans DJ, Lewis SR, Kourbeti IS, Smith AF. Automated monitoring compared to standard care for the early detection of sepsis in critically ill patients. Cochrane Database Syst Rev. 2018 Jun 25;6:CD012404

4. 4.JM.Ferreras, D.Judez, et al. Implementation of an Automatic Alarms System for Early Detection of Patients With Severe Sepsis. Enferm Infecc Microbiol Clin. 2015 Oct;33(8):508-15.doi: 10.1016/j.eimc.2015.01.002.Epub 2015 Feb 18.

5. 5. V Herasevich 1, B Afessa, C G Chute, O Gajic. Designing and Testing Computer Based Screening Engine for Severe sepsis/septic Shock. AMIA Annu Symp Proc. 2008 Nov 6;966.

6. 6. M. H Hooper1, L.Weavind, et al. Randomized Trial of Automated, Electronic Monitoring to Facilitate Early Detection of Sepsis in the Intensive Care unit*.Crit Care Med. 2012 Jul;40(7):2096-101.doi: 10.1097/ cCM.0b013e318250a887.

7. 7. C. A Umscheid 1, J. Betesh, et al. Development, Implementation, and Impact of an Automated Early Warning and Response System for Sepsis. J Hosp Med. 2015 Jan;10(1):26-31.doi: 10.1002/jhm.2259.Epub 2014 Sep 26.

8. 8. J. L Guidi 1, K. Clark, et al. Clinician Perception of the Effectiveness of an Automated Early Warning and Response System for Sepsis in an Academic Medical Center. Ann Am Thorac Soc. 2015 Oct;12(10):1514-9. doi:10.1513/ AnnalsATS.201503-1290C

9. 9. M. Faisal 1, D. Richardson, et al, Computer-aided National Early Warning Score to Predict the Risk of Sepsis Following Emergency Medical Admission to Hospital: A Model Development and External Validation Study. CMAJ. 2019 Apr 8;191(14):E382-E389.doi: 10.1503/cmaj.181418.

\section{4}

Causes of nutrition deficit during immediate postoperative period after free flap surgery for cancer of the head and neck

J. Nurkkala'; S. Lahtinen²; M. Vakkala²; T. Kaakinen²; J. Liisanantti ${ }^{2}$

${ }^{1}$ University of Oulu, Oulu, Finland; ${ }^{2}$ Department of anesthesiology, University of Oulu, Oulu, Finland; ${ }^{3}$ Study group of surgery, anesthesiology and intensive care, Oulu University, Medical Research Center, Oulu, Finland, Finland

\section{Correspondence: J. Nurkkala}

Intensive Care Medicine Experimental 2020, 8(2): 000154

Introduction: Underfeeding and malnutrition are known to be associated with impaired short and long-time recovery after major surgery inpatients admitted to ICU [1-3]. Although head and neck cancer (HNC) free flap surgery may have a substantial impact on normal eating, chewing and swallowing, there is a lack of studies contemplating the adequacy of nutrition during the immediate postoperative period after HNC surgery [4-5].

Objectives: The aim of the present study was to evaluate the adequacy of nutrition after free flap surgery for HNC during the immediate in-hospital recovery and to discover factors associated with inadequate nutrition delivery.

Methods: This single-center retrospective cohort study included 218 HNC patients who underwent free flap surgery in Oulu University Hospital, Finland between the years 2008 and 2018. Nutrition delivery methods, the adequacy of nutrition and complication rates were evaluated during the first 10 postoperative days.

Results: A total of $131(60.1 \%)$ patients reached nutritional adequacy of $60 \%$ of calculated individual demand during the follow-up period. According to multivariate analysis, nutrition inadequacy was associated with higher ideal body weight (OR 1.11 [1.04-1.20]) whereas adequate nutrition was associated with higher number of days with oral food intake (OR 0.79 [0.67-0.93]). In the univariate model tracheostomy, bilateral neck dissection and postoperative surgical complications were associated to poor postoperative nutrition adequacy.

Conclusion: Inadequate nutrition is common after HNC free flap surgery. The present results suggest that more adequate nutrition delivery might be obtained by the early initiation of oral food intake and close monitoring of nutrition support.

\section{Reference(s) and grant ackowledgment(s)}

1. [5] McAuley D, Barry T, McConnell K, Smith J, Stenhouse J. Early feeding after free flap reconstruction for oral cancer. Br.J.Oral Maxillofac.Surg. 2015 Sep;53(7):618-20.

2. [4] Parhar HS, Durham JS, Anderson DW, Rush B, Prisman E. The association between the Nutrition-Related index and morbidity following head and neck microsurgery. Laryngoscope 2019 Mar 6.

3. [3] Talwar B, Donnelly R, Skelly R, Donaldson M. Nutritional management in head and neck cancer: United Kingdom National Multidisciplinary Guidelines. J.Laryngol.Otol. 2016 May;130(S2):S32-40.

4. [2] Singer P, Blaser AR, Berger MM, Alhazzani W, Calder PC, Casaer MP, et al. ESPEN guideline on clinical nutrition in the intensive care unit. Clin.Nutr. 2019 Feb;38(1):48-79.

5. [1] McClave SA, Taylor BE, Martindale RG, Warren MM, Johnson DR, Braunschweig $C$, et al. Guidelines for the Provision and Assessment of Nutrition Support Therapy in the Adult Critically III Patient: Society of Critical Care Medicine (SCCM) and American Society for Parenteral and Enteral Nutrition (A.S.P.E.N.). JPEN J.Parenter.Enteral Nutr. 2016 Feb;40(2):159-211.

6. Application for travel and participating costs for ESICM LIVES 2020 congress has been send to the Finnish medical association (FMA).

\section{2}

Alternative Substrates in the Critically III Subject (ASICS): Protocol for a Feasibility Study

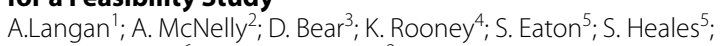

H. Montgomery ${ }^{6}$; ZA. Puthucheary ${ }^{2}$

${ }^{1}$ Dietetics, Adult critical care unit, The Royal London Hospital, London, United Kingdom; ${ }^{2}$ Adult critical care unit, The Royal London Hospital, London, United Kingdom; ${ }^{3}$ Critical care, St Thomas' Hospital, London, United Kingdom; ${ }^{4}$ Critical care, Bristol Royal Infirmary, Bristol, United Kingdom; ${ }^{5}$ Developmental biology \& cancer, UCL Great Ormond Street Institute of Child Health, London, United Kingdom; ${ }^{6}$ Centre for human health \& performance, University College London, London, United Kingdom

Correspondence: A. McNelly

Intensive Care Medicine Experimental 2020, 8(2): 000192

Introduction: Aggressive muscle wasting occurs early in critical illness. It is associated with greater duration of mechanical ventilation, and intensive care unit (ICU) and/or hospital stays, and frequent longlasting functional impairment. No known interventions prevent this wasting.

Ketone bodies, produced by the liver from fatty acids in response to starvation, can act as metabolic fuels for muscle or other peripheral tissues, and as signalling molecules.

Increasing ketone body production by altering nutrition composition - providing it is feasible, safe and tolerated by ICU patients - may mitigate early muscle loss in critical illness by acting as a more efficient energy source, improving related outcomes.

Objectives: To perform a randomised controlled feasibility study to determine whether:

i) ICU patients during early critical illness can be recruited to and retained in a study of ketogenic enteral feed administration, and whether

ii) this feed significantly raises plasma and urine ketone body levels while being safe and well-tolerated.

Methods: A two centre feasibility study conducted over 30 months. Patients will be recruited (with consultee agreement) within 48 hours of ICU admission and randomised to either ketogenic or standard enteral feed over a 10-day period. Data will be collected during the study period, and 3, 6 and 12 months post ICU-discharge.

Inclusion criteria: (i) $>18$ years old (ii) prescribed enteral nutrition (iii) mechanically ventilated $\geq 48$ hours (iv) likely to remain on ICU $\geq 5$ days and (v) likely to survive $\geq 10$ days (vi) multi-organ failure (SOFA score $>2)$.

Exclusion criteria: (i) existing primary neuromyopathy/significant neurological impairment (ii) uni- or bilateral lower limb amputation (iii) requiring sole/supplemental parenteral nutrition (iv) need for specialist nutrition (v) patients with known inborn errors of metabolism. 
Intervention: Modular ketogenic enteral feed will be reconstituted on the ICU for each patient for 10 days, as prescribed by dietitians to meet nutritional energy targets: medium chain triglycerides (Betaquik ${ }^{\circledR}$, Vitaflo) increasing from $40 \%$ to $80 \%$ during the study period, plus long chain triglycerides (Fresubin $5 \mathrm{kcal}^{\circledR}$, Fresenius Kabi) to keep total fat at $80 \%$ of energy input; $15 \%$ protein (Renapro ${ }^{\circledR}$, Stanningley Pharma), provided minimum protein requirements are met; and 5\% carbohydrate (Maxijul ${ }^{\circledR}$, Nutricia). The contribution of energy from other sources. e.g. propofol and dextrose (including additional dextrose used as drug diluents) will be taken into account.

Sample size: 24 ICU patients receiving randomised feed for 10 days or up to ICU discharge: allowing for a $34 \%$ dropout rate, up to 37 eligible patients will be required. Site-specific data and past trial experience suggest that recruitment will take 15 months.

We will assess feasibility of patient follow-up at 3, 6 and 12 months post-ICU discharge, and the views of ICU nurses on study safety, practicality and acceptability. Levels of ketone bodies and other metabolites will be determined, as well as cost-effectiveness comparisons between the two feed regimens.

Results: Nine patients have been recruited so far with no feed-related adverse effects reported.

Conclusion: If using ketogenic feed in ICU patients is shown to be safe and feasible, it may mitigate muscle loss in early critical illness, providing a new way to improve function and well-being in ICU survivors. ClinicalTrials.gov NCT04101071.

\section{Reference(s) and grant ackowledgment(s)}

1. This abstract presents independent research funded by the National Institute for Health Research (NIHR) under its Research for Patient Benefit (RfPB) Programme (Grant Reference Number PB-PG-0317-20006). The views expressed are those of the author(s) and not necessarily those of the NIHR or the Department of Health and Social Care.

\section{1}

Prevalence of Hypophosphatemia in the ICU - preliminary results of an international one-day point prevalence survey

M. Berger ${ }^{1}$; O. Appelberg ${ }^{2}$; A. Reintam Blaser ${ }^{3}$; C. Ichai ${ }^{4}$ O. Joannes-Boyau ${ }^{5}$; M. Casaer ${ }^{6}$; S. Schaller ${ }^{7}$; J. Starkopf ${ }^{8}$

${ }^{1}$ Service of Adult Intensive Care \& Burns, Lausanne University Hospital -CHUV, Lausanne, Switzerland; ${ }^{2} \mathrm{Cu}$, University of Tartu, Tartu, Estonia; ${ }^{3}$ Department of Anaesthesiology and Intensive Care, University of Tartu, Tartu, Estonia; ${ }^{4}$ Réanimation polyvalente, Hospital Pasteur, Nice, France; ${ }^{5}$ SAR SUD, Hopital Magellan, CHU Bordeaux, Pessac, France; ${ }^{6}$ Laboratory of Intensive Care Medicine, Academic Department of Cellular and Molecular Medicine, KU Leuven, Leuven, Belgium; ${ }^{7}$ Department of Anesthesiology and Surgical intensive Care, Charité - Universitätsmedizin Berlin, Berlin, Germany; ${ }^{8}$ Department of Anaesthesiology and Intensive Care, Tartu University Hospital, Tartu, Estonia

Correspondence: $M$. Berger

Intensive Care Medicine Experimental 2020, 8(2): 000251

Introduction: Hypophosphatemia (HypoP) is frequent in intensive care unit (ICU) settings and may affect up to $35 \%$ of admissions. Severe hypophosphatemia is associated with organ dysfunction and mortality. Its true prevalence and its proportional relationship to refeeding syndrome remain largely unknown.

Objectives: The aim was to learn the point-prevalence of HypoP defined as blood phosphate $(\mathrm{P})<0.8 \mathrm{mmol} / \mathrm{l}$ on one particular day (D-Day) at international level while gathering information about the internal ICU policies/guidelines for the management of HypoP among international ICUs.

Methods: This point-prevalence survey was conducted with Ethical approval by the Section of Metabolism, Endocrinology and Nutrition of ESICM and endorsed by ESICM, : each site had one D-Day during the week 11/2020: the primary endpoint was the prevalence of blood $\mathrm{P}<$ $0.8 \mathrm{mmol} / \mathrm{l}$. Data were collected on ICU (number of beds, frequency of $P$ analysis, treatment strategies) and patients (collected for hypoP patients only): age, days in ICU, nutritional therapy, renal replacement therapy (CRRT)). Data are presented as number (percentage) for categorical and median [IQR1;3] for continuous variables.

Results: 59 ICUs from 23 countries participated and 4 ICUs were paediatric: 40/59 ICUs were mixed medico-surgical, (see Table); 20 sites did measure P daily, 7 ICUs stated "never". Out of 881 patients present on D-Day, 675 (76.6\%) patients had P determined, and 99 (15\%) presented hypoP, with 48 (48.5\%) patients with severe hypoP $<0.65 \mathrm{mmol} /$; $50 \%$ of the hypoP were documented within the first 5 days in the ICU. Age was $62[52 ; 71]$ years. HypoP was most frequent in medical patients. HypoP was not associated with feeding (enteral in $52 \%$, parenteral in $19 \%)$, but with being on CRRT $(p=0.045)$

\begin{tabular}{|c|c|c|c|c|}
\hline \multirow[b]{2}{*}{ ICU information } & \multirow[b]{2}{*}{ Total } & Small ICL & Middle ICU & Large ICU \\
\hline & & $\begin{array}{r}(<12 \\
\text { beds })\end{array}$ & (13-40 beds) & ( $>40$ beds $)$ \\
\hline ICU profile & 59 & 20 & 37 & 2 \\
\hline Mixed & 40 & 10 & 28 & 2 \\
\hline Medical & 8 & 6 & 2 & 0 \\
\hline Cardio-Surgical & 11 & 4 & 7 & 0 \\
\hline $\begin{array}{l}\text { Total patients in the ICU on } \\
\text { D-Day }\end{array}$ & 881 & 156 & 647 & 78 \\
\hline Total patients with $\mathrm{P}$ analysi & $675(77 \%)$ & $102(65 \%)$ & $500(77 \%)$ & $73(94 \%)$ \\
\hline Patients with $\mathrm{P}<0.8 \mathrm{mmol} / \mathrm{l}$ & $99(15 \%)$ & $12(12 \%)$ & $75(15 \%)$ & $12(16 \%)$ \\
\hline $\mathrm{P}<0.65 \mathrm{mmol} / 1$ & 48 & 31 & 40 & 1 \\
\hline $\begin{array}{l}\text { Phosphate measure } \\
\text { frequency: }\end{array}$ & & & & \\
\hline At least once daily & $21(35.5 \%)$ & & 15 & 1 \\
\hline $3 \times$ per week & $21(35.5 \%)$ & & 13 & 1 \\
\hline never & $7(12 \%)$ & 4 & 3 & 0 \\
\hline
\end{tabular}

Conclusion: The point-prevalence survey shows that HypoP is present at least in $15 \%$ of ICU patients, and in half of them is observed during the first 5 ICU days. Half of the patients had a severe hypoP. This number is an underestimation since systematic measurements occur only in $3 / 4$ of patients, while $12 \%$ of ICUs never monitor this electrolyte. Moreover the study was done in ICUs with metabolic interest.

\section{1}

Effect of High-Protein Nutrition in Critically III Patients:

A Retrospective Cohort Study

G. Suzuki ${ }^{1}$; R. Ichibayashi ${ }^{1}$; M. Honda

${ }^{1}$ Critical care center, Toho University Medical Center Omori Hospital, Ota City, Japan

Correspondence: G. Suzuki

Intensive Care Medicine Experimental 2020, 8(2): 000261

Introduction: Early provision of a high-protein nutrition improves the prognosis of patients in intensive care units (ICUs). However, high protein intake increases blood urea nitrogen (BUN). No study has compared outcomes according to protein intake, and the clinical significance of changes in BUN ( $\triangle B U N)$ in ICU patients is unclear. Here, we investigated the association of high protein intake with outcomes and BUN and assessed the clinical significance of $\triangle B U N$.

Objectives: This study aimed to investigate the effect of high-protein provision on the outcomes and BUN, and to investigate the clinical significance of $\triangle B U N$.

Methods: This was a single-center retrospective cohort study. Between 1 January 2016 and 30 September 2019, 295 ICU patients received enteral nutrition for at least 3 days while undergoing mechanical ventilation. After applying the exclusion criteria of an age of $<18$ years, gastrointestinal disease, maintenance dialysis, renal replacement therapy after admission, kidney transplantation, and death within 7 days of commencing enteral nutrition, 206 patients remained.

Participants were divided into those receiving $>1.2 \mathrm{~g} / \mathrm{kg} /$ day of protein (highprotein group; $\mathrm{n}=111$ ) and those receiving $\leq 1.2 \mathrm{~g} / \mathrm{kg} /$ day of protein (non-high-protein group; $n=95$ ). The groups were 
balanced by propensity score matching. The primary endpoint was 28-day mortality, and the secondary endpoints were 90-day mortality, length of ICU stay, number of ventilator-free days in the first 28 days, and $\triangle B U N$.

Results: The high-protein group had significantly lower 28- and 90-day mortality and significantly greater $\triangle B U N$, including after propensity score matching. $\triangle B U N$ might not be associated with outcomes.

Conclusion: Provision of $>1.2 \mathrm{~g} / \mathrm{kg} /$ day of protein may be associated with lower mortality of tube-fed and mechanically ventilated patients. Furthermore, while high protein intake may be associated with higher BUN, these changes may not be adversely associated with outcomes.

\section{Reference(s) and grant ackowledgment(s)}

1. 1. Tian F, Wang X, Gao X, et al: Effect of initial calorie intake via enteral nutrition in critical illness: a meta-analysis of randomised controlled trials. Crit Care 2015; 19:180. 2. Elke G, Wang M, Weiler N, et al: Close to recommended caloric and protein intake by enteral nutrition is associated with better clinical outcome of critically ill septic patients: secondary analysis of large international nutrition database. Crit Care 2014; 18:R29. 3. McClave SA, Saad MA, Esterle M, et al: Volume-based feeding in the critically ill patient. J Parenter Enteral Nutr 2015; 39:707-712. 4. McCall M, Cahill N, Murch L, et al: Lessons learned from implementing a novel feeding protocol: results of a multicenter evaluation of educational strategies. Nutr Clin Pract 2014; 29:510-517. 5. Heyland DK, Cahill NE, Dhaliwal R, et al: Enhanced proteinenergy provision via the enteral route in critically ill patients: a single center feasibility trial of the PEP uP protocol. Crit Care 2010; 14:R78. 6. Long CL: Energy balance and carbohydrate metabolism in infection and sepsis. Am J Clin Nutr 1977; 30:1301-1310. 7. Jensen GL, Mirtallo J, Compher C, et al: Adult starvation and disease-related malnutrition: a proposal for etiologybased diagnosis in the clinical practice setting from the International Consensus Guideline Committee. Clin Nutr 2010; 29:151-153. 8. Allingstrup MJ, Esmalizadeh N, Wilkens Knudsen A, et al: Provision of protein and energy in relation to measured requirements in intensive care patients. Clin Nutr 2012; 31:462-468. 9. McClave SA, Taylor BE, Martindale RG, et al: Guidelines for the provision and assessment of nutrition support therapy in the adult critically ill patient: Society of Critical Care Medicine (SCCM) and American Society for Parenteral and Enteral Nutrition (ASPEN). JPEN J Parenter Enteral Nutr 2016; 40:159-211. 10. Singer P, Blaser AR, Berger MM, et al: ESPEN guideline on clinical nutrition in the intensive care unit. Clin Nutr 2019; 38:48-79. 11. Wolfe RR, Goodenough RD, Burke JF, et al: Response of protein and urea kinetics in burn patients to different levels of protein intake. Ann Surg 1983; 197:163-171. 12. Shaw JH, Wildbore M, Wolfe RR: Wole body protein kinetics in severely septic patients. The response to glucose infusion and total parenteral nutrition. Ann Surg 1987; 205:288-294. 13. Larsson J, Lennmarken C, Martensson J, et al: Nitrogen requirements in severely injured patients. Br J Surg 1990; 77:413-416. 14. Ishibashi N, Plank LD, Sando K, et al: Optimal protein requirements during the first 2 weeks after the onset of critical illness. Crit Care Med 1998; 26:1529-1535. 15. Strack van Schijndel RJ, Weijs PJ, Koopmans RH, et al: Optimal nutrition during the period of mechanical ventilation decreases mortality in critically ill, long-term acute female patients: a prospective observational cohort study. Crit Care 2009; 13:R132. 16. Sevette A, Smith RC, Aslani A, et al: Does growth hormone allow moreefficient nitrogen sparing in postoperative patients requiring parenteral nutrition? A double-blind, placebo-controlled trial. Clin Nutr 2005; 24:943-955. 17. Tamura T, Yatabe T, Yokoyama M, et al: Current status of studies about optimal composition of amino acid and protein for ICU patients: systematic review. Anaesth Intensive Care 2014; 42:806-807. 18. Weijs PJ, Stapel SN, de Groot SD, et al: Optimal protein and energy nutrition decreases mortality in mechanically ventilated, critically ill patients: a prospective observational cohort study. JPEN J Parenter Enteral Nutr 2012; 36:60-68. 19. Dickerson RN: Estimating energy and protein requirements of thermally injured patients: art or science? Nutrition 2002; 18:439-442. 20. Frankenfield D: Energy expenditure and protein requirements after traumatic injury. Nutr Clin Pract 2006; 21:430-437. 21. Hoffer $\mathrm{L}$, Bistrian BR: What is the best nutritional support for critically ill patients? Hepatobiliary Surg Nutr 2014; 18:701. 22. Frank H, Graf J, Amann-Gassner U, et al: Effect of short-term high-protein compared with normal-protein diets on renal hemodynamics and associated variables in healthy young men. Am J Clin Nutr 2009; 90:1509-1516. 23. Arihan O, Wernly B, Lichtenauer M, et al: Blood urea nitrogen (BUN) is independently associated with mortality in critically ill patients admitted to ICU. PLoS One 2018; 13:e0191697. 24.
Larson J, Ljljedahl SO, Schildt B, et al: Metabolic studies in multiple injured patients. Clinical features, routine chemical analysis and nitrogen balance. Acta Chir Scand 1981; 147:317-324. 25. Schoenfeld DA, Bernard GR: Statistical evaluation of ventilator-free days as an efficacy measure in clinical trials of treatments for acute respiratory distress syndrome. Crit Care Med 2002; 30:1772-1777. 26. Austin PC: An introduction to propensity score methods for reducing the effects of confounding in observational studies. Multivariate Behav Res 2011; 46:399-424. 27. Stuart EA: Matching methods for causal inference: A review and a look forward. Stat Sci 2010; 25:1-21. 28. Arabi YM, Tamim HM, Dhar GS, et al: Permissive underfeeding and intensive insulin therapy in critically ill patients: a randomized controlled trial. Am J Clin Nutr 2011; 93:569-577. 29. Krishnan JA, Parce PB, Martinez A, et al: Caloric intake in medical ICU patients: consistency of care with guidelines and relationship to clinical outcomes. Chest 2003; 124:297-305. 30. Rice TW, Mogan S, Hays MA, et al: Randomized trial of initial trophic versus full-energy enteral nutrition in mechanically ventilated patients with acute respiratory failure. Crit Care Med 2011; 39:967-974. 31. Rice TW, Wheeler AP, Thompson BT, et al: Initial trophic vs full enteral feeding in patients with acute lung injury: the EDEN randomized trial. JAMA 2012; 307:795-803. 32. Ahrens CL, Barletta $J$ J, Kanji S, et al: Effect of low-calorie parenteral nutrition on the incidence and severity of hyperglycemia in surgical patients: a randomized, controlled trial. Crit Care Med 2005; 33:2507-2512. 33. McCowen KC, Friel C, Sternberg $\mathrm{J}$, et al: Hypocaloric total parenteral nutrition: effectiveness in prevention of hyperglycemia and infectious complications: a randomized clinical trial. Crit Care Med 2000; 28:3606-3611.

2. None declare

\section{3}

Hypermetabolism: Does it affects prognosis in critical patients? Indirect Calorimetry as an instrument of research

G. Sousa'; I. Mendes ${ }^{2}$; L. Tavares ${ }^{1}$; R. Carvalho ${ }^{2}$; M. Henriques ${ }^{1}$; H. Costa ${ }^{1}$ ${ }^{1}$ Intensive Care, Hospital do Divino Espírito Santo, Ponta Delgada, Portugal; ${ }^{2}$ Endocrinology and nutrition, Hospital do Divino Espírito Santo, Ponta Delgada, Portugal

Correspondence: G. Sousa

Intensive Care Medicine Experimental 2020, 8(2): 000313

Introduction: Energy expenditure (EE) evaluation in Intensive Care Unit (ICU) patients is very challenging. Critical illness is characterized by great variability in the $\mathrm{EE}$, which is influenced by the disease itself and the effects of treatment. Indirect calorimetry (IC) is currently the gold standard to measure EE in ICU patients. However, calorimeters are not widely available and predictive formulas (PF) are still commonly used, which may lead to under or overfeeding and its deleterious consequences.1,2 Important metabolic changes occur and catabolism becomes prominent in critically ill patients.3,4 However, both hyper and hypometabolism are observed.5 Hypermetabolism is a condition mediated by the immune system, which can be affected by damaged tissue, pathogenic microorganisms, and the entry of their toxins into the bloodstream, as well as the body's response, with hormones and cytokines release. Due to this situation, hypermetabolic patients often have higher mortality rates compared to metabolically normal patients. 6,7

Objectives: To assess the incidence of hypermetabolism and to compare clinical outcomes between hypermetabolic and normometabolic patients in ICU with EE measured by IC.

Methods: Single-centre, retrospective, observational study including mechanically ventilated patients admitted in ICU between August 2018 and February 2020. EE was measured by IC. Basal Energy Expenditure (BEE) was estimated by the Harris-Benedict equation $(\mathrm{HB})$. According to their metabolic state (EE/BEE $\times 100)$, patients were divided in hypermetabolic $(\geq 130 \%)$ and normometabolic $(<130 \%)$. To determine the limits of agreement between $\mathrm{EE}$ and $\mathrm{BEE}$, we performed a Bland-Altman (BA) analysis. Baseline characteristics, severity criteria, and main diagnosis were compared. The primary outcome considered was 30-day mortality. Other outcomes were evaluated, such as the length of stay (LOS) in ICU, LOS in hospital, and length of invasive ventilation. 
Results: Of the 55 ICU patients included, median age was $62,0[52,0$ $72,0]$ years. BA showed a mean $-411 \pm 554 \mathrm{Kcal} / \mathrm{d}$ for EE and BEE. Seventeen patients $(30,9 \%)$ were in hypermetabolic group with a significantly higher proportion of male patients $(88,2$ vs $11,8 \% ; p=0,04)$, a lower BMI $(26,1[23,0-27,7]$ vs $27,7[24,7-33,8] \mathrm{Kg} / \mathrm{m} 2 ; \mathrm{p}=0,04)$, a higher proportion of patients admitted due to medical conditions $(76,5$ vs $47,4 \%, p=0,04)$, and due pneumonia $(41,2$ vs $15,8 \% ; p=$ $0,04)$. We did not find any statistically significant difference between the two groups, neither in 30-day mortality nor in the other outcomes compared.

Conclusion: There was no statistically significant difference between the two groups in all the clinical outcomes considered. However, we concluded that hypermetabolic patients were predominantly males, had a lower BMI, and had a higher proportion of admissions due to medical conditions and pneumonia.

\section{Reference(s) and grant ackowledgment(s)}

1. 1Choban P, Dickerson R, Malone A, Worthington P, Compher C. A.S.P.E.N. Clinical Guidelines. Journal of Parenteral and Enteral Nutrition. 2013;37(6):714-744.

2. 2Frankenfield D, Ashcraft C, Galvan D. Prediction of Resting Metabolic Rate in Critically III Patients at the Extremes of Body Mass Index. Journal of Parenteral and Enteral Nutrition. 2012;37(3):361-367.

3. 3Chioléro R, Revelly J, Tappy L. Energy metabolism in sepsis and injury. Nutrition. 1997;13(9):45-51

4. $4 \mathrm{McClave} \mathrm{SA}$, Snider HL. Understanding the metabolic response to critical illness: factors that cause patients to deviate from the expected pattern of hypermetabolism. New Horiz. 1994; 2:139-46.

5. 5Fontaine E, Müller M. Adaptive alterations in metabolism: practical consequences on energy requirements in the severely ill patient. Current Opinion in Clinical Nutrition and Metabolic Care. 2011;14(2):171-175.

6. 6Selberg O, Bottcher J, Tusch G, Pichlmayr R, Henkel E, Muller M. Identification of high- and low-risk patients before liver transplantation: A prospective cohort study of nutritional and metabolic parameters in 150 patients. Hepatology. 1997;25(3):652-657.

7. 7Wu C, Wang X, Yu W, Tian F, Liu S, Li P et al. Hypermetabolism in the Initial Phase of Intensive Care Is Related to a Poor Outcome in Severe Sepsis Patients. Annals of Nutrition and Metabolism. 2015;66(4):188-195.

\section{2}

\section{Diarrhea: Interventions, Consequences and Epidemiology} in the Intensive Care Unit: The DICE-ICU Prospective Multicenter

\section{Cohort Study}

J. Dionne'; L. Mbuagbaw²; J. Devlin ${ }^{3}$; M. Duprey ${ }^{3}$; R. Cartin-Ceba ${ }^{4}$.

J. Muscedere ;M. Alshahrani ; W. Szczeklik7 ; J. Tsang ${ }^{8}$; A. Takaoka9;

K. Sullivan ${ }^{10}$; K. Borowska ${ }^{11}$;W. Serednicki ${ }^{7}$; W. Alhazzani ${ }^{1}$; P. Moayyedi ${ }^{12}$.

D. Armstrong ${ }^{12}$; L. Thabane ${ }^{2} ;$ R. Jaeschke ${ }^{8} ;$ T. Karachi $^{8} ;$ D. Cook ${ }^{1}$

'Department of medicine/department of health research methods, evidence and impact, McMaster University, Hamilton, Canada; ${ }^{2}$ Department of health research methods, evidence and impact, McMaster University, Hamilton, Canada; ${ }^{3}$ Bouve college of health sciences, Northeastern University, Boston, United States of America; ${ }^{4}$ Critical care, Mayo Clinic Hospital, Phoenix, United States of America; ${ }^{5}$ Critical care, Queen's University, Kingston, Canada; ${ }^{6}$ Emergency and critical care, Imam Abdulrahman Bin Faisal University Hospital, Dammam, Saudi Arabia; ${ }^{7}$ Department of anesthesiology and intensive care, Jagiellonian University, Kraków, Poland; ${ }^{8}$ Department of medicine, division of critical care, McMaster University, Hamilton, Canada; ${ }^{9}$ Critical care, St. Joseph's Healthcare Hamilton, Hamilton, Canada; ${ }^{10}$ Department of medicine, division of cardiology, McMaster University, Hamilton, Canada; ${ }^{11}$ Department of intensive care, Jagiellonian University, Kraków, Poland; ${ }^{12}$ Department of medicine, division of gastroenterology, McMaster University, Hamilton, Canada

Correspondence: J. Dionne

Intensive Care Medicine Experimental 2020, 8(2): 000352

Introduction: Diarrhoea is a common concern in the intensive care unit (ICU) and incidence varies from $2-95 \%$ in previous studies. This variation is due to lack of a consistent definition of diarrhoea and scientific inattention to this clinical problem. The prevalence, predisposing factors, and prognosis of diarrhoea during critical illness have not been well defined in prospective studies.

Objectives: To determine the incidence (using different diagnostic criteria), risk factors, management modifications, and clinical outcomes associated with diarrhoea in critically ill adults.

Methods: Design, Setting and Participants: We prospectively enrolled consecutive adults admitted to 12 Intensive Care Units (ICU), in 4 countries, during a 10 -week period. We excluded patients admitted to the ICU for $<24$ hours.

Main Outcome: The primary outcome was diarrhoea incidence, using the World Health Organization (WHO) definition, and the Bristol Stool Chart (Bristol) and Bliss Stool Classification System (Bliss), and risk factors. Secondary outcomes included Clostriodiodes difficile-associated diarrhoea (CDAD), ICU and hospital length of stay and mortality.

Analysis: We used logistic regression to evaluate 12 potential risk factors for diarrhoea, and Cox regression to estimate the association between diarrhoea and morbidity and mortality. All models were adjusted for age, sex and APACHE II scores.

Results: Among 1109 patients age 61.4 [17.49] mean [standard deviation] years, APACHE-II 18.8 [7.98], 981(88.5\%) medical patients, 645 $(58.2 \%)$ mechanically ventilated, diarrhoea was common (WHO 73.8\%, 95\% confidence interval [Cl] 71.1-76.6; Bristol 53.5\%, 95\%Cl 46.7-50.4; and Bliss $37.7 \%, 95 \% \mathrm{Cl} 34.9-40.4)$. Independent risk factors for diarrhoea (WHO Definition) included enteral nutrition (OR 2.43, 95\% Cl 1.70-3.48), suppository use (OR $1.73,95 \% \mathrm{Cl} 1.28-2.35)$ and number of antibiotic days (OR 1.10, 95\% Cl 1.05-1.14). Female sex (OR 0.71, 95\% $\mathrm{Cl} 0.52-0.95)$ and opioid use (OR $0.32,95 \% \mathrm{Cl} 0.22-0.48)$ were associated with a lower risk of diarrhoea. Diarrhoea resulted in modifications to patient management including: enteral nutrition held or changed, stool softener or prokinetic held, rectal appliance used, or $C$. difficile test ordered (OR 10.9; 95\% Cl 5.74-21.03). CDAD prevalence in the ICU was 85/1109 (7.7\%). Among the 99 patients with diarrhea tested for CDAD, 23/99 were positive, for a CDAD ICU incidence of 23/ 1109 (2.1\%). Diarrhoea was associated with a longer length of stay in the ICU (HR 2.62, 95\% Cl 2.23-3.06; $\mathrm{p}<0.001)$ and hospital (HR 1.72; 95\% Cl $1.46-2.01 ; \mathrm{p}<0.001)$ and increased hospital mortality (HR 2.12; $95 \% \mathrm{Cl}$ $1.61-2.78 ; p<0.001)$.

Conclusion: Diarrhoea is common during critical illness; incidence varies by diagnostic definition. Several risk factors are modifiable. Diarrhoea is associated with increased morbidity and mortality.

\section{Reference(s) and grant ackowledgment(s)}

1. Funding: This work was supported by peer-review grants from the Hamilton Regional Medical Associates, Hamilton Health Sciences Department of Medicine, Physicians Services Incorporated of Ontario, and the Canadian Association of Gastroenterology. Career Awards were from Physicians Services Incorporated of Ontario (J Dionne) and the Canadian Institutes for Health Research (D Cook).

\section{5}

Evaluation of nutritional practices in the critical care patient: Does nutritional support really influence outcomes?

JC. Lopez-Delgado'; L. Servia-Goixart ${ }^{2}$; T. Grau-Carmona ${ }^{3}$; J. Trujillano ${ }^{4}$; L. Bordejé5; E. Mor-Marco ; P. Vera-Artazcoz'; C. Lorencio-Cardenas ${ }^{8}$; L. Macaya-Redin ${ }^{9}$; E. Portugal-Rodriguez ${ }^{10}$; JF. Martinez-Carmona ${ }^{11}$; J. Marin-Corral ${ }^{12}$; D. Monge-Donaire ${ }^{13}$; B. Llorente-Ruiz ${ }^{14}$;

R. Iglesias-Rodriguez ${ }^{15}$; C. Gonzalez-Iglesias ${ }^{16}$; M. Zamora-Elson ${ }^{16}$;

C. Seron-Arbeloa ${ }^{17}$; A. Sanchez-Miralles ${ }^{18}$; B. Vila-Garcia ${ }^{19}$

${ }^{1}$ Intensive care, Hospital Universitari de Bellvitge, L'Hospitalet de Llobregat (Barcelona), Spain; ${ }^{2}$ ntensive care, University Hospital Arnau de Vilanova, Lleida, Spain; ${ }^{3}$ Intensive care, Hospital 12 de Octubre, Madrid, Spain; ${ }^{4}$ Intensive care unit, University Hospital Arnau de Vilanova, Lleida, Spain; Intensive care unit, Hospital Germans Trias i Pujol, Badalona, Spain; ${ }^{6}$ Intensive care, Hospital Germans Trias i Pujol, Badalona, Spain; ${ }^{7}$ Intensive care, Hospital Sant Pau, Barcelona, Spain; ${ }^{8}$ Intensive care, Hospital Josep Trueta, Girona, Spain; ${ }^{9}$ Intensive care, Complejo Hospitalario de Navarra, Pamplona, Spain; ${ }^{10}$ Intensive care, Hospital Clínico, Valladolid, Spain; ${ }^{11}$ Intensive care, Hospital Regional Universitario de Málaga, Málaga, Spain: ${ }^{12}$ Critical care department, IMIM, Barcelona, Spain; ${ }^{13}$ Intensive care, Hospital Virgen De La Concha, Zamora, Spain; ${ }^{14}$ Intensive care, Hospital Univer- 
sitario Príncipe de Asturias, Alcala de Henares, Spain; ${ }^{15}$ Intensive care, Hospital General de Granollers, Granollers, Spain; ${ }^{16}$ Intensive care, Hospital de Barbastro, Barbastro, Huesca, Spain; ${ }^{17}$ Intensive care, Hospital General San Jorge, Huesca, Spain; ${ }^{18}$ Intensive care, Hospital Universitari Sant Joan d'Alacant, Sant Joan d'Alacant, Spain; ${ }^{19}$ Intensive care, Infant Cristina University Hospital, Parla, Spain

Correspondence: J.C. Lopez-Delgado

Intensive Care Medicine Experimental 2020, 8(2): 000385

Introduction: The positive influence of nutritional support is underrecognized and nutrition is considered an adjunctive more than a primary therapy itself.

Objectives: The main aim of the present study was to evaluate the influence of nutritional support in outcomes in the critical care setting. Methods: Prospective observational multicenter study. Demographic, comorbidities, cause of admission, nutritional evaluation, type and characteristics of nutritional support (during the whole nutritionaldelivery or at least the first 14 days of nutritional support), and outcomes, with special attention in 28-day mortality, were registered in a database. Confounders, such as disease severity, type of patient and the length of nutritional support among others, were considered for final statistical approach (NCT:03634943).

Results: 639 patients were included with a $25.6 \%$ of mortality. Nonsurvivors showed older age, higher comorbidities, higher SOFA score $(6.6 \pm 3.3 \mathrm{vs} 8.4 \pm 3.7 ; \mathrm{P}<0.001)$ and nutritional risk $(3.8 \pm 2.1 \mathrm{vs} 5.2 \pm 1.7 ; \mathrm{P}$ $<0.001$ ) on admission. Nutritional support did no differ among groups, except a trend towards lower mean protein intake in nonsurvivors. Non-survivors suffer from higher needs on vasopressor therapy $(70.4 \% v s 83.5 \% ; \mathrm{P}=0.001)$ and renal replacement therapy $(12.2 \%$ vs $23.2 \% ; \mathrm{P}=0.001)$. Multivariate analysis showed that older age (HR:1.023;95\% Cl:1.008-1.038; $\mathrm{P}=0.003)$, higher SOFA Score (HR:1.096;95\% Cl:1.036-1.160; $\mathrm{P}=0.001$ ), higher NUTRIC Score (HR:1.136;95\% Cl:1.025-1.259; $\mathrm{P}=0.015$ ), the need of parenteral nutrition (PN) after the initiation of enteral nutrition (EN) (HR:2.368;95\% $\mathrm{Cl}: 1.168-4.798 ; \mathrm{P}=0.017$ ), mean $\mathrm{Kcal} / \mathrm{Kg} /$ day (HR:1.057;95\% Cl:1.015$1.101 ; \mathrm{P}=0.008$ ), and mean $\mathrm{g}$ protein $/ \mathrm{Kg} /$ day $(\mathrm{HR}: 0.507 ; 95 \% \mathrm{Cl}: 0.263-$ $0.977 ; P=0.042$ ) were factors associated with mortality.

Conclusion: Elderly, higher average of organ failure and nutritional risk are nutritional related factors that may be associated with higher mortality. Patients who need of PN after initiation of EN may represent a high mortality risk subgroup due to illness severity and problems in giving appropriate nutritional support. The mean caloric and protein delivery may play a key role in outcomes.

\section{Reference(s) and grant ackowledgment(s)}

1. Lew CCH, Yandell R, Fraser RJL, Chua AP, Chong MFF, Miller M. Association Between Malnutrition and Clinical Outcomes in the Intensive Care Unit: A Systematic Review. JPEN J Parenter Enteral Nutr. 2017;41:744-758. doi: 10.1177/0148607115625638.

2. Preiser JC, Ichai C, Orban JC, Groeneveld AB. Metabolic response to the stress of critical illness. Br J Anaesth. 2014;113:945-54. doi: 10.1093/bja/ aeu187.

3. The present research was supported by SENPE (The Spanish Society of Metabolism \& Nutrition)

\section{6}

The influence of Pharmaconutrition in the ICU: Does it impact outcomes?

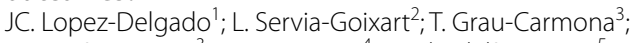

C. García-Fuentes ${ }^{3}$; E. Mor-Marco ${ }^{4}$; JL. Flordelís Lasierra ${ }^{5}$;

S. Aldunate-Calvo ${ }^{6}$; R. Gastaldo Simeón ${ }^{7}$; I. Martínez De Lagrán Zurbano ${ }^{8}$;

JC. Yebenes-Reyes ${ }^{\text {; }}$ E. Navas-Moya ${ }^{10}$; A. Martín-Luengo ${ }^{11}$; EM. Menor

Fernandez ${ }^{12}$; C. Leon-Cinto ${ }^{13}$; L. Mateu Campos ${ }^{14}$; M. Gero-Escapa ${ }^{15}$;

L. Sanchez-Ales ${ }^{16}$; E. Portugal-Rodriguez ${ }^{17}$; JC. Montejo-Gonzalez ${ }^{3}$;

C. Lorencio-Cardenas ${ }^{18}$

${ }^{1}$ Intensive care, Hospital Universitari de Bellvitge, L'Hospitalet de Llobregat (Barcelona), Spain; ${ }^{2}$ Intensive care, University Hospital Arnau de Vilanova, Lleida, Spain; ${ }^{3}$ Intensive care, Hospital 12 de Octubre, Madrid, Spain; ${ }^{4}$ Intensive care, Hospital Germans Trias i Pujol, Badalona, Spain;
${ }^{5}$ Intensive care, Hospital Universitario Severo Ochoa, Leganés, Spain; ${ }^{6}$ Intensive care, Complejo Hospitalario de Navarra (CHN), Pamplona, Spain; ${ }^{7}$ Intensive care unit, Hospital de Manacor, Manacor, Spain; ${ }^{8}$ Intensive care unit, Hospital de Mataró, Mataró, Spain; ${ }^{9}$ Intensive care, Hospital de Mataró, Mataró, Spain; ${ }^{10}$ Intensive care, Hospital Mútua de Terrassa, Terrassa, Spain; ${ }^{11}$ Intensive care, Hospital Universitario Rio Hortega, Valladolid, Spain; ${ }^{12}$ Intensive medicine, Hospital Álvaro Cunqueiro, Estrada de Clara Campoamor, Vigo, Spain, Vigo, Spain; ${ }^{13}$ Intensive care, Hospital Royo Villanova, Zaragoza, Spain; ${ }^{14}$ Intensive care unit, Hospital General Universitario de Castellón, Castellón de la Plana, Spain; ${ }^{15}$ Intensive care, Hospital Universitario de Burgos, Burgos, Spain; ${ }^{16}$ Intensive care, Hospital de Terrassa, Terrassa, Spain; ${ }^{17}$ Intensive care, Hospital Clínico, Valladolid, Spain; ${ }^{18}$ Intensive care, Hospital Josep Trueta, Girona, Spain

Correspondence: J.C. Lopez-Delgado

Intensive Care Medicine Experimental 2020, 8(2): 000386

Introduction: There is a gap between the theoretical benefits of pharmaconutrition and the moderate benefit in clinical practice in the Intensive Care Unit (ICU).

Objectives: The aim of the present research was to evaluate the impact of immunonutrition formulas (IMN) with standard enteral formulas in outcomes during the ICU stay.

Methods: National multicenter prospective observational study (37 hospitals) from April to June 2018, conducted in ICUs throughout Spain (NCT Registry: 03634943). Patient characteristics, calorie-protein dose, life support needs and mortality were evaluated. Statistical differences were analyzed according to the administration of IMN formulas and the type of patient.

Results: 525 patients who received enteral nutrition (EN) were included in the analysis, of which 406 (77\%) received only EN. 16.57\% of the patients received IMN formulas. The age was $61.33 \pm 15.01$ years, BMI: $27.86 \pm 6.06 \mathrm{Kg} / \mathrm{m} 2$, the majority were medical patients (68\%), malnutrition was $38.58 \%$ (SGA) and the mean nutritional risk was $4.17 \pm 2.14$ (NUTRIC Score). The 28 -day mortality was $26.1 \%$ and the IMN formulas were not associated with lower mortality compared to the other formulas when comparing the subgroup of survivors with non-survivors.

However, a lower need for vasopressor support (75.6\% vs. $60.66 \%$; OR: $0.490 ; 95 \% \mathrm{Cl}: 0.260-0.910 ; \mathrm{P}=0.023)$ and continuous renal replacement therapies (OR: $0.130 ; 95 \% \mathrm{Cl}: 0.010-0.650 ; \mathrm{P}=0.049$ ) was observed in those patients who received IMN formulas compared with standard formulas. The use of IMN formulas was associated with a higher average protein delivery during the nutritional therapy $(0.74$ $\pm 0.34 \mathrm{vs} 0.9 \pm 0.31 \mathrm{~g} / \mathrm{Kg} / \mathrm{d} ;$ OR: $6.230 ; 95 \%$ Cl: $2.590-15.540 ; \mathrm{P}<0.001$ ).

Conclusion: We showed lower need of vasopressors and continuous renal replacement therapies during ICU stay with the use of IMN formulas, which may be associated with their modulatory effect over inflammatory response. A higher protein delivery may be provided with IMN formulas in the ICU.

Reference(s) and grant ackowledgment(s)

1. Braga M., et al. Cliinical evidence for pharmaconutrition in major elective surgery. JPEN. 2013.

2. The present research $w$ as supported by SENPE (The Spanish Society of Metabolism \& Nutrition)

000467

Thyroid levels in ICU. More than sick euthyroid?

J. Nikhilesh'; A. Thakur ${ }^{2}$; V. Joshi ${ }^{3}$

${ }^{1}$ Dept of critical care medicine, CHL Hospital, Indore, India; ${ }^{2}$ Dept of critical care, CHL hospital, Indore, Madhya Pradesh, India, India; ${ }^{3}$ Dept of critical care services, Shalby Hospitals, Indore, Madhya Pradesh, India, India

Correspondence: J. Nikhilesh

Intensive Care Medicine Experimental 2020, 8(2): 000467

Introduction: Background: In critically ill patients thyroid levels have a significant role in terms of recovery from critical illness however, their replacement has not been shown to confer mortality/outcome 
benefit in subsets with lower hormone levels. This communication attempts to delineate the outcomes/mortality correlations in patients with abnormalities of thyroid levels getting admitted to an intensive care unit (ICU).

Objectives: Comparison of mortality outcomes in patients of sepsis with abnormalthyroid levels and no underlying thyroid disorders getting admitted to ICU.

Setting: 60 bedded mixed ICUs of tertiary care units.

Methods: All patients getting admitted with Sepsis from Jan 2019- till date were included and their baseline samples of TSH/T3/T4 were collected. Data was collected with a focus on demographics, SOFA scores, Length of stay (LOS) and mortality. Patients with hypoalbuminemia, preexisting thyroid disorders and receiving Amiodarone were excluded. Death/discharge from ICU were considered as end points. Statistical analysis was done using SPSS version 23. Chi square test was used to delineate association with mortality.

Results: Ninety patients were included ( $n=90$, M: F-58:32). Age of the cohort was $50 \pm 17$ years (Range-22-85). SOFA scores were 11.8 \pm 3.2 (Range-6-18). Length of stay was $6.9 \pm 4.3$ days (Range-1-20) and in hospital mortality was $37.8 \%(n=34)$. Thyroid hormone levels were

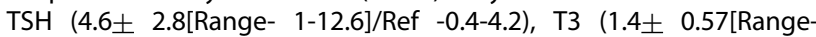
0.6-2.5]/ Ref-0.4-2.4) and T4 (7+2.6[Range-2.2-11.8]/Ref-6.09-12.23) respectively. On statistical analysis in isolation both low TSH levels and Low T3 levels were significant predictors of mortality $[0.0006 \mathrm{P}$ and $0.0001 \mathrm{P}]$. Combination of these variables did not improve the association and was statistically not significant

Conclusion: Low TSH and T3 levels at baseline predict mortality in sepsis cohorts. However, whether the relationship would remain sacrosanct in patients with hypoalbuminemia and patients receiving Amiodarone remains to be seen. We will need bigger subsets with more variables to enhance our knowledge levels for the same.

\section{9}

Nutritional status and response of long stayer COVID-19 patients upon admission to intensive care differ from non-COVID patients - preliminary data

O. Pantet ' D. Favre'; M. Charrière ${ }^{2}$; A. Schneider ${ }^{1}$; M. Berger ${ }^{1}$

${ }^{1}$ Service of Adult Intensive Care \& Burns, Lausanne University Hospital, Lausanne, Switzerland; ${ }^{2}$ Nurition clinique - icu, Lausanne University Hospital, Lausanne, Switzerland

Correspondence: $\mathrm{M}$. Berger

Intensive Care Medicine Experimental 2020, 8(2): 000559

Introduction: The COVID pandemic has resulted in a high number of intensive care (ICU) admissions, generally The COVID pandemic has resulted in a high number of intensive care (ICU) admissions, generally for respiratory distress. While research has focused on respiratory and infectious characteristics, little is known about their metabolic and nutritional characteristics compared to other categories of patients.

Objectives: The aim was to compare COVID patients' status and metabolic response to persistently critically ill patients $(\mathrm{PCI})$ defined as $>10$ days in ICU

Methods: Prospective observational study in consecutive cohorts of long stay patients on mechanical ventilation and COVID-19 patients were analysed. Variables: demographic data, severity score (SAPS2), Nutritional risk screening (NRS) score, C-reactive protein (CRP). Prealbumin values determined on admission (adm) and D6-9 (delta pre-albumin was calculated between admission and D6-9 values), nutritional management, length of stay and outcome. Nutritional recommendations were: energy target $20 \mathrm{kcal} / \mathrm{kg}$ during first week (then adapted according to indirect calorimetry measurements for LSP, no calorimetry for COVID-19), protein $1.2 \mathrm{~g} / \mathrm{kg}$, early enteral nutrition privileged (time to feeding recorded). The confined dieticians worked remotely. Statistics: median (interquartiles), percentage.
Results: Altogether 50 LST and 54 COVID-19 mechanically ventilated patients were included. Characteristics are shown in Table. The two cohorts were similar in terms of age, gender distribution ( $74 \%$ men) and disease severity; however, COVID patients were significantly heavier. Median NRS was 5 in both groups, several points coming from reduced eating during last days in COVID patients, which was less frequent in $\mathrm{PCl}$ cohort. Mortality did not differ. Enteral nutrition was initiated earlier in COVID: on Day 1 in $50 \%$ vs $20 \%$, on Day $287 \%$ vs $33 \%$, and Day3 $94 \%$ vs $53 \%(p<0.0001)$ respectively. Combined EN and parenteral feeding was only required on rare occasions $(<2 \%)$. Admission prealbumin was significantly lower in COVID vs $\mathrm{PCl}$, unrelated to NRS score, and poorly correlated to CRP $(\mathrm{r} 2=0.082)$. Prealbumin increase (delta $0.19 \mathrm{~g} / \mathrm{l}$ ) was significantly higher in COVID patients, but also in survivors of both groups, where difference over time was $0.03(0 ; 0.09)$ $g / l(p<0.0001)$. Protein data are in prepartion.

\begin{tabular}{|l|l|l|l|}
\hline & PCI (n=50) & COVID $(\mathbf{n = 5 4})$ & P value \\
\hline Age $(\mathrm{yrs})$ & $61(53 ; 73)$ & $61(56 ; 71)$ & 0.822 \\
\hline Weight & $77(63 ; 90)$ & $85(75 ; 98)$ & 0.0001 \\
\hline BMI $(\mathrm{kg} / \mathrm{m} 2)$ & $25.5(21.4 ; 31.7)$ & $27.7(25.7 ; 33.4)$ & 0.0036 \\
\hline SAPS2 score & $41(33 ; 59)$ & $40(33 ; 46)$ & 0.703 \\
\hline Length ICU stay $(\mathrm{d})$ & $26.1(21.0 ; 38.0)$ & $18.7(10.4 ; 27.7)$ & 0.0002 \\
\hline Prealbumin Adm. g/L & $0.095(0.07 ; 0.14)$ & $0.070(0.06 ; 0.09)$ & 0.014 \\
\hline Delta prealbumin g/L & $0.025(0.01 ; 0.06)$ & $0.19(0.09 ; 0.32)$ & $<0.0001$ \\
\hline Mortality & $20.0 \%$ & $15.4 \%$ & 0.541 \\
\hline
\end{tabular}

Conclusion: Mechanically ventilated COVID patients were heavier both groups presenting a similar nutritional risk. Prealbumin values on admission were significantly lower and reverted faster to normal values, which might be due to a faster clearance of inflammation and related catabolism, but maybe also to nutrition. Early enteral feeding was significantly more frequent in COVID patients, reflecting the lesser impact of a single respiratory organ failure on $\mathrm{Gl}$ tract.

Reference(s) and grant ackowledgment(s)

1. No conflict of interest to declare

\section{9}

Impact of beta-Hydroxy-beta-Methylbutyrate (HMB) on muscle loss in critically ill patients. A RCT

M. Viana'; F. Becce' ${ }^{2}$; S. Schmidt ${ }^{2}$; G. Bagnoud'; M. Berger ${ }^{1}$; NE. Deutz ${ }^{3}$

${ }^{1}$ Adult Intensive Care, Lausanne University Hospital, Lausanne, Switzerland; 'Radiology, Lausanne University Hospital, Lausanne, Switzerland, ${ }^{3}$ Department of health and kinesiology, Texas A\&M University, College Station, United States of America

Correspondence: M. Viana

Intensive Care Medicine Experimental 2020, 8(2): 000599

Introduction: The rapid decline of muscle mass and function in mechanically ventilated critically ill patients is associated with poor outcome, and prolonged impairment in physical function. The loss of lean body mass (LBM) is rapid and massive being close to $20 \%$ in one week. Nutrition only partly attenuates muscle loss. HMB, a metabolite of leucine, has been shown to substantially reduce loss of muscle mass in older adult healthy and sick subjects but has not been tested in ICU patients.

Objectives: The study aimed at testing the impact of HMB on LBM using ultrasound assessment and on amino acid metabolism.

Methods: RCT in 37 critically ill patients (ITT), on mechanical ventilation, with full treatment and a functional gastro- intestinal tract. Intervention: Enteral HMB 2*1.5 g/day versus placebo from day 4 to day 30 after admission. Primary outcome: muscle mass loss (ultrasound of thigh on days 4 and 15 to determine \% change of skeletal muscle index (SMI). On days 4 and 15, amino acid metabolism was investigated with 
the pulse administration of a mixture of stable amino acid tracers. Plasma enrichments were measured by LC-MS/MS, and areas under the curve (AUC) were calculated to determine whole body production and interconversions of several amino acids. Patients were separated in two groups at day 4 based on net protein breakdown (netPB: conversion of phenylalanine to tyrosine): low (lower than lower limit of the $95 \% \mathrm{Cl}$ ) and high. Stats by 2 factor ANOVA (Graphpad Prism 8.4).

Results: Critically ill patients with age $65 \pm 15$ years and SAPSII 47 \pm 12 were studied. Seven patients were studied only at day 4 . SMI decreased significantly $(p<0.001)$ in both groups, without difference between HMB group or placebo. However, patients with a lowPB who received HMB had a smaller SMI loss compared to placebo (mean difference $-3.98(95 \% \mathrm{Cl}-6.29$. to $-1.68 ; \mathrm{p}=0.0155)$ and mean interaction difference $-5.87(95 \% \mathrm{Cl}-10.48$ to $-1.26 ; \mathrm{p}=0.0148)$ ).

Conclusion: In a heterogeneous group of severe ICU patients, HMB did not significantly reduced muscle loss. Although, post hoc analysis showed that HMB can reduce muscle loss in patients with the lowest netPB.

\section{Reference(s) and grant ackowledgment(s)}

1. Deutz NEP, Thaden JJ, Ten Have GAM, Walker DK, Engelen MPJK. Metabolic phenotyping using kinetic measurements in young and older healthy adults. Metabolism 2018:78:167-78.

2. English KL, Paddon-Jones D. Protecting muscle mass and function in older adults during bed rest. Curr Opin Clin Nutr Metab Care 2010;13:34-9.

3. Excellence fellowship of the Swiss Dpt of foreign affairs (DFAE)

4. ESPEN fellowship

\section{7}

Hypovitaminosis D in ICU patients: incidence and association with poor outcome

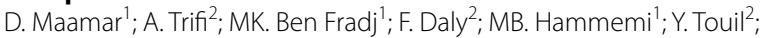
C. Abdennebi ${ }^{2}$; M. Feki' ${ }^{1}$ 'S. Abdellatif²; S. Ben Lakhal ${ }^{2}$

${ }^{1}$ Laboratory of biochemistry, Hospital la Rabta, Tunis, Tunisia; ${ }^{2}$ nntensive care unit, Hospital la Rabta, Tunis, Tunisia

Correspondence: A. Trifi

Intensive Care Medicine Experimental 2020, 8(2): 000757

Introduction: Patients in intensive care units (ICUs) are at high risk of unfavorable outcomes. Considering the role of vitamin $D(V i t D)$ in cardiovascular and immune functions, Vit D deficiency could affect ICU patients' outcomes.

Objectives: To evaluate Vit D status and its predictive value for outcome in ICU patients.

Methods: prospective cohort study that included all adult patients admitted to the medical ICU of la Rabta Hospital from January 2016 to December 2018. Non inclusion criteria were: Sepsis, cancer, renal or liver failure, surgery within 3 months; immunotherapy or vitamin supplements within 6 months; pregnant or lactating, discharge or death within $48 \mathrm{hr}$ of admission were the exclusion criteria. Plasma 25 -hydroxyvitamin $\mathrm{D}(25(\mathrm{OH}) \mathrm{D})$ was assessed by immunoassay. The primary outcome was the occurrence of the following major adverse events (combined MAE): the need for a central venous catheter (CVC), organ failure, and septic shock. Secondary outcomes were the occurrence of at least one ICU-acquired infection (combined ICU-AI), septic shock, organ failure, other individual adverse events, and ICU mortality. Multivariate Cox regression analyses were performed to test the associations of low $25(\mathrm{OH})$ D levels with poor outcomes.

Results: A total of 169 ICU patients were followed during ICU stay. Around $75 \%$ of patients had $25(\mathrm{OH})$ D levels $<12 \mathrm{ng} / \mathrm{ml}$. During their ICU stay, 114 patients presented MAE, 85 patients presented an ICUacquired infection, and 22 patients died. The comparative distribution of plasma 25 -hydroxyvitamin $\mathrm{D}(25(\mathrm{OH}) \mathrm{D})$ in ICU patients according to selected outcomes are displayed in the attached figure.

Plasma 25(OH) D levels $<12 \mathrm{ng} / \mathrm{ml}$ were associated with higher risk of major adverse events, Hazard ratio [95\% Cl], $4.47[1.77,11.3], p=0,020$, and ICU-acquired infection, $2.67[1.01,7.42], p=0,049$, but not with increased risk of ICU mortality.

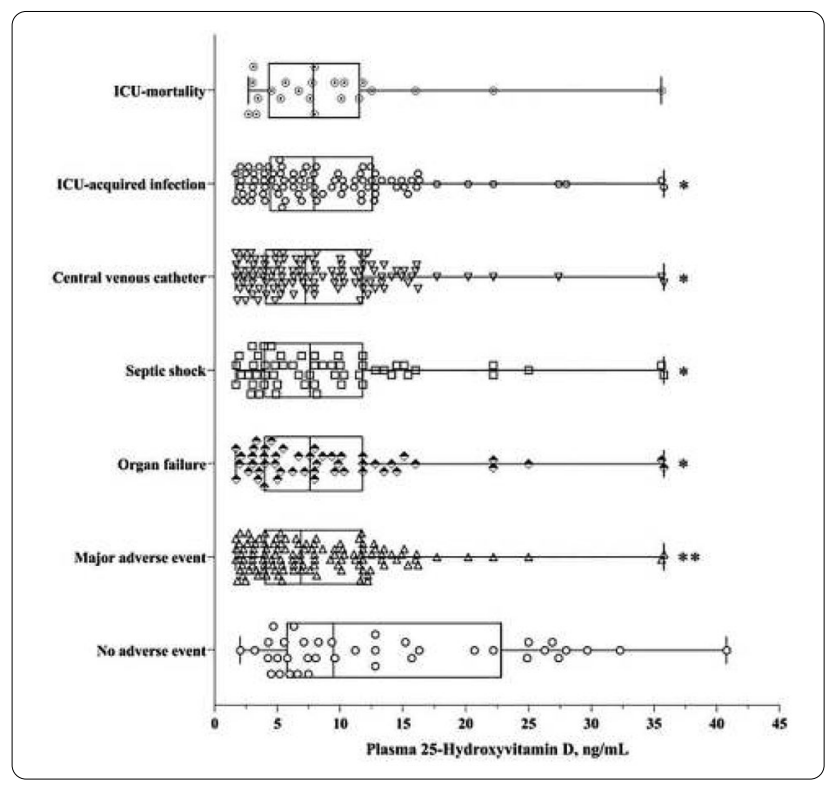

Conclusion: Hypovitaminosis D is common in ICU patients. Results of the present study show that low plasma $25(\mathrm{OH}) \mathrm{D}$ levels are associated with increased risk of unfavorable outcomes. Additional research is needed to investigate the impact of Vit D status and effect of Vit $D$ supplementation in ICU patients.

\section{0}

The prevalence of vitamin D deficiency and outcome in children with severe sepsis or septic shock

L. Kongkiattikul ${ }^{1}$; R. Leelasukharom ${ }^{2}$; R. Samransamruajkit ${ }^{1}$

${ }^{1}$ Pediatrics, King Chulalongkorn Memorial Hospital, Chulalongkorn University, Bangkok, Thailand; ${ }^{2}$ Pediatrics, King Chulalongkorn Memorial Hospital, Bangkok, Thailand

Correspondence: L. Kongkiattikul

Intensive Care Medicine Experimental 2020, 8(2): 000760

Introduction: Vitamin $D$ is an essential nutrient and hormone that plays role in calcium homeostasis and bone health. In the last two decades, vitamin $D$ is known to promote innate and adaptive immunity. It may play a role in modulating the outcome of sepsis.

Objectives: This study was conducted to describe the prevalence of vitamin $D$ deficiency in pediatric patients with severe sepsis or septic shock and to evaluate the correlation between vitamin $D$ level and severity of illness.

Methods: This is cross-sectional study. Populations were children aged 30 days to 18 years who were diagnosed with severe sepsis or septic shock from August 2018 to May 2019. We collected demographic data, principal diagnosis, 25-Hydroxyvitamin D (25-OHD) level, 1,25-Dihydroxyvitamin $\mathrm{D}(1,25-\mathrm{OHD})$ level, severity, and clinical outcomes of the disease.

Results: Nineteen patients were included. The median age was 21 months, most of them have normal nutritional status (17/19). Source of infection were pneumonia (6), urinary tract infection (2), catheter related blood stream infection (1), and others (10). The prevalence of $25-\mathrm{OHD}$ deficiency $(<30 \mathrm{ng} / \mathrm{ml})$ and 1,25-OHD deficiency $(<19.9 \mathrm{pg} /$ $\mathrm{ml}$ ) in severe sepsis and septic shock patients were $57.9 \%$ and $10.5 \%$ respectively. All patients were survived. Vitamin D deficiency (25$\mathrm{OHD}$ and $1,25-\mathrm{OHD}$ ) was not correlated with length of stay in pediatric intensive care unit (25-OHD: $r=0.076, p=0.756 ; 1,25-\mathrm{OHD}$ : $r=0.170, p=0.486$ ), and vasoactive inotropic score (25-OHD: $r=$ $0.20, p=0.408 ; 1,25-\mathrm{OHD}: \mathrm{r}=0.077, \mathrm{p}=0.755)$. Subgroup analysis of patients who did not received antibiotics before enrollment, $25-\mathrm{OHD}$ 
deficiency had higher risk of positive blood culture compare to the group with normal 25-OHD level (odds ratio $=2.75,95 \% \mathrm{Cl} 1.26,6.01$, $\mathrm{p}=0.017)$ and $1,25-\mathrm{OHD}$ level had statistically significant inverse correlation with PRISM III score $(r=-0.527, p=0.036)$ (figure 1$)$.

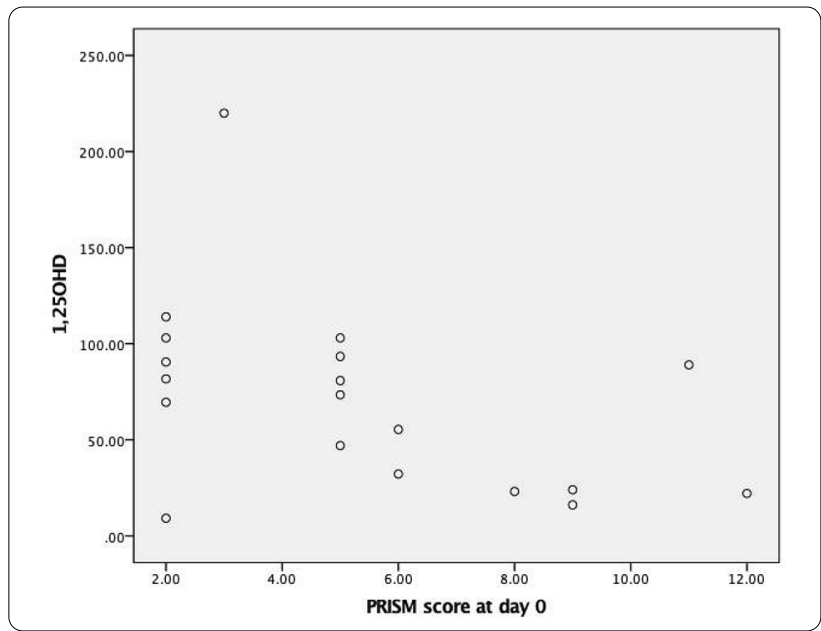

Conclusion: Our findings suggested that there may be a correlation between vitamin D deficiency and severity of illness in pediatric patients with severe sepsis and septic shock. Further study with larger sample size should be performed.

\section{Reference(s) and grant ackowledgment(s)}

1. 4. McNally JD, Menon K, Chakraborty P, Fisher L, Williams KA, Al-Dirbashi OY, et al. The Association of Vitamin D Status with Pediatric Critical Illness. Pediatrics. 2012; 130(3):429.

2. 3. Nguyen HB, Eshete B, Lau KHW, Sai A, Villarin M, Baylink D. Serum 1,25-Dihydroxyvitamin D: An Outcome Prognosticator in Human Sepsis. PLOS ONE. 2013; 8(5): e64348.

3. 2. Upala S, Sanguankep A, Permpalung. Significant association between vitamin $D$ deficiency and sepsis: a systematic review and meta-analysis. BMC Anesthesiology. 2015; 15:84.

4. 1. Udani S. The Role of Vitamin D in Sepsis: A review. Journal of Pediatric Critical Care. 2015; 2:33-6

5. Rachadapisaek Sompote Fund, Chulalongkorn University, Bangkok, Thailand

\section{7}

"Profile of a postoperative bariatric patient admitted to our Intensive Care Unit and its post-ICU metabolic control."

R. Mancha Salcedo'; FL. PEREZ CABALLERO' ${ }^{1}$; MS. Pereira Gama' ${ }^{1}$; S. Godoy Boraita'; I. Peña Luna'; M. Diaz-Tavora Luque'; MF. BenitezMorillo ${ }^{1}$ Unidad de cuidados intensivos, Hospital de Mérida, Mérida, Spain Correspondence: F.L. PEREZ CABALLERO

Intensive Care Medicine Experimental 2020, 8(2): 000777

Introduction: Obesity is defined by the World Health Organization as an excessive accumulation of fat in the body of people that can be harmful to their health, measured through a body mass index (BMI) $\geq 30 \mathrm{~kg} / \mathrm{m}^{2}$, cataloged as a chronic and identified disease in children, adolescents and adults, being recognized as the epidemic of the $21 \mathrm{st}$ century.

There are approximately 500 million obese adults and 42 million obese children under the age of five worldwide, hence the importance of their prevention, diagnosis and early treatment, to avoid subsequent comorbidities.

Obese patients present a high vital risk, since severe obesity is associated with cardiovascular, respiratory, metabolic, osteoarticular, digestive pathologies and even with the increase in some types of malignancies.
Surgery and bariatric procedures continue to be the most effective sustained weight loss option for patients with morbid obesity, highlighting among its main objectives to maximize weight loss and maintain nutritional health while preventing micronutrient deficiencies and loss of lean body mass.

Although it is an innovative and effective procedure, it is not exempt from post-surgical complications. It is essential to previously exhaust the resources of prevention and medical treatment, although most of these patients are not capable of maintaining a weight loss with psychological, dietary or pharmacological treatment.

Objectives: Main: To determine the profile of bariatric surgery patients admitted to our Intensive Care Unit (ICU) between May-2018 and November-2019 and its post- ICU metabolic control.

Secundary: To analyze the frequency of comorbidities, preoperative and postoperative characteristics, analytical results, ICU evolution, score on risk scales (APACHE, SOFA and SAPS II). To assess the degree of linear correlation between the BMI and the age of the patients, with the scores obtained on the risk assessment scales.

Methods: Observational, descriptive, prospective and correlational study in bariatric postoperative patients admitted to our ICU in 18 months, using a database of 40 patients, with medical and surgical history, anthropometric data, analytical results, type of surgery, postoperative treatments, and scores on the risk scales, in addition to a correlational analysis to determine the degree of linear relationship between BMI and age, with the risk scales, as well as the comparison of means of its post-ICU metabolic control.

Results: Among the 40 patients, the mean age was 42 years, women $(77.5 \%)$, with an average BMI of obesity type $3(46.55 \mathrm{kgm}-2)$. The most frequent type of surgery was gastric sleeve $(70 \%)$. Highlighting sleep apnea-hypopnea syndrome (SAHS) with CPAP $(62.5 \%)$, trauma pathologies (37.5\%), genitourinary and digestive (both 35\%), hypertension (30\%) and anxiety/depression (27, 5\%). Complications (surgical) were found only in $2.5 \%$ of patients, with a mortality rato of $0 \%$.

Performing the comparison of means through T-Student for related samples, we obtain a statistically significant decrease during the first six months after discharge from the ICU in LDL ( $p$ 0.018), HbA1c ( $p$ $<0.001)$ and baseline blood glucose $(p<0.001)$.

Upon their arrival in the ICU, prophylactic antibiotherapy was performed in $100 \%$ of them and anticoagulation in $75 \%$; being $10 \%$ of the airway difficult. $95.1 \%$ were admitted intubated, with an extubation time of 5 hours, and a 1-day stay. Analgesia was performed with Remifentanil (75\%), with a low incidence of complications $(0.02595 \%$ CI). 55\% required Sugammadex, and $40 \%$ weaning was performed with a T-tube only, and associated with CPAP in $32.5 \%$.

The high probability of average death is impressive: APACHE II of 19.95, SOFA 7.58 and SAPS II 55.25. We determined the level of correlation between $\mathrm{BMI}$ and age with the risk scales, obtaining a moderate correlation $r=0.414$ ( $p$ 0.008) between APACHE II and age; moderate $r=0.508$ ( $p$ 0.001) between SOFA and SAPS II; and low, $r=0.353$ ( $p$ 0.028 ) between SOFA and BMI.

Conclusion: The strongest associated variables were SAHS (with (PAP), traumatic, genitourinary and digestive pathologies, in women, obese type III, with high scores on risk scales, with a favorable evolution, with a minimum incidence of complications, and an adequate post-ICU metabolic control (LDL, $\mathrm{HbAc1}$ and baseline blood glucose), hence the importance that the postoperative period of these patients should be performed in the ICU.

Reference(s) and grant ackowledgment(s)

1. Rubio-Almanza M, Cámara-Gómez R, Hervás-Marín D, Ponce-Marco JL, Merino-Torres JF. Cardiovascular risk reduction over time in patients with diabetes or pre-diabetes undergoing bariatric surgery: data from a singlecenter retrospective observational study. BMC Endocr Disord. 2018;18(1):90. Published 2018 Nov 28. doi:10.1186/s12902-018-0317-4

2. Frigolet ME, Dong-Hoon K, Canizales-Quinteros S, Gutiérrez-Aguilar R. Obesity, adipose tissue, and bariatric surgery. Obesidad, tejido adiposo y cirugía bariátrica. Bol Med Hosp Infant Mex. 2020;77(1):3-14. doi:10.24875/ BMHIM.19000115

3. Deutsch L, Ben Haim L, Sofer Y, Gluck N, Santo E, Fishman S. Long-term effects of proximal small bowel exclusion by duodenal-jejunal bypass liner 
on weight reduction and glycemic control in diabetic patients. Surg Obes Relat Dis. 2018;14(10):1561-1569. doi:10.1016/j.soard.2018.07.022

4. SAPUNAR JORGE, ESCALONA ALEX, ARAYA A. VERÓNICA, AYLWIN CARMEN GLORIA, BASTÍAS MARÍA JULIANA, BOZA CAMILO et al . Rol de la cirugía bariátrica/metabólica en el manejo de la diabetes mellitus 2. Consenso SOCHED/SCCBM. Rev. méd. Chile [Internet]. 2018 Dic [citado 2020 Jun 28]; 146(10): 1175-1183.

\section{3}

Illusory movements improved basal metabolic rate in patients with metabolic failure

B. Bakalar ${ }^{1}$; R. Zajicek ${ }^{2}$; LM. Grünerova ${ }^{3}$

${ }^{1}$ Department of Anaesthesiology and Critical Care, 3rd Faculty of Medicine UK, Prague, Czech Republic; ${ }^{2}$ Department of bUrns, 3rd Faculty of Medicine UK, Prague, Czech Republic; ${ }^{3}$ Department of Physiotherapy and Rehabilitation, 3rd Faculty of Medicine UK, Prague, Czech Republic

Correspondence: B. Bakalar

Intensive Care Medicine Experimental 2020, 8(2): 000903

Introduction: Large burns are the cause of extensive skeletal muscle devastation which contributes to the risk of metabolic failure [1]. Illusory movements are a relatively new method of physiotherapy, which through functional proprioceptive stimulation (FPS) enables the integration and networking of muscle units and has a neurotrophic effect on the cerebral cortex [2]. However, illusory movements haven't been studied in the critically ill yet. How they influence human's metabolism is not known.

Objectives: Six male patients with large deep burns (> $30 \%$ total body surface area) in the late acute phase of burn disease, median age 53 years, median day since admission 96 days, median APACHE II score 19. They suffered from prolonged catabolism and poor healing. Their basal metabolic rate (BMR) was about $32 \%$ (means) below their calculated BMR.

Methods: We rehabilitated the patients with illusory movements for 30 minutes twice a day, for 14 days, along with standard rehabilitation. After two weeks, FPS was stopped, and patients received standard rehabilitation only. Nitrogen balance was calculated daily. On the day 1,15 , and 30, we measured BMR with indirect calorimetry, insulin resistance and glucose disposal, muscle strength, and diameter of $\mathrm{m}$. rectus femoris measured with ultrasound.

Results: In all six patients, BMR increased significantly compared to baseline ( $p<0.01$ ), with a (non-significant) trend to improve nitrogen balance and muscle strength. 14 days after the end of FPS, BMR decreased to the level of calculated BMR, but remained significantly increased from baseline $(p<0.05)(T a b .1)$. No adverse side effects were observed in any patient. All of them survived and were discharged from the hospital.

Table 1. Basal Metabolic Rate in study subjects

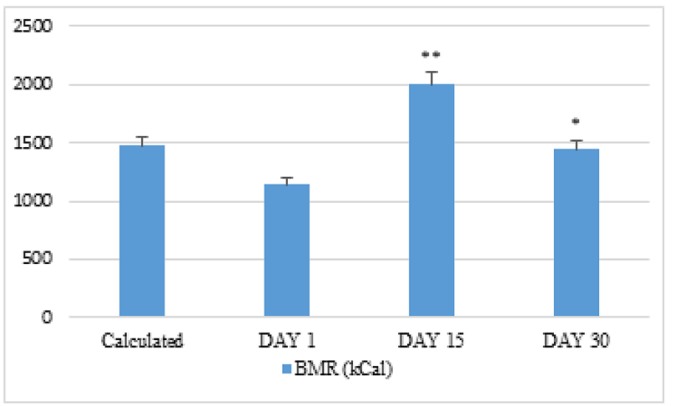

All values are group means $\pm \mathrm{SE}, * \mathrm{p}<0.05, * * \mathrm{p}<0.01$
Conclusion: Illusory movements improved decreased BMR in six patients with metabolic failure. It is not known whether the observed increase in energy expenditure is a quintessential feature of FPS, or if it was specific to patients with hypometabolism.

\section{Reference(s) and grant ackowledgment(s)}

1. 1. Singer, Mervyn MD. Metabolic failure. Crit Care Med 2005;33(12):S539-S542. 2. Roll R, Kavounoudias A, Albert F, et al. Illusory movements prevent cortical disruption caused by immobilization. NeuroImage. 2012;62(1):510-519.

\section{4}

Role of Capillary Blood Ketone Assay in Diagnosis and Management of DKA in PICU in resource limited settings M. Shahzad'; A. Haque ${ }^{2}$; M. Sadiq ${ }^{2}$

${ }^{1}$ Pediatric ICU, The Indus Hospital, Karachi, Pakistan; ${ }^{2} \mathrm{Picu}$, The Indus Hospital, Karachi, Pakistan

Correspondence: $M$. Shahzad

Intensive Care Medicine Experimental 2020, 8(2): 000964

Introduction: Diabetic ketoacidosis (DKA) is the most common lifethreatening metabolic-endocrine emergency in children. The current criterion for the diagnosis of DKA is characterized by a triad of hyperglycemia (blood glucose $\geq 200 \mathrm{mg} / \mathrm{d}$, metabolic acidosis (venous $\mathrm{pH}<7.3$, or serum $\mathrm{HCO} 3<15 \mathrm{mEq} / \mathrm{l}$ ) and ketonemia (blood $\beta$-hydroxybutyrate $\geq 3 \mathrm{mmol} / \mathrm{L}$ ) or moderate to large ketonuria(1). In DKA, $\beta$-hydroxybutyrate $(\mathrm{BOH} B)$ is the major ketone body and its concentration increased to 4-10 times.(2) Urine ketone assay has several potential limitations such as delayed voiding delay the diagnosis and unnecessarily prolonged insulin infusion because of continued excretion of ketoacid in the urine.(3) The current literature strongly support the use of capillary blood BHOB in the establishing diagnosis as well as response to treatment of DKA both in children and adults. (4) (5) There is a paucity of data available its use in the management of DKA in PICU. The objective of this study to assess the clinical and statistical correlation of capillary blood $\beta-\mathrm{OHB}$ level with the traditional standard acid variables like $p \mathrm{H}, \mathrm{HCO}, \mathrm{AG}$, and $\mathrm{BD}$ in the diagnosis and resolution of ketoacidosis in the management of DKA in PICU in resource limited settings.

Objectives: To affirm the Role of Capillary Blood Ketone Assay in Diagnosis and Management of DKA in PICU in resource limited settings.

Methods: We retrospectively reviewed electronic medical record of children (aged 2 mo-16yrs) with DKA from January 2018 to January 2020 admitted in PICU of The Indus Hospital. All children received the treatment according to standard protocol. Capillary blood BOHB were measured on admission and q 4-6 hourly along with blood glucose. All parameters were recorded on a structured sheet.

Results: Among 1080 admissions, 26 patients (2.4\%) were diagnosed as DKA. The mean age was $9.8 \pm 3.8$ years and females were $54 \%$. Half patients were newly diagnosed with 1DDM. Mild, moderate, and severe cases of DKA were 19\%, 50\%, and 31\% respectively. The median time to resolution of acidosis was 17 (10-39) hours. At the time of resolution of acidosis, the correlation between capillary BOHB and blood $\mathrm{pH}(\mathrm{r}=0.11, p=0.56), \mathrm{HCO} 3(\mathrm{r}=0.37, p=0.86), \mathrm{AG}(\mathrm{r}=0.37, p=0.06)$, and $\mathrm{BD}(r=0.04, p=0.82)$ was noted. Hyperchloremia was present in $61.5 \%$. Five patients (19.2\%) developed AKI that recovered. There was a strong clinical correlation of BHOB with standard acid variables of DKA management.

Conclusion: Bedside capillary blood BOHB is a simple, inexpensive, point-of-care test that helps in the diagnosis and treatment of DKA especially in resource-limited settings for avoiding unnecessary delays.

\section{Reference(s) and grant ackowledgment(s)}

1. 1. Wolfsdorf Jl, Glaser N, Agus M, Fritsch M, Hanas R, Rewers A, et al. ISPAD Clinical Practice Consensus Guidelines 2018: Diabetic ketoacidosis and the hyperglycemic hyperosmolar state. Pediatric diabetes. 2018;19:155-77. 2. Wallace T, Matthews D. Recent advances in the monitoring and management of diabetic ketoacidosis. Qjm. 2004;97(12):773-80. 3. Misra S, Oliver N. Utility of ketone measurement in the prevention, diagnosis and management of diabetic ketoacidosis. Diabetic Medicine. 2015;32(1):14-23. 4. 
Klocker A, Phelan H, Twigg S, Craig M. Blood $\beta$-hydroxybutyrate vs. urine acetoacetate testing for the prevention and management of ketoacidosis in Type 1 diabetes: a systematic review. Diabetic medicine. 2013;30(7):818 24. 5. Brooke J, Stiell M, Ojo O. Evaluation of the accuracy of capillary hydroxybutyrate measurement compared with other measurements in the diagnosis of diabetic ketoacidosis: a systematic review. International journal of environmental research and public health. 2016;13(9):837.

2. No grant funded

\section{5}

Copeptin is not a major determinant of stress-induced hyperglycemia: a prospective study

L. R. Henrique ; D. C. Moreira ${ }^{2}$; T. Viecelii ${ }^{3}$; A. F. Schaeffer ${ }^{1}$; P. Bellaver ${ }^{4}$;

C. B. Leitão ${ }^{2}$; T. Rech ${ }^{5}$

${ }^{1}$ School of medicine, Universidade Federal do Rio Grande do Sul, Porto Alegre, Brazil; 2 Endocrine division, Hospital de Clínicas de Porto Alegre, Porto Alegre, Brazil; ${ }^{3}$ Internal medicine division, Hospital de Clínicas de Porto Alegre, Porto Alegre, Brazil; ${ }^{4}$ Critical care division, Hospital de Clínicas de Porto Alegre, Porto Alegre, Brazil; ${ }^{5}$ Intensive care unit, Hospital de Clínicas de Porto Alegre, Porto Alegre, Brazil

Correspondence: A.F. Schaeffer

Intensive Care Medicine Experimental 2020, 8(2): 000995

Introduction: Changes in homeostasis activate the hypothalamicpituitary axis and induce an adaptive hypercatabolic state (1), which is harmful if persistent (2). Copeptin, an equimolar indicator of serum antidiuretic hormone (ADH), has been associated with a higher mortality of critically ill patients (3). However, alterations in copeptin levels over time during an acute stress are not known.

Objectives: The aim of the present study is to investigate the correlation of copeptin levels with glycemic parameters in critically ill patients.

Methods: From June 2019 to October 2019, critically ill adult patients were prospectively enrolled and followed for 90 days. Plasma copeptin levels were quantified during the first 24 hours of intensive care unit (ICU) admission (T1), $24 \mathrm{~h}$ after the study entry (T2), and $48 \mathrm{~h}$ after the study entry (T3). Blood glucose and glycated hemoglobin ( $\mathrm{HbA} 1 \mathrm{c})$ values were measured at study entry. ICU and hospital mortality, 30 and 90-day mortality, need for mechanical ventilation (VM), need for renal replacement therapy (RRT), time on VM and on RRT, length of stay in the ICU and hospital, and readmission to the ICU were evaluated.

Results: A total of 104 patients admitted to the ICU were included. Overall mortality was $40.4 \%(n=42)$. Weak inverse correlations were detected between blood glucose and copeptin T2 $(r=-0.23, p=0.03)$, and glycemic gap $(r=-0.25, p=0.03)$ and SHR $(r=-0.24, p=0.03)$ and copeptin T3. No associations were identified between copeptin values at T1 and T2 with clinical outcomes. However, copeptin values at T3 were significantly higher in survivors than in non-survivors at hospital discharge (561 pg/mL [370 to 856] vs $300 \mathrm{pg} / \mathrm{mL}$ [231 to 693], $\mathrm{p}=0.015)$, at 30 days (581 pg/mL [387 to 865$] \mathrm{vs} 299 \mathrm{pg} / \mathrm{mL}$ [231 to 690], $\mathrm{p}=0.02$ ) and at 90 days (517 pg/mL [380 to 884] vs $492 \mathrm{pg} / \mathrm{mL}$ [295 to 698], $p=0.03$ ). Copeptin values at T1 and T2 were not different between survivors and non-survivors.

Conclusion: Weak inverse correlations were found between copeptin levels and glycemic parameters, suggesting that copeptin is not a major factor in the induction of hyperglycemia during critical illness. Besides, copeptin values at the third ICU day were significantly higher in survivors than in non-survivors.

\section{Reference(s) and grantackowledgment(s)}

1. (1) Van den Berghe, G., de Zegher, F., \&amp; Bouillon, R. Acute and Prolonged Critical Illness as Different Neuroendocrine Paradigms 1. The Journal of Clinical Endocrinology \& Metabolism, 1998.

2. (2) Rothwell PM, Lawler PG. Prediction of outcome in intensive care patients using endocrine parameters. Crit Care Med. 1995.

3. (3) Koch A, Yagmur E, Hoss A, Buendgens L, Herbers U, Weiskirchen R, Koek $\mathrm{GH}$, Trautwein C, Tacke F. Clinical relevance of copeptin plasma levels as a biomarker of disease severity and mortality in critically ill patients. J Clin Lab Anal. 2018.
4. This work was supported by Fundo de Incentivo à Pesquisa e Ensino (FIPE), Hospital de Clínicas de Porto Alegre (project number 2019-0304).

5. DC and CBL have received scholarships from Conselho Nacional de Desenvolvimento Científico e Tecnológico (CNPq; PQ-1D).

001032

Diabetic Ketoacidosis : Do We Have to Give Too Many Intravenous Fluid ?

N. H. BRAHMI'; R. Soedono ${ }^{1}$

${ }^{1}$ Anesthesiology and Intensive Care, Fakultas Kedokteran Universitas Indonesia, Jakarta, Indonesia

Correspondence: N.H. BRAHMI

Intensive Care Medicine Experimental 2020, 8(2): 001032

Introduction: Approach consideration in DKA are intravenous fluid, correction of hyperglycemia with insulin, electrolytes disturbances, acid base balance and treatment to precipitating condition. Many patients with DKA were given fluid more than 2 litre in the initial 4 hours developed into lung edema and longer period of hospitalization.

Objectives: To evaluate the outcome between patients in who were given more than 3 litre in the initial 4 hours of treatment to patients who were given maintenance fluid dose in emergency room

Methods: We had 6 patients diagnosed with DKA, in their 30's and 40 's. They are obese type II diabetic with oral hypoglycemia medication. Before admission, all of them reported suffered from infection: CAP, UTI, gastrointestinal infection. There was no sign of nausea, vomitus, or dehydration. All of them in mild hypotension with normal serum sodium level. There were 2 group, 1 group, the liberal fluid, were given more than 3 liter $\mathrm{NaCl} 0,9 \%$ (7 to $14 \mathrm{ml} / \mathrm{kg} / \mathrm{hour}$, with initial boluses 1 litre $\mathrm{NaCl}$ 0,9\% before within an hour) in the early 4 hours phase of the treatment, the other only given 1 litre initial boluses $\mathrm{NaCL}$ $0,9 \%$ within an hour then followed by maintenance fluid $25-30 \mathrm{ml} /$ $\mathrm{kg} / \mathrm{d} \mathrm{NaCl} 0,9 \%$. All of them were given insulin intravenously depend on their glucose status hourly, then we maintained the glucose level to $150-200 \mathrm{mg} / \mathrm{dL}$, deescalation antibiotics broad spectrum, continuous monitored hemodynamics and level of consciousness. We also documented fluid balance, urin output every hour, and acid base, electrolite, and ketonuric status every 12 hour. Fluid challenge test was given if urine output not reached target,and will be given additional fluid to reach normorehydration status. If there is imbalance electrolyte present, we corrected it with supplement potassium intravenously if needed.

Results: In both groups, DKA were resolved within first 24 hours. All the patients regain their consciousness, the blood glucose were reach the target. In the liberal fluid, 1 patient developed lung edema, so we need to intubate him, 1 patient with positive balance without clinical deteoritation, and the other one recovered well. The 2 nd group, none had lung edema, but 1 patient was given fluid challenge test once and responded well so we gave extra $500 \mathrm{ml} \mathrm{NaCl} 0,9 \%$ within 3 hours to total maintenance fluid. 5 out of 6 were able to be stepped down to the ward, while 1 patient from the liberal fluid stepped up to intensive care unit.

Conclusion: DKA occurred in the obese diabetic type II patient precipitated by infection, resolved well within 24 hours with deescalation technique on antibiotics, insulin therapy, and maintenance dose of fluid.

Reference(s) and grant ackowledgment(s)

1. Gosmanov AR, Gosmanova EO, Dillard-Cannon E. Management of adult diabetic ketoacidosis. Diabetes Metab Syndr Obes. 2014;7:255-264. Published 2014 Jun 30. doi:10.2147/DMSO.S50516

2. Adrogué HJ, Barrero J, Eknoyan G. Salutary Effects of Modest Fluid

Replacement in the Treatment of Adults With Diabetic Ketoacidosis: Use in Patients Without Extreme Volume Deficit. JAMA. 1989;262(15):2108-2113. doi:10.1001/jama.1989.03430150076029

3. ABBAS E. KITABCHI, PH.D., M.D., and BARRY M. WALL, M.D., Management of Diabetic Ketoacidosis. Am Fam Physician. 1999 Aug 1;60(2):455-464 


\section{2}

Glycaemic control predicts mortality in non-diabetic, critically ill patients with COVID-19

S. Lockhart ${ }^{1}$; B. Petrisor ${ }^{1}$; A. Usman ${ }^{1}$; H. Griffiths ${ }^{1}$; L. Heales ${ }^{2}$; V. Bansiya ${ }^{3}$;

R. Mahroof ${ }^{1}$; A. Conway-Morris ${ }^{4}$

'John farman Intensive Care Unit, Cambridge University Hospitals NHS Foundation Trust, Cambridge, United Kingdom; ${ }^{2}$ Department of Anaesthetics \& Intensive Care Medicine, Cambridge University Hospitals NHS Foundation Trust, Cambridge, United Kingdom; ${ }^{3}$ Department of Diabetes \& Endocrinology, Cambridge University Hospitals NHS Foundation Trust, Cambridge, United Kingdom; ${ }^{4}$ Division of Anaesthesia, Department of Medicine, University of Cambridge, Cambridge, United Kingdom

Correspondence: S. Lockhart

Intensive Care Medicine Experimental 2020, 8(2): 001082

Introduction: Analysis of glycaemia in non-critically ill patients with COVID-19 has suggested that hyperglycaemia is associated with poor outcomes in patients with and without diabetes. However, to our knowledge there has not been a longitudinal assessment of glycaemic control and mortality in critically ill patients with COVID-19.

Methods: Retrospective observational study of contemporaneous electronic medical records of patients admitted to our intensive care unit (ICU) for $>24$ hours with COVID-19 during the months of March and April 2020. Glycaemic control was assessed relative to the target range of $4-10 \mathrm{mmol} / \mathrm{L}$ by three related metrics: estimated time in range $4-10 \mathrm{mmol} / \mathrm{L}$, percentage of $B G$ measurements between 4 and $10 \mathrm{mmol} / \mathrm{L}$, and mean BG.

Results: 54 patients met inclusion criteria, 1 patient had incomplete BG data and was omitted from our analysis. Median time in range was $86 \%$, similar to previous studies. Hypoglycaemia was rare with 4 patients experiencing mild hypoglycaemia (BG $<4 \mathrm{mmol} / \mathrm{L}$ ) and 2 patients experiencing biochemically severe hypoglycaemia (BG $<2.5$ $\mathrm{mmol} / \mathrm{L}$ ). In the whole cohort we did not find a difference in any glucose metric between those discharged alive from ICU or those who died in ICU. However, we did observe an interaction between diabetes status and ICU outcome with respect to mean blood glucose $(P<0.05)$, suggesting that the relationship between glycaemia and mortality may depend on diabetes status. Indeed, the percentage of BG measurements in range, time in range and mean blood glucose were significantly different in ICU survivors and non-survivors in the cohort without diabetes ( $N=42, \mathrm{P}<0.05$ for all metrics) with better glycaemic control observed in ICU survivors. In univariate logistic regression analyses mean $\mathrm{BG}(\mathrm{OR}=0.44,95 \% \mathrm{Cl} 0.19-0.84, \mathrm{P}=0.02)$ and $\mathrm{BG}$ measurements in range $(\mathrm{OR}=1.05,95 \% \mathrm{Cl} 1.00-1.11, \mathrm{P}=$ 0.04) predicted discharge alive from ICU with better glycaemic control positively associating with survival. Interestingly, both mean BG and BG measurements in range had superior predictive ability for ICU mortality to BMl, steroid use, sex and cross-sectional measurements of CRP, Troponin and D-Dimer in univariate analyses. To determine the additive predictive effect of glycaemia to the dominant risk factor for COVID-19 mortality, age, we examined the effect of glycaemia (BG measurements in range) in a model adjusted for age. The model explained $22 \%$ of the variance of the data, compared to $11 \%$ forage alone with a similar effect size for glycaemia as was seen in the univariate analysis (OR $1.05 \mathrm{Cl}: 1.00-1.11)$.

Conclusion: Here we demonstrate, for the first time, an association between glycaemia and mortality in critically ill non-diabetic patients with COVID-19. Given the high prevalence of hyperglycaemia in critically ill patients with COVID-19 further assessment of the interaction between hyperglycaemia and ICU outcomes in diabetic and non-diabetic patients is warranted.

\section{Reference(s) and grant ackowledgment(s)}

1. SL is supported by an Academic Clinical Fellowship from the National Institute of Health Research

2. ACM is supported by a Wellcome Trust grant (WT 2055214/Z/16/Z)
001093

Ability of NUTRIC and Modified NUTRIC score to predict ICU mortality in critically ill COVID-19 patients

A. Liberti' ; E. Piacentino ${ }^{1}$; G. Catzula ${ }^{1}$; E. Clemenza ${ }^{1}$; M. Umbrello ${ }^{1}$; S. Muttini ${ }^{1}$ ${ }^{1}$ Uo anestesia e rianimazione, Ospedale San Carlo Borromeo, ASST Santi Paolo e Carlo, MILANO, Italy

Correspondence: M. Umbrello

Intensive Care Medicine Experimental 2020, 8(2): 001093

Introduction: International guidelines recommend the assessment of nutrition status before the initiation of nutritional support. NUTrition Risk in the Critically ill (NUTRIC) score has been proposed to quantify the risk of adverse outcomes that may be modified by artificial nutrition. A "modified NUTRIC score" (mNUTRIC) has been introduced without IL-6 levels. An unprecedented number of relatively young and previously healthy patients has been admitted to the ICU during the recent COVID-19 outbreak. These patients often experienced a dysregulated inflammatory response, potentially leading to a cytochine storm and multiple organ damage. In this population, IL-6 has been measured to assess the inflammatory status, allowing the calculation of NUTRIC and mNUTRIC scores.

Objectives: To assess whether NUTRIC score is a reliable nutritional risk assessment tool in patients with COVID-19-related acute respiratory failure and to compare the prognostic performance of NUTRIC and mNUTRIC score.

Methods: Single centre, retrospective cohort study in critically ill patients with COVID-19-related acute respiratory failure. Patients with NUTRIC or mNUTRIC score $\geq 5$ were considered at high nutritional risk. The prognostic performance of NUTRIC and mNUTRIC score were compared, as well as their discriminative abilities for ICU-mortality.

Results: 42 patients were enrolled: $34(79 \%)$ males, age $62 \pm 9$, BMI 27.6 \pm 4.2. Average NUTRIC score: $2.5 \pm 1$; mNUTRIC score: $2.6 \pm 1.1$.

ICU mortality was $40.5 \%$; all non-survivors had mNUTRIC score $\leq 4$ (low nutritional risk); only one non-survivor had a high nutritional risk (NUTRIC Score $\geq 5$ ). Non-survivors were older ( $67.5 \pm 6.7$ vs. $59.0 \pm 9.9$ years, $\mathrm{p}=0.0036)$, had higher IL-6 blood levels $(152[78 ; 264]$ vs. 53 [12; 109], $\mathrm{p}=0.0202)$ and a higher NUTRIC score $(3[2 ; 3]$ vs. $2[2 ; 3]$ points, $p=0.0425)$. mNUTRIC score was not different between survivors and non-survivors $(p=0.0743)$.

In multivariate logistic analysis, NUTRIC score was significantly related with ICU mortality (OR 2.49 [95\% Cl 1.02-6.10]). The discriminative ability of NUTRIC score for ICU mortality was (AUROC) 0.67 [0.525-0.826], while that of mNUTRIC-score was 0.66 [0.514-0.817], $\mathrm{p}=0.4109$.

Conclusion: Despite a relatively low nutritional risk, NUTRIC score seems to identify a subpopulation of critically ill, COVID-19 patients who may benefit from nutritional support. The prognostic performance of NUTRIC and mNUTRIC score is comparable in patients with high inflammatory status as in COVID-19.

\section{Reference(s) and grant ackowledgment(s)}

1. Rahman A, et al. Identifying critically-ill patients who will benefit most from nutritional therapy: Further validation of the "modified NUTRIC" nutritional risk assessment tool. Clin Nutr. 2016:35:158-162.

\section{8}

Ultrasonography skeletal muscle assessment predicts worse outcome in critically ill patients under mechanical ventilation: observational cohort study

B. Cardoso Gomes'; B. De Freitas²; M. Jacques³; D. Toledo ${ }^{4}$; D. Santos²; F. Do Amaral'; R. Dib'2; JM. Silva²

${ }^{1}$ Departamento de Medicina Integrada, Universidade Federal do Paraná, Curitiba, France; ${ }^{2}$ cu, Hospital Israelita Albert Einstein, São Paulo, Brazil ${ }^{3}$ Psicologia, UniCesumar - Curitiba Campus, Curitiba, Brazili; ${ }^{4}$ Medicina, USP - University City, São Paulo, Brazil

Correspondence: B. Cardoso Gomes

Intensive Care Medicine Experimental 2020, 8(2): 001128

Introduction: Survivors of critical illness experience significant skeletal muscle wasting that may predict clinical outcome. Ultrasound (US) is a noninvasive method that can measure muscle quadriceps muscle layer thickness (QMLT) at the bedside. 
Objectives: The objective of this study was to determine muscle wasting (assessed using US) in critically ill patient over a period of 7 days and the relationship between loss of muscle quadriceps thickness and hospital survival probability in critically ill patient under mechanical ventilation.

Methods: The data were entered into an electronic database (Microsoft Excel; Microsoft, Redmond, Washington) and analyzed using SPSS V.26. Data collection was halted after the successful inclusion of 70 patients. Demographic data were expressed as the means $\pm S D$, medians and percentiles or percentages. This study involved patients $\geq$ 18 years of age admitted to the intensive care unit (ICU) who needed mechanical ventilation for at least 48 hours. The QMLT at the twothirds-thigh was quantified using bedside US with a $12 \mathrm{MHz}$ linear transducer. Measurements of the first (D1), third (D3) and seventh (D7) days were performed of QMLT (mean \pm SD) was displayed for left and right legs.

Results: Ultrasound quadriceps thickness measurements were performed on 74 critically ill patients. The mean age was $62.3 \pm 19.5$ years, $54.1 \%$ male, BMI $25.5 \pm 4.6 \mathrm{~kg} / \mathrm{m} 2$, SAPS 3 was $55.2 \pm 17.2$ and NRS $3.2 \pm 1.0$. Overall, the percentage muscle thickness declined at the right leg in $15 \%(95 \% \mathrm{Cl}, 10.5 \%$ to $19.4 \%)$, and $12.7 \%(95 \% \mathrm{Cl}, 9.1 \%$ to $16.3 \%)$ at the left leg assessed by the US occurred from the first to the seventh day. The decline in muscle thickness at the right leg and left leg were higher among non-survivors compared to survivors at all time points. In right leg $2.1 \pm 0.7 \mathrm{~cm}$ in survivor vs $1.7 \pm 0.5 \mathrm{~cm}$ nonsurvivor at D1, $1.9 \pm 0.7 \mathrm{~cm}$ in survivor vs $1.6 \pm 0.4 \mathrm{~cm}$ nonsurvivor at $\mathrm{D} 3,1.8 \pm 0.6 \mathrm{~cm}$ in survivor vs $1.4 \pm 0.4 \mathrm{~cm}$ nonsurvivor at $D 7 ; G L M-P=0.01$. In left leg $2.1 \pm 0.7 \mathrm{~cm}$ in survivor vs $1.7 \pm 0.4 \mathrm{~cm}$ nonsurvivor at $\mathrm{D} 1,2.0 \pm 0.8 \mathrm{~cm}$ in survivor vs $1.7 \pm 0.5 \mathrm{~cm}$ nonsurvivor at $\mathrm{D} 3,1.8 \pm 0.6 \mathrm{~cm}$ in survivor vs $1.5 \pm 0.5 \mathrm{~cm}$ nonsurvivor at D7; GLM- P $=0.04$. ROC analysis showed that a cutoff of decline in muscle thickness of less than or equal to $1.64 \mathrm{~cm}$ at day 7 can predict survival, $A U C=0.7$ ( $95 \% \mathrm{Cl} 0,582$ to 0,801$)$. Thus, in cox regression after adjusting for SAPS 3 and gender, the probability of patients remaining in the mechanical ventilation was higher with $\leq 1.64 \mathrm{~cm}$ at day 7 of loss of thigh muscle thickness, the hazard was 2.1 times $(95 \% \mathrm{Cl} 1.1-3.8)$ higher than their counterparts with $p$ value 0.017 . The same occurred $\leq 1.64 \mathrm{~cm}$ at day 7 of loss of thigh muscle thickness about ICU survival probability; $\mathrm{HR}=3.7(95 \%$ $\mathrm{Cl} 1.2$ to 11.5$)$ and hospital survival probability; $\mathrm{HR}=4.5(95 \% \mathrm{Cl} 1.5$ to 13.7).

Conclusion: Among these critically ill patients, muscle wasting occurred during the first week of critical illness under mechanical ventilation. The patients who lost the most muscle was those with the worst survival. Decline in muscle thickness was associated with in-hospital mortality. USG has a potential to identify patients at risk of worse in-hospital outcomes.

\section{Reference(s) and grant ackowledgment(s) \\ 1. 13. Tillquist M,Kutsogiannis DJ, Wischmeyer PE, et al. Bedside ultrasound is apractical and reliable measurement tool for assessing quadriceps muscle layer thickness. JPEN Journal of parenteral and enteral nutrition. 2014;38(7):886-890. \\ 2. Parry SM, El-Ansary D, Cartwright MS, et al. Ultrasonography in the intensive care setting can be used to detect changes in the quality and quantity of muscle and is related to muscle strength and function. Journal of critical care. 2015;30(5):1151e1159-1114}

\section{5}

Retrospective audit on the use of hypertonic saline in the management of acute symptomatic hyponatraemia in hospitalised patients

B. Nicklas ${ }^{1}$; D. Sharma ${ }^{1}$; V. Mishra

'Biochemistry, Royal Liverpool and Broadgreen University Hospitals NHS Trust, Prescot Street, Liverpool, UK, Liverpool, UK, United Kingdom

Correspondence: $\mathrm{D}$. Sharma

Intensive Care Medicine Experimental 2020, 8(2): 001185

Introduction: We introduced the hospital policy on the management of severe symptomatic hyponatraemia (SSH), with hypertonic saline
(HS) of $1.8 \%$ and $5 \%$ saline in 2017 . This is one of the few UK centres to offer this treatment, therefore the evidence is limited. The policy was based on European and American guidelines (1)(2).

Objectives: To study the adherence, and effectiveness, of this guideline in managing patients with SSH.

Methods: Patients were identified using the Biochemistry tracking system between 01/01/18 until 14/05/20. The terms - Hypona, sod, Hypertonic, $5 \%, 1.8 \%$ a were searched. The laboratory result system was used to identify hyponatraemic $(<130 \mathrm{mmol} / \mathrm{L})$ patients at the time they were logged onto the tracking system. Clinical notes were crossmatched for the use of $1.8 \%$ and $5 \%$ saline for those with sodium $<130 \mathrm{mmol} / \mathrm{L}$. 12 patients were identified.

The following data was collected using hospital records: age, gender, location, fluid status, presentation, symptoms after HS adminsitration, volume and type of fluids. Sodium was recorded; prior to and during presentation, after HS saline, at 24, 48 hours and at discharge or death. Cause of death (if applicable), complications of HS and lenght of admission after treatment were also noted. This was compared to the 2017 current hospital guideline

\begin{tabular}{l|l|r|}
\hline Standard 1: patients treated with hypertonic saline should be symptomatic of hyponatraemia: & $9 / 12^{1}$ & $75 \%$ \\
\hline Standard 2a: Severe Symtomatic Hyponatraemia should be treated with 5\% Saline: & $0 / 9$ & $0 \%$ \\
\hline Standard 2b: Moderately Symtomatic Hyponatraemia should betreated with 1.8\% Saline: & $2 / 2$ & $100 \%$ \\
\hline Standard 2c: Serum Na rise S5 with hypertonic saline: & $5 / 12^{2}$ & $42 \%$ \\
\hline Standard 2d: Clinical improvement of symptoms after HS: & $9 / 11^{3}$ & $82 \%$ \\
\hline Standard 2e: HS administered as per the protocol: & $9 / 12^{4}$ & $75 \%$ \\
\hline Standard 2f: Normal saline administered after hypertonic saline & $10 / 12^{5}$ & $83 \%$ \\
\hline Standard 3a: $\leq 10 \mathrm{~mm}$ ol rise in 24 Hours: & $5 / 12^{6}$ & $42 \%$ \\
\hline Standard 3b: $\leq 18 \mathrm{~mm}$ ol rise in 48Hours: & $7 / 12^{7}$ & $58 \%$ \\
\hline
\end{tabular}

\section{Results:}

Standard 1: 11 patients had symptoms of hyponatraemia. They were confusion (6), somnolence (5), seizure (4), vomiting (3). Other sympotms included muscle twitching, fatigue and nausea. SSH was suspected in 2/11 patients, but it was not the primary diagnoses. One patient was asymptomatic- the only patient with chronic hyponatraemia.

Standard 2a/b: All patients identified were treated with $1.8 \%$ saline. None with $5 \%$ saline.

Standard 2c: $7 / 12$ patients overcorrected (there should be a $<5 \mathrm{mmol} / \mathrm{L}$ increase following HS). In $4 / 7$ there was inappropriate application of the guideline; HS was given despite improvement of symptoms or after the $5 \mathrm{mmol} / \mathrm{L}$ target had been achieved.

An average volume of $481 \mathrm{ml}$ (range $100-1250 \mathrm{ml}$ ) HS was given over an average of $3 \mathrm{~h} 53 \mathrm{~m}$. This increased sodium by mean of $6.4 \mathrm{mmol} / \mathrm{L}$ (range 2-12) by the end of treatment with HS.

Standard 2d: 9/11 patients had atleast partial or full improvement in the symptoms at the end of treatment with $1.8 \%$ saline. In 2/11 whose symptoms were secondary to other causes (paranoia/ hallucinations), did not have improvement of symptoms after HS.

Standard 2e: 9/12 patients had HS administered as per the recommended guideline calculation for rate. 2 patients were hypervolaemic and were treated with a slower infusion to prevent overload. 1 patient was treated using $100 \mathrm{mls} 1.8 \%$ saline for no clear reason.

Standard 2f: 2 patients were clinically hypervolaemic - NS was avoided due to risk of overload. 1 patient had a $9 \mathrm{mmol}$ increase after 24 hours, $(<10 \mathrm{mmol}$ increase advised).

Standard 3a/b: There was $7 / 12$ who overcorrected at 24 hours, and $5 / 12$ at 48 hours (should be $<10 \mathrm{mmol} / \mathrm{L}$ and $<18 \mathrm{mmol} / \mathrm{L}$, respectively). The mean increase in serum sodium at 24 and 48 hours were $12.25 \mathrm{mmol} / \mathrm{L}$ (range $7-21$ ) and $17.25 \mathrm{mmol} / \mathrm{L}$ (range $10-26$ ) respectively. $11 / 12$ patients eventually normalised (>130 $\mathrm{mmol} / \mathrm{L}$ ) their serum sodium. 1 patient self discharged before this. The mean lenght of time to achieve this was 6.5 days.

In addition: 3 patients died. No deaths were attributed to treatment with $1.8 \%$ saline. Specifically, no patients developed central pontine myelinolysis

Conclusion:

1. Our audit showed symptomatic improvement in moderate and SSH, following HS use. 
2. Patients with symptoms due to other causes, did not show improvement in symptoms following $\mathrm{HS}$. Before administrating $\mathrm{HS}, \mathrm{SSH}$ should be the most likely diagnoses.

3. 5\% Saline was not adminstered, it should be removed from the guidelines $1.8 \%$ can safely be used in severe and moderate cases.

4. The guideline was inappropriately followed for cases where there was overcorrection following HS. It should be clearly stated that HS can be stopped once there is symptomatic improvement, even if this occurs prior to the $5 \mathrm{mmol} / \mathrm{L}$ target

5. The increase in serum sodium at 24 hours and 48 hours should be $<10 \mathrm{mmol}$ and $<18 \mathrm{mmol}$ from baseline. This includes administeration of HS followed by NS.

6. No adverse events were identified from treatment with HS or from overcorrection.

\section{Reference(s) and grant ackowledgment(s)}

1. (1) Spasovski G, Vanholder R, Allolio B, Annane D, Ball S, Bichet D, Decaux G, Fenske W, Hoorn E, Ichai C, et al. Clinical practice guideline on diagnosis and treatment of hyponatraemia. European Journal of Endocrinology. 2014;170:G1-G47. doi: 10.1530/EJE-13-1020

2. (2) Verbalis JG, Goldsmith SR, Greenberg A, Korzelius C, Schrier RW, Sterns $\mathrm{RH}$, Thompson CJ. Diagnosis, evaluation, and treatment of hyponatremia: expert panel recommendations. American Journal of Medicine. 2013;126:S1-S42. doi: 10.1016/j.amjmed.2013.07.006

\section{8}

Adiposity, but not leptin, protects against critical illness induced muscle wasting and weakness

W. Vankrunkelsven ${ }^{1}$; S. Derde ; S. Vander Perre ; M. Boone ; G. Van den

Berghe ; L. Langouche

${ }^{1}$ Department of cellular and molecular medicine, laboratory of intensive care medicine, Katholieke Universiteit Leuven, Leuven, Belgium

Correspondence: W. Vankrunkelsven

Intensive Care Medicine Experimental 2020, 8(2): 000348

Introduction: Critical illness-induced weakness is prevalent and associated with increased morbidity and mortality. Interestingly, obesity attenuates both muscle wasting and weakness.1,2 We here aimed to investigate whether the adipokine leptin mediates this protective effect.

Methods: In a catheterized, antibiotic-treated, fluid-resuscitated mouse model of sepsis, evoked by cecal ligation and puncture, impact of leptin availability was compared in obese and lean mice. In four groups of septic mice ( $n \geq 11$ survivors per group) - diet induced obese (Ob-DIO), leptin-deficient obese (Ob-KO), placebo-treated lean (Ln) and leptin-treated lean (Ln-lep) mice - body composition (MRI), specific muscle force (ex vivo contractibility in $\mathrm{mN} / \mathrm{mm} 2$ per mass $E D L$ ) and plasma markers of lipolysis and inflammation were measured.

Results: Prior to sepsis, plasma leptin was undetectable in $\mathrm{Ob}-\mathrm{KO}$, and higher in Ob-DIO (12.4[8.2-15.1]ng/ml) than Ln (1.8[0.9-3.0]ng/ml) $(p$ $<.0001)$. Ob-DIO and Ob-KO were similar in body weight $(\mathrm{p}=.5)$ but $46 \%$ heavier than lean mice. Ob-KO $(22.4 \pm 2.6 \mathrm{~g})$ had more fat mass than Ob-DIO $(19.3 \pm 1.8 \mathrm{~g})$ and $\operatorname{Ln}(2.7 \pm 1.2 \mathrm{~g})$, but less lean mass (19.3 $\pm 1.8 \mathrm{~g})$ than Ob-DIO $(25.8 \pm 2.2 \mathrm{~g})$ or $\operatorname{Ln}(24.8 \pm 2.1 \mathrm{~g})(\mathrm{p}<.0001$ for all $)$. After 5 days of sepsis, mortality was highest in Ob-KO $(53 \%)(p \leq .06)$ but was comparable in Ln (39\%), Ln-lep (36\%) and Ob-DIO (23\%). Plasma leptin was undetectable in Ob-KO, higher in Ob-DIO (15.4[12.6$19.4] \mathrm{ng} / \mathrm{ml})$ and Ln-lep (34.7[14.7-135.4]ng/ml) than $\operatorname{Ln}(0.2[0.1-0.7]$ $\mathrm{ng} / \mathrm{ml})(\mathrm{p}<.0001)$. Loss of lean mass loss was $2 x$ more pronounced in lean than in obese mice ( $p<.0005)$, but not affected by leptin availability in lean $(\Delta 4.2 \pm 1.6 \mathrm{~g} \mathrm{Ln}$ and $3.3 \pm 2.0 \mathrm{~g} \mathrm{Ln}$-lep, $\mathrm{p}=.2)$ or obese $(\Delta 1.8 \pm 1.1 \mathrm{~g} \mathrm{Ob}-\mathrm{DIO}$ and $1.3 \pm 1.1 \mathrm{~g} \mathrm{Ob}-\mathrm{KO}, \mathrm{p}=.2)$. Functionally, Ob$\mathrm{DIO}$ and Ob-KO mice maintained their specific muscle force $(\mathrm{p} \geq .1)$ up to healthy levels $(98.8 \%$ and $116.5 \%$ of healthy control, respectively), whereas $\mathrm{Ln}$ and Ln-Lep mice suffered from reduced specific muscle force $(77.9 \%$ and $65.4 \%$ of healthy control, respectively, $\mathrm{p} \leq$ $.01)$, again not affected by leptin availability ( $p \geq .2)$. In contrast to muscle, fat mass loss was $2 x$ more pronounced in obese than in lean $(p<$ $.0001)$, greatest in Ob-DIO $(\Delta 4.2 \pm 0.8 \mathrm{~g} \mathrm{Ob-DIO}$ and $3.2 \pm 0.7 \mathrm{~g} \mathrm{Ob}-\mathrm{KO}$, $\mathrm{p}=.0002)$, but was not affected by leptin in lean $(\Delta 1.9 \pm 0.5 \mathrm{~g} \mathrm{Ln}$ and $2.1 \pm 0.8 \mathrm{~g}$ Ln-lep, $\mathrm{p}=$ =.4). In accordance, both $\mathrm{Ob}-\mathrm{DIO}$ and $\mathrm{Ob}-\mathrm{KO}$ mice had increased plasma free fatty acids, glycerol and peak 3-hydroxybutyrate concentrations compared to Ln and Ln-lep $(p \leq .01)$. Both obese groups showed an attenuated inflammatory response compared to both Ln and Ln-lep, evident by a 50\% lower plasma TNFa increase (p $<$.005).

Conclusion: Not increased leptin availability, but adiposity attenuated critical illness induced muscle wasting and weakness. A more efficient mobilisation of fat stores and ketogenesis, as shown previously2, but also a blunted inflammatory response might play a mediating role.

\author{
Reference(s) and grant ackowledgment(s) \\ 1. 2. Goossens et al, Crit Care 2019 \\ 2. 1. Goossens C et al. JCSM 2017 \\ 3. Study funded by FWO (G.0C78.17N) and ERC (AdvG 2017-785809)
}

\section{5}

Bio-electrical impedance analysis (BIA) in healthy volunteers in various conditions by various observers with various devices: what matters?

I. Myatchin ${ }^{1}$; A. Minini ${ }^{1}$; M. Diltoer ${ }^{1}$; MLNG. Malbrain

${ }^{1}$ Intensive care unit, University Hospital Brussels (UZB), Jette, Belgium

Correspondence: I. Myatchin

Intensive Care Medicine Experimental 2020, 8(2): 000425

Introduction: To examine 1) inter-device, inter- and intra-observer variability of BIA measurements and 2) effect of fluid load on BIA measurements in healthy adult volunteers.

Methods: We studied two groups of ten healthy adults (women:men $=1: 1$ in each group). In the first group, three observers performed in each participant three tests on the right and three tests on the left side with two BIA devices (BioScan Touch i8 (Maltron Int. Ltd) and Nutrilab (Akern SRL)). In the second group, BIA tests were done in each participant before and after (immediately - $30 \mathrm{~min}-60 \mathrm{~min}-120 \mathrm{~min}$ later) the fluid load (i.e., drinking of $0.5 \mathrm{~L}$ or $1 \mathrm{~L}$ of bottled still water). TBW (total body water), ECW (extracellular water), ICW (intracellular water), ECW/ICW, BCM (body cell mass), FM (fat mass), FFM (fat-free mass), plasma and interstitial fluid volume, and $\mathrm{PhA}$ (phase angle at $50 \mathrm{kHz}$ ) were compared.

Results: 1) No significant differences between BIA parameters while tested by the same observer. Significant differences were seen in all BIA parameters between BIA devices $(p<0.001)$. In tests obtained with BioScan, no significant interobserver difference was seen, while significant $(p=0.004)$ and almost significant $(p=0.091$ and 0.06$)$ differences between observers were found with Nutrilab. 2) Water load led to significant change in ECW, ICW, ECW/ICW, interstitial fluid, PhA ( $p<$ $0.001)$ and $B C M(p=0.002)$ with time, which was most pronounced in the measurements taken as latest.

Conclusion: 1) BIA measurements show low intraobserver variability. With BioScan, inter-observer variability was low as well, while with Nutrilab it was significantly high. Various BIA devices reproduce significantly different results. 2) After fluid load, ECW, ICW, interstitial fluid, $\mathrm{PhA}$ and $\mathrm{BCM}$ changed significantly with time.

\section{8}

Induction of sustained simultaneous hyperlactatemia and hypoglycemia in a porcine model

S. De Falco ; MC. Paleari ${ }^{1}$; J. Fumagalli²; A. Rigoli ${ }^{1}$; O. Biancolilli²; F. Gori²; M. Battistin²; D. Dondossola ${ }^{3}$; C. Lonati ; G. Grassellii; A. Pesenti';

M. Nijsten ${ }^{5}$; A. Zanella ${ }^{1}$

${ }^{1}$ Department of pathophysiology and transplantation, University of Milan, Milano, Italy; ${ }^{2}$ Department of anesthesiology, Fondazione IRCCS Ca' Granda Ospedale Maggiore Policlinico, Milano, Italy; ${ }^{3}$ Liver transplant and general surgery unit, Fondazione IRCCS Ca' Granda Ospedale Maggiore Policlinico, Milano, Italy; ${ }^{4}$ Center for preclinical research, Fondazione IRCCS Ca' Granda Ospedale Maggiore Policlinico, Milano, Italy; ${ }^{5}$ Department of intensive care medicine, University Medical Center Groningen, Groningen, Netherlands

Correspondence: S. De Falco

Intensive Care Medicine Experimental 2020, 8(2): 000778 
Introduction: Both physiological theory (1) and anecdotal observations (2) support the principle that most mammalian cells may sustain their metabolism using lactate instead of glucose. Combined hyperlactatemia and hypoglycemia (HLHG) may constitute a novel treatment for various diseases, including cancer and malaria. However, induction, maintenance and reversal of HLHG may be challenging and leading to profound derangement of the electrolyte and acid-base equilibrium.

Objectives: To develop a porcine model of HLHG by extracorporeal administration of lactic acid (HLa) and hyperinsulinemic clamping.

Methods: Five female swine $(36.0 \pm 6.5 \mathrm{~kg})$, received an oral dose of $1000 \mathrm{mg}$ metformin before anesthesia induction and connection to an extracorporeal circuit for HLa administration (3). Target lactatemia level was 8-10 mmol/L. Once lactatemia achieved $7.5 \mathrm{mmol} / \mathrm{L}$, deep hypoglycemia (target glycaemia: $23-31 \mathrm{mg} / \mathrm{dL}, 1.3-1.7 \mathrm{mmol} / \mathrm{L}$ ) was induced and maintained with hyperinsulinemic clamp, i.e. continuous infusion of insulin and concentrated glucose. Hypoglycemic counterresponses were inhibited by infusion of labetalol and octreotide. Respiratory rate (RR) was adjusted targeting an arterial pCO2 of $40 \mathrm{mmHg}$. After 4 hours of stable HLHG, normal glycaemia and lactatemia were restored. Numerous blood samples were obtained for gas and biochemistry. Data are reported as mean \pm standard deviation.

Results: The figure shows the time course of lactatemia and glycaemia. The initial target lactatemia $(8.3 \pm 1.2 \mathrm{mmol} / \mathrm{L})$ was achieved by infusing a total amount of $190.8 \pm 82.2 \mathrm{mmol}$ of HLa over $48 \pm 22$ minutes. Subsequently, hypoglycemia was obtained in $44 \pm 18$ minutes by infusing $81.6 \pm 53.2 \mathrm{IU}$ insulin. In this phase hyperlactatemia $(8.9 \pm 1.1$ $\mathrm{mmol} / \mathrm{L}$ ) was maintained by infusing $267.1 \pm 41.5 \mathrm{mmol}$ of $\mathrm{HLa}$, while a total amount of $20.0 \pm 8.0 \mathrm{mcg}$ octreotide and $16.2 \pm 7.9 \mathrm{mg}$ labetalol was administered. HLHG was maintained for $260 \pm 23$ minutes with a glycaemia of $25 \pm 4 \mathrm{mg} / \mathrm{dL}$ requiring low dose insulin continuous infusion $(0.4 \pm 0.5 \mathrm{UI} / \mathrm{h})$. In order to avoid serum glucose dropping below $18 \mathrm{mg} / \mathrm{dL}, 50 \%$ glucose administration was required in all animals at an average rate of $5.1 \pm 2.7 \mathrm{~g} / \mathrm{h}$, for a total infused dose of $23.7 \pm 10.1 \mathrm{~g}$. Labetatol infusion was performed at a rate of $26 \pm 22 \mathrm{mg} / \mathrm{h}$ for a total labetalol dose $124.5 \pm 99.4 \mathrm{mg}$. After the HLHG phase, $20.7 \pm 12.1 \mathrm{~g}$ of glucose over $72 \pm 24$ minutes were required to restore normoglycemia ( $>60 \mathrm{mg} / \mathrm{dL}$ ). No major hemodynamic derangements occurred. A mild positive fluid balance was observed, but not exceeding $1 \mathrm{~L}$. RR was increased from $20.4 \pm 3.3 \mathrm{bpm}$ at baseline to a maximum of $29.8 \pm 3.8$ $\mathrm{bpm}$ and returned to $19.6 \pm 2.6$ bpmafter restoration of normal glucose and lactate levels. Minimum values of arterial $\mathrm{pH}$ and bicarbonate were $7.25 \pm 0.09$ and $19.3 \pm 3.4 \mathrm{mmol} / \mathrm{L}$, respectively. Biomarkers of hepatic, renal and myocardial damage did not increase throughout the experimental procedures.

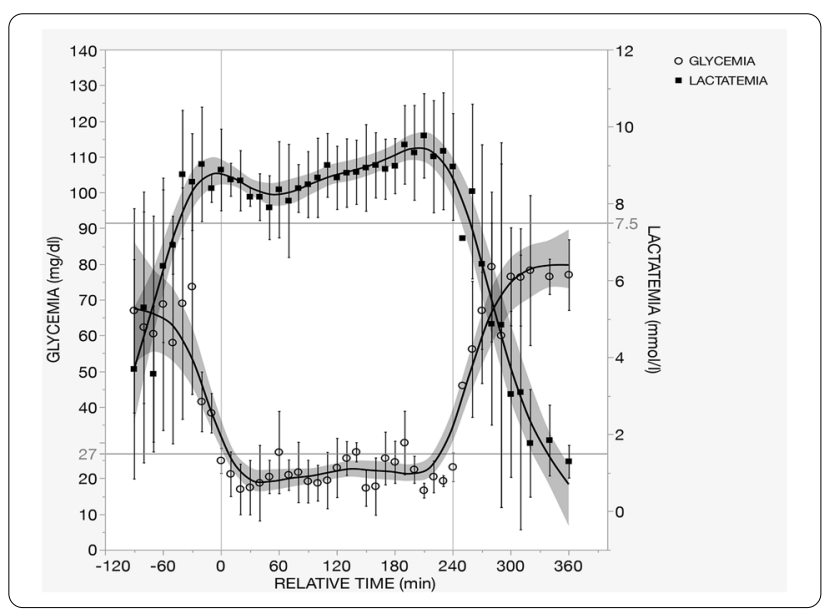

Conclusion: In a large mammal model, induction, maintenance and reversal of HLHG was feasible and without major hemodynamic or metabolic derangements. Further studies will be required to evaluate safety and possible therapeutic effects of HLHG.

\author{
Reference(s) and grant ackowledgment(s) \\ 1. Zanella A. et al. Intensive Care Med 2009 Aug;35(8):1484-7 \\ 2. Oldenbeuving G. et al. Anaesth Intensive Care 2014 Jul;42(4):507-11 \\ 3. Veneman T. et al. Diabetes 1994 Nov;43(11):1311-7 \\ 4. Part of these studies were supported by Laca Medical.
}

\section{9}

Urinary titin is a novel biomarker of muscle atrophy

in non-surgical critically ill patients: a two-center prospective observational study

N. Nakanishi'; R. Tsutsumi ; K. Hara ${ }^{2}$; T. Takashima'; E. Nakataki'; T. Itagaki'; M. Matsuo ${ }^{4}$ : J. Oto ${ }^{5}$ : H. Sakaue ${ }^{2}$

${ }^{1}$ Emergency and critical care medicine, Tokushima University Hospital, Tokushima, Japan; 'Department of nutrition and metabolism, Tokushima University Graduate School of Biomedical Sciences, Tokushima, Japan; ${ }^{3}$ Critical care medicine, Tokushima Prefectural Central Hospital, Tokushima Japan; ${ }^{4}$ Research center for locomotion biology, Kobe Gakuin University, Kobe, Japan; ${ }^{5}$ Emergency and disaster medicine, Tokushima University Hospital, Tokushima, Japan

Correspondence: N. Nakanishi

Intensive Care Medicine Experimental 2020, 8(2): 000009

Introduction: Skeletal muscle atrophy is common in critically ill patients. Although monitoring of muscle mass is important, biomarkers associated with muscle atrophy have not been reliably identified. Titin is a spring-like protein in muscle, and has become measurable as a marker of muscle breakdown. We hypothesized that urinary titin is a useful biomarker for monitoring muscle atrophy in critically ill patients.

Objectives: We conducted a two-center prospective observational study to investigate titin level and its association with muscle atrophy in critically ill patients.

Methods: We recruited adult patients who were expected to remain in the intensive care unit (ICU) for more than 5 days. We excluded postoperative patients due to the increased titin level by surgery. Urine sample was collected on days $1,2,3,5$, and 7 . The urinary $\mathrm{N}$-terminal fragment of titin was measured using an enzyme-linked immunosorbent assay (ELISA) kit. We corrected titin with urinary creatinine (normal range: $1-3 \mathrm{pmol} / \mathrm{mg} \mathrm{Cr}$ ). In order to assess muscle atrophy, rectus femoris cross-sectional area and diaphragm thickness were measured with ultrasound on days $1,3,5$, and 7 . The primary outcome was the relationship between cumulative titin level and the atrophy rate of rectus femoris muscle. Secondary outcomes included the relationship between urinary titin level and the change of diaphragm thickness, the incidence of ICU-acquired weakness (ICU-AW), ICU mobility scale (IMS), and mortality in the ICU.

Results: Fifty-six patients ( 33 males and 23 females; mean age, 72 \pm 13 years) were included at 232 titin measurements. The APACHE II score was 25 (19-29) and the length of ICU stay was 6 (4-9) days. The titin level was 27.9 (16.8-59.6), 47.6 (23.5-82.4), 46.6 (24.4-97.6), 38.4 (23.6-83.0), and $49.3(27.4-92.6) \mathrm{pmol} / \mathrm{mg} \mathrm{Cr}$ on days $1,2,3,5$, and 7 , respectively. The cumulative titin level was significantly associated with the atrophy of rectus femoris muscle on day $3(P<.01)$, day $5(P$ $<.01)$, and day $7(P=.03)$, although titin was not associated with the change of diaphragm thickness $(P=0.54)$. Furthermore, the cumulative titin level was associated with ICU-AW $(P=0.01)$ and mortality in the ICU $(P=0.02)$, but not with IMS $(P=0.18)$.

Conclusion: In non-surgical critically ill patients, the urinary titin level was increased approximately 10-30 times the normal level. The increased titin level was associated with lower limb muscle atrophy, incidence of ICU-AW, and ICU mortality.

\section{5}

Systemic involvement and epidemiology of diabetic patients admitted for Acute Coronary Syndrome

A. M. González González; A. M. Garcia Bellon; M. Cano-Garcia; M. De

Mora-Martin

${ }^{1}$ Cardiology, Regional Hospital of Malaga, Málaga, Spain

Correspondence: A.M. González González

Intensive Care Medicine Experimental 2020, 8(2): 000165 
Introduction: Diabetes Mellitus(DM) is a chronic disease, which is associated with a marked increase in cardiovascular morbidity and mortality

Objectives: Our aim was to analyze the clinical and metabolic control, as well as the degree of systemic affection in this group of patients (p) Methods: An observational analytical prospective study including 161 diabetic patients, consecutively admitted to the Intensive Care Unit with acute coronary syndrome (ACS).

Results: Mean age at diagnosis:69.1 years \pm 9.2. Male:63,2\%.Years of evolution of DM $11.9 \pm 2.3$. Ambulatory treatment:23 $\mathrm{p}(14.3 \%)$ diet; $\quad 44 p(27.3 \%)$ oral antidiabetic(OAD)monotherapy,32p(19.9\%) combined OAD,24p (14.9\%)OAD+insulin and 38 p(23.6\%),insulin. Average weekly controls: $7 \pm 3$.Monitoring was performed by $72 \%$ general practioner; Endocrinology 13\%, 8\% without followup and $6.8 \%$ by other specialists;with 2 annual reviews of average. During their hospitalization, the mean glycemic level was: before breakfast:115.8 $\pm 37 \mathrm{mg} / \mathrm{dl}$; before lunch:209.2 $\pm 63 \mathrm{mg} / \mathrm{dl}$ and before dinner: $187 \pm 70 \mathrm{mg} / \mathrm{dl}$. Mean Glycosylated hemoglobin level was $7.5 \%$. Regarding macrovascular affection:previous heart disease:74.5\% (22.9\% type angina, 37.9\% acute myocardial infarction,other heart disease 13.7\%).Previous stroke: $17 p$ (10.5\%).37p intermittent claudication (23.5\%).Pedal pulses present in $43.5 \%$,weak in $42,8 \%$ and absent in $13.6 \%$.Microvascular affection:retinopathy $32 \mathrm{p}(19.8 \%)$.Nephropathy $39 p(24.2 \%)$.Neuropathy in $34 p(21.1 \%)$.History of lower limb ulcers in $15 p$ (9.3\%). Monofilament examination:sensitivity $6 / 6$ in $56.5 \%, 5 / 6$ in $11.1 \%, 4 / 6$ in $8.7 \%, 3 / 6$ in $13.6 \%, 2 / 6$ in $4.9 \%, 1 / 6$ in $2.5 \%, 0 / 6$ in $3.1 \%$. Conclusion: The clinical profile of diabetic patients admitted in our Unit corresponds to a middle-aged man, with insulin regimen and improvable metabolic control.Cardiovascular complications were found in a remarkable percentage.Therefore,it must be highlighted the importance of a strict control of blood glucose levels in these patients to slow the progression of the disease.

\section{4}

The impact of volume therapy (VT) with $\mathrm{NaCl} 0.9 \%$ vs Ringer lactate as a part of complex intensive therapy (CIT) for early rebalancing of the diabetic patient

I. Camerzan'; C. Gutu-Bahov'

"Intensive Care Unit, Municipal Clinical Hospital "Sfanta Treime", Chisinau, Moldova; ${ }^{2}$ Professor department intensive care unit, Municipal Clinical Hospital "Sfinta Treime", Chișinău, Moldova

Correspondence: I. Camerzan

Intensive Care Medicine Experimental 2020, 8(2): 000714

Introduction: Rapid crystalloid fluid loading in ketoacidosis is essential to restore the patient to normal metabolic state. The main objective of the CIT is to identify triggers and to fix it as soon as possible.

Objectives: To evaluate DKA's triggers; to appreciate the role of early VT with $\mathrm{NaCl} 0.9 \%$ vs Ringer lactate on acid-base balance values and time spent in ICU for patients with DKA.

Methods: A retrospective study (conducted in the hospital "Sfanta Treime", Chisinau, during the 2018-2019 years) based on 64 critical ill patients (mean of age $35.68 \pm 3.45$ years, $P=0.204$ ) who needed VT, mechanical ventilation (MV), acid-base balance and glycemic correction in 24 hours. Patients were divided in 2 groups. Group I $(n=34)-$ patients received $\mathrm{NaCl} 0.9 \%$ and group II $(n=30)$ - Ringer lactate as VT. In these patients were appreciated clinical manifestation, BP (blood pressure), HR (heart rate), PICCO parameters, acid-base balance (ABB), glycemia, lactate, anionic gap (AnGap), blood osmolarity.

Results: According to this study triggers for DKA were: neglected insulin therapy regimen $n=28(43,75 \%)$, wrong diet $n=6(9,37 \%)$, inflammatory process $n=30(46,87 \%)$. At admission in ICU in group I were found a higher APACHE II and SOFA score (group I-APACHE II 16p \pm 2.1 , SOFA $14 p \pm 1.9$ vs group II - APACHE II $14 p \pm 2.3$, SOFA $12 \pm 2.0$ ) and at the end of the treatment - a shorter hospital stay (group I-3.4 days vs group II-4.8 days). Also, in the group I was observed a faster improvement of the ABB values (lactate $=8.2-3.5 \mathrm{mmol} / \mathrm{l}$, glycemia $=31-16$ $\mathrm{mmol} / \mathrm{l}$, blood osmolarity - $275-290 \mathrm{mmol} / \mathrm{kg}$, AnGap $12-16 \mathrm{mmol} / \mathrm{l})$ vs group II (lactate $=8.2-4.5 \mathrm{mmol} / \mathrm{l}$, glycemia $=30-18 \mathrm{mmol} / \mathrm{l}$, blood osmolarity - 285-900 mmol/kg, AnGap $14-18 \mathrm{mmol} / \mathrm{l})$. Mortality in group I was 5.88\%; $P=0,012$ vs. group II-6.66\%; $P=0,023$.

Conclusion: Clear identifying of the DKA's triggers and early VT with $\mathrm{NaCl} 0.9 \%$ given to critical ill patients showed a faster improvement in $A B B$ values, shorter hospital stay and reduced mortality rate.

\section{9}

Professionals of an Intensive Care Unit's perception of dysphagia in critically ill patients

Santana-Cabrera'; J. Alcaraz Jimenez'; J. Rico-Rodriquez'; JD. Martin

Santana ${ }^{2}$; B. N. Santana-Lopez ${ }^{1}$

${ }^{1}$ Intensive care unit, Maternal and child Hospital, Las Palmas de Gran Canaria, Spain; ${ }^{2}$ Economics, Universidad de las Palmas de Gran Canaria, Las Palmas de Gran Canaria, Spain

Correspondence: B.N. Santana-Lopez

Intensive Care Medicine Experimental 2020, 8(2): 000799

Introduction: Oropharyngeal dysphagia and its consequences are problems that seem frequent among critically ill patients, although this question has yet to be resolved, since its incidence in this patient profile is not clearly known.

Objectives: To study the perception of dysphagia among ICU physians and nurses.

Methods: Cross-sectional, prospective, descriptive and quantitative study through a personal, self-administered survey, which has been translated from a reference work and adapted to our environment.

Results: 102 questionnaires were fullfiled. Almost $80 \%$ of ICU professionals consider dysphagia as a problema. In addition, more than $60 \%$ of the interviewees believe that dysphagia prolongs the length of stay in the ICU of the patients and almost half believe that dysphagia increases morbidity and mortality (46.1\%). However, no screening is done (65.7\%), but when this is accomplished, it is through a test meal (38.2\%).

On the other hand, they believe that the main risk factors for developing dysphagia during admission are prolonged intubation, ICU admission for acute or pre-existing neurological disease. Finally, regarding the complications produced by dysphagia, a large majority of professionals point to aspiration pneumonia as the main one.

Conclusion: The staff perceive that dysphagia is a problem, although it is not very frequent, it can have an impact on the patient's morbidity and mortality. Furthermore, being determined of the need to improve their level of awareness, we believe that it is necessary to implement standardized screening protocols, guided by experts from multidisciplinary areas and focused on early suspicion, diagnosis and treatment of dysphagia.

\section{Reference(s) and grant ackowledgment(s)}

1. Zuercher P, Moret CS, Dziewas R, Schefold JC. Dysphagia in the intensive care unit: epidemiology, mechanisms, and clinical management. Crit Care 2019;23(1):103. doi: http://dx.doi.org/10.1186/s13054-019-2400-2.

2. Skoretz SA, Flowers HL, Martino R. The incidence of dysphagia following endotracheal intubation: a systematic review. Chest. 2010;137(3):665-73. doi: http://dx.doi.org/10.1378/chest.09-1823.

\section{1}

Impact of liver support systems in acute-on-chronic liver failure: uncertainty remains - a systematic review and network meta-analysis

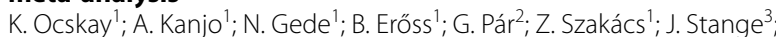
S. Mitzner ${ }^{3}$; P. Hegyi ${ }^{1}$; Z. Molnar

${ }^{1}$ Institute for Translational Medicine, University of Pécs Medical School, Pécs, Hungary; ${ }^{2}$ Division of gastroenterology, fisrt department of medicine, University of Pécs Medical School, Pécs, Hungary; ${ }^{3}$ Department of medicine, University of Rostock, Rostock, Germany

Correspondence: K. Ocskay

Intensive Care Medicine Experimental 2020, 8(2): 000441 
Introduction: Although several devices were tested in randomized controlled trials and are in use currently, the role of artificial and bioartificial liver support systems (ALSS) in acute-on-chronic liver failure (ACLF) is still controversial. There is a lack of evidence for survival benefit, therefore most international societies do not advise the routine use of these devices in ACLF. Network meta-analyses enable indirect comparison of interventions without the need for head-to-head trials, therefore may produce additional evidence.

Objectives: Our aim was to perform the first network meta-analysis comparing different liver support systems to each other and to standard medical therapy (SMT) in patients with ACLF.

Methods: The protocol was registered with PROSPERO (CRD42020155850). The systematic search was conducted in MEDLINE, CENTRAL, Scopus, Embase and Web of Science databases. Selection and data extraction was performed in duplicate and a Bayesian network meta-analysis of randomized controlled trials was conducted. Risk ratios and $95 \%$ credible intervals $(\mathrm{Crl})$ were calculated and interventions were ranked by their posterior probability via calculating the surface under cumulative ranking (SUCRA) curve values. SUCRA values range from 0 to $100 \%$. The higher the SUCRA value, and the closer to $100 \%$, the higher the likelihood that a therapy is in the top rank or one of the top ranks. Version 2 of the Cochrane risk-of-bias tool for randomized trials (RoB 2) and a modified GRADE approach were used for the assessment of risk of bias and quality of evidence.

Results: The systematic search yielded 2774 records. Twenty-three studies were included in the qualitative synthesis from which sixteen were incorporated in the meta-analysis, using MARS ${ }^{\circledR}$, Prometheus ${ }^{\circledR}$, $\mathrm{ELAD}^{\circledR}$, plasma exchange $(\mathrm{PE})$ and BioLogicDT ${ }^{\circledR}$. Overall $(\mathrm{OS})$ and transplant-free (TFS) survival were assessed at 1 and 3 months. PE significantly improved 3-month OS compared to SMT (RR 0.74, Crl: 0.6-0.94) and ranked first on the cumulative ranking curves in three out of four outcomes. Other comparisons did not reach statistical significance. ELAD ranked first before PE for 1-month TFS. MARS performed better in the analyses for OS than for TFS. Quality of evidence was very low except for PE considering 1 month OS and both TFS outcomes with moderate certainty. Hepatic encephalopathy, laboratory parameters and safety were assessed in the systematic review.

Conclusion: This is the first network meta-analysis evaluating the effects of all available and tested artificial and bioartificial liver support therapies on survival in ACLF.Plasma exchange seemed to be the most beneficial for this patient population at present, but based on the low quality of evidence more and better-designed trials are needed refining evidence for already existing options and introducing new devices.

\section{Reference(s) and grant ackowledgment(s)}

1. Human Resources Development Operational Programme Grant, Grant Number: EFOP-3.6.2-16-2017-0006 - LIVE LONGER is co-financed by the

European Union (European Regional Development Fund) within the framework of Programme Széchenyi 2020.

2. The project titled "GINOP-2.3.2-15-2016-00048 - STAY ALIVE" is co-financed by the European Union (European Regional Development Fund) within the framework of Programme Széchenyi 2020.

\section{1}

Outcome of Patients with Liver Cirrhosis Referred to Critical Care in a District General Hospital

N. Pervez ${ }^{1}$; P. Sarkar ${ }^{1}$; S. Jankowski ${ }^{1}$; J. Dixon ${ }^{1}$

${ }^{1}$ Intensive care unit st helier hospital, Epsom and St Helier University Hospitals, London, United Kingdom

Correspondence: N. Pervez

Intensive Care Medicine Experimental 2020, 8(2): 000601

Introduction: Chronic liver failure is frequently a terminal event unless there is a specific and potentially reversible precipitant such as variceal haemorrhage. Increasing organ failure carries a poor prognosis with renal failure having a "particularly high mortality".

It has also been well-established that patients with or without a specific and potentially reversible precipitant have a mortality in excess of
$89 \%$ when three or more organs (including the liver) have failed. Most available literature is from tertiary liver centres (1).

\section{Objectives:}

1. Investigate the ICU-mortality of patients with liver cirrhosis referred to ICU in a district general hospital

2. Assess the mortality within one year

Methods: Data was collected over a one year period from 1st April 2018 to 31st March 2019 by retrospectively reviewing patient notes, Wardwatcher ${ }^{\circledR}$, and ICU referrals audit data.

Patients were classed as having liver cirrhosis if they had documented biopsy or radiological evidence of liver cirrhosis before referral to ICU and had been reviewed in a hepatology clinic for management of chronic liver disease.

Results: 39 patients were referred to ICU with liver cirrhosis: 21 were admitted to ICU, 7 improved following an endoscopy, 2 were transferred to a specialist centre, 7 required level 1 care and 1 required end of life care.

15 patients were admitted to ICU due to decompensated liver cirrhosis, 6 were admitted for other reasons (e.g pneumonia). ICU mortality was $87 \%$ in the decompensated liver disease group and $93 \%$ at 1 year. All patients with cirrhosis admitted to ICU for other reasons survived ICU however 1-year mortality was 33\%. 1-year mortality was 19\% in the patients not admitted to ICU.

Conclusion: Patients with liver cirrhosis have a high mortality regardless of the reason for ICU admission. Most patients admitted to ICU with decompensated liver cirrhosis as their main problem died; no patients with liver cirrhosis died in ICU if their primary reason for admission was not related to liver cirrhosis.

We recommend that patients with liver cirrhosis should be assessed on an individual basis and be considered for ICU admission if there is a potentially reversible cause for their presentation. We advise wardbased teams to discuss with regional specialist centres at an early stage to assess whether an episode of decompensation is reversible (e.g. Transjugular Intrahepatic Portosystemic Shunt). Daily consultant reviews of patients could avoid late referrals, improve the rate of timely interventions and avoid the need for critical care.

\section{Reference(s) and grant ackowledgment(s)}

1. 1. Das V, Boellw PY, Galbois A, Guidet B, Maury E, Carbonell N, Moreau R, Offenstadt G. Cirrhotic patients in the medical intensive care unit: early prognosis and long term survival. Crit Care Med 2010;38(11):2108-16.

\section{1}

Hemoadsorption in liver failure - Subgroup analysis of the CytoSorb International Registry

K. Ocskay'; D. Tomescu²; A. Faltlhauser ${ }^{3}$; D. Jacob ${ }^{4}$; S. Friesecke ${ }^{5}$;

M. Malbrain ${ }^{6}$; K. Kogelmann; R. Bogdanski ${ }^{8}$; F. Bach'; H. Fritz ${ }^{10}$; A. Hartjes ${ }^{11}$;

A. Kortgen ${ }^{12}$; J. Soukup ${ }^{13}$; S. Utzolino ${ }^{14}$; M. Van Tellingen ${ }^{15} ; \mathrm{K}^{\text {. Träger }}{ }^{16}$;

Z. Molnar'; U. Schumacher ${ }^{17}$; F. Brunkhorst ${ }^{18}$

${ }^{1}$ Institute for Translational Medicine, University of Pécs Medical School, Pécs, Hungary; ${ }^{2}$ Anesthesiology and intensive care, Fundeni Clinical Institute, București, Romania; ${ }^{3}$ Medizinische klinik i, Weiden Clinic, Weiden in der Oberpfalz, Germany; ${ }^{4}$ Klinik für anaesthesiologie und intensivtherapie, University Hospital Magdeburg, Magdeburg, Germany; ${ }^{5}$ Klinik und poliklinik für innere medizin $b$, Universitätsmedizin Greifswald, Greifswald, Germany; ${ }^{6}$ Intensive care unit, University Hospital Brussels (UZB), Jette, Belgium; ${ }^{7}$ Klinik für anästhesiologie und intensivmedizin, Klinikum Emden - Hans-Susemihl-Krankenhaus, Emden, Germany; ${ }^{8}$ Klinik für anästhesiologie, Klinikum rechts der Isar der Technischen Universität München, München, Germany; ${ }^{9}$ Klinik für anästhesiologie, intensiv-, notfallmedizin, transfusionsmedizin und schmerztherapie, Evangelisches Krankenhaus Bielefeld, Bielefeld, Germany; ${ }^{10}$ Klinik für anaesthesiologie und intensivmedizin, Hospital Martha-Maria Halle-Dölau, Halle (Saale), Germany; ${ }^{11}$ Anästhesie, intensiv- und palliativmedizin, Krankenhaus der Barmherzigen Schwestern Ried, Ried im Innkreis, Austria; ${ }^{2}$ Klinik für anästhesiologie und intensivmedizin, Universitätsklinikum Jena, Jena, Germany; ${ }^{13} \mathrm{~K}$ linik für anästhesiologie, intensivtherapie und palliativmedizin, Hospital Carl-Thiem-Klinikum Cottbus, Cottbus, Germany; ${ }^{14}$ Abteilung allgemein- und viszeralchirurgie, Universitätsklinikum Freiburg, Freiburg im Breisgau, Germany; ${ }^{15}$ Intensive care, De Tsjongerskâns, Heerenveen, 
Netherlands; ${ }^{16}$ Kardioanasthesiologie, University Hospital Of UIm, UIm, Germany; ${ }^{17}$ Zentrum für klinische studien, Universitätsklinikum Jena, Jena, Germany; ${ }^{18}$ Department of anesthesiology and intensive care medicine, Universitätsklinikum Jena, Jena, Germany

Correspondence: K. Ocskay

Intensive Care Medicine Experimental 2020, 8(2): 000861

Introduction: CytoSorb (CytoSorbents, USA) is a relatively new extracorporeal blood purification device. It was primarily used in septic shock and other hyperinflammatory conditions for removing inflammatory mediators, preventing the harmful effects of cytokine storm. Acute liver failure is a severe, devastating condition that usually necessitates intensive care. Data from the International CytoSorb Registry shows that Cytosorb is often used in liver failure to prevent the development of multiple organ failure.

Objectives: We report on the biggest case-series to date on CytoSorb use in liver failure as a subgroup from the International Cytosorb Registry.

Methods: The International CytoSorb Registry (NCT02312024) prospectively collects data from adult patients who underwent CytoSorb treatment. No exclusion criteria are defined. Data was recorded at baseline, up to 24 hours before treatment (T1), up to 24 hours after the last CytoSorb therapy (T2) and at discharge from hospital or mortality onelectronic case report forms. Descriptive statistical methods were used. Changes were calculated form T1 and T2 values using t-test as well as a linear model for selected variables. $95 \%$ confidence intervals (CI) were provided.

Results: From May 2015 until November 2019, 930 patients were entered into the Registry from 9 countries by 42 centres. In 89 cases the stated indication for therapy was liver failure, being the third most frequent indication after sepsis and pre-emptive use during cardiac surgery. In this subgroup the mean age was 48.2 years and the majority of patients were male (56.2\%). Patients received a median of 2 treatments (i.e.: 2 adsorbers), with a median overall treatment duration of 43 hours. The median time of use per adsorber was one day and time between treatments was less than 1 hour (median).

More than $90 \%$ of patients were alive at the termination of CytoSorb therapy. Forty-seven patients died in the ICU and 3 more during hospitalization, which corresponds to a $57.5 \%$ in-hospital mortality. Attending physicians observed improvement in 62 cases (70.4\%). No change was registered in 15 cases (17\%) and in 4 patients the condition worsened. SOFA scores hardly changed (T2-T1). Changes in CRP and PCT were not significant. Serum total bilirubin (TBIL) showed a significant reduction (T2-T1; mean [CI]: -61.7 [-92.7 to -30.8$]$ ). Regarding safety, device-related serious adverse events were not reported.

Conclusion: We report on the largest case series on CytoSorb application in patients with liver failure. The therapy proved safe, reduced TBIL significantly and resulted in subjective improvement in the patient's condition due to CytoSorb treatment in $70 \%$ of the cases. These encouraging results underline the need for randomized controlled trials assessing CytoSorb in liver failure.

\section{Reference(s) and grant ackowledgment(s)}

1. The CytoSorb ${ }^{\circledR}$ Registry is funded by CytoSorbents Europe $\mathrm{GmbH}$ with unrestricted grants. The Center for Clinical Studies at Jena University Hospital provides all necessary services in the field of data and project management. This study was funded by "GINOP-2.3.2-15-2016-00048 - STAY ALIVE" co-financed by the European Union (European Regional Development Fund) within the framework of Programme Széchenyi 2020, and by the Human Resources Development Operational Programme Grant, Grant Number: EFOP 3.6.2-16-2017-00006 - LIVE LONGER which is co-financed by the European Union (European Regional Development Fund) within the framework of Programme Széchenyi 2020.
000900

Is acute liver injury more prevalent in critically ill patients with Covid-19 infection and respiratory failure?

L. Tong ${ }^{1}$; HD. Aya ${ }^{1}$; D. Padman²; M. Hamilton ${ }^{1}$; A. Rhodes ${ }^{2}$; S. Leaver ${ }^{3}$

${ }^{1}$ General intensive care unit, St George's Hospital, London, United Kingdom; ${ }^{2}$ Anaesthesia and intensive care medicine, St George's University Hospitals NHS Foundation Trust, London, United Kingdom; ${ }^{3}$ Research lead critical care directorate, St George's Hospital, London, United Kingdom

Correspondence: $L$. Tong

Intensive Care Medicine Experimental 2020, 8(2): 000900

Introduction: Several studies have identified abnormal liver function tests (ALFT) during the clinical course of Covid-19. [1, 2] Acute liver injury (ALI) in Covid-19 patients may be caused by direct viral effects, systemic inflammatory response, drug toxicity or haemodynamic factors. There is currently no literature comparing incidences and characteristics of liver dysfunction between critically ill patients with and without Covid-19.

Objectives: To assess whether infection with Covid-19 has any impact on liver function in critically ill patients with respiratory failure who are infected with Covid-19 and is relevance for clinical outcomes.

Methods: This is retrospective cohort study of patients admitted to an intensive care unit (ICU) over 5 months. Laboratory data related to liver pathology and function (Alanine transferase (ALT), alkaline phosphatase (ALP), bilirubin, albumin, prothrombin time) was collected at day 1, 7 and 14 of admission. ALFT are defined as a rise in ALT/ALP greater than the normal limit, whereas ALI is defined as a rise in ALT/ ALP by $\geq 3$ times the upper limit of normal. Any ALFT or ALI cases were classified into hepatocellular, cholestatic, or mixed liver injury. Categorical data was assessed with $x^{2}$ test and odds ratio (OR). The MannWhitney $U$ test was used to assess continuous variables between patients infected with Covid-19 (as confirmed by positive viral PCR swab) and those who were not. $P$ values lower than .05 were considered statistically significant.

Results: There were 256 admissions into intensive care during the study period. 132 (51.6\%) was infected with Covid-19. ALFT was present in $80(60.6 \%)$ of Covid-19 patients; $90 \%$ had a hepatocellular pattern, $10 \%$ had a mixed pattern and $2 \%$ was cholestatic. ALFT was present in 55 (44.4\%) non-Covid-19 patients ( $p=.009$, OR $1.9,95 \% \mathrm{Cl}$ $1.2,3.2$ ). ALI was observed in $10.1 \%$ of non-Covid patients and $10.7 \%$ of Covid patients ( $p=.87$ ). At day 1 of admission, Covid-19 patients had a median ALT of $44(28,78)$ compared to non-Covid-19 patients with a median of $29(18,58 ; p<.001)$. At day 7 , Covid-19 patients had a median albumin of 17 (14-19) compared to $19.5(16,24, p=.01)$ in non-Covid-19 patients. On day 14, there was no significant differences in distribution of liver biochemistry or prothrombin time between the two cohorts. Within the Covid-19 group, there are no significant differences between patients with or without ALFT or ALI in 28-day mortality, requirement of renal replacement therapy, duration of mechanical ventilation or duration of vasoactive support

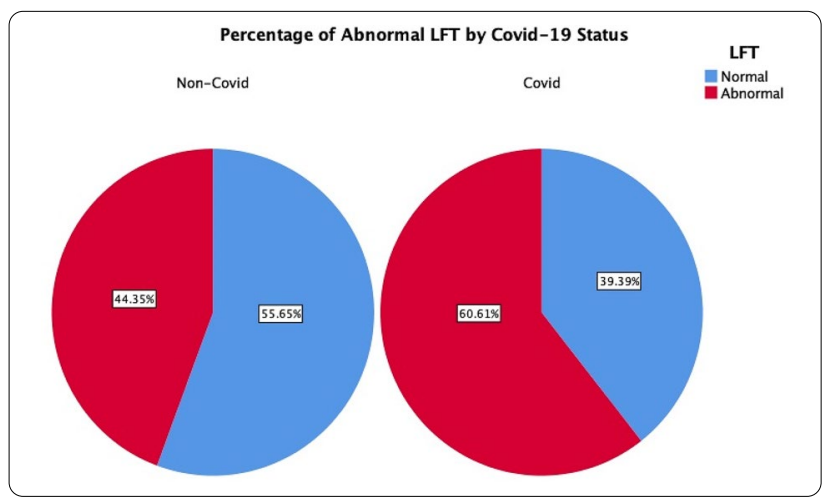


Conclusion: ALFT is more prevalent in Covid-19 patients compared to other patients with respiratory failure, and mainly with a hepatocellular pattern. Covid-19 patients may display poorer liver synthetic function in early stages of the disease $(<14$ days). There is no impact on clinical outcomes.

\section{Reference(s) and grant ackowledgment(s)}

1. 2. Cai Q, Huang D, Yu H, Zhu Z, Xia Z, Su Y, Li Z, Zhou G, Gou J, Qu J, Sun Y, Liu Y, He Q, Chen J, Liu L, Xu L. COVID-19: Abnormal liver function tests. Journal of Hepatology. 2020; 1 -9.

2. 1. Garrido I, Liberal R, Macedo G. Review article: COVID-19 and liver disease- what we know on 1st May 2020. Aliment Pharmacol Ther. 2020. doi: 10.1111/apt.15813.

3. None

\section{8}

Outcomes for patients with acute-on-chronic liver failure admitted to Oxford ICU

T. Grant ${ }^{1}$;. Mcclelland ${ }^{2}$; D. Clarke ${ }^{3}$; J. Bentley ${ }^{4}$; A. Ellis ${ }^{4}$; A. Dobra ${ }^{5}$; G. Barker ${ }^{6}$ ${ }^{1}$ Oxford school of anaesthesia, John Radcliffe Hospital, Oxford, United Kingdom; ${ }^{2}$ Foundation school, John Radcliffe Hospital, Oxford, United Kingdom; ${ }^{3}$ ntensive care medicine, John Radcliffe Hospital, Oxford, United Kingdom; ${ }^{4}$ Adult intensive care unit, John Radcliffe Hospital, Oxford, United Kingdom; ${ }^{5}$ Adult critical care services, Churchill Hospital, Oxford, United Kingdom; ${ }^{6}$ Intensive care medicine and anaesthesia, John Radcliffe Hospital, Oxford, United Kingdom

Correspondence: T. Grant

Intensive Care Medicine Experimental 2020, 8(2): 001008

Introduction: Acute-on-chronic liver failure (ACLF) was defined in the CANONIC study by acute development of one or more major complication of decompensated liver cirrhosis, plus organ failure and high risk of short-term mortality. ACLF is common, affecting approximately $30 \%$ of hospitalised cirrhotic patients and may develop in patients without previousdecompensation. Precipitants include infection, alcoholic hepatitis and surgery, but in more than $40 \%$ of patients no precipitating event is identified. Clinical management focuses on treatment of a precipitant, whilst addressing the characteristic systemic inflammation and physiological disturbance of the critically ill patient with liver disease. Given the high risk of short-term mortality, much of the literature relates to the use of prognostic scores to contextualise the degree of ALCF organ failure and arguably the utility of critical care admission; with the CLIF-C ACLF score shown to have greater predictive accuracy in ACLF than other cirrhotic liver disease scoring systems.

Objectives: This study retrospectively identified patients with ACLF, admitted to our general adult critical care service (ICU), in order to review their admitting aetiology, level of organ support and survival. In addition, we sought to apply a range of available prognostic scores for cirrhotic liver failure to determine their efficacy.

Methods: The Intensive Care National Audit and Research Centre (ICNARC) database was searched for patients admitted to ICU at Oxford University Hospitals (OUH), between 1st April and 31st December 2019, with a diagnosis code of: liver cirrhosis, acute alcoholic hepatitis, encephalopathy, portal hypertension, or gastrointestinal/variceal haemorrhage. Also identified were cirrhotic patients with sepsis or acute renal failure by necessary association with these codes.

Review of these patient records identified patients with ACLF; including their clinical data, admission reason, survival to unit and hospital discharge, and 28- and 90-day mortality. CLIF-C organ failure, UKELD and Child-Pugh scores were calculated for time of critical care admission and, given their association with predicted mortality for a given score, compared between survivors and non-survivors with the aim of comparing prognostic ability. CLIF-C was also calculated with 48 hours post-admission data, to give delta-CLIF scores.

Results: 21 patients (M:F/13:8, median age 51 [38-76] years), were admitted to ICU due to ACLF - though this specific diagnosis was rarely made.
Stated admission cause included: remaining intubated post-endoscopy for upper gastrointestinal (UGI) haemorrhage (9/21, 43\%), vasopressor treatment $(5 / 21,24 \%)$, invasive monitoring $(4 / 21,19 \%)$, reduced conscious level $(2 / 21,9.5 \%)$, palliative care $(1 / 21,4.5 \%)$. Commonest aetiology was gastrointestinal (GI) bleeding; variceal (7/21, $33 \%)$, non-variceal $(1 / 21,4.5 \%)$, lower GI $(2 / 21,9.5 \%)$. Other causes included bacterial infection $(3 / 21,14 \%)$, acute alcoholic hepatitis $(3 / 21,14 \%)$, ischaemic bowel $(1 / 21,4.5 \%)$. In four cases, no clear precipitant for ACLF was identified (4/21, 19\%).

$81 \%(17 / 21)$ were admitted with CLIF-C ACLF grade 3, 19\% (4/21) admitted with ACLF grade 2; giving a median CLIF-C ACLF prognostic score of 68 [IQR 11.5], which, for the median age of 51 years, would convey $85 \%$ predicted mortality at 1 month and $95 \%$ at 3 months.

Actual mortality at 48 -hours was $48 \%(10 / 21)$, at ICU discharge, $67 \%$ (14/21), and hospital discharge, 28 day- and 90 -day mortality rates were the same at $71 \%(15 / 21)$. Of $11 / 21$ patients alive at 48 hours, median delta-CLIF organ failure score was 0 [IQR 2].

Median CLIF organ failure, UKELD and Childs-Pugh scores were compared between those patients alive at 28/90 days and those who had died: CLIF, 13 vs. 15 ( $P=.31$ ); UKELD, 58 vs. 59 ( $P=.33$ ); Childs-Pugh, 11 vs. $12(P=.15)$.

Conclusion: Studied mortality was lower than predicted by the most well-validated prognostic score available in this context (CLIF-C ACLF). Possibly due to small sample size, the difference in severity between prognostic scores in survivors and non-survivors was not statistically significant - nor was the CLIF-C score shown to have superior prognostic ability. Unchanged average delta-CLIF scores was likely a result of the advanced degree of organ failure on admission, which is recognised to correlate with slower improvement in this context. Further prospective study of ACLF scores and outcomes, ideally in both referrals and admissions to critical care, are needed in order to understand the potential role for prognostic scoring in guiding critical care intervention.

\section{Reference(s) and grant ackowledgment(s)}

1. Gustot et al (2014) Short-term (28-day) clinical course and transplant-free mortality in acute-on-chronic liver failure (ACLF); evidence for reversibility of ACLF (a study from the CANONIC database), P476, Journal of Hepatology;60:5228

2. CLIF-C ACLF score and expected mortality rates, European Foundation for the Study of Liver Failure, online: https://www.efclif.com/scientific-activity/ score-calculators/clif-c-aclf [accessed 29/06/20]

3. Bernal et al (2015) Acute-on-chronic liver failure, Lancet;386:1576-87

4. Jalan et al (2014) Development and validation of a prognostic score to predict mortality in patients with acute-on-chronic liver failure, Journal of Hepatology;61:1038-1047

5. Moreau et al (2013) Acute-on-Chronic Liver Failure Is a Distinct Syndrome That Develops in Patients With Acute Decompensation of Cirrhosis, Gastroenterology; 144:1426-1437

6. Nil grant acknowledgement required.

\section{2}

Aneurysmal subarachnoid hemorrhage: A decade of surgical intensive care experience

N. Shaikh ${ }^{1}$ K. Adnan ${ }^{2}$; K. Mohsin²; A. Aisha ${ }^{2}$

${ }^{1}$ Hamad Medical Corporation, Doha, Qatar; ${ }^{2}$ Neurosurgery, Hmc, Doha, Qatar

Correspondence: N. Shaikh

Intensive Care Medicine Experimental 2020, 8(2): 000012

Introduction: Spontaneous subarachnoid hemorrhage (SAH) is one of the significant aetiologies for stroke. SAH causes higher morbidity, mortality with loss of productivity resulting in increased disease burden. A few studies about SAH are from the region and the epidemiological features of $\mathrm{SAH}$ and aneurysmal subarachnoid hemorrhage (aSAH) have not been comprehensively studied before in Qatar.

Objectives: Aim of our study was to describe the epidemiological profile of patients with SAH and aSAH in the State of Qatar 
Methods: A retrospective chart review was performed for all spontaneous subarachnoid hemorrhage and/or ruptured aneurysm cases that were consecutively admitted to Hamad General Hospital (600 bedded tertiary care facility) during the period January 1, 2007 until December 31, 2016. A quantitative analysis of demographics, clinical characteristics, diagnostic findings, interventions and overall mortality was done. Data was entered in SPSS version 18 . Chi square and student $t$ tests were used to compare between the groups. $P$ value of $<0.05$ was considered stastically significant.

Results: A total of 323 patients were included in the study composed of both aneurysmal and non-aneurysmal subarachnoid hemorrhage. Mean age at presentation was $47.4 \pm 12.2$ years. Men comprised $68.7 \%$ of the cases. $86.6 \%$ of the patients presented with acute onset headache. 217 patients had 1 aneurysm and 32 patients had multiple aneurysms. ACOMA (Anterior communicating artery) aneurysm was the most common aneurysm. Non Aneurysmal Subarachnoid hemorrhage occurred in 74 patients (22.9\%) and was more common in men. $23.7 \%$ of the patients underwent microsurgical clipping of the aneurysm and $52.6 \%$ of the patients underwent coiling of the aneurysm. Overall mortality was less in WFNS 1 and $2 \mathrm{SAH}$ than in higher grades $(28.6 \%$ vs $71.4 \%) .69$ out of a total of 323 patients died within 1 month post ictus, accounting for an overall mortality rate of $21.2 \%$ in our study.

Conclusion: The annual incidence of aneurysmal SAH in Qatar is on the rise. Males had a higher incidence of aSAH. ICA aneurysms have been found to be more common in Qatari women which may have a genetic basis. Lower WFNS grades of aSAH was associated with better prognosis. The overall mortality associated with aSAH in Qatar has declined over the last 3 years.

\section{Reference(s) and grant ackowledgment(s)}

1. Nogueira G. J. Spontaneous Subaraehnoid Haemorrhage and Ruptured Aneurysms in the Middle East. A Myth Revisited. ActaNeurochir (Wien) (1992): 114:20-25

2. Donkor ES. Stroke in the Century: A Snapshot of the Burden, Epidemiology, and Quality of Life. Stroke Research and Treatment 2018; 2018: 1-10 3. None

\section{4}

Status epilepticus: a retrospective observational study of current practice and outcomes at a single UK tertiary centre neurocritical care unit

B. Scally ${ }^{1}$; A. Boyle ${ }^{1}$; M. Blackstock

${ }^{1}$ Intensive care unit, western general hospital, NHS Lothian, Edinburgh, United Kingdom

Correspondence: B. Scally

Intensive Care Medicine Experimental 2020, 8(2): 000034

Introduction: Evidence for the treatment of refractory and superrefractory status epilepticus in intensive care is mostly based on case reports and small case series; there is a strong imperative to publish larger cohorts and share data on clinical outcomes.

Objectives: To describe the demographics, management, and outcomes of patients with status epilepticus requiring critical care support at a single UK tertiary centre neurocritical care unit.

Methods: A retrospective observational study of all consecutive patients admitted with status epilepticus to intensive care for level two or level three organ support at the Western General Hospital, Edinburgh over a five year period (Jan 1st 2014 - Dec 31st 2019).

Results: During the study period, there were 141 admissions to intensive care from 133 unique patients with a confirmed diagnosis of status epilepticus. $56 \%$ of patients were male; mean average age at admission was 51 years; and mean average length of stay was nine days. The mean average APACHE II score for the study population was 16 corresponding to a predicted non-operative mortality of $25 \%$. The actual observed mortality in our study population was only $15 \%$. Mortality was higher among patients admitted following out-of-hospital cardiac arrest or neurosurgical procedures and lower for patients with a pre-existing diagnosis of any epilepsy syndrome. The drug treatments most frequently prescribed for each step of management were as follows: (1) benzodiazepines; (2) phenytoin; (3) levetiracetam; (4) midazolam infusion; and (5) phenobarbitone or thiopentone coma. Overall, $74 \%$ of patients required general anaesthesia and mechanical ventilation. $46 \%$ of patients received formal EEG testing and $10 \%$ were diagnosed with super-refractory status epilepticus treated with formal burst suppression. The observed mortality among patients with superrefractory status epilepticus was $29 \%$.

Conclusion: Mortality in patients admitted to intensive care with status epilepticus is higher following out-of-hospital cardiac arrest and neurosurgical procedures. More than $70 \%$ of patients in super-refractory status epilepticus at our site survived to hospital discharge.

\section{7}

Association of Different Transcranial Doppler-Derived Indices of Cerebrovascular Dynamics with Vasospasm and Delayed Cerebral Ischemia in Patients suffering from Subarachnoid Hemorrhage

V. Papaioannou ${ }^{1}$; C. Budohoski ${ }^{2}$; M. Placek ${ }^{3}$; P. Smielewski ${ }^{4}$; M. Czosnyka ${ }^{5}$ ${ }^{1} \mathrm{ICU}$, Democritus University of Thrace, Alexandroupoli, Greece; ${ }^{2}$ Neurosurgery, Neurosciences Unit R3, Cambridge, United Kingdom; ${ }^{3}$ Brain physics laboratory, Department of clinical neurosciences, division of neurosurgery, Cambridge, United Kingdom; ${ }^{4}$ Brain physics laboratory, Department of clinical neurosciences, division of neurosurgery, Cambridge, United Kingdom; ${ }^{5}$ Department of clinical neurosciences, brain physics laboratory, division of neurosurgery, University of Cambridge, Cambridge, United Kingdom

Correspondence: $\vee$. Papaioannou

Intensive Care Medicine Experimental 2020, 8(2): 000127

Introduction: Delayed cerebral ischemia (DCl) constitutes a major complication following subarachnoid hemorrhage $(\mathrm{SAH})$ and is partly associated with vasospasm (VS) and impaired cerebral autoregulation. Different surrogate markers of cerebral blood flow (CBF), such as non-invasive transcranial doppler (TCD), are frequently used to monitor development of VS, as well as integrity of autoregulation. In this respect, different physiological parameters can be estimated, such the dynamic index of autoregulation Mxa, which is a moving correlation coefficient between spontaneous fluctuations of cerebral blood flow velocity (CBFV) and arterial blood pressure (ABP). A positive correlation signals disturbed autoregulation. Other indices include cerebral arterial time constant (tau) that reflects arterial filling time distal to insonation vessel and critical closing pressure $(\mathrm{CrCP})$, which estimates the theoretical lowest value of ABP that is needed for maintaining CBF. Objectives: We investigated how VS affects different TCD-derived indices of cerebrovascular dynamics in patients suffering from SAH. Secondarily, we explored the potential differences of such VS-associated changes between subgroups of patients with and without DCI, in both temporal and spatial settings.

Methods: A retrospective analysis was performed using recordings from $32 \mathrm{SAH}$ patients, admitted to the Neurocritical Care Unit at Addenbrooke's Hospital, in Cambridge, UK, diagnosed with VS through bilateral TCD measurements. Patients were divided in 2 groups, depending on development of $\mathrm{DCl}(\mathrm{DCl}$ group, $\mathrm{N}=19$ vs non$\mathrm{DCl}$ group, $\mathrm{N}=13$ ). Cerebral autoregulation was estimated using the index Mxa. We also measured tau as the product of arterial resistance (Ra) and compliance ( $\mathrm{Ca}$ ) and estimated $\mathrm{CrCP}$, as the intercept point of a regression line between systolic and diastolic $A B P$ plotted along the $x$ axis and systolic and diastolic CBFV plotted along the $y$ axis.

Results: In the whole population, VS caused shortening of tau (ipsilateral to spasm: $0.17 \pm 0.08$ vs before spasm: $0.25 \pm 0.17 \mathrm{sec}, \mathrm{p}=0.04$ ), decrease in CrCP (ipsilateral to spasm: $9.69 \pm 23.28$ vs before: $27.23 \pm$ $23.31 \mathrm{mmHg}, \mathrm{p}=0.01$ ) and interhemispheric asymmetry with lower values on ipsilateral side $(p<0.01)$. Ipsilateral CrCP during VS was negatively correlated with $\mathrm{Mxa}(\mathrm{r}=-0.43, \mathrm{p}=0.01)$ and positively correlated with tau $(r=0.74, p<0.001)$. In patients with $D C l, V S$ caused increase in Mxa (ipsilateral to spasm: $0.36 \pm 0.18$ vs before: $0.26 \pm 0.23, p=0.04$ ) decrease in CrCP (ipsilateral to spasm: $6.61 \pm 24.5$ vs before: $17.24 \pm$ $19.4 \mathrm{mmHg}, p=0.04)$ and lower values on ipsilateral side $(p<0.01)$. 
Conclusion: VS was associated with reduced tau and $\mathrm{CrCP}$ in both temporal and spatial assessments, in all patients with SAH. Impaired autoregulation and shortened tau correlated with decreased CrCP. VS in patients who developed $\mathrm{DCl}$, reduced $\mathrm{CrCP}$ (temporal and spatial effect) signifying vasodilatation, and increased Mxa, reflecting disturbed autoregulation.

\section{Reference(s) and grant ackowledgment(s)}

1. 7. Budohoski K, Czosnyka M, Kirkpatrick P, Smielewski P, Steiner L, Pickard J. Clinical relevance of cerebral autoregulation following subarachnoid hemorrhage. Nat Rev Neurol 2013; 9(3): 152-63.

\section{5}

A safe strategy for early weaning and tracheostomy avoidance in cervical spinal cord injury: a single-center experience

M. Guarnieri' ; F. Fossi²; F. Pozziं'; F. Curto ${ }^{2}$; A. Chieregato ${ }^{2}$

${ }^{1}$ Department of medicine and surgery, university of Milano bicocca, Milan, Italy; ${ }^{2}$ Department of neurointensive care, ASST Great Metropolitan Niguarda, Milano, Italy

Correspondence: M. Guarnieri

Intensive Care Medicine Experimental 2020, 8(2): 000145

Introduction: High cervical spinal cord-injured (C-SCI) patients experience severe disability and are at risk of ventilator-dependency and tracheostomy $(1,2)$. In the acute phase, they need invasive ventilator support. Conventional weaning parameters such as $\mathrm{P}$ 0-1, pressure muscleindex (PMI)(3) and negative inspiratory force (NIF) are not always informative in $\mathrm{C}-\mathrm{SCl}$, because most of them will not match the conventional targets and still undergo successful weaning. The global incidence of tracheostomy in $\mathrm{C}-\mathrm{SCl}$ varies widely among the different studies, and is described up to $60 \%$, with a higher risk in males, AIS A grade, upper C-SCl, high injury severity score (ISS), GCS $\leq 8$, thoracic injury, and patients with respiratory complications(4). Some studies suggest that the use of non-invasive mechanical ventilation (NIMV) and mechanically cough assist (MAC) can allow extubation without further complications(5).

Objectives: To retrospectively assess the safety and efficacy of an aggressive respiratory approach with early extubation coupled with NIMV, MAC, and intensive nursing assistance on $\mathrm{C}-\mathrm{SCl}$ patients ( $\mathrm{C} 3$ to C6 SCl).

Methods: This was a single-center retrospective study. Clinical records of the patients with spinal cord injury from January 2018 to December 2019 were revised. All the patients with cervical spinal cord injury were included, with no limits of age or comorbidities.

Standard management of quadriplegic patients in our department is based on aggressive respiratory management, early extubation, NIMV and MAC performed regularly at least 4 times a day; the clearance of respiratory secretions is also facilitated by alternated side positioning. Results: Twenty-five patients with acute cervical spinal cord injury were admitted to our department in the study period. Age was $39 \pm 17$ years and $72 \%$ of them were male. Full data of the initial population are shown in Table 1. Twenty-four of them were traumatic, 1 ischemic. Median ISS in traumatized patients was 25 and most of the patients $(80 \%)$ were isolated cervical traumas. The full data of the initial population are shown in table 1.

All patients except 2, were intubated, only 3 patients (12\%) were tracheostomized. Five patients (20\%) failed an extubation trial, only 1 of them underwent a tracheostomy. In one of the tracheostomized patients, our protocol was not applicable because it came from another hospital three months after the trauma. Among patients who failed the extubation trial, 3 came from other hospitals, 2 were treated in our institution from the beginning. The extubation failure rate in patients admitted to our institution first was $8 \%$. One patient was diagnosed with pneumonia, while another patient experienced complete atelectasis of a pulmonary lobe, treated with bronchoscopy. The incidence of tracheostomy was different according to the level of lesion, being (50\%) in C3 lesion, $28 \%$ in C4 lesion and 0 in the lower levels. ASIA A patients underwent tracheostomy in the $8 \%$ of cases, ASIA B in the $28 \%$, while no ASIA C patient received a tracheostomy. At 1-year follow-up (when available) no new tracheostomy has been performed, no urgent re-admission to ICU occurred for any reason. The intubation rate at admission was $23 / 25$ (92\%). An extubation trial was performed in $21 / 25$ patients ( $84 \%)$.

\begin{tabular}{|c|c|c|}
\hline Age & & $39 \pm 17$ \\
\hline \multicolumn{3}{|l|}{ Level of lesion } \\
\hline & $\mathrm{C} 3$ & $2(8 \%)$ \\
\hline & C4 & $7(28 \%)$ \\
\hline & $\mathrm{C} 5$ & $10(40 \%)$ \\
\hline & $\mathrm{C} 6$ & $6(24 \%)$ \\
\hline \multirow[t]{4}{*}{ ASIA } & & \\
\hline & $\mathrm{A}$ & $12(48 \%)$ \\
\hline & B & $7(28 \%)$ \\
\hline & $\mathrm{C}$ & $6(24 \%)$ \\
\hline Traumatic injury & & $24(96 \%)$ \\
\hline ISS & & $27 \pm 4.5$ \\
\hline
\end{tabular}

Conclusion: We present a C-SCI group of patients, with a lower rate of tracheostomy compared to current evidence $(1,4,6,7)$, and a low rate of respiratory complications, such as pneumonia or atelectasis. Our data suggest that an aggressive weaning program tailored to specific patient's needs could lead to a higher rate of respiratory autonomy without side effects.

\section{Reference(s) and grant ackowledgment(s)}

1. 1. Childs BR, Moore TA, Como JJ, Vallier HA. American Spinal Injury Association Impairment Scale Predicts the Need for Tracheostomy After Cervical Spinal Cord Injury. Spine 2015;40(18):1407-13.

2. 2. Selvarajah S, Hammond ER, Haider AH, et al. The burden of acute traumatic spinal cord injury among adults in the united states: an update. J Neurotrauma 2014;31(3):228-38

3. 3. Foti G, Cereda M, Banfi G, Pelosi P, Fumagalli R, Pesenti A. End-inspiratory airway occlusion: a method to assess the pressure developed by inspiratory muscles in patients with acute lung injury undergoing pressure support. Am J Respir Crit Care Med 1997:156(4 Pt 1):1210-6.

4. 4. Wang Y, Guo Z, Fan D, et al. A Meta-Analysis of the Influencing Factors for Tracheostomy after Cervical Spinal Cord Injury. Biomed Res Int 2018;2018:5895830

5. 5. Bach JR, Gonçalves MR, Hamdani I, Winck JC. Extubation of patients with neuromuscular weakness: a new management paradigm. Chest 2010;137(5):1033-9.

6. 6. Flanagan CD, Childs BR, Moore TA, Vallier HA. Early Tracheostomy in Patients With Traumatic Cervical Spinal Cord Injury Appears Safe and May Improve Outcomes. Spine 2018;43(16):1110-6.

7. 7. Higashi T, Eguchi H, Wakayama Y, Sumi M, Saito T, Inaba Y. Analysis of the risk factors for tracheostomy and decannulation after traumatic cervical spinal cord injury in an aging population. Spinal Cord 2019;57(10):843-9.

\section{7}

Comparative analysis of serum iron in patients with favorable and unfavorable outcomes of the treatment of traumatic brain injury

D. Markevich

${ }^{1}$ Department of Anesthesiology and Resuscitation, Mogilev City Emergency Hospital, Mogilev, Belarus

Correspondence: $D$. Markevich

Intensive Care Medicine Experimental 2020, 8(2): 000147

Introduction: TraumaTraumatic brain injury (TBI) is considered as a generalized process in which a systemic inflammatory response syndrome often develops [1]. Iron is a co-factor of metalloproteases and its metabolism is actively changing with multiple organ dysfunction $[2,3]$. Monitoring changes of serum iron is very interesting as a prognostic criterion of treatment outcome in patients with head injury [4]. 
Objectives: To study the dynamics of serum iron in patients with head injury in the postoperative time and its potential in predicting treatment outcome.

Methods: A prospective study was hold from 01.05.2018 to 31.12.2019. The ethics committee received permission for this study. 76 adult patients ( 13 women and 63 men) who needed surgical treatment of traumatic brain injury wereincluded the study. The age of the patients was $54(43.5 ; 60)$ years. 45 patients underwent general anesthesia, and 31 patients underwent combined general anesthesia and scalp block with using small volumes (0.5-1 $\mathrm{ml}$ for one nerve) $0.75 \%$ ropivacaine. General anesthesia was total intravenous anesthesia using propofol and fentanyl. In 3-6 months after craniotomy the Glasgow Outcome Scale (GOS) was taken for rating the outcome of treatment in patients with head injury. The treatment outcome was unfavorable 1-3 points by GOS(1 - patient's death, 2 - vegetative state, 3 - severe disability).The treatment outcome was favorable at 4 or 5 points by GOS( 4 - the possibility of self-care, 5 - full recovery). Two groups of patients were formed. Group 1 included 46 patients with a favorable outcome, and group 2 included 30 patients (of whom 17 patients (56.7\%) died) patients with an unfavorable outcome.

The groups of patients did not differ in age, weight, sex, method of anesthesia. Patients of groups 1 and 2 significantly reliably differed at the volume of intracranial hematomas, the intraoperative bleeding and estimated Glasgow Coma Scale (in group $1-14(11 ; 15)$ in group 2 - $7(6 ; 10)), p<0.05$.

Serum iron was compared between groups 1 and 2. A BA 400 Biosystems biochemistry analyzer (Spain) was used for analysis of serum iron. The reference values of serum iron was $11.6-31.3 \mu \mathrm{mol} / \mathrm{l}$. Blood sampling was performed 1-2 hours before the operation, and further in $11(6 ; 17)$ hours on the 1 st day after the operation, then in $35(29 ; 40)$ hours on the 2nd day, in $60(52 ; 65)$ hours on 3 day, after $108(100 ; 113)$ hours on the 5 th day, in $155(148 ; 161)$ hours on the 7 th day and in 226 $(220 ; 233)$ hours on the 10th day after craniotomy.

Statistical analysis was performed by Statistica 8.0 and Microsoft Excel 2007. The normal distribution was checked using the Shapiro-Wilk's test. Nonparametric methods were used to test the significance of statistical hypotheses. Descriptive statistics are presented as medians and lower and upper quartiles Me (LQ; $\cup Q)$. The hypothesis of a statistically significant difference in the independent groups was tested using the Mann-Whitney test. The determination of serum iron as an unfavorable classifier of outcome was evaluated using logistic regression with the construction of ROC-curves and calculating the area(AUC), sensitivity (Se) and specificity (Sp).

Results: Significant differences in the serum iron between patients of groups 1 and 2 were revealed on the 5th, 7th and 10th days after the operation. Before surgery, the serum iron in patients of group 1 was $13.5(5 ; 17) \mu \mathrm{mol} / \mathrm{l}$, in group 2 it was $7.7(6.5 ; 14.3) \mu \mathrm{mol} / \mathrm{l}, \mathrm{p}=0.82$. On the 1st day after the operation, in group 1 the iron was 6.6 (4.2; $13.7)$, and in group 2 it was $7.5(5.5 ; 13.2) \mu \mathrm{mol} / \mathrm{l}, \mathrm{p}=0.48$; on 2 th day after surgery in group $1-3.9(2.8 ; 6.6)$, and in group $2-4.9(2.8 ; 8.1)$ $\mu \mathrm{mol} / \mathrm{l}, \mathrm{p}=0.61$; on 3 th day in group 1 - $5.1(2.7 ; 7.6)$, and in group 2 $3.1(2.3 ; 5.6) \mu \mathrm{mol} / \mathrm{l}, \mathrm{p}=0.12$; on 5 th day after surgery in group $1-6.1$ (2.6; 14.5), and in group $2-2.5(1.9 ; 5.2) \mu \mathrm{mol} / \mathrm{l}, \mathrm{p}=0.02$; on 7 th day in group $1-7.3(4 ; 13.5) \mu \mathrm{mol} / \mathrm{l}, \mathrm{p}=0.02$; on the 10 th day after craniotomy in group 1 the iron was $7.3(3.6 ; 12.3)$, and in group 2 - 3.6 (1.9; 5.7) $\mu \mathrm{mol} / \mathrm{l}, \mathrm{p}=0.03$.

Using logistic regression, a statistically significant difference was revealed between patients of groups 1 and 2 in serum iron on 5th and 10thdays after the operation for an unfavorable outcome. On 5th day: AUC 0.71 , sensitivity (Se) $70.6 \%$, specificity (Sp) $-65.2 \%$. On 10th day: AUC 0.73 , Se $75 \%$, Sp - 60\%.

Conclusion: 1. Patients with an unfavorable outcome of treatment's $\mathrm{TBI}$ on the 5th, 7th and 10th days after craniotomy revealed significantly lower level of serum iron than in patients with a favorable outcome.

2. Lower value serum iron starting 5th day after craniotomy can be considered a predictor of an unfavorable outcome in the treatment of patients with TBI.

\section{Reference(s) and grant ackowledgment(s)}

1. 1. Tsentsiper LM, Dryagina NV, Rumyantseva MV, Aybazova MI, Nazarov RV, Ulitin AY, Kondratev AN. Neurohumoral response in acute period of severe traumatic brain injury. Russian journal of anaesthesiology and reanimatology. 2017; 62(3):205-209. doi: 10.18821/0201-7563-2017-62-3-205-209. (in Russian). 2. Matskevich VS, Kizimenko AN. Features of microelements exchange in critically ill children. Novosti Khirurgii. 2019; 27(3):328-336 doi: https://dx.doi.org/10.18484/2305-0047.2019.3.328. (in Russian). 3. Belatar B, Elabidi A, Barkiyou M, Faroudi MEl, Eljaoudi R, Lahlou L, Kabbaj S, Maazouzi W. The influence of heavy metals and trace elements on comatose patients with severe traumaticbraine injury in the first week of admission. J Toxicol. 2018:7252606. doi:10.1155/2018/7252606. 4. Nisenbaum EJ, Novikov DS, Lui YW. The presence and role of iron in mild traumatic brain injury: an imaging perspective. Journal of neurotrauma. 2014; 31: 301-307. doi: 10.1089/ neu.2013.3102.

\section{7}

\section{Malnutrition in patients with ischemic stroke}

A. Gritsan ; N. Dovbysh

${ }^{1}$ Anaesthesiology and intensive care, Krasnoyarsk State Medical University, Krasnoyarsk Regional Clinical Hospital, Krasnoyarsk, Russia

Correspondence: A. Gritsan

Intensive Care Medicine Experimental 2020, 8(2): 000157

Introduction: It is known that patients with ischemic stroke may have nutritional deficiency. However, the frequency of its occurrence, primarily at the beginning of the disease and the possible connection with mortality remain issues of discussion.

Objectives: To assess the incidence of malnutrition in patients with ischemic stroke at the beginning of the disease and its relationship with mortality in these patients.

Methods: An observational study was conducted of patients with IS admitted to the NICU within 1 randomly selected month. The criterion for exclusion from the study was the presence of a terminal stage of cancer. A total of 84 patients were included in the study, of which 61 (73\%) were women and $23(27 \%)$ were men. The age of the patients is $71.5[68.0 ; 80.8]$ years. All patients on admission were evaluated on the Nutritional Risk Screening (NRS) scale. Estimated height, weight of patients, calculated body mass index (BMI). Data are presented as median and $25 \%$ and $75 \%$. Statistical analysis was performed using the $x 2$ criterion.

Results: The weight of patients at admission was $76.5[66.3 ; 88.0] \mathrm{kg}$, body mass index (BMI) - 27.5 [26.8; 28.5]. When assessing the presence of risk factors for malnutrition according to the NRS 2002, the only risk factor was hospitalization in the intensive care unit. In the final assessment, in 57 (68\%) patients, the total score was more than 3 due to the following parameters - age over 70 years and the presence on the APACHE II scale of more than 10 points. From these $16(19 \%)$ patients were diagnosed with mild malnutrition due to a decrease in the absolute number of lymphocytes. Moderate and severe malnutrition were not diagnosed in these patients.

$12(14.3 \%)$ patients died. Of these, 7 (43.8\%) patients died in the group of patients with mild malnutrition. It is significantly higher than in the group of patients without malnutrition $(x 2=14.013, p=0.001)$.

Conclusion: Patients with ischemic stroke have at admission a low risk of malnutrition assessed according to the INR 2002. The presence of mild malnutrition in patients with IS is due to the age of patients older than 70 years and the presence of an APACHE II score of more than 10 points. Mortality in patients with mild malnutrition is significantly higher than in patients without malnutrition.

\section{Reference(s) and grant ackowledgment(s)}

1. No 
000198

A Retrospective Review of Intensive Care Admissions with Acute Ischaemic Stroke in an Irish Tertiary Referral Centre

D. Edgeworth"; O. Nichoileann 1; D. O'leary²; G. Curley' ; RA. O'leary ${ }^{1}$

${ }^{1}$ Anaesthesiology \& Critical Care, Beaumont Hospital, Dublin, Ireland;

${ }^{2}$ School of medicine, University College Dublin, Dublin, Ireland

Correspondence: $\mathrm{D}$. Edgeworth

Intensive Care Medicine Experimental 2020, 8(2): 000198

Introduction: Acute Ischaemic Stroke (AIS) remains a significant cause of morbidity and mortality worldwide. With increasingly advanced techniques for reperfusion and prevention of secondary injury, the role of intensive care management in AIS is expanding. Beaumont Hospital, Dublin is the national neurosurgical and interventional neuroradiology referral centre in the Republic of Ireland with 17 Intensive Care beds. There were 2531 ICU admissions across all categories during this time period.

Objectives: The study sought to review the number and nature of admissions, baseline characteristics, interventions and outcomes of patients with AIS and compare these to international data.

Methods: A retrospective review of admissions from January 2017 to July 2019 was performed using standard data points collected by the National Office of Clinical Audit (NOCA). Approval for retrieval and assessment of data was granted by NOCA. Patients with a primary admission diagnosis of AIS were included. Other causes of cerebrovascular accident including subarachnoid haemorrhage were excluded. Eighty-seven pre-determined data points were examined. Statistical analysis was performed in RStudio, Verision 1.1.456. Results are presented as percentages, mean/median and 95\% Confidence Interval as appropriate.

Results: 68 patients were admitted in this period with a primary diagnosis of AIS. 34\% were female. Mean age was 60 years $(95 \% \mathrm{CI} 58.8$ 61.2). The MCA territory was implicated in $38 \%$ cases.

$16 \%$ of patients presented locally. Referring hospitals ranged from 5.6 to 243 kilometres in distance from our centre.

Thrombectomy was performed in 36 patients (52\%). The majority of patients $(89.7 \%)$ were mechanically ventilated. The median mechanical ventilation days was 4 (range 1-22 days). Tracheostomy was performed in seven patients. The majority of patients in this group had posterior circulation involvement. $43 \%$ of patients were treated for hyperglycaemia.

Median Intensive Care length of stay was 6 days (range 1-26 days). 63\% of patients survived to ICU discharge. Middle Cerebral Artery decompression was performed in 16 patients (24\%). This group of patients had a lower median age, higher rates of stroke-associated pneumonia and a similar survival to ICU discharge. Increasing age was associated with reduced survival to ICU discharge (OR 0.94; $\mathrm{Cl} 0.90-0.99 ; \mathrm{P}=0.02$ ). Other variables such as body mass index, hyperglycaemia, mechanical ventilation days and ICU length of stay did not reach statistical significance. This may be reflective of the relatively small study numbers.

Conclusion: This data confirms the high acuity and treatment burden posed by this evolving group of patient. Rates of mechanical ventilation, pneumonia and ICU mortality were similar to data previously reported (1). Increasing age was predictive of ICU mortality.

\section{Reference(s) and grant ackowledgment(s)}

1. Kirkman M, Citerio G, Smith M. The intensive care management of acute ischemic stroke: an overview. Intensive Care Med(2014) 40:640-653

\section{0}

Purine metabolites, magnesium, corticosteroid and thyroid hormones, lethal outcome in acute cerebrovascular pathology

E. Oreshnikov'; S. Oreshnikova'; A. Oreshnikov²

${ }^{1}$ Anaesthesiology \& intensive care, Chuvash State University named I.N.Ulyanova, Cheboksary, Russia; ${ }^{2}$ Internal medicine, Chuvash State University named I.N.Ulyanova, Cheboksary, Russia

Correspondence: E. Oreshnikov

Intensive Care Medicine Experimental 2020, 8(2): 000270
Introduction: We study the possibility of predicting the onset of death with the help of various hormonal and metabolic indicators in the acute period of cerebral stroke.

Methods: In 626 adult patients in the acute period of cerebral stroke (regardless of its type, variant, etc.) who were treated in the intensive care unit, along with generally accepted instrumental and laboratory tests, in cerebrospinal fluid and venous blood samples colorimetric determination of adenine, guanine, hypoxanthine, xanthine, uric acid, magnesium was carried out, enzyme-linked immunosorbent assay for cortisol and dehydroepiandrosterone, thyroid-stimulating hormone, thyroxine and triiodothyronine.

Results: The most specific factors were: increased concentration of uric acid in the blood serum on the 1 st and 3rd day of stroke $(0.89$ and 0.98 ), combination of an increased content of uric acid in blood serum and cerebrospinal fluid on the $1 \mathrm{st}$ and $3 \mathrm{rd}$ day of a stroke $(0.90$ and 0.97); reduced guanine in cerebrospinal fluid (0.84) on the 3rd day of stroke; increased content of free fractions of triiodothyronine and thyroxine on the $1 \mathrm{st}$ day of stroke $(0.86$ and 0.76$)$. The relative risk of a fatal outcome is high when a stroke of increased concentration in the blood serum of cortisol is detected on the 1st and 3rd day (3.25 and 7.39); increased content of hypoxanthine (2.49), xanthine (2.49) and uric acid (3.53) on the 3rd day of stroke, a combination of increased serum uric acid and cerebrospinal fluid (2.71). The highest chances of a fatal outcome when a stroke is detected on the 1st day of a stroke are elevated concentrations of cortisol and free thyroxine in blood serum (OR 4.98 and 2.59, respectively), uric acid in cerebrospinal fluid (OR 2.79); when a stroke concentration of adenine (OR 2.61), guanine (OR 3.75), hypoxanthine (OR 4.55), xanthine (OR 3.60), urine is detected in the blood serum on the 3rd day of a stroke acid (OR 20.0), cortisol (OR $10.4)$, a combination of high uric acid in blood serum and cerebrospinal fluid (OR 23.3).

Conclusion: In patients in the acute period of cerebral stroke (regardless of its type), indicators of the purine spectrum, stress-adaptive hormonal status are highly informative factors in predicting the onset of a fatal outcome. The most prognostically "powerful" of the studied parameters is the blood level of uric acid.

\section{2}

Purine metabolites, magnesium, corticosteroid and thyroid hormones, letal outcome in acute cerebral ischemia: diagnostic and prognostic aspects

E. Oreshnikov'; S. Oreshnikova ; A. Oreshnikov

${ }^{1}$ Anaesthesiology \& intensive care, Chuvash State University named I.N.Ulyanova, Cheboksary, Russia; ${ }^{2}$ Internal medicine, Chuvash State University named I.N.Ulyanova, Cheboksary, Russia

Correspondence: E. Oreshnikov

Intensive Care Medicine Experimental 2020, 8(2): 000272

Introduction: We study the possibility of predicting the onset of a fatal outcome using various hormonal and metabolic parameters in acute cerebral ischemia.

Methods: In 402 adult patients in the acute period of cerebral ischemic stroke, who were treated in the intensive care unit and intensive care unit of the angioeurological department, along with generally accepted instrumental and laboratory tests, colorimetric measurements of adenine, guanine, hypoxanthine, xanthan were carried out in samples of cerebrospinal fluid and venous blood uric acid, magnesium, malondialdehyde. Based on the concentration ratio of uric acid / xanthine, xanthine / hypoxanthine, uric acid / hypoxanthine, the activity of the first and second stages of the xanthine oxidase reaction and the total xanthine oxidase activity were calculated. Enzyme immunoassays examined the levels of cortisol and dehydroepiandrosterone, thyroid stimulating hormone, thyroxine and triiodothyronine. 
Results: The most sensitive predictors of lethal outcome of ischemic stroke were: increased xanthine concentration on the 1st and 3rd day of stroke ( 0.83 and 0.76$)$, reduced xanthine oxidase activity (in the second stage of the xanthine oxidase reaction - 0.61). Among the most specific such factors: an increased concentration of uric acid in the blood serum on the 1 st and 3rd day of a stroke (0.91 and 0.98), a combination of an increased content of uric acid in the blood serum and cerebrospinal fluid on the 1 st day of a stroke (0.93). The relative risk of death is highest when a stroke concentration of uric acid in the blood serum is detected on the 3 rd day (3.93). The highest chances of a fatal outcome when a stroke is detected on the 3rd day of an elevated blood serum uric acid (OR 21.5), adenine (OR 3.55), guanine (OR 3.27); a combination of increased serum uric acid and cerebrospinal fluid (OR 3.37), increased serum uric acid (OR 2.87), decreased xanthine oxidase activity in the second stage of the xanthine oxidase reaction on the 1st day of stroke (OR 2,67).

Conclusion: In patients with acute cerebral ischemia in the acute period of cerebral ischemic stroke, the most prognostically "powerful" lethal outcome factors are uric acid level and xanthine oxidase activity.

\section{4}

Spontaneous Subarachnoid Hemorrhage: Impact of Demographic, Clinical and Monitoring Data in Patient Outcome

D. Cruz ; J. Fernandes ${ }^{2}$; E. Monteiro ${ }^{2}$

${ }^{1}$ Faculty, Faculty of Medicine of Porto University, Porto, Portugal; ${ }^{2}$ Intensive care medicine department, São João Universitary Hospital Center, Porto, Portugal

Correspondence: D. Cruz

Intensive Care Medicine Experimental 2020, 8(2): 000294

Introduction: Subarachnoid Hemorrhage $(\mathrm{SAH})$ is a medical emergency and the most frequent etiology is a ruptured aneurysm. It remains a major cause of morbidity and mortality due to the initial insult but mainly as a result of secondary brain injury (SBI). Multimodal Monitoring provides an individualized care at bedside, giving tools to guide therapeutic interventions, preventing or minimizing SBI.

Objectives: To identify possible factors with impact in outcome in patients admitted with spontaneous SAH.

Methods: A retrospective case-series study that included all patients with SAH admitted to a Neurocritical Unit (NCCU) of a tertiary hospital at intensive care level between the first january 2018 and the 30th june 2019. Primary outcome was defined by Glasgow Outcome Scale at discharge from NCCU and at 28,90 and 180 days after the event. Results: Forty-six patients ( 29 females) were included. Median age was 55.5 years (50.0-63.3). In 41 patients an aneurysm was identified. The most frequent aneurysm locations were Anterior Communicating Artery and Middle Cerebral Artery, in 11 (23.9\%) and 9 (19.6\%) cases, respectively. The median length of stay in the NCCU at intensive care level was 20.5 (4.8-31.0)days. Fourteen (30.4\%) patients were submitted to neuroradiology procedure. No patient submitted to neuroradiology procedure had a bad outcome at six months. At all moments of follow-up, Glasgow Coma scale (GCS) at admission, Hunt \& Hess $(\mathrm{H} \& \mathrm{H})$ scale, duration of invasive ventilation (IMV) and delayed cerebral ischemia (DCI) had an association with patients' outcome $(\mathrm{p}<$ $0.05)$. The three deaths $(6.5 \%)$ recorded occurred during NCCU stay: two patients with severe SAH (GCS of 3, Fisher scale of 4 and H\&H scale of $\mathrm{V}$ ) and a third patient who developed many complications (hydrocephalus, re-bleeding and vasospasm requiring intra-arterial administration of verapamil), despite admission with GCS of 15, Fisher scale of 4 and $\mathrm{H} \& \mathrm{H}$ of II.

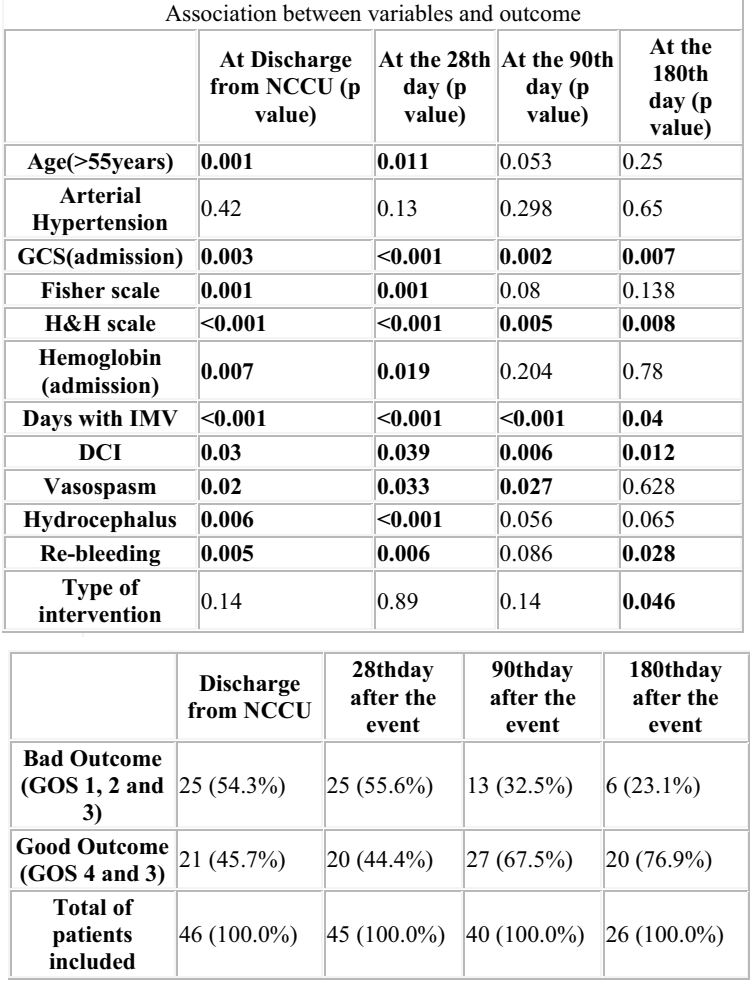

Conclusion: SAH related complications were common and associated with worse outcomes, therefore treatment approach should be focused on its avoidance and prompt resolution. Endovascular treatment had better outcomes at six months than surgical intervention.

Reference(s) and grant ackowledgment(s)

1. Molyneux AJ, Birks J, Clarke A, Sneade M, Kerr RSC. The durability of endovascular coiling versus neurosurgical clipping of ruptured cerebral aneurysms: 18 year follow-up of the UK cohort of the International Subarachnoid Aneurysm Trial (ISAT). The Lancet. 2015;385(9969):691-7.

2. Fujii M, Yan J, Rolland WB, Soejima Y, Caner B, Zhang JH. Early brain injury, an evolving frontier in subarachnoid hemorrhage research. Transl Stroke Res. 2013;4(4):432-46.

000407

Reliability of the SDASH score for predicting shunt dependency after aneurysmal subarachnoid hemorrhage: external validation study

A. Herraiz'; R. García-Armengol'2; M. Misis ${ }^{1}$; P. Puyalto ${ }^{3}$; A. Rodríguez ${ }^{2}$; JF. Julián ${ }^{4}$; AC. Pérez ${ }^{3}$; F. Brugada-Bellsolà2; j. Rimbau²

${ }^{1}$ Intensive care unit, Hospital Germans Trias i Pujol, Badalona, Spain; ${ }^{2} \mathrm{Neu}$ rosurgery, Hospital Germans Trias i Pujol, Badalona, Spain; ${ }^{3}$ Radiology, Hospital Germans Trias i Pujol, Badalona, Spain; ${ }^{4}$ Surgery, Hospital Germans

Trias i Pujol, Badalona, Spain

Correspondence: A. Herraiz,

Intensive Care Medicine Experimental 2020, 8(2): 000407 
Introduction: Chronic hydrocephalus $(\mathrm{CH})$ is a common and poten tially modifiable complication of spontaneous subarachnoid hemorrhage (SAH) requiring prompt treatment for optimal outcomes. Risk factors for $\mathrm{CH}$ have been extensively investigated, but reliable scores to predict shunt dependency after aneurysmal SAH (aSAH) are scarce. A newly developed shunt dependency in aSAH (SDASH) score based on the combination of acute hydrocephalus (aHP), Hunt and Hess grade, and Barrow Neurological Institute (BNI) score was found to be a reliable tool for predicting shunt-dependent hydrocephalus (SDHC). This prediction model, however, was based on a single-center retrospective population.

Objectives: The present study was designed to validate the SDASH score in an external cohort of aSAH patients.

Methods: The study population included 214 of 249 (85.9\%) patients with aSAH admitted to our hospital (2016-2019). In each patient, the SDASH score, the Hijdra score, the BNI score, the chronic hydrocephalus ensuing from SAH score (CHESS), the Graeb, and the modified Graeb score (mGS) were calculated, and the reliability of these models were compared. Logistic regression analysis was used to determine the reliability of the SDASH score, and the area under the receiver operating characteristics (ROC) curve (AUC) to assess the discrimination capacity of the model.

Results: 40 patients (18.7\%) developed SDHC. Older age, high Hunt and Hess grade, high grade of the WFNS classification scale, EVD placement, ventricular hemorrhage, CSF daily output and higher bicaudate index, correlated significantly with an increased rate of ventriculoperitoneal shunt insertion. The AUC for SDASH score was 0.816 . The SDASH score reliably predicted SDHC in aSAH (odds ratio of 2.93; $95 \%$ confidence interval 1.99-4.31; $p<0.001$ ). No statistically significant differences were found between SDASH score and the other predictive scores.

Conclusion: The SDASH score was found to be a reliable tool for predicting SDHC and could be used in daily practice. The SDASH score may be added to the list of other scores such as CHESS, Graeb, or mGS which may help to early management of hydrocephalus in aSAH.

\section{Reference(s) and grant ackowledgment(s)}

1. Erixon HO, Sorteberg A, Sorteberg W, Eide PK. Predictors of shunt dependency after aneurysmal subarachnoid hemorrhage: results of a single-center clinical trial. Acta Neurochir (Wien) 156:2059-2069, 2014

2. Diesing D, Wolf S, Sommerfeld J, Sarrafzadeh A, Vajkoczy P, Dengler NF. A novel score to predict shunt dependency after aneurysmal subarachnoid hemorrhage. J Neurosurg 128:1273-1279, 2018.

3. Czorlich P, Mende KC, Vettorazzi E, Regelsberger J, Westphal M, Schmidt NO. Validation of the modified Graeb score in aneurysmal subarachnoid hemorrhage. Acta Neurochir (Wien) 157:1867-1872, 2015.

4. Bretz JS, Von Dincklage F, Woitzik J, Winkler MKL, Major S, Dreier JP, et al. The Hijdra scale has significant prognostic value for the functional outcome of Fisher grade 3 patients with subarachnoid hemorrhage. Clin Neuroradiol 27:361-369, 2017

5. Alaraj A, Hussein AE, Esfahani DR, Amin-Hanjani S, Aletich VA, Charbel FT. Reducing length of stay in aneurysmal subarachnoid hemorrhage: a three year institutional experience. J Clin Neurosci 42:66-70, 2017.

6. None.

\section{7}

Delayed cerebral ischemia associated risk factors in patients with subarachnoid hemorrhage in a neurotraumatic ICU

C. Sánchez Ramírez'; CF. Lübbe Vázquez'; J. López Pérez'; JL. Vicente

Arranz ${ }^{1}$; C. Vázquez Pineda'; A. Padrón Mujica'; JM. Ríos Bort';

J. Garriga Segarra'; J. García-Biosque Rodríguez ${ }^{1}$; C. Rodriguez Mata ${ }^{1}$.

P. Saavedra-Santana²; S. Ruiz-Santana

'Intensive care medicine, University Hospital of Gran Canaria Dr. Negrin, Las Palmas de Gran Canaria, Spain; ${ }^{2}$ Mathematics and informatics deparment, University of Las Palmas:, Las Palmas de Gran Canaria, Spain

Correspondence: $C$. Sánchez Ramírez

Intensive Care Medicine Experimental 2020, 8(2): 000427
Introduction: Delayed cerebral ischemia (DCl) is a major contributor to the high fatality and morbidity case rates of aneurysmal subarachnoid hemorrhage (SAH). About $30 \%$ of the SAH patients develop DCI but it is difficult to predict which patients will develop it.

Objectives: To assess the development of DCl risk factors in patients with $\mathrm{SAH}$, admitted in a Neurotraumatic ICU.

Methods: Prospectively collected data of patients admitted from October, 2013 to December 2019 to a 10-bed Neurotraumatic ICU. We analyzed: main diagnosis at admission; demographic data including sex and race; neurological data (clinical examination, pupils reactivity and size, and Glasgow Coma Score: GCS; localization and aneurysm size; presence of intracranial hematoma $(\mathrm{ICH})$; presence and volume of intraventricular bleeding; days to develop vasoespam; development of DCl; Fisher scale, Modified Fisher scale (MFS), Hunt and Hess scale (HHS), Word Federation of Neurosurgeons (WFNS) scale; presence of vasoespasm in doppler orarteriography; delayed of admission in ICU; treatment of the aneurysm; complications, including infections; Glasgow Outcome Scale (GOS) at ICU discharge and 6 months after ICU discharge and several other risk factors. A univariate analysis of $\mathrm{DCl}$ was performed. To obtain a predictive rule for the $\mathrm{DCl}$, a model for the prediction was obtained using the classification and regression trees (CART) procedure [1]. CART classifies data using a sequence of if-then rules. The basis of the decision tree algorithms is the binary recursive partitioning of the data. The most discriminative variable is first selected to partition the data set into child nodes. The splitting continues until some stopping criterion is reached. At each terminal node, the probability of $\mathrm{DCl}$ was estimated as the proportion of patients belonging to that node that developed the event. The tree was constructed according to the following algorithm: in the first stage, the tree grows until all cases are correctly classified, and in the second stage, we used the tenfold cross-validation method of successive pruning [1]. Finally, the tree that minimized the error measurement (deviance) was chosen. For this predictor the corresponding ROC curve was obtained and the AUC was estimated by means of a $95 \% \mathrm{Cl}$. Results: One hundred forty two SAH patients were collected, 32 $(22,5 \%)$ of them developed DCl. Demographic data and types of admission are shown in Table $1 \mathrm{a}$ and $1 \mathrm{~b}$.

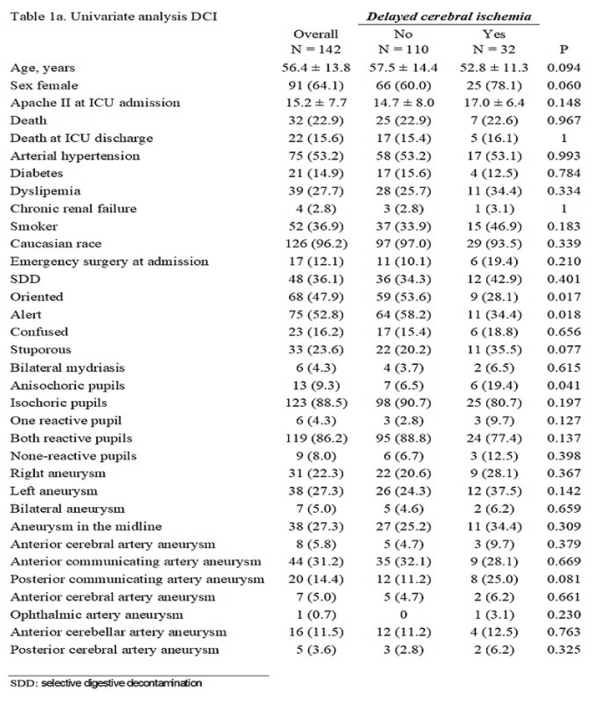




\begin{tabular}{|c|c|c|c|c|}
\hline \multirow[t]{2}{*}{ Table 1b. Univariate analysis DCI } & \multirow[b]{2}{*}{$\begin{array}{l}\text { Overall } \\
\mathrm{N}=142\end{array}$} & \multicolumn{2}{|c|}{$\begin{array}{l}\text { Detayert cere brat } \\
\text { ischemia }\end{array}$} & \multirow[b]{2}{*}{ p } \\
\hline & & $\begin{array}{c}\text { No } \\
N=110\end{array}$ & $\begin{array}{c}\text { Yes } \\
N=32\end{array}$ & \\
\hline Basilar artery aneurysm & $12(8.6)$ & $10(9.3)$ & $2(6.2)$ & \\
\hline $\begin{array}{l}\text { Posterior inferior cerebellar artery } \\
\text { aneurysm }\end{array}$ & $8(5.8)$ & $7(6.5)$ & $1(3.1)$ & 0.682 \\
\hline Carotid aneurysm & $10(7.2)$ & $5(4.7)$ & $5(15.6)$ & \\
\hline Multiple aneur & $23(16.6)$ & $15(13.9)$ & $8(25.8)$ & 0.115 \\
\hline Aneurysm clipping & $24(17.0)$ & $16(14.7)$ & $8(25.0)$ & 0.172 \\
\hline Lumbar drainage & $2(1.4)$ & $1(0.9)$ & $1(3.1)$ & 0.404 \\
\hline Embolization of the aneurysm & $83(58.9)$ & $61(56.0)$ & $22(68.8)$ & 0.196 \\
\hline Embolization and surgery treatment & $2(1.4)$ & $1(0.9)$ & $1(3.1)$ & 0.404 \\
\hline Conservative treatment & $25(17.7)$ & $22(20.2)$ & $3(9.4)$ & 0.159 \\
\hline Decompressive craniectomy & $6(4.3)$ & $4(3.7)$ & $2(6.2)$ & 0.618 \\
\hline Intraoperative aneurysm rupture & $6(4.3)$ & $3(2.8)$ & $3(9.4)$ & 0.130 \\
\hline External ventricular device & $67(48.2)$ & $45(42.1)$ & $22(68.8)$ & 0.008 \\
\hline Stroke & $38(26.8)$ & $14(12.7)$ & $24(75.0)$ & c \\
\hline Cerebrospinal fluid fistula & & & & \\
\hline Hydroceph & 6104 & & $18(56.2)$ & 0.092 \\
\hline $\mathrm{MV}>7$ days & $48(33,8)$ & $33(30.0)$ & $15(46.9)$ & 0.076 \\
\hline Frontal ICH & $25(17.7)$ & $20(18.4)$ & $5(15.6)$ & 0.723 \\
\hline Temporal ICH & $22(156)$ & $20(12.4)$ & $2(6.2)$ & 0.163 \\
\hline Suboural hematoma & $14(9.9)$ & $12(11.0)$ & $2(6.2)$ & 0.737 \\
\hline Vasoespasm_doppler & 3212 & $15(14.7)$ & $(56.7)$ & $<.001$ \\
\hline Vasoespasm_Ateriographgy & $29(223)$ & $11(11.0)$ & $18(60.0)$ & $<.001$ \\
\hline $\mathrm{AC}$ & $10(7.0)$ & $9(8.2)$ & $1(3.1)$ & 0.456 \\
\hline Rebleedng 72 hours & $7(4.9)$ & $4(3.6)$ & $3(9.4)$ & 0.190 \\
\hline & $14(10.1)$ & & (12.5) & 0.738 \\
\hline Fisher scale & $3(3-4)$ & $3.5(3-4)$ & $(3-4)$ & 727 \\
\hline Fisher modfied scale & $4(3-4)$ & $3(2-4)$ & $4(4-4)$ & 002 \\
\hline Hunt and Hess scale & $\begin{array}{l}2(1-4) \\
21-4\end{array}$ & $\begin{array}{l}2(1-3) \\
3(1-4)\end{array}$ & $\begin{array}{l}3(1,5-4) \\
4(2-45)\end{array}$ & 0.027 \\
\hline $\begin{array}{l}\text { WFNNSCCde } \\
\text { APACHE vasospasm }\end{array}$ & $\begin{array}{l}2(1-4) \\
16(9-18)\end{array}$ & $\begin{array}{c}2(1-4) \\
14(4-18)\end{array}$ & $\begin{array}{l}4(2-4.5) \\
17(12-18)\end{array}$ & $\begin{array}{l}0.025 \\
0.234\end{array}$ \\
\hline 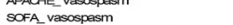 & $\begin{array}{c}3(19-8) \\
3(1-8)\end{array}$ & $\begin{array}{l}74(4-10) \\
3(0.5-6)\end{array}$ & $3(1.5-8.5)$ & \\
\hline ICUreadrissionthy & $11(2-24)$ & & $12(9-20)$ & \\
\hline GCSon site & & $15(13-15)$ & $14(12-15)$ & o.1 \\
\hline ocsinemerg & $14(9-15)$ & $14(1$ & $12(8$ & 0.157 \\
\hline ocsatioua & $11(4-15)$ & $14(4-15)$ & $8(4-14)$ & \\
\hline
\end{tabular}

Anterior communicating (Aco) artery aneurysms were the most frequently found and most of them were located in the left side. . The most frequent $\mathrm{DCl}$ patient complications were stroke 24 (75\%) and hydrocephalus $18(56.2 \%)$. GOS at ICU discharge was 4 and, six months after ICU discharge, was 5 in patients with SAH. Patients who developed DCI had GOS at ICU discharge of 4 and it was also 4 six months later. Also $19(59,37 \%)$ had GOS > 3 at ICU discharge. Thirty two (22.9\%) SAH patients died. Seven (22.6\%) DCl patients died. There was not statistical significant difference in mortality in $\mathrm{DCl}$ vs $\mathrm{SAH}$ patients. Classification tree showed:HHS 1 has a high probability of not having DCI.MFS $>3$ and HHS $>1$ have probabilities of around $30 \%$ of DCl.

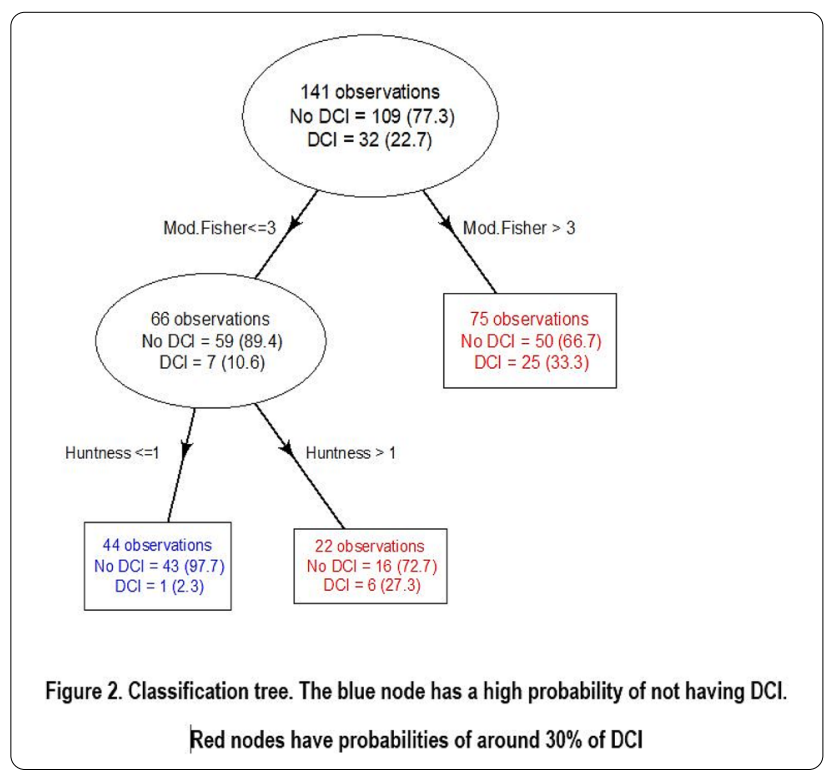

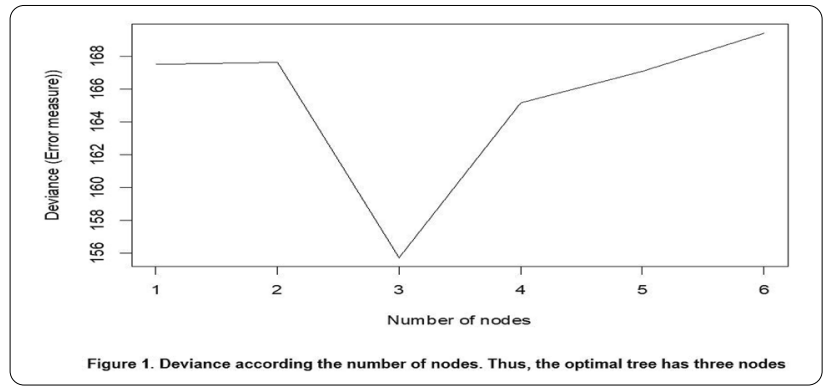

Conclusion: Our data show $22,5 \%$ of our studied patients had DCI. GOS reflected low disability of DCI patients both at ICU discharge and six months later. Classification tree showed: HHS 1 has a high probability of not having $\mathrm{DCl}$. MFS $>3$ and HHS $>1$ have probabilities of around $30 \%$ of $\mathrm{DCl}$. Finally, mortality was not significantly greater in the studied patients.

Reference(s) and grant ackowledgment(s)

1. [1] Breiman L, Freidman JH, Olshen RA, Stone CJ (1984) Classification and regression trees. Wadsworth, Belmon

\section{5}

Disability associated risk factors in patients with subarachnoid hemorrhage in a neurotraumatic ICU

C. Sánchez Ramírez ; CF. Lübbe Vázquez¹; J. López Pérez ; ; J. Vicente Arranz ; ; A. Padrón Mujica'; C. Vázquez Pineda'; JM. Ríos Bort';

J. Garriga Segarra'; J. García-Biosque Rodríguez ${ }^{1}$; C. Rodriguez Mata ${ }^{1}$; P. Saavedra-Santana ${ }^{2}$; S. Ruiz-Santana'

${ }^{1}$ Intensive care medicine, University Hospital of Gran Canaria Dr. Negrin, Las Palmas de Gran Canaria, Spain; ${ }^{2}$ Mathematics and informatics deparment, University of Las Palmas:, Las Palmas de Gran Canaria, Spain

Correspondence: $C$. Sánchez Ramírez

Intensive Care Medicine Experimental 2020, 8(2): 000445

Introduction: Aneurysmal subarachnoid hemorrhage $(\mathrm{SAH})$ is a worldwide health burden with serious outcomes such as permanent disability. It is important to learn the disability risk factors and identify those potentially preventable, to reduce them.

Objectives: To assess disability risk factors in patients admitted with subarachnoid hemorrhage (SAH), admitted in a neurotraumatic ICU.

Methods: Prospectively collected data of patients admitted from October, 2013 to December 2019 to a 10-bed Neurotraumatic ICU. We analyzed: main diagnosis at admission; demographic data, including sex and race; neurological data (clinical examination, pupils reactivity and size, and Glasgow Coma Score (GCS); location and aneurysm size; presence of intracranial hematoma $(\mathrm{ICH})$; presence and volume of intraventricular bleeding; days to develop vasoespam; development of delayed cerebral ischemia (DCI); Fisher scale, modified Fisher scale, Hunt and Hess scale, Word Federation of Neurosurgeons (WFNS) scale; presence of vasoespasm in doppler or arteriography; delayed of admission in ICU; treatment of the aneurysm; complications, including infections; Glasgow Outcome Scale (GOS) at ICU discharge and 6 months after ICU discharge and several other risk factors. Disability was defined as GOS $\leq 3$. In order to identify those factors that maintain independent association with disability, a multivariate logistic regression analysis were performed. It was considered significant if $p$ $\leq .05$.

Results: Among $152 \mathrm{SAH}$ admitted patients, 58 (38.15\%) out of 94 patients had GOS $\leq 3$. Univariate analysis is shown in Table $1 \mathrm{a}$ and $1 \mathrm{~b}$ 


\begin{tabular}{|c|c|c|c|}
\hline \multirow[t]{2}{*}{ Table1a. Univariate anañysis SAH disability } & \multicolumn{2}{|c|}{ GOS at discharge from ICU } & \multirow[b]{2}{*}{$\mathrm{p}$} \\
\hline & $\begin{array}{c}>3 \\
N=94\end{array}$ & $\begin{array}{c}\leq 3 \\
N=58\end{array}$ & \\
\hline Age, years & $53.8 \pm 13.4$ & $61.3 \pm 14.4$ & 0.001 \\
\hline Sex female & $61(64.9)$ & $39(67.2)$ & 0.767 \\
\hline Apache II at ICU admission & $11.5 \pm 6.1$ & $21.6 \pm 5.8$ & $<.001$ \\
\hline Death & $3(3.3)$ & $32(56.1)$ & $<.001$ \\
\hline Death after ICU discharge & $0(0.0)$ & $24(42.1)$ & $<.001$ \\
\hline Hospital death & $3(3.3)$ & $8(14.3)$ & 0.022 \\
\hline Arterial hypertension & $45(48.4)$ & $33(56.9)$ & 0.309 \\
\hline Diabetes & $11(11.8)$ & $10(17.2)$ & 0.35 \\
\hline Dyslipemia & $26(28.0)$ & $15(25.9)$ & 0.778 \\
\hline Chronic renal failure & $3(3.2)$ & $1(1.7)$ & 1 \\
\hline Neoplasm & $3(3.2)$ & $2(3.5)$ & 1 \\
\hline Malnutrifion & $0(0.0)$ & $1(1.7)$ & 0.384 \\
\hline Smoker & $34(36.6)$ & $21(36.2)$ & 0.965 \\
\hline Caucasian race & $85(95.5)$ & $51(98.1)$ & 0.652 \\
\hline \multicolumn{4}{|l|}{ Number of platelets at ICU admission $\times 10^{3}$} \\
\hline $\begin{array}{l}\text { Emergency surgery at admission } \\
\text { Previous surner }\end{array}$ & $\begin{array}{l}8(8.7) \\
6(65)\end{array}$ & $\begin{array}{c}11(19.0) \\
1(1.7)\end{array}$ & $\begin{array}{l}0.066 \\
0.49\end{array}$ \\
\hline SDD & $19(22.4)$ & $34(60.7)$ & $<.001$ \\
\hline Oriented & $61(65.6)$ & $11(19.0)$ & $<.001$ \\
\hline Alert & $65(69.9)$ & $16(27.6)$ & $<.001$ \\
\hline Confused & $14(15.1)$ & $10(17.2)$ & 0.721 \\
\hline Stuporous & $10(11.0)$ & $24(41.4)$ & $<.001$ \\
\hline Bilateral mydniasis & $1(1.1)$ & $6(10.5)$ & 0.014 \\
\hline Anisochoric pupils & $5(5.6)$ & $10(17.5)$ & 0.021 \\
\hline Isochoric pupils & $83(93.3)$ & $43(75.4)$ & 0.002 \\
\hline One reactive pupil & $3(3.4)$ & $4(7.0)^{\prime}$ & 0.434 \\
\hline Both reactive pupils & $79(89.8)$ & $45(79.0)$ & 0.07 \\
\hline None-reactive pupils & $4(5.9)$ & $6(11.5)$ & 0.327 \\
\hline Right aneurysm & $15(16.3)$ & $17(31.5)$ & 0.032 \\
\hline Left aneurysm & $26(28.3)$ & $17(31.5)$ & 0.68 \\
\hline Bilateral aneurysm & $6(6.5)$ & $1(1.8)$ & 0.254 \\
\hline Aneurysm in the midline & $28(30.4)$ & $10(18.5)$ & 0.113 \\
\hline Anterior cerebral artery aneurysm & $4(4.4)$ & $5(9.1)$ & 0.3 \\
\hline Anterior communicating artery aneurysm & $27(29.7)$ & $18(31.6)$ & 0.806 \\
\hline Posterior communicating artery aneurysm & $17(18.5)$ & $7(13.0)$ & 0.385 \\
\hline Anterior cerebral artery aneurysm & $4(4.3)$ & $3(5.6)^{\prime}$ & 0.71 \\
\hline Ophthalmic artery aneurysm & $1(1.1)$ & $0(0.0)$ & 1 \\
\hline Middle cerebral artery aneurysm & $10(10.9)$ & $7(13.0)$ & 0.703 \\
\hline Posterior Cerebral Artery aneurysm & $1(1.1)^{\prime}$ & $4(7.4)^{\prime}$ & 0.063 \\
\hline
\end{tabular}

\begin{tabular}{|c|c|c|c|}
\hline \multirow[t]{2}{*}{ Table $1 b$. Univariate analysis SAH disability } & \multicolumn{2}{|c|}{ GOS at discharge from ICU } & \multirow[b]{2}{*}{$\mathrm{p}$} \\
\hline & $\begin{array}{l}>3 \\
N=94\end{array}$ & $\begin{aligned} & \leq 3 \\
N & =58\end{aligned}$ & \\
\hline Basilar Artery aneurysm & $10(10.9)$ & $2(3.7)$ & 0.211 \\
\hline Posterior inferior cerebellar artery aneurysm & $2(2.2)$ & $6(11.1)$ & 0.052 \\
\hline Arteriovenous malformation & $10(10.9)$ & $3(5.6)$ & 0.373 \\
\hline Carotid Carotid aneurysm & $5(5.4)$ & $6(11.1)$ & 0.33 \\
\hline Multiple aneurysm & $12(13.2)$ & $11(20.0)$ & 0.274 \\
\hline Aneurysm clipping & $12(13.0)$ & $15(26.3)$ & 0.041 \\
\hline Thrombolysis & $1(1.1)$ & $1(1.8)$ & 1 \\
\hline Lumbar drainage & $2(2.2)$ & $0(0.0)$ & 0.524 \\
\hline Embolization of the aneurysm & $53(57.6)$ & $33(57.9)$ & 0.973 \\
\hline Embolization and surgery treatment & $1(1.1)$ & $2(3.5)$ & 0.558 \\
\hline Conservative treatment & $19(20.9)$ & $8(13.8)$ & 0.274 \\
\hline Decompressive craniectomy & $0(0.0)$ & $7(12.1)$ & 0.001 \\
\hline Intraoperative aneurysm rupture & $4(4.3)$ & $3(5.3)^{\prime}$ & 1 \\
\hline Died after treatment & $0(0.0)$ & $2(3.5)$ & 0.145 \\
\hline External ventricular device & $28(31.5)$ & $42(75.0)$ & $<.001$ \\
\hline Stroke & $16(17.4)$ & $24(42.9)$ & $<.001$ \\
\hline Cerebrospinal fluid fistula & $4(4.3)$ & $1(1.8)$ & 0.344 \\
\hline Hydrocephalus & $23(25.3)$ & $41(73.2)$ & $<.001$ \\
\hline MV $>7$ days & $12(13.0)$ & $39(69.6)$ & $<.001$ \\
\hline $\mathrm{ICH}$ & $13(14.3)$ & $26(45.6)$ & $<.001$ \\
\hline ICH_FRONTAL & $12(13.5)$ & $8(14.0)$ & $<.001$ \\
\hline Temporal ICH & $9(10.1)$ & $14(24.6)$ & 0.019 \\
\hline Subdural hematoma & $8(9.0)$ & $7(12.3)$ & 0.019 \\
\hline Vasoespasm_doppler & $23(27.1)$ & $10(19.2)$ & 0.523 \\
\hline Vasoespasm_Arteriographgy & $19(22.6)$ & $10(19.6)$ & 0.298 \\
\hline Internal carotid artery aneurysm & $2(2.2)$ & $10(18.2)$ & 0.68 \\
\hline $\mathrm{DGl}$ & $19(21.1)$ & $13(25.0)$ & 0.001 \\
\hline RRT & $1(1.1)$ & $1(1.9)$ & 1 \\
\hline Rebleeding 72 hours & $2(2.2)$ & $5(9.1)$ & 0.103 \\
\hline Ventriculitis & $7(8.0)$ & $7(12.7)$ & 0.35 \\
\hline SOFA_ICU admission & $1.0(0.0-3.0)$ & $7.0(4.0-10.0)$ & $<.001$ \\
\hline Fisher scale & $3.0(2.0-4.0)$ & $4.0(3.2-4.0)$ & $<.001$ \\
\hline Fisher modified scale & $3.0(2.0-4.0)$ & $4.0(3.0-4.0)$ & $<.001$ \\
\hline Hunt and Hess scale & $1.0(1.0-2.0)$ & $4.0(2.0-5.0)$ & $<.001$ \\
\hline WFNS scale & $1.0(1.0-2.0)$ & $4.0(2.0-5.0)$ & $<.001$ \\
\hline APACHE_vasospasm & $12.0(5.0-17.0)$ & $17.5(16.8-20.0)$ & 0.001 \\
\hline SOFA_vasospasm & $2.0(1.0-3.0)$ & $8.0(5.8-10.0)$ & $<.001$ \\
\hline ICU re-admission by vasospasm & $8.0(0.0-20.0)$ & $12.0(5.0-24.0)$ & 0.07 \\
\hline GCS on site & $15.0(14.0-15.0)$ & $13.0(6.0-14.5)$ & $<.001$ \\
\hline GCS in emergency room & $15.0(13.0-15.0)$ & $8.5(6.0-13.0)$ & $<.001$ \\
\hline GCS at ICU admission & $15.0(8.8-15.0)$ & $5.5(3.0-8.0)$ & $<.001$ \\
\hline
\end{tabular}

Twenty four SAH patients (16.1\%) died at ICU discharge. Decompresive craniectomy was performed in 7 (4.7 \%) SAH patients, all of them with GOS $\leq 3$. The independent risk factors associated with disability (GOS $\leq 3)$ were: Apache II at ICU admission per unit OR $(95 \% \mathrm{Cl}) 1.547$ (1.229; 1.948); number of platelets $\times 103$ at ICU admission OR : 112 (5.4; 2304); SDD OR: 0.064 (0.008; 0.538); stupor OR: 6.479 (1.223; 34.336); isochoric pupils OR: 0.05 (0.004; 0.652); hydrocephalus OR: 6.708 $(1.359 ; 33.103)$ and MV $>7$ days OR: 36.861 (3.90; 340.497) (Table 2).

\begin{tabular}{|c|c|c|c|}
\hline & P-value ${ }^{*}$ & $\mathrm{AC}^{*+}$ & Odd-Ratio (95\% a) \\
\hline Apache II at ICU admission per unit & $<0.001$ & 98.3 & $1.547(1.229 ; 1.948)$ \\
\hline Number of platelets at ICU admission $\times 10^{\circ}$ & $<0.001$ & 77.2 & $112(5.4 ; 2304)$ \\
\hline SOD & 0.004 & 73.8 & $0.064(0.008 ; 0.538)$ \\
\hline Stupor & 0.020 & 71.1 & $6.479(1.223 ; 34.336)$ \\
\hline Isochoric pupils & 0.009 & 72.5 & $0.05(0.004 ; 0.652)$ \\
\hline Hydrocephalus & 0.013 & 71.9 & $6.708(1.359 ; 33.103)$ \\
\hline MN $>7$ days & $<0.001$ & 80.2 & $36.861(3.990 ; 340.497$ \\
\hline
\end{tabular}

Conclusion: In SAH patients admitted to a neurotraumatic ICU, risk factors that were independently associated with disability were: Apache II at ICU admission per unit, number of platelets at ICU admission, SDD, stupor, isochoric pupils, hydrocephalus and MV > 7 days.

\section{8}

Risk factors associated with disability and mortality in patients in a neurotraumatic ICU with decompressive craniectomy six months after discharge. A seven year study

C. Sánchez Ramírez ; CF. Lübbe Vázquez ; ; LDM. Díaz Suárez²; C. Vázquez Pineda'; J. Vicente Arranz'; A. Padrón Mujica'; C. Rodriguez Mata';

P. Saavedra-Santana ${ }^{3}$; S. Ruiz-Santana ${ }^{1}$

${ }^{1}$ Intensive care medicine, University Hospital of Gran Canaria Dr. Negrin, Las Palmas de Gran Canaria, Spain; ${ }^{2}$ Intensive care unit, University Hospital of Gran Canaria Dr. Negrin, Las Palmas de Gran Canaria, Spain; ${ }^{3}$ Mathematics and informatics deparment, University of Las Palmas:, Las Palmas de Gran Canaria, Spain

Correspondence: C. Sánchez Ramírez

Intensive Care Medicine Experimental 2020, 8(2): 000448

Introduction: Second level therapeuticactions for controlling intracranial hypertension $(\mathrm{ICH})$ proposed by the European Brain Injury Foundation include barbiturates, moderate hypothermia and decompressive craniectomy (DC), but outcomes are controversial. Our aim was to evaluate the factors associated with disability and mortality after ICU discharge in patients undergoing DC.

Objectives: To assess the factors associated with disability and mortality, after ICU discharge, in DC patients after 7 years.

Methods: Prospective study of patients admitted from January 1 , 2013 to March 2020 who required DC. DC was performed due ICH refractory to medical treatment. We analyzed: main admission diagnosis; demographic data; neurological data (clinical examination and Glasgow Coma Score: GCS); hypotension type of craniectomy and DC complications; Rankin scale, and Glasgow outcome scale (GOS) at 30, 60 days after ICU admission, at ICU discharge and 6 months after ICU discharge; preoperative serum lactate levels; hypo and hyperglycemia; application of mannitol or hypertonic saline solution before and after DC; leukocytes and platelets previous and after DC and other factors related to prognosis. Univariate analysis of disability (Rankin $>3$ ) and mortality in ICU, 60 days after ICU admission and 6 months after ICU discharge were performed. Statistical significance was set at $p \leq 0.05$.

Results: Thirty five DC patients were collected. Demographic data and types of admission are shown in Table 1 


\begin{tabular}{|c|c|}
\hline Table 1. & N 35 \\
\hline Age years, $n$ (IQR) & $47.1(34.9-55.3)$ \\
\hline Female, $\mathrm{n}(\%)$ & $22(62.9)$ \\
\hline Dislypemia, $\mathbf{n}(\%)$ & $225(19-26)$ \\
\hline Diabetes, $\mathrm{n},(\%)$ & $4(11.4)$ \\
\hline Hypertension, n (\%) & $6(17.1)$ \\
\hline Antiplatelet therapy, $n(\%)$ & $2(5.7)$ \\
\hline APACHE-II, $n,( \pm S D)$ & $22.14 \pm 4.88$ \\
\hline TBI on admission, $n(\%)$ & $13(37.1)$ \\
\hline \multicolumn{2}{|l|}{ Diagnosis on admission } \\
\hline Subarachnoid hemorrhage, $n(\%)$ & $17(48.6)$ \\
\hline Stroke Malignant middle cerebral artery, $n(\%)$ & $10(28.6)$ \\
\hline Tumor, $\mathrm{n}(\%)$ & $3(8.6)$ \\
\hline Acute Subdural Hemorrhage & $14(40)$ \\
\hline Mortality & 12 \\
\hline ICU, $\mathrm{n}(\%)$ & $6(17.1)$ \\
\hline Hospital, n (\%) & $2(5.7)$ \\
\hline Upon discharge from hospital, $\mathbf{n}(\%)$ & $4(11,4)$ \\
\hline Highest GCS, in ICU & $14(9 ; 15)$ \\
\hline GOS at ICU discharge & $3(3-3)$ \\
\hline GOS 30 days after ICU admission & $3(3-4)$ \\
\hline GOS 60 days after $D C / 6$ months after ICU discharge & $3(2.5-4)$ \\
\hline Rankin at CU discharge & $4(3-5)$ \\
\hline Rankin 60 days after ICU admission & $4(3-5)$ \\
\hline Rankin 6 months after ICU discharge & $4.5(2-5.75)$ \\
\hline
\end{tabular}

Most DC were subarachnoid hemorrhages (SAH) 17 (48.6\%) and 75\% of them were hemicranial. The most frequent complications were reoperation due to complications ( $42,9 \%$ of them) and hydrocephalus $(42,9 \%)$. Six patients died at ICU discharge $(17,1 \%), 4(66,6 \%)$ of them were SAH. Rankin score at ICU discharge was 4 and GOS was 3. Rankin score at 6 months after ICU discharge was 4,5 and GOS was 3. Obliteration of basal cisterns was found to be a significant risk factor associated with disability, six months after ICU discharge (Table 2)

Table 2. Disability six months after discharge

\begin{tabular}{|c|c|c|c|}
\hline & \multicolumn{2}{|c|}{ Disability } & \multirow[b]{2}{*}{$\mathrm{P}$} \\
\hline & $\begin{array}{l}\text { No: Rankin<3 } \\
N=7\end{array}$ & $\begin{array}{c}\text { Yes: Rankin }=3 \\
N=28\end{array}$ & \\
\hline Age, (years) & $51.1(38-58.7)$ & $47.1(33.9-55.2)$ & 0.594 \\
\hline Male sex & $4(57.1)$ & $18(64.3)$ & 1 \\
\hline APACHE\| & $25.5(22.8-26.8)$ & $22(19-25.8)$ & 0.103 \\
\hline Diabetes melitus & 0 & $4(14.3)$ & 0.562 \\
\hline Hypertension & $2(28.6)$ & $4(14.3)$ & 0.576 \\
\hline Dysliperria & $1(14.3)$ & $4(14.3)$ & 1 \\
\hline Tumnor & $1(16.7)$ & $2(7.1)$ & 0.453 \\
\hline SAH & $4(57.1)$ & $13(46.4)$ & 0.691 \\
\hline Ttoantiagregantes & $1(14.3)$ & $1(3.6)$ & 0.365 \\
\hline Stroke Malignant middle cerebral artery & $1(14.3)$ & $9(32.1)$ & 0.644 \\
\hline Acute subdural hematama & $2(33.3)$ & $12(42.9)$ & 1 \\
\hline Obliteration of besal cistems & 0 & $12(42.9)$ & 0.049 \\
\hline Non evacuated hematama & $2(33.3)$ & $5(17.9)$ & 0.58 \\
\hline Focal contusicn with edema and expensivity & $3(50.0)$ & $10(35.7)$ & 0.653 \\
\hline Evacuated Injury & $6(30.5)$ & $3(25.0)$ & 1 \\
\hline$T B$ & $4(57.1)$ & $9(33.3)$ & 0.387 \\
\hline Pre Hosptal Enddtracheal intubation I & $3(42.9)$ & $7(25.0)$ & 0.381 \\
\hline Emergency Endotracheal intubation & $1(14.3)$ & $11(39.3)$ & 0.380 \\
\hline Endotrachead intubation at surgery & $2(28.6)$ & $6(21.4)$ & 0.648 \\
\hline Transfusion previous $D C$ & 0 & $8(29.6)$ & 0.424 \\
\hline Seazures before $D C$ & 0 & $4(14.8)$ & 1 \\
\hline Midriasis bilateral arreactiva previous aCD & 0 & $2(7.4)$ & 1 \\
\hline Both reactiv pupils previous $D C$ & $6(100.0)$ & $20(71.4)$ & 0.297 \\
\hline One reactiv pupil previous $D$ & 0 & $4(14.3)$ & 1 \\
\hline None reactiv pupis previous $D C$ & $1(14.3)$ & $2(7.1)$ & 0.499 \\
\hline Extracraneal injured & 0 & $3(11.1)$ & 1 \\
\hline Midine shitt, $\infty \mathrm{CT}$ at admission & $1(0-8)$ & $5(0-8.8)$ & 0.601 \\
\hline GCSat admission & $8(4-12.5)$ & $10(6-14)$ & 0.565 \\
\hline GCSat ICU admission & $3(3-4)$ & $3.0(3.0-6.5)$ & 0.773 \\
\hline Number of platelets, after $D C \times 10^{3}$ & $2 \pi 7(252-298)$ & $217(182-272)$ & 0.102 \\
\hline
\end{tabular}

Mortality 6 months after discharge was significantly associated with bilateral pupillary reactivity prior to $\mathrm{DC}$, female sex and the presence of focal contusion with edema and expansivity (Table 3 ).

Table 3.Mortality risk factors six months after DC

\begin{tabular}{|c|c|c|c|}
\hline & \multicolumn{2}{|c|}{ Exitus } & \multirow[b]{2}{*}{$P$} \\
\hline & $\begin{array}{c}N o \\
N=23\end{array}$ & $\begin{array}{c}\text { Yes } \\
\mathrm{N}=12\end{array}$ & \\
\hline Age (years) & $44(30-55)$ & $47(40-56)$ & 0.302 \\
\hline Fenale Sex & $17(80.0)$ & $5(41.7)$ & 0.050 \\
\hline APACHE & $22(18-25)$ & $24(22-27)$ & 0.082 \\
\hline Diabetes melitus & $1(5.0)$ & $2(16.7)$ & 0.54 \\
\hline Hypertension & $5(25.0)$ & $1(8.3)$ & 1 \\
\hline Dyslipemia & $3(15.0)$ & $2(16.7)$ & 1 \\
\hline Tuma & $1(5.3)$ & $2(16.7)$ & 0.543 \\
\hline ASH & $10(52,6)$ & $7(58.3)$ & 0.314 \\
\hline Sroke Melignant middle cerebral artery & $6(30.5)$ & $4(33.3)$ & 0.696 \\
\hline Acute subdural hematoma & $10(526)$ & $4(33.3)$ & 0.293 \\
\hline Oditeration of bessal cistems & $7(36.8)$ & $4(33.3)$ & 1 \\
\hline Focal contusion with edema and expansivity & $11(526)$ & $2(16.7)$ & 0.050 \\
\hline Evacuated Injury & $2(10.5)$ & $3(25.0)$ & 0.35 \\
\hline TB & $10(52,6)$ & $3(25.0)$ & 0.274 \\
\hline Both reactivpupils previous DC & $21(94.7)$ & $5(41.7)$ & 0.002 \\
\hline None reactiv pupls previous $D C$ & $1(5.0)$ & $2(16.7)$ & 0.540 \\
\hline Pre Hospital Endatracheal intubation & $8(25.0)$ & $2(16.7)$ & 0.683 \\
\hline Emergency Enddrached intubetion & $7(35.0)$ & $5(41.7)$ & 0.724 \\
\hline Endotracheal intubation at surgery & $5(25.0)$ & $3(25.0)$ & 1 \\
\hline Transfusion previous $D C$ & $4(21.1)$ & $3(27.3)$ & 0.481 \\
\hline Seazures before $D$ & $1(5.3)$ & $3(27.3)$ & 0.126 \\
\hline Midine shift, $\propto$ CT at admission & $2(0-5.5)$ & $7(0.5-9.5)$ & 0.193 \\
\hline GCSat admission & $10(3.5-14.5)$ & $11(6-14)$ & 0.82 \\
\hline GCSat ICUadnission & $3(3-6)$ & $3(3-4.2)$ & 0.875 \\
\hline
\end{tabular}

alues are medians $(\mid C R)$ and frequencies (\%), DC: clecompresive craniectomy;: SHA subahacnoidea hemonage

Conclusion: DC patients showed low ICU mortality $(17,14 \%)$ Rankin and GOS reflected moderate / severe disability of these patients, at ICU discharge. Rankin and GOS also showed disability, sixty days and 6 months after ICU discharge. Obliteration of basal cisterns was significantly associated with disability 6 months after ICU discharge. Mortality, 6 months after discharge, was significantly associated with female sex, bilateral pupillary reactivity prior DC and presence of focal contusion with edema and expansivity.

000571

Management and Outcomes of Cerebral Vasospasm after Aneurysmal Subarachnoid Haemorrhage in the National Neurosurgical Centre in Ireland

P. Wiseman ${ }^{1}$; C. Larkin ${ }^{1}$

${ }^{1}$ Intensive Care Medicine, Beaumont Hospital, Dublin, Ireland

Correspondence: P. Wiseman

Intensive Care Medicine Experimental 2020, 8(2): 000571

Introduction: Cerebral vasospasm continues to account for high morbidity and mortality in patients following aneurysmal subarachnoid haemorrhage (SAH).[1] Recent advances in neuroimaging have led to improved detection of vasospasm in this cohort but, despite many years of research, choice of hypertensive therapies remains highly variable between neurosurgical centres.[2]

Objectives: Demonstrate the morbidity caused by vasospasm in patients admitted to Beaumont Hospital, the national neurosurgical centre in Ireland, following aneurysmal SAH.

-Examine current practices and outcomes in treatment of vasospasm in the national centre.

Methods: We identified all patients admitted with aneurysmal SAH over a three year period from February 2016 to February 2019. We conducted a retrospective chart analysis examining demographics, clinical features and outcome data. A subgroup who developed clinically 
significant vasospasm was further analysed to examine management and outcomes.

Results: 532 patients with aneurysmal SAH were identified, of which 68 developed significant vasospasm. Patients who presented with a higher Fisher grade were more likely to develop vasospasm $(p<0.05)$. A significant increase in morbidity was shown in patients who developed cerebral vasospasm versus those who did not, with median Glasgow Outcome Score (GOS) at three months of 3 versus 4, respectively $(\mathrm{p}<0.05)$. The 68 patients with vasospasm stayed a combined 783 days in ICU, with mean LOS of 11.5 days (range 1-38 days). Regarding hypertensive treatment of vasospasm, 27 patients (39.7\%) were treated with noradrenaline (NA) alone and 35 (51.5\%) with NA plus vasopressin. There was no difference between the two groups in grade of presentation (Fisher or WFNS), MAP targets set or the percentage of days MAP targets were met. Of note however, worse GOS at three months occurred in those who received double agent hypertensive therapy vrs NA alone $(p<0.05)$. In addition, of those treated with NA plus vasopressin, $28 / 35(80 \%)$ developed hyponatraemia, versus $8 / 27(<30 \%)$ of those treated with NA alone $(p<0.01)$

Conclusion: Cerebral vasospasm after $\mathrm{SAH}$ is a significant cause of morbidity for patients in the national neurosurgical centre in Ireland. Vasospasm also causes a significant burden due to duration of ICU stay. Hypertensive therapies used in vasospasm are not benign treatments. Despite equal MAP control in both groups, patients treated with NA and vasopressin had significantly worse outcomes than those treated with NA alone, with associated high rates of severe hyponatraemia. This may call into question the benefit of the addition of vasopressin in this cohort. There are no consensus guidelines regarding treatment of vasospasm. Ideally, well designed, multicentre randomised control trials are required to evaluate the optimal treatment of this patient cohort.

\section{Reference(s) and grant ackowledgment(s)}

1. 1. Diringer, M.N. and A.R. Zazulia, Aneurysmal Subarachnoid Hemorrhage: Strategies for Preventing Vasospasm in the Intensive Care Unit. Semin Respir Crit Care Med, 2017

2. 2. Li, K., et al., A Review of the Management of Cerebral Vasospasm After Aneurysmal Subarachnoid Hemorrhage. World Neurosurg, 2019. 3. N/A

\section{7}

The effect of early fluid balance on vasospasm occurrence in Spontaneous Subarachnoid Hemorrhage patients on neuro-ICU R. Varudo'; A. Mota'; E. Pereira ${ }^{3}$; C. Dias

'Intensive care department, Garcia de Orta Hospital, Almada, Portugal; ${ }^{2}$ Intensive care department, Central Hospital, Funchal, Portugal; ${ }^{3}$ Neurointensive care unit, São João Universitary Hospital Center, Porto, Portugal Correspondence: R. Varudo

Intensive Care Medicine Experimental 2020, 8(2): 000627

Introduction: The ultimate goal of therapy of spontaneous Subarachnoid Hemorrhage (sSAH), after acute phase, is to prevent secondary cerebral injury. Induced hypertension, hypervolemia and hemodilution (triple $\mathrm{H}$ ) therapy have been used to increase cerebral blood flow. Recently, the physiological effects of components of triple-H therapy have been questioned. Hypervolemia may result in potentially life-threatening complications, like pulmonary and cerebral edema. Optimal fluid management after $\mathrm{SSAH}$ is variable throughout different centers but maintaining euvolemia is what is recommended by ESICM consensus 2018, through vigilant assessment of fluid balance (FB) along with arterial blood pressure. A positive FB has been associated with worse functional outcomes at 3 months but has not been widelyassessed during the critical period when patients are most likely to develop symptomatic vasospasm.

Objectives: To assess the impact of early FB in the occurrence of vasospasm in patients (pts) with sSAH.

Methods: Retrospective single-center study that includes pts admitted in a neuro-ICU with SSAH, during a period of 2 years. Data were collected during the first 10-days of ICU. We hypothesized that an early more positive FB, particularly at 48 hours, may influence the development of vasospasm in the following days. All reported $p$ values are two-tailed, with a $p$ value of 0.05 indicating statistical significance. Receiver operating characteristic (ROC) curve and area under curve (AUC) were calculated, a cutoff point of the cumulative fluid balance at day 2 was identified and two groups were formed: (i) pts with cumulative $\mathrm{FB}$ at day $2>1.5$ Litres (L) and (ii) pts with $\mathrm{FB}<1.5 \mathrm{~L}$. Multivariable logistic regression was used to adjust for potential confounders. Analyses were performed with the use of SPSS ${ }^{\circledR}$ software v22 and MedCalc ${ }^{\circledR}$ v19.2.3

Results: A total of 83 pts were included, $57 \%$ male, with a mean age of $56 \pm 12.1$ years. Hunt-Hess was IV-V in 21 pts $(25 \%)$ and Modified Fisher scale was III-IV in 70 pts (84\%). Only $7(8,4 \%)$ did not developed sonographic vasospasm criteria during the 10-day period. The mean FB at day 2 was $1.6 \pm 1.3 \mathrm{~L}$ and it was significantly higher in pts who developed vasospasm after 2 nd day (vasospasm: 1.8L [IQR 1.2]; no vasospasm: 1.1L [IQR 1.5]; $p=0.007$ ). A ROC curve was calculated (Fig. A) and the AUC was 0.675 ( $p=0.007 ; \mathrm{Cl} 95 \% 0.1-0.8)$. Kaplan-Meier curve (Fig. $B$ ) demonstrated that pts with less positive FB on 2 nd day had lower occurrence of vasospasm. A cumulative FB at 2 nd day $>1.5 \mathrm{~L}$ was associated with increased odds of vasospasm occurrence from the 4th to the 7th day. After adjustment for possible confounders, FB $>1,5 \mathrm{~L}$ on the 2 nd day remains an independent predictor of vasospasm occurrence from 4th to 6th-day [day 4: adjusted (adj) OR 5.3,95\% CI 1.4-19.9; day5: adj OR 6.9, 95\% Cl 1.7-28.4; day 6: adj OR 6.1, 95\% Cl 1.5-24.0].

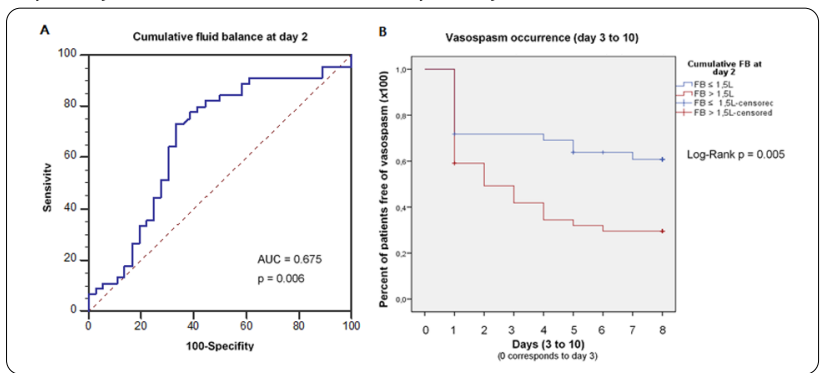

Conclusion: The main finding of this study is that a high FB in $\mathrm{SSAH}$ pts, in the first 48 hours after admission, is an independent predictor of vasospasm occurrence.

Reference(s) and grant ackowledgment(s)

1. 7. Sakr Y. et al. Poor outcome is associated with less negative fluid balance in patients with aneurysmal subarachnoid hemorrhage treated with prophylactic vasopressor-induced hypertension. Ann. Intensive Care (2016) 6:25

2. 6. Rass V. et al. Fluid Intake But Not Fluid Balance Is Associated With Poor Outcome in Nontraumatic Subarachnoid Hemorrhage Patients. Critical Care Medicine 2019

3. 5. Oddo M. et al. Fluid therapy in neurointensive carepatients: ESICM consensus and clinical practice recommendations. Intensive Care Med. 2018

4. 4. Kissoon N. et al. Positive Fluid Balance Is Associated With Poor Outcomes in Subarachnoid Hemorrhage. Journal of Stroke and Cerebrovascular Diseases, 2015: pp 1-7

5. 3. Martini R. et al. The association between fluid balance and outcomes after subarachnoid hemorrhage. Neurocrit Care (2012) 17:191-198

6. 2. Velly L. et al. Anaesthetic and ICU management of aneurysmal subarachnoid haemorrhage. Eur J Anaesthesiol 2015; 32:168-176

7. 1. Vergouw L. et al. High Early Fluid Input After Aneurysmal Subarachnoid Hemorrhage: Combined Report of Association With Delayed Cerebral Ischemia and Feasibility of Cardiac Output-Guided Fluid Restriction. Journal of Intensive Care Medicine 2020, Vol. 35(2) 161-169

\section{1}

Neurologic outcomes in Spontaneous Subarachnoid Hemorrhage patients and vasoreactivity-is there a relationship?

A. Mota' ; R. Varudo ${ }^{2}$; E. Pereira ${ }^{3}$; C. Dias

${ }^{1}$ Intensive care department, Central Hospital, Funchal, Portugal; ${ }^{2}$ Intensive care department, Garcia de Orta Hospital, Almada, Portugal; ${ }^{3}$ Neurointensive care unit, São João Universitary Hospital Center, Porto, Portugal 
Correspondence: R. Varudo

Intensive Care Medicine Experimental 2020, 8(2): 000631

Introduction: Cerebral blood flow is compromised following spontaneous subarachnoid hemorrhage ( $\mathrm{SSAH})$, not only due to the early consequences of the acute bleeding, namely intracranial hypertension, but also due to late complications such vasospasm and vasoreactivity impairment in response to acute changes either in oxygen or carbon dioxide levels.

Objectives: To assess the vasoreactivity response to oxygen and carbon dioxide after sSAH and its impact on neurological outcome.

Methods: Retrospective single-center study that includes patients (pts) admitted in a neuro-ICU with SSAH diagnosis, from January 2017 to December 2018. Patients' demographics, clinical grading of SAH, Glasgow Coma Scale (GCS) at admission and at hospital discharge, Glasgow Outcome Scale (GOS) at 6 months and gasometric parameters during the first 10 days were recorded. Categorical variables are presented as frequencies and percentages and were compared with the use of Fisher's exact test or chi-square test. Continuous variables were expressed as means and standard deviations, or medians (Mdn) and interquartile ranges (IQR) and compared with the use of Student's t-test or Mann-Whitney U-test. All reported $p$ values are two-tailed, with a $p$ value of 0.05 indicating statistical significance. Multivariable logistic regression was used to adjust for potential confounders. Analyses were performed with the use of SPSS ${ }^{\circledR}$ software v22.

\begin{tabular}{|c|c|c|c|c|}
\hline Predictors & & Adjusted OR (95\% Cl) & & $P$ value \\
\hline APACHE II & $1+$ & & $0.94(0.65-1.5)$ & 0.72 \\
\hline SAPS \| & i & 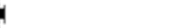 & $1.04(0.90-1.19)$ & 0.62 \\
\hline GCS & $1+4$ & & $0.86(0.59-1.25)$ & 0.43 \\
\hline \multicolumn{5}{|l|}{ Hunt \& Hess } \\
\hline I-II & 中 & 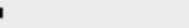 & 1.00 & \\
\hline III & $\longmapsto$ & -1 & $0.15(0.01-4.34)$ & 0.27 \\
\hline IV-V & $\longmapsto$ & $=$ & $5.23(0.12-224.36)$ & 0.39 \\
\hline \multicolumn{5}{|l|}{ Fisher } \\
\hline I-II & 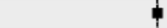 & 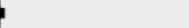 & 1.00 & \\
\hline III & & $\square$ & $23.80(1.04-544.48)$ & 0.05 \\
\hline IV & $\longmapsto$ & $=\quad-1$ & $1.19(0.07-20.66)$ & 0.90 \\
\hline Vasospasm & . & . & & \\
\hline No & 中 & 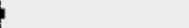 & 1.00 & \\
\hline Yes & $\longmapsto$ & $=$ & $1.64(0.06-42.61)$ & 0.77 \\
\hline VMI' (time) & & 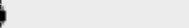 & $0.99(0.92-1.08)$ & 0.89 \\
\hline $\mathrm{PaCO}_{2}$ & & -1 & $1.73(1.15-2.60)$ & 0.01 \\
\hline $\mathrm{PaO}_{2}$ & 中 & 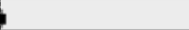 & $1.02(0.98-1.06)$ & 0.31 \\
\hline & 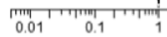 & $\left.\right|_{10} \mid+m_{100}$ & & \\
\hline
\end{tabular}

Results: A total of 83 pts with sSAH were included, $57 \%$ male, with a mean age of $56 \pm 12$ years. At admission, mean GCS was $12 \pm 4$ but with $18,6 \%(n=16)$ of pts presented GCS 3-8; at hospital discharge the mean of GCS was $14 \pm 3$ with a decrease in number of patients with GCS $\leq 8(n=6,7 \%)$. The difference between GCS at the discharge and at admission was $1.3 \pm 3.5$ and each increase in $1 \mathrm{mmHg}$ in $\mathrm{PaO} 2$ was associated with an increase of 0.31 points in that difference. About $55 \%$ of pts $(n=47)$ had GOS 5 at 6 months, 17 pts $(19,8 \%)$ had poor neurologic outcome (GOS 1-3) and 5 pts died.

During the first ten days, median of $\mathrm{PaCO} 2$ and $\mathrm{PaO} 2$ are significantly higher in the group of pts with GOS 1-3 at 6 months (PaCO2: 40.3, IQR 4.2; PaO2: 143.9, IQR 52.8) than in pts with GOS 4-5 (PaCO2:35.8, IQR 4.0; $\mathrm{PaO} 2$ 103.1, IQR 44.1) (PaCO2: $\mathrm{p}<0.001 ; \mathrm{PaO} 2: \mathrm{p}=0.017)$. Each additional increment of $1 \mathrm{mmHg}$ in $\mathrm{PaCO} 2$ was associated with a $49 \%$ $(95 \% \mathrm{Cl} 1.2-1.9)$ increase in the odds of having a GOS 1-3. After adjustment for potential confounders (Fig. 1), PaCO2 (adjusted OR 1.99, 95\% $\mathrm{Cl}$ 1.17-3.37), but not $\mathrm{PaO} 2$, remains an independent predictor of poor neurologic outcome.

Conclusion: In our study $\mathrm{PaCO} 2$ was an independent predictor of poor neurologic outcomes in patients with $\mathrm{SSAH}$ with significantly higher levels in pts with GOS 1-3 at 6 months. $\mathrm{PaO} 2$ was associated with an increase in the difference between GCS at the discharge and at admission.

Reference(s) and grant ackowledgment(s)

1. 3. Solaiman O, Singh JM: Hypocapnia in aneurysmal subarachnoid hemorrhage: incidence and association with poor clinical outcomes. J Neurosurg Anesthesiol 2013, 25:254-261

2. 2. Matsuda M, Shiino A, Handa J: Sequential changes of cerebral blood flow after aneurysmal subarachnoid haemorrhage. Acta Neurochir (Wien) 1990, 105:98-106

3. 1. Foreman P. et al. Hypocapnia as a poor prognostic factor in aneurysmal subarachnoid hemorrhage. Medical Gas Research 2013,3:25

\section{1}

Decompressive Craneotomies in Traumatic Brain Injury: The Intensivist's point of view

F. Ortuño-Andériz'; ME. Fuentes-Ferrer²; F. Rascón-Ramírez ${ }^{3}$;

C. Galbán-Malagón'; A. Palazón-Blanco ; ; M. Calle-Romero;

AL. Blesa-Malpica ${ }^{1}$

${ }^{1}$ Critical Care Unit, Hospital Clinico Universitario San Carlos, Madrid, Spain; ${ }^{2}$ Preventive medicine department, Hospital Clinico Universitario San Carlos, Madrid, Spain; ${ }^{3}$ Neurosurgery department, Hospital Clinico Universitario San Carlos, Madrid, Spain

Correspondence: $\mathrm{F}$. Ortuño-Andériz

Intensive Care Medicine Experimental 2020, 8(2): 000641

Introduction: The usefulness of Decompressive Craniotomy (DC) after a Traumatic Brain Injury (TBI) is not clear, so it is impossible to prepare Guidelines with adequate evidence. Furthermore, there are no early clinical or radiological findings before the development of refractory Intracranial Hypertension that appropriately indicate a DC surgery.

Objectives: To perform a score with early clinical and radiological findings after a TBI that identify the patients who in their subsequent evolution are going to undergo DC.

Methods: Observational study of a retrospective cohorts of patients who, after a TBI, enter the Neurocritical Section of the Intensive Care Unit of our hospital for a period of 5 years (2014-2018). Main event of the study: DC. Detection of clinical (GCS, mydriasis, shock) and radiological (CT) criteria and generation of all possible models with significant, clinically relevant and easy to detect early variables. Selection of the one with the lowest Bayesian Information Criterion (BIC) and Akaike Information Criterion (AIC) values for the creation of the score. Calibration and internal validation of the score using the Hosmer-Lemeshow and a bootstrapping analysis with 1,000 re-samples respectively.

Results: 37 DC were performed in 153 patients who were admitted after a TBI. The resulting final model was:

\begin{tabular}{|l|l|l|l|}
\hline Dependent variable: CD & Odds Ratio & $\mathrm{p}$ & $95 \% \mathrm{CI}$ \\
\hline Cerebral Midline Deviation & 6,35 & 0,0001 & $2,37-17,04$ \\
\hline $\begin{array}{l}\text { TCE according to GCS (mild/moderate- } \\
\text { severe)) }\end{array}$ & 4,32 & 0,025 & $1,20-15,50$ \\
\hline \begin{tabular}{l} 
Ventricular collapse \\
\hline
\end{tabular} & 5,27 & 0,002 & $1,89-14,74$
\end{tabular}

Area under ROC Curve: 0.84 (95\% IC 0.78-0.91). Hosmer-lemeshow $\mathrm{p}=0.71$. A value of 1 was assigned to each of the factors. A score value of 2 had a very high Sensitivity and Specificity (greater than 78\%) with acceptable Positive and Negative Predictive Value to detect patients who were going to need a DC. The developed score detected well those patients who were going to need an early (primary) DC after a TBI $(2.5+/-0.5)$ but not those who would need it in a later stage of their disease (secondary) $(1.7+/-0.8)$. However, it seems to advice us about the patients who, although not requiring a primary DC are likely to need it later in their evolution (secondary DC vs do not require DC $1.7+/-0.8$ vs $1+/-0.7 ; p=0.002$ ).

Conclusion: We have developed a prognostic score using early clinical-radiological criteria that, in our environment, detects with good 
sensitivity and specificity those patients who, after a TBI, will require a DC.

\section{Reference(s) and grant ackowledgment(s)}

1. 1.- Bratton SL, Chestnut RM, Ghajar J, et al. Guidelines for the management of severe traumatic brain injury. J Neurotrauma 2007; 24: Suppl 1: S14-S44. 2.- Sahuquillo J, Arikan F. Decompressive craniectomy for the treatment of refractory high intracranial pressure in traumatic brain injury. Cochrane Database Syst Rev. 200625 (1): 1-41. (updated May 2008) 3.- Menon DK. Cerebral protection in severe brain injury: physiological determinants of outcome and their optimisation. Br Med Bull 1999; 55: 226-58. 4.- Bor-SengShu E, Figueiredo EG, Amorim RLO, et al. Decompressive craniectomy: a meta-analysis of influences on intracranial pressure and cerebral perfusion pressure in the treatment of traumatic brain injury. J Neurosurg. Sep 2012;117(3):589-596. PMID: 22794321 . 5.- Eberle BM, Schnuriger B, Inaba K, Gruen JP, Demetriades D, Belzberg H. Decompressive craniectomy: surgical control of traumatic intracranial hypertension may improve outcome. Injury Sep 2010; 41 (9): 894-898. 6.- Bohman LE, Schuster JM. Decompressive craniectomy for management of traumatic brain injury: an update. Curr Neurol Neurosci Rep. Nov 2013; 13 (11): 392. 7.- Huang X, Wen L. Technical considerations in decompressive craniectomy in the treatment of traumatic brain injury. Int J Med Sci. 2010; 7 (6): 385-390. 8.- Ragel BT, Klimo P, Jr., Martin JE, Teff RJ, Bakken HE, Armonda RA. Wartime decompressive craniectomy: technique and lessons learned. Neurosurg Focus. May 2010; 28 (5): 1-10 9.- Quinn TM, Taylor JJ, Magarik JA, Vought E, Kindy MS, Ellegala DB. Decompressive craniectomy: technical note. Acta Neurol Scand. Apr 2011; 123 (4): 239-244. 10.- Chibbaro S, Tacconi T. Role of decompressive craniectomy in the management of severe head injury with refractory cerebral edema and intractable intracranial pressure. Our experience with 48 cases. Surgical Neurology 2007; 68: 632-638 11.- Guidelines for the Management of Severe Traumatic Brain Injury. Brain Trauma Foundation. 4th Edition. September 2016. Braintrauma .org 12.- Cooper J, Jeffrey V, Rosenfeld M.D. et al for the DECRA Trial Investigators and the Australian and New Zealand Intensive Care Society Clinical Trials Group. Decompressive Craniectomy in Diffuse Traumatic Brain Injury. N Engl J Med 2011; 364 (16): 1493-1502 13.- Jia-Wei Wang, Jin-Ping Li, Ying-Lun Song, Ke Tan, Yu Wang, Tao Li, Peng Guo, Xiong Li, Yan Wang, Qi-Huang Zhao. Decompressive craniectomy in neurocritical care. J Clin Neurosci. 2016; 27: 1-7 14.- Hutchinson PJ, Kolias AG, Timofeev IS, et al, for the RESCUEicp Trial Collaborators. Trial of decompressive craniectomy for traumatic intracranial hypertension. New Eng J Med 2016; 375: 1119-1130. 15.- Shah A, Almenawer S, Hawryluk G. Timing of Decompressive Craniectomy for Ischemic Stroke and Traumatic Brain Injury: A Review. Frontiers in Neurology. 2019; 10 (11): 1-12 16.- Geeraerts T, Velly L, Abdennour L, Asehnoune K, Audibert Ge, Bouzat P et al for the French Society of Anaesthesia, Intensive Care Medicine (Sociéte' francaise d'anesthésie et de réanimation [SFAR]) in partnership with Association de neuro-anesthésie-réanimation de langue francaise (Anarlf) the French Society of Emergency Medicine (Société Francaise de Médecine d'urgence (SFMU), the Société francaise de neurochirurgie (SFN), Groupe francophone de réanimation et d'urgences pédiatriques (GFRUP), Association des anesthésistes-réanimateurs pédiatriques d'expression francaise (Adarpef). Management of severe traumatic brain injury (first 24 hours). Anaesth Crit Care Pain Med 2018;37 (2): 171-186. 17.- Ji-Yao Jiang, Wei Xu, Wei-Ping Li, Wen-Hui Xu, Jun Zhang, Ying-Hui Bao, Yu-Hua Ying, and Qi-Zhong Luo. Efficacy of Standard Trauma Craniectomy for Refractory Intracranial Hypertension with Severe Traumatic Brain Injury: A Multicenter, Prospective, Randomized Controlled Study. Journal of Neurotrauma 2005; 22 (6): 623628. 18.- Taylor A, Butt W, Rosenfeld J, Shann F, Ditchfield M, Lewis E, et al. A randomized trial of very early decompressive craniectomy in children with traumatic brain injury and sustained intracranial hypertension. Childs Nerv Syst 2001; 17 (3): 154-62. 19.- Qiu W, Guo C, Shen H, Chen K, Wen L, Huang $\mathrm{H}$, et al. Effects of unilateral decompressive craniectomy on patients with unilateral acute post-traumatic brain swelling after severe traumatic brain injury. Crit Care 2009; 13 (6): R 185. 20.- Van Essen TA, de Ruiter GC, Kho $\mathrm{KH}$, Peul WC. Neurosurgical treatment variation of traumatic brain injury: evaluation of acute subdural hematoma management in Belgium and The Netherlands. J Neurotrauma 2017; 34: 881-89. 21.- Compagnone C, Murray GD, Teasdale GM, et al, and the European Brain Injury Consortium.
The management of patients with intradural post-traumatic mass lesions: a multicenter survey of current approaches to surgical management in 729 patients coordinated by the European Brain Injury Consortium. Neurosurgery 2005; 57: 1183-92, discussion 1183-92. 22.- Ghajar J, Hariri RJ, Narayan RK, lacono LA, Firlik K, Patterson RH. Survey of critical care management of comatose, head-injured patients in the United States. Crit Care Med 1995; 23: 560-67. 23.- Sahuquillo J, Dennis JA. Decompressive craniectomy for the treatment of high intracranial pressure in closed traumatic brain injury. Cochrane Database of Systematic Reviews 2019, Issue 12. Art. No.: CD003983.

\section{2}

Role of surgical intervention and anticoagulation

after intracranial hemorrhage in patients with LVAD devices: a review of the literature

A. Kastoris'; A. Papastylianou ${ }^{1}$; C. Efseviou ${ }^{1}$; L. Palazis

${ }^{1}$ Intensive Care Unit, Nicosia GeneralHospital, Nicosia, Cyprus

Correspondence: A. Kastoris

Intensive Care Medicine Experimental 2020, 8(2): 000732

Introduction: The risk of intracranial hemorrhage $(\mathrm{ICH})$ in patients with left ventricular assist devices is high. Surgical intervention and reversal of anticoagulation is not without risk. There is limited, published information regarding the surgical management of LVAD patients and often decision making is difficult, requiring a multidisciplinary approach.

Objectives: We aim to review the relevant literature regarding surgical and medical management of intracranial hemorrhage in patients with LVAD and present a case from our ICU.

Methods: A systematic review of the literature was undertaken using the key words "intracranial hemorrhage" and "left ventricular assist device" in the Scopus, PubMed, and Google Scholar databases. Published case reports and series written in English were included with adult patients. Abstracts were not included in the review. Data was extracted regarding the number of surgical interventions performed to total number of patients reported, and the type of ICH and surgical procedures performed. Data was also extracted regarding preoperative and postoperative anticoagulation as well as the outcome of the surgical patients. A case of a young patient from our ICU is also presented.

Results: In total 9 studies (7 case series, 2 case reports) were included in our review regarding a total of 292 patients with intracranial bleeding. Surgery was undertaken in $49(16.7 \%)$ patients with surgery ranging between $5.7 \%$ and $40 \%$ in the case series patients. Type of bleeding, surgery performed, anticoagulation and outcome parameters are summarized in Table 1 . Spontaneous intraparenchymal hematomas (IPH) were more common and presented with the worst outcomes throughout all the included studies. No significant correlation was established between elevated INR and the incidence nor severity of $\mathrm{ICH}$. Immediate thromboembolic events were not encountered in any patients. Interestingly decision for surgical treatment was not specified in most studies. Three studies indicated that surgical intervention provided no improvement in outcome although it was not linked to increased mortality. Several limitations exist due to the heterogeneity of data provided and the fact that often little data was given regarding surgical intervention patients.

A 29-year-old female, with LVAD placement one-month prior was admitted to our ICU due to a spontaneous large left sided IPH. The patient's INR upon admission was 1.6. Immediate extensive left side craniectomy and hematoma removal was performed. Re initiation of anticoagulation commenced 5 days after craniectomy following stable CT scan imaging of the hematoma with argatroban infusion due to a history of HIT. APTT values of 1.5 to 2 times initial aPTT were aimed (45 to 60 seconds). The patient was successfully discharged from the hospital 2 months after ICU admission with a GOS of 4, on warfarin, to a rehabilitation center. 


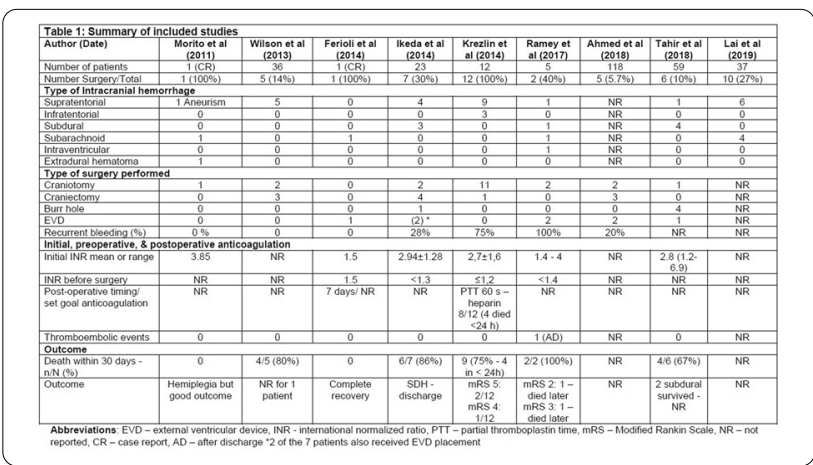

Conclusion: Early surgical treatment with craniectomy in large IPH that present a midline shift and large volumes may be lifesaving. Medical anticoagulation may be withheld from patients with LVAD treatment for a certain period without thromboembolic events. A possible bridge to re-initiation of warfarin in patients presenting with HIT or heparin resistance may be achieved with argatroban infusion with set aPTT levels of 1.5 to 2 times initial values according to our experience. The current literature is lacking regarding effective surgical and medical management and further research is of great necessity.

\section{Reference(s) and grant ackowledgment(s)}

1. Ahmed, M. M., Rahman, M., Neal, D., Aranda Jr, J. M., \& Klodell, C. T. (2018). Ventricular Assist Device Patients Have Different Clinical Outcomes and Altered Patterns of Bleeding with Intracranial Hemorrhage. Asaio Journal, 64(4), e55-e60.

2. Ferioli, S., York, J., Ringer, A., \& Bonomo, J. (2014). Spontaneous subarachnoid hemorrhage and acute hydrocephalus in a patient with a Left Ventricular Assist Device (LVAD). Interdisciplinary Neurosurgery, 1(1), 8-10,

3. Ikeda, D. S., Bansal, S., Shaw, A., Marlin, E., Sai-Sudhakar, C. B., \& Sauvageau, E. (2014). Acute operative intervention for intracranial hemorrhage in adult patients with ventricular assist device therapy associated with fatal outcomes. Int I Surg Res Pract, 1, 013.

4. Krenzlin, H., Rosenthal, C., Wolf, S., Vierecke, J., Kowski, A., Hetzer, R., \& Czabanka, M. (2014). Surgical treatment of intraparenchymal hemorrhage during mechanical circulatory support for heart-failure-a single-centre experience. Acta neurochirurgica, 156(9), 1729-1734.

5. Wilson, T. J., Stetler, W. R., Al-Holou, W. N., Sullivan, S. E., \& Fletcher, J. J. (2013). Management of intracranial hemorrhage in patients with left ventricular assist devices. Journal of neurosurgery, 118(5), 1063-1068,

6. Lai, G. Y., Devlin, P. J., Kesavabhotla, K., Rich, J. D., Pham, D. T., Potts, M. B., \& Jahromi, B. S. (2019). Management and outcome of intracranial hemorrhage in patients with left ventricular assist devices. Journal of neurosurgery, 1.

7. Morito, H., Nishimura, T., Ando, M., Kinoshita, O., Hisagi, M., Imai, H., \& Ono, M. (2012). Successful treatment of cerebral hemorrhage using computed tomography angiography in a patient with left-ventricular-assist device. Journal of Artificial Organs, 15(1), 90-93.

8. Ramey, W. L., Basken, R. L., Walter, C. M., Khalpey, Z., Lemole Jr, G. M., \& Dumont, T. M. (2017). Intracranial hemorrhage in patients with durable mechanical circulatory support devices: institutional review and proposed treatment algorithm. World neurosurgery, 108, 826-835.

9. Tahir, R. A., Rotman, L. E., Davis, M. C., Dupépé, E. B., Kole, M. K., Rahman, M., .. \& Walters, B. C. (2018). Intracranial hemorrhage in patients with a left ventricular assist device. World neurosurgery, 113, e714-e721.

\section{9}

Prediction for the need of tracheostomy in patients with large vessel occlusion stroke beeing treated with meachanical thrombectomy

I. Maier' ; K. Schramm'; D. Behme²; MN. Psychogios'; J. Liman

${ }^{1}$ Neurology, University Medicine Goettingen, Göttingen, Germany; ${ }^{2} \mathrm{Neu}-$ roradiology, University Medicine Göttingen, Göttingen, Germany; ${ }^{3}$ Neuroradiology, University Hospital of Basel, Basel, Switzerland

Correspondence: I. Maier

Intensive Care Medicine Experimental 2020, 8(2): 000869
Introduction: Patients with large vessel occlusion stroke (LVOS) eligible for mechanical thrombectomy (MT) are at risk for stroke- and nonstroke related complications resulting in the need for tracheostomy (1). Evidence suggest that early tracheostomy might exhibit distinct advantages over continued orotracheal intubation, ventilation and sedation (2).

Objectives: To investigate clinical-, periinterventional- and imaging factors to predict the need for tracheostomy in major stroke patients undergoing MT.

Methods: Prospectively derived data from patients with LVOS and MT being treated in a large, academic neuro-ICU between 2014 and 2018 were analyzed in this monocentric study. Predictive value of peri- and postinterventional factors, stroke imaging and pre-stroke medical history were investigated for their potential to predict tracheostomy during ICU-stay using logistic regression models.

Results: From 635 LVOS-patients treated with MT, 40 (6.3\%) underwent tracheostomy during their neuro-ICU stay. Patients receiving tracheostomy were younger (71 (62-75) vs. $77(66-83), p<0.001)$, had a higher National Institute of Health Stroke Scale (NIHSS) at baseline (18 (15-20) vs. $15(10-19), p=0.009)$ as well as higher rates of hospital acquired pneumonia (HAP) $(39(97.5 \%)$ vs $224(37.6 \%), p<0.001)$, failedextubation $(15(37.5 \%)$ vs $19(3.2 \%), p<0.001)$, sepsis $(11(27.5 \%)$ vs. $16(2.7 \%), p<0.001)$, symptomatic intracerebral hemorrhage $(\mathrm{sICH})$ (5 (12.5\%) vs. $22(3.9 \%), p=0.026)$ and decompressive hemicraniectomy (DH) (19 (51.4\%) vs. $21(3.8 \%), p<0.001)$. In multivariate logistic regression analysis, HAP (OR $21.1(\mathrm{Cl}, 2.7-163.3), \mathrm{p}<0.003)$, Sepsis (OR $5.6(1.8-18), p=0.003)$, failed extubation (OR $8.9(3.2-24.5), p<0.001$ ) and $\mathrm{DH}(\mathrm{OR} 9.1(3.5-23.3), \mathrm{p}<0.001)$ remained as strongest predictors for tracheostomy. Patients with later timepoint of tracheostomy had longer ICU length of stay $(r=0.384, p=0.030)$. There was no association between time to tracheostomy and clinical outcome (NIHSS at discharge: $r=0.125, p=0.461 ; m R S$ at 90 days: $r=-0.179, p=0.403$ ).

Conclusion: Patients with LVOS undergoing MT are at high risk to require tracheostomy if extubation after the intervention fails, $\mathrm{DH}$ is needed and severe infectious complications occur in the acute phase after ischemic stroke. These factors are likely to be useful for the indication and timing of tracheostomy to reduce overall sedation and shorten ICU length of stay.

\section{Reference(s) and grant ackowledgment(s)}

1. (2) Bösel J, Schiller P, Hook Y, et al. Stroke-related Early Tracheostomy versus Prolonged Orotracheal Intubation in Neurocritical Care Trial (SETPOINT): a randomized pilot trial. Stroke. 2013;44:21-8

2. (1) Alonso A, Ebert AD, Kern R, et al. Outcome Predictors of Acute Stroke Patients in Need of Intensive Care Treatment. Cerebrovasc Dis. 2015;40:10-7 3. Not applicable

\section{8}

Spectral $p$ roperties of the EEG during recovery of consciousness after acute brain injury

M. Napolitani ${ }^{1}$; M. Colombo ${ }^{2}$; F. Curto ${ }^{1}$; L. Tinti ${ }^{3}$; S. Beretta ${ }^{3}$; G. Citerio ${ }^{4}$;

M. Rosanova²; S. Sarasso²; A. Chieregato'

${ }^{1}$ Department of neurointensive care, Niguarda Hospital, ASST Grande ospedale metropolitano, Milano, Italy; ${ }^{2}$ Department of biomedical and clinical science "I.sacco", University of Milan, Milano, Italy; ${ }^{3}$ Department of neurology, san gerardo hospital asst monza, University of Milano-Bicocca, Milano, Italy; ${ }^{4}$ School of medicine and surgery, University of Milano-Bicocca, Monza, Italy

Correspondence: M. Napolitani

Intensive Care Medicine Experimental 2020, 8(2): 000878

Introduction: EEG slowing (a relative increase of slow frequency and decrease of high-frequency power) is a common feature associated with loss of consciousness. We previously described how the EEG power spectrum slope ( $1 / f$ decay) is steeper when consciousness is lost (e.g. during propofol and xenon anesthesia) and flatter when consciousness is preserved despite similar behavioral unresponsiveness (e.g. during ketamine anesthesia). The Spectral Exponent $\beta$ describes how steep is the decay of the power spectral density over frequencies 
( $P S D=f-\beta$, i.e. the EEG slowing)1. Similarly to both sleep and pharmacologically induced conditions, coma due to acute brain injury is typically associated with EEG slowing, whereas exit from a coma is associated with a recovery of fast frequencies and low amplitude EEG activity typical of normal wakefulness.

Objectives: We tested the hypothesis that the recovery of consciousness after acute brain injury is paralleled by a progressive flattening of the PSD decay.

Methods: We collected 107 longitudinal EEG at different days from injury from 22 patients (mean 4,6 EEG for each patient, IQR 3 - 6) with acute brain injury with transient loss of consciousness and good recovery (functional communication at discherge). Patients are divided into two subgroups based on etiology: post anoxic groups $(n=14)$ and generic acute brain injuries (different etiologies but post anoxic, $n=8$ ). Recordings with any anesthetics or status epilepticus were excluded. We obtained the Spectral Exponent (SE) for each EEG. We then estimated if the modifications of the $\beta$ values were linearly predictable by two variables: time form injury and a clinical feature expressed by the Glasgow Coma Scale (GCS). A mixed-model effect was used to estimate whether the variation of $\beta$ was dependent across subjects to either time post injury or the GCS, beyond the effects observable within each subject.

Results: For both groups we found a significant effect of the two independents variables on the Spectral Exponent $\beta$ : $\beta$ decreases significantly as a function of the time after injury ( $<<0.005$ for both groups) and as a function of GCS ( $p<0.0001$ for both groups).

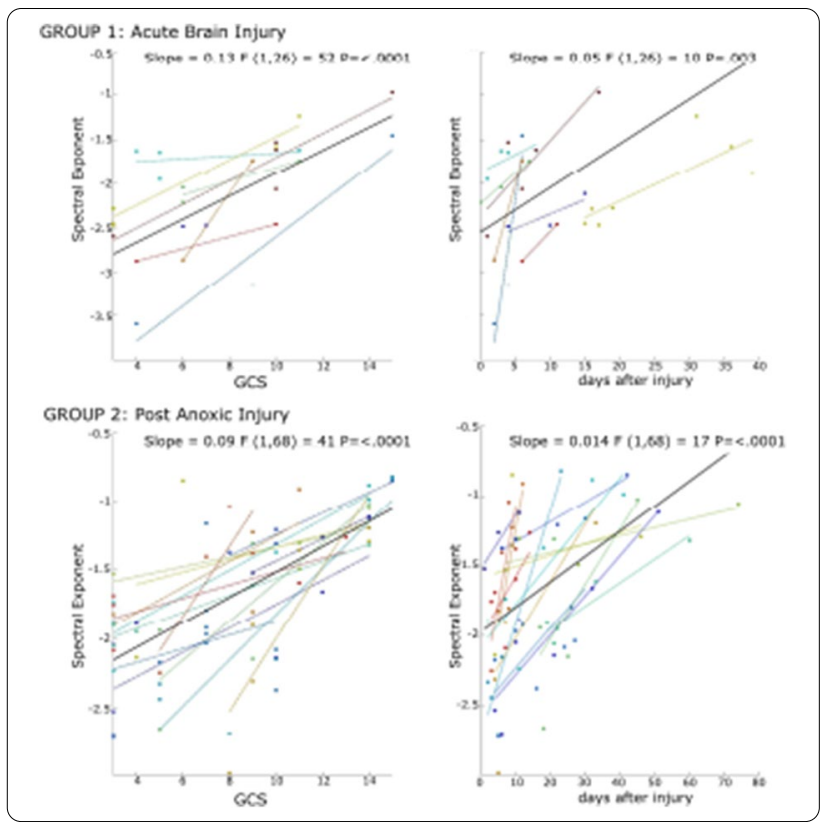

Figure: each coloured dot represents the value of the spectral exponent colour-coded for patients who recovered from coma (acute brain injury, Group 1; post anoxic, group 2). Regression lines are represented with colour coding for patients. Bold black line represents the mixed model effects: slope, F Fischer and $p$ value are reported for the two variables of each group.

Conclusion: Together with the traditional clinical evaluation, the Spectral Exponent $\beta$ seems to be a reliable measure for tracking brain state transitions leading to the recovery of consciousness after acute brain injury. The theoretical background about the genesis of the slow rhythms in disorders of consciousness provides a framework for the clinical use of the spontaneous EEG in objectively monitoring brain states behind clinical signs. The Spectral Exponent may help clinicians to identify the underlying brain processes that steer from slow rhythms to a recovery of more balanced EEG activity patterns able to support consciousness.
Reference(s) and grant ackowledgment(s)

1. Colombo, Michele Angelo, Martino Napolitani, Melanie Boly, Olivia Gosseries, Silvia Casarotto, Mario Rosanova, Jean-Francois Brichant, et al. «The Spectral Exponent of the Resting EEG Indexes the Presence of Consciousness during Unresponsiveness Induced by Propofol, Xenon, and Ketamine». Neurolmage 189 (01 2019): 631-44. https://doi.org/10.1016/j. neuroimage.2019.01.024.

\section{7}

The prognostic role of lactate concentrations after aneurysmal subarachnoid hemorrhage

N. Ndieugnou Djangang ${ }^{1}$;. Ramunno ${ }^{1}$; A. Izzi ${ }^{1}$; A. Garufi'; M. Menozzi';

D. Diaferia'; L. Peluso' ; C. Prezioso ${ }^{1}$; M. Talamonti ${ }^{1}$;. Jean-Louis ${ }^{1}$.

J. Creteur ${ }^{1} ;$ FS. Taccone ${ }^{1}$; E. Bogossian ${ }^{1}$

${ }^{1}$ Intensive Care, Hospital Erasme, Bruxelles, Belgium

Correspondence: $N$. Ndieugnou Djangang

Intensive Care Medicine Experimental 2020, 8(2): 000937

Introduction: The value of serial lactate measurements in patients after SAH has not been well studied. The aim of this study was to assess the prognostic role of serial lactate measurements on survival and neurological outcomes in aneurysmal subarachnoid hemorrhage (aSAH) patients.

Methods: We reviewed data of all patients admitted to our Department with the diagnosis of aSAH from January 01, 2007 to March 31, 2019. Arterial blood lactate levels were routinely obtained at least once a day. We collected the highest lactate level daily during the first 5 days. We reported hospital mortality and 3-month unfavorable neurological outcome (UO) defined as a Glasgow Outcome Scale of 1-3.

Results: Of a total of 456 patients, $64 \%$ were female, with a median age of 54 [46-63] years. Hospital mortality occurred in $158(35 \%)$ patients and UO in $209(46 \%)$ patients. The highest lactate level was on admission (2.1 [1.2-3.4] $\mathrm{mmol} / \mathrm{L})$ and then progressively decreased until day 5. Patients with UO had significantly higherlactate levels over time than the other patients, in particular on the day of admission (2.9 [1.9-4.1] vs. 1.5 [0.9-2.6] $\mathrm{mmol} / \mathrm{L} ; \mathrm{p}<0.01)$. In a multivariable analysis, lactate levels measured on admission were significantly associated with UO (OR 1.25 [Cl95\% 1.0-1.6]; $p=0.049$ ).

Conclusion: Initial lactate levels have prognostic implications in $\mathrm{SAH}$ patients; their value in conjunction with other variables should be evaluated in larger cohorts.

\section{4}

Super Refractory Status Epilepticus in children and adolescents

S. Ilia ${ }^{1}$; C. Stefanogianni'; M. Miliaraki'; P. Briassoulis ${ }^{1}$; M. Raissaki ${ }^{2}$;

I. Zaganas ${ }^{3}$; G. Briassoulis

${ }^{1}$ Pediatric intensive care unit, medical school, University of Crete, Heraklion, Greece; ${ }^{2}$ Radiology department, medical school, University of Crete, Heraklion, Greece; ${ }^{3}$ Neurology department, medical school, University of Crete, Heraklion, Greece

Correspondence: M. Miliaraki

Intensive Care Medicine Experimental 2020, 8(2): 000984

Introduction: Refractory and super-refractory status epilepticus (SE) in children are associated with high morbidity and mortality. Refractory SE (RSE) is defined as persistent seizures that fail to respond to antiepileptics and is only controlled with continuous infusions of anesthetic solutions, while super-refractory SE (SRSE) persists after induction of anaesthetics or leads to rebounds after withdrawal of anaesthesia $(1,2)$. Thorough imaging, metabolic, immunological and genetic investigations are often necessary to identify the cause.

Objectives: The present study aims to describe characteristics of status epilepticus in children and adolescents, focusing on RSE and SRSE.

Methods: This is a retrospective, single-centre study of SE, RSE, and SRSE cases treated in the pediatric ICU of the University Hospital of Heraklion from 2009 to 2019.

Results: A total of 93 hospitalisations for SE, concerning 86 patients ( $50 \%$ females), were included in the study. The median age of the 
patients at first SE was 5 years (IQR 1.86-9.5). 63 admissions (67.7\%) were due to RSE and 15 to SRSE (16.1\%). Compared to idiopathic SE $(53,8 \%)$, which predominated in the new-onset RSE (NORSE) $(p<0.001)$, acute symptomatic $(60 \%)$ and febrile infection-related Epilepsy Syndrome (FIRES) (88.9\%) more often evolved to SRSE ( $p=0.04)$. The likelihood of abnormal EEG or CT did not differ between SE, RSE, and SRSE, while abnormal MRI findings were most commonly observed in the group of SRSE $(p=0.023)$. When the initial investigation was inconclusive, a definitive diagnosis was reached by genetic testing (7.5\%), or brain biopsy, followed by viral detection with PCR (7.7\%).Higher incidence of autoimmune, mitochondrial diseases, and FIRES characterized SRSE compared to SE or RSE $(p=0.017)$. NORSE or FIRES were diagnosed in $25 \%$ of cases, most commonly presenting as SRSE $(53 \%)$ rather than RSE (24\%) or SE (0\%) ( $p<0.001)$. In RSE/SRSE, immunomodulatory treatments $(p=0.05)$ and ketogenic diet $(p=0.005)$ were more frequently used. Also, in RSE/SRSE, weaning off of anaesthetics was prolonged $(p<0.001)$, and mechanical ventilation duration and ICU/ hospital length of stay $(p<0.001)$ were increased. Mortality was $3.2 \%$ and concerned younger patients $<5$ years $(p=0.013)$. The prognosis was particularly poor in patients with NORSE or FIRES or with acute symptomatic SE and progressive encephalopathy $(p<0.001)$.

Conclusion: A high percentage of pediatric SE episodes evolve to RSE or SRSE, which is characterized by a high incidence of FIRES, NORSE, autoimmune and mitochondrial diseases. Further research in this area is necessary and evidence-based protocols for the management of SRSE need to be established.

\section{Reference(s) and grant ackowledgment(s)}

1. 1. Vasquez, A., Farias-Moeller, R. \& Tatum, W. Pediatric refractory and superrefractory status epilepticus. Seizure 68, 62-71 (2019).

2. 2. Dubey, D., Kalita, J. \& Misra, U. K. Status epilepticus: Refractory and superrefractory. Neurol. India 65, S12-S17 (2017).

\section{0}

Delay in treatment and incidence of complications in patients with subarachnoid hemorrhague

A. Fuentes Calatayud' ;. Pino Sanchez ${ }^{2}$; F. Villagran Ramirez ${ }^{2}$; LF. Guerrero ${ }^{3}$ ${ }^{1}$ Intensive care, University Hospital Complex of Granada, Granada, Spain; ${ }^{2}$ Intensive care unit, Hospital Virgen de las Nieves, Granada, Spain; ${ }^{3}$ Neurocritical and trauma intensive care unit, University Hospital Complex of Granada, Granada, Spain

Correspondence: A. Fuentes Calatayud

Intensive Care Medicine Experimental 2020, 8(2): 000990

Introduction: Subarachnoid hemorrhage treatment has been changed over the last years because of the improvement of Interventionist Radiologist technology, but has it changes the mortality and the incidence of complications?

Objectives: To study the differences in complications, mortality and hospital stay between the patients admitted in ICU suffering subarachnoid hemorrhage (SAH) before and after the Interventionist Radiologist team $24 \mathrm{~h}$ disponibility started.

Methods: We performed a cohorts study including all patients admitted to ICU because of SAH since 1996 until 2019. We divided them into two groups (group 1: before; group 2: after the team started), since the admission to ICU until hospital discharge. We took sex, age, initial symptoms (GSC, H\&H, Fisher, WFNS), APACHE II. We studied location of aneurysm, type of treatment and delay, hospital length of stay and mortality.

Statistical analysis: basic descriptive statistics. Mann-Whitney $U$ test and T-student for continuous variables between groups, as appropriate; chi-square for qualitative variables. We considered statistically signification $\mathrm{p}<0.05$.

Results: 1233 patients were analyzed, $59.1 \%$ women. 1111 in group 1 and 122 in group 2. There were not significants differences between groups in sex, age $(56,2 \pm 14,5)$, APACHE II $(13.1 \pm 8,6)$, location of aneurysm or initial symptoms (measured by GSC, H\&H and WFNS). Delay in treatment, either endovascular or surgical, has been significantly reduced in group $2(6,05 \pm 9,3$ vs $1,34 \pm 1,86)$, as well as incidence of rebleeding and brain stroke. It has been shown a trend to reduce the incidence of vasospasm. There have been no significantly differences in mortality or hospital length of stay.

Conclusion: After the start of a specific program for endovascular treatment disponibility, delay on treatment and incidence of complications have been reduced, even though we have not found differences in mortality and hospital length of stay yet.

\section{7}

Functional outcome of patients with aneurysmal subarachnoid hemorrhage admitted to Neurocritical Care Unit

J. Fernandes ${ }^{1}$; D. Cruz ${ }^{2}$; E. Monteiro ${ }^{3}$;. Maia ${ }^{3}$; E. Pereira ${ }^{3}$; C. Dias ${ }^{3}$; JA. Paiva ${ }^{1}$ Intensive care medicine department, São João University Hospital Center, Porto, Portugal; ${ }^{2}$ Faculty of medicine, University of Porto, Porto, Portugal; ${ }^{3}$ Neurocritical care unit, intensive care medicine department, São João University Hospital Center, Porto, Portugal

Correspondence: J. Fernandes

Intensive Care Medicine Experimental 2020, 8(2): 000997

Introduction: Aneurysmal subarachnoid hemorrhage (ASAH) is a sudden and infrequent acute brain injury. Despite fatality rates tending to improve in the last years, preventing or minimizing secondary brain lesion remains a challenge with significant impact on outcomes.

Objectives: Characterization of the ASAH population and identification of clinicaland therapeutic predictors of outcome.

Methods: Retrospective analysis of all adult patients admitted from 1st January 2018 to 31st December 2019 at a Neurocritical Care Unit of an University Hospital in Portugal, who underwent treatment for ruptured cerebral aneurysms (endovascular coil embolization or surgical clip intervention). The primary outcome was unfavorable functional outcome defined by Glasgow Outcome Scale (GOS) of 1, 2 or 3 at 28th day, 3rd and 6th month of follow-up.

Results: Sixty-five patients were included, 43 females (66.2\%), with a median age of 55 (47.5-66.5) years, 35 (53.8\%) had pre-ictus hypertension and 19 smoking habits (29.2\%). All except two patients had a previous Modified Rankin Score (mRS) of 0 or 1. Initial Glasgow Coma Score (GCS) inferior to 8 points was observed in $14(21.5 \%)$, Hunt and Hess score of 4 or 5 in $25(38.5 \%)$ and Fisher score of 3 or 4 in 58 (89.2\%). Forty-two patients (64.6\%) were submitted to surgical clipping and 23 (35.4\%) underwent endovascular coiling, with similar clinical severity in both groups. Regarding complications associated with ASAH, 42 (64.6\%) had Doppler documented vasospasm $(66.7 \%$ submitted to intra-arterial infusion of verapamil), $26(40.0 \%)$ developed delayed cerebral ischemia, 23 (35.4\%) hydrocephalus and $7(10,8 \%)$ rebleeding. ICU-mortality was $10,8 \%$. Among the patients with a registered medical follow-up, mortality was $9.4 \%$ (6 of 64$)$ at 28th day, $14.0 \%$ (8 of 57 ) at 3rd month and $16.7 \%$ (8 of 48 ) at 6th month. Analyzing the patients with a previous mRS of 0 or 1 , an unfavorable GOS was observed in $53.2 \%$ (33 of 62 ) at 28th day, in $36.4 \%$ (20 of 55 ) at 3rd month and in $26.1 \%$ ( 12 of 46 ) at 6 th month. At 3rd month there was a significant difference in patient's outcome comparing the two therapeutic approaches $(45.9 \%$ vs. $16.7 \%, p=0,03)$, favoring endovascular coiling, but it was not observed at 28th day and 6th month. In final multivariate logistic regression models, adjusted for age, two independent predictors of unfavorable functional outcome were identified at the 3 follow-up periods considered: lower initial GCS (28th day: OR $0.49,95 \% \mathrm{Cl} 0.21-0.76$; 3rd month: OR $0.76,95 \% \mathrm{Cl} 0.61-0.92 ; 6$ th month: OR $0.67,95 \% \mathrm{Cl} 0.48-0.94)$ and development of delayed cerebral ischemia (28th day: OR $14.75,95 \% \mathrm{Cl} 2.28-95.38$; 3rd month: OR $16.08,95 \%$ Cl 2.82-91.83; 6th month: OR $91.38,95 \%$ Cl 3.26-2564.56); hydrocephalus was also a predictor of poor outcome at 28th day (OR $10.60,95 \% \mathrm{Cl} 1.37-82.11$ ), no other variables persisted in the models after adjustment.

Conclusion: ASAH mortality rates remain high. In this non-selective ASAH location, surgical or endovascular methods had similar outcomes at 28th day and 6th month of follow-up. Lower GCS and delayed cerebral ischemia are independent predictors of unfavorable functional outcome. 


\section{Reference(s) and grant ackowledgment(s)}

1. Ironside N, Buell TJ, Chen CJ, et al. High-Grade Aneurysmal Subarachnoid Hemorrhage: Predictors of Functional Outcome. World Neurosurg. 2019;125:e723-e728.

2. Nastasovic T, Milakovic B, Stosic M, et al. Predictors of unfavourable outcome in aneurysmal subarachnoid haemorrhage. Neurol Neurochir Pol. 2019;53(6):421-427.

3. Diringer MN, Bleck TP, Claude Hemphill J III, et al; Neurocritical Care Society. Critical care management of patients following aneurysmal subarachnoid hemorrhage: recommendations from the Neurocritical Care Society's multidisciplinary consensus conference. Neurocrit Care. 2011:15(2):211-240

4. Molyneux AJ, Kerr RS, Yu LM, Clarke M, Sneade M, Yarnold JA, et al. International subarachnoid aneurysm trial (ISAT) of neurosurgical clipping versus endovascular coiling in 2143 patients with ruptured intracranial aneurysms: a randomised comparison of effects on survival, dependency, seizures, rebleeding, subgroups, and aneurysm occlusion. Lancet. 2005;366(9488):809-17.

\section{6}

Are anosmia or headaches in critically ill COVID-19 patients an early indicator of neurological complications?

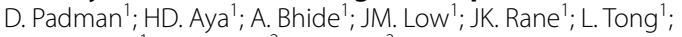

M. Hamilton '; A. Rhodes ${ }^{2}$; S. Leaver ${ }^{3}$

${ }^{1}$ Intensive care, St George's Hospital, London, United Kingdom; ${ }^{2}$ Anaesthesia and intensive care medicine, St George's University Hospitals NHS Foundation Trust, Londres, United Kingdom; ${ }^{3}$ Research lead critical care directorate, St George's Hospital, London, United Kingdom

Correspondence: D. Padman

Intensive Care Medicine Experimental 2020, 8(2): 001016

Introduction: On the frontline of the battle against coronavirus disease 2019 (COVID-19) the importance of identifying symptoms early and understanding their clinical relevance is crucial. Up to $36 \%$ of Covid 19 patients exhibit some form of neurological symptoms, such as anosmia and headaches on presentation $(1,2)$. However, it is not known whether patients presenting with neurological symptoms are at higher risk of neurological complications in an intensive care unit (ICU).

Objectives: This study aims to assess whether critically ill patients presenting with neurological symptoms are at higher risk of subsequent neurological sequelae in critically ill patients infected with Covid-19.

Methods: This is a retrospective observational study of PCR positive Covid-19 patients admitted to ICU in a single tertiary hospital. Anosmia and headache were considered neurological symptoms at presentation (3). Neurological complications, including stroke and intracranial bleeding, were diagnosed using a combination of clinical evidence and consultant level neuroradiology review of computer tomography (CT) imaging (4). Chi-square statistic with risk estimation was used to assess association between categorical variables. $P$ values lower than 0.05 were considered significant.

Results: 132 patients were included in the study. 14 (10.6\%) presented with headache and 11 (8.3\%) with anosmia. In terms of complications, $21(15.9 \%)$ had a intracranial haemorrhage, and $3(2.3 \%)$ also had a stroke. Among 21 patients who had neurological complications in ICU, 3 patients presented with headache $(21.4 \%)$ and 2 patients had anosmia on presentation (18.2\%). These associations are neither significant for headache $(p=0.55$, OR $1.5,95 \% \mathrm{Cl} 0.4,6.0)$ nor for anosmia $(p=0.83)$

Conclusion: Anosmia or headache at presentation is not associated with higher incidence of neurological complications in critically ill patients infected with Covid-19.

\section{Reference(s) and grant ackowledgment(s)}

1. 4) Mahammedi, A., Saba, L., Vagal, A., Leali, M., Rossi, A., Gaskill, M., Sengupta, S., Zhang, B., Carriero, A., Bachir, S., Crivelli, P., Paschè, A., Premi, E., Padovani,

A. and Gasparotti, R. (2020). Imaging in Neurological Disease of Hospitalized COVID-19 Patients: Anltalian Multicenter Retrospective Observational Study. Radiology, p.201933.
2. 3) Klopfenstein, T., Kadiane-Oussou, N.J., Toko, L., Royer, P.-Y., Lepiller, Q., Gendrin, V. and Zayet, S. (2020). Features of anosmia in COVID-19. Médecine et Maladies Infectieuses.

3. 2) Vaira, L.A., Salzano, G., Deiana, G. and De Riu, G. (2020). Anosmia and Ageusia: Common Findings in COVID-19 Patients. The Laryngoscope.

4. 1) Mao, L., Wang, M., Chen, S., He, Q., Chang, J., Hong, C., Zhou, Y., Wang, D., Li, Y., Jin, H. and Hu, B. (2020). Neurological Manifestations of Hospitalized Patients with COVID-19 in Wuhan, China: a retrospective case series study.

\section{9}

Withholding and withdrawal of life support in mechanically ventilated stroke patients

E. de Montmollin ${ }^{1}$; C. Schwebel ${ }^{2}$; C. Dupuis ${ }^{3} ;$ M. Garrouste-Orgeas ${ }^{4}$; D. Da Silva ${ }^{5}$; E. Azoulay ${ }^{6}$; V. Laurent ${ }^{7}$; G. Thiéry ${ }^{8}$; A. Grinea ${ }^{1}$; G. Marcotte ${ }^{9}$ J. Oziel ${ }^{10} ;$ M. Gainnier ${ }^{11} ;$ S. Siami ${ }^{12}$; J. Reignier ${ }^{13}$; B. Sztrymf ${ }^{14}$; C. Adrie ${ }^{15}$; S. Ruckly ${ }^{16}$; R. Sonneville ${ }^{1}$; JF. Timsit ${ }^{1}$

${ }^{1}$ Medical and infectious Intensive Care Unit, Bichat-Claude Bernard Hospital, Paris, France; ${ }^{2}$ Intensive care unit, Chu Grenoble Alpes, La Tronche, France; ${ }^{3}$ Intensive care unit, CHU Gabriel-Montpied, Clermont-Ferrand, France; ${ }^{4}$ Medical unit, Hôpital Franco-Britannique, Levallois-Perret, France; ${ }^{5}$ Intensive care unit, Hopital Delafontaine, Saint-Denis, France; ${ }^{6}$ Réanimation médicale, Hôpital Saint-Louis, Paris, France; ${ }^{7}$ Intensive care unit, C.H. de Versailles, Le Chesnay, France; ${ }^{8}$ Intensive care unit, Hospital Nord, Saint-Priest-en-Jarez, France; ${ }^{9}$ Intensive care unit, Hospital Édouard Herriot, Lyon, France; ${ }^{10}$ Intensive care unit, Avicenne Hospital (AP-HP), Bobigny, France; ${ }^{11}$ Intensive care unit, Hospital Timone, Marseille, France; ${ }^{12}$ Intensive care unit, Hospital Center Sud Essonne -, Étampes, France; ${ }^{13}$ Médecine intensive réanimation, Nantes University Hospital Hotel-Dieu, Nantes, France; ${ }^{14} \mathrm{Ap}$-hp, Hôpital Antoine-Béclère Ap-Hp, Clamart, France; ${ }^{15}$ Service d'explorations fonctionnelles, Cochin Hospital, Paris, France; ${ }^{16}$ Umr 1137, INSERM, Paris, France

Correspondence: $\mathrm{E}$. de Montmollin

Intensive Care Medicine Experimental 2020, 8(2): 001049

Introduction: The determinants of decisions to limit life support (withholding or withdrawal) in ventilated stroke patients have been poorly investigated.

Objectives: We sought to describe the incidence, timing, and factors associated with life support limitation in critically ill patients, with either ischemic or hemorrhagic stroke, requiring mechanical ventilation.

Methods: In a prospective multicenter observational cohort (20052016), we evaluated limitation of life support in ventilated stroke patients and in a non-brain-injured population and identified factors associated with this limitation using Fine and Gray competing risk models.

Results: We identified 373 stroke patients (ischemic, $n=167$ (45\%); hemorrhagic, $n=206(55 \%))$, and 5683 non-brain-injured patients Decisions to limit life support were taken in $41 \%$ of ischemic stroke cases (vs. non-brain-injured patients, sHR 3.59 [95\% Cl 2.78-4.65]) and in $33 \%$ of hemorrhagic stroke cases (vs. non-brain-injured patients, sHR 3.9 [95\% Cl 2.97-5.11]). Time from ICU admission to the first limitation was longer in ischemic than in hemorrhagic stroke (5 [3 - 9] vs 2 [1 - 6] days, $p<0.01$ ). Limitation of life support preceded ICU death in $70 \%$ of ischemic strokes and $45 \%$ of hemorrhagic strokes $(p<0.01)$. Life support limitation in ischemic stroke was associated with a vertebrobasilar location (vs. anterior circulation, sHR, 1.61 [95\% Cl 1.01 - 2.59]) and in hemorrhagic stroke, it was associated with an age $>70$ years (2.29 [1.43 - 3.69]), a Glasgow score<8 (2.15 [1.08 - 4.3]) and a lower nonneurologic admission SOFA score (per point, 0.89 [0.82 - 0.97]).

Conclusion: In ventilated stroke patients, decisions to limit life support are more than 3 times more frequent than in non-brain injured patients, with different timing and associated risk factors between ischemic and hemorrhagic strokes. 


\section{6}

\section{Timing of Pharmacological Thromboprophylaxis}

Administration in Traumatic Brain Injury: A Systematic Review and Meta-Analysis:- The systematic review lite method

A. Chung ${ }^{1}$; S. Hand ${ }^{1}$; C. Hogg ${ }^{1}$; T. Lanerolle ${ }^{1}$; C. Li ; ; M. Steinruecke ;

P. Wahi-Singh ${ }^{1}$; A. Jamjoon ${ }^{2}$;. A. Anderson ${ }^{3}$; J. Rhodes ${ }^{4}$

${ }^{1}$ Medical school, The University of Edinburgh, Edinburgh, United Kingdom;

${ }^{2}$ Department of neurosurgery, NHS Lothian, Edinburgh, United Kingdom;

${ }^{3}$ Critical care, anaesthesia and pain medicine, NHS Lothian, Edinburgh,

United Kingdom; ${ }^{4}$ Critical care, anaesthesia and pain medicine, University

of Edinburgh and NHS Lothian, Edinburgh, United Kingdom

Correspondence: J. Rhodes

Intensive Care Medicine Experimental 2020, 8(2): 001056

Introduction: Pharmacological thromboprophylaxis (PT) may be effective in reducing the incidence of DVT following TBI (Carney et al., 2017). However, there is a reluctance to commence it due to concerns about the risk of intracranial haematoma $(\mathrm{ICH})$ expansion and contusion enlargement (Geerts et al., 1996). PT is therefore often delayed, exposing patients to potential risks and complications of DVT. Appropriate timing to start pharmacological thromboprophylaxis (PTP) in TB patients is unclear. Our aim is to conduct a systematic review to assess the safety of PTP administration before and after 72 hours.

Objectives: 1 -To produce an updated systematic review on the safety and efficacy of early verses late PTP in TBI.

2-To explore the feasibility of a rapid low-cost systematic review process, "systemic review lite" used to direct future research efforts.

Methods: This project was undertaken by medical students over a 10-week student selected module. PubMed and Embase were searched for cohort studies or randomised control trials comparing early versus late administration of PTP in TBI patients. "Early" was defined as $<72$ hours and "late" as $\geq 72$ hours. Primary outcomes were defined as VTE diagnosis and ICH progression.

In the first filter studies were excluded if full text or English translation were not available. Non-human studies and studies on neonates and children were excluded. Authors were not contacted for missing data and individual references were not individually hand searched and cross checked for relevant publications. In the second filter full text articles were assessed and excluded if they included alternat time frames or lacked appropriate comparators. Data was extracted to Review manager 5.3 (Cochrane Collaboration 2014). Quality was assessed by 2 independent reviewers using the Newcastle-Ottawa Scale.

Results: After the second pass filter full text articles were reduced to 10 after alternate time frames and lack of appropriate comparators were considered.

Meta-analysis revealed no statistically significant difference in either VTE diagnosis (Odds ratio $0.74[95 \% \mathrm{Cl} 0.52,1.05]$ ) or $\mathrm{ICH}$ progression (Odds ratio 0.85 [95\% Cl 0.38, 1.89]) for early verses late PTP. Subgroup analysis of high-quality studies demonstrated a decrease in VTE diagnosis with early PTP (Odds ratio 0.63 [95\% Cl $0.42,0.95] \mathrm{p}=0.03$ ). In studies of severely injured patients $(\mathrm{GCS}<=8$ or $\mathrm{AIS}>=3)$, early PTP was associated with a decrease in VTE incidence (Odds ratio 0.53 [95\% $\mathrm{Cl} 0.39,0.71] \mathrm{p}=<0.0001$. Mortality risk showed no difference between early and late groups.

Conclusion: This review suggests that early PTP can reduce the risk of VTE. However, possible the confounding effect of only administering early PTP to patients with stable brain injuries cannot be discounted.

This systemic review lite format proved to be feasible, the student group delivering clear results in a short time frame that serves as a useful update to previous reviews (Chelladurai et al., 2013; Jamjoom and Jamjoom, 2013).

\section{Reference(s) and grant ackowledgment(s)}

1. Jamjoom, A. A. B. and Jamjoom, A. B. (2013) 'Safety and efficacy of early pharmacological thromboprophylaxis in traumatic brain injury: systematic review and meta-analysis.', Journal of neurotrauma. United States, 30(7), pp. 503-511. doi: 10.1089/neu.2012.2584.

2. Geerts, W. H. et al. (1996) 'A comparison of low-dose heparin with lowmolecular-weight heparin as prophylaxis against venous thromboembolism after major trauma.,' The New England journal of medicine. United States, 335(10), pp. 701-707. doi: 10.1056/NEJM199609053351003.

3. Denson, K. et al. (2007) 'Incidence of venous thromboembolism in patients withtraumatic brain injury.', American journal of surgery. United States, 193(3), pp. 380-384. doi: 10.1016/j.amjsurg.2006.12.004.

4. Chelladurai, Y. et al. (2013) 'Venous thromboembolism prophylaxis in patients with traumatic brain injury: A systematic review', F1000Research. Faculty of 1000 Ltd, 2. doi: 10.12688/f1000research.2-132.v1.

5. Carney, N. et al. (2017) 'Guidelines for the Management of Severe Traumatic Brain Injury, Fourth Edition', Neurosurgery, 80(1), pp. 6-15. doi: 10.1227/ NEU.0000000000001432.

6. Dr Rhodes is supported by a National Health Service Research Scotland Clinician Fellowship.

\section{1}

Viscoelastic Coagulation Results:in Patients with Moderate to Severe Traumatic Brain Injury:- early results from the Traumatic Brain Injury Associated Radiological Deep Vein Thrombosis Incidence and Significance (TARDIS) Study

M. Ulla ${ }^{1}$; A. Gordon ${ }^{1}$; J. Rhodes ${ }^{2}$

${ }^{1}$ Adult intensive care unit, St Mary's Hospital, Imperial College NHS Trust, London, United Kingdom; ${ }^{2}$ Critical care, anaesthesia and pain medicine, University of Edinburgh and NHS Lothian, Edinburgh, United Kingdom

Correspondence: J. Rhodes

Intensive Care Medicine Experimental 2020, 8(2): 001071

Introduction: Patients with traumatic brain injury (TBI) may present with several clotting abnormalities, both pro and anticoagulant, involving complex mechanisms such as the acute traumatic coagulopathy (ATC) and delayed coagulopathy secondary to hemodilution (Brohi et al., 2003; Maegele et al., 2017).

Viscoelastic testing of coagulation allows near patient assessment of coagulation profiles. However, there is a paucity of data specifically in patients with traumatic brain injury.

Methods: TARDIS is a prospective, multi centre, observational pilot study to investigate the incidence of deep venous thrombosis (DVT) after TBI, using compression ultrasound. Patients admitted to an intensive care unit within 72 hours of injury are eligible. As an additional sub study, viscoelastic testing performed using ether rotational thromboelastometry (ROTEM) or thromboelastography (TEG) as per the manufacturers' instructions. These results were compared with conventional laboratory coagulation testing from the same day.

Data is described as mean (standard error of the mean) and median (interquartile range). Proportions were analysed by Chi square, $p<0.05$ being considered significant.

Results: 21 patients with moderate to severe TBI (mean GCS 6.2 SEM $0.6)$ had ROTEM INTEM $(n=8)$ or TEG $(n=13)$ data available. The mean age was 52.9 years (SEM 4.8). The majority were male $(\mathrm{N}=16,76 \%)$. Marshal scores were $1, n=1(4.8 \%), 2, n=9(42.9 \%), 3, n=1(4.8 \%), 4$, $\mathrm{n}=4$ (19.1\%), 5, $\mathrm{n}=5$ (23.8\%) and $6, \mathrm{n}=1$ (4.8\%). Three (14.3\%) patients were taking anticoagulant medication prior to injury. Two (9.5\%) received coagulation factors and 8 (38.1\%) were given Tranexamic acid.

The mean activated partial thromboplastin time was 31.8 seconds (SEM 1.1), within our normal laboratory range (26 - 36 seconds). Median prothrombin time (PT) was slightly prolonged at 14.8 seconds (IQR 14.0 - 16.1) compared with the laboratory range (10.5 - $13.5 \mathrm{sec}$ onds). Similarly, the fibrinogen concentration was slightly increased at $4.6 \mathrm{~g} / \mathrm{l}$ (SEM 0.3) compared to the reference range (1.5-4.0 g/l).

We found evidence of abnormal viscoelastic parameters in 7 or 21 patients. Three (14.3\%) had a Clotting Time/R prolonged and 6 (29.0\%) had a prolonged clot formation time/K. Alpha angle was reduced in 4 (19.1\%) and maximum clot firmness/maximum amplitude reduced in $3(14.3 \%)$. None of the patients with ROTEM/TEG abnormalities where taking anticoagulants prior to injury. Two had been given Tranexamic acid and 2 coagulation factors. Comparing laboratory PT with viscoelastic parameters significantly more patients had a PT greater than 
reference range than had an abnormal ROTEM or TEG profile, 18 $(86.0 \%)$ versus $7(33.3 \%) p=0.002$.

Conclusion: Within 72 hours of TBI we found evidence of impairment of coagulation in conventional and a near patient viscoelastic tests of coagulation function. This abnormality was significantly more frequent in the PT than ROTEM/TEG profiles.

\section{Reference(s) and grant ackowledgment(s)}

1. Maegele, M. et al. (2017) 'Coagulopathy and haemorrhagic progression in traumatic brain injury: advances in mechanisms, diagnosis, and management., The Lancet. Neurology. England, 16(8), pp. 630-647. doi: 10.1016/ S1474-4422(17)30197-7.

2. Brohi, K. et al. (2003) 'Acute Traumatic Coagulopathy', Journal of Trauma. J Trauma, 54(6), pp. 1127-1130. doi: 10.1097/01.TA.0000069184.82147.06.

3. Dr Rhodes is supported by a National Health Service Research Scotland Clinician Fellowship.

4. TARDIS is supported by a grant from the National Institute of Academic Anaesthesia and the British Journal of Anaesthesia

\section{6}

\section{Impact of phosphatemia variability in neurologic outcomes} in patients with spontaneous subarachnoid hemorrhage

\section{R. Varudo ; A. Mota ${ }^{2}$; E. Pereira ${ }^{3}$; C. Dias}

'Intensive care department, Garcia de Orta Hospital, Almada, Portugal; ${ }^{2}$ Intensive care department, Central Hospital, Funchal, Portugal; ${ }^{3}$ Neurocritical care unit, intensive care department, São João Universitary Hospital Center, Porto, Portugal

Correspondence: $\mathrm{R}$. Varudo

Intensive Care Medicine Experimental 2020, 8(2): 001086

Introduction: Electrolyte disturbances, such as dysnatremia, hypokalemia and hypomagnesemia, are frequently observed during the acute period after spontaneous subarachnoid hemorrhage (SSAH). However, the data concerning hypophosphatemia after $\mathrm{SSAH}$ are scarce.

Objectives: To analyze the frequency of phosphate disturbance in sSAH patients (pts), daily phosphate repletion and to acess the influence on the outcomes comparing with pts with no $\mathrm{SSAH}$.

Methods: All pts with sSAH admitted from January 2017 to December 2018 in the Neurocritical Care Unit were enrolled in our cohort. Control group includes critically ill non-neurologic pts admitted in a general ICU in the same ICU Department. Serum phosphate levels and daily phosphate repletion were collected on the first 10 days after admission. The primary endpoint was neurologic outcome, using Glasgow Outcome Scale at 6 months (GOS-6M) and Glasgow Coma Scale at ICU discharge (GCS-ICUd). Outcomes analysis were made using mean phosphate level during the first 10 days. We performed multiple linear regression, adjusted for age, GCS at admission, APACHE II, phosphate repletion (and Hunt-Hess and Modified Fisher scale for SSAH group) to assess the effect of phosphatemia in outcomes. Analysis were performed with SPSS ${ }^{\circledR}$ software v22.

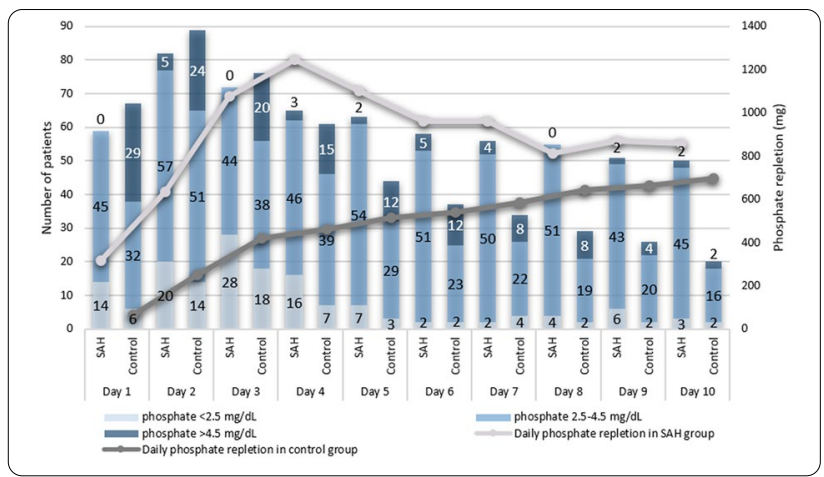

Results:

83 pts with $\mathrm{SSAH}$ and 92 controls, with mean age of $56 \pm 12.1$ and $64 \pm 13.1$ years, respectively. Pts with sSAH had increased odds of having hypophosphatemia at admission as compared with controls (OR $3.2,95 \% \mathrm{Cl} 1.1-8.9, \mathrm{p}=0.029)$ and none presented with hyperphosphatemia. Mean phosphate level was lower in SSAH patients compared to controls $(3.1 \pm 0.4$ vs $3.9 \pm 1.3, p<0.001)$ as well as daily levels. Hypophosphatemia was more frequent in SSAH pts, mostly in the first 4 days (figure 1) and 52 pts (63\%) had at least one day with hypophosphatemia (35 in control group, $p<0.002$ ). Median phosphate dose repletion was higher in sSAH pts [696 (1045) vs 100 (284), p<0.001].

\begin{tabular}{|c|c|c|c|c|}
\hline \multirow[b]{2}{*}{ Independent variables } & \multicolumn{2}{|l|}{ GCS-ICUd } & \multicolumn{2}{|l|}{ GOS-6M } \\
\hline & $\beta_{\text {stand }}(95 \% \mathrm{Cl})$ & $p$ value & $\beta_{\text {stand }}(95 \% \mathrm{Cl})$ & $p$ value \\
\hline Age (years) & $-0.53-0.06$ to 0.03 ) & 0.54 & $-0.11(-0.03$ to 0.01$)$ & 0.24 \\
\hline GCS at admission & $0.64(0.22$ to 0.70$)$ & $<0.001$ & 0.36 (0.000 to 0.196$)$ & 0.05 \\
\hline APACHE II & $-0.44(-0.33$ to -0.09$)$ & 0.001 & $-0.31(-0.10$ to -0.009$)$ & 0.02 \\
\hline Hunt \& Hess & $0.38(0.15$ to 1.66$)$ & 0.02 & $-0.01(-0.32$ to 0.30$)$ & 0.95 \\
\hline Fisher & $-0.01(-0.61$ to 0.56$)$ & 0.93 & $-0.12(-0.39$ to 0.09$)$ & 0.21 \\
\hline Phosphate level (mg/dl) & 0.24 (0.43 to 2.87 ) & 0.01 & $0.13(-0.17$ to 0.83$)$ & 0.19 \\
\hline Phosphate repletion (mg) & 0.25 (0.000 to 0.002$)$ & 0.01 & $0.21(0.000$ to 0.001$)$ & 0.03 \\
\hline
\end{tabular}

\begin{tabular}{|c|c|c|c|c|}
\hline \multirow[b]{2}{*}{ Independent variables } & \multicolumn{2}{|l|}{ GCS-ICUd } & \multicolumn{2}{|l|}{ GOS-6M } \\
\hline & $\beta_{\text {stand }}(95 \% \mathrm{Cl})$ & $p$ value & $\beta_{\text {stand }}(95 \% \mathrm{Cl})$ & p value \\
\hline Age (years) & $0.08(-0.06$ to 0.11$)$ & 0.49 & $-0.10(-0.04$ to 0.02$)$ & 0.36 \\
\hline GCS at admission & $0.19(-0.03$ to 0.49$)$ & 0.08 & $-0.10(-0.14$ to 0.05$)$ & 0.33 \\
\hline APACHE II & $-0.03(-0.15$ to 0.12$)$ & 0.80 & $-0.11(-0.07$ to 0.03$)$ & 0.38 \\
\hline Phosphate level (mg/dL) & $-0.396(-2.49$ to -0.69$)$ & 0.001 & $-0.39(-0.87$ to -0.23$)$ & 0.001 \\
\hline Phosphate repletion (mg & $0.096(-0.002$ to 0.006$)$ & 0.42 & $0.08(-0.001$ to 0.002$)$ & 0.49 \\
\hline
\end{tabular}

In SSAH pts, phosphate level and phosphate repletion (table 1) were independently associated with GCS-ICUd $\left(R^{2} 58 \%, R^{2}\right.$ adj $\left.54 \%\right)$; pts with hypophosphatemia at admission had lower GCS-ICUd compared with pts with normophosphatemia $(12.1 \pm 3.4$ vs $14.1 \pm 2.4, p=0.005)$. sSAH pts with lower GOS-6M had hypophosphatemia at admission and those who died had lower mean phosphate level ( $2.8 \pm 0.8$ vs $3.1 \pm 0.4)$

In multivariable analysis, phosphate repletion was significantly associated $(\beta 0.21,95 \% \mathrm{Cl} 0.000-0.001)$ with GOS-6M ( $R^{2} 52 \%$, $R^{2}$ adj $\left.47 \%\right)$, but not mean phosphate level (table 1). In control group, lower mean phosphate level was associated with higher GOS-6M $\left(R^{2} 27 \%, R^{2}\right.$ adj $22 \%)$ and higher GCS-ICUd $\left(R^{2} 25 \%, R^{2}\right.$ adj $\left.21 \%\right)$; there was not an association between phosphate repletion and outcomes (Table 2).

Conclusion: Pts with SSAH had lower mean phosphate levels and required a significantly higher daily phosphate replacement to achieve normal values, compared with non-neurologic critically ill pts. sSAH pts need cautious phosphate repletion, as hypophosphatemia may be associated with poor neurologic outcomes. Further studies are warranted to better understand the role of phosphorus in the pathophysiology of $\mathrm{SSAH}$, namely regarding brain metabolism, kidney-brain links and outcomes.

Reference(s) and grant ackowledgment(s)

1. Junttila E, Koskenkari J, Ala-Kokko T. Hypophosphatemia after nontraumatic intracranial hemorrhage. Acta Anaesthesiol Scand. 2017;61(6):641-649.

2. Alimohamadi M, Saghafinia M, Alikhani F, Danial Z, Shirani M, Amirjamshidi A. Impact of electrolyte imbalances on the outcome of aneurysmal subarachnoid hemorrhage: A prospective study. Asian J Neurosurg. 2016;11(1):29-33.

3. Wartenberg KE, Schmidt JM, Claassen J et al. Impact of medical complications on outcome after subarachnoid hemorrhage. Crit. Care Med.34(3),617-623; quiz 624 (2006).

4. Qureshi Al, Suri MF, Sung GY et al. Prognostic significance of hypernatremia and hyponatremia among patients with aneurysmal subarachnoid hemorrhage. Neurosurgery50(4),749-755; discussion 755-756 (2002). 


\section{6}

Mechanical thrombectomy in acute ischemic stroke. Most

frequent results and complications in our unit

E. Simón Polo'; JM. Jimenez Vizuete ${ }^{1}$; C. Palau Marti ${ }^{2}$

${ }^{1}$ Anaesthesiology, Complejo Hospitalario Universitario de Albacete, Calle Hermanos Falco, Albacete, Spain, Albacete, Spain; ${ }^{2}$ Anaesthesiology, Critical Care and Pain Therapy Service, Consorci Hospital General Universitari de València, València, Spain

Correspondence: E. Simón Polo

Intensive Care Medicine Experimental 2020, 8(2): 001106

Introduction: In developed countries, ischemic stroke is one of the leading causes of disability and the second leading cause of death, $85 \%$ of them being of cardioembolic or atherothrombotic origin. In recent years, endovascular treatment in this type of patient has shown superiority over systemic fibrinolysis.

Objectives: The objective of this study is to analyze the results and associated complications after endovascular treatment in patients with acute ischemic stroke admitted to our Reanimation Unit.

Methods: An observational retrospective study was carried out. All patients with acute ischemic stroke undergoing mechanical thrombectomy who were admitted to the Reanimation Unit in February 2015 and December 2018 were included. The following variables were selected as study variables: demographic (age and sex), clinics (NIHSS at admission, cardiovascular risk), type of anesthesia performed (general or sedation), neurological complications during your stay, and infectious complications (respiratory and urinary). Statistical analysis was performed using the SPSS 25.0 program. Continuous variables were described using the mean. Categorical variables are described by absolute and relative frequencies.

Results: A total of 224 patients were collected. $90 \%$ of the patients who underwent endovascular treatment remained admitted to the Reanimation Unit. The mean age was $69.3 \% .43 .3 \%$ were men and $56.7 \%$ women. Cardiovascular risk factors: Hypertension described in $73 \%$, diabetes mellitus $32 \%$, dyslipidemia $45 \%$, personal history of ischemic heart disease $13.6 \%$, intermittent claudication $5.7 \%$, tobacco use $19 \%$, history of SAHS $5 \% .27 .4 \%$ consumed oral anticoagulants and $28.1 \%$ had AF among their antecedents, de novo arrhythmia was diagnosed in the emergency room in $11 \%$. In $91 \%$ of the cases described, there was no previous stroke. Stroke mechanism: cardioembolic $71 \%$, atherothrombotic $22.8 \%$, undetermined origin $22.3 \%$, infrequent $6.7 \%$. In $36 \%$ of cases, the artery predominantly affected was the middle cerebral artery in segment M1. The least frequently affected artery is the vertebral artery, with $2.3 \%$ of cases. The median time from clinical presentation to the start of endovascular treatment was $160 \mathrm{~min}$ utes. Neurological assessment was performed using the NIHSS scale: on arrival at the Emergency Department, the mean was 17 points, with no variations after the procedure. After 24 hours it is at 8 points. The mean at discharge was 9 points. The type of anesthesia performed was general in $96 \%$ while sedoanalgesia was performed in $4 \%$. VTE was indicated for failure of fibrinolysis with IVr-t-PA in 8 patients (13.3\%). Infectious complications: $38 \%$ of patients develop urinary tract infection; in $43 \%$ respiratory infections (pneumonia associated with mechanical ventilation, nosocomial pneumonia, tracheobronchitis). Percutaneous tracheostomy was required in $13 \%$. The average stay in our unit is 3 days.

Conclusion: Ischemic stroke is a medical emergency that requires urgent intensive treatment and strict postoperative control. Bladder catheterization or orotracheal intubation prior to the procedure can be risk factors for the development of serious infections that can compromise the patient's life. Performing sedoanalgesia as an anesthetic procedure can be useful in minimizing these risks, reducing hospital stay, reducing costs and improving the patient's prognosis.
Reference(s) and grant ackowledgment(s)

1. 3. Shan W et al. General Anesthesia may have Similar Outcomes with Conscious Sedation in Thrombectomy Patients with Acute Ischemic Stroke: A Real-World Registry in China. Eur Neurol. 2018; 80(1-2):7-13.

2. 2. Valent A, Lukaszewicz AC, Labeyrie MA, Payen D. Increased middle cerebral artery Doppler velocities after stroke thrombectomy performed under general anaesthesia: A pilot monocentric retrospective study. Anaesth Crit Care Pain Med. 2018.

3. 1. Viña Soria Let al. Results and functional outcomes of acute ischemic stroke patients who underwent mechanical thrombectomy admitted to intensive care unit. Med Intensiva. 2018;42(5):274-282

4. To ESCIM to give us the opportunity to publish this paper

001153

The Risk of Stroke after Acute Myocardial Infarction in Patients with and without Aatrial fibrillation

C.H. Chiang ${ }^{1}$; W.T. Hung ${ }^{2}$; W.C. Huang ${ }^{3}$; K.C. Linn ${ }^{2}$; C.C. Cheng ${ }^{1}$; F.Y. Kuo'; G.Y. Mar ${ }^{2}$

${ }^{1}$ Critical care medicine and cardiovascular center, Kaohsiung Veterans General Hospital, Kaohsiung, Taiwan; ${ }^{2}$ Critical care medicine, Kaohsiung Veterans General Hospital, Kaohsiung, Taiwan; ${ }^{3}$ Critical care medicine and cardiovascular center, Kaohsiung Veterans General Hospital, Taipei, Taiwan

Correspondence: W.C. Huang

Intensive Care Medicine Experimental 2020, 8(2): 001153

Introduction: Both acute myocardial infarction (AMI) and atrial fibrillation (AF) are risks for stroke. The risk of stroke after AMI may be different in patients with or without AF. The aim of this study was to evaluate the impact of AF on stroke in patients after first AMI.

Methods: SettingRetrospective nationwide cohortstudy.

ParticipantsA total of 170,472 patients who had the primary diagnosis of first AMI between 2000 and 2012. Among them, 8,530 with AF was identified. Propensity score matching technique was used to match 8,530 patients without AF with similar ages and genders.

Primary and secondary outcome measuresAll patients were followed until stroke or 31 December 2012, whichever occurred first. Kaplan-Meier cumulative survival curves were constructed to compare stroke between AMI patients with and without AF.

Results: Overall, 12-year stroke rate was higher in patients with AF than without AF (log rank P-value $<0.001$ ), including in different genders, agesor intervention subgroups. In patients with $A F$, those withpreexisted AF have higherstroke rates in male gender, agebelow65 years, and with interventionsubgroups thanthose with new-onset AF. In cox proportional-hazard regression analysis, AF was an independent risk factor for stroke after first AMI (hazard ratio, 1.67; 95\% confidence interval, 1.5-1.87).

Conclusion: AF significantly increased stroke risks after first AMI.In patients with $A F$, those with preexisting $A F$ have higher stroke risks in male genders, ages below 65 years, and with interventions than those with new-onset $\mathrm{AF}$.

\section{8}

Transcranial Doppler Emboli Monitoring for Infective Endocarditis G. Huang ${ }^{1}$; L. Johnson ${ }^{2}$; J. Peacock Jr. ${ }^{3}$; C. Tegeler ${ }^{2}$; K. Davis ${ }^{4}$; A. Sarwal ${ }^{2}$ ${ }^{1}$ Internal medicine, University of California, Los Angeles, Los Angeles, United States of America; ${ }^{2}$ Neurology, Wake Forest Baptist Medical Center, Winston-Salem, United States of America; Internal medicine, Wake Forest Baptist Medical Center, Winston-Salem, United States of America; ${ }^{4}$ Pharmacy, Wake Forest Baptist Medical Center, Winston-Salem, United States of America

Correspondence: $L$. Johnson

Intensive Care Medicine Experimental 2020, 8(2): 000288 
Introduction: Ischemic stroke can occur in $20-55 \%$ of patients with infective endocarditis (IE) with 75\% occurring during the first 2 weeks of treatment. CT or MRI brain can diagnose the sequelae of stroke but transcranial Doppler (TCD) can document active embolization. We undertook a retrospective review of our patient cohort and a systematic review of literature to assess the role of TCD in early diagnosis and management of ischemic stroke in IE.

Methods: Retrospective chart review and literature review.

Results: We found 89 patients with stroke caused by IE at our institution from December 2011 to April 2018. TCDs were obtained on 26 patients; 16 were abnormal for cerebrovascular abnormalities. Only 4 patients had 30-minute emboli monitoring performed, of which one revealed emboli. We found 3 studies investigating the role of TCDs in IE that showed promise in its use as a predictive tool in stroke risk stratification.

Conclusion: Presence of embolization in the form of high intensity transient signals (HITS) detected on TCDs can be used for early diagnosis of IE, assessing efficacy of antibiotic therapy, and stratification of stroke risk in IE. This can aid further research into testing preventative interventions for reducing stroke burden in IE, such as earlier valvular surgery or vacuum-assisted vegetation extraction.

Reference(s) and grant ackowledgment(s)

1. Wolf $\mathrm{O}$, Heider P, Heinz M, et al. Microembolic signals detected by transcranial Doppler sonography during carotid endarterectomy and correlation with serial diffusion-weighted imaging. Stroke 2004;35:e373-5.

2. Ferro JM, Fonseca AC. Infective endocarditis. Handb Clin Neurol 2014;119:75-91.

3. Murdoch DR, Corey GR, Hoen B, et al. Clinical presentation, etiology, and outcome of infective endocarditis in the 21st century: the international collaboration on endocarditis-prospective cohort study. Arch Intern Med 2009;169:463-73.

4. Vicent L, Saldivar HG, Bouza E, et al. Prognostic implications of a negative echocardiography in patients with infective endocarditis. Eur J Intern Med 2018;52:40-8.

5. Habib G, Derumeaux G, Avierinos J-F, et al. Value and limitations of the Duke criteria for the diagnosis of infective endocarditis. J Am Coll Cardiol 1999;33:2023-9.

6. Li JS, Sexton DJ, Mick N, et al. Proposed modifications to the Duke criteria for the diagnosis of infective endocarditis. Clin Infect Dis 2000;30:633-8.

7. Wang A, Gaca JG, Chu VH. Management considerations in infective endocarditis: a review. JAMA 2018:320:72-83.

8. Cahill TJ, Baddour LM, Habib G, et al. Challenges in infective endocarditis. J Am Coll Cardiol 2017:69:325-44.

9. Alexandrov AV, Sloan MA, Tegeler $\mathrm{CH}$, et al. Practice standards for transcranial Doppler (TCD) ultrasound. Part II. Clinical indications and expected outcomes. J Neuroimaging 2012;22:215-24.

10. Tsivgoulis G, Alexandrov AV, Sloan MA. Advances in transcranial doppler ultrasonography. Current Neurology and Neuroscience Reports 2009;9:46-54.

\section{4}

\section{Neurocritical patients - can we predict outcome?}

R. Ferreira de Almeida'; R. Pereira ${ }^{2}$; H. Costa ${ }^{3}$; L. Vicente ${ }^{2}$; A. Binnie ${ }^{4}$;

P. Perez'; J. Moreno'; D. Nuñez ${ }^{1}$

${ }^{1}$ Intensive care departement, Centro Hospitalar e Universitário do Algarve, Faro, Portugal; ${ }^{2}$ Internal medicine service, Centro Hospitalar e Universitário do Algarve, Faro, Portugal; ${ }^{3}$ Cardiology service, Centro Hospitalar e Universitário do Algarve, Faro, Portugal; ${ }^{4}$ Critical care, William Osler Health System, Toronto, Canada

Correspondence: R. Ferreira de Almeida

Intensive Care Medicine Experimental 2020, 8(2): 000304

Introduction: Neurocritical care is a subspecialty of critical care medicine that focusses on the evaluation, diagnosis, and management of patients with acute life-threatening central nervous system diseases. $(1,2)$ Because of their complexity, neurocritical patients needs specific continuous monitorization, to allow their recovery.
Objectives: 1. Characterize neurocritical Intensive Care Unit (ICU) patients admitted in 2019 and to correlate neurocritical care variables with Glasgow outcome Scale at ICU discharge and 6 months after discharge.

2. Finding the most significative variables in order to build a model that best predict outcome.

Methods:

An observational retrospective study included 105 neurocritical patients in our intensive care unit (ICU) during the year of 2019. Collected variables included age, gender, diagnoses, severity scores (APACHE, SAPS, initial SOFA), in local Glasgow coma score (GCS), hospital admission GCS, Marshall, Hunt \& Hess scale, Fisher scale, National institute of Health stroke scale (NIHSS), intracranial pressure (ICP) at ICU admission, time with ICP $>24$ in the first $72 \mathrm{~h}$ after ICU admission, mean ICP during ICU stay, doppler variables in the first $24 \mathrm{~h}$ of ICU stay (middle cerebral artery (MCA) blood velocity and pulsatility index) and Glasgow Outcome Scale (GOS) at ICU discharge and 6 months after discharge.We included also the time (minutes) between local to emergency departement (ED) admission and time between ED and ICU.

We performed a Multiple Regression Analysis using Akaike's Information Criterion (AIC) to identify the most relevant variables that best explain outcome.

Results: One hundred and five neurocritical patients were included: traumatic brain injury (TBI) (61), aneurism (31) of which 27 had subarachnoid haemorrage, stroke (11) and arteriovenous malformation (2). In patients with TBI $84,8 \%$ were male, mean age of 45,7 years, $80,4 \%$ present moderate to severe TBI, $68,4 \%$ present Marshall scores of 3 and above. $52 \%$ with inital sofa score of 7 and above.

In patients with aneurism, 72,4 were female, mean age of 57,8 years, mean hospital admision GCS of $10,42 \%$ hunt \&hess of 2 and above, 47 $\%$ with initial sofa score of 7 and above.

The best model for predicting GOS at ICU discharge included 5 variables: Sex, Age, initial SOFA Score (at admission), admition GCS, and Time between ED and ICU:

-Associated with best outcome : male sex, younger age, higher admition GCS, more Time ED-ICU.

-Associated with worse outcome :female sex, older age, lower admition GCS, high SOFA score.

The adjusted R 2 for this model was $40 \%$ (accounts for $40 \%$ of variation in outcome)with a p-value $<1.6 \times 10^{\wedge} 9$.

Conclusion: It was not surprise for us that high admition GCS and younger age were associated with best outcome. However we were not expected that more time between ED-ICU leads to better outcome. The male sex appears to preform better than female sex. Those facts will be a good discussion points.

The focus of this work was characterization of our neurocritical care population. The identification of a new predictive model presents an interesting hypothesis for future validation.

Reference(s) and grant ackowledgment(s)

1. Sekhon MS, Gooderham P, Toyota B, Kherzi N, Hu V, Dhingra VK, et al. Implementation of Neurocritical Care Is Associated with Improved Outcomes in Traumatic Brain Injury. Can J Neurol Sci. 2017:44(4):350-7.

2. Korbakis G, Bleck T. The Evolution of Neurocritical Care. Crit Care Clin. 2014;30(4):657-71.

000314

Continuous Heart Rate Variability and Electroencephalography Monitoring in Severe Acute Brain Injury: A Preliminary Study

H. Lee'; JM. Koo'; SB. Jeon'; KS. Lee ${ }^{2}$

${ }^{1}$ Neurology, Asan Medical Center, Seoul, Republic of Korea; ${ }^{2}$ Neurology, The Catholic University of Korea Seoul St. Mary's Hospital, Seoul, Republic of Korea

Correspondence: $\mathrm{H}$. Lee

Intensive Care Medicine Experimental 2020, 8(2): 000314

Introduction: Reductions in heart rate variability (HRV) are associated with poor outcomes in patients with severe acute brain injury. However, it is unknown if the changes in HRV precede neurological 
deterioration in such patients, as an early indicator of brain-heart network dysfunction.

Objectives: We examined the changes in HRV measured by electrocardiography (ECG) in patients who experienced neurological deterioration after severe acute brain injuries, and analyzed the correlation between HRV and the qualitative and quantitative variables of electroencephalography (EEG).

Methods: Thirteen EEG channels and one channel of simultaneouslyacquired ECG were segmented in $120 \mathrm{~s}$ epochs and used to calculate ten HRV features (standard deviation of the normal-normal intervals, root mean square of the difference in successive intervals, very low frequency power, low frequency power, high frequency power, low frequency/high frequency power ratio, SD along the transverse axis of the Poincaré plot [SD1], SD along the longitudinal axis of the Poincaré plot [SD2], SD2/SD1 ratio, and approximate entropy) and three quantitative EEG features (suppression ratio, asymmetry index, and alpha/ delta band power ratio). Raw EEGs of epochs were also reviewed using standardized terminology and graded according to its background activities and ictal-interictal continuum patterns.

Results: The neurophysiological features of 25 patients with severe acute brain injury due to various causes were analyzed. The HRV and EEG features showed progressive and significant changes in the 48-hour period prior to neurological deterioration. Notably, the suppression ratio and background severity grade of the EEG showed significantly negative correlations with all HRV features.

Conclusion: Integration of HRV monitoring with continuous EEG monitoring may enable timely identification of progressive secondary brain injury before the development of irreversible injury.

\section{Reference(s) and grant ackowledgment(s)}

1. Schmidt JM, Sow D, Crimmins M, Albers D, Agarwal S, Claassen J, et al. Heart rate variability for preclinical detection of secondary complications after subarachnoid hemorrhage. Neurocrit Care 2014;20:382-9.

2. Gollwitzer S, Groemer T, Rampp S, Hagge M, Olmes D, Huttner HB, et al. Early prediction of delayed cerebral ischemia in subarachnoid hemorrhage based on quantitative EEG: A prospective study in adults. Clinical Neurophysiology 2015;126:1514-23.

3. Heart rate variability. Standards of measurement, physiological interpretation, and clinical use. Task Force of the European Society of Cardiology and the North American Society of Pacing and Electrophysiology. Eur Heart J 1996:17:354-81.

\section{1}

Intracranial pressure management in acute brain-injured patients: baseline characteristics of the Synapse-ICU Centers C. Bonetti' ; F. Elli ${ }^{1}$; F. Graziano'; G. Isernia ${ }^{1}$; G. Palio ${ }^{2}$; C. Robba ${ }^{3}$; P. Rebora'; S. Galimberti'; A. Vargiolu'; G. Citerio

${ }^{1}$ School of medicine and surgery, University of Milano-Bicocca, Milan, Italy; ${ }^{2}$ Department of intensive care, Clinica Santa Isabel, Universidad de Buenos Aires, Buenos Aires, Argentina; ${ }^{3}$ Department of anesthesia and intensive care, San Martino Policlinico Hospital, IRRCS for Oncology, Genova, Italy; ${ }^{4}$ Neurointensive care, department of emergency and intensive care, Ospedale San Gerardo di Monza, Monza, Italy

Correspondence: $\mathrm{C}$. Bonett

Intensive Care Medicine Experimental 2020, 8(2): 000501

Introduction: Increased intracranial pressure (ICP) is one of the major clinical complications of acute brain injuries (ABIs) and correlates with poor outcome. ICP monitoring (ICPm) is the most common neuromonitoring modality used in intensive care units (ICUs). However, no evidence-based recommendations on ICP management are present in the current literature. Practice about indications of ICPm is variable in high- (HICs) and in low-middle-income countries (LMICs), where data is scarce or inconsistent.

Objectives: Aim is to quantify practice variations in ICPm in HICs and LMICs and in TBI and non-TBI patients.

Methods: The SYNAPSE-ICU is a prospective, observational, international cohort study (NCT03257904) focused on describe the current practice of ICPm using a worldwide sample. Centre Data
Characteristics Form has been submitted to each center enrolled in the study to assess the standard intensive care practice in $\mathrm{ABI}$ patients. Information about the institution and ICP monitoring and management were indicated.

Results: Worldwide, 156 sites distributed in 41 countries participated in the study. The Center Form was completed by $97.4 \%$ of sites. Based on World Bank List of Economies Classification (2019-2020), 110 (72.4\%) of centers were localized in HICs and 42 (27.6\%) in LMICs. Over two thirds of the centers have an academic affiliation (69.7\%). A specialist neurocritical ICU was available in $31.6 \%$ of centers, whereas in the majority of them (68.4\%) ABI patientswere admitted to other ICUs (mixed, surgical, medical). In $99.3 \%$ of sites patients were monitored $24 / 7$ by medical staff, and the most represented nurse:patient ratio was $1: 2(54.0 \%)$.

Overall, $72.4 \%$ of centers routinely used ICPm in TBI patients, and approximately half of them routinely monitored ICP in SAH and ICH. A protocol/policy for ICP treatment in TBI, SAH and ICH was available in $63.8 \%, 49.3 \%$ and $48.0 \%$ of centers, respectively.

Regarding the availability of neuromonitoring devices, $95.4 \%$ of ICUs declared to have an intracranial catheter to monitor ICP. At least one additional non-invasive neuromonitoring technique (transcranial doppler, optic nerve sheath diameter ultrasound, EEG, pupillometry and NIRS) was available in most of centers both in HICs (91.8\%) and in LMICs (90.5\%), whereas an additional invasive neuromonitoring device (brain tissue oxygen and temperature, cerebral microdialysis, regional cerebral blood flow, deep/cortical EEG electrodes) was usable in $49.1 \%$ of $\mathrm{HIC}$ and in $26.2 \%$ of LMIC centers.

Neuroimaging modality most frequently used was CT scan $(98.6 \%$ of ICUs), while an advanced technique (MRI, CT angiography, CT perfusion, and PET) was available in $59.3 \%$ of centers.

Conclusion: A great center variability in ICPm of ABI patients was observed worldwide. The results stress the need of evidence-based recommendations regarding ICPm, in particular in LMICs, where resource limitation may preclude ICPm utilization.

Reference(s) and grant ackowledgment(s)

1. The study received an award from the ESICM, and it is inserted in the ESICM research portfolio

000682

Near-infrared spectroscopy in brain dead neurocritical care patients

F. Romagnosi ${ }^{1}$; E. Milani ${ }^{1}$; M. Taiana' ${ }^{2}$ C. Crippa ${ }^{1}$; C. Allegri' ${ }^{1}$ S. Danese'; C. Alfier ${ }^{1}$; V. Pellegrini ${ }^{1}$; L. Casartelli ${ }^{1}$; P. Zanatta ${ }^{1}$

${ }^{1}$ Unit of anaesthesia and intensive care $\mathrm{a}$, Hospital University Integrated trust of Verona, Verona, Italy; ${ }^{2}$ Unit of anaesthesia and intensive care b, Hospital University Integrated trust of Verona, Verona, Italy

Correspondence: F. Romagnosi

Intensive Care Medicine Experimental 2020, 8(2): 000682

Introduction: Does near-infrared spectroscopy (NIRS) is a reliable index of brain oxygenation? NIRS is generally used as a non-invasive tool to assess regional cerebral oxygen saturation ( $\mathrm{rSO}$ ) to prevent ischemic brain damage. Albeit its use is widespread in the neurocritical care field[1], its real role in accurately detect ischemic insults and low cerebral flow is still a matter of debate mainly because of a lack of information on the NIRS algorithm utilized by each manufacturer and the low-resolution depth of the elliptical path of the optical spectrophotometry $[2-4,6]$.

Objectives: Our purpose is to detect the cerebral oxygen saturation of the forehead in a clinical model of a diffuse ischemic brain injury, such as brain-dead patients.

Methods: We retrospectively analyzed adult neurocritical care patients who evolved into brain death between January 2019 and March 2020. NIRS was sampled with the $03^{\mathrm{TM}}$ (Masimo, Irvine, CA) and SensmartTM X-100 (Nonin Medical, Inc., Plymouth, Minnesota, US) system for the total duration of 20 minutes after the patient had been declared brain dead, according to the Italian national criteria/ guidelines. 
Results: A total of 40 brain dead patients were studied (median age 72 IQR[55-79], 35\% intracranial hemorrhage, 35\% traumatic brain injury, $15 \%$ ischemic stroke, $7 \%$ hypoxic brain injury post-cardiac arrest, $3 \%$ subarachnoid hemorrhage from brain aneurysm rupture, $5 \%$ others). Among them, despite the presence of clinical, neurophysiological and neuroradiological criteria of brain death (assessed with brainstem reflexes, electroencephalography, and CT angiography), we recorded a median $\mathrm{rSO} 2$ value of $72 \%$ (IQR [63-75]). The $90 \%$ of the total series $(36 / 40)$ have experienced a normal $\mathrm{rSO} 2$ values (i.e., $\mathrm{rSO} 2>50 \%)$ and only $10 \%$ (4 patients) showed $\mathrm{rSO} 2$ values below $50 \%$, among whom two patients presented concomitant severe cardiac dysfunction. A left-sided decompressive craniectomy was performed in two patients, in which we observed the left $\mathrm{rSO} 2$ value much higher than contralateral (i.e., both with delta $\mathrm{rSO} 2>20)$. Moreover linear correlation was found between $\mathrm{rSO} 2$ and $\mathrm{SaO} 2(\mathrm{r}=-0,393 ; p<0,02)$.

Conclusion: In the experimental model of global brain ischemia such as brain death, the current NIRS technology does not mirror the real state of the brain oxygenation; indeed a normal rSO2 values were found in our analysis (72\%, IQR[63-75]) These data sustain the low sensitivity of the NIRS monitoring in measure faithfully the correct oxygenation status beyond the forehead[5, 6]. More studies are needed to better understand the limits and reliability of NIRS algorithms in detecting brain ischemia.

\section{Reference(s) and grant ackowledgment(s)}

1. 1. Vinciguerra L, Bösel J (2017) Noninvasive Neuromonitoring: Current Utility in Subarachnoid Hemorrhage, Traumatic Brain Injury, and Stroke. Neurocritical Care 27:122-140. https://doi.org/10.1007/s12028-016-0361-8

2. 2. Francony G, Bouzat P, Payen J-F (2012) Monitorage par spectroscopie de proche infrarouge en neuroréanimation. Annales Françaises d'Anesthésie et de Réanimation 31:e133-e136. https://doi.org/10.1016/j.annfar.2012.04.017

3. 3. Green MS, Sehgal S, Tariq R (2016) Near-Infrared Spectroscopy: The New Must Have Tool in the Intensive Care Unit? Semin Cardiothorac Vasc Anesth 20:213-224. https://doi.org/10.1177/1089253216644346

4. 4. Yu Y, Lu Y, Meng L, Han R (2016) Monitoring cerebral ischemia using cerebral oximetry: pros and cons. J Biomed Res 30:1-4. https://doi.org/10.7555/ JBR.30.20150096

5. 5. Davie SN, Grocott HP (2012) Impact of Extracranial Contamination on Regional Cerebral Oxygen Saturation: A Comparison of Three Cerebral Oximetry Technologies. Anesthesiology 116:834-840. https://doi.org/10.1097/ ALN.0b013e31824c00d7

6. 6. Zanatta P, Forti A Effectiveness of NIRS to sample the frontal brain cortex in all cardiac surgery patients. MINERVA ANESTESIOLOGICA 2

7. The author declares that the research was conducted in the absence of any commercial or financial founding, excluding a potential conflict of interest.

\section{9}

Acquiring competencies in cerebral ultrasonography: a pragmatic study

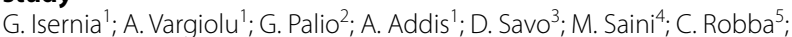

F. Graziano ${ }^{6}$; R. Bertuetti ${ }^{7}$; R. Vaschetto ${ }^{8}$; G. Citerio

${ }^{1}$ School of medicine and surgery, University of Milano-Bicocca, Monza, Italy; ${ }^{2}$ Department of intensive care, Clinica Santa Isabel, Universidad de Buenos Aires, Buenos Aires, Argentina; ${ }^{3}$ Neurointensive care unit, San Gerardo Hospital, ASST-Monza, Monza, Italy; ${ }^{4}$ Intensive care unit, Desio Hospital, ASST-Monza, Monza, Italy; ${ }^{5}$ Department of anaesthesia and intensive care, IRCCS AOU San Martino, Genova, Italy; ${ }^{6}$ School of medicine and surgery, university of milano - bicocca, italy, Bicocca bioinformatics biostatistics and bioimaging center b4, Monza, Italy; ${ }^{7}$ Department of anesthesia, intensive care and emergency medicine, Spedali Civili University Hospital, Brescia, Italy; ${ }^{8}$ Department of translational medicine, Università Degli Studi Del Piemonte Orientale, Novara, Italy

Correspondence: G. Isernia

Intensive Care Medicine Experimental 2020, 8(2): 000959

Introduction: Echography is becoming a diffuse, economic and noninvasive tool used in general ICU (Intensive care unit) while is less utilized for exploring the brain, protected by the skull. However, it could have multiple applications in the study of cerebral vessels anatomy and from its important role in the diagnosis of acute intracranial pathologies (1). Skills to be acquired have been recently described in a Consensus (2). No information about the utility of practical training are available.

Objectives: The aim of this project is to evaluate the improvement in the use of ultrasonography and TCCD (transcranial colour-coded duplex) diagnostic methods after a dedicated training course.

Methods: In December 2019, 31 participants (3 intensivists and 28 residents) naïve to the technique attended for two days the SONIC (Sonography in Neuro-Intensive Care) theoretical-practical course at University of Piemonte Orientale. Specialists in this field described the use of ultrasonography and TCCD in a large spectrum of clinical conditions, followed by practical hands-on sessions on volunteers and in ICU.

Before and after the training, all participants answered to a 51 multiple-choice online questionnaire and were required to evaluate a set of brain echographic images. A paired t-test was applied to evaluate the improvement.

Results: The median score obtained in the multiple-choice question was respectively 27 (pre) and 36 (post).

The paired t-test applied, showed a significant difference between the mean performance scores pre and post training course $(p<0.001)$. The mean difference, between pre and post scores, was equal to 10.19 points (95\% confidence interval $=7.95-12.43$ ).

The median score obtained in the images' evaluation was 4 (maximum score of 5).

Conclusion: The analysis conducted on the scores obtained before and after the theoretical-practical course showed how useful could be a targeted approach to the correct use of TCD ultrasonography improving personal skills about it.

According to the Consensus (2) we could consider an upgrade of the participants' level to basic-plus/pre-advance.

Reference(s) and grant ackowledgment(s)

1. 2. Care N, Robba C, Poole D, Citerio G, Taccone FS, Rasulo FA. Brain Ultrasonography Consensus on Skill Recommendations and Competence Levels Within the Critical Care Setting. 2019; DOI: 10.1007/s12028-019-00766-9

2. 1. Robba C, Goffi A, Geeraerts T, Cardim D, Via G, Czosnyka M, et al. Brain ultrasonography: methodology, basic and advanced principles and clinical applications. A narrative review. Intensive Care Med [Internet]. 2019; Available from: https://doi.org/10.1007/s00134-019-05610-4

\section{9}

Brain Oxygen Tension Over the First 24 Hours of Monitoring, Predicts Intensive Care Survival in Adult Patients with Traumatic Brain Injury

K. Overend'; T. Anderson ${ }^{2}$; J. Rhodes ${ }^{3}$

${ }^{1}$ Medical school, The University of Edinburgh, Edinburgh, United Kingdom; ${ }^{2}$ Critical care, anaesthesia and pain medicine, NHS Lothian, Edinburgh, United Kingdom; ${ }^{3}$ Critical care, anaesthesia and pain medicine, University of Edinburgh and NHS Lothian, Edinburgh, United Kingdom

Correspondence: J. Rhodes

Intensive Care Medicine Experimental 2020, 8(2): 000969

Introduction: Optimal management and monitoring of traumatic brain injury (TBI) remains uncertain. Intracranial pressure (ICP) monitoring lacks high quality evidence and does not prevent cerebral hypoxia (Stiefel et al., 2006; Carney et al., 2017) . Robust evidence for the use of brain oxygen tension (PbtO2) monitoring is also lacking, but preliminary work is promising and suggests it may improve outcomes. In a prior study, we found that early PbtO2 improvement, or low hypoxic load over the first 24 hours, were associated with ICU survival (Rhodes, Chandrasekaran and Andrews, 2016).

Objectives: In a second independent cohort of patients with moderate to severe $\mathrm{TBI}$, to validate the observation that exceedingly early changes in $\mathrm{PbtO} 2$ are associated with ICU survival.

Methods: A retrospective analysis was performed on prospectively collected data from a cohort of 60 TBI patients at a single centre focusing on the first 24 hours of monitoring. Minute-to-minute ICP, cerebral 
perfusion pressure (CPP) and $\mathrm{PbtO} 2$ values were recorded via an invasive cerebral monitor on to a bedside laptop running ICU pilot software (CMA, Sweden). The relationship of $\mathrm{PbtO} 2 \mathrm{vs}$. time was described as a Spearman correlation coefficient for each patient. The percentage of time that $\mathrm{PbtO} 2$ was $<20 \mathrm{mmHg}$ was calculated. Demographic and baseline characteristics were collected. The primary outcome was ICU survival.

Comparisons were made using paired or unpaired T tests and Wilcoxon signed rank test or Mann-Whitney U test. ICU survival was analysed using logistic regression.

Results: Demographics were well matched, except age, which was significantly higher in non-survivors (43 years [IQR $26.3-555.0$ ] vs. 60 years [IQR $49.3-72.8] \mathrm{p}=0.01$ ). Survivors demonstrated significant increases in PbtO2 over the first 24 hours, $(18.9 \mathrm{mmHg}$ [IRQ 12.8 to $23.1]$ vs. $25.6 \mathrm{mmHg}$ [21.6 to 30.6$], \mathrm{p}<0.001$, which was not seen in nonsurvivors (16.3 mmHg [IRQ 8.5 to 32.3 ] vs. $18.3 \mathrm{mmHg}$ [16.9 to 21.8], $\mathrm{p}=0.898$. Survivors experienced a significantly higher $\mathrm{PbtO} 2$ over 24 hours than non-survivors $(25.3 \mathrm{mmHg}$ [22.1 to 26.4$]$ vs. $18.4 \mathrm{mmHg}$ [17.3 to 20], $\mathrm{p}<0.001$ ). Age (odds ratio 0.95 , [ $95 \% \mathrm{Cl} 0.90$ to 1.00 ] $\mathrm{p}=0.032$ ), the correlation coefficient between $\mathrm{PbtO} 2$ and time (odds ratio 5.24 [95\% Cl 1.02 to 26.96$] p=0.047$ ) and percentage hypoxia in the first 24 hours (odds ratio 0.98 [ $95 \% \mathrm{Cl} 0.96$ to 1.00$] \mathrm{p}=0.033$ were significantly associated with ICU survival. When the data from this cohort were combined with the original cohort $(n=25)$, these findings were preserved.

Conclusion: In patients with moderate to severe TBI undergoing invasive multimodality monitoring, data from $\mathrm{PbtO} 2$ monitoring as early as the first 24 hours of monitoring is associated with survival. An improving $\mathrm{PbtO} 2$ with time and low cumulative hypoxia were significantly associated with ICU outcome. The findings of the original observational study are upheld. What remains unclear is if this association with survival occurs spontaneously or is the result of goal directed therapy.

\section{Reference(s) and grant ackowledgment(s)}

1. Rhodes JK, Chandrasekaran S, Andrews PJ. Early changes in brain oxygen tension may predict outcome following severe traumatic brain injury. Vol. 122, Acta Neurochirurgica, Supplementum. 2016.

2. Stiefel MF, Udoetuk JD, Spiotta AM, Gracias VH, Goldberg A, Maloney-Wilensky $\mathrm{E}$, et al. Conventional neurocritical care and cerebral oxygenation after traumatic brain injury. JNeurosurg. 2006 Oct; 105(4):568-75.

3. Carney N, Totten AM, O'Reilly C, Ullman JS, Hawryluk GWJ, Bell MJ, et al. Guidelines for the Management of Severe Traumatic Brain Injury, Fourth Edition. Neurosurgery. 2017 Jan 1;80(1):6-15.

4. Dr Rhodes is supported by a National Health Service Research Scotland Clinician Fellowship

\section{4}

Velocity Time Integral (VTI) in Trans-Cranial Doppler (TCD) in healthy controls $(C)$ versus ICU patients $(P)$ for detection of cerebral blood flow (CBF) abnormalities

J. Richecoeur'; D. Combaux'; N. Verrier'; A. Sagnier ${ }^{1}$; H. Kowalik';

D. Boisbault' ${ }^{1}$; R. Mercier'; R. De Bock' ; C. Caplin ${ }^{1}$; C. Soulignac ${ }^{1}$; D. Luis ${ }^{1}$

${ }^{1}$ Mixed icu, Hospital Center Simone Veil De Beauvais, Beauvais, France

Correspondence: J. Richecoeur

Intensive Care Medicine Experimental 2020, 8(2): 001064

Introduction: Fewstudies report the relation between functional brain alterations during and after ICU stay and abnormalities of CBF displayed on TCD. Using VTI as hemodynamic parameter is unusual for evaluation of $\mathrm{CBF}(1)$

\section{Objectives:}

The purpose of this preliminary study is to compare the values of VTI of healthy Controls $(\mathrm{C})$ versus ICU (P) with usual parameters (i,e systolic $(\mathrm{Vs})$, diastolic $(\mathrm{Vd})$ and mean velocities $(\mathrm{Vm})$, resistance (RI) and pulsatility (PI) indexes.(2).

Methods: $50 \mathrm{C}$ and $160 \mathrm{P}$ were consecutively included during a 6 months period (August 2019 to May 2020). Velocities and VTIMCA were sampled using TCD at the middle cerebral artery (MCA, M1 segment). Left ventricular outflow (VTIAo) was assessed by transthoracic echocardiography.

Demographic data, reasons for admission in ICU, SAPS, sedative, vasopressor use and ventilator support were recorded as well MAP, Vs, Vd, $\mathrm{Vm}, \mathrm{Pl}, \mathrm{RI}$.

Data are shown as medians (interquartile:25-75).Ttest or non-parametric test (if appropriate) were use. $P<0,05$ was considered as significant. Results: Sex ratio(M/F): 2,5 in P with SAPS II: 57 (41-69) and 1 in C. Age: 58 years (51-69) in $\mathrm{P}$ vs 40 years (30-52) in $C$.

Reasons for admission were sepsis: 71 (47 septic shocks), cardiac arrests: 19 cardiogenic shocks: 11, neurologic disorders: 12, others: 27 . 210 measurements were realized respectively under: mechanical ventilation: 183, vasopressor support: 101 and sedative drugs: 116.Table1 shows relevant data.

\begin{tabular}{|llllll|l|}
\hline \multicolumn{3}{l}{$\mathrm{C}(\mathrm{n}=50)$} & \multicolumn{2}{l|}{$\mathrm{P}(\mathrm{n}=160)$} & $\mathrm{p}$ \\
\hline MAP mmHg & 95,00 & $(89,00-98,00)$ & 80,00 & $(73,00-92,00)$ & $<0,001$ \\
\hline VTIAo cm & 21,50 & $(19,50-30,00)$ & 18,00 & $(15,00-21,50)$ & $<0,001$ \\
\hline VTIMCA cm & 51,75 & $(44,00-58)$ & 34,80 & $(26,40-42,00)$ & $<0,001$ \\
\hline Vd cm/s & 40,30, & $(35,38-46,20)$ & 32,06 & $(23,52-44,00)$ & $<0,015$ \\
\hline Vm cm/s & 58,50 & $(54,70-66,15)$ & 52,00 & $(40,65-65,90)$ & $<0,007$ \\
\hline RI & 0,55 & $(0,50-0,59)$ & 0,63 & $(0,52-0,69)$ & $<0,001$ \\
\hline PI & 0,86 & $(0,73-1,01)$ & 1,09 & $(0,84-1,30)$ & $<0,001$ \\
\hline VTIMCA/VTIAo & 2,52 & $(2,15-3,09)$ & 1,90 & $(1,43-2,44)$ & $<0,001$ \\
\hline HF*VTIMCA/BSA & 1876 & $(1712-2219)$ & 1542 & $(1229.9-$ & $<0,001$ \\
\hline HF*VTIAo/BSA & 759 & $(682-919)$ & 817 & $(639-1016)$ & $\mathrm{NS}$ \\
\hline
\end{tabular}

$\mathrm{HF}=$ Heart Frequency. BSA=Body Surface Area

There was a weak correlation between VTIMCA and VTIAo in P (not found in C).

However, VTIMCA and VTIAo were markedly dependent when VTIAMCA/ VTIAo ratio was $<1,5$ in $\mathrm{P}$ with marked $\mathrm{PI}$ and Vd abnormalities. (Spearman, $r=0,82, p<0,001$ ).

This ratio highlighted the coupling between VTIMCA and VTIAo. Low VTIAo correlated to low VTIMCA. P with vasopressors had higher VTIAMCA/ VTIAo ratio.

In addition, VTIMCA $<35 \mathrm{~cm}$ was more accurate than $\mathrm{Pl}>1.3$ and $\mathrm{VD}<25 \mathrm{~cm} / \mathrm{s}$ for the detection of abnormal neuropsychological behavior (respectively AUC: $0.81 ; 0.541 ; 0.345$ )

Conclusion: CBF could be estimate using VTIMCA and a hemodynamic index as HF*VTIMCA/BSA. The VTIMCA/ VTIAo ratio could be a tool to detect coupling. Low VTIMCA should do assess a VTIAo measurement. Further studies are needed to evaluate the clinical pertinence of the TCD in general ICUs.

\section{Reference(s) and grant ackowledgment(s)}

1. Tegeler, C.H., Crutchfield, K., Katsnelson, M., Kim, J., Tang, R., Passmore Griffin, L., Rundek, T. and Evans, G. (2013), Transcranial Doppler Velocities in a Large, Healthy Population. Journal of Neuroimaging, 23: 466-472. doi:10.1111/j.1552-6569.2012.00711.x

2. Pierrakos, C., Antoine, A., Velissaris, D. et al. Transcranial doppler assessment of cerebral perfusion in critically ill septic patients: a pilot study. Ann. Intensive Care 3, 28 (2013). https://doi.org/10.1186/2110-5820-3-28

3. Special thanks to the paramedic staff and residents

\section{4}

\section{Automated pupillometry can identify unreactive} electroencephalographic background

L. Peluso'; M. Talamonti' ; L. Ferlini²; N. Ndieugnou Djangang ${ }^{1}$;

E. Bogossian ${ }^{1}$; M. Menozzi ' ; J. Creteur'; J J. Vincent ${ }^{1}$; N. Gaspard²;

FS. Taccone

${ }^{1}$ Department of intensive care, Hopital Erasme, Bruxelles, Belgium; ${ }^{2}$ Department of neurology, Hopital Erasme, Bruxelles, Belgium

Correspondence: $L$. Peluso

Intensive Care Medicine Experimental 2020, 8(2): 001144

Introduction: Electroencephalography (EEG) is one of the most used non-invasive monitoring methods in critically ill patients. However, EEG interpretation requires the presence of an experienced 
neurophysiologist and is time-consuming. The aim of this study was to evaluate whether automated pupillometer (AP)-derived variables might help to assess the degree of cerebral dysfunction in critically ill patients.

Methods: Prospective study conducted in the Department of Intensive Care of Hôpital Erasme in Brussels. Pupillary assessments were done using the AP in three subgroups of patients concomitantly monitored with continuous EEG: 1) Post-anoxic brain injury; 2) Primary brain injury; 3 ) Others (i.e. sepsis, liver failure ...). An independent neurologist blinded to patient's history and AP results scored the degree of encephalopathy and reactivity on EEG using a standardized scale. The mean value of Neurologic Pupil Index (NPi), pupillary size, constriction rate, constriction and dilation velocity (CV and DV) and latency for both eyes were reported using the NPi ${ }^{\circledR}-200$ (Neuroptics, Laguna Hills, CA, USA).

Results: We included 214 patients (mean age 60 years, 55\% male). EEG tracings were categorized in: mild $(n=111,52 \%)$, moderate $(n=65$, $30 \%)$ or severe $(n=16,8 \%)$ encephalopathy; burst/suppression $(n=19$, $9 \%)$ or suppression $(n=3,1 \%)$. A total of $38(18 \%)$ EEG were classified as unreactive. We found a significant difference in all pupillometry variables among different EEG categories; an unreactive EEG was associated with lower NPi, pupil size, pupillary reactivity, CV and DV and a higher latency than reactive recordings. DV and sedation were independently predictors of unreactive EEG (OR 47.68 [5.65-402.76]; $\mathrm{p}<0.01$ and 6.67 [2.49-17.86]; $\mathrm{p}<0.01$, respectively). In particular, DV values had an AUC of $0.85[0.78-0.91 ; p<0.05]$ to predict the presence of unreactive EEG; in subgroups analyses, area under the curve (AUC) of DV to predict unreactive EEG was 0.70 [0.55-0.86; $\mathrm{p}<0.01]$, $0.91[0.82-1.00 ; p<0.01]$ and $0.95[0.88-1.00 ; p<0.01]$ for post-anoxic, primary brain injury and others, respectively.

Conclusion: This study suggests that DV assessment via the AP might effectively identify unreactive EEG background, in particular in nonanoxic critically ill patients.

\section{2}

PBtO2 vs $\mathrm{pBtO2} / \mathrm{paO2}$ ratio. Which is the best predictor

of outcome in patients with acute brain injury?

T. Schizodimos' ; E. Siomos' ; P. loannidis ${ }^{1}$; I. Karageorgiou' ${ }^{1}$ P. Poulios ${ }^{1}$;

N. Kapravelos ${ }^{1}$; C. lasonidou ${ }^{1}$

'B icu, General Hospital "G. Papanikolaou", Thessaloniki, Greece

Correspondence: T. Schizodimos

Intensive Care Medicine Experimental 2020, 8(2): 001162

Introduction: Neurocritical care patients with acute brain injury (ABI) are at risk of developing intracranial hypertension which is associated with poor outcome. Monitoring of intracranial pressure (ICP) and other additional parameters, such as brain tissue pO2 (PbtO2), and brain oxygenation ratio ( $\mathrm{PbtO} 2 / \mathrm{PaO} 2$ ratio), can assess the severity of $\mathrm{ABI}$ and guide treatment. Thus, it may help predict and improve outcomes of these patients.

Objectives: To investigate the relationship between the type of outcome and parameters measured using an intraparenchymal monitoring catheter ( $\mathrm{PbtO} 2$ and $\mathrm{PbtO} 2 / \mathrm{PaO} 2$ ratio) in patients with $\mathrm{ABI}$.

Methods: An observational study was performed in a Greek ICU from July 2018 to May 2020, including 34 patients with ABI (13 Traumatic brain injury, 14 Subarachnoid hemorrhage, 5 Intracerebral hemorrhage, 2 Ischemic stroke) and ICP/PbtO2 monitoring. GCS on admission, APACHE II score, ICP, $\mathrm{PbtO} 2$ and $\mathrm{PbtO} 2 / \mathrm{PaO} 2$ ratio during the first 24 hours were recorded. We ensured normal ICP $(<22 \mathrm{mmHg})$ with appropriate treatment measures in all patients. We considered as critical thresholds of $\mathrm{PbtO} 2<15 \mathrm{mmHg}$ and $\mathrm{PbtO} 2 / \mathrm{PaO} 2$ ratio $<$ 0.15 . Patient outcome was determined as favorable (patient had good recovery or moderate disability) or unfavorable (patient died, or was in a vegetative state or had severe disability) using the Extended Glasgow Outcome Scale (GOS-E) at 6 months after ABI. Simple logistic regression models were fitted to the data.

Results: Included 34 patients had a median age of 57 (IQR 15) years, median ICU admission GCS 7 (IQR 4.75) and mean APACHE II score 16.91 (SD \pm 5.25 ). An external ventricular drain was inserted in 19 patients (55.9\%) and surgery before ICU admission occurred in 20 patients (58.8\%). A positive association of mean $\mathrm{PbtO} 2$ on day 1 with type of outcome is detected. The odds for favorable outcome are 1.06 times higher or $6 \%$ increased for every $\mathrm{mmHg}$ that $\mathrm{PbtO} 2$ is increased but this is not significant $(p=0.149, p>0.05,95 \% \mathrm{Cl}$ is $0.98-1.15$ and includes 1) (Table 1). In our small sample, natural logarithm of mean $\mathrm{PbtO} 2 / \mathrm{PaO} 2$ ratio on day 1 is positively related with type of outcome. Regarding 0.1 increments in natural logarithm of $\mathrm{PbtO} 2 / \mathrm{PaO} 2$ ratio the odds for favorable outcome are 1.045 times or $4.5 \%$ higher. This relationship is not significant though $(\mathrm{p}=0.296, \mathrm{p}>0.05,95 \% \mathrm{Cl}$ is 0.68-3.55 and includes 1) (Table 1). Mean $\mathrm{PbtO} 2$ and $\mathrm{PbtO} 2 / \mathrm{PaO} 2$ ratio values for favorable and unfavorable outcome are recorded in Table 2.

Table 1. Association of outcome with catheter-based monitoring parameters.

$\begin{array}{lll}\text { Variable } & \text { OR }(\mathbf{9 5 \%} \text { CI }) & \text { p-value } \\ \text { Mean } \mathrm{PbtO} 2 \text { on day 1 } & 1.06(0.98,1.15) & 0.149,>0.05 \\ \text { Mean } \mathrm{PbtO} 2 / \mathrm{PaO} 2 \text { on day } 11.55(0.68-3.55) & 0.296,>0.05 \\ & 1.045 \text { for } 0.1 \text { increments } & \end{array}$

Table 2. Means of catheter-based monitoring parameters according to outcome.

$\begin{array}{lll}\text { Variable } & \text { Favorable outcome } & \text { Unfavorable outcome } \\ \text { Mean } \mathrm{PbtO} 2 \text { on day } 1 & 18.6 & 12.58 \\ \mathrm{Mean} \mathrm{PbtO} 2 / \mathrm{PaO} 2 \text { on day } 10.158 & 0.112\end{array}$

Conclusion: In addition to ICP, monitoring and normalizing parameters, such as $\mathrm{PbtO} 2$ and $\mathrm{PbtO} 2 / \mathrm{PaO} 2$ ratio could help improve outcome in patients with $\mathrm{ABI}$. Mean $\mathrm{PbtO} 2$ and $\mathrm{PbtO} 2 / \mathrm{PaO} 2$ ratio on day 1 had similar performance in these patients but $\mathrm{PbtO} 2 / \mathrm{PaO} 2$ ratio was inferior to $\mathrm{PbtO} 2$ alone in our small sample. There remains a need for more, high quality trials in patients with $A B I$.

\section{Reference(s) and grant ackowledgment(s)}

1. 1. Roh D, Park S. Brain Multimodality Monitoring: Updated Perspectives. Curr Neurol Neurosci Rep. 2016;16(6):56.

2. 2. Dellazizzo L, Demers SP, Charbonney E, et al. Minimal PaO2 threshold after traumatic brain injury and clinical utility of a novel brain oxygenation ratio. $J$ Neurosurg. 2018;1-9.

\section{7}

In Situ Simulation for ITU Nursing Staff: No Haemorrhage Too Big; No Timeframe Too Small

L. Bezzina ${ }^{1}$; A. Mohamed ${ }^{1}$; S. Javier ${ }^{1}$; P. Walker ${ }^{1}$; C. Curtin ${ }^{1}$

${ }^{1}$ Medical education, Queen's Hospital, Romford, United Kingdom

Correspondence: $L$. Bezzina

Intensive Care Medicine Experimental 2020, 8(2): 000187

Introduction: The Barking, Havering, Redbridge University Trust (BHRUT) simulation team, ITU team and transfusion team collaborated in an attempt to improve knowledge among ITU nurses around local Massive Haemorrhage $(\mathrm{MH})$ guidelines [1] through simulation, to great effect in a mere 1-hour whilst on ITU.

Methods: The simulation team ran a high-fidelity in-situ simulation of an unexpected massive haemorrhage in an ITU patient, on the intesive care unit. The doctor in the scenario managed the patient's airway during the ongoing massive haematemesis. The transfusion team were aware of the simulation and attended, when called, to simulate real life.

Questionnaires were administered pre and post simulation to gauge:

1. Knowledge about $\mathrm{MH}$ protocols in general [2]

2. Confidence levels with regards to MH guidelines specific to the Trust

3. Confidence levels around what constitutes a MH

4. Confidence levels around the key initial steps in escalating a patient with a $\mathrm{MH}$ 
Results: A 5-point Likert scale was used to assess the key learning objectives for the session, where 5 was the most positive outcome and 1 was the least positive outcome:

1. Prior to simulation, ITU nurses had an average score 2.0 when asked about their general knowledge on Massive Haemorrhage protocols, this improved to 4.3 after simulation

2. Perceived confidence levels with regards to following the Trusts' flow diagram on managing a $\mathrm{MH}$ improved from an average score of 1.8 to 4.0 after participants were involved in simulation.

3. ITU nurses felt much more confident in identifying a $\mathrm{MH}$ as a result of partaking in the scenario; 100\% of nurses were able to identify a $\mathrm{MH}$ after simulation, compared with only $25 \%$ being able to name one parameter that constitutes a $\mathrm{MH}$ prior to the simulation.

4. $100 \%$ of nurses knew the two most important steps to take in the event of a $\mathrm{MH}$ before simulation, but valued the opportunity to take those steps in real-time during the simulation.

Conclusion: Haemorrhage following trauma is the leading cause of death in young adults in the UK [3], haemorrhage following childbirth remains the third leading cause of direct maternal death in the UK [4] and acute upper gastrointestinal bleeds (AUGIB), a common in-hospital emergency, and has a case mortality of $10 \%$, according to the Joint UK Blood Transfusion and Tissue transplant Services [5]. Thus, it's not uncommon for our ITU staff to be faced with massive haemorrhage and hence it made this particular emergency an important target for simulation.

A mere 1-hour of in-situ simulation, centred around very specific massive haemorrhage learning objectives, proved an extremely effective way to impart learning during working hours to prepare ITU nurses for the inevitable day when they are faced with a real major bleed. Here we have demonstrated considerable improvements in knowledge and confidence with regards to management of massive haemorrhage following only 60 minutes of simulation.

\section{Reference(s) and grant ackowledgment(s)}

1. 5. Joint UK Blood Transfusion and Tissue transplant Services . 7.3.5: Acute upper gastrointestinal bleeding. https://www.transfusionguidelines.org/ transfusion-handbook/7-effective-transfusion-in-surgery-and-criticalcare/7-3-transfusion-management-of-major-haemorrhage.

2. 4. Royal College of Obstetrics \& Gynaecologists. RCOG release: Excessive bleeding after childbirth remains one of the leading causes of maternal deaths https://www.rcog.org.uk/en/news/rcog-release-excessive-bleedingafter-childbirth-remains-one-of-the-leading-causes-of-maternal-deaths/.

3. 3. Bozzette A, Aeron-Thomas A. Reducing trauma deaths in the UK. Lancet. 2013 Jul 20;382(9888):208

4. 2. National Institute of Health and Care Excellence, NICE. Major Haemorrhaging In Hospital. https://pathways.nice.org.uk/pathways/trauma/majorhaemorrhaging-in-hospital\#content=view-node:nodes-haemorrhageprotocols

5. 1. Barking Havering \& Redbridge University Trust. BHRUT Massive Haemorrhage Flow Diagram . https://www.bhrhospitals.nhs.uk/ (accessed). [link to be inserted when available].

\section{7}

\section{Develop a removal of central access device label to standardize} care in Intensive Care Unit

N.W. Chu'; W.Y. Kwok

${ }^{1}$ Intensive care unit, Prince of Wales Hospital, Hong Kong, Hong Kong

Correspondence: N.W. Chu

Intensive Care Medicine Experimental 2020, 8(2): 000297

Introduction: Many adult critical ill patients have central access devices (CAD) that includes central venous catheter (CVC), continuous renal replacement therapy (CRRT) catheter, or femoral arterial line during their acute phase of illness in the intensive care unit (ICU) for invasive monitoringof physiological status and treatment responsiveness. Bleeding is the most likely complication to occur after removal of the CAD when applied direct pressure to the puncture site is not adequate to achieve haemostasis. This kind of complication can be fatal especially if the larger size (such as 13FR) CRRT catheters are used.
Therefore, a Continuous Quality Improvement team has developed a removal of central access devices label aimed to provide quick clinical guide to bedside ICU nurses for the removal of CAD.

Objectives:

1. To deliver safe and standardize care to ICU patients during the removal of CAD

2. To prevent bleeding after removal of CAD

Methods: Wide staff consultations were made about the various kinds of method used to remind nurses on important clinical steps of the removal of CAD. Finally, a removal of central access device label was developed. The label reinforced the following critical actions for the removal of these line / catheter: 1. Application of direct pressure to the CAD exit site after the line has been removed for at least 10 minutes, 2. Re-checking of the removal site 5 minutes later after direct pressure was stopped for any bleeding / haematoma, 3. Removal of all central venous \& arterial lines as early as possible while the patient was waiting to transfer out to general ward, 4 . Checking the integrity of removed lines to avoid any retention of lines material, 5. Enhancement of nursing clinical handover through standardization of nursing documentation

Promotion of the project: 1. Staff education during briefing, 2. Eye catching smart point posted in the common staff areas of the unit, 3 . Ad hoc compliance check during daily round

Results: The project was implemented in May 2016. Every year, the Intensive Care Unit of Prince of Wales Hospital has around 1800 admissions of which 450 CVC lines and CRRT catheters were removed. A regular surprise audit on removal of CAD and implementation of the label was $100 \%$ compliance. Moreover, there were no incidences of immediate bleeding.

However, there were 2 reported cases of delay bleeding after removal of CRRT catheters due to the patient's underlying illness in 2018. Therefore, a second check was made 15 to 30 minutes after direct pressure was stopped in order to close this loophole. As of December 2019, there were no immediate or delay bleeding cases reported.

To evaluate the effectiveness of the project, a staff survey was also conducted with questionnaires distributed to 80 ICU nurses (76\%). Totally, $65(81 \%)$ completed questionnaires were returned with the result analysed as follows:

Table 1. Result of the staff survey

\begin{tabular}{|l|l|l|l|l|l|}
\hline Questions & $\begin{array}{l}\text { Strongly } \\
\text { Agree }\end{array}$ & Agree & Neutral & Disagree & $\begin{array}{l}\text { Strongly } \\
\text { Disagree }\end{array}$ \\
\hline $\begin{array}{l}\text { Q1. The removal of CAD label } \\
\text { can provide quick clinical } \\
\text { guide to prevent bleeing } \\
\text { complication after removal of } \\
\text { CAD }\end{array}$ & $18.6 \%$ & $69.5 \%$ & $8.5 \%$ & $3.4 \%$ & $0 \%$ \\
\hline $\begin{array}{l}\text { Q2. The removal of CAD label } \\
\text { can remind you to prevent } \\
\text { bleeding complication after } \\
\text { removal of CAD }\end{array}$ & $18.6 \%$ & $67.8 \%$ & $10.2 \%$ & $3.4 \%$ & $0 \%$ \\
\hline $\begin{array}{l}\text { Q3. The removal of CAD label } \\
\text { can help the prevention of } \\
\text { bleeding complication }\end{array}$ & $15.3 \%$ & $55.9 \%$ & $22.0 \%$ & $6.8 \%$ & $0 \%$ \\
\hline $\begin{array}{l}\text { Q4. The removal of CAD label } \\
\text { can facilitate your }\end{array}$ & $30.5 \%$ & $62.7 \%$ & $5.1 \%$ & $1.7 \%$ & $0 \%$ \\
\hline $\begin{array}{l}\text { documentation and clinical } \\
\text { handover to nurses of next } \\
\text { shift and general ward nurses }\end{array}$ & & & & & \\
\hline
\end{tabular}

Data in Table 1 shows that there was positive response on the project from the staff survey. Although there was $71.2 \%$ of the staff stated agree and strongly agree on the label which can help the prevention of bleeding complication, over $80 \%$ of the staff $(88.1 \%, 86.4 \%$ and $93.2 \%$ respectively) agreed that the label can provide quick clinical guide and remind nurses to prevent bleeding complication after removal of CAD and also can facilitate their documentation and clinical handover. Hence, the result of staff survey showed that the removal of CAD label can provide quick clinical guide to prevent bleeding complication after removal of CAD. 
Conclusion: Development of a removal of central access devices label has demonstrated its' effectiveness in preventing post removal bleeding complication in ICU. Nurses also feedback to enhance clinical documentation and handover as a biproduct of the project.

\section{Reference(s) and grant ackowledgment(s) \\ 1. Hospital Authority Head Office. Advanced Nursing Standards for Patient Care Short Term Non-Tunneled Central Venous Access Device Removal. Hong Kong: Hospital Authority; 2017. Document No. HAHO-COC-NS-NUR-100-11-V01. \\ 2. Special thanks to all nursing team of Intensive Care Unit, Prince of Wales Hospital, Sha Tin, Hong Kong}

\section{2}

Effectiveness and Safety of Early Rehabilitation for Patients with Acute Brain Impairment in Intensive Care Unit

S. Andreichenko ${ }^{1}$; M. Bychinin ${ }^{1}$; T. Klypa ${ }^{1}$

${ }^{1}$ Intensive care unit, Federal Research and Clinical Centre, FMBA of Russia, Moscow, Russia

Correspondence: $M$. Bychinin

Intensive Care Medicine Experimental 2020, 8(2): 000422

Introduction: More data has been recently acquired about effectiveness of early rehabilitation in the Intensive Care Unit. However, the data on the safety of this technique for patients with acute brain impairment $(A B I)$ is still being discussed [1, 2].

\section{Objectives:}

To assess the effectiveness and safety of early rehabilitation of $A B I$ patients in the Intensive Care Unit (ICU).

Methods: The inclusion criteria were $\mathrm{ABI}$ following a brain infarction, intracerebral hematoma, or subarachnoid hemorrhage. Criteria of an exclusion were a short rehabilitation course ( $\leq 7$ days), deeply decreased level of consciousness ( $\leq 7$ points on Glasgow coma scale), multi organ decompensated failure. All patients $(n=42)$ were assigned to the Early Rehabilitation Group (ERG) if it started 1-2 day from $A B I$ onset, or to the Delayed Rehabilitation Group (DRG) if it started after 2 nd day. All patients were given a daily mobilization complex taking into consideration their mobility and tolerance of physical activity. Clinical and laboratory indicators were analyzed, including the frequency of depression and deliria, the length of hospital stay and the time of mechanical ventilation. The development of serious complications during mobilization and mortality were also recorded. The data is presented as absolute values (frequency in percent), average (standard deviation), or median (25-75th percentile) depending on the type and allocation of the data.

Results: In group ERG $(n=15)$ mobilization started on 2nd (1-2) day on average, while in group DRG $(n=27)$ only on 7th (4-12) day ( $p<$ 0.001 ). The groups were comparable in degree of consciousness disorder and organ dysfunction. All patients had severe disabilities $(\geq 4$ points on the modified Renkin scale). No differences in groups were found as for the duration of mechanical ventilation, hospital admissions and mortality. The frequency of both delirium $(p=0.024)$ and depression $(p=0.040)$ was lower in the ERG group. The length of hospital stay in the Intensive Care Unit was higher in the DRG group than in the ERG group (27 (21-34) and 19 (10-27), respectively; $p=0,042)$. At the time of discharge, the incidence of severe disability decreased in both groups (to $87 \%$ in group ERG and to $78 \%$ in group DRG, $p=$ 0.482 ). There were no serious complications during mobilization in both groups.

Conclusion: Early rehabilitation can be effectively and safely applied for patients with $A B I$. The use of early rehabilitation for patients with acute cerebral deficiency reduces the frequency of mental disorders and the time in the Intensive Care Unit, which creates the basis for favorable long-term outcomes.

\section{Reference(s) and grant ackowledgment(s)}

1. 1. Zivi I, Valsecchi R, Maestri R, Maffia S, et al. Early Rehabilitation Reduces Time to Decannulation in Patients With Severe Acquired Brain Injury: A Retrospective Study. Frontiers in Neurology [Internet]. Frontiers Media SA; 2018 Jul 10;9. 2. Avert Trial Collaboration group. Efficacy and safety of very early mobilisation within $24 \mathrm{~h}$ of stroke onset (AVERT): a randomised controlled trial. The Lancet [Internet]. Elsevier BV; 2015 Jul;386(9988):46-55

\section{9}

Extensive use of prone positioning in COVID-19 patients with ARDS

F. Binda ${ }^{1}$; F. Marelli'; A. Galazzi'; E. Vinci'; I. Adamini ${ }^{1}$; D. Laquintana ${ }^{2}$ 'Intensive care unit, Fondazione IRCCS Ca' Granda Ospedale Maggiore Policlinico, Milano, Italy; ${ }^{2}$ Direction of healthcare professions, Foundation IRCCS Ca' Granda Ospedale Maggiore Policlinico, Milano, Italy

Correspondence: $\mathrm{F}$. Binda

Intensive Care Medicine Experimental 2020, 8(2): 000439

Introduction: Italy was one of the most affected country at the beginning of COVID-19 pandemic with the highest number of deaths in Europe mostly concentrated in the Lombardy region. Critically ill patients with COVID-19 required intensive care unit (ICU) admission due to the development of acute respiratory distress syndrome (ARDS). Prone positioning as rescue therapy can support respiratory function in patients with severe ARDS although the feasibility of the extensive use depends from the personnel expertise.

Objectives: To report the experience with the respiratory management and extensive use of prone positioning in COVID-19 patients admitted to the ICU hub of a tertiary-level hospital in Lombardy region.

Methods: All patients with laboratory-confirmed SARS-CoV-2 infection and subsequently admitted to ICU of Foundation IRCCS $\mathrm{Ca}^{\prime}$ Granda Ospedale Maggiore Policlinico (Milan), between February 23 and May 31, 2020, were enrolled in this retrospective consecutive case-series study. The recorded date included: age, sex, mode of respiratory support (non-invasive or invasive mechanical ventilation), use of prone positioning, mortality and ICU discharge.

Results: Of the 128 patients included, 121 required endotracheal intubation and prone positioning was used in $46 \%(59 / 128)$ as rescue therapy. The other results are summarized in the table.

\begin{tabular}{|c|c|c|c|c|c|c|}
\hline Characteristics & All pts. & $\begin{array}{l}\text { Age 20- } \\
39\end{array}$ & $\begin{array}{l}\text { Age 40- } \\
49\end{array}$ & $\begin{array}{l}\text { Age 50- } \\
59\end{array}$ & $\begin{array}{l}\text { Age 60- } \\
69\end{array}$ & $\begin{array}{l}\text { Age } \geq \\
70\end{array}$ \\
\hline N (\%) & $\begin{array}{l}128 \\
(100 \%)\end{array}$ & $11(9 \%)$ & $\begin{array}{l}23 \\
(18 \%)\end{array}$ & $\begin{array}{l}36 \\
(28 \%)\end{array}$ & $\begin{array}{l}48 \\
(37 \%)\end{array}$ & $10(8 \%)$ \\
\hline Median age ( & $\begin{array}{l}59(49- \\
65)\end{array}$ & $\begin{array}{l}34(29- \\
39)\end{array}$ & $\begin{array}{l}46(44- \\
47)\end{array}$ & $\begin{array}{l}55(52- \\
58)\end{array}$ & $\begin{array}{l}64(62- \\
66)\end{array}$ & $\begin{array}{l}74(72- \\
77)\end{array}$ \\
\hline $\operatorname{Sex}($ & $96(75 \%)$ & $8(73 \%)$ & $\begin{array}{l}17 \\
(74 \%)\end{array}$ & $\begin{array}{l}28 \\
(78 \%)\end{array}$ & $\begin{array}{l}37 \\
(77 \%)\end{array}$ & $6(60 \%)$ \\
\hline Prone & $59(46 \%)$ & $5(45 \%)$ & $8(35 \%)$ & $\begin{array}{l}17 \\
(47 \%)\end{array}$ & $\begin{array}{l}26 \\
(54 \%)\end{array}$ & $3(30 \%)$ \\
\hline ECMO support & $6(5 \%)$ & $2(18 \%)$ & $3(13 \%)$ & $1(3 \%)$ & $0(0 \%)$ & $0(0 \%)$ \\
\hline ICU discharge & $92(72 \%)$ & $8(73 \%)$ & $\begin{array}{l}20 \\
(87 \%)\end{array}$ & $\begin{array}{l}28 \\
(78 \%)\end{array}$ & $\begin{array}{l}32 \\
(67 \%)\end{array}$ & $4(40 \%)$ \\
\hline ICU m & $36(28 \%)$ & $3(27 \%)$ & $3(13 \%)$ & $8(22 \%)$ & $\begin{array}{l}16 \\
(33 \%)\end{array}$ & $6(60 \%)$ \\
\hline $\begin{array}{l}\text { ICU lenght of stay } \\
\text { (IQR) }\end{array}$ & $14(8-27)$ & $\begin{array}{l}13(3- \\
15)\end{array}$ & $\begin{array}{l}27(20- \\
38)\end{array}$ & $\begin{array}{l}17(10- \\
31)\end{array}$ & $\begin{array}{l}18(9- \\
30)\end{array}$ & $\begin{array}{l}11(8- \\
26)\end{array}$ \\
\hline
\end{tabular}

Conclusion: Prone positioning is part of the strategies available to intensive medicine for ARDS treatment in COVID-19 patients. During COVID-19 pandemic era, extensive use of prone positioning as rescue therapy can be applied using a specific protocol to support the staff decisions, limit the occurence of complications and avoid excessive nursing staff workload.

\section{3}

Perspectives of ICU patients and relatives on competencies for expert ICU nurses

C. Jones ${ }^{1}$; R. Endacott ${ }^{2}$; K. Iliopoulou ${ }^{3}$; M. Peskett ${ }^{4}$; P. Ramsay ${ }^{5}$

${ }^{1}$ Research, ICUsteps Peer Support Charity, London, United Kingdom;

${ }^{2}$ School of nursing and midwifery, University of Plymouth, Plymouth, 
United Kingdom; ${ }^{3}$ Nursing, King's College London, London, United Kingdom; ${ }^{4}$ None, ICUsteps Peer Support Charity, London, United Kingdom; ${ }^{5}$ School of nursing and health sciences, University of Dundee, Dundee, United Kingdom

Correspondence: $C$. Jones

Intensive Care Medicine Experimental 2020, 8(2): 000523

Introduction: One output from the INACTIC collaboration (1) is a set of core competencies for advanced practice ICU nurses across Europe. Some European countries, such as the UK (2), have identified such competencies, however these roles are rare across the rest of Europe. The INACTIC competencies were developed with an expert panel of 184 ICU nurses from 20 countries; however it is important to verify these with patients and family members with experience of intensive care.

Objectives: The aim of the study was to examine the views of recovered ICU patients and relatives regarding the INACTIC core competencies.

Methods: Three Focus Groups were conducted in the UK ( $n=12$ participants) and Greece ( $n=5$ participants) to discuss a lay version of the competencies.

Results: The feedback from across the focus groups confirmed the inclusion of statements about personal attributes, such as 'patient advocacy' or 'having the confidence to defend your opinion about a patients' care' and described this as the 'essence of the expert role'. There was a cultural difference with the Greek focus group: because of very restricted family visiting relatives felt encouraging their participation in patient care was not possible.

Conclusion: The perspectives of patients and family members largely aligned with the professional consensus of the expert panel, in particular related to personal attributes of the expert ICU nurse. Cultural differences in ICU experience highlight the changes that some ICUs would need to make for the competencies to be embedded.

Reference(s) and grant ackowledgment(s)

1. 1. https://www.esicm.org/education/inactic/

2. 2. The National Education and Competency Framework for Advanced Critical Care Nurses. March 2008. https://www.ficm.ac.uk/sites/default/files/ National\%20Education\%20\%26\%20Competence\%20Framework\%20for\%20 ACCPs.pdf accessed March 2020

3. Thank you to all the ICU patients and relatives who helped with the study. This study was co-funded by the Erasmus + programme of the European Union.

\section{8}

Family-centred care in Intensive Care Units: a cross-sectional survey in German-speaking countries in Europe

M. Brauchle'; P. Nydahl'; G. Pregartner ${ }^{3}$; M. Hoffmann ${ }^{4}$; MM. Jeitziner ${ }^{5}$ Department for Anesthesia and Intensive Care Medicine, Landeskrankenhaus Feldkirch, Feldkirch, Austria; ${ }^{2}$ Department of anaesthesiology and intensive care medicine, Universitätsklinikum Schleswig-Holstein, Campus Kiel, Kiel, Germany; ${ }^{3}$ Institute for medical informatics, statistics and documentation, Medical University of Graz, Graz, Austria; ${ }^{4}$ Division of endocrinology and diabetology/research unit for safety in health/ gm-rm, Medical University of Graz, University Hospital Graz, Graz, Austria; ${ }^{5}$ Department of intensive care medicine, University Hospital Bern, Bern, Switzerland

\section{Correspondence: $M$. Brauchle}

Intensive Care Medicine Experimental 2020, 8(2): 000588

Introduction: To provide insights into visiting policies of Intensive Care Units (ICUs), especially for children and adolescents, and familycentred care in German-speaking countries (Austria, Germany, Luxembourg and the German-speaking part of Switzerland).

Methods: Online survey with leading clinicians from all adult, agemixed, or paediatric ICUs from German-speaking countries $(n=1,943)$. Results: In total, maximal $19.8 \%(n=385)$ of clinicians answered the survey. Most respondents were physicians $(n=213,57.3 \%)$. Open visiting times were reported by $36.3 \%(n=117)$, with significant differences between paediatric ICUs (79.2\%), adult (21.3\%), and age-mixed ICUs $(41.2 \%)$ ICUs $(p<001)$. More than two thirds of clinicians stated that their ICUs had no age restrictions for children as visitors $(n=221$, $68.4 \%$ ). Most frequently implemented family-centred care activities were open visitation times and information' dissemination. Clinicians reported concerns about children's traumatization, risk of infection, and workload as major barriers. Arguments for family-centred care were evidence, education, and communication.

Conclusion: One third of participating clinicians reported providing open visiting policies in their ICUs. Two thirds of the respondents' ICUs allow children as visitors without age restrictions, with even higher proportions in paediatric ICUs. The majority of respondents reported to offer family-centred care in their ICUs, although they stated concerns about risks and debatable benefits.

\section{Reference(s) and grant ackowledgment(s)}

1. MB received a grant from the German Society of Critical Care Medicine to conduct this study. PN received a delirium-research grant from the German Society of Critical Care Medicine and Philips. All other authors report to have no conflicts. These institutions and companies were not responsible for or involved in designing, conducting, or analysing this study.

2. 1. Craft $M$, Cohen $M$, Titler $M$, Dehammer M.Experiences in childrenof critically ill parents: a time of emotional disruption and needfor support. Crit Care Nurs Q 1993;16:64-71. 2. Vandijck DM, Labeau SO, Geerinckx CE, De Puydt E, Bolders AC,Claes B, et al. An evaluation of family-centered care services and organization of visiting policies in Belgian intensive care units: a multicenter survey. Heart Lung 2010;39:137-46. 3. Knutsson S, Samuelsson I, Hellström AL, Bergbom I. Children's expe-riences of visiting a seriously ill/ injured relative on an adult intensive care unit. J Adv Nurs 2008;61:154—62. 4. Knutsson S, Bergbom I: Children's thoughts and feelings related to visiting critically ill relatives in an adult ICU: A qualitative study. Intensive Crit Care Nurs 2016; 32: 33-41 5. Olsen KD, Dysvik E, Hansen BS. The meaning of family members'presence during intensive care stay: a qualitative study. Intensive Crit Care Nurs 2009;25:190_8. 6. Kleinpell R, Heyland D, Lipman J, Sprung C, Levy M, et. al. Patient and family engagement in the ICU: Report from the task force of the World Federation of Societies of Intensive and Critical Care Medicine, Journal of Critical Care, 2018 vol: 48 pp: 251-256

\section{9}

The Development Of A Teaching Package For Airway Pressure Release Ventilation (APRV)

N. Paver ${ }^{1}$

${ }^{1}$ Intensive Care, Royal Devon and Exeter Hospital (Wonford), Exeter, United Kingdom

Correspondence: N. Paver

Intensive Care Medicine Experimental 2020, 8(2): 000589

Introduction: APRV is a pressure controlled mode of ventilation that delivers an almost continuous positive pressure with intermittent, time-cycled, short releases at a lower pressure while spontaneous breathing is encouraged. It is based on the open lung approach (Stock and Down 1987). Few UK ICUs have a consistent experience of APRV as it is primarily used as a rescue mode of ventilation where others modes have failed (Zhou et al 2017; Swindon et al 2020). On our ICU APRV use has increased over the past 5 years, with a significant increase since 2017.

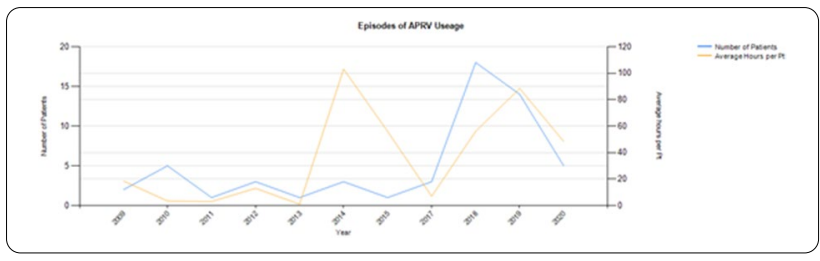

Critical care nurses play a vital role in the care and management of patients requiring mechanical ventilation. The Nursing and Midwifery 
code of conduct specifies nurses must work within the limits of their competence and that the necessary training should be completed before carrying out any new role. Our ICU currently has no teaching package or written guideline for the setup and management of APRV. Methods: A MDT working group was established to deliver the project. 5 ICU's around the region were surveyed and no unit had an APRV teaching package. A questionnaire regarding knowledge and confidence in the use of APRV was sent out to all members of nursing staff. A teaching package was designed starting with small pilots of teaching to a multi-disciplinary team on an adhoc basis. The teaching package will be cascaded via the Bath University Hospital Tea Trolley Teaching method (Hawkes 2018), enabling larger numbers of staff to be educated in a timelier manner. The efficacy of the teaching program will be assessed by a further questionnaire sent out after the teaching has been attended.

Results: The baseline questionnaire was sent to 77 members of staff and generated 46 responses (58.4\%). $91 \%$ of staff completing had cared for a patient receiving APRV and $66 \%$ of those report no prior knowledge of APRV. Of the 46 responses 45 expressed the need for education in the free text section. 20 members of staff received teaching as part of the pilot, achieving 14 post teaching questionnaire responses $(70 \%)$

How would you rate the content of the teaching package?

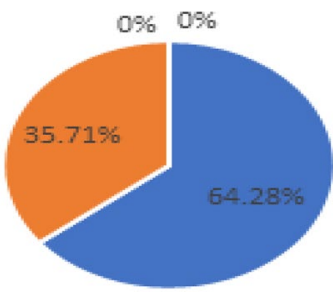

$$
\begin{aligned}
& \text { - Excellent } \\
& \text { - Good } \\
& \text { - Average } \\
& \text { - Poor }
\end{aligned}
$$

\section{Following the teaching would you be happy in caring for a patient receiving APRV?}

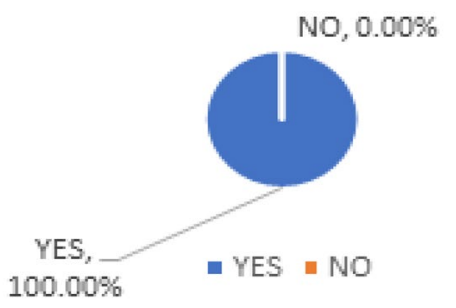

Conclusion: APRV use is increasing and the first survey showed a lack of knowledge or experience/confidence in its use. This has been addressed by the creation of a teaching package, and the pilot data has shown a positive outcome to both the delivery and content. Staff confidence/knowledge of APRV will be re-evaluated again once the teaching package has been delivered. The results of this will be available for presentation at the conference.

\section{Reference(s) and grant ackowledgment(s)}

1. All authors have declared no financial support was received from any organization for this submitted work

2. Zhou, Y., Jin, X., Lv, Y., Wang, P., Yang, Y., Liang, G., Wang, B. and Kang, Y., 2017. Early application of airway pressure release ventilation may reduce the duration of mechanical ventilation in acute respiratory distress syndrome. Intensive care medicine, 43(11), pp.1648-1659
3. Swindon J, Sampson C and Howaston A (2020) Airway Pressure Release Ventilation, BJA Education 20(3) 80-88

4. Stock, M.C., Downs, J.B. and Frolicher, D.A., 1987. Airway pressure release ventilation. Critical care medicine, 15(5), pp.462-466.

5. Nursing and Midwifery Council (2015) The Code: Professional standards of practice and behaviour for nurses, midwives and nursing associates, available at https://www.nmc.org.uk/

6. Joyce, M.F., Berg, S. and Bittner, E.A., 2017. Practical strategies for increasing efficiency and effectiveness in critical care education. World journal of critical care medicine, $6(1)$, p.1.

7. Hawkes, N., 2018. The BMJ Awards 2018: Education Team of the Year. BMJ, 361, p.k1519.

\section{5}

A National Evaluation of the Advanced Critical Care Practitioner Role within the United Kingdom

S. Diamond-Fox ${ }^{1}$; D. Waeland ${ }^{2}$; C. Boulanger²; J. Poynter²; S. Gardner²;

A. Platt ${ }^{3}$

${ }^{1}$ Periops \& critical care and faculty of health \& life sciences, Newcastle upon Tyne Hospitals NHS Foundation Trust \& Northumbria University, Newcastle upon Tyne, United Kingdom; ${ }^{2}$ Advanced critical care practitioner working group, The Faculty Of Intensive Care Medicine, London, United Kingdom; ${ }^{3}$ Department of nursing, midwifery and health, Northumbria University, Coach Lane Campus (East), Newcastle upon Tyne, United Kingdom

Correspondence: S. Diamond-Fox

Intensive Care Medicine Experimental 2020, 8(2): 000595

Introduction: Advanced Critical Care Practitioners (ACCPs) are now well integrated within critical care (CC) workforces across England, Scotland and Wales. As such, ACCPs have been acknowledged within landmark publications for their contribution to the delivery of CC services1-3. There have been numerous key initiatives that have advanced the development of the role, including national curriculums/pathways for training and continued professional development 4-6. Despite now being well embedded within the CC workforce, only local quality-assurance datasets that have evaluated the perceived effectiveness and/or safety perceptions surrounding the introduction of the ACCP role have been published7-10.

Objectives: The study had several objectives: 1) Evaluate perceptions surrounding the ACCP role within the CC workforce; 2) Assess the level at which current qualified ACCPs were perceived to be working towards the expectations of the role described by the Department of Health (DoH) and the Faculty of Intensive Care Medicine (FICM)4-5; 3) Evaluate whether the introduction of the ACCP role had any negative impact on Medical, Nursing and/or Allied Healthcare Professional (AHP) training; 4) Identify areas of concerns regarding interprofessional working and 5) Assess the scope for further development of the role.

Methods: Previous work conducted at a local level informed the objective and design of this study7. We designed and distributed an online anonymous electronic survey via OnlineSurveys.com to multiple centres within England, Scotland and Wales via multiple CC networks. A link to the survey was hosted on the FICM website. All professional groups that had experience of workingdirectly with ACCPs were eligible to take part in the survey. ACCPs were excluded from participating. Institutional ethical decision tools did not deem this project to require independent peer review.

Results: 591 clinicians responded to the survey during the 6-week period study period. The majority of respondents ( $n=150,25 \%$ ) were Medical Consultants, followed closely by Staff Nurses ( $n=141$, 23.9\%). Respondents ranged across all regions in which ACCPs are currently known to be in post. Results demonstrated overall positive outcomes for all objectives (Fig. 1). Qualitative analysis of free text data is ongoing, however initial thematic analysis identified several themes; improved workforce stability, enhanced patient safety, variations in level of training, deskill in other members of the workforce, limited career progression. 


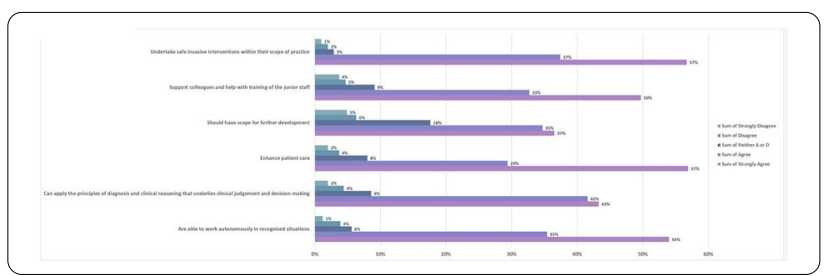

Conclusion: The ACCP role continues to develop across England, Scotland and Wales. This survey has identified that overall respondents perceive the introduction of the ACCP role to be positive and that current qualified ACCPs meet the expectations set by the DoH and the FICM, and should be given scope to develop their roles further. However, a number of areas have been identified amongst respondents which warrants further exploration to inform effective and sustainable future development of the ACCP role.

\section{Reference(s) and grant ackowledgment(s)}

1. 3. The Faculty of Intensive Care Medicine and The Intensive Care Society (2019) Guidelines for the Provision of Intensive Care Services - edition 2. Available at: https://ics.ac.uk/ICS/ICS/Pdfs/GPICS_2nd_Edition.aspx

2. 7. Thomas I \& Colling S (2019) A Quality Assurance Survey to Evaluate the Perceptions of the Multi-Disciplinary Team on the Role of the Advanced Critical Care Practitioner (ACCP). Intensive Care Society State of the Art 2017 Abstracts. (2018). Journal of the Intensive Care Society, 19(2_suppl), 1-162. https://doi.org/10.1177/1751143718772957

3. 8. Denton G, Green L, Palmer M, et al. The provision of central venous access, transfer of critically ill patients and advanced airway management.: Are advanced critical care practitioners safe and effective? Journal of Intensive Care Medicine 2018; Oct 20;(3):248-254

4. 9. Williams C, Bennett E \& Bromage N. Advanced Critical Care Practitioners - Practical experience of implementing the Advanced Critical Care Practitioner Faculty of Intensive Care Medicine Curriculum in a London Critical Care Unit: Response letter. Journal of the Intensive Care Society 2019; Feb 11.

5. 10. Manning N, Beard L, Snelson C, Sachdeva R, Whitehouse T, Knowles M, Smith F G \& Veenith T. Impact of Advanced Critical Care Practitioner led analgesia service in Critical Care Unit patients after multiple rib fractures. Intensive Care Society State of the Art 2019 Abstracts. Journal of the Intensive Care Society. 2020;21(2_suppl):1-225. doi:10.1177/1751143720915029

6. 6. The Faculty of Intensive Care Medicine (2020) Advanced Critical Care Practitioners CPD \& Appraisal Pathway v2.2 2020 - https://www.ficm. ac.uk/accps/cpd-and-appraisal

7. 5. The Faculty of Intensive Care Medicine (2015) Curriculum for Training Advanced Critical Care Practitioners. Available at: https://www.ficm. ac.uk/accps/curriculum

8. 4. Department of Health (2008) The National Education and Competence Framework for Advanced Critical Care Practitioners. Available at: www.ficm.ac.uk\%2Fsites\%2Fdefault\%2Ffiles\%2FNational\%2520Educati on\% $2520 \% 2526 \% 2520$ Competence $\% 252$ Framework\% 2520 for $\% 2520$ ACCPs.pdf\&usg=AOvVaw1vYNwK3iUEiP-4ybZzK2LN

9. 2. The Faculty of Intensive Care Medicine and The Intensive Care Society (2015) Guidelines for the Provision of Intensive Care Services - edition 1. Available at: https://www.ficm.ac.uk/standards-research-revalidation/ guidelines-provision-intensive-care-services-v2

10. 1. The Faculty of Intensive Care Medicine and The Intensive Care Society (2013) Core Standards for Intensive Care Units. Available at: www.ficm.ac.uk\%2Fsites\%2Fdefault\%2Ffiles\%2FCore \%2520Standards\%2520for\%2520ICUs\%2520Ed.1\%2520(2013). pdf\&usg=AOvVaw3sRkD4Cm-XwGJoPCu6XnFp
000737

BUILDING AND SUPPORTING A TRAINEE NURSE PRACTITIONER PROGRAM. Grace Banks and Charlotte Tivy-Jones Great Ormond Street Hospital, Cardiac Intensive Care, Advanced Clinical Practitioners

B. Grace ${ }^{1}$

${ }^{1}$ Cardiac Intensive Care, Great Ormond Street Hospital, London, United Kingdom

Correspondence: $\mathrm{B}$. Grace

Intensive Care Medicine Experimental 2020, 8(2): 000737

Introduction: The success of advanced nurse practitioners in intensive care is widely documented. Advanced Clinical practitioners (ACP) are highly skilled, experienced and competent practitioners. In Intensive Care (ICU) there is not only a need for university qualification and achievment but a specialist training program specific to ICU to run alongside. The ACPs on this $\mathrm{CICU}$ have set up an innovative program for training; obtaining and documenting competency and supporting trainee nurse practitioners. The 'specialist core training' program. This program runs paralel to university education, complimenting learning but focusing on core ICU skills and development. Core training can be individualised to learner needs but has central achievment stages throughout the 3 year training period. This specialist core training includes; speciality skills and education, involvment in providing MDT teaching, leadership skills, research focus groups and competency/ pathway document. The aim is to enhance care delivery, specialise ICU training, complement foraml university training, cover the 4 pillars of ACP and support/improve the transition from training into ACP roles. Ultimatly the goal, on completion of this specialist program, is a trainee who can function successfully as an ACP on a medical rotation, initiate and lead change projects, be innovative and forward focused.

Objectives: The aim of this PICU specialty core training is to compliment general ACP training and allow PICU ACPs to have a sound foundation, knowledge and competence in their chosen specialist area. Our hypothesis is that the trainee experience, multidisciplinary team (MDT) experience and patient care and safety will be enhanced. We would consider this comprehensive program to be reproducible within other PICU environments.

Methods: PICU requires specialist knowledge and competence these skills can be taught alongside ACP training. Developing PICU specialist core training has required MDT collaboration including; trainee ACPs, anaesthetic consultants, ICU consultants, established ACPs and consideration of national guidance. We have developed a comprehensive program that runs alongside the formal ACP program that will map career progression from induction through to qualification. Thus providing a transparent vision/expectation as to how their career should progress.

Results: The project has been very successful. Feedback from current trainee ACPs highlights that this more robust program will help with training into such specialty ACP roles. The program covers 4 pillars of ACP, specialist skills, specialist education, MDT learning and clinical leadership and managment training.

Trainee Feedback 'The specialist training I would say is extensive, we have to be able to prove we can make reliable and safe judgements, planning our own caseloads and are also able to adapt swiftly to changing situations.'

Conclusion: This program has enhanced the training our trainee ACPs recieve and supports the formal university learning. Has already enhanced the trainee ACP, MDT and patient experience. We would like to consider a national application for this program across UK PICU. 
Reference(s) and grant ackowledgment(s)

1. Brown, A., Christie, L., Higgerson, R., Mikhailov, T., Stojadinovic, B. and Forbes, M. (2012) A national survey of PICU nurse practitioners - opportunities to standardize practices and optimize service delivery. Pediatric Nursing. 28 (5), pp. 249-250. Crowe, S. (2014) A role for nursepractitioners in the ICU: Advocating for change. Canadian Association Of Critical Care Nursing, 25 (3), pp. 26- 29. Davies, J., Bickell, F. and Tibby, S. (2010) Attitudes of paediatric intensive care nurses to development of a nurse practitioner role for critical care transport. Journal Of Advanced Nursing, 67 (2), pp. 317-326. Krista, J., Haatainen, K., Meretoja, R. and Pietila, A. (2014) Advanced practice nursing roles: the phases of the successful role implementation process. International Journal of Caring Sciences, 7 (3), pp. 946-954

\section{7}

\section{Structural dimensions of the training needs of intensive care} nurses

Y.G. Santana-Padilla'; M.D. Bernat-Adell²; L. Santana-Cabrera';

T. Linares-Pérez ${ }^{3}$

${ }^{1}$ Intensive care unit, Maternal and child Hospital, Las Palmas de Gran Canaria, Spain; ${ }^{2}$ Nursing department, Jaume I University, Castelló de la Plana, Spain; ${ }^{3}$ Cs cueva torres, Gerencia de Atención primaria de Gran Canaria, Las Palmas de Gran Canarias, Spain

Correspondence: Y.G. Santana-Padilla

Intensive Care Medicine Experimental 2020, 8(2): 000807

Introduction: Intensive care nurses develop specialized and specific care for which extensive and continuous training is necessary(1).Several scientific associations have developed different tools to standardize minimum training requirements. The European Federation of Critical Care association has developed a series of areas where the different competences of nurses are developed, these areas are: clinical, professional, management, educational and development (2).These competences, as different authors name, must be developed through specific training that must focus on the own needs of nurses throughout their professional continuum and the healthcare organization requires (3).

Objectives: Identify the different dimensions focused on the training needs of intensive care nurses in Spain and the nurses' perception of them.

Methods: Descriptive cross-sectional study by means of an electronic questionnaire to 84 intensive care units. The questionnaire was previously validated by a brain trust. The survey consisted of 66 items using a Likert scale from 1 to 10 . Statistical analysis was performed by exploratory factor analysis (EFA) through polychoric correlations with the Factor Analysis 10.9.02 program and inferential statistics with the spss 20.0 program.

Results: The sample consisted of 568 nurses. The EFA determined 13 dimensions of care, which explains a total percentage of explained variance of 64,219 and a Cronbach's alpha of 0.934 . The dimensions detected were: Skills in critical patient care, Communication and clinical safety, Nursing knowledge and clinical reasoning, Reception plans for novice nurses, Specific and continuous training of staff nurses, Excellence in care, Evaluation of critical patient, tools and technology, health management, application of support measures, decision-making and coping at the end of life, measures to improve care, motivation to continue training, impact of training.

Significant differences were found in the assessment of these training needs among the nurses with the longest years of experience as a nurse $(p=0.004)$ as an ICU nurse $(p=0.021)$, the nurses with official postgraduate degree $(p=0.001)$ and the rest of the nurses.

Nurses with a higher academic degree and greater work experience as nurses value more positively the training needs and the need to have previous training requirements as the Jonckheere tepstra test ( $p=$ $0.035)$ and Chi-Square $(p=0.016)$ demonstrate.

Nurses from smaller hospital also value more positively 6 of the dimensions detected with $\mathrm{p}$ values between $0.002-0.036$ compared to ICU nurses from large centers (> 500 beds).

Conclusion: The perception of the training needs of nurses are influenced by intrinsic and extrinsic factors. Among the intrinsic factors the following stand out: the total work experience and in the ICU as a nurses and their own academic degree. Within external factors, the size of the hospital center influences more than the size of the intensive care unit itself.

\section{Reference(s) and grant ackowledgment(s)}

1. 3. Linn AC, Caregnato RCA, Souza EN de. Clinical simulation in nursing education in intensive therapy: an integrative review. Rev Bras Enferm. 2019;72(4):1061-70. Disponible en: 10.1590/0034-7167-2018-0217

2. 2. Waters D, Kokko A, Strunk H, Georgiou E, Hadjibalassi M, Satosek D, et al. EfCCNa Competencies for European Critical Care Nurses. 2013.

3. 1. Lakanmaa R. Competence in intensive and Critical Care Nursing - development of a basic assessment scale for graduatinn nursing students. Turku, Finland; 2012

4. The study has the approval of the research committee of the Jaume I University.

5. This study has been self-financed by the principal investigator. No subventions or donations have been received.

\section{0}

Multi-infusion with integrated multiple pressure sensing allows earlier detection of line occlusions

F. Doesburg ${ }^{1}$; R. Oelen ${ }^{1}$; J. Xu²; M. Renes ${ }^{1}$; M. Nijsten ${ }^{1}$

${ }^{1}$ Department of critical care, University Medical Center Groningen, Groningen, Netherlands; ${ }^{2}$ Institute of engineering, Hanze University Groningen, Groningen, Netherlands

Correspondence: F. Doesburg

Intensive Care Medicine Experimental 2020, 8(2): 000810

Introduction: Occlusions of intravenous (IV) tubing can prevent vital and time-critical medication or solutions from being delivered into the bloodstream of patients receiving IV therapy. At low flow rates $(\leq 1$ $\mathrm{ml} / \mathrm{h}$ ) the alarm delay (time to an alert to the user) can be up to 2 hours using conventional pressure threshold algorithms. In order to reduce alarm delays we developed and evaluated the performance of three new real-time occlusion detection algorithms that exploit the trend in pressure changes.

Methods: Bench-tested experimental runs were recorded in triplicate at rates of $1,2,4,8,16$, and $32 \mathrm{ml} / \mathrm{h}$. Each run consisted of 10 minutes of non-occluded infusion followed by a period of occluded infusion of 10 minutes or until an occlusion alarm. The first algorithm based on binary logistic regression tried to detect occlusions based on the pump's administration rate $(\mathrm{Q}(\mathrm{t}))$ and pressure sensor readings $(\mathrm{P}(\mathrm{t}))$. The second algorithm continuously checked whether the actual variation in the pressure (P-SD-actual) exceeded a threshold of 2 standard deviations (SD) above the baseline pressure. When a pump detected an occlusion using either the regression or SD algorithm, a third algorithm correlated the pressures of multiple pumps to detect the presence of a shared occlusion. The algorithms were evaluated using the six bench-tested single-pump occlusion scenarios and seven multipump co-occlusion scenarios (i.e. with flow rates of $1+1,1+2,1+4$ $1+8,1+16$, and $1+32 \mathrm{ml} / \mathrm{h}$ respectively). Alarm delay (minutes) was the primary performance measures.

Results: In the single-pump occlusion scenarios the overall mean $\pm S D$ alarm delay of the regression and SD algorithms were $(1.8 \pm 0.8 \mathrm{~min})$ and $(0.4 \pm 0.2 \mathrm{~min})$, respectively. Compared to our local alarm threshold of $400 \mathrm{mmHg}(7.4 \pm 7.5 \mathrm{~min})$ this corresponds to a mean time reduction of $52 \pm 34 \%(p=0.003)$ and $90 \pm 6 \%(p=0.001)$, respectively. In the multi-pump scenarios a correlation $>0.8$ between multiple pump pressures after initial occlusion detection by the regression or $\mathrm{SD}$ algorithms had a mean \pm SD alarm delay of $0.4 \pm 0.2 \mathrm{~min}$. No occlusions were missed in the single-pump and multi-pump scenarios by any of the algorithms.

Conclusion: Both the regression and SD algorithms were able to significantly reduce alarm delays compared to conventional pressure limit based detection. Coupled with a correlation algorithm co-occlusions could reliably be detected far more rapidly than with the conventional technique. The latter may be used to localize the segment of tubing in which the occlusion occurs. 


\section{6}

Mobilization in the evening to prevent and treat delirium in critically ill patients: the MENTAL random-ized, controlled trial P. Nydahl'; D. McWilliams'; N. Weiler ${ }^{3}$; C. Borzikowsky ${ }^{4}$; F. Howroyd²; A. Brobeil ${ }^{5}$; R. Von Haken ${ }^{6}$; M. Kott ${ }^{7}$

${ }^{1}$ Nursing Research, Universitätsklinikum Schleswig-Holstein, Campus Kiel, Kiel, Germany; ${ }^{2}$ Critical care rehab team, Queen Elizabeth Hospital Birmingham, Birmingham, United Kingdom; ${ }^{3}$ Klinik für anästhesiologie und operative intensivmedizin, Universitätsklinikum Schleswig-Holstein, Campus Kiel, Kiel, Germany; ${ }^{4}$ Institute of medical informatics and statistics, Universitätsklinikum Schleswig-Holstein, Campus Kiel, Kiel, Germany; ${ }^{5}$ Department of critical care, Heidelberg University, Heidelberg, Germany; ${ }^{6}$ Department of anaesthesiology, Mannheim University Hospital, Mannheim, Germany; ${ }^{7}$ Department of anaesthesiology and intensive care medicine, Universitätsklinikum Schleswig-Holstein, Campus Kiel, Kiel, Germany

Correspondence: P. Nydahl

Intensive Care Medicine Experimental 2020, 8(2): 000816

Introduction: Delirium is a common complication in patients on Intensive Care Units (ICU). Intervetions such as mobilization are effective in prevention and treatment of delirium, but recommended during daytime.

Objectives: The objective of this study was to prove the effect of mobilization in the evening to prevent and reat ICU patients from delirium

Methods: The design was a multicentric, randomized, controlled trial in 5 mixed ICUs over a period of 2 weeks. Patients in the interventiongroup were mobilized onto the edge of the bed or more between 9:00-11:00 p.m. Patients in the control group received usual care. Primary outcome parameter was the duration of a delirium. Secondary parameters were delirium incidence, mortality, duration of mechanical ventilation (MV) and stay for 28 days follow-up, feasibility, and safety

Results: In summary, $\mathrm{n}=69$ patients could be analysed (intervention $\mathrm{n}=36$, control $\mathrm{n}=33$ ). Mobilization in the evening did reduce duration of existing delirium (Median (Interquartil Range) 1.5 (1-2.7) vs. 2 (1-2) days, albeit not statistically significant. Length of MV or days in ICU and hospital, and mortality were not significantly different. In an unplanned post-hoc analysis, mobilization in the evening prevented patients from delirium in the first four days ( $n=6(16.7 \%)$ vs. $n=13$ (39.4\%), $p=0.032$ ), with a number needed to treat of 5 . The intervention was feasible in $71.7 \%$ of eligible patients, with $13.6 \%$ mobilization-related safety events.

Conclusion: In a mixed ICU population, mobilization in the evening did not show a reduction in duration of delirium, MV or length of stay, but a possible reduction in the incidence of early onset delirium.

\section{Reference(s) and grant ackowledgment(s)}

1. Haley MN, Casey P, Kane RY, Darzins P, Lawler K. Delirium management: Let's get physical? A systematic review and meta-analysis. Australasian journal on ageing. 2019.

2. McWilliams D, Jones C, Atkins G, Hodson J, Whitehouse T, Veenith T, et al. Earlier and enhanced rehabilitation of mechanically ventilated patients in critical care: A feasibility randomised controlled trial. Journal of Critical Care. 2018:3(44):407-12

3. Schaller SJ, Anstey M, Blobner M, Edrich T, Grabitz SD, Gradwohl-Matis I, et al. Early, goal-directed mobilisation in the surgical intensive care unit: a randomised controlled trial. Lancet. 2016;388(10052):1377-88.

4. American-Psychiatric-Association, editor. Diagnostic and Statistical Manual of Mental Disorders. Fifth Edition ed: American Psychiatric Association, Arlington; 2013.

5. Devlin JW, Skrobik Y, Gelinas C, Needham DM, Slooter AJC, Pandharipande PP, et al. Clinical Practice Guidelines for the Prevention and Management of Pain, Agitation/Sedation, Delirium, Immobility, and Sleep Disruption in Adult Patients in the ICU. Crit Care Med. 2018;46(9):e825-e73.

6. This is work is funded by the Delirium Research Prize of the German Interdisciplinary Association for Intensive Care and Emergency Medicine (DIVI) and the Philips company. Both entities did not have any influence in designing, conducting, analysing, interpreting, or writing the study.
000829

Identification of the training needs of ICU nurses through a Delphi technique

Y.G. Santana-Padilla'; M.D. Bernat-Adell ${ }^{2}$; L. Santana-Cabrera ${ }^{1}$

T. Linares-Pérez ${ }^{3}$; R.F. Acosta-Rodriguez ${ }^{4}$; J. Alemán-González

${ }^{1}$ Intensive care unit, Maternal and child Hospital, Las Palmas de Gran Canaria, Spain; ${ }^{2}$ Nursing department, Jaume I University, Castelló de la Plana, Spain; ${ }^{3}$ Cs cueva torres, Gerencia de Atención primaria de Gran Canaria, Las Palmas de Gran Canarias, Spain; ${ }^{4}$ Surgery ward, Maternal and child Hospital, Las Palmas de Gran Canaria, Spain

Correspondence: Y.G. Santana-Padilla

Intensive Care Medicine Experimental 2020, 8(2): 000829

Introduction: The perspective of training needs is an element focused on daily clinical practice.The difficulties and opportunities detected in the day to day can be useful to assess and evaluate which elements of improvement are applicable.lt is necessary to extract the experiences of nursing professionals in a field as specific as intensive care, to later develop elements that can help us generate training that provides safe and quality care by ICU nurses.

Objectives: Generate a questionnaire to analyze the training needs of nurses in the ICU.

Methods: Delphi technique modified with an extensive semi-structured pre-interview based on the recommendations of the European Federation of critical care nurse association and digitally recorded. The panel of experts consisted of 15 nurses with three or more years of experience as an ICU nurse in a polyvalent adult unit of the Complejo Hospitalario Insular Materno-Infantil. It took three rounds for the expert group to reach a consensus.

The scale used for its evaluation was a Likert of 10 points, where 1 were always unimportant values and 10 were very important values.Items with a mean score greater than or equal to 6.1 and a median greater than 7 were included.The diffusion of the questionnaire was telematic with a period of 15 days for each of the phases.

Statistical analysis was performed using the SPSS statistical program version 20.0

Results: In the preparatory round, 92 questions were developed by the research team from the interviews previously carried out with the expert panel.

In addition, text-type comments were allowed to assess the questions asked by the experts.

In the first phase, the 92 questions were sent and due to the experts' assessment, 14 items were withdrawn, leaving the assessment document at 78 items. In the second phase the 78 items were sent, and the text comments made in phase 1 . In this second phase, text comments were not received on the questions or related to their drafting.

The questionnaire with 66 questions was forwarded for evaluation in the third phase.This questionnaire was distributed as follows: clinical setting ( 18 items), professional setting ( 14 items), management setting (16 items), educational and development setting (6 items), and training activities ( 12 items).

The third round was evaluated by experts and subsequently analyzed to assess the generated construct.The reliability of the scale was assessed by Cronbach's alpha, whose result was 0.908

Conclusion: The questionnaire generated following the steps of the Delphi technique allows us to reliably explore the training needs of nurses in the ICU. The experts included a large amount of care distributed throughout the questionnaire. The expertsespecially included questions aimed at maintaining a continuous and permanent training program

\section{Reference(s) and grant ackowledgment(s)}

1. Landeta J, Barrutia J, Lertxundi A. Hybrid Delphi: A methodology to facilitate contribution from experts in professional contexts. Technol Forecast Soc Change. 2011;78(9):1629-41. Available in: 10.1016/j.techfore.2011.03.009

2. Keeney S, Hasson F, McKenna H. The Delphi Technique in Nursing and Health Research. John Wiley \& Sons Ltd, editor. Oxford: Wiley/Blackwell (10.1111); 2011.198 p. Available in: 10.1002/9781444392029

3. Santana-Padilla YG, Santana-Cabrera L, Bernat-Adell MD, Linares-Pérez T, Alemán-González J, Acosta-Rodríguez RF. Training needs detected by 
nurses in an intensive care unit: a phenomenological study. Enferm Intensiva. 2019;30(4):181-91. Available in: 10.1016/j.enfi.2019.05.001

4. The study has the approval of the Ethics and Research Committee of the Jaume I University.

5. The study was self-funded by the principal investigator

\section{3}

Continuity of care for ICU patients'

M. Ashkenazy ${ }^{1}$

${ }^{1}$ Icu, Hadassah Medical Center, Hebrew university, Jerusalem, Israel

Correspondence: S. Ashkenazy

Intensive Care Medicine Experimental 2020, 8(2): 000863

Introduction: The recovery from prolonged Intensive Care Unit (ICU) hospitalization involves physical and psychological sequela that influence patients' recovery from complicated illness. Patients and families deal with the transition from high dependency care of the ICU to a less intense environment of the regular ward where nurses cope with multiple complicated patients. A new function was suggested and implemented, dealing with the need for continuity of care for such patients. The function, "Follow up ICU" was implemented in various hospitals, each including varying specific roles and responsibilities. I discuss the implementation of immediate intrinsic follow up (during hospitalization) in Israel, addressing specific characteristics of the unique hospital environment and role distribution between the different wards. Special attention was given to patient-family centered care for the post ICU patient. Utilization and benefits were evaluated using surveys with medical stuff

Objectives: Evaluate utility and gain from establishing intrinsic ICU follow up in an Israeli hospital, as implemented by a dedicated nursing function assisted with other medical services.

Methods: During 2019 - 2020 follow-up nursing was implemented, with additional medical support functions. A survey comparing pre and post-prior assessment of the quality of care by ward nurses was conducted. services in hospital adopting the patient-family centered care for improving recovery from acute illness.

Results: ICU follow-up team, led by a nurse and assisted by a dietician, social worker, physiotherapist, and a physician created a patient-specific plan of care. The plan was implemented starting before releasing from ICU and continuing as the patient was transferred to the ward. During 2019 the process was implemented in support of 200 patients. A mean per-patient number of post ICU hospitalization was $3.5 .44 \%$ of the visits involved respiratory assessments, 34\% involved mobility and wound care, and $39 \%$ post ICU syndrome caregiver-patient conversations. Survey with ward nurses indicated mainly that: post-ICU follow up support led to improved critical thinking and improved quality of care for post-ICU patients, as special attention was dedicated to unique treatment aspects. Dedicated guidance and support for the ward nurses led to significant improvement of specific needed nursing skills which in turn assist in increased quality of care. Two prominent examples include: care for tracheostomy patients and dealing with post-ICU syndrome. Overall $90 \%$ of interviews indicated high benefit due to improved communication between the ICU unit and the ward.

Conclusion: Following the establishment of an intrinsic post-ICU followup unit quality of care as evaluated by ward nurses improved significantly.

\section{Reference(s) and grant ackowledgment(s)}

1. Meyer, J., Brett, S.J. \& Waldmann, C., (2018). Should ICU Clinicians follow patients after ICU discharge? Yes. Intensive Care Medicine. https ://doi. org/10.1007/s00134- 018-5117-9

2. Haraldsson, H., Christensson, L., Conlon, L., (2015).The experience of ICU patients during follow up sessions - A qualitative study. Intensive and Critical Care Nursing. 31, 223-231.
000880

Preliminary findings from a qualitative study of nurses'

experiences towards physical restraint use in Intensive Care Unit

S. Mattiussi ${ }^{1}$; L. Ndroqi ${ }^{1}$; D. Caruzzo ${ }^{1}$; M. Danielis ${ }^{1}$

${ }^{1}$ Department of medical sciences, University of Udine, Udine, Italy

Correspondence: $M$. Danielis

Intensive Care Medicine Experimental 2020, 8(2): 000880

Introduction: The application of physical restraints remains a common and unchallenged practice in the Intensive Care Units (ICUs) in many countries worldwide. However, if on the one hand nurses consider physical restraints as an unavoidable practice in ICU, on the other some physical adverse effects, such as skin breakdown and functional decline, and psychological outcomes, such as delirium and post-traumatic stress disorders, have been documented. To date, there is little understanding of physical restraint use from the perspective of ICU nurses in Italy.

Objectives: To assess the knowledge, the attitudes, and the practice of intensive care nurses about physical restraints use.

Methods: A qualitative study following a phenomenological approach was carried out between September and October 2019. Semi-structured face-to-face and audio-recorded interviews were carried out in three general Italian ICUs. All interviews were audio-recorded and transcribed verbatim. Each interview lasted approximately 20 minutes. Data were analysed with a content analysis method.

Results: 11 nurses were interviewed, mainly female $(n=6,63.6 \%)$ and with an average age of 36 years. A preliminary data analysis produced 53 initial categories, 11 subthemes, and 3 main themes. The main themes included: a) The decision-making process (patients' characteristics, organizational features, decision maker's role, and nursesspecific factors); b) The use of physical restraints (rationale, methods, outcomes, and alternative approaches); and c) The experiences of nurses (nurses' lived experiences, and ethical dilemmas).

Conclusion: According to the findings, the antecedent for an almost routine use of physical restraints in ICUs is the need to ensure patient safety. Although known by nurses, the alternative approaches to limit this practice were not implemented.

\section{Reference(s) and grant ackowledgment(s)}

1. Perez, D., Peters, K., Wilkes, L., \& Murphy, G. (2019). Physical restraints in

intensive care-An integrative review. Australian critical care, 32(2), 165-174.

\section{4}

The rapid response and utilisation of the advanced critical care practitioner group in a large critical care service based at the epicentre of the UK COVID 19 outbreak

S. Sears-Aizenberg1; I. Salako1; K. Latchem¹; N. Gardner'; N. Lushpay ${ }^{1}$;

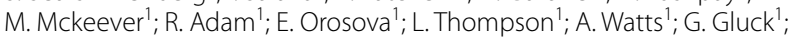

N. Gray'; A. Czapran ${ }^{1} ;$ T. Best ${ }^{1}$; T. Hurst ${ }^{1}$; P. Hopkins ${ }^{2}$

${ }^{1}$ King's critical care, King's College Hospital, London, United Kingdom;

${ }^{2}$ Critical Care, King's College Hospital, London, United Kingdom

Correspondence: P. Hopkins

Intensive Care Medicine Experimental 2020, 8(2): 000904

Introduction: King's College Hospital in South East London was one of the acute hospitals most affected by the SARS-CoV2 pandemic. The institution treated more than 2000 patients with COVID 19 pneumonitis, 192 of whom required a critical care admission. The hospital expanded its critical care capacity from 69 to 196 ICU beds during the peak of the outbreak and was one the busiest critical care services in the UK with COVID 19 critical illness. Patients in our institution also had high severity COVID 19 due to the high prevalence of acute renal failure in $50 \%$ of our ICU admissions.

A key component of the response of King's Critical Care was provided by the Advanced Critical Care Practitioners (ACCPs). The ACCP program, within King's Critical Care, was launched in 2017 using the Faculty of Intensive Care Medicine curriculum delivered over two years (FICM, 2008). This first cohort consisted of seven intensive care nurses, 
two critical care paramedics, and two intensive care physiotherapists, with a combined total of over 100 years of intensive care unit (ICU) experience. 2 ACCPs were provided on each 12 hour shift. Here, we assess the contribution, performance and effectiveness of this team to the COVID 19 response through use of the Critical Care Informatics System and database. Specifically, the contribution of ACCPs to invasive interventions, proning (Gattinoni, 2020) and intra-hospital transfer were examined.

Objectives: To assess the utilisation and effectiveness of the ACCP group during the COVID 19 pandemic in a critical care service at the epicentre of the United Kingdom Outbreak.

Methods: Appropriate service improvement approvals were gained. The ICCA (Intellivue Critical Care \& Anaesthesia, Philips HealthCare) database was queried for ACCP interventions (lines, intubations, proning procedures) during the COVID 19 pandemic. (Microsoft SQL Server Management Studio 17). The ICCA complications audit table was also queried. The ACCP active directory role was queried to ensure anonimity of healthcare professionals and no patient identifiers are reported.

Results: Within the ICCA dataset, the ACCP group were involved in the assessment and management of 177/192 patients admitted with COVID 19. They performed 86 central lines; 74 arterial lines; 29 vascaths and assisted 59 intubations across the Emergency Department; Critical Care and acute medical wards within our institution during the pandemic response. Additionally the ACCP group were the main providers of PICC line insertion. No immediate adverse events were reported. The ACCP group were tasked with the challenge of setting up and delivering a prone service for patients with oxygenation failure due to ARDS. Throughout their training they had been signed off as airway competent and able to perform stable intubated transfers, empowering them to lead and orchestrate in total 64 prone positions with only 2 adverse events and zero events leading to immediate patient harm. The ACCP team independently performed 56 intra-hospital patient transfers, with only two adverse events and no direct patient harm. The interventional procedures performed by ACCPs had less complications recorded than those performed by physicians and higher documentation compliance (eg seldinger wire out confirmation/use of check-lists). Finally, the ACCPs played a key role in developing and delivering simulation training for the resuscitation (including rapid-sequence intubation, transfer and proning) of critically ill patients with COVID 19.

Conclusion: The 2020 COVID 19 pandemic confirmed the critical role that the ACCP group play within the critical care service at our institution. The inherent strengths of the ACCP team, such as geopolitical awareness; awareness of protocols; a willingness to follow protocols; longevity as a team (no rotation); high technical skills led to the ability of this team to rapidly adapt at a time of unprecedented operational and clinical strain. Specifically, the ACCP group were able to bridge the communications between strained medical and nursing teams, enabling structured and systematic task-based management of patients with severe COVID 19 illness in very difficult clinical settings with ad hoc organ support systems and critical supply chain pressures. Further work is planned, to use these operational and clinical observations from the ACCP response during the COVID 19 pandemic, to further develop this service. One key goal will be to explore independent working from ACCPs.

\section{Reference(s) and grant ackowledgment(s)}

1. Gattinoni L, Chiumello D, Caironi P, Busana M, Romitti F, Brazzi L, et al. COVID-19 pneumonia: different respiratory treatments for different phenotypes? Intensive Care Med. 2020 Jun:46(6):1099-102.

2. The National Education and Competence Framework for Advanced Critical Care Practitioners. :61. 2008. Faculty Of Intensive Care Medicin

\section{2}

\section{Early tracheostomies in COVID-19 patients: A retrospective case} review of early rehabilitation effect on modified CPAx score

C. Hellyarr'; E. Clibbens ${ }^{2}$; K. Dalby ${ }^{3}$

${ }^{1}$ Anaesthetics, Princess Alexandra Hospital, Harlow, United Kingdom; ${ }^{2}$ Physiotherapy, Princess Alexandra Hospital, Essex, United Kingdom; ${ }^{3}$ Physiotherapy, Princess Alexandra Hospital, Harlow, United Kingdom
Correspondence: $\mathrm{C}$. Hellyar

Intensive Care Medicine Experimental 2020, 8(2): 000912

Introduction: COVID-19 can cause acute critical illness requiring intubation and mechanical ventilation. Early rehabilitation in the Intensive care unit (ICU) may prevent some complications of critical illness such as ICU-Acquired Weakness (ICU-AW) and may lead to improved functional status[1]. In the United Kingdom (UK), tracheostomy for patients with COVID-19, is recommended to be considered 10-14 days after tracheal intubation[2]. However, earlier tracheostomy may be associated with reduced sedation, early rehabilitation and therefore improved functional outcomes[3].

Objectives: To assess whether rehabilitation outcomes were affected by early tracheostomy in patients with COVID-19 admitted to an ICU.

Methods: A retrospective review of all adults admitted in a single centre district general ICU in the UK with a diagnosis of COVID-19 who were mechanically ventilated and had a tracheostomy. Patients were excluded who were transferred to other institutions for ICU capacity reasons or who died during their ICU admission.

Data was collected from patient records and physical morbidity measured using a modified Chelsea Critical Care Physical Assessment Tool (CPAx) score[4]. This was calculated for each patient at the time of tracheostomy, when active rehabilitation commenced and on discharge to the ward and home.

A 2-tailed Mann-Whitney $U$ test statistic assuming an alpha value of 0.05 was then calculated and data for tracheostomies performed before and after 7 days compared.

Results: Tracheostomies were performed on 11 mechanically ventilated patients within a median 5 days (IQR range 3.5-8.5). Median time to start active rehabilitation was 4 days (3.5-8.5) after tracheostomy. The median modified CPAx score on the first day of active rehabilitation was 9/45 (8.5-10.5); on step-down to a ward it was 28/45 (15-35); and on discharge it was 45/45 (44-45). 10 patients (91\%) were discharged from hospital directly to their home.

When comparing tracheostomies performed before and after 7 days, there was a statistically significant difference $(p<0.05)$ in: length of critical care admission (median 17 vs 31 days); days on sedation (median 5 vs 21.5 days); days on controlled ventilation (median 6 vs 23 days); total hospital length of stay (median 27 vs 53 days); and modified CPAx score on step down to the ward (median $34 / 45$ vs $12 / 45$ ).

Conclusion: In this single centre case series early tracheostomy in COVID-19 patients who survived their ITU admission was associated with improved modified CPAx scores and functional outcomes.

\section{Reference(s) and grant ackowledgment(s)}

1. Sutt A, Tronstad O, Barnett A, Kitcheman S and Fraser J (2020) 'Earlier tracheostomy is associated withan earlier return to walking, talking and eating' Australian Critical Care 33 (213-218)

2. Sosnowski K, Lin F, Mitchell M and White $H$ (2015) 'Early rehabilitation in the intensive care unit: an integrative literature review'Australian Critical Care $28(216-225)$

3. McGrath B, Ashby N, Birchall M et al (2020) 'Multidisciplinary guidance for safe tracheostomy care during the COVID-19 pandemic: the NHS National Patient Safety Improvement Programme (NatPatSIP)'Anaesthesia 10

4. Corner E, Soni N, Handy J, Brett S (2014) 'Construct validity of the Chelsea critical care physical assessment tool: an observational study of recovery from critical illness' Critical Care 18(2) R55

\section{7}

An Observational Pilot Study to Evaluate Predictors and Risk Factors Influencing Extubation Readiness

T. Lea'; H. Wu' ${ }^{2}$ P. Katy ${ }^{2}$

${ }^{1}\left\{\right.$ street_address\}, Birmingham, United Kingdom; ${ }^{2}$ Physiotherapy, Queen

Elizabeth Hospital Birmingham, Birmingham, United Kingdom

Correspondence: $T$. Lea

Intensive Care Medicine Experimental 2020, 8(2): 000967 
Introduction: Extubation failure is associated with $42 \%$ increase in mortality and doubles Intensive Care Unit (ICU) length of stay (Menon et al 2012). There is a $10-20 \%$ re-intubation rate despite a spontaneous breathing trial (Thille et al 2013). The current evidence base outlines key factors that predict risk of extubation failure, such as; rapid shallow breathing Index (Yang and Tobin 1991), suction frequency (Miu 2013) and respiratory secretion abundance (Salam et al 2004). Currently at a large West Midlands teaching hospital, physiotherapists are not involved in the decision making process for extubation readiness on ICU.

Objectives: 1 . To evaluate if newly developed evidence based tool predicts extubation readiness

2. To evaluate risk factors that predict extubation failure.

Methods: Data was collected for all patients admitted to the adult ICU due for extubation from February 2019 until September 2019. Exclusion criteria were palliative patients and patients tracheotomised with no trial for extubation. Data was collected within one hour prior to extubation using an extubation readiness tool. This was devised using evidence based key factors that can help predict success or failure. Extubation failure was defined by the requirement for reintubation within one week or if the patient died.

Each risk factor from the assessment tool was categorised as high or low risk for extubation failure for each patient. Pass and failure rates for each risk factor were established. Data was analysed using the Fisher exact test.

Results: A total of 18 patients met inclusion criteria and were included in the analysis, of which 7 were scored as high risk based on the assessment tool. Patients deemed high risk were significantly more likely to fail extubation ( $43 \%$ vs $0 \%, p<0.05)$, with no patients deemed low risk requiring reintubation. The most common risk factors associated with failure were respiratory rate, secretion load, MIP and Po1:MIP ratio. (See Table 1)

Table 1: Results of significant risk factors

\begin{tabular}{|l|l|l|l|}
\hline Risk Factors & N -number & $\begin{array}{l}\text { Low risk (actual } \\
\text { failure) }\end{array}$ & $\begin{array}{l}\text { High risk (actual } \\
\text { failure) }\end{array}$ \\
\hline Ventilator days & 18 & $9(1)$ & $9(2)$ \\
\hline Trauma & 18 & $4(1)$ & $14(2)$ \\
\hline Respiratory rate & $\mathbf{1 8}$ & $\mathbf{6}(\mathbf{0})$ & $\mathbf{1 2}(\mathbf{3})$ \\
\hline $\begin{array}{l}\text { Secretion volume } \\
\text { Rapid shallow } \\
\text { breathing Index } \\
\text { (RSBI) }\end{array}$ & $\mathbf{1 8}$ & $\mathbf{9}(\mathbf{0})$ & $\mathbf{9}(\mathbf{3})$ \\
\hline P01 & 18 & $15(1)$ & $3(2)$ \\
\hline $\begin{array}{l}\text { Maximal } \\
\text { Inspiratory pressure } \\
\text { (MIP) }\end{array}$ & 18 & $10(1)$ & $8(2)$ \\
\hline P01:MIP ratio & $\mathbf{1 8}$ & $\mathbf{1 2}(\mathbf{0})$ & $\mathbf{6}(\mathbf{3})$ \\
\hline Peak cough flow & 18 & $8(1)$ & $\mathbf{9}(\mathbf{3})$ \\
\hline White blood cells & 18 & $10(1)$ & $10(2)$ \\
\hline CRP & 18 & $1(0)$ & $8(2)$ \\
\hline Pneumonia & 18 & $14(1)$ & $17(3)$ \\
\hline
\end{tabular}

Conclusion: The extubation readiness tool was effective in identifying patients at high risk for extubation failure. The tool demonstrated $100 \%$ successful extubation in patients identified as being in the low risk category. The four most significant risk factors that predict extubation success or failure are respiratory rate, secretion volume, maximal inspiratory pressure and P01:MIP.

Future projects will aim to recreate the tool using the factors that were deemed most significant and effective in predicting success or failure, and increasing the sample size. Limitations of this study were that the trial took a long period of time to recruit to secondary to balancing a clinical caseload alongside non-clinical work and difficulties in consistently identifying with the medical team which were the planned extubations each day.

\section{Reference(s) and grant ackowledgment(s)}

1. Yang, K. and Tobin, M., 1991. A Prospective Study of Indexes Predicting the Outcome of Trials of Weaning from Mechanical Ventilation. New
England Journal of Medicine, 324(21), pp.1445-1450. https://www.nejm. org/doi/full/10.1056/NEJM199105233242101

2. Salam, A., Tilluckdharry, L., Amoateng-Adjepong, Y. and Manthous, C., 2004. Neurologic status, cough, secretions and extubation outcomes. Intensive Care Medicine, 30(7), pp.1334-1339. https://doi.org/10.1007/ s00134-004-2231-7

3. Salam, A., Tilluckdharry, L., Amoateng-Adjepong, Y. and Manthous, C., 2004. Neurologic status, cough, secretions and extubation outcomes. Intensive Care Medicine, 30(7), pp.1334-1339. https://doi.org/10.1007/ s00134-004-2231-7

4. Salam, A., Tilluckdharry, L., Amoateng-Adjepong, Y. and Manthous, C., 2004. Neurologic status, cough, secretions and extubation outcomes. Intensive Care Medicine, 30(7), pp.1334-1339. https://doi.org/10.1007/ s00134-004-2231-7

5. Salam, A., Tilluckdharry, L., Amoateng-Adjepong, Y. and Manthous, C., 2004. Neurologic status, cough, secretions and extubation outcomes. Intensive Care Medicine, 30(7), pp.1334-1339. https://doi.org/10.1007/ s00134-004-2231-7

6. Salam, A., Tilluckdharry, L., Amoateng-Adjepong, Y. and Manthous, C., 2004. Neurologic status, cough, secretions and extubation outcomes. Intensive Care Medicine, 30(7), pp.1334-1339. https://doi.org/10.1007/ s00134-004-2231-7

7. Salam, A., Tilluckdharry, L., Amoateng-Adjepong, Y. and Manthous, C., 2004. Neurologic status, cough, secretions and extubation outcomes. Intensive Care Medicine, 30(7), pp.1334-1339. https://doi.org/10.1007/ s00134-004-2231-7

8. Salam, A., Tilluckdharry, L., Amoateng-Adjepong, Y. and Manthous, C., 2004. Neurologic status, cough, secretions and extubation outcomes. Intensive Care Medicine, 30(7), pp.1334-1339. https://doi.org/10.1007/ s00134-004-2231-7

9. Salam, A., Tilluckdharry, L., Amoateng-Adjepong, Y. and Manthous, C., 2004. Neurologic status, cough, secretions and extubation outcomes. Intensive Care Medicine, 30(7), pp.1334-1339. https://doi.org/10.1007/ s00134-004-2231-7

10. Menon, N., Joffe, A., Deem, S., Yanez, N., Grabinsky, A., Dagal, A., Daniel, S. and Treggiari, M., 2012. Occurrence and Complications of Tracheal Reintubation in Critically III Adults. Respiratory Care, 57(10), pp.15551563. http://rc.rcjournal.com/content/respcare/57/10/1555.full.pdf

\section{1}

Necessity and Invention During the CoVID-19 Pandemic: Introducing Dedicated Family Liaison to Intensive Care

A. Carpenter ${ }^{1}$; K. Dafter ${ }^{1}$

${ }^{1}$ Intensive Care, Royal Devon and Exeter Hospital (Wonford), Exeter, United Kingdom

Correspondence: A. Carpenter

Intensive Care Medicine Experimental 2020, 8(2): 000981

Introduction: CoVID-19 has severely challenged communication between intensive care unit (ICU) staff and patients' families, limiting information sharing, whilst also denying patients and their loved ones mutually supportive interaction.[1-3] Consequential reliance on lesseffective telephone, video or e-mail communication.[2] is complicated by personal protective equipment and infection control requirements within clinical areas. Concurrently, many staff have been required to step back from direct patient contact due to risk to their own health. [4] Addressing these issues, our ICU introduced a new family liaison (FL) role. Two team members shielding from direct patient contact were utilized to establish this new service aimed at ensuring ongoing family centeredcare through regular, consistent contact between relatives, clinicians, and patients[3-5] and relieving pressures on direct care staff through reducing time burdens of family communication and associated administration. Key activities include facilitating videocalls, collating personal 'This is Me' information for new admissions, family updates and 'check-ins,' organizing 'garden visits' with a relative and coordinating bereavement support.

Objectives: To evaluate the impact and possible longer-term benefit of the reallocation of staff to a new family liaison role during the CoVID-19 pandemic. 
Methods: We conducted a retrospective audit of documented family phone communications, direct visits and video-calls, for all patients on ICU for one week periods in February (prior to CoVID restrictions), March (during restriction, before FL introduction) and May (restriction in place, FL established). A web-based survey of was also conducted utilizing scaled, multiple-choice responses and free text comments.

\begin{tabular}{l|c|c|c|c|c}
\multirow{2}{*}{ Week } & Total No. ICU & Patient-days (sum & \multicolumn{3}{|c}{ Mean communications per patient } \\
\cline { 4 - 6 } & $\begin{array}{l}\text { Patients (with } \\
\text { of days each patient } \\
\text { CoVID-19) }\end{array}$ & on during week) & Phone & Visits & Video \\
\hline $9^{\text {th }}$ Feb & $23(0)$ & 78 & 2.47 & 2.35 & 0 \\
\hline $\mathbf{2 9}^{\text {th }}$ March & $15(10)$ & 55 & 4.8 & 0 & 0.13 \\
\hline $3^{\text {rd }}$ May & $18(6)$ & 56 & 5.72 & 0.06 & 1.56 \\
\hline
\end{tabular}

Results: Of 109 ICU staff invited, 50 (45.9\%) responded to the survey to date. $100 \%(n=50)$ indicated FL has improved consistency of family communication, $94 \%(n=47)$ reported it as helpful during end-of-life care and $84 \%(n=42)$ that FL reduced stress of communication. $54 \%$ $(n=27)$ reported less telephone time and $98 \%(n=49)$ that video calls improved patient and family care. $42 \%(n=20)$ found FL made it easier to build relationships with families, $35 \%(n=17)$ no change, and $23 \%$ $(n=11)$ found it harder ( 2 non-respondents). $84 \%(n=42)$ thought it would be valuable to continue the FL role beyond the current pandemic situation. The survey generated 137 comments (mean 15.2 per question) for further analysis.
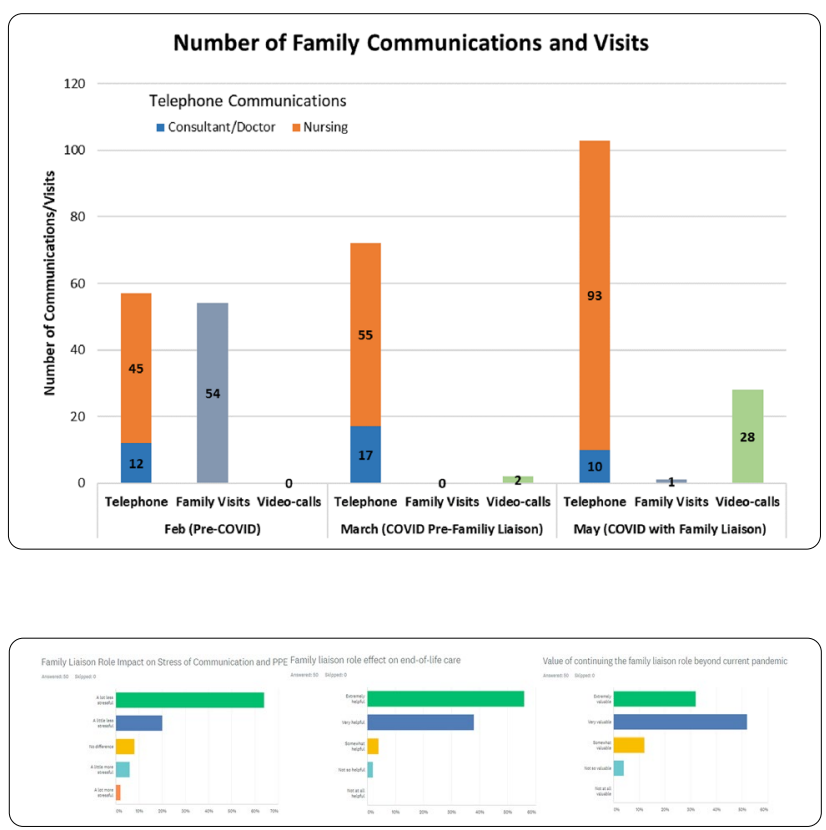

Conclusion: Innovative use of staff at risk from direct patient to create a FL role appears to have increased volume of documented phone and video contacts with patients' families. FL has been well received by colleagues, improving consistency of communication, helping endof-life care, reducing stress and time on the phone. We hope to present further insight regarding impact and interest in continuing the FL through analysis of comments and further examination of evolving functions since the roles' introduction.

\section{Reference(s) and grant ackowledgment(s)}

1. The authors would like to acknowledge the work of Carole Boulanger, Katherine Dafter and Rachel Reason for the initial development and introduction of this role.

2. 5. Ludmir J, Netzer G, Family-Centered Care in the Intensive Care Unit What Does Best Practice Tell Us? Seminars in Respiratory and Critical Care Medicine 2019; 40 (5): 648-654. https://doi.org/10.1055/s-0039-1697957.
3. 4. Carter C, Notter J. COVID-19 disease: a critical care perspective. Clinics in integrated Care 2020; Vol 1, July, https://doi.org/10.1016/j. intcar.2020.100003

4. 3. Akgün, KM, Shamas TL, Feder SL, Schulman-Green D. Communication strategies to mitigate fear and suffering among COVID-19 patients isolated in the ICU and their families. Heart \& Lung 2022, article in press, https://doi. org/10.1016/j.hrtIng.2020.04.016.

5. 2. Marra A, Buonanno P, Vargas M, lacovazzo C, Ely EW, Servillo G. How COVID-19 pandemic changed our communication with families: losing nonverbal cues. Critical Care 2020; 24:297, published online 5th June 2020, https://doi.org/10.1186/s13054-020-03035-w.

6. 1. Hart JL, Turnbull AE, Oppenheim IM, Courtright KR. Family-Centered Care During the COVID-19 Era. Journal of Pain and Symptom Management 2020; in press, corrected proof, April 22nd 2020, https://doi.org/10.1016/j. jpainsymman.2020.04.017.

\section{3}

\section{Mortality Associated to Late-Onset sepsis in the NICU}

E. Afonso ; K. Smets ${ }^{2}$; E. Verstraete ${ }^{3}$; S. Blot ${ }^{4}$

${ }^{1}$ Internal medicine department, Faculty of Medicine and Health Sciences, Ghent University, Ghent, Belgium; ${ }^{2}$ Neonatal intensive care unit, Ghent University Hospital, Gent, Belgium; ${ }^{3}$ Havenzate care centre, Medical departement, Veurne, Belgium; ${ }^{4}$ Internal medicine department, Ghent University Hospital, Gent, Belgium

Correspondence: $\mathrm{E}$. Afonso

Intensive Care Medicine Experimental 2020, 8(2): 001003

Introduction: Neonatal late-onset-sepsis occurs after 72 hours of life $(1,2)$. The relationship between late-onset-sepsis and mortality can be confounded by other events that are part of NICU journey, thus the true effect on mortality of this complication is difficult to assess (3).

Objectives: The aim of this study was to assess the impact of lateonset-sepsis on mortality in critically ill infants.

Methods: Single-centre retrospective cohort study of neonates admitted to the NICU for $\geq 72$ hours in a 10-year period (2002-2011). Lateonset-sepsis is defined according to National Institute of Child Health and Human Development criteria (2). Multivariate logistic regression analysis was used to assess risk factors for mortality.

Results: Of all admissions, 6.9\% developed late-onset-sepsis (Table 1). Infants with late-onset-sepsis had higher mortality, lower gestational age, lower birthweight, longer NICU stay, spent more days mechanically ventilated and on parenteral nutrition. Furthermore, initiation of enteral nutrition was delayed by 7 days in infants who had late-onset-sepsis.

Independent risk factors for mortality included gestational age $<=28$ weeks $(\mathrm{OR}=9.2, \mathrm{Cl} 3.8-22.3)$, periventricular leukomalacia $(\mathrm{OR}=4.3, \mathrm{Cl}$ 2.7-6.98), late-onset-sepsis ( $\mathrm{OR}=2.6, \mathrm{Cl} 1.5-4.2)$, abdominal surgery $(\mathrm{OR}=2.3, \mathrm{Cl} 1.1-4.7)$ and chronic lung disease (OR 1.97, $\mathrm{Cl}$ 0.966-4.0).

\begin{tabular}{llll}
\hline & No Late-onset-sepsis & Late-onset-sepsis & p-value \\
\hline N/ total (\%) & $4558 / 4928(93.1 \%)$ & $340 / 4928(6.9 \%)$ & $<0.05$ \\
Gestational age weeks & $35(32-38)$ & $32(28-38)$ & $<0.05$ \\
Birthweight & $2410(1740-3200)$ & $1715(1100-2840)$ & $<0.05$ \\
Gender (male) & $2690(58.6 \%)$ & $195(57.4 \%)$ & 0.64 \\
\hline Length of NICU stay (in days) & $13(8-24)$ & $42(21-75)$ & $<0.05$ \\
\hline Mechanical ventilation days & $0(0-3)$ & $4(0-12)$ & $<0.05$ \\
Respiratory Distress Syndrome & $2546(55.5 \%)$ & $229(67.4 \%)$ & $<0.05$ \\
Chronic Lung Disease 28 days & $254(5.5 \%)$ & $87(25.6 \%$ & $<0.05$ \\
Systemic corticoids & $308(6.7 \%)$ & $72(21.6 \%)$ & $<0.05$ \\
\hline Total parenteral nutrition days & $7(1-12)$ & $20(12-32)$ & $<0.05$ \\
Start enteral feeds & $3(1-7)$ & $10(5-17)$ & $<0.05$ \\
\hline Periventricular leukomalacia & $229(5 \%)$ & $52(15.3 \%)$ & $<0.05$ \\
\hline Mortality & $232(5.1 \%)$ & $34(10 \%)$ & $<0.05$ \\
\hline
\end{tabular}

Table 1 - Demographics and risk factors when comparing infants who didn't develop late-onset-sepsis with infants who developed late-onset-sepsis. Continuous variables expressed in median (IQR) and dichotomous variables expressed in $\mathrm{n}(\%)$.

Conclusion: Infants who developed late-onset-sepsis had a higher mortality and a longer, more eventful NICU stay. After adjustment for major risk factors, late-onset-sepsis appeared an independent risk factor for mortality. 
Reference(s) and grant ackowledgment(s)

1. 1. Auriti C, Maccallini A, Di Liso G et al. (2003) Risk factors for nosocomial infections in a neonatal intensive-care unit. J Hosp Infect 53 (1): 25-30.

2. 2. Stoll BJ, Hansen N, Fanaroff AA, et al. (2002). Late-onset sepsis in very low birth weight neonates: the experience of the NICHD Neonatal Research Network. Pediatrics 110: 285-291

3. 3. Verstraete EH, De Coen K, Vogelaers D et al (2015) Risk Factors for Health Care-Associated Sepsis in Critically III Neonates Stratified by Birth Weight. Pediatr Infect Dis J 34(11):1180-6.

\section{4}

Introducing a physiotherapy follow-up service on transition

from ICU to the ward: A pilot study (2020)

Fiona'; A. Waqaar'; J. Oldhams'; D. McWilliams

${ }^{1}$ Critical care rehab team, Queen Elizabeth Hospital Birmingham, Birming ham, United Kingdom

Correspondence: $\mathrm{H}$. Fiona

Intensive Care Medicine Experimental 2020, 8(2): 001044

Introduction: It is well recognized that the transfer from Intensive Care Units (ICU) to the ward is a highrisk transition which causes challenges for staff and anxiety for patients (1). National Guidance for rehabilitation after critical illness (2), recommends a structured handover process for patients and appropriate multidisciplinary follow-up on transition from ICU to the ward. A previous project identified ongoing rehabilitation on the ward is often inconsistent leading to delays in ongoing recovery.

Objectives: To assess the feasibility of a physiotherapy follow-up service for patients transitioning from ICU to the ward.

Methods: Patients admitted to a large UK based ICU between December 2018 and February 2019 were recruited to the study. Inclusion criteria included patients under the care of a specialist critical care rehabilitation team, discharged from ICU with a Manchester Mobility Score (MMS) of less than 5 (i.e. unable to mobilise) and considered to have rehab potential.

On transfer to the ward, patients were continued to be reviewed daily by the ICU physiotherapy team for one week. The physiotherapists provided ongoing structured rehabilitation and communication with the patient, their relatives and facilitated a gradual handover to the receiving ward therapy team during this transition period. Primary outcome was changes in MMS, with secondary measures of patient and staff satisfaction.

Results: 13 patients were included in the pilot. Rehabilitation was delivered consistently during the trial period, with patients reviewed by the ICU physiotherapy team for a mean of 6 days on transition to the ward.

During this time, the median MMS scores increased from 4 (i.e. able to stand with assistance) at ICU discharge, to 6 (i.e. mobilising up to 30 metres).

Patient feedback was extremely positive. Patients valued the continuity of care and felt supported during the step down in care from ICU. Positive feedback was also received from ward staff who felt more supported in the handover process and able to deliver seamless care. However, as the follow-up team was based in ICU, they did face practical challenges during the pilot; including coordination of rehabilitation sessions and communication across multiple wards.

Conclusion: Although a small pilot study, the results demonstrate that the physiotherapy ward follow-up service was feasible to introduce. The pilot study demonstrated an improvement in mobility scores with ongoing structured, physiotherapy rehabilitation. Patient and staff feedback was positive, although the service requires good lines of communication to support timetabling of rehabilitation and team working.

Reference(s) and grant ackowledgment(s)

1. [2] National Institute for Health and Care Excellence (2019) Rehabilitation after critical illness in adults, Clinical Guideline [CG83

2. [1] Häggström, M., \& Bäckström, B. (2014). Organizing safe transitions from intensive care. Nursing research and practice, 2014, 175314. https://doi. org/10.1155/2014/175314
001060

The effect of guidelines implementation combined with simulation training on the course of complications during operation of central venous access devices

E. Beresten ${ }^{1}$; E. Korneeva'; U. Loaisa²; S. Averyanov'; O. Pimenova ${ }^{3}$;

V. Selivanov'; V. Shchukin ${ }^{1}$; A. Satsuk ${ }^{4}$

${ }^{1}$ Anestesiology, Dmitry Rogachev National Research Center of Pediatric Hematology, Oncology and Immunology, Moscow, Russia; ${ }^{2}$ Methodological accreditation center, Dmitry Rogachev National Research Center of Pediatric Hematology, Oncology and Immunology, Moscow, Russia ${ }^{3}$ Management, Dmitry Rogachev National Research Center of Pediatric Hematology, Oncology and Immunology, Moscow, Russia; ${ }^{4}$ General medical department, Dmitry Rogachev National Research Center of Pediatric Hematology, Oncology and Immunology, Moscow, Russia

Correspondence: $\mathrm{E}$. Beresten

Intensive Care Medicine Experimental 2020, 8(2): 001060

Introduction: Providing a patient with a reliable venous access device is a key factor determining the effectiveness of therapy in pediatric oncology, hematology and immunology. In practice nurses resolve issues related to the usage of central venous access devices.

Objectives: The aim of the study was to determine the impact of implementation of central venous access devices (CVAD) guidelines combined with simulation training of nursing staff on the change in the numbers of infectious and occlusal complications in patients with central vascular access.

Methods: There was a retrospective and prospective analysis of the operational peculiarities of 3357 venous access devices installed to 2453 patients (1336 males, 1117 females) aged from 1 month to 18 years over a January 2018 to December 2020 period.

In 2017 the National Research Center of Pediatric Hematology, Oncology and Immunology approved and implemented guidelines for the operation of central venous access devices. In 2018 the record of all complications during the operation of central venous access devices was started. In 2019 simulation training for nurses was introduced; by the end of 2019106 people had been trained. The main outcomes were the assessment of complications during the operation of vascular devices.

Results: In 20181618 central venous catheters were installed, in 2019 - 1739 ones. During the year after the implementation of the CVAD guidelines the following issues were recorded: infectious complications $-3.2 \%(n=52)$, lumen occlusion unrelated to thrombosis $-1.8 \%$ $(n=30)$, catheter-related thrombosis - 12.2\% $(n=199)$, which in $7.4 \%$ $(n=121)$ of patients led to catheter damage during attempts to restore patency. In 2019 given the additional simulation training the following issues were revealed: infectious complications - $2.6 \% \quad(n=46)$, lumen occlusion unrelated to thrombosis $-1.72 \%(n=30)$, catheterrelated thrombosis $-13.7 \%(n=239)$, catheter damage $-4.6 \%(n=81)$ of patients.

In the group of patients where the guidelines and simulation training were used, the frequency of catheter damage was statistically significantly lower, Pearson's chi-square test showed 11.791, $\mathrm{p}<0.001$, OR $1.65495 \%, \mathrm{Cl} 1.238-2.211$

A total of 120,059 catheter-wearing days were recorded in 1203 patients in 2018, and 148.327 catheter-wearing days in 1250 patients in 2019.

Conclusion: An analysis of the data showed that the simulation training of nurses in conditions of compliance with the guidelines does not practically affect the frequency of thrombotic and infectious complications, which is most likely related to many additional factors in the pathogenesis of these complications. However due to the simulation training under our conditions the number of catheter damages when restoring catheter patency during the development of intraluminal thrombosis decreased.

The experience of the National Research Center of Pediatric Hematology, Oncology and Immunology has shown that the implementation of simulation training in addition to the usage of the guidelines had reliably reduced the number of damages of central venous access devices. 
001120

Preliminary results from an observational study on the incidence of diarrhoea and constipation in Intensive Care Unit

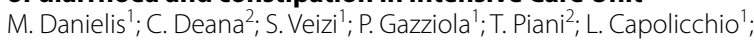
F. Gigante ; L. Castriotta ${ }^{3}$; E. Mattiussi ${ }^{1}$

${ }^{1}$ Department of medical sciences, University of Udine, Udine, Italy ${ }^{2}$ Department of anesthesia and intensive care, Azienda Sanitaria Universitaria Friuli Centrale, Udine, Italy; ${ }^{3}$ Institute of hygiene and clinical epidemiology, Azienda Sanitaria Universitaria Friuli Centrale, Udine, Italy

Correspondence: M. Danielis

Intensive Care Medicine Experimental 2020, 8(2): 001120

Introduction: Gastrointestinal motility disorders are frequent events in Intensive Care Unit (ICU). A number of factors increase the frequency of diarrhoea and constipation among critically ill patients, and a number of interventions can prevent them. Given the relevance of these two phenomena, further epidemiological studies areneeded.

Objectives: To determine the incidence of diarrhoea and constipation among critically ill patients in ICU.

Methods: An observational study was carried out in three general Italian ICUs with a total if 28 beds. The present investigation was prospective in design, with the analysis of medical records and the direct observation of patients admitted during the period from September and October 2019. Chi-squared and Wilcoxon tests were used to compare factors associated with diarrhoea and constipation. Statistical analysis was performed using the $S A S^{\circledR}$ software. The study protocol was endorsed by the Regional Ethics Committee of the Friuli Venezia Giulia region (CEUR-2019-Os-170).

Results: Fifty-three patients were enrolled in the study, mostly males $(n=37 ; 69.8 \%)$, with an average age of 66.6 years (SD 13.4) and a BMI of 26.8 . For about a half of the sample $(n=29 ; 54.7 \%)$ the main diagnosis reported at time of admission was organ failure (especially respiratory). Diarrhoea affected the patients in 10/53 (18.9\%) cases within 72 hours after admission in ICU, and in 19/53 (35.8\%) during the whole ICU-stay period. The incidence of diarrhoea in patients who received antibiotics and prokinetics was $18 \%$ and $37.5 \%$ respectively, with no differences with patients who received no antibiotics $(p=0.37)$ and no prokinetics $(p=0.78)$. Among patients who received laxatives, the incidence of diarrhoea was $100 \%$, compared with those received no laxatives (13.6\%; $p=0.001)$. Against, constipation occurred in 26/53 (49.1\%) patients within 72 hours after admission in ICU, and in 4/27 $(14.8 \%)$ on the sixth day. There was no difference between constipated and no constipated patients in terms of opiate and curare use, mechanical ventilation, and hypotension. Remifentanil administration was associated with increased constipation $(p=0.001)$.

Conclusion: Diarrhoea and constipation are very common among critically ill patients in ICU. Diarrhoea was associated with the use of laxatives, and constipation was associated with the use of remifentanil.

\section{Reference(s) and grant ackowledgment(s)}

1. Warren, D., \& Kent, B. (2019). Determining the impact of a bowel management protocol on patients and clinicians' compliance in cardiac intensive care: A mixed-methods approach. Journal of Clinical Nursing, 28(1-2), 89-103.

2. Hay, T., Bellomo, R., Rechnitzer, T., See, E., Abdelhamid, Y. A., \& Deane, A. M. (2019). Constipation, diarrhea, and prophylactic laxative bowel regimens in the critically ill: a systematic review and meta-analysis. Journal of Critical Care, 52, 242-250.

\section{1}

Critical patient Safety: transition of care in the Nursing Handover

L. C. A. Leite ; C. Carvalho

'Serviço de medicina intensiva 1, Centro Hospitalar Universitário do Algarve, Faro, Portugal

Correspondence: L. C. A. Leite

Intensive Care Medicine Experimental 2020, 8(2): 001171
Introduction: The handover/handoff process takes place on a daily basis in all health care institutions, accounting for a significant portion of the work time of health professionals, moments and processes that are sometimes neglected and undervalued, although they are considered critical to patient safety.

Objectives: • Introduce a semi-structured approach, evidence-based, in the Nursing handover in a level III ICU.

Methods: Running between December 2017 and March 2018 in a level III UCI, based on project work methodology (1), a semi-structured evidenced-based approach was introduced, adapted to the context combining the ISBAR (Identification, Situation, Background, Assessment, Recommendations) with the primary approach of the critical patient $A B C D E$ (Airway, Breathing, Circulation, Disability and Exposition). It was divided into mandatory items, mandatory reference in all handover moments, and optional items related to the specific clinical situation.

This strategy was assessed through direct observation of the Nursing Handover, with prior obtaining an informed consent, and applying a questionnaire to assess the perception of the Nursing team, after the implementation of the semi-structured evidenced-based approach, with regard to knowledge held on patient safety and the usefulness, satisfaction and sensitive and specific gains to the form.

Results: 100 Handover moments were observed. All nurses gave their consent for observation and records made during these moments. Conformity related to the items referring to the ISBAR mnemonic at the observed times was $89.5 \%$, ranging from $100 \%$ (Identification and Evaluation) to $70 \%$ (Situation). The compliance related to items referring to the primary $A B C D E$ assessment at the time observed varied from $84 \%$ (exposure) and 99\% (Airway and Ventilation), with an average of $92.25 \%$. An average of $75.65 \%$ of mandatory items was referenced, ranging from $50 \%$ (fluid balance) to $94 \%$ (Mechanical Ventilation/Oxigen setings)

With regard to the questionnaire, using a Likert Scale (1-10), a high perception of better organization and systematization $(7,67)$ of the Nurse's Handover is notorious, more efficient $(7,5)$ and of better quality $(7,16)$, with the constructed semi-structured instrument being considered useful $(7,7)$, contributing to the increase of satisfaction $(7,4)$ of the Nursing team at this fundamental moment to providing excellent care. These results are transversal to the literature found, with regard to organization and systematization (2-4) efficiency and quality $(3,5)$ and, finally, related to the global satisfaction and usefulness of structuring the care transition $(3,6-7)$.

Conclusion: There was little knowledge about the subject of patient safety, effected by a relative lack of knowledge in relation to the essential documents that guide this subject, highlighting the improvement of this knowledge after implementation. There was a high rate of compliance, based on the semi-structured tool built for this purpose, in relation to the mandatory reference items, the contents contained in the ISBAR mnemonic and included in the primary assessment, using the ABCDE approach. It is also clear a high perception on the part of the Nursing team, after the use of the semi-structured tool, with regard to improving the organization, systematization, efficiency, quality and satisfaction with the passage of the Nursing shift, with agreement on the part of the referred team the usefulness of the tool created and adapted.

Reference(s) and grant ackowledgment(s)

1. (7) Randmaa, M., Mårtensson, G., Swenne, C. \& Engstrom, M. (2014). SBAR improves communication and safety climate and decreases incident reports due to communication erros in an anaesthetic clinic: a prospective intervention study. BMJ Open, 21(4). doi: 10.1136/bmjopen-2013-004268

2. (6) Emlet, L., Al-Khafaji, A., Kim, Y., Venkataraman, R., Rogers, P. \& Angus, D. (2012) Trial of shift scheduling with standardized sign-out to improve continuity of care in intensive care units. Critical Care Medicine; 40(12), 3129-3134 doi: 10.1097/CCM.0b013e3182657b5d

3. (5) Moon, T., Gonzales, M., Woods, A. \& Fox, P. (2016). Improving the quality of the operating room to intensive care unit handover at an urban teaching hospital through a bundled intervention. Journal of Clinical Anesthesia, 21, 5-12. doi: 10.1016/j.jclinane.2016.01.001. 
4. (4) Yang, Jian-Gua \& Zhang, Jun (2016). Improving the postoperative handover process in the intensive care unit of a tertiary teaching hospital. Journal of Clinical Nursing, 25 (7-8), 1062-1072. doi: 10.1111/jocn.13115.

5. (3) Smeulers, M., Dolman, C., Atema, D., Dieren, S., Maskant, J. \& Vermeulen, H. (2016). Safe and effective nursing shift handover with NURSEPASSAn interrupted time series. Applied Nursing Research, 32, 199-205. doi:10.1016/j.apnr.2016.07.010.

6. (2) Graan, S., Botti, M., Wood, B., Redley, B. (2015). Nursing handover from ICU to cardiac Ward: Standardised tools to reduce safety risks. Australian Critical

7. (1) Issel, L. (2014). Health Program Planning and Evaluation: a practical, sys-

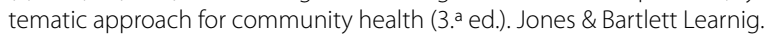

\section{2}

Use of the vasoactive-ventilation-renal score in pediatric heart failurecases as a predictor of an extended intensive care unit stay S. J. Choi $^{1}$

${ }^{1}$ Asan Medical Center, Seoul, Republic of Korea

Correspondence: S.J. Choi

Intensive Care Medicine Experimental 2020, 8(2): 000052

Introduction: It has been reported that the vasoactive ventilation renal (VVR) score predicts the outcomes of cardiac surgery in children. Objectives: We investigated whether the VVR score on admission predicts the length of stay (LOS) in the pediatric intensive care unit (PICU) in children diagnosed with heart failure (HF).

Methods: Medical records were reviewed for 96 pediatric HF cases between January 2010 and December 2016. A prolonged LOS (PLOS) was defined by a longer stay than the median duration for the surviving cases.

Results: The median LOS in our current patient series was 12 days. The VIS $(14.1[7.4,24.3]$ vs $8.5[5.0,18.3], p=0.018), \operatorname{VVR}(27.8[11.6,36.2]$ vs $11.1[5.5,20.7], \mathrm{p}<0.001)$, lactic acid level $(\mathrm{mmol} / \mathrm{L})(2.8[1.2,5.3]$ vs 1.8 $[1.1,2.5], p=0.020)$, and B-type natriuretic peptide concentration (BNP, $\mathrm{pg} / \mathrm{mL})(2340.0[1234.8,6836.8]$ vs $1478.5[529.8,4532.8], \mathrm{p}=0.036)$ were significantly different between the prolonged LOS versus the control group. The use of a mechanical ventilator and extracorporeal membrane oxygenation (ECMO) were associated with PLOS (odds ratios: 10.82 and 3.61, respectively). The AUCs of the VIS, VVR score, lactic acid level, and BNP for evaluating the association with mechanical ventilator or ECMO use were $0.682(p=0.006), 0.823(p=0.000), 0.683$ $(p=0.006)$, and $0.783(p=0.000)$, respectively. In multivariable logistic regression analysis, the VVR score was the only parameter that was significant for predicting PLOS.

Conclusion: The VVR score on admission to the PICU is predictive for mechanical ventilator or ECMO use, which correlates with a PLOS. The VVR score at PICU admission in children with HF is therefore a useful LOS marker.

\section{Reference(s) and grant ackowledgment(s)}

1. Whellan DJ, Zhao X, Hernandez AF, et al. Predictors of hospital length of stay in heart failure: findings from Get With the Guidelines. J Cardiac Fail 2011;17:649-56

2. Kogan A, Preisman S, Bar A, et al. The impact of hyperlactatemia on postoperative outcome after adult cardiac surgery. J Anesth 2012;26(2):174-8

3. Andersen LW, Holmberg MJ, Doherty M, et al. Postoperative Lactate Levels and Hospital Length of Stay After Cardiac Surgery. J Cardiothorac Vasc Anesth 2015;29(6):1454-60

4. Miletic KG, Spiering TJ, Delius RE, et al. Use of a novel vasoactive-ventilationrenal score to predict outcomes after paediatric cardiac surgery. Interact Cardiovasc Thorac Surg 2015;20(3):289-95

5. Miletic KG, Delius RE, Walters HL 3rd, et al. Prospective Validation of a Novel Vasoactive-Ventilation-Renal Score as a Predictor of Outcomes After Pediatric Cardiac Surgery. Ann Thorac Surg 2016;101(4):1558-63

6. Scherer B, Moser EA, Brown JW, et al. Vasoactive-ventilation-renal score reliably predicts hospital length of stay after surgery for congenital heart disease. J Thorac Cardiovasc Surg 2016;152(5):1423-9.e1

7. Cashen K, Costello JM, Grimaldi LM, et al. Multicenter Validation of the Vasoactive-Ventilation-Renal Score as a Predictor of Prolonged
Mechanical Ventilation After Neonatal Cardiac Surgery. Pediatr Crit Care Med 2018;19(11):1015-23

8. Dickstein K, Cohen-Solal A, Filippatos G, et al. ESC Guidelines for the diagnosis and treatment of acute and chronic heart failure 2008: the Task Force for the Diagnosis and Treatment of Acute and Chronic Heart Failure 2008 of the European Society of Cardiology. Developed in collaboration with the Heart Failure Association of the ESC (HFA) and endorsed by the European Society of Intensive Care Medicine (ESICM). Eur Heart J 29:2388-442

9. Not applicable.

\section{9}

Anthropometric methods for assessing nutritional status

in a child of one year old with viral diarrhea

Y. Erpuleva'; M. Gadzhimaeva'; D. Levina'

${ }^{1}$ Pediatrics and infections diseases, Sechenov University, Moscow, Russia

Correspondence: M. Gadzhimaeva

Intensive Care Medicine Experimental 2020, 8(2): 000059

Introduction: Young children with viral diarrhea lose their body weight very quickly, and consequently their anthropometric indicators sharply decrease, and malnutrition increases up to cachexia. Thereby, it is necessary to perform a short-term assessment of the nutritional status of the child, in order to select nutritional supportrationally. The purpose of our study was to assess the nutritional disorder in children on the first year of life with moderate to severe acute intestinal infection of viral etiology based on anthropometric research methods.

Methods: We examined 12 children with moderate to severe acute intestinal infection of viral etiology. Anthropometric methods for assessing nutritional status were used: mass-growth indicators, caliperometry with an assessment of the skin-fat fold of the triceps at baseline and a week after admission. Evaluation of the indicators was carried out according to the calculation formula: body mass index $(\mathrm{BMI}=$ Weight / Height2 $(\mathrm{kg} / \mathrm{m} 2))$, centile tables. Used tools: caliper, centimeter tape, table scales.

Results: The analysis of the data revealed that by the time of admission to the hospital $5(41 \%)$ of 12 children showed a decrease in BMI by $10 \%$, and $3(25 \%)$ children showed a decrease in BMI by $20 \%$. One week later BMI was restored to normal. When assessing the skin-fat fold of the triceps, it decrease was found to be 1-2 mm from the norm in all studied children (100\%), and was regarded as a mild degree of malnutrition due to indicators less than the 50-th percentile. By the 5 th day of the ongoing nutritional support, the fold was restored to the physiological norm (50th percentile).

Conclusion: Anthropometric methods for assessing nutritional status in children with viral diarrhea on the first year of life is found to be easy and helpfully.

\section{5}

Underweight and Organ Dysfunction in Critically III Children

P. Hong-Zhu'; A. Marroquín"; J. Silva-Gburek';. Desai'; S. Rodriguez";

T. Fogarty'; L. Shekerdemian ${ }^{1} ;$ J. A. Coss-Bu ${ }^{1}$

${ }^{1}$ Department of pediatrics, section of critical care, Baylor College of Medicine, Texas Children's Hospital, Houston, United States of America

Correspondence: J.A. Coss-Bu

Intensive Care Medicine Experimental 2020, 8(2): 000365

Introduction: Underweight on admission to the pediatric intensive care unit (PICU) has been associated with outcome. The Pediatric Sequential Organ Failure Assessment (pSOFA) score has been reported as predictor of outcome in critically ill children.

Objectives: The study aim was to assess the effect of organ dysfunction and nutritional status on outcomes of children admitted to PICU.

Methods: Retrospective study of children admitted to the PICU at Texas Children's Hospital (Jan-Dec 2016) with a length of stay (LOS) $\geq 3$ days; nutritional status assessed by weight for age (WFA: underweight), z-scores by WHO and CDC. Malnutrition (MAL) defined as mild, and moderate-severe if $z$-scores were $<-1,<-2$, respectively. Hospital and PICU LOS, risk of mortality (ROM) by PIM2, need of 
mechanical ventilation (MV) on admission to PICU, and mortality collected. Organ dysfunction (OD) was defined as a pSOFA score $>5$, and collected on days $1 \& 3$.

Results: A total of 638 children $(367 \mathrm{M} / 271 \mathrm{~F})$ were included; age 2.8 yr. (0.6-11; median (25-75th IQR)). 513 (80.4\%) children required MV on admission, had PIM2 ROM (\%), 3.1 (0.9-4.7), PICU LOS, 6.4 days (4.411), Hospital LOS, 17 days (10-31); and PSOFA score on days 1 \& 3 of 5 (4-7) and $5(3-6)(p<0.0001$, Wilcoxon Signed Rank Test) for a prevalence of organ dysfunction of $45 \%$ and $38 \%$, respectively. WFA z-score of $-0.63 \pm 1.97$ (SD) and the prevalence of underweight was $35.6 \%$; moderate to severe underweight was associated with organ dysfunction; odds ratio $(\mathrm{OR})(95 \% \mathrm{Cl}) 1.62(1.10-2.39)(\mathrm{p}<0.05)$. Children with malnutrition or $\mathrm{OD}$ and children with malnutrition and $\mathrm{OD}$ vs. normal children were associated with need of MV on admission to the PICU and with mortality; $2.29(1.51-3.47)(p<0.0001), 7.26$ (3.04-17.35) $(p<0.0001)$; respectively; and $5.04(1.73-14.69)(p<0.005), 4.02(1.15-$ $14.04)(p<0.05)$; respectively. A total of 36 children died for a hospital ortality rate of $5.64 \%$.

\begin{tabular}{|l|l|l|l|}
\hline & PIM2 ROM (\%) & PICU LOS Days & $\begin{array}{l}\text { Hospital LOS } \\
\text { Days }\end{array}$ \\
\hline Normal (n=229) & $1.40(0.80-4.07)$ & $5.60(3.87-8.82)$ & $15(10-27)$ \\
\hline $\begin{array}{l}\text { MAL or OD } \\
(\mathrm{n}=304)\end{array}$ & $3.18(0.91-4.98)$ & $6.97(4.72-11.2)$ & $17(11-34)$ \\
\hline MAL + OD (n=105) & $3.81(1.19-$ & $7.87(4.92-$ & $21(11-39)^{* *}$ \\
\hline & $6.39)^{*}$ & $12.1)^{*}$ & \\
\hline
\end{tabular}

Values are median (25th-75th IQR); ${ }^{*} \mathrm{p}<0.0001,{ }^{* *} \mathrm{p}<0.05$ by Kruskal-Wallis

Conclusion: Organ dysfunction and underweight were commonly found on children admitted to the PICU. The presence of either or both conditions was associated with higher risk of mortality, need of ventilatory support, longer PICU and hospital LOS, and mortality.

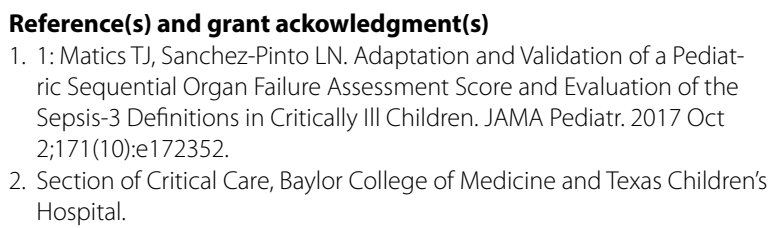

\section{3}

Time course of altered DNA-methylation evoked by paediatric critical illness and by early administration of parenteral nutrition in the PICU

I. Verlinden ${ }^{1}$; F. Guiza Grandas'; I. Derese ${ }^{1}$; P. Wouters ${ }^{1}$; K. Joosten².

S. Verbruggen²; G. Van den Berghe'; I. Vanhorebeek ${ }^{1}$

${ }^{1}$ Department and laboratory of intensive care medicine, KU Leuven, Leuven, Belgium; ${ }^{2}$ Department of paediatrics and paediatric surgery, Erasmus MC-Sophia", Rotterdam, Netherlands

Correspondence: I. Verlinden

Intensive Care Medicine Experimental 2020, 8(2): 000723

Introduction: A genome-wide DNA-methylation study previously identified de novo alterations in leukocyte DNA of critically ill children at paediatric intensive care unit (PICU) discharge, which offered a biological basis for the impaired long-term neurocognitive development of these patients.1 Early use of parenteral nutrition (early-PN) in the PICU, as compared with omitting PN in the first week (late-PN), explained the differential methylation of $23 \%$ of the affected CpGsites. It remained unclear how fast these changes in DNA-methylation took place and how they evolved over time in PICU.

Objectives: We documented the time course of altered DNA-methylation in PICU and the impact hereon of early nutritional management. Methods: We selected 36 early-PN and 36 late-PN patients, matched for age, type (admission diagnosis) and severity of illness (PeLOD score), infection upon admission, gender, height, weight and educational level of the parents and 42 age and gender matched healthy children. We quantified DNA-methylation on day 3, 5 and 7 for the 147 CpG-sites of which methylation was normal upon PICU admission in this subset, and altered by critical illness at PICU discharge (EPIC BeadChip). For each time point, we determined how many of the CpG-sites were differentially methylated in critically ill as compared with healthy children, applying ANOVA and FDR 0.05. Next, for each CpG-site, we performed within-patients time course analyses of the methylation status, to identify any patterns within the time series based on paired t-test $p$-value and direction of change from admission to day 3 and beyond. For the CpG-sites affected by early-PN on the last PICU day, earlier changes in DNA methylation status -and patterns hereof- were compared for early-PN and late-PN patients.

Results: Methylation in patients differed from healthy children for $64.6 \%$ of the 147 CpG-sites on day 3, for $72.8 \%$ on day 5 and for $90.5 \%$ on day 7. Rapid demethylation from admission to day 3 occurred for $76.2 \%$ of the CpG-sites, of which $67.9 \%$ remained equally demethylated or partially remethylated and $32.1 \%$ further demethylated beyond day 3. From admission to day 3,19.7\% of the CpG-sites became hypermethylated, of which, beyond day 3, 34.5\% remained equally hypermethylated or partially demethylated again and $65.5 \%$ further hypermethylated. For $4.1 \%$ of the CpG-sites, changes only appeared beyond day 3 . Of the $\mathrm{CpG}$-sites affected by early-PN versus late-PN at PICU discharge, 38.9\% were already differentially methylated by day 3 , another $25.0 \%$ by day 5 and another $13.9 \%$ by day 7 . Conclusion: Critical illness- and early-PN-induced changes in DNAmethylation occurred mainly within 3 days. Most abnormalities were at least partially maintained or got worse with longer time in PICU. Interventions targeting aberrant DNA-methylation changes should be initiated early.

\section{Reference(s) and grant ackowledgment(s) \\ 1. 1Guïza et al. Lancet Respir Med 2020 \\ 2. Methusalem;ERC;IWT;Sophia Research Foundation;Agis \\ Zorginnovatie;ESPEN}

\section{5}

Anaesthetic management of tracheo-oesophageal fisula with pulmonary atresia, pda with single ventricle with tetrology of fallot

M. singh ${ }^{1}$; A. Kumar ${ }^{2}$

${ }^{1}$ ANAESTHESIA AND CRITICAL CARE, London North West University Healthcare NHS Trust, London, United Kingdom; ${ }^{2}$ Anaesthesia and critical care, Lady Hardinge Medical College, New Delhi, India

Correspondence: $M$. singh

Intensive Care Medicine Experimental 2020, 8(2): 000875

Introduction: Esophageal atresia (EA) with or without trachea-esophageal fistula is a common neonatal emergency which may be associated with major life incompatible cardiac abnormalities1. Survival of such neonate depends on stabilization and proper resuscitation followed by postoperative care.

Objectives: Keywords- Esophageal atresia, trachea-esophageal fistula, single ventricle, PDA, VSD

Methods: Case Report:

We came across a 4 days old male baby, with diagnosis of EA and distal TEF, posted for right thoracotomy with primary repair under GA. On preoperative evaluation baby had respiratory distress, frothing from mouth \& was hemodynamically unstable on dopamine with oxygen saturation (SpO2) of $85 \%$ on room air. Intraoperatively surgery was uneventful but baby not able to maintain $\mathrm{SpO} 2$ even after ligation of fistula. At time of reversal of neuromuscular blockade, he had active movements, was maintaining SpO2 of $88 \%$ with $100 \%$ O2 \& on dopamine. After extubation he maintained $\mathrm{SpO} 2$ of $85 \%$ on nasal prongs when shifted to PACU. But after 3 hours of surgery, his SpO2 started falling \& developed persistent cyanosis, so trachea was intubated with chest tube insertion on right side done suspecting pneumothorax or hemothorax or pleural effusion which were subsequently ruled out, 
emergency $2 \mathrm{D}$ echocardiography showed large VSD, pulmonary atresia, PDA, single ventricle with Tetrology of Fallot. He was started on PGE1, Alprostadil \& dobutamine because of worsening hemodynamic instability shifted to NICU but was not able to maintain SpO2>75\% with mechanical ventilation. His general physical condition deteriorated over next 2 days and died due to respiratory failure and cardiac arrest.

Results: Discussion and Conclusion:

EA- TEF is a major congenital anomaly and when it is associated with life incompatible anomalies like single ventricle along with PDA, VSD and Pulmonary atresia,it becomes a nightmare and a real challenge to both pediatric surgeon andanaesthesiologis1,4.Success in survival of neonate with EA-TEF is attributed to improved neonatal care, anesthesia and better understanding of neonatal physiology.But unfortunately our institution is not well equipped with emergency cardiac back up which if would have been there, then after successful ligation of trachea-esophageal fistula, our 4 days old neonate could have been saved, who died of respiratory failure and cardiac arrest.

Conclusion: This case emphasizes the importance of considering headache \&/or visual changes developing during postpartum period - a prodrome for LPE even in normotensive patients. Early diagnosis with subsequent initiation of anticonvulsants \& antihypertensive can prevent severe complications.

\section{Reference(s) and grant ackowledgment(s)}

1. 4. Walker A, Stokes M, Moriarty A. Anesthesia for major general surgery in neonates with complex cardiac defects. Paediatr Anaesth 2009:19:119-25.

2. 3. Hiroaki K, Koonosuke N, Takeshi H, et al. Unilateral pulmonary agenesis with tracheoesophageal fistula: A case report. J Pediatr Surg 1995; 30:1523-5.

3. 1. John JH. Atresia and tracheoesophageal fistula. In: Richard EB, Robert MK, Hal BJ, eds. Nelson textbook of pediatric. 16th ed. Philadelphia: W.B. Saunders, 2000; 1122-3.

4. 2. White MC. Approach to managing children with heart disease for noncardiac surgery. Paediatr Anaesth 2011;21:522-9

\section{5}

Isolated pathogens and their trends on antimicrobial resistance in a university pediatric intensive care unit: a decade-long longitudinal study

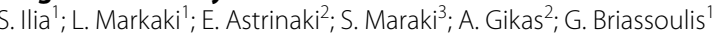

${ }^{1} \mathrm{PICU}$, University of Crete, Heraklion, Greece; ${ }^{2}$ Infection control committee, University Hospital, Heraklion, Greece; ${ }^{3}$ Microbiology laboratory, University Hospital, Heraklion, Greece

Correspondence: S. Ilia

Intensive Care Medicine Experimental 2020, 8(2): 001045

Introduction: Longitudinal data on isolated pathogens and trends of antimicrobial resistance (AMR) in pediatric intensive care units (PICUs) are worldwide limited.

Objectives: To determine the most common microorganisms cultured from biological samples along with AMR trends over the years 2008-2019 in a university PICU.

Methods: Data from the Infection Control Committee electronic database were retrospectively exported and analyzed.

Results: Out of 2,127 positive cultures, 1,598 were analyzed (529 duplicates). Overall, Gram (-) microorganisms (972, 60.8\%) predominated, mainly represented by Pseudomonas aeruginosa (365, 37.6\%). Out of Gram (+) pathogens (475, 29.7\%) coagulase-negative Staphylococci (CoNS) $(280,58.9 \%)$ were most commonly found. Fungal positive cultures were infrequently reported $(151,9.5 \%)$. CoNS increased markedly over the years, compared to other Gram $(+)$ pathogens $(p=0.003)$ and were the most consistently identified isolates in blood cultures $(p<0.001)$. Gram (-) bacteria resistance rates are shown in Table 1.

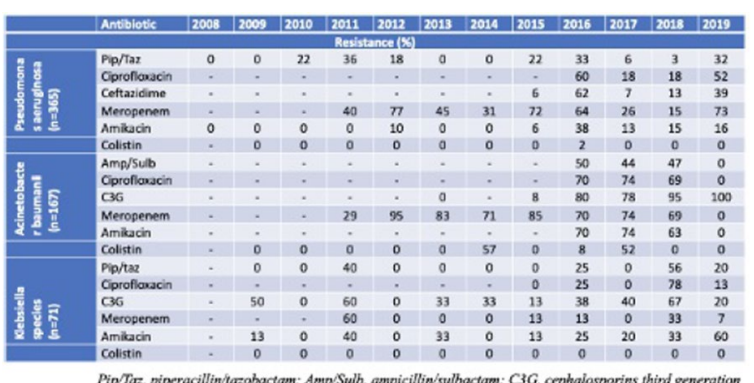

Conclusion: Gram-negative bacteria were the most commonly cultured pathogens, mainly represented by P.aeruginosa. Although their incidence did not change over a decade, the high carbapenem and third-generation cephalosporin resistance rates stress the need for strict implementation of infection control bundles and AMR stewardship interventions.

\section{Reference(s) and grant ackowledgment(s)}

1. Karakonstantis S, Kritsotakis El, Gikas A. Pandrug-resistant Gram-negative bacteria: a systematic review of current epidemiology, prognosis and treatment options. The Journal of antimicrobial chemotherapy. 2020 Feb 1;75(2):271-82.

2. Levy M, Bonacorsi S, Naudin J, Caseris M, Thebault E, Mariani-Kurkdjian P, et al. Multidrug-resistant bacterial carriage and related healthcare-associated infections in a pediatric intensive care unit: a 6 -year prospective study. Intensive care medicine. 2019 Jul:45(7):1037-9.

3. European Centre for Disease Prevention and Control. Factsheet for experts - Antimicrobial resistance 2020 [cited 202028 Februray]. Available from: https://w w w .ecdc.europa.eu/en/antimicrobial-resistance/facts/ factsheets/experts.

001167

Patent ductus arteriosus (PDA) management: a therapeutic strategy in newborns on vv- ECMO support

S. Bianzina ${ }^{1}$; R. Tedeschi²; S. Pezzato ${ }^{1}$; S. Buratti'; A. Moscatelli

${ }^{1}$ Neonatal and Pediatric Intensive Care Unit, G. Gaslini Hospital, Genoa, Italy; ${ }^{2}$ Anesthesia and intensive care, University of Bari, Bari, Italy

Correspondence: S. Bianzina

Intensive Care Medicine Experimental 2020, 8(2): 001167

Introduction: There is some evidence supporting the manipulation of DA patency in newborns affected by pulmonary hypertension, but choosing the appropriate timing for PDA closure is still a challenge.

Objectives: Aim of our study was to evaluate the haemodynamic effects of PDA closure during vv-ECMO support.

Methods: We evaluated newborns affected by severe acute respiratory failure secondary to pulmonary hypertension on $\mathrm{vv}-\mathrm{ECMO}$ and with PDA, admitted to our ICU between May 2017 and July 2018. We collected haemodynamic parameters (MAP, HR, SpO2, NIRS, urinary output), blood gasanalysis, biochemical data, respiratory and inotropic support at 24 hours pre and 48 hours post-PDA closure (documented by echocardiography).

Results: We analyzed 7 newborns ( 2 males and 5 females), with mean gestational age of 36.4 weeks and mean body weight of $2.9 \mathrm{~kg} .5$ out of 7 patients were affected by congenital diaphragmatic hernia, 1 by meconium aspiration syndrome and 1 by hemolytic disease of the newborn with hydrops. In 3 cases we obtained pharmacological PDA closure (with ibuprofen iv), while 4 patients underwent surgical approach. Comparing post vs pre-PDA closure parameters, we found 
a significant reduction $(p<0.05)$ in lactate levels $(14 \pm 7.8$ vs $20.5 \pm 13$ $\mathrm{mg} / \mathrm{dl})$ and epinephrine dose $(0.05 \pm 0.1 \mathrm{vs} 0.08 \pm 0.1 \mathrm{mcg} / \mathrm{kg} / \mathrm{min})$. We also detected a trend in increasing of MAP $(58.7 \pm 11.8$ vs $53.3 \pm 15.2$ $\mathrm{mmHg}$ ) and decreasing of milrinone $(0.4 \pm 0.33 \mathrm{vs} 0.44 \pm 0.32 \mathrm{mcg} / \mathrm{kg} /$ $\mathrm{min})$ and iNO (9.3 \pm 6.1 vs $12.2 \pm 6.9 \mathrm{ppm}$ ) support, but with no statistical significance. There were no differences, instead, in SpO2 $(95.6 \pm 2.1$ vs $92.4 \pm 9.7 \%)$, NIRS (61.8 \pm 9.1 vs $61.9 \pm 8.1 \%)$ and urinary output $(6.3 \pm 2.7 \mathrm{vs} 7 \pm 4 \mathrm{ml} / \mathrm{kg} / \mathrm{h})$.

Conclusion: During the early phase of severe pulmonary hypertension, PDA is important to ensure the unloading of right ventricle and a better systemic perfusion. With the progressive lowering of pulmonary vascular resistances, instead, PDA closure leads to an improvement of systemic perfusion indexes (lactates) and a reduction of inotropic drugs.

\section{4}

Perioperative risk factors for ischemic stroke after isolated coronary artery bypass surgery

F. Ampatzidou'; C. Mavromanolis²; A. Vlahou²; K. Diplaris²; O. Ananiadou²; A. Baddour ${ }^{2}$; A. Madesis'; G. Drossos ${ }^{2}$

${ }^{1}$ Cardiothoracic icu, G.Papanikolaou Hospital, Thessaloniki, Greece; ${ }^{2}$ Cardiothoracic surgery, G.Papanikolaou Hospital, Thessaloniki, Greece

Correspondence: F.Ampatzidou

Intensive Care Medicine Experimental 2020, 8(2): 000134

Introduction: Stroke after coronary artery bypass surgery (CABG), is a serious complication with an incidence of $1.3 \%$ and a reported mortality of $20 \%$. Identification of intraoperative and early postoperative pathogenetic mechanisms is important for developing preventive strategies

Objectives: To evaluate perioperative factors related to postoperative stroke in coronary artery bypass surgery (CABG

Methods: All patients who underwent CABG procedure with the use of cardiopulmonary bypass from June 2012 to February 2020 and were complicated with postoperative ischemic stroke, were retrospectively evaluated. The following perioperative factors were compared between stroke patients-Group A, and control group B: Female gender, history of stroke, current smoking, asymptomatic carotid disease $(>50 \%$ stenosis), diabetes mellitus, cardiopulmonary bypass time(CPB),low cardiac output syndrome(LCOS) and postoperative atrial fibrillation

Chi square test was used for statistical analysis

Results: From a total of 2339 CABG patients (37 off pump procedures were excluded), 43 were complicated with postop ischemic stroke Results are shown in Table 1

\begin{tabular}{|l|l|l|l|}
\hline & Group A & Control group & \\
& $\mathrm{n}=43$ & $\mathrm{n}=2296$ & \\
\hline Age & $65,6 \pm 9$ & $64,9 \pm 9.5$ & \\
\hline Female (n, \%) & $11(25,6 \%)$ & $320(13,9 \%)$ & 0,04 \\
\hline History of stroke & $8(18,6 \%)$ & $214(9,3 \%)$ & 0,06 \\
\hline Smoking & $12(27,9 \%)$ & $834(36,5 \%)$ & 0,28 \\
\hline Carotid disease & $10(23,7 \%)$ & $286(12,5 \%)$ & 0,04 \\
\hline Diabetes & $22(51,2 \%)$ & $889(38,7 \%)$ & 0,11 \\
\hline CPB $>120$ min & $13(30,2 \%)$ & $406(17,7 \%)$ & 0,03 \\
\hline LCOS & $7(16,3 \%)$ & $142(6,1 \%)$ & 0,03 \\
\hline Post-op AF & $24(55,8 \%)$ & $670(29,2 \%)$ & $<0,01$ \\
\hline
\end{tabular}

Conclusion: Female gender, carotid disease, CPB time $>120 \mathrm{~min}$ LCOS and postoperative atrial fibrillation are factors with statistical significant relationship with postoperative ischemic stroke after CABG

\section{Reference(s) and grant ackowledgment(s)}

1. Gaudino M. Stroke After Coronary Artery Bypass Grafting and Percutaneous Coronary Intervention: Incidence, Pathogenesis, and Outcomes. J Am Heart Assoc. 2019 Jul 2;8(13):e013032
000136

Time interval between myocardial infarction and elective coronary artery bypass surgery: Impact on postoperative morbidity and mortality

F. Ampatzidou'; C. Mavromanolis²; K. Diplaris²; N. Mihail'²; A. Stratou²; N. Antonopoulos 2 ; T. Karaiskos ${ }^{2} ;$ G. Drossos ${ }^{2}$

${ }^{1}$ Cardiothoracic icu, G.Papanikolaou Hospital, Thessaloniki, Greece; ${ }^{2}$ Cardiothoracic surgery, G.Papanikolaou Hospital, Thessaloniki, Greece

Correspondence: F. Ampatzidou

Intensive Care Medicine Experimental 2020, 8(2): 000136

Introduction: Surgical revascularization is the only therapeutic choice in many patients with coronary artery disease. The optimal timing of coronary artery bypass grafting (CABG) after myocardial infarction (MI) remains unclear

Objectives: To investigate if time interval between myocardial infarction and elective CABG has any impact on early postoperative morbidity and mortality

Methods: All patients with a history of myocardial infarction, who underwent $C A B G$ procedure with the use of cardiopulmonary bypass from June 2012 to February 2020, were retrospectively evaluated. Exclusion criteria were : Rescue CABG and off pump procedures. Based on time distance from myocardial infarction(MI) patients were divided in : $\mathrm{MI}<1$ month (Group A) and MI > 1 month (Group B) The following factors were compared between the groups: post-op use of Intra-aortic Balloon Pump (IABP), use of Levosimedan for inotropic support atrial fibrillation (AF), acute kidney injury $(\mathrm{AKI})$, prolonged mechanical ventilation ( $>24$ hours), perioperative myocardial infarction (PMI) and mortality. We also compared patients with $\mathrm{Ml}<1$ week (Group C) with the rest of the cohort (Group D). Age, gender, Euro score II were similar between the groups. Chi square test was used for the statistical analysis

Results: Results are shown in Tables 1 and 2

\begin{tabular}{|l|l|l|l|}
\hline & Group A & Group B & P value \\
& $\mathrm{n}=467$ & $\mathrm{n}=635$ & \\
\hline Use of IABP (n,\%) & $57(12,2 \%)$ & $45(7,1 \%)$ & 0,004 \\
\hline Levosimendan (n,\%) & $49(10,5 \%)$ & $43(6,8 \%)$ & 0,03 \\
\hline AF(n, \%) & $150(32,1 \%)$ & $201(31,7 \%)$ & 0,87 \\
\hline AKI(n,\%) & $50(10,7 \%)$ & $94(14,6 \%)$ & 0,1 \\
\hline Prolonged ventilation & $17(3,6 \%)$ & $20(3,1 \%)$ & 0,65 \\
\hline (n,\%) & & & \\
\hline PMI(n, \%) & $5(1,1 \%)$ & $8(1,3 \%)$ & 0,8 \\
\hline Mortality (n, \%) & $13(2,8 \%)$ & $8(1,3 \%)$ & 0,07 \\
\hline
\end{tabular}

Table 1

\begin{tabular}{|l|l|l|l|}
\hline & Group C & Group D & P value \\
& $\mathrm{n}=81$ & $\mathrm{n}=1021$ & \\
\hline Use of IABP $(\mathrm{n}, \%)$ & $14(17,3 \%)$ & $88(8,6 \%)$ & 0,01 \\
\hline Levosimendan(n,\%) & $11(13,6 \%)$ & $81(7,9 \%)$ & 0,08 \\
\hline Prolonged vent & $9(11,1 \%)$ & $28(2,7 \%)$ & $<0,01$ \\
\hline$(\mathrm{n}, \%)$ & & & \\
\hline PMI $(\mathrm{n}, \%)$ & $4(4,9 \%)$ & $9(0,9 \%)$ & 0,01 \\
\hline Mortality $(\mathrm{n}, \%)$ & $4(4,9 \%)$ & $17(1,7 \%)$ & 0,04 \\
\hline
\end{tabular}

Conclusion: In patients who underwent CABG during the first month from myocardial infarction IABP and Levosimendan were more common required. Patients with time interval of 1 week had higher mortality, while use of IABP was more frequent. Perioperative myocardial infarction and prolonged mechanical ventilation were also more frequent 
Reference(s) and grant ackowledgment(s)

1. E. Nichols.Optimal Timing From Myocardial Infarctionto Coronary Artery

Bypass Grafting on Hospital Mortality Ann Thorac Surg 2017;103:162-71

\section{8}

Prevalence of postoperative atelectasis in patients who had minimally invasive cardiac surgery through a right mini-thoracotomy

N. Ishizuka' ${ }^{1}$;. Santanda ${ }^{1}$; K. Ishii ${ }^{1}$; Y. Norisue ${ }^{1}$

'Intensive care medicine, Tokyo Bay Urayasu Ichikawa Medical Center, Urayasu, Japan

Correspondence: N. Ishizuka

Intensive Care Medicine Experimental 2020, 8(2): 000148

Introduction: Postoperative atelectasis after cardiac surgery with median sternotomy, occurring 32 to $68 \%$ [1] [2] [3], is commonly considered as a cause of postoperative hypoxia. On the other hand, re-expansion pulmonary edema (RPE), rather than atelectasis, is frequently considered as a cause of postoperative hypoxia after minimally invasive cardiac surgery (MICS) with right mini-thoracotomy although the reported incidence of RPE is only $2.1 \%$ [4]. There is no previous study reporting the incidence of postoperative atelectasis in patients who had MICS with right mini-thoracotomy.

Objectives: The aim of this study is to assess the incidence rate of postoperative atelectasis in patients who underwent a right mini-thoracotomy MICS.

Methods: We retrospectively investigated the incidence rate of postoperative atelectasis in patients who underwent a right minithoracotomy MICS between January 2017 and December 2018 in our hospital. Postoperative chest X-rays were reviewed by two independent doctors and diagnosis of atelectasis was made only when two doctors agreed. We included only atelectasis defined by collapse or incomplete expansion of the right lung or part of the right lung and excluded pulmonary edema without volume loss of the lung field.

Results: One hundred fifty patients underwent right mini-thoracotomy MICS during the period. We excluded 3 patients who had reoperation on the same day because of complications of the first surgery and we included total of 147 patients. The mean age was 59.1 years and $57 \%$ were male. 48 patients $(32.7 \%)$ were diagnosed with atelectasis on the operated side. Cohen's kappa inter-evaluator reliability was 0.637 . Most conducted surgery was mitral valve surgery (82 patients, $55.8 \%)$. RPE was detected only in six patients (4.1\%).

Patients with postoperative atelectasis were older (mean age; 64.8 vs $58.9, P=0.035$ ) than patients without postoperative atelectasis. There was no statistical difference in the incidence of atelectasis between aortic valve surgery and mitral valve surgery $(37.2 \%$ vs $30.5 \%, \mathrm{P}=$ 0.29).

Conclusion: We detected about $30 \%$ postoperative atelectasis on the operated side after right mini-thoracotomy MICS. Atelectasis is a common postoperative complication not only in cardiac surgery with median sternotomy but also in MICS with right mini-thoracotomy. Atelectasis should be included in the differential diagnosis for postoperative hypoxemia after MICS with right mini-thoracotomy because postoperative atelectasis can be rapidly improved with active physical therapy.

\section{Reference(s) and grant ackowledgment(s)}

1. 1) Niyayeh Saffari NH, et al. Frequency Rate ofAtelectasis in Patients Following Coronary Artery Bypass Graft and Its Associated Factors at Mazandaran Heart Center in 2013-2014. Glob J Health Sci. 2015;7:97-105.

2. 2) Foghsgaard $S$, et al. Minimally invasive aortic valve replacement reduces atelectasis in cardiac intensive care. Acute Card Care. 2009;11:169-172.

3. 3) Vargas FS, et al. Influence of atelectasis on pulmonary function after coronary artery bypass grafting. Chest. 1993;104:434-437.

4. 4) Irisawa $Y$, et al. Re-expansion pulmonary oedema after minimally invasive cardiac surgery with right mini-thoracotomy. Eur J Cardiothorac Surg. 2016:49:500-505.
000167

SIM, RECORD, REPLAY, REPEAT Model: Putting lessons from simulation \& simulation debrief into practice within 15 minutes

P. Walker ${ }^{1}$; S. Peck'; L. Bezzina ${ }^{1}$; A. Mohamed ${ }^{1}$; C. Curtin

${ }^{1}$ Medical education, Queen's Hospital, Romford, United Kingdom

Correspondence: L. Bezzina

Intensive Care Medicine Experimental 2020, 8(2): 000167

Introduction: The literature, qualitative feedback and personal experience tells us that simulation provides a highly effective platform for learning owed to its' fully immersive design which aims to replicates substantial aspects of real life for a medical professional [1] but without putting patients at risk. Yet it is hard to quantify if it is more effective than the traditional didactic equivalents and if it is, as we suspect it is, to what degree [2]. Secondly, it remains almost impossible to evaluate whether the clear technical and non-technical learnings, that take place during a full day of simulation, are put in to practice upon an individual's return to work.

Here we describe a simulation design which enable the learning from simulation and the simulation debrief to be put into practice immediately within an individuals' workplace, with their usual colleagues, in the same morning. This is likely to be a superior model for in-situ simulation going forward. Barking, Havering and Redbridge University Trust (BHRUT) hold anaesthetic and theatre clinical governance meetings on a monthly basis and thus, all theatre activity is stopped across site for an entire morning. On these mornings theatre staff are asked to come in as normal and hence this presents a perfect opportunity for training. Objectives: We aim to demonstrate that learning and knowledge through the proposed SIM, RECORD, REPLAY, REPEAT process is more likely to be retained as a result of making errors and correcting errors in consecutive simulations, respectively, within their work environment and with their usual team.

Methods: The simulation team, the junior on-call anaesthetist, the consultant anaesthetist on-call and a full cohort of theatre staff including, ODPs, scrubs nurses and anaesthetic nurses were involved in an in-situ simulation scenario of anaphylaxis on induction.

The simulation was recorded on the 'SMOTS ${ }^{\mathrm{TM}}$ In-Situ System' cameras with playback SMOTS technology [3]. The simulation ran for 10-minutes in a functional theatre and the debrief that followed took place immediately afterwards in the neighbouring empty theatre. Staff first watched their performance back and then debriefed their performance, stating which technical and non-technical factors they felt they underperformed in and how these could be corrected. Staff were then given the opportunity to take part in an identical simulation, retaining the same roles, in an endeavour to improve aspects of their performance that they identified as suboptimal.

Results: Result collection is ongoing at time of writing: Through this work we hope to demonstrate that a second playback and debrief of a second consecutive identical in-situ simulation will provide evidence of significant improvements in performance. More importantly, we hope to demonstrate that learning and knowledge through this process is more likely to be retained as a result of making errors and correcting errors in consecutive simulations, respectively.

Conclusion: We put the 'SIM, RECORD, REPLAY, REPEAT' process described here forward as a superior model for in-situ simulation training because of the obvious advantages of the conditions (no theatre lists, same workplace, same team) together with being able to action both technical and non-technical learnings with immediate effect. We postulate that learnings in these conditions are more likely to make it into the working environment as a result of the usual team being involved and bearing witness to improvements over a short period of time.

We believe this model can be extrapolated to ITU and other areas of medicine and we would encourage staff and managers to look to create space for this model of learning owed to the clear benefits for patient safety.

\section{Reference(s) and grant ackowledgment(s)}

1. 3. The smots ${ }^{\mathrm{TM}}$ Insitu system (SC742HD3); http://www.scotiauk.com/products/smots/products/packngo/index.html 
2. 2. Shapiro MJ, Morey JC, Small SD, Langford V, Kaylor CJ, Jagminas L, et al. Simulation based teamwork training for emergency department staff: does it improve clinical team performance when added to an existing didactic teamwork curriculum? Qual Saf Health Care. 2004;13:417-21.

3. 1. Lateef F. Simulation-based learning: Just like the real thing. J Emerg Trauma Shock. 2010 Oct;3(4):348-52.

\section{0}

Cost-effectiveness of vitamin C suppletion in CABG: a prospective, single centre randomised clinical trial

E. Annaert ${ }^{1}$; N. Van Der Vekens ${ }^{1}$; A. Kalmar ${ }^{1}$; H. Vanoverschelde ${ }^{1}$; S. Allaert ${ }^{1}$; J. Heerman ${ }^{1}$

${ }^{1}$ Anesthesiology, Maria Middelares, Gent, Belgium

Correspondence: E. Annaert

Intensive Care Medicine Experimental 2020, 8(2): 000210

Introduction: Vitamin $C$ has been investigated for a wide variety of uses within intensive care due to its anti-oxidative properties $(1,2,3)$. Research regarding its potential for the prevention of postoperative atrial fibrillation (POAF) after cardiac surgery is ongoing but results remain inconsistent $(4,5,6)$. Due to a broad intracellular anti-oxidant effect, several postoperative pathological effects might be mitigated. Taking into account the low cost of vitamin C, it might be more valuable to investigate a pharmaco-economic benefit in addition to an effect on POAF.

Objectives: Our primary goal was to investigate the pharmaco-economic impact of perioperative supplementary oral ascorbic acid intake in patients undergoing coronary artery bypass graft surgery (CABG). Secondary endpoints were the effects on the length of stay in both the intensive care unit (ICU) and the hospital and the incidence of POAF.

Methods: After IRB approval, patients planned for CABG who met the inclusion criteria (in cardiac sinus rhythm preoperatively and not taking supplementary vitamin C independently) were randomized into a placebo group and an intervention group. The medication was given orally, 1 gram twice a day, starting from 5 days preoperatively until 10 days postoperatively. The used medication, length of hospital and ICU stay and development of POAF in ICU were recorded. We report the preliminary results with a calculated target sample size of 75 patients. Significant differences were assessed using an independent T-test and a Mann-Whitney $U$ test.

Results: Of the 119 screened patients, 68 patients completed the study. Except for BMI $(p=0.029)$, there was no statistically significant difference in baseline characteristics. (Table 1) The median pharmacoeconomic cost was significantly lower $(P=0.009)$ in the vitamin $C$ group ( $€ 1636)$ than in the placebo group (€1687). There was no significant difference in the incidence of POAF or length of stay. (Table 2)

Table 1. Demographics

\begin{tabular}{|l|l|l|}
\hline & Vitamin C & Placebo \\
\hline Age (Mean; Standard Deviation) & $66(10)$ & $66(8)$ \\
\hline Male/Female (Count) & $24 / 6$ & $31 / 7$ \\
\hline BMI (Mean; Standard Deviation) & $25,9(3,1)$ & $28,0(4,5)$ \\
\hline
\end{tabular}

Table 2. Results

\begin{tabular}{|c|c|c|c|}
\hline & Placebo & Vitamin C & P-value \\
\hline $\begin{array}{l}\text { Pharmaco economic } \\
\text { expenditure (median; } \\
\text { min-max) }\end{array}$ & $\begin{array}{l}€ 1687 \\
(€ 1299-€ \\
3662)\end{array}$ & $\begin{array}{l}€ 1636 \\
(€ 663-€ \\
1892)\end{array}$ & 0.009 \\
\hline $\begin{array}{l}\text { Length of hospital } \\
\text { stay (days) (Median; } \\
\text { min-max) }\end{array}$ & $7(5-14)$ & $7(5-9)$ & $>0.05$ \\
\hline $\begin{array}{l}\text { Length of ICU stay } \\
\text { (hours) (Median; min- } \\
\text { max) }\end{array}$ & $36(8-93)$ & $28(21-68)$ & $>0.05$ \\
\hline POAF incidence & $7 / 38(18.4 \%)$ & $7 / 30(23.3 \%)$ & $>0.05$ \\
\hline
\end{tabular}


The postoperative complications are shown Table 1. Although no differences were observed in acute kidney injury between the EFB and non-EFB $(P=0.36)$, the cardiovascular complications postoperatively were higher in EFB group. Also, mortality rates were statistically different in EFB (10\%) and in non-EFB (3.9\%) groups $\mathrm{P}=0.02$.

Table 1. Postoperative outcomes

\begin{tabular}{|c|c|c|c|c|c|c|}
\hline & \multicolumn{3}{|c|}{ Original Cohort } & \multicolumn{3}{|c|}{ Matched Cohort } \\
\hline & $\begin{array}{l}\text { Non-EFB } \\
(n=257)\end{array}$ & $\begin{array}{l}\text { EFB } \\
(n=329)\end{array}$ & $\mathbf{P}$ & $\begin{array}{l}\text { Non-EFB } \\
(n=180)\end{array}$ & $\begin{array}{l}\text { EFB } \\
(n=180)\end{array}$ & $\mathbf{P}$ \\
\hline $\begin{array}{l}\text { On ICU } \\
\text { postoperatively } \\
\text { Composite }\end{array}$ & & & & & & \\
\hline $\begin{array}{l}\text { cardiovascular, } \mathrm{n} \\
(\%)\end{array}$ & $190(34.4 \%)$ & $270(45,7 \%$ & 0.0 & $063(35.0 \%)$ & $96(53.3 \%)$ & 0.00 \\
\hline $\begin{array}{l}\text { intra-aortic } \\
\text { balloon pump } \\
\text { requirement, } \mathrm{n} \\
(\%)\end{array}$ & $12(2,2 \%)$ & $39(6.6 \%)$ & & $03(1.7 \%)$ & $11(6.1 \%)$ & 0.02 \\
\hline $\begin{array}{l}\text { Acute kidney } \\
\text { injury }(\%)\end{array}$ & 209 (37. 9) & $207(35 \%)$ & & $261(33.9)$ & $53(29.4 \%$ & 0,36 \\
\hline $\begin{array}{l}\text { ICU LOS (d), } \\
\text { median (IQ 25- } \\
75 \text { ) }\end{array}$ & $2(1-4)$ & $2(1-4)$ & & $93(1-4)$ & $2(1-4)$ & 0.29 \\
\hline $\begin{array}{l}\text { Hospital LOS } \\
\text { (d), median (IQ } \\
25-75)\end{array}$ & $17(11-26)$ & $16(9-25)$ & & $\begin{array}{l}0 \begin{array}{l}15(9- \\
23.75)\end{array} \\
0\end{array}$ & $\begin{array}{l}22(13- \\
30.75)\end{array}$ & 0.01 \\
\hline $\begin{array}{l}\text { Hospital } \\
\text { mortality rate }\end{array}$ & $53(9,0 \%)$ & $66(12 \%)$ & 0.1 & $07(3.9 \%)$ & $18(10.0 \%)$ & 0.02 \\
\hline
\end{tabular}

Legends: EFB - Excessive fluid balance. ICU - intensive care unit. All values between parentheses represent percentile $25-75$ values observed.

Conclusion: Patients undergoing off-pump coronary artery bypass with excessive intraoperative fluid balance have more complications and higher hospital mortality, but the same incidence of early acute kidney injury.

\section{Reference(s) and grant ackowledgment(s)}

1. Romagnoli S, Rizza A, Ricci Z. Fluid Status Assessment and Management During the Perioperative Phase in Adult Cardiac Surgery Patients. Journal of cardiothoracic and vascular anesthesia. 2016;30(4):1076-84.

2. Guarnieri M, De Gasperi A, Gianni S, Baciarello M, Bellini V, Bignami E. From the Physiology to the Bedside: Fluid Therapy in Cardiac Surgery and the ICU. Current Anesthesiology Reports. 2019;9(3):248-56.

3. We would like to thank all the teams from the two hospitals for their help in collecting data.

\section{8}

Postoperative blood pressure deficit and acute kidney injury

in early postoperative period after a liver transplantation

I. Saez de la Fuente'; J. Saez De La Fuente'; M. Isaias'; G. Judith'; Q. Nerea'; Z. Molina-Collado'; JA. Sanchez-Izquierdo'; JC. Montejo Gonzales ${ }^{1}$

${ }^{1}$ Critical care, University Hospital 12 de Octubre, Madrid, Spain; ${ }^{2}$ Pharmacy, Hospital Ramón y Cajal, Madrid, Spain

Correspondence: I. Saez de la Fuente

Intensive Care Medicine Experimental 2020, 8(2): 000308

Introduction: Acute kidney injury (AKI) is a significant complication in the care of patients with end-stage liver disease (ESLD) after liver transplantation (LT). In the present study, we addressed the question of whether those with AKI had a greater difference (deficit) between premorbid and within-ICU hemodynamic pressure-related parameters compared to those without AKI.

Methods: Prospective observational study including all patients admitted to the ICU after liver transplantation over a 3-years study period. Demographic variables (sex, age, cause of transplantation and severity scores), hemodynamic variables, and those related to graft function and postoperative complications were recorded. Patients were classified according to the development of AKI.

We obtained premorbid and ICU, time-weighted average values for hemodynamic pressure-related parameters (systolic [SAP], diastolic
[DAP], and mean arterial pressure [MAP];central venous pressure [CVP], mean perfusion pressure [MPP], and diastolic perfusion pressure [DPP]) and calculated deficits in those values.

Data statistical analysis was done using SPSS software. Quantitative variables are expressed as mean with standard deviation (SD) and qualitative variables as absolute and relative frequencies. The statistical significance of the proportion comparison was addressed by the chi-square test, Mann Whitney U test or Student's t-Test with a statistical significance $p<0.05$.

Results: During the study period, 100 patients were admitted to the ICU after a liver transplantation (80\% male) with a median age of 56 (IQR 50-61). The main causes of the liver transplant were alcoholism (58\%), hepatocellular carcinoma $(45 \%)$ and hepatitis $C$ virus infection (43\%). Median MELD score was 13 (IQR 9-19); Median ICU stay was 5 days (IQR 4-9), with an overall ICU mortality of $8 \%$.

Fifty nine patients presented AKI, which was associated with a significantly higher ICU stay ( 4 vs $6, p=0.005$ ), higher hospital stay ( 13 vs 20 , $\mathrm{p}=0.005$ ) and higher ICU mortality (0 vs $8, p=0.001)$.

All achieved pressure-related values were similar between patients with or without AKI progression. However, a significantly greater increase in CVP was observed in patients with AKI (0 vs $27 \%, p=0,039)$. Conclusion: Acute Kidney Injury after liver transplantation is a frecuent complication wich is associated with worse outcomes. The hypervolemia defined as an early increase in CVP could be a modifiable factor.

\section{Reference(s) and grant ackowledgment(s)}

1. Saito S, Uchino S, Takinami M, et al. Postoperative blood pressure deficit and acute kidney injury progression in vasopressor-dependent cardiovascular surgery patients. Critical Care (2016) 20:74

2. DellaVolpe J, Al-Khafaji A. Acute Kidney Injury Before and After Liver Transplant. Journal of Intensive Care 2019;34(9):687-695

3. Durand F, Francoz C, Asrani S.K, et al. Acute Kidney Injury After Liver Transplantation. Transplantation 2018;102: 1636-1649.

4. None

000387

Influence of nutritional status in outcomes of cardiac surgery

J. C. Lopez-Delgado1; RE. Avila-Espinoza²; G. Muñoz-Del Rio²;

M. Martinez-Medan²; N. Latorre-Feliu²; C. Sanz-Mellado²; S. Gonzalez-Del

Hoyo $^{2}$; D. Berbel-Franco 2 ; G. Fernandez-Olivares ${ }^{3}$

${ }^{1}$ Intensive care, Hospital Universitari de Bellvitge, L'Hospitalet de Llobregat (Barcelona), Spain; ${ }^{2}$ Intensive care, Hospital Universitari de Bellvitge, Barcelona, Spain; ${ }^{3}$ Statistics, IDIBELL Institut d'Investigació Biomèdica de Bellvitge, L'Hospitalet de Llobregat, Spain

Correspondence: J.C. Lopez-Delgado

Intensive Care Medicine Experimental 2020, 8(2): 000387

\section{Introduction:}

Nutritional status influences periorperative outcomes in patients with heart failure and cardiac surgery

Objectives: The aim of our study was to assess the influence of nutritional status on the results and prognosis of patients undergoing cardiac surgery.

\section{Methods:}

Prospective observational study from 2011 to 2015. Preoperative nutritional status was assessed using the Prognosis Nutritional Index (PNI). PNI was calculated as $10 \times$ serum albumin $(\mathrm{g} / \mathrm{dL})+0.005 \times$ total lymphocyte count (per $\mathrm{mm} 3$ ).Preoperative and intraoperative variables, postoperative complications, and hospital and long-term mortality were collected in a local database.

Results: 1279 patients were included: mean age 67 (59-73.43) years; 68.49\% (876) were men; BMI: 27.3 (24.9-30) $\mathrm{kg} / \mathrm{m}^{2}$; APACHE II: 12 (9-15). $54.03 \%$ (691) were valve surgeries and $40.27 \%$ (515) bypass surgeries. Hospital mortality was $6.8 \%$ (87). The patients were classified according to the nutritional status based on PNI values in three subgroups:

Normal 64.8\% (829), Moderate 20\% (256) and Severe 15.2\% (194). 
Univariate analysis between subgroups identified higher postoperative complications and mortality in the subgroups with the worst PNI. The multivariate analysis showed a longer mean hospital stay (24 (1633 ) vs 29.5 (21.75-41) days; OR: $1.015 ; 95 \% \mathrm{Cl}: 1.006-1.024 ; \mathrm{P}=0.001)$ in the moderate subgroup when compared to the normal one. Likewise, a higher

degree of renal dysfunction was observed (93 (77-115) vs 113 (83169) $\mathrm{umol} / \mathrm{L}$ of creatinine; OR: $1.003 ; 95 \% \mathrm{Cl}: 1.001-1.005 ; \mathrm{P}=0.005)$, a greater mean hospital stay (24 (16-33) vs 33 (24-49) days; OR: 1.022; $95 \% \mathrm{Cl}: 1.009-1.034 ; \mathrm{P}<0.001)$ and a longer time in mechanical ventilation (14 (8-20) vs 18 (10-68) hours; OR: 0.998; 95\% Cl: 0.996-0.999; $\mathrm{P}=0.01$ ) in the subgroup of patients with severe $\mathrm{PNI}$ compared to the normal subgroup.

Conclusion: A higher PNI may be associated with greater postoperative complications (i.e., renal and respiratory) after cardiac surgery, as well as a longer hospital stay in our population.

\section{Reference(s) and grant ackowledgment(s)}

1. Shirakabe A., et al. The prognostic impact of malnutrition in patients with severely decompensated acute heart failure, as assessed using the Prognostic Nutritional Index (PNI) and Controlling Nutritional Status (CONUT) score. Heart Vessels. 2018.

2. Lopez-Delgado JC., et al. Nutrition in Adult Cardiac Surgery: Preoperative Evaluation, Management in the Postoperative Period, and Clinical Implications for Outcomes. J Cardiothorac Vasc Anesth. 2019.

3. N/A

\section{9}

\section{Blood volume and preload responsiveness; a post hoc analysis} of a randomized controlled trial

A. Lindén-Sønders $\varnothing^{1}$; S. Statkevicius ${ }^{2}$; J. Bonnevier ${ }^{2}$; P. Bentzer ${ }^{1}$

${ }^{1}$ Department of anaesthesiology and intensive care, Helsingborg Hospital, Helsingborg, Sweden; ${ }^{2}$ Department of intensive and perioperative care, Skåne University Hospital Lund, Lund, Sweden

Correspondence: A. Lindén-Sønders $\varnothing$

Intensive Care Medicine Experimental 2020, 8(2): 000579

Introduction: Signs of inadequate cardiac output in the post-operative phase after major surgery is often treated with fluid in order to optimize preload [1]. Given that overly aggressive fluid treatment is likely to worsen outcome, prediction of fluid responsiveness by dynamic indices should be used in patients with circulatory instability to identify those likely to respond to fluid therapy with an increase in cardiac output [2]. However, preload is dependent not only on blood volume but also on venous tone meaning that fluid responsiveness may be related to a lower than normal venous tone rather than hypovolemia [3]. If so, this could imply that a pharmacological increase in venous tone can be a more rational approach to increase preload in some preload responsive patients.

Objectives: To assess the prevalence of hypovolemia in a cohort of patients likely to be preload responsive.

Methods: Data from the randomized controlled trial AIR was analyzed [4]. The AIR trial included post-operative patients after major abdominal surgery and was approved by the regional ethical vetting board in Lund, Sweden.

Inclusion criteria for the present analysis were post-operative patients with signs of hypoperfusion: a) $\mathrm{ScvO} 2<70 \%$, b) lactate $>2 \mathrm{mmol} / \mathrm{L}$, c) urine output $<0,5 \mathrm{ml} / \mathrm{kg}$ in the hour prior to inclusion or d) systolic BP $<100 \mathrm{mmHg}$ or MAP $<55 \mathrm{mmHg}$. In spontaneously breathing postoperative patients the passive leg raising test appear to be the most useful predictor of preload responsiveness $[5,6]$. A positive passive leg raising test was used to identify patients likely to be preload responsive and was defined as an increase in pulse pressure $(\Delta \mathrm{PP})$ of $>9 \%$, measured through an arterial line [7].

Plasma volume(measured using 125I-albumin) and hematocrit (measured spectrophotometrically) was used to calculate blood volume (BV). Normal BV was estimated based on length, weight and gender $[8,9]$. Patients were considered hypovolemic if the measured BV was at least $10 \%$ lower than the predicted normal BV, as previously defined
[10]. We defined hypervolemia as at least $10 \%$ higher BV than the predicted normal value. Data are presented as median (interquartile range) or as proportions.

Results: A total of 40 patients were included in the analysis. Baseline characteristics can be seen in Table 1.

Median blood volume in the cohort was 4051 (3373-5275) $\mathrm{ml}$ or 55 $(49-64) \mathrm{ml} / \mathrm{kg}$. A total of $43 \%(17 / 40)$ of the patients were hypovolemic, $32 \%(13 / 40)$ were euvolemic and $25 \%(10 / 40)$ were hypervolemic (Figure 1). A correlation analysis showed no correlation between blood volume and $\triangle \mathrm{PP}$ after PLR (Spearman, $\mathrm{R}=-0.109[95 \% \mathrm{Cl}-0.414-0.219$, $\mathrm{P}=0.505)$.

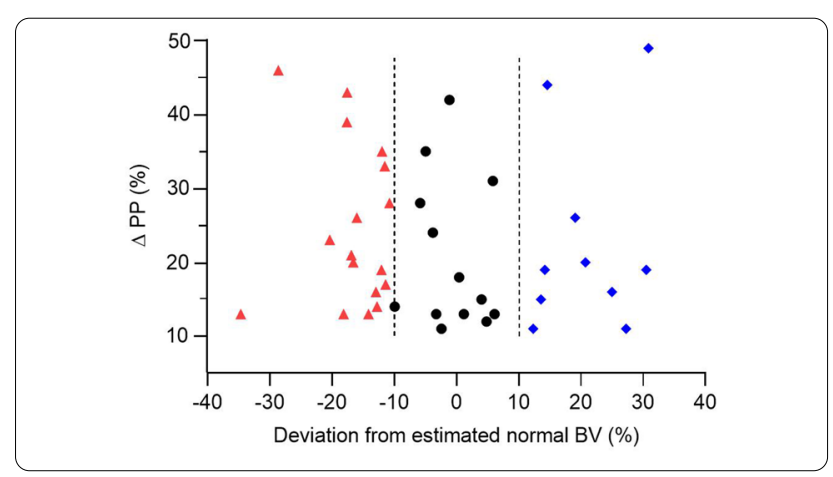

Table 1 - Patient demographics

\begin{tabular}{|l|l|}
\hline Age, $y r s$ & $67(54-73)$ \\
\hline Weight, $\mathrm{kg}$ & $71(62-85)$ \\
\hline Height, $\mathrm{cm}$ & $167(163-176)$ \\
\hline ASA & $2(2-3)$ \\
\hline POSSUM physiology score & $15(13-17)$ \\
\hline Revised Cardiac Index Score & $1(1-1)$ \\
\hline Duration of surgery, $\mathrm{min}$ & $402(329-480)$ \\
\hline Duration of anesthesia, min & $506(439-585)$ \\
\hline Peroperative bleeding, $\mathrm{ml}$ & $600(263-1000)$ \\
\hline Peroperative diuresis, $\mathrm{ml} / \mathrm{kg} / \mathrm{h}$ & $0.6(0.4-1.0)$ \\
\hline Peroperative crystalloids, $\mathrm{ml}$ & $4325(3963-5250)$ \\
\hline Peroperative colloid, $\mathrm{ml}$ & $725(500-1000)$ \\
\hline
\end{tabular}

Conclusion: A large fraction of hemodynamically unstable post-operative patients likely to be preload responsive are not hypovolemic and in these patients a pharmacological increase in venous tone may be a more rational approach to increase preload than further intravenous fluid administration.

\section{Reference(s) and grant ackowledgment(s)}

1. 10. Stephan, F., et al., Clinical evaluation of circulating blood volume in critically ill patients-contribution of a clinical scoring system. $\mathrm{Br}$ J Anaesth, 2001. 86(6): p. 754-62.

2. 9. Wennesland, R., et al., Red cell, plasma and blood volume in healthy men measured by radiochromium ( $\mathrm{Cr} 51)$ cell tagging and hematocrit: influence of age, somatotype and habits of physical activity on the variance after regression of volumes to height and weight combined. J Clin Invest, 1959. 38(7): p. 1065-77.

3. 8. Brown, E., et al., Red cell, plasma, and blood volume in the healthy women measured by radiochromium cell-labeling and hematocrit. J Clin Invest, 1962. 41: p. 2182-90.

4. 7. Preau, S., et al., Passive leg raising is predictive of fluid responsiveness in spontaneously breathing patients with severe sepsis or acute pancreatitis. Crit Care Med, 2010. 38(3): p. 819-25.

5. 6. Monnet, X., P. Marik, and J.L. Teboul, Passive leg raising for predicting fluid responsiveness: a systematic review and meta-analysis. Intensive Care Med, 2016. 42(12): p. 1935-1947. 
6. 5. Bentzer, P., et al., Will This Hemodynamically Unstable Patient Respond to a Bolus of Intravenous Fluids? Jama, 2016. 316(12): p. 1298-309.

7. 4. Statkevicius, S., et al., Albumin infusion rate and plasma volume expansion: a randomized clinical trial in postoperative patients after major surgery. Crit Care, 2019. 23(1), 191. doi:10.1186/ s13054-019-2477-7

8. 3. Martin, G.S., et al., Perioperative Quality Initiative (POQI) consensus statement on fundamental concepts in perioperative fluid management: fluid responsiveness and venous capacitance. Perioperative medicine (London, England), 2020. 9: p. 12-12.

9. 2. Navarro, L.H., et al., Perioperative fluid therapy: a statement from the international Fluid Optimization Group. Perioper Med (Lond), 2015. 4: p.

10. 1. Jammer I, Creagh-Brown B (2019) Squeeze. European Society of Anesthesiology. https://www.esahq.org/uploads/media/ESA/Files/ Research/Squeeze\%20protocol\%2010.pdf Accessed 10 May 2020.

11. Region Skåne (\#18401), the Anna and Edwin Berger Foundation, Sweden and the Birgit and Henry Knutsson Foundation, Sweden.

\section{9}

Influence of Infusion Therapy on severity of Intestinal Failure

M. F. NAKADE ${ }^{1}$; V. V. Endler ${ }^{2}$; M. V. Petrova ; A. V. Butrov ${ }^{1}$; R. Mohan ${ }^{1}$;

V. A. Kozlov ${ }^{3}$

${ }^{1}$ Department of anesthesiology and intensive care, Rossiysky universitet druzhby narodov «RUDN University», Moscow, Russia; ${ }^{2}$ Intensive care unit, Federal research and clinical center of intensive care medicine and rehabilitology, Moscow, Russia; ${ }^{3}$ Department of general surgery, City Hospital named after V.V.Vinogradov, Moscow, Russia

Correspondence: M.F. NAKADE

Intensive Care Medicine Experimental 2020, 8(2): 000679

Introduction: Most important problem of early post- operative period post major abdominal surgeries is Intestinal Failure (IF) which if unmanaged properly might lead to Abdominal Compartment Syndrome, ultimately causing Multiorgan Failure. Postoperative IF is suppression of motor-evacuation function, alteration of small bowel digestive, food intake capability, developing critical water-electrolyte imbalance, circulatory hypoxia of bowel wall, dysbacteriosis with proximal microbial colonization of bowels, accompanied by changes in acid-base balance. Main reason is inadequate infusion therapy during perioperative period. Liberal infusion therapy might aggravate visceral edema of ischemic bowels, while as insufficient infusion therapy might aggravate splanchnic ischemia

Objectives: Improving result of surgical treatment of patients post emergency abdominal surgery with selection of perioperative infusion therapy strategies, optimal for postoperative restoration of intestinal function

Methods: Research work included 60 patients (28 male, 32 female, average age $56.5 \pm 14$ years) post emergent abdominal surgeries, divided into 2 groups. In group $1(n=29)$ perioperative infusion therapy included balanced ionic solutions. 2nd group $(n=31)$ balanced ionic solutions was combined with synthetic colloids. For investigation of intestinal function in postoperative period, complex assessment of gastrointestinal tract condition, including physical examination, measuring of intra-abdominal pressure (IAP), ultrasound visualization of condition of intestinal wall as well as absorptive function of gastrointestinal tract, laboratory monitoring, perioperative infusion therapy was recorded, monitoring of vital functions

Results: The correlation reviewed was strong significant $(r=1.000$, $\mathrm{p}=0.01$ ) between the amount of infusion therapy and stage of IF. Day 1 \& subsequent two days, patients in both groups showed IF 3rd stage. Signs of bowel function recovery were observed by Day 4 . Correlation analysis, strong significant correlation between stage of IF and lumen diameter of small bowel $(r=0.931)$, intestinal wall thickness $(r=0.940)$, IAP $(r=0.998, P=0.01)$. The IAP as parameter with highest $r$ compared to other parameters was considered prognostic index for the IF dynamics, used to determine tactics of infusion therapy. In group 2, significant correlation was found between intraoperative infusion volume and IAP $(r=0.417)$, demonstrating that both excessive \& insufficient infusion load results in increased IAP on Day 1. Investigation of influence of infusion therapy quality on intestinal failure stage discovered that inclusion of a third generation hydroxyethyl starch infusion solution (patients of group 2) lowered infusion therapy influence on the bowels condition. Analysis of influence of infusion therapy quality on IAF and IF severity did not find significant differences between use and non-use of colloids as a part of infusion therapy; nevertheless, a trend towards more favorable course of IF in patients of group 2 was noted. Hence, the presence of colloids as a part of infusion therapy can be considered influence on IF severity

Conclusion: The amount of intraoperative infusion therapy has direct effect on postoperative intestinal function. Optimization and administration of oriented infusion therapy in postoperative periods leads to early regression of intestinal failure

\section{2}

Fluid administration guided by inferior vena cava ultrasound before spinal anaesthesia may reduce post procedural hypotension rate. A randomizedtrial

G. Bona'; M. Biggiogero ${ }^{2}$; M. Favre ${ }^{3}$; M. Roncador ${ }^{4}$; A. Saporito ${ }^{5}$; S. Ceruti ${ }^{6}$

${ }^{1}$ Internal medicine department, Clinica Luganese Moncucco, Lugano, Switzerland; ${ }^{2}$ Clinical research unit, Clinica Luganese Moncucco, Lugano, Switzerland; ${ }^{3}$ Anesthesia, Hôpitaux Universitaires de Genève (HUG), Genève, Switzerland; ${ }^{4}$ Internal medicine department, University Hospital of Zürich, Zürich, Switzerland; ${ }^{5}$ Anesthesia, EOC Ente Ospedaliero Cantonale, Bellinzona, Switzerland, Bellinzona, Switzerland; ${ }^{6}$ Critical care department, Clinica Luganese Moncucco, Lugano, Switzerland

Correspondence: S. Ceruti

Intensive Care Medicine Experimental 2020, 8(2): 000692

Introduction: Arterial hypotension following spinal anaesthesia is a common issue in clinical practice, possibly leading to major complications in emergency settings [1]. To prevent this, standard administration of intravenous fluids is usually performed. Inferior vena cava (IVC) ultrasound [2] and passive leg raising test (PLRT) [3] are effective methods in guiding fluid therapy in non-critical patients.

Objectives: Aim of the study was to evaluate IVC ultrasound and PLRT guided volaemic optimisation compared to standard of care in patients undergoing spinal anaesthesia, in order to decrease postanaesthesia hypotension rate and excessive fluid administration.

Methods: This prospective, controlled, randomised trial included 474 consecutive patients, allocated in a block of six into a control group, an IVC-ultrasound group and a PLRT group. Inclusion criteria were adult patients of both sexes, evaluated as American Society of Anaesthesiology (ASA)-risk class I to III 4, undergoing an elective intervention under spinal anaesthesia. Patients from the three groups received the same standard non-invasive monitoring.

Control group: According to standard of care, volaemic filling status was not checked prior to the procedure. Post-spinal anaesthesia hypotensive patients were treated according to standard protocol and clinical situation with crystalloid replacement and vasoactive drugs.

IVC-ultrasound group: IVC collapsibility index (IVC-CI) of $36 \% 5$ was chosen as the cut-off level to differentiate responsive (with IVC-CI $>36 \%$ ) or unresponsive patients (IVC-CI $<36 \%$ ) to fluid administration. In case of responsive pattern, $500 \mathrm{ml}$ of crystalloid infusion over 20 minutes was administered.

PLRT group: Before and after the PLRT, etCO2 analysis was performed; patients with an etCO2 increasing more than $12 \%$ from baseline were interpreted as responsive 3 and received crystalloid infusion.

Primary outcome was the hypotension rate reduction after spinal anaesthesia. Secondary outcomes were fluid amount administered, the amount of vasoactive drug used and the time needed to perform the whole anaesthetic procedure. 
Results: An $11 \%$ reduction rate in hypotension ( $p=0.047$ ) was observed between the IVCUS group and the control group. A $2 \%$ reduction rate in hypotension $(p=0.428)$ was observed between the PLRT and the control group. Total fluid amount administered was greater in the IVCUS than in the control group $(593 \mathrm{ml}$ versus $453 \mathrm{ml}$, $\mathrm{p}=0.014$ ), but selectively administered, according to patient's volaemic status. Total amine consumption was $36 \%$ in control group, $16 \%$ in IVCUS group and $22 \%$ in PLRT group. Procedure time was increased in the IVCUS group (48 $\mathrm{min}$ ) compared to control group (46 $\mathrm{min}$ ) and PTLR group (40 min), $p=0.35$.

Conclusion: IVCUS-guided fluid replacement was a method able to generate a significant reduction in post-spinal arterial hypotension rate.

\section{Reference(s) and grant ackowledgment(s)}

1. J. Zhao, G Wang. Inferior Vena Cava Collapsibility Index is a Valuable and Non-Invasive Index for Elevated General Heart End-Diastolic Volume Index Estimation in Septic Shock Patients. Med Sci Monit 2016 Oct 20:22:3843-3848.

2. NJ Hackett, GS De Oliveira, UK Jain, JY Kim. ASA class is a reliable independent predictor of medical complications and mortality following surgery. Int J Surg 2015 Jun; 18:184-90

3. M.I. Monge García, A. Gil Cano, M. Gracia Romero, R. Monterroso Pintado, V. Pérez Madueño, J.C. Díaz Monrové. Non-invasive assessment of fluid responsiveness by changes in partial end-tidal $\mathrm{CO} 2$ pressure during a passive leg-raising manoeuvre. Ann Int Care 2012;2:9

4. E. Zöllei, V. Bertalan, A. Németh, P. Csábi, I. László, J. Kaszaki et al. Noninvasive detection of hypovolemia or fluid responsiveness in spontaneously breathing subjects. BMC Anesthesiol 2013;13(1):40.

5. RL Carpenter, R.A. Caplan, D.L. Brown, C. Stephenson, R. Wu. Incidence and risk factors for side effects of spinal anesthesia. Anesthesiology 1992;76(6):906-16.

6. No funding was received for this study

\section{9}

Central venous to arterial $\mathrm{CO} 2$ difference (DCO2) assisted goal directed hemodynamic management during major surgerya randomized controlled trial

L. Kumar ${ }^{1}$; PK. Das ${ }^{2}$; S. Tripathy ${ }^{3}$

${ }^{1}$ Anesthesia \& critical care, AllMS, Bhubaneswar, India; ${ }^{2}$ Assistant professor, anesthesia \& critical care, AllMS, Bhubaneswar, India; ${ }^{3} \mathrm{Md}$, dnb,edic additional professor, dept. of anesthesia and critical care, All India Institute of Medical Sciences, Bhubaneswar, Bhubaneswar, India

Correspondence: $\mathrm{S}$. Tripathy

Intensive Care Medicine Experimental 2020, 8(2): 000859

Introduction: Conventional goal-directed therapy(GDT) as used in perioperative \& critical care to optimize resuscitation, has had mixed outcomes. (Gelman 2020) The venous-to-arterial partial pressure of $\mathrm{CO} 2$ difference (DCO2) reflects oxygen supply to demand adequacy and has been suggested to improve resuscitation in sepsis. (Mallat et al. 2016)(Nassar and Mallat 2019) We hypothesized that a DCO2 guided GDT protocol would decrease morbidity after major surgeries. Objectives: To compare the incidence of postoperative organ dysfunction, length of ICU, and hospital stay in patients undergoing major surgeries when managed by a goal-directed DCO2 aided protocol versus usual care.

Methods: This was a double-blind randomized controlled trial (RCT) permitted by the institutional ethical committee and registered prospectively (CTRI/2018/07/014987). Conducted from July 2018 to May 2020 in an 800 bedded university teaching center with 16 multispecialty theatres and 14 bedded intensive care unit. $100 \mathrm{ASA} 1 / 2$ patients undergoing elective major surgeries and planned for arterial and central venous catheters were randomized (computer-generated random numbers) and allocated (sealed opaque envelopes). In both groups, arterial and venous blood samples were collected second hourly (or earlier in the intervention group (I) if changes were made). Fluid management was based on a prespecified protocol that used pulse pressure variation (PPV), central venous saturation (SCVO2), hemoglobin
$(\mathrm{Hb})$, and DCO2 levels to guide GDT in group I. Dobutamine infusion was started if $\mathrm{DCO} 2$ was $>6 \mathrm{mmHg}$, other parameters being normal. (Figure1). Control group C was provided care as per unit protocols. Patient demographics, details of the surgery, duration, hemodynamics, fluid balance, vasopressors and inotropes used, blood gas reports, and ICU parameters like APACHE and S071OFA scores were noted for $48 \mathrm{hrs}$ after surgery. Organ dysfunction was defined as an increase in SOFA score from the preoperative value. Appropriate statistical tests were applied for sample size calculation and comparing the outcomes.

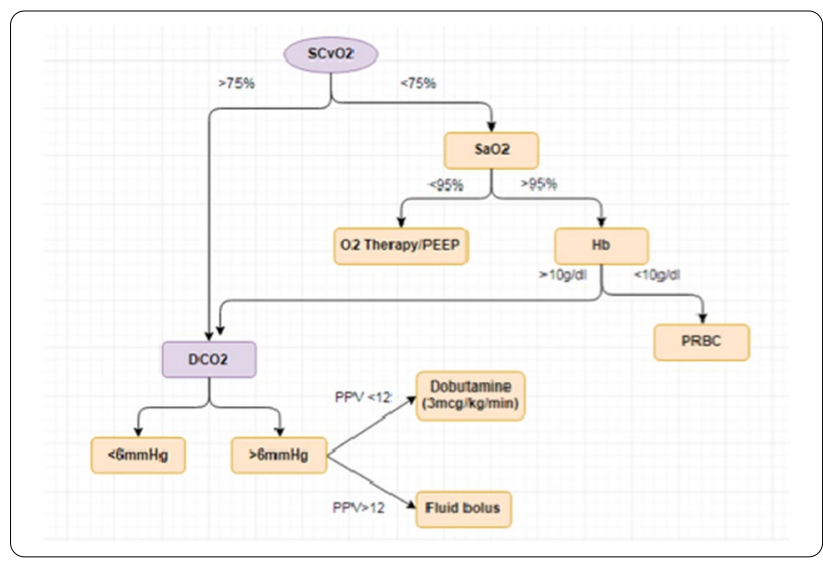

Results: Data were analyzed from 44 patients in each group. Patients were similar between the groups. The majority of the patients underwent oncosurgery $(64 \%)$, followed by neurosurgery $(16 \%)$ and urosurgery $(10 \%)$. Length of ICU stay in group I $(M=1.52$, SD 0.82$)$ was significantly less than group $C(M=2.18, S D=1.08, t=3.21, p=.002)$. The incidence of complications and the length of hospital stay were comparable between the groups.

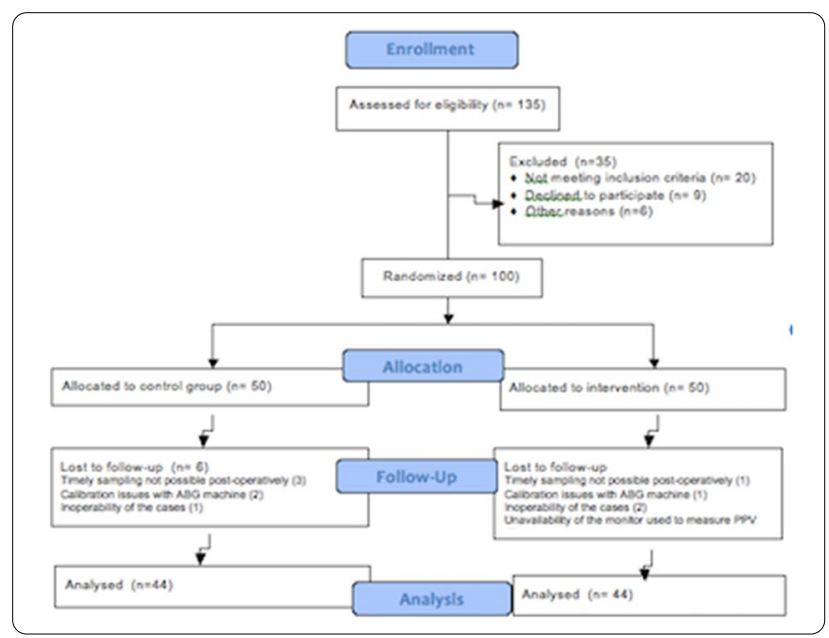

Conclusion: Conclusion- In this RCT a protocolized approach of DCO2 guided goal-directed therapy in patients undergoing elective major surgery, resulted in a significantly decreased length of ICU stay. The concept of using DCO2 seems both elegant and cost-effective in major surgeries (where arterial and central lines are already planned) and may prove of benefit in the era of enhanced recovery after surgery. Better powered multicentric studies in patients with comorbidities may demonstrate a difference in organ dysfunction and other outcomes in the future. 


\section{Reference(s) and grant ackowledgment(s)}

1. Gelman S (2020) Is goal-directed haemodynamic therapy dead? Eur. J. Anaesthesiol. 37:159-161 Mallat J, Lemyze M, Meddour M, et al (2016) Ratios of central venous-to-arterial carbon dioxide content or tension to arteriovenous oxygen content are better markers of global anaerobic metabolism than lactate in septic shock patients. Ann Intensive Care 6:1-9. https://doi.org/10.1186/s13613-016-0110-3 Nassar B, Mallat J (2019) Usefulness of venous-to-arterial partial pressure of $\mathrm{CO} 2$ difference to assess oxygen supply to demand adequacy: Effects of dobutamine. J Thorac Dis 11:S1574-S1578.

2. Mallat J, Lemyze M, Meddour M, et al (2016) Ratios of central venous-toarterial carbon dioxide content or tension to arteriovenous oxygen content are better markers of global anaerobic metabolism than lactate in septic shock patients. Ann Intensive Care 6:1-9.

3. Gelman S (2020) Is goal-directed haemodynamic therapy dead? Eur. J. Anaesthesiol. 37:159-161

4. The costs incurred were borne by the institute. No extramural grants are involved.

\section{4}

Surgical Prophylaxis for preoperative MDR colonization: our ICU

\section{experience}

A. Lerose ; G. Cappellazzo ${ }^{1}$; D. Cigolini²; RP. Boetti ${ }^{1}$; M. Taiana²;

V. Schweiger ${ }^{1}$; L. Gottin ${ }^{3}$; E. Polati ${ }^{1}$; K. Donadello ${ }^{1}$

${ }^{1}$ Anesthesia and intensive care b unit, University of Verona, AOUI-University Hospital Integrated Trust of Verona, Verona, Italy; ${ }^{2}$ Anesthesia and intensive care b unit, AOUI-University Hospital Integrated Trust of Verona, Verona, Italy; ${ }^{3}$ Cardio-thoracic anesthesia and intensive care unit, University of Verona, AOUI-University Hospital Integrated Trust of Verona, Verona, Italy Correspondence: K. Donadello

Intensive Care Medicine Experimental 2020, 8(2): 000894

Introduction: MDR colonization is a major issue for perioperative and intensive care medicine frequently resulting in life-threatening infections.

Objectives: Within our ICU admissions, the aim of this study was to evaluate MDR colonized patients undergoing surgery and their postoperative course compared to non colonized patients.

Methods: We retrospectively analyzed all consecutive patients undergoing surgery in our hospital for a period of 21 months (July 2017April 2019) that required ICU admission on POD 0. We then compared MDR colonized (rectal and/or pharyngeal swab) patients (MDR+) to non colonized patients (MDR-) for post operative complications, ICU and hospital LOS and mortality. We also investigated the impact of targeted perioperative antibiotic therapy compared to empiric antibiotic prophylaxis (https://www.save.veneto.it/home-sanitari/).

Results: 540 patients were included in the study (352 men, 65\%), mean age $68 \pm 12$ years; they underwent either elective $(n=477,88 \%)$ or emergency $(n=63,12 \%)$ surgery; $234(44 \%)$ were submitted to pancreatic surgery, $137(25 \%)$ to hepato-biliary surgery, $100(19 \%)$, to intestinal resection and the rest to other abdominal/vascular surgery; they were admitted to ICU on POD 0 for scheduled ( $n=461,85 \%$ ) or unscheduled intensive PO monitoring.

143 pts were pre operatively MDR+ (27\%), 54 (38\%) of whom were either Klebsiella Pneumoniae Carbapenemasis Producer or Enterococcus Faecium VRE colonized and 115 (80\%) were ESBL colonized. ICU LOS was significantly higher in MDR+ compared to MDR- $(8.02 \pm 13.8$ vs $2.39 \pm 3.4$ days, $p<0.05)$. Hospital mortality occured in - patients, were MDR+, - of them in the ICU. Complication rate and type were significantly higher in MDR+ patients $(97,68 \%)$ compared to MDR- $(118$, 30\%).
$88 \mathrm{MDR}+$ patients (52\%) received targeted pre-operative antibiotic therapy compared to standard prophylaxis but, despite a trend, this did not influence their outcome significantly.

Conclusion: Pre-operative surveillance swab positivity correlates with both ICU LOS and post-operative mortality. Targeted perioperative antibiotic prophylaxis may modify patient's outcome and this should be routinely evaluated. More studies are needed to further investigate the possibile therapeutic options in MDR+ patients undergoing surgery.

\section{0}

Goal Directed Fluid Therapy Post Orthotopic Liver

Transplantation: A Systematic Review

T. Joseph ${ }^{1}$; F. Froghi ${ }^{1}$; D. Martin ${ }^{1}$; B. Davidson

${ }^{1}$ Ucl division of surgery and interventional sciences,

Royal Free Hospital, London, United Kingdom

Correspondence: $T$. Joseph

Intensive Care Medicine Experimental 2020, 8(2): 000910

Introduction: Orthotopic liver transplantation (OLT) is the only effective treatment for end stage liver disease (ESLD) [1]. Advanced liver cirrhosis has numerous pathophysiological consequences that leads to difficulty in controlling fluid balance. The OLT procedure also has significant haemodynamic consequences [2]. Goal-directed fluid therapy (GDFT) has been shown to reduce postoperative complications in the general surgical population [3] but the value of post-operative GDFT has not been established in patients with liver cirrhosis undergoing OLT.

Methods: A systematic review was conducted on GDFT post OLT by searching EMBASE, MEDLINE and PubMed databases. Human studies from $01 / 01 / 1980$ to $10 / 06 / 2020$ investigating goal directed fluid therapy in the post-operative period after OLT were included. We excluded animal experimental studies, those not reported in English language, and non-OLT studies (eg living donor hepatectomy) and studies that did not directly investigate a haemodynamic variable.

Results: Of the 533 studies identified by the search terms 522 were excluded for lack of relevance or were duplicates. Of the remaining 11, 9 were excluded (non-OLT surgery (1), no haemodynamic variable monitored (7) and one transfusion study). One RCT and one cohort analysis evaluated GDFT in the immediate (0-72 hours) postoperative setting of liver transplantation (110 participants).

Of the two studies Martin et al (2019) reported a randomised, controlled feasibility trial of GDFT post OLT. In this study GDFT was based on SV optimisation in the first 12 hours post operatively. Controls received standard of care without the use of cardiac output measurement. SV optimisation was achieved by repeated boluses of $250 \mathrm{ml}$ crystalloid until a $<10 \%$ increase in SV was seen. It was demonstrated that delivering GDFT immediately post-OLT is safe and feasible. The GDFT group had significantly higher volumes of crystalloid infusion. Although there were signals of reduced length of ICU and hospital stay thestudy was not powered to demonstrate a clinical benefit. [4] Reydellet et al (2014) conducted a retrospective analysis of patients undergoing OLT before and after introduction of a GDFT protocol. The GDFT protocol was based on several haemodynamic parameters Haemodynamic optimisation intraoperatively and in the first 48 hours postoperatively was achieved with drugs or fluids. A transfusion protocol with $\mathrm{Hb}>8 \mathrm{~g} / \mathrm{dl}$ or $\mathrm{ScvO} 2>70 \%$ as the targets was also used. The control was physician guided fluid therapy prior to introduction of the protocol. Contrary to the COLT study, the protocol group in this cohort received significantly less crystalloid volume. The study also 
demonstrated a significant reduction in post-operative ileus and duration of mechanical ventilation in the protocol group. [5]

Conclusion: The two studies suggest clinical benefit but were not adequately powered to demonstrate efficacy. An adequately powered effectiveness study of GDFT following liver transplant is required.

\section{Reference(s) and grant ackowledgment(s)}

1. 3 Som A, Maitra S, Bhattacharjee S, Baidya DK. Goal directed fluid therapy decreases postoperative morbidity but not mortality in major non-cardiac surgery: a meta-analysis and trial sequential analysis of randomized controlled trials. Journal of Anesthesia. 2017;31(1):66-81

2. 1 Møller S, Henriksen JH, Bendtsen F. Extrahepatic complications to cirrhosis and portal hypertension: Haemodynamic and homeostatic aspects. World Journal of Gastroenterology. 2014;20(42):15499-517

3. 4 Reydellet L, Blasco V, Mercier M-F, Antonini F, Nafati C, Harti-Souab K, et al. Impact of a goal-directed therapy protocol on postoperative fluid balance in patients undergoing liver transplantation: A retrospective study. Annales Françaises d'Anesthésie et de Réanimation. 2014;33(4):e47-54.

4. 5 Martin D, Koti R, Gurusamy K, Longworth L, Singh J, Froghi F, et al. The cardiac output optimisation following liver transplant (COLT) trial: a feasibility randomised controlled trial. HPB. 2019

5. 2 Rudnick MR, Marchi L De, Plotkin JS, Rudnick MR, Marchi L De, Plotkin JS. Hemodynamic monitoring during liver transplantation : A state of the art review. 2015;7(10):1302-11

\section{1}

Standardized US upper airway evaluation for difficult airway prediction: a little more evidence? A monocentric, prospective, cohort, blinded study

A. Bianchini ${ }^{1}$; R. D'andrea ${ }^{2}$; L. Querci ${ }^{3}$; F. Castaldo ${ }^{3}$; C. Laici ${ }^{1}$; F. Scuppa ${ }^{4}$;

C. Serra ${ }^{5}$; S. Cavicchi ${ }^{2}$; E. Nardi ${ }^{6}$; A. Zanoni ${ }^{2}$; A. Siniscalchi ${ }^{1}$

${ }^{1}$ Department of anesthesia and intensive care, department of organ deficiencies and transplants, S.Orsola-Malpighi Hospital, Bologna, Italy; ${ }^{2}$ Anesthesia and intensive care unit, S.Orsola-Malpighi Hospital, Bologna, Italy; ${ }^{3}$ Anesthesia and intensive care residence, Università di Bologna, Via Zamboni, Bologna, Metropolitan City of Bologna, Italy, Bologna, Italy; ${ }^{4}$ Cardiology residence, Università di Bologna, Via Zamboni, Bologna, Metropoli$\tan$ City of Bologna, Italy, Bologna, Italy; ${ }^{5}$ Department of organ deficiencies and transplants, S.Orsola-Malpighi Hospital, Bologna, Italy; ${ }^{6}$ Statistic department, S.Orsola-Malpighi Hospital, Bologna, Italy

\section{Correspondence: $L$. Querci}

Intensive Care Medicine Experimental 2020, 8(2): 000941

Introduction: The management and safety of the airway during general anesthesia or in a critical environment is one of the most important skills of an anesthesiologist or an intensive care physician. Inadequate airway protection or insufficient oxygenation of the patient is associated with an overall increase in mortality and morbidity. The primary goal is the early identification of the Difficult Airways (DA) to reduce or eliminate potential risks linked to incorrect management. The identification of DA is, generally, based on clinicalanamnestic evaluation with low sensitivity and specificity. Ultrasound evaluation has been recently proposed as a useful, non-invasive, and cost-effective tool. Many ultrasonographic parameters have been studied to identify a diagnostic tool to predict difficult laryngoscopy, but no useful cut-off range was at present validated to define a standard parameter, mainly due to the lack of a systematic scan approach in ultrasound neck examination. Among sonographic parameters, the increased thickness of soft tissues anterior to the epiglottis (STTe) was emerging as one of the most reliable independent predictors of difficult laryngoscopy and mask ventilation. In our usual clinical practice, we have highlighted that the anteroposterior and longitudinal scanning allows us to obtain a systematically standardized and reproducible measurement of STTe.
Objectives: This study aimed to analyze the use of a standardized ultrasound approach to identify a DA with specific research of predictive parameters of difficult intubation (DI) and difficult ventilation (DV) combined with clinical-anamnestic evaluation. The aim was, therefore, to verify whether these parameters are useful in order to help creating an airway plan in the operating room, but also a critical care setting. Another possible use of these tools could be the selection of patients who need a protection extubation.

Methods: Prospective, observational, cohort, single-blind study. The study was conducted at the University of Bologna S.Orsola-Malpighi Hospital and approved by the Independent Ethics Committee. Eligibility criteria were consenting adult patients requiring general anesthesia and orotracheal intubation. The patients were consecutively enrolled (from September 2017 to October 2018) during the preoperative anesthesia evaluation. Exclusion criteria were emergency surgeries, rapid-sequence induction, uncooperative or pregnant patients, patients with a history of difficult intubation, or with difficult laryngoscopy and/or with indication to bronchoscopy. The clinical design is divided into two different phases: the preoperative phase (phase 1) and the intraoperative phase (phase 2). Evaluations carried out in phase 1 and in phase 2 were performed by different anesthesiologists at different times (blinded study). In phase 1 clinical-anamnestic evaluation and ultrasound were performed. The intraoperative phase was conducted by expert anesthesiologists (more than 5 years of experience). General anesthesia was conducted according to a standard protocol. DI was evaluated using the Cormack-Lehane score.

Results: As statistical estimation, 253 patients were enrolled $(50 \%$ male, range 18-88 years old). Direct laryngoscopy was classified as difficult $(C L>3)$ in 15 patients $(6 \%), 10$ were predicted by the preoperative clinical evaluation, while the remaining 5 cases $(2,1 \%)$ had not been anticipated. The ultrasound parameter predictive of DI was the thickness of the soft tissues anterior to the epiglottis in hyperextension of the head and protrusion of the tongue (STTep). The ROC curve analysis identified a cut off of STTep equal to a value of $2.50 \mathrm{~cm}$ above which we can predict difficulties in intubation (specificity $55.9 \%$, sensitivity $73.3 \%$; AUC $0.649, \mathrm{Cl} 0.537-0.762 ; \mathrm{p}=0.05$ ). This cut-off value is confirmed even better in the group of patients with difficult laryngoscopy (Cormack-Lehane $\geq 3$ even after BURP) in which it results the same specificity (55.9\%) but greater sensitivity (87.5\%; AUC 0.726; $\mathrm{p}=0.03$ ). In addition, we verified the predictive role of the US in identifying the borderline difficult laryngoscopy $(C L 2 e)$. We found 23 having a CL 2e: STTep was verified both before $(p=0.001)$ and after BURP $(p=0.001)$. No statistically significant differences were found between the two groups (easy vs difficult) for other US parameters for DI. STTep was, also, the best predictor for DV.

Conclusion: The use of ultrasound has found in recent decades a wide application as a diagnostic and a therapeutic tool, specifically at present airways ultrasound evaluation is an important tool to predict a DI. Standardized ultrasound assessment of the STTep may be useful to predict "complex difficult" direct laryngoscopy and difficult intubation and to identify a DI not foreseen by the conventional clinical parameters (clinically unexpected or unexpected DI). Preoperative identification of DA permit to better organize patient's management in the perioperative and the pre-procedure identification of DA permit to better choose the airway strategy. Further studies are necessary to validate the parameters found and correlate them to the preoperative clinical evaluation, improving patient safety, and allowing the best use ofresources. US airway evaluation could find a place in scores for DA prediction.

Reference(s) and grant ackowledgment(s)

1. Cattano $D$ et al. Risk factors assessment of the difficult airway: an Italian survey of 1956 patients. Anesth Analg 2004; 99:1774-9.

2. Combes X, Jabre P, Jbeili C, Leroux B, Bastuji-Garin S, Margenet A, Adnet F, Dhonneur G. Prehospital standardization of medical airway 
management: incidence and risk factors of difficult airway. Acad Emerg Med. 2006 Aug;13(8):828-34. Epub 2006 Jun 28.

3. Lundstrøm LH, Møller AM, Rosenstock C, Astrup G, Wetterslev J. High body mass index is a weak predictor for difficult and failed tracheal intubation: a cohort study of 91,332 consecutive patients scheduled for direct laryngoscopy registered in the Danish Anesthesia Database. Anesthesiology. 2009 Feb; 110(2):266-74. doi: 10.1097/ ALN.0b013e318194cac8.

4. Lee A, Fan LT, Gin T, Karmakar MK, Ngan Kee WD. A systematic review (meta-analysis) of the accuracy of the Mallampati tests to predict the difficult airway Anesth Analg. 2006 Jun:102(6):1867-78.

5. Crawley SM, Dalton AJ. Predicting the difficult airway. Continuing Education in Anaesthesia Critical Care \& Pain 2015 Oct; 15(5):253-257.

6. Roth D, Pace NL, Lee A, Hovhannisyan K, Warenits AM, Arrich J, Herkner H. Airway physical examination tests for detection of difficult airway management in apparently normal adult patients. Cochrane Database of Systematic Reviews 2018, Issue 5. Art. No.: CD008874. DOI: 10.1002/14651858.CD008874.pub2.

7. Practice Guidelines for Management of the Difficult airway of the American Society of Anesthesiologists, Inc. Lippincott Williams \& Wilkins. Anesthesiology 2013; 118:251-70.

8. American Society of Anesthesiologists Task Force of the Difficult Difficult Airway; Practice guidelines for management of the difficult airway: an updated report by the American Society of Anaesthesiologists task force on management of difficult airway. Anaesthesiology 2003; 98:1269-77.

9. CookTM, Woodall N, Frerk C. Major complications of airway management in the UK: result of The Fourth National Audit Project of the Royal College of Anesthesists and the Difficult Airway Society. Part 1: anaesthesia. BR J Anaesth 2011; 106:617-31.

10. Asai T, Koga K, Vaughan RS. Respiratory complications associated with tracheal intubation and extubation. BR J Anaesth 1998; 80:767-75.

11. None to declare.

\section{1}

When bladders and bowels don't mix - post-operative ileus following radical cystectomy

L. Alvarez-Belon'; C. Andrew²; N. Boyer ${ }^{3}$; J. Zekavica'; Z. Maha'; E. Potter ${ }^{4}$ ${ }^{1}$ Intensive care, Royal Surrey County Hospital, Guildford, United Kingdom; ${ }^{2}$ Anaesthesia, King's College Hospital, London, United Kingdom; ${ }^{3}$ Intensive care unit, Royal Surrey County Hospital, Guildford, United Kingdom; ${ }^{4}$ Intensive Care Unit, Royal Surrey County Hospital, Guildford, France

Correspondence: E. Potter

Intensive Care Medicine Experimental 2020, 8(2): 001011

Introduction: In patients who have undergone a radical cystectomy, delayed return of bowel function is to be expected. However, a cohort of patients $(10-40 \% 1)$ go on to develop the complication of post-operative paralytic ileus (POI), which can negatively impact outcomes2. Although the causative factors of $\mathrm{POI}$ are often complex3, identification of potential risk factors could prove useful in the management of these patients in order to improve patient outcomes.

Objectives: To:

* establish the incidence of ileus in patients having radical cystectomies

* compare length of stay and mortality between those that had and did not have ileus

* identify features that correlate with developing ileus.

Methods: This was a single-centre retrospective, observational study carried out at the Royal Surrey County Hospital. We reviewed the electronic and paper medical records, drug charts, anaesthetic charts and operative notes of all patients who had radical cystectomies and ileal conduits in 2018.
Results: 72 patients were identified from a surgical registry. The incidence of ileus was 34\% (24/72). Full notes including intra-operative notes were only available for 61 patients.

\begin{tabular}{|l|l|l|l|}
\hline & $\begin{array}{l}\text { Delayed return } \\
\text { to normal bowel } \\
\text { function and } \\
\text { ileus }\end{array}$ & $\begin{array}{l}\text { Normal post- } \\
\text { operative bowel } \\
\text { function }\end{array}$ & $\begin{array}{l}\text { Statistical } \\
\text { significance }\end{array}$ \\
\hline Hospital length of stay & $8.5(6-21)$ & $6(5-7)$ & $\mathrm{p}<0.0001$ \\
\hline Mortality n (\%) & $6 / 24(25 \%)$ & $0 / 47(0 \%)$ & $\mathrm{p}<0.0001$ \\
\hline $\begin{array}{l}\text { Intrathecal diamorphine } \\
\text { (mcg) }\end{array}$ & $700(500-1000)$ & $800(500-800)$ & $\mathrm{p}=\mathrm{n} . \mathrm{s}$. \\
\hline $\begin{array}{l}\text { First } 48 \text { hrs post-op po } \\
\text { morphine equivalent dose } 61(37-108) \\
\text { (mg) }\end{array}$ & $45(25-114)$ & $\mathrm{p}=\mathrm{n} . \mathrm{s}$. \\
\hline $\begin{array}{l}\text { Intra-operative iv fluids } \\
\text { (ml) }\end{array}$ & $2000(2000-$ & $2000(1688-$ & $\mathrm{p}=\mathrm{n} . \mathrm{s}$. \\
\hline $\begin{array}{l}\text { Highest intra-operative } \\
\text { lactate (mmol/L) }\end{array}$ & $2500)$ & $2500)$ & $\mathrm{p}=\mathrm{n} . \mathrm{s}$. \\
\hline $\begin{array}{l}\text { Parenteral nutrition, } \mathrm{n} \\
(\%)\end{array}$ & $6 / 24(25 \%)$ & $0 / 47(0 \%)$ & $\mathrm{p}<0.0001$ \\
\hline
\end{tabular}

Conclusion: Delay to return to normal bowel function including postoperative ileus was associated with a significant increase in hospital length of stay, increased requirement for parenteral nutrition and significantly increased mortality rates. This is a complex, multifactorial post-operative complication and we were unable to find any factors that were associated with ileus in this study. Further prospective research is required to establish if there are causative factors which may be optimised to prevent this post-operative complication.

Reference(s) and grant ackowledgment(s)

1. Svatek RS, Fisher MB, Williams MB, Matin SF, Kamat AM, Grossman HB, Nogueras-Gonzales GM, Urbauer DI, Dinney CP. Age and body mass index are independent risk factors for the development of postoperative paralytic ileus after radial cystectomy. Journal of Urology. 76 (6) 2010

2. Chang SS, Cookson MS, Baumgartner RG, Wells N, Smith JA. Analysis of early complications after radical cystectomy: results of a collaborative care pathway. The journal of urology. Vol 167. 2002

3. Kim MJ, Min GE, Yoo KH, Change SG, Jeon SH. Risk factors for postoperative ileus after urologic laparoscopic surgery. Journal of the Korean Surgical Society. 2011. 80: 384-389.

\section{9}

Volume status and fluid management in the early postpartum period

A. Ronenson ${ }^{1}$; E. Shifman²; A. Kulikov ${ }^{3}$; S. Sitkin ${ }^{4}$

${ }^{1}$ Head of the department of anesthesiology and intensive care, Tver Regional Perinatal Center, Tver, Russia; ${ }^{2}$ Anesthesia and intensive care, Moscow Regional Clinical Research Institute named M.F. Vladimirsky, Moscow Russia; ${ }^{3}$ Anesthesia and intensive care, Ural State Medical University, Yekaterinburg, Russia; ${ }^{4}$ Head of the department of anesthesiology and intensive care, Tver State Medical University, Tver, Russia

Correspondence: A. Ronenson

Intensive Care Medicine Experimental 2020, 8(2): 000069

Introduction: Major changes occur in central hemodynamic during early postpartum period: redistribution of fluid from the interstitial space into the bloodstream, which increases the blood volume circulating and cardiac output. All these changes are aimed at stabilizing the patient in the postpartum period with blood loss. However, blood volume circulating during pregnancy and postpartum period remains unknown and studies on its results come to the conclusion about its great variability. The aim of this study was to evaluate the postpartum 
volume status of puerperas with blood loss $10-20 \%$ of blood volume circulating.

Methods: Thirty puerperas after a elective cesarean section with spinal anesthesia, underwent ultrasound evaluation of the heart andinferior vena cava immediately after surgery, 3 and 6 hours.

Results: The volume of blood loss measured by the gravimetric method was $914 \pm 410 \mathrm{ml}$, which amounted to 10 to $20 \%$ of the blood volume circulating, calculated by the formula $100 \mathrm{ml}$ * weight before pregnancy $(\mathrm{kg})$. The ejection fraction of the left ventricle, measured immediately after surgery, 3 and 6 hours, was $55.5 \%$ (95\% confidence interval 51.9 - 61.6), 58.2\% (95\% Cl 59.4 - 61.2) and 55.4\% (95\% Cl 53.5 - 59.9), respectively. The Friedman test ( $x 2$ ) showed the absence of a statistically significant difference of the ejection fraction $(p=0.692)$. Inferior vena cava collapsibility index showed a statistically significant decrease in the measurement periods: $35.5 \%(95 \% \mathrm{Cl} 22.5$ - 44.8), $20.5 \%$ (95\% Cl 14.6 - 28.5), $14.4 \%$ (95\% Cl 12.2 - 25.6), respectively, the Friedman test $(x 2) p=0.036$. A statistically significant increase of the end-diastolic volume of the left ventricle was also noted: $73.0 \mathrm{ml}(95 \%$ Cl 66.3 - 76.7), $81.2 \mathrm{ml}(95 \% \mathrm{Cl} 72.9$ - 87.3), $89.8 \mathrm{ml}(95 \% \mathrm{Cl} 80.4$ - 96.7), respectively, the Friedman test $(x 2) p=0.021$.

Conclusion: Blood loss $10-20 \%$ of blood volume circulating or more than $1000 \mathrm{ml}$ does not always require replacement fluid management, redistribution of fluid volume from the interstitial space increases blood volume circulating within 3-6 hours after delivery. Further studies of the volume status are needed.

\section{2}

Fluid responsiveness assessment in Cardiac Surgery patients. Superior Vena cava respiratory variability vs Mini fluid challenge B. Chekroud'; C. Gandon'; P. BURTIN'; JY. Bigeon'; A. Roussiaux'; N. Ducrocq

'Anesthésie et réanimation, Clinique du Millénaire, Montpellier, France Correspondence: P. BURTIN

Intensive Care Medicine Experimental 2020, 8(2): 000752

Introduction: Fluid responsiveness assessment is the cornerstone of fluid therapy in both the ICU and the operating room $(1,2)$.The use of dynamic indices (fluid challenge or the cardiopulmonary interaction) has proved to be the most effective method and these tests have been included in guidelines (3).Nevertheless, fluid responsiveness in cardiac surgery (CS) patients is weakly investigated. A recent study has reported that in ICU patients the repiratory variability of the superior vena cava (SVC) performed better than other tests (4).

Objectives: The routine use of a TEE probe in all our CS patients allowed us to investigate in a prospective observational study the performance of the respiratory variation of the VCS (Delta VCS) and mini fluid challenge ( $F C 100)$ as a predictive indice for fluid response.

Methods: All patients were included after CPB and chest closure while still under general anesthesia. A fluid infusion was performed in all patients by infusion of the CPB residual volume treated by a cell saver (500-600 ml;hematocrit $>50 \%$ ). The cell saver bag was infused sequentially and hemodynamic measures were obtained after $100 \mathrm{ml}$ (FC 100), $250 \mathrm{ml}$ (FC250) and $500 \mathrm{ml}$ (FC 500). We recorded demographic and procedural data. At baseline and at each stage of the $F C$ we recorded: SV, VTI, HR, BP, E/A ratio, E/E' ratio, S'Ann wave, the diameter of SVC during inspiration and expiration. SVC diameter was measured during QRS and T wave of the ECG. We calculated the respiratory variation index (\%) of SVC as (Exp VCS Diam - Insp VCS Diam)/ Exp VCS Diam for QRS wave, T wave and absolute variation. Patients were classified as Fluid responders(RESP) if SV increased by $+15 \%$ after FC500 or Non Responders (NRESP). Groups were compared by KHI2 and Student $t$ tests. ROC curves were built for FC 100, Delta VCS QRS, Delta VCS T and Delta VCS absolute $(p<0.05)$
Results:

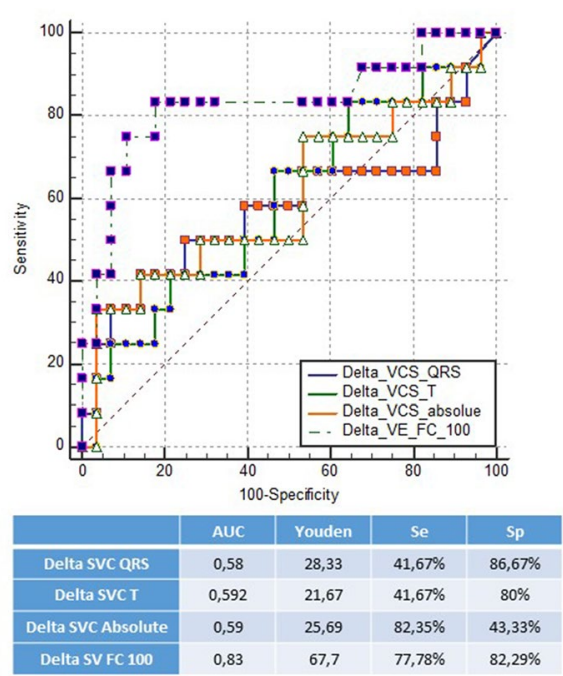

Fig 1: ROC curves comparisons for the 4 dynamic indices tested $(p<0.036)$

49 patients were included : M 39 (79.6\%), CABG 51\%, AVR 27\%, MVR $8 \%$, Other 14\%; mean age $67+/-11.5$; mean Euroscore 23.48 / 3.16\%; mean LVEF 53.9 +/- 11,4. 31 patients (63,3\%) were NRESP and 18 $(36,7 \%)$ were RESP. There was no difference for RESP / NRESP groups for LVEF (RESP : $51.2 \%$ vs NRES : $56.6 \%$ ) CPB duration (RESP : $71.2 \mathrm{~min}$ vs NRES : $68.6 \mathrm{~min}$ ), Cross clamp time (RESP : $57 \mathrm{~min}$ vs NRES : $54.4 \mathrm{~min}$ ), baseline E/A (RESP : $1.49+/-0.24$ vs NRES : $1.19+/-0.19$ ), baseline E/e (RESP : $7.86+/-1.28$ vs NRES : $8.54+/-1.37$ ). Delta SVC QRS and delta SVC T were higher in the RESP group but the difference did not reach significance : (RESP : $13.67 \%$ vs NRES : $7.84 \%$ and RESP : $13.89 \%$ vs NRES : $9.91 \%$ respectively). Groups were significantly different only for delta SV after FC100 (RESP : $11.4+/-3.08 \%$ vs NRES : $2.54+/-0.57 \%$; $95 \mathrm{C}$ 0.05918 to 0.21458 ). ROC curves are shown in fig 1. The highest AUC is obtained by FC100 (AUC $0.829 ; 95 \mathrm{Cl} 0.677$ to 0.929 ) and significantly higher than the SVC derived indices $(p<0.036)$.

Conclusion: The main findings of our study are : First, in CS up to $63 \%$ of the patients may be unresponsive to $\mathrm{FC}$ and this is similar septic shock patients. Second, the SVC derived indices perform poorly in predicting fluid responsiveness and that is not consistent with the results obtained in shock patients (4). The influence of the specificities of CS patients (type of anesthesia, cardioplegia and CPB, chest drainage system) are discussed. Third, the good performance of the mini fluid challenge ( $F C 100)$ makes it a method of choice in post CPB CS patients.
Reference(s) and grant ackowledgment(s)
1. 4 - Am J Respir Crit Care Med; 195 : 1022-1032
2. 3 - Eur. J. Anaesthiol 2016;33:488-521
3. 2 - Anesthesiology 2017;127:450-456
4. 1 - Anesthesiology 2011;115:455-6

\section{0}

Is there preservation of micro- and macro-vascular coherence following liver resection surgery delivered within an ERAS pathway with GDFT?

E. Potter' ${ }^{1}$ J. Mcvey ${ }^{2}$; L. Forni ${ }^{3}$; B. Creagh-Brown ${ }^{1}$

${ }^{1}$ Intensive care and perioperative medicine, Royal Surrey County Hospital, Guildford, United Kingdom; ${ }^{2}$ Biochemical sciences, University Of Surrey, Guildford, United Kingdom; ${ }^{3}$ Intensive care, Royal Surrey County Hospital, Guildford, United Kingdom

Correspondence: E. Potter

Intensive Care Medicine Experimental 2020, 8(2): 000820 
Introduction: It has been demonstrated that persistently decreased microvascular blood flow is associated with mortality in the critical care population.1,2 Microvascular blood flow is a potentially important therapeutic target, that may diverge from macrovascular parameters leading to a loss of haemodynamic coherence. 3 In our centre we use a low central venous pressure (CVP) anaesthetic for liver resection surgery to decrease intra-operative blood loss 4 with subsequent goal-directed fluid therapy (GDFT). This can be considered as a model of shock and resuscitation. These patients were studied to assess peri-operative microvascular flow and relation to macrovascular parameters.

Objectives: To establish:

- changes in microvascular blood flow peri-operatively in liver resection surgery

- relationship between microvascular blood flow and macrovascular parameters

- relationship between microvascular blood flow following GDFT to post-operative complications

Methods: This was a single-centre prospective observational study (REC 14/NE/1150) enrolling patients undergoing elective liver resection surgery. Microvascular blood flow, measured using the MicroScan device (MicroVision Medical, Amsterdam) and physiological measurements were taken at pre-specified time points throughout the peri-operative period ( $\mathrm{T} 1$, awake on day of surgery pre-operatively, T2 following induction of anaesthesia, T3 on exposure of the liver, T4 completion of liver resection prior to fluid resuscitation, T5 arrival at ICU, T6 completion of goal directed fluid therapy (GDFT)). Mortality rates, post-operative morbidity survey (POMS) and Clavien-dindo classification were used to assess post-operative complications.

Results: A total of 55 data sets were included in the analyses, 28 male and 27 female, mean age was 58 years (S.D. 13) and BMI $28.1 \mathrm{~kg} /$ $\mathrm{m} 2$ (S.D. 5.4), $18 \%$ were smokers, $13 \%$ were diabetic and $76 \%$ had neoadjuvant chemotherapy.

CVP was decreased successfully during anaesthesia from $7 \mathrm{mmHg}$ (IQR $4.7-10)$ at $\mathrm{T} 2$ to $3 \mathrm{mmHg}(\mathrm{IQR} 0.7-5)$ at $\mathrm{T} 4(\mathrm{p}<0.0001)$. There was a statistically significant decrease in mean arterial pressure (MAP); $74 \mathrm{mmHg}$ (IQR $65-88)$ at T2 to $64 \mathrm{mmHg}(58-74)$ at $\mathrm{T} 4(\mathrm{p}=0.0002)$. This was accompanied by a rise in heart rate from a median of $67 \mathrm{bpm}$ (IQR $60-77$ ) to $73 \mathrm{bpm}$ (IQR 64-87) at T4 ( $\mathrm{p}=0.013)$. A subset of 18 patients had records of cardiac output measured by oesophageal doppler, this was not suitable for use in awake patients so was only recorded at $\mathrm{T} 2$, $\mathrm{T} 3$ and T4. There was no significant difference between cardiac index (Cl) at time 2 and time 4; median $\mathrm{Cl} 2.55 \mathrm{~L} / \mathrm{min} / \mathrm{m} 2(1.88-3.48)$ and $2.00 \mathrm{~L} / \mathrm{min} / \mathrm{m} 2(1.80-2.30)$ respectively.

Following completion of GDFT (T6) there was no significant difference in heart rate compared to baseline (T1). However, MAP at T6 remained significantly lower than baseline (T1); $100 \mathrm{mmHg}(88-110)$ vs 77 (7389), $\mathrm{p}<0.0001$.

Microvascular flow measurements decreased post-operatively and this fall was sustained after completion of GDFT as detailed below.

Table 1 - Microvascular flow (results displayed as median (IQR), Kruskal-Wallis was used to determine significance of variation between groups)

\begin{tabular}{|l|l|l|l|l|}
\hline Parameter & T1 & T5 & T6 & p \\
\hline \multirow{2}{*}{ MFI } & 2.78 & 2.5 & 2.58 & \\
& $(2.42-2.98)$ & $(1.94-2.75)$ & $(2.08-2.83)$ & \\
\hline \multirow{2}{*}{$\mathbf{s P P V}$} & 95.6 & 66.6 & 65.1 & \\
& $(87.4-99.8)$ & $(58.5-66.7)$ & $(61.3-66.8)$ & \\
\hline \multirow{2}{*}{$\mathbf{s P V D}$} & 4.85 & $5.9(4.4-7.8)$ & 5.77 & \\
& $(3.69-5.9)$ & & $(4.91-7.2$ & $\mathrm{p}<0.0001$ \\
\hline
\end{tabular}

There was an increase in proportion of perfused large blood vessels (IPPV); $66.7(34.1-90.9)$ at T1 vs $99(91-100)$ at T6, $\mathrm{p}<0.0001$ density (tPVD).
There was no significant correlation between MAP and the microvascular parameters MFI, sPPV or SPVD at any time point using Pearson and Spearman correlation coefficients as appropriate. There was no association with microvascular flow and post-operative morbidity or mortality.

Conclusion: Microvascular blood flow decreased post-operatively and this was sustained despite completion of GDFT and restitution of macrovascular parameters. There was evidence of shunting with a decrease in small perfused blood vessel density and increase in large perfused blood vessel density from baseline to completion of GDFT. GDFT was insufficient to restore post-operative sublingual microvascular perfusion.

Reference(s) and grant ackowledgment(s)

1. 2. De Backer D, Donadello K, Sakr Y, Ospina-Tascon G, Salgado D, Scolletta S, Vincent JL: Microcirculatory alterations in patients with severe sepsis: impact of time of assessment and relationship with outcome. Crit Care Med.41(3):791-799, 2013.

2. 1. Sakr Y, Dubois M-J, De Backer D, Creteur J, Vincent J-L: Persistent microcirculatory alterations are associated with organ failure and death in patients with septic shock*. Crit Care Med.32(9):1825-1831, 2004.

3. 4. Jones RM, Moulton CE, Hardy KJ. Central venous pressure and its effect on blood loss during liver resection. Br J Surg 1998;85(8):1058-60. (In eng). DOI: 10.1046/j.1365-2168.1998.00795.x.

4. 3. Arnemann P, Seidel L, Ertmer C. Haemodynamic coherence - The relevance of fluid therapy. Best Pract Res Clin Anaesthesiol 2016;30(4):419-427. (In eng). DOI: 10.1016/j.bpa.2016.11.003.

\section{8}

Hyperglycemia and predisposing risk factors in patients after cardiac surgery

S. Dimopoulos ${ }^{1}$; M. Georgopoulou ${ }^{1}$; N. Rouvali ${ }^{1}$; C. Kidi ${ }^{1}$; M. Mavraki ${ }^{1}$;

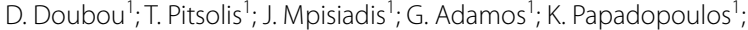
S. Nanas ${ }^{2}$; A. Karabinis ${ }^{1}$

${ }^{1}$ Icu, Onassis Cardiac Surgery Center, Kallithea, Greece; ${ }^{2}$ Icu, Evaggelismos General Hospital, Athina, Greece

Correspondence: S. Dimopoulos

Intensive Care Medicine Experimental 2020, 8(2): 001018

Introduction: High blood glucose levels are frequently shown in patients post-cardiac surgery patients that might affect prognosis.

Objectives: To evaluate postoperative blood glucose trend levels and predisposing risk factors in cardiac surgery patients, during their stay in intensive care unit (ICU).

Methods: 210 consecutive post-cardiac surgery patients, admitted to ICU, participated in this prospective clinical study. Blood glucose levels were measured by patients' admittance to ICU and afterwards every 2-3 hours daily, until their discharge or for up to seven days (if discharged later). An existing ICU protocol for blood glucose control was applied. Patients were classified by mean blood glucose level during their stay in ICU.

Results: $56 \%$ of the patients had mean blood glucose levels 140 $180 \mathrm{mg} / \mathrm{dl}, 25 \%$ above $180 \mathrm{mg} / \mathrm{dl}$ and $19 \%$ below $140 \mathrm{mg} / \mathrm{dl} .36 \%$ of the participants had mean blood glucose levels above $180 \mathrm{mg} / \mathrm{dl}$ during the first and second day of ICU treatment and 30\% during the third day. Multivariate logarithmic regression analysis has shown history of Diabetes mellitus, Euroscore II and pre-operative white blood cells count as independent predictors of post-cardiac surgery mean blood glucose levels above $180 \mathrm{mg} / \mathrm{dl}$. Patients with mean blood glucose levels above $180 \mathrm{mg} / \mathrm{dl}$ post-cardiac surgery had greater duration of mechanical ventilation support $(\mathrm{p}=0.019)$.

Conclusion: According to our study, a high percentage of cardiac surgery patients admitted to ICU exhibit blood glucose levels $>180 \mathrm{mg} /$ $\mathrm{dl}$, at the immediate postoperative period despite standard treatment protocol. Diabetes mellitus, Euroscore II and pre-operative white blood cells count were independent predictors of hyperglycemia. Appliance of a further bundle for optimization of blood glucose levels is deemed necessary. 
16. Poisoning/Toxicology /Pharmacology > Poisoning/Toxicology / Pharmacology

\section{6}

Incidence Pattern of Neurological Diseases after Carbon

Monoxide Poisoning: Retrospective Longitudinal Study in South Korea from 2012 to 2018

H. H. Kim ${ }^{1}$; C. Sangchun ${ }^{1}$

${ }^{1}$ Emergency Medicine, Ajou University Hospital, Suwon, Republic of Korea Correspondence: H.H. Kim

Intensive Care Medicine Experimental 2020, 8(2): 000006

Introduction: Neurological sequelae after carbon monoxide (CO) poisoning has recently become a critical issue. Epidemiologic data of delayed neurological sequelae such as incidence, time of development, and duration vary widely in the literature.

Objectives: We aim at analyzing nationwide claims data from South Korea for incidence patterns of neurological disorders in survivors of carbon dioxide poisoning.

Methods: A national cohort was abstracted from database that includes the patients diagnosed with carbon monoxide poisoning from January 1st, 2007 to December 31st, 2018. We investigated the whole diagnostic codes for nervous system diseases. The codes were retrieved from the claims data generated during the patients' initial visits to an inpatient or an outpatient after the diagnosis of T58, carbon monoxide poisoning.

Results: Among the total of 26,778 patients, 18,720 (69.9\%) patients were diagnosed with nervous system diseases after the carbon monoxide poisoning. The most common disease was disorders of sleep initiating and maintaining $(n=701,3.74 \%)$, followed by tension-type headache $(n=477,2.55 \%)$, and anoxic brain injury $(n=406,2.17 \%)$. Admission to the intensive care unit after carbon monoxide poisoning was associated with subsequent development of nervous system diseases. The cumulative hazard ratio was 2.25 ( $95 \% \mathrm{Cl}, 2.07$ to 2.44 , $\mathrm{p}<0.05)$.

Conclusion: In poisoned patients with carbon monoxide, it needs to consider that nervous system diseases followed after carbon monoxide poisoning have relatively early peak incidence patterns. Nervous system diseases after carbon monoxide poisoning had different patterns of incidence depending on whether the patient had been admitted to intensive care unit or not.

\section{Reference(s) and grantackowledgment(s)}

1. Kim H, Choi S, Park E, Yoon E, Min Y, Lampotang S. Serum markers and development of delayed neuropsychological sequelae after acute carbon monoxide poisoning: anion gap, lactate, osmolarity, S100B protein, and interleukin-6. Clin Exp Emerg Med. 2018;5(3):185-91.

2. Burch R. Migraine and Tension-Type Headache: Diagnosis and Treatment. Med Clin North Am. 2019;103(2):215-33.

3. Kahriman A, Zhu S. Migraine and Tension-Type Headache. Semin Neurol. 2018;38(6):608-18.

4. Hoffman RS, Howland MA, Lewin NA, Nelson LS, Goldfrank LR. 125 Carbon monoxide: McGraw-Hill Education / Medical 2014.

5. Choi YR, Cha ES, Chang SS, Khang YH, Lee WJ. Suicide from carbon monoxide poisoning in South Korea: 2006-2012. J Affect Disord. 2014;167:322-5

6. Hampson NB. U.S. Mortality Due to Carbon Monoxide Poisoning, 1999-2014. Accidental and Intentional Deaths. Ann Am Thorac Soc. 2016;13(10):1768-74.

7. Spina V, Tomaiuolo F, Celli L, Bonfiglio L, Cecchetti L, Carboncini MC. A Case of Carbon Monoxide-Induced Delayed Neurological Sequelae Successfully Treated with Hyperbaric Oxygen Therapy, N-Acetylcysteine, and Glucocorticoids: Clinical and Neuroimaging Follow-Up. Case Rep Neurol Med. 2019;2019:9360542.

8. Annane D, Chevret S, Jars-Guincestre C, Chillet P, Elkharrat D, Gajdos P, et al. Prognostic factors in unintentional mild carbon monoxide poisoning. Intensive Care Med. 2001;27(11):1776-81
9. Pepe G, Castelli M, Nazerian P, Vanni S, Del Panta M, Gambassi F, et al. Delayed neuropsychological sequelae after carbon monoxide poisoning: predictive risk factors in the Emergency Department. A retrospective study.

10. This research did not receive any specific grant from funding agencies in the public, commercial, or not-for-profit sectors.

16. Poisoning/Toxicology/Pharmacology > Poisoning/Toxicology / Pharmacology

000057

Inorganic arsenic suppresses human neutrophil extracellular traps in vitro

M. M. Islam ; N. Takeyama ${ }^{1}$

${ }^{1}$ Emergency and Critical Care Medicine, Aichi Medical University School of Medicine, Nagakute, Aichi, Japan

Correspondence: M.M. Islam

Intensive Care Medicine Experimental 2020, 8(2): 000057

Introduction: Arsenic, a group 1 carcinogen classified by International Agency for Research on Cancer, is a major environmental toxicant and pollutant, which has caused health concern all over the world. The Agency for Toxic Substances and Disease Registry also enlisted arsenic as number one in their ranking of substance priority list 2017. Neutrophils are the vanguard of host innate immune system against invading microbial pathogens and several diseases. Neutrophil extracellular traps (NETs) have been disclosed as an essential aspect of human innate immunity. NETs are comprised of meshwork of chromatin fibers decorated with antimicrobial proteins like myeloperoxidase (MPO), neutrophil elastase (NE) and cathepsin G [1]. The function of NETs is to capture, immobilize and kill intruding gram negative and gram positive bacteria, fungi, parasites as well as invading microorganisms extracellularly [1]. The influence of arsenic on human NET formation, however, was not well established.

Objectives: This study aimed to evaluate the effects of acute low dose (20 ng/ml) NaAsO2 exposure on innate defense mechanisms regarding NETs and phagocytosis in vitro.

Methods: Isolated polymorphonuclear neutrophils (PMN) from peripheral blood of healthy human volunteers were incubated with $\mathrm{NaAsO} 2$ for $12 \mathrm{~h}$ and phorbol myristate acetate was used to induce NET formation. Effect of arsenic exposure on PMN functions including NETs release, phagocytosis, bacterial killing and reactive oxygen species (ROS) production were assessed. NETs formation was quantified by measuring cell-free extracellular DNA (cf-DNA), MPO-DNA and NE-DNA which are characteristic of NET. Cell-free DNA (cf-DNA) was measured using the Quant-iT PicoGreen dsDNA assay kit. MPO- and NE-DNA were measured by sandwich ELISA. ROS production was measured in live cells by a technique that converts $2^{\prime}, 7^{\prime}$-dichlorofluorescin diacetate which was oxidized to a fluorescence dye 2', 7'-dichlorofluorescein. Extracellular killing of bacteria by NETs was evaluated by co-culture of Escherichia coli and neutrophils in the presence of a phagocytic inhibitor cytochalasin D.

Results: In vitro inorganic arsenic exposure to human PMN significantly dampens the release of cf-DNA, MPO-DNA and NE-DNA which are the main components of NETs. Either capacity of phagocytosis and bacterial killing were also reduced after acute arsenic exposure.

Conclusion: We conclude that low dose of inorganic arsenic will disturb PMN function including NETs and phagocytosis. Our results open new doors for understanding the detailed mechanisms of arsenic intoxication on human innate immunity.

Reference(s) and grant ackowledgment(s)

1. 1. Brinkmann V, Reichard U, Goosmann C et al (2004) Neutrophil extracellular traps kill bacteria. Science 303: 1532-1535

16. Poisoning/Toxicology /Pharmacology > Poisoning/Toxicology /

Pharmacology 
000138

Comparison between mask oxygen therapy and nasal high-flow oxygen therapy using tracheal oxygen concentration measurement model

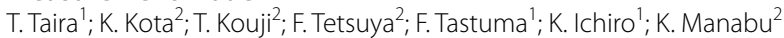
${ }^{1}$ Intensive care medicine,emergency medicine, University of the Ryukyus Hospital, Nishihara, Japan; ${ }^{2}$ Intensive care medicine, anesthesia, University of the Ryukyus Hospital, Nishihara, Japan

Correspondence: T. Taira

Intensive Care Medicine Experimental 2020, 8(2): 000138

Introduction: An effective treatment for carbon monoxide poisoning is the elimination of blood carbon monoxide by the administration of high oxygen concentration. Hyperbaric oxygenation therapy has the highest concentration of oxygen; however, medical institutions without hyperbaric oxygenation chambers also often treat patients with carbon monoxide poisoning. If hyperbaric oxygenation therapy is unavailable, the first-line treatment is a $100 \%$ oxygen mask; of late, another oxygen administration method in use is the nasal high-flow (NHF) therapy. However, no previous study has verified the type of oxygen administration method that can deliver higher oxygen concentration to the body. Here, we present a comparison of $100 \%$ intratracheal oxygen administration by oxygen mask or by NHF, using an original tracheal oxygen concentration measuring model.

Objectives: To compare the tracheal oxygen concentration of $100 \%$ oxygen administered by mask or by NHF using a special intratracheal oxygen measuring model.

Methods: A Michigan Instruments artificial lung was connected to a Hamilton ventilator $\mathrm{C} 1$ and a human model of intubation practice. The measuring device of oxygen concentration with INOflo, manufactured by Mallinckrodt pharmaceuticals, was inserted into the trachea of the human model to measure the oxygen concentration. The lung tidal volume and the minute volume were adjusted by the Hamilton $\mathrm{C} 1$. The minute volume was set to $5 \mathrm{~L}$ and $10 \mathrm{~L}$; the flow rate of the oxygen mask was set to $6 \mathrm{~L}, 10 \mathrm{~L}$, and $15 \mathrm{~L}$; and the flow rate of the NHF was set to $10 \mathrm{~L}, 20 \mathrm{~L}$, and $40 \mathrm{~L}$. For each of these, the measurement was performed four times.

Results: The model tracheal oxygen concentration results were as follows. Under the $100 \%$ oxygen by mask and $5 \mathrm{~L}$ of minute ventilation conditions, when the oxygen flow rates were set to $\mathrm{L}, 10 \mathrm{~L}$, and $15 \mathrm{~L}$, the average oxygen concentrations were $58.75 \%, 63.75 \%$, and $68.75 \%$, respectively. When the minute ventilation was set to $10 \mathrm{~L}$, these values were $39.75 \%, 46.5 \%$, and $53.75 \%$, respectively. In NHF, 5L of minute ventilation, when the oxygen flow was $10 \mathrm{~L}, 20 \mathrm{~L}$, and $30 \mathrm{~L}$, the average oxygen concentrations were $96.5 \%, 97 \%$, and $97.5 \%$, respectively; and for the $10 \mathrm{~L}$ of minute ventilation, for the $10 \mathrm{~L}, 20 \mathrm{~L}$, and $30 \mathrm{~L}$ flow rates, the average values were $91.75 \%, 95 \%$, and $97 \%$, respectively.

Conclusion: The oxygen concentration in the human model's trachea was higher with NHF oxygen administration, regardless of the ventilation minute volume and oxygen flow rate. These results suggested that for patients with carbon monoxide poisoning, NHF may reduce carbon monoxide in a shorter time than oxygen administration via a mask.

\section{Reference(s) and grant ackowledgment(s)}

1. Maggiore SM, Idone FA, Vaschetto R, Festa R, Cataldo A, Antonicelli F, et al. Nasal high-flow versus venturi mask oxygen therapy after extubation,

Effects on oxygenation, comfort, and clinical outcome. American Journal of Respiratory and Critical Care Medicine, 2014;190(3):282-288.

2. Weaver LK. Carbon monoxide poisoning. New England Journal of Medicine, 2009;360(12);1217-1225.

3. There is no grant to acknowledge.

16. Poisoning/Toxicology /Pharmacology > Poisoning/Toxicology /

Pharmacology
000190

Acute brain lesion on magnetic resonance imaging as predictive factor of delayed neurological sequelae in carbon monoxide poisoning

S. Nah'; S. Han ${ }^{1}$

${ }^{1}$ Emergency Department, Soonchunhyang University Bucheon Hospital, Bucheon, Republic of Korea

Correspondence: S. Nah

Intensive Care Medicine Experimental 2020, 8(2): 000190

Introduction: Carbon monoxide (CO) is a colorless, odorless toxic gas produced by incomplete combustion of carbon-based fuel and material. Because of these characteristics, many patients cannot recognize that they are poisoned and in mild cases, diagnosis and treatment are often delayed because symptoms do not appear immediately. In CO poisoning, the brain and heart which are vulnerable to ischemia are damaged due to the decline of oxygen transport. Development of delayed neurological sequelae (DNS) defined as any neurological symptoms that newly developed within 6 weeks after $\mathrm{CO}$ poisoning. Preventing DNS is a major goal of treating acute $\mathrm{CO}$ poisoning, but to our knowledge there are no reliable tools for assessing the probability of these sequelae.

Objectives: To find out whether acute brain lesions on diffusionweighted imaging are relevant to subsequent development of DNS after acute $\mathrm{CO}$ poisoning.

Methods: This registry-based observational study was conducted at a university hospital in Korea, between January 1, 2017, and December 31,2018 . Of 254 patients (aged $\geq 18$ ) with acute CO poisoning, 154 patients $(60.63 \%)$ who underwent diffusion-weighted imaging were considered for the study. Patients who refuse magnetic resonance imaging (MRI) $(n=40)$, discharge against medical advice $(n=6)$, follow up loss $(n=32)$, persistent neurological symptoms at discharge $(n=8)$ and refuse hyperbaric oxygen therapy $(n=14)$ were excluded. Patients were scheduled for MRI within 2 days of visiting hospital. If two or more of the univariate factors retained in the multivariate analysis were highly correlated, then only one factor was selected for further modeling to avoid collinearity before modeling. (Table 1).

Table 1. Factors associated with the development of delayed neurologic sequelae

\begin{tabular}{|c|c|c|c|c|c|c|}
\hline & \multicolumn{2}{|l|}{$\begin{array}{l}\text { Univariable } \\
\text { analysis }\end{array}$} & \multicolumn{4}{|c|}{ Multivariable analysis } \\
\hline & \multirow[b]{2}{*}{$\begin{array}{l}\text { OR }(95 \% \\
\text { CI) }\end{array}$} & \multirow[b]{2}{*}{$\begin{array}{l}\mathrm{P}- \\
\text { value }\end{array}$} & \multicolumn{2}{|l|}{ Model 1} & \multicolumn{2}{|l|}{ Model 2} \\
\hline & & & $\begin{array}{l}\text { OR }(95 \% \\
\text { CI) }\end{array}$ & \begin{tabular}{|l|} 
P- \\
value
\end{tabular} & $\begin{array}{l}\text { OR }(95 \% \\
\text { CI) }\end{array}$ & $\begin{array}{l}P \text { - } \\
\text { value }\end{array}$ \\
\hline Headache & $\begin{array}{l}0.438(0.096- \\
1.992\end{array}$ & 0.285 & & & & \\
\hline CRP & $\begin{array}{l}1.055(0.964- \\
1.155)\end{array}$ & 0.245 & & & & \\
\hline \multirow[t]{2}{*}{ Troponin I } & $1.164(0.894-$ & 0.259 & & & & \\
\hline & 1.516) & & & & & \\
\hline GCS & $\begin{array}{l}0.853(0.771- \\
0.944)\end{array}$ & 0.002 & $\begin{array}{l}0.864(0.760- \\
0.983)\end{array}$ & 0.026 & $\begin{array}{l}0.849(0.745- \\
0.967)\end{array}$ & 0.014 \\
\hline $\begin{array}{l}\text { Exposure } \\
\text { time, min }\end{array}$ & $\begin{array}{l}1.001(1.000- \\
1.002)\end{array}$ & 0.038 & $\begin{array}{l}1.002(1.001- \\
1.004)\end{array}$ & 0.010 & $\begin{array}{l}1.003(1.001- \\
1.004)\end{array}$ & 0.005 \\
\hline $\begin{array}{l}\text { Abnormal } \\
\text { MRI }\end{array}$ & $\begin{array}{l}3.804(1.624- \\
8.911)\end{array}$ & 0.002 & $\begin{array}{l}2.242(0.864- \\
5.821)\end{array}$ & 0.097 & & \\
\hline $\begin{array}{l}\text { White } \\
\text { matter } \\
\text { lesion }\end{array}$ & $\begin{array}{l}8.057(2.448- \\
26.516)\end{array}$ & 0.001 & & & $\begin{array}{l}6.741(1.843- \\
24.660)\end{array}$ & 0.004 \\
\hline
\end{tabular}

OR, odds ratio; CI, confidence interval; CRP, C-reactive protein; GCS, glasgow coma scale; MRI, magnetic resonance imaging 
Results: Of the 154 included patients, acute brain lesions on diffusionweighted imaging were observed in 49 patients (31.8\%). Among 154 patients, DNS occurred in 30 patients (19.5\%). In the multivariable logistic regression analysis, abnormal MRI was associated with DNS in model 1 (odds ratio [OR], 2.24; 95\% Confidence interval [CI]), 0.864$5.821 ; P=0.097)$. In model 2 , the acute brain lesions on MRI in white matter was notably associated with development of DNS (OR, 6.741; $95 \% \mathrm{Cl}, 1.843-24.660 ; \mathrm{P}=0.004)$. And Glasgow coma scale and exposure time were also related to subsequent development of DNS after CO poisoning in model 1,2 .

Conclusion: Acute brain lesions on MRI in white matter was notably associated with the development of DNS. Diffusion-weighted imaging during the acute phase of $\mathrm{CO}$ poisoning may predict the risk of developing subsequent neurologic deficit.

\section{Reference(s) and grant ackowledgment(s)}

1. 4. Myers RA, Snyder SK, Emhoff TA. Subacute sequelae of carbon monoxide poisoning. Ann Emerg Med. 1985;14(12):1163-7.

2. 3. Acute carbon monoxide poisoning and delayed neurological sequelae: a potential neuroprotection bundle therapy. Neural Regen Res. 2015;10(1):36.

3. 2. O'donnell P, Buxton P, Pitkin A, Jarvis L. The magnetic resonance imaging appearances of the brain in acute carbon monoxide poisoning. Clinical radiology. 2000;55(4):273-80

4. 1. Guzman JA. Carbon monoxide poisoning. Crit Care Clin. 2012;28(4):537-48

16. Poisoning/Toxicology/Pharmacology > Poisoning/Toxicology / Pharmacology

\section{2}

\section{Impact of Hydrogen Selenide against Oxidative Stress in Human} Hepatocytes

M. Kuganesan'; N. Arulkumaran ${ }^{1}$; A. Kleyman ${ }^{1}$; M. Singer ${ }^{1}$; A. Dyson ${ }^{1}$

'University college london, Bloomsbury Institute of Intensive Care Medicine, London, United Kingdom

Correspondence: A. Dyson

Intensive Care Medicine Experimental 2020, 8(2): 000442

Introduction: Selenium, an essential micronutrient incorporated into antioxidant 'selenoproteins', has been extensively trialled as a putative therapy in critical illnesses. However, when administered in its oxidised form, selenite, it exhibits varying degrees of success [1]. Importantly, the ability of any molecule to undergo 'redox' reactions depends on its oxidation status. We previously showed that the reduced form of selenium, selenide, acts both as an antioxidant and a metabolic modulator via transient inhibition of mitochondrial cytochrome $C$ oxidase [2]. Here we assessed the effects of a basic selenide salt (NaHSe) against oxidative stress in human hepatocytes.

Methods: HepG2 human hepatocytes were grown to $80 \%$ confluence under standard cell culture conditions. Sodium hydrogen selenide (NaHSe) was synthesized as described [3]. Cells were incubated with 0.3 or $1 \mu \mathrm{M}$ NaHSe (as therapeutic concentrations) for 1 hour. Oxidative stress was initiated by addition of hydrogen peroxide $(\mathrm{H} 2 \mathrm{O} 2 ; 500$ $\mu \mathrm{M}$ ); cells were incubated for a further 24 hours. Cell viability (in tetrazolium chloride $[1 \% \mathrm{w} / \mathrm{v}]$ stained cells) or mitochondrial membrane potential (MMP; in tetramethylrhodamine methyl ester [TMRM] incubated [ $25 \mathrm{nM}$ for $30 \mathrm{~min}$ ] cells) were measured using spectrophotometry and flow cytometry, respectively.

Results: $\mathrm{H} 2 \mathrm{O} 2$ decreased cell viability and mitochondrial membrane potential and these could be reversed by NaHSe treatment (Fig 1).

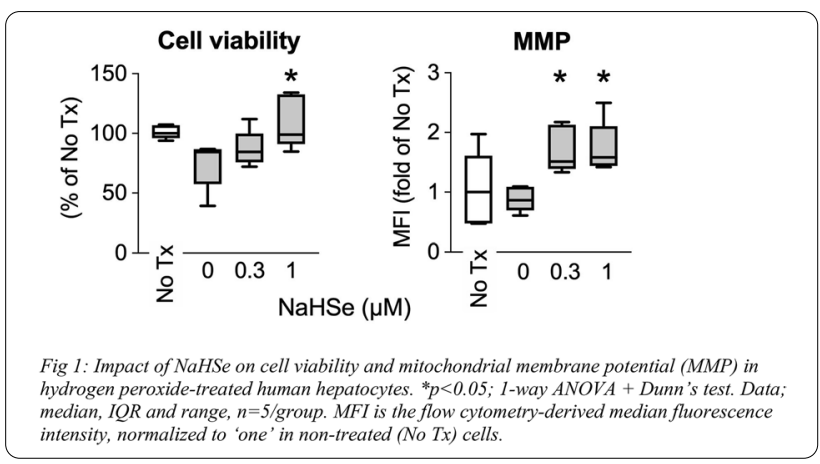

Conclusion: Treatment withthe reduced form of selenium, hydrogen selenide, protected against oxidative stress in human hepatocytes. This was associated with a clear elevation in mitochondrial membrane potential. The ability of selenide to confer protection against oxidative pathologies via a mitochondrial mechanism of action (and the importance of its oxidation status) will be subject to further investigation.

Reference(s) and grant ackowledgment(s)

1. Zhao et al, Medicine (Baltimore). 2019; 98:e15473.

2. [2] Samra et al, Intensive Care Med Exp. 2019; 7:000883.

3. [3] Klayman and Griffin. J. Am. Chem. Soc. 1973; 95:197-199.

16. Poisoning/Toxicology /Pharmacology > Poisoning/Toxicology /

Pharmacology

\section{4}

Selenium Therapy: Impact of Oxidation Status on Antioxidant Selenoprotein Expression

W. Smith ${ }^{1}$; M. Kuganesan ${ }^{1}$; R. Tidswell ${ }^{1}$; M. Singer ${ }^{1}$; A. Dyson ${ }^{1}$

${ }^{1}$ University college london, Bloomsbury Institute of Intensive Care Medicine, London, United Kingdom

Correspondence: A. Dyson

Intensive Care Medicine Experimental 2020, 8(2): 000444

Introduction: Selenium, an essential micronutrient, is incorporated into numerous 'selenoprotein' oxidoreductase enzymes that impact on cellular redox status in health and disease. As a putative antioxidant therapy in critical illness states, selenium has been administered solely as the oxidised form, selenite, with varying success [1]. However, the ability of any molecule to undergo 'redox' reactions depends on its oxidation status, and this may impact on its utility as a therapy [2]. We assessed the ability of both the oxidized selenium product, selenite, and the reduced form, selenide, to modify selenoprotein expression in human hepatocytes.

Methods: HepG2 human hepatocytes were grown to $80 \%$ confluence under standard cell culture conditions. Cells were plated and incubated with either sodium hydrogen selenide (NaHSe; $30 \mathrm{nM}$ synthesized as described [3]), sodium selenite $\left(\mathrm{Na}_{2} \mathrm{SeO}_{3} ; 30 \mathrm{nM}\right)$ or no treatment (cell medium) for 24 hours. L-cysteine $(0.1 \mathrm{mM})$ was used as a positive control (to decrease selenoprotein synthesis) as it interferes with selenocysteine, a crucial intermediate. Protein expression of glutathione peroxidase-1 (GPX-1) and thioredoxin reductase-1 (TxR-1) was determined by Western blot analysis. These selenoproteins were chosen as they are ubiquitously expressed, readily abundant, and 
crucially involved in the detoxification of inorganic and organic peroxides, respectively.

Results: The oxidized selenium form, selenite, used in critically ill patients as an antioxidant therapy, negatively impacted on antioxidant selenoprotein expression (Fig 1). By contrast, exposure to the reduced form, selenide, showed increased abundance of the two enzymes studied.

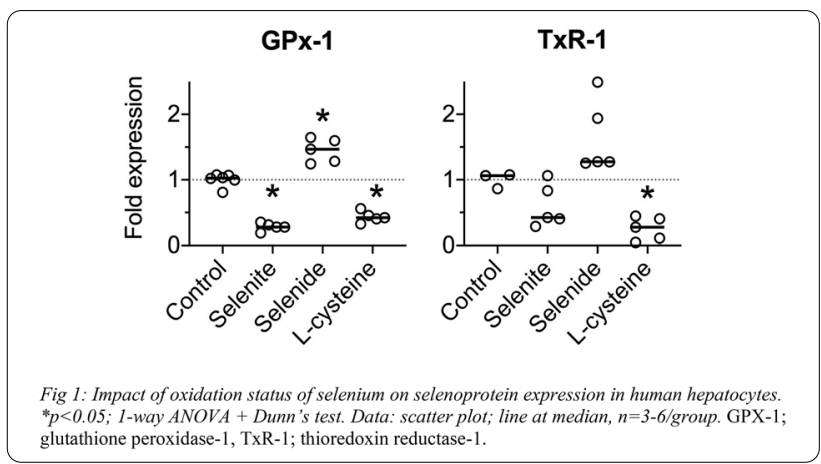

Conclusion: The oxidation status of selenium-based therapies on selenoprotein expression revealed divergent results. This demonstrates that oxidation status can impact on efficacy, and suggests that targeted administration of reduced selenium forms may yield more favourable results.

Reference(s) and grant ackowledgment(s)

1. [1] Zhao et al. Med. Baltim., 2019; 98:15473.

2. [2] Kuganesan et al. Intensive Care Med. Exp., 2019; 7:71.

3. [3] Klayman \& Griffin. J. Am. Chem. Soc., 1973; 95:197-199.

16. Poisoning/Toxicology/Pharmacology > Poisoning/Toxicology /

Pharmacology

\section{8}

Organ and system substitution techniques to treat poisonings

C. Palau Marti '; S. Moliner Velázquez' ; R. Rubio Haro ${ }^{1}$; C. Ferrer Gómez E. Mateo Rodríguez'; J. De Andrés Ibáñez

${ }^{1}$ Anaesthesiology, Critical Care and Pain Therapy Service, Consorci Hospital General Universitari de València, València, Spain

Correspondence: C. Palau Marti

Intensive Care Medicine Experimental 2020, 8(2): 000948

Introduction: $3-14 \%$ ICU admissions are due to poisonings. The mortality rate is $2,1 \%$. The treatment for acute poisonings requires: precociousness, vital functions support, metabolic support and symptomatic treatment, reducing or stopping the absorption of the poison, an antidote-based treatment and measures to increase the elimination of the toxic (intestinal and renal excretion as well as extracorporeal techniques).

Objectives: The aim of this review is to establish the organ and system substitution techniques currently available and how they were used to treat poisonings in our ICU during the 2015-2018 period.

Methods: Study authorized by the ethics committee in the centre. In the 2015-2018 period there were 7941 admissions to the ICU. 31 of these were poisonings $(0,39 \%)$. The organ and system substitution techniques consist in: 1. Renal replacement techniques (hemodialysis, continuous renal replacement techniques and peritoneal dialysis),
2. Plasmapheresis and plasma exchange, 3. Hemoperfusion and 4. Exchange transfusion. The following extracorporeal organ support techniques are also available: 1. Extracorporeal liver support (MARS: molecular adsorbent recirculating system), and 2. Extracorporeal membrane oxygenation (ECMO).

Results: In our series, $64 \%$ were men and the average age was 44 years old. The most common cause was medication-related poisoning (benzodiazepines). Ethanol was the main addictive drug. The measures taken in our ICU have been continuous renal replacement therapy in one occasion (lithium poisoning).

Conclusion: In spite of the fact that the ICU admissions rate is low, it is necessary to be familiar with the organ and system substitution techniques in order to apply the best one in each case. It is equally important to know about the pharmacokinetic characteristics of those toxics that can be expelled using these extracorporeal techniques.

Reference(s) and grant ackowledgment(s)

1. Weiner L, Mazzeffi MA, Hines EQ, Gordon D, Herr DL, Kim HK. Clinical utility of venoarterial-extracorporeal membrane oxygenation (VA-ECMO) in patients with drug-induced cardiogenic shock: a retrospective study of the Extracorporeal Life Support Organizations'ECMO case registry. Clin Toxicol (Phila). 2020;58(7):705-710. doi:10.1080/15563650.2019.1676896

2. Ghannoum M, Gosselin S. Enhaced poison elimination in Critical Care. Adv Chronic Kidney Dis. 2013 Jan;20(1):94-101

16. Poisoning/Toxicology/Pharmacology > Poisoning/Toxicology / Pharmacology

\section{9}

A QIP on Naloxone Administration: Do Staff Need a Push to Give a Bolus in an Emergency?

D. Wirth ${ }^{1}$; C. Briggs ${ }^{1}$; P. Beddoes ${ }^{1}$; T. Smith ${ }^{1}$

${ }^{1}$ Anaesthetics department, Whipps Cross University Hospital, London, United Kingdom

Correspondence: D. Wirth

Intensive Care Medicine Experimental 2020, 8(2): 001079

Introduction: Naloxone, an opioid antagonist, is used for the management of opioid toxicity, presenting with respiratory depression, miosis and/or decreased consciousness. This is a medical emergency occurring in different settings: theatre recovery, maternity or general surgical wards. Urgent naloxoneadministration is vital and can be given IV, SC or IM at an initial dose of $400 \mathrm{mcg}(1)$. Naloxone is initially given as a push bolus, and locally is prepared as $400 \mathrm{mcg}$ in $1 \mathrm{ml}$ ampoules (2). We recognised that familiarity with naloxone at our institution was low. Lack of knowledge and familiarity is a known contributor to drug error(3). Use of cognitive aids can facilitate timely implementation of treatment in emergencies such as opioid toxicity(4).

\section{Objectives:}

- To assess staff familiarity and confidence with naloxone.

- To assess knowledge regarding naloxone administration.

Methods: An anonymous staff survey was designed to assess familiarity and confidence with naloxone use and was distributed to theatre recovery, surgical wards and maternity. Later a poster (Figure 1) was designed and placed on control drug (CD) cupboards to serve as an accessible cognitive aid in an emergency. A safety alert was emailed to guide staff on how to manage suspected opiate toxicity. We re-surveyed to assess improvement in knowledge and confidence of naloxone use. 
$27 / 05 / 2020$

\section{NALOXONE FOR SUSPECTED OPIATE TOXICITY}

SUSPECT IF:
- Pinpoint pupils
- Decreased conscious level
- $R R<8$

\begin{tabular}{|c|}
\hline ACTION: \\
\hline $\begin{array}{ll}\text { - } & \text { Give naloxone } \\
\text { - } & \text { Give } 15 L \text { L } 02 \text { NRB } \\
\text { - } & \text { Call doctor } \\
\text { - } & \text { Consider } 2222 \text { call }\end{array}$ \\
\hline
\end{tabular}

\begin{tabular}{|l|}
\hline NALOXONE ADMINISTRATION: \\
- Undiluted $400 \mathrm{mcg}(1 \mathrm{ml})$ bolus \\
- IV preferable (same dose SC or \\
IM if no IV access)
\end{tabular}

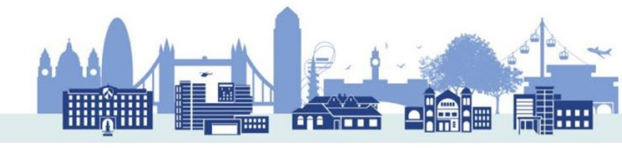

Results: Our initial survey found low confidence in naloxone administration with $79 \%$ of respondents either unwilling or needing a nomogram to administer it. This was unsurprising as most had not used naloxone in the preceding year. On re-survey, we found improved confidence, with an increase in those either confident or very confident from $20 \%$ to $40 \%$ and fewer people unwilling to use naloxone (17\% to $12 \%)$. Staff selecting the correct dose as per trust protocol increased by $31 \%$, from $41 \%$ to $72 \%$ ( $p=0.02 \mathrm{Cl} 4.5 \%-51.9 \%$ ).

Conclusion: Use of naloxone is rare and staff at our institution are unfamiliar with it, demonstrated here by a lack of confidence and knowledge of correct dose. $30 \%$ of medication errors relate to gaps in knowledge or application of knowledge of a drug(3). However, in opiate toxicity, prompt, accurate administration of naloxone is imperative, yet human factors such as stress and communication breakdown in an emergency scenario may further inhibit ability to correctly administer treatment.

A simple and low-cost cognitive aid positioned on CD cupboards combined with a safety alert via email improved accuracy and confidence with naloxone use.

In critical care, doctors drill for rare emergencies though simulation (sim), but key members of the MDT such as nurses and midwives receive less sim-based training. We have demonstrated an increase in confidence and accuracy of naloxone administration with simple, cost-effective measures. Further work could examine the sustainability of these improvements and implement sim-based training to further improve MDT performance.

\section{Reference(s) and grant ackowledgment(s)}

1. 4 - Hall, C., Robertson, D., Rolfe, M. et al. 2020 Do cognitive aids reduce error rates in resuscitation team performance? Trial of emergency medicine protocols in simulation training (TEMPIST) in Australia. Hum Resour Health 18,1

2. 3 - Lesar, T.S., Briceland, L. and Stein, D.S., 1997. Factors related to errors in medication prescribing. Jama, 277(4), pp.312-317.

3. 2 - Medusa. 2017. NHS Injectable Medicines Guide - Naloxone Hydrochloride. Available at: Barts Health NHS Trust intranet. Accessed: June 2020
4. 1 - NICE. 2019. British National Formulary - Naloxone Hydrochloride. Available at: https://bnf.nice.org.uk/drug/naloxone-hydrochloride. html\#indicationsAndDoses. Accessed: June 2020

\section{4}

Post-discharge psychological symptoms in critical care survivors who had been difficult to sedate

D. Gil Castillejos ${ }^{1}$; L. Rico Rubio ${ }^{2}$; C. Ferre Grau²; MA. De Gracia Ballarín”; M. Bodí'; A. Sandiumenge

${ }^{1}$ Intensive and Critical Care Unit, Hospital Universitari de Tarragona Joan XXIII, Tarragona, Spain; ${ }^{2}$ Department of nursing, Rovira i Virgili University, Tarragona, Spain; ${ }^{3}$ Medical trasplant coordinator, Vall d'Hebron University Hospital, Barcelona, Spain

Correspondence: D. Gil Castillejos

Intensive Care Medicine Experimental 2020, 8(2): 000004

Introduction: Patients admitted to an intensive care unit (ICU) have a potentially fatal physical condition which also has an intense psychological impact

The emotional impact of this experience can have negative repercussions during the patient's recovery, as evidenced by the fact that anxiety, depression and post-traumatic symptoms are more prevalent among critical care survivors than in the general population.

The use of analgesia-based sedation, or analgosedation, is therefore a common and necessary practice in ICUs, the aim being to achieve an optimum level of comfort and pain relief for patients, to reduce their anxiety and disorientation, and to allow them to rest and sleep without interfering with medical and nursing care. However, the use of analgosedation is not free of complications, not least as its overuse can hamper the conscious processing of events experienced in the ICU. In addition, patients who undergo prolonged sedation may become difficult to sedate and require increasing doses, a scenario that has been associated with longer ICU stays and more complications during admission. In this respect, analgosedation may increase the likelihood of severe psychological sequelae among critical care survivors. Moreover, patients who prove difficult to sedate during an ICU admission experience more periods of over- and under-sedation as it is a challenge to keep them within the target range of conscious sedation (Richmond Agitation-Sedation Scale score of $-2,-1,0$ ).

Objectives: The aim of the present study was to determine the incidence of anxiety, depression and PTSD among critical care survivors and to explore the impact of difficult sedation on the frequency and intensity of these symptoms.

Methods: Descriptive and prospective analysis of analgosedation and psychological symptoms in patients who required $>24 \mathrm{~h}$ of mechanical ventilation during their ICU admission. We compared complications, clinical outcomes and psychological sequelae according to whether or not patients had been difficult to sedate. Descriptive analysis and multivariate logistic regression were performed.

Results: Data were obtained for 195 patients, of whom 30\% experienced difficult sedation. Difficult to sedate patients were younger $(52.2 \pm 16.4$ vs. $61.4 \pm 14.1$ years; $p=.001$ ), less critically ill (APACHE II score $22.1 \pm 9.9$ vs. $26.4 \pm 8.7 ; \mathrm{p}=.002$ ) and more likely to engage in harmful use of alcohol $(p=.001)$ and to have used psychoactive/ psychotropic drugs during the previous three months. They also spent longer in the ICU ( $28.1 \pm 25.8$ vs. $17.6 \pm 15.4$ days; $p=.001)$ and a greater percentage of time on mechanical ventilation $(61.1 \pm 15.4 \mathrm{vs}$. $51.4 \pm 18.8 ; p=.001)$. At 1-month follow up, $87.7 \%$ of difficult-to-sedate patients reported anxiety (vs. $45.4 \% ; p=.001$ ), with the corresponding figures at 3 and 6 months being $75.4 \%$ (vs. 29\%; $p=.001$ ) and $70.8 \%$ (vs. $23.7 \%$; $p=.001$ ). Depression was reported by $82.4 \%$ (vs. $43.1 \%$; $p=.001$ ), $66 \%$ (vs. $33.9 \% ; p=.001$ ) and $60.4 \%$ (vs. $27.2 \% ; p=.001$ ) of difficult-to-sedate patients at 1, 3 and 6 months, respectively. At I month, $28.1 \%$ of these patients (vs. $11.5 \% ; p=.007$ ) reported symptoms of PTSD.

Conclusion: Critical care patients who undergo prolonged analgosedation may become difficult to sedate, and this can have a negative impact on their subsequent emotional wellbeing. It is therefore crucial to implement multidisciplinary sedation protocols based on 
algorithms that encourage collaborative sedation and weaning. The early identification of patients who are likely to experience difficult sedation, coupled with strategies for preventing it, are essential for minimizing the impact of this complication, both in the short and long term. It is also important to ensure that adequate attention is paid not only to the physical but also the psychological needs of critical care patients, since both aspects will impact their subsequent recovery process.

\section{Reference(s) and grant ackowledgment(s)}

1. Estébanez MB, Alonso MA, Sandiumenge A, Jimenez M. Sedación prolongada en Unidades de Cuidados Intensivos. Med Intensiva.2008;32:19-30.

2. Jacka MJ, Mitchell N, Perez-Parada J. Incidence and prevalence of anxiety, depression, and post-traumatic stress disorder among critical care patients, families, and practitioners. Anesthesia \& Intensive Care Medicine 2016:1(1):1-4.

3. Contreras Moreno A, Palacios-Espinosa X. Contribuciones de la Psicología al manejo interdisciplinario del paciente en Unidad de Cuidados Intensivos (UCI). Rev. Univ. Ind. Santander Salud. 2014;46(1):47-60.

4. Guze SB. Diagnostic and Statistical Manual of Mental Disorders, 4th ed. (DSM-IV). Am J Psychiatry [Internet]. 1995 Aug;152(8):1228-1228. Available from: http://psychiatryonline.org/doi/abs/10.1176/ajp.152.8.1228

5. Gil B, Ballester R, Gómez S, Abizanda R. Afectación emocional de los pacientes ingresados en una unidad de cuidados intensivos. Rev Psicopatología y Psicol Clínica. 2013;18(2):129-38.

\section{0}

Delirium after on-pump cardiac surgery: a single centre descriptive study

H. Stragier ${ }^{1}$; D. Mesotten ${ }^{1}$; M. Vander Laenen ${ }^{1}$; W. Boer ${ }^{1}$; T. Fivez ${ }^{1}$ ${ }^{1}$ Department of anesthesiology, intensive care and pain therapy, Ziekenhuis Oost-Limburg A. V., Genk, Belgium

Correspondence: $\mathrm{H}$. Stragier

Intensive Care Medicine Experimental 2020, 8(2): 000120

Introduction: Cardiac surgery requiring cardiopulmonary bypass (CPB) has been associated with neurocognitive impairment, called the postperfusion syndrome. Delirium is the most common form of neurocognitive impairment and has been linked with increased morbidity and mortality. It has been suggested that micro-emboli during CPB cause this postperfusion syndrome

Objectives: We aimed to assess whether the duration of CPB is the major factor in delirium after cardiac surgery.

Methods: This single centre retrospective study included 235 patients, admitted to the ICU after cardiac surgery with CPB between 01-012019 and 31-12-2019. Delirium was defined as patients having been administered the antipsychotic drugs haloperidol or quetiapine, either intravenously or orally, at least once during ICU-stay. Patient characteristics and drugs were retrieved directly from the electronic medical records. Risk factors for delirium after cardiac surgery were analysed: age, type of surgery, Euroscore, APACHE-II score. Data were presented as median and IQR.

Results: Patients underwent arcus ( $n=10), C A B G(n=85), C A B G / v a l v e$ $(n=36)$, valve $(n=51)$, mitral valve $(n=44)$ and multiple valve surgery $(n=9) .24 / 235(10 \%)$ received antipsychotics during ICU-stay. ICU-stay and hospital stay were longer in patients with delirium (median 5, IQR 4-7.5 and median 17.5, IQR 11-40.5)than in patients without (median 3, IQR 3-5 and median 11, IQR 9-17)(both $p<0.004$ ). Median duration of CPB did not differ between patients with delirium (135.5, IQR 105.75$203)$ and those without $(150$, IQR $115-193)(\mathrm{p}=0.90)$. Age, type of surgery, Euroscore, APACHE-Il score also did not differ between patients with or without delirium.

Conclusion: In our centre, duration of CPB was not associated with incidence of delirium. However, both length of stay at the ICU and in the hospital were significant prolonged in cardiac surgery patients with delirium. This findings underline the clinical importance of delirium both on short term and long term outcome.
Reference(s) and grant ackowledgment(s)

1. Su L, Yan Y, Huang W et al. Risk factors for intensive care unit delirium after cardiac operation. Zhonghua Wei Zhong Bing Ji Jiu Yi Xue. 2019 Feb;31(2):165-171

2. Brown CH Delirium in the cardiac surgical ICU.Curr Opin Anaesthesiol. 2014 Apr;27(2):117-22

000290

Evaluation of pain scales and outcome in critically ill patients of a Greek ICU

S. C. Kotoulas'; S. Kalliontzis²; M. Romanopoulou²; K. Manika;

S. Anissoglou ${ }^{2}$; P. Kontou ${ }^{1}$

${ }^{1} A^{\prime}$ icu, G. Papanikolaou Hospital, Thessaloniki, Greece; ${ }^{2} \mathrm{lcu}$, Theagenio Hospital, Thessaloniki, Greece; ${ }^{3}$ Pulmonary department, G. Papanikolaou Hospital, Thessaloniki, Greece

Correspondence: P. Kontou

Intensive Care Medicine Experimental 2020, 8(2): 000290

Introduction: Critically ill patients experience pain during Intensive Care Unit (ICU) stay, which must be assessed by valid scales since it affects various outcomes.

Objectives: To evaluate painful procedures in critically ill patients and to investigate their effect as well as the role of analgesia in the outcome.

Methods: We included 28 mechanically ventilated patients who were subjected to $5.71 \pm 4.29$ painful (turning, endotracheal suction, physiotherapy, central venous catheter placement) and non-painful (oral care, dressing change) procedures. We measured their pain level before, during and after the stimuli by using the Behavioral Pain Scale (BPS) and the Critical care Pain Observation Tool (CPOT). We also recorded their vital signs (systolic and mean blood pressure, heart rate and respiratory rate) at the same timepoints. We analyzed the correlation of our measurements as well as the use of bolus analgesia with the duration of mechanical ventilation, the ICU stay and the survival of the patients.

Results: There were statistically significant differences in the pain scores and vital signs between the different timepoints (before-during and during-after) for all procedures. The differences in the values of BPS and CPOT scales in the aforementioned timepoints were significantly correlated with the respective differences in the values of vital signs. Regarding the type of the procedure, it was shown that physiotherapy influenced more than the others the change of both pain scales. The use of bolus analgesia during the procedure compared to the non-use predicted both less days of mechanical ventilation (17.94 \pm 12.73 vs $25.02 \pm 21.19, p=0.015)$ and less days of ICU stay (18.54 \pm 12.82 vs $25.56 \pm 20.92, p=0.016)$. The higher difference in the BPS before and during the stimuli was correlated with more days of mechanical ventilation $[\mathrm{B}(95 \% \mathrm{Cl})=3.640(1.001-6.280), \mathrm{p}=0.007]$ and more days of ICU stay $[\mathrm{B}(95 \% \mathrm{Cl})=3.645(1.035-6.254), \mathrm{p}=$ 0.006]. As far as the outcome, the higher difference in the CPOT during and after the stimuli and the non-use of bolus analgesia related to increased chance of death $[\mathrm{OR}(95 \% \mathrm{Cl})=1.492(1.107-2.011), \mathrm{p}=$ 0.009 and $\mathrm{OR}(95 \% \mathrm{Cl})=2.626(1.013-6.806), \mathrm{p}=0.047$ respectively].

Conclusion: Increased level of patients' pain during various procedures in the ICU, as assessed by the BPS and CPOT scales, is related to worse outcomes while the use of bolus analgesia during these procedures might improves the outcomes.

Reference(s) and grant ackowledgment(s)

1. None

000306

Delayed adverse events after sedation for hematologic procedure in pediatric patients

H. Koh ${ }^{1}$; L. Nuri ${ }^{1}$; M. Kwanguck ${ }^{1}$

${ }^{1}$ Anesthesiology and pain medicine, The Catholic University of Korea Seoul St. Mary's Hospital, Seoul, Republic of Korea

Correspondence: $\mathrm{H}$. Koh

Intensive Care Medicine Experimental 2020, 8(2): 000306 
Introduction: While procedures and treatments are performed, sedation is undergone not only for reducing patient's anxiety and pain but also for facilitating the performance. Although there are various sedatives in pediatrics, most of them are not officially approved. The choice and doses of drugs are different case by case and also the responses of them are inconsistentwith expectation. For this reason, careful management are particularly necessary. Usually, until the early recovery period in post-anesthetic care unit (PACU) after sedation, adverse events can be observed through careful monitoring. However, it is difficult to identify delayed complications (after 2hours) and also few studies introduced.

Objectives: In this study, we investigated the delayed adverse events that may occur in pediatrics after sedation. We would like to find out the most common adverse reactions and their period. And based on these evidences, how to deal with delayed responses takes a message in the future.

Methods: The subjects were pediatric patients ( 3 to 18 year-old) who had undergone sedation for procedures related to hemato-oncology examinations such as bone marrow biopsy, aspiration, spinal tap or both. The adverse events ere consisted of 20 categories and they were recorded after sedation by their parents. Patients were divided in two groups; patients who were visited to day care center (DCC) (group A) and admitted in ward (group B). They had repeated these procedures periodically. With Fentanyl $1.5 \mathrm{mcg} \mathrm{kg-1}$ as analgesics, three type sedatives were used in order; propofol: ketamine 3:1 combination (type A), 4:1 combination (type B) and propofol single use $1.5 \mathrm{mg} \mathrm{kg}^{-1}$ (type C). The occurrence time interval was set at two hour intervals including 30 minutes recovery room period as session one, total 13 sessions. The beginning and ending time of adverse events were also recorded. These categories were based on adverse event of sedative drugs and others specific events were recorded separately.

Results: A total of 168 Patients were participated, group A was 103 patients and group B was 65 patients. Adverse symptoms were showed mostly within 24 hour; group A was $34.95 \%$ (36/103) and group B was $51.56 \%$ (33/64). Among 20 items, most common adverse events were nausea $(13.59 \%, 14 / 103)$ in group $A$ and drowsiness $(26.56 \% ; 17 / 64)$ and dizziness $(23.44 \% ; 15 / 64)$ in group B. The incidence was not related to the type of procedure or the combination of sedatives. For hemostasis and intrathecal chemotherapy, during this time, patients were lying down on recovery and intensively monitored, adverse events in group A were showed distribution of various time session $(32.8 \% ; 21 / 64$ in recovery room) but those of group $B$ were mostly occupied in this period $(74.4 \% ; 32 / 43)$. More than $50 \%$ of adverse events occurred within 6 hours. In particular, group B rarely occurred after 12 hours $(2 \% ; 1 / 43)$. Adverse events were disappeared within a day except four cases in group A $(6 \% ; 4 / 64)$. All adverse events were not required any treatment, refusal of next sedation or need for change of sedatives.

Conclusion: Although there are various sedatives in pediatrics, most of them are not officially approved. The choice and doses of drugs are different case by case and also the responses of them are inconsistent with expectation. The overall trend and degree of risk could be predicted through this study on delayed adverse reactions to sedatives. Though various adverse responses were developed, most of them disappeared within 24 hours spontaneously.

Based on these results, we could consider that sedation with propofol and ketamine should be safe sedatives to reduce anxiety and facilitate sedation safely even when continuous intensive monitoring and management is impossible. Apart from studies of delayed adverse responses in pediatric patients, future studies seek to identify and address how to reduce the adverse reactions and related variables.

\section{Reference(s) and grant ackowledgment(s)}

1. Individualizing propofol dosage: a multivariate linear model approach. J Clin Monit Comput. 2014;28(6):525-36

2. The safety, feasibility, and acceptability of patient-controlled sedation for colonoscopy: prospective study. Hong Kong Med J. 2004;10(2):84-8

3. There were no interests and financial supports.

4. Adverse events of procedural sedation and analgesia in a pediatric emergency department. Annals of Emergency Medicine. 1999;34(4):483-491

\section{2}

Risk factors associated with the development of delirium in a polyvalent ICU

B. Lobo Valbuena'; L. López de la Oliva Calvo; R. Molina'; A. Abella Alvarez'; I. Torrejón”; DD. Varillas²; F. Gordo

${ }^{1}$ Intensive care unit, H. U del Henares, Madrid, Spain; ${ }^{2}$ Unidad de apoyo a la investigación, facultad de medicina, Universidad Francisco de Vitoria, Madrid, Spain

Correspondence: B. Lobo Valbuena

Intensive Care Medicine Experimental 2020, 8(2): 000392

Introduction: Delirium is a severe neuropsychiatric disorder of organic origin characterized by the appearance of alterations in both consciousness and cognitive functions. The development of delirium is associated with multiple complications: increased mortality, longer duration of mechanical ventilation, higher rate of reintubation, and increased hospital stay. Since effective treatment has proven troublesome, prophylactic strategies become paramount.

Objectives: Analysis of the risk factors associated with the development of delirium in a polyvalent ICU.

Methods: Prospective observational study on a cohort of ICU patients admitted from $1 / 10 / 2016$ to $1 / 05 / 2019$; those requiring transfer to another centre were excluded. Patients were classified into two groups, according to whether they developed delirium or not, and the following variables were analysed: sex, age, frailty, reason for admission, comorbidities, SAPS 3, SOFA, specific management measures and development of organic failure(s) (OF). Statistical analysis between subgroups was carried out: Pearson's Chi-square test or Mann-Whitney's $U$ to find significant differences, and uni- and multivariate logistic regression analysis to quantify existing associations.

Results: 1534 patients were admitted during the indicated time, 72 excluded. Ninety-three developed delirium (incidence 6.3\%). After obtaining the initial data, we performed a multivariate analysis (table 1) including significant variables of the univariate analysis (with $\mathrm{p}<0.1$ ), and finally, recursive partitioning using Chaid tree, observing (a) a higher incidence of delirium in patients who presented more than 2 OF (20.4\%) and, (b) in patients with less than 2 OF, higher incidence in patients above 74 years (8.6\%). Patients who developed delirium had longer ICU and hospital length-of-stays and a higher rate of readmission, without differences in mortality.

Table 1. Multivariate analysis
\begin{tabular}{ll|}
\hline VARIABLE & OR $($ CI 95\%) \\
\hline Age $(>74)$ & $2,1(1,3-3,5)$ \\
\hline Coma as reason for admission & $2,5(1,07-5,8)$ \\
\hline More tan 2 OF & $4,9(2,9-8,2)$ \\
\hline Invasive mechanical ventilation & $1,9(1,1-3,3)$ \\
\hline Confirmed isolation & $2,4(1,2-4,6)$ \\
\hline Continuous neuromuscular blockade & $7,2(2-26,3)$ \\
\hline
\end{tabular}

Conclusion: The highest risk observed for developing delirium clustered in patients who presented more than 2 OF and patients over 74 years old. The detection of patients at high risk for developing delirium could imply a change in management and an improvement in the quality of care.

\section{Reference(s) and grant ackowledgment(s)}

1. 4. Zaal IJ, Devlin JW, Peelen LM, Slooter AJ (2015). A systematic review of Risk Factors for Delirium in the ICU. Crit Care Med 43:40-7.

2. 3. Salluh JI, Wang H, Schneider EB, Nagaraja N, Yenokyan G, Damluji A, Serafim RB, Stevens RD (2015). Outcome of delirium in critically ill patients: Systematic review and meta-analysis. BMJ 350:h2538.

3. 2. Ely EW, Shintani A, Truman B, SperoffT, Gordon SM, Harrell FE Jr, Inouye SK, Bernard GR, Dittus RS (2004). Delirium as a predictor of mortality in mechanically ventilated patients in the intensive care unit. JAMA 291:1753-62. 
4. 1. Ely EW, Siegel MD, Inouye SK (2001). Delirium in the Intensive Care Unit: an under-recognized syndrome of organ dysfunction. Seminars Respir Crit Care Med 22:115-6.

\section{6}

\section{Delirium in the Critical Care}

R. Boot ${ }^{1}$; F. Howroyd ${ }^{2}$; E. Felton ${ }^{2}$; T. Veenith

${ }^{1}$ Critical care, Queen Elizabeth Hospital Birmingham, Birmingham, UnitedKingdom; ${ }^{2}$ Critical care rehab team, Queen Elizabeth Hospital Birmingham, Birmingham, United Kingdom

\section{Correspondence: $\mathrm{R}$. Boot}

Intensive Care Medicine Experimental 2020, 8(2): 000396

Introduction: Delirium is a common often misdiagnosed, unrecognised and misunderstood condition in the critical care setting (Page et $\mathrm{al}, 2009)$ that has been associated with increased mortality, morbidity and cognitive dysfunction (Leutz et al, 2010). Delirium has implications for increasing cost to the National Health Service as it is linked to prolonged ventilation, and ICU stay which exposes the patient to associated risks as well as increased hospital stay (Mehta et al, 2015, Zhang et al, 2013, Gleason et al 2015).

Objectives: The aim of this study was to identify our prevalence of delirium in our critically ill patient population and assess our compliance with using CAM-ICU (Confusion Assessment Method for the Intensive Care Unit), the gold standard screening tool for identifying patients with delirium in the critical care setting (Gelinas et al, 2018 NICE, 2010).

Methods: A prospective cohort study was undertaken in a 75 bedded UK critical care unit, between August 2018 to December 2018. Patients were reviewed consecutively for 2 weeks between 14:00 and 16:00hrs in which a Richmond Agitation and Sedation Score (RASS) was completed. For patients that were found to have a RASS score $>-1$, CAMICU was completed. 106 Patients were excluded (106 patients) due to a length of stay less than 2 days, a previous known cognitive dysfunction, non-English speaking or were $<18$ years old.

Results: 217 patients were identified in the 2 week period. A RASS score was completed on 111 patients, of which 24 percent of patients were found to be CAM-ICU positive at least once during their critical care stay, $61 \%$ were had a negative CAM-ICU and $14 \%$ were unable to be assessed for delirium as remained with a RASS $<1$ throughout the study. Higher rates of CAM-ICU positive were found in patients admitted to the neuro and cardiothoracic speciality areas, $50 \%$ and $32 \%$ respectively. CAM positive score were more likely to be seen in males and in emergency admissions. No differences in ICNARC or APACHE II scores were identified between the 2 groups. The compliance with CAM-ICU was poor, out of 311 opportunities identified; CAM-ICU was only completed on 52 occasions (19\%). Of the CAM positive group, only 14 percent had been screened for delirium. Patients that were found to be CAM-ICU positive had long lengths of ITU stay and longer lengths of hospital stay. This cohort was less likely to be discharge back to their usual place of residence.

Conclusion: Delirium in critical care is effecting a quarter of our patients yet continues to be under diagnosed. Further work is required to increase compliance with CAM-ICU screening in an effort to identify patients at risk and implement intervention to minimise the long-term effects associated with ICU delirium.

\section{Reference(s) and grant ackowledgment(s)}

1. Page, V. Navarange, S. Gama, S. McAuley, D. (2009) Routine delirium monitoring in a UK critical care unit. Critical Care. Vol 13 Issue 1.

2. National Institute for Health and Care Exceelence (2010) Delirium: Prevention, diagnosis and management: Clinical Guideline. Viewed online at https://www.nice.org.uk/guidance/cg103/resources/delirium-preventiondiagnosis-and-management-pdf-35109327290821 on 25/07/2019.

3. Mehta S, Cook D, Devlin JW, Skrobik Y, Meade M, Fergusson D, Herridge M, Steinberg M, Granton J, Ferguson N, et al. Prevalence, risk factors, and outcomes of delirium in mechanically ventilated adults. Crit Care Med. 2015;43(3):557-66.

4. Leutz, A. Heymann, A. Radtke, F. Chenitir, C. Neuhaus, U. Nachigall, I. von Dossow, V. Marz, S. Eggers, V. Heinz, A. Wernecke, K. Spies, C. (2010) Difference assessment tool for intensive care delirium; which score to use. Critical Care Medicine. Vol 38 Issues 2 pp409-418.

5. Gleason LJ, Schmitt EM, Kosar CM, Tabloski P, Saczynski JS, Robinson T, Cooper Z, Rogers SO Jr, Jones RN, Marcantonio ER, et al. Effect of delirium and other major complications on outcomes after elective surgery in older adults. JAMA Surg. 2015;150(12):1134-40.

\section{8}

Efficacy of delirium pharmacological treatment in ICU patients: a systematic review

M. S. Manciola; LS. Sancho; JC. Ribeiro; JGR. Ramos'; SF. Da Guarda;

RDH. Passos ${ }^{1}$; ALN. Gobatto ${ }^{1}$

${ }^{1}$ Critical Care Medicine, Hospital São Rafael, Salvador, Brazil

Correspondence: M. S. Manciola

Intensive Care Medicine Experimental 2020, 8(2): 000558

Introduction: Delirium is a common condition in the intensive care unit (ICU), notably among the elderly, and is associated with poor outcomes. Pharmacological therapy is often used for its treatment, however, its efficacy has not yet been established.

Objectives: To investigate whether pharmacological therapy is effective in reducing delirium-related outcomes among ICU adult pacients. Methods: A systematic review of randomized clinical trials was conducted. For the review of literature, we searched MEDLINE and EMBASE from January 1st 1980 through February 4th 2020 for parallel-group clinical trials testing pharmacological treatment for delirium. Inclusion criteria were randomized clinical trials of adult participants with delirium in the ICU. The exclusion criterion was trials including patients on antipsychotic treatment for pre-existing disorder. Title/abstract screening and full-text screening were independently conducted by two authors. In case of disagreement, a third reviewer was consulted. The primary outcome was the number of days with delirium. Secondary outcomes were delirium-free days, reduction in delirium scale scores, ICU and hospital length of stay, ICU and hospital mortality, mortality in longest period reported, mechanical ventilation-free days, incidence of coma, coma-free days and adverse outcomes. Quality assessment and data extraction were performed by two authors. Meta-analysis was not conducted due to between-study heterogeneity.

Results: From 1055 articles identified by the search strategy, seven fulfilled the inclusion criteria and were analyzed, comprising 1244 patients. The following drugs were analyzed: haloperidol, morphine sulfate, quetiapine, olanzapine, rivastigmine and dexmedetomidine. Significantly shorter duration of delirium in the intervention group was found in two studies (28.6\%). Greater reduction in delirium severity among patients receiving intervention was found in one study $(14,3 \%)$. More ventilator-free days in the intervention group was reported in one study (14,3\%). Also, longer ICU length of stay in patients receiving intervention was found in one study $(14,3 \%)$, and higher mortality in the intervention group was reported in one study (14,3\%), although not significant (RR: 2.78 IC 95\%, 0.958-8.051). Four studies $(57,1 \%)$ reported the number of patients with adverse effects. See the attached figures for detailed data.

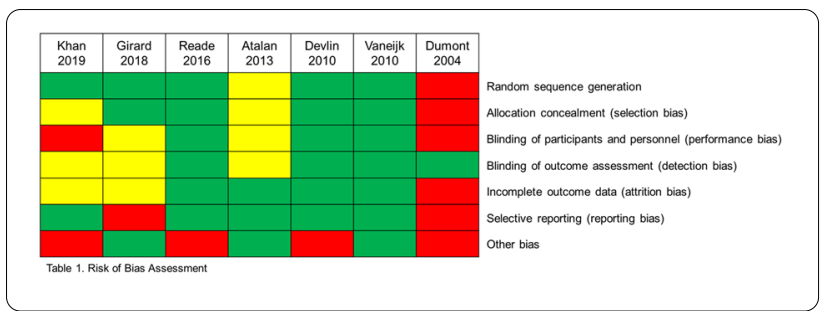



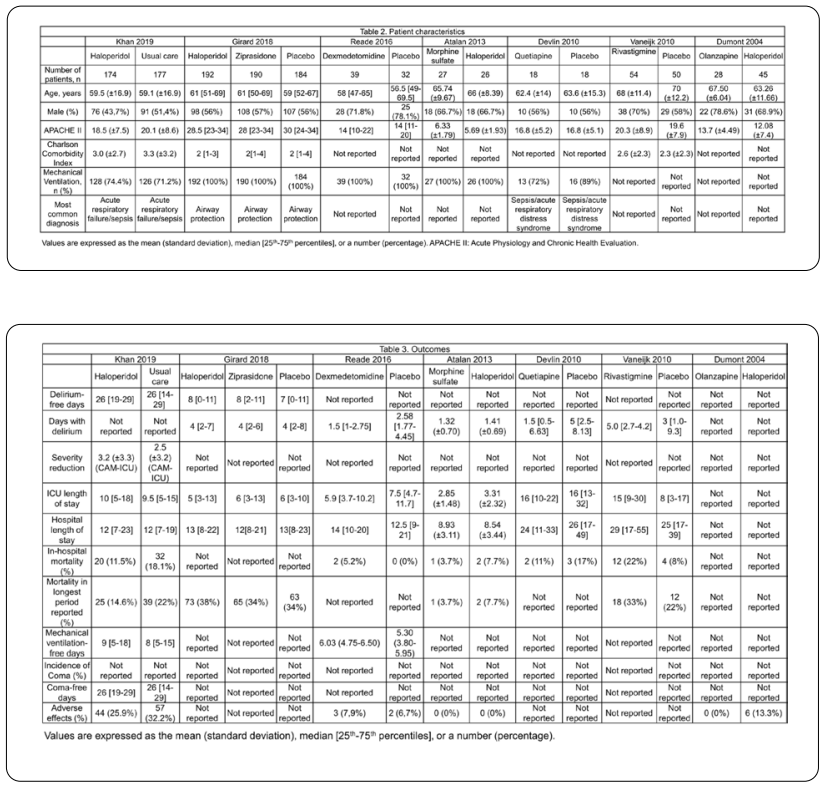

Conclusion: This systematic review concluded that pharmacological approach to delirium in the ICU may reduce duration of delirium. However, high heterogeneity between studies and their risk of bias compromise quality of evidence and analysis. Future assessment by more homogeneous trials with lower bias are required for solid conclusions.

\section{Reference(s) and grant ackowledgment(s)}

1. Higgins Julian P T et al. The Cochrane Collaboration's tool for assessing risk of bias in randomised trials BMJ 2011; 343:d5928.

2. Cook IA:Guideline Watch: Practice Guideline for the Treatment of Patients With Delirium. Arlington, VA: American Psychiatric Association, 2004.

3. Inouye SK, Westendorp RG, Saczynski JS. Delirium in elderly people. Lancet 2014; 383(9920):911-22.

4. The authors declare that they have no competing interests.

\section{5}

\section{Psychoactive drug use after hospital discharge following a critical} illness: A retrospective cohort study

L. Burry'; N. Tabbara'; J. Mailman ${ }^{3}$; HT. Wang ${ }^{4}$; M. Eisa ${ }^{2}$; D. Smart ${ }^{5}$; R. Pinto ${ }^{6}$; H. Wunsch ${ }^{6}$; L. Rose ${ }^{7}$; D. Williamson ${ }^{8}$

${ }^{1}$ Critical care, Mount Sinai Hospital, Toronto, Canada; ${ }^{2}$ Pharmacy, Mount Sinai Hospital, Toronto, Canada; ${ }^{3}$ Pharmacy, Saskatchewan Health Authority, Regina, Canada; ${ }^{4}$ Critical care, Maisonneuve-Rosemont Hospital, Montréal, Canada; ${ }^{5}$ College of medicine, University of Saskatchewan, Saskatoon, Canada; ${ }^{6} \mathrm{Criti}$ al care, Sunnybrook Health Sciences Centre, Toronto, Canada; ${ }^{7}$ Nursing \& midwifery, King's College London, London, United Kingdom; ${ }^{8}$ Pharmacy, Hôpital du Sacré-Coeur de Montreal, Montréal, Canada

Correspondence: L. Burry

Intensive Care Medicine Experimental 2020, 8(2): 000575

Introduction: Psychoactive drugs such as benzodiazepine/non-benzodiazepine sedatives and antipsychotics are routinely prescribed for sedation, agitation, and delirium in the intensive care unit (ICU). Although intended for short-term use, recent studies suggest these drugs may not be stopped on ICU discharge and continued at hospital discharge in some patients.

Objectives: Among ICU patients discharged alive, 1) determine if invasive mechanical ventilation (iMV) $\geq 48$ hours is associated with new (i.e. initiated in the ICU) psychoactive drug use on hospital discharge; 2 ) to identify factors predicting the number of new psychoactive drugs, adjusting for potential confounders and clustering by site.
Methods: We conducted a multicentre retrospective cohort study of adult patients admitted to 3 academic ICUs during one year to characterize continuation of psychoactive drugs initiated in the ICU at hospital discharge. Data collected included demographics, severity of illness, clinical management, and drug exposures. Multivariable logistic and Poisson models were constructed to address primary and secondary objectives, respectively.

Results: Data collection has been completed for 1111 ICU patients that survived to hospital discharge. At discharge, $12.3 \%$ were discharged on one, $3.3 \%$ two, and $<1 \%$ on three new psychoactive drugs; $83.5 \%$ were discharged without a new psychoactive drug. Psychoactive drug use prior to hospital admission and $\mathrm{iMV} \geq 48$ hours increased the odds of continuing a newly initiated psychoactive drug in the ICU on hospital discharge (adjusted odds ratio (aOR) 1.5, 95\% confidence interval $(\mathrm{Cl}) 1.0,2.1$ and aOR $2.4,95 \% \mathrm{Cl} 1.6,3.4$ respectively) while female sex decreased the odds (aOR $0.7,95 \% \mathrm{Cl} \mathrm{0.5,0.9)}$. We found females were discharged on fewer new psychoactive drugs (adjusted rate ratio (aRR) $0.7,95 \% \mathrm{Cl} 0.5,0.9)$ whereas patients started on antipsychotics or non-benzodiazepine sedatives while in the ICU were discharged on a greater number of psychoactive drugs (aRR $2.5,95 \% \mathrm{Cl}$ $1.9,3.3$ and aRR $1.7,95 \% \mathrm{Cl} 1.2,2.5$ respectively).

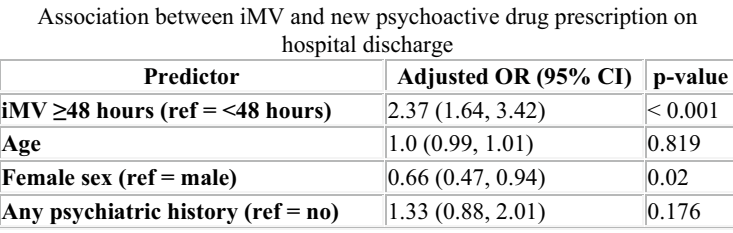

Association between iMV and new psychoactive drug prescription on hospital discharge

\begin{tabular}{|c|c|c|}
\hline Predictor & Adjusted OR (95\% CI) & p-value \\
\hline Alcohol use disorder $($ ref $=$ no $)$ & $1.04(0.63,1.74)$ & 0.861 \\
\hline Illicit drug use disorder $($ ref $=$ no $)$ & $1.32(0.71,2.43)$ & 0.378 \\
\hline Smoker $($ ref $=$ no $)$ & $1.04(0.69,1.55)$ & 0.863 \\
\hline Psychoactive drug PTA $($ ref $=$ no $)$ & $1.47(1.0,2.14)$ & 0.047 \\
\hline Admission category (ref $=$ medical) & $0.97(0.68,1.4)$ & 0.848 \\
\hline SOFA score & $1.0(0.95,1.05)$ & 0.995 \\
\hline
\end{tabular}

Abbreviations: $\mathrm{OR}=$ odds ratio $\mathrm{CI}=$ confidence interval, $\mathrm{iMV}=$ invasive mechanical ventilation; ref $=$ reference; $\mathrm{PTA}=$ prior to admission; $\mathrm{SOFA}=$ sequential organ failure assessment .

Conclusion: Preliminary analyses suggest iMV $\geq 48$ hours and psychoactive drug use prior to hospitalization are associated with continuation of an ICU-initiated psychoactive drug at hospital discharge. Furthermore, antipsychotics and non-benzodiazepine sedatives initiated in the ICU impact the number of prescribed psychoactive drugs on discharge. The final results will be used to inform the development of a quality improvement initiative to optimize medication reconciliation of psychoactive drugs initiated in critically ill patients.

\section{Reference(s) and grant ackowledgment(s)}

1. Scales DC, Fischer HD, Li P, et al. Unintentional continuation of medications intended for acute illness after hospital discharge: A population-based cohort study. J Gen Intern Med 2016;31 (2):196-202.

2. Rowe AS, Hamilton LA, Curtis RA, et al. Risk factors for discharge on a new antipsychotic medication after admission to an intensive care unit. J Crit Care 2015;30(6):1283-6.

\section{3}

Adjunctive acetaminophen for preventing postoperative delirium in surgical patients: a systematic review and meta-analysis H. Yonekura'; Y. Nakamori'; ; S. Taito ${ }^{2}$

'Department of Clinical Anesthesiology, Mie University Hospital, Tsu, Japan; ${ }^{2}$ Department of clinical practice and support, Hiroshima University Hospital, Hiroshima, Japan

Correspondence: $\mathrm{H}$. Yonekura

Intensive Care Medicine Experimental 2020, 8(2): 000823 
Introduction: Postoperative delirium is one of a common complication after major surgeries and associated with increased morbidity and mortality, prolonged mechanical ventilation, longer lengths of ICU or hospital stays, increased cost of care, and worse long-term cognitive function. Therefore, pharmacologic intervention to prevent postoperative delirium is of particular importance for surgical patients. Acetaminophen is commonly used to as adjuvant analgesics in surgeries and may reduce inflammatory response and decrease opioid consumption in surgical patients. Previous studies demonstrated that acetaminophen analgesia may decrease the incidence of postoperative delirium.

Objectives: The aim of this study was to determine the effects of adjunctive acetaminophen analgesia on postoperative delirium.

Methods: We searched randomized controlled trials (RCTs) in databases including MEDLINE, EMBASE, Cochrane Library, the National Institute of Health Clinical Trials Register, the World Health Organization International Clinical Trials Registry Platform, and the reference lists of retrieved articles from inception date to March 2020. The primary outcome was the incidence of postoperative delirium. The protocol has been registered with the International Prospective Register of Systematic Reviews (PROSPERO) registration number CRD42019145666.

Results: Out of 840 reports, 29 RCTs met the inclusion criteria and were appropriate for our meta-analysis. Of 29 RCTs, only 3 RCTs reported the primary outcome. The pooled results of included clinical trials indicated that acetaminophen significantly decreased the incidence of postoperative delirium: pooled risk ratio was 0.38 [95\% confidence interval $(\mathrm{Cl}) 0.18-0.80 ; 12=0 \% ; n=263]$. The certainty of the body of evidence was judged as moderate using the GRADE methodology.

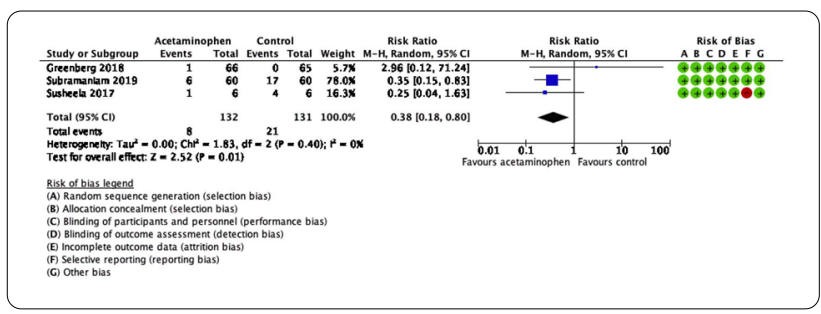

Conclusion: The results of this systematic review and meta-analysis indicated that administration of acetaminophen was associated with decreased incidence of postoperative delirium.

\section{1}

Delirium in Critical Care Unit

S. Shahzad ${ }^{1}$; S. Pesian

${ }^{1}$ Critical Care Medicine, Stepping Hill Hospital, Hazel Grove, United Kingdom; ${ }^{2}$ Critical care unit, Walsall Manor Hospital, Walsall, United Kingdom

Correspondence: $\mathrm{S}$. Shahzad

Intensive Care Medicine Experimental 2020, 8(2): 000851

Introduction: Delirium has paramount morbidityand mortality burden; and requires early diagnosis and prompt treatment.

Objectives: We introduced a delirium tool including RASS scoring system first time in our trust in 2016 based on an audit at that time, best practice and NICE recommendations. To ensure the effective usage of these important tools we conducted a prospective audit to capture our compliance.

Methods:

- Location: ITU/HDU

- Time Frame: 4 months

- Data Collection: A questionnaire designed according to NICE recommendations that collected data regarding risk factors, indicators, preventive measures and diagnosis using RASS and CAM-ICU.
- Data Source: Information from patients, nursing staff, patients notes, and nursing sheets.

Results: Results were analyzed according to NICE guidelines.

- Risk factor assessment: $91 \%$ of patients were at high risk for delirium on admission to ITU.

- Indicators of Delirium: Out of 4 major indicators of delirium defined by NICE, $77 \%$ of the patients had at least 1 of these indicators present.

- Interventions to prevent Delirium:

- To prevent cognitive dysfunction, not a single patient had all the simple preventive measures that are recommended by NICE.

- Mobilization was done only in $44 \%$ of the patients and out of this only $17 \%$ had walking mobilization (ventilated patients were excluded).

- Pain scores were recorded only in $37 \%$ patients. 2 patients were currently in pain, but pain scores were not documented.

- Sleep disturbance was found in $55 \%$ of the patients.

- RASS score hourly and CAM ICU once a shift is the key to diagnose delirium according to NICE. Results showed that hourly RASS was done only in $28 \%$ and CAM ICU only in $14 \%$.

Conclusion: Delirium has paramount morbidity and mortality issues. Our audit highlighted several areas of potential improvement. As part of an action plan, formal and informal teaching sessions were arranged that showed positive feedback. Next step is to reaudit to assess quality improvement.

000917

Methadone as a rescue drug to control difficult sedoanalgesia in critical ill patients ventilated due to ARDS in SARS-CoV-2 infection

R. Fernández Tobar' ${ }^{1}$ M. Pérez Torres ${ }^{1}$ : B. Castiñeiras Amor ${ }^{1}$. C. Arenas Mazarrota'; MA. Romera Ortega ${ }^{1}$; C. Chamorro Jambrina

'Intensive Care Unit, Puerta de Hierro Majadahonda University Hospital, Majadahonda, Spain

Correspondence: R. Fernández Tobar

Intensive Care Medicine Experimental 2020, 8(2): 000917

Introduction: To investigate de efficacy of enteral methadone (MET) to control difficult sedoanalgesia in critical ill patients ventilated due to ARDS in SARS-CoV-2 infection.

Methods: We analyze retrospectively case reports who received MET during the Pandemia. Descriptive analyses were used to describe demographic, clinical and evolutionary trends of the patients and sedative, analgesic and neuromuscular blockade doses five days before and after MET. Adverse effects were also described. Difficult sedoanalgesia was defined as difficulties to weaning from mechanical ventilation (MV) due to agitation and delirium while reducing sedatives and/ or analgesics (group A); pain due to opiate withdrawal (group B) or nonoptimal patient ventilator interaction despite high dose of sedatives/analgesics (group C).

Results: 13 patients met the inclusion criteria and constituted the study population. Median age was $64 \pm 9$ years and 12 were male. With a median of VM of $49 \pm 16$ days from the first MET dose, 7 patients were included as group $A, 3$ group $B$ and 3 group $C$, respectively.

Five days before starting MET, all the patients had a median cumulative IV morfine dose of $589 \pm 332 \mathrm{mg} .11$ patients were receiving propofol with a median dose $15.443 \pm 11.189 \mathrm{mg} .5$ patients were also receiving midazolam (median dose $589 \pm 261 \mathrm{mg}$ ) and 5 patients needed cisatracurium (median $1.753 \mathrm{mg} \pm 1.706 \mathrm{mg}$ ) too.

MET initial dose (enteral route) was $45 \pm 23 \mathrm{mg}$. 
Five days after MET treatment, 9 patients could reduce opiate treatment in a $68 \% \pm 17 \%$ of the initial dose. 4 patients could also decrease propofol in a $68 \% \pm 27 \% .3$ of 5 patients that were taking midazolam reduced $51 \pm 31 \%$ the initial dose. All patients that were receiving cistracurium decreased doses in $43 \pm 31 \%$.

Regarding difficult sedoanalgesia, 3 patients of group A could progress in the weaning from MV, the other 4 patients could reduce sedatives and analgesics but couldn't progress from MV because of respiratory conditions. All group B patients could control the pain and all patients in group $C$ reached optimal patient ventilator interaction while reducing analgesics and sedatives.

Hypotension was observed in 3 patients after the first MET dose and crystalloids and vasoactive were needed. No other adverse effects were reported.

Conclusion: We concluded that in patients with difficult sedoanalgesia, methadone is effective to reduce doses of sedatives and analgesics and help weaning from MV.

\section{8}

\section{Recalling ICU - Short term impact of dexmedetomidine}

N. Pereira'; N. Fernandes ${ }^{1}$; I. Jesus Pereira ${ }^{2}$; C. C. Dias ${ }^{3}$; G. Campello ${ }^{4}$

${ }^{1}$ Serviço de medicina intensiva 2, Centro Hospitalar Universitário do Algarve - Hospital de Portimão, Portimão, Portugal; ${ }^{2}$ Departamento de emergência, urgência e cuidados intensivos, Centro Hospitalar Universitário do Algarve - Unidade Faro, Faro, Portugal, Portugal; ${ }^{3}$ Department of community medicine, information and health decision, Faculty of Medicine of Oporto, Porto, Portugal; ${ }^{4}$ Unidade de cuidados intensivos, Centro Hospitalar do Tâmega e Sousa, Penafiel, Portugal

Correspondence: P. Nuno

Intensive Care Medicine Experimental 2020, 8(2): 000938

Introduction: Critical illness is often related to the development of brain disfunction, which can present itself in several ways, either on the short or the long term.

Up to $80 \%$ of the critical illness survivors present with some form of brain disfunction either during the acute episode or when evaluated soon after hospital discharge, and in some patients even years after the acute episode.

Evidence points to the possible improvement in cognitive prognosis when a2 adrenergic agonists are used in the critically ill. The sedation pattern of these agents is quite different from other sedatives, in which patients can easily be aroused and their cognitive performance in psychometric tests is usually preserved.

The main purpose of this work was to investigate the relationship between the use of dexmedetomidine in the critically ill and some dimensions of the post intensive care syndrome.

Methods: Data was collected retrospectively from the individual medical records of patients admitted to a Mixed Intensive Care Unit (ICU) during the year of 2017. Collected data focused on the results of the inquiries done during the follow-up outpatient clinic usually held between the third and sixth months after hospital discharge. The applied formularies were the Anxiety and Depression Scale, the ICU - Post Traumatic Stress Syndrome (PTSS) questionnaire and the ICU Memories questionnaire.

Results: A total of 318 patients were admitted to the ICU, $64 \%$ were male. Most of the patients (72\%) were admitted for medical reasons and $64 \%$ received mechanical ventilation. The mortality rate was $26 \%$. Dexmedetomidine was used in $26 \%$ of the patients, usually as part of a combined sedation strategy. Only 123 patients (39\%) attended the follow-up outpatient clinic.

No significative difference was found in the group of patients under dexmedetomidine when considering the Anxiety and Depression Scale or the ICU - PTSS questionnaire. When the ICU Memories questionnaire was applied, we observed that patients seemed prone torecall more memories when under the use of dexmedetomidine, interestingly both pleasant and unpleasant ones.

Conclusion: Our study has some limitations, namely the fact that only a moderate percentage of patients attended the outpatient clinic, possibly explained by the fact that our ICU population assists many foreigners as it is located in a touristic region. Still it allows us to conclude that patients who receive dexmedetomidine are more aware of their surrounding environment, more cooperative and less susceptible to some adverse effects from the sedative medications.

\section{Reference(s) and grant ackowledgment(s)}

1. Cruickshank M, Henderson L, MacLennan G, et al. Alpha-2 agonists for sedation of mechanically ventilated adults in intensive care units: a systematic review. Health Technol Assess. 2016

2. Rawal G, Yadav S, Kumar R. Post-intensive Care Syndrome: an Overview. J Transl Int Med. 2017

3. Honarmand K, Lalli RS, Priestap F, et al. Natural History of Cognitive Impairment in Critical IIIness Survivors: A Systematic Review. Am J Respir Crit Care Med. 2020

\section{3}

Level and duration of sedation and sedation agents before and after implementation of pain, agitation and delirium guideline $-\mathrm{a}$ before and after study

H. Birgisdóttir ${ }^{1}$; RJ. Jónasdóttir

${ }^{1}$ Faculty of nursing, University of Iceland; Landspitali - The National University Hospital of Iceland, Reykjavík, Iceland

Correspondence: $\mathrm{H}$. Birgisdóttir

Intensive Care Medicine Experimental 2020, 8(2): 000943

Introduction: Deep sedation can have negative consequences for Intensive Care Unit (ICU) patients such as delirium, prolonged mechanical ventilation, and longer ICU and hospital stay.

Objectives: To compare the level and duration of sedation, sedative agents, and clinical outcomes of ICU patients before and after implementation of Pain, Agitation, and Delirium (PAD) guideline.

Methods: A before and after study design. Eligible patients were mechanically ventilated, 18 years and older, with 24 hours or longer ICU stay, of mixed ICU patient population in two, single-center ICUs. Data were collected over six weeks, from 1st December 2017 - 12th January 2018, before the PAD implementation and over six weeks, from 1st May - 15th June 2019, four months after completion of the PAD implementation. The sedation level was measured for the first three days of the patients' stay with the Richmond Agitation Sedation Scale (RASS). Sedation duration and types of sedation agents were collected through the patients' stay.

Results: There were 101 patients included, 52 in the before group and 49 in the after group. The two groups were comparable in age, gender, and reason for ICU admission. The after group had a lower APACHE II score compared to the before group $(p=0.01)$. The level of sedation was -3 (median) on the RASS scale in both groups the first three days of the ICU stay $(p=0.18)$. Patients in the before group were sedated 41 hours vs. 20 hours (median) in the after group, without difference between the groups after adjusting for background variables $(p=0.22)$. Seven types of analgesia and morphine-based continuous sedation were used, in various combinations, from none to six in the before group and the after group. Propofol was the most common sedative in both groups $(92 \%$ vs. $90 \%, p=0.66)$, and the duration of propofol sedation was shorter in after group (30 vs.16 hours, $p=0.04$ ). More patients in the after group were sedated with one drug compared to patients in the before group ( $37 \%$ vs. $17 \%, \mathrm{p}=0.03$ ) and fewer patients in the after group were sedated with a combination of two drugs than patients in the before group $(12 \%$ vs. $29 \%, p=0.04)$. The difference between the groups in duration of mechanical ventilation (median, 36 vs. 16 hours, $p=0.44$ ), and length of ICU stay (median, 6.2 vs. 4.9 days, $p=0.44$ ), and hospital stay (median, 12.6 vs. 10.3 days, $\mathrm{p}=0.46$ ) stay was not significant.

Conclusion: The level of sedation was moderate (RASS -3) before and after the PAD guideline implementation without a difference in sedation duration. The depth of sedation, the number of sedation agents administered, and the PAD guideline implementation needs further investigation. 
Reference(s) and grant ackowledgment(s)

1. Shehabi, Y., Bellomo, R., Kadiman, S., Ti, L. K., Howe, B., Reade, M. C. Kalyanasundaram, G. (2018). Sedation intensity in the first 48 hours of mechanical ventilation and 180-day mortality: A multinational prospective longitudinal cohort study. Critical Care Medicine, 46(6), 850-859. doi:10.1097/CCM.0000000000003071

2. Ng, S. Y., Phua, J., Wong, Y. L., Kalyanasundaram, G., Mukhopadhyay, A., Lim, D., .. Ti, L. K. (2020). Singapore SPICE: Sedation practices in intensive care evaluation in singapore - A prospective cohort study of the public healthcare system. Singapore Medical Journal, 61(1), 19-23. doi:10.11622/ smedj.2019055

3. Devlin, J. W., Skrobik, Y., Gélinas, C., Needham, D. M., Slooter, A. J. C., Pandharipande, P. P., . . Alhazzani, W. (2018). Executive summary: Clinical practice guidelines for the prevention and management of pain, Agitation/Sedation, delirium, immobility, and sleep disruption in adult patients in the ICU. Critical Care Medicine, 46(9), 1532-1548. doi:10.1097/CCM.0000000000003259

4. Aragón, R. E., Proaño, A., Mongilardi, N., De Ferrari, A., Herrera, P., Roldan, R., Checkley, W. (2019). Sedation practices and clinical outcomes in mechanically ventilated patients in a prospective multicenter cohort. Critical Care, 23(1) doi:10.1186/s13054-019-2394-9

5. Landspitali University Hospital Research Fund

\section{5}

Epidemiological profile, clinical features and incidence of delirium during the first three days of ICU admission in Hospital Virgen de las Nieves (2019)

I. Guzmán Adum¹; L. Hidalgo Manchado ; R. Diaz Contreras ${ }^{1}$

${ }^{1}$ Intensive care unit, Hospital Universitario Virgen de las Nieves, Granada, Spain

Correspondence: I. Guzman

Intensive Care Medicine Experimental 2020, 8(2): 000975

Introduction: Delirium is a disturbance of consciousness with changes in cognition. It manifests as a constellation of symptoms with acute onset and a fluctuating course. Delirium is extremely common in the ICU especially amongst mechanically ventilated patients and it has prognostic implications ( 2.5 fold increase in short term mortality and 3.2 in 6months). Three subtypes have been recognized: hyperactive, hypoactive, and mixed. It is frequently undiagnosed unless specific diagnostic instruments are used. The CAM-ICU is the most studied and validated diagnostic tool.

Objectives: - To calculate the incidence of delirium during the first 72 hours of ICU admission.

- To use Confusion Assessment Method Scale (CAM- ICU) to diagnosed delirium in the ICU.

- To describe the main features and causes of admission in the ICU of the patients that developed delirium in the ICU, compared to the ones that didn't.

Methods: Observational, prospective and descriptive study that includes patients that entered the $\mathrm{UCl}$ between October 15th and November 15th, 2019. We performed the CAM-ICU scale at the admission and daily for the first 72 hours or until they were discharged to hospitalization ward, required orotracheal intubation or died. We excluded patients that required orotracheal intubation at admission and those who had mental disorders or central nervous system disorders that made impossible to evaluate delirium with CAM-ICU scale. The collected data was analyzed with SPSS and evaluated in terms of descriptive statistics. We used the mean and standard deviation for quantitative variables and absolute and relative frequencies for qualitative variables.

Results: 102 patients were included, the mean age was 68 ( \pm 14 years), 39 (38.9\%) patients were previously at the hospitalization ward. The incidence of delirium of those patients that werepreviously in the hospitalization ward was $64.10 \%$ (25 patients). The global incidence of delirium in the first 72 hours in the ICU was $28.4 \%$, during the first day in the ICU the incidence was $15.69 \%$, during the second day was $22.56 \%$, and the third day was $11.76 \%$. The disorders that were more strongly related with the development of delirium in the ICU were: Acute coronary syndrome (50\%), decompensated cirrhosis (19\%), sepsis (16\%), upper gastrointestinal bleeding (7\%)) and patients with transcatheter aortic-valve implantation (5\%).

Conclusion: - Delirium is a frequent complication in the ICU, even in the first 72 hours, and without the presence of another factors related with delirium such as illness severity, mechanical ventilation and sedation.

- The incidence of delirium in this study is higher (28 cases per 100 patients) than the one described in other studies (11 cases per 100 patients).

- The disease that was most associated with the presence of delirium was acute coronary syndrome. This suggest the need of performing studies that include patients earlier and with less stay in the ICU.

- The use of a specific scale to diagnosed delirium in the ICU is a useful tool to diagnosed it in the ICU.

\section{Reference(s) and grant ackowledgment(s)}

1. van Eijk MM, van Marum RJ, Klijn IA, de Wit N, Kesecioglu J, Slooter AJ: Comparison of delirium assessment tools in a mixed intensive care unit. Crit Care Med 2009, 37(6):1881-1885.

2. Ely EW, Margolin R, Francis J, May L, Truman B, Dittus R, Speroff T, Gautam S, Bernard GR, Inouye SK: Evaluation of delirium in critically ill patients: validation of the Confusion Assessment Method for the Intensive Care Unit (CAM-ICU). Crit Care Med 2001, 29(7):1370-1379.

3. Roberts B, Rickard CM, Rajbhandari D, Turner G, Clarke J, Hill D, Tauschke C, Chaboyer W, Parsons R: Multicentre study of delirium in ICU patients using a simple screening tool. Aust Crit Care 2005, 18(1):6. 8-9, 11-14 passim.

\section{4}

Delirium and risk factors in patients after cardiac surgery

S. Dimopoulos ${ }^{1}$; N. Rouvali ${ }^{1}$; M. Georgopoulou ${ }^{1}$; C. Kidi ${ }^{1}$; M. Mavraki ${ }^{1}$

E. Spiropoulou ${ }^{1}$; K. Petsios ${ }^{1}$; L. Samartzis ${ }^{2}$; V. Tsagkari ${ }^{1}$;. Kriaras

E. Lyberopoulou'; S. Nanas ${ }^{2}$; A. Karabinis

${ }^{1}$ Icu, Onassis Cardiac Surgery Center, Kallithea, Greece; ${ }^{2} \mathrm{Icu}$, Evaggelismos

General Hospital, Athina, Greece

Correspondence: S. Dimopoulos

Intensive Care Medicine Experimental 2020, 8(2): 001004

Introduction: Delirium is a condition characterized by an acute cognitive decline, a fluctuating mental status, disturbance of consciousness, inattention, or disorganized thinking. It is a well-recognized adverse prognostic index in Intensive Care Unit patients that is associated with increased morbidity, mortality, and the development of long-term neurocognitive deficits.

Objectives: To evaluate the presence of delirium, using a well validated scale the "Confusion Assessment Method for the ICU", in postcardiac surgery patients and to identify possible risk factors which contribute in the development of delirium.

Methods: Our cohort consisted of 202 consecutive post-cardiac surgery patients admitted to ICU. All patients were evaluated for delirium using the CAM-ICU scale. According to this scale, patients developed an acute onset of cognitive decline or a fluctuating mental status, disturbance of consciousness, inattention, or disorganized thinking were diagnosed with delirium.

Results: Patients (mean age: $66 \pm 13$ years old) had a prevalence of delirium of $29.2 \%$. Patients with delirium were 4 years older than nondelirium subjects $(p=0.002)$, while there were no gender differences between the two groups.

Prevalence of chronic kidney disease in study cohort was significantly higher in delirium patients (12\%) compare to non-delirium subjects $(4 \%)(p=0.040)$.

The in-hospital mortality risk using Euroscore II score was 1,67\% in the study cohort, while patients with delirium scored significantly higher Euroscore II compared with the non-delirium ones $(2.32 \%$ versus $1.38 \%$, respectively, $\mathrm{p}<0.001$ ).

The median duration of anesthesia-sedation was 360 minutes, while patients presented with delirium had prolonged anesthesia-sedation duration compared to no delirium patients $(p<0.001)$.

These patients had also prolonged mechanical ventilation (median duration in patients with delirium was 1230 minutes versus 717 
minutes in non-delirium patients, $\mathrm{p}<0.001)$ and a longer ICU stay (3210 versus 1440 minutes, $p<0.001$ ).

According to ROC curve analysis, variables that predicted delirium were duration of anesthesia-sedation, age, Euroscore II, urea, lactic acid and intravenous vasoactive medications.

Moreover, in a multivariate logistic regression analysis it was shown that age, Euroscore II and duration of anesthesia-sedation were all independent predictors of delirium.

Conclusion: According to our study the prevalence of delirium in ICU after cardiac surgery was high. Age, prolonged duration of anesthesia and Euroscore II score represent independent risk factors of delirium. Delirium was associated with prolonged mechanical ventilation and ICU stay.

\section{9}

Relationship between ICU Acquired Weakness and Cognitive Deterioration: the muscle's endocrine activity hypothesis

I. PREVIGLIANO'; C. Foster'; A. Cuenca²; MB. Leiva²; GM. Roldán ${ }^{2}$

${ }^{1}$ Intensive care, Hospital Fernández, Buenos Aires, Argentina; ${ }^{2}$ Physical

therapy, Universidad Maimónides, Buenos Aires, Argentina

Correspondence: I. PREVIGLIANO

Intensive Care Medicine Experimental 2020, 8(2): 001019

Introduction: There is evidence of the muscle/brain interaction in several cognitive disorders due to the neurotrophic factors released by muscle endocrine, paracrine and autocrine activity. We didn't found any research on this topic in Post Intensive Care Syndrome (PICS).

Objectives: Our hypothesis was to determinate if there is any relationship between cognitive impairment after critical illness (CIACI) and ICU Acquired Weakness (ICUAW).

Methods: Setting: teaching hospital, medical and surgical ICU. Maimonides Universty ethic commitee aproved the protocl and waived informed consent. Inclusion criteria: >18yo, ARDS, sepsis or septic shock, APACHEII $>14$, Clinical Frailty Scale, Delirium $>48$ h, mechanical ventilation $>4 \mathrm{~d}$, ICU stay $>7$ days, use of NMB or steroids, vasopressors. CAM-ICU and RASS (daily), Medical Research Council (MRC) scale and Spanish version of Montreal Cognitive Assessment (MoCA) were the evaluation tools. MRC $<18$ diagnoses ICUAW and MoCA $>26 \mathrm{CIACl}$. Statistical analysis: IBM SPSS Macintosh 25.0. Descriptive statistics. Chi squared or Fischer exact test.

Results: From April 1st to September 30th, 92 patients were admitted. Of them 28 fulfilled IC. 13 (53.6\%) were female, mean age (), in table 1 mean values are expressed. $75 \%$ presented delirium. At ICU discharge $64,3 \%$ presented ICUAW and $60.7 \mathrm{CIACl}$. There was a significant associ-

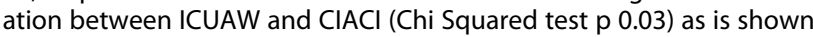
in figure 1.

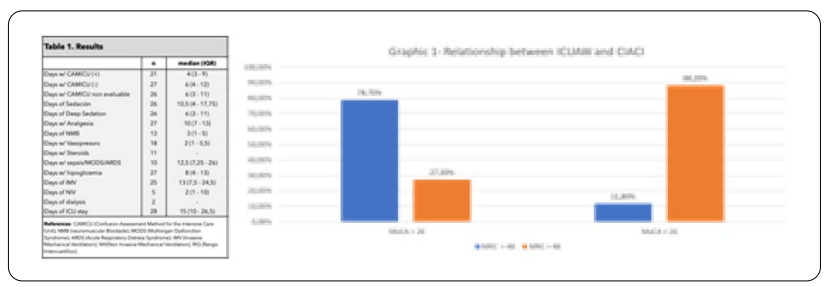

Conclusion: There was a significative association between ICUAW and $\mathrm{CIACl}$ in this PICS population. According to our pathophysiological hypothesis our data opens a window for myokines and neurotrophic factors measurements and for the evaluation of the efficacy of multimodal neurotrophic factors preparations.

\section{2}

Loss of mobility and independence in patients admitted for SARS

\section{COV-2}

L. UGALDE JAUREGUI'; R. PradaOsorio ${ }^{1}$; MP. Eugenio Robaina';

L. Santana-Cabrera²; JD. Martin Santana ${ }^{3}$; JC. Martin Gonzalez

${ }^{1}$ ICU, Complejo Hospitalario Universitario Insular Materno Infantil, Las Palmas de Gran Canaria, Spain; ${ }^{2}$ Intensive care unit, Maternal and child Hospital, Las Palmas de Gran Canaria, Spain; ${ }^{3}$ Economics, Universidad de las Palmas de Gran Canaria, Las Palmas de Gran Canaria, Spain

Correspondence: L. UGALDE JAUREGUI

Intensive Care Medicine Experimental 2020, 8(2): 001052

Introduction: Muscle weakness is a frequent entity in patients admitted to the ICU. Its most common presentations are polyneuropathy and critical patient myopathy, or a combination of the two. In addition, SARS COV-2 requires prolonged invasive ventilatory support and the use of neuromuscular blockers, favoring its appearance. Prevention has an important role in reducing the loss of functionality at ICU discharge.

Objectives: 1-Value the degree of functionality and independence of patients admitted for SARS COV-2 at ICU discharge.

2- Identify the risk factors associated with the loss of functionality and independence during admission.

Methods: Retrospective observational study conducted in a multipurpose ICU with 30 beds. All the patients admitted for hypoxemic respiratory failure secondary to SARS-COV-2 during the months of March - May 2020 were collected. Demographic data, days of stay in the ICU and risk factors associated with acquired ICU weakness were recorded, including the time of start of physical therapy after admission. The Barthel index was used to calculate the functionality and degree of independence of all patients, both at admission and at discharge from the ICU. In the statistical analysis, the Pearson correlation coefficient was used for the quantitative variables and the mean comparison test for qualitative variables.

Results: During the study period, 27 patients were admitted due to SARS COV-2 infection, of which $5(18.51 \%)$ were exitus, for which a total of 22 patients were included in the study. All entered with a score on the Barthel index of 100 points, while the mean score on the Barthel index at discharge was $37.05 \pm 16.67$. In the association test carried out, we found a high correlation of the loss of functionality at discharge from the ICU with the variables: Days of admission to the ICU $(p=0.000)$, Days of mechanical ventilation: $(p=: 0.000)$, Days with sedoanalgesia and neuromuscular block: $(p=: 0.000)$, Days with delirium: $(p=: 0.000)$, Physiotherapy during the ICU stay: $(p=0.000)$, Days of initiation of physical therapy: $(p=0.000)$. 
BARTHEL

\begin{tabular}{|c|c|c|c|}
\hline RISK FACTORS & Media & D.T. & $\begin{array}{l}r \\
(p)\end{array}$ \\
\hline DAYS IN ICU & --- & --- & $\begin{array}{l}-0,811 \\
(0,000)\end{array}$ \\
\hline VM DAYS & --- & --- & $\begin{array}{l}-0,804 \\
(0,000)\end{array}$ \\
\hline
\end{tabular}

HEMODYNAMIC INSTABILITY:

Yes

No

$\begin{array}{cccc}63,00 & 6,71 & & 7,690 \\ & & --- & \\ 29,41 & 8,99 & & (0,000)\end{array}$

DAYS WITH BZD

$\begin{array}{llll} & -- & -- & (0,000) \\ \text { DAYS WITH PROPOFOL } & & & -0,583 \\ & -- & -- & (0,004) \\ \text { DAYS WITH OPIATES } & & & -0,833\end{array}$

DAYS WITH OPIATES $\quad---\quad---\quad(0,000)$

$\begin{array}{lllll}\text { NEUROMUSCULAR BLOCKERS: } & 63,00 & 6,71 & & \\ \text { Yes } & 29,41 & 8,99 & & (0,000)\end{array}$

No

$\begin{array}{llll}\text { DAYS WITH NEUROMUSCULAR } & & & -0,796 \\ \text { BLOCKERS: } & --- & -- & (0,000) \\ & & & -0,716 \\ \text { DAYS WITH DELIRIUM } & --- & --- & \end{array}$

PHYSIOTHERAPY:

Yes

No

DAYS OF WAITING TO PHYSIOTHERAPY:

that predict the likelihood of delirium development in different patients can help in setting priorities with regard to the use of delirium preventive measures, especially in situations of limited resources such as COVID infection.

Objectives: 1.Evaluate the correlation between the E-Predeliric and Predeliric values when they are recoded into four categories of risk of developing delirium (very low, low, moderate and high risk).

2.Evaluate the correlation between the predicted probability of delirium according to the E-Predeliric and Predeliric models with the actual rate of delirium developed in patients admitted to the ICU for SARS COV 2.

Methods: Retrospective observational study carried out in a multipurpose ICU with 30 beds. All patients admitted for SARS COV2 infection were collected during the months of March to May 2020. The E-PREDELIRIC was calculated in all patients at the time of ICU admission and the PRE-DELIRICa24 hours after admission, classifying patients into four risk groups according to the result (very low, low, moderate and high risk). We quantified the incidence of delirium after sedation was removed. The presence of agitation, maladjustment (in the absence of pain or ventilatory problems) and the need for administration of dexmedetomidine or an antipsychotic drug was considered delirium. And finally the results were compared with the actual rate of delirium observed in these patients.

Results: During the period from March to May 2020, a total of 27 patients were admitted to our service with the diagnosis of SARS COV 2, with an average age of $65.81 \pm 9.72$. There is a high correlation between the percentage values of E-Predeliric and Predeliric $(r=$ $0.608, p=0.01$ ). This result is corroborated with the association test between both variables when they are recoded in four categories. There were no statistically significant differences in the percentage value of E-Predeliric and the patient's state of real delirium ( $t=1,481$, $p=0.151)$. Their mean values are very similar $(38.95 \%$ and $33.50 \%$ for the patients who suffered delirium versus those who did not, respectively). The opposite occurs with Predeliric $(t=5,460, p=0.00)$, with the mean values being $85.68 \%$ and $41.00 \%$. These results are corroborated with the association test performed between the patient's actual state of delirium and these two scales coded in its four states.

Conclusion: 1.The E-Predeliric and Predeliricscores allow patients to be classified according to the risk of developing delirium in 4 groups, in a homogeneous manner, regardless of the score used.

2.The predicted risk at admission according to the E-Predeliric, in relation to the observed delirium rate, shows that the risk of delirium is underestimated, while the $24 \mathrm{~h}$ after admission calculatedPredeliric is better correlated with the actual incidence observed.

001148

Analysis of patients suffering critical care delirium in an Intensive Care Unit in the North West of England

J. Manalayil'; AN. Thomas ${ }^{1}$

${ }^{1}$ Critical Care, Salford Royal NHS Foundation Trust (Formerly Hope Hospital), Manchester, United Kingdom

Correspondence: J. Manalayil

Intensive Care Medicine Experimental 2020, 8(2): 001148

Introduction: Identifying patients with delirium is essential for the prevention, treatment and management of the condition; this requires a structured assessment that can be done using the CAM-ICU methodology (Miranda et al 2018). We set a standard of recording twice daily CAM-ICU assessments in patients' electronic records (EPR). Access to these records has allowed us to audit compliance to our standards and to review outcomes of different types of delirium and review the frequency with which patients meet different stages of the assessment.

Objectives: To retrospectively assess 1-year mortality of patients with hyperactive and hypoactive delirium, to assess compliance with delirium assessments and to review the frequency with which patients met different components of theCAM-ICU.

Methods: The review was conducted in a 32 bedded general and neurosciences unit. EPR records (Sunrise Clinical Manager) of the CAMICU assessments from August 2018-August 2019 were exported to Excel files. Patients were categorised as who were delirium positive (with or without agitation), or negative but with or without cognitive

Introduction: The incidence of delirium in patients admitted to ICU due to SARS COV-2 infection is very high according to the different registers. The use of validated scores such as Predeliric and E-predeliric 
impairment. Cognitive impairment was defined as an acute change from a patient's normal mental state. These patients can then be dived into those with inattention errors using the Letters Attention Test, or with disorganized thinking using straightforward Yes/No questions and commands to follow.

Records were linked to the patients' Intensive Care National Audit and Research Centre (ICNARC) records to investigate reason for admission, co-morbidities, social history, length of stay and outcome. On six occasions we provided feedback to nurses about the number of assessments by individual nurses and with information about the assessments.

Results: There were a total of 2728 admissions (2408 total patients, $57 \%$ male, mean age 57 (SD 18 years)) in the ICNARC records. 1358 patients had 10,530 recorded CAM-ICU assessments, of these 1191 had full ICNARC records and were reviewed in more detail ( 6 patients with delirium had ICNARC details filled out from EPR). 214 (18\%) had at least one positive assessment, of the remaining 977 patients, 82 had with cognitive impairment and 895 had no cognitive impairment, the remaining 167 remained in a coma. Of 10,530 assessments, 66 were excluded as incomplete, 7,243 were not deeply sedated. 989 had an acute change from their baseline mental state, out of which 775 had $>2$ inattention errors on assessment (CAM-ICU positive). The remaining 199 patients had 0-2 inattention errors (CAM-ICU negative). Of the 775, 246 were calm and alert; 199 had 2 or more points for disordered thinking. Patients with delirium had a longer length of hospital and ICU stay (LOS) compared to those without delirium (median (IQR) ICU LoS CAM-ICU positive 9 (4-18) days, negative with cognitive impairment 4 (2-8) days, without 3 (2-6) days. Patients with delirium required more organ support, had a higher mortality (ICU: $14 \%$ vs $7 \%$ vs $9 \%$ ) and were more likely to be male, their mortality rate increased from $13 \%$ at 30 days to $22 \%$ at one year. Those that died were older, had more comorbidities and had worse functional status than those that survived. In all patients, $23 \%$ had an assessment completed once per shift and $62 \%$ once per day. In patients with a positive assessment, $65 \%$ had investigations to identify a cause, $42 \%$ required some form or restraint and $65 \%$ had cognitive impairment at unit discharge. Those suffering from hypoactive delirium were older (mean 60 years, SD 18 years) and more likely to die (1-year mortality of $26 \%$ ).

Conclusion: Electronic records of delirium assessments can be used to audit compliance with standards for delirium management. Even with staff feedback, only $62 \%$ of patients had daily assessments. The study provides further evidence that patients with delirium are more likely to be male, have a higher mortality (particularly with hypoactive delirium), need more support, and continue to die in the 12 months after their ICU stay.

\section{Reference(s) and grant ackowledgment(s)}

1. Ely, E., 2004. Delirium as a Predictor of Mortality in Mechanically Ventilated Patients in the Intensive Care Unit. JAMA, 291 (14), p.1753.

2. Herling, S., Greve, I., Vasilevskis, E., Egerod, I., Bekker Mortensen, C., Møller, A., Svenningsen, H. and Thomsen, T., 2018. Interventions for preventing intensive care unit delirium in adults. Cochrane Database of Systematic Reviews,

3. Kalish, V., Gillham, J. and Unwin, B., 2014. Delirium in older persons: evaluation and management. Am Fam Physician, 90(3), pp.150-8.

4. Nice.org.uk. 2020. Overview | Delirium: Prevention, Diagnosis And Management | Guidance | NICE. [online] Available at: <https://www. nice.org.uk/guidance/cg103/> [Accessed 25 July 2020].

5. Page, V., Navarange, S., Gama, S. and McAuley, D., 2009. Routine delirium monitoring in a UK critical care unit. Critical Care, 13(1), p.R16.

6. Pandharipande, P., Girard, T., Jackson, J., Morandi, A., Thompson, J., Pun, B., Brummel, N., Hughes, C., Vasilevskis, E., Shintani, A., Moons, K., Geevarghese, S., Canonico, A., Hopkins, R., Bernard, G., Dittus, R. and Ely, E., 2013. Long-Term Cognitive Impairment after Critical IIIness. New England Journal of Medicine, 369(14), pp.1306-1316.

7. Pisani, M., Kong, S., Kasl, S., Murphy, T., Araujo, K. and Van Ness, P., 2009. Days of Delirium Are Associated with 1-Year Mortality in an Older Intensive Care Unit Population. American Journal of Respiratory and Critical Care Medicine, 180(11), pp.1092-1097.

8. Salluh, J., Wang, H., Schneider, E., Nagaraja, N., Yenokyan, G., Damluji, A., Serafim, R. and Stevens, R., 2015. Outcome of delirium in critically ill patients: systematic review and meta-analysis. BMJ, 350(may19 3), pp.h2538-h2538.

9. Thomason, J., Shintani, A., Peterson, J., Pun, B., Jackson, J. and Ely, E., 2005. Critical Care, 9(4), p.R375.

10. Miranda, F., Arevalo-Rodriguez, I., Díaz, G., Gonzalez, F., Plana, M. Zamora, J., Quinn, T. and Seron, P., 2018. Confusion Assessment Method for the intensive care unit (CAM-ICU) for the diagnosis of delirium in adults in critical care settings. Cochrane Database of Systematic Reviews.

000220

Is pupilary dilation reflex (PDR) a valuable tool for measuring pain in ICU patients compared to behavioral pain scale (BPS)?

Y. López De Audícana Jiménez De Aberásturi' ; N. Parraza Diez²; A. Vallejo de la Cueva ${ }^{3} ;$ A. Quintano Rodero ${ }^{3} ;$ K. Pelegrín Gaspar ${ }^{3} ;$ MB. Bengoetxea Ibarrondo ${ }^{4}$; MJ. Rodriguez Borrajo ${ }^{3}$; I. Rodriguez Trujillo ${ }^{3}$; C. Rodriguez Nuñez $z^{3}$ I. Arce Gómez $z^{3}$

${ }^{1}$ College of nursing, University of Basque Country, Vitoria-Gasteiz, Spain; ${ }^{2}$ Bioaraba, institute of health research, Statistics and Methodology Unit, Vitoria-Gasteiz, Spain; ${ }^{3}$ Intensive care unit, University Hospital of Araba, Vitoria-Gasteiz, Spain; ${ }^{4}$ Intensive care unit, University Hospital of Cruces, Bilbao, Spain

Correspondence: A. Vallejo de la Cueva

Intensive Care Medicine Experimental 2020, 8(2): 000220

Introduction: Pupilometry is a simple and non invasive technique that allows to asses nociceptive responds to stimulus through pupillary dilation reflex (PDR) objective measurement.

Objectives: To evaluate diagnostic efficacy of PDR compared to BPS scale.

Methods: ICU patients over 18 years, under intravenous sedoanalgesic drugs, mechanically ventilated, basal BPS of 3, RASS between -1 and -4 . Exclusion criteria: use of paralyzing drugs, neuromuscular diseases, polyneuropathy, pupillary damage, GCS $<6$, clonidine, dexmedetomidine, tramadol, ketamine, adrenalin, calcioantagonist, noradrenaline $>0.6$ or dobutamine $>10 \mathrm{mg} / \mathrm{kg} / \mathrm{min}$. It will be assessed pain response with BPS and PDR in pain absence, pain stimulus of 10,2030 and $40 \mathrm{~mA}$ and with tracheal aspiration. PDR measurements : Pupilometer AlgiScan ${ }^{\circledR}$. Presence of pain was considered as BPS $>3$. It will be preformed a diagnostic PDR test vs BPS globally and separated for each painful stimulus or not. Significance as $p<0.05$. Approved by Basque Ethics Committee (CEIm-E).

Results: We obtained an AUC of 0,885 (IC 0,830-0,940). PDR value of best diagnostic efficiency was $11,5 \%$, with a sensitivity of $89,8 \%$ (Cl $95 \% 78,2-95,6)$ and specificity of 78,4\% (Cl 95\% 70,6-84,5) (Table 1).

Table 1. Diagnostic efficiency of different PDR cut points (global measurements).

\begin{tabular}{|l|l|l|l|l|}
\hline & PDR 11,5\% & PDR 7,5\% & PDR 5,5\% & PDR 3.5\% \\
\hline $\begin{array}{l}\text { Sensitivity } \\
\text { (\%) }\end{array}$ & $89,8(78,2-$ & $89,8(78,2-$ & $93,9(83,5-$ & $95,9(86,3-$ \\
\hline Specificity & $95,6)$ & $95,6)$ & $97,9)$ & $98,9)$ \\
\hline (\%) & $84,5)$ & $70,4)$ & $59,8)$ & $46,5)$ \\
\hline PPV(\%) & $60,3(48,8-$ & $46,8(37,0-$ & $41,4(32,7-$ & $36,2(28,4-$ \\
\hline NPV(\%) & $70,7)$ & $56,8)$ & $50,7)$ & $44,7)$ \\
\hline FP(\%) & $95,5(89,8-$ & $94,4(87,5-$ & $95,8(88,5-$ & $96,2(87,2-$ \\
\hline FN (\%) & $21,6(15,5-$ & $97,6)$ & $98,6)$ & $99,0)$ \\
\hline Accuracy & $19,4)$ & $45,7)$ & $56,9)$ & $61,9(53,5-$ \\
(\%) & $81,4(7,4,4-21,8)$ & $10,2(4,4-21,8)$ & $6,1(2,1-16,5)$ & $4,1(1,1-13,7)$ \\
\hline Odds ratio & $86,4)$ & $69,9(62,9-$ & $62,8(55,6-$ & $53,6(46,3-$ \\
\hline PPC & $81,8(11,5-$ & $76,1)$ & $69,5)$ & $60,6)$ \\
\hline NPC & $4,15(2,9-5,8)$ & $2,4(1,9-3,06)$ & $1,9(1,6-2,3)$ & $1,6(1,4-1,8)$ \\
\hline & $0,13(0,06-0,3)$ & $0,16(0,07-0,4)$ & $0,12(0,04-0,3)$ & $0,1(0-0,4)$ \\
\hline
\end{tabular}


In $20 \mathrm{~mA}$ stimulus we found the best results on efficiency diagnostic parameters, with an AUC of $0,853(0,7-1,0)$ and a sensitivity of $100 \%$ $(34,2-100)$ and a specificity of $75,9 \%(57,9-87,8)$ for the cut point of PDR $=11,5 \%$ (Table 2).

Conclusion: PDR valuesover $11,5 \%$ indicate pain with a $90 \%$ of sensitivity and $80 \%$ of specificity.

A pupillary response over $11,5 \%$ during $20 \mathrm{~mA}$ stimulus could predict the need of analgesics during tracheal aspiration. Further studies will be needed to confirm.

\section{Reference(s) and grant ackowledgment(s)}

1. Paulus J, Roquilly A, Beloeil $\mathrm{H}$, et al. Pupillary reflex measurement predicts insufficient analgesia before endotracheal suctioning in critically ill patients. Crit Care Lond Engl. 2013;17(4):R161

2. Guglielminotti J, Grillot N, Paule M, et al. Prediction of movement to surgical stimulation by the pupillary dilatation reflex amplitude evoked by a standardized noxious test. Anesthesiology. 2015;122(5):985-93.

3. Li D, Miaskowski C, Burkhardt D, Puntillo K. Evaluations of physiologic reactivity and reflexive behaviors during noxious procedures in sedated critically ill patients. J Crit Care. septiembre de 2009;24(3):472.e9-13.

4. Devlin JW, Skrobik Y, Gélinas C, Needham DM, Slooter AJC, Pandharipande PP, et al. Clinical Practice Guidelines for the Prevention and Management of Pain, Agitation/Sedation, Delirium, Immobility, and Sleep Disruption in Adult Patients in the ICU. Crit Care Med. 2018;46(9):e825-73.

5. Pupilometer AlgiScan, provided by Prhoinsa

6. Basque Government

\section{5}

Analysis of the response to pain in intensive care unit (ICU) comparing the behavior pain scale (BPS) versus the pupillary dilation reflex (PDR) according to level of severity, sedation and age

Y. López De Audícana Jiménez De Aberásturi'; A. Vallejo de la Cueva²; N. Parraza Diez ${ }^{3}$; A. Quintano Rodero²; AA. Margüello Fernández z' P. Garcia Domelo²; A. Muniozguren Puertas ${ }^{2}$; MB. Bengoetxea Ibarrondo ${ }^{4}$; E. Cardo Castro $^{4}$; V. Sanz Porras ${ }^{2}$

${ }^{1}$ College of nursing, University of the Basque Country., Vitoria-Gasteiz, Spain; ${ }^{2}$ Intensive Care unit, University Hospital of Araba, Vitoria-Gasteiz, Spain; ${ }^{3}$ Bioaraba, institute of health research, Statistics and Methodology Unit, Vitoria-Gasteiz, Spain; ${ }^{4}$ Intensive care unit, University Hospital of Cruces, Bilbao, Spain

Correspondence: A. Vallejo de la Cueva

Intensive Care Medicine Experimental 2020, 8(2): 000295

Introduction: Pain assessment in the non-communicative patient in critical care units remains under continuous evaluation. Clinical complexity of the critically ill patient could establish behavior responsiveness to pain.

Objectives: To determine the pain response in critically ill patients by comparing two tools: the behavior pain scale (BPS) and the pupillary dilation reflex (PDR), establishing the demographic (age) and clinical factors (severity level-APACHE II, and sedation level-Bispectral Index scale-BIS) which may interfere with their response.

Methods: ICU patients over 18 years, under intravenous sedoanalgesic drugs, mechanically ventilated, basal BPS of 3 , RASS between -1 and -4 . Exclusion criteria: use of paralyzing drugs, neuromuscular diseases, polyneuropathy, pupillary damage, GCS $<6$, clonidine, dexmedetomidine, tramadol, ketamine, adrenalin, calcioantagonist, noradrenaline $>$ 0.6 or dobutamine $>10 \mathrm{mg} / \mathrm{kg} / \mathrm{min}$. It will be assessed pain response with BPS and PDR in pain absence, pain stimulus of 10,20 30 and 40 $\mathrm{mA}$ and with tracheal aspiration (TA) PDR measurements: Pupilometer AlgiScan ${ }^{\circledR}$. Presence of pain was considered as BPS $>3$. Categorical variables as percentages \% and compared using $\mathrm{X} 2$ o Fisher, continuous variables median IQR and compared using two samples T-test. Multivariate analysis was performed using a logistic regression model showed as odds ratios (OR) and $95 \%$ confidence intervals (Cl). $\mathrm{P}<, 05$. Approved by Basque Ethics Committee (CElm-E)

Results: 183 measurements on 31 patients. Demographic characteristics; mean $\pm S D$ : age $62,4 \pm 17,1$; APACHE II 21 $\pm 8,3$; BIS $63 \pm 19,5$; RASS
$-3,4 \pm 1,8$. Percentage: Female 38,7; Admission: ARF 25,8; Post-surgery 32,2; Sepsis 29,1; Trauma 12,9.

Only in 49 measurements (26.7\%) of them presented pain (BPS $\geq 4$ ), with median of PDR of $28 \%$ (17-48), vs 134 where not experience pain $(\mathrm{BPS}<4)$ with a median of $5 \%(2-11)(\mathrm{p}<0.001)$.

In the bivariate analysis, an age over 65 , a higher level of severity (APACHE II >20) or a higher level of sedation (BIS $\leq 40$ ) did not predict a lower likelihood of having pain measured for BPS (BPS $\geq 4$ ) (Table 1). Similarly, age above 65 was not related to lower PDR. In contrast, BIS $\leq 40$ and APACHE $>20$ were both significantly associated with lower PDR (Table 2).

Table 1. Behavior of BPS according to level of BIS, age and APACHE II.

\begin{tabular}{|c|c|c|c|c|c|}
\hline & \multicolumn{2}{|c|}{ BPS $<4$} & \multicolumn{2}{|c|}{$B P S \geq 4$} & \multirow[b]{2}{*}{ p } \\
\hline & $\mathbf{n}$ & $\%$ & n & $\%$ & \\
\hline $\mathrm{BIS} \leq 40$ & 20 & $14,9 \%$ & 5 & $10,2 \%$ & \multirow{2}{*}{0,41} \\
\hline BIS $>40$ & 114 & $85,1 \%$ & 44 & $89,8 \%$ & \\
\hline $\operatorname{Edad} \leq 65$ & 70 & $52,2 \%$ & 25 & $51 \%$ & \multirow{2}{*}{0,884} \\
\hline Edad $>65$ & 64 & $47,8 \%$ & 24 & $49 \%$ & \\
\hline APACHE $\leq 20$ & 57 & $45,2 \%$ & 26 & $57.8 \%$ & \multirow{2}{*}{0,149} \\
\hline APACHE $>20$ & 69 & $54.8 \%$ & 19 & $42.2 \%$ & \\
\hline
\end{tabular}

Table 2: Behavior of PDR according level of BIS, age and APACHE II

\begin{tabular}{|l|l|l|l|}
\hline & n & P50 (P25-P75) & p \\
\hline BIS $\leq \mathbf{4 0}$ & 25 & $3(2-10,5)$ & 0,020 \\
\hline BIS $>\mathbf{4 0}$ & 158 & $9(3,75-21)$ & \\
\hline Edad $\leq \mathbf{6 5}$ & 95 & $9(3-22)$ & 0,242 \\
\hline Edad $>\mathbf{6 5}$ & 88 & $7(3-19)$ & 0,029 \\
\hline APACHE $\leq \mathbf{2 0}$ & 83 & $11(4-29)$ & \\
\hline \hline APACHE $>\mathbf{2 0}$ & 88 & $6,5(3-17)$ & \\
\hline
\end{tabular}

In the multivariate analysis adjusted by APACHE and BIS the OR in adjusted and non adjusted model was $1,095(1,062-1,130)(p<0,001)$.

Conclusion: Higher PDR values are related to painful response according to BPS.

The response to pain is independent of the level of sedation, age and severity of the patients.

\section{Reference(s) and grant ackowledgment(s)}

1. Rouche O, Wolak-Thierry A, et al. Evaluation of the depth of sedation in an intensive care unit based on the photo motor reflex variations measured by video pupillometry. Ann Intensive Care. 2013;3(1):5

2. Li D, Miaskowski C, Burkhardt D, et al. Evaluations of physiologic reactivity and reflexive behaviors during noxious procedures in sedated critically ill patients. J Crit Care. 2009:24(3):472.e9-13.

3. Devlin JW, Skrobik Y, Gélinas C, et al. Clinical Practice Guidelines for the Prevention and Management of Pain, Agitation/Sedation, Delirium, Immobility, and Sleep Disruption in Adult Patients in the ICU. Crit Care Med. 2018;46(9):e825-73.

4. Basque Government

5. Pupilometer AlgiScan, provided by Prhoinsa. 
000137

Scleroderma renal crisis - an unusual diagnosis in a district general hospital

C. Peers ${ }^{1}$; M. Cooper ${ }^{1}$; S. Sonia'; F. Bond

${ }^{1}$ Intensive Care Unit, Princess OfWales Hospital, Bridgend, United Kingdom

Correspondence: $C$. Peers

Intensive Care Medicine Experimental 2020, 8(2): 000137

Introduction: We report a patient who presented with pulmonary oedema, hypertension and progressive renal failure. Recent investigations for systemic sclerosis had been performed but results were still awaited on admission. Systemic Sclerosis is a rare disease with an incidence of two to ten cases per million of the population. Scleroderma Renal Crisis, with an incidence of $5 \%$ in patients with diffuse disease, is a rare complication of a rare disease.

Methods: This 70-year-old lady presented with pulmonary oedema and acute kidney injury. A diagnosis of Systemic Scleroderma was considered due to presence of dermatological changes (fig.1), hypertension, presence of right heart strain on transthoracic echocardiography and rapidly progressive renal failure. Scleroderma renal crisis became the primary diagnosis following positive biochemical tests.

After immediate resuscitation and initiation oftreatment with the angiotensin converting enzyme inhibitor, Captopril, the patient was transferred to the regional renal unit for continued management. The patient's renal failure worsened and she became dialysis dependant. She also developed left ventricular failure and worsening pulmonary hypertension. Despite prompt diagnosis of a rare condition and aggressive resuscitation, the patient did not survive her hospital admission.

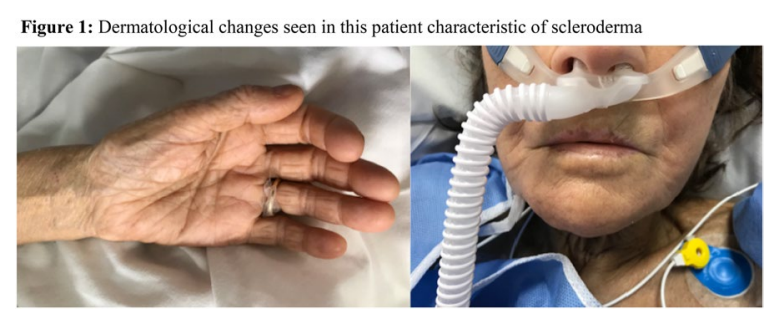

Results: An immunological screen was sent with key positive findings including positive CENP B, PM-Scl100, anti-nuclear AB screen and P-ANCA screen pattern. Echocardiogram demonstrated left ventricular hypokinesia with an ejection fraction of $35 \%$ and moderate pulmonary hypertension. Urine dipstick showed protein +++ , blood +++ . Conclusion: Acute renal failure is not always secondary to septic shock in a patient with sepsis. This case demonstrates the importance of considering rare causes of acute renal failure in the intensive care setting.

\section{1}

ARDS as debut of Pulmonary Sarcoidosis: Two case report

C. Saito'; JR. Fernández Villanueva²

${ }^{1}$ Medicina Intensiva, Complejo Hospitalario Santiago de Compostela, Santiago de Compostela, Spain; ${ }^{2}$ Critical care unit, Hospital Clínico Universitario de Santiago de Compostela, Santiago de Compostela, Spain

Correspondence: $C$. Saito

Intensive Care Medicine Experimental 2020, 8(2): 000701

Introduction: Sarcoidosis is a mutisystemic granulomatous disorder of unknown etiology that most commonly affects the lung and lymphatic system. It is characterized pathologically by the presence of noncaseating granulomas in involved organs. The estimated prevalence is 10 to 20 per 100,000 population. In approximately on-half the disease is detected incidentally by radiographic abnormalities prior to the development of symptoms, which includes fever, cough, dyspnea and chest pain.
Objectives: We present two cases of Sarcoidosis which had lately diagnosed because of their debut as acute respiratory distress syndrome (ARDS)

Methods: A 52 year old man and a 62 year old woman, both presented as fever and dyspnea of approximately two weeks of evolution, the chest radiography at presentation revealed extensive bilateral intersticial infiltrates. On physical examination: tachypnea with normal skin coloration and fever, no skin, eye or joint lesions. Severe hypoxemia and on auscultation "velcro-like" crackles in bibasilar areas.

Results: Complete blood count, electrolytes and rena and liver test were within normal limits. At day +6 and +4 of adminission, respectivelly, they both presented poor respiratory income and required both orothraqueal intubation and connection to mechanic ventilation Both thoracic CT showed with bilateral extensive multifocal infiltrates with significant mediastinal and bilateral hiliar lymphadenopathy. Empirical treatment with intravenous antiobiotics and, in the first case, antituberculostatic treatment, was started, because of the appearance of positive Mantoux test. Fibreoptic bronchoscopy with bronchoalveolar lavage $(B A L)$ were performed in both cases and revealed no evidence of PCP, acid fast bacilli, fungi, or malignancy. No other causes were found.

Conclusion: Due to this results, initiation of corticosteroids and pulmonary biopsy was performed in both cases. In the first case, endoscopic ultrasound guided needle aspiration was performed and in the second case transbronchial lung biopsy. Histopathologic detection of noncaseating granulomas, compatibility clinical and radiographic manifestations and exclusion of other diseases, concluded in the diagnosis of pulmonary sarcoidosis. By days +2 and +4 of initiation with corticosteroids, they both presented notably improvement in respiratory status and radiograph images.

Reference(s) and grant ackowledgment(s)

1. Gera K, et al. Acute alveolar sarcoidosis presenting with hypoxaemic respiratory failure. BMJ Case Rep 2014.

2. Sabrina Arondi, Alberto Valsecchi, Andrea Borghesi, and Stefano Monti. Pulmonary sarcoidosis presenting with acute respiratory distress syndrome. Ann Thorac Med. 2016 Jan-Mar; 11(1): 79-81.

3. Adi Leiba, Sara Apter, Merav Leiba, Michael Thaler and Ehud Grossman. Acute Respiratory Failure in a Patient with Sarcoidosis and Immunodeficiency_An Unusual Presentation and a Complicated Course. Lung (2004) 182:73-77

4. F Sabbagh, C Gibbs, L S Efferen. Pulmonary sarcoidosis and the acute respiratory distress syndrome (ARDS). Thorax 2002;57:655-656

\section{0}

Study of three clinical variables as a grading prognosis for acute severe pancreatitis

A. Muñoz-Cantero'; D. Pérez-Civantos'; JD. Jiménez-Delgado².

M. Cidoncha-Gallego²; P. Nieto-Sánchez¹; J. Pérez-Bazaga ${ }^{1}$

${ }^{1}$ Critical Care Unit, Complejo Hospitalario Universitario de Badajoz, Badajoz, Spain; ${ }^{2}$ Critical care unit, Don Benito-Villanueva Hospital, Don Benito, Spain

Correspondence: A. Muñoz-Cantero

Intensive Care Medicine Experimental 2020, 8(2): 000890

Introduction: The assessment of severity at the initial medical examination of serious acute pancreatitis plays an important role in introducing adequate early treatment and the transfer of patients to the ICU. Different early prognostic scores have been proposed but many are not sharply enough for many practitioners.

Objectives: To study the correlation of imaging using TC severity score against SOFA score plus serum lactate and the first 24 hours fluid balance as a predictor score for severe acute pancreatitis.

Methods: A lineal retrospective, two centers study was carried out. We enrolled all consecutive patients with acute pancreatitis admitted to the ICU of two Hospitals (a teaching tertiary center and a small county Hospital).

Univariate analysis was performed to identify the parameters which showed some correlation with the mortality. We used a square-Chi 
model for qualitative parameters and T-Student for quantitative. We also performed a multivariate analysis using independent significant $\left(p^{<} 0.05\right)$ parameters in univariate analysis.

We evaluated the area under the ROC curve (AUC) for significant singles parameters in a logistic regression model. Finally, we constructed a model using the analysis of three parameters altogether like an early predictor of the severity of acute pancreatitis. To assess the diagnostic performance of the model, we compared the AUC-ROC with each selected single parameter. The SPSS 22.0 program was used for statistical analysis.

Results: 64 patients were enrolled in the study. All data expressed as median and standard deviation. Age was $60,4+/-14$. Sequential Organ Failure Assessment (SOFA) score at ICU admission was $3.3+1$ 3.1, Acute Physiology and Chronic Health Evaluation (APACHE-II) score were $11.6+/$ - 5.8. We also analyze parameters like Fluid Balance during the first 34 hours, $3.2+/-2.5$ litres, and serum lactate $2.0+/-1.4$ $\mathrm{mmol} / \mathrm{l}$. The aetiology of acute pancreatitis was: gallstones $(60.9 \%)$ alcoholism (14.0\%), TGC (1.6\%), and other causes (20.4\%). ICU Mortality was $30.2 \%$. Ten patients required surgical treatment and we found a significant correlation between mortality and the need for surgery ( $p$ $0.001)$. In the same case SOFA score ( $\left.p^{<} 0.0001\right)$, serum lactate, initial fluid balance, ICTS and the sum of SOFA Score + Lactic + Fluid Balance were significantly associated with ICU mortality. For the early prediction of the severity, we used a three feet analysis model that was constructed using the SOFA score, Fluid Balance, and serum lactate on the first 24 hours of ICU admission. Our model showed a trend of a higher AUC (0.8892) than that of every single parameter (SOFA score, 0.766 ; Fluid Balance, 0.822; lactate, 0.852, ICTS, 0.447).

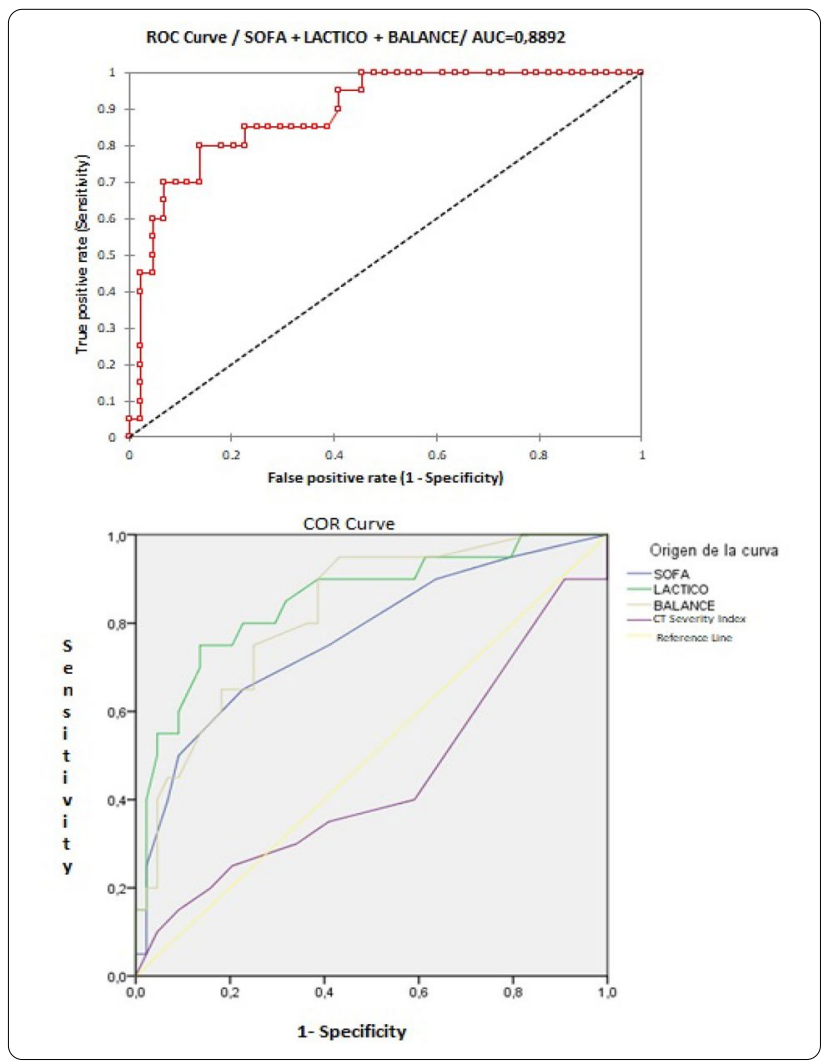

Conclusion: We can conclude saying that probably the sum of three clinical parameters like initial Fluid Balance plus Serum Lactate plus SOFA score is superior to TC severity score as an early prediction of mortality for severe pancreatitis. These preliminary results should need to be confirmed in further studies with a larger number of patients.
Reference(s) and grant ackowledgment(s)

1. Imrie CW. Prognostic indicators in acute pancreatitis. Can J Gastroenterol 2003:17:325-328

2. Vriens PW, van de Linde P, Slotema ET, Warmerdam P E, PJ Breslau.Computed To-mography Severity Index Is an EarlyPrognostic Tool for Acute Pancreatitis. J Am Coll Surg 2005;201:497-502.

3. None

\section{4}

Regular immunosuppression does not lead to worse outcomes in critically ill patients infected with Covid-19

JK. Rane'; HD. Aya2; A. Bhide²; JM. Low'; D. Padman'; A. Rhodes³;

M. Hamilton'2 S. Leaver ${ }^{4}$

${ }^{1}$ Intensive care unit, St George's Hospital, London, United Kingdom; ${ }^{2} \mathrm{Gen}$ eral intensive care unit, St George's Hospital, London, United Kingdom ${ }^{3}$ Anaesthesia and intensive care medicine, St George's University Hospitals NHS Foundation Trust, Londres, United Kingdom; ${ }^{4}$ Research lead critical care directorate, St George's Hospital, London, United Kingdom

Correspondence: J.K. Rane

Intensive Care Medicine Experimental 2020, 8(2): 001054

Introduction: Immunosuppressed Covid-19 patients were predicted to have poorer outcomes (1-3), however what contributes to these worse outcomes remain unknown.

Objectives: To assess whether immunosuppression on presentation is a predictor of poor outcomes in critically ill patients admitted with Covid-19 infection in an intensive care unit (ICU) and to identify principle determinants of the poorer outcomes.

Methods: Immunosuppressed patients were defined as patients on regular immunosuppressive treatments. PCR positive Covid-19 patients at St George's Hospital ICU from 01/03/20 to 15/05/20 were included in this observational study. Primary outcome was the odds ratio (OR) of mortality at 28-days after ICU admission. Disease severity was assessed as secondary end points, such as ICU length of stay (LOS), duration of mechanical ventilation (MV), duration of cardiovascular (CV) support, and the need for renal replacement therapy (RRT). A univariate analysis was performed with Chi square statistic, and a logistic and linear regression model was used for the multivariable analysis. Medians were compared with Mann-Whitney $\mathrm{U}$ test. $\mathrm{P}$ values greater that 0.05 were considered significant.

Results: A total of 132 adult Covid-19 patients were included in the study, out of which 15 (11.4\%) were immunosuppressed. $6(40 \%)$ of immunosuppressed patients died at 28 days (Figure1), compared to 64 (54.7\%) in the immunocompetent group (OR $1.8 ; 95 \% \mathrm{Cl} 0.6,5.4 ; p=$ 0.28.). Immunosuppression in Covid-19 patients did not affect LOS (12 days in immunosuppressed group $(5,22)$ vs 11 days $(5,17) p=0.8)$, the requirement of RRT ( $26.7 \%$ vs $37.6 \%$, OR $1.6,95 \% \mathrm{Cl} 0.48,5.5, p=0.4)$ length of $\operatorname{MV}(6$ days $(0,22)$ vs 9 days $(3,16), p=0.8)$ or duration of $C V$ support ( 1.5 days $(0,8.5)$ vs. 4 days $(0,9), p=0.3)$.

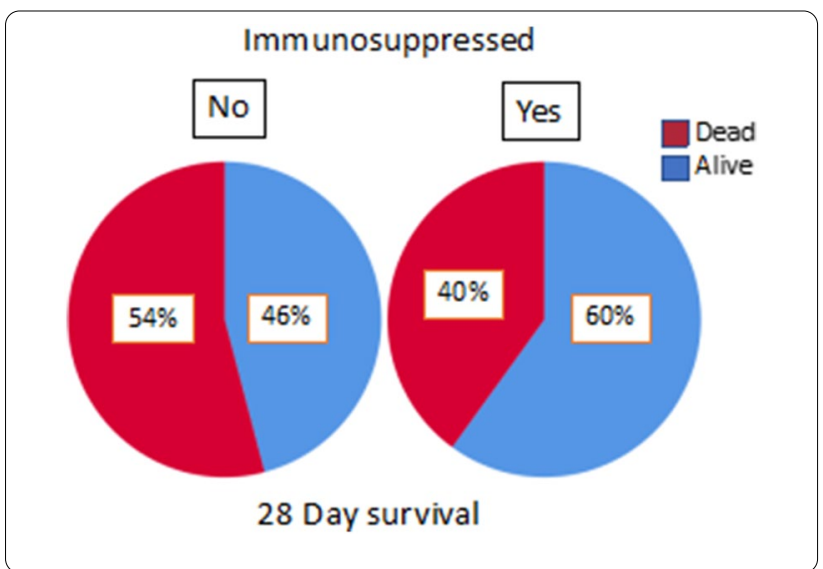


Conclusion: Immunosuppression on presentation neither increases 28-day mortality nor pre-disposes patients to severe Covid-19 complications.

\section{6}

Effects of dexmedetomidine on platelet P-selectin expression in patients with diabetes in the postoperative intensive care unit S. Kawamoto ${ }^{1}$;. Murata ${ }^{1}$; E. Kusudo ${ }^{1}$; K. Fukuda ${ }^{1}$

${ }^{1}$ Department of anesthesia, Kyoto University Hospital, Kyoto, Japan

Correspondence: K. Shuji

Intensive Care Medicine Experimental 2020, 8(2): 0000016

Introduction: Diabetes is a risk factor for various postoperative complications. One of the most serious of these complications is thromboembolism such as myocardial infarction and cerebral infarction, in which enhanced platelet a2-adrenoceptor function may play a key role (Ref. 1). Currently, many patients admitted to the postoperative intensive care unit (ICU) are sedated long-term by continuous intravenous infusion of the a2-adrenoceptor sedative dexmedetomidine. Therefore, it is extremely important to examine changes in platelet functions in patients with diabetes due to differences in anesthetics and sedatives, and to establish an optimal approach to perioperative management for prevention of postoperative bleeding and thromboembolism.

Objectives: The aim of this study is to analyze the effects of dexmedetomidine on expression of P-selectin, a platelet activation marker, by flow cytometry using peripheral blood samples collected before and after surgery from patients with and without diabetes who were admitted to the ICU after major surgery.

Methods: Informed consent was obtained from subjects and the protocol was approved by the ethics committee (R1109). The subjects were patients with $(n=5)$ and without $(n=4)$ diabetes who entered the ICU after subtotal esophagectomy or pancreatectomy. The exclusion criteria were age $<20$ years; abnormal platelet count, platelet function, or coagulation ability before surgery; treatment with antiplatelet drugs or anticoagulants up to 2 weeks before surgery; intraoperative blood transfusion, and intraoperative treatment with drugs that might affect platelet function. Blood samples were collected from arterial pressure lines before the start of surgery and immediately after admission to the ICU after surgery. Platelet-rich plasma was stimulated by ADP with dexmedetomidine $(0-100 \mathrm{ng} / \mathrm{ml})$ at room temperature for $30 \mathrm{~min}$. Samples were coincubated with PerCP-labeled anti-CD61 antibody and PE-labeled anti-CD62P (P-selectin) antibody, and P-selectin expression levels were assessed by flow cytometry. Data from before to after surgery were compared by t-test. All other data were compared by one-way ANOVA, followed by a Dunnett post hoc test. $P<0.05$ was considered to indicate significance in all analyses.

Results: In patients without diabetes, dexmedetomidine increased P-selectin expression in a dose-dependent manner, with no significant difference between pre- and postoperative data (Fig. 1). In patients with diabetes, P-selectin expression was higher than in patients without diabetes at all concentrations of dexmedetomidine before and after surgery (Fig. 2). P-selectin expression in patients with diabetes was enhanced in a dexmedetomidine dose-dependent manner, similarly to patients without diabetes, but was significantly higher after surgery than before surgery.

These results show that the P-selectin expression level in patients with diabetes is higher before and after surgery, compared to that in patients without diabetes, which indicates that platelets from patients with diabetes are activated and have a tendency for thrombosis. Our previous studies on healthy individuals showed that dexmedetomidine enhanced P-selectin expression in a concentration-dependent manner (Ref. 2). This is consistent with the results of this study and indicates that this tendency does not change after surgical invasion. In patients without diabetes, dexmedetomidine had similar levels of platelet activation before and after surgery, whereas patients with diabetes had significantly increased P-selectin expression after surgery. This suggests that the sensitivity of a2-adrenoceptors may be enhanced or the number of a2-adrenoceptors may increase due to surgical invasion in platelets of patients with diabetes.
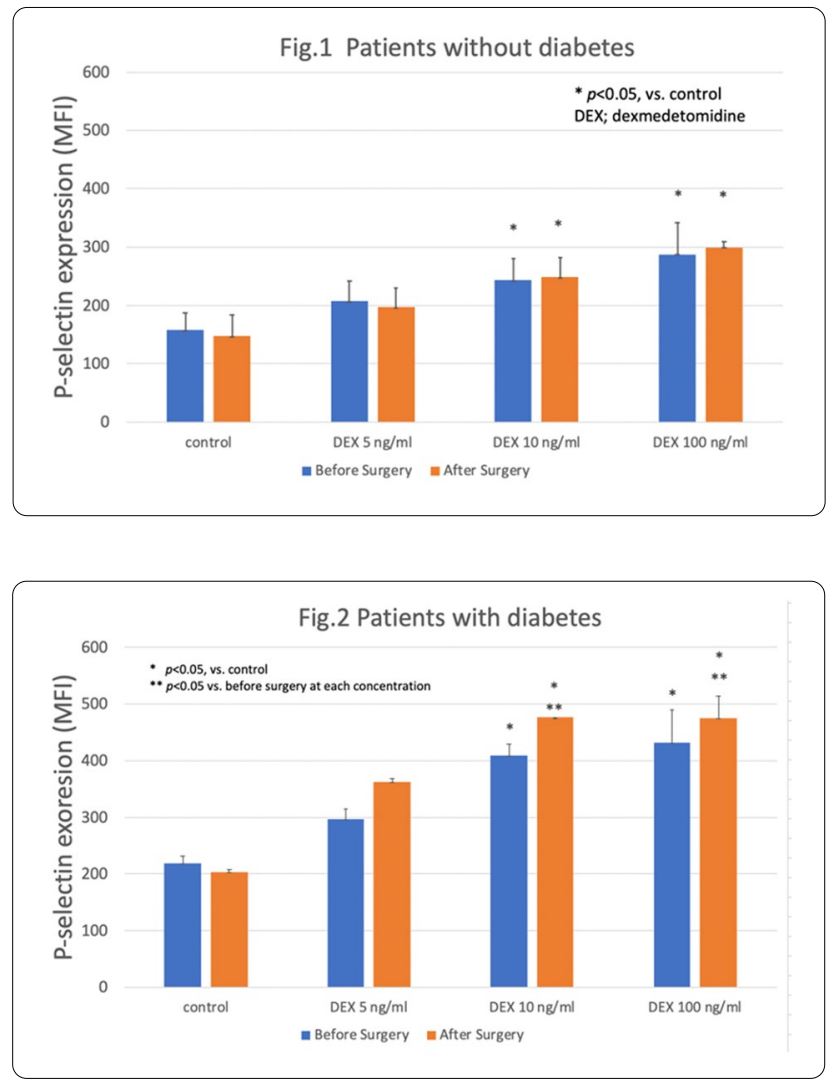

Conclusion: The function of platelet a2-adrenoceptor may be enhanced postoperatively and platelets may be activated more strongly by dexmedetomidine in patients with diabetes compared to patients without diabetes.

\section{Reference(s) and grant ackowledgment(s)}

1. 1. Lahiri P. et al. Insensitivity to the alpha2-adrenergic receptor blocker yohimbine hydrochloride and occurrence of spontaneous platelet macroaggregation (SPMA) in diabetes. 2005 Platelets 16,111-5

2. Grant-in-Aid for Scientific Research (KAKENHI) :17K16730

3. 2. Kawamoto $S$. et al. Bidirectional effects of dexmedetomidine on human platelet functions in vitro. 2015 Eur J Pharmacol 766, 122-8

\section{7}

Application of rotational thromboelastometry to assess hypercoagulability in COVID-19 patients

N. Van Veenendaal' ; T. Scheeren ${ }^{2}$; K. Meijer ${ }^{3}$; P. Van Der Voort ${ }^{4}$

${ }^{1}$ Department of critical care / department of anaesthesiology, University Medical Center Groningen, Groningen, Netherlands; ${ }^{2}$ Department of anaesthesiology / department of critical care, University Medical Center Groningen, Groningen, Netherlands; ${ }^{3}$ Department of hematology, University Medical Center Groningen, Groningen, Netherlands; ${ }^{4}$ Department of critical care, University Medical Center Groningen, Groningen, Netherlands

Correspondence: $\mathrm{N}$. Van Veenendaal

Intensive Care Medicine Experimental 2020, 8(2): 000417

Introduction: Coronavirus disease 2019 (COVID-19 is associated with coagulation abnormalities leading to numerous thromboembolic complications. Conventional coagulation tests show trends of coagulopathy, but reflect only limited parts of the coagulation cascade. 
Rotational thromboelastrometry $\left(\right.$ ROTEM $\left.^{\circledR}\right)$ is a digitized modification of thromoboelastography reported to be useful in identifying hypercoagulable conditions.

Objectives: The aim of this study was to evaluate ROTEM ${ }^{\circledR}$ profiles of COVID-19 patients in identifying hypercoagulable states.

Methods: Patients admitted to the Intensive Care Unit with COVID-19 were included in this prospective observational study. Blood samples were collected for ROTEM ${ }^{\circledR}$ thomboelastometry. Coagulation patterns were analyzed using extrinsic and intrinsic rotational thromboelastometry activators (respectively EXTEM and INTEM). The following variables were recorded and analyzed: clotting time (CT), clotting formation time (CFT), clot firmness' amplitude after 5 minutes (A5) and after 10 minutes (A10), and maximum clot firmness (MCF). The ROTEM ${ }^{\circledR}$ results were compared with conventional coagulation parameters, which were collected as part of standard clinical care. Hypercoagulability was defined as a reduction in CFT and increase in MCF.

Results: Forty-seven COVID-19 patients were included, of which $81 \%$ were male. The median age was 63 years (range 29-79), and the median BMI was $28.8 \mathrm{~kg} / \mathrm{m} 2$ (range 24.4-48.4). The median length of stay at the ICU at the time of blood sampling for ROTEM ${ }^{\circledR}$ was 8 days (range 1-25). All patients required mechanical ventilation.

Mean ROTEM ${ }^{\circledR}$ clot formation time (CFT) was shortened in EXTEM (45.3 s; normal 46-149 s) and INTEM (45.6 s, normal 62-130 s). Mean maximum clot firmness (MCF) was increased with all three activators (EXTEM, 77.3 mm, normal 55-72 mm; INTEM $72.9 \mathrm{~mm}$, normal 51-69 $\mathrm{mm}$; FIBTEM $32.9 \mathrm{~mm}, 6-21 \mathrm{~mm}$ ).

Conventional coagulation analyses showed the typical coagulopathy pattern with increased fibrinogen (mean $7.2 \mathrm{~g} / \mathrm{L}$, SD $1.5 \mathrm{~g} / \mathrm{L}$ ), prolonged PT (15.3 s, SD $2.0 \mathrm{~s})$, and prolonged APTT (34.7 s, SD $8.7 \mathrm{~s})$. Mean platelet count was slightly increased (404 109/L, SD 154 109/L). Conclusion: ROTEM $^{\circledR}$ confirmed an overall hypercoagulable state of COVID-19 patients admitted to the ICU.

\section{8}

Prospective evaluation of critically ill patients with therapeutic plasma change in medical intensive care unit

M. Caliskan ' ; K. Gundogan²; S. Temel'; EN. KARAOGLU'; M. Arslan';

M. Yıldırım ${ }^{\text {; }}$ M. Cetin ${ }^{1}$; L. Kaynar ${ }^{3}$; M. Sungur ${ }^{4}$

${ }^{1}$ Department of internal medicine, Erciyes University, Kayseri, Turkey; ${ }^{2}$ Department of internal medicine, division of intensive care, Erciyes University, School of medicine, Kayseri, Turkey; ${ }^{3}$ Division of hematology, department of internal medicine, Erciyes University, Kayseri, Turkey; ${ }^{4}$ Division of critical care medicine, department of internal medicine, Erciyes University School of Medicine, Kayseri, Turkey, Kayseri, Turkey

Correspondence: M. C.ALISKAN

Intensive Care Medicine Experimental 2020, 8(2): 000418

Introduction: Therapeutic plasma exchange (TPE), the use of which has been expanding in recent years, is a nonspecific extracorporeal treatment method and is used in many immunological and toxicological diseases.

Objectives: The aim of this study is to determine demographic and clinical characteristics of critically ill patients in the intensive care unit (ICU) with TPE

Methods: : This study was performed prospectively in ICU. Patients in need of ICU, bigger than 18 years old and with TPE indication were included in the study.

Results: We enrolled 31 patients. A total of 207 sessions of TPE were performed. The mean age was $46 \pm 18$ years. The median APACHE II score was 20 (min:5- max:40). The most common reason for ICU admission were $56 \%$ microangiopathic hemolytic anemia (MAHA), 13\%
Crimean-Congo Hemorrhagic Fever (CCHF) and 10\% Guillain-Barre Syndrome, respectively. There were significant difference between survivors and non survivors APACHE II (survivors $17 \pm 7$, non survivors $25 \pm 9, p=0.02$ ) and GC score (survivors:12 (min:3-max:15), non survivors:8 (min:3-max:15), $\mathrm{p}=0.08$ ). Length of ICU stay was 12 (min-max: $1-86$ ) days. ICU mortality was $48 \%$.

Conclusion: As a result of this study, the most common TPE indication was determined as MAHA in the ICU. The mean age of patients undergoing TPE was lower than the general ICU patient profile. Nearly half of the patients died. Scoring systems such as APACHE II score and GKS maybe effective in determining the mortality of these patients.

\section{Reference(s) and grant ackowledgment(s)}

1. 2. Reeves HM, Winters JL. The mechanisms of action of plazma exchange. British Journal of Haematology, 2014, 164:342-351.

2. 1. Kaynar L, Altuntas F, Aydogdu I, et al. Therapeutic plasma exchange in patients with neurologic diseases: retrospective multicenter study. Transfus Apher Sci 2008;38: 109-15

000741

Incidence of Venous Thromboembolism in critically ill COVID-19 patients

S. Boyd'; E. O'connor ${ }^{2}$; D. Collins'; I. Martin-Loeches ${ }^{3}$

${ }^{1}$ Anaesthesia and intensive care medicine, St James hospital, Dublin, Ireland; ${ }^{2}$ Anaesthesia and intensive care, St James hospital, Dublin, Ireland; ${ }^{3}$ School of medicine, Trinity College Dublin, Dublin, Ireland

Correspondence: S. Boyd

Intensive Care Medicine Experimental 2020, 8(2): 000741

Introduction: COVID-19 is a novel Coronavirus that is currently responsible for the Global Pandemic of 2019-2020. It has been reported that up to $25 \%(1)$ of hospitalized patients with COVID-19 develop VTE (Venous Thromboembolism) and this figure can be as high as $31 \%$ in ICU patients with COVID-19(2). We aimed to establish theincidence rate of VTE in COVID-19 patients admitted to ICU compared to patients admitted with Seasonal Influenza and mechanically ventilated without both influenza and COVID-19.

Methods: We included 38 patients that were admitted to the ICU at SJH with COVID between the 31/03/2020 - 13/04/2020. We also included the last 38 patients that had been admitted to ICU with Seasonal Influenza, as a comparison group. These patients were admitted between 06/02/2016 - 22/03/2020, as there were fewer cases per year. We used a control group consisting of the last 37 patients that had been admitted to the ICU and required Mechanical Ventilation for greater than $48 \mathrm{hrs}$ for a non-respiratory reason.

Each patient's Electronic Patient Record was reviewed retrospectively. We recorded each patient's background and co-morbidities, whether they developed VTE or not and reviewed the radiological evidence. We also looked at each patient's Coagulation profile on admission and how they were anticoagulated in ICU. We used SPSS 26.0 to analyse the data.

Results: SAPSII scores and SOFA scores were significantly higher in patients without both influenza and COVID-19 compared to influenza and COVID-19 respectively (Table 1)The incidence rate of VTE in the COVID group was $13.2 \%$ (DVT 5.3\%, PE 10.5\%). The Rate of VTE in the Seasonal Influenza group was $15.8 \%$ (DVT 13.2\%, PE 2.6\%). The Rate of VTE in the Non-respiratory Group was 8.1\% (DVT 8.1\%, PE 2.7\%). There was no significant difference between the groups ( $p 0.5$ ).

All patients that went on to develop VTE had received prophylactic anticoagulation before diagnosis, and all received therapeutic anticoagulation thereafter. 


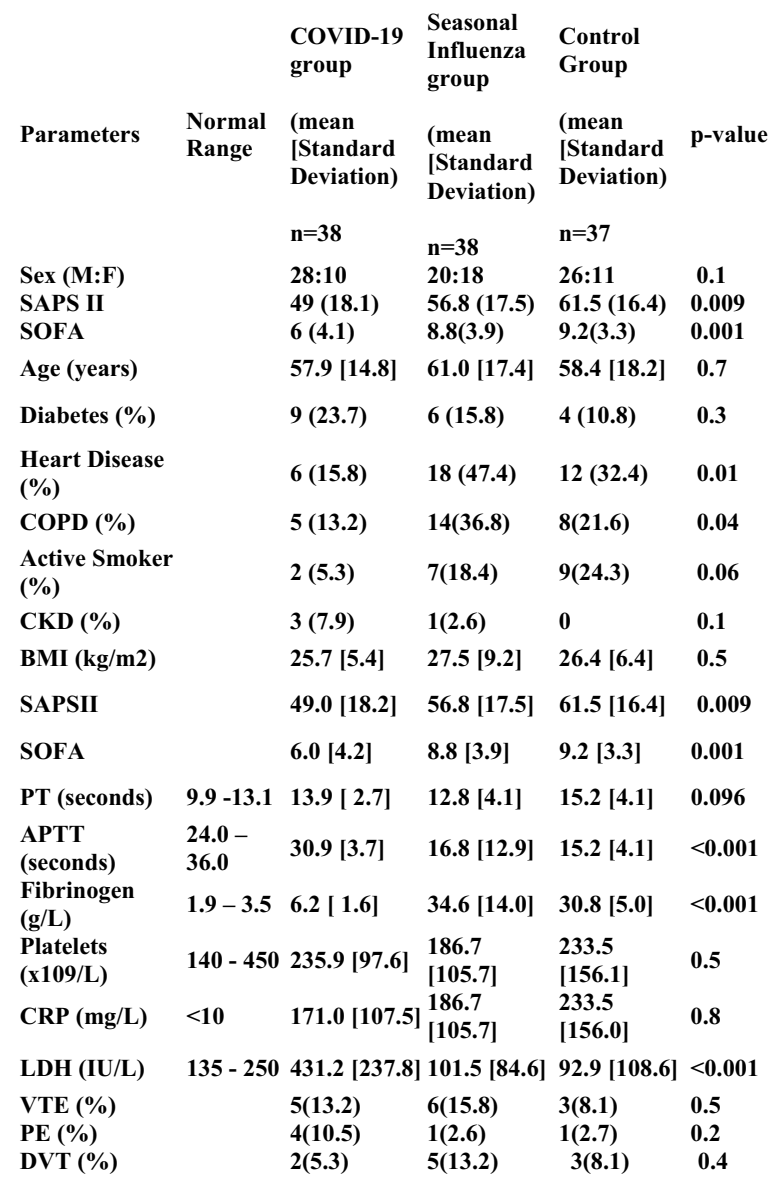

Conclusion: There was no significant difference in the rates of VTE between ICU patients with COVID-19 compared to Influenza, in our cohort. Yet, VTE still occurred despite prophylactic anticoagulation and remains a pertinent differential to keep in mind.

\section{Reference(s) and grant ackowledgment(s)}

1. 1. Yao Y, Cao J, Wang Q, Liu K, Luo Z, Yu K, et al. D-dimer as a biomarker for disease severity and mortality in COVID-19 patients: a case control study. Crit Care Emerg Med. 2020. Available from: https://doi.org/10.21203/ rs.3.rs-20850/v1.

2. 2. Klok FA, Kruip MJ, van der Meer NJ, Arbous MS, Gommers DA, Kant KM, et al. Incidence of thrombotic complications in critically ill ICU patients with COVID-19. Thromb Res. 2020 Apr:S0049-3848(20)30120-1.

3. We would like to thank all of the staff in ICU and the entire hospital throughout this difficult period.

\section{7}

COVID-19 is associated with catheter-related thrombosis in critically ill patients: a multicenter case-control study

JM. Smit'; JE. Lopez Matta ${ }^{2}$; R. Vink ${ }^{3}$; MCA. Müller ${ }^{4}$; KF. Choi ${ }^{1}$; FEHP. Van

Baarle; ; APJ. Vlaar'; FA. Klok ${ }^{5}$; MV. Huisman ${ }^{5}$; CV. Elzo Kraemer';

ARJ. Girbes ${ }^{1}$; DJ. Van Westerloo ${ }^{2}$; PR. Tuinman ${ }^{1}$

'Intensive Care, Amsterdam UMC, locatie VUmc, Amsterdam, Netherlands; ${ }^{2}$ Intensive care, Leiden University Medical Center (LUMC), Leiden, Netherlands; ${ }^{3}$ Intensive care, Tergooi - locatie Hilversum, Hilversum, Netherlands; ${ }^{4}$ Intensive care, Amsterdam UMC, locatie AMC, Amsterdam, Netherlands; ${ }^{5}$ Internal medicine, Leiden University Medical Center (LUMC), Leiden, Netherlands

Correspondence: J.M. Smit

Intensive Care Medicine Experimental 2020, 8(2): 000857
Introduction: Since its emergence in December 2019, the coronavirus disease 2019 (COVID-19) epidemic rapidly progressed into a pandemic. A noticeable observation in critically ill patients affected by COVID-19 pneumonia is the high incidence of thrombotic complications, especially that of pulmonary embolism. Importantly, the majority of patients admitted to the ICU receive a central venous catheter of which a considerable proportion develops catheter-related thrombosis. When considering COVID-19 coagulopathy to cause an overall hypercoagulable state rather than mainly a local pro-thrombotic state in the pulmonary circulation, the prevalence of catheter-related thrombosis is presumably higher in COVID-19 patients than in nonCOVID-19 patients with a central venous catheter.

Objectives: The aim of this study was to investigate whether COVID19 is associated with catheter-related thrombosis.

Methods: This was a multicenter case-control study conducted at four intensive care units. Adult critically ill patients with an indwelling central venous catheter of the internal jugular, subclavian or femoral vein were included. Patients were excluded if catheter indwellingtime was less than 48 hours. All patients underwent a compression and duplex ultrasound examination of the cannulated vein. Cases and controls were patients with and without catheter-related thrombosis, respectively. Primary outcome was the association of COVID-19 with catheter-related thrombosis. Patients were considered to have COVID19 pneumonia if they had a positive polymerase chain reaction assay for SARS-CoV-2 and had corresponding abnormalities on thoracic imaging.

Results: Ultrasound examination was performed in 87 intensive care unit patients. Catheter indwelling-time was less than two days or was unknown in five patients. A total of 82 patients were included in the analysis, of whom 18 were diagnosed with catheter-related thrombosis $(22 \%)$. The majority was male $(80.5 \%)$ and median age was 63.0 [IQR: $54.5,70.0]$ years. The overall prevalence of COVID-19 was $76 \%$ $(62 / 82)$ and $94 \%(17 / 18)$ among the cases. The crude odds ratio for catheter-related thrombosis in patients with COVID-19 versus controls was 7.2 (95\% Cl: 1.32, 130). The odds ratio for catheter-related thrombosis adjusted for anticoagulation dosage and catheter indwellingtime in critically ill COVID-19 patients was 18.3 (95\% Cl: $2.31,410)$.

Conclusion: COVID-19 is highly associated with catheter-related thrombosis, suggesting a diffuse hypercoagulable state rather than mainly a local pro-thrombotic state in the pulmonary circulation leading to pulmonary immunothrombosis.

\section{Reference(s) and grant ackowledgment(s)}

1. Levi M, Thachil J, Iba T, Levy JH. Coagulation abnormalities and thrombosis in patients with COVID-19. Lancet Haematol. Published online May 2020:S2352302620301459. doi:10.1016/S2352-3026(20)30145-9

2. Helms J, Tacquard C, Severac F, et al. High risk of thrombosis in patients with severe SARS-CoV-2 infection: a multicenter prospective cohort study. Intensive Care Med. Published online May 4, 2020. doi:10.1007/ s00134-020-06062-x

3. Timsit J-F, Farkas J-C, Boyer J-M, et al. Central Vein Catheter-Related Thrombosis in Intensive Care Patients: Incidence, Risks Factors, and Relationship With Catheter-Related Sepsis. Chest. 1998;114(1):207-213. doi:10.1378/ chest.114.1.207

4. Klok FA, Kruip MJHA, van der Meer NJM, et al. Incidence of thrombotic complications in critically ill ICU patients with COVID-19. Thromb Res. 2020;191:145-147. doi:10.1016/j.thromres.2020.04.013

5. No funding was received for this study

\section{2}

\section{Platelet function and kinetics during CPB}

M. Törnudd'; ;. Alfredsson²; JP. Kvitting ${ }^{3}$; S. Ramström ${ }^{4}$; S. Berg ${ }^{5}$

${ }^{1}$ Department of Cardiothoracic and Vascular Surgery and Department of Medical and Health Sciences, University hospital, Linköping, Sweden; ${ }^{2}$ Department of cardiology and department of medical and health sciences, University hospital, Linköping, Sweden; ${ }^{3}$ Department of cardiothoracic surgery, Oslo University Hospital, Oslo, Norway; ${ }_{i}^{4}$ Cardiovascular research centre, school of medical sciences, Örebro University, Örebro, Sweden; ${ }^{5}$ Department of cardiothoracic and vascular and department 
of medical and health sciences, Linkoping University Hospital, Linköping, Sweden

Correspondence: M. Törnudd

Intensive Care Medicine Experimental 2020, 8(2): 000862

Introduction: The use of cardio-pulmonary bypass (CPB) during cardiac surgery is associated with bleeding complications. Alterations in platelet function has bearing on the risk of bleeding. The effect of CPB on the actual number and function of platelets is not fully understood. Objectives: The aim of this study was to analyse the kinetics and function of platelets during and immediately after weaning from $C P B$.

Methods: Thirty-nine elective coronary artery bypass grafting (CABG) patients were studied with impedance aggregometry (Multiplate) and blood count. Samples were taken before anaesthetic induction (Baseline), 30 minutes after start of CPB (22 of the patients) (CPB30), after weaning from CPB before protamine administration (EndCPB) and five minutes after protamine (After Protamine). Adenosine diphosphate (ADP) was used as activator and aggregation was expressed as the area under the curve (AUC). The number of platelets was corrected for haemodilution by using haemoglobin as marker and expressed as number of platelets $\left(\times 10^{9} / \mathrm{L}\right)$ divided by haemoglobin concentration $(\mathrm{g} / \mathrm{L})$. Statistics used were paired t-test followed by Bonferroni correction for multiple comparisons. Data are expressed as mean \pm standard deviation.

Results: Mean CPB time was $85.1 \pm 21.3$ minutes (range 46-138 minutes). The number of platelets corrected for dilution was not reduced during CPB. Mean number of platelets divided by concentration of haemoglobin was at baseline $1.7 \pm 0.4 * 10^{9} / \mathrm{g}$. At $30 \mathrm{~min}$ of CPB it was almost identical at $1.7 \pm 0.3 * 10^{9} / \mathrm{g}$ and after weaning from CPB increased to $1.9 \pm 0.4 * 10 \% \mathrm{~g}(\mathrm{p}<0.05)$. Five minutes after protamine it decreased to $1.7 \pm 0.5 * 10^{9} / \mathrm{g}$ compared to before protamine $(p<0.001)$. With impedance aggregometry, mean AUC for platelets activated with ADP was $77.1 \pm 26.3$ at baseline. After 30 minutes of CPB, AUC was reduced to $49.3 \pm 24.7(p<0.001)$. After weaning from $C P B$, mean AUC had increased to $68.5 \pm 28.4$. Five minutes after protamine, AUC decreased to $58.9 \pm 29.3$ ( $p<0.05$ compared to before protamine).

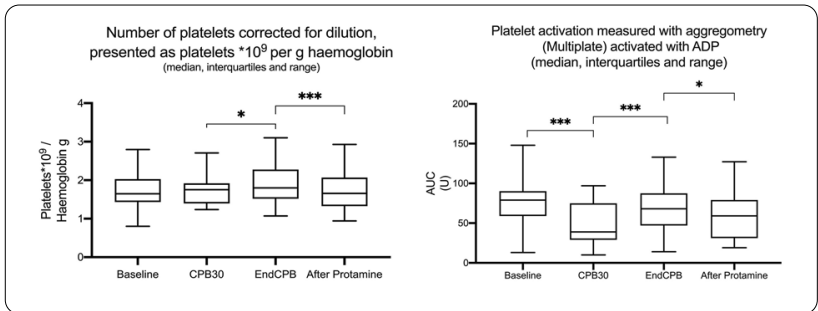

Conclusion: Platelet numbers corrected for dilution were not decreased and platelets were not consumed during CPB. Initially during CPB platelet aggregability was reduced but returned to baseline levels at the end of CPB. After administration of protamine platelets were consumed as expected. The reversal of heparin with protamine seems however to impair platelet function. These effects of CPB and protamine on platelet function can be of significance for the bleeding problems seen in cardiac surgery.

\section{4}

Targeted anticoagulation in Covid 19 patients based on $\mathrm{d}$ dimers: a retrospective study of Anti Xa versus APTTr monitoring in patients on systemic heparin infusions to establish superiority and patient outcomes

K. Bhalsod'; ;. Husselbee ; R. Matthews ${ }^{1}$; R. Carroll' ;. Stumpfle ${ }^{1}$

${ }^{1}$ Intensive care, Hammersmith Hospital, London, United Kingdom

Correspondence: $\mathrm{K}$. Bhalsod

Intensive Care Medicine Experimental 2020, 8(2): 000924
Introduction: COVID-19 patients with elevated d dimers have a higher mortality rate(1), which is reduced when treated with heparin(2), resulting in its recommendation for hospitalised patients.(3) However the type and dose of heparin and heparin infusion monitoring (Anti Xa versus APTTr (Activated Partial Thromboplastin Time Ratio)) remains under discussion $(3,4,5)$.

Methods: We undertook a single centre, retrospective observational service evaluation examining COVID-19 positive critical care admissions receiving heparin infusions (based on d dimer $>3000 \mathrm{ng} / \mathrm{ml}$ ). Two groups of patients were identified: the first group had heparin monitoring via APTTr whilst the second by Anti Xa. We compared the two groups over 2 separate 10 day periods, assessing whether either method of heparin monitoring would affect the following endpoints:

1. The mean rate of heparin infusion

2. Number of monitoring blood tests sent (Anti Xa assay or APTTr)

3. Number of infusion changes made, as a surrogate marker for nursing interventions

4. The mean Anti Xa and APTTr values as a surrogate marker for adequate anticoagulation

5. Clotting and bleeding episodes.

\section{Results:}

Our sample groups comprised 18 patients monitored with APTTR and 21 patients monitored with Anti Xa. The table below describes results obtained.

Table 1: To compare Anti Xa vs APTTr monitoring in 2 separate defined 10 day periods

\begin{tabular}{|c|c|c|c|}
\hline Variable & Anti Xa group & APTTr group & P-Value * \\
\hline $\begin{array}{l}\text { Total number of } \\
\text { patients }\end{array}$ & 21 & 18 & $t^{2}$ \\
\hline $\begin{array}{l}\text { Mean heparin } \\
\text { infusion rate } \\
(\mathrm{U} / \mathrm{kg} / \mathrm{hr})\end{array}$ & 18.46 & 16.31 & 0.09 \\
\hline $\begin{array}{l}\text { Mean number of } \\
\text { monitoring blood } \\
\text { tests per patient per } \\
24 \mathrm{hrs}(\boldsymbol{S D})\end{array}$ & $\begin{array}{l}1.67 \\
( \pm 0.66)\end{array}$ & $\begin{array}{l}1.88 \\
( \pm 0.78)\end{array}$ & 0.38 \\
\hline $\begin{array}{l}\text { Mean number of } \\
\text { heparin infusion } \\
\text { adjustments per } \\
\text { patient per } 24 \mathrm{hrs}(S D)\end{array}$ & $\begin{array}{l}0.68 \\
( \pm 0.39)\end{array}$ & $\begin{array}{l}0.79 \\
( \pm 0.36)\end{array}$ & 0.37 \\
\hline Mean test** value & 0.41 (Target 0.3- & 2.00 (Target $1.5-$ & {[} \\
\hline$(S D)$ & $\begin{array}{l}0.7) \\
( \pm 0.17)\end{array}$ & $\begin{array}{l}2.5) \\
( \pm 0.42)\end{array}$ & \\
\hline $\begin{array}{l}\text { Total number of } \\
\text { patients in each group } \\
\text { on RRT (CVVHDF) }\end{array}$ & 12 & 15 & - \\
\hline $\begin{array}{l}\text { Mean number of RRT } \\
\text { filter changes per } \\
24 \mathrm{hrs}(S D)\end{array}$ & $\begin{array}{l}0.15 \\
( \pm 0.24)\end{array}$ & $\begin{array}{l}0.26 \\
( \pm 0.32)\end{array}$ & 0.34 \\
\hline $\begin{array}{l}\text { Mean RRT filter } \\
\text { lifespan (hrs) }(S D)\end{array}$ & $\begin{array}{l}72.2 \\
( \pm 37.1)\end{array}$ & $\begin{array}{l}70.7 \\
( \pm 36.9)\end{array}$ & 0.91 \\
\hline $\begin{array}{l}\text { Total number of VTE } \\
* * *\end{array}$ & 1 & 2 & 0.47 \\
\hline $\begin{array}{l}\text { Total number of } \\
\text { bleeding } \\
\text { complications } * * *\end{array}$ & 2 & 0 & 0.19 \\
\hline
\end{tabular}

Conclusion: Overall, Anti Xa monitoring is at least comparable to APTTr monitoring in critically unwell COVID-19 patients. Our data suggests that Anti Xa monitoring may be more favourable (improved filter lifespan, less labour intensive and less blood test monitoring), however this needs further investigation.

Heparin remains the mainstay of treatment of covid-19 patients and given the ongoing pandemic, urgent studies are required to establish the optimum method of heparin monitoring in critical care patients.

Reference(s) and grant ackowledgment(s)

1. 5. Connors I \& Levy J. COVID-19 and its implications for thrombosis and anticoagulation. Blood. 2020;135(23):2033-40

2. 4. Thachil J. The versatile heparin in COVID-19. J Thromb Haemost. 2020;18:1020-22. 
3. 3. Kollias A et al. Thromboembolic risk and anticoagulant therapy in COVID-19 patients: emerging evidence and call for action. British Journal of Haematology. 2020;189:846-7

4. 2. Tang, $\mathrm{N}$ et al. Anticoagulation treatment is associated with decreased mortality in severe coronavirus disease 2019 patients with coagulopathy. Thromb Haemost. 2020;18:1094-99.

5. 1. Zhou F et al. Clinical course and risk factors for mortality of adult inpatients with COVID-19 in Wuhan, China: a retrospective cohort study. The Lancet. 2020;395:1054-62.

\section{2}

Acute pulmonary embolism in critically ill COVID-19 patients

F. Ricottilli ${ }^{1}$; PA. Gevenois²; L. Peluso ${ }^{1}$; Z. Pletschette ; O. Lheureux ${ }^{1}$; A. Brasseur ${ }^{1}$; A. Garufi ${ }^{1}$; M. Talamonti ${ }^{1}$; S. Motte ${ }^{3}$; L. Nobile ${ }^{1}$; D. Grimaldi ${ }^{1}$; J. Creteur ${ }^{1}$; JL. Vincent ${ }^{1}$; FS. Taccone ${ }^{1}$

${ }^{1}$ Department of intensive care, Hopital Erasme, Université Libre de Bruxelles (ULB), Bruxelles, Belgium; ${ }^{2}$ Department of radiology, Hopital Erasme, Université Libre de Bruxelles (ULB), Bruxelles, Belgium; ${ }^{3}$ Department of vascular medicine, Hopital Erasme, Université Libre de Bruxelles (ULB), Bruxelles, Belgium

Correspondence: F. Ricottilli

Intensive Care Medicine Experimental 2020, 8(2): 001062

Introduction: COVID-19 patientstypically have a prothrombotic status and acute pulmonary embolism (PE) may be a cause of persistent hypoxemia or sudden death. However, the incidence of PE has not been well reported.

Methods: Prospective institutional database of critically ill adult mechanically ventilated COVID-19 patients, conducted between March 10 and April 20, 2020. Patients were eligible if they underwent a computed tomography pulmonary scanner angiography (CTPA), as part of the routine management in case of persistent hypoxemia or respiratory deterioration. The primary endpoint of this study was the occurrence of $P E$; the impact of standard thromboprophylaxis (i.e. subcutaneous enoxaparin $4000 \mathrm{IU}$ once daily) or high-regimen thromboprophylaxis (i.e. subcutaneous enoxaparin 4000 IU twice daily or therapeutic unfractioned heparin) on PE occurrence was also analyzed.

Results: Of 42 mechanically ventilated COVID-19, 36 underwent CTPA after a median of 7 [4-9] days since ICU admission and 13 [10-16] days since the onset of symptoms. Twelve $(33 \%)$ patients were diagnosed of $P E$, which was bilateral in 5 patients and localized in the right lung in 7 patients. Ten of the patients with PE (83\%) and 11 of the other 24 patients (46\%) received standard thromboprophylaxis (OR 3.57 [1.0913.47]; $p=0.04)$. $D$-dimers on the day of CTPA had a predictive accuracy of 0.90 (95\% Cls $0.78-1.00)$ for APE. Two patients with PE (17\%) and 10 patients without PE (43\%) died at ICU discharge (OR 0.28 [0.051.35]; $p=0.26)$.

Conclusion: One third of COVID-19 mechanically ventilated patients have a pulmonary embolism visible on CTPA. Optimal diagnostic and prophylaxis strategies need to be further assessed.

\section{4}

Comparison of injury patterns among cyclists with alcohol consumption

D. Jeong ${ }^{1}$; T. Kim ${ }^{1}$

${ }^{1}$ emergency, Samsung Medical Center, Irwon-ro, Irwon-dong, Gangnam-gu, Seoul, South Korea, Seoul, Republic of Korea

Correspondence: $\mathrm{D}$. Jeong

Intensive Care Medicine Experimental 2020, 8(2): 000084

Introduction: As the cycling population grows, lack of public awareness of cycling while under the influence of alcohol has become an issue. The purpose of this study was to investigate the association between alcohol consumption and bicycle-related injuries such as traumatic brain injuries and EMR-ISS.

Methods: We conducted a retrospective analysis using data collected from the Korean Emergency Department-based Injury In-depth
Surveillance (EDIIS) database from 20 emergency departments during 2011-2016. Study subjects had sustained bicycle-related injuries at the age of over 18 years. Covariates included mechanism, place, and time of injury. Outcomes were traumatic brain injury (TBI) incidence and severe trauma EMR-ISS ${ }^{3} 25$. Effects of alcohol consumption on these outcomes were analyzed, and differences in effects were determined using logistic regression.

Results: Of 24,297 individuals, 1,912 had alcohol-related bicycle injuries, which led to a higher proportion of single-vehicle injury incidents (alcohol $63.7 \%$ vs. non-alcohol $46.4 \%, p<0.001$ ). The alcohol group had a higher rate of TBI (alcohol 11.5\% vs. non-alcohol $4.6 \%, p<0.001$ ) and severe EMR-ISS (alcohol $23.1 \%$ vs. non-alcohol $11.7 \%, p<0.001$ ). TBI (odds ratio [OR], 2.72; 95\% confidence interval [CI], 2.33-3.16) and EMR-ISS (OR, 2.26; 95\% Cl, 2.01-2.53) showed a significant association with alcohol. Head and neck injuries were higher in the alcohol group (alcohol 73.7\% vs. non-alcohol 40.0\%, $p<0.001$ ).

Conclusion: Our study showed an association of alcohol consumption with higher incidence of traumatic brain injuries and severe EMRISS. Education should focus more on the association between cycling under alcohol influence and injury severity.

\section{Reference(s) and grant ackowledgment(s)}

1. No potential conflict of interest relevant to this article was reported. No financial disclosures were reported by the authors of this paper.

2. Airaksinen N, Luthje P, Nurmi-Luthje I. Cyclist Injuries Treated in Emergency Department (ED): Consequences and Costs in South-eastern Finland in an Area of 100000 Inhabitants. Annals of advances in automotive medicine Association for the Advancement of Automotive Medicine Annual Scientific Conference. 2010;54:267-74

3. Li G, Baker SP, Smialek JE, Soderstrom CA. Use of alcohol as a risk factor for bicycling injury. Jama. 2001;285(7):893-6.

4. Crocker P, Zad O, Milling T, Lawson KA. Alcohol, bicycling, and head and brain injury: a study of impaired cyclists' riding patterns R1. The American journal of emergency medicine. 2010;28(1):68-72.

5. Kim T, Jung KY, Kim K, Yoon H, Hwang SY, Shin TG, et al. Protective effects of helmets on bicycle-related injuries in elderly individuals. Injury prevention : journal of the International Society for Child and Adolescent Injury Prevention. 2018.

6. Hwang SH, Ahn MR, Han KD, Lee JH. The Prevalence of "Drinking and Biking" and Associated Risk Factors: The Korea National Health and Nutrition Examination Survey. Journal of Korean medical science. 2017;32(9):1396-400.

7. Kim YJ, Seo DW, Lee JH, Lee YS, Oh BJ, Lim KS, et al. Trends in the incidence and outcomes of bicycle-related injury in the emergency department: A nationwide population-based study in South Korea, 2012-2014. PloS one. 2017;12(7):e0181362.

8. Shah S, Sinclair SA, Smith GA, Xiang H. Pediatric hospitalizations for bicycle-related injuries. Injury prevention : journal of the International Society for Child and Adolescent Injury Prevention. 2007;13(5):316-21.

9. Ministry of the Interior and Safety, 2017 Bicycle usage. 2018. https://www.mois.go.kr/synap/popup/synapFileViewOpen. do?atchFileld=FILE_00081790_y7NgVI\&fileSn=0 (accessed 13 Nov 2019).

10. Institute TKT. Report of cycling infrastructure by local government, 2014. 2015

11. Orsi C, Ferraro OE, Montomoli C, Otte D, Morandi A. Alcohol consumption, helmet use and head trauma in cycling collisions in Germany. Accident; analysis and prevention. 2014;65:97-104.

\section{2}

Consideration of usage criteria of fibrinogen concentrate for severe traumatic brain injury

G. Fujiwara

${ }^{1}$ Neurosurgery, Kyoto Prefectural University of Medicine - Kawaramachi

Campus, Kyoto, Japan

Correspondence: G. Fujiwara

Intensive Care Medicine Experimental 2020, 8(2): 000102 
Introduction: Traumatic coagulopathy is often observed in patients with severe traumatic brain injury (sTBI). The association between TBI and coagulopathy is well established. Earlier transfusion with high blood product ratios is recommended for severely injured patients, and fibrinogen is known as one of the most important coagulation factor in the trauma field. Fibrinogen concentrate (FC) is widely used for severe trauma patient and its effectiveness is gradually recognized. However, FC for STBI is less reported and usage criteria is not established. We started to use FC since 2019 after approval of clinical ethics committee and aggressively administer to multiple trauma patients. We aimed to identify predicting factors of fibrinogen declining in order to set the criteria of FC usage for sTBI.

Methods: We retrospectively reviewed successive adults STBI in our hospital between January 2017 and December 2019. We compared factors between two groups (high fibrinogen group: fibrinogen value is less than $150 \mathrm{mg} / \mathrm{dL} 3-6$ hours after arrival, and low fibrinogen group: fibrinogen value is $150 \mathrm{mg} / \mathrm{dL}$ or more $3-6$ hours after arrival). We researched vital signs, labolatory data, findings of computed tomography $(\mathrm{CT})$ and analyze predictingfactor.

Results: 50 patients were enrolled. 35 patients archieved fibrinogenvalue 150 or more, and 15 patients did not. D-dimer on arrival above $50 \mu \mathrm{g} / \mathrm{mL}$, skull fracture and depressive skull fracture were strong predicting factors of low fibrinogen group.

Conclusion: Low fibrinogen group should be administered FC. Some labolatory data of Coagulation abnormality demonstrated low fibrinogen as reported. Likewise, skull fracture and especially depressive skull fracture reflect severe coagulopathy in this study and patients without skull fracture is enough treated by using only fresh frozen plasma. Skull fracture has possitility of provoking tissue factors and predicting sevrere coagulopathy.

\section{4}

\section{Risk factors for pulmonary embolism among patients} with trauma: a nested case-control study in Japan

H. Iriyama ${ }^{1}$; T. Abe ${ }^{2}$; A. Komori' ;. Kainoh'; Y. Kondo ${ }^{3}$; T. Naito

'Department of general medicine, Juntendo University, 2 Chome-1-1 Hongo, Bunkyo City, Tokyo, Japan, Bunkyo City, Japan; ${ }^{2}$ Health services research and development center, University of Tsukuba, Tsukuba, Japan; ${ }^{3}$ Department of emergency and critical care medicine, Juntendo University Urayasu Hospital, Urayasu, Japan

\section{Correspondence: $\mathrm{H}$. Iriyama}

Intensive Care Medicine Experimental 2020, 8(2): 000254

Introduction: Pulmonary embolism (PE) is a major complication and crucial disease in patients with trauma. Previous studies have been scarce to investigate risk factors for PE in patients with trauma.

Objectives: Our aim was to investigate risk factors for PE among patients with trauma.

Methods: This is a nested case-control study using the Japan Trauma Data Bank (JTDB) from 2004 to 2017. The JTDB is a nationwide trauma registry in Japan. We included patients who had blunt or penetrate trauma; aged $\geq 16$ years old; had Injury Severity Scores (ISSs) $\geq 9$; and survived for more than 2 days after hospital admission. A nested case-control study (1:5) was conducted using propensity score (PS) matching. The variables included in the PS matching were age, gender, mechanism of injury, vital signs at emergency department, ISSs, and ward type. Then, we conducted a conditional logistic regression analysis to identify risk factors for PE in the matched cohort. The primary outcome was occurrence of PE during hospital stay. The explanatory variables were selected based on prior studies and univariate analysis, such as comorbidities, abbreviated injury scale (AIS), transfusion, central vein catheter placement, and surgical procedures.

Results: Of those who were registered in the JTDB database, 719 patients with PE and 3,595 patients without PE were selected by PS matching. The median age was 73 (interquartile range (IQR): 55-84), and $57 \%$ was female. The mean ISSs was 10 (IQR: 9-19). Patients with $\mathrm{PE}$ had upper and lower extremity open fracture more frequently than those without PE (7.9 vs. $4.6 \%$ [p <0.001], 27.7 vs. $7.7 \%$ [p <0.001], respectively). Patients with PE had central vein catheter placement and surgical procedure more frequently than those without PE (7.0 vs. $2.9 \%$ [p $<0.001], 86.6$ vs. $57.1 \%$ [p $<0.001$ ], respectively). In-hospital mortality was higher in patients with PE than in those without PE (5.8 vs. $3.4 \%[p=0.003]$ )

In the multivariate logistic regression model, lower extremity open fracture (odds ratio (OR) [95\% confidence interval (CI)], 3.42 [2.65$4.41])$, central vein catheter placement (OR [95\% Cl], 2.41 [1.55-3.73]), and surgical procedure $(\mathrm{OR}[95 \% \mathrm{Cl}, 5.34$ [4.04-7.06]) were independently associated with PE.

Conclusion: Patients with trauma complicated with PE had high inhospital mortality. Lower extremity open fracture, central vein catheter placement, and surgical procedures could be risk factors for PE among patients with trauma.

\section{5}

Assessment of Gut Barrier Functions in Intensive Care Unit With Critical Trauma Patients: A pilot study

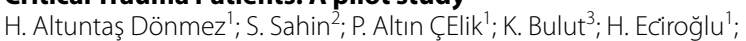

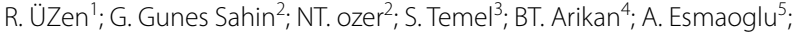
M. Sungur ${ }^{6} ;$ K. Gundogan ${ }^{7}$

${ }^{1}$ Medical biology, Erciyes University, Kayseri, Turkey: ${ }^{2}$ Deparment of health sciences institute, division of clinical nutrition, Erciyes University, Kayseri, Turkey; ${ }^{3}$ nternal medicine, Erciyes University, Kayseri, Turkey; ${ }^{4}$ General surgery, Erciyes University, Kayseri, Turkey; ${ }^{5}$ Department of anesthesiology and reanimation, Erciyes University, School of medicine, Kayseri, Turkey; ${ }^{6}$ Division of critical care medicine, department of internal medicine, Erciyes University School of Medicine, Kayseri, Turkey, Kayseri, Turkey; ${ }^{7}$ Department of internal medicine, division of intensive care, Erciyes University, School of medicine, Kayseri, Turkey

Correspondence: S. Sahin

Intensive Care Medicine Experimental 2020, 8(2): 000335

Introduction: In critically ill patients after trauma, the intestine can remain a vulnerable organ. Gastrointestinal system dysfunction is common in these patients. Intestinal damage occurs in about $50 \%$ of critical patients.

Objectives: In this study, it was aimed to investigate the intestinal barrier functions in adult critical patients who were followed up in the intensive care unit (ICU) due to trauma.

Methods: This study was performed prospectively. The study included 29 critical patients bigger than 18 age who were admitted to the ICU for 5 days or more due to trauma, and 18 healthy people of similar age and gender as the control group. Claudin, occludin, tricellulin and junctional adhesion molecule 1 (JAM-1) levels in the protein structure of the blood samples taken from patients to evaluate the intestinal barrier functions were measured using ELISA kits.

Results: The median age of patients was 35 (min:18- max:66) years. The GCS of the patients was 11 (min:3-max:15), and the APACHE II score was $11 \pm 1$. The most common reason for ICU admission was traffic accidents (75\%). A statistically significant difference was found between the baseline, 2nd day, 5th day, and 10th day plasma levels between patients with claudin, occludin and tricellulin levels $(p<0.001$, $\mathrm{p}<0.001, \mathrm{p}<0.05$ respectively). An increase in claudin and occludin levels was found in patients' baseline plasma samples compared to controls $(p<0.01)$. This increase in plasma claudin levels of patients decreased gradually on the 2 nd day, 5 th day and 10 th day $(p<0.01)$. However, plasma occludin levels of patients increased on the 2 nd and 5 th days $(p<0.01)$ then decreased on the 10th day $(p<0.01)$. The plasma tricellulin levels of the patients increased on the $2 \mathrm{nd}$ and 5 th days compared to baseline ( $p<0.01$ and $p<0.05$, respectively), then decreased on the 10th day $(p<0.05)$. Length of ICU stay was 15 (min:5max:39) days. ICU mortality was $13 \%$.

Conclusion: As a result, it was determined that gut barrier functions were impaired in adult critical patients who were followed up in the ICU due to trauma. We can suggest that claudin and occludin protein levels can be used as markers in these patients. 
Reference(s) and grant ackowledgment(s)

1. Li H, Chen Y, Huo F, Wang Y, Zhang D. Association between acute gastrointestinal injury and biomarkers of intestinal barrier function in critically ill patients, BMC Gastroenterol.; 17: 45. (2017).

2. Btaiche, I. F., Chan, L. N., Pleva, M., \& Kraft, M. D. (2010). Critical illness, gastrointestinal complications, and medication therapy during enteral feeding in critically ill adult patients. Nutrition in Clinical Practice, 25(1), 32-49.

3. Erciyes University Scientific Research Committee TOA-2019-8633

\section{0}

Dynamic effect of age and Systolic Blood Pressure on severe trauma patients prognosis

C. A. Francois-talpaert ${ }^{1}$;A. Talpaert ${ }^{2}$; E. Achtade Ach $^{3}$ M. Raux ${ }^{3}$

${ }^{1}$ Anesthesiology and critical care department, C.H.U. Charleroi ISPCC, Charleroi, Belgium; ${ }^{2}$ Data science, $X$, Versailles, France; ${ }^{3}$ Département d'anesthésie réanimation, AP-HP, Groupe Hospitalier Universitaire APHP-Sorbonne Université, site Pitié-Salpêtrière, Paris, France

Correspondence: C.A. FRANCOIS-TALPAERT

Intensive Care Medicine Experimental 2020, 8(2): 000350

Introduction: Systolic arterial blood pressure (SBP) increases with age. We aimed at testing the combination of SBP and age on mortality after trauma.

Methods: This retrospective Real World Evidence (RWE) monocentric study included all adult patients from a French level- 1 trauma center. A Generalized Additive Model (GAM) was created to predict 30 daymortality based on age and SBP. The gray zone technique served to determine SBP thresholds. Subgroup analysis focused on Injury Severity Score (ISS).

Results: Among 2655 patients, 717 (27\%) were older than 50 yo. Median ISS was 13 [6 - 25], average mortality was $11.5 \%$ [10.3\% $12.7 \%$ ]. Older patients had higher ISS, higher mortality ( $22 \%$ vs. $7 \%$, $p<0.001)$; more so when ISS $\geq 26$. Threshold effects on mortality appeared as SBP $\leq 90 \mathrm{mmHg}$, but none specific to ISS subsets. Risk appeared as SBP $\geq 130 \mathrm{mmHg}$, increased when combined with ISS $\geq 26$, reduced with ISS $\leq 15$. Computation led to no SBP threshold depending on age; in particular not $110 \mathrm{mmHg}$ for 50 yo.

Conclusion: We confirmed the threshold of SBP below $90 \mathrm{mmHg}$, and effects above $130 \mathrm{mmHg}$ with severe ISS. Older age and lower SBP combined increased mortality. Computation did not prove $110 \mathrm{mmHg}$ should be used as a threshold for patients older than 50 yo.

Reference(s) and grant ackowledgment(s)

1. none

\section{3}

Impact of frailty on traumatic brain injury outcome. Data

\section{from CENTER-TB}

G. Palio ${ }^{1}$; G. Isernia²; F. Graziano ${ }^{3}$; C. Robba ${ }^{4}$; G. Belelli; MG. Valsecchi ${ }^{3}$;

S. Galimberti ${ }^{6}$; G. Citerio ${ }^{7}$

${ }^{1}$ Department of intensive care, clinica santa isabel, University of Buenos Aires, Capital Federal, Argentina; ${ }^{2}$ School of medicine and surgery, University of Milano - Bicocca, Milan, Italy; ${ }^{3}$ Bicocca bioinformatics biostatistics and bioimaging center b4, School of Medicine and Surgery, University of Milano - Bicocca, Milan, Italy; ${ }^{4}$ Department of anaesthesia and intensive care, IRCCS AOU San Martino, Genova, Italy; ${ }^{5}$ Acute geriatric unit, asst-monza, University of Milano - Bicocca, Monza, Italy; ${ }^{6}$ Bicocca bioinformatics biostatistics and bioimaging center b4, School of Medicine and Surgery, University of Milano - Bicocca, Milano, Italy; ${ }^{7}$ School of medicine and surgery, University of Milano-Bicocca, Milano, Italy

Correspondence: $G$. Palio

Intensive Care Medicine Experimental 2020, 8(2): 000363

Introduction: Frailty can be defined as a multidimensional syndrome characterized by organic and functional impairments associated with a decrease in physiological reserves. Current evidence suggests that frailty is associated with unfavourable outcome among the geriatric population exposed to a stressor event. No information is available about the impact of frailty on long term outcomes in the subpopulation of TBI patients.

Objectives: The aim of our study is to create a reliable index to describe frailty among patients admitted to ICU with a diagnosis of TBI and to assess its association with the 6 months neurological outcome expressed in terms of Extended Glasgow Outcome Scale (GOSE<5) and mortality at 6 months.

Methods: The study was conducted using the data extracted from the CENTER-TBI database v2.1 with Neurobot v2.6. Patients' information were collected at ICU admission and used to build a frailty index (FI) as a compound of accumulated deficits. Thirty-five variables assessing health status at baseline were used to develop the FI. These features included chronic medical conditions, previous TBI, laboratory findings, number and type of drugs, alcohol or toxic agents abuse. For any subject, the FI was calculated as the sum of the reported deficits divided by the totality of evaluated items, expressed in percentage; $\mathrm{FI}(\%)$ is related to an incremental factor of $\cong 2$ points.

A Logistic regression model was performed to estimate the contribution of the FI to explain the 6-month outcomes and it was adjusted for the IMPACT core variables (i.e. age, pupillary reactivity and GCS motor). The model was also performed on the group of elderly subjects ( $\geq 65$ years old).

Results: 1754 participants were included in this study $(n=425$ subjects $\geq 65$ years old). The median age was 49 [IQR: 29; 65.75] and sex prevalence of males (72.6\%). The median baseline value of the FI overall was 8.82 (I-III quartiles 3.44-14.71; range 0.00 to 54.54) and 14.82 (I-III quartiles 9.09-23.33; range 0.00 to 42.8 ) considering the subset of elderly subjects.

Frailty significantly increased the risk of unfavourable GOSE at 6 months, with an odds ratio (OR) of 1.03 (95\% Cl 1.01-1.04, p-value $<0.001)$ and also the risk of mortality, with an OR of $1.004(95 \% \mathrm{Cl}$ $1.002-1.006, p$-value $=0.001)$. Considering only elderly subjects, there was no evidence that frailty increased the risk of unfavourable outcome $(O R=1.03,95 \% \mathrm{Cl}=0.99-1.08, p$-value $=0.204)$ and mortality $(\mathrm{OR}=1.001,95 \% \mathrm{Cl}=0.997-1.006, \mathrm{p}$-value $=0.541)$.

Conclusion: In this study we developed a FI for the specific application on TBI patients. This index was built from data collected from a large cohort of TBI patients characterized by a wide age spectrum. Our results indicated a significant increased risk of unfavourable neurologic outcomes in patients with high $\mathrm{FI}$ score, regardless of age and other IMPACT variables.

\section{Reference(s) and grant ackowledgment(s)}

1. CENTER-TBI (clinicaltrials.gov NCT02210221) was supported by the European Union 7th Framework program (EC grant 602150).

2. Searle, S.D., Mitnitski, A., Gahbauer, E.A. et al. A standard procedure for creating a frailty index. BMC Geriatr 8, 24 (2008). https://doi. org/10.1186/1471-2318-8-24

\section{6}

Efficacy and Safety of Tranexamic Acid in Acute Traumatic Brain Injury: A Systematic Review and Meta-analysis of Randomized Controlled Trials

K. Al Lawati ${ }^{1}$; S. Sharif'; S. Al Maqbali²; H. Al Rimawi²; A. Petrosoniak;

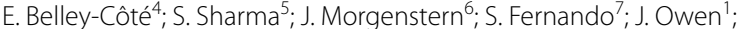
M. Zeller ; D. Quinlan²; W. Alhazzani ${ }^{2}$; B. Rochwerg ${ }^{10}$

${ }^{1}$ Medicine, division of emergency medicine and critical care, McMaster University, Hamilton, Canada; ${ }^{2}$ Medicine, division of emergency medicine, McMaster University, Hamilton, Canada; ${ }^{3}$ Medicine, division of emergency medicine, University of Toronto - St. George Campus, Toronto, Canada; ${ }^{4}$ Medicine, McMaster University, Hamilton, Canada; ${ }^{5}$ Medicine and surgery, division of critical care and neurosurgery, McMaster University, Hamilton, Canada; ${ }^{6}$ Family and community medicine, division of emergency medicine, University of Toronto - St. George Campus, Toronto, Canada; ${ }^{7}$ Emergency medicine and critical care, University of Ottawa, Ottawa, Canada; ${ }^{8}$ Medicine, division of hematology and thromboembolism, McMaster University, Hamilton, Canada; ${ }^{9}$ Department of medicine/ department of health research methods, evidence and impact, McMaster University, Hamilton, Canada; ${ }^{10}$ Critical care, McMaster University, Hamilton, Canada 
Correspondence: S. Sharif

Intensive Care Medicine Experimental 2020, 8(2): 000866

Introduction: Tranexamic Acid (TXA) is used for a number of indications, including trauma, based on evidence that it decreases bleeding and improves survival. Its role in acute traumatic brain injury (TBI) is less certain, although a recent large randomized controlled trial (RCT) suggested it may improve head-injury related deaths. We conducted a systematic review and meta-analysis, including this latest RCT, to investigate the efficacy and safety of TXA in acute TBI.

Methods: We performed a comprehensive search of MEDLINE, PubMed, EMBASE, CINHAL, ACPJC, Google Scholar, and unpublished sources from inception until June 24th, 2020 for randomized controlled trials (RCTs) examining the role of TXA in adults with acute TBI. We pooled data using the inverse variance method and random effect model and report summary estimates using relative risk (RR) for dichotomous outcomes and mean difference (MD) for continuous outcomes, both with corresponding $95 \%$ confidence intervals (Cls). For outcomes reported at multiple end-points, we used the longest duration of follow-up for analysis. We assessed individual study risk of bias using a modified Cochrane risk of bias tool and certainty of evidence using GRADE methodology.

Results: We included nine RCTs ( $n=14,846$ patients). Pooled results demonstrate that TXA has no effect on mortality (RR $0.95 ; 95 \% \mathrm{Cl}$ 0.88 to 1.02 ; absolute risk reduction [ARR] $1.0 \%$; $95 \% \mathrm{Cl} 2.4 \%$ reduction to $0.4 \%$ increase, moderate certainty), no difference in disability as assessed by the Glasgow Outcome Scale (GOS) (MD, -0.16 points; $95 \% \mathrm{Cl}-0.57$ to 0.25 ; moderate certainty) or disability based on proportion of patients with a GOS score ${ }^{3} 4$ (RR $0.87 ; 95 \% \mathrm{Cl}, 0.57$ to 1.32 $0.4 \% \mathrm{ARR} ; 95 \% \mathrm{Cl} 1.3 \%$ reduction to $1.0 \%$ increase; very low certainty) compared to those that did not receive TXA. TXA may reduce hematoma expansion on subsequent imaging (RR $0.78 ; 95 \% \mathrm{Cl} 0.59$ to 1.04 ARR $3.2 \%, 95 \% \mathrm{Cl} 6.0 \%$ reduction to $0.6 \%$ increase); however, this was based on low certainty evidence, limited by imprecision. Risks of adverse events including pulmonary embolism, deep vein thrombosis, stroke, and seizure (all moderate certainty) were similar between those receiving and those not receiving TXA.

Conclusion: In patients with acute TBI, TXA probably has no effect on mortality or disability. TXA may decrease hematoma expansion on subsequent imaging; however, this outcome is of unclear benefit to patients. Use of TXA is probably not associated with an increased risk of adverse events.

\section{4}

Patients with resuscitation associated trauma have worse 30 -days mortality and worse $\mathbf{3 0}$ days neurological status

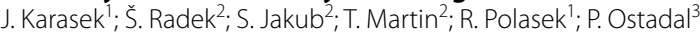

${ }^{1}$ Cardiology, Hospital Liberec, Liberec, Czech Republic; ${ }^{2} 3$ rd medical faculty, Charles University, Prague, Czech Republic, ${ }^{3}$ Cardiovascular center, Na Homolce Hospital, Prague, Czech Republic

Correspondence: J. Karasek

Intensive Care Medicine Experimental 2020, 8(2): 000934

Introduction: CPR related injuries were not properly observed since were established new guidlines for resuscitation (CPR) 2015 with stronger recomandation for bystader and topless CPR. Data were not objectivised by any scale in effort to establish seriousness of injury. Objectives: To determine impact of trauma associated with resuscitation, describe their frequency and seriousness and their influence on morbidity and mortality of patients after OHCA (Out of Hospitals Cardiac Arrest).

Methods: Analyse of data from prospective registry of OHCA from regional Cardiac Arrest Center (CAC). Trama patients were identified with clinical suspection and subsequently with imagine methods (CT, $\mathrm{X}$ ray, sonography), trauma as cause of arrest was no included. To determine seriousness we used Abbreviated injury scale (AIS) and summary of all injuries with New injury severity score (NISS).
Results: In study were included 231 patients after OHCA admitted to ICU. $30(13 \%)$ had relevant CPR related trauma established with clinical suspection and confirmed with imagine methods. In baseline characteristics was no difference in gender $(76,7 \%$ of men in TRAUMA group vs. $78,1 \%$ in nonTRAUMA group, $p=0,82$ ), no difference in age ( $65 \pm 13$ years in TRAUMA group vs. $62 \pm 13$ years in nonTRAUMA group, $p=0,38$ ), no difference in inicial shockable rythm (57\% in TRAUMA group vs. $73,1 \%$ in nonTRAUMA group, $p=0,08$ ) and no difference in witnessed arrest (83\% in TRAUMA group vs. $87 \%$ in nonTRAUMA group, $p=0,58$ ). There was no differences in duration of bystander CPR $(9,3 \pm 4,4 \mathrm{~min}$ in TRAUMA group vs. median $10 \mathrm{~min}$, IQR 25-75:5-10 in nonTRAUMA group, $p=0,77$ ), but total duration of CPR untill ROSC was signifficant longer in TRAUMA group (median 25, IQR 25-75:14,5-30 min in TRAUMA group vs. median 18, IQR 25-75:10$25 \mathrm{~min}$ in nonTRAUMA group, $\mathrm{p}=0,03$ ). There was no differences in ventilation days (mean $6 \pm 5$ days in TRAUMA group vs. median $4, \mathrm{IQR}$ 25-75:1-7 days in nonTRAUMA group, $p=0,35$ ) and ICU stay (median 6, IQR 25-75:1-15 days in TRAUMA group vs. median 7, IQR 25-75:3-12 days in nonTRAUMA group, $p=0,84$ ). Cardiac cause of arrest was signifficant higher in nonTRAUMA group (37\% in TRAUMA group vs. $71 \%$ in nonTRAUMA group, $\mathrm{p}=0,0003$ ) and also coronarography was provided more frequent in nonTRAUMA group (37\% in TRAUMA group vs. $64 \%$ in nonTRAUMA group. $p=0,005)$, but without difeerence in frequency of revascularisation ( $28 \%$ in TRAUMA group vs. $43 \%$ in nonTRAUMA group, $p=0,11) .30$ days mortality was signifficant worse in TRAUMA group (66\% in TRAUMA group vs. $38 \%$ in nonTRAUMA group, $\mathrm{p}=0,005$ $\mathrm{RR} 2,76,95 \% \mathrm{Cl} 1,4-5,6)$ and 30 days good neurological outcome (CPC 1,2 ) also worse in TRAUMA group (vs. $56 \%$ in nonTRAUMA group, $\mathrm{p}=0,0014, \mathrm{RR} 3,5,95 \% \mathrm{Cl} 1,6-7,7)$. In TRAUMA group was lung contusion present by $16,7 \%$, pneumothorax by $36,7 \%$, liver injury by $13,3 \%$ and spleen injury by $6,7 \%$ of patients. Ribs fractures were presented by $60 \%$ and sternum fractures by $20 \%$ of patients. Mean of fracutred ribs was $9 \pm 5,2$ ribs. Median of AIS was 3 (IQR 25-75) 2-4. Median of NISS was 11,5 (IQR 25-75) 7-19,25.

Conclusion: Trauma is present by $13 \%$ of OHCA patients with ROSC. There is no difference in age, gender, initial shockable rythm and bystander CPR between TRAUMA and nonTRAUMA group, but resuscitation is signifficant longer in TRAUMA group. In TRAUMA group we have observed worse 30-days mortality and good neurological outcome.

Reference(s) and grant ackowledgment(s)

1. no refferneces to abstract

2. no grant support

001168

Quality assessment for in-hospital management of massive hemorrhage

R. Munoz-Calahorro'; JA. Barea-Mendoza'; C. Mudarra-Reche";

C. García-Fuentes ${ }^{1}$; M. Chico-Fernández ${ }^{1}$

${ }^{1}$ Medicina intensiva, University Hospital 12 de Octubre, Madrid, Spain

Correspondence: R. Munoz-Calahorro

Intensive Care Medicine Experimental 2020, 8(2): 001168

Introduction: Massive hemorrhage in trauma is a diagnostic and therapeutic challenge. An early approach to hemorrhagic shock by transfusion of blood products and damage-control resuscitation is essential to avoid lethal pentad and death.

Objectives: To assess the quality of care in traumatic massive bleeding in a Level 1 Trauma Centre by analyzing two indicators: early activation of the massive transfusion protocol (MTP) and optimal transfusion of packed red blood cells (pRBC).

Methods: A retrospective single-center cohort study was obtained from two prospective databases of University Hospital 12 de Octubre (Madrid): the Severe Traumatic Disease registry and the MTP database in a 6-year period of time (2013-2019).

Study subjects: Traumatic patients who were admitted in the ICU and required transfusion of ten or more $\mathrm{pRBC}$ in the first 24 hours of admission (defining criteria of massive hemorrhage, $\mathrm{MH}$ ). 
Variables of interest:

- Early diagnostic sensitivity (S): Percentage of activation of MTP in patients with traumatic MH. TheTrauma-Associated-Severe-Haemorrhage (TASH) Score, the Injury-Severity-Score (ISS) and the percentage of FAST were retrospectively calculated in the false-negative (FN) subgroup.

- Optimal transfusion of pRBC: According to scientific literature, three cohorts were described according to hemoglobin $(\mathrm{Hb})$ at 24 hours of admission. Thepercentage of under-transfusion (UT) ( $\mathrm{Hb}<8 \mathrm{mg} / \mathrm{dl}$ ) and over-transfusion (OT) ( $\mathrm{Hb}>11.9 \mathrm{mg} / \mathrm{dl}$ ) was studied.

Data analysis: SPSS Statistics 24.0.0. Variable expression: mean and standard deviation.

Results: Descriptive statistics

First, a descriptive analysis of the MH cohort $(n=145)$ was performed. The mean age was $40.7 \pm 17$ years, with male predominance (76.6\%). The mean ISS was $42.8 \pm 16$, highlighting a $72.4 \%$ prevalence of hemorrhagic shock coagulopathy and an overall mortality of $43.4 \%$.

Deaths were due to exsanguination $(38 \%, n=24)$, intracranial hypertension $(36.5 \%, n=23)$, multiple organ dysfunction syndrome $(12.7 \%$, $\mathrm{n}=8)$ and limitation of therapeutic effort $(6.3 \%, \mathrm{n}=4)$.

Regarding the mechanisms involved, the most relevant were precipitation $(n=45)$, sharp objects injury $(n=23)$, automobile accident $(n=$ $21)$, motorcycle accident $(n=17)$ and pedestrian injury $(n=16)$.

FAST was performed in $71.7 \%$ of the cases. Therapeutic arteriography was performed in $28.8 \%$ of the cases. $81.3 \%$ underwent damage control surgery.

Diagnostic sensitivity

MTP was activated in 135 out of the 145 patients with MH criteria (S = 93.1\%).

In FN $(\mathrm{n}=10)$, the mean TASH score was $12.4 \pm 3$ and the mean ISS was $31.7 \pm 12$.

$30 \%(\mathrm{n}=3)$ presented a TASH score $<=9$ (MH pre-test probability $<5 \%$, all presenting with bleeding during damage control surgery requiring intraoperative transfusion $>6 \mathrm{pRBC}$.

In the remaining cases $(n=7)$ the TASH score was $>13(\mathrm{MH}$ pre-test probability range $28-35 \%$ ), with FAST being performed in $57 \%$ of cases $(\mathrm{n}=4)$

Transfusion quality

$68.7 \%$ of patients were in the optimal transfusion cohort, while $8.9 \%$ were in the UT cohort and $22.5 \%$ in the OT cohort. Mortality was respectively $25.6 \%, 50 \%$ and $24 \%$, without statistically significant differences in subgroup analysis $(p=0.243)$.

Conclusion: The diagnostic sensitivity was similar to that achieved by the $\mathrm{MH}$ prediction scores, considered the gold-standard. Nonetheless, FN analysis shows the need to optimize early identification.

The transfusion target was suboptimal in one third of patients. Specific transfusion protocols need to be created for severe traumatic disease with intermediate pre-test probability of $\mathrm{MH}$.

\section{Reference(s) and grant ackowledgment(s)}

1. Brockamp T, Nienaber U, Mutschler M, Wafaisade A, Peiniger S, Lefering R et al. Predicting on-going hemorrhage and transfusion requirement after severe trauma: a validation of six scoring systems and algorithms on the TraumaRegister DGU ${ }^{\circledR}$. Critical Care. 2012;16:R129. doi: 10.1186/cc11432

2. Spahn D, Bouillon B, Cerny W, Duranteau J, Filipescu D, Hunt B et al. The European guideline on management of major bleeding and coagulopathy following trauma: fifth edition. Critical Care. 2019;23:98. doi:10.1186/ s13054-019-2347-3

3. Zielinski M, Wilson G, Johnson P, Polites S, Jenkins D, Harmsen W et al. Ideal Hemoglobin Transfusion Target for Resuscitation of Massive Transfusion Patients. Surgery. 2016;160(6):1560-1567. doi:10.1016/j.surg.2016.05.022

\section{3}

\section{Impact of protocoled nursing care on quality performance} measures in patients with severe trauma

N. Matsumoto'; M. Sato ${ }^{1}$; M. Toyodome ${ }^{1}$;. Shimoikeda'; T. Sawai ${ }^{1}$. M. Murai ${ }^{1}$; J. Yoshimura²; K. Yamakawa²; S. Fujimi

${ }^{1}$ Department of nursing, Osaka General Medical Center, Osaka, Japan ${ }^{2}$ Division of trauma and surgical critical care, Osaka General Medical Center, Osaka, Japan
Correspondence: N. matsumoto

Intensive Care Medicine Experimental 2020, 8(2): 000143

Introduction: Trauma remains the leading cause of death all over the world. To better exploit the trauma care system, precise diagnosis of the injury site and prompt control of bleeding are essential. Here, we created a nursing protocol for initial medical care for trauma.

Objectives: The aim of this study was to evaluate the impact of protocoled nursing care for trauma on measures of quality performance.

Methods: This was a retrospective historical control study, consisted of consecutive severe trauma patients (Injury Severity Score $>16$ ). People were divided into two groups: protocoled group (from April 2017 to March 2019) and control group (from April 2014 to March 2017). We set the primary endpoint as mortality for bleeding. The secondary endpoints included time allotted from arrival to start of CT scan and surgery, administration rate of several drugs (sedations, painkillers, preoperative antibiotics, and tranexamic acid). For the statistical analysis, continuous variables were expressed as median (interquartile range) and were compared by Wilcoxon rank sum tests given a nonnormal distribution of the data.

Results: We included 152 patients in the study: 84 in the control group before the introduction of the protocol, 68 in the protocoled group. As a primary endpoint, the mortality for bleeding was similar between two groups (5\% in the control group and 5\% in the protocoled group). As a secondary endpoint, the time to CT initiation [Group A 11 (7-16) min vs Group B 7 (5-10) min; $p<0.001]$, and emergency procedure [Group A 41 (33-56) min vs group B 29(25-43); p <0.001] were shortened by the protocol introduction. Furthermore, the administration rates of sedations, painkillers, preoperative antibiotics, and tranexamic acid were increased in the protocoled group compared with the control group.

Conclusion: Although the mortality as a patient-oriented outcome was not affected, improved quality of medical care by nursing protocol introduction may be suggested in this analysis.

\section{7}

Limitation of life-sustaining treatment (LLST) in ICU: 16 years of experience

M. Iturbe Rementería ; N. Ruiz Cañas ${ }^{1}$; JA. Urturi Matos ${ }^{1}$; S. Castaño Avila²; I. Saralegui Reta

${ }^{1}$ Icu, AUO - Santiago Apóstol, Vitoria-Gasteiz, Spain; ${ }^{2}$ Intensive care unit, University Hospital of Araba, Vitoria-Gasteiz, Spain

Correspondence: $M$. Iturbe

Intensive Care Medicine Experimental 2020, 8(2): 000097

Introduction: care at the end of life of patients who die; includes the shared decision-making between health professionals and family members, the communication and registration of agreements.

Objectives: to analyze the LLST procedure from 1-1-2004 to 12-312019 (16 years), the evolution of recommendations and improvement actions.

Methods: retrospective descriptive study

Results: During the analyzed period, 12422 patients were admitted to the ICU, 1329 of whom died (10.69\% mortality). LLST has been agreed in $10 \%$ of the admitted patients and in $81^{\prime} 46 \%$ of the deceased (range $73-94 \%$ ). The opinion of the nursing staff evaluated through a survey detected training needs and interest in participating in decision-making and information for family members. The few conflicts registered have been resolved by postponing the decision and encouraging the participation of other specialists involved. The procedure has improved with: a) the use of a specific sheet of medical orders on LLST b) its subsequent computerization to be recorded in the electronic medical record c) Terminal Extubation procedure d) continuing training on Bioethics and LLST of health personnel. The opinion of the patients' relatives who died after LLST is collected through semistructured interviews (in the study phase). The LLST experience facilitated the launch of the Controlled Asystole Donation procedure. More than 100 training and outreach sessions have been held, not only for 
healthcare personnel but also forpatient associations. Care and treatment planning (Advance Directives) have been promoted to try to improve decision-making through specific programs for the chronically ill, interviewing more than 500 patients and family members.

Conclusion: LLST is a common procedure, with established, published and improved recommendations. It allows palliative care to be integrated into the field of intensive medicine.

\section{Reference(s) and grant ackowledgment(s)}

1. Holzapfel L, Demingeon G, Oiralla B: A four-step protocol for limitation of treatment in terminal care. An observational study in 475 intensive care unit patients. Intensive Care Med 2002; 28:1309-1315.

2. The SUPPORT Principal Investigators: A controlled trial to improve care for seriously ill hospitalized patients. The study to understand prognoses and preferences for outcomes and risks for treatments (SUPPORT). JAMA 1995; 274:1591-98.

3. Saralegui I, Poveda Y, Martín A, Balciscueta G, Martínez S, Pérez C et al: LifeSustaining-Treatment Limitation in ICU: a well established and improved practice with critical patients. Intensive Care Med 2009; 35(Suppl 1):S262.

4. Azoulay E, Timsit JF, Sprung CL: Conflicus Study Investigators and for the Ethics Section of the European Society of Intensive Care Medicine.

Prevalence and factors of intensive care conflicts. The Conflicus study. Am J Respir Crit Care Med 2009; 180(9):853-860.

5. Saralegui I, Monzón JL, Martín MC: Instrucciones previas en medicina Intensiva [Advance directives in Intensive Care Medicine]. Med Intensiva 2004; 28(5):256-261

6. Winter B, Cohen S: ABC of intensive care. Withdrawal of treatment. BMJ 1999; 319(7205):306-308.

\section{5}

End of Life Sedation with Intravenous Morphine and Midazolam: A Guideline for the Medical High Dependency Unit in Aberdeen Royal Infirmary

K. Hudson'; S. Roberts ${ }^{1}$; C. Kaye ${ }^{1}$

${ }^{1}$ Intensive Care Unit, Aberdeen Royal Infirmary, Aberdeen, United Kingdom

Correspondence: $\mathrm{K}$. Hudson

Intensive Care Medicine Experimental 2020, 8(2): 000935

Introduction: Sedation with morphine and midazolam (M\&M) is often used at the end of life (EoL) to relieve symptoms during the terminal phase of a patient's illness. While the medications used can control these symptoms, excessive doses hasten death. Therefore, doses should be titrated carefully, based on the patient's condition. In Medical High Dependency Unit (MHDU), a prescription for Intravenous (IV) infusion of M\&M would be written for a patient approaching EoL and nursing staff would titrate dosing independently based on their clinical judgment. Only 33\% of nursing staff always felt comfortable titrating the infusion rate and $67 \%$ wanted more guidance on dose titration. It was also felt that a patient's infusion rate could vary dependent on the nurse looking after them.

Objectives: The aim was to implement clear guidance for IV M\&M infusions into MHDU by February 2020 and for it to be used in $90 \%$ of IV M\&M infusions. This would;

-provide guidance for nursing staff to tailor the infusion rate to the individual

-protect nursing staff from any legal uncertainty.

Methods: Existing practice was reviewed by surveying nursing staff and collecting data from the last 10 patients who received an IV M\&M infusion from May to July 2019. A guideline was created for MHDU using the Respiratory Distress Observation Scale(1) and the Critical Care Pain Observation Tool(2) to provide a validated scoring system to guide dose titration. Small group teaching with both nursing and medical staff was organised for education. Data was then collected for 10 subsequent patients from October 2019 to February 2020 and analysed. Qualitative data via anonymous feedback forms was collected from staff after implementation.

Results: All patients received IV M\&M infusions according to the guideline $(n=10)$. The average maximum dose infused was reduced (morphine: pre-intervention $4.75 \mathrm{mls} / \mathrm{hr}$, post-intervention $2.40 \mathrm{ml} / \mathrm{hr}$; midazolam: pre-intervention $4.75 \mathrm{mls} / \mathrm{hr}$, post-intervention $3.20 \mathrm{mls} /$ $\mathrm{hr}$ ). The average rate of infusion was reduced (morphine: pre-intervention $3.87 \mathrm{mls} / \mathrm{hr}$, post-intervention $2.28 \mathrm{mls} / \mathrm{hr}$; midazolam: pre-intervention $3.87 \mathrm{mls} / \mathrm{hr}$, post-intervention $2.33 \mathrm{mls} / \mathrm{hr}$ ). The average time between starting IV M\&M and death was not significant (pre-intervention $3.09 \mathrm{hrs}$, post-intervention $2.99 \mathrm{hrs}$ ). Documented reasons behind dose adjustments increased from $6 \%$ to $81 \%$. All staff felt more comfortable titrating M\&M when using the guideline $(n=10)$. In one case, the scoring system within the guideline led to a patient not receiving any IV M\&M and subsequently surviving to discharge.

Conclusion: The guideline was widely appreciated by the nursing staff and therefore used in all patients who required IV M\&M. Using the guideline showed a reduction in the dose of M\&M administered, without affecting the time to death. The guideline gave more confidence behind titrating dose to patient symptoms and the results also gave reassurance that the doctrine of double effect was not being taken advantage of via administration of these drugs.

\section{Reference(s) and grant ackowledgment(s)}

1. Gelinas C, Fillion L, Puntillo KA, Viens C, Fortier M. Validation of the Critical Care Pain Observation Tool in adult patients. The American Journal of Critical Care. 2006;15(4):420-427

2. Campbell ML, Templin T, Walch J. A respiratory distress observation scale for patients unable to self-report dyspnea. Journal of Palliative Medicine. 2010;13(3):285-290

\section{9}

Evaluation of the differences between suitable vs. discarded livers obtained from control cardiac death donors managed with normothermic regional perfusion

S. Alcántara Carmona ${ }^{1}$; M. Pérez Redondo ${ }^{1}$; H. Villanueva Fernández ${ }^{1}$; D. Ballesteros Sanz'; J. Duerto ÁLvarez²; C. Pardo Rey²; A. Ortega López';

F. Del Río Gallegos²; JJ. Rubio Muñoz ${ }^{2}$

${ }^{1}$ Intensive care, Hospital Puerta de Hierro-Majadahonda, Majadahonda, Spain; ${ }^{2}$ nntensive care, Hospital Clinico Universitario San Carlos, Madrid, Spain

Correspondence: S. Alcántara Carmona

Intensive Care Medicine Experimental 2020, 8(2): 000249

Introduction: Normothermic regional perfusion (NRP) in controlled donation after circulatory death is becoming a popular method due to the favorable results of the grafts procured under this technique.

Objectives: To study the differences between livers suitable vs. livers discarded for transplantation obtained from controlled circulatory death donors (CDCD) managed with NRP.

Methods: Retrospective study (February 2017/December 2019). We studied all CDCD managed by the mobile NRP team of the Autonomous Community of Madrid (Spain) in which the liver was evaluated for transplantation. The following data were recovered: 1) CDCD demographics: age, sex, ICU length of stay and reason for limitation of life sustain therapies (LLST); 2) warm ischemia times (WIT) defined as the interval between systolic arterial pressure $<60 \mathrm{mmHg}$ to commencement of NRP; 3) NRP duration and 4) biochemical parameters (Figure 1) at the start and end of NRP together with the differences between final - initial NRP values.

Results: During the period studied the mobile NRP team assisted in the management of $55 \mathrm{CDCD}$. Liver evaluation was performed in 32 (58.2\%) cases. The liver was deemed suitable for transplantation in 19 (59.4\%) and discarded 13 (40.6\%). We found no differences between suitable vs. discarded livers regarding: age [60(48-64) vs. 63(55-65) years], sex [9(47.4\%) vs. 5(38.5\%) males], ICU length of stay [8(4-14) vs. 6(4-13.5) days] or main reason for LLST [catastrophic brain injury: $19(100 \%)$ vs. $11(84.6 \%)]$. Suitablelivers had lower WIT [11(7-15) vs. $15.5(12-19) \mathrm{min} ; \mathrm{p}<0.03]$ and longer NRP times [90(73-110) vs. 63(3189) min;p<0.02]. We found no differences in the biochemical parameters analyzed (Figure 1). The main reason for organ rejection was the existence of an unfavourable visual assessment ( $n=12 ; 92.3 \%)$. One liver was rejected after a renal carcinoma was found during the 
laparotomy. None of the livers were discarded due to a transaminase elevation during NRP.

Conclusion: In our series we found no differences between suitable vs. discarded livers in CDCD managed with NRP. An unfavourable visual assessment was the main reason for organ rejection. In order to improve the ratio of suitable livers coming from $C D C D$ and managed with NRP, it might be necessary to search for more objective strategies that might include a microscopical evaluation of the organ and/or newer biochemical markers.

\section{2}

Determining the impact of timing and of clinical factors during end-of-life decision-making in potential controlled donation after circulatory death donors

A. Kotsopoulos ${ }^{1}$; N. Jansen ${ }^{2}$; P. Vos ${ }^{3}$; M. Witjes ${ }^{4}$; M. Volbeda ${ }^{5}$; J. Epker ${ }^{6}$; H. Sonneveld ${ }^{7}$; K. Simons ${ }^{8}$; E. Bronkhorst ${ }^{9}$; H. Van Der Hoeven ${ }^{4}$; F. Abdo ${ }^{4}$

${ }^{1}$ Intensive care, Elisabeth TweeSteden Hospital, Tilburg, Netherlands; ${ }^{2}$ Research, Dutch Transplant Foundation, Leiden, Netherlands; ${ }^{3}$ Intensive care, Erasmus University Medical Center, Tilburg, Netherlands; ${ }^{4}$ Intensive care, Radboud University Medical Center, Nijmegen, Netherlands; ${ }^{5}$ Intensive care, UMCG University Medical Center, Groningen, Netherlands; ${ }^{6}$ Intensive care, Erasmus University Medical Center, Rotterdam, Netherlands; ${ }^{7}$ Intensive care, Isala Hospital, Zwolle, Netherlands; ${ }^{8}$ Intensive care, Jeroen Bosch Hospital, s'Hertogenbosch, Netherlands; ${ }^{9}$ Department of health evidence, Radboud University Medical Center, Nijmegen, Netherlands

Correspondence: A. Kotsopoulos

Intensive Care Medicine Experimental 2020, 8(2): 000492

Introduction: Controlled donation after circulatory death (CDCD) occurs after a decision to withdraw life-sustaining treatment (WLST) and subsequent family approach and approval for donation. We currently lack data on factors that impact the decision-making process on WLST and whether time from admission to family approach, influences family consent rates. Such insights could be important in improving the clinical practice of potential CDCD donors.

Objectives: The main aim of our study was to assess timing and patient characteristics that are associated with the decision-making process leading to the initiation of end-of-life care, in a large multicenter prospective study of potential CDCD donors. A second objective was to explore the influence of this timing in family approach on consent to organ donation.

Methods: In a prospective multicenter observational study, we evaluated the impact of timing and of the clinical factors during the end-oflife decision-making process in potential CDCD donors. Characteristics and medication use, of 409 potential CDCD donors admitted to the intensive care units (ICU), were assessed.

Results: End-of-life decision-making was made after a mean time of $97 \mathrm{~h}$ after ICU admission and mostly during the day. Intracranial hemorrhage or ischemic stroke and a high APACHE IV score were associated with a short decision-making process. Preserved brainstem reflexes, high Glasgow Coma Scale scores or cerebral infections were associated with longer time to decision-making. Our data also suggest that the organ donation request could be made shortly after the decision to stop active treatment and consent rates were not influenced by day- or nighttime or by the duration of the ICU stay.

Conclusion: In summary, our data provide valuable insights on the largely overlooked topic of end-of-life decision-making in potential organ donors and show that early prognostication occurs in certain patient categories more often. Although prognostication is more difficult in the acute setting, our data show that if the treating team believes decision-making about WLST is possible early after hospital admission, this will not necessarily have a negative effect on the consent rates to organ donation.

\section{7}

Organ Donation within an Intensive Care Unit in Portugal

C. Roriz ; D. Castro ; F. Sequeira ${ }^{1}$; H. Fernando ${ }^{1}$; O. Gomes ${ }^{1}$; L. Pereira

${ }^{1}$ Serviço de medicina intensiva, Centro Hospitalar de Leiria, Leiria, Portugal Correspondence: $C$. Roriz

Intensive Care Medicine Experimental 2020, 8(2): 000867

Introduction: Organ and tissue transplantation is often the only treatment for end stage organ failure. In 2017, Portugal ranked second among 82 member states which reported to the global observatory on donation and transplantation with 34.1 donors per million inhabitants. The development of organ support systems in recent decades led to the ability to artificially maintain organ viability, leading to the concept of brain death. Brain death is the irreversible cessation of cerebral and brain stem function; characterized by the absence of electrical activity in the brain, blood flow to the brain, and brain function as determined by clinical assessment of responses. The brain death diagnosis and organ donor treatment are primordial given the importance of achieving a better quality of organs available for transplant. Organ donor treatment represents a crucial part of the donation process being the ultimate goal to obtain as many viable organs as possible by donor.

Objectives: The purpose of this study is to characterize potential organ donors admitted to a 10-bed combined medical-surgical intensive care unit of a Portuguese hospital, identify reasons for non-donation, length of stay in the intensive care unit and to determine the number and type of donated organs.

Methods: A retrospective cohort study of all the potential organ donor admissions in a 10-bed combined medical-surgical intensive care unit of a Portuguese hospital between January 2018 and May 2020 and characterize demographic and clinical variables as well as the number of harvest organs.

Results: In the period of 2 years and 5 months 26 potential organ donors of whom 15 were effective donors, were identified and admitted to the intensive care unit, representing $3.7 \%$ of all intensive care unit admissions. Among potential donors the mean age was 60.6 and the majority were men $(61.5 \%)$. With respect to the effective donors, the mean age was 59.5 and $73.3 \%$ were men. Hemorrhagic stroke accounted for $73 \%$ of brain deaths, anoxia and ischemic stroke were the cause of brain death in $26 \%$ of the patients $(13.5 \%$ each type of lesion). Among the non-effective donors, the reasons for non-donation were refusal by the national coordination transplantation related with patient comorbidities in 4 patients, non-evolution to brain death in 2 patients and evidence of sepsis in 5 patients. The most common comorbidity of the potential donors was arterial hypertension (69.2\%). The mean time from ICU admission to the diagnosis of brain was 56 hours, and the mean time from ICU admission to organ harvesting was 66 hours. The most frequently collected organs were the kidneys $(93.3 \%)$ followed by the liver $(66.7 \%)$ and the most common collected tissue was cornea in $53.3 \%$ of the patients.

Conclusion: At the time of the study, potential organ donors were mostly men, over fifty years old, had arterial hypertension as comorbidity and died of brain death follow hemorrhagic stroke. The kidneys and liver were harvested in most cases.

Reference(s) and grant ackowledgment(s)

1. Global Observatory on Donation, International Report on organ donation and transplantation activities executive summary, accessed on 29 July 2020, <http://www.transplant-observatory.org/ download/2017-activity-data-report/> 
2. Organ donation within the intensive care unit: A retrospective audit. Aus Crit Care . 2020 Mar;33(2):167-174.

3. Organ donation: the reality of an intensive care unit in Portugal. Rev Bras Ter Intensiva. 2018;30(2):201-207

\section{3}

A machine learning based model development for hypotension event predictor in ICU patients

B. Lee

${ }^{1}$ VHS medical center, Seoul, Republic of Korea

Correspondence: $\mathrm{B}$. Lee

Intensive Care Medicine Experimental 2020, 8(2): 000253

Introduction: In the ICU, hypotension is one of the most critical event to reduce patient mortality If we can predict the hypotension early, patient can take safer medical care. We developed the machine learning model for hypotension predictor(systolic blood pressure $<60$ ) (5hours later) from the ICU data of 1968 patients between January and December 2018 in veterans' health service medical center at South Korea. In the ICU, hypotension is one of the most critical event to reduce patient mortality If we can predict the hypotension early, patient can take safer medical care. We developed the machine learning model for hypotension predictor(systolic blood pressure <60) (5hours later) from the ICU data of 1968 patients between January and December 2018 in veterans' health service medical center at South Korea.

Objectives: To develop the novel technique to predict short term hypotension event predictor in ICU with machine learning analysis

Methods: A total of 1968 patient stayed in ICU of veterans' health service medical center between January and December 2018 were enrolled and their electrical medical data including hourly recorded vital signs, clinical manifestations, demographic features were collected for analysis. Keras (version 1.2.0, backend engine: tensorflow 1.0) was used to generate multi perceptron neural networking model for modelling.

Results: Multi perceptron neuronal model with Keras was successfully generated from patient's data. The prediction rate of the model was $97.4 \%$ and no overfitting was observed during model generation. The AUC value from ROC curve was 0.996 for the generated model. To compile multi perceptron learning model, adam optimizer was used Conclusion: Multi perceptron learning algorithm could be very useful to develop new hypotension prediction model. Further study is necessary to evaluate the validity of new short-term hypotension prediction model.

\section{1}

\section{Predicting fluid balance change in septic patients}

during deresuscitation

R. Ahlsdorf'; A. Rahman¹; Y. Chang ; A. Peine ${ }^{2}$; E. Ghosh'; L. Martin²

${ }^{1}$ Connected care and personal health, Philips Research North America, Cambridge, United States of America; ${ }^{2}$ Klinik für operative intensivmedizin und intermediate care, University Hospital RWTH Aachen, Aachen, Germany

Correspondence: $\mathrm{E}$. Ghosh

Intensive Care Medicine Experimental 2020, 8(2): 000291

Introduction: Fluid overload resulting from suboptimal fluid management is common in critically ill patients and associated with adverse outcomes. Appropriate fluid management strategies to relieve fluid overload following the initial resuscitation period (the "deresuscitation phase") are largely variable in clinical practice - potentially as a result of the heterogeneity in disease progression and numerous other factors, such as age and premorbidities. There is a need to better understand patients' progression in the deresuscitation phase and to accurately predict strategies in fluid balance management with the goal of guiding fluid therapy to achieve optimal fluid balance.

Objectives: We extracted fluid balance and physiological factors in the deresuscitation phase from a large cohort of patients with sepsis in a retrospective electronic health records database and built a machine learning model to predict fluid balance change periodically after patients had been stabilized.

Methods: We used MIMIC-III, a publicly available critical care dataset from Beth Israel Deaconess Medical Center, Boston, MA. Our cohort consisted of adult ICU patients with sepsis (based on the Sepsis-III criteria) who received vasopressors and/or inotropes during resuscitation. We excluded patients with Do Not Resuscitate status, cardiac surgery and burn patients. End of resuscitation phase was defined as the time of last vasopressor/ inotrope administration. Clinical data including, demographics, vitals, labs, fluid inputs and outputs, interventions and medications were extracted for the time period starting from end of resuscitation to ICU discharge. Data was segmented into 4-hour blocks. Fluid balance was calculated for each block and the change (increase, no change or decrease) within a block evaluated. We trained a 3-class logistic regression model to predict the change of fluid balance in the next block using the extracted clinical data in the current block. Model was evaluated using 6-fold cross validation and top predictors were calculated.

Results: A total of 2,565 ICU admissions were included in our cohort which was divided into 62,465 4-hour blocks. The mean area under receiver operator curve (AUROC) for predicting fluid balance increase was 0.78 , mean AUROC for predicting no change in fluid balance was 0.71 and mean AUROC for predicting fluid balance decrease was 0.77 . Conclusion: We trained a model to predict fluid balance change during deresuscitation in our cohort. The model performs reasonably well in distinguish patients with no change in fluid balance, a decrease, and an increase in fluid balance. Additionally, we identified top predictors for fluid balance change, which agree with known physiology. The top positive correlates for fluid balance decrease are being on CRRT and cumulative output. Conversely, being on CRRT is a negatively correlated with fluid balance increase. Therefore our model uses clinically meaningful features to distinguish between the different classes.

\section{8}

Hiding in Plain Sight: SaO2/FiO2 as the Superior Hypoxic Measure B. Lee'; G. Singh²; J. Bartock'; S. Patel ${ }^{1}$

${ }^{1}$ Critical Care Medicine, Cooper University Health Care, Camden, United States of America; ${ }^{2}$ Critical care medicine, Piedmont Columbus Regional, Columbus, United States of America

Correspondence: $\mathrm{B}$. Lee

Intensive Care Medicine Experimental 2020, 8(2): 000368

Introduction: $\mathrm{PaO} 2$ to $\mathrm{FiO} 2$ ratio $(\mathrm{P} / \mathrm{F})$ is used to assess the degree of hypoxemia adjusted for oxygen requirements. The Berlin definition of Acute Respiratory Distress Syndrome (ARDS) includes P/F as a diagnostic criterion. $P / F$ is invasive and cost-prohibitive for resourcelimited settings. Pulse Oximetric Saturation ( $\mathrm{SpO} 2)$ is noninvasive, continuous, and pervasive. Previous work suggests that $\mathrm{SpO} 2$ to $\mathrm{FiO} 2$ ratio (S/F) correlates with P/F and can be used as a surrogate in ARDS (1). It is unclear whether S/F can be used to identify intensive care (ICU) patients that are at high risk (2). We hypothesize that S/F is non-inferior to $\mathrm{P} / \mathrm{F}$ as a predictive feature in the critically ill.

Objectives: To demonstrate the non-inferiority of $S / F$ to $P / F$ in the prediction of ICU mortality.

Methods: We extracted data from the elCU Collaborative Research Database. The dataset included seven variables ( 6 independent and 1 dependent) and 141,535 values (Table 1). Mortality was the dependent variable for our prediction models. Exploratory data analysis was performed in Python. Missing data was imputed with Sklearn Iterative Imputer. We split the six independent variables into a train and test set with an 80/20 distribution. We scaled the data using the Sklearn Min Max Scaler. The mortality outcome (death) was rare $(136,451$ alive versus 8,184 died). We used a Gradient boosting decision tree algorithm variant called LightGBM as our model. We used AUC as our metric for model performance. Feature importance assessed using ELI5 and SHAP Python libraries. SHAP values reflect the contribution of the feature in the reduction of prediction error (3). ELI5 uses the permutation 
of featurevalues and calculates the change in error. Feature value permutation leads to a large rise in prediction error.

Results: LightGBM demonstrates good classification performance with a AUC of 0.782 (Figure 1). S/F had the strongest average contribution to predicting mortality based upon the mean calculated SHAP value (Figure 2). S/F value permutation had the largest increase in prediction error, thus it ranks as the strongest feature (Figure 3 ).

\begin{tabular}{|l|rrrrrr|}
\hline \multirow{4}{*}{ Table 1 } & \multicolumn{7}{|c|}{ count } & mean & std & min & $\max$ \\
\cline { 2 - 8 } & Age & 141535.0 & 62.23 & 16.46 & 18.0 & 89.00 \\
& PaO2 & 141535.0 & 108.73 & 43.26 & 9.0 & 636.00 \\
& FIO2 & 141535.0 & 52.22 & 13.43 & 21.0 & 100.00 \\
& SaO2 & 141535.0 & 93.05 & 8.00 & 0.0 & 100.00 \\
& P/F & 141535.0 & 225.69 & 61.15 & 17.0 & 1992.00 \\
& S/F & 141535.0 & 183.68 & 43.78 & 0.0 & 476.19 \\
\hline
\end{tabular}
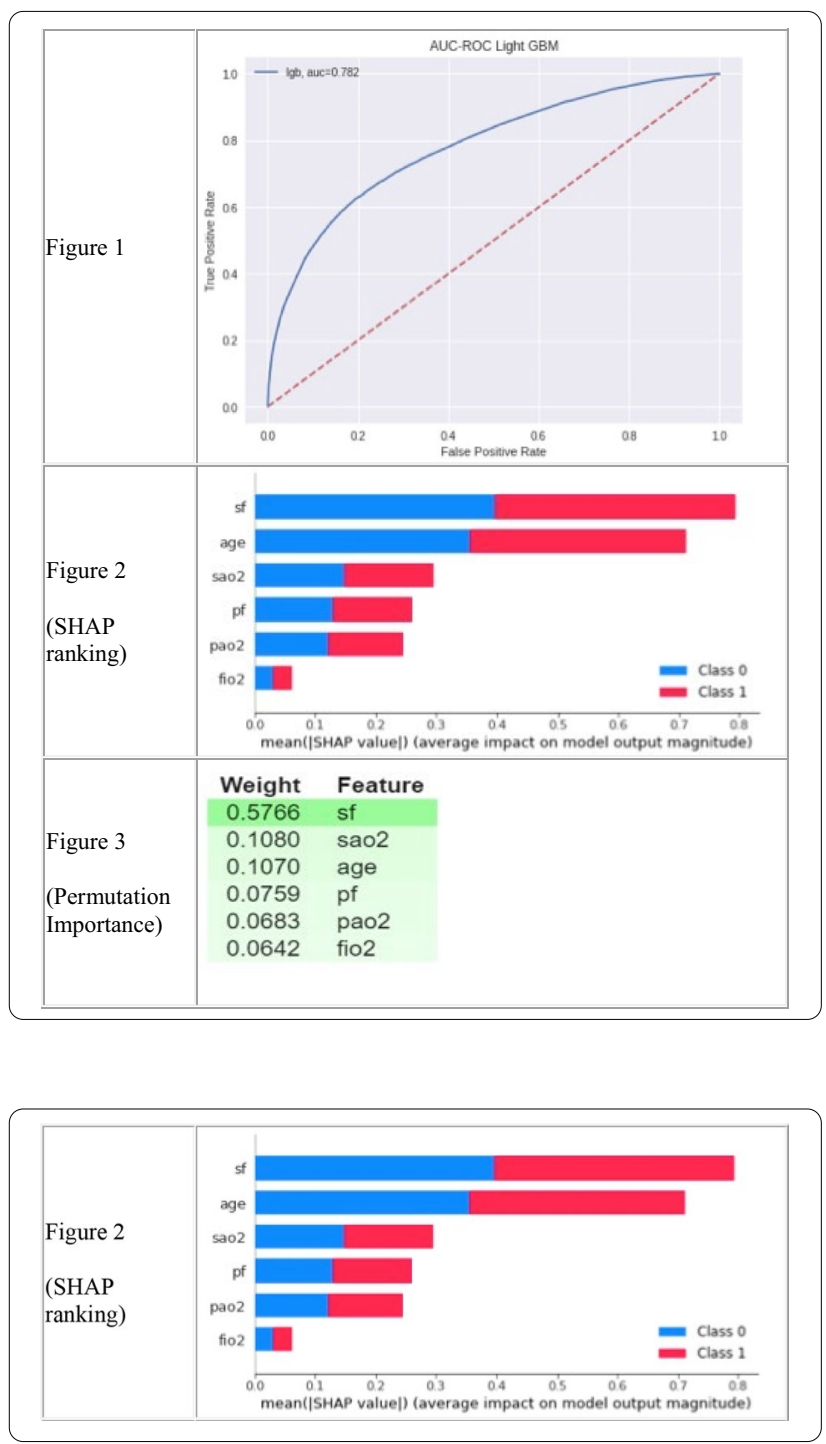

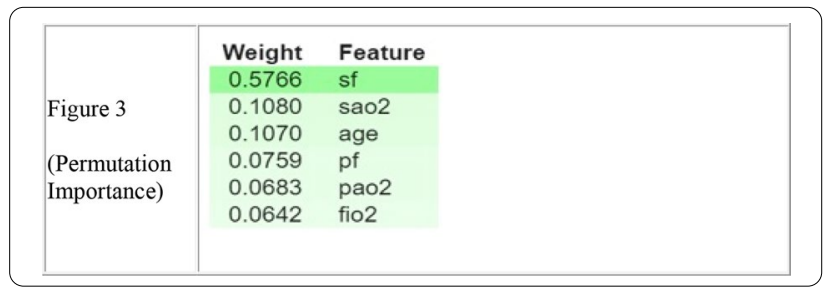

Conclusion: $\mathrm{S} / \mathrm{F}$ was a stronger predictor of mortality as compared to $\mathrm{P} / \mathrm{F}$ based upon feature importance evaluation of our data. Our study is hypothesis-generating and a prospective evaluation is warranted.

Reference(s) and grant ackowledgment(s)

1. Rice TW, Wheeler AP, Bernard GR, Hayden DL, Schoenfeld DA, Ware LB. Comparison of the $\mathrm{SpO} 2 / \mathrm{FIO} 2$ ratio and the $\mathrm{PaO} 2 / \mathrm{FIO}_{2}$ ratio in patients with acute lung injury or ARDS. Chest. 2007;132(2):410-7.

2. KwackWG, Lee DS, Min H, Choi YY, Yun M, Kim Y, et al. (2018) Evaluation of the $\mathrm{SpO} 2 / \mathrm{FiO} 2$ ratio as a predictor of intensive care unit transfers in respiratory ward patients for whom the rapid response system has been activated. PLOS ONE 13(7): e0201632.

3. Blagus, R., Lusa, L. SMOTE for high-dimensional class-imbalanced data. BMC Bioinformatics 14, 106 (2013).

000372

Clinical characteristics of critically ill patients with SARS COV 2

Infection

S. Moreno Cano ${ }^{1}$; GB. Bárbara ${ }^{1}$; B. Diez Del Corral' ; M. Perez Ruiz' ; A. Breval Flores ${ }^{1}$ : A. Estella

${ }^{1}$ University hospital of jerez., Intensive care unit, Jerez, Spain

Correspondence: S. Moreno Cano

Intensive Care Medicine Experimental 2020, 8(2): 000372

Introduction: SARS COV 2 pandemic infection has led to an increase in hospital admissions and in particular in Intensive Care Units (ICU) in last months.

Objectives: To report epidemiological characteristics and medical history of patients admitted in ICU with SARS COV 2 infection, and to describe clinical features and outcome of these patients.

Methods: Retrospective, single-center case series of consecutive critically ill patients admitted in ICU of a community hospital with severe pneumonia caused by SARS COV 2. Origin prior admission in ICU, APACHE II and SOFA at admission, medical history, age, gender, invasive mechanical ventilation requirement, time of mechanical ventilation, ICU length of stay, radiological pattern, procalcitonin and C-reactive protein levels, and mortality were collected. Statistical analysis: Data were analyzed by SPSS 18 and quantitative variables were expressed as a mean \pm standard deviation.

Results: A total of twenty patients were admitted within a monthin an ICU with 17 beds; a capacity that had to be increased. At present three patients are currently still admitted to ICU. Mortality was 30\%. $15 \%$ were admitted from the Emergency room, 15\% from other hospitalsand $70 \%$ from a medical ward. $80 \%$ required mechanical ventilation and most of these patients were ventilated in prone position at admission, $93,75 \%$. Haemofiltration was indicated in $20 \%$ and was related with higher mortality. Hypertension was the most common preexisting medical condition, $50 \%$ in deceased patients and $28,6 \%$ in survivors. 
Table shows clinical characteristics based on mortality.

\begin{tabular}{lll}
$\begin{array}{lll}\text { SARS COV 2 } \\
\text { Respiratory Infection }\end{array}$ & Survival n:14 & Exitus n:6 \\
Age (years) & $60 \pm 8,4$ & $65,67 \pm 6,86$ \\
Gender (male/female) & $64,3 / 35,7$ & $66,6 \% / 33,3 \%$ \\
Body mass index & $28,14 \pm 4,71$ & $31,64 \pm 5,53$ \\
Time from onset symptoms & $9,64 \pm 4,74$ & $12 \pm 5,6$ \\
to ICU admission (days) & \\
APACHE II at admission & $10 \pm 4,85$ & $11 \pm 2,36$ \\
SOFA at admission & $3,21 \pm 2,11$ & $4 \pm 1,09$ \\
ICU lenght of stay (days) & $13,7 \pm 10,78$ & $20,17 \pm 10,3$ \\
Creatinine/Urea at ICU & $0,84 \pm 0,28 / 44 \pm 44$ & $1,25 \pm 0,24 / 86,3 \pm 50,02$ \\
admission (mg/dl) & \\
PCR (mg/l)/PCT (ng/ml) & $154,64 \pm 190,02 / 0,47 \pm 0,64183,2 \pm 157 / 0,45 \pm 0,34$ \\
$\begin{array}{l}\text { Fibrinogen mg/dl } \\
\text { D Dimer ng/ml }\end{array}$ & $641 \pm 280,9$ & 685 \\
CONCLUSION. Obesity and hypertension were risk factors associated with severe cases of \\
SARS COV 2 infection. \\
\multicolumn{2}{l}{$\begin{array}{l}\text { Renal failure, and the need for haemofiltration and mechanical ventilation were associated } \\
\text { with anincreased mortality. }\end{array}$}
\end{tabular}

Conclusion: Obesity and hypertension were risk factors associated with severe cases of SARS COV 2 infection.

Renal failure, and the need for haemofiltration and mechanical ventilation were associated with anincreased mortality.

\section{4}

\section{Determinants of Mortality Due to Hyperglycemia}

\section{among Neurocritical Care Patients Using Machine Learning}

C. Lu'; AR. Arévalo²; E. J. Owen³; P. Mathur" A. Morca ${ }^{5}$

${ }^{1}$ Department of biomedical informatics, Harvard Medical School, Boston, United States of America; ${ }^{2}$ Idmec, IDMEC - Instituto de Engenharia Mecânica (Pólo IST), Lisboa, Portugal; ${ }^{3}$ Department of pharmacy, Barnes-Jewish Hospital, St. Louis, United States of America; ${ }^{4}$ Anesthesiology, Cleveland Clinic, Cleveland, United States of America; ${ }^{5}$ Internal medicine, Anamur State Hospital, Anamur, Turkey

Correspondence: P. Mathur

Intensive Care Medicine Experimental 2020, 8(2): 000574

Introduction: Several randomized clinical trials have suggested that different patient populations, such as medical or surgical critically ill patients, may benefit from different glucose targets [1,2]. While normoglycemia is often preferred in neuro-critically ill patients, the effects of hyper- and hypoglycemia on these patients is less clearly defined and optimal glycemic targets remain uncertain.

Objectives: We hypothesize that glycemic fluctuations may be associated with higher mortality amongst all neurocritically-ill patients, and set out to answer this question using an observational ICU dataset.

Methods: This analysis utilized the elCU Collaborative Research Database (elCU-CRD) [3]. For the 13448 adult patients diagnosed with primary central neurological disease, the following features were extracted: blood glucose measurements (finger-stick glucometer and serum values), age, diabetes status, and Acute Physiology And Chronic Health Evaluation (APACHE) IV score. Logistic regression (LR) was estimated to predict mortality during the first 24 hours of admission.

Results: When comparing characteristics for patients who survived versus those who expired(1,302,9.7\%), survivers were younger $(61.5 \mathrm{y}$ vs $65.8 y ; p<0.001)$, were more likely to have a surgical admission (14.4\% vs 9.2\%; $p<0.001)$, and have lower APACHE IV scores (48.7 vs 80.0; $\mathrm{p}<0.001)$. Additionally, survivors had lower mean glucose values (134.3 mg/dL vs $155.2 \mathrm{mg} / \mathrm{dL} ; \mathrm{p}<0.001)$ and a lower glucose variability $(-1.8$ vs $-4.0 ; p=0.001)$.
Table 1. Logistic regression results

\begin{tabular}{|c|c|c|c|}
\hline Covariate & $\begin{array}{l}\text { Adjusted } \\
\text { Odds } \\
\text { Ratio } \\
\text { (AOR) }\end{array}$ & $\begin{array}{l}95 \% \\
\text { Confidence } \\
\text { Interval for } \\
\text { AOR }\end{array}$ & $\begin{array}{l}p \text { - } \\
\text { values }\end{array}$ \\
\hline APACHE score (per point increase) & 9.14 & $8.62-9.70$ & $<0.001$ \\
\hline Diabetes & 0.64 & $0.61-0.67$ & $<0.001$ \\
\hline Mean glucose (per mg/dL increase) & 2.48 & $2.35-2.62$ & $<0.001$ \\
\hline $\begin{array}{l}\text { Mean } \Delta \text { glucose (per } \mathrm{mg} / \mathrm{dL} \\
\text { increase) }\end{array}$ & 0.82 & $0.79-0.86$ & $<0.001$ \\
\hline
\end{tabular}

The LR model achieved AUROC of 0.84 in predicting mortality by day 1 of ICU admission(Table 1). Predictor variables include diabetes status, APACHE IV score, mean glucose levels and change in glucose levels within the first 24 hours of admission.

Conclusion: Older age, higher APACHE IV score, lack of diabetes, higher mean glucose levels and greater decrease in glucose levels were associated with higher mortality within 24 hours of ICU admission in neurocritically-ill patients.

Reference(s) and grant ackowledgment(s)

1. The elCU Collaborative Research Database, a freely available multi-center database for critical care research. Pollard TJ, Johnson AEW, Raffa JD, Celi LA, Mark RG and Badawi O. Scientific Data (2018). DOI: 10.1038/sdata.2018.178. Available at: https://www.nature.com/articles/sdata2018178

2. NICE-SUGAR Study Investigators. Intensive versus conventional glucose control in critically ill patients.New England Journal of Medicine 360, 12831297 (2009).

3. Van den Berghe, G. et al. Intensive Insulin Therapy in Critically III Patients. New England Journal of Medicine 345, 1359-1367 (2001).

4. The work of Aldo Arévalo was supported by the PhD scholarship PD/ BD/114107/2015 from FCT under MIT-Portugal Program - Bioengineering Systems focus area.

\section{4}

Comparison of errors of death certificate related to poisoning between issued in emergency department and beyond emergency department

C. H. Park ${ }^{1}$

${ }^{1}$ University of Ulsan college of Medicine, Ulsan University Hospital, Ulsan, France

Correspondence: C.H. Park

Intensive Care Medicine Experimental 2020, 8(2): 000704

Introduction: We were to investigate the errors of death certificate (DC) issued after death associated with poisoning and compare the errors of DCs depending on the place of issue between the emergency department (ED) and beyond ED

Methods: We reviewed the DC related to poisoning issued in one training hospital for 9 years. The errors of DCs were categorized into major and minor errors depending on the influence for process of choosing the cause of death.

Results: Seventy-nine DCs related to poisoning were reviewed. Fourty-three DCs were issued in emergency department (ED) and thirty-six in beyond ED. Total number of errors of the DCs were 3.7 with 0.4 for major errors and 3.3 for minor errors. The errors of DCs issued at the ED are less than beyond ED (3.3 vs 4.4). Incorrect manner of death, unacceptable cause of death and mode of dying as an underlying cause of death were common major errors. Incorrect time interval from date of onset to death, incorrect other significant conditions and no record for date of onset were common minor errors.

Conclusion: The total number of errors was less in DCs issued in ED than beyond ED. The most common major error and minor error of DCs related to poisoning were incorrect manner of death and incorrect time interval from date of onset to death. 
000709

Predicting lethal courses in intensive care patients with COVID-19 using a machine learning system trained on data of intensive care patients with non-COVID-19 viral pneumonia

G. Lichtner; F. Balzer²; S. Haufe ${ }^{3}$; N. Giesa ${ }^{3} ;$ F. Schiefenhövel2;

M. Schmieding ${ }^{2}$; C. Jurth ${ }^{1}$; W. Kopp ${ }^{4}$; A. Akalin ${ }^{4}$; SJ. Schaller ${ }^{1}$;

S. Weber-Carstens ${ }^{1}$; C. Spies ${ }^{1}$; F. von Dincklage ${ }^{1}$

${ }^{1}$ Klinik für anästhesiologie m.s. operative intensivmedizin, Charité - Universitätsmedizin Berlin, Berlin, Germany; ${ }^{2}$ Einstein center digital future, Einstein Center Digital Future, Berlin, Germany; ${ }^{3}$ Klinik für neurologie mit experimenteller neurologie, Charité - Universitätsmedizin Berlin, Berlin, Germany; ${ }^{4}$ Bioinformatics platform, Berlin Institute for Medical Systems Biology, Berlin, Germany

Correspondence: F. von Dincklage

Intensive Care Medicine Experimental 2020, 8(2): 000709

Introduction: One of the challenges of the COVID-19 pandemic is the lack of experience with the disease and its clinical courses in intensive care patients. Based on first data, specific models have been developed to predict lethal courses in COVID-19 intensive care patients (1). However, it is yet unclear whether lethal courses of COVID-19 patients actually differ from lethal courses in patients with other viral pneumonia.

Objectives: The objective of this project was to analyze whether a machine learning system trained on data of intensive care patients with non-COVID-19 viral pneumonia is also able to predict lethal courses in intensive care patients with COVID-19.

Methods: After approval by the local ethics committee, we trained a machine learning system to predict lethal courses in intensive care patients with non-COVID-19 viral pneumonia. To focus on predictions early enough to allow for adjusting treatment, we based the model on clinical data that was available at the time window of $96 \mathrm{~h}-120 \mathrm{~h}$ before the death of patients in contrast to patients without a lethal course at time windows matched in regard to duration since ICU admission. Therefore, we analyzed all data available in the electronic databases of Charité - Universitätsmedizin Berlin for 463 intensive care patients (including 193 lethal courses) with non-COVID-19 viral pneumonia. From these data, we extracted 163 clinical parameters of which we selected 10 parameters, based on clinical expertise supported by univariate analyses and bivariate covariance matrices. Using the data of the 10 selected parameters, we trained a gradient-boosted decision tree models by supervised learning using the open source software library XGBoost. Then we applied the resulting prediction model to predict lethal courses in 94 COVID-19 intensive care patients (including 34 lethal courses) and compared the predictive performance of our model with that of established clinical scores.

Results: The ROC-AUC to predict lethal courses in COVID-19 patients based on our model trained on non-COVID-19 patients was 0.86 [95\%$\mathrm{Cl}$ : 0.78-0.94] and thus outperforms the established clinical scores SOFA (0.80 [95\%-Cl: 0.71-0.89]), SAPS2 (0.73 [95\%-Cl: 0.62-0.84]) and APACHE2 (0.61 [95\%-Cl: 0.49-0.73]).

Conclusion: Our machine learning system trained on data of intensive care patients with non-COVID-19 viral pneumonia shows a good performance for the prediction of lethal courses in COVID-19 intensive care patients. This suggests that despite all extraordinary features of COVID-19 intensive care patients the clinical parameters that indicate lethal courses widely match those of patients with other viral pneumonia.

\section{Reference(s) and grant ackowledgment(s)}

1. (1) Wynants $L$ et al. Prediction models for diagnosis and prognosis of covid-19 infection: systematic review and critical appraisal. BMJ 2020 Jun 3; 369:m2204.
001223

AKI in ICU patients admitted for COVID-19: Prevalence and Risk Factors

A. Marchiset ${ }^{1}$; V. Serazin²; O. Ben Hadj Salem¹; C. Pichereau'; L. Lima Da

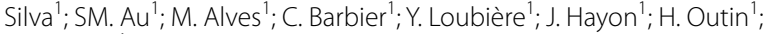

M. Jamme

${ }^{1}$ Médecine intensive réanimation, Hospital Center Intercommunal Poissy/Saint-Germain-En-Laye., Poissy, France; ${ }^{2}$ Laboratoire de biologie médicale, Hospital Center Intercommunal Poissy/Saint-Germain-En-Laye., Poissy, France

Correspondence: $M$. Jamme

Intensive Care Medicine Experimental 2020, 8(2): 001223

Introduction: Main feature of the COVID-19 outbreak is an acute respiratory failure linked with diffuse alveolar damages. There are reports of increased rates of acute kidney injury (AKI) ranging widely from $5 \%$ to $36 \%$ of hospitalized patients and rised up to $52.2 \%$ in ventilated ICU patients. Mechanism, risk factors and outcome of AKI related to COVID-19 are not fully understood.

Methods: We conducted a observational study to assess risk factors of AKI in patients admitted in ICU for COVID-19. All patients adults admitted in our ICU with COVID-19 confirmed by positive SARS-CoV2 RTPCR or COVID-19 serology between 01/03/2020 and 30/04/2020 were included. We collected all clinical and biological data within the 14 first days following admission in ICU. Primary outcome was severe AKI was defined by stage $\geq 2$ according the KDIGO definition. Statistical analysis was carried out by using a multivariate time-dependent Cox cause-specific proportional hazard model. Missing values of covariates in the multivariable model were handled bymultiple imputations with chained equations. All tests were two-sided, with $P$ values of 0.05 or less denoting statistical significance.

Results: Forty-nine patients were included in the study population and represented of 442 daily measurement points. Median age of patients was 70 [58-74] including 40 (81.6\%) men and 9 (18.4\%) women. Invasive mechanical ventilation were realized in $43(87.8 \%)$ patients and vasopressors used in 37 (75.5\%) patients. AKI occurred in $19(38.8 \%)$ patients in a median time of 6 [3-8] days following ICU admission. Time-dependant Cox cause specific model showed that hemodynamic SOFA score ( $\mathrm{CSH}=1.52$ [1.06-2.16], $\mathrm{p}=0.02)$, arterial pCO2 (1.27 [1.06-1.53] per increase of $\left.5 \mathrm{~cm} \mathrm{~d} \mathrm{~d}^{\prime} \mathrm{H} 2 \mathrm{O}\right)$ and arterial base excess $(0.88$ [0.78-0.97] per increase of $1 \mathrm{mmol} / \mathrm{L})$ was associated with AKI. A statistical trend was observed with expiratory positive pressure (1.15 [0.99-1.34] per increase of $1 \mathrm{~cm} \mathrm{d'} \mathrm{H} 2 \mathrm{O}, \mathrm{p}=0.05)$.

Conclusion: Our findings show that severe AKI in a common complication observed in ICU patients admitted for COVID-19. Respiratory status with EPP levels, hypercapnia, metabolic acidemia and hemodynamic deficiency are associated with higher risk of AKI in COVID-19 patients. Impact of mechanical ventilation, with EPP levels, and severity of patient's respiratory state, highlighted by respiratory acidosis, are novel risk factors of AKI in ICU patients. Further studies are needed to confirm those findings and understand underlying mechanism of this pulmonary-kidney interaction.

\section{1}

Acute kidney injury in critically ill COVID-19 patients: incidence and outcome

W. Zarrougui ${ }^{1}$; N. Bejaoui ${ }^{1}$; M. Alibenali ${ }^{1}$; N. Elbeki ${ }^{1}$; F. Lambiotte ${ }^{1}$

${ }^{1}$ Intensive care unit, Hospital Jean Bernard, Valenciennes, France

Correspondence: W. Zarrougui

Intensive Care Medicine Experimental 2020, 8(2): 001281

Introduction: In December 2019, coronavirus disease 2019 (COVID19) emerged in Whuan, China and had rapidly spreading worldwide, to become the most global health issue nowadays. Kidneys are one of the extra pulmonary organs frequently affected by this infection. Acute kidney injury $(\mathrm{AKI})$ is a well-recognized risk factor which increased the mortality rate in critically ill patients with sepsis. 
Objectives: The aim of this study was to determine the frequency and risk factors of AKI in COVID-19 critically ill patients as well as to establish clinical and prognosis profile.

Methods: We retrospectively reviewed the charts of consecutive critically ill patients with confirmed COVID-19 infection between Mars 11 and Mai 15, 2020 in Valenciennes regional hospital. The study collected clinical features at admission, severity of illness, ICU course, the occurrence and management of AKI. The diagnosis of AKI was based on kidney disease improving global outcomes (KDIGO) classification. Univariate and multivariate regression analyses were performed to identify factors independently associated with AKI, and to determine the risk of mortality secondary to AKI

Results: A total of 77 confirmed COVID-19 patients were admitted to the ICU during the study period. Among them $37(48.05 \%)$ presented an acute renal failure. Their main characteristics were: mean age, $64.67 \pm 11.02$; male, 29(78.4\%); mean $\mathrm{BMl}, 31.97 \pm 6.7 \mathrm{~kg} / \mathrm{m}^{2}$; mean Charlson index, $3.03 \pm 1.99 ; 4(10.8 \%)$ patients with medical history of chronic kidney disease; mean SAPSII score, $39.78 \pm 11.28$; mean SOFA score, 5.56 2.93; invasive mechanical ventilation, 32(86.5\%); vasopressors, 33(89.2\%); hydrocortisone, 14(37.8\%). Renal replacement therapy (RRT) was required in 24(64.9\%) patients and the most used modality was an intermittent hemodialysis in 10 patients (41.7\%). Continuous veno-venous hemodialysis was used in 3(12.5\%) patients and $9(37.5 \%)$ patients had received both modalities of hemodialysis. Mortality rate was $21(56.8 \%)$ and the mean length of ICU stay was $19.46 \pm 17.72$ days. For ICU survivors, only 4 patients had their ad integrum renal function restored and no patient need dialysis after hospital discharge.

Univariate then multivariate regression analysis identified the AKI as an independent risk factor for mortality in COVID-19 patients (OR, $17.74 ; 95 \% \mathrm{Cl}[4.22-74.52], \mathrm{p} \quad 0.000$ ), and yielded the following variables as independently associated to AKI: age $>65$ years (OR, 3.77; 95\% CI[1.19-11.91], p 0.024), septic shock (OR, 5.54; 95\% CI[1.49-20.5], $\mathrm{p} 0.01$ ) and the use of muscles blocker agents (OR, 4.26; 95\% CI[1.3413.5], p 0.014).

Conclusion: The present study among COVID-19 critically ill patients identified acute kidney injury as independent factor associated with mortality and showed that the incidence of acute renal failure, increasing in patients aged more than 65 years, presented septic shock and had received muscle blocker agents during their ICU stay.

\section{2}

Risk factors associated with acute renal failure in critically ill patients

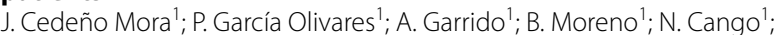

C. Ramirez' ; M. Sancho '; J. Peral' ; S. Arenal' ; JM. Gomez ; P. Oliver ${ }^{1}$

'Intensive care unit, H.G.U Gregorio Marañón, Madrid, Spain

Correspondence: P. García Olivares

Intensive Care Medicine Experimental 2020, 8(2): 001342

Introduction: The cause of acute renal failure is often multifactorial with pre-existing co-morbidities further increasing the risk.

Objectives: To analyze the risk factors related to acute renal failure (ARF) in critically ill patient.

Methods: Prospective, observational study performed on patients admitted to ICU of HGU Gregorio Marañón between NovemberDecember 2019. Epidemiological data, ARF risk factors, patient clinical characteristics and renal function data, were collected. ARF was defined according to AKIN, RIFLE and KDIGO scores.

Descriptive data were reported as means with standard deviation (SD) for normally distributed continuous variables, medians with interquartile range (IQR) for non-normally distributed variables, and as percentages for categorical data. Univariate analysis was performed by means of Chi-square (RR) for the qualitative variables, and simple logistic regression (OR) for the quantitative variables. The risk factors related to acute renal failure were obtained by means of a multiple logistic regression analysis.

Results: Ninety-five patients included. $56 \%$ were male, mean age was $60 \pm 17$ years and Charlson comorbidity index 2 points (1-4).
The main risk factors for ARF were hypertension $45 \%$, dyslipidaemia $41 \%$, peripheral vascular disease $34 \%$, chronic heart failure $18 \%$, diabetes mellitus $16 \%$ and arrhythmias $13 \%$. Reasons for admission: neurological $30 \%$, respiratory $26 \%$ and $20 \%$ infectious pathology. Severity scores: APACHE II $19 \pm 8$, SOFA $7 \pm 4$, the $52 \%$ of patients needed mechanical ventilation and $51 \%$ vasoactive therapy. $34 \%$ of patients developed ARF during admission, according to the following scales: $14 \%$ stage $\mathrm{I} /$ risk, $8 \%$ stage II/damage, $12 \%$ stage III/failure. $33 \%$ of patients with ARF required renal replacement therapy. The Inhospital mortality was $29 \%$, with an average of 11 days of hospital stay (6-29).

In the univariate analysis, the variables related to ARF were: age (OR $1.05 ; 95 \% \mathrm{Cl} 1.01-1.08$ ), diabetes (RR 5.27; 95\% Cl 1.62-17.17), chronic heart failure (RR 3.64; $95 \% \mathrm{Cl} 1.23-10.76$ ), arrhythmias (RR 4.92; 95\% $\mathrm{Cl} 1.35-17.87$ ), infectious pathology (RR 4.80; $95 \% \mathrm{Cl} 1.66-13.89$ ), APACHE II (OR $1.21 ; 95 \% \mathrm{Cl} 1.12-1.32)$, SOFA (OR 1.58; 95\% Cl 1.291.94) and vasoactive therapy (RR $3.98 ; 95 \% \mathrm{Cl} 1.54-10.29$ ). In the multivariate analysis, on a maximum model including the previously described, diabetes (OR 14.90; 95\% Cl 2.95-75.28) and SOFA (OR 1.45; $95 \% \mathrm{Cl} 1.13-1.86$ ) were the only variables independently associated with acute renal failure.

Conclusion: There is a high incidence of acute renal failure in critically ill patients. The only factors independently associated with the development of acute renal failure were diabetes and SOFA.

\section{3}

NephroCheck test to help assess the risk of acute kidney injury in trauma patients

S. De Rosa'; M. Greco²; G. Golino'; A. Rigobello'; G. Pierbellini'; P. Navalesi3 C. Ronco ${ }^{4}$; V. Danzi

${ }^{1}$ Department of anesthesia and intensive care unit, San Bortolo Hospital Of Vicenza, Vicenza, Italy; ${ }^{2}$ Anesthesia and intensive care, Humanitas Research Hospital, Milano, Italy; ${ }^{3}$ Department of anesthesia and intensive care unit, University of Padua, Padova, Italy; ${ }^{4}$ Department of nephrology, dialysis, and transplantation, San Bortolo Hospital Of Vicenza, Vicenza, Italy Correspondence: S. De Rosa

Intensive Care Medicine Experimental 2020, 8(2): 001373

Introduction: Acute kidney injury is frequently observed in trauma patients. A rise in serum creatinine and a fall in urine output are consequences of much earlier injury to the most sensitive part of tubular cells located at the proximal tubule. The $\mathrm{G} 1$ cell cycle inhibitors tissue inhibitor metalloproteinase-2 (TIMP-2) and insulin-like growth factorbinding protein 7 (IGFBP7) have been identified as novel biomarkers for the prediction of moderate to severe acute kidney injury (AKI) risk.

Objectives: The aim of the present study was to investigate the course of two cell-cycle arrest urinary biomarkers compared to serum creatinine in trauma patients.

Methods: A bedside test known as NephroCheck measures two urinary parameters: IGFBP7 and TIMP-2. The test is based on a sandwich immunoassay technique. The final test output, labeled AKIRisk, is shown as a numeric result. We conducted a cohort study, measuring [TIMP2]-[IGFBP7] levels in trauma patients admitted to the ICU and classified the patients as NephroCheck (NC) (+) or NC (-) according to [TIMP-2]-[IGFBP7] values and AKI (+) or AKI (-) according to Kidney Disease: Improving Global Outcomes (KDIGO) criteria

Results: 45 patients developed AKI (29.4\%.) 29 patients (19.9\%) developed stage $1 \mathrm{AKI}, 16$ patients (10.5\%) stage 2-3 AKI. RRT was used in 5 patients. In patients with no AKI at admission, AKI at 24h was associated with higher nephrocheck values at admission (median AKIrisk 3.49 (2.33-3.73) in stage 2-3 AKI vs 0.35 (0.14-0.82) in no AKI, $p=0.011)$ but not with creatinine values at admission $(0.99 \mathrm{mg} / \mathrm{dl}(0.9-1.03)$ in stage $2-3 \mathrm{AKI}$ vs $0.79 \mathrm{mg} / \mathrm{dl}(0.66-0.99))$. There was no difference in nephrocheck values at admission or creatinine values at admission in patients with AKI was present at ICU admission. In patients with AKI, there was no association between Nephrocheck and progression or regression of $A K I$ ( $p 0.4$ for progression and $p=0.2$ for regression of AKI).We defined 4 groups according to levels of biological and functional markers 


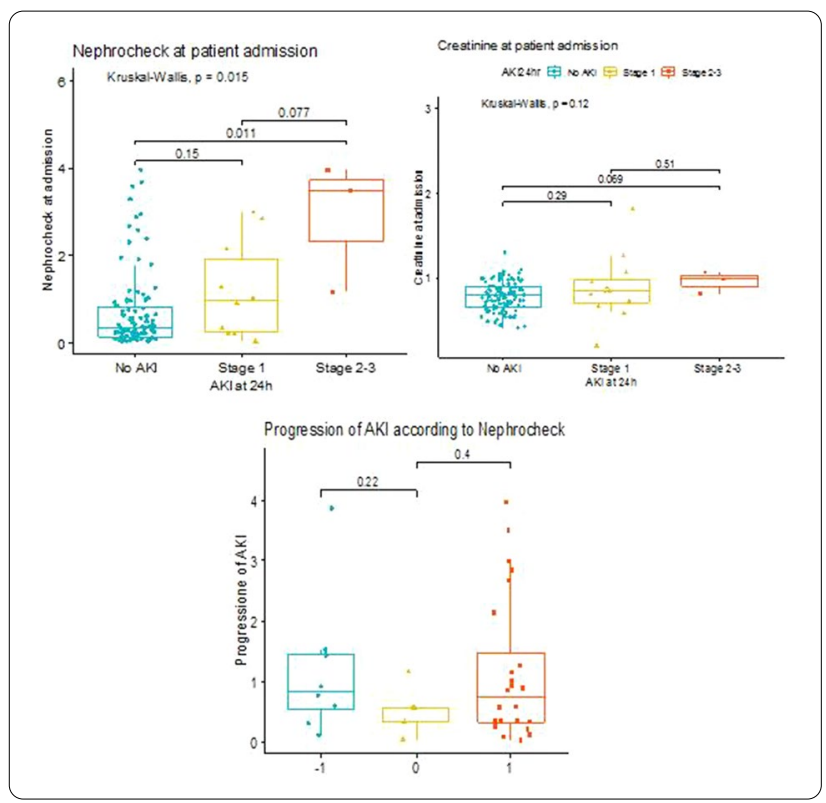

Conclusion: Markers of acute tubular stress/damage such as TIMP-2 and IGFBP7 (cell cycle arrest biomarkers identified as NC are useful in the risk assessment for AKI with high ability to identify patients likely to develop stage $2 / 3 \mathrm{AKI}$ within $24 \mathrm{hrs}$. NC may help to identify patients with tubular damage in trauma patients that may or may not evolve into a clinically manifest syndrome

\section{Reference(s) and grant ackowledgment(s)}

1. Burmeister DM, Gómez BI, Dubick MA. Molecular mechanisms of traumainduced acute kidney injury: Inflammatory and metabolic insights from animal models. Biochim Biophys Acta Mol Basis Dis. 2017;1863(10 Pt B):2661-2671

2. Lisowska-Myjak B. Serum and urinary biomarkers of acute kidney injury. Blood Purif. 2010;29(4):357-365.

3. None

\section{5}

Management of renal dysfunction in COVID-19 patients admitted to the ICU

JM. Martín Cano ${ }^{1}$; M. Pérez Ruiz' ; B. Diez Del Corral' ${ }^{\text {; J. Villegas }}{ }^{1}$

${ }^{1}$ University hospital of jerez., Intensive care unit, Jerez, Spain

Correspondence: B. Diez Del Corral

Intensive Care Medicine Experimental 2020, 8(2): 001405

Introduction: Diffuse alveolar damage and acute respiratory failure are the main features of COVID-19 patients admitted to the ICU, although it may also cause damage to other organs such as the kidneys. Many of these patients with SARS CoV2 infection develop acute renal failure, and their prognostic results are worse than in those who do not develop it. More attention should be paid to comorbidities in these patients, that can be related to the infection course.

Objectives: - To analyze renal support therapies and the need of other support devices used in patients admitted to the ICU with the diagnosis of SARS-CoV-2 infection who develop renal dysfunction.

- To study the association of this organic failure with morbidity and mortality.

Methods: This retrospective observational study was conducted in the medico-surgical Intensive Care Unit of Jerez de la Frontera Hospital. All the patients admitted to the ICU, with the diagnosis of SARS-CoV-2 infection, during the study period (March-June 2020) were included. Personal history, demographic, analytical and clinical variables, as well as severity and mortality scores were collected. Statistical analyses were performed using SPSSV22 software.
Results: Eleven patients, predominantly male $(72.7 \%)$, with a mean age of $63.55 \pm 7.53 \mathrm{SD}$, were included. With regard to the severity scales, mean SOFA value at admission was 4.27 points \pm 2 SD and APACHE II 11.45 points $\pm 4.2 \mathrm{SD}$, with SOFA mean value on the day of the diagnosis of ARF of 9.1 points $\pm 3.2 \mathrm{DS}$. When analyzing CVRF, it should be noted that these patients had a high BMI with mean values of $31.93 \pm 4.68 \mathrm{SD} \mathrm{cm} / \mathrm{m} 2$. These patients required IMV and prone position in $100 \%$ of the cases, requiring $81.8 \%$ to perform a tracheostomy and $90.9 \%$ hemodynamic support with norepinephrine. $63.7 \%$ (7) required CRRT, being CVVH the predominant modality of choice $(100 \%)$. With regard to the indications of renal support, the most frequent was oligoanuria $57.1 \%$ (4) followed by metabolic or ionic alterations in $28.6 \%$ (2) and refractory shock $14.3 \%$ (1). The most frequently venous access used was the right femoral one (42.9\%) compared to the right jugular venous access used in $28.6 \%$ of cases (2). Heparin sodium was the anticoagulant of choice in $100 \%$ of the patients who required CRRT. When analyzing the scales of renal damage, we found that the day the ARF was diagnosed they presented: AKIN I 45.5\%, II $18.2 \%$, III 36.4\%; RIFLE R 45.5\%, I 9\%, F 45.5\%. Mortality was very high, reaching $63.7 \%(7)(p<0.05)$ and in those who required CRRT was $100 \%(\mathrm{p}<0.05)$, despite of improving renal function according to creatinine criteria in $71.4 \%$ (5).

Conclusion: - In our series of cases, renal failure and the use of CRRT were independently associated with worst prognostic results.

- Those who developed ARF had a significantly higher BMI and required more organic support than those who did not develop it.

\section{5}

Incidence of renal failure in patients with SARS_CoV 2 infection admitted to the ICU

JM. Martín Cano ${ }^{1}$; M. Pérez Ruiz' ; B. Diez Del Corral' ${ }^{\text {; J. Jillegas }}{ }^{1}$

${ }^{1}$ University hospital of jerez., Intensive care unit, Jerez, Spain

Correspondence: B. Diez Del Corral

Intensive Care Medicine Experimental 2020, 8(2): 001415

Introduction: COVID-19 disease is mainly characterized by acute respiratory failure and diffuse alveolar damage, but it may also cause damage to other organs such as the kidneys. Many of these patients develop acute renal failure, and may have a worseprognosis.

Objectives: - To look-over the incidence of kidney failure in COVID-19 patients admitted to the ICU of a second-level hospital.

- To analyze if there are differences between patients with kidney failure and those who do not develop this complication.

- To determine whether the development of renal failure is related to mortality.

Methods: This retrospective observational study was conducted in a medico-surgical Intensive Care Unit of a second-level hospital. All the patients, with positive PCR for SARS-CoV2, admitted to the ICU during the study period (March-June 2020) were included. Personal history, demographic, analytical and clinical variables, as well as severity and mortality scores were collected. Statistical analyses were performed using SPSSV22 software.

Results: Twenty patients, predominantly male, $63 \%$ (13), with a mean age of $61.8 \pm 8.2 \mathrm{SD}$ and BMI $28.9 \mathrm{~cm} / \mathrm{m} 2 \pm 4.9 \mathrm{SD}$, were included. With regard to the severity scales, we found a mean SOFA value at admission of 3.6 points \pm 2.1 SD and APCHE II $10.25 \pm 4.1$ SD. The most prevalent comorbidities were HTN 45\% (9); DLP 40\% (8); DM 20\% (4). The average ICU stay was 23.5 days. $55 \%$ (11) of the patients developed renal failure with a mean ICU stay of 7.8 days. $63.7 \%$ (7) required CRRT. The average SOFA on the day of the diagnosis of ARF was 9.1 points \pm 3.2DS, presenting AKIN I 45.5\%, II 18.2\%, III 36.4\%; RIFLE R $45.5 \%$, I 9\%, F 45.5\%. We found significant differences, between the total series and the patients who developed renal failure, in the following variables: $\mathrm{BMI}(31.9 \mathrm{~cm} / \mathrm{m} 2 \mathrm{vs} 25.2 \mathrm{~cm} / \mathrm{m} 2)$, Creatinine $(1.2 \mathrm{mg} /$ dl vs $0.8 \mathrm{mg} / \mathrm{dl}), \mathrm{pH}$ (7.03 vs 7.31$)$, bicarbonate ( $19.7 \mathrm{mmol} / \mathrm{L}$ vs 25.3 $\mathrm{mmol} / \mathrm{L})$ at admission or during the ICU stay $(\mathrm{p}<0.05)$. With regard to the mortality, we found that patients who developed ARF had a much higher mortality, $63.7 \%$ (7), compared to those who did not have this 
organic failure $(35 \%)(p<0.05)$. Those who required CRRT had a mortality of $100 \%(7)(p<0.05)$.

Conclusion: - Patients admitted to our unit, infected with SARS-CoV 2 , developed kidney failure in a high percentage.

- The presence of ARF is associated with a higher mortality compared to those who do not develop it.

- Renal failure in COVID-19 patients who require continuous renal replacement therapies reach $100 \%$ mortality in our series.

\section{6}

\section{MR-proAdrenomedullin in critically ill patients with COVID-19} requiring renal replacement therapy

K. Roedl ${ }^{1}$; D. Jarczak'; M. Fischer ${ }^{1}$; M. Haddad ${ }^{2}$; O. Boenisch ${ }^{1}$; G. De Heer ${ }^{1}$; C. Burdelski ${ }^{1}$; D. Frings ${ }^{1}$; B. Sensen ${ }^{1}$; M. Karakas ${ }^{3}$; S. Kluge ; A. Nierhaus ${ }^{1}$

${ }^{1}$ Department of intensive care medicine, University Medical Center Hamburg-Eppendorf, Hamburg, Germany; ${ }^{2}$ Institute of clinical chemistry and laboratory medicine, University Medical Center Hamburg-Eppendorf, Hamburg, Germany; ${ }^{3}$ Department of general and interventional cardiology, University Heart Center Hamburg-Eppendorf, Hamburg, Germany Correspondence: K. Roedl

Intensive Care Medicine Experimental 2020, 8(2): 001426

Introduction: A novel coronavirus (SARS-CoV-2) caused a worldwide pandemic and human health emergency. Worldwide coronavirus disease 2019 (COVID-19) has led to a large number of hospital and ICU admissions. Critically ill patients with COVID-19 suffer from high morbidity and mortality. About $20 \%$ of critically ill COVID-19 patients need renal replacement therapy (RRT). Data on early prediction of acute kidney injury and RRT requirement are lacking. Mid-regional pro-adrenomedullin (MR-proADM), is an early marker of endothelial dysfunction predicting organ dysfunction in septic shock.

Objectives: The aim of this study was to investigate MR-proADM for prediction of RRT in critically ill patients with COVID-19.

Methods: We conducted a single-center observational study and consecutively included all adult patients with microbiologically confirmed COVID-19 treated at our department of intensive care medicine. Patient characteristics, severity of disease, course of the disease and ICU mortality were assessed. MR-proADM together with other biomarkers of inflammation was measured as part of clinical routine.

Results: Overall we could identify 64 critically ill patients with COVID19. 49 patients $(77 \%)$ were male, median age was $62.5(54-73)$ years. $47(73 \%)$ patients required mechanical ventilation. 25 (39\%) patients had severe ARDS, and veno-venous extracorporeal membrane oxygenation was established in 10 patients. 50 (78\%) patients needed vasopressor therapy. 29 (45\%) patients required RRT during the ICUstay. The median time from admission on ICU to start of RRT was 2 (1 9) days. MR-proADM on admission was significantly higher in patients requiring RRT ( 2.491 vs. $1.230 \mathrm{nmol} / \mathrm{l} ; \mathrm{p}=0.002$ ). ROC curve analysis showed that MR-proADM served as predictor of requirement for RRT with an AUC of 0.68 ( $95 \% \mathrm{Cl}: 0.54-0.81 ; \mathrm{p}=0.02)$. Multivariate logistic regression showed that MR-proADM was an independent predictor (OR: $4.02,95 \% \mathrm{Cl} 1.13$ to $14.27, \mathrm{p}<0.05$ ) for the requirement of RRT.

Conclusion: MR-proADM was able to independently predict RRTrequirement in critically ill patients with COVID-19 upon admission to the ICU.

\section{2}

\section{Impact of Chloremia Levels on Renal prognosis in Intensive Care} Unit Patients

M. Jamme ; A. Robert ${ }^{2}$; Z. Massy²; B. Stengel ${ }^{3}$; M. Essig²; G. Geri ${ }^{4}$

${ }^{1}$ Médecine intensive Réanimation, Hospital Center Intercommunal Poissy/ Saint-Germain-En-Laye., Poissy, France; ${ }^{2}$ Néphrologie, Ambroise Paré Hospital (AP-HP), Boulogne-Billancourt, France; ${ }^{3}$ Cesp, equipe epidemiologie clinique, Inserm, Villejuif, France; ${ }^{4}$ Médecine intensive réanimation, Ambroise Paré Hospital (AP-HP), Boulogne-Billancourt, France

Correspondence: $M$. Jamme

Intensive Care Medicine Experimental 2020, 8(2): 001462
Introduction: Acute kidney injury (AKI) is one of the most common complication observed in ICU patients. Besides classical risk factors associated with AKI occurrence, several studies have found a protective renal effect favoring the use of balanced solution. This could have been explained by the deleterious effects of hyperchloremia acidosis induced by the administration of a highly concentrated solution in chlorine.

Objectives: We aimed to assess the association of chloremia $[\mathrm{Cl}]$ with AKI occurrence.

Methods: We included all adults patients admitted to a tertiaty ICU between June 1st2006 and June 13th2019. AKI was defined by KDIGO stage $\geq 2$. Hypo and hyperchloremia were defined as $[\mathrm{Cl}]<95 \mathrm{mmol} / \mathrm{L}$ and $>110 \mathrm{mmol} / \mathrm{L}$, respectively. We assessed the relationship between chloremia and AKI occurrence within ICU stay as follows: Cox cause specific model using the first chloremia at ICU admission and timedependant Cox cause specific model using all chloremia recorded within ICU stay before AKI occurrence.

Results: We included 4,908 patients. Median age was 65 [51-76] years with mostly males (58.1\%). At ICU admission, sepsis was observed in $63.2 \%$, acute respiratory failure in $47.2 \%$ and shock in $37.5 \%$. AKI occurred in $896(18.2 \%)$ patients in a median time of 4 [2-9] days. 388 (7.9\%) patients died in ICU without AKI. Using first chloremia at ICU admission, hypochloremia and hyperchloremia were both associated with AKI with Cox cause specific hazard ratio (CSH) of respectively 1.40 (95\% Cl [1.07-1.09], $\mathrm{p}=0.01$ ) and 1.60 (95\%Cl [1.09-2.39], $\mathrm{p}=0.002$ ). Considering all chloremias before AKI as time dependent covariate, the statistical association was still observed but stronger with hyperchloremia (CSH $=2.39$ [1.75-3.27], $\mathrm{p}<0.001$ ) than hypochloremia (CSH $=1.86$ [1.49-2.33], $\mathrm{p}=0.02$ ).

Conclusion: Abnormal chlore levels was associated with AKI occurrence in a large cohort of ICU patients. While both hypochloremia and hyperchloremia were associated with AKI, hyperchloremia seemed to be strongly associated with renal outcome.

Reference(s) and grantackowledgment(s)

1. MJ was granted by OpenHealth Institute

\section{5}

D-dimer as a Predictor of Acute Kidney Injury in the Covid-19

Patient Cohort

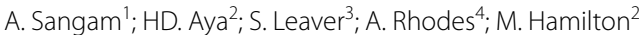

${ }^{1}$ Critical Care Medicine, St George's Hospital, London, United Kingdom; ${ }^{2}$ General intensive care unit, St George's Hospital, London, United Kingdom; ${ }^{3}$ Research lead critical care directorate, St George's Hospital, London, United Kingdom; ${ }^{4}$ Intensive care unit, St George's Hospital, London, United Kingdom

\section{Correspondence: A. Sangam}

Intensive Care Medicine Experimental 2020, 8(2): 001555

Introduction: From the Covid-19 pandemic of 2020 it is evident that this new condition, which largely presents to critical care as respiratory failure is, in fact, a multisystem entity $(1,2$,$) . Although data interpreta-$ tion thus far has identified clinical indices, such as elevated D-dimer, that are associated with increased likelihood of critical care admission or an increased mortality (3), less is currently understood about how such markers can prognosticate specific organ failures such as acute kidney injury, and the need for specific critical care interventions such as renal replacement therapy.

Objectives: Our aim was to assess whether a persistently elevated D-Dimer is predictor of acute kidney injury (AKI) in critically ill patients admitted with Covid-19 infection.

Methods: This is a retrospective, observational study examining the medical records of all adult patients admitted to critical care at St George's Hospital in South London between 1st March 2020 and 31st May 2020. The D-dimer level was recorded at critical care admission, at day 7 and day 14. AKI was defined as per the AKIN guidelines(4). Requirement of renal replacement therapy (RRT) and mortality after 28 days of admission were secondary outcomes. Proportions were compared using chi-squared statistic, and odds ratios are reported with $95 \%$ confidence interval (CI). 
Results: From 132 patients, D-Dimer was measured in 95 (72\%), with a median value of $1294(503,5520) .24 .2 \%$ had a normal D-Dimer on admission, $49.5 \%$ had a D-Dimer between $500-6000,26.3 \%$ had a D-Dimer greater than 6000 (the maximum laboratory value). 16 (69.6\%) patients with a normal D-Dimer on admission had AKI compared to $63(87.5 \%)$ patient with elevated D-Dimer $(p=0.045$, OR 2.4 , $95 \% \mathrm{Cl} 1.02,5.8) .9$ (39\%) patients with a normal D-Dimer on admission required RRT compared to 25 (34.7\%) patients with elevated D-Dimer $(p=0.7) .9$ (39\%) patients with normal D-Dimer on admission died compared to $43(60 \%)$ in the group of elevated D-Dimer $(p=0.084)$. At day 7,71 patients remained in the ICU: 2 of $5(40 \%)$ patients with normal D-Dimer had AKI, compared with 51 of 66 (77.3\%) with elevated D-Dimer $(p=0.065)$. At day 14, 51 patients remained in the ICU: 1 of $4(25 \%)$ patients with normal D-Dimer had AKI compared to 31 of 47 $(66 \%)$ with elevated D-Dimer $(p=0.10)$

Conclusion: An elevated D-dimer on admission, but not a persistent elevation, is a predictor of AKI in critically ill patients with Covid-19.

\section{Reference(s) and grant ackowledgment(s)}

1. 4. Mehta RL, Kellum JA, Shah SV, et al. Acute kidney injury network: report of an initiative to improve outcomes in acute kidney injury Crit Care. 2007

2. 3. Zhang $L$, Yan $X$, Fan $Q$, et al. D-dimer levels on admission to predict inhospital mortality in patients with Covid-19. J Thromb Haemost, 2020;18(6): 1324-1329

3. 2. Tang N, Li D, Wang X, Sun Z. Abnormal coagulation parameters are associated with poor prognosis in patients with novel coronavirus pneumonia. $J$ Thromb Haemost. 2020;18(4):844-847

4. 1. Guan WJ, Ni ZY, Hu Y, et al. Clinical Characteristics of Coronavirus Disease 2019 in China. N Engl J Med. 2020.

\section{5}

\section{Early diagnosis of Acute Kidney Injury in Critically III patients} D. Paz

'Intensive Care Unit, Coyoacan, Mexico City, CDMX, Mexico, Mexico Correspondence: D. Paz

Intensive Care Medicine Experimental 2020, 8(2): 001615

Introduction: The incidence of acute kidney injury in critically ill patients is gradually increasing, it indicates a poor prognosis with higher morbidity and mortality. Failure on acute diagnosis may delay the onset of an adequate therapy which may cause an unfavorable outcome, such as the need for renal replacement therapy, generating high costs to the health system. The validation of new methods that identify the presence of acute kidney injury in its early stages is needed.

Objectives: To demonstrate which is the earliest marker for the diagnosis of acute kidney injury in critically ill patients, including creatinine, cystatin C, urinary sediment and N- GAL.

Methods: A prospective, longitudinal and descriptive study is presented, with data obtained from patients over 18 years of age admitted to Angeles Lomas Hospital Intensive Care Unit, between October 2019 and July 2020.

\begin{tabular}{|c|c|c|c|c|}
\hline Caracteristics & Total $(n=20)$ & AKI present $(n=12)$ & AKI absent $(n=8)$ & p Value \\
\hline$\overline{~ S e r u m ~ C r e a t i n i n e ~}(\mathrm{mg} / \mathrm{dL})$ & $1.32 \pm .5$ & $1.58 \pm .6$ & $0.92 \pm .2$ & $<0.009$ \\
\hline N-GAL (ng/dL) & $191 \pm 207.4$ & $274.1 \pm 232.3$ & $63.3 \pm 48.3$ & $<0.024$ \\
\hline Cistatina C (ml/min) & $65.8 \pm .24 .8$ & $56.7 \pm 21.3$ & $79.5 \pm 24.7$ & $<0.042$ \\
\hline Cilinders in urine & & & & $<0.036$ \\
\hline Absent & $15(75 \%)$ & $7(46.6 \%)$ & $8(53.3 \%)$ & \\
\hline Present & $5(25 \%)$ & $5(100 \%)$ & $0(0 \%)$ & \\
\hline
\end{tabular}

SD. Pvalue for the comparison between IPA present vs absent.

Results: Serum creatinine and cylinders in urine had similar capacity to detect cases of AKI (baseline sensitivity $57.1 \%$, specificity $100 \%$ and sensitivity $41.7 \%$, specificity $100 \%$, 24 hours sensitivity $58.3 \%$, specificity $100 \%$ and sensitivity $57.1 \%$, specificity $100 \%$ respectively).

\begin{tabular}{|c|c|c|c|c|}
\hline Caracteristics & Total $(n=20)$ & AKI present $(n=14)$ & AKI absent $(n=6)$ & p Value \\
\hline Serum Creatinine (mg/dL) & $1.5 \pm .9$ & $1.9 \pm 1$. & $0.86 \pm .181$ & $<0.027$ \\
\hline $\mathrm{N}-\mathrm{GAL}(\mathrm{ng} / \mathrm{dL})$ & $295 \pm 298$ & $393.2 \pm 307.6$ & $65.8 \pm 41.9$ & $<0.020$ \\
\hline Cistatina C ( $\mathrm{ml} / \mathrm{min})$ & $60.7 \pm .28 .3$ & $50.7 \pm 25$ & $84.1 \pm 22$ & $<0.011$ \\
\hline Cilinders in urine & & & & $<0.015$ \\
\hline Absent & $12(60 \%)$ & $6(50 \%)$ & $6(50 \%)$ & \\
\hline Present & $8(40 \%)$ & $8(100 \%)$ & $0(0 \%)$ & \\
\hline
\end{tabular}

Cystatin C clearance showed better sensitivity (75\% and $78.6 \%$ ) but its specificity was reduced when detecting the mild and moderate cases of AKI (62.5\% and $83.3 \%)$. N-GAL showed the best sensitivity $(83.3 \%$ and $85.7 \%$ ) and specificity (100\% in both scenarios).
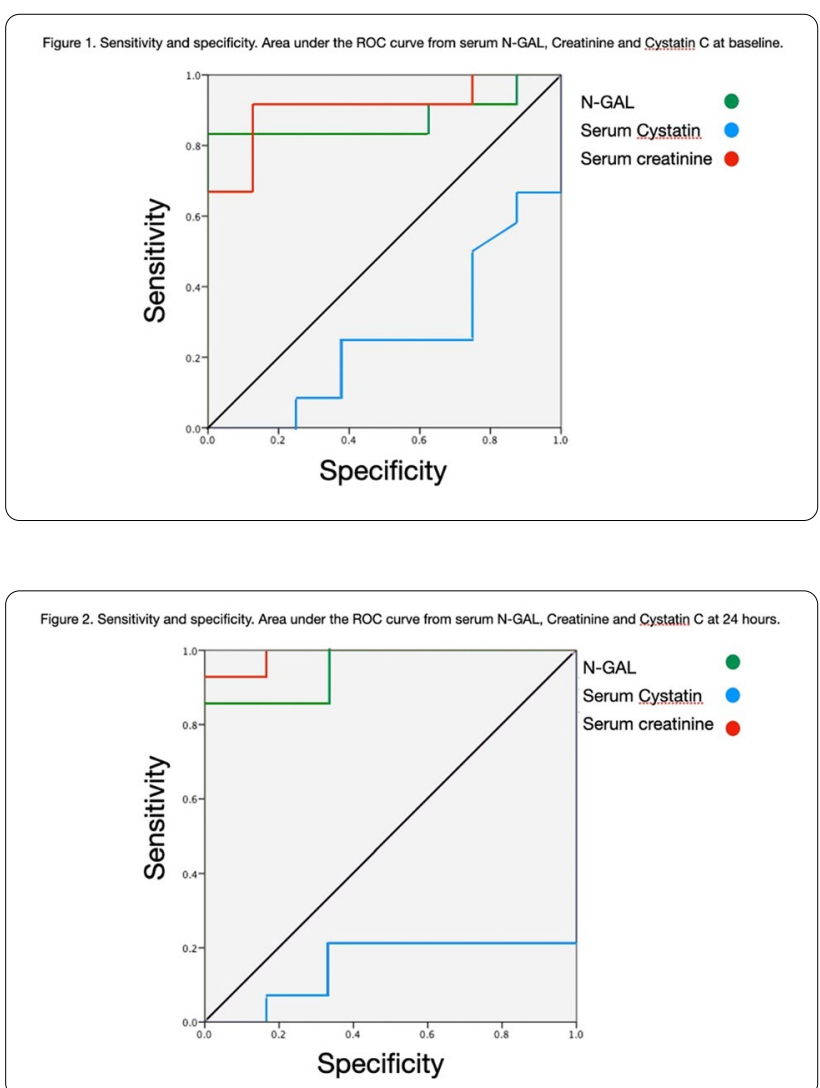

Conclusion: In this study we found that N-GAL is the most reliable biomarker for the diagnosis and prognosis of acute kidney injury in critically ill patients, compared to serum creatinine, cystatin C clearance and urinary sediment. Therefore, NGAL should be considered for the assesment on critically ill patients with or at risk of AKI. Future prospective and larger studies are required to validate its clinical utility as an early and accurate predictor of AKI.

\section{Reference(s) and grant ackowledgment(s)}

1. Schrezenmeier E V., Barasch J, Budde K, WesthoffT, Schmidt-Ott KM. Biomarkers in acute kidney injury - pathophysiological basis and clinical performance. Acta Physiol. 2017;219(3):554-572. doi:10.1111/ apha. 12764

2. Bianca G, Raluca F, Veres M, et al. Plasma Neutrophil Gelatinase Associated Lipocalin (NGAL) - Early Biomarker for Acute Kidney Injury in Critically III Patients. J Crit Care Med. 2015;1 (4):154-161. doi:10.1515/ jccm-2015-0023 
3. Ralib AM, Nanyan S, Nor MBM. Dynamic changes of plasma neutrophil gelatinase-associated lipocalin predicted mortality in critically ill patients with systemic inflammatory response syndrome. Indian J Crit Care Med. 2017;21(1):23-29. doi:10.4103/0972-5229.198322

4. Zhang J, Han J, Liu J, Liang B, Wang X, Wang C. Clinical significance of novel biomarker NGAL in early diagnosis of acute renal injury. Exp Ther Med. 2017;14(5):5017-5021. doi:10.3892/etm.2017.5150

5. Beker BM, Corleto MG, Fieiras C, Musso CG. Novel acute kidney injury biomarkers: their characteristics, utility and concerns. Int Urol Nephrol. 2018;50(4):705-713. doi:10.1007/s11255-017-1781-x

6. Kashani K, Cheungpasitporn W, Ronco C. Biomarkers of acute kidney injury: The pathway from discovery to clinical adoption. Clin Chem Lab Med. 2017;55(8):1074-1089. doi:10.1515/cclm-2016-0973

7. Hoste E, Bihorac A, Al-Khafaji A, et al. Identification and validation of biomarkers of persistent acute kidney injury: the RUBY study. Intensive Care Med. 2020;1. doi:10.1007/s00134-019-05919-0

8. Di Somma S, Magrini L, De Berardinis B, et al. Additive value of blood neutrophil gelatinase-associated lipocalin to clinical judgement in acute kidney injury diagnosis and mortality prediction in patients hospitalized from the emergency department. Crit Care. 2013;17(1):1-13. doi:10.1186/cc12510

9. Giudice AD. Acute Kidney Injury in the Elderly: Epidemiology, Risk Factors and Outcomes. Journal of Nephrology \& Therapeutics. 2012;02(06).

10. Andreucci M, Faga T, Pisani A, Perticone $M$. The ischemic/nephrotoxic acute kidney injury and the use of renal biomarkers in clinical practice. European Journal of Internal Medicine. 2017;39:1-8

11. I thank Alfredo Sierra MD, Jean Paul Vazquez MD and Erick Vidal MD for their support. Thanks for letting me be part of your ICU group at Angeles Lomas Hospital.

\section{2}

\section{Acute Kidney Injury in acutely ill patients hospitalized}

\section{for COVID-19 disease}

M. Greco'; S. De Rosa ${ }^{2}$; G. Villa ${ }^{3}$; S. Spano ${ }^{1}$; PF. Caruso ${ }^{4}$; G. Angelotti ${ }^{5}$;

M. Cecconi $^{6}$

${ }^{1}$ Department of anesthesia and intensive care, Humanitas Clinical and Research Center-IRCCS, Humanitas University, Rozzano, Italy; ${ }^{2}$ Department of anesthesia and intensive care unit, San Bortolo Hospital Of Vicenza, Vicenza, Italy; ${ }^{3}$ Health sciences department, section of anesthesiology, intensive care and pain medicine, Careggi, Florence, Italy; ${ }^{4}$ Department of anesthesia and intensive care, Humanitas Clinical and Research Center-IRCCS, Rozzano, Italy; ${ }^{5}$ Department of anesthesia and intensive care, Humanitas Clinical and Research Center-IRCCS, Milano, Italy; ${ }^{6}$ Anaesthesia and intensive care units, Humanitas Clinical and Research Center-IRCCS, Humanitas University, Rozzano, Italy

\section{Correspondence: M. Greco}

Intensive Care Medicine Experimental 2020, 8(2): 001622

Introduction: Since February 2020, an outbreak of Novel Coronavirus disease (COVID-19) developed in Lombardy, Italy. COVID-19 primarily manifest as acute respiratory illness with bilateral interstitial pneumonia, however it can affect several organs, including the kidney. The exact mechanism of kidney involvement is unclear, and may be related with cytokine storm, direct cellular damage due to the virus, or prerenal AKI due to dehydration. The incidence and characteristics of AKI in acutely ill patients are not known.

Objectives: To assess the incidence and characteristics of $\mathrm{AKI}$ in acutely ill patients admitted to general wards in a large teaching hospital

Methods: We retrospectively collected data from all patients admitted to Humanitas Research Hospital for COVID19 infection, from February 2020,22 to April 2020. Study obtained IRB approval. Data were anonymized at extraction from EHR. AKI was defined according to KDIGO criteria, using both urinary output and creatinine values. When baseline creatinine values were not available, the lowest intra-hospital creatinine value was used as baseline. Data are described as medianIQR or frequency-percentage. Chi square test or Kruskal-Wallis test are used for comparison.
Results: A total of 544 patients were included in the analysis, with a median age of 66 (IQR 53-76, range 22-93). 353/544 (64.9\%) patients were male. $219 / 544$ patients (40.2\%) developed AKI during hospital stay according to KDIGO criteria, with $78 / 544$ patients (14.3\% reaching stage 2-3 AKI)

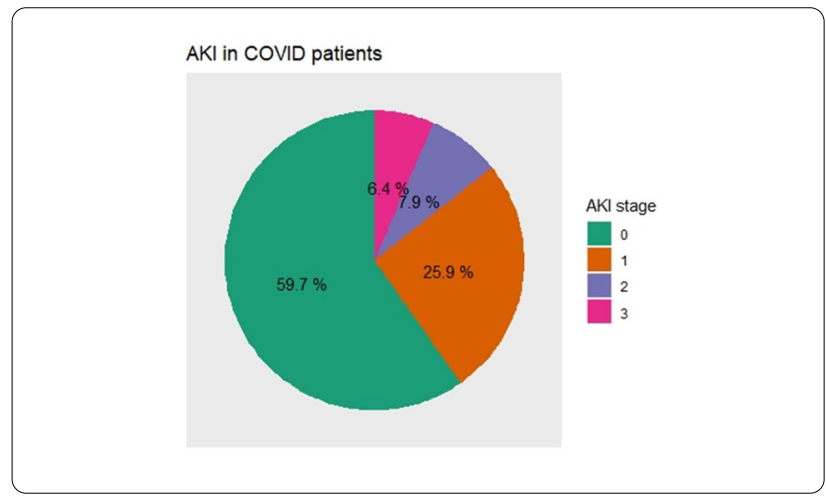

Patients with AKI were older than patients without AKI. (71 (62-79 years) vs 61 (49-72) years, $\mathrm{p}<0.001)$ Patients with AKI had a two-fold increase in the risk of death (66 patients $(30.1 \%)$ vs 47 patients (14.5\%), $\mathrm{p}<0.001$ ). Median LOS in the total population was 8 days (IQR 5-14). LOS increased according to AKI stage from 0 (3-10) days in patients with no AKI, to 12 (8-20) in patients with AKI stage 1, to 18 (11-26) days in stage $2-3$ ( $p<0.001$ in each comparison).

Stages of AKI according to hospital admission day are reported in

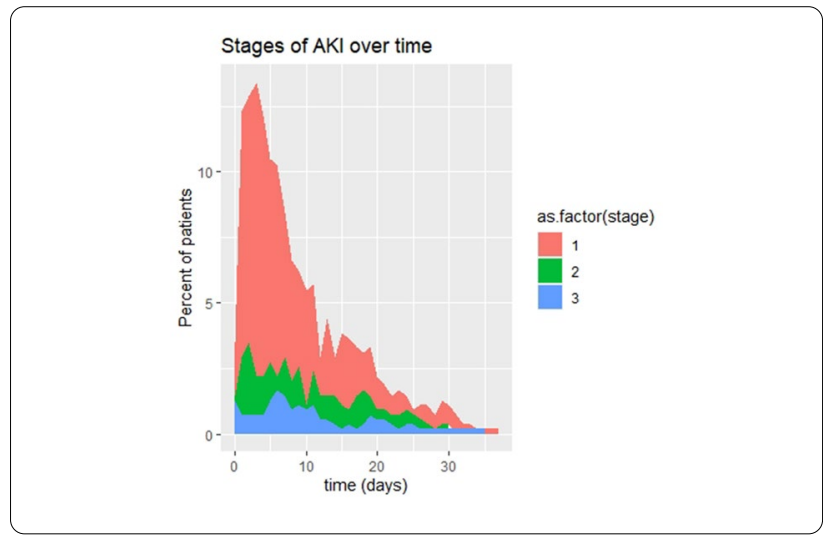

The cumulative percentage of AKI, according to hospital admission day, is reported in 3 .

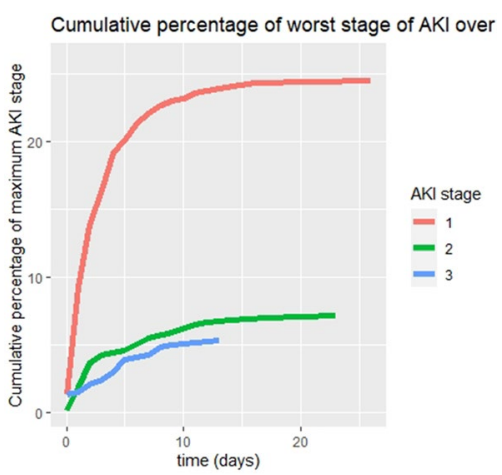


Conclusion: AKI is frequent in acutely ill patients with COVID-19 infection admitted to hospital, and leads to worse outcome, including increased mortality and prolonged hospital stay.

\section{Reference(s) and grant ackowledgment(s)}

1. Kidney Disease: Improving Global Outcomes (KDIGO) Acute Kidney Injury Work Group. KDIGO Clinical Practice Guideline for Acute Kidney Injury. Kidney inter., Suppl. 2012; 2: 1-138

2. Naicker S, Yang CW, Hwang SJ, Liu BC, Chen JH, Jha V. The Novel Coronavirus 2019 epidemic and kidneys. Kidney Int. 2020;97(5):824-828.

\section{9}

Right ventricular changes in critically ill COVID-19 patients are associated with the need for renal replacement therapy

C. Moxey ${ }^{1}$; J. Powys-Lybbe'; O. Blackford ${ }^{1}$; L. Tong ${ }^{1}$; O. Valencia'; J. Aron ${ }^{1}$ 'General intensive care unit, St George's Hospital, London, United Kingdom

Correspondence: P.L. James

Intensive Care Medicine Experimental 2020, 8(2): 001639

Introduction: Patients with COVID-19 who need renal replacement therapy (RRT) have higher mortality[1]. Acute kidney injury is multi-factorial[2], in the early stages inflammatory mediators reduce systemic and peripheral vascular resistance, in the later stages multisystem involvement due to micro-emboli and venous congestion are injurious. We hypothesize a hyperdynamic RV or a dilated RV could be seen, respectively, on echocardiography during these stages.

Objectives: To determine if there a hyperdynamic RV, of RV dilatation is associated with RRT in critically ill COVID-19 patients.

Methods: We performed an observational study of patients admitted to intensive care unit (ICU) in a tertiary hospital across a three-week period. Only patients with 2 swab PCR confirmed COVID-19 were included in the study. RV function was assessed using tricuspid annular plane systolic excursion (TAPSE) and right ventricular S prime (RV $\mathrm{S}^{\prime}$ ) during their admission. RV was recorded as normal or dilated by qualitative method. We used fisher's exact test and odds ratio (OR) to determine the association between these variables and the need for RRT. ROC curve analysis was used to determine the best cut-off for continuous variables.P-Value of less than 0.05 is considered statistically significant.

Results: A total of 49 COVID-19 positive patients were included. Of these patients $36 \%(n=18)$ required RRT during their admission.

TAPSE readings were available for 46 / 49 patients, including all 18 RRT patients. Using a cut-off of $26 \mathrm{~mm}$ or more, 9 out of 20 patients (45\%) needed RRT. 17 out of 26 patients $(65.4 \%)$ with a TAPSE $<26 \mathrm{~mm}$ did not need RRT. There was no significant association between TAPSE and RRT $(p=0.47)$.

RVS' readings were available for $44 / 49$ patients, including $16 / 18$ RRT patients. Using a cut off of $16 \mathrm{~mm}$ or more, 14 patients out of 30 patients (46.7\%) needed RRT. 12 out of 14 patients $(85.7 \%)$ with an RV $\mathrm{S}^{\prime}<16 \mathrm{~mm}$ did not need RRT. P-value $=0.049$. OR $5.25(1.00-27.61)$. Assessment on RV dilatation was recorded on all 49 patients. 38 of 49 patients $(78 \%)$ had severe RV dilatation of whom 17 required RRT. 10 out 11 patients $(90.9 \%)$ with a normal size RV did not need RRT. $(p<0.038)$ OR $8.1(0.94-69.69)$.

Conclusion: In ICU patients with Covi19 pneumonitis, a patient with a severely dilated RV or an RVS' greater than $16 \mathrm{~mm}$ on echocardiography is more likely to need Renal Replacement therapy.

\section{Reference(s) and grant ackowledgment(s)}

1. Intensive Care National Audit and research Centre'ICNARC report on COVID-19 in critical care 24 July 2020' www.icnarc.org

2. No grant was accepted for this study

\section{1}

Glomerular filtration rate assessment in critically ill patients

J. Cedeño Mora ${ }^{1}$; B. Moreno ${ }^{1}$; P. García Olivares ${ }^{1}$; A. Garrido ;

P. Santa-Teresa1'; JM. Gomez ; C. Sotillo ${ }^{1}$; S. Arenal'; J. Peral' E. Bermejo

López

${ }^{1}$ Intensive care unit, H.G.U Gregorio Marañón, Madrid, Spain

Correspondence: P. García Olivares

Intensive Care Medicine Experimental 2020, 8(2): 001301

Introduction: Difficulties associated with the evaluation and monitoring of glomerular filtration rate in critically ill patients are well known. Objectives: To analyse the concordance between the estimated glomerular filtration rate (GFR) obtained by means of the application of formulas according to serum creatinine, compare to those according to serum cystatin.

Methods: Prospective, observational study carried out in ICU admitted patients, during the months of November-December 2019. General data and renal function analysis data were collected: creatinine $(\mathrm{Cr})$ and glomerular filtration rate were estimated by MDRD 4-IMDS (GFRCr); Cystatin (Cs) and glomerular filtration rate were estimated by CKD-EPI (GFRCs)

Quantitative variables were expressed as mean (SD) or median (IQR) and qualitative variables as percentages. The concordance between the GFR values obtained by both methods was estimated by the interclass correlation coefficients (ICCs $<0.5$ bad, ICCs 0.51-0.70 moderate, ICCs $0.71-0.90$ good and ICCs $>0.90$ excellent) and by Bland Altman plot (quantification of the mean difference between the two methods with 2DS with $95 \% \mathrm{CI}$ ). In view of the lower reliability of the MDRD method for high GFR, we performed a general analysis and another for low GFR (GFR $<60 \mathrm{ml} / \mathrm{min})$.

Results: Eighty-five pairs of measurements were obtained in $95 \mathrm{ICU}$ admitted patients. General data: male $56 \%$, age $64 \pm 14$ years, weight $73 \pm 18 \mathrm{Kg}$, height $166 \pm 9 \mathrm{~cm}, 90 \%$ white race. Renal function values: Cr $1.26 \pm 1.47 \mathrm{mg} / \mathrm{dl} ;$ GFRCr $93.07 \pm 55.72 \mathrm{ml} / \mathrm{min}$, Cs $1.32 \pm 0.76 \mathrm{mg} / \mathrm{l}$ GFRCs $67.90 \pm 32.33 \mathrm{ml} / \mathrm{min} ; 29 \%$ of patients had $\mathrm{GFRCr}<60 \mathrm{ml} / \mathrm{min}$ and $39 \%$ GFRCs $<60 \mathrm{ml} / \mathrm{min}$.

In the general analysis of data, a moderate agreement was obtained between the two methods of GFR (ICCs $0.54 ; 95 \% \mathrm{Cl} 0.19-0.73)$, with a significant difference between the means $(24.32 ; 95 \% \mathrm{Cl} 11.86-36.78 ; \mathrm{p}$ $<0,001$ ) (Figure 1)

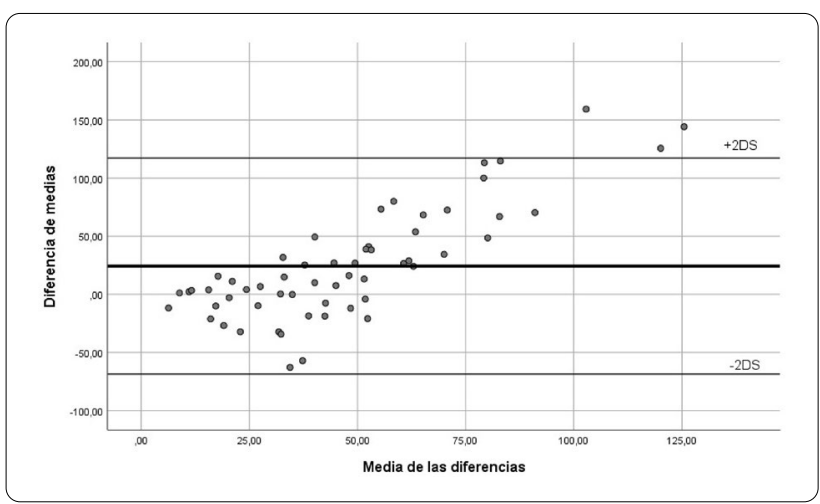

Performing the same analysis when GFR $<60 \mathrm{ml} / \mathrm{min}$, a good concordance between the two methods was obtained (ICCs $0.78 ; 95 \% \mathrm{Cl} 0.38$ 0.92), with a non-significant mean difference $(-5.91 ; 95 \% \mathrm{Cl}-14.57-2.74$; $\mathrm{p}=0.13$ ) (Figure 2) 


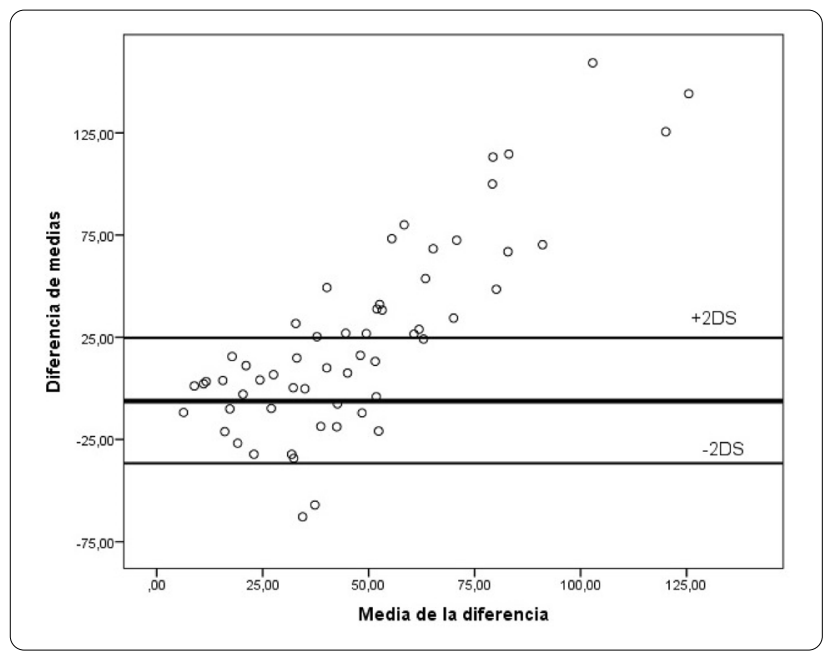

Conclusion: Waiting to determine its clinical application, our study showed a good agreement between the values of glomerular filtration estimated by creatinine and cystatin when there is impairment renal function.

\section{5}

Relation of Kidney function at the admission into ICU with mortality and develpmetn of multiorganic failure in patients with Coronavirus Infection

R. Pérez Manrique'; M. Orantes Pozo ${ }^{2}$; M. Guerrero Marin²;

E. Aguilar-Alonso ${ }^{3} ;$ T. Amat Serna²

${ }^{1}$ Medicina intensiva, Hospital Público De Montilla, Montilla, Spain;

${ }^{2}$ Medicina intensiva, Hospital of Jaen, Jaén, Spain; ${ }^{3}$ Medicina intensiva, Hospital Cabra, Cabra, Spain

Correspondence: $C$. Aranda Martínez

Intensive Care Medicine Experimental 2020, 8(2): 001455

Introduction: To assess in admitted patients to the ICU by coronavirus the influence on mortality and also at the development of multiorgan failure of renal function evaluated with the numbers of urea at the moment of admission to assess how coronavirus influence to the patients admitted by this ill at ICU on mortalityand also on the development.

Methods: Prospective cohort study of patients with coronavirus admitted to the ICU at theHospital Universitario de Jaén, Hospital San Agustín de Linares and the Hospital InfantaMargarita de Cabra.Continuous variables are expressed as median (25th percentile, 75th percentile) andqualitative variables with absolute and relative frequencies. $U$ Mann Whitney was usedfor comparison of continuous variables and X2 for qualitative variables. P \&lt;0.05 wasconsidered as statistically significant.

Results: We have studied 63 admitted patients AT ICU by coronavirus INFECTION, 43 of themAT Jaén hospital ICU, 11 at Cabra hospital and 8 at Linares hospital.The average age was $58(37,62)$. The evaluated severity with APACHE II was $12(9,17)$ pointsand the same evaluation but using SOFA was $6(4,8)$ points at the first day. $63,5 \%(n=40)$ required tracheal intubation on their first day and $82,5 \%(n=52)$ during the entire ICU stay.Mortality in the ICU was a percent of 38 and (meanwhile) at the hospital was 39,7Those patients who deceased were older than the survivors, $64(60,72)$ vs $60(52,70)$ years old,but the differences weren't statistically significant (e.s) $(p=0.16$.). These ones also showedmost acutely according to Apache II, $18(14,23)$ vs 14 $(12,18)$ pointa $(p<0,005)$, and a higherSOFA at the admission $7(4,8)$ vs $6(3,8)$ points, but without being statistically significant $(p=0.09)$.
The Who died show higher urea at the admission $63(34,59)$ vs $45(34,59) \mathrm{mg}$, $(p=0.051)$ although the differences weren't s.s. Then, we classified the patientsaccording to whether the urea was higher than 70 upon admission to the ICU and his relationto the mortality. The 46 patients $(73 \%)$ whose urea were less than 70 showed a mortality of $30.43 \%$ and those that had a higher or equal urea than $70 \quad n=17$, $26.98 \%)$ the mortality was $64.70 \%$, $(p=0.003)$

On the third day, we evaluated the severity with SOFA and observed that the patients whodied presented a higher SOFA 8 (7.9) vs 6 (3.8) points, $(p=0.021)$.When The relation between SOFA at the moment of the admission and SOFA on the third daywas evaluated we could see that a correlation existed $(r=0.588, p<0.001)$. SOFA on the thirdday was higher in patients with Urea greater than 709 (8.12) vs 8 (7.11), (p $=0.007$ )

When SOFA on the third day of the admission was studied using multiple linear regression itwas noticed that there was a relation with both SOFA at the day of admission $(B=0.548 \pm 0.126, p<0.001)$ and urea at the moment of the admission $(B=0.019+0.003, p=0.033), R=0.629$. Cuando se estudio con Regresión lineal múltiple el SOFA al tercer día se relacionó con el SOFAal ingreso $(B=0.548+0.126, p<0.001)$ y con la Urea al ingreso $(B=0.019+0.003, p=0.033), R=0.629$.

Conclusion: In ICU patients admitted for coronavirus infection, a higher urea at the admission is related tothe mortality and is linked to a later worsening., as it\&\#39; s showed by an increase in theSOFA score on the third day compared to that which they had at admission in those patientswith higher Urea numbers.

\section{5}

Acute kidney injury in patients with COVID-19 admitted to an ICU in a second level Hospital in Madrid

L. Castro-Bournissen ' ${ }^{1}$ F. Dos Santos Alvernia ${ }^{1}$; A. Alvarez-Mendez'; P. Perez Fernandez ${ }^{1}$; A. Del Caño Garcia ${ }^{1}$; M. Lopez-Gil'; M. Mas Lodo '; D. Varillas

Delgado'; N. Franco Garrobo

${ }^{1}$ Intensive care unit, Hospital Universitario de Móstoles, Móstoles, Spain; ${ }^{2}$ Unidad de apoyo a la investigación, facultad de medicina, Universidad Francisco de Vitoria, Madrid, Spain

Correspondence: L. Castro-Bournissen

Intensive Care Medicine Experimental 2020, 8(2): 001525

Introduction: Critically ill patients with COVID-19 infection frequently have kidney involvement with clinical presentation ranging from mild renal failure to progressive acute kidney injury (AKI) necessitating renal replacement therapy (RRT). We describe our experience during the peak of the COVID-19 pandemic.

Methods: Retrospective cohort study of patients suffering from COVID-19 infection admitted to the ICU during the peak of COVID-19 pandemic from March to June 2020. We collected data from electronic medical records on demographics, Simplified Acute Physiology Score III (SAPS III), comorbidities, treatments received, need for RRT, use of low molecular weight heparin, length of ICU admission and mortality in ICU.

Results: 82 patients diagnosed with COVID-19 admitted to ICU. Mean age was $63.06(+/-10.036)$ and the majority $(76.8 \%)$ were men. $68.3 \%$ patients (56) suffered AKI and 18 of those $(32.1 \%)$ required RRT, the majority of them through continuous therapy (94.4\%).

Comorbidities included hypertension (48.2\%), diabetes (37.5\%), chronic respiratory condition (asthma or COPD) 17.9\%, obesity (defined by BMI > 30) $21.4 \%$, ischaemic miocardiopathy $9.1 \%$ and $5.4 \%$ patients with some level of immunosuppression (all causes). No patients with autoimmune disorders within our population.

Patients with AKI received the following COVID-specific treatment according to our Hospital protocol: $98.2 \%$ of patients received Hidroxycloroquine, $91.1 \%$ Lopinavir/Ritonavir, $73.2 \%$ steroids (Metilprenisolone or Dexametasone), 58.9\% Tocilizumab, 33.9\% Beta-Interferon, 10.7\% Baricitinib and 3.6\% Anakinra. 
Mean SAPS III score was $57.96(+/-8.6)$. Mean ICU length stay was 23.06 days (+/- 21.46$)$ and mortality $71.4 \%$, (OR 1.471; 1.022-2.117, $\mathrm{p}$ 0.018 ) against $41 \%$ mortality of patients without renal failure.

Conclusion: The proportion of critically ill patients with COVID-19 suffering from AKI was significantly higher in our population (68.3\%) than figures published in the literature (20 to $40 \%)$. AKI has been considered a marker of disease severity and a negative prognostic factor for survival. In our cohort, mortality rate in patients with AKI was considerably higher than mortality in patients without AKI. RRT rate, however, was similar to the literature published $(21,9 \%)$. Even though comorbidities were more frequently found in the AKI group of patients, multivariate logistic regression analysis failed to reveal an association. Furthermore, no association was found between AKI and COVID 19-specific treatment received.

\section{4}

\section{A preliminary study on TIMP2 and IFGBP7 urinary biomarker}

\section{in COVID-19 patients at ICU admission}

M. Greco ; S. De Rosa ${ }^{2}$; S. Spano ${ }^{1}$; F. Baccanelli'; R. Aceto ${ }^{3}$; M. Cecconi ${ }^{1}$

${ }^{1}$ Department of anesthesia and intensive care, Humanitas Clinical and Research Center-IRCCS, Humanitas University, Rozzano, Italy; ${ }^{2}$ Department of anesthesia and intensive care unit, San Bortolo Hospital Of Vicenza, Vicenza, Italy: ${ }^{3}$ Department of anesthesia and intensive care, Humanitas Clinical and Research Center-IRCCS, Rozzano, Italy

\section{Correspondence: M. Greco}

Intensive Care Medicine Experimental 2020, 8(2): 001634

Introduction: The novel severe acute respiratory coronavirus 2 (SARSCoV-2) is the cause of COVID-19 disease. COVID-19 disease has a wide spectrum of manifestations, from asymptomatic individuals, to acute illness with respiratory failure requiring hospitalization, to critical illness requiring ICU admission and mechanical ventilation. While COVID-19 disease mostly affects the lung, Acute Kidney Injury (AKI) is frequent in patients with SARS-CoV-2 infection, specifically in patients admitted to critical care.

Immunoassay test based on the urinary concentration of two biomarkers, tissue inhibitor of metalloproteinases-2 (TIMP-2) and insulin-like growth factor-binding protein-7 (IFGBP7), have been proposed and validated for AKI in other settings. No data are available on TIMP-2 and IFGBP7 urinary concentration in COVID-19 patients with critical illness.

Objectives: To investigate whether TIMP-2 and IGFB7P urinary biomarkers at ICU admission are related with the risk of AKI in patients admitted to ICU for COVID-19 disease

Methods: We conducted a preliminary prospective cohort study, enrolling subsequent patients admitted to COVID-ICU in Humanitas Research Hospital, due to COVID-19 disease. Study obtained IRB approval. Informed consent was obtained according to local regulations. Urinary levels of TIMP-2 and IGFB7P biomarkers were measured at ICU admission (AKIrisk score by NephroCheck ${ }^{\circledR}$ Test), and after $12 \mathrm{~h}$, $24 \mathrm{~h}$ and $48 \mathrm{~h}$ from ICU admission. AKI was defined according to KDIGO criteria.

Data are described as median-IQR or frequency-percentage. Chi square test or Kruskal-Wallis test are used for comparison.

Results: A total of 13 patients were included in the study. Patients were mostly male $(11 / 13,83 \%)$, with a median age of 59 (IQR 45-67). 5 patients (38\%) had no previous disease, while the most frequent comorbidity was diabetes (3 patients, 23).11 patients underwent mechanical ventilation (84.6\%).

A total of 9 patients developed AKI stage $2 / 3$ (69\%). $78 \%$ of patients needed diuretics during ICU stay, but none of these 13 patients underwent CRRT.

Urinary biomarkers (AKlrisk) values according to time of measurement are reported in Figure 1

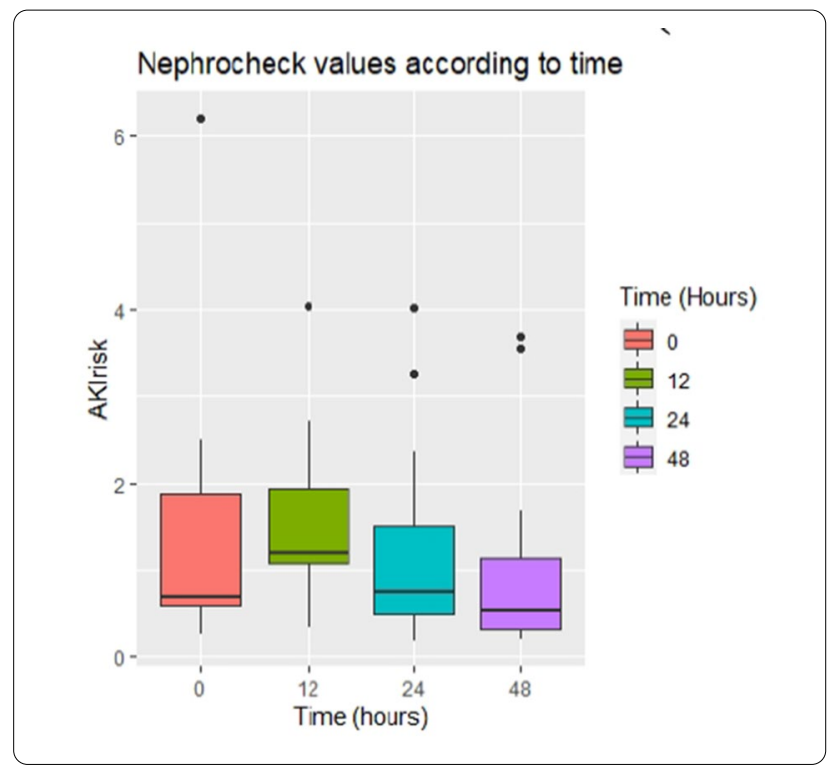

AKlrisk values according to AKI stage and time of measurement are reported in figure 2 . .Only AKIrisk value at $12 \mathrm{~h}$ were significantly associated with AKI stage $2-3(p=0.03)$

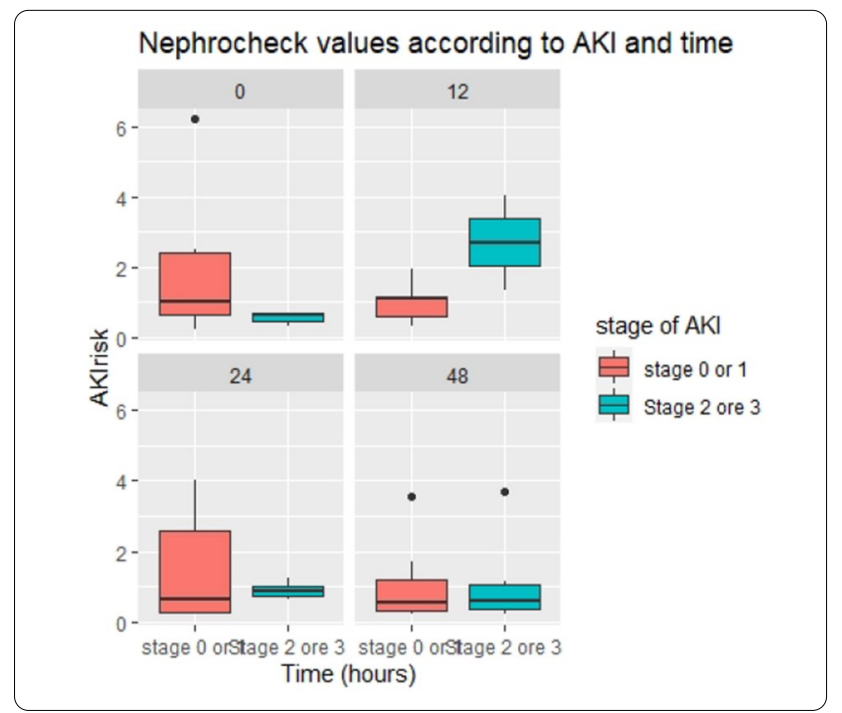

Conclusion: In this pilot study, TIMP-2 and IGFB7P are generally elevated in patients admitted to ICU for COVID-19 disease, and present wide variability. This may be partly explained by the large variability in ICU admission time, according to the onset of symptoms and to the first day of hospitalization. Only TIMP2-IGFB7P urinary values at $12 \mathrm{~h}$ from ICU admission were correlated with AKI stage 2-3.

\section{Reference(s) and grant ackowledgment(s)}

1. Kidney Disease: Improving Global Outcomes (KDIGO) Acute Kidney Injury Work Group. KDIGO Clinical Practice Guideline for Acute Kidney Injury. Kidney inter., Suppl. 2012; 2: 1-138, 
001312

Unfractionated Heparin with Adequate Anti-Xa Activity

Monitoring is Associated with Improved Filter Set Longevity Among Critically III COVID-19 Patients Undergoing Renal

Replacement Therapy

R. Spencer'; R. Appleton ${ }^{1}$; E. Black ${ }^{1}$

'Department of Critical Care, Queen Elizabeth University Hospital, Glasgow, United Kingdom

Correspondence: R. Spencer

Intensive Care Medicine Experimental 2020, 8(2): 001312

Introduction: Approximately $29 \%$ of all critically ill COVID-19 patients in Scotland required renal replacement therapy (RRT), and requirement for RRT is associated with greater mortality(1). Usual local protocol for anticoagulation to facilitate continuous RRT uses citrate, allowing anticoagulation of the filter circuit only without systemic anticoagulation of the patient. However, early in the pandemic it was noted that filtration circuits were clotting unusually quickly, perhaps related to the well documented procoagulant state of severe COVID19. Therefore, a new protocol was devised using systemic unfractionated heparin. Ordinary monitoring of this would be activated partial thromboplastin time (APTT), however this was altered due to international evidence that it is unrepresentative of true coagulation state among COVID-19 patients $(2,3)$. Therefore, anti-Xa activity monitoring was used, targeting a range of 0.3-0.7 initially, increased to 0.5-0.7 later. Objectives:

1. To assess the impact of different forms of anticoagulation on continuous haemodiafiltration filter set longevity among COVID-19 patients, with particular attention paid to the effect of anti-Xa activity levels on longevity. 2. To assess the frequency of adverse events associated with systemic unfractionated heparin.

Methods: Patients admitted to our ICU whose primary diagnosis was 'COVID-19 confirmed/suspected' and who underwent at least one day of RRT were identified. Data were collected retrospectively regarding filter set longevity, the reason for stopping/changing filter set, method of anticoagulation, anti-Xa activity along with patient-level data on length of stay, mortality and adverse events. The data were analysed with regard to whether systemic heparinisation was associated with longevity, whether anti-Xa monitoring affected this and frequency of recorded adverse events related to heparin. Data were also analysed to identify any association between filter set longevity and patient outcome.

Results: In total, 144 filter sets were used in 21 COVID-19 patients. Citrate anticoagulation was used in $27(19 \%)$ and heparin in the remaining 117. The reason for stopping/changing filter set was clotting of the set in 88 of these (61.1\%). Median longevity in sets that clotted was 10 hours (range $0-87$ ) and very positively skewed. No statistically significant difference in longevity was observed when comparing sets using citrate to all sets using heparin anticoagulation (median 9 vs 10 hours, $p=0.27$ ). We then compared three groups: citrate, heparin with no anti-Xa levels/first anti-Xa level less than 0.3, and heparin with the first anti-Xa level greater than 0.3 . There was significantly greater longevity in sets with the first anti-Xa greater than 0.3 compared to the other two groups (median 30 hours vs 10 hours vs 9 hours, $p=0.012$ ). When analysing for an anti-Xa target range greater than 0.5 , the median longevity was 35 hours, a further significant improvement ( $p=0.022$, see chart).

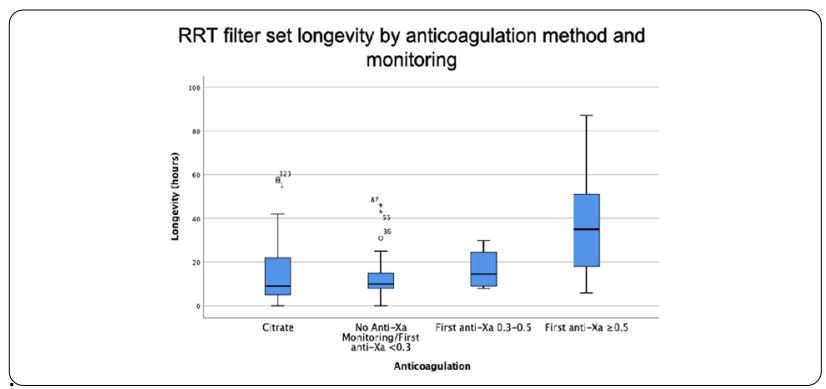

A significant positive correlation was observed within filter sets between the percentage time spent with anti-Xa levels $>0.3$ and longevity $(p=0.007)$. Two adverse events related to heparin (i.e. bleeding) were recorded, one fatal. Both occurred while anti-Xa levels were within the range $0.3-0.7$. No significant association was seen between patients' average filter set longevity and their outcomes or severity scoring on admission to ICU.

Conclusion: This study provides evidence that the use of anti-Xa activity monitoring is useful in improving filter set lifespan among COVID19 patients undergoing continuous RRT, while using unfractionated heparin without anti-Xa monitoring is no better than citrate anticoagulation. The effect is best seen with anti-Xa levels at a level greater than 0.5. However, the risk of adverse bleeding events related to heparin is a significant one and anti-Xa monitoring does not appear to be protective against this in our cohort.

\section{Reference(s) and grant ackowledgment(s)}

1. 1. Scottish Intensive Care Society Audit Group. Scottish Intensive Care Society Audit Group Report on COVID-19. Public Health Scotland. July 2020

2. 2. Bowles, Platton, Yartey et al. Correspondence: Lupus anticoagulant and abnormal coagulation tests in patients with COVID-19. The New England Journal of Medicine 2020.

3. 3. Panigada, Bottino, Tagliabue et al. Hypercoagulability of COVID19 patients in intensive care unit: A report of thromboelastography findings and other parameters of haemostasis. J Thromb Haemost. 2020;18:1738-1742

\section{9}

Combined blood purification in patients with septic shock after neurosurgical interventions

A. Burov ${ }^{1}$; T. Abramov' ${ }^{1}$ D. Korotkov'; NS. Kostritca ${ }^{2}$; IA. Savin

${ }^{1} \mathrm{ICU}, \mathrm{N}$. N. Burdenko National Medical Research Center of Neurosurgery, Moscow, Russia; ${ }^{2}$ Medical student, M. V. Lomonosov Moscow State University, Moscow, Russia

Correspondence: A. Burov

Intensive Care Medicine Experimental 2020, 8(2): 001529

Introduction: Sepsis and septic shock in patients with primary brain injury can lead to or aggravate the existing neurologic symptoms due to cytokine release impairing the hematoencephalic barrier. For such patients standard therapy may be insufficient for fast multiorgan failure (MOF) regress and hemodynamic instability correction. Blood purification is a potentially reasonable option for patients with septic shock not responding to standard therapy.

Objectives: The objective of the present study is to evaluate the effectiveness of the combined blood purification (CBP) in neurosurgical patients with septic shock.

Methods: We present 6 neurosurgical patients $29-82$ y.o. with septic shock. All of them received standard therapy according to the Surviving Sepsis Campaign guidelines. CBP therapy was started within 10 hours after septic shock diagnosis in 4 patients and within 20 hours - in the other 2 patients. The procedure description: CVVHDF mode on Gambro Prismaflex. Increased adsorption capacity filters (AN69ST (5 patients) and Oxiris (1 patient)) action was amplified by CytoSorb adsorber for all patients. Systemic anticoagulation was performed with heparin in 2 patients, regional citrate anticoagulation - in 3 patients; 1 patient was not anticoagulated. The duration of the procedure was 24 hours.

We observed the procedure's influence on the vasopressor requirements, MOF (by SOFA score), PCT, CRP, IL- 6 and lactate levels.

Results: In the course of CBP we observed clinical improvement in all patients. In 3 patients clinical improvement (MOF regress and lactate level decrease) was stable; in other 3 patients - transient.

Median NE demand for all patients reduced from $0,885 \mu \mathrm{g} / \mathrm{kg} / \mathrm{min}$ $(0,18-2,13)$ before CBP to $0,19 \mu \mathrm{g} / \mathrm{kg} / \mathrm{min}(0-1,64)$ in 24 hours and to $0,16 \mu \mathrm{g} / \mathrm{kg} / \mathrm{min}(0-3,6)$ in 72 hours after CBP initiation (Figure 1). At the same time, we observed lactate level reduction from $3,25 \mathrm{mmol} / \mathrm{l}$ 
$(2-9,8)$ before CBP to $2,05 \mathrm{mmol} / \mathrm{l}(1,8-7,9)$ in 24 hours $(\mathrm{p}<0,05)$ and to $1,8 \mathrm{mmol} / \mathrm{l}(0,7-5,8)$ in 72 hours after CBP initiation (Figure 2).

Mean SOFA score decreased from 14 (11-18) before CBP to 11 (4-17) in 24 hours $(p<0,05)$ and to $9(4-18)$ in 72 hours after CBP initiation (Figure 3).

Mean PCT level decreased from $97.77 \mathrm{ng} / \mathrm{ml}(5.32$ - >200) before CBP to $25.93 \mathrm{ng} / \mathrm{ml}(1.43-86.62)$ in 24 hours $(\mathrm{p}<0,05)$ and to $22.8 \mathrm{ng} / \mathrm{ml}$ (3.1-101.45) in 72 hours after CBP initiation (Figure 4). Mean CRP level decreased from $138,5 \mathrm{mg} / \mathrm{l}(34,7-257,3)$ before CBP to $102,2 \mathrm{mg} / \mathrm{l}$ $(35,4-254,4)$ in 48 hours and to $68 \mathrm{mg} / \mathrm{l}(28,2-298,1)$ in 72 hours after CBP initiation (Figure 5). Mean IL-6 level decreased from $896 \mathrm{pg} / \mathrm{ml}$ (56-32280) before CBP to $249 \mathrm{pg} / \mathrm{ml}(19.46-9142)$ in 6 hours and to $80.5 \mathrm{pg} / \mathrm{ml}(20.98-6352)$ in 24 hours after CBP initiation (both $\mathrm{p}<0,05)$ (Figure 6).

3 patients with stable clinical improvement had shock reversal; in 2 of them we observed consciousness level increase. 2 Patients with transient clinical improvement died in 72 and 74 hours after CBP initiation due to cerebral herniation in the setting of malignant brain oedema, 1 patient died at 26 hours after the procedure start due to cardiac insufficiency progress after pulmonary embolism.

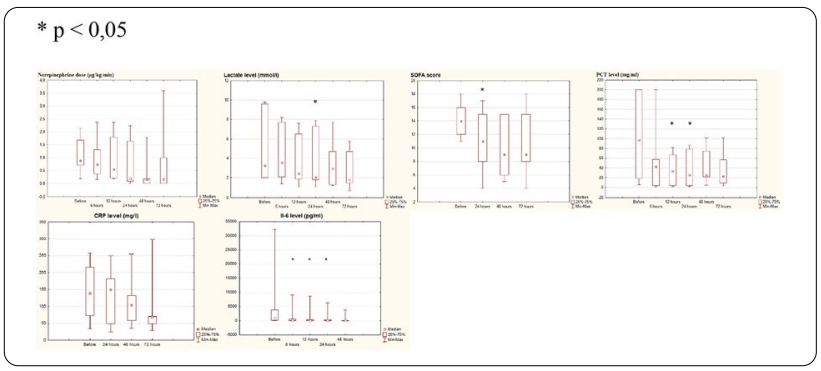

Conclusion: We observed that CBP in combination with standard intensive care contributed to overall clinical improvement in all patients (stable or transient). Among the key changes were the significant MOF regress, lactate, PCT and IL- 6 level decrease and not statistically significant vasopressor demand reduction. The use of CBP in septic shock patients after neurosurgical interventions may be rational. Further studies on the method efficacy and safety in neurosurgical patients with septic shock are required.

\section{1}

Concentrations of fentanyl before and after initiation of continuous veno-venous hemodialysis in septic patients with acute kidney injury - a prospective observational study

K. Polok' ; I. Nowak-Kózka' ; J. Fronczek' ; J. Górka' ; W. Szczeklik ${ }^{1}$

${ }^{1}$ Intensive care and perioperative medicine, Jagiellonian University Medical College, Kraków, Poland

Correspondence: $\mathrm{K}$. Polok

Intensive Care Medicine Experimental 2020, 8(2): 001541

Introduction: Acute kidney injury (AKI) complicates sepsis in 40-50\% of cases and often necessitates introduction of continuous renal replacement therapy (CRRT) which significantly affects pharmacokinetics of multiple medications commonly used in the intensive care unit. Association between use of CRRT and fentanyl concentration, particularly in the critical care setting, has not been well described yet. Objectives: To compare concentrations of fentanyl before and during continuous veno-venous hemodialysis with regional citrate anticoagulation (RCA-CVVHD) in septic patients with AKI.

Methods: We enrolled patients at the of $>35$ and $<85$ years with severe sepsis or septic shock and clinical indications for CRRT due to $\mathrm{AKI}$, according to KDIGO recommendations. RCA-CVVHD was initiated according to the local practice. It was performed using commercially available equipment and solution, with anticoagulation performed using $4 \%$ trisodium citrate and $\mathrm{CaCl} 2$ solution. All CRRT settings were identical for every participant at the beginning of procedure and were subsequently adapted based on the patient's clinical status. Fentany was administered via a continuous intravenous infusion with a dosage equal to $100 \mu \mathrm{g} / \mathrm{h}$. The dosing of fentanyl was not changed after RCA-CVVHD was initiated. Fentanyl concentrations were measured in seven predefined timepoints both before and after the initiation of RCA-CVVHD with the use of high-pressure liquid chromatography tandem mass spectrometry with an electrospray ionization source.

Results: Fourteen patients at the median age of 59.5 (IQR 45.0-64.0) years, among whom 9 were males $(64.3 \%)$, were enrolled in the study. The concentration of fentanyl was similar before and after initiation of RCA-CVVHD in the entire cohort $(0.535$ vs $0.581[\mathrm{ng} / \mathrm{ml}], \mathrm{p}=0.14)$ as well as in the subgroups of patients with residual diuresis $(0.729$ vs. $0.804[\mathrm{ng} / \mathrm{ml}], \mathrm{p}=0.50)$ and in patients without residual diuresis $(0.430$ vs. $0.559[\mathrm{ng} / \mathrm{ml}], \mathrm{p}=0.21$ ).

Conclusion: There were no significant differences in fentanyl levels before and after initiation of RCA-CVVHD, irrespective of residual diuresis status. Results of presented study suggest a limited usefulness of fentanyl concentration monitoring in this population.

\section{3}

\section{Use of Continuous Renal Replacement Therapy} with hemoadsorption (Oxiris ${ }^{\circledR}$ ) in patients with severe acute respiratory failure due to COVID-19 and associated renal failure M. Gonzalez-Fernandez'; N. Quílez-Trasobares²; A. Lesmes'; JA.

Barea-Mendoza ${ }^{3}$; JA. Sanchez-Izquierdo ${ }^{4}$; Z. Molina-Collado²

${ }^{1}$ Intensive care, Hospital 12 De Octubre, Madrid, Spain; ${ }^{2}$ Intensive care, University Hospital 12 de Octubre, Madrid, Spain; ${ }^{3}$ Intensive care medicine, Hospital 12 De Octubre, Madrid, Spain; ${ }^{4}$ Critical care, University Hospital 12 de Octubre, Madrid, Spain

Correspondence: N. Quílez-Trasobares

Intensive Care Medicine Experimental 2020, 8(2): 001543

Introduction: The use of continuous renal replacement therapy with hemoadsorption (Oxiris ${ }^{\circledR}$ ) in patients with severe acute respiratory failure due to COVID-19 and associated renal failure, may modify the systemic inflammatory response associated with this disease based on cytokine clearance.

Objectives: Describe the characteristics of our serie, the therapy applied, and assess the impact of hemoadsorption on the outcomes of our patients.

Methods: Prospective, unicentric, observational study in patients admitted to the ICU from march to april 2020 due to SARS-CoV-2 infection with acute kidney injury (AKI) and need for Continuous Renal Replacement Therapy (CRRT) in which hemoadsorption with Oxiris ${ }^{\circledR}$ membrane is set up. We analyze epidemiology, severity scores, respiratory status and inflammatory markers before and after therapy, dose and complications of CRRT, ICU lenght of stay and mortality. Statistical analysis is performed with SPSS system.

Results: 128 patients were analized; $33 \%$ of them presented acute kidney injury, $18 \%$ required CRRT and in 11\% (14 patients) was associated with hemoadsorption: median age of 62 years and male predominance $(86 \%)$ were included, $57 \%$ of them were hypertensive, $43 \%$ obese and $21 \%$ chronic pneumopaths. The worst SOFA at $24 \mathrm{~h}$ of admission was 5 (IQR: 4-7), the worst PaFi was 105 (IQR: 66-120) and the worst compliance was $45 \mathrm{ml} / \mathrm{cmH}_{2} \mathrm{O}$ (IQR: 40-52). Neuromuscular blockade and prone position were used in $93 \%$ of them and veno-venous ECMO was applied in one case. The main indication of CRRT was homeostasis $(78 \%)$ and fluid balance $(14 \%)$. The initial dose employed was $35 \mathrm{ml} / \mathrm{kg}$ (IQR: 35-40), final $30 \mathrm{ml} / \mathrm{kg}$ (IQR 30-45) and median duration of $72 \mathrm{~h}$. The anticoagulation system used was: $86 \%$ citrate, $9 \%$ nothing and $5 \%$ heparin, without any case of citrate accumulation. We detected several complications, all of them in mild degree: persistence of metabolic acidosis $86 \%$, hypothermia $50 \%$, hyperkalemia $23 \%$, metabolic alkalosis $14 \%$, hypophosphoremia $9 \%$ hypomagnesemia and hypokalemia $5 \%$. No statistically significant differences were found between inflammatory markers (PCR, PCT, ferritin, lymphocytes, fibrinogen), SOFA or PaFi pre and post-CRRT due to the small sample size, althought they were found between pre and post-CRRT D-dimer $(p<0.05)$. ICU lenght of stay was 12 days (IQR 6-18) and ICU mortality rate was $50 \%$. 
Conclusion: In our serie, CRRT with associated hemoadsorption has been useful and safe technique in the management of acute renal failure in COVID-19 patients. This therapy could lower D-dimer levels.

\section{Reference(s) and grant ackowledgment(s)}

1. Lau SKP, Lau CCY, Chan KH, Li CPY, Chen H, Jin DY, et al. Delayed induction of proinflammatory cytokines and suppression of innate antiviral response by the novel Middle East respiratory syndrome coronavirus: Implications for pathogenesis and treatment. J Gen Virol. 2013;94(PART 12):2679-90.

2. Xu X, Han M, Li T, Sun W, Wang D, Fu B, et al. Effective Treatment of Severe COVID-19 Patients with Tocilizumab.

3. Malard B, Lambert C, Kellum JA. In vitro comparison of the adsorption of inflammatory mediators by blood purification devices.Intensive Care Med Exp. 2018 May 4;6(1):12. https://doi.org/10.1186/s40635-018-0177-2.

4. Liu Y, Chen X, Wang D, et al. Hemofiltration Successfully Eliminates Severe Cytokine Release Syndrome Following CD19 CAR-T-Cell Therapy. J Immunother 2018:41:406-410

5. Friesecke S, Träger K, Schittek GA, et al. International registry on the use of the CytoSorb ${ }^{\circledR}$ adsorber in ICU patients Study protocol and preliminary results. Med Klin Intensivmed Notfmed. 2017 Sep 4. https://doi. org/10.1007/s00063-017-0342-5. [Epub ahead of print].

\section{3}

Management of a Metformin-Associated Lactic Acidosis

by Different Modalities of Renal Replacement Therapy

K. Van Laethem ${ }^{1}$; B. Dewulf'; M. Bourgeois ${ }^{1}$

${ }^{1}$ Intensive Care, AZ Sint-Jan, Brugge, Belgium

Correspondence: K. Van Laethem

Intensive Care Medicine Experimental 2020, 8(2): 001553

Introduction: The optimal treatment modality for a metformin lactate acidosis (MALA) remains controversial [1]. Though intermittent haemodialysis (IHD) is considered superior in correcting acidemia and clearing metformin, continuous renal replacement therapy (CRRT) is still used frequently due to a proposed better hemodynamic tolerability and less logistic implications [1-4].

Objectives: Does the fear of an increased risk of hemodynamic perturbation outweighs the proven benefit of a more effective clearance? In this paper we illustrate a significant difference in clearance between both modalities of RRT with a pharmacokinetic time course of metformin levels.

Methods: This trial is a retrospective, observational study. From July 2019 to December 2019, we identified all patients who were admitted to the ICU of the Sint-Jan Hospital in Bruges with the diagnosis of severe MALA requiring RRT. The metformin plasma levels where monitored with an interval of three hours. RRT was discontinued as soon as the clinical condition and renal function improved sustainably.

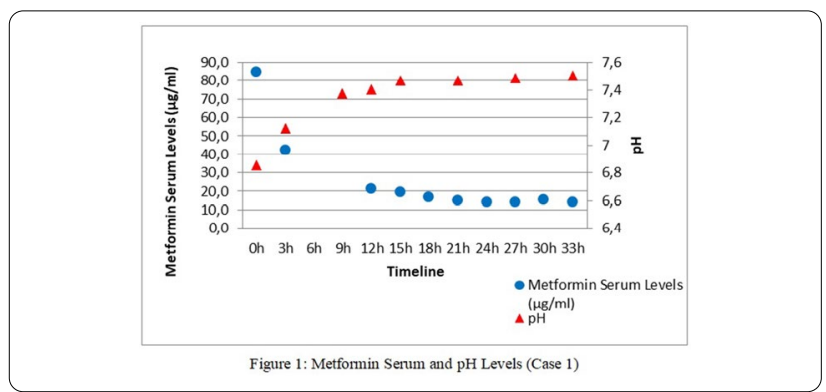

Results: Our trial population consists of 2 patients. The first patient underwent IHD (Qb $345 \mathrm{ml} / \mathrm{min}$, Qd $500 \mathrm{ml} / \mathrm{min}$ ) during $4 \mathrm{~h}$ after which CVVH (Qb $120 \mathrm{ml} / \mathrm{min}$, Qe $40 \mathrm{~mL} / \mathrm{kg} / \mathrm{h}, 50 \%$ pre-dilution and $50 \%$ post-dilution with Hemosol BO replacement fluid) was started. Metformin concentrations were dramatically reduced in the first $4 \mathrm{~h}$ and metabolic acidosis was corrected. The mean rate of metformin elimination was $12 \% / \mathrm{h}$ with IHD and $5 \% / \mathrm{h}$ with CVVH (Fig. 1).

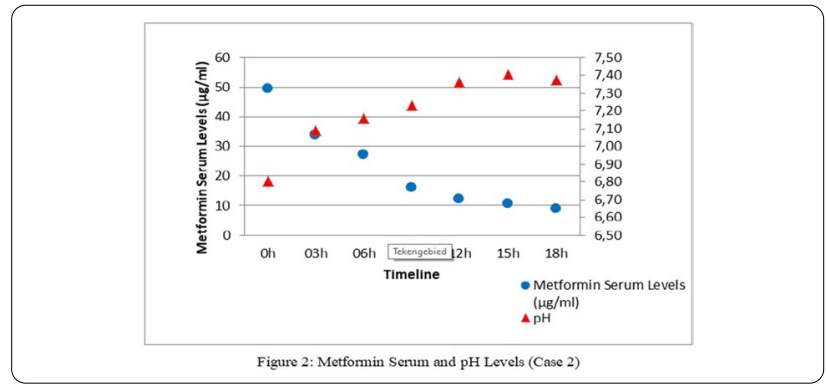

The other patient immediately received CVVHDF (Qb $120 \mathrm{ml} / \mathrm{min}$, Qe $55 \mathrm{ml} / \mathrm{kg} / \mathrm{h}, 66 \%$ pre-dilution and $33 \%$ post-dilution with Hemosol BO replacement fluid, Qd of $2000 \mathrm{ml} / \mathrm{h}$ ) with a gradual normalisation of the metabolic acidosis and metformin concentrations in the first $24 \mathrm{~h}$. The mean rate of metformin elimination was $2.4 \% / \mathrm{h}$ (Fig. 2).

\begin{tabular}{|c|ccc|}
\hline & Case 1 & Case 2 & Mean \pm SD \\
\hline $\begin{array}{c}\text { Type } \\
\text { IHD or CVVH }\end{array}$ & IHD / CVVH & CVVHDF & - \\
\hline $\begin{array}{c}\text { Metformin clearance } \\
\text { Rate of elimination (\%/h) }\end{array}$ & $12 / 5$ & 2,4 & $7,2 \pm 4,8$ \\
\hline $\begin{array}{c}\text { Outcomes } \\
\text { ICU length of stay (days) } \\
\text { Survival to discharge } \\
\text { Discharge at home }\end{array}$ & 3 & 17 & $10 \pm 7$ \\
Yes & Yes & Yes & - \\
\hline \multicolumn{4}{|c|}{ Table 1: RRT and outcomes } \\
\hline
\end{tabular}

The IHD was hemodynamically well tolerated, while the second patient was highly vasopressor dependent during the first $20 \mathrm{~h}$ of CVVHDF. Both our patients had a favourable outcome (Table 1).

Conclusion: CRRT and IHD are equivalent dialysis strategies regarding outcome for the ICU patient with AKI [5]. Arguments in favor of CRRT are a presumed better hemodynamic tolerability and its potential for gradual fluid removal. On the other hand, IHD is more flexible and cost-effective, offers the possibility to minimize anticoagulation and removes small solutes more efficiently [5]. Our study illustrates that IHD was more efficient to restore the metabolic homeostasis and eliminate metformin. It's our opinion that IHD is the preferred modality of RRT in MALA, despite the presence of hypotension. There is no need for net ultrafiltration and the hemodynamic instability is most likely induced by the extreme metabolic derangements related to metformin [5-6]. However, further studies are needed to determine the indication, timing and modalities of RRT and the potential role of hybrid therapies such as sustained low-efficiency daily dialysis in patients with MALA.

Reference(s) and grant ackowledgment(s)

1. 1. Yeh HC, Ting IW, Tsai CW, Wu JY, Kuo CC. Serum lactate level and mortality in metformin-associated lactic acidosis requiring renal replacement therapy: a systematic review of case reports and case series. BMC Nephrol. 2017;18(1):229.

2. 2. Peters N, Jay N, Barraud D, Cravoisy A, Nace L, et al. Metformin-associated lactic acidosis in an intensive care unit. Crit Care. 2008:12:R149.

3. 3. Arroyo AM, Walroth TA, Mowry JB, Kao LW. The MALAdy of metformin poisoning: is CVVH the cure? Am J Ther. 2010;17:96-100.

4. 4. Seidowsky A, Nseir S, Houdret N, Fourrier F. Metformin-associated lactic acidosis: a prognostic and therapeutic study. Crit Care Med. 2009;37:2191-2196

5. 5. Vanholder R, Van Biesen W, Hoste E, Lameire N. Pro/con debate: Continuous versus intermittent dialysis for acute kidney injury: a never-ending story yet approaching the finish. Crit Care. 2011 Jan 28;15(1):204.

6. 6. Calello DP, Liu KD, Wiegand TJ, et al. Extracorporeal Treatment for Metformin Poisoning: Systematic Review and Recommendations From the 
Extracorporeal Treatments in Poisoning Workgroup. Crit Care Med 2015; 43:1716-30.

\section{6}

Initial evaluation of an on-line register for the control of renal depuration techniques: DialyReg

M. Gonzalez-Fernandez'; N. Quílez-Trasobares²; JA. Barea-Mendoza³; JA. Sanchez-Izquierdo ${ }^{4}$; MD. Arias-Verdu ${ }^{5}$; J. Barrueco-Francioni ${ }^{5}$; G.

Seller-Pérez $z^{5}$; M. Herrera-Gutiérrez ${ }^{6}$

${ }^{1}$ Intensive care, Hospital 12 De Octubre, Madrid, Spain; ${ }^{2}$ Intensive care, University Hospital 12 de Octubre, Madrid, Spain; ${ }^{3}$ Intensive care medicine, Hospital 12 De Octubre, Madrid, Spain; ${ }^{4}$ Critical care, University Hospital 12 de Octubre, Madrid, Spain; ${ }^{5}$ nntensive care, Hospital Carlos Haya, Málaga, Spain; ${ }^{6}$ Intensive care, Regional Hospital of Malaga, Málaga, Spain

Correspondence: N. Quílez-Trasobares

Intensive Care Medicine Experimental 2020, 8(2): 001556

Introduction: On-line databases have grown as an alternative method for storing and analysing scientific data, respecting confidentiality, providing security and simplicity of use. Based on the use of an international database, initial evaluation of an on-line database for the followup of renal depuration techniques is presented.

Objectives: Analize the initial results in a multicenter register which allows a continuos follow up of the patients with Continuous Renal Replacement Therapy (CRRT) with the objective of detect and solve the problems in their implementation.

Methods: Observational, prospective, multicenter and descriptive studio, by the database DialyReg, which one has the caractheristic of a database with a little number of variables, making it easier to fill. REDCap is an on-line database which has the legal garantees regarding security and confidentiality, feasibility and an easy access, with a possibility of data analize in real time. Statistical analysis is performed with SPSS system and it has got the bioethical approve.

Results: In 2019, 159 patients has been included with the main admission diagnosis of medical illness(47\%), postoperative $(26 \%)$, sepsis (24\%) and trauma (5\%). The principal indication for the theraphy was internal homeostasis (43\%), oliguria (24\%), hemodynamics (16\%) and hydric disbalance (15\%). The median of the number of catheter per patient was 1 (IQR1-1) with a median of duration by 6 days (IQR 3-9). The $82 \%$ of the catheters were coaxial dual lumen, and the preferential vascular acces was internal femoral vein (58\%). The main complications derived by the theraphy were hematoma (2\%), infection (2\%) and trombosis (1\%). Median of the hemodialysis sets was 3 (IQR 2-6), with a median of therapy set by 22 hours (IQR 12-48 h). The intitial theraphy dosis was set in $30 \mathrm{ml}$ per kilogram (IQR 20-40 ml) and dynamic adjustment was perform in the $68 \%$ of the sample. The main motive for discontinuation of the theraphy was recovery $(58 \%)$, exitus (29\%) and intermittent renal replacement (8\%). Discontinuation was: planned $(37 \%)$,end of the treatment $(18 \%)$ or a dyalisis set coagulation (36\%). The main complications derived by the therapy were hypophosphoraemia(15\%), hypotermia(12\%), metabolic acidosis, hypokalemia and hypocalcemia (6\% respectively). 53\% of the patients were discharged from the hospital while $40 \%$ died in the ICU and $6.5 \%$ during hospitalization.

Conclusion: The DialyReg register is a feasible tool, affordable for a real time control of the results of dyalisis theraphy in our units. It could be usefull for the maintenance of security programs in these thecniques.

\section{Reference(s) and grant ackowledgment(s)}

1. Paul A Harris, Robert Taylor et al.Research electronic data capture (REDCap) a metadata-driven methodology and workflow process for providing translational research informatics support.J Biomed Inform . 2009 Apr;42(2):37781. https://doi.org/10.1016/j.jbi.2008.08.010. Epub 2008 Sep 30.
001582

Impact of therapy characteristics in terms of efficacy, safety, and complications due to citrate use versus other anticoagulation modalities in Continuous Renal Replacement Therapy

N. Quílez-Trasobares; M. Gonzalez-Fernandez ; JA. Barea-Mendoza²;

JA. Sanchez-Izquierdo 3 ; MD. Arias-Verdu ${ }^{4}$; J. Barrueco-Francioni ${ }^{4} ; G$. Seller-Pérez ${ }^{4}$; M. Herrera-Gutiérrez ${ }^{5}$

${ }^{1}$ Intensive care, University Hospital 12 de Octubre, Madrid, Spain; ${ }^{2}$ Intensive care medicine, University Hospital 12 de Octubre, Madrid, Spain; ${ }^{3}$ Critical care, University Hospital 12 de Octubre, Madrid, Spain; ${ }^{4}$ Intensive care, Hospital Carlos Haya, Málaga, Spain; ${ }^{5}$ Intensive care, Regional Hospital of Malaga, Málaga, Spain

Correspondence: N. Quílez-Trasobares

Intensive Care Medicine Experimental 2020, 8(2): 001582

Introduction: Citrate-based renal purification therapy is increasing its use over other conventional anticoagulation modalities, providing evidence of a safe and effective alternative. For this purpose we would like to analyse the impact of complications due to the use of citrate versus other anticoagulation modalities in Continuous Renal Replacement Therapy (CRRT).

Objectives: Comparison between citrate anticoagulation with other modalities in terms of efficacy and safety.

Methods: Prospective multicenter observational cohort study based on the DialyReg registry, with the Clinical research ethics committee (CEIC) approval from both hospitals. We compare therapy doses, clot filter loss and common complications in patients undergoing Continuous Renal Replacement Therapy (CRRT) in 2019. Statistical analysis is performed with SPSS system.

Results: Data from 159 patients corresponding to 690 haemofilters are analysed. The distribution in anticoagulation systems was: heparin $(35.8 \%)$, citrate $(29 \%)$, and without anticoagulation $(44.5 \%)$. The initial dose of therapy was similar in citrate group (30 ml/kg, IQR 25-35) with respect to the heparin and without anticoagulation groups. Dynamic adjustment was performed more frequenty in the citrate group (84\%) than in heparin (69\%) and without anticoagulation groups (66\%) $(p<0.05)$. The final therapy dose was lower with citrate $(20 \mathrm{ml} / \mathrm{kg}$, IQR 20-30) versus heparin (30 ml/kg, IQR 20-35) and without anticoagulation $(25 \mathrm{ml} / \mathrm{kg}$, IQR 15-35), $(\mathrm{p}<0.05)$. Median duration of the set was higher in citrate group ( $55 \mathrm{~h}, \mathrm{IQR} 24-72)$ versus $23 \mathrm{~h}$ in heparine group (IQR 12-48) and $12 \mathrm{~h}$ in no anticoagulation group (IQR 12-31) $(p<0.05)$. Coagulation of the system had a higher incidence with heparin $(46.7 \%)$ than with citrate $(29.5 \%)(p<0.05)$. Complications associated with the technique were observed: hypothermia (19\% citrate vs $9 \%$ rest), hypokalemia (18\% citrate vs $3 \%$ rest) and hypomagnesemia $(6.6 \%$ citrate vs $2 \%$ rest) $(p<0.05)$; although these findings observed in higher proportion in citrate group are attributable to the learning curve of one of the participating hospitals. Following proper use of the technique and the use of phosphate dialysis fluids, complications are similar to the other types of therapy. Citrate anticoagulation was also analyzed in the subgroup of hepatopathic patients ( 27 patients:52\% cirrhosis, 37\% liver transplantation, 7\% acute liver failure, and $4 \%$ acute-on-chronic failure). Heparin was used in $39 \%$, citrate in $21 \%$, and no anticoagulation was used in $38 \%$ of the sample. No statistically significant differences were found in terms of complications due to therapy, however there was a lower frequency of citrate use in hepatopathic patients than in non -hepatopathic ones (19\% vs. 35\%) $(\mathrm{p}<0.05)$.

Conclusion: Citrate anticoagulation increases the duration of the haemofilters and allows better adjustment of the therapy dose in our series, possibly being more cost-effective. It is a safe anticoagulation system once the learning curve is covered.

\section{Reference(s) and grant ackowledgment(s)}

1. Wu MY, Hsu YH, Bai CH, et al. Regional citrate versus heparin anticoagulation for continuous renal replacement therapy: a meta-analysis of randomized controlled trials. Am J Kidney Dis 2012;59(6):810-818.

2. Oudemans-van Straaten HM, Ostermann M. Bench-to-bedside review: Citrate for continuous renal replacement therapy, from science to practice. Crit Care 2012;16:249. 
3. Pinnick RV, Wiegmann TB, Diederich DA. Regional citrate anticoagulation for hemodialysis in the patients at high risk for bleeding. N Engl J Med 1983;308(5):258-61.

4. Shaikh A Nurmohamed et al.Continuous venovenous haemofiltration with citrate-buffered replacement solution is safe and efficacious in patients with a bleeding tendency: a prospective observational study. BMC Nephrol. 2013 Apr 18;14:89. https://doi.org/10.1186/1471-2369-14-89.

5. Pasquale De Vico,Valentina Messino,et al.Safety and efficacy of citrate anti-coagulation continuous renal replacement therapies in post-cardiac surgery patients with liver dysfunction.Ther Apher Dial. 2015 Jun;19(3):2728. https://doi.org/10.1111/1744-9987.12280. Epub 2015 Feb 6.

\section{9}

Inflammatory response trajectory is associated with increased failure to wean neuromuscular blockade in ventilated COVID-19 patients

C. McCue ${ }^{1}$; S. Norman ${ }^{1}$; C. Macdonald ${ }^{1}$; R. Cowan

${ }^{1}$ Intensive care, Glasgow Royal Infirmary, Glasgow, United Kingdom

Correspondence: C. McCue

Intensive Care Medicine Experimental 2020, 8(2): 001189

Introduction: The global COVID-19 pandemic resulted in a significant proportion of hospitalised patients developing ARDS requiring mechanical ventilation(1). Prominent features of COVID-19 are a large inflammatory response with significant pyrexia and moderate-severe ARDS often requiring prolonged ventilation(2). Our unit uses an evidenced based protocol to manage patients with ARDS which includes lung protective ventilation, prone positioning and paralysis with neuromuscular blockade(3). Our clinical experience using this protocol to manage COVID-19 patients was that we encountered multiple episodes of failure to wean paralysis after an initial apparent improvement in oxygenation and respiratory mechanics. We hypothesised that this failure may be more likely if there was evidence of significant ongoing inflammatory response. We sought to analyse if ongoing significant inflammation as determined by pyrexia and rising CRP was associated with failure to wean paralysis.

Methods: Data was collected on ventilated patients admitted with COVID-19 pneumonia from March to May 2020. For each patient on each day ventilated we collected $\mathrm{P} / \mathrm{F}$ ratio, mandatory vs spontaneous ventilation, whether paralysis was weaned successfully, peak temperature and CRP. Pyrexia was defined as temperature $>380 \mathrm{C}, \mathrm{CRP}$ was compared to the previous 24 hour's result to determine trajectory. We routinely use continuous atracurium infusions titrated to effect for paralysis. Statistical analysis was performed on Real Stats for Excel.

Results: Over the time period; 51 patients with COVID-19 pneumonia were ventilated in our ICU. We excluded patients whose medical records were unavailable $(n=1)$ or those who didn't complete their entire ventilated stay in our ICU $(n=7)$. All were followed up until ICU discharge. As of June 17th 2020, 4 patients remained in hospital with $59 \%$ of patients (44) discharged home. Over the study period, there were 125 attempts at weaning paralysis of which 32 were successful. The median number of days receiving paralysis was 6 . In pyrexial patients with rising CRP, $11 \%$ of attempts to wean were successful compared to $34 \%$ of attempts in apyrexial patients with falling CRP $(p=0.048)$.

\begin{tabular}{|c|c|}
\hline Demographia & Result \\
\hline Male & $70-2$ \\
\hline Median age (range) & $57(37-72)$ \\
\hline ICU mortality & 3: \\
\hline $\begin{array}{l}\text { Median ICU length of } \\
\text { stav Irangel }\end{array}$ & $19(2-72)$ \\
\hline
\end{tabular}

Table I. Demographics and outcomes

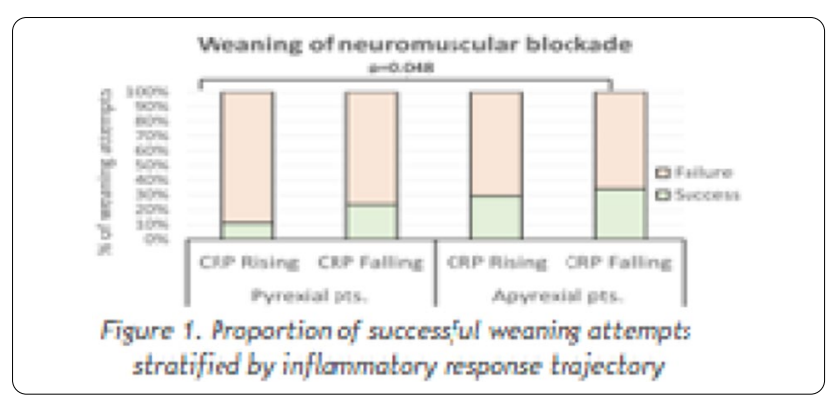

Conclusion: To our knowledge there are no other reports of neuromuscular blockade management during the COVID-19 pandemic. We have seen high rates of failure weaning paralysis in our patient cohort, but improved success rates when there is evidence of improving inflammatory response.

Reference(s) and grant ackowledgment(s)

1. 3. Papazian L, Forel JM, Gacouin A, Penot-Ragon C, Perrin G, Loundou A, et al. Neuromuscular blockers in early acute respiratory distress syndrome. N Engl J Med. 2010;363(12):1107-16.

2. 2. Bhatraju PK, Ghassemieh BJ, Nichols M, et al. Covid-19 in critically ill patients in the Seattle region — case series. N Engl J Med. https://doi. org/10.1056/nejmoa2004500.

3. 1. Docherty AB, Harrison EM, Green CA, et al. Features of 16,749 hospitalised UK patients with COVID-19 using the ISARIC WHO Clinical Characterisation Protocol. medRxiv 2020; published online April 28. https://doi. org/10.1101/2020.04.23.20076042 (preprint).

\section{0}

Prevalence and outcomes of acute hypoxaemic respiratory failure in Wales: the PANDORA-WALES study

M. Kopczynska ${ }^{1}$; B. Sharif ${ }^{1}$; R. Pugh ${ }^{2}$; I. Otahal ${ }^{3}$; P. Havalda ${ }^{3}$; W. Groblewski ${ }^{3}$. C. Lynch"; D. George ${ }^{5}$; J. Sutherland ; M. Murdoch ; M. Pandey ${ }^{7} ;$ M. Morgan $;$; A. Hatalyak ${ }^{8}$; P. Jones ${ }^{8}$; C. Thorpe ${ }^{9}$; R. Jones ${ }^{10}$; J. Villar ${ }^{11}$; T.

Szakmany

${ }^{1}$ Department of anaesthesia, intensive care and pain medicine, division of population medicine, Cardiff University, Cardiff, United Kingdom; ${ }^{2}$ Anaesthetic department, Betsi Cadwaladar University Health Board, Bodelwyddan, United Kingdom; ${ }^{3}$ Anaesthetic department, Hywel Dda University Health Board, Haverfordwest, United Kingdom; ${ }^{4}$ Anaesthetic department, Cwm Taf Morgannwg UHB, Swansea, United Kingdom; ${ }^{5}$ Anaesthetic department, Betsi Cadwaladar University Health Board, Wrexham, United Kingdom; ${ }^{6}$ Anaesthetic department, Swansea Bay UHB, Swansea, United Kingdom; ${ }^{7}$ Anaesthetic department, Cardiff \& Vale University Health Board, Cardiff, United Kingdom; ${ }^{8}$ Anaesthetic department, Aneurin Bevan UHB, Newport, United Kingdom; ${ }^{9}$ Anaesthetic department, Betsi Cadwaladar University Health Board, Bangor, United Kingdom;

${ }^{10}$ Anaesthetic department, Cwm Taf Morgannwg UHB, Bridgend, United Kingdom; ${ }^{11}$ Ciber de enfermedades respiratorias, Instituto de Salud Carlos III, Las Palmas de Gran Canaria, Spain

Correspondence: M. Kopczynska

Intensive Care Medicine Experimental 2020, 8(2): 001220

Introduction: Although there are few previous published observational studies examining the incidence and mortality of patients with acute respiratory failure and ARDS, there are no studies specifically assessing the epidemiological characteristics, patterns of ventilation and clinical outcomes in patients with acute hypoxemic respiratory failure (AHRF) in the current era of lung protective ventilation. Understanding the factors associated with AHRF outcomes could lead to development of effective interventions and improvement of patient care. 
Objectives: The aim of the study was to identify the intensive care unit (ICU) mortality for patients suffering from AHRF. Secondary aim was analysis of factors influencing patient mortality and investigation of the parameters of ventilatory management as well as the use of rescue therapies for hypoxemia.

Methods: This was a multi-centre prospective study carried out in nine hospitals in Wales, UK, over two 2-month periods. All patients admitted to ICU were screened for AHRF and followed-up until discharge from the ICU or hospital. Data was collected from patient charts on patient demographics, reason for ICU admission, methods of AHRF diagnosis, physiological and laboratory results, treating team management and complications during the ICU stay.

Results: We recruited 197 patients in our study. Fifty-nine (29.9\%) were non-survivors, 101 (51.3\%) were survivors and data was missing for $37(18.8 \%)$. Inotropic support $(p=0.03)$, re-intubation $(p=0.04)$, pneumonia $(p=0.002)$ and increased SOFA score $(p=0.04)$ were associated with mortality. The majority of patients $(87.8 \%)$ did not receive the optimal tidal volumes of ventilation (VT), recommended as 6-7 ml/ $\mathrm{kg}$ predicted body weight (PBW), with only $13.35 \%$ of all VTmax and $27.98 \%$ of VTmin measurements within the optimal range. $77.90 \%$ VTmax and $23.69 \%$ VTmin measurements had values above $7 \mathrm{ml} /$ $\mathrm{kg}$ PBW. Rescue therapies were not used in the vast majority of the patients. Muscle paralysis was used only for $21.3 \%$ and recruitment manoeuvres for $17.8 \%$ of patients, whereas prone positioning and more invasive technologies such as ECMO were used in less than $1 \%$ of patients.

Conclusion: AHRF is associated with high mortality despite increased awareness and recognition of this condition. Despite advances of care, mechanical ventilation and use of adjunctive measures is still not optimal for the vast majority of patients.

\section{1}

Prone Positioning as a Treatment for Hypoxaemia in Patients with Severe Respiratory Failure Secondary to SARS-CoV-2

K. Richmond ${ }^{1}$; A. Laurie ${ }^{2}$; S. Stott ${ }^{2}$

${ }^{1}$ Anaesthesia, Aberdeen Royal infirmary, Aberdeen, United Kingdom: ${ }^{2}$ Critical care, Aberdeen Royal Infirmary, Aberdeen, United Kingdom

Correspondence: $\mathrm{K}$. Richmond

Intensive Care Medicine Experimental 2020, 8(2): 001231

Introduction: The global pandemic of SARS-CoV-2 resulted in a large cohort of patients requiring critical care support for severe respiratory failure (SRF) (1). Prone positioning (PP) is a well-established technique for improving oxygenation in patients with SRF (2).

Objectives: To describe our experience of PP as a management strategy for patients with hypoxaemia admitted to the intensive care unit (ICU) in Aberdeen Royal Infirmary (ARI) with SRF due to SARS-CoV-2 infection.

Methods: Patients admitted to the ARI ICU between March and May 2020 with respiratory failure and confirmed SARS-CoV-2 infection were identified. Retrospective clinical and demographic data were gathered from electronic notes, observation charts and the local incident reporting system. Pa02/Fi02 ratio (P/F) change was calculated using the last value prior to PP and the maximum value during PP.

Results: 46 patients with confirmed SARS-CoV-2 infection were identified. 16 patients underwent proning during their ICU admission. Demographic data is shown in table 1 . There were 52 discrete proning episodes; the maximum number of times a single patient was proned was 12. In 33 episodes the P:F ratio prior to proning placed the patient in the category of severe ARDS. The mean time from admission to first PP was 4.1 days (0-11). Average prone episode duration was 14.6 hours (2-19.5). All prone episodes bar one involved invasive mechanical ventilation via an endotracheal tube or tracheostomy. Mode of ventilation when prone comprised $56 \%$ volume control and $44 \%$ pressure control. Average $\mathrm{P} / \mathrm{F}$ ratio prior to $\mathrm{PP}$ was $13.4 \mathrm{kPa}$ compared to $24.5 \mathrm{kPa}$ during PP. In all proning episodes the P/F ratio improved with a mean percentage increase of $86 \%$ (8.7-197.5) (figure 1). The percentage increase for prone episodes involving volume control ventilation was $89 \%$ compared to $84 \%$ for those involving pressure control ventilation. Complications associated with PP included cardiac arrest on proning ( 2 cases), brachial plexus injury ( 1 case) and facial pressure injury ( 3 cases). ICU mortality was $37.5 \%$ for those with prone episodes and $30 \%$ in those patients not proned.

Table 1 Demographic data for PP patients and those not proned. Values expressed as mean unless otherwise stated.

\begin{tabular}{|c|c|c|c|}
\hline & Proned & Not pronec & $P$ value \\
\hline Age, years & 61.5 & 55 & 0.06 \\
\hline BMI & 29.4 & 28.6 & 0.60 \\
\hline morbidity $(\%)$ Hypertension & 50 & 40.7 & 0.44 \\
\hline omorbidity (\%) Diabetes Mellitus & 43.8 & 14.8 & 0.03 \\
\hline ICU LOS, days & 20.6 & 6.7 & 0.0004 \\
\hline Mortality (\%) & 37.5 & 30 & 0.60 \\
\hline
\end{tabular}

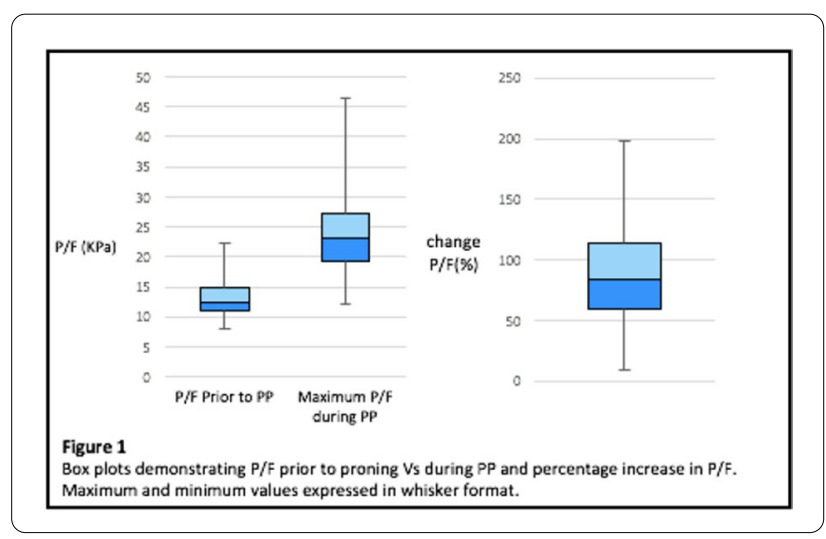

Conclusion: Based on our experience, for patients with moderate to SRF secondary to SARS-CoV-2, PP appears to improve oxygenation. The mode of ventilation does not seem to influence change in oxygenation. PP may be associated with increased risk of pressure damage, nerve injury and haemodynamic instability including cardiac arrest.

Reference(s) and grant ackowledgment(s)

1. 2. Sud, S., Friedrich, J., Adhikari, N. et al. Effect of prone positioning during mechanical ventilation on mortality among patients with acute respiratory distress syndrome: a systematic review and meta-analysis. CMAJ (2014); 186 (10): 381-390.

2. 1. Bamford, P., Bentley, A., Dean, J. et al 2020. ICS Guidance for Prone Positioning of the Conscious COVID Patient 2020. Intensive Care Society. Viewed July 2020. https://icmanaesthesiacovid-19.org/news/ics-guidance-forprone-positioning-of-the-conscious-covid-patient-2020.

\section{8}

The use of inhaled Iloprost in COVID-19 ARDS patients: an observational study at the Royal Free Hospital

Z. Zhao'; J. Yeats ${ }^{2}$; B. O'farrell ${ }^{3} ;$ K. Dhadwal ${ }^{3}$;. T. Banerjee ${ }^{3}$; A. Adlakha ${ }^{3}$

${ }^{1}$ Emergency department, Derriford Hospital, London, United Kingdom; ${ }^{2} U \mathrm{cl}$ medical school, Royal Free Hospital, London, United Kingdom; ${ }^{3}$ Intensive care unit, Royal Free Hospital, London, United Kingdom

Correspondence: $Z$. Zhao

Intensive Care Medicine Experimental 2020, 8(2): 001238

Introduction: Acute respiratory distress syndrome (ARDS) is a form of acute, inflammatory lung injury with increased vascular permeability that results in respiratory failure (1). The outbreak of coronavirus disease 2019 (COVID-19) caused by the severe acute respiratory syndrome coronavirus 2 (SARS-CoV-2) has triggered a rapid increase in the number of ARDS cases worldwide. The current best practice for management of ARDS includes low tidal volume ventilation (2) and prone positioning where appropriate. lloprost is a prostacyclin which, in nebulised form, is licensed for treatment of primary pulmonary hypertension (3); its actions have been hypothesised to reduce V/Q mismatch in ARDS. 
Objectives: This study aims to observe the current practice of using inhaled iloprost in patients with refractory ARDS at the Royal Free Hospital Intensive Care Unit, and evaluate its use in mechanically ventilated patients with COVID-19 related ARDS.

Methods: Ten COVID-19 positive patients with confirmed severe ARDS as per the Berlin criteria (1), and multi-organ failure were observed. lloprost nebuliser solution was given every 4 hours at a dose of 5 micrograms initially, with stepwise increments of 5 micrograms to a maximum of 20 micrograms. Changes in oxygenation were monitored using the $\mathrm{PaO} 2 / \mathrm{FiO} 2$ ratio (P/F ratio) from arterial blood gas ( $\mathrm{ABG}$ ) measurements. Measurements were recorded from 24 hours prior to the first dose to 24 hours after the last dose was administered. Data were analysed with linear regression and Pearson's $r$ correlation coefficient using SPSS. Mean arterial pressure was used to monitor for safety. Results: Ten patients were followed (table 1). lloprost treatment was given for 3 days on average. The average follow-up time was 106 hours per patient with 303 time points sampled. The correlation in the 24 hours prior to initiating iloprost was $-0.225(p=0.67)$ (figure 1). The correlation between P/F ratio and time in the first 24 hours after iloprost was started was $0.146(p=0.2)$ (figure 2$)$. No adverse drug reactions were reported. Two patients died during the study and four died in the days after iloprost was stopped.

Table 1: Patient demographics and outcomes

\begin{tabular}{|c|c|}
\hline Cohort & $n=10$ \\
\hline Age & $53.9(36-65)$ \\
\hline $\begin{array}{l}\text { Sex } \\
\text { Female } \\
\text { Male }\end{array}$ & $\begin{array}{l}5 \\
5\end{array}$ \\
\hline $\begin{array}{l}\text { Ethnicity } \\
\text { White } \\
\text { Black } \\
\text { Asian } \\
\text { Other }\end{array}$ & $\begin{array}{l}3 \\
4 \\
3 \\
0\end{array}$ \\
\hline Days in ICU prior to starting on iloprost & $12.2(1-34)$ \\
\hline $\begin{array}{l}\text { Comorbidities } \\
\text { Cardiovascular } \\
\text { Respiratory } \\
\text { Renal } \\
\text { Liver } \\
\text { Metastasis } \\
\text { Immunocompromised }\end{array}$ & $\begin{array}{l}7 \\
0 \\
1 \\
0 \\
0 \\
0\end{array}$ \\
\hline $\begin{array}{l}\text { Pulmonary embolism } \\
\text { None } \\
\text { Clinically suspected } \\
\text { Diagnosed by CTPA } \\
\text { - Trunk } \\
\text { - Segmental } \\
\text { - Subsegmental }\end{array}$ & $\begin{array}{l}3 \\
3 \\
0 \\
0 \\
3\end{array}$ \\
\hline No. of days on iloprost & $2.95(1-10)$ \\
\hline $\begin{array}{l}\text { Adverse drug reactions } \\
\text { Anaphylaxis } \\
\text { Any other reactions }\end{array}$ & $\begin{array}{l}0 \\
0\end{array}$ \\
\hline Hypotension & 0 \\
\hline $\begin{array}{l}\text { Vasopressors } \\
\text { Noradrenaline }\end{array}$ & 7 \\
\hline $\begin{array}{l}\text { Steroids } \\
\text { Methylprednisolone }\end{array}$ & 1 \\
\hline $\begin{array}{l}\text { Other drug trials } \\
\text { IV epoprostenol } \\
\text { Remdesivir } \\
\text { Enoximone } \\
\text { Tocilizumab } \\
\text { Magnesium sulphate }\end{array}$ & $\begin{array}{l}2 \\
2 \\
1 \\
2 \\
1\end{array}$ \\
\hline Proning & 6 \\
\hline $\begin{array}{l}\text { Death } \\
\text { Whilst on iloprost } \\
\text { After iloprost trial }\end{array}$ & $\begin{array}{l}2 \\
4\end{array}$ \\
\hline
\end{tabular}
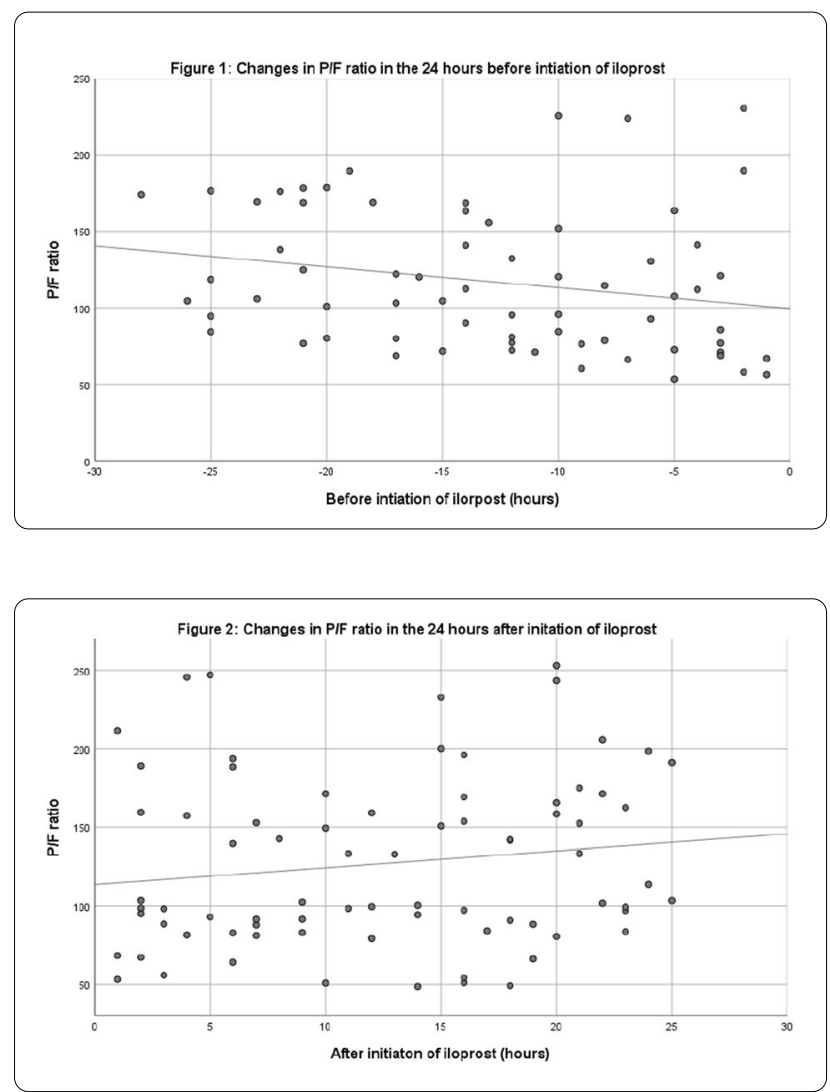

Conclusion: The overall effect of nebulised iloprost was inconclusive. There were possible short-term improvements in oxygenation during treatment but there was no evidence that inhaled iloprost conferred a survival benefit. Inhaled iloprost was well tolerated with no safety concerns. The findings reported are in keeping with current literature however the study lacked statistical power to draw conclusions that may influence practice $(4,5)$. Inhaled iloprost may offer a potentially useful treatment intervention in the management of COVID-19 related ARDS and appears to be safe even in patients with multiple co-morbidities. Further, more powerful studies are needed to determine the precise clinical value of iloprost in ARDS and COVID-19 related ARDS.

Reference(s) and grant ackowledgment(s)

1. 4. Pacheco J, Arnold H, Skrupky L, Watts P, Micek ST, Kollef MH. Predictors of outcome in 216 subjects with ARDS treated with inhaled epoprostenol. Respir Care 2014;59(8):1178-85.

2. No grants were offered or received for this study.

3. 5. Alhazzani W, Møller MH, Arabi YM, Loeb M, Gong MN, Fan E, et al. Surviving Sepsis Campaign: guidelines on the management of critically ill adults with Coronavirus Disease 2019 (COVID-19). Intensive Care Med 2020 May 1;46(5):854-87.

4. 1. Ranieri VM Rubenfeld GD, Thompson BT, Ferguson ND, Caldwell E, Fan E, et al. Acute respiratory distress syndrome: The Berlin definition. JAMA 2012 Jun 20; 307(23):2526-33.

5. 2. Brower RG, Matthay MA, Morris A, Schoenfeld D, Thompson BT, Wheeler A. Ventilation with lower tidal volumes as compared with traditional tidal volumes for acute lung injury and the acute respiratory distress syndrome. N Engl J Med 2000 May 4;342(18):1301-1308.

6. 3. Gomberg-Maitland M Olschewski H. Prostacyclin therapies for the treatment of pulmonary arterial hypertension. Eur Respir J 2008;31: 891-901. 
001290

Normalization of laboratory parameters in critically-ill COVID-19 survivors

IT. Geldigitti ${ }^{1}$; B. Halacli ${ }^{1}$; B. Er ${ }^{1}$; M. Yildirim¹; TK. Sahin ${ }^{2}$; MY. Pektezel ${ }^{1}$; G. Tok ; B. Erdemir ${ }^{1}$; AM. Vural ${ }^{1}$; LG. Koc Yamanyar ${ }^{1}$; E. Ortac Ersoy ${ }^{1}$; S. ÖCal' A. Topeli ${ }^{1}$

${ }^{1}$ Department of internal medicine, division of intensive care medicine, Hacettepe Universtity Faculty of Medicine, Ankara, Turkey; ${ }^{2}$ Department of internal medicine, Hacettepe Universtity Faculty of Medicine, Ankara, Turkey

Correspondence: I.T. Geldigitti

Intensive Care Medicine Experimental 2020, 8(2): 001290

Introduction: Laboratory parameters in COVID-19 regarding disease severity have been extensively evaluated [1, 2]. However, duration of their normalization is unknown.

Objectives: To determine the normalization time and sequence of laboratory parameters in critically-ill COVID-19 survivors.

Methods: Medical records of COVID-19 patients, admitted to ICU between March 29th and June 1st 2020 and discharged till July 18th 2020 were examined. For each laboratory parameter, normalization date was accepted as the first day of the normal laboratory result series following two or more abnormal consecutive results. $\mathrm{PaO} 2 / \mathrm{FiO} 2$ (P/F) of $\geq 200 \mathrm{mmHg}$ was accepted as improved value for oxygenation. Results: A total of 21 patients were enrolled in the study. Patient characteristics, normalization and treatment details are demonstrated in table. P/F improved first, and D-dimer normalized last. The sequence of normalization is demonstrated in figure.

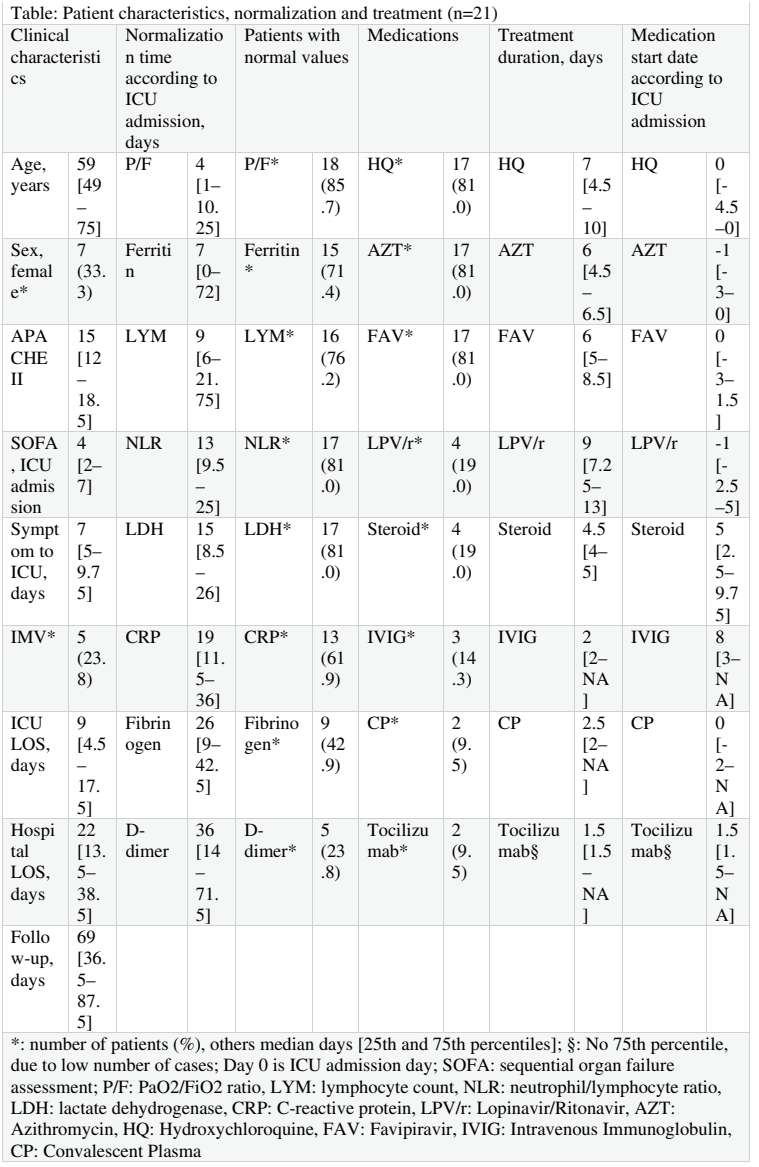

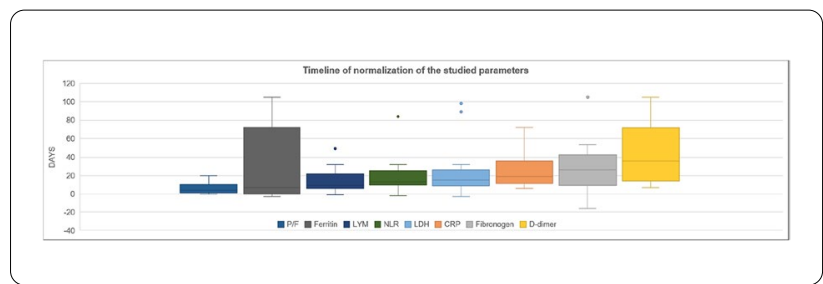

Conclusion: In critically-ill COVID-19 survivors, oxygenation improves before inflammatory parameters, and D-Dimer normalizes last, suggesting prolonged coagulopathy.

\section{Reference(s) and grant ackowledgment(s)}

1. Skevaki C, Fragkou PC, Cheng C, et al (2020) Laboratory characteristics of patients infected with the novel SARS-CoV-2 virus. J Infect. https://doi. org/10.1016/j.jinf.2020.06.039.

2. Velavan TP, Meyer CG (2020) Mild versus severe COVID-19: Laboratory markers. Int J Infect Dis IJID Off Publ Int Soc Infect Dis 95:304-307. https://doi. org/10.1016/j.ijid.2020.04.061.

001297

Prone positioning in non-intubated critically-ill patients with COVID-19 pneumonia

B. Erdemir ${ }^{1}$; IT. Geldigitti ${ }^{1}$; M. Yildirim¹' B. Er ${ }^{1}$; MY. Pektezel ${ }^{1}$; G. Tok ; S. Ozen ${ }^{1}$; ND. Bulut Yuksel'; B. Halacli ${ }^{1}$; E. Ortac Ersoy ${ }^{1}$; S. ÖCall ; A. Topeli ${ }^{1}$

${ }^{1}$ Department of internal medicine, division of intensive care medicine, Hacettepe University Faculty of Medicine, Ankara, Turkey

Correspondence: $\mathrm{B}$. Erdemir

Intensive Care Medicine Experimental 2020, 8(2): 001297

Introduction: Patients with COVID-19 pneumonia are at major risk for acute respiratory distress syndrome (ARDS). Prone positioning (PP) has many benefical effects on pulmonary physiology and improves oxygenation in patients with ARDS.

Objectives: We aimed to investigate the effects of PP in non-intubated patients with COVID-19 related pneumonia.

Methods: In this retrospective, single-center, before-after study we enrolled spontaneously breathing patients with confirmed diagnosis of COVID-19 pneumonia who received PP between March 21th and July 5th, 2020. We collected baseline data of demographic variables, SOFA score, APACHE II Score, oxygen therapy methods (as nasal cannula, simple face mask, non-rebreather mask) and non-invasive ventilation. We compared $\mathrm{PaO} 2, \mathrm{SpO} 2, \mathrm{FiO} 2, \mathrm{PaO} 2 / \mathrm{FiO} 2, \mathrm{SpO} 2 / \mathrm{FiO} 2$, respiratory rate (RR) before $\mathrm{PP}$, during $\mathrm{PP}$ and 1 hour after return to supine position. The main outcome is improvement in oxygenation. For statistical analysis of related repeated measures the Friedman test was used. Results are expressed as median (IQR).

Results: Fifteen patients (13 males) were enrolled in the study. Age was 67 (59-75), APACHE II and SOFA Scores were 15 (12-19) and 3 (3-5) As seen in Table $\mathrm{PaO} 2, \mathrm{SpO} 2, \mathrm{FiO} 2, \mathrm{SpO} 2 / \mathrm{FiO} 2$ and $\mathrm{RR}$ improved during PP compared to baseline. Overall 3 patients were intubated in whom $\mathrm{PaO} 2 / \mathrm{FiO} 2$ improved less than 15\% during PP. Six of 12 patients had $>15 \%$ improvement in $\mathrm{PaO} 2 / \mathrm{FiO} 2$ during PP. There was not any improvement in $\mathrm{PaO} 2 / \mathrm{FiO} 2$ after $\mathrm{PP}$ except 1 patient. Two intubated patients died.

\begin{tabular}{|l|l|l|l|l|}
\hline $\mathrm{n}=15$ & before PP & during PP & after PP & p value \\
\hline $\mathrm{PaO} 2, \mathrm{mmHg}$ & $63.9(11.4)$ & $73.9(13.6)$ & $66.4(19.2)$ & 0.02 \\
\hline $\mathrm{SpO} 2, \%$ & $91(1.9)$ & $93.9(2.3)$ & $91.4(1.5)$ & $<0.0001$ \\
\hline $\mathrm{FiO} 2, \%$ & $43.6(18.2)$ & $41.7(17.7)$ & $40(14.3)$ & 0.032 \\
\hline $\mathrm{PaO} 2 / \mathrm{FiO} 2, \mathrm{mmHg}$ & $179.9(56.3)$ & $197.5(64.1)$ & $167(46.7)$ & 0.06 \\
\hline $\mathrm{SpO} 2 / \mathrm{FiO} 2, \%$ & $236.6(73)$ & $251(72.4)$ & $248.6(65.2)$ & $<0.0001$ \\
\hline $\mathrm{RR}$, breaths per min & $30.8(5.4)$ & $25.5(4.5)$ & $25.6(6.1)$ & $<0.0001$ \\
\hline
\end{tabular}


Figure: Each line represents one patient and $\mathrm{PaO} 2 / \mathrm{FiO} 2$ (A), $\mathrm{SpO} 2 / \mathrm{FiO} 2$ (B), $\mathrm{RR}$ (C) changes before, during, after PP demonstrated in graphics.
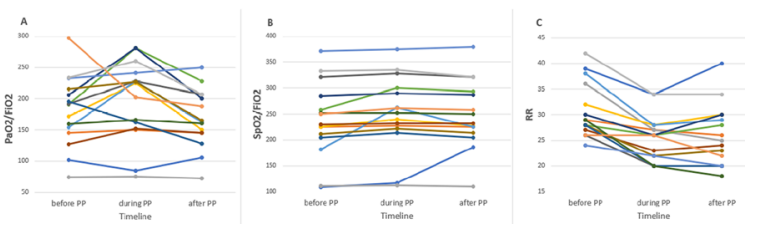

Conclusion: PP improves oxygenation and RR in patients with COVID19 pneumonia.

\section{Reference(s) and grant ackowledgment(s)}

1. Elharrar X, Trigui Y, Dols A-M, et al (2020) Use of Prone Positioning in Nonin tubated Patients With COVID-19 and Hypoxemic Acute Respiratory Failure. JAMA 323:2336-2338. https://doi.org/10.1001/jama.2020.8255.

2. Coppo A, Bellani G, Winterton D, et al (2020) Feasibility and physiological effects of prone positioning in non-intubated patients with acute respiratory failure due to COVID-19 (PRON-COVID): a prospective cohort study. Lancet Respir Med 0: https://doi.org/10.1016/S2213-2600(20)30268-X.

\section{5}

Covid-19-related acute respiratory distress syndrome: different phenotypes of lung water and pulmonary vascular permeability and relationship with mortality. The PiCCOVID study

R. Shi ${ }^{1}$; C. Lai ${ }^{1}$; JL. Teboul ${ }^{1}$; M. Dres ${ }^{2}$; N. De Vita ${ }^{3}$; T. Pham¹; V. Bonny ${ }^{2}$; J.

Mayaux ${ }^{2}$; R. Vaschetto ${ }^{3} ;$ A. Beurton ${ }^{2} ;$ X. Monnet $^{1}$

${ }^{1}$ Médecine intensive réanimation, Bicetre Hospital AP-HP, Le Kremlin-Bicêtre, France; ${ }^{2}$ Service de pneumologie et réanimation médicale, University Hospitals Pitié Salpêtrière - Charles Foix, Paris, France; ${ }^{3}$ Anestesia e terapia intensiva, University Hospital Maggiore della Carita, Novara, Italy

Correspondence: $\mathrm{R}$. SHI

Intensive Care Medicine Experimental 2020, 8(2): 001305

Introduction: Previous studies have described the respiratory mechanics of Covid-19 related acute respiratory distress syndrome (ARDS) and different phenotypes. However, their characteristics regarding transpulmonary thermodilution variables, such as extravascular lung water (EVLW) and pulmonary vascular permeability, have been rarely described. Considering these parameters are important to fluid management strategy, our study aimed to describe EVLW and pulmonary vascular permeability in Covid-19-related ARDS and compared them with a historical control group of patients with non Covid19 ARDS.

Objectives: To describe the characteristics of extravascular lung water index (EVLWi) and pulmonary vascular permeability index (PVPI) in Covid-19 ARDS patients. To investigate the relationship of EVLWi and PVPI with mortality and to compare these patients to a historical cohort of patients with non-Covid-19 ARDS.

Methods: In this observational multicentre study performed in three ICUs, we included patients with mild to severe Covid-19 ARDS and monitored by transpulmonary thermodilution. Demographic and relevant clinical data were collected. Transpulmonary thermodilution variables were collected at least once a day for a maximum of 14 days. We selected values corresponding to the maximum value of EVLWi (EVLWimax) and of PVPI (PVPImax) per day. Other hemodynamic values, the ratio of arterial partial pressure of oxygen ( $\mathrm{PaO} 2)$ over $\mathrm{FiO} 2$ $(\mathrm{PaO} 2 / \mathrm{FiO} 2)$ and other respiratory parameters were recorded at the time when the transpulmonary thermodilution values of interest were assessed.

Results: From March 13th to May 10th, 45 ARDS patients with confirmed Covid-19, monitored by transpulmonary thermodilution were included. On day 1, EVLWi was 17 (13-19) mL/kg and PVPI was 3.4 (2.64.2). EVLWimax and PVPImax was reached on 3 (1-4) and 2 (1-3) days, respectively. EVLWimax was higher in non-survivors than in survivors
(24 (19-28) vs. 18 (16-25) mL/kg, respectively, $\mathrm{p}=0.03)$. PVPImax was also elevated in non-survivors than in survivors (4.9 (3.9-6.0 vs. 3.8 (3.44.7), respectively, $\mathrm{p}=0.03$ ). Nevertheless, 4 of 27 patients with $\mathrm{PaO} 2 /$ FiO2 $\leq 100 \mathrm{mmHg}$ exhibited moderately increased EVLWi ( $\leq 15 \mathrm{~mL}$ ) $\mathrm{kg}$ ) and PVPI (<4). Compared to non Covid-19 ARDS patients, Covid19 patients had similar values of EVLWimax (22 (17-27) vs. 20 (14-26), respectively) and PVPImax (4.5 (3.6-5.4) vs. 3.9 (3.0-5.5), respectively). At multivariate analysis of the merged cohorts of ARDS patients with and without Covid-19, EVLWimax (odds ratio $(\mathrm{OR})=1.06,95 \%$ confidence interval (CI) [1.01-1.10]), PVPImax (OR=1.23, 95\% Cl [1.01-1.49]), and the Covid-19 status $(\mathrm{OR}=2.73,95 \% \mathrm{Cl}$ [1.17-6.37]), were independent factors of 28-day mortality.

Conclusion: Covid-19 ARDS is characterized by elevated EVLWi and PVPI, which do not differ from non-Covid-19 ARDS. The maximal values of these variables are associated with the outcome. Covid-19 ARDS seems to be an independent risk factor for mortality.

\section{0}

Prone Positioning in a COVID-19 ARDS Cohort- A Retrospective Analysis on Oxygenation and Ventilation

C. Mcgarrigle; ; M. Ahmed ; B. Doyle ; I. Medan

${ }^{1}$ Anaesthesia and intensive care medicine, Tallaght University Hospital, Dublin, Ireland

Correspondence: C. McGarrigle

Intensive Care Medicine Experimental 2020, 8(2): 001310

Introduction: An acute respiratory disease, caused by a novel coronavirus (SARS-CoV-2), the coronavirus disease 2019 (COVID-19) has spread throughout the world. COVID-19 induces a severe inflammatory response causing acute respiratory distress syndrome (ARDS) and respiratory failure. The management of ARDS is primarily supportive which includes ventilatory and non-ventilatory management. Due to acute lung parenchymal injury, ventilation often proves difficult and rescue measures are required, which include prone position ventilation. In previous studies, prone position ventilation has shown dramatic improvement in oxygenation and ventilation but no statistically significant difference in mortality and ICU length of stay.

Objectives: To Assess the effects of prone positioning on ventilation and oxygenation of patients with COVID-19 ARDS.

Methods: Following ethical approval, data was collected from both written and electronic records (IntelliSpace Critical Care and Anaesthesia information system). All patients admitted to Tallaght University Hospital's Intensive Care Unit (ICU) over a four month period between February 2020 to June 2020 with a Covid19 positive viral PCR and who were subsequently intubated, ventilated and proned were included in this study. Self proning, non-intubated patients were excluded. Clinical data was extracted at four periods around each proning session; immediately prior to prone positioning (R1), two hours post prone positioning (R2), one hour pre-supination (R3) and one hour post supination (R4).

Data was collected and analysed using Microsoft Excel.

Results: In total, 16 patients $(4 \mathrm{~F}, 12 \mathrm{M})$ were intubated and proned. Five patients were of Asian origin, three of African Origin and eight were Caucasian. The mean age was 51 and the median body mass index (BMI) was $31.5 \mathrm{~kg} / \mathrm{m}^{2}$. 14 patients (87.5\%) survived and were discharged from ICU. Significant cardiovascular comorbidities were present in the two non-surviving patients. 77 proning session took place in total. The mean day of proning post intubation was day 2 , with an average of 4.8 sessions per patient, lasting a mean of 17.1 hours. The mean $\mathrm{PaO} 2 / \mathrm{FiO} 2$ ratio (PFR) prior to the first proning session was 17 (11-29) versus the mean PFR after the patient's final proning session of 26 (15-46).

A PFR increase of $>30 \%$ was present in $44 \%$ (34/77) of sessions immediately post proning (R1 vs R2) vs 64\% (49/77) found after a full proning session (R1 vs R3), suggesting an improvement in oxygenation with time. Comparing the PFR in the supine position pre and post proning sessions, an improvement was present in $68 \%$ of cases (R1vsR4).

Where end tidal $\mathrm{C} 02$ levels were accurately recorded, a reduction in the End tidal-Arterial CO2 gradient was present in $68 \%(45 / 66)$ of 
sessions after proning (R1 vs R3) with a median change of $-0.3 \mathrm{kPa}$, suggesting a reduction alveolar dead space.

Conclusion: Prone positioning of patients with ARDS caused by COVID-19 appears to improve the oxygenation and ventilation in a similar manner compared to ARDS attributed to other causes.

\section{Reference(s) and grant ackowledgment(s)}

1. 7. Mure M, Martling CR, Lindahl SG. Dramatic effect on oxygenation in patients with severe acute lung insufficiency treated in the prone position. Crit Care Med 1997;25:1539-44. [Crossref] [PubMed].

2. 6. Guérin C, Reignier J, Richard JC, et al. Prone positioning in severe acute respiratory distress syndrome. N Engl J Med 2013;368:2159-68. [Crossref] [PubMed]

3. 5. Mancebo J, Fernandez R, Blanch $L$, et al. A multicenter trial of prolonged prone ventilation in severe acute respiratory distress syndrome. Am J Respir Crit Care Med 2006;173:1233-9. [Crossref] [PubMed].

4. 4. Guerin C, Gaillard S, Lemasson S, et al. Effects of systematic prone positioning in hypoxemic acute respiratory failure. JAMA 2004;292:2379-87. [Crossref] [PubMed].

5. 3. Richter T, Bellani G, Scott Harris R, et al. Effect of prone position on regional shunt, aeration, and perfusion in experimental acute lung injury. Am J Respir Crit Care Med 2005;172:480-7. [Crossref] [PubMed].

6. 2. Gattinoni L, Pelosi P, Vitale G, et al. Body position changes redistribute lung computed-tomographic density in patients with acute respiratory failure. Anesthesiology 1991;74:15-23. [Crossref] [PubMed].

7. 1. Cornejo RA, Diaz JC, Tobar EA, et al. Effects of prone positioning on lung protection in patients with acute respiratory distress syndrome. Am J Respir Crit Care Med 2013;188:440-8. [Crossref] [PubMed]. 8. Nil.

\section{8}

Covid-19 Critical ill patients in Santiago de Compostela. Case series

B. Lence ; C. Rivero

${ }^{1}$ Intensive Care Unit, Santiago Clinic Hospital, Santiago de Compostela, Spain

\section{Correspondence: $B$. Lence}

Intensive Care Medicine Experimental 2020, 8(2): 001338

Introduction: Our experience with Covid-19 critical ill patients in Santiago de Compostela during spring 2020.

Objectives: To learn about what we did and how we did it, getting new perspectives and ideas as next steps to improve.

Methods: We identified 23 patients with severe acute respiratory syndrome that needed ICU admission. Clinical data were reviewed of medical records. The data reported here are those through March 12 to June 26. Each patient had the whole hospital stay of follow-up.

Results: We admitted 23 patients with SARS-CoV19 in ICU. The average age was 65 years old, being the youngest 42 years old and the oldest 80 years old. 19 of those patients were men and only 4 were female.

All patients reported were admitted for respiratory failure, 35\% needed mechanical ventilation in the admission moment, finally requiring the $80 \%$ of the patients mechanical ventilation (during the ICU stay). The initial approach for $52 \%$ (12) of the patients were high flow nasal cannulas, failing to meet the oxygen needs and requiring in 10 of them to iniciate mecanichal ventilation. In 14 patients (78\%) prono theraphy brought benefits. The maximum time on mecanichal ventilation was 43 days, with and average amount of 14 days, being the longest 53 days. Most of the initial chest $x$-ray during the hospital admission showed consolidation in $4 / 4$ lung fields, whilst those $x$-rays that initially showed diffuse infiltration and only consolidation in $1 / 4$ or $2 / 4$ lung fields deteriorated quickly on the following hours. The initial chest $x$-ray affectation at the ICU admission was not related to the oxygen support needed.

Concerning the treatment:
- All of the patients in this ICU were treated with hydroxychloroquine and lopinavir/ritonavir, with disparities on how long were administered (between 5 and 15 days).

- Only 1 patient had hypotension and needed vasopressors during the ICU admission.

- $87 \%$ (20) of the patients received corticosteroid therapy. 9 with dexametasone, 10 with methylprednisolone and 1 with hydrocortisone.

- Regarding anticoagulant therapy, all the 23 patients received Low-Molecular-Weiht-Heparin in different doses $(0.5 \mathrm{mg} / \mathrm{kg} /$ day; $1 \mathrm{mg} / \mathrm{kg} /$ day; - $1,5 \mathrm{mg} / \mathrm{kg} /$ day; $1 \mathrm{mg} / \mathrm{kg} / 12 \mathrm{~h}$ ). More information must be addressed to determine an accurate dosing.

- $56 \%$ of the patients received tocilizumab when IL-6 levels exceeded $40 \mathrm{UI} / \mathrm{L}$.

- Only $21 \%$ of them required continuous renal replacement therapy.

Futhermore, the highest ICU occupancy rates were on the first week of April, yet mantaining capacity to admitt more patients.

Conclusion: During the SARS-CoV19 pandemic, 23 patients were admitted to ICU with the common admission criteria of hypoxemic respiratory failure leading to mechanichal ventilation. Most of this patients received corticosteroid therapy and this could play a significant role regarding to mortality: $83 \%$ of our patients survived.

Reference(s) and grant ackowledgment(s)

1. Maatman T, Jalali F, Feizpour C, et al. Routine venous trhomboembolism profhyliaxis may be inadequate in the hypercoagulable state of severe coronavirus disease. Critical Care Medicine 2020.

2. Pavan K, Bijan J, Michelle Nichols, et al. Covid-19 in critically ill patients in the Seattle Region - Case series. NEJM March 30, 2020.

3. Yang X, Yu Y, Xu J, et al. Clinical course and outcomes of critically ill patients with SARS-CoV-2 pneumonia in Wuhan, China: a single centered, retrospective, observational study. LAncet respir Med 2020 February 24.

4. World Health Organization. Novel coronavirus - China. Juanary 12, 2020

\section{7}

COVID-19 in a critical care unit: several characteristics and mortality analysis of patients with severe pneumonia due to SARS-COV-2

M. Aseguinolaza Lizarazu'; I. Eguibar Villimar'; M. Diez Bengoechea'; L. Vidaur Tello'; T. Ormazabal Zabala'; R. Sebastian Alda ${ }^{1}$

${ }^{1}$ Critical care, Hospital Universitario Donostia, San Sebastián-Donostia, Spain

Correspondence: M. Aseguinolaza Lizarazu

Intensive Care Medicine Experimental 2020, 8(2): 001347

Introduction: In the face of the SARS-COV-2 pandemic, the intensive care units (ICU) have been involved in a dynamic of hard work by a little known disease, but which has caused serious illness and has involved the use of many health resources. The aim of this study is to show our experience with this kind of patients.

Objectives: The objective of this study is to analyse demographics, clinical and analytical characteristics, complications and health care resources consumption in a cohort of patients with a diagnosis of severe COVID-19 infection admitted to an ICU of a University Hospital. Secondary objective is to analyse factors related with mortality in his group of patients.

Methods: This is a retrospective observational study including all the critically ill patients admitted to ICU with COVID-19 severe infection. Patients were included during the COVID-19 pandemic, starting in March 2020. The analyzed demographic variables included age, sex, comorbidities and severity at admission measured by APACHE II and SOFA scores. Analytic variables studied were those related to prognosis in other studies such as lactate dehydrogenase (LDH), creatine kinase (CK), Ferritine, D-Dimer, C-reactive protein (CRP) or 
procalcitonin (PCT). Health care resource consumption was measured by the days of mechanical ventilation and ICU stay. Quantitative variables are expressed as mean \pm standard deviation and the qualitative ones as absolute and relative frequencies. The comparison between quantitative variables was made by student- $t$ test and qualitative variables by chi squared test for a $95 \%$ confidence interval.

Results: A total of 60 patients were admitted to our department for severe pneumonia due to SARS-COV-2 and 43 of them (72\%) were male. The average age has been 66.5 years, indeed, age has been mortality related factor. Each patient has been admitted to the ICU for 23 days on average. Among all the patients, $82 \%$ were intubated and connected to mechanical ventilation (MV) at admission, and this MV has gone on for 19+-11 days, increasing morbidity and mortality, and resource consumption. Overall mortality has been $23.3 \%$, especially up to the age of 60 (under the age of 60 , only one patient died amongst 16). High blood pressure $(p=0.03)$, obesity $(p<0.01)$, APACHE at admission $(p=0.04)$ and SOFA at admission $(p=0.02)$ have been related to higher mortality as well. Although they have not been statistically significant, it seems that the elevation of LDH, CRP, PCT, ferritin and D-dimer are associated with greater death rate.

\begin{tabular}{|c|c|c|c|}
\hline \multicolumn{4}{|c|}{ Demographic variables } \\
\hline \multicolumn{2}{|c|}{ Patients $(\mathrm{n}=60)$} & \multicolumn{2}{|r|}{ Results } \\
\hline \multicolumn{2}{|l|}{ Mean age [p25 -p75] } & \multicolumn{2}{|c|}{$66,5[58-71,75]$} \\
\hline \multicolumn{2}{|l|}{ Sex: Male, Female (\%) } & \multicolumn{2}{|c|}{$43(72 \%) / 17(28 \%)$} \\
\hline \multicolumn{2}{|c|}{ ICU stay in days [p25-p75] } & \multicolumn{2}{|c|}{$23\left[\begin{array}{ll}14,5 & 30,75]\end{array}\right.$} \\
\hline \multicolumn{2}{|c|}{ Mortality (\%) } & \multicolumn{2}{|c|}{$14(23,3 \%)$} \\
\hline \multicolumn{2}{|l|}{$<50(\mathrm{n}=7)$} & \multicolumn{2}{|l|}{0} \\
\hline \multicolumn{2}{|l|}{$51-60(\mathrm{n}=9)$} & \multicolumn{2}{|l|}{$1(11 \%)$} \\
\hline \multicolumn{2}{|l|}{$61-70(n=26)$} & \multicolumn{2}{|l|}{$6(23 \%)$} \\
\hline \multicolumn{2}{|l|}{$71-80(n=18)$} & \multicolumn{2}{|l|}{$7(39 \%)$} \\
\hline \multicolumn{2}{|l|}{ MV at admission $\mathrm{n}(\%)$} & \multicolumn{2}{|l|}{$49(82 \%)$} \\
\hline \multicolumn{2}{|c|}{ APACHE score / SOFA score } & \multicolumn{2}{|c|}{$12 \pm 4,6 / 5,1 \pm 2,5$} \\
\hline \multicolumn{2}{|c|}{ MV days } & \multicolumn{2}{|c|}{$19 \pm 11$} \\
\hline \multicolumn{4}{|c|}{ Univariate analysis of mortality } \\
\hline & Dead $(\%)$ & Survivors (\%) & $\mathbf{p}$ \\
\hline APACHE score & 14,2 & 11,3 & 0,04 \\
\hline SOFA score & 6,4 & 4,7 & 0,02 \\
\hline CRP at admission & 209,9 & 190,6 & 0,56 \\
\hline PCT at admission & 0,6 & 0,37 & 0,08 \\
\hline $\begin{array}{l}\text { LDH / D dimer / } \\
\text { ferritine }\end{array}$ & $\begin{array}{l}625 / 2786 / \\
3061\end{array}$ & $\begin{array}{l}478 / 5837 / \\
1750\end{array}$ & $\begin{array}{l}0,053 / 0,058 / \\
0,06\end{array}$ \\
\hline
\end{tabular}

Conclusion: Patients admitted to ICU with severe COVID-19 infection have a great mortality with an important health care resource consumption. In this group of patients age, high blood pressure and obesity have been associated with mortality. $\mathrm{LDH}$, ferritine,..... are higher at admission in patients who died.

\section{1}

\section{COVID-19 in critically ill solid organ transplantation recipients:} Our experience

F. Onieva Calero ${ }^{1}$; R. Carmona Flores'; P. Carmona Sánchez'; MC. López'; S. Carcel Fernandez ${ }^{1}$; JC. Pozo Laderas's; C. De La Fuente Martos ${ }^{1}$

${ }^{1}$ Intensive Care Unit, Hospital Universitario Reina Sofia, Córdoba, Spain; ${ }^{2}$ Intensive care medicine, Hospital Universitario Reina Sofía, Córdoba, Spain, Spain

\section{Correspondence: F. Onieva Calero}

Intensive Care Medicine Experimental 2020, 8(2): 001371

Introduction: COVID-19 has emerged as a rapidly spreading communicable disease affecting people worldwide. A particular concern exists regarding the vulnerability of patients who have been immunosuppressed prior or during this pandemic. Data on COVID 19 in solid organ transplantation (SOT) that required intensive care are scarce.

Objectives: Describe our mortality in SOT recipients that have been infected with COVID-19 and have required intensive care.

Methods: Case series of adults (age $>18$ years) SOT recipients from the Intensive Care Unit (ICU) of a third level hospital in southern Spain with a positive test for COVID-19 between March 24, 2020 and May 29, 2020. The data were extracted from a database of 42 patients that have required admission in our ICU with a positive reversed-transcriptase PCR of COVID-19.

Results: 3 patients were admitted with a median age of 53 years. Our patients were a kidney-pancreas recipient, a kidney recipient, and a lung recipient. They had a median APACHE score of 14, SOFA score of 9 , and a Brescia scale of 8 . The most frequently found comorbidity was chronic kidney failure. Two patients were in treatment with domiciliary corticoids, two patients with mycophenolate, and three patients with tacrolimus. At the admission time they had a leukocyte median of $2640 \mathrm{~mm} 3$, a lymphocyte median of $430 \mathrm{~mm} 3$, a CD4 median of 440 $\mathrm{mm} 3$, a lgG median of $438 \mathrm{mg} / \mathrm{dL}$, a IL-6 median $46.2 \mathrm{pg} / \mathrm{mL}$, and a C-Reactive Protein median of $100.7 \mathrm{mg} / \mathrm{dL}$. In all of these patients airway isolation were required, with a median of invasive mechanical ventilation (IMV) of 8 days. One patient received High Flow Nasal Cannula (HFNC) support that failed. Two patients suffered renal graft dysfunction, one of them required renal replacement therapies. Three patients received Hydroxychloroquine, two patients received Lopina$\mathrm{vir} /$ Ritonavir, one patient received Interferon and one patient received Immunoglobulin. In all of these patients, mycophenolate and tacrolimus were discontinued, maintaining methylprednisolone at $1 \mathrm{mg} / \mathrm{kg}$. One patient was co-infected with CMV and received empirical antibiotherapy. One patient had secondary bacterial infections during the ICU stay, one Ventilator-Associated Pneumonia and one Catheter-Associated Bacteremia. The three patients were alive at the ICU discharge, and at the hospital discharge. The mean of ICU stay was 10 days, and hospital stay of 26 days.

Conclusion: In our setting, all patients with COVID-19 and TOS survived. These data suggest that the prognosis associated with critical illness due to COVID-19 in SOT recipients may not be as poor as previously reported, and that being a SOT recipient does not indicate worse mortality.

\section{Reference(s) and grant ackowledgment(s)}

1. Pereira MR, Mohan S, Cohen DJ, et al. COVID-19 in solid organ transplant recipients: Initial report from the US epicenter. Am J Transplant. 2020; https ://doi.org/10.1111/ajt.15941.

\section{4}

Feasibility and efficacy of prone position combined with CPAP in COVID-19 patients with acute hypoxemic respiratory failure K. Belkhouja'; S. Alzahrani ${ }^{1}$; N. Al-Shalhoub ${ }^{1}$; T. Negm ; M. Darwish'; MW. Pathan ${ }^{1}$; MM. Lone ${ }^{1}$; S. Omer ${ }^{1}$; H. Al-Sharif ${ }^{1}$

${ }^{1}$ Intensive care department, King Fahad Armed Forces Hospital, Jeddah, Saudi Arabia

\section{Correspondence: K. Belkhouja}

Intensive Care Medicine Experimental 2020, 8(2): 001374

Introduction: The COVID-19 pandemic has led to rapidly saturation of resources and the availability of ICU bed. Prone positioning is strongly recommended in intubated patients with severe acute respiratory distress syndrome. Recently, small case series showed feasibility and improvement in oxygenation in awake COVID-19 patients placed in the prone position with noninvasive ventilation (NIV). It is unknown if the proning with NIV can reduce the rate of intubation.

Objectives: To evaluate the feasibility, the safety and efficacy of prone positioning with continuous positive airway pressure (CPAP) in COVID19 patients with acute hypoxemic respiratory failure.

Methods: This prospective observational cohort study was conducted, from Mars 25, 2020, to September 24, 2020, in King Fahad armed forces hospital, Kingdom of Saudi Arabia. All adult patients with confirmed COVID-19 pneumonia and developed acute hypoxemic 
respiratory failure defined by $\mathrm{PaO} 2 / \mathrm{FiO} 2$ less than $200 \mathrm{mmHg}$, and required $\mathrm{CPAP}$, were included in this trial, if they fulfill the inclusions criteria. Demographic, clinical, laboratory, radiological, ventilation setting, and treatment data were collected and analyzed. The patient will be included in one of the two groups, CPAP with proning (prone group) or CPAP without proning (supine group). The supine group included either patient refused proning or who used CPAP in the ward. The primary outcome was the rate of tracheal intubation. The secondary outcomes were, the rate of ICU mortality, the length of stay, and the rate of health care workers (HCWs) infected by SARS-CoV2.

Results: In this preliminary analysis, a total of 79 COVID-19 patients were included, 31 patients in the prone group and 48 patients in the supine group. The two groups were similar on term of baseline characteristics, general management and ventilatory support. In the prone group there was significant increase of $\mathrm{PaO} 2 / \mathrm{FiO} 2$ during proning compared to supine setting. The general acceptance of proning was from moderate to well and no complications or events were observed during proning periods. The rate of intubation was $6.5 \%$ (2 patients) in the prone group and $25 \%$ (12 patients) in the supine group ( $P=0.035)$. Five patients died in the supine group versus one patient in the prone group $(P=0.395)$. One nurse caring for a patient in the supine group was infected with SARS-CoV2.

Conclusion: In COVID-19 patients with acute hypoxemic respiratory failure, the use of prone position combined with CPAP, is feasible, safe and decrease the rate of tracheal intubation.

\section{Reference(s) and grant ackowledgment(s)}

1. We are indebted to the doctors, nurses and respiratory therapists with the Intensive Care Medicine Service who provides care for the patients included in the study and to all the frontline staff of COVID-19 patients.

\section{2}

\section{Comparison of Critically-III COVID-19 and Influenza Patients} with Acute Respiratory Failure

M. Yildirim ${ }^{1}$; B. Halacli'; MY. Pektezel' ; B. Er' ; DA. Ozbek²; G. Tok ; B. Erdemir $^{1}$; C. Balci Altin ${ }^{1}$; G. Atceken ${ }^{1}$; E. Ortac Ersoy ${ }^{1}$; S. ÖCal ${ }^{1}$; A. Topeli

${ }^{1}$ Division of intensive care medicine, department of internal medicine, Hac ettepe University Faculty of Medicine, Ankara, Turkey; ${ }^{2}$ Department of internal medicine, Hacettepe University Faculty of Medicine, Ankara, Turkey Correspondence: M. Yildirim

Intensive Care Medicine Experimental 2020, 8(2): 001382

Introduction: Although respiratory virus infections are generally selflimiting, they are one of the leading causes of community-acquired pneumonia, acute respiratory failure (ARF), sepsis and death as we have experienced from miserable pandemics in the last two decades. Objectives: To compare the clinical characteristics and prognosis of COVID-19 and influenza patients with ARF admitted to intensive care unit (ICU).

Methods: Patients with laboratory confirmed COVID-19 who were admitted due to ARF to our ICU between 20th March and 23th June 2020 and patients with laboratory confirmed influenza who were admitted due to ARF between 1st January 2015 and 1st March 2020 were retrospectively evaluated. Clinical characteristics and outcomes of patients were compared.

Results: 36 COVID-19 patients and 59 influenza patients were evaluated and 34 COVID-19 and 55 influenza patients who presented with ARF due to viral pneuomonia were included in this study. Age, gender, SOFA scores, duration of symptom onset to hospital and ICU admission were similar between two groups. Diabetes, chronic lung disease and cardiac diseases were more frequent in influenza patients. ECOG, Clinical Frailty Scale $\odot$ (CFS), APACHE II score of influenza patients were worse than COVID-19 patients. Invasive mechanical ventilation requirement was higher in influenza group, while admission $\mathrm{PaO} 2 /$ $\mathrm{FiO} 2$ ratio was similar. Frequency of ARDS on admission was higher in COVID-19 patients, but mild ARDS was seen more in COVID-19 patients. Prone positioning was utilized more in COVID-19 patients. Bacterial co- and superinfection, sepsis and acute kidney injury (AKI) were encountered more in influenza group. ICU and hospital mortality in COVID-19 patients were significantly lower than patients with influenza ( $24 \%$ vs $47 \%, \mathrm{p}=0.025$ ) (Table).

\begin{tabular}{|c|c|c|c|c|}
\hline & All $(\mathbf{n = 8 9})$ & $\begin{array}{l}\text { COVID } \\
(n=34)\end{array}$ & $\begin{array}{l}\text { Influenza } \\
(\mathbf{n}=55)\end{array}$ & p value \\
\hline Age* (years) & $63(55-77)$ & $63(55-76)$ & $62(51-77)$ & 0.970 \\
\hline Male gender, n (\%) & $51(57)$ & $22(65)$ & $29(53)$ & 0.267 \\
\hline \multicolumn{5}{|l|}{ Comorbidities, n (\%) } \\
\hline Hypertension & $38(43)$ & $12(35)$ & $26(47)$ & 0.260 \\
\hline Cardiac disease & $25(28)$ & $4(12)$ & $21(38)$ & 0.007 \\
\hline Diabetes & $23(26)$ & $4(12)$ & $19(35)$ & 0.017 \\
\hline Malignancy & $20(23)$ & $4(12)$ & $16(29)$ & 0.057 \\
\hline Chronic lung disease & $16(18)$ & $2(6)$ & $14(26)$ & 0.019 \\
\hline Chronic kidney disease & $6(7)$ & $0(0)$ & $6(11)$ & 0.079 \\
\hline ECOG* & $2(1-3)$ & $1(0-2)$ & $2(1-3)$ & 0.002 \\
\hline CFS@ $\odot *$ & $4(3-6)$ & $3(1-5)$ & $5(3-7)$ & $<0.001$ \\
\hline APACHE II & $18(14-25)$ & $15(12-24)$ & $19(15-27)$ & 0.031 \\
\hline $\mathrm{PaO} 2 / \mathrm{FiO} 2$ ratio $\dagger$ & $\begin{array}{l}162(108- \\
235)\end{array}$ & $\begin{array}{l}153(110- \\
233)\end{array}$ & $180(102-250)$ & 0.670 \\
\hline ARDS, n (\%) & $65(73)$ & $31(91)$ & $34(62)$ & 0.002 \\
\hline Mild ARDS & $13(20)$ & $10(32)$ & $3(9)$ & 0.018 \\
\hline IMV, n (\%) & $51(57)$ & $15(44)$ & $36(66)$ & 0.048 \\
\hline Prone position, n (\%) & $19(21)$ & $16(47)$ & $3(6)$ & $<0.001$ \\
\hline Co-infection, n (\%) & $27(30)$ & $6(18)$ & $21(38)$ & 0.041 \\
\hline Superinfection, n (\%) & $44(49)$ & $10(29)$ & $34(62)$ & 0.003 \\
\hline Sepsis, n $(\%) \dagger$ & $30(34)$ & $6(18)$ & $24(44)$ & 0.012 \\
\hline AKI, n (\%)† & $49(55)$ & $13(38)$ & $36(66)$ & 0.012 \\
\hline 28th day mortality, n (\%) & $25(28)$ & $6(18)$ & $19(35)$ & 0.085 \\
\hline $\begin{array}{l}\text { ICU and Hospital } \\
\text { mortality, } \mathbf{n}(\%)\end{array}$ & $34(38)$ & $8(24)$ & $26(47)$ & 0.025 \\
\hline ICU LOS* (days) & $12(6-26)$ & $14(6-24)$ & $13(7-32)$ & 0.720 \\
\hline Hospital LOS* (days) & $23(13-39)$ & $22(13-37)$ & $24(13-42)$ & 0.571 \\
\hline
\end{tabular}

*median[IQR], $\dagger$ On admission, Text in bold in p value indicated statistical significance.

Conclusion: The prognosis of influenza patients with ARF followed in ICU seems to be worse than COVID-19 patients.

Reference(s) and grant ackowledgment(s)

1. Huang, C., Wang, Y., Li, X. et al. Clinical features of patients infected with 2019 novel coronavirus in Wuhan, China. Lancet. 2020; 395: 497-506.

2. https://www.who.int/emergencies/diseases/novel-coronavirus-2019, accessed in 29.06.2020.

\section{2}

The impact of ethnicity and gender on outcome in patients admitted to Intensive Care for management of COVID-19 M. Wilson ${ }^{1}$; T. Drewé2 ; S. Dilke'; J. Borkowski'; B. Moore ${ }^{3}$; G. Musollari ${ }^{3}$; S. Bakare $^{4}$

${ }^{1}$ Intensive care, London North West University Healthcare NHS Trust, London, United Kingdom; ${ }^{2}$ Infectious disease epidemiology, Imperial College London, London, United Kingdom; ${ }^{3}$ Medical schooi, Imperial College London, London, United Kingdom; ${ }^{4}$ Anaesthetic and Intensive Care Medicine, Northwick Park Hospital, London, United Kingdom

Correspondence: M. Wilson

Intensive Care Medicine Experimental 2020, 8(2): 001402

Introduction: Northwick Park Hospital supplies a diverse population of at least $40 \%$ black, Asian and minority ethnic groups (BAME). During the COVID19 pandemic, the Intensive Care Unit (ICU) experienced an unprecedented demand for beds, leading to the declaration of a critical incident on 19th March demanding a rapid expansion and restructure.

Recent analyses of COVID19 transmission and susceptibility have shown that the BAME population is disproportionately affected by both a high infection rate and increased mortality from COVID19. These inferences have been attributed to both socioeconomic disparity and occupational hazard. Similarly, an association has been found between the male sex and the severity of COVID19 infection. 
This study investigates these associations. The large sample size provided within a single clinical setting provides valuable insights into BAME disparity in order to optimise future treatment of COVID19.

Methods: We performed a retrospective cohort study of all the patients admitted to ICU at NWP with COVID19 between 01/03/2020 and 31/05/2020. Data were collected from patient notes, local histopathology databases and the ICNARC database. These included baseline characteristics (ethnicity, date of birth, sex and comorbidities), baseline $\mathrm{P} / \mathrm{F}$ ratio $(\mathrm{PaO} 2 / \mathrm{FiO} 2)$, organ support requirements and mortality. Statistical analyses were performed in R. We used logistic regression to determine predictors of mortality and differences between BAME and white patients.

Results: 264 patients were admitted to the ICU with COVID19, of which $83.3 \%$ of these identified as BAME. $80.3 \%$ of patients were male. Stepwise regression showed that increasing age, and male sex, were the significant predictors of low P/F at admission. Logistic regression analyses showed that pre-morbid state (OR 1.67, 95\% Cl 1.22-2.32), BAME (OR 4.92, 95\% Cl 1.77-17.70) and age (OR 1.04, 95\% Cl 1.011.06) were significantly associated with requiring haemodialysis, but as expected, only P/F on admission was significantly associated with likelihood of intubation (OR $0.96,95 \% \mathrm{Cl} 0.94-0.99)$. Regression analyses on admission parameters demonstrated that age (OR $1.05,95 \% \mathrm{Cl}$ 1.02-1.08), pre-morbid state (OR 1.47, $95 \% \mathrm{Cl} 1.07-2.05)$, and low PF (OR $0.97,95 \% \mathrm{Cl} 0.94-0.99)$ were significantly associated with death. Adjusting regression for intra-ICU parameters showed that age (OR $1.05,95 \% \mathrm{Cl} 1.02-1.08$ ) and haemodialysis (OR $3.00,95 \% \mathrm{Cl} 1.67-5.44)$ were the statistically significant predictors of death. Ethnicity was not associated with mortality in the ICU.

Conclusion: While BAME patients are over-represented in ICU admissions for COVID19, the BAME population had parity in severity of ARDS on admission and mortality. Pulmonary capacity at admission was negatively affected by being male and by increasing age, in keeping with national and international data.

Being of BAME ethnicity was significantly associated with haemodialysis requirement on ICU, which is something to be considered during a second wave of the pandemic.

\section{1}

Comparison of clinical features and prognosis factors in patients with acute respiratory syndrome related to SARS-COV2 versus other respiratory virus

M. Bueno'; M. De Alba'; R. León ${ }^{1}$; J. Rodriguez ${ }^{1}$; C. López ; JM. Dueñas; JM. Dueñas ${ }^{1}$; E. Torres ${ }^{1}$; D. Bautista ${ }^{1}$; JC. Robles ${ }^{1}$; C. De La Fuente-Martos ${ }^{1}$ Intensive Care Unit, Hospital Reina Sofía, Córdoba, Spain

Correspondence: $M$. Bueno

Intensive Care Medicine Experimental 2020, 8(2): 001411

Introduction: Coronavirus disease 2019 (COVID19) is caused by severe acute respiratory syndrome coronavirus 2 (SARS-COV2) and represents the causative agent of potentially fatal disease that is of great global public health concern. About $5 \%$ are critical infections characterized by acute respiratory distress syndrome (ARDS). There is scare information about clinical characteristics and prognosis in these patients.

Objectives: The aims of this study are: (I) compare demographic and clinical characteristics of COVID19 versus others respiratory virus. (II) Analyze outcome.

Methods: Retrospective cohort study of all adults admitted to Tertiary Hospital in Spain from October 2018 to 15th June 2020. We evaluated all adults admitted to Intensive Care Unit (ICU) with confirmed SARSCOV-2 virus infection and other respiratory virus with acute respiratory failure. Main variable was COVID19 aetiology. We evaluate overall mortality in ICU and at 60 day of admission. Exposure variables were: demographics, previous conditions, clinical characteristics, severity of illness, pharmacologic, support therapy and outcome. For categorical variables we applied chi square test (fisher when needed) and for continuous t de student or U Mann-Whitney test when needed. We allied Kaplan Meier Survival analysis and log rank test for overall 60 day mortality and aetiology.

Results: During the study period there were included a total of 82 patients: 42 patients with confirmed COVID19 versus 40 patients with other respiratory virus infection and acute respiratory failure. Clinical characteristics of COVID19 cohort were: median age was 60.6, majority were males $64 \%$. The most frequent comorbidity was hypertension (54\%), followed by obesity (33\%). 3 Patients with solid organ trasplantation were included. $64 \%$ of patients with COVID19 needed mechanical ventilation and $21 \%$ renal replacement therapy. In comparison with those with other respiratory virus infection, COVID19 patients had less severity of illness, SOFA at admission ( 5 vs $7, p=0.04)$, APACHE II (12.7 vs $20, p=0.001$ ). Mortality at 60 days was $26.1 \%(11)$ in COVID19 and 22.5 in other respiratory virus infection. Survival Kaplan Meier analysis of overall mortality at 60 days and unfavorable did not showed differences between COVID19 and no COVID19 cohort $(p=0.74)$; at this time there were still admitted in ICU 7\% of COVI19 patients.

Conclusion: COVID19 patients had more age and less APACHEII and SOFA at admission in ICU than those with other respiratory virus infection. Overall mortality at 60 day was not statiscally higher in COVID19 (log rank $p=0.74$ ). Taking into account that $7 \%$ of COVID19 were still admitted, prognosis variables at longer time (ej.: overall motality at 6 months) may be more precise to evaluate prognosis in COVID-19 patients.

\section{Reference(s) and grant ackowledgment(s)}

1. Grasselli G, Zangrillo A, Zanella A, Antonelli M, Cabrini L, Castelli A, et al. Baseline Characteristics and Outcomes of 1591 Patients Infected with SARSCoV-2 Admitted to ICUs of the Lombardy Region, Italy. JAMA - J Am Med Assoc. 2020;1-8.

2. Yang X, Yu Y, Xu J, Shu H, Xia J, Liu H, et al. Clinical course and outcomes of critically ill patients with SARS-CoV-2 pneumonia in Wuhan, China: a single-centered, retrospective, observational study. Lancet Respir Med. 2020;2600(20):1-7.

\section{4}

Ventilation and pulmonary hemodynamics in patients

A. Becker ${ }^{1}$; F. Seiler ; RM. Muellenbach²; G. Danziger ' ; S. Mang ; ; A. Omlor ${ }^{1}$; C. Jentgen ${ }^{1}$; M. Kamphorst ${ }^{1}$; H. Wehrfritz ${ }^{1}$ C. Lotz ${ }^{3}$;. Mertke ${ }^{4}$; H. Wilkens ${ }^{1}$; R. Bals ${ }^{1}$; PM. Lepper ${ }^{1}$

${ }^{1}$ Inner medicine - pneumology, allergology and intensive care medicine University of Homburg, Homburg, Germany; ${ }^{2}$ Anaesthesiology and critical care, Campus Kassel of the University of Southampton, Kassel, Germany; ${ }^{3}$ Anaesthesiology and critical care medicine, Medizinische Fakultät der Julius-Maximilians-Universität Würzburg, Würzburg, Germany; ${ }^{4}$ Anaesthesiology, critical care medicine and pain medicine, University of Homburg, Homburg, Germany

Correspondence: A. Becker

Intensive Care Medicine Experimental 2020, 8(2): 001414

Introduction: It has been suggested that COVID-19-associated severe respiratory failure (CARDS) might differ from usual acute respiratory distress syndrome (ARDS) due to failing auto-regulation of pulmonary vessels and higher shunt.

Objectives: To investigate pulmonary hemodynamics in patients with CARDS compared to patients with ARDS due to other causes.

Methods: Retrospective analysis of ten CARDS patients treated on our ICU in 04/2020 and comparison of the data to matched controls with ARDS $(n=10)$ that were treated on our ICU from $01 / 2014$ to $08 / 2019$ and for whom pulmonary artery catheter data were available.

Results: COVID-19 patients had similar ventilation characteristics and showed similar $\mathrm{PO} 2 / \mathrm{FiO} 2$ ratio at a higher degree of hypercapnia as compared to ARDS patients as the estimated $\mathrm{CO} 2$ production was significantly higher $(p<0.05)$. Cardiac output was higher in CARDS vs ARDS patients (mean $8.1 \pm 1.7 \mathrm{~L} / \mathrm{min}$ vs. $5.4 \pm 1.5 \mathrm{~L} / \mathrm{min} ; \mathrm{p}<0.05$ ). COVID-19 patients had similar pulmonary artery pressure but significantly lower pulmonary vascular resistance. Shunt fraction was similar in CARDS compared to ARDS (mean $26 \% \pm 13 \%$ vs. $23 \% \pm 15 \%$, $p>0.05$ ) and was in both groups correlated with hypoxemia, as was VD/VT (CARDS $46 \% \pm 20 \%$ vs. ARDS $43 \% \pm 18 \%$; $p>0.05$ ). The arterio-venous pCO2 difference (DpCO2) was elevated (CARDS $5.5 \pm$ $2.8 \mathrm{mmHg}$ vs. $4.7 \pm 1.1 \mathrm{mmHg} ; \mathrm{p}>0.05)$ as was venous-arterial $\mathrm{CO} 2$ gap to arterial-venous oxygen content difference $(\mathrm{P}(\mathrm{v}-\mathrm{a}) \mathrm{CO} 2 / \mathrm{C}(\mathrm{a}-\mathrm{v}) \mathrm{O} 2)$ ratio (CARDS mean $2.2 \pm 1.5$ vs. ARDS $1.7 \pm 0.8 ; p>0.05$ ), indicating 
global microcirculatory dysfunction in CARDS. AaDO2 was similar in both groups $(p>0.05)$.

Conclusion: Respiratory failure in COVID-19 patients differs only slightly from ARDS in ventilation characteristics and pulmonary hemodynamics mainly due to increased $\mathrm{CO} 2$ production. Our data indicate microcirculatory dysfunction. More data needs to be collected to assure these findings and gain more pathophysiological insights in COVID-19 and respiratory failure.

\section{Reference(s) and grant ackowledgment(s)}

1. 10. Acute Respiratory Distress Syndrome N, Brower RG, Matthay MA, et al. Ventilation with lower tidal volumes as compared with traditional tidal volumes for acute lung injury and the acute respiratory distress syndrome. N Engl J Med 2000;342:1301-8.

2. 9. Sinha P, Fauvel NJ, Singh S, Soni N. Ventilatory ratio: a simple bedside measure of ventilation. Br J Anaesth 2009:102:692-7.

3. 8. Roza AM, Shizgal HM. The Harris Benedict equation reevaluated: resting energy requirements and the body cell mass. Am J Clin Nutr 1984;40:168-82.

4. 7. Ferguson ND, Fan $E$, Camporota $L$, et al. The Berlin definition of ARDS: an expanded rationale, justification, and supplementary material. Intensive Care Med 2012;38:1573-82

5. 6. Beiderlinden M, Kuehl H, Boes T, Peters J. Prevalence of pulmonary hypertension associated with severe acute respiratory distress syndrome: predictive value of computed tomography. Intensive Care Med 2006:32:852-7.

6. 5. Ashbaugh DG, Bigelow DB, Petty TL, Levine BE. Acute respiratory distress in adults. Lancet 1967;2:319-23.

7. 4. Zapol WM, Snider MT. Pulmonary hypertension in severe acute respiratory failure. N Engl J Med 1977;296:476-80.

8. 3. Varga Z, Flammer AJ, Steiger P, et al. Endothelial cell infection and endotheliitis in COVID-19. Lancet 2020;395:1417-8.

9. 2. Marini JJ, Gattinoni L. Management of COVID-19 Respiratory Distress. JAMA 2020

10. 1. Grasselli G, Zangrillo A, Zanella A, et al. Baseline Characteristics and Outcomes of 1591 Patients Infected With SARS-CoV-2 Admitted to ICUs of the Lombardy Region, Italy. JAMA 2020.

\section{7}

Neutrophil Extracellular Traps in SARS-CoV2 related pneumonia in ICU patients: the NETCOV2 study

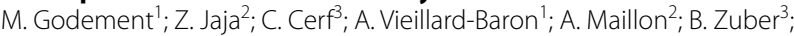
V. Bardet ${ }^{2} ;$ G. Geri

${ }^{1}$ Haut de seine, Icu, Ambroise Paré Hospital (AP-HP), Boulogne-Billancourt, France; ${ }^{2}$ Haut de seine, Hematology, Ambroise Paré Hospital (AP-HP), Boulogne-Billancourt, France; ${ }^{3}$ Haut de seine, Icu, Hospital Foch, Suresnes, France

Correspondence: M. Godement

Intensive Care Medicine Experimental 2020, 8(2): 001427

Introduction: Pathogenesis of severe acute respiratory syndrome coronavirus 2 (SARS-CoV-2) is poorly understood. Neutrophil extracellular traps (NETs) have been involved in infection-related acute respiratory distress syndrome. The role of NETs in SARS-CoV-2 related pneumonia in unknown.

Objectives: Investigate the association between the quantification of blood NETs at ICU admission and the respiratory failure evolution towards refractory hypoxemia in ICU patients with SARS-COV2 related pneumonia.

Methods: Prospective bicentric study including consecutive patients with SARS-CoV2 related pneumonia admitted in intensive care unit. NETs blood levels were quantified in blood samples at day- 1 and day- 3 by flow cytometry (using myeloperoxidase [MPO] and citrullinated histones [Cit-H3] markers). The primary outcome was the association between the day-1 blood level of NETs and the number of days with refractory hypoxemia defined by a $\mathrm{PaO} 2 / \mathrm{FiO} 2$ ratio $<100 \mathrm{mmHg}$.

Results: 58 patients (median age $62[54,69]$, mostly male $(75.9 \%)$ ) were included in the analysis. The median number of days with severe hypoxemia $(\mathrm{PaO} 2 / \mathrm{FiO} 2<100 \mathrm{mmHg}$ ) was $4[2,6]$ days and day-28 mortality was $27.6 \%(n=16)$. In multivariable negative binomial regression, MPO+ NETs were negatively associated with the number of days with severe hypoxemia within 7 days $(0.84[0.73,0.97])$, while neither single positive Cit-H3+ NETS nor double-positive NETS were significantly associated with the primary outcome. The blood level of NETS was lower at day-1 than at day-3 in patients who died (32.6 [22.3, $70.3]$ vs. $56.7[23.3,105.6] \mathrm{p}<0.001,0.37[0.0,1.45]$ vs. $2.2[0.0,4.3]$ $\mathrm{p}<0.001$ and $4[1.2,7.6]$ vs. $6.8[1.8,15.0] \mathrm{p}<0.001$ for MPO+, $\mathrm{Cit}-\mathrm{H} 3+$ and double-positive NETS, respectively).

Conclusion: The blood level of NETs at day-1 was negatively associated with the number of days with severe hypoxemia in patients admitted to the intensive care unit for SARS-CoV2 related pneumonia. This could suggest a functional deficit of neutrophils in these patients that require further investigation.

\section{4}

Susceptibility and severity of COVID-19 in the ABO-blood group system in a single tertiary centre

A. M. Karimi ' ${ }^{1}$ PJ. Masters ${ }^{2}$; E. Riddell ${ }^{1}$; J. Ramzi ${ }^{1}$; R. Mahroof ${ }^{1}$; A.

Conway-Morris

${ }^{1}$ Intensive care, Addenbrooke's Hospital, Cambridge, United Kingdom ${ }^{2}$ Acute medicine, Addenbrooke's Hospital, Cambridge, United Kingdom

Correspondence: A.M. Karimi

Intensive Care Medicine Experimental 2020, 8(2): 001444

Introduction: Understanding the behaviour of Severe Acute Respiratory Syndrome CoronaVirus 2 (SARS-CoV-2) in precipitating the spectrum of Coronavirus disease (COVID-19) has been the scientific challenge of 2020. Genomewide association analysis has suggested an association with the $A B O$ blood-group system; with blood group $A$ being more susceptible, while blood group $O$ seemingly conferring a protective effect in the development COVID-19.

\section{Objectives:}

1. To compare the ABO blood-group of patients admitted with COVID-19 to that of the general population in the UK.

2. To investigate whether any $A B O$ blood groups correlated with more severe illness.

Methods: Contemporaneously recorded data from our electronic medical record system (Epic $\left.{ }^{\circledR}, \mathrm{WI}, \mathrm{USA}\right)$ was used to retrospectively analyse $A B O$ and clinical data of patients admitted to our hospital with PCR positive SARS-CoV-2 for a 6 week period starting April 2020. Data included APACHE II calculations, whether patients received mechanical ventilation, renal replacement therapy or developed thrombotic complications. The peak sustained $\mathrm{FiO} 2$ in ICU patients was deemed if stable for 2 or more hours.

Results: Blood group data was available in 92 hospital patients, 52 of whom went to ICU. The distribution of blood groups was similar to that seen in the background population (Table 1). Overall mortality was non-significantly lower in patients with blood group $\mathrm{O}$ compared to patients with blood group A; $16 \%(95 \% \mathrm{Cl} 6-35 \%)$ vs $39 \%(95 \% \mathrm{Cl}$ $20-61 \%) p=0.09$ by chi-squared. Comparison of illness severity markers between the two largest groups (A \& O) in ICU did not reveal any obvious indication that blood group A patients had greater severity of illness to explain their apparent worse outcomes (Table 2).

Table 1: Distribution of blood groups amongst COVID-19 patients admitted

\begin{tabular}{|c|c|c|c|c|}
\hline $\begin{array}{c}\text { ABO } \\
\text { Blood } \\
\text { Group }\end{array}$ & $\begin{array}{c}\text { ICU } \\
\text { Cohort } \\
(\mathbf{n = 5 2})\end{array}$ & $\begin{array}{c}\text { Ward } \\
\text { Cohort } \\
(\mathbf{n = 4 0 )}\end{array}$ & $\begin{array}{c}\text { Combined Ward } \\
\text { and ICU patients } \\
(\mathbf{n = 9 2})\end{array}$ & $\begin{array}{c}\text { Background } \\
\text { population rate }\end{array}$ \\
\hline A & $18(35 \%)$ & $13(33 \%)$ & $31(34 \%)$ & $38 \%$ \\
\hline B & $7(13 \%)$ & $4(10 \%)$ & $11(12 \%)$ & $10 \%$ \\
\hline O & $25(48 \%)$ & $23(58 \%)$ & $48(52 \%)$ & $48 \%$ \\
\hline AB & $2(4 \%)$ & $0(0 \%)$ & $2(2 \%)$ & $3 \%$ \\
\hline
\end{tabular}


Table 2: Severity of illness markers amongst blood group A and O ICU patients

\begin{tabular}{|c|l|l|}
\hline \multicolumn{3}{|c|}{ patients } \\
\hline Parameter & \multicolumn{1}{|c|}{ A (n=18) } & \multicolumn{1}{|c|}{ O (n=25) } \\
\hline Median APACHE II score (range) & $14(3-21)$ & $15(6-30)$ \\
\hline Median age (range) & $63(34-77)$ & $64(17-83)$ \\
\hline Median peak sustained FiO2 (range) & $0.72(36-100 \%)$ & $0.70(35-100 \%)$ \\
\hline \% Mechanically ventilated (95\% CI) & $78 \%(55-91 \%)$ & $84 \%(65-94 \%)$ \\
\hline$\%$ Received renal replacement & $44 \%(25-66 \%)$ & $40 \%(23-59 \%)$ \\
\hline$\%$ Developed DVT/PE & $6 \%(1-26 \%)$ & $16 \%(6-35 \%)$ \\
\hline
\end{tabular}

Conclusion: $\mathrm{ABO}$ blood groups in our population were similar to the background population in the UK and did not appear to predict ICU admission. There was a trend towards increased mortality in patients with blood group A amongst those admitted to ICU but this was not associated with the severity of illness markers examined. The finding of increased mortality in patients with blood group $A$ is consistent with a recent large genome-wide association study; the mechanism of this increase in mortality, and implications are currently unclear.

\section{Reference(s) and grant ackowledgment(s)}

1. Ellinghaus, D et al. (June 17, 2020) Genomewide Association Study of Severe Covid-19 with Respiratory Failure. New England Journal of Medicine. https://doi.org/10.1056/nejmoa2020283.

2. UK blood group distribution, NHS Blood and Transplant. https://www.blood .co.uk/why-give-blood/blood-types/ (accessed 29/7/20).

\section{1}

Management of acute respiratory failure due to SARS-CoV-2 pneumonia using a standard of care ventilatory treatment and individualized pharmacological strategy

J. Lastra'; P. Fernández'; N. Pavez'; B. Nahuelpan²; G. Ojeda ${ }^{3}$; J. Barbosa ${ }^{3}$; R. Colima'2; R. Abud ${ }^{2}$; P. Sanchez ${ }^{2}$; F. Urrutia ${ }^{2}$; L. Ferreira ${ }^{2}$

${ }^{1}$ Departamento de medicina interna, University of Concepción - Hospital Regional de Concepcion, Concepción, Chile; ${ }^{2}$ Intensive care unit, Regional Clinical Hospital of Concepción, Concepción, Chile; ${ }^{3}$ Departamento de medicina interna, University of Concepción, Concepción, Chile

Correspondence: N. Pavez

Intensive Care Medicine Experimental 2020, 8(2): 001501

Introduction: Severe ARDS due to SARS-CoV-2 infection has been a huge challenge to the health systems. Many coadjuvant therapies have been proposed to manage the disease, such as antivirals to reduce viral load, interleukin inhibitors, and early steroids to decrease the inflammatory response, or the use of convalescent plasma to enhance the immune system. Since the evidence of the use of these treatments was scarce, and the pharmacology availability was limited, we decided to apply our standard ARDS protocol, based on the limitation of tidal volume, best PEEP titration, sequential application of neuromuscular blockade and prone positioning, and fluid restriction, leaving extracorporeal oxygenation as rescue therapy for refractory hypoxemia and to administer selected therapies according to different patient profiles: thrombotic or inflammatory profile, based on clinical and laboratory results.

Objectives: To present the results of a protocol on severe respiratory failure due to COVID-19 in the management of patients with severe and critical pneumonia, with selective use of novel therapies.

Methods: Observational clinical study. We analyzed prospectively from March 21st to July 21st, 2020 all patients admitted with severe and critical COVID-19. We describe demographic, clinical, and laboratory characteristics. The main outcomes were hospital stay and hospital mortality. Data were analyzed by descriptive statistics, distribution was evaluated according to the Shapiro-Wilk test, and were expressed according to mean \pm SD and/or median and p25-75, as appropriate. Results: Hundred thirty-eight patients were included (age 55 years $\pm 13.8,60$ women $(43.5 \%), \mathrm{PaO} 2 / \mathrm{FiO} 2$ at admission was $148 \pm 34,8$. The number of symptomatic days before hospitalization was $7.8 \pm 3,9$. SARS-CoV-2 virus was identified in all patients. Mechanical ventilation in 128 patients. Of them, $66,4 \%$ received NMB, prone positioning in 67 (52\%). Use of high-dose steroids for a high inflammatory profile in 6 patients. 21 patients were fully anticoagulated due to a high thrombotic profile. No other pharmacological therapies were used, and no patients were connected to ECMO. Tracheostomy use in $13 \%$. The median time in MV and ICU were 12 [9 - 17] and 15 [10 - 20] days, respectively. In hospital and 28 -days mortality was $9,4 \%$.

Conclusion: A protocolized management of acute respiratory failure using validated and easy to implement therapies allows satisfactory outcomes. The use of standardized protocols on severe patients and health policies to control infection spreading may act synergistically in the care of communities during pandemics.

\section{Reference(s) and grant ackowledgment(s)}

1. Fan E, Brodie D, Slutsky AS. Acute Respiratory Distress Syndrome: Advances in Diagnosis and Treatment. JAMA. 2018;319(7):698-710. https://doi. org/10.1001/jama.2017.21907

2. Rismanbaf A. Potential Treatments for COVID-19; a Narrative Literature Review. Arch Acad Emerg Med. 2020;8(1):e29. Published 2020 Mar 21.

3. Bedford J, Enria D, Giesecke J, et al. COVID-19: towards controlling of a pandemic. Lancet. 2020;395(10229):1015-1018. https://doi.org/10.1016/s0140 $-6736(20) 30673-5$

\section{0}

Effect of Prone Positioning on the outcome of patients with ARDS: a retrospective study

C. Carvalho ${ }^{1}$; R. Amaral ${ }^{1}$; I. Milet ${ }^{1}$

${ }^{1}$ Intensive medicine, Centro Hospitalar De Trás-Os-Montes E Alto Douro, E.P.E., Vila Real, Portugal

Correspondence: $C$. Carvalho

Intensive Care Medicine Experimental 2020, 8(2): 001530

Introduction: Previous studies have proven that prone positioning optimizes lung recruitment and ventilation-perfusion matching, resulting in an improvement of gas exchange. However, reliable predictors of prone positioning response and the effect on ARDS morbidity and mortality remains to be determined.

Objectives: Evaluate the outcome of patients with ARDS with prone positioning.

Methods: Observational retrospective study on Acute Respiratory Distress Syndrome patients, according to Berlin criteria, mechanically ventilated from January 2017 to December 2019. Patients were divided into those who underwent prone positioning $(n=54)$ and those who did not $(n=35)$. Data were extracted from medical records. Results: 89 ARDS patients were included (mean age of $60,1 \pm 13,7$ years, 62 male/27 female). Severity scores were calculated at admission and mean results were 20,3 77,06 for Acute Physiology, Age, Chronic Health Evaluation (APACHE) II, 47,2 $\pm 12,7$ for Simplified Acute Physiology Score (SAPS) II, and 9,46 $\pm 3,26$ for inicial Sequencial Organ Failure Assessment (SOFA). No statistically significant differences in background characteristics were observed. The main cause of ARDS was pneumonia ( $n=57$ cases, $64,0 \%$ ). Moderate ARDS in $47,1 \%$ of the cases and severe ARDS in $32,6 \%$.

We found a mean improvement in partial pressure of oxygen to inspired fraction of oxygen in the prone group of $14 \%$ vs supine group $(p<0,001)$. After adjustment for other covariates: the severity of ARDS, primary or secondary ARDS, the length of stay in the ICU and the length of hospital stay we did not find a significantly difference in mortality between the two groups. The mortality of patients with severe ARDS at the time of discharge from the intensive care unit, was $34,9 \%$ in the prone and $28,6 \%$ in the supine group ( $\mathrm{RR}, 0,75 ; 95 \% \mathrm{Cl}, 0,121$ $4,701: P=0,571)$, while their 60 -days mortality was $46,8 \%$ and $28,6 \%$, respectively ( $R R, 1,06 ; 95 \% \mathrm{Cl} 0,31-13,1: \mathrm{P}=0,670)$.

Conclusion: Although limited by its retrospective nature, small sample size and heterogeneous ARDS etiologies: our study shows that prone positioning contributed to improve the blood oxygenation in patients with moderate and severe hypoxemia, however, does not improve survival in patients with ARDS. 
Reference(s) and grant ackowledgment(s)

1. Pintado, M, R De Pablo, M Trascasa, J Milicua, and M Sánchez-garcía. "Compliance-Guided versus FiO 2 -Driven Positive-End Expiratory Pressure in Patients with Moderate or Severe Acute Respiratory Distress Syndrome According to the Berlin Definition"41, no. 5 (2017).

2. Cardinal-fernández, P, E Correger, J Villanueva, and F Rios. "Distrés Respiratorio Agudo : Del Síndrome a La Enfermedad" 40, no. 3 (2016).

3. Mora-arteaga, J A, O J Bernal-ramírez, and S J Rodríguez. "Efecto de La Ventilación Mecánica En Posición Prona En Pacientes Con Síndrome de Dificultad Respiratoria Aguda . Una Revisión Sistemática y Metanálisis" 39, no. 6 (2015).

4. Bloomfield, R, Noble Dw, and A Sudlow. "Prone Position for Acute Respiratory Failure in Adults (Review)," no. 11 (2015). https://doi.org/10.1002/14651 858.CD008095.pub2.www.cochranelibrary.com.

\section{0}

Severe COVID-19: description of the first patients admitted to an Intensive Care Unit in Porto, Portugal

A. Martins ${ }^{1}$; M. Mouro ${ }^{1}$; J. Caldas ${ }^{1}$; A. Silva-Pinto ${ }^{1}$; AS. Santos ${ }^{1}$; S. Xerinda1; A. Ferreira ${ }^{1}$; P. Figueiredo ${ }^{1}$; A. Sarmento ${ }^{1}$; L. Santos ${ }^{1}$

${ }^{1}$ Serviço de Doenças Infecciosas, Centro Hospitalar e Universitário de São João, Porto, Portugal

Correspondence: A. Martins

Intensive Care Medicine Experimental 2020, 8(2): 001550

Introduction: Coronavirus disease 2019 (COVID-19) is an emerging disease that frequently has a selflimited course, but $15 \%$ patients develop pneumonia that requires supplemental oxygen and 5\% progress to critical illness with respiratory and organ dysfunctions (1).

Objectives: To describe the characteristics and outcomes of COVID-19 patients admitted to an Infectious Diseases Intensive Care Unit (ICU) in Porto, Portugal.

Methods: Adults with confirmed SARS-CoV-2 infection admitted to the Infectious Diseases ICU of Hospital São João, between March 13 and July $28 / 2020$ and treated with oxygen supplementation or invasive mechanical ventilation (IMV) were included. Demographic and clinical data were collected.

Results: Fifty-seven patients were included (median age 72 years [IQR, 55-78], 63.2\% male). One patient still remains in the ICU, 7 were transferred to other ICUs after intubation for IMV and 39 were discharged. Comorbidities were identified in 53 (93\%) cases, most frequently hypertension (63.2\%) and diabetes mellitus (35.1\%). Median time from symptoms onset to admission was 8 days (IQR, 4-12) and the most common were fever $(78.9 \%)$, dyspnea $(57.9 \%)$ and dry cough $(56.1 \%)$. Thirty-one $(54.4 \%)$ patients received IMV while 18 $(69.2 \%)$ of the remaining were treated with high flow nasal cannula. Between IMV and non-IMV patients no significant differences were seen in age, sex, comorbidities or respiratory symptoms. Differences between IMV and non-IMV patients lymphocytes, AST, ferritin and D-dimers mean values were $-397 \times 10^{\wedge} 6 / \mathrm{l}(95 \% \mathrm{Cl}-619 ;-208), 59 \mathrm{U} / \mathrm{I}$ (95\% Cl 28;84), $1095.3 \mathrm{ng} / \mathrm{ml}(95 \% \mathrm{Cl} 383.9 ; 2029)$ and $3.1 \mathrm{ug} / \mathrm{ml}(\mathrm{Cl}$ $95 \% 1.86 ; 5.33)$, respectively. The mean lowest $\mathrm{PaO} 2 / \mathrm{FiO} 2$ ratio was 94.09 (95\% Cl 80.33;107.85) in IMV and 180.84 (95\% Cl 136.77;224.91) in non-IMV patients. IMV parameters, mean $( \pm S D)$, included compliance $39.87 \pm 15.09 \mathrm{ml} / \mathrm{cmH} 20$, PEEP $12.48 \pm 2.27 \mathrm{cmH} 2 \mathrm{O}$, plateau pressure $25.3 \pm 4.1 \mathrm{cmH} 2 \mathrm{O}$ and driving pressure $13.61 \pm 3.87 \mathrm{cmH} 2 \mathrm{O}$. IMV duration was $17 \pm 9.03$ days, eighteen (78.2\%) patients needed prone positioning, two CVVHF and two other ECMO-VV. Besides respiratory failure, cardiovascular $(40 \%)$ and renal $(26 \%)$ were the most common dysfunctions, and nineteen (38\%) patients had bacterial superinfection. Cardiovascular dysfunction and bacterial superinfection were significantly different between the two groups $(p<0.001)$. Hydroxychloroquine (HCQ) was given in 23 cases (46\%) and HCQ plus azithromycin in 15 cases (30\%), with no side effects. Eleven patients (22\%) were given corticoids, one tocilizumab and two remdesivir. Overall mortality was $20 \%$ (10 patients), including five IMV patients (20.8\%) and five with no indication to escalate intensive care due to subjacent condition. No readmissions were registered.

Conclusion: Although COVID-19 is a new disease that remains largely unknown, critically ill patients may potentially recover if early and appropriate intensive care is provided. Our cohort allowed us to offer medical and human support to all patients admitted.

\section{Reference(s) and grant ackowledgment(s)}

1. (1) Phua J, Weng $L$, Ling $L$, et al. Intensive care management of coronavirus disease 2019 (COVID-19): challenges and recommendations. Lancet Respir Med 2020; published online April 6. https://doi.org/10.1016/S2213 $-2600(20) 30161-2$

\section{4}

Plasma lipid mediator concentrations are linked with disease severity in COVID-19 patients

J. Clarke'; F. Palmas ${ }^{2}$; E. Cifuentes ${ }^{2}$; R. Colas ${ }^{2}$; A. Keogh ${ }^{1}$; G. Curley ${ }^{1}$; J. Dalli ${ }^{2}$ ${ }^{1}$ Department of anaesthesia and critical care medicine, Royal College of Surgeons in Ireland, Beaumont Hospital, Dublin, Ireland; ${ }^{2}$ Lipid mediator unit, Queen Mary University of London, London, United Kingdom

Correspondence: J. Clarke

Intensive Care Medicine Experimental 2020, 8(2): 001564

Introduction: The factors that lead to critical illness in individuals infected with SARS-CoV-2 are not completely understood and the development of severe disease does not seem to be solely related to viral load and could involve an excessive inflammatory response. One aspect of the immune response that has, to date, been greatly understudied in COVID-19 patients is whether lipid mediator production is dysregulated with increasing disease severity. These molecules, which are produced from essential fatty acids, constitute a central part of the immune regulatory mechanisms activated during infections and are responsible for initiating and amplifying inflammation as well as for its termination.

Objectives: We objected to characterise the profile of lipid mediators in patients with COVID-19 in order to identify whether lipid mediator production is dysregulated with increasing disease severity and identifying the pathways that may become disrupted in COVID-19 infections.

Methods: We collected plasma from 41 patients with polymerase chain reaction confirmed SARS-CoV 2 infection that were admitted to Beaumont Hospital, Ireland between the 16th of March and the 1st of May 2020. We categorized the patients into two main groups, severe and non-severe disease. Patients were categorized as severe if they required invasive mechanical ventilation in the intensive care unit. We defined non-severe as those who were managed at ward level with supplemental oxygen or non-invasive ventilation. Employing a liquid chromatography tandem-mass spectrometry-based approach we then quantified lipid mediators from the four main bioactive metabolomes that include the classic eicosanoids and the omega-3 fatty acidderived pro-resolving mediators. These molecules were identified and quantified in accordance with established criteria.

Results: Eight samples were excluded as they did not meet quality control criteria. Using partial least square discriminant analysis we found that overall plasma lipid mediator concentrations were distinct between the severe group when compared with the non-severe group. Further analysis demonstrated that mediators from the leukotriene $(\mathrm{LT})$ metabolome, including the potent chemoattractants LTD4 and LTB4, as well as the pro-resolving mediator Resolvin D1 (RvD1) were upregulated with patients with non-severe disease when compared with those with severe disease. Statistical assessment of lipid mediator concentrations demonstrated a significant downregulation of mediators from the LT metabolome in plasma from patients in the non-severe group when compared with those in the severe group. We also found that the concentrations of the 22-hydroxy-Maresin 1 $(22-\mathrm{OH}-\mathrm{MaR} 1)$, the further metabolite of the pro-resolving mediator Maresin 1, were significantly upregulated in plasma from patients in the severe group. Notably, we did not observe significant differences in plasma prostaglandin concentrations, including PGE2 and PGD2, between the two groups. This observation can be attributed to the fact that all patients received paracetamol. We observed an increase, albeit not reaching statistical significance of the Thromboxane B2 in patients in the severe group which is in line with the increased incidence of thrombosis in these patients. In order to designate the best 
subset of lipid mediators as predictors of disease severity, least absolute shrinkage and selection operator regression analysis was used. This analysis identified 4 lipid mediators, LTD4, 20-OH-LTB4, RvD1 and $22-\mathrm{OH}-\mathrm{MaR} 1$ that gave the strongest predictive value.

Conclusion: These results indicate that increased disease severity in patients with COVID-19 was linked with a failure to engage lipid mediator biosynthesis. They also suggest that the use of therapeutics that interfere with lipid mediator production and /or actions should be considered with caution given that it may exacerbate disease severity. The present study was limited to one hospital and evaluated lipid mediator concentrations in 33 patients. Despite these limitations the present findings suggest that plasma lipid mediator concentrations may also be useful markers for evaluating the efficacy of therapeutics in rectifying host immune responses during COVID-19 infections.

\section{0}

\section{Percutaneous tracheostomy in 46 COVID-19 patients admitted} to the ICU: safety and reliability

M. Emily'; L. Gobbi²; I. Fabbri'² P. Giaccaglia'; D. Morri²; L. Bernabè2; S.

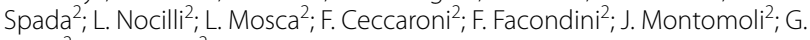
Nardi'; A. Potalivo ${ }^{2}$

${ }^{1}$ Anesthesia and intensive care unit, Marche Polytechnic University Faculty of Medicine, Ancona, Italy; ${ }^{2}$ Anaesthesia and intensive care, Infermi Hospital, AUSL della Romagna, Rimini, Italy

Correspondence: J. Montomoli

Intensive Care Medicine Experimental 2020, 8(2): 001600

Introduction: COVID-19 outbreak has led to an unprecedented need for mechanical ventilation (MV). Tracheostomy might help ease airway management and early weaning of the patients. However, it has not been widely used since it exposes physicians at high risk of contracting COVID-19 infection.

Objectives: We aim to describe the cohort of the 46 patients who underwent percutaneous tracheostomy during ICU-stay at our unit.

Methods: We retrieved from the electronic medical records data from all COVID-19 patients undergoing tracheostomy during ICU admission at the Rimini Hospital in the period 2/3/2020-29/4/2020.

Results: Among the 68 patients that received MV and admitted to the ICU, tracheostomy was performed in 46 patients $(66 \%) .76 \%(n=35)$ were males and the median age was 68 (range 32-80). Median SOFA score at admission was 7 (3-18). All tracheostomy were percutaneous (Griggs or percutwist). Median ICU length of stay at the day of tracheostomy was 11 days (1-22) and the median P/F ratio before procedure was 172 (67-298). Five complications were recorded, none during the procedure, two bleeding from the stoma, two stoma infections, and one patient experienced accidental decannulation with subsequent cardiac arrest. All complications were treated successfully including the patient with accidental decannulation that survived and was discharged from ICU. ICU-mortality rate was $32.6 \%(n=15)$ with a median ICU length of stay of 23 (7-108). Decannulation was performed before ICU discharge in all of ICU survivors and the median length of open tracheostomy was 17 (3-78). Only one patient was still admitted to the ICU at the 30/07/2020 after 132 days of ICU admission. By following the ICU safety protocol, no healthcare worker involved in the procedures has been infected.

Conclusion: Our results showed that percutaneous tracheostomy may be safely performed in severe COVID-19 patients allowing a better airways management in patients that require long ICU stay.

\section{Reference(s) and grant ackowledgment(s)}

1. Sommer DD, Engels PT, Weitzel EK, et al. Recommendations from the CSO-HNS taskforce on performance of tracheotomy during the COVID-19 pandemic. J Otolaryngol Head Neck Surg. 2020;49(1):23. Published 2020 Apr 27. https://doi.org/10.1186/s40463-020-00414-9.

2. Takhar A, Walker A, Tricklebank S, et al. Recommendation of a practical guideline for safe tracheostomy during the COVID-19 pandemic. Eur Arch Otorhinolaryngol. 2020;277(8):2173-2184. https://doi.org/10.1007/s0040 5-020-05993-x.

3. David AP, Russell MD, El-Sayed IH, Russell MS. Tracheostomy guidelines developed at a large academic medical center during the COVID-19 pandemic. Head Neck. 2020;42(6):1291-1296. https://doi.org/10.1002/ hed.26191.

4. McGrath BA, Ashby N, Birchall M, et al. Multidisciplinary guidance for safe tracheostomy care during the COVID-19 pandemic: the NHS National Patient Safety Improvement Programme (NatPatSIP) [published online ahead of print, 2020 May 12]. Anaesthesia. 2020;https://doi.org/10.1111/ anae.15120. https://doi.org/10.1111/anae.15120.

5. McGrath BA, Brenner MJ, Warrillow SJ, et al. Tracheostomy in the COVID19 era: global and multidisciplinary guidance. Lancet Respir Med. 2020;8(7):717-725. https://doi.org/10.1016/s2213-2600(20)30230-7.

\section{6}

Improved ROX Index Following Awake Prone Positioning in COVID-19

R. Khorasanee ; A. Isted ${ }^{1}$; R. Sykes ${ }^{2}$; C. Cox²; A. Bhalla²; B. Rose ${ }^{1}$

${ }^{1}$ Critical Care, University Hospital Lewisham, Lewisham High Street, London, UK, London, United Kingdom; '2Department of critical care, University Hospital Lewisham, London, United Kingdom

Correspondence: $\mathrm{R}$. Khorasanee

Intensive Care Medicine Experimental 2020, 8(2): 001626

Introduction: Awake Prone Positioning is an increasingly widespread treatment modality for hypoxaemic respiratory failure in patients with COVID-19. We sought to evaluate whether this technique could improve ROX index - a non-invasive composite measure of oxygenation and work of breathing shown to predict need for intubation in patients with acute respiratory distress syndrome (ARDS).

Methods: In this prospective cohort study, adult patients with COVID19 pneumonia requiring supplemental oxygen were recruited at a district general hospital in London. Baseline characteristics were collected and ROX index calculated as the peripheral oxygen saturation (SpO2) divided by the product of the fractional concentration of inspired oxygen (FiO2) and the respiratory rate (RR). Patients were instructed or assisted into prone position and ROX index recalculated at 15 to 60 minutes and again at 120 to 240 minutes where tolerated. Results: In a cohort of 12 patients, ROX index improved significantly from a median of 3.61 (IQR $3.00-5.16$ ) to 4.58 (IQR $3.83-5.77$ ) after 15 to 60 minutes $(p=0.012)$ and in the seven patients that maintained prone position, they experienced a further increase in ROX index to 8.00 (IQR $4.86-11.73$ ) at 120 to 240 minutes $(p=0.028)$.

Conclusion: Awake prone positioning was feasible and significantly improved ROX index, which has previously been shown to predict need for intubation in ARDS. Safe avoidance of intubation of COVID19 patients would be beneficial to avoid harms from invasive ventilation and to optimize usage of scarce hospital resources in both high and low income countries. ROX Index is a simple composite measure of respiratory parameters that may be valuable in assessing patients when access to blood gas analysis is limited by availability or by patient numbers. Further work is needed to delineate potential risks and outcome benefits of this technique and to clarify its optimal mode of delivery, and to establish whether changes in ROX Index in COVID19 are associated with patient relevant outcomes.

\section{Reference(s) and grant ackowledgment(s)}

1. Sartini C, Tresoldi M, Scarpellini P, et al. Respiratory Parameters in Patients With COVID-19 After Using Noninvasive Ventilation in the Prone Position Outside the Intensive Care Unit. JAMA Published Online First: 15 May 2020. https://doi.org/10.1001/jama.2020.7861.

2. Elharrar $X$, Trigui Y, Dols A-M, et al. Use of Prone Positioning in Nonintubated Patients With COVID-19 and Hypoxemic Acute Respiratory Failure. JAMA Published Online First: 15 May 2020. https://doi.org/10.1001/ jama.2020.8255.

3. Roca O, Caralt B, Messika J, et al. An index combining respiratory rate and oxygenation to predict outcome of nasal high-flow therapy. Am J Respir Crit Care Med Published Online First: 2019. https://doi.org/10.1164/ rccm.201803-0589oc. 


\section{8}

Viral respiratory infections and ARDS in the pre-Covid19 age, a small case series

R. Amaral' ; C. Carvalho ${ }^{1}$; B. Costa'; H. Santos ${ }^{1}$; A. Pais ${ }^{1}$; J. Carvalho

${ }^{1}$ Intensive Medicine, Centro Hospitalar De Trás-Os-Montes E Alto Douro,

E.P.E., Vila Real, Portugal

Correspondence: R. Amaral

Intensive Care Medicine Experimental 2020, 8(2): 001628

Introduction: Acute Respiratory Disease Syndrome has one of the core topics of intensive medicine due to its relative high incidence, and important morbidity and mortality. Since Covid 19 disease emerged all literature is focusing on this new entity. Through discussions about this respiratory disease some have questioned although Berlin criteria for ARDS are met and because the lung compliance is not the most common in usual ARDS if this not truly ARDS.

Objectives: To report on our experience with viral respiratory infections causing ARDS in 2019, pre-covid19.

Methods: Retrospective analysis of the clinical information of patients admitted in intensive care with diagnosis of ARDS according to Berlin criteria, and laboratory specimens of viral colonization of airway.

Results: We obtained 7 patients with diagnosis of ARDS due to viral pneumonia. Most of the patients were male $(n=4)$, median age 62 (first quartile (1Q) 52 and third quartile (3Q) 74). Severe ARDS was diagnosed in 2 patients median partial arterial oxygen pressure inspired oxygen fraction quotient ( $\left.\mathrm{PaO} 2 / \mathrm{FiO}^{2}\right)$ of 80,5 (10 77,75 and 30 83,25); moderate ARDS in 3 patients median $\mathrm{PaO} 2 / \mathrm{FiO} 2142(1 \mathrm{Q}$ 136,5 and $3 \mathrm{Q}$ 149); mild ARDS in 2 patients median PaO2/FiO2 226 (1Q 214 and 30 238). Invasive mechanical ventilation was necessary in $42,9 \%$ of patients $(n=3)$, the other 4 patients were managed exclusively with non-invasive ventilation. Median days of mechanical ventilation were $11(1 \mathrm{Q} 6$ and $3 \mathrm{Q} 15,5)$ in invasive and 3 (1Q 1 and 3Q 8,75) in non-invasive ventilation. Median positive end expiratory pressure was $8(1 \mathrm{Q} 8$ and $3 \mathrm{Q} 10)$. The 2 patients with severe ARDS were manged with invasive mechanical ventilation and prone positioning. Identified respiratory virus were: rinovirus, adenovirus, influenzae $A$ and $B$, parainfluenzae, coronavirus 229 and metapneumovirus. Co-bacterialinfection was observed in 1 patient, identified agents were Moraxella and Haemophilus. Antibiotics were administered to all patients $(n=7)$ and $58,7 \%(n=6)$ were treated with anti-virals. Corticosteroids were administered to 5 patients $(71,4 \%)$. Severity scores were: APACHE II 21 (1Q 16 and $3 Q$ 22,5); SAPS II 40 (1Q 36,5 and 3Q 40,4); SAPS 359 (1Q 59 and $3 Q 70)$; SOFA at admission 7 (1Q 6,5 and 3Q 8). Mean length of stay in intensive care was 11 (1Q 5,5 and $3 Q$ 13) days and in hospital was $21(1 \mathrm{Q} 13$ and $3 \mathrm{Q} 21,5)$ days. Hospital mortality was the same of net mortality: 2 patients $(28,6 \%)$ - with severe ARDS.

Conclusion: Although with limitations due to its retrospective nature, this study presents our reality and exposure to ARDS due to viral pneumoniae. All our patients were medicated with antibiotics, although only 1 patient had confirmed bacterial infection. The majority of patients received corticosteroids. Outcome was linked to disease severity and comorbidities. Applied PEEP levels were lower than does presented by ARDSnet suggesting normal compliance.

\section{Reference(s) and grant ackowledgment(s)}

1. Yaseen M. Arabi, Robert Fowler and Frederick G. Hayden; Critical care management of adults with community-acquired severe respiratory viral infection; Intensive Care Med (2020) 46:315-328.

2. The National Heart, Lung, and Blood Institute ARDS Clinical Trials Network; Higher versus Lower Positive End-Expiratory Pressures in Patients with the Acute Respiratory Distress Syndrome; N Engl J Med 2004; 351:327-336..

\section{8}

Lung Ultrasound abnormalities as a diagnostic adjunct for COVID-19 pneumonitis: a pilot study

J. Powys-Lybbe'; L. Tong ${ }^{1}$; C. Moxey ${ }^{1}$; O. Valencia'; H. Aya'; J. Aron ${ }^{1}$

${ }^{1}$ General intensive care unit, St George's Hospital, London, United Kingdom

Correspondence: P.L. James

Intensive Care Medicine Experimental 2020, 8(2): 001638
Introduction: Covid-19 pneumonitis has caused a pandemic surge in Europacross the globe, with almost 700,000 dead at the time of writing [1]. Current diagnosis of Covid-19 is based on PCR detection of surface antigens [2], however radiological features of Covid-19 infection have been reported and use as adjuvant diagnostic tool [3]. We hypothesise that lung ussultrasound (LUS) can also identify a characteristic pattern associated with Covid-19 infection.

Objectives: We aimed to assess and describe a lung ultrasound pattern associated with COVID-19 pneumonitis.

Methods: We performed a prospective observational study on patients admitted to the ICU in a tertiary hospital during a three-week period. A 6-point Lung ultrasound assessment on all new admissions, scoring each region 0 to 3 (as per FUSIC guidance [4]). The primary outcome was the likelihood ratio (LR) of cumulative lung scores at admission to ICU with COVID-19 diagnosis, confirmed by at least two COVID-19 antigen-positive swabs, using fishers exact test. ROC curve analysis will be used to establish best threshold for continuous variables. P-Value of less than 0.05 is considered statistically significant.

Results: From 69 patients admitted during the study period, 61 were included with a complete US assessment. 43/61 (62\%) had a positive antigen COVID-19. Cumulative scores ranged from 0 to 14 to both groups. A cut off of $>6$ was used to discriminate the two populations. $34 / 43$ patients $(79 \%)$ who had a score $>6$ were diagnosis of COVID19. $17 / 18$ patients $(94.4 \%)$ who scored 6 of under were negative for COVID-19. Positive Predictive Value $0.97(0.85-0.99)$. Negative Predictive Value $0.65(0.46-0.81)$. Accuracy 0.84 . Area under the Curve 0.87 OR 64.2 (7.51 - 549.4). Sensitivity $79 \%(0.65-0.89)$ specificity $94 \%$ (0.74-0.99)

Conclusion: A cumulative score greater than 6 on 6-point Lung Ultrasound is a useful screening tool for COVID-19 pneumonitis.

Reference(s) and grant ackowledgment(s)

1. 1. Focused Ultrasound in Intensive Care (FUSIC): FUSIC COVID info [internet]. London: Intensive Care Society Available from: https://www.ics.ac.uk/ICS/ Pdfs/FUSIC_DOCS/FUSIC_COVID19_info.aspx.

2. S. Kulkarni'Point-of-care lung ultrasound in intensive care during the COVID-19 pandemic'Clinical Radiology 13 May 2020. https://doi. org/10.1016/j.crad.2020.05.001.

3. Linda J Carter et. al 'Assay Techniques and Test Development for COVID-19 Diagnosis' ACS Cent Sci. 2020 May 27; 6(5): 591-605.https://doi.org/10.1021/ acscentsci.0c00501.

4. European Centre For Disease Prevention and Control.

001611

The role of Brixia score to assess severity of lung damage and management of ventilatory support in severe COVID-19 patients

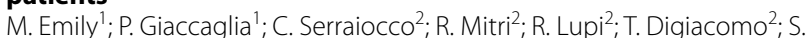
Cicconi $^{2}$; R. D'angelo²; E. Cavagna ${ }^{3}$; G. Nardi²; A. Potalivo ${ }^{2}$; J. Montomoli ${ }^{2}$. F. Facondini ${ }^{2}$

${ }^{1}$ Anesthesia and intensive care unit, Marche Polytechnic University Faculty of Medicine, Ancona, Italy; ${ }^{2}$ Anaesthesia and intensive care, Inferm Hospital, AUSL della Romagna, Rimini, Italy: ${ }^{3}$ Dept of radiology, Ospedale "Infermi" di Rimini, Rimini, Italy

Correspondence: J. Montomoli

Intensive Care Medicine Experimental 2020, 8(2): 001611

Introduction: Adequate ventilation strategy is crucial in patients affected by COVID-19. The Brixia score could help categorizing patients.

Objectives: Our purpose was to observe Brixia score values and the outcome of different ventilation strategies.

Methods: Hospitalized COVID-19 patients at Rimini Hospital requiring noninvasive ventilation (NIV) or invasive mechanical ventilation (IMV) between February 26 and April 18, 2020 were enrolled in this observational cohort study and followed up to 60 days after hospital admission. The Brixia-score assesses the extent of lung damage in COVID patients dividing each lung into 3 sectors on the transverse plane; to each of the obtained six sectors a score is assigned, ranging from 0 (no alteration) to 3 (interstitial-alveolar infiltrates). The overall score may 
range from 0 to 18 and it was reported by the radiologist at the time of the chest x-ray.

Results: 112 patients were treated with NIV and/or IMV. Among those, $46(41.1 \%)$ were treated only with NIV, 25 (22.3\%) underwent IMV after a NIV trial, and 41 (36.6\%) were treated only with IMV. The Brixia score among patients with a successful NIV trial was higher than among patients that failed it although it did not reach the statistical significance $(12.1 \pm 2.6$ vs $10.9 \pm 3.7$, p-value 0.12$)$. Brixia score was obtained at the time of endotracheal intubation among patients that failed a NIV trial and among patients that were treated with IMV without a NIV (13.8 \pm 2.8 vs $13.8 \pm 2.5$, p-value 0.99). Patients who failed the NIV trial had a Brixia score of $10.8 \pm 3.5$ before the NIV which worsened to 13.6 $\pm 2.7(p=0.002)$ when tracheal intubation was performed.

Conclusion: Although the Brixia score was able to identify patients with a more severe degree of interstitial-alveolar infiltrates it has some limitation when used to guide the decision of the ventilatory strategy to adopt with COVID-19 patients.

\section{Reference(s) and grant ackowledgment(s)}

1. Maroldi R., Gerevini A., Zuccolotto P. Data mining and machine learning for the development of risk-predicting models in patients with Covid-19 pneumonia, based on the chest x-ray Brixia-severity score and lab tests. https:// bodai.unibs.it/covid19brixia/.

2. Borghesi A, Zigliani A, Masciullo R et al. Radiographic severity index in COVID-19 pneumonia: relationship to age and sex in 783 Italian patients. La radiologia medica. 2020; 125 (461-464). https://doi.org/10.1007/s1154 7-020-01202-1.

3. Zhou F, Yu T, Du R, et al. Clinical course and risk factors for mortality of adult inpatients with COVID-19 in Wuhan, China: a retrospective cohort study. The Lancet. 2020;395(10229):1054-1062. https://doi.org/10.1016/s0140 $-6736(20) 30566-3$

4. Yang X, Yu Y, Xu J, et al. Clinical course and outcomes of critically ill patients with SARS-CoV-2 pneumonia in Wuhan, China: a single-centered, retrospective, observational study. Lancet Respir Med. February 2020. https://doi. org/10.1016/s2213-2600(20)30079-5

5. ICNARC - Report on COVID-19 in critical care 22 May 2020. https://www. icnarc.org/Our-Audit/Audits/Cmp/Reports. Accessed May 27, 2020.

6. Grasselli G, Zangrillo A, Zanella A, et al. Baseline Characteristics and Outcomes of 1591 Patients Infected With SARS-CoV-2 Admitted to ICUs of the Lombardy Region, Italy. JAMA. April 2020. https://doi.org/10.1001/ jama.2020.5394.

7. Richardson S, Hirsch JS, Narasimhan M, et al. Presenting Characteristics, Comorbidities, and Outcomes Among 5700 Patients Hospitalized With COVID-19 in the New York City Area. JAMA. April 2020. https://doi. org/10.1001/jama.2020.6775..

\section{5}

Tracheal button as a safely artificial airway removal protocol during COVID 19 PANDEMIA in patients with percutaneous tracheostomy in ICU

O. Moreno Romero ; MT. Cruces Moreno ; M. Muñoz Garach ; P.

Fernandez Morales

'Intensive Care Unit, Hospital Universitario Clínico San Cecilio, Granada, Spain

Correspondence: $\mathrm{O}$. Moreno Romero

Intensive Care Medicine Experimental 2020, 8(2): 001275

Introduction: decannulation is an importan step for our critical patients with a tracheostomy during the weaing process and it's failure suposes a higher morbility, mostly in terms of bronchoaspiration, specially in the time of isolation during Covid-19 pandemia, where the reponse to an emergency inside the box could be prolonged.

Objectives: First, to analyze the use of tracheal button during an artificial airway removal protocol in patients with tracheostomy and isolation with Covid-19 pneumonia in ICU. Second, to describe our complications, if present.

Methods: A prospective 3 month-study of all patients admitted to our ICU, which reconverted from usual 20 to a 40-bed unit, with COVID19 pneumonia who needed invasive mechanical ventilation (IMV), a percutaneous tracheostomy and a tracheal button during a maximum of 24 hours inmediately after decanulating. Variables analyzed: Patient data (sex, age, SAPS II), complications of tracheal button, and from all patients (mean days of IMV, mean time of ICU stay and ICU mortality rate).

Results: We treated 49 patients with COVID-19 pneumoniae: men 35 (71\%). Mean age 60,2 years (32-78). SAPS II of 50,8 (39-68). 44 patients underwent IMV (89\%), $28(63 \%)$ needed a percutaneous tracheostomy for weaning (22 (78\%) men). In 9 patients (32\%) we applied a tracheal button. Mean time of MV of 21,3 days (17-36). Global mortality rate Covid-19 patients in ICU 20\%(10 patients), all mechanically ventilated: 6 as a limitation of therapeutic effort and 4 as multiple organ disfunction/septic shock, none after removing artificial airway. Complication of tracheal button was mostly discomfort which needed removing it before 16 hours in 2 patients ( $25 \%$ ).

Conclusion: The aplication of tracheal button is a safe technique of removing defenitively artificial airway in isolated patients with Covid-19 infection. Discomfort was seen in $25 \%$ and needed an early removal. No serious complication was seen, although our sample is small.

\section{0}

Percutaneous Tracheostomy in ICU in the time of COVID 19

Pandemia

O. Moreno Romero'; I. Cruz Valero'; J. Tejero Aranguren'; M. Muñoz

Garach

${ }^{1}$ Intensive care unit, Hospital Universitario Clínico San Cecilio, Granada, Spain

Correspondence: $\mathrm{O}$. Moreno Romero

Intensive Care Medicine Experimental 2020, 8(2): 001280

Introduction: Percutaneous tracheostomy is an essential procedure for weaning in patients with SARS and invasive mechanical ventilation specially to minimize complications due to weakness of prolonged ventilation

Objectives: First, to describe the caracteristics of all percutaneous tracheostomies performed in our patients with new Covid-19 infection admitted to our ICU and performed by intensive care physicians. Second, to analyze the infection rate of the ICU workers after implementing these technique.

Methods: A prospective 3 month-study of all patients admitted to our ICU, which reconverted from usual 20 to a 40-bed unit, with COVID-19 pneumonia who needed invasive mechanical ventilation (IMV) and a percutaneous tracheostomy for weaning. All tracheostomies were performed by 2 intensive care physicians and 1 nurse, all with a "personal protection equipment" (PPE) and a percutaneous tracheostomy kit. Variables analyzed: patient data (sex, age, SAPS II), mean days of IMV, number of tracheostomies and the day it was performed, complications, mean time of ICU stay, mortality rate. Secondly, describe our ICU staff infection rate before and after initiating tracheostomies.

Results: We treated 49 patients with COVID-19 pneumoniae: men 35 (71\%). Mean age 60,2 years (32-78). SAPS II of 50,8 (39-68). 44 patients underwent IMV (89\%), $28(63 \%)$ needed a percutaneous tracheostomy for weaning (22 (78\%) men). Mean day of tracheostomy 11 (6-16). Mild haemorrage as complication in $4(14 \%)$. Global mortality rate Covid19 patients in ICU 20\%(10 patients), all mechanically ventilated: 6 as a limitation of therapeutic effort and 4 as multiple organ disfunction/ septic shock. Mean time of ICU stay in ventilated patients, 20,8 days (5-43). Global mortality rate in ICU 19,6\%(10 patients). None of the staff was infected after initiating this technique. Infection rate during first period: 8 workers from a total of 135 (6\%): 3 doctors, 5 nurses.

Conclusion: A percutanoeus tracheostomy in ICU during covid-19 infection can be performed safely for the patient and our workers with only $6 \%$ of infection rate in workers and none after initiating the technique. $64 \%$ needed tracheostomy as a weaing protocol with a mean day of perform of 11 days. Our ICU mortality rate was $19 \%$. 
001287

Optimal Timing of Tracheostomy Insertion - Mechanical

Ventilation Requirements

C. Cox ; N. Bandla

${ }^{1}$ Critical Care, University hospital of north Midlands, Stoke-on-Trent, United Kingdom

Correspondence: C. Cox

Intensive Care Medicine Experimental 2020, 8(2): 001287

Introduction: Percutaneous Tracheostomy on Intensive Care is an established practice [1]. Timing of Tracheostomy with regards to the duration of ICU stay is well researched [2]. But the optimal timing of Tracheostomy with regards to the degree of ventilatory support is not well researched. The French Tracheostomy guidelines [3], suggest that the patients that are severely hypoxemic $(\mathrm{PaO} 2 / \mathrm{FiO} 2<13.3 \mathrm{Kpa}$ with a PEEP of $>10 \mathrm{~cm} \mathrm{H2O}$ ) are not suitable for an airway procedure as this can significantly worsen their clinical situation. The general consensus among the ICU clinicians is that it is safe to perform a Tracheostomy when $\mathrm{FiO} 2$ is $<50 \%$ with a PEEP $<10 \mathrm{~cm} \mathrm{H} 2 \mathrm{O}$.

Objectives: Our aim was to determine the optimal time of Tracheostomy in relation to the degree of ventilatory support. It was observed that patients have suffered episodes of desaturation and increased ventilatory requirements following the procedure, demanding a review into the clinical practice. We reviewed the pre-procedural ventilatory requirements and post procedural ventilatory requirements at 6 , 12,24 and 48 hours after the procedure.

Methods: We performed a retrospective analysis of the mechanical ventilation data in a university hospital adult intensive care unit and a major trauma centre over a 3-month period. The review is performed using the electronic notes of patients that underwent a percutaneous tracheostomy.

Results: A total of 51 patients were eligible to be reviewed. The mean age is 59 years with an age range of 20-81 years. Out of hospital cardiac arrest, Community Acquired Pneumonia, Traumatic Brain Injury were the three main admission diagnoses. Poor neurological recovery and facilitation of respiratory weaning were the two main indications for Tracheostomy. The mean day for insertion was day 9 of mechanical ventilation.

24 hours prior to tracheostomy insertion the mean PEEP among 51 patients was 7 and the mean PF ratio was $32 \mathrm{Kpa}$. There were deviations from the general safety consensus e.g.: one patient with a PEEP of 14 and another patient with a PF ratio of $17 \mathrm{kpa}$ at the time of procedure.

Following tracheostomy, ventilator settings and oxygenation were reviewed at 6 hours, 12 hours, 24 hours and 48 hours. There was no significant deterioration in the PF ratios and the degree of ventilatory support among our patients at 6 hours, 12 hours, 24 hours and 48 hours after the procedure. Although, several episodes of short-lasting hypoxia and mucus plugging were noticed.

Conclusion: The retrospective analysis of the ventilatory data in a population of 51 mechanically ventilated patients revealed that percutaneous tracheostomy is reasonably safe with a PEEP of $<10 \mathrm{~cm} \mathrm{H} 2 \mathrm{O}$ and $\mathrm{FiO} 2$ of $<50 \%$, when performed by experienced clinicians.

\section{Reference(s) and grant ackowledgment(s)}

1. Trouillet, J.L., Collange, O., Belafia, F. et al. Tracheotomy in the intensive care unit: guidelines from a French expert panel. Ann. Intensive Care 8, 37 (2018).

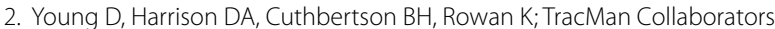
Effect of early vs late tracheostomy placement on survival in patients receiving mechanical ventilation: the TracMan randomized trial. JAMA. 2013 May 22;309(20):2121-9. https://doi.org/10.1001/jama.2013.5154.

3. Mehta C, Mehta Y. Percutaneous tracheostomy. Ann Card Anaesth. 2017;20(Supplement):S19-S25. https://doi.org/10.4103/0971-9784.197793.
001460

A single centre retrospective cohort study of the survival differences associated with the timing of tracheostomy in patients ventilated for more than $\mathbf{1 4}$ days

T. Gordon ${ }^{1}$; A. Waite ${ }^{1}$; N. Main ${ }^{1}$

${ }^{1}$ Critical Care, Royal Liverpool University Hospital, Liverpool, United Kingdom

Correspondence: $T$. Gordon

Intensive Care Medicine Experimental 2020, 8(2): 001460

Introduction: Tracheostomy is often performed to aid weaning from mechanical ventilation but the timing of tracheostomy has not been shown to confer a mortality benefit [1]. As practice in our department tends towards conventionally late tracheostomies, we aimed to determine whether there was a difference in outcomes for patients ventilated for $>14$ days, who i) underwent tracheostomy on or before day 14 of their ICU admission; ii) underwent tracheostomy after 14 days; or iii) had prolonged intubation without tracheostomy.

Methods: We retrospectively reviewed the notes of patients admitted to the Royal Liverpool University Hospital ICU who were mechanically ventilated for $>14$ days between January 2015 and January 2020. Patients admitted with a tracheostomy already in situ were excluded. Data collected included demographic information, APACHE II score, ICU length of stay (LOS), day of tracheostomy, and mortality on ICU and hospital discharge. The primary outcome was survival to hospital discharge. Patients who had a tracheostomy on or before day 14 are referred to as the 'earlier' group, whilst patients who had a tracheostomy after day 14 are referred to as the 'later' group. Statistical analysis was performed in IBM SPSS 25.0 using X2 tests (Fisher's Exact Test where appropriate), Kruskal-Wallis or Mann-Whitney U tests.

Results: In total 246 patients were ventilated for $>14$ days. Of these patients, 91 (37.0\%) had an earlier tracheostomy, 66 (26.8\%) underwent later tracheostomy and 89 patients $(36.2 \%)$ were not tracheostomised. Median day of tracheostomy in the earlier group was day 11 (IQR 8-13) and in the later group day 18.5 (IQR 16.0-21.3, p<0.001). There was no difference in age or APACHE II scores between the two tracheostomy groups. Patients with a later tracheostomy had a longer LOS (later tracheostomy median LOS 35.8 days [IQR 30.8-48.0] vs earlier tracheostomy median LOS 28.0 days [IQR 22.5-42.30] vs no tracheostomy median LOS 23.6 days [IQR 18.5-29.2], $\mathrm{p}<0.001$ ) and more days of mechanical ventilation (later tracheostomy median 31 days [IQR 24.0-41.3] vs earlier tracheostomy 25 days [IQR 19.0-34.0] vs no tracheostomy 19 days [IQR $16.0-23.5], \mathrm{p}<0.001)$. In the earlier tracheostomy group, 73 patients $(80.2 \%)$ survived to hospital discharge; in the later group, 41 patients (62.1\%) survived; and of those who did not undergo tracheostomy, 48 patients $(53.9 \%)$ survived $(p<0.001)$.

Conclusion: Patients who were ventilated for $>14$ days had a better rate of survival to hospital discharge when a tracheostomy was performed within the initial 14 days of their ICU admission compared to later during their admission or not at all. Our study is limited by its retrospective nature and there may be confounding factors influencing the poorer outcomes in those patients who were tracheostomised after 14 days, not represented by the data collected. Further research is indicated to assess the impact of earlier tracheostomy on patientfocused outcomes such as ability to engage in rehabilitation.

Reference(s) and grant ackowledgment(s)

1. 1. Young D, Harrison DA, Cuthbertson BH, Rowan K: Effect of early vs late tracheostomy placement on survival in patients receiving mechanical ventilation: the TracMan randomized trial. JAMA 2013, 309(20):2121-2129. 


\section{8}

Resuming oral feeding in Tracheostomised COVID-19 patients admitted to Tertiary Specialist Cardiothoracic Intensive Care Unit N. Maistry'; N. Adam²; W. Gemma' S. Ethan²; S. Hannah²

${ }^{1}\left\{\right.$ street_address\}, London, United Kingdom; ${ }^{2}$ Physiotherapy, Brunel University London, London, United Kingdom

\section{Correspondence: N. Maistry}

Intensive Care Medicine Experimental 2020, 8(2): 001498

Introduction: Data on the functional outcomes of patients surviving an intensive care unit (ICU) admission for COVID-19 is sparse [1]. It is widely recognised that Speech and Language Therapists (SLT) have an essential role within ICU to support patients achieving optimal communication and swallowing function post critical care [2]. Patients diagnosed with COVID-19 were intubated and ventilated for prolonged periods, increasing their risk of post-extubation dysphagia [1]. Objectives: To evaluate the mean and median duration of commencing a normal diet in patients with COVID-19 who were tracheostomised and to compare this with data collected on tracheostomised patients referred to SLT in 2018, who were admitted to the same intensive care unit at a Tertiary Specialist Cardiothoracic centre.

Methods: During the period March to June 2020 (inclusive), SLT was referred and provided intervention to 30 tracheostomised COVID-19 patients. Data was contemporaneously collected regarding intubation, extubation, tracheostomy insertion and every SLT intervention, including diet changes.

This data was compared to data collected over a 5-month period in 2018 , in which 31 cardiothoracic or cardio-respiratory tracheostomised patients were referred and seen by SLT.

This data was analysed retrospectively, in terms of the time taken to resume a normal diet post tracheostomy insertion.

Results: A total of 25 COVID-19 patients were seen for dysphagia intervention, by SLT, post tracheostomy. There were 22 male and mean age was 52 . The mean time taken for tracheostomy patients to commence a normal diet was 13.2 days (Median 13.5).

This was compared to data from 2019, when 21 patients were seen for dysphagia intervention post tracheostomy insertion, by SLT. There were 8 males and the mean age was 61 . These patients had a mean time to commence a normal diet of 14 days (Median 13.1).

Conclusion: These are small but comparable patient samples, that show that there does not appear to be a significant difference in the length of time taken to commence a normal diet in tracheostomised patients with COVID-19 patients, when compared to the "normal" cohort of patients seen at a Tertiary Cardiothoracic Centre in London, despite the differences in the mean age of the sample groups.

\section{Reference(s) and grant ackowledgment(s)}

1. 3. Intensive Care Society. The Faculty of Intensive Care Medicine. Guidelines for the Provision of Intensive Care Services. Edition 2. June 2019 [online]. Accessed 27/7/20

2. 2. McGrath, B A.; Wallace, Sarah. The UK National Tracheostomy Safety Project and the role of speech and language therapists Otolaryngology \& Head \& Neck Surgery 2014 22:3:181-187.

3. 1. Frajkova, Z et al. (2020) 'Post intubation dysphagia during COVID-19 outbreak-Contemporary review'. Dysphagia..

\section{7}

Early Tracheotomy in COVID-19 Patients is a safe Procedure for both, Patients and Physicians: a retrospective analysis

T. Mertke1; G. Danziger²; S. Mang²; A. Becker²; C. Metz²; A. Raddatz; A.

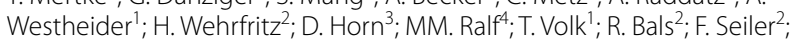
ML. Philipp

${ }^{1}$ Anaesthesiology, critical care medicine and pain medicine, Saarland University Hospital, Homburg, Germany; ${ }^{2}$ Inner medicine - pneumology, allergology and intensive care medicine, Saarland University Hospital, Homburg, Germany; ${ }^{3}$ Department of for oral and cranio-maxillofacial surgery, Saarland University Hospital, Homburg, Germany; ${ }^{4}$ Anaesthesiology and critical care, Kassel of the University of Southampton, Kassel, Germany Correspondence: $M$. Thilo

Intensive Care Medicine Experimental 2020, 8(2): 001527
Introduction: Guidelines for long-term ventilation agree that tracheotomy has several benefits for patient treatment [1]. However, during the coronavirus disease-19 (COVID-19) pandemic it is widely seen as an unsafe procedure with a high risk of infection $[2,3]$. However, as mentioned by Piccin et al., theoretical considerations might speak in favour of early tracheotomies also in COVID-19 patients, such as earlier and safer weaning in order to free ICU capacity as soon as possible and a reduced need for analgosedation, the drugs which were running out in many regions, including ours [4]. Most of these considerations are of theoretical nature, little clinical data is available on tracheotomy in COVID-19 patients. In our retrospective study, we evaluated tracheotomies performed on 16 COVID-19 patients to assess the risk of infection for HCWs, adverse events and the time, patients spend on a ventilator.

Methods: We evaluated tracheotomies performed on 16 COVID-19 patients as there is considerable disagreement if and how to implement tracheotomies in COVID-19 patients. All tracheotomies were performed bedside according to our standard protocol for dilatation tracheotomy. Tracheotomies were performed by experienced ICU physicians and trainees under supervision. Four Patients were on ECMO support during tracheotomy. Personal protective equipment (PPE) consisting of a protective gown, a N95 (FFP3) facial mask, goggles, visor and double layer of gloves was used in all procedures. Patients or legal representatives gave informed consent to the procedure. Infections on HCW were monitored by frequent nasopharyngeal swabs. Results: Patients were in mean 61 years old (median age $60 \pm 7$ years; $15 / 16$ male), mean BMI was $31.3 \mathrm{~kg} / \mathrm{m}^{2}$ (median $29.6 \pm 6.9 \mathrm{~kg} / \mathrm{m}^{2}$ ). Most frequent pre-existing medical condition was arterial hypertension. All procedures were percutaneous dilatation tracheotomies (PDT). Mean time between intubation and tracheotomy was 8.35 days, (median $7.5 \pm 5$ days). Mean time of mechanical ventilation after tracheotomy was 30 days (median; SD $24.5 \pm 15.9$ days). There was no procedure related complication or death due to tracheotomy. Mortality in COVID-19 patients undergoing tracheotomy was 6 out of 16 (37.5\%). No SARS-CoV2 infections were recorded in tracheotomy-performing healthcare workers (HCW) or other team members.

Conclusion: Tracheotomy in COVID-19 patients is almost identical to patients without COVID-19. The only difference is the PPE during the intervention. Tracheotomy is a safe procedure in COVID-19 for both patients and HCW under PPE. Given the shortness in ventilators and medication that are reported from some areas, it might be feasible for safe and forward patient management during COVID-19.

\section{Reference(s) and grant ackowledgment(s)}

1. [4] Piccin O, Albertini R, Caliceti U, et al.; Early experience in tracheotomy and tracheotomy tube management in Covid-19 patients; Am J Otolaryngol. 2020 May 7:102535.

2. [3] Tran K, Cimon C, Severn M, et al.; Aerosol Generating Procedures and Risk of Transmission of Acute Respiratory Infections to Healthcare Workers: A Systematic Review; PLoS One. 2012;7(4):e35797.

3. [2] Hiramatsu M, Nishio N, Ozaki M, et al.: Anesthetic and surgical management of tracheotomy in a patient with COVID-19, Auris Nasus Larynx; 2020 Apr 18:S0385-8146(20)30082-1.

4. [1]Mehta AB, Syeda SN, Bajpayee L, et al.: Trends in tracheotomy for mechanically ventilated patients in the United States. Am J Respir Crit Care Med. 2015 Aug 15;192(4):446-54

\section{2}

Evaluation of a modified tracheostomy technique in ventilated COVID-19 patients: is it safe for patients and staff?

K. Liatsikos' ; L. Skelly ; L. Ayres ${ }^{1}$; E. O'callaghan; J. Rodrigues ${ }^{1}$; B. Borgatta'; S. Mcdougall ${ }^{2} ;$ G. Dempsey

${ }^{1}$ Intensive care, Aintree University Hospital, Liverpool, United Kingdom; ${ }^{2}$ Intensive care, The Walton Centre, Liverpool, United Kingdom

Correspondence: $\mathrm{K}$. Liatsikos

Intensive Care Medicine Experimental 2020, 8(2): 001602

Introduction: During the Covid-19 pandemic an unprecedented number of patients have required intensive care unit (ICU) admission and prolonged ventilation, posing a significant threat to availability 
of resources. Early tracheostomy could benefit this cohort in terms of reduction of duration of mechanical ventilation, length of ICU stay (LOS), delirium and laryngeal injury [1]. However, professional societies advocated the delay of tracheostomies or discouraged them altogether given the expected increase in risk of aerosol-generating procedures (AGP) [2-3]. We performed tracheostomy in an effort to deal with predicted pandemic related resource shortages and to reduce ICU weakness and hospital stay.

Objectives: To compare patient characteristics, tracheostomy complications and evidence of viral transmission to staff.

Methods: Prospective, single centre, service evaluation project. We set out a multidisciplinary approach to tracheostomy insertion, both surgical and percutaneous, the principal aim of which was staff safety. Selection for tracheostomy criteria were defined as: $\mathrm{FiO} 2 \leq 50 \%$, positive end-expiratory pressure (PEEP) $\leq 10$ for at least $24 \mathrm{hrs}$ prior to procedure, unready for trial of extubation and likely need for prolonged ventilation. We performed modified surgical and percutaneous tracheostomies aiming to reduce aerosolisation. This involved personal protective equipment (PPE) as per Public Health England (PHE) guidelines with sterile gown and gloves donned over the PPE. The modified percutaneous technique included using 3 drapes and use of muscle relaxation/paralysis and ventilation pauses. Following tracheostomy, we documented complications and outcomes. We surveyed the operators regarding evidence of infection.

Results: Tracheostomy was performed in 33 out of the 74 patients that were admitted in our ICU. 14 were percutaneous and 19 were surgical. Patients were predominantly male $(63.6 \%)$, median age was 64 (IQR:58-68). Tracheostomy was performed after 7.5 days (median, IQR:5-12) from intubation and after 10 days (median IQR:7-14) of symptoms prior to ITU admission. We have not seen a significant difference in tracheostomy cuff leak nor wound infection/breakdown associated with either insertion technique. There was an increase in $\mathrm{PaO2}: \mathrm{FiO} 2$ ratio from 23.1 (median, IQR:19-26) prior to the procedure to 26 (median, IQR: 19-35) after. Mortality was $20.6 \%$ (7 patients). 26 patients $(78.8 \%$ ) have been discharged home. There were no episodes of absence of staff with COVID-19 like illness and operators performing tracheostomy all had negative antibodies.

Conclusion: We have demonstrated that earlier tracheostomy in stable COVID-19 pneumonitis patients, with modifications to insertion technique, is feasible. We have not seen increased complication rates or instances of staff infection with COVID-19 during the study period. Earlier insertion during the pandemic may allow for improved resource allocation and reduction in upper airway complications related to prolonged oro-tracheal intubation.

\section{Reference(s) and grant ackowledgment(s)}

1. 3.Chao TN, Braslow BM, Martin ND, Chalian AA, Atkins JH, Haas AR, et al. Tracheotomy in Ventilated Patients With COVID-19. Ann Surg. 2020.

2. 2.ViRUS 9: High risk of aerosolization or droplet spread from tracheostomy in patients with COVID-19 when ventilator or CPAP circuit disconnected, Faculty of Intensive care medicine.

3. 1. Andriolo BNG, Andriolo RB, Saconato H, Atallah ÁN, Valente O. Early versus late tracheostomy for critically ill patients. Cochrane Database of Systematic Reviews 2015, Issue 1. Art. No.: CD007271. https://doi.org/10.1002/14651 858.cd007271.pub3.

\section{1}

\section{Percutaneous tracheostomy in COVID 19 patients admitted} in Intensive care unit for invasive ventilation

S. Danndhiganaahalli'; N. Bandla²; P. Matheswaran ${ }^{3}$; C. Mcmullenclare ; M. Lynch $^{1}$

${ }^{1}$ Intensive care Medicine, University Hospital of North Midlands, Newcastle-under-Lyme, United Kingdom; ${ }^{2}$ Critical care, University Hospital Of North Midlands, Stoke-on-Trent, United Kingdom; ${ }^{3}$ Anaesthetics and Intensive care medicine, University hospitals of North midlands, Stoke-on-Trent, United Kingdom

Correspondence: S. Danndhiganaahalli

Intensive Care Medicine Experimental 2020, 8(2): 001621
Introduction: The Covid-19 pandemic has placed an extraordinary burden on critical care medicine globally. Supportive care continues to be mainstay of treatment.1 At the time of the study two thirds of Covid -19 patients who required critical care in the UK had mechanical ventilation within $24 \mathrm{hrs}$ of admission.2 Due to changing guidelines, there was little evidence on the appropriate mode (surgical vs bedside) of tracheostomy for patients admitted to Intensive care unit .Some studies suggested that early tracheostomy was associated with better outcomes 3 than late tracheostomy.4.

Objectives: This is a prospective observational study looking at possibility of doing percutaneous tracheostomy in Covid19 patients admitted in a tertiary hospital intensive care unit requiring invasive mechanical ventilation.

Methods: The present study involved review of covid-19 patients who underwent tracheostomy during their stay in our intensive care unit from 18th of March 2020 to 29 th of April 2020.(6 weeks). Data related to demographic parameters, duration of ventilation with Endotracheal tube, day and type of tracheostomy from time of ICU admission and outcome were reviewed.

Results: 20 patients underwent planned tracheostomy during the study duration with 15 males (75\%) and 5 female patients (25\%). The overall mean age was $55.1 \mathrm{yrs}$; for males 57.13 and for females $49.20 \%$ had normal BMI, $20 \%$ pre obese and $60 \%$ were obese. Mean days of ventilation with ET tube was $13.15+/$ - 6.53. Earliest tracheostomy was done on day 8 of ICU admission and latest was on day 30 of ICU admission. Median day of tracheostomy was Day 14.11 (55\%) patients underwent percutaneous tracheostomy in the ICU while 9 (45\%) patients underwent surgical tracheostomy in operation theatre . Of the 9 surgical tracheostomy patients, 2 were difficult percutaneous tracheostomy that were subjected to surgical tracheostomy. 8 patients were discharged to ward, 9 patients continue to be ventilated and 3 patients died during the study.

Conclusion: This study shows that percutaneous tracheostomy can be safely done on Covid 19 patients in an Intensive care unit with adequate planning and preparation. Nevertheless, a surgical tracheostomy should be considered when necessary.

\section{Reference(s) and grant ackowledgment(s)}

1. 4)Young D, Harrison D A, Cuthbertson B H, Rowan K; TracMan Collaborators.Effect of early vs late tracheostomy placement on survival in patients receiving mechanical ventilation: the TracMan randomized trial JAMA 2013309202121-2129.

2. 3) Griffiths J, Barber VS, Morgan L, Young J D. Systematic review and metaanalysis of studies of the timing of tracheostomy in adult patients undergoing artificial ventilation BMJ 2005330(7502):1243.

3. 2) Mahase E. Covid-19: most patients require mechanical ventilation in first 24 hours of critical care. BMJ. 2020 Mar 24;368:m1201.

4. 1) NHS England guidelines.

\section{5}

Diuretics for fluid overload in critically ill patients. The multicenter randomized-controlled IRIHS study

R. Cinotti ${ }^{1}$; J. Lascarrou ${ }^{2}$; M. Azais ${ }^{3}$; G. Colin ${ }^{4}$; J. Quenot ${ }^{5}$; P. Mahé1; A.

Gaultier $^{6} ;$ K. Asehnoune $;$; J. Reignier ${ }^{8}$

${ }^{1}$ Anesthésie-réanimation, C.H.U. de Nantes, Nantes, France; ${ }^{2}$ Mir, C.H.U. de Nantes, Nantes, France; ${ }^{3}$ Mir, Centre Hospitalier Départemental Vendée, Boulevard Stéphane Moreau, La Roche-sur-Yon, France, La Roche-sur-Yon, France; ${ }^{4}$ Medical surgical intensive care unit, Hospital Center Departmental Vendée, La Roche-sur-Yon, France; ${ }^{5}$ Mir, University Hospital F.mitterand Dijon-Bourgogne, Rue Paul Gaffarel, Dijon, France, Dijon, France; ${ }^{6}$ Biostatistics, C.H.U. de Nantes, Nantes, France; ${ }^{7}$ Service d'anesthésie-réanimation, Chu Nantes-Hotel Dieu, Nantes, France; ${ }^{8}$ Médecine intensive réanimation, Nantes University Hospital Hotel-Dieu, Nantes, France

Correspondence: R. Cinotti

Intensive Care Medicine Experimental 2020, 8(2): 001435

Introduction: Fluid overload has been associated with increased morbidity and mortality in critically ill patients. 
Objectives: To assess the efficacy and tolerance of a rationalized diuretic strategy to treat fluid overload after shock in patients undergoing mechanical ventilation.

Methods: The IRIHS study (NCT 02345681) was a single-blind, randomized-controlled study performed in 4 intensive care units (ICUs) in France, from May 5th 2015 to February 2nd 2019. Patients were eligible in case of fluid overload defined as in-ICU weight increase $\geq 3 \%$, mechanical ventilation $\geq 24$ hours, and hemodynamic stabilization (vasopressors drugs discontinuation $>6$ hours; dobutamine $\leq 10 \mu \mathrm{g}$ $\mathrm{kg}-1 . \mathrm{mn}-1$ ). Patients were randomized into diuretic group (intravenous furosemide, twice a day, maximum daily dose of $250 \mathrm{mg}$ from randomization until successful extubation), or a control group without furosemide. The primary outcome was the fluid balance, defined as weight variation, from randomization to successful extubation. The main secondary outcome was the safety of diuretic.

Results: 171 patients were randomized, 5 patients who did not satisfy the inclusion criteria were excluded and thus, 166 included in analysis: 77 in the diuretic and 89 in the control group. The primary outcome was available in $144(84.2 \%)$ patients. In the complete cases analysis, fluid balance was $1.4[-2.5 ; 4.5] \mathrm{kg}$ in the diuretic group and 6.4 $[0.5 ; 11.2] \mathrm{kg}$ in the control group $(p<0.001)$. In the multiple imputation analysis, fluid balance was significantly decreased in the diuretic group $(\beta=-4.895 \% \mathrm{Cl}[-7.3 ;-2.5], \mathrm{p}<0.001)$. Eleven (14\%) patients died in ICU in the diuretic group, and $16(18 \%)$ patients in the control group $(p=0.5)$. The rates of kidney failure were respectively $55(63.2 \%)$ and $55(73.3 \%)$ in the diuretic and the control group $(p=0.2)$.

Conclusion: In this multicenter randomized-controlled study, diuretics decreased the fluid overload of critically ill patients as compared to a no-diuretic policy.

\section{Reference(s) and grant ackowledgment(s)}

1. Mehta S, Burry L, Cook D, et al. Daily sedation interruption in mechanically ventilated critically ill patients cared for with a sedation protocol: a randomized controlled trial. JAMA. 2012;308(19):1985-1992.

2. Perner A, Haase N, Guttormsen AB, et al. Hydroxyethyl starch 130/0.42 versus Ringer's acetate in severe sepsis. N Engl J Med. 2012;367(2):124-134. https://doi.org/10.1056/nejmoa1204242.

3. Acheampong A, Vincent J-L. A positive fluid balance is an independent prognostic factor in patients with sepsis. Crit Care. 2015;19(1):774. https:// doi.org/10.1186/s13054-015-0970-1.

4. French ministry of health (Appel d'Offre La Roche sur Yon-Nantes, 31 January 2014)

\section{7}

Use of lung recruitment and individualized PEEP setting in the ventilatory management of C-ARDS

J. A. Sanchez Giralt' ; M. San Baron ${ }^{1}$; M. Trigueros Genao ; J. Lázaro Gonzalez'; B. Muñoz Molina'; S. Portillo Sánchez'; P. Patiño Haro'; B. Abad Santamaría'; A. Canabal Berlanga ${ }^{1}$; F. Suarez Sipmann ${ }^{1}$

1 Intensive care medicine, Hospital de La Princesa, Madrid, Spain

Correspondence: F. Suarez Sipmann

Intensive Care Medicine Experimental 2020, 8(2): 001447

Introduction: Lung protective ventilation is the cornerstone of the ventilatory management of COVID-29 related ARDS (C-ARDS). Lung recruitment maneuvers (RM) and their related optimum PEEP setting are frequently used as adjuvant interventions. However, as lung collapse does not seem to be an important pathophysiologic component, these interventions may be less effective in C-ARDS.

Objectives: To study the role of RM and individualized PEEP settings as adjuvant lung protective interventions in the management of ventilated C-ARDS patients. We specifically analyzed the obtained PEEP levels and the effects on lung mechanics.

Methods: Retrospective analysis of all registered RM followed by a decremental PEEP titration performed in C-ARDS patients performed during the peak of the COVID pandemia in a teaching tertiary hospital ICU. Maneuvers were registered either by electrical impedance tomography (EIT- Enlight 1800,Timpel) or a sequential automated recruitment maneuver (Servo-u, Maquet CC). We compared individual PEEP levels determined during a decremental PEEP trial by global $(\mathrm{Cg})$ or regional (Creg) compliance. Cg was defined by the maximal global compliance and Creg by the point of minimum overdistension and collapse as measured by EIT(1). We also analyzed differences in PEEP individualization between supine and prone positioning. After checking for normality of the data distributions means were compared using t-test for independent variables.

Results: The analysis includes a total of 90 registered RM-PEEP titrations ( 60 in supine and 30 in prone) in 19 patients (4.6 RM per patient). Recruitment pressures (mean \pm SD) were $48 \pm 4 / 33 \pm 4 \mathrm{cmH} 2 \mathrm{O}$ (inspiratory pressure/PEEP). Obtained individualized PEEP levels were $10.5 \pm 2.2 \mathrm{cmH} 2 \mathrm{O}$ for $\mathrm{Cg}$ and $8.8 \pm 2,3 \mathrm{cmH} 2 \mathrm{O}$ for Creg $(\mathrm{p}<0.05)$.

Respiratory system compliance, plateau and driving pressures improved after RM and PEEP setting from $37.1 \pm 14.2$ to $42.5 \pm 13.8 \mathrm{ml} /$ $\mathrm{cmH} 2 \mathrm{O} \mathrm{p}<0.001 ; 24.9 \pm 3.6$ to $22.7 \pm 3.1 \mathrm{cmH} 2 \mathrm{O} \mathrm{p}<0.001$ and $13.8 \pm 3.1$ to $11.5 \pm 2.8 \mathrm{cmH} 2 \mathrm{O} p<0.01$, respectively. There were no differences between supine and prone position in individualized PEEP levels (Cg $10.6 \pm 3.2$ vs $10.2 \pm 3.33$ and Creg $8.9 \pm 3.1$ vs $8.6 \pm 3.6)$ and respiratory system compliance was better in supine than in prone $(41.9 \pm 13.8$ vs $42.8 \pm 16.8 p<0.05)$ respectively. At the determined $\mathrm{Cg}$ and Creg PEEP levels lung protective ventilation resulted in a percentage overdistension of $10 \pm 7.9$ and $4.5 \pm 3.4 \%$ and collapse of $y$ $3.4 \pm 3.6$ and $4.5 \pm 2,5 \%$ of the total lung respectively as measured by EIT. Small changes in the applied PEEP levels resulted in important changes in the percentage overdistension and/or collapse.

Conclusion: Lung recruitment and individualized PEEP titration was an effective adjuvant lung protective intervention. It allowed to adjust and minimize PEEP in each individual patient and at different stages during the evolution. The individualized PEEP levels according to Creg were lower with less overdistension but more collapse than the ones according to $\mathrm{Cg}$.

\section{Reference(s) and grant ackowledgment(s)}

1. 1. Costa ELV, Borges JB, Melo A, Suarez Sipmann F, Toufen C, Böhm SH, et al. Bedside estimation of recruitable alveolar collapse and hyperdistension by electrical impedance tomography. Intensive Care Med. 2009 Mar 3:35(6):1132-7.

2. None.

\section{7}

Impact of frailty, age and comorbidities on the need for invasive ventilation in critically ill patients with COVID-19

BW. Johnston ${ }^{1}$; S. White'; DO. Hamilton '; E. Morgan'; A. Ross²; M.

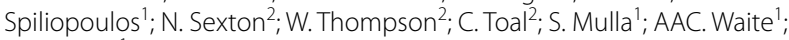
ID. Welters

${ }^{1}$ Intensive care unit, Royal Liverpool University Hospital, Liverpool, United Kingdom: ${ }^{2}$ Acute medicine, Royal Liverpool University Hospital, Liverpool, United Kingdom

Correspondence: A. Waite

Intensive Care Medicine Experimental 2020, 8(2): 001457

Introduction: In line with NHS England guidance [1], during the first wave of the COVID-19 pandemic where appropriate all patients admitted to hospital, irrespective of COVID-19 status, were assessed for frailty, using the Rockwood Frailty Scale as part of a holistic assessment.

In this retrospective cohort study, we aimed to assess the impact of frailty, age and comorbidities in patients with COVID-19 requiring invasive ventilation.

Methods: We retrospectively reviewed case notes of all patients admitted to the Royal Liverpool University Hospital Accident and Emergency (A\&E) Department, between March 23rd and April 23rd 2020 with a positive COVID-19 RT-PCR result. Routine data, including demographic data, comorbidity data and Rockwood Frailty Score, were extracted. We assessed the predictive value of age, comorbidities and Rockwood Frailty Scores for disease progression to requiring invasive ventilation. Receiver operating curves were generated to illustrate sensitivity and specifity for each variable to predict the need for invasive ventilation.

Results: Patients with a diagnosis of COVID-19 who progressed to invasive ventilation were younger with a median age of 58.5 [53.23; 
$59.81]$ years compared to $72[58.25 ; 81]$ years $(p<0.001)$. The majority of patients invasively ventilated had a Rockwood Frailty Score $<3$ $(85 \%)$. Patients who required invasive ventilation were less likely to suffer from comorbidities including COPD, asthma, hypertension, chronic kidney disease, hepatic disease, diabetes mellitus, atrial fibrillation, malignancy or hypercholesteraemia and were less likely to have a history of myocardial infarction or stroke $(p<0.01)$

Area under the curve for age was $74.2 \%$, and $87.1 \%$ for Rockwood Frailty Score. For comorbidities, AUCs were between $47 \%$ and $59 \%$, indicating a low predictive value.

Conclusion: Patients presenting to our A\&E Department with a higher Rockwood Frailty Score were less likely to be admitted to ICU and to be invasively ventilated. Similarly, the age of patients on ICU was significantly lower than for patients presenting to A\&E who had confirmed COVID-19. Our results are in line with large databases [2] that demonstrate that during the COVID-19 pandemic frail patients and those requiring support in their activities of daily living have been less frequently admitted to ICU compared to patients with viral pneumonia in non-pandemic situations. During pandemics, shortness of ventilators may become a reality, supporting the need for careful planning and triaging of patients requiring invasive ventilation to identify those who will need scarce resources early during their hospital admission.

\section{Reference(s) and grant ackowledgment(s)}

1. [1] https://www.nice.org.uk/guidance/ng159/resources/covid19-rapid -guideline-critical-care-in-adults-pdf-66141848681413.

2. [2] https://www.icnarc.org/Our-Audit/Audits/Cmp/Reports.

\section{5}

Length of ICU stay, type of preintubation and postintubation support and outcomes in mechanically ventilated COVID19 patients admitted in Catalan ICUs (UCIsCAT study group)

J. Mancebo'; JR. Masclans'; R. Ferrer Roca'; A. Mas ${ }^{4}$; P.Vera ${ }^{5}$; R. Mañez ; J.

Trenado $^{7}$; R. Fernandez ${ }^{8}$; JM. Sirvent Calvera $;$ M. Martínez ${ }^{10}$; M. Ibarz ${ }^{11}$; E. Sandoval ${ }^{12}$; P. Garro ${ }^{13}$; JL. Lopera ${ }^{14} ;$ M. Bodíl ${ }^{15} ;$ JC. Yebenes-Reyes ${ }^{16} ; C$. Triginer $^{17}$; I. Vallverdú ${ }^{18} ;$ A. Baró Serra ${ }^{19} ;$ F. Bodi $^{20}$

${ }^{1}$ Intensive care, Hospital de la Santa Creu i Sant Pau, Barcelona, Spain; ${ }^{2}$ Intensive care dpt, Hospital del Mar, Barcelona, Spain; ${ }^{3}$ Intensive care dpt/ sodir research group, Vall d'Hebron University Hospital, Barcelona, Spain; ${ }^{4}$ Intensive care dpt, Hospital de Sant Joan Despí Moisès Broggi, Sant Joan Despí, Spain; ${ }^{5}$ Intensive care dpt, Hospital de la Santa Creu i Sant Pau, Barcelona, Spain; ${ }^{6}$ ntensive care dpt, Hospital Universitari de Bellvitge, L'Hospitalet de Llobregat, Spain; ${ }^{7}$ Intensive care dpt, Mútua Terrassa University Hospital, Terrassa, Spain; ${ }^{8}$ Intensive care dpt, Althaia, Xarxa Assistencial Universitària de Manresa, Manresa, Spain; ${ }^{9}$ Intensive care dpt, Hospital Universitari de Girona Dr Josep Trueta, Girona, Spain; ${ }^{10}$ Intensive care dpt, Hospital General De Cataluña, Sant Cugat del Vallès, Spain; ${ }^{11}$ Intensive care dpt, Hospital Universitari Sagrat Cor - Grup Quirónsalut, Barcelona, Spain; ${ }^{12}$ Cardiovascular surgery, Hospital Clínic de Barcelona, Barcelona, Spain; ${ }^{13}$ Intensive care dpt, Hospital General de Granollers, Granollers, Spain; ${ }^{14}$ Intensive care dpt, Hospital Universitari de Vic (Consorci Hospitalari de Vic), Vic, Spain; ${ }^{15}$ Intensive care dpt, Hospital Universitari de Tarragona Joan XXIII, Tarragona, Spain; ${ }^{16}$ Intensive care dpt, Hospital de Mataró, Mataró, Spain; ${ }^{17}$ Intensive care dpt, Igualada Hospital, Igualada, Spain; ${ }^{18}$ Intensive care dpt, Hospital Universitari Sant Joan de Reus, Reus, Spain; ${ }^{19}$ Intensive care dpt, Hospital de Santa Caterina, Salt, Spain; ${ }^{20}$ Intensive care dpt, Hospital de Sant Pau i Santa Tecla, Tarragona, Spain

Correspondence: J. Mancebo

Intensive Care Medicine Experimental 2020, 8(2): 001535

Introduction: The surge of patients with COVID19 severe acute hypoxemic respiratory failure has put resources at their limits.

Objectives: To describe how severely hypoxemic patients were managed with noninvasive and invasive respiratory support in the Catalan ICU network between 15 March and 15 April.

Methods: Consecutive patients admitted between 15 March and 15 April in 26 ICUs, who tested positive for SARS-Cov-2 (RT-PCR in a nasopharingeal swab) and had severe acute hypoxemic respiratory failure, presenting with bilateral infiltrates in the chestX-ray and requiring invasive mechanical ventilation. Follow-up time was til ICU discharge.
Study was approved by the Ethics Committee of the coordinating center (HospitalUniversitari Sant Pau, Barcelona).

Results: A total of 1388 patients required invasive mechanical ventilation and overall mortality rate was 35\% (491/1388). Most common single modes of oxygenation before intubation were facemask with oxygen reservoir in $39 \%$ patients $(538 / 1388)$, of whom 182 died (34\%), and high flow nasal cannula in $22 \%$ patients (308/1388), of whom 88 died ( $29 \%) ; p=0.12$ for mortality differences.

Once intubated, the most frequent ventilator mode was volume assistcontrol ventilation and was used in 1236/1388 patients (89\%). The most frequently used ventilators were conventional ICU machines in $66 \%$ patients $(915 / 1388)$, and anesthesia machines in $12 \%$ patients (169/1388). The anesthesia machines were used when conventional ICU ventilators were lacking. Patients ventilated with ICU machines had an ICU mortality of $34 \%$ (308/915) whereas those ventilated with anesthesia machines had a $47 \%$ mortality $(79 / 169), p=0.002$. ECMO was employed in 31 patients (2,2\%), 28 ECMO VV and 3 ECMO VA. Tracheostomies were performed in $28 \%$ patients $(524 / 1388)$, of whom $26 \%(137 / 524)$ died.

\begin{tabular}{|c|c|c|c|c|}
\hline Length of stay and $c$ & $\begin{array}{l}\text { All } \\
(\mathrm{n}=1388)\end{array}$ & $\begin{array}{l}\text { Alive } \\
(\mathrm{n}=897)\end{array}$ & Dead (491) & $\begin{array}{l}\text { p (alive } v s \\
\text { dead) }\end{array}$ \\
\hline $\begin{array}{l}\text { Hospital stay pre-ICU, } \\
\text { days }\end{array}$ & $2.38 \pm 3.5$ & $2.25 \pm 3.2$ & $2.62 \pm 3.8$ & ns \\
\hline ICU stay, days & $20.1 \pm 14.9$ & $22.2 \pm 15.5$ & $16.4 \pm 12.7$ & $<0.001$ \\
\hline $\begin{array}{l}\text { Hospital admission to } \\
\text { intubation, days }\end{array}$ & $2.74 \pm 3.5$ & $2.6 \pm 3.2$ & $3.02 \pm 3.97$ & $<0.05$ \\
\hline $\begin{array}{l}\text { ICU admission to } \\
\text { intubation, days }\end{array}$ & $0.47 \pm 1.2$ & $0.48 \pm 1.07$ & $0.43 \pm 1.51$ & ns \\
\hline $\begin{array}{l}\text { Duration of mechanical } \\
\text { ventilation, days }\end{array}$ & $17.5 \pm 13.8$ & $18.3 \pm 14.2$ & $16.02 \pm 12.7$ & 0.003 \\
\hline $\begin{array}{l}\text { Intubation to } \\
\text { tracheostomy, days }\end{array}$ & $13.8 \pm 6^{*}$ & $13.5 \pm 6.1 * *$ & $14.5 \pm 5.7 * * *$ & ns \\
\hline
\end{tabular}

$* \mathrm{n}=524, * * \mathrm{n}=387, * * * \mathrm{n}=137$

Conclusion: COVID19 mechanically ventilated patients consumed a substantial amount of resources, were often tracheostomized and their ICU length of stay was prolonged.

\section{Reference(s) and grant ackowledgment(s)}

1. Other contributors: J-A.Santos, J.Baldirà, A-J.Betbesé, M.Izura, I.Morán, J-C. Suárez, L.Zapata, N.Rodríguez, M.Torrens, A.Cordón, C.Gomila, M.Flores, A.Segarra, M.Morales, L.Mateo, M.Martos, P.Saludes, J-R.Cervelló, M.Valencia, F.Roche, G.Masdeu, B. Cancio, S.Hernández-Marín, C.Pedrós, P.Sebastian, X.Saiz, M.Morales, A.Huerta, E.Esteban, S.Benito, C.Barberà, J.Echevarría, P.Santigosa, D.Tognetti, C.González.

\section{8}

Ventilation related characteristics of COVID-19 patients classified by clinical phenotype

C. Díaz-Rodríguez ; P. Blanco-Schweizer ${ }^{1}$; J. Sánchez-Ballesteros ${ }^{1}$; R. Herrán-Monge ; AM. Prieto-Lamo ; ML. Fernández-Rodríguez¹; D. Pérez-Torres ${ }^{1}$; I. Canas-Pérez ; JÁ. Berezo-García1; JJ. Sanz-Hernán1; V. Fraile-Gutiérrez ; AM. Olmos-Linares ${ }^{1}$; C. Cuenca-Rubio ${ }^{1}$; A Martín-Luengo ${ }^{1}$; GJ. Posadas-Pita ${ }^{1}$; PA. Merino-García'; MM. García-García;

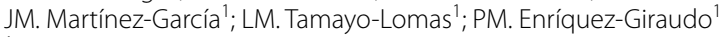
${ }^{1}$ Servicio de medicina intensiva, Hospital Universitario Río Hortega, Valladolid, Spain

Correspondence: $C$. Díaz-Rodríguez

Intensive Care Medicine Experimental 2020, 8(2): 001538

Introduction: SARS-CoV-2 infection leads in the most severe cases to a profound respiratory failure that requires admission to the intensive care unit (ICU) and mechanical ventilation. Two different phenotypes have been proposed: $\mathrm{H}$ (HIGH elastance-right to left shunt ratio-lung weight-recruitability) and L (LOW elastance-VA/Q ratio-lung weightrecruitability) (ref 1 . Gattinoni). 
Objectives: To describe the incidence, clinical differences and outcomes of phenotype $\mathrm{H}$ and $\mathrm{L}$ patients with SARS-CoV-2 infection (COVID-19) who are admitted in an ICU of a third-level hospital with extracorporeal membrane oxygenation (ECMO) support capacity.

Methods: We retrospectively collected and analysed data from all COVID-19 confirmed patients, admitted to the ICU of a third-level hospital in Spain between 15th February and 15th June 2020. We categorised patients into two phenotypes, $\mathrm{L}$ and $\mathrm{H}$, and compared their clinical and epidemiological variables as well as ventilation related parameters. The data are expressed as mean \pm standard deviation, median \pm interquartile range, and compared using T-test, Wilcoxon rank test, Chi-square test and Fisher's exact test as appropriate.

Results: A total of 87 COVID-19 confirmed patients were included out of which 79 (93\%) received invasive mechanical ventilation. $77 \%$ males, mean age $60 \pm 12$, median BMI $27 \mathrm{Kg} / \mathrm{m}^{2}$ (IQR 25-32), mean APACHE-II $17 \pm 6.13$ patients needed VV-ECMO support (61\% survival). Overall hospital mortality was 38\%. Comorbidities: hypertension $30 \%$, diabetes $14 \%$, ischemic heart disease $5 \%$, asthma $8 \%$, COPD $3 \%$.

The incidence of $\mathrm{H}$ phenotype was $40 \%$, $\mathrm{L}$ phenotype $32 \%$ and in $28 \%$ of the patients it was not possible to determine. Comparison of ventilation related parameters between both groups are expressed in Table 1.

\begin{tabular}{|c|c|c|c|}
\hline & $\begin{array}{l}\text { H-Phenotype } \\
(\mathrm{n}=32) \\
(\text { mean } \pm \text { sd) } \\
\text { (median, IQR) }\end{array}$ & $\begin{array}{l}\text { L-Phenotype } \\
(\mathbf{n}=25) \\
(\text { mean } \pm \text { sd) } \\
(\text { median, IQR) }\end{array}$ & $\mathbf{p}$ \\
\hline PaO2/FiO2 day 1 & $171(114,216)$ & $161(134,209)$ & $>0.05$ \\
\hline PaO2/FiO2 day 3 & $195(146,263)$ & $200(144,236)$ & $>0.05$ \\
\hline $\mathrm{PaO} 2 / \mathrm{FiO} 2$ day 5 & $176(156,240)$ & $189(142,218)$ & $>0.05$ \\
\hline $\mathrm{PaO2} / \mathrm{FiO2}$ day 10 & $184 \pm 68$ & $217 \pm 73$ & $>0.05$ \\
\hline PEEP day $1(\mathrm{cmH2O})$ & $15 \pm 3$ & $11 \pm 2$ & $<0.01$ \\
\hline PEEP day $5(\mathrm{cmH2O})$ & $10 \pm 3$ & $11 \pm 2$ & \\
\hline $\begin{array}{l}\text { Days of mechanical } \\
\text { ventilation }\end{array}$ & $15(8,30)$ & $11(6,24)$ & $>0.05$ \\
\hline $\begin{array}{l}\text { Tidal volume/ideal weight } \\
\text { day } 1(\mathrm{ml} / \mathrm{kg})\end{array}$ & $7(6,7)$ & $7 / 6,7)$ & $>0.05$ \\
\hline $\begin{array}{l}\text { Tidal volume/ideal weight } \\
\text { day } 10(\mathrm{ml} / \mathrm{kg})\end{array}$ & $7(6,8)$ & $8(7,8)$ & $<0.05$ \\
\hline Use of prone position (\%) & 68 & 60 & $>0.05$ \\
\hline Number of prone sessions & $2.6 \pm 2$ & $2.8 \pm 1$ & $>0.05$ \\
\hline Mortality ICU (\%) & 33 & 29 & $>0.05$ \\
\hline
\end{tabular}

Conclusion: Out of all admitted patients, only $72 \%$ of them could be classified into one of the proposed phenotypes.

Patients with $\mathrm{H}$ phenotype were treated with a higher PEEP level on the first day of mechanical ventilation, but with a similar tidal volume. Tidal volume was significantly higher in L phenotype patients on day 10.

No differences in $\mathrm{PaO} 2 / \mathrm{FiO} 2$ ratio, days of mechanical ventilation and the use of prone position was observed between groups.

\section{Reference(s) and grant ackowledgment(s)}

1. Luciano Gattinoni, Davide Chiumello, Pietro Caironi, Mattia Busana, Federica Romitti, Luca Brazzi; Luigi Camporota.COVID 19 pneumonia: different respiratory treatments for different phenotypes?Intensive Care Med (2020) 46:1099-1102.

2. ARDS Definition Task Force, Ranieri VM, Rubenfeld GD, Thompson BT, Ferguson ND, Caldwell E, Fan E, Camporota L, Slutsky AS. Acute respiratory distress syndrome: the Berlin Definition. JAMA. 2012 Jun 20;307(23):2526-33. https://doi.org/10.1001/jama.2012.5669.

\section{3}

Use of inhaled nitric oxide as an adjuvant therapy in C-ARDS

JA. Sanchez Giralt'; M. San Baron'; M. Trigueros Genao'; J. Lázaro Gonzalez'; B. Muñoz Molina'; S. Portillo Sánchez'; P. Patiño Haro'; B. Abad Santamaría ${ }^{\prime}$; A. Canabal Berlanga'; F. Suarez Sipmann ${ }^{1}$

'Intensive Care Medicine, Hospital de La Princesa, Madrid, Spain

Correspondence: F. Suarez Sipmann
Intensive Care Medicine Experimental 2020, 8(2): 001563

Introduction: COVID-29 related ARDS (C-ARDS) has specific pathophysiologic features that include a relatively higher compliance as non C-ARDS of similar severity and a pulmonary vascular thrombosis diathesis together with a severe early hypoxemia. Given these features inhaled nitric oxide (iNO) could have a potential adjuvant role in the ventilatory management of C-ARDS.

Objectives: To study the effects of iNO on the relative change in pulmonary perfusion and on gas exchange in C-ARDS.

Methods: Retrospective analysis C-ARDS patients with severe ARDS treated with iNO delivered via the SoKINOXTM device (Air Liquide). The relative distribution of pulmonary blood flow before and after the administration of iNO was determined by EIT (Enlight, 1800 Timpel). Perfusion data were obtained by means of a bolus infusion of $10 \mathrm{ml}$ of hypertonic (7.6\%) Saline solution during a brief apnea period maintaining the same PEEP level(1). Ventilator settings before and after iNO were maintained unchanged so that no changes in the regional distribution of ventilation were expected. At least one blood gas before and after iNO, although at different time periods in relation to the start of iNO therapy, were obtained. Due to the low number of patients and measurments no statistical analysis was performed in this descriptive study.

Results: A total of 8 patients were included in the analysis. After a few dose response trials performed in the first patients average lowest iNO dose resulting in the maximum change in perfusion was $15 \mathrm{ppm}$. Changes in the relative distribution of ventilation were $<1 \%$ for all studied regions. The response to iNO was very variable both in terms of the distribution of perfusion and gas exchange with no clear defined pattern. Only four patients responded according to oxygenation with an average increase in $\mathrm{PaO} 2 / \mathrm{FiO} 2$ of $40.5 \pm 17.7 \%$. When analyzing the changes in the distribution of perfusion in vertical gravitational axis, iNO predominantly shifted perfusion to the ventral (non-dependent) lung half in 3 patients $(13 \pm 6.95 \%$ shift) and to the dorsal (dependent) lung half in 5 patients $(8.4 \pm 4.1 \%$ shift). This corresponded to a decrease of $5.5 \pm 4.4 \%$ in $\mathrm{PaO} 2 / \mathrm{FiO} 2$ and an increase of $13.7 \pm 17.7 \%$ in $\mathrm{PaCO} 2$ and an increase of $29.9 \pm 28.3 \%$ in $\mathrm{PaO} 2 / \mathrm{FiO} 2$ and a decrease of $2.3 \pm 14.5 \%$ in $\mathrm{PaCO} 2$ in those predominantly shifting perfusion to ventral and to dorsal regions respectively.

Conclusion: The response to iNO in C-ARDS patients was highly variable and required higher than usual doses. In responding patients iNO caused a redistribution of pulmonary blood flow to dorsal nondependent regions.

Reference(s) and grant ackowledgment(s)

1. 1. Borges JB, Suarez Sipmann F, Böhm SH, Tusman G, Melo A, Maripuu E, et al. Regional lung perfusion estimated by electrical impedance tomography in a piglet model of lung collapse. Journal of Applied Physiology. 2012 Jan;112(1):225-36.

\section{8}

Ventilation related characteristics of COVID-19 patients admitted to ICU and extended-ICU during SARS-CoV-2 pandemic

C. Díaz-Rodríguez ${ }^{1}$; P. Blanco-Schweizer ${ }^{1}$; ML. Fernández-Rodríguez ${ }^{1}$; AM. Prieto-Lamo'; R. Herrán-Monge'; J. Sánchez-Ballesteros'; D. Pérez-Torres'; I. Canas-Pérez'; JÁ. Berezo-García'; JJ. Sanz-Hernán'; C. Cuenca-Rubio ${ }^{1}$; AM. Olmos-Linares ${ }^{1}$; V. Fraile-Gutiérrez' ${ }^{1}$; GJ. Posadas-Pita'; PA. Merino-García'; A. Martín-Luengo'; JM. Martínez-García'; AF. Muriel-Bombin ${ }^{1}$; LM. Tamayo-Lomas'; PM. Enríquez-Giraudo"

${ }^{1}$ Servicio de medicina intensiva, Hospital Universitario Río Hortega, Valladolid, Spain

Correspondence: C. Díaz-Rodríguez

Intensive Care Medicine Experimental 2020, 8(2): 001568

Introduction: Patients with the most severe forms of SARS-CoV-2 infection develop a profound respiratory failure that, during the global pandemic, has forced hospitals to increase their ICU-bed capacity. Spaces with ICU-level monitoring, such as post-anesthesia care units or operating rooms have been used as temporary ICU beds (extended-ICU). Moreover, collaboration between professionals in the 
departments of critical care, anesthesiology and other medical specialties has been necessary in order to attend all critical care patients. (ref. 1,2).

Objectives: We aim to determine the differences in ventilation related variables between patients admitted to the ICU and patients admitted to the extended-ICU in a third-level hospital in Spain between 15th February and 15th June 2020.

Methods: We retrospectively collected and analysed data from all COVID-19 confirmed patients admitted to a third-level hospital in Spain and needed intensive cares, between 15th February and 15th June 2020. We categorised the patients into two groups: admitted to ICU and admitted to extended-ICU. We compared ventilation related parameters between groups. The data are expressed as mean \pm standard deviation, median \pm interquartile range, and compared using T-test, Wilcoxon rank test, Chi-square test and Fisher's exact test as appropriate.

Results: A total of 87 COVID patients are included, out of which 79 (93\%) received invasive mechanical ventilation. $77 \%$ males, aged $60 \pm 12, \mathrm{BMI} 27 \mathrm{Kg} / \mathrm{m}^{2}$ (IQR 25-32). Mean APACHE-II $17 \pm 6.13$ patients needed VV-ECMO support (61\% survival). Overall hospital mortality was $38 \%$. Comorbidities: hypertension $30 \%$, diabetes $14 \%$, Ischemic heart disease $5 \%$, asthma $8 \%$, COPD 3\%.

Ventilation related parameters are expressed in Table 1.

Table1

\begin{tabular}{|c|c|c|c|}
\hline Variable & $\begin{array}{l}\text { ICU } \\
(n=45)\end{array}$ & $\begin{array}{l}\text { Extended-ICU } \\
(\mathrm{n}=42)\end{array}$ & $\mathbf{P}$ \\
\hline $\begin{array}{l}\text { Mechanical ventilation } \\
\text { free days }\end{array}$ & $1(0,4)$ & $0(0,2)$ & $<0.05$ \\
\hline $\begin{array}{l}\text { Tidal volume/ideal body } \\
\text { weight day } 1(\mathrm{ml} / \mathrm{kg})\end{array}$ & $7(6,8)$ & $7(6,8)$ & $>0.05$ \\
\hline $\begin{array}{l}\text { Tidal volume/ideal body } \\
\text { weight day } 5(\mathrm{ml} / \mathrm{kg})\end{array}$ & $7(6,8)$ & $7(6,8)$ & $>0.05$ \\
\hline Tidal volume/ideal body & $8(7,8)$ & $7(6,8)$ & $>0.05$ \\
\hline \multicolumn{4}{|l|}{ weight day $10(\mathrm{ml} / \mathrm{kg})$} \\
\hline $\mathrm{PaO2} / \mathrm{FiO} 2$ day 1 & $167(109,221)$ & $185(142,222)$ & $>0.05$ \\
\hline $\mathrm{PaO2} / \mathrm{FiO} 2$ day 5 & $183(128,240)$ & $190(147,232)$ & $>0.05$ \\
\hline $\mathrm{PaO2} / \mathrm{FiO2}$ day 10 & $205 \pm 79$ & $167 \pm 69$ & $<0.05$ \\
\hline $\begin{array}{l}\text { Driving pressure day } 1 \\
\text { (cmH2O) }\end{array}$ & $12(10,14)$ & $14(12,20)$ & $<0.05$ \\
\hline $\begin{array}{l}\text { Driving pressure day } 5 \\
(\mathrm{cmH2O})\end{array}$ & $12 \pm 3$ & $15 \pm 4$ & $<0.05$ \\
\hline $\begin{array}{l}\text { Driving pressure day } 10 \\
\text { (cmH2O) }\end{array}$ & $12(10,14)$ & $14(12,20)$ & $>0.05$ \\
\hline $\begin{array}{l}\text { Plateau pressure day } 1 \\
(\mathrm{cmH2O})\end{array}$ & $23 \pm 3$ & $26 \pm 4$ & $<0.05$ \\
\hline $\begin{array}{l}\text { Plateau pressure day } 5 \\
(\mathrm{cmH2O})\end{array}$ & $23 \pm 3$ & $25 \pm 3$ & $>0.05$ \\
\hline $\begin{array}{l}\text { Plateau pressure day } 10 \\
(\mathrm{cmH2O})\end{array}$ & $22 \pm 4$ & $23 \pm 7$ & $>0.05$ \\
\hline $\begin{array}{l}\text { Use of pre-intubation non- } \\
\text { invasive ventilation }(\%)\end{array}$ & 17 & 23 & $>0.05$ \\
\hline Extubation failure (\%) & 5 & 7 & $>0.05$ \\
\hline
\end{tabular}

Conclusion: Patients admitted to the extended-ICU had less mechanical ventilation free days.

Patients treated in extended-ICU had worse oxygenation, expressed as $\mathrm{PaO} 2 / \mathrm{FiO} 2$ ratio than patients treated in ICU.

Patients admitted to extended-ICU had higher driving pressure and higher plateau pressure during the first days of treatment, when compared to patients admitted to ICU.

There were no differences between groups in the use of pre-intubation non-invasive ventilation or in the extubation failure.

\section{Reference(s) and grant ackowledgment(s)}

1. 2. Maves RC, Jamros CM, Smith AG. Intensive care unit preparedness during pandemics and other biological threats. Crit Care Clin. 2019;35:609-618.
2. 1. Kelly M. Griffin,1 Maria G. Karas,2 Natalia S. Ivascu,3 and Lindsay Lief 1. Hospital Preparedness for COVID-19: A Practical Guide from a Critical Care Perspective. Am J Respir Crit Care Med. 2020 Jun 1; 201(11): 1337-1344.

\section{9}

Impact of Early Invasive Mechanical ventilation on Day 60 mortality for the COVID-19 patients with acute hypoxemic respiratory failure, a multicenter cohort study of the OUTCOMEREA Network

C. Dupuis ${ }^{1}$; E. de Montmollin²; D. Goldgran-Toledano ${ }^{3}$; C. Schwebel ${ }^{4}$; J. Reignier ${ }^{5}$; M. Neuville; U. Moreno $^{7}$; S. Siami ${ }^{8}$; S. Ruckly ${ }^{9}$; C. Alberti ${ }^{10}$; B. Mourvillier ${ }^{11}$; S. Bailly ${ }^{12}$; K. Grapin ${ }^{13}$; V. Laurent ${ }^{14}$; N. Buetti ${ }^{15}$; M. Gainnier ${ }^{16}$; B. Souweine ${ }^{13}$; JF. Timsit ${ }^{17}$

${ }^{1}$ Medecine Intensive Reanimation, CHU Gabriel-Montpied, Clermont-Ferrand, France; ${ }^{2}$ Medical and infectious Intensive Care Unit, Bichat-Claude Bernard Hospital, Paris, France; ${ }^{3}$ Médecine intensive et réanimation, Intercommunal Hospital Group Le Raincy Montfermeil, Montfermeil, France; ${ }^{4}$ Médecine intensive réanimation, C.H.U de Grenoble, La Tronche, France ${ }^{5}$ Médecine intensive réanimation, Nantes University Hospital Hotel-Dieu, Nantes, France; ${ }^{6}$ Réanimation, Hospital Foch, Suresnes, France; ${ }^{7}$ Inserm umrs 1138 team 22, Paris-Sorbonne University, Paris, France; ${ }^{8}$ Réanimation, Hospital Center Sud Essonne -, Étampes, France; ${ }^{9}$ Umr 1137, INSERM, Paris, France; ${ }^{10}$ Epidemiology, Assistance Publique - Hôpitaux de Paris, Hôpital Robert Debré, Paris, France; ${ }^{11}$ Médecine intensive et réanimation polyvalente, CHU Reims - Champagne Clinic, Reims, France; ${ }^{12}$ Laboratoire hp2, Chu Grenoble Universitaire, La Tronche, France; ${ }^{13}$ Médecine intensive et réanimation, CHU Gabriel-Montpied, Clermont-Ferrand, France; ${ }^{14}$ Réanimation, C.H. de Versailles, Le Chesnay, France; ${ }^{15}$ Inserm umr 1137, Paris Diderot University, Paris, France; ${ }^{16}$ Intensive care unit, Hospital Timone, Marseille, France; ${ }^{17}$ Réanimation médicale et infectieuse, Bichat-Claude Bernard Hospital, Paris, France

Correspondence: $C$. DUPUIS

Intensive Care Medicine Experimental 2020, 8(2): 001569

Introduction: Approximately 5\% of coronavirus disease 2019 (COVID19) are admitted to intensive care unit (ICU) for acute hypoxemic respiratory failure (AHRF). Whether invasive mechanical ventilation (IMV) should be used as first line therapy over non-invasive ventilation (NIV), high flow nasal cannula (HFNC), continuous positive airway pressure (CPAP) and oxygen by mask, is debated.

Objectives: The aim of the study was to assess a non-biased effect of Early-IMV in COVID induced AHRF on day- 60 mortality.

Methods: Data from consecutive COVID-19 patients admitted in 11 ICUs of the French OutcomeReaTM network were prospectively collected. Patients with AHRF $(\mathrm{PaO} 2 / \mathrm{FiO} 2<300 \mathrm{mmHg})$ within 2 days of ICU admission and not referred from another ICU were included. The study compared day- 60 mortality between patients who were administered IMV within 48 hours of ICU admission (Early IMV group) and patients who did not (non-Early IMV group). We used a Cox proportional-hazard model with ponderation with inverse probability of Early IMV weighting (IPTW) to model the risk of death at day 60 (HR > 1 in favor of death).

Results: Of the 306 included patients, the median age (IQR) age was of 61 (52-69) years, 241 were males (78.8\%), $175(57.2 \%)$ had at least one comorbidity; the median SAPSII and PaO2/FiO2 were $33(25-44)$ and 133 (96-186) $\mathrm{mmHg}$, repsectively. On admission, 105 (34.4\%) patients received IMV, 26 (8.4\%) NIV, 122 (39.8\%) HNFC, 21 (6.8\%) CPAP, and $32(10.4 \%)$ oxygen by mask. Ultimately, the Early and non-Early IMV groups included 149 and 157 patients; and Day-60 mortality was $40.9 \%$ and $22.9 \%$, respectively. Between the Early and non Early IMV group the rates of ICU-acquired pneumonia and bacteremia were 71 vs $29(P<0.01)$ and 15 vs $8(P<0.01)$; the median ICU length of stay 15 $(10-22)$ vs $8(5-15)$ days $(P<0.01)$, respectively. In the IPTW analysis, day- 60 mortality remained higher in the Early than in the non-Early IMV group (IPTWsHR=1.78, $\mathrm{Cl} 95 \%, 1.16$ to $2.71, \mathrm{p}<0.01$ ). Similar results were observed in a sensitive analysis using Multivariate Cox survival model (Table). 


\begin{tabular}{|llll|}
\hline Variables & HR & Cl 95\% & pvalue \\
\hline Early IMV & 1.67 & {$[1.04-2.67]$} & 0.03 \\
Time from symptoms to ICU admission & 0.75 & {$[0.48-1.19]$} & 0.22 \\
Chronic cardiac failure & 1.76 & {$[1.11-2.78]$} & 0.02 \\
Chronic respiratory failure & 2.06 & {$[1.19-3.59]$} & 0.01 \\
Chronic renal failure & 1.59 & {$[0.88-2.87]$} & 0.12 \\
Immunosuppression & 2.11 & {$[1.1-4.04]$} & 0.03 \\
Age (per 1 year increase) & 1.05 & {$[1.02-1.07]$} & $<0.01$ \\
SOFA without respiratory & 1.2 & {$[0.75-1.91]$} & 0.44 \\
$T^{\circ}>39^{\circ} \mathrm{C}$ & 1.81 & {$[1.15-2.85]$} & 0.01 \\
Lymphocytes < 800 & 1.4 & {$[0.9-2.17]$} & 0.13 \\
\hline IVV: Invasive mechanical ventilation; SOFA: SequentialOrgan Failure Assessment
\end{tabular}

Conclusion: In ICU patients admitted with COVID-19 -induced acute hypoxemic respiratory failure, Early-IMV increases day-60 mortality and should not be used as first line respiratory support.

\section{5}

Breath-synchronized electrical stimulation of the expiratory muscles in mechanically ventilated patients: a randomized controlled pilot study and pooled analysis

A. Jonkman 1;. Frenzel'; E. Mc Caughey³; A. Mclachlan; J. Schouten²; PR. Tuinman ${ }^{5}$; J. Butler ${ }^{3}$; L. Heunks ${ }^{6}$

${ }^{1}$ Intensive Care Volwassenen, Amsterdam UMC, locatie VUmc, Amsterdam, Netherlands; ${ }^{2}$ Intensive care, Radboud University Medical Center Nijmegen, Netherlands; ${ }^{3}$ Neuroscience research australia, NeuRA, Sydney, Australia; ${ }^{4}$., Liberate Medical, Crestwood, United States of America; ${ }^{5}$ Intensive care, Amsterdam UMC, locatie VUmc, Amsterdam, Netherlands; ${ }^{6}$ Intensive care volwassenen, Amsterdam UMC, Amsterdam, Netherlands Correspondence: A. Jonkman

Intensive Care Medicine Experimental 2020, 8(2): 001575

Introduction: Expiratory muscle weakness leads to difficult ventilator weaning. Maintaining their activity with functional electrical stimulation (FES) may therefore improve outcome. We studied feasibility and efficacy of breath-synchronized expiratory muscle FES in a mixed ICU ("Holland study") and pooled data with our previous work [1] ("Australian study") to analyze effects in a larger and more heterogeneous group.

Methods: Holland: Twenty patients with contractile response to FES received active or sham expiratory muscle FES (30-min, twice-daily, five days/week until weaned). Endpoints were respiratory muscle thickness, feasibility, and ventilation duration. Pooled: Data on clinical endpoints from the Holland and Australian studies were combined $(\mathrm{N}=40)$, including ventilation duration and ICU length-of-stay. Plasma cytokines (day 0,3 ) were analyzed to study the effects of expiratory muscle FES on systemic inflammation.

Results: Holland: 272 sessions were performed (sham/active: 103/169, compliance: 91.1\%). No FES-related serious adverse events were reported. Respiratory muscle thickness was not different over time and between groups. Pooled: At day 3, there was a between-group difference in total expiratory muscle thickness favoring the active group (treatment difference (95\% confidence interval); $2.25(0.34,4.16) \mathrm{mm}$, $P=0.02$ ). Plasma cytokine levels indicated that early FES did not induce systemic inflammation. Median ventilation duration and ICU length-of-stay were 10 vs. $52(P=0.07)$, and 12 vs. $54(P=0.03)$ days for the active vs. sham group. $3 / 20$ patients died in the active group, vs. $9 / 20$ in the sham group $(P=0.08)$.

Conclusion: Expiratory muscle FES is feasible in selected ICU patients. This might be a promising intervention within a respiratory muscleprotective ventilation strategy. A next step would be to test whether this approach could improve weaning outcome.

\section{Reference(s) and grant ackowledgment(s)}

1. [1] McCaughey EJ, Jonkman AH, Boswell-Ruys CL, McBain RA, Bye EA, Hudson AL, Collins DW, Heunks LMA, McLachlan AJ, Gandevia SC, Butler JE (2019) Abdominal functional electrical stimulation to assist ventilator weaning in critical illness: a double-blinded, randomised, sham-controlled pilot study. Crit Care 23 (1):261. https://doi.org/10.1186/s13054-019-2544-0. 2. The study was partly sponsored by a research grant from Liberate Medical.
001195

Influence of an expiratory brake on the expiratory impedance in a model of mild acute respiratory distress syndrome

M. Pellegrini ${ }^{1}$; PS. Mariotti ${ }^{2}$; A. Larina ${ }^{1}$; G. Perchiazzi ${ }^{1}$

${ }^{1}$ Department of surgical sciences, anesthesia and intensive care medicine, Uppsala University, Uppsala, Sweden; ${ }^{2}$ Medical school, University of Bari Aldo Moro, Bari, Italy

Correspondence: M. Pellegrini

Intensive Care Medicine Experimental 2020, 8(2): 001195

Introduction: Optimized external expiratory resistances improve lung mechanics, reduce tidal expiratory flow limitation (EFL) and prevent expiratory lung collapse in injured lungs (1).

EFL is associated with an increased mortality in acute respiratory distress syndrome (ARDS) patients $(2,3)$. To detect and avoid EFL can have important consequences on patients outcome.

Whether EFL can be detected by electrical impedance tomography is still unknown.

Objectives: EFL is expected to affect regional expiratory flow and consequently regional variation of expiratory electrical impedance. We hypothesized that changes in regional expiratory flow and EFL, consequent to different expiratory resistances and positive end-expiratory pressure (PEEP) levels, can be detected as changes in expiratory electrical impedance of the thorax.

Methods: Ten anesthetized and mechanically ventilated pigs underwent lung lavages to reach mild ARDS with a PO2/FIO2 ratio of $250 \mathrm{mmHg}$ at PEEP of $5 \mathrm{cmH} 2 \mathrm{O}$. During the experiment the pigs breathed spontaneously during continue positive airway pressure. Three PEEP levels $(12,3$ and $0 \mathrm{cmH} 2 \mathrm{O})$ and four external expiratory resistances were tested (labeled as $\mathrm{R} 0, \mathrm{R} 1, \mathrm{R} 2, \mathrm{R} 3$ ). The electrical impedance signal was simultaneously acquired. The impedance signal was analyzed breath-by-breath at 5 time-points of the expiratory phase (end-inspiration, first, second, third and fourth quartile of the expiration) and separately for dependent and not-dependent lung regions.

Results: In absence of additional expiratory resistance (R0), a significant difference in electrical impedance was observed in non-dependent lung regions at different PEEP levels. High PEEP corresponded to a slower decrease in impedance throughout expiration.

Applying additional expiratory resistances (R1-R3), the differences in electrical impedance related to PEEP disappeared and showed a trend closer to the one observed at high PEEP and RO.

Conclusion: A change in the time course of the expiratory impedance at PEEP $0 \mathrm{cmH} 2 \mathrm{O}$ was interpreted as sign of expiratory flow limitation. Addition of expiratory resistances contrasts the appearance of this pattern. Electrical impedance tomography can indirectly detect phenomena of expiratory flow limitation in injured lungs during spontaneously breathing ventilation.

\section{Reference(s) and grant ackowledgment(s)}

1. 1) Pellegrini M. et al. AJRCCM 2020 Mar 9. https://doi.org/10.1164/ rccm.201909-1690oc.

2. 2) Yonis H. et al. AJRCCM 2018; 198:131-134.

3. 3) Junhasavasdikul D. et al. Chest. 2018 Oct; 154(4):948-962.

4. European Society of Intensive Care Medicine grant (NEXT Grant Award 2017).

5. Swedish Heart-Lung Foundation (20170531).

\section{9}

A Novel System of "Split Ventilation" Using Electronically

Controlled Valves Can Be Used to Deliver Modified Lung

Protective Ventilation in a Porcine Model of Acute Respiratory

Distress Syndrome

J. Clarke1; P. Geoghegan '; G. Hogan'; A. Keogh'; H. Marsh'; G. Curley

${ }^{1}$ Department of anaesthesia and critical care medicine, Royal College

of Surgeons in Ireland, Beaumont Hospital, Dublin, Ireland

Correspondence: J. Clarke

Intensive Care Medicine Experimental 2020, 8(2): 001369

Introduction: During surges in demand for ventilator capacity, demand may outstrip supply. Split ventilation has been suggested as a 
temporising measure for individual hospitals to cope with such surges in demand. Optimising split ventilation may be important in the ongoing pandemic of SARS-CoV-2 and the associated high prevalence of acute respiratory distress syndrome. We developed a low cost electronically controlled system for delivering split ventilation, provisionally termed the Combiventilate system.

Objectives: 1) To demonstrate that an electronically controlled split ventilation system could be used to delivery independent volumecontrolled ventilation to two test lungs under conditions of varying resistance, compliance and leak. 2) To demonstrate that in a porcine model of acute respiratory distress syndrome, the Combiventilate system could deliver independent modified lung protective ventilation. 3) To show that in a porcine model of ARDS, markers of ventilator induced lung injury were similar in Cobiventilate ventilated animals and single ventilated animals. 4) To demonstrate that with minimal training, critical care staff could independently operate the Combiventilate system in simulated clinical scenarios.

Methods: We performed benchtop testing of the Combiventilate system using linear test lungs to ensure it's ability to provide differential ventilation and accommodate for changes in respiratory mechanics. We established a porcine model of acute respiratory distress syndrome by administering intrabronchial hydrochloric acid. We compared ventilation using the Combiventialte system to traditional individual ventilation in both injured and uninjured animals $(n=30)$. Groups were; individually ventilated uninjured $(n=5)$, individually ventilated injured $(n=5)$, Combiventilate managed uninjured $(n=10)$ and Combiventilate managed injured $(n=10)$.

We compared between group differences in the ability to deliver lung protective ventilation and meet gas exchange targets over a period of 6 hours of mechanical ventilation. We also compared immunological and histological markers of ventilator induced lung injury between groups. We performed benchtop simulations of clinical scenarios to verify that, with minimal training, clinical staff could set up and operate the Combiventilate system under conditions simulating common clinical scenarios.

Results: The Combiventilate system was able to deliver individualised volume-controlled ventilation in benchtop testing with linear test lungs. In a porcine model of ARDS, the Combiventilate system was able to facilitate similar adherence to modified lung protective ventilation as compared to individual traditional ventilation. Immunological and histological markers of ventilator induced lung injury were similar in the Combiventilate managed groups compared to the traditionally ventilated groups. In benchtop simulations, clinical staff were able to set up and operate the Combiventilate system with minimal training. Conclusion: Performance of the Combiventilate system in benchtop testing and in a large animal model of ARDS supports the ability to deliver modified lung protective ventilation. Similar degrees of ventilator induced lung injury as observed in the Combiventilate group as compared to the individually ventilated group. These data are promising and support further efforts to develop and investigate this system for use in humans as a temporising measure when surge demand exceeds capacity for indivualised ventilation.

\section{Reference(s) and grant ackowledgment(s)}

1. An Enterprise Ireland Innovation Partnership Feasibility Project grant was awarded to the group to perform this work.

\section{7}

Documentation of Plateau Pressures and the incidence of High Plateau Pressures in Mechanically Ventilated Patients with ARDS secondary to COVID-19

L. O'Gorman'; G. Murphy'; C. Power ${ }^{1}$

'Intensive care medicine, Tallaght University Hospital, Dublin, Ireland Correspondence: L. O'Gorman

Intensive Care Medicine Experimental 2020, 8(2): 001187

Introduction: Elevated Plateau Pressures in mechanically ventilated patients with ARDS have previously been shown to increase the time needed for liberation from mechaincal ventilation, increase the lenght of ICU stay and increase hospital mortality $(1,2)$. International guidelines for the management of ARDS, including ARDS secondary to COVID-19, reflect these findings and recommend maintaining plateau pressures of less than $30 \mathrm{cmH} 2 \mathrm{O}$ (3).

Objectives: 1. To assess how frequently Plateau Pressures were recorded in mechanically ventilated patients admitted with ARDS secondary to COVID-19.

2. To investigate the incidence of elevated plateau pressures in mechanically ventilated patients with ARDS secondary to COVID-19.

Methods: This was a retrospective audit of all patients with ARDS secondary to COVID-19 admitted to the ICU over a three month period (06/03/2020 - 06/06/2020) who required intubation and mechanical ventilation. Data was collected from patient's medical notes, including both paper charts as well as digital documentation. Data was also collected from patient's flow charts. Data was only collected on days during which the patient was mechanically ventilated on a Pressure Regulated Volume Control (PRVC), Pressure Control (PC) or Volume control (VC) mode of ventilation. Elevated plateau pressures were defined as a pressure greater than or equal to $30 \mathrm{cmH} 2 \mathrm{O}$.

Results: 24 patients were admitted to the ICU with ARDS secondary to COVID-19 during this time period. They spent a cumulative total of 428 days intubated and ventilated in ICU during this time. The total number of days spent on a PRVC, PC or VC mode of ventilation was 262. This was broken down as 250 days on PRVC, 8 days on PC and 4 days on VC. In total, plateau pressures were recorded on $47 \%$ of days while on a PRVC, PC or VC mode of ventilation (124 of the 262 days). There was a documented contraindication to recording plateau pressures on 2 out of the 262 days. Of the recorded plateau pressures, $29.8 \%$ were greater than or equal to $30 \mathrm{cmH} 20$ (37 of the 124 measurements).

Conclusion: We found that for mechanically ventilated patients with ARDS secondary to COVID-19, plateau pressures were not being measured routinely and were frequently found to be above the threshold limit recommended by international guidelines. This could have implications for patient's length of ICU stay, duration of mechanical ventilation as well as hospital mortality.

\section{Reference(s) and grant ackowledgment(s)}

1. 1. Yasuda H, Nishimura T, Kamo T, Sanui M, Nango E, Abe T, Takebayashi T, Lefor AK, Hashimoto S. Optimal plateau pressure for patients with acute respiratory distress syndrome: a protocol for a systematic review and metaanalysis with meta-regression. BMJ Open. 2017 May 29;7(5):e015091.

2. 2. Villar J, Martin-Rodriguez C, Dominguez-Berrot AM, Fernandez L, Ferrando C, Soler JA, et al. A quantile analysis of plateau and driving pressures: effects on mortality in patients with acute respiratory distress syndrome receiving lung-protective ventilation. Crit Care Med. 2017:45(5):843-50.

3. 3. (2020) 'Management of critical COVID-19: acute respiratory distress syndrome (ARDS)', in World Health Organization (ed.) Clinical Management of COVID-19, pp. 23.

\section{8}

Flexible Fiberoptic Bronchoscopy performed by intensive care physicians in patients under Invasive Mechanical Ventilation

O. Moreno Romero ${ }^{1}$; M. Muñoz Garach ${ }^{1}$; A. Carranza Pinel ${ }^{1}$

${ }^{1}$ Intensive care unit, Hospital Universitario Clínico San Cecilio, Granada, Spain

Correspondence: $\mathrm{O}$. Moreno Romero

Intensive Care Medicine Experimental 2020, 8(2): 001278

Introduction: Flexible fiberoptic bronchoscopy (FOB) has become an important tool for the diagnostic and management of complications in critically ill ventilated patients, specially since ICU physicians can performe this technique with low incidence of complications.

Objectives: First, to describe the etiology and complications of performing a flexible fiberoptic bronchoscopy (FOB) by intensive care physicians in patients under invasive mechanical ventilation (IMV) in our intensive care unit. Secondly, to check whether there was a significant modification of treatment due to the results of FOB.

Methods: Retrospective study in a 24 month period (from january 2018 until december 2019) of all FOB performed by intensive care physicians in our patients under IMV. Variables analyzed: data of patients 
(sex, age, APACHE II), cause for IMV (primary respiratory, septic shock, cardiovascular, neurological), cause for implementing the bronchoscopy (atelectasis in US, suspected orotracheal tube obstruction, evaluation of tracheostomy, suspected tumor, diagnostic bronchoalveolar lavage), complications, change in treatment.

Results: In 24 months we performed 167 FOB in 397 patients under IMV. Male 55\%. Mean age $58 \pm 16,3$ y. Mean APACHE II $19 \pm 8$. Cause of IMV: primary respiratory 135 (34\%), septic shock 118 (30\%), neurological $45(11,3 \%)$, cardiovascular $44(10 \%)$, other $55(13,7 \%)$. Causes: atelectasis $48(28,7 \%)$, orotracheal tube obstruction $11(6,6 \%)$, evaluation of tracheostomy $25(15 \%)$, suspected tumor $7(4,2 \%)$, diagnostic BAL $76(45,5 \%)$. Complications: mild haemorrage $16(9,6 \%)$, supraventricular tachicardya $8(4,8 \%)$, transient hypoxemia $12(7 \%)$, transient bronchospasm $8(4,8 \%)$. A significative change in treatment was implemented in 56 (33\%). No mortality related to FOB.

Conclusion: The performing of flexible bronchoscopy in patients under IMV by intensive care physicians is a safe technic in our units. Complications are mild and in aproximately $25 \%$ of patients. A change in treatment was made in $33 \%$, mostly due to microbiological findings in BAL, diagnostic of tumors and limitation of therapeutic effort.

\section{7}

Contrafluran as an alternative scavenger for NO and NO2 in ICU

R. Knafelj ${ }^{1}$; V. Z. '; A. Kokove

${ }^{1}$ Univesity medical center ljubljana, MICU, Ljubljana, Slovenia

Correspondence: R. Knafelj

Intensive Care Medicine Experimental 2020, 8(2): 001327

Introduction: Inhaled Nitric oxide (iNO) is used during mechanical ventilation as salvage therapy for refractory hypoxemia in ARDS, as (selective) pulmonary vasodilator in pulmonary hypertension and in right heart failure. At therapeutic levels for adults (up to $30 \mathrm{ppm}$ ) residual NO and its metabolite NO2 are released in the surrounding from the ventilator expiratory port and must be scavenged. With prolonged use ( $>12 \mathrm{~h}$ ) dedicated NO/NO2 scavenger filter causes additional resistance due to moisture accumulation and leads to expiratory flow limitation. Sevoflurane demonstrates numerous advantages over intravenous sedation and is preferred sedation agent in our ICU.

Objectives: Since volatile agents used for sedation in ICU also require scavenging we tested NO scavenging capacity of commonly used Contrafluran ${ }^{\circledR}$ filter.

Methods: Breathing circuit (Elisa 800VIT, Löwenstein Medical, Germany) with NO delivery/measurement system (NO-A, EKU Elektron$\mathrm{ikm} \mathrm{GmbH}$ ), Anaconda (Sedana Medical, Sweden) and Contrafluran (ZeoSys Medical, $\mathrm{GmbH}$ ) was assembled according to manufacturer's recommendation and hospital standards. All measurements were performed during volume control ventilation (Vt $450 \mathrm{~mL}$, FiO2 0.5, PEEP 10 , RR $20 / \mathrm{min}$ ) first without sevoflurane co-administration and then with sevoflurane at a rate of $10 \mathrm{~mL} / \mathrm{h}$. In 20 benchmark tests concentrations of $\mathrm{NO}$ and $\mathrm{NO} 2$ were measured in different parts of the breathing circuit (Figure 1). Statistical analysis was performed using Student t-test (Numbers, MacIntosh).

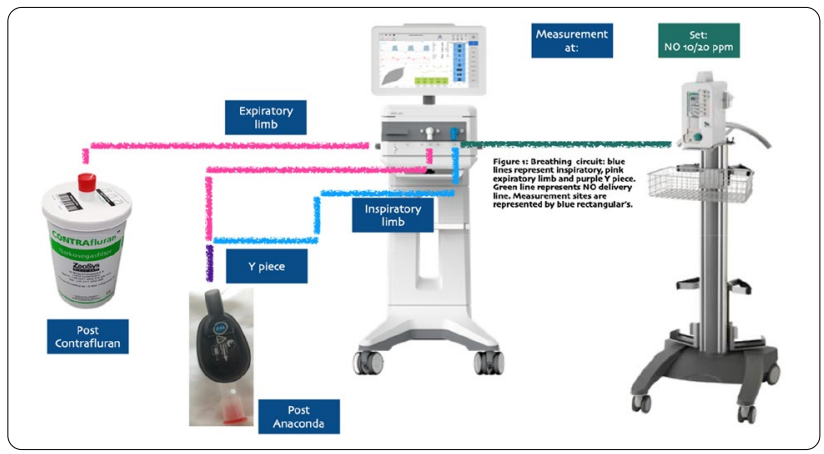

Results: In table:

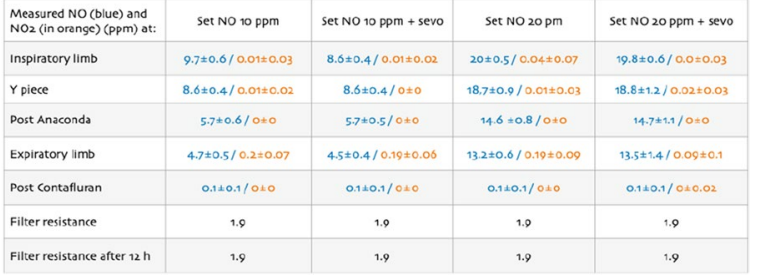

Conclusion: iNO concentration varies within the breathing circuit as a consequence of a Flow-by, mixture of inspired and expired gasses at $Y$ piece and scavenging effect of Anaconda. Special caution must be practiced when interpreting the results of measured iNO values. Addition of sevoflurane does not interfere with $\mathrm{NO} / \mathrm{NO} 2$ measurements. Contrafluran filter is an excellent alternative to standard scavenger filter for $\mathrm{NO} / \mathrm{NO} 2$, scavenging $>99.91 \% \mathrm{NO}$ and $100 \% \mathrm{NO} 2$ at 10 and $20 \mathrm{ppm}$ respectively. There was no increase in respiratory system resistance and intrinsic PEEP formation over time, while using Contrafluran filter.

Reference(s) and grant ackowledgment(s)

1. Goslar T, Knafelj R. Does anaconda scavenge iNO? Mechanical ventilation during CPR. 37th International Symposium on Intensive Care and Emergency Medicine (part 2 of 3).Critical Care 2017, 21 (Suppl 1):57; P276. R. Knafelj, P. Kordis. Co-administration of nitric oxide and sevoflurane using anaconda. P374 Critical Care201620(Suppl 2):94.

2. None.

001392

Implementation of protective lung ventilation and its association with mortality, Focus on driving pressure

Y. Michael ${ }^{1}$; Z. Goldik'; A. Eden

${ }^{1}$ Department of anesthesiology, critical care \& pain medicine, Lady Davis Carmel Medical Center, Haifa, Israel

Correspondence: $Y$. Michael

Intensive Care Medicine Experimental 2020, 8(2): 001392

Introduction: Lung protective ventilation (LPV) strategies should guide ventilatory care throughout the ICU stay. We evaluated whether mechanical ventilation followed LPV guidelines throughout the mechanical ventilation period, with emphasis on the driving pressure $(\Delta \mathrm{P})$ and their association with 28 and 90 day mortality.

Methods: Retrospective study included 124 patients admitted to ICU in the Lady Davis Carmel medical center, Haifa, Israel, during 2019, who were mechanically ventilated for at least 48 hours. Ventilatory data was collected every 10 minutes for up to 28 days of mechanical ventilation in the ICU. We investigated the degree of compliance with the LPV guidelines and its association with mortality at 28 and 90 day post discharge.

Results: Analysis of the data per patient (mean $\pm \mathrm{SD}$ ) revealed $\Delta \mathrm{P}$ of $15.5 \pm 3.6 \mathrm{cmH} 2 \mathrm{O}, \mathrm{Vt}$ of $7.6 \pm 1.4 \mathrm{ml} / \mathrm{kgpbw}$ and end inspiratory pressure of $23.2 \pm 4.5 \mathrm{cmH} 2 \mathrm{O}$. The median prevalence per patient of $\Delta \mathrm{P}>14 \mathrm{cmH} 2 \mathrm{O}$ was $58 \%$ of their ventilation period, $\mathrm{Vt}>8 \mathrm{ml} / \mathrm{Kgpbw}$ was $38 \%$ and of end inspiratory pressure $>30 \mathrm{cmH} 2 \mathrm{O}$ was $2 \%$ of the ventilation period. Fifty-one patients $(41 \%)$ died within 28 day and 56 patients $(45.2 \%)$ within 90 day post discharge. Mean $\Delta \mathrm{P}$ and amount of time spent with $\Delta \mathrm{P}>14 \mathrm{cmH} 2 \mathrm{O}$ were significantly correlated with mortality at 28 day post discharge from ICU: $\mathrm{OR}=1.13,95 \% \mathrm{Cl}(1.02-$ 1.26), $\mathrm{p}=0.021$; and, $\mathrm{OR}=1.02,95 \% \mathrm{Cl}(1.003-1.03), \mathrm{p}=0.015$ respectively. At 90 day, the time spent with DP $>14 \mathrm{cmH} 2 \mathrm{O}$ (more than $50 \%$ of measurements) was associated with increased mortality $\mathrm{OR}=2.58$ $95 \% \mathrm{Cl}(1.2-5.4), \mathrm{p}=0.012$, but the mean $\Delta \mathrm{P}$ was not. An increase in the frequency of elevated $\Delta \mathrm{P}$ (quartiles) was associated with increased mortality (figure). No correlation was found between either Vt or end inspiratory pressure and mortality. 


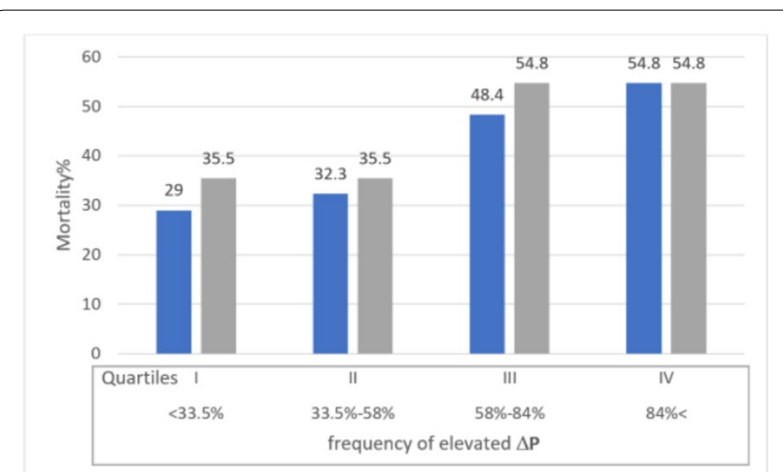

Figure: Mortality in relation to the frequency of measurements of elevated DP. Blue bars 28 day mortality $(\mathrm{P}=0.018)$ and Grey bars 90 day mortality $(\mathrm{P}=0.054)$

Conclusion: LPV was not always implemented, it is especially shown with the high frequency of elevated $\Delta \mathrm{P}$ values. $\Delta \mathrm{P}$ was significantly associated with mortality regardless of whether the patient had ARDS or not. To validate these results, further studies are needed.

Reference(s) and grant ackowledgment(s)

1. 4. Amato, M. B. P. et al. Driving pressure and survival in the acute respiratory distress syndrome. N. Engl. J. Med. 372, 747-55 (2015).

\section{6}

PRONE VENTILATION IN POSITIVE PATIENTS FOR SARS-CoV2

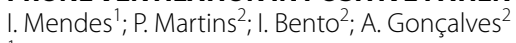

${ }^{1}$ Medicina interna, Hospital Beatriz Angelo, Loures, Portugal; ${ }^{2}$ Medicina intensiva, Hospital Beatriz Ângelo, Loures, Portugal

Correspondence: P. Martins

Intensive Care Medicine Experimental 2020, 8(2): 001476

Introduction: The COVID-19 pandemic has seen a surge of patients with acute respiratory distress syndrome (ARDS) in intensive care units. Although early reports suggested that COVID-19-associated ARDS has distinctive features that set it apart from historical ARDS, emerging evidence indicates that the respiratory system mechanics of patients with ARDS, with or without COVID-19, are broadly similar.1 Prevalence of ARDS among COVID-19 patients has been reported to be up to 17\%.2 Among the introduced treatment methods for management of ARDS patients, prone position can be used as an adjuvant therapy for improving ventilation in these patients.3.

Objectives: The aim of this study was to describe our experience with prone position in SARS-CoV2 positive patients. We also compared the patients who survived with those who died to understand if there were any 28-day mortality predictors.

Methods: We retrospectively studied the patients who tested positive for SARS-CoV2 admitted in Hospital Beatriz Ângelo (HBA) ICU from April to June of 2020. Demographic data, comorbidities, severity scoring scales, $\mathrm{PaO} 2 / \mathrm{FiO} 2$, days on mechanical ventilation, number and hours of prone position, complications, days at Intensive Care Unit and deaths were extracted from medical records and were submitted to statistic description and analysis.

Results: There were 62 patients positive for SARS-CoV2 admitted in HBA ICU from April to June of 2020, and 39 were mechanically ventilated. Twenty patients (32\%) were placed in prone position, $65 \%$ were males. The mean age was 63 years old (minimum 31 and maximum 83). In relation to the severity criteria, the medium APACHE II score was 13 points and SAPS II was 37 points.
Half of the patients (10) registered a severe ARDS (P/F $\leq 100)$ when admitted in the ICU and had had symptoms for 8 days before IOT. In six patients there was a HNFC trial, without success.

These 20 patients fulfilled on average 5 periods of prone ventilation and the mean of prone ventilation hours were 94 . The patients were ventilated for 32 hours on average before prone position. The first prone position lasted a mean of 20 hours.

These patients were intubated on average 15 days. Fifty percent were extubated for NIV and in 5 patients was performed a tracheostomy. There were complications like accidental extubation (2), exteriorization of a central venous catheter (1), facial edema (1) and pressure ulcers (5).

These patients have been in the ICU for 20 days on average, with a 20 percent mortality rate in the ICU plus $5 \%$ in the hospital $<28$ days.

When we compare the patients that survived with those who died, the latter were older (60 years vs 71), had worse SAPS II (34 vs 46) and APACHE II (12 vs 16), more days of symptoms until intubated (7 vs 12) and hours of ventilation until prone position (25 vs 53 ). On the other hand, we found that patients that survived had an inferior initial $p / f$ ratio (118 vs 170).

Conclusion: Even before SARS-CoV2 pandemic we knew ARDS is an inflammatory process, with long hospitalization and significant mortality. From our experience, this study seems to show that the sooner the patients are intubated and placed in prone position, the best the outcome. Further research is needed.

\section{Reference(s) and grant ackowledgment(s)}

1. 3. Ghelichkhani P, Esmaeili M. Prone Position in Management of COVID-19 Patients; a Commentary. Arch Acad Emerg Med. 2020; 8(1): e48.

2. 2. Chen $N$, Zhou M, Dong Xuan et al Epidemiological and clinical characteristics of 99 cases of 2019 novel coronavirus pneumonia in Wuhan, China: a descriptive study. Lancet 2020; 395: 507-13.

3. 1. Fan E, Beitler JR, Brochard L et al. COVID-19-associated acute respiratory distress syndrome: is a different approach to management warranted? Lancet Respir Med 2020, July 6.

\section{0}

Can lung protective ventilation with lower driving pressures lead to better survival in COVID 19 related ARDS ? A prospective single center observational cohort from Turkey

C. Kirakli'; B. Acar Çinleti ${ }^{1}$; S. Yildirim ${ }^{1}$; S. Saygili ${ }^{1}$; E. Senel ${ }^{1}$; Ö. Ediboğlu ${ }^{1}$

${ }^{1}$ Intensive Care Unit, University of Health Sciences, Dr. Suat Seren Chest

Diseases and, Surgery Training Hospital, Izmir, Turkey

Correspondence: C. Kirakli

Intensive Care Medicine Experimental 2020, 8(2): 001490

Introduction: The main respiratory dynamics of invasively ventilated COVID-19 patients are still not well known.

Objectives: In this prospective cohort we aimed to observe the characteristics of the respiratory system of COVID-19 patients under invasive mechanical ventilation and evaluate their relationship with mortality.

Methods: Patients admitted to our ICU with COVID-19 (confirmed SARS-CoV-2 infection in nasal or pharyngeal swab specimens) and invasively ventilated for more than 24 hours were enrolled. Demographics and laboratory values were recorded at baseline. Respiratory variables such as tidal volume, plateau pressure, PEEP, static compliance, driving pressure were recorded daily under passive conditions and median values were analyzed. Data are expressed as median (IQR) of numbers (\%).

Results: 48 patients ( 33 male) were enrolled between 01 March 2020 and 01 July 2020. All patients had ARDS. Median age was 64 years (5872). Mortality was $60 \%$ at day 28 . Survivors were significantly younger than nonsurvivors. Plateau pressure, driving pressure and static compliance significantly differ between survivors and nonsurvivors. Other laboratory values were comparable. (Table 1). 


\begin{tabular}{|c|l|l|l|}
\hline \multicolumn{4}{|c|}{ Characteristics of patients } \\
\hline Variable & \multicolumn{1}{|l|}{ Nonsurvivors } & \multicolumn{1}{|c|}{ Survivors } & \multicolumn{1}{|c|}{ p value } \\
\hline Age, years & $66(62-73)$ & $61(46-67)$ & $\mathbf{0 . 0 2 1}$ \\
\hline APACHE II & $21(15-33)$ & $19(11-21)$ & 0.06 \\
\hline SAPS & $53(38-79)$ & $47(40-56)$ & 0.26 \\
\hline PaO2/FiO2 & $140(96-190)$ & $136(92-229)$ & 0.84 \\
\hline Vt/kg, ml & $8(7-8)$ & $7(6-9)$ & 1 \\
\hline Pplateau, cmH2O & $31(28-33)$ & $28(24-30)$ & $\mathbf{0 . 0 1}$ \\
\hline PEEP, cmH2O & $11(8-14)$ & $10(9-12)$ & 0.73 \\
\hline Pdriving. $\mathbf{c m H 2 O}$ & $20(14-23)$ & $15(12-18)$ & $\mathbf{0 . 0 0 8}$ \\
\hline Cst, $\mathbf{m l} / \mathbf{c m H 2 O}$ & $29(21-36)$ & $37(30-50)$ & $\mathbf{0 . 0 0 8}$ \\
\hline
\end{tabular}

Conclusion: COVID 19 related ARDS seems to have similar characteristics as other forms of ARDS. Lung protective ventilation with low plateau and driving pressures may be related with lower mortality.

\section{7}

Prediction model of endotracheal obstruction, in patients with severe pneumonia due to COVID 19, analyzing ventilatory parameters using intelligent computation

G. Pérez-Acosta'; J. Navarro-Mesa ${ }^{2}$; L. Santana-Cabrera' ; J. Blanco-Lopez C. Suárez-Araujo ${ }^{3}$; J. Martín-González

'ICU, C.H.U. Insular-Materno Infantil, Las Palmas de Gran Canaria, Spain; ${ }^{2}$ Idetic, Universidad de las Palmas de Gran Canaria, Las Palmas de Gran Canaria, Spain; ${ }^{3}$ luctc, Universidad de las Palmas de Gran Canaria, Las Palmas de Gran Canaria, Spain

Correspondence: $\mathrm{G}$. Pérez-Acosta

Intensive Care Medicine Experimental 2020, 8(2): 001617

Introduction: Patients admitted to the ICU with severe COVID-19 pneumonia present clinical complications during their admission, such as endotracheal obstruction due to accumulation of secretions. This problem often requires urgent endotracheal tube change and manual ventilation, both of which are health risk procedures for the generation of aerosols and constitute a potential life-threatening risk for the patient.

The model based on Machine Learning $(\mathrm{ML})$ techniques and intelligent computing, using the signals produced by the respirators, constitute fundamental tools for the extraction of common patterns related to obstruction, and for the creation of prognostic and therapeutic prediction systems.

Objectives: To study the possibility of predicting the appearance of endotracheal obstruction in patients with COVID-19 pneumonia, using neuronal computing techniques, integrating the different respiratory variables during hospital admission into a model.

Generate a predictive model that follows the time course of patients with COVID-19 pneumonia undergoing mechanical ventilation and predict the appearance of endotracheal obstruction well in advance.

Methods: Descriptive, observational study using data mining in 22 patients with Pneumonia due to COVID-19, where 16 of them presented an episode of endotracheal obstruction during their admission to the IMU. Compilation of the respiratory variables Peak Pressure, Plateau Pressure, Medium Pressure, Resistance and Compliance, collected at least every 15 minutes during the entire intensive care admission. The recording of signals during the hours prior to the development of the obstruction is studied, representing the signals collected synchronously and in parallel. To develop a neural computing model, using data mining techniques, to analyze the existence of specific temporal patterns that help early detection of endotracheal obstruction, hours before it occurs.

Results: Based on the designed predictive model, changes in the respiratory mechanical signals collected by the system during admission to the ICU that precede the development of endotracheal obstruction are detected, with an average anticipation of 47 hours.

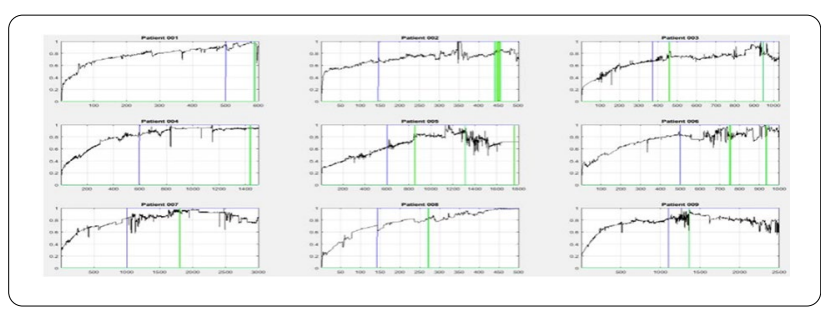

The model's output (black line) provides information about lung mechanics, from patient admission to endotracheal obstruction (green line). Image 1 shows common patterns of changes in lung mechanics prior to obstruction. The blue line indicates the start of abnormal changes in the registry, thus indicating the start time of the alert against possible obstruction. In the studied patients, changes in the pulmonary mechanics are observed with an average 47 hours before the episode of endotracheal obstruction.

Conclusion: We demonstrate that the application of computational intelligence techniques and data mining on lung mechanical signals studied, show promising results in the early detection of endotracheal obstruction.

This is of great value for the prevention of this complication during ICU admission, being useful in reducing the mortality of COVID-19 patients and reducing biosanitary risk.

\section{Reference(s) and grant ackowledgment(s)}

1. Clinical course and outcomes of critically ill patients with SARS-CoV-2 pneumonia in Wuhan, China: a single-centered, retrospective, observational study. Yang X, Yu Y, Xu J, et al. Clinical course and outcomes of critically ill patients with SARS-CoV-2 pneumonia in Wuhan, China: a single-centered, retrospective, observational study SO. Lancet. 2020

2. Pérez Acosta G, Santana L Traqueobronquitis necrotizante con obstrucción del tubo endotraqueal en pacientes COVID-19. Revista Clínica Española. DOI: 10.1016/j.rce.2020.05.002

3. Ian Goodfellow, Yoshua Bengio, Aaron Courville. "Deep Learning". MIT Press. 2016. http://www.deeplearningbook.org.

4. Amisha, Paras Malik, Monika Pathania, and Vyas Kumar Rathau. Overview of artificial intelligence in medicine. J Family Med Prim Care. 2019 Jul; 8(7): 2328-2331. https://doi.org/10.4103/jfmpc.jfmpc_440_1.

5. None.

\section{3}

Prediction model of endotracheal obstruction, in patients with severe pneumonia due to COVID 19, analyzing clinical parameters using intelligent computing

G. Pérez-Acosta ${ }^{1}$; J. Navarro-Mesa ${ }^{2}$; J. Blanco-Lopez ; C. Suárez-Araujo ${ }^{3}$; L. Santana-Cabrera'; J. Martín-González ${ }^{1}$

${ }^{1}$ ICU, C.H.U. Insular-Materno Infantil, Las Palmas de Gran Canaria, Spain; ${ }^{2}$ Idetic, Universidad de las Palmas de Gran Canaria, Las Palmas de Gran Canaria, Spain; ${ }^{3}$ luctc, Universidad de las Palmas de Gran Canaria, Las Palmas de Gran Canaria, Spain

Correspondence: G. Pérez-Acosta

Intensive Care Medicine Experimental 2020, 8(2): 001633

Introduction: The current COVID-19 pandemic has caused a high percentage of patients who require critical management due to acute hypoxemic respiratory failure with the need for mechanical ventilation. This situation implies a high vital risk in addition to possible respiratory complications such as obstruction of the endotracheal tube.

This complication occurs suddenly from the first week of admission, it is a medical emergency due to the impossibility of ventilation of the patient and implies a high risk of contagion for the health workers due to exposure to aerosols.

Data mining and Artificial Intelligence (Al) techniques are a fundamental tool to create patient profiles, study their common characteristics and help predict the evolutionary development of this disease.

Objectives: Design and develop a computational predictive model to study the evolution of patients with COVID-19 pneumonia with 
mechanical ventilation. Analyzing the clinical variables that help predict the development of endotracheal obstruction over time.

Methods: We recorded the clinical variables (heart rate, systolic blood pressure, diastolic pressure, mean pressure, oxygen saturation, and temperature) of all patients at least every 15 minutes during the entire intensive care admission. The variables were The set of variables were studied, represented in graphs synchronously and in parallel.

Through data mining and the development of neural networks, a computational model was generated that helps early detection of endotracheal obstruction.

Results: In our unit, of the 22 patients with COVID-19 Pneumonia who required mechanical ventilation, $72.7 \%$ had at least one episode of endotracheal obstruction. $100 \%$ of these events took place after 7 days of admission.

The predictive model detects changes in the clinical variables studied that precede the endotracheal obstruction with an average advance of 42 hours.

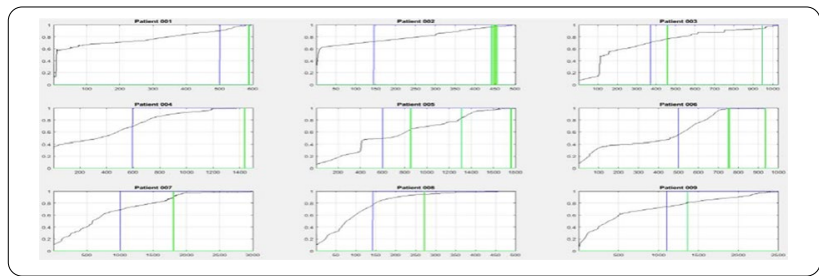

In the Image 1, the changes observed in the clinical variables precede the development of endotracheal obstruction (green line). It shows common patterns of disturbances in the studied signals prior to obstruction. The blue line shows the start of abnormal registry changes indicating the time.

In the studied patients, clinical changes are observed with an average 42 hours before the endotracheal obstruction.

Conclusion: The use of signal recording of clinical variables, studied techniques using data mining and RNN techniques, show promising results in the early detection of endotracheal obstruction, being of great value for the prevention of this complication during ICU admission, reducing mortality of the Covid-19 patients as well as the risk for the health workers.

\section{Reference(s) and grant ackowledgment(s)}

1. Trevor Hastie, Robert Tibshirani, Jerome Friedman. The Elements of Statistical Learning Data Mining, Inference, and Prediction. Springer Series in Statistics book series (SSS). 2009. DOI https://doi.org/10.1007/978-0-387-84858 $-7$.

2. Ian Goodfellow, Yoshua Bengio, Aaron Courville. "Deep Learning". MIT Press. 2016. http://www.deeplearningbook.org.

3. Pérez Acosta G, Santana L Traqueobronquitis necrotizante con obstrucción del tubo endotraqueal en pacientes COVID-19. Revista Clínica Española. DOI: 10.1016/.r.re.2020.05.002

4. Clinical course and outcomes of critically ill patients with SARS-CoV-2 pneumonia in Wuhan, China: a single-centered, retrospective, observational study. Yang X, Yu Y, Xu J, et al. Clinical course and outcomes of critically ill patients with SARS-CoV-2 pneumonia in Wuhan, China: a single-centered, retrospective, observational study SO. Lancet. 2020

5. None.

\section{4}

CPAP in prehospital patients with dyspnoea: implications during COVID-19 pandemic in Portugal. Analysis of an ambulance staffed nurse

P. Vasconcelos ${ }^{1}$; J. Lourenço' ; LM. Ladeira'; F. Barros' ${ }^{1}$; S. Rodeia ${ }^{1}$; A.

Correia'; S. Nelson²; F. Rato

'Departamento de emergência médica, Instituto Nacional de Emergência Médica, Lisboa, Portugal; ${ }^{2}$ Serviço de anestesiologia, Hospital Professor Doutor Fernando da Fonseca, E.P.E, Amadora, Portugal

Correspondence: J. Lourenço

Intensive Care Medicine Experimental 2020, 8(2): 001214
Introduction: Continuous Positive Air Pressure (CPAP) treatment is used when approaching patients with dyspnoea in the prehospital (PH) setting1. Despite being one of the best treatment strategies for this clinical condition, CPAP is an aerosol generating procedure. Therefore brought insecurity to the emergency PH teams and challenges to maintain its application. In order to promote its safe use the Department of Emergency Medicine (DEM) of the National Institute for Medical Emergency (INEM), modified the procedure promoting security.

Objectives: To analyse prehospital CPAP use in two homologous periods, before and during the COVID-19 pandemic.

Methods: A case series of CPAP used by Lisbon Immediate Life Support ambulance (SIV- ambulance staffed nurse), before and during COVID-19 pandemic will be compared. For this purpose, patients records between 1st of March and 30th July 2019 and the same period in 2020 were extracted from the ITEAMS clinical record (INEM Tool for Emergency Alert System), concerning the application of dyspnoea protocol3,4 where CPAP is recommended.

Results: Between 1st of March and 30th July 2019, dyspnoea protocol was applied in 121 patients, resulting in 11 uses of CPAP $(9 \%)$. For the same period in 2020 (COVID-19 pandemic period), dyspnoea protocol was applied in 80 patients, resulting in 4 CPAP applications (5\%). However, it should be considered that at the beginning of the pandemic in Portugal the use of CPAP was discouraged. Only after 28th of April 2020, when a safer method of CPAP was suggested, nurses felt comfortable and secure to use this treatment.

Between 1st of May and 30th July 2020, dyspnoea protocol was applied to 39 patients, resulting in 3 CPAP uses $(7.7 \%)$, compared to the same period in 2019 when dyspnoea protocol was applied in 66 patients, resulting in 4 CPAP uses (6\%).

Conclusion: The use of CPAP in PH is essential to maintain quality of care for patients with dyspnoea. Its use was decreased at the beginning of the pandemic in Portugal but measures were implemented promoting the safe use of CPAP. The results demonstrate the maintenance and even an increase in the use of CPAP compared to the same period in 2019 (7.7\% in 2020 vs 6\% in 2019). Until now, no staff member of the SIV Lisboa ambulance had COVID-19. This analysis refers to one out of the $40 \mathrm{SIV}$ ambulances with nurses in Portugal, which is not sufficient to national extrapolation of data.

\section{Reference(s) and grant ackowledgment(s)}

1. 1. Schwerin DL, Goldstein S. EMS, Prehospital CPAP Devices. StatPearls Publ. 2019. https://www.ncbi.nlm.nih.gov/books/NBK470429/. Accessed July 23, 2020

2. 2. Departamento de Emergência Médica/Instituto Nacional de Emergência Médica. Orientação Técnica 11/2020 DEM: Oxigenoterapia, Suporte Respiratório e SAV, Em Pandemia COVID-19.

3. 3. Departamento de Emergência Médica/Instituto Nacional de Emergência Médica. Protocolo Ambulância SIV: Dispneia.; 2018.

4. 4. Departamento de Emergência Médica/Instituto Nacional de Emergência Médica. Protocolo Ambulância SIV: Dispneia Em Pandemia COVID-19.; 2020.

5. 5: Departamento de Emergência Médica/Instituto Nacional de Emergência Médica. Orientação Técnica 03/2020 DEM: Atuação Perante Suspeita de Doença COVID-19.

001261

Predicting the response to high flow nasal cannula as first-line respiratory support in the patients with pneumonia covid-19 and acute respiratory failure

M. De Alba Aparicio ${ }^{1}$; S. Carcel Fernandez ${ }^{1}$; C. López Martin ${ }^{1}$; M. Porras Pantojo ${ }^{1}$; C. De La Fuente Martos ${ }^{1}$; JM. Serrano Simón ${ }^{1}$

${ }^{1}$ Intensive Care Unit, Hospital Universitario Reina Sofia, Córdoba, Spain

Correspondence: M. De Alba Aparicio

Intensive Care Medicine Experimental 2020, 8(2): 001261

Introduction: Experience from the use of high-flow nasal oxygen (HFO) in acute hypoxemic respiratory failure (AHRF) (1), indicates that this method of respiratory support may reduce the invasive mechanical ventilation requirement of patients with COVID-19, depending on the degree of $\mathrm{V} / \mathrm{Q}$ matching, and may be an appropriate therapy when conventional oxygen therapy does not provide sufficient respiratory 
support. However, its application is controversial, particularly for the risk of aerosolization.

Objectives: To describe the characteristics and identify prognostic factors in response to the application of HFO as first line of respiratory support of patients Covid-19 with AHFR.

Methods: Analysis of prospectively collected data of 41 patients with pneumonia Covid-19, confirmed by RT-PCR assay, admitted to our polyvalent ICU of terciary hospital (34 bed), from 22 March to 5 May 2020. Patients with moderate AHRF (PaO2/FiO2 100-200 at admission) were included. Excluded patients were $<18$, immediate mechanical ventilation (MV) criteria, hypercapnia or given $\mathrm{HFO}$ as a tool to weaning from MV. The HFO was applied using Philips $\mathrm{V}^{\circ} 0^{\circledR}$ ventilator and Fisher \& Paykel ${ }^{\circledR}$ MR850 device, flow 40-60 L/min, FiO2 for SpO2 $\geq$ $92 \%$. We compared baseline characteristics, physiological, prognosis parameters, and Rox index in the first $12 \mathrm{~h}$, as ratio of $\mathrm{SpO} 2 / \mathrm{FiO} 2$ to respiratory rate (2), according to success or failure HFO. For statistical analysis we used U-Mann-Whitney or Fisher test as appropriate. Prognosis of success of HFO was calculated as sensitivity and specificity, relative risk (RR) $(95 \% \mathrm{Cl})$ and $\mathrm{ROC}$ curves for ROX index. The literature determines the cut-off point at $12 \mathrm{~h}$ at 4,88 .

Results: Among 41 patients with AHRF Covid-19 admitted to the ICU, $20(48.78 \%)$ were intubated on admission. Altogether 21 (51.22\%) patients were eligible for HFO, 15 (71.43\%), were defined as success which was regarded as no escalation to non-invasive ventilation, MV ECMO or death within 28 days after commencement of HFO. Characteristics patients are shown in Table 1, the two group were well matched, only SOFA scores shown significant difference in failure HFO. Overall ICU mortality: 11 (26.83\%), MV vs HFO: 8 (19.5\%) vs $3(7.3 \%)$ $(p=0.015)$. Parameters related to outcome see Table 2 . ROC analysis for ROX index showed an AUC of 0.889 ( $95 \% \mathrm{Cl} 0.697-0.988)$. A cutoff $\geq 5.57$ was associated with the best index for success, sensivity of 0.93 ( $95 \% \mathrm{Cl} 0.75-0.91)$, and specificity of 0.83 ( $95 \% \mathrm{Cl} 0.41-0.98)$, PPV of 0.94 and NPV 0.83. RR 6 (95\% Cl 1.47-24.42).

\begin{tabular}{|c|c|c|c|c|c|}
\hline \multicolumn{2}{|l|}{ Parameter } & $\begin{array}{l}\text { Overall } \\
(\mathrm{N}=21)\end{array}$ & Success ( $N=15)$ & Failure $(N=6)$ & $P$ \\
\hline \multirow{2}{*}{\multicolumn{2}{|c|}{$\begin{array}{l}\text { Age, years } \\
\text { Sex. Male, } N(\%)\end{array}$}} & $61(48.5-73)$ & $54(39.75-67)$ & $62(50-73)$ & 0.761 \\
\hline & & $12(93 \%)$ & $9(75 \%)$ & $3(25 \%)$ & 0.523 \\
\hline \multicolumn{2}{|c|}{ Body mass index, $\mathrm{kg} / \mathrm{m}^{2}$} & $\begin{array}{l}29.38(26.70- \\
30.99)\end{array}$ & $\begin{array}{l}29.38(26.73- \\
31.14)\end{array}$ & $\begin{array}{l}28.71(25.02- \\
33.48)\end{array}$ & 0.785 \\
\hline \multicolumn{2}{|c|}{ APACHE II score } & $10(7.5-15)$ & $11(6.75-12.75)$ & $\begin{array}{l}12.5(7.75- \\
21.25)\end{array}$ & 0.319 \\
\hline \multicolumn{2}{|c|}{$\begin{array}{l}\text { Sequential Organ Failure } \\
\text { Assessment score }\end{array}$} & $3(3-4)$ & $3(2-4)$ & $6(3.75-7)$ & $0.006^{*}$ \\
\hline \multicolumn{2}{|c|}{$\begin{array}{l}\text { Time between 1st symptoms and } \\
\text { ICU admission, days }\end{array}$} & $14(9-23)$ & $15(10-24)$ & $\begin{array}{l}10.50(1.75- \\
17)\end{array}$ & 0.149 \\
\hline \multicolumn{2}{|c|}{$\begin{array}{l}\text { Time between PCR and ICU } \\
\text { admission, day }\end{array}$} & $5(2-7)$ & $5(1.5-7)$ & $4(2-8.5)$ & 0.965 \\
\hline \multicolumn{2}{|c|}{$\mathrm{PaO}_{2} / \mathrm{FiO}_{2}, \mathrm{mmHg}$} & $155.67 \pm 45.65$ & $162.99 \pm 36.85$ & $139.80 \pm 61.65$ & 0.253 \\
\hline \multirow{2}{*}{\multicolumn{2}{|c|}{$\begin{array}{l}\mathrm{PCO}_{2}, \mathrm{mmHg} \\
\mathrm{Aa}-\mathrm{DO}_{2}, \mathrm{mmHg}\end{array}$}} & $39.67 \pm 6.44$ & $39.38 \pm 5.47$ & $40.40 \pm 9.23$ & 0.826 \\
\hline & & $\begin{array}{l}372.8(319.63- \\
381.24)\end{array}$ & $\begin{array}{l}\begin{array}{l}371.55(312.13- \\
379.05)\end{array} \\
\end{array}$ & $\begin{array}{l}377.8(366.55- \\
423.45)\end{array}$ & 0.167 \\
\hline \multirow[t]{2}{*}{$\begin{array}{l}\text { Main } \\
\text { Comorbidities }\end{array}$} & \begin{tabular}{|l|} 
Arterial \\
hypertension
\end{tabular} & $11(57,9 \%)$ & $8(42,1 \%)$ & $3(15,8 \%)$ & 0.506 \\
\hline & \begin{tabular}{|l} 
Respiratory \\
\end{tabular} & $5(26.3 \%)$ & $3(15.8 \%)$ & $2(10,5 \%)$ & 0.520 \\
\hline
\end{tabular}

Table 2. Outcome of the 21 patients covid-19 with HFO.

\begin{tabular}{|l|l|l|l|l|}
\hline Parameter & Overall $(\mathrm{N}=21)$ & Success $(\mathrm{N}=15)$ & Failure $(\mathrm{N}=6)$ & $\mathrm{P}$ \\
\hline Duration HNFO, days & $3(2-5)$ & $4(3-5)$ & $2(1.75-2.25)$ & $0.007^{*}$ \\
\hline Duration of stay ICU, days & $6(3-9)$ & $4(3-7)$ & $8.75(6.75-18.75)$ & $0.026^{*}$ \\
\hline ICU mortality, $\mathrm{n}(\%)$ & $3(14.3 \%)$ & 0 & $3(50 \%)$ & $0.025^{*}$ \\
\hline Rox index 12h & $6.60(5.48-9.85)$ & $7.84(6.0-10.89)$ & $4.88(3.53-6.48)$ & $0.007^{*}$ \\
\hline
\end{tabular}

Conclusion: In our sample of Covid-19 patients, the Rox index at $12 \mathrm{~h}$ allowed us to predict with accuracy the response to HFO with a cut value of 5.57, similar to the cut value describe in the literature for other causes of moderate AHRF. SOFA index value could be helpful in selecting the patients who could benefit from HFO. Careful selection of patients to whom HFO is applied as first-line treatment is necessary, since those who fail and delay intubation have high mortality and higher stay in ICU. Data need to be confirmed in wider population.

Reference(s) and grant ackowledgment(s)

1. 1. Frat JP, et al. High-flow oxygen through nasal cannula in acute hypoxemic respiratory failure. N Engl J Med. 2015.
2. 2. Roca O, Messika J, Caralt B, García-de-Acilu M, Sztrymf B, Ricard JD, Masclans JR. Predicting success of high-flow nasal cannula in pneumonia patients with hypoxemic respiratory failure: The utility of the ROX index. Journal of Critical Care 35 (2016) 200-205.

001277

High-flow nasal cannula as a resource on treatment of acute hypoxemic respiratory failure in COVID-19

S. Lafayette'; A. Rosa'; P. Azevedo'; C. Gomes'; M. Knibel'² WT. Nakasato ${ }^{3}$ ${ }^{1}$ Physiotherapy, Hospital São Lucas Copacabana, Rio de Janeiro, Brazil; ${ }^{2}$ Education center, Hospital São Lucas Copacabana, Rio de Janeiro, Brazil ${ }^{3}$ Medical director, Hospital São Lucas Copacabana, Rio de Janeiro, Brazil

Correspondence: A. Rosa

Intensive Care Medicine Experimental 2020, 8(2): 001277

Introduction: COVID-19 pandemic is an international health problem. Declared a worldwide pandemic by World Health Organization (WHO) in February 2020, the main complication causes severe acute respiratory syndrome (SARS-CoV-2) progressing to hypoxemia, requiring high oxygen fractions and invasive ventilation. The growing number of patients requiring invasive ventilatory support and the limited number of mechanical ventilator overload of the health systems. Alternative ventilatory support strategies are need for these individuals with COVID-19.

High-flow nasal cannula (HFNC) get attention as an alternative resource of ventilatory support for patients with severe hypoxemia.

Objectives: The primary outcome this study was to evaluate the proportion of patients that HFNC avoided tracheal intubation.

Methods: We realized a retrospective observational study of the patients that received HFNC as ventilatory support for treatment of acute respiratory failure because COVID-19.

Results: This study analyzed a total of 16 patients who used high flow nasal cannula (HFNC) for treatment of hypoxemic acute respiratory failure on patients with COVID-19, between the patients, we analyzed a subgroup of 5 patients who received combined therapy. For the purposes of this we classified combined therapy as HFNC and noninvasive ventilation (NIV). The rate of success for avoid tracheal intubation was $69 \%$ on HFNC group and $100 \%$ on combined therapy group, the average time of use of therapy was 4 days versus 6 days respectively, we also analyzed the time of duration on hours that was $92,8(S D \pm 64,7)$ hours versus 134,4 (SD $\pm 84,2$ ) hours respectively. This study verified that the most of patients presented the worst radiologic pattern but doesn't seem influenced on outcome.

Conclusion: High-flow nasal cannula was feasible, and it seems to be an efficient in avoid tracheal intubation in patients with COVID-19. The effect of HFNC in COVID-19 survival should be further investigated in a large prospective study.

Reference(s) and grant ackowledgment(s)

1. Nishimura M (2019) High-Flow Nasal Cannula Oxygen Therapy Devices. Respir Care 64:735-742. https://doi.org/10.4187/respcare.06718.

2. Drake MG (2018) High-Flow Nasal Cannula Oxygen in Adults: An Evidence-based Assessment. Ann Am Thorac Soc 15:145-155. https://doi. org/10.1513/AnnalsATS.201707-548FR.

3. Agarwal A, Basmaji J, Muttalib F, et al (2020) High-flow nasal cannula for acute hypoxemic respiratory failure in patients with COVID-19: systematic reviews of effectiveness and its risks of aerosolization, dispersion, and infection transmission. Can J Anesth/J Can Anesth. https://doi.org/10.1007/ s12630-020-01740-2.

4. Lai C-C, Shih T-P, Ko W-C, et al (2020) Severe acute respiratory syndrome coronavirus 2 (SARS-CoV-2) and coronavirus disease-2019 (COVID-19): The epidemic and the challenges. Int J Antimicrob Agents 55:105924. https:// doi.org/10.1016/j.ijantimicag.2020.105924.

5. Thanks, the physiotherapy team at the São Lucas Copacabana Hospital - Rio de Janeiro - Brazil, we worked bravely and tirelessly to promote recover of patients with COVID-19. 


\section{9}

Face mask CPAP to Treat Acute Hypoxemic Respiratory Failure in COVID-19 Patients: characteristics and outcomes

K. Belkhouja'; W. Algarni ${ }^{1}$; A. Alansari ${ }^{1}$; B. Aljedaani ${ }^{1}$; A. Hassan ${ }^{1}$; M.

Darwish ${ }^{1}$ : S. Omer ${ }^{1}$;H. Al-Sharif

${ }^{1}$ Intensive care department, King Fahad Armed Forces Hospital, Jeddah, Saudi Arabia

\section{Correspondence: K. Belkhouja}

Intensive Care Medicine Experimental 2020, 8(2): 001399

Introduction: Acute hypoxemic respiratory failure and ARDS are common in COVID-19. The benefit of continuous positive airway pressure (CPAP), and associated risks of viral transmission through aerosolization, are unclear. Discouraging noninvasive ventilation (NIV) in the COVID-19 patient may increase the need for intubation and induce increased morbidity and mortality and led to rapidly saturation of resources and the availability of ICU bed. Some studies suggest that helmet CPAP can represent an effective alternative to improve the hypoxemia and decrease the risk of droplets' air-dispersion.

Objectives: To evaluate, the safety and efficacy of face mask CPAP in COVID-19 patients with acute hypoxemic respiratory failure.

Methods: Retrospective descriptive observational cohort study was conducted, from Mars 25, 2020, to July 28, 2020, in King Fahad armed forces hospital, Kingdom of Saudi Arabia. We included all adult patients with confirmed COVID-19 pneumonia and developed acute hypoxemic respiratory failure defined by $\mathrm{PaO} 2 / \mathrm{FiO} 2$ less than $300 \mathrm{mmHg}$ and required CPAP. We collected baseline data on demographics, clinical presentation, laboratory, arterial blood gas, ventilation setting, and the outcomes, mainly the rate of tracheal intubation, the rate of Health care workers (HCWs) contaminated by SARS-CoV2, the length of stay and the rate of mortality. The failure of CPAP was defined by the requirement of tracheal intubation. We determined by multivariate analysis, the independent predictive factors of CPAP failure.

Results: Of the 86 patients included in the study, the median (IQR) age was 62 (54-70) years and 58 (67.3\%) were male. 78 patients (90.7\%) had at least one comorbidity. The median (IQR) of SOFA score and APACHE II score were 4 (3-5) and 9 (7-13) respectively. The median time from first symptom to CPAP was 7.0 days. The median level of CPAP and $\mathrm{FiO} 2$ were $10 \mathrm{cmH} 2 \mathrm{O}$ and 0.5 , respectively. The median (IQR) $\mathrm{PaO} 2 / \mathrm{FiO} 2$ was 143 (120-169) and 13 patients $(15.1 \%)$ had severe acute respiratory distress syndrome. The prone position with CPAP was performed in 31 patients (36\%). The methylprednisolone was administered to 70 patients (81.4\%) and empiric antibiotics to 83 patients (96.5\%). The rate of failure of CPAP was $17.4 \%$ (15 patients) among them 3 patients was intubated within the first 24 hours of admission. The SOFA score at admission more than 4 [OR, $4.63 ; 95 \% \mathrm{Cl}, 1.11-$ 19.33; $\mathrm{P}=0.035$ ) and $\mathrm{PaO} 2 / \mathrm{FiO} 2$ at admission less than $140 \mathrm{mmHg}$ $(\mathrm{OR}, 11.22 ; 95 \% \mathrm{Cl}, 2.44-51.61 ; \mathrm{P}=0.002)$, were independent risk factors of CPAP failure whereas the proning during CPAP (OR, 0.067; 95\% $\mathrm{Cl}, 0.01-0.41 ; \mathrm{P}=0.004$ ) was a protective factor. One nurse caring for a patient was infected with SARS-CoV2. The median (IQR) length of stay was 9 (7-12) days and 6 patients (7\%) had died.

Conclusion: The face mask CPAP as respiratory support for COVID-19 pneumonia with acute hypoxemic respiratory failure, is effective and safe. The rate of CPAP failure is less than $18 \%$ and the 3 predictive factors of failure were, SOFA score at admission, $\mathrm{PaO} 2 / \mathrm{FiO} 2$ at admission and the use of prone position during CPAP.

\section{Reference(s) and grant ackowledgment(s)}

1. We are indebted to the doctors, nurses and respiratory therapists with the Intensive Care Medicine Service who provides care for the patients included in the study and to all the frontline staff of COVID-19 patients.
001495

CPAP helmet vs Invasive respiratory support in COVID-19 acute respiratory distress syndrome in elder patients: a monocentric retrospective study

M. Napolitani ${ }^{1}$; F. Stefanini ${ }^{2}$; L. Bastia ${ }^{2}$; S. Russo ${ }^{3}$; I. Cuevas Cairo ${ }^{4}$; A. Pezzi ${ }^{5}$

${ }^{1}$ Department of neurointensive care, Niguarda Hospital, ASST Grande ospedale metropolitani, Milan, Italy; ${ }^{2}$ Department of intensive care, Niguarda Hospital, ASST Grande ospedale metropolitano, Milano, Italy; ${ }^{3}$ Department of biomedical and clinical science "I.sacco", University of Milan, Milano, Italy; ${ }^{4}$ Department of intensive care, San Carlo Borromeo Hospital, Milano, Italy; ${ }^{5}$ Department of intensive care, Ospedale Bassini, Cinisello Balsamo, Italy

Correspondence: M. Napolitani

Intensive Care Medicine Experimental 2020, 8(2): 001495

Introduction: The recent COVID-19 pandemic has been dramatically aggressive in the region of Lombardy: the overwhelming number of patients who needed intensive care during the peak of the health emergency, exceeded the available resources. This crisis led to a revision of the usual admission criteria to the ICU: patients with a higher expectation of survival had priority access to ICU. Outside the ICU, the highest ventilatory care was CPAP helmet (up to $12 \mathrm{cmH} 20$ PEEP and FiO2 0.8). Looking backward to those daunting weeks, we noticed some anecdotal cases who survived in medical wards despite severe COVID acute distress respiratory syndrome (C-ARDS) without mechanical ventilation (MV).

Objectives: Hence, we retrospectively compared mortality rates between elder C-ARDS patients treated with MV vs CPAP helmet.

Methods: We retrospectively analyzed the outcomes of patients with severe C-ARDS. Two groups were made: first, CPAP group with elder patients (age 70-79) treated with CPAP helmet (with no access to ICU) with a refractory hypoxemia $(P / F<150$ for more than $48 \mathrm{~h}$ regardless the administration of CPAP helmet); second MV groups, made of patients assisted with an endotracheal tube (ETT) and ventilated with positive pressures, aged 55-69, and elder ones, aged 70 -78. Additionally, we collected the maximal d-dimer values per each patient before and after the placement of the ETT.

Results: We hospitalized 493 patients with covid respiratory distress syndrome. CPAP group $(n=22)$ had a lethality rate of $50 \%$. The lethality of the MV group aged 55-69 $(n=31)$ was $52 \%$, while about the elder CMV group $(n=13) 77 \%$ died. We found a significant increase ( $p$ value $<0.05$ ) in plasmatic d-dimer after CMV begun: mean values pre - ETT $(n=15) 8362 \mu \mathrm{g} / \mathrm{dl}(\mathrm{Cl} \pm 5987)$; mean values post - EET $(\mathrm{n}=30)$ $21846 \mu \mathrm{g} / \mathrm{dl}(\mathrm{Cl} \pm 10781)$.

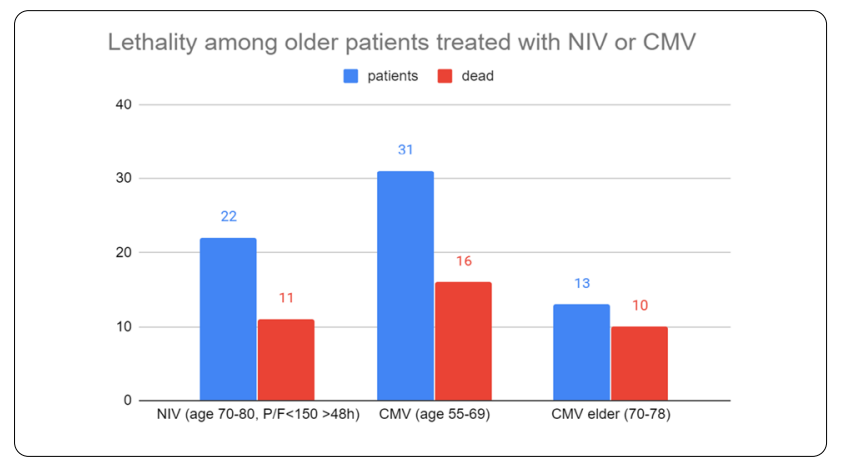

Conclusion: Among patients with C-ARDS, we found only a slight difference in mortality between the population admitted to ICU (receiving $\mathrm{MV}$ ) and the population not admitted (receiving CPAP helmet). Considering the thrombophilic condition caused by SARS COV2 infection, we raise the question if an aggressive invasive respiratory approach is advisable compared to a more gradual approach. However, we can not exclude that, in spite of similar hypoxemia, the ICU patients had a more severe clinical picture liable for the worse outcome. We acknowledge the limitations of our sample, but our results are interesting in light of a "less is more" strategy. 
001578

The impact of different levels of non-invasive ventilation on hemodynamics in healthy subjects

D. Vanhonacker ${ }^{1}$; J. Jonckheer ${ }^{1}$; E. De Waele

${ }^{1}$ Intensive care, UZ Brussel, Jette, Belgium

Correspondence: D. Vanhonacker

Intensive Care Medicine Experimental 2020, 8(2): 001578

Introduction: Non-invasive ventilation (NIV) consists of respiratory support without the need for intubation. NIV has been used in a plethora of clinical situations such as acute pulmonary oedema, acute respiratory failure in patients with chronic pulmonary disease and obstructive sleep apnoea (1).

The application of positive pressure in the thoracic cavity has both positive and negative hemodynamic effects. It impairs the venous return to the right heart and the blood flow to the pulmonary arteries (reduced RV preload, increased afterload) but aids the flow of blood to and from the left ventricle (increased LV preload, decreased afterload) (2).

Objectives: The aim of this pilot trial is to investigate the impact of different levels of NIV on hemodynamics in a group of 5 healthy subjects. Methods: The study protocol was approved by the ethical committee of UZ Brussel (B.U.N. 143201940980) and registered at clinicaltrials.gov (NCT04178694).

NIV (V60, Philips) was administered with an oro-nasal mask at an expiratory positive airway pressure (EPAP) of $4 \mathrm{cmH} 2 \mathrm{O}$ and a pressure support (PS) of $0 \mathrm{cmH} 2 \mathrm{O}$. The subjects were allowed to adapt to the treatment for 5 minutes. Then, PS was gradually increased by $2 \mathrm{cmH} 2 \mathrm{O}$ every 2 minutes until an inspiratory positive airway pressure (IPAP) of $12 \mathrm{cmH} 2 \mathrm{O}$ had been reached and held for another 2 minutes.

Non-invasive hemodynamic monitoring (Nexfin ${ }^{\circledR}$ ) was used to monitor a range of hemodynamic parameters.

We analysed our data by simple linear regression with Graphpad Prism ${ }^{\circledR}$.

Results: Although the IPAP increased, the heart rate $(\mathrm{HR})$ of the test subjects did not significantly rise (slope $0.189 ; R^{2} 0.57 ; p$ 0.085). However, the diastolic blood pressure (DBP) significantly rose (slope 0.507 ; $\mathrm{R}^{2}$ 0.68; $\mathrm{p}$ 0.043), the systolic (SBP) and mean arterial blood pressure (MAP) also went up but not significantly (slope $0.832 ; R^{2} 0.60 ; p 0.070$ and slope $\left.0.536 ; R^{2} 0.65 ; p 0.054\right)$.

The stroke volume index (SVI) dropped significantly at higher IPAP levels (slope -0.164; $\left.R^{2} 0.82 ; p 0.014\right)$, the cardiac index $(\mathrm{Cl})$ diminished slightly but the change was not significant (slope $-0.005 ; R^{2} 0.52 ; p$ 0.108).

Both the systemic vascular resistance index (SVRI), a surrogate for afterload, and the $\mathrm{dP} / \mathrm{dt}$, which is a measure for LV contractility as it represents the ratio of pressure change in the LV during the isovolumetric contraction period, increased but not by a statistically significant amount (slope 20.42; $R^{2} 0.65 ;$ p 0.053 and slope $13.44 ; R^{2} 0.65 ; p$ $0.051)$.

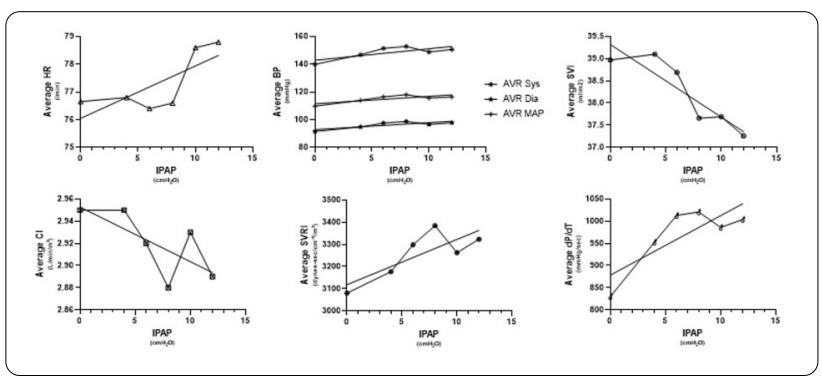

Conclusion: This pilot trial suggests that NIV induces hemodynamic changes in healthy volunteers. These changes appear to be related to the amount of IPAP applied.

Following a decline in venous return, a decreased stroke volume and cardiac output may trigger baroreceptors in the cardiovascular system. The activation of these receptors could augment sympathetic tone, resulting in a blood pressure rise, an increase in HR, SVR and contractility.

A follow-up trial on a larger population is needed to confirm and validate these results.

Reference(s) and grant ackowledgment(s)

1. 2. Luecke, T. and Pelosi, P. (2005). Clinical review: Positive end-expiratory pressure and cardiac output. Critical Care, 9(6), p.607.

2. 1. Cross, A.M. (2012). Non-invasive ventilation in critical care. Internal Medicine Journal, 42, pp.35-40.

001595

\section{CPAP for COVID - is it beneficial?}

Z. Campbell ${ }^{\text {; }}$ L. Alvarez-Belon²; L. Bullman; N. Boyer ${ }^{1}$; R. Pierce ; D.

Cottam ${ }^{1}$; M. Cox ${ }^{3}$; J. Jones ${ }^{3}$; M. Zuleika ${ }^{1}$; E. Potter ${ }^{4}$; L. Forni ${ }^{2}$

${ }^{1}$ Intensive care unit, Royal Surrey County Hospital, Guildford, United Kingdom; Intensive care, Royal Surrey County Hospital, Guildford, United Kingdom; ${ }^{3}$ Medicine, Royal Surrey County Hospital, Guildford, United Kingdom; ${ }^{4}$ Intensive Care Unit, Royal Surrey County Hospital, Guildford, France

Correspondence: E. Potter

Intensive Care Medicine Experimental 2020, 8(2): 001595

Introduction: The optimal management strategy for respiratory support in covid-19 remains unclear. A low threshold for invasive ventilation in patients with moderate to severe respiratory failure has been advocated by some. The use of NIV in MERS, a similar coronavirus, had a high risk of failure. In addition, the hypothetical risk of patient selfinflicted lung injury (PILI) raised concerns that CPAP may be detrimental in Covid-19. However, use of CPAP avoids associated morbidity of invasive ventilation and is less burdensome on healthcare resources.

Objectives: Primary: To establish the success of CPAP for respiratory failure secondary to covid-19; defined as avoidance of invasive ventilation, liberation from CPAP and discharge from hospital in those appropriate for escalation of care.

Secondary: 1. To establish incidence of intubation and ventilation following CPAP and compare mortality rates for patients for those that were invasively ventilated on admission to ICU compared to those that had a period of CPAP prior to invasive ventilation. 2. To identify associated factors of those patients on CPAP that required intubation and ventilation.

Methods: A single centre retrospective observational study of all patients managed with CPAP for respiratory failure secondary to Covid-19 from 10th March to 21st May 2020. Patients positive for SARS CoV2 RNA and those with a clinical diagnosis of covid requiring respiratory support were included. Further respiratory parameters were collected from a subset of 45 patients admitted between 16th March to the 3rd April 2020.

Results: 37 of 61 patients (60\%) who received CPAP and were appropriate for escalation of care were managed successfully on CPAP alone. No patients that were appropriate for escalation died without escalation of care. Of those that required invasive ventilation $(n=24)$ mortality rates in those that had prolonged CPAP prior to invasive ventilation was not increased compared to those intubated immediately. In those where CPAP was the ceiling of care, outcome was poor; mortality rate $58 \%$ (19 of 33$)$.

Table 1: Outcome of patients suitable for escalation who received CPAP

\begin{tabular}{|l|l|}
\hline Outcome & Survival n (\%) \\
\hline CPAP only, $\mathbf{n = 3 7}$ & $37(100 \%)$ \\
\hline $\begin{array}{l}\text { Invasive ventilation following }<\mathbf{4 8} \mathbf{~ h r} \\
\text { CPAP, } \mathbf{n = 8}\end{array}$ & $3(38 \%)$ \\
\hline $\begin{array}{l}\text { Invasive ventilation following }>\mathbf{4 8 h r} \\
\text { but }<\text { 7 days of CPAP, } \mathbf{n = 1 1}\end{array}$ & $5(45 \%)$ \\
\hline $\begin{array}{l}\text { Invasive ventilation following }>\mathbf{7} \\
\text { days of CPAP, } \mathbf{n = 5}\end{array}$ & $4(80 \%)$ \\
\hline $\begin{array}{l}\text { Immediate invasive ventilation } \\
\text { without CPAP, } \mathbf{n}=\mathbf{8}\end{array}$ & $5(63 \%)$ \\
\hline $\begin{array}{l}\text { All patients managed initially with } \\
\text { CPAP who were appropriate for } \\
\text { escalation, } \mathbf{n = 6 1}\end{array}$ & $54(89 \%)$ \\
\hline
\end{tabular}


Table 2: Respiratory parameters of those who progressed to invasive ventilation compared to those that were managed successfully on CPAP alone.

$\mathrm{SF}=$ oxygen saturations $/$ fraction inspired oxygen. $\mathrm{ROX}=$ ratio of pulse oximetry/FIO2 to respiratory rate

\begin{tabular}{|c|c|c|c|}
\hline Variable & $\begin{array}{l}\begin{array}{l}\text { Invasive } \\
\text { ventilation }\end{array} \\
\text { Median [IQR] }\end{array}$ & $\begin{array}{l}\text { Successful } \\
\text { CPAP } \\
\text { Median [IQR] }\end{array}$ & p (Wilcox) \\
\hline SF pre CPAP & 120 [116-152] & 153 [120-160] & 0.08 \\
\hline ROX index pre CPAP & $5.28[3.54-6.22]$ & $5.94[5.32-7.15]$ & 0.12 \\
\hline $\begin{array}{l}\text { Change in SF during } \\
\text { CPAP }\end{array}$ & $30[9.58-55.88]$ & $\begin{array}{l}40.83[9.17- \\
49.93]\end{array}$ & 0.94 \\
\hline $\begin{array}{l}\text { Change in ROX during } \\
\text { CPAP }\end{array}$ & $1.2[-0.16-1.83]$ & $1.8[0.35-4.16]$ & 0.23 \\
\hline Days on CPAP & $1.5[1-3.5]$ & 3 [1.25-5.75] & 0.23 \\
\hline
\end{tabular}

$S: F$ ratios and ROX index were significantly lower in patients trialled on CPAP that went on to require invasive ventilation.

Conclusion: Patients with Covid-19 that present with respiratory failure could be managed with an initial trial of CPAP in a well controlled and monitored area. It is likely that a third of these will progress to needing invasive ventilation, but two thirds are likely to escape the need for intubation and ventilation. This has a tremendous impact on resource allocation, ICU bed days, length of stay, and complications associated with intubation and ventilation. SF ratio and ROX index may play a role in differentiating between patients that are likely to improve with CPAP alone and those that will require invasive ventilation.

\section{Reference(s) and grant ackowledgment(s)}

1. Gattinoni L, Chiumello D, Rossi S. COVID-19 pneumonia: ARDS or not? Crit Care 2020;24:154

2. Alraddadi BM, Qushmaq I, Al-Hameed FM et al. Noninvasive ventilation in critically ill patients with Middle East respiratory syndrome. Influenza Other Respir Viruses. 2019 Jul;13(4):382-390. Remove [x].

3. Roca O, Messika J, Caralt B, Garcia-de-Acilu M, Sztrymf B, Ricard JD, et al. Predicting success of high-flow nasal cannula in pneumonia patients with hypoxemic respiratory failure: the utility of the ROX index. J Crit Care. 2016; $35: 200-5$

\section{1}

Non-invasive positive pressure ventilation in COVID-19 respiratory failure: a single-center experience

M. Ferreira'; F. Kikuta'; R. Silva1; P. Rezende ${ }^{1}$; D. Novaes ${ }^{1}$; C. Dias ${ }^{1}$; R. Ferreira $^{2}$; J. Ramalho ${ }^{\text {; }}$ B. Villarinho ${ }^{1}$; R. Lima

${ }^{1}$ Intensive care unit, Hospital Samaritano, Rio de Janeiro, Brazil; ${ }^{2}$ Cardiology, Hospital Samaritano, Rio de Janeiro, Brazil

Correspondence: R. Ferreira

Intensive Care Medicine Experimental 2020, 8(2): 001601

Introduction: Available data suggests that $5-20 \%$ of patients with Coronavirus Disease-2019 (COVID-19) develop acute hypoxemic respiratory failure (AHRF) (1). Guidelines have given a weak recommendation for non-invasive positive pressure ventilation (NIPPV) as a preventive strategy for avoiding intubation in this scenario (2). As such, the procedure should only be considered in selected COVID-19 patients with AHRF and without major organ dysfunction.

Objectives: To assess the effect of NIPPV on the rates of invasive mechanical ventilation (IMV) and in-hospital mortality in a series of patients with COVID-19 and AHRF.

Methods: Consecutive patients from a single center with confirmed COVID-19 admitted between March 1st and July 20th, 2020 were retrospectively analyzed. Patients were stratified according to an 8-point ordinal scale recommended by the World Health Organization for use in clinical research among hospitalized patients with COVID-19, and only those with severe disease (score $\geq 5$ ) were included (3). Clinical variables and the respiratory support strategy were correlated to IMV requirement and in-hospital mortality. Categorical data were expressed by percentages and continuous variables were represented by the median with the 25 th to 75 th percentile interquartile range (IQR). Multivariate logistic regression models were performed to determine independent associations. A p value $<0.05$ was considered significant.

Results: Nine two patients were included, of which $61.9 \%$ were male, with a median age of 72 years (IQR 60.5-84). Significant comorbidities were hypertension (48.9\%), diabetes $(25 \%)$, cardiovascular disease (CVD) $(10 \%)$ and obesity (10\%). Sixty nine (75\%) patients received NIPPV for a median of 5 days (IQR 1-13.5), of which $44(63.8 \%)$ did not require IMV. NIPPV was significantly associated with a lower frequency of IMV $(36.2 \%$ vs $87 \%, p<0.001)$, even when adjusted for age, sex, hypertension, diabetes, obesity and underlying cardiovascular disease (OR 0.08 [95\% Cl 0.02-0.3], $\mathrm{p}<0.001$ ). The overall mortality rate was $32.6 \%(n=30)$, and was lower in those treated with NIPPV $(26.1 \%$ vs $52.2 \%, p=0.02)$. In the multivariate analysis, NIPPV remained associated with lower mortality (OR 0.25 [95\% Cl 0.08-0.84], p<0.001), whereas age $\geq 75$ years was predictive of death (OR $12.2[95 \% \mathrm{Cl} 3.4-$ 43.1], $\mathrm{p}<0.001)$.

Conclusion: In a series of patients with COVID-19 and AHRF, NIPPV was significantly associated with a lower frequency of IMV and in-hospital mortality. Identifying patients who may benefit from NIPPV and determining predictors of failure from the intervention are key factors to adequately allocate resources and improve clinical outcomes.

Reference(s) and grant ackowledgment(s)

1. 1. Cummings $M$, Baldwin M, Abrams D, Jacobson S, Meyer B, Balough E et al. Epi-demiology, clinical course, and outcomes of critically ill adults with COVID-19 in New York City: a prospective cohort study. The Lancet. 2020;395(10239):1763-1770

2. 2. Rochwerg B, Brochard L, Elliott M, Hess D, Hill N, Nava S et al. Official ERS/ ATS clinical practice guidelines: noninvasive ventilation for acute respiratory failure. Eu-ropean Respiratory Journal. 2017:50(2):1602426.

3. 3. [Internet]. Who.int. 2020 [cited 30 July 2020]. Available from: https://www. who.int/blueprint/priority-diseases/key-action/COVID-19_Treatment_Trial _Design_Master_Protocol_synopsis_Final_18022020.pdf.

4. None to report.

\section{1}

High Flow Oxygen Therapy in the ICU - A Secondary Care Center Experience

R. Passos ; F. Cardoso ${ }^{1}$; D. Alves ${ }^{1}$; M. Romano ${ }^{2}$; F. Silva ${ }^{2}$; J. Braga ${ }^{1}$; R. Corga ${ }^{1}$; J. Caldeiro ${ }^{1}$

${ }^{1}$ Intensive medicine, Hospital Santa Luzia - Ulsam, Viana do Castelo, Viana do Castelo, Portugal; ${ }^{2}$ Internal medicine, Hospital Santa Luzia - Ulsam, Viana do Castelo, Viana do Castelo, Portugal

Correspondence: F. Cardoso

Intensive Care Medicine Experimental 2020, 8(2): 001651

Introduction: High-flow oxygen therapy via nasal canula (HFNC) is a technique that delivers heated and humidified oxygen through short nasal prongs at high flow rates. Its great tolerability and positive results of its use in pneumonia, immunocompromised patients and ventilatory weaning have highlighted it as a first-line option for some of these conditions. Recent data show that it's the only non-invasive ventilatory technique that doesn't increase death space during ventilatory support, allowing for continuous air renewal, minimizing the risk of hypercapnia. HNFC allows to oxygenate critical patients with isolated hypoxemia, preventing intubation alllowing the lung to heal. It is the only device that oxygenates critical patients without compromising communication or feeding. It's benefical effect on the epithelial lining makes it an option even in chronic obstructive respiratory disease.

Our study illustrates a 14 months experience in a secondary care center, since HNFC acquisition. The team based their strategies on the local protocol that relied on starting with maximum temperature, $\mathrm{O} 2$ Flow and $\mathrm{FiO} 2$, and decrease support after 2 hours.

Methods: We performed a retrospective study with a descriptive analysis of HFNC's application in our Secoundary Care Center Intensive Care Unit (ICU), from February 2019 through June 2020.

Results: In 14 months a total of 33 patients were treated with HNFC. The mean age was 63.5 years and $63.6 \%$ of the patients were male. The average duration of HNFC was 2 days. The major condition for its 
use was acute respiratory failure (72.7\%). In $18.2 \%$ it was used for ventilatory weaning. In 3 cases it was applied in patients with a do-notintubate order. The most common diagnosis was community acquired pneumonia (54.2\%). HNFC was successful in $51.5 \%$ of the cases, with pneumonia being the most common successful condition $(58.8 \%$ of the successful cases). In the cases of failure, $25 \%$ had COVID-19 pneumonia, and $31.3 \%$ presented with associated multiorgan disfunction. $50 \%$ needed invasive mechanical ventilation, while $25 \%$ proceeded successfully with non-invasive ventilation (NIV) with positive pressure. When HNFC was used for ventilatory weaning, $66.7 \%$ had a successful outcome.

Conclusion: Literature on HFNC includes an heterogenous group of patients with diverse clinical diagnosis. It is increasingly applied in adults with hypoxemic respiratory failure with diverse etiologies. As observed in our report, diverse studies present pneumonia as the most common cause of respiratory failure in patients selected for HFNC. Some studies assessed the need of intubation after a HFNC trial and found that only $30 \%$ required intubation after failure of HFNC. In our study the "failure rate" was approximately $48.5 \%$. HNFC delayed use in patients with advanced stage disease and multiorgan disfunction might explain these results. The development of studies to determine which group of patients benefit the most from HFNC is imperative. The $25 \%$ patients with HFNC failure that progressed successfully to NIV raise the question whether they had a limitation for the technique that was not initially identified by the physician. The possibility of avoiding intubation in patients with severe hypoxemia worths the trial, even in cases with uncertain predicted responsiveness. It is fundamental to watch closely the evolution of these patients because, besides its benefits, HNFC shall not delay intubation when it is needed.

03. AIDS, hematologic-oncologic issues in the ICU > AIDS, hematologic-oncologic issues in the ICU.

\section{5}

Critically ill patients with hematological malignancies in the ICU

Y. Cardenas ${ }^{1}$; SA. Prieto Garces ${ }^{1}$; D. Rodriguez-Feria ${ }^{2}$; LV.

Mendoza-Montenegro ${ }^{2}$; MC. Peñaloza-Mayorga ${ }^{3}$; GE. Quintero-Vega ${ }^{2}$

${ }^{1}$ Medicina critica y cuidado intensivo, Fundacion Santa Fe de Bogota University Hospital, Bogotá, Colombia; ${ }^{2}$ Hematología, Fundacion Santa Fe de Bogota University Hospital, Bogotá, Colombia; ${ }^{3}$ Facultad de medicina, Universidad de los Andes, Bogotá, Colombia

Correspondence: S.A. Prieto Garces

Intensive Care Medicine Experimental 2020, 8(2): 001285

Introduction: Patients with cancer are at risk of being admitted to the intensive care unit (ICU) with a mortality rate similar to that of the general ICU population (30-65\%) (1). Prognosis factors are described for general ICU patients, while there is scarce information about such factors in critically ill patients with hematological malignancies. Hence, to provide better care for our patients, more information is needed.

Objectives: To describe the characteristics of patients with hematologic malignancies (HM) admitted to the ICU, and to determine the association between already known prognostic factors and mortality in our population.

Methods: A descriptive-analytical, cross-sectional study was done. Adult patients with HM admitted to the ICU from Jan 2008 - Dec 2018 at Hospital Universitario Fundación Santa Fe de Bogotá, Colombia, were enrolled. Demographics, disease stage, disease-specific parameters, laboratory results, hospital course, and outcomes were gathered from medical charts and analyzed using SPSS ver. 25.

Results: From the 185 patients enrolled, most were men (56.8\%), the mean age was 63 years, and ICU admission was mostly due to cardiovascular causes (17.9\%). Lymphoma (43.2\%) and multiple myeloma(MM) (28.1\%) were the most frequent hematologic diagnosis, and the majority were diagnosed recently (49.7\%). More than half $(\sim 65 \%)$ were receiving acute treatment, either induction therapy $(45 \%)$ or rescue therapy $(18.3 \%)$. Around $29,1 \%$ of patients required vasopressors and $25,4 \%$ required mechanical ventilation, with a mean duration of 3.34 (1-21), and 5.45 (1-26) days respectively. Overall mortality was $31.3 \%$, divided into ICU mortality of $21,6 \%$ and in hospital mortality of $9,7 \%$.
Subgroup analysis for HM type showed a difference in mortality rates. Lymphoma patients had greater mortality $(41.3 \%)$, followed by acute leukemias (29.3\%), and MM (24.1\%). Nevertheless, this difference was not statistically significant. On the other hand, mortality was more common in patients undergoing induction $(24.48 \%)$ and rescue (22.41\%) chemotherapy.

The bivariate analysis showed that male sex (OR:2.18,p:.022), previous diagnosis of non-hematological malignancy (OR:2.17,p:043), neutropenia on admission (OR:2.11,p:.038), disseminated intravascular coagulation (OR:4.49,p:.012), alveolar bleeding, (OR:9,86,p:.03), requirement of mechanical ventilation (OR:5.99,p:<.001) and transfusion (OR:3.39,p:<.001) were associated with general mortality.

Binomial logistic regression showed that diabetic patients (B:1,638;OR $2,68 ; p=, 034)$, vasopressor requirement $(B: 2,83 ;$ OR 8,$31 ; p=<, 001)$ and dialysis requirement $(\mathrm{B}: 2,93 ; \mathrm{OR} 8,602 ; \mathrm{p}=, 001)$ were strongly associated with ICU mortality.

Conclusion: Mortality rates in our patients were similar to those already described, albeit on the lower end. Lymphoma patients and those in induction therapy had greater mortality compared to others. Also, hematological causes were the most common cause of admission for deceased patients. Lastly, in our population, the main predictors of mortality were diabetes, use of vasopressors and hemodialysis requirement.

\section{Reference(s) and grant ackowledgment(s)}

1. Freedman N. Prognosis of cancer patients in the intensive care unit. Post TW, ed. UpToDate. Waltham, MA: UpToDate Inc. https://www.uptodate.com (Accessed on May 15, 2020)

03. AIDS, hematologic-oncologic issues in the ICU > AIDS, hematologic-oncologic issues in the ICU.

\section{6}

Characteristics of oncohematological patients admitted to the ICU of the Virgen de las Nieves Hospital in 2018-2020

L. Hidalgo Manchado 1; A. Sánchez González; I. Guzmán Adum; R. De La Chica Ruano

${ }^{1}$ Intensive care unit, Hospital Universitario Virgen de las Nieves, Granada, Spain

Correspondence: I. Guzman

Intensive Care Medicine Experimental 2020, 8(2): 001576

Introduction: The prognosis of malignant hematological diseases has changed in the past few years, making it increasingly frequent the admission of these patients to the ICU; but controversy continues regarding the real benefit of ICU admission in the long term.

Objectives:

- To describe the baseline characteristics of hematological patients admitted to our ICU.

- Identify factors likely associated with higher mortality in these patients.

- Description of mortality according to the main causes of ICU admission and comparison between them.

Methods: Observational, retrospective and analytical study that includes oncohematological patients admitted to the ICU between April 2018 and 2020. A database was created in SPSS with the baseline characteristics of the patients (demographic, comorbidities, underlying disease, transplantation of hematopoietic precursors, neutropenia), cause of ICU admission, cause of death, respiratory support and decision to limit life support treatment. They were then evaluated in terms of descriptive statistics with frequencies for qualitative variables and median, interquartile range for quantitative; Comparisons between groups were made using the Student's t test and chi square respectively.

Results: Of the 60 patients included, $25 \%$ were admitted in 2018, $46.7 \%$ in 2019 and $28.3 \%$ in 2020 so far, of which $28(46.7 \%)$ are women and $32(53.3 \%)$ men. Base disease: lymphomas 18 (30\%), leukemias $36(60 \%)$, myelomas $3(5 \%)$, others $3(5 \%)$, relationship with mortality $(p=0.54)$. Prior to admission, $3(5 \%)$ had received autoHSCT, $24(40 \%)$ 
alloHSCT and 32 (53.3\%) had not yet been transplanted; association with mortality $(p=0.59) .39(65 \%)$ developed neutropenia, with no direct relationship to mortality ( $p=0.63)$. The causes of ICU admission were: respiratory failure (56.67\%), septic shock (26.67\%) and loss of consciousness $(11.6 \%)$, with no difference in mortality between them $(p=0.6)$. Regarding respiratory support, $45(75 \%)$ required NIMV/ HNFO and $33(55 \%)$ IMV, the latter with higher mortality $(p=0.003)$. Total mortality in ICU 38 (63.3\%), significantly higher than overall unit mortality (18.3\%), $p=0.001$. LTE was decided in 24 patients (40\%).

\section{Conclusion:}

- The admissions of oncohematological patients in the ICU are increasing in the last years.

- The characteristics that were significantly related to a higher mortality were: age, invasive mechanical ventilation and SOFA score.

- The underlying disease, the cause for admission to the ICU, the HSCT, the presence of neutropenia and the requirement for NIMV did not present a statistically significant relationship with the mortality of these patients.

- ICU mortality in oncohematological patients, regardless of the cause of admission, is higher than the overall mortality in the ICU.

\section{0}

Early Carboxyhaemoglobin (COHb) Blood Concentration May Predict Outcome in Patients with Out of Hospital Cardiac Arrest (OOHCA)

T. Bucknall'; O. Valencia ${ }^{2}$; G. Zilahi ${ }^{3}$

${ }^{1}$ Foundation year doctor, St Peter's Hospital, Lyne, United Kingdom; ${ }^{2}$ Cardiovascular sciences research centre, St George's, University of London, London, United Kingdom; ${ }^{3}$ Intensive care, St George's Hospital, London, United Kingdom

\section{Correspondence: $T$. Bucknall}

Intensive Care Medicine Experimental 2020, 8(2): 001340

Introduction: Outcome prediction after Out of Hospital Cardiac Arrest $(\mathrm{OOHCA})$ remains challenging with high cost and resource burden. Multimodal approach has been adapted in most hospitals as advised by the ESICM and ERC (1). Carbon Monoxide (CO) has recently gained interest as a molecule with wide ranging physiological properties (2). The potential for novel pharmacological intervention is vast and exciting $(3,4)$. Most notably, CO plays an important role in apoptosis related cell death with ischaemic-reperfusion brain injury (5-7). We proposed that early $\mathrm{CO}$ measurment, using $\mathrm{COHb}$ as a surrogate marker, correlates with outcome in our OOHCA patient population.

Objectives: We retrospectively analysed Arterial Blood Gas (ABG) data to measure $\mathrm{COHb}$ blood concentrations in patients admitted from $\mathrm{OOHCA}$ for an initial 72-hr period. We evaluated $\mathrm{COHb}$ with regard to our long term follow up outcomes.

Methods: Retrospective data for 94 patients who were admitted to our Cardiothoracic Intensive Care Unit (CTICU) after OOHCA during 2016. Data collected included: ABG measurements for the $72 \mathrm{hr}$ period, demographic, and prognostic scores (Intensive Care National Audit \& Research Centre (INARC) and Acute Physiology and Chronic Health Evaluation (APACHE II) and outcome measures (Death and cerebral performance score (CPC) in hospital and at 3-, 6- and 12-month intervals).

Univariate analyses of categorical and continuous data were performed to determine differences between Patients who died or remained alive following OOHCA. Tabulations of dichotomous or categorical variables for comparisons using Chi-square Test. The distribution of continuous variables was assessed for normality with Shapiro-Wilk Test, and Independent Samples Test or Mann-Whitney $\mathrm{U}$ Tests were used to compare both groups. The Receiver Operating Characteristic Curve analysis was required for survival, along with Multiple Logistic Regression analyses and graphical methods. Friedman Repeated Measures Analysis of Variance on Ranks was needed and Pairwise Multiple Comparison Procedures (Tukey Test) was used to isolate the group of samples that differ from the others.
Results: 94 patients were analysed, and the overall survival was $56 \%$. $73 \%$ of the patients were male and median age was 65 years. Median age ( $\mathrm{p}$ : $<0.001)$, mean ICNARC score ( $\mathrm{p}:<0.001)$, median APACHE II score ( $\mathrm{p}:<0.001)$, median lowest $\mathrm{pH}(\mathrm{p}:<0.001)$, and median lowest $\mathrm{COHb}$ concentrations in the first 5 hours ( $\mathrm{p}$ : 0.032 ) were significantly different in the surviving population. Initial 5 Hour Median $\mathrm{COHb} \geq$ $0.975 \%$ had a sensitivity of 0.77 and specificity of 0.49 to predict survival in our OOHCA patient population with the AUC of $63 \%$.

Conclusion: Initial $\mathrm{COHb}$ blood concentration measurements are predictive of overall survival in patients presenting to hospital with OOHCA. This may be explained by the cytoprotective effects of $\mathrm{CO}$ in ischaemic-reperfusion brain injury, but further larger scale evaluation of the association is planned and required.

Reference(s) and grant ackowledgment(s)

1. 7. Mahan VL, Zurakowski D, Otterbein LE, Pigula FA. Inhaled Carbon Monoxide Provides Cerebral Cytoprotection in Pigs. Meisel A, editor. PLoS ONE. 2012 Aug 7;7(8):e41982

2. 6. Qiao L, Zhang N, Huang J, Yang X. Carbon monoxide as a promising molecule to promote nerve regeneration after traumatic brain injury. Med Gas Res. 2017;7(1):45.

3. 5. Schallner N, Romão CC, Biermann J, Lagrèze WA, Otterbein LE, Buerkle $\mathrm{H}$, et al. Carbon Monoxide Abrogates Ischemic Insult to Neuronal Cells via the Soluble Guanylate Cyclase-cGMP Pathway. Koch K-W, editor. PLoS ONE. 2013 Apr 8;8(4):e60672.

4. 4. Kim H-H, Choi S. Therapeutic Aspects of Carbon Monoxide in Cardiovascular Disease. Int J Mol Sci. 2018 Aug 13;19(8):2381.

5. 3. Ulbrich F, Hagmann C, Buerkle H, Romao CC, Schallner N, Goebel U, et al. The Carbon monoxide releasing molecule ALF-186 mediates antiinflammatory and neuroprotective effects via the soluble guanylate cyclase $\beta 1$ in rats' retinal ganglion cells after ischemia and reperfusion injury. J Neuroinflammation [Internet]. 2017 Dec [cited 2017 Oct 11];14(1). Available from: http://jneuroinflammation.biomedcentral.com/articles/10.1186/s1297 4-017-0905-7.

6. 2. Heinemann SH, Hoshi T, Westerhausen M, Schiller A. Carbon monoxide - physiology, detection and controlled release. Chem Commun. 2014;50(28):3644-60.

7. 1. Nolan JP, Soar J, Cariou A, Cronberg T, Moulaert VRM, Deakin CD, et al. European Resuscitation Council and European Society of Intensive Care Medicine Guidelines for Post-resuscitation Care 2015. Resuscitation. 2015 Oct; $95: 202-22$

8. This project was initially started as part of a Student Selected Component during undergraduate studies at St George's, University of London.

\section{4}

Out-of-hospital cardiac arrest (OHCA) during the COVID-19 pandemic in Doha, Qatar

B. Albaroudi '; O. Albaroudi'; F. Mohammed ${ }^{1}$; T. Salam ${ }^{1}$; AM. Ahmad ${ }^{1}$; A. Abdulrahamn ${ }^{2} ; \mathrm{H}$. Tim

${ }^{1}$ Emergency department, Hamad Medical Corporation, Doha, Qatar; ${ }^{2}$ Cardiology department, Hamad Medical Corporation, Doha, Qatar

Correspondence: B. Albaroudi

Intensive Care Medicine Experimental 2020, 8(2): 001574

Introduction: OHCA is a common disease with a high mortality with the commonest cause being ischaemic heart disease(1). Survival ranges from around $1-3 \%$ to $20-25 \%$, suggesting that high quality care can have significant impact on survival(2). One small study reported a survival of $8.1 \%$ for Qatar(3). The COVID-19 pandemic caused by SARS-CoV-2, initially reported in China in December, 2019, and subsequently announced as a global pandemic by WHO on March 11, 2020. The first case of SARS-CoV-2 reported in Qatar was on 29th of February. More than 90000 cases of COVID-19 have been reported in Qatar since February 2020, with more than 145 deaths as of mid-July, 2020. The risk of viral transmission from patients in OHCA to health care workers saw changes in practice recommended in international guidelines. Cardiopulmonary resuscitation (CPR) and endotracheal intubation according to the centers for disease control and prevention $(C D C)$ are aerosolizing procedures and should be performed using full personal protective equipment (PPE), moreover few hospitals told 
their healthcare worker not to perform CPR or ventilation in patient suspected to have Covid-19 diagnosis unless they are wearing full PPE $(5,6)$. Public behavior also changed due to either the restrictions on public movement to reduce viral transmission or the fear from coming to hospital with COVID-19 cases with many countries reporting falling in ED attendances (7). In Qatar all outpatient clinics moved on line with possible difficulties for the patients to obtain medications and investigations, arguably reducing the quality of care provided. Our ED saw only small falls in public attendance and the cardiac catheterization rate remained stable. All these previously listed factors and changes have unknown consequences on the incidence and survival of OHCA with possible direct or indirect impact on the quality of care. Previous publications have highlighted an increased incidence of OHCA and reduced survival during the Covid-19 pandemic. Given these differences, we collected data on the incidence and survival of OHCA in our population.

Methods: Date collection: We developed a dedicated Covid-19 OHCA guideline and set up a dedicated team to teach this in our ED. In order to ensure rapidly emerging knowledge and to be translated into clinical care we set up a dedicated team to collect data on non-traumatic OHCA management and outcomes. We met weekly and adjusted our care according to local data, international guidelines and emerging knowledge on both transmission risk and treatment. The data in this report was collected using PAROS (Pan Asian Resuscitation Outcomes Study) which relay on Ustein complaint data. The Ustein criteria are a standardized data set used internationally to collect data on OHCA(4). Data was collected prospectively during Covid-19 outbreak from mid-March till mid-June 2020 (12 weeks period). Retrospective data was collected from the electronic medical records in the same period extending from mid-March till mid-June 2019 (12 weeks period) using the same template. Both periods data (2019 and 2020) was analyzed for the following: incidence, location of arrest, gender, age, first assessed rhythm, survival to hospital admission, survival to hospital discharge. Comparison graphs were established to show the deference between 2019 and 2020 of the same yearly periods. Setting: Qatar has a population of 2.8 million with around $90 \%$ being non-nationals and a median age of 32.3 years. Hamad General hospital emergency department (ED) is the largest ED in Doha, Qatar, with average ED visit $>1000$ patients per day. It is co-located with a dedicated cardiac hospital and receives $>90 \%$ of cardiac arrests in Qatar. There is $24 / 7 \mathrm{PCl}$ and an active ECMO service. eCPR was ceased during the pandemic to preserve the resource for Covid-19 related respiratory failure. Local policy sees around two thirds of OHCA transported to hospital. The project was registered along departmental guidelines with corporation level approval.

Results: The total number of non-traumatic OHCA during COVID-19 outbreak period in 2020 was 92 cases compared to 69 cases in nonoutbreak period in 2019. The average weekly incidence of OHCA for outbreak period and the non-outbreak period were 7 and 5.3 respectively. survival to hospital admission (SHA) and Survival to hospital discharge (SHD) were in outbreak period $(28.3 \%)$ and $(11 \%)$ respectively, compared to the non-outbreak period where SHA (43.5\%) and SHD (22\%).

Conclusion: Two reports from Europe showed an increase the OHCA incidence during COVID outbreak. The first one in Lombardy, Italy (8) and the second one in Paris, France (9). There is an increase in OHCA incidence during COVID-19 outbreak in Qatar. This result might be partly related to the effect of COVID-19 outbreak. Unusual unexpected situation like Outbreak or a crisis might have an impact on health care system by direct or indirect ways. healthcare provider training and population education might mitigate that effect.

\section{Reference(s) and grant ackowledgment(s)}

1. https://www.moph.gov.qa/english/mediacenter/News/Pages/NewsDetail s.aspx?ltemld=83. https://www.cdc.gov/coronavirus/2019-ncov/hcp/infec tion-control-recommendations.html. https://www.who.int/countries/qat/ en/. https://www.worldometers.info/world-population/qatar-population/.

https://www.scri.edu.sg/crn/pan-asian-resuscitation-outcomes-study-paros -clinical-research-network-crn/about-paros/.

2. Nolan JP, Hazinski MF, Billi JE, Boettiger BW, Bossaert L, de Caen AR, et al. Part 1: Executive summary: 2010 International Consensus on Cardiopulmonary
Resuscitation and Emergency Cardiovascular Care Science With Treatment Recommendations. Resuscitation. 2010;81 Suppl 1:e1-25.

3. Sasson C, Rogers MA, Dahl J, Kellermann AL. Predictors of survival from out-of-hospital cardiac arrest: a systematic review and meta-analysis. Circ Cardiovasc Qual Outcomes. 2010;3(1):63-81.

4. Irfan FB, Bhutta ZA, Castren M, Straney L, Djarv T, Tariq T, et al. Epidemiology and outcomes of out-of-hospital cardiac arrest in Qatar: A nationwide observational study. Int J Cardiol. 2016;223:1007-13.

5. Perkins GD, Jacobs IG, Nadkarni VM, Berg RA, Bhanji F, Biarent D, et al. Cardiac arrest and cardiopulmonary resuscitation outcome reports: update of the Utstein Resuscitation Registry Templates for Out-of-Hospital Cardiac Arrest: a statement for healthcare professionals from a task force of the International Liaison Committee on Resuscitation (American Heart Association, European Resuscitation Council, Australian and New Zealand Council on Resuscitation, Heart and Stroke Foundation of Canada, InterAmerican Heart Foundation, Resuscitation Council of Southern Africa, Resuscitation Council of Asia); and the American Heart Association Emergency Cardiovascular Care Committee and the Council on Cardiopulmonary, Critical Care, Perioperative and Resuscitation. Circulation. 2015;132(13):1286-300.

6. Mahase E, Kmietowicz Z. Covid-19: Doctors are told not to perform CPR on patients in cardiac arrest. BMJ. 2020;368:m1282.

7. DeFilippis EM, Ranard LS, Berg DD. Cardiopulmonary Resuscitation During the COVID-19 Pandemic: A View From Trainees on the Front Line. Circulation. 2020;141(23):1833-5.

8. Pfefferbaum B, North CS. Mental Health and the Covid-19 Pandemic. New England Journal of Medicine. 2020.

9. The authors received no financial support for the research.

\section{0}

Landiolol vs Esmolol on hemodynamic response during weaning of post-operative ICU patients with heart failure

G. Koukoulitsios ${ }^{1}$; G. Maglaras ${ }^{1}$; M. Anifanti ${ }^{1}$; A. Koutivas ${ }^{1}$;. Ionna'; I. Tsoni ${ }^{1}$. S. Bakouli ${ }^{1}$; K. Tsikritsaki1'; C. Mandila ${ }^{1}$; S. Antonopoulou ${ }^{1}$; V. Koutsoukou ${ }^{1}$; V. Salma'; A. Kalogeromitros ${ }^{1}$

${ }^{1}$ ICU, General Hospital of Athens "G. Gennimatas", Athina, Greece

Correspondence: G. Koukoulitsios

Intensive Care Medicine Experimental 2020, 8(2): 001190

Introduction: Weaning and tracheal extubation of post-operative ICU patients is a stressful procedure which triggers the sympathetic nervous system leading to potential deterioration of cardiac performance in patients with left ventricular dysfunction. This study was conducted to compare the efficacy and safety of Landiolol, a novel ultra-shortacting-beta-1 blocker with those of Esmolol in cardiovascular response during the extubation of patients with heart failure.

Methods: Thirty-nine patients with heart failure [LVEF (\%): $36.6 \pm 7.6$, NYHA Class III/IV (n): 32/9] were randomly divided into two groups: Landiolol group of 19 patients and Esmolol group of 20 patients. Landiolol infusion at the rate of $4 \mu \mathrm{g} / \mathrm{Kg} / \mathrm{min}$ and Esmolol infusion at the rate of $100 / \mathrm{Kg} / \mathrm{min}$ was started as soon as the procedure of weaning began. Heart rate (HR) systolic arterial pressure (SAP) diastolic arterial pressure (DAP) mean arterial pressure (MAP) and cardiac rhythm were recorded every minute up to 30 min after the extubation of trachea.

Results: Mean age, LVEF, NYHA Class, Acute Physiology and Chronic Health Evaluation II and Sequential Organ Failure Assessment score were similar between the two groups. Landiolol produced a more rapid and substantial decrease in HR than Esmolol $(-40 \pm 20$ vs $-30 \pm 16 \mathrm{bpm}$ ) without any deterioration of hemodynamics. Significant reduction of MAP was recorded in the Esmolol group.

Conclusion: Landiolol more rapidly and more potent reduced the HR than Esmolol. Significant reduction of MAP was recorded only in the Esmolol group.

\section{4}

Is hypercapnia in pulmonary embolism a sign of poor prognosis? M. Benlabed"; S. Benlabed ${ }^{2}$; R. Gaudy ${ }^{3}$; S. Nedjari ${ }^{4}$; F. Ouanes ${ }^{5}$; A. Ladjouze ${ }^{6}$ ${ }^{1}$ Anesthesiology and Intensivecare, Lille University, Lille, France; ${ }^{2}$ Erasme hospital, Université Libre de Bruxelles, Bruxelles, Belgium; ${ }^{3}$ Anesthesiology and intensivecare, Lille Catholic University, Lille, France; ${ }^{4}$ Anesthesiology, Algiers university, Alger Centre, Algeria, Algeria; ${ }^{5}$ Anesthesiology, Algiers 
unversity, Algiers, Algeria; ${ }^{6}$ Anesthesiology, Algiers University, Algiers, Algeria

Correspondence: $M$. Benlabed

Intensive Care Medicine Experimental 2020, 8(2): 001254

Introduction: Massive pulmonary embolism is a life-threatening complication of the patient admitted in intensive care unit (ICU) and the consecutive hemodynamic instability or cardiogenic shock is sometimes accompanied by unusual blood gas alterations. If hypoxemia and hypocapnia are usual, hypercapnia in embolic disease is rarely reported in the literature and is the result of increased dead space ventilation due to extensive embolic vascular obstruction.

Objectives: So the aim of this study was to hypothesize that the presence and persistence of hypercapnia during pulmonary embolism would be a marker of severity and of poor prognosis.

Methods: we retrospectively collected data from a group of 15 ICU patients (group 1) who presented hypercapnic pulmonary embolisms from 2017 to 2019 and compared them with those of a group of 15 patients (group 2) presenting pulmonary embolisms with normocapnia or hypocapnia, between 2012 and 2015. The number of patients with hypercapnic pulmonary embolisms being rare, we compared them with an identical number of patients presenting pulmonary embolisms with normocapnia or hypocapnia. The patients of the 2 groups were aged $68-+7$ years, weighing $75+-6 \mathrm{~kg}$, without previous cardiac or pulmonary pathology. The patients of the 2 groups were in spontaneous ventilation and breathed oxygen between 6 to $10 \mathrm{l} / \mathrm{min}$. On admission to ICU, we monitored and recorded systolic arterial pressure (SAP) with a catheter inserted in radial left artery and evaluated with echocardiography, cardiac index $(\mathrm{Cl})$ and systolic pulmonary arterial pressure.(SPAP).We measured blood gases, lactate,BNP from the arterial wave line and finally we evaluated ICU mortality in each group.

Results: .Statistical analysis was performed using Student 's t test. Results were expressed as mean +-standard deviation.

We observed that $\mathrm{PcO} 2(\mathrm{mmHg})$ in the group of patients presenting hypercapnic pulmonary embolism (group 1) was obviously different than in the group of embolism with hypocapnia (group 2), respectively $51.41+-4.10$ vs $28.86+-1.18$. $\mathrm{P}<0.0001$. However, Po2 $(\mathrm{mmHg})$ was not different between group 1 and group 2,respectively $74.33+-4.8$ vs76.33+-1.87 $\mathrm{P}<0.14$. SAP $(\mathrm{mmHg})$ was in the group 1 significantly different of SAP of the group 2 , respectively $79.2+-8.3$ vs $102.06+-5.73$ $P<0.0001$. Similarly, SPAP $(\mathrm{mmHg})$ was different between group 1 and group 2 respectively $55.46+-2.50$ vs $44.8+-1.78$. $\mathrm{P}<0.0001$. Cl was different between group 1 and group 2 , respectively $1.85+-0.09$ vs $2.28+-$ $0.14 \mathrm{P}<0.0001$. The value of $\mathrm{BNP}(\mathrm{Pg} / \mathrm{ml})$ was more elevated in group 1 than in group 2 respectively $2888.33+-827$ vs $1722+-291 \mathrm{P}<0.0001$. Similarly, Blood lactate concentration $(\mathrm{mmol} / \mathrm{l})$ was higher in group 1 than in group2,respectively $4.78+-1.14$ vs $3.69+-0.77 . P<0.005$.ICU mortality,despite any therapeutic, was higher in the group 1 than in the group 2 respectively $50 \%$ vs $40 \%$.

Conclusion: We can observe that massive pulmonary embolisms with hypercapnia seem to have a worse prognosis than those which are,accompanied by hypocapnia or normocapnia.

The persistence of hypercapnia in a patient with previously healthy lungs indicates that $50 \%$ or more of the lung is underperfused and prompts the use of either thrombolytic therapy or surgical intervention.

\section{Reference(s) and grant ackowledgment(s)}

1. The RIETE investigators. Systolic blood pressure and mortality in acute symptomatic pulmonary embolism. International Journal of Cardiology.302,2020.

2. A.Bouchama et al. Refractory hypercapnia complicating massive pulmonary embolism.Am Rev Resp Dis. 138,466-468,1988.
001289

Upper Gastrointestinal Bleeding (UGIB) in Latin America: Demographics, management and the role of AIMS65 predicting ICU mortality

SA. Prieto Garces ${ }^{1}$; FS. Terán-Ibarra²; YE. Caballero-Diaz²; H.

Gómez-Velasco ${ }^{2}$; LM. Sabogal-Angel ${ }^{2}$; LC. García-Medina²; A.

Gómez-Aldana33. M. ÁLvarez-Gaviria ${ }^{4}$

${ }^{1}$ Medicina critica y cuidado intensivo, Fundacion Santa Fe de Bogota University Hospital, Bogotá, Colombia; ${ }^{2}$ Facultad de medicina, Universidad de los Andes, Bogotá, Colombia; ${ }^{3}$ Gastroenterología, Fundacion Santa Fe de Bogota University Hospital, Bogotá, Colombia; ${ }^{4}$ Cardiología, Fundacion Santa Fe de Bogota University Hospital, Bogotá, Colombia

Correspondence: S.A. Prieto Garces

Intensive Care Medicine Experimental 2020, 8(2): 001289

Introduction: Upper gastrointestinal bleeding (UGIB) is an emergency requiring prompt management to achieve favorable outcomes(1). It presents with hematemesis and/or melena (1), and may be followed by hemodynamic instability. Hemorrhagic gastritis and peptic ulcer disease make up for $75 \%$ of UGIB etiologies, while esophageal varices, esophagitis, duodenitis, andMallory-Weiss syndrome represent the remaining causes ( 2 ).

However, in our population, knowledge about the epidemiological characteristics is scarce. Therefore, to correctly utilize our limited health resources, information regarding patients in our territory is needed.

Objectives: The objective is to characterize UGIB patients admitted into the intensive Care Unit (ICU) in a Latin American hospital and to determine the presence of prognostic factors associated with ICU mortality, in-hospital mortality and rebleeding.

Methods: An analytical, cross-sectional study, was carried out. UGIB patients admitted to the ICU between Jun/2013-Jun/2018 in a hospital in Bogotá, Colombia were enrolled. Demographics, comorbidities, clinical presentation, laboratory results, management, predictive scores, clinical course, complications and outcomes were retrieved and analyzed using SPSS ver.25. Descriptive analysis was done alongside a correlation matrix and binomial regression using outcomes such as rebleeding, ICU mortality and in hospital mortality, with a statistical significance of $p<0.05$. Rebleeding risk was according to Forrest classification.

Results: The study enrolled 321 patients, $62 \%$ were male and average age was 66.11 years. At admission, $75.7 \%$ were diagnosed with UGIB black stools were present in $67 \%$ of patients, hematemesis in $31.8 \%$, hematochezia in $17.1 \%$, and ground-like vomit in $16.8 \%$. Also, a significant number presented hypovolemic shock on admission (20.5\%). Notably, 26.2\% patients received chronic antiplatelet therapy, and $17.8 \%$ were anticoagulated.

For management, $99.1 \%$ received proton pump inhibitors and endoscopy was performed in $94.1 \%$ of patients. The main findings were peptic ulcer (44.9\%), gastroduodenal erosions (17.4\%), and esophagitis (10\%). During endoscopy, $32.1 \%$ of patients underwent sclerotherapy, and $19.6 \%$ varicose veins clip ligation. Around $62.9 \%$ required red blood cell transfusion and $10 \%$ had intrahospital re-bleeding. Anticoagulation/anti-aggregation was restarted 6.34 days on average. Lastly, ICU mortality was $8 \%$ and average ICU stay was 4.93 days.

Logistic regression concluded that ICU mortality increases 7.39 times for males, 26 times if there are dementia, lung and/or liver comorbidities, 8.5 times if the person is anticoagulated and 5 times with each point of the AIMS65 (all of the above with a $p=<0,05$ ).

Conclusion: The demographic characteristics of UGIB patients in our hospital were similar to those reported on global literature. Also, rebleeding affects $10 \%$ of patients, a value lower than those reported (3). Lastly, this is the first study in south American literature that shows the usefulness of AIMS65 predicting ICU mortality of UGIB patients. 
Reference(s) and grant ackowledgment(s)

1. 3. Maggio D, Barkun AN, Martel M, Elouali S, Gralnek IM, Investigators TR, Predictors of Early Rebleeding after Endoscopic Therapy in Patients with Nonvariceal Upper Gastrointestinal Bleeding Secondary to High-Risk Lesions. Canadian Journal of Gastroenterology. 2013;27(8):454-8.

2. 2. Farfán $Y$, Restrepo A, Molano J. La hemorragia digestiva alta en la Unidad de Cuidados Intensivos. [Internet]. Rev Col Gastroenterol. 2006 [cited 2020]. Available from: http://www.scielo.org.co/scielo.php?script = sci_artte xt\&amp;pid = S0120-99572006000200013lng =en.

3. 1. Saltzman J. Approach to acute upper gastrointestinal bleeding in adults [Internet]. 2019 [cited 2019]. Available from: https://www-uptodate-com. ezproxy.uniandes.edu.co:8443/contents/approach-to-acute-upper-gastr ointestinal-bleeding-in-

\section{5}

Risk of venous thromboembolic disease in critically ill patients with severe COVID-19 pneumonia

C. Palmada; D. Gill; L. Chiscano-Camón; M. García-Reyes²; C. Marrero²; M. De-Nadal' ${ }^{3}$; S. Bellmunt ${ }^{2}$; R. Ferrer ${ }^{1}$; J. Ruíz-Rodriguez ${ }^{1}$

${ }^{1}$ Intensive Care, Vall d'Hebron University Hospital, Barcelona, Spain; ${ }^{2}$ Vascular and endovascular surgery department, Vall d'Hebron University Hospital, Barcelona, Spain; ${ }^{3}$ Anesthesiology, Vall d'Hebron University Hospital, Barcelona, Spain

Correspondence: C. Palmada

Intensive Care Medicine Experimental 2020, 8(2): 001385

Introduction: Patients admitted to the Intensive Care Unit (ICU) for coronavirus disease 2019 (COVID-19) present an inflammatory state that conditions a high thrombotic risk. There are several studies about thrombotics events in patients with COVID-19 in ICU but they are limited in size and without report all thrombotic events.

Objectives: To determine the incidence of venous thromboembolic disease (VTE) in critically ill patients with COVID-19 and the correlation with D-dimer levels.

Methods: A prospective study of critically ill patients with severe SARS-CoV-2 pneumonia. Inclusion criteria: severe SARS-CoV-2 pneumonia confirmed by PCR and admission to the ICU. Exclusion criteria: ECMO, pregnant woman, puerperium, age $<18$ years. The study included all patients admitted to the ICU who met the inclusion criteria and none of the exclusion on 15-16 April 2020. Ultrasound screening with venous compression bilateral in lower extremities was performed. Asymptomatic and symptomatic VTE was recorded [pulmonary thromboembolism (PD), deep vein thrombosis (DVT)] throughout the ICU stay, patient characteristics, blood test results, and results with 30 days follow-up. Categorical data are expressed as frequency (\%). The quantitative data are expressed as mean (standard deviation) or as median (interquartile range 25-75). Categorical variables were compared using the chi-square test and Fisher's exact test. Continuous variables were compared with the T Student test or the Mann-Whitney $U$ test. The association between D-Dimers and VTE has been calculated using the area below the curve (AUROC). Approved by the Ethics Committee of Clinical Research (PR (AG) 213/2020).

Results: On 15-16 April 2020, 118 patients [ $77 \%$ male, age mean 59.37 (10.20) years, BMI 30.5 (4.74), 100 (84.74\%) with invasive mechanical ventilation] were studied. D-Dimer on the day of the ultrasound screening was 1575 (703.5-2963) ng/mL. VTE was prevalence of 30.5\% (36/118). At 30 days of follow-up, the cumulative incidence of VTE was 35\% (41/118): 31 DVT, 5 PD, 5 DVT + PD. There were 17 (14\%) hemorrhages (4 major and 14 minor). The VTE was not more frequent in ventilated patients. Mortality was $13.6 \%$ (16 patients) without difference depending on the existence of thrombosis ( 5 patients in patients with VTE and 11 in patients without VTE, p ns). Elevation of the D-Dimer above $1500 \mathrm{ng} / \mathrm{ml}$ is related to thromboembolic phenomena (AUROC $0.77, \mathrm{p}<0.0001,95 \% \mathrm{Cl} 0.62-0.82$ ), sensitivity $73 \%$ and specificity $60 \%$. Conclusion: Patients admitted to the ICU for severe SARS-CoV-2 pneumonia are at high risk of VTE. The elevation of D-Dimers is related to VTE.
Reference(s) and grant ackowledgment(s)

1. Julien Nahum, MD et al. Venous Thrombosis Among Critically III Patients With Coronavirus Disease 2019 (COVID-19). JAMA Network Open. 2020;3(5):e2010478. https://doi.org/10.1001/jamanetworkopen.2020.10478.

\section{4}

A second course of ventricular assist device support in refractory cardiogenic shock: experience in a third level hospital

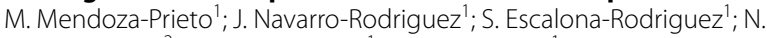
Palomo-Lopez ${ }^{2}$; Y. Corcia-Palomo ${ }^{1}$; L. Martin-Villen ${ }^{1}$

${ }^{1}$ Critical care unit, Hospital Universitario Virgen del Rocío, Sevilla, Spain; ${ }^{2}$ Critical care, Harefield Hospital, Harefield, United Kingdom

Correspondence: M. Mendoza-Prieto

Intensive Care Medicine Experimental 2020, 8(2): 001434

Introduction: The availability of different mechanical circulatory support devices (MCSDs) allows physicians to provide the most appropriate support to a wide range of clinical conditions.

Objectives: To analyze the different types of MCSDs used in our Intensive Care Unit (ICU). Analyze the clinical characteristics of the patients who had removed their first device support and required a second device course.

Methods: Retrospective observational study from 2013 to 2019 in a third level ICU with heart transplantation program. We included all patients who required more than one device implantation. We examined demographic data, cause for VAD implantation, type of first (VAD1) and second (VAD2) device used, days of VAD support, ICU length of stay, in-hospital and ICU mortality. Absolute number and frequency described qualitative variables and median and interquartile range (IR) described quantitative variables.

Results: A total of 22 patients were included, 16 were male $(72.7 \%)$, with a median age of 53.50 (34.25-60.50). The main reason for more than one device placement was post myocardial infarction cardiogenic shock $10(45.5 \%)$ follow by descompensated chronic cardiomyopathy 6 (27.3\%), postcardiotomy shock 1 (4.5\%), non-ischemic cardiogenic shock 1 (4.5\%), acute myocarditis 1 (4.5\%), electrical storm $1(4.5 \%)$, right ventricular disfunction 1 (4.5\%) and ischemic cardiomyopathy $1(4.5 \%)$. VAD1 as bridge to transplant was performed in 11 patients (50\%), bridge to decision in $8(36.4 \%)$ and to recovery in $3(13.6 \%)$. Different types of VAD implanted are shown in table 1. An average of 7 days of VAD1 support was reported (4.50 - 11.50[1]) and 7.5 days for VAD2 support (4.75 - 13.25). A second device placement was needed due to clinical deterioration as bridge to recovery 17 (77.3\%), decision 3 (13.6\%) and transplant 2 (9.1\%). Median ICU stay of 32.50 days ( $16.50-44.75)$. ICU and hospital mortality of $36.4 \%$.

\begin{tabular}{|l|l|l|}
\hline Table 1. Types of VAD implanted. & VAD1 & VAD2 \\
\hline & $(\mathrm{n}=22)$ & $(\mathrm{n}=22)$ \\
\hline Central VA-ECMO & $2(9.1)$ & $5(22.7)$ \\
\hline Peripheral femoro-femoral VA-ECMO & $8(36.4)$ & $5(22.7)$ \\
\hline Peripheral femoro-axilar VA-ECMO & $4(18.2)$ & $3(13.6)$ \\
\hline Right Centrimag & & $3(13.6)$ \\
\hline Left Centrimag & $5(22.7)$ & $3(13.6)$ \\
\hline EXCOR & $2(9.1)$ & \\
\hline EXCOR + Centrimag D & & $1(4.5)$ \\
\hline Biventricular Centrimag & $1(4.5)$ & \\
\hline VV ECMO & & $2(9.1)$ \\
\hline
\end{tabular}

Conclusion: In our cohort, post myocardial infarction cardiogenic shock was the main cause for placing more than one VAD. Femorofemoral VA-ECMO was the most frequent device used. A second device placement increased to $59.1 \%$ the percentage of patients recovered. 


\section{9}

ECMO as a bridge to recovery: progress over the last decade

J. Navarro-Rodriguez ${ }^{\text {; }}$ M. Mendoza-Prieto ${ }^{1}$; S. Escalona-Rodriguez ${ }^{\text {; }}$ N.

Palomo-Lopez ${ }^{2}$; Y. Corcia-Palomo ${ }^{1}$; L. Martin-Villen ${ }^{1}$

${ }^{1}$ Critical care unit, Hospital Universitario Virgen del Rocío, Sevilla, Spain;

${ }^{2}$ Critical care, Harefield Hospital, Harefield, United Kingdom

Correspondence: M. Mendoza-Prieto

Intensive Care Medicine Experimental 2020, 8(2): 001449

Introduction: Veno-arterial extracorporeal membrane oxygenation (VA-ECMO) is a temporary mechanical circulatory support (MCS) used as rescue therapy in patients with acute refractory cardiogenic shock. Its use as a bridge to recovery has been increasing over the last decade.

Objectives: To describe epidemiological and clinical features of patients with acute heart failure (AHF) admitted to our ICU that required $V A-E C M O$ implantation as a bridge to recovery.

Methods: A retrospective study was performed from January 2011 to December 2019 in a third-level cardiovascular ICU. All patients with AHF that required $\mathrm{VA}-\mathrm{ECMO}$ as a bridge to recovery were included. We collected demographic data, cardiovascular risk factors such as smoking, alcoholism, hypertension and diabetes mellitus, AHF etiology, ICU length of stay, ECMO cannulation techniques, days on ECMO, clinical outcomes and complications after ECMO implantation. Qualitative variables were described by absolute number and frequency; quantitative variables by median and interquartile range.

Results: A total of 40 patients were included, median age was 51.50 (40.25-60.75), 24 were males (60\%). Smoking habits 21 (52.5\%), alcoholism 6 (15\%), hypertension 19 (47.5\%) and diabetes mellitus 8 (20\%). Past medical history of heart disease was detected in $26(65 \%)$ patients being heart valve disease the most frequent in 8 patients $(20 \%)$, followed by ischemic 5 (12.5\%), congenital 4 (10\%), idiopathic dilated 3 (7.5\%), hypertrophic $2(5 \%)$ and restrictive cardiomyopathy 1 (2.25\%). Peripheral femoro-femoral cannulation was the most common technique in $22(55 \%)$ patients, central ECMO in $10(25 \%)$ and peripheral femoro-axilar in 8 (20\%). Median time on ECMO was 6 days (3-11.25). Main causes of acute heart failure are shown in Table 1 . The most frequent complications were pericannula bleeding 11 (27.5\%) and mediastinal bleeding 14 (35\%). Ventilator-associated pneumonia was reported in 15 patients (37.5\%) under ECMO support and bacteriemia in $13(30 \%)$. Clinical outcomes are shown in Table 2. Median length of stay in ICU and in Hospital was 18 (7-38) and 51 (25-76) days respectively. Hospital mortality was $40 \%$.

Table 1

\begin{tabular}{|l|l|}
\hline Etiology AHF $(\mathrm{n}=40)$ & $\mathrm{n}(\%)$ \\
\hline Postcardiotomy shock & $11(27.5)$ \\
\hline Ischemic cardiomyopathy & $7(17.5)$ \\
\hline Right ventricle dysfunction & $9(22.5)$ \\
\hline Acute myocarditis & $5(12.5)$ \\
\hline Acute transplant rejection & $3(7.5)$ \\
\hline Primary graft dysfunction & $2(5)$ \\
\hline Non-ischemic chronic cardiomyopathy & $2(5)$ \\
\hline Tachycardiomyopathy & $1(2.5)$ \\
\hline
\end{tabular}

Table 2. Clinical outcomes.

\begin{tabular}{|l|l|}
\hline & n $(\%)$ \\
\hline Recovery and weaned & $20(50)$ \\
\hline Heart transplant & $2(5)$ \\
\hline Second MCS device & $2(5)$ \\
\hline Overall ICU mortality & $16(40)$ \\
\hline
\end{tabular}

Conclusion: ECMO is widely used in refractory cardiogenic shock as a bridge to recovery. Although the indications of implantation have increased in the last years, ECMO is not free of risk, being infections and bleeding the most frequent complications.

Reference(s) and grant ackowledgment(s)

1. 2. Rao Prashant, Khalpey Zain, Smith Richard, Burkhoff Daniel, Kociol Robb D. Venoarterial Extracorporeal Membrane Oxygenation for Cardiogenic Shock and Cardiac Arrest. Circulation: Heart Failure. 1 September 2018;11(9):e004905

2. 1. Rousse $N$, Juthier F, Pinçon C, Hysi I, Banfi C, Robin E, et al. ECMO as a bridge to decision: Recovery, VAD, or heart transplantation? International Journal of Cardiology. 6 de mayo de 2015;187:620-7.

\section{0}

Infected endocarditis surgery in patients older than $\mathbf{7 0}$ years

VA. Hortigüela Martín; R. Hernandez Estefanía; G. Aldamiz Echevarria Del Castillo $^{2}$; E. Rosas Carvajall ; JJ. Paez Vargas ${ }^{1}$; AM. Ioan'; S. Sánchez Bernal ${ }^{1}$; M. Sanchez Casado ${ }^{3}$; C. Pérez Calvo ${ }^{1}$

${ }^{1}$ Intensive care medicine, Hospital Universitario Fundación Jiménez Díaz, Madrid, Spain; ${ }^{2}$ Cardiac surgery, Hospital Universitario Fundación Jiménez Díaz, Madrid, Spain; ${ }^{3}$ Intensive care medicine, Virgin Health Hospital, Toledo, Spain

Correspondence: V.A. Hortigüela Martín

Intensive Care Medicine Experimental 2020, 8(2): 001450

Introduction: Despite the differential characteristic of older patients, the results are not worse than in younger patients, so clinical decisions in these cases should be individualized.

Objectives: To assess the clinical, evolutive and prognostic characteristics of patients older than 70 years who underwent cardiac surgery for infective endocarditis.

Methods: We evaluated endocarditis patients from two hospital centers (Fundación Jimenez Diaz and Hospital Rey Juan Carlos in Madrid) operated by the same surgical team between 2010 and 2019.

Patients were divided in two groups according to age: GROUP 1 $\leq 70$ years and GROUP $2>70$ years. Any value of $p<0.05$ is considered significant.

Results: We evaluated 103 patients, 50 of them older than 70 years $(48.5 \%)$. Comparing the two groups ( $\leq 70$ years vs $>70$ years) we obtained:.

Demographic data: Male sex (83\% vs 50\%; $p<0.001)$ and age $(58.77 \pm$ 9.36 vs $77.16 \pm 4.49 ; \mathrm{p}>0.001)$.

Personal history: smoking ( $26.4 \%$ vs. $10.2 \% ; p=0.036)$, alcohol/ drugs $(7.5 \%$ vs. $0 \% ; p=0.05)$, arterial hypertension $(45.3 \%$ vs. $71.4 \%$; $\mathrm{p}=0.008)$, COPD ( $17 \%$ vs. $10.2 \% ; \mathrm{p}=0.320)$, Stroke $(18.9 \%$ vs. $38 \%$; $p=0.031)$, hypothyroidism $(5.7 \%$ vs. $16.3 \% ; p=0.083)$, very reduced mobility (Barthel index <50) $(17 \%$ vs $30 \%$; $p=0.118)$, non invasive mechanical ventilation ( $28.3 \%$ vs $12.2 \%$; $p=0.045)$, previous cardiac surgery $(18,9 \%$ vs $55.1 \%$; $p<0.001)$, coronary artery disease $(7.5 \%$ vs $12.2 \% ; p=0.425)$

Disease data: pre-surgery inotropic (34\% vs. $14.3 \%$; $p=0.021$ ), moderate or severe LV dysfunction (20.8\% vs. $10.2 \%$; $p=0.143)$, arrhythmias (22.6\% vs $40.8 \%$; $p=0.048)$, cardiogenic shock $(20.8 \%$ vs $6.1 \%$; $p=$ $0.032)$, prosthetic dysfunction ( $20.8 \%$ vs $51 \%$; $p=0.001)$, emergency surgery $(18,9 \%$ vs $2 \% ; p=0.01)$, pre-intervention antibiotic (7.45 \pm 7.15 vs $12.45 \pm 10.99$ days; $p=0.007$ ) and days between diagnosis and surgery (5.05 \pm 5.89 vs. $7.91 \pm 8.05 ; p=0.44)$, type of surgery (mitral valve $30.2 \%$ vs. $44.9 \%$; aortic valve $39.6 \%$ vs. $28.6 \%$; mitral valve and aortic $18.9 \%$ vs $20.4 \% ; p=0.362$ ) and logistical EUROSCORE ॥ $(25.37 \pm 21.23$ vs $37.04 \pm 20.50 ; p=0.006)$.

Complications: atrial fibrilation ( $17 \%$ vs. $6.1 \% ; p=0.089)$, renal failure requiring temporary hemodialisis (18.9\% vs. $8.2 \%$; $p=0.116$ ) mechanical ventilation $>96$ hours $(17 \%$ vs. $2 \%$; $p=0.011)$, ICU admission days (13.22 \pm 19.44 vs. $7.75 \pm 9.88 ; p=0.08)$, hospytal discharge mortality ( $13.2 \%$ vs. $16 \% ; p=0.688)$

Conclusion: There is a great number of elderly patients in our serie, predominantly male, smokers, alcoholic, with arterial hypertension, previous Stroke, and non invasive mechanical ventilation compared to younger ones. The orders present more pre-intervention complications with need os vasoactives and cardiogenic shock, arrhythmias, 
prosthetic dysfunction, EUROSCORE II log and longer mechanical ventilation times.

\section{1}

Prognostic value of Troponin increase in the first days of ICU admission due to Coronavirus

M. Rojas Amezcua'; M. Guerrero Marin²; MC. Molina De la torre 3; JA. Camacho Pulido ${ }^{4}$; C. Colmenero Aguilar ${ }^{2}$

${ }^{1}$ Medicina intensiva, Hospital Cabra, Cabra, Spain; ${ }^{2}$ Medicina intensiva, Hospital of Jaen, Jaén, Spain; Intensive care, Hospital of Jaen, Jaén, Spain; ${ }^{4}$ Medicina intensiva, Hospital Universitario "San Agustín", Linares, Spain

Correspondence: $C$. Aranda Martínez

Intensive Care Medicine Experimental 2020, 8(2): 001461

Introduction: To asses in those patients admitted on the ICU for coronavirus the relationbetween mortality and some prognostic ratings evaluated at the admission and on the thirdday.

Methods: Prospective cohort study of patients with coronavirus admitted to the ICU at the HospitalUniversitario de Jaén, Hospital San Agustín de Linares and the Hospital Infanta Margarita deCabra.Continuous variables are expressed as median (25th percentile, 75th percentile) andqualitative variables with absolute and relative frequencies. $U$ Mann Whitney was used forcomparison of continuous variables and X2 for qualitative variables. Discrimination wasassessed with the area under RO curve $\mathrm{P}$ \& It $; 0.05$ was considered as statistically significant (s.s).A specifically "R" program "randomForestSRC" was used to impute missing values.

Results: We have studied 52 admitted patients at ICU by coronavirus infection, 41 of them at Jaénhospital ICU, 11 at Cabra hospital.51 of the 52 patients were alive on the third day and they were evaluated. The average agewas $62(58,71)$ years old. APACHE II at the admission was $12(8.5,16)$ points and SOFA $6(3.8)$ points. SOFA on the third day was 7 $(3.5,9)$ points and the SOFA difference between the firstand third day was $0(-2.0)$ points.

There weren't the value of troponin in 10 of the patients (less than $20 \%$ of the sample)and it was input by statistics methods. Ultrasensitive troponin at the admission was $20(10,42)$ and on the third day was $19(9,49)$. In 23 patients $(46 \%)$ the determinations carried out on days 1 and 3 were elevated. In 23 patients (45.09\%) the determinations on the first and third dayswere elevated.

Patients who deceased $(n=19)$ showed higher number of troponin at the admission butthe differences weren't s.s. $26(14,42)$ vs $17(10,42)$, $(p=0.47)$ and on the third day $35(19,54)$ vs $13(8,31),(p=0.029)$. $31.58 \%$ of the 28 patients without troponin increment on the first andthird day, died, and $68.42 \%$ of the 23 patients in whom it was increased in the three days $(p=0.009)$.

Those patients who died $(n=19)$ had more SOFA at the admission 7 $(4,8)$ vs $6(3,7)$ points $(p=0.20)$ but without being significant the differences. However, differences were statisticallysignificant on the third day in regard to SOFA $8(7,9)$ vs $6(3,8)$ points $(p=0.003)$ and thedifferences between the first and the third day of SOFA $-1(-3.0)$ vs 0 $(-1.25,1)$ points $(p=0.007)$. Diifferences regarding APACHE II were also significant $14(12,18)$ vs $10(8,15)$ points $(p=0.011)$.

For a better display of prognostic capacity of SOFA on the third day, patients wereclassified according to SOFA, if was less than 7 points $(n=22)$ with a $9 \%$ of mortality or if was 7 points or more $(n=29)$ with $59 \%$ of mortality $(p<0.0029)$.

Multivariate analysis with logistic regression shows that hospital mortality is relatedwith SOFA higher than or equal to 7 points OR on the third day: $12.78(2.81,94.14)$ with anincrease of troponin on the first day. On the first day, OR: $4.09(1.05,17.79)$. Thediscrimination of the exemplar, evaluated with the area under ROC curve was $0.82(0.72$, 0.93 ).From the 22 patients studied ( $43 \%$ of the sample) with SOFA lower than 7 points and withoutan increase of troponin, just 2 of them died (9\%).

Conclusion: In ICU patients, admitted for coronavirus, on the third day afteradmission to the ICU, most are alive and the increase of troponins on the first and third day isassociated with higher mortality. And if we add, to the said before, high SOFA on the third day,it allows us to select a group of patients with low mortality.
001473

Coronary artery bypass surgery in the last eight years

VA. Hortigüela Martín' ; A. Santos ${ }^{1}$; R. Hernandez Estefanía ${ }^{2}$; E. Rosas Carvajal'; AM. Ioan'; A. Heredero Jung²; A. Donado Miñambres²; JJ.

Paez Vargas ${ }^{1}$; MP. Calderon Romero²; S. Sánchez Bernal ${ }^{1}$; JM. Milicua ${ }^{3}$; G. Aldamiz Echevarria Del Castillo²; C. Pérez Calvo ${ }^{1}$

${ }^{1}$ Intensive care medicine, Hospital Universitario Fundación Jiménez Díaz, Madrid, Spain; ${ }^{2}$ Cardiac surgery, Hospital Universitario Fundación Jiménez Díaz, Madrid, Spain; ${ }^{3}$ lcu, Hospital Universitario Fundación Jiménez Díaz, Madrid, Spain

Correspondence: V.A. Hortigüela Martín

Intensive Care Medicine Experimental 2020, 8(2): 001473

Introduction: Despite appearance of other less invasive therapeutic alternatives for the treatment of coronary artery disease, surgery continues to be a safe technique with very good results in selected patients.

Objectives: Describe clinical and evolutive characteristics of the coronary artery bypass surgery.

Methods: Retrospective and descriptive study of the patients operated of coronary artery disease from January 2012 to June 2020, in two centers by the same surgical and Intensive Care Unit (ICU) team (Hospitales Fundación Jiménez Díaz y Rey Juan Carlos from Madrid).We collected demographics parameters (age, sex), EUROSCORE II, personal history of coronary artery intervention (interventional or surgery), type of surgery (urgent or not), surgical times, post-operative complications, length of stay on ICU and hospital, ICU re-entry and mortality. We made a quartile analysis according to the stay in ICU by hours and got four homogeneous groups on number, sex and age of the patients: GROUP $1=<24 \mathrm{H}$; GROUP $2=24-48 \mathrm{H}$; GROUP $3=48-72 \mathrm{H}$; GROUP $4=>72 \mathrm{H}$.

Results: 724 patients. $84 \%$ males. Medium age of $65,5 \pm 10$ years. Medium EUROSCORE 2 was $4.31 \pm 5.50 .19 .75 \%$ had previous coronary artery intervention. Medium length of stay in ICU was $66 \pm 82$ hours, $10 \pm 8$ days on hospitalization floor and global hospital time was $14 \pm 9$ days. The total mortality was $1.5 \%$. Comparing the groups:

\begin{tabular}{|c|c|c|c|c|c|}
\hline & GROUP 1 & GROUP 2 & GROUP 3 & GROUP 4 & $\mathbf{p}$ \\
\hline $\begin{array}{l}\text { ICU stay } \\
\text { (hours) }\end{array}$ & $<24$ & $24-48$ & $48-72$ & $>72$ & \\
\hline $\mathbf{N}$ & 183 & 270 & 118 & 153 & \\
\hline Males (\%) & 87 & 83 & 86 & 82 & 0.502 \\
\hline $\begin{array}{l}\text { Previous CI } \\
(\%)\end{array}$ & 23 & 17 & 28.8 & 13.7 & 0.0007 \\
\hline Urgent Surgery & 0 & 0.3 & 0.8 & 9.8 & $<0.001$ \\
\hline $\begin{array}{l}\text { Surgery with } \\
\text { EC }\end{array}$ & 79.2 & 81.1 & 85.6 & 85.7 & 0.494 \\
\hline $\begin{array}{l}\text { EUROSCORE } \\
\text { II }\end{array}$ & $\begin{array}{l}2.28 \\
(1.42-3.65)\end{array}$ & $\begin{array}{l}2.34 \\
(1.33-3.96)\end{array}$ & $\begin{array}{l}2.25 \\
(1.40-4.87)\end{array}$ & $\begin{array}{l}4.29 \\
(1.94-9.92)\end{array}$ & $<0.001$ \\
\hline EC time (hours) & $96(77-114)$ & $99(76-120)$ & $\begin{array}{l}101(85- \\
125)\end{array}$ & $\begin{array}{l}105(83- \\
126)\end{array}$ & 0.0840 \\
\hline $\begin{array}{l}\text { Ischemia time } \\
\text { (hours) }\end{array}$ & $60(48-80)$ & $61(49-75)$ & $62(50-80)$ & $64(46-84)$ & 0.949 \\
\hline MV time(hours) & $4(3-5)$ & $5(3-7)$ & $6(4-11)$ & $14(6-61)$ & $<0.001$ \\
\hline $\begin{array}{l}\text { Complications } \\
\text { (\%) }\end{array}$ & 16.39 & 20 & 31.4 & 67 & $<0.001$ \\
\hline $\begin{array}{l}\text { Cardiogenic } \\
\operatorname{shock}(\%)\end{array}$ & 0.5 & 1.1 & 0.8 & 6.5 & 0.002 \\
\hline IABP need $(\%)$ & 0 & 0.4 & 2.5 & 13.7 & $<0.001$ \\
\hline PMI (\%) & 0.5 & 1.1 & 1.6 & 5.8 & 0.002 \\
\hline ARF (\%) & 0.5 & 1.9 & 4.2 & 20.9 & $<0.001$ \\
\hline $\begin{array}{l}\text { ERRT need } \\
(\%)\end{array}$ & 0 & 0.4 & 2.5 & 7.1 & $<0.001$ \\
\hline Bleeding (\%) & 0.5 & 0 & 5.1 & 7.8 & $<0.001$ \\
\hline Floor stay & $7(6-8)$ & $7(7-8)$ & $8(7-10)$ & 13(9-18 & $<0.001$ \\
\hline \multicolumn{6}{|l|}{ (days) } \\
\hline $\begin{array}{l}\text { Total stay } \\
\text { (days) }\end{array}$ & $10(8-14$ & $10(8-14)$ & $11(8-16$ & $18(13-23)$ & $<0.001$ \\
\hline Mortality (\%) & 0.5 & 1.1 & 0 & 4.5 & 0.005 \\
\hline
\end{tabular}


Conclusion: Coronary artery bypass surgery in our series has few complications, short ICU and hospital stay times and low mortality, considering that we have not a specific cardiologic ICU. Patients who require urgent surgery and spend in ICU $>72 \mathrm{~h}$, have worse results.

\section{4}

\section{Hypertension and antihypertensive treatment in patients} with Covid19. Mortality analysis

I. Guzmán Adum'; L. Hidalgo Manchado'; R. Diaz Contreras'; R. De La Chica Ruano

${ }^{1}$ Intensive care unit, Hospital Universitario Virgen de las Nieves, Granada, Spain

Correspondence: I. Guzman

Intensive Care Medicine Experimental 2020, 8(2): 001624

Introduction: Since Covid19 was described, almost 17 million cases and more than 665000 deaths have been reported. Different theories regarding the increased mortality in patients diagnosed with high blood pressure that IECAs/ARAll as antihypertensive treatment have been postulated, the evidence of which has changed.

Objectives:

- To describe the general characteristics of the patients with Covid19 admitted in the ICU of the Virgen de las Nieves Hospital.

- To describe the association between mortality and the history of hypertension.

- To describe the relationship between the use of antihypertensives acting on the renin-angiotensin-aldosterone system and the mortality of patients with Covid19.

Methods: Observational, retrospective and analytical study that includes the 58 patients admitted to ICU by Covid19 between March and July 2020. A database was created in SPSS with the demographic characteristics of patients, comorbidities, antihypertensive treatment, SOFA at admission, APACHEIl in the first 24 hours and type of respiratory support. They were subsequently evaluated in terms of descriptive statistics with frequencies, means, and standard deviation; Mortality-based comparisons were made using Student's T-test for quantitative variables and chi squared for qualitative variables.

Results: Of the 58 patients included, $79 \%$ were men and $21 \%$ women. Their mean age was 60 years $(+/-11)$, their main comorbidities were: hypertension (54\%) $23(39.66 \%)$ of whom were treated with ACEi/ ARB, diabetes mellitus (35\%), obesity (56\%), COPD $(28 \%)$, renal failure (9\%). SOFA at admission $6.79(+/-2.68)$ with significant association with mortality $\mathrm{p}=0.006$. APACHE II in the first $24 \mathrm{~h}$ was $15.9(+/-6.68)$ association with significant mortality $\mathrm{p}=0.002$. IMV since admission $32(55 \%)$ and combination of HFNO and then IMV $17(29 \%)$, unrelated to mortality. ICU mortality $33 \%$, hospital mortality $39 \%$. Mortality of hypertensive patients $24.13 \%(p=0.23)$ and of those taking ACEI/ARB is $13.79 \%(p=1)$.

\section{Conclusion:}

- More than half of the patients admitted to the ICU for Covid19 are hypertensive, however this risk factor does not represent a significant increase in their mortality. Most of them also associate other cardiovascular risk factors.

- In accordance with what is described in the medical literature, hypertensive patients treated with drugs that act on RAAS do not present a higher mortality compared to those who take other types of antihypertensive medication.
001233

Incidence of New Onset Supraventricular Arrhythmias in Septic

Shock Patients and Its Impact on Outcome

S. Hegazy ${ }^{1}$; T. Helmy²; A. Salam²

${ }^{1}$ critical care medicine department, Alexandria Faculty of Medicine, Alexandria, Egypt; ${ }^{2}$ Critical care medicine, Alexandria Faculty of Medicine, Alexandria, Egypt

Correspondence: S. Hegazy

Intensive Care Medicine Experimental 2020, 8(2): 001233

Introduction: Sepsis is frequently associated with new-onset supraventricular arrhythmias (NOSVA). The identified predictors of SVA in sepsis include advanced age, renal failure, preexisting cardiomyopathy, history of hypertension, high SOFA score, and previous calcium channel blocker treatment.

Objectives: The aim of this study is to prospectively assess the prevalence of NOSVA during septic shock, identify the associated factors, and to evaluate its impact on hemodynamics and outcome of patients. Methods: This prospective observational study enrolled 60 critically ill and mechanically-ventilated patients admitted to the critical care medicine department at Alexandria University Hospitals, screened with continuous 12-lead ECG for the occurrence of NOSVA, and assessed for hemodynamic tolerance and prognosis.

Results: The first group (SVA Group) included 24 patients who developed NOSVA. The second group (SR Group) consisted of 36 patients who didn't develop NOSVA. The deterioration in mean arterial pressure increased the prevalence of inotrope indication in SVA group. Serum creatinine was significantly higher in SVA group. Also serum calcium and magnesium were significantly lower in SVA group. Patients with SVA group had higher rate of mortality within 7 days from admission and higher duration on mechanical ventilation but the difference from SR group didn't reach the level of statistical significance.

Conclusion: New onset SVA is frequent and poorly tolerated during septic shock, and is associated with higher rates of inotrope use. Hypomagnesemia, hypocalcemia and elevated serum creatinine are considered as potential co-factors or causes for the development of NOSVA in septic shock patients.

\section{6}

Improving the Documentation of Emergency Anaesthesia in the ICU and Emergency Department

C. Docherty ${ }^{1}$; E. Kerr ${ }^{1}$; K. Bennett

${ }^{1}$ Critical Care, NHS Lanarkshire, Wishaw, United Kingdom

Correspondence: $C$. Docherty

Intensive Care Medicine Experimental 2020, 8(2): 001206

Introduction: Emergency anaesthesia is frequently performed in ICU and Emergency Departments for critically ill patients. However, it has been demonstrated that there is a higher incidence of complications when performing emergency anaesthesia in these environments compared to the operating theatre. In our department we anecdotally noted that documentation of emergency anaesthesia was frequently of poor quality or had missing information. During the course of an ICU admission patients may require reintubation and details (such as grade of intubation, equipment and drugs used) regarding the initial procedure are essential for safe planning.

Objectives: The aim of our quality improvement project was to improve the documentation of emergency anaesthesia in the ICU and Emergency Department by implementation of a sticker.

Methods: A retrospective audit was performed of 40 consecutive patients who were intubated and ventilated in the emergency department or ICU. 17 key points for documentation were identified and data was collected as to whether or not these were documented: indication, intubator, airway assistant, monitoring, laryngoscope, airway, grade, tied at lips, manoeuvres, pre-oxygenation, apnoeic ventilation, adjuncts, opiate, anaesthetic agent, neuromuscular blocker, emergency drugs and critical incidents.

Following the audit an emergency anaesthesia sticker was introduced to act as an aide memoire and to improve efficiency of documentation. These were located in the ICU and emergency department. A 
further prospective audit of 40 consecutive patients was performed to determine whether or not there was an improvement.

Results: Prior to the introduction of the sticker, the audit confirmed that documentation was inadequate. Use of monitoring was documented in $23 \%$ of cases and pre-oxygenation in $18 \%$. Choice of laryngoscope was documented in $80 \%$ of cases, airway used in $78 \%$ and adjuncts in $23 \%$. Of note in $13 \%$ of cases there was no documentation of drugs used either in the notes or on the kardex and $44 \%$ made no reference to whether or not any critical incidents occurred.

Following the implementation of the sticker uptake on use was $68 \%$ (27/40 cases). Where the sticker was used, documentation was noted to be improved. $100 \%$ documented monitoring, pre-oxygenation, laryngoscope, airway and drugs. 70\% documented use of adjuncts and $93 \%$ documented whether or not a critical incident occurred.

Conclusion: Accurate and thorough documentation of emergency anaesthesia is not only important for medico-legal purposes but is also crucial to ensure that any future intubations are prepared for and managed as safely as possible. Implementation of an emergency anaesthesia sticker is a simple and cost effective intervention and this quality improvement project demonstrated that it delivers a significant improvement in the documentation of emergency anaesthesia in the ICU and emergency department. Further education within the department will be aimed at improving the uptake of use of the sticker.

\section{Reference(s) and grant ackowledgment(s)}

1. 1. Cook TM et al. Major complications of airway management in the UK: results of the 4th national audit project of the Royal College of Anaesthetists and the Difficult Airway Society. Part 2 Intensive care and the emergency department. British Journal of Anaesthetics 2011.

\section{4}

Formulation and Implementation of a COVID-19 Modified Percutaneous Tracheostomy Checklist to Ensure Safety of Healthcare Workers and to Promote Good Medical Record Keeping

S. Ng ; M. Watanabe'; J. Mandeville' ; P. Shanmugasundaram¹; A.

Manoras ${ }^{1}$; T. Ali

${ }^{1}$ Intensive care department, Stoke Mandeville Hospital, Aylesbury, United Kingdom

\section{Correspondence: S. Ng}

Intensive Care Medicine Experimental 2020, 8(2): 001234

Introduction: COVID-19 is considered an airborne pathogen by the WHO and Public Health England in the context of aerosol generating procedures. During the 2002 to 2004 SARS epidemic, healthcare workers were shown to be at a significantly increased risk of infection(1), with tracheal intubation posing the highest risk for transmission(2). Therefore, amid the current pandemic additional precautions have been introduced to minimise the risk of SARS-CoV-2 transmission.

Medical record keeping plays an integral role in delivering safe patient care $(3,4)$. Despite this, documentation is often given a low priority when additional demands are placed on doctors.

In an effort to improve patient and staff safety, and to promote a consistent procedural approach and good quality documentation among doctors, we implemented a modified COVID-19 percutaneous tracheostomy checklist and procedure note.

Objectives: This project aimed to assess the improvement in quality of documentation of percutaneous tracheostomy procedures performed by the ICU team after the implementation of the checklist.

Methods: We undertook retrospective audits of the documentation of percutaneous tracheostomy procedures on ICU during the whole of $2019(n=14)$ and during the COVID-19 period up to June 2020 $(n=11)$.
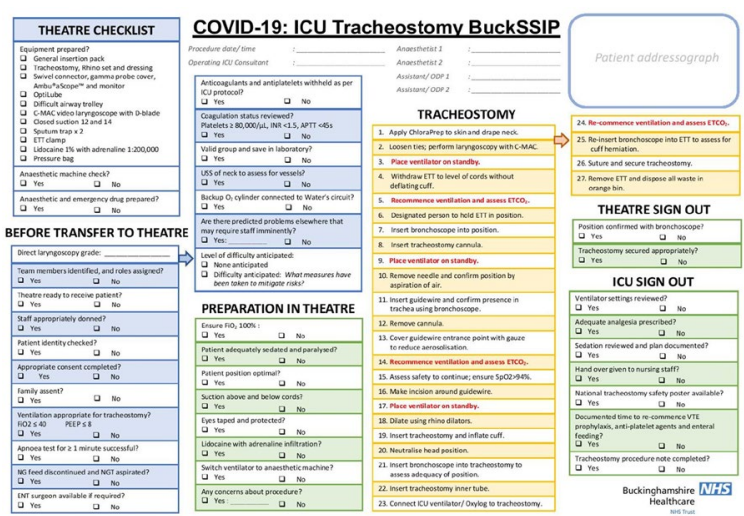

Following the first audit, we formulated and introduced a modified COVID-19 percutaneous tracheostomy checklist. The checklist not only includes 'Sign In', 'Time Out' and 'Sign Out' but also highlights key steps in the updated procedure guidelines, which were created through repeated simulation sessions. This formed part of a COVID-19 Tracheostomy Local Safety Standards for Invasive Procedures (LocSSIPs) to promote a more consistent technical approach amongst operating doctors.

Results: The initial audit from 2019 in our Trust demonstrated a poor quality of documentation. This issue was successfully addressed with implementation of the COVID-19 percutaneous tracheostomy checklist and procedure note. The second audit reported a significant improvement: $100 \%$ of percutaneous tracheostomy procedures were documented using the designated form, versus $50 \%$ in the first audit; $100 \%$ of equipment used were also recorded, up from $57.14 \%$ in 2019. A consistent improvement was also observed in the documentation of patient consent, post-operative instructions, and overall quality of documentation. Furthermore, doctors and theatre staff reported a reduction in concern when remembering the additional steps made necessary for personal protection.

Conclusion: The implementation of a checklist and procedure note not only helped to provide a consistent structured approach to the procedure and to documentation but also reduced anxiety among the healthcare workers. We strongly feel that the quality of documentation is a consistently overlooked element of good quality patient care and we find the improvement observed, even in the face of increased technical and human factors complexity, very encouraging.

Reference(s) and grant ackowledgment(s)

1. 1. Kamming, D., Gardam, M. and Chung, F., 2003. I. Anaesthesia and SARS. British Journal of Anaesthesia, 90(6), pp.715-718.

2. 2. Tran, K., Cimon, K., Severn, M., Pessoa-Silva, C. and Conly, J., 2012. Aerosol Generating Procedures and Risk of Transmission of Acute Respiratory Infections to Healthcare Workers: A Systematic Review. PLoS ONE, 7(4), p.e35797.

3. 3. Care Quality Commission. Guidance for Providers. Regulation 17: Good Governance. [Internet]. Cqc.org.uk. 2020 [cited 27 July 2020]. Available from: https://www.cqc.org.uk/guidance-providers/regulations-enforcement/ regulation-17-good-governance\#guidance.

4. 4. Good Medical Practice [Internet]. General Medical Council; 2013 [cited 30 July 2020]. Available from: https://www.gmc-uk.org/-/media/documents/ good-medical-practice—english-20200128_pdf-51527435.pdf.

\section{7}

Scaling a UK District General Hospital (DGH) Critical Care Unit by $400 \%$ during the COVID 19 Pandemic

R. Loveless'; A. Falek' ;. Samuels'; A. Myers ${ }^{1}$

${ }^{1}$ Intensive care unit, East Surrey Hospital, Redhill, United Kingdom

Correspondence: R. Loveless

Intensive Care Medicine Experimental 2020, 8(2): 001237

Introduction: East Surrey Hospital is a DGH in South East England that serves a population of 535,000 and has 700 acute beds(1). The Critical 
Care Unit has 8 level 3 beds and 8 level 2 beds. The unit is staffed by 10 consultants, 16 junior doctors (50\% airway trained), and 84 nurses ( $1 / 3$ have less than 2 years ICU experience). The trust has 17 ICU ventilators (including 4 transfer ventilators) and 29 anaesthetic machines offering only basic mandatory ventilation. COVID 19 required planning to accommodate an increase in patients with severe respiratory failure requiring mechanical ventilation.

Objectives: We aimed to increase level 3 bed capacity safely by $400 \%$. Methods: We identified 6 elements of our organisation requiring attention in order to facilitate rapid escalation in ICU capacity.

1. Footprint.

A 22 bed medical ward was converted into an additional ICU. At peak this unit had 17 ventilated patients. Theatre recovery was used for non-COVID patients.

\section{Equipment.}

17 ICU ventilators were supplemented by 21 anaesthetic machines. Anaesthetic machine gas analysers were used as EtCO2 monitors ran out. Haemofiltration units were rotated between patients in 12 hour sessions. Midazolam sedation was used as supplies of propofol were compromised.

\section{Infection Control.}

Full PPE was used in all critical care zones during the pandemic. A patient gym became a donning area and temporary partitions created a one way system for doffing. Acquiring PPE was challenging nationwide, but the procurement department was proactive ensuring adequate supply.

\section{Staffing}

An emergency rota was created that provided $24 / 7$ consultants on site, with 9 ICU doctors at all times. 10 consultants and 24 trainees worked a 1 in 4 pattern, with 6 anaesthetic consultants joining critical care. Doctors with previous experience were redeployed to ICU. Anaesthetic consultants staffed 2 MERIT teams 24/7 for emergency intubations and transfers. 151 nurses were redeployed from Theatres and surgical wards. Patients were divided into "zones" of 3-4 patients, with 1 ICU nurse leading 3-4 non-ICU trained nurses. Physiotherapists, consultant and trainee surgeons formed proning teams.

\section{Communication.}

Two way radios allowed communication between units, doctors, charge nurses and the emergency department. A temporary bespoke electronic patient record (COVID SitRep) was designed using FileMaker(TM) and was used for patient tracking, handover and audit. A family liaison team updated each patient's family daily.

\section{Clinical Management Protocols.}

Protocols for airway management, CT transfers, admission investigations and proning ensured patients received the highest level of care. Results: We had a maximum of 33 ventilated patients at any one time, across 3 geographically distant critical care units. Over the pandemic we treated 59 confirmed and 34 suspected COVID-19 patients in critical care, and performed 10 non-clinical transfers of ventilated patients. Our mortality for ventilated COVID 19 patients was $29 \%$.

Conclusion: During the COVID 19 pandemic we delivered safe, effective care at $400 \%$ capacity. Using a robust education and management structure for new nurses, redesigning rotas to increase staffing and creating protocols using national guidance(2) ensured safe expansion. Our outcomes compare favourably with other institutions in the UK (ICNARC mortality of ventilated COVID 19 patients is $48 \%(3)$ ). Feedback from patients, staff and families was positive. Structures are in place for psychological support for staff and patients, which aim to mitigate the effects of an unprecedented time.

\footnotetext{
Reference(s) and grant ackowledgment(s)

1. 3. Intensive Care National Audit and Research Centre. ICNARC Report on COVID-19 in Critical Care 24th July 2020. https://www.icnarc.org/DataServic es/Attachments/Download/af7be2d4-bdcd-ea1 1-9127-00505601089b.

2. 2. Intensive Care Society/Royal College of Anaesthetists/AAGBI/FICM. Information, guidance and resources supporting the understanding and management of coronavirus (Covid 19). https://icmanaesthesiacovid-19. org.

3. 1. Surrey and Sussex NHS Trust website. https://surreyandsussex.nhs.uk/ about-us/.
}

001243

Family communications - lessons learned during the Covid-19 outbreak

S. Dunin De Skrzynno'; E. Gravell'; L. Davies ${ }^{1}$; M. Cuartero ${ }^{1}$

${ }^{1}$ St. Mary's Intensive Care Unit., Imperial College Healthcare NHS Trust, London, United Kingdom

Correspondence: S. Dunin De Skrzynno

Intensive Care Medicine Experimental 2020, 8(2): 001243

Introduction: The importance of good communication in an Intensive Care Unit (ICU) is being increasingly recognised.[1] Effective communications improves families' trust in the medical team clinical ability and decision making, as well as their own psychological well being.[2].

The COVID19 pandemic is an unprecedented health crisis which has reshaped our way of practicing medicine and communicating with our patients and families. The growth rate of the COVID19 virus led to restrictions and subsequently banning of all visitors to hospital wards and ICU. At the height of the pandemic, communication between medical staff and families could only be through phone or video calls.

Objectives: To assess communication with families at the height of the pandemic in a UK based intensive care unit. This was measured against standards set by senior nursing staff and our consultant body prior to the pandemic. Standards set were for patient families to be contacted by nursing staff daily, and medical staff every 48 hours.

Methods: We conducted a retrospective case note review of all patients admitted to the Adult Intensive Care Unit (AICU) or Recovery Intensive Care Unit (RICU) between the 16th and 31st of March 2020 at St Mary's hospital. Only COVID19 positive, or clinically suspected cases were included in this study.

Family Communication notes, Nursing documentation as well as Daily Documentation notes were reviewed on the critical care software system IntelliSpace Critical Care and Anaesthesia (ICCA).

Key clinical characteristics of patients were recorded including age, length of ICU admission, if mechanical ventilation was used, level of care on admission, and whether any End of Life (EoL) care was in place. Documented family discussions were reviewed and classified as either: First meeting within 48 hours, Update or Follow-up, Clinical deterioration or DNACPR discussion. The date and clinical staff leading each communication was recorded.

Results: A total of 32 patients were admitted with positive or clinically suspected COVID19, and 331 communications were recorded. The mean length of stay in ICU was 17.3 days, and the mean age was 54 years old. Mechanical ventilation was used in $29(91 \%)$ of our cohort. 12 (38\%) patients died in the unit, 15 (47\%) were discharged to a ward. Among our cohort, $16(50 \%)$ had a DNACPR in place, and 10 (31\%) had an EoL care pathway in place.

Nursing staff met our standard of contacting families daily in $1(3 \%)$ patient, and doctors of contacting families every 48 hours in $6(18 \%)$ patients. $5(18 \%)$ patients had no documented family discussions with a doctor, $3(9 \%)$ had no documented conversation with a nurse and $2(6 \%)$ had no documented discussions with any healthcare professional.

Family discussions within 48 hours of admission were made in 25 $(78 \%)$ of our cohort, with nursing staff initiating $18(72 \%)$ of these conversations. The median time to a first documented discussion with family for nurses was 1 day, and 4 days for doctors.

Junior Doctors led 8/10 (80\%) of documented DNACPR discussions, and 25/58 (43\%) Clinical Deterioration discussions. Consultant discussions were held with families in 2/10 (20\%) patients on an End Of Life care pathway.

Conclusion: At the height of the pandemic, nurses and doctors did not meet the standards set for family communication. Nursing staff initiated family discussions within 48 hours of admission, and Junior Doctors were leading difficult discussions with families. Agreed guidelines on the regularity of family communication during a pandemic, and the level of Nurse/Doctor expected to communicate needs to be developed. Further training is needed to support Junior Doctors in conducting difficult discussions if a further spike is to occur. 
Reference(s) and grant ackowledgment(s)

1. 2. Lilly CM, De Meo DL, Sonna LA, et al. An intensive communication intervention for the critically ill. Am J Med. 2000;109(6):469-475. https://doi. org/10.1016/s0002-9343(00)00524-6.

2. 1. Rubenfeld, G.D. and Randall Curtis, J. (2001). End-of-life care in the intensive care unit: A research agenda. Critical Care Medicine, 29(10), pp.2001-2006.

\section{6}

Tracheostomy in Intensive Care: Delays between decision and procedure

A. Gupta'; J. Gosling

${ }^{1}$ Anaesthesia and Intensive Care, Chelsea and Westminster NHS trust, London, United Kingdom; ${ }^{2}$ Anaesthesia, West Middlesex University Hospital, Isleworth, United Kingdom

Correspondence: A. Gupta

Intensive Care Medicine Experimental 2020, 8(2): 001246

Introduction: Tracheostomy is usually performed for patients with prolonged stays on mechanical ventilation and/or embarking upon a slow wean from mechanical ventilation.

Objectives: The aims of this audit were to;identify the length of, and reasons for, delays in performing tracheostomy once a decision made to proceed and to determine the adequacy of documentation of delays.If a delay in carrying out tracheostomy is encountered, whether planned or unforeseen, the reason for delay should be documented for each day tracheostomy is delayed beyond 2 days after a decision. The two standards employed in this audit are therefore; $75 \%$ of patients should receive a tracheostomy within 2 days of decision to proceed and if tracheostomy is delayed beyond 2 days, $100 \%$ of the days in question should have a documented reason for the delay.

Methods: Retrospective review of patients undergoing either percutaneous or surgical tracheostomy during ICU stay between September 2015 and September 2016. We recorded 1)technique of tracheostomy 2) number of days between decision to perform tracheostomy (classed as Day 0) and the procedure itself, where there were more than 2 days between decision and procedure, the number of days for which a reason for the delay was documented.

Results: A total of 21 tracheostomy episodes were recorded during the audit period, of which 12 were percutaneous and 9 surgical. 2 patients had unsuccessful percutaneous attempts followed by successful surgical tracheostomies.Overall, $12 / 21$ patients $(57 \%)$ had their tracheostomies performed within 2 days of the decision to proceed. Only 1 additional patient had procedure within 3 days of decision; the remaining 8 patients waited 4 or more days.

There was no significant difference between the delays in the percutaneous and surgical groups, although both patients with a delay of 8 days required surgical tracheostomies.

$\begin{array}{lll} & \text { Mean } & \text { Median } \\ \text { Overall } & 3.0 & 2 \\ \text { Percutaneous } & 2.3 & 2 \\ \text { Surgical } & 4.1 & 4.5\end{array}$

Table 1 - Days Delay (by type of procedure)

The reasons documented were:

\begin{tabular}{l|}
\hline Liver USS \\
\hline ENT review required $(x 2)$ \\
\hline ENT not available $(x 2)$ \\
\hline Atrial fibrillation causing compromise \\
\hline Coagulopathy requiring reversal \\
\hline Anaesthetist not available \\
\hline Family discussion \\
\hline
\end{tabular}

Table 2 - Documented reasons for delays in tracheostomy

Conclusion: The unit did not meet the standards for either timely performance or documentation of reasons for delays, with rates of $57 \%$ (standard $75 \%$ ) and $18 \%$ (standard 100\%) respectively. Possible reasons for this were availability of experienced anaesthetic and ENT .Clinical limitations e.g. coagulopathies or anti-coagulant treatment, or a general instability and low reserve to tolerate procedure. It was difficult to comment on the appropriateness of delays by a sizeable minority of patients because of the poor standard of documentation. Possible reasons for incomplete documentation include insufficient time on ward round or at the end of shifts, lack of or misunderstanding of reasons for delays and attributing little importance to such documentation.

Reference(s) and grant ackowledgment(s)

1. Veenith T, Ganeshamoorthy S, Standley T, Carter J, Young P. Intensive care unit tracheostomy: a snapshot of UK practice. Int Arch Med. 2008 Oct 25;1(1):21. https://doi.org/10.1186/1755-7682-1-21.

2. Kluge S, Baumann HJ, Maier C, Klose H, Meyer A, Nierhaus A, Kreymann G. Tracheostomy in the intensive care unit: a nationwide survey. Anesth Analg. 2008 Nov;107(5):1639-43. https://doi.org/10.1213/ane.0b013e318188b818.

3. Not applicable.

\section{9}

Short-term mortality of patients $\geq \mathbf{8 0}$ years old admitted to European intensive care units - a prospective cohort study J. Fronczek ; H. Flaatten ${ }^{2}$; B. Guidet ${ }^{3}$; K. Polok'; BY. Andrew ${ }^{4}$; D. De Lange ${ }^{5}$; J. Fjølner ; J. Górka'; C. Jung ${ }^{7} ;$ K. Kusza ${ }^{8}$; S. Leaver ${ }^{9}$; RP. Moreno ${ }^{10}$; R. Owczuk ${ }^{11} ;$ A. Rhodes ${ }^{12}$; W. Szczeklik ${ }^{1}$

${ }^{1}$ Intensive care and perioperative medicine, Jagiellonian University Medical College, Kraków, Poland; ${ }^{2}$ Department of anaesthesia and intensive care, Haukeland University Hospital, Bergen, Norway; ${ }^{3}$ Réanimation Médicale, Hôpital Saint-Antoine, Paris, France; ${ }^{4}$ Department of anesthesiology, Duke University, Durham, United States of America; ${ }^{5}$ Department of intensive care medicine, Utrecht University, Utrecht, Netherlands; ${ }^{6}$ Department of intensive care, Aarhus University Hospital, Aarhus, Denmark; ${ }^{7}$ Dep. of cardiology, pulmonology and angiology, University Hospital of Düsseldorf, Düsseldorf, Germany; ${ }^{8}$ Department of anaesthesiology and intensive therapy, Poznan University of Medical Sciences, Poznań, Poland; ${ }^{9}$ Research lead critical care directorate, St George's Hospital, London, United Kingdom; ${ }^{10}$ Unidade de cuidados intensivos neurocríticos, Hospital de São José, Centro Hospitalar de Lisboa Central, Nova Médical School, Lisbon, Portugal; ${ }^{11}$ Department of anaesthesiology and intensive therapy, Medical University of Gdańsk, Gdańsk, Poland; ${ }^{12}$ Anaesthesia and intensive care medicine, St George's University Hospitals NHS Foundation Trust, Londres, United Kingdom

Correspondence: J. Fronczek

Intensive Care Medicine Experimental 2020, 8(2): 001259

Introduction: Limited evidence indicates that mortality rates of elderly patients treated in the intensive care unit (ICU) vary widely across Europe.

Objectives: The aim of this study was to evaluate the variation in short-term mortality among patients $\geq 80$ years old admitted to European ICUs.

Methods: We used data from two multicenter prospective cohort studies which enrolled very old intensive care unit patients (VIPs) between the years 2016 and 2019 in 20 European countries. The primary outcome was mortality within 30-days from admission to the ICU. Nine clinical variables were adjusted for in multilevel logistic 
regression models. Country was included in the model as a random effect and categorized healthcare expenditure per capita $(<2500 \$$, 2500-5000\$, >5000\$) was a country-level variable.

Results: The median age in the sample of 8257 consecutive patients was 84 years (IQR: 81-86) and the median Sequential Organ Failure Assessment score was equal to 6 points (IQR: 4-10). Crude mortality in participating countries ranged from $10.6 \%$ to $50.0 \%$ in the ICU and from $21.9 \%$ to $54.4 \%$ in 30-day follow-up. After adjusting for patient-level variables, the proportion of individual variation in mortality attributable to systematic differences between countries was equal to $3 \%$ at discharge from the ICU and $2 \%$ in 30-day follow-up. Lower healthcare expenditure per capita was associated with higher ICU mortality, but we did not find evidence that a similar relationship exists in 30-day follow-up.

Conclusion: Systematic differences between patients, not countries, largely explain the variation in short-term mortality among European countries. Lower healthcare expenditure is associated with higher mortality in the ICU, but the direction and magnitude of this relationship is less certain in 30-day follow-up.

\section{5}

\section{Beta-D-Glucan testing in the ICU: A single-centre experience}

DO. Hamilton'; S. Waite'; A. Howard²; I. Welters

${ }^{1}$ Critical Care, Royal Liverpool and Broadgreen University Hospitals NHS Trust, Liverpool, United Kingdom; ${ }^{2}$ Department of microbiology, Royal Liverpool and Broadgreen University Hospitals NHS Trust, Liverpool, United Kingdom

Correspondence: D.O. Hamilton

Intensive Care Medicine Experimental 2020, 8(2): 001265

Introduction: Invasive fungal disease is a common cause of morbidity and mortality in Intensive Care Units (ICU) worldwide [1]. A BetaD-glucan (BDG) test has a high negative predictive value and can be used to exclude invasive fungal disease in appropriate populations $[1,2]$.

Objectives: To describe the prevalence and clinical outcomes of patients treated with empirical antifungals and to identify how BDG testing is utilised in the ICU at the Royal Liverpool University Hospital, a tertiary academic teaching hospital.

Methods: We performed a retrospective observational study of all patients treated with anti-fungal medication between December 2018-December 2019 utilising medical notes, microbiological data and prescription charts. Patients treated for oral or genital candidiasis or with prophylactic antifungals were excluded.

Financial estimates were made by analysing the prescriptions of patients with no positive fungal culture, excluding those with haematological malignancy or oesophageal perforation, for whom empirical anti-fungal use is appropriate, and assuming an eight-day turnaround time for testing.

Results: One-hundred-and-six patients received anti-fungal medication over 107 admissions to ICU during the specified time period. Sixty-two (58\%) admissions were male, the mean age was 56 and 63\% were admitted from surgical specialties, most commonly Upper Gastrointestinal or Hepato-pancreato-biliary. The inpatient mortality was 28.9\%.

Fifty-seven (53\%) patients had a positive fungal culture, 3.9\% of the 1480 admissions in the study period. The most common species cultured was Candida albicans $(\mathrm{n}=25)$, followed by Candida glabrata $(n=15)$. The most commonly infected sites were: intra-abdominal fluid $(n=25)$; blood culture $(n=11)$; and urine $(n=6)$. There were four blood stream infections due to central venous catheters. The median number of days patients were treated with antifungals was 16 [IQR 11-28].

Of the 49 patients with no positive fungal culture, only $6(12 \%)$ had a BDG test sent, which were all negative. The mean number of days for a BDG result to be available was $8.3 \pm 3.3$ days. We estimate that applying BDG testing to appropriate patient cohorts would save 142 days of anti-fungal use and approximately $€ 12,300$ per year.

Conclusion: It is common for no fungal species to be identified in patients who are started on empirical antifungals. BDG testing is under-utilised in our ICU and improving its uptake and speed are important measures for both antifungal stewardship and fiscal management.

\section{Reference(s) and grant ackowledgment(s)}

1. [1] - British Society for Medical Mycology best practice recommendations for the diagnosis of serious fungal diseases. Schelenz et al. Lancet Infect Dis 2015; 15: 461-74.

2. [2]- $\beta-D-g l u c a n$ assay for the diagnosis of invasive fungal infections: a metaanalysis. Karageorgopoulos et al. Clin Infect Dis 2011; 52: 750-70. 3. Nil.

\section{0}

'Training for ICU Crash Course (TICC)': Rapid, modifiable cross-skilling course for staff redeployed to Intensive Care Units in response to the COVID-19 pandemic

D. Sureshkumar'; V. Blackburn ${ }^{1}$; E. Hardman ${ }^{1}$

${ }^{1}$ Anaesthetics/ICU, Whittington Hospital, London, United Kingdom

Correspondence: $D$. Sureshkumar

Intensive Care Medicine Experimental 2020, 8(2): 001270

Introduction: The COVID-19 pandemic has seen a unique response arise in hospitals, one feature of which has been the rapid redeployment of healthcare workers of all stratifications to intensive care units (ICUs) across the country. This posed a sudden and unfamiliar challenge of a high-acuity environment to many staff, who have had little or no prior ICU experience. In the Whittington Hospital ICU, staff expressed uncertainty about assessing and managing patients, but opportunities for bedside teaching were limited due to workload, staffing ratios and communication challenges due to personal protective equipment (PPE). This teaching programme was designed to deliver high quality, relevant information for navigating ICU, appropriate to different healthcare backgrounds of staff recruited at short notice. The aim was to provide basic but thorough cross-skilling swiftly, in a structured and standardized way, to enhance confidence amongst newly deployed staff and to improve patient safety in an unprecedented situation ${ }^{1}$.

Objectives: The overall objective was to increase the competence of as many staff as possible - both medical and auxiliary - who were redeployed to the Whittington ICU. Further aims were to improve staff confidence in assessing and managing an intensive care patient by $25 \%$ following delivery of 'Training for ICU Crash Course' ('TICC') programme, and provide with the relevant learning supplementation appropriate to level. Lastly, to provide bedside checklist resources for basic management as an aide-memoir, for improved communication under difficult circumstances and amongst unfamiliar teams.

Methods: TICC comprised of a standalone session delivered as a tutorial with accompanying slides away from the bedside so staff did not have responsibility for clinical care and were not hindered by wearing PPE. It consisted of a set of slides using a standardised systems (ABCDE) approach which was tailored to the audience background, and augmented by practical demonstration of simple equipment such as arterial lines, three way taps and giving sets. Participants were all healthcare workers with 6 months or less experience in Intensive Care, or any current ICU recruit who felt that they would benefit from attending.

The learning supplement consisted of two stratified checklists appropriate to level (one targeted at doctors, one at auxiliary staff), given as handouts at the end, in the format corresponding to the course. The bedside resource was a further corresponding laminated checklist, with a wipe-clean tick box function, for use in PPE on the unit. It was designed to improve coordination, efficiency and safety in the patient's multi-person team by keeping track of jobs and assessments, again according to the system taught.

Feedback including confidence levels by system, usefulness of content and format was collected immediately before and after teaching by an anonymous questionnaire. Further feedback was obtained several weeks later by an online questionnaire to assess retention and confidence. 
Results: The course was delivered to 47 members of staff who were working in the Whittington Hospital ICU including doctors (foundation doctor level to medical registrars), registered nurses, physiotherapists, healthcare assistants, medical and nursing students, other healthcare workers (e.g. speech and language therapists, podiatrists). Of the students, 74\% (35/47) had no previous ICU experience. The topics that people identified as being the most anxious about were airway and breathing. Immediately after the course, confidence levels in assessing an ICU patient were improved by $39 \%$, increasing from a mean score of $2.5 / 5$ to $3.5 / 5$. 2 months following the course, participants confidence levels were $36 \%$ greater than prior to the course. Students reported feeling most confident in assessing breathing, and the most useful topics were determined to be either airway, or the whole session A-I. Qualitative feedback on the usefulness of the learning materials distributed post-tutorial was highly positive, with a constructive influence on team coordination.

Conclusion: The teaching session was designed to be both achievable and rapid to implement, while still being fully modifiable as to be appropriate for a variety of audiences. It was successful in this aim and in providing a basic standardised structure for novice workers on ICU. Increased confidence levels of healthcare staff were reported in high risk areas (e.g breathing assessment) which is a vital skill for safely managing patients with COVID-19 pneumonitis. In particular, qualitative feedback suggested it helped to maintain safety standards and team co-ordination when ICU nursing ratios were low. This course has the potential to rapidly cross-skill staff from a variety of backgrounds to improve staff confidence and wellbeing, team co-ordination and efficiency. Ultimately improving patient safety in rapid response to a novel, chaotic and highly challenging situation.

\section{Reference(s) and grant ackowledgment(s)}

1. N/A.

2. 1. Brzezinski, M. et al.(2019). Time-efficient, goal-directed and evidencebased teaching in the ICU, Current Opinion in Anaesthesiology, 32 (2) p136-143.

001271

Tele-assisted Safety Huddle implementation during COVID-19 outbreak is associated with positive health care workers perceptions and reduction in hospital mortality for critically ill patients

DT. Noritomi': AP. Mikulenas ${ }^{1}$ : C. Chrispino ${ }^{2}$ : CN. Roderjan². PTMCA. Rocha'; JP. Maciel ${ }^{3}$; SCG. Da ${ }^{2}$; NS. Nascimento ${ }^{4}$; WT. Nakasato ${ }^{5}$

${ }^{1}$ Quality and patient safety department, Rede Impar, Sao Paulo, Brazil; ${ }^{2} \mathrm{Crit}-$ ical care deparment, Hospital São Lucas Copacabana, Rio de Janeiro, Brazil; ${ }^{3}$ Quality and patient safety department, Hospital São Lucas Copacabana, Rio de Janeiro, Brazil; ${ }^{4}$ Head of nurse, Hospital São Lucas Copacabana, Rio de Janeiro, Brazil; ${ }^{5}$ Medical director, Hospital São Lucas Copacabana, Rio de Janeiro, Brazil

Correspondence: $D$. Noritomi

Intensive Care Medicine Experimental 2020, 8(2): 001271

Introduction: The COVID 19 pandemic brought a significant increase in workload for healthcare workers (HCW) around the world (1). This fact was especially relevant in the ICU where adverse events could be caused by this burden. The Safety Huddle $(\mathrm{SH})$ is a strategy designed to mitigate this risk (2), mostly focusing on increase in situation awareness, communication skills and psychological safety for HCW. It has not been tested in the current COVID-19 scenario.

Hospital Sao Lucas is a 210-bed general private hospital in the city of Rio de Janeiro - Brazil. Before the pandemic, the hospital had $75 \mathrm{ICU}$ beds. After the beginning of COVID-19 in the city of Rio de Janeiro, the number of ICU beds was increased to 100 beds, of which 79 beds dedicated exclusively to COVID-19 patients. As part of the Quality and Safety department improvement initiatives, two-tier safety huddle was implemented in a 60 bed ICU.
Objectives: The aim of the present study is to test if the feasibility, HCW impact and clinical outcomes associated with SH implementation in the context of COVID-19 pandemic.

Methods: The first level SH was leaded by the shift nurse and involved the bedside team of the corresponding shift. The second level was conducted with representants of each ICU (senior doctor or nurse), heads of nurse, respiratory therapy, medical director and quality and safety. Most of participants were in remote contact with each other, including the leader of the 2 nd tier.

An anonymous electronic survey consisting of perceptions was launched for HCW, 5 weeks after the beginning of the safety huddle.

A before and after analysis was performed for the critically ill patients. Outcome for COVID 19 was retrieved from the EMR, using current definitions.

Results: Safety huddles were performed daily, including weekends, from 7 thMay, until now. The duration was approximately $10 \mathrm{~min}$ in the fist level and 60 minutes in the second level.

Three hundred and twenty (35\%) two out of $906 \mathrm{HCW}$ answered the survey of whom 181 (56\%) RN and nurse assistants, 87 (27\%) respiratory therapists and $54(17 \%)$ other professionals (including 4 physicians). The main findings are described in the Table 1.

$\begin{array}{ll}\text { Question } & \begin{array}{l}\text { Agreement } \\ \text { rate }\end{array} \\ \begin{array}{l}\text { Do you believe that Safety Huddle brings positive results? } \\ \text { Do you believe that Safety Huddle has a positive effect in } \\ \text { your daily routine? }\end{array} & 97 \% \\ \begin{array}{l}\text { Do you feel comfortable in sharing your perceptions and } \\ \text { doubts during the Safety Huddle? }\end{array} & 92 \% \\ \begin{array}{l}\text { Do you believe that structural improvements were } \\ \text { implemented because of the Safety Huddle? }\end{array} & 89 \% \\ \begin{array}{l}\text { Table 1. Proportion of respondents in a HCW survey concerning the perceptions of Safety Huddle } \\ \text { M }\end{array}\end{array}$

From 16th March 2020 to 15th July, HSL discharged 452 patients, of whom 255 (56\%) stayed in the ICU for some time. The main clinical outcomes, before and after the beginning of $\mathrm{SH}$ are described in table 2 .

$\begin{array}{llll}\begin{array}{l}\text { Characteristics and } \\ \text { clinical outcome }\end{array} & \begin{array}{l}\text { Before } \mathrm{SH}(\mathrm{n}= \\ \text { 91 patients })\end{array} & \begin{array}{l}\text { After } \mathrm{SH} \\ \text { patients })\end{array} & \mathrm{n}=164 \\ \text { Age - years }( \pm \text { SD }) & 69.0 & 70.2 & 0.59 \\ \text { SAPS3 - points }( \pm \text { SD) } 58.4 & 57.7 & 0.70 \\ \text { Use of MV - n }(\%) & 55(60) & 96(58) & 0.76 \\ \text { Hospital LOS - days } & 14.6 & 26.0 & 0.03 \\ \text { Hospital death n }(\%) & 46(50) & 49(30) & <0.01 \\ \text { SMR }( \pm \text { SD) } & 1.51 & 1.00 & <0.01\end{array}$

Conclusion: Safety Huddle is feasible during COVID-19 pandemic crisis and is associated with positive perception among healthcare workers and better clinical outcomes for critically ill patients.

Reference(s) and grant ackowledgment(s)

1. Improving Situation Awareness to Reduce Unrecognized Clinical Deterioration and Serious Safety Events. Patrick W. Brady, Stephen Muething, Uma Kotagal, Marshall Ashby, Regan Gallagher, Dawn Hall, Marty Goodfriend, Christine White, Tracey M. Bracke, Victoria DeCastro, Maria Geiser, Jodi Simon, Karen M. Tucker, Jason Olivea, Patrick H. Conway and Derek S. Wheeler. Pediatrics January 2013, 131 (1) e298-e308; DOI: https://doi. org/10.1542/peds.2012-1364.

2. Nursing Activities Score is increased in COVID-19 patients Lucchini A, Giani M, Elli S, Villa S, Rona R, Foti G.. Intensive Crit Care Nurs.2020 https:// doi.org/10.1016/j.iccn.2020.102876. 2. Improving Situation Awareness to Reduce Unrecognized Clinical Deterioration and Serious Safety Events. Patrick W. Brady, Stephen Muething, Uma Kotagal, Marshall Ashby, Regan Gallagher, Dawn Hall, Marty Goodfriend, Christine White, Tracey M. Bracke, Victoria DeCastro, Maria Geiser, Jodi Simon, Karen M. Tucker, Jason Olivea, Patrick H. Conway and Derek S. Wheeler. Pediatrics January 2013, 131 (1) e298-e308; DOl: https://doi.org/10.1542/peds.2012-1364. 


\section{6}

Improving wellbeing and reducing anxiety among core trainees in ICU via feedback led intervention

A. Holmes ; B. Greatorex 2

${ }^{1}$ Anaesthetics and Intensive Care, NHS Highland, Inverness, United Kingdom; ${ }^{2}$ Icu, NHS Highland, Inverness, United Kingdom

Correspondence: $A$. Holmes

Intensive Care Medicine Experimental 2020, 8(2): 001346

Introduction: Prioritising staff wellbeing improves the health of staff and patients 1 . In the latest GMC survey almost $25 \%$ of trainees reported burnout2. This can be particularly prevalent for trainees working in critical care3. A key contributor to anxiety is uncertainty4, which renders core anaesthetic trainees vulnerable as they transition into intensive care. In contrast to anaesthetic practice - which sets a standard using the initial assessment of competence 5 - there is no benchmark for trainees starting in ICU. Trainees may assume high levels of responsibility with little experience and distant senior support out of hours.

Objectives: To identify the anxieties experienced by trainees prior to starting on the ICU on-call rota, to gain their perspective on what could have reduced these anxieties, and to use this information to deliver targeted training to alleviate these concerns for future trainees. To assess the impact of our intervention.

Methods: A survey was sent to all trainees who had completed core anaesthetic training within our hospital over the past two years, detailing their experiences of transitioning onto the on-call ICU rota in CT2. The results were assessed and a targeted one-day ICU boot-camp was developed to address the areas in which trainees had felt vulnerable. This was delivered to two trainee cohorts over two years prior to them going on-call. Surveys were distributed before and after the bootcamp to assess changes in anxiety and perceived preparedness. A pre and post-course quiz was included to provide objective information on improvements in knowledge. Further information was collected on the on-going concerns of trainees and how they felt these could be addressed.

Results: Our initial survey highlighted that trainees had not felt adequately prepared for transitioning onto the on-call rota, with preparedness being scored as $3.8 / 10(0-6.5 ; n=7)$. Specific issues highlighted were variation in length of shadowing in ICU (0-4 weeks; mean 1.1); and inconsistencies in experience during shadowing. $86 \%$ of trainees stated that they would have liked a standardised introduction to ICU. After implementation of our boot-camp, 100\% of trainees reported reduction in anxiety and increase in preparedness for transitioning onto the ICU on-call rota $(n=7)$. Test scores improved from $51 \%$ to $87.5 \%$. Feedback has highlighted a need for additional simulated critical incidents and more real-life exposure to ICU referrals.

Conclusion: Understanding the anxieties of trainees is invaluable in improving their experience. A simple survey can be a useful way to gain insight and to design relevant interventions. From our initial work, we designed a simple yet effective ICU training day that improved trainee performance and confidence. Based on our ongoing feedback we plan to design a database of simulation scenarios to facilitate teaching, and to implement a CT1 bleep for trainees to carry prior to their ICU block alerting them to ICU referrals so they can attend in a supernumerary capacity.

\section{Reference(s) and grant ackowledgment(s)}

1. 5. Royal College of Anaesthetists. CCT in Anaesthetics: Annex B Core Level Training. Edition 2. [Internet]. 2010 [cited Jul 2020]. Available from: https:// www.rcoa.ac.uk/sites/default/files/documents/2019-08/TRG-CCT-ANNEX B.pdf.

2. 4. Grupe DW, Nitschke JB. Uncertainty and anticipation in anxiety: An integrated neurobiological and psychological perspective. Nat Rev Neurosci. [Internet]. 2013 [cited Jul 2020]; 14(7): 488-501. Available from: https://www. ncbi.nlm.nih.gov/pmc/articles/PMC4276319/.

3. 3. Larsson J, Sanner M. Doing a good job and getting something out of it: on stress and well-being in anaesthesia. BJA. [Internet]. 2010 [cited Jul 2020]; 105(1): 34-37. Available from: https://academic.oup.com/bja/artic le/105/1/34/306500.
4. 2. General Medical Council. National Training Surveys 2019: Initial Findings Report. [Internet]. 2019 [cited Jul 2020]. Available from: https://www. gmc-uk.org/-/media/gmc-site-images/about/national-training-surveys-initi al-findings-report20190705 2.pdf.

5. 1. General Medical Council. Caring for Doctors Caring for Patients: How to transform UK healthcare environments to support doctors and medical students to care for patients. [Internet]. 2019 [cited Jul 2020]. Available from: https://www.gmc-uk.org/-/media/documents/caring-for-doctors-carin g-for-patients_pdf-80706341.pdf.

6. None.

\section{8}

VCreate: a new communication tool during COVID-19 within adult critical care

A. Thomson ${ }^{1}$; H. Jordan²; M. Wheelhouse'; J. Tait ${ }^{3}$; A. Graham'; S. Rodgers ${ }^{4}$. C. Mcculloch ${ }^{3}$; L. Mackinnon ${ }^{3}$; J. Tocher ${ }^{4}$; K. Nunn ${ }^{1}$; A. Abu-Arafeh ; E.

Wilson $^{3}$

${ }^{1}$ Western general hospital, intensive care medicine, NHS Lothian, Edinburgh, United Kingdom; ${ }^{2}$ Intensive care medicine, NHS Lothian, Edinburgh, United Kingdom; ${ }^{3}$ Royal infirmary of edinburgh, intensive care medicine, NHS Lothian, Edinburgh, United Kingdom; ${ }^{4}$ University of edinburgh, NHS Lothian, Edinburgh, United Kingdom

Correspondence: $A$. Thomson

Intensive Care Medicine Experimental 2020, 8(2): 001388

Introduction: The COVID-19 pandemic brought unprecedented challenges to critical care units across the world. Significant restrictions on hospital visiting hugely impacted the way in which we communicate and interact with families. Effective communication, usually in the form of family meetings and bedside updates, improves family understanding, clinical decision making and the psychological wellbeing of family members. 1 We found that the absence of face-to-face communication heightened anxiety and distress for families and the critical care team. A lack of physical presence resulted in many families struggling to comprehend the severity of their relatives' condition. In particular, end of life discussions was extremely difficult to cope with In order to improve communication and patient care we implemented a new secure method of asynchronous video communication called vCreate ${ }^{\circledR}$ (Windsor, Berkshire).

Methods: This study was conducted in two critical care units in Edinburgh, during and slightly after the acute phase of the COVID-19 peak in the UK. vCreate has been used in the UK neonatal critical care setting but was an entirely new concept for adult critical care. It provides a means to send regular visual updates via pre-recorded videos and photos to document patient progress, both positive and negative. This virtual connection has enabled us to record and share key moments in a patient's journey: videos prior to intubation, treatments such as proning and has been used by our physiotherapy and rehabilitation team. In addition, we have developed vCreate to allow relatives to send personal videos and photos to be shared with the patient. To evaluate the impact of vCreate and its acceptability as a new communication tool, a family survey was conducted.

Results: 36 patients and their families have benefitted from the service with 163 videos and 111 photos being sent. The initial responses from 10 families who used the service is illustrated in Figure 1. Of these 10 families, 9 strongly agreed that using vCreate improved their connection with their relative, 7 strongly agreed that vCreate improved communication and 8 strongly agreed that vCreate helped them to understand their relatives' situation. 


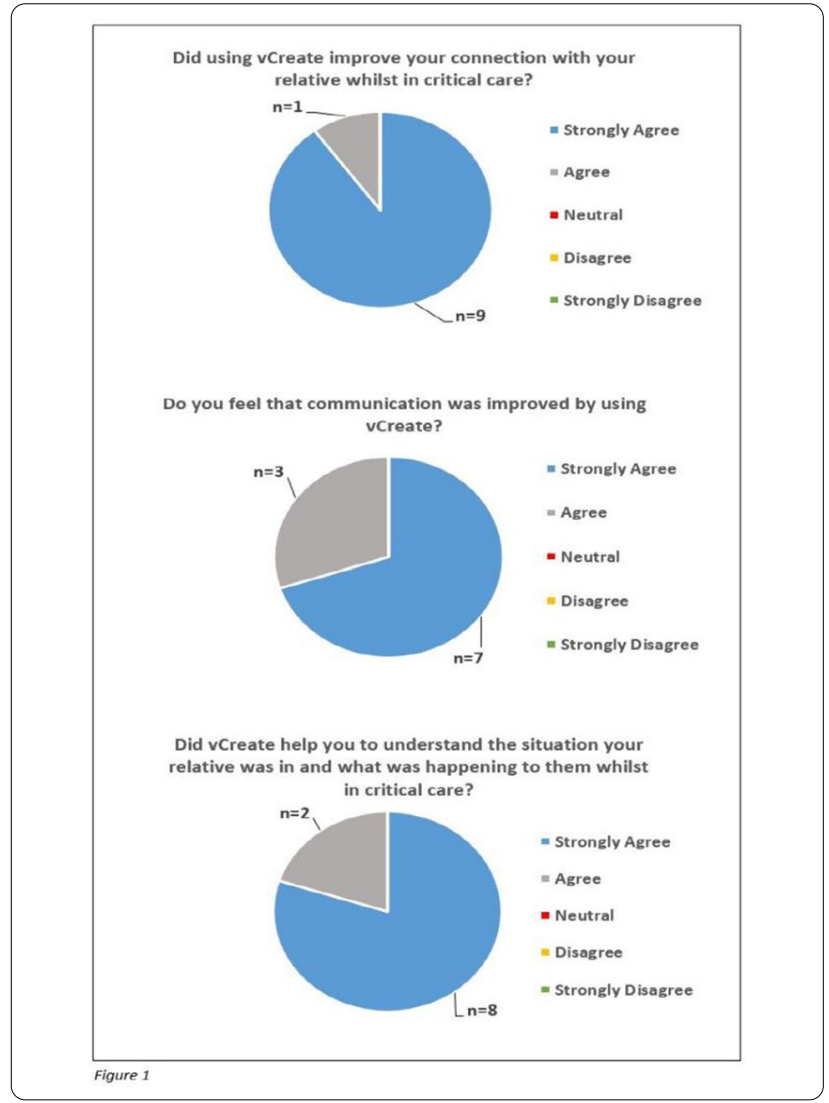

All 10 families responded that they would recommend the use of vCreate to other families.

Conclusion: Initial feedback has been overwhelmingly positive and has shown vCreate to be an effective additional communication tool, improving family and patient connection, communication and understanding of the patient's condition and prognosis. A full evaluation is underway to determine the overall impact of video communication with families, using vCreate. Based on this initial success we will continue to use this technology. Both hospitals are tertiary referral centres, and so vCreate could provide an invaluable communication link for many patient groups whose families live remotely, even once hospital visiting returns to normal.

\section{Reference(s) and grant ackowledgment(s)}

1. 1. Pauldine R, Dorman T. Communication with families in ICU. ICU Management \& Practice 6 (2), URL:https://healthmanagement.org/c/icu/issueartic le/communication-with-families-in-the-icu (2006, accessed 15 July 2020).

\section{8}

\section{Use of a Proning Protocol in Intensive Care during the COVID-19}

\section{Pandemic}

S. Qandil ${ }^{1}$; F. Holbrook'; R. Medlock ${ }^{1}$;. Ali ${ }^{2}$

${ }^{1}$ Intensive Care, Buckinghamshire Healthcare NHS Trust, Stoke Mandeville, United Kingdom; ${ }^{2}$ Intensive care department, Stoke Mandeville Hospital, Aylesbury, United Kingdom

Correspondence: S. Qandil

Intensive Care Medicine Experimental 2020, 8(2): 001408

Introduction: Prone positioning has been used since the 1970's and is well recognised to provide benefit in patients with acute respiratory distress. 1, 2 The PROSEVA trial demonstrated the significant mortality benefits of prone positioning when used within 36 hours of ICU admission with severe ARDS. 3 Robust evidence for proning in COVID-19 infection is not yet published. However, it has been observed as a useful therapeutic tool.

Protocols can optimise and standardise clinical practice, during the COVID-19 pandemic period protocols may help maintain good clinical practice. 4,5 In our District General Hospital we have developed a protocol specific to proning and supinating intubated COVID-19 patients. Objectives: Ascertain how frequently proning was utilised during a four-month period across our trust. We also aimed to identify any complications related to proning that occurred during this time period.

Methods: We reviewed all electronic and paper records of any patient admitted to our ICUs between 01/03/20 - 30/06/20. We also reviewed incident report forms for this time period.

Results: Seventy-Two patients tested positive for COVID-19 during this period. Patient demographics include mean age of 60.6 years old, range 21 to 86 years, $49(68 \%)$ of all patients were male. Twenty-eight (39\%) patients with COVID-19 died. Twenty-six patients (36\%) were proned during their admission. Fifty-five proning attempts were made, ranging from one to six times per patient, $50 \%$ of these patients were only proned once. For those that were proned, the average length of ICU admission was 26.4 days vs 8 days for non-proned patients. Fortytwo percent of the patients that were proned did not survive, compared to $37 \%$ of the patients that were never proned.

There were five complications attributed to proning - this was $19.2 \%$ of proned patients, and $9.1 \%$ of all proning attempts. This included one shoulder subluxation and one brachial plexus injury. Optic neuropathy/retinal haemorrhage were identified in two further patients. One patient developed pressure sores on their ear and clavicle.

Conclusion: Patients that were proned were more likely to die from COVID-19, this likely indicates the disease severity. Fewer patients experienced complications than those identified in previous studies. $2,6,7$ Meticulous use of our proning protocol helped to minimise the risk of the most common complications and is in line with national guidance and standards 6 . A clear protocol also facilitated teams crossskilling from other specialties into an unfamiliar environment.

\section{Reference(s) and grant ackowledgment(s)}

1. 7. Gattinoni L, Tognoni G, Pesenti A, et al. Effect of prone positioning on the survival of patients with acute respiratory failure. New England Journal of Medicine. 2001: 345(8); 568-573.

2. 6. The Intensive Care Society. Guidance For: Prone Positioning in Adult Critical Care. November 2019. Faculty of Intensive Care, London.

3. 5. Ortiz-Reyes Lea. Increased efficacy and safety of enteral nutrition support with a protocol (ASNET) in Non-critical patients: A randomized Controlled Trial. Journal of the Academy of Nutrition and Dietetics. 2018: 188(1); 52-61.

4. 4. Mahmoud LZ, AR, Thompson, BB \& Wendell, LC. Outcomes of protocolised analgesia and sedation in a neurocritical care unit. Brain Injury. 2018: 32 (7); 941-947.

5. 3. Guerin C. et al (PROSEVA Study Group). Prone Positioning in Severe Acute Respiratory Distress Syndrome. New England Journal of Medicine. 2013: 368; 2159-2168.

6. 2. Kallet RH. A Comprehensive Review of Prone Position in ARDS. Respiratory Care. 2015: 60(11); 1660-1687.

7. 1. Piehl MA, Brown RS. Use of extreme position changes in acute respiratory failure. Critical Care Medicine 1976: 4(1); 13-14.

8. None.

\section{9}

ICU bed occupancy in Ireland during COVID-19 surge; impact on ICU mortality

R. Dwyer'; B. Moran'; F. Treanor ${ }^{1}$; M. Baggot ${ }^{1}$; T. Mitchell'2 P. Flanagan²; J. O'donnell'2; K. O'donnell ${ }^{2}$

${ }^{1}$ NOCA, RCSI, Dublin, Ireland; ${ }^{2} H p s c$, Dept of Health, Dublin, Ireland

Correspondence: $\mathrm{R}$. Dwyer

Intensive Care Medicine Experimental 2020, 8(2): 001409 
Introduction: ICU bed capacity is relatively low in Ireland ( 255 beds at baseline, 5.2 ICU beds/100,000 population). The COVID-19 pandemic had the potential to overwhelm ICU capacity leading to a high mortality rate. We documented (i) ICU bed occupancy in Ireland during the surge in COVID activity in March - May 2020 and (ii) mortality for COVID-19 patients in ICU in Ireland up to July 6th 2020. We referenced this to mortality in ICU in the UK in the same period as documented by the Intensive Care National Audit and Research Centre, UK (ICNARC)1. Methods: The National Office of Clinical Audit (NOCA) collected data on ICU bed occupancy during March-May 2020. Data were entered on a web portal by all ICU's twice daily, with support and validation by NOCA. A Census documented ICU bed capacity (i) on March 13th, at baseline and (ii) on May 1st, after the expansion of capacity to cope with COVID-19.

The Health Protection Surveillance Centre (HPSC) published weekly data on outcomes in COVID patients admitted to ICU in Ireland. ICNARC publish data for the United Kingdom (UK) (except Scotland) weekly (1).

Results: ICU bed capacity in Ireland increased by 39\% from 255 at baseline to 354 beds at peak surge capacity. The peak number of patients in ICU during this period was 255 and the peak number of COVID patients in ICU was 176. Numbers of non-COVID patients decreased significantly, to a low point of just 62 patients.

Thus total numbers of patients in ICU in Ireland remained less than the baseline number of ICU beds. This was due to (i) a smaller number of COVID patients admitted to ICU than expected and (ii) a major decrease in non-COVID patients in ICU. Numbers of patients in ICU nationally remained significantly below the total number of beds available after the expansion of capacity for COVID.

ICU mortality for COVID patients in Ireland was lower than that reported in other countries, including the UK (Table 1). This was noted for (i) all ICU admissions with COVID and (ii) for ICU patients undergoing invasive ventilation.

\begin{tabular}{|c|c|c|}
\hline & Ireland & $\mathbf{U K}(1)$ \\
\hline $\begin{array}{l}\text { COVID-19 ICU admissions / } \\
\text { population }\end{array}$ & & \\
\hline Age on admission (yrs, median) & 60 & 60 \\
\hline $\begin{array}{l}\% \text { of ICU admissions invasively } \\
\text { ventilated } \quad 66 \%\end{array}$ & $72 \%$ & \\
\hline $\begin{array}{l}\text { ICU mortality - all COVID admissions to ICU } \\
\% \quad 40 \%\end{array}$ & 17 & \\
\hline $\begin{array}{l}\text { Hospital mortality }- \text { all COVID admissions to } \\
\text { ICU } 20 \%\end{array}$ & & \\
\hline $\begin{array}{l}\text { ICU mortality in COVID patients invasively } \\
\text { ventilated } 26 \%\end{array}$ & & \\
\hline
\end{tabular}

Table 1. Outcomes of (i) all COVID admissions to ICU and (ii) COVID patients who underwent invasive ventilation

Conclusion: The demand for ICU beds remained within the available capacity because of (i) fewer COVID admissions to ICU, relative to the UK and (ii) decreased numbers of non-COVID patients in ICU. This was associated with a lower ICU mortality rate than the rate reported for the UK which has a comparable healthcare system.

\section{Reference(s) and grant ackowledgment(s)}

1. (1) ICNARC (2020). ICNARC report on COVID-19 in critical care (3 July 2020). ICNARC, London, UK.

\section{2}

Organization and Rebuild the New Intensive Care Unit for COVID-19 in a Secondary, Public Provincial Hospital

YJ. Cho'; J. Yoon²; JW. Jang ${ }^{3}$; HK. Yang ${ }^{4}$; B. Ko ${ }^{5}$; MJ. Song ${ }^{1}$; SY. Lim'; SK. Lim6 ${ }^{1}$ Internal medicine (pulmonary and critical care medicine), Seoul National University Bundang Hospital, Seongnam-si, Republic of Korea; ${ }^{2}$ Nursing (medicial intensive care unit), Seoul National University Bundang Hospital, Seongnam-si, Republic of Korea; ${ }^{3}$ Nursing (education team), Gyeonggi Provincial Medical Center Ansung Hospital, Ansung-si, Republic of Korea;
${ }^{4}$ Nursing (intensive care unit), Gyeonggi Provincial Medical Center Ansung Hospital, Ansung-si, Republic of Korea; ${ }^{5}$ Internal medicine, Gyeonggi Provincial Medical Center Ansung Hospital, Ansung-si, Republic of Korea; ${ }^{6}$ Internal medicine (infectious diseases), Gyeonggi Provincial Medical Center Ansung Hospital, Ansung-si, Republic of Korea

Correspondence: Y.J. Cho

Intensive Care Medicine Experimental 2020, 8(2): 001412

Introduction: Organization and Rebuild intensive care unit (ICU) during pandemic case surge such as COVID-19 is essential for preventing the collapse of healthcare system and excessive damage.

Objectives: We developed new ICU for COVID-19 in the secondarylevel, non-teaching, public provincial hospital.

Methods: The administration of Gyeonggi province in the Republic of Korea raised the fund for reconstructing ICU and equipping medical resources for COVID-19, such as high flow nasal cannula (HFNC), mechanical ventilator (MV), critical care ultrasound system, continuous renal replacement machines, etc. Collaborating the tertiary-level, teaching, public central hospital, one intensivist, and ICU nurse master prepared the educational plan including simulation workshop, device demonstration.

Results: After two weeks of preparedness, the confirmed COVID-19 critically ill patients from the long term care facilities were admitted to the new ICU due to respiratory failure enough requiring HFNC or MV support. Invasive monitoring via arterial lines and maintaining critical care medication via central venous lines were done by collaboration dispatched one intensivist with local house staffs. Currently, five COVID-19 critically ill patients were admitted, two discharged with survival, one transported to the tertiary hospital, remained still admitted in the new ICU.
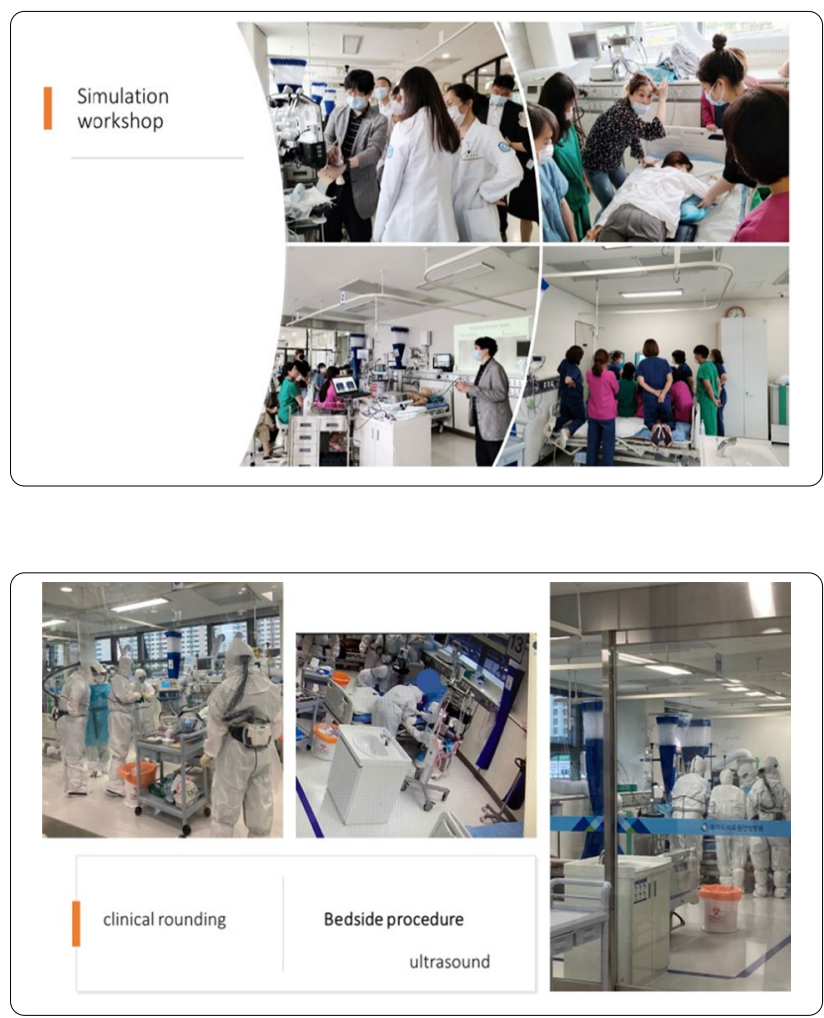

Conclusion: We reported the first experience of the organization and rebuild the new ICU for COVID-19 via collaboration in one public sector. 
Reference(s) and grant ackowledgment(s)

1. Managing ICU surge during the COVID-19 crisis: rapid guidelines. Intensive Care Med (2020) 46:1303-1325.

\section{6}

Activity carried out by the ICU without walls (ICUWW) team in a 400-bed hospital in Lleida, Spain during 2019

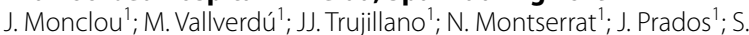

Iglesias ${ }^{1}$; S. Carvalho Brugger ${ }^{1}$; M. Miralbés Torner ${ }^{1}$; JG. Jiménez²; J. Caballero-López ${ }^{2}$

${ }^{1}$ Intensive Care Unit, University Hospital Arnau de Vilanova, Lleida, Spain; ${ }^{2}$ Intensive care department, University Hospital Arnau de Vilanova, Lleida, Spain

\section{Correspondence: J. Monclou}

Intensive Care Medicine Experimental 2020, 8(2): 001436

Introduction: Show the results for the year 2019; from the ICUWW team; which is a reactive and proactive team that performs assessment, assistance, monitoring and procedures activities for critical/ semi-critical patients outside the scope of the Intensive Care Unit (ICU). Responsible for the early detection and treatment of those who are admitted and present clinical deterioration regardless of where they are, the ICUWW is also in charge of monitoring the patients assessed in the previous 24 hours by the ICU on-call team and it also follow fragile patients after discharge from the ICU. We the results we want to evaluate if it is justified the implementation of this team.

Methods: Descriptive study from January to December 2019; that shows the results of the evaluations made by the ICUWW. Variables are described as means, \pm standard deviation or percentage. Chi square (X2) and Mann-Whitney test for continuous variables were used to compare categorical variables.

Results: In 2019, a total of 1,289 patients were treated by the ICUWW team, who underwent 2,059 interventions, 798 were men (61.9\%) and 491 (38.1\%) women, with an average age of 61.7 years. The average attention time was 158 minutes, with a follow-up of 1 to 12 days (average 1.26). 553 patients $(42.9 \%)$ had medical pathology, 624 (48.4\%) surgical and $112(8.7 \%)$ trauma. $25.21 \%$ of the patients were evaluated in the Emergency Department, $15.4 \%$ in the Digestive Surgery service, and the rest in other services. In $54.4 \%$ of the occasions the attention was in response to a direct call; $6.9 \%$ follow-up was of patients assessed in the previous on-call team and who had not been admitted to the ICU; $17.1 \%$ of the evaluations were for CVC placement; $3.8 \%$ follow-up of fragile patients after ICU discharge. $30.2 \%$ of the care resulted in admission to the ICU, 24.7\% were attended for management and $33.5 \%$ did not require admission, due to lack of criteria or due to proper management of patients at risk. $5.35 \%$ underwent limitation of the therapeutic effort (LTE) after evaluation. Of the fragile patients who were followed-up at discharge, $8,8 \%$ required readmission to the ICU.

Conclusion: With the number of patients evaluated and the number of interventions made (active and reactive); continuing with ICUWW team for our hospital or a similar hospital is justified.

\section{6}

Optimising family communication during a pandemic: do video calls have a role?

S. Jeyabraba'

${ }^{1}$ Critical care, University Hospital Lewisham, London, United Kingdom

Correspondence: S. Jeyabraba

Intensive Care Medicine Experimental 2020, 8(2): 001446

Introduction: This survey aims to evaluate whether communication with families was affected by the coronavirus (COVID-19) pandemic and whether video call software mitigated these difficulties.

In 2011 the NHS Outcome Framework recognised "ensuring people have a positive experience of care" as a key domain of quality. In critical care, family members play a crucial role in the care and decision making process. Meeting the emotional and practical needs of family members is essential to delivering good quality care. Family Satisfaction in the Intensive Care Unit FS-ICU has been developed and validated for assessing families' satisfaction with care and communication. The coronavirus pandemic has placed unforeseen constraints on all aspects of critical care including communication with patients' family members. Due to the need to limit coronavirus spread, family visitations have been limited if not absent across the country. In order to allow families to communicate with their inpatient relatives, we acquired tablet computers with the TouchAway application. Furthermore, doctors on our ICU made a daily phone call to each family.

Methods: An anonymous, retrospective survey was sent to all families affected by the visitor restrictions during the coronavirus period from early March until the present. The questionnaire utilized was based on the FS-ICU questionnaire, and was modified to focus on communication with doctors and include questions regarding video call use between families and patients.

Results: 24 responses were received. Half of the cohort who responded to the survey had access to video calling. The answers to each domain have been categorised into satisfied and not satisfied and presented in the table below.

\begin{tabular}{|l|l|l|}
\hline Domain & Satisfied & Not satisfied \\
\hline Frequency of communication & $83(20 / 24)$ & $17(4 / 24)$ \\
\hline $\begin{array}{l}\text { Ease of getting information and adequate } \\
\text { explanations }\end{array}$ & $88 \%(21 / 24)$ & $12(3 / 24)$ \\
\hline Honesty of information & $96 \%(23 / 24)$ & $4 \%(1 / 24)$ \\
\hline Consistency of information & $79 \%(19 / 24)$ & $21 \%(5 / 24)$ \\
\hline Ease of video calling & $91 \%(10 / 11)$ & $9 \%(1 / 11)$ \\
\hline Frequency of video calls & $67 \%(8 / 12)$ & $33 \%(4 / 12)$ \\
\hline Video calls help to stay connected & $\begin{array}{l}100 \% \\
(12 / 12)\end{array}$ & $0 \%(12 / 12)$ \\
\hline
\end{tabular}

Table 1: Results by selected domain, divided by satisfaction

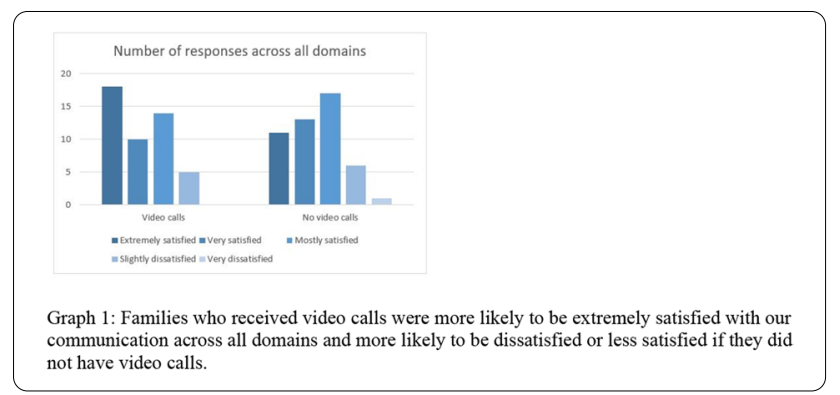

We also included an opportunity in the survey for free text comments regarding improving our communication and the use of video calls. Many families were very positive; "daily phone call from ICU for update was a lifeline for my family" and that doctors were thorough and honest with them regarding their relative's condition. There was also feedback that some families did not receive consistent phone calls particularly around time of admission.

Families were very grateful for the opportunity to video call, which helped them appreciate how unwell their relative was and helped them feel connected with their relative and the staff. They commented the application was easy to set up. Families who were not able to use video calls, also said that they would have liked to use this service and all agreed that even outside of pandemic restrictions, it would be a useful addition.

Conclusion: This survey has shown that during a pandemic, we can effectively communicate with patients' relatives. The utilisation of video calls in critical care has been a welcome addition to our services while families are unable to visit their loved ones. The future benefits of using this technology remain enormous; particularly during further COVID-waves. 
Reference(s) and grant ackowledgment(s)

1. Molokhia A, Khaliq W. Br J Anaesth. 2020

2. Visitor Guidance NHS England April 2020

3. Wright $S$ et al. ICNARC Free V1:3, 2014.

4. Heyland et al. Crit Care Med 30:1413-1418, 2002.

5. Departmemt of Health. The NHS Outcomes Framework 24-28, 2010.

\section{2}

An observational cohort study of physiological parameters that predict the need for mechanical ventilation and intensive care unit admission in patients admitted to hospital with due to SARS-CoV-2 infection

BW. Johnston ${ }^{1}$; C. Toal ${ }^{2}$; E. Morgan ${ }^{1}$; S. Mulla ${ }^{1}$; A. Ross ${ }^{2}$; N. Sexton²; M. Spiliopoulos'; W. Thompson²; S. Waite'; S. White ${ }^{2}$; AAC. Waite'; ID. Welters ${ }^{1}$ Intensive care unit, Royal Liverpool University Hospital, Liverpool, United Kingdom; ${ }^{2}$ Acute medicine, Royal Liverpool University Hospital, Liverpool, United Kingdom

\section{Correspondence: A. Waite}

Intensive Care Medicine Experimental 2020, 8(2): 001452

Introduction: The COVID-19 pandemic poses massive strains on healthcare systems worldwide. In a recent report from the UK, approximately $17 \%$ of hospitalised patients required intensive care unit (ICU) admission. Efficient triage of patients with COVID-19 at the time of hospital admission will support local resource planning. There is an urgent need for prediction models that use commonly available clinical measurements to estimate the risk of patients requiring respiratory support and ICU admission.

We aimed to compare physiological observations between patients who require invasive mechanical ventilation and those who do not using the first available data after presentation of patients to our Accident and Emergency Department to allow rapid triaging.

Methods: We retrospectively reviewed case notes of all patients admitted to the Royal Liverpool University Hospital between March 23rd and April 23rd 2020 with a positive COVID-19 RT-PCR result. The chosen time period reflects the peak of the pandemic in North West England. Routine clinical data including demographic data and initial admission physiological data were extracted. We assessed the predictive value of admission physiological parameters including heart rate, oxygen saturations, required fraction of inspired oxygen ( $\mathrm{FiO} 2$ ), mean arterial pressure, temperature and National Early Warning Score (NEWS) by generating Receiver Operator Characteristics.

Results: 455 RT-PCR positive COVID patients were included in our analysis. In univariate analysis, patients requiring mechanical ventilation had lower admission oxygen saturation ( $87 \%$ vs $93 \%, p<0.001$ ), higher respiratory rate ( 27 vs $22 \mathrm{bpm}, \mathrm{p}<0.001$ ), lower estimated P/F ratio (46 vs 35, p<0.001) and were more tachycardic (102 vs 93 bpm, $\mathrm{p}<0.001)$. Mean arterial blood pressure was not significantly different (93 vs $90 \mathrm{mmHg}$, p:0.44). Temperature was comparable between groups ( 37 vs $37.50 C, p=0.55$ ). When physiological parameters were combined into the NEW score, patients requiring mechanical ventilation had a significantly higher NEWS ( 6 vs $4 p<0.001$ ). Following logistic regression, oxygen saturations and estimated $P / F$ ratio predicted need for mechanical ventilation with an area under the curve of $77 \%$ and $73 \%$ respectively.

Conclusion: Our study identifies a number of physiological parameters which predict the need for invasive mechanical ventilation. Physiological markers associated with hypoxia, including lower admission oxygen saturations, higher respiratory rate and lower estimated $\mathrm{P} / \mathrm{F}$ ratio show potential to predict the need for invasive ventilation. Patients with COVID-19 were relatively haemodynamically stable with comparable mean arterial pressures between patients requiring mechanical ventilation and those who do not. In the UK it is common practice to combine physiological parameters into the NEWS, however, for patients with COVID-19 the NEWS was not superior in predicting those patients requiring mechanical ventilation.
001466

Decision-making on escalation of treatment to invasive ventilation: A critical review of referrals during the COVID-19 pandemic

M. Crews ${ }^{1}$; E. Morgan ; A. Ardeshana1; BW. Johnston ${ }^{1}$; AAC. Waite ${ }^{1}$; ID. Welters $^{1}$

${ }^{1}$ Intensive care unit, Royal Liverpool University Hospital, Liverpool, United Kingdom

Correspondence: A. Waite

Intensive Care Medicine Experimental 2020, 8(2): 001466

Introduction: Invasive ventilation to treat respiratory failure may become a scarce resource during pandemics, necessitating critical review to allocate ventilators and intensive care beds to patients likely to benefit most from this intervention.

Demographic characteristics and differences in functional state between patients who were declined escalation to invasive mechanical ventilation compared to those who were admitted to critical care for this intervention during the COVID-19 pandemic have not been well described so far.

In this retrospective cohort study we analysed data from 83 patients who were referred to critical care specialists with the question as to whether they should be admitted to critical care for invasive ventilation in case of clinical deterioration.

Methods: Data for all patients admitted to Royal Liverpool University Hospital between March 23rd and April 23rd 2020 with a positive COVID-19 RT-PCR result who were referred to and reviewed by a consultant intensivist were analysed. Outcome of the critical care review was documented as "suitable for escalation to invasive ventilation if required" or "ward level care only". Frailty scores, comorbidities, demographic data and laboratory results were reviewed for the day of hospital admission. Univariate statistics were performed using $\mathrm{X}^{2}$ tests for categorical and Mann-Whitney-U test or T-test for independent samples for metric data.

Results: 83 patients were included in the analysis. 53 patients were assessed as suitable for escalation to invasive ventilation, while treatment was limited to ward level care in 31 patients. Patients in whom escalation was deemed appropriate had lower Rockwood frailty scores $(2.5 \pm 1.3)$ compared to patients declined for escalation of care $(4.3 \pm 1.4, p<0.001)$. Patients offered escalation were significantly younger $(63.8 \pm 11.5$ years) than patient who were not offered escalation $(54.0 \pm 12.2$ years, $p<0.001)$. Markers of infection on admission to hospital did not differ between patient groups, indicating that the severity of infection did not influence decision making. Ischemic heart disease, COPD, malignancies, liver disease and chronic kidney disease were documented significantly more often for patients deemed unsuitable for escalation of care $(p<0.001)$. In contrast, comorbidities that showed no significantly different distribution between groups included diabetes, asthma and hypertension.

Conclusion: Patient factors that influenced decision making of critical care specialists as to whether treatment offered should include invasive ventilation included frailty, comorbidities such as heart, kidney or liver disease and patient age. Although not directly investigated in this study, age correlated with the occurrence of these comorbidities and with frailty. Mortality of COVID-19 increased with age. Further research is required to fully understand the decision-making process for offering escalation of care in pandemic situations with limited critical care capacity.

\section{0}

The ICU without walls (ICUWW) team. A reactive and proactive service, created for the care of critically ill patients outside the ICU J. Monclou'; M. Vallverdú1; JJ. Trujillano ${ }^{1}$; N. Montserrat ${ }^{1}$; J. Prados ${ }^{1}$; S. Iglesias ${ }^{1}$; S. Carvalho Brugger ; M. Miralbés Torner ${ }^{1}$; JG. Jiménez ${ }^{2}$; J. Caballero-López ${ }^{2}$

${ }^{1}$ Intensive Care Unit, University Hospital Arnau de Vilanova, Lleida, Spain; ${ }^{2}$ Intensive care department, University Hospital Arnau de Vilanova, Lleida, Spain

Correspondence: J. Monclou

Intensive Care Medicine Experimental 2020, 8(2): 001480 
Introduction: The ICUWW team is conformed by intensivist doctors who carry out activities from 8 am to $8 \mathrm{pm}$, accompanied by a nurse with training in critical patients from 10 am to $5 \mathrm{pm}$, Monday through Friday. Its activity has 2 components. The first is that of the ICU, which is reactive to any need of the hospital (care of critically ill patients, assistance in CPR, assessment of multiple trauma patients, etc..) and other that is proactive; which focuses on the follow-up of fragile patients after discharge from the ICU or who have previously been evaluated by on-call doctors or by the ICUWW team.

Objectives: Show some comparative data about the care provided by the ICUWW team for the years 2018 and 2019, in a hospital of 400 beds in Lleida, Catalunya, Spain.

Methods: Descriptive study from January 2018 to December 2019; that shows the results of the evaluations made by the ICUWW. Variables are described as means, \pm standard deviation or percentage. Chi square (X2) and Mann-Whitney test for continuous variables were used to compare categorical variables.

Results: In 2018 the ICUWW attended 932 patients, with a total of 1496 interventions. $63 \%$ were men and $37 \%$ women, with an average age of 61 years. The average time of attention was 81 minutes with one average follow-up of 1.36 days. $52 \%$ had medical pathologies, $38.2 \%$ surgical, and $9.8 \%$ traumatic. The $29.7 \%$ were assessed at the emergency department, $13.2 \%$ at the digestive surgery unit, and the rest in other areas. $49.3 \%$ of the attention was made by a direct call; $5.6 \%$ was a follow-up of patients assessed in the previous ICU night shift, 11,4\% required central venous catheter placement, $5.7 \%$ were the follow-up of fragile patients after the ICU discharge. The $17.2 \%$ required admission to ICU, $13.5 \%$ did not require admission. $8.5 \%$. underwent limitation therapeutic effort (LTE). Of the patients that required follow-up $(\mathrm{N}=193), 20.7 \%$ needed more than 2 days; $14 \%$ admission to ICU, and the rest avoided the admission. Only $(\mathrm{N}=53) 24.5 \%$ of the patients with a follow-up after the discharge from ICU required re-entry.

In 2019, a total of 826 patients were treated by the ICUWW team, who underwent 2,060 interventions; 289 patients with scheduled admissions were also evaluated and 174 CVC insertions were performed. $61.9 \%$ were men and $38.1 \%$ women; with a mean age of 61.7 years old. The average attention time was 158 minutes, with an average followup of 1.26 days. $42.9 \%$ had medical, $48.4 \%$ surgical, and $8.7 \%$ trauma pathology. $25.2 \%$ of the patients were evaluated in the emergency department, $15.4 \%$ in the digestive surgery unit, and the rest in other services. $30.2 \%$ required admission to the ICU, $24.7 \%$ were attended for management and support and $33.5 \%$ did not require admission. $5.35 \%$ underwent therapeutic effort limitation (LTSV) after evaluation. Of the frail patients who underwent follow-up at ICU discharge ( $\mathrm{N}: 45)$, $8.9 \%$ required readmission to the ICU.

Conclusion: With these data, we show the evolution of the ICUWW. Our goal is to improve the team. Try to make better monitoring of fragile patients, and identify critically ill patients earlier. Those are the task for the next years.

\section{4}

\section{Audit of Twice Daily Consultant Reviews on the Intensive Care} Units at Buckinghamshire Healthcare NHS Trust

E. Leadbeater ${ }^{1}$; M. Taquet ; N. Fry ${ }^{1}$; T. Ali

${ }^{1}$ Stoke mandeville hospital, Buckinghamshire Healthcare NHS Trust, Stoke Mandeville, United Kingdom

Correspondence: $\mathrm{E}$. Leadbeater

Intensive Care Medicine Experimental 2020, 8(2): 001484

Introduction: This audit retrospectively repeats a closed loop on an audit assessing adherence to the GPICS standards of $100 \%$ patients receiving twice-daily consultant review (1). An initial audit was carried out before trust wide implementation of a daily management proforma, which contains a pre-printed section for evening ward rounds. The loop was closed after implementation, showing a modest. As staff have become more used to the proforma, the audit was repeated to re-assess documentation.

Methods: A retrospective case notes review was carried out using 'Evolve', an electronic record of scanned handwritten notes. All patients across both sites (site A and site B) were audited over pre-selected days. The days were chosen such that a selection of consultants would be reviewed; 1 day a week, 8 days apart between 2nd December 2018 and 7 th January 2019. This allowed for approximately 2 years since the previous data collection, during which, the proforma had been used exclusively. A patient was deemed to have been reviewed by a consultant if the consultant themselves had documented an assessment or if a doctor had documented the consultant name into the notes. Data were recorded using a simple spreadsheet. Slightly differently to the previous audit, we reviewed whether a specific bed space in the ICU had been reviewed as opposed to a particular patient.

Results: During the assessment period, a total of 87 patient episodes were assessed. An episode was either a morning or an afternoon review. Notes were not available for 14 of those episodes on Evolve. These were included for the data analysis as 'consultant review not documented'. Where a bedspace was empty or a patient was stepped down, no episode was recorded.

Prior to implementation of the proforma, trust-wide $24 \%$ patients had a twice daily consultant review documented, immediately post introduction, $35 \%$ patients had a twice daily review and two years later, $33 \%$ patients had a twice daily review.

1. 29 patients had two documented consultant reviews daily (25 site A 4 site B)

2. 25 patients had documented morning reviews only ( 21 site A 4 site B).

3. 7 patients had documented evening reviews only ( 6 site A 1 site B).

4. 26 patients had no documented reviews (16 site A 10 site B).

This table compares \% patients with documented consultant reviews at both sites for morning and afternoon episodes pre and post introduction of the proforma and the repeat audit.

\begin{tabular}{|l|l|l|l|}
\hline SITE A & Pre & Post 1 & Post 2 \\
\hline Twice & $21 \%$ & $45 \%$ & $37 \%$ \\
\hline None & $17 \%$ & $20 \%$ & $24 \%$ \\
\hline AM & $57 \%$ & $33 \%$ & $31 \%$ \\
\hline PM & $5 \%$ & $2 \%$ & $8 \%$ \\
\hline SITE B & Pre & Post 1 & Post 2 \\
\hline Twice & $31 \%$ & $11 \%$ & $21 \%$ \\
\hline None & $4 \%$ & $21 \%$ & $53 \%$ \\
\hline AM & $65 \%$ & $68 \%$ & $21 \%$ \\
\hline PM & $0 \%$ & $0 \%$ & $5 \%$ \\
\hline Overall & Pre & Post 1 & Post 2 \\
\hline Twice & $24 \%$ & $35 \%$ & $33 \%$ \\
\hline None & $14 \%$ & $21 \%$ & $30 \%$ \\
\hline AM & $59 \%$ & $43 \%$ & $29 \%$ \\
\hline PM & $3 \%$ & $1 \%$ & $8 \%$ \\
\hline
\end{tabular}

Conclusion: The GPICS standards of $100 \%$ of patients having twice daily documented consultant reviews has not been met long after introduction of the ward round proforma. Trust wide, the number of twice-daily reviews have decreased from $35-33 \%$. There has been an improvement across both sites for evening reviews.

The reason for the drop may be as simple as the consultant presence on the ward round not being documented.

Recommended actions:

- departmental presentation of audit findings to encourage more meticulous documentation.

- to further encourage use of the daily management sheet on ward rounds.

- inclusion of the proforma as part of the paperwork pack when a patient is admitted to the unit.

- interviewing staff using the proforma regarding potential difficulties in its use.

\section{Reference(s) and grant ackowledgment(s)}

1. 1. Guidelines for Provision of Intensive Care Services (GPICS) 3.1.1.5 https:// www.ficm.ac.uk/sites/default/files/GPICS\%20-\%20Ed.1\%20\%282015\%29_0. pdf. 
001494

Catastrophic health expenditure in an Indian ICU

for the management of severe sepsis

P. Murthy'; J. Gopaldas ${ }^{1}$

${ }^{1}$ Critical Care Medicine, Manipal Hospital Old Airport Road, Bengaluru, India

Correspondence: P. Murthy

Intensive Care Medicine Experimental 2020, 8(2): 001494

Introduction: The rapidly increasing burden of sepsis in India and also the availability of advanced care options have profound impacts on health care costs for patients and their families. High out-of-pocket expenditure, lack of insurance, and low government expenditure create a vicious cycle, leading to household impoverishment.

Objectives: We aim to address the financial implications of sepsis and septic shock management in a tertiary care ICU. We also plan to study the factors influencing financial toxicity and catastrophic health expenditure.

Methods: Prospective data from 23 patients who underwent ICU admission for sepsis and septic shock between January 2019 and July 2019 were collected and analyzed. The time frame for consideration of expenses, including all preoperative investigations, was from the first hospital visit to the day of discharge. Socioeconomic status was assessed using Kuppaswamy Scale [1] and financial burden was assessed using the Comprehensive Score for Financial Toxicity(COST) [2].

Results: Overall mortality in the study was $40 \%$ consistent with APACHE II mortality (adjusted for 6 patients who required emergency surgery). The proportion of patients who had partial or full insurance coverage was $50 \%$. The average Kuppuswamy score for the study population is 2.5 (consistent with the middle-income group). Financial toxicity was noted in all irrespective of socioeconomic class. It was significantly higher in lower and middle-income groups than the highincome groups(Table 1).

Table 1: Comparison of socio-economic status and COST Score

\begin{tabular}{|l|l|l|l|}
\multicolumn{4}{|c|}{ Table 1: Comparison of socio-economic status and COST Score } \\
\hline $\begin{array}{l}\text { Socioeconomic } \\
\text { class(Kupusswamy Score) }\end{array}$ & $\begin{array}{l}\text { Number of } \\
\text { patients }\end{array}$ & $\begin{array}{l}\text { COST Score } \\
(\text { Mean } \pm \text { SD) }\end{array}$ & $\begin{array}{l}\text { p- } \\
\text { Value }\end{array}$ \\
\hline $\begin{array}{l}\text { Class 1 and 2 (High and upper- } \\
\text { middle) }\end{array}$ & 11 & $20.9 \pm 4.79$ & 0.003 \\
\hline $\begin{array}{l}\text { Class 3 and 4 (Lower middle } \\
\text { and Low) }\end{array}$ & 12 & $20.9 \pm 4.79$ & \\
\hline
\end{tabular}

Conclusion: Thus to conclude, a major financial burden was seen in most patients treated for severe sepsis and was highest in low and middle-income groups. Recoverable illness like sepsis needs financial support programs/insurance schemes like the ones seen with cancer care.

\section{Reference(s) and grant ackowledgment(s)}

1. 2. de Souza JA, Yap BJ, Wroblewski K, Blinder V, Araújo FS, Hlubocky FJ, Nicholas LH, O'Connor JM, Brockstein B, Ratain MJ, Daugherty CK, Cella D. Measuring financial toxicity as a clinically relevant patient-reported outcome: The validation of the Comprehensive Score for financial Toxicity (COST). Cancer. 2017 Feb 1;123(3):476-484

2. 1. Wani RT. Socioeconomic status scales-modified Kuppuswamy and Udai Pareekh's scale updated for 2019. J Family Med Prim Care. 2019 Jun;8(6):1846-1849.

3. Nil.

\section{6}

Basic Life Support Training amid the COVID-19 pandemic: perspectives from the UK's largest ERC course

A. O'Donnell ${ }^{1}$; H. Percivall ; B. Stanley ${ }^{1}$; T. Burton ${ }^{1}$; N. Coffin ${ }^{2}$; J. Hulme ${ }^{3}$; J. Alderman ${ }^{4}$; A. Owen ${ }^{4}$

${ }^{1}$ Medicine, University of Birmingham, Birmingham, United Kingdom; ${ }^{2}$ Anaesthesia and critical care, Russells Hall Hospital, Birmimgham, United Kingdom; ${ }^{3}$ Anaesthesia and critical care, Sandwell and West Birmingham
NHS trust, Birmingham, United Kingdom; ${ }^{4}$ Anaesthesia and critical care, Queen Elizabeth Hospital Birmingham, Birmingham, United Kingdom

Correspondence: A. O'Donnell

Intensive Care Medicine Experimental 2020, 8(2): 001526

Introduction: The Basic Life Support (BLS) course run by Resuscitation for Medical Disciplines (RMD) Birmingham at the University of Birmingham uses a peer to peer teaching model, teaching approximately 1000 healthcare students each year (1). The importance of face-to-face training needs to be balanced with limiting the risk to educators and students and maintaining an environment in which the risk of transmission of SARS-CoV 2 is as low as possible. We therefore describe the modifications that have been introduced to our BLS course; the largest European Resuscitation Council (ERC) certified course in the United Kingdom.

Objectives: This project aimed to investigate the adaptations made to the ongoing academic course (2020-2021) to ensure high quality BLS education is delivered in a safe environment.

Methods: Our course's faculty and student committee produced a COVID-19 strategy using a modified Delphi approach. Amendments to our course's usual running were authored iteratively and shared electronically. Modifications were added at each stage until the group achieved consensus.

Strategic planning utilised guidelines from the ERC and World Health Organisation (WHO) to develop an adapted BLS course for the COVID19 era $(2,3)$.

Results: Broadly, our group's strategy was to reduce face-to-face contact where possible, and where impractical, to take steps to minimise risks to trainers and participants.

Data from our prior work (publication under review) suggests that face-to-face teaching outperforms distance learning methods to reteach BLS in healthcare students from their second year of study onwards. Some aspects of our curriculum can be delivered remotely - including all lectures and seminars where no practical training is delivered.

The WHO clearly encourages strict hand hygiene and limited social contact (by reducing group sizes), and the ERC suggests remote training may be effective for professionals requiring BLS training $(2,3)$. Process management and crowd flow through buildings was also analysed and systems were introduced to enable large numbers of peoples to pass through the educational environment for training and testing without reduction in social distancing and maintaining a low total number of participants in the building at any time.

Conclusion: BLS education at this large-scale ERC BLS course in the UK has been adapted to reduce the risk of COVID-19 transmission, maintaining the use of face to face teaching as the most effective teaching method in our cohort. Such teaching will incorporate additional safety measures with the view to reduce transmission risk of COVID-19, whilst maintaining a high standard of BLS education.

Reference(s) and grant ackowledgment(s)

1. 3. European Resuscitation Council. European Resuscitation Council COVID19 Guidelines. 2020 [Cited July 2020]. [internet'. Available from: https:// www.erc.edu/sites/5714e77d5e615861f00f7d18/content_entry5ea884fa4c 84867335e4d1ff/5ea886ae4c84867421e4d1f8/files/ERC_covid19_pages section6.pdf?1588257412.

2. 2. World Health Organisation. Coronavirus disease (COVID-19) advice for the public. 2020 [Cited April 2020]. [internet]. Available from: https://www.who. int/emergencies/diseases/novel-coronavirus-2019/advice-for-public.

3. 1. Harvey P, Higenbottam CV, Owen A, Hulme J, Bion JF. Peer-led training and assessment in basic life support for healthcare students: Synthesis of literature review and fifteen years practical experience. Resuscitation. 2012;83(7): 894-899.

4. NA 
001547

Healthcare stakeholders' perceptions and experiences of factors affecting the implementation of critical care telemedicine (CCT): qualitative evidence synthesis

K. Iliopoulou'; A. Xyrichis

${ }^{1}$ Florence nightingale faculty of nursing, midwifery and palliative care, King's College London, London, United Kingdom

Correspondence: $\mathrm{K}$. Iliopoulou

Intensive Care Medicine Experimental 2020, 8(2): 001547

Introduction: Critical care telemedicine (CCT) has long been used to expand the delivery of best care to critically ill patients located in geographically distant areas[1]. During the COVID-19 pandemic, those health systems with CCT appeared better prepared to respond to the pandemic [2]. However, several challenges remain for CCT to be successfully implemented [3].

Objectives: To undertake a qualitative evidence synthesis of healthcare stakeholders' perceptions and experiences of factors affecting the implementation of CCT, with a view hypothesising about factors more likely to foster implementation.

Methods: To undertake a qualitative evidence synthesis of healthcare stakeholders' perceptions and experiences of factors affecting implementation of $\mathrm{CCT}$, with a view hypothesising about factors more likely to foster implementation.Included studies were imported into Nvivo (QSR International Ltd) for coding. Data synthesis followed the BestFit framework approach, guided by the Consolidated Framework for Implementation Research (CFIR) [4]. Additional themes not captured by CFIR were classified under a separate theme. The GRADE-CERQual [5] approach was used to summarise confidence in the review findings. Conflicts throughout the process were resolved through team discussion.

Results: Thirteen studies were identified, published between 2008 and 2019, and conducted in North America. Most studies $(n=12)$ included bedside and hub staff, directors and hospital administrators; one study $(n=1)$ included family members. Most studies collected data through interviews and observations $(n=10)$, while three provided qualitative data through open-ended survey questions.

Analysis and synthesis of evidence identified twenty review findings. Most mapped under CFIR's domains of intervention characteristics, inner setting and individual characteristics. The most prominent findings related to networks and communication, knowledge and beliefs about the intervention, and interactions between hub and bedside teams.

ICU stakeholders, including patients and family members, acknowledged CCT's potential for improving patient safety and quality of care regarding standardisation of practice, early recognition of patient deterioration and continuous data collection; and valued the potential of allowing patients to remain in their community. Hospital staff also highlighted the mentoring role of CCT for junior staff at the bedside. Barriers to implementation stemmed from CCT's disruptiveness within the everyday workflow, and additional work imposed on bedside staff, such as relaying patient information to the hub team. The presence of a camera was reported as a risk to patient confidentiality and staff autonomy. The absence of ongoing dialogue and engagement by leadership were perceived as major barriers.

Hospital staff suggested adequate training and rehearsing of the processes relating to the use of CCT prior to its implementation as important facilitators. Both bedside and hub clinicians reported that they wanted their needs to be heard throughout the process, allowing time for them to accept and normalize CCT in their daily workflow.

While bedside clinicians valued prompt access to critical care expertise enabled by the CCT, they were concerned about the hub team interfering with their decisions over patient treatment. The conflict between bedside and hub clinicians was reported, in some instances, raising issues of disrespect and animosity. Effective communication featured by trust, teamness, and a sense of professional community were important enablers to the implementation of CCT.

Conclusion: We concluded with high or moderate confidence in the evidence contributing to several of these review findings. Further research, especially in contexts other than North- America, with different cultures, norms and practices will strengthen the evidence base for the implementation of CCT internationally.

\section{Reference(s) and grant ackowledgment(s)}

1. 1. Xyrichis A, Mackintosh NJ, Terblanche M, ..., \& Sandall J. Healthcare stakeholders' perceptions and experiences of factors affecting the implementation of critical care telemedicine (CCT): qualitative evidence synthesis. COCHRANE DB SYST REV. 2017; 11. | 2. Ohannessian R, Duong TA, Odone A. Global telemedicine implementation and integration within health systems to fight the COVID-19 pandemic: a call to action. JMIR public health and surveillance. 2020; 6(2): e18810. | 3. Mackintosh N, Terblanche M, Maharaj $\mathrm{R}, \ldots$, \& Magalhaes JH. Telemedicine with clinical decision support for critical care: a systematic review. Syst Rev. 2016; 5 (1):176. | 4. Damschroder LJ, Aron DC, Keith RE, ... \& Lowery JC. Fostering implementation of health services research findings into practice: a consolidated framework for advancing Implementation Sci. Implementation Sci. 2009; 4(1):50.| 5. Noyes J, Booth A, Lewin S, ..., \& Tunçalp Ö. Applying GRADE-CERQual to qualitative evidence synthesis findings-paper 6 : how to assess relevance of the data. Implementation Sci. 2018 Jan;13(1):4.

2. Review co-authors: Mackintosh N, Bench S, Philippou J, Terblanche M, Sandall J.

\section{6}

COVID-19 ICU Daily Review Sheet

T. Baumer'; E. Godfrey'; P. Jones ${ }^{2}$

${ }^{1}$ Intensive care, Royal Gwent Hospital, Newport, United Kingdom; ${ }^{2}$ Department of intensive care and anaesthesia, Royal Gwent Hospital, Newport, United Kingdom

Correspondence: T. Baumer

Intensive Care Medicine Experimental 2020, 8(2): 001606

Introduction: The first patient admitted to our intensive care unit (ICU) with COVID-19 at the Royal Gwent Hospital (RGH), Newport, Wales was on the 9th March 2020. RGH ICU went into surge capacity on the 28th March, remaining well beyond normal occupancy for 40 days. During this time additional doctors were redeployed from the anaesthetic and surgical departments to cover an emergency ICU rota. Objectives: A quality improvement project was undertaken to introduce a "COVID-19 ICU Daily Review Sheet" that could be completed during daily consultant-led ward-rounds for each patient. The aim was to promote standardisation of care and completion of comprehensive daily assessments, in a time-effective manner, during a chaotic and stressful pandemic environment. A further aim was to assist junior doctors with minimal ICU experience, such as those being redeployed, with structuring reviews of patients.

Methods: Between the 13th and 14th April, pre-intervention measurements were taken of daily ward-rounds $(n=32)$ from the hospital notes regarding junior preparatory review prior to senior review; full systematic patient review; and an "ICU-bundle" review: antibiotics, lines, drug chart, gastroprotection, venous-thromboembolism (VTE). The "COVID-19 ICU Daily Review Sheet" was introduced on the 15th April after emailing staff and making sheets available on the ICU. Post-intervention, between the 25th April and 1st May (7 days), wardrounds $(n=119)$ were re-assessed.

Results: Following its introduction, the "COVID-19 ICU Daily Review Sheet" was used on $69 \%$ (82 of 119) of ward rounds overall between the 25th April and the 1st May. Pre-intervention 31\% (10/32) of patients had a pre-emptive junior doctor review and 56\% (18/32) received a full systematic review. Post-intervention this had risen to $65 \%(77 / 119)$ and $68 \%(81 / 119)$ respectively. "ICU-bundle" documentation before/after the intervention was as follows: antibiotics $(34 \% / 45 \%)$, lines $(28 \% / 30 \%)$, drug chart $(0 \% / 28 \%)$, gastroprotection $(3 \% / 48 \%)$, VTE (13\%/27\%).

Conclusion: The "COVID-19 ICU Daily Review Sheet" had high uptake, appearing to encourage more comprehensive ward-rounds and documentation. The sheets appeared to promote a culture of 'near to normal levels of care' despite being in surge capacity. The wide-spread uptake suggests that the sheets were equally, if not more, time-efficient than free-hand documentation. We would reintroduce the sheet 
in the event of a second wave as a useful aid in this complex cohort of patients.

\section{9}

\section{Antibiotic stewardship practices in ICUs worldwide: data} from the DIANA study

K. Vanderbiest ${ }^{1}$; L. De Bus ' ; P. Depuydt ; ${ }^{1}$ A. Tabah² ; G. Dimopoulos ${ }^{3}$; J. Garnacho-Montero ${ }^{4}$ M. Leone ${ }^{5}$; J. Lipman ${ }^{6}$; JA. Paiva $;$; J. Schouten ${ }^{8}$; JF. Timsit ${ }^{9}$; J. Roberts ${ }^{10}$; JR. Zaharr ${ }^{11}$; J. De Waele

${ }^{1}$ Intensive care, Ghent University Hospital, Gent, Belgium; ${ }^{2}$ Intensive care unit, Redcliffe and Caboolture Hospitals, Brisbane, Australia; ${ }^{3}$ Department of critical care, University Hospital Attikon, Athens, Greece; ${ }^{4}$ Servicio medicina intensiva, hospital universitario virgen macarena, Sevilla, Spain; ${ }^{5}$ Department of anesthesiology and intensive care medicine, University Hospital of Marseille, Marseille, France; ${ }^{6}$ Department of intensive care medicine, the university of queensland centre for clinical research, The University of Queensland, Brisbane, Australia; ${ }^{7}$ Emergency and intensive care department, Centro Hospitalar de São João, Porto, Portugal; ${ }^{8}$ Intensive care, Radboud University Medical Center, Nijmegen, Netherlands; ${ }^{9}$ Intensive care medicine department, Assistance Hopitaux Publique de Paris, Paris, France; ${ }^{10}$ The university of queensland centre for clinical research, department of intensive care medicine, The University of Queensland, Brisbane, Australia; ${ }^{11}$ Microbiology, infection control unit, University of Paris, GH Paris Seine Saint- Denis, APHP, Paris, France

Correspondence: $\mathrm{K}$. Vanderbiest

Intensive Care Medicine Experimental 2020, 8(2): 001609

Introduction: Antibiotic stewardship programmes (ASPs) aim to improve antibiotic use and patient outcomes and to lower antimicrobial resistance (AMR) rates. Despite growing international commitment and the availability of antibiotic stewardship (AS) implementation guidelines, differences in local practices still may exist.

Objectives: This study aimed to (1) assess current AS practices in intensive care units (ICU) worldwide, and (2) to explore characteristics of AS-focused centers vs. others.

Methods: Data of the DIANA study (DetermInants of Antimicrobial use aNd de-escalAtion in critical care), a multicentre international prospective observational cohort study, were used. Details of ICU and hospital characteristics, background AMR levels, as well as different aspects of AS practices, including the use of antibiotic de-escalation (ADE), were collected from participating centers. AS-focused centers were defined as centers where (1) local guidelines on antibiotic therapy were available, (2) ASP team members were easily accessible for consultation and/or multidisciplinary AS meetings were held on a regular basis with presence of at least 2 out of 3 of the following team members other than the intensivist: infectious diseases (ID) specialist, medical microbiologist and pharmacologist, and (3) microbiology samples were processed daily. Descriptive statistics were used to compare ICUs in AS-focused centers vs. other centers and a 2-proportion two-sided Z-test was used to explore for differences in ADE proportions between both groups.

Results: Data from 144 ICUs were analyzed. Local guidelines on antibiotic treatment were available in 94 ICUs (65\%). A scheduled multidisciplinary AS meeting with the intensivist plus at least an ID specialist, a medical microbiologist or a pharmacologist, was organized in $28 \%$, $23 \%$ and $22 \%$ of the ICUs respectively. In $69 \%$ of the ICUs, microbiology samples were processed on a daily basis. Consequently, fortynine ICUs (34\%) were categorized as AS-focused centers. AS-focused centers were located more often in the Americas and Oceania, compared to Asia and Europe $(64 \%, 67 \%, 18 \%$ and $38 \%$ respectively). Country economy status (high income vs. low and middle income) and hospital status (teaching and non-teaching hospitals) were comparable. Median bed capacity in AS-focused centers and other centers was 17 vs. 11 beds. A closed ICU setting was present in 37\% of AS-focused centers, vs. $26 \%$ of the other centers. Hospital AMR levels were lower in AS-focused centers compared to other centers. A statistically higher proportion of patients underwent ADE in ICUs located in an AS-focused center, compared to those admitted in other centres $(p<0.001)$.
Conclusion: This study showed that only 1 out of 3 ICUs fulfilled the set criteria of AS-focused center, but these did not have a typical geographical or organisational profile. The observed AMR levels were lower and the ADE rates higher in AS-focused centers.

\section{Reference(s) and grant ackowledgment(s)}

1. De Bus L, Depuydt P, Steen J, Dhaese S, De Smet K, Tabah A, et al. Antimicrobial de-escalation in the critically ill patient and assessment of clinical cure: the DIANA study. Intensive Care Med. 2020 Jul;46(7):1404-17.

2. Kallen MC, Binda F, ten Oever J, Tebano G, Pulcini C, Murri R, et al. Comparison of antimicrobial stewardship programmes in acute-care hospitals in four European countries: A cross-sectional survey. Int J Antimicrob Agents. 2019 Sep;54(3):338-45.

3. Barlam TF, Cosgrove SE, Abbo LM, MacDougall C, Schuetz AN, Septimus EJ, et al. Executive Summary: Implementing an Antibiotic Stewardship Program: Guidelines by the Infectious Diseases Society of America and the Society for Healthcare Epidemiology of America. Clin Infect Dis. 2016 May 15;62(10):1197-202

4. This study received no grant.

\section{1}

Surging forward: junior doctors' experiences from the COVID-19 frontline

N. Kelly ${ }^{1}$; C. Holt ${ }^{1} ;$ H. Yusuf ${ }^{1}$

${ }^{1}$ Critical Care, Guy's and St Thomas' NHS Foundation Trust, London, United Kingdom

Correspondence: N. Kelly

Intensive Care Medicine Experimental 2020, 8(2): 001631

Introduction: The COVID-19 pandemic has placed enormous strain on healthcare systems worldwide requiring rapid reorganisation of services. Necessary expansion of Intensive Care (ICU) capacity mandated mass redeployment to support existing ICU staf.

Objectives: The primary end point of this study was the impact of the pandemic on career aspirations, specifically likelihood of pursuing further experience or training in ICM. Other outcomes explored include qualitative data on experiences during the pandemic surge and perceived learning outcomes during this challenging period.

Methods: The study was conducted as a single centre, observational cross-sectional analysis of survey data. Data were collected at Guy's and St Thomas's NHS Foundation Trust, a 1100 bed hospital, regional High Consequences Infectious Diseases (HCID) and ECMO centre. An online questionnaire was offered to all doctors via electronic mailing lists at the end of the 'redeployment' period. Data were analysed using $\mathrm{R}$ (version 4.0.0, http://www.r-project.org).

Results: 115 doctors completed the survey; 17 already worked on ICU, 41 were redeployed to ICU and 57 worked in other departments during the pandemic. Most respondents $(44.8 \%, 26 / 58)$ worked on ICU for 2-3 months. Roughly half of those working on ICU during the pandemic had at least 1 month post-graduate ICU experience $(48.3 \%$, 28/58). We found no difference in our primary endpoint of likelihood to pursue further employment in ICU in any of the groups surveyed. That being said as shown in Figure 1, whilst as a cohort there is no consistent shift, on an individual level there is flux in both directions in all groups. Follow up phone calls are ongoing and we hope to present further data on the experiences underlying these individual changes in career aspiration. Our secondary findings showed the majority of trainees deployed to ICU (72.5\%) felt they had learnt new skills during their redeployment useful to their parent specialty compared to those not deployed to ICU (29.9\%). Canvassing the three words to best describe their experience on ICU the top four words selected were teamwork (67.2\%), difficult (44.8\%), supportive $(44.8 \%)$ and camaraderie $(36.2 \%)$. 


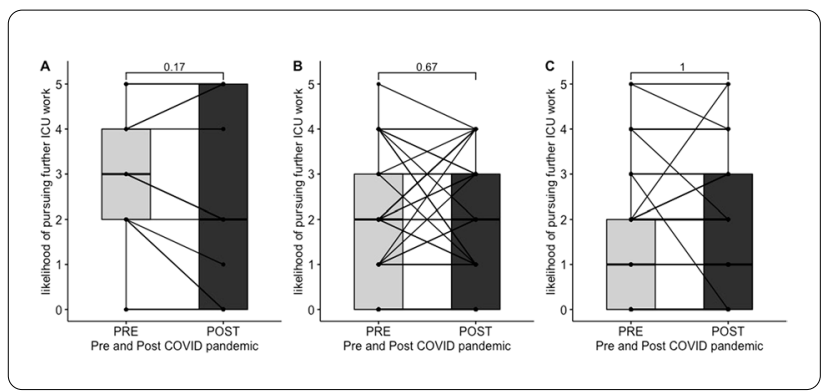

Conclusion: The COVID-19 pandemic was an unprecedented national healthcare crisis that rapidly evolved over a short period of time, requiring the mass redeployment of junior doctors under difficult circumstances. However it also presented a unique opportunity for doctors from many specialties to work together towards a collective goal. Despite not resulting in a consistent shift towards or against pursuing further training in ICU, our results support the assertion that those deployed to ICU in particular felt they had learned new skills and become part of a cohesive team rising to the challenges faced.

\section{2}

Burnout experience in the ICU staff of the City Clinical

\section{Hospitals in Ukraine}

M. Grynovska'; A. Wong ${ }^{2}$

${ }^{1}$ Anesthesia and intensive care, Ivano-Frankivsk National Medical University and Regional University Teaching Hospital, Ivano-Frankivsk, Ukraine; ${ }^{2}$ Critical care, Royal Surrey County Hospital, Guildford, United Kingdom

Correspondence: M. Grynovska

Intensive Care Medicine Experimental 2020, 8(2): 001642

Introduction Burnout (BO) is reaching epidemic levels in the medical population with prevalence reported around or over $50 \%{ }^{1}$. Its presence is associated with poor patient safety outcomes ${ }^{2}$. Burnout is a threat to the profession of critical care medicine, with high prevalence rates across critical care provider disciplines ${ }^{3}$. In Ukraine, specifically, the concept of BO in ICM remains poorly understood and is largely under recognized, or dismissed. We decided to continue investigating the reality of $\mathrm{BO}$ experience in our country.

Objectives To assess the $\mathrm{BO}$ experience and identify associated risk factors in the ICU of City Clinical Hospitals in Ukraine.

Methods The focus of the study was the frontline ICU staff of two different city clinical hospitals. The final sample size was nurses $(n=99)$ and doctors $(n=113)$. Data collection was performed with the help of the Maslach Burnout Inventory (MBI) survey. The BO rate was assessed across various ICU types, seniority, gender and age groups.

Results The response rate was $72 \%$. The overall prevalence of BO in ICUs was $69 \%$ (nurses $71.2 \%$, doctors $66.4 \%$ ). There was no difference on $\mathrm{BO}$ subscales between doctors and nurses $(95 \% \mathrm{Cl}, \mathrm{P}>0.05) \mathrm{We}$ also found no statistically significant difference in the incidence of $\mathrm{BO}$ between doctors and nurses $(P>0.05)$. Our study showed no difference $(P>0.05)$ in the incidence of BO in doctors across ICU type, seniority level, gender or age group. In contrast, there was a difference in the incidence of $\mathrm{BO}$ in nurses across the ICU type $(\mathrm{P}<0.05)$.

Conclusions BO prevalence was notably high in both ICU nurses and doctors. Our findings indicate that doctors and nurses are equally at risk of a $\mathrm{BO}$ experience. The study showed no clear association between any of the risks and the incidence of BO in doctors. With regard to nurses, the ICU type proved to be a factor in the incidence of burnout. The nurses of neurosurgery and burn ICUs were most affected possibly due to demanding workload and severity of the patients in these units. Limitations of our study include a relatively small number of respondents that prevents from drawing conclusions in the context of a country/healthcare system. Further research is warranted in hospitals across the country to determine $\mathrm{BO}$ rates and risk factors. We expect our study raise awareness about the reality of $\mathrm{BO}$ in Ukraine. We hope obtained data will contribute to better understanding of $\mathrm{BO}$ experience and will help us explore the existing opportunities to mitigate its impact and restore fulfillment among ICM professionals.

\section{References and Grant acknowledgments}

1. Shanafelt TD et al. Mayo Clinic Proceedings. 2015: 90(12):1600-1613.

2. Hall LH et al. PLoS One. 2016;11(7):e0159015.

3. Kerlin MP, McPeake J, Mikkelsen ME. Burnout and Joy in the Profession of Critical Care Medicine. Crit Care 2020;24:98.

I would like to thank ESICM NEXT Mentoring and my mentor Adrian Wong for supervision and guidance.

\section{9}

The effect of ventilator associated pneumonia on the length of stay in patients with traumatic brain injury in the Intensive Care Unit

G. Koukoulitsios'; M. Anifanti '; K. Tsikritsaki' ; I. Tsoni' ${ }^{1}$; S. Bakouli ${ }^{1}$; I. Ionna'; G. Maglaras'; A. Koutivas' ${ }^{1}$; C. Mandila'; S. Antonopoulou'; V. Koutsoukou'; V. Salma'; A. Kalogeromitros

'ICU, General Hospital of Athens "G. Gennimatas", Athina, Greece

Correspondence: G. Koukoulitsios

Intensive Care Medicine Experimental 2020, 8(2): 001199

Introduction: In our study we describe the effect of Ventilator Associated Pneumonia (VAP) on the on the length of stay (LOS) in patients with traumatic brain injury (TBI) in the Intensive Care Unit (ICU).

Methods: From December 2016 until January 2020, 156 patients, 96 males and 60 females with TBI requiring mechanical ventilation more than 48 hours $\mathrm{GCS} \leq 8$ were studied. The mean age was 43 years. On admission to the ICU they had body mass index $27 \pm 6 \mathrm{~kg} / \mathrm{m}^{2}$ and APACHE II score $20 \pm 2$. Out of 156 patients the 54 had co morbid medical illness.

Results: VAP occurred in 69 out of 156 TBI patients There was no difference in predominance as far as gender was concerned. VAP was significantly associated to patients requiring longer mechanical ventilation 18 days \pm 2 and longer sedation 14 days \pm 2 . Patients with co morbid medical illness and VAP were associated with a significantly greater degree of non neurological organ system dysfunction, though there was no difference in frequency of VAP development compared to the patients without co morbid medical illness. Although VAP was not associated with increased hospital mortality, patients who developed VAP had a longer duration of mechanical ventilation (28 versus 10 days, $p<0.0001$ ) and longer ICU lengths of stay (31 versus 12 days, $\mathrm{p}<0.0001$.

Conclusion: The effect of VAP in patients with TBI is high; increasing the mechanical ventilation duration and the ICU length of stay; however, does not affect the prognosis and does not seem to be associated with a significantly increased risk of mortality though increases the mechanical ventilation duration.

\section{1}

Seasonal variation and weather changes in admissions to critical care with aneurysmal subarachnoid haemorrhage 2000 - 2019 K. Massie ${ }^{1}$; S. Stott ${ }^{2}$

'Physiotherapy, Aberdeen Royal Infirmary, Aberdeen, United Kingdom; ${ }^{2}$ Critical Care, Aberdeen Royal Infirmary, Aberdeen, United Kingdom

Correspondence: $\mathrm{S}$. Stott

Intensive Care Medicine Experimental 2020, 8(2): 001211 
Introduction: Several reports have suggested that the incidence of aneurysmal subarchnoid haemorrhage (SAH) varies seasonally with peaks in autumn and/or winter (1) and recently have suggested that weather effects may be related (2).

Objectives: To see if admissions to critical care (CC) with SAH were seasonal and if weather factors were related over a twenty year period. Methods: Our patient record database was searched for patients with an admission diagnosis of SAH between January 2000 and December 2019. Data for age, sex, Glasgow coma score (GCS) at presentation, length of unit and hospital stay, unit and hospital outcome and ultimate discharge destination were retrieved. Weather data for the local health board region were obtained from online weather sources. Variables of monthly maximum, minimum and average monthly temperature and maximum, minimum and average atmospheric pressure were obtained.

Results: 357 patients were identified. 63\% were female. Median age 56 years (range 18-86, IQR 17). Median time to CC admission 0 days (range 0-46, IQR 1). Median length of stay 1.8 days (range 0.1-30.5, IQR 3.7). $61 \%$ died before hospital discharge, $37.5 \%$ died in critical care. $15.9 \%$ were discharged to home or normal residence. Median APACHE II score 18 (range 4-40).

There was a seasonal variation of admissions to critical care with a peak occurring in October (figure 1.). There was no correlation with maximum, minimum, average monthly or monthly changes in temperature $(r=-0.10783,-0.07064,-0.11881,-0.02244$ respectively), or with maximum, minimum, average monthly or monthly change in atmospheric pressure $(r=0.088,0.449,0.009,0.002$ respectively). There was no significance difference in number of deaths according to month of admission $(p=0.9)$

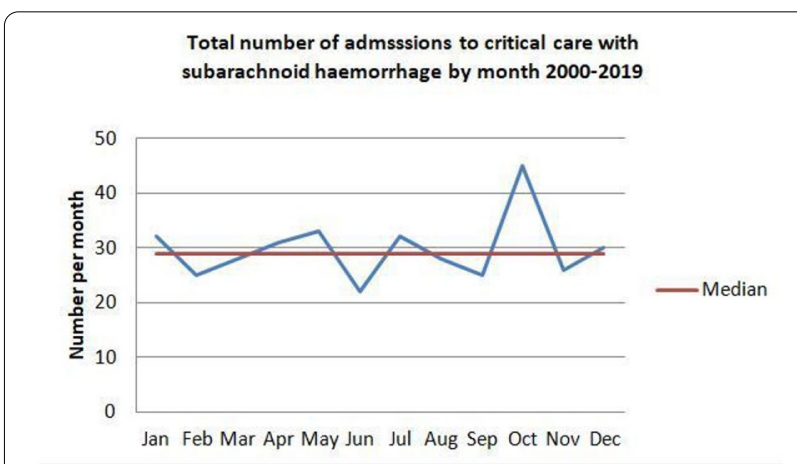

Figure 1. Total number per month of SAH admitted to critical care 2000-2019

Conclusion: We have shown that there is a seasonal variation in the incidence of SAH being admitted to critical care in the North of Scotland. We have not been able to explain this in relation to changes in temperature or atmospheric pressure.

\section{Reference(s) and grant ackowledgment(s)}

1. 2. Examination of seasonal variations in the incidence of subarachnoid hemorrhage. TetsuhisaYamada, YoshihiroNatori. Interdisciplinary Neurosurgery, Volume 19, March 2020, https://doi.org/10.1016/j.inat.2019.100581.

2. 1. A 5 -year retrospective study assessing the association between seasonal and meteorological change and incidence of aneurysmal subarachnoid haemorrhage Hughes MA; Grover PJ; Butler CR; Elwell VA; Mendoza ND. British Journal of Neurosurgery. 24(4):396-400.

\section{2}

\section{Prospective analysis of gastrointestinal bleeding critically ill} patients in intensive care unit

M. Yıldırım ; M. Aslann; S. Temel'; M. Caliskan³ ; NK. Elif²; K. Gundogann ; M. Sungur ${ }^{5}$

${ }^{1}$ Internal medicine, Erciyes Üniversitesi Tıp Fakültesi Hastaneleri, Kayseri, Turkey; ${ }^{2}$ Internal medicine, Erciyes University, Kayseri, Turkey; ${ }^{3}$ Deparment of internal medicine, Erciyes University, Kayseri, Turkey; ${ }^{4}$ Department of internal medicine, division of intensive care, Erciyes University, School of medicine, Kayseri, Turkey; ${ }^{5}$ Division of critical care medicine, department of internal medicine, Erciyes University School of Medicine, Kayseri, Turkey, Kayseri, Turkey

Correspondence: M. Yıldırım

Intensive Care Medicine Experimental 2020, 8(2): 001242

Introduction: To investigate the demographic, clinic and laboratory features of the critically ill patients with gastrointestinal bleeding (GIB) in Intensive Care Unit (ICU).

Methods: This study was performed prospectively in ICU. Patients aged 18 years and older, who had hospitalized for at least 24 hours, with gastrointestinal bleeding, were included the study.

Results: A total of 86 patients were enrolled the study. The mean age was $65 \pm 14$ years. The APACHE II score was 13 (2-41). Glasgow Coma Scale (GCS) was $13 \pm 4$ and SOFA score was 3 (0-16). Glasgow-Blatchford risk score was 12 (2-18) and Rockall risk score was 6 (1-10). The most common co-morbid diseases were hypertension (44\%), diabetes mellitus (27\%), and chronic liver disease (27\%). The most frequent predisposing factor for GIB were chronic liver disease (22\%) and thrombocytopenia (21\%). The anatomic location of bleeding was mostly in the esophagus (35\%) and stomach (24\%). Endoscopic intervention was performed $42 \%$ of patients during ICU follow-up. The length of ICU stay was 5 days (min: 2-max: 28). The ICU mortality rate was $19 \%$. APACHE II, SOFA and Glasgow-Blatchford scores were higher in the patients who died, compared to survivor patients $(p<0.05)$.

Conclusion: As a result of this study, chronical liver disease and thrombocytopenia were found the most common predisposing factor of GIB. The most common localization of bleeding was the stomach and esophagus regions. Approximately half of the patients were treated endoscopically. APACHE II, SOFA and Glasgow-Blatchford scores were higher in patients who died compared to the survivors.

001272

A demographic analysis of admissions to high dependency with COVID-19 at the Queen Elizabeth University Hospital,

Glasgow

R. Best' ${ }^{1}$ : R. Hart

${ }^{1}$ Critical Care, Queen Elizabeth University Hospital, Glasgow, United Kingdom

Correspondence: $\mathrm{R}$. Best

Intensive Care Medicine Experimental 2020, 8(2): 001272

Introduction: During the 2020 COVID-19 pandemic, critical care departments across the United Kingdom expanded capacity to care for unprecedented numbers of critically ill patients. The Queen Elizabeth University Hospital in Glasgow (QEUH) is relatively unusual in its separation of high dependency (level 2) and intensive care (level 3) patients within the critical care cohort. Therefore the opportunity was taken to investigate the demographics of the COVID-19 positive patients presenting to high dependency (HDU) during the pandemic and to explore whether these demographics were associated with either HDU or hospital outcome.

Methods: All patients with COVID-19 presenting to HDU at QEUH were identified. Data on age, gender, comorbidities, length of HDU stay, augmented care period (ACP), discharge destination, unit outcome and hospital outcome were collected. Deprivation quintile was calculated using the Scottish Index of Multiple Deprivation (SIMD) according to the patient's home postcode.

The comorbidities that were assessed were diabetes $(1,2)$, cardiovascular disease (excluding hypertension) (1-3), hypertension (1-3), chronic respiratory disease $(1,3)$, renal disease (4) and obesity (5). These comorbidities were chosen as early studies had identified them as comorbidities that might affect infection rate and/or outcome in patients with COVID-19.

Data were analysed to determine whether age, gender, deprivation status or the presence of certain comorbidities were associated with HDU outcome (improved or deteriorated) and hospital outcome (survived to discharge or died in hospital). 
Results: Between 24/03/20 and 25/05/20, there were 74 admissions of 69 patients to HDU at the QEUH. Together they amassed 307 patient days in HDU: 13 days at ACP level 3; 230 at level 2; 54 at level 1; and 10 at level 0. Age ranged from 27-76 years (mean 57, IQR 50-65) and 51 patients were male (74\%). The majority of patients admitted lived in areas of high deprivation. Length of HDU stay ranged from 1-16 days (median 3, IQR 2-5).

Of the 69 patients, 23 had none of the pre-determined comorbidities (33\%); 16 had diabetes mellitus (23\%); 8 had cardiovascular disease (12\%); 11 had hypertension (16\%), 13 had chronic respiratory disease (19\%); 4 had renal disease (6\%); and 31 were obese (55\%). Twenty-four patients were multimorbid (35\%), as described as having two or more of the aforementioned diagnoses.

Of the 74 admissions, 42 patients improved and were discharged to the ward (57\%); 29 patients deteriorated and were admitted to intensive care (39\%); and three patients deteriorated but were not escalated (4\%). Of the 69 patients who were admitted to HDU, 51 survived hospital and were discharged home (74\%).

In analysis, none of the demographic or comorbidity variables were associated with HDU or hospital outcome.

Conclusion: During the covid pandemic an unusually sick cohort of patients were admitted to HDU at the QEUH, the majority of whom survived to hospital discharge. However, none of the prior identified risk factors for poor outcome were found to be reliable in our cohort. This should be borne in mind in the event of a second wave of the pandemic when making decisions regarding critical care escalation.

\section{Reference(s) and grant ackowledgment(s)}

1. 1. Report of the WHO-China Joint Mission on Coronavirus Disease 2019 (COVID-19). 16-24 February 2020.

2. 2. Chen N, Zhou M, Dong X et al. Epidemiological and clinical characteristics of 99 cases of 2019 novel coronavirus pneumonia in Wuhan, China: a descriptive study. Lancet 395:507-513. 2020.

3. 3. Dong X, Cao Y, Lu X et al. Eleven faces of coronavirus disease 2019 . Allergy. 2020.

4. 4. Henry B and Lippi G. Chronic kidney disease is associated with severe coronavirus disease 2019 (COVID-19) infection. International Urology and Nephrology. 2020

5. 5. Simmonet A, Chetboun M, Poissy J et al. High prevalence of obesity in severe acute respiratory syndrome coronavirus-2 (SARS-CoV-2) requiring invasive mechanical ventilation. Obesity. 2020.

6. No funding was obtained for this project.

\section{4}

Factors predicting mortality in critically ill COVID-19 patients W. Zarrougui ' ;. Saint Leger ; ; M. Alibenali ${ }^{1}$; H. Boughanmi ${ }^{1}$; N. Elbeki ; F. Lambiotte

${ }^{1}$ Intensive care unit, Hospital Jean Bernard, Valenciennes, France

Correspondence: $W$. Zarrougui

Intensive Care Medicine Experimental 2020, 8(2): 001274

Introduction: Since the last week of December, 2019, a massive public health crisis has been rapidly spreading from China to the rest of the world, caused by a newly identified coronavirus named SARS-CoV-2. This rapidly evolving infection affected mainly the respiratory system and can lead to fatal morbidity and mortality.

Objectives: The aim of this study was to describe clinical characteristics and to identify the factors associated with ICU mortality in critically ill COVID-19 patients.

Methods: A retrospective cohort study was performed in the medical ICU of Jean Bernard hospital in Valenciennes, France, including all consecutive critically ill patient with confirmed COVID-19 infection between Mars 11 and Mai 15, 2020. A confirmed case of COVID19 was defined by a positive RT-PCR (upper or lower respiratory tract specimens). Data were collected by reviewing the electronic medical charts. Were collected: clinical features at admission, severity of illness and ICU course. Univariate and multivariate analysis regression analyses were performed to identify factors independently associated to mortality. Statistical seizure and analyses were performed using the statistical software package SPSS 24.0.

Results: Among 685 COVID-19 patients hospitalized in Valenciennes regional hospital during the study period, $77(11.2 \%)$ were admitted to the ICU. Their general characteristics were: age, $64.67 \pm 11$ years with ages ranging from 24 to 89 years; male, 52(67.5\%); mean $\mathrm{BMI}$, $31.27 \pm 9.05 \mathrm{~kg} / \mathrm{m}^{2}$; Charlson comorbidity index, $3.03 \pm 1.99$; duration of symptoms before ICU admission, $9 \pm 4.1$ days; the mean SAPSll score, $36.11 \pm 10.33$; the mean SOFA score, $4.56 \pm 2.46$; invasive mechanical ventilation (IMV), 57(74\%) with median duration, 17[926.25] days; the median of peep and compliance during first 3 days were respectively $12[12-16] \mathrm{cmh} 2 \mathrm{O}$ and $36[30-45] \mathrm{ml} / \mathrm{cmH} 2 \mathrm{O}$; the most CT- lung opacification percentage at admission [50\%-75\%], 23(29.9\%); Vasopressors, 45(72.7\%); hydrocortisone, 23(29.9\%); hydroxychloroquine, 29(37.7\%); ECMO, 4(5.1\%); tracheostomy, 10(13\%); thromboembolism complications, 19(24.6\%); acute kidney injury (AKI), 34(44.2\%); renal replacement therapy, 20(26\%); median length of ICU stay, 17[6-26] days; mortality rate, 3.8\%.

Univariate analysis showed significant association between mortality and the following factors: age $>65$ years $(p=0.03)$, Charlson index $(p=0.032)$, IMV at admission $(p=0.032)$, AKI $(p=0.000)$, and ventilator free days $(p=0.000)$.

Multivariate logistic regression analysis has identified only 3 variables as independently associated with ICU mortality: Age(OR, 1.06 95\%CI[1.00-1.13], p 0.048), AKI(OR, 17.74; 95\%CI[4.22-74.52], p 0.000), and length of ICU stay(OR, 0.94; 95\%CI[0.91-0.98], p 0.011).

Conclusion: In this non selected consecutive COVID-19 population in a medical ICU, the age, acute kidney injury and the length of ICU stay, demonstrated acceptable predictive values for the in-hospital mortality of critically ill patients with COVID-19.

001282

Decline in functional status after ICU discharge of COVID-19 patients

W. Zarrougui ${ }^{1}$; N. Bejaoui ${ }^{1}$; F. Sedrati ${ }^{1}$; N. Elbeki' ; F. Lambiotte ${ }^{1}$ Intensive care unit, Hospital Jean Bernard, Valenciennes, France Correspondence: $W$. Zarrougui

Intensive Care Medicine Experimental 2020, 8(2): 001282

Introduction: In December 2019, cases of COVID-19 infection were reported and this novel virus spread rapidly to touch every corner of the world within weeks. COVID-19 affects mainly the respiratory system and would result in acute respiratory distress syndrome, requiring ICU admission and prolonged mechanical ventilation. For ICU survivors, recovery may be characterized by a marked compromise between physical and psychological health after ICU discharge which may decrease quality of life.

Objectives: The objective of our study was to evaluate the incidence and risk factors of post ICU physical impairment of COVID-19 patients. Methods: A retrospective study was performed in the medical ICU of Jean Bernard hospital in Valenciennes, France, included all consecutive critically ill patients with confirmed COVID-19 infection between Mars 11 and Mai 15, 2020. The study collected clinical features at admission, severity of illness, ICU course, and acute management procedures. Univariate and multivariate regression analyses were performed to identify factors independently associated with physical declines. The statistical analyses were performed using the SPSS 24.0.

Results: A total of 77 patients were included: 52 male $(67.5 \%)$, mean age, $64.67 \pm 11$ years; mean BMl, $31.27 \pm 9.05 \mathrm{~kg} / \mathrm{m}^{2}$; mean Charlson index, $3.03 \pm 1.99$; mean SAPSII score, $36.11 \pm 10.33$; mean SOFA score, $4.56 \pm 2.46$; invasive mechanical ventilation (IMV), 57(74\%) with median duration, 17[9-26.25] days; vasopressors, 45(72.7\%); hydrocortisone, 23(29.9\%); neuromuscular blockers agents, 46 (59.7\%); ECMO, 4(5.1\%); tracheostomy, 10(13\%); median length of ICU stay, 17[6-26] days; mortality rate, $33.8 \%$. Twenty-one (42\%) of ICU survivors were transferred to rehabilitation ward with mean length of stay, $19.35 \pm 9.45$ days. On the day of hospital discharge, 14(28\%) patients kept persistent physical impairment.

Univariate analysis identified several factors associated to rehabilitation needs after ICU stay: age, p 0.006; use of corticosteroids and 
neuromuscular blockers agents with $p$ value respectively 0.000 and 0.006; duration of IMV, p 0.006 and duration of ICU stay, p 0.000. In multivariate analysis two predictors were independently associated to rehabilitation needs: the age (OR, 1.104; 95\%CI[1.00-1.22], p 0.051) and duration of ICU stay (OR, 1.11; 95\%CI[1.01-1.22], p 0.023).

Univariate analysis identified the age $(p=0.038)$, neuromuscular blockers agents $(p=0.009)$ and medical history of diabetes $(p=$ $0.026)$ as factors associated with prolonged stay (>14 days) in rehabilitation ward.

Conclusion: Critically ill patients who survive COVID-19 infection frequently experience important and long-lasting physical impairments. In fact, identified factors associated with persistent physical disabilities after ICU discharge may offer physicians the opportunity to facilitate adequate screening and to develop preventive measures to improve health-related quality of life of critical illness survivors.

\section{9}

\section{Descriptive analysis of critically ill patients admitted} to a third-level hospital in covid-19 pandemic

P. García Olivares ${ }^{1}$; A. Garrido '; J. Cedeño Mora'; C. Mata ${ }^{1}$; J. Cui' ; P. Oliver ${ }^{1}$; G. Castañeda'; C. Alvarez'; N. Cango ${ }^{1}$; JM. Gomez

${ }^{1}$ Intensive Care Unit, H.G.U Gregorio Marañón, Madrid, Spain

Correspondence: P. García Olivares

Intensive Care Medicine Experimental 2020, 8(2): 001299

Introduction: COVID-19 is a potentially fatal disease, with high contagiousness, that has become a global public health problem.

Objectives: The aim of this study was to describe the characteristics of patients admitted to the critical care units of a third level hospital during the COVID-19 pandemic.

Methods: Prospective, observational study performed in critically ill Covid-19 patients admitted to H.G.U Gregorio Marañón during March-April 2020. We collected epidemiological data, comorbidities, presentation symptoms, relevant clinical data, organic support measures used, complications during stay, administered treatments and outcome.

Descriptive analysis was express as means (SD) for normally distributed quantitative variables, medians (IQR) for non-normally distributed variables, and as percentages for categorical data.

Results: One hundred and fifty patients were included, $70 \%$ were male, mean age was 61 years (49-70). Charlson Comorbidity Index 1 pts (0-3) and the most frequent comorbidities observed were: Obesity $(\mathrm{MCl} \geq 30) 53 \%$, hypertension $53 \%$, diabetes $18 \%$, dyslipidemia $47 \%$, cardiovascular disease $56 \%$, chronic respiratory disease $26 \%$ and immunodeficiency $10 \%$. The elapsed time from the onset of the clinical picture to hospital admission was $6 \pm 4$ days, being the most common symptoms: fever $90 \%$, cough $70 \%$, shortness of breath $60 \%$, myalgia $46 \%$ and headache $7 \%$. The delay until ICU admission was $2 \pm 1$ days and the main clinical characteristics at admission were: APACHE II $17 \pm 5$, SOFA $6 \pm 3, \mathrm{PaO} 2 / \mathrm{FiO} 2$ ratio $102 \pm 45$, mechanical ventilation $70 \%$, High-flow oxygen therapy $27 \%$ and requiring immediate prone-position ventilation $57 \%$ of patients. During ICU stay, $88 \%$ of patients needed mechanical ventilation, with an average time of 15 days (8-25), 74\% prone-position ventilation, with a median of 2 sessions (0-5), $64 \%$ neuromuscular blockers and $37 \%$ of patients underwent tracheostomy for respiratory support removal. Vasopressor therapy was required by $63 \%$ of patients, $30 \%$ developed acute renal failure and $10 \%$ needed renal replacement therapy. During ICU stay, infectious complications (ventilator-related pneumonia $28 \%$ and catheter-related bacteraemia 24\%) were the most frequent, followed by delirium $30 \%$, pneumothorax $10 \%$ and clinical thromboembolism $7 \%$. Regarding the treatment administered: $99 \%$ of patients received antibiotics, 99\% Ritonavir/Lopinavir, 97\% Hidroxichloroquine, 76\% Tocilizumab, $60 \%$ Beta-Interferon and $22 \%$ Remdesivir. Of all the patients, $76 \%$ received corticosteroids at some time during hospital stay. The ICU mortality was $38 \%$, with mean stay of 24 days (10-32). In-hospital mortality was $40 \%$, with mean stay of 36 days (17-53). The main causes of mortality were multiple organ dysfunction syndrome $47 \%$, and refractory hypoxemia $40 \%$.
Conclusion: There was a significant mortality and a large number of complications in critically ill Covid-19 patients. A high percentage of patients needed respiratory support and prone-position ventilation. Prolonged ICU and hospital stay was required.

\section{9}

Reducing post-traumatic stress disorder after ICU discharge with the IPREA3 program for discomfort reduction in the ICU: A stepped wedge cluster-randomized controlled study, the PTSD-REA study

P. Kalfon'; K. Baumstarck'; B. Mauchien'; J. Audibert'; MA. Geantot ${ }^{3}$; J. Crozon-Clauzel ${ }^{4} ;$ E. Azoulay ${ }^{5}$; S. Deparis-Dusautois ${ }^{6} ;$ N. Revel $^{7} ;$ J. Pottecher $^{8}$; A. Monsel ${ }^{9}$; S. Ehrmann ${ }^{10}$; A. Loundou ${ }^{2}$; W. El-Hage ${ }^{11}$; P. Auquier $^{2}$

${ }^{1}$ Réanimation, C.H. de Chartres, Le Coudray, France; ${ }^{2}$ Ceress, unité de recherche ea3279, Aix-Marseille University, Marseille, France; ${ }^{3}$ Département d'anesthésie réanimaiton, Chu De Dijon, Dijon, France; ${ }^{4}$ Réanimation polyvalente, Hôpital Edouard Herriot, Hospices Civils de Lyon, Lyon, France; ${ }^{5}$ Réanimation médicale, Hôpital Saint-Louis, Paris, France; ${ }^{6}$ Réanimation, Troyes Hospital, Troyes, France; ${ }^{7}$ Réanimation, Hospital Pasteur $\mathrm{CHU}$ de Nice, Nice, France; ${ }^{8}$ Anesthésie - réanimation chirurgicale, Hôpital de Hautepierre - Hôpitaux Universitaires de Strasbourg, Strasbourg, France: ${ }^{9}$ Réanimation chirurgicale, University Hospitals Pitié Salpêtrière - Charles Foix, Paris, France; ${ }^{10}$ Médecine intensive réanimation, Chru Hôpitaux De Tours, Tours, France; ${ }^{11}$ Psychiatrie d'adultes, addictologie, et umr inserm u 1253 - ibrain - psychiatrie neuro-fonctionn, Chru Hôpitaux De Tours, Tours, France

Correspondence: P. Kalfon

Intensive Care Medicine Experimental 2020, 8(2): 001309

Introduction: Critically ill patients in intensive care units (ICUs) are exposed to stressful conditions and experience discomfort from multiple sources, such as the environment or related to care provided in the $\mathrm{ICU}$, depending on the care organizations and patient health status. This discomfort may have short-term and long-term consequences for ICU survivors, such as various degrees of anxiety and/or depression or post-traumatic stress disorder (PTSD) (1) which may affect patient's quality of life. Reducing discomfort in the ICU has therefore become a major issue. IT has already been demonstrated that the implementation of a tailored multicomponent program for discomfort prevention in adult ICUs (named the IPREA3 program): 1) may decrease the selfperceived discomfort in general adult ICU survivors of at the end of their ICU stay, according the results of the IPREA3 study, a cluster-randomized controlled study (2); 2) may reduce substantial PTSD symptoms assessed 1 year after ICU discharge, according the results of the AQVAR study, a prospective cohort study inluding patients who were exposed and unexposed to the IPREA3 program (3). These promising results justify the need for a confirmatory study using a randomized design, the PTSD-REA study.

Methods: The PTSD-REA study is a stepped wedge cluster-randomized interventional study involving 18 French ICUs. The cluster level comprises the ICUs, which are the units of randomization. Patients hospitalized in the ICUs comprise the individual level.

The intervention is the implementation of the IPREA3 program, a tailored multicomponent program consisting of assessment of ICUrelated self-perceived discomforts, immediate and monthly feedback to the healthcare team, and site-specific tailored interventions. The whole duration of the inclusion period will be 15 months and patients will be followed-up 1 year after ICU discharge. During the first month of the inclusion period, no ICU is exposed to the IPREA3 program. Every following month, 2 new ICUS of the 18 participating ICUs are randomized to the application of IPREA3 until the end of the inclusion period, leading to 9 steps. We considered 3 periods, a control period, an unvariable 5-month learning period for each ICU, and an interventional period during which the IPREA3 program will be applied during more than 5 months until the end of the inclusion period. Patients will be included only during the control period and the interventional period. For each participating ICU, the durations of the control period and the interventional period range from 1 to 9 months, according the randomization date for crossing over to the IPREA3 program. 
The eligible patients are patients who survived an ICU stay of at least 3 calendar days with an affiliation to a social security scheme. The exclusion criteria include death prior to the 1-year follow-up after the index ICU hospitalization, patients under trusteeship, patients with cognitive incapacity, and patients who did not understand French sufficiently to complete the questionnaires exploring psychiatric morbidity. All participants provided written informed consent. We planned to include 4500 patients during the study, 2250 patients in each group.

The primary outcome measure is the presence of substantial PTSD symptoms at one year after ICU discharge assessed from the posttraumatic stress-disorder checklist (PCL-5) which is a 20-item selfreport measure for PTSD symptoms according the DSM-5 (Diagnostic and Statistical Manual of Mental Disorders). The secondary outcomes are the following : the ICU stay's duration, the number of days with mechanical ventilation, the overall score of discomfort assessed from the IPREA3 questionnaire, the duration of hospital stay after ICU discharge. The other secondary outcomes will be asseseed at 1 year after ICU discharge: the number of symptoms in categories $A, B, C, D$ and $E$, respectively, assessed from the PCL-5, the scores of the sub-scales $A$ and D, respectively, of the questionnaire HAD-S (Hospital and Anxiety Depression Scale), the score obtained from the World Health Organization Quality of Life (WHOQOL-BREF), the number of emergency room visits, hospitalizations, and consulations with a psychologist or psychiatrist during the past year since ICU discharge, return to own home, and occupational status.

Results: The inclusion period started on November 1, 2020, but was interrupted on March 19, 2020 due to the consequences of the global COVID-19 pandemic on the functionning of French ICUs, when 1252 patients had already been included. It is planned to resume the inclusions in the ICUs on September 1, 2020. The provisional date for ending the inclusions without further interruption due to the COVID-19 pandemic is now July $31,2021$.

Conclusion: Despite the interruption of the PTSD-REA study due to the COVID-19 pandemic, more than a thousand patients in the control group could be already included. The results of the PTSD-REA study are eagerly expected to confirm that applying the IPREA3 program during the ICU stay may reduce substantial PTSD symptoms at 1 year after ICU discharge in general adult ICU survivors.

\section{Reference(s) and grant ackowledgment(s)}

1. 1. Davydow DS, Gifford JM, Desai SV, Needham DM, Bienvenu OJ. Posttraumatic stress disorder in general intensive care unit survivors: a systematic review. Gen Hosp Psychiatry. 2008:30:421-434.

2. 2. Kalfon P, Baumstarck K, Estagnasie P, Geantot MA, Berric A, Simon G, Floccard B, Signouret T, Boucekine M, Fromentin M, Nyunga M, Sossou A, Venot $M$, Robert R, Follin A, Audibert J, Renault A, Garrouste-Orgeas M, Collange O, Levrat Q, Villard I, Thevenin D, Pottecher J, Patrigeon RG, Revel N, Vigne C, Azoulay E, Mimoz O, Auquier P, group IS. A tailored multicomponent program to reduce discomfort in critically ill patients: a cluster-randomized controlled trial. Intensive Care Med. 2017;43:1829-1840.

3. This research was financially supported by a grant from the Programme de Recherche sur la Performance du Système de Soins, 2018, PREPS-18-0812, funded by the French Ministry of Health.

4. 3. Kalfon P, Alessandrini M, Boucekine M, Renoult $S$, Geantot MA, DeparisDusautois S, Berric A, Collange O, Floccard B, Mimoz O, Julien A, Robert R, Audibert J, Renault A, Follin A, Thevenin D, Revel N, Venot M, Patrigeon RG, Signouret T, Fromentin M, Sharshar T, Vigne C, Pottecher J, Levrat Q, Sossou A, Garrouste-Orgeas M, Quenot JP, Boulle C, Azoulay E, Protocole PTSDREA - PREPS 2018 - version 6.0 2019-04-03 89/89 Baumstarck K, Auquier P, Group I-AS. Tailored multicomponent program for discomfort reduction in critically ill patients may decrease post-traumatic stress disorder in general ICU survivors at 1 year.

\section{6}

\section{Upper gastro-intestinal bleeding in VA ECMO patient:} a retrospective cohort study

\section{J. Stern'; D. Claire' ${ }^{2}$.JF. Timsit ${ }^{3}$; R. Sonneville}

${ }^{1}$ Intensive care, Bichat-Claude Bernard Hospital, Paris, France; ${ }^{2}$ Paris, Medical and infectious Intensive Care Unit, Bichat-Claude Bernard Hospital,
Paris, France; ${ }^{3}$ Intensive care medicine department, Assistance Hopitaux Publique de Paris, Paris, France

Correspondence: J. stern

Intensive Care Medicine Experimental 2020, 8(2): 001316

Introduction: Veno-arterial Extracorporeal Membrane Oxygenation(VA ECMO) is used in cases of cardiogenic shock or refractory cardiac arrest. Upper gastrointestinal bleeding (UGIB) is a common complication in intensive care patients. Few data are available for patients on VA ECMO.

Objectives: The objective of our study was to assess the incidence, risk factors and attributable morbidity and mortality of UGIB in this population.

Methods: We conducted a retrospective cohort study in our medical intensive care unit (ICU) at Bichat Hospital (APHP-Paris). All patients admitted between 2014 and 2017 who had a VA ECMO were included. A survival model with death as a competitive risk was used to determine the risk factors for UGIB.

Results: 257 patients were included, 48 patients (18.7\%) presented UGIB. These patients had a lower SAPS II score and a lower lactate level at the setting up of ECMO (respectively $51[41 ; 67.5]$ vs $60[44 ; 80] p=$ $0.05 ; 4 \mathrm{mmol} / \mathrm{L}[2.4 ; 8.1$ ] vs $5.6 \mathrm{mmol} / \mathrm{L}[2.7 ; 10.3], \mathrm{p}=0.04)$ a longer length of stay in ICU and mechanical ventilation duration (respectively 40.5 days $[19 ; 82]$ vs 15 days [6;26]; $p<0.01$ and 32 days $[17.5 ; 45]$ vs 9 days $[5 ; 18] ; \mathrm{p}<0.01)$. UGIB occurred in median at 18 days $[7 ; 43]$. The most frequently found lesions on endoscopy were peptic ulcers. In multivariate analysis a BMI (body mass index) > 30 (HR 2.46 [1.22; 4.97], $\mathrm{p}=0.01$ ), diabetes (HR 2.91 [1.48; 5.72]; $\mathrm{p}<0.01)$ and a history of cerebrovascular disease (HR 3.18 [1.19; 8.47]; $p=0.02$ ) were associated with a risk of developing UGIB. UGIB was associated with a reduced risk of mortality (HR 0.53 [0.34; 0.83]; $p=0.01$ ).

Conclusion: The incidence of UGIB is high in the population of patients on ECMO VA (18.7\%). The decrease in the risk of mortality in this population could be explained by a lower severity of patients on admission, allowing survival long enough to develop UGIB. Obesity, diabetes, and a history of cerebrovascular disease appear to be linked to the risk of developing UGIB and could be witnesses to damage to pre-existing splanchnic microcirculation.

\section{1}

Applying RYAN mortality prediction model in patients with severe burns

MK. Belba'; A. Aleksi'; I. Nezha'; A. Belba²

${ }^{1}$ Anesthesia and intensive care, Mother Teresa Hospital, Tirana, Albania; ${ }^{2}$ Anesthesia and intensive care, KU Leuven University Hall, Leuven, Belgium Correspondence: M.K. Belba

Intensive Care Medicine Experimental 2020, 8(2): 001331

Introduction: Many prognostic scoring models have been devised to predict mortality risk in burn patients. Ryan mortality prediction model is a system that predicts mortality taking into account three major risk factors: age 60 years or over, the burned surface area (BSA) of at least $40 \%$, and the presence of an inhalation injury. An increasing number of risk factors (0-3) is associated with an increasing mortality rate. This formula predicts $0.3 \%, 3 \%, 33 \%$, and approx. $90 \%$ mortality when, respectively, zero, one, two, or three risk factors are present [1]. Objectives: The aim of this presentation is to apply this prediction model in two cohort burn patients hospitalized in ICU and to evaluate the observed deaths with the predicted ones.

Methods: The study retrospectively analyzes the data of all burn patients admitted in the ICU of the Service of Burns and Plastic Surgery of the UHC in Tirana, Albania in 1998 to 2008 and 2009 to 2019.

Results: Overall mortality during 2009 to 2019 was improved : 6.89\% (116 deaths in 1684 patients) compared with 1998 to 2008 which was $10.5 \%$ ( 255 deaths in 2337 patients). The observed mortality was $0.09 \%, 5.61 \%, 35.23 \%$ and $81.4 \%$ when, respectively, zero, one, two or three risk factors were present and there is statistical significance for the difference between two decades(table 1). We performed the comparison of the full model (Age, BSA and presence of Inhalation Injury) against a null through Nominal Regression. $[\times 2(3)=488.808$, 
$p<0.001]$. The Goodness of Fit test was used to affirm that the data gathered fit the distribution form the population $[\times 2(694)=617.914$, $\mathrm{p}=.982]$. The likelihood Ratios confirmed all the predictors of the model to be significant $(p<0.001)$.

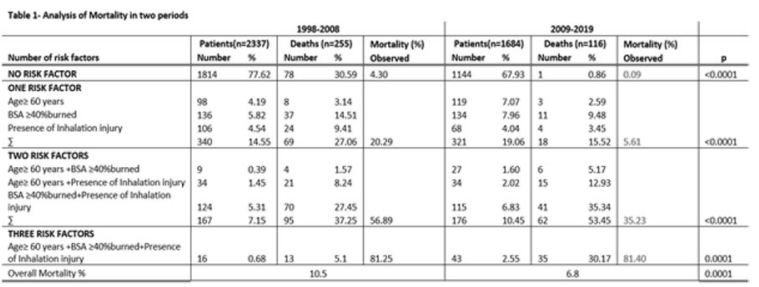

Conclusion: Observed mortality in patients with one and two risk factors is improved but when are present the three risk factors mortality is higher. The data derived from this study confirm the strength of the Ryan model.

\section{Reference(s) and grant ackowledgment(s)}

1. [1] Ryan CM, Schoenfeld DA, Thorpe WP, Sheridan RL. Objective estimates of the probability of death from burn injuries. N Engl J Med. 1998;38(6):362366. https://doi.org/10.1056/nejm199802053380604.

\section{6}

Evaluate efficacy of tele-yoga on multiple outcomes after a period of critical illness

L. Orwelius'; T. Jaarsma²; L. Klompstra²; I. Thylen²; A. Strömberg ${ }^{2}$

${ }^{1}$ street_address\}, Linköping, Sweden; ${ }^{2}$ Department of health, medicine and caring sciences, Linköping University, Linköping, Sweden

Correspondence: $L$. Orwelius

Intensive Care Medicine Experimental 2020, 8(2): 001336

Introduction: Medical yoga, a form of Kundalini Yoga with movements, breathing exercises and meditation has been found to be associated with improved regulation of the sympathetic nervous system and hypothalamic-pituitary-adrenal system. Yoga may improve a number of biological measure (cortisol, systolic blood pressure, resting heart rate, high frequency heart rate variability, fasting blood glucose, cholesterol and low density lipoprotein) (1) as well as patient reported outcome measures e.g. health-related quality of life in various patient populations (e.g. anxiety, depression, stress-related disorders, cardiovascular disease, neurological conditions and cancer). (2-4). This indicates that Medical yoga can be used as an instrument for patients with prolonged disorders after critical illness such as post-intensive care syndrome (PICS) or heart failure. However, for severely ill persons it can be difficult to leave the home and attend regular yoga classes.

Objectives: To develop a feasibility- and pilot tested home-based tele-yoga intervention.

Methods: The tele-yoga includes live yoga sessions twice a week led by a certified instructor and individual daily yoga practice supported by an app. The mHealth tool used is a tablet with Internet connection that contains a video communication ability for the yoga sessions and an app with instructions (text, pictures, and sound files) on how to selfperform medical yoga daily. The video communication app (ZOOM) and yoga app, installed on a tablet with a sim card and 4G surf, were tested for feasibility together with nine persons from a local cardiac patient organization. Further, a pilot study with 10 participants with heart failure (five intervention and five controls) were conducted to test the design of a planned RCT study.

Results: The feasibility testing resulted in minor modifications, and most technical problems were related to sound. Therefore, the yoga instructor changed to a more advanced microphone in the pilot study and a more detailed checklist for using the technology was developed. In the pilot study the design and technology worked sufficiently. The RCT study is now ongoing aiming to recruit 200 participants with post intensive care or heart failure randomized to receive medical yoga remotely at home (teleyoga) for 12 weeks or individualized training to the same extent. The primary hypothesis is that medical tele-yoga significantly increases physical function, health-related quality of life and mental well-being in people after a period of critical illness compared to a control group receiving individualized exercise advice. The secondary hypothesis is that medical yoga improves sleep, heart rate, blood pressure, biomarkers for stress and inflammation and cognition in people after a period of critical illness compared to a control group receiving individualized exercise advice.

Conclusion: The tele-yoga intervention was feasible after minor adjustments and the RCT design was successfully tested in the pilot study. The ongoing RCT study will evaluate efficacy of tele-yoga on multiple outcomes after critical illness.

Reference(s) and grant ackowledgment(s)

1. 4. Desveaux, Lee A, Goldstein R, Brooks D. Yoga in the Management of Chronic Disease: A Systematic Review and Meta-analysis. Med Care 2015 Jul;53(7):653-61

2. 3. Carmer H, Lauche R, LKlose P, Lange S, Langhorst J, Dobos G. Yoga for improving health-related quality of life, mental health and cancer-related symptoms in women diagnosed with breast cancer. Cochrane Database Syst Rev 2017 Jan 3;1(1):CD010802.

3. 2. Carmer H, Anheyer D, Lauche R, Dobos G. A systematic review of yoga for major depressive disorders. J Affect Disord 2017 Apr 15:213:70-77.

4. 1.Pascoe MC, Thompson DRT, Ski CF. Yoga, mindfulness-based stress reduction and stress-related physiological measures: A meta-analysis. Psychoneuroendocrinology 2017;86:152-168.

\section{1}

Mortality associated with early acute renal failure in critically ill

patients

A. Garrido'; J. Cedeño Mora'; P. García Olivares'; B. Moreno ; G.

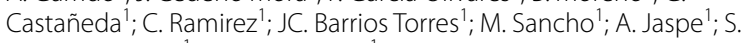

Casanova Prieto $^{1}$; S. Ramos-Mejía ${ }^{1}$

${ }^{1}$ Intensive Care Unit, H.G.U Gregorio Marañón, Madrid, Spain

Correspondence: P. García Olivares

Intensive Care Medicine Experimental 2020, 8(2): 001341

Introduction: Acute renal failure is very common in the critically ill patients and it is associated with increased mortality.

Objectives: To determine the prognostic impact of the early acute renal failure (ARF) in critically ill patients.

Methods: Prospective, observational study conducted during the months of November and December 2019. Epidemiological data, reason for admission, severity scores, organic support and renal function data, were collected in all patients admitted to ICU. The ARF was defined according to AKIN, RIFLE and KDIGO scores in the first 24 hours of admission.

In descriptive analysis, qualitative variables were expressed in percentages and quantitative variables in means (SD) or medians (IQR). The univariate analysis was performed using Chi-square (RR) for qualitative variables and simple logistic regression (OR) for quantitative variables. The prognostic impact of ARF was determined by means of a multiple logistic regression analysis (OR), adjusted for different confounding factors.

Results: We included 95 patients. Epidemiological profile: males 56\%, age $60 \pm 17$ years, some comorbidity $52 \%$. Reason of admission: neurological pathology $30 \%$, respiratory $26 \%$ and infectious $20 \%$. Severity scores: APACHE II 18 (13-25), SOFA 6 (4-8). Mechanical ventilation was required in $52 \%$ of patients and $51 \%$ needed vasoactive therapy. The three scores used determined ARF in $34 \%$ of patients (14\% stage I/risk, $8 \%$ stage II/damage, $12 \%$ stage III/failure), $33 \%$ of them required renal replacement therapy. Mortality was $29 \%$ and average stay was 11 days (6-29).

In the univariate analysis, the patients with early ARF had higher mortality $(50 \%$ vs $17.5 \%, p=0,001)$. For each stage of ARF there was an increase in mortality (stage I/risk $23 \%$, stage II/damage $50 \%$, stage III/ failure $82 \%, p=0.001$ ). 
In the multivariate analysis (adjusted for age, comorbidities, reason of admission, severity scores, mechanical ventilation and vasoactive therapy), early ARF was an independent prognostic factor for mortality (OR $6.28 ; 95 \% \mathrm{Cl} 1.28-30.99)$. Each stage of ARF increased the risk of death: stage I/risk (OR 1.39; 95\% Cl 0.33-5.90), stage II/damage (OR 4.64; 95\% Cl 1.01-21.44), stage III/failure (OR 20.86; 95\% Cl 3.95-110.27).

Conclusion: Early acute renal failure was an independent prognostic factor for mortality. The risk of death increased with each stage proposed in the scores used.

\section{6}

The Role of FOUR Score in Intensive Care Mortality Prediction

T. Ozturk' ; C. Bor'; K. Demirag ; M. Uyar

${ }^{1}$ Anesthesiology and intensive care, Ege University Hospital, Izmir, Turkey Correspondence: K. Demirag

Intensive Care Medicine Experimental 2020, 8(2): 001356

Introduction: Glasgow Coma Scale which predicts ICU mortality is being criticized due to difficulty in verbal assessment in tracheally intubated patients. FOUR Score (Full Outline of Unresponsiveness Full Outline of Unresponsiveness) includes eye movements, motor response, brainstem reflexes and breathing pattern and it is speculated as a more reliable score. BIS (Bispectral index score) gives numerical data to monitor neurological status. We aimed to compare the FOUR score with GCS and BIS to predict mortality.

Methods: Between August 2015 and April 2016, all unconcious adult patients were monitored by BIS in the first 24-h of admission after hemodynamic and metabolic stabilisation. GCS and FOUR scores were assessed by the same physician. Patients younger than 18 , concious, paralyzed or sedated patients were excluded. Demographics, ICU and hospital stay and mortality were recorded.

Results: 65 patients included (32 males). Mortality was 83\% (54/65 patients). Average age, BMI, mean arterial pressure, APACHE II score, duration of ICU and hospital stay differed between the survivors and non-survivors. In predicting mortality, the sensitivity and spesificity of GCS, FOUR and BIS scores were $83.3 \% / 63.6 \%, 83.3 \% / 54.5 \%$ and $90.7 \% / 63.6 \%$ respectively. The cut-off values of GCS, FOUR and BIS for predicting mortality were 6,8 and 60 , respectively. The correlation between BIS/FOUR and between BIS/GCS score was similar (0.797 vs 0.764).

Conclusion: In this patient population with advanced age and high APACHE II score, GCS $\leq 6$, FOUR score $\leq 8$ and BIS value $\leq 60$ were related with poor prognosis. When compared with BIS score, the prognostic values of GCS and FOUR score were similar and can be used interchangeably.

\section{Reference(s) and grant ackowledgment(s)}

1. Okasha AS, Fayed AM, Saleh AS. The FOUR Score Predicts Mortality, Endotracheal Intubation and ICU Length of Stay After Traumatic Brain Injury. Neurocrit Care. 2014;21:496-504

2. None

\section{9}

\section{Description of the specific cause of death in $\mathbf{2 8}$ critically ill}

\section{COVID-19 patients}

S. Guarino ${ }^{1}$ : E. Mosconi ${ }^{1}$; C. Angeloni ${ }^{1}$; G. Girotti ${ }^{1}$; L. Tassinari ${ }^{1}$; A. Parini ${ }^{1}$; R. Carnevale' ; B. Capatti ${ }^{1}$; F. Facondini ${ }^{1}$; A. Potalivo '; G. Nardi'; J. Montomoli ${ }^{1}$ ${ }^{1}$ Anaesthesia and intensive care, Infermi Hospital, AUSL della Romagna, Rimini, Italy

Correspondence: J. Montomoli

Intensive Care Medicine Experimental 2020, 8(2): 001359

Introduction: Existing studies have looked at the risk factors for death among COVID-19 patients admitted to the intensive care unit (ICU). However, few is known about the actual process of death such as the precise role of secondary bacterial infections, cardiac or kidney failure. Objectives: The present study aimed to describe specific causes of death among COVID-19 patients at our ICU to better understand physiopathological pathway of the disease and to guide diagnostic and therapeutic decision.
Methods: Medical charts of all COVID-19 patients died at the ICU of Rimini Hospital between 20/02/2020 and 30/06/2020 were retrieved. Information regarding laboratory test, imaging findings, microbial culture and daily not in the medical records were reviewed to define the final process that lead to death (according to WHO guidelines). Severity of organ failure was estimated by the SOFA score. Finally, we classified patients according to the clinical conditions that contributed to the dying process including the proportionality of care and unexpected deaths.

Results: Among 71 patients admitted to ICU in the study period, 28 (39.4\%) died during the ICU admission (median ICU stay: 15 days (range: 0-88)). Respiratory failure was the leading cause of death in $16(57.1 \%)$ patients. Pneumonia-COVID related was identified in 10 (35.7\%) deceased patients with a bacterial superinfection present in 4 of those. Six (21.4\%) patients developed ventilator associated bacterial pneumonia prior to death and in 3 of those COVID-related pneumonia was not improving while in 1 case severe lung fibrosis had developed leading to impossible weaning from the ventilators (ICU length of stay: 88 days). Bacterial infections were the main cause of death in 7 (25\%) patients and in 6 of them COVID-related pneumonia was improving. Finally, 5 (17.9\%) patients death was related to other causes: 1 fulminant myocarditis, 1 cerebral hemorrhage, 1 hemorrhagic shock, 1 myocardial infarction, and one disseminated neoplasm. In 3 cases deaths was unexpected while in 5 patients withdrawal of treatment was decided. The median SOFA score increased from 6.5 (3-11) the day of ICU admission to 8 (4-18) 3 days before death, and to 11 (3-18) the day of death.

Conclusion: The course of critically ill COVID-19 patients is heterogeneous. Bacterial infections played an important role in the dying process and a systematic approach to infection vigilance could help to prevent some deaths especially in a contest where inflammatory markers might have been masked by use of steroids or immunosuppressive therapies.

\section{Reference(s) and grant ackowledgment(s)}

1. Grasselli G, Greco M, Zanella A, et al. Risk Factors Associated With Mortality Among Patients With COVID-19 in Intensive Care Units in Lombardy, Italy. JAMA Intern Med. Published online July 15, 2020. https://doi.org/10.1001/ jamainternmed.2020.3539.

2. Wendel Garcia PD, Fumeaux T, Guerci P, et al. Prognostic factors associated with mortality risk and disease progression in 639 critically ill patients with COVID-19 in Europe: Initial report of the international RISC-19-ICU prospective observational cohort. EClinicalMedicine. Published online July 2020:100449. https://doi.org/10.1016/j.eclinm.2020.100449.

3. Vincent IL, Taccone FS. Understanding pathways to death in patients with COVID-19. Lancet Respir Med. 2020 May; 8(5): 430-432. https://doi. org/10.1016/s2213-2600(20)30165-x.

4. INTERNATIONAL GUIDELINES FOR CERTIFICATION AND CLASSIFICATION (CODING) OF COVID-19 AS CAUSE OF DEATH. https://www.who.int/ classifications/icd/Guidelines_Cause_of_Death_COVID-19.pdf?ua =1 (30/07/2020)

5. Onder G, Rezza G, Brusaferro S Case-fatality rate and characteristics of patients dying in relation to COVID-19 in Italy. JAMA. 2020; (published online March 23.) https://doi.org/10.1001/jama.2020.4683.

\section{0}

The impact of Speech and Language Therapy Dysphagia Intervention with COVID-19 patients at a Tertiary Cardiothoracic Centre

S. Stagnell ${ }^{1}$; N. Maistry

${ }^{1}$ Speech and Language Therapy, Royal Brompton Hospital, London, United Kingdom

Correspondence: $\mathrm{S}$. Stagnell

Intensive Care Medicine Experimental 2020, 8(2): 001360

Introduction: The increased risk of dysphagia and laryngeal injury in patients following intubation is widely described in the literature [2]. Patients diagnosed with COVID-19 were intubated and ventilated for 
prolonged periods and subsequently presented with profound weakness and confusion, all of which contribute to increased risk of postextubation dysphagia [1, 3]. The presence of Speech and Language Therapy (SLT) within the MDT, to provide early dysphagia assessment $[1,4]$, is essential.

Objectives: To review the impact on SLT dysphagia intervention using a validated outcome measure with all COVID-19 patients referred to SLT at the Royal Brompton Hospital.

Methods: During the period between March to June 2020 (inclusive), SLT were referred and treated 42 of 136 COVID-19 patients admitted to the Royal Brompton Hospital (RBH). One patient solely received voice intervention and six were repatriated prior to completing their dysphagia treatment; and so these patients were excluded from data analysis.

Impact was measured using 'The Royal Brisbane Hospital Outcome Measures (RBHOM)'. This is a validated numerical scale, which shows progress from Nil-by-Mouth (NBM) to full oral intake [5]. This outcome measure was scored following initial assessment and repeated on discharge.

Results: A total of 35 patients, including 23 with tracheostomy with and without mechanical ventilation, received SLT dysphagia intervention. These are interim findings and data collection is still being collated.

Interim data analysis has shown that an average of eight days of SLT dysphagia intervention resulted in patients having an average five point increase on the RBHOM. Only one patient remained NBM with a gastrostomy on discharge whilst all others returned to full oral intake. From the data collected thus far, $97 \%$ of patients went from NBM to safely returning to oral intake.

Conclusion: Whilst this is a small sample of patients and data collection is ongoing, results are strongly showing that SLT dysphagia intervention has a direct impact on improving outcome measures for COVID-19 patients.

\section{Reference(s) and grant ackowledgment(s)}

1. 5. Ward, F.C \& Conrow A.-L (1999) 'Validity, reliability and responsivity of the Royal Brisbane Hospital Outcome Measure for Swallowing'. Asia Pacific Journal of Speech, Language and Hearing. Vol 4. Issue 2. [Published online 2013]

2. 4. Intensive Care Society. The Faculty of Intensive Care Medicine. Guidelines for the Provision of Intensive Care Services. Edition 2. June 2019 [online]. Accessed 23/6/20.

3. 3. Frajkova, Z et al. (2020) 'Post intubation dysphagia during COVID-19 outbreak-Contemporary review'. Dysphagia.

4. 2. Brodsky, M et al (2018) 'Laryngeal Injury and Upper airway symptoms after oral endotracheal intubation with mechanical ventilation during critical care: A systematic review.' Critical Care. Vol 46 (12) 2010-2017.

5. 1. Brodsky, M et al (2020) 'Post-extubation dysphagia: a problem needing multidisciplinary efforts'. Intensive Care Medicine. Vol 46 (1).

\section{1}

Short-term physical and mental health outcome of critically-ill COVID-19 patients after discharge from intensive care unit

A. Topeli ${ }^{1}$; B. Halacli'; E. Ortac Ersoy ${ }^{1}$; S. Kilic ${ }^{1}$; S. ÖCal'

'Division of intensive care medicine, department of internal medicine, Hacettepe University Faculty of Medicine, Ankara, Turkey

Correspondence: A. Topeli

Intensive Care Medicine Experimental 2020, 8(2): 001381

Introduction: Critically-ill patients with COVID-19 have a prolonged intensive care unit (ICU) course and their short- and long-term health outcome are unknown.

Objectives: To report short-term physical, nutritional, cognitive, psychological impairment, and quality-of-life in ICU survivors with COVID19, 1 month after ICU discharge.

Methods: Patients who stayed in ICU for $>7$ days between March 21 2020 and May 1, 2020 were included. Patients were evaluated in the follow-up visit within 1st month after ICU discharge, where general performance, nutritional status, 6-minute-walking test (6MWT), muscle function, cognitive, psychological and quality-of-life assessments were performed. Related samples were compared by WilcoxonSigned-Rank test and results are presented as median (IQR).

Results: Out of 30 patients with COVID-19 who were admitted to ICU, 11 patients ( 7 males, 4 females) with a median age of 60 (52-75) were evaluated. Their admission APACHE II score was 15 (12-18). ICU length of stay was 13 (9-23) and hospital length of stay was 23 (17-33) days. $\mathrm{PaO} 2 / \mathrm{FiO} 2$ improved at ICU discharge as compared to admission values $(260(217-300)$ vs $158(146-227) ; p=0.03)$. Five patients received invasive mechanical ventilation. ECOG performance and Clinical Frailty Scale $\odot$ scores were worse during the follow-up visit than their baseline $(0(0-1)$ vs $0(0-0), p=0.03$ and $2(1-3)$ vs $1(1-2), p=0.008$, respectively). Compared to baseline they lost $7.9 \%$ (5.8-11.8) of their weight at ICU discharge and weight loss at follow-up visit was 6.5\% (2.4-12.4) $\mathrm{kg}$. Predicted distance walked during 6MWT in 10 patients was $82.2 \%$ (72.4-88.8) and 6 patients reported dyspnea at rest. The handgrip strength test was below normal in 4 patients. ICU-acquired weakness according to Medical Research Council was present in 5 patients at ICU discharge, whereas it improved in the follow-up visit compared to baseline (54 (51-58) vs 48 (42-52), $p=0.005)$. The Mini-mental test was abnormal in 2 patients. Three patients had anxiety, 1 had borderline depression and 1 had post-traumatic stress disorder. Six patients had moderate-severe pain and 6 patients reported sleep problems. The physical-component score of Short Form-36 was low in 8, the mentalcomponent score was low in 4 patients.

Conclusion: Short-term outcome of critically-ill COVID-19 patients 1 month after ICU discharge was poor. Long-term outcome should be evaluated in more number of patients.

\section{Reference(s) and grant ackowledgment(s)}

1. 1. Huang Y, Tan C, Wu J, et al. Impact of coronavirus disease 2019 on pulmonary function in early convalescence phase. Respir Res. 2020;21 (1):163. Published 2020 Jun 29. https://doi.org/10.1186/s12931-020-01429-6.

\section{9}

Meeting the need for Post ICU care of COVID -19

patients- A multi-professional response model for follow up and rehabilitation

A. Ersson ${ }^{1}$; P. Ackermark ${ }^{2}$

${ }^{1}$ Anaesthesia and Intensive Care, Region Sormland, Nykoping, Sweden; ${ }^{2}$ Rehabilitation and pain medicine, Region Sormland, Nykoping, Sweden

Correspondence: A. Ersson

Intensive Care Medicine Experimental 2020, 8(2): 001389

Introduction: The Covid-19 pandemic has put the need for ICU rehabilitation in bright light due to extreme strain on ICU capacity. During the pandemic, the Sormland region became one of the heaviest targeted areas in Sweden with 57 patients/100.000 inhabitants needing intensive care. To meet the anticipated need for ICU rehabilitation, a multi professional taskforce was formed to design and institute a rehabilitation program encompassing all phases of the clinical course from the ICU to hospital discharge and the following time in outpatient care.

ICU patients frequently develop sequel after their ICU stay with longstanding reduction in HRQoL. These impairments, described as PICS (Post Intensive Care Syndrome), involves both cognitive and neuromuscular dysfunction. Early ICU adapted rehabilitation and use of PICS preventive bundles have shown favourable effects on long time outcome and individually tailored aftercare in ICU step down and rehabilitation units has been advocated. Compared to the normal ICU setting, Covid -19 patients are cared for in a most unfavourable ICU milieu which expose them extensively to virtually all known risk factors for PICS including separation from relatives, use of heavy sedation and neuromuscular blockers in addition to the severe respiratory injury and biomedical stress. 
In this paper we describe the development of the rehab program. Collection of outcome data are in progress and are not presented at this stage.

Objectives:

- To design and implement a follow up and rehabilitation program for COVID-19 patients.

- Within the program collect data on clinical outcome, PREM and PROM, later to be used to describe longitudinal outcome trajectories.

Methods: During multiple interprofessional workshops, a patient/ family centred rehabilitation program was developed and instituted in clinical practice. ICU follow up clinics were used for program coordination and for data collection. The program was organized in inhospital and outpatient components. A multi professional rehab team was formed and evaluation was done using pre set clinimetrics. A "stepdown unit" was formed were extubated ICU patients could deescalate respiratory and residual organ support parallel with escalating physio and occupational therapy. At discharge from the stepdown unit, all patients were triaged by a specialist in rehab medicine to individual trajectories.

Results: Between 200311- 200719 a total of 143 patients were treated in the ICU. At the start of the pandemic, an already developed ICU early rehab program was expediently initiated. Thus, an early in-ICU rehab program was available at ICU admission.

The complete Rehab-program was developed during the first three weeks of the pandemic and was operational in April from when all ICU patients were discharged to the stepdown facility. The individual course of the patients is currently monitored by the rehab team at 2, 6 and 12 months scheduled Post ICU clinics. Currently, the 2-month clinics and data collection are in progress.

Conclusion: The urgent demand for post ICU rehabilitation resulting from the COVID-19 pandemic could be rapidly met by the expedient institution of a complete rehab program. This was possible due to the work of an interprofessional response team, endorsed by the hospital board, were ICU and rehab competencies collaborated. This program, catalysed by the pandemic, satisfies a long-experienced need for an organised rehab program for ICU patients.

\section{2}

\section{Post Intensive Care Syndrome: Prevalence 5 years after ICU} admission. A partial report

SA. Prieto Garces'; LC. García-Medina²; E. Noguera-Alfonso ${ }^{3}$; Y. Cardenas ${ }^{1}$ ${ }^{1}$ Medicina critica y cuidado intensivo, Fundacion Santa Fe de Bogota University Hospital, Bogotá, Colombia; ${ }^{2}$ Facultad de medicina, Universidad de los Andes, Bogotá, Colombia; ${ }^{3}$ Salud mental, Fundacion Santa Fe de Bogota University Hospital, Bogotá, Colombia

Correspondence: S.A. Prieto Garces

Intensive Care Medicine Experimental 2020, 8(2): 001422

Introduction: Post Intensive Care Syndrome (PICS) is an entity that affects patients cognitively, physically and emotionally after being managed in the Intensive Care Unit (ICU) (1). These sequalae impact the quality of life (QOL) of ICU survivor patients and can persist after 5 years (2). The diagnosis of PICS, same as other pathologies such as Delirium, can be challenging and make it difficult to determine its exact prevalence (Rawal 2017). Given its impact on the QOL of patients, it is necessary to stablish its prevalence in order to generate awareness of it and the strategies available to prevent it.

Objectives: The objective is to determine the prevalence of PICS 5 years after admission to the ICU of a Latin American Hospital in Bogotá, Colombia. Also, to characterize the patients that were admitted during that same year.

Methods: A cross-sectional, descriptive study was done. Patients 18 years old or older admitted into the ICU during 2015 were enrolled. Demographics, comorbidities, diagnostics, severity scores, ICU stay,
Hospital stay, days of vasopressor and invasive mechanical ventilation, complications and contact information were gathered. To evaluate PICS, the Post Intensive Care Syndrome Questionnaire (PICSq) was used (3). This tool is a Likert style questionnaire consisting of 18 questions that evaluate the cognitive, physical and emotional spheres (6 questions per sphere). The answers can be graded as "never0 ","sometimes-1","often-2" and "always-3", thus, giving it a range of 0-54 points. A score of 23 or more is indicative of the development of PICS. Furthermore, A value equal to or superior to 6,9 and 7 in the cognitive, physical and mental spheres respectively, indicate severe compromise. This tool was previously validated for Spanish language in our institution. Data gathered was analyzed using SPSS ver. 25.

Results: A total of 1434 patients were admitted to the ICU on 2015. At this moment, 119 medical charts have been reviewed. Same event mortality was $21,8 \%$, (26/119), making our available population 93 patients. Most $(63,8 \%)$ were male and mean age was 64,5 years. Average ICU stay and Hospital stay were 5,7 days and 22,2 days respectively. Vasopressors were used on $38,7 \%$ of patients with an mean duration of 2 days. Invasive mechanical ventilation was used on $25,8 \%$ of patients with a mean duration of 1,9 days. A total of 23 patients $(24,7 \%)$ died along the course of the 5 years after ICU admission, $18,2 \%$ died the first year, $3,2 \%$ on the second year, $1,07 \%$ on the third year, $3,2 \%$ on the fourth year and none died on the fifth year. This left us with 70 eligible patients for applying the PICSq.

Out of the 70 eligible patients, 47 (67\%) had changed contact information or were not available. The PICSq was applied to 23 patients $(32,8 \%)$ and the results were as following: The average score was 10,2. Only 3 patients had scores above the cut off value of 23 , giving PICS a prevalence of $13,04 \%$. The mean score for the cognitive component was 2,56, for the physical component was 5,3 and for the mental component was 2,3. According to the cut off values, out of the 23 patients evaluated, $13,04 \%$ had severe cognitive impairment, $26 \%$ had severe physical impairment and $8,6 \%$ had severe mental compromise. Lastly, for those that did not meet the criteria for PICS, around $20 \%$ of patients suffered from severe physical compromise.

Conclusion: The partial results of this study show that PICS affects around $13 \%$ of our population, with severe physical compromise being the most common affection for both those diagnosed with PICS and those that did not meet the criteria. The process of gathering information needs to continue in order to stablish the real prevalence of PICS in our population. Lastly, a better strategy to follow up patients is needed in the ICU.

\section{Reference(s) and grant ackowledgment(s)}

1. 3. Jeong YJ, Kang J. Development and validation of a questionnaire to measure post-intensive care syndrome. Intensive Crit Care Nurs. 2019;55:102756. https://doi.org/10.1016/j.iccn.2019.102756.

2. 2. Colbenson G, Johnson A, Wilson M. Post-intensive care syndrome: impact, prevention, and management. Breathe [Internet]. 2019 [cited 7 January 2020];15(2):98-101. Available from: https://www.ncbi.nlm.nih.gov/pmc/artic les/PMC6544795/.

3. 1. Rawal, G., Yadav, S., \& Kumar, R. (2017). Post-intensive Care Syndrome: an Overview. Journal of translational internal medicine, 5(2), 90-92. https://doi. org/10.1515/jtim-2016-0016.

001459

Prevalance And Burden Of Hepatobilier Injury In Pulmonary ICU Patients

E. Macit Aydın'; Z. çınar ; A. Mammadova²; N. Navruzvai²; S. Kodalak²; G. Gursel ${ }^{3}$

${ }^{1}$ Department of anaesthesiology and reanimation, division of critical care, Gazi University Faculty of Medicine, Anaesthesiologist, fellow of critical care, Ankara, Turkey; ${ }^{2}$ Department of pulmonary critical care medicine, Gazi University Faculty of Medicine, Resident of Pulmonary Diseases, Ankara, Turkey; ${ }^{3}$ Department of pulmonary critical care medicine, Gazi University Faculty of Medicine, Professor of Respiratory Medicine, Ankara, Turkey

Correspondence: E. Macit Aydın

Intensive Care Medicine Experimental 2020, 8(2): 001459 
Introduction: Hepatobilier injury $(\mathrm{HBI})$ is very frequent in ICU patients and associated with poor outcome. Pulmonary ICU patients are vulnarable to $\mathrm{HBI}$ reasons like hypoxemia, pulmonary hypertension, right heart failure and frequent ICU infections and antibiotic usage. But there is limited data about HBI's prevalance, type, risk factors and impact on mortality in Pulmonary ICU patients.

Objectives: The aim of this study was to assess frequency, risk factors, types, severity and effect on outcome of $\mathrm{HBI}$ in critically ill patients.

Methods: A retrospective observational cohort study was performed in an University hospital pulmonary ICU and all patients who admitted last 5 years were included in the study. All potential risk factors for HBI like sepsis, hypoxia, congestion, medications and nutrition routes, were recorded from the patient files. Hepatobilier injury and severity are defined according to the classification of the European and American guidelines for 2017 and 2019. The value of R for HBI and the type of damage (hepatocellular, cholestatic and mix) calculated according to RUCAM system. All potential risk factors were compared with univariate analysis (t test, Chi square test, Mann Whitney $\mathrm{U}$ test). Risk factors for $\mathrm{HBI}$, mortality, length of stay in ICU were investigated with logistic regression analysis.

Results: 478 patients were included in the study and there were $\mathrm{HBI}$ in the 207 (43\%) of them. Most frequent ICU admission reasons were, community-acquired pneumonia $(21 \%)$, respiratory failure $(18 \%)$ and COPD (18\%). The mean age of the patients was $70 \pm 15,55 \%$ of them were male, mean APACHE II score was $20 \pm 8$, and mean SOFA score was $4 \pm 3$.

$\mathrm{HBI}$ developed median 5 days before ICU admission in the $71 \%$ of patients and 7 days after ICU admission in the $29 \%$ of patients, during the worst period of $\mathrm{HBl}, 39 \%$ of the patients were at stage $1,33 \%$ at stage $2,15 \%$ at stage 3 , and $14 \%$ at stage $4 \mathrm{HBI}$ was reversible in $77 \%$ of the patients. According to R coefficient HBI was considered as cholestatic (54\%), mixed (29\%) and hepatocellular (16\%) patterns. DILI was thought to be present in $42 \%$ (86) of the patients with HBI. Mortality was higher in $\mathrm{HBI}$ group also ( $39 \%$ vs $6 \%$ ).

Risk factors for $\mathrm{HBI}$ as $(\mathrm{OR}(95 \% \mathrm{Cl}), \mathrm{p})$ were TPN: $2.3(1.3-4)$, p: 0.004, enteral nutrition: 2.3 (1.5-3.7), p: 0.0001, APACHE II score on admission: 1.1 (1.06-1.14), p: 0.001, use of clarithromycin: 2.3 (1.2-4.4), p: 0.01, and immunosuppression: 2.6 (1.1-6.3), p: 0.032 . HBI increased mortality risk four-fold in this study population(3.9(2-8), p:o.0o1). and was not risk factor for the increased length of ICU stay more than ten days. Liver function results of patients with hepatobiliary injuries are given in the table.

\begin{tabular}{|c|c|}
\hline \multicolumn{2}{|c|}{ Table. Laboratorx results of patients with Hepatobilier injury } \\
\hline AST HBI $I_{\max } *$ & $406 \pm 1165$ \\
\hline ALT HBI max $*$ & $251 \pm 747$ \\
\hline 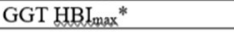 & $141 \pm 222(\mathrm{n}: 179)$ \\
\hline ALP HBI max $_{\text {ax }} *$ & $208 \pm 308(n: 198)$ \\
\hline LDH HBI $_{\max } *$ & $1426 \pm 3547(\mathrm{n}: 73)$ \\
\hline Total bilirubin $\mathrm{HBI}_{\max } *$ & $1.5 \pm 2.5(\mathrm{n}: 200)$ \\
\hline Direct bilirubin HBI $\mathrm{Hax}_{\max }$ & $0.8 \pm 1.7(\mathrm{n}: 203)$ \\
\hline \multicolumn{2}{|c|}{$\begin{array}{l}\text { HBL:Hepatobilier iniury, AST: Aspartate aminotransferase, ALT:Alanine } \\
\text { aminottansferase, GGI:Gamma glutamyl transferase, ALP:Alkaline phosphatase, } \\
\text { LDH:Lactate dehydrogenase } \\
\text { "Dean } \pm \text { SD }\end{array}$} \\
\hline
\end{tabular}

Conclusion: In this study population, $43 \%$ of the patients developed mostly cholestatic type HBI, despite many of them had mild-modarete stage and reversible disease it was an independent risk factor for ICU mortality.

\section{Reference(s) and grant ackowledgment(s)}

1. EASL Clinical Practical Guidelines on the management of acute (fulminant) liver failure. J Hepatol. 2017 May;66(5):1047-1081.

2. Paul Y. Kwo, Stanley M. Cohen, Joseph K. Lim. ACG Clinical Guideline: Evaluation of Abnormal Liver Chemistries. Am J Gastroenterol 2017; 112:18-35.
001500

Outcomes of critically ill patients with acute mesenteric ischemia in a French intestinal stroke center

A. Tran Dinh'1; B. Guignier'; E. Charbit²; D. Bouzid'; A. Nuzzo²; M. Ronot ${ }^{2}$; L. Maggiori ${ }^{3}$; K. Peoc'h'ㄹ B. Lortat-Jacob ${ }^{1}$; S. Tanaka ${ }^{1}$; L. Rebibo'; A. Roussel'; Y. Castier ${ }^{1}$; S. Dahmani ${ }^{4}$; C. Paugam-Burtz'; P. Montravers' ${ }^{1}$; O. Corcos² ${ }^{2}$ E. Weiss ${ }^{2}$

${ }^{1} 75$, Bichat-Claude Bernard Hospital, Paris, France; ${ }^{2} 92$, Hospital Beaujon AP-HP, Clichy, France; ${ }^{3} 75$, Hospital _ Saint-Louis Ap-Hp, Paris, France; ${ }^{4} 75$, Hôpital Robert-Debré Ap-Hp., Paris, France

Correspondence: A. Tran Dinh

Intensive Care Medicine Experimental 2020, 8(2): 001500

Introduction: Acute mesenteric ischemia (AMI) is a life-threatening disease. In 2016, the first French national intestinal stroke center was set up (SURVI, Beaujon and Bichat Hospitals, AP-HP, France); it is based on a multidisciplinary and multimodal management of life- and gut-saving strategy, focusing on intestinal viability that is achieved through (1) early AMI recognition, (2) urgent medical protocol, and (3) digestive revascularization and necrotic bowel resection if required.

Objectives: Our objectives were to describe the prognosis in the intensive care unit (ICU) of patients with AMI admitted in the intestinal stroke center, and to analyze factors associated with ICU mortality.

Methods: An observational retrospective analysis of a prospective database was conducted from February 2016 to December 2018, which consecutively included all patients with AMI admitted in ICU in the intestinal stroke center. Collected data were patients' demographic characteristics, past medical history, mechanisms of AMI, intra-operative findings and ICU hospitalization. Data are presented as median [IQR] or number (percentage). Univariate analyses of factors associated with ICU mortality were performed using Chi-squared tests and Mann-Whitney $\mathrm{U}$ tests. Multivariate exact logistic regression was performed on clinical relevant factors that were significantly associated with ICU mortality in univariate analyses $(p<0.05)$. Statistical significance was considered for $p$-value $<0.05$.

Results: Seventy patients (age 65 [54-76] years, $61 \%$ of male, SOFA 4 [2-6], SAPS II 42 [30-53]) were admitted to ICU with AMI, mainly due to superior mesenteric artery occlusion (87\%). Sixty patients (91\%) underwent initial urgent laparotomy. Bowel resection was performed in 32 patients $(50 \%)$, and 7 patients (10\%) had extensive bowel necrosis beyond therapeutic possibilities. Forty-one patients (64\%) had endovascular $(n=17)$ or open $(n=24)$ initial revascularization of the ischemic gut. Thirty patients (43\%) required one or more revision surgeries.

Median ICU length of stay and duration of mechanical ventilation were 6 [2-14] days and 2 [0-5] days respectively. Forty-seven patients (67\%) had acute kidney injury. On ICU discharge, 20 patients (29\%) had short bowel syndrome and 44 patients $(63 \%)$ required exclusive parenteral nutrition.

Seventeen patients (24\%) died in ICU. Factors associated with ICU mortality in univariate analysis were age $\geq 80$ years old, chronic hypertension, signs of hepatic infarction on CT scan, SAPS II on admission, delta SOFA score between admission and day $1>0$, duration of mechanical ventilation and acute kidney injury.

Delta SOFA score $>0$ (OR 9.0, 95\%Cl [1.8-69.5]) and chronic hypertension (OR 7.7, 95\% CI [1.8-42.4]) were independently associated with ICU mortality in multivariate analysis.

Conclusion: We describe for the first time the prognosis of critically ill patients with $\mathrm{AMI}$ in an intestinal stroke center in France. The $24 \%$ ICU mortality rate suggests that intestinal stroke center might improve the outcomes of ICU patients with AMI.

\section{5}

Baseline characteristics and outcomes of critically-ill COVID-19 patients: A multi-center study in Turkish intensive care units

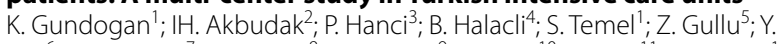
Bilir $^{6}$; FT. Bozkurt'; F. Yildirim ${ }^{8}$; RC. Yuksel'; L. Talan ${ }^{10}$; G. Elay ${ }^{11}$; G. Guven ${ }^{12}$; I. Kara ${ }^{13}$; S. Yilmaz ${ }^{14}$; S. Sari ${ }^{15}$; T. Akbas ${ }^{16}$; BA. Cinleti ${ }^{17}$; NA. Ayhan ${ }^{18}$; A. Topeli ${ }^{4}$ ${ }^{1}$ Department of internal medicine, division of intensive care, Erciyes University, School of medicine, Kayseri, Turkey; ${ }^{2}$ Intensive care unit, Pamuk- 
kale University Hospital, Denizli, Turkey; ${ }^{3}$ ntensive care unit, Minisrty of Health, Erzurum State Hospital, Erzurum, Turkey; ${ }^{4}$ Division of intensive care medicine, department of internal medicine, Hacettepe Universtity Faculty of Medicine, Ankara, Turkey; Intensive care unit, Ministry of Health, Yenimahelle Educating and Traninh Hospital, Ankara, Turkey; ${ }^{6}$ Intensive care unit, Ministry of Health, Kartal Lutfi Kırdar Educating and Training Hospital, İstanbul, Turkey; ${ }^{7}$ Intensive care unit, University of Health Sciences, Mehmet Akif Inan Hospital, Şanlıurfa, Turkey; ${ }^{8}$ Intensive care unit, Ministry of Health, Diskapi Educating and Training Hospital, Ankara, Turkey; ${ }^{9}$ Intensive care unit, Ministry of Health, Kayseri Educating and Training Hospital, Kayseri, Turkey; ${ }^{10}$ Division of intensive care medicine, Ankara University Faculty of Medicine Department of Internal Medicine, Ankara, Turkey: ${ }^{11}$ Department of internal medicine, division of intensive care, Gaziantep University, School of medicine, Kayseri, Turkey; ${ }^{12}$ Intensive care unit, Minisrty of Health, Tokat State Hospital, Tokat, Turkey; ${ }^{13}$ Intensive care unit, Selçuk University, School of medicine, Kayseri, Turkey; ${ }^{14}$ Intensive care unit, University of Health Sciences, Kütahya, Turkey; ${ }^{15}$ Intensive care unit, Ministry of Health, Omer Halisdemir Educating and Training Hospital, Niğde, Turkey; ${ }^{16}$ Department of internal medicine, division of intensive care, Duzce University, School of medicine, Düzce, Turkey: ${ }_{i}^{17}$ Intensive care unit, University of Health Sciences, Dr.Suat Seren Hospital, Izmir, Turkey; ${ }^{18}$ Department of internal medicine, division of intensive care, Cukurova University, School of medicine, Adana, Turkey

Correspondence: K. Gundogan

Intensive Care Medicine Experimental 2020, 8(2): 001505

Introduction: Critically-ill patients with COVID-19 have been proposed to have poor prognosis.

Objectives: To evaluate baseline characteristics and outcomes of patients with COVID-19 requiring ICU admission in Turkish ICUs.

Methods: Critically-ill patients admitted to 26 ICUs from 23 Hospitals in Turkey from 10 March to 11 June 2020 were evaluated in this descriptive observational study. Results are presented as median (IQR) and counts (\%).

Results: A total of 426 confirmed COVID-19 patients were included. The median age of the population was 67 (57-76) years. There were $254(59.6 \%)$ male and $172(40.4 \%)$ female patients. The most frequent comorbidity was hypertension $217(50.9 \%)$, followed by diabetes mellitus 131 (30.8\%), cardiac diseases 107 (25.1\%), chronic respiratory diseases $82(19.2 \%)$, cancer $46(10.8 \%)$ and chronic renal failure 16 (3.8\%). Duration from start of symptoms till ICU admission was 6 (4-10) days. Admission APACHE II score was 17 (12-25) and SOFA score was 4 (3-7). Admission PaO2/FiO2 was 148 (102-206) and SpO2/FiO2 196 (147-240). Admission lymphocyte count was 860 (560-1240) per mm3, CRP was 109.3 (57.7-188.5) mg/L, D-Dimer was 1120 (547-2970) ng/mL and ferritin was 491 (197-958) $\mathrm{ng} / \mathrm{mL}$. Invasive mechanical ventilation was applied in 231 (54.2\%) patients and vasopressors in 163 (37.3\%) patients. Hydroxychloroquine was used in $304(71.4 \%)$, antibacterial treatment in 205 (48.1\%), azitromycin in 196 (46.0\%), favipiravir in 162 (38.0\%), oseltamivir in 158 (37.1\%), convalescent plasma in 51 (12.0\%), tocilizumab in $41(9.6 \%)$, corticosteroids in $30(7.0 \%)$, intravenous immunoglobulin in $10(3.3 \%)$, lopinavir/ritonavir $9(2.1 \%)$, cytokine/ endotoxin removal $9(2.1 \%)$, plasma exchange in $5(1.2 \%)$ patients. ICU length of stay was 7 (3-14) and ICU mortality was 215 (50.5\%). Conclusion: Multi-center study from Turkish ICUs revealed that more than half of critically-ill COVID-19 patients were older than 65 years of age, invasive mechanical ventilation was applied in more than half of patients and ICU mortality rate was high.

\section{Reference(s) and grant ackowledgment(s)}

1. 1. Grasselli G, Zangrillo A, Zanella A, et al. Baseline Characteristics and Outcomes of 1591 Patients Infected With SARS-CoV-2 Admitted to ICUs of the Lombardy Region, Italy. JAMA. 2020;323(16):1574-1581. https://doi. org/10.1001/jama.2020.5394.

2. We are grateful to all authors of COVID-19 Study Group Investigators of the TuRkish Intensive Care Studies-Network (TRICS-Net).

3. We are grateful to Omega CRO, Ankara, Turkey for their support in the conduction of this study.
001540

Intensive care support in cancer patients: a retrospective study in an oncological center

M. Leitão' ; T. Vieira²; B. Pereira ${ }^{3}$; F. Faria ${ }^{4}$

'Oncologia Médica, Instituto Português de Oncologia do Porto, Porto, Portugal; ${ }^{2}$ Intensive care, São João Universitary Hospital Center, Porto, Portugal; ${ }^{3}$ Intensive care, Ipo, Porto, Portugal; ${ }^{4}$ Intenisve care, Instituto Português de Oncologia do Porto Francisco Gentil, Porto, Portugal

Correspondence: M. Leitão

Intensive Care Medicine Experimental 2020, 8(2): 001540

Introduction: Cancer is the second global cause of death and incidence is increasing. Mortality of ICU cancer patients has dropped, yet prognostication and patient selection remain to be adjusted. The severity of acute illness could be more important to predict short-term survival than the underlying type or stage of cancer.

Objectives: To determine the outcome of patients with solid tumors in ICU, the usefulness of SAPS 2 and APACHE II as prognostic tools and identify poor outcome factors in the oncologic population.

Methods: We enrolled patients with solid malignancies in active systemic treatment admitted in an oncologic intensive care unit due to medical complications in a 3-year period. Clinical data were collected retrospectively.

Results: In our sample $(n=57)$, gastrointestinal cancers were the most frequent $(14,25 \%)$ followed by lung and breast (9 each, 16\%). The cancer was stage IV in 36 patients (64\%), 78\% received chemotherapy in the 30 days prior and half was of palliative intent; 6 patients had received immunotherapy. Infection, acute respiratory failure and hematological disturbances were the most common problems at admission (75\%, $77 \%$ and $82 \%), 39 \%$ of cases were classified as multi-organic disfunction. Major ICU interventions included invasive mechanical ventilation (53\%) and vasopressor support (40\%). ICU and hospital mortality were $25 \%$ and $44 \%$. Six month mortality was $60 \%$. Stage IV cancer, ECOG performance status, hematologic disturbances and infection were not independently related to higher mortality. APACHE II and SAPS2 scores showed a positive correlation with mortality in the ICU. APACHE II and SAPS2 score distributions showed statistically significant relation to mortality $(p=0.017)$.

Conclusion: Stage IV cancer was not an independent risk factor for acute mortality, confirming the results of other published works. Most of the patients that survived the hospital stay were alive in 6 months. APACHE II and SAPS2 are often used in cancer patients, although they were never validated in this population. In our work, these scores reasonably predict ICU mortality, but were far from perfect tests.

Conclusion: Patients receiving systemic treatment for cancer should be considered for ICU admission like other severely ill patients without cancer. Larger prospective studies are needed to understand prognostic factors and refine predictive scores in this special population. Individual evaluation of patient characteristics, oncological disease and treatment response are essential. Dialogue between oncologists and intensive care specialists is paramount in the decision making process of this population.

001544

Brachial plexopathy secondary to prone positioning, an aetiology to consider during the COVID-19 pandemic

A. Truelove'; A. Baldwin ${ }^{2}$; R. Shirley ${ }^{3}$; T. Ali ${ }^{4}$

${ }^{1}$ Critical Care Medicine, Stoke Mandeville Hospital, Aylesbury, United Kingdom; ${ }^{2}$ Intensive care, Stoke Mandeville Hospital, Aylesbury, United Kingdom; ${ }^{3}$ Plastic surgery, Stoke Mandeville Hospital, Aylesbury, United Kingdom; ${ }^{4}$ Intensive care department, Stoke Mandeville Hospital, Aylesbury, United Kingdom

Correspondence: A. Truelove

Intensive Care Medicine Experimental 2020, 8(2): 001544

Introduction: With the ongoing Coronavirus disease 2019 (COVID-19) pandemic, increased numbers of patients are being admitted to intensive care units (ICU) worldwide. Many guidelines recommend early utilisation of the prone position ('proning') following intubation in this patient population, owing to the evidence that this position improves 
lung mechanics and gas exchange. 1 The increased utilisation of proning in patients where sedation and muscle relaxants have been used creates the hypothetical risk of precipitating brachial plexus injuries, for which the terms 'Prone Position Plexopathy' has been coined.2 The objective of this paper is to report a case and a review of the relevant literature.

A 36 year old gentleman presented to the emergency department with an 8 day history of progressive shortness of breath. His past medical history was limited to a high body mass index (BMI) and a previous traumatic brain injury (TBI) in 2002. He was found to be significantly hypoxic despite maximal oxygen therapy, and was brought to the critical care unit for ventilation and proning directly. He initially improved with airway pressure release ventilation (APRV) and frequent proning; however, he developed a ventilator-associated pneumonia (VAP) and deteriorated, and was transferred for veno-venous ECMO (VV-ECMO). On his return, he was noted to have new right upper limb weakness despite a computed tomography (CT) scan of his head showing no new signs; on examination he was unable to abduct his right shoulder and he had MRC grade 2 of the right Bicep, with generalised bilateral weakness. On nerve conduction studies and electromyography of his right upper limb several weeks after a successful discharge, he was found to have an underlying moderately severe lesion of the upper trunk of the right brachial plexus from which the large majority of nerve fibres to the right suprascapular, musculocutaneous and axillary nerves are derived, with relative sparing of the right middle and lower trunks, and posterior and medial cords, confirming the neurological findings.

Methods: A systematic literature search was conducted on PubMed using the search strategy: ("prone position*") AND ("brachial plexus injur*" OR "brachial plexopathy" OR "nerve injury"), which identified 34 articles that were subsequently screened. The resulting seven full-text articles were assessed for eligibility. Five studies, of which three were case reports and two were case series, were included.3-7.

Results: Twenty-one cases of brachial plexopathy, or impending brachial plexopathy, secondary to proning were identified. The most common reason for proning was for spinal surgery $(n=18)$. This was followed by thoracoscopic oesophagectomy $(n=1)$, debridement of necrotising fasciitis $(n=1)$ and, indeed, the use of prone positioning in a patient with ARDS in an intensive care unit setting $(n=1)$. Only one case within the dataset underwent proning as it is described in the use for patients with ARDS secondary to COVID-19, and this was the patient who was proned for up to 12 hours a day in ICU as part of the management of ARDS. All of the plexopathies identified had almost entirely resolved within ten months, with two patients experiencing residual sensory deficits.3-7.

Conclusion: injury secondary to proning during the COVID-19 pandemic, and the second case in an ICU patient proned for the management of ARDS. Brachial plexopathy is a pathology to consider in patients who have undergone proning in an ICU environment as part of their treatment of ARDS secondary to COVID-19 who show signs of focal neurological deficits of the upper limb upon removal of paralysis and sedation; and as the COVID-19 pandemic continues, adaptations such as modifying SSKIN (a five step model for pressure ulcer prevention) during proning and using scoring systems in critical care follow up will be needed to improve outcomes.

\section{Reference(s) and grant ackowledgment(s)}

1. N/A.

2. Saiwai H, Okada S, Kawaguchi Kl, Saito T, Hayashida M, Matsushita A, et al. Prone position surgery for a professional sumo wrestler with thoracic ossification of the posterior longitudinal ligament resulting in intraoperative brachial plexus injury by hypertrophic pectoral muscles. J Clin Neurosci 2019;63:227-30.

3. Aisu Y, Hori T, Kato S, et al. Brachial plexus paralysis after thoracoscopic esophagectomy for esophageal cancer in the prone position: A thoughtprovoking case report of an unexpected complication. Int J Surg Case Rep 2019:55:11-14

4. Schwartz DM, Drummond DS, Hahn M, Ecker ML, Dormans JP. Prevention of positional brachial plexopathy during surgical correction of scoliosis. J Spinal Disord 2000;13:178-82.
5. Anderton JM, Schady W, Markham DE. An unusual cause of postoperative brachial plexus palsy. Br J Anaesth 1994;72:605-7.

6. Goettler CE, Pryor JP, Reilly PM. Brachial plexopathy after prone positioning. Crit Care 2002;6:540-42.

7. QuickT, Brown H. A commentary on Prone Position Plexopathy during the COVID-19 pandemic. British Orthopaedic Association: The Transient Journal of Trauma, Orthopaedics and the Coronavirus, 2020. https://www.boa.ac.uk/ policy-engagement/journal-of-trauma-orthopaedics/journal-of-traum a-orthopaedics-and-coronavirus/a-commentary-on-prone-position-plexo pathy.html [Accessed July 17, 2020].

8. Meng L, Qiu H, Wan L, Yuhang A, Zhanggang X, Qulian G, et al. Intubation and Ventilation amid the COVID-19 Outbreak: Wuhan's Experience. Anesthesiology 2020 [Published online ahead of print].

\section{5}

A single centre retrospective observational study: Clinical characteristics and outcomes of 57intensive care patients with Covid-19 in South London

B. Shurovi ${ }^{1}$; A. Isted ${ }^{2}$

${ }^{1}$ Critical Care, University Hospital Lewisham, London, United Kingdom ${ }^{2}$ Critical care, University Hospital Lewisham, Lewisham High Street, London, UK, London, United Kingdom

Correspondence: $\mathrm{B}$. Shurovi

Intensive Care Medicine Experimental 2020, 8(2): 001645

Introduction: Novel Coronavirus (SARS-Coronavirus-2: SARS-CoV-2) or Coronavirus Disease 19 (COVID-19) emerged in Wuhan, China in December 2019 and it was declared a global pandemic in March 2020.

Objectives: To describe the epidemiological characteristics and clinical manifestations of patients with COVID-19 in South-East London Region.

Methods: A single centre retrospective observational study was completed comprising patients with laboratory confirmed diagnosis of Covid-19 in a period between 12th March and 3rd April. Data was collected on patient characteristics, clinical manifestations, laboratory results, radiology findings and management in Intensive Care Unit (ICU). Moreover, described some of the complications that were noted in the COVID-19 cohort.

Results: 57 patients (68\% male) were followed up for 23 days. The most common co-morbidities were hypertension (54\%), obesity (53\%) and type2diabetes (32\%). The most common clinical manifestations were shortness of breath (88\%), fever (\%88) and cough $(84 \%)$, which was initially noted 8 days prior to ICU admission. The typical radiology findings seen in COVID-19 patients were bilateral infiltrates on chest X-ray. Laboratory findings that were common in majority of patients were lymphopenia with raised white cell count, raised C-reactive protein and raised ferritin. $21 \%$ of the patients required renal replacement therapy due to acute kidney injury. Invasive mechanical ventilation (IMV) was required by $93 \%$ of the cohort; however, initially the unit did not try non-invasive ventilation for COVID-19 patients. $\mathrm{PaO} 2 / \mathrm{FiO} 2$ ratio illustrated appropriately the biochemical picture of acute respiratory distress syndrome. At the end of the follow-up period for this study, 35\% had died, $28 \%$ had been discharged from hospital, $9 \%$ was stepped down to the ward from ICU and $28 \%$ remained in ICU. The average ICU stays in this cohort was 15 days. Mortality was correlated with age, $\mathrm{PaO} 2 / \mathrm{FiO} 2$ ratio, ventilator settings, peak ferritin and neutrophil count over the first 72hours of IMV.

Some of the complications that were seen in this cohort: eight patients developed pneumomediastinum, surgical emphysema and pneumothoraces, one patient had a ST-elevation myocardial infarction requiring $\mathrm{PCl}$ and one had a micro-haemorrhage/subarachnoid haemorrhage $(8 \mathrm{~mm})$ and one patient developed posterior reversible encephalopathy syndrome (PRES).

Conclusion: This retrospective observational study described the clinical characteristics and outcomes of a South-East London cohort. It described few of the complications that were seen in this cohort, which could be co-related to COVID-19. Moreover, it reports factors that are correlated with mortality for patients with COVID-19. 
001293

\section{Fluid management in acute burn injuries}

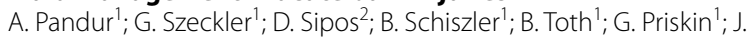

Betlehem ${ }^{1}$; B. Radnai'

${ }^{1}$ Institute of Emergency Care and Pedagogy of Health, University of Pécs Faculty of Health Sciences, Pécs, Hungary; ${ }^{2}$ University of pecs, Doctoral School of Health Sciences, Pécs, Hungary

Correspondence: A. Pandur

Intensive Care Medicine Experimental 2020, 8(2): 001293

Introduction: In severly burnt patients, it is important to focus on fluid resuscitation, not just pain relief, because a burnt patient loses a big amount of fluid.

Objectives: Our study's purpose is to find out how much fluid did these patients get during the prehospital care.

Methods: We included those patients who suffered an at least $20 \%$ second, third degree burn, and their fluid resuscitation started in the prehospital phase. We did our study with document analysis. We checked the documents from 2008.01.01. to 2018.12.31. With the hepl of including and exluding criterias we could include 104 people $(n=104)$. For data analysis we used Microsoft Word and Excel 2016 and SPSS version 25,0 . For statistical tests, we used correlation, Chisquare test and independent T-test.

Results: In this study there were 70 male $(67,3 \%)$ and 34 female $(32,7 \%)$ patients. The average age was 56,59 years. Patients suffered an average $31,90 \%$ burn. In the prehospital care these people got averagely $1315,84 \mathrm{ml}$ fluid. The $32,7 \%$ of the patients died during hospital care. We found that TBSA (total burn surface area) correlate significantly with surviving $(p<0,01)$. Surviving also correlate with almost every complications significantly $(p<0,001)$. We also found that Evans formula is more sensitive than Parkland formula, and there is a significant difference between real fluid resuscitation and recommended fluid resuscitation by the formulas $(p<0,001)$. We confirmed that Parkland formula decreases occurence of complications. It was also clear that patients got more than twice as many fluid prehospitaly than Parkland formula would provided.

Conclusion: Evans formula is more sensitive and it would drive patients fluid management better, but in prehospital care it is impracticable due to lack of equipment. There is more chance of complications if the patients got not the right amount fluid what they're needed. Finally patients got more than twice as many fluid as it was recomended by Parkland formula in the guidline, so it is clear the prehospital medical providers didn't work as the guidline would recommend.

\section{7}

Validity of APACHE II and SAPS II scores as predictors of early global mortality at $\mathbf{3}$ months from sepsis

MJ. Palavras ${ }^{1}$; C. Faria'; M. Cabral' ; F. Santos ${ }^{1}$; J. Gonçalves ${ }^{1}$; A. Araújo ${ }^{3}$; L. Pereira $^{3}$

'Department of internal medicine, Centro Hospitalar de Leiria (CHL), Hospital de Santo André (HSA), Leiria, Portugal; ${ }^{2}$ Department of cardiology, Centro Hospitalar de Leiria (CHL), Hospital de Santo André (HSA), Leiria, Portugal; ${ }^{3}$ Intensive care unit, Centro Hospitalar de Leiria (CHL), Hospital de Santo André (HSA), Leiria, Portugal

Correspondence: M.J. Palavras

Intensive Care Medicine Experimental 2020, 8(2): 001407

Introduction: APACHE (Acute Physiology and Chronic Health Evaluation) II and SAPS (Simplified Acute Physiology Score) II have become a necessary tool to describe ICU (intensive care unit) populations and to predict differences in mortality. Both are calculated in the first 24 hours of hospitalization, in which higher levels correspond to more serious diseases and a higher risk of death. Sepsis is a major cause of morbidity in ICU, it is estimated that about half of the patients have a nosocomial infection and are, therefore, at a higher risk of death.

Objectives: The aim of this study was to determine the comparative efficacy of different scoring system (APACHE II and SAPS II scores) in assessing the prognosis of critically ill patients that were admitted to the ICU by sepsis in a tertiary hospital and compare them for prediction of the outcome in these patients in early mortality (up to 3 months).

Methods: We performed a retrospective review of five hundred and thirty three patients that were admitted to the ICU from January 2018 to December 2019. We included all patients (medical or surgical patients) over the age 16 years old, and we selected those with the first diagnosis of "sepsis". Patient selection and information collection were obtained through medical records. It was calculated the predictive mortality by APACHE II and SAPS II and also the early mortality. Group comparisons between survivors and non-survivors from sepsis were performed using the Student- $T$ test for continuous variables and Pearson chi-square test for categorical variables. A p-value less than 0.05 was considered significant. Statistical analysis was performed using SPSS software version 25.0 (IBM, Armonk, NY, USA).

Results: Out of 533 patients, 71 had sepsis (13\%). Of these 71 patients, $36(50,7 \%)$ died up to 3 months. Average APACHE II and SAPS II scores in all patients were $23,5 \pm 8,1(p=0,02)$ and $52,9 \pm 17,7(p<0,01)$ respectively, and they were significantly different between survivors and non-survivors. A statistically significant positive correlation was established between the values of SAPS II and APACHE II $(r=0.76$; $p<0.01$ ). By the examination of validity and classification accuracy by ROC curve, we conclude that: SAPS II had AUC $>0,662$ and APACHE II had AUC $>0,639$. Both can predict the risk of mortality in these patients, but SAPS II seems to be slightly more accurate. We also observed that elderly patients were expected to have a worse prognosis. The average age of survivors was $64,5 \pm 13,8$ years and for nonsurvivors 75, $2 \pm 8,8$ years $(p<0,01)$.

Conclusion: Both APACHE and SAPS II were accurate tools to describe and predicted mortality caused by sepsis. There was no significant difference in the clinical values of SAPS II and APACHE II and taking in consideration the high correlation between both, only one, could be enough to make a mortality prediction.

Reference(s) and grant ackowledgment(s)

1. Harrison DA, Welch CA, Eddleston JM. The epidemiology of severe sepsis in England, Wales and Northern Ireland, 1996 to 2004: secondary analysis of a high quality clinical database, the ICNARC Case Mix Programme Database. Crit Care 2006; 10:R42.

2. Iftikhar Haider Naqvi, Khalid Mahmood, Syed Ziaullaha, Syed Mohammad Kashif, and Asim Sharif. Better prognostic marker in ICU- APACHE II,SOFA or SAP II!. Pakistan Journal of Medical Sciences. Bi- Monthly 2016.

3. Capuzzo M, Valpondi V, Sgarbi A, et al. Validation of severity scoring systems SAPS II and APACHE II in a single-center population. Intensive Care Med 2000; 26:1779.

4. Escarce JJ, Kelley MA. Admission source to the medical intensive care unit predicts hospital death independent of APACHE II score. JAMA 1990; 264:2389.

5. Ho KM, Dobb GJ, Knuiman M, et al. A comparison of admission and worst 24-hour Acute Physiology and Chronic Health Evaluation II scores in predicting hospital mortality: a retrospective cohort study. Crit Care 2006; 10:R4.

001463

Validity of APACHE II or SAPS II as predictors of the hospital length of stay in multi-trauma patients: the analysis of a Portuguese ICU in a tertiary hospital

C. Faria ${ }^{1}$; MJ. Palavras'; M. Cabral' ; F. Santos ${ }^{1}$; J. Gonçalves ${ }^{1}$; A. Araujo ${ }^{3}$; L.

Pereira $^{3}$

${ }^{1}$ Department of internal medicine, Centro Hospitalar de Leiria $(\mathrm{CHL})$, Hospital Santo André (HSA), Leiria, Portugal; ${ }^{2}$ Department of cardiology, Centro Hospitalar de Leiria (CHL), Hospital Santo André (HSA), Leiria, Portugal; ${ }^{3}$ Intensive care unit, Centro Hospitalar de Leiria (CHL), Hospital de Santo André (HSA), Leiria, Portugal

Correspondence: $C$. Faria

Intensive Care Medicine Experimental 2020, 8(2): 001463

Introduction: APACHE (Acute Physiology and Chronic Health Evaluation) II and SAPS (Simplified Acute Physiology Score) II are the two 
main tools to evaluate patients in an intensive care unit (ICU), 24 hours after admission, regarding the predictable intrahospital rate of death. In multi-trauma patients, previous reviews showed that SAPS II was valid to predict intrahospital death in these patients when compared to APACHE II. However, the correlation of these scores with the hospital length of stay was not demonstrated.

Objectives: The aim of this study was to verify if there is a correlation between the main score systems used in a Portuguese tertiary hospital ICU and the hospital length of stay (LOS) in multi-trauma patients.

Methods: This retrospective review evaluated the admitted patients in an ICU during two years, from January 2018 to December 2019, through the consultation of clinical records, calculated predictive mortality by APACHE II and SAPS II, as well as the hospital length of stay, including the time spent in ICU. We obtained a population of 533 patients from which we selected the ones admitted with "multitrauma" as first diagnosis.

Group comparisons between both scores and LOS were performed using the Pearson correlation. A linear regression model was applied. A p-value less than 0.05 was considered statistically significant. Statistical analysis was performed using SPSS software version 25.0 (IBM, Armonk, NY, USA).

Results: Out of 533 patients, 27 had multi-trauma (5.06\%). These 27 patients were $53.6 \pm 18.1$ years old, from which $6(22.2 \%)$ were female and $21(77.8 \%)$ were male. The hospital LOS was $47.2 \pm 24.3$ days. Average APACHE II and SAPS II scores were $13.9 \pm 6.0$ and $33.0 \pm$ 12.7 , respectively. Pearson correlation showed us that there is a correlation between SAPS II and LOS $(p=0.021)$ that was not verified to APACHE II $(p=0.188)$. Therefore, it was possible to apply a simple linear regression model correlating the SAPS II and the hospital LOS which concluded that each unity added to SAPS II represented an increase of 0.42 days to the LOS. This linear regression model explains the LOS variation by $6 \%(p=0.028)$.

Conclusion: A correlation between SAPS II and the hospital length of stay was verified with a statistic significance that was not verified with APACHE II. It is possible to assume that the calculation of SAPS II in multi-trauma patients could predict the approximated hospital length of stay, which is a remarkable predictor to clinicians to established the plan for these patients. Further studies on this topic are needed to confirm the association between SAPS II and the hospital length of stay.

\section{Reference(s) and grant ackowledgment(s)}

1. Sardinha, D., Sousa, R., Damiani L. Risk factors for the mortality of trauma victims in the intensive care unit. Intensive and Critical Care Nursing 2015; 31:76-82. https://doi.org/10.1016/j.iccn.2014.10.008

2. Moreno R, Morais P. Outcome prediction in intensive care: results of a prospective, multicenter, Portuguese study. Intensive Care Med 1997;23:177186. https://doi.org/10.1007/s001340050313.

3. Korkmaz Toker M, Gülleroğlu A, Karabay AG, Biçer IG, Demiraran Y. SAPS III or APACHE IV: Which score to choose for acute trauma patients in intensive care unit?. Ulus Travma Acil Cerrahi Derg 2019;25:247-252. https://doi. org/10.5505/tites.2018.22866.

\section{2}

The value of APACHE II and SAPS II as predictors of intrahospital mortality in critical patients with Acute Kidney Injury

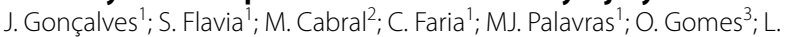
Pereira $^{3}$

${ }^{1}$ Department of internal medicine, Centro Hospitalar de Leiria (CHL), Hospital Santo André (HSA), Leiria, Portugal; ${ }^{2}$ Cardiologia, Hospital Santo Andre - Hospital Distrital De Leiria, Leiria, Portugal; ${ }^{3}$ Serviço de medicina intensiva, Centro Hospitalar de Leiria, Leiria, Portugal

Correspondence: J. Gonçalves

Intensive Care Medicine Experimental 2020, 8(2): 001522

Introduction: APACHE (Acute Physiology and Chronic Health Evaluation) II and SAPS (Simplified Acute Physiology Score) II are commonly used scoring systems to calculate the severity of illness in intensive care patients. Both are calculated in the first 24 hours of hospitalization and can predict the risk of intrahospital mortality. Acute Kidney Injury (AKI) is frequent and has high morbidity and mortality rates, which determines an enormous importance in the context of ICU (Intensive Care Unit).

Objectives: The aim of this study is to investigate the capacity of main score systems to predict intrahospital mortality of patients with AKI, in a portuguese tertiary hospital ICU.

Methods: We performed a retrospective review that evaluated the admitted patients in an ICU during two years, from January 2018 to December 2019, through the consultation of clinical records and calculated predictive mortality by APACHE II and SAPS II. From the 535 patients included, those with AKI at admission were selected, regardless of the main diagnosis, according to Acute Kidney Injury classification (AKIN).

Group comparisons between survivors and non-survivors from AKI were performed using the Student-T test for continuous variables and Pearson chi-square test for categorical variables. A p-value less than 0.05 was considered significant. Statistical analysis was performed using SPSS software version 25.0 (IBM, Armonk, NY, USA).

Results: Out of 535 patients, 272 patients (50.8\%) had AKI and 263 patients $(49.4 \%)$ had not. The mortality rate was $37.9 \%$ and $21.7 \%$, respectively, with significant difference between them $(p<0.01)$. Patients with AKI were mainly males (57\%). There is no difference between the mortality rate and gender $(p=0,06)$. Out of 272 patients with AKI, 169 survived (62.1\%) and 103 died (37.9\%). The average age of survivors was $68.93 \pm 14.59$ years and of non-survivors group was $74.14 \pm 11.82$ years. The mean SAPS II score in the survivors group was $53.60 \pm 19.24$ while in the non-survivors group was $68.08 \pm$ $19.83(p<0,01)$. The mean APACHE II score was $25.11 \pm 9.36$ and $30.98 \pm 8.84$, respectively $(p<0,01)$. Conserning creatinine and urea levels in both groups of patients, there was no difference neither in mean creatinine levels $(p=0.72)$ neither in mean urea levels $(p=0.34)$. APACHE II and SAPS II are valid predictors of intrahospital mortality in critical patients with Acute Kidney Injury at admission. Furthermore, non-survivor patients showed a higher age mean than survivors, as expected. Despite our assumptions, the main renal function analytical parameters showed no influence in the outcome of the patients with Acute Renal Injury.

Conclusion: APACHE II and SAPS II are valid predictors of intrahospital mortality in critical patients with Acute Kidney Injury at admission. Furthermore, non-survivor patients showed a higher age mean than survivors, as expected. Despite our assumptions, the main renal function analytical parameters showed no influence in the outcome of the patients with Acute Renal Injury.

\section{Reference(s) and grant ackowledgment(s)}

1. Le Gall JR, Lemeshow S, Saulnier F. A new Simplified Acute Physiology Score (SAPS II) based on a European/North American multicenter study [published correction appears in JAMA 1994 May 4;271(17):1321]. JAMA. 1993;270(24):2957-2963. https://doi.org/10.1001/jama.270.24.2957.

2. Bouchard J, Acharya A, Cerda J, et al. A Prospective International Multicenter Study of AKI in the Intensive Care Unit. Clin J Am Soc Nephrol. 2015;10(8):1324-1331. https://doi.org/10.2215/cjn.04360514.

3. Gong Y, Ding F, Zhang F, Gu Y. Investigate predictive capacity of in-hospital mortality of four severity score systems on critically ill patients with acute kidney injury. J Investig Med. 2019;67(8):1103-1109. https://doi.org/10.1136/ jim-2019-001003.

\section{Comparison of 3 risk prediction scores for Covid-19 in a UK} hospital

A. Garg ${ }^{1}$; F. Wyn-Griffiths' ${ }^{1}$ S. Hiou' ; F. Hossain ${ }^{2}$

${ }^{1}$ Anaesthetics, Walsall Manor Hospital, Birmingham, United Kingdom; ${ }^{2} R \& d$, Walsall Manor Hospital, Birmingham, United Kingdom

Correspondence: A. Garg

Intensive Care Medicine Experimental 2020, 8(2): 001524 
Introduction: As the Covid-19 pandemic has spread around the world, several new scores have been released to predict risk of critical illness or mortality among Covid-19 patients. None of these scores have been validated in the UK population.

Objectives: We attempted to compare 3 scores - COVID-GRAM [1], HNC-LL [2] and CURB-65 scoring systems in assessing accuracy for predicting critical illness or in-patient mortality among Covid-19 patients at our institution.

Methods: We collected data on 429 patients with RT-PCR confirmed Covid-19 infection identified from the local ISARIC CCP and hospital databases, admitted during March and April 2020. Our endpoints were 1). Critical illness defined as Deaths and/or ICU admission 2). In-patient mortality.

Results: The incidence of inpatient mortality and progression to critical illness in our cohort was $32.1 \%$ and $38.7 \%$ respectively. COVIDGRAM tool showed the highest accuracy in predicting critical illness (AUC $0.647 ; 95 \% \mathrm{Cl} 0.600$ to 0.692 ) and inpatient mortality (AUC 0.695; $95 \% \mathrm{Cl} 0.649$ to 0.739 ). There was no significant difference in accuracy of AUC between CURB-65 and COVID-GRAM (p-0.9074) for inpatient mortality but both were better than HNCLL. ( $p-0.0415$ and 0.0112$)$. HNCLL showed lowest accuracy in predicting both the endpoints of interest.

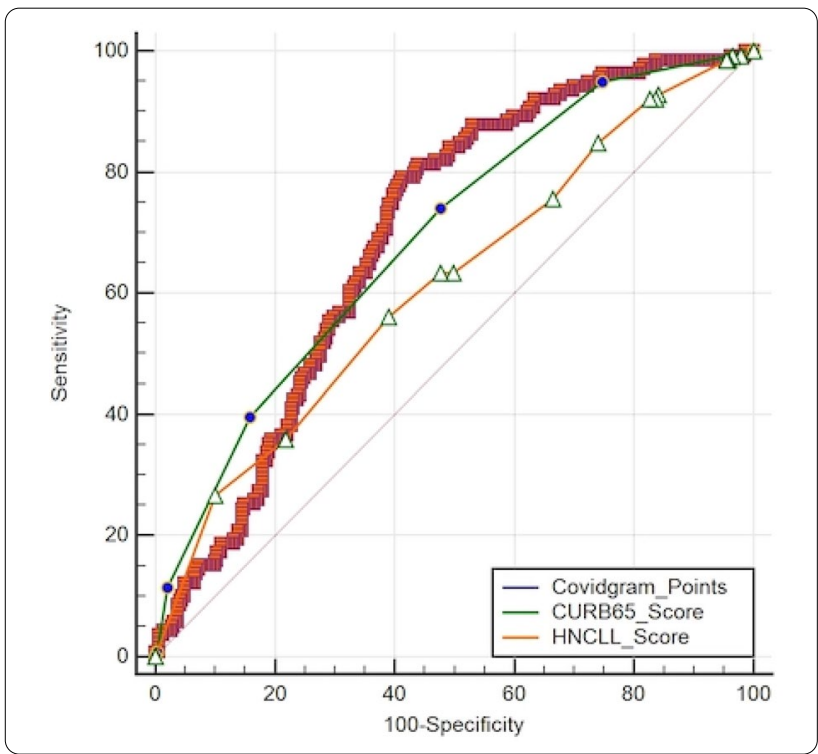

Conclusion: While COVID-GRAM was most accurate in predicting critical illness and inpatient mortality from COVID 19, it was only marginally higher than CURB65. We recommend the continued use of CURB 65 as convenient and easy to use tool in predicting complications and mortality from COVID 19.

\section{Reference(s) and grant ackowledgment(s)}

1. 2). Xiao L et al. Development and validation of the HNC-LL score for predicting the severity of coronavirus disease 2019. EBioMedicine; doi.org/https:// doi.org/10.1016/j.ebiom.2020.102880.

2. 1). Liang $W$, Liang $H$, Ou L et al. Development and Validation of a Clinical Risk Score to Predict the Occurrence of Critical Illness in Hospitalized Patients With COVID-19. JAMA Intern Med. https://doi.org/10.1001/jamai nternmed.2020.2033.

3. Not applicable.
001239

Acceptability of Non-Invasive Eye Tracking in Intensive Care Unit: A Cross MDT Questionnaire

A. Al-Hindawi ${ }^{1}$; F. El-Hibri'; M. Vizcaychipi

${ }^{1}$ Intensive care unit, Chelsea and Westminster Hospital, London, United Kingdom

Correspondence: A. Al-Hindawi

Intensive Care Medicine Experimental 2020, 8(2): 001239

Introduction: Eye tracking has a long history in diagnosis of neuropsychiatric and neurodevelopmental disorders, specifically in schizophrenia and autism [1]-[4]. It has recently been advocated for as a method for inferring attention as a surrogate marker of delirium but has been hampered due to lack of technological solutions available for the Intensive Care Unit (ICU) environment [5]. To that extent, we have developed a non-invasive, end of the bed, eye tracker that automatically tracks the eye movements and the gaze of a patient without instrumentation or calibration.We sought to understand the acceptability of this new monitoring method from the multidisciplinary team across a single centre prior to deployment in a clinical feasibility trial.

Objectives: To ascertain the:

1. Practical issue with current delirium assessment methods.

2. Acceptability of automating delirium detection.

3. Acceptability of non-invasive eye tracking in intensive care.

Methods: A Google Form of qualitative and quantitative questions was made ascertaining the views of delirium assessment and the views on the automation of delirium detection. A picture of the system as setup in an ICU bed-space was then shown with an explanation of its function (Figure 1). Open questions were asked about their opinion prior to closed questions of specific interest.

$R$ version 4.0.1 was used for analysis.

Results: A wide range of MDT roles were gathered $(n=29,65 \%$ nurses, $21 \%$ doctor, $3 \%$ dietician, 3\% pharmacist, 3\% critical care technologist, $3 \%$ patient care coordinator).Nurses found that current delirium assessment methods were laborious, often confusing to perform and are only formalising their intuition but welcomed automation (82\%). The majority $(92 \%)$ of responders welcomed the use of eye tracking as a method of delirium detection. The majority thought that the setup would not interfere with their day to day clinical activities with the patient $(75 \%)$, their families (80\%), and other members of the MDT (98\%).Qualitatively, a theme of avoiding "clutter" around the bedspace was highlighted. The end of the bed camera marker $(a 10 \times 10 \mathrm{~cm}$ plastic code) was highlighted as a potential point of delirium 'generation'.Of interest, the theme of privacy and was not highlighted.

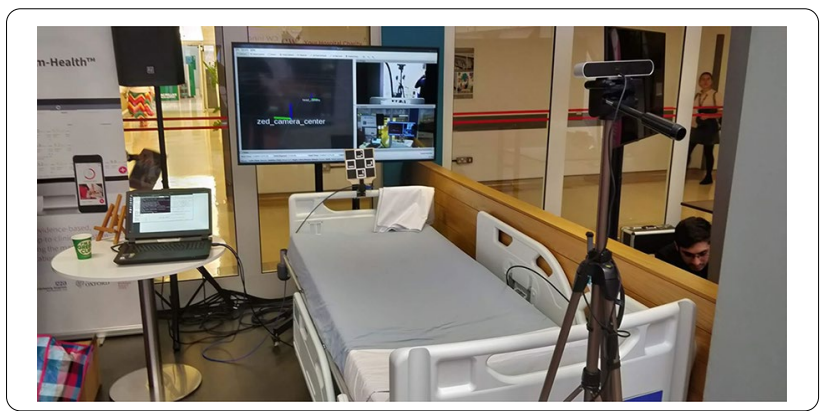

Conclusion: Non-invasive eye tracking as a method of delirium assessment is generally well accepted in AICU across the MDT. Specific care is to be taken to avoid adding further clutter around an already busy AICU bed-space.

Reference(s) and grant ackowledgment(s)

1. [5] C. Exton and M. Leonard,'Eye tracking technology: a fresh approach in delirium assessment?', Int. Rev. Psychiatry, vol. 21, no. 1, pp. 8-14, 2009, doi: $10.1080 / 09540260802675106$ 
2. [4] S. Wang et al.,'Atypical Visual Saliency in Autism Spectrum Disorder Quantified through Model-Based Eye Tracking', Neuron, vol. 88, no. 3, pp. 604-616, 2015, https://doi.org/10.1016/j.neuron.2015.09.042.

3. [3] S. M. Mavadati, H. Feng, A. Gutierrez, and M. H. Mahoor, 'Comparing the gaze responses of children with autism and typically developed individuals in human-robot interaction', IEEE-RAS Int. Conf. Humanoid Robots, vol. 2015-Febru, pp. 1128-1133, 2015, https://doi.org/10.1109/human oids.2014.7041510.

4. [2] J. Heinzle, E. A. Aponte, and K. E. Stephan, 'Computational models of eye movements and their application to schizophrenia', Curr. Opin. Behav. Sci., vol. 11, pp. 21-29, 2016, https://doi.org/10.1016/j.cobeha.2016.03.008.

5. [1] S. A. Beedie, D. M. St.Clair, and P. J. Benson, 'Atypical scanpaths in schizophrenia: Evidence of a trait- or state-dependent phenomenon?', J. Psychiatry Neurosci., vol. 36, no. 3, pp. 150-164, 2011, https://doi.org/10.1503/ jpn.090169.

\section{5}

\section{APACHE II and SAPS II as death predictors in intensive care} surgical patients

F. Santos ${ }^{1}$; M. Cabral²; J. Gonçalves ${ }^{3}$; C. Faria ${ }^{3}$; MJ. Palavras ${ }^{1}$; N. Ferreira ${ }^{4}$; L. Pereira $^{4}$

${ }^{1}$ Department of internal medicine, Centro Hospitalar de Leiria (CHL), Hospital Santo André (HSA), Leiria, Portugal; ${ }^{2}$ Department of cardiology, Centro Hospitalar de Leiria (CHL), Hospital Santo André (HSA), Leiria, Portugal; ${ }^{3}$ Department of internal medicine, Centro Hospitalar de Leiria $(\mathrm{CHL})$, Hospital de Santo André (HSA), Leiria, Portugal; ${ }^{4}$ Department of intensive care, Centro Hospitalar de Leiria (CHL), Hospital Santo André (HSA), Leiria, Portugal

\section{Correspondence: F. Santos}

Intensive Care Medicine Experimental 2020, 8(2): 001515

Introduction: Intensive Care Unit (ICU) delivers care through a multidisciplinary approach. In order to predict the probability of death we have some severity indices to use, such as Acute Physiology and Chronic Health Evaluation II (APACHE II) and Simplified Acute Physiology Score (SAPS II). They were initially designed for sepsis patients, but nowadays they are used as mortality predictors in ICU patients of all etiologies.

Objectives: The aim of the study was to analyze if APACHE II and SAPS II are feasible scores to predict mortality in surgical patients admitted to ICU, given that both were not originally designed for these kind of patients.

Methods: The authors designed a retrospective study, considering all the patients admitted to the ICU during a two year period (from January 2018 until December 2019). The APACHE II and SAPS II scores were analyzed in order to understand in which cases these scores are more accurate. All the patients over 16 years old were included. The patients admitted for a period inferior to 24 hours were excluded.

The statistical analysis was made using SPSS software version 205.0. T-student test was used to compare survivals and non survivals patients in different time-lines and ROC curve to understand the validity of the scores in surgical patients.

Results: We analyzed 533 patients, 155 of them admitted in ICU as surgical patients. 74 women and 81 men, with median age of 68.6 $( \pm 17.1)$. With an APACHE II mean of $18.9( \pm 9.3)$ and SAPS II mean $45.6( \pm 20.1) .113$ were survivors and 42 were non-survivors, (mortality rate of $27 \%$ ). The survivors presented APACHE II score mean of 16 and a SAPS II mean score of $40(p<0.01)$. The non-survivors presented a mean APACHE II score of 25 and a mean SAPS II score of $61(p<0.01)$. ROC curves showed that both scores were good predictors of mortality in ICU, showing a AUC 0.782 for APACHE II $(p<0.01)$ and 0.782 $(p<0.01)$ for SAPS II. Between the non-survivors $(n=42), 69 \%(n=29)$ died at the ICU admission and $12 \%(n=5)$ during hospital admission after ICU discharge. 19\% $(n=8)$ died at home before 3 months after ICU discharge.

For predicting mortality during hospital admission after ICU discharge, mean APACHE II score of $21.4( \pm 12)(p=0.14)$ and mean SAPS II of $57.6( \pm 17.5)(p>0.01)$ for APACHE II and SAPS II did not show feasible results to predict mortality at home during 3 months after ICU discharge ( $p=0.64$ for APACHE II and $p=0.16$ for SAPS II).
The patients were divided on scheduled surgical ones $(n=40)$ and non-scheduled surgical patients $(n=115)$. In scheduled surgical patients the mortality rate was $12.5 \%(n=5)$, with APACHE II and SAPS II scores predicting death in these patients showing no accuracy at all ( $p=0.07$ and $p=0.54$, respectively). In the non-scheduled surgical patients the mortality rate was $32 \%(n=37)$; in this patients both scores showed to be good mortality predictors in this group (APACHE II with $\mathrm{p}<0.01$, SAPS II with $\mathrm{p}<0.01$ ).

Conclusion: The results showed that APACHE II and SAPS II are both good mortality predictors scores in surgical patients admitted in ICU. SAPS II was better in predicting mortality during hospital admission after ICU discharge than APACHE II.

Both showed valid results to predict death during ICU admission and early death ( $<3$ months).

Both showed accurate results to predict mortality in non-scheduled surgical patients but not on scheduled surgical ones.

\section{Reference(s) and grant ackowledgment(s)}

1. Khwannimit B, Geater A. A comparison of APACHE II and SAPS II scoring systems in predicting hospital hospital hospital mortality in Thai adult intensive care units. J Med Assoc Thai. 2007;90(4):643-52.

2. Yalçın, M., Gödekmerdan, E., Tayfur, K., Yazman, S., Ürkmez, M., \& Ata, Y. (2019). The APACHE II Score as a Predictor of Mortality After Open Heart Surgery. Turkish journal of anaesthesiology and reanimation, 47(1), 41-47. https://doi.org/10.5152/TJAR.2018.44365.

3. Sakr Y, Krauss C, Amaral AC, et al. Comparison of the performance of SAPS II, SAPS 3, APACHE II, and their customized prognostic models in a surgical intensive care unit. Br J Anaesth. 2008;101(6):798-803. https://doi. org/10.1093/bja/aen291.

\section{6}

The exposure of radiation in young trauma patients treated in the Intensive Care Unit (ICU)

G. Koukoulitsios ${ }^{1}$; M. Anifanti ${ }^{1}$; K. Tsikritsaki ${ }^{1}$; I. Tsoni ${ }^{1}$; S. Bakouli ${ }^{1}$; I. Ionna ${ }^{1}$; G. Maglaras'; A. Koutivas ' ;. Mandila'; S. Antonopoulou' ; V. Koutsoukou'; V. Salma'; A. Kalogeromitros ${ }^{1}$

"ICU, General Hospital of Athens "G. Gennimatas", Athina, Greece

Correspondence: $G$. Koukoulitsios

Intensive Care Medicine Experimental 2020, 8(2): 001186

Introduction: Young trauma patients undergo repeat radiologic imaging during initial trauma evaluation and ICU stay trough high radiation exposure of these patients is suspected studies are scarce. In this regard we retrospectively calculated ionizing radiation exposure of young ICU trauma patients due to medical imaging.

Methods: We reviewed records of $<40$ year old ICU trauma patients with lengths of stay (LOS) $>20$ days treated within the past 18 months. All imaging studies involving ionizing radiation were recorded. A Typical Effective Dose (TED) per type of study was assumed based on the literature. TED's incurred by plain radiographs (XR), computed tomographic (CT) scans and fluoroscopic studies (FL) were added to calculate a Cumulative Effective Dose (CED) per patient. CED's were compared to established limits of annual radiation exposure. The total medical radiation detriment was calculated using the formula $0.073 X C E D / S v$. Numbers given represent mean \pm sem.

Results: 36 patients (male:34 mean age $26.8 \pm 1.1$ years; mean los $48.6 \pm 7.7$ days; mean Injury Severity Score ISS:32.7 \pm 1.7$)$ underwent 1311 XRs ( $36.4 \pm 2.7 /$ patient), 248 CT-scans (6.9 $\pm 0.5 /$ patient), and 25 FL's ( $0.8 \pm 0.2$ /patient; 1 limb angiography, 23 fluoroscopy guided bone fixation procedures, 1 diaphragm fluoroscopy). The mean CED/patient was $40.5 \pm 2.8 \mathrm{mSv}$. Partial CED's attributable to XR, CT and FL were $2.5 \pm 0.3,37.3 \pm 2.8$ and $0.81 \pm 0.15 \mathrm{mSv} /$ patient or $8 \pm 2 \%, 89 \pm 3 \%$ and $3 \%$ of total CED respectively. The mean detriment due to medical exposure to ionizing radiation in our study was $0.00296 \pm 0.0002$.

Conclusion: The mean CED exceeded international and national (environmental:1 $\mathrm{mSv}$ occupational:20 $\mathrm{mSv}$ ) annual radiation exposure limits. CT scans accounted for the bulk of CED. Studies designed to investigate the short-term benefits vs long-term sequellae of X-ray 
imaging studies performed in ICU patient are needed especially individuals at child-bearing ages with long life-expectancies.

\section{8}

Current views and understanding in an anaesthetic department surrounding power injection of intravenous contrast via central venous catheters for $\mathrm{CT}$

E. Caine ${ }^{1}$; D. Horner

${ }^{1}$ Anaesthetics, Charing Cross Hospital, London, United Kingdom

Correspondence: E. Caine

Intensive Care Medicine Experimental 2020, 8(2): 001288

Introduction: During the COVID 19 pandemic, the anaesthetic team was often involved in the transfer of patients from intensive care (ICU) to computerized tomography (CT). With the associated thromboembolic events in COVID patients, often these scans were CT pulmonary angiograms (CTPAs) and therefore required higher intravenous (IV) contrast flows. Often these patients had been on ICU for a long period of time, had no peripheral access and only a central venous catheter (CVC) line in situ. We came across a huge variation in clinical practice with CVC lines being used for power injecting contrast. Some radiographers would not allow the CVC line to be used and asked for a peripheral cannula to be inserted. Others were happy for the CVC line to be used, as long as the accompanying doctor connected the power injector to the CVC line took responsibility for it. This variety in practice led us to research current understanding and experience with injecting IV contrast down a CVC line via a power injector and to review the literature and guidance surrounding it. We then ran a teaching session for the anaesthetic department and reassessed these views after this session.

Objectives: 1. To find out current understanding and experience in the radiology and anaesthetic department surrounding power injecting IV contrast down a CVC line.

2. If required, to run a teaching session to help develop an understanding of the issues and guidance surrounding administering IV contrast in patients with CVC lines but no peripheral access.

3. To find out whether further education has changed understanding or practice.

Methods: We sent out a short questionnaire via SurveyMonkey to both the anaesthetists $(n=28)$ and to the radiographers $(n=27)$. This was to find out the current understanding, experiences and practices of both anaesthetists and radiographers. We then offered a teaching session to the anaesthetic department on the equipment used in CT, the CVC lines used at the hospital, the flows of IV contrast required for certain CT scans and how Poiseuille's Law links pressure in the lines to its length, gauge, and viscosity of the contrast. We also reviewed the current literature looking at CVC lines used with power injectors. We then did a second questionnaire for the anaesthetists $(n=11)$ which looked at whether their views and practice had changed.

Results: Results showed that $85 \%$ of radiographers were not comfortable with IV contrast being given via the CVC line. Despite this, around half anaesthetists who answered the questionnaire have been asked by the radiographer to connect the power injector to the CVC line. $100 \%$ anaesthetists found the teaching useful with $91 \%$ anaesthetists feeling informed about the issues surrounding giving IV contrast via a CVC line compared to $9 \%$ prior to teaching.

$100 \%$ anaesthetists said that this teaching would change their practice, with $36 \%$ anaesthetists likely to give IV contrast via through a CVC line compared to $64 \%$ prior to teaching.

Conclusion: There is currently no UK national guidance on injection of IV contrast via a CVC line. In the United States, the American Committee or Radiology has recently released guidance suggesting that for power injection of IV contrast via CVC lines the radiologist should consult the manufacturers guidance (American College of Radiology, 2020). Some hospitals have local policies (UCSF, 2020; Royal Marsden NHS Foundation Trust Policy, 2017) but this is not something that we currently have implemented at our hospital.

Seeing as there is no formal guidance nationally, and few hospitals have formal policies implemented, often it comes down to individual risk analysis as to whether IV contrast can be injected via a CVC line.
Our data showed that few anaesthetists were well informed on these issues prior to teaching.

Anaesthetists and intensivists are often involved in the transfer of unwell patients. As a result, they are often are involved in decision making such as whether or not IV contrast can be injected down a central line. Interestingly, following teaching, the number of anaesthetists who would power inject IV contrast via a CVC line nearly halved. This quality improvement project shows that with education on this subject, anaesthetists may change their practice. $100 \%$ of anaesthetists said that they would like a formal policy in place in order to help with the decision-making process. This is something we hope to create and implement in the future in concordance with the radiology department.

\section{Reference(s) and grant ackowledgment(s)}

1. ACR Committee On Drugs And Contrast Media. 2020. [pdf] American College of Radiology. Available at: < https://www.acr.org/-/media/ACR/files/ clinical-resources/contrast_media.pdf>.

2. POLICY FORTHE INSERTION AND CARE OF CENTRAL VENOUS ACCESS DEVICES (CVAD) IN HOSPITAL. 2017. [pdf] Royal Marsden NHS Foundation Trust Policy. Available at: < https://www.nice.org.uk/guidance/mtg34/resou rces/policy-for-the-insertion-and-care-of-central-venous-access-devicescvad-in-hospital-royal-marsden-nhs-ft-pdf-4481503169>.

3. UCSF Department of Radiology and Biomedical Imaging. 2020. Vascular Access And Use Of Central Lines And Ports In Adults. [online] Available at: < https://radiology.ucsf.edu/patient-care/patient-safety/contrast/iodin ated/vascular-access-adults>.

4. No Grant

\section{8}

Point-of-care ultrasound for COVID-19 pneumonia patients in the ICU

Z. Bitar ; M. Shamsah²; O. Bamasood ${ }^{2}$; O. Maadrani ${ }^{1}$; H. Al-Foudari ${ }^{3}$ ${ }^{1}$ Critical care unit, New Ahmadi hospital KOC -

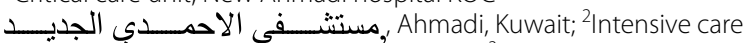
department, Adan Hospital, Hadiya, Kuwait; ${ }^{3}$ Head of intensive care department and anesthesia, Adan Hospital, Hadiya, Kuwait

Correspondence: Z. Bitar

Intensive Care Medicine Experimental 2020, 8(2): 001328

Introduction: Point-of-care ultrasound (POCUS) has a major role in the management of patients with acute hypoxic respiratory and circulatory failure and guides hemodynamic management. There is scarce literature on POCUS assessment characteristics in COVID-19 pneumonia with hypoxic respiratory failure.

Methods: The study is an observational, prospective, single-center study conducted in the intensive care unit of Adan General Hospital from May 1 st, 2020, to June 25, 2020. The study included adults suspected to have COVID-19 transferred to the intensive care unit (ICU) with fever or suspected respiratory infection. Patients were transferred to the ICU after reverse transcriptase-polymerase chain reaction (RT$\mathrm{PCR}$ ) testing. A certified intensivist in critical care ultrasound who was blinded to the RT-PCR results, performed the lung ultrasound and echocardiology within 12 hours of the patient's admission to the ICU. We calculated the E/e', E/A ratio, left ventricular ejection fraction EF, IVC diameter, RV size and systolic function. We performed ultrasound in 12 chest areas.

Results: Of 92 patients with suspected COVID-19 pneumonia, 77 (84\%) cases were confirmed.

In the group of patients with confirmed COVID-19 pneumonia, echocardiographic findings showed normal E/e', deceleration time (DT), and transmittal E/A ratio in comparison to the non-COVID19 patients (P .001 for both). The IVC diameter was $<2 \mathrm{~cm}$ with $>50 \%$ collapsibility in 62 (81\%) patients with COVID-19 pneumonia; a diameter of $>2 \mathrm{~cm}$ and $<50 \%$ collapsibility in all patients, with a $P$ value of 0.001 , was detected among those with non-COVID-19 pneumonia. There were 3 cases of myocarditis with poor EF (5.5\%), severe RV dysfunction was seen in 9 cases (11.6\%), and 3 cases showed RV thrombus. 


\begin{tabular}{|c|c|c|c|c|}
\hline & $\begin{array}{l}\text { Confirmed } \\
\text { COVID-19 } 77 \\
\text { cases }(84.4 \%)\end{array}$ & $\begin{array}{l}\text { Non-COVID- } \\
1915 \text { cases } \\
(15.5 \%)\end{array}$ & $\begin{array}{l}\text { Total } 92 \\
\text { cases }(\%)\end{array}$ & P value \\
\hline \multicolumn{2}{|c|}{ Median age (IQR, years)53 (82-36) } & $68(25-80)$ & & \\
\hline Male & $64(83 \%)$ & $7(46 \%)$ & $71(77 \%)$ & 0.005 \\
\hline \multicolumn{5}{|l|}{ Medical history } \\
\hline IHD & $9(11 \%)$ & $10(66 \%)$ & $19(20 \%)$ & $<0.0001$ \\
\hline $\mathrm{CABC}$ & $2(2.6)$ & $8(53 \%)$ & $10(10 \%)$ & 0.001 \\
\hline Hypertension & $20(25 \%)$ & $12(80 \%)$ & $32(34 \%)$ & 0.002 \\
\hline Diabetes mellitus & $25(32 \%)$ & $14(100 \%)$ & $39(42 \%)$ & $<0.0001$ \\
\hline COPD & $3(3 \%)$ & $3(20 \%)$ & $2(2 \%)$ & 0.02 \\
\hline $\begin{array}{l}\text { Chronic renal } \\
\text { impairment }\end{array}$ & $25(32 \%)$ & $10(66 \%)$ & $10(66 \%)$ & 0.015 \\
\hline Cancer & 0 & $1(6 \%)$ & $1(1 \%)$ & 0.091 \\
\hline \multicolumn{5}{|l|}{$\begin{array}{l}\text { Status on admission to } \\
\text { ICU }\end{array}$} \\
\hline Acute MI & $2(2.6)$ & $10(10 \%)$ & $12(13)$ & 0.001 \\
\hline Acute PE & $9(11.6)$ & 0 & & 0.186 \\
\hline $\begin{array}{l}\text { Duration of symptoms } \\
\text { (median in days) }\end{array}$ & $5(2-10)$ & $2(3-4)$ & & \\
\hline Hypoxemia & All & All & $\begin{array}{l}77 \\
(100 \%)\end{array}$ & 0.02 \\
\hline \multirow{2}{*}{$\begin{array}{l}\mathrm{PO} 2 / \mathrm{FiO} 2 \text { (mean) } \\
\mathrm{HFNC}\end{array}$} & 145 & 226 & & 0.026 \\
\hline & $35(71 \%)$ & $2(33 \%)$ & $50(64 \%)$ & \\
\hline IV & $21(27 \%)$ & $4(26 \%)$ & $25(27 \%)$ & 0.002 \\
\hline \multirow{2}{*}{$\begin{array}{l}\text { Facemask } \\
\text { SOFA score (mean) }\end{array}$} & $17(22 \%)$ & $2(13 \%)$ & $19(20 \%)$ & \\
\hline & 7.7 & 6 & & 0.39 \\
\hline \multicolumn{5}{|l|}{ Echocardiogram } \\
\hline $\mathrm{E} / \mathrm{A}$ & 1.1 & 1.8 & & 0.001 \\
\hline DT & 252 & 151 & & $<0.0001$ \\
\hline $\mathrm{E} / \mathrm{E}$ & 9.7 & 19 & & $<0.0001$ \\
\hline LVEF & 66 & 55 & & 0.01 \\
\hline $\mathrm{IVC}<2 \mathrm{~cm}$ & $62(80)$ & 0 & & $<0.0001$ \\
\hline IVC $>2 \mathrm{~cm}$ & $15(19.4)$ & 100 & & $<0.0001$ \\
\hline \multicolumn{5}{|l|}{ US chest finding } \\
\hline Aeration score & 27 & 21 & & 0.018 \\
\hline subpleural consolidati & 13 & 1 & & $<0.0001$ \\
\hline
\end{tabular}

Conclusion: POCUS plays an important role in bedside diagnosis, hemodynamic assessment and management of patients with acute hypoxic respiratory and circulatory failure in patients with COVID-19 pneumonia.

\section{Reference(s) and grant ackowledgment(s)}

1. 3. Johri AM, Galen B, Kirkpatrick JN, Lanspa M, Mulvagh S, Thamman R. ASE Statement on Point-of-Care Ultrasound during the 2019 Novel Coronavirus Pandemic. J Am Soc Echocardiogr. 2020;33(6):670-673.

\section{3}

Diagnostic performance of transthoracic and transesophageal echocardiography to predict cardiac tamponade after cardiac surgery

AJ. De Bie ; B. Van Straten ${ }^{1}$; L. Van Kessel'; L. Otterspoor ${ }^{1}$

${ }^{1}$ Intensive care, Catharina Ziekenhuis, Eindhoven, Netherlands

Correspondence: L. Otterspoor

Intensive Care Medicine Experimental 2020, 8(2): 001393

Introduction: Cardiac tamponade is a severe complication after cardiac surgery. Echocardiography, both transthoracic (TTE) and transesophageal (TEE), may help to identify cardiac tamponade in postoperative period, but its diagnostic value is still questioned and has not been studied in large cohorts.

Methods: From January 2011 to March 2017, 8.974 adult patients underwent cardiac surgery in the Catharina hospital Eindhoven, a tertiary referral cardiothoracic center. A retrospective study was conducted in which patients with suspected cardiac tamponade were included. Sensitivity and specificity were calculated by comparing echocardiographic findings with surgical confirmation of tamponade. Patients in whom a re-exploration was not performed and recovered without intervention were considered not having tamponade.
Results: 511 patients (male $=65 \%$, median age 66 years) were included in whom 427 echocardiograms were performed. The following patient characteristics differed between patients who underwent a TTE or TEE: percentage of emergency surgery (13 vs $35 \%$ ), EuroSCORE (mean (SD) 9 (11) vs 18 (22)), and duration extracorporeal circulation (mean (SD) 115 (69) vs 171 (101) minutes). Cardiac tamponade was confirmed at re-exploration in 189 patients, while 278 patients were considered to be negative. In-hospital mortality of the re-intervention group was $14 \%$. The diagnostic value for TTE comprises a sensitivity of $64 \%$ ( $95 \% \mathrm{Cl} 53-75)$, a specificity of $96 \%$ (95\% Cl 92-98), a positive predictive value (PPV) of $81 \%(95 \% \mathrm{Cl} 69-89)$, and a negative predictive value (NPV) of $90 \%(95 \% \mathrm{Cl} 86-93)$. Regarding TEE, the diagnostic value a sensitivity of $50 \%(30-70)$, a specificity of $79 \%(59-91)$, a PPV of $68 \%(44-86)$, and a NPV of $63 \%$ (45-78). The area under the ROC curve for TTE was $0,80(p<0.001)$, and for TEE $0.64(p=0.072)$.

Conclusion: In patients within 4 weeks after cardiac surgery, TTE has a high specificity and negative predicting value to detect cardiac tamponade. These diagnostic values were lower for TEE, which might be caused by a selection bias based on severity of disease.

001491

Long-term pulmonary sequelae of Covid-19 in critically ill patients with severe interstitial pneumonia

S. Miori' ; S. Lassola'; FL. Gatti' ${ }^{2}$ A. Sanna'; AA. Colombo ${ }^{3}$; A. Cucino ; M. Zardin'; D. Penzo'; G. Pedrotti ${ }^{3}$; S. Magnoni'

${ }^{1}$ Anesthesia and intensive care, OSPEDALE S. CHIARA APSS, Trento, Italy; ${ }^{2}$ Radiology, OSPEDALE SANTA CHIARA APSS, Trento, Italy; ${ }^{3}$ Anesthesia and intensive care, OSPEDALE SANTA MARIA DEL CARMINE APSS, Rovereto, Italy

Correspondence: S. Magnoni

Intensive Care Medicine Experimental 2020, 8(2): 001491

Introduction: The long-term sequelae after Covid-19 severe disease are largely unknown. In particular, late radiological and clinical evolution have not investigated yet.

Objectives: The aims of this study is to describe morphological abnormalities of "late" lung CT scan in relationship to disease severity and clinical evolution in a small cohort of Covid-19 critically ill patients with severe interstitial pneumonia.

Methods: We retrospectively analyzed the data of 24 Sars-Cov- 2 positive patients with severe interstitial pneumonia admitted to the intensive Care Unit in a large regional hospital in Northern Italy, in March-April 2020, with a "late" lung CT scan performed for clinical reason not earlier than 2 weeks after hospital admission. CT scans were analyzed by two experienced radiologists and classified according to morphological abnormalities previously described in ARDS subjects based on the Fleischner Society Glossary, including 1) ground glass opacities 2) consolidation 3) fibrotic pattern with interlobular and intralobular thickening or cyst walls and 4) decreased attenuation, which includes emphysema and small airways disease. Six patients had two CT scan (i.e. admission and follow up CT scan) for longitudinal analysis. Clinal data were electronically recorded and retrospectively collected in an electronical data base.

Results: Patients were $22 \mathrm{M}$ and $2 \mathrm{~F}$, the median age was 66 ( $\min 39$, max 79). The median time from symptom onset to ICU admission was 5 days (range 4-8). 9 patients (37.5\%) underwent immediate intubation and mechanical ventilation (MV) and were directly admitted to ICU, whereas the majority $(62,5 \%)$ received prior non invasive ventilation (NIV). The median duration of MV was 24 days (range 18-34). One patient was treated with NIV and never MV. Patients were ventilated according to protective strategies with TV of 6-8 $\mathrm{ml} / \mathrm{kg}$, plateau < $20 \mathrm{mmHg}$. Hypoxemia was extremely severe as indicated by median $\mathrm{PaO} 2 / \mathrm{FiO} 2$ (PF) before intubation of 99 (range 81-143), which ameliorated by day 1 (134; range 101-178), to day 5 (146; range 137-180), day 10 (183; range 147-211) and day 15 (191; 147-251); Kruskal-Wallis test, $\mathrm{p}=0.015$. PEEP levels decreased significantly over time, from day 1 (mean PEEP $=12$, range 12-14), to day 5 (mean $P E E P=12$; range 10-14) day 10 (men $P E E P=10$, range 9-12) and day 15 (mean 9.5, range 7-10); Kruskal-Wallis test, $p<0.001$. Rescue therapies were frequently used: prone position in $16(66 \%)$ and NO in $3(12 \%) .7$ patients (25\%) died in 
ICU. The median length of ICU stay was 27 days (range 19-34). Surviving patients were discharged home $(n=13)$ or to a rehabilitation facility $(n=4)$. Length of hospital stay (LOS) was 51 days (range 34-68). CT scan were performed at a median time of 36 days (range 19-55) after hospital admission. Ground glass opacities $(n=20)$, classical morphological abnormalities of the early phase, and reticular pattern (22), a typical late abnormality, were both the most frequent pattern. Parenchymal consolidation $(n=19)$, bronchiectasis $(n=17)$ and emphysema $(n=13)$ were also frequent. Longitudinal analysis $(n=6)$ shows reticular pattern in all subjects, both in early and late scans (resembling early fibrosis in these latter). Notably, decrease attenuation pattern could only be observed in late scans, except in one patient with few bronchiectasis on admission.

Conclusion: In a selected cohort of patients with severe Covid-19 disease requiring prolonged ventilation and hospital stay, follow up CT scans show severe lung damage with predominant reticular pattern, resembling early fibrosis, emphysema and small airways disease (i.e. bronchiectasis). These abnormalities are likely the consequences of the disease and not related to previous lung pathology. Long-term follow up studies will be necessary to determine the clinical and morphological evolution of criticall pulmonary Covid-19 disease, to provide new preventive and therapeutic strategies.

\section{2}

A prospective observational trial study comparing diaphragmatic ultrasound for prediction of weaning failure in critically ill patients

J.P. Lasso-Perdomo ${ }^{1}$; A. Vargas-Villanueva ${ }^{1}$; C. Santacruz Herrera ${ }^{1}$

${ }^{1}$ Departament of critical and intensive care medicine, Fundacion Santa Fe de Bogota University Hospital, Bogotá, Colombia

Correspondence: A. Vargas-Villanueva

Intensive Care Medicine Experimental 2020, 8(2): 001492

Introduction: Weaning failure from mechanical ventilation is associated with an increased risk of morbidity and mortality. Different diaphragmatic ultrasound parameters have been studied. However, there are many gaps in our understanding of the discriminatory capacity to predict weaning failure.

Methods: A prospective study in a heterogeneous cohort of patients in intensive care units. The diaphragmatic ultrasound was used to determine the diaphragmatic inspiratory excursion (DE), time to peak inspiratory amplitude of the diaphragm (TPIAdia,), diaphragmatic excursion velocity (DE/TPIAdia, $\mathrm{cm} / \mathrm{sec}$ ), and diaphragm thickening fraction $(\Delta t \mathrm{tdi} \%)$. The measures were performed at the end of a spontaneous breathing trial (SBT) and compared with the extubation outcome. Also, the rapid shallow breathing index (RSBI) was calculated. Weaning failure was defined as the inability to maintain spontaneous breathing for at least $72 \mathrm{~h}$, without any form of ventilatory support.

Results: The study included 43 patients. Median age was 72 years [58.0-77.5], APACHE II score was 18.0 [9.0-23.0], and RSBI previous to the SBT was 37 [24.0-48.5]. Patients who failed the SBT (30\%) had a lower median vital capacity $(12.4[11.5-14.1(\mathrm{~mL} / \mathrm{Kg})]$ vs. $21.3[15.6-$ $23.6] ; \mathrm{p}=0.05), \mathrm{DE}(\mathrm{cm})(1.8[1.6-2.1]$ vs. $1.50[1.3-1.8] ; \mathrm{p}=0.03)$, and $\Delta$ tdi\% (29.0\% [21.0\%-35.0\%] vs. $39.0 \%$ [28.2\% - 47.5\%; $p=0.02)$. [E1] RSBI had the highest discriminatory capability (AUC-ROC 0.76). Among DUS measures, $\triangle$ tdi\% had the highest AUC-ROC $(0.71)$, followed by DE (0.70), and DE/TPIAdia (0.54). Using both, the DE/TPIAdia and RSCI the discriminatory capacity was improved and associated with a successful weaning trial (AUC-ROC 0.77). DE/TPIAdia $(\mathrm{cm} / \mathrm{sec})$ alone was not able to discriminate patients who failed the SBT (1.40 [1.10-1.80] vs. 1.55 [1.22- 1.80]; $p=0.69$ )

Conclusion: Combining the used of DE/TPIAdia and RSBI leads to a discriminatory capability improventfor detecting patients at high risk of extubation. This combined measure was similar to RSBI alone.
001577

Ultrasound of Accessory Respiratory Muscles in the Patients admitted to the Intensive Care Unit

L. Atlagic '; S. Kvolik'; T. Turk²; I. Harsanji Drenjancevic

${ }^{1}$ Anesthesiology and icu, Osijek University Hospital; Medical Faculty, Osijek, Croatia; ${ }^{2}$ Radiology, Osijek University Hospital; Medical Faculty, Osijek, Croatia

Correspondence: S. Kvolik

Intensive Care Medicine Experimental 2020, 8(2): 001577

Introduction: Patients with severe respiratory diseases usually use accessory respiratory muscles. In that specific patients sternocleidomastoid and trapezius muscle may be hypertrophic than in control intensive care unit (ICU) population. That specific muscles may have different ratio as compared to the quadriceps femoris muscle, which is usually an indicator of nutritional status and overall skeletal muscle status.

Objectives: The aim of this study is to compare the diameter of accessory respiratory muscles in patients with respiratory diseases and patients without such diseases and compare these muscles with the quadriceps femoris muscle in the patients with and without chronic respiratory diseases. We aimed to determine whether there is a correlation between the ultrasound-measured thickness of accessory respiratory muscles and patients age, hemoglobin, body mass index (BMI), duration of mechanical ventilation (MV) in the ICU, and patients' outcome in the ICU.

Methods: Ethics committee approval was obtained for this study and patients or their legal representatives gave written informed consent. A total of 50 subsequent patients admitted into the ICU were examined. Patients of both genders were included, with and without chronic respiratory diseases, including patients using mechanical ventilation. The study was performed using a Mindray TE7 ultrasound machine with the depth of the linear probe at $70-\mathrm{mm}$ and gain of $90-\mathrm{dB}$; settings remained unchanged during the study. The probe was set at the musculoskeletal ultrasound mode. Measurements of the right $\mathrm{m}$. sternocleidomastoideus (SCM), the right trapezius muscle, and the right quadriceps femoris muscle (vastus lateralis, vastus intermedius, vastus medialis, and rectus femoris; MQF) were taken at the admission into ICU, three times in each patient. The final value was the average of three measurements. Laboratory measurements, a duration of $\mathrm{MV}$, and survival were registered.

Results: Out of 50 patients examined, there were 15 patients with previously recognized chronic pulmonary diseases, and 48 patients were mechanically ventilated. Patients with chronic pulmonary diseases have a significantly thinner SCM than patients without chronic pulmonary diseases ( $79 \pm 13$ vs. $92 \pm 17 \mathrm{~mm}$; Student's t test $P=0.007)$, and it was it was thinner in relation to SCM than in patients without chronic lung disease(SCM/MQF $16,9 \pm 3.5 \%$ vs. $19.8 \pm 5.3 \% ; P=0.03$ ). No differences in other muscles was observed. A significant negative correlation was observed between patients age and MQF $(r=-0.359$, $\mathrm{P}=0.01), \mathrm{BMI}$ and MQF $(\mathrm{r}=0.338 ; \mathrm{P}=0.02)$ and MQF and patients' hemoglobin $(r=0.458, P=0.001)$. Treatment outcomes were not affected by the thickness of accessory respiratory muscles.

Conclusion: The thickness of the SCM was lower in the patients with chronic pulmonary diseases than in patients without such diseases. The importance of accessory respiratory muscle thickness for the respiratory function in the ICU may be better evaluated after subsequent measurements and whether elasticity of the muscles was evaluated.

\section{Reference(s) and grant ackowledgment(s)}

1. Wilhelm EN, et al. Age (Dordr) 2014;36:9625.

2. Nijholt, W. et al. Clinical Nutrition ESPEN, 30, 152-158,

3. VIF 2017-MEFOS-16 supported by Faculty of Medicine, J. J. Strossmayer University of Osijek, Croatia.
Reference(s) and grant ackowledgment(s)

1. No grant acknowledgment. 


\section{4}

Failure of focused echocardiography to differentiate pulmonary embolism in COVID-19 offers potential role for lung ultrasound

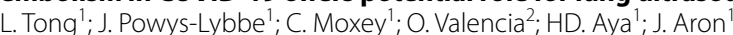
${ }^{1}$ General intensive care unit, St George's Hospital, London, United Kingdom; ${ }^{2}$ Cardiovascular sciences research centre, St George's, University of London, London, United Kingdom

Correspondence: $L$. Tong

Intensive Care Medicine Experimental 2020, 8(2): 001594

Introduction: A high incidence of pulmonary embolus (PE) has been reported in patients infected with Covid-19 [1]. When computed tomography (CT) is not available, transthoracic echocardiography (TTE) has a high specificity for the diagnosis of PE [2]. However, raised pulmonary pressures associated with Covid-19 can create similar echocardiographic changes to a PE [3]. Lung ultrasound (LUS) can identify PE associated wedge infarcts and may have a place in diagnosis when other investigations fail [4].

Objectives: To evaluate the role of focused echocardiography and lung ultrasound in the diagnosis of PE in patients with COVID-19 pneumonitis.

Methods: We performed a prospective observational study of Covid19 patients admitted into intensive care during a 3-week period. Patients received focused echocardiography (looking at RV dilatation, TRVMax, TAPSE and RVS') and 6-point lung ultrasound (giving each region a score of 0 to 3 [5]). On day 1 and 5 of admission, cumulative lung score was also calculated using ROC curve analysis for the best cut-off threshold for $\mathrm{PE}$. Individual lung regions were reviewed to determine if a score 3 (wedge infarct) was associated with PE. Covid-19 was confirmed by viral PCR swabs. Fisher exact test or and odds ratio (OR) were used to determine association between variables. $P$ values less than 0.05 is considered statistically significant.

Results: 49 Covid-19 positive patients were admitted to our intensive care units during the study period. 16/49 (37.2\%) patients had confirmed PE. We did not find any statistical difference in focused echocardiography between the two groups. RV dilatation was seen in 15/38 (60.5\%) patients with PE and absent in 9/11 (81.8\%) non-PE patients $(p=.19)$. TAPSE of $26 \mathrm{~mm}$ or more was seen in 5/20 (25\%) PE patients and $<26 \mathrm{~mm}$ in $15 / 26(57.7 \%)$ non-PE patients $(p=.22)$. RVS' $>16 \mathrm{~mm}$ was seen in 10/30 (33.3\%) patients with PE and absent in 8/14 (57.1\%) patients without PE $(p=.54)$. TRV Max of $30 \mathrm{mmHg}$ or more was seen in $12 / 28(42.9 \%)$ patients with a PE and $<30 \mathrm{mmHg}$ in $10 / 13(76.9 \%)$ patients without PE $(p=.22)$.

43/49 had complete 6-point lung ultrasound scores. At day 1 of admission, average cumulative lung scores did not differ significantly between patients with or without PE (9.3 (SD 2.8) vs 8.7 (SD 3.4); $p=.6$ ). At day 5 of admission, a cumulative lung score of $\geq 7$ was shown to be significantly associated with presence of PE ( $p=.03$, OR $5.83,95 \% \mathrm{Cl}$ 1.06-32.02). Individually, left upper lobe score of $3(p=.012)$ and left lateral and score of $3(p=.038)$ were significantly associated with presence of PE.

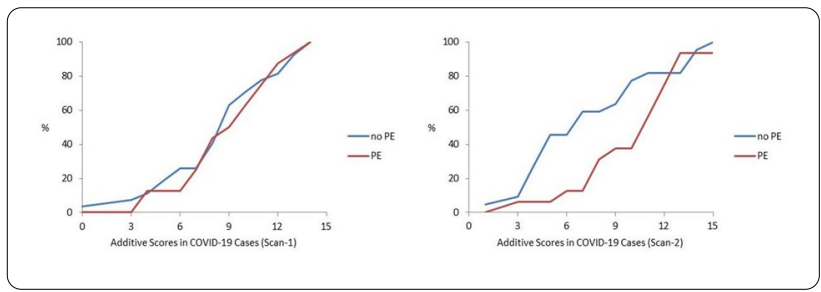

Conclusion: Focused echocardiography is not useful in the diagnosis of PE in COVID-19. Under these circumstances lung ultrasound has diagnostic value.

\section{Reference(s) and grant ackowledgment(s)}

1. 1. Bompard F, Monnier H, Saab I, Tordjman M, Abdoul H, Fournier L, Sanchez

O, Lorut C, Chassagnon MR. Pulmonary embolism in patients with Covid-19 pneumonia. Eur Respir J 2020. https://doi.org/10.1183/13993003.01365 $-2020$
2. 2. Fields MJ, Davis J, Girson L, Au A, Potts J, Morgan CJ, Vetter I, Riesenberg LA. Transthoracic echocardiography for diagnosing pulmonary embolism: A Systematic Review and Meta-Analysis. J Am Soc Echocardiogr 2017; 30(7): 714-723.

3. 3. Szekely Y, Lichter Y, Taieb P, Banai A, Hochstadt, Merdler I, Oz AG, Rothschild E, Baruch G, Peri Y, Arbel Y, Topilsky Y. Spectrum of Cardiac Manifestations in COVID-19: A systematic echocardiographic study. Circulation 2020; 142: 342-353.https://doi.org/10.1161/circulationaha.120.047971.

4. 4. Squizzato A, Rancan E, Dentali F, Bonzini M, Guasti L, Steidl L, Mathis G, Ageno W. Diagnostic accuracy of lung ultrasound for pulmonary embolism: a systematic review and meta-analysis. J Thromb Haemost 2013; 11: 1269-1278.

5. 5. Focused Ultrasound in Intensive Care (FUSIC): FUSIC COVID info [internet]. London: Intensive Care Society Available from: https://www.ics.ac.uk/ICS/ Pdfs/FUSIC_DOCS/FUSIC_COVID19_info.aspx.

6. No grants were received.

\section{9}

Diagnostic value of quantitative computed tomography

\section{in patients with COVID-19 pneumonia}

S. Tsarenko ${ }^{1}$; L. Davydova ${ }^{2}$; D. Dolotova ${ }^{3} ;$ Y. Andreev ${ }^{4}$; A. Andreev ${ }^{1} ;$ E.

Filimonova'; M. Lysenko'; A. Gavrilov ${ }^{3}$

${ }^{1}$ Intensive care, Moscow City Clinical Hospital № 52, Moscow, Russia; ${ }^{2}$ Intensive care, Moscow city clinical hospital №52, Moscow, Russia; ${ }^{3}$ Skobeltsyn institute of nuclear physics, Moscow State University, Moscow, Russia; ${ }^{4}$ Faculty of fundamental medicine, Moscow State University, Moscow, Russia

Correspondence: A. Andreev

Intensive Care Medicine Experimental 2020, 8(2): 001599

Introduction: In March, 2020 - June 2020 Central and East Europe had a period of the response to COVID-19 pandemic. Disease severity of pneumonia on admission can be assessed by various scales, including PORT, PSI, CURB-65, CRB-65 and SMRT-CO. Some of them include laboratory findings but none of them include the amount of lung lesion on CT, which is important to assess COVD-19 pneumonia. The most frequent types of lesions on CT for COVID-19 pneumonia are ground-glass opacity (GGO) and consolidation. Lesion volumes can be determined by quantitative CT. Therefore, it is important to analyze the relationship between quantitative CT parameters and clinical and laboratory findings.

Objectives: To evaluate the sensitivity of COVID-Multivox program in estimation of severity of lung injury in COVID-19 patients and to assess the correlation between quantity of involved lung parenchyma, types of CT-findings and laboratory findings.

Methods: 76 patients with COVID-19 were retrospectively reviewed from March 15, 2020 to March 31, 2020. Laboratory data and CT findings on admission were analyzed. CT quantitative parameters, including GGO volume, consolidation volume, total lesion volume, percentage of GGO, and consolidation) were calculated. Relationships between the CT findings and laboratory data were statistically estimated (Spearman rank correlation coefficient was used).

Results: There were $56.6 \%$ men of the 76 people examined. The most common laboratory markers of COVID-19 were an increase in the level of C-reactive protein, observed in 72 (94.7\%) patients, an increase in LDH, noted in $62(81.6 \%)$ patients, and lymphopenia, which was noted in 40 patients (52.6\%). CT-findings revealed total lesion volume $11,2 \%$ $54,2 \%$ (mean 29,4\%), with a GGO volume $9,4 \%-49,2 \%$ (mean $28,5 \%$ ) and a consolidation volume $0,3-1,8 \%$ (mean $0,7 \%$ ).

A correlation was revealed between $\mathrm{LDH}$ and the total volume of the affected lung tissue, the total percentage of lesions by the type of ground glass, the percentage of consolidation ( $r s=0.610, r s=0.628$, $r s=0.316 ; p<0.01$, respectively), slightly less correlation of the level of C-reactive protein with similar volumes ( $\mathrm{rs}=0.497, \mathrm{rs}=0.468$, $\mathrm{rs}=$ $0.410 ; p<0.01$ ), as well as a inverse correlation between the total volume of the lesion and the level of lymphocytes ( $r s=-0.251, p<0.05$ ). Conclusion: There is a strong correlation of the percentage of lung tissue lesion, detected by quantitative computed tomography, with 
the level of LDH and C-reactive protein. Quantitative lungs CT may be used not only to estimate the degree of the lung damage, but also to assess the severity of the systemic inflammatory response for patients with COVID-19.

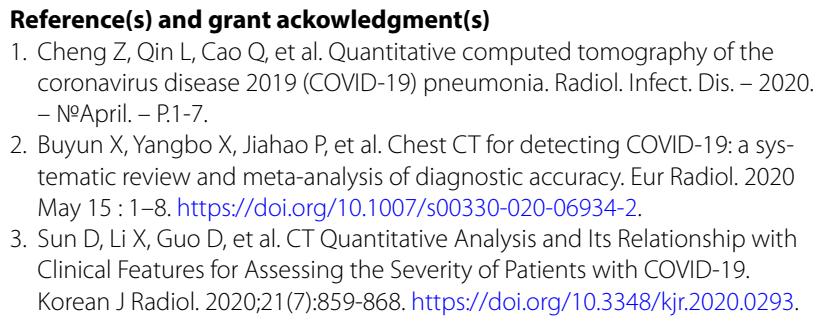

\section{5}

The current state of critical care ultrasound training in Ukraine

\section{Grynovska'; A. Wong ${ }^{2}$}

${ }^{1}$ Anesthesia and intensive care, Ivano-Frankivsk National Medical University and Regional University Teaching Hospital, Ivano-Frankivsk, Ukraine; ${ }^{2}$ Critical care, Royal Surrey County Hospital, Guildford, United Kingdom

Correspondence: M. Grynovska

Intensive Care Medicine Experimental 2020, 8(2): 001635

Introduction Ultrasound (US) is increasingly recognized as an essential tool in the ICU setting. The safety and utility of bedside ultrasonography by an appropriately trained ICU physician has now been well demonstrated ${ }^{1}$. Despite the worldwide routine use of ultrasound in ICUs, a large number of ICU physicians remain unacquainted with US techniques. Following the 2011 international expert statement, the training of every ICU physician should incorporate basic level of critical care ultrasonography (CCUS) ${ }^{2}$. We decided to continue investigating the evolving CCUS paradigm of ICU physician skills and competencies in our country.

Objectives To summarize knowledge on the current use of CCUS and attitudes towards its utilization in the ICUs of two city hospitals.

Methods Our study focused on the ICU physicians $(n=113)$ of two city hospitals. Data collection was performed with the help of an electronic survey.

Results The response rate of the survey was $78 \% .64 \%$ of the respondents were young specialists. The majority (56\%) had no previous experience in CCUS and only $45.5 \%$ were aware of the locally organized training programs. The most useful CCUS modalities reported were TTE and TEE, pleural/ lung US, vascular US for access, nerve block, FAST and vascular US-DVT. All of the respondents recognized the existence of barriers to CCUS training, citing lack of formal training and financial costs as the most significant ones. Although In Ukraine CCUS still remains the prerogative of the on-call radiologists, it was not perceived as a training barrier.

Conclusions While Ukraine is lacking a formal CCUS training program, there is an unmet demand for it in the ICU. Our survey has demonstrated a prevailing lack of US skills among the young ICU physicians. At the same time there was a general consensus on list of core US modalities that should be incorporated in such training as well as on the barriers that prevent ICU physicians from mastering them. The young demographics of the study has also highlighted the potential for international training programs and initiatives. Due to the limited number of respondents in our survey, further study across hospitals is required to fill in the knowledge gap on the country-specific state of CCUS training. We expect our ongoing study to be the call for action among the ICU physicians to stimulate the activity of the locally organized training programs. In future we hope to start a cross-country conversation on the challenges of CCUS standards implementation and the possible ways forward.
001637

The value of radiological features as a screening tool in Covid-19 infection

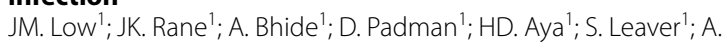

Rhodes ; M. Hamilton ${ }^{1}$

${ }^{1}$ General intensive care unit, St George's Hospital, London, United Kingdom

Correspondence: J.M. Low

Intensive Care Medicine Experimental 2020, 8(2): 001637

Introduction: SARS-CoV-2 has spread rapidly from first recognition in December 2019. Radiological findings on chest radiograph (CXR) and computerised tomography (CT) are often non-specific, most commonly consistent with atypical pneumonia, with predominant bilateral, peripheral and basal involvement [1,2]. Current recommendations advise that $\mathrm{CT}$ imaging should be reserved for the evaluation of complications and should not be employed as a diagnostic tool for Covid-19 [3,4,]. As an alternative, CXRs are often the first-line imaging modality employed, and there is paucity in the literature regarding how this correlates with definitive reverse transcriptase polymerase chain reaction (RT-PCR) testing.

Objectives: To describe radiological findings on CXR and CT imaging in patients with acute respiratory failure caused by SARS-Cov-2 infection and to investigate whether classical radiological features of SARSCov-2 infection on CXR are reliable as a diagnostic tool.

Methods: This was a single centre retrospective observational study. Patients admitted to Intensive Care Units (ICUs) in a tertiary teaching hospital from March to May 2020 with a CXR and a RT-PCR test were included. Baseline and serial CXRs and, where available, CT scans were reviewed and findings categorised under pre-specified headings. Reports of radiological features as classic Covid-19 were noted, according to the British Society of Thoracic Imaging (BSTI) grading templates[5]. These CXRs were deemed to fulfil the criteria of lower lobe and peripheral predominant multiple opacities that are bilateral, and given the BSTI code CVCX1. A chi-square statistic was used for the univariate analysis, and estimation of predictive values. A p-value less than 0.05 was considered statistically significant.

Results: 134 patients were included in the study. 109 (79\%) had a positive PCR for Covid-19. On review of the CXRs[sL1] of the Covid19 positive cohort, $42.4 \%$ (45) of patients had alveolar consolidation (26.6\% lobar, $73.3 \%$ bilateral), and $89.6 \%$ (95) had hazy increased opacity ( $90.5 \%$ bilateral, $9.5 \%$ Lobar) which was associated with a positive PCR $(p=0.02)$. Of the 44 patients who had a CT scan, 35 had a positive PCR. Within these 35 CT reports, 10 had ground-glass opacity (2 central, 8 peripheral), 4 had enlarged lymph nodes, 3 had pulmonary embolisms or pleural effusions, 2 had architectural distortion and there was 1 example each of air bronchogram, vascular thickening and fibrous stripes (figure 1). Classic radiological features of Covid-19 were associated with a positive PCR (c2 (1) 7.9, $p=0.005$ ), with a sensitivity of $65.1 \%$, specificity of $64.3 \%$, positive predictive value of $87.3 \%$ and negative predictive value of $32.7 \%$.

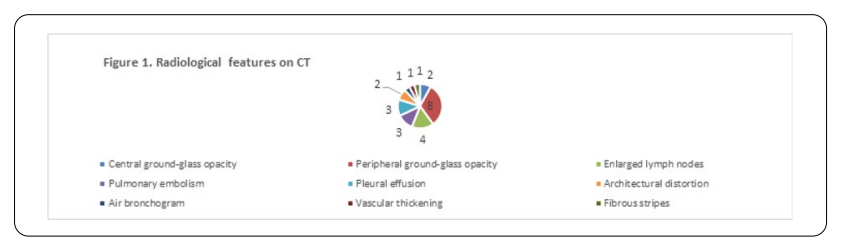

Conclusion: Covid-19 infection typically presents with bilateral hazy increased opacity on CXR, and less frequently bilateral alveolar consolidation. Classic radiological features of Covid-19 infection can be useful as a screening tool. 
Reference(s) and grant ackowledgment(s)

1. 5. Baseline CXR Proforma. Available at: https://www.bsti.org.uk/covid-19resources/covid-19-bsti-reporting-templates/ [accessed 25th June 2020].

2. 4. RCR position on the role of CT in patients suspected with COVID-19 infection. The Royal College of Radiologists, 12 March 2020. Available at: https:// www.rcr.ac.uk/college/coronavirus-covid-19-what-rcr-doing/clinical-infor mation/role-ct-chest/role-ct-patients [accessed 25th June 2020].

3. 3. ACR Recommendations for the Use of Chest Radiography and Computed Tomography (CT) for Suspected COVID-19 Infection. American College of Radiology, 11 March 2020. Available at: https://www.acr.org/Advocacy-andEconomics/ACR-Position-Statements/Recommendations-for-Chest-Radio graphy-and-CT-for-Suspected-COVID19Infection [accessed 25th June 2020].

4. 2. Kanne J, Little B, Chung J, Elicker B, Ketai L. Essentials for Radiologists on COVID-19: An Update-Radiology Scientific Expert Panel. (2020) Radiology. https://doi.org/10.1148/radiol.2020200527.

5. 1. Kooraki S, Hosseiny M, Myers L, Gholamrezanezhad A. Coronavirus (COVID-19) Outbreak: What the Department of Radiology Should Know. (2020) Journal of the American College of Radiology : JACR. https://doi. org/10.1016/j.jacr.2020.02.008

\section{0}

Innovative collaborative project for training in Point of care Ultrasound in Intensive care medicine and acute specialities

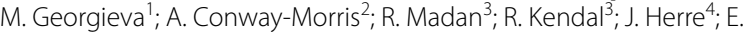
Marini ${ }^{5} ;$ K. Patel $^{6}$

${ }^{1}$ Intensive Care, Addenbrooke's Hospital, Cambridge, United Kingdom; ${ }^{2}$ Department of anaesthetics \& intensive care medicine, Cambridge University Hospitals NHS Foundation Trust, Cambridge, United Kingdom ${ }^{3}$ Emergency department, Addenbrooke's Hospital, Cambridge, United Kingdom; ${ }^{4}$ Respiratory medicine, Addenbrooke's Hospital, Cambridge, United Kingdom; ${ }^{5}$ Anaesthetics, Addenbrooke's Hospital, Cambridge, United Kingdom; ${ }^{6}$ Acute medicine, Addenbrooke's Hospital, Cambridge, United Kingdom

Correspondence: M. Georgieva

Intensive Care Medicine Experimental 2020, 8(2): 001640

Introduction: Point of care ultrasound (POCUS) is an important diagnostic and monitoring tool in ICU. The ICU at Addenbrooke's Hospital in Cambridge, UK is running a successful educational POCUS program for the last 6 years. Focused ultrasound is widely used also by the emergency medicine and acute medicine physicians in our institution with a separate certification body and educational program.

Objectives: In 2019 an innovative collaborative project for training in POCUS was established by the ICU, emergency medicine and acute medicine focused ultrasound leads. The aim of the project was to provide equal quality of training and governance of the POCUS amongst the acute specialities.

Methods: A two day course Point of care ultrasound for acute specialities (POCAS) was launched covering the requirements for certification of the three acute specialities. The certification bodies were approached for course approval and CPD points. The course was aimed for acute specialty physicians ST3 to consultant level. The administration of the course and the venue were provided by the Postgraduate Medical Centre. The course consisted of lectures and practical sessions covering focused lung, heart, abdominal, and vascular ultrasound. The faculty was represented by the POCUS mentors in the acute specialities and consultant cardiologist, respiratory physician and radiologist experts in the ultrasound field. The learners were offered POCUS educational mentorship program run by the same faculty at Addenbrooke's Hospital.
Results: Two courses were run in 2019- April and December edition. The April edition was attended by 23 learners and the December one by 24 . The spring edition had more equal attendance spread amongst the three specialities while the winter one had minimal attendance from the emergency medicine. It can be explained by deanery required parallel course running for this speciality just before our course. Most of the attendees were doctors in training. The consultant attendance was 2 for the first edition and 3 for the second one. The courses received detailed feedback evaluating the course as a valid method to acquire theoretical knowledge and basic practical skills in POCUS. Interestingly the lectures run by the acute specialities ultrasound leads were valued more than the ones delivered by the experts appraising the simplicity of explanation and focused approach. The acute medicine attendees rated the vascular access practical session very high which can represent less experience in their field in placement of lines under US guidance. The most valued session was the one with video cases of ultrasound imaging of real patients, their interpretation and application to the clinical management of the patients. The mentorship educational program was established and we are training 12 learners of which 5 got already certified in POCUS.

Conclusion: Point of care ultrasound is a powerful tool in the diagnosis and management of the acutely unwell patients. We have established a training system in POCUS amongst the acute specialities that guarantee high quality of training offered to all the learners regardless of their primary speciality and adequate governance of the educational process.

\section{1}

FACTORS ASSOCIATED WITH MORTALITY IN PATIENTS ADMITTED AS SARS-CoV-2 PNEUMONIA

B. Muñoz Molina'; J. Lázaro Gonzalez; M. Triqueros Genao; S. Portillo Sánchez ; I. Magaña Bru ; I. Temprano García'; C. Alvargonzález Riera ; L.

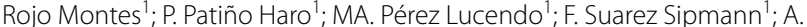
Canabal Berlanga ${ }^{1}$

${ }^{1}$ ICU, Hospital de La Princesa, Madrid, Spain

Correspondence: $\mathrm{B}$. Muñoz Molina

Intensive Care Medicine Experimental 2020, 8(2): 001221

Introduction: COVID related severe respiratory syndrome (SARS CoV-2) is associated to a high mortality. Several risk factors have been identified, such as hypertension, obesity, advanced age or male gender.

Objectives: We wanted to study the presence of such factors in our population, and assess if there existed a relation with the mortality in patients admitted in our Intensive Care Unit as pneumonia caused by SARS-CoV-2.

Methods: Retrospective, observational and analytical study, on patients diagnosed with SARS-CoV-2 pneumonia admitted to the ICU of a tertiary hospital, between March and June 2020.

We collected demographic, clinical and therapeutical variables including: age, gender, ethnicity, previous medical conditions and treatment, presenting symptoms, need of mechanical ventilation, treatment received, complications (neurological, musculoskeletal, cardiac, abdominal, renal, nosocomial infection, haemorrhagic thrombotic) and mortality.

We used $x 2$ test and Fisher's exact test, regression study, considering statistical significance when $\mathrm{p}<0.05$.

Results: A total of 101 patients were included in the analysis, with a global mortality of $26.7 \%$. 69 were men, with a mortality of $24.6 \%$ among them, and 32 were women, with a mortality of $31.2 \%$. 
Subgroup analysis identified female gender and Hispanic ethnicity as risk factor for increased mortality. 13 women were Hispanic (40.6\%), mortality within that subgroup $53.8 \%, p=0.049$.

In studied cohort most of the previously identified risk factors weren't associated to an increased mortality, not even obesity, in $36.6 \%$ of our patients, regression study: OR $-2.4, \mathrm{p}=0.017, \mathrm{Cl}[(-5.4)-(-0.5)]$.

Presenting symptoms were dyspnoea and hypoxemia in $82.2 \%$ and $83.2 \%$, respectively. Within the first 24 hours, 69 patients $(68.3 \%)$ needed invasive mechanical ventilation, and 29 (28.7\%) non-invasive, without relation with their mortality. Of those 29 patients, during admission 7 (24.1\%) required intubation.

Regarding treatment received, prolonged neuromuscular blockade (43 patients, $42.6 \%, p=0.03$ ) was the only therapeutical intervention related to mortality. A persistently positive PCR test on 30th day occurred in 7 patients $(6.9 \%)$ and was significantly related to mortality $(\mathrm{p}=0.01)$.

Regarding complications, only renal failure (23 patients (22.8\%) presented impaired renal function, with or without needing renal replacement therapy) had a significative relation with mortality $(p=0.04)$.

In the regression study, there was also a relation with mortality in use of kidney replacement therapy (4\% of cohort), $\mathrm{p}=0.03, \mathrm{OR} 2.17, \mathrm{Cl}$ [0.04-0.9], and prolonged neuromuscular blockade (42.6\%), $\mathrm{p}=0.02$, OR 2.29, Cl [0.06-0.8]).

Conclusion: In our cohort, Hispanic female patients were more prone to die. The extended use of neuromuscular blockade, possibly due to the presence of a more severe respiratory failure, and the persistence of Coronavirus detection on the 30th day of admission, indicating continued viremia, were related with mortality. Among complications, the one most associated with mortality and worse prognosis was renal failure.

\section{8}

Clinical Characteristics and Outcomes of 187 Critically III Patients With COVID-19

J. Azevedo

${ }^{1}$ Intensive care unit, Hospital São Domingos, São Luís, Brazil

Correspondence: J. Azevedo

Intensive Care Medicine Experimental 2020, 8(2): 001248

Introduction: Although Brazil is the second country in the world in number of cases and deaths by COVID-19 there are limited information describing clinical characteristics and outcomes of the patients requiring treatment in Brazilian intensive care units.

Objectives: To describe the clinical characteristics and outcomes of patients with COVID-19 requiring treatment in an intensive care unit in northeastern Brazil.

Methods: Retrospective observational study of 187 consecutive patients with confirmed diagnosis of COVID-19 admitted to a 35-bed ICU at a tertiary hospital in the state of Maranhão, Brazil, from March 27 to June 15, 2020. We collected demographic and clinical-laboratory data, therapeutic interventions including management of respiratory failure, complications and mortality.

Results: Of the 187 patients included in the study, the median age was 64 years (IIQ 51-75), $122(65,2 \%)$ were male and 163 patients $(87,1 \%)$ had at least one chronic disease, the most common being arterial hypertension (100 patients, 53,4\%) and diabetes melitus (86 patients, $45,9 \%$ ). Upon arrival to the ICU, patients had a median $\mathrm{PaO} 2 / \mathrm{FIO} 2$ of 195 (IIQ 136-300). Thirty-seven patients (19.8\%) were treated with non-invasive respiratory support while $150(80.2 \%)$ underwent invasive mechanical ventilation. Seventy-four patients (39.6\%) underwent hemodialysis and one patient was treated with ECMO. Seventy-nine patients $(42,2 \%)$ died during the ICU stay. Mortality among the 150 patients undergoing invasive mechanical ventilation (77 patients, $51.3 \%$ ) was significantly higher than that of the 37 patients undergoing non-invasive respiratory support $(5,4 \%), p<0.0001$. Among the 74 patients (39.6\%) who had a bacterial infectious complication, mortality (38 patients, 51.3\%) was significantly higher compared to the 110 patients who did not have a secondary infection (38 patients, $34.5 \%$ ); $\mathrm{p}=0.001$

Conclusion: This case series describes the clinical characteristics and outcomes of 187 critically ill patients with COVID-19 treated in a tertiary hospital in northeastern Brazil.

001255

Mechanisms of antibiotic resistance of microorganisms in patients with severe respiratory infections in intensive care unit V. Ziamko ${ }^{1}$

'Viciebsk State Order of Peoples' Friendship Medical University, Viciebsk, Belarus

Correspondence: $\vee$. Ziamko

Intensive Care Medicine Experimental 2020, 8(2): 001255

Introduction: It has been known about possibility of microorganisms to create specific multi-layered structures called biofilms. Non-cellular deoxyribonucleic acid actively participates in regulation of properties of biofilms. Thus, in biofilms transfer of genetic information including genes responsible for sensitivity to antibacterial drugs occurs much more often than in single-living bacterial cells. However, despite the involvement of extracellular deoxyribonucleic acid in adhesive processes and intercellular interactions, its role has not been fully understood.

Methods: 238 isolates isolated from sputum and pharynx of 175 patients during 2016-2019 were studied. Patients were divided into two groups: the 1st group of 139 people $(79,4 \%)$ had severe respiratory infections, the 2 nd of 36 people $(20,6 \%)$ - respiratory infections of moderate severity.

Results: A method was developed for determining percentage of deoxyribonucleic acid in microbial community using 4'6-diamidino2-phenylindole dihydrochloride. Average age of the 1st group was higher than the second $(p<0,05)$. Pseudomonas aeruginosa had the largest mass of biofilm and percentage of deoxyribonucleic acid in group $1,48,25[30,5-70,1] \mathrm{mcg} / \mathrm{ml}$ and $5,21[2,17-7,67] \%, \mathrm{p}=0,04$. A strong relationship was found between percentage of deoxyribonucleic acid in Pseudomonas aeruginosa and severity of disease, $r=$ $0,73, p<0,05$. The incidence of adverse outcomes in isolating antibiotic resistant isolates was higher than in antibiotic sensitive $(p<0,05)$. Analysis of results made it possible to propose fatal outcome when mass of microbial biofilm is $>47,5 \mathrm{mcg} /$ well and percentage of deoxyribonucleic acid is $>2,33 \%(p<0,01)$.

Conclusion: Method for determining percentage of deoxyribonucleic acid in biofilm has been proposed. With age there is a decrease in immune system which contributes to adherence of more pathogenic, antibiotic resistant microflora which has high biofilm weight and deoxyribonucleic acid percentage leading to disease progression and death.

\section{Reference(s) and grant ackowledgment(s)}

1. Surveillance of antimicribial resistance in Europe [Electronic resource] : annu. rep. of the Europ. Antimicrobial Resistance Network (EARS-Net), 2016 // European Centre for Disease Prevention and Control : an agency of the Europ. Union. - Mode of access: http://ecdc.europa.eu/sites/porta 
I/files/documents/AMR-surveillance-Europe-2016.pdf - Date of access: 27.01.2020

\section{6}

\section{Lysozyme activity in serum of patients with severe bacterial} pneumonia

V. Ziamko

${ }^{1}$ Viciebsk State Order of Peoples' Friendship Medical University, Viciebsk, Belarus

Correspondence: $\vee$. Ziamko

Intensive Care Medicine Experimental 2020, 8(2): 001266

Introduction: In modern concepts of pathogenesis of inflammatory diseases, much attention is paid to nonspecific humoral factors of immune system, including lysozyme.

Objectives: To create a simpler and a cheaper way of evaluating lysozyme activity in biological fluids and analyse its level in patients with severe bacterial pneumonia.

Methods: A complex examination of 56 patients with severe bacterial pneumonia and 50 practically healthy people was performed. Blood was taken from the ulnar vein before meals into sterile test tubes.

Results: We have created a simpler and a cheaper way of evaluating lysozyme activity in biological fluids, which demands little time and has clear evaluation criteria. The invention relates to medicine, namely to laboratory diagnosis. From cell walls of Micrococcus lysodeikticus culture ATCC 4698 peptidoglycan substrate was prepared, and the activity of lysozyme was calculated by the formula obtained after constructing calibration curve for lysozyme, which showed the dependence of the concentration of lysozyme and the optical density of the solution of Congo red. The positive effect of the proposed method was that the substrate for the production of reaction was prepared once and could be used for a long time. The way was easy and simple in play. The sensitivity of this method to lysozyme was $0.06 \mathrm{mg} / \mathrm{ml}$. This is enough to determine immunodeficiency states, because the content of lysozyme in biological fluids varies according to various data in a wide range from 0.2 to $28 \mathrm{mg} / \mathrm{ml}$ in normal.

It was found that the lysozyme activity in the serum was lower in patients with severe bacterial pneumonia (116.0; $56.5-160.1 \mathrm{mcg} /$ $\mathrm{ml})$ in comparison with practically healthy people $(246,0 ; 183,6-305,7$ $\mathrm{mcg} / \mathrm{ml})$.

Conclusion: There was a statistically significant decrease of nonspecific humoral resistance in patients with severe bacterial pneumonia.

\section{Reference(s) and grant ackowledgment(s)}

1. Antimicrobial activity of lysozyme isoforms: key molecular features/M.

Derde [et al.] // Biopolymers. - 2017. - Vol. 107, № 12. - P. 1-11.

\section{4}

\section{Antimicrobial resistance in the critically ill: new perspectives} for the future?

S. Branco Ribeiro ; A. Krystopchuk' ; S. Castro ${ }^{2}$; J. Moreno ${ }^{3}$; D. Nuñez ${ }^{4}$

${ }^{1}$ Emergency and intensive care medicine department, Algarve University Hospital Centre, Faro, Portugal; ${ }^{2}$ Serviço de medicina intensiva 1, Centro Hospitalar Universitário do Algarve, Faro, Portugal; ${ }^{3}$ Intensive care departement, Centro Hospitalar e Universitário do Algarve, Faro, Portugal, Portugal; ${ }^{4}$ Intensive care unit, Centro Hospitalar Universitário do Algarve - Faro, Faro, Portugal

\section{Correspondence: S. Branco Ribeiro}

Intensive Care Medicine Experimental 2020, 8(2): 001294
Introduction: The ongoing emergence of antimicrobial resistance is considered a major threat for public health. Mainly due to its extremely vulnerable population of critically ill patients, the unique nature of the intensive care unit (ICU) environment makes this a focus for the emergence and spread of many antimicrobial resistant pathogens. However, and despite the total burden of multi-drug resistant (MDR) bacteria in the broader community remains a blind spot, it now seems that a growing number of patients are already colonised or infected with MDR microorganisms on arrival in the ICU.

Objectives: To investigate the relationship between MDR bacterial infections, patient provenience and mean hospital stay before ICU admission.

Methods: This was a retrospective study performed in a mixed medical and surgical ICU at Faro Hospital, Portugal, between January and December 2019. During the study period, 464 patients were admitted to the ICU. Data from a total of 104 patients were collected after applying exclusion criteria. Patient demographics, clinical details and microbiological data were recorded and analysed.

Results: One hundred and four patients were included in this study. They were predominantly male $(n=67,64.4 \%)$, with a mean age of 61.4 years, a mean APACHE II of 21, and mainly admitted due to urgent surgical causes $(n=50,48.1 \%)$. Overall, 171 organisms were isolated from this patients. The most frequent infection sites were urinary $(26.7 \%)$, respiratory $(25.3 \%)$, bloodstream $(18.8 \%)$ and intraabdominal (16.3\%). Most bacterial isolates were Gram-negative (59.1\%). Escherichia coli was the main genus identified, of which $27.3 \%$ were MDR. Staphylococcus aureus represented $40.4 \%$ of all Gram-positive isolates, with $33.3 \%$ methicillin-resistance. Prevalence of MDR bacteria was $24.2 \%$, of which the most frequent were Escherichia coli (16.2\%), Staphylococcus aureus (13.5\%), Pseudomonas aeruginosa (13.5\%), Enterobacter aerogenes (13.5\%) and Klebsiella pneumoniae (10.8\%). Interestingly, MDR bacteria were isolated in patients coming predominantly from the Emergency Department (ED) $(n=62,59.6 \%)$, with a mean hospital stay before ICU admission of 3,3 days. Infections by MDR bacteria were also associated with an increased hospital length of stay.

Conclusion: In this study we noted that critically ill patients infected with MDR bacteria were admitted predominantly from the ED, with a mean hospital stay before ICU admission of 3,3 days. These findings suggest they could be already colonised or infected with MDR pathogens on arrival to the ICU, and make us think about having to reconsider the usual empiric coverage strategies used in several patients. Future studies are required to prove this changing pattern of antimicrobial resistance and its growing tendency of occurrence in the community. Additional efforts to increase the effectiveness of infection control programs, knowledge of local epidemiologies of resistance and antimicrobial stewardship strategies are essential to make progress in this problematic matter.

Reference(s) and grant ackowledgment(s)

1. 5. MacVane SH. Antimicrobial Resistance in the Intensive Care Unit: A Focus on Gram-Negative Bacterial Infections, J Intensive Care Med. 2017 Jan;32(1):25-37. https://doi.org/10.1177/0885066615619895.

2. 4. Campion M, Scully G. Antibiotic Use in the Intensive Care Unit: Optimization and De-Escalation, J Intensive Care Med. 2018 Dec;33(12):647-655. https://doi.org/10.1177/0885066618762747.

3. 3. Montrucchio G, Sales G, Corcione S, De Rosa FG, Brazzi L. Choosing wisely: what is the actual role of antimicrobial stewardship in Intensive Care Units? Minerva Anestesiol. 2019 Jan;85(1):71-82. https://doi.org/10.23736/s0375 $-9393.18 .12662-9$

4. 2. Kollef MH, Bassetti M, Francois B et al. The intensive care medicine research agenda on multidrug-resistant bacteria, antibiotics, and stewardship, Intensive Care Med. 2017 Sep;43(9):1187-1197. https://doi. org/10.1007/s00134-017-4682-7 
5. 1. Brusselaers et al. The rising problem of antimicrobial resistance in the intensive care unit. Annals of Intensive Care. 2011, 1:47. https://doi. org/10.1186/2110-5820-1-47.

\section{8}

The age and comorbidities, are independent risk factors for mortality in critically ill covid-19 patients?

P. García Olivares ${ }^{1}$; J. Cedeño Mora ${ }^{1}$; A. Garrido ${ }^{1}$; J. Cui ${ }^{1}$; P. Oliver ${ }^{1}$; C. Mata ${ }^{1}$; G. Castañeda ${ }^{1}$; N. Cango ${ }^{1}$; C. Alvarez ${ }^{1}$; S. Arenal

${ }^{1}$ Intensive Care Unit, H.G.U Gregorio Marañón, Madrid, Spain

Correspondence: P. García Olivares

Intensive Care Medicine Experimental 2020, 8(2): 001298

Introduction: Since the start of the COVID-19 pandemic, age and chronic diseases have been described as important risk factors related to patient prognosis.

Objectives: To assess whether the age and comorbidity of COVID-19 critical patients are independent risk factors related to mortality.

Methods: Prospective, observational study carried out in patients admitted to HGU Gregorio Marañón critical care units during the COVID-19 pandemic. Demographic and clinical data, comorbidities, severity risk scores, complications during stay, administered treatments and outcome, were collected.

Descriptive analysis was expressed as mean (SD) or median (RIC) for quantitative variables and as percentages for qualitative variables. Univariate analysis was performed using Chi-square (RR) for qualitative variables and a simple logistic regression analysis (OR) for quantitative variables. Multiple logistic regression analysis, adjusted for severity, was carried out to determine if the age and comorbidity were independently factors related to mortality.

Results: We included 150 patients, $71 \%$ were male, mean age was $58 \pm 14$ years. Charlson comorbidity Index was $1(0-3), 53 \%$ presenting obesity and 53\% hypertension (comorbidities not included in that index). Severity risk scores: APACHE II 16 (12-20), SOFA 6 (4-8). 88\% of patients required mechanical ventilation and $74 \%$ prone-position. $63 \%$ of patient required vasoactive drugs, $30 \%$ developed renal failure and $10 \%$ required continuous renal replacement therapy. Mortality in ICU was $38 \%$, with the following age distribution: < 40 years $10 \%$, $41-50$ years $30 \%, 51-60$ years $32 \%, 61-70$ years $52 \%$ and $71-80$ years $48 \%$. The univariate analysis showed that age (range) OR $1.54 ; 95 \%$ $\mathrm{Cl} 1.17-2.02$, Charlson index $1.44 ; 95 \% \mathrm{Cl} 1.17-1.78$ and hypertension RR $2.07 ; 95 \% \mathrm{Cl} 1.05-4.05$, were related to an unfavourable prognosis, but not obesity (BMI >30) RR $1.17 ; 95 \% \mathrm{Cl} 0.60-2.25$. When the age was analysed by ranges, after the sixth decade the risk of death increased significantly: $61-70$ years RR $9.44 ; 95 \% \mathrm{Cl} 1.91-46.66$ and $71-80$ years RR 8.05; $95 \% \mathrm{Cl} 1.62-39.91$.

In the multivariate analysis, adjusted for severity (APACHE II, SOFA and mechanical ventilation), obesity and hypertension, both age OR 1.46; 95\% Cl 1.04-2.04 and Charlson Comorbidity Index OR 1.24; 95\% CI 1.02-1.56 remained as independent risk factors for mortality.

Conclusion: In our experience, both age and comorbidity, expressed as the Charlson index, were independent risk factors associated with mortality in critically ill COVID-19 patients.
001321

A Retrospective Analysis of Unit Acquired Infections in a West London Adult Intensive Care Unit During the COVID-19 Pandemic H. Wilson ${ }^{1}$; R. Snooks ${ }^{1}$; H. Said ${ }^{1}$; M. Popescu ${ }^{1}$

${ }^{1}$ Intensive Care Unit, West Middlesex University Hospital, Isleworth, United Kingdom

Correspondence: $\mathrm{H}$. Wilson

Intensive Care Medicine Experimental 2020, 8(2): 001321

Introduction: Unit acquired blood infections are commonly used as a quality indicator. Intensive care units report such data quarterly and this is collected by ICNARC(1).

West Middlesex University Hospital (WMUH) has a high turnover AICU, with 199 and 167 admissions for quarters 1 and 2 respectively in 2019 . From April to December 2019, WMUH had reported 0 unit acquired infections. Nationally, an increase in secondary bacterial infections in COVID ICU patients has been reported(2). Studies have reported 50\% of COVID patients who died had secondary bacterial infections(3).

Objectives: To compare the incidence of unit acquired infections during the COVID-19 pandemic with a typical pre-pandemic period.

Methods: We included 74 patients over a 55-day period. Patients were confirmed COVID positive from throat or tracheal aspirate swab PCR. Data was extracted from the ICE reporting system. Unit acquired infections were deemed to be positive blood cultures greater than 48 hours after admission.

Results: Thirteen patients had a unit acquired infection. There were 16 positive blood cultures in total, as some had more than one positive culture during admission. Cases are broken down by organism, see Figure 1. Average length of time from admission to positive blood culture was 14.4 days. The length of stay for all 74 COVID positive ICU patients was 12.6 days compared to 25.8 days for those who developed unit acquired infection. 32 out of 74 COVID patients died (43.2\%). 7 of the 13 patients with a positive blood culture died (53.8\%).

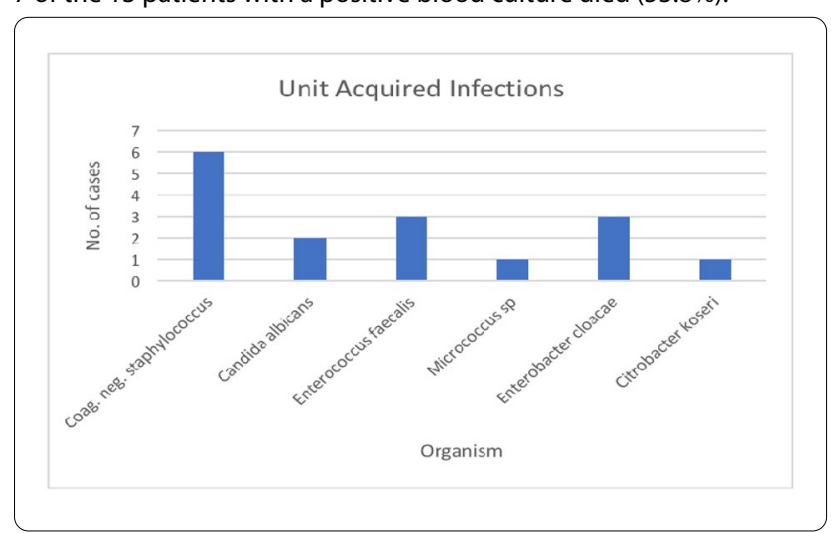

Conclusion: We have demonstrated an unexpected increase in unit acquired infections during the COVID pandemic. $17.5 \%$ of patients developed a unit acquired infection, compared to $0 \%$ over a similar time period in 2019.

This is almost certainly multifactorial, with the average length of stay and exposure to invasive procedures a significant risk factor in developing infection (1). During the height of the pandemic, WMUH AICU 
was expanded from an 11-bed unit with a maximum of 6 level 3 beds to 35 level 3 beds.

We can speculate that increasing the nursing ratio from standard 1:1 to 1:4.5 patients, as seen on an international level (3), along with multiple proned patients, made it challenging to maintain usual strict infection control measures.

In particular, proned patients with CVCs sited in the internal jugular vein may have been at a greater risk of contamination from organism containing oro-pharygeal secretions.

Additionally, lines were inserted in closed spaces whilst wearing heavy PPE, making sterility challenging. Once in situ lines could be contaminated when disconnecting during patient turning, as reported at other ICUs (2). These patients were also physiologically unstable, receiving multiple courses of antibiotics and iatrogenic immuno-suppression with steroids. It is therefore not unreasonable that this group of patients developed secondary infection.

\section{Reference(s) and grant ackowledgment(s)}

1. 1. Sturdy A, Basarab M, Cotter M, Hager K, Shakespeare D, Shah N, Randall P, Spray D, Arnold A. Severe COVID-19 and Healthcare Associated Infections on the ICU: Time to Remember the Basics? Journal of Hospital Infection. June 2020; 105(4): 593-595.

2. 2. Edgeworth J, Batra R, Wulff J, Harrison D. Reductions in Methicillinresistant Staphylococcus aureus, Clostridium difficile Infection and Intensive Care Unit-Acquired Bloodstream Infection Across the United Kingdom Following Implementation of a National Infection Control Campaign. Clinical Infectious Diseases. June 2020; 70(12): 2530-2540.

3. 3. Zhou F, Yu T, Du R, Fan G, Liu Y, Liu Z et al. Clinical course and risk factors for mortality of adult inpatients with COVID-19 in Wuhan, China: a retrospective cohort study. Lancet. March 2020; 395(10229): 1054-1062. 4. Not applicable.

\section{9}

Obesity is not associated with a different immune response and disease course in critically ill COVID-19 patients

E. Kooistra'; A. De Nooijer²; W. Claassen'; I. Grondman²; N. Janssen²; M. Netea $^{2}$; F. Van De Veerdonk'; H. Van Der Hoeven'; M. Kox' : P. Pickkers 'Intensive care, Radboud University Medical Center, Nijmegen, Netherlands; ${ }^{2}$ Internal medicine, Radboud University Medical Center, Nijmegen, Netherlands

Correspondence: $\mathrm{E}$. Kooistra

Intensive Care Medicine Experimental 2020, 8(2): 001339

Introduction: Obesity appears to be an independent risk factor for ICU admission and a severe disease course in COVID-19 patients (1). An aberrant inflammatory response and impaired respiratory function have been suggested as underlying mechanisms $(2,3)$. Today, it is not known whether obesity is only a risk factor for COVID-19 susceptibility and for the need of intensive care, or whether it also influences the outcome of the patients once admitted on intensive care units.

Objectives: We investigated whether obesity is associated with differences in inflammatory, respiratory, and clinical outcome parameters in critically ill COVID-19 patients.

Methods: Sixty-seven COVID-19 ICU patients were divided into obese (BMI $\geq 30 \mathrm{~kg} / \mathrm{m} 2, \mathrm{n}=18$ ) and non-obese (BMl $<30 \mathrm{~kg} / \mathrm{m} 2, \mathrm{n}=49$ ) groups. Plasma concentrations of interleukin (IL)-6, interferon gammainduced protein (IP)-10, monocyte chemoattractant protein (MCP)-1, and granulocyte colony-stimulating factor (G-CSF) were determined from ICU admission until 10 days afterwards, and routine laboratory and clinical parameters were collected.

Results: BMI was 32.6 [31.2-34.5] and 26.0 [24.4-27.7] kg/m2 in the obese and non-obese group, respectively. Apart from temperature, which was significantly lower in obese patients (38.1 [36.9-38.9] vs. $38.7[38.0-39.5]{ }^{\circ} \mathrm{C}, \mathrm{p}=0.02$ ), there were no between-group differences on ICU admission. Plasma cytokine concentrations declined over time ( $p<0.001$ for all), but no differences between obese and non-obese patients were observed. The kinetics of clinical inflammatory parameters and respiratory mechanics were also similar in both groups. Finally, no differences in time on ventilator (22 [16-40] vs. 27 [14-40] days, $p=0.34)$, ICU length of stay $(25[17-40]$ vs. 29 [15-40] days, $\mathrm{p}=0.49)$, or 40 -day mortality $(17 \%$ vs. $24 \%, \mathrm{p}=0.76)$ between obese and non-obese patients were apparent (Fig. 1).

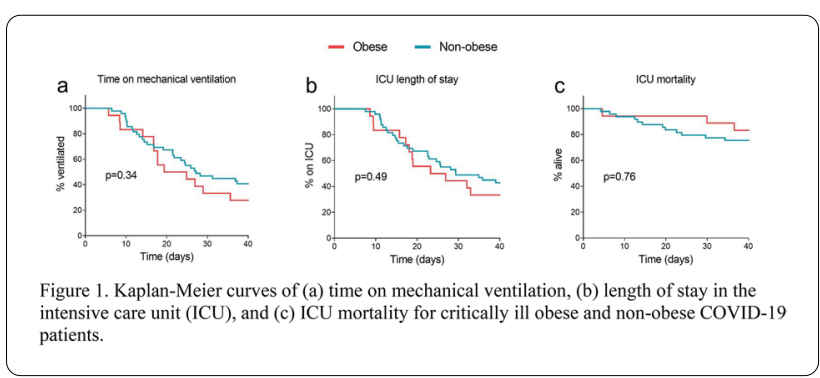

Conclusion: In COVID-19 patients requiring mechanical ventilation in the ICU, obesity is not related to a different immunological response, unfavourable respiratory mechanics, or impaired outcome.

Reference(s) and grant ackowledgment(s)

1. 3. Kruglikov IL, Scherer PE. The role of adipocytes and adipocyte-like cells in the severity of COVID-19 infections. Obesity (Silver Spring). 2020.

2. 2. Littleton SW, Tulaimat A. The effects of obesity on lung volumes and oxygenation. Respir Med. 2017;124:15-20.

3. 1. Simonnet A et al. High prevalence of obesity in severe acute respiratory syndrome coronavirus-2 (SARS-CoV-2) requiring invasive mechanical ventilation. Obesity (Silver Spring). 2020.

4. This study was funded by the participating departments.

\section{3}

\section{Anakinra treatment in critically ill COVID-19 patients}

E. Kooistra ${ }^{1}$; N. Waalders' ${ }^{1}$ I. Grondmann2; N. Janssen²; A. De Nooijer'² M. Netea ${ }^{2}$; F. Van De Veerdonk'; E. Ewalds ${ }^{3}$; H. Van Der Hoeven ${ }^{1}$; M. Kox; P. Pickkers $^{1}$

${ }^{1}$ Intensive Care, Radboud University Medical Center, Nijmegen, Netherlands; ${ }^{2}$ Internal medicine, Radboud University Medical Center, Nijmegen, Netherlands; ${ }^{3}$ Intensive care, Bernhoven, Uden, Netherlands

Correspondence: $\mathrm{E}$. Kooistra

Intensive Care Medicine Experimental 2020, 8(2): 001343

Introduction: The clinical spectrum of SARS-CoV-2 infection ranges from asymptomatic cases to severe pneumonia and viral sepsis leading to organ dysfunction and high mortality (1). A subset of critically ill COVID-19 patients develop a hyperinflammatory state. Anakinra, a recombinant interleukin-1 receptor antagonist, is known to be effective in several hyperinflammatory diseases. Recently, small studies suggest that anakinra improve respiratory function and clinical outcome in COVID-19 patients (2-4).

Objectives: We investigated the effects of anakinra on inflammatory parameters and clinical outcomes in critically ill, mechanically ventilated COVID-19 patients with clinical features of hyperinflammation.

Methods: In this prospective cohort study, 21 critically ill COVID19 patients treated with anakinra were compared to a group of 39 
critically ill COVID-19 patients receiving standard care. Serial data of clinical inflammatory parameters and concentrations of several circulating cytokines were aligned on start day of anakinra in the treatment group, and median start day of anakinra in the control group. Analysis were performed from day -10 to +10 relative to alignment day. Clinical outcomes were analyzed during 28 days. Additionally, a sensitivity analysis was performed in propensity score matched groups.

Results: Patient characteristics and clinical parameters on ICU admission were similar between groups. Due to bias by indication, plasma levels of ASAT $(p=0.0002)$, ferritin $(p=0.009)$, and temperature $(p=0.001)$ were significantly higher in the anakinra group on alignment day. Following treatment, no differences in the kinetics of circulating cytokines were observed between both groups. Decrease of temperature $(p=0.03)$, white blood cell counts $(p=0.02)$, and plasma levels of ferritin $(p=0.003)$, procalcitonin $(p=0.001)$, creatinine $(p=0.01)$, and bilirubin $(p=0.007)$ was more pronounced in the anakinra group (Fig. 1 a-f respectively). No differences in clinical outcomes were observed between groups. The propensity-matched analysis confirmed these results.

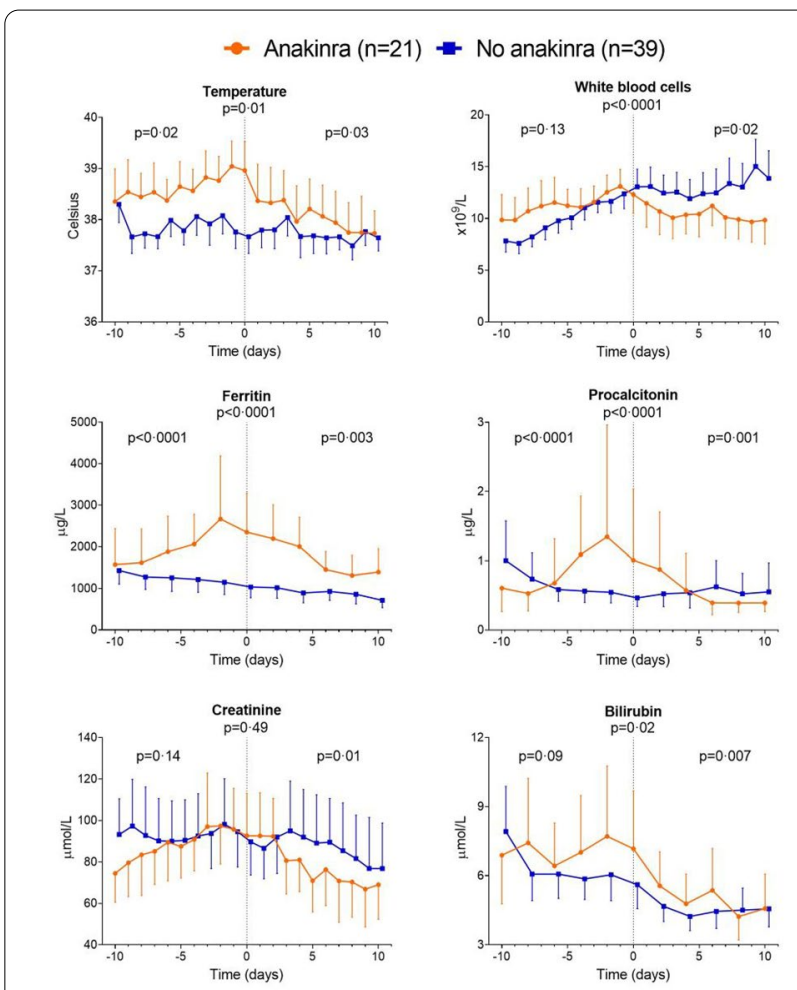

Figure 1. Differences in kinetics of laboratory inflammation parameters between anakinra and control group. Geometric means $+95 \%$ CI.

Conclusion: Anakinra is effective in treating clinical signs of hyperinflammation that may promote organ dysfunction in critically ill COVID19 patients.

\section{Reference(s) and grant ackowledgment(s)}

1. 4. Dimopoulos $\mathrm{G}$ et al. Favorable anakinra responses in severe COVID-19 patients with secondary hemophagocytic lymphohistocytosis. Cell Host Microbe. 2020.

2. 3. Huet et al. Anakinra for severe forms of COVID-19: a cohort study. The Lancet Rheumatology. 2020.
3. 2. Cavalli et al. Interleukin-1 blockade with high-dose anakinra in patients with COVID-19, acute respiratory distress syndrome, and hyperinflammation: a retrospective cohort study. Lancet Rheumatol. 2020;2(6):e325-e31.

4. 1. Zhou F et al. Clinical course and risk factors for mortality of adult inpatients with COVID-19 in Wuhan, China: a retrospective cohort study. Lancet. 2020;395(10229):1054-62.

5. Funded by the participating departments.

\section{9}

Comparison of procalcitonin levels according to pathogen type and gram staining in bloodstream infection in ICU

O. Kaynarca'; M. Uyar' ; C. Bor ${ }^{3}$; K. Demirag ${ }^{2}$

${ }^{1}$ Anesthesiology and intensive care, Ege University Medical Faculty Hospital, İzmir, Turkey; ${ }^{2}$ Anesthesiology and intensive care, Ege University Hospital, Izmir, Turkey; ${ }^{3}$ Anesthesiology and intensive care, Ege Üniversitesi Tıp Fakültesi, İzmir, Turkey

Correspondence: $O$. Kaynarca

Intensive Care Medicine Experimental 2020, 8(2): 001349

Introduction: Procalcitonin (PCT) is a biochemical marker that can increase in hours in the presence of bacterial infection, which may assist in the early recognition of bacteremia and sepsis with a high specificity and sensitivity. Studies conducted in recent years reveal that the PCT level in plasma varies according to the type of pathogen causing the infection and the gram staining feature. In our study, the relationship between the type of causative pathogen and PCT level in patients diagnosed with bloodstream infection was investigated with a retrospective observational study design.

Methods: 208 infection epizode from 137 ICU patients who were diagnosed as bloodstream infection between January 2016 and February 2018 were included in the study. The highest fever, leukocyte, CRP and procalcitonin values in 24-h before and 24-h after the blood culture, infectious agent, APACHE II score, use of antibiotic when blood culture was taken, duration of ICU stay and 30-day survival was recorded using the data in the electronic patient file.

Results: Patients were divided into four groups as gram positive, gram negative, fungus and multiple agents according to the culture results. No significant difference was observed between the groups for fever, leukocyte and CRP levels, while procalcitonin level was found higher in gram negative infections ( $p<0.0001$ ). Procalcitonin cut-off value for gram negative infection was $5.95 \mathrm{ng} / \mathrm{ml}$ (sensitivity $54 \%$, specificity $81 \%$ ). It has been shown that procalcitonin and CRP levels correlate with APACHE II score, but the correlation coefficient of procalcitonin value is higher. The relationship between PCT and CRP levels with 30-d mortality was found significant, with a procalcitonin cut-off value was $4.99 \mathrm{ng} / \mathrm{ml}$ (sensitivity $66.7 \%$ specificity $75 \%$ ), while CRP cut-off value $19.01 \mathrm{mg} / \mathrm{L}$ (sensitivity $62.4 \%$ and specificity $67 \%$ ). No significant difference in PCT levels was found between the patients who used antibiotics and those who did not when culture was taken. There was also no significant relationship between the duration of ICU stay and the PCT level.

Conclusion: In conclusion, we think that procalcitonin is a marker to help predict the causative agent of infection and determine the probability of mortality in patients with bloodstream infection.

\section{Reference(s) and grant ackowledgment(s)}

1. Yan ST, Sun LC, Jia HB, Gao W, Yang JP, Zhang GQ. Procalcitonin levels in bloodstream infections caused by different sources and species of bacteria. American Journal of Emergency Medicine 2017;25:579-583.

2. Jensen JU, Heslet L, Jensen TH, Espersen K, Steffensen P, Tvede M. Procalcitonin increase in early identification of critically ill patients at high risk of mortality. Crit Care Med. 2006 Oct;34(10):2596-602. 


\section{5}

\section{Necrotizing Vasculitis in Covid-19: watch out for bowel} perforation

T. Persiano'; F. Grulli'; F. Bonomi². P. Cornara ${ }^{2}$; A. Casazza ${ }^{3}$; E. Bellazzi ${ }^{3}$. D. Ciprandi ${ }^{3}$; R. Preda ${ }^{3}$; MP. Storti ${ }^{3}$; R. Vanzino ${ }^{3}$; ST. Mediani'; A. Coci ${ }^{5} ;$ L. Carnevale $^{3}$

${ }^{1}$ Scuola di specialità medicina di emergenza e urgenza, The University of Pavia, Pavia, Italy; ${ }^{2}$ Scuola di specialità anestesia e rianimazione, The University of Pavia, Pavia, Italy; ${ }^{3}$ Anestesia e Rianimazione Vigevano, ASST di Pavia, ospedale di Vigevano, Vigevano, Italy; ${ }^{4} U$ oc cure palliative, ASST di Pavia, ospedale di Vigevano, Vigevano, Italy; ${ }^{5}$ Anatomia patologica, ASST di Pavia, ospedale di Vigevano, Vigevano, Italy

Correspondence: A. Casazza

Intensive Care Medicine Experimental 2020, 8(2): 001355

Introduction: Despite most common SARS-CoV-2 infection clinical findings are related to respiratory apparatus, Covid-19 may affect other organs than lungs such as liver, bowel and kidneys [1]. Commonly, in fact, patients suffer for abdominal and gastro-enteric symptoms[2]. We report the first case, to our knowledge, of a Covid-19 patient affected by ascending colon perforation due to a Pseudomembranous Colitis (PC) in absence of Clostridium Difficile (CD) toxins, but with SARS-CoV-2 identification in gastric fluid.

Methods: In April 2020, a 73-years-old man came in our ED with fever and dyspnea for $24 \mathrm{~h}$. His remote medical history revealed ischemic heart disease, hypertension and NIDDM. Chest X-ray showed right basal consolidation and bilateral congestion. A RT-PCR test for Covid19 was negative. The patient was admitted with the diagnosis of CAP and heart failure and treated with antibiotics and diuretics. Ten days later, respiratory symptoms and radiological findings worsening leaded to perform a new nasopharyngeal swab for Covid-19 that resulted positive. The patient needed CPAP ventilation and hydroxychloroquine, azithromycin and LMWH administration were started. Five days later, he developed fever and diarrhea: on physical examination abdomen is quite manageable. Piperacillin/tazobactam was started empirically. An abdominal CT scan showed a parietal ascending colon thickening and intra-peritoneal air collection. Right hemicolectomy with ileostomy for perforation has been performed. After surgery, he was admitted to our ICU. The anatomopathological examination revealed a PC. Diffuse tissue edema, acute inflammation and necrotizing vasculitis with fibrin thrombi and intravascular neutrophils infiltration were identified (Fig.1). Samples from ileostomy and stool in rectal ampoule were negative for CD toxins. A PCR test for SARSCoV-2 performed on gastric fluid was positive. After improvement, the patient was discharged from ICU to a low intensity ward.

Fig.1
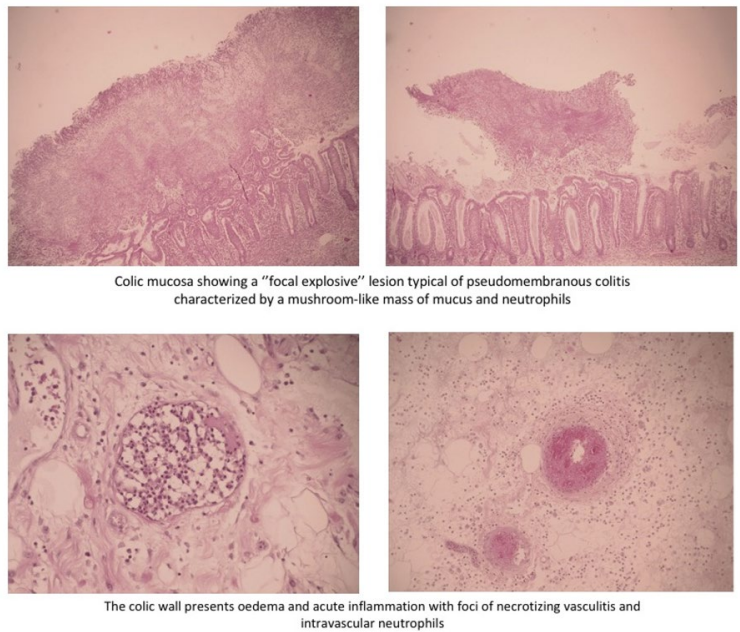

Results: Necrotizing vasculitis is commonly found in Covid-19 patients in several organs [3]. Some authors suggest enteric involvement in up to $50 \%$ of Covid-19 patients, but it's not yet completely understood. ACE-2 receptor is expressed by intestinal epithelium: mouse models suggested that ACE- 2 receptor modification due to infection can cause bowel inflammation and colitis [4]. In this case, clinical findings were typical and patient had several predisposing factors for CD colitis. Since $C D$ tests were always negative and the patient improved without $C D$ specific therapies, we supposed viral infection related necrotizing vasculitis as pseudomembranous colitis and perforation cause. Anatomopathological findings and viral RNA detection in gastric fluid support our hypothesis.

Conclusion: Many COVID 19 patients present GI symptoms together with respiratory ones. Gastro-enteric involvement may be due to enteric viral replication. CD negative PC due to a necrotizing vasculitis, in our case, may be a SARS-CoV-2 infection complication too.

\section{Reference(s) and grant ackowledgment(s)}

1. D'Amico, D. C. Baumgart, S. Danese, L. Peyrin-Biroulet. Diarrhea During COVID-19 Infection: Pathogenesis, Epidemiology, Prevention, and Management. Clinical Gastroenterology and Hepatology 2020;18(8):1663-1672.

2. 3. Roncati L, Ligabue G, Fabbiani L, Malagoli C, Gallo G, Lusenti B, Nasillo V, Manenti A, Maiorana A. "Type 3 hypersensitivity in COVID-19 vasculitis." Clinical immunology. 2020. https://doi.org/10.1016/j.clim.2020.108487.

3. 2. Bruni A, Garofalo E, Zuccalà V, Currò G, Torti C, Navarra G, De Sarro G, Navalesi P, Longhini F, Ammendola M. Histopathological findings in a COVID-19 patient affected by ischemic gangrenous cholecystitis. World Journal of Emergency Surgery. 2020. https://doi.org/10.1186/s13017-020-00320-5.

4. 1. Huang C, Wang Y, Li X, Ren L, Zhao J, Hu Y, et al. Clinical features of patients infected with novel coronavirus in Wuhan, China. Lancet. 2020;395:497-506

\section{1}

A cross-sectional study of personal protective equipment use in a UK intensive care unit during the COVID-19 pandemic

M. Chotalia'; JE. Alderman'; K. Hook ${ }^{2}$; I. Ewington ${ }^{2}$; AM. Johnston ${ }^{2}$; D. Balthazor $^{2}$; J. Patel ${ }^{1}$

${ }^{1}$ Birmingham acute care research group, University of Birmingham, Birmingham, United Kingdom; ${ }^{2}$ Critical care, Queen Elizabeth Hospital Birmingham, Birmingham, United Kingdom

Correspondence: M. Chotalia

Intensive Care Medicine Experimental 2020, 8(2): 001361

Introduction: Healthcare workers are at high risk of contracting Severe Acute Respiratory Syndrome Coronavirus-2 (SARS-CoV-2) pneumonia from either infected patients or staff [1]. Whilst effective use of personal protective equipment (PPE) is essential, sourcing adequate supplies has been a global challenge. [2].

Objectives: To perform a cross-sectional study at two time points during the COVID-19 pandemic to estimate the type and quantity of PPE being used in the intensive care unit.

Methods: This was a single-centre, prospective study at the Queen Elizabeth Hospital Birmingham. PPE use was in line with Public Health England guidance for ICUs [3] and all staff entering the COVID-19 ICU wore Level-3 PPE. Redeployed non-clinical military and outpatient hospital staff were trained as donning and doffing officers to ensure staff followed the PPE protocols. Donning officers recorded the type of PPE used every time a staff member entered the ICU. This was documented for each day, over two sampling periods (19th-30th March and 18th-28th April) on one of our three dedicated COVID-19 ICUs. Continuous data is presented as mean ( \pm SEM) and compared using a Student's t-test. Categorical data is presented as \% and analysed using a chi squared test. A p value of $<0.05$ determined statistical significance. Results: Over the total period of data collection, 5,066 donning episodes were recorded. The daily mean number of patients was $22( \pm 2)$ and staff working on the area was $101( \pm 6)$. The daily mean number of donning episodes was $300( \pm 26)$, equating to approximately 3 episodes per healthcare worker or 14 episodes per patient each day. 
$86 \%$ of donning episodes were with an FFP3 face mask and $14 \%$ used a powered respirator.

In the second study period, the number of staff and patients increased, as did the number of donning episodes (see Table 1). A greater frequency of donning episodes relative to the number of staff on the unit was also observed ( 3.4 vs 2.4 episodes per staff member per day) and a higher proportion of staff wore FFP3 masks rather than powered respirators (FFP3 mask use: $89.3 \%$ in 2 nd period vs $77.4 \%$ in 1 st period; $\mathrm{p}<0.0001)$.

Eleven percent of nursing staff were on leave due to illness each week. Staff sickness rates were equivalent between the two periods of data collection (10.6\% vs $10.5 \%)$. Non-clinical nursing staff (working in administrative roles) had a sickness rate of $5.4 \%$ over the same study period.

\begin{tabular}{|l|l|l|l|l|}
\hline & $\begin{array}{l}\text { Daily average } \\
\text { (all) }\end{array}$ & $\begin{array}{l}\text { Daily average } \\
(1 \mathrm{st})\end{array}$ & $\begin{array}{l}\text { Daily average } \\
(\text { nd) }\end{array}$ & pvalue \\
\hline Patients (n) & $22( \pm 2)$ & $16( \pm 2)$ & $27( \pm 1)$ & $<0.0001$ \\
\hline $\begin{array}{l}\text { Healthcare Worker } \\
(\mathrm{n})\end{array}$ & $101( \pm 6)$ & $84( \pm 4)$ & $116( \pm 8)$ & 0.004 \\
\hline Donning episodes & $300( \pm 26)$ & $199( \pm 6)$ & $390( \pm 21)$ & $<0.0001$ \\
\hline $\begin{array}{l}\text { Donning episodes } \\
\text { per HCW }\end{array}$ & 3 & 2.4 & 3.4 & N/A \\
\hline FFP masks (n) & $257( \pm 27)$ & $154( \pm 9)$ & $348( \pm 22)$ & $<0.0001$ \\
\hline $\begin{array}{l}\text { Powered respirators } \\
(\mathrm{n})\end{array}$ & $43( \pm 3)$ & $45( \pm 5)$ & $42( \pm 5)$ & 0.80 \\
\hline Gloves (n) & $321( \pm 25)$ & $244( \pm 12)$ & $398( \pm 22)$ & $<0.0001$ \\
\hline Surgical gowns (n) & $300( \pm 26)$ & $199( \pm 6)$ & $390( \pm 21)$ & $<0.0001$ \\
\hline Visors / goggles (n) & $257( \pm 27)$ & $154( \pm 9)$ & $348( \pm 22)$ & $<0.0001$ \\
\hline
\end{tabular}

Conclusion: Vast quantities of PPE were used on a daily basis during the UK-peak of the COVID-19 pandemic to maintain the safety of staff and patients. Whilst the number of healthcare workers increased between the two periods, PPE use relative to the number of staff was also greater. This may be explained by the introduction of additional support teams to deliver interventions as patient numbers grew (proning, line insertion et cetera). FFP3 masks were more frequently used compared to powered respirators.

Compared to prior reports [1] a lower rate of illness in healthcare workers was observed in our study, and sickness rates in clinical nursing staff were only slightly higher than their non-clinical colleagues. This indicates a lower rate of healthcare worker infection - which could in part be explained by the PPE strategy used in our ICU.

In conclusion, PPE use and preference changed over two time periods in one area of a single ICU during the COVID-19 pandemic. As equipment is scarce but in high demand, healthcare departments should be encouraged to regularly audit PPE use to better estimate clinical need.

Reference(s) and grant ackowledgment(s)

1. [1] International Council of Nurses. ICN calls for data on healthcare worker infection rates and deaths. Available from https://www.icn.ch/news/icncalls-data-healthcare-worker-infection-rates-and-deaths (accessed 6 May 2020).

2. [2] Livingston E, Desai A, Berkwits M. Sourcing personal protective equipment during the COVID-19 pandemic. JAMA. 2020; 323(19): 1912-14.

3. [3] 3. Public Health England. COVID-19 persona protective equipment (PPE). Available from www.gov.uk/government/publications/wuhan-novel-coron avirus-infection-prevention-and-control/covid-19-personal-protective -equipment-ppe (accessed 28 May 2020).

\section{8}

Epidemiology of COVID-19 ICU admissions - ethnicity and deprivation

T. Baumer'; E. Phillips ${ }^{1}$; A. Dhadda'; T. Szakmany ${ }^{2}$

${ }^{1}$ Intensive Care, Royal Gwent Hospital, Newport, United Kingdom; ' 2 Department of anaesthesia and intensive care, Royal Gwent Hospital, Newport, United Kingdom

Correspondence: $T$. Baumer
Intensive Care Medicine Experimental 2020, 8(2): 001368

Introduction: SARS-CoV-2 infection, originating from Wuhan, China is the underlying cause of COVID-19 disease [1]. The first known patient with COVID-19 disease was admitted to our intensive care unit (ICU) at the Royal Gwent Hospital (RGH), Newport, Wales on the 9th March.

Objectives: In this case-series analysis we describe the epidemiology of the first wave of COVID-19, focusing on the interplay between ethnicity and social deprivation, by using data from the first 60 days at the RGH ICU.

Methods: Our prospective service evaluation recorded anonymised data on patient age; sex; BMI; postcode; ethnicity; Welsh Index of Multiple Deprivation (WIMD) score [2]; need for any assistance with daily activities; APACHE II score; time from hospital admission to ICU, and ICU mortality. Background data on ethnicity for the hospital catchment area was obtained from the UK Census data 2011 (Office for National Statistics).

Results: The Black, Asian and Minority Ethnic (BAME) population, while being only $5 \%$ of the background population, represented $35 \%$ of ICU COVID-19 admissions (18 of 52) and 35\% of deaths (6 of 17). Within the BAME group, $72 \%$ (13 of 18) of patients were from geographical areas representing the $20 \%$ most deprived (lowest quintile) in Wales, versus $27 \%$ of Caucasians ( 9 of 33). When looking at deaths by ethnicity, more occurred within the lowest deprivation quintile in the BAME group (4 of 6 deaths) compared to the Caucasian group (3 of 11).

Conclusion: We found that patients from the BAME group are at significantly higher risk of ICU admission and death from COVID-19, despite an overwhelming Caucasian background population (95\%). We observed a striking interaction between deprivation and ethnicity, which may be multifactorial, including greater prevalence of comorbidities; increased community spread; genetic polymorphisms; or other unidentified factors [3-5]. These factors need further investigation and evaluation.

\section{Reference(s) and grant ackowledgment(s)}

1. 5. Docherty $A B$, Harrison EM, Green $C A$, et al. Features of 16,749 hospitalised UK patients with COVID-19 using the ISARIC WHO Clinical Characterisation Protocol. MedRxiv (2020). doi.org/https://doi.org/10.1101/2020.04.23.20076 042.

2. 4. Lu N, Yang Y, Wang Y, et al. ACE2 gene polymorphism and essential hypertension: an updated meta-analysis involving 11,051 subjects. Molecular Biology Reports (2012). 39: 6581-6589.

3. 3. Centre for Evidence Based Medicine. BAME COVID-19 DEATHS - WHAT DO WE KNOW? RAPID DATA \& EVIDENCE REVIEW: 'HIDDEN IN PLAIN SIGHT' (2020). www.cebm.net/wp-content/uploads/2020/05/BAME-COVID-Rapid -Data-Evidence-Review-Final-Hidden-in-Plain-Sight-compressed.pdf [accessed 21/05/2020].

4. 2. Welsh Index of Multiple Deprivation. The Welsh Government - Statistics and Research (2019). Available at: https://wimd.gov.wales/ [accessed on 21/05/2020].

5. 1. Huang C, Wang Y, Li X, et al. Clinical features of patients infected with 2019 novel coronavirus in Wuhan, China. The Lancet (2020). 395: 497-506.

\section{8}

Therapeutic Plasma Exchange Followed by Convalescent Plasma Therapy in COVID-19

V. Jaiswal'; M. Marashi²; M. Raouf ${ }^{3} ;$ M. Gupta ${ }^{4}$

${ }^{1}$ ICU, Mediclinic City Hospital Health care City branch, Dubai, United Arab Emirates; ${ }^{2}$ Hematology, Mediclinic City Hospital Health care City branch, Dubai, United Arab Emirates; ${ }^{3}$ Pathology and genetics, Dubai Blood Donation Center, Dubai, United Arab Emirates; ${ }^{4}$ Obstetric and gynaecology, JTS Medical Centre, dubai, United Arab Emirates

Correspondence: $V$. Jaiswal

Intensive Care Medicine Experimental 2020, 8(2): 001378

Introduction: Convalescent plasma containing virus specific antibodies has been used before and was associated with reduced mortality during Spanish flu, SARS, and H1N1 influenza A pandemics. 
Therapeutic plasma exchange (TPE) has shown some benefits for cytokine clearance in sepsis with multiorgan failure in the past and in 2009 H1N1 influenza A pandemic. We combined both therapies together for COVID-19 treatment.

Objectives: To evaluate safety and efficacy of therapeutic plasma exchange followed by convalescent plasma therapy in critically ill patients with severe acute respiratory syndrome corona virus 2 (SARSCOV-2) infections.

Methods: 6 patients with laboratory-confirmed COVID-19 and acute respiratory distress syndrome (ARDS) were treated with one session TPE followed by $500 \mathrm{ml}$ convalescent plasma transfusion in addition to standard treatment who met the following criteria: severe pneumonia with rapid progression and life threatening illness (PAO2/FIO2 $<300$ and mechanical ventilation, shock, organ failure). The study was conducted at Mediclinic City Hospital, Dubai, UAE from April 20, 2020 to June 20,2020. Outcomes measured were death from any cause, time to negative SARS-COV-2 PCR, time to removal of mechanical ventilation after intervention, clinical (body temperature, Sequential Organ Failure Assessment (SOFA) score, PAO2/FIO2), biochemical (ferritin, D-dimer, CRP, LDH, procalcitonin) and radiological parameters before and after therapy. Mechanical ventilation days, ICU length of stay, hospital length of stay were also measured.

Results: Patients were in the age range of 36-65 years, 4 males and two females. All patients were receiving mechanical ventilation, Methylprednisone and therapeutic anticoagulation. All the patients survived and were discharged home. Time to negative SARS-COV-2 RT PCR was 1-18 days and time to removal of mechanical ventilation was $2-41$ days after intervention. Out of six, 4 patients were off mechanical ventilation within 7 days of therapy. 3 out of 6 patients had normal body temperature, 5 had improved PAO2/FIO2 within $48 \mathrm{hrs}$. SOFA score improved by 2 points within 48 hrs in 3 patients and within 5 days in another 2 patients. All the biochemical parameters improved within 48 hrs except D- dimer which worsened in 3 patients after therapy. Chest $X$ ray showed improvement in pulmonary lesions within 48 hrs and significant improvement by day 7 . Mechanical ventilation days, ICU and hospital length of stay was 8-42, 10-42 and 20-60 days respectively.

Conclusion: Critically ill Covid-19 patients with ARDS treated with therapeutic plasma exchange followed by convalescent plasma in addition to standard therapy showed improvements in clinical, laboratory, and virological parameters. Although this uncontrolled limited case series had very good success rate, further evaluation in clinical trials is necessary in order to corroborate the findings.

\section{Reference(s) and grant ackowledgment(s)}

1. 1. https://www.who.int/docs/default-source/coronaviruse/situation-repor ts/20200713-covid-19-sitrep-175.pdf?sfvrsn = d6acef25_2 2. Wang D, $\mathrm{Hu}$ B, Hu C, et al. Clinical characteristics of 138 hospitalized patients with 2019 novel coronavirus-infected pneumonia in Wuhan, China. JAMA. 2020; 323(11):1061-1069. https://doi.org/10.1001/jama.2020.1585 3. Wu Z, McGoogan JM. Characteristics of and important lessons from the coronavirus disease2019 (COVID-19) outbreak in China: summary of a report of 72314 cases from the Chinese Center for Disease Control and Prevention. JAMA. 2020;323 (13):1239-1242. https://doi.org/10.1001/ jama.2020.2648 4. BMJ 2020; 370 doi: https://doi.org/10.1136/bmj.m2648 5. Beigel JH, Tomashek KM, Dodd LE, et al. ACTT-1 Study Group Members. Remdesivir for the treatment of Covid-19-preliminary report. N Engl J Med. Published online May 22, 2020. https://doi.org/10.1056/nejmoa2007 764 6. Shen C, Wang Z, Zhao F, et al. Treatment of 5 Critically III Patients With COVID-19 With Convalescent Pasma. JAMA. 2020;323(16):1582-1589. https ://doi.org/10.1001/jama.2020.4783 7. Duan K, Liu B, Li C, et al. Effectiveness of convalescent plasma therapy in severe COVID-19patients. Proc Natl Acad Sci U S A. 2020;117(17): 9490-9496. https://doi.org/10.1073/ pnas.2004168117 8. Wiersinga WJ, Rhodes A, Cheng AC, Peacock SJ, Prescott HC et al. Pathophysiology, Transmission, Diagnosis, and Treatment of Coronavirus Disease 2019 (COVID-19): A Review. JAMA. Published online July 10, 2020. https://doi.org/10.1001/jama.2020.12839 9. Luke TC, Kilbane EM, Jackson JL, Hoffman SL.Meta-analysis: convalescent blood products for Spanish influenza pneumonia: a future H5N1 treatment? Ann Intern Med.2006;145(8):599-609.https://doi.org/10.7326/0003-4819-145-8-20061 0170-00139 10. Cheng Y,Wong R, Soo YO, et al. Use of convalescent plasma therapy in SARS patients in Hong Kong. Eur J Clin Microbiol Infect Dis. 2005;24(1):44-46. https://doi.org/10.1007/s10096-004-1271-9 11. Hung IF, To KK, Lee CK, et al. Convalescent plasma treatment reduced mortality in patients with severe pandemic influenza A (H1N1) 2009 virus infection. Clin Infect Dis.2011;52(4):447-456.https://doi.org/10.1093/cid/ciq10 6 12. Zhang B, Liu S, Tan T, et al. Treatment with convalescent plasma for critically ill patients with severe acute respiratory syndrome coronavirus 2 infection. Chest. Published online May 18, 2020.https://doi.org/10.1016/j. chest.2020.03.039 13. Rajendran K, Krishnasamy N,Rangarajan J, Rathinam J, Natarajan M, Ramachandran A. Convalescent plasma transfusion for the treatment of COVID-19: Systematic review. J Med Virol. 2020;19.https:// doi.org/10.1002/jmv.25961 14. Keith P, Day M, Perkins L, Moyer L, Hewitt $\mathrm{K}$, Wells A. A novel treatment approach to the novel coronavirus: an argument for the use of therapeutic plasma exchange for fulminant COVID-19. Crit Care. 2020;24:128 15. Chang JC. Sepsis and septic shock: endothelial molecular pathogenesis associated with vascular rmicrothrombotic disease. Thromb J. 2019;17:10. 16. Zhuplatov Sergey B, Zhuplatov llya S. Removal of Endotoxins and Cytokines by Plasmapheresis Filtration with Plasma Exchange Therapy (TPE) Could Benefit Patients with Covid-19 in Critical Condition. J Anest\&Inten Care Med. 2020; 10(5): 555798. https:// doi.org/10.19080/jaicm.2020.10.555798 17. Xu X, Han M, Li T, et al. Effective treatment of severe COVID-19 patients with tocilizumab. Proc Natl Acad Sci U S A. 2020;117:10970- 5. 18. Busund R, Koukline V, Utrobin U, et al. Plasmapheresis in severe sepsis and septic shock: a prospective, randomized, controlled trial. Intensive Care Med. 2002;28:1434-9. 19. Rimmer E, Houston BL, Kumar A, et al. The efficacy and safety of plasma exchange in patients with sepsis and septic shock: a systematic review and meta-analysis. Crit Care. 2014;18(6):699. 20 .Faryal Khamis, Ibrahim Al-Zakwani, et al. Therapeutic Plasma Exchange in Adults with Severe COVID-19 Infection.: S1201-9712(20)30499-9 DOI: https://doi.org/10.1016/j.jijd.2020.06.064 Reference: IJID 4369 21. Recommendations for Investigational COVID-19 Convalescent Plasma. US FDA. Published May 1, 2020. https://www.fda.gov/ vaccines-blood-biologics/investigational-newdrug-ind-or-device-exemp tion-ide-process-cber/recommendations-investigational-covid-19-conva lescent-plasma 22. Zhang XY, Ye XW, Feng DX, Han J, Li D, Zhang C (2011) Hemophagocytic lymphohistiocytosis induced by severe pandemic influenza A (H1N1) 2009 virus infection: A case report. Case Report Med: 3. 23. Shen C, Chen J, Li R, et al. A multimechanisticantibody targeting the receptor binding site potently cross-protects against influenza B viruses. Sci Transl Med. 2017;9(412):eaam5752. 24. Shen C, ZhangM, Chen Y, et al. An IgM antibody targeting the receptor binding site of influenza B blocks viral infection with great breadth and potency. Theranostics. 2019;9(1):210-231. 25. Wang C, LiW, Drabek D, et al A human monoclonal antibody blocking SARS-CoV-2 infection. bioRxiv. March 12, 2020. https://doi.org/10.1101/2020.03.11.98795 8 26. Kogelmann K, Jarczak D, Scheller M, Drüner M. Hemoadsorption by CytoSorb in septic patients: a case series. Crit Care. 2017;21:74. 2. None.

\section{7}

The influence of blood group on COVID-19 infection in our Intensive Care Unit

FL. PEREZ CABALLERO' ; M. Diaz-Tavora Luque ${ }^{1}$; I. Peña Luna ${ }^{1}$; R. Mancha Salcedo'; MS. Pereira Gama'; S. Godoy Boraita'; MF. Benitez Morillo' ${ }^{1}$ UNIDAD DE CUIDADOS INTENSIVOS, Hospital de Mérida, Mérida, Spain Correspondence: F.L. PEREZ CABALLERO

Intensive Care Medicine Experimental 2020, 8(2): 001387

Introduction: On March 2020, World Health Organization (WHO) declared COVID-19, a disease caused by the SARS-COV2 virus, as a pandemic. Some studies have highlighted advanced age, male sex, and other comorbidities as cardiovascular diseases, diabetes, and pulmonary diseases, increase the risk and severity of the COVID-19.

There have been few molecular level hypotheses raised for the variable susceptibility to disease and vulnerability to severe disease. Landsteiner's ABO carbohydrate moieties are genetically inherited. Some reports suggest a correlation between $\mathrm{ABO}$ blood group, cardiovascular diseases, and cancers, as well as typing and susceptibility to certain infections, including COVID-19 infection. 
Only few clinical studies demonstrated that there exists a relationship between infection risk and the blood groups, and how blood group $\mathrm{O}$ had a negative predictive effect, associated with a lower risk of infection and mortality, and the blood group A was correlated with a severe pulmonary damage.

Objectives: Main: To analize a group of COVID-19 patients admitted to our Intensive Care Unit (ICU) between March-2020 and June-2020 by their blood group and Rh factor.

Secundary: To determine the frequency of main comorbidities, analytical results, ICU evolution, treatment needed, ventilation characteristics, and score on risk scales.

Methods: Observational, descriptive, retrospective study in COVID19 patients admitted to our ICU in 3 months, using a database of 14 patients, with previous defined blood group and Rh factor, and medical history, analytical results (at the admission, 2 days, 4 days, 6 days and 8 days later), ICU evolution, treatment needed (vasoactive drugs and COVID-19 drugs), ventilation characteristics and scores on the risk scales (APACHE, SOFA and SAPS II).

Results: The clinical characteristics of 14 patients included according to their blood group, were: main age of patients 63 (44-78) and the percentage of female patients was $57 \%$. The most frequently detected blood group was type $\mathrm{O}$ with $57 \%$ amongst the COVID-19 patients. This was followed by group type A with $43 \%$. Rh factor more detected was positive with $93 \%$, being statistically significant the relation between female gender and positive Rh factor 54\% ( $p$ 0.011).

An important history of cardiovascular diseases was determined among these patients, highlighting high blood pressure $50 \%$ type $O$ and $50 \%$ type $A$; obesity $50 \%$ type $O$ and $37,5 \%$ type $A$, dyslipidemia $66 \%$ type $O$ and $12,5 \%$ type, without statistically significant.

No important difference was found in the analytical results at the admission, 2 days, 4 days, 6 days and 8 days later; just highlight the main D-Dimer levels at the admission in ICU was substantially more elevated in type A $(4342 \mathrm{ng} / \mathrm{ml})$ than O $(1429 \mathrm{ng} / \mathrm{ml})$.

Group type $A$ had highest needs of vasoactive drugs: Noradrenaline 100\% (62\% Group type 0), Dobutamine 50\% and Adrenaline 16\% (both $0 \%$ in Group type 0), and also a $100 \%$ needed glucocorticoid treatment (62\% Group type 0).

The use of renal replacement therapy was prescribed for $50 \%$ of patients in Group type A, compared to. $12,5 \%$ in Group type 0, as well as the need of percutaneous tracheostomy (same percentages).

The ICU evolution was fateful in Group type A, with $50 \%$ of death compared to $37,5 \%$ in Group type 0 , without statistically significant differences. Likewise, the ICU evolution was worse in negative Rh factor patient.

Fibrinolytic therapies such as rtPA, or bronchodilator therapies with nitric oxide, were used for the treatment of refractory cases, in $33 \%$ of Group typ A, and in the negative Rh factor patient, unlike $7 \%$ positive $\mathrm{Rh}$ factor patients, being statistically significant $(\mathrm{p} 0,017)$.

No differences in mechanical ventilation was found between both groups.

Finally, in relation to the score on risk scales, SAPS- II after the admission of patients with positive Rh factor, had a mean of 34,63, statistically significant $(p 0,012)$; we did not detect differences in SOFA or APACHE scales after the admission.

Conclusion: Group type $\mathrm{O}$ and positive $\mathrm{Rh}$ factor were the most frequent in our COVID-19 patients, being statistically significant the relation between female gender and SAPS-II score after admission with positive Rh factor. Although Group type A patients were less numerous, they needed more vasoactive drugs, refractory treatments and had worse ICU evolution.

\section{Reference(s) and grant ackowledgment(s)}

1. Latz CA, DeCarlo C, Boitano L, et al. Blood type and outcomes in patients with COVID-19 [published online ahead of print, $2020 \mathrm{Jul}$ 12]. Ann Hematol. 2020;1-6. https://doi.org/10.1007/s00277-020-04169-1.

2. Zietz M, Tatonetti NP. Testing the association between blood type and COVID-19 infection, intubation, and death. Preprint. medRxiv. 2020;2020.04.08.20058073. Published 2020 Apr 11. https://doi. org/10.1101/2020.04.08.20058073.

3. Göker H, Aladağ Karakulak E, Demiroğlu H, et al. The effects of blood group types on the risk of COVID-19 infection and its clinical outcome [published online ahead of print, 2020 Jun 4]. Turk J Med Sci. 2020;50(4):679-683. https ://doi.org/10.3906/sag-2005-395.

001391

Predictors of the severe disease of patients admitted to an ICU with COVID-19

T. Klypa'; M. Bychinin ${ }^{1}$; I. Mandel1'; S. Andreichenko ; A. Minec ${ }^{1}$; N.

Kolyshkina ${ }^{2}$; A. Troitsky ${ }^{3}$

${ }^{1}$ Intensive care unit, Federal Research and Clinical Centre, FMBA of Rus sia, Moscow, Russia; ${ }^{2}$ Laboratory medicine, Federal Research and Clinica Centre, FMBA of Russia, Moscow, Russia; ${ }^{3}$ Chief executive officer, Federal Research and Clinical Centre, FMBA of Russia, Moscow, Russia

Correspondence: $M$. Bychinin

Intensive Care Medicine Experimental 2020, 8(2): 001391

Introduction: Providing an efficient care to the patients of the most severely affected category - ICU patients - has become one of the serious problems appearing in the COVID-19 pandemics [1]. A typical patient's clinical portrait in ICUs of COVID centers is very similar in different countries, however, the key to improve the treatment results for critically ill patients has not yet been found [2].

Objectives: The aim of the study is to identify predictors of the severe pneumonia caused by the SARS-CoV-2 virus in patients admitted to an intensive care unit (ICU) of the COVID-center of the Federal Research Clinical Center.

Methods: In this report, we describe the clinical, laboratory and instrumental data of 70 patients admitted to the ICU, and discuss the found predictors of the severe COVID-19 pneumonia course.

Results: The mortality in the ICU was $48.9 \%$, the mortality among the patients required mechanical ventilation was $57.9 \%$. The following factors have been determined which contribute to the development of the severe course of the disease and to the risk of the mortality: male gender $(p=0.021)$, age older than 70.5 years $(p=0.014)$, initial lymphocytopenia of lower than $0.98 \times 109 / \mathrm{l}(\mathrm{p}=0.014)$, neutrophil to lymphocyte ratio of higher than $7.75(p=0.004)$, D-dimer level of higher than $0.85 \mu \mathrm{g} / \mathrm{ml}(\mathrm{p}=0.001)$, IL-6 of higher than $184.7 \mathrm{pg} / \mathrm{ml}(\mathrm{p}=0.011)$ procalcitonin of higher than $0.22 \mathrm{ng} / \mathrm{ml}(\mathrm{p}=0.009)$, hyperglycemia of higher than $9 \mathrm{mmol} / \mathrm{l}$, signs of myocardial damage (high-sensitive troponin $\mathrm{T}$ of higher than $22 \mathrm{pg} / \mathrm{ml}(\mathrm{p}=0.005)$, echocardiography data) and a severe vitamin D deficiency (lower than $9.9 \mathrm{ng} / \mathrm{ml}, \mathrm{p}=0.044$ )

Conclusion: Clinical features of the patients change in course of pandemia. These are influenced by changes in treatment approaches and new discoveries in disease pathophysiology. Above mentioned predictors of severe course of disease is partly modifiable and we are able to influence them and perhaps achieve better results in treatment of severe patients with COVID-19.

\section{Reference(s) and grant ackowledgment(s)}

1. 2. Petrilli CM, Jones SA, Yanget J, al. Factors associated with hospitalization and critical illness among 4,103 patients with COVID-19 disease in New York City. Posted Content. 2020. doi: 10.1101/ 2020.04.08.20057794.

2. 1. Zhou F, Yu T, Du R, et al. Clinical course and risk factors for mortality of adult inpatients with COVID-19 in Wuhan, China: a retrospective cohort study. Lancet. 2020;395(10229):1054-1062. https://doi.org/10.1016/s0140 $-6736(20) 30566-3$

001394

Characteristics and outcome of severe COVID-19 patients: experience of tertiary hospital of Jeddah

S. Omer ${ }^{1}$; A. Khatib ${ }^{1}$; M. Darwish ${ }^{1}$; H. Almejally ${ }^{1}$; A. Alzahrani ${ }^{1}$; MM. Lone ${ }^{1}$; K. BELKHOUJA ${ }^{1} ;$ H. Al-Sharif ${ }^{1}$

${ }^{1}$ Intensive care department, King Fahad Armed Forces Hospital, Jeddah, Saudi Arabia

Correspondence: $\mathrm{K}$. BELKHOUJA

Intensive Care Medicine Experimental 2020, 8(2): 001394 
Introduction: The COVID-19 is considered as the major pandemic that we have experienced in the last century. Therefore, the management of the critically ill patients with COVID-19 is extremely important. The need for intensive care might differ according to institutions or even countries, depending on the demand and supply ranging from $5 \%$ to $32 \%$. ICU mortality due to COVID-19 varies from $16 \%$ to $78 \%$ according to different studies. As of July 28th, 2020, a total of over 270000 COVID-19 cases and 2800 deaths have been reported in Saudi Arabia. No data available concerning the characteristics and outcomes of severe COVID-19 patients in the Kingdom.

Objectives: To describe the demographic and clinical characteristics and report our management and the outcomes of patients admitted for severe COVID-19.

Methods: Retrospective descriptive observational cohort study was conducted, from Mars 25, 2020, to July 28, 2020, in King Fahad armed forces hospital, Kingdom of Saudi Arabia. We included all adult patients with confirmed severe COVID-19 and required ICU admission or ICU management outside ICU. We collected baseline data on demographics, clinical presentation, laboratory, arterial blood gas, ventilation setting, the management, and the outcomes.

Results: Of the 110 patients included in the study, 52 (47.3\%) were admitted in ICU and the remaining patients were followed by ICU team in the ward. The median (IQR) age was 62 (54-70) years and 74 $(67.3 \%)$ were male. 97 patients $(88.2 \%)$ had at least one comorbidity, mainly diabetes mellitus (70\%) and hypertension (65.5\%). The median (IQR) of SOFA score and APACHE II score were 4 (3-5) and 9 (7-13) respectively. The median time from first symptom to ICU team management was 7.0 days. All patients had acute respiratory failure and 34 patients $(30.9 \%)$ had combined others organ damage. The lymphopenia less than $1.0 \times 109 / \mathrm{L}$, D-dimer more than $1 \mathrm{mcg} / \mathrm{mL}$ and high ferritin occurred in $49 \%, 54.5 \%$ and $85.5 \%$ respectively. The respiratory support was needed for 90 patients $(81.8 \%)$, among them 86 patients $(78.2 \%)$ received continuous positive airway pressure (CPAP). Invasive mechanical ventilation within the first 24 hours of admission occurred for 8 patients (7.3\%). Moderate to severe acute respiratory distress syndrome (ARDS) was observed in $71.8 \%$ of the patients and the median (IQR) PaO2/FIO2 was 152 (123-191). The median FIO2 was $45 \%$ (IQR, 35\%-60\%). Awake proning with CPAP was performed for 31 patients $(28.2 \%)$. All patients had negative fluid balance during at least the first 3 days of ICU team management. Methylprednisolone was administered to 78 patients $(71 \%)$ and empiric antibiotics to 106 patients (96.4\%). During hospital stay, 20 patients $(18.2 \%)$ required tracheal intubation and the median (IQR) length of stay was 8 (5-11) days. Among the 110 patients, three were still in the ICU, and $8(7.5 \%)$ had died.

Conclusion: In this single center case series of severe COVID-19, all patients had acute respiratory failure and the majority needed respiratory support mainly by CPAP and the rate of mortality was low, less than $8 \%$.

\section{Reference(s) and grant ackowledgment(s)}

1. We are indebted to the doctors, nurses and respiratory therapists with the Intensive Care Medicine Service who provides care for the patients included in the study and to all the frontline staff of COVID-19 patients.

\section{7}

Descriprive Study of hemocultives in patients admitted to the ICU COVID-19 from march to may 2020

D. García Huertas ; P. Fernandez Florido1; I. Guzmán Adum¹; J. Machado Casas

${ }^{1}$ Intensive care unit, Hospital Universitario Virgen de las Nieves, Granada, Spain

\section{Correspondence: I. Guzman}

Intensive Care Medicine Experimental 2020, 8(2): 001397

Introduction: Catheter-related bloodstream infection (CRBSI) and other infections have beenfrequent in patients admitted in ICU for
Covid -19. However, many cultures collected withsuspected infectionhave been negative or contaminated.

Objectives: We aim to analyze the microbiological isolates from blood cultures in patients admitted for Covid-19 and Catheter-related bloodstream infection (CRBSI) in our Unit during the months of the Covid-19 pandemic.

Methods: Retrospective, descriptive, observational study of the microbiological isolates in blood cultures and catheter tips in patients admitted for Covid-19 from March to May 2020 in the ICU of a thirdlevel hospital. The data we used was obtained from the ENVIN-HELICS registry. The following were analyzed: demographic data, number of blood cultures extracted, number of positive blood cultures, percentage of Gram positive, Gram negative and fungal organisms, number and incidence density of CRBSI, hospital mortality and ICU mortality. Results: 60 patients were included, $48(80 \%)$ were men and 12 women (20\%), median APACHE II score 14.1 points, median age 60.47 years. Some blood culture was extracted from 51 patients (90\%), 31 of them (53.4\%) had the blood culture positive. In total, 163 blood cultures were performed: 73 were positive (45\%) and 90 negative (55\%). Of the positive results, $80 \%$ correspond to Gram positive microorganisms, $9 \%$ to Gram negative and $9 \%$ to fungi. The most frequent microorganism was coagulase- negative Staphilococcus (34\%).

69 catheter tips were removed. In 21 (30.4\%) microorganisms were isolated: gram positive $60 \%$, gram negative $17 \%$ and fungi $23 \%$. The remaining $69.6 \%$ were negative. There were 12 CRBSI in a total of 1285 days of central venous catheter (9.34 bacteremia/1000 days of central venous catheter). Utilization ratio $=1.02$

ICU mortality was $33 \%$ and hospital mortality $38.98 \%$.

Conclusion: Our study reported a high number of blood cultures sent and catheters removed with suspected infection. Although most blood cultures were negative, there is a high rate of Gram-positive isolated and catheter-related infections, probably due to the high number of devices used and the severity of the disease. Despite these data mortality in our ICU is similar to that described in the literature.

This study makes us think that in an unknown Scenario and with exceptional protection measures, we cannot forget "zero projects" as a measure to lower infection rates and to establish improvement points in ICUs.

\section{4}

Endotracheal tube obstruction in COVID-19 critical ill patients: complications

SH. De Miguel Martín ${ }^{1}$; AM. Leal Micharet ${ }^{1}$; JD. Czapka Mital'; M. Aviles

Garcia'; MT. Cubells Romeral'; I. Pozuelo Echegaray'; LM. Mateus

Sanabria'; VA. Benitez Ferreiro'; A. Gamo De Maeyer'

${ }^{1}$ Icu, General Hospital Villalba, Collado Villalba, Spain

Correspondence: S.H. De Miguel Martín

Intensive Care Medicine Experimental 2020, 8(2): 001424

Introduction: SARS-CoV 2 (COVID-19) critical ill patients with pneumonia requiring mechanical ventilation (MV) frequently present endotracheal tube (ET) obstruction episodes. ET obstruction and ET emergent change procedure may increase the risk of Intensive Care Unit (ICU) complications with prognostic implications.

Methods: Retrospective observational trial. Patients with confirmed SARS-CoV-2 (COVID-19) pneumonia requiring mechanical ventilation longer than 48 hours between March 6th and May 6th 2020 were included. In patients with ET obstruction and ET emergent change, the following events were recorded: new onset ventilator associated pneumonia (VAP), pneumothorax and mortality related to ET change. Intubation days, ICU-stay and mortality were compared between both groups (patients with ET obstruction vs patients without ET obstruction)

Results: 49 COVID-19 patients with pneumonia required MV. Two patients died in the next 48 hours and were excluded. 47 patients were included. Mean age was $61,7(+/-1,6)$ years old, $35(74,4 \%)$ male. Mean APACHE 2 was 14,4 (+/- 0,8), mean SAPS $234,1(+/-1,3)$. Global mortality was $29,7 \%$. Mean ICU-stay was $29(+/-2,6)$ days and mean intubation days $24(+/-2,4)$. In 28 patients $(59,5 \%)$ ET was changed at least once. Mean ET change day was $11,1(+/-1,3)$. Among ET change 
group, $14,3 \%$ had pneumothorax; $60,7 \%$ developed VAP in the follow 72 hours, $58,8 \%$ of which had new germ isolation. After comparing both groups, patients who needed ET change stayed longer intubated $(28,4$ vs $17,7, p<0,05)$ and admitted in ICU $(34,5$ vs 21 days, $p<0,05)$. There were not significant differences in mortality. One patient died $(3,6 \%)$ secondary to a ET change.

Conclusion: More than half SARS-CoV 2 intubated critical ill patients presented ET obstructions. These episodes may increase the risk of developing complications such as VAP and pneumothorax, which determine longer intubation period and ICU-stay. Strategies headed for limiting and preventing ET obstruction episodes may decrease morbidity during MV, having an impact in ICU-stay.

\section{9}

Impact of Remdesivir in the outcome of SARS-CoV-2 infection patients: a comparative study with supportive care

J.P. Cidade' ; I. Castro ${ }^{1}$; R. Morais' ; P. Moniz'; 'V. Costa ${ }^{1}$; L. Morais ${ }^{1}$; P. Fidalgo'; A. Tralhão ${ }^{1}$; C. Paulino ; D. Nora ${ }^{1}$; B. Valerio ${ }^{1}$; V. Mendes ${ }^{1}$; L. Coelho ${ }^{1}$; C. Tapadinhas'; P. Povoa

'Polyvalent Intensive Care Unit, Hospital São Francisco De Xavier, Lisboa, Portugal

Correspondence: J.P. Cidade

Intensive Care Medicine Experimental 2020, 8(2): 001439

Introduction: The recent SARS-CoV-2 pandemic has motivated the evaluation of several therapeutic agents as treatment for the coronavirus disease 19 (Covid-19). Remdesivir has been shown to be superior to placebo in shortening the time to recovery in adults hospitalized with Covid-19(1).

Objectives: After the early access program implementation in Portugal, we began to treat Covid-19 patients with remdesivir according to the published criteria. We aimed to determine the impact of remdesivir in the outcomes (ICU length of stay, SOFA score improvement and adapted clinical eight-category ordinary WHO scale (ACECOWS) improvement, at ICU discharge date) of patients admitted to ICU with SARS-CoV-2 pneumonia, as compared to supportive care (SC) (with no use of off-label drugs). Secondary objective was to determine the impact of remdesivir in the duration of mechanical ventilation of Covid-19 patients and ICU-mortality and hospital mortality of both groups.

Methods: We performed a single center retrospective cohort study. All adult patients admitted in ICU with SARS-CoV-2 pneumonia were eligible for the study. SARS-CoV-2 pneumonia was diagnosed using clinical and radiologic and microbiological criteria. Statistical assessment of the outcomes was made between remdesivir-treated group vs SC group.

Results: 43 patients (mean age 61 years, 65\% males) were included in the analysis (36 in SC group and 7 in the remdesivir group). The ICU length of stay ( 8.8 days vs 12.1 days, $p=0.408$ ) and duration of mechanical ventilation ( 4.9 days vs 8.1 days, $p=0.41$ ) were lower in the remdesivir-treated group. In addition, remdisivir-treated group was associated with greater SOFA score improvement $(p=0.036)$ and greater ACECOWS improvement $(p=0.030)$ at ICU discharge. Till now, no deaths were registered in the remdesivir-treated group, either during ICU or hospital stay in our cohort.

Conclusion: Despite our small cohort, remdesivir seems to be superior to SC in the treatment of Covid-19 patients, with a faster and more marked clinical improvement at ICU discharge.

\section{Reference(s) and grant ackowledgment(s)}

1. [1] Beigel JH, Tomashek KM, Dodd LE, et al. Remdesivir for the treatment of Covid-19 - preliminary report. N Engl J Med. https://doi.org/10.1056/ nejmoa2007764.
001471

Characteristics of positive blood cultures over time in a cohort of COVID-19 patients in intensive care in Glasgow, Scotland C. McCue ; S. Pybus' 2 M. Macleod ${ }^{2}$; M. Murphy²; K. Puxty ${ }^{1}$

${ }^{1}$ Intensive care, Glasgow Royal Infirmary, Glasgow, United Kingdom; ${ }^{2}$ Microbiology, Glasgow Royal Infirmary, Glasgow, United Kingdom

Correspondence: C. McCue

Intensive Care Medicine Experimental 2020, 8(2): 001471

Introduction: During the UK peak of the COVID-19 pandemic, many intensive care units expanded bed capacity rapidly. In our own unit, our bed capacity peaked at $258 \%$, with 37 days spent above $100 \%$ capacity. Combined with this; redeployment of staff from other areas and use of personal protective equipment understandably leads to concerns about increased rates of hospital acquired infection, or crosscontamination between patients. We sought to examine our own experience through analysis of blood stream infections.

Methods: We identified all patients admitted to our intensive care between March and May 2020 with COVID-19 pneumonia. Data was collected on demographics and outcome from our local critical care database Wardwatcher, and positive microbiology from blood cultures obtained peripherally or from invasive lines.

We categorised positive results into pathogenic organisms, likely contaminants, and low pathogenicity organisms associated with nosocomial infection. To review results over time, we categorised into first 48 hours, 3 to 7 days, 7 to 21 days, and after 22 days of ICU stay. Analysis was performed in Excel.

Results: From March to May 2020, we admitted 52 patients with COVID-19 pneumonia of whom $96 \%$ were invasively ventilated. $71 \%$ of patients were male, median age 57 , median length of stay 16 days and overall ICU mortality was $27 \%$. All ventilated patients had arterial and central venous catheters (CVC) placed, with those experiencing longer stays having lines replaced if concerns about possible line infection. Overall we documented 61 positive blood culture results of which $16 \%$ were pathogenic (Klebsiella pneumoniae, and Candida albicans), the majority of these grew from samples from CVCs and represent 3 separate patient episodes (6\%). 31\% were nosocomial associated organisms (Enterobacter species, one vancomycin resistant enterococcus faecium), again the majority from CVC samples.

Over time, we saw the majority of positive blood cultures occurring after 7 days, with both pathogenic and nosocomial organisms peaking between 7 to 22 days (see Fig 1).

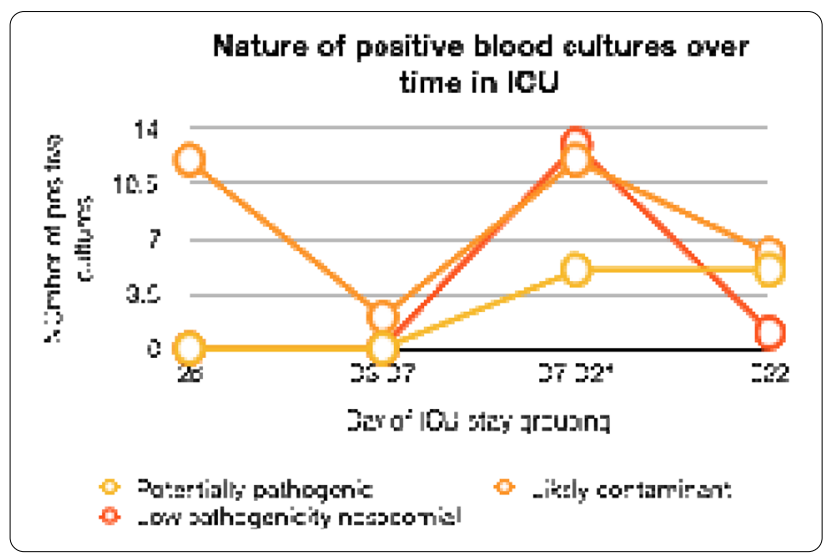

Conclusion: Our results demonstrate similar rates of pathogenic blood stream infections compared to published literature of $8 \%(1)$, the majority of which occurred after 7 days in ICU, similar to a recently published cohort from UK(2). This concurs with other published evidence on the need for focus on infection control measures to reduce morbidity in this patient cohort when faced with the unique challenges associated with a pandemic. 
Reference(s) and grant ackowledgment(s)

1. 2. Sturdy A., Basarab M., Cotter M., et al. Severe COVID-19 and healthcareassociated infections on the ICU: time to remember the basics? J Hosp Infect. 2020 Aug; 105(4): 593-595.

2. 1. Rawson T.M., Moore L.S.P., Zhu N., et al. Bacterial and fungal co-infection in individuals with coronavirus: a rapid review to support COVID-19 antimicrobial prescribing. Clin Infect Dis. 2020.

\section{4}

Impact of Biofire ${ }^{\circledR}$ FilmArray ${ }^{\circledR}$ on the Management of Patients with Severe Respiratory Infections in the ICU of a Provincial Hospital in Northern Greece

I. Chouris ${ }^{1}$; K. Marmanidou'; V. Radu ${ }^{1}$; M. Stougianni ${ }^{1}$; A. Pappa-Konidari ${ }^{2}$ D. Lagonidis ${ }^{1}$

${ }^{1}$ Intensive care unit, Giannitsa General Hospital, Giannitsa, Greece; ${ }^{2}$ Professor, 1st laboratory of microbiology, Medical School AUTH, Thessaloniki, Greece

\section{Correspondence: I. Chouris}

Intensive Care Medicine Experimental 2020, 8(2): 001474

Introduction: Respiratory infection is a frequent reason for ICU admissions or complications. It is a condition associated with substantial morbidity and mortality. Multiple organ failure is often observed in this setting. Timely management and appropriate antibiotic selection are of paramount importance in order to achieve favorable outcomes. Biofire ${ }^{\circledR}$ FilmArray ${ }^{\circledR}$ Pneumonia Panel Plus is a multiplex PCR platform that may rapidly identify the pathogen. It may detect 18 bacterial and 8 viral pathogens commonly associated with CAP, as well as 7 antibiotic resistance genes.

Objectives: We examine the impact of the use ofBiofire ${ }^{\circledR}$ FilmArray ${ }^{\circledR}$ in the management of ICU patients with severe respiratory infections requiring mechanical ventilation in a six bed general ICU of a provincial hospital in Northern Greece.

Methods: It is a retrospective observational study of the impact of the use of Biofire ${ }^{\circledR}$ FilmArray ${ }^{\circledR}$ in the treatment of patients with severe respiratory infections and respiratory insufficiency requiring mechanical ventilation during one year. The parameters analyzed included: demographics, microbiological and routine laboratory data, use of antibiotics, clinical outcome. Also, we defined two groups, depending on whether Biofire ${ }^{\circledR}$ FilmArray ${ }^{\circledR}$ was tested within 48 hours from admission or not.

Results: A total of 19 patients were included in the study. In 13 of them, Biofire ${ }^{\circledR}$ FilmArray ${ }^{\circledR}$ was positive. Ten tests revealed the presence of multiple pathogens; in 5 tests, 9 "fastidious" (i.e. difficult to grow without specific conditions) microbes were detected, (Haemophilous inf, Streptococcus pne), 6 were positive for viruses (Influenza A, Rhinovirus, Parainfluenza), 8 tests identified 12 common ICU pathogens (Acinetobacter, Klebsiella, Pseudomonas), in two cases Legionella was diagnosed and in one case Moraxella. Routine cultures yielded negative results in most of these cases $(84,6 \%$ bronchial secretions, $76,9 \%$ pharyngeal). The test results led to antibiotic treatment modification in $76,9 \%$. In 4 samples the presence of antibiotic resistance genes was noted and appropriate antibiotics were selected.

Conclusion: The introduction and integration of Biofire ${ }^{\circledR}$ FilmArray ${ }^{\circledR}$ in the clinical practice, had a decisive impact in the treatment of the patients. This was demonstrated not only in minimizing the time to obtain the results, but also when considering that the routine cultures collected on admission to the ICU often yielded negative results. Thus, Biofire ${ }^{\circledR}$ FilmArray ${ }^{\circledR}$ provided a valid identification of the pathogen and, at the same time, allowed for a prompt de-escalation of the broad-spectrum antibiotics initially administered. In conjunction with the economic implications of this practice, sparing unnecessary antibiotic usage may be beneficial for both the patients and the antibiotic resistance patterns of the nosocomial flora.

\section{Reference(s) and grant ackowledgment(s)}

1. • Lee SH, Ruan SY, Pan SC, Lee TF, Chien JY, Hsueh PR. Performance of a multiplex PCR pneumonia panel for the identification of respiratory pathogens and the main determinants of resistance from the lower respiratory tract specimens of adult patients in intensive care units. J Microbiol Immunol Infect. 2019;52(6):920-928. https://doi.org/10.1016/j.jmii.2019.10.009.

2. • Buchan BW, Windham S, Balada-Llasat JM, et al. Practical Comparison of the BioFire FilmArray Pneumonia Panel to Routine Diagnostic Methods and Potential Impact on Antimicrobial Stewardship in Adult Hospitalized Patients with Lower Respiratory Tract Infections. J Clin Microbiol. 2020;58(7):e00135-20. Published 2020 Jun 24. https://doi.org/10.1128/ jcm.00135-20.

001487

Do common CONTACT ISOLATION CRITERIA PREDICT THE CARRIER STATE OF MULTIDRUG-RESISTANT MICROORGANISMS IN CRITICALLY ILL PATIENTS?

L. López-Rodríguez; ; D. Vasco²; D. Manzano²; F. Palacios²; JA. Lorente ${ }^{3}$ ${ }^{1}$ Intensive Care, Universtiy Hospital of Getefe, Madrid, Spain; ${ }^{2}$ Intensive care, University Hospital of Getafe, Madrid, Spain; ${ }^{3}$ Intensive care., University Hospital of Getafe. CIBER of Respiratory Diseases. European University, Madrid, Spain

Correspondence: L. López-Rodríguez

Intensive Care Medicine Experimental 2020, 8(2): 001487

Introduction: Despite the widespread use of contact isolation criteria $(\mathrm{CIC})$ in critically ill patients, their ability to predict the carrier state of multidrug-resistant microorganisms (MDRO) has not been well defined.

Objectives: To determine the ability of commonly used $\mathrm{CIC}$ to predict the carrier state of MDRO in critically ill patients.

Methods: Patients admitted consecutively to the Intensive Care Unit (ICU) were studied. Inclusion criteria were all patients admitted from July 2018 to December 2019. Exclusion criterion was previous ICU admission.

Pharyngeal and rectal swabs were collected at admission and weekly for epidemiological surveillance cultures. The presence of any of the following CIC was registered: (1) hospital admission for longer than 6 days over the previos 3 months; (2) admission in a chronic health care facility; (3) known MDRO carrier; (4) antibiotic treatment for more than 6 days in the previous month; (5) receiving hemodialysis or peritoneal dialysis; (6) diagnosis of a chronic condition associated with colonization by MDRO; (7) transferred from another institution.

Sensitivity (Sn), specificity (Sp), positive predictive value (PPV) and negative predictive value (NPV) to predict the carrier state by MDRO were calculated for each of the criteria. Values are percentage and median (p25, p75). WASPSS (Wise Antimicrobial Stewardship Support System) and SPSS V25.0 were used for the analysis. The local Ethics Committee approved the study.

Results: We studied 1,156 patients (age 70 [61-78] years, SAPS III 55.5 [47-66] points, ICU non survivors 42 [26.6\%], women 50 [31.6\%]) of whom $158(13.7 \%)$ were isolated for having at least one of the CIC. Carrier state was confirmed by culture of the first surveillance sample in 40 patients. The 7 criteria were present, respectively, in $123(12 \%), 11$ (1\%), 21 (2\%), 76 (7\%), 6 (1\%), $15(1 \%)$ and 24 (2\%). For each criteria considered individually, Sn was $<50 \%$ (except for criterion 1: Sn 67\%) and PPV $<30 \%$ (except criterion 3, PPV 52\%). Each criteria considered individually had Es and NPV > 90\%. At least one of the CIC was found in 158 (15\%) patients (Sn 80\%, Es 88\%, PPV 20\%, NPV 99\%).

Conclusion: Individual CIC, in general, have a very low $\mathrm{Sn}$ and a high $\mathrm{Sp}$ for the detection of MDRO carrier state. None of the $\mathrm{CIC}$ is useful enough to determine the carrier state, since Se and PPV (which should be interpreted with caution as it depends on the prevalence) are low. The use of a set of $7 \mathrm{CIC}$ presents a moderate Se for the detection of MDRO carrier state.

\section{Reference(s) and grant ackowledgment(s)}

1. Djibré M, Fedun S, Le Guen P, Vimont S, Hafiani M, Fulgencio JP, Parrot A, Denis M, Fartoukh M. Universal versus targeted additional contact precautions for multidrug-resistant organism carriage for patients admitted to an intensive care unit. Am J Infect Control. 2017 Jul 1;45(7):728-734.

2. FEDER. B2017/BMD-3727-EXOHEP-CM 
001493

Effect of antibiotic de-escalation on the murine microbiota and immune system under environmental antimicrobial resistance pressure: recreating ICU environmental pressure in a laboratory mouse model

I. Lakbar'; M. Ben-Khedher²; J. Delerce²; JM. Rolain²; F. Bregeon²; M. Leone ${ }^{3}$ ${ }^{1}$ Anesthesiology and intensive care medicine, University Hospital De Toulouse, Toulouse, France; ${ }^{2}$ Aix-marseille university, ird, aphm, mephi, Mediterranean Infection, Marseille, France; ${ }^{3}$ Department of anesthesiology and intensive care medicine, University Hospital of Marseille, Marseille, France

Correspondence: I. Lakbar

Intensive Care Medicine Experimental 2020, 8(2): 001493

Introduction: De-escalation consists in the reappraisal of anti-microbial therapy as soon as antimicrobial susceptibility testing results are available, and may be achieved through replacing broad-spectrum antibiotics by narrow-spectrum drugs, or stopping components of an antibiotic combination. No study has been performed so far to explore this strategy on animal models, and its effect on microbial diversity and resistance genes in the microbiota and on the immune system remains unknown.

Objectives: Here, we aimed to study the effect of antibiotic deescalation versus non de-escalation on a microbiota exposed to environmental anti-microbial resistance pressure, both on microbial diversity and resistance genes in the microbiota, but also on the immune system.

Methods: Mice were divided in three groups, whether they received amoxicillin, imipenem, or imipenem followed by amoxicllin. The ICU environment was recreated by co-housing antibiotic-treated mice with mice previously contaminated with carbapenemase-carrying E.coli. Stools were cultured in selective media supplemented with cefotaxime, ertapenem and colistin, and the stool DNA was extracted for PCR and whole metagenomics analysis. Blood cytokines were measured by ELISA.

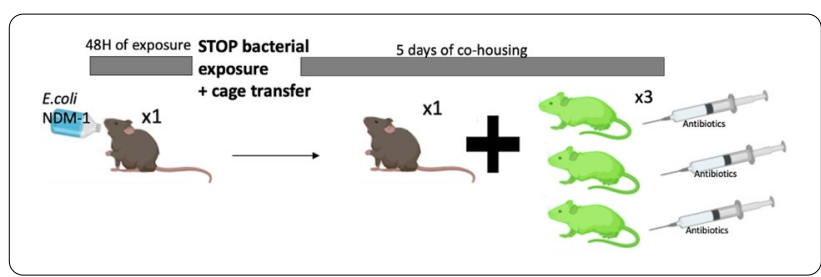

Results: Stool culture revealed the carriage of carbapenemase-positive E.coli, confirmed by PCR in 7/8 mice in the imipenem group, 5/10 mice in the de-escalation group and 7/9mice in the amoxicillin group $(p>0.05)$. Metagenomics analysis showed no difference in diversity and in antimicrobial resistance genes between groups. ELISA tests are still ongoing.

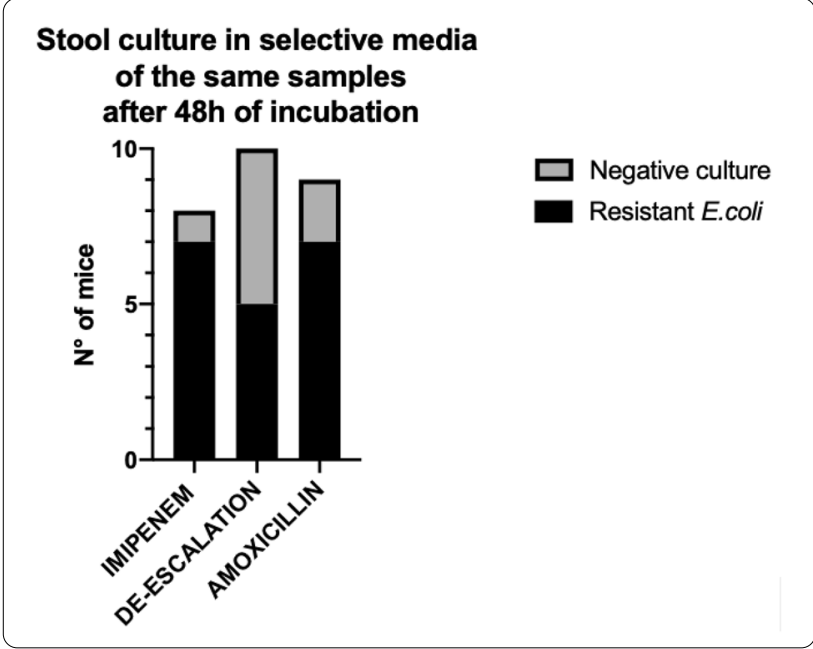

Conclusion: There were no difference between groups in terms of antimicrobial resistance emergence, as confirmed by culture, PCR and metagenomics techniques. Antibiotic de-escalation does not prevent the acquisition of resistance genes compared to narrow or broadspectrum antibiotic regimen.

\section{Reference(s) and grant ackowledgment(s)}

1. Lakbar I, De Waele JJ, Tabah A, Einav S, Martin-Loeches I, Leone M. Antimicrobial De-Escalation in the ICU: From Recommendations to Level of Evidence. Adv Ther. 2020;37(7):3083-3096. https://doi.org/10.1007/s1232 5-020-01390-2.

2. European Society of Intensive Care (ESICM), Levi-Montalcini Biomedical science Award.

3. Groupe Pasteur Mutualité, Bourse de Recherche Villa M.

\section{9}

Serum ACE "1" levels in critically ill patients with COVID-19 disease

DJ. Kirkin ${ }^{1}$; A. Gardner'; S. Rodriguez-Villar²

${ }^{1}$ Anaesthetics, King's College Hospital, London, United Kingdom; ${ }^{2}$ Intensive care medicine, King's College Hospital, London, United Kingdom

Correspondence: D.J. Kirkin

Intensive Care Medicine Experimental 2020, 8(2): 001509

Introduction: Genetic similarities with SARS-CoV-1, and subsequent biophysical data, suggested a role for Angiotensin Converting Enzyme 2 (ACE2) in the SARS-CoV-2 invasion of pneumonocytes1,2,3. Subsequently, other proteins constituting the Renin-Angiotensin-Aldosterone System (RAAS) have been scrutinised for their relevance to the COVID-19 pathology. Such proteins may be pharmacological targets benefitting outcomes, or biomarkers of disease severity.

Angiotensin Converting Enzyme (ACE) performs a key role within the RAAS, cleaving angiotensin 1 to angiotensin 2 (ANG-2). ACE is prevalent in the pulmonary epithelium, and thus equally ANG-2. ANG-2 is a potent vasoconstrictor, and associated with inflammation, fibrosis and oxidative stress4. It has been implicated in COVID-19 disease severity, raised ANG-2 has been reported in cases of COVID-195 and in mice studies of ARDS is associated with severe lung injury6.

While much literature focusses on the regulatory functions of ACE2 on ANG-2, we considered a potential role for measuring serum ACE to monitor disease severity. We hypothesised that unknown pathological mechanisms would modify serum ACE levels, given an increase in ANG-2. We were unclear whether this may be through a negative or positive feedback mechanism.

Objectives: To perform opportunistic isolated serum ACE levels on COVID-19 patients admitted to our Intensive Care Unit (ICU), during a 1 -month period, to determine whether it was objectively altered during the disease state. This pilot study would justify future, systematic investigation.

Methods: Within the analysis period we tested 28 patients admitted to our District General Hospital ICU, London. Severe Acute Respiratory Syndrome Coronavirus 2 infection had been diagnosed by reversetranscriptase polymerase-chain-reaction for 26 patients and 2 with compelling radiographic evidence. Data on ACE-inhibitor use was not available, though it is likely no patient used them within 7 days of testing.

Results: Of the patients tested, 26 had a normal serum ACE level (8-52 IU/L) representing $93 \%$ of the sample. One patient's level was objectively low $(<5 \mathrm{IU} / \mathrm{L})$, and another high $(78 \mathrm{IU} / \mathrm{L})$. The overall cohort mortality was $32 \%$. Of note, both objectively abnormal patients died.

Conclusion: ANG-2 is regulated in its production by $A C E$, and removal by ACE-2. Any increase in ANG-2 activity may be resultant from an excess of the former, and/or a deficiency in the latter. Given the largely normal serum ACE amongst the population, it further implicates a downregulation of ACE-2 in the pathophysiology of severe COVID-19. Murine and in vitro studies of SARS-CoV-1 observed decreased ACE-2 expression following its interaction with the viral spike protein7, 8. 
ACE does not appear to be reliably modified during the COVID-19 disease process for use as a biomarker, or to justify future biochemical analysis; an important negative finding for ongoing investigations into the relevance of the RAAS pathway to COVID-19. Robust investigation of the changes to ANG-2 regulation, particularly relating to ACE-2, is required.

\section{Reference(s) and grant ackowledgment(s)}

1. 1. Lu R, Zhao X, Li J, et al. Genomic characterisation and epidemiology of 2019 novel coronavirus: implications for virus origins and receptor binding Lancet. 2020;395(10224):565-574.

2. 2. Hoffmann M, et al. SARS-CoV-2 cell entry depends on ACE2 and TMPRSS2 and is blocked by a clinically proven protease inhibitor. Cell, 181 (2020), pp. 271-280.

3. 3. Zhou $P$, Yang $X L$, Wang $X G$, et al. A pneumonia outbreak associated with a new coronavirus of probable bat origin. Nature. 2020;579(7798):270-273.

4. 4 South AM, Diz DI, Chappell MC. COVID-19, ACE2, and the cardiovascular consequences. Am J Physiol Heart Circ Physiol. 2020;318(5):H1084-H1090.

5. 5. Liu Y, Yang Y, Zhang C, et al. Clinical and biochemical indexes from 2019$\mathrm{nCoV}$ infected patients linked to viral loads and lung injury. Sci China Life Sci. 2020;63(3):364-374.

6. 6. Imai Y, Kuba K, Rao S, et al. Angiotensin-converting enzyme 2 protects from severe acute lung failure. Nature. 2005;436(7047):112-116.

7. 7. Imai Y, Kuba K, Penninger JM. The discovery of angiotensin-converting enzyme 2 and its role in acute lung injury in mice. Exp Physiol. 2008;93(5):543-548

8. 8. Glowacka I, Bertram S, Herzog P, et al. Differential downregulation of ACE2 by the spike proteins of severe acute respiratory syndrome coronavirus and human coronavirus NL63. J Virol. 2010;84(2):1198-1205.

\section{1}

\section{Is there A RELATIONSHIP BETWEEN THE CARRIER STATE} OF MULTIDRUG-RESISTANT MICROORGANISMS AND MORTALITY IN CRITICALLY ILL PATIENTS?

L. López-Rodríquez'; D. Manzano ; D. Vasco ; F. Palacios'; ; J. Lorente

${ }^{1}$ Intensive Care, University Hospital of Getafe, Madrid, Spain; ${ }^{2}$ Intensive care., University Hospital of Getafe. CIBER of Respiratory Diseases. European University, Madrid, Spain

Correspondence: L. López-Rodríguez

Intensive Care Medicine Experimental 2020, 8(2): 001511

Introduction: The implementation of isolation measures in critically ill patients with multidrug-resistant microorganisms (MDRO) is resource consuming. However, whether patients who are carriers of MDRO have a higher mortality is not well established.

Objectives: To determine whether the carrier state (CS) of MDRO in critically ill patients is associated with mortality.

Methods: Patients admitted consecutively to Intensive Care Unit (ICU) from July 2018 to December 2019 were studied. Inclusion criteria were patients who met any of the following contact isolation criteria: (1) hospital admission for longer than 6 days over the previos 3 months; (2) admission in a chronic health care facility; (3) known MDRO carrier; (4) antibiotic treatment for more than 6 days in the previous month (5) receiving hemodialysis or peritoneal dialysis; (6) diagnosis of a chronic condition associated colonization by MDRO; (7) transferred from another institution. Exclusion criterion was previous ICU admission. Demographic variables and SAPS III were recorded. Survivors (SUR) and non survivors (NONSUR) were compared using a univariate analysis (Chi-square test) and a multivariate logistic regression analysis, adjusting for SAPS III score. Values are percentage and median $(p 25, p 75)$. The strength of the association was measured by the odds ratio (OR) and its $95 \%$ confidence interval $(\mathrm{Cl})$. The WASPSS (Wise Antimicrobial Stewardship Support System) and SPSS V25.0 were used. The local Ethics Committee approved the study.

Results: We studied 192 patients, NONSUR 52 (27.1\%), CS 66 (34.4\%). NONSUR, as compared to SUR were older (75.5 [65.0-79.0] vs. 69.0 [58.3-77.8] years, $p=0.074)$, had a higher SAPS III score (64, 5 [51.575.8 ] vs. 54.0 [45.0-62.0], $\mathrm{p}=0.001$ ), had a lower proportion of women
(17.5\% vs. $82.5 \%, p=0.037)$, and a similar proportion of patients with CS (18 [34.6\%] vs. 48 [34.3\%], $p=0.548$. In univariate analysis, the mortality of CS versus non CS was not different (18 of 66 [27.3\%] vs. 34 of 126 [27.0\%], $p=0.548$ ). In multivariate analysis, after adjusting for SAPS III score, CS was not significantly associated with mortality (OR 1,$171 ; 95 \% \mathrm{Cl} 0.574-2.388, \mathrm{p}=0.665$ ).

Conclusion: MDRO carrier state is not associated with increased mortality in critically ill patients.

Reference(s) and grant ackowledgment(s)

1. Djibré M, Fedun S, Le Guen P, Vimont S, Hafiani M, Fulgencio JP, Parrot A, Denis M, Fartoukh M. Universal versus targeted additional contact precautions for multidrug-resistant organism carriage for patients admitted to an intensive care unit. Am J Infect Control. 2017 Jul 1:45(7):728-734.

2. FEDER. B2017/BMD-3727-EXOHEP-CM.

\section{6}

INFECTIONS IN PATIENTS WITH COVID-19 AND ACUTE

\section{RESPIRATORY FAILURE requiring MECHANICAL VENTILATION}

L. López-Rodríguez'; F. Palacios'; D. Vasco'; D. Manzano'; JA. Lorente

${ }^{1}$ Intensive care, University Hospital of Getafe, Madrid, Spain; ${ }^{2}$ Intensive care., University Hospital of Getafe. CIBER of Respiratory Diseases. European University, Madrid, Spain

Correspondence: $L$. López-Rodríguez

Intensive Care Medicine Experimental 2020, 8(2): 001516

Introduction: A more detailed knowledge of infectious complications in critically ill patients with COVID-19 may help the implementation of preventive measures and improve survival.

Objectives: To determine the prevalence of infections and the most frequent pathogens involved, and its impact on mortality, in patients with COVID-19 and acute respiratory failure requiring invasive mechanical ventilation.

Methods: We studied all patients admitted to our Intensive Care Unit (ICU) with the diagnosis of viral pneumonia due to SARS-CoV-2 and requiring invasive mechanical ventilation from March 8 to May 1 , 2020. Patients transferred to another facility were excluded. The end point was survival status on day 60 after ICU admission. All patients were treated with selective digestive decontamination (oral and enteral neomycin, tobramycin and nystatin). Data are median (p25p75) or $\mathrm{n}$ and percentage. Data were compared by the Chi square test or the Fisher exact test, as appropriate, or by the Mann- Whitney $\mathrm{U}$ test. Association between independent variables and survival status was analyzed by multivariate regression analysis adjusted by the SPAS III score, and measured by the odds ratio (OR) and its $95 \%$ confidence interval $(\mathrm{Cl})$. A p value $<0.05$ was considered statistically significant. The local Ethics Committee approved the study.

Results: We admitted 118 patients during the study period, of whom 6 were transferred to another facility. Of 112 patients, age was 67 (5962) years, 79 (70.5\%) were male, SAPS III score was 50 (46-55), 53 (47\%) had had at least one infection, and $41(37 \%)$ had died by day 60 . The prevalence of any infection was $(48 \%, 46 \%)$, primary bacteremia $(11 \%$, $10 \%)$, secondary bacteremia $(8 \%, 7 \%)$, catheter-related bacteremia $(18 \%, 27 \%)$, urinary tract infection (UTI) $(21 \%, 12 \%)$, for survivors and non survivors, respectively ( $p>0.3$ for all comparisons). Only ventilator associated pneumonia (VAP) differed in survivors and non survivors (4 of 71 [6\%] versus 8 of 41 [20\%], respectively, $p=0.029$; adjusted odds ratio $4.47,95 \% \mathrm{Cl} 1.11 ; 18.02$ )

The most common pathogens in all isolates were: for primary bacteremia $(n=12$ isolates), S. faecalis $6(50 \%)$, other grampositive cocci 6 $(50 \%)$; for secondary bacteremia $(n=8$ isolates), gramnegative bacilli $4(50 \%)$, S. faecalis $3(38 \%)$; for catheter-related bacteremia $(n=24$ isolates), S. epidermidis $12(50 \%)$, S. faecalis $7(29 \%)$; for VAP $(n=21$ isolates), S. aureus $5(24 \%)$, gramnegative bacilli $5(24 \%), \mathrm{S}$. faecalis 2 (10\%); for UTI (23 isolates), S. faecalis 9 (39\%), gramnegative bacilli 8 (25\%).

Conclusion: Infections are very prevalent in patients with COVID-19 and acute respiratory failure. VAP is strongly associated with survival. 
$\mathrm{S}$. faecalis and a variety gramnegative bacilli, along with $\mathrm{S}$. aureus in the case of VAP, are the most prevalent microorganisms isolated in these patients. Measures aimed at pneumonia prevention could improve survival of critically ill patients with COVID-19.

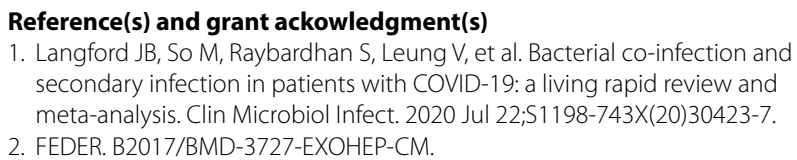

\section{7}

SARS-Cov2 pneumonia: Highlights from our experience

I. Guzmán Adum'; A. López Fernández'; J. Machado Casas '; J. Castaño Pérez

${ }^{1}$ Intensive care unit, Hospital Universitario Virgen de las Nieves, Granada, Spain

Correspondence: I. Guzman

Intensive Care Medicine Experimental 2020, 8(2): 001517

Introduction: Since Covid19 was first described, almost 17 million cases and more than 665,000 deaths have been reported; therefore it is considered an emergency situation in public health of international interest. It is postulated that many characteristics of the most affected population have direct relationship with mortality.

Objectives: - To describe the main aspects related to the increased mortality of patients admitted with Covid19 in the ICU of the Virgen de las Nieves Hospital.

- Describe the differences between the characteristics and evolution of the patients admitted in our ICU and those published by other centers. Methods: Observational, retrospective and analytical study that includes the patients admitted to the ICU with Covid19 between March and July 2020. A database was created in SPSS with the demographic characteristics of the patients, comorbidities, APACHE II in the first $24 \mathrm{~h}$, laboratory tests related to inflammation and coagulation and type of respiratory support. Data was evaluated in terms of descriptive statistics with frequencies, means and standard deviation for variables with normal distribution and median with interquartile range for non-parametric distributions; Comparisons between groups according to mortality were made using the Student's T test for quantitative variables and chi-square for qualitative variables.

Results: Of the 58 patients included, $79 \%$ were men and $21 \%$ women. Their mean age was 60 years $(+/-11)$, their main comorbidities were: hypertension (54\%), diabetes mellitus (35\%), obesity (56\%), COPD (28\%), kidney failure (9\%). ICU mortality 33\%, hospital mortality $39 \%$. SCID score mean was $1.94(+/-1.3)$. Analytically, the difference between the ferritin on the first and seventh day (delta ferritin) stands out with an average of $306(+/-2190)$, the comparison of delta ferritin between the living and the dead had a $\mathrm{p} 0.029$. Patients exclusively with IMV were $32(54 \%)$ and combination of HFNO+IMV $17(29 \%)$ average hours of HFNO prior to IMV was 6 (2-23), days of IMV 19 (1026), APACHE II in patients with combined MV $12(+/-4)$ and exclusive IMV $15(+/-4)$ with a $\mathrm{p}=0.007, \mathrm{pO} / \mathrm{FiO} 2$ in patients with combined VM 148 (+/- 54), exclusive IMV $120(+/-45) \mathrm{p}=0.04$, mortality of patients with combined VM $25 \%$ and exclusive VM $30 \%(p=0.16)$.

Conclusion: -Delta ferritin and not the ferritin peak is independently associated with mortality. The lower the ferritin clearance capacity is, the higher the mortality.

-Patients who received HFNO prior to IMV had similar mortality to those with IMV since the admission, contrary to what was postulated regarding self-inflicted lung injury. We believe that these findings are related to the fact that patients with the combined approach had significantly lower APACHE II and higher pO2/FiO2.

-Despite what has been published regarding the importance of coagulopathy, according to the diagnostic scale for sepsis induced coagulopathy, our patients did not present coagulation disorders.
001518

Outbreak of New Delhi metallo- $\beta$-lactamase (NDM)-1

Carbapenemase-Producing Enterobacteriaceae during Covid pandemic in ICU of a second level Hospital

L. Castro-Bournissen ; F. Dos Santos Alvernia'; A. Alvarez-Mendez ; P. Perez

Fernandez'; A. Del Caño Garcia'; M. Lopez-Gil ${ }^{1}$; M. Mas Lodo'; D. Varillas

Delgado²; N. Franco Garrobo

${ }^{1}$ Intensive care unit, Hospital Universitario de Móstoles, Móstoles, Spain; ${ }^{2}$ Unidad de apoyo a la investigación, facultad de medicina, Universidad Francisco de Vitoria, Madrid, Spain

Correspondence: L. Castro-Bournissen

Intensive Care Medicine Experimental 2020, 8(2): 001518

Introduction: Carbapenemase-producing Enterobacteriaceae (CPE) can cause healthcare-associated infections with high mortality rates.

Objectives: We analised an outbreak of NDM carbapenemase in an increased capacity (35 beds) usual 12-bed ICU during the COVID-19 pandemic peak in Madrid.

Methods: Retrospective cohort study of patients suffering from SARS CoV-2 infection admitted to the ICU and other areas reconverted into ICU in Hospital de Mostoles in Madrid, Spain, during the peak of COVID-19 pandemic from March to June 2020. We collected data from electronic medical records on demographics, Simplified Acute Physiology Score III (SAPS III),comorbidities, type of ICU where the patient was admitted, treatments received prior to the carbapenemase outbreak, length of ICU admission and mortality in ICU.

Results: 13 (15.85\%) patients over 82 critically-ill patients with SARS CoV-2 infection admitted to ICU suffered from carbapenemase infection $(69 \%)$ or colonisation (31\%). Mean age was 61 and $84.6 \%$ were men. All patients except one had been admitted first to an ICU-converted area (92\%), including operating theatre, before moving, eventually, to our ICU.

COVID 19-specific treatment, administered according to our Hospital protocol, included Ceftriaxone and Azithromicin in $92.3 \%$ of the 13 patients, only one received Ceftriaxone alone; $100 \%$ received Lopinavir/Ritonavir, Hidroxychloroquine and steroids (Metilprenisolone or Dexametasone), 53\% Tocilizumab and Baricitinib and 15\% Anakinra and Beta-Interferon. All 13 patients had received, during ICU admission, broad-spectrum antibiotics. Mean length of stay in ICU was 24,2 days. Carbapenemase infection included $38.5 \%$ pneumonia, $15.4 \%$ tracheobronchitis and one UTI. All patients were treated with Ceftazidime-Avibactam plus Aztreonam as in vitro synergy was proven in the Hospital Lab. Mean SAPS III score 60.1 and mortality 38.5\%.

Conclusion: A significant number of our critically ill SARS CoV-2 patients suffered from different multidrug resistant bacterial infections. $13(15.85 \%)$ patients of the entire cohort of 82 developed NDM- 1 carbapenemase. They had a high mortality rate despite directed antibiotic therapy. Several factors may have contributed to the appearance of this outbreak in our Unit: high antibiotic selection pressure and extensive use of immunosuppressant therapy, workload burden, deficient implementation of infection control protocols generally in place in Spanish ICUs most possibly associated to working areas not usually used as ICUs (open wards and operating theatres, health professionals unfamiliar with protocols) and lack of active surveillance cultures.

\section{9}

Secondary infection in mechanically ventilated, critically ill-patients with COVID-19

P. Sánchez Díaz'; P. Molinero Sander ; R. De La Oliva'; P. Serrano'; A. Suarez-de-la-Rica²; E. Maseda ${ }^{2}$; I. Falces Romero ${ }^{3}$

${ }^{1}$ Department of anesthesiology and surgical critical care, Hospital Universitario La Paz, Madrid, Spain; ${ }^{2}$ Department of Anesthesiology and Surgical Critical Care, Hospital La Paz-madrid, Madrid, Spain; ${ }^{3}$ Department of microbiology and parasitology, Hospital Universitario La Paz, Madrid, Spain

Correspondence: P. Sánchez Díaz

Intensive Care Medicine Experimental 2020, 8(2): 001519

Introduction: Secondary infection in critically ill patients with COVID19 is poorly understood. It is challenging to discriminate between 
isolated COVID-19 viral and extra bacterial or fungal infection and there is paucity of data, mainly in critically ill patients (1). We investigated the secondary infection rate in COVID-19 critically ill patients, risk factors for infection and mortality.

Methods: We performed a retrospective analysis of critically ill COVID19 patients admitted to the Critical Care Unit (CCU) of the Hospital Universitario La Paz (Madrid, Spain), a 1300- bed teaching hospital, between 16 March 2020 and 17 May 2020. In that period a total of 3002 patients with COVID-19 were admitted to hospital, and our unit had a total of 83 beds during the pandemic period. All patients that needed invasive mechanical ventilation were included. We recorded patient demographic data, microbiology data and incidence of secondary infection during CCU stay, including ventilator associated pneumonia (VAP) and nosocomial bacteremia (primary and secondary), according to Center for Disease Control (CDC) criteria.

A total of 107 patients with a mean age 62.210.6 years were included. Incidence of secondary infection during CCU stay was $43.0 \%$ (46 patients), including nosocomial bacteremia (32.1\%, 34 patients) and $\operatorname{VAP}(32.7 \%, 35$ patients).

Results: In univariate analysis, age and coronary arterial disease were related to development of secondary infection. Age remained as an independent predictor of secondary infection in multivariate analysis (OR 1.053, 95\% Cl 1.010-1.098, $p=0.015$ ). Nor the use of systemic corticosteroids and tocilizumab was related to secondary infection.

Overall hospital mortality by 30 June 2020 was $68.2 \%$. Mortality was higher in older patients (mean age 65.08 .1 vs. 56.112 .8 years, $\mathrm{p}=0.001$ ), in patients infected by antimicrobial resistant pathogens $(94.1 \%$ vs. $63.3 \%, p=0.012)$ and in case of secondary infection $(82.6 \%$ vs. $57.3 \%, p=0.006$ ). Corticosteroids and tocilizumab administration was not related to mortality. Age and infection by antimicrobial resistant pathogens were independent predictors of mortality in multivariate analyses $(\mathrm{OR}=1.091,95 \% \mathrm{Cl} 1.039-1.145, \mathrm{p}<0.001$; and $\mathrm{OR}=8.742,95 \% \mathrm{Cl} 1.069-71.485, \mathrm{p}=0.043$, respectively).

The incidence of secondary infection in critically ill COVID-19 patients in our study is high $(43.9 \%)$, higher than reported by Ferrando et al (4), with and incidence of respiratory bacterial infection and bacteremia of $27.15 \%$ and $26.24 \%$, respectively, and Yang et al (5) (incidence of pneumonia and bacteremia of $11.5 \%$ and $2 \%$, respectively). Feng et al declared an incidence of secondary infection of $34.4 \%$ in the more severe subgroup of COVID-19 patients (6). Our cohort includes only patients under invasive mechanical ventilation, what may explain partially these differences. An important finding of our study is the lack of relationship of corticosteroids and tocilizumab use and development of infections and mortality, in contrast to findings published by Sommers et al, with a lower mortality rate but a higher infection occurrence with tocilizumab use (10). Ferrando et al also showed no association between corticoids and tocilizumab use and mortality (4). To our knowledge, this is the first study that focus on secondary nosocomial infections in mechanically ventilated, critically ill patients with COVID-19. Our study has several limitations: the retrospective nature of the analysis; the lack of invasive modalities to help diagnose VAP (bronchoalveolar lavage) due to infection control measures; and the absence of data regarding antibiotic treatment since appropriate empiric antibiotic treatment affects mortality.

Conclusion: In conclusion, our data suggest that the incidence of secondary infection is very high in critically-ill patients with COVID-19. Infections by antimicrobial resistant pathogens may have a significant impact in prognosis. We cannot rule out that infections by resistant pathogens was simply a marker of severity. Further studies on larger populations are needed to investigate secondary infection in critically ill COVID-19 patients.

\section{Reference(s) and grant ackowledgment(s)}

1. Rawson TM, Moore LSP, Zhu N, et al. Bacterial and fungal co-infection in individuals with coronavirus: A rapid review to support COVID-19 antimicrobial prescribing [published online ahead of print, 2020 May 2]. Clin Infect Dis. 2020;ciaa530. https://doi.org/10.1093/cid/ciaa530.

2. Torpy JM, Lynm C, Glass RM. JAMA patient page. Ventilator-associated pneumonia. JAMA. 2007;297(14):1616. https://doi.org/10.1001/ jama.297.14.1616.
3. Maseda E, Ramírez S, Picatto P, et al. Critically ill patients with community-onset intraabdominal infections: Influence of healthcare exposure on resistance rates and mortality. PLoS One. 2019;14(9):e0223092. https ://doi.org/10.1371/journal.pone.0223092.

4. Ferrando C, Mellado-Artigas R, Gea A et al. Patient characteristics, clinical course and factors associated to ICU mortality in critically ill patients infected with SARS-CoV-2 in Spain: a prospective, cohort, multicentre study. Rev Esp Anestesiol Reanim. 2020. https://doi.org/10.1016/j.redar 2020.07.003.

5. Yang $X, Y u Y, X u$ J, et al. Clinical course and outcomes of critically ill patients with SARS-CoV-2 pneumonia in Wuhan, China: a singlecentered, retrospective, observational study [published correction appears in Lancet Respir Med. 2020 Apr;8(4):e26]. Lancet Respir Med. 2020;8(5):475-481. https://doi.org/10.1016/s2213-2600(20)30079-5.

6. Feng Y, Ling Y, Bai T, et al. COVID-19 with Different Severities: A Multicenter Study of Clinical Features. Am J Respir Crit Care Med. 2020;201(11):1380-1388. https://doi.org/10.1164/rccm.202002-0445oc.

7. Hughes S, Troise O, Donaldson H, Mughal N, Moore LS. Bacterial and fungal coinfection among hospitalised patients with COVID-19: A retrospective cohort study in a UK secondary care setting [published online ahead of print, 2020 Jun 27]. Clin Microbiol Infect. 2020;S1198743X(20)30369-4. https://doi.org/10.1016/j.cmi.2020.06.025.

8. Langford BJ, So M, Raybardhan S, et al. Bacterial co-infection and secondary infection in patients with COVID-19: a living rapid review and meta-analysis [published online ahead of print, $2020 \mathrm{Jul}$ 22]. Clin Microbiol Infect. 2020;S1198-743X(20)30423-7. https://doi.org/10.1016/j. cmi.2020.07.016.

9. Guillon A, Hiemstra PS, Si-Tahar M. Pulmonary immune responses against SARS-CoV-2 infection: harmful or not? [published online ahead of print, 2020 Jul 17]. Intensive Care Med. 2020;1-4. https://doi. org/10.1007/s00134-020-06170-8.

10. Somers EC, Eschenauer GA, Troost JP, et al. Tocilizumab for treatment of mechanically ventilated patients with COVID-19 [published online ahead of print, 2020 Jul 11]. Clin Infect Dis. 2020;ciaa954. https://doi. org/10.1093/cid/ciaa954.

\section{4}

Preliminary Safety and Efficacy Evaluation of Inhaled 13C-Urea in Subjects with Pneumonia: Phase 1, Open-Label Study

J. Baca ${ }^{1}$; N. Parchim²; J. Femling ${ }^{1}$; R. Taylor ${ }^{1}$; S. Bussman ${ }^{1}$; L. Candelaria ${ }^{1}$; T. Norii ; M. Moyer ${ }^{3}$; R. Laliberte ${ }^{3}$; S. Patel ${ }^{3}$; R. Nowak ${ }^{3}$

${ }^{1}$ Emergency medicine, UNM Hospital, Albuquerque, United States of America; ${ }^{2}$ Internal medicine, The University of New Mexico, Albuquerque, United States of America; ${ }^{3}$ Emergency medicine, Henry Ford Hospital, West Grand Boulevard, Detroit, MI, USA, Albuquerque, United States of America

\section{Correspondence: N. Parchim}

Intensive Care Medicine Experimental 2020, 8(2): 001534

Introduction: Rapid identification of pneumonia-causing organisms is challenging in the emergency department setting. Delayed identification of organisms results in improper antibiotic selection and administration. A point-of-care, nebulized urea breath test for pneumonia caused by urease-producing bacteria could aid in proper antibiotic selection, but the safety of nebulized urea has not yet been established. We report initial safety outcomes and study feasibility in an ongoing investigation of this test. We further combine sputum and oropharengeal microbiome data to assess the potential utility of the inhaled urea breath testing.

Methods: We enrolled a pre-defined safety cohort of 15 emergency department subjects with radiographic and clinical evidence of pneumonia, and who were planned for outpatient treatment. Subjects in this cohort completed inhalation of a nebulized preparation of $50 \mathrm{mg} \mathrm{13C}$-urea dissolved in $3 \mathrm{~mL}$ of sterile water. Breath samples were collected before and after inhalation of 13C-urea and analyzed for exhaled 13CO2. Adverse events (AEs) were recorded from time of enrollment until completion of telephone follow-up at 48-96 hours post nebulization. Exhaled $13 \mathrm{CO} 2$ was measured before and after 13C-urea nebulization to calculate a normalized change from baseline. Student's T-test was used to determine significance of change over 
baseline. We further enrolled 54 subjects admitted for inpatient treatment of pneumonia in a broader safety and feasibility cohort. Forty-six patients completed telephone follow up. Tracheal aspirate and saliva samples were isolated from these patients for microbiome analysis.

Results: Initially, we screened 257 subjects for the study and excluded 242 for clinical signs of severe disease (Mild, self-limited cough was noted in 3 cases and nausea in one case after administration of 13C-urea. One episode of syncope was noted but was determined to be an exacerbation of a pre-existing condition and not related to the study product. From both cohorts, eight patients were determined to have significantly elevated exhaled $13 \mathrm{CO} 2$ and were found in post-hoc chart review to have high suspicion for bacterial pneumonia including elevated procalcitonin levels or bilateral pneumonia on imaging.

Conclusion: Mild cough was common after urea administration, though all subjects were able to complete nebulization. There were no reports of clinical deterioration related to the study drug. This study establishes a preliminary safety profile of inhaled 13C-urea in subjects with pneumonia. We noted significantly elevated exhaled13CO2 in eight subjects and are currently analyzing sputum microbiome data to ascertain the potential utility of the inhaled urea breath test.

\section{Reference(s) and grant ackowledgment(s)}

1. None

2. AVISA Pharmaceuticals.

\section{7}

Performance characterization of a new tracheostomy tube with subglottic suction port

U. Borg ${ }^{1}$; C. Cobb ${ }^{1}$; K. Bull

${ }^{1}$ Medical affairs, Medtronic Surgical Solutions, Boulder, United States of America

\section{Correspondence: $U$. Borg}

Intensive Care Medicine Experimental 2020, 8(2): 001537

Introduction: Introduction: Tracheostomy is a common procedure performed for the management of upper and lower airway conditions. There is evidence that secretions draining from the upper airways can collect on the top of the tracheostomy tube cuff and become a risk for tracheal aspiration resulting in infections [1]. Reports in the literature describes posterior tracheal wall injury from the subglottic suction port [2,3]. A new tracheostomy product (Voyager, Medtronic Boulder USA) has a suction port just above the cuff intended for evacuation of secretions collecting on top of the cuff. The new tracheostomy cannula has the suction port located in the lateral wall of the tube body. The purpose of this study was to characterize the suction performance and tracheal wall injuries in an animal model during mechanical ventilation.

Methods: Methods: 16 porcine underwent tracheostomy and received the Voyager tracheostomy tube. Eight animals (Group 1) had normal secretion viscosity (500centiPoise) liquid and 8 animals (Group 2) had thick simulated mucous (5000centiPoise) liquid infused at $3 \mathrm{ml} /$ hour on top of the cuff. The subglottic space was suctioned every 15 minutes for the 4 hour duration of the study using the subglottic suction port. The suction method was 3 cycles of 15 seconds on and 10 seconds off at $100 \mathrm{cmH} 2 \mathrm{O}$ negative pressure (Ohio Medical Push-To$\mathrm{Set}^{\mathrm{TM}}$ Vacuum Regulator, OHIO Medical, Gurnee, USA)[4]. Fluoroscopic images and data collection were performed through 4 hours following placement of the device. At the completion, the trachea was removed with the Voyager tracheostomy tube in situ and the site of the suction lumen exposed. Changes in the tracheal wall was assessed through macroscopic observations and given scores of 0-4 where 0 is no injury and 4 is necrosis[5].

Results: Results: Both groups had equal amounts of secretions infused on top of the cuff, Group1 $12.1 \mathrm{ml}$ and Group $211.9 \mathrm{ml}(\mathrm{p}<0.29)$ and in both groups secretion was recovered, Group $10 \mathrm{ml}$ and Group $28 \mathrm{ml}$ $(p<0.33)$. Damage to the trachea from the suction port, Group 1 had 1 animal scored as 2 and 3 as 1 and Group 2 had 3 animals scored as 1. No secretions were seen below the cuff in any Group.
Conclusion: Conclusion: There were no technical issues with the subglottic suction system and the suction lumen was patent during the experiment. The suction performance was similar between the groups with slightly less recovery of the 5000 centiPoise fluid as could be expected. Most animals in both Groups had no tracheal injury (0) or mild erythema (1) at the site of the suction port. One animal in Group 1 had edema (2) at the site of the suction port. The Voyager tracheostomy tube design with the suction port in the lateral wall allows for adequate removal of subglottic secretions. Only clinical studies will completely elucidate the long-term effect of the new design.

\section{Reference(s) and grant ackowledgment(s)}

1. 1. Kollef M, Skubas NJ, Sundt TM A Randomized Clinical Trial of Continuous Aspiration of Subglottic Secretions in Cardiac Surgery Patients CHEST 1999; 116:1339-1346 2. Vallés J, Millán S, Díaz E, Castanyer E, Gallardo X, MartínLoeches I, Andreu M, Prenafeta M, Saludes P, Lema J, Batlle M, Bacelar N, Artigas A. Incidence of airway complications in patients using endotracheal tubes with continuous aspiration of subglottic secretions. Ann Intensive Care. 2017 Nov 2;7(1):109. 3. Harvey RC, Miller P, Lee JA, Bowton DL,

MacGregor DA Potential Mucosal Injury Related to Continuous Aspiration of Subglottic Secretion Device Anesthesiology 2007; 107:666-9 4. Smulders K, van der Hoeven H,Weers-Pothoff I, Vandenbroucke-Grauls, C. A randomized clinical trial of intermittent subglottic secretion drainage in patients receiving mechanical ventilation. CHEST 2002; 121:858-862 5. Seguin P, Perrichet H, Le Pabic E, Launey Y, Tiercin M, Corre R, Brinchault G, Laviolle B Effect of Continuous versus Intermittent Subglottic Suctioning on Tracheal Mucosa by the Mallinckrodt TaperGuard Evac Oral Tracheal Tube in Intensive Care Unit Ventilated Patients: A Prospective Randomized Study Indian J Crit Care Med. 2018 Jan; 22(1): 1-4.

2. Authors are employees of Medtronic Medical Affairs.

3. Study sponsored by Medtronic Respiratory Interventions, Boulder CO USA.

001554

Managing critically ill patient in a French General hospital during a large COVID-19 outbreak

JY. Mootien'; V. Pointurier'; E. Virot'; L. Jandeaux'; G. Labro'; A. Dureau'; C. Ionescu' ; C. Mathien ${ }^{1}$; L. Pinto ${ }^{1}$;Y. Rabouel'; A. Poiidevin ${ }^{1}$; K. Kuteifan ${ }^{1}$

${ }^{1}$ Medical icu, GHRMSA, Mulhouse, France

Correspondence: J.Y. Mootien

Intensive Care Medicine Experimental 2020, 8(2): 001554

Introduction: The city of Mulhouse, situated in the Alsace region, has been one of the largest European clusters of SARS-CoV-2 infection after a gathering of more than 2000 people during several days. The Groupe Hospitalier de la Région de Mulhouse Sud-Alsace (GHRMSA) is a hospital complex with a total capacity of 2612 beds including 20 medical ICU beds and 16 surgical ICU beds respectively, covering the needs of about 480,000 people.

Objectives: The first patient was admitted in our medical ICU on March 2nd for severe acute respiratory syndrome and during the following first week 18 patients was admitted for the same reason. Our bed capacity was rapidly saturated. According to the occurring trend, quick interventions and innovations appeared to be more than essential so as to handle the expected coming tsunami.

Methods: During the first week Non - COVID -19 patients were transferred to the surgical ICU so as to admit only COVID -19 patients in our unit but by the end of the first week our medical ICU bed capacity was saturated. During the coming weeks, scheduled surgical and medical activities have had to be cancelled. The cardiac surgery ICU ( 4 beds) was transformed to a COVID-19 ICU, 16 additional beds were added in five operative and post-operative rooms and the surgical ICU was converted in a sole COVID-19 ICU too. During the third week a national as well as an international cooperation was set up in order to transfer ICU patients to other cities, regions and countries by air or by train so as to cope with the massive influx of critical patients. Finally on the fourth week patients were transferred to a 30-bed field military intensive care hospital deployed on the hospital car park by the French military medical service: I'Élément Militaire de Réanimation du Service de Santé 
des Armées (EMRSSA). This stood as an unprecedented experience in France.

Results: More than 340 critically ill patients have been admitted in our general hospital where more than 1500 patients have been hospitalized up to now. More than 140 critically ill patients have been admitted in our medical ICU and about $50 \%$ of them have been transferred to another hospital. 47 patients have been admitted in the field military intensive care hospital deployed on the hospital car park.

Conclusion: The organization set up so as to cope with the massive influx of critically ill patient will stand as an unprecedented experience in this setting. ICU medical and non-medical staffs working hand in hand with other colleagues and hospital administrators so as to take quick decision leaving behind frontiers and barriers provided a way to our will.

\section{Reference(s) and grant ackowledgment(s)}

1. Reference Kuteifan, K., Pasquier, P., Meyer, C. et al. The outbreak of COVID-19 in Mulhouse. Ann. Intensive Care 10, 59 (2020). https://doi.org/10.1186/ s13613-020-00677-5.

\section{9}

Descriptive analysis of the samples of bronchial aspirate culture in patients admitted with COVID-19 between March - May 2020 D. García Huertas ${ }^{1}$; P. Fernandez Florido ${ }^{1}$; A. Fuentes Calatayud ${ }^{2}$; CJF. Machado ${ }^{3}$

${ }^{1}$ Intensive care unit, University Hospital Complex of Granada, Granada, Spain; ${ }^{2}$ Intensive care, University Hospital Complex of Granada, Granada, Spain; Intensive care unit, Hospital Virgen de las Nieves, Granada, Spain Correspondence: A. Fuentes Calatayud

Intensive Care Medicine Experimental 2020, 8(2): 001589

Introduction: A significant number of patients admitted in ICU for Covid-19 have had bacterial isolatesin respiratory samples. Many of these isolates correspond to colonizations, but other patients havedeveloped bacterial superinfections during their evolution in the ICU, such as ventilator-associatedpneumonia (VAP). Data extracted from the ENVIN-HELICS registry.

Objectives: Our objective was to analyze the number of positive bronchial aspirates performed,the number of bronchial aspirates with a positive result, the rate of VAP and the most frequentmicroorganisms. Methods: Descriptive, observational, and retrospective study of bronchial aspirates that wereperformed in patients admitted with Covid19 during the months of March to May 2020 in the ICU of athird-level hospital.

Results: 49 patients on mechanical ventilation were included, of which $36(62 \%)$ had a bronchialaspirate culture withdrawn during their ICU stay. In 28 of them (77\%) it was positive. In total, 90 bronchial aspirates were performed. 74 microorganisms were isolated: $11(15 \%)$ were Gram positivemicroorganisms, 35 (57\%) Gram negative and 28 (38\%) fungi.

Conclusion: In patients admitted for coronavirus, the bacterial superinfection rates have been high, probably due to the severity of the patients and a high rate of use of MV. Most are caused by Gramnegative and fungi.

The data from our study, show us that even though we are facing a new virus and we are working indifferent conditions than usual, we should not forget the project \&quot;Zero pneumonia\&quot; as prevention ofVAP.

\section{2}

Cardiovascular events after community-acquired pneumonia: a systematic review and meta-analysis of observational studies A. Tralhão'; P. Povoa ${ }^{2}$

${ }^{1}$ Polyvalent Intensive Care Unit, Hospital de São Francisco Xavier, CHLO, Lisbon, Portugal; ${ }^{2}$ Polyvalent intensive care unit, Hospital de São Francisco Xavier, CHLO, Lisbon, Portugal, Lisboa, Portugal

Correspondence: $\mathrm{A}$. Tralhão

Intensive Care Medicine Experimental 2020, 8(2): 001592
Introduction: Acute cardiovascular disease after community-acquired pneumonia (CAP) is a well-accepted complication for which definitive treatment strategies are lacking. These complications share some common features but have distinct diagnostic and treatment approaches.

Objectives: To determine the incidence of overall and individual cardiac complications after CAP and its impact on short-term mortality (< 90 days).

Methods: Systematic review of published studies (Medline) of adult non-immunocompromised patients with clinical and radiological evidence of CAP for whom outcomes were explicitly reported, followed by a meta-analysis of proportions, using the double-arcsine transformation and a random-effects model. Meta-regression analysis by year of publication was further performed.

Results: From 1984 to November 2019, thirty-nine studies were accrued, involving 92,188 patients, divided by setting (inpatients versus outpatients) and clinical severity (low risk versus high risk). Overall cardiac complications occurred in 14\% (95\% confidence interval (CI) 9.6-19), acute coronary syndromes in $4.5 \%(95 \% \mathrm{Cl} 2.9-6.5)$, heart failure in $9.2 \%$ (95\% Cl 6.7-12), arrhythmias in 7.2\% (95\% Cl 5.6-9.0) and stroke in $0.71 \%(95 \% \mathrm{Cl} 0.1-3.9)$ of pooled inpatients. During this period, meta-regression analysis suggests that the incidence of overall and individual cardiac complications is decreasing. After adjusting for confounders, cardiovascular events taking place after CAP independently increase the risk for short-term mortality (range of odds-ratio: 1.4-5.5).

Conclusion: Despite a reduction in the last decades, the incidence of cardiac complications after CAP remains high. Our findings highlight the need for efective, large trial based, preventive and therapeutic interventions in this important patient population.

001593

Descriptive study of urine cultures in patients admitted to the ICU with COVID-19 during the months of March-May 2020

P. Fernandez Florido ; A. Fuentes Calatayud²; D. García Huertas ${ }^{1}$; CJF. Machado ${ }^{3}$

${ }^{1}$ Intensive care unit, University Hospital Complex of Granada, Granada, Spain; ${ }^{2}$ Intensive care, University Hospital Complex of Granada, Granada, Spain; ${ }^{3}$ Intensive care unit, Hospital Virgen de las Nieves, Granada, Spain

Correspondence: A. Fuentes Calatayud

Intensive Care Medicine Experimental 2020, 8(2): 001593

Introduction: Bacterial superinfections have been a very frequent finding in patients admitted to theICU diagnosed with COVID-19, some of them associated with devices, highlighting thecatheter associated urinary tract infection (CA-UTI).

Objectives: Our objective with this abstract is to analyze the results of urine cultures in patientsdiagnosed with COVID-19 admitted in UCI. Methods: Descriptive, observational, and retrospective study of urine cultures that werewithdrawn in patients admitted with COVID19, between March and May 2020 in theUCl of a third-level hospital. We analyze : demographic data, number of urine cultureswithdrawn, number of positive urine cultures, percentage of gram positive, gramnegative and fungi and incidence density of CA-UTI.

Results: We included 60 patients, $48(80 \%)$ were men and 12 women (20\%), median APACHEII score 14.1 points, median age 60.47 years. In 40 patients $(68.9 \%)$ a urine culturewas withdrawn, with a total number of urine cultures of 77 samples. The number ofpatients with a positive results were $18(31 \%)$, and the number of positives urineculture were 22 (28.5\%); negative results were 55 (71.4\%). Regarding themicrobiological isolates, in 5 urine cultures (22.7\%) gram positives were isolated, beingall of them E. faecalis. In 4 positive urine cultures (18.1\%), gram negatives wereisolated, being all of them E. coli, 2 of wich were ESBL. Also striking is the highpercentage of fungal isolates, in a total of 13 urine cultures (59\%). 11 of them were C.albicans, and the remaining 2 were C. glabrata.Of the 22 urine cultures with positive results, 14 of them were considered colonizationsor contaminants, while 8 of them were considered bacterial superinfections. The totalnumber of urinary catheterization days were 1118 , with a total of 7.16 urinary infectionsfor every 1000 days of urinary catheterization. 
Conclusion: Our study shows a high percentage of urine cultures with negative results. Amongpositive results, the majority of isolates have been considered colonization orcontamination, and not superinfection. These data can be explained by the presence ofdifferent confounding factors in these patients, since they are complex patients withhigh severity and extensive use of devicesAlthough most cultures are negative or contaminated, the urinary infection rate isabove the results obtained in other series of patients not admitted for COVID-19 in ourunit. This reminds us that although we work in different conditions than usual and witha new disease, we must not forget the prevention measures of CA-UTI Zero project.

\section{2}

COVID-19 and Bloodstream infections in ICU

A. L. Rios ${ }^{1}$; N. R. ; A. D. ; A. Marques ${ }^{1}$; P. Castelões ${ }^{1}$

${ }^{1}$ Medicina intensiva, Centro Hospitalar de Vila Nova Gaia/Espinho, Vila Nova de Gaia, Portugal

Correspondence: A. L. Rios

Intensive Care Medicine Experimental 2020, 8(2): 001612

Introduction: The coronavirus disease 2019 (COVID-19) is an emerging health threat.(1).

Sepsis, respiratory failure, acute respiratory distress syndrome, heart failure, and shock are commonly observed in these critically ill patients.(2).

Bloodstream infections (BSI) in recent pre-COVID19 studies has a reported rate between 3,5 and 5\% (3)(4). However Xiaochen Li et al found a rate of BSI of $14,1 \%$ in severe COVID-19 committed patients from Tongji Hospital. (5).

Objectives: To describe the rate and the pathogens involved in BSI in critically-ill COVID-19 patients, and to explore its impact in ICU outcomes.

Methods: Retrospective study of all critically-ill COVID-19 patients admitted in the ICU in a tertiary hospital in Northern Portugal.

Primary end-points: incidence of BSI, agent identification and primary infection site.

Secondary end-points: association with co-morbidities and admission scores of organ dysfunction. Evaluation of impact of BSI on ICU length of stay(LOS), organ-support requirements and mortality.

Results: Between March 17th and May 10th, 36 COVID-19 patients were admitted to our ICU, with median APACHE II 18,0 (IQR 10,75) and median SAPSII 42,0 (IQR 20,5). Median LOS was 16 days (IQR 16,5). 12 (33.3\%) patients developed a BSI during ICU stay, with Klebsiella pneumoniae being the most frequently isolated pathogen $(8,4 \%)$, followed by Pseudomonas aeruginosa $(5,6 \%)$ and MSSA $(5,6 \%)$. As for primary source, respiratory infection was the most frequent, accounting for $19,4 \%$ of all cases. Overall mortality was $25 \%(n=9)$.

Severity of comorbidities, as measured by the Charlson Comorbidity Index, age and gender were not associated with the occurrence of BSI. Higher SAPS II score at admission was significantly associated with the occurrence of BSI (45 vs 34; $p=0,03)$. Patients who developed BSI during ICU stay had more severe respiratory insufficiency, as measured by the $\mathrm{PO} 2 / \mathrm{FiO} 2$ ratio at admission (136 VS $171.5 ; \mathrm{p}=0,049$ ).

There was no correlation in need for invasive mechanical ventilation or pronning maneuvers; however there was an association with longer duration of mechanical ventilation $(p=0,002)$, need for traqueostomy and duration of vasopressor requirement $(p=0,029)$. Patients with $B S I$ also had longer ICU LOS (27 VS 14 days; $p<0,001)$. Regarding treatment options, there was an association between corticoid use and BSI, even with low doses used for shock management.(OR 9,8; Cl 95\%).

There was no impact on mortality.

Conclusion: In this sample, COVID-19 patients were more susceptible to BSI, which resulted in greater ICU LOS and greater length of invasive mechanical ventilation.

Main study limitations are the sample size and retrospective nature.

\section{Reference(s) and grant ackowledgment(s)}

1. Li X, Xu S, Yu M, et al. Risk factors for severity and mortality in adult

COVID-19 inpatients in Wuhan [published online ahead of print, 2020
Apr 12]. J Allergy Clin Immunol. 2020;S0091-6749(20)30495-4. https://doi. org/10.1016/j.jaci.2020.04.006.

2. Prowle JR, Echeverri JE, Ligabo EV, et al. Acquired bloodstream infection in the intensive care unit: incidence and attributable mortality. Crit Care. 2011;15(2):R100. https://doi.org/10.1186/cc10114.

3. European Centre for Disease Prevention and Control. Incidence and attributable mortality of healthcare-associated infections in intensive care units in Europe, 2008-2012. Stockholm: ECDC; 2018.

4. Zhou F, Yu T, Du R, et al. Clinical course and risk factors for mortality of adult inpatients with COVID-19 in Wuhan, China: a retrospective cohort study. Lancet. 2020;395(10229):1054-1062. https://doi.org/10.1016/s0140 -6736(20)30566-3.

5. World Health Organization. Coronavirus disease 2019 (COVID-19): situation report 44. Published March 4, 2020. Accessed March 16, 2020. https://www. who.int/docs/default-source/coronaviruse/situation-reports/20200304-sitre p-44-covid-19.pdf?sfvrsn = 783b4c9d_2.

\section{0}

Gastrointestinal complications in patients admitted to the icu with SARS-Cov 2 infection. Our experience in the current pandemic

B. Llorente Ruiz'; R. Molina Montero'; E. Serrano Yebenes²; M. Rascon Risco ${ }^{3}$; L. Alcazar Sanchez-Elvira'; A. Acha Aranda'; M. Trascasa Muñoz'; E. Lopez Ramos ${ }^{1}$; J. Higuera Lucas ${ }^{1}$; Y. Ortiz De Zarate'; C. Serrano ${ }^{1}$; A. Ruiz García ; R. Arraoz Illanes ${ }^{1}$

${ }^{1}$ Intensive care unit, Hospital Príncipe de Asturias, Alcalá de Henares, Spain ${ }^{2}$ General surgery, Hospital Príncipe de Asturias, Alcalá de Henares, Spain ${ }^{3}$ Radiodiagnostic, Hospital Príncipe de Asturias, Alcalá de Henares, Spain

Correspondence: $\mathrm{B}$. Llorente Ruiz

Intensive Care Medicine Experimental 2020, 8(2): 001620

Introduction: COVID-19 infection has been characterized by respiratory manifestations. After the experience of the current pandemic, it has been shown that the profile of manifestations of this infection is not limited exclusively to the respiratory system.

Objectives: To analyze the gastrointestinal manifestations of COVID19 infection in patients.

Methods: A retrospective observational study was conducted in a ICU of a second level hospital. We collected demographic variables, severity scales on admission, comorbidities, analytical data, treatment received, and mortality. Statistical analysis was performed with IBMâSPSS Statistics 25.

Results: 101 patients were admitted requiring transfer 26 due to the absence of beds in our Unit, so the sample of the present study is made up of 75 patients. Demographic variables, comorbidities, severity scales on admission to the ICU and treatment received are shown in Table 1. All were admitted with the diagnosis of severe respiratory failure secondary to atypical pneumonia due to SARS-CoV-2 without any clear digestive manifestation. The most frequent digestive complications are shown in Table 2. In the analysis by their group we found that age and obesity are associated with an increase risk of Ogilvie syndrome; those with a history of hypertension and cardiovascular disease more frequently developed diarrhea, lower gastrointestinal bleeding (LGB) and cholestasis; and dyslipidemic patients more frequently developed ischemic hepatitis. Regarding the treatment received, the use of lopinavir/ritonavir and tocilizumab was associated with an increase in cases of cytolysis; corticotherapy with the development of cytolysis and cholestasis; and interferon with constipation. It should be noted that in patients who developed AKI during their ICU admission had a higher incidence of mesenteric ischemia, ischemic hepatitis, LGB and cytolysis. When analyzing the inflammatory parameters, the determination of D-Dimer was significantly higher in patients with mesenteric ischemia, ischemic hepatitis and diarrhea; levels of IL- 6 were higher in those with cytolysis, diarrhea, ischemic hepatitis, mesenteric ischemia, and adinamic ileus; ferritin levels in patients with cholestasis, LGB and ischemic hepatitis; CRP in patients with diarrhea and ischemic hepatitis; and finally patients with cholestasis and cytolysis presented more severe lymphopenia. It is relevant 
in our sample that the patients who suffered mesenteric ischemia, diarrhea, cholestasis and cytolysis had a higher mortality than those who did not present these gastrointestinal complications.

\begin{tabular}{|c|c|c|c|}
\hline $\operatorname{Sex}(\precsim)$ & 67,6 & Age (years) & 59,66 \\
\hline Obesity (\%) & 80,3 & Hypertension (\%) & 43,4 \\
\hline Dyslipemia (\%) & 48,7 & DM (\%) & 22,4 \\
\hline Bronchopathy (\%) & 29,9 & & \\
\hline APACHE II & 16,83 & SOFA & 6,8 \\
\hline $\begin{array}{l}\text { Mechanical ventilation } \\
(\%)\end{array}$ & 100 & $\begin{array}{l}\text { Neuromuscular blockers } \\
(\%)\end{array}$ & 80,3 \\
\hline AKI (\%) & 46,1 & Vasoactive support (\%) & 98,7 \\
\hline Lopinavir/ritonavir (\%) & 90,8 & Darunavir/cobicistat (\%) & 11,8 \\
\hline Tocilizumab (\%) & 92.1 & Chloroquine (\%) & 92.1 \\
\hline Corticosteroids (\%) & 80.3 & Beta-interferon (\%) & 40.8 \\
\hline Enteral feeding (\%) & 94,6 & Parenteral nutrition (\%) & 43,2 \\
\hline
\end{tabular}

\begin{tabular}{|l|l|l|l|}
\hline \multicolumn{4}{|c|}{ Table 2: Gastrointestinal manifestations in COVID-19 infection } \\
\hline Cytolysis (\%) & 67,6 & Cholestasis (\%) & 64,9 \\
\hline Adynamic ileus (\%) & 37,8 & Diarrhea (\%) & 28,4 \\
\hline Constipation (\%) & 14,9 & Ogilvie syndrome (\%) & 10,8 \\
\hline Ischemic hepatitis (\%) & 5,4 & $\begin{array}{l}\text { Mesenteric ischemia } \\
\text { (\%) }\end{array}$ & 4,1 \\
\hline $\begin{array}{l}\text { Low digestive bleeding } \\
\text { (LDB) (\%) }\end{array}$ & 4,1 & $\begin{array}{l}\text { Upper digestive } \\
\text { bleeding (UDB) (\%) }\end{array}$ & 1,4 \\
\hline Collecistitis (\%) & 0 & Pancreatitis (\%) & 0 \\
\hline
\end{tabular}

Conclusion: Despite the fact that the reason for admission was pulmonary involvement by SARS-CoV-2, the development of various gastrointestinal disorders was observed in our cohort, with an increase in mortality in certain cases. The development of ARF increases the risk of Gl complications as well as the use of lopinavir/ritonavir, tocilizubam and corticosteroids. Inflammatory parameters can be useful to identify patients at risk of $\mathrm{Gl}$ manifestations.

\section{5}

Point of Care Ultrasound is highly predictive in the diagnosis of COVID-19

J. Powys-Lybbe'; C. Moxey ; O. Blackford'; L. Tong ' ; O. Valencia'; G. Zilahi'; H. Aya ${ }^{1}$; J. Aron ${ }^{1}$

${ }^{1}$ General intensive care unit, St George's Hospital, London, United Kingdom

Correspondence: P.L. James

Intensive Care Medicine Experimental 2020, 8(2): 001625

Introduction: The COVID-19 pandemic continues to threaten populations across the world [1]. Delay in the diagnosis of COVID-19 can cause exponential harm. Lung ultrasound can quickly identify a COVID-19 phenotype as interstitial syndrome of the lung [2]. Combined with focused echocardiogram to rule out left ventricular (LV) impairment as a cause, it can be used to quickly identify patients highly likely to have COVID-19.

Objectives: We aimed to assess a describe a system for the diagnosis of COVID-19 by focused heart and lung ultrasound.

Methods: We performed a prospective observational study on all patients admitted to a tertiary hospital Intensive Care Unit over a three-week period. Each patient received a point of care ultrasound (POCUS) scan consisting of focused echocardiogram and 6-point lung ultrasound (using FUSIC guided scoring system [3]). Patients were diagnosed with COVID-19 using two swab positive PCR. The primary outcome was the association between COVID-19 diagnosis and phenotype of COVID-19 defined by two category 2 regions on Lung ultrasound (interstitial syndrome) in the absence of LV impairment. We used fisher's-exact test for comparison of the two groups. A p-value of less than 0.05 was considered significant.

Results: Of 69 patients admitted to ICU, 61 patients were eligible for inclusion in our study with adequate POCUS. 43 patients (70\%) were swab positive for COVID-19, 18 were negative. 33 out of $43(76.7 \%)$ swab positive patients had a positive COVID-19 phenotype on focused ultrasound, $10 / 43(23.3 \%)$ did not. $16 / 18$ (88.9\%) swab negative patients had a negative COVID 19 phenotype on focused ultrasound, $2 / 18(11.1 \%)$ had a positive phenotype. P-value $=0.000002$. Area under the Curve $0.83(\mathrm{Cl} 0.71-0.94)$. Accuracy $0.8(0.71-0.91)$. Positive Predictive value $0.94(0.81-0.98)$. Negative Predictive Value 0.62 (0.43 - 0.78). Likelihood ratio of 6.91 (Cl $1.85-25.8)$.

Conclusion: Focused lung ultrasound is highly predictive of COVID19 when looking for Interstitial syndrome of the lung and excluding patients with LV failure.

Reference(s) and grant ackowledgment(s)

1. European Centre for Disease Prevention and Control.

2. Focused Ultrasound in Intensive Care (FUSIC): FUSIC COVID info [internet].

London: Intensive Care Society Available from: https://www.ics.ac.uk/ICS/ Pdfs/FUSIC DOCS/FUSIC COVID19_info.aspx.

3. S. Kulkarni 'Point-of-care lung ultrasound in intensive care during the COVID-19 pandemic'Clinical Radiology 13 May 2020. https://doi. org/10.1016/j.crad.2020.05.001.

001627

Thrombocytosis in COVID-19 patients admitted to the ICU

M. Fazzi ${ }^{1}$; M. Nijsten ${ }^{1}$

${ }^{1}$ Department of critical care, University Medical Center Groningen, Groningen, Netherlands

Correspondence: M. Nijsten

Intensive Care Medicine Experimental 2020, 8(2): 001627

Introduction: Some of the most severe aspects of COVID-19 may be related to expression of interleukin-6 (IL-6). IL-6 is a key mediator of thrombopoiesis and thrombocytosis in acute and chronic inflammation [1]. COVID-19 patients may thus manifest IL-6 induced thrombocytosis.

Objectives: Examine the time course of platelet counts (PLC) in a cohort of COVID-19 patients admitted to our ICU and compare this time course with that of non-COVID ICU-survivors and non-survivors from a large published cohort [2]. Since COVID-19 patients have relatively long ICU-stays we used only non-COVID patients with an ICUstay of $\geq 14$ days.

Methods: Over the first 30 ICU days median daily PLC in COVID-19 patients were compared with non-COVID hospital survivors and nonCOVID hospital non-survivors.

Results: A total of 90 COVID-19 patients were compared with 2682 non-COVID-19 ICU-survivors (S) and 1149 non-COVID-19 non-survivors (NS) who had an ICU-stay of $\geq 14$ days. A total of 89,000 PLC were analyzed. Median PLC in COVID-19 patients followed a markedly different time course from both S and NS non-COVID patients. COVID-19 patients had a higher PLC at admission: $29510^{9} / \mathrm{L}$ vs $17510^{9} / \mathrm{L}$ and 172 $10^{9} / \mathrm{L}$ in $\mathrm{S}$ and NS respectively $(P<0.001)$. Subsequently the nadir in PLC observed between ICU day 1 and 5 in S and NS alike was absent in COVID-19 patients. The rise in PLC observed in non-COVID-19 during the second ICU week was also observed in COVID-19 patients, although the maximal PLC in COVID-19 patients was higher: 486 $10^{9} /$ Lcompared to $36610^{9} /$ Land $24910^{9} / \mathrm{L}$ in $\mathrm{S}$ and NS respectively $(P<0.001)$. In contrast to $S$ and NS non-COVID patients, even after 30 days median PLC was still above the reference range (150 to 350 $10^{9} / \mathrm{L}$ ) in COVID-19 patients.

Conclusion: COVID-19 patients display a pronounced thrombocytosis during the first ICU month compatible with the thrombopoietic action of IL-6. Ongoing trials with IL-6 receptor inhibitors in may establish the causality of IL- 6 in thrombocytosis in COVID-19 patients.

\section{Reference(s) and grant ackowledgment(s)}

1. [2] Alkozai EM, Mahmoodi BK, Decruyenaere J, Porte RJ, Oude Lansink - Hartgring A, Lisman T, Nijsten MW. Systematic comparison of routine 
laboratory measurements with in-hospital mortality: ICU-Labome, a large cohort study of critically ill patients. Clin Chem Lab Med 2018;56:1140-1151. 2. [1] Nijsten MW, Hack CE, Helle M, ten Duis HJ, Klasen HJ, Aarden LA. Interleukin- 6 and its relation to the humoral immune response and clinical parameters in burned patients. Surgery 1991;109:761-7.

\section{4}

Comparison of time courses of albumin and total protein levels in COVID and non-COVID patients: evidence of marked gamma-globulin production in addition to acute phase proteins in COVID-19

L. Hulshof ${ }^{1} ;$ M. Nijsten ${ }^{1}$

${ }^{1}$ Department of critical care, University Medical Center Groningen, Groningen, Netherlands

Correspondence: $M$. Nijsten

Intensive Care Medicine Experimental 2020, 8(2): 001644

Introduction: COVID-19 patients display several responses related to severe and sustained inflammation. Interleukin-6 (IL-6) is considered to be a key mediator among the cytokines released during COVID-19. One of the actions of IL- 6 is the stimulation of polyclonal immunoglobulin production by B-cells [1].

Objectives: Analyze the secondary components of the acute phase response as can be derived from routine laboratory measurements. Over a 1 month period we compared the time course of albumin, total protein and fibrinogen in COVID-19 patients with that observed in non-COVID ICU patients. We also specifically looked at the difference between total protein and albumin as a reflection of the (immuno-) globulin fraction.

Methods: From a large database of ICU-patients and laboratory data built in our center for the ICU-Labome study [2], we selected only patients with a prolonged ICU-stay (i.e. $\geq 14$ days). This was done for better comparison with the COVID-19 patients who tend to have relatively long ICU stays. We compared COVID-19 patients (C) with nonCOVID hospital survivors ( $\mathrm{nC}-\mathrm{S}$ ) and non-COVID non-survivors (nC-NS). All available fibrinogen, albumin and total protein levels were analyzed. To estimate the non-albumin proteins, albumin was subtracted from total protein. Median levels are reported and comparisons were performed with non-parametric tests.

Results: The nC-S and nC-NS groups consisted of 2682 and 1149 patients respectively. These were compared with 90 COVID-19 ICU patients treated till May 2020. 3400 and 129,000 laboratory measurements were used. At ICU-admission, fibrinogen levels were already considerably higher $(P<0.001)$ in COVID-19 patients: $7.6 \mathrm{~g} / \mathrm{L} \mathrm{com-}$ pared with 2.5 and $2.9 \mathrm{~g} / \mathrm{L}$ in $\mathrm{nC}-\mathrm{S}$ and $\mathrm{nC}-\mathrm{NS}$. Only after 2 weeks of ICU admission fibrinogen in COVID had decreased to levels similar to $\mathrm{S}$ and NS. The time course of albumin in COVID-19 patients was similar to that nC-S. Total protein levels were much higher at admission in COVID-19 than either $\mathrm{nC}-\mathrm{S}$ and $\mathrm{nC}-\mathrm{NS}(\mathrm{P}<0.001)$, with admission levels of $61 \mathrm{~g} / \mathrm{L}$ vs 50 and $50 \mathrm{~g} / \mathrm{L}$ respectively, with only limited subsequent changes. The estimated non-albumin fraction at admission in COVID-19 was $34 \mathrm{~g} / \mathrm{L}$ compared to 23 and $25 \mathrm{~g} / \mathrm{L}$ in $\mathrm{nC}-\mathrm{S}$ and $\mathrm{nC}-\mathrm{NS}$ $(P<0.001)$. The estimated non-albumin fraction stayed 9 to $10 \mathrm{~g} / \mathrm{L}$ higher than $\mathrm{nC}-\mathrm{S}$ and $\mathrm{nC}-\mathrm{NS}$ over the observation period $(\mathrm{P}<0.001)$ and rose to $40 \mathrm{~g} / \mathrm{L}$ at day 30 .

Conclusion: In addition to the well-described components of the acute phase response in COVID-19 such as elevated CRP and fibrinogen, there is a pronounced and sustained rise in total protein that points to the production of other acute phase proteins or gammaglobulins. This response is markedly different from that seen in other critically ill patients. Ongoing trials with IL-6 receptor inhibitors may indicate if IL- 6 responsible for these peculiar reactions.

\section{Reference(s) and grant ackowledgment(s)}

1. [2] Alkozai E et al. Systematic comparison of routine laboratory measurements with in-hospital mortality: ICU-Labome, a large cohort study of critically ill patients. Clin Chem Lab Med 2018.

2. [1] Kishimoto T. The biology interleukin-6. Blood 1989.

3. None.
001649

Intensive Care Unit during the COVID-19 pandemic

F. Cardoso ${ }^{1}$; R. Passos ${ }^{1}$; D. Alves ' ${ }^{1}$ P. Moura ${ }^{1}$; R. Corga ${ }^{1}$; J. Caldeiro ${ }^{1}$

${ }^{1}$ Intensive Medicine, Hospital Santa Luzia - Ulsam, Viana do Castelo, Viana do Castelo, Portugal

Correspondence: F. Cardoso

Intensive Care Medicine Experimental 2020, 8(2): 001649

Introduction: In December 2019, the first cases of respiratory pathology (COVID-19) caused by the new coronavirus (SARS-CoV-2) were reported in Wuhan. Since then, COVID-19 has spread exponentially throughout the world, having been recognized by the World Health Organization (WHO) as an international public health emergency on January 30, 2020. On March 11, 2020, the WHO has officially declared the COVID-19 pandemic.

In our country the first case of COVID-19 was confirmed on March 2, with causalities starting on March 18.

The differences between countries or different Intensive Care Units (ICU) are still under investigation for the disparities in regional admissions and the severity of illness. This illustrates how natural evolution of COVID-19 and its prognosis are still not fully understood.

Conscient that there is an important impact of public health restriction and confinement in local evolution and transmission, there is relevancy in mentioning that National Emergency State was declared on March 18, and a national calamity on May 3, resulting in closure of not essential public stores.

There was an adjustment of our ICU organization, with the creation of a separate cohort, named COVID ICU exclusively dedicated to these patients.

Objectives: Characterization of the population of patients infected with SARS-Cov-2 admitted to Intensive Care Unit at a university affiliated hospital, during the COVID-19 pandemic. Analysis of a district hospital experience during an outbreak (from preparation to the results).

Methods: This is a retrospective and descriptive study that includes only data from the usual clinical practice. All positive COVID-19 patients admitted to the ICU from March 2, 2020 to June 26 were included. Patients were followed from admission to discharge from the ICU.

Results: Over the last 3 months, coincidentally with the national spike and the so-called "first wave" of cases, there was an adjustment of the ICU organization, with the creation of a separate cohort exclusively dedicated to this patients.

With a total of 169 patients admitted to our hospital, we had 13 admissions ( 12 patients, but one readmitted).

The mean age of the patients was 74 years, and $66.6 \%$ were men. Of the 12 patients, 2 had no symptoms but had known risk contact and presented with silent hypoxemia. Length of stay varied from 1 to 25 days.

Laboratory results showed the usual highly elevated c- reactive Protein levels and lactate dehydrogenase.

At admission, patients had a mean $\mathrm{PaO} 2 / \mathrm{FiO} 2$ ratio of 169 , with a minimum of 52. Five had mechanical ventilation since the admission with an average of 14 days of ventilation.

Patients who developed an ARDS (5 patients) were treated according to the recommendations.

All patients were treated with hydroxychloroquine following the recommendations in force until the hospitalization.

Four of the 12 patients didn't survive (33.3\%).

Conclusion: The pandemic has tested public health measures as the functioning and responsiveness of health services worldwide. The ICUs, in the role of manager of the critical patient, have a particularly important role in the approach of these patients considering the risk of flooding ICU during outbursts, and the consciousness that intensive care support is crucial for the surviving of severe cases. The lack of knowledge and the limit of resources are factors of added difficulty in the approach of patients with COVID - 19. The variability between centers is still not understood. The lack of evidence has originated different strategies around the world. 


\section{0}

\section{It is hard to predict the effect of a fluid bolus}

O. Wall' ${ }^{1}$; S. Cutuli'; L. Luccheta ${ }^{3}$; A. Wilson ${ }^{4}$; G. Eastwood ${ }^{5}$; A. Lipka-Falck J. Mårtensson?7 D. Törnberg ${ }^{8}$; C. Svensén ${ }^{9}$; E. Joelsson-Alm ${ }^{9}$; R. Bellomo ${ }^{5}$; M. Cronhjort ${ }^{9}$

${ }^{1}$ Department of Clinical Science and Education, Karolinska Institutet, Solna, Sweden, Stockholm, Sweden; ${ }^{2}$ Department of anesthesiology and intensive care, Università Cattolica del Sacro Cuore, Facoltà di Medicina e Chirurgia, Roma, Italy; ${ }^{3}$ Department of anaesthesia, San Raffaele Hospital, Milano, Italy; ${ }^{4}$ Adult critical care, Manchester Royal Infirmary, Manchester, United Kingdom; ${ }^{5}$ Department of intensive care, Austin Hospital, Melbourne, Australia; ${ }^{6}$ Department of anaesthesia and intensive care, Södersjukhuset, Stockholm, Sweden; ${ }^{7}$ Department of physiology and pharmacology, section of anaesthesia and intensive care medicine, Karolinska Institute, Stockholm, Sweden; ${ }^{8}$ Department of clinical sciences danderyds sjukhus, Karolinska Institute, Stockholm, Sweden; ${ }^{9}$ Department of clinical science and education, Karolinska Institutet, Södersjukhuset, Stockholm, Sweden Correspondence: $\mathrm{O}$. Wall

Intensive Care Medicine Experimental 2020, 8(2): 001260

Introduction: Fluid bolus therapy (FBT) is our most common ICU intervention [1], however there is little agreement on when and how this therapy should be used. FBT is often needed to maintain organ perfusion, but excess fluid has been associated with increased longterm morbidity [2-5]. Also, there are indications that the response to fluid is usually smaller than anticipated [6,7]. What triggers are used and what would constitute a significant response vary. Therefore, we conducted a prospective clinical trial in Sweden and Australia to describe accuracy and precision of clinical expectation compared to actual response to FBT.

Objectives: To describe the accuracy and precision of clinicians' expectations of the hemodynamic effect of fluid boluses (FB) on physiological variables at completion of a FB and after one hour. . To investigate triggers for FBT and to what extent clinicians' expectations are met.

Methods: We evaluated 77 ICU patients at Austin Hospital, Melbourne Australia and Södersjukhuset, Stockholm Sweden. Patients were included if they were planned to receive a FB of $\geq 250 \mathrm{ml}$ of resuscitation fluid over $\leq 30$ minutes. The treating physician then completed a questionnaire asking for primary and secondary triggers for FBT from a list. Also, they were asked what physiological responses to the FB they expected at the end of the FB and one hour later.

Clinicians' responses were then compared to the measured values at these time-points. Data was collected directly from the electronical monitoring systems and the electronic medical records regarding physiological variables, lab values etc. Levels of vasopressors and sedation was collected to statically correct for these.

Results: 18 patients were recruited in Australia and 59 in Sweden. 46 were males and 31 females. Mean SOFA score was 8.2. Mean bolus size (SD) was $352.0 \mathrm{ml}$ (107.6) over 22 minutes (11.8). 57.1\% of boluses were Ringer's Acetate and $42.9 \%$ Albumin $40-50 \mathrm{~g} / \mathrm{L} .54 .5 \%$ of patients had infusions of noradrenaline and $37.7 \%$ had infusions of propofol. Hypotension was the most common trigger for a FB (46.8\%), followed by poor urine output $(20.8 \%)$. The most common secondary triggers for a FB were tachycardia and poor urine output (each 16.9\%).

At the end of the FB, $25 \%$ of expectations were within $10 \%$ of measured mean arterial pressure (MAP), and $52.1 \%$ within $10 \%$ of measured heart rate (HR).

One hour later, $37.5 \%$ of expectations were within $10 \%$ of measured MAP, and $39.2 \%$ within $10 \%$ of measured $H R$, and $8 \%$ of expectations were within $10 \%$ of measured urine output.

The mean difference between expected and measured MAP (SD) was $9.1 \mathrm{mmHg}$ (8.7), the difference in HR (SD) $7.1 \mathrm{bpm}(8.9)$, the difference in urine output (SD) $44 \mathrm{ml}(52.6)$ and the difference in cardiac index (Cl) (SD) $0.32(0.30)$ at the end of the bolus.

$33 \%$ of patients had an increase in MAP $>10 \%$ at the end of a fluid bolus, and $29.3 \%$ at $1 \mathrm{~h}$, whereas only $13 \%$ had an increase in $\mathrm{Cl}>10 \%$ at end of bolus but $45.5 \%$ after $1 \mathrm{~h}$ and $6.6 \%$ had a decrease in HR $>10 \%$ at end of bolus and $10.4 \%$ after $1 \mathrm{~h}$.
Correction for levels of sedation or pressors led to no significant change of these values.

Conclusion: The most common reasons for administering a FB was to improve MAP and urine output, followed by resolving tachycardia. Clinicians' expectations were only accurate in $25-51 \%$ of cases and were often not met, with a mean difference of $9 \mathrm{mmHg}$ between expected and measured MAP. Further research might be needed into how FBs are used in the ICU and what expectations we have of them, so that we might choose to use them more judiciously.

\section{Reference(s) and grant ackowledgment(s)}

1. Olof Wall was supported by Region Stockholm (combined clinical residency and $\mathrm{PhD}$ training program).

2. Finfer S, Liu B, Taylor C, Bellomo R, Billot L, Cook D, Du B, McArthur C, Myburgh J, (2010) Resuscitation fluid use in critically ill adults: an international cross-sectional study in 391 intensive care units. Crit Care 14: R185.

3. Boyd JH, Forbes J, Nakada TA, Walley KR, Russell JA, (2011) Fluid resuscitation in septic shock: a positive fluid balance and elevated central venous pressure are associated with increased mortality. Crit Care Med 39: 259-265.

4. Murphy CV, Schramm GE, Doherty JA, Reichley RM, Gajic O, Afessa B, Micek ST, Kollef MH, (2009) The importance of fluid management in acute lung injury secondary to septic shock. Chest 136: 102-109.

5. Sadaka F, Juarez M, Naydenov S, O'Brien J, (2014) Fluid resuscitation in septic shock: the effect of increasing fluid balance on mortality. J Intensive Care Med 29: 213-217.

6. Vincent JL, Sakr Y, Sprung CL, Ranieri VM, Reinhart K, Gerlach H, Moreno R, Carlet J, Le Gall JR, Payen D, (2006) Sepsis in European intensive care units: results of the SOAP study. Crit Care Med 34: 344-353.

7. Glassford NJ, Eastwood GM, Bellomo R, (2014) Physiological changes after fluid bolus therapy in sepsis: a systematic review of contemporary data. Crit Care 18: 696.

8. Bihari S, Prakash S, Bersten AD, (2013) Post Resusicitation Fluid Boluses in Severe Sepsis or Septic Shock: Prevalence and Efficacy (Price Study). Shock 40: 28-34.

\section{2}

\section{Co-Infection in ICU Patients with COVID 19}

G. Crowe'; B. Doyle'; J. Fennell'; G. Fitzpatrick ${ }^{1}$

${ }^{1}$ Anaesthesia and Intensive Care, Tallaght Hospital, Dublin, Ireland; ${ }^{2}$ Department of microbiology, Tallaght Hospital, Dublin, Ireland

Correspondence: $\mathrm{G}$. Crowe

Intensive Care Medicine Experimental 2020, 8(2): 001262

Introduction: Severe Acute Respiratory Syndrome Coronavirus 2 (SARS-CoV-2) has spread across the globe. Among infected patients,causes mild respiratory disease in most have a mild disease course, however a significant minority require hospitalisation. with Of those hospitalised, between $5-15 \%$ of those in hospital requiringe admission to the intensive care unit (ICU) 1 .

Secondary infection is well described in ICU patients with other viral respiratory illnesses such as influenza2, Severe Acute Respiratory Syndrome (SARS)3 and Middle East Respiratory Syndrome (MERS) 4, and may lead to increased mortality. Although data is limited, it is likely that SARS-CoV-2 predisposes to co-infection. ICU admission and organ support such as mechanical ventilation are risk factors for infection, independent of COVID-19. Furthermore, many established pre-morbid conditions such as diabetes mellitus and obesity which pre-dispose to co-infection in ICU have also been described as risk factors for severe SARS-CoV-2 disease1. Finally, patients with severe COVID-19 appear to have significant immune dysregulation, with lymphopaenia, abnormal levels of inflammatory cytokines and features of T cell immune "exhaustion" 5 . This abnormal immune response and associated lung damage can may leave patients vulnerable to bacterial proliferation.

Objectives: 1. Characterisation of the co-infections in SARS-CoV-2 patients admitted to Tallaght Hospital ICU.

2. Description of the effect of co-infection on patient outcomes.

Methods: This is a single centre retrospective observational study of patients admitted to a single ICU with COVID-19 from January to June 
2020. Patients were followed until discharge from ICU. Patient demographics, microbiology, clinical/pharmacological treatment were recorded until discharge from ICU. The primary analysis compared patients with bacterial co-infection to those without. recorded from paper and electronic chartCo-infection was defined as any positive bacterial culture during ICU admission requiring antimicrobial treatment. Proportions and continuous variables were compared using Fisher's exact test and independent samples $t$ testing respectively. Results: Twenty-five patients were admitted during the study period. Thirteen (52\%) developed co-infection. Baseline demographics and co-morbidities were similar between patientsgroups. Organism's identified as co-infections are detailed in Table 1. A highly resistant ESBL producing Klebsiella pneumoniae was isolated in 3 patients. Staphylococcus aureus pneumonia was identified in the respiratory tract of 3 patients. Two patients who underwent prolonged mechanical ventilation developed Stenotrophomonas maltophila. MRSA and VRE were identified in 3 and 2 patients respectively. There were no cases of $C$. difficile colitis, despite extensive use of empiric cephalosporins (68\%) amongst the patients. Patients who developed co-infection spent a significantly longer time mechanically ventilated and on vasopressor therapy than those who did not. ICU length of stay was also prolonged in co-infected patients (Table 2).

\begin{tabular}{|c|c|c|c|}
\hline \begin{tabular}{|l} 
Respiratory \\
\end{tabular} & & Blood & \\
\hline MRSA & 3 & S. Epidermidis & 1 \\
\hline P. Aeruginosa & 1 & ESBL producing $K$. Pneumoniae & 1 \\
\hline S. Epidermidis & 1 & Urine & \\
\hline ESBL producing $K$. Pneumoniae & 2 & ESBL producing K. Pneumoniae & 2 \\
\hline Methicillin Sensitive Staphylococcus Aureus & 2 & Resistant Organisms on Screening & \\
\hline S. Maltophila & 3 & MRSA & 3 \\
\hline E. Cloacae & 1 & VRE & 4 \\
\hline K. Aerogenes & 1 & & \\
\hline Chroseobacteriur & 1 & Clostridium Difficile Colitis & 0 \\
\hline
\end{tabular}

\begin{tabular}{|lllll|}
\hline Table 2: Outcomes & Co-Infection & No Co-Infection & All & p-value \\
\hline ICU Length of Stay (Mean, SD) & $22.38(12.31)$ & $12.83(4.00)$ & $17.3(10.33)$ & 0.0174 \\
Mechanical ventilation (No., \% total) & $13(100 \%)$ & $11(92 \%)$ & $24(96 \%)$ & 0.480 \\
Days intubated (Mean, SD) & $22(12.75)$ & $10.08(4.08)$ & $16.28(11.22)$ & 0.005 \\
Days on vasopressor support (Mean, SD) & $12.77(10.26)$ & $5.58(4.52)$ & $9.32(8.69)$ & 0.036 \\
Days on renal replacement therapy (Mean, SD) & $2.69(6.18)$ & $1.33(4.62)$ & $2.04(5.42)$ & 0.543 \\
Proned & $8(62 \%)$ & $6(50 \%)$ & $14(56 \%)$ & 0.700 \\
Death & $1(8 \%)$ & $3(25 \%)$ & $4(16 \%)$ & 0.322 \\
\hline
\end{tabular}

Conclusion: Co-infection increases duration of mechanical ventilation, ICU stay and vasopressor dependence in ICU patients with COVID-19.

Reference(s) and grant ackowledgment(s)

1. 5. Moon C. Fighting COVID-19 exhausts T cells. Nat Rev Immunol. 2020;20(5):277.

2. 4. Arabi YM, Al-Omari A, Mandourah Y, et al. Critically III Patients With the Middle East Respiratory Syndrome: A Multicenter Retrospective Cohort Study. Crit Care Med. 2017;45(10):1683-1695.

3. 3. Peiris JS, Chu CM, Cheng VC, et al. Clinical progression and viral load in a community outbreak of coronavirus-associated SARS pneumonia: a prospective study. Lancet. 2003;361(9371):1767-1772.

4. 2. Rice TW, Rubinson L, Uyeki TM, et al. Critical illness from 2009 pandemic influenza A virus and bacterial coinfection in the United States. Crit Care Med. 2012;40(5):1487-1498.

5. 1. Guan WJ, Ni ZY, Hu Y, et al. Clinical Characteristics of Coronavirus Disease 2019 in China. N Engl J Med. 2020;382(18):1708-1720.
001263

Global final diastolic volume indexed as an independent predictor of critical care mortality

MC. González'; D. Masevicius²; A. Dubin ${ }^{3} ;$ C. Lovesio $^{4}$

${ }^{1}$ Intensive Care, Sanatorio Parque, Rosario, France; ${ }^{2}$ Emergentología, Hospital otamendi, AAB, Argentina; ${ }^{3}$ Cátedra de farmacología aplicada, Universidad Nacional de La Plata, Facultad de Ciencias Médicas, La Plata, Argentina; ${ }^{4}$ Intensive care, Sanatorio Parque, Rosario, Argentina

Correspondence: M.C. González

Intensive Care Medicine Experimental 2020, 8(2): 001263

Introduction: Septic cardiomyopathy is a serious complication in the course of sepsis. A prognostic association between patients who, in the context of impaired cardiac contractility, maintain increased diastolic volumes has been described by different authors. Furthermore, the emergence of various minimally invasive technologies has allowed access to different indicators of hemodynamic status that have become relevant in recent years.

Objectives: The objective of our work was to determine if the global final diastolic volume (VDFG) and the global ejection fraction (FEG) could be associated with a worse prognosis in septic patients with hemodynamic failure, and if the association between systolic dysfunction due to septic cardiomyopathy and dilatation of cardiac chambers had a better prognosis in that population.

Methods: Aperture measurement was included retrospectively for all patients requiring minimally invasive hemodynamic monitoring using the transpulmonary thermodilution technique with the Ed $1000 \mathrm{EV}$ equipment.

Age, sex, height, weight, Apache II, SOFA, diagnosis, cardiac index, indexed global final diastolic volume, indexed systolic volume, extravascular pulmonary water index, pulmonary vascular permeability index, central venous pressure, variability of systolic volume were measured, index of indexed systemic vascular resistance, mean arterial pressure, heart rate. The patients were subsequently classified into patients with impaired contractility and increased or decreased diastolic volumes.

A univariate analysis was performed with the student $t$ test for continuous variables and $\mathrm{chi}^{2}$ for categorical variables. Subsequently, the variables were included in a multivariate analysis to test their association with mortality.

Results: After multivariate analysis, the only factor that was independently associated with mortality was overall final diastolic volume. Those patients had no associated contractility disorders.

\begin{tabular}{|c|c|c|c|}
\hline & & SURVIVOR & NOT SURVIVOR \\
\hline $\mathbf{N}$ & 63 & 33 & 30 \\
\hline Edad (años) & $58 \pm 18$ & $61 \pm 19$ & $55 \pm 17$ \\
\hline Sexo Femenino (n) & 24 & 52 & 11 \\
\hline Apache II & $24 \pm 5$ & $25 \pm 5$ & $23 \pm 6$ \\
\hline SOFA & $8 \pm 2$ & 72 & 82 \\
\hline VDFGI $\left(\mathrm{ml} / \mathrm{m}^{2}\right)$ & $659 \pm 170$ & $596 \pm 166$ & $716 \pm 159$ \\
\hline VSI (ml/lat/m²) & $32 \pm 11$ & $30 \pm 10$ & $33 \pm 12$ \\
\hline $\operatorname{ELWI}\left(\mathbf{m l} / \mathbf{m}^{2}\right)$ & $11 \pm 5$ & $12 \pm 4,8$ & $12 \pm 5$ \\
\hline IPVP & $2,5 \pm 1,3$ & $2,8 \pm 1,6$ & $2,2 \pm 0,9$ \\
\hline IC $\left(\mathrm{L} / \mathrm{min} / \mathrm{m}^{2}\right)$ & $3,2 \pm 1,2$ & $2,8 \pm 1,2$ & $3,5 \pm$ \\
\hline FEG (\%) & $20 \pm 7$ & $20 \pm 7,6$ & $20 \pm 7,3$ \\
\hline PVC (mmHg) & $12 \pm 8$ & $10 \pm 7$ & $13 \pm 8$ \\
\hline $\begin{array}{l}\text { IRVS(dinas-s- } \\
\mathbf{m}^{2} / \mathbf{c m}^{2} \text { ) }\end{array}$ & $1994 \pm 1263$ & $2007 \pm 1400$ & $1979 \pm 1170$ \\
\hline VVS (\%) & $12 \pm 6$ & $13 \pm 5,6$ & $12 \pm 6,5$ \\
\hline Talla (cm) & $170 \pm 9$ & $170 \pm 8$ & $172 \pm 11$ \\
\hline Peso (kg) & $81 \pm 20$ & $77 \pm 18$ & $85 \pm 22$ \\
\hline TAM (mmHg) & $75 \pm 20$ & $76 \pm 22$ & $74 \pm 18$ \\
\hline FC (lpm) & $95 \pm 27$ & $98 \pm 23$ & $93 \pm 23$ \\
\hline
\end{tabular}

Conclusion: In this cohort of critically ill patients with shock and multiorgan failure, the only independent predictor associated with mortality was the index global final diastolic volume. Patients with high end 
diastolic volumes were more likely to survive, although this was not related to disorders of cardiac contractility.

\section{Reference(s) and grant ackowledgment(s)}

1. 1) Surviving Sepsis Campaign: International Guidelines for Management of Severe Sepsis and Septic Shock, 2012. Intensive Care Med (2013) 39:165-228 2) Elisa Estenssoro; Vanina S. Kanoore Edul ; Cecilia I. Loudet; Javier Osatnik; Fernando G. Ríos; Daniela N. Vázquez. Predictive Validity of Sepsis-3 Definitions and Sepsis Outcomes in Critically III Patients: A Cohort Study in 49 ICUs in Argentina,Crit Care Med 2018; XX:00-00 3) HeyndrickX G, Millard R, Mcritchie R Maroko E, and Vatner S. Regional Myocardial Functional and Electrophysiological Alterations after Brief Coronary Artery Occlusion in Conscious Dogs. The Journal of Clinical Investigation Volume 56 October 1975@978-985 4) Alain Rudiger; Mervyn Singer, Mechanisms of sepsis-induced cardiac dysfunction. (Crit Care Med 2007; 35:1599-1608) 5) GustavoA. Ospina-Tascón, RicardoL. Cordioli, Jean-Louis Vincent. What type of monitoring has been shown to improve outcomes in acutely ill patients? Intensive Care Med (2008) 34:800-820 6) Monnet and Teboul Critical Care (2017) 21:147. Transpulmonary thermodilution: advantages and limits. 7) Monnet et al. Precision of the transpulmonary thermodilution Measurements. Critical Care 2011, 15:R204 8) Combes A, Berneau JB, Luyt CE, Trouillet JL. Estimation of left ventricular systolic function by single transpulmonary thermodilution. Intensive Care Med. 2004:30(7):1377-83 9) Jabot J, Monnet X, Lamia B, Chemla D, Richard C, Teboul JL. Cardiac function index provided by transpulmonary thermodilution behaves as an indicator of left ventricular systolic function. Crit Care Med. 2009;37:2913-8 10) Mathieu Jozwiak, MD; Serena Silva, MD; Romain Persichini, MD; Nadia Anguel, MD; David Osman, MD; Christian Richard, MD; Jean-Louis Teboul, MD, PhD; Xavier Monnet, MD, PhD. Extravascular lung water is an independent prognostic factor in patients with acute respiratory distress síndrome. Crit Care Med 2013; 41:472-480 11) Parker M, Shelhamer J, Bacharach S, Green M, Natanson C, Frederick T, Damske B, Parrillo J. Profound but Reversible Myocardial Depression in Patients with Septic Shock, Annals of Internal Medicine. 1984;100:483-490. 12) Persistent Preload Defect in Severe Sepsis Despite Fluid Loading. Franc ois Jardin, MD; Thierry Fourme, MD; Bernard Page, MD; Yann Loubie`res, MD; Antoine Vieillard-Baron, MD; Alain Beauchet, MD; and Jean-Pierre Bourdarias, MD. CHEST 1999; 116:1354-1359 13) Naik M, Diamond G, Pai T, Soffer A, Siegel R Correspondence of Left Ventricular Ejection Fraction Determinations From Two-Dimensional Echocardiography, Radionuclide Angiography and Contrast Cineangiography Am Coil Cardiol 1995;25:937-42) 14) Manuel Ignacio Monge García1*, Zhongping Jian2, Jos J. Settels2, Charles Hunley3, Maurizio Cecconi4,. Determinants of left ventricular ejection fraction and a novel method to improve its assessment of myocardial contractility. Feras Hatib2 and Michael R. Pinsky5 Ann. Intensive Care (2019) 9:48.

\section{4}

Effects of combined intravenous immunoglobulins and vitamin C for the treatment of severe Community acquired pneumonia M. Benlabed ${ }^{1}$; S. Benlabed ${ }^{2}$; R. Gaudy ${ }^{3}$; S. Nedjari ${ }^{4}$

${ }^{1}$ Anesthesiology and Intensivecare, Lille University, Lille, France; ${ }^{2}$ Erasme hospital, Université Libre de Bruxelles, Bruxelles, Belgium; ${ }^{3}$ Anesthesiology and intensivecare, Lille Catholic University, Lille, France; ${ }^{4}$ Anesthesiology, Algiers university, Alger Centre, Algeria, Algeria

Correspondence: $M$. Benlabed

Intensive Care Medicine Experimental 2020, 8(2): 001284

Introduction: The use of intravenous immunoglobulins (IVIG) as adjunctive therapy for the treatment of infections, and particularly pneumonia, is controversial but there is less controversy for intravenous vitamin C (IVvit-C) associated with antibiotics to treat sepsis since the study of Fowler et al. published in JAMA 2019. However, we never observed in the literature the combination of IVIG and IVvit-C for treatment of severe sepsis or community acquired pneumonia.

Objectives: So the objective of our study was to assess the administration of IVIG and IVvit-C in patients with severe community acquired pneumonia and to evaluate the effectiveness of this association in terms of outcome including morbidity and mortality. So, we hypothesized that the combination of IVIG and IVvit-C would be more effective than IVIG alone or placebo.

Methods: We conducted a prospective observational study from march 2018 to march 2020 and enrolled a total of 45 patients suffering of severe hypoxemic community acquired pneumonia and under invasive mechanical ventilation(IMV). They were $60+-13$ years old and presented the same apache II score. All the patients received the first of dose of antibiotics in the emergency room and were infused with norepinephrine. We randomized the patients in 3 groups.

A first group of 15 patients receiving IVIG $1 \mathrm{~g} / \mathrm{kg}$ the first day, $0.5 \mathrm{~g} / \mathrm{kg}$ the next two days and IV-vitC $50 \mathrm{mg} / \mathrm{kg} / 6 \mathrm{~h}$ during $96 \mathrm{~h}$, administered 2 hours after the first dose of IVIG.

A second group of 15 patients receiving immunoglobulins alone at the same dose as previously described.

And finally a third group receiving placebo alone. We performed a blood level of vit- $C$ and immunoglobulins $(\mathrm{IG})$ at $96 \mathrm{~h}$ in each group. We recorded for each group: SOFA score day1 and day4, duration of vasopressors, duration of invasive mechanical ventilation (IMV), ICU stay, dosage of immunoglobulins and vit-C and finally ICU mortality. Results: Statistical analysis was performed using Student 's t test. Results were expressed as mean +- standard deviation.

SOFA score day 1 was not different between the 3 groups and calculated at 4 but SOFA score day4 was, respectively, in the IVIG-vitC group significantly lower than in the IVIG group and placebo group(4.53+0.74 vs $7.33+-0.72$ vs $12.13+-0.74) p<0.0001$. Similarly, duration of IMV was respectively shorter in IVIG-vitC group than in IVIG and placebo groups. (6.76+-0.71vs9.86+-0.29vs12.73+-0.59) $p<0.0001$. Duration of vasopressors(days) was shorter respectively in IVIG-vitC group than IVIG and placebo group (1.96+-0.12vs 3.03+-0.22vs5.06+-0.17) $\mathrm{p}<0.001$. ICU length of stay was also shorter in IVIG-vit-C group than in IVIG and placebo groups. (11.93+-0.45vs15.86+-0.51vs19.73+-0.7) $p<0.0001$. Blood levels of immunoglobulins at $96 \mathrm{~h}$ were obviously and respectively lower in the placebo group than in the IVIG-vitC and IVIG group(4.99 $\mathrm{g} / \mathrm{l}+-0.63$ vs14.66 $\mathrm{g} / \mathrm{l}+-0.89$ vs $14,8 \mathrm{~g} / \mathrm{l}+-0.86$ ) $\mathrm{P}<0.0001$. Blood levels of vit $C$ were low in the 3 groups before administration $(99 \mu \mathrm{mol} / \mathrm{l}+-2.2)$ and reach at $96 \mathrm{~h}$ a value of $703 \mu \mathrm{mol} / \mathrm{l}+-3.2$ in the IVIG-vitC-group. ICU mortality was significantly lower in the IVIG-vit-C group than in the IVIG group and placebo group, respectively $26 \%, 40 \%$ and $66 \%$.

Conclusion: The administration of high dose of IVIG combined with IV vit-C improved SOFA score day4 increases the ventilator free days, ICU free days, and decreases ICU mortality compared with immunoglobulins alone or placebo. Early infusion of this therapeutic associated with antibiotics for the treatment of severe community acquired pneumonia improves outcome. A large Randomized Controlled Trial is needed to confirm these preliminary results.

Reference(s) and grant ackowledgment(s)

1. A.A.Fowler et al.Effect of Vitamin C Infusion on Organ Failure and Biomarkers of Inflammation and Vascular Injury in Patients With Sepsis and Severe Acute Respiratory Failure: The CITRIS-ALI Randomized Clinical Trial .JAMA 2019

2. Alejandria MM. et al. Intravenous immunoglobulin for treating sepsis and septic shock. Cochrane Database Syst Rev 2000: CD001090.

3. B.J.Fisher et al. Attenuation of sepsis induced organ injury in mice by Vitamin C. JPEN 2014

\section{0}

Post-surgical bacteremia results by Acinetobacter Baumannii carbapenem resistant and colistin sensitive in critically ill patients T. Amat Serna ${ }^{1}$; C. Aranda Martínez²; I. Machuca Sánchez ${ }^{3}$; J. De La Torre Cisneros $^{3} ;$ JC. Robles Arista ${ }^{4}$

${ }^{1}$ Medicina intensiva, Hospital of Jaen, Jaén, Spain; ${ }^{2}$ Medicina Intensiva, Hospital of Jaen, Jaén, Spain; ${ }^{3}$ Enfermedades infecciosas, Hospital Universitario Reina Sofia, Córdoba, Spain; ${ }^{4}$ Medicina intensiva, Hospital Universitario Reina Sofia, Córdoba, Spain

Correspondence: C. Aranda Martínez

Intensive Care Medicine Experimental 2020, 8(2): 001390 
Introduction: To study the evolution and mortality of postsurgical bacteremia due to Acinetobacter baumannii resistant to carbapenems and sensitive to colistin in the critically ill patient.

Methods: Retrospective, multicenter cohort study. We analyze postsurgical patients (previous surgery 30 days) who had monomicrobial bacteremia due to Acinetobacter baumannii carbapenemas resistant and sensitive-colistin. We use logistic regression for multivariate analysis. The main variable was mortality on day 14 and 30 after the diagnosis of bacteremia.

Results: Of the total of patients, 41 (35\%) presented postoperative bacteremia detected during the study time, $61 \%$ men with a mean age of $61 \pm 11$, APACHE II at admission $23 \pm 9$ and Charlson index $3 \pm 2$. This type of Bacteremia was more frequent in patients undergoing abdominal surgery: 18 patients (44\%). Bacteremia appeared on day +12 after initial surgery ( 8 vs. 26 ). Most frequent comorbidities: diabetes mellitus $15(36 \%)$ and chronic kidney failure $8(20 \%)$. The most frequent type of bacteremia was secondary (33/41): pneumonia associated with mechanical ventilation $49 \%(20 / 41)$ and peritonitis $32 \%(13 / 41) .71 \%$ of the patients presented septic shock. In this group of patients, 29 required renal replacement therapy. The treatment administered was: monotherapy with colistin $73 \%$ (30/41) and combination therapy with tigecycline-colistin in $27 \%(11 / 41)$. Mortality at 14 days was $32 \%(13 / 41)$ and at 30 days it was $63 \%(26 / 41)$. There is no statistically significant difference between mortality in postoperative bacteremia and non-postoperative bacteremia.

Conclusion: Post-surgical bacteremia in our patients is similar with that of non-postoperative bacteremia. Empirical treatment with colistin did not reduce 30-day mortality after this type of bacteremia. Mortality at day 14 and day 30 is similar to that which occurs in nonpostoperative bacteremia.

\section{6}

Etiology of ventilator-associated pneumonia after orotracheal tube obstruction in critically ill patients with SARS-CoV 2

AM. Leal Micharet ${ }^{1}$; SH. De Miguel Martín ${ }^{1}$; JD. Czapka Mital ${ }^{1}$; MT. Cubells Romeral'; LM. Mateus Sanabria'; I. Pozuelo Echegaray ${ }^{1}$; M. Aviles Garcia'; VA. Benitez Ferreiro'; A. Gamo De Maeyer ${ }^{1}$

${ }^{1}$ Icu, General Hospital Villalba, Collado Villalba, Spain

Correspondence: A.M. Leal Micharet

Intensive Care Medicine Experimental 2020, 8(2): 001396

Introduction: Ventilator-associated pneumonia (VAP) is a serious complication in critically ill patients. The usual etiology of these pneumonias is usually similar in the same complexity units.

Objectives: To determine whether the isolates in pneumonia that were observed in the following $72 \mathrm{~h}$ after an episode of orotracheal tube (OTT) obstruction directly compared to those common in VAP.

Methods: Single-center retrospective study. Patients with confirmed SARS-COV 2 pneumonia (COVID-19) who required mechanical ventilation (VM) for more than 48 hours from March 6 to May 6 in the Intensive Care Unit (ICU) in a medium complexity hospital. The microbiological isolates of those patients who required one or more changes of OTT and developed a VAP in the following $72 \mathrm{~h}$ were recognized.

Results: 47 patients were included. Two patients who died in the first 36 hours of admission with bacterial pneumonia associated with SARS-VOC 2 were excluded. The mean age was 61.7 years, 35 (74.4\%) men and $12(25.5 \%)$ women. The average APACHE 2 was 15 , the average SAPS 2 was $35.6 .60 \%$ of patients affected a VAP in the following 72 hours. The isolates found were Enterobacter 5 (29.4\%), E. faecalis 3 (17.6\%), methicillin sensitive $S$. aureus $3(17.6 \%)$, methicillin resistant $S$. aureus (MRSA) 2 (11.8\%), Pseudomonas aeruginosa 2 (11.8\%), Candida spp 1 (5.9\%), Others 1 (5.9\%).

Conclusion: The development of VAP in the first 72 hours after OTT obstruction has been a frequent finding in COVID patients. The most frequently isolated microorganism was Enterobacter with a higher percentage than that found in published series, followed by E. faecalis, which also has a higher than expected presence. Germs more commonly related to VAP, such as Pseudomonas and SAMR, have presented in the usual proportions for patients intubated in the ICU.
001464

Differential characteristic in surgical infective endocarditis based on valve microbiological findings

VA. Hortigüela Martín ${ }^{1}$; R. Hernandez Estefanía 2 ; G. Aldamiz Echevarria Del Castillo $^{2}$; E. Rosas Carvajal'; JJ. Paez Vargas'; AM. Ioan ${ }^{1}$; S. Sánchez Bernal'; Á. Vidal González'; M. Sanchez Casado ${ }^{3}$; C. Pérez Calvo

${ }^{1}$ Intensive care medicine, Hospital Universitario Fundación Jiménez Díaz, Madrid, Spain; ${ }^{2}$ Cardiac surgery, Hospital Universitario Fundación Jiménez Díaz, Madrid, Spain; ${ }^{3}$ Intensive care medicine, Virgin Health Hospital, Toledo, Spain

Correspondence: V.A. Hortigüela Martín

Intensive Care Medicine Experimental 2020, 8(2): 001464

Introduction: Positive culture of the heart valve could be associated with severity and condition worse prognosis of the disease.

Objectives: To assess if surgical patients with endocarditis and microbiological isolation in the infected valve, present any clinical or evolutive differences from the others patients.

Methods: We evaluated endocarditis patients from two hospital centers (Fundación Jimenez Diaz and Hospital Rey Juan Carlos in Madrid) operated by the same surgical team between 2010 and 2019.

Patients were divided in two groups according to the microbiological findings in the infected valve: GROUP 1 with a negative culture (NC) and GROUP 2 with a positive culture (PC). Any value of $p<0.05$ is considered significant.

Results: 103 patients were included, 30 with PC (29.4\%).

Comparing the groups (NC vs PC) we observe:

Personal history: alcohol-drugs ( $2.8 \%$ vs. $6.7 \% ; p=0.357)$, mellitus diabetes ( $27.8 \%$ vs. $43.3 \% ; p=0.126)$, arterial hypertension $(54.2 \%$ vs. $66.7 \% ; p=0.244)$, dyslipidemia ( $43.1 \%$ vs. $63.3 \% ; p=0.062)$, COPD ( $9.7 \%$ vs. $23.3 \% ; p=0.069)$, renal failure $(26.4 \%$ vs. $56,7 \% ; p=0.004)$, previous cardiac surgery ( $31.9 \%$ vs $46.7 \% ; p=0.159)$, paroxistic atrial fibrillation ( $4.2 \%$ vs $13.3 \%, p=0.095)$ permanent Atrial fibrillation (23, $6 \%$ vs. $13.3 \% ; p=0.242)$, coronary artery disease ( $8.3 \%$ vs. $13.3 \% ; p=$ $0.439)$, cardiogenic shock $(12.5 \%$ vs. $16.7 \% ; p=0.577)$, recent urological handling ( $2.8 \%$ vs. $13.3 \% ; p=0.039)$, cancer ( 0 vs. $6.7 \% ; p=$ $0.027)$, bicuspid valve ( $12.5 \%$ vs. $3.3 \% ; p=0.156)$.

Disease data: vegetations $>15 \mathrm{~mm}(19.4 \%$ vs $31 \% ; p=0.213)$, preintervention antibiotic time $(10.36 \pm 9.90$ vs $8.56 \pm 8.38$ days; $p=$ 0.386), Logistic EUROSCORE II $(29.20 \pm 22.45$ vs $35.56 \pm 19.38 ; p=$ 0.178 ) and type of surgery (mitral valve $38.9 \%$ vs $33.3 \%$, aortic valve $30.6 \%$ vs $43.3 \%$, mitral and aortic valve ( $22.2 \%$ vs. $13.3 \%) ; p=0.551)$. Evolutive data: intubation time (189.86 \pm 1047.94 vs $187.40 \pm 365.55$ (hours); $\mathrm{p}=<0.001)$, ICU stay time $(6.61 \pm 10.59$ vs $12.76 \pm 16$, 26 days; $\mathrm{p}=0.026)$, tracheostomy ( $8.3 \%$ vs. $16.7 \%)$, pleural effusion $(4.2 \%$ vs. $10 \% ; p=0.254)$, reoperation because bleeding $(5.6 \%$ vs. $13.3 \% ; p=0.183$ ), postcardiotomy shock ( 0 vs $6.7 \% ; p=0.027$ ), renal failure $(26.4 \%$ vs $40 \% ; p=0.173)$, lower gastrointestinal bleeding (0 vs $10 \% ; p=0.006)$, liver failure ( $1.4 \%$ vs. $10 \% ; p=0.041)$, polyneuropathy $(1.4 \%$ vs. $16.7 \% ; p=0.003)$, pericarditis ( 0 vs. $6.7 \% ; p=0.027)$ and state of death at hospital discharge (13.9\% vs. $16.7 \%$; $p=0.718)$.

Conclusion: Patients with positive culture in infected valve have higher incidence of Renal failure, recent urological handling and previous cancer. They present longer intubation and ICU stay times and more complications: cardiogenic shock, upper gastrointestinal bleeding, liver failure, severe polyneuropathy, and postoperative pericarditis. However, we did not find significant differences before surgery or in mortality at hospital discharge.

\section{5}

Relation between the age and the severity assessed by SOFA upon the admission to the ICU of the patients with coronavirus infection

JJ. Rios Toro'; LB. Yaguez Mateos²; C. Aranda Martínez³; M. Guerrero Marin $^{2}$; M. Ruiz Garcia ${ }^{4}$

${ }^{1}$ Medicina intensiva, Serrania de Ronda Emergency Hospital, Ronda, Spain; ${ }^{2}$ Medicina intensiva, Hospital of Jaen, Jaén, Spain; ${ }^{3}$ Medicina Intensiva, Hospital of Jaen, Jaén, Spain; ${ }^{4}$ Intensive care, Hospital of Jaen, Jaén, Spain Correspondence: $C$. Aranda Martínez

Intensive Care Medicine Experimental 2020, 8(2): 001465 
Introduction: To evaluate the influence of the age and multiorganic fail of patients at themoment of being admitted at the ICU for coronavirus on the mortality.

Methods: Prospective cohort study of patients with coronavirus admitted to the ICU at theHospital Universitario de Jaén, Hospital San Agustín de Linares and the Hospitallnfanta Margarita de Cabra.Continuous variables are expressed as median (25th percentile, 75th percentile) andqualitative variables with absolute and relative frequencies. $U$ Mann Whitney was usedfor comparison of continuous variables and X2 for qualitative variables. P \&lt;0.05 wasconsidered as statistically significant (s.s.).

Results: We have studied 63 admitted patients AT ICU by coronavirus INFECTION, 44 of them to theJaén hospital ICU, 11 at Cabra hospital and 8 at Linares hospital. The average age was $58(37,62)$. The evaluated severity with APACHE II was $12(9,17)$ pointsand the same evaluation but using SOFA was $6(4,8)$ points at the first day. $63,5 \%(n=40)$ required tracheal intubation on their first day and $82,5 \%(n=52)$ during the entire ICU stay.Mortality in the ICU was a percent of 38 meanwhile in the hospital was 39,7Those patients who deceased were older than the survivors, $64(60,72)$ vs $60(52,70)$ years old, but the differences weren't statistically significant (s.s) $(p=0.16$.). These ones also showed mostacutely according to Apache II, $18(14,23)$ vs $14(12,18)$ points $(p<$ $0,005)$, and a higher SOFA atthe admission $7(4,8)$ vs $6(3,8)$ points, but without being statistically significant $(p=0.09)$.

Later, we classified the severity evaluated with the SOFA and age in intervals for a betterevaluation of the relation of both variables with age and in suspicion that the relation was notlinear.Patients were classified according to the SOFA upon admission as 7 or more points $(n=32,51.6 \%)$ and another group if it was less than 7 points $(n=30$, $48.4 \%$ ). In the 32 patients withSOFA at admission of less than 7 points, 9 died $(28.1 \%)$ and in the 30 with SOFA of 7 or morepoints, 16 died $(53.3 \%)(p=0.043)$.

The patients were classified according to the age, over 60 years $(\mathrm{n}=$ $40,64.5 \%)$ or less or equalto 60 years ( $n=22,35.5 \%$ ). Mortality in those over 60 years old was higher, $47.5 \%$ vs. $27.3 \%$ but the differences were not s.s. $(p=0.12)$. Then, the influence of the age on mortality was analyzed according to the severity evaluatedwith the SOFA at admission. And it was observed that the mortality of patients with SOFA of 7or more points was high in the two age groups, $55.6 \%$ of 9 patients aged 60 years or youngerand $52.4 \%$ of 21 with ages over 60 years, without s.s. differencesThen the influence of the age to the mortality.

Nevertheless, in patients with less severity (SOFA lower than 7 points) mortality was high inthose who were older than 60 years old (42.18\% of 119 patients) but in youth people wasmuch lower $(7.7 \%$ of 13 patients) ( $p=0.033$ ).SOFA of the 13 patients with SOFA less than 7 points and age less than or equal to 60 yearswas ( 2 points: $15.4 \%, 3$ points:30.8\%, 4 points:30.8\% and 6 points: $23.1 \%$ ) and $69.2 \%$ requiredintubation.

Conclusion: In ICU patients admitted for coronavirus infection, the ICU evaluation of SOFAand age allows identification of a group of patients with lower mortality than the rest. They arealso those with low SOFA (less than 7 points) and age less than or equal to 60 years. Mortalityin this group was less than $10 \%$ in our series. In the rest of the patient groups, with high SOFAregardless of age and low SOFA but with age over 60 years, mortality is much higher than theaforementioned group.

\section{8}

Use of human albumin solution in septic and non-septic critically ill patients

Y. H.Y. Chong ${ }^{1}$; A. A. C. Waite' ${ }^{1}$ I. D. Welters

${ }^{1}$ Intensive care unit, Royal Liverpool University Hospital, Liverpool, United Kingdom

Correspondence: A. Waite

Intensive Care Medicine Experimental 2020, 8(2): 001468

Introduction: Albumin is known to be associated with outcomes in critically ill patients, but the use of human albumin solution (HAS) has a less clear role. As a mortality benefit has not been demonstrated in large clinical trials, HAS is currently reserved for particular patient cohorts and otherwise its use remains clinician dependent.

We have previously described 7-day trends of serum albumin in septic and non-septic critically ill patients [1]. In this study we aimed to evaluate the use of human albumin solution (HAS) in the same population, and to assess the effect of HAS administration on outcomes.

Methods: We retrospectively assessed the records of 755 patients admitted to the Royal Liverpool University Hospital Intensive Care Unit (ICU) between 2008 and 2014, who had seven days of albumin data available. Sepsis was identified in 513 patients $(67.9 \%)$ of patients. The overall mortality rate at day 28 was $20.3 \%$. We collected demographic data, including age, sex and APACHE II scores, 28 day mortality rates. We also noted the degree of hypoalbuminaemia during the first 7 days of ICU admission, and the first day patients received HAS. IBM SPSS 25.0 was used for statistical analysis; X2 tests or Fisher's Exact test were used for categorical data, and Student's t-test or Mann-Whitney $U$ test were used for continuous variables.

Results: Data for HAS administration was available for 719 of the 755 patients. 64 patients received HAS (8.9\%). 4.4\% (10/226) of non-septic patients and $11 \%(54 / 493)$ of septic patients received HAS. Amongst septic patients, HAS was more commonly administered to patients with albumin levels $<20 \mathrm{~g} / \mathrm{L}$ ( 47 patients, $87.0 \%$ vs 7 patients, $13.0 \%$ of patients with serum albumin $20-34 \mathrm{~g} / \mathrm{L}, \mathrm{p}=0.001$ ); and most patients were given HAS during the first 4 days of their admission (43 patients, 79.6\%). There was no significant difference in APACHE II score or age between septic patients who did or did not receive HAS, but there was a difference in the minimum albumin level, which was lower in patients who received HAS $(p<0.01)$. We found higher mortality rates amongst septic patients who received HAS compared to those who did not $(p=0.006)$.

Conclusion: We found that septic patients were more likely to receive HAS than non-septic patients; and the majority of septic patients received HAS during the first 4 days of their ICU admission. Higher mortality rates were noted amongst septic patients receiving HAS in our study, though they also had lower minimum levels of albumin. More research is required for the development of evidence-based recommendations to guide HAS administration to septic critically ill patients during the resuscitative and de-resuscitative phases of their ICU admission.

\section{Reference(s) and grant ackowledgment(s)}

1. [1] 40th International Symposium on Intensive Care \& Emergency Medicine. Critical Care 2020, 24(1):87.

\section{9}

Long-term outcomes in septic shock patients: an observational study

I. Semproni'; M. Barbera'; E. Antonucci

"Intermediate care unit, Ospedale "Guglielmo da Saliceto", Piacenza, Italy

Correspondence: $\mathrm{E}$. Antonucci

Intensive Care Medicine Experimental 2020, 8(2): 001479

Introduction: Septic shock is a well-known clinical condition associated to high mortality rate and impaired quality of life. Some studies showed that age, comorbidities, functional status and the type of pathogen may affect the prognosis of septic patients 1 . Although many studies have examined short-term outcomes in septic shock, long-term outcomes in this field have been poorly investigated in the literature.

Objectives: We hypothesized that septic shock patients show poor long-term outcomes such as high rates of mortality, hospital readmission and condition of frailty at the discharge.

Methods: We performed a retrospective analysis, reviewing all patients admitted for septic shock in our Intermediate Care Unit (IMCU) from January 1st 2016 to December 31th 2019. The inclusion criteria were age $\geq 18$ years old; diagnosis at IMCU admission of septic shock (according to SEPSIS-3 criteria) requiring norepinephrine (or other vasopressors) for 24 hours at least. We collected demographic 
characteristics and we evaluated long-term outcomes such as rates of mortality at 28 days, 6 months and 1 year; rate of hospital readmission in the first year and condition of frailty at the first discharge from the hospital (i.e. rate of bedridden patients; pressure ulcers; percutaneous endoscopic gastrostomy/nasogastric tube; urinary catheter).

Results: We reviewed 113 patients and $79 / 113$ respected the inclusion criteria. Demographic characteristics are shown in the Table 1. 39/79 $(49,4 \%)$ patients died at 28 days from the hospital admission; other $18 / 79(22,8 \%)$ patients died at 6 month while a single patient died at 1 year (see Figure 1). Total mortality rate at 1 year was 59/79 (73,4\%) patients. We found a total of 17 hospital readmissions (8/17 in Intensive Care Unit; 4/17 in IMCU; 5/17 in general wards). About condition of frailty, $3 / 79(3,8 \%)$ patients showed percutaneous endoscopic gastrostomy/nasogastric tube; $29 / 79(36,7 \%)$ showed urinary catheter; $10 / 79(12,7 \%)$ showed pressure ulcers; $27 / 79(34,1 \%)$ patients were bedridden.

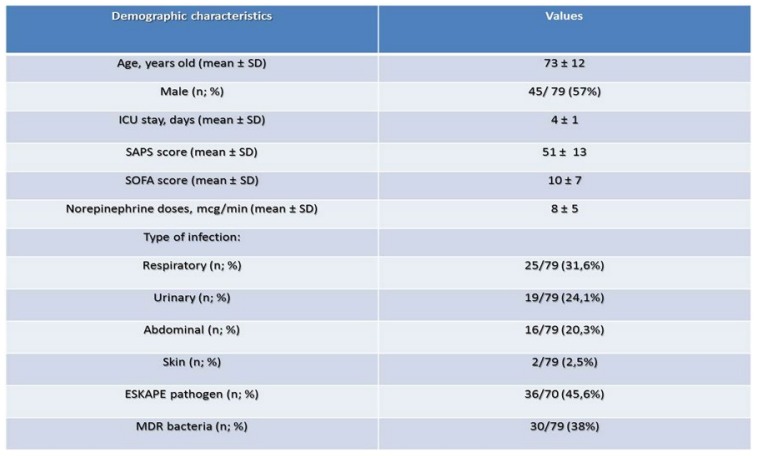

Table 1 - Demographic characteristics

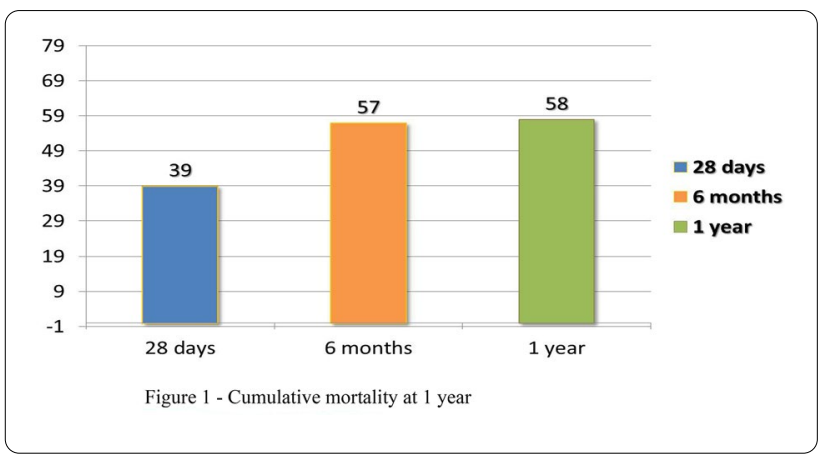

Conclusion: In our observational study, septic shock patients showed poor long-term outcomes with high rates of mortality, hospital readmission and condition of frailty at the discharge.

\section{Reference(s) and grant ackowledgment(s)}

1. 1. J Francisco, I Aragão, T Cardoso. Risk factors for long-term mortality in patients admitted with severe infection. BMC Infect Dis . 2018 Apr 5;18(1):161.

\section{3}

\section{Does Procalcitonin testing support decision making} regarding change of antibiotics, initiation of high-dose steroids or immunomodulation in critically ill patients with severe COVID-19?

L. Skelly; DO. Hamilton ${ }^{2}$; J. Folb³ ; A. Hall ${ }^{2}$; I. Welters ${ }^{2}$; S. Selladurai ${ }^{1}$

${ }^{1}$ Critical care unit, Aintree University Hospital NHS Trust, Liverpool, United Kingdom; ${ }^{2}$ Critical care unit, Royal Liverpool and Broadgreen University
Hospitals NHS Trust, Liverpool, United Kingdom; ${ }^{3}$ Microbiology, Royal Liverpool and Broadgreen University Hospitals NHS Trust, Liverpool, United Kingdom

\section{Correspondence: L. Skelly}

Intensive Care Medicine Experimental 2020, 8(2): 001513

Introduction: COVID-19 infection can range from asymptomatic infection to life-threatening illness. Severe disease commonly manifests as Adult Respiratory Distress syndrome (ARDS) (1) but may also cause immune dysregulation (2), which may be difficult to distinguish from superimposed bacterial infection. Procalcitonin (PCT) is a biomarker that is released in response to bacterial infection and strongly correlates with severity (3). COVID-19 itself does not appear to increase PCT levels (4). Therefore, a raised PCT in these patients could identify a superimposed bacterial infection $(5,6)$ and guide appropriate initiation and cessation of antimicrobial therapy (7).

Methods: We included adult ICU patients with proven or highly suspected COVID-19 infection who had a PCT sent as part of clinical care. Demographic, clinical and laboratory data were obtained from medical records. The Median and Interquartile Range (IQR) were calculated for White Cell Count (WCC), neutrophils, C-reactive protein (CRP) and PCT. Mann-Whitney-U test was performed to compare patients who died with those who survived. Spearman correlation coefficient was used to demonstrate correlation of PCT results with the other inflammatory markers.

Results: Forty PCT tests were performed on 26 patients, of which eight died. Mean age was $57.8 \pm 12.5$ years with a mean length of stay of $26.8 \pm 19.0$ days. PCT testing was performed for a variety of reasons: "fever" for 14 tests; "clinical deterioration" in eight tests; "comparison with previous test" for six tests; "difficult wean" for three tests; and "to stop antibiotics" for one test. There was no documentation for eight requests.

PCT levels correlated with WCC $(r=0.515, p=0.007)$, lymphocyte count and CRP levels $(r=0.423, p=0.044)$. Survivors had significantly lower PCT results $(0.57 \mathrm{ng} / \mathrm{mL}, \mathrm{QQR}: 3.95)$ than patients who died ( $2.82 \mathrm{ng} / \mathrm{mL}$, IQR: 7.35, $\mathrm{p}=0.047$ ). Similarly, CRP was lower in survivors (109 mg/L, IQR: 3.62 versus $270 \mathrm{mg} / \mathrm{L}$, IQR: $186.5 \mathrm{p}=0.003)$. A change in treatment was made following 9 of $40(22.5 \%)$ test results. On three occasions, antibiotics were commenced, antibiotics were stopped on one occasion and a change in antibiotics occurred on five occasions. High-dose steroids were started in six patients after PCT results had become available.

Conclusion: Our results, albeit in a small population, confirm the previously described correlation between PCT and traditional inflammatory markers, WCC and CRP. PCT levels were significantly higher in non-survivors from COVID-19.

Although treatment was changed after nearly a quarter of PCT tests, it remains unclear on how many occasions treatment would have been changed based on WCC and CRP alone. Further research is warranted to fully elucidate the usefulness of procalcitonin in patients with COVID-19 for initiation of immunomodulatory therapy, high-dose steroids, diagnosis of superimposed bacterial infection or change in antibiotic regimen.

\section{Reference(s) and grant ackowledgment(s)}

1. Nothing to Declare.

2. 1) Xu et al. Acute respiratory failure in COVID-19: is it "typical" ARDS? Crit Care. 2020. 6:24:198.

3. 2) Giamarellos-Bourboulis E et al. Complex Immune Dysregulation in COVID-19 Patients with Severe Respiratory Failure. Cell Host and Microbe. 2020; 27 (6): 992-1000

4. 3) Mehanic $S$ et al. The Importance of Serum Procalcitonin in Diagnosis and Treatment of Serious Bacterial Infections and Sepsis. Mater Sociomed. 2013 Dec; 25(4): 277-281.

5. 4) Guan W, et al. Clinical Characteristics of Coronavirus Disease 2019 in China. N Engl J Med 2020.

6. 5) Diagnosis and monitoring of sepsis: procalcitonin testing. NICE. 2014

7. 6) Becker $\mathrm{K}$ et al. Procalcitonin assay in systemic inflammation, infection, and sepsis: Clinical utility and limitations. Crit Care Med 2008; 36;941-52.

8. 7) Jin et al. Procalcitonin: Uses in the Clinical Laboratory for the Diagnosis of Sepsis. Laboratory Medicine. 2010. 41;173-77. 


\section{1}

Prognostic value of APACHE II in patients admitted to the ICU for Coronavirus

LB. Yaguez Mateos ${ }^{1}$; AJ. Pontes Moreno²; C. Aranda Martínez ${ }^{3}$; M. Ruiz Bailén ${ }^{4}$; T. Amat Serna ${ }^{1}$

${ }^{1}$ Medicina intensiva, Hospital of Jaen, Jaén, Spain; ${ }^{2}$ Medicina intensiva, Hospital Universitario "San Agustín", Linares, Spain; ${ }^{3}$ Medicina Intensiva, Hospital of Jaen, Jaén, Spain; ${ }^{4}$ Intensive care, Hospital of Jaen, Jaén, Spain Correspondence: C. Aranda Martínez

Intensive Care Medicine Experimental 2020, 8(2): 001531

Introduction: Evaluation of the relation between APACHE II and mortality on patients admitted tothe ICU for Coronavirus.

Methods: Prospective cohort study of patients with coronavirus admitted to the ICU at theHospital Universitario de Jaén, Hospital San Agustín de Linares and the Hospital InfantaMargarita de Cabra.Continuous variables are expressed as median (25th percentile, 75th percentile) andqualitative variables with absolute and relative frequencies. $U$ Mann Whitney was usedfor comparison of continuous variables and X2 for qualitative variables. Discriminationwas evaluated with the area under the RO curve. P \&lt;0.05 was considered asstatistically significant (e.s).

Results: We have studied 52 admitted patients AT ICU by coronavirus INFECTION, 41 of themAT Jaén hospital ICU, 11 at Cabra hospital. The average age was $63(58,71)$. The evaluated severity with APACHE II was $12(9,16)$ pointsand the same evaluation but using SOFA on the first day was $6(3,8)$ points. $62,5 \%(n=32)$ required tracheal intubation on their first day and $78,8 \%(n=41)$ during the entire ICU stay. $9(17,3 \%)$ of them didn't manifest any of the evaluated records (cirrhosis, cardiac, renal,respiratory, neurological, oncological, hematological or surgical pathology) and 43 patients $(82.7 \%)$ showed 1 or more records.Mortality in the ICU was a percent of 38,5 and (meanwhile) at the hospital was 38,5\%.Deceased patientes had higher APACHE II $14(12,18)$ vs 10 $(8,15$ points, $(p=0.007))$, and biggersofa on the day of the admission, but without statistically significant differences $7(4,8)$ poinstvs $6(3,7)$ points $(p=0.227)$. Predicted mortality by APACHE II according to the original equation was $16 \%$ and thatobserved was less than expected, specifically $38.5 \%$.

Conclusion: On ICU patienes admitted for coronavirus, the severity assessed by APACHE Ilis bigger in those who die than in those whos survive. Anyway, APACHE II statistics of these illpeople are not very high, being the observed mortality higher than predicted for patients withthe same APACHE II score and admitted to the ICU for acute respiratory distress.

\section{1}

Clinical and Biological evaluation of patients with septic shock according to their immunosuppression state

A. Cortes Herrera1; JC. Ruiz-Rodríguez²; M. Pérez-Carrasco³; A. Ruiz ; JJ. González ; P. Povoa ${ }^{5}$ : R. Ferrer Roca

${ }^{1}$ Intensive care department, Vall d'Hebron University Hospital, Barcelona, Spain; ${ }^{2}$ Intensive Care Department, Hospital Vall d'Hebron, Barcelona, France; ${ }^{3}$ Intensive care, Vall d'Hebron University Hospital, Barcelona, Spain ${ }^{4}$ Microbiology department, Hospital Vall d'Hebron, Barcelona, Spain; ${ }^{5}$ Polyvalent intensive care unit, São Francisco Xavier Hospital, CHLO, Lisbon, Portugal

Correspondence: A. Cortes Herrera

Intensive Care Medicine Experimental 2020, 8(2): 001581

Introduction: Biological evaluation of septic shock.

Objectives: To describe and analyse the biological profile, risk of development of multiorgan dysfunction (MODS), and mortality of critically ill patients with septic shock and a previous state of immunosuppression (IS).

Methods: We performed a retrospective analysis of prospectively collected data of patients with respiratory, abdominal and urinary septic shock, according to their immunocompetent (IC) or immunosuppressive (IS) state (1). We include patients identidied through the in-hospital activation of Sepsis Code (SC) and admitted in the ICU in septic shock. Samples were stored in the Sepsis Bank between 2017 and 2018.

We analysed the demographic variables, acute severity scores (APACHE II), development of multiorgan dysfunction (MODS) defined by a Sequential Organ Failure Assessment (SOFA) score $>9$, in-hospital and ICU mortality, the vital status at day 28 and the biological profile through the measurement of cytokine levels (IL-1, IL-2 RA, IL-6, IL8 IL-10, IL-12, INF, TNFa), and inflammatory biomarkers (PCT, CRP, MRproADM).Categorical data were expressed as proportions, and quantitative data were expressed as mean (SD) or median (interquartile range), according to data distribution. The comparison of qualitative data was performed using the Chi-square test. The comparison of quantitative was performed using the Mann Whitney $U$ test or the Student $t$ test. The Institutional Ethical Committee approved the present study (CEIC (PR (AG) 11/2016, PR (AG) 336/2016)), and the patients or their representatives signed the informed consent.

Results: During the study period, 758 SC activations were registered; a total of 76 patients fulfilled the inclusion criteria. Fifty-one patients $(67.1 \%)$ were men, age 62 (14.5) years, the mean of SOFA $9.2(2.5)$ and the APACHE 23.5 (6.9). 41 of patients (53.9\%), were considered IS, mainly by solid organ transplant under immunosuppressive treatment (26.9\%). The most frequent focus of infection was respiratory $(39.5 \%)$ In-hospital mortality was $28.9 \%$, and ICU mortality was $18.4 \%$. $63.2 \%$ developed MODS during the index episode of ICU admission. The ICU mortality, 28-day mortality and in-hospital mortality of IS vs. IC groups were $31.7 \%$ vs . $2.8 \%$ ( $p=0.01$ ), $29.3 \%$ vs. $0 \% p<0.01,41.4 \%$ vs $14.2 \%$ $(p=0.08)$, respectively.In-hospital mortality was $41.4 \%$ vs $14.2 \%$ ( $p=0.08$ ) in the IC group and IS group, respectively. ICU Mortality was $31.7 \%$ vs $.2 .8 \%$ ( $p=0.01)$. 28 -day mortality was $(29.3 \%$ vs. $0 \%$ $\mathrm{p}<0.01$ ), in the IC group and the IS group, respectively. There were no significant differences in the development of MODS $(56.3 \%$ vs. $43.8 \%$ $\mathrm{p}=0.38$ ) between both groups. There were not significant differences in the biological profiles between IC and IS patients. We obtained the following plasma levels of cytokines in both groups: IL1 $\beta$ (11.8 [6] vs. [12.1 [3.8] pg/mL p=0.67), IL2RA (2610[91] vs. 3310.3 [461] pg/mL p= $0.21)$, IL6 (2618 [45.4] vs. 2399.2 [11] pg/mL $p=0.78)$, IL8 (165.9 [77] vs. 158 [22] pg/mL p=0.84), IL10 (291.1 [12] vs. 199.5 [13] pg/mL p= $0.20)$, IL12 (24[3.8] vs. 31.6 [1.8] pg/mL $p=0.11)$, INF (41.6 [9.5] vs. 47.2 [5.7] $\mathrm{pg} / \mathrm{mL} \mathrm{p}=0.97$ ), TNFa (153.7 [4.8] vs. 200.3 [3.8] pg/mL p=0.85); inflammatory reactants: PCT (7.6 [3.9] vs.16[9] $\mathrm{ng} / \mathrm{mL} \mathrm{p}=0.83), \mathrm{CRP}$ (19.9 [3] vs.28 [1.9] ng/mL p=0.16), MR-proADM (5.3 [3.1] vs. 6.6 [4.1] $\mathrm{nmol} / \mathrm{L} \mathrm{p}=0.84$ ).

Conclusion: We found an association between the IS state of critically ill patients with septic shock and mortality; however, it was not associated with increased risk for the development of MODS. Besides it seems that IS state was not associated with a different inflammatory response (cytokine levels and biomarkers). There may be other factors related to the prognosis in these patients.

Reference(s) and grant ackowledgment(s)

1. Violaine T et al. Sepsis Severe or Septic Shock Outcome According to Immune Status and Immunodeficiency Profile. CHEST 2014; 146(5): 1205 $-1213$.

001587

Influence of the neutropenia in the prognostic of patient with septic shock in the ICU

A. Cortes Herrera'; JC. Ruiz-Rodríguez²; M. Pérez-Carrasco33; A. Ruiz'; JJ. González'; R. Ferrer Roca'; P. Povoa

${ }^{1}$ Intensive care department, Vall d'Hebron University Hospital, Barcelona, Spain; ${ }^{2}$ Intensive Care Department, Hospital Vall d'Hebron, Barcelona France; ${ }^{3}$ Intensive care, Vall d'Hebron University Hospital, Barcelona, Spain; ${ }^{4}$ Microbiology department, Hospital Vall d'Hebron, Barcelona, Spain; ${ }^{5}$ Polyvalent intensive care unit, São Francisco Xavier Hospital, CHLO, Lisbon, Portugal

Correspondence: A. Cortes Herrera

Intensive Care Medicine Experimental 2020, 8(2): 001587 
Introduction: Biological evaluation of septic shock.

Objectives: To describe the impact of the Neutropenia (NT) in the biological profile, development of Multiorgan Failure (MOF), and mortality in critically ill patients with septic shock (SS).

Methods: We performed a retrospective analysis of prospectively collected data of patients with respiratory, abdominal,and urinary SS and evaluated the presence of NT at the admission and the first $48 \mathrm{~h}$. We include patients identified through the in-hospital activation of Sepsis Code (SC) and admitted in the ICU in septic shock. We analysed the demographic variables, acute severity scores (APACHE II), development of MOF defined by a (SOFA score) $>9$, day 28 mortality, the presence of NT $(1.0 \times 109$ /litre $) 1$ and the biological profile through the measurement of cytokine levels (IL-1, IL-2 RA, IL-6, IL8 IL-10, IL-12, INF, TNFa), and inflammatory biomarkers (PCT, CRP, MR-proADM).

Results: 758 SC activations were registered with a total of 76 septic shock patients. Fifty-one patients (67.1\%) were men, age $62(14.5)$ years, the mean of SOFA 9.2 (2.5) and APACHE 23.5 (6.9). Respiratory was more frequent focus (39.5\%) of infection. 41 patients $(53.9 \%)$, presented a state of immunosuppression, mainly by solid organ transplant (26.9\%). 18 patients (23.7\%) presented NT, at $48 \mathrm{~h}, 4(22.2 \%)$ have recovered and 12 (75\%) have persisted. 28 days mortality was $19.7 \%$ and $63.2 \%$ developed MOF during the index episode of ICU admission. There were no significant differences in the development of MOF (27.1\%vs. $17.9 \% \mathrm{p}=0.26)$, and 28 days mortality [68.2\% vs $.31 .8 \%$ $(p=0.21)]$, between Non-Neutropenic (NN) and Neutropenic (NT) group respectively. The assessment of the biologic profile showed no significant differences between NN and NT group: IL1 $\beta$ (13.8 [6] vs. [13.1 [2.8] pg/mL $p=0.63)$, IL2RA (2691.5[93] vs. 3311.3 [467] pg/mL $\mathrm{p}=0.24)$, IL6 (2818.3 [35.4] vs. 2344.2 [12] pg/mL p=0.78), IL8 (165.9 [77] vs. 158.4 [21] pg/mL $p=0.84)$, IL10 (251.1 [11] vs. 199.5 [13] pg/ $\mathrm{mL} \mathrm{p}=0.19)$, IL12 (23.9 [3.8] vs. 31.6 [1.9] $\mathrm{pg} / \mathrm{mL} \mathrm{p}=0.13)$, INF (42.6 [9.5] vs. $47.8[5.7] \mathrm{pg} / \mathrm{mL} \mathrm{p}=0.95)$, TNFa (173.7 [4.8] vs. $190.5[3.8] \mathrm{pg} /$ $\mathrm{mL} \mathrm{p}=0.84$ ); inflammatory biomarkers: PCT (7.5 [3.9] vs.14.5 [10] ng/ $\mathrm{mL} p=0.84$ ), CRP (19.9 [3] vs.25.7 [1.9] $\mathrm{ng} / \mathrm{mL} p=0.19$ ), MR-proADM (5.3 [3.2] vs. $6.6[4.7] \mathrm{nmol} / \mathrm{L} p=0.84)$. There were no significant differences in the persistent NT in the inflammatory profile.

Conclusion: Septic shock patients with neutropenia and persistent neutropenia, not presented a significantly difference in the mortality, developing of MOF. In addition, the degree of neutropenia does not influence the levels of cytokines and biomarkers.

\section{6}

\section{Disordered collagen metabolism is associated with elevated}

\section{plasma high sensitive cardiac troponin $\mathrm{T}$ in septic shock}

B. Bollen Pinto' ; A. Herpain ${ }^{2}$; L. Brunelli ${ }^{3}$; K. Bendjelid ${ }^{4}$; R. Pastorelli ${ }^{3}$; M.

Ferrario

'Anesthesiology, Hôpitaux Universitaires de Genève (HUG), Genève, Switzerland; ${ }^{2}$ Department of intensive care, Université Libre de Bruxelles, Bruxelles, Belgium; ${ }^{3}$ Laboratory of mass spectrometry, Istituto di Ricerche Farmacologiche Mario Negri IRCCS, Milano, Italy; ${ }^{4}$ Intensive care medecine, Hôpitaux Universitaires de Genève (HUG), Genève, Switzerland ${ }^{5}$ Department of electronics, information and bioengineering, Politecnico di Milano, Milan, Italy

Correspondence: M. Ferrario

Intensive Care Medicine Experimental 2020, 8(2): 001276

Introduction: Release of cardiac troponins (cT) can occur when myocytes are damaged by a variety of conditions, such as exposure to toxins, inflammation and necrosis. Sepsis is frequently associated with release of cT and the levels of cTs are independently associated with in-hospital and short-term mortality after sepsis [1].

Objectives: The objective of the work was to investigate associations between circulating high-sensitive cardiac troponin T (hs-cTnT) and quantitative mass spectrometry-based metabolomics profile of plasma and cardiac tissues in an animal model of septic shock. This is an ancillary study, the data from the animal experiments were previously published [2,3].

Methods: Septic shock (SS) was induced in anesthetized, instrumented and ventilated adult swines by polymicrobial peritonitis (6 SS and 3 sham pigs). Metabolites were quantified in the myocardium and in plasma with quantitative targeted metabolomics. Circulating hsCTnT was measured using the Elecsys ${ }^{\circledR}$ assay. Fold-change in hs-cTnT from baseline and after full resuscitation (fluids and vasopressors) was used to stratify the animals. Univariate and multivariate analyses were performed.

Results: No sign of myocardial infarction or contractility reduction was detected in this animal model. Hs-cTnT wasn't significantly different between SS and sham animals at each time point, but hs-cTnT foldchange was higher in septic versus sham animals. In harvested heart tissues, proline and glycine levels were significantly higher in the high hs-cTnT fold-change group, i.e. in those animals with a more severe degree of myocardial injury. By stratifying animals on the basis of hscTnT fold-change, proline and hydroxyproline were among the first ranked circulating metabolites in multivariate models to explain the severity of myocardial injury.

Conclusion: Evidence of myocardial injury occurs in the early phase of shock, as indicated by the hs-cTnT fold-changes in the animal study. As collagen is an abundant reservoir for proline and hydroxyproline, we may infer that sepsis-associated myocardial injury could be associated with collagen destruction and/or dysregulated metabolism. In a longitudinal study [4] markers of collagen metabolism were increased in patients with sepsis. The authors showed that serum increasing collagen propeptide levels were associated with the development of multiple organ failure and death. Our results reinforce the concept that collagen degradation could be one of the important mechanisms that contributes to myocardial injury secondary to septic shock.

\section{Reference(s) and grant ackowledgment(s)}

1. [4] Gäddnäs, F., et al. (2009). Markers of collagen synthesis and degradation are increased in serum in severe sepsis: a longitudinal study of 44 patients. Crit. Care 13, 1-10.

2. [3] Ferrario, M., et al. (2019). The systemic alterations of lipids, alanineglucose cycle and inter-organ amino acid metabolism in swine model confirms the role of liver in early phase of septic shock. Front. Physiol. 10, $1-13$

3. [2] Carrara, M., et al. (2019). A Mathematical Model of dP/dt Max for the Evaluation of the Dynamic Control of Heart Contractility in Septic Shock. IEEE Trans. Biomed. Eng. 66, 2719-2727.

4. [1] Vasile, V. C., et al. (2013). Elevated Cardiac Troponin T Levels in Critically III Patients with Sepsis. Am. J. Med. 126, 1114-1121.

5. This work was supported by the EU FP7 Health Programme, ShockOmics project, Grant \#602706.

001560

Effects of Parenteral Lipid Emulsions on Mesenteric Hypoperfusion, Oxidative Stress and Organ Damage in Experimental Mouse Sepsis Model

G. Oz'; E. Ozcelebi'; AY. Gocmen ${ }^{1}$; C. Sokmensuer ${ }^{2}$; S. Yildirim²; A. Topeli ${ }^{3}$. AB. Iskit ${ }^{1}$

${ }^{1}$ Department of medical pharmacology, Hacettepe University Faculty of Medicine, Ankara, Turkey; ${ }^{2}$ Department of pathology, Hacettepe University Faculty of Medicine, Ankara, Turkey; ${ }^{3}$ Division of intensive care medicine, Department of internal medicine, Hacettepe University Faculty of Medicine, Ankara, Turkey

Correspondence: A. Topeli

Intensive Care Medicine Experimental 2020, 8(2): 001560

Introduction: In sepsis, enteral feeding is avoided or trophic enteral feeding is recommended especially in the early period when hemodynamics is impaired. There are no clear recommendations in the international nutritional guidelines for parenteral nutrition and lipid content in sepsis, largely due to lack of evidence.

Objectives: Multiple organ injury due to free radicals and decreased mesenteric blood flow are the consequences of septic shock. We investigated and compared whether different Parenteral Lipid Emulsions could attenuate the deleterious effects of endotoxin, such as mesenteric hypoperfusion, inflammatory oxidative damage and multiple organ damage in a mouse model of sepsis. 
Methods: Swiss-albino mice (20-40 g) were administered subcutaneously sterile saline $(\mathrm{NaCl} 0.9 \%, \mathrm{w} / \mathrm{v})$, fish oil (Omegaven), soybean oil (Kabiven) or olive oil (Oliclinomel) in $50 \mathrm{ml} \mathrm{kg-1} \mathrm{dosage} 10$ min after they have received E.coli endotoxin (LPS, O55:B5, $10 \mathrm{mg} \mathrm{kg-1)}$ ) or its solvent sterile saline. Four hours later, mice were anaesthetized, mesenteric blood flow values were measured, spleen and liver weight/body weight ratios were determined and the organs were examined histopathologically. Oxidative stress index was also calculated according to total antioxidant status (TAS) and total oxidant status (TOS) measurements which were done from tissue homogenates and serum by ELISA assay (Oxidative stress index (OSI) $=$ TOS/TAS). Results are expressed as mean \pm SE. Statistical analysis was performed with one-way ANOVA or Kruskal-Wallis.

Results: Endotoxin decreased mesenteric blood flow ( $\mathrm{ml} \mathrm{min-1,} \mathrm{saline:}$ $3.02 \pm 0.10$; endotoxin: $1.44 \pm 0.10 ; n=12, P<0.05$ ), increased the weight of liver (g per kg body weight, saline: $47.51 \pm 1.05$; endotoxin: $56.46 \pm 1.46 ; n=12, P<0.05$ ) and spleen ( $\mathrm{g}$ per kg body weight, saline: $4.41 \pm 0.17$; endotoxin: $8.92 \pm 0.80 ; n=12, P<0.01$ ) while it inflicted significant histopathological injury to both organs. Although fish oil emulsion treatment abrogated the decrease in mesenteric artery blood flow, none of the parenteral lipid emulsions reversed the wet weight gain in liver and spleen. Histopathological examination of the liver revealed portal inflammatory reaction, intrasinusoidal congestion, intrasinusoidal lymphoreticular reaction, hepatocellular injury, ballooning degeneration and congestion was observed in spleen in endotoxin-treated mice. Lipid emulsions, at the doses used, lead to histopathologically harmful effects in the liver and spleen of the control group (saline-saline). Fish oil emulsion treatment has abolished some of the deleterious effects of endotoxin only in liver. OSI was increased in liver, kidney, lung, spleen and serum after endotoxin challenge. The protective effects of some emulsions were observed only in kidney and spleen.

Conclusion: The main finding of this study is that fish oil emulsions containing high omega-3 fatty acids abrogate the decrease in mesenteric blood flow and prevent liver damage in the experimental sepsis model. All of the lipid emulsions, including fish oil, may cause organ damage in healthy animals, even in protective doses.

\section{Reference(s) and grant ackowledgment(s)}

1. Fell GL, Nandivada P, Gura KM, Puder M. Intravenous Lipid Emulsions in Parenteral Nutrition. Adv Nutr. 2015; 6(5): 600-610. https://doi.org/10.3945/ an.115.009084.

2. None.

\section{2}

\section{Role of citrulline and intestinal fatty acid binding protein} in evaluation of acute gastrointestinal injury and dysfunction in septic patients

MA. Tekes ; M. Durmaz²; SK. Erdemir²; L. Talan³ ; BI. Erguder ${ }^{2}$; ND. Altintas ${ }^{3}$

${ }^{1}$ Department of internal medicine, Ankara University Faculty of Medicine, Ankara, Turkey; ${ }^{2}$ Department of biochemistry, Ankara University Faculty of Medicine, Ankara, Turkey; ${ }^{3}$ Department of intensive care, Ankara University Faculty of Medicine Department of Internal Medicine, Ankara, Turkey Correspondence: M.A. Tekes

Intensive Care Medicine Experimental 2020, 8(2): 001562

Introduction: Citrulline and intestinal fatty acid binding protein (iFABP) are proposed as biomarkers to detect acute gastrointestinal injury which is commonly unrecognized in critically ill.

Objectives: To evaluate role of citrulline and iFABP to predict acute gastrointestinal dysfunction (AGD) in critically ill septic patients admitted to a medical ICU.

Methods: Critically ill patients admitted to a medical ICU with sepsis were evaluated for AGD in accordance with ESICM recommendations. Patients with chronic renal disease were excluded. Blood samples for citrulline and iFABP were collected between 48-72 hours of admission. Patients were grouped as with or without AGD. Healthy controls were included from outpatient clinics.

Results: A total of 51 patients and 51 controls were included in the study. Mean age of patients was 70.1 years. There were 19 males (37.3\%). All were medical admissions. Mean APACHEll score was 24.8 . Mean SOFA score was 8.2. Acute renal injury developed in 41 (80.4\%) of patients. Citrulline levels in septic patients were significantly lower than the controls (11.6 vs $13.0 ; p=0.004)$. There was a moderate, positive correlation between citrulline and iFABP in septic patients ( $r: 0.567, p<0.001)$. AGD score was higher in patients with hypocalcemia $(p=0.016)$ and hyperthermia $(p=0.049)$. Patients with higher AGD score were less likely to attain nutritional goals $(p<0.001)$. When patients with and without AGD were compared, no significant difference was found for citrulline and iFABP. Citrulline and iFABP levels were not associated with mortality.

Conclusion: This study with critically ill medical patients have shown that citrulline and iFABP levels alone may not be adequate to detect acute gastrointestinal injury presenting as a component of multiorgan dsyfunction syndrome.

\section{Reference(s) and grant ackowledgment(s)}

1. Gaël Piton, Gilles Capellier. Curr Opin Crit Care 2016 22(2):152-60.

2. Annika Reintam Blaser, Manu L. N. G. Malbrain, Joel Starkopf, et al. Intensive Care Med 2012(38):384-94

3. Grant was received from 'Ankara Tiplılar Vakfı' for kits.

\section{7}

Vitamin D levels on admission to predict ICU mortality in patients with Covid-19

M. Bychinin ${ }^{1}$;. Klypa ${ }^{1}$;. Mandel'; S. Andreichenko ${ }^{1}$; A. Minec ${ }^{1}$; N.

Kolyshkina ${ }^{2}$; A. Troitsky ${ }^{3}$

${ }^{1}$ Intensive care unit, Federal Research and Clinical Centre, FMBA of Russia, Moscow, Russia; ${ }^{2}$ Laboratory medicine, Federal Research and Clinical Centre, FMBA of Russia, Moscow, Russia; ${ }^{3}$ Chief executive officer, Federal Research and Clinical Centre, FMBA of Russia, Moscow, Russia

Correspondence: $M$. Bychinin

Intensive Care Medicine Experimental 2020, 8(2): 001377

Introduction: Vitamin D deficiency has been associated with an increased risk of respiratory infections such as respiratory syncytial virus infection, tuberculosis and influenza [1, 2]. Sufficient vitamin D level may decrease the risk of death from COVID-19 [3]. However, the vitamin D level in patients with COVID-19, its optimal cut-off value to predict mortality has not been evaluated yet.

Objectives: The aim of the present study was to evaluate vitamin $D$ level in patients on admission to intensive care unit (ICU) as a predictor of mortality in COVID-19 infection.

Methods: The retrospective study was conducted in Federal Research and Clinical Centre. 40 adult patients were enrolled from April 06 , 2020 to April 27, 2020. Blood samples were collected within 24 hours after admission to ICU to perform routine laboratory tests and level of 25 -hydroxyvitamin $\mathrm{D}(25(\mathrm{OH}) \mathrm{D})$. Continuous and categorical variables were presented as mean \pm standard deviation or median (Inter-quartile range), as appropriate. The optimal vitamin D cut-off point was evaluated by receiver operator characteristic (ROC) curve.

Results: In present study 40 patients of $67 \pm 16.5$ years were enrolled, $13(32.5 \%)$ of them were males. The average $25(\mathrm{OH}) \mathrm{D}$ level on admission was $12(9 ; 15) \mathrm{ng} / \mathrm{ml}$.

A significant difference in vitamin $D$ level between the dead patients $(n=13)$ and the survivors $(n=27)$ was revealed $9[7.35 ; 11.5]$ and 14 [10; 16], respectively, $\mathrm{p}=0.004$ (the Mann-Whitney test). The area under the ROC curve was 0.79 (95\% confidence interval (CI) [0.62; $0.95]), p=0.004$. The optimum cut-off value for vitamin D was $9.45 \mathrm{ng} /$ $\mathrm{ml}$. The predictive accuracy of mortality in patients with $25(\mathrm{OH}) \mathrm{D}$ of less than $9.45 \mathrm{ng} / \mathrm{ml}$ had a sensitivity of $85 \%$ and a specificity of $62 \%$, figure 1. 


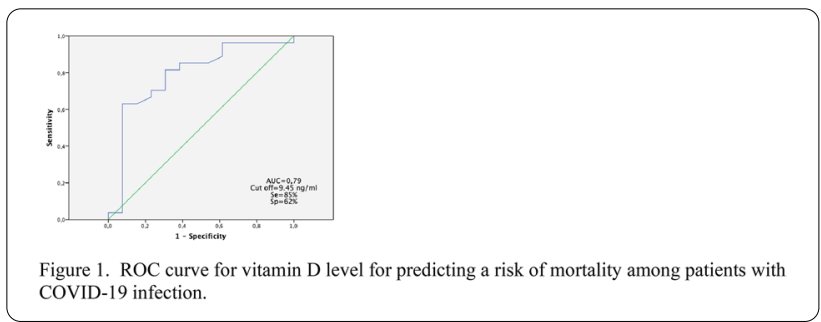

Patients with vitamin D deficiency $(25(\mathrm{OH}) \mathrm{D}$ of $\leq 9.45 \mathrm{ng} / \mathrm{ml})$ had a 7-fold higher risk of death compared with $25(\mathrm{OH}) \mathrm{D}$ levels $>9.45 \mathrm{ng} / \mathrm{ml}$ (Odds Ratio 7.04, 95\% Cl [1.6-30.9]).

Conclusion: ICU COVID patients had deficiency of vitamin D. 25(OH)D level on admission less than $9.45 \mathrm{ng} / \mathrm{mL}$ could predict in-hospital mortality in patients with COVID-19. Interventional study is warranted to elucidate whether vitamin $D$ supplementation is useful for treatment of COVID-19.

\section{Reference(s) and grant ackowledgment(s)}

1. 1. Berry D.J., Hesketh K., Power C. Vitamin D status has a linear association with seasonal infections and lung function in British adults. Br J Nutr. 2011;106:1433-1440.

2. 2. Cannell J.J., Vieth R., Umhau J.C. Epidemic influenza and vitamin D. Epidemiol Infect. 2006;134:1129-1140.

3. 3. Grant WB, Lahore H, McDonnell SL, et al. Evidence that vitamin D supplementation could reduce risk of influenza and COVID-19 infections and deaths. Nutrients. 2020;12(4):988

\section{4}

Nutricional characteristics of Covid-19 mechanical ventilated patients in Virgen de las Nieves hospital's ICU

T. Rodríguez Fernández De Simón ' ; L. Hidalgo Manchado'; D. García Huertas'; A. Fuentes Calatayud²; AB. Garcia Ruano ${ }^{3}$; F. Manzano Manzano ${ }^{1}$ ${ }^{1}$ Intensive care unit, University Hospital Complex of Granada, Granada, Spain; ${ }^{2}$ Intensive care, University Hospital Complex of Granada, Granada, Spain; ${ }^{3}$ Clinical analyses, University Hospital Complex of Granada, Granada, Spain

\section{Correspondence: L. Hidalgo}

Intensive Care Medicine Experimental 2020, 8(2): 00384

Introduction: Covid 19 infection is an emerging disease that has become a worldwide pandemic. Some patients develop a severe pneumonia that evolves in acute respiratory distress syndrome and therefore in a long stay in ICU.

Objectives: To describe the main nutritional characteristics of mechanical ventilated patients with covid 19 pneumonia admitted to Virgen de Las Nieves Hospital's ICU.

Methods: We perform a retrospective observational study with 50 patientes that required mechanical ventilation (MV) in ICU.

Results: Among the 50 patients, $76 \%$ were men, the mean age was $60+/-10$ years, APACHE at the admission in ICU was $14.7+/-6$, and the mean of MV were $22+/-17$ days. Mortality rate in ICU was $36 \%$, without significant differences between men and women when they required $\mathrm{MV}(\mathrm{p}<0.26, \mathrm{Cl} 0.11-1.79)$.

Enteral nutrition was given to $97 \%$ of patinets, while only $12.2 \%$ received parenteral nutrition. Protein intake was $44 \%$ of daily recommended $(0.57 \mathrm{~g} / \mathrm{kg} /$ day) and caloric intake was $59.9 \%(1198+/-$ $355 \mathrm{kcal} /$ day). Mean IMC was 30.4 .

MRC (muscle power assestment scale) at ICU exit was $>48$ in $78 \%$ of the patients, which implies a lack of intensive care unit-adquired weakness.

Caloric intake was a protective factor against mortality $(p<0.99, \mathrm{Cl}$ 0.994-0.999). Meanwhile, poor glycemic control was an independent risk factor for mortality ( $p<0.03, \mathrm{Cl} 1.00-1.03)$.
Table 1: Sample's baseline characteristics

\begin{tabular}{|l|l|l|}
\multicolumn{4}{c|}{ Table 1: Sample's baseline characteristics } \\
\hline & $\begin{array}{c}\text { Total } \\
\text { (n) }\end{array}$ & \multicolumn{1}{|c|}{ Percentage \% } \\
\hline Sex & 50 & $\begin{array}{l}\text { Male 76\% }(\mathrm{n}=38), \text { Female 24\% } \\
(\mathrm{n}=12)\end{array}$ \\
\hline Diabetes & 19 & $38 \%$ \\
\hline Dyslipidemia & 21 & $42 \%$ \\
\hline $\begin{array}{l}\text { Hypertriglicerydemia TG }>500 \\
\text { mg/dl }\end{array}$ & 9 & $18.8 \%$ \\
\hline ICU mortality & 18 & $36 \%$ \\
\hline Hospital mortality & 20 & $40 \%$ \\
\hline Enteral nutrition & 40 & $97 \%$ \\
\hline Parenteral nutrition & 5 & $12.2 \%$ \\
\hline
\end{tabular}

Table 2: Sample's baseline characteristics

\begin{tabular}{|l|l|l|}
\hline \multicolumn{3}{c}{ Table 2: Sample's baseline characteristics } \\
\hline Age & Total (n) & \multicolumn{1}{c|}{ Mean + SD } \\
\hline BMI & 50 & $60+/-10.7$ years \\
\hline Apache & 50 & $30.4+/-4.3$ \\
\hline SOFA & 50 & $14.7+/-6$ \\
\hline MV & 50 & $7.8+/-2.7$ \\
\hline Neuromuscular blokade & 50 & $22.1+/-17.5$ days \\
\hline Prone position & 50 & $6.3+/-5.2$ days \\
\hline Corticoisteroids & 50 & $2.94+/-4$ days \\
\hline Trigliceryds first 48 hours & 49 & $6+/-5.1$ days \\
\hline Trigliceryds first week & 48 & $335+/-242 \mathrm{mg} / \mathrm{dL}$ \\
\hline Caloric intake & 46 & $445.6+/-263$ \\
\hline Protein intake & 38 & $54.9+/-20 \%$ \\
\hline Blood sugar & 37 & $44.4+/-20.1 \%$ \\
\hline MRC & 50 & $161.6+/-46.35 \mathrm{mg} / \mathrm{dL}$ \\
\hline & 30 & $52.2+/-10.4$ \\
\hline
\end{tabular}

Table 3: Multivariable analysis with Cox regression; dependant variable:

\begin{tabular}{|l|l|c|}
\multicolumn{1}{c|}{ mortality } & p \\
\hline Vlood sugar & \multicolumn{1}{|c|}{ HR } & 0.003 \\
\hline Caloric intake & 1.023 (CI 1.008-1.039) & 0.017 \\
\hline
\end{tabular}

Conclusion: An aproppiate nutrition in ICU allowed better results in patients with covid 19 infection.

- Mortality rate was higher in patients with hyperglucemia and lower in patients with higher caloric intake.

- Hypertriglyceridemia is frequent in patients with covid 19 pneumonia in MV (18\% of patients with TG $>500 \mathrm{mg} / \mathrm{dL})$.

- The incidence of intensive care unit-adquired weakness in patients with covid 19 infection was $22 \%$ (MRC < 48).

001395

Utility of NUTRIC Score in postoperative of pancreatic surgery oncological, assessment of entry in our Intensive Care Unit and Mortality

T. Amat Serna ${ }^{\prime}$; C. Aranda Martínez²; A. Pérez Alonso³; E. Pérez Mesa ; LB. Yaguez Mateos'; RF. R. ${ }^{4}$

${ }^{1}$ Medicina intensiva, Hospital of Jaen, Jaén, Spain; ${ }^{2}$ Medicina Intensiva, Hospital of Jaen, Jaén, Spain; ${ }^{3}$ Cirugía general, University Hospital Complex of Granada, Granada, Spain; ${ }^{4}$ Intensive care, Hopsital Neurotraumatológico Universitario, Jaen, Spain

Correspondence: C. Aranda Martínez

Intensive Care Medicine Experimental 2020, 8(2): 001395 
Introduction: Relate nutritional status with the presence of complications and mortality in patients undergoing pancreatic oncological surgery admitted to our Intensive Care Unit (ICU).

Methods: Retrospective cohort study of patients undergoing pancreatic oncological surgery at the University Hospital of Jaén, admitted to the ICU for postoperative management from December 2017 to December 2019. Nutritional risk was evaluated using the NUTRIC(NUTrition RIsk in Critically ill) score. A descriptive analysis was performed and multivariate analysis.

Results: 47 patients, 29 were male. The median age was 69 (43-86) years, APACHE II 12 (5-29), SOFA score 6 (1-15), Charlson index 3 (2-9) and Nutric score 4 (2-9). Median days of ICU admission was 2 days (1-37). Comorbidities: $55 \%$ arterial hypertension, $36 \%$ diabetes mellitus, $11 \%$ atrial fibrillation, $8 \%$ ischemic heart disease, $6 \%$ COPD and $4 \%$ chronic kidney disease.

32 patients had pancreatic neoplasia. In $87 \%$ (36/41) the type of surgery performed was cephalic duodenopancreatectomy. Complications intraoperative: $36 \%$ (17/47) hemodynamic instability, 23\% (11/47) bleeding intraoperative and of these 5 required polytransfusion. Surgical complications: 7 Suture dehiscence and 6 pancreatic fistula. 16 patients had a NUTRIC of high risk and in 13 of them early parenteral nutrition was started.

Six patients re-admitted in the ICU and 4 of them died on re-admission, median of re-entry days was 6 (2-15) from ICU discharge. ICU mortality was $12 \%$ and hospital mortality of $23 \%$.

In the multivariate analysis we observed a statistically significant relationship between the high nutritional risk (NUTRIC score 5-9): history of chronic kidney disease (p.044; OR $0.31(0.20-0.48)$ ), ICU readmission (p.044; OR $0.31(0.20-0.48)$ and ICU Mortality (p.001; OR 0.26 (0.16-0.44).

Conclusion: We have observed that a high-risk nutritional status can relate to the mortality of our sample, in addition readmissions of these patients were more frequent. A multidisciplinary team has been created management of these patients with the aim of improving the state nutritional and consequently prognosis and morbidity and mortality.

\section{7}

Creatine trials in Critically III patients: review of literature

J. Deen

${ }^{1}$ Intensive Care, ZNA Middelheim, Antwerpen, Belgium

Correspondence: J. Deen

Intensive Care Medicine Experimental 2020, 8(2): 001467

Introduction: Many critically ill patients experience a long rehabilitation course due to prolonged ventilatory support and inflammatory state. Creatine has a pivotal role in ATP formation and increasing muscular performance, when combined with resistance training (1). Supplementation might enhance the recovery of the critically ill patient.

Objectives: A review of the literature assessing the possible roles of creatine supplementation in rehabilitation in critical illness.

Methods: A PubMed search was conducted, only articles in English, on adult humans were withheld. Case reports were excluded. Cochrane database was searched. Ongoing trials in the ICU were assessed. (clinicaltrials.gov, controlled-trials.com).

Results: 42 articles were withheld: no trials in the ICU were found. Only 18 of these were clinical trials. Cochrane reviews were found on creatine in cardiovascular disease and (neuro)muscular disorders.

Conclusion: The muscular performance-enhancing effect of creatine supplementation is preserved in age related sarcopenia(2), disuse muscle wasting(3), cachexia(4) and muscular disorders(5), but only when combined with resistance training. No clear benefit was found in neuromuscular(6), mitochondrial(7) or motor neuron disease(8). Creatine's effect on ICU acquires weakness will depend on whether myopathy or polyneuropathy predominate. Creatine's free-radical scavenging and post-ischemic protein synthesis enhancing properties might attenuate the muscular effects of systemic inflammatory conditions(9).

Both pulmonary and cardiac dysfunction might impair rehabilitation tolerance. Creatine supplementation does not ameliorate
COPD patient's quality of life or exertional performance(10)., yet the underlying pathophysiology of COPD is different than that of respiratory weakness after prolonged ventilation. A reduction of dyspnea and orthopnea in heart failure have been noted after months of creatine intake(11). Cognitive performance, neuroprotection and post-traumatic stress disorder have been improved after creatine administration(12).

Meta-analysis confirms the safety and low incidence of side effects(13). Meta-analysis could not show any changes in plasma creatinine(14), nor urea excretion. The effect on urinary output is unclear, but kidney function can be tested by biomarker or urinary albumin excretion. A decreased endogenous creatine synthesis in chronic kidney disease might require supplementation(15).

There are no studies into creatine supplementation in the ICU, only two reviews advocate its further study $(16,17)$. Other creatine supplementation studies in specific populations are often lacking in patients and are relatively few in number. Dosing regimens vary amongst studies making comparison harder. Creatine supplementation might have multiple positive effects on rehabilitation of critically ill patients when combined with resistance training. Studies establishing its applications and safety in the ICU are needed.

Reference(s) and grant ackowledgment(s)

1. 1. Kreider RB, Kalman DS, Antonio J, Ziegenfuss TN, Wildman R, Collins R, et al. International Society of Sports Nutrition position stand: Safety and efficacy of creatine supplementation in exercise, sport, and medicine. J Int Soc Sports Nutr. 2017;14(1):1-18. 2. Dolan E, Artioli GG, Pereira RMR, Gualano B. Muscular Atrophy and Sarcopenia in the Elderly: Is There a Role for Creatine Supplementation? Biomolecules. 2019 Oct;9(11). 3. Hespel P, Op't Eijnde B, Van Leemputte M, Ursø B, Greenhaff PL, Labarque V, et al. Oral creatine supplementation facilitates the rehabilitation of disuse atrophy and alters the expression of muscle myogenic factors in humans. J Physiol. 2001 Oct;536(Pt 2):625-33. 4. Sakkas GK, Schambelan M, Mulligan K. Can the use of creatine supplementation attenuate muscle loss in cachexia and wasting? Curr Opin Clin Nutr Metab Care. 2009 Nov;12(6):623-7. 5. Kley RA, Tarnopolsky MA, Vorgerd M. Creatine for treating muscle disorders. Cochrane Database Syst Rev. 2013;2013(6). 6. Wadman Rl, van der Pol WL, Bosboom WM, Asselman F-L, van den Berg LH, lannaccone ST, et al. Drug treatment for spinal muscular atrophy types II and III. Cochrane database Syst Rev. 2020 Jan; 1 (1):CD006282. 7. Pfeffer G, Majamaa K, Turnbull DM, Thorburn D, Chinnery PF. Treatment for mitochondrial disorders. Cochrane database Syst Rev. 2012 Apr;2012(4):CD004426. 8. Pastula DM, Moore DH, Bedlack RS. Creatine for amyotrophic lateral sclerosis/motor neuron disease. Cochrane database Syst Rev. 2012 Dec;12:CD005225. 9. Kitzenberg D, Colgan SP, Glover LE. Creatine kinase in ischemic and inflammatory disorders. Clin Transl Med. 2016 Dec;5(1):31. 10. Al-Ghimlas F, Todd DC. Creatine supplementation for patients with COPD receiving pulmonary rehabilitation: a systematic review and meta-analysis. Respirology. 2010 Jul;15(5):785-95. 11. Horjus DL, Oudman I, van Montfrans GA, Brewster LM. Creatine and creatine analogues in hypertension and cardiovascular disease. Cochrane database Syst Rev. 2011 Nov;2011 (11):CD005184. 12. Andres RH, Ducray AD, Schlattner U, Wallimann T, Widmer HR. Functions and effects of creatine in the central nervous system. Brain Res Bull. 2008 Jul;76(4):329-43. 13. Kim HJ, Kim CK, Carpentier A, Poortmans JR. Studies on the safety of creatine supplementation. Amino Acids. 2011 May;40(5):1409-18. 14. de Souza E Silva A, Pertille A, Reis Barbosa CG, Aparecida de Oliveira Silva J, de Jesus DV, Ribeiro AGSV, et al. Effects of Creatine Supplementation on Renal Function: A Systematic Review and Meta-Analysis. J Ren Nutr Off J Counc Ren Nutr Natl Kidney Found. 2019 Nov;29(6):480-9. 15. Post A, Tsikas D, Bakker SJL. Creatine is a Conditionally Essential Nutrient in Chronic Kidney Disease: A Hypothesis and Narrative Literature Review. Nutrients. 2019 May; 11(5). 16. Wandrag L, Brett SJ, Frost G, Hickson M. Impact of supplementation with amino acids or their metabolites on muscle wasting in patients with critical illness or other muscle wasting illness: A systematic review. J Hum Nutr Diet. 2015;28(4):313-30. 17. Weitzel LRB, Sandoval PA, Mayles WJ, Wischmeyer PE. Performance-enhancing sports supplements: Role in critical care. Crit Care Med. 2009;37(SUPPL. 10).

2. None. 
001572

\section{Albumin Is Not a Buffer in Human Plasma}

M. Krbec ${ }^{1}$; K. Koudelkova ${ }^{1}$; F. Duska

${ }^{1}$ Department of anaesthesia and intensive care medicine, Third Faculty of Medicine, Charles university and FNKV University Hospital, Prague, Czech Republic

Correspondence: $M$. Krbec

Intensive Care Medicine Experimental 2020, 8(2): 001572

Introduction: Physiology textbooks consider albumin to be an important buffer in human plasma and blood. (1) According to traditional definition buffers are substances which by their presence in solution increase the amount of acid or alkali that must be added to cause unit change in pH. (2) Contrarily, Stewart's quantitative physicochemical theory predicts that weak acids such as albumin actually cause $\mathrm{H}+$ to change more rapidly with addition of acid (defined as change in strong ion difference or pCO2). (3) However, this theory has not yet been experimentally verified.

Objectives: Our aim was to prepare an experiment to assess which of the aforementioned theories better describes the effect of albumin on $\mathrm{pH}$ in human plasma.

Methods: We used lyophylized albumin from human serum, sodium chloride, sodium bicarbonate and water to prepare 5 solutions of artificial plasma with varying concentrations of albumin $(10,30,50,70$ and $90 \mathrm{~g} / \mathrm{L})$. Strong ion difference of all samples was $39-41 \mathrm{mEq} / \mathrm{L}$ and osmolarity was 252-284 mOsmol/l. Using CO2 tonometer, we exposed the samples to varying levels of $\mathrm{pCO} 2(2-16 \mathrm{kPa})$ at $37^{\circ} \mathrm{C}$. The $\mathrm{pCO} 2$ and corresponding $\mathrm{pH}$ were immediately measured using standard blood gas analyser. At least 15 measurements were performed on each sample. In physiological $\mathrm{pH}$ range the dependency of $\mathrm{H}+$ on pCO2 in plasma is linear, therefore the buffer power of each solution was estimated according to slope of the $\mathrm{H}+/ \mathrm{pCO} 2$ line. To increase biological plausibility of the model the whole process was than reproduced with addition of red blood cells (RBC) of a healthy volunteer thus creating solutions resembling whole blood.

Results: As shown in the figure, increasing the concentration of albumin caused the solution to be more acidic (observed as shift to higher $\mathrm{H}+$ concentrations). At the same time, increasing concentration of albumin caused the $\mathrm{H}+$ to change more rapidly (observed as steeper $\mathrm{H}+/$ pCO2 line). The same phenomenon was observed in presence of $\mathrm{RBC}$.

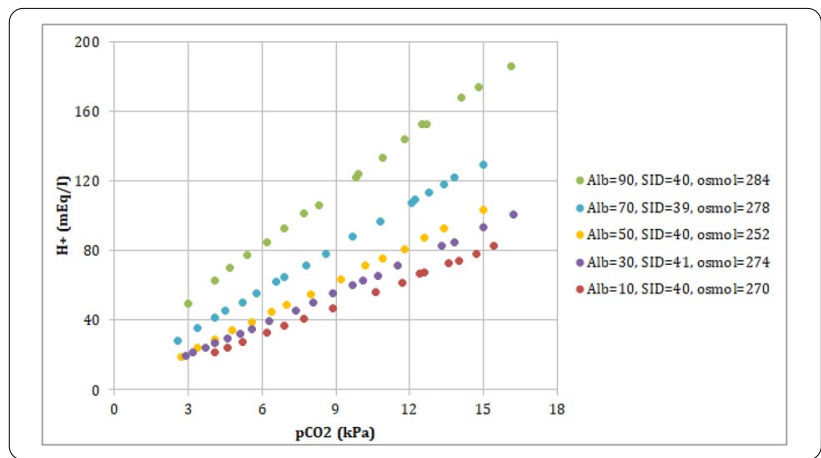

Conclusion: The results of our experiment confirm Stewart's predictions that albumin, as a weak non-volatile acid, does not make the solution more resistant to $\mathrm{pH}$ changes, but rather sets a baseline value from which the $\mathrm{pH}$ changes of the solution are derived. Despite being recognized by Stewart's approach enthusiasts, this fact is not well known by majority of physiologists and clinicians. Consequently, patients with hypoalbuminaemia and respiratory failure might be less prone to acidifying effects of $\mathrm{CO} 2$ accumulation, but clinical relevance of this phenomenon needs further investigation.
Reference(s) and grant ackowledgment(s)

1. 1. BARRETT, K., BROOKS, H., BOITANO, S., \& BARMAN, S. (2010). Ganong's review of medical physiology. 23rd edition. McGraw-Hill Companies. ISBN 978-0-07-160568-7.

2. 2. VAN SLYKE, D. D. (1922). On the measurement of buffer values and on the relationship of buffer value to the dissociation constant of the buffer and the concentration and reaction of the buffer solution. J. Biol. Chem. 52, $525-570$

3. 3. KELLUM, J. A., \& ELBERS, P.W. G. (2009). Stewart's Textbook of Acid-Base. 2nd edition. AcidBase.org. ISBN 978-1-4092-5470-6.

\section{2}

\section{Dysnatraemia in patients with COVID-19}

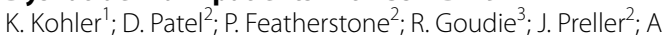
Conway-Morris ${ }^{4}$

${ }^{1}$ Division of anaesthesia, Department of medicine, University of Cambridge, Cambridge, United Kingdom; ${ }^{2}$ Intensive Care, Addenbrooke's Hospital, Cambridge, United Kingdom; ${ }^{3}$ Department of biostatistics, University of Cambridge, Cambridge, United Kingdom; ${ }^{4}$ Department of anaesthetics \& intensive care medicine, Cambridge University Hospitals NHS Foundation Trust, Cambridge, United Kingdom

Correspondence: D. Patel

Intensive Care Medicine Experimental 2020, 8(2): 001442

Introduction: Hypernatraemia in COVID-19 patients is multi-factorial. Known reasons include increased insensible losses due to pyrexia, tachypnoea, and use of diuretics to achieve negative fluid balance. However, it is possible that COVID-19 independently causes hypernatraemia through an undetermined pathway.

Objectives: To determine the prevalence and potential associations of hypernatraemia in COVID-19 patients admitted to acute medical wards compared to intensive care (ICU).

Methods: Data including sodium ( $\mathrm{Na}$ ), base excess (BE), haematocrit, use of diuretics and renal replacement therapy were extracted for 230 COVID-19 patients admitted to a ward and 70 COVID-19 patients admitted to a single ICU. COVID-19 status was confirmed by PCR.

Results: The mean Na for ICU patients was 143, for ward patients 139 $(p<0.001)$ (Fig.1a). Very severe dysnatraemia $(\mathrm{Na}>160 / \mathrm{Na}<120)$ was seen in $10.0 \% / 2.9 \%$ of ICU patients and $1.3 \% / 0.9 \%$ of ward patients, whilst severe dysnatraemia $(\mathrm{Na}>150 / \mathrm{Na}<130)$ was seen in $48.6 \% /$ $25.7 \%$ of ICU patients and $5.7 \% / 10.9 \%$ of ward patients respectively. This effect was largely independent of diuretic use (Fig.1b). There was a weak positive correlation firstly between base excess and sodium (adjusted R2 0.14), and secondly between urea-creatinine elevation ratio (UCR) and sodium (adjusted R2 0.14) (Fig.1C).

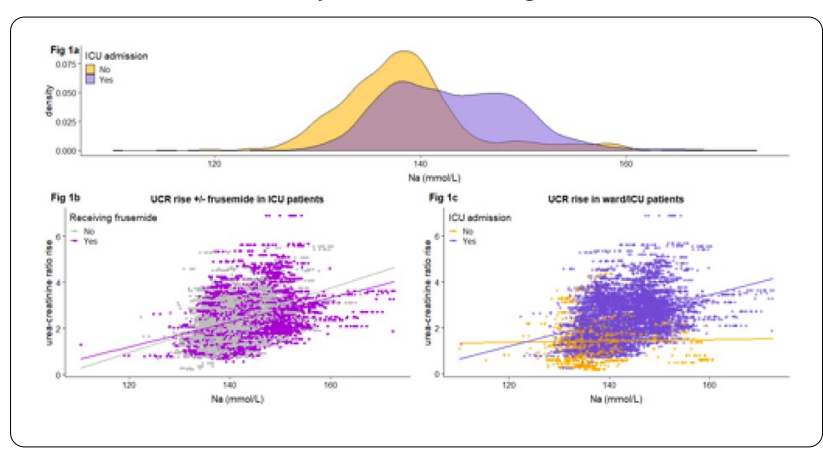

Conclusion: Dysnatraemia was common, especially amongst critically ill patients, and this was more likely to be hyper- rather than hyponatraemia. Although there is a correlation between elevated sodium and markers of dehydration, which was mostly seen in critically ill patients, these did not completely explain the degree of hypernatraemia seen. We hypothesise that dysnatraemia may be a consequence of COVID-19 specific sodium retention, and that this is more pronounced with increasing severity of illness. 
001591

A descriptive study of nutritional treatment of patients affected by SARS-CoV-2 admitted in the ICU of the Consorcio Hospital General Universitario from Valencia in Spain

C. Palau Marti'; R. Rubio Haro'; L. Alós Zaragoza' ; I. Cobo Ciurlizza'; P. Solis Albamonte'; J. Gandia Llopis''; J. De Andrés Ibáñez'; C. Ferrer Gómez'

${ }^{1}$ Anaesthesiology, Critical Care and Pain Therapy Service, Consorci Hospital

General Universitari de València, València, Spain

Correspondence: $C$. Palau Marti

Intensive Care Medicine Experimental 2020, 8(2): 001591

Introduction: Up until today, the acute patient affected by COVID 19 disease presents with a severe acute inflammatory disease, mainly affecting the respiratory system. The severely acute patients need admission to Intensive Care Units (ICU), in order to receive respiratory support, for at least 48 hours. Due to this complications, COVID 19 is related to high nutritional requirements and at the same time the need to avoid volume overload. Moreover, patients affected by COVID 19 , need muscular relaxation and prone positions maneuvers as part of the treatment, as well as the administration of rather unusual medication via nasogastric tube. Additionally, this unusual medication, is rarely used in ITU and has higher risk of interaction with other medication. On the basis of the above, the acute patient affected by SARS-CoV-2 infection has higher risk of malnutrition, with all the consequences associated with it. This patients might benefit from a nutritional protocol specific for ITU patients.

Objectives: To describe and analyse if patients affected by SARSCoV-2 admitted in the ICU of the Consorcio Hospital General Universitario from Valencia (CHGUV) during the pandemic period from February to May 2020 reached the $80 \%$ of the nutritional requirements obtained by weight.

Methods: Observational, descriptive, retrospective and longitudinal design to evaluate the accomplish of estimated nutritional requirements in positive COVID-19 patients admitted to the ICU of the CHGUV.

Results: A sample of 29 patients was obtained. $24.1 \%$ started enteral nutrition early. $28.6 \%$ of the patients reached the nutritional requirements before day 10 , while $35.7 \%$ did not reach the nutritional requirements at any time. $6.9 \%$ reached nutritional requirements before day 15 and $17.2 \%$ before day $20.12 .6 \%$ was not evaluable because oral tolerance was not possible. Furthermore, $10.34 \%$ received complementary parenteral nutrition, $33.3 \%$ for failing to meet the requirements.

Conclusion: In our study, data collection such as height and weight was not accurate enough, so the exact BMI was unknown. Besides, the pandemic situation provoked a different behaviour and more difficulties to accomplish the nutritional standards. That means, our patients were no table to get the nutritional requirements. Finally, it is very important to create new protocols to help us to understand our context and improve it.

\section{Reference(s) and grant ackowledgment(s)}

1. Oliveira V et al. Rev Assoc Med Bras 2016; 62(3): 287-293.

2. Saez de la Fuente I et al. JPEN 2014;40:250-5.

3. Dustin D Linn et al. Intensive Crit Care Nurs. 2015; 31:38-43.

4. Jin et al. Mil Med Res. 2020 Feb 6;7(1):4.

5. Singer $P$ et al. Clinical Nutrition 2019; 38:48-79.

\section{7}

Muscle relaxation does not reduce $\mathrm{CO} 2$ production

in mechanically ventilated COVID patients

M. Renes'; M. Nijsten ${ }^{1}$

${ }^{1}$ Department of critical care, University Medical Center Groningen, Groningen, Netherlands

Correspondence: M. Nijsten

Intensive Care Medicine Experimental 2020, 8(2): 001607

Introduction: COVID-19 respiratory failure frequently causes severe pulmonary gas exchange disturbances. Neuromuscular blockade
(NMB) can be employed to improve ventilatory mechanics. However, it may also be benificial to reduce oxygen consumption although there are conflicting reports $[1,2]$.

Objectives: To assess the shortterm effect of NMB on metabolism reflected by carbon dioxide minute production $\left(\mathrm{VCO}_{2}\right)$ as measured by mechanical ICU ventilators.

Methods: Most of our ICU ventilators report $\mathrm{VCO}_{2}$ measurements (Evita Drager V500, Evital Drager XL, Maquet Servo-I, Maquet Servo$U)$. Our electronic health record records device measurements at one minute intervals. From all $(n=94)$ initial ICU admissions to our ICU during the first wave of the COVID-19 pandemic in the Netherlands (March 19th, 2020 until May 1st, 2020) we analyzed $\mathrm{VCO}_{2}$ measurements in the 3 hour time window ending 30 minutes before the start of the first administration of continuous NMB and compared those with $\mathrm{VCO}_{2}$ measurements in the 3 hour time window starting 30 minutes after the start of continous NMB. We excluded all patients ventilated less than 12 hours before or 4 hours after initiation of NMB, or patients who received any dose of NMB in the 12 hours before start of continuous NMB. Before and after $\mathrm{VCO}_{2}$ difference were compared using paired samples Student t-test. Rocuroniumbromide was used exclusively for NMB.

Results: Our selection criteria identified 17 patients out of 94 tested for inclusion. 12(70\%) were male with a mean \pm SD age of $62.8 \pm 8.8$ years. Mean weight and BMI were $94.1 \pm 16.3 \mathrm{~kg}$ and $30.1 \pm 4.7 \mathrm{~kg} / \mathrm{m}^{2} .12(70 \%)$ patients survived until hospital discharge. Mean VCO was $260 \pm 65 \mathrm{~mL} / \mathrm{min}(2.79 \pm 0.68 \mathrm{~mL} / \mathrm{kg} / \mathrm{min})$ before initiation of NMB and $264 \pm 66 \mathrm{~mL} / \mathrm{min}(2.82 \pm 0.65 \mathrm{~mL} / \mathrm{kg} / \mathrm{min})$ after $(\mathrm{p}=0.413)$.

Conclusion: In this cohort of mechanically ventilated COVID-19 patients the initiation of continuous NMB using rocuronium did not significantly change $\mathrm{VCO}_{2}$. Therefore, it is unlikely that initiation of NMB has significant effects on $\mathrm{O}_{2}$-consumption and energy expenditure.

\section{Reference(s) and grant ackowledgment(s)}

1. [1] J Lemson, JJ Driessen, JG van der Hoeven. The effect of neuromuscular blockade on oxygen consumption in sedated and mechanically ventilated pediatric patients after cardiac surgery. Intensive Care Med 2008 Dec;34(12):2268-72

2. [2] Marik PE, Kaufman D. The effects of neuromuscular paralysis on systemic and splanchnic oxygen utilization in mechanically ventilated patients. Chest 1996 Apr;109(4):1038-42.

3. None.

\section{8}

Prospective investigations of patients demographic and clinical characteristics with chronic liver disease in intensive care unit M. Aslan ${ }^{1}$; EN. Karaoglu' ; M. Caliskan'; M. Yildirim'; S. Temel2; M. Sungur²; K. Gundogan ${ }^{2}$

${ }^{1}$ Department of internal medicine, Erciyes University, School of medicine, Kayseri, Turkey; ${ }^{2}$ Department of internal medicine, Division of intensive care, Erciyes University, School of medicine, Kayseri, Turkey

Correspondence: K. Gundogan

Intensive Care Medicine Experimental 2020, 8(2): 001588

Introduction: The aim of this study is to prospectively evaluate demographic and clinical parameters of patients with critical chronic liver disease (CLD) followed in the intensive care unit (ICU).

Methods: Patients with CLD who were admitted to the ICU and older than 18 years old were included in this study. The demographic and clinical information of the patients, the etiology of CLD, the indications for hospitalization, and the mortality status were investigated.

Results: A total of 75 patients were included in the study. The mean age was $66 \pm 13$ years. The most common reason for ICU admission was gastrointestinal (GIS) bleeding (34\%) and hepatic encephalopathy (32\%). The most frequent causes of CLD were hepatitis C (24\%) and hepatitis B (20\%). Mean APACHE II score was $15 \pm 7$. The median SOFA score of the patients was 5 (min: 0 max: 16).Mean MELD score of all patients was $18 \pm 8$. Mechanical ventilation was needed $27 \%$ of patients during ICU period. A total of $24 \%$ patients received 
vasopressor treatment. Acute kidney injury (AKI) was developed in $33 \%$ of patients. We determined new site infections in 23 patients (31\%). Cancer as additional co-morbidity ( $\beta$ (SE): 0.711, OR (low, up): 9.874 (2.451-39.783), $\mathrm{p}=0.001)$ and increased age ( $\beta$ (SE): $0.032 \mathrm{OR}$ (low, up): 1.096 (1.029-1.167), $p=0.004$ ) was determined as an independent risk factor for ICU mortality. ICU mortality was $27 \%$.

Conclusion: As a result of this study, the most common cause of CLD was hepatitis $C$ and the most frequent reason for hospitalization was Gl bleeding. Cancer and older age were determined independent risk factor for mortality with critical chronic liver disease patients in ICU.

\section{2}

Risk factors associated with disability and mortality at six months after ICU dicharge, in traumatic brain injury patients with refractory intracranial hypertension and decompressive craniectomy in a neurotraumatic ICU

Y. Rivero Perdomo '; C. Sánchez Ramírez ; CF. Lübbe Vázquez ; A. Padrón Mujica $^{1}$; J. López Pérez ${ }^{1}$; P. Saavedra-Santana ${ }^{2}$; S. Ruiz-Santana ${ }^{1}$

${ }^{1}$ Intensive care medicine, University Hospital of Gran Canaria Dr. Negrin, Las Palmas de Gran Canaria, Spain; ${ }^{2}$ Mathematics and informatics deparment, University of Las Palmas:, Las Palmas de Gran Canaria, Spain

Correspondence: $C$. Sánchez Ramírez

Intensive Care Medicine Experimental 2020, 8(2): 001192

Introduction: Patients with severely head-injured have worst outcomes. Moderate and severe disability occurred around a sixth of these patients. Moreover, about $33 \%$ of them do not survive and the remaining patients usually end up with persistent vegetative state. Decompressive craniectomy (DC) is recommended as a second level treatment of Traumatic Brain Injured (TBI) patients with refractory intracranial hypertension (ICH). The outcome of DC still remains controversial.

Objectives: TTo analyze factors related to disability and mortality at 6 months after ICU discharge, in TBI patients with refractory ICH with DC. Methods: A retrospective study was performed in TBI patients admitted to the ICU from May 1, 2009 to December 31, 2018, who had DC after a TBI with refractory $\mathrm{ICH}$. Demographic variables and risk factors associated with mortality were analyzed. demographic data; neurological data (clinical examination and Glasgow Coma Score: GCS); hypotension type of craniectomy and DC complications; Rankin scale, and Glasgow outcome scale (GOS at ICU discharge and 6 months after ICU discharge and 30 days after it; hypo and hyperglycemia; application of mannitol or hypertonic saline solution before and after DC and other related factors. The percentages were compared with the $\mathrm{X} 2$ test or Fisher's exact test, the means with the t-test and the medians with the Wilcoxon test for independent data. A multidimensional logistic analysis of mortality was performed 6 months after ICU discharge.

Results: Twenty eight out of $230 \mathrm{TBI}$ and 105 polytrauma patients, with associated severe TBI, were included. Hospital mortality was $28.6 \%$ and ICU mortality was $10.7 \%$. Factors associated with mortality that showed statistically significant differences $(p<0.05)$ were among others (Table $1 a$ and $1 b)$ : age $(p=0.002)$, APACHE II $(p=0.006)$, Higher GCS at ICU admission ( $p=0.013)$, midline displacement on cranial CT, GOS and Rankin Scale, both at ICU discharge $(p=0.005$ and $p=0.001$, respectively) and 30 days after ICU discharge $(p=0.004$ and $p=0.005$, respectively). GOS values were 3 at ICU discharge and 30 days after it Rankin was 5 at ICU discharge and 30 days after it was 3 . Factors that were independently associated with mortality, at 6 months after ICU discharge, were diabetes mellitus, hypertension, orotracheal intubation in the prehospital setting, no reactive pupils before and reactive after DC (Table 2). The most common observed complications were nosocomial pneumonia (67.9\%), extraaxial hematoma at the surgical site (57.1\%) and hydrocephalus (39.3\%).

Conclusion: Patients with TBI and ICH refractory to medical treatment and undergoing DC, had a low mortality rate at ICU discharge, but with severe disability.Factors independently associated with mortality at 6 months after ICU discharge were diabetes mellitus, arterial hypertension, orotracheal intubation in the prehospital setting, none reactive pupils before DC and both reactive pupils after DC.

\begin{tabular}{|c|c|c|c|c|}
\hline & $\begin{array}{l}\text { Overall } \\
\mathrm{N}=28\end{array}$ & $\begin{array}{l}\text { Alive } \\
\mathrm{N}=20\end{array}$ & $\begin{array}{l}\text { Exitus } \\
N=8\end{array}$ & p \\
\hline Age, years & $42.0 \pm 17.1$ & $36.0 \pm 14.1$ & $56.9 \pm 15.5$ & 0.002 \\
\hline Male sex & $24(85.7)$ & $19(95.0)$ & $5(62.5)$ & 0.058 \\
\hline APACHE II & $21.5 \pm 6.2$ & $19.7 \pm 4.9$ & $27.3 \pm 6.8$ & 0.008 \\
\hline GCS in site & $9.5(5.0-14.0)$ & $7.5(4.8-12.0)$ & $14.0(8.8-14.2)$ & 0.113 \\
\hline GCS in emergency room & $6.0(3.0-10.5)$ & $5.0(3.0-8.5)$ & $6.5(6.0-14.0)$ & 0.160 \\
\hline GCS at ICU admission & $3.0(3.0-3.0)$ & $3.0(3.0-3.0)$ & $3.0(3.0-3.0)$ & 0.830 \\
\hline Higher GCS at ICU admission & $11(8-14)$ & $12(10-15)$ & $8(3-10)$ & 0.013 \\
\hline Diabetes mellitus & $3(11.1)$ & 0 & $3(42.9)$ & 0.012 \\
\hline Arterial hypertension & $6(22.2)$ & $2(10.0)$ & $4(57.1)$ & 0.024 \\
\hline Dyslipemia & $2(7.4)$ & $1(5.0)$ & $1\{14.3\rangle$ & 0.459 \\
\hline $\begin{array}{l}\text { Endotracheal irtubation previous } \\
\text { Hospital }\end{array}$ & $11(39.3)$ & $11(55.0)$ & 0 & 0.010 \\
\hline $\begin{array}{l}\text { Endotracheal irtubation in emergency } \\
\text { room }\end{array}$ & $12(42.9)$ & $6(30.0)$ & $6(75.0)$ & 0.044 \\
\hline $\begin{array}{l}\text { Endotracheal intubation at ICU } \\
\text { admission }\end{array}$ & $2(7.1)$ & $1(5.0)$ & $1\langle 12.5\rangle$ & 0.497 \\
\hline $\begin{array}{l}\text { Endotracheal intubation in operating } \\
\text { room }\end{array}$ & $2(7.1)$ & $2(10.0)$ & 0 & 1 \\
\hline Bilateral ameactive midriasis previous $\mathrm{DC}$ & $2(7.1)$ & $2(10.0)$ & 0 & 1 \\
\hline Both reactive pupils previous DC & $16(57.1)$ & $14(70.0)$ & $2(25.0)$ & 0.044 \\
\hline None reactive pupils previous $D C$ & $5(17.9)$ & $1(5.0)$ & $4(50.0)$ & 0.015 \\
\hline bilateral ameactive Misriasis post DC & $2(7.1)$ & 0 & $2(25.0\rangle$ & 0.074 \\
\hline Both reactive pupils post DC & $20(71.4)$ & $17(85.0)$ & $3(37.5)$ & 0.022 \\
\hline None reactive puplis post DC & $6(21.4)$ & $2(10.0)$ & $4(50.0)$ & 0.038 \\
\hline
\end{tabular}

\begin{tabular}{|c|c|c|c|c|}
\hline & $\begin{array}{l}\text { Overall } \\
\mathrm{N}=28\end{array}$ & $\begin{array}{l}\text { Alive } \\
\mathrm{N}=20\end{array}$ & $\begin{array}{l}\text { Exitus } \\
N=8\end{array}$ & $P$ \\
\hline SAH & $16(57.1)$ & $12(60.0)$ & $4(50.0)$ & 0.6910 \\
\hline Diftuse axonal injury & $2(7.1)$ & $2(10.0)$ & 0 & 1 \\
\hline $\begin{array}{l}\text { Obiteration of basal cisterns previous } \\
\text { DC }\end{array}$ & $18(64.3)$ & $12(60.0)$ & $6(75.0)$ & 0.669 \\
\hline Evacuated Injury & $4(14.3)$ & $3(15.0)$ & $1(12.5)$ & 1 \\
\hline $\begin{array}{l}\text { Focal contusion with edema and } \\
\text { expansivity }\end{array}$ & $25(89.3)$ & $18(90.0)$ & $7(87.5)$ & 1 \\
\hline Epidural hematoma & $7(25.0)$ & $5(25.0)$ & $2(25.0)$ & 1 \\
\hline Hyperthermia post DC & $27(96.4)$ & $20(100.0)$ & $7(87.5)$ & 0.286 \\
\hline Osmolar therapy & $26(92.9)$ & $19(95.0)$ & $7(87.5)$ & 0.497 \\
\hline Exitus in ICU & $3(10.7)$ & - & $3(37.5)$ & . \\
\hline $\begin{array}{l}\text { Midiline displacement on cranial CT at } \\
\text { admission }\end{array}$ & $7.5(2.8-10)$ & $5(0-8.2)$ & $12(9-16)$ & 0.002 \\
\hline $\begin{array}{l}\text { Midline displacement on cranial CT } \\
\text { previous DC }\end{array}$ & $9(4.8-11.4)$ & $6(3.8-10)$ & $15.5(11.9-18.2)$ & 0.001 \\
\hline $\begin{array}{l}\text { Midline displacement on cranial CT } \\
\text { post DC }\end{array}$ & $0(0-2.0)$ & $0(0-0)$ & $4.5(1-7.2)$ & 0.014 \\
\hline GOS at ICU discharge & $3(2-3)$ & $3(3-3.2)$ & $2(1-3)$ & 0.005 \\
\hline GOS 30 days affer ICU discharge & $3(2.5-5)$ & $4(3-5)$ & $2(2-2)$ & 0.004 \\
\hline Rankin at ICU discharge & $5(4-5)$ & $4(2-5)$ & $5(5-6)$ & 0.001 \\
\hline Rankin 30 after ICU discharge & $3(2-5)$ & $2.5(2-4)$ & $5(5-5)$ & 0.005 \\
\hline Hospital stay days & $68(50-89)$ & $68(54-89)$ & $69(11-95)$ & 0.703 \\
\hline ICU stay days & $26(18-35)$ & $26(22-34)$ & $24(10-42)$ & 0.741 \\
\hline $\begin{array}{l}\text { Days from craniectomy to } \\
\text { carranioplasty }\end{array}$ & $67(46-82)$ & $67(49-80)$ & $68(9-94)$ & 0.819 \\
\hline
\end{tabular}




Table 2. Multivariate analysis of mortality 6 months after ICU discharge
\begin{tabular}{|l|c|c|c|c|}
\hline & $\begin{array}{c}\text { ALIVE } \\
\mathrm{N}=20\end{array}$ & $\begin{array}{c}\text { EXITUS } \\
\mathrm{N}=\mathbf{8}\end{array}$ & $\mathbf{P}$ & OR (95\% Ci) \\
\hline Diabetes Mellitus & 0 & $3(42.9)$ & 0.012 & 0 \\
\hline Arterial hypertension & $2(10.0)$ & $4(57.1)$ & 0.024 & $10.49(1.37-111)$ \\
\hline $\begin{array}{l}\text { Orotracheal intubation in the prehospital } \\
\text { setting }\end{array}$ & $11(55.0)$ & $0(0.0)$ & 0.01 & $0(0-0.60)$ \\
\hline None reactive pupils previous DC & $1(5.0)$ & $4(50.0)$ & 0.015 & $16.36(1.66-483)$ \\
\hline Both reactive pupils post DC & $17(85.0)$ & $3(37.5)$ & 0.022 & $0.12(0.02-0.87)$ \\
\hline
\end{tabular}

\section{7}

Retrospective analysis of the practice of anti-seizure prophylaxis in traumatic brain injury

S. Premaratne ${ }^{1}$; T. Mehesrey ${ }^{1}$; E. Neyens ${ }^{1}$; N. Chakraborty ${ }^{1}$; S. Pacheco ${ }^{1}$; M Chapman ${ }^{1}$

${ }^{1}$ Critical Care Medicine, Sunnybrook Health Sciences Centre, Toronto, Canada

\section{Correspondence: S. Premaratne}

Intensive Care Medicine Experimental 2020, 8(2): 001257

Introduction: Significant variability exists among physicians, in the prophylactic use of anticonvulsants in severe Traumatic Brain Injury (TBI).

Objectives: The purpose of this study was to evaluate the degree of compliance to the Brain Trauma Foundation guidelines in the administration of anti-epileptic prophylaxis and to determine the incidence of post traumatic seizures (PTS) in a cohort of critically ill patients with TBI.

Methods: A retrospective analysis of adult patients with traumatic brain injury, admitted to a level III Intensive Care Unit (ICU) over a period of 4 years, was carried out. Details on the demographic data, severity of injury, CT severity grades, use of EEG monitoring and the administration of anti epileptic prophylaxis were determined. The incidence of post-traumatic seizures was compared between the groups of patients who received anti-epileptic prophylaxis and those who did not.

Results: 562 patients were included in the study. Anti-seizure prophylaxis was given to $77 \%$ of the patients with severe TBI. Serum phenytoin levels were measured in only a minority of patients (13.1\%) and $34 \%$ of them were at a sub-therapeutic level. Standard EEG monitoring was performed in $26 \%$ of patients and epileptiform activity was found in only $3 \%$ of them. Continuous EEG monitoring was employed in only $0.5 \%$ of the study population. $13.3 \%$ of the study population developed post traumatic seizures (PTS). The incidence of early PTS was $7.3 \%$ and that of late PTS was 3.6\%. Post-traumatic seizures occurred at a significantly higher rate in the group of patients who received antiepileptic prophylaxis ( $20.2 \%$ vs $3.1 \%, p=0.000)$.

Conclusion: Post-traumatic seizures occur despite the prophylaxis with anti-epileptics. Administration of phenytoin should be guided with serial measurements of serum phenytoin levels and continuous EEG monitoring.

\section{Reference(s) and grant ackowledgment(s)}

1. Carney N, Totten AM, O'Reilly C et al. Guidelines for the Management of Severe Traumatic Brain Injury, Fourth Edition. Neurosurgery; 2017;80(1):6-15

2. Chang BS, Low enstein DH. Practice parameter: Antiepileptic drug prophylaxis in severe traumatic brain injury. Report of the quality standards subcommittee of the American Academy of Neurology. Neurology 2003;1(2):10-16
3. Debanham S, Sabit B, Saluja RS et al. A critical look at phenytoin use for early post traumatic seizure prophylaxis. Can J Neurol Sci 2011:38:896-901.

4. Huijben JA, Volovici V, Cnossen MC et al. Variation in general supportive and preventive intensive care management of traumatic brain injury: a survey in 66 neurotrauma centers participating in the Collaborative European NeuroTrauma Effectiveness Research in Traumatic Brain Injury (CENTER-TBI) study. Critical Care (2018) 22:90 https://doi.org/10.1186/ s13054-018-2000-6.

5. Inglet S, Baldw in M, Quinones AH et al. Seizure prophylaxis in patients w ith traumatic brain injury: A single centre study. Cureus 8(8): e753. https://doi.org/10.7759/cureus.753.

6. Jacka MJ, Zygun D. Survey of Management of Severe Head Injury in Canada. Can J Neurol Sci 2007; 34: 307-312

7. Kruer RM, Harris LH, Goodw in H et al. Changing trends in the use of seizure prophylaxis after traumatic brain injury: A shift from phenytoin to Levetiracetam. Journal of Critical Care 2013;28:883.e9-883.e13.

8. Sundararajan K, Milne D, Edw ards S et al. Anti-seizure prophylaxis in critically ill patients $w$ ith traumatic brain injury in an intensive care unit. Anaesth Intensive Care 2015:43(5):646-651.

9. Temkin NR, Dikmen SS, W ilensky AJ et al. A randomized double blind study of phenytoin for the prevention of post traumatic seizures. N Eng J Med 1990;323(8):497-502.

10. Zaman A, Dubiel R, Driver $S$ et al. Seizure prophylaxis guidelines follow ing traumatic brain injury: An Evaluation of Compliance. J Head Trauma Rehabil. 2017 Mar/Apr;32(2):E13-E17. https://doi.org/10.1097/htr.00000 00000000243

\section{1}

Prognosis, clinical and demographic characteristics of critical patients with spontaneus intracraneal hemorrage

S. Ramiro González ; R. Prieto Jurado ${ }^{2}$; MJL. Serrano ${ }^{3}$; LF. Guerrero ${ }^{4}$

${ }^{1}$ Intensive Care Unit, Virgen de las Nieves Hospital, Granada, Spain; ${ }^{2}$ Intensive Care, Hospital Virgen de las Nieves, Granada, Spain; ${ }^{3}$ Intensive care, Hospital Virgen de las Nieves, granada, Spain; ${ }^{4}$ Neurocritical and trauma intensive care unit, University Hospital Complex of Granada, Granada, Spain

Correspondence: R. Prieto Jurado

Intensive Care Medicine Experimental 2020, 8(2): 001291

Introduction: Spontaneus intracraneal hemorrage $(\mathrm{SICH})$ is the second most frequent type of cerebrovascular accident with an incidence of $10-15 \%$ and a high morbidity and mortality worldwide. To differentiate patients at higher risk of mortality may improve its management and result in better outcomes.

Objectives: To describe the characteristics of patients with $\mathrm{SICH}$ as well as treatment strategies and mortality.

Methods: Observational, descriptive and prospective study of 1321 patients who were admitted to a neurocritical ICU between March 1996 and March 2020. We analyzed demographic and clinical characteristics (Glasgow Coma Scale (GSC), Intracraneal Hemorrage Score $(\mathrm{ICH})$, hydrocephalus and pupils). The volume and location of the hematoma, intraventricular extension, treatment strategy and mortality were analyzed too.

Results: Mean age was 58,8 years (SD 15,1 ) and $62,6 \%$ were males with a mean ICU stay of 7,2 $\pm 9,3$ days and hospital stay of 25,7 \pm 35,1 days. Mean GSC score at ICU admissión was 6 (SD 3) with 8 points or less in a $66,3 \%$ of patients and mean ICH score was 2,37 (SD 1,38). Only 1 patient $(0,1 \%)$ was admitted with an ICH score of 6 . Most of patients $(73 \%)$ presented with normal pupillary reflex and only $11 \%$ were admitted with nonreactive bilateral mydriasis. Mean hematoma volume was $54,6 \mathrm{cC}$ (SD 51,1) and the most frequent location was supratentorial (78,3\%), being basal ganglia specifically the most prevalent $(40,8 \%)$. Intraventricular hemorrage developed in $70,7 \%$ but less than half $(45,1 \%)$ suffered from hydrocephalus. $22,8 \%$ of patients were under surgical treatment and an external ventricular drainage was placed in $14,8 \%$ of patients. ICU mortality was $45,4 \%$ and hospital mortality was higher $(50,5 \%)$.

Conclusion: Patient with $\mathrm{SICH}$ who is admitted at our neurocritical ICU is usually a male in his sixties with high risk of mortality (high bleeding 
volume with low GSC score). Major mortality rate happened at ICU during the acute phase and this results are consistent with that published in the literature.

\section{Reference(s) and grant ackowledgment(s) \\ 1. 1. Hemphill JC 3rd, Bonovich DC, Besmertis L, Manley GT, Johnston SC. The ICH score: a simple, reliable grading scale for intracerebral hemorrhage. Stroke. 2001; 32(4): 811-97. 2. Feigin VL, Lawes CM, Bennett DA, Barker-Collo SL, Parag V. Worldwide stroke incidence and early case fatality reported in 56 population-based studies: a systematic review. Lancet Neurol. 2009; 8(4): 355-69. 3. van Asch CJ, Luitse MJ, Rinkel GJ, van der Tweel I, Algra A, Klijn CJ. Incidence, case fatality, and functional outcome of intracerebral haemor- rhage over time, according to age, sex, and ethnic origin: a systematic review and meta-analysis. Lancet Neurol. 2010; 9(2): 167-76.}

\section{5}

\section{Clinical characteristics and initial management strategy} as predictors of outcome in patients with severe non-traumatic subarachnoid hemorrhage, a single center experience P. Ioannidis'; E. Siomos' ; T. Schizodimos ${ }^{1}$; A. Kosmas ${ }^{1}$; E. Lazoudi'; P. Poulios ${ }^{1}$; I. Karageorgiou'; C. lasonidou

'B icu, General Hospital "G. Papanikolaou", Thessaloniki, Greece Correspondence: $\mathrm{P}$. loannidis

Intensive Care Medicine Experimental 2020, 8(2): 001345

Introduction: Non-traumatic subarachnoid hemorrhage $(\mathrm{SAH})$ is a disease with high mortality and disability rates. 1 Identification of risk factors and initial management decisions may affect outcome.2.

Objectives: To investigate the association of clinical characteristics and early interventions with outcome in patients with severe nontraumatic $\mathrm{SAH}$.

Methods: This study is an observational, retrospective, single-center study conducted in a level III intensive care unit (ICU) of a tertiary referral hospital in Greece. Population of the study was comprised of 29 patients that were admitted in the ICU between July 2018 and February 2020 with the diagnosis of non-traumatic SAH. Outcome was assessed with the Glasgow Outcome Scale Extended (GOS-E) at 28 days, 3 months and 6 months after admission. A favorable outcome was defined as a GOS-E score of 5 or higher at last available assessment. The clinical parameters that were evaluated as potential predictors of outcome were age, gender, Acute Physiology And Chronic Health Evaluation (APACHE) II score and Glascow Coma Scale (GCS) on admission. Initial treatment and monitoring options under investigation were external ventricular drainage (EVD) catheter, surgery before ICU admission and intracranial pressure (ICP) monitoring. Fisher's exact test was used for comparison and univariate logistic regression models were fitted to the data.

Results: We present our results as preliminary because of limited sample size. Mean age of participants was $62.3( \pm 8.5)$ years and the majority $(72.4 \%)$ were females. Median GCS on admission was 6 (IQR $5)$ indicating severity and mean APACHE II score was 20.34 ( \pm 5.38$)$. $A$ trend of age being negatively associated with favorable outcome was observed $(p=0.126, p>0.05$, OR $0.87,95 \% C l 0.73,1.04)$. Likewise, increased APACHE II score on admission exhibited a negative impact on favorable outcome $(p=0.224, p>0.05$, OR $0.86,95 \% \mathrm{Cl} 0.67-1.1)$. Increased GCS on admission had a significant positive relationship with favorable outcome $(p=0.02, p<0.05$, OR $1.74,95 \% C l 1.09,2.27)$. In our population male gender was related with unfavorable outcome but that was not significant ( $p=0.9, p>0.05$, OR $0.86,95 \% \mathrm{Cl} 0.08,9.69$ ). Regarding interventions, surgery for SAH during admission was associated with a trend towards favorable outcome $(p=0.483, p>0.05$, OR $2.36,95 \% \mathrm{Cl} 0.21,25.91)$. In the majority of our patients $(86.2 \%)$ EVD was placed so EVD was not related with type of outcome $(p=1$, $p>0.05)$. ICP monitoring was beneficial regarding outcome $(p=0.55$, $p>0.05$ ).

Conclusion: In our population of patients with non-traumatic SAH, severe clinical presentation assessed with decreased GCS and increased APACHE II score were associated with unfavorable outcome.
Younger patients and females showed a trend toward more favorable outcome. EVD was not related with outcome and ICP monitoring exhibited a positive effect on outcome of patients with SAH. Early surgery was beneficial in our population but that was not significant. As our cohort of patients increases, we aim to clarify our results and study more specific subgroups of patients.

\section{Reference(s) and grant ackowledgment(s)}

1. Daou BJ, Koduri S, Thompson BG, Chaudhary N, Pandey AS. Clinical and experimental aspects of aneurysmal subarachnoid hemorrhage. CNS Neurosci Ther. 2019;25(10):1096-1112. https://doi.org/10.1111/cns.13222.

2. Rosengart AJ, Schultheiss KE, Tolentino J, Macdonald RL. Prognostic factors for outcome in patients with aneurysmal subarachnoid hemorrhage. Stroke 2007;38(8):2315-2321. https://doi.org/10.1161/strokeaha.107.484360.

3. None.

\section{8}

Characteristics and results of two cohorts of patients with spontaneus intracraneal hemorrage

MJL. Serrano'; S. Ramiro González²; R. Prieto Jurado ${ }^{3}$; A. Iglesias Santiago ${ }^{4}$; LF. Guerrero ${ }^{5}$

${ }^{1}$ Intensive care, Hospital Virgen de las Nieves, Granada, Spain; ${ }^{2}$ Intensive Care Unit, Virgen de las Nieves Hospital, Granada, Spain; ${ }^{3}$ Intensive Care, Hospital Virgen de las Nieves, granada, Spain; ${ }^{4}$ Critical care department, Hospital Virgen de las Nieves, Granada, Spain; ${ }^{5}$ Neurocritical and trauma intensive care unit, University Hospital Complex of Granada, Granada, Spain

Correspondence: R. Prieto Jurado

Intensive Care Medicine Experimental 2020, 8(2): 001348

Introduction: Stroke's incidence has decreased by $42 \%$ in developed countries in the last 4decades due to improvements in health prevention. Conversely, morbidity andmortality have not suffered possitive changes despite the efforts.

Objectives: To compare the characteristics and results of 2 series of patients withspontaneus intracraneal hemorrage $(\mathrm{SICH})$ and to analyze the differences interms of severity, treatment strategies and mortality.

Methods: Observational and prospective study which compares 2 temporal cohorts of patients with SICH. The first one consists in 1208 patients recruited between March 1996 and June 2016 and the second one consists in 113 patients recruited between March 2018 and March 2020. Both groups were admitted to a neurocritical ICU. Variables analyzed were clinicial variables, $\mathrm{SICH}$ chracteristics, treatment strategy and mortality. Statistic analysis with Chi-squared test for cualitative variables and Student's T-distribution for cuantitative variables.

Results: Age was significantly higher in the second cohort of patients $(61,8 \pm 11,4$ years) with respect to the first one $(58,6 \pm 15,3$ years $)$ $\mathrm{p}=0.007$ without differences in sex between them. Regarding severity scores; APACHE-II and APACHE-III were significantly lower in the second cohort $(p=0.003$ for APACHE-II, $p=0.001$ for APACHE-III) but for SAPS-II score difference was not significant $(44 \pm 22$ cohort $1,42 \pm 19$ cohort $2, p=0.229)$. Mean ICU stay in the first cohort was $7,2 \pm 9,5$ days and $7,3 \pm 7,4$ days in the second one $(p=0.108)$. Mean hospital stay was 25,6 $\pm 35,6$ days in the first cohort and 26,9 \pm 29,9 days in the second one $(p=0.096)$. Both groups had similar SICH characteristics in terms of volume $(p=0.096)$, location $(p=0.169)$, intraventricular hemorrage $(p=0.354)$ and hidrocephalus $(p=0.189)$. No differences were found as well in treatment strategy. Surgical intervention was $21,9 \%$ in the first cohort and $32,7 \%$ in the second one $(p=0.08)$. Ventricular drainage was placed in $14,4 \%$ of the first and $18,6 \%$ of the second cohort $(p=0.231)$. Regarding mortality there were not statistically significant differences in ICU mortality $(46,1 \%$ first cohort, $38,1 \%$ second cohort, $\mathrm{p}=0.102$ ) or hospital mortality $(51,2 \%$ first cohort, $42,5 \%$ second cohort, $p=0.075)$.

Conclusion: We can conclude that the characteristics of the patients with $\mathrm{SICH}$ have been similar over time except age that is higher in the most recent cohort. Results are similar in both groups in terms of 
ICU/Hospital stay, treatment strategy and mortality. Despite failing to achive statistically significant differences, there is a statistical trend in the second cohort of patients to lower ICU/Hospital mortality as well as to higher surgical intervention and ventricular drainage placement as treatment options.

\section{7}

Incidence of Ventilatory Associated Events (VAEs) in patients with severe Traumatic Brain Injury (TBI) and association with Thromboelastography (TEG) abnormalities

M. Ulla'; I. Corrales²; D. Banach²; M. Berardino'; A. Gordon²; J. Rhodes ${ }^{3}$ ${ }^{1}$ Anaesthesia and intensive care, Citta Della Salute e Della Scienza University Hospital, Turin, Italy; ${ }^{2}$ Adult intensive care unit, St Mary's Hospital, Imperial College NHS Trust, London, United Kingdom; ${ }^{3} \mathrm{Critical}$ care, anaesthesia and pain medicine, University of Edinburgh and NHS Lothian, Edinburgh, United Kingdom

Correspondence: J. Rhodes

Intensive Care Medicine Experimental 2020, 8(2): 001437

Introduction: Deep Venous Thrombosis (DVT) is common in patients with $\mathrm{TBI}$ and correlates with poor outcome, increased number of days of mechanical ventilation and length of admission [1,2]. An increased number of VAEs has been described in these patients. The TARDIS (Traumatic Brain Injury Associated Radiological Deep Vein Thrombosis Incidence and Significance) study has been designed to investigate incidence (via compression ultrasound) of DVT in patients with moderate/severe TBI who require ventilation. The association between DVT and acute lung injury (ALI), VAEs, ICU length of stay and short or longterm survival and disability outcomes are secondary objectives. Severe trauma patients, including TBI, present coagulation abnormalities with increased morbidity and mortality [3]. Conventional coagulation tests have many limits while TEG displayed a potential diagnostic and prognostic role [4].

Methods: Following consent, ventilatory associated events (new x-ray infiltrates, worsening of oxygenation or increased secretions, signs of inflammation, need to commence patients on antibiotics with or without microbiological confirmation) were recorded to 10 days post injury in ventilated patients with brain trauma admitted to intensive care unit, as part of the TARDIS study protocol. A compression ultrasound scan (USS) of both legs was performed and repeated on alternate days up to Day 10 from injury; clinical, ventilatory and laboratory variables were collected daily. TEG was performed at admission, as per the manufacturers' instructions. Clinical and viscoelastic variables were correlated with the incidence of VAE. Data is described as mean (standard error of the mean) and median (interquartile range) as appropriate. Proportions were analysed by $\mathrm{t}$-student and Chi square test, $\mathrm{p}<0.05$ are considered significant.

Results: Preliminary data from a single Institution are included. Six out of $15(40 \%)$ patients developed VAE during the first 10 days of admission. No significant correlation was observed between gender, age, GCS and Marshall scores at admission and VAEs.

\begin{tabular}{|c|c|c|c|c|}
\hline Variables & $\begin{array}{l}\begin{array}{l}\text { Overall } \\
(n=15)\end{array} \\
\text { (n) }\end{array}$ & $\begin{array}{l}\text { VAEE } \\
(n=6)\end{array}$ & $\begin{array}{c}\text { NO VAE } \\
(n=8)\end{array}$ & Pvalue \\
\hline$K \quad \underset{\substack{0,8-2,1 \\
>2,1}}{2}$ & $\begin{array}{l}6(42,86 \%) \\
8(57,14 \%)\end{array}$ & $\begin{array}{l}2(33.33 \%) \\
4(66,67 \%)\end{array}$ & $\begin{array}{l}4(50.00 \%) \\
4(50,00 \%)\end{array}$ & 0.62 \\
\hline $\begin{array}{c}\text { Alpha } 63.78 \\
<63\end{array}$ & $\begin{array}{l}5(35,71 \%) \\
9(64,29 \%)\end{array}$ & $\begin{array}{l}1(16,67 \%) \\
5(83,33 \%)\end{array}$ & $\begin{array}{l}4(50 \%) \\
4(50 \%)\end{array}$ & 0,30 \\
\hline$K>2,1$ and Alpha $<63$ & $8(57,14 \%)$ & $4(66,67 \%)$ & $4(50 \%)$ & 0,62 \\
\hline
\end{tabular}

TEG displayed increased $\mathrm{K}$ and reduced Alpha angle values (see Table). Mean values were altered in both groups, but contingency test showed a high percentage of patients with VAEs with prolonged K $(66 \%)$ and reduced a (83\%). Four out of 6 patients $(66 \%)$ had both values altered.

Conclusion: VAEs are frequent in our cohort of patients with TBI requiring mechanical ventilation. Despite no statistical significance, our data suggest that a hypocoagulable profile recorded via TEG seems associated with increased risk of developing VAEs. This work requires further confirmation and is continuing in the TARDIS study.

\section{Reference(s) and grant ackowledgment(s)}

1. 1. Denson K, Morgan D, et al. (2007) Incidence of venous thromboembolism in patients with traumatic brain injury. American journal of surgery 193: 380-383; discussion 383-384 2. Skrifvars MB, Bailey M, et al. (2016) Venous thromboembolic events in critically ill traumatic brain injury patients. Intensive Care Med 3. Brohi K, Singh J, Heron M, Coats T. Acute traumatic coagulopathy. J Trauma 2003;54:1127-30 4. Samuels J, Moore E, et al. Severe traumatic brain injury is associated with a unique coagulopathy phenotype. Journal of Trauma and Acute Care Surgery. DEC 2018.

2. Dr Rhodes is supported by a National Health Service Research Scotland Clinician Fellowship.

3. TARDIS is supported by a grant from the National Institute of Academic Anaesthesia and the British Journal of Anaesthesia.

\section{8}

Catheter associated ventriculitis potential "triggers", incidence and microbial etiology in the neurocritical care setting: a single-center experience

M. Sileli' ; A. Kosmas ' ${ }^{1}$ E. Lazoudi'; P. Poulios ${ }^{1}$; I. Karageorgiou' ${ }^{1}$ L.

Ntemourtsidis'; D. Dragoumis ${ }^{2}$; N. Kapravelos ${ }^{1}$; C. lasonidou'

'B icu, General Hospital "George Papanikolaou", Thessaloniki, Greece, Greece; ${ }^{2}$ Neurosurgery department, General Hospital "G. Papanikolaou", Thessaloniki, Greece, Thessaloniki, Greece

Correspondence: $M$. Sileli

Intensive Care Medicine Experimental 2020, 8(2): 001448

Introduction: In the neurocritical care setting catheter associated ventriculitis (CAV) is a frequent complication associated with increased morbidity and mortality. Precipitating risk factors are still under discussion.

Objectives: To investigate potential CAV "triggers" and secondary to record for microbial etiology and incidence in our cohort.

Methods: Single center study on adult neurocritical care patients requiring urgent EVD insertion (January 2018 - April 2020). Exclusion criteria were: prior hospitalization, CNS infection or hydrocephalus and CSF leakage. All catheters were antibiotic impregnated connected to a drainage system placed in the operating theatre. Periprocedural antibiotics were given in all. Dressing change performed every 2 days. CSF samples were obtained from the EVD as clinically indicated. CAV defined as CSF: positive culture \pm pleocytosis \pm hypoglycorrhachia (IDSA guidelines 2017). Patients were divided into two groups (non CAV/CAV). Demographics, Charlson comorbidity index (CCI) score, GCS, APACHE II, neurosurgical intervention, EVD related data, CSF sampling count, CAV incidence, bacteria cultured, intraventricular antibiotic use and concurrent infection were recorded. Student t-test or Mann Whitney U test used for quantitative data and the Pearson chisquared test for qualitative data as necessary. The level of significance was set at $P<0.05$.

Results: We retrieved 52 medical records with 76 EVD insertion procedures for a total of $804 \mathrm{EVD}$ days (mean age $61.65 \pm 11.51$ years). The indication was TBI (5.7\%), aSAH (63.5\%) and ICH/IVH (30.8\%). CAV confirmed in 9 patients (17.3\%). Each of them experienced only one episode of EVD infection while $3(33.33 \%)$ had a co-infection. CAV rate estimated as 11.2/1000 days. Gram stain had findings in 3 of them (33.3\%). Major CSF pathogens were: Acinetobacter baumannii $(n=5$, $55.6 \%)$, coagulase-negative staphylococci $(n=2,22.2 \%)$, Enterococcus species $(n=1,11.1 \%)$ and Klebsiella oxytoca $(n=1,11.1 \%)$. Intraventricular antibiotics used as an adjunct in 5 (55.6\%).

Baseline characteristics (gender, age, CCI, GCS) and indication of EVD insertion were similar in both groups $(p>0.05)$. Univariate analysis showed that CAV patients received more EVD catheters $(p<0.001)$ and had longer total drainage duration (13.93vs22.77; $p=0.016)$ but retention of the drainage system $>10$ days was not correlated with increased risk of infection $(p=0.81)$. Indeed in most CAV patients 
$(6 / 9 ; 66.6 \%)$ infection documented during the time period of the first inserted EVD. Among other tested variables potential "triggers" were neurosurgical intervention $(p=0.01)$, CSF sampling $>1$ time $(p<0.001)$ and the simultaneous presence of intraparenchymal ICP monitoring devices $(p=0.021)$.

Conclusion: Neurosurgical operation and recurrent sampling, but not shunting duration, were factors associated with EVD infections. Interestingly enough, concurrent existence of intraparenchymal ICP monitoring devices seems to have a correlation with the presence of CAV in our cohort. These results are in agreement with previous studies and confirm the need to focus on minimizing potential "triggers" in order to overcome the CAV obstacle in neurointensive care patients.

\section{Reference(s) and grant ackowledgment(s)}

1. 1. Dorresteijn K, Brouwer M, Jellemaa K, Van de Beek D. Bacterial external ventricular catheter-associated infection. Expert Rev Anti Infect Ther 2020;18(3):219-229

2. None.

\section{3}

Cell index as an early predictor of external ventricular device related infections in hemorrhagic CSF: a single institution experience

M. Sileli ${ }^{1}$; A. Kosmas ${ }^{1}$; E. Lazoudi ${ }^{1}$; L. Ntemourtsidis ${ }^{2}$; E. Siomos ${ }^{1}$; F. Sifaki ${ }^{1}$; I. Karageorgiou'; P. Poulios'; N. Kapravelos ${ }^{1}$; C. Iasonidou

'B icu, General Hospital "George Papanikolaou", Thessaloniki, Greece, Greece; ${ }^{2}$ Neurosurgery department, General Hospital "G. Papanikolaou", Thessaloniki, Greece, Thessaloniki, Greece

Correspondence: M. Sileli

Intensive Care Medicine Experimental 2020, 8(2): 001453

Introduction: Positive CSF culture is the cornerstone of EVD related infections (ERI) diagnosis. However it is time consuming while prompt antimicrobial therapy is crucial. Several conventional CSF parameters have supplementarily used but their diagnostic accuracy especially in case of hemorrhagic CSF is uncertain. Under these limitations the cell index, a formula to correct for blood contamination, and the CSF/ serum glucose ratio have been proposed as alternatives.

Objectives: To evaluate and compare the utility of cell index over the $\mathrm{CSF} /$ serum glucose ratio in early diagnosis of ERI in patients with hemorrhagic CSF.

Methods: We reviewed data of consecutive adult neurocritical patients who received an EVD in an emergency setting from January 2018 to April 2020. We enrolled patients with raised clinical suspicion for ERI in whom at least one CSF sample and complete blood count send for analysis at the same day. Samples were obtained from the EVD system. Patients who excluded were those who had prior hospitalization or CNS infection. All included patients received antimicrobial impregnated EVD catheters at the operating theatre and protocol based antimicrobial prophylaxis as well with no further antibiotic exposure until CSF sampling. ERI was defined based on CSF positive cultures. We assigned patients into two groups according to presence or absence of ERI. Data concerning demographics, indication of EVD insertion, GCS, Charlson Comorbidity Index (CCI) and APACHE II were collected. Peripheral blood (WBC, RBC, glucose) and CSF (WBC, RBC, glucose, culture) cytobiochemical/microbiological analyses at the time of clinical suspicion of ERI were retrieved. We calculated for each sample the cell index [(CSF leukocytes/CSF erythrocytes)/(blood leukocytes/blood erythrocytes)] and the CSF/serum glucose ratio. Categorical variables were tested using a chi-square test and continuous variables using the Student $t$ test or Mann Whitney $U$ test. The level of significance was set at $\mathrm{P}<0.05$. The predictive ability of the CSF tests of interest was estimated using an area under the receiver operating characteristic curve (AUC-ROC) analysis.

Results: 28 patients met study criteria (mean age $62.21 \pm 11.59$; females $60.71 \%) .19$ of them were classified as non ERI whereas 9 as ERI (32.14\%). Indications for drain placement were TBI (1vs0), aSAH (11vs7) and ICH/IVH (7vs2). No differences were observed in baseline characteristics (gender, age, GCS, CCI, APACHE II) or in indication of EVD insertion between the groups ( $p>0.05$ ). In patients with ERI the median cell index was 15.14 vs 0.61 for the rest of patients $(p<0.001)$. No statistical difference was noted in CSF/serum glucose ratio when comparing ERI and non ERI patients ( 0.53 vs 0.76 respectively; $p=0.058$ ). Discriminatory power of cell index was excellent (AUC:0,927; 95\%Cl:0,763-0,991; $\mathrm{p}<0001$ ) in confirming ERI at the time of clinical suspicion whereas that of the CSF/serum glucose ratio was fair (AUC:0,722; 95\%Cl: 0,522-0,874; $p=0.069$ )

Conclusion: In the context of hemorrhagic CSF, cell index calculation had an excellent diagnostic accuracy in confirming ERI in our cohort as compared to the widely used CSF/serum glucose ratio. Our results extend knowledge from previous studies. Further research should be carried out in order to incorporate this costless index in every day clinical practice.

Reference(s) and grant ackowledgment(s)

1. 1. Dorresteijn $K$, Jellema K, Van de Beek D and Brouwer M. Factors and measures predicting external CSF drain-associated ventriculitis: A Review and Meta-Analysis. Neurology 2019;93(22):964-972

2. None.

\section{3}

Diagnostic experience of patients with stroke mimic in a Latin American University Hospital

JD. Villalobos-Ibarra'; A. Vargas-Villanueva'; JA. Carrizosa

${ }^{1}$ Departament of critical and intensive care medicine, Fundacion Santa Fe de Bogota University Hospital, Bogotá, Colombia

Correspondence: A. Vargas-Villanueva

Intensive Care Medicine Experimental 2020, 8(2): 001483

Introduction: One-third of the patients that present to the emergency room with stroke symptoms are stroke mimics (SM) (1), leading to stroke code activation, several diagnostic tests required, unnecessary treatment, and high costs to the health system before the diagnosis of a stroke mimic can be confirmed (2).

Objectives: This study aimed to describe the patient's characteristics, risk factors, diagnostic tests, and types of mimic presenting to a university hospital at Bogota, Colombia.

Methods: This was a single-center cross-sectional study of patients admitted to the emergency room with stroke symptoms and further diagnosis of SM. Data were obtained from January 2017 to March 2020 from a clinical database. Patients excluded if the stroke code was not activated or the admission at the emergency room (ER) was not due to stroke symptoms. FABS and Telestroke score for SM prediction were calculated.

Results: The study included 111 patients, being women $54.1 \%$ of them. The clinical characteristics on admission to the ER were similar between men and women. The mean time between the onset of symptoms and admission at ER was $4.7+/-8.5$ hours, and the meantime of stroke code activated was $2.1+/-2$ hours. The most common disease in medical history was hypertension $(49.5 \%)$, followed by dyslipidemia (19.8\%) and cancer (15.3\%). In most patients, the diagnosis of mimic was made between 1 and 4 hours from the ER admission (64.9\%), MRI was the most frequent first neuroimage that allows the identification of SM (57.7\%). Only $4.5 \%$ of cases did not require a neuroimage at ER because the diagnosis of SM was predominant over stroke. The mean score of Telestroke and FABS score was $15.8+/-6.2$ and $2.1+/-0.6$, respectively, with a high probability of being a mimic according to both scores in $84.7 \%$ and $99.1 \%$ of cases, respectively. The most common etiology of mimic among women were migraine (18.3\%) followed by a transient ischemic attack (16.7\%) and conversion disorder $(11.7 \%)$ with no cases of conversion disorder among men. The most common cause of SM among both sexes was also migraine (15.3\%). Administration of tissue plasminogen activator was done in $0.9 \%$ of patients, being only men.

Conclusion: Both FABS and Telestroke seem to easily stratify patients with a high probability of having an SM at the ER. This pathology is 
most common in women and predominantly confused with migraine symptoms. It is crucial to identify the population at risk of SM to reduce the unnecessary administration of stroke treatments.

\section{Reference(s) and grant ackowledgment(s)}

1. (1) Merino JG, Luby M, Benson RT, Davis LA, Hsia AW, Latour LL, et al. Predictors of acute stroke mimics in 8187 patients referred to a stroke service. $J$ Stroke Cerebrovasc Dis 2013;22:e397-403.

2. (2) Goyal N, Male S, AI Wafai A, Bellamkonda S, Zand R. Cost burden of stroke mimics and transient ischemic attack after intravenous tissue plasminogen activator treatment. J Stroke Cerebrovasc Dis 2015;24:828-33.

3. No grant acknowledgment.

\section{7}

Diagnosis of Meningitis in Neurocritical patients - a retrospective analysis

E. Coelho' ; L. Costa ${ }^{1}$; P. Costa ${ }^{2}$; JE. Oliveira ${ }^{1}$; A. Maia-Gonçalves ${ }^{1}$; L. Lencastre

1 Unidade de Cuidados Intensivos Polivalente, Hospital de Braga, Braga, Portugal; ${ }^{2}$ Life and health sciences research institute (icvs), School of Medicine - University of Minho, Braga, Portugal

Correspondence: $\mathrm{E}$. Coelho

Intensive Care Medicine Experimental 2020, 8(2): 001497

Introduction: The diagnosis of meningitis is a clinical challenge in intensive care, even more in neurocritical patients. This population has an increased risk of meningitis, related to cerebral damage, surgical intervention and use of intracranial devices.

However, the classical clinical diagnosis of meningitis is compromised in neurocritical patients, due to impaired neurological status, and difficult valuation of cerebrospinal fluid (CSF).

Objectives: To describe and analyse the determinant factors to diagnose meningitis in neurocritical patients: risk factors, interventions/ intracranial devices, clinical signs and CSF analysis.

Methods: Retrospective analysis of adult neurocritical patients admitted in an intensive care unit (ICU) from a 3-year period in which meningeal infection was suspected and CSF analysis was done.

Results: Twenty-seven surgical patients were included, with a mean age of 49 years old. Seventy percent of the patients were male. The ICU mortality was $11 \%$. Meningitis was diagnosed in more than $65 \%$ of the sample. There were no significant association between any risk factor, intervention/intracranial device or clinical sign and the diagnosis of meningitis. When analysing the CSF, there was a significant association between cell counting and the diagnosis of meningitis, with a mean cell counting of $122 / \mathrm{mL}$ vs $923 / \mathrm{mL}(\mathrm{p}<0.05)$.

Conclusion: Classical signs of meningitis are of little use in neurocritical intensive care patients. Risk factors and interventions or the use of intracranial devices may be an alert to doctors but were not significantly associated with the diagnosis. The most crucial parameter to better diagnosis of meningitis is the cell number of CSF analysis.

\section{Reference(s) and grant ackowledgment(s)}

1. Ortiz, O, Garcia, H, Ramirez, F, et al, Development of a prediction rule for diagnosing postoperative meningitis: a cross-sectional study, Journal of Neurosurgery, 2018

2. Tunkel, AR, Hasbun, R, Bhimraj, A, et al, 2017 Infectious Diseases Society of America's Clinical Practice Guidelines for Healthcare-Associated Ventriculitis and Meningitis, Clinical Infectious Diseases, 2017.

3. Tunkel, AR, Clinical features and diagnosis of acute bacterial meningitis in adults, Post TW, ed. UpToDate. Waltham, MA: UpToDate Inc. Accessed January 2019.
001546

Evaluation of the effect of early administrated neuroprotective drug on treatment results in patients after traumatic brain injuryPILOT trial

K. Kojder ${ }^{1}$; K. Jarosz ${ }^{1}$; A. Agata ${ }^{2}$

${ }^{1}$ Anesthesiology and intensive care, Pomeranian Medical University, Szczecin, Poland; ${ }^{2}$ Anesthesiolog and intensive care, University Hospital 1 Szczecin, Szczecin, Poland

Correspondence: K. Kojder

Intensive Care Medicine Experimental 2020, 8(2): 001546

Introduction: TBI (Traumatic Brain Injury:traumatic brain injury) is associated with one of the highest rate of deaths and setbacks in the recovery and return to life in society. The latest reports of the medical literature emphasized the need for further research in terms of methods for treatment of patients with TBI. One of the drugs with positive impact on prognosis in this group of patients is cerebrolysin. This drug is a component produced from a homogeneous, purified pig brain, containing different protein molecules of low molecular weight, which have neuroprotective and neurotrophic effects in vitro and in vivo. In the existing literature there are no reports describing the effect of early cerebrolysin administration in patients with $\mathrm{TBI}$, in correlation with neuropsychological tests and neuroimaging results.

Objectives: Evaluation of the impact of early administration of neuroprotective drug (cerebrolysin) on outcome of patients after a TBI, who were treated neurosurgically and under the conditions of the ICU .

Methods: 10 patients were included in the study according to inclusion criteria: TBI diagnosis (subarachnoid, epidural, or subdural hemorrhage) in patients, who were qualified for neurosurgical treatment (decompressive craniectomy,implantation of ventricular drainage) age 40-65y, GCS <8, time from the injury less than $6 \mathrm{~h}$. After randomisation patients were assigned to two groups (Group 1early drug adminstration, Group 2 control group). After the qualification the neuroprotective drug was administrated in Group 1 (Cerebrolysin intravenous infusion $\mathbf{5 0} \mathbf{~ m l}$ for 21 days, first dose in first 6 hours after the injury). After the neurosurgical treatment the patients of both groups were hospitalized in the ICU where standard intensive care was provided in both groups. The condition of the patients was monitored by neuroimaging (Computed Tomography), and neuropsychological tests (Barthel scale: evaluation of the efficiency of the patient, Mini -Mental State Examination :the scale of assessment of cognitive disorders).

Results: The results of the study contain the results of general outcome of the patients, GOC, the neuroimaging and psychological tests results.

Conclusion: The effects of early administration of neuroprotective drug (cerebrolysin) in correlation with neuroimaging results and neuropsychological test results of patients after TBI need further research on larger group.

Reference(s) and grant ackowledgment(s)

1. 3/ L. Marshall et al. Head injury: the recent past, present, and future. Neurosurgery 2000: 47/3, 546-561

2. 2/ P. Konig Cerebrolysin in traumatic brain injury- a pilot study of a neurotrophic and neurogenic agent in the treatment of acute traumatic brain injury. J. Neurol. Neurochir. Psyciatr. 2006, 3: 12-20.

3. $1 / \mathrm{N}$. Bornstein et al. Accelerated recovery from acute brain injuries: clinical efficacy of neurotrophic treatment in stroke and traumatic brain injuries. Drugs of Today 2012: 48, 43- 61.

4. Presented PILOT study was funded by University Hospital nr 1 in Szczecin with Everpharma partial participation in neuroprotective drug (Cerebrolysin) costs. 
001558

Functional situation of patients who enter the ICU by Traumatic Brain Injury at 3-4 years of follow-up

T. Amat Serna ${ }^{1}$; C. Aranda Martínez²; M. Delange Van Der Kroff ${ }^{3}$; M. Guerrero Marin ${ }^{1}$; M. Prieto-Palomino ${ }^{4}$

${ }^{1}$ Medicina intensiva, Hospital of Jaen, Jaén, Spain; ${ }^{2}$ Medicina Intensiva, Hospital of Jaen, Jaén, Spain; ${ }^{3}$ Intensive care, Hospital Comarcal Axarquia, Vélez-Málaga, Spain; ${ }^{4}$ Intensive care, Hospital Carlos Haya, Málaga, Spain Correspondence: C. Aranda Martínez

Intensive Care Medicine Experimental 2020, 8(2): 001558

Introduction: To study in patients admitted to the ICU with traumatic brain injury (TBI), the long-term functional situation (3-4 years) and analyze whether the evaluation one year after admission in ICU reflects what your situation will be in the future.

Methods: Prospective cohort study of patients with TBI admitted to the ICU of the Regional Hospital of Malaga during the years 2004 to 2008 collection of data at hospital admission and subsequent follow-up with telephone call to evaluate functional situation. GOS (Glasgow Outcome Scale) was used to assess the functional situation. X 2 was used for proportion comparison and $p<0.05$ was considered statistically significant. Results: A total of 531 patients, age 35 (22.56) years, APACHE II 17 (13.23) points, Glasgow 7 (4.10) points, were included in the study. Mortality in the ICU was $25 \%$ and in hospital mortality was $28.6 \%$.

Of the 379 patients admitted to the hospital, follow-up could be performed at one year in 363 patients and their functional situation was as follows: GOS 1 (deceased): 38 (10.5\%), GOS 2: 15 (4.1\%), GOS 3: 98 (27\%), GOS 4: 99 (27.3\%) and GOS 5: $113(31.1 \%)$.

At 3-4 years, a new follow-up of patients was performed and 445 patients ( 86 missing) could be evaluated. The classification according to the Glasgow Outcome Scale shows that 181 patients (171 per year and 10 thereafter) had died (GOS 1). At 1 year, $40.2 \%$ of the 445 patients evaluated had good functional status (Normal (GOS 5) or Disfunction but Self-sufficient (GOS 4)). At 3-4 years, 45\% $(\mathrm{N}=200)$ had good functional status (GOS-4-GOS 5) and 64 (14.38\%) of the patients followed and who had not died had a poor functional status (Vegetative (GOS 2) or Disfunction and not Self-Sufficer (GOS 3)). Among the patients who had no died, only 1 patient had worsened their functional status between the year and 3-4 years and 65 patients had improved their functional status $(14.6 \%$ of the total 445 patients evaluated per year and $23.7 \%$ of the patients who had not died per year and who were followed at 3-4 years).

\begin{tabular}{|c|l|l|l|l|l|l|}
\hline \multicolumn{9}{|c|}{ GOS 3rd-4th years } \\
\hline GOS 1st year & 1 & 2 & 3 & 4 & 5 \\
\hline $\mathbf{1}$ & 171 & - & - & - & - \\
\hline $\mathbf{2}$ & 0 & 10 & 1 & 0 & 0 \\
\hline $\mathbf{3}$ & 5 & 0 & 52 & 25 & 2 \\
\hline $\mathbf{4}$ & 2 & 0 & 0 & 43 & 37 \\
\hline $\mathbf{5}$ & 3 & 0 & 0 & 1 & 93 \\
\hline & 181 & 10 & 54 & 68 & 132 \\
\hline $\mathbf{X}=\mathbf{1 2 0 0}, \mathbf{p}<\mathbf{0 . 0 0 1}$ & & & & & \\
\hline
\end{tabular}

Conclusion: In patients admitted to the ICU with TBI, the year-end evaluation of the functional situation does not reflect what their functional situation will be in the future. And these patients show an improvement in their functional situation between the periods of 1 year and 3-4 years in a fairly high group of patients.

\section{7}

A correlation of gastrointestinal motility disorders and acute brain injury in the patients admitted to the ICU in one year period F. Kunovac'; S. Kvolik'; N. Nenad'; T. Turk'ㄹ A. Cicvaric'; D. Muzevic ${ }^{3}$ ${ }^{1}$ Anesthesiology and icu, Osijek University Hospital; Medical Faculty, Osijek, Croatia; ${ }^{2}$ Radiology, Osijek University Hospital; Medical Faculty, Osijek, Croatia; ${ }^{3}$ Neurosurgery, Osijek University Hospital; Medical Faculty, Osijek, Croatia Correspondence: S. Kvolik

Intensive Care Medicine Experimental 2020, 8(2): 001567
Introduction: Gastrointestinal motility (GI) disorders were frequently observed in the patients with neurodegenerative diseases and in the critically ill patients. The incidence of both gastroparesis and constipation in the neurosurgical patients in the intensive care units (ICU) was rarely reported and was not correlated with brain swelling in the area of the vagal nuclei.

Objectives: The aim of this cross-sectional study was to evaluate the incidence of gastroparesis, constipation and stress ulcers in neurosurgical patients, and the correlation between acute brain injury in the area of the vagal nerve and GI motility disorders.

Methods: An institutional ethics committee approval was obtained prior to the study. Electronic database and medical records of 207 neurosurgical patients hospitalized at ICU of the University Hospital Centre Osijek from November 2018 to November 2019 were analyzed. Demographic data, total opioid consumption per patient, drugs used for $\mathrm{Gl}$ stimulation, and brain CT images were correlated with gastroparesis and constipation. A subgroup of 69 mechanically ventilated (MV) patients who stayed in the ICU for $\geq 4$ days was investigated up to the day 15. Total opioids were calculated as morphine equivalents (ME). Brain lesions involving the vagal nuclei area was graded by neuroradiologist as follows: 1 no edema, 2 edema present, 3 edema with midline shift. Gastroparesis and constipation were considered if delayed emptying without evidence of mechanical obstruction was present.

Results: A total of 113 emergency and 94 elective patients were admitted in the ICU. In the subgroup of $69 \mathrm{MV}$ patients $(63$ emergency and 6 elective) who stayed $\geq 4$ days in the ICU brain edema was registered in $57(82.6 \%)$ patients. Gastroparesis was observed in $48(69.6 \%)$, constipation in $67(97.1 \%)$ and stress ulcers in $4(5.8 \%)$ of MV patients. During their ICU stay patients had GI stimulation for 463 days (39\%), and had stool in 306 days (25\%) out of 1183 ICU days. The first stool, despite stimulation was registered later in patients with brain edema compared to those who did not have edema $(7.0 \pm 3.1$ vs $3.5 \pm 2.6$ days; $P=0.012$ ). Patients with swelling in the vagal nuclei area had common paroxysmal hypertensive episodes and needed more opioids for sedation as compared to patients without edema $(12.7 \pm 12.1$ vs. $46.2 \pm 35.9 \mathrm{ME} ; \mathrm{P}<0.001)$.

Conclusion: Patients with brain edema had more Gl dysmotility disorders than patients without edeam. Randomized controlled studies comparing different sedation protocols, opioid vs. nonopioid may more accurately confirm an occurrence of Gl dysmotility disorders, and their correlation with brain edema, and opioid and non-opioid sedation in the ICU.

Reference(s) and grant ackowledgment(s)

1. Patejdl R, et al. Neurol Res. 2017;39(11):959-64. Heinonen T, et al.

Aust Crit Care. 2020;33(2):151-154. Meyfroidt G, et al. Lancet Neurol. 2017;16(9):721-729.

2. Institutional projects VIF2015-MEFOS-16, VIF2016-MEFOS-15 and VIF 2017-MEFOS-16.

001570

Synocarotid syndrome in carotid angioplasty with stenting

R. Polishchuk'; Y. Ryabinkina'; M. Piradov'; I. Luneva'

${ }^{1} \mathrm{Icu}$, Research Center of Neurology, Moscow, Russia, Russia

Correspondence: I. Luneva

Intensive Care Medicine Experimental 2020, 8(2): 001570

Introduction: Stroke is the most important medical and social problem. One of the primary prevention methods is transluminal balloon angioplasty with stenting of the internal carotid arteries (ICA TBA) for stenosing atherosclerosis as the most common cause of ischemic stroke. A significant risk of surgery is carotid sinus syndrome (SCS).

Objectives: To determine of the frequency and timing of the development of SCS to ensure its personalized prevention and treatment 
in patients with atherosclerotic stenosis of the internal carotid arteries when performing TBA.

Methods: The prospective study included 120 patients with atherosclerotic carotid stenosis, who underwent TBA. The indications for surgical treatment were "symptomatic" stenoses of $50 \%$ or more and "asymptomatic" stenoses of $70 \%$ or more. All patients underwent invasive (Blood Preassure [BP]) and non-invasive monitoring (BP, heartbeat $[\mathrm{HB}], \mathrm{sPO} 2$, respiratoty rate $[\mathrm{RR}])$ during and after surgery. The diagnosis of SCS was established according to the generally accepted classification.

Results: SCS developed in $70 \%$ of cases of ICA TBA $(n=84)$, significantly more often in men - 71.4\% $(n=60)$ than in women $-28.6 \%$ $(n=24),(p<0.05)$. The median age was $68(44: 91)$. The predominant type of SCS was mixed than cardio-inhibitory or vasodepressor $(p<0.05)$.It took place in $63 \%$ of cases and was characterized by the almost simultaneous occurrence of sinus bradycardia (or asystole) and hypotension. In a little more than half of cases, $54.7 \%(n=29)$ SDS developed in response to balloon dilatation, in $11.3 \%(n=6)$ patients within 1 hour after dilatation, and in $34 \%(n=18)$ - in more distant terms, on average after 6 hours (from 2 to 11 hours). The cardio-inhibitory type was observed in $20 \%$ of cases $(n=17)$ and in the majority of patients $82 \%(n=14)$ manifested sinus bradycardia both during balloon dilatation and within the first hour after it. Atrioventricular [AV] block of I and II degrees took place in 2 patients (11.7\%). Asystole for more than 3 seconds without loss of consciousness was noted in 1 patient for balloon dilatation (6.3\%). The vasodepressor type was observed in $17 \%$ of cases $(n=14)$. In half of cases - $50 \%(n=7)$ arterial hypotension occurred immediately on balloon dilatation, in 5 patients - within the first hour after dilatation, and in 2 patients - at more distant periods (after 2 and 5 hours).

Conclusion: The work performed showed that three out of four patients with elective surgical endovascular intervention on the internal carotid arteries develop SCS, which is most often manifested by the almost simultaneous development of arterial hypotension and sinus bradycardia. In some cases, surgery is accompanied by cardiac arrest. These symptoms can occur not only during balloon dilatation, but also hours after surgery. This requires maximum awareness and preparedness of the anesthesiologist-resuscitator to provide the necessary assistance to patients, including the correct maintenance of blood pressure and heart function in order to prevent stroke and myocardial infarction.

\section{Reference(s) and grant ackowledgment(s)}

1. Acute Hemodynamic Changes During Carotid Artery Stenting/Farrell O. Mendelsohn, MD, Neil J. Weissman, MD, Robert J. Lederman, MD, James J. Crowley, MD, John L. Gray, MD, Harry R. Phillips, MD, Mark J. Alberts, MD, Richard L. McCann, MD, Tony P. Smith, MD, and Richard S. Stack, MD. THE AMERICAN JOURNAL OF CARDIOLOGYT. - 1998: 82, pp. 1077-1081.

2. Ueber mechanische Reizung des Nervus vagus beim Menschen. Czermak, Johann N. 1879. pp. 779-787

3. Carotid sinus syndrome. O.L. Bockeria, A.V. Sergeev. Annals of Arrhythmology, 2015: 12(2), pp. 106-113.

\section{5}

Critically ill patients with encephalitis in ICU

I. Luneva ${ }^{1}$; R. Polishchuk ; Y. Ryabinkina ${ }^{1}$; M. Piradov ${ }^{1}$

${ }^{1}$ Icu, Research Center of Neurology, Moscow, Russia

Correspondence: I. Luneva

Intensive Care Medicine Experimental 2020, 8(2): 001585

Introduction: Encephalitis is a severe pathology of the nervous system that is difficult to diagnose and treat. Currently, there are a number of limitations for verifying the etiology of encephalitis. The most common symptom is a change in behavior, a decrease in the level of consciousness up to coma, epileptic seizures, and focal neurological pathology. Often, with viral encephalitis, there is a hemorrhagic transformation of the inflammation focus, interstitial edema in the area of the focus, often with a displacement of the mid-stem structures, which may require decompression craniotomy.

Objectives: To investigate the peculiarities of diagnosis and treatment of encephalitis of various etiologies.

Methods: We observed 5 patients with encephalitis of various etiology. All patients underwent MRI of the brain at admission and in the medical examination. To exclude myelitis, MRI of the cervical spinal cord was performed. Also, all patients underwent a general study of cerebrospinal fluid, PCR of cerebrospinal fluid (CSF) for the presence of DNA viruses and intrathecal synthesis of oligoclonal antibodies. Also, taking into account the volume and level of lesion of the foci, all patients underwent EEG, and a number of patients underwent blood tests for the presence of antibodies to NMDA receptors.

Results: In the neurological status, all patients had behavioral changes, and later a decrease in the level of consciousness to coma in 4 patients and 1 patient developed mutism. Upon further examination, the following etiology of encephalitis was established: in 2 cases, herpetic encephalitis (PCR for Herpes simplex virus types 1 and 2 was positive), in 1 case, bacterial ventriculomeningoencephalitis (PCR for Listeria monocytogenes was positive), in 1 patient, necrotizing encephalitis associated with Influenza virus, and 1 case - multiple sclerosis (intrathecal synthesis of oligoclonal antibodies was determined in the (SF). In the general analysis of CSF, all patients had an increase in protein and cytosis. EEG in 3 patients out of 5 revealed epileptiform activity, which required the appointment of anticonvulsant therapy. All patients received glucocorticosteroid therapy and antiviral therapy 3 out of 5, antibiotic therapy in 1 patient. IVL was performed in 4 out of 5 patients. 1 patient with herpetic encephalitis underwent decompression trepanation, 1 patient received external ventricular drainage. The outcome in all patients is favorable. (fig MRI).

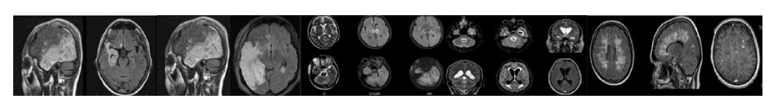

Conclusion: Thus, MRI and CSF exsamination are necessary diagnostic methods for detecting encephalitis and verifying its etiology. Also, neurosurgical treatment can improve the outcome of patients with progressive ventriculitis or laterally displaced encephalitis.

\section{6}

Asses functional situation at year of patients with Traumatic Brain Injury (TBI)

T. Amat Serna ${ }^{1}$; C. Aranda Martínez²; M. Delange Van Der Kroff ${ }^{3}$; M.

Guerrero Marin ${ }^{1}$; M. Prieto-Palomino ${ }^{4}$

${ }^{1}$ Medicina intensiva, Hospital of Jaen, Jaén, Spain; ${ }^{2}$ Medicina Intensiva, Hospital of Jaen, Jaén, Spain; ${ }^{3}$ Intensive care, Hospital Comarcal Axarquia Vélez-Málaga, Spain; ${ }^{4}$ Intensive care, Hospital Carlos Haya, Málaga, Spain

Correspondence: $C$. Aranda Martínez

Intensive Care Medicine Experimental 2020, 8(2): 001566

Introduction: Study in patients who are discharged from the Intensive Care Unit (ICU) with a diagnosis of traumatic brain injury (TBI), if the situation neurological evaluated with the Glasgow coma Scale at discharge from ICU and other variables known at admission and during their stay at ICU are related to the situation functional at one year evaluated with the Glasgow Outcome Scale (GOS).

Methods: Prospective cohort study of patients with TBI admitted to the ICU of the Regional Hospital of Malaga during the years 2004 to 2008 , data collection at hospital admission and monitoring of the functional situation with a phone call. Descriptive analysis using programme $R$.

Results: A total of 531 patients, age 35 (22.56) years, APACHE I| 17 (13.23) points, Glasgow on admission 7 (4.10) points were included in the study. 398 patients from the total of those admitted were discharged to the ward ( 6 of them to other hospitals). Follow-up at one year was made of 491 patients, 34 were lost to follow-up. 177 patients died from hospital admission (133 in the ICU, 19 in the hospital ward and 19 in the following year). Using the " $R$ " program, through the 
"randomForestSRC" program and the function "Vimp" we have studied the variables with the highest relationship with the dependent variable "Functional situation at one year", classified as good or bad situation. The most related variables in order of importance were: Glasgow Coma Scale score at discharge from ICU, Age, APACHE II, Glasgow Coma Scale Score at ICU a mission. The following table shows the relationship with these variables categorized in intervals.

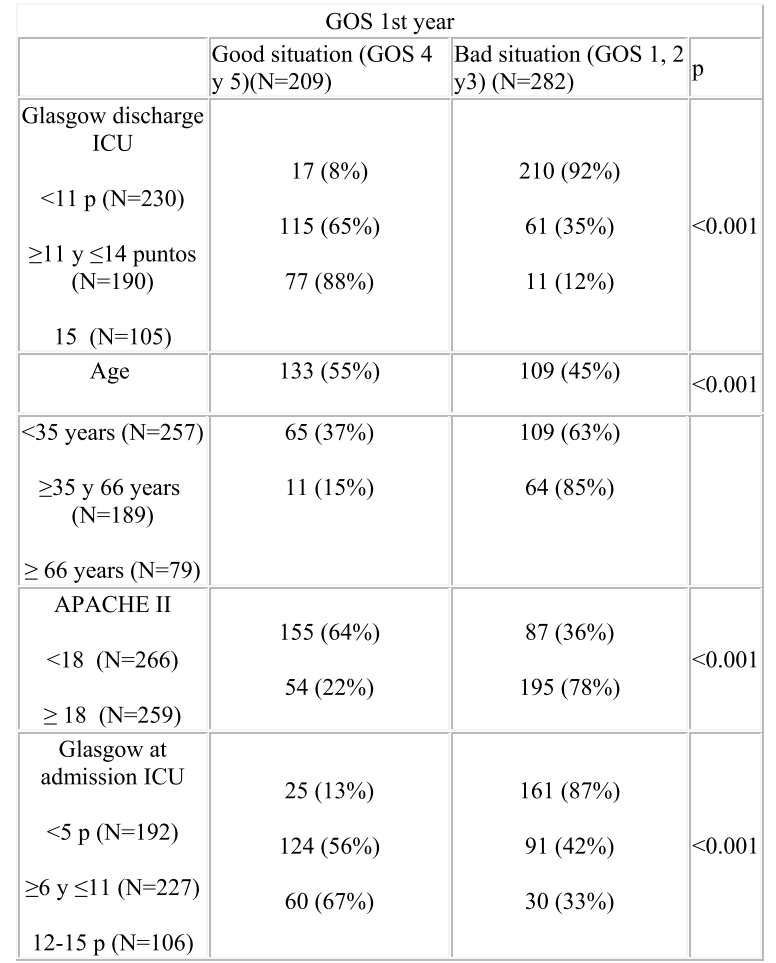

With these variables it was possible to create a predictive model with "randomForestSRC" with a discrimination evaluated with the Area under the ROC curve 0.818 (0.73-0.90).

Conclusion: The patients with the best functional situation at one year are patients who have a Glasgow score on admission to the ICU greater than or equal to 6 points, with an age of less than 66 years, an APACHE II score of less than 18 points and a Glasgow discharge at discharge of ICU greater than or equal to 11 points. We can observe that in those patients who are discharged from the ICU with a Glasgow score of less than 11 in 92\%, the functional situation is poor at one year (death, vegetative state or severe disability).

\section{0}

Trans cranial doppler (TCD) in Covid-19 patients (C-19 P) admitted in Intensive Care Unit shows marked abnormalities of the cerebral blood flow (CBF)

J. Richecoeur ${ }^{1}$; N. Verrier ${ }^{1}$; D. Combaux ${ }^{1}$; A. Sagnier ${ }^{1}$; R. Mercier ${ }^{1}$; R. De Bock ; C. Caplin ${ }^{1}$; C. Soulignac ${ }^{1}$; M. Toutoundjian ${ }^{1}$; D. Luis ${ }^{1}$

${ }^{1}$ Mixed icu, Hospital Center Simone Veil De Beauvais, Beauvais, France

Correspondence: J. Richecoeur

Intensive Care Medicine Experimental 2020, 8(2): 001250

Introduction: Neuropsychological dysfunction is reported in Covid-19 patients (C-19 P) (1).

This could be explained by CBF alterations.

TDC was performed in C-19 P to explore CBF and was compared to healthy control subjects (C).

Methods: $29 \mathrm{C}-19 \mathrm{P}$ and $50 \mathrm{C}$ were included during a two months period (March to May 2020). Doppler samples were set at the middle cerebral artery (M1 segment of MCA). Transthoracic echocardiography was used to record Velocity Time Integral at the left ventricular outflow tract (VTIAo).

Demographic data, reasons for admission in ICU, SAPS, sedative, vasopressor use and ventilator support were recorded as well as Mean Arterial Pressure (MAP), Systolic (Vs), diastolic (Vd) and mean velocities, (Vm), Pulsatility (PI) and Resistivity Indexes (RI). Velocity Time Integral MCA (VTIMCA) was sampled using TCD. Data were shown as median (interquartile: $25-75$ ). T test or non-parametric test (if appropriate) were use. $P<0,05$ was considered as significant.

Results: Reason for admission in C-19 P was ARDS. The main comorbidities were HTA, diabetes and obesity.

Sex ratio(M/F): 20/9 in C-19 P with SAPS II =47(38-62) and 26/24 in C Age: 62 years (53-67) in C-19 P vs 40 years (30-52) in C. All patients but 2 had invasive respiratory and vasopressor support and sedative drugs.

Results are shown in table 1

\begin{tabular}{lllllll|}
\hline & \multicolumn{2}{l}{$\mathrm{C}(\mathrm{n}=50)$} & \multicolumn{2}{l|}{$\mathrm{C}-19 \mathrm{P}(\mathrm{n}=29)$} & $\mathrm{p}$ \\
\hline MAP mmHg & 95,00 & $(89,00-98,00)$ & 86 & $(77,00-, 96,00)$ & $<0,001$ \\
\hline VTIAo cm & 21,50 & $(19,50-30,00)$ & 21,17 & $(18,00-25,88)$ & $<0,001$ \\
\hline VTIMCA cm & 51,75 & $(46-58)$ & 34,85 & $(31,23-41,00)$ & $<0,001$ \\
\hline Vd cm/s & 40,30 & $(35,00-50,00)$ & 27,70 & $(21-37,25)$ & $<0,03$ \\
\hline Vm cm/s & 58,50 & $(54,70-66,15)$ & 50,70 & $(40-55,55)$ & $<0,001$ \\
\hline RI & 0,55 & $(0,500-0,58)$ & 0,66 & $(0,64-0,74)$ & $<0,001$ \\
\hline PI & 0,86 & $(0,73-1,01)$ & 1,24 & $(1,00-1,34)$ & $<0,0001$ \\
\hline VTIMCA/VTIAo & 2,51 & $(2,15-3,09)$ & 2,15 & $(1,91-2,64)$ & $<0,001$ \\
\hline HF*VTIMCA/BSA & 1876 & $(1712-2219)$ & 1422 & $(1164-1858)$ & $<0,045$
\end{tabular}

CF: Heart Frequency, BSA: Body Surface Area

All TCD classical parameters were altered.

The low values of VTIMCA/VTIAo ratio and HF*VTIMCA/BSA (might be a surrogate of the CBF for the later) showed an alteration of the CBF. For 15 C-19P, VTIMCA/VTIAo ratio was $<1,5$ and suggested a depressed $\mathrm{CBF}$ autoregulation independently of CO. (Spearman: $R 2: 0,62 \mathrm{p}<0,001)$.

$13 \mathrm{C}-19 \mathrm{P}$ had delirium during the weaning and two had a fatal ischemic stroke.

Conclusion: CBF autoregulation in Covid -19 patients was markedly altered. Alteration of the vasomotor tone thought to be linked to endothelium dysfunction. The persistence of CBF abnormalities must be evaluated as well as the neuro-psychological and cognitive impact on the following.

Reference(s) and grant ackowledgment(s)

1. 1.Kotfis et al. Covid-19: ICU delirium management during SAR-CoV-2 pandemic. Critical care (2020). 24:176.

\section{3}

Correlation between intracranial pressure (ICP) and optic nerve sheath diameter (ONSD) measured by CT in patients with severe traumatic brain injury (TBI)

K. Muradyan ${ }^{1}$; O. Sosnovskaya ${ }^{1}$; A. Turkin²; A. Oshorov' I. Savin ${ }^{1}$; Z. Veliev; A. Burov ${ }^{3}$

${ }^{1}$ Icu, Nmits Neyrokhirurgii Im. Akademika N.n. Burdenko, Moskva, Russia; ${ }^{2}$ Radiology, Nmits Neyrokhirurgii Im. Akademika N.n. Burdenko, Moskva, Russia; ${ }^{3}$ ICU, Burdenko Nerosurgical, Moscow, Russia

Correspondence: K. Muradyan

Intensive Care Medicine Experimental 2020, 8(2): 001433

Introduction: One of the promising methods of non-invasive ICP assessment in the acute period of traumatic brain injury is the ONSD assessment based on CT data [1, 2, 3]. The advantages of the method are simplicity, relative availability, speed and reproducibility of ONSD assessment $[5,6,7]$.

Objectives: The aim of the study was to assess the correlation between ONSD measured according to CT data, and invasive ICP value in patients in the acute period of severe TBI. 
Methods: A retrospective analysis of 49 patients with severe TBI was made. Among them 38 were males and 11 females. Average age was $34+/-12$ years, GCS (Glasgow coma scale) score - $5.7+/-1.8$. Twentynine $(59 \%)$ patients were operated, 17 of them underwent decompressive craniectomy. All victims were admitted to the ICU in the acute period of TBI: $2+/-1$ days from the moment of injury. Upon admission, all patients underwent brain CT scan and invasive monitoring of ICP. ONSD was assessed at a distance of $3 \mathrm{~mm}$ from the posterior pole of the eyeball $[3,7,8]$. Statistical processing was performed using the StatSoft STATICTICA 7.0 package.

Results: A significant correlation was obtained between ONSD and the ICP value at the time of ICP sensor $(r=0.39 ; r 2=0.15 ; p<0.01)$ implantation. The correlation between the maximum, average ICP value for the entire monitoring period and ONSD did not reach a significant level ( $p>0.05)$, what can be explained by the influence of therapeutic and neurosurgical methods on the ICP value used for intracranial hypertension correction. There was a significant difference in ONSD in the groups of patients with normal and high ICP ( $Z=$ $-2.366 ; p=0.017$ )

Conclusion: In our research significant correlation between ONSD and ICP in the acute period of severe TBI was established. There is a further need for a more deeper analysisof the relationship between ONSD and intracranial hypertension duration, the role of aggressive methods for ICP correction [1, 4], as well as the prognostic value of ONSD in patients with various types of acute cerebral injury $[4,9,10]$.

\section{Reference(s) and grant ackowledgment(s)}

1. 10. Barret Rush, MD, Andrew Wormsbecker-2016. Optic nerve sheath diameter on computed tomography not predictive of neurological status post-cardiac arrest. Vancouver, Canada.

2. 9. David Skoloudık, Roman Herzig - 2010. Distal enlargement of the optic nerve sheath in the hyperacute stage of intracerebral haemorrhage. Olomouc, Czech Republic.

3. 8. Michael Vaiman, Paul Gottlieb - 2014. Quantitative relations between the eyeball, the optic nerve, and the optic canal important for intracranial pressure monitoring. Tel Aviv, Israel.

4. 7. Heidi Harbison Kimberly, Vicki E Noble - 2008. Using MRI of the optic nerve sheath to detect elevated intracranial pressure. Boston, US.

5. 6. Heidi Harbison Kimberly, MD, Sachita Shah - 2008. Correlation of Optic Nerve Sheath Diameter with Direct Measurement of Intracranial Pressure. Boston, US

6. 5. M. Steinborn1, J. Fiegler - 2010. High Resolution Ultrasound and Magnetic Resonance Imaging of the Optic Nerve and the Optic Nerve Sheath: Anatomic Correlation and Clinical Importance. NY, US.

7. 4. Lisa Romagnuolo MDa, Vivek Tayal MD - 2005. Optic nerve sheath diameter does not change with patient position. NC, US.

8. 3. S H. C. Hansen, K. Helmke - 1996. The subarachnoid spaces surrounding the optic nerves. An ultrasound study of the optic nerves sheath. Hamburg, Germany.

9. 2. A. Ballantyne, G. O'Neill - 2002. Observer variation in the sonographic measurement of optic nerve sheath diameter in normal adults. Glasgow, UK.

10. 1. Nancy Carney PhD, Annette M. Totten PhD- 2016. Guidelines for the Management of Severe Traumatic Brain Injury, Brain Trauma Foundation.
Introduction: Anemia incidence in neurocritical pathologies like subarachnoid hemorrhage (SAH) and traumatic brain injury (TBI) is around 30 and $46 \%$ respectively $(1,2)$. Anemia is an important cause of secondary neurologic injury due to hypoxia(3). Despite its impact, the thresholds for red blood cell (RBC) transfusion in clinical practice guidelines are only based on hemoglobin values, with no clear recommendation in many cases (4-7).

Objectives: To identify the paraclinical parameters used by health care personal to decide the best moment to transfuse critical care patients with SAH or TBI.

Methods: Data were obtained from December 2017 to December 2019 through a google forms survey distributed by email and published at the "Asociación Colombiana de Medicina Crítica y Cuidado Intensivo (AMCI)" and Latin American Brain Injury Consortium (LABIC) web pages. The questionnaire used a format of multiple-choice and open-ended questions to assess socio-demographic information, literature support in which the contestants based their decisions, safe number of RBC units to transfuse in less than 24 hours, and optimal range of paraclinical tests variables appraising hemoglobin $(\mathrm{Hb})$ brain tissue oxygen tension (PbtO2), jugular venous oxygen saturation (SjvO2) and arterio-jugular differences of $\mathrm{CO} 2$ (A-VYCO2). The entire dataset was stored in a spreadsheet (xlsx) gathering a total of 90 responses.

Results: The majority of responses came from intensivists (59\%) with ages between $25-40$ years old (55.4\%). The countries involved in this study were Colombia (22\%) and Mexico (11.1\%). The type of intensive care unit where the responders work, was predominantly a mixed unit in $88.2 \%$, with disponibility of blood bank in $85.1 \%$ of cases. The majority of the participants (72 and $76.6 \%$ ) agree with less than 2 RBC units as a secure limit for blood transfusion in less than 24 hours for patients with TBI and SAH, respectively. Predominantly, there is no perception of medical literature guidance to conceal blood cell transfusion in patients with TBI $(66.6 \%)$ or SAH $(78.8 \%)$. The most common response at the moment to determine the optimal range of $\mathrm{Hb}$ for TBI and SAH was $9.1-10.0 \mathrm{~g} / \mathrm{dL}(31.1 \%, 34.44 \%)$ (Fig.1), being this parameter used alone the most common referent to start RBC transfusion (57\% for $\mathrm{TBI}$ and $60 \%$ for $\mathrm{SAH})$. The prevailing values to start a RBC transfusion in patients for TBI and SAH were $<7 \mathrm{~g} / \mathrm{dL}$ for $\mathrm{Hb}(38.8 \%$ and $33.3 \%)$; $16-20 \mathrm{mmHg}$ for PbtO2 (14.4\% and $14.4 \%) ;<55 \%$ for SjvO2 (33.3\% and $32.2 \%)$ and $>8$ for A-VYCO2 $(22.2 \%$ and $16.6 \%)$ respectively. The majority of respondents use $\mathrm{Hb}$ alone for transfusion of RBC (Table 1).

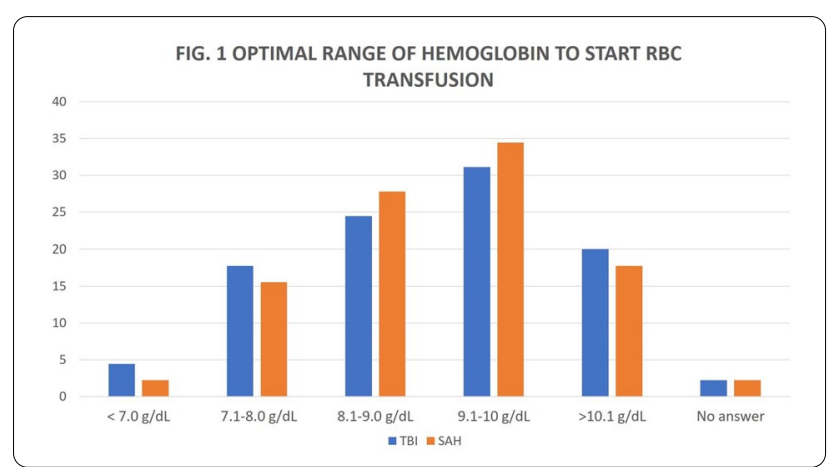

\section{9}

Perception assessment of blood transfusion criteria

in neurocritical care patients

J. Barrero ${ }^{1}$; A. Vargas-Villanueva ${ }^{2}$; JN. Carreño ${ }^{1}$

${ }^{1}$ Departament of critical and intensive care medicine, Fundacion Santa Fe de Bogota University Hospital, Bogotá, Colombia; ${ }^{2}$ Critical care department, Fundacion Santa Fe de Bogota University Hospital, Bogotá, Colombia

Correspondence: A. Vargas-Villanueva

Intensive Care Medicine Experimental 2020, 8(2): 001499 
TABLE 1. USE OF MULTIMODAL NEUROMONITORING TO START RBC TRANSFUSION

\begin{tabular}{|c|c|c|c|c|}
\hline$\overline{\text { VARIABLE }}$ & SAH (n) & SAH (\%) & $T B I(n)$ & TBI (\%) \\
\hline A-VYCO2 & 3 & $3,3 \%$ & 3 & $3,3 \%$ \\
\hline $\mathrm{Hb}$ & 54 & $60,0 \%$ & 52 & $57,8 \%$ \\
\hline PbtO2 & 7 & $7,8 \%$ & 6 & $6,7 \%$ \\
\hline SjvO2 & 3 & $3,3 \%$ & 3 & $3,3 \%$ \\
\hline $\mathrm{Hb}+\mathrm{A}-\mathrm{VYCO} 2$ & 1 & $1,1 \%$ & 0 & $0,0 \%$ \\
\hline $\mathrm{Hb}+\mathrm{PbtO2}$ & 1 & $1,1 \%$ & 4 & $4,4 \%$ \\
\hline $\mathrm{Hb}+\mathrm{Sj} \mathrm{j} \mathrm{O2}$ & 6 & $6,7 \%$ & 4 & $4,4 \%$ \\
\hline $\mathrm{PbtO} 2+\mathrm{SjvO} 2$ & 1 & $1,1 \%$ & 2 & $2,2 \%$ \\
\hline SjvO2+A-VYCO2 & 1 & $1,1 \%$ & 0 & $0,0 \%$ \\
\hline $\mathrm{Hb}+\mathrm{PbtO} 2+\mathrm{A}-\mathrm{VYCOZ}$ & 1 & $1,1 \%$ & 0 & $0,0 \%$ \\
\hline $\mathrm{Hb}+\mathrm{PbtO} 2+\mathrm{Sj} v \mathrm{O} 2$ & 1 & $1,1 \%$ & 4 & $3,3 \%$ \\
\hline $\mathrm{Hb}+\mathrm{SjvO2}+\mathrm{A}-\mathrm{VYCO} 2$ & 3 & $3,3 \%$ & 2 & $2,2 \%$ \\
\hline $\mathrm{Pbto} 2+\mathrm{SjvO} 2+\mathrm{A}-\mathrm{VYCO} 2$ & 0 & $0,0 \%$ & 1 & $1,1 \%$ \\
\hline $\mathrm{Hb}+\mathrm{Pbto}+\mathrm{Sj}$ jVO2+A-VYCO2 & 6 & $6,7 \%$ & 1 & $8,9 \%$ \\
\hline No answer & 0 & $2,2 \%$ & 1 & $1,1 \%$ \\
\hline
\end{tabular}

Conclusion: It is important to promote the assessment of other paraclinical tests instead of just $\mathrm{Hb}$ to achieve a multimodal neuromonitoring for RBC transfusion in neurocritical care patients. This will allow us to understand more precisely the brain's metabolic and perfusion

\section{Reference(s) and grant ackowledgment(s)}

1. (1) Ayling OGS, Ibrahim GM, Alotaibi NM, Gooderham PA, Loch Macdonald R. Anemia after aneurysmal subarachnoid hemorrhage is associated with poor outcome and death. Stroke. 2018;49(8):1859-65.

2. (2) Lelubre C, Bouzat P, Crippa IA, Taccone FS. Anemia management after acute brain injury. Crit Care 2016:20:1-11.

3. (3) Lazaridis C. Cerebral oxidative metabolism failure in traumatic brain injury: "Brain shock." J Crit Care 2017;37:230-233.

4. (4) Edlow BL, Samuels O. Emergency Neurological Life Support: Subarachnoid Hemorrhage. Neurocrit Care. 2017;27:116-23.

5. (5) American College of Surgeons. Best Practices in the Management of Traumatic Brain Injury. Table of Contents. 2015;(January):1-29. Available from: https://www.facs.org/ /media/files/quality programs/trauma/tqip/ traumatic brain injury guidelines.ashx.

6. (6) Kowalski RG, Claassen J, Kreiter KT, Bates JE, Ostapkovich ND, Connolly ES, et al. After Subarachnoid Hemorrhage. 2013;291(7):1-4.

7. (7) Lawton MT, Vates GE. Subarachnoid hemorrhage. N Engl J Med. 2017;377(3):257-66 status and therefore to improve patients' outcomes.

their knowledge of respiratory failure, ARDS and proning. They then attended a proning education session where they were given a short lecture followed by a teaching session on how to prone and deprone a patient safely.

After the session the same knowledge questionnaire was given out. This allowed us to directly compare their knowledge and see if our intervention was successful.

Results: On average each staff member had proned around one patient in the past 12 months.

They also felt before the session that it would take around 30 minutes to prone someone and their main concerns were tube displacement, patient position, risk of injury to patient, line displacement and patient stability.

There were ten knowledge questions. All showed an improvement after the session. When the ten questions were averaged, the percentage of correct answers improved from $55.6 \%$ to $85.5 \%$.

The staff's confidence to prone a patient, using a scale out of 10 improved from 4.0 to 8.9 .

The staff's knowledge of the local protocol, using a scale out of 10 , improved from 3.2 to 10.

Conclusion: Staff knowledge of respiratory failure, ARDS and proning was greatly increased following the session.

Staff confidence to perform the procedure was also greatly improved. After the success of these education sessions. We are looking to run further sessions on proning and other important aspects of ICU care.

The timing of this study was very fortuitus seeing as proning has become such an essential part of managing COVID-19 patients. Having done and taught the staff in our ICU before the pandemic started, it allowed the busy team to focus on other important and vital aspects of ICU care.

\section{Reference(s) and grant ackowledgment(s)}

1. 1. Chertoff J. Why is prone positioning so unpopular? Journal of Intensive Care. 2016 Nov 25:4(1):70.

2. 2. Guérin C, Reignier J, Richard J-C, Beuret P, Gacouin A, Boulain T, et al. Prone Positioning in Severe Acute Respiratory Distress Syndrome. New England Journal of Medicine. 2013 Jun 6;368(23):2159-68.

3. 3. Ventilation with Lower Tidal Volumes as Compared with Traditional Tidal Volumes for Acute Lung Injury and the Acute Respiratory Distress Syndrome. New England Journal of Medicine. 2000 May 4;342(18):1301-8.

4. 4. Bloomfield R, Noble DW, Sudlow A. Prone position for acute respiratory failure in adults. Cochrane Database Syst Rev. 2015 Nov 13;(11):CD008095.

001235

Building emotional capital through the staff huddles in Covid19 times: An appreciative inquiry

S. lucena-amaro ${ }^{1}$; H. Schroeder²; S. Schwarze ${ }^{1}$; P. Subramanian ${ }^{1}$; J.

Whittaker ${ }^{1}$; P. Fitch ${ }^{1}$

${ }^{1}$ Adult critical care unit, Barts Health NHS Trust, The Royal London Hospital, United Kingdom; ${ }^{2}$ Schroeder forbes, Schroeder Forbes Ltd, Welwyn Garden City, United Kingdom

Correspondence: $\mathrm{S}$. lucena-amaro

Intensive Care Medicine Experimental 2020, 8(2): 001235 to be due to the additional need for resources to carryout, and risk of adverse events (1). However, the ARDSNET trial in 2000 and PROSEVA trial in 2013, both showed mortality benefit for patients without serious adverse outcomes $(2,3)$. The lack of harm was also confirmed by a recent Cochrane review in 2015 (4). In 2019, our remote district general ICU felt that proning should be used more frequently, and an education session was set up to try and improve the multidisciplinary teams' knowledge and ability to carry it out.

Objectives: Improve the knowledge of respiratory failure and acute respiratory distress syndrome (ARDS) amongst the ICU multidisciplinary team.

Teach all members of the multidisciplinary team how to prone and deprone a patient safely.

Empower the team to highlight when they feel a patient is deteriorating and requires proning.

Methods: The teaching sessions were commenced in October 2019. A pre attendance questionnaire was given to the attendees to test
Introduction: The Royal London Hospital is a major teaching hospital in East London (UK). The Adult Critical Care Unit (ACCU) comprises 44 beds, staffed by 210 nurses. During the Covid-19 pandemic, critical care services doubled in capacity. Critical care nurse to patient ratios were dramatically altered. At the same time, over 300 non-critical care nurses were redeployed into the department to accommodate this increased demand for critical care beds.

Objectives: The primary aim of this project was to offer nurses a collective reflection and processing space to enhance post pandemic wellbeing support. The secondary aim was to capture learning about processes that supported effective care delivery. Factors contributing to positive and effective working practices would inform preparedness for a potential second surge. 
Methods: An Appreciative Inquiry model was used to guide small group reflective conversations (1). Critical care and redeployed nurses were invited to take part. Groups were distributed according to their clinical role. All the sessions were facilitated by an external coach, a clinical psychologist and senior staff from the ACCU. A thematic analysis approach was used to identify emerging themes.

Results: The sessions allowed staff the opportunity to discuss difficult experiences from working in the Covid19 pandemic. Fifty nurses from each staff group participated in these discussions, which generated clear descriptions of what promoted effective working and patient safety. The views from the two different nursing groups about what they valued were complementary. Nurses reported an increased emotional load during Covid-19 with anxiety levels often higher at the beginning of the shift. These resulted from feeling overwhelmed and fearful of not being able to cope.

The staff huddle at the start of the shift emerged as a key event to enable effective working and promote nurses' psychological safety, although this was not consistently used. Redeployed nurses lacked the supportive structures of their familiar workplaces and therefore valued the welcoming and team working environment created by the ITU nurses.

Outcomes from these results include the development of a huddle template and education strategies for promoting staff wellbeing.

Conclusion: Appreciative Inquiry proved a valuable and accessible intervention for nursing staff to acknowledge the achievements and contributions during the Covid19 pandemic. The staff huddle emerged as a vital part of staff wellbeing. It promoted effective working relationships, which in turn, are likely to impact positively on care delivery and patient experience. Ongoing work will focus on how to deliver further sessions more widely to other staff and training.

\section{Reference(s) and grant ackowledgment(s)}

1. Keefe MR, Pesut D. Appreciative inquiry and leadership transitions. J Prof Nurs. 2004;20(2):103-109.

2. This project received no specific grant from any funding agency in the public, commercial, or not-for-profit sectors.

\section{7}

\section{Are you reading what I'm writing in the notes? Why not?}

N. Campbell ${ }^{1}$; N. Duffy²; K. Surman ${ }^{2} ;$ S. Godlee ${ }^{3}$; S. Mcguire ${ }^{4}$; C. Carle ${ }^{4} ;$ Y. Yang ${ }^{5} ;$ K. Ripullone ${ }^{5}$; P. Nadya ${ }^{6} ;$ T. Baker $^{7}$

${ }^{1}$ Department of respiratory medicine, West Suffolk NHS Foundation Trust, Bury St Edmunds, United Kingdom; ' $D$ Department of surgery, North West Anglia NHS Foundation Trust, Huntingdon, United Kingdom; ${ }^{3}$ Department of medicine, West Suffolk NHS Foundation Trust, Bury St Edmunds, United Kingdom; ${ }^{4}$ Department of anaesthesia and critical care medicine, North West Anglia NHS Foundation Trust, Peterborough, United Kingdom; ${ }^{5} \mathrm{Jvf}$ intensive care unit, Cambridge University Hospitals NHS Foundation Trust, Cambridge, United Kingdom; ${ }^{6}$ Faculty of divinity, Cambridge University, Cambridge, United Kingdom; 7 Department of anaesthesia, Cambridge University Hospitals NHS Foundation Trust, Cambridge, United Kingdom

Correspondence: $\mathrm{N}$. Campbell

Intensive Care Medicine Experimental 2020, 8(2): 001317

Introduction: In the ICU, nursing notes represent the patient's narrative at the extremes of illness and are used to support decisions regarding the escalation or withdrawal of treatment. Their importance has recently been dramatically highlighted in a seminal paper that revealed that nursing notes can and do predict patients' probability of survival on ICU1.

Objectives: This is a multi-centre service evaluation investigating the nursing and medical attitudes towards nursing notes. We wanted to investigate how nurses perceived the attitudes of, and frequency with which doctor colleagues read their entries into the patients notes and if there were differences in attitudes across the different training hierarchies of nurses and doctors. In addition we wanted to characterise the perceived reasons that nurses attributed to this lack of attention.
Methods: This project used an anonymous web-based questionnaire that was sent to nurses and doctors in four ICUs in the East of England. Nurses reported their grade, how frequently they believed that doctors read their entries into the patients notes (Always/often/ sometimes/rarely/never) and aspects of their notes they thought were important to the doctors. For the purpose of analysis, the frequency of note reviewing was divided into generally positive responses (always/ often/sometimes) and generally negative responses (rarely/never). Doctors reported their grade, the frequency with which they read nursing notes and their views on nursing note contents and their utility. Responses were collected from 47 doctors and 120 nurses. Nurses were stratified by grade into five groups; Group 1 ( $<5$ years nursing experience), Group 2 ( $>5$ years experience), Group 3 (Sisters), Group 4 (CCOT and nurse specialists) and Group 5 (Ward managers and Matrons). Doctors were stratified by staff hierarchical grade into four groups (Foundation, SHO, Registrars and Consultants).

Results: Analysis of 121 nursing staff responses revealed differences in the perceived frequency that nurses reported that their doctor colleagues reviewed their notes. The frequency with which nurses believed doctors reviewed nurses entries appeared to vary with the grade of the nurse, with $66 \%$ of Group 4 nurses reporting positively. The most negative responses were that of the most senior nurses in Group 5 with $100 \%$ of responses falling into the negative response category. Analysis of the 47 doctors also revealed hierarchical differences, with doctors in training having more 'generally positive' results than consultants.

$80 \%$ of nurses thought this was due to doctors not having enough time/not being interested in the content/not finding them useful. $49 \%$ of doctors made similar reports.

Conclusion: Nursing notes appear not to be perceived as serving their function to facilitate effective communication between allied healthcare professionals. Writing notes reduces the time spent on direct clinical tasks, consideration for how to improve this needs to be made.

\section{Reference(s) and grant ackowledgment(s) \\ 1. Waudby-Smith, I. E. R. et al. (2018) 'Sentiment in nursing notes as an indica- tor of out-of-hospital mortality in intensive care patients', PLOS ONE. Edited by P. van Bogaert. Public Library of Science, 13(6), p. e0198687. https://doi. org/10.1371/journal.pone.0198687. 2. None.}

001334

Functional Electrical Stimulation-Assisted Cycle Ergometry for Mechanically Ventilated Patients: Randomised Controlled Trial with Six Months Follow Up

P. Waldauf ; N. Hruskova ${ }^{2}$; B. Blahutova ${ }^{3}$; J. Gojda ${ }^{4}$; T. Urban ${ }^{5}$; A. Krajcova ${ }^{4}$. M. Fric ${ }^{1}$; K. Jiroutkova ${ }^{1}$; K. Rasova ${ }^{2}$; F. Duska ${ }^{1}$

${ }^{1}$ Department of anaesthesia and intensive care medicine, Intensivist, Prague, Czech Republic; ${ }^{2}$ Department of rehabilitation medicine, Physiotherapists, Prague, Czech Republic; ${ }^{3}$ Department of rehabilitation medicine, Medical student, Prague, Czech Republic; ${ }^{4}$ 2nd department of internal medicine, Physician, Prague, Czech Republic; ${ }^{5}$ Department of anaesthesia and intensive care medicine, Biochemist, Prague, Czech Republic

Correspondence: P. Waldauf

Intensive Care Medicine Experimental 2020, 8(2): 001334

Introduction: Functional electrical stimulation-assisted cycle ergometry (FESCE) enables in-bed leg exercise independently of patients' volition. We hypothesised that early use of FESCE-based intensified rehabilitation improves physical function in survivors of critical care after 6 months.

Methods: We enrolled mechanically ventilated adults estimated to need $>7$ days of ICU stay into an assessor-blinded single centre randomised controlled trial to receive either FESCE-based protocolised or standard rehabilitation that continued up to day 28 or ICU discharge.

Results: We randomised in 1:1 ratio 150 patients (Age $61 \pm 15$ years, APACHE II $21 \pm 7$ ) at a median of 21 (IQR 19-43) hours after admission to ICU. Mean rehabilitation dose delivered to intervention vs. 
control group was $80 \pm 35$ vs. $56 \pm 15$ mins per day, $p<0.01$. At six months $42(56 \%)$ and $46(61 \%)$ were alive in interventional vs. control groups $(p=0.51)$ and their physical component summary of SF36 (primary outcome) was not different [50 (IQR 21-69) vs. 49 (IQR 49-76); $\mathrm{p}=0.26]$. At ICU discharge, there were no differences in the ICU length of stay, functional performance, rectus femoris cross-sectional area or muscle power despite daily nitrogen balance $0.6(95 \% \mathrm{Cl} 0.2-$ 1.0) $\mathrm{g}$ less negative in intervention group. There were more ICP elevations in intervention group ( 1.5 vs. 0 ICP elevations/day, $\mathrm{p}=0.018$ ) and significantly worse mental component summary score of SF-36 after 6 months [53 $(95 \% \mathrm{Cl} 45 ; 61)$ vs. $64(95 \% \mathrm{Cl} 57 ; 72), \mathrm{p}=0.018]$.

Conclusion: Early delivery of FESCE-based protocolised rehabilitation to ICU patients does not improve physical functioning at 6 months in survivors and may cause harm.

\section{2}

\section{Early mobilization as a facilitator of successful weaning} from mechanical ventilation in patients with COVID-19

A. Rosa'; S. Lafayette ${ }^{1}$; P. Azevedo ${ }^{1}$; C. Gomes'; M. Knibel ${ }^{2}$; WT. Nakasato ${ }^{3}$

${ }^{1}$ Physiotherapy, Hospital São Lucas Copacabana, Rio de Janeiro, Brazil; Education center, Hospital São Lucas Copacabana, Rio de Janeiro, Brazil ${ }^{3}$ Medical director, Hospital São Lucas Copacabana, Rio de Janeiro, Brazil

Correspondence: A. Rosa

Intensive Care Medicine Experimental 2020, 8(2): 001532

Introduction: Coronavirus disease (COVID-19) is a pandemic of international concern, cause severe respiratory failure and require invasive mechanical ventilation.

Early mobilization (EM) in the ICU is safe, feasible, and reduces the frequency and severity of complications in critical illness patients. Weakness is a frequent problem in ICU, even more evident in patients undergoing mechanical ventilation, patients with COVID-19 usually needs mechanical ventilation.

We purpose evaluate early mobilization as a facilitator of successful weaning from mechanical ventilation and post-extubation outcome in patients with SARS-CoV-2 pneumonia.

Objectives: We purpose evaluate early mobilization as a facilitator of successful weaning from mechanical ventilation and post-extubation outcome in patients with SARS-CoV-2 pneumonia.

Methods: This was a observational retrospective analysis performed form medical records of patients with SARS-CoV-2 pneumonia who required invasive mechanical ventilation (IMV) for more than 24 hours, and, after that, having passed the spontaneous breathing trial. They were divided into two groups: the first group that underwent early mobilization during the IMV period and the second group receive only passive range of motion (PROM). We defined as early mobilization the following conducts: sitting on the edge of bed, sitting out of bed, active standing, passive standing with a tilt table, and walking during IMV.

Results: We analyzed a total of 40 patients who were submitted tracheal extubation. Patients were divided in two groups, EM group 21 patients and PROM group 19 patients, with average mean age 56,1 $(S D \pm 16,03)$ and $66,3(S D \pm 12,91)$ respectively, the SOFA score mean was 4,52 (SD $\pm 2,60$ ) versus $6,32(\mathrm{SD} \pm 2,71)$, and the SAPS III was $53,29$ (SD $\pm 9,76$ ) versus 57,11 (SD $\pm 9,24)$, the duration of mechanical ventilation was $10,38(S D \pm 4,48)$ versus $10,42(S D \pm 6,02)$ respectively.

The primary outcome was rate of failed extubation that was in the EM group 2/21 (10\%) and PROM group 7/19 (35\%) (OR 0,18 [IC 0,03-1,01]), the rate of mortality was the second outcome, the EM group presented (0/21) $0 \%$ versus (4/19) $20 \%$ respectively.

Conclusion: Early mobilization was feasible, safe and maybe associated with a better outcome for extubated critical patients. Early mobilizations can be associated with lower mortality in COVID-19 patients, probably due the improve muscle strength that these patients develop.

The relation between early mobilization and successful weaning of mechanical ventilation in patients with COVID-19 should be further investigated in a large prospective study, that can measure objectively the force at the time of extubation.
Reference(s) and grant ackowledgment(s)

1. Ali NA, O'Brien JM, Hoffmann SP, et al (2008) Acquired Weakness, Handgrip Strength, and Mortality in Critically III Patients. Am J Respir Crit Care Med 178:261-268. https://doi.org/10.1164/rccm.200712-18290C.

2. Harapan H, Itoh N, Yufika A, et al (2020) Coronavirus disease 2019 (COVID19): A literature review. Journal of Infection and Public Health 13:667-673. https://doi.org/10.1016/j.jiph.2020.03.019.

3. Hodgson CL, Stiller K, Needham DM, et al (2014) Expert consensus and recommendations on safety criteria for active mobilization of mechanically ventilated critically ill adults. Crit Care 18:658. https://doi.org/10.1186/s1305 4-014-0658-y

4. Miranda Rocha AR, Martinez BP, Maldaner da Silva VZ, Forgiarini Junior LA (2017) Early mobilization: Why, what for and how? Med Intensiva 41:429-436. https://doi.org/10.1016/j.medin.2016.10.003.

5. Rose $L$ (2015) Strategies for weaning from mechanical ventilation: A state of the art review. Intensive and Critical Care Nursing 31:189-195. https://doi. org/10.1016/j.iccn.2015.07.003.

6. Schweickert WD, Hall J (2007) ICU-Acquired Weakness. Chest 131:15411549. https://doi.org/10.1378/chest.06-2065.

7. Thanks, the physiotherapy team at the São Lucas Copacabana Hospital - Rio de Janeiro - Brazil, we worked bravely and tirelessly to promote recover of patients with COVID-19.

\section{0}

Endotracheal cuff pressure variation in subglottic devices: a prospective observational study

I. Comisso"; A. Qualizza'; SG. Di²

${ }^{1}$ Anesthesia and Intensive Care, Azienda Sanitaria Universitaria Integrata di Udine, Udine, Italy; ${ }^{2}$ Emergency department - latisana hospital, Azienda Sanitaria Universitaria Integrata di Udine, Udine, Italy

Correspondence: I. Comisso

Intensive Care Medicine Experimental 2020, 8(2): 001580

Introduction: Subglottic devices, i.e. endotracheal tube (ETT) and tracheostomic cannula (CT) are used in critical care both to provide mechanical ventilation and to isolate airways from the gastrointestinal tract. A correct cuff pressure management is required while caring for critical care patients, in order to avoid ischemic damages coming from an excessive pressure, and to prevent the inhalation risk related to insufficient cuffing. Several studies investigated the influence of mobilization procedures on the cuff pressure, whilst the effects of other procedures (such as endotracheal suctioning) were rarely.

Objectives: The aim of this study was to determine the cuff pressure variations determined by caring interventions, in order to define an ideal cuff pressure monitoring interval and to highlight the procedures most frequently involved in generating cuff pressure out of recommended ranges $(20-30 \mathrm{cmH} 2 \mathrm{O})$.

Methods: Study design and setting. A prospective observational study was conducted within the three ICU of the Anesthesia and Intensive Care Department at the University Hospital of Udine, Italy.

Population. Patients carrying ETT or CT, aged $\geq 18$ admitted to ICU between December 2019 and February 2020.

Variables. Cuff pressure was checked before and after caring procedures (changes in position, general hygiene care, oral care, tracheal suctioning, fibre-optic bronchoscopy, device's dressing). Other patients and devices variables were recorded.

Statistical analysis. Data were analyzed using the software GraphPad Prism 8.0 (GraphPad Software, San Diego, CA, USA). Descriptive measures were used to describe sample charachteristics. Moreover, pressure variation after the procedures was calculated, and the percentage of inadequate (i.e. exceeding the range $20-30 \mathrm{mmHg}$, as recommended from the available literature) values was determined. Two-tailed rank Wilcoxon test was used to describe periprocedural pressure variation. Fisher exact test was used to determine associations between the independent variables "need for pressure adjustment prior to the procedure", "lenght of intubation $\geq 5$ days", "body temperature $\geq 37.5^{\circ} \mathrm{C}$ ", and the dependent variable "inadequate cuff pressure". Kruskal-Wallis test was used to describe cuff pressure variations during the days after 
patients enrollment in the study. Statistical significance was considered when $\mathrm{p}<0.05$.

Results: 81 patients (58 males, 72\%) were enrolled for observation. Globally, 601 paired observations (i.e. before and after the procedure) were conducted. Median number of observations per patient was 4 (range 1-35). Cuff pressure was out of recommended range in 351 $(58 \%)$ observations prior procedure performance and in $178(30 \%)$ after procedures. Median cuff pressure variation when pre-procedural adjustment was not required was $-4 \mathrm{~cm} \mathrm{H} 2 \mathrm{O}$ (range $-20 ; 8 \mathrm{~cm} \mathrm{H} 2 \mathrm{O}$ ). Median cuff pressure variation when pre-procedural adjustment was required was $-6 \mathrm{~cm} \mathrm{H} 2 \mathrm{O}$ (range $-23 ; 10 \mathrm{~cm} \mathrm{H} 2 \mathrm{O}$ ). Post-procedural cuff pressure was out of recommended range after postural change in $58 \%$ (7/12) of observations, after body hygiene in 31\% (50/163) of observations, in $27 \%$ (33/123) of observations after oral care hygiene, in $28 \%$ (37/132) of observations after tracheal suctioning, in 33\% (6/18) of observations after fibre-optic bronchoscopy, in 23\% (45/193) of observations after ETT/CT dressing. Post-procedural cuff pressure reduction occurred in 510 (85\%) of observations; particularly, in 62 observations cuff pressure values were $\leq 15 \mathrm{~cm} \mathrm{H} 2 \mathrm{O}$, and in 3 observatons values were $5 \mathrm{~cm} \mathrm{H} 2 \mathrm{O}$. Median pressure decrease was $6 \mathrm{cmH} 2 \mathrm{O}$ (range -23;-1 $\mathrm{cmH} 2 \mathrm{O}$ ). In 65 (11\%) observations cuff pressure had no variations and in $26(4 \%)$ it increased. Median pressure increase was $6 \mathrm{cmH} 2 \mathrm{O}$ (range $1-10 \mathrm{cmH} 2 \mathrm{O}$ ). Paired two tail Wilcoxon test revealed a statistically significant decrease for all paired observations (median difference -5 $\mathrm{cmH} 2 \mathrm{O} ; \mathrm{p}<0.0001$ ) and for single procedures. Fisher test highlighted that the risk for inadequate cuff pressure significantly decreased when pre-procedural adjustment was required (RR 0.58, 95\% IC 0.45-0.74; $p<0.0001$ ). Duration of intubation $\geq 5$ days resulted in a lower risk for inadequate post-procedural cuff pressure, although the association was not statistically significant (RR $0.78,95 \%$ IC $0.60-1.00 ; p=0.595$ ), whilst body temperature $\geq 37.5^{\circ} \mathrm{C}$ slightly increased the risk for postprocedural inadequate cuff pressure $(\mathrm{RR}=1.104,95 \% \mathrm{IC} 0.81-1.49 ; \mathrm{p}=$ $0.58)$.

Conclusion: In most observations, a pressure reduction was observed after a procedure was performed, and the amount of procedures generating significant out-of-range pressures was not negligible. Therefore, cuff pressure should always be checked before and after each procedure generating potential cariations. Further studies with larger samples might highight the role of rarely investigated procedures (such as fibre-optic bronchoscopy and body hygiene) and other risk factors related to patient in generating inadequate cuff pressures.

\section{Reference(s) and grant ackowledgment(s)}

1. Nseir, S., Brisson, H., Marquette, C.H., Chaud, P., Di Pompeo, C., Diarra, M., Durocher, A. (2009) Variations in endotracheal cuff pressure in intubated critically ill patients: prevalence and risk factors. Eur J Anesthesiol 26 (3), 229-34.

2. Beccaria, L.M., Doimo, T.M.A., Polletti, N.A.A., Barbosa, T.P., Da Silva, C.D.C., Werneck, A.L. (2017) Tracheal cuff pressure change before and after the performance of nursing care. Rev Bras Enferm 70 (6), 1145-50.

3. Hockey, C.A., van Zundert, A.A., Paratz, J.D. (2016) Does objective measurement of tracheal tube cuff pressures minimize adverse effects and maintain accurate cuff pressures? A systematic review and meta-analysis. Anaesth Intensive Care 44 (5), 560-70.

4. Lizy, C., Swinnen, W., Labeau, S., Poelaert, J., Vogelaers, D., Vandewoude, K., Dulhunty, J., Blot, S. (2014) Cuff pressure of endotracheal tubes after changes in body position in critically ill patients treated with mechanical ventilation. American Journal of Critical Care 23 (1), 1-8.

\section{6}

IVAPP (ICU APP) - a support to patients and relatives, before during and after the time at ICU

E. Åkerman ${ }^{1}$; U. Malmstrom¹; M. Tennback'; H. Laurinen-Ovelius ${ }^{1}$; S. Cheviakova ${ }^{1}$; AM. Sundfors ${ }^{1}$; C. Vonknorring ${ }^{1}$; M. Carlsson ${ }^{1}$

${ }^{1}$ Anesthesiology and intensive care, Karolinska University Hospital, Stockholm, Sweden

Correspondence: $\mathrm{E}$. Åkerman

Intensive Care Medicine Experimental 2020, 8(2): 001616
Introduction: Patients and relatives have an increased need for information about what happens before, during and after a critical illness and the time at ICU. The knowledge can bring a sense of security and safety and also an opportunity to be more involved in the patient care, and also to be able to get back to the information and read it again during the care The most common form to inform is an information booklet, but there are limitations as i.e. updating and availability. Today's technology gives an increased possibility for accessibility of information which can help the patients and relatives during the critical illness and recovery.

Objectives: to create an easily accessible, interactive web-application (IVAPP) with information that covers the time of care, before-duringand after ICU.

Methods: An interprofessional group with a physiotherapist, intensive care nurses, assistant nurses, physicians and management staff was formed. To collect and create content in the IVAPP, a search for current data, as existing information, research and web-sites was done. Contact was also made with various support functions and organizations for their input. Photographs from the ICU and equipment were taken to supplement the written text. Former ICU patients and relatives were invited at two times to review the draft and give feedback on what information they would appreciated to get during their ICU care. The final version was qualitative reviewed by the ICU director and nursing manager before publishing.

The IVAPP was implemented in October 2019 and the information about the release was spread in Swedish national broadcasting, newspapers, in Karolinska Hospital ICUs and at open meetings for staff and patients. Also, all the care units that the ICU patients often stays before and/or after their ICU care was informed about the app.

Results: The feedback was positive both from staff, patients and relatives. The information obtained in the cellphone filled the needs, it was accessible for all, even to those that could not be in the ICU and it could be accessed on demand. The information could reduce anxiety, aid preparing for discharge from the ICU and hospital and individualize patient care. Statistics showed that "general information about the ICU"," to come home", "what happened during a day in the ICU", "working in ICU" and "', were the most visited sides.

Conclusion: IVAPP has been developed in an interprofessional collaboration together with patients and relatives. IVAPP is easily accessible and can give relatives and patients the opportunity to easily find information about intensive care before, during and after the care period.

\section{5}

Echocardiographic parameters and mortality in pediatric sepsis: a systematic review and meta-analysis

V. La Rosa ${ }^{1}$; C. Grasso' ${ }^{1}$ S. Tigano ${ }^{1}$; M. Leonardi ${ }^{2}$; F. Perna ${ }^{2}$; E. Tringali² F. Oliveri'; F. Sanfilippo ${ }^{2}$ : M. Astuto ${ }^{2}$

${ }^{1}$ School of anaesthesia and intensive care, University Hospital "G. Rodolico", Catania, Italy; ${ }^{2}$ Department of anesthesia and intensive care, A.O.U. "Policlinico-Vittorio Emanuele", Catania, Italy

Correspondence: $\mathrm{V}$. La Rosa

Intensive Care Medicine Experimental 2020, 8(2): 001445

Introduction: The International Pediatric Sepsis Consensus defined septic shock as sepsis syndrome complicated with cardiovascular organ dysfunction, with myocardial and/or circulatory failure being leading causes of death. Indeed, septic shock is often characterized also by myocardial depression. The evaluation of cardiac function is important to detect early deterioration that can have important implications for treatment and prognostication. Critical Care Echocardiography (CCE) is an established noninvasive tool for the assessment of hemodynamic changes in adult patients with shock, and its use is increasing in the pediatric critically ill patient too. Several parameters describing left and right ventricular (LV and RV, respectively) function in septic newborns or children have been studied, from the most conventional ones to the use of Tissue Doppler Imaging (TDI) and strain echocardiography (STE). Therefore, we conducted a systematic-review in order to describe the echocardiography parameters most studied so far, and subsequently we performed a meta-analysis aiming at 
investigating the prognostic value of echocardiography parameters and mortality in pediatric patients with sepsis.

Objectives: We conducted a systematic review and meta-analysis to investigate the prognostic value of echocardiographic parameters and mortality in pediatric septic patients.

Methods: Data Sources: MEDLINE, PubMed and EMBASE (last update April 5th, 2020). Study Selection: observational studies conducted on pediatric sepsis providing echocardiographic parameters according to mortality. Data Extraction: Echocardiography data were divided in those describing left ventricular (LV) systolic or diastolic function, right ventricle (RV) function and strain echocardiography parameters. Data were entered dividing studies in two subgroups: newborns and children. Analysis is reported as standardized mean difference (SMD) and $95 \%$ confidence interval $(\mathrm{Cl})$.

Results: We included data from 14 articles ( $\mathrm{n}=5$ newborns, $\mathrm{n}=98$ children). The Fractional Shortening was the most commonly reported variables ( 11 studies, $\mathrm{n}=555$ patients) and was not associated with mortality (SMD 0.22, 95\%Cl[-0.02, 0.47]; $\mathrm{p}=0.097, \mathrm{I} 2=28 \%$ ). No association with mortality was seen also for LV ejection fraction ( 9 studies, $\mathrm{n}=417 ; \mathrm{SMD} 0.06,95 \% \mathrm{CI}[-0.27,0.40] ; \mathrm{p}=0.72,12=51 \%), \mathrm{s}^{\prime}(4$ studies, $\mathrm{n}=178 ; \mathrm{SMD}-0.01,95 \% \mathrm{CI}[-0.34,0.33] ; \mathrm{p}=0.97,12=0 \%)$, and myocardial performance index (4 studies, $\mathrm{n}=199$; SMD $-0.37,95 \% \mathrm{CI}[-1.01$, $0.26] ; p=0.25,12=65 \%)$. Regarding LV diastolic function, we found a significant association of mortality with E/e' ratio (4 studies, $n=189$, SMD $-0.45,95 \% \mathrm{CI}[-0.80,-0.10] ; \mathrm{p}=0.01,12=0 \%$ ) or e' wave (3 studies, $\mathrm{n}=159 ; \mathrm{SMD} 0.49,95 \% \mathrm{CI}[0.13,0.85] ; \mathrm{p}=0.008, \mathrm{I}=0 \%)$. No association was found between E/A ratio ( 6 studies, $\mathrm{n}=273$; SMD $0.28,95 \% \mathrm{CI}[-$ $0.42,0.99] ; p=0.43,12=81 \%)$ and $s^{\prime}$ measured at tricuspid annulus ( 3 studies, $n=148 ;$ SMD $-0.18,95 \% \mathrm{CI}[-0.53,0.17] ; \mathrm{p}=0.32,12=0 \%)$. Only few studies were conducted with strain echocardiography.

Conclusion: We found no association between mortality of septic pediatric patients and parameters evaluating LV systolic function and RV function. A significant association was found between mortality and higher E/e' or lower $\mathrm{e}^{\prime}$, indicating a possible influence of worsened LV diastolic function. These findings are preliminary due to the high clinical heterogeneity of the studies included.

\section{Reference(s) and grant ackowledgment(s)}

1. 1. Singer M, Deutschman CS, Seymour CW, et al. The Third International Consensus Definitions for Sepsis and Septic Shock (Sepsis-3). JAMA : the journal of the American Medical Association 2016;315(8):801-810. 2. Fleischmann-Struzek C, Goldfarb DM, Schlattmann P, et al. The global burden of paediatric and neonatal sepsis: a systematic review. The Lancet Respiratory medicine 2018;6(3):223-230. 3. Watson RS, Carcillo JA. Scope and epidemiology of pediatric sepsis. Pediatric critical care medicine : a journal of the Society of Critical Care Medicine and the World Federation of Pediatric Intensive and Critical Care Societies 2005;6(3 Suppl):S3-5. 4. Kawasaki T. Update on pediatric sepsis: a review. Journal of intensive care 2017:5:47. 5. Liu L, Oza S, Hogan D, et al. Global, regional, and national causes of child mortality in 2000-13, with projections to inform post-2015 priorities: an updated systematic analysis. Lancet 2015;385(9966):430-440. 6. Odetola FO, Gebremariam A, Freed GL. Patient and hospital correlates of clinical outcomes and resource utilization in severe pediatric sepsis. Pediatrics 2007;1 19(3):487-494. 7. Watson RS, Carcillo JA, Linde-Zwirble WT, et al. The epidemiology of severe sepsis in children in the United States. American journal of respiratory and critical care medicine 2003;167(5):695701. 8. Weiss SL, Fitzgerald JC, Pappachan J, et al. Global epidemiology of pediatric severe sepsis: the sepsis prevalence, outcomes, and therapies study. American journal of respiratory and critical care medicine 2015;191(10):1147-1157. 9. Kaur G, Vinayak N, Mittal K, et al. Clinical outcome and predictors of mortality in children with sepsis, severe sepsis, and septic shock from Rohtak, Haryana: A prospective observational study. Indian journal of critical care medicine : peer-reviewed, official publication of Indian Society of Critical Care Medicine 2014;18(7):437-441. 10. WHO Executive Board (EB140/12). Improving the prevention, diagnosis and clinical management of sepsis. 2017. https://apps.who.int/gb/ebwha/ pdf_files/WHA70/A70_R7-en.pdf? ua =1 (accessed on May 17, 2020). 11. Goldstein B, Giroir B, Randolph A. International pediatric sepsis consensus conference: definitions for sepsis and organ dysfunction in pediatrics. Pediatric critical care medicine : a journal of the Society of Critical Care Medicine and the World Federation of Pediatric Intensive and Critical Care
Societies 2005;6(1):2-8. 12. Raj S, Killinger JS, Gonzalez JA, et al. Myocardial dysfunction in pediatric septic shock. The Journal of pediatrics 2014;164(1):72-77 e72. 13. Sanfilippo F, Orde S, Oliveri F, et al. The Challenging Diagnosis of Septic Cardiomyopathy. Chest 2019;156(3):635636. 14. Weiss SL, Balamuth F, Hensley J, et al. The Epidemiology of Hospital Death Following Pediatric Severe Sepsis: When, Why, and How Children With Sepsis Die. Pediatric critical care medicine : a journal of the Society of Critical Care Medicine and the World Federation of Pediatric Intensive and Critical Care Societies 2017;18(9):823-830. 15. Weiss SL, Peters MJ, Alhazzani W, et al. Surviving Sepsis Campaign International Guidelines for the Management of Septic Shock and Sepsis-Associated Organ Dysfunction in Children. Pediatric critical care medicine : a journal of the Society of Critical Care Medicine and the World Federation of Pediatric Intensive and Critical Care Societies 2020;21(2):e52-e106. 16. Davis AL, Carcillo JA, Aneja RK, et al. American College of Critical Care Medicine Clinical Practice Parameters for Hemodynamic Support of Pediatric and Neonatal Septic Shock. Critical care medicine 2017;45(6):1061-1093. 17. International consensus statement on training standards for advanced critical care echocardiography. Intensive care medicine 2014;40(5):654-666. 18. Vieillard-Baron A, Millington SJ, Sanfilippo F, et al. A decade of progress in critical care echocardiography: a narrative review. Intensive care medicine 2019;45(6):770-788. 19. NICE. National Institute for Health and Care Excellence (2016) Sepsis: recognition, diagnosis and early Management (NICE Guideline 51). https ://www.nice. org.uk/guida nce/ng51 (accessed on May 17, 2020). [cited Available from: 20. Negrine RJ, Chikermane A, Wright JG, et al. Assessment of myocardial function in neonates using tissue Doppler imaging. Archives of disease in childhood Fetal and neonatal edition 2012;97(4):F304-306. 21. Sanfilippo F, Scolletta S, Morelli A, et al. Practical approach to diastolic dysfunction in light of the new guidelines and clinical applications in the operating room and in the intensive care. Annals of intensive care 2018;8(1):100. 22. Szymanski C, Levy F, Tribouilloy C. Should LVEF be replaced by global longitudinal strain? Heart (British Cardiac Society) 2014;100(21):1655-1656. 23. Vieillard-Baron A. Septic cardiomyopathy. Annals of intensive care 2011;1 (1):6. 24. Ho CY, Solomon SD. A clinician's guide to tissue Doppler imaging. Circulation 2006;113(10):e396-398. 25. Sanfilippo F, Corredor C, Arcadipane A, et al. Tissue Doppler assessment of diastolic function and relationship with mortality in critically ill septic patients: a systematic review and meta-analysis. BJA: British Journal of Anaesthesia 2017;1 19(4):583-594. 26. Sanfilippo F, Corredor C, Fletcher N, et al. Erratum to: Diastolic dysfunction and mortality in septic patients: a systematic review and meta-analysis. Intensive care medicine 2015. 27. Sanfilippo F, Corredor C, Fletcher N, et al. Diastolic dysfunction and mortality in septic patients: a systematic review and meta-analysis. Intensive care medicine 2015. 28. Sanfilippo F, Corredor C, Fletcher N, et al. Left ventricular systolic function evaluated by strain echocardiography and relationship with mortality in patients with severe sepsis or septic shock: a systematic review and meta-analysis. Critical care 2018;22(1):183. 29. Huang SJ, Nalos M, McLean AS. Is early ventricular dysfunction or dilatation associated with lower mortality rate in adult severe sepsis and septic shock? A meta-analysis. Critical care 2013;17(3):R96. 30. Liberati A, Altman DG, Tetzlaff J, et al. The PRISMA statement for reporting systematic reviews and meta-analyses of studies that evaluate health care interventions: explanation and elaboration. Journal of clinical epidemiology 2009;62(10):e1-34. 31. Luo D, Wan X, Liu J, et al. Optimally estimating the sample mean from the sample size, median, mid-range, and/or mid-quartile range. Statistical methods in medical research 2018;27(6):1785-1805. 32. Wan X, Wang W, Liu J, et al. Estimating the sample mean and standard deviation from the sample size, median, range and/or interquartile range. BMC medical research methodology 2014;14:135. 33. Wells G, Shea B, O'Connell D, et al. The Newcastle-Ottawa Scale (NOS) for assessing the quality of nonrandomized studies in meta-analyses. http://www.ohri.ca/programs/clinical_epidemiolo gy/oxford.htm. 34. Sankar J, Das RR, Jain A, et al. Prevalence and outcome of diastolic dysfunction in children with fluid refractory septic shock-a prospective observational study. Pediatric critical care medicine : a journal of the Society of Critical Care Medicine and the World Federation of Pediatric Intensive and Critical Care Societies 2014;15(9):e370-378. 35. Jain A, Sankar J, Anubhuti A, et al. Prevalence and Outcome of Sepsis-induced Myocardial Dysfunction in Children with 'Sepsis' With' and 'Without Shock'-A Prospective Observational Study. Journal of tropical pediatrics 2018;64(6):501-509. 36. Abdel-Hady HE, Matter MK, El-Arman MM. Myocardial dysfunction in neonatal sepsis: a tissue Doppler imaging study. 
Pediatric critical care medicine : a journal of the Society of Critical Care Medicine and the World Federation of Pediatric Intensive and Critical Care Societies 2012;13(3):318-323. 37. Baranwal AK, Deepthi G, Rohit MK, et al. Longitudinal Study of CPK-MB and Echocardiographic Measures of Myocardial Dysfunction in Pediatric Sepsis: Are Patients with Shock Different from Those without? Indian journal of critical care medicine peer-reviewed, official publication of Indian Society of Critical Care Medicine 2020;24(2):109-115. 38. El-Zayat RS, Shalaby AG. Mitral Annular Plane Systolic Excursion as a Predictor of Mortality in Children With Septic Shock. Pediatric critical care medicine : a journal of the Society of Critical Care Medicine and the World Federation of Pediatric Intensive and Critical Care Societies 2018;19(9):e486-e494. 39. Fahmey SS, Hodeib M, Refaat K, et al. Evaluation of myocardial function in neonatal sepsis using tissue Doppler imaging. The journal of maternal-fetal \& neonatal medicine : the official journal of the European Association of Perinatal Medicine, the Federation of Asia and Oceania Perinatal Societies, the International Society of Perinatal Obstet 2019:1-5. 40. Li J, Ning B, Wang Y, et al. The prognostic value of left ventricular systolic function and cardiac biomarkers in pediatric severe sepsis. Medicine 2019;98(13):e15070. 41. Tomerak RH, El-Badawy AA, Hussein G, et al. Echocardiogram done early in neonatal sepsis: what does it add? Journal of investigative medicine : the official publication of the American federation for Clinical Research 2012;60(4):680-684. 42. Williams FZ, Sachdeva R, Travers CD, et al. Characterization of Myocardial Dysfunction in Fluid- and Catecholamine-Refractory Pediatric Septic Shock and Its Clinical Significance. Journal of intensive care medicine 2019;34(1):17-25. 43. Wu JR, Chen IC, Dai ZK, et al. Early Elevated B-Type Natriuretic Peptide Levels are Associated with Cardiac Dysfunction and Poor Clinical Outcome in Pediatric Septic Patients. Acta Cardiologica Sinica 2015;31(6):485-493. 44 Domico M, Liao P, Anas N, Mink RB. Elevation of Brain Natriuretic Peptide Levels in Children With Septic Shock. Pediatr Crit Care Med. 2008 Sep;9(5):478-83. 45. Carmona F, Manso PH, Silveira VS, et al. Inflammation, myocardial dysfunction, and mortality in children with septic shock: an observational study. Pediatric cardiology 2014;35(3):463-470. 46. Patel MD, Mariano K, Dunbar T, et al. Cardiac Dysfunction Identified by Strain Echocardiography Is Associated With Illness Severity in Pediatric Sepsis. Pediatric critical care medicine : a journal of the Society of Critical Care Medicine and the World Federation of Pediatric Intensive and Critical Care Societies 2020;21(4):e192-e199. 47. Saini SS, Kumar P, Kumar RM. Hemodynamic changes in preterm neonates with septic shock: a prospective observational study*. Pediatric critical care medicine : a journal of the Society of Critical Care Medicine and the World Federation of Pediatric Intensive and Critical Care Societies 2014;15(5):443-450. 48. Haileselassie B, Su E, Pozios I, et al. Strain Echocardiography Parameters Correlate With Disease Severity in Children and Infants With Sepsis. Pediatric critical care medicine : a journal of the Society of Critical Care Medicine and the World Federation of Pediatric Intensive and Critical Care Societies 2016;17(5):383390. 49. Basu S, Frank LH, Fenton KE, et al. Two-dimensional speckle tracking imaging detects impaired myocardial performance in children with septic shock, not recognized by conventional echocardiography. Pediatric critical care medicine : a journal of the Society of Critical Care Medicine and the World Federation of Pediatric Intensive and Critical Care Societies 2012;13(3):259-264. 50. Huang S, Sanfilippo F, Herpain A, et al. Systematic review and literature appraisal on methodology of conducting and reporting critical-care echocardiography studies: a report from the European Society of Intensive Care Medicine PRICES expert panel. Annals of intensive care 2020;10(1):49. 51. Dragulescu A, Mertens L, Friedberg MK. Interpretation of left ventricular diastolic dysfunction in children with cardiomyopathy by echocardiography: problems and limitations. Circulation Cardiovascular imaging 2013;6(2):254-261. 52. Okumura K, Slorach C, Mroczek D, et al. Right ventricular diastolic performance in children with pulmonary arterial hypertension associated with congenital heart disease: correlation of echocardiographic parameters with invasive reference standards by high-fidelity micromanometer catheter. Circulation Cardiovascular imaging 2014;7(3):491-501. 53. Dallaire F, Slorach C, Hui W, et al. Reference values for pulse wave Doppler and tissue Doppler imaging in pediatric echocardiography. Circulation Cardiovascular imaging 2015;8(2):e002167. 54. Cantinotti M, Lopez L. Nomograms for blood flow and tissue Doppler velocities to evaluate diastolic function in children: a critical review. Journal of the American Society of Echocardiography: official publication of the American Society of Echocardiography 2013;26(2):126-141. 55. Eidem BW, McMahon CJ, Cohen RR, et al. Impact of cardiac growth on Doppler tissue imaging velocities: a study in healthy children. Journal of the American Society of Echocardiography : official publication of the American Society of Echocardiography 2004;17(3):212221. 56. Nagueh SF, Smiseth OA, Appleton CP, et al. Recommendations for the Evaluation of Left Ventricular Diastolic Function by Echocardiography: An Update from the American Society of Echocardiography and the European Association of Cardiovascular Imaging. European heart journal cardiovascular Imaging 2016 Dec;17((12)):1321-1360.

\section{0}

Critical care admission after elective surgery: A retrospective study

C. Roriz ; D. Castro ${ }^{2}$; F. Sequeira ${ }^{2}$; H. Fernando 2 ; O. Gomes ${ }^{2}$; L. Pereira ${ }^{2}$

${ }^{1}$ Intensive Care Unit, Centro Hospitalar de Leiria, Leiria, France; ${ }^{2}$ Serviço de medicina intensiva, Centro Hospitalar de Leiria, Leiria, Portugal

Correspondence: $C$. Roriz

Intensive Care Medicine Experimental 2020, 8(2): 001380

Introduction: Elective intensive care unit (ICU) admission represents an important part of all ICU admissions. Critical care admission may be necessary for surgical patients requiring organ support or invasive monitoring in the perioperative period. Planed postoperative admissions aim to prevent complications arising in the postoperative period, reducing the risk of a poor outcome. Several prognostic indices have been used to predict the outcome of surgical critically ill patients. Among them, acute physiology and chronic health evaluation II (APACHE II) and simplified acute physiology score 2 (SAPS 2) are broadly used. Moreover, biological markers such as C-reactive protein (CRP), albumin, and blood lactate levels correlate with the degree of inflammation during the immediate postoperative phase and could be used as independent predictors.

Objectives: The purpose of this study is to demographically and clinically analyze all the elective surgical admissions between January 2018 and June 2020 and also to evaluate patients outcomes comparing the different predictive values of prognostic indices and biological markers in the outcome of 62 surgical patients admitted to the ICU in the postoperative phase.

Methods: A retrospective cohort study of all elective surgical admissions in a ten-bedded combined medical-surgical intensive care unit of a Portuguese hospital between January 2018 to June 2020. Comparation between albumin levels were performed using Man-Whitney U-test.

Results: In the period from January 2018 to June 2020, 62 patients were elective admitted to our ICU. The mean patient's age was 67.9 (19-88), being $50 \%$ of them men (31). Among the elective admissions the majority were after whipple procedure, representing $67.7 \%$ of all admissions. The median Charlson score was 5 points [0-11 points]. The median ICU length of stay was 3 days [2-4 days] and the median time with mechanical ventilation was 2 days [1-3 days], being that 34 patients needed less than 24 hours of mechanical ventilation. Regarding the prognostic indices the median SAPS 2 was 30 [23-43.3] and the median APACHE II was 13 [8.8-19]. As to biological markers the median admission C-reactive protein was $58 \mathrm{mg} / \mathrm{dL}$ [17.3-101.7] and the median admission lactate was 2,6 [2.1-3.3]. When analyzing albumin, the patients submitted to whipple procedure demonstrate a significant lower ICU admission albumin levels with a median of $26 \mathrm{~g} /$ $\mathrm{dL}$ than the patients undergone to other types of procedures with a median of $30 \mathrm{~g} / \mathrm{dL}$ (P 0.004). As for the evaluation of sepsis during the length of ICU stay, 5 patients $(8,1 \%)$ develop sepsis. According to the acute kidney injury classification (AKIN) $11,3 \%$ had a stage 1 acute kidney injury, $6,5 \%$ a stage 2 , and $1,6 \%$ a stage 3 . None of the patients analyzed needed renal replacement therapy. The overall ICU mortality was 3,2\%, the hospital mortality was $6,5 \%$ and 49 patients were still alive after a 6 month follow up, representing $79 \%$ of patients.

Conclusion: This retrospective study conducted in a 10-bed intensive care unit shows that critical care admitted patients after elective surgery were mainly submitted to a whipple procedure and have a median charlson score of 5 . The median length of stay in the ICU was 
less than 4 days and the median mechanical ventilation time was 4 days. Regarding the prognostic indices we found low APACHE II and SAPS 2 values reflecting the fact that those patients were electively admitted and that most of them didn't develop any complication during the perioperative phase or during the hospital stay. We also found that postoperative albumin levels were low mainly in the group of patients submitted to a whipple procedure, probably reflecting the magnitude of surgery and the associated stress response. In addition, overall mortality was low in this patient population.

\section{Reference(s) and grant ackowledgment(s)}

1. Hübner M, Mantziari S, Demartines N, Pralong F, Coti-Bertrand P, Schäfer M. Postoperative Albumin Drop Is a Marker for Surgical Stress and a Predictor for Clinical Outcome: A Pilot Study. Gastroenterol Res Pract. 2016;2016:8743187.

2. Basile-Filho A, Lago AF, Menegueti MG, Nicolini EA, Rodrigues LAB, Nunes RS, et al. The use of APACHE II, SOFA, SAPS 3, C-reactive protein/albumin ratio, and lactate to predict mortality of surgical critically ill patients: a retrospective cohort study. Medicine (Baltimore) 2019;98(26):e16204.

3. The International Surgical Outcomes Study group Global patient outcomes after elective surgery: prospective cohort study in 27 low-, middle- and high-income countries. Br J Anaesth 2016;117:601-9.

\section{9}

Predictors of mortality and outcomes of acute surgical admissions: a restrospective study

D. Castro ${ }^{1}$; C. Roriz' ; F. Sequeira ${ }^{1}$; N. Ferreira' ${ }^{1}$ L. Linhares ${ }^{1}$; L. Pereira

${ }^{1}$ Serviço de Medicina Intensiva, Centro Hospitalar de Leiria, Leiria, Portugal Correspondence: D. Castro

Intensive Care Medicine Experimental 2020, 8(2): 001419

Introduction: Acute surgical patients account for a significant percentage of admissions in an Intensive Care Unit (ICU). Outcomes of these surgical patients must be assessed and several clinical scores are available to guide clinicians. Acute Physiology And Chronic Health Evaluation II (APACHE II) and Simplified Acute Physiology Score 2 (SAPS 2) have been used to predict the outcome of surgical critically ill patients. However, none of these scores have a $100 \%$ sensitivity or $100 \%$ specificity. Most of these patients are septic and serum lactate level is considered a sensitive marker for sepsis or septic shock reflecting a cell-based metabolism. C-reactive protein (CRP), albumin, CRP/ Albumin ratio and blood lactate levels correlate with the degree of inflammation during postoperative care. These and potentially others biological parameters could be used as independent predictors of poor outcomes.

Objectives: The aim of this study is to compare prognostic value of two indices (APACHE II and SAPS 2 scores) and biological markers in the outcome of acute surgical patients admitted in ICU in the immediate postoperative care.

Methods: An observational retrospective study of all acute surgical admissions into our ICU in the immediate postoperative care from January, 1st to December, 31st 2018 and had a ICU length of stay longer than 24 hours. Data concerning the diagnosis, comorbidities and demographics were documented in medical records. The biological markers CRP, albumin, serum blood lactate, N-terminal pro-B type natriuretic peptide (NT Pro-BNP), leukocytes, platelets and Glomerular Filtration Rate were collected during the first 24 hours after patient's admission and prognosis indices (APACHE II and SAPS 2) were recorded. Patients were divided into survivors and non-survivors. Comparations between survivors and non-survivors were performed using the Student-T test or Man-Whitney U-test for quantitative variables (parametric and non-parametric variables, respectively) and Pearson Chi-square test for qualitative variables.

Results: Sixty surgical patients admitted into our ten-bed ICU in the immediate postoperative care were retrospectively studied. Patients were divided into survivors $(n=33)$ and non survivors $(n=27)$ as decribed. The ICU and overall in-hospital mortality were 30\% and $15 \%$, respectively. Non-survivors were older than survivors (76 vs 69 yeats old, $p=0.024$ ) and males died more than females (males $63 \%$ vs $36,4 \%, p=0.042$ ). Comorbidities were performed by Charlson's score and non-survivors had a statistically higher Charlson's score (score 5 vs $3, p=0.001)$. There were no statistic differences in ICU length of stay between groups. APACHE II ( 27 vs $19, p<0.001)$, SAPS ( 61 vs 46 , $p<0.001)$, serum lactate $(4,8$ vs 3,$2 ; p=0.015)$ were significantly higher in the non-survivors group. Glomerular Filtration Rate in ICU admission was lower in non-survivors $(17,4$ vs 56,$3 ; p=0.001)$ and the need of Renal Replacement Therapy was higher in this group $(29,6$ vs $9,1 \%$; $p=0,043$ ). No differences were found between groups in days of mechanical ventilation, ratio CRP/Albumin, CRP, Albumin, NT-proBNP, leukocytes count, hemoglobin or blood transfusions. No differences were found between two groups regarding sepsis in admission.

Conclusion: Mortality rates in acute surgical patients are still very high in Intensive Care Unit and several scores of severity of critically ill patients can predict their mortality like APACHE II and SAPS 2 . In our study these scores are higher in non-survivors patients and can be associated with greater severity of the disease and their higher in-hospital mortality. Serum lactate levels are related with mortality and can be associated with tissue hypoxia and elevated inflammation during postoperative care. Glomerular Filtration Rate is lower in non-survivors, reflecting acute kidney injury and renal failure with the need of Renal Replacement Therapy. Studies have showned that sepsis is frequently complicated by acute kidney injury which increases the mortality risk.

\section{Reference(s) and grant ackowledgment(s)}

1. Gayat E, Cariou A, Deye N, et al. Determinants of long-term outcome in ICU survivors: results from the FROG-ICU study. Crit Care. 2018;22(1):8. Published 2018 Jan 18. https://doi.org/10.1186/s13054-017-1922-8.

2. Basile-Filho A, Lago AF, Menegueti MG, et al. The use of APACHE II, SOFA, SAPS 3, C-reactive protein/albumin ratio, and lactate to predict mortality of surgical critically ill patients: A retrospective cohort study [published correction appears in Medicine (Baltimore). 2019 Jul;98(29):e16675]. Medicine (Baltimore). 2019;98(26):e16204. https://doi.org/10.1097/md.0000000000 016204.

3. Lee CW, Kou HW, Chou HS, et al. A combination of SOFA score and biomarkers gives a better prediction of septic AKI and in-hospital mortality in critically ill surgical patients: a pilot study. World J Emerg Surg. 2018;13:41. Published 2018 Sep 10. https://doi.org/10.1186/s13017-018-0202-5.

4. Jung C, Wernly B, Muessig JM, et al. A comparison of very old patients admitted to intensive care unit after acute versus elective surgery or intervention. J Crit Care. 2019;52:141-148. https://doi.org/10.1016/j. jcrc.2019.04.020.

001512

Intraoperative hemodynamic changes as a risk factor for postoperative complications in patients undergoing elective laparoscopic colorectal surgery

V. Panafidina'; I. Shlyk ${ }^{1}$

${ }^{1}$ Anesthesiology and intensive care, Pavlov First State Medical University of St. Petersburg, Sankt-Peterburg, Russia

Correspondence: $\vee$. Panafidina

Intensive Care Medicine Experimental 2020, 8(2): 001512

Introduction: Despite the fact that laparoscopic surgery seems to be safe in high-risk surgical patients, it is associated with significant hemodynamic changes. Patients with colorectal malignances often have a number of cardiovascular and other comorbidities, and severe hemodynamic fluctuations could be a risk factor for postoperative morbidity. Thus, we hypothesis that hemodynamic changes during elective laparoscopic colorectal surgery might be associated with postoperative complications development.

Objectives: The aim of the study is to analyze potentially modifiable risk factors related to hemodynamic changes during laparoscopic surgery in patients with colorectal malignances.

Methods: A single-center retrospective study was performed in a university clinic in Saint-Petersburg, Russia. We analyzed 100 medical 
recordings of patients undergoing elective colorectal surgery in 20172019. The analysis considered systolic blood pressure (SBP) changes, changes of mean arterial pressure (MAP), intraabdominal perfusion pressure (IPP), incidence of tachy- or bradycardia during operation time, and complications Clavien-Dindo Grade III-V. Univariate and multivariate analyses were performed in SPSS Statistics 20.0 to identify risk factors for postoperative complications.

Results: Postoperative complications occurred in 16 patients (16\%). These patients had significantly more episodes of SBP drop from baseline, MAP $<65 \mathrm{~mm} \mathrm{Hg}$, IPP $<65 \mathrm{~mm} \mathrm{Hg}$ during pneumoperitoneum. SBP lowering, MAP, IPP were significant risk factors for postoperative complications $(p<0,001)$. In multivariate analysis, SBP lowering (OR $1,535,95 \% \mathrm{Cl} 1,095-2,152, \mathrm{p}=0,013)$ and IPP (OR 0,75, 95\% Cl 0,611$0,932, p=0,009$ ) were independent risk factors for postoperative complications.

Conclusion: Our results may suggest that severe hemodynamic fluctuations could be related to postoperative complications in patients undergoing elective laparoscopic colorectal surgery.

\section{3}

Incidence of major cardiovascular complications after vascular surgery during 1-year follow-up - a prospective cohort study K. Polok' ; J. Fronczek'; P. Alonso-Coello'; J. Górka'; B. Biccard ${ }^{3}$; E.

Duceppe $^{4}$; P. Devereaux $;$; D. Ashbridge Scott ${ }^{5}$; M. Chan ${ }^{6}$; W. Szczeklik

${ }^{1}$ Intensive care and perioperative medicine, Jagiellonian University Medical College, Kraków, Poland; ${ }^{2}$ Centro cochrane iberoamericano, Instituto de Investigación Biomédica (CIBERESP-IIB Sant Pau), Barcelona, Spain; ${ }^{3}$ Department of anaesthesia and perioperative medicine, University of Cape Town and New Groote Schuur Hospital, Cape Town, South Africa; ${ }^{4}$ Population health research institute, McMaster University and Hamilton Health Sciences, Hamilton, Canada; ${ }^{5}$ Leeds teaching hospitals, NHS Trust and University of Leeds, Leeds, United Kingdom; ${ }^{6}$ Faculty of medicine, Chinese University of Hong Kong, Hong Kong, Hong Kong

Correspondence: $\mathrm{K}$. Polok

Intensive Care Medicine Experimental 2020, 8(2): 001623

Introduction: Vascular surgeries are associated with a particularly high risk of postoperative complications due to a high number of comorbidities in this population as well as increased risk of perioperative bleeding and hypotension associated with these procedures.

Objectives: To assess the incidence of major cardiovascular complications in 1-year follow-up after vascular surgeries.

Methods: This is a substudy of the Vascular Events In Noncardiac Surgery Patients Cohort Evaluation Study (VISION), in which patients aged $\geq 45$ years undergoing non-cardiac surgeries and hospitalized at least overnight were enrolled. In this large, international prospective cohort study, each patient had postoperative troponin measurement to assess the association between troponin level and postoperative outcomes. Additionally, patients or their relatives were contacted 30 days and 1 year after the procedure to assess the incidence of complications in short- and long-term observation.

Results: Study group consisted of 2641 patients undergoing vascular surgeries. There were $2047(77.5 \%)$ males and the mean age accounted to 68.2 (SD 9.8) years. The most common comorbidities were hypertension (75.5\%), peripheral artery disease $(67.5 \%)$ and coronary artery disease $(38.0 \%)$. During the 1-year follow-up 223 patients died (8.4\%), 243 patients had myocardial infarction (9.2\%), 35 patients suffered a stroke (1.3\%), 192 patients underwent amputation (7.3\%), 68 patients developed congestive heart failure $(2.6 \%)$ and 18 patients had non-fatal cardiac arrest $(0.7 \%)$.

Conclusion: Major cardiovascular complications were common among patients undergoing vascular surgeries in the long-term observation. The most common complications were myocardial infarction, death and amputation.

\section{7}

Perioperative Diabetes Insipidus: Report of two unusual cases S. Nissar'; S. Nawaz

${ }^{1} \mathrm{Sicu}$, Hamad Medical Corporation, Doha, Qatar; ${ }^{2}$ Medical Education, Hamad Medical Corporation, Doha, Qatar
Correspondence: S. Nawaz

Intensive Care Medicine Experimental 2020, 8(2): 001227

Introduction: Diabetes insipidus (DI) is a clinical disorder of water metabolism in the human haemostasis, commonly resulting from the deficiency of antidiuretic hormone $(\mathrm{ADH})$ or from renal insensivity to $A D H$. DI manifests by the patient passing diluted copious amount of urine.1 DI is classified as central or nephrogenic and pregnancy induced, in central DI, posterior pituitary gland fails to secret $\mathrm{ADH}$. Nephrogenic $\mathrm{DI}$ is when the renal receptors do not respond to $A D H$. Pregnancy induced $D I$ is when placenta consumes most of the secreted ADH, leading to ADH deficency. $2 \mathrm{DI}$ is common after the pituitary gland surgeries. 3 There are a few case reports of DI after general surgical procedures. 4 Although polyuria is common in the post-operative period, it is important to diagnose DI to prevent severe dehydration and life-threatening electrolyte imbalance. We report two unusual cases of DI during the perioperative period requiring hydration and intravenous desmopressin.

Methods: Case Reports.

Results: Case1: A 49 years old healthy male was diagnosed with cervical vertebral disc prolapse (C5-6), planned for cervical discectomy and cage fusion. General anaesthesia was induced with etomidate and fentanyl, trachea was intubated under the effect of rocuronium, in addition patient received ketamine and cefazolin during induction. Anesthesia was maintained by propofol and remifentanil target controlled infusion $(\mathrm{TCl})$. Foley's catheter was in place and he was pouring 50 and $70 \mathrm{ml}$ of urine per hour. His hemodynamics were stable. In the 2nd hour of surgery he became polyuric and passed 700 to $800 \mathrm{ml}$ diluted urine in next 2 hours until the surgical procedure was over. He was started on $50 \mathrm{ml} /$ hour of $0.45 \%$ saline and shifted to post anaesthesia care unit. He remained polyuric and keep passing 400 and $300 \mathrm{ml}$ of diluted urine next 2 hours. He developed hypernatremia, renal functions were with in normal range and urine specific gravity was $<1005$, diagnosed to have central diabetes insipidus and given intravenous (IV) 2 micrograms ( $\mu$ grams) of desmopressin. For the next hours his urine became concentrated and urine output dropped to 50,60 and $70 \mathrm{ml} /$ hour. He was transferred to high dependency unit (HDU), remained stable, from there discharged home and followed up in neurosurgical outpatient clinics.

Case 2: A 36-year-old healthy male presented with on and off headache. Computer tomography (CT) and MRI (magnetic resonance tomography) diagnosed to have 3rd ventricular cyst. He underwent right frontal craniotomy and excision of ventricular cyst. Anesthesia was induced with propofol and remifentanil, trachea was intubated under the effect of rocuronium. He received cefazolin during the induction of anaesthesia. Anesthesia was maintained with remifentanil and propofol infusion. Patient remained hemodynamically stable. His urine output remained 50 to $70 \mathrm{ml} /$ hour during the surgical period. Patient was transferred to the surgical intensive care unit (SICU) for further monitoring. In the SICU during the 1st and 2 nd hour he passed $600 \mathrm{ml}$ and $700 \mathrm{ml}$ of diluted urine respectively. His serum sodium jumped to $148 \mathrm{mmol} /$ litre, renal functions were normal and urinary specific gravity was $<1005$. Patient was started on $100 \mathrm{ml} /$ hour of $0.45 \%$ saline and desmopressin $2 \mu$ gram was administrated intravenously. From the next hour his urine became concentrated and urine output dropped to 50 to $100 \mathrm{ml} /$ hours. Patient remained hemodynamically stable and serum sodium became normal. He was transferred to the ward the next day and from there discharged home. Followed up in the neurosurgical outpatient clinic without any issues.

Conclusion: : The occurrence of DI in above mentioned surgeries is very rare. Both surgical procedure and anaesthesia medications can cause perioperative DI.

\section{Reference(s) and grant ackowledgment(s)}

1. 1. Saifan C, Nasr R, Mehta S, Acharya PS, Perrera I, Faddoul G et al. diabetes insipidus: A clinical challenging diagnosis with new drug therapy. ISRN Nephro 2013; 2013:1-7 2. Kalra S, Zargar AH, Jain SM, Sethi B, Chowdhury $\mathrm{S}$, Singh AK et al. diabetes insipidus. The other diabetes. Ind J Endocrinol Metab 2016; 20(1):9-21 3. Hirotsugu M, Ryuji N, Hiroshi H. Nephrogenic diabetes mellitus during the Tran sphenoidal pituitary adenectomy. J neurosurg Anesth 2016; 28(1):79-80 4. Yamashita H, Ishimaru M, Yanaguchi H, 
Yamauchi $\mathrm{H}$, Sugiura A et al. Massive post-operative polyuria following total gastrectomy Anesthesia 2006;20(1):36-9 5. Magbri A, El- Magbri E, Hershit S. hypernatremia and central diabetes insipidus following neurosurgical procedure after trauma. Arch Pathol Clin Res 2017:1;5-8 6. Edate S, Albanese A: Management of Electrolyte and Fluid Disorders after Brain Surgery for Pituitary/Suprasellar Tumours. Horm Res Paediatr 2015; 83:293-301 7. Kaplan L, Bronstein Y, barzilay Y, Hasharoni A, Finkelstein J. Canal expensive laminectomy in the management of cervical sponylotic myelopathy. IMAJ 2006; 8:548-22 8. Pinto FCG, Chavantes MC, Fonoff ET, Teixeira MJ. Treatment of colloid cysts of the third ventricle through neuroendoscopic Nd:YAG laser stereotaxis. Arq. Neuro-Psiquiatr2009;67;1082-87 9. Soo J, Gray J, Manecke G. propofol and diabetes insipidus. J Clin Anesth 2014;26(8): 679-83 10. Kassebaum N, Hairr J, Goldsmith W, John Barwise J, Pandharipande PM. Diabetes insipidus associated with propofol anesthesia. Journal of Clinical Anesthesia (2008) 20, 466-468 11. Gaffar S Jonathan P. Eskander, Burton D. Beakley Brian P. McClure, Amenta P, Pierre N. A case of central diabetes insipidus after ketamine infusion during an external to internal carotid artery bypass. J Clin Anesthesia 2017; 36:72-75.

\section{6}

Comparison of dynamic parameters of fluid responsiveness based on the analysis of arterial waveform in high-risk surgical patients B. Cenkova ${ }^{1}$; M. Chobola ${ }^{1}$; V. Sramek ${ }^{1}$; P. Suk ${ }^{1}$

${ }^{1}$ Department of anaesthesiology and intensive care, St. Anne's University Hospital, Brno, Czech Republic

Correspondence: P. Suk

Intensive Care Medicine Experimental 2020, 8(2): 001486

Introduction: Dynamic indices of fluid responsiveness (e.g. pulse pressure variation - PPV and stroke volume variation - SVV) differ between hemodynamic monitors, which use proprietary algorithms, and vary even over a short period of time.

Objectives: The aim is to compare the stability and predictive value for fluid responsiveness of PPV and SVV measured by various minimally invasive devices in high-risk patients undergoing general surgery.

Methods: In this prospective study, clinically indicated fluid challenges (FC; $500 \mathrm{ml}$ of balanced crystalloid administered over 10 minutes) in patients ventilated with tidal volume of $8 \mathrm{ml} / \mathrm{kg}$ were monitored. Increase in cardiac output (CO) $\geq 15 \%$ defined fluid responders. Two minutes of arterial pressure waveform was recorded with simultaneous video recording of hemodynamic monitors - GE Carescape B650 (measuring PPV), FloTrac/Vigileo (SVV) and LidCO Rapid (PPV and SVV) before and after the FC. All devices used the same arterial line and waveform; arterial line flushing was used for synchronization with the video. Analysis was limited to intervals with regular rhythm and good waveform quality. Up to 17 values were read from the video in $5 \mathrm{~s}$ intervals and reference PPV was calculated from the stored arterial pressure waveform at the same timepoints offline. Stability of the parameter was assessed by calculating the range and standard deviation (SD) of the index over observed period up to $80 \mathrm{~s}$ before FC. Receiver operating characteristic (ROC) analysis and a correlation of the index with change in $\mathrm{CO}$ were used to evaluate the predictive value of the indices.

Results: Twenty three patients were analysed; 14 of 60 fluid challenges were excluded due to changes in vasoactive drugs, patient's position change, arrhythmia or artefacts during the FC. Stability was assessed by variation of parameters - mean values of range (and SD) were 3.8 (1.0) \% for reference PPV, 1.8 (0.5) \% for GE PPV, 10.8 (3.0) $\%$ for LiDCO PPV, 8.3\% (2.4) for LiDCO SVV and 2.3 (0.9) \% for Vigileo SVV. Mean value of the indexes during 80 -second interval before FC was used to calculate the area under the ROC curve (AUROC), optimal threshold, correlation with $\Delta C O(r)$ and specificity/sensitivity for threshold values of $10 \%$ and $13 \%$, which are presented in the table. Mostly, an average value of the index had better AUROC than minimal or maximal observed values within the observed period.

\begin{tabular}{|c|l|l|l|l|l|l|}
\hline index & median & AUROC & threshold & \multicolumn{1}{|c|}{ r } & spec/senz@10\% & spec/senz@13\% \\
\hline $\begin{array}{c}\text { ref. } \\
\text { PPV }\end{array}$ & $8.0 \%$ & 0.692 & $9.4 \%$ & 0.511 & $74 / 58$ & $85 / 37$ \\
\hline $\begin{array}{c}\text { GE } \\
\text { PPV }\end{array}$ & $7.8 \%$ & 0.722 & $7.5 \%$ & 0.553 & $73 / 50$ & $89 / 39$ \\
\hline $\begin{array}{c}\text { LiDCO } \\
\text { PPV }\end{array}$ & $13.6 \%$ & 0.734 & $13.5 \%$ & 0.472 & $42 / 95$ & $64 / 74$ \\
\hline $\begin{array}{c}\text { LiDCO } \\
\text { SVV }\end{array}$ & $10.7 \%$ & 0.739 & $12.2 \%$ & $0.51258 / 76$ & $75 / 53$ \\
\hline $\begin{array}{c}\text { Vigileo } \\
\text { SVV }\end{array}$ & $9.6 \%$ & 0.703 & $10.2 \%$ & $0.42567 / 68$ & $81 / 53$ \\
\hline
\end{tabular}

Conclusion: LiDCO displayed higher fluctuation of indices than the other monitors. Although a significant span of threshold values was observed, the predictive value of PPV and SVV from all the monitors was comparable. Despite ideal conditions, the diagnostic ability of all parameters was only moderate and lower than expected.

16. Poisoning/Toxicology /Pharmacology > Poisoning/Toxicology / Pharmacology.

\section{3}

Metformin associated lactic acidosis: a case series of $\mathbf{2 0}$ patients

R. Amaral ${ }^{1}$; H. Santos ${ }^{1}$; C. Carvalho ${ }^{1}$; J. Carvalho ${ }^{1}$; B. Costa ${ }^{1}$; A. Pais ${ }^{1}$

${ }^{1}$ Intensive Medicine, Centro Hospitalar De Trás-Os-Montes E Alto Douro, E.P.E., Vila Real, Portugal

Correspondence: R. Amaral

Intensive Care Medicine Experimental 2020, 8(2): 001323

Introduction: Metformin associated lactic acidosis (MALA) has been reported as from 1977, but full understanding of this entity has yet to be achieved. It is the major toxicity of metformin with high attributed mortality rate. In the most severe cases intensive care admission and renal replacement therapy (RRT) are required.

Objectives: To report on our experience with the MALA patient's characteristics, severity scores, supportive measures required in the last 5 years.

Methods: Retrospective analysis of the clinical information of patients admitted in intensive care level 2 and 3 with main or additional diagnosis of MALA from 2015 to 2019.

Results: We obtained 20 patients of which $13(65,0 \%)$ had principal diagnosis of MALA. Most of the patients were female $(n=11)$, mean age $75,9 \pm 5,9.14$ patients $(70,0 \%)$ were admitted on level 3 care. Antibiotics were administered to $60,0 \%(n=12)$ of patients with only $41,6 \%$ of those $(n=5)$ having clinically confirmed infection. Initial lactate levels were 12,6 $\pm 3,5$ and 24 h lactate levels were 3,2 $\pm 3,3$. $60,0 \%$ of patients required renal replacement therapy $(n=12)$. The RRT modalities were: continuous veno-venous hemodiafiltration in $33,3 \%(n=4)$, continuous veno-venous hemofiltration in 33,3\% $(n=4)$ and conventional haemodialysis in $33,3 \%(n=4)$. Invasive mechanical ventilation was necessary in $25,0 \%$ of patients. Severity scores were: APACHE II 25,5 \pm 7,3; SAPS II 50,9 $\pm 11,5$; SAPS $369,2 \pm 16,2$; SOFA at admission 9,0 $\pm 3,8$. Mean length of stay in intensive care was 3,5 \pm 4,2 days and in hospital was 16,0 $\pm 9,1$ days. Hospital mortality was the same of net mortality: 2 patients $(10,0 \%)$.

Conclusion: Although with limitations due to its retrospective nature, known under-report of the condition in matter, and varied concurrent pathologies of patients reported, this study presents our reality while treating MALA. We confirmed high use of antibiotics compared to clinically confirmed infection, something referred in the literature. Continuous veno-venous renal replacement was used in the majority of the patients requiring renal replacement. Patients with diagnosis of MALA present with high severity scores but have low mortality in our experience.

\section{Reference(s) and grant ackowledgment(s)}

1. Diane P. Calello et al, Extracorporeal Treatment for Metformin Poisoning: Systematic Review and Recommendations From the Extracorporeal Treatments in Poisoning Workgroup, Critical Care Medicine 2015. 
2. Lalau, J-D et al, Metformin-associated lactic acidosis (MALA): Moving towards a new paradigm; Diabetes Obes Metab. 2017;19:1502-1512; https ://doi.org/10.1111/dom.12974.

3. Metformin-associated lactid acidosis: current perspectives on causes and risk, BMJ 2015; https://doi.org/10.1016/j.metabil.2015.10.014.

4. Angioi et al. Metformin associated lactic acidosis: a case series of 28 patients treated with sustained low-efficiency dialysis (SLED) and long-term follow-up. BMC Nephrology (2018) 19:77; https://doi.org/10.1186/s1288 2-018-0875-8.

5. None.

16. Poisoning/Toxicology /Pharmacology > Poisoning/Toxicology / Pharmacology.

\section{3}

Impact of Mental Health Assessment on Intensive Care Stay in Drug Overdose - A Retrospective Observational Study

A. Jayasinghe 1 ; J. Collins ${ }^{1}$

${ }^{1}$ Broomfield hospital, MEHT NHS Trust, Chelmsford, United Kingdom

Correspondence: A. Jayasinghe

Intensive Care Medicine Experimental 2020, 8(2): 001423

Introduction: Studies on characteristics, outcome and clinical burden of Intensive Care Unit (ICU) admissions following drug overdose are scarce despite the significant health and social impact caused.

Methods: A retrospective observational study was carried out from 2015 to 2019 in an ICU of a district general hospital in Essex, UK with the aim of identifying the demographic and clinical characteristics and factors affecting delay in discharge of the patients admitted to ICU following drug overdose.

Results: Total number of admissions with illicit or prescription drug overdose over the last 5 years were 202 (4.3\% of total admissions). $56 \%$ were females. Mean age was 38.7 years and patients in the age range of 30-39 years had the highest number of admissions $(n=51$, 25.2\%). 74.2\% were known patients with mental health disorders and $49.5 \%$ had a previous history of self-harm. 25 were readmissions which comprised $12.43 \%$ of total admissions. Psychoactive medications were the commonest drugs used. Average ICU stay was 2.85 days (Median 1.8 days). $93.7 \%$ were discharged and mortality was $4.4 \%$. The majority were home discharges (43.5\%). All home discharges were reviewed by the mental health team (MHT) except 11 self-discharges. All ward discharges $(30.7 \%)$ were referred to $\mathrm{MHT}$ to be reviewed in ward. Delay in discharge from ICU for more than 6 hours since being declared 'Medically Fit for Discharge' was identified as a late discharge. 40.5\% of admissions got a delay in discharge. Average delay in discharge was 1.05 days and it ranged from 8 - 96 hours. $72 \%$ of the delays occurred due to late assessment by MHT to ensure mental fitness for safe home discharge or in arranging a mental health ward bed if not fit for discharge. Reasons for delay and the frequency are shown in the following table.

\begin{tabular}{|l|l|l|}
\hline Reason for delay & Number of cases & Percentage \\
\hline Delay in review by mental health team & 43 & $53.4 \%$ \\
\hline $\begin{array}{l}\text { Delay in transfer to a mental health ward } \\
\text { bed }\end{array}$ & 16 & $19.5 \%$ \\
\hline Delay in getting a ward bed & 23 & $28 \%$ \\
\hline
\end{tabular}

Notably the duration of ICU stay had a rising trend over the years along with a proportional increase in delay in discharge as shown below.

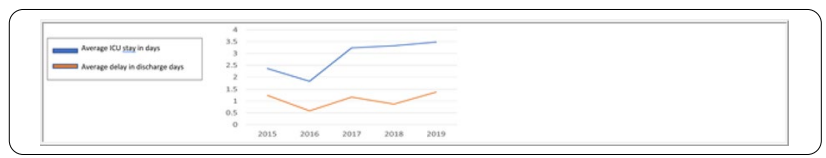

Conclusion: Drug overdose continues to be a growing public health concern worldwide. Importantly, younger generation is mainly affected, most are preventable and many require ICU care. A striking proportion of our study; $75 \%$ had mental health issues and $50 \%$ had a previous history of self-harm. Readmission rate was high, highlighting the importance of psychological support these patients continues to require more. Hence review by MHT is essential before a safe discharge. However, a significant percentage $(40.5 \%)$ had a late discharge, longest being 96 hours. The main reason was a delay in MHT's review and arranging further psychological care. Patients who faced delays awaiting MHT were otherwise medically fit for direct discharge to home and did not require inward care.

Drug overdose admissions accounted for an average of 115.1 ICU bed days per year. Out of this, 42.42 ICU beds days (36.8\%) were occupied due to the delay in discharge. Since the estimated daily cost of a level 2 ICU bed at our institute is 800 pounds, 33,936 pounds/year can be saved by optimising the barriers to prompt discharge from ICU. These may include increasing MHT staff and their availability in ICU and prioritizing ward bed allocation to patients stepping down from ICU.

\section{Reference(s) and grant ackowledgment(s)}

1. [1] J Orsinia, N Dina, et al. Clinical and epidemiological characteristics of patients with acute drug intoxication admitted to ICU. journal of community hospital internal medicine perspectives, 2017 vol. 7, no. 4,202-207. (https://doi.org/10.1080/20009666.2017.1356189) [2] D Clark, DB Murray, D Ray. Epidemiology and outcomes of patients admitted to critical care after self-poisoning. JIC 2011; Volume 12, Number 4 [3] "National statistics on drug misuse - England, 2019" published in November 2019 https://digit al.nhs.uk/data-and-information/publications/statistical/statistics-on-drugmisuse/2019 [4] M. A. Cretikos, M. J. A. Parr. Drug Related Admissions to Intensive Care: The Role of Illicit Drugs and Self Poisoning. Critical Care and Resuscitation 2003; 5: 253-257 [5] V. Athavale, C Green, et al. Characteristics and outcomes of patients with drug overdose requiring admission to Intensive Care Unit. Australasian Psychiatry 2017, Vol 25(5) 489 -493 (https://doi. org/10.1177/1039856217706824) [6] Morrow RL, Basset K, et al. Outcomes associated with hospital admissions for accidental opioid overdose in British Columbia: a retrospective cohort study. BMJ 2019;9: e025567. (https ://doi.org/10.1136/bmjopen-2018-025567) [7] M Fendricha, J Beckera, et al. Psychiatric symptoms and recent overdose among people who use heroin or other opioids: Results from a secondary analysis of an intervention study. Addictive Behaviors Reports:Volume 10, December 2019, 100212. (https:// doi.org/10.1016/j.abrep.2019.100212).

2. Dr. V Bharadhvaj, Audit lead, ICU-Broomfield Hospital.

16. Poisoning/Toxicology /Pharmacology > Poisoning/Toxicology / Pharmacology.

\section{6}

Measured creatinine clearance and its impact on vancomycin levels in critically ill COVID-19 patients

K. Selles ; J. Van Der Mast ${ }^{2}$; M. Volbeda ${ }^{1}$; M. Renes ${ }^{1}$; S. Berger ${ }^{3} ;$ D. Touw ${ }^{2}$; W. Bult $^{2} ;$ M. Nijsten ${ }^{1}$

${ }^{1}$ Department of critical care, University Medical Center Groningen, Groningen, Netherlands; ${ }^{2}$ Dpt of clinical pharmacy and pharmacology, University Medical Center Groningen, Groningen, Netherlands; ${ }^{3}$ Dt of nephrology, University Medical Center Groningen, Groningen, Netherlands

Correspondence: $M$. Nijsten

Intensive Care Medicine Experimental 2020, 8(2): 001506

Introduction: Renal function changes in COVID-19 patients admitted to the ICU are still poorly delineated. Sole use of plasma creatinine only allows for determination of estimated glomerular filtration rate (eGFR), whereas combined with $24 \mathrm{~h}$ urinary creatinine excretion, direct determination of measured creatinine clearance ( $\mathrm{mCC}$ ) is possible.

Objectives: Our goal was to assess renal function with $\mathrm{mCC}$ in critically ill COVID-19 patients and also assess its relationship with vancomycin dosing.

Methods: Consecutive COVID-19 patients admitted to our ICU until May 2020 were analyzed over the first 30 ICU days. Vancomycin was administered as a loading dose followed by continuous infusion with dose adjustments based on therapeutic drug monitoring (TDM) with a target vancomycin plasma concentration between 20 and $25 \mathrm{mg} / \mathrm{L}$. Plasma creatinine, eGFR, $\mathrm{mCC}$ as well as vancomycin concentrations 
were regularly obtained. We defined augmented renal clearance as an $\mathrm{mCC}>130 \mathrm{~mL} / \mathrm{min} / 1.73 \mathrm{~m}^{2}$ [1]. Our current vancomycin TDM algorithms do not use $\mathrm{mCC}$ but are based on weight and eGFR.

Results: We evaluated 90 patients $(73 \%$ male), with a mean $( \pm S D)$ age of $62 \pm 10$ years. Unadjusted hospital mortality was $21 \%$. Mean weight, BMI and BSA were $90 \pm 16 \mathrm{~kg}, 28.5 \pm 4.6 \mathrm{~kg} / \mathrm{m}^{2}$ and $2.07 \pm 0.20 \mathrm{~m}^{2}$. In total $1063 \mathrm{mCC}$ measurements were available. In 31 (34\%) patients an $\mathrm{mCC}<30 \mathrm{ml} / \mathrm{min}$ was observed at any time and 11 of these patients required renal replacement therapy (RRT). In patients not requiring RRT the mean $\mathrm{mCC}$ was $112 \pm 48 \mathrm{ml} / \mathrm{min}$. Augmented renal clearance was observed in 39 patients (43\%) for a total of 194 (18\%) mCC measurements.

Vancomycin was prescribed for 35 episodes in 30 (33\%) patients with a mean loading dose of $1724 \pm 574 \mathrm{mg}(19.0 \pm 4.9 \mathrm{mg} / \mathrm{kg})$, and a mean maintenance dose of $2268 \pm 886 \mathrm{mg}(25.3 \pm 10.8 \mathrm{mg} / \mathrm{kg})$. 181 vancomycin levels were determined with a mean concentration of $22.7 \pm 6.7 \mathrm{mg} / \mathrm{L}$ with $17(57 \%)$ patients having at least 2 measurements below $20 \mathrm{mg} / \mathrm{L}$. Daily vancomycin dose was more correlated $(P<0.01)$ with $\mathrm{mCC}\left(R^{2}=0.66\right)$ compared to eGFR $\left(R^{2}=0.43\right)$.

Conclusion: Although we observed renal failure in our cohort of COVID-19 patients treated in the ICU, a considerable subgroup showed augmented renal clearance when MCC was employed, likely explaining the failure to reach therapeutic vancomycin levels. Determining mCC in COVID-19 patients has therefore clear benefits over the use of eGFR.

\section{Reference(s) and grant ackowledgment(s)}

1. [1] Bilbao-Meseguer I, et al. Augmented renal clearance in critically ill patients: A systematic review. Clin Pharmacokinet 2018

2. None

16. Poisoning/Toxicology /Pharmacology > Poisoning/Toxicology / Pharmacology.

\section{8}

Tramadol metabolism and systemic inflammation after major abdominal surgery: a prospective observational study

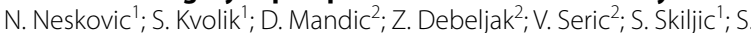
Marczi $^{3}$

${ }^{1}$ Anesthesiology and icu, Osijek University Hospital; Medical Faculty, Osijek, Croatia; ${ }^{2}$ Department for clinical and laboratory diagnostics, Osijek University Hospital; Medical Faculty, Osijek, Croatia; ${ }^{3}$ Transfusion mediicne, Osijek University Hospital; Medical Faculty, Osijek, Croatia

Correspondence: S. Kvolik

Intensive Care Medicine Experimental 2020, 8(2): 001618

Introduction: A systemic inflammation may affect tramadol metabolism within first 24 postoperative hours.

Objectives: The aim of this study was to examine concentrations of tramadol, O-demethyltramadol (ODT) and $\mathrm{N}$-demethyltramadol (NDT) in patients admitted to the intensive care unit (ICU) after major abdominal surgery in the patients with and without criteria for systemic inflammation.

Methods: For this study Ethics committee approval was obtained and all the patients wrote informed consent. In between of 50 postsurgical patients, a group of 47 patients who had both CYP2D6 gene polymorphism analyses and tramadol and it's metabolites measured were analyzed. The development of a systemic inflammation was assessed based on preoperative levels of CRP, PCT, leukocyte count, plasma cholinesterase activity (ChE), arterial blood gas, and postoperative clinical indicators. Postoperatively, patients received $100 \mathrm{mg}$ of tramadol IV. every 6 hours for the next 24 hours. Tramadol, ODT and NDT levels were determined 1, 2 and 4 hours after the first dose, and before the second, third and fifth doses of tramadol by high-performance liquid chromatography. Pain was assessed before and 30 minutes after tramadol administrations.

Results: According to CYP2D6 polymorphism there were 2 poor (PM), 22 intermediate (IM), 22 extensive (EM) and 1 ultrafast metabolizer (UM). Tramadol effectively reduced pain in all patients except PM. The highest levels of ODT were measured in the EM and IM, 97.4
(52.5-139) and 51.7 (25.7-93.5) $\mu \mathrm{g} / \mathrm{L}$, respectively $(P=0.03)$. ODT levels in all measurements were higher in EM compared to IM and PM, $P<0.05$. ChE activity of $£ 4244 \mathrm{U} / \mathrm{L}$ was a cut-off value in prediction of systemic inflammation. There was a statistically significant increase in NDT levels in IM and EM patients with low ChE activity (LChE group, $\mathrm{n}=18$ ) compared to patients with normal ChE activity ( $\mathrm{NChE}$ group $\mathrm{n}=25)$. The ranges were from $11.58(4.2-21.9)$ to $59.31(18.5-92.6)$ $\mu \mathrm{g} / \mathrm{L}$ vs. 3.93 (3.5-5.6) to $26.31(17.4-48.9) \mu \mathrm{g} / \mathrm{L}$ in LChE and NChE, respectively. Genetically determined difference in ODT levels between IM and EM no longer existed in states of systemic inflammation in all measurements. Despite these differences, tramadol was effective in patients who were $\mathrm{EM}$ and $\mathrm{IM}$ in reducing pain at all measurement points $(P<0.001)$, but not in 2 patients who were categorized as poor metabolizers $(P>0.05)$.

Conclusion: The results of this study confirm that CYP2D6 polymorphism is a major factor in O-demethylation of tramadol. In the systemic inflammation characterized by low ChE activity there is a significant shift in the tramadol metabolism towards $\mathrm{N}$-demethylation. Further studies should evaluate whether tramadol dose may be reduced in the patients with systemic inflammation.

\section{Reference(s) and grant ackowledgment(s)}

1. VIF2016-MEFOS-15 and VIF 2017-MEFOS-16 supported by Faculty of Medicine, J. J. Strossmayer University of Osijek, Croatia.

\section{6}

Sedation practices made worse by COVID 19

K. Fok'; S. Jarvis'; G. Saville

${ }^{1}$ Critical Care Unit, Royal Cornwall Hospital, Truro, United Kingdom

Correspondence: K. Fok

Intensive Care Medicine Experimental 2020, 8(2): 001256

Introduction: Sedation is an integral practice in intensive care units (ICU). Higher sedation requirements were observed in patients with COVID 19 [1]. However, the negative impact of oversedation in the intensive care setting is well established. Patients are at risk of a higher morbidity, increased length of stay, longer time on mechanical ventilation, increased nosocomial infections, haemodynamic compromise and neurological complications such as delirium to name a few [2-5].

Objectives: This is a retrospective analysis of sedation practice preand during the COVID 19 pandemic at the Royal Cornwall Hospital, a district general hospital in the United Kingdom. IV sedation agents were reviewed for rapid escalation or for exceeding maximum recommended doses.

Methods: All patients receiving continuous IV sedation in January 2020 (pre-COVID 19) and April 2020 (during the COVID 19 pandemic) were screened. Those who had $>24$ hour sedation were included. Their charts were reviewed for breaching the maximum doses according to local protocol or the British National Formulary (Table 1) or increasing $>=50 \%$ in 4 hours. A notes review was carried out around the time of a breach for any documented rationale. The number of adjuvant sedative medications a patient received in the duration of their ICU admission was also recorded. This included oral clonidine, intermittent doses of benzodiazepines (IV and oral), haloperidol and quetiapine.

Table 1. Sedative agents and defined maximum doses
\begin{tabular}{|l|l|}
\hline Sedative Agent & Maximum dose \\
\hline Propofol & $4 \mathrm{mcg} / \mathrm{kg} / \mathrm{hr}$ \\
\hline Alfentanil & $5 \mathrm{mcg} / \mathrm{kg} / \mathrm{hr}$ \\
\hline Remifentanil & $0.5 \mathrm{mcg} / \mathrm{kg} / \mathrm{hr}$ \\
\hline Midazolam & $100 \mathrm{mcg} / \mathrm{kg} / \mathrm{hr}$ \\
\hline Clonidine & $4 \mathrm{mcg} / \mathrm{kg} / \mathrm{hr}$ \\
\hline
\end{tabular}


Results: Prior to the COVID-19 pandemic 6/16 (37.5\%) of patients had a sedation breach. This is compared to $9 / 12(75 \%)$ of patients during the pandemic of which half (6) were patients who did not have COVID 19. Of these, 4/6 (66.7\%) of the non COVID 19 and 5/6 (83.3\%) of the COVID 19 patients had sedation breaches. Breaches recorded were predominantly in exceeding maximum infusion rates. Pre-COVID 19, there were only 2 patients breached for rate increases $>=50 \%$ versus 5 patients who exceeded maximum rate infusions. During- COVID 195 patients recorded rate increases $>=50 \%$ compared to 7 who exceeded maximum infusion rates.

Propofol was the main agent for which sedation breaches occurred in both cohorts -before COVID 19 5/15 (33.3\%) and 8/10 (80\%) during the panedemic. The main reason for edation breaches was difficult ventilation in both cohorts (37.5\% pre CVOID 19 and $42.5 \%$ during). Adjuvant sedative medications were used in 9/16 patients pre- COVID 19 and $9 / 12$ patients during COVID 19. During COVID 19 the patients received 3 or more adjuvant agents 6/9 compared to pre-COVID 19 2/9.

Conclusion: The sedation of patients at this ICU was less compliant to guidance and experienced more sedation infusion rate increases $>=50 \%$ during the COVID 19 pandemic in keeping with experience elsewhere that they had a higher sedation need. However, these patients also received a larger number of adjuvant sedative medications. This could also relate to a change in sedation practices by preemptive concerns about difficult to sedate patients or other factors such as routine ICU medication stock levels.

Although there were fewer sedation breaches pre-COVID 19, over a third of patients still received high doses of sedation in normal times. This suggests more can be done to optimise sedation administration through a multimodal approach which addresses education and ergonomic clinical environments. Recommendations implemented at this centre include: training for staff new to the ICU environment, an easily accessible "sedation user guide" which to highlight common clinical considerations for sedative choice and encourages staff to review why sedation requirement may have changed, a standardised whiteboard to record sedation targets and infusion rates, and once per shift reminders to check sedative infusion rates $A$ review is due in the autumn of 2020 subsequent to the adoption of these practices.

\section{Reference(s) and grant ackowledgment(s)}

1. Hanidziar, D et al. Anaesthesia \& Analgesia 131(1): e40-e41, 2020.

2. Nseir S et al. Critical Care 14(2):R30. (223), 2010.

3. Watson PL et al Critical Care Medicine 36(12):3171-3177, 2008.

4. Pandharipande $P$ et al. Anesthesiology104(1):21-26, 2006.

5. Kollef MH et al. Chest 114(2):541-548, 1998.

\section{8}

\section{Pain analysis in an Intensive Care Unit}

C. Mata Martínez'; C. Sotillo'; J. Cedeño Mora'; G. Alberto' ${ }^{1}$; B. Moreno Rivero ${ }^{1}$; S. Casanova Prieto'; E. Bermejo López ${ }^{1}$; C. Herrera Alonso ${ }^{1}$; C. Diez Saenz'; JE. Guerrero Sanz

${ }^{1}$ Intensive Care Medicine, Gregorio Marañón Hospital, Madrid, Spain

Correspondence: C. Mata Martínez

Intensive Care Medicine Experimental 2020, 8(2): 001258

Introduction: Management of pain in critical patients is one of the most important part of the treatment and it is necessary to improve it in order to get better prognosis.

Objectives: To describe prevalence of pain detection and analgesic prescriptions in patients admitted to an ICU of a terciary hospital.

Methods: Weekly registration for 1 month in 2 consecutive years (2018-2019) of: type of admission, treatment phase, analgesic regimen and assessment of pain through Visual Analogue Scale (VAS), Behaviour Indicators of Pain and Richmond Agitation Sedation Scale (RASS), as appropriate; as well as pharmacological non-pharmacological interventions.

Results: In a total of 184 patients, of which $56.5 \%$ of them were males; respiratory failure $(45.7 \%)$, followed by neurological deterioration (32.6\%) and shock (17.4\%) were main causes for admission. $58.7 \%$ of the patients were awake and able to self-report pain, and the rest were under sedation or non-collaborative. Of all of them, $69 \%$ required mecanical ventilation (MV) (invasive MV $54.9 \%$ and non invasive MV $14.1 \%$ ) and $20.7 \%$ had tracheostomy.

Attending to analgesic regimen, $99.5 \%$ of the patients had established analgesic regimen: $29.3 \%$ of them had a fixed regimen without adjuvant therapy, $13.6 \%$ were only prescribed in case of pain, and $56.5 \%$ had a fixed regimen plus adjuvant therapy during pain episodes. and rescues. $66.8 \%$ of the patients had up to 2 medications for pain and the rest of them, had more than 3 medicationes.

Attending to registration of pain episodes: In every patient it was recorded at least once per day. In $1.6 \%$ it was only registered once a day, in $20.7 \%$ twice a day; in $54.3 \%$ three times per day, and $13.4 \%$ four or more times per day. There was also a explicit reference of that in in $79.3 \%$ of nursery registration during mornings, $78.3 \%$ during afternoon, and $72.3 \%$ during night, as well as in $80 \%$ of medical comments. According to the degree of pain, $44 \%$ of the patients did no show pain $36 \%$ of them showed mild pain (VAS 1-3), 13.5\% showed moderate pain (VAS 4-6) and $6.5 \%$ had episodes of severe pain (VAS> 7). Over $20 \%$ of episodes of moderate or severe pain (VAS> 4), $97 \%$ of them were treated and solved.

Regarding non-pharmacological measures, calm environment was detected in $86 \%$ of the records, adequate light in $97.8 \%$, proper sound in $77.2 \%$ and pleasant temperature in $90.2 \%$ of them. Every patient had postural changes. $81 \%$ of them were movilized to chair, and only $25 \%$ of the patients who required physical therapy, received it. In comparison between the 2 years, differences were only observed in the highest registration of pain episodes by nursing staff $(68 \%$ vs. $85 \%$ p0.03). Attending analgesic regime, the one prescribed just in case of pain, was related to more pain episodes.

Conclusion: We consider pain awareness and registration are well established in our Unit; although it is still needed to minimice episodes of moderate or severe pain and to improve physical therapy in our patients.

\section{Reference(s) and grant ackowledgment(s)}

1. E. Celis-Rodríguez, C. Birchenall, M.A. de la Cal, G. Castorena Arellano, A. Hernández, D. Ceraso, et al. Guía de práctica clínica basada en la evidencia para el manejo de la sedoanalgesia en el paciente adulto críticamente enfermo. Med Intensiva, 37 (2013), pp. 519-574.

2. Barr, G.L. Fraser, K. Puntillo, E.W. Ely, C. Gélinas, J.F. Dasta, et al. Clinical practice guidelines for the management of pain, agitation, and delirium in adult patients in the intensive care unit. Crit Care Med, 41 (2013), pp. 263-306.

\section{1}

Actigraphy in Traumatic Brain Injury patients in the Intensive Care Unit: a feasibility study

M. Saavedra-Mitjans' ; S. Van Der Maren ${ }^{1}$; N. Gosselin'; IS. Cherifa ${ }^{1}$; AJ. Frenette $^{2}$; C. Arbour'; L. Burry ${ }^{3}$; S. Mehta ${ }^{4}$;V. Williams'; F. Bernard ${ }^{5}$; D. Williamson ${ }^{2}$

${ }^{1}$ Research centre, Hôpital du Sacré-Coeur de Montreal, Montréal, Canada, ${ }^{2}$ Pharmacy, Hôpital du Sacré-Coeur de Montreal, Montréal, Canada; ${ }^{3}$ Pharmacy, Mount Sinai Hospital, Toronto, Canada; ${ }^{4}$ Critical care, Mount Sinai Hospital, Toronto, Canada; ${ }^{5}$ Critical care, Hôpital du Sacré-Coeur de Montreal, Montréal, Canada

Correspondence: M. Saavedra-Mitjans

Intensive Care Medicine Experimental 2020, 8(2): 001311

Introduction: Agitation is a common behavior in intensive care patients following acute traumatic brain injury (TBI), which can prolong recovery of TBI patients due to delayed mechanically ventilation weaning and/or mobilization. Actigraphy, which measures rest-wake rhythms using movements with an accelerometer, has been used as an alternative to polysomnography to estimate sleep and to measure physical activity in outpatient settings. In the ICU setting, actigraphy has been used for the same purposes, but more studies are needed as it has only been studied in patients in whom analgosedation has been 
discontinued. Our hypothesis is that actigraphy could be a useful tool to monitor agitation and sleep-wake cycles in TBI patients.

Objectives: The primary study objective was to evaluate the feasibility of using wrist actigraphs in critically ill TBI patients who were receiving analgosedation. The feasibility aims were to measure recruitment rate, to describe actigraphy recording duration, to assess missing activity count per day, and to identify impediments to its use.

Methods: We prospectively recruited adult patients admitted to the ICU of Hôpital du Sacré-Coeur de Montréal with acute TBI and abnormal CT scan within 48 hours of ICU admission. Exclusions criteria included: spinal cord injury, prior history of TBI, major neurological or psychiatric disease, high risk of short-term mortality, expected stay $<48$ hours. Actiwatch-Spectrum actigraphs (Philips/Respironics, Murrysville, PA) were placed in patients' non-paralyzed wrists after ICU admission and study inclusion. Reasons for not using actigraph were documented. Actigraphy data such as the amount of missing data and total recording time were analysed.

Results: Between September 2018 and July 2019, 47 patients were considered eligible and 30 patients were recruited. Median age was 64.5 years (IQR 41.3 ) and $73 \%$ were males. TBls were severe, moderate and mild complex in $30 \%, 43 \%$ and $27 \%$ of cases, respectively. One patient was discharged before actigraph was placed and four patients had less than $24 \mathrm{~h}$ of recorded data. These 5 patients were excluded from analyses. A total of 214 completed days of actigraphy data were recorded. The mean number of completed days per patient was 6.5 (SD 5.1), with a minimum of 1 completed day and a maximum of 27 . The mean missing activity count per day was 20.3 minutes (SD 81.7) and $89 \%$ of the days had less than 30 minutes of missing data per day. Actigraphs were removed for surgery or exams outside the ICU, as well as to download recorded data. Electronic interferences between ICU equipment, tissue oedema and neuromuscular blocker use were found as barriers on the use of actigraphy.

Conclusion: This study supports the feasibility of actigraphy use to monitor agitation and sleep-wake cycles in TBI patients during their stay in the ICU. However, specific aspects of analgosedated TBIpatients should be considered when monitoring patients.

\section{Reference(s) and grant ackowledgment(s)}

1. Schwab KE, To AQ, Chang J, Ronish B, Needham DM, Martin JL, et al. Actigraphy to Measure Physical Activity in the Intensive Care Unit: A Systematic Review. J Intensive Care Med. 2019:885066619863654.

2. Nichols JF, Morgan CG, Sarkin JA, Sallis JF, Calfas KJ. Validity, reliability, and calibration of the Tritrac accelerometer as a measure of physical activity. Med Sci Sports Exerc. 1999;31 (6):908-12.

3. Van de Water AT, Holmes A, Hurley DA. Objective measurements of sleep for non-laboratory settings as alternatives to polysomnography-a systematic review. J Sleep Res. 2011;20(1 Pt 2):183-200.

4. Reznik ME, Schmidt JM, Mahta A, Agarwal S, Roh DJ, Park S, et al. Agitation After Subarachnoid Hemorrhage: A Frequent Omen of Hospital Complications Associated with Worse Outcomes. Neurocrit Care. 2017;26(3):428-35.

5. Schwab KE, Ronish B, Needham DM, To AQ, Martin JL, Kamdar BB. Actigraphy to Evaluate Sleep in the Intensive Care Unit. A Systematic Review. Ann Am Thorac Soc. 2018;15(9):1075-82.

6. M. Saavedra-Mitjans is supported by a Fundación Alfonso Martín Escudero research scholarships in universities or centres abroad.

7. D. Williamson is supported by a Fonds de recherche du Québec - Santé (FRQ-S) clinical scientist career grant.

\section{5}

First report from SEDACONDA - A phase III multicenter randomized controlled trial evaluating the efficacy and safety of isoflurane via AnaConDa for sedation in invasively ventilated patients

A. Meiser ; T. Volk' ; J. Wallenborn²; U. Günther ${ }^{3}$;. Becher ${ }^{4}$; H. Bracht ${ }^{5}$; K. Schwarzkopf ${ }^{6}$; A. Faltlhauser ${ }^{7}$; R. Knafelj ${ }^{8}$; S. Thal ${ }^{9}$; M. Bellgardt ${ }^{10}$; P. Sackey ${ }^{11}$

${ }^{1}$ Klinik für Anästhesiologie, Intensivmedizin und Schmerztherapie, Saarland University Hospital, Homburg, Germany; ${ }^{2}$ Anästhesie und inten- sivmedizin, Helios Klinikum Aue, Aue, Germany; ${ }^{3}$ Universitätsklinik für anästhesiologie/intensiv-/notfallmedizin/schmerztherapie, Hospital Oldenburg, Oldenburg, Germany; ${ }^{4}$ Klinik für anästhesiologie und operative intenismedizin, Universitätsklinikum Schleswig-Holstein, Campus Kiel, Kiel, Germany; ${ }^{5}$ Klinik für anästhesiologie, University Hospital Of Ulm, Ulm, Germany; ${ }^{6}$ Anästhesiologie und intensivmedizin, Klinikum Saarbrücken, Saarbrücken, Germany; ${ }^{7}$ Medizinische klinik i, Weiden Clinic, Weiden in der Oberpfalz, Germany; ${ }^{8}$ Univesity medical center Ijubljana, MICU, Ljubljana Slovenia; ${ }^{9}$ Klinik für anästhesiologie, Witten/Herdecke University, Witten, Germany; ${ }^{10}$ Anesthesiology, Catholic Hospital Bochum - St. Josef-Hospital, Bochum, Germany; ${ }^{11}$ Chief medical officer, sedana medical ab vendevägen 87, 13282 danderyd, Karolinska Institute, Department of physiology and pharmacology, Stockholm, Sweden

Correspondence: A. Meiser

Intensive Care Medicine Experimental 2020, 8(2): 001315

Introduction: Patients in need for sedation during mechanical ventilation are at risk for adverse effects of currently used intravenous sedatives, such as long and unpredictable wake-up times, development of delirium, propofol infusion syndrome or life-threatening bradycardia. A number of publications and increasing clinical use indicate that inhaled isoflurane for sedation may be a good option. This study was performed with the aim to evaluate the efficacy and safety of isoflurane via the AnaConDa (SEDACONDA).

Objectives: To compare the efficacy and safety of SEDACONDA with propofol as the sole sedative regimen for up to 48 hours in invasively ventilated patients in a phase III randomized controlled registration trial.

Methods: After ethical approval (Ärztekammer des Saarlandes, 11/17), German and Slovenian competent authority approval and trial registration (Eudra CT\#: 2016-004551-67), 300 adult invasively ventilated patients with clinical need for sedation were recruited in 23 study sites in Germany and Slovenia. Written informed consent was obtained from the patient or legal representative according to national laws and regulations. Patients were randomized to either SEDACONDA (Sedana Medical, Danderyd, Sweden) or intravenous propofol, until extubation or for up to $48+/-6$ hours, whichever was first. Sedation efficacy was assessed as the proportion of time within RASS- 1 to RASS -4 without rescue sedation (primary outcome measure). Wake-up times after stop of sedation, opioid dosing over time, percentage of time with spontaneous breathing, ventilator-free and ICU-free days in the first 30 days, adverse events and 30 day mortality were also monitored. For the primary outcome, the non-inferiority margin for isoflurane was predefined to $15 \%$ relative the mean proportion of time in target RASS for propofol.

Results: Between July 2017 and January 2020, 301 patients were included. The percentage of time patients were sedated in the target RASS range without rescue sedation was similar for the SEDACONDA and propofol groups (mean percentage of time [CI] 90.7 [86.8-94.6\%] vs $91.1[87.2,95.0])$. Thus, the lower CI of SEDACONDA was above the non-inferiority margin (77.5\%). Secondary outcome measures are under statistical evaluation and will be presented during the congress. Conclusion: The preliminary results indicate that SEDACONDA is efficacious as a primary sole sedative, in the same efficacy range as propofol. Further evaluation of study data and future studies will answer clinically important questions regarding other effects of isoflurane for long-term sedation of critically ill patients.

\section{8}

Experienced Delirium in the Intensive Care Unit: A Mixed-methods Study

K. la Cour '; CB. Mortensen '; LM. Poulsen ${ }^{1}$; M. Oxenbøll-Collet ${ }^{2}$; I. Egerod ${ }^{3}$; O. Mathiesen ${ }^{1}$; N. Andersen-Ranberg'

${ }^{1}$ Department of anaesthesiology, intensive care unit, Zealand University Hospital, Køge, Denmark; ${ }^{2}$ Department of anaesthesiology, intensive care unit, Rigshospitalet, Copenhagen, Denmark; ${ }^{3}$ Department of neuroanaesthesiolgoy, Rigshospitalet, København, Denmark

Correspondence: $\mathrm{K}$. la Cour

Intensive Care Medicine Experimental 2020, 8(2): 001438 
Introduction: Delirium is under-recognized, misdiagnosed and undertreated. Research focusing on associations, prevention and treatment of delirium has increased tremendously in the last decade, but still little is known about patient's experience of delirium. Up to $80 \%$ of intensive care unit (ICU) survivors remember their delirium. Delirium has been described as highly unpleasant and distressing with vivid hallucinations and scary delusions. Delirium has been linked to subsequent cognitive dysfunction, psychological sequelae and posttraumatic stress disorder. Knowledge on how delirium is perceived by patients has the potential to improve recognition, management and treatment of delirium.

Objectives: This study investigates how ICU patients recall and experience delirium. We aim to identify predictors of delirium recall and interventions that might reduce distress and symptom severity related to delirium. We aim to understand recollections of delirium and thereby highlight the importance of early recognition and guide future management to relieve patient distress related to delirium.

Methods: This study has a mixed-methods design involving both quantitative measurements and qualitative assessment. Subjects were adult critically ill patients that were CAM-ICU positive during ICU stay. We conducted interviews after ICU discharge when patients were delirium-free assessed by 'rapid clinical test for delirium' (4AT). The interviews where semi-structured and took place at the general ward or patient home. Interviewees used an interview-guide to gather detailed information on recall, experiences and various symptoms related to ICU delirium. The interviews were transcribed and thematically analysed by the research team.

Results: To date sixteen of twenty-five patients have been interviewed. A univariate analysis comparing patients with and without memory of delirium using background characteristics as co-variates (age, admission type, SMS-ICU and delirium motor subtype) will be performed. At present, an $81 \%$ of participants remember some part of their delirium during their ICU stay. Fourteen out of sixteen participants found their delirium experience to be very distressing, including two patients who did not remember their ICU stay at all. Twelve participants remembered being confused/disoriented, eleven remembered sleep disturbance and nine had one or more clear memories of hallucinations and/or delusions. Patients tend to experience delirium as a mix of fact and fiction where realistic events merge with delusions and frightening hallucinations. Final results of the thematic and statistical analysis will be presented at the congress.

Conclusion: The conclusion will be presented at the congress.

\section{Reference(s) and grant ackowledgment(s)}

1. Ely EW, Siegel MD, Inouye SK. Delirium in the intensive care unit: an underrecognized syndrome of organ dysfunction. Semin Respir Crit Care Med. 2001;22(2):115-126

2. Van Rompaey B, Van Hoof A, van Bogaert P, Timmermans O, Dilles T. The patient's perception of a delirium: A qualitative research in a Belgian intensive care unit. Intensive \& critical care nursing. 2016;32:66-74.

3. Breitbart W, Gibson C, Tremblay A. The delirium experience: delirium recall and delirium-related distress in hospitalized patients with cancer, their spouses/caregivers, and their nurses. Psychosomatics. 2002;43(3):183-194.

4. O'Malley G, Leonard M, Meagher D, O'Keeffe ST. The delirium experience: a review. Journal of psychosomatic research. 2008;65(3):223-228.

5. Pandharipande PP, Girard TD, Jackson JC, et al. Long-term cognitive impairment after critical illness. The New England journal of medicine. 2013:369(14):1306-1316.

6. Innovation Fund Denmark.
001503

Impact of the opioid use in the ICU in patients with ARDS secondary to SARS-CoV-2 in analgesic treatment at hospital discharge

M. Pérez Torres ${ }^{1}$; C. Arenas Mazarrota ; B. Castiñeiras Amor'; R. Fernández Tobar'; M. Carretero Rodrigo'; MA. Romera Ortega'; C. Chamorro Jambrina

${ }^{1}$ Intensive Care Unit, Puerta de Hierro Majadahonda University Hospital, Majadahonda, Spain

Correspondence: M. Pérez Torres

Intensive Care Medicine Experimental 2020, 8(2): 001503

Introduction: Administration of opioids to patients during hospitalization can induce its chronic consumption. Analgo-sedation strategies based on 1st analgesia, 2nd sedation, can cause the administration of high doses of opioids.

Objectives: Assess the impact of opioid use during ICU admission in patients mechanically ventilated for ARDS secondary to SARS-CoV-2, as well as factors modifiable in their discharge treatment in this subgroup of patients.

Methods: Prospective study approved by the Hospital's Ethics and Research Committee. This includes all patients admitted to the ICU between March and May 2020 for ARDS secondary to SARS-CoV-2 who were discharged from the ICU and subsequently from the hospital. Demographic factors and clinical data related to admission to the ICU and hospitalization were analyzed. The cumulative dose of opiates is expressed in $\mathrm{mg}$ of iv morphine $(10 \mathrm{mg}$ morphine iv $=100 \mu \mathrm{g}$ fentanyl iv $=100 \mu \mathrm{g}$ remifentanil $=20 \mathrm{mg}$ oral methadone). Data expressed in mean ( \pm standard deviation), median (IQ25-IQ75) or with frequency and percentage. To compare means, the Mann-Whitney $\mathrm{U}$ test was used.

Results: 54 patients are included, 41 males, with mean age 62 years $( \pm 12$ ), stay included median ICU of 13 days (8-25) and 14 days (8-20) in the hospitalization. APACHE II $16.9( \pm 4.6)$, SOFA at income $6.6( \pm$ 2.1). No patient received opioids prior to admission. In ICU, patients received continuous infusion opioids for a median of 216 hours (133348), and a cumulative median dose of $2910 \mathrm{mg}$ (969-7892). At discharge from the ICU, in 16 patients (30\%), they prescribed opiates ( 6 iv morphine if needed, $5 \mathrm{IV}$ prescribed morphine and 5 transdermal fentanyl). Four patients were discharged from the hospital with opiates, all with fentanyl transdermal already prescribed for discharge from the ICU. The average cumulative dose of opiates administered in ICU in patients discharged from the hospital with opiates was $12,450 \mathrm{mg}$, and $4,883 \mathrm{mg}$ in those without opiate prescribed at discharge ( $U$ of Mann-Whitney 45.00, $p=0.07$ ).

Conclusion: $7 \%$ of survivors were discharged from the hospital with opiates, all with transdermal fentanyl. In $80 \%$ of patients discharged from the ICU with fentanyl transdermal, this was maintained at hospital discharge. A study with a larger number of patients is necessary to assess whether the total dose of opiates during ICU admission influences their maintenance at hospital discharge.

\section{5}

Factors associated with chronic opioid use in patients admitted to the ICU for ARDS secondary to SARS-CoV-2

M. Pérez Torres' ${ }^{1}$; C. Arenas Mazarrota'; B. Castiñeiras Amor'; R. Fernández Tobar' $^{1}$ E. Bueso-Inchausti García'; MA. Romera Ortega ${ }^{1}$; C. Chamorro Jambrina

${ }^{1}$ Intensive Care Unit, Puerta de Hierro Majadahonda University Hospital, Majadahonda, Spain

Correspondence: M. Pérez Torres

Intensive Care Medicine Experimental 2020, 8(2): 001545

Introduction: Administration of opioids to patients during hospitalization can induce its chronicconsumption. Analgo-sedation strategies based on 1st analgesia, 2nd sedation,can cause the administration of high doses of opioids.

Objectives: Assess the impact of opioid use during ICU admission in mechanically ventilated patients with ARDS secondary to SARSCoV-2. In addition, we studied the presence of chronic analgesic 
consumption, the quality of life perceived three months after hospital discharge, and its possible relationship with the administration of opioids during admission to the ICU.

Methods: Prospective study approved by the Hospital's Ethics and Research Committee. This includes all patients admitted to the ICU between March and May 2020 for ARDS secondary to SARS-CoV-2 who were discharged from the ICU and subsequently from the hospital. Demographic factors and clinical data related to admission to the ICU and hospital ward are analyzed. The cumulative dose of opioids is expressed in $\mathrm{mg}$ of iv morphine (10 $\mathrm{mg}$ iv morphine $=100 \mu \mathrm{g}$ iv fentanyl $=100 \mu \mathrm{g}$ remifentanil $=20 \mathrm{mg}$ oral methadone). Three months after hospital discharge, a phone call is made to assess the chronic consumption of analgesics drugs, assess the perceived quality of life and the existence or not of chronic pain. Information is collected on the usual analgesic treatment and will be verified by reviewing the electronic medical record and MUP (single prescription module in the Community of Madrid). For the evaluation of quality of life, the Euroqol EQ-5D questionnaire will be used. In patients who answer $>1$ to item number 4 of this questionnaire (existence of pain), the evaluation of pain will be complemented with the Latineen index. Data expressed as mean ( \pm standard deviation), median (IQ25-IQ75) or with frequency and $\%$. The Mann-Whitney $U$ test was used to compare means.

Results: 32 patients are included, $69 \%$ male, with a mean age of 62 years ( \pm 11 ), median ICU stay of 9 days (7-16) and 11 days (7-14) in hospitalization. APACHE II $16.8( \pm 4)$, SOFA at income $6.8( \pm 2.1)$. No patient received opioids prior to admission. In the ICU, patients received continuous infusion opiates for a median of 170 hours (116223), and a median cumulative dose of $1447 \mathrm{mg}$ (672-4960). At discharge from the ICU, opiates were prescribed in 6 patients (19\%) (3 prescribed iv morphine and 3 iv morphine if needed). No patient was discharged from the hospital with opioid treatment. At 3 months (phone call made $93 \pm 2$ days after hospital discharge), only 1 patient received opioid treatment with tramadol, while $14(44 \%)$ received non-opioid analgesics (paracetamol, metamizole and/or NSAIDs). The mean cumulative dose of opioids administered in the ICU in patients who received analgesics 3 months after hospital discharge was $4141 \mathrm{mg}$, and $2416 \mathrm{mg}$ in those who had no chronic use (MannWhitney $\mathrm{U} 94.00, \mathrm{p}=0.22$ ). The results of the EQ-5D questionnaire are shown in Table 1, as well as the relationship between the average doses of opiads consumed depending on the scores on quality of life. $50 \%$ of patients reported some pain or discomfort 3 months after hospital discharge (EQ-5D pain $>1$ ); in them, the mean Latineen index was $5.9( \pm 4), 56 \%$ consumed analgesics but only 1 patient consumed opioid analgesia.

Table 1

\begin{tabular}{|c|c|c|c|c|}
\hline \multirow{3}{*}{$\begin{array}{l}\text { Euroqol EQ- } \\
5 \mathrm{D}\end{array}$} & \multirow{2}{*}{\multicolumn{2}{|c|}{ Scores $\mathrm{n}(\%)$}} & \multirow{3}{*}{ in ICU } & \\
\hline & & & & \multirow{2}{*}{$\begin{array}{l}\text { Mann-Whitney U } \\
\text { test (p) }\end{array}$} \\
\hline & & & & \\
\hline $\begin{array}{l}\text { EQ-5D } \\
\text { Mobility }\end{array}$ & $\begin{array}{l}1 \\
>1\end{array}$ & $\begin{array}{l}25(78 \%) \\
7(22 \%)\end{array}$ & $\begin{array}{l}2720 \mathrm{mg} \\
4780 \mathrm{mg}\end{array}$ & $57,00(0.16)$ \\
\hline EQ-5D Cares & $\begin{array}{l}1 \\
>1\end{array}$ & $\begin{array}{l}32 \\
(100 \%)\end{array}$ & $\begin{array}{l}3171 \mathrm{mg} \\
-\end{array}$ & - \\
\hline $\begin{array}{l}\text { EQ-5D } \\
\text { Activities }\end{array}$ & $\begin{array}{l}1 \\
>1\end{array}$ & $\begin{array}{l}21(66 \%) \\
11(34 \%)\end{array}$ & $\begin{array}{l}2571 \mathrm{mg} \\
4316 \mathrm{mg}\end{array}$ & $90,00(0.31)$ \\
\hline EQ-5D Pain & $\begin{array}{l}1 \\
>1\end{array}$ & $\begin{array}{l}16(50 \%) \\
16(50 \%)\end{array}$ & $\begin{array}{l}2257 \mathrm{mg} \\
4084 \mathrm{mg}\end{array}$ & $94,00(0,20)$ \\
\hline $\begin{array}{l}\text { EQ-5D } \\
\text { Anxiety }\end{array}$ & $\begin{array}{l}1 \\
>1\end{array}$ & $\begin{array}{l}19(59 \%) \\
13(41 \%)\end{array}$ & $\begin{array}{l}2546 \mathrm{mg} \\
4083 \mathrm{mg}\end{array}$ & $100,00(0,36)$ \\
\hline
\end{tabular}

Conclusion: There is no relationship between the doses of opioids administered in the ICU and their maintenance at hospital discharge or chronic consumption. Neither is there a relationship with different aspects of perceived quality of life at 3 months or with the existence of chronic pain. However, a study with a larger number of patients is necessary to adequately assess these findings.

001557

High doses of propofol and isoflurane in Covid-19 patients with and without ECMO - First clinical experiences

J. Speltz' ; A. Kermad'; D. Guy ${ }^{2}$; M. Thilo ${ }^{1}$; T. Volk' ; PM. Lepper ${ }^{2}$; A. Meiser ${ }^{1}$

${ }^{1}$ Klinik für anästhesiologie, intensivmedizin und schmerztherapie, Saarland University Hospital, Homburg, Germany; ${ }^{2}$ Internal medicine - pneumology, allergology and intensive care medicine, Saarland University Hospital, Homburg, Germany

Correspondence: J. Speltz

Intensive Care Medicine Experimental 2020, 8(2): 001557

Introduction: Large numbers of Covid-19 patients simultaneously needed invasive mechanical ventilation in many countries, leading to shortages of ICU beds and intravenous sedatives [1,2]. Some report that ventilated CoV19 patients need high doses of sedatives and neuromuscular blocking agents (NMBA) [3]. For those patients, inhaled sedation with isoflurane (ISO) applied with the AnaConDa system (Sedana Medical, Danderyd, Sweden) may be beneficial $[4,5]$.

Objectives: We investigated dosing of propofol (PRO) and ISO, in Covid-19 patients in need for deep sedation with and without extracorporeal membrane oxygenation (ECMO) treatment.

Methods: In a chart review, we analysed the patients' needs of PRO, ISO, use of co-sedatives, NMBAs, opioids, documented sedation scores (Richmond Agitation Sedation Scale, RASS), minute ventilation (MV) and ECMO treatment during the first 30 days of treatment in our ICU as long as the patients were in need for deep sedation. Sedation days were classified according to the sedative used (PRO, ISO, or both). All parameters were averaged on a daily basis, and compared between PRO and ISO days with Student's t-Tests for parametric data, or $\mathrm{Chi}^{2}$-tests for frequencies.

Results: 19 consecutive Covid-19 patients, aged $64 \pm 10$ years, admitted to the ICU with acute respiratory failure in need for invasive mechanical ventilation and deep sedation were evaluated. Eight patients had been sedated with isoflurane during $7.1 \pm 7.7$ days, all but one had received propofol on $9.1 \pm 8.2$ days. 9 patients received ECMO treatment for $14 \pm 4$ days during this time.

In total, 305 sedation days were recorded, of which PRO had been used on 164 days, and ISO on 100 days. On 41 days, both drugs had been used. These days were excluded from further analysis.

During PRO days, high doses of PRO were recorded. PRO infusion rates (IR) were $8.9 \pm 2.8 \mathrm{ml} / \mathrm{h}$ corresponding to $214 \pm 67 \mathrm{ml} / 24 \mathrm{~h}$. On $24 \%$ of PRO days, mean doses higher than $3.5 \mathrm{mg} / \mathrm{h} / \mathrm{kg}$ predicted body weight (PBW, $22^{*}$ height $[\mathrm{m}]^{2}$ ) were used, and on $12 \%$, the recommended maximum dose of $4 \mathrm{mg} / \mathrm{h} / \mathrm{kg}$ PBW was exceeded. Under PRO compared to ISO, NMBAs (PRO vs ISO: $24 \%$ vs $11 \%, \mathrm{P}=0.010$ ) as well as co-sedatives ( $32 \%$ vs $9 \%, P=0.001$, mainly clonidine: $26 \%$ vs $7 \%, \mathrm{P}=0.001$ ) were used more frequently and sufentanil doses were higher ( $5.7 \pm 1.2$ vs $4.0 \pm 1.2 \mathrm{~mL} / \mathrm{h}, \mathrm{P}=0.029)$. RASS scores, as judged by nursing staff, indicated deeper sedation under ISO $(-2.9 \pm 1.4$ vs $-3.5 \pm 0.9, \mathrm{P}=0.001)$.

ISO-IR were $4.8 \pm 2.2 \mathrm{ml} / \mathrm{h}$ corresponding to $115 \pm 53 \mathrm{ml} / 24 \mathrm{~h}$ with a mean end-tidal concentration (ET) of $1.07 \pm 0.37 \mathrm{Vol} \%$. ET correlated poorly with the IR, negatively with the MV, and best with the ratio IR divided by MV. As the MV during ECMO is reduced, IR were significantly lower during ECMO days ( $3.5 \pm 1.4 \mathrm{vs} 7.1 \pm 3.1 \mathrm{ml} / \mathrm{h}, \mathrm{P}=0.001)$.

Conclusion: Inhaled sedation with isoflurane is an interesting alternative to propofol in invasively ventilated CoV19 patients in need for deep sedation. High doses of both drugs are needed. Propofol use was associated with polypharmacy, and higher doses of opioids. Isoflurane 
consumption is reduced under ECMO treatment. These data may guide stockpiling of sedatives in the future.

\section{Reference(s) and grant ackowledgment(s)}

1. 5. Jabaudon M, Boucher P, Imhoff E et al. Sevoflurane for Sedation in Acute Respiratory Distresss Syndrome. A Randomized Controlled Pilot Study. American Journal of Respiratory and Critical Care Medicine. 2016; 195:792-800.

2. 4. Ferrando C, Aguilar G, Piqueras L et al. Sevoflurane, but not propofol, reduces the lung inflammatory response and improves oxygenation in an acute respiratory distress syndrome model: A randomised laboratory study. Eur J Anaesthesiol. 2013:30(8):455-63.

3. 3. Jerath A, Ferguson ND, Cuthbertson B. Inhalational volatile-based sedation for COVID-19 pneumonia and ARDS [Internet]. Intensive Care Medicine. Springer Berlin Heidelberg; 2020. Available from: https://doi.org/10.1007/ s00134-020-06154-8.

4. 2. Guarascio F. EU scrambles to buy intensive care drugs to tackle COVID shortages. Reuters. July 8, 2020. Available from: https://www.reuters.com/ article/us-health-coronavirus-eu-patients/eu-scrambles-to-buy-intensivecare-drugs-to-tackle-covid-shortages-idUSKBN2492D5.

5. 1. FDA Drug Shortages. Current and Resolved Drug Shortages and Discontinuations Reported to FDA. April 10, 2020. Available from: https://www. accessdata.fda.gov/scripts/drugshortages/dsp_ActivelngredientDetails. $\mathrm{cfm} ? \mathrm{Al}=$ Propofollnjectable Emulsion\&st $=c$.

\section{3}

Withdrawal Syndrome in the Intensive Care Unit

MY. Pektezel' ; S. ÖCall'; IT. Geldigitti'; M. Yildirim'; G. Tok ${ }^{1}$; B. Erdemir ${ }^{1}$; B. Halacli'; E. Ortac Ersoy'; A. Topeli

${ }^{1}$ Department of internal medicine, Division of intensive care medicine, Hacettepe University Faculty of Medicine, Ankara, Turkey

Correspondence: M.Y. Pektezel

Intensive Care Medicine Experimental 2020, 8(2): 001443

Introduction: Withdrawal Syndrome (WS) is a common problem in the intensive care unit (ICU). Recognizing WS could be difficult due to lack of a sensitive tool avaliable at the bedside in adult ICUs. Neuropsychiatric disorders accompanying patients being weaned from sedo-analgesia is another crucial point challenging WS diagnosis.

Objectives: We aimed to validate Withdrawal Assessment Tool-1 (WAT-1) in terms of detecting WS in adult ICU.

Methods: Patients $>18$ years old admitted to Hacettepe University Medical ICU between March 2019 and March 2020, who were intubated and received sedo-analgesics for $>72$ hours, were prospectively evaluated. Patients with neuro-psychiatric disorders were excluded. WS was defined according to DSM-5 criteria. Both WAT- 1 and DSM-5 assessments were performed by a neurologist. Primary outcome was to define the correlation between WAT-1 and DSM- 5 with respect to WS diagnosis whereas secondary outcome was to compare patients with WS with those without WS.

Results: WS occured in $28(70 \%)$ out a total of 40 patients included in the study. Patient characteristics are seen in Table. All patients survived. Sensitivity and specificity $(95 \% \mathrm{Cl})$ of WAT-1 were found to be $0.78(0.59-0.91)$ and $1.00(0.70-1.00)$, respectively. Age ( $<50$ years) was the only independent variable predicting WS when adjusted for ARDS, pulmonary edema, and midazolam dose (Odd Ratio [95\% Cl]: 8.97 [1.03-77.50], $p=0.04$ )

\begin{tabular}{|c|c|c|c|}
\hline Total, $n=40$ & WS $(+), \mathbf{n}=\mathbf{2 8}$ & WS (-), $n=12$ & p \\
\hline Age* (years) $^{*}$ & $50 \pm 20$ & $67 \pm 18$ & 0.006 \\
\hline Gender (F), n (\%) & $17(61)$ & $6(50)$ & 0.532 \\
\hline SOFA** & $5[0-10]$ & $2[0-7]$ & 0.280 \\
\hline APACHE-II** & $23[8-38]$ & 14 [0-29] & 0.212 \\
\hline Hypertension, n (\%) & $21(75)$ & $12(100)$ & 0.089 \\
\hline Diabetes Mellitus, n (\%) & $18(64)$ & $8(67)$ & 0.880 \\
\hline COPD, n (\%) & $9(32)$ & $6(50)$ & 0.281 \\
\hline Coronary Artery Disease, n (\%) & $6(21)$ & $6(50)$ & 0.070 \\
\hline Chronic Kidney Disease, n (\%) & $6(21)$ & $4(33)$ & 0.421 \\
\hline ARDS, n (\%) & $12(43)$ & O (0) & 0.007 \\
\hline Sepsis, n (\%) & $11(39)$ & $8(67)$ & 0.112 \\
\hline Acute Kidney Injury, n (\%) & $10(36)$ & $4(33)$ & 0.882 \\
\hline Shock, n (\%) & $8(29)$ & $4(33)$ & 0.763 \\
\hline COPD Execerbation, $\mathbf{n}(\%)$ & $7(25)$ & $4(33)$ & 0.587 \\
\hline $\begin{array}{l}\text { Cardiogenic Pulmonary Edema, } \\
\text { n (\%) }\end{array}$ & $2(7)$ & $6(50)$ & 0.002 \\
\hline CRRT, n (\%) & $2(7)$ & $0(0)$ & 0.481 \\
\hline IMV duration*, (day) & $5 \pm 3$ & $5 \pm 2$ & 0.920 \\
\hline Midazolam dose*,$(\mathrm{mg} / \mathrm{h})$ & $2.9 \pm 1.2$ & $4.4 \pm 0.8$ & 0.020 \\
\hline Fentanyl dose*, (mcg/h) & $98 \pm 41$ & $126 \pm 32$ & 0.221 \\
\hline $\begin{array}{l}\text { Noradrenaline dose*, } \\
(\mathrm{mcg} / \mathrm{kg} / \mathrm{min})\end{array}$ & $0.04 \pm 0.04$ & $0.07 \pm 0.03$ & 0.562 \\
\hline Delirium Development, n(\%) & $62(93)$ & $6(26)$ & 0.002 \\
\hline ICU LOS*, (days) & $9 \pm 6$ & $13 \pm 9$ & 0.163 \\
\hline Hospital LOS*, (days) & $13 \pm 7$ & $31 \pm 31$ & 0.02 \\
\hline
\end{tabular}

${ }^{*}$ mean $\pm S D,{ }^{*}$ median[IQR], F: Female

Text in bold shows statistical significance.

Conclusion: WAT-1 has been demonstrated to have a very good sensitivity and specificity to detect WS at the bedside in adult critically-ill patients who received prolonged sedo-analgesics. Age $<50$ years was the only independent variable predicting WS.

\section{Reference(s) and grant ackowledgment(s)}

1. Cammarano WB, Pittet JF, Weitz S, Schlobohm RM, Marks JD. Acute withdrawal syndrome related to the administration of analgesic and sedative medications in adult intensive care unit patients. Crit Care Med 1998; 26(4): 676-84.

2. No grant

\section{5}

Acute pancreatitis in intensive care unit patients: A retrospective study

F. Sequeira' ; L. Linhares' ; D. Castro ${ }^{1}$; C. Roriz ; L. Pereira ${ }^{1}$

${ }^{1}$ Medicina intensiva, Hospital Santo Andre - Hospital Distrital De Leiria, Leiria, Portugal

Correspondence: $F$. Sequeira

Intensive Care Medicine Experimental 2020, 8(2): 001325

Introduction: Acute pancreatitis (AP) is an inflammatory condition, the worldwide incidence is 13 to $45 / 100,000$. Severe acute pancreatitis (SAP) is associated with serious morbidity and mortality and these patients require intensive care unit (ICU) admission. Factors predicting mortality and morbidity need to be identified for improving outcome. Objectives: The aim of our study was evaluation of demographic and outcome of patients with SAP admitted to our ICU from June 2018 to June 2020. The secondary objective was to identify the factors responsible for adverse outcome.

Methods: Retrospective analysis of patients with SAP admitted to the ICU between june 2018 and june 2020.

Results: Eleven SAP patients were admitted to ICU (54.5\% female, mean age 64.3). 
The most common aetiologie were gallstones $(72.7 \%)$ and hypertriglyceridemia (18.2\%).

The severity of AP as depicted by Ranson's score showed mean score of 3.2 at admission, and 3.5 after 48 hours. On admission to ICU, the median Acute Physiology and Chronic Health Evaluation (APACHE) II score was 22.3 and the median Computed Tomography Severity Index (CTSI) was 3 (range 2-4).

During the ICU stay, $72 \%$ of patients required mechanical ventilatory support, ranging in duration from 1 to 35 days; $90.9 \%$ developed acute kidney injury (AKI) and $18.2 \%$ required continuous renal replacement (CRR); 72.7\% needed vasopressor support.

A significant number of patients (54.5\%) tolerated early enteral nutrition via either a nasogastric tube or a nasojejunal tube, while total parenteral nutrition (TPN) was used in $18.2 \%$ of patients.

The disease course was complicated with pancreatic abscess (45.5\%), abdominal compartment syndrome (18.2\%) and pseudo cyst formation (9\%). Surgical intervention was required in $50 \%$ of these patients. The median length of stay in ICU and hospital were 13 (ranged from 1 to 74 ) and 25 (ranged from 1 to 110) days, respectively.

ICU mortality and hospital mortality were $45 \%$ and $9 \%$, respectively. Mean APACHE II score of these patients was 28.7. All these patients had AKI and two patients who needed RRT died.

Conclusion: Acute pancreatitis remains a challenging disease for intensive care medicine and this retrospective data showed high mortality of $A P$ requiring $I C U$ admission.

Predicting the outcome of ICU patients with AP remains a difficult task despite the availability of scoring systems. In our study, the APACHE II score was higher between non-survivors. The mean of Ranson score in our study population was 3.2 and 3.5 at and after 48 hours of admission, which has a predicted mortality of 11 to $15 \%$, so the predictive value of this scoring system is questionable in our study. Although AKI itself was not an independent risk factor of adverse outcome in our study, patients who need CRR died.

The authors wish to highlight the need to early severity assessment and management for those patients at risk of SAP.

\section{4}

Macrovascular Thrombosis in COVID-19

S. Tetlow'; A. Segiet-Swiecicka²; R. O'sulluvan'; C. Brathwaite-Shirley'; K. Kalb $^{1}$; S. O'halloran'; B. Regan ${ }^{1}$; P. Roberts ${ }^{1}$

'Department of critical care, Queen Elizabeth Hospital, London, United Kingdom; ${ }^{2}$ Department of coronary artery disease and cardiac rehabilitation, National Institute of Cardiology, Warsaw, Poland

Correspondence: S. Tetlow

Intensive Care Medicine Experimental 2020, 8(2): 001204

Introduction: Macrovascular thrombi are a common complication of COVID-19. Risk factors for COVID-related thrombi are not well understood (1).

Objectives: To examine the incidence of macrovascular thrombi in patients admitted to the intensive care unit (ICU) with COVID-19, and to explore factors associated with thrombi in COVID-19.

Methods: We retrospectively enrolled all patients aged $>18$ admitted to ICU between $1 / 3 / 2020$ and 30/4/2020 with a laboratory-confirmed diagnosis of COVID-19 $(n=93)$. 1 patient was excluded as an incidental diagnosis of COVID-19 admitted to ICU for post-operative monitoring. Data was extracted from health records using a standardised data collection tool. Patients were followed up to $24 / 5 / 20$.

Results: The median age was 57 (IQR 48 - 62), 70 (76.1\%) were male. The population was diverse; 37 (44.6\%) were Caucasian, $26(31.3 \%)$ were Black, 18 (21.7\%) were Asian, 2 (2.4\%) were of mixed ethnicity and in 9 cases ethnicity was not recorded. Median duration of illness prior to hospital admission was 7 (IQR 4 - 9) days.

Cardiovascular risk factors were common; 44 (47.8\%) were hypertensive, $30(32.6 \%)$ were obese and $30(32.6 \%)$ were diabetic. Fourteen $(15.2 \%)$ patients had a cardiovascular condition, $10(10.9 \%)$ had chronic respiratory disease, $13(14.1 \%)$ had a baseline eGFR $<60 \mathrm{ml} /$ min, and 5 (5.4\%) had known cancers. Several patients used coagulation-modifying drugs; 9 (9.8\%) took antiplatelet agents, and one was anticoagulated with warfarin.
All patients received anticoagulation during their admission. Treatment dose anticoagulation was given to 22 (23.9\%), whilst 70 (76.1\%) received only prophylactic anticoagulation. The median duration of COVID-19 symptom onset to thromboprophylaxis initiation was 8 days (IQR 6 - 10).

Macrovascular thrombi were detected in $22(23.9 \%)$ of the population. Of these, 7 (33.3\%) were pulmonary emboli, and 7 (33.3\%) were venous thrombi. There were 2 strokes, 2 arterial occlusions, 1 myocardial infarction, 1 renal vein thrombus and 1 ventricular thrombus (fig 1). During the same period, 8 (8.7\%) patients developed significant haemorrhages; 4 had an upper gastrointestinal bleed, 2 an intracranial haemorrhage, and there was one pulmonary and one urinary tract haemorrhage.

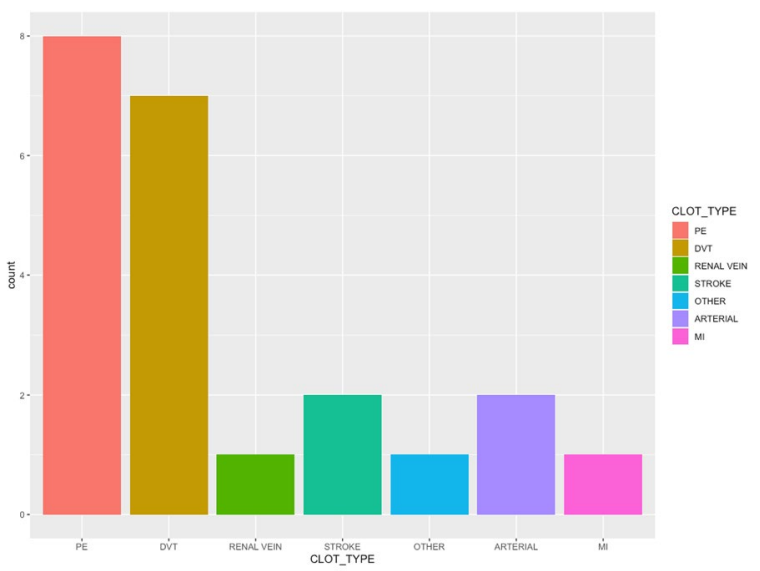

Fig 1: Diagnosed thrombi in the study population

On univariate analysis, there was a non-statistically significant reduction in the incidence of major thrombi in Black (OR 0.336, 95\% Cl $0.085-1.111, p=0.090$ ) and Asian patients (OR $0.231,95 \% \mathrm{Cl} 0.033$ $0.986, p=0.076$ ) compared to Caucasian patients. APTT ratio prolongation $>1.8 \mathrm{x}$ was more frequent in patients not developing thrombi $(p=0.032)$, whilst Fibrinogen levels $<1.8 \mathrm{~g} / \mathrm{L}$ occurred more often with thrombosis $(p=0.015)$.

Conclusion: Critically ill patients with COVID-19 face high incidence of thrombotic complications despite anticoagulation. Low levels of fibrinogen are associated with thrombi. Clinicians must remain vigilant to the development of thrombi even in anticoagulated patients.

\section{Reference(s) and grant ackowledgment(s)}

1. 1: Klok FA, Kruip MJHA, van der Meer NJM, et al. Incidence of thrombotic complications in critically ill ICU patients with COVID-19. Thrombosis Research 2020; 191: 145

2. The authors received no grants or funding for this work.

\section{0}

Blood and blood component transfusion in a tertiary care intensive care unit

R. Sinha' ${ }^{1}$ D. Roxby ${ }^{1}$; M. Teague ${ }^{2}$; P. Hakendorf ${ }^{3}$

${ }^{1}$ Molecular medicine and pathology, Flinders University, Bedford Park, Australia; ${ }^{2}$ Haematology, Flinders Medical Centre, Bedford Park, Australia; ${ }^{3}$ Epidemiology, Flinders Medical Centre, Bedford Park, Australia

Correspondence: $\mathrm{R}$. Sinha

Intensive Care Medicine Experimental 2020, 8(2): 001330

Introduction: Blood transfusions are common in critically ill patients; 30 to $45 \%$ patients receive red cell transfusions of, $10-30 \%$ plasma transfusions and $10-20 \%$ platelet transfusions during their ICU stay. 
Each of the studies that have reported on plasma and platelet use and outcomes in ICU has reported separately for each blood product type. There are limited studies that have reported on the transfusion of red cells, plasma, platelets and cryoprecipitate concurrently in ICU.

Objectives: The primary outcomes of the study were to examine the prevalence of red cells and multicomponent transfusion during ICU stay.

Methods: A retrospective study of 5783 patients enrolled in the INFORM trial including 2332 patients that were admitted at the Flinders Medical Centre ICU between April 2012 and October 2015 was performed. Total red cells (RC) and blood products transfused at any point between ICU admission and discharge were analysed leading to three specific categories - RC only transfusion (RC-only), MCT and non-transfusion (NT). Multicomponent transfusion included RC-MT [RC co-transfusion with plasma (FFP), platelets (PLT) and cryoprecipitate (CRYO) or combination of components without RC (MT). The common types of RC-MCT included RC+FFP, RC+ PLT, RC+FFP+PLT and $\mathrm{RC}+\mathrm{FFP}+\mathrm{PLT}+\mathrm{CRYO}$.

Results: Of the $2726 \mathrm{ICU}$ admissions analysed, $1323(48.5 \%, 95 \% \mathrm{Cl}$ 46.6-50.4) received no transfusion (NT), 660 (24.2\%, 95\% Cl 22.6-25.9) received MCT and $743(27.7 \%, 95 \% \mathrm{Cl} 25.6-28.9)$ received RC-only during ICU stay. Besides trauma (33.7\%) and haematology admissions (31.8\%), half or more than half of the admissions in each clinical groups did not receive a transfusion during ICU stay. RC-MT ranged from $9.2 \%$ to $45.6 \%$ across all clinical groups, highest being in haematology (41.5\%) and gastroenterology (GIT, 32.1\%) admissions (Figure 1). Amongst all types of RC-MT, RC +FFP+PLT (36.1\%) was the commonest type, followed by RC+FFP (27.3\%), RC +PLT (16.5\%) and $\mathrm{RC}+\mathrm{FFP}+\mathrm{PLT}+\mathrm{CRYO}$ (16.5\%). RC-MCT varied across clinical groups, with $61.5 \%$ of metabolic admissions received RC+FFP, 30.8\% of sepsis admissions received $\mathrm{RC}+\mathrm{PLT}$ and $26.3 \%$ of GIT admissions received $\mathrm{RC}+\mathrm{FFP}+\mathrm{PLT}+\mathrm{CRYO}$ (Figure 2).

Fig. 1 \& 2 - Percentage of non-transfused and transfused admissions including transfusedadmissions with types of multi-component transfusion for various admitting ICU diagnoses

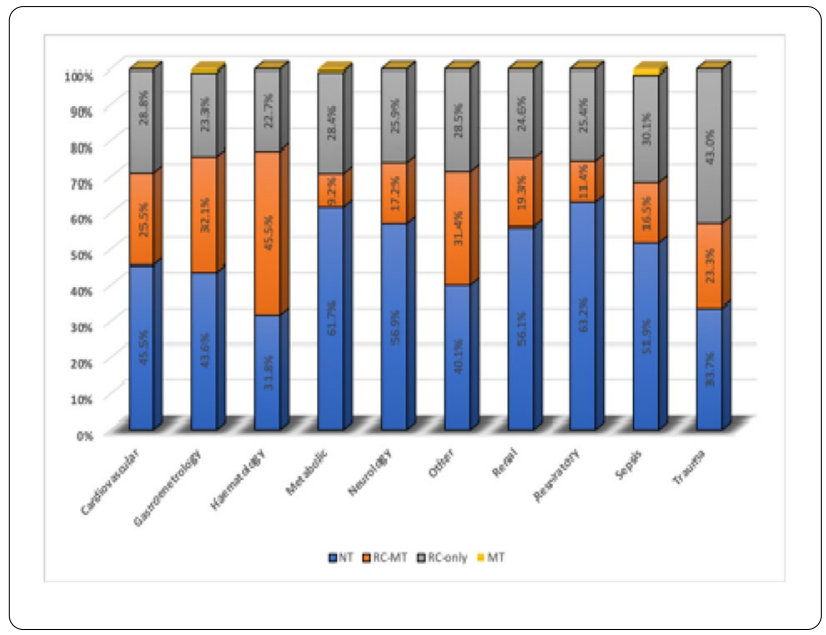

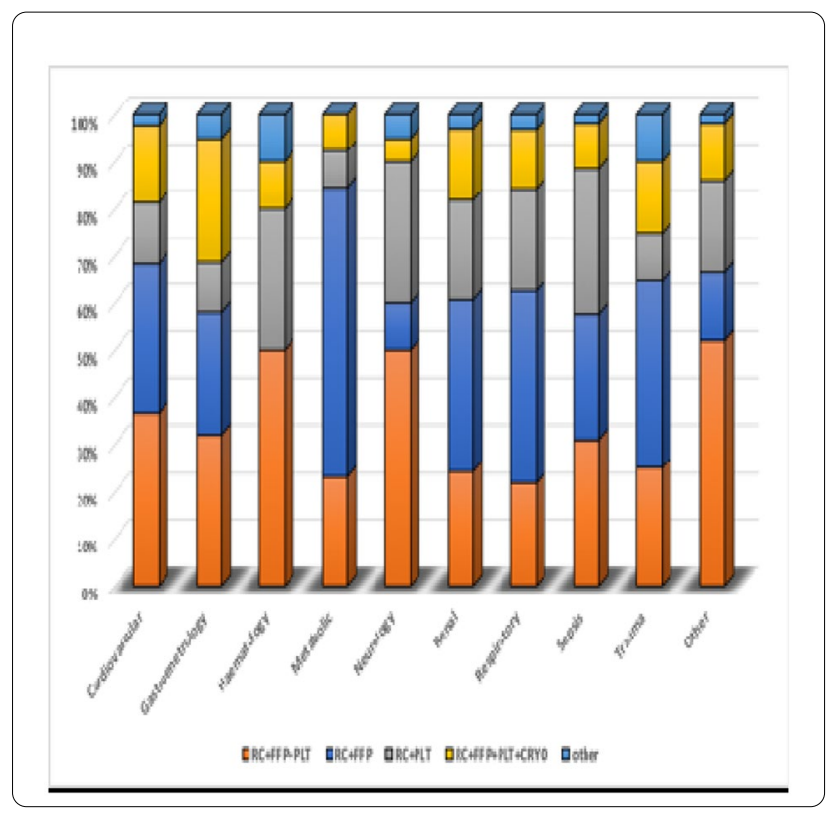

Conclusion: Almost half of ICU admissions were transfused. Fifty percent of those transfused received red cells and the other half received multicomponent transfusion. Multicomponent transfusion including the various types of multicomponent transfusion varied between clinical groups. Further studies are required to study outcomes in patients receiving multicomponent transfusion in ICU.

\section{Reference(s) and grant ackowledgment(s)}

1. Vasu BK, R Sinha, S Verghese. Thrombocytopenia and guideline-evaluated appropriateness of platelet transfusion in a tertiary care intensive care unit 2016;11: 102-9.

2. Stanworth SJ, TS Walsh, RJ Prescott, et al. A national study of plasma use in critical care: clinical indications, dose and effect on prothrombin time. Critical care (London, England) 2011;15: R108-R.

3. auzier F, D Cook, L Griffith, et al. Fresh frozen plasma transfusion in critically ill patients. Crit Care Med 2007;35: 1655-9.

4. Vincent JL, JF Baron, K Reinhart, et al. Anemia and Blood Transfusion in Critically III Patients. JAMA 2002;288: 1499 - 507

5. Corwin H, A Gettinger, R Pearl, et al. The CRIT Study: anemia and blood transfusion in the critically ill - current clinical practice in the United States. Crit Care Med 2004;32: 39 - 52

001379

Coagulation parameters as predictors of mortality in critically ill COVID-19 patients

D. Adrião ${ }^{1}$; R. Neto'; R. Pombal ${ }^{2}$; AL. Rios ${ }^{1}$; H. Gomes². É. Costa'; M.

Carvalho'; P. Castelões

${ }^{1}$ Intensive care department, Centro Hospitalar Vila Nova de Gaia/Espinho, Vila Nova de Gaia, Portugal; ${ }^{2}$ Immunohemotherapy department, Centro Hospitalar Vila Nova de Gaia/Espinho, Vila Nova de Gaia, Portugal

Correspondence: $\mathrm{D}$. Adrião

Intensive Care Medicine Experimental 2020, 8(2): 001379

Introduction: Coronavirus disease 2019 (COVID-19), caused by SARS CoV-2, has recently emerged in Wuhan, China, and rapidly spread worldwide [1]. Apart from respiratory failure, coagulopathy is a common abnormality in COVID-19 patients and is associated with poor prognosis [2, 3]. Regardless of increasing evidence of coagulation dysfunction, the data available for the most severe patients is still scarce.

Objectives: To describe the coagulation parameters of critically ill COVID-19 patients and evaluate its association with mortality.

Methods: Observational retrospective study of coagulation parameters and outcomes of all COVID-19 patients admitted to a polyvalent 
intensive care unit (ICU) of a Portuguese tertiary hospital between March 15th and May 10th 2020.

Results: Thirty-six patients required ICU admission from a total of 264 COVID-19 patients admitted to our hospital. Median age was 62 [58.3; 65.8] years, 23 (63.9\%) were men and median APACHE II score and SAPS II were $18[13.0 ; 23.7]$ and $42[31.0 ; 51.5]$, respectively. Twentynine $(80.6 \%)$ patients had chronic diseases, mainly arterial hypertension $(n=21,58.3 \%)$, diabetes $(n=11,30.6 \%)$, respiratory disease $(n=7,19.4 \%)$ and coronary heart disease $(n=5,13.9 \%)$. Median ICU length of stay was $16[6.8 ; 22.3]$ days and the overall mortality was $25 \%$ $(n=9)$. All patients received at least prophylactic doses of low molecular weight heparin. The coagulation parameters were analyzed from admission to day 14 of ICU stay. No difference in ICU stay was found between survivors and non-survivors ( 17 vs 14 days, $p=0.494$ ) and none of the coagulation parameters at admission were correlated with ICU length of stay. Non-survivors had significantly higher D-dimer levels at admission ( $2.89 \mathrm{vs} 1.47 \mathrm{mg} / \mathrm{mL}, \mathrm{p}=0.04$ ), and longer prothrombin time (PT) and activated partial thromboplastin time (APTT) at day 10 (17.67 vs $16.43 \mathrm{sec}, p=0.016$ and 38.07 vs $33.30 \mathrm{sec}, p=0.046$, respectively). Despite high fibrinogen levels at admission (mean $675 \pm 156 \mathrm{mg} / \mathrm{dL}$ ) and during ICU stay, no statistical significance was found between the two groups. Sepsis-Induced Coagulopathy (SIC) and International Society on Thrombosis and Haemostasis Disseminated Intravascular Coagulation (DIC) scores were significantly higher in non-survivors at day 10 ( 3 vs $2, p=0.006$ and 4 vs $2, p=0.001$ ). Furthermore, $44.4 \%(n=4)$ of non-survivors and $16.6 \%(n=4)$ of survivors met the SIC criteria, but only one (11.1\%) non-survivor and 2 (7.4\%) survivors matched the ISTH DIC score. From these, only one patient actually developed clinical DIC.

Conclusion: At admission D-dimer was positively correlated with mortality. By the late ICU stay, non-survivors revealed significantly prolonged PT and APTT. The occurrence of DIC was rare. It seems that COVID-19 coagulopathy might not be equal to the known sepsis coagulopathy, so DIC diagnostic criteria applied to this specific disease should be reviewed. Further studies are needed to clarify the effective prognosis value of coagulation parameters in critically ill COVID-19 patients.

\section{Reference(s) and grant ackowledgment(s)}

1. 1. Zhou F, YuT, Du R, et al. Clinical course and risk factors for mortality of adult inpatients with COVID-19 in Wuhan, China: a retrospective cohort study. Lancet. 2020;395(10229):1054-1062. https://doi.org/10.1016/s0140 $-6736(20) 30566-3$.

2. 2. Tang N, Li D, Wang X, Sun Z. Abnormal coagulation parameters are associated with poor prognosis in patients with novel coronavirus pneumonia. J Thromb Haemost. 2020;18(4):844-847. https://doi.org/10.1111/jth.14768.

3. 3. Joly, B.S., Siguret, V. \& Veyradier, A. Understanding pathophysiology of hemostasis disorders in critically ill patients with COVID-19. Intensive Care Med (2020). https://doi.org/10.1007/s00134-020-06088-1.

\section{3}

\section{Prevalence of DVT amongst a critically ill population} with COVID-19

A. Neill'; R. Turner'; S. O'sullivan'; B. Marsh²

${ }^{1}$ Critical Care, The Mater Misericordiae University Hospital, Dublin, Ireland; ${ }^{2}$ Anaesthesia, The Mater Misericordiae University Hospital, Dublin, Ireland Correspondence: A. Neill

Intensive Care Medicine Experimental 2020, 8(2): 001383

Introduction: Coronavirus Infectious Disease 2019 (COVID-19) is reported to be associated with a high rate of venous thromboembolism (VTE). Most published reports to date (1-3) are retrospective, do not systematically screen for deep venous thrombosis (DVT) and suggest a higher frequency of pulmonary embolism (PE) than DVT.

Objectives: To determine the rate of DVT in critically ill patients with COVID-19. The study is ongoing and the results of the first 15 patients are reported here.
Methods: We conducted a prospective, observational trial to examine rates of DVT in patients COVID-19 admitted to the critical care unit of a large tertiary hospital. We enrolled consecutive patients with COVID19 confirmed by polymerase chain reaction (PCR) testing. The screening test was compression ultrasound (CUS) of the proximal veins of both legs performed by any of 3 advanced intensive care medicine trainees all of whom had prior experience in CUS for DVT. The leg veins were compressed every $2 \mathrm{~cm}$ from the common femoral vein at the inguinal ligament until the trifurcation in the popliteal fossa. A non-compressible vein was considered positive for DVT. Any positive scans were reviewed by several investigators. Positive results were communicated to the treating physician. Scans were performed every $72 \mathrm{hrs}$ up until 30 days or until the patient left critical care.

The primary outcome was the rate of DVT by CUS.

Results: We enrolled 15 patients with confirmed COVID-19 between 2nd April and 10th May 2020. Baseline characteristics are shown in Table 1.

Table 1.

\begin{tabular}{|l|l|}
\hline Age & 60 \\
\hline Ventilated at admission & $66 \%(10 / 15)$ \\
\hline D-dimer at admission (median) & 3.24 \\
\hline Fibrinogen at admission (median) & 6.25 \\
\hline $\begin{array}{l}\text { Platelet count at admission (median) } \\
\begin{array}{l}\text { Therapeutic anticoagulation at } \\
\text { enrolment }\end{array}\end{array}$ & 235 \\
\hline
\end{tabular}

No patient had a clinical suspicion for DVT prior to enrolment. All patients were on either therapeutic or prophylactic anticoagulation. At the time of study, COVID-19 patients were being treated with a higher dose of or prophylactic enoxaparin than typical critical care patients at a dose of $0.5 \mathrm{mg} / \mathrm{kg}$ twice daily.

We found a single DVT in the popliteal vein of a ventilated patient at time of enrolment to the study. 4 other patients were diagnosed with VTE during the study, 3 with PE and one with an upper limb DVT. Further enrollment is currently halted due to significant drop off in COVID-19 prevalence in Ireland at the time of writing.

Conclusion: In this ongoing study the rate of DVT in critically ill patients was low with only 1 of the 15 patients having a lower limb DVT throughout their critical care stay, though it is notable this occurred in the setting of higher dose prophylaxis.

However, the observed $20 \%$ rate of PE despite this prophylaxis may represent thrombosis in situ or immunothrombosis rather than embolism from a distal venous site.

\section{Reference(s) and grant ackowledgment(s)}

1. Middeldorp S, Coppens M, van Haaps TF, Foppen M, Vlaar AP, Müller MCA et al. Incidence of venous thromboembolism in hospitalized patients with COVID-19. J Thromb Haemost. 2020 May 5; 14888.

2. Helms J, Tacquard C, Severac F, Leonard-Lorant I, Ohana M, Delabranche X, et al. High risk of thrombosis in patients with severe SARS-CoV-2 infection: a multicenter prospective cohort study. Intensive Care Med. 2020 May 4;173:4030-10.

3. Klok FA, Kruip MJHA, van der Meer NJM, Arbous MS, Gommers DAMPJ, Kant $\mathrm{KM}$, et al. Incidence of thrombotic complications in critically ill ICU patients with COVID-19. Thrombosis Research. 2020 Apr 10:1-11.

001410

Novel oral anticoagulants (NOACS): Preoperative use and postoperative hemorrhagic complications in cardiac surgical patients

F. Ampatzidou'; O. Drosos²; N. Mihail'3 ; C. Mavromanolis ${ }^{3}$; M. Ganidou ${ }^{4}$; A. Anastasiadis ${ }^{4}$; T. Asteri ${ }^{5}$; G. Drossos ${ }^{3}$

${ }^{1}$ Cardiothoracic icu, G.Papanikolaou Hospital, Thessaloniki, Greece; ${ }^{2}$ University of cambridge, University of Cambridge, Cambridge, United Kingdom; ${ }^{3}$ Cardiothoracic surgery, G.Papanikolaou Hospital, Thessaloniki, Greece; 
"Blood bank department, General Hospital of Thessaloniki "George Papanikolaou", Thessaloniki, Greece; ${ }^{5}$ Cardiothoracic anesthesia, G.Papanikolaou Hospital, Thessaloniki, Greece

Correspondence: F. Ampatzidou

Intensive Care Medicine Experimental 2020, 8(2): 001410

Introduction: Novel oral anticoagulants (NOACs) represent first-line treatment for venous thromboembolism and prevention of stroke in nonvalvular atrial fibrillation. Bleeding complications have been reported in NOACS users during the postoperative period.

Objectives: Aim of the study is to identify if there is any correlation between postoperative hemorrhagic complications and pre-operative use of novel oral anticoagulants (NOACS) in cardiac surgical patients. Methods: All patients who underwent elective cardiac surgical procedures with the use of cardiopulmonary bypass from January 2019 to March 2020 were evaluated. Patients were divided in 3 categories: Group A consisted of patients with preop use of NOACS : Apixaban(Eliquis), Dabigadran(Pradaxa) and Rivaroxaban (Xarelto). Group B consisted of patients with preop use of vitamin K antagonists and Group C consisted of patients without preop use of any anticoagulant. NOACS were discontinued 3-5 days before surgery. The following perioperative factors were compared: sternal reopening because of hemorrhage, transfusion with at least 1 unit of platelets, transfusion with at least 1 unit of Fresh Frosen Plasma, transfusion with more than 3 units of red blood cells and no transfusion at all. Decisions for transfusion was based on lab results and rotational thromboelastometry ROTEM. Chi square test was used for statistical analysis.

Results: A total of 581 patients were evaluated. First we compared Group A ( $n=41$ - 7 Apixaban,24 Rivaroxaban and 13 Dabigadran) with Group C. Results are shown in table 1

\begin{tabular}{|l|l|l|l|}
\hline & $\mathrm{A}(\mathrm{n}=41)$ & $\mathrm{C}(\mathrm{n}=528)$ & P value \\
\hline Resternotomy & $4(9,8 \%)$ & $19(3,6 \%)$ & 0,07 \\
\hline PLT & $5(12,2)$ & $73(13,8 \%)$ & 0,65 \\
\hline FFP & $7(15,9 \%)$ & $37(7)$ & $\mathbf{0 , 0 3}$ \\
\hline >3 RBC & $8(8,2 \%)$ & $91(17,2 \%)$ & 0,87 \\
\hline No transfusion & $13(29,5 \%)$ & $131(24,8 \%)$ & 0,48 \\
\hline
\end{tabular}

Table 1

Comparison between drugs revealed statistical significant correlation between use of Dabigadran with resternotomy (20/559 -3,6\% versus $3 / 13-23,1 \% \mathrm{p}<0,005)$.

We also compared Groups A and B. Results are shown in table 2.

\begin{tabular}{|l|l|l|l|} 
& $\mathrm{A}(\mathrm{n}=41)$ & $\mathrm{B}(\mathrm{n}=9)$ & P value \\
\hline Resternotomy & $4(9,8 \%)$ & $0(0 \%)$ & 0,33 \\
\hline PLT & $5(12,2)$ & $2(22,2 \%)$ & 0,43 \\
\hline FFP & $7(15,9 \%)$ & $1(11,1 \%)$ & 0,65 \\
\hline >3 RBC & $8(8,2 \%)$ & $1(11,1 \%)$ & 0,55 \\
\hline No transfusion & $13(29,5 \%)$ & $2(22,2 \%)$ & 0,77 \\
\hline
\end{tabular}

Table 2

Conclusion: Preoperative use of NOACS correlates significantly with higher incidence of transfusions with FFP after elective cardiac surgical procedures. Dabigadran use correlates with higher incidence of resternotomy because of hemorrhage. No difference in hemorrhagic complications were found between use of NOACS and use of Vitamin K antagonists.

\section{Reference(s) and grant ackowledgment(s)}

1. Hassan K. Bleeding Complications After Use of Novel Oral Anticoagulants in Patients UndergoingCardiac Surgery Ann Thorac Surg 2018;105:702-8.
001470

Mortality analysis of trauma-related major hemorrhage

in a European regional referral hospital

I. Castelo 1 U. Amaia²; M. Alberto ${ }^{3} ;$ T. Gontzal $^{4}$

${ }^{1}$ Department of anesthesiology and critical care medicine. trauma intensive care unit, Hospital Universitario Cruces, Barakaldo, Spain; ${ }^{2}$ Department of hematology, Hospital Universitario Cruces, Barakaldo, Spain; ${ }^{3}$ Department of anesthesiology and critical care medicine, Hospital Universitario Cruces, Barakaldo, Spain; ${ }^{4}$ Department of anesthesiology and critical care medicine. trauma intensive care unit, Hospital Universitario Cruces, Barakaldo, Spain

Correspondence: I. Castelo

Intensive Care Medicine Experimental 2020, 8(2): 001470

Introduction: Between the ages of 1 and 4 years and the ages of 15 and 34 years, trauma is the single most common cause of death in Europe1. Major uncontrolled hemorrhage after trauma remains the leading cause of preventable death after severe traumatic injury2. Any improvement in the quality of care provided to these patients could have a great impact on healthcare systems, reducing associated medical and social costs.

Objectives: The aim of this study was to determine the epidemiology of trauma-related mayor hemorrhage at our institution and to identify modifiable factors associated with mortality.

Methods: A retrospective analysis of prospectively collected data of adult trauma patients who activated the "Massive Hemorrhage Protocol" (MHP) at Cruces University Hospital (HUC) and received at least one unit of Red Blood Cells (RBC) from 15th February 2017 to 4th September 2019, was conducted. HUC is a trauma regional referral Hospital in the Basque Country (Northern Spain), for an estimated population of 1 million.

Results: A total of 32 patients met the inclusion criteria. All patients were critically injured, with median Injury Severity Score of 34 (IQR 25-43) and with median admission systolic blood pressure of 95 (IQR 70-117) $\mathrm{mmHg}$. Median age was 47 (IQR 38-74) years, and $28 \%$ of the patients were 65 years or older. The proportion of male patients was $59 \%$. Regarding the trauma mechanism, most of our patients presented blunt trauma (up to $84 \%$ ).

Patients received a median of 2 (IQR 0-4) units of Fresh Frozen Plasma (FFP), 5 (IQR 4-9) units of RBC, 0 (IQR 0-1) units of platelets (1:2.5:0 median ratio), and a median of 2 (IQR 0-4) grams of Fibrinogen concentrate. In $41 \%$ of patients resuscitation was TEG-guided.

We observed an overall mortality of $44 \%$, with $64 \%$ of the deaths within the first $24 \mathrm{~h}$ after admission. Exsanguination due to uncontrolled hemorrhage below the diaphragm was the cause $100 \%$ of the early deaths in our study population.

The median lactate level and base excess (BE) on admission were 5.2 (IQR 3.0-11.8) $\mathrm{mmol} / \mathrm{L}$ and -9 (IQR -13.98 - -6.45) $\mathrm{mmol} / \mathrm{L}$, respectively. Median International Normalized Ratio (INR) and fibrinogen level on admission were 1.5 (IQR 1.3-1.85) and 189 (IQR 146-264) mg/dl. In the group of patients that survived $>24 \mathrm{~h}$ after admission and damage control resuscitation was completed; lactate, BE, INR and fibrinogen values were corrected (median Lactate $1.2 \mathrm{mmol} / \mathrm{L}$, median $\mathrm{BE}$ $-0.5 \mathrm{mmol} / \mathrm{L}$, median INR 1.2, median Fibrinogen $331 \mathrm{mg} / \mathrm{dl}$ ).

No statistically significant differences were found between mortality and gender, age, blood product consumption or ISS; however, increased lactate or INR on admission, and the need of damage control surgery where associated to higher mortality rates.

Conclusion: Although a damage control resuscitation strategy probed to be highly effective for critically injured patients (median ISS 34) with adequate hemorrhage control, exsanguination due to uncontrolled hemorrhage below the diaphragm was the cause of $100 \%$ of the early deaths in our series.

According to a recent joint statement from the American College of Surgeons Committee on Trauma, the American College of Emergency Physicians, the National Association of Emergency Medical Services Physicians and the National Association of Emergency Medical Technicians regarding the clinical use of Resuscitative Endovascular BalIoon Occlusion of the Aorta (REBOA) in civilian trauma systems; this device may be used for traumatic life-threatening hemorrhage below 
the diaphragm in patients in hemorrhagic shock who are refractory to resuscitation. This indication resemble the main cause of preventable death identified in our study.

The development of a local REBOA protocol and a quality assurance program could be recommended to reduce early trauma-related mayor hemorrhage mortality of in our setting.

\section{Reference(s) and grant ackowledgment(s)}

1. Bulger E, Perina D, Qasim Z, Beldowicz B, Brenner M, Guyette F et al. Clinical use of resuscitative endovascular balloon occlusion of the aorta (REBOA) in civilian trauma systems in the USA, 2019: a joint statement from the American College of Surgeons Committee on Trauma, the American College of Emergency Physicians, the National Association of Emergency Medical Services Physicians and the National Association of Emergency Medical Technicians. Trauma Surgery \& Acute Care Open. 2019;4(1):e000376.

2. Spahn D, Bouillon B, Cerny V, Duranteau J, Filipescu D, Hunt B et al. The European guideline on management of major bleeding and coagulopathy following trauma: fifth edition. Critical Care. 2019;23(1).

3. Ec.europa.eu. (2019). Accidents and injuries statistics - Statistics Explained. [online] Available at https://ec.europa.eu/eurostat/statisticsexplained/ index.php?title $=$ Accidents_and_injuries_statistics\&oldid $=452017$ \#Death s_from_accidents.2C_injuries_and_assault [Accessed 22 Nov. 2019].

\section{0}

Experience of thromboprophylaxis of patients with COVID-19 disease across two Intensive Care Units in the West of Scotland A. Taylor ; ; F. Mcmorrow ${ }^{2}$; A. Allison ${ }^{3}$; R. Hart'; ;. Black'; J. Allan²

${ }^{1}$ intensive care, Queen Elizabeth University Hospital, Glasgow, UK, United Kingdom; ${ }^{2}$ Intensive care, University Hospital Crosshouse, Crosshouse, United Kingdom; ${ }^{3}$ Intensive care, Queen Elizabeth University Hospital, Glasgow, United Kingdom; ${ }^{4} \mathrm{Critical}$ care, Queen Elizabeth University Hospital, Glasgow, United Kingdom; ${ }^{5}$ Department of critical care, Queen Elizabeth University Hospital, Glasgow, United Kingdom

\section{Correspondence: A. Taylor}

Intensive Care Medicine Experimental 2020, 8(2): 001520

Introduction: The incidence of venous thrombo-embolism (VTE) in intensive care patients is between $5-15 \%$ (1). Fixed dosing of once daily low molecular weight heparin (LMWH) is routinely used to mitigate this when appropriate. Target dosing is of $0.5 \mathrm{mg} / \mathrm{kg}$ but this is often not achievable at extremes of body weight. It is not routine practice currently to measure anti-xa levels for assessing attainment of therapeutic levels.

Objectives: During the COVID 19 pandemic it became quickly apparent these patients were presenting profoundly pro-thrombotic and hyper-inflammatory, often with d-dimers in tens of thousands and with a high incidence of VTE. After consultation with haematology colleagues we changed our practice of once daily (OD) to twice daily (BD) dosing of $\mathrm{LMWH}$ in response, and sought to ensure no increase in patient harm or significant bleeding complications.

Methods: Data was collected for two hospital sites in the West of Scotland: Queen Elizabeth University Hospital and University Hospital Crosshouse. A total of 84 patients admitted over a 9 weight period from March 2020 requiring ventilation were reviewed. The regime and dose of LMWH, incidence of VTE and outcome were recorded. Dose adjustment was made for those with renal impairment and extremes of body weight. Anti-xa levels were not routinely measured but were noted when taken. We recorded patients actual weight which allowed calculation of $\mathrm{mg} / \mathrm{kg}$ dosing. An excel document was used for recording patient data. Patients admitted on treatment dose anti-coagulation and those who were on renal replacement therapy and heparin only throughout their admission were excluded.
Results: During the weeks $1-3$ prior to practice change $100 \%$ of patients admitted were commenced on OD LMWH. Practice change was introduced in week 4, and from week 5-9 there is a 95\% compliance rate with either changing from $\mathrm{OD}$ to $\mathrm{BD}$ dosing, or commencing on $\mathrm{BD}$ dosing. Our sample size is too small to show statistical significance but we see a fall in mortality in the weeks following change and trend to lower incidence of confirmed VTE.

Actual dose was calculated for each of our patients with an accurately recorded weight. We found $63 \%(\mathrm{~N}=30)$ of those who died in the QEUH were on an equivocal dose of $<0.5 \mathrm{mg} / \mathrm{kg}$ daily.

Anti-xa levels were not routinely measured in patients on OD LMWH however when checked in patients moved to BD dosing levels were between 0.12-0.39.

We did not find any increase in significant bleeding complications or evidence of patient harm from this change.

\begin{tabular}{|c|c|c|c|c|c|c|c|}
\hline Week & $\begin{array}{l}\text { OD } \\
\text { LMWH }\end{array}$ & $\begin{array}{l}\text { OD- } \\
>\mathrm{BD} \\
\mathrm{LMWH}\end{array}$ & $\begin{array}{l}\text { BD } \\
\text { LMWH }\end{array}$ & $\begin{array}{l}\text { VTE } \\
\text { I confirmed }\end{array}$ & $\begin{array}{l}\text { Discharged } \\
\text { home }\end{array}$ & Died & $\begin{array}{l}\text { Total no of } \\
\text { admissions }\end{array}$ \\
\hline $\begin{array}{c}1 \\
16 / 03 / 2020\end{array}$ & 4 & 0 & 0 & 0 & 2 & 2 & 4 \\
\hline $\begin{array}{c}2 \\
23 / 03 / 2020\end{array}$ & 9 & 6 & 0 & 4 & 6 & 9 & $\begin{array}{l}15(1 \\
\text { exluded })\end{array}$ \\
\hline $\begin{array}{c}3 \\
30 / 03 / 2020\end{array}$ & 17 & 2 & 0 & 6 & 5 & 14 & $\begin{array}{l}19(2 \\
\text { excluded) }\end{array}$ \\
\hline $\begin{array}{c}4 \\
06 / 04 / 2020\end{array}$ & 5 & 6 & 4 & 2 & 5 & 10 & $\begin{array}{l}15(2 \\
\text { excluded })\end{array}$ \\
\hline $\begin{array}{c}5 \\
13 / 04 / 2020\end{array}$ & 0 & 1 & 3 & 1 & 1 & 3 & 4 \\
\hline $\begin{array}{c}6 \\
20 / 04 / 2020\end{array}$ & 0 & 1 & 6 & 4 & 6 & 1 & 7 \\
\hline $\begin{array}{c}7 \\
27 / 04 / 2020\end{array}$ & 1 & 1 & 5 & 1 & 4 & 3 & $\begin{array}{l}7(1 \\
\text { excluded })\end{array}$ \\
\hline $\begin{array}{c}8 \\
04 / 05 / 2020\end{array}$ & 0 & 1 & 1 & 0 & 2 & 0 & 2 \\
\hline $\begin{array}{c}9 \\
11 / 05 / 2020\end{array}$ & 0 & 0 & 1 & 0 & 0 & 1 & 1 \\
\hline
\end{tabular}

Conclusion: We have shown the ability to rapidly induce a change in practice across two Intensive Care Units in the West of Scotland. We have not shown any evidence of increase in harm. We have highlighted a variable patient specific dose in this pro-thrombotic patient cohort already at increased risk of significant VTE. It may be in the future we should consider altering our practice further. Dose adjustment for underlying condition, renal function, weight and specific drug half-life with routine measurement of anti-xa levels may allow a more patient specific, targeted approach. This could further help reduce VTE incidence, not just in COVID 19 patients but in all critically ill patients receiving VTE prophylaxis.

Reference(s) and grant ackowledgment(s)

1. 3. Farkas, J. PulmCrit - mythbusting $40 \mathrm{mg}$ enoxaparin daily for DVT prophylaxis in critical illness. Accessed July 2020. https://emcrit.org/pulmc rit/40-enoxaparin/.

2. 2. Cui S, Chen S, Li X, Liu S, Wang F. Prevalence of venous thromboembolism in patients with severe novel coronavirus pneumonia. J Thromb Haemost. 2020

3. 1. Helms J, Tacquard C, Severac F, Leonard-Lorant I, Ohana M, Delabranche X, Merdji H, Clere-Jehl R, Schenck M, Fagot Gandet F, et al. High risk of thrombosis in patients in severe SARS-CoV-2 infection: a multicenter prospective cohort study. Intensive Care Med. 2020. 
001542

AntiXa determination as a guide for anticoagulation in COVID-19 patients in our hospital

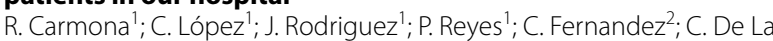
Fuente Martos

${ }^{1}$ Intensive care unit, Hospital Universitario Reina Sofia, Córdoba, Spain; ${ }^{2}$ Haematology unit, Hospital Universitario Reina Sofia, Córdoba, Spain

Correspondence: R. Carmona

Intensive Care Medicine Experimental 2020, 8(2): 001542

Introduction: Many studies are beginning to study the relationship between COVID-19 infected patients and an increased risk of prothrombotics. So far, all clinical guidelines suggest starting prophylactic anticoagulation in this type of patient, but we do not really know what is the optimal dose to avoid bleeding complications and at the same time decrease the risk of thrombosis. We hypothesize that monitoring of the antiXa factor may serve to guide anticoagulant therapy, either at prophylactic or therapeutic doses in patients infected with SARS-CoV-2.

Methods: This is a prospective descriptive study in which we included 11 patients infected with COVID19 admitted to the Intensive Care Unit at a tertiary hospital in southern Spain. Variables such as comorbidity, renal function, BMI, initial and final dose of low molecular weight heparin, and laboratory parameters such as factor VIII coagulant activity, Von Willebrand antigen (VW Ag), ristocetin cofactor Von Willebrand's (VWRCo), antithrombin, fibrinogen, D-dimer were collected, as well as bleeding and thrombotic complications.

The following data were collected for each patient: demographics, previous conditions: comorbidities, obesity, Charlson comorbidity index, clinical characteristics, severity of illness (APACHE II and SOFA score), renal clearance, the need for renal replacement therapy (RRT), and cardiovascular risk factors (hyperlipidemia, hypertension, diabetes, ischemic cardiopathy). In addition, initial enoxaparin dose and subsequent goal dose, renal function, and all anti-factor Xa levels were collected. Major and minor bleeding events, Heparine-Induced Thrombocytopenia (HIT) and thrombotics events rule out by $\mathrm{CT}$, were analysed. All patients received at least standard doses thromboprophylaxis (enoxaparine $40 \mathrm{mgr} / 24 \mathrm{~h}$ ).

Results: $54 \%$ of our patients needed to increase the dose of enoxaparin to reach levels of AntiXa in the prophylactic range (AntiXa 0.3-0.7 $\mathrm{lU} / \mathrm{ml})$. Only 2 were maintained with the same initial dose (enoxaparine $40 \mathrm{mgr} / 24 \mathrm{~h}$ and $100 \mathrm{mgr} / 24 \mathrm{~h}$ respectively).We performed computed tomography (CT) to rule out thrombosis, and only one of our patients presented jugular thrombosis. 3 of our patients presented bleeding events that required blood product transfusion.

Conclusion: We found that conventional prophylactic dose might not be enough for prophylactic anticoagulation in patients with severe pneumonia and ARDS COVID-19 in the Intensive Care Unit. Most of the time (54\%), we needed increasingly higher doses os enoxaparine (up to $100 \mathrm{mg}$ per day) to range prophylactic dose. The study concludes with the need to determine the AntiXa factor in all patients infected with COVID 19 as a guide for anticoagulant therapy or prophylaxis, probably due to the proinflammatory state. Although the sample of our observation is small, we consider a highly relevant topic that has not yet been reported in patients with COVID-19. The importance of the findings will serve as a basis for studies with a larger sample. To further investigate the significance of these findings, we suggest studying these data in depth in future controlled clinical trails.
001583

Low incidence of thrombotic events in SARS-CoV-2 mechanically ventilated anticoagulated patients

A. Perot ${ }^{1}$; B. Misset ${ }^{2}$; P. Massion ${ }^{3}$; S. Piret ${ }^{4}$; AF. Rousseau ${ }^{1}$; P. Wiesen ${ }^{1}$; S Robinet $^{1}$; B. Lambermont ${ }^{4}$; P. Morimont ${ }^{5}$; D. Ledoux ${ }^{3}$; JL. Canivet ${ }^{1}$; N. Layios $^{1}$

${ }^{1}$ Department of intensive care, University Hospital Liege, Liège, Belgium; ²Department of Intensive Care, CHU de Liège, Avenue de L'Hòpital, Liège, Belgium, Liège, Belgium; ${ }^{3}$ Department of intensive care, Chu De Liège, Liège, Belgium; ${ }^{4}$ Intensive care, University Hospital Liege, Liège, Belgium; ${ }^{5}$ Intensive care, Chu De Liège, Liège, Belgium

Correspondence: N. Layios

Intensive Care Medicine Experimental 2020, 8(2): 001583

Introduction: Early after the beginning of the Covid-19 pandemic, reports of fatal pro-thrombotic complications of the disease led some experts to advocate the use of systemic anticoagulation in critically ill SARS-CoV-2 patients. As a consequence, an institutional algorithm, stratified on severity, was implemented at the Liege University Hospital.

Objectives: The objective of this retrospective study is to highlight the incidence of thromboembolic or hemorrhagic events in critically ill mechanically ventilated patients with SARS-CoV2 who were all treated with systemic anticoagulation, either prophylactic or therapeutic. The primary endpoint was to compare all-cause mortality between the 2 regimens of anticoagulation. The secondary endpoint was to compare the incidence of thrombotic events (deep venous thrombosis, pulmonary embolism, myocardial infarction, mesenteric ischemia, hepatic ischemia, stroke) and hemorrhagic events between the 2 groups of patients.

Methods: Between March 14th and June 1st, 2020, all consecutive patients with SARS-CoV-2 hospitalized in the 7 ICUs at the Liege University Hospital were included in this study. The Institutional Review Board waived the need for consent to use prospectively collected clinical data and the study was appointed the serial number 2020-214. The exclusion criteria included: the lack of use of mechanical ventilation and of anticoagulation. All patients received either prophylactic (enoxaparin $0.5 \mathrm{mg} / \mathrm{kg} /$ day) or therapeutic (enoxaparin $1 \mathrm{mg} / \mathrm{kg}$, twice a day) anticoagulation. All patients were sampled for D-dimers, platelets, Fibrinogen upon admission. Calculation of DIC score (ISTH criteria) and severity scores (SOFA, SAPS2) was acquired upon admission for all patients. Arterial and venous thrombotic events led to a diagnostic workup upon clinical suspicion.

Results: Seventy-three patients were included in the final analyses. Demographic and clinical data are shown in Table 1. Mortality was not different between the 2 groups: $19 / 49$ (38.8\%) in the prophylactic group versus $7 / 24(29.2 \%)$ in the therapeutic group. Moreover, there was no difference in the incidence of thrombotic or hemorrhagic events between the 2 groups. Median platelet counts were normal and DIC was predominantly non-overt. However, fibrin monomers taken from $49 \%$ of patients and D-Dimers, which should consolidate DIC scoring, were statistically higher in the prophylactic group in which three thrombotic events occurred (3/73, 4.1\%). Of note, the latter incidence is substantially lower than in recent reports thus validating a systemic anticoagulation in Covid-19 patients(1, 2).

Finally, while not conferring a mortality benefit as suggested by a recent study, the therapeutic regimen appeared safe. 
Table 1: Demographic, clinical and biological characteristics of anticoagulated SARS-CoV-2 patients $(\mathrm{N}=73)$

\begin{tabular}{|l|l|l|l|}
\hline All & $\begin{array}{l}\text { Therapeutic } \\
\text { anticoagulation n=24 } \\
(32.9 \%)\end{array}$ & $\begin{array}{l}\text { Prophyl actic } \\
\text { anticoagulation }\end{array}$ & P value \\
(N=73) & $147.1 \%)$ & \\
\hline Sex : Male & $14(58.3 \%)$ & $36(73.5 \%)$ & $0.191 *$ \\
\hline Age (years) & $64(57-67)$ & $64(56-72)$ & $0.402^{* * *}$ \\
\hline $\begin{array}{l}\text { Death (N) (\%) } \\
\text { Thrombotic } \\
\text { events (N) (\%) }\end{array}$ & $7(29.2 \%)$ & $19(38.8 \%)$ & $0.421 *$ \\
\hline $\begin{array}{l}\text { Hemorragic } \\
\text { events (N) (\%) }\end{array}$ & $1(4.17 \%)$ & $3(6.1 \%)$ & $0.546^{* *}$ \\
\hline $\begin{array}{l}\text { DIC score (ISTH) } \\
\text { Fibrin Monomer }\end{array}$ & $2(2-3)$ & $3(6.1 \%)$ & $1.00^{* *}$ \\
\hline $\begin{array}{l}\text { detected (N) (\%) } \\
\text { DDimers (mg/L) }\end{array}$ & $0.93(0.68-1.41)$ & $1.98(0.95-4.55)$ & $0.006 * * *$ \\
\hline $\begin{array}{l}\text { Platelets } \\
\left(\mathrm{x} 10^{3} / \mathrm{mm}^{3}\right)\end{array}$ & $1 .(146-254)$ & $201(146-271)$ & $0.822 * * *$ \\
\hline
\end{tabular}

* Chisquare test

** Fisher exact test

*** rank sum test

Age, DIC score, DDimers, platelets median (IQR)

Conclusion: Taken together, these findings are concordant with previous reports in emphasizing the fact that Covid-19 coagulopathy, while overlapping with many well characterized coagulopathies does not perfectly match any of them(3). They also confirm that systemic anticoagulation is mandatory in critically ill mechanically ventilated patients.

\section{Reference(s) and grant ackowledgment(s)}

1. 1. Helms, J., Tacquard, C., Severac, F. et al. High risk of thrombosis in patients with severe SARS-CoV-2 infection: a multicenter prospective cohort study. Intensive Care Med 46, 1089-1098 (2020). 2. Wichmann, D., Sperhake, J.P., Lutgehekmann, M. et al. Autopsy Findings and Venous Thromboembolism in Patients With COVID-19. Ann Intern Med 2020 May 6. pii: 2765934. https ://doi.org/10.7326/m20-2003. 3. Iba, T., Levy, J.H., Connors, J.M. et al. The unique characteristics of COVID-19 coagulopathy. Crit Care 24, 360 (2020. 2. None

\section{3}

Incidence and mortality of thrombotic complications ICU patients with COVID-19

B. Llorente Ruiz'; A. Acha Aranda ; I. Guitierrez Jomarron²; L. Castilla García²; R. Molina Montero'; M. Rascon Risco33; L. Alcazar Sanchez-Elvira. M. Trascasa Muñoz ; C. Serrano ; A. Ruiz García

${ }^{1}$ Intensive care unit, Hospital Príncipe de Asturias, Alcalá de Henares, Spain; ${ }^{2}$ Hematology, Hospital Príncipe de Asturias, Alcalá de Henares, Spain; ${ }^{3}$ Radiodiagnostic, Hospital Príncipe de Asturias, Alcalá de Henares, Spain Correspondence: B. Llorente Ruiz

Intensive Care Medicine Experimental 2020, 8(2): 001603

Introduction: In COVID-19 patients hyperinflamation, immobilisation, hypoxia and vascular dysfunction may predispose to both venous and arterial thromboembolism. Describing these complications is necessary in order to adjust the intensity of thromboprophylaxis specially in critically ill patients who are at increased thrombotic risk.

Objectives: To identify predictive factors and determine the frequency of thrombotic complications in ICU COVID-19 patients receiving pharmacological thromboprophylaxis.

Methods: Descriptive retrospective study. Statistical analysis was performed with IBMâSPSS Statistics 25.

Results: 77 patients with confirmed COVID-19 hospitalized in the ICU of our second level hospital were screened between March 6, 2020 and May 6, 2020. All patients were adults diagnosed with respiratory failure and undergoing mechanical ventilation. Demographics, comorbidities, severity score,inflammatory markers, medical and antithrombotic treatment were collected. All these data were analyzed between patients with thrombotic episodes and those who did not, establishing the incidence of thrombosis, the type, the diagnosis used, and analyzed the significance of the risk factors.

Of the 77 patients included in the study, $64.9 \%$ were male. The average age is $58 \pm 11$ years. At admission, the mean APACHE II was $17 \pm 17.32$. $40.3 \%$ had a history of HT, $70 \%$ chronic lung disease, $67.5 \%$ DM, $45.5 \%$ $\mathrm{DL}$ and $74 \%$ obesity, with CKD being present in one of the patients and $7.8 \%$ previous thrombotic events. $15.5 \%$ were oncologic patients. Regarding anti-thrombotic treatment with $\mathrm{LMWH}, 55.8 \%$ received thromboprophylaxis, $16.9 \%$ adjusted the dose to weight/renal function, $15.6 \%$ received intermediate doses of prophylaxis $(1 \mathrm{mg} / \mathrm{kg} / \mathrm{day})$ and $10.4 \%$ anticoagulant doses. The incidence of venous thromboembolism was $32.5 \%$ of the total with DVT being $32 \%$ ( 3 with distal; 2 with distal and proximal; 1 with bilateral; 1 other type) and pulmonar thromboembolism in $76 \%$ (6 bilateral; 8 with segmental; 2 with lobar; 3 with massive). Note that, the means of the inflammation parameters were significantly higher in the patients with thrombotic complications compared to those who did not suffer them (D-dimer $63,3 \pm 124 \mathrm{vs} 16 \pm 21 \mathrm{ng} / \mathrm{ml}, \mathrm{p}=0,001 ; \|-62311 \pm 2767 \mathrm{vs} 924 \pm 1175$, $P=0,001 ; P C R 246 \pm 122$ vs $215 \pm 117, p=0,016 ;$ Ferritina $6012 \pm 2014$ vs $1827 \pm 1560 \mathrm{ng} / \mathrm{ml}, \mathrm{p}=0,012 ; \mathrm{LDH} 708 \pm 388 \mathrm{vs} 1344 \pm 1552 \mathrm{ng} / \mathrm{ml}$, $\mathrm{p}<0,01)$. Paradoxically, mortality among thrombosed patients is not significantly higher.

Conclusion: In conclusion, despite systematic thrombosis prophylaxis, the $32,5 \%$ incidence of thrombotic complications in these patients is remarkably high and well comparable to the VTE incidence in other patient categories, but mortality mong these patients is not significantly higher. D-dimer, IL-6, Ferritina, LDH and PCR level-guided more aggressive thromboprophylaxis regimens using higher doses of heparin seem to be a good instrument in critically ill patients but more prospective studies are needed in this regard.

\section{4}

Association between ROTEM hypercoagulable profile and outcome in a cohort of severely ill COVID-19 patients under mechanical ventilation

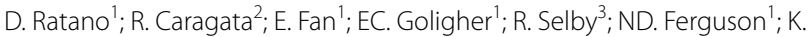
Karkouti'; 'SA. Mccluskey²

${ }^{1}$ Department of medicine, University health network, Interdepartmental Division of Critical Care Medicine, University of Toronto, Toronto, Canada; ${ }^{2}$ Department of anesthesia and pain medicine, Toronto General Hospital, University Health Network, University of Toronto, Toronto, Canada; ${ }^{3}$ Department of laboratory medicine and medicine, University Health Network, University of Toronto, Toronto, Canada

Correspondence: $D$. Ratano

Intensive Care Medicine Experimental 2020, 8(2): 001604

Introduction: Recently published data show that COVID-19 is characterized by a hypercoagulable ROTEM profile and decreased fibrinolysis[1]. Multiple reports suggest that severe COVID-19 infection is associated with an increased thromboembolic risk[2,3]. There are limited data associating a hypercoagulable ROTEM profile and outcomes in the literature. A hypercoagulable ROTEM profile could help identify patients at risk of worse outcome and help target a study population for enhanced anticoagulation therapy or other COVID-19 therapy.

Objectives: To determine if early hypercoagulability on ROTEM is associated with a higher risk of thromboembolic complications or worse outcome, in a population of mechanically ventilated COVID-19 patients transferred to a tertiary ARDS/ECMO referral center.

Methods: All COVID-19 patients receiving mechanical ventilation at our center between April 3 and June 15,2020 were assessed with ROTEM. Testing performed included at least ExTEM and FibTEM. Patients were classified as hypercoagulable $(\mathrm{HC})$ or not $(\mathrm{nHC})$ using the 
following criteria from ROTEM: EXTEM clot formation time (E_CFT) < $40 \mathrm{sec}$, alpha (a) angle $>79^{\circ}$ and, either ExTEM or FibTEM maximum clot firmness (E_MCF or F_MCF) $>70 \mathrm{~mm}$ or $>24 \mathrm{~mm}$, respectively. Outcomes, assessed at discharge from our ICU, included thromboembolic events (TE: composite of deep vein thrombosis, pulmonary embolism and ischemic stroke), acute kidney injury (AKI: AKIN 4 stage $>2$ with or without dialysis[4]), duration of mechanical ventilation, requirement for ECMO, and death. Difference between groups was determined using the chi-square or Fisher-exact test for categorical data and a t-test for continuous variables respectively, using $\mathrm{p}<0.05$ as significant.

Results: Of 59 patients included, 31 were hypercoagulable $(\mathrm{HC})$ on admission. Mean (SD) age was 52(14), and SOFA score on admission was high 15(2). The severity of disease was similar in both groups especially for the cardiac and respiratory SOFA components (Table 1). The composite outcome of TE was not different between the groups (HC 9/31, nHC 6/28, p=0.7). The composite renal outcome was not different (HC 18/31 vs $\mathrm{nHC} 16 / 28)$. Mortality rate $(29 \%$, HC 9 vs $\mathrm{nHC}$ 8 ), and the duration of ventilation in survivors (HC 22 vs $\mathrm{nHC} 22$ days) were similar in both groups. Requirement for ECMO support was similar (HC 8 vs $n H C 12, p=0.27$ ). Patients were tested with daily viscoelastic tests and, neither fibrinolysis nor DIC were documented. All patients received TE prophylaxis and 34 received therapeutic intravenous heparin for ECMO $(n=20)$ or TE treatment $(n=14)$. Initial ROTEM were conducted on therapeutic heparin in 22 cases ( $12 \mathrm{HC}, 10 \mathrm{nHC}$ )

Table 1:

\begin{tabular}{|c|c|c|c|c|}
\hline Variable & $\begin{array}{l}\text { Overall } \\
(n=59)\end{array}$ & $\begin{array}{l}\text { Non } \\
\text { hypercoagulable } \\
(\mathrm{n}=28)\end{array}$ & $\begin{array}{l}\text { Hypercoagulable } \\
(\mathrm{n}=31)\end{array}$ & p-value \\
\hline Age [years] & $52( \pm 14)$ & $51( \pm 15)$ & $53( \pm 14)$ & 0.48 \\
\hline Sex [male] $(\%)$ & $44(76)$ & $20(74)$ & $24(77)$ & 0.80 \\
\hline $\begin{array}{l}\text { BMI [kg/m2] } \\
\text { (IQR) }\end{array}$ & $30.8(8.3)$ & 30.3 & 31.3 & 0.64 \\
\hline SOFA score & $14( \pm 2)$ & $15( \pm 2)$ & $14( \pm 3)$ & 0.83 \\
\hline $\operatorname{Resp}>3$ & $58 / 58$ & $27 / 27$ & $31 / 31$ & \\
\hline Cardiac $>3$ & $42 / 58$ & $20 / 28$ & $22 / 31$ & \\
\hline E_CFT [sec] & $55(16)$ & $68(13)$ & $43(6)$ & $<0.001$ \\
\hline $\mathbf{E}_{-} \propto\left[^{\circ}\right]$ & $79(3)$ & $76(3)$ & $83(1)$ & $<0.001$ \\
\hline E_MCF [mm] & $75(6)$ & $71(5)$ & $79(4)$ & $<0.001$ \\
\hline F_MCF [mm] & $43(12)$ & $37(13)$ & $47(9)$ & 0.001 \\
\hline
\end{tabular}

Conclusion: Our data suggest that in a population of mechanically ventilated patients with severe COVID-19, an early hypercoagulable profile on ROTEM is not associated with an increased risk of thromboembolic events, AKI, prolonged ventilation, ECMO or death during their ICU stay.

\section{Reference(s) and grant ackowledgment(s)}

1. 1. Pavoni V, Gianesello L, Pazzi M, Stera C, Meconi T, Frigieri FC. Evaluation of coagulation function by rotation thromboelastometry in critically ill patients with severe COVID-19 pneumonia. J Thromb Thrombolysis 2020;50(2):281-6. 2. CRICS TRIGGERSEP Group (Clinical Research in Intensive Care and Sepsis Trial Group for Global Evaluation and Research in Sepsis), Helms J, Tacquard C, et al. High risk of thrombosis in patients with severe SARS-CoV-2 infection: a multicenter prospective cohort study. Intensive Care Med 2020;46(6):1089-98. 3. Klok FA, Kruip MJHA, van der Meer NJM, et al. Incidence of thrombotic complications in critically ill ICU patients with COVID-19. Thrombosis Research 2020;191:145-7. 4. Mehta RL, Kellum JA, Shah SV, et al. Acute Kidney Injury Network: report of an initiative to improve outcomes in acute kidney injury. Crit Care 2007;11(2):R31.

2. Dr Ratano is supported by fellowship grants from the SICPA Foundation, the Livio-Glauser Foundation and the Lausanne University Hospital, Lausanne Switzerland. Dr Ratano is also the recipient of the 2020 Paul Blanc Bursary (Bourse Paul Blanc) of the Vaud Academic Society (Société Académique Vaudoise), Lausanne Switzerland.
001613

Incidence and risk factors associated with bleeding complications in COVID patients

A. Acha Aranda'; B. Llorente Ruiz'; I. Guitierrez Jomarron²; L. Castilla García ${ }^{2}$; R. Molina Montero'; M. Rascon Risco ${ }^{3}$; L. Alcazar Sanchez-Elvira ${ }^{1}$. E. Lopez Ramos '; J. Higuera Lucas'; M. Trascasa Muñoz'; C. Serrano ; A. Ruiz García'; Y. Ortiz De Zarate

${ }^{1}$ Intensive care unit, Hospital Príncipe de Asturias, Alcalá de Henares, Spain; ${ }^{2}$ Hematology, Hospital Príncipe de Asturias, Alcalá de Henares, Spain; ${ }^{3}$ Radiodiagnostic, Hospital Príncipe de Asturias, Alcalá de Henares, Spain Correspondence: B. Llorente Ruiz

Intensive Care Medicine Experimental 2020, 8(2): 001613

Introduction: In COVID-19 patients,coagulopathy is frequently reported and is a significant poor prognosis factor. There is particularly high frequency of thromboembolic events, so the use of heparin was associated with reduced mortality in COVID-19.

Objectives: In order to optimize the treatment in these patients, the objective of our study was to analyze the incidence of bleeding complications and what factors were most associated with them.

Methods: We made a descriptive retrospective study with adult patients with confirmed COVID-19 hospitalized in the ICU of our second level hospital between March 6, 2020 and May 6, 2020. Statistical analysis was performed with IBMâSPSS Statistics 25 .

Results: All were diagnosed with respiratory failure and required invasive mechanical ventilation.We collect demographics, comorbidities, severity score (APACHE, SOFA, SAPS II), medical and antithrombotic treatment,an extensive study of coagulation, mortality and average ICU stay. We try to establish the incidence and importance of bleeding complications analyzing the importance of risk factors associated with them.Our sample included 77 patients. Most of them (64.9\%) were male. The average age was $58 \pm 11$ years. At admission, the mean APACHE II was $17 \pm 17.32$. $40.3 \%$ had a history of HT, $70 \%$ chronic lung disease, $67.5 \% \mathrm{DM}, 45.5 \% \mathrm{DL}$ and $74 \%$ obesity, with CKD being present in one of the patients and $7.8 \%$ previous thrombotic events. $15.5 \%$ were oncologic patients. Regarding anti-thrombotic treatment with $\mathrm{LMWH}, 55.8 \%$ received thromboprophylaxis, $16.9 \%$ adjusted the dose to weight/renal function, $15.6 \%$ received intermediate doses of prophylaxis $(1 \mathrm{mg} / \mathrm{kg} /$ day) and $10.4 \%$ anticoagulant doses. The incidence of bleeding complications $(11,68 \%, 8$ patients) compared with that of thrombosis $(32,5 \%)$ was low. However thrombotic complications were not significantly associated with higher mortality, while hemorrhagic complications were. There were,three patients with cerebral hemorrhage, 2 with digestive hemorrhage, 1 with hemoptysis, 1 with peritracheostomy bleeding and one with muscular hematoma. Of these, 2 patients had LMWH at anticoagulant doses, 2 with intermediate doses $(1 \mathrm{mg} / \mathrm{kg}), 3$ with prophylactic doses and one with a dose adjusted for renal function. Only 1 received antifibrinolytic treatment. Comparing the data between patients with hemorrhages and those without SOFA on admission $(6,84 \pm 18 v s 6,5 \pm 3,6 p=0,003)$ was the only risk factor that was significantly associated. Clarify, that 3 of them had had concomitant major whirlwind events.

Conclusion: Therefore we can conclude that although hemorrhagic complications have a lower incidence than thrombotic, their mortality is much higher and that it is difficult to establish possible risk factors that help us predict them. It is important to emphasize in order to optimize thrombotic event prophylaxis that heparin doses were not significantly associated with bleeding complications, but we require further studies with a larger sample to try to establish more risk factors that are associated.

\section{1}

CABARET: Clinical implicAtions of Bromelain Application in buRn patiEnTs

S. Provinciael ${ }^{1}$; K. Colpaert ${ }^{1}$; S. Monstrey ${ }^{2}$

${ }^{1}$ Critical care, Ghent University Hospital, Ghent, Belgium; ${ }^{2}$ Plastic surgery, Ghent University Hospital, Ghent, Belgium

Correspondence: S. Provinciael

Intensive Care Medicine Experimental 2020, 8(2): 001191 
Introduction: Bromelain-based enzymatic debridement of burns is a relatively new technique, though it has already shown promising results on wound healing, with limiting blood loss and a lower probability of skin transplantation.

However, a higher degree of inflammation was clinically suspected in patients receiving bromelain. This study examines the association of using bromelain and the initiation of an inflammatory reaction.

Methods: A retrospective survey was performed on the database of burn patients in the universitary hospital of Ghent (Belgium), during the period of $05 / 2016-06 / 2019$, with a total of 52 included patients. Inflammatory parameters were studied before and after the application of bromelain. The parameters include vital signs (blood pressure, heart rate, temperature), laboratory findings (such as WBC (white blood cell count), CRP (C-reactive protein), coagulation, renal function, fibrinogen, hematocrit, lactate and blood glucose) and therapeutic measurements (such as use of oxygen, inotropic agents and vasopression). Sequential Organ Failure Assessment (SOFA) Score was additionally determined.

Results: A statistical difference $(p<0.05)$ was seen in temperature, tension, heart rate, WBC, CRP and coagulation. We observed a higher temperature, hypotension, tachycardia, a higher CRP, a prolonged coagulation and a lower hematocrit. The white blood cell count was significantly lower after application of bromelain. No significant difference was seen in the other variables, neither in SOFA score.

Conclusion: Significant differences of augmented temperature, hypotension, tachycardia, elevated CRP and prolonged coagulation were noticed after the application of bromelain, which suggest that bromelain may induce inflammation. Further research is needed in order to determine a clear causal relationship between the application of bromelain and the induced inflammatory response with the application of standardised prospective study protocol.

\section{9}

Elevated monocyte distribution width in trauma: a rapidly available biomarker of severity

A. Marcos-Morales ${ }^{1}$; JA. Barea-Mendoza ${ }^{1}$; C. Cueto-Felgueroso ${ }^{2}$; S.

Gómez-Rojas $^{3}$; G. Pérez-Segura ${ }^{3}$; C. García-Fuentes ${ }^{1}$; M. Chico-Fernández ${ }^{1}$

1 Intensive care department, Hospital Doce de Octubre, Madrid, Spain; 2Biochemistry department, Hospital Doce de Octubre, Madrid, Spain; ${ }^{3}$ Hematology department, Hospital Doce de Octubre, Madrid, Spain

Correspondence: A. Marcos-Morales

Intensive Care Medicine Experimental 2020, 8(2): 001229

Introduction: Different acute critical illnesses such as sepsis or severe traumatic injury share some kind of inflammatory response (1), in which monocytes play an active role (2). Changes in cell volume parameters are related to cell activation, and one of these parameters is the monocyte distribution width (MDW), which is the standard deviation of monocyte volume.

Recently, an elevated monocyte distribution width (MDW) has been found to differentiate patients with sepsis from those with a less severe infection (3-4).

We hypothesized that an elevated MDW would also occur in some trauma patients, especially in those with increased severity and a worse prognosis.

Objectives: The aim of this study was to evaluate the association between MDW value on admission of politraumatized patients, with trauma severity and prognosis.

Methods: This study took place in a trauma ICU in Madrid. Patients admitted with traumatic injury between August and November 2019 were included, in which the MDW was measured in the first whole blood sample on the UniCel ${ }^{\circledR}$ DxH 800 Coulter $^{\circledR}$ Cellular Analysis System. No patients were excluded. They were classified according to MDW values (greater or less than $20 \mathrm{U}$ ). We compared for each group: injury severity score (ISS), hemodynamic instability, organ failures, length of stay and mortality. For statistical analysis we used SPSS software, version 20

Results: We included 87 patients with a median age of 43 years (interquartile range IQR $27-52$ ), $77 \%$ were men. Half of the patients presented with severe trauma, with a median ISS of 17 (IQR 11-25). Severe traumatic brain injury occurred in $18 \%$ of patients, $20 \%$ had severe thoracic trauma, $17 \%$ moderate-to-severe orthopedic trauma, and $15 \%$ moderate-to-severe abdominal trauma. Demographic variables were well balanced between MDW defined groups: those with a MDW $\geq 20 \mathrm{U}$ represented $43 \%$ of patients, median age was 45 years (IQR 31-61), and median ISS was 17 (IQR 13-27). High MDW patients were more likely to present hemodynamic instability on admission $(77 \%$ vs $23 \%, p=0,013)$, as well as acute renal failure during ICU stay ( $80 \%$ vs $20 \%, p=0,017$ ). These results were still observed after adjusting for age, ISS and metabolic acidosis on admission (base deficit of $4 \mathrm{mmol} / \mathrm{L}$ or more). A MDW $>20 \mathrm{U}$ was associated with hemodynamic instability on admission with an OR of 3,8 (IC 95\% 1,09-12; $p=0,03)$, with failure of two organs or more with an OR of 12 (IC 95\% 1,2-123; $p=0,034$ ), and with acute kidney injury (AKI) with an OR of 17 (IC 95\% 1,5-202; $p=0,023$ ). We found no association between an elevated MDW and mortality of length of stay.

Conclusion: In trauma patients, a MDW $\geq 20 \mathrm{U}$ on admission was independently associated with greater hemodynamic instability and a greater risk of multiorgan failure, especially AKI.

The monocyte distribution width is a parameter available from the complete blood count. It could be useful in predicting severity in the initial evaluation of trauma patients.

\section{Reference(s) and grant ackowledgment(s)}

1. 1) Johansson PI, Stensballe J, Ostrowski SR. Shock induced endotheliopathy (SHINE) in acute critical illness - a unifying pathophysiologic mechanism [published correction appears in Crit Care. 2017 Jul 13;21(1):187]. Crit Care. 2017;21(1):25. Published 2017 Feb 9. https://doi.org/10.1186/s1305 4-017-1605-5

2. 2) Swystun $L L$, Liaw PC. The role of leukocytes in thrombosis. Blood. 2016;128(6):753-762. https://doi.org/10.1182/blood-2016-05-718114.

3. 3) Crouser ED, Parrillo JE, Seymour CW, et al. Monocyte Distribution Width: A Novel Indicator of Sepsis-2 and Sepsis-3 in High-Risk Emergency Department Patients. Crit Care Med. 2019;47(8):1018-1025. https://doi. org/10.1097/ccm.0000000000003799.

4. 4) Crouser ED, Parrillo JE, Seymour C, et al. Improved Early Detection of Sepsis in the ED With a Novel Monocyte Distribution Width Biomarker. Chest. 2017;152(3):518-526. https://doi.org/10.1016/j.chest.2017.05.039.

001319

Femoral veno-arterial carbon dioxide tension difference following severe trauma as organ perfusion index

M. Werner'; B. Bergis ${ }^{1}$; PE. Leblanc' ; L. Wildenberg ${ }^{1}$; J. Duranteau'; B. Vigué $^{\prime}$; A. Harrois'

${ }^{1}$ Anesthesiology and intensive care, Bicetre Hospital AP-HP, Le Kremlin-Bicêtre, France

Correspondence: M. Werner

Intensive Care Medicine Experimental 2020, 8(2): 001319

Introduction: Veno-arterial carbon dioxide tension difference ( $\triangle \mathrm{PCO} 2)$ is inversely related to cardiac output. No study has ever assessed $\triangle \mathrm{PCO} 2$ as a marker of hemodynamic status following severe trauma.

Objectives: The aim of this study was to assess femoral $\triangle \mathrm{PCO} 2(\triangle \mathrm{PCO} 2$ fem) ability to predict the need of emergency erythrocyte transfusion following severe trauma.

Methods: This is a prospective, observational study, conducted in a French level I trauma center. Patients admitted to the trauma resuscitation room following severe trauma with an Injury Severity Score (ISS) $>15$, who had arterial and venous femoral catheters inserted were included. $\triangle \mathrm{PCO} 2 \mathrm{fem}$ and arterial lactatemia were measured over the first 48 hours of admission. $\triangle \mathrm{PCO} 2$ fem and lactate abilities to predict erythrocyte transfusion during the first six hours of admission and emergency hemostatic procedure have been assessed using receiver operating characteristics curve.

Results: 42 trauma patients were included in the study. Median ISS was 27 (22-32). 22 patients (48\%) received at least one red blood cell unit and 17 patients $(40 \%)$ had a hemostatic procedure performed during the first six hours of admission. Mean overall $\triangle \mathrm{PCO} 2 \mathrm{fem}$ at 
admission was $9.5 \pm 6.3 \mathrm{mmHg}$ and median lactatemia was 2.2 (1.54) $\mathrm{mmol} / \mathrm{l}$. $\triangle \mathrm{PCO} 2 \mathrm{fem}$ was significantly higher in patients who were transfused than in those who were not transfused $(12 \pm 7 \mathrm{mmHg}$ vs $7 \pm 3 \mathrm{mmHg}, p=0.018$ ). The best threshold to predict red blood cell transfusion in the first six hours of admission was $8.1 \mathrm{mmHg}$ and the best threshold to predict emergency hemostatic procedure was $5.8 \mathrm{mmHg}$. Lactatemia was not predictive of red blood cell transfusion or hemostatic procedure in the first six hours of admission.

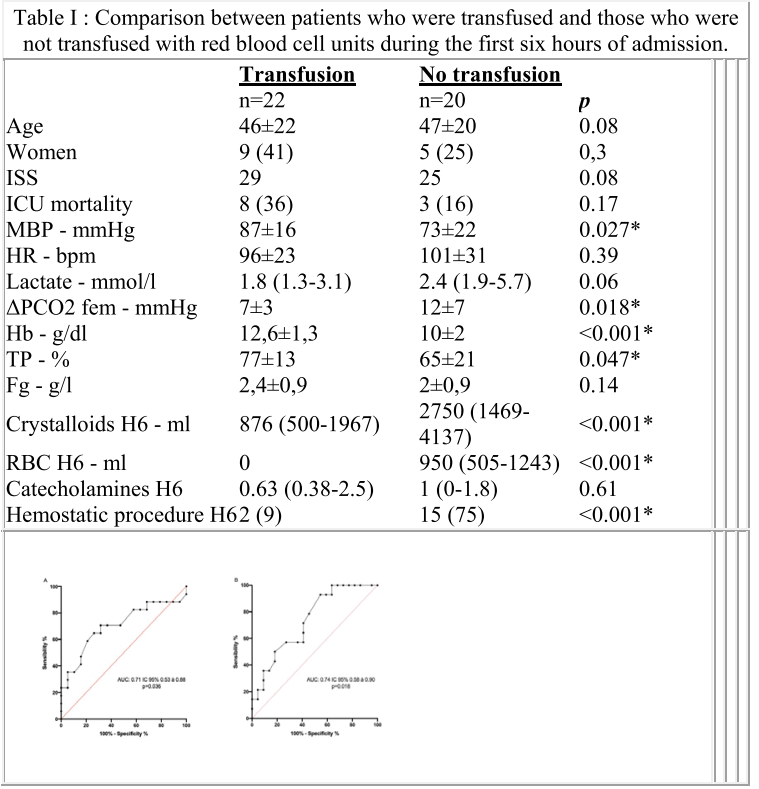

Conclusion: After severe trauma, $\triangle \mathrm{PCO} 2$ fem at hospital admission predicts the need for red blood cell transfusion and emergency hemostatic procedure. $\triangle \mathrm{PCO} 2 \mathrm{fem}$ on admission has the potential to stratify patients at risk of severe bleeding following trauma.

\section{Reference(s) and grant ackowledgment(s)}

1. Van der Linden P, Rausin I, Deltell A, Bekrar Y, Gilbart E, Bakker J, et al. Detection of tissue hypoxia by arteriovenous gradient for $\mathrm{PCO} 2$ and $\mathrm{pH}$ in anesthetized dogs during progressive hemorrhage. Anesth Analg. févr 1995;80(2):269-75

2. Fox EE, Holcomb JB, Wade CE, Bulger EM, Tilley BC. Earlier Endpoints Are Required for Hemorrhagic Shock Trials among Severely Injured Patients. Shock. mai 2017:47(5):567-73.

3. Raux M, Le Manach Y, Gauss T, Baumgarten R, Hamada S, Harrois A, et al. Comparison of the Prognostic Significance of Initial Blood Lactate and Base Deficit in Trauma Patients: Anesthesiology. mars 2017;126(3):522-33.

4. Zhang $\mathrm{H}$, Vincent JL. Arteriovenous differences in PCO2 and $\mathrm{pH}$ are good indicators of critical hypoperfusion. Am Rev Respir Dis. oct 1993;148(4 Pt 1):867-71

\section{7}

A Definite Rib Fracture Pathway in Major Trauma Patients Reduces Critical Care Admissions

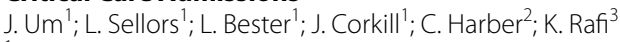

${ }^{1}$ Major Trauma, Queen's Medical Centre, Nottingham, United Kingdom;

${ }^{2}$ Anaesthetics, Queen's Medical Centre, Nottingham, United Kingdom;

${ }^{3}$ Critical care, Queen's Medical Centre, Nottingham, United Kingdom

Correspondence: J. Um

Intensive Care Medicine Experimental 2020, 8(2): 001417
Introduction: Queen's Medical Centre (QMC) is a regional major trauma centre in East Midlands of England, serving the population of 4.8 million people. A significant proportion of patients admitted to Major Trauma Ward (MTW) have chest wall injuries.

A trust-wide guideline on management of blunt chest trauma and rib fractures was published in July 2018 to help identify patients with multiple rib fractures who would benefit from an early administration of a thoracic epidural, which is the gold standard of analgesia in those with rib fractures in QMC.

Objectives: This study aims to look at the incidence of escalation to Critical Care (Level 2 \& 3 care) from Major Trauma Ward (Level 1 care) in patients with $\geq 3$ rib fractures since the introduction of the blunt chest trauma and rib fractures guideline.

Methods: List of patients who were admitted to MTW directly from Emergency Department with $\geq 3$ rib fractures between July 2018 and June 2019 was obtained from Trauma Audit and Research Network (TARN) database. Data such as patient demographics, number of rib fractures, whether it was isolated chest injury, a form of analgesia including rib open reduction and internal fixation (ORIF), reason for delay in administration of an appropriate analgesia (including anticoagulant use), escalation to Critical Care, total length of hospital stay were collected and analysed.

Results: A total of 176 patients were admitted to MTW with $\geq 3$ rib fractures over one year period. 2 patients were excluded due to insufficient data.

The mean age was 64.5. 44 (25.3\%) had an isolated chest wall injury. The mean number of ribs fractured was 5.7. The mean length of hospital stay was 9.9 days.

$89(51.1 \%)$ received an oral analgesia and $85(48.9 \%)$ received a regional block. 7 (4\%) had a rib ORIF.

Out of 85 with a regional block, $81(91.0 \%)$ received a thoracic epidural, $3(3.5 \%)$ received a morphine PCA and $1(1.2 \%)$ received a paravertebral block.

In terms of escalation, 10 out of 174 (5.7\%) were escalated from MTW to Critical Care. 7 were on oral analgesia (for non-respiratory reasons) and 3 on thoracic epidural. All of these 3 epidural patients were escalated for respiratory deterioration. 2 patients had a delay in epidural insertion; one due to deranged clotting due to liver cirrhosis and the other due to being on DOAC.

Conclusion: Early administration of a thoracic epidural in rib fracture patients enhances respiratory function and minimises the risk of respiratory complications. The result showed that only $3(1.7 \%)$ patients were escalated to Critical Care due to respiratory deterioration over one year period. Having a definite rib fracture pathway for analgesia minimises the incidence of admission to Level 2 and 3 care. This potentially increases the cost effectiveness and allows appropriate utilisation of resources in the critical care environment.

\section{2}

Brain-heart interactions: primary cardiac injury in patients with traumatic brain injury

J. Lenstra ${ }^{1}$; R. Wiersema ${ }^{2}$; M. Nijsten ${ }^{2}$; ICC. Van Der Horst ${ }^{2}$; J. Van Der Naalt ${ }^{1}$ ${ }^{1}$ Neurology, University Medical Center Groningen, Groningen, Netherlands; ${ }^{2}$ Critical care, University Medical Center Groningen, Groningen, Netherlands

Correspondence: J. Lenstra

Intensive Care Medicine Experimental 2020, 8(2): 001482

Introduction: Cardiac mimicry have been described in patients with subarachnoid hemorrhage(1), which presumably is caused by an elevated sympathetic tone with excessive catecholamine release. This phenomenon has not been extensively studied in patients with traumatic brain injury (TBI). Recently we observed a high incidence of electrocardiographic (ECG) abnormalities in patients with severe TBI. (2) However, primary cardiac injury as cause of ECG abnormalities was not determined systematically by echocardiography.

Objectives: In this study we evaluated brain-heart interactions in TBI patients admitted to the intensive care unit (ICU) with echocardiographic images systematically obtained within $24 \mathrm{~h}$ after ICU admission. We aimed to determine the incidence of right ventricle 
dysfunction, since it is most at risk due its anatomical position during thoracic injury(3), and their association with brain injury severity in comparison to a trauma-control group.

Methods: Prospective observational study of trauma patients ( $\geq 18$ years) admitted to the ICU of the University Medical Center Groningen between April 2015-July 2019 (SICS-I and SICS-II study(4)). Primary outcome was the incidence of right ventricular dysfunction; right ventricular systolic excursion (RV S') (abnormal $\leq 9.5 \mathrm{~cm} / \mathrm{s}$ ) and tricuspid annular peak systolic excursion (TAPSE) (abnormal $<17 \mathrm{~mm}$ ). Secondary outcome was the association with brain injury severity scored according to the Glasgow Coma Scale.

Results: In total, 109 patients were included, median (IQR; interquartile range) age was 57 years (38-68), and 69\% were male. The 30-day mortality rate was $14 \%$. Brain injury severity groups were as follows: trauma-control $(20 \%)$, mild- $(35 \%)$, moderate- $(11 \%)$, and severe TBI (34\%). Overall RV $\mathrm{S}^{\prime}$ was abnormal in $5 \%$ of patients with a median (IQR) of $14.0 \mathrm{~cm} / \mathrm{s}(11-16)$ and TAPSE was abnormal in $10 \%$ with a median IQR of $22.2 \mathrm{~mm}$ (18.2-25.0). No differences were observed between RV $S^{\prime}$ and TAPSE with brain injury severity groups $(p=0.866$ and $p=0.589$, respectively).

Conclusion: Right ventricular cardiac function remains adequate in patients with traumatic brain injury admitted to the ICU and is not associated with brain injury severity. Despite often observed ECG abnormalities in patients with severe $\mathrm{TBI}$, our data underscores that ECG abnormalities more likely reflect the secondary effect of brain injury (i.e. cardiac mimicry) rather than primary cardiac dysfunction.

\section{Reference(s) and grant ackowledgment(s)}

1. 1. Sommargren, CE. (2002). Electrocardiographic abnormalities in patients with subarachnoid hemorrhage. Am J Crit Care 11, 48-56.

2. 2. Lenstra, JJ., Kuznecova-Keppel Hesselink, L., La Bastide-van Gemert, S., Jacobs, B., Nijsten, MWN., van der Horst, ICC., van der Naalt, J. Brain-heart interactions: electrocardiographic abnormalities as secondary effect of severe traumatic brain injury. Submitted to Neurocritical Care.

3. 3. Kaye P, O'Sullivan I. Myocardial contusion: emergency investigation and diagnosis. Emerg Med J 2002; 19:8-10.

4. 4. Wiersema, R., Castela Forte, J. N., Kaufmann, T., de Haas, R. J., Koster, G., Hummel, Y. M., Koeze, J., Franssen, C. F. M., Vos, M. E., Hiemstra, B., Keus, F., van der Horst, I. C. C. Observational Study Protocol for Repeated Clinical Examination and Critical Care Ultrasonography Within the Simple Intensive Care Studies. J. Vis. Exp. (143), e58802, https://doi.org/10.3791/58802 (2019). 5. No

\section{1}

The evolution in anaesthetic practice of trauma rapid sequence induction (RSI) in a major tertiary hospital during its transition to a major trauma centre

A. Abdelhadi ; P. Jonetzko ${ }^{1}$; L. Weekes ${ }^{1}$

${ }^{1}$ Department of anaesthetics, University Hospitals Plymouth NHS Trust, Plymouth, United Kingdom

Correspondence: A. Abdelhadi

Intensive Care Medicine Experimental 2020, 8(2): 001561

Introduction: Major trauma is the leading cause of death among children and young adults with more than 16000 annual deaths in the UK Following advances in trauma care from armed conflicts and excessive mortality and morbidity from inadequacies in delivery of trauma care in the UK, a centralised trauma network system was introduced.

University Hospitals Plymouth was designated as a major trauma centre in 2012 with 4 district general satellite trauma units in the South West of England. We analysed various RSI methods used during the transition and incidence of complications and vasopressor use in trauma RSI.

Objectives:

- Evaluate the change in practice of trauma RSI during transition to a major trauma centre between 2012 and 2019.

- Compare clinical practice to existing RSI standard operating procedure (SOP) during the transition period 2012-2019.
- Review complications associated with different induction agents and vasopressor use in trauma RSI.

Methods: Patients were identified from UK's Trauma Audit \& Research Network (TARN) database. We selected trauma cases across three years encompassing the transition period (2012, 2015 and 2019). Data was collected retrospectively based on TARN data base and hospital records. Patients intubated pre-hospitally and transfers from trauma units were included.

Results: We analysed 120 patients in total, only those with complete set of data were included in the final analysis $(n=68)$. In our study group, blunt trauma was the main mechanism of injury (95.5\%) and $85 \%$ of RSIs were performed in patients with an injury severity score $>15$. Only $11 \%$ of RSIs were done by non-anaesthtists and ketamine/ midazolam was used for those. Table 1 details induction agents used.

Table 1: RSI induction agents

\begin{tabular}{|l|l|l|l|l|l|l|}
\hline & Propofol & Ketamine Thiopental & Etomidate & Midazolam $\begin{array}{c}\text { No } \\
\text { Induction } \\
\text { agent }\end{array}$ \\
\hline $\mathbf{2 0 1 2}$ & $79 \%$ & $8.5 \%$ & $12.5 \%$ & $0 \%$ & & \\
\hline $\mathbf{2 0 1 5}$ & $53 \%$ & $35 \%$ & $6 \%$ & $6 \%$ & $0 \%$ & \\
\hline $\mathbf{2 0 1 9}$ & $48 \%$ & $41 \%$ & $0 \%$ & & $4 \%$ & $7 \%$ \\
\hline
\end{tabular}

Rocuronium largely replaced suxamethonium for muscle relaxation. It was used for half RSIs in 2015, with suxamethonium only being used once in 2019.

Hypotension (systolic blood pressure $<90$ ) was the main complication $(24.5 \%)$, and has largely occurred with propofol $(87 \%)$ but also seen with ketamine (13\%). $40 \%$ of propofol inductions in patients with traumatic brain injury (TBI) required vasopressor use.

Conclusion: The incorporation of major trauma centre RSI protocols required a transition from individual anaesthetist-centred practice to a multidisciplinary trauma team based approach.Whilst adaptation of rocuronium as the preferred muscle-relaxant occurred ahead of updating the trauma RSI SOP in 2018, propofol remained as the induction agent of choice despite SOP suggesting ketamine use. This might be due to most RSIs being delivered by anaesthetists who use propofol more regularly. This may not necessarily worsen outcomes for non-TBI; however, it may do so in traumatic brain injuries where hypotension commonly occurred with propofol.Overall incidence of hypotension was similar to other published data, and the selection of $\mathrm{RSI}$ drugs is comparable to RSI practice in the UK.

Reference(s) and grant ackowledgment(s)

1. 1. Trauma Audit and Research Network, www.tarn.ac.uk.

2. 2. Findlay G, Martin I, Carter S., et al. Trauma: Who Cares?; A report of the National Confi dential Enquiry into Patient Outcome and Death.NCEPOD 2007

3. 3. Hylands $M$, Toma A, Beaudoin N, et al. Vasopressor use following traumatic injury: protocol for a systematic review. BMJ Open 2017.

4. 4. Heffner A, Swords D, Kline J, Jones A. The frequency and significance of postintubation hypotension during emergency airway management. J Crit Care. 2012.

5. 5. Sajayan A, Wicker J, Ungureanu N, et al. Current practice of rapid sequence induction of anaesthesia in the UK - a national survey. Br J Anaesth. 2016.

\section{0}

Thoracic Trauma: first steps into a new score

M. Romano ${ }^{1}$; R. Passos²; F. Cardoso²; D. Alves²; J. Braga²; R. Corga²; J. Caldeiro $^{2}$

${ }^{1}$ Internal medicine, Hospital Santa Luzia - Ulsam, Viana do Castelo, Viana do Castelo, Portugal; ${ }^{2}$ Intensive medicine, Hospital Santa Luzia - Ulsam, Viana do Castelo, Viana do Castelo, Portugal

Correspondence: $F$. Cardoso

Intensive Care Medicine Experimental 2020, 8(2): 001650 
Introduction: Thoracic trauma is a common admission problem to the emergency department, with a wide variety of presentations. Trauma patients may be difficult to access, especially with blunt lesions, considering that the extent of the visible lesions may underestimate its severity. Several scoring systems are commonly used to evaluate severity in these patients, and have been proposed to predict the development of acute respiratory distress syndrome (ARDS), however there is no global acceptance for a single scoring system. Our goal is to design a new score. Combining the Lung Ultrasound Score sectorial points, we hope to enhance sensitivity for severe (more diffuse) lesions. While preparing this new scoring system to be ultrasound based, we decided first to retrospectively evaluate its usefulness reviewing our patient data and their Computarized Tomography (CT) scan, knowing beforehand which have developed such complication.

Methods: We conduct a retrospective, consecutive review of the medical records of patients who were admitted to the ICU with thoracic trauma who were managed at our institution between september 2019 and june 2020. Inclusion criteria were: thoracic trauma, admission in ICU, arterial blood gasometry at admission, and thoracic CT scan within the first 12 hours. The score used accessed each patient by gasometry, presence of pneumothorax and hemothorax, and contusion/consolidation extent, ranging from 0 to 20 . Then we analyzed the association between the proposed score and evolution to ARDS, need for mechanical ventilation and in-hospital mortality.

Results: 19 patients met the final inclusion criteria. The minimum score was 4 and the maximum 12. In this study we noticed that all patients that didn't evolved to ARDS (from mild to severe) had a score equal or inferior to 7 . The same cut-off was noticed to the need of mechanical ventilation. From the 19 patients enrolled, only one died and was one of the 3 patients with the highest score (12).

Conclusion: The present study aims to evaluate all thoracic trauma patients admitted to an ICU for a period of 10 months and evaluate the validity of a new score as a predictor of in-hospitality mortality and/ or evolution to ARDS, trying to find cut-off above or under which one could decide ICU admission. The authors have in mind the handicaps of these study: 1 ) the few patients enrolled, 2) the retrospective evaluation, 3) the use of CT. Nevertheless, it was important to notice a clear cut-off for the less ill patients, in spite of their admission in ICU. This emphasizes the need for further research in this area, with a greater number of patients for a longer time. The authors of the study aim for future testing the score using thoracic ultrasonography at admission. This point of care exam would be faster, safer (innocuous to the patient) and cheaper. We add that the operator dependency is easily resolved with basic training and experience.

\section{6}

Communication and Humanization in End-of-life COVID-19 ICU patients

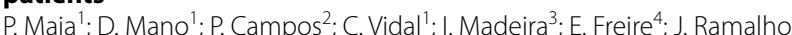
${ }^{1}$ Intensive Care Medicine, Centro Hospitalar Universitário do Porto, Porto, Portugal; ${ }^{2}$ Unidade de cuidados intensivos polivalente, Centro Hospitalar e Universitário do Porto, Porto, Portugal; ${ }^{3}$ Anesthesiology, Centro Hospitalar Universitário do Porto, Porto, Portugal; ${ }^{4}$ Palliative care, Centro Hospitalar Universitário do Porto, Porto, Portugal

Correspondence: $P$. Maia

Intensive Care Medicine Experimental 2020, 8(2): 001216

Introduction: The rapid COVID-19 pandemic spreading, associated with insufficient resources, changed the focus of health care professionals (HCP) from caring with the best quality to preserving as many lives as possible. End-of-life (EOL) procedures (1) implemented in the ICU were changed to minimize the use of scarce personal protective equipments (PPE) and the risk to disseminate SARS-CoV-2 infection among HCP and families of ICU patients. Portuguese authorities implemented restrictions following state of emergency declaration (2): visits were forbidden, EOL decision meetings with families were cancelled, communication was restricted to a physician in charge call to one family member once or twice daily. In addition, institutional religious support was withheld. As many patients admitted to the ICU were previously in a good medical condition they had not a living will and, though they were awake, conversations with them about EOL issues were short or nonexistent due to severe hypoxia: therefore their will was mostly unknown. Communication of EOL decisions is traditionally variable (3): however, in such conditions, the extent of the impact of these measures on communication and humanization is unpredictable.

Objectives: To describe decision making, communication practices and palliative measures in EOL COVID-19 patients at an university hospital intensive care department.

Methods: Data from all COVID-19 patients admitted to the ICU from 11-3 to 29-4-2020 were studied. No interventions were given to patients. Institutional approval was obtained [2020.084(067-DEFI/068CE) - TRILIPA 19(TRiage, LImitation of therapy and PAlliative care in COVID 19 patients)].

Results: 51 patients were enrolled ( $67 \%$ male, mean age 65 yo) with a median SOFA score on ICU admission of 7, a median Charlson Comorbidity Index of 4 and median Frailty Score of 3; median LOS in ICU was 8 days. $42(82 \%)$ patients were awake on ICU admission.

$21(41 \%)$ patients died in the ICU, 27 had decisions to limit life sustaining treatments (LST); none patient had a living will; no information about patient's wishes was asked. Although only 2 patients had limitation of LST on admission, neither the patient or the family participated on its discussion. Decisions to either withholding or to withdrawing therapy were communicated to the family before death in $22 \%$ of the cases. Nurses participated in $8 \%$ of EOL decisions. Palliative care measures included sedoanalgesia (100\%) to guarantee that patients died gently and tranquilly. There were no visits (100\%) for the dying patients.

Conclusion: Procedures related to EOL care of the dying patient in the ICU were changed during COVID 19 pandemic, consequently humanization in EOL was not protected and these patients were not equally treated in rights and dignity. The psychological and sociological effects of the civil liberties and human rights restrictions on both the families and caregivers of the dead and HCP are to be determined.

\section{Reference(s) and grant ackowledgment(s)}

1. 1. Maia P et al. Intensive Care Med 2007;33(S2): S200.

2. 2. https://www.dgs.pt/directrizes-da-dgs/orientacoes-e-circulares-infor mativas/orientacao-n-0092020-de-11032020-pdf.aspx.

3. None.

4. 3. Cohen S et al. Intensive Care Med (2005) 31:1215-1221.

\section{5}

End-of-life practices in COVID-19 ICU patients: The TRILIPA 19 study

D. Mano'; P. Campos²; C. Vidal'; J. Ramalho'; I. Madeira ${ }^{3}$; M. Bertao ${ }^{4}$; P. Vita';

P. Maia

${ }^{1}$ Intensive care medicine, Centro Hospitalar Universitário do Porto, Porto, Portugal; ${ }^{2}$ Unidade de cuidados intensivos polivalente, Centro Hospitalar e Universitário do Porto, Porto, Portugal; ${ }^{3}$ Anesthesiology, Centro Hospitalar Universitário do Porto, Porto, Portugal; ${ }^{4}$ Palliative care, Centro Hospitalar Universitário do Porto, Porto, Portugal

Correspondence: $\mathrm{P}$. Maia

Intensive Care Medicine Experimental 2020, 8(2): 001295

Introduction: Though End-of-life pratices (EOLP) are variable [1] and change over time [2]. The COVID-19 pandemic brought new dilemmas to ICU physicians concerning triage, limitation of life-sustaining therapy (LLST) and humanization. Many decisions were left to the ICU staff alone (sometimes to the physician in charge alone), being the patient or the family unable to participate in the decision making process. Documentation, which is frequently poor [3], may be even more challenging under these circunstances.

Objectives: To describe EOLP in COVID-19 patients at an university hospital intensive care department.

Methods: All COVID-19 patients admitted to the ICU from 11-3-2020 to 29-4-2020 were enrolled in a retrospective observational study. Institutional approval was obtained [2020.084(067-DEFI/068-CE) 
- TRILIPA 19 (TRiage, LImitation of therapy and PAlliative care in COVID-19 patients)] with a waiver of informed consent. LLST definitons used were: "Do-not-resuscitate (DNR) order" when the order to DNR was prescribed and documented; "failed CPR" when death occurred despite cardiopulmonary resuscitation (CPR) efforts, "withholding life support" (WH) when a decision was made not to start or increase a lifesustaining intervention like cardiopulmonary resuscitation (WH_CPR), vasopressors and/or inotropes (WH_Vas), invasive mechanical ventilation (WH_IMV), renal replacement therapy (WH_RRT) or extracorporeal membrane oxygenation (WH_ECMO); "withdrawing life support" (WD) when a decision was made to actively stop a life-sustaining intervention presently being given, maintaining only closed circuit ventilation to protect health care workers from the risk of contamination.

Results: 51 patients were enrolled ( $67 \%$ male, mean age 65 yo, median LOS in ICU 8 days): on ICU admission, the median SOFA score was 7 , the median Charlson Comorbidity Index (CCl) was 4 and the median Frailty Score was 3. The ICU mortality was $41 \%(n=21)$ and the hospital mortality was $49 \%(n=25)$. At admission a decision to WH was made in 2 patients (WH_IMV). During ICU stay, 25 decisions of LLST in 20 patients were taken: DNR in 1 patient, WH_RRT in 1 patient, WH ECMO in 2 patients, WH_CPR in 6 patients and WD in 15 patients. Only 1 patient who had LLST survived to the hospital stay. Death occured after WH treatment in 8 patients (38\%) and after WD treatment in 13 (62\%). Reasons for a LLST were multi-organ failure or devastating brain injury. $74 \%$ of the LLST decisions were partially documented.

\begin{tabular}{|l|l|l|l|}
\hline Patients & $\begin{array}{l}\text { With LLST at } \\
\text { admission }(\mathbf{n}=\mathbf{2})\end{array}$ & $\begin{array}{l}\text { With LLST } \\
\text { during ICU stay } \\
(\mathbf{n = 2 0})\end{array}$ & $\begin{array}{l}\text { Without LLST } \\
(\mathbf{n = 2 9 )}\end{array}$ \\
\hline SOFA at admission & 3 & 8 & 7 \\
\hline $\begin{array}{l}\text { SOFA at day of } \\
\text { decision }\end{array}$ & 4 & 11 & NA \\
\hline CCI & 6 & 4 & 3 \\
\hline Frailty score & 4 & 3 & 2 \\
\hline Lenght of symptoms & 4 & 6 & 6 \\
\hline LOS & 3 & 10 & 1 \\
\hline
\end{tabular}

Conclusion: All COVID-19 patients died after a LLST due to multiorgan failure or devastating brain injury. Though these patients had low prevalence of comorbidities and low level of functional disability, none was considered for CPR. LLST decisions were insufficiently documented.

\section{Reference(s) and grant ackowledgment(s)}

1. 2. Sprung $C L$ et al. JAMA. 2019:322:1-12.

2. 3. Spronk PE et al. Anesthesia \& Analgesia. 2009;109(3): 841-846.

3. None.

4. 1. Sprung CL et al. JAMA. 2003;290:790-797.

\section{2}

Narrowing the gap: donation-centered measures and end-of-life care to increase organs for transplantation, availability, a successful experience

C. Palau Marti ; C. Ferrer Gómez ${ }^{1}$; S. Moliner Velázquez ; ;. Piolatti Luna²; R. Rubio Haro'; JM. Blanco Rodríguez'; J. De Andrés Ibáñez'

${ }^{1}$ Anaesthesiology, Critical Care and Pain Therapy Service, Consorci Hospital General Universitari de València, València, Spain; ${ }^{2}$ Anaesthesiology, critical care and pain therapy service, Hospital Clínico de Valencia - Servicio de Pediatría, València, Spain

Correspondence: C. Palau Marti

Intensive Care Medicine Experimental 2020, 8(2): 001552

Introduction: Spain has developed a succesful transplant activity, fundamentally thanks to deceased organ donation, reaching 46.9 million per million inhabitants in 2017. Since 2017, the General Consortium University Hospital of Valencia (GCUHV), a tertiary hospital, has collaborated in the increase and development of such activity and has included in its protocols for patients that meet the criteria for potential donors the option to donate when the responsible medical team changes its therapeutic objetective towards donation-centered comfort measures and end-of-life care.

Objectives: To describe the number and characteristics of patients who deceased in our hospital and were candidates for organ donation in asystole from December 2017 to May 2019.

Methods: Descriptive and retrospective study including patients who deceased in our hospital and were candidates for organ donation in asystole. Variables such as age, intensive care stay in days, cause of death and removed organs were retrieved.

Results: A total of 13 asystolic donors were collected from December 2017 to May 2019 at this tertiary hospital. Of these, 11 were neurocritical patients and 2 were patients recovered from cardiac arrest. The average age was 60.3 years (40-69) and mean intensive care stay was 7.38 days (3-17). Among the removed organs, it is worth mentioning that kidneys from all 13 patients, livers from 9 of them and a total of 3 pancreas were successfully implanted.

Conclusion: Despite a significant increase in the availability of organs for transplantation in recent years, a gap between supply and demand still persists. The development of new alternative means of organ donation is necessary in order to assure the availability of organs for transplantation. The program was initially very cautious, but its easily improvable. We expect a significant increase in the number of donations for the coming years.

\section{Reference(s) and grant ackowledgment(s)}

1. Tang JX, Na N, Li JJ, Fan L, Weng RH, Jiang N. Outcomes of Controlled Donation After Cardiac Death Compared With Donation After Brain Death in Liver Transplantation: A Systematic Review and Meta-analysis. Transplant Proc. 2018;50(1):33-41. https://doi.org/10.1016/j.transproceed.2017.11.034.

2. Dos Santos MJ, Leal de Moraes E, Santini Martins M, et al. Trend Analysis of Organ and Tissue Donation for Transplantation. Transplant Proc. 2018;50(2):391-393. https://doi.org/10.1016/j.transproceed.2017.11.075.

\section{1}

Machine learning to predict ICU admission in septic patients: exploring new strategies to anticipate clinical deterioration

PF. Caruso ; G. Angelotti ; M. Greco ; C. Ciccone ; N. Stomeo ; ${ }^{1}$ M. Cecconi ${ }^{1}$ ${ }^{1}$ Anesthesia and intensive care, Humanitas Research Hospital, Milano, Italy; ${ }^{2}$ Anesthesia and intensive care, Humanitas Research Hospital, Milan, Italy Correspondence: P.F. Caruso

Intensive Care Medicine Experimental 2020, 8(2): 001351

Introduction: Early intervention in sepsis have been shown to be crucial in preventing the transition into septic shock and reducing mortality in this population. Early recognition can be challenging, and many scores have been created to help physicians anticipate clinical deterioration (e.g. qSOFA or NEWS). Main limitations are lack in external validity and inclusion of few parameters only. We propose to predict clinical deterioration by considering all the variables systematically recorded by electronic health records (EHR). With the advent of new techniques in healthcare settings like machine learning, we aim to build tailored-to-patients scores that will improve the ability to anticipate and prevent intensive care admission.

Objectives: Predicting ICU admission in septic patients using machine learning models.

Methods: Our cohort included all the patients with ICD-9 codes for sepsis (995.92) and septic shock (785.52) that were admitted from standard ward to in intensive care units (ICU), among all patients hospitalized in 2018 in Humanitas Research Hospital. Data were anonymized at extraction from repository. Demographics and vital signs available in general wards were extracted from EHR, including heart rate, respiratory rate, peripheral oxygen saturation, temperature and peripheral blood pressure. We divided the features in data windows and the mean of each vital sign was calculated and retained for each block. We began the iterative process of building machine learning models (Logistic Regression and Random Forest Classifier) to 
predict the admission in ICU, using a train-test set division and k-fold cross validation. We trained the models with the $72 \mathrm{~h}$ hours data before ICU admission for cases and $72 \mathrm{~h}$ prior the worst mean arterial pressure for controls (as it was considered the most critical moment in those who were not admitted to ICU), considering age, gender, heart rate, respiratory rate, peripheral oxygen saturation, temperature and the trend of systolic, diastolic and mean arterial pressures.

Results: The cohort comprehends 105 patients of which 15 were admitted to ICU (14\%). The median age for those admitted in ICU was statistically significantly lower (69.5[68-79] vs 77 [69-85], p-value < $0.01]$ ) and most patients admitted in ICU were males (12 vs 3 , $\mathrm{p}$-value $=0.20$ ). The best cross-validated logistic regression model had an AUROC of 0.77, while for random forest the AUROC was 0.74 . In logistic regression, male gender, heart rate and respiratory rate were positively correlated, while age, diastolic blood pressure and oxygen saturation were reversely correlated.

Conclusion: In this first preliminary study, we demonstrated that machine learning models might be an innovative solution to predict intensive care admission in septic patients before clinical deterioration using data derived from EHR Further studies on larger cohorts and wider types of data, like laboratory values, should be performed to assess the real potential of machine learning models.

\section{Reference(s) and grant ackowledgment(s)}

1. 10. Falini S, Angelotti G, Cecconi M. ICU management based on big data. Curr Opin Anaesthesiol. 2020;33(2):162-169. https://doi. org/10.1097/aco.0000000000000834.

2. 9. Raith EP, Udy AA, Bailey M, et al. Prognostic Accuracy of the SOFA Score, SIRS Criteria, and qSOFA Score for In-Hospital Mortality Among Adults With Suspected Infection Admitted to the Intensive Care Unit. JAMA. 2017;317(3):290-300. https://doi.org/10.1001/jama.2016.20328.

3. 8. Goulden R, Hoyle MC, Monis J, et al. qSOFA, SIRS and NEWS for predicting inhospital mortality and ICU admission in emergency admissions treated as sepsis. Emerg Med J. 2018;35(6):345-349. https://doi. org/10.1136/emermed-2017-207120.
4. 7. Cecconi M, De Backer D, Antonelli M, et al. Consensus on circulatory shock and hemodynamic monitoring. Task force of the European Society of Intensive Care Medicine. Intensive Care Med. 2014;40(12):17951815. https://doi.org/10.1007/s00134-014-3525-z.

5. 6. Shankar-Hari M, Phillips GS, Levy ML, et al. Developing a newdefinition and assessing newclinical criteria for Septic shock: For the third international consensus definitions for sepsis and septic shock (sepsis-3). JAMA - J Am Med Assoc. 2016;315(8):775-787. https://doi. org/10.1001/jama.2016.0289.

6. 5. Seymour CW, Liu VX, Iwashyna TJ, et al. Assessment of clinical criteria for sepsis for the third international consensus definitions for sepsis and septic shock (sepsis-3). JAMA - J Am Med Assoc. 2016;315(8):762-774. https://doi.org/10.1001/jama.2016.0288.

7. 4. Rajkomar A, Dean J, Kohane I. Machine learning in medicine. N Engl J Med. 2019;380(14):1347-1358. https://doi.org/10.1056/nejmra1814259.

8. 3. Rhodes A, Evans LE, Alhazzani W, et al. Surviving Sepsis Campaign: International Guidelines for Management of Sepsis and Septic Shock: 2016. Vol 43. Springer Berlin Heidelberg; 2017. https://doi.org/10.1007/ s00134-017-4683-6.

9. 2. Shamout F, Zhu T, Clifton L, et al. Early warning score adjusted for age to predict the composite outcome of mortality, cardiac arrest or unplanned intensive care unit admission using observational vital-sign data: A multicentre development and validation. BMJ Open. 2019;9(11):1-7. https://doi.org/10.1136/bmjopen-2019-033301.

10. 1. Cecconi M, Evans L, Levy M, Rhodes A. Sepsis and septic shock. Lancet. 2018;392(10141):75-87. https://doi.org/10.1016/s0140 $-6736(18) 30696-2$

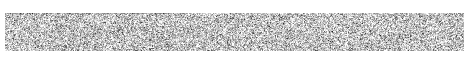

\section{Publisher's Note}

Springer Nature remains neutral with regard to jurisdictional claims in published maps and institutional affiliations.
Ready to submit your research? Choose BMC and benefit from:

- fast, convenient online submission

- thorough peer review by experienced researchers in your field

- rapid publication on acceptance

- support for research data, including large and complex data types

- gold Open Access which fosters wider collaboration and increased citations

- maximum visibility for your research: over 100M website views per year

At BMC, research is always in progress.

Learn more biomedcentral.com/submissions 